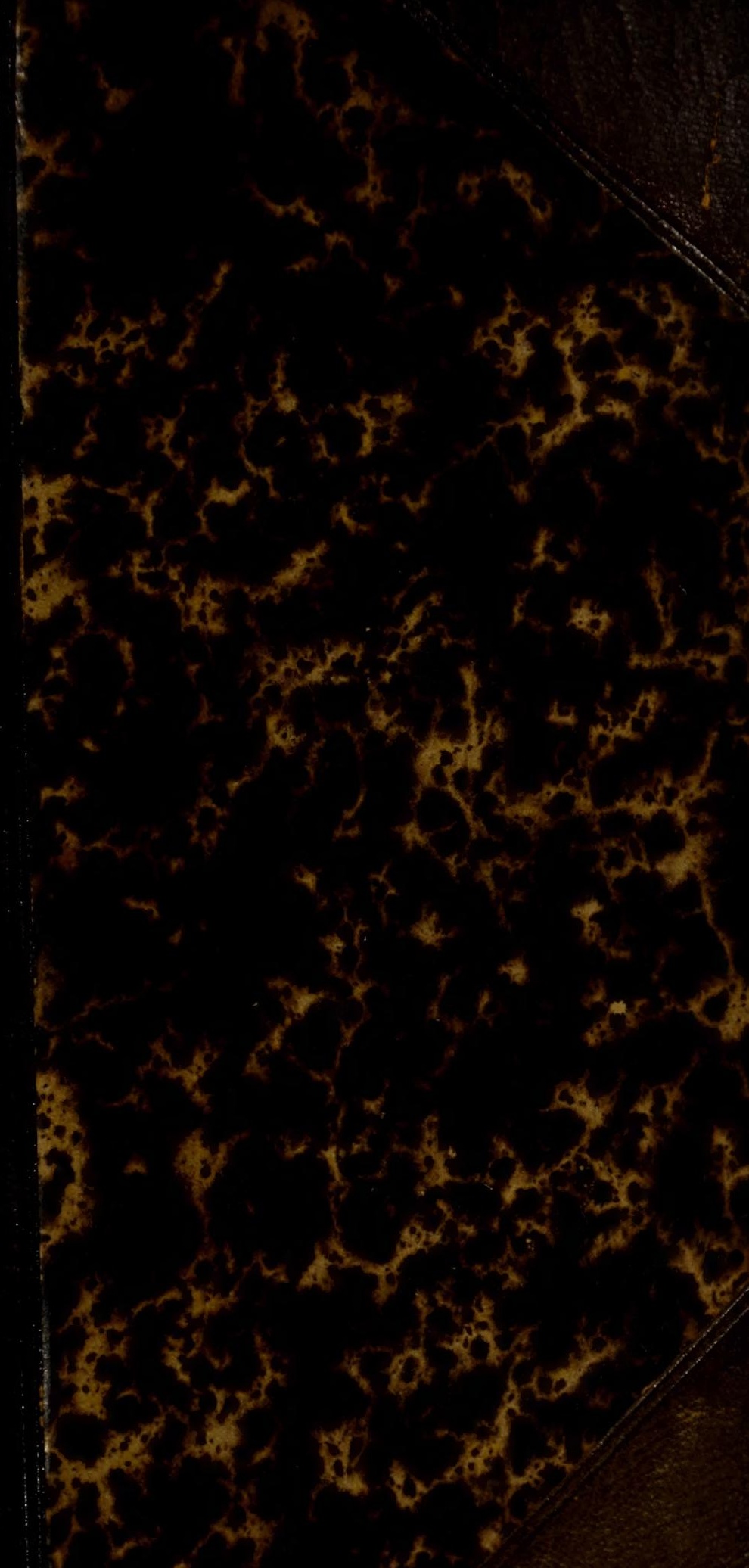


D) $>3 \rightarrow 50355$

Case

Shelf.

No.

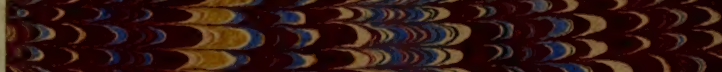
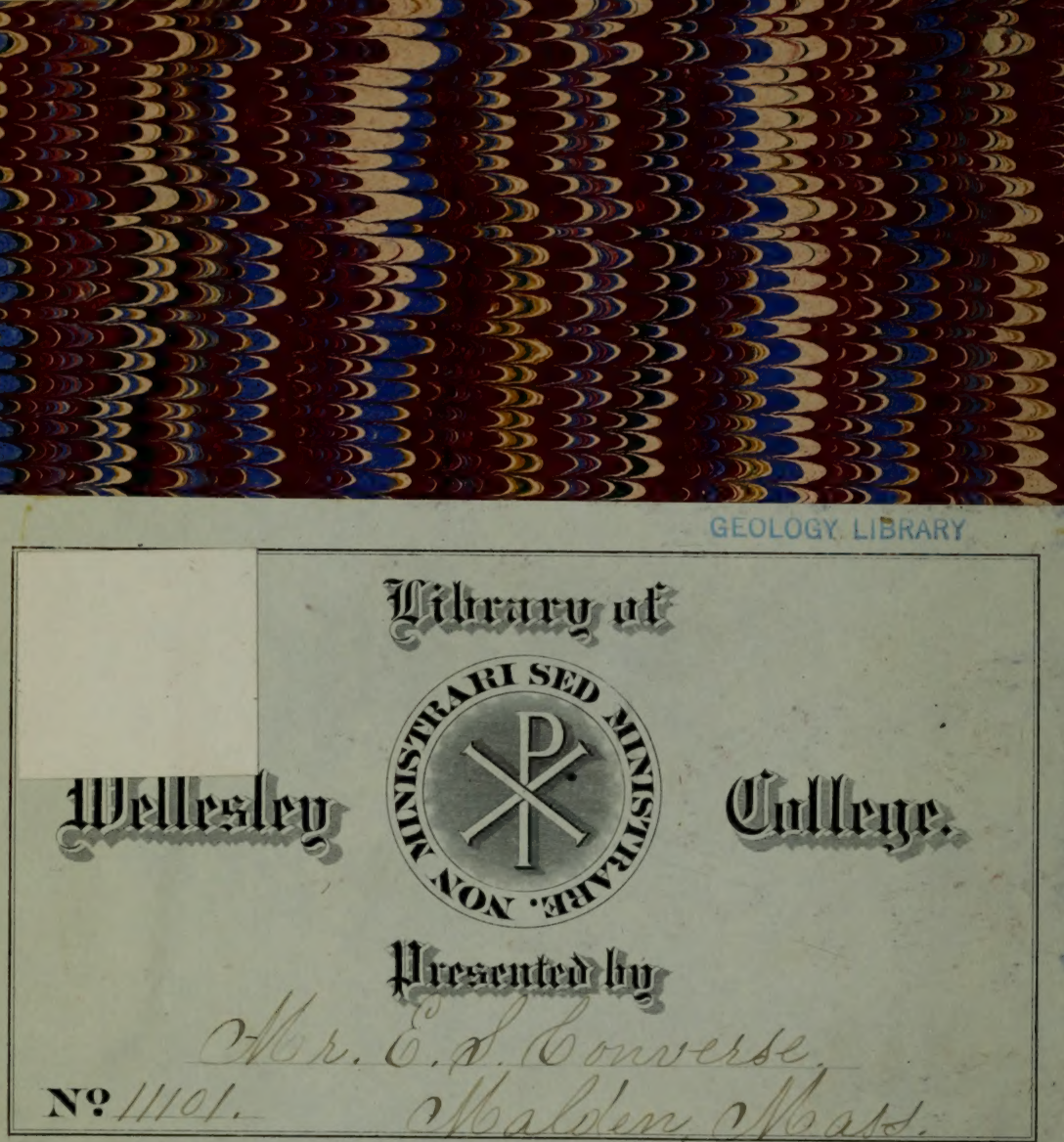
)

( ) 35353

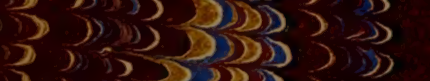

3) D 3 गD

3 3. 32020

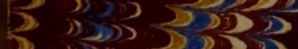

3

3

,

3,3
3,3
3

$3,3,3$

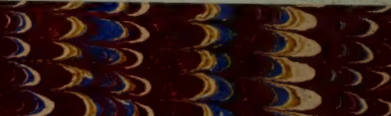
(i) $3 \sum_{3}>3$ $3 \rightarrow 3$ $\lim _{3}, 3$

$\int_{3}$

5i) $>2$

(2) 25

20

23

$\int_{3}$
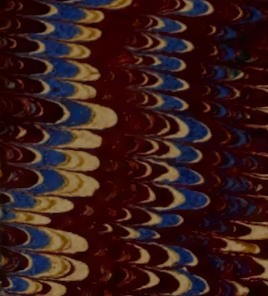

\section{$\int_{3}$}
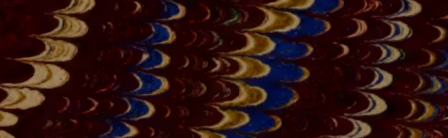

325

D)

D)

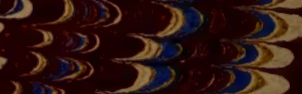

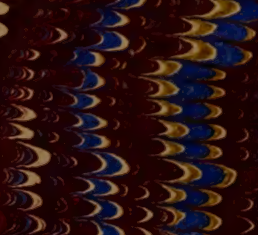

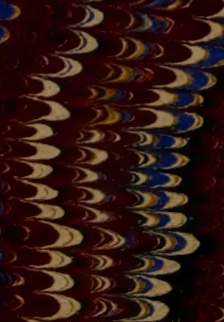




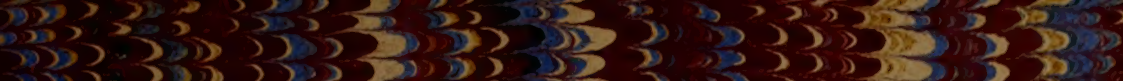

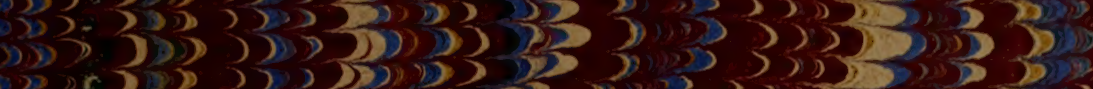

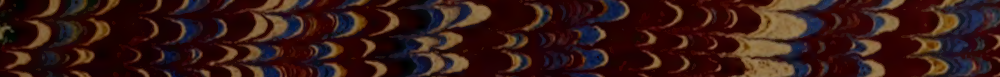

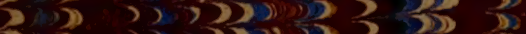

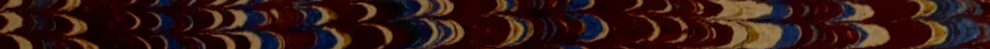

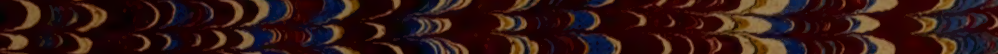

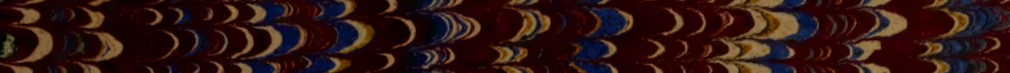

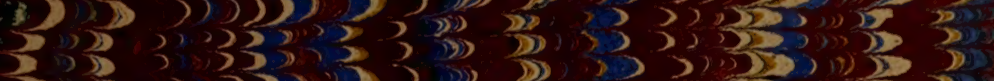

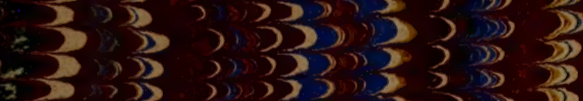

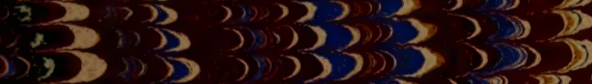

$$
3\{3,5,3,15
$$
7)

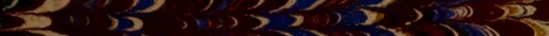
$3>2,2,12$ $3,3,2,3,1$ )

D> $\rightarrow>$

325

32023

33203

D) DD?

(3)

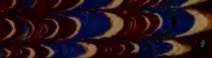

(2)

$32 \rightarrow 3$

$33,3,35$

3

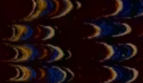

$\rightarrow$

$\sum_{i \rightarrow \infty} \rightarrow 3$

)

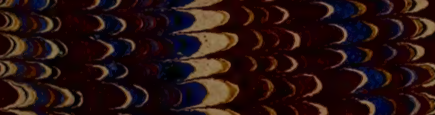
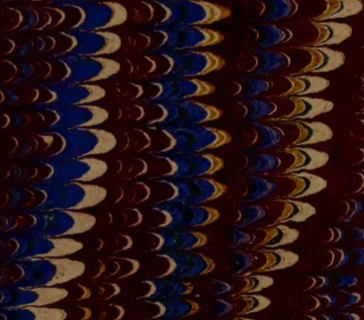

$>(3)$

$3>2>?$

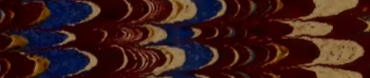

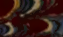

3

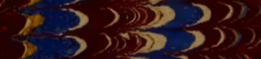

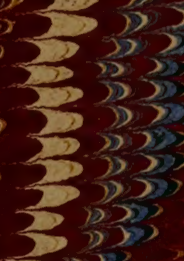

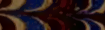

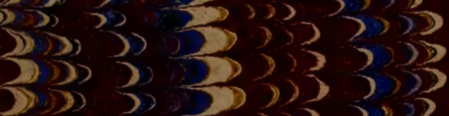

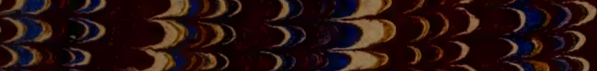

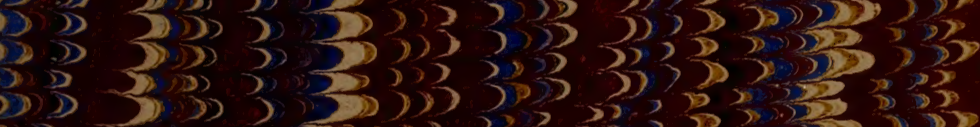

). $>>3>3$

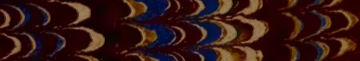

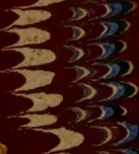

3 (ग)

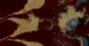

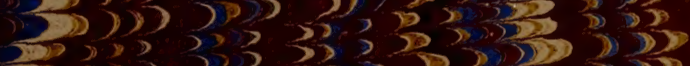

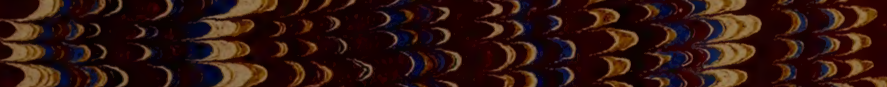

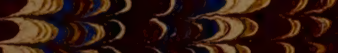

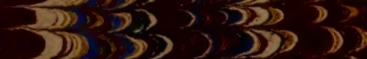
$35 \pi .23>203$ 530,59503020 35233,29

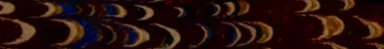
$33(, 0), D>D$

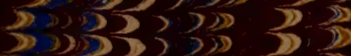

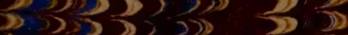

$2353 \rightarrow 302$

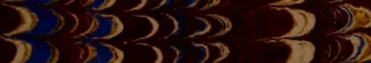

$3,2,5>3)>2$

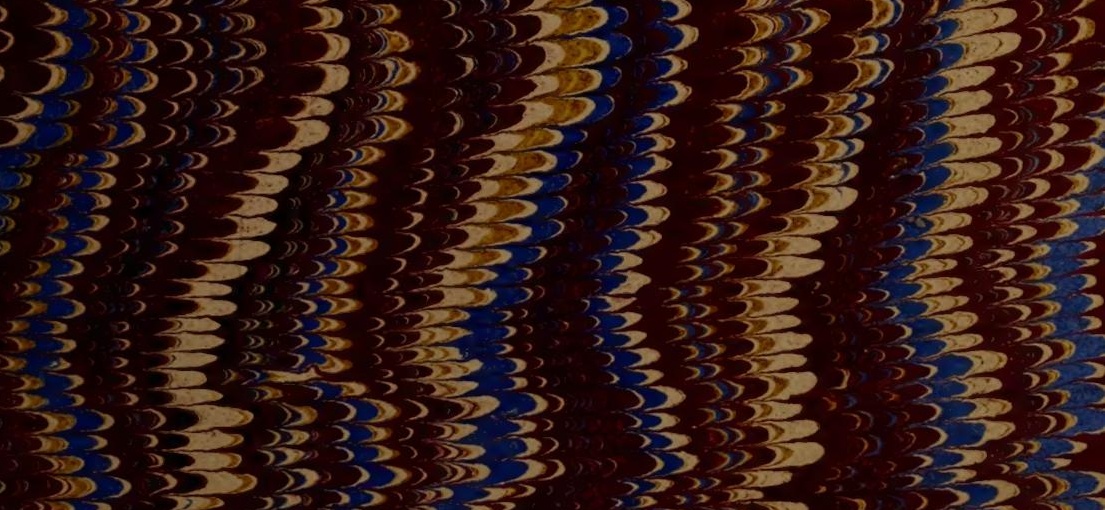

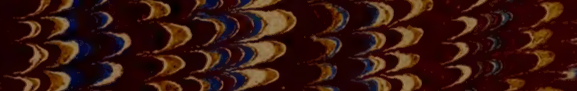

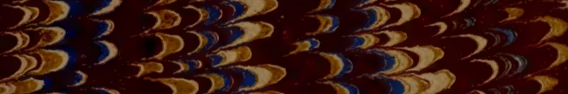

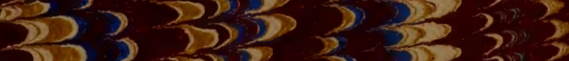

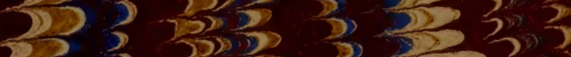

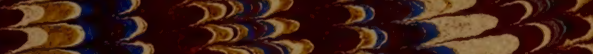
23,20 320

D) 9,2 3

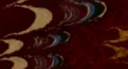
2 





\title{
INDAX PHLIEONTOLOGICLS
}

ODER

\author{
ÜBERSICHT DER BIS JETZT BEKANNTEN
}

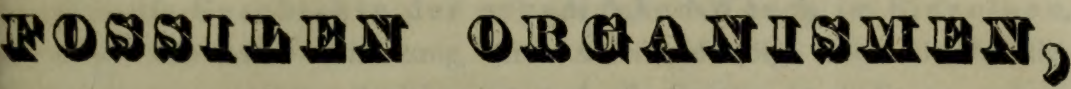

UNTER MITWIRKUNG

DER HH. PROF. W. R. GÖPPERT UND WTEM. v, MTYYR

BRARBBITET VON

Dr. H: G: BRoNN.

Bweite Abtheilumg.

B. Enumerator palaeontologicus:

Systematische Zusammenstellung

und

geologische Entwickelungs-Gesetze der organischen Reiche.

\section{STUTTGART.}

E. Schweizerbart'sche Verlagshandlung und Druckerei. $18+9$. 


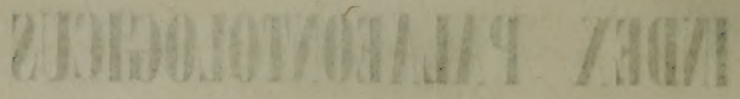

Axan

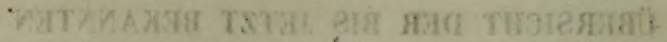

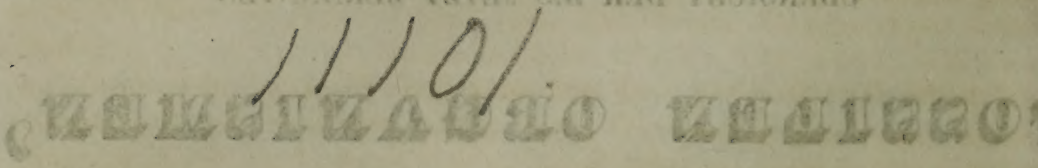

wAIm)

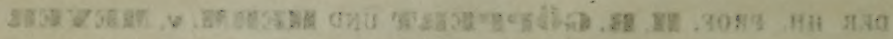

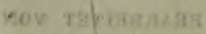

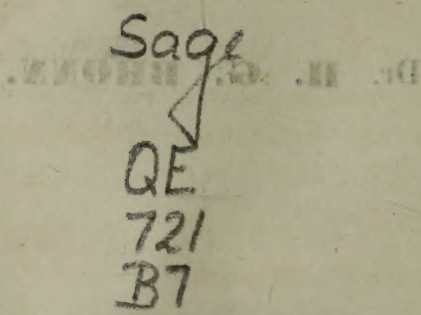

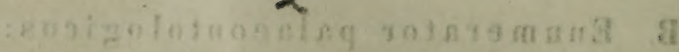

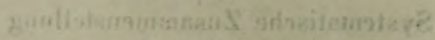

. buts

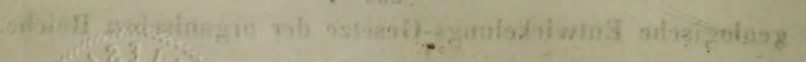

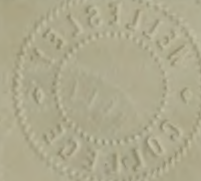

\section{J月A.0T? ITE}

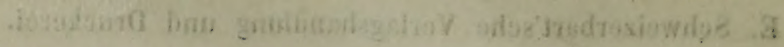
Qtisi, 


\section{Die Geschichte des Erscheinens der einzelnen organischen Wesen auf der Erd-Oberfläche.}

Um die Geschichte der organischen Wesen im Einzelnen, die Frage von ihrer Verbreitung, ihrer Aufeinanderfolge oder Gleichzeitigkeit, ihrer gegenseitigen Beziehungen und alle Gesetze zu studieren, welche in deren Auftreten und Verschwinden auf der Erd-Oberfläche gewaltet haben, müssen wir Schicht um Schicht die ganze Erd-Rinde sorgfältig durchforschen und aus den darin eingeschlossenen organischen Resten die Familien, Genera, Arten früherer Lebenwesen nach der jedesmaligen Bildungs-Zeit jener Erd-Schichten zu bestimmen suchen.

Diese Aufgabe indessen unterliegt sehr grossen Schwierigkeiten, 1) hinsichtlich der speziellen Bestimmung und der Vergleichung der von verschiedenen Personen an verschiedenen Orten bestimmten organischen Reste selbst; 2) hinsichtlich der Parallelisirung und Gruppirung der GebirgsSchichten, welche sie einschliessen; 3) hinsichtlich der bis jezt nur geringen Ausdehnung unserer Forschungen über alle Schichten der Erd-Rinde in Vergleich zu deren ganzer Erstreckung.

a. Um die organischen Reste nach Geschlechtern und Arten zu bestimmen und sie unter sich und mit den lebenden Wesen vergleichen zú können, müssen wir die Kenntnisse der Zoologen und Botaniker zu Hülfe nehmen. Wenn es indessen bei lebenden Wesen schon schwierig ist, wiederzuerkennen, was ein andrer Naturforscher an fremdem Orte vor uns beschrieben und etwa abgebildet hat, vorausgesetzt selbst, dass uns diese Beschreibungen und Abbildungen alle zugänglich sind, so wird die Schwierigkeit doppelt gross bei dem meist fragmentarischen und unvollkommenen Züstande der fossilen Wesen. Bald wirḍ man für gleichartig halten, was verschieden ist, noch öfter als etwas Neues bestimmen, was Andere schon beschrieben haben. Da es nun kaum möglich ist, alle Werke sich zu verschaffen und nachzuschlagen, worin solche Beschireibungen bereits enthalten sind, und da verschiedenen Arbeiten der Art oft sehr verschiedene naturhistorische Systeme zu Grund gelegt worden sind, so müssen diese Schwierigkeiten noch zunehmen und insbesondere die Namen der von verschiedenen Autoren aufgestellten Arten sich sehr mehren. Um nun aus der vorhandenen grossen 
Menge von Namen die wirklichen Arten und die ihnen vorzugsweise gebührenden Benennungen leichter heraus zu finden, haben wir in einer Vorarbeit (s. die Einleitung S. I ff.) alle uns bekannten Namen alphabetisch und systematisch in einen Nomenclator palaeontologicus zusammengestellt, um dadurch unsre den historisch wissenschaftlichen Faden festhaltenden Untersuchungen nicht zu unterbrechen. Aber diese Zusammenstellung ist selbst nur das augenblickliche Resultat der Ausbeute unsrer Litteratur, und bei den wirbellosen Thieren insbesondere erscheint eine Menge von Arten-vertretenden Namen, die bei einer monographischsorgfältigen Behandlung des Gegenstandes, welche indessen melıre Jahrzehnde erfordern würde, noch mit andern zusammenfallen müssten. Auf diese Weise kennt man in der That bri den Wirbel-losen Thieren vielleicht ein Fünftel aller Arten weniger, als die in nachfolgender Tabelle aufgezählten Namen andeuteu würden.

b. Was die Zusammenstellung der fossilen Arten in die einzelnen GebirgsSchichten betrifft, so genügt es für unsern $Z$ weck, wenn wir uns auf die Annahme einer mässigen Anzahl von Gebirgs.Abschnitten beschränken, jene etwa, welche man gewölnnlich Formationen oder zuweilen Systeme nennt. Die Annahme einer grössern Zahl von Unterabtlieilungen könnte dem Geologen wohl wünschenswerth seyn, würde uns aber nicht nur nöthigen, die tabellarische übersichtliche Form der Zusammenstellung aufzugeben, sondern auch die schen ohnediess grossen Schwierigkeiten der Parallelisirung an verschiedenen Orten vorkommender Schichten in gleichem Grade vermehren. Wir sind indessen jedenfalls genöthigt, über die in den folgenden Tabellen angenommenen Gebirgs-Abtheilungen einige Rechenschaft zu geben, indem wir hinsicl:tlich des Details noch auf eine besondere geologische Folio-Tabelle verweisen.

Im Ganzen wird man die mit a, b bis $x$, y bezeichneten Gebirgs-Abschnitte schon aus ihrer Aufeinanderfolge leicht begreifen; ihnen reiht sich noch eine Rubrik z an, um die Arten bezeichnen zu können, welche auch lebend noch vorkommen. Das Alter einer fossilen Art wird immer durch Wiederholung des entsprechenden Buchstabens hinter ihrem Namen in der Tabelle angegeben. Indessen: doch nicht jede Ru!rik, nicht jeder Buchstabe bezeichnet eine chronologisch selbstständige Gebirgs - Abtheilung, sondern einige (wie h, s, ü, v) sind vorhanden, um solche Bildungen aufzunehmen, welchen zur Zeit noch kein sicherer Platz in den übrigen angewiesen werden konnte, um nicht etwa die Ergebnisse der andern auf eine fühlbare Weise zu verfälschen. So stehen die „MuschelkalkGebilde von St. Cassian" in der Rubrik h beisammen, bis es entschieden seyn wird, ob dieselben wirklich als chronologisch selbstständige Bildungen zwischen Zechstein und Muschelkalk eingeschaltet werden müssen (vielleicht besser nach i), oder ob sie nur eine andere „Facies" des Muschelkalks darstellen. Die Oolithen-Reihe von Lias bis an den Kimmeridge-clay haben wir nicht weiter unterabtheilen können, weil in der Bezeichnung und Bestimmung der Glieder bei den Autoren zu viele Unsicherheit herrscht. Wir hatten anfangs eine Grenze zwischen Oxford-Thion und Coral-rag gelegt, mussten sie aber nach weit vorgeschrittener Arbeit aufgeben, weil eine allzugrosse Quote der Arten in beiden zugleich angezeigt wird. Doch werden wir in wichtigeren Fällen den 
Unter-Jura in $n^{1} n^{2} n^{3} n^{4} n^{5} \quad\left(n^{12345}\right.$ ) zerlegen, wo $n^{1}$ die schwankenden Schichten mit Trigonia navis, $\mathrm{n}^{2}$ den Unteroolith, $\mathrm{n}^{3}$ den Mitteloolith, $\mathrm{n}^{4}$ den Kelloway-rock und Oxford-Thon, $\mathbf{n}^{5}$ den Koral-rag und weissen Jura bis an den Kimmeridge-clay bedeutet. Dann haben wir das Wälder.Gebilde (p) noch abgesondert gehalten, welches einige Geologen nur als die lacustre Facies des Neocomien betrachten. Mit dem Neocomien q ist Frtron's Untergrünsand verbunden, aber dessen Grü nsand von Blackdown mit dem Grünsand r zusammen geworfen, obschon dersolbe nach Firtos's eigner Ansicht die ga nze Grünsand-Reihe in sich begreift: daher kann es geschehen, dass hiu und wieder eine Art in Grünsand $r$ angegeben erscheint, welche nur dem Neocomien q zusteht. Eben dahin (q) musste ein grosser Theil des bisher sogen. Französischen Grïnsandes gebracht werden. Überhaupt bietet der Grünsand an meisten Schwierigkeiten dar, indem die Eugländer ausser den schon bezeichneten Verschiedenheiten den Grünsand nochmals in unteren und oberen unterschieden, weleher letzte der gewöhnlichen Kreide-Glauconie und einem Theil des gewöhnlichen Grünsandes der Frauzosen entspricht, die beide zu s gehören, aber nicht immer näher bezeichnet zu werden pflegen. Eben so vicle Schwierigkeiten machen diese Bildungen in Deutschland. Es ist daher nicht möglich gewesen, hier Alles richtig und sicher einzuorduen. Insbesondere mögen manche Arten in $r$ stehen, die nur in s gehörten. - Zwischen Kreide und Untertertiär-Bildungen haben wir einige Nummuliten-Gest eine eingeschaltet, deren Stelle nicht ganz sicher ist, und welche zuweilen gemengte Fossilien zu enthalten scheinen. Im südlichen Frankreich stehen dieselben demungeachtet den Tertiär-Bildungen so entschieden nahe, dass wir kein Bedenken getragen haben, deren Vorkommnisse mit letzten zu vereinigen. Dagegen sagt Agassiz von den Fischen des Monte Bolca, den man sonst (in Ermangelung fast aller andern fossilen Reste von da) für untertertiär anzusehen gewöhnt ist, dass seine Fische sich mehr denen der Kreide annäherten, als deuen der Eocen-Bildungen. - Die Molasse $v$ ist von den mittelund den ober-tertiären Gebilden getrennt, weil ihre Stelle noch immer ungewiss, ihre Wirbelthiere mehr den tiefurn und ihre Konchylien melır den hühern Vorkommnissen entsprechend siud. Ihr haben wir jene Braunkohle a - Ablagerungen im nordwestlichen Deutschland angeschlossen, von welchen man weiss, dass sie unter dem ober-tertiären Muschr-Sande ruhen, und den Bernstein. Als mitteltertiär haben wir dagegen jene Braunkohlen-Lager in mitteln und südlichen Deutschlande betrachtet, welche fern von sicheren obertertiären Bildungen in der Nähe von mitteltertiären vorkommen; beide Braunkohlen bleiben auf diese Weise doch unmittelbare Nachbarn; während jene im Pariser Becken der ïtern Tertiär-Abtheilung grösstentheils anheim fallen, die wir nach Elig de Beaunont's Ansicht (so weit uns bekannt) bis zum obern Meeres-Sandstein (ausschliessend) angenommen hatten. Doch hat dieser eine bei weitem grössere Anzahl von Arten mit den eocenen Bildungen desselben Beckens gemein, als mit allen miocenen Gebilden zusammengenommen; wir werden daher seine Reste durch ein besonderes Zeichen (ii in der Rubrik u) kenntlich machen. Endlich wollen wir durch $\mathbf{v}$ in der Rubrik $\mathbf{v}$ diejenigen mikroskopischen Thierchen bezeichnen,

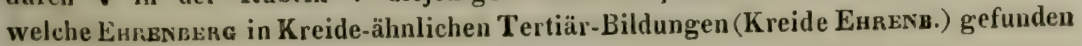


hat. - Hinsichtlich des Steinkohlen-Gebirgs haben wir noch nachtriglich zu bemerken, dass, wie man bald sehen wird, ihm eine viel zu grosse Anzahl Arten gemeinsam mit dem Devon-Systeme zugetheilt wird. Fast alle diese Angaben rühren von Phiclips her, der sie nach seiner Geology of Yorleshire im Kohlen-Kalke, nach seinen Palaeozoic Fossils im Devon-Kalke gefunden hat, ohne Auskunft darüber zu bieten.

c. Der Schwierigkeiten, welche aus der geographischen Beschränktheit unsrer Kenutnisse über die einzelnen Gebirgs - Schichten entspingen, zeigen sich nicht bei der Zusammentragung der Detail-Beobachtungen, sondern erst danu, wenn man aus diesen allgemeinere Resultate ziehen will. Wir haben uns beschränkt, das geographische Vorkommen der fossilen Reste ebenfalls nur im Grossen in dic erste Rubrik unsrer systematischen Tabelle einzutragen. $\mathbf{E}$ bedentet Europa, $\mathbf{\Phi}, \mathbf{M}, \mathbf{U}$ bedeuten aSien, aFrica, aMerica, aUstralien, und die diesen Buchstaben rechts angefügten Ziffern 1, 2, 3, 4 bezeichmen der Reihe nach die nördliche kalte, die nördliche gemässigte, die heisse und die südliche gemässigte Zone. Da fast alle Angaben in $\mathbf{E}^{2}$ fallen, so hat man dieses Zeichen, als sich von selbst verstehend, gewóhnlich weggelassen und nur dann ausdrücklich gesetzt, wo es mit einem andern zusammen steht.

4

*

Wo in unsrer Tabelle gleich hinter dem Namen einer Familie Zahlen stehen, da bedeuten sie die in ihr bekannten fossilen Genera und Spezies, erste in grössern, letzte in kleinern Ziffern angegeben; gleich hinter Genus-Namen bedeuten Ziffern die fossilen Arten; - die Zahlen aber am Ende der Zeilen und so viel möglich unter der Rubrik $\mathrm{z}$ bedeuten beziehungsweise die lebend bekannten Genera und Spezies; - ein 0 hier und dort drückt aus, dass solche nicht bekannt sind, ein $\infty$ aber, dass deren Anzahl nicht näher bestimmt sey; $\smile$ heisst viele, $\frown$ wenige.

Nur in wenigen Fällen ist es möglich gewesen, alle fossilen Arten eines Geschlechtes in systematischer Ordnung (ohne Rücksicht auf geologische Verbreitung) aufeinander folgen zu lassen. Zuweilen ist es nur mit denen einer oder einiger Formationen geschehen. Bei den wirbellosen Thieren sind meistens die Arten eines Genus nur nach den Formationen, und in diesen nicht weiter geordnet. Sind die Arten weder geologisch noch alphabetisch eingereihet, 80 können sie als systematisch georinet betrachtet werden. 


\section{VEGETARILA.}

Cl. I. Plantae cellulares, p. 5.

Cl. I1. Plantae vasculares, p. 11.

I. MONOCOTYLEDONES, p. 11.

II. DICOTYLEDONES, p. 37.

SUPPLEMENTUM PLANTARUM, p. 61.

APPENDIX: ORgana PLANTARUM ELEMENTARIA, p. $7 /$. 


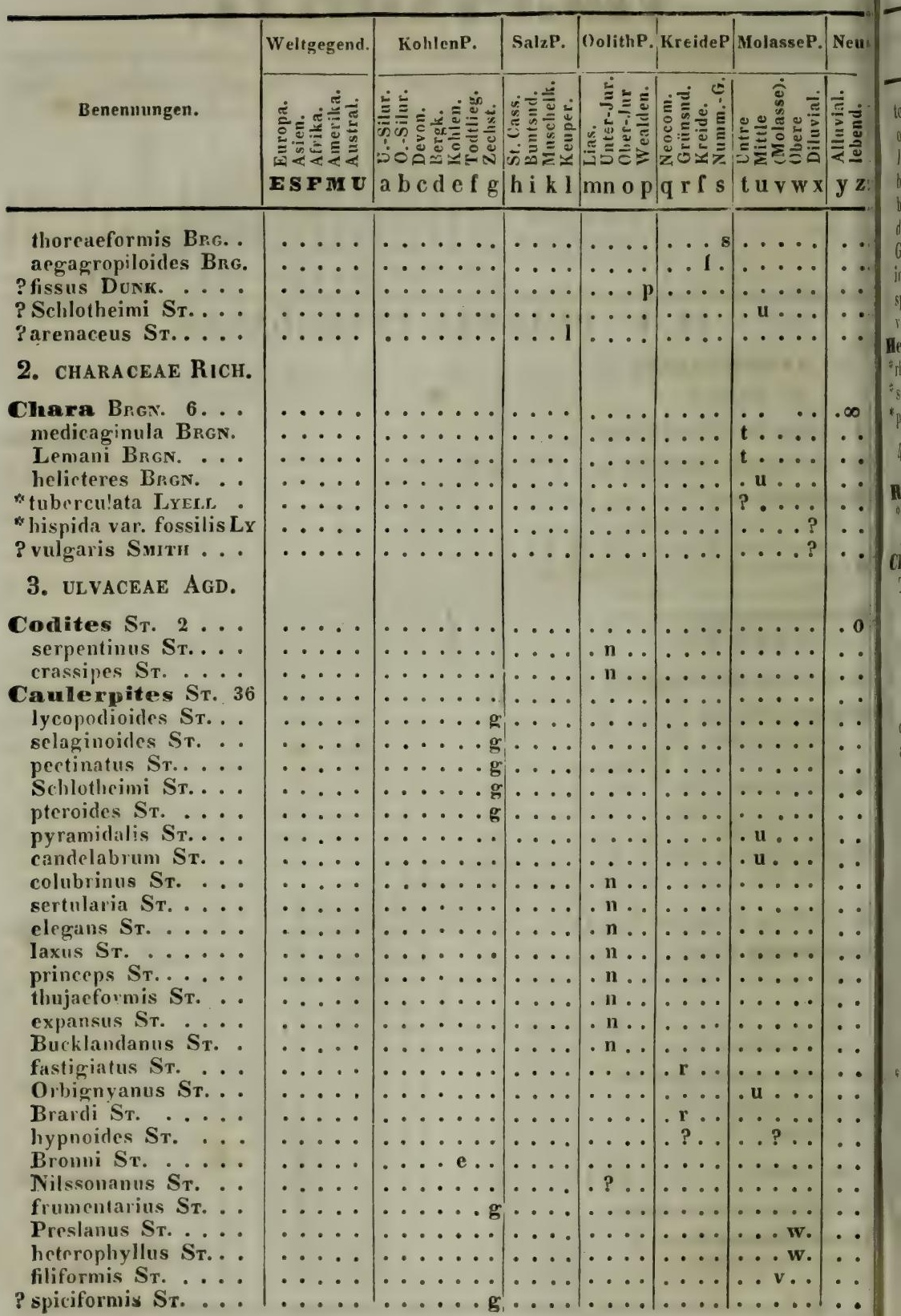




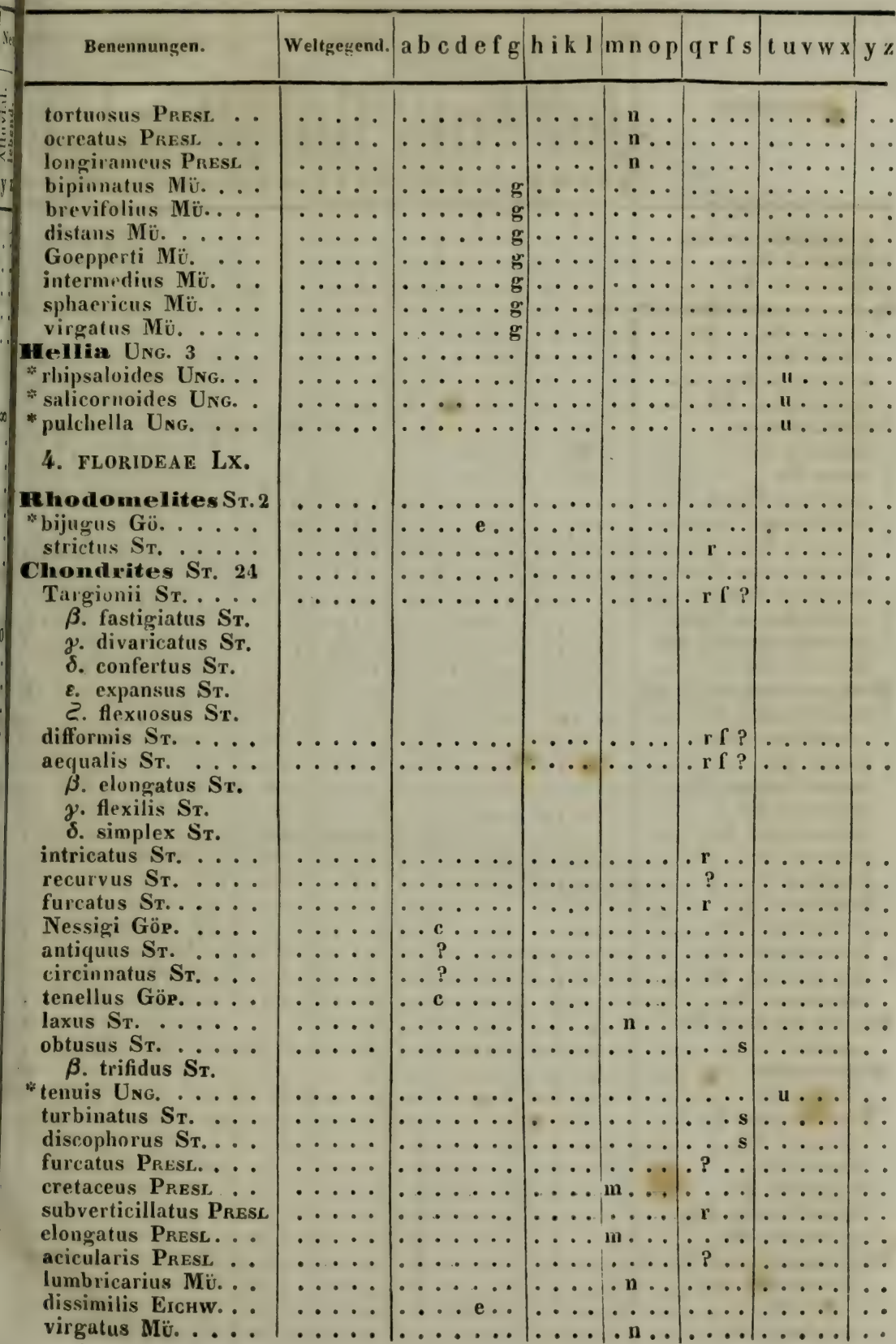




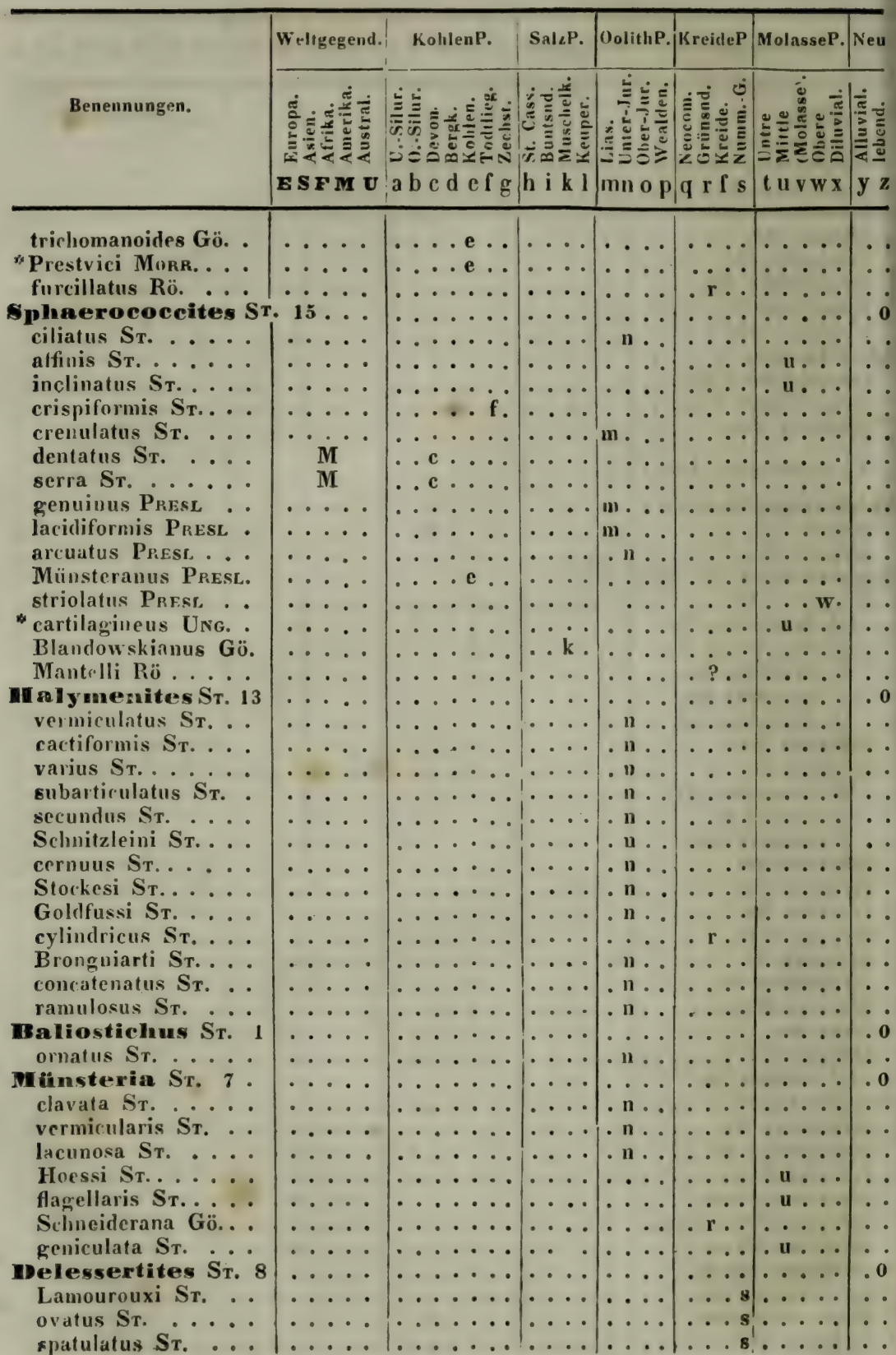




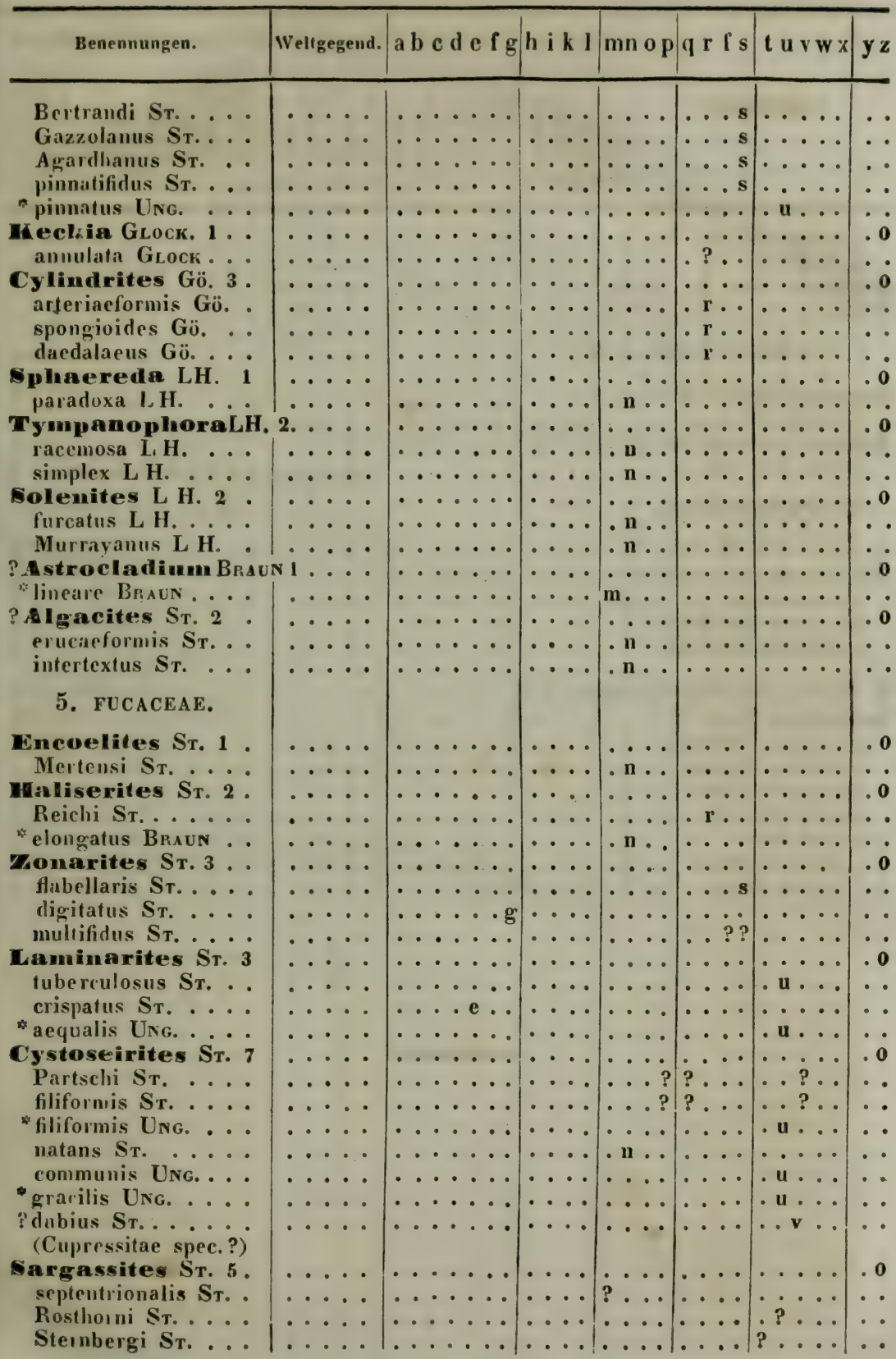




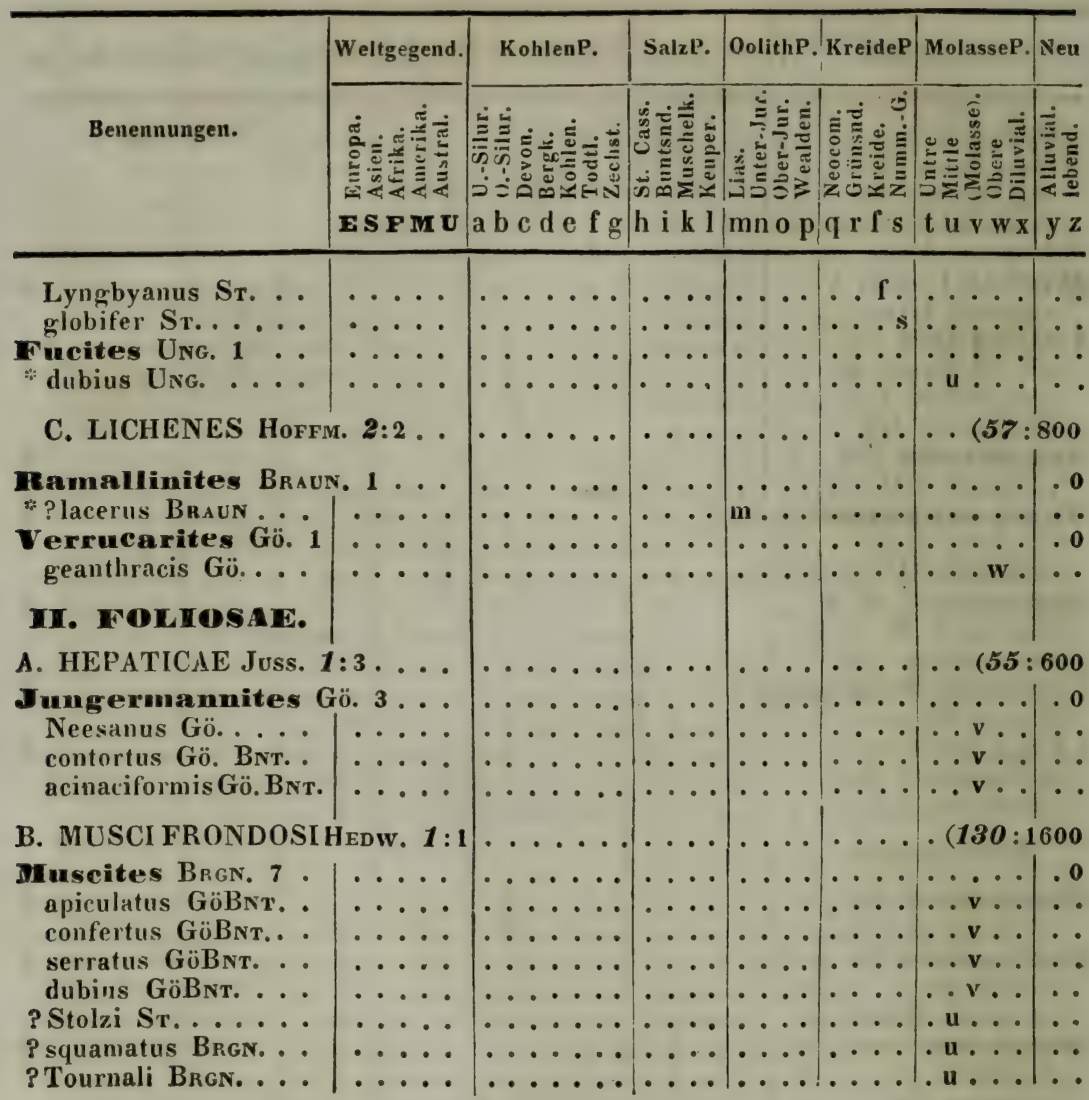




\begin{tabular}{|c|c|c|c|c|c|c|c|}
\hline & Weltgegend. & KohlenP. & SalzP. & OolithP. & KreideP & Molasse P. & Neu \\
\hline Benennungen. & 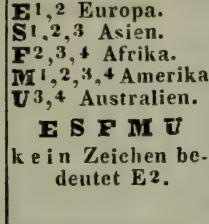 & 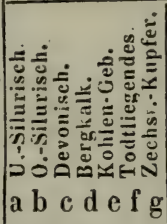 & 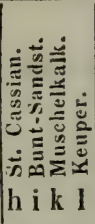 & 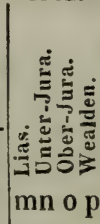 & 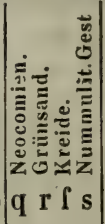 & 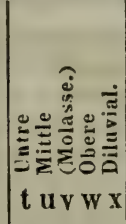 & 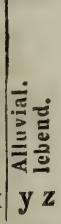 \\
\hline
\end{tabular}

\section{Cl. II. Plantae VASCULARES.}

\section{MONOCOTYLEDONES.}

\section{A. CRYPTOGAMAE.}

1. equisetaceae DC. $6: 84 \ldots$

Colramites Succ. 50 tenuissimus Gö.

dilatatus $\mathbf{G o ̈ .}$

remotissimus Gö. $\therefore$ transitionis Gö. . . . stigmarioides Gö... tuberculatus Gö. . . Voltzi Brign...... aequalis ST...... *affinis Gutb. ....

? alternansGerm.K AULF. approximatus Scheth. articulatus GutB. bistriatus ST.

Brongniarti ST.... cannaeformisSchLoth. Cisti Brgn. ..... concentricus ST. . Cottaanus Sr.. . . cruciatus ST. . . . decorafus Schloтн.. difformis ST.

? dubius ArT. Dirri Gutb. ...... elongatus Sr. . . gigas Bron. ..... inaequalis L H. . . infractus Gutb. ... ornatus ST. . . . . pachyderma .....

* Petzholdti Gutb. ranosus ART. .... regularis $S_{T}$.
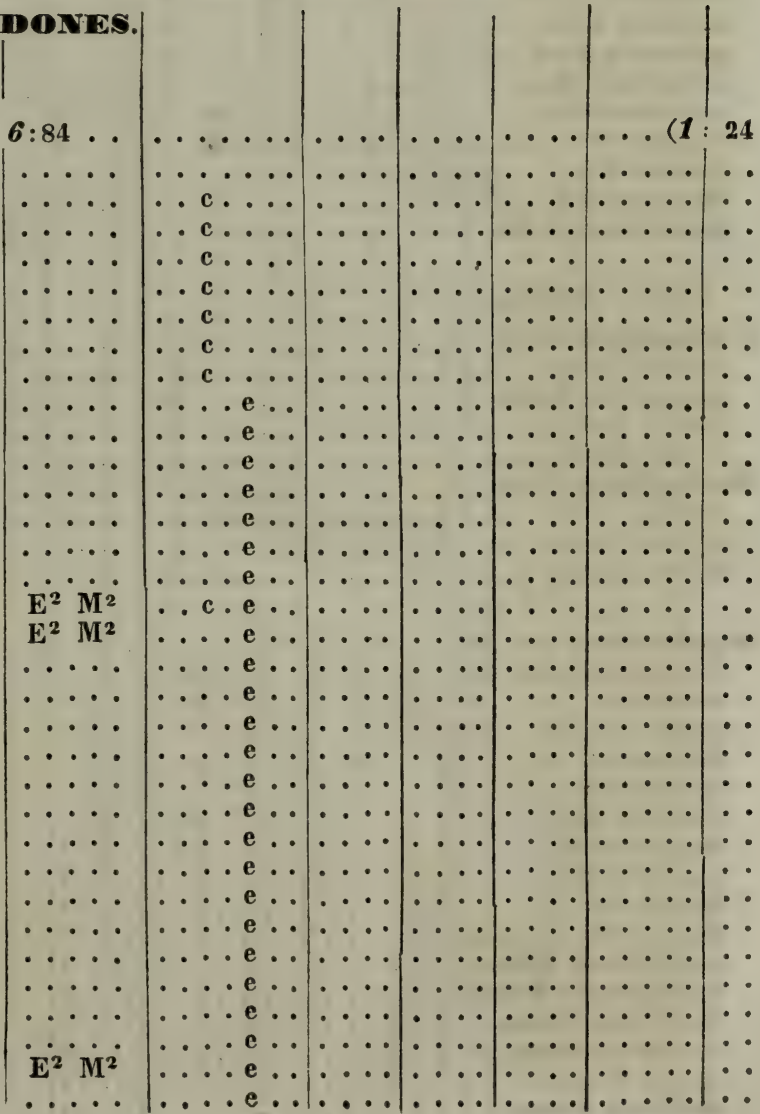
Steinhaueri ST.

Suckowi Brgn. sulcatus GutB.

*tripartitus GutB. tumidus ST.

tuberculosus GutB.. undulatus $\mathbf{S}$.

varians $S_{T}$.

verticillatus $\mathbf{L} \cdot \mathbf{H}$. cellulosus KuT.

trigonus KUT. .

columella Kur.

irregularis KuT.

a renaceus $\mathbf{J}$ ig.

lineatus $\mathbf{s}$.

Jaegeri ST.

Mougeoti LH.

Lehmannauus Gö.

Dubiae affinitatis:

Calamitea Cotra. 4 bistriata Cotra ... concentrica Cotra lineata Coтts.... . striata Corra

Medullosa Cotтs. 3 elegans Cotra ... porosa Cotta.... stellata Corta....

Equisetites Sr. 24 infundibuliformis ST. dubius $\mathbf{S}_{\mathrm{T}}$. columnaris $\mathbf{S}$ T. Schoenleini St.... radiatus $\mathrm{S}$. mirabilis ST. Minsteri ST. conicus $S$ T.

Bronni Sr.

Meriani St. moniliformis Prest. Roessertanus Presl Hoefleanus PresL. . cuspidatus Presl. acutus PresL elongatus Prest

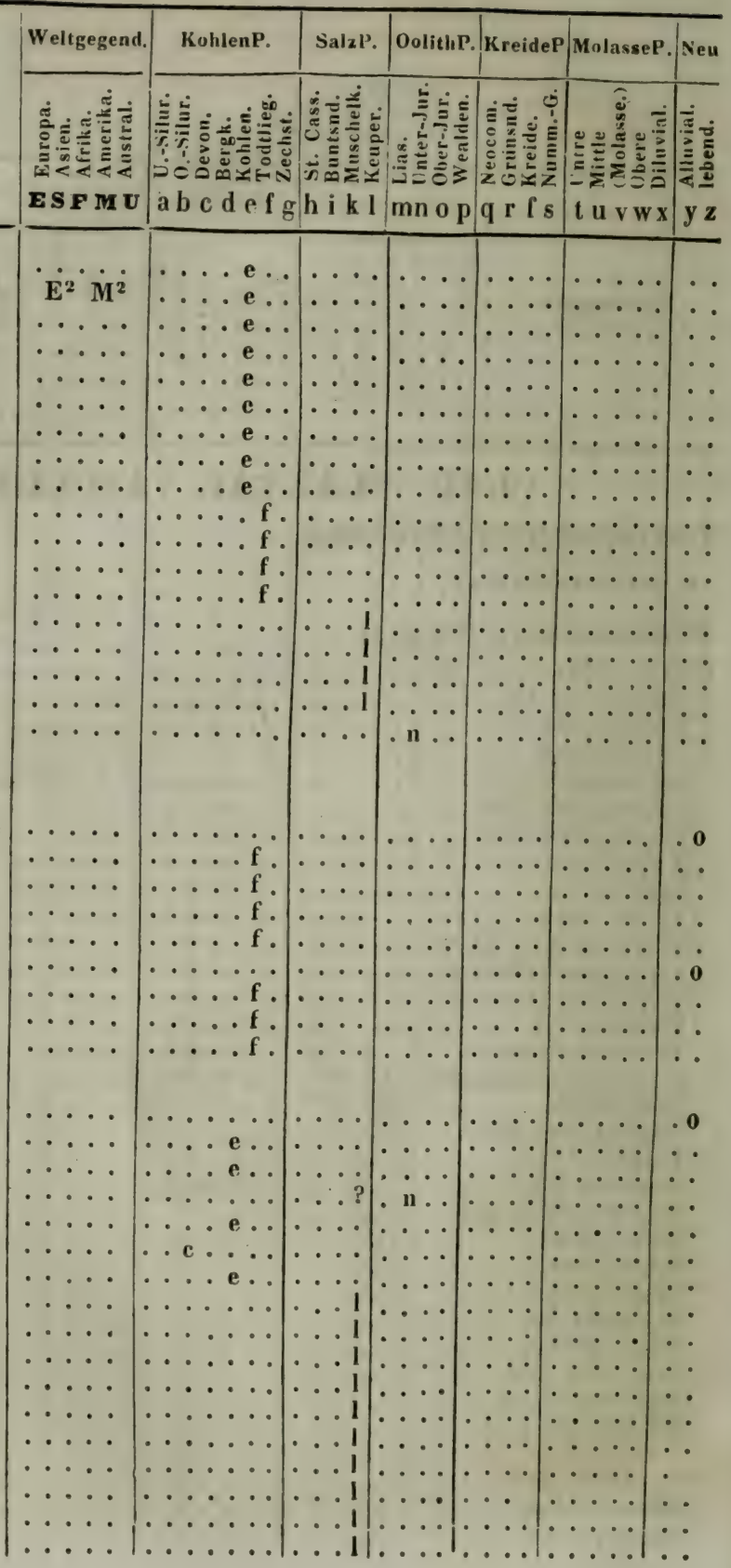




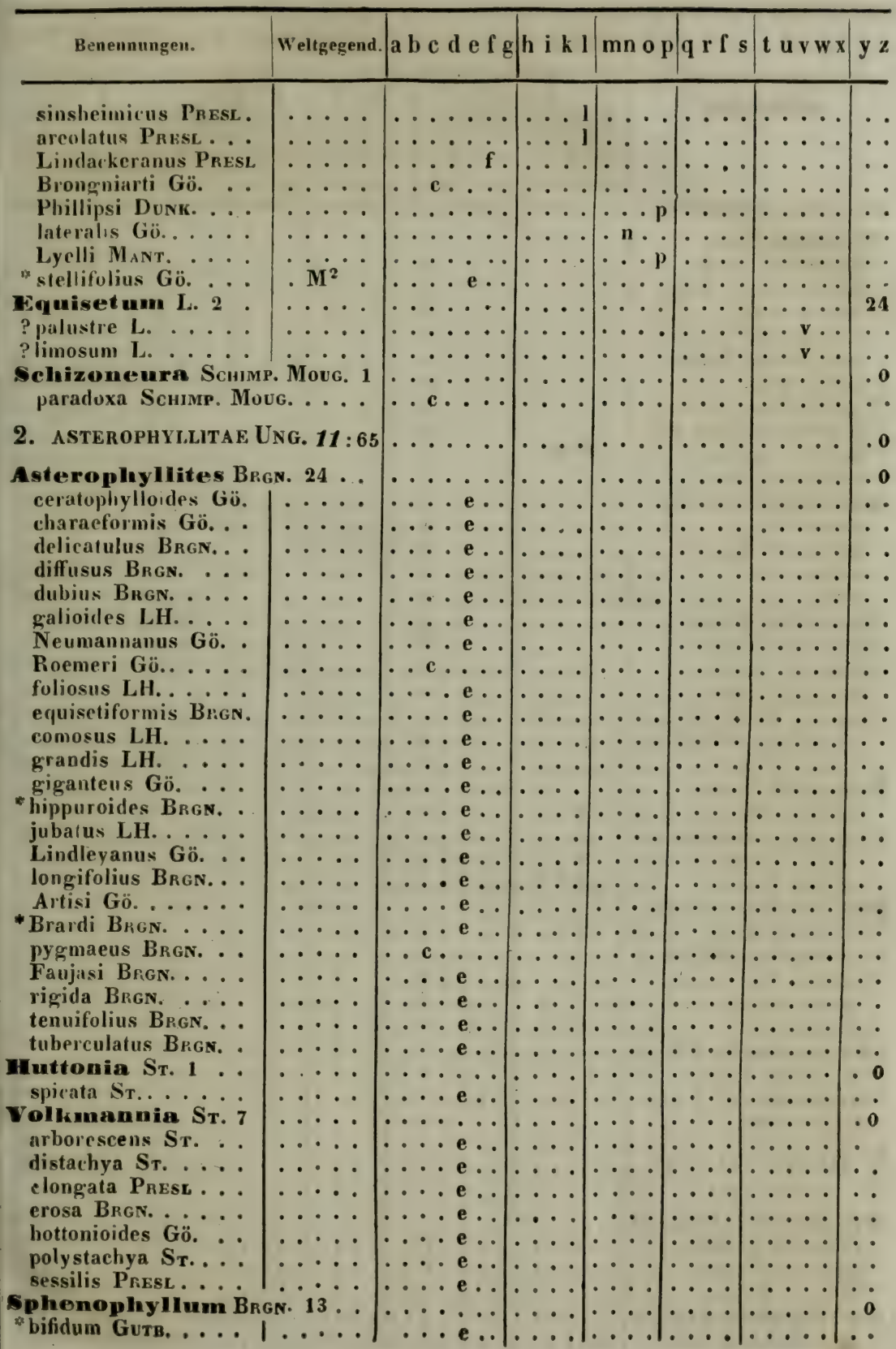




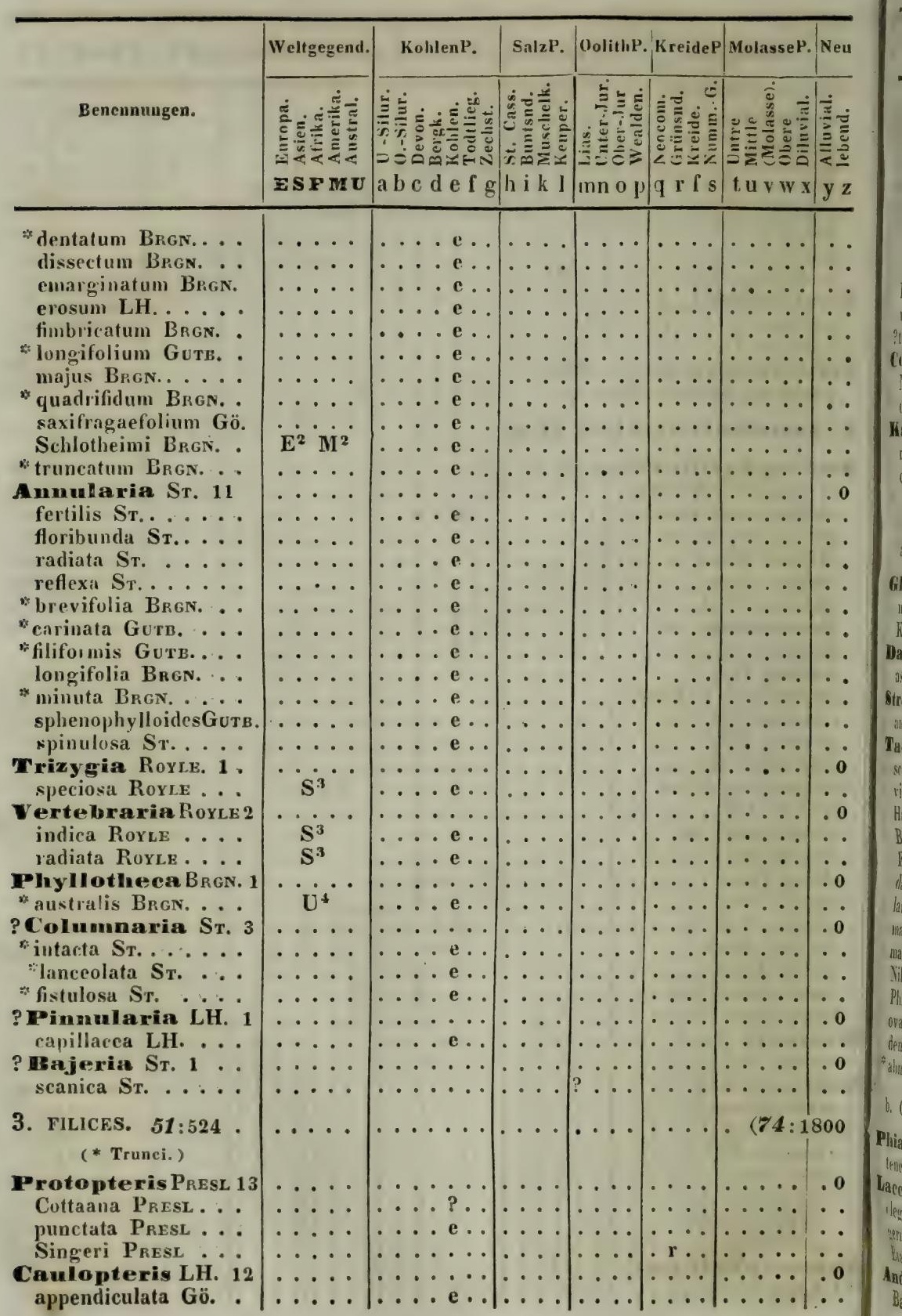




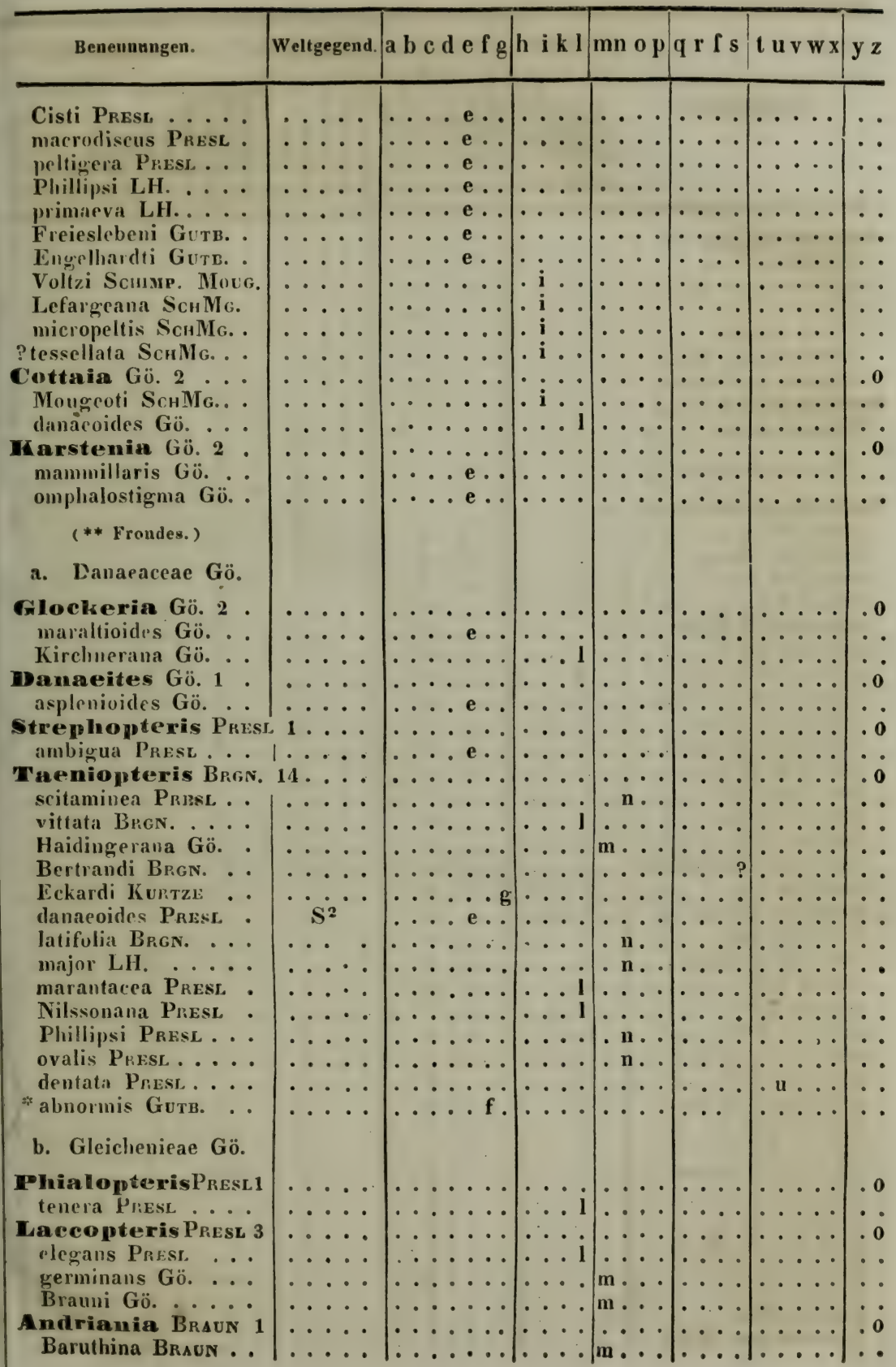




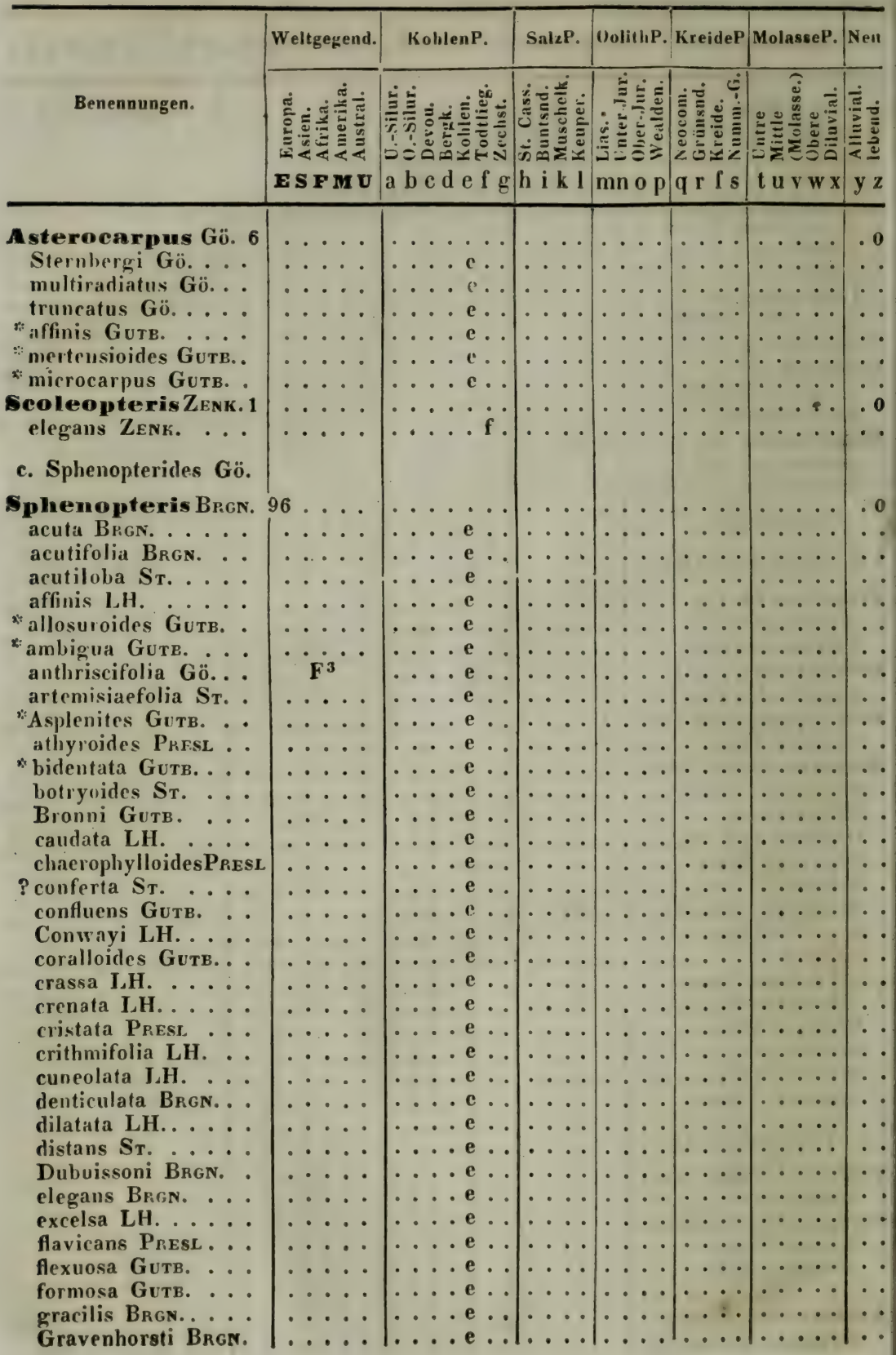




\begin{tabular}{|c|c|c|c|c|c|c|c|}
\hline 1. & end. & e $f \mathrm{~g}$ & 1 & $\mathrm{~m}$ & s $s$ & $\mathbf{x}$ & $\mathbf{z}$ \\
\hline 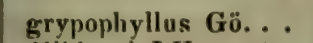 & $\cdots$ & 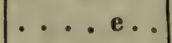 & & & & $\cdots \cdots$ & \\
\hline & .... & ....e. & $\cdots$ & 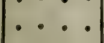 & $\ldots$ & $\ldots \ldots$ & \\
\hline RGX. & $\ldots$ & ...e. & $\cdots \cdot$ & $\cdots \cdot$ & $\ldots$ & $\ldots \ldots$ & \\
\hline$\cdots$ & $\cdots \cdot$ & $\ldots$ e.. & $\cdots \cdots$ & $\cdots \cdot$ & $\ldots$ & $\ldots$. & \\
\hline$\cdots$ & $\cdots \cdots$ & $\cdots \mathbf{e} \cdots$ & $\cdots \cdots$ & - & $\ldots$ & $\ldots \ldots$ & \\
\hline la & $\ldots \ldots$ & ....e. & $\cdots$ & & 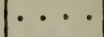 & $\cdots \cdot$ & \\
\hline la & • & $\cdots \mathbf{e}$ & $\cdots$ & 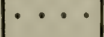 & $\cdots$ & $\cdots \cdot$ & \\
\hline$\cdots$ & 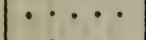 & $\cdots \mathbf{e} \cdot \cdot$ & & $\cdot \bullet$ & $\cdots \cdot$ & $\cdots \cdots$ & \\
\hline le & - & •.e. & & & & & \\
\hline li & & e... & $\because$ & & 1. & - & \\
\hline $\begin{array}{l}\mathbf{L} \\
\mathbf{L}\end{array}$ & $\because \ddot{0}$ & $\mid \begin{array}{ll}\cdots \\
\cdots & \cdots\end{array}$ & $\cdots$ & 1. & . & $\cdots$ & \\
\hline $\begin{array}{l}\text { L } \\
\text { ly }\end{array}$ & $\cdots$ & $\therefore$ e. & $\because \cdots$ & $:$ & $\therefore$ & $\because$ & \\
\hline Di. . & .... & $\ldots$.... & $\ldots$ & $\ldots$ & $\ldots$ & $\ldots$ & \\
\hline . . & $\cdots \cdot$ & .... & $\cdots \cdot$ & $\cdots \cdot$ & $\ldots$ & $\ldots .$. & \\
\hline тв. & $\cdots \cdots$ & $\ldots$ e. & $\cdots \cdots$ & $\cdots \cdot$ & $\ldots$ & $\ldots$ & \\
\hline & $\cdots \cdots$ & $\ldots$ e. & . ... & $\cdots \cdot$ & $\cdots \cdot$ & $\ldots \ldots$ & \\
\hline$\therefore$ & $\cdots \cdot$ & $\ldots$ e... & $\cdots \cdots$ & $\ldots \ldots$ & $\ldots$ & $\ldots \ldots$ & \\
\hline & $\cdot \cdots \cdot$ & $\ldots$ e... & $\cdots \cdots$ & $\cdots \cdot$ & $\cdots \cdot$ & $\ldots \ldots$ & \\
\hline B. & $\cdots \cdot$ & $\ldots$ e & $\cdots \cdots$ & $\cdots \cdot$ & $\cdots$ & $\ldots \ldots$ & \\
\hline$\cdot$ & $\cdots \cdot \cdot$ & 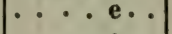 & $\cdots \cdots$ & $\cdots \cdot \cdot$ & $\cdots$ & $\cdots \cdots$ & \\
\hline . & $\cdots \cdot$ & $\ldots$ e. & $\cdots \cdot$ & $\cdots \cdot$ & $\cdots \cdots$ & $\cdots \cdots$ & \\
\hline$\therefore$ & .. & . e.. & $\cdots \cdots$ & $\cdots \cdot$ & $\cdots \cdot$ & $\cdots \cdots$ & \\
\hline . & - & . & -1 & $\cdots \cdots$ & . & $\cdots \cdots$ & \\
\hline$\therefore$ & : & : & : & $\therefore$ & . & $\cdots$ & \\
\hline$\therefore$ & … & $\because \cdots$ & $\therefore \ldots$ & $\cdots$ & $\cdots \cdots$ & $\left|\begin{array}{c}\cdots \\
\cdots\end{array}\right|$ & \\
\hline$\because$ & .... & $\ldots$ e. & $\ldots$ & $\ldots$ & $\ldots$ & $\ldots .$. & \\
\hline$\therefore$ & ... & $\ldots$ e. & . & $\cdots \cdot$ & $\ldots$ & $\ldots .$. & \\
\hline$\ldots$ & $\cdots$ & $\ldots$ e... & $\cdots \cdot \cdot$ & $\cdots \cdot$ & $\ldots$ & $\ldots \ldots$ & \\
\hline & $\cdots \cdots$ & $\ldots$ e... & $\cdots \cdot$ & $\cdots$ & $\cdots$ & $\ldots .$. & \\
\hline$\therefore$ & $\cdots \cdot$ & $\ldots$ e... & $\cdots \cdot$ & $\cdots \cdot$ & $\ldots$ & $\ldots$ & \\
\hline r. . . & . . & $\ldots$ e... & $\cdots \cdot$ & $\cdots \cdots$ & $\cdots \cdot$ & $\ldots \ldots$ & \\
\hline$\cdots$ & $\cdots \cdots$ & $\ldots$ e. & $\cdots \cdot$ & $\cdots \cdot$ & $\ldots$ & $\ldots .$. & \\
\hline$\cdots$ & $\cdots \cdot$ & $\ldots$ e. & $\cdots$ & $\cdots \cdot$ & $\cdots \cdot$ & $\ldots \ldots$ & \\
\hline$\cdots$ & $\cdots$ & . e.. & $\cdots$ & $\cdots \cdots$ & $\cdots$ & $\cdots \cdots$ & \\
\hline$\therefore$ & $\cdots \cdots$ & . e e . & $\cdots$ & $\cdots$ & $\cdot$ & $\ldots \ldots$ & \\
\hline SL. . & $\cdots \cdot \cdot$ & . e . & $\cdots \cdots$ & $\cdots \cdots$ & - & $\ldots \ldots$ & \\
\hline iv. . & $\cdots \cdots$ & . e. & $\cdots$ & $\cdots$ & - & $\cdot \cdot$ & \\
\hline$\cdots$ & $\cdots \cdot$ & - e.. & : & 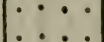 & • & $\cdots$ & \\
\hline & - & . & $\therefore 1$ & & & $\cdots$ & \\
\hline PrEsL. & $\cdots$ & & $\because 1$ & & $\therefore \cdots$ & $\therefore \cdots$ & \\
\hline RESL. . & $\cdots$ & . & . . 1 & $\cdots$ & $\cdots \cdot$ & $\ldots .$. & \\
\hline ESL & $\cdots \cdot$ & . & & $\cdots \cdot$ & . & $\cdots \cdots$ & \\
\hline Gö. & & & & & & & \\
\hline$\therefore$ & $\cdots \cdots$ & $\cdots \cdots \cdots$ & $\cdots \cdots$ & $\mathrm{m} \cdots$ & $\cdots$ & $\cdots \cdots$ & \\
\hline & & & & & & $\cdots \cdot$ & \\
\hline 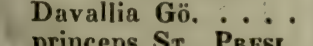 & & & & $\int_{m}^{m}$ & & •. & \\
\hline$\therefore$ & •. & $\cdots$ & $\cdots \cdots$ & 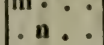 & $\cdots \cdots$ & $\cdots$ & \\
\hline ILI. & $\cdots \cdots$ & $\cdots$ & $\cdots \cdot$ & . & & & \\
\hline to & & & & & & & \\
\hline
\end{tabular}




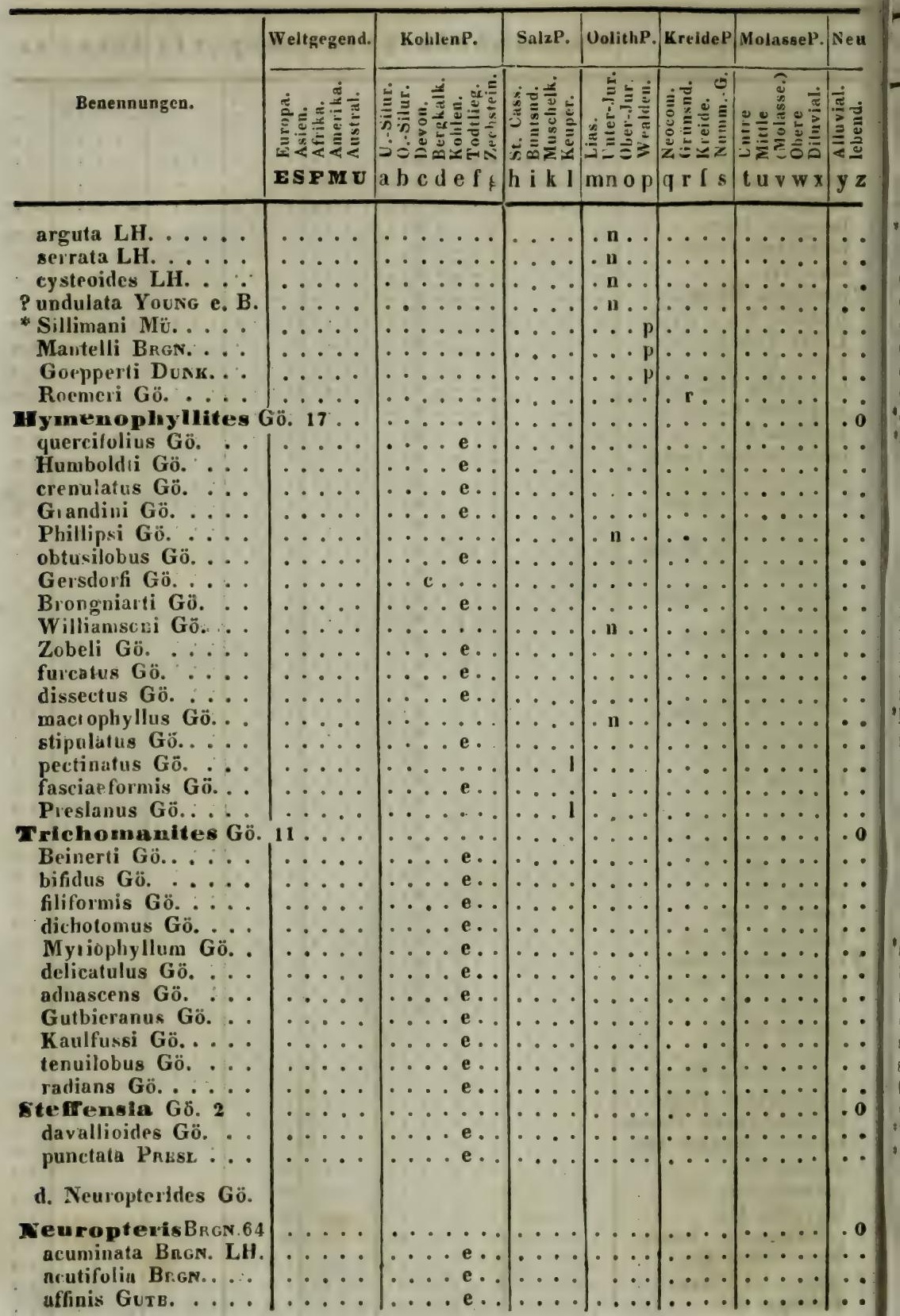


alpina Sr. angustifolia BRGN. attenuata $\mathbf{S}_{\mathrm{T}}$. auriculata $S_{T}$.

Brongniarti $\mathbf{S}_{\mathrm{T}}$.

* conformis Eicuw.

Cisti Brgn. conferta ST. confluens GutB. conjugata Gö. cordata BRGN. crenulata BrgN. decurrens $S_{T}$.

- dichotoma Fiscr.

dickebergensisHoffm. distans $\mathbf{S}_{\mathrm{T}}$.

flexuosa ST. ..... gigantea $\mathrm{ST}$.

Grangeri BugN. heterophylla Sr. ingens $\mathrm{LH}$.

lanceolata String. Lindleyana ST. lubifolia Preston. Loshi LH. macrophylla BHGN.

* Martini ST. microphylla BRGN. mirabilis Rost oblongata ST. obliqua Gö. obovata ST. ovata HofrM. plicata $\mathrm{S}_{\mathrm{T}}$. rotundifolia BRGN. rubescens Presc

* salicifolia Fisch. Scheuchzeri Brgn. serrata $\mathrm{S}_{\text {T. }}$. Soreti BrgN. smilacifolia ST. . subcrenulata Rost. tenuifolia ST. thymifolia Sr. . . .

* Wangenheimi Frsch. * pinnatifida Gutb. grandifolia Scurmp. . Dufresneyi Brgn. elegans BrGN. imbricata Sснімp. MG. intermedia Scнімp.

\begin{tabular}{|c|c|c|c|c|c|c|}
\hline & (2) & & & ・・・ & $\cdots$ & \\
\hline & - e & $\cdots$ & $\cdots$ & $\cdots \cdot$ & $\cdots$ & \\
\hline & ..e & - & $\cdots$ & $\cdots$ & $\cdots$ & \\
\hline & ...e & - & $\cdots$ & $\cdots$ & $\cdots \cdots$ & \\
\hline s & $\ldots$ & $\cdots$ & $\cdots$ & $\cdots$ & $\cdots \cdots$ & \\
\hline - & ・ & & & $\cdots \cdot$ & $\cdots$ & \\
\hline - & $\cdots$ & . & $\cdots$ & • . • & $\cdots \cdots$ & \\
\hline • & - & & - & & & \\
\hline • & 0 & & : & $\dot{.}$ & & \\
\hline 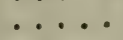 & . & . & .. & . & . & \\
\hline - & $\ldots$ & $\cdots$ & .. & $\cdots$ & $\ldots$ & \\
\hline 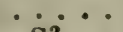 & ...e & $\cdots$ & . . & $\ldots$ & $\cdots$ & \\
\hline $\mathbf{S}^{2} \ldots$ & $\cdots$ & - • • & $\ldots$ & $\cdots$ & $\ldots$ & \\
\hline • & $\cdots$ & $\cdots \cdot$ & $\ldots$ & $\cdots \cdots$ & $\cdots$ & \\
\hline : & ...e & $\cdots$ & $\cdots$ & $\cdots \cdots$ & $\cdots$ & - \\
\hline 2. & $\ldots \mathbf{e}$ & $\cdots$ & $\cdots$ & $\cdots$ & . . & \\
\hline$\bullet$ & ..e... & $\cdots$ & $\cdots$ & $\cdots \cdots$ & $\cdots$ & \\
\hline$\cdots$ & ...e & $\cdots$ & $\cdots$ & $\cdots \cdots$ & $\cdots$ & \\
\hline$\cdots$ & $\cdots \mathbf{e}$ & $\cdots$ & $\cdots$ & $\cdots$ & $\cdots \cdots$ & \\
\hline : & $\cdots$ & . & $\cdots$ & $\cdot \cdot$ & $\cdots$ & \\
\hline • & $\cdots$ & $\cdots$ & $\cdots$ & - & ${ }^{\circ}$ & \\
\hline . & $\cdots$ & & $\cdots$ & $\cdot$ & $\because$ & 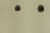 \\
\hline$E^{2} \cdot M^{2}$ & - c e & • • & $\therefore \cdots$ & $\ldots$ & .... & \\
\hline$\cdots$ & ... e . & $\cdots$ & $\cdots$ & $\ldots$ & $\ldots$ & 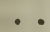 \\
\hline - & ...e & - & $\cdots$ & $\cdots \cdots$ & $\cdots \cdot$ & \\
\hline $\mathbf{M}^{2}$ & $\ldots$ & $\cdots$ & $\ldots$ & $\cdots$ & $\ldots$ & \\
\hline - & ...e & $\cdots$ & $\cdots$ & $\cdots$ & $\cdots$ & \\
\hline$\cdots$ & $\cdots$ & $\cdots$ & $\cdots$ & $\cdot$ & $\cdots \cdot$ & \\
\hline & . & $\cdots$ & $\cdots$ & $\cdot$ & $\cdots \cdot$ & \\
\hline & $\cdots$ & $\cdots$ & $\cdot$ & - & $\cdots$ & \\
\hline$\cdots$ & - . & - & $\cdot$ & $\cdot$ & $\cdots$ & \\
\hline - & $\cdots$ & • & : & $\dot{\bullet}$ & $\therefore$ & \\
\hline & $\ldots A, \ldots$ & $\cdots$ & . . . & $\cdots$ & $\cdots \cdots$ & \\
\hline $\mathbf{S}^{2}$ & . e e & $\ldots$ & $\ldots$ & $\ldots$ & $\ldots$ & $\cdot$ \\
\hline - & ... e & $\cdots$ & $\cdots$ & $\cdots$ & $\cdots$ & $\cdot$ \\
\hline$\cdots$ & $\ldots e$ & $\cdots$ & . & - & $\ldots$ & \\
\hline$\cdots$ & $\cdots \mathbf{e}$ & . & $\cdots$ & . & $\ldots \ldots$ & \\
\hline$\cdots$ & - & $\cdots$ & $\cdots$ & - & - & \\
\hline 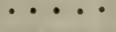 & $\cdots \mathbf{e}$ & • & & - $15-2$ & $\cdots$ & \\
\hline & 6 & & $\because$ & & $\cdots \cdots$ & \\
\hline$S^{2}$ & $\ldots$ e . & $\cdots \cdots$ & $\ldots$ & $\cdots$ & $\ldots$ & \\
\hline$\cdot$ & $\ldots \ldots f$ & & $\ldots$ & $\cdots$ & $\ldots \ldots$ & . \\
\hline & $\cdots$ & & • & . & $\cdots \cdots$ & \\
\hline & - & i... & - & . & $\ldots \ldots$ & \\
\hline & 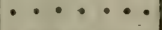 & & & - & $\cdots \cdots$ & \\
\hline & $\bullet$ & & & $\cdots$ & $\because$ & \\
\hline & & & & & & \\
\hline
\end{tabular}
Voltzi BrgN. 


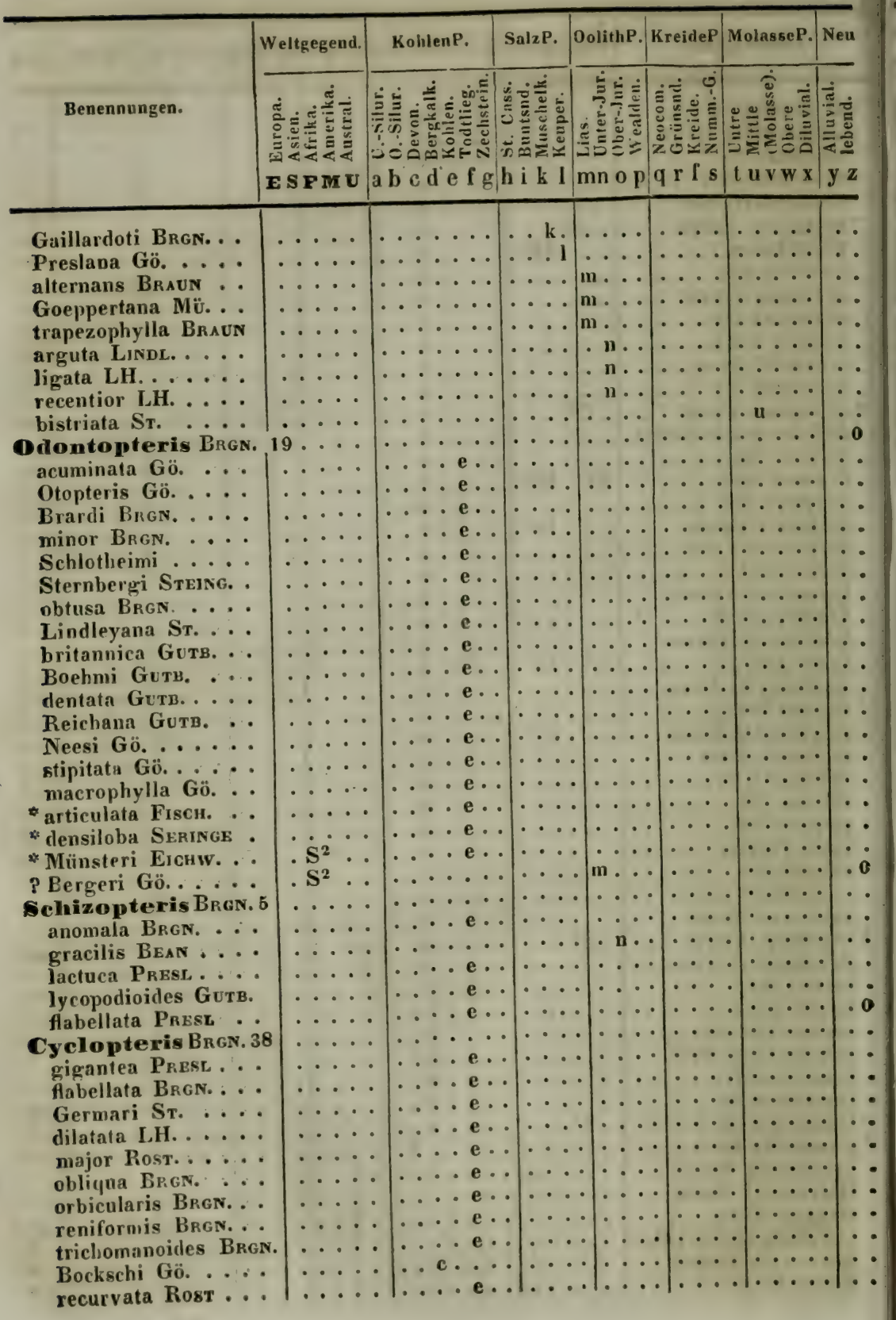


alpina Presr. .... amplexicaulis GutB.

Beani LH. .

otopteroides Gö.

crassa Prese

cuneata Presc

inaequalis Gutr.

Sternbergi Gutr.

terminalis GutB.

varians GuxB. .

Villiersi Sr......

heterophylla Gö. adiantoides Presc

frondusa Gö. ...

Murchisoni Presl. nervosa Gö.

oblata LindL. ....

oblongifolia Presr.

obovata PresL

polyphylla Presr.

* semiflabelliformis Morr. tenuifolia Gö.

triloba Gö.

Huttoni Sr.

dissecta Gö.

digitata BrGN.

* Fischerana Gö....

Dietyopteris GutB. 1

Brongniarti Gutr.

NoeggerathiaSt.10

foliosa St.

flabellata LH. ....

Beinertana Gö.

aequalis Gö.

distans $\mathbf{G o ̈}$.

Kutorgai Gö.

obliqua Gö.

ovata Gö.

Puschana Gö.

pusilla Gö.

e. Pecopterides Gö.

a. Nervis secundariis anostomosantibus.

Ctenis LH. 1 .

falcata LH.

Clossopteris Brinis angustifolia BrgN.

Brownana Brgn.

? coriacea Sterisg.

SagenopterisPresl4 rhoifolia Prest

elongata $\mathrm{MÜ.}$.

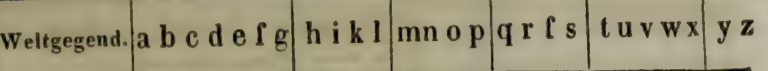

\begin{tabular}{l|l|l|l|l|}
\hline &
\end{tabular}




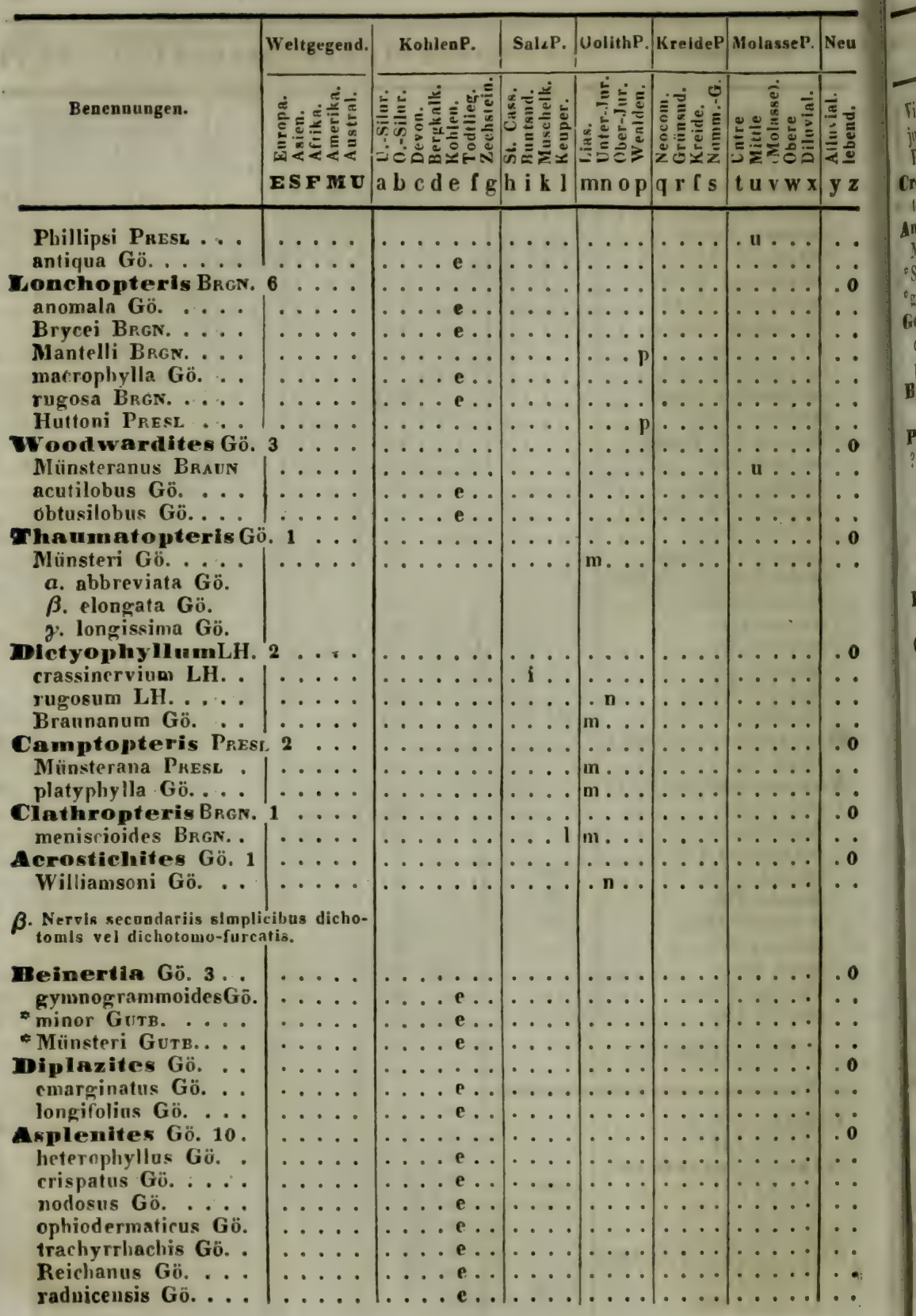




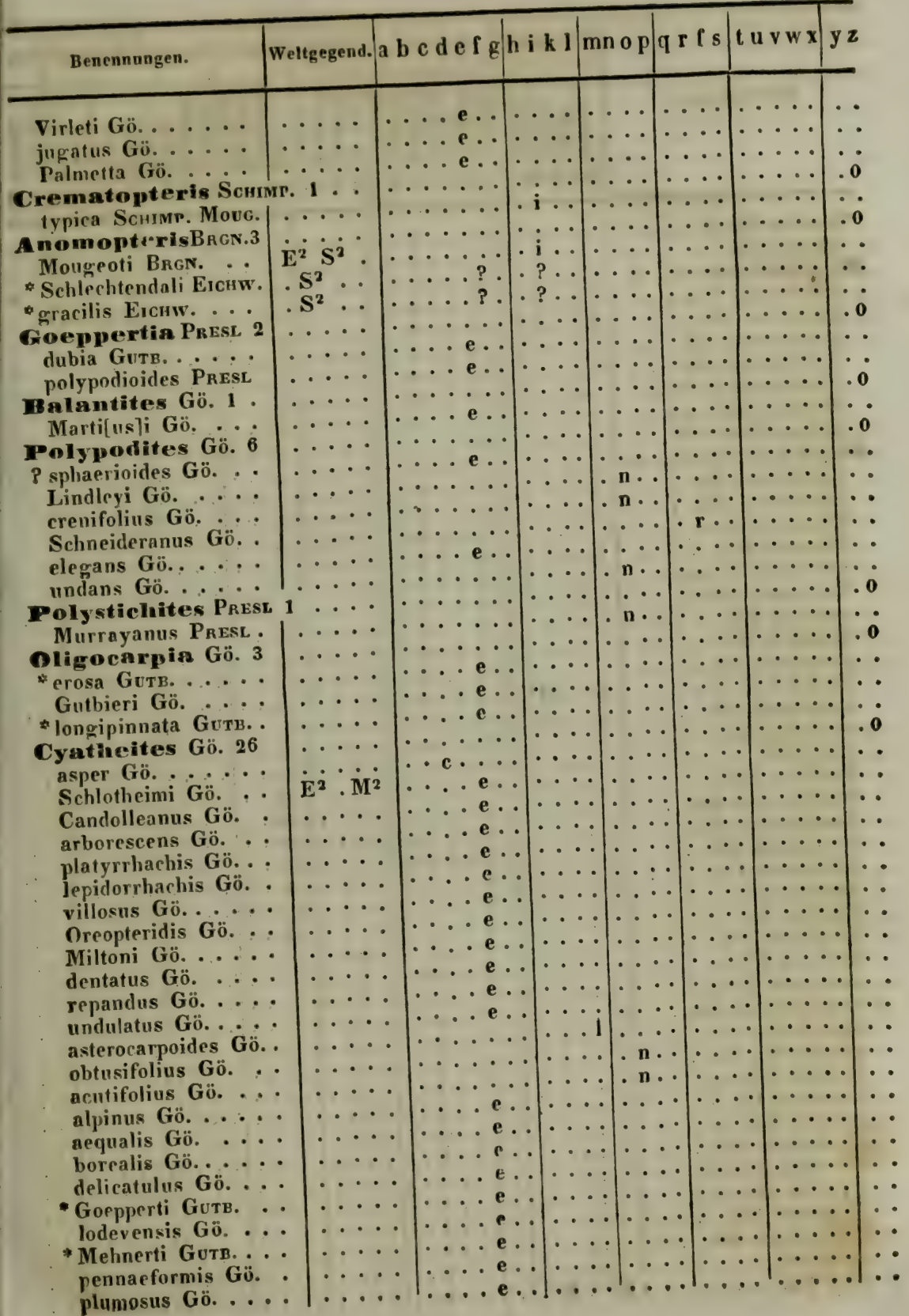




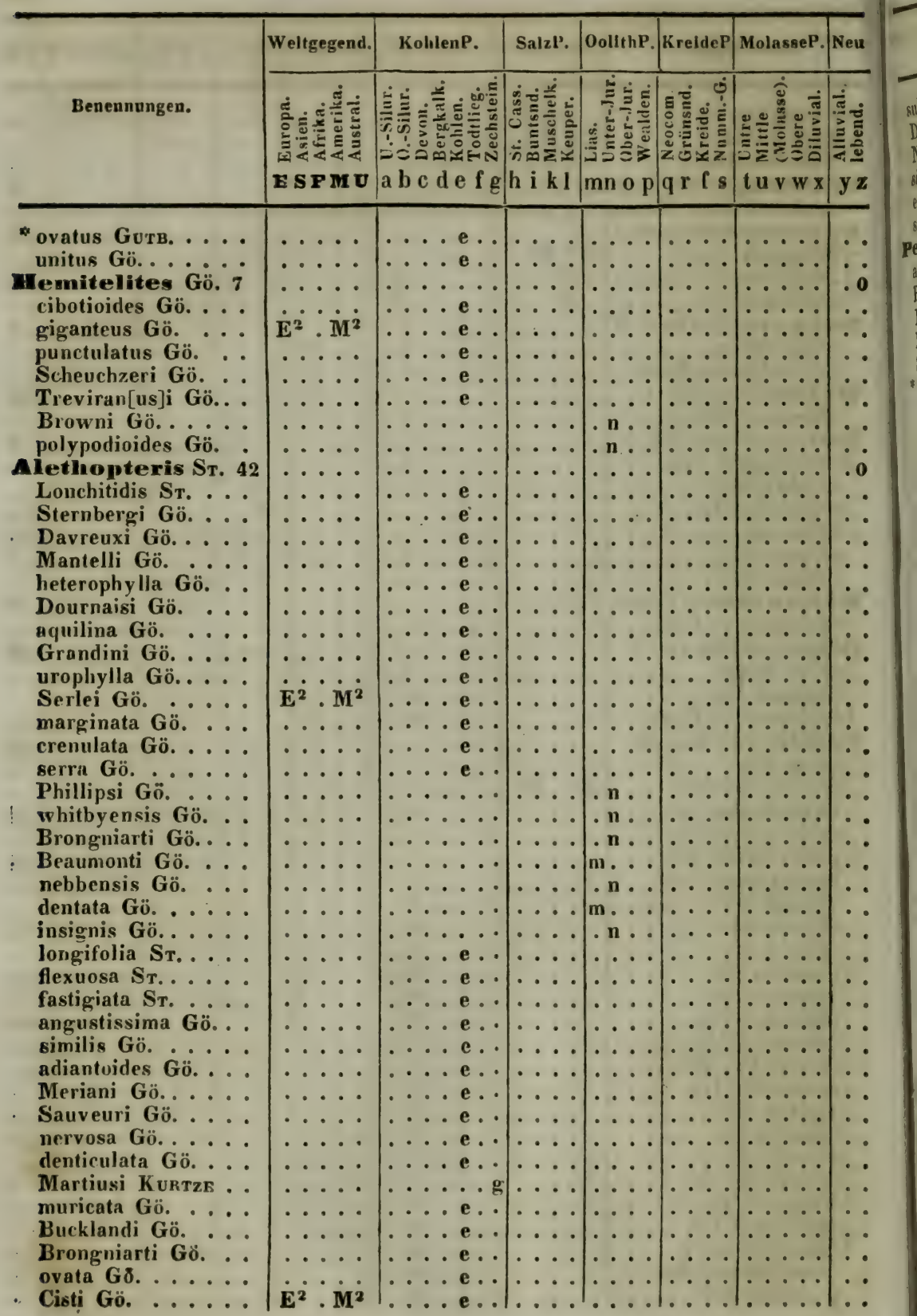




\begin{tabular}{|c|c|c|c|c|c|c|c|}
\hline & egend. & e $f g$ & h i k 1 & $\mathrm{p}$ & s & $\mathbf{w} \mathbf{x}$ & 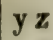 \\
\hline sulz & 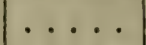 & & 1.8 & $0^{\circ}+2$ & & & \\
\hline Gö. . . . & $\cdots \cdot$ & $\ldots$... & $\ldots$ & $\ldots$ & .... & .... & \\
\hline Nestlerana Gö. ... & $\cdots \cdot$ & $\ldots$ e... & $\cdots \cdot$ & $\cdots$ & $\ldots$ & $\cdots \cdots$ & \\
\hline$\therefore \cdots$ & $\cdots \cdots$ & $\ldots$ e... & $\cdots$ & $\ldots$ & $\ldots$ & $\cdots \cdots$ & \\
\hline$\cdot \quad \cdots$ & $\ldots \ldots$ & $\ldots$. & $\cdots \cdot$ & $\cdots$ & $\cdots$ & $\ldots$ & \\
\hline$\cdots$ & - & $\ldots$ e & - & $\cdots \cdot$ & $\cdots$ & $\cdots \cdots$ & \\
\hline RGN. 60 & - & $\cdots \cdot \cdot$ & $\cdots$ & $\cdots \cdot$ & $\cdots$ & $\cdots \cdots$ & .0 \\
\hline N... & $\cdots \cdot$ & . e.. & - & $\cdots$ & $\cdots$ & $\cdots$ & \\
\hline$\cdots$ & $\cdots \cdot$ & $\cdots \cdots$ & $\cdots \cdot$ & $\cdots \cdot$ & $\cdots$ & $\cdots \cdots$ & \\
\hline$\cdot$ & $\cdots \cdots$ & $\cdots$ e $\ldots$ & $\cdots \cdot$ & $\cdots \cdots$ & $\cdots$ & $\cdots \cdots$ & \\
\hline$\cdots$ & .0 .0 & $\ldots$ e... & $\cdots$ & $\cdots \cdot$ & $\cdots$ & $\cdots \cdots$ & \\
\hline N. . . . & & . e . & $\cdots$ & $\cdots \cdots$ & $\cdots$ & $\cdots$ & \\
\hline ua $\mathbf{S}_{\mathrm{T}} \ldots \ldots$ & & - & & $\cdots \cdots$ & $\cdots$ & $\cdots$ & \\
\hline$\therefore \ldots$. & & e.. & • & - 1 & $\cdots$ & $\cdots$ & \\
\hline$\cdots$ & & $\cdots$ & $\cdots$ & $\cdots \cdots$ & $\cdots$ & $\cdots$ & \\
\hline$\cdots \cdots$ & $\begin{array}{l}\cdots \\
\cdots\end{array}$ & $\begin{array}{cc}\cdots & \ldots \\
\cdots & \mathrm{e}\end{array}$ & - & $\begin{array}{l}\cdots \\
\cdots \\
\cdots\end{array}$ & $\cdots$ & $\cdots$ & \\
\hline . & $\begin{array}{l}\cdots \\
\cdots\end{array}$ & $\cdots$ & $\because$ & $\left|\begin{array}{c}\cdots \\
\cdots \\
\cdots\end{array}\right|$ & $\cdots$ & $\cdots$ & \\
\hline$\cdots$ & ... & $\ldots$. . & $\ldots$ & $\ldots$ & $\therefore \cdots$ & $\cdots$ & \\
\hline$\ldots$ & $\ldots$. & .... & $\ldots$ & $\ldots$ & $\ldots$ & $\ldots \ldots$ & \\
\hline T. . . & $\cdots$ & $\ldots \ldots$ & $\ldots$ & $\ldots$ & $\ldots$ & $\ldots \ldots$ & \\
\hline$\ldots$ & $\cdots$ & $\ldots$ e $\ldots$ & $\ldots$ & $\ldots$ & $\ldots$ & $\ldots .$. & \\
\hline ... & $\cdots \cdot \cdot$ & $\ldots \mathbf{e} \ldots$ & $\cdots$ & $\ldots$ & $\ldots$ & $\ldots$. & \\
\hline & $\cdots \cdot$ & $\ldots \mathbf{e} \ldots$ & - & • & $\cdots$ & $\cdots \cdots$ & \\
\hline ชo. . . & $\cdots \cdot$ & $\ldots$ e $\ldots$ & $\because \cdots$ & $\cdots$ & $\ldots$ & $\cdots \cdots$ & \\
\hline$\cdots$ & $\cdots \cdots$ & $\ldots$ e $\ldots$ & $\cdots \cdot$ & $\cdots$ & $\cdots$ & $\cdots \cdots$ & \\
\hline Yö. . . & $\cdots$ & $\ldots \ldots \mathbf{e}$ & $\cdots \cdot$ & $\cdots \cdots$ & $\cdots$ & $\cdots \cdots$ & \\
\hline$\therefore$ & .. & $\cdot \mathbf{e} \cdots$ & $\ldots$ & $\cdots \cdots$ & $\cdots$ & $\cdots \cdots$ & \\
\hline Gö. . & $\cdots \cdots$ & $\ldots \ldots$ & $\cdots \cdots$ & $\cdots \cdots$ & $\cdots$ & $\cdots$ & \\
\hline$\cdots$ & $\cdots$ & $\ldots \ldots$ & $\cdots$ & $\cdots \cdots$ & $\cdots$ & $\cdots \cdots$ & \\
\hline$\cdots$ & $\cdots$ & $\ldots \ldots$ & $\cdots \cdots$ & $\cdots \cdots$ & $\cdots$ & $\cdots \cdots$ & \\
\hline$\cdots$ & . & $\cdots \cdots$ & $\cdots$ & $\cdots \cdots$ & $\cdots$ & $\cdots \cdots$ & \\
\hline ró. $\ldots$ & $\bullet \cdot \cdot \cdot$ & $\mid \begin{array}{ll}\cdots & \mathbf{e} \\
\cdots & \mathbf{e}\end{array}$ & $\cdots \cdots$ & $\cdots \cdots$ & $\begin{array}{l}\cdots \\
\cdots\end{array}$ & $\cdots$ & \\
\hline folia Fisch. & $\ldots$ & $\ldots$ & $\cdots$ & $\cdots$ & $\ldots$ & $\ldots$ & \\
\hline 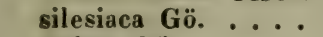 & $\ldots$ & $\ldots \ldots$ & $\cdots$ & $\ldots$ & $\ldots$ & ..... & \\
\hline$\cdots$ & $\cdots \cdot$ & $\ldots \ldots$ & $\ldots$ & $\ldots$ & $\ldots$ & $\cdots \cdots$ & \\
\hline .... & $\cdots \cdots$ & $\ldots$ e $\ldots$ & $\cdots$ & $\ldots$ & $\ldots$ & $\ldots \ldots$ & \\
\hline . & $\cdots \cdot$ & $\ldots$ e & $\cdots$ & $\cdots$ & $\cdots$ & $\ldots \ldots$ & \\
\hline$\cdots \cdot$ & $\cdots \cdot$ & $\ldots \mathbf{e} \ldots$ & $\cdots$ & $\cdots \cdot$ & $\cdots$ & $\cdots \cdots$ & \\
\hline ... & $\cdots$ & $\ldots$ e $\ldots$ & $\cdots$ & $\cdots \cdots$ & $\cdots \cdots$ & $\cdots \cdots$ & \\
\hline Gutb. & $\cdot$ & $\ldots$ e... & $\cdots$ & $\cdots \cdots$ & $\cdots$ & $\cdots \cdots$ & \\
\hline c & & & & - & & - & \\
\hline ESL . . & $\ldots \mathbf{U}$ & ...e. & $\cdots$ & 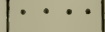 & & - & \\
\hline . & $\cdots \cdot$ & ... & $\ldots$ & & · & . 0 & \\
\hline SI. . & & - e & $\cdots$ & & $\cdots$ & . & \\
\hline$\ddot{0}$ & & . e .. & $\cdots$ & & & & \\
\hline 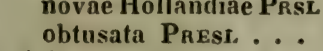 & . & . e .. & & $\cdots$ & $\ldots$ & $\cdots \cdots$ & \\
\hline$* 8$ & & .. & & & $\cdots \cdots$ & $\ldots \ldots$ & \\
\hline${ }^{*} \mathbf{L}$ & $\mathbf{M}^{2}$ & . & & $\cdots \cdot \cdot$ & . & $\cdots \cdots$ & \\
\hline LE . & & & & & & & \\
\hline & & & & & & & \\
\hline
\end{tabular}




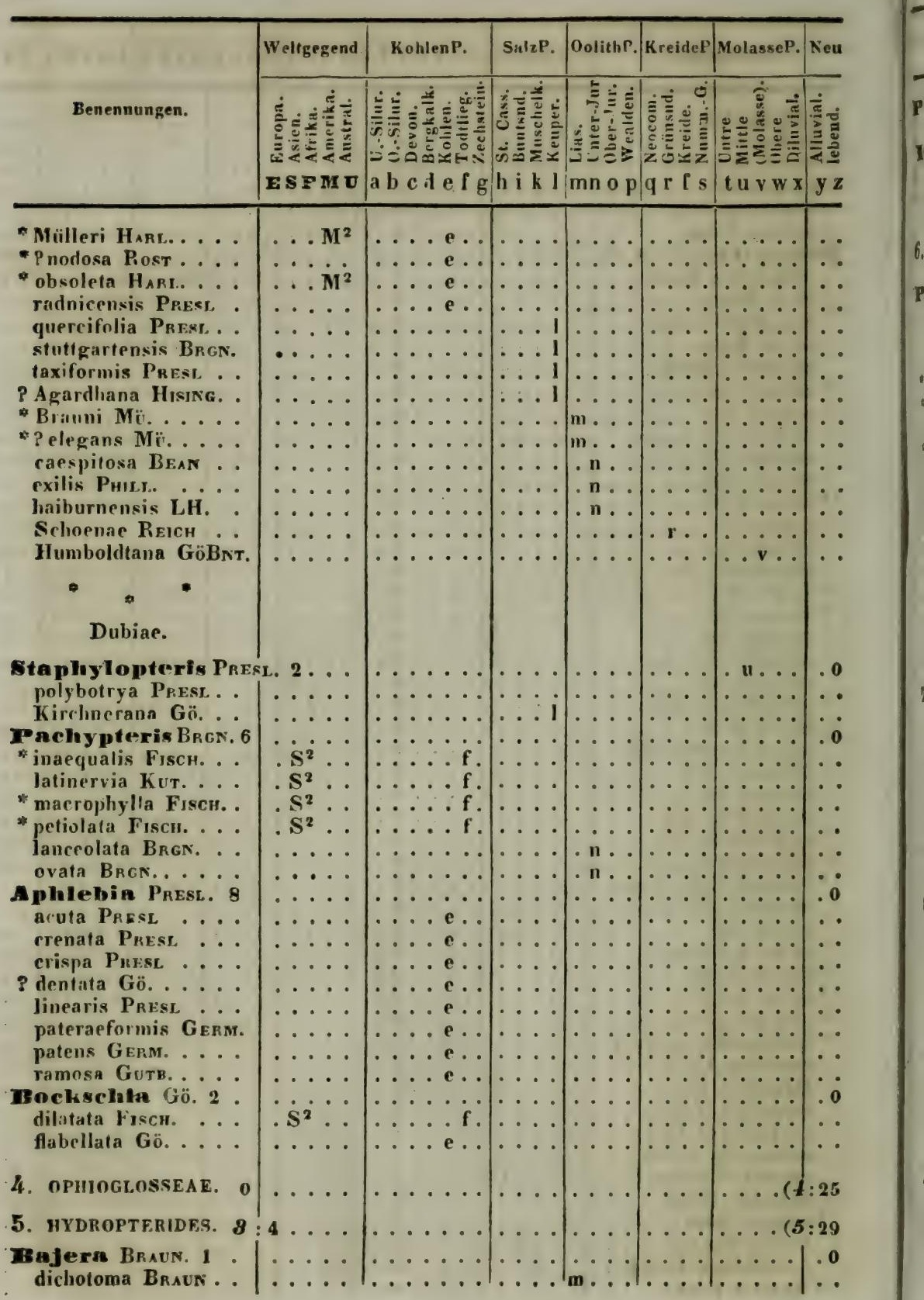


\begin{tabular}{l|l|l|l|l|l|l|l|}
\hline Benennungen. & Weltgegend. a b c d e f g h i k l mn op & q r c s & t u v w x & y $z$
\end{tabular}

Pilularites Gö. 1 . Brauni Gö.

Isoetites Gö. 2 crociformis $\mathrm{Mi}$.

Münsteri Gö.

6. pSaronieat Uno.

Psaronius Cotra. 13 infarctus Ung. helmintholithus UNG. scolecolithus Ung.

* medullosus Ung.

- radiatus Une. Asterolithus Cotra.

* Aacunosus Urg.

* Augustodunersis Ung.

- simplex Unc. intertextus Corta. dubius Corda cyatheaeformis CORDA parkeriaeformisCORDA

1.

$-\circ$

Dubiae affinitatis.

Tubienulis Cotta. 5 primarius Cotra Solenites Cotra. ramosus Cotta dubius Cotra.

7. stigmarieae Ung. Gö. 3:10 : 18

Stigmaria Brgn. 7 ficoides Gö.

$\beta$. undulata Gö. . : भ. reticulata Gö.

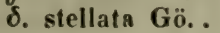

c. sigillarioides $\mathbf{G o ̈ . ~}$

* intermedia BrgN.

- minima BrgN.

* regularis Bren.

* rigida Brgn.

* tuberculosa BRgN.

* stellata Егснw.

Areistrophylimingö. stignariaeforme $\mathbf{G}$. minutum Gö.

Didymopleyilum Gö. Schottini Gö.
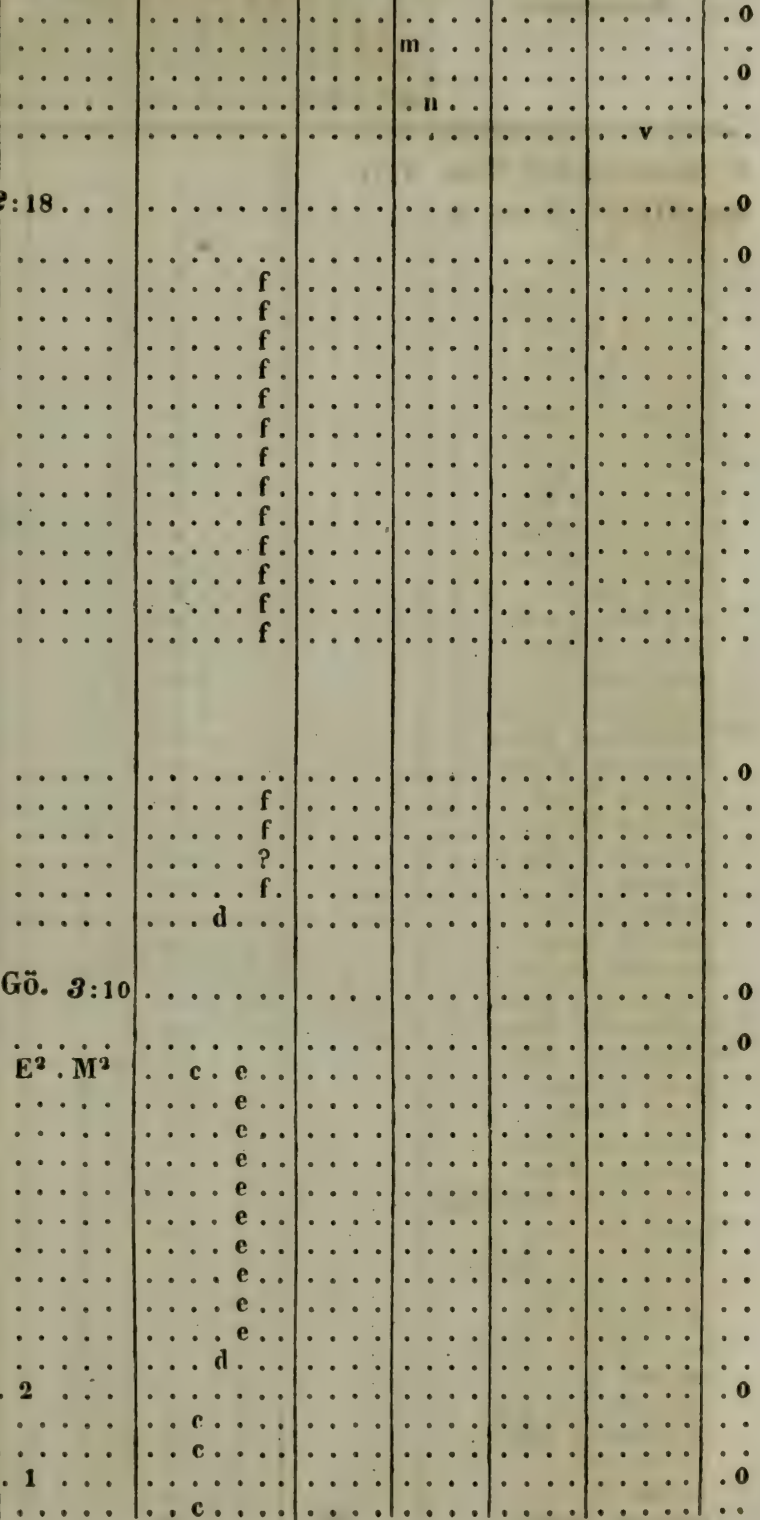


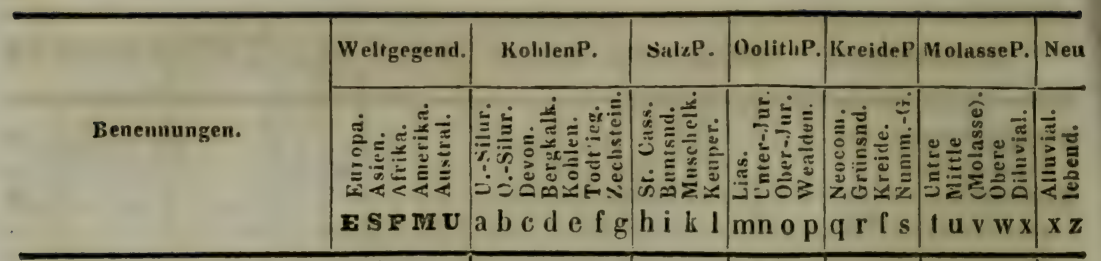

8. Sigillarizae UNG.

Gigillaria Bron. 71

* affinis Kön.

alternans $\mathbf{L H}$.

alveolaris Bron.

angusta BRGN.

Bublayei BrgN.

Brardi BRgN

Brochanti Brgn.

canaliculata Bron.

catenulata $\mathbf{L H}$.

cyclostigma $\mathbf{G o ̈ . ~}$

Candollei BrgN.

contracta BugN.

Cortei Brgn.

cuspidata BrgN.
Davreuxi BrgN.

decora Gö.

Defrancei Bren.

Deutschana BrgN.

Dournaisi BrgN.

elegans BrGN.

elliptica $B_{R G N}$.

elo:?gata Brigr.

flexuosa $\mathbf{L H}$.

fibrosa BrGN.
gracilis BRGN.

Graeseri BrGN. .

hippocrepis Brgs.

intermedia Bran.

* irregularis Ser.

Knorri BHGN.

laevis Brgv.

leioderma $\mathrm{BRGN}$.

laevigata BP.GN. .

lepidodendrifoliaBrGN

*lineolaris SER.

mammillaris BRGN.

? marineria Gö.

Mentzelana Gö. .

microstigma BRGN.

minima Brge.

monostachya $\mathrm{LH}$.

Murchisoni LH.

nodosa Gö.

notata BRGN.

obliqua BrgN.

$1: 71$ 


\begin{tabular}{|c|c|c|c|c|c|c|c|}
\hline Bene & eltgegend. & a b c d e f g & k 1 & $m n$ op & q $r$ f s & $t u v w x$ & $y^{2}$ \\
\hline & & & & & & $=x^{-5}$ & \\
\hline & $\cdots$ & $\ldots$ e. & & & $\cdots$ & $\cdots \cdots$ & \\
\hline$\cdots$ & $\cdots \cdot$ & $\ldots$ e. & $\cdots \cdot$ & & $\cdots$ & $\cdots \cdots$ & \\
\hline$\cdots$ & $\cdots \cdot$ & $\ldots$ e... & $\cdots$ & $\cdots$ & $\cdots$ & $\cdots \cdots$ & 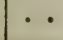 \\
\hline$\dot{x}$ & $\cdots \cdot$ & $\cdots$ e. & $\cdots \cdot$ & $\ldots$ & & $\cdots \cdots$ & \\
\hline N. & .. & $\begin{array}{lll}\cdots & \ldots \\
\cdots & \text { e. } \\
\cdots & \text { e. }\end{array}$ & & . & & $\cdots$ & \\
\hline$\because$ & $\cdots$ & $\therefore$ e & $\therefore \ldots$ & $\cdots \cdots$ & $\ldots \ldots$ & $\cdots$ & \\
\hline$\cdots$ & $\ldots \mathrm{M}$ & $\ldots$ e... & $\ldots$ & $\ldots$ & $\ldots$ & $\ldots$ & \\
\hline . & $\cdots \cdot$ & $\ldots$ c... & $\cdots \cdot$ & $\cdots \cdot$ & $\cdots \cdot$ & $\ldots \ldots$ & \\
\hline $\mathrm{RGN}$. & $\cdots \cdots$ & . e.. & $\cdots \cdot$ & $\cdots \cdot$ & $\cdots \cdot$ & $\ldots$ & - \\
\hline • & . & $\ldots$ e... & $\cdots \cdot$ & $\cdots \cdot$ & $\cdots$ & $\cdots \cdots$ & \\
\hline$\cdots$ & $\cdots$ & $\ldots$ e... & $\cdots$ & $\cdots$ & $\cdots$ & $\cdots$ & \\
\hline$\cdots$ & $\ldots \mathbf{M}_{2}$ & $\ldots$ e & $\cdots \cdots$ & $\cdots$ & $\cdots \cdot$ & $\cdots \cdots$ & \\
\hline$\cdots$ & $\cdots \cdot$ & $\cdots \mathbf{c} \cdots$ & $\cdots$ & $\cdots \cdot$ & $\cdots$ & $\cdots$ & \\
\hline$\cdots$ & -. & . e.. & $\cdots \cdot$ & $\cdots \cdot$ & $\cdots$ & $\cdots$ & $\cdots$ \\
\hline N. & $\dot{\mathrm{E}}^{2} \cdot \dot{\mathrm{M}}^{2}$ & 1 & $\cdots \cdots$ & $\therefore$ & $\because$ & $\because$ & $\therefore$ \\
\hline GN.. & $\ldots$ & e... & $\cdots$ & $\cdots$ & $\because$ & : & \\
\hline & ... & . e.. & ... & $\ldots$ & $\ldots$ & $\ldots \ldots$ & \\
\hline & $\cdots \cdot$ & $\ldots c \ldots$ & $\ldots$ & $\ldots$ & $\ldots$ & $\ldots$ & $\cdots$ \\
\hline GN. . & $\cdots$ & $\ldots$ e... & $\cdots$ & $\ldots$ & $\ldots$ & $\ldots$ & . \\
\hline$\cdots$ & $\cdots \cdots$ & $\ldots$ e. & $\cdots$ & $\cdots$ & $\ldots$ & $\cdots$ & \\
\hline$\cdots$ & $\cdots$ & $\ldots$ e. & $\cdots$ & $\cdots$ & $\cdots$ & $\cdots \cdots$ & \\
\hline - & $\cdots$ & . c . . & $\cdots$ & $\cdots$ & $\cdots \cdots$ & $\cdots \cdots$ & 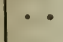 \\
\hline - & & & & & & - & - \\
\hline$A E D$ & 2: & & & $\ldots$ & . & $\ldots . .5$ & 180 \\
\hline Psi & & & 1 & $\cdots$ & $\cdots$ & - & .0 \\
\hline & - & & . & $m \ldots$ & $\cdots$ & $\ldots .$. & $\cdots$ \\
\hline *ro & . & $\cdots \cdots$ & $\cdots$ & $\mathrm{m} \cdots$ & $\ldots$ & $\ldots \ldots$ & $\cdots$ \\
\hline N.28 & $\cdots$ & $\ldots \ldots$ & $\cdots \cdot$ & $\cdots$ & $\ldots$ & $\ldots$. & .0 \\
\hline$\cdots$ & & $\cdot \cdot$ & $\cdots$ & $\cdot \cdot$ & & $\cdots$ & - \\
\hline$\cdots \cdot$ & & $\cdots$ & $\cdots$ & $\cdots$ & $\cdot$ & $\cdots$ & \\
\hline$\cdots$ & & - & $\cdots$ & $\cdot \mathbf{n} \cdot \cdot$ & $\cdot$ & $\cdots$ & \\
\hline$\cdots \cdot$ & & $\cdots$ & $\cdot$ & 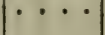 & • & $\cdots$ & \\
\hline$\cdots$ & $\therefore$ & $:$ & $\therefore$ & - 1 & $\dot{.}$ & $\because$ & \\
\hline$\cdots$ & $\begin{array}{l}\cdots \\
\cdots\end{array}$ & $\begin{array}{ll}\cdots \\
\cdots\end{array}$ & $\cdots$ & $\cdots \cdots$ & $\cdots$ & $\mid \begin{array}{c}\cdots \\
\cdots\end{array}$ & \\
\hline Gö. & .... & $\ldots \ldots$ & $\ldots$ & $\ldots$ & $\ldots$ & $\ldots \ldots$ & \\
\hline$\therefore$ & & $\ldots$ e & $\ldots \cdot$ & $\ldots$ & $\ldots$ & $\ldots$ & \\
\hline e & E. . M. & $\ldots$. e. & $\ldots$ & $\cdots$ & $\cdots$ & . & \\
\hline & $\cdots \cdot$ & $\ldots$ e... & $\cdots$ & $\cdots \cdots$ & $\cdots$ & $\cdots \cdot$ & \\
\hline es Brgn. & & $\ldots$ e... & & $\cdots \cdots$ & $\cdots$ & $\cdots \cdots$ & . \\
\hline is NiLs. & & $\cdots \cdot$ & $\ldots 1$ & $\ldots$ & $\ldots$ & $\ldots$ & . \\
\hline$\cdots$ & $\cdots$ & $\ldots \mathbf{e}$ & $\cdots$ & $\cdots \cdot$ & $\cdots$ & $\cdots \cdots$ & $\cdots$ \\
\hline$\cdots$ & & - e & & $\cdots \cdot$ & $\cdots$ & . & \\
\hline & $\cdots$ & & 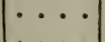 & $\cdots$ & $\cdots$ & $\cdots$ & $\cdots$ \\
\hline B & $\because 5$ & . & $\dot{1}$ & & & ? & $\cdots$ \\
\hline MORR & • & $\cdot \mathrm{e}$ & • & $\cdots$ & $\cdot$ & $\cdots$ & \\
\hline & & & & & & & \\
\hline
\end{tabular}




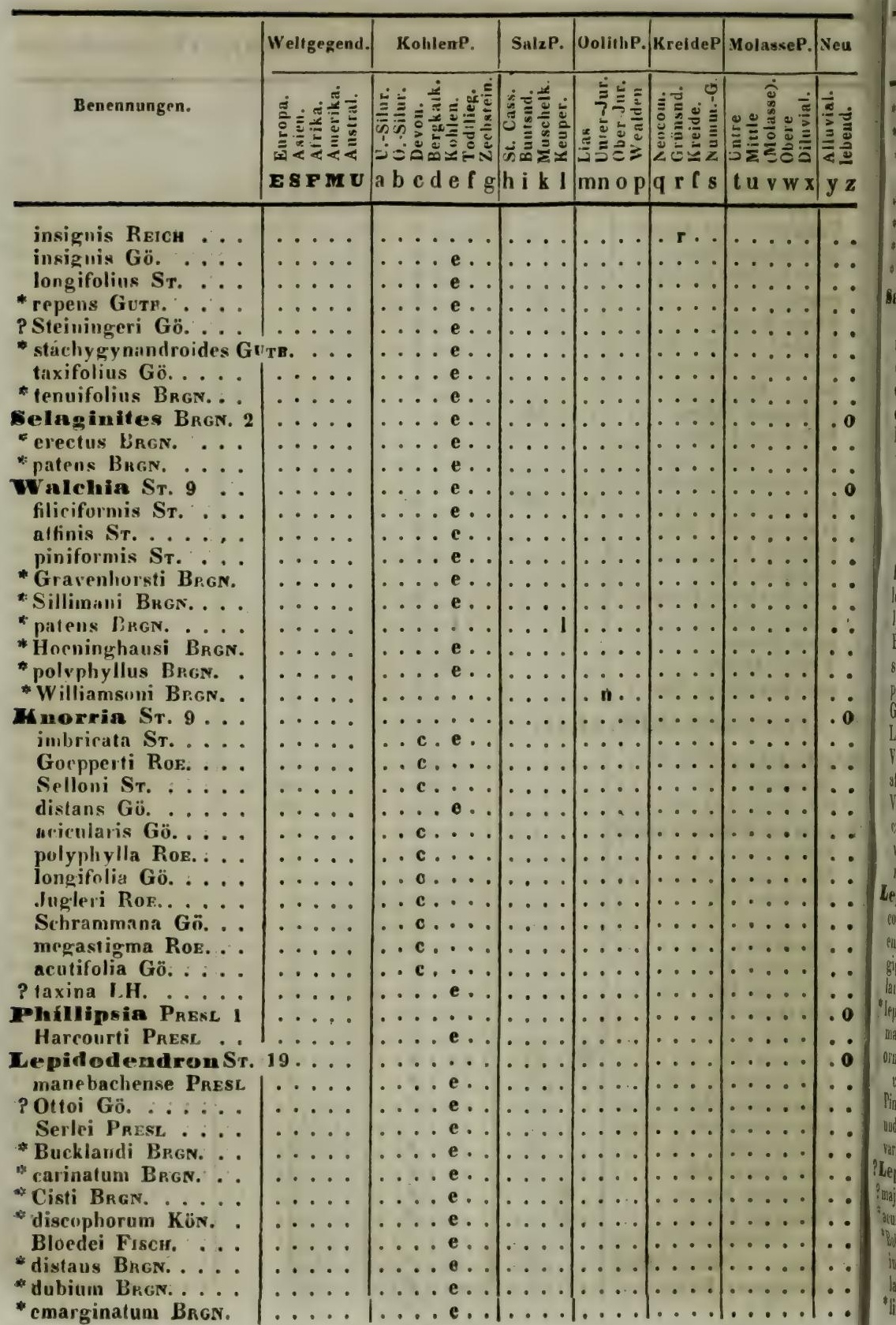




\begin{tabular}{|c|c|c|c|c|c|c|c|}
\hline Ben & end. & $a b$ & $k 1$ & $\mathrm{mn}$ op & 8 & $\mathbf{w} \mathbf{x}$ & $y z$ \\
\hline$\cdots$ & & & & & & & \\
\hline$\ldots$ & • & - & 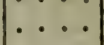 & $\ldots$ & $\cdots \cdot$ & $\ldots \ldots$ & \\
\hline osum Brgr & $\cdots \cdot \cdot$ & $\ldots$ e & . 1 & $\cdots \cdot$ & $\cdots \cdot$ & $\ldots$ & \\
\hline ulosum $\mathbf{R}$ & · & . e $\ldots$ & $\cdots$ & $\cdots$ & $\cdots \cdot$ & $\cdots \cdots$ & - \\
\hline GN. . & . & & & $\cdots \cdots$ & & & \\
\hline GN. & - & - $\mathbf{e}$ & & & & $\cdots$ & \\
\hline$\cdots$ & $\cdots$ & $\begin{array}{ll}\cdots \mathbf{e} \\
\cdots & \mathbf{e}\end{array}$ & - & $\cdots \cdots$ & $\cdots$ & $\cdots \cdots$ & \\
\hline $\begin{array}{l}1 \text { BRGN. . . } \\
\text { riat Brge }\end{array}$ & $\cdots \cdots$ & $\ldots \ldots$ & $\cdots$ & 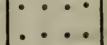 & $\cdots$ & $\cdots$ & \\
\hline . & $\cdots \cdot$ & $\ldots$ e $\ldots$ & $\cdots$ & $\ldots$ & $\ldots$. & $\ldots \ldots$ & . \\
\hline$\cdots \cdot$ & $\cdots \cdots$ & $\ldots$ e. & $\cdots \cdot$ & $\ldots$ & $\ldots$ & $\ldots \ldots$ & 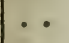 \\
\hline$\cdots$ & $\cdots \cdot$ & $\ldots$ e $\ldots$ & $\cdots \cdot$ & $\cdots$ & $\ldots$ & $\ldots$ & \\
\hline L $\cdots$ & $\cdots \cdot$ & $\ldots$ e. & $\cdots \cdots$ & $\cdots$ & $\cdots$ & $\cdots \cdots$ & \\
\hline$\cdots$ & & $\ldots$ e & $\ldots \circ$ & $\cdots \cdots$ & $\cdots$ & $\cdots$ & \\
\hline s. . . & & - & $\cdots$ & $\cdots$ & $\cdots$ & $\cdots$ & \\
\hline$\cdots \cdot$ & & & & & $\cdot \cdot$ & $\cdots$ & \\
\hline$\cdots$ & & & • & - & $\cdot$ & $\cdots$ & \\
\hline o & & $\mathbf{e}$ & & $\cdots$ & $\cdot$ & $\cdots$ & \\
\hline$\therefore$ & $\because$ & $\mid \begin{array}{ll}\cdots \\
\cdots & \mathrm{e}\end{array}$ & $\cdots$ & $\left|\begin{array}{c}\cdots \\
\cdots\end{array}\right|$ & $\cdots$ & $\cdots$ & \\
\hline$\therefore$ & . & ........ & $\ldots$. & $\ldots$ & $\ldots$ & 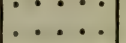 & \\
\hline$\cdots$ & $\cdots \cdot$ & $\ldots$ e $\ldots$ & $\cdots$ & $\ldots$ & $\ldots$. & $\ldots \ldots$ & \\
\hline ö. . & $\cdots \cdots$ & $\ldots$ e $\ldots$ & $\cdots$ & $\cdots$ & $\ldots$ & $\ldots \ldots$ & . \\
\hline$\cdots$ & $\cdots \cdot$ & $\ldots \ldots$ e... & $\ldots \ldots$ & $\ldots$ & $\ldots$. & $\ldots \ldots$ & 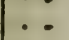 \\
\hline & $\cdots \cdot$ & $\ldots \mathbf{c} \ldots$ & $\cdots$ & $\ldots$. & $\ldots$ & $\ldots$ & \\
\hline$\cdots$ & . & $\ldots c \ldots$ & $\mid \cdots \cdots$ & $\ldots$. & $\ldots$ & $\ldots$ & \\
\hline L. & $\cdots \cdot$ & $\ldots$ e... & $\ldots$ & $\ldots$. & $\ldots$ & $\ldots$ & \\
\hline . & $\cdots \cdot$ & $\ldots \ldots$ & $\cdots$ & $\ldots$. & $\ldots$ & $\ldots \ldots$ & \\
\hline RESL & $\cdots$ & $\ldots \ldots$ & $\cdots$ & $\ldots$ & $\cdots$ & $\cdots \cdots$ & \\
\hline . & $\cdots \cdot$ & $\cdots \mathbf{e}$ & $\cdots$ & $\cdots \cdots$ & $\cdots \cdots$ & $\cdots \cdots$ & \\
\hline $8 \mathbf{s}$ & - & . e .. & $\cdots$ & $\cdots \cdots$ & $\cdots$ & $\cdots$ & \\
\hline & • & ....e. & $\cdot$ & $\cdots$ & $\cdots \cdots$ & - & \\
\hline$\cdots$ & $\cdot$ & $\begin{array}{l}\ldots \\
\cdots e\end{array}$ & $\cdot$ & $\cdots$ & $\cdots \cdots$ & $\cdots$ & \\
\hline$\dot{B} \dot{B r G}$ & 1. $11 \ldots$ & e & & $\cdots$ & $\cdots$ & $\because$ & $.08-2>$ \\
\hline $\begin{array}{l}\text { ostrobus Brgt } \\
\text { us LH. . . . }\end{array}$ & $\ldots \ldots$ & $\ldots \ldots$ e... & $\mid \begin{array}{ll}\cdots \\
\cdots & \cdots \\
\cdots\end{array}$ & $\left|\begin{array}{c}\cdots \\
\cdots\end{array}\right|$ & $\cdots \cdots$ & $\cdots \cdots$ & $\cdot 0$ \\
\hline GN. . & $\ldots$ & $\ldots \ldots$ & $\ldots$ & $\ldots$. & $\ldots$ & $\cdots \cdots$ & \\
\hline$\cdots$ & $\cdots$ & . e. & & $\ldots$ & $|\cdots \cdot|$ & $\cdots \cdots$ & \\
\hline$\cdots$ & $\cdots$ & - e & & $\cdots \cdot \mid$ & $\cdots \cdots$ & $\ldots \ldots$ & \\
\hline atus Gutb. & - & e.. & - & $\cdots \cdots$ & $\cdots$ & $\cdots \cdots$ & • \\
\hline . . & & e & & $\cdots$ & & $\cdots \cdots$ & \\
\hline 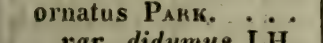 & • & e e. & & $\cdots \cdots$ & $\cdots$ & $\cdots \cdots$ & \\
\hline LH. & & $e$ & & $\because$ & $\because$ & & \\
\hline . & & e & & & & & \\
\hline$\cdots$ & & e. & & & & . & \\
\hline $\operatorname{lnm} B$ & N. 7 . & . . & & $\ldots$ & & .... & - \\
\hline . & $\cdots \cdots$ & $\ldots . e$ & & $\cdots \cdot$ & $\therefore \cdots$ & $\ldots \ldots$ & \\
\hline$\cdot$ & $\cdots \cdot$ & & & $\cdots$ & $\cdots \cdot$ & $\cdots \cdots$ & \\
\hline$* 1$ & $\cdot \cdot$ & - & ・ & & & $\cdots \cdots$ & . \\
\hline . . & & & & & & $\cdots$ & \\
\hline & & & & & & & \\
\hline
\end{tabular}




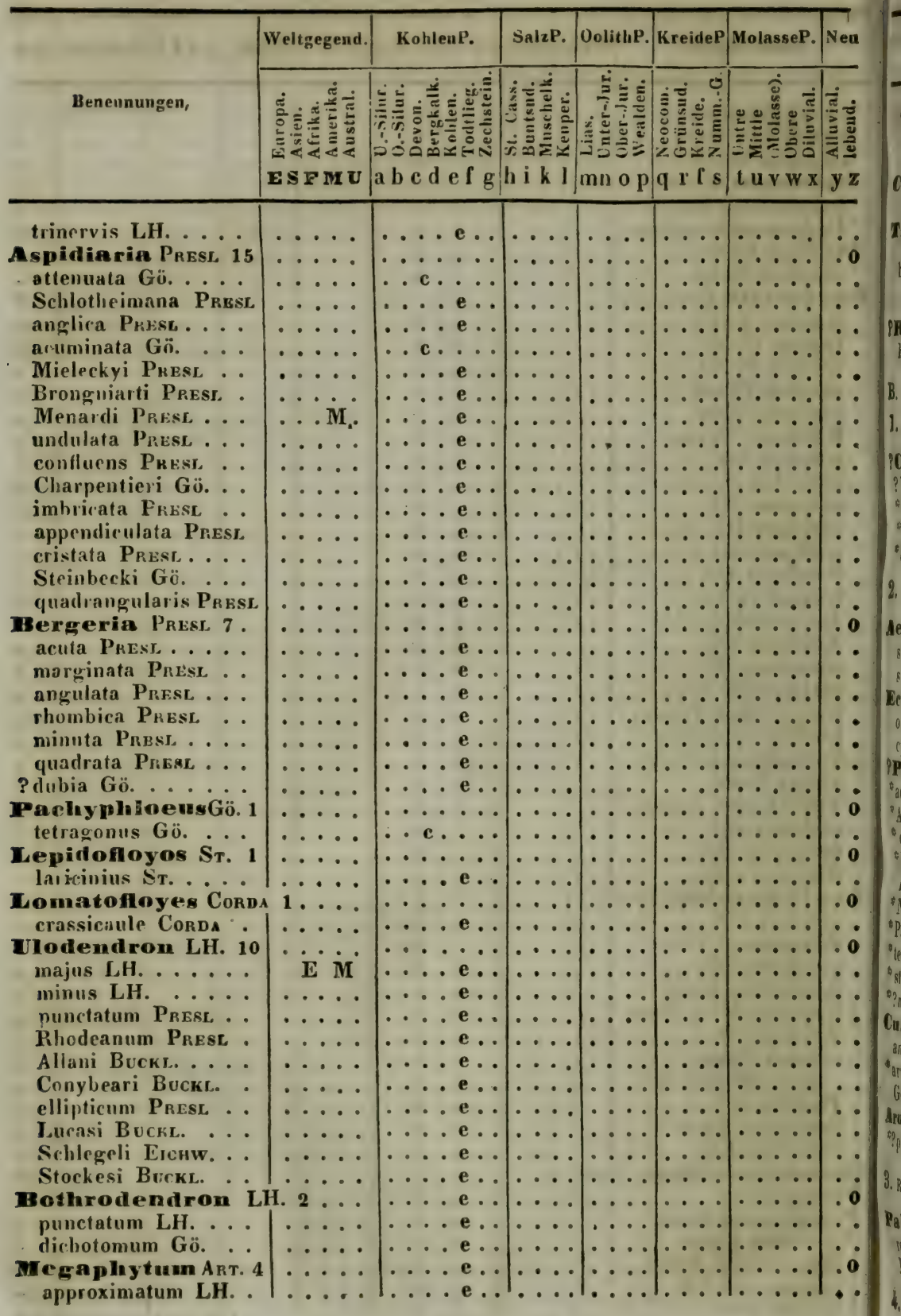




\begin{tabular}{|c|c|c|c|c|c|c|c|}
\hline n. & Itgegend. & $\mathrm{fg}$ & & $p$ & $s$ & & . \\
\hline & & & & & $\cdots$ & & \\
\hline & $\cdots \cdots$ & $\ldots$ e. & & $\ldots$ & $\ldots$ & $\ldots \ldots$ & . \\
\hline · & $\cdots \cdot$ & $\ldots \ldots$ & $\cdots$ & $\ldots$ & $\ldots$ & $\ldots$. & . \\
\hline $\begin{array}{c}1 \\
\ldots\end{array}$ & $\cdots \cdots$ & $\cdots \cdots \cdots$ & & $\cdots$ & $\cdots$ & $\cdots \cdots$ & .0 \\
\hline $\begin{array}{l}\text { Tithy man } \\
\text { (an hujus }\end{array}$ & $1 \cdots$ & $\cdots$ & $\because$ & $\left|\begin{array}{c}\cdots \\
\cdots\end{array}\right|$ & $\left|\begin{array}{c}\cdots \\
\cdots\end{array}\right|$ & $\mid \cdots \cdots$ & $\ddot{0}$ \\
\hline $\begin{array}{r}\text { biformis } \\
\text { Dul }\end{array}$ & $\cdots$ & e.. & . & $\ldots$. & . & . & - \\
\hline rgia Cot & TA 1 . & & $\ldots$ & $\ldots$ & $\ldots$ & $\ldots \ldots$ & .0 \\
\hline . & . & & & $\cdots$ & $\cdot$ & $\cdots$ & .0 \\
\hline B. $\mathrm{MC}$ & 8 & & & & & & \\
\hline 1. $\mathrm{c}$ & 1. & . & . & $\ldots$ & $\ldots$ & . . $(66:$ & 00 \\
\hline $\mathrm{C}$ & & & & $\cdot$ & $\cdots$ & $\cdots$ & \\
\hline & & & & $\cdot$ & $\cdots$ & - & . \\
\hline & & & & $\cdots$ & $\cdots$ & $\cdots$ & • \\
\hline $\begin{array}{l}\text { S BraUN. } \\
\text { BraUN } \cdots\end{array}$ & $\because$ & $\cdots \cdots \cdots$ & & $\begin{array}{l}\mathrm{m} \cdot \cdots \\
\mathbf{m} . \cdots\end{array}$ & $\left|\begin{array}{c}\cdots \\
\cdots\end{array}\right|$ & $\cdots$ & \\
\hline NEAE JuSS. & 5 & & & & & $(250: 2$ & \\
\hline Aet & . & 1 & & & & & \\
\hline & & . & . $1 \ldots$ & $\ldots$ & . & $\ldots$ & $\cdot$ \\
\hline & . & & $1 \ldots$ & $\ldots$ & $\ldots$ & $\ldots$ & \\
\hline BrGN & v. $2 \ldots$ & $\cdots$ & . & $\cdots$ & $\ldots$ & $\ldots .$. & .0 \\
\hline & $\ldots$ & & . i .. & $\ldots$ & $\ldots$ & $\ldots$. & . \\
\hline & - & $\cdots$ & . i . . & $\ldots$ & $\ldots$ & $\cdots$ & \\
\hline 10 & $\ldots \ldots$ & - & $\ldots$ & $\cdots$ & $\cdots$ & $\ldots \ldots$ & $\cdot c$ \\
\hline$\cdots$ & - & $\cdots$ & $\cdots$ & $\cdots$ & $\cdots$ & $\cdots$ & - \\
\hline . & - & & $\cdots$ & $\cdots$ & $\cdots$ & $\cdots$ & \\
\hline$\cdots$ & • & & - & $1 \cdot$ & $\cdot \cdot$ & $\cdots$ & \\
\hline$\cdots$ & & e.. & $\cdots$ & : & $\therefore$ & $\because$ & \\
\hline . & $\ldots$ & $\ldots$ & $\ldots$ & $\mathbf{m} \ldots$ & $\cdots$ & $\because$ & \\
\hline N .. & ... & $\ldots \ldots$ & $\ldots$ & $m \ldots$ & $\ldots$ & $\therefore$ & \\
\hline ... & $\cdots$ & $\ldots$ e.. & $\ldots$ & $\ldots$ & $\ldots$ & $\cdots$ & - \\
\hline ... & $\ldots$ & $\ldots$ e... & $\ldots$ & $\ldots$ & $\cdots$ & $\cdots$ & \\
\hline$\cdots$ & $\cdots \cdot$ & $\ldots$ e.. & $\cdots$ & $\ldots$ & $\cdots$ & $\cdots$ & \\
\hline 3 & $\cdots \cdot$ & $\cdots$ & $\ldots$ & $\cdots$ & $\cdots$ & $\cdots$ & $\cdots$ \\
\hline & & & • & . & & $\mathbf{w} \cdot$ & • \\
\hline B.. & & & & & & $\cdots$ & 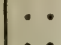 \\
\hline$\therefore$ & $\ldots$ & & $\ldots$ & $\left|\begin{array}{c}\cdots \\
\cdots \\
\cdots\end{array}\right|$ & $\begin{array}{l}\mathbf{r} \\
\cdots \\
\cdots\end{array}$ & $\left|\begin{array}{cc}\cdots & \cdots \\
\cdots & \cdots\end{array}\right|$ & $\ddot{\infty}$ \\
\hline tres $\mathrm{L}$. & $\ldots$ & $\cdots$ & $\cdots$ & $\cdots$ & $\cdots$ & $\therefore \mathbf{v}$ & .2 \\
\hline CEAE BARTL. & 2 & & & & & $\ldots(18$ & \\
\hline $\mathbf{P e}$ & & & & & & & \\
\hline M & & & & & & & \\
\hline RTL.0 & & & & & & & \\
\hline
\end{tabular}




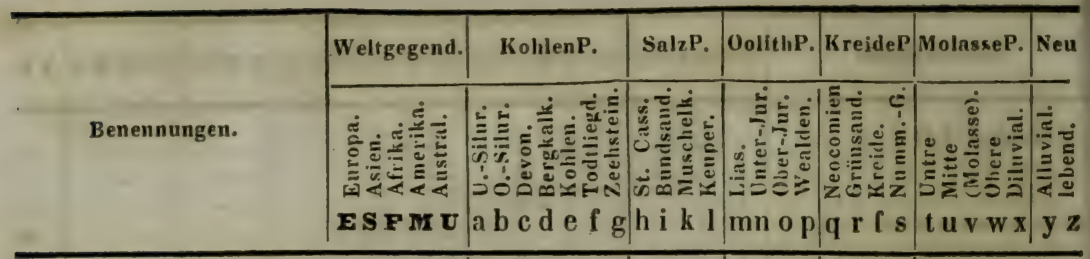

5. Xyrideae Kunth. 0

6. Cammelinaceae Brwn. 0 .

7. NAJADEAE Juss, $7: 20 \ldots$

Zosterites Bren. 8 Oŕbignyana BrgN. .

Bellovisiana [?] BrgN. elongatus BRGN.

lineatus BrGN.

Agardhanus BrgN. .

* taeniaeformis BRGN.

* enervis Brgn. . . . . marinus Ung.

Caulinites Brgn. 4 parisiensis Brgn. . radobojensis UNG. nodosus UNG.

ambiguus Uing. ...

Mraximinna Ung. I Meneghinii Ung. ..

Ruppia Ung. 1 ... (Ruppites Gö.) pannonica Urg. . . .

malochloris Ung. 1 cymodoceoides UNG.

Potannogeton (ites)

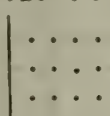

* geniculatus At. Braun Tritonis Unc. ..... najadum Ung.

Potanophyllites.1 multinervis BRgN.

Dubiae (1):

Carpolithes thalictroides BrGN. . a. Websteri

$\beta$. parisiensis

8. PodostemeanRich. 0

9. JUNCA GiNeAE Rich, 0

10. alismaceae Rich, 0

11. вutomeae Rich, 0

12. orontiaceae Bartl. 0

13. callacea Bartu. 1:1 ..

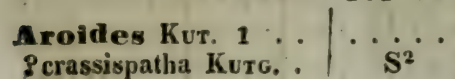

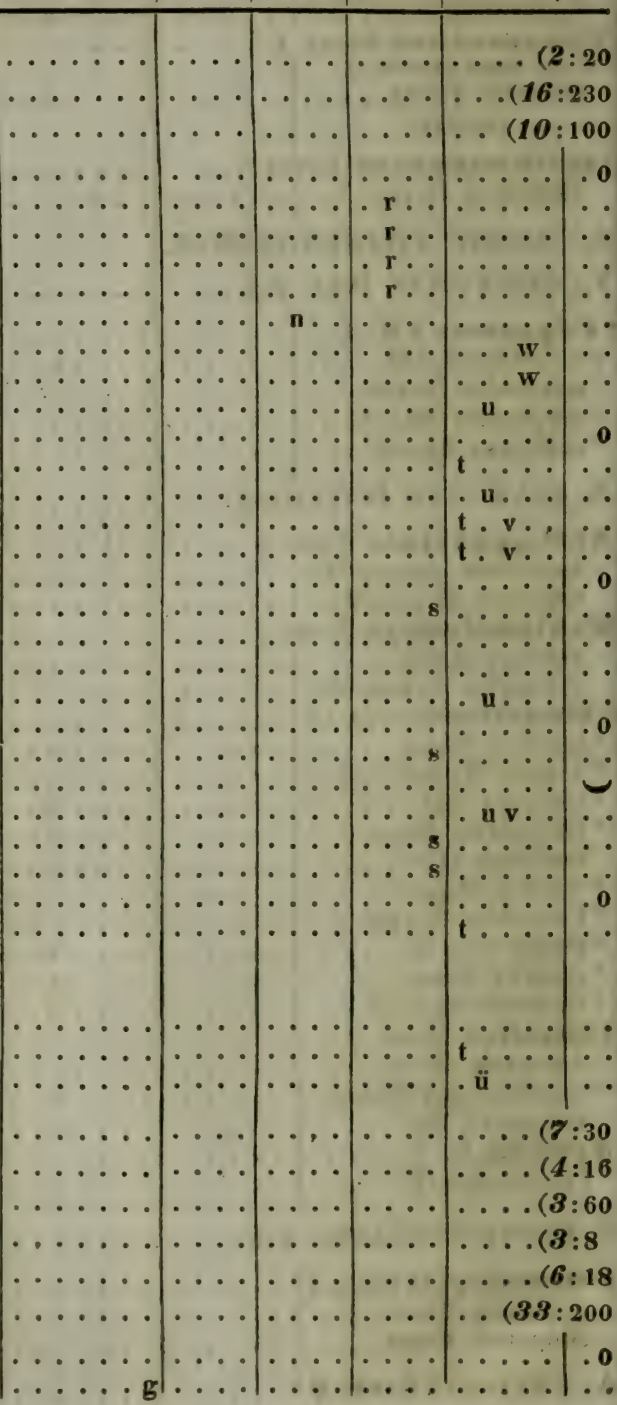




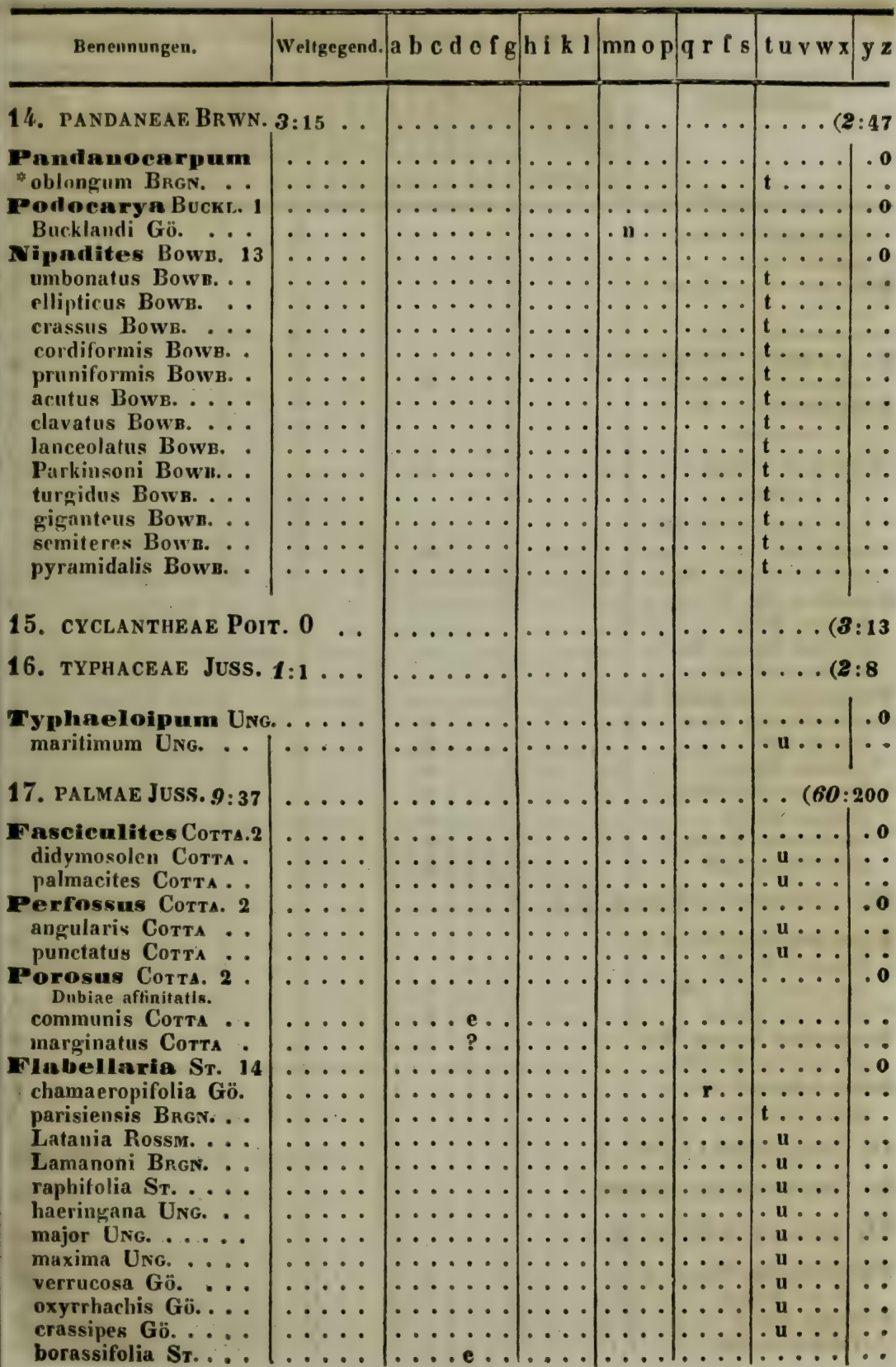




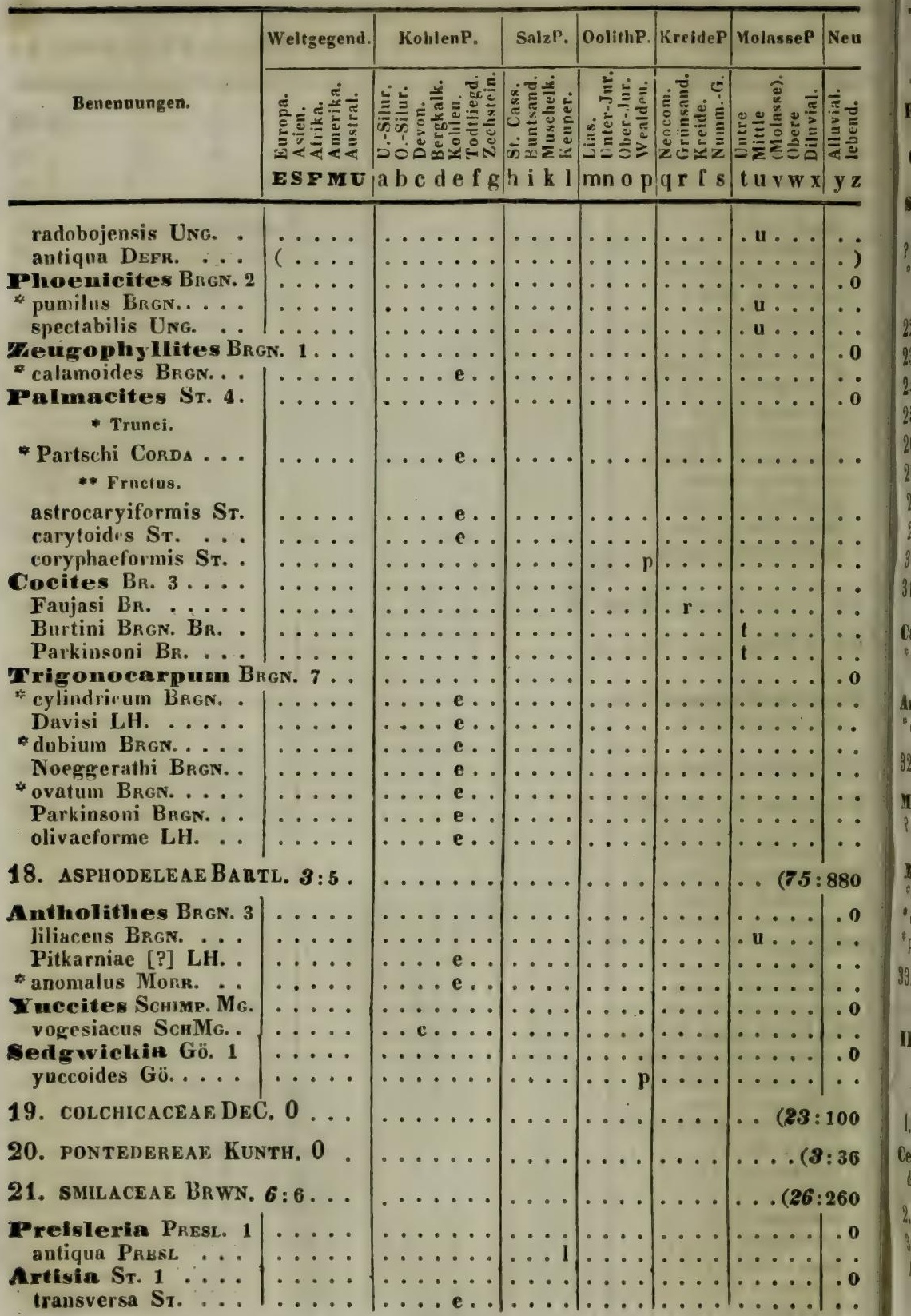




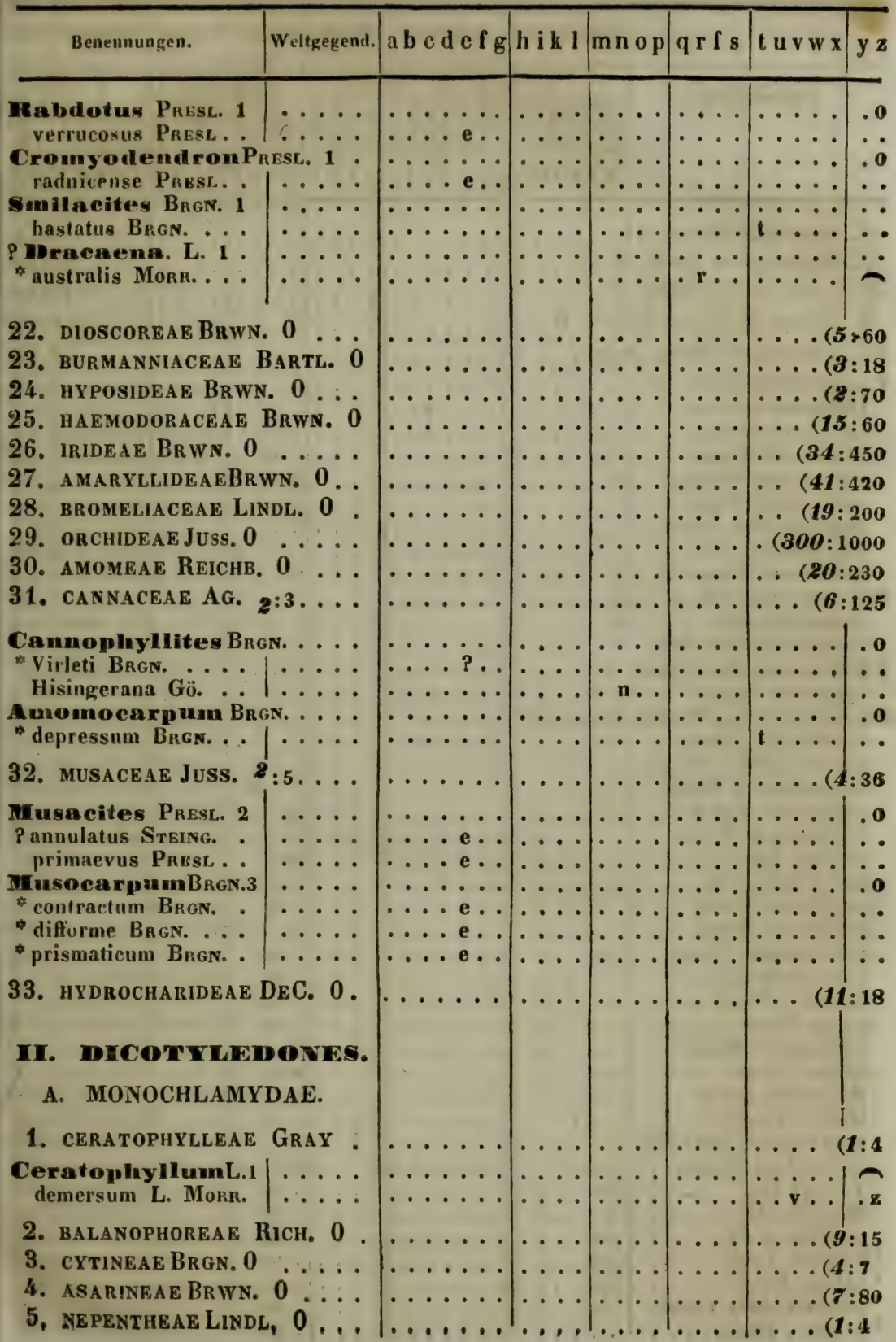




\begin{tabular}{|c|c|c|c|c|c|c|c|}
\hline & Weltgegend. & KohlenP. & SalzP. & OolithP. & KreideP & MolasseP. & Neu \\
\hline Benennungen. & 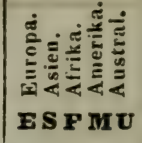 & 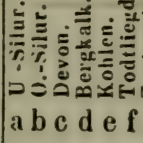 & 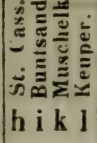 & 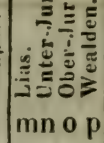 & $\left|\begin{array}{ll}\mid \\
q \\
q\end{array}\right|$ & 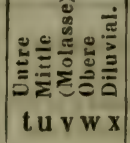 & 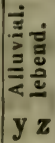 \\
\hline
\end{tabular}

6. Tacceae Presl. 0

7. Saurureae Rich. 0

8. PIPERACEAE Rich. 0

9. Chlorantheak BRwn. 0 . 10. cycadeae Pers. 9:87.

Cyeadites Brgn. 8 columnaris Press involutus Presc . Bucklandi Press . eylindricus Morr. salicifolius Press. angustifolius PresL Brongniarti RoE. . . pectinatus BERG.

Raumeria Gö. 2 Schulzana Gö. Reichenbachana Gö. Calamoxylon Cord cycadeum CORDA

EramitesBrgn.,Gö.,Presl(pars)30 Cordai St., Presl megalophyllus PresL microphyllus Press. pygmaeus Morr .. Brongniarti Prest. distans Prest. .... lanceolatus LH. undulatus PresL .. petiolatus ZENK. . . whitbyensis Press. Crantzanus Gö. falcatus Prese gigas Morr. ..... Schmiedeli PresL Bechei Brgn. .... Bucklandi BRgN. lagotis BRGN. .... vogesiacus Scнiмp.Mg. brevifolius Braun Youngi Gö. Mantelli Gö. gramineus Gö.

- Feneoni Brgn. - patens BRgr. - pennaeformis BrgN.

* elegans Brgs.

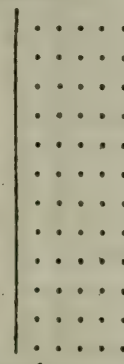

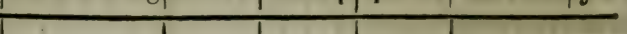

$(2: 3$

( $4: 12$

(4:360

$(4: 19$

$(3: 24$

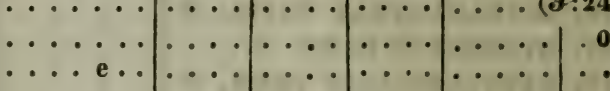

. . $\mathrm{e}$.

. . . .

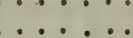

- 


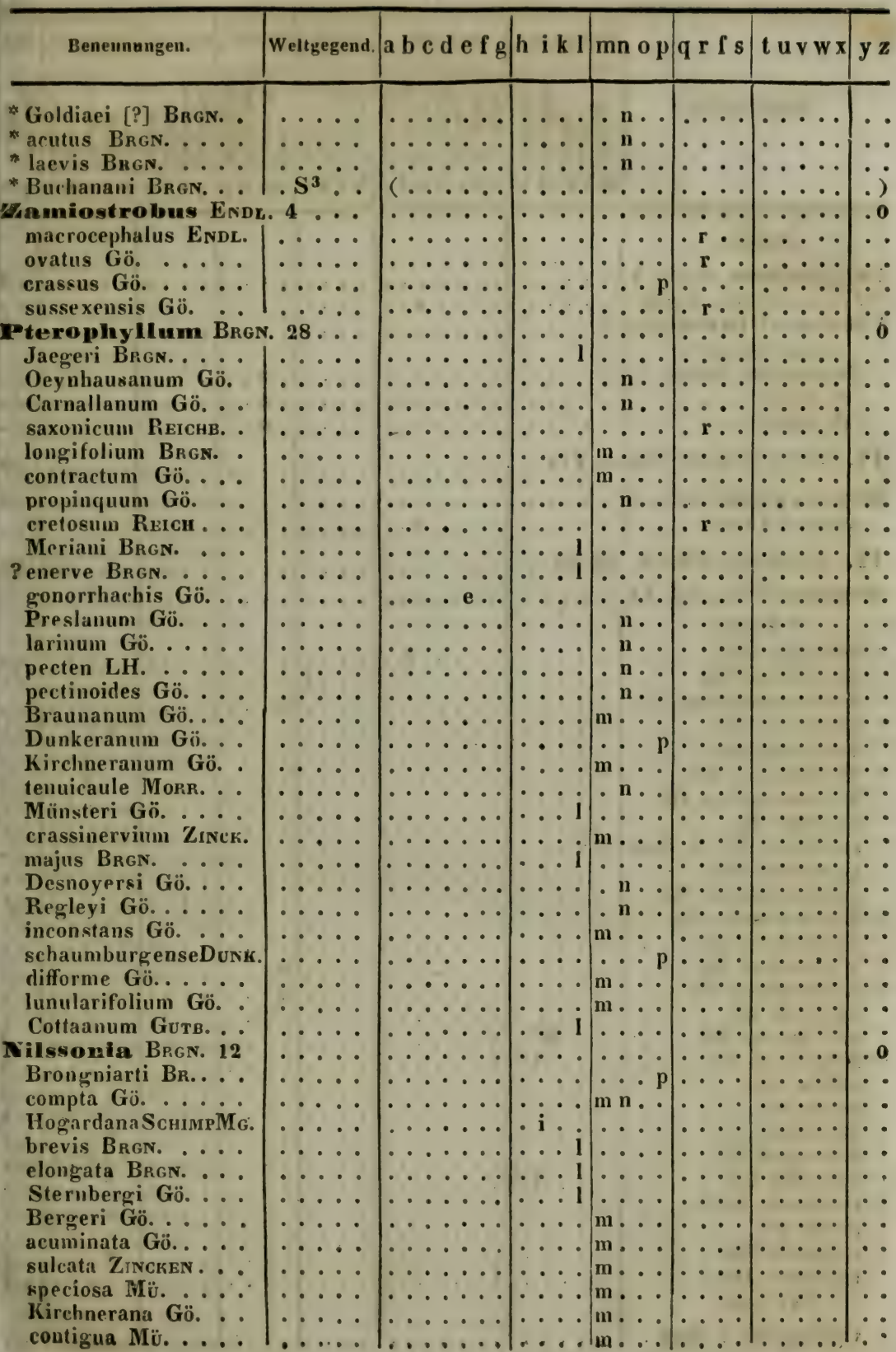




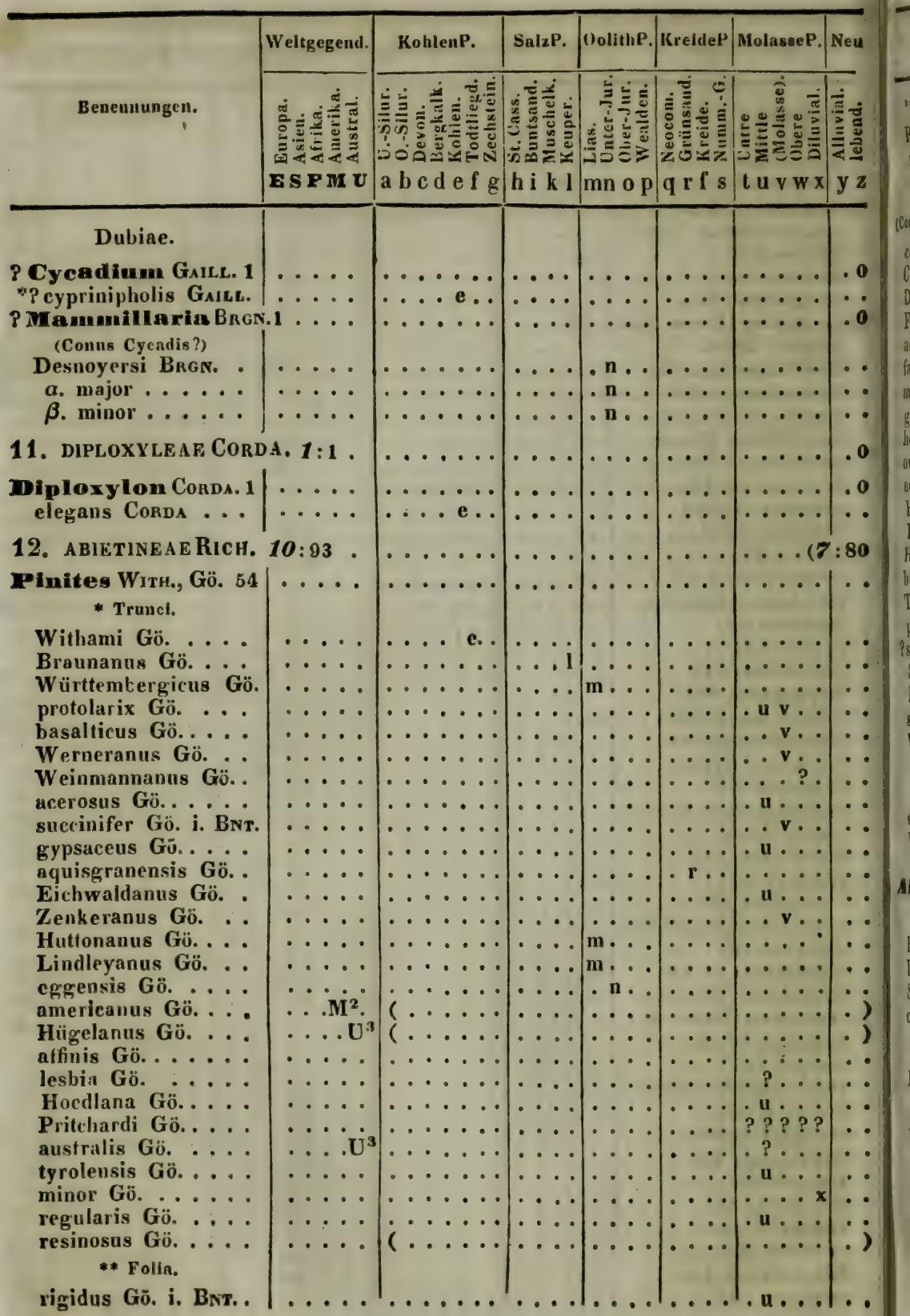




\begin{tabular}{|c|c|c|c|c|c|c|c|}
\hline Benennungen. & Weltgegend. & $|a b c d e f g|$ & h i k I & mn op & $q r$ is & $t u v w x$ & yz \\
\hline \multicolumn{8}{|l|}{ *** Amenta maxcula. } \\
\hline $\begin{array}{l}\text { Roessertanus Press } \\
\text { microstacliys Presi }\end{array}$ & $\ddot{\cdots} \dot{ }$ & & $\cdots 1$ & $\cdots$ & $\cdots$ & $\cdots \cdots$ & $\because$ \\
\hline microstacliys PRI & $\cdots \cdot \cdot$ & $\cdots$ & $\cdots 1$ & $\cdots \cdots$ & $\cdots \cdots$ & $\cdots \cdots$ & - \\
\hline \multicolumn{8}{|l|}{ (Conites Sx., Strobilltes LH.) } \\
\hline cauariensis Gö.... & $\dot{\cdots}$ & & $\cdots \cdots$ & $\ldots$ & $\cdots \cdots$ & $|\cdots w \cdot|$ & \\
\hline Gö............. & $\cdots \cdots$ & 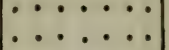 & $\cdots \cdots$ & $\cdots \cdots$ &. & $\mathbf{w} \cdot$ & \\
\hline cei Gö. . . . & $\ddot{\cdots} \cdot$ & $\because \cdots \cdots$ & 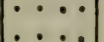 & $\cdots \cdots$ & $\cdot$ & $\cdots$ & \\
\hline $\begin{array}{l}\text { Faujasi Gö. . . . . } \\
\text { armatus Gö. . . . }\end{array}$ & $\because \cdots$ & $\ldots$... & $\left|\begin{array}{c}\cdots \\
\cdots\end{array}\right|$ & $\left|\begin{array}{c}\cdots \\
\cdots \\
\cdots\end{array}\right|$ & $\left|\begin{array}{c}\cdots \\
\cdots\end{array}\right|$ & $\cdots \cdots$ & $\because$ \\
\hline is Gö...... & $\cdots \cdots$ & $\ldots \ldots$ & $\ldots$ & $\ldots$ & $\ldots$ & u... & \\
\hline ชö. . . & $\ldots \ldots$ & $\ldots \ldots$ & $\ldots$. & $\ldots$ & $\ldots$ & $t \ldots$. & \\
\hline$\cdots$ & $\cdots \cdots$ & $\cdots \cdots \cdots$ & $\cdots$ & $\ldots$ & $\ldots$ &. $\mathbf{u} \ldots$ & \\
\hline$\cdots$ & $\ldots \ldots$ & $\cdots \cdots$ & $\mid \cdots$ & $\ldots$ & $\cdots \cdots$ &. $\mathbf{u} \ldots$ & \\
\hline$\therefore$ & $\cdots \cdots$ & $\cdots \cdots$ & $\cdots \cdots$ & $\cdots \cdots$ & $\ldots$ & u... & \\
\hline Gö. . & $\cdots \cdot$ & $\cdots \cdots \cdots$ & $\cdots$ & $\ldots$ & $\cdots$ & u $\ldots$ & - \\
\hline j. & $\cdots \cdots$ & $\cdots \cdots \cdot$ & $\cdots \cdots$ & $\cdots \cdot$ & $\cdots \cdots$ & $\cdots \ldots$ & \\
\hline$\cdots$ & $\cdots \cdot \cdot$ & $\cdots \cdots$ & $\cdots \cdot$ & $\cdots$ & $\cdots \cdots$ & $\cdot \mathbf{u} \cdots$ & \\
\hline$\cdots$ & $\cdots \cdot \cdot$ & $\ldots \ldots$. & $\cdots \cdots$ & $\ldots$ & $\cdots \cdots$ & $\cdot \mathbf{u} \ldots$ & 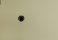 \\
\hline$\dot{x}$ & $\cdots \cdots$ & $\cdots \cdots$ & $\cdots$ & $\ldots$ & $\cdots \cdot$ & . ? . . & - \\
\hline BNT. & $\cdots \cdot \cdot$ & $\cdots \cdots \cdots$ & $\cdots \cdots$ & $\cdots \cdots$ & $\cdots \cdot$ & $\cdots \mathbf{v} \cdot \cdots$ & \\
\hline$\cdots$ & - & $\cdots$ & $\cdots \cdots$ & $\cdots \cdots$ & $\cdots$ & $\cdots$ & \\
\hline$\cdots$ & & $\cdots$ & $\cdots \cdots$ & $\cdots \cdots$ & $\cdots$ & $\because \cdots$ & . \\
\hline Gö̈. & $\therefore$ & $\cdots$ & $\cdots \cdots$ & $\cdots \cdots$ & $\cdots$ & $\cdots$ & \\
\hline ̛ัö. . & $\cdots \bullet \cdot$ & $\cdots \cdots$ & $\cdots \cdots$ & $\cdots \cdots$ & 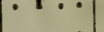 & $\because \cdots$ & \\
\hline$\cdots$ & $\ddot{\cdots} \cdot$ & $\cdots \cdots \cdots$ & $\cdots \cdots$ & $\cdots \cdots$ & $\cdots \cdots$ & $\cdot u \cdots$ & \\
\hline Woodwardi Gö. . . & & & $\cdots \cdots$ & $\cdots \cdots$ & $\cdots \cdots$ & $\cdots w \cdot$ & \\
\hline \multicolumn{8}{|l|}{ Dubiae. } \\
\hline elo & ・・ & $\cdots$ & & $\mathrm{m} \ldots$ & $\cdots$ & $\cdots$ & \\
\hline MG. & - & $\cdots$ & . i & $\cdots \cdots$ & $\cdots \cdots$ & $\cdots \cdots$ & \\
\hline $\begin{array}{c}\text { Abietites NiLs., Gö. } 6 \\
\text { Folia. }\end{array}$ & 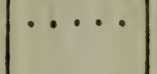 & $\cdots \cdots$ & - & $\cdots$ & $\cdots \cdots$ & $\cdots \cdots$ & - \\
\hline$\cdots$ & $\because$ & $\cdots \cdot$ & & $|\cdots \cdots|$ & . $\mathbf{r}$. & $\cdots \cdot$ & \\
\hline$\cdot$ & $\therefore$ & $\cdot$ & $\cdots$ & $\cdots \mathbf{p}$ & $\cdots \cdot$ & $\cdots$ & \\
\hline Sternbergi Nrrs. . & $\cdots$ & & $\cdot \mathbf{n} \cdot \cdot$ & $\cdots \cdots$ & $\cdots \cdots$ & - & \\
\hline $\begin{array}{l}\text { obtusifulius Gó } \\
\text { ** Flores masc }\end{array}$ & & & $\cdots \cdots$ & $\mid \cdots \cdots$ & - & - & \\
\hline $\begin{array}{c}\text { Reichanus GöBNT. } \\
\text { *** Strobili. }\end{array}$ & $\cdots \cdot$ & $\cdots \cdots \cdots$ & $\cdot 1$ & $\cdots \cdots$ & $\cdots \cdots$ & $\cdots \mathbf{v} \cdot \cdots$ & 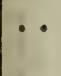 \\
\hline BNT. & $\cdots \cdots$ & $\ldots \ldots$ & $\cdots$ & $\cdots \cdots$ & $\cdots \cdots$ & $\cdots \mathbf{v} \cdot \cdots$ & -・ \\
\hline & $\cdots \cdot \cdot$ & & $\cdots \cdots$ & $\cdots$ & $\cdot \mathbf{r} \cdot$ & $\cdots$ & \\
\hline g & $\cdots$ & & $\cdots$ & $\cdots \cdots$ & & $\mathbf{w} \cdot 1$ & $\because$ \\
\hline 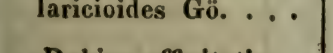 & & & $\cdots \cdots$ & $\cdots$ & & $\cdots$ & \\
\hline & & & & & & & \\
\hline ? lenticulosus Rossm. & $\cdots \cdots$ & & & $|\cdots \cdots|$ & $\left|\begin{array}{l}\ldots \\
\ldots\end{array}\right|$ & & $\dot{0}$ \\
\hline
\end{tabular}




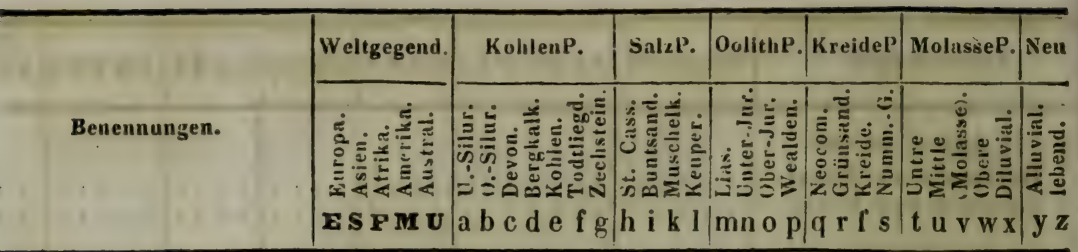

Elate Ende. 1 austriaca UNG.

\section{SteinhameriaPresi3} subglobosa Presc. oblonga Presc .

Cunningluanites PresL 3 . dubius Presc oxycedrus Presi.

* sphenolepis Braus.

AraucaritesSt.Gö.13

* Trunci.

ambiguus Gö.....

Brandlingi Gö. ... carbonarius Gö... . .

- keuperanus Gö.... . medullaris Gö. ... Sterubergi Gö..... stigmolithus Gö. . Tchihatcheffauus Gö. Withami Gö.

\section{*** Folia.}

peregrinus LH. . . . Sternbergi Gö. . . Reichenbachi Gö... . *** Strobill.

Goepperti ST.

Pissadendron ENoL. primaevum Ung. .. antiquum UNG.

DammaritesPresl2 albens Presc crassipes Gö.

Alberti[i]a SchMG. 4 latifolia SснMg. elliptica SснMg. Brauni SchMg. . . . speciosa SснMg.
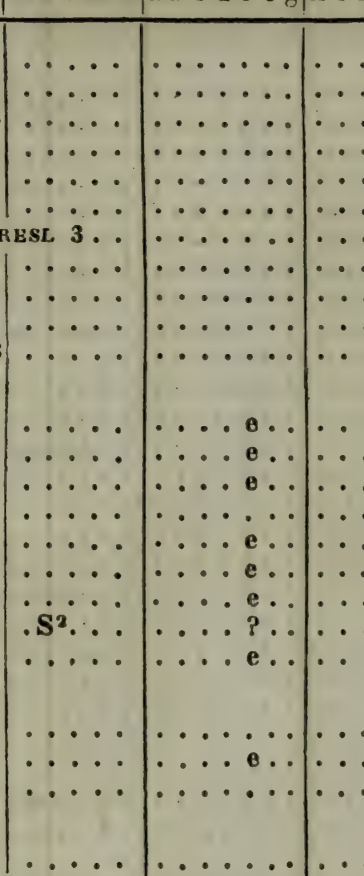


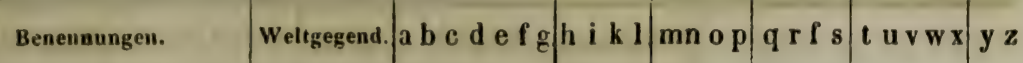

Cupressinites BowB. 13

globosus Bow в.

elongatus BowB.

recurvatus Bow B...

subfusiformis Bow B.

curtus Bow B.

Ccmptoni BowB. .

thuioides Bowв. .

crassus Bow B.

subangularis Bowв. corrugatus BowB... sulcatus Bows.

semiplotus Bow в.

tessellatus Bowв...

Cupressites Br. 5 .

Bockanus GöBnr...

Linkanus GöBrr...

Brougniarti Gö.

Hardí Gö.

Ullmanni BR.

Taxodium Rich.

- Oeningense Al.Braun

"distichum Rich.

Traxodites Prest 5 Münsteranus PresL tenuifolius PresL. dubius Press.

flabellatus Gö.

europaeus Gö.

Thuites Brgn. 11 . Breynanus GöBNT. . KlinsmannanusGöBNT Kleinanus GöBNr. Rapoldanus GöBNT. Mengeanus GöBNr. . callitrina UNG. salicornioides UNG. . inbricatus DuNk.. . gramineus ST.

- gracilis Gö.

* Langsdorfi Gö.

Thuioxylum UNG. 5 * juniperiuum UNG.. * arceuthicum UNG.

- ambiguum UNG....

* arctannulatum Ung.

Voltzia Brgn, 3 acutifolia Brgn.. heterophylla Bran. Phillipsi LH.

BrachyphyllumBagn. is. mammillare BRGN.

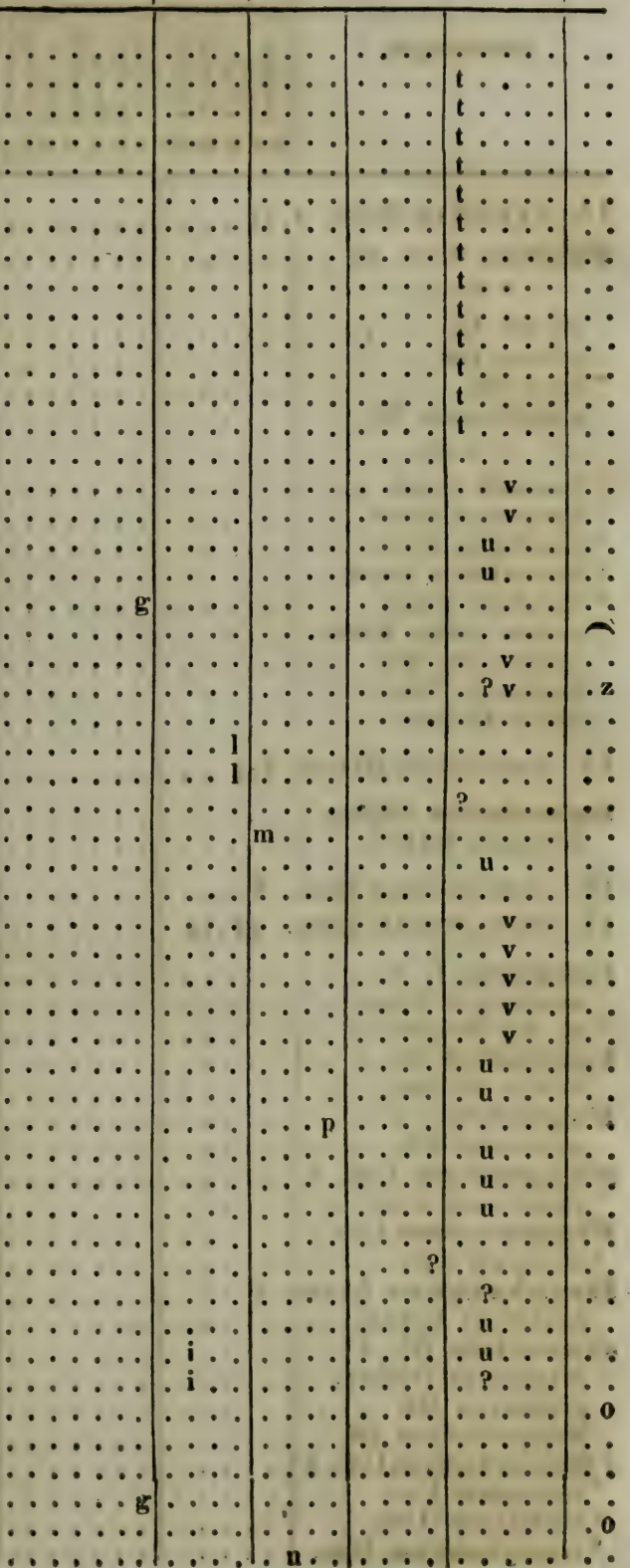




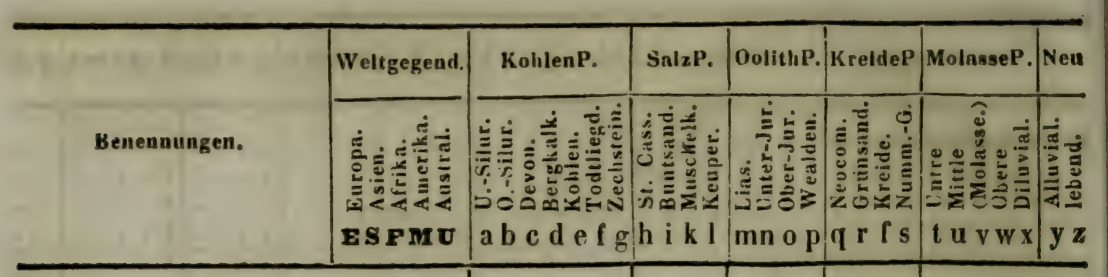

14. Taxineae Rich. $1: 13 \ldots$

Traxites BRGN. 13 - Trunel.

Ayckei Gö. priscus Grö.

- tener Gö. scalariformis Gö. ** Folia.

"Tournali Brgn. . . . * Langsdorfi Brgn. . . * tenuifolius BrgN. . . " diversifolius Brgn. .

- acicularis BrgN.

- podocarpoides Brgn. carbonarius Mü. . . Rosthorni UNG.... affinis GöBNT.

15. GNetacear Buvme
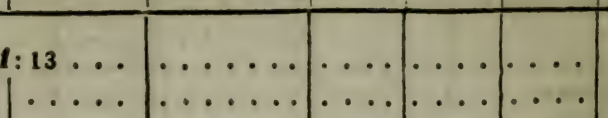

Ephedrites GöBNr. Johnanus GöBNT.

16. casuarineae Mirb. 17. cupuliferae Rich.

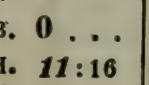

Carpinus L. 2 ... macroptera BRGN.

* sp. Ar. Braun ...

Carpinites Gö. 1 . dubius GöBкт.

Fagites Gö. 1 ... gypsace us Gö.

Phegominm UNG. 1

vasculoxum Ung. .

Plataninium UNG. 1

* acerinum UNG.

Dryobalanis Lando. basalticus Lande.

Quercitew Gö. 3 Meyeranus GüBNr. primaevus Gö. Kefersteinauns Gô. .

P Quercintunn UNG. 3 - sabulosum Urig.

* austriacum UNe.

* transylvanjcum UNG.

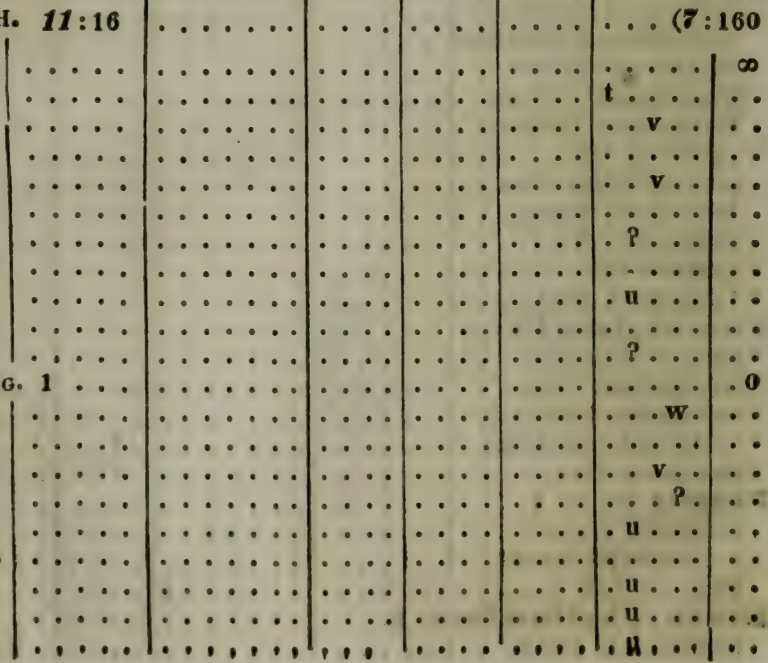




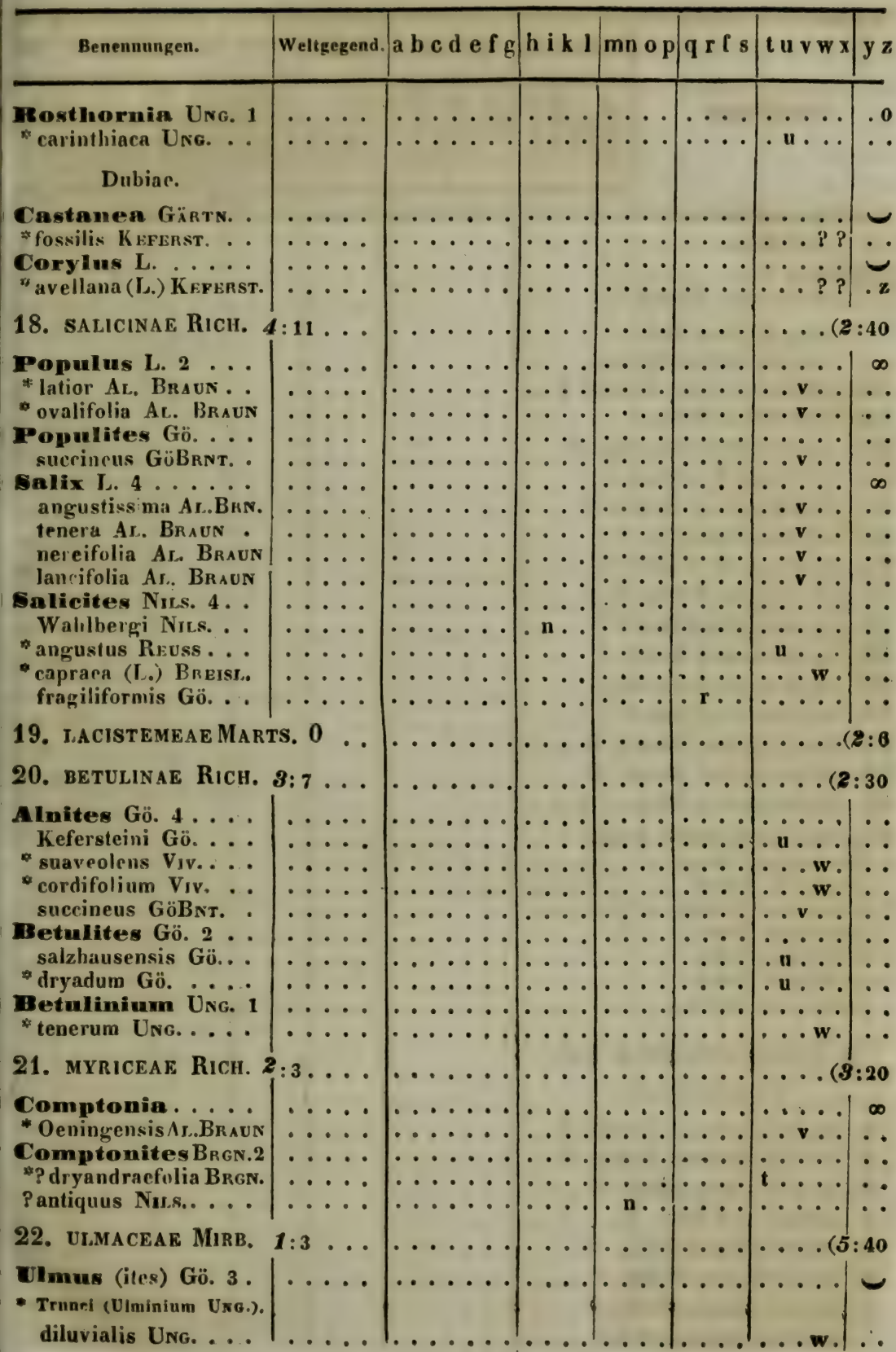




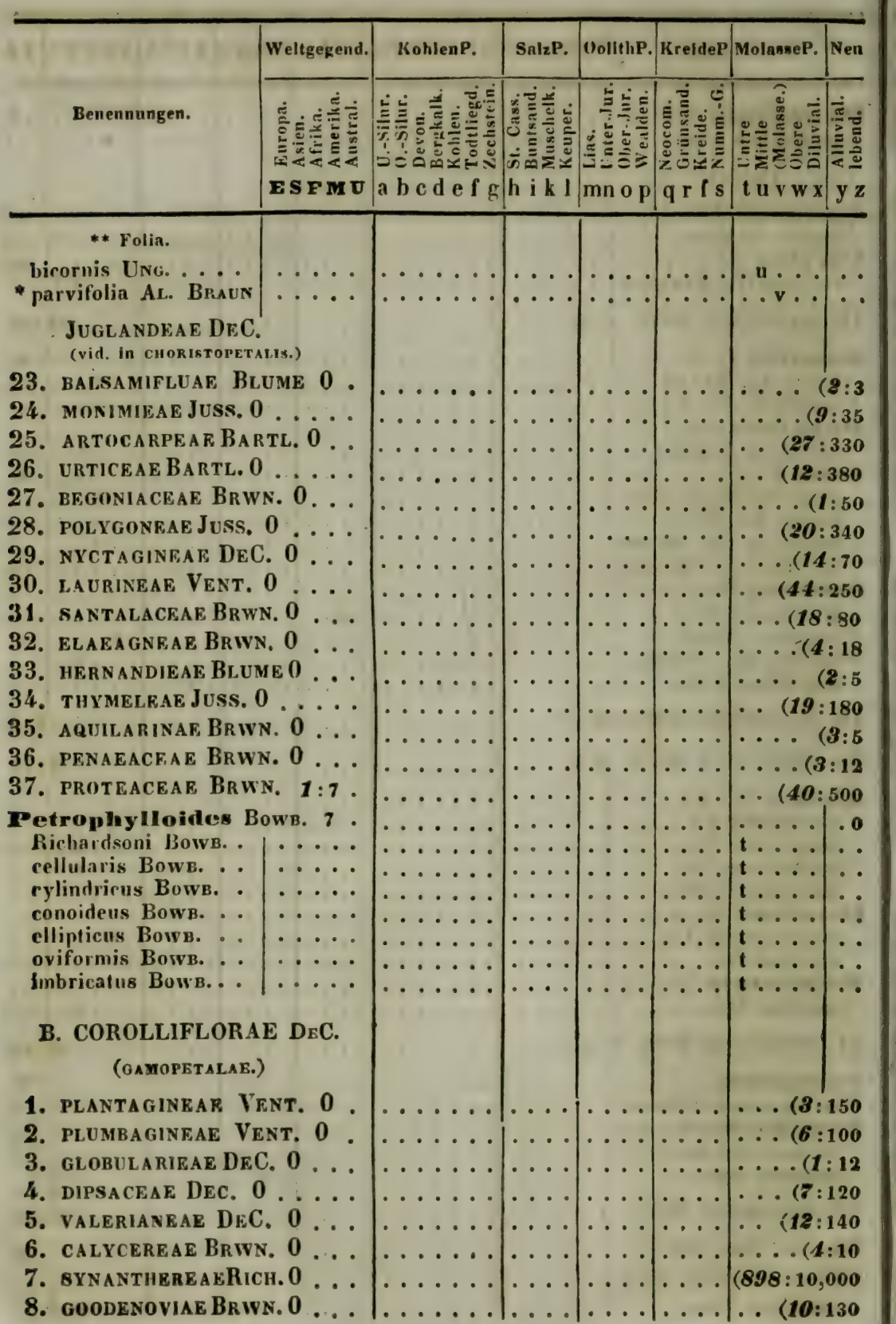




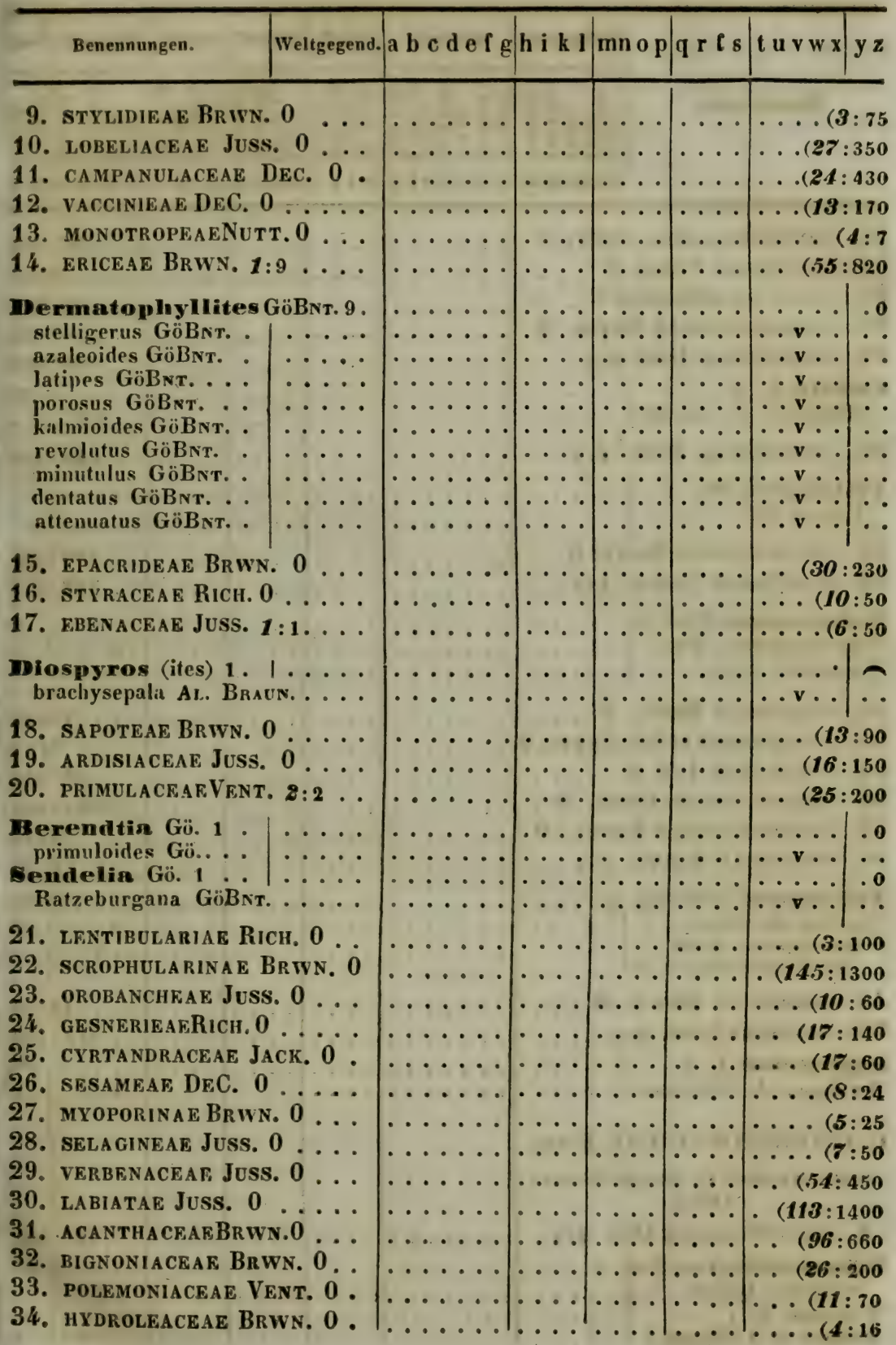




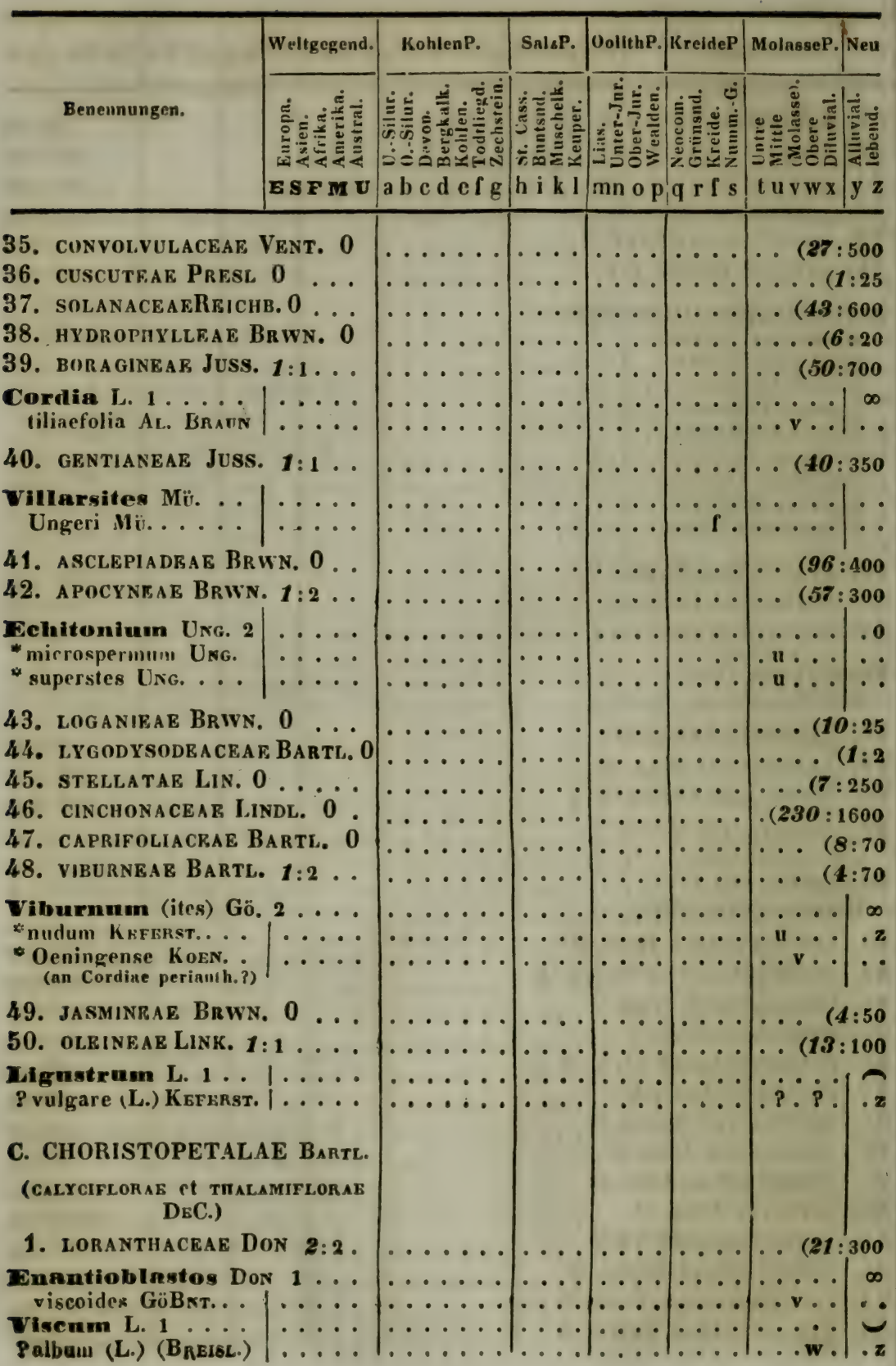




\begin{tabular}{|l|l|l|l|l|l|l|l|}
\hline Benennungen. & Weltgegend. a b c d e f g & h i k l & mn o p & q r f s & t u v w x & y z \\
\hline
\end{tabular}

2. UMbelliferaE JuSs, 1:1.

Piampinellites Ung. 1

"zizioides UNG.

3. araliaceae Juss. 0 ...

4. hEDERACEAEPERLEB 0

5. hamamelidfae Brwn. $1: 1$

Yiquidamber L. 1

Europaeum Ar.Braun

6. Berberidear Vent. 0

7. MenispermeaE Juss. 0

8. MYRISTICEAE BRWN. 0

9. anonaceae Rich. 0

10. Magnoliaceae DeC. 0 ..

11. Dilleniaceae DeC. 0 ..

12. paeoniaceae Bartl. 0 .

13. RANUNCULACEAK Juss, 0

14. СAвоMBEAE Rich. 0

15. Nelumboneae Bartl. 0

16. NYMPHAEACEAE BARTL. 1: 1

Nymphacites Sr. 1

* Arethusae ST.

17. TREMANDREAE Brwn. 0..

18. Polygaleae Juss. 0 ...

19. Datischae Brwn. 0

20. Resedaceae DeC. 0

21. Fumariaceae Dec. 0

22. papaverachae DeC, 0 .

23. CRUCIFERAE JUSS. 0

24. capparideat Vent. 0

25. SAMYDEAE GÄRTN. 0

26. HOMALINEAE BRWN, 0

27. PAPAY Y CEAE Marts. 0

28. PASSIFloREaE Juss. 0

29. MALESHERBIACEAE DoN 0

30. Turveracear DeC. 0 ...

31. LOASEA JuSS. 0

32. cucurbitacean JuSs. 1:1

\begin{tabular}{c|cc} 
Cucumites Bowr. 1 & $\ldots .$. \\
variabilis Bows. . . & $\ldots .$.
\end{tabular}

33. escallonieae Brivn. 0 .

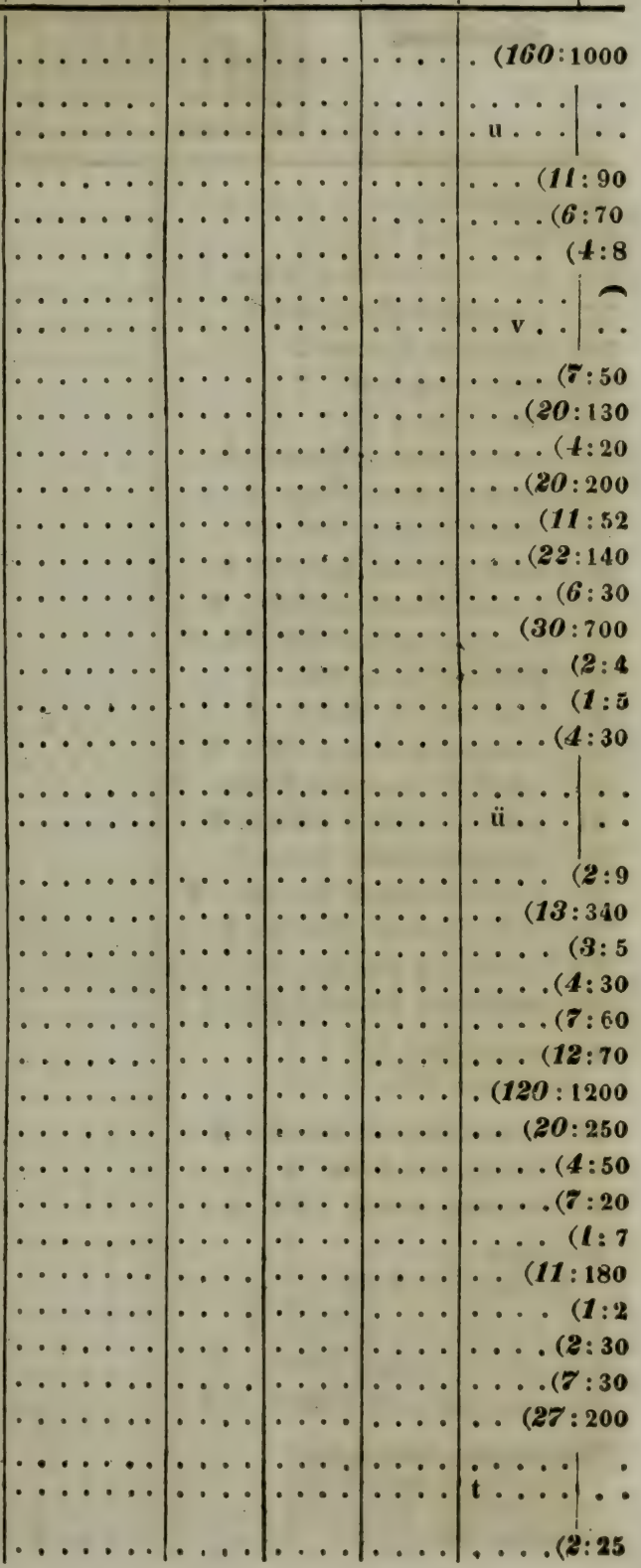




\begin{tabular}{|c|c|c|c|c|c|c|c|}
\hline & Weltgegend. & Kohlen P. & SnlzP. & |NolithP. & KreideP & Molasse P. & Neu \\
\hline Benennungen. & 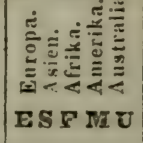 & 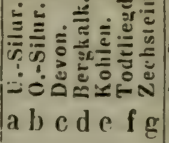 & 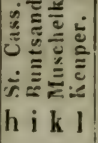 & 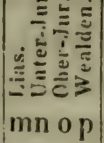 & 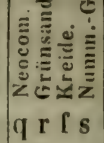 & 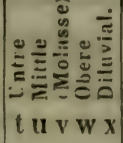 & 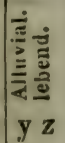 \\
\hline
\end{tabular}

34. grossularieae DeC. 0 . 35. NOPALEAE DEC. 0 36. flacovrtianeae Rich, 0 . 37. MARCGRAVIEAE JUSS. 0 ... 38. BIXINEAE KUNTH 0 39. cistineae DeC. 0 40. violarieas DeC. 0 41. SAUVAgesieae BartL. $0 \ldots$ 42. SARRACENIEAE TURP. 0 43. Droseraceav DeC. 0 44. Parnassieae Reichi. 0 . 45. TAMARISCINEAE DESV. 0 . 46. FRANKENIACEAE St. HiL. 0 47. hyfericinea De DeC. $0 \ldots$ 48. Garcinieae Bartl. 0 ... 49. Chenopodiaceae DeC. 0 . 50. amaranthaceae Brwn. 0 51. phytolacceae Brwn. 0 . 52. SClerantheae LiNk 0 . 53. paronychieae St. HiL. 0 54. Portulaceae Bartl. 0 . 55. alsineare Bartl. 0 56. Sileneae Bartl. $1: 1 \ldots$ Cucubalites Gö. 1 Goldfussi Gö.

57. Nitrariace,ae Linde. 0 . 58. Ficoldeae Juss. 0 59. Crassulaceae DeC. $1: 1$. Eedites Gerv. 1 . ? Rabenhorsti Gein. . 60. Sa xifrageae Vent. 0 61. cunoniaceae Brwn. 0.. 62. HALORAGEAE BRWN. 1:2 .

Myriophyllites St. 2 . . . . (an Calamit. radices?) capillifolius UNG... ? dubius ST.

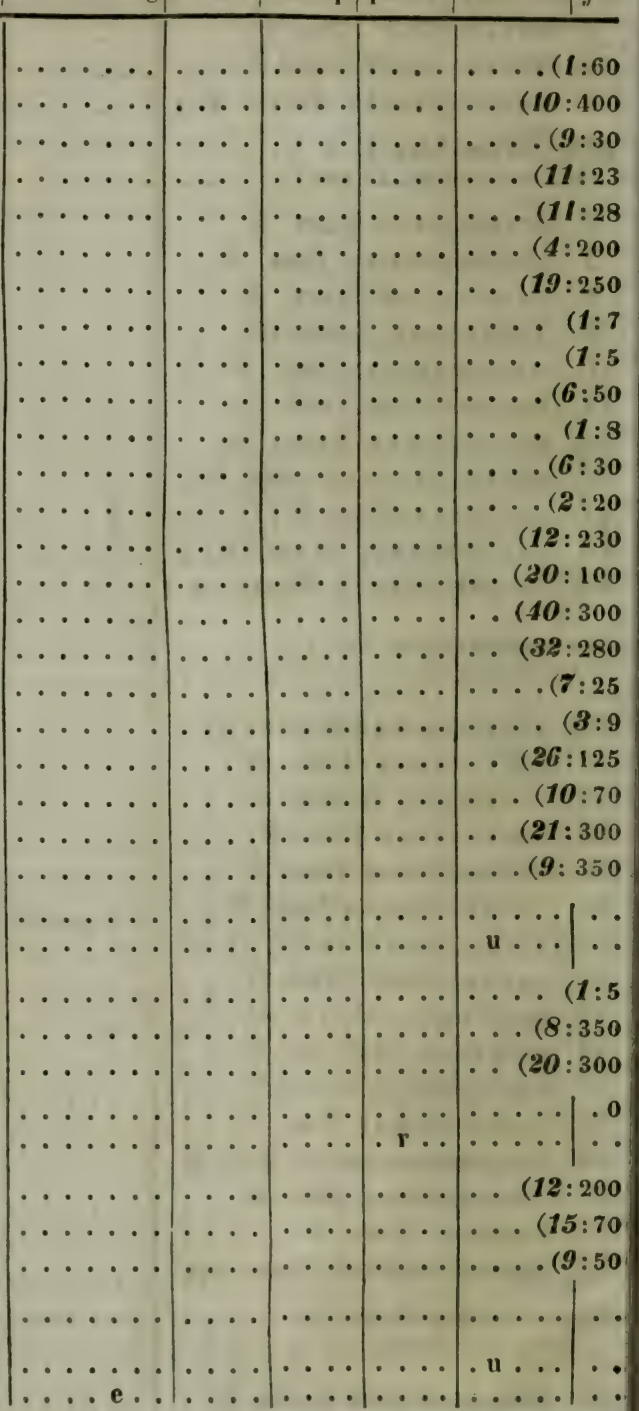




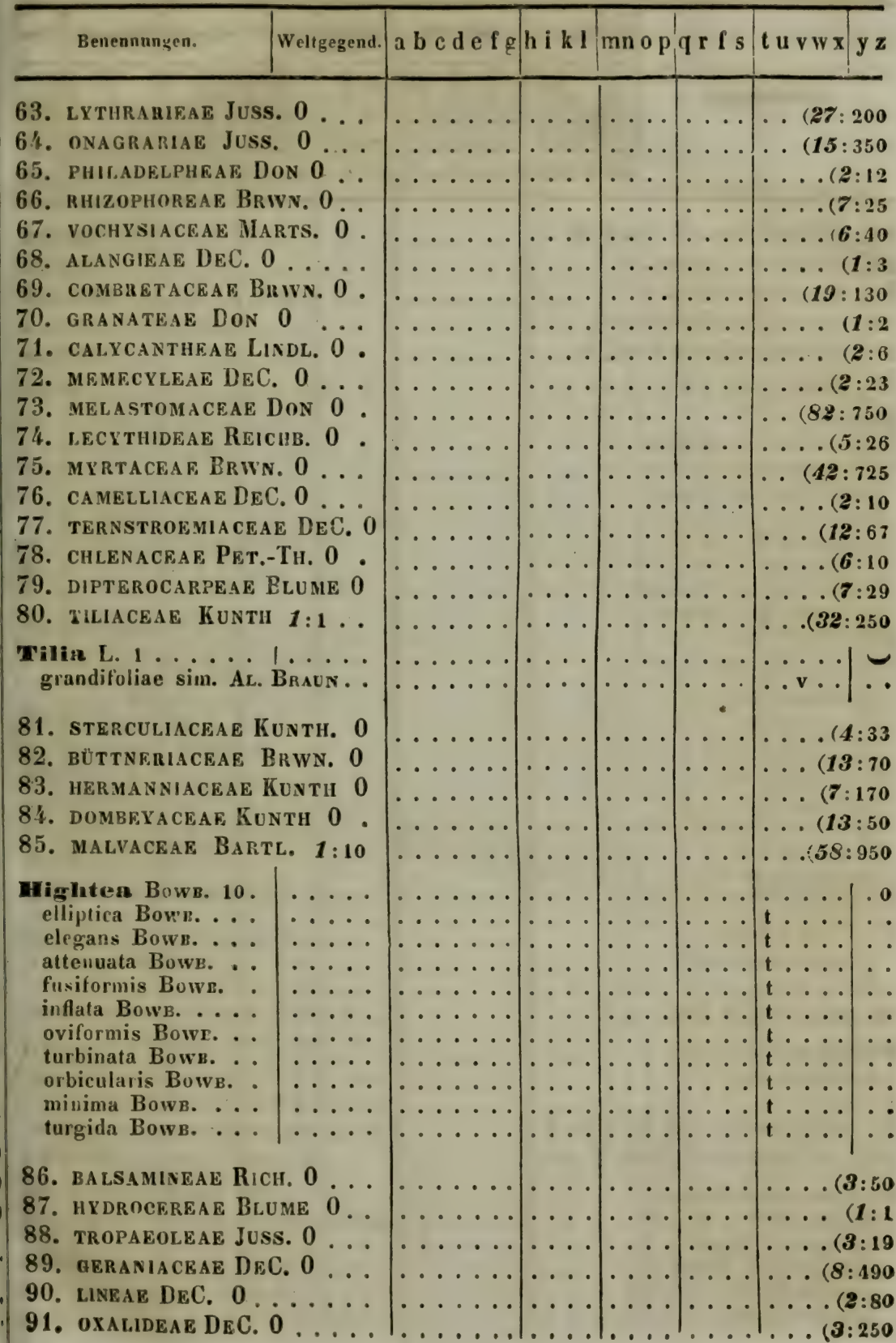




\begin{tabular}{|c|c|c|c|c|c|c|c|}
\hline & Weltgegend. & KohlenP. & SalzP. & OolithP. & KreideP & MolasseP. & Neu \\
\hline Beneunungen. & 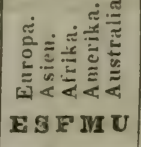 & 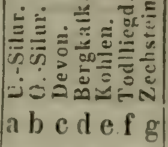 & 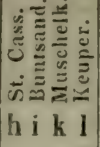 & 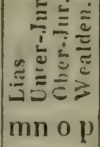 & 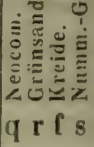 & 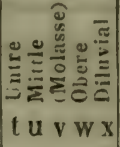 & 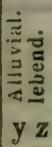 \\
\hline
\end{tabular}

92. sarmentaceae Vent. 0 . 93. LeEAcFae Bartl. 0

94. MELIACEAE JUSS. 0

95. Cenreleae Brwn. 0

96. MalpighiaceaE Juss. 0

97. ACERINEAE DEC.

Acer L. 7

* productum Ac. Braun

* protensum? Al.Braun

* trilobatum Al. Braun

* vitifolium As. Bpasen

?campestre (L.) AL. Br. ß3. Neguncho.

"trifoliatum As. Braun

* radiatum As. Braun

Acerites NILS. 9 .

* Trunci (Acerinium Vig.)

* danubialis Gö. ** Folia.

tricuspidatus Gö. . .

* Langrdurfi Gö.

* ficifolius Viv.

* monspessulanus(L.)V

* elongatus Viv.

*integerrimas Vav.

cretaceus Nirs.

*** Fructus.

campylopteris Gö.

98. coriarieae DeC. $1: 1 \ldots$

Coriaria (ites) Gö.

? myrtifolia Vıv.

99. ERYTHROXYLFAF KUNTH. 0 100. SAPINDACEAE JUSS. 2:15

Cuganoides Bowb. 8

lobatus Bown.

corvegatus Bowr.

subangulatus BowB.

grandis Buwr.

tumidus Bow в.

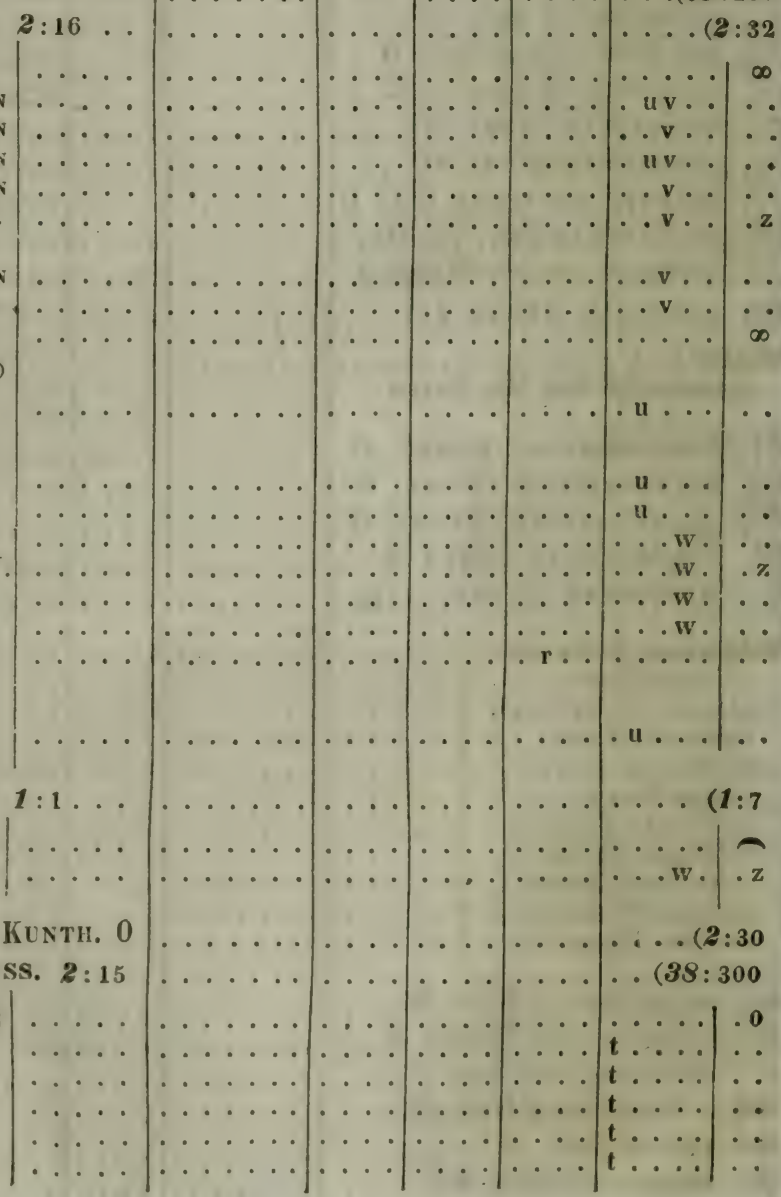

$4: 250$

(2: 14

$(27: 110$

. $(8: 19$

$25: 250$

(2: 32 


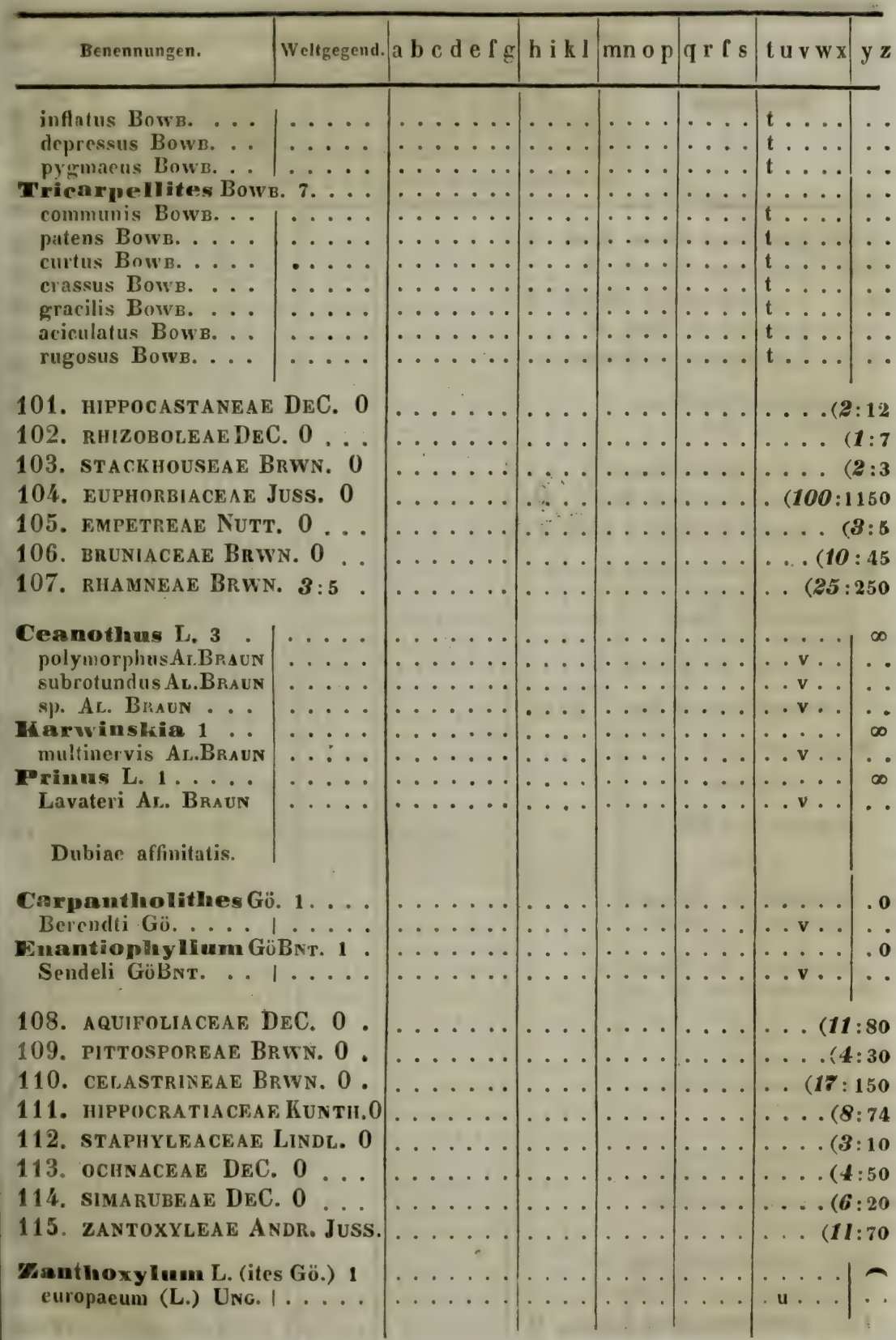




\begin{tabular}{|c|c|c|c|c|c|c|c|}
\hline & Weltgegend & KolilenP. & SulzP. & Oolithr. & KreideP & MolasseP. & Neu \\
\hline Benennungen. & 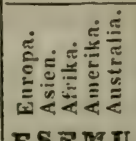 & 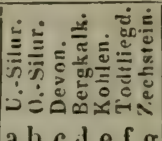 & 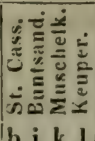 & 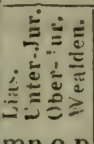 & 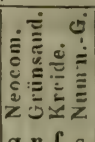 & 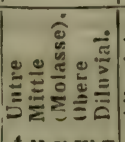 & 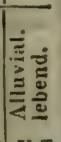 \\
\hline & ESETO & a Dcolg & h i k I & $\mathrm{mn} 0 \mathrm{p}$ & 1 r I s & t u v w x & $y z$ \\
\hline
\end{tabular}

116. diosmeae Andr. Juss. 0 117. rutaceae Bartl. 0 118. zygophylleae BrWn. 0 119. olacineae Mirb, 0 120. aURantiaceaeCorrea 1:1 wetherellia Bowb. 1

121. A MYRIDEAE BRWN. 0 . . 122. connaracear Brwn. 0 123. Terebinthaceae Juss. $1: 3$

Thus L. (Rhoites Gö.) 4 .... Pyrrhae Uns..... . Rhadamanti UNG. stygium UnG. . . . . punctatum AL. Braun

124. JuglandeAE DeC. $2: 15$

Juglans $\mathrm{L}$.

* Fructus.

cinerea (L.) Br...

** Folia.

falcifolia Az. Brave Jatifulia Ac. Braun . B. (Carya?) acuninata Ar. Brats

Juglandites $\mathrm{S}_{\mathrm{T}}$. 11 costatus Presi, ... minor Prist. elegaus Gö. Hagenanus GöBnt. salinarum ST. .... ventricusus ST. ... laevigatus Brgn... Schweirg ri GëBvt. nux-taurimensis Gö.. rostratus Gö.

125. POMaceae Linde. 0 126. rosaceae Spens 0 127. SANGUISORBEAE LINDL. 0

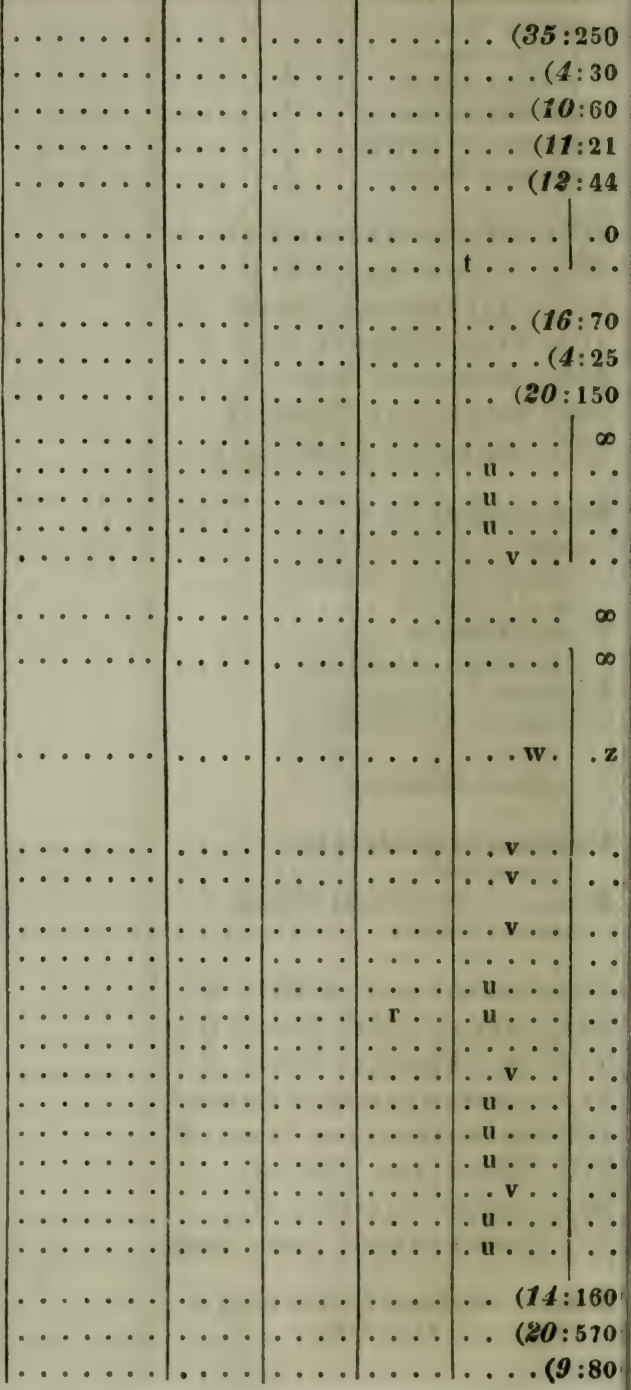


128. spiraeaceae Kunth 0

129. AMygdaleae Barti. 0 .

130. chrysobalaneae BrWN. 0

131. Neuradeae DeC. 0

132. MORINGEAE BRIVN. 0

133. Leguminosae JUSS. $13: 58$

Cytisus L. 2 .

"OeningensisAL. Braun

*? Lavateri Al. Braun

Dolichites Ung. 1

" europaeus UNG...

IDesmodites UNG. 1

"radobojensis Ung.

Cxleditschia L. (ites Gö.) 1

" poducarpa AL. Braun

Phacites Вrot.Culla 2

*alpinus Brot. Colga

? gothlandic.usW AHLENB.

Faboidea bowb. 25 longiusrula Bowe. crassa BowB. crassicutis Bow B. . . planidorsa BowB. symmetrica Bowb. piana Bowв. . marginata Bowb. . . semicurvilinearis BwB. larga Bows.

complanata Bowв. subdisca Bowb. oblonga Bowв. ovata Bows. ventricosa Bowb. robusta Bown. pinguis Bowb. subrobusta Bowe. planimeta Bowr. quadrapes Bowb. bifalcis Bowe. tenuis Bowr. subtenuis Bnwв. rostrata Bows. doliformis BowB. aruta Bow B.

Leguminosites Bowe. 18 subovatus Bowb. erassus Bowb. elegans Bowb. rotundatus BowB. longissimus Buwb.
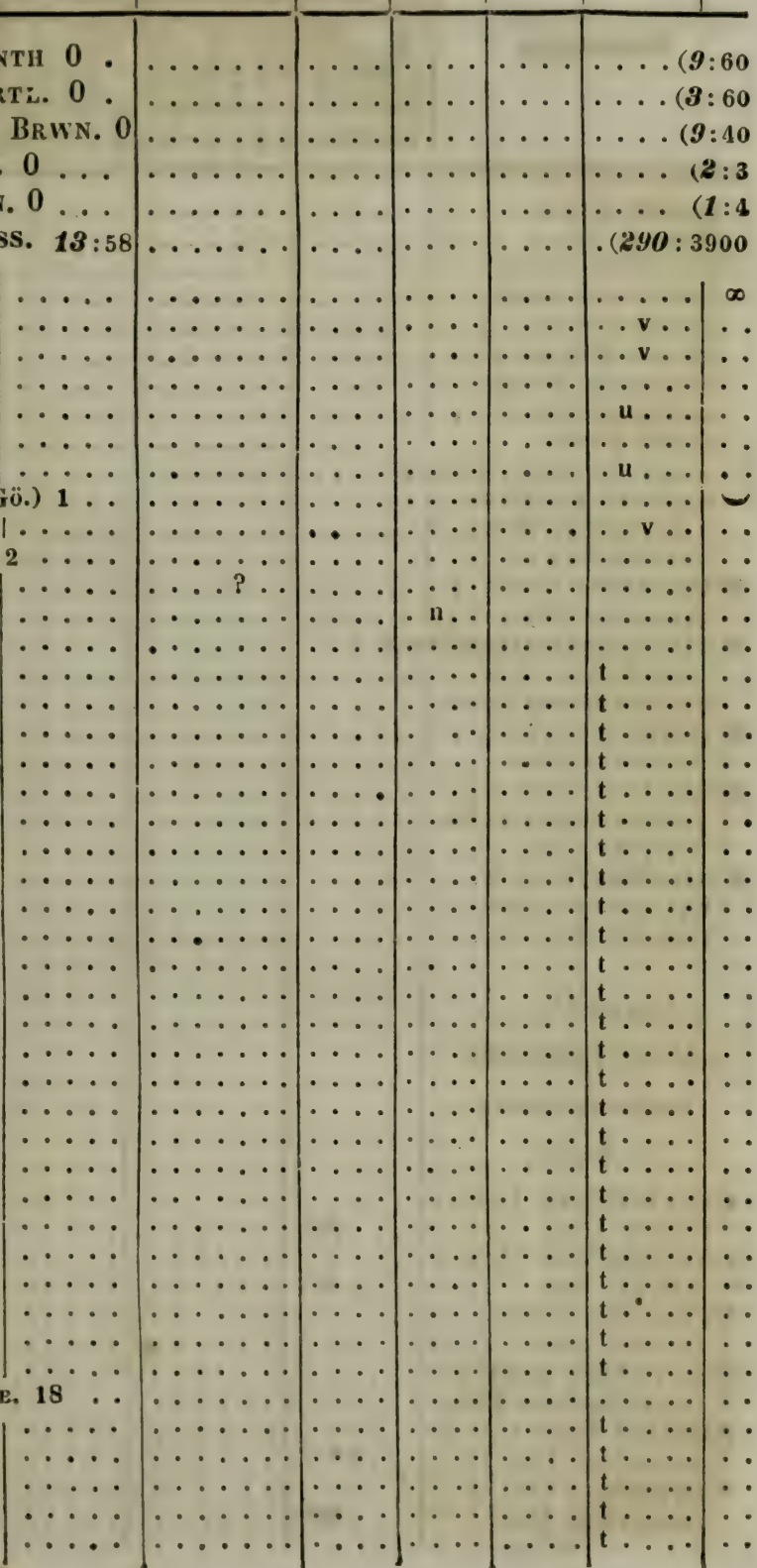


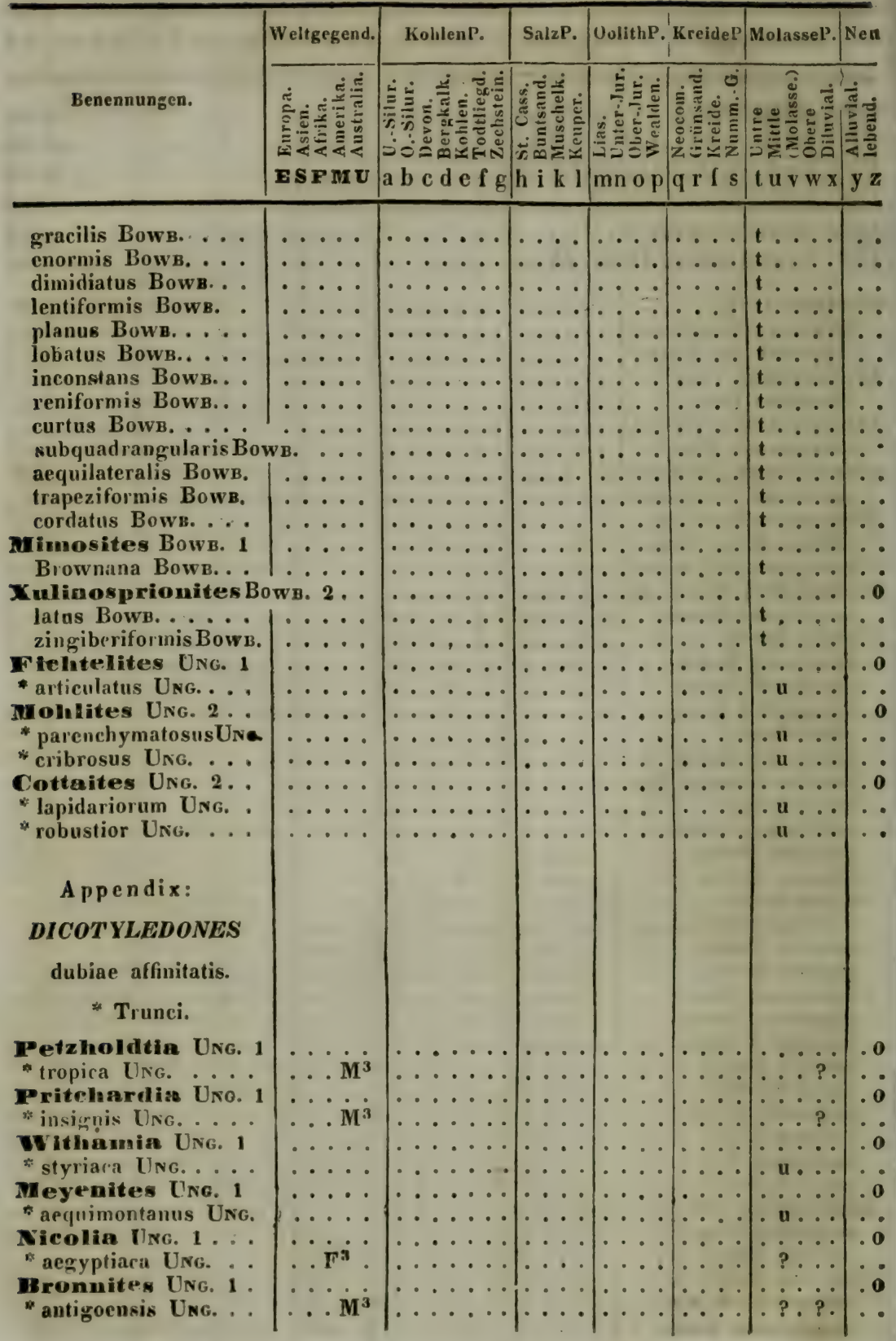




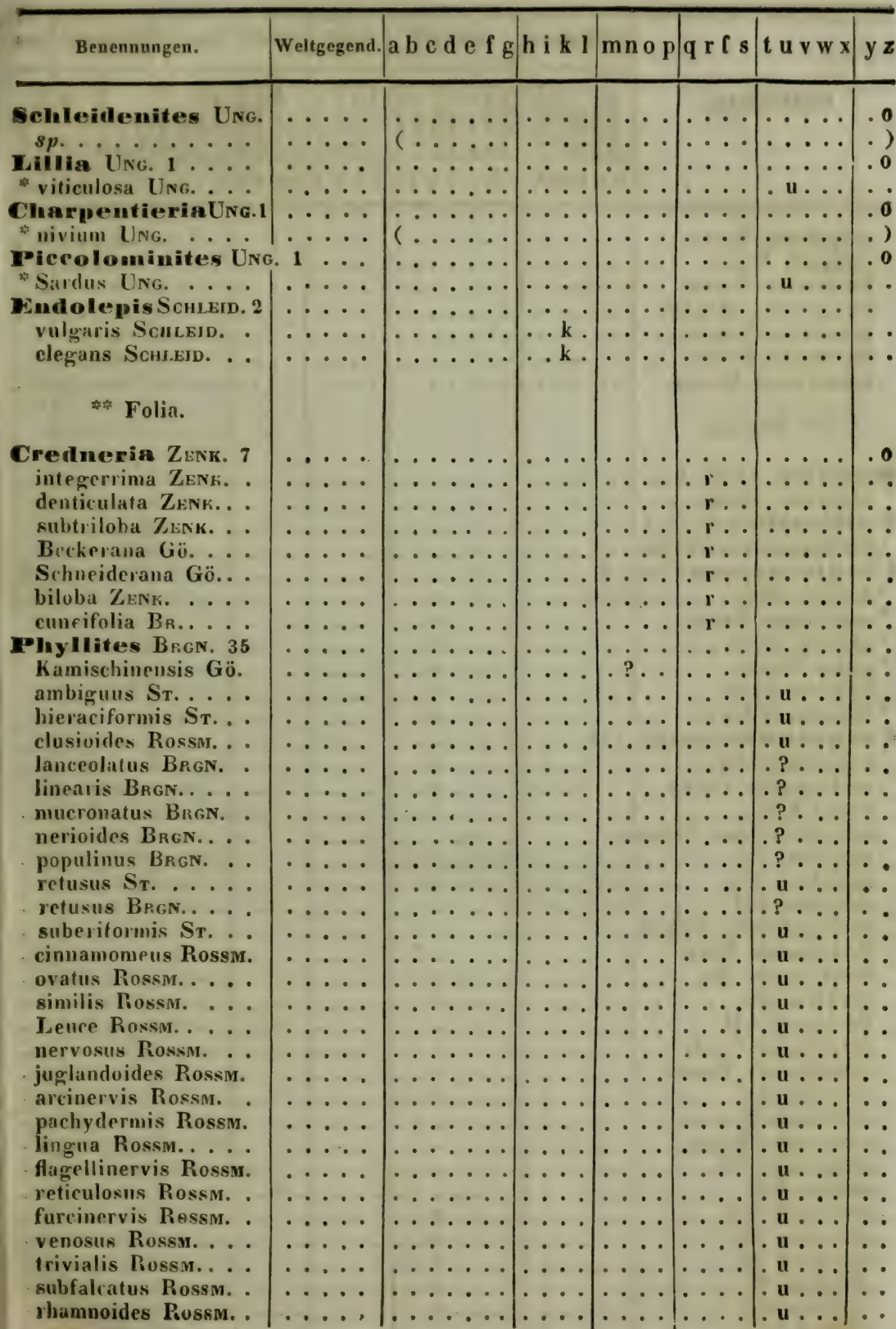




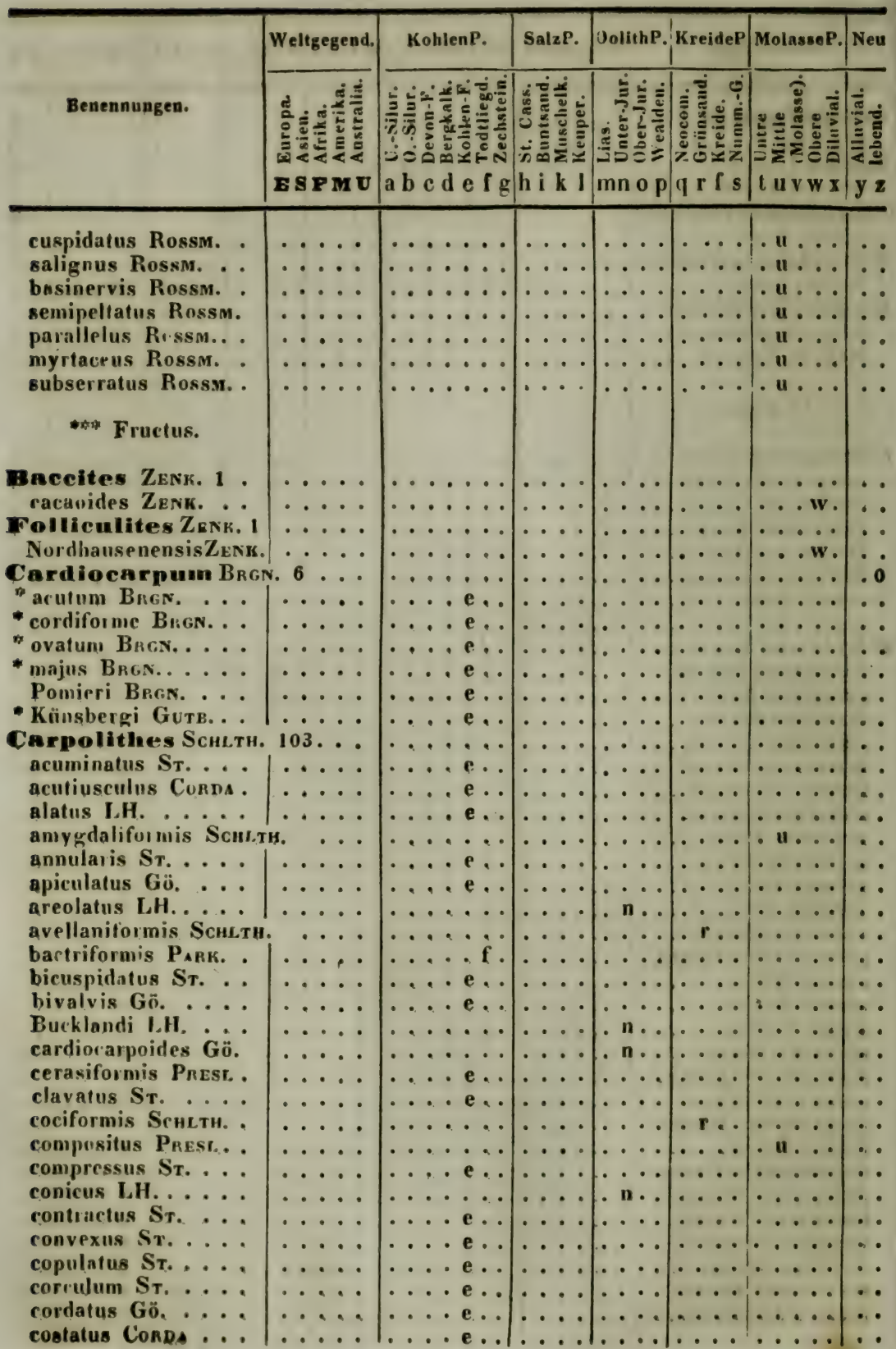




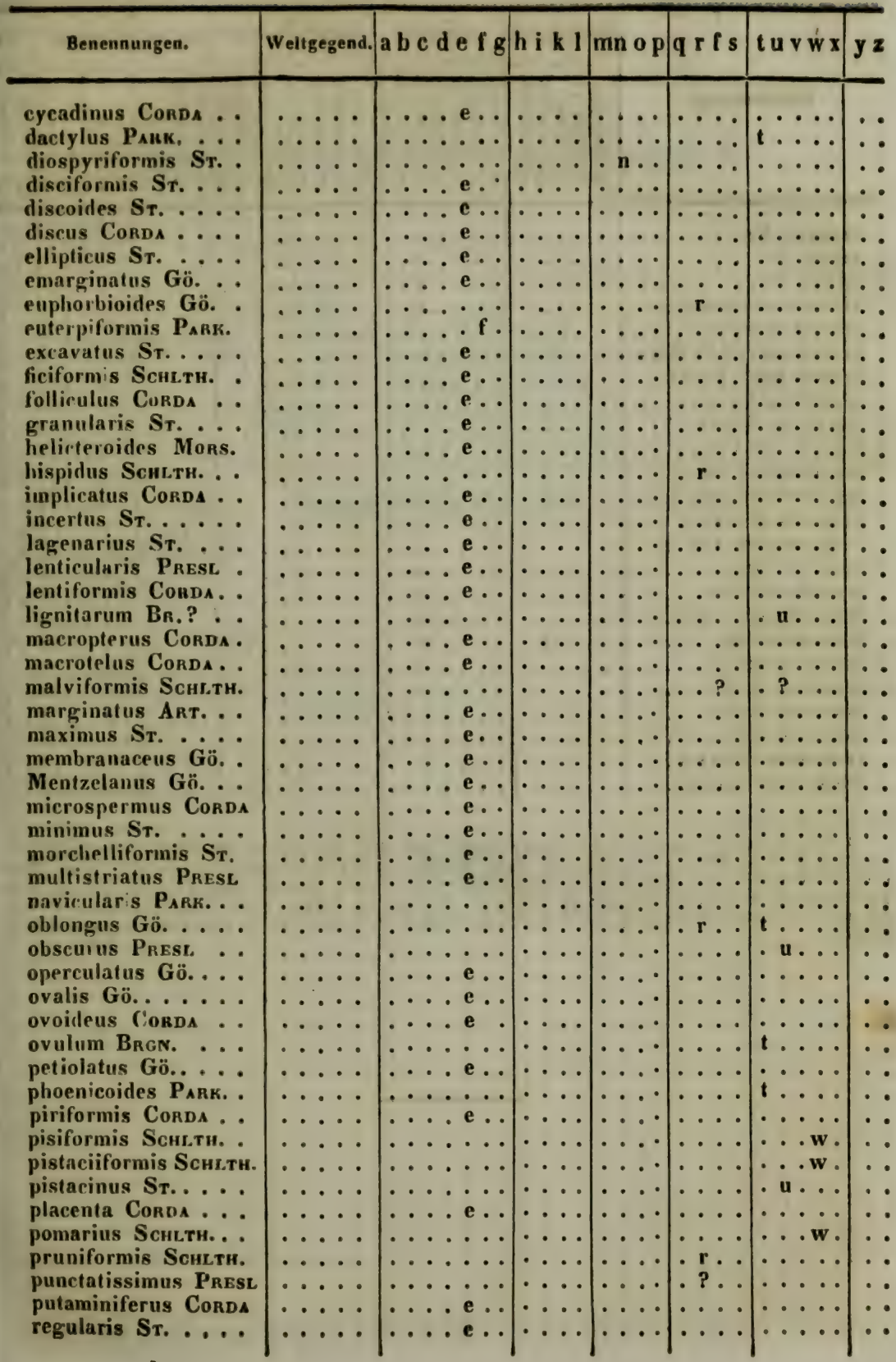




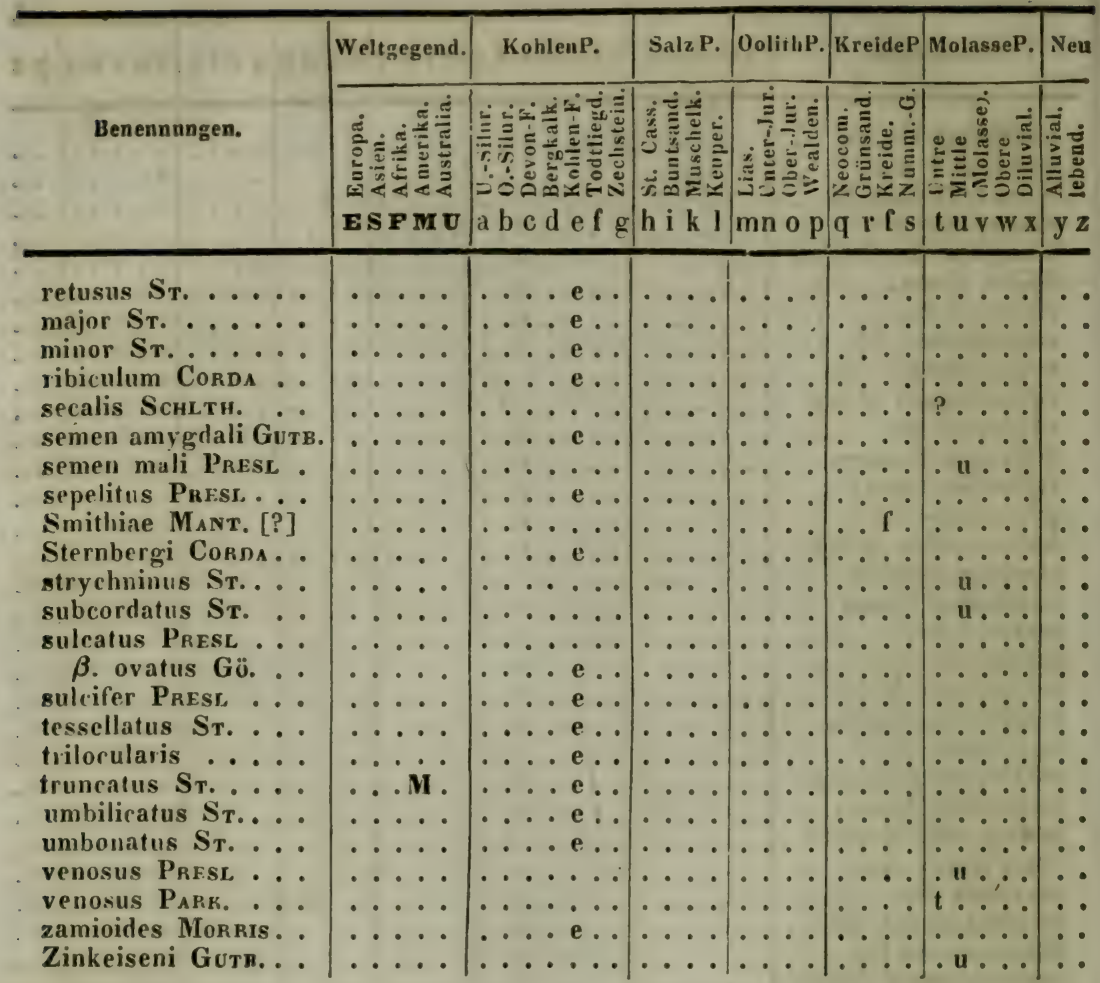

1 Die während des Abdrucks dieser Bogen von Hrn. Az. Bratr im Jahrbuch bekannt gemachten Öuinger PHanzen, welche Hr. Prof. Göpper nicht mehr bearbeiten konnte, sind zwar von mir noch in dieses Verzeichniss eingeschaltet, die Namen aber uach meinem im zoologischen Theiledurchgefiihrten Grundsatze so wenig als möglich zu ändern, unverïndert belassen, da ieh sie nicht in Übereinstimnung mit den übrigen Namen verwandter Arten abzuändern unternehnen wollte. BR, 


\section{Supplenentum plantarum ':}

\begin{tabular}{|c|c|c|c|c|c|c|c|}
\hline & IVeltgegend. & KohlenP. & SalzP. & OolithP. & KreideP & Molasse $P$. & $\mathrm{Ne}$ \\
\hline Benennungen. & 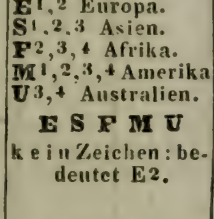 & 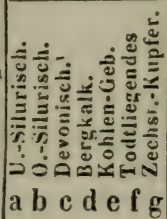 & 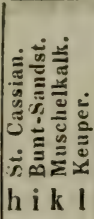 & 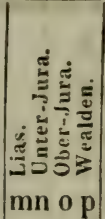 & 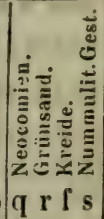 & 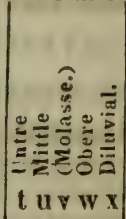 & $\frac{z}{\bar{z}}$ \\
\hline
\end{tabular}

\section{Cl. I. Plantae cellulares.}

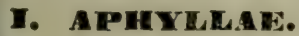

A. FUNGI (p. 5).

3.GASTEROMYCETESFR.

Xylomites ( $p \quad 5)$. irregularis Gö. ....

B. ALGAE Roth (p. 5)

1. Confervoldeak AgH.

Confervites (p. 5)

"Bilinicus Ung.

2. charachae Rich.

Chara (p.6) ....

" prisca Ung.

5. FuCACEAE Lx.

Cystoseirites (p. 9) Helli UNG.

Algae dubia affinitatis.

Fucus L...... subtilis Eichiv. ... taeniola Eichiv.... .

Fucoides HarL. 2 . Brongniarti Hall. . Alleghanniensis Harc.

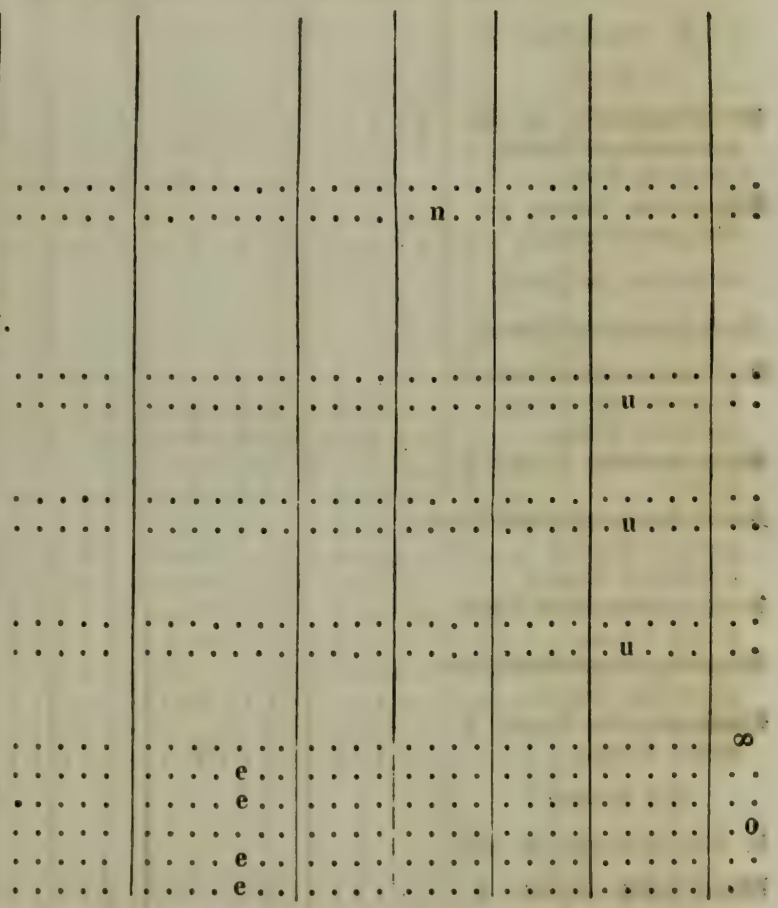

1 Seit dem Abdruck der vorigen Bogen ișt über eill Jąhp vergangen und daher dieser Nachtring nüthig geworden (1846 im Mai). 


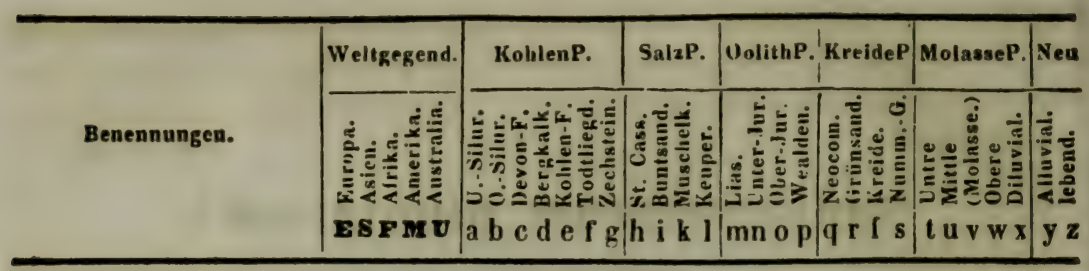

\section{Cl. II. PLANTAE VAsculares.}

\section{F. MONGCAT THEDONDS.}

A. CRYPTOGAMAF.

1. nQuisetaceak DC.

Calmaiteg (p. 11). Hoprensis His. ... variolatus $\mathbf{G o ̈ . ~}$

Fquisetum (p. 13)

* Brauni Ung.

*? stelliferum HARL.

3. FILICES.

* Trunci.

Protopteris (p. 14) microrrhiza Corda. - neonata Urg.

ZTippeía Corda, 1. disticha Coros.

* Stipites vel petioll.

Rachiopterides CoRDa.

Delenopteris Cond.2 Radnizensis Corda. involuta Conds...

Gyropteris Corda. 1 crassa .......

Anachoropteris Conds. 2 . pulchra CoRda ... rutundata Сондs ...

Ptilorrhachis Corda. 1 ... dubia Corda .... $1 \ldots \ldots$

Diplophacelus Corda. 1 .. arboreus Conda... $\ldots . .$.

Culopteris Corda. 1 dubia Corda

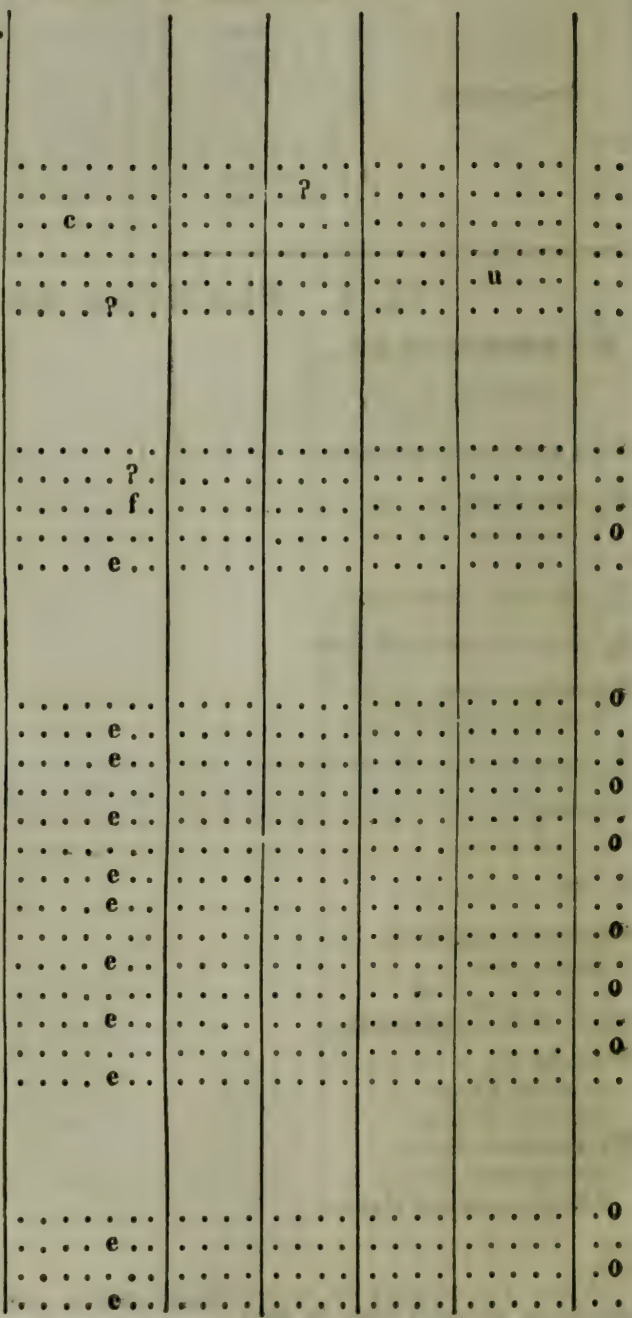

b. Gleichenieae (p. 15)

Alavieia Conda. 1 pulcherrima Conna.

Chorionopteris Corda. i.. gleichenioides Conda |..... 


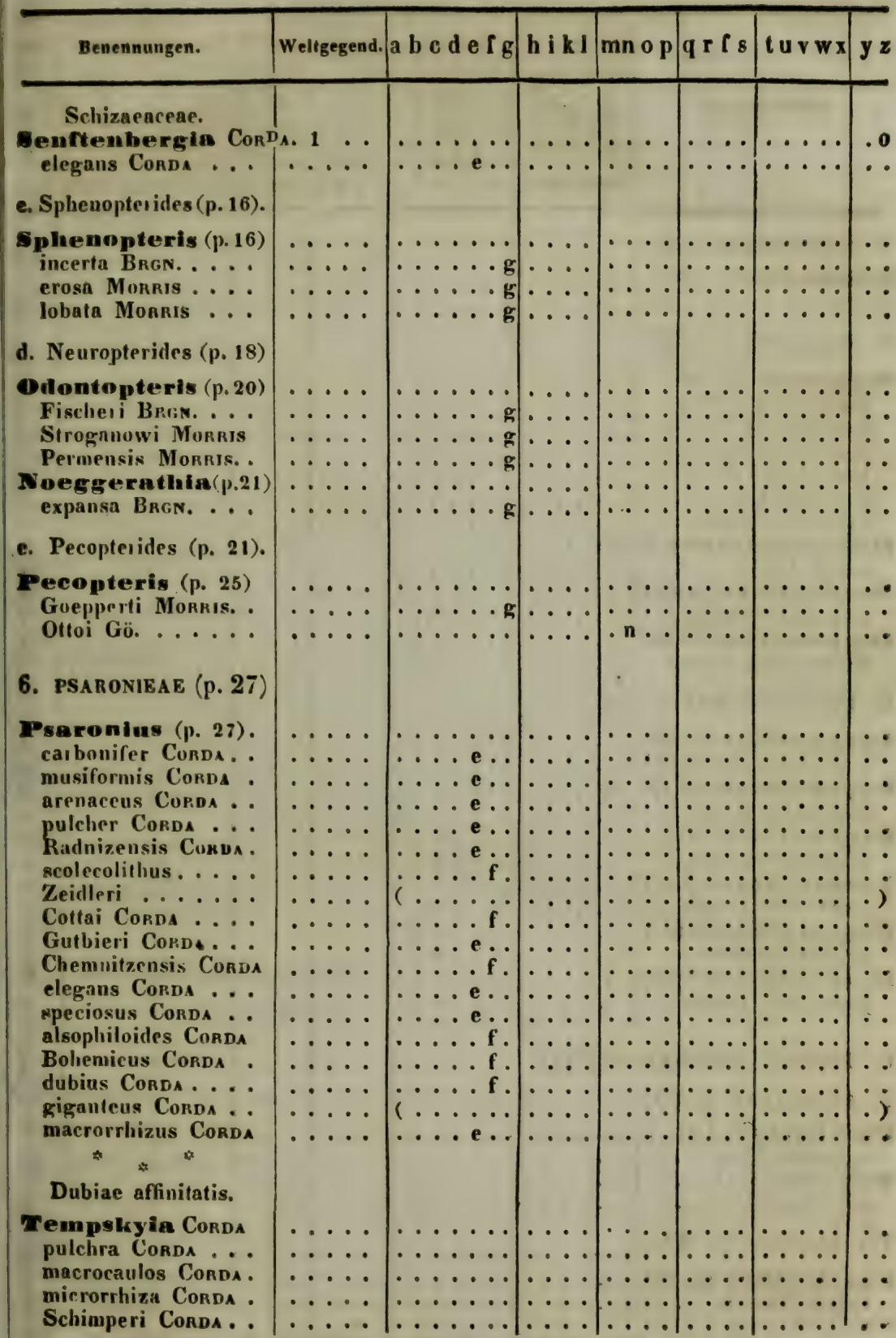


Benennungen.

Stigmaria (p. 27).

? conferta Corda...

8. sigillarieae (p.28;

Sigillaria (p. 28). diploderma Corisa.

Rlyytidolepis Conda.

Arzisensis [?] Corda ichthyolepis Corna.

parallela UNG.

"? clypeata SANDB. .

mhytidophioyos Corda. 1

tenuis Conton

MyelopithysCoRDA.1

medullosa Corda. .

Diplotegium Corda. Brownanum CoRda

9. Lycopodiaceae (p. 29).

Tycopodites ( $p .29$ )

* hexagonus Brschoff 1 . elongatum Brgn. .

Sagenaria (p. 31). fusiformic Conda. .

Lomatofloyos p.32) crassicaule Conda. .

Teptoxylunirorda.1 geminum Conda ... ....

Meterangium CoRDa. $1 \cdots$ paradoxum Corda $\mid \ldots \ldots$

B. MONOC. PHANEROGAMAE.

2. GRAMINEAE (p. 33)

Bambusium Ung. 1

* sepultum Uns.

Triticum L. 1 ... $s p$. Braun ....

17. PALMAE (p. 33).

Wasciculites (p. 33) Cottai Ung.?

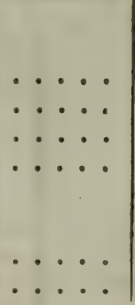

\begin{tabular}{|c|c|c|c|c|}
\hline Kolı & SalzP. & OolithP. & KreideP & MolasseP. \\
\hline 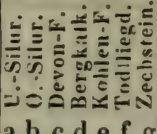 & 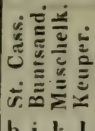 & 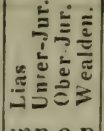 & 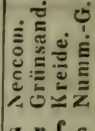 & 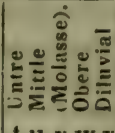 \\
\hline b c & i l I & inn op & I r f s & W X \\
\hline
\end{tabular}

1 Dic ron mir frïher unter Lycopoditcs Meyeranus aufgefulhrte Art ist zu streichen. Sie gehört zil Pecopteris Ottoi.

GöpP. 


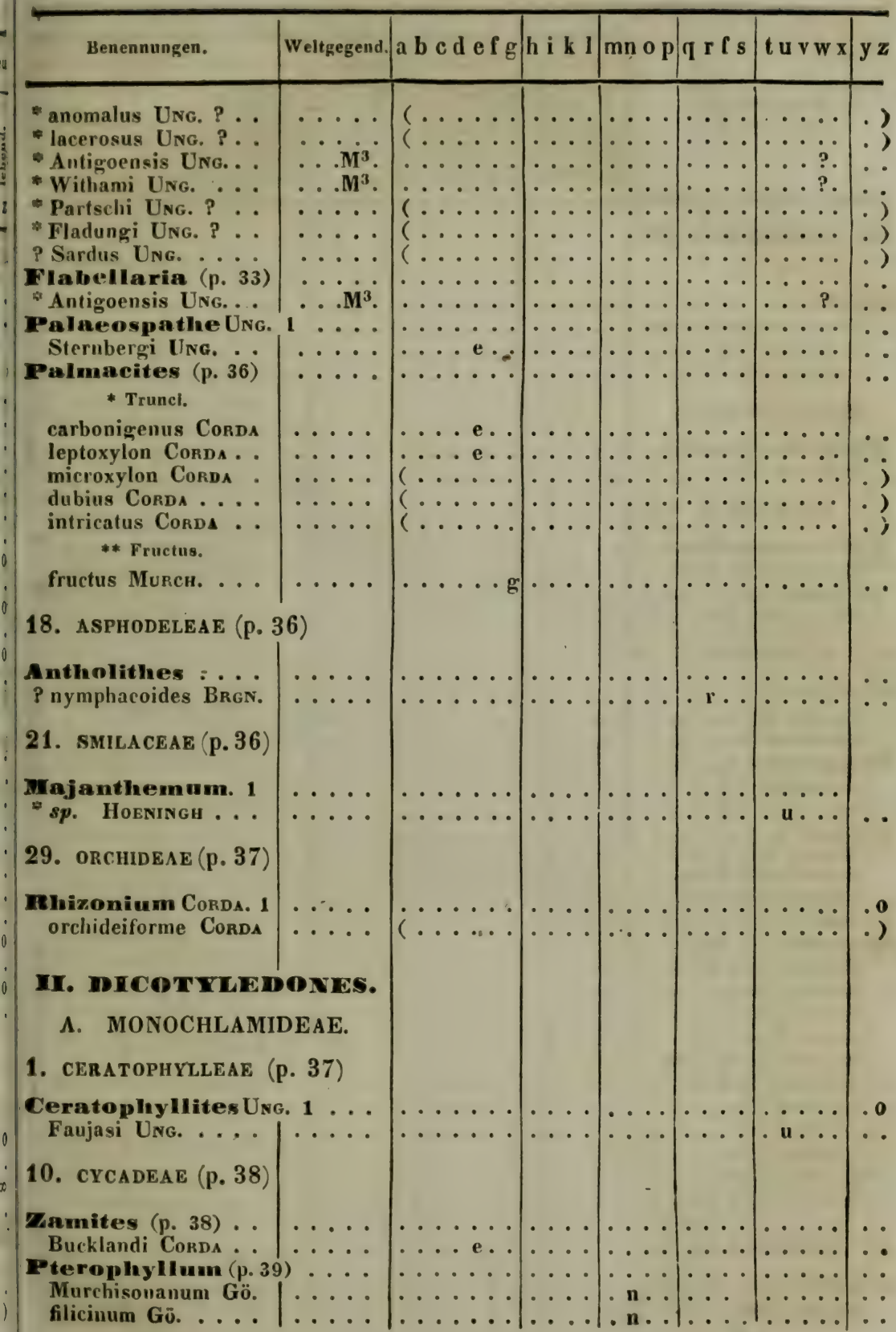




\begin{tabular}{|c|c|c|c|c|c|c|c|}
\hline & Weltgegend. & KohlenP. & SalzP. & WolithP. & KreideP & MolasseP. & Neu \\
\hline Benennungen. & 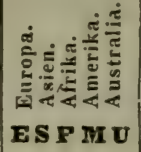 & 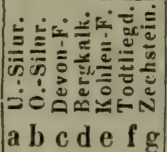 & 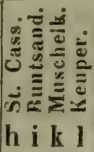 & 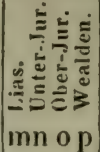 & 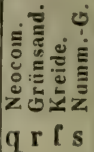 & 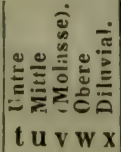 & 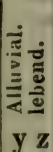 \\
\hline
\end{tabular}

12. ABIETINEAE (p.40) - Trunci.

Pinites (p. 40) ... Goeppertanus Schleid jurassicus Gö. pertinax Gö. .

Dubiue affinitatis.

Palaeocedrus Ung. 1 * exstincta UNG.

14. TAXINEAE (p. 44)

potocarpusHerit. 3

macrophyllus Linoc.

Salisburyia Sмитн. 1

* adiantuides Ung.

17. CUPUliferae (p. 44)

Carpiness (p. 44) grandis UNG. ... betuloides Ung.

Carpinites (p. 44). gypsaceus Gö.... arenaceus Gö.

\section{Fagus L.}

(Fagites Gö., p. 44.)

Deucalionis Ung.. rastaneifolia Ung. Atlantica Ung.

Quercus L. 11 ... Palacococrus Ung. . Bilinira Ung. .... Berra UNG. aspera UNe...... Hamadryadum UNG. chlorophylla Ung. Daphnes Ung. elaena Ung. . lignitum UNo. Drrmeja Ung. Mediterranea Uru.

18. SALiCINAE (p. 45)

Poopulus L. (p. 45) crenata Ung.

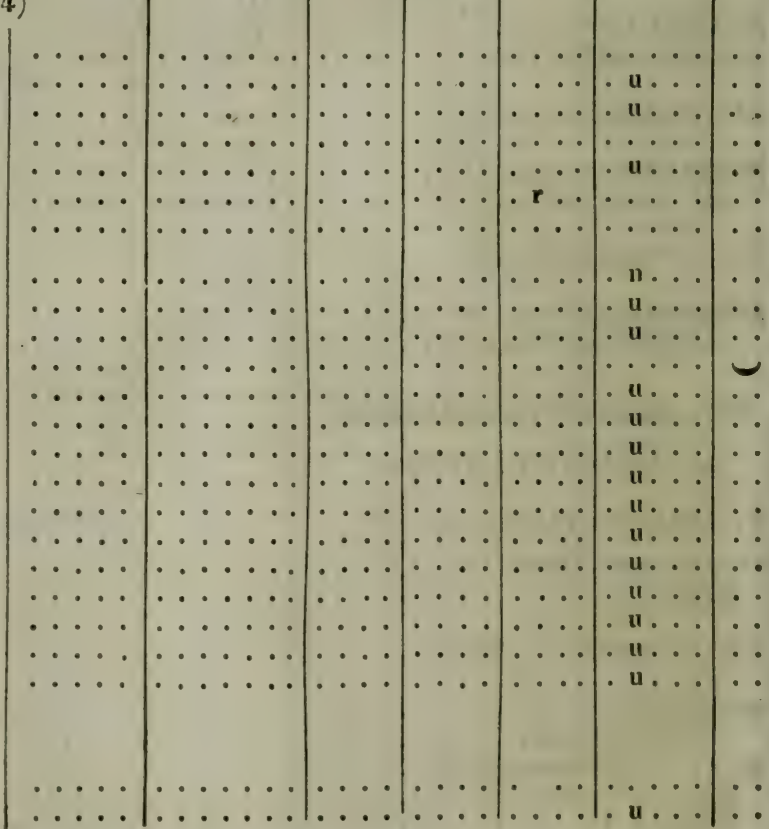




\begin{tabular}{|l|l|l|l|l|l|l|l|}
\hline Benennungen. & Weltgegend. a b c d e f g h i k l & mn o p & q r f s & t u v w x & y z \\
\hline
\end{tabular}

* Acoli Ung.

* gigas Ung.

* Lence Ung.

* Phaetonis Ung.

sp. BRGN.

20. BETUliNaE (p. 45$)$

Alnites Gö. (p. 45) gracilis UNG. ... nostratum UNG. . . .

Retulites Gö. (p. 45) macropterus UNG.

Betulinium UNG. (p. 45)

Parisiense Ung.

21. MYRICEAE (p. 45)

Comptomia (p. 45) grandifolia Ung... breviloha BRGN.

Myriea L. 6 .... (Myricites Gö)

* quercina Ung.

* inundata Ung.

* banksiifolia UNG. . .

* Haeringana Ung. . .

* aruminata UNG.

* Iongifulia Ung. ...

22. ULMACEAE (p. 45)

Ulanus ( $p .45)$

$$
\text { - Folia. }
$$

Bronni Ung.

longifolia Ung.... prisca Ung. plurinervia UNG. .. quercifolia UNG. . zelkovaefolia UNG.

23. BALSAMIFLUAE (p. 46)

Liquidambar (p.49) $s p$. FАUJ.

25. ARTOCARPEAE (p. 46)

Ficus Tournef. 2

* hyperborea Unc.

" pertusa Nichols. . PLAtaneae Lestib. Plataninium Ung. 1 - acerinum UNG.

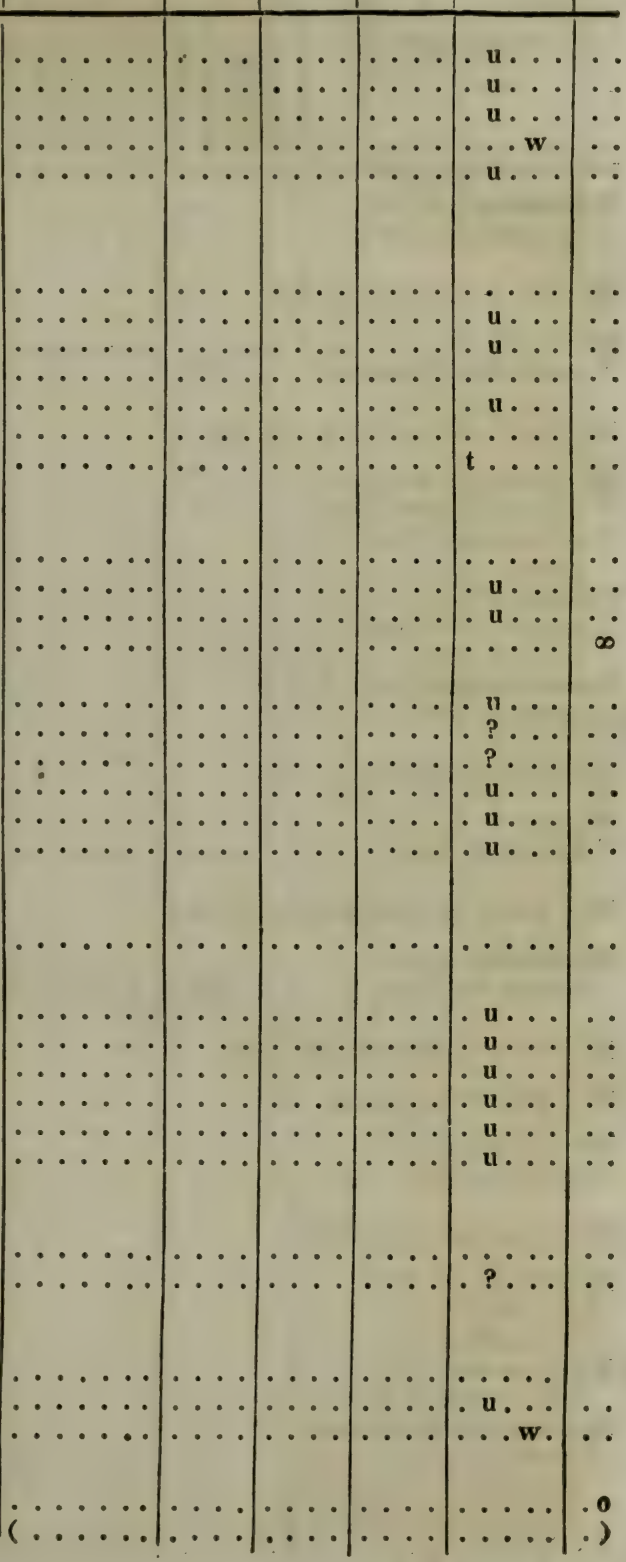




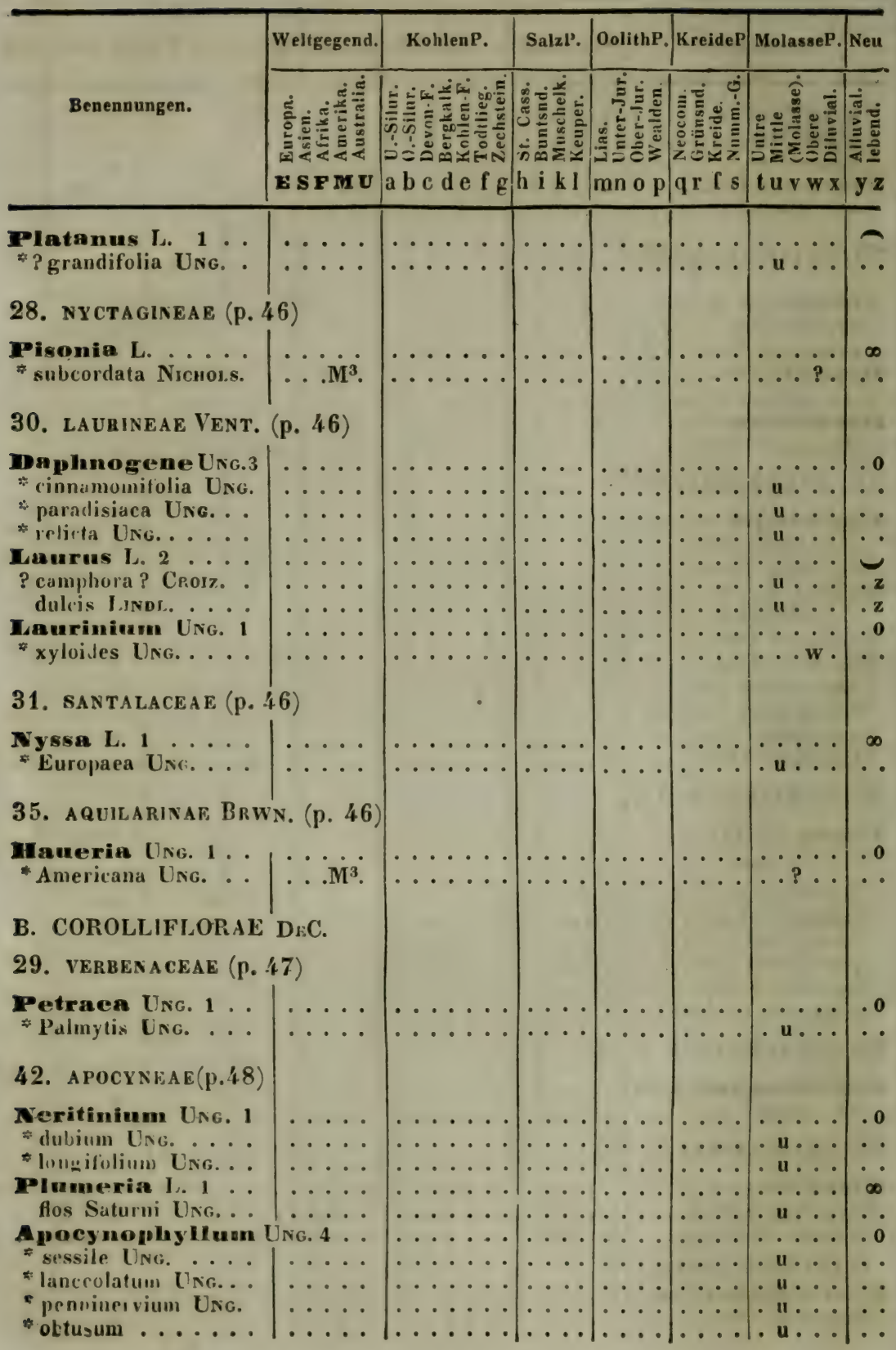




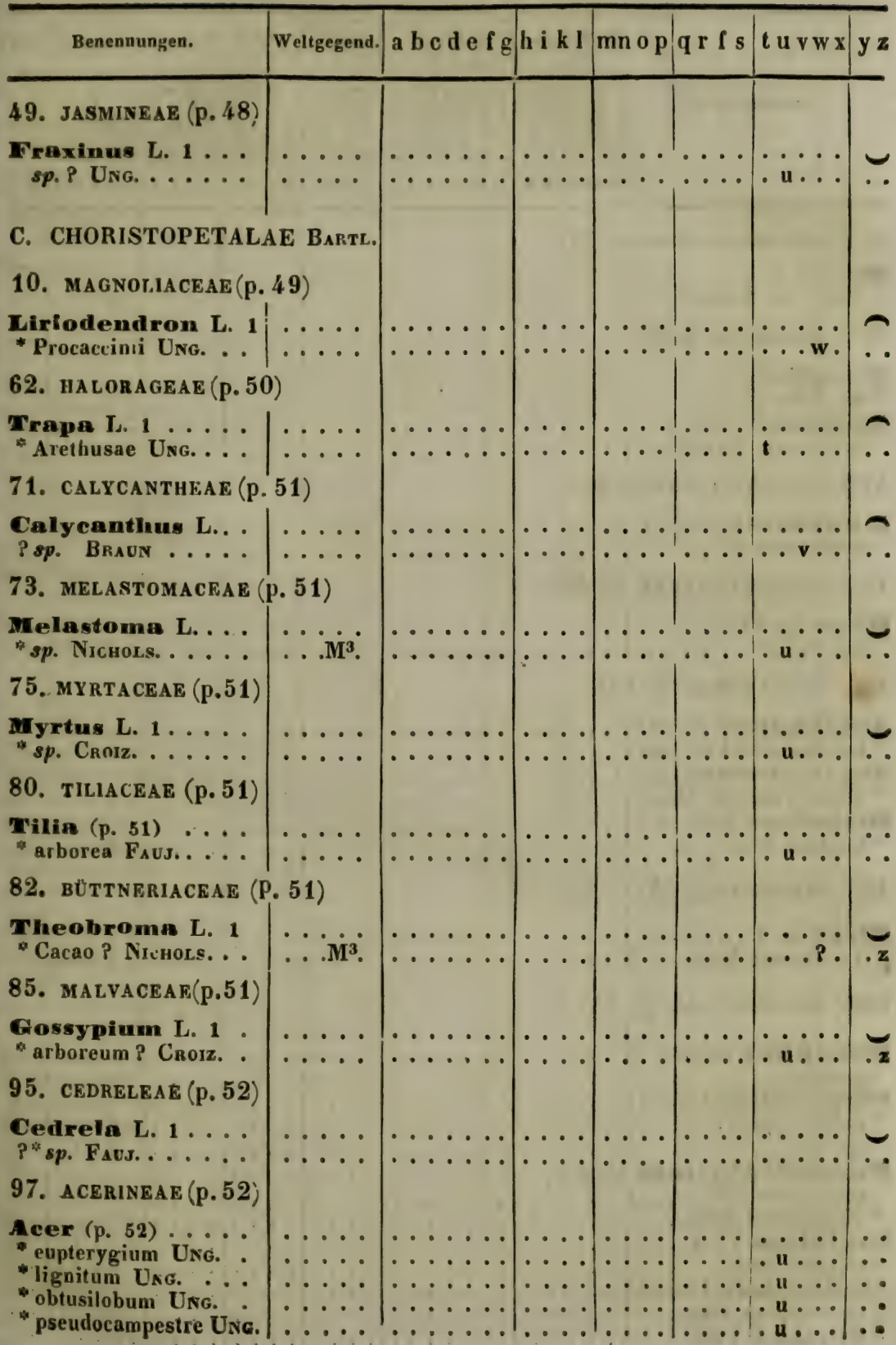




\begin{tabular}{|c|c|c|c|c|c|c|c|}
\hline & Weltgegend. & KohlenP. & SalzP. & ()olithP. & KreideP & MolasseP. & Neu \\
\hline Benenuungen. & 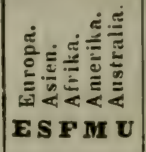 & 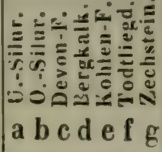 & 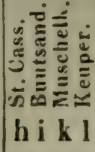 & 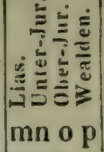 & 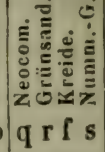 & 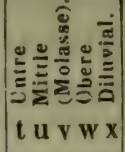 & 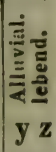 \\
\hline
\end{tabular}

104. EUPHORBIACEAE (p. 53)

\#uxus L. 1 ......

107. RHAMNEAE (p.53)

zizyphus

* $s p$. FAI'J. .

Rleaenus $; \cdots$

termiualis BraUN..

120. AURANTIACEAE (p. 54)

Mlipsteinia Ung. 1

* medullaris Urig. . .

123. Terebinthaceate (p. 54)

Tehog (p. 54)

*sp. FaUJ.

124. JUGLANDEAE (p. 54)

Juglandites $(p .54) \mid \ldots \ldots$

* Bergamensis C'riverdi . . . .

Juglandinium Ung. 1

* Mediterianeum Ung.

mirbelites UNG. 1

"Lesbius Ung. . . . .

125. POMACEAE (p.54)

Prixus L. 1

* Theobroma UNG.

133. LeguminOSAe (p. 55)

Dolichitex (p. 55)

* maximus. . . . .

Placeolites Unc. 2

* cassiaefolius UNG.

* $s p$. Ung.

Desmodophyllum Ung. 2 .

* adoptivum UNG.

- viticinoides UNG...

Frythrina L. 1 .

* sepulta Ung.

Adelocercis Ung. 2

* Radubojana UNG.

* Prevaliana UNG.

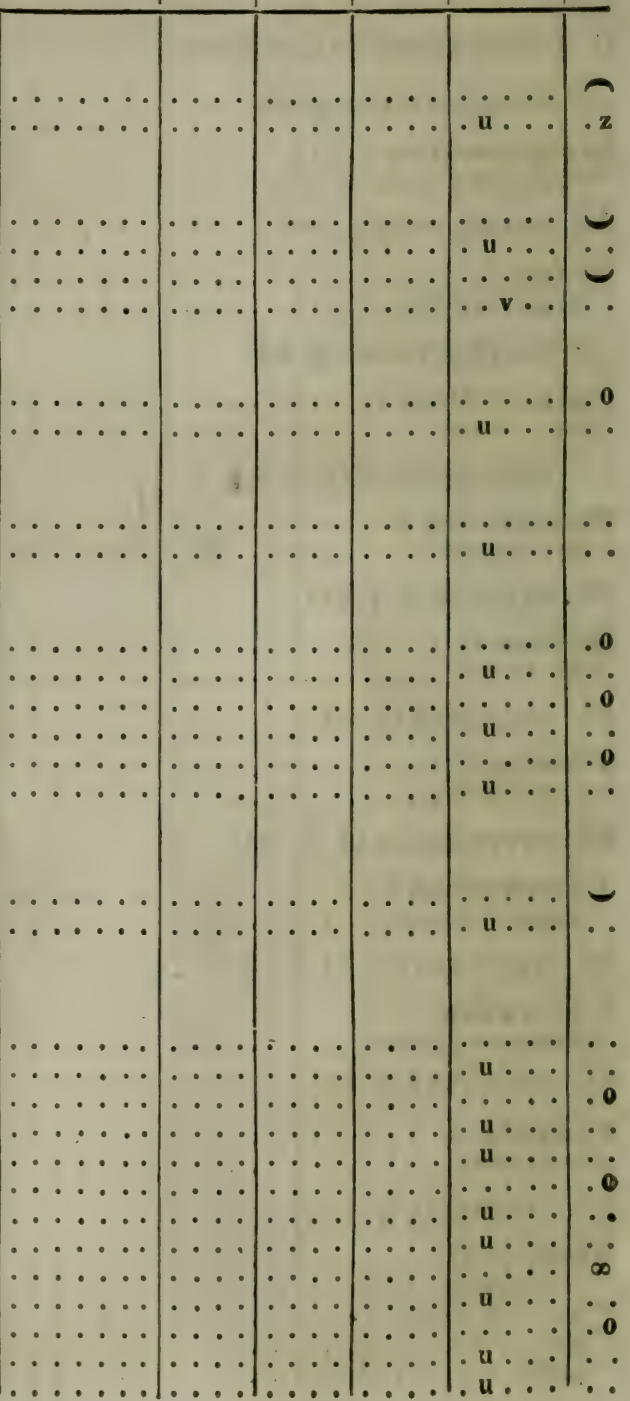




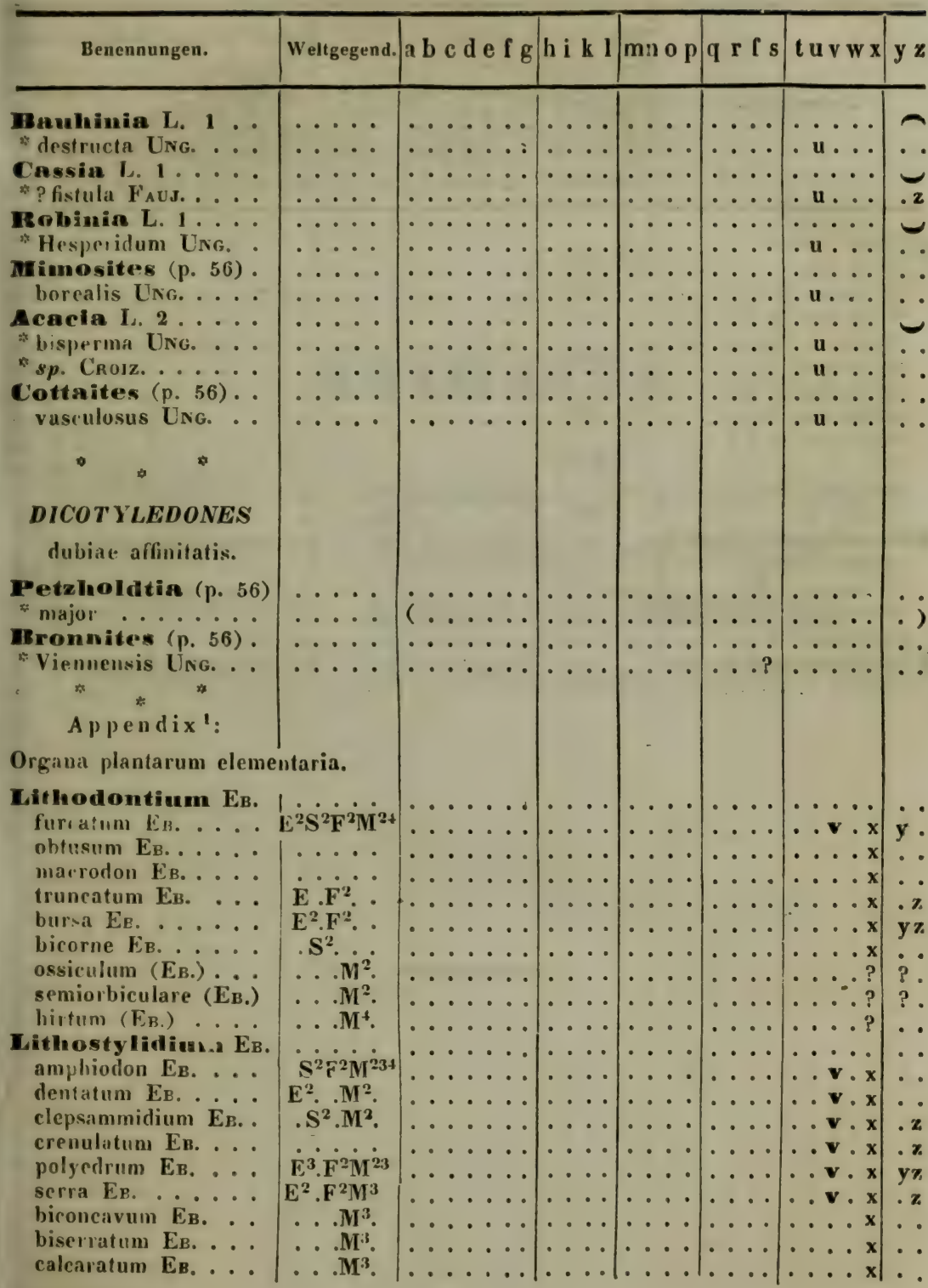

1 Ich habe geglaubt, wegen der Übersicht der geographisch-geologischen Verbreitung diesen Theil noch beifigen un nüssen, da Hr. GöpPERT seinen Inhalt wahrscheinlich nnr fïr den numenklitorischen Theil bestimmt hatte. Die Entfernung unserer Wohnorte hat niclit niehr ge. statiet, mit ihm desshalb zu verkehren.

BR. 


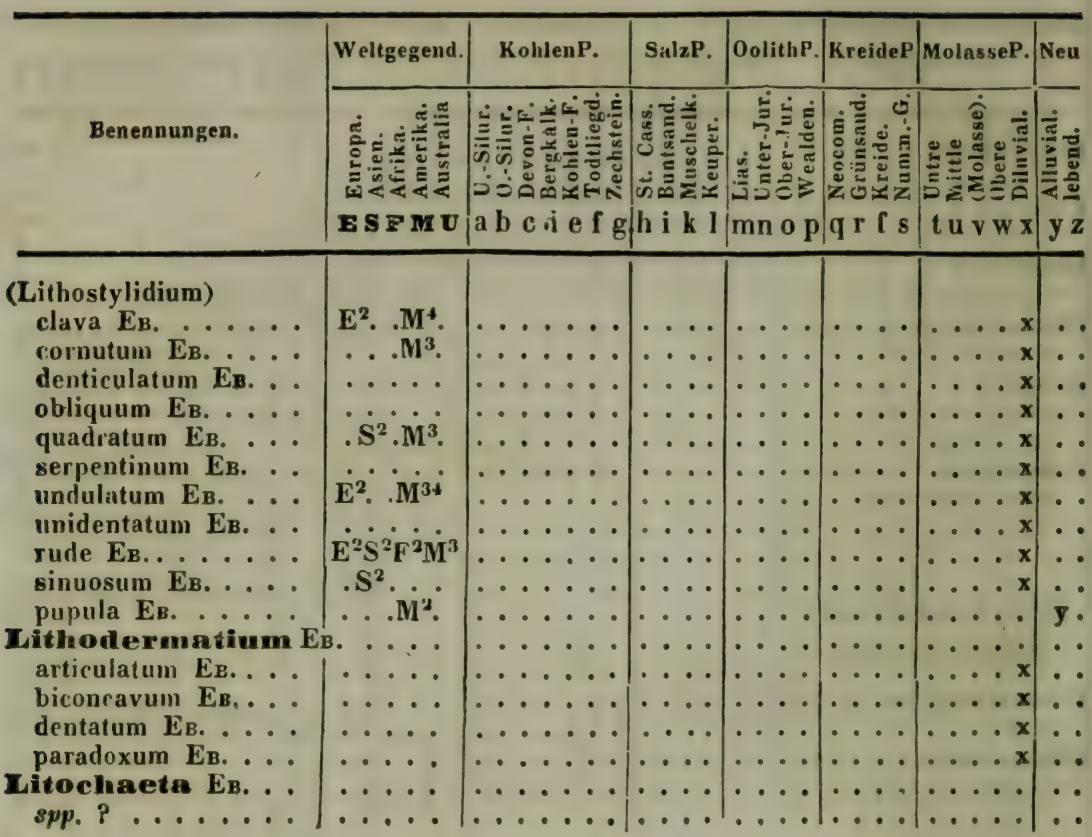




\section{ANIMAEIA.}

SUBREGNUM I. PHYTOZOA: PFLANZENTHIERE. SUBREGNUM II. MALACOZOA: WEICHTHIERE. SUBREGNUM III. ENTOMOZOA: KERB'THIERE. SUBREGNUM IV. SPONDYLOZOA: WIRBELTHIERE. 


\section{ANTMATA.}

Für den zoologischen Theil dieses Verzeiclınisses bis an die Reptilien habe ich Erläuterungen vorauszusenden, die theils in der stattgefundenen Vertheilung der Arbeit an verschiedene Verfasser, theils in dem mohr als einjährigen ZeitVerlauf seit dem Abdruck des botanischen Theils (mit Ausschluss des eben dadurch nöthig gewordenen Surpleneutes) ihren Grund haben '. Es deuten an:

t vor den Art-Namen: dessen Nichtberechtigung auf Beibehaltung in der systematischen Nomenklatur, in so ferne derselbe ein todt-geborener, d. h. indem er weder begleitet worden ist von einer genügenden Beschreibung, Diagnose oder Abbildung, noch die durch ihn bezeichnete Spezies durch beigefügte Synonyme kenntlich geworden ist. Namen dieser Art sind übrigens meistens nur im Nomenclator aufgenommen worlen, wo niclst irgend eine Wahrscheinlichkeit vorhanden gewesen, dass sie wirklich eiue neue, sonst noch nicht benannte Art bezeichmen und dass diese Art vom Autor noch genauer bekannt gemacht werden wird. Diess ist besonders der Fall mit den Art-Namen, welche in den vollständigeren Gor.pfuss'schen (bei v. Deches), Defrance'schen (in Dictionnaire), Schiothemm'schen und Münsten'schen Verzeichnissen aufgefülırt, aber weder dort noch in deren anderen Werken mit näherer Bezeichnung versehen worden sind.

? vor dem Art-Namen drückt Zweifel aus, ob diese Art zu dem Genus gehöre, unter welchem er steht; ist es ein Synonym: ob or zu derjenigen Ât gehöre, unter welche er cingrreihet worden ist. Ein solcher Zweifel hätte freilich noch weit öfter ausgedrückt werden können; das? rührt meistens schon vom Autor her.

o vor dem Art-Namen drückt die Gewissheit aus, dass dic Art nicht zu dem Genus gehöre, wo sie steht.

? hinter dem Art-oder hinter den Autor-Namen drürkt Unsicherheit in Bezug auf jenen oder diesen aus.

$q$ und $f$ sind öfters mit ${ }^{1}$ und $z$ bezeichnet worden. $q^{1}$ und $q^{2}$ bedeuten D'Orbigny's Terrain ncocomien und Terrain aptien (Thon mit Plicatula); $\boldsymbol{f}^{2}$ ist D'OrBigny's Craie chloritée mit Gryphara columba (in Deutschland oft als Grüusand bezeichnet und öfters als solcher mit $r$ in unserer Tahelle eingetragen); $f^{2}$ die daraut liegende weisse Kreide aller Autoren, d'Orbignv's Terrain senonien. fo $f c, f^{d}$ bedeuten d'Orbigny's zwcite bis vierte PudistenZone, welche noch alle in dessen $\mathbf{f}^{\prime}$ (chloritische Kreide) gehören, und daher in Deufschland oft zum Grünsand gerechnet werden; die erste RudistenZone ist in $q^{2}$.

Die Rubrik $\mathbf{s}$ ist von nun an aus der Kreide.Periode in die Molasse-Periode versetzt worden, weil es sich inzwischen herausgestelit hat, dass nicht nur das hauptsïrhlichste der dahin eingereiheten Nummuliten-Gesteine, nämlich das von Monle-Bolca, sondern auch fast alle übrigen, dirse letzten ungeachtet einiger (je 1-2-3) darin eingestrcut gefundener Kreide-Versteinerungen, zum EocenGebirge gehöre॥. Nur hinsichtlich des Kressenberger Gesteines und seiner Äquivalente in Baiern bleibt mir in diesem Augenblicke norlh $Z$ weifel übrig. Auch scheint in der Krim ein ganz allmählicher Übergang aus s in t Statt zu finden. - Der Monte Bulca ist von jetzt a $\mathrm{n}$ bleibend in die Spalte $t$ als $\tau$ aufonommen. Kommt eine Art in mehren Schichten zugleich vor, so wird ihr Hauptvorkommen öfters durch einen Buchstaben aus fetterer Schrift als die übrigen anged eutet.

1 Vor allem Anderen muss jedoch auf den Druckfehler 5.3 anfmerksam gemacht werden, no statt s s als Zeichen der Kreide zweimal $f$, $f$ gelesen werden muss, wic es in der darauf folgeuden Tabelle überall richtig gebraucht worden ist. 


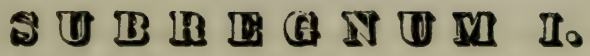

\section{PH YTOZOA: PFLANZENTIIIERE.}

Cl. 1. PSEUdOZOA de Br., p. 77.

Cl. II. AMORPHOZOA dE BL., p. 79.

CI. III. POLYgastrica Eb.

Cl. IV. POLYPI L.

Cl. V. ENTOzOA Rod.

Cl. VI. ACA LEPHAE Cuv.

Cl. VII. ECHINOdermata Cuv. 



\begin{tabular}{|c|c|c|c|c|c|c|c|}
\hline & Weltgegend. & KohlenP. & SalzP. & UolithP. & $\begin{array}{l}\text { Krei- } \\
\text { deP. }\end{array}$ & MalasseP. & Neu \\
\hline Benennungen. & 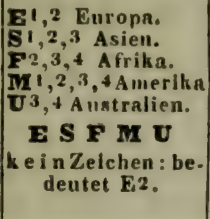 & 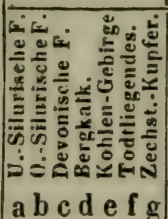 & 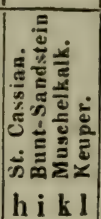 & 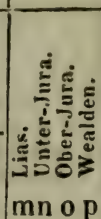 & 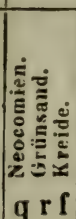 & 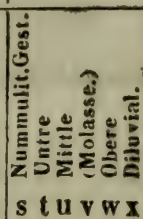 & 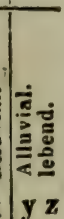 \\
\hline
\end{tabular}

\section{Cl. I. PSEUDOZOA de BLV. '}

\section{NEMATOPHYTA BLv.}

(CONFERVAe et naviculae.) (vdtr. inter Vegetabilia et Polygastrica.)

\section{CALCLP TTA BLv.}

1. FUCOIDEAE BLV.

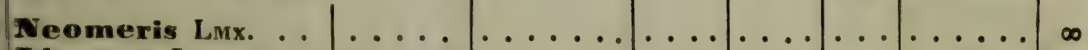

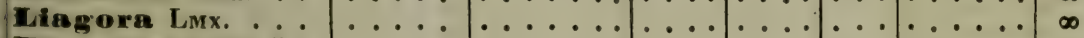

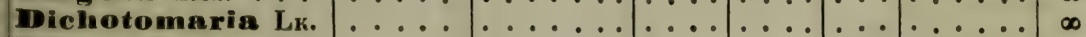

Wdotea Lmx. $1 \ldots . \ldots \ldots \ldots \ldots$

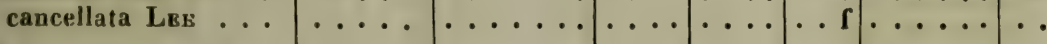

2. corallinae Blu.

Polyphysa LK. ... ..... Acetabulum Tournf, $1 \ldots$ antiquun DRFR.... $\ldots .$.

Gàlaxaura Lax. . . . . . Nesaen Lmx. (Penicillus Lк.). . Amphirhor Lrx. . 1 ..... Malimeda Lmx.(Flabellaria Lk.) Jania LMx. ..........

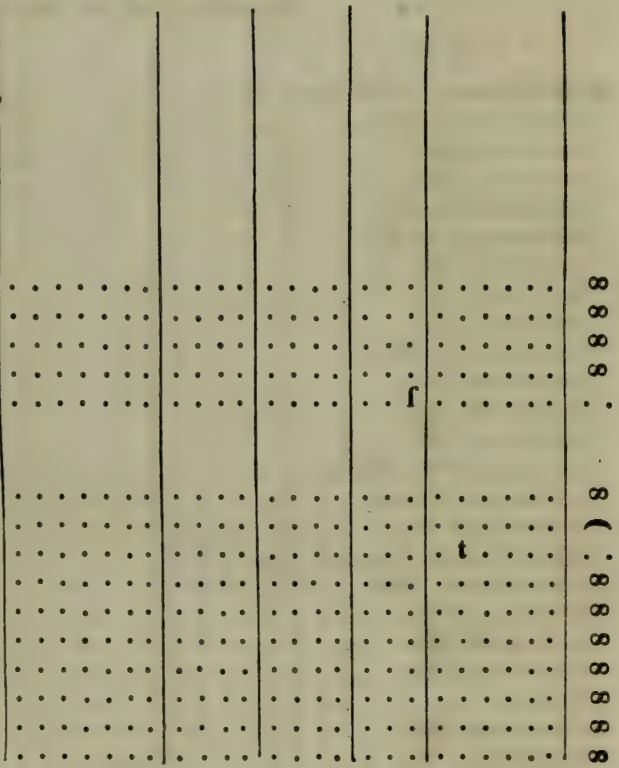
Corallina (Lк.) Lmx. $\ldots \ldots$
Cymopolia Lmx. . . . .

1 Nach dem heutigen Stande der Wissenschaft gehörte diese ganze Abtheilung zu den $P$ fI a wen. Zur Zeit, als ich mich mit Hrn. Prot. Göppers in die Arbeit theilıe, waltete noch Zweifel darüber; desshalb erscheint sie hier. 


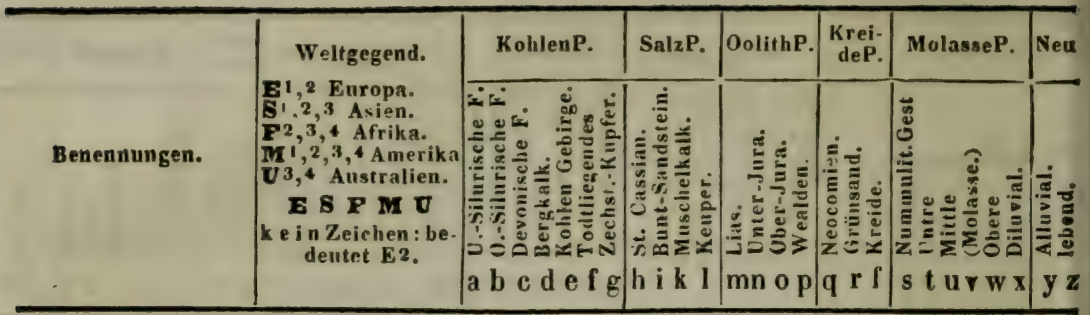

\section{Cl. II. AMORPhOZOA de BLv. ${ }^{1}$.}

\section{(sPoNgIA LIN, - SEE-SCHW ̈̈мMе.)}

- Stirpes.

Achilleum Schweigg. 29 ...

verrucosum Mü. .

subcariosum Mü.

rugosum Mü.

granulosum Mü.

milleporatum Mü...

patellare Mü.....

Waltheri Mü.....

Faundeli Mü......

radiciforme Mü. ...

obscurum Mü.....

voluta Wissm. . . .

reticulare Mü......

Ppolymorphum KLPST.

poraceum KL.ss. . . tuberosum Mü.... cheirotonum GF. . rancellatum Mü. costatum Mü..... morrhella GF. ... muricatum GF. ... Rocmeri n.

?truncatum GF. fungiforme GF. . . auriforme Rok. deforme RoE. . $\because$. globosum HAG.... perreticulatum Porte. cariosum GF. .... rugosum Revss...

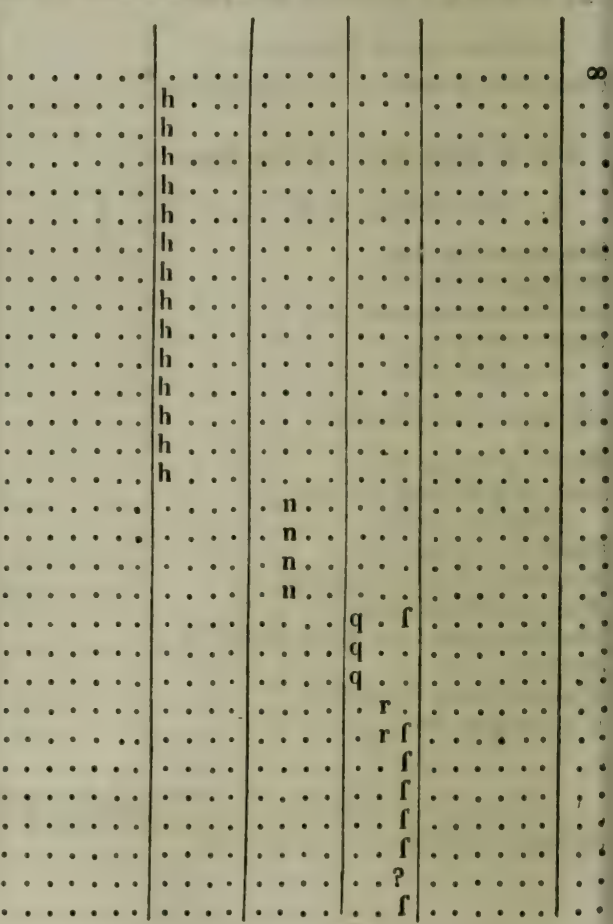

1 Auch die Amorphozoen scheinen eher den Pflanzen als den Thieren anzugeliören. Von den Zoologen wie von den Botanikern zurückgestossen schwanken sie zwischen beiden NaturReichen. 


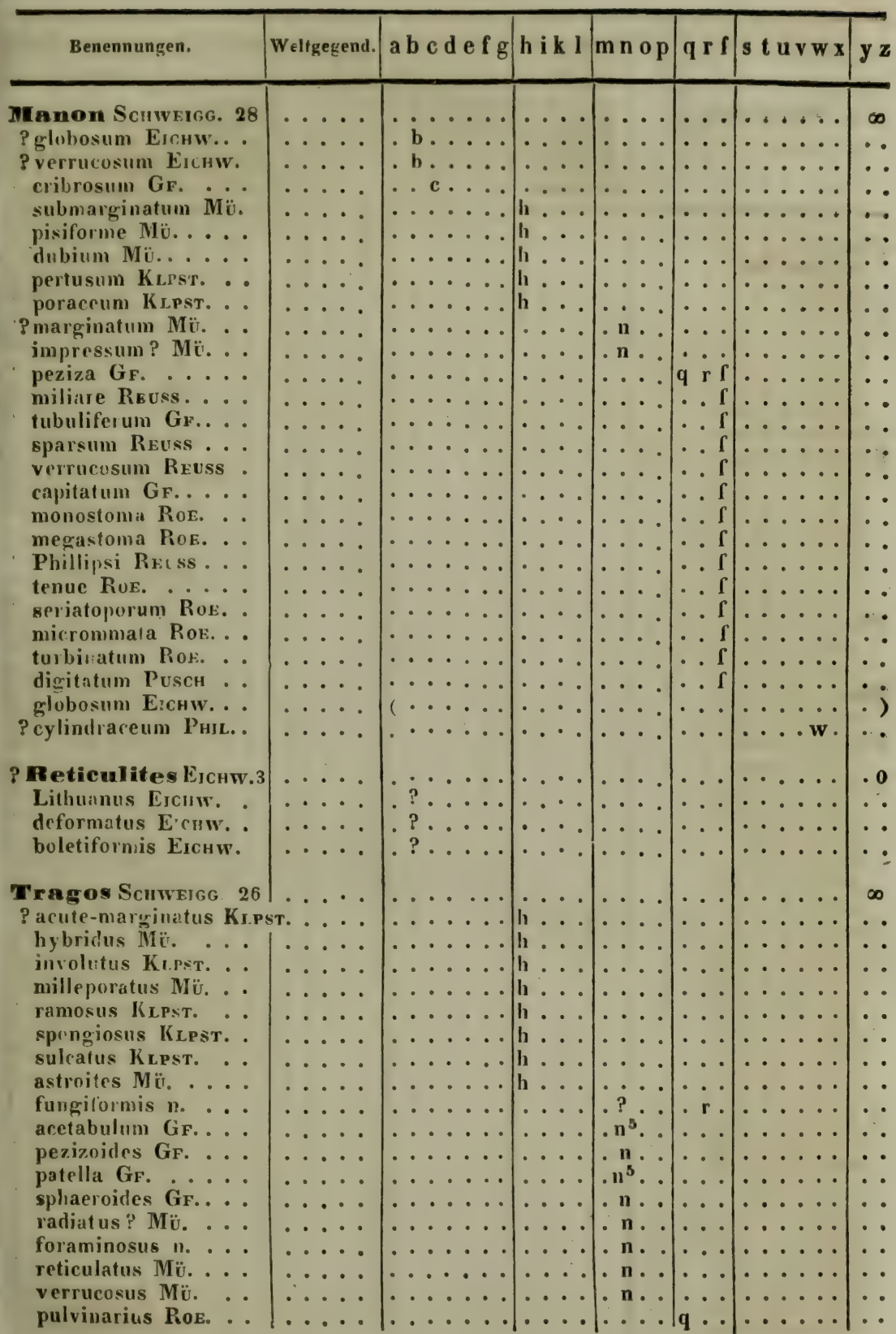




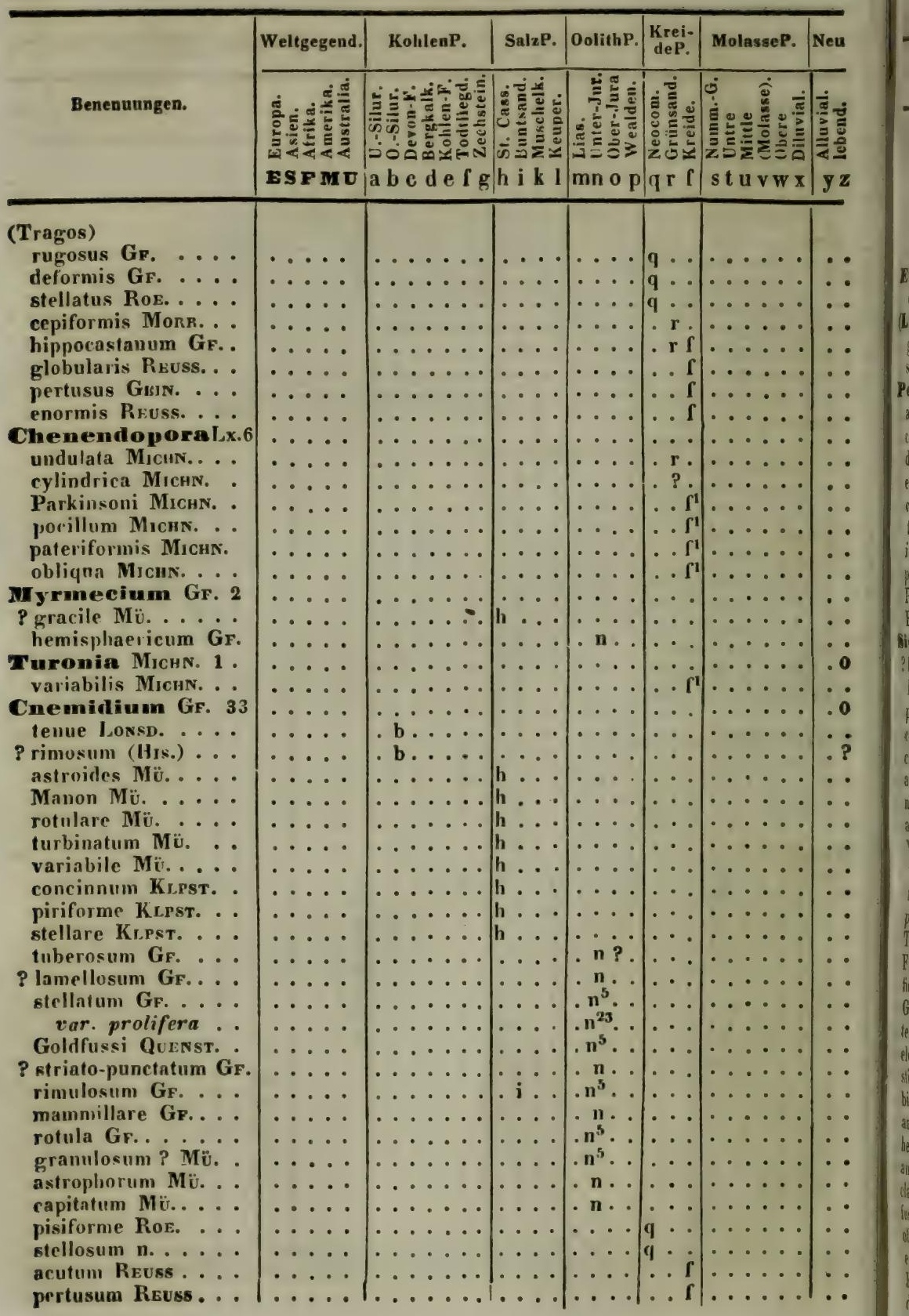




\begin{tabular}{|c|c|c|c|c|c|c|c|}
\hline Ber & Weitge & a b c & h i k l & $\mathrm{mn} 0 \mathrm{p}$ & q I & s t u v w X & \\
\hline 3. & . & & & & & 1 & \\
\hline um МiснN... . & $\cdots \cdot$ & $\ldots . \cdots$ & & 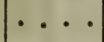 & . . $f^{\prime}$ & $\cdots \cdots \cdots$ & \\
\hline 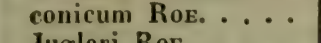 & $\cdots \cdot$ & $\cdots \cdot \cdots$ & . . . & $\cdot \cdot$ & - $f$ & $\cdots \cdots$ & \\
\hline ROE... & ... & ... • & ... & - $\cdot$ & - $f$ & $\cdots \cdot \cdot \cdot$ & \\
\hline es $\mathbf{G E}_{\mathrm{E}}$ & $\bullet \bullet \cdot$ & $\cdots \cdot \cdot \cdot$ & $\cdots \cdot$ & $\cdots \cdot$ & . & •. • & \\
\hline ise & $\cdots \cdot \cdot$ & $\cdots \cdots \cdot$ & $\cdots \cdot$ & $\cdots \cdot$ & & $\cdots \cdots \cdot$ & \\
\hline $\begin{array}{l}\text { Lx.) Michis. } 1 \\
\text { a Michn. }\end{array}$ & $\cdots \cdot$ & $\cdots \cdot \cdot$ & . . . & & & $\cdots \cdots$ & \\
\hline norea $\left.I_{\text {x. }}\right){ }_{2}$ & $\begin{array}{l}\cdots \\
\cdots\end{array}$ & $\begin{array}{l}\cdots \\
\bullet \cdot \\
\bullet\end{array}$ & $\ddot{\cdot}$ & $\begin{array}{ll}n^{3} \cdot \cdots \\
.\end{array}$ & & $\cdots \cdots$ & \\
\hline еа MгснN. . & ... & $\ldots \ldots$ & & . $\mathbf{n}^{3}$ & $1 \cdot$ & $\dot{\bullet}$ & \\
\hline Mic & $\cdots \cdot \cdot$ & $\cdots \cdots$ & & $\ldots$ & . & $\cdot$ & \\
\hline BEN.10 & - $\cdot$ & $\ldots \ldots$ & & $\cdots$ & & $\cdots$ & \\
\hline . & $\cdot \cdot \cdot$ & - & . . . & $\cdots \cdot$ & & $\cdots \cdot$ & \\
\hline - & $\cdot \cdot \cdot$ & $\cdots \cdots$ & $\cdot \cdot$ & $\cdots \cdot$ & • & $\cdots \cdots$ & \\
\hline • & $\cdots \cdot$ & 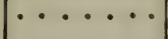 & - & $\cdots \cdot$ & - & $\cdots \cdots$ & \\
\hline - & • • • & . . & $\cdots$ & $\cdots \cdot$ & & $\cdots \cdots$ & \\
\hline$\dot{\bullet}$ & $\cdots$ & - & & $\cdots \cdot$ & . & $\cdots \cdot$ & \\
\hline um Bé. & $\begin{array}{l}\cdots \cdots \\
\cdots \cdots\end{array}$ & $\dot{*}$ & & $\begin{array}{ll}\cdots \cdots \\
\cdots\end{array}$ & $\dot{\bullet}$ & $\cdot \bullet \cdot \bullet$ & \\
\hline - & $\cdots$ & ... & & ... & & $\cdots \cdots$ & \\
\hline - & $\cdots \cdot$ & $\cdots \cdots$ & . & - & - & 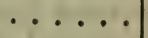 & \\
\hline Mic & $\cdots \cdot$ & $\cdots \cdots$ & & $\cdots \cdot$ & & $\cdot \therefore \cdot$ & \\
\hline 30 & $\cdot \cdot \cdot$ & $\cdots \cdots \cdot$ & & & & $\cdots$ & $\infty$ \\
\hline ides & $\cdots \cdot$ & $\cdots \cdots \cdots$ & $\cdot \cdot$ & - $\mathbf{n} \cdot \cdot$ & 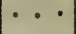 & $\cdots \cdots$ & \\
\hline • & $\cdots \cdot$ & $\cdots \cdot$ & & . $\mathbf{u}^{3} \cdot$ & & $\cdots \cdot \cdot$ & \\
\hline - & $\cdots \cdot$ & • • • & & . & & $\cdots \cdot$ & \\
\hline$\dot{\bullet}$ & $\begin{array}{l}\cdot \cdot \cdot \\
\bullet \cdot\end{array}$ & . & & $\cdot \cdots$ & & . & \\
\hline - & $\begin{array}{l}\cdots \\
\cdots \\
\cdots\end{array}$ & $\cdot \cdot$ & & 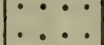 & $\dot{\cdot}$ & $\cdot \cdots$ & \\
\hline s. & - & $\ldots \ldots$ & & . . . & & 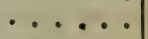 & \\
\hline - & $\cdots \cdot$ & $\ldots \ldots$ & & $\cdots \cdot$ & . & - & \\
\hline - & $\cdots \cdot$ & $\cdots \cdots$ & & ... & & $\ldots \ldots$ & 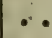 \\
\hline$\cdots$ & $\cdot \cdot \cdot$ & $\cdot \cdot$ & & - & & - • & \\
\hline - $\cdot$ & & $\cdots \cdot$ & - & •. & & $\cdots$ & \\
\hline - $\cdot$ & & - $\cdot$ & & & - r r & $\cdots \cdot$ & \\
\hline$\cdots$ & • & • & & • & & $\cdots$ & \\
\hline$\cdots$ & - & 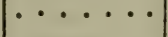 & & & $\cdot f_{1}^{1}$ & $\cdots \cdots$ & \\
\hline 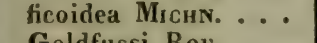 & & & & - & & $\cdot \bullet \cdot$ & \\
\hline . . & & $\cdots \cdot$ & & • $\bullet$ & - & $\cdots$ & \\
\hline$\cdots$ & & $\cdot \cdot$ & & - & & $\cdots$ & $\bullet$ \\
\hline$\cdots$ & $\cdot$ & $\cdots$ & $\cdots$ & • & - $\cdot$ & $\cdots$ & \\
\hline . & & 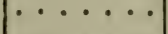 & & 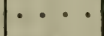 & I & - & \\
\hline . . & - & 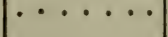 & & 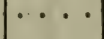 & I & - $\cdot$ & \\
\hline$\varepsilon_{-1}$ & & & & & & $x^{2}+$ & \\
\hline EUss . & - & - & & • & 1 & • • & \\
\hline $\mathbf{B} \cdot \cdot$ & & & & & & 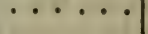 & \\
\hline$\cdots \cdot$ & 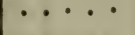 & ( & & r & & $\cdots$ & • \\
\hline nis $\mathrm{L}$ & & & & & & 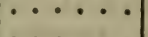 & \\
\hline Dis. & 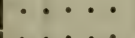 & 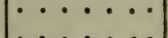 & & & & 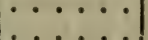 & 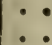 \\
\hline . & & & & & $\ldots \mathbf{I}$ & 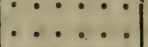 & \\
\hline 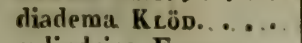 & $\bullet$ & & $\cdots$ & & $\cdots 1$ & $\cdots$ & \\
\hline ylindrice & & & & & & & \\
\hline
\end{tabular}




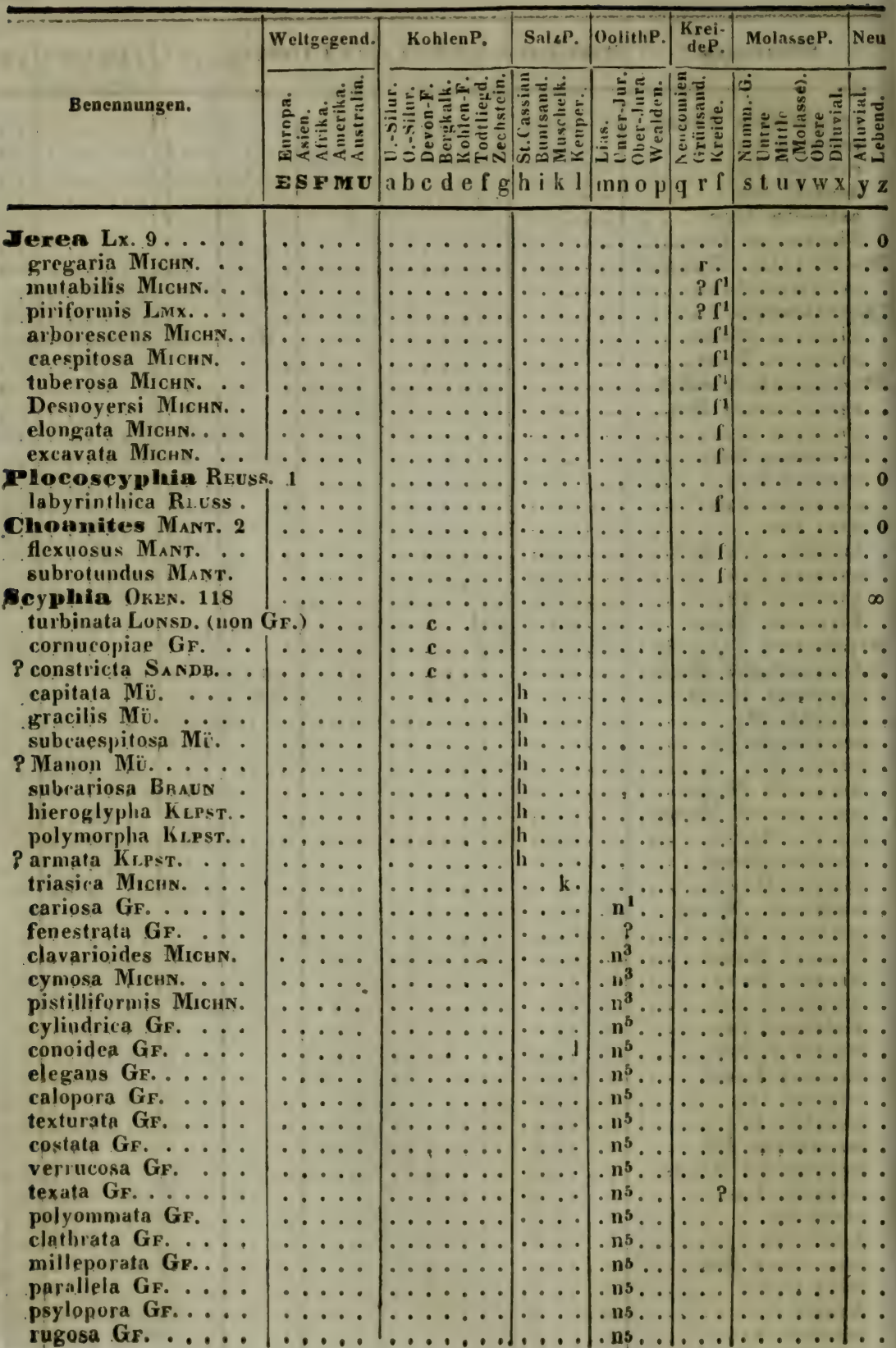




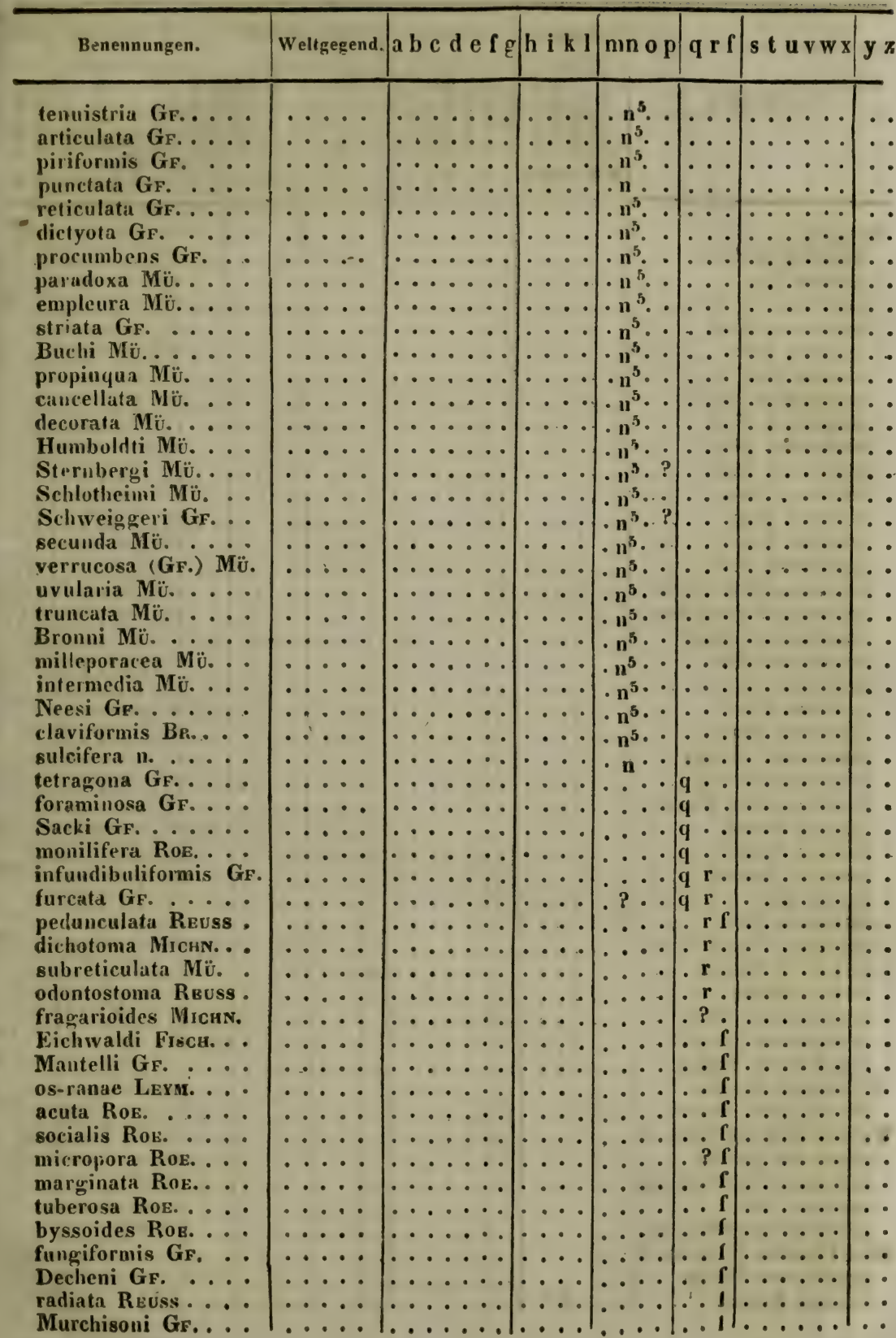




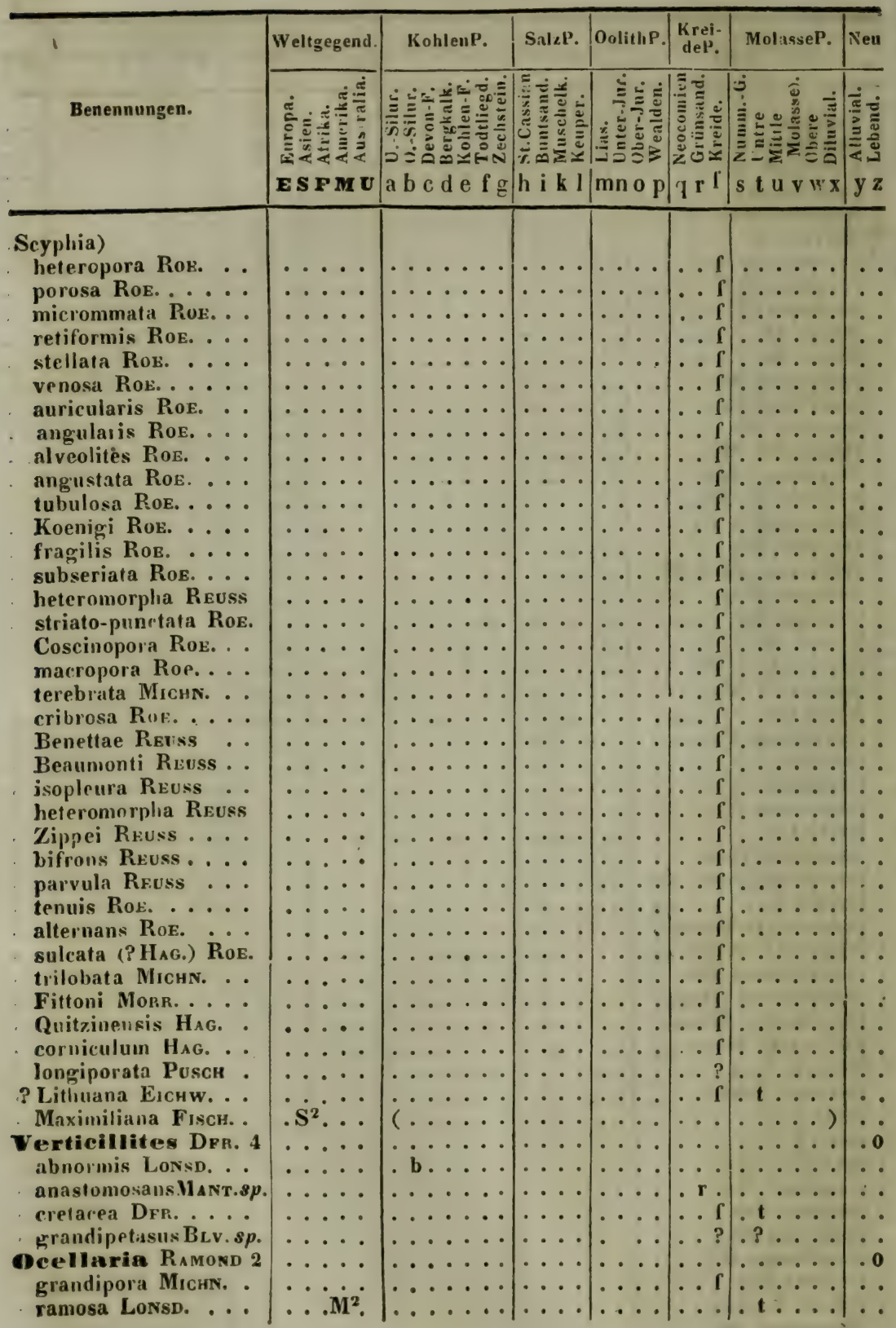




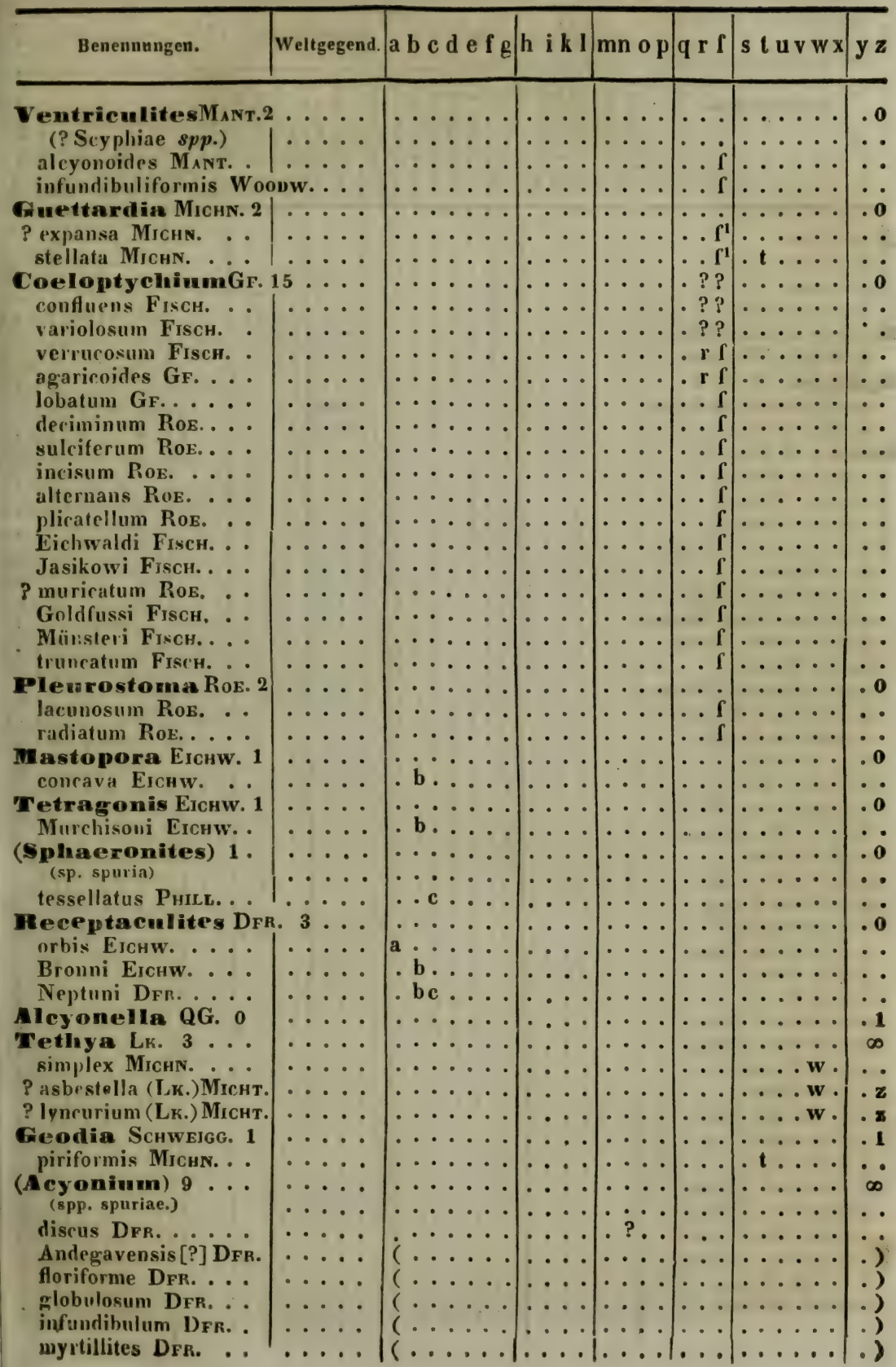




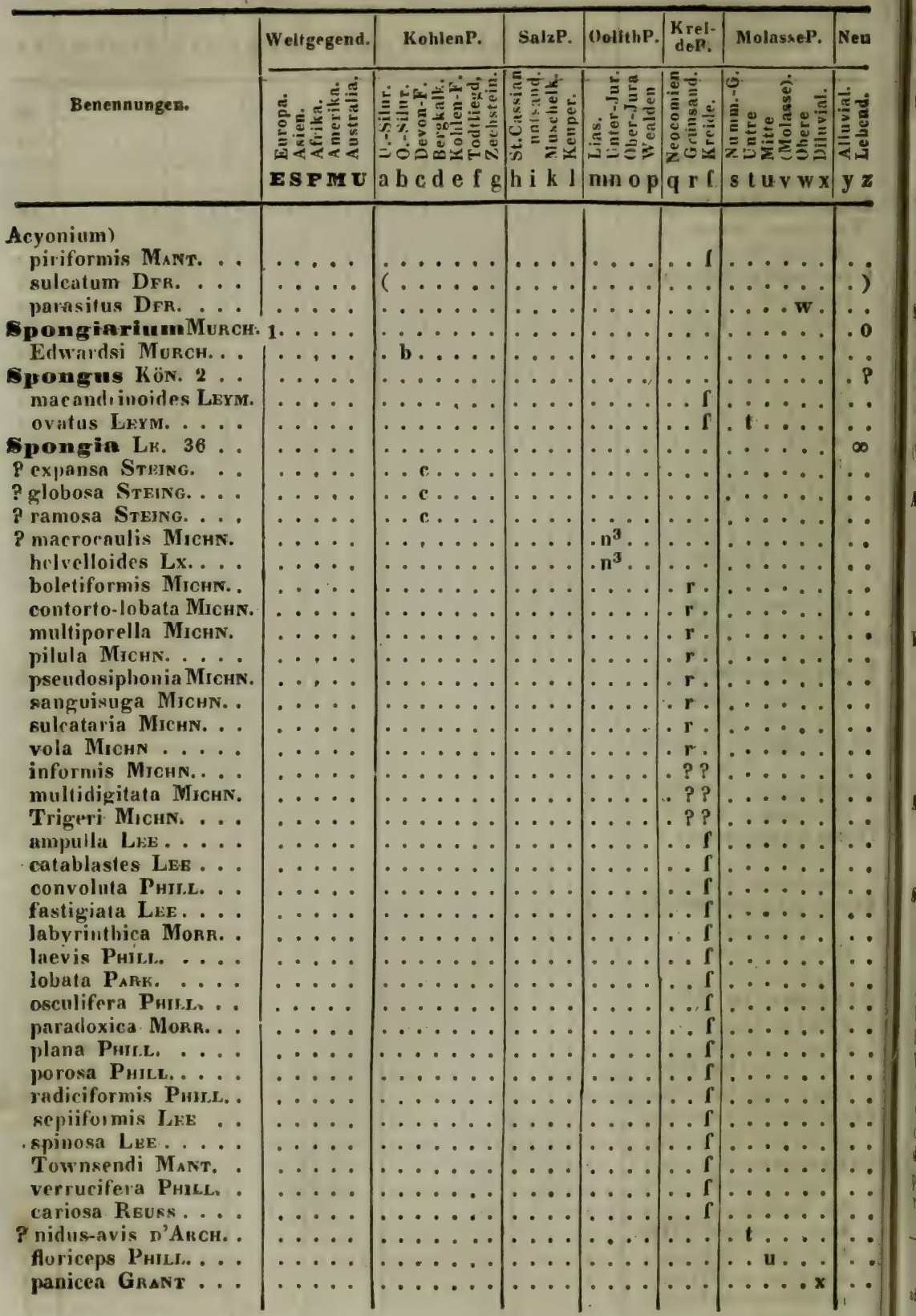




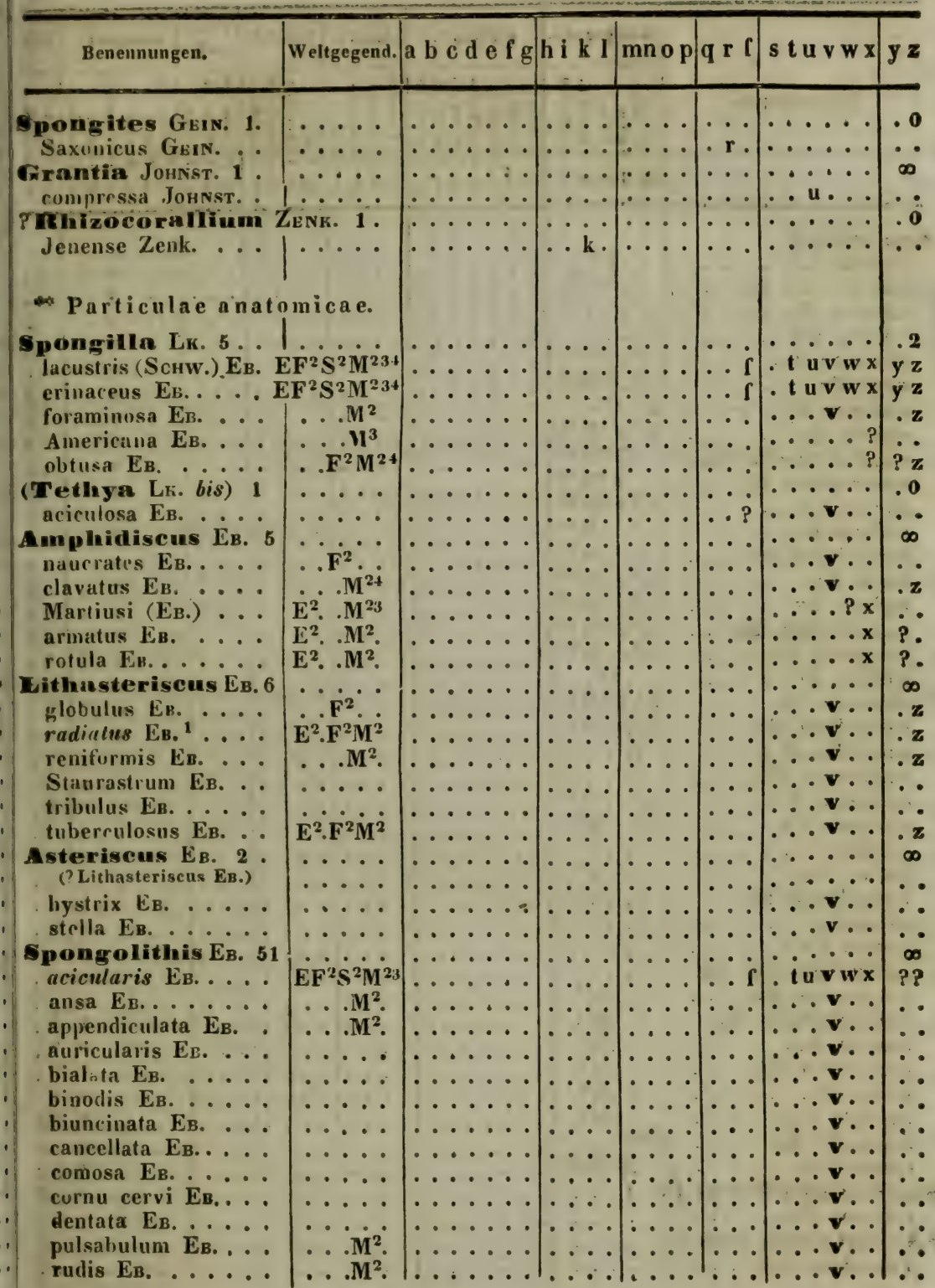

1 Lithasterisci et Spongolithis species, quarum nomina litteris obliquis impressa sunt, jam ad Spongillarum species, eademque plerumque complures ad unam, referuitur. 


\begin{tabular}{|c|c|c|c|c|c|c|c|}
\hline & Weltgegend. & KohlenP. & SalzP. & WolithP. & $\begin{array}{r}\text { Krei- } \\
\text { deP. }\end{array}$ & MolasseP. & Neu \\
\hline Benent & 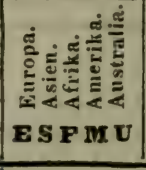 & 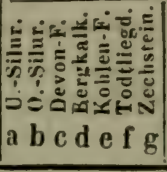 & 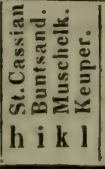 & 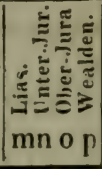 & 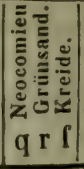 & 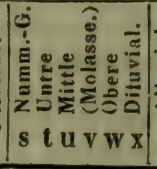 & 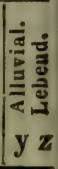 \\
\hline (his) & & & & & & & \\
\hline $\begin{array}{c}\text { Eв. } \ldots \\
\text { ulata Ев. }\end{array}$ & $\ldots \mathbf{F}^{2} \cdot \dot{M}^{2}$ & & & & $\cdots$ & $\cdots \mathbf{v}$ & \\
\hline$E_{B} \ldots \ldots$ & $\ldots$ & $\ldots \ldots$ & $\cdots$ & $\ldots$. & $\cdots$ & $\begin{array}{l}\cdots \mathbf{v} \\
\cdots \mathbf{v}\end{array}$ & \\
\hline Ев. . . . & $\dot{E}^{2} \dot{M^{2}}$ & & $\cdots$ & $\cdots \cdots$ & $\cdots$ & $\cdots \mathbf{v}$ & \\
\hline${ }_{a}^{A}$ & $\mathrm{E}^{2} \cdot \mathrm{F}^{2} \mathrm{M}^{2}$ & $\cdots$ & $\because \dot{ }$ & & $\mid \begin{array}{l}\cdots \\
\cdots\end{array}$ & $\begin{array}{l}\cdots \mathbf{v} \cdot \mathbf{x} \\
\cdots \mathbf{v} \cdot .\end{array}$ & \\
\hline$\cdots$ & $\mathrm{E}^{2} \cdot \mathbf{F}^{2}$ & . & $\cdots$ & $\cdots$ & $\cdots$ & $\ldots v$ & $\begin{array}{l}\cdot z \\
.7\end{array}$ \\
\hline is $\ddot{\mathrm{EB}}$. & $\left|\begin{array}{c}E^{2} \cdot F^{2} M^{234} \\
. F^{2} M^{24}\end{array}\right|$ & & $\cdots$ & $\cdots$ & $\cdots$ & $\cdots v ? x$ & $? z$ \\
\hline Eв. . . & $\therefore \mathbf{F}^{2} \mathbf{M}^{24}$ & $\therefore$ & $\cdots$ & $\begin{array}{ll}\cdots \\
\cdots \\
\cdots\end{array}$ & $\cdots$ & $\begin{array}{l}\cdots \mathbf{v} \cdot \cdot \\
\ldots \mathbf{v} .\end{array}$ &. $\mathbf{z}$ \\
\hline$\cdots$ & $\mathrm{E}^{2} \cdot \mathrm{F}^{2}$ & $\cdots$ & $\ldots$ & $\ldots$ & $\cdots$ & $\cdots \mathbf{v} \cdot ?$ & $\dot{p}_{z}^{z}$ \\
\hline & $\cdots M^{24}$ & $\cdots$ & $\cdots$ & $\cdots \cdot$ & $\cdots$ & $\cdots \mathbf{v} \cdot$ & $\cdot \mathbf{z}$ \\
\hline & $\dot{\mathrm{E}}^{2} \cdot \mathbf{F}^{2} \mathbf{M}^{234}$ & • $\cdots$ & $\cdots$ & $\left|\begin{array}{cc}\cdots & \cdots \\
\cdots & \cdots\end{array}\right|$ & $\cdots$ & $\begin{array}{l}\cdots v \\
\cdots v \cdot x\end{array}$ & - $z$ \\
\hline & $E^{2} \cdot F^{2} \cdot l^{4}$ & $\cdots \cdots \cdots$ & $\cdots \cdots$ & $\cdots \cdots$ & $\cdots$ & $\begin{array}{l}\cdots v \cdot x \\
\cdots v \cdot x\end{array}$ & $\begin{array}{l}\cdot \mathrm{z} \\
. \mathrm{z}\end{array}$ \\
\hline Ев. . . & $E^{2} \cdot \mathbf{F}$ & $\cdots \cdots$ & $\ldots$ & $\cdots$ & $\ldots$ & ..v. $\mathrm{x}$ &.$z$ \\
\hline$\cdots$ & $\mathrm{E}^{2} . \mathrm{M}^{24}$ & $\cdots \cdots$ & $\cdots$ & $\cdots \cdot$ & $\ldots$ & ...x.$?$ & $? z$ \\
\hline & $\dot{\mathrm{E}^{2} \cdot \mathrm{F}^{2}} \dot{\mathrm{M}}^{24}$ & $\cdots \cdots$ & $\cdots$ & $\cdots \cdot$ & $\cdots$ & $\cdots \mathbf{v} \cdot$ & 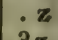 \\
\hline$\cdots$ & $E^{2} \ldots M^{4}$ & $\ldots$ & $\cdots \cdot$ & $\cdots$ & $\because \cdots$ & $\therefore \mathbf{v} \cdot ?$ & $\begin{array}{l}82 \\
.2\end{array}$ \\
\hline B. . &.$S^{2} \ldots$ & $\cdots \cdots$ & $\ldots$ & $\cdots \cdot \cdot$ & $\cdots$ & $\ldots \ldots x$ & . \\
\hline$\cdots$ & $\mathrm{v}^{23}$ & $\cdots \cdots$ & $\cdots$ & $\cdots$ & $\ldots$ & $\cdots \ldots x$ & \\
\hline & $\mathrm{E}^{2} \cdot \mathrm{M}^{3}$ & $\cdots \cdots$ & $\cdots$ & $\cdots \cdot$ & $\cdots$ & $\cdots x x$ & ?. \\
\hline$\cdots$ & . . . & $\ldots \ldots$ & $\cdots$ & $\cdots \cdot$ & $\cdots$ & $\cdots \cdots x$ & - \\
\hline$\cdots$ & ... $\mathbf{M}^{2}$ & .......... & $\begin{array}{ll}\cdots \\
\cdots\end{array}$ & $\cdots \cdots$ & $\cdots$ & $|\cdots \cdots x|$ & - \\
\hline anea Eв.... & ... & $\ldots \ldots$ & $\cdots$ & $\cdots$ & $\cdots$ & $\cdots \cdot x$ & \\
\hline$\ldots$ & $\ldots \ldots$ & $\ldots \ldots$ & $\ldots$ & $\cdots \cdots$ & $\cdots$ & 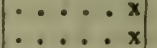 & \\
\hline & $\cdots$ & $\ldots \ldots$ & $\ldots$ & $\ldots$. & $\ldots$ & 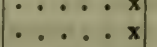 & \\
\hline a E. & & $\cdots \cdots$ & $\ldots$ & $\ldots$ & $\ldots$ & $\ldots x \mathrm{x}$ & \\
\hline ylu Ев. . . & $\ldots \mathbf{M}^{2}$ & $\ldots \ldots$ & $\ldots$ & $\cdots \cdot$ & $\cdots$ & $\ldots . . x$ & \\
\hline nsis EB. & $\ldots \mathrm{M}^{2} \mathrm{U}^{3}$ & $\ldots$ & $\cdots$ & $\cdots \cdot \cdot$ & $\cdots$ & ....? & ? \\
\hline в. & $\cdots \mathbf{M}^{2}$ & $\ldots$ & $\cdots$ & $\cdots \cdot$ & $\cdots$ & $\ldots \ldots ?$ & \\
\hline B. & & $\cdots$ & $\cdots$ & $\cdots \cdot$ & $\cdots$ & …? & \\
\hline . & 2 & $\cdots$ & $\begin{array}{l}\cdots \\
\cdots\end{array}$ & $\cdots$ & 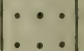 & {$\left[\begin{array}{lll}\cdots & \cdots & \text { ? } \\
\cdots & \cdots & \text { ? }\end{array}\right]$} & $\begin{array}{l}? \\
? \mathrm{z}\end{array}$ \\
\hline is $\mathrm{F}$ & $E^{2}$ & $\cdots$ & $\cdots \cdots$ & $\ldots$. & $\cdots$ & $\ldots . . x$ & $\mathrm{z}$ \\
\hline & & $\cdots \cdots$ & $\cdots$ & $\cdots \cdots$ & $\cdots$ & $\ldots \ldots ?$ & $? \mathrm{z}$ \\
\hline :a: $>$ & • & $\cdots \cdots \cdots$ & $\cdots \cdot$ & $\cdots \cdot$ & $\cdots$ & $\cdots \cdots \cdot$ & .0 \\
\hline D'Авсн. 1 & & $\ldots \ldots$ & $\cdot$ & $\cdots$ & 0 & $\cdots \mathbf{v} \cdot$ & i \\
\hline 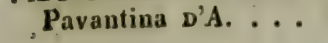 & & & & & & tu $\ldots$ & \\
\hline
\end{tabular}




\begin{tabular}{|c|c|c|c|c|c|c|c|}
\hline & Weltgegend. & KohlenP. & SalxP. & OolithP. & $\begin{array}{l}\text { Krei- } \\
\text { deP. }\end{array}$ & MolasseP. & Neu \\
\hline Benennungen. & 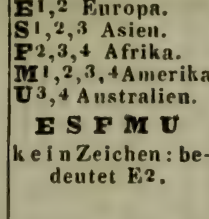 & 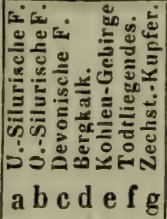 & 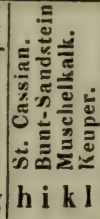 & 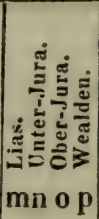 & 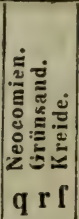 & 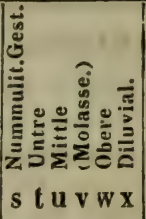 & 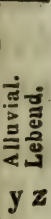 \\
\hline
\end{tabular}

\section{Cl. III. POLYGASTRICA, Magenthierchen Eв. ${ }^{1}$.}

(INFUSORIA L., pars.)

\section{A. ANENTERA Eb.}

1. Monadina EB. 1:1 ... (corpora mollia)

Monas .............

Dunali JoLY .... I...

2. CRyptomonadina EB. 0 . (corpora mollis)

3. volvocina EB. 0 .... (corpora mollia)

4. vibrionia Eb. 0 (corpora mollia)

5. closterina EB. 0 .... (corpora mollia)

6. astasiaen Eb. $0 \ldots .$. (corpora mollia)

7. dinobryina Eb. $0 \ldots \ldots$ (corpora mollia)

8. амовваеа Ев. 0 ..... (corpora mollia)

9. arcellina EB. 2:4 ... (corpora pleraque mollia)

Difnugia Eв. 2 .. areolata Ев. .... enchelys $\mathbf{E}_{B .} . \ldots$

Arcella EB. 2 ...

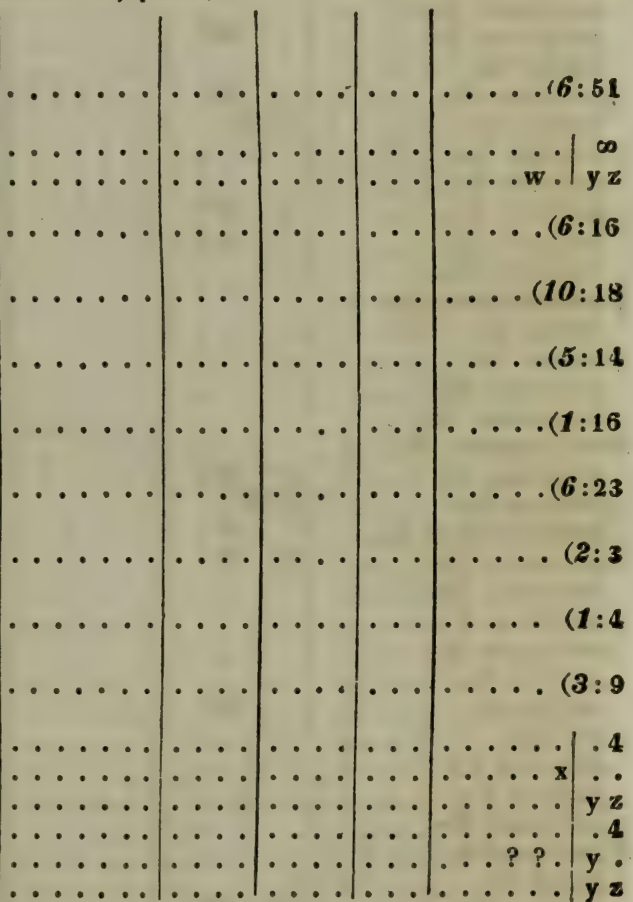

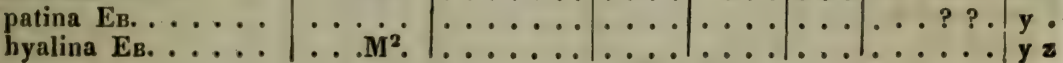

I Ehrenberg fragmenta tantum laborum sworum edldit; inde s a e pe dubium manet, quae species fossiles adhue vivant; multaeque species ipsae, aliorum affinitates, loci, synonyma latent. Revera itaque specierum fossiliu m numerus indicato longe major adhuc vivit. - Etiam numeri specierum solo viventium valde augendi essent. 


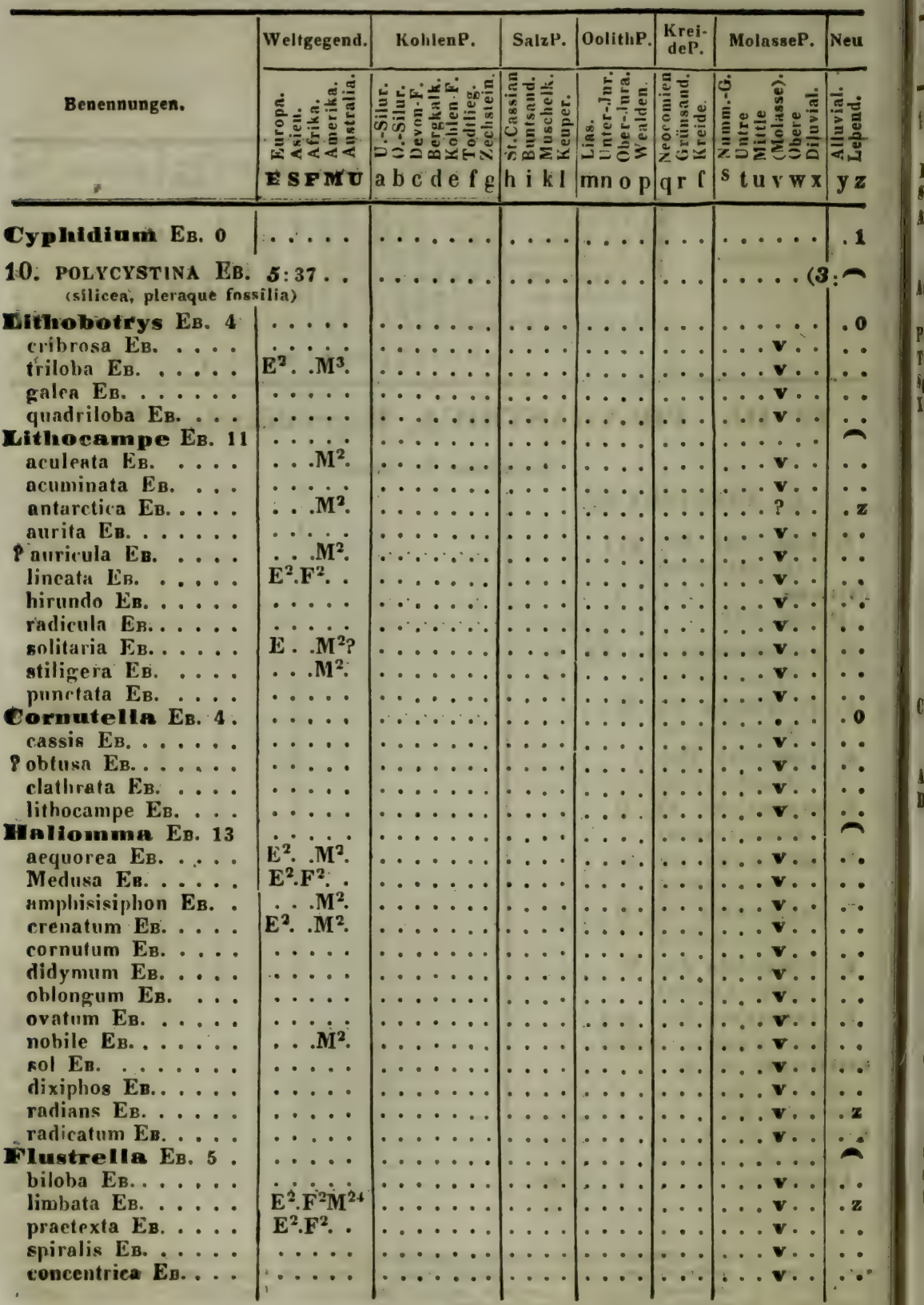




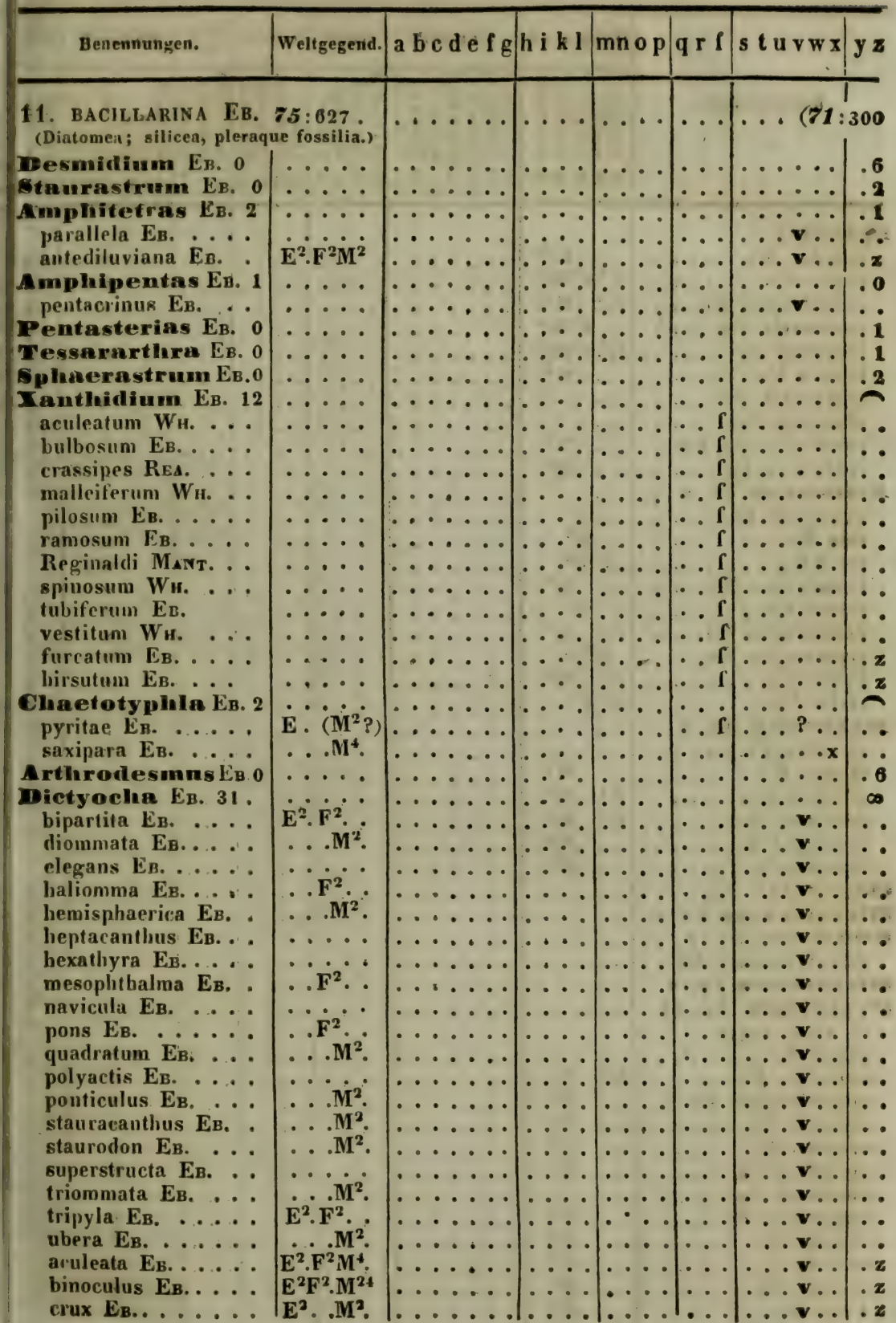




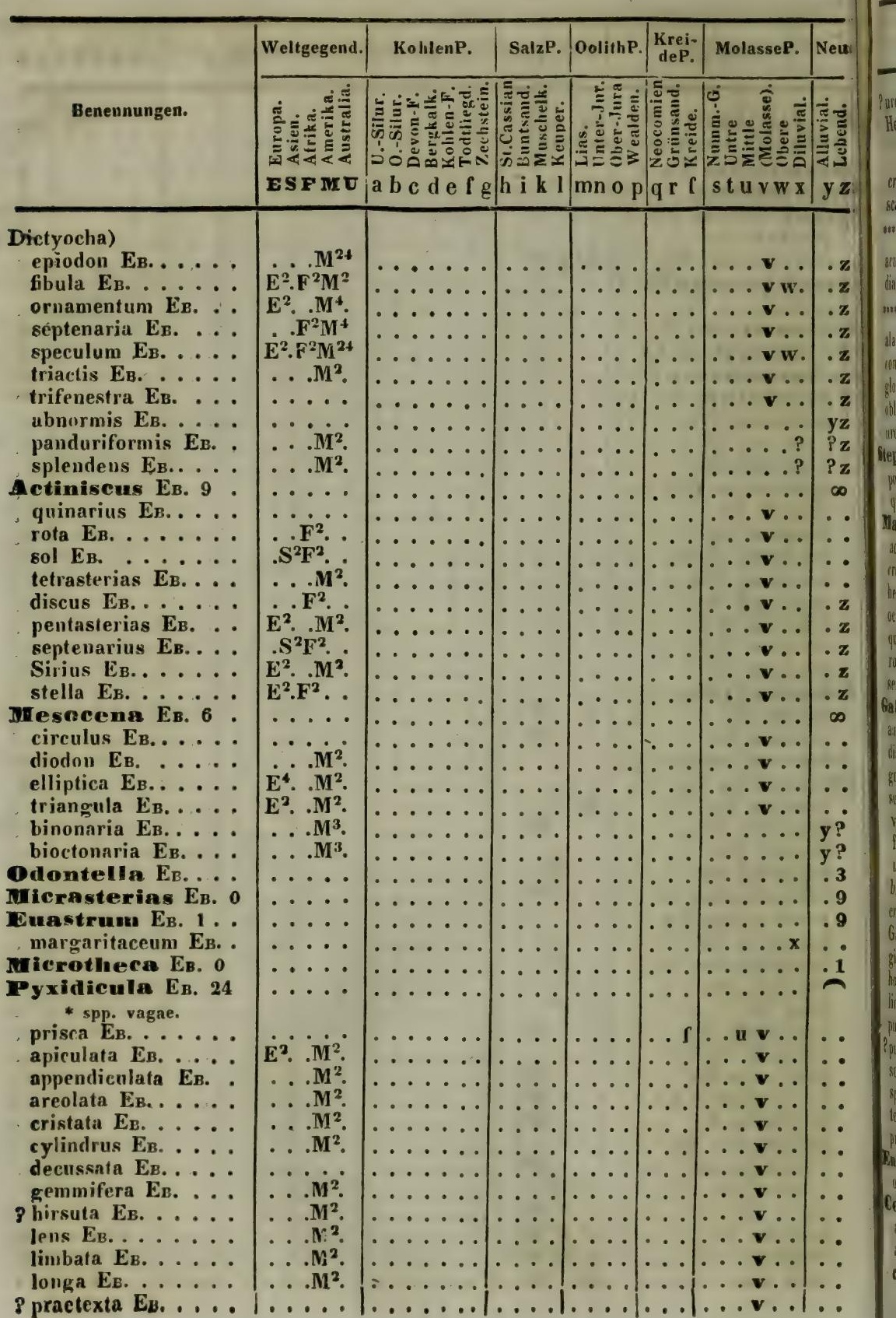




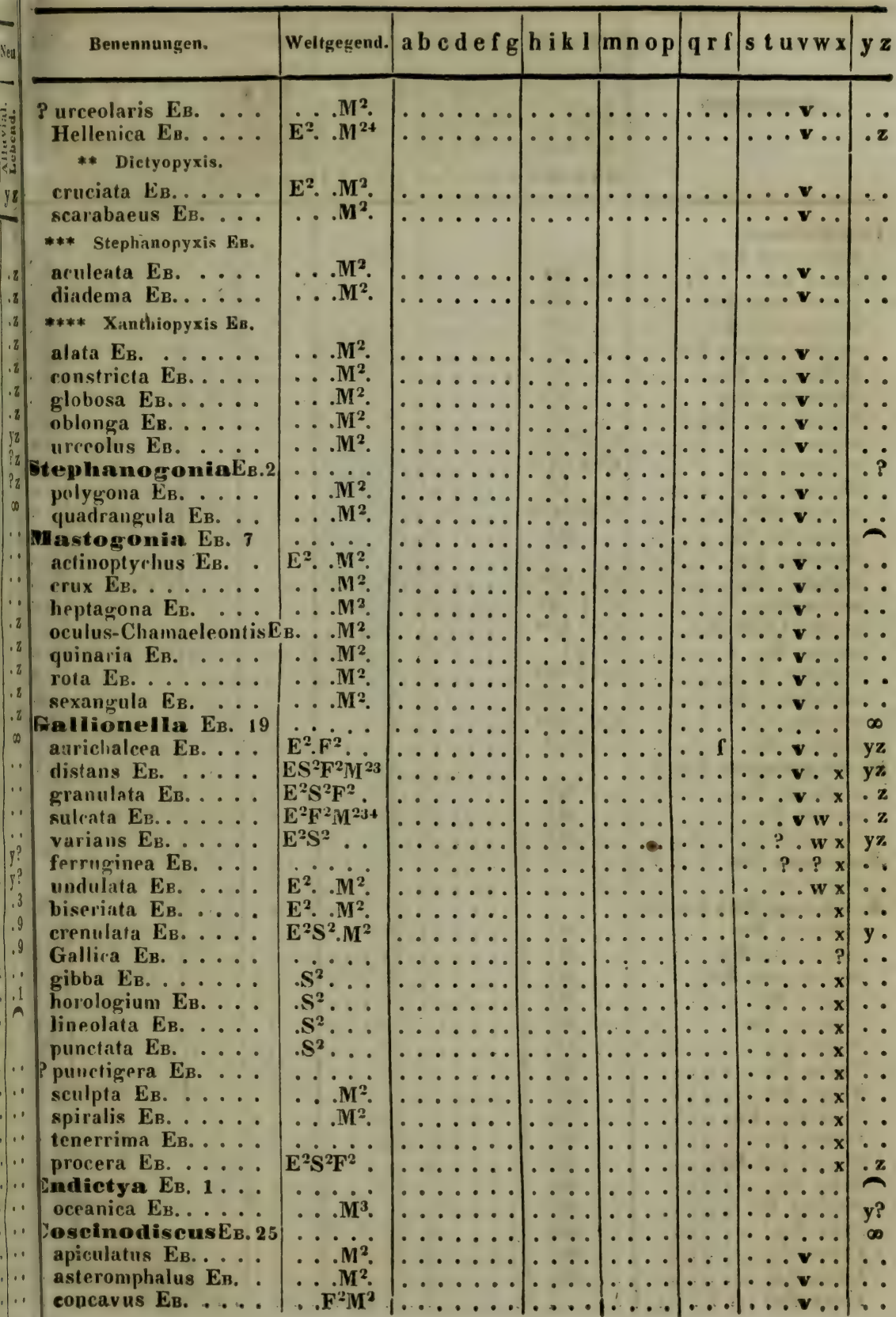




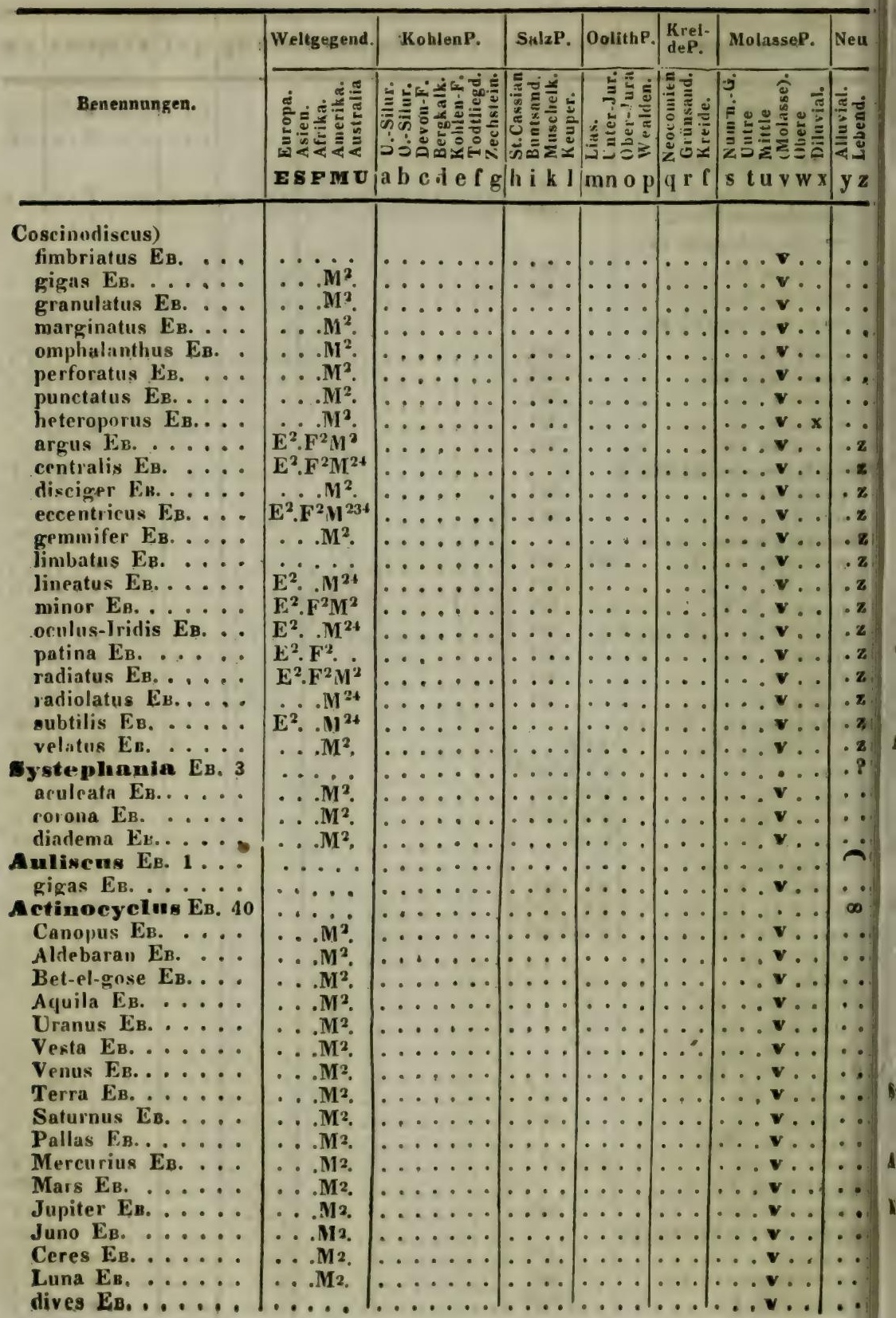




\begin{tabular}{|c|c|c|c|c|c|c|c|}
\hline Benennungen. & Weltgegend. & $\mathbf{a} \mathbf{b}$ & h $\quad$ i $\quad$ k l l & mnop & $q \times f$ & stuvw & $y^{2}$ \\
\hline $\mathbf{v}$ & & & & & & & \\
\hline arius Eв. . & & & & & $\cdots$ & & \\
\hline s E. . . . & & $\ldots \ldots$ & $\ldots$ & $\cdots \cdots$ & $\cdots$ & $\ldots \mathbf{v}$ & \\
\hline E. & • & $\ldots \ldots$ & $\cdots$ & & $\cdots$ & & \\
\hline в. . & & $\ldots$ & I. & & & $\ldots \mathbf{v}$ & \\
\hline B. $\cdot \dot{\text { Ens. }}$ & & & & & - & & $\because$ \\
\hline $\begin{array}{l}\text { irius } \mathbf{E}_{B .} \\
\text { is E. E. . }\end{array}$ & & . & 1. & & & 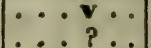 & \\
\hline $\begin{array}{l}\text { rius } \text { Eв. . }^{\text {rius Eв. . }}\end{array}$ & $\therefore \dot{M}^{2}$ & $\cdots$ & $\dot{\cdot}$ & & & 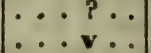 & $z$ \\
\hline sedenarius? Ee. & & $\ldots$ & $\ldots$ & 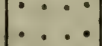 & 1 & $\begin{array}{l}\cdots \mathbf{v} \cdots \\
\ldots \mathbf{v}\end{array}$ & $\cdot z$ \\
\hline s Ев... & $\mathbf{E}^{2}$ & $\ldots \ldots$ & $\ldots$ & $\therefore \cdots$ & 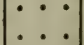 & . & $\mathbf{z}$ \\
\hline b & $\mathbf{E}^{2} .1$ & $\ldots \ldots$ & $\ldots$ & $\ldots$ & 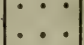 & $\left|\begin{array}{cc}\cdots & \cdots \\
\cdots & \cdots\end{array}\right|$ & z \\
\hline 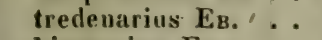 & & $\ldots \ldots$ & $\ldots$ & $\ldots$ & $\therefore$ & $\ldots \mathbf{v}$ & .2 \\
\hline Ев. ... & $\mathrm{E}^{2}$ & $\ldots \ldots$ & $\cdots \cdots$ & $\ldots$ & . & $\ldots \mathbf{v}$ &. $\mathrm{z}$ \\
\hline Ев. & $E^{2}$ & $\cdots$ & & & $\ldots$ & $\ldots \mathbf{v} \cdots$ & $\cdot \mathrm{z}$ \\
\hline B. $\therefore$. & & & & & $\cdots$ & $\ldots \mathbf{v} \ldots$ & $\cdot \mathbf{z}$ \\
\hline Eв. & & $\cdots$ & $\cdot$ & $\ldots$ & $\cdots$ & $\ldots \mathbf{v} \cdots$ &. $\mathrm{z}$ \\
\hline us EB. . . & & $\ldots \ldots$ & $\cdots \cdot$ & $\ldots$ & $\cdots$ & $\ldots \mathbf{v} \mathbf{w}$ & $\cdot x$ \\
\hline$\cdots$ & & $\cdots \cdots$ & $\cdots$ & $\cdots$ & $\cdots$ & $\cdots \mathbf{v}$ & $\mathrm{z}$ \\
\hline b & & $\ldots \ldots$ & $\cdots$ & $\cdots \cdot$ & $\cdots$ & . &. $\mathrm{z}$ \\
\hline$\therefore$ & & $\cdots$ & $\cdots$ & $\cdots$ & $\cdots$ & $\cdots \mathbf{v} \cdot$ & $\cdot z$ \\
\hline$\cdots$ & $\mathrm{E}$ & . & $\because$ & $\cdots$ & & . & $i^{x}$ \\
\hline iscus $E_{B} .3$ & & $\cdots \cdots$ & $\cdots$ & $\left|\begin{array}{c}\cdots \\
\cdots \\
\cdots\end{array}\right|$ & & $\because$ & ? \\
\hline $\mathbf{s}$ & & $\ldots \ldots$ & $\ldots$ & $\ldots$ & $\cdots$ & $\ldots \mathbf{v}$ & \\
\hline $\mathbf{U}$ & 2 & $\ldots \ldots$ & $\ldots$ & $\ldots$ & $\ldots$ & $\ldots \mathbf{v}$ & \\
\hline . . & $\therefore \mathrm{M}^{2}$ & $\ldots \ldots$ & $\ldots$ & $\ldots$ & $\ldots$ & $\ldots \ldots$ & $y ?$ \\
\hline Eв.14 & & $\ldots \ldots$ & $\ldots$ & $\cdots \cdot$ & $\cdots$ & $\ldots \ldots$ & $\infty$ \\
\hline$\ldots$ & & $\ldots, \ldots$ & $\ldots$ & $\ldots$ & $\cdots$ & $\ldots \nabla$ & . \\
\hline$\therefore$ & . & $\ldots \ldots$ & $\cdots$ & $\cdots \cdot$ & . . & $\ldots \mathbf{v} \ldots$ & \\
\hline ... & $\therefore$ & - & $\cdots$ & $\cdots \cdot$ & $\cdots$ & $\cdots$ & , \\
\hline$\cdots$ & $\ldots$ & $\ldots \ldots$ & $\ldots$ & $\ldots$ & $\cdots$ & $\ldots \mathbf{v}$ & . \\
\hline Ев... & $\ldots$ & $\cdots \cdots$ & $\ldots$ & $\ldots$ & $\ldots$ & $\ldots \mathbf{v} \cdots$ & $\cdot$ \\
\hline$\cdots$ & & $\ldots \ldots$ & $\ldots$ & $\ldots$ & $\cdots$ & $\ldots \cdot \mathbf{v}$, & \\
\hline 3. & & $\cdots$ & $\cdots$ & $\cdots \cdot$ & $\cdot \cdot$ & $\cdots \mathbf{v} \cdots$ & \\
\hline s & . & - & $\cdots$ & $\cdots \cdot$ & $\cdot \cdot$ & $\cdots \mathbf{v} \cdots$ & $\mathbf{z}$ \\
\hline de & .1 & $\cdot$ & $\cdot$ & $\cdots \cdot$ & $\cdot$ & $\cdots$ & $\mathbf{z}$ \\
\hline $\mathbf{E}_{1}$ & $\mathrm{E}^{2} \cdot \mathrm{F}^{2} \mathrm{M}^{2}$ & . & $\cdot$ & $\cdots \cdot$ & $\cdots$ & $\cdots \cdot$ & - $z$ \\
\hline$E_{B} \cdots$ & & $\cdots$ & 1. & $\cdots$ & $\cdot \cdot 1$ & $\cdots$ & \\
\hline s Eв. . . & $\ldots$ & & • & $\cdots$ & $\cdots$ & $\bullet$ & \\
\hline $\begin{array}{l}\text { Irius Eв. } \\
\text { denarius Eв. }\end{array}$ & 2 & - & $\cdot$ & $\cdots \cdots$ & $\cdots$ & $\because$ & \\
\hline EB.2 & & $\because$ & & $\ldots \ldots$ & $\cdots$ & $\ldots \ldots$ & 0 \\
\hline & . & $\ldots \ldots$ & $\ldots$ & $\ldots$ & $\therefore$ & $\cdots \dot{v} \cdot$ & . \\
\hline . & $\mathrm{M}^{2}$ & $\cdots \cdots$ & $\cdots \cdots$ & $\cdots \cdot$ & $\cdots$ & $\cdots v$ & \\
\hline EB. 1 & & $\cdots$ & - & $\cdots \cdot$ & $\cdots \cdot$ & $\cdots$ & 0 \\
\hline dica EE. & $\bullet$ & & - & & & $\cdots$ & \\
\hline 1 a EB. 67 . & & & & & & & \\
\hline E & $\mathbf{L}^{*}$ & & & $\cdots$ & $\cdots$ & $\because \cdots \cdot \mathrm{x}$ & $\cdots$ \\
\hline tata & & & $\cdot, \cdot$ & & 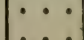 & & \\
\hline curysoma $\mathbf{E}$, . & & $\cdots$ & $\cdots$ & $\cdots \cdots$ & - & $\ldots v$ & \\
\hline
\end{tabular}




\begin{tabular}{|c|c|c|c|c|c|c|c|}
\hline & d. & ?. & $12 \mathrm{P}$. & OolithP. & & P. & \\
\hline Benennungen. & 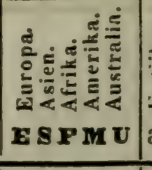 & 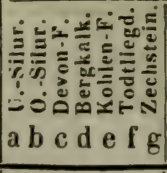 & 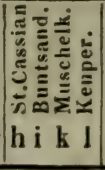 & 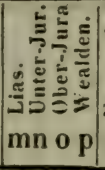 & 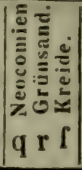 & 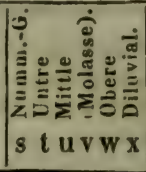 & \\
\hline 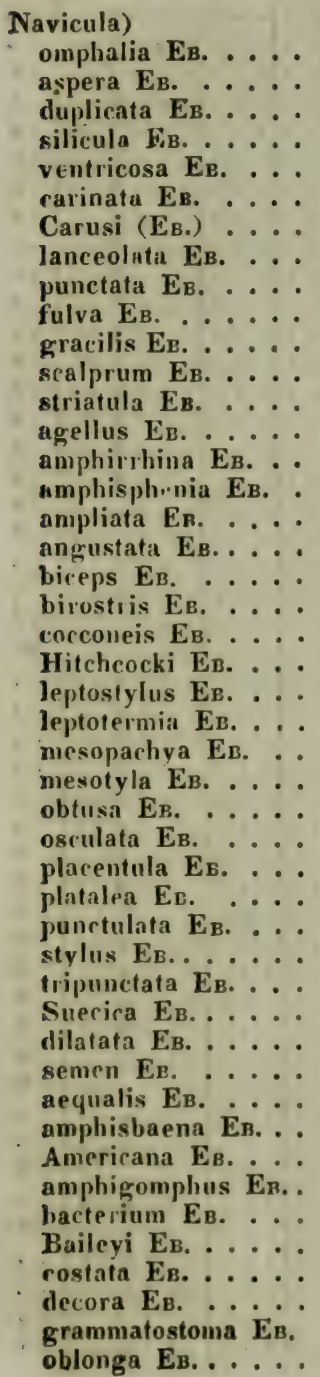 & 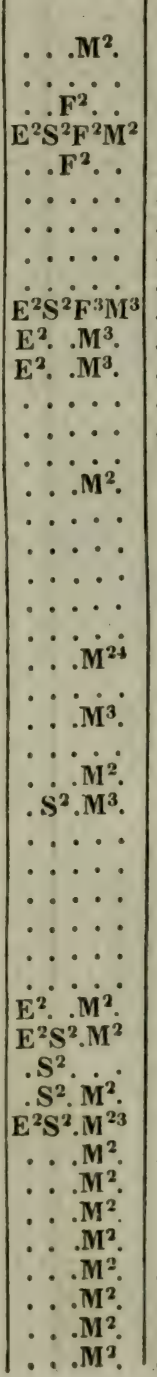 & 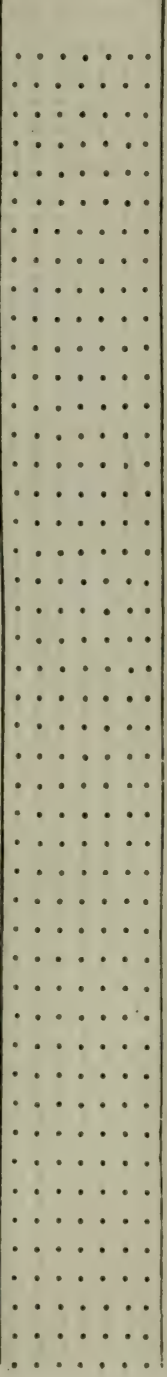 & $\begin{array}{c}\cdots \\
\cdots \\
\cdots \\
\cdots \\
\cdots \\
\cdots \\
\cdots \\
\cdots \\
\cdots \\
\cdots \\
\cdots \\
\cdots \\
\cdots \\
\cdots \\
\cdots \\
\cdots \\
\cdots \\
\cdots \\
\cdots \\
\cdots\end{array}$ & 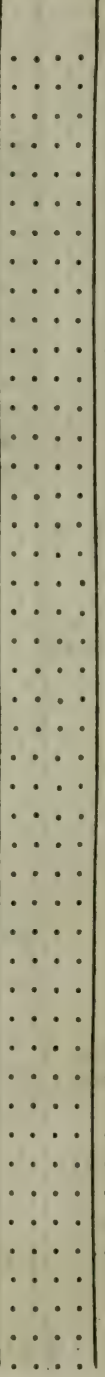 & 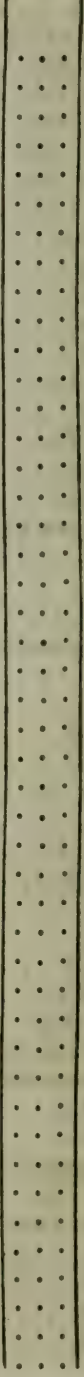 & 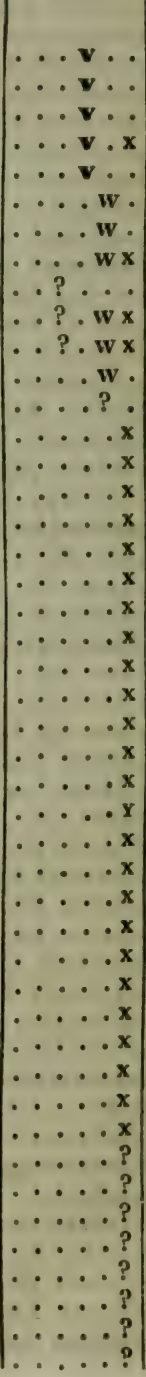 & y \\
\hline
\end{tabular}




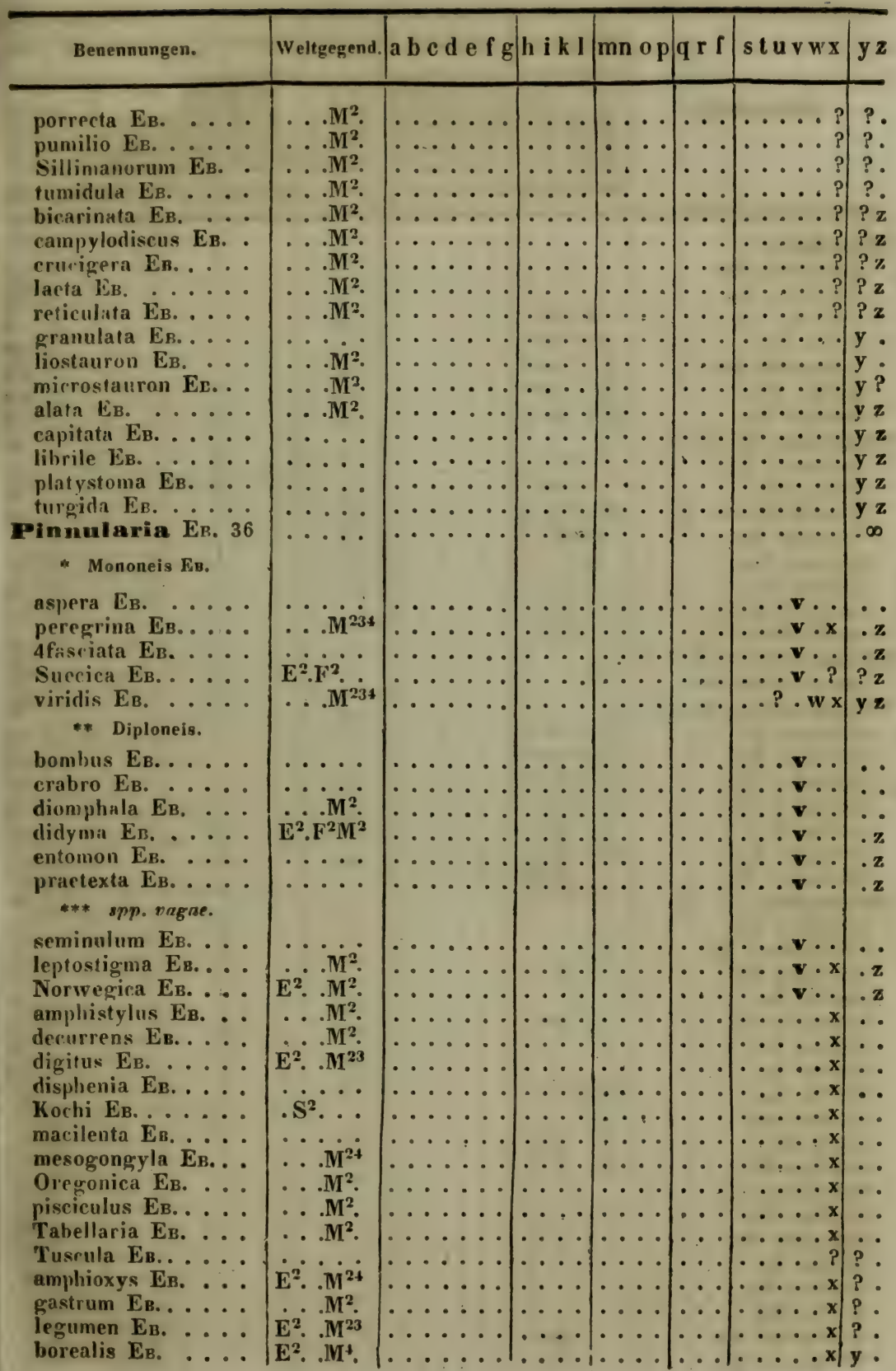




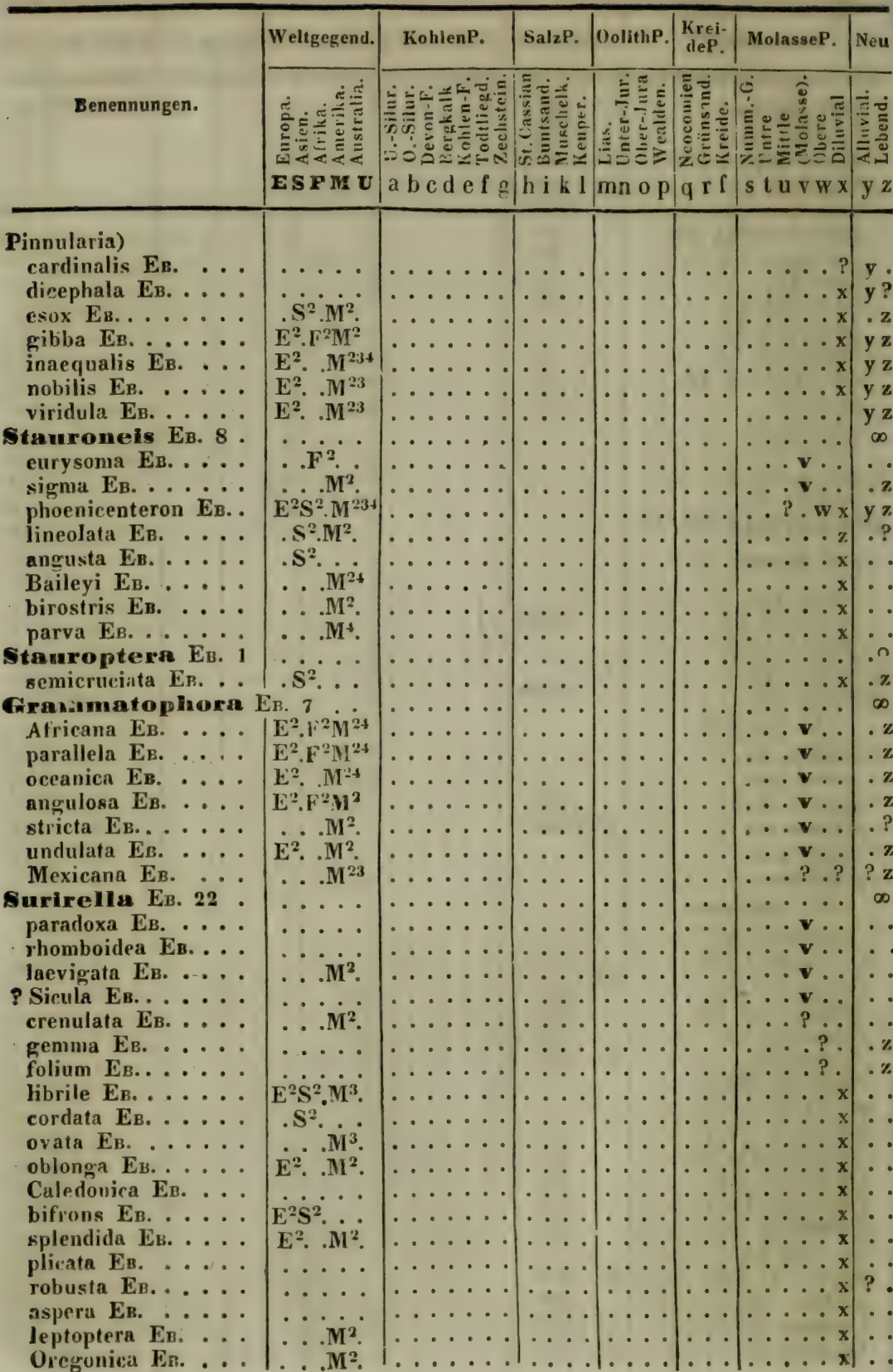




\begin{tabular}{|c|c|c|c|c|c|c|c|}
\hline t. & & $\mathrm{fg}$ & & & \ & $w \mathbf{x}$ & $\mathbf{y}$ \\
\hline & & & & & & .. $x$ & \\
\hline 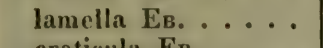 & & & $\cdots$ & & $\cdots$ & $\ldots \ldots x$ & . $z$ \\
\hline & & & & $\cdot$ & & & $y x$ \\
\hline 1 & $\dot{\mathrm{I}}^{3}$ & & - & $\cdots$ & . & $\because \cdots$ & $n$ \\
\hline n Ев. 1 & & & & 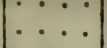 & & $\cdots$ & $\ddot{?}$ \\
\hline & & & & $\cdot$ & & $\cdots$ & \\
\hline $\mathbf{n}$ & - & $\cdots$ & 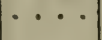 & $\cdots \cdot$ & . & $\cdots \cdots$ & \\
\hline 1 & & & & $\cdots$ & $\cdots$ & $\cdots \mathbf{v} \cdots$ & \\
\hline B. 4 & & & & & . • & v.. & \\
\hline$\therefore$ & 2 & & 1. & - 1 & & $\therefore$ &.$?$ \\
\hline & 2. & $\ldots$ & . & $\cdots$ & $\cdots$ & $\cdots$ & \\
\hline . & & $\ldots$ & . & $\cdots \cdots$ & & $\begin{array}{ll}\cdots \mathbf{v} \\
\cdots \\
\cdots\end{array}$ & \\
\hline & & $\cdots$ & $\cdots$ & $\ldots$ & $\therefore$ & $\begin{array}{l}\cdots \mathbf{v} \\
\cdots \mathbf{v}\end{array}$ & \\
\hline Ев. 6 & & $\cdots$ & $\cdot$. & $\cdots \cdot$ & $\ldots$ & $\ldots \ldots$ & \\
\hline . & - & $\ldots$ & 1. & $\ldots$. & $\ldots$ & $\cdots \mathbf{v}$ & \\
\hline$\cdots$ & . & $\ldots \ldots$ & $\ldots$ & $\cdots$ & $\ldots$ & $\therefore$ v. & \\
\hline$\cdots$ & - & $\ldots \ldots$ & $\ldots$ & $\cdots$ & $\cdots$ & $\therefore \mathbf{v}$ & \\
\hline & . & $\ldots \cdots$ & $\cdots$ & $\cdots$ & - & .. $\mathbf{v} \ldots$ & \\
\hline 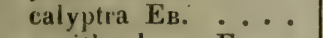 & $\cdots$ & $\cdots \cdots$ & $\cdots \cdot \cdot$ & $\cdots \cdot$ & $\cdots$ & $\ldots \mathbf{v} \ldots$ &. $\mathrm{z}$ \\
\hline B. . & $\cdots$ & $\cdots$ & 1 & $\cdots \cdot$ & $\bullet^{\circ}$ & $\ldots \mathbf{v}$. & $\mathrm{z}$ \\
\hline Ев. 0 & & $\cdots \cdots$ & . & $\cdots \cdots$ & $\cdots$ & $\ldots \ldots$ & . n \\
\hline$\cdots$ & & $\cdots \cdots$ & - & $\cdots \cdot$ & $\cdots$ & $\ldots \ldots$ & $n$ \\
\hline 5 & $\cdots$ & $\cdots \cdots \cdots$ & $\cdots \cdot$ & $\cdots \cdots$ & $\cdots$ & $\cdots \cdots \cdots$ & 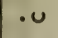 \\
\hline & & & $\cdots$ & $\cdots \cdot$ & $\cdots$ & $\cdots \mathbf{v} \ldots$ & \\
\hline & & $\cdots$ & ${ }^{\circ}$ & $\cdots \cdot$ & $\cdots$ & $\cdots$ & \\
\hline & - & . & $\cdots$ & $\cdots \cdots$ & - & $\cdots$ & $\mathbf{z}$ \\
\hline $\begin{array}{l}\text { R } \\
G\end{array}$ & $\dot{E^{2}}$ & - & $\cdots$ & $\cdots \cdot$ & . $\cdot$ & $\cdot$ & . $x$ \\
\hline Ев. 1 & E. & . & & & & $\cdots$ & $\mathbf{y}$ \\
\hline Ев. 1 & & & • 1 & $\ldots$ & • & $\cdots$ & \\
\hline is $\mathrm{E}_{\mathbf{B}}$. & 2. & . & $\cdots$ & $\ldots$ & • & $\cdots \cdots$ & .0 \\
\hline . & & $\ldots$ & . & $\ldots$ & - & ... & \\
\hline & $\cdots$ & . & . & $\cdots$ & - & $\ldots \mathbf{v} \ldots$ & - \\
\hline Ita Ев. 5 . & & . & - & $\cdots$ & 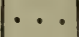 & $\cdot \cdot$ & .0 \\
\hline$\cdots$ & • & $\cdots \cdots$ & $\ldots$ & $\ldots$ & $\cdots$ & $\ldots \mathbf{v} \ldots$ & \\
\hline & . & $\ldots \ldots$ & $\cdots \cdot$ & $\cdots \cdot$ & $\because$ & $\ldots \mathbf{v} \ldots$ & \\
\hline & & $\cdot$ & $\cdots$ & $\cdots \cdot$ & . & $\ldots \mathbf{v} \ldots$ & \\
\hline & & & - & $\cdots$ & • & $\ldots \mathbf{v} \ldots$ & \\
\hline S & - & $\cdot$ & & $\cdots$ & & $\cdots \mathbf{v} \cdots$ & \\
\hline taEs.4 & & & & & & & \\
\hline 8 & & & & & & - & \\
\hline-5 & • & & & & & ... & \\
\hline & $\cdots$ & & & • & & $\because$ & \\
\hline & $\cdots$ & & & & & $\because$ & .0 \\
\hline EB. & & & ・. & $\cdots \cdots$ & • & $\cdots \mathbf{v} \cdot \cdot$ & \\
\hline Eв. 2 & & & & - & - & & \\
\hline 1 & & 然 & $\cdots$ & . & 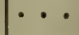 & $\cdots$ & - \\
\hline 7 & & & & & & & \\
\hline
\end{tabular}




\begin{tabular}{|c|c|c|c|c|c|c|c|}
\hline & Weltgegend. & KolıtenP. & SalzP. & OolithP. & $\begin{array}{l}\text { Krel- } \\
\text { deP. }\end{array}$ & MolasseP. & Neu \\
\hline Benennungen. & 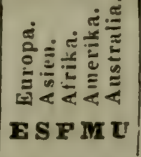 & 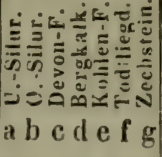 & 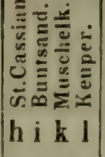 & 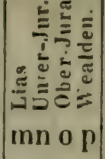 & 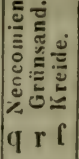 & 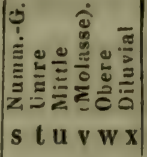 & 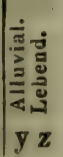 \\
\hline
\end{tabular}

Dunotia En. 52 cretae $\mathrm{F}_{\mathbf{B}}$.... Hellenica Ев. . . . ocellata EB. comta EB. diodon Eв. gibba Ев. monodon Ев. zebra Ев. Westermanni EB... amphidicranon Eв. . depressa Ев. dizyga Eв.

formica $\mathrm{E}_{B}$..... gibberula EB. icosodon EB. longicornis EB. . . Luna Ев. nonaria EB. parallela $\mathbf{E}_{B}$ quaternaria Ев. quinaria EE. sella EB.

P semilunaris EB. senaria Es. sima Ев.

textricula EB. tridentula EB. ventralis $E_{B}$. biceps Ев. bidens $\mathrm{E}_{B}$. $\dot{\mathrm{E}}^{2} \dot{\mathrm{M}^{2}}$ uncinata EB. zebrina Es. praerupta EB. diadema Ев. endecaodon $\mathbf{E}_{\mathbf{B}}$. faba Ев. noilosa EB. pentodon Ев. serra EB. tetraodon Eв. triodon Ев. turgida Ев. granulata EB. amphioxys Eв. bioctonaria Eв.
decaodon EB.

$\mathbf{E}^{2} \cdot \dot{M}^{2}$ $\mathrm{E}^{2} \mathrm{~S}^{2} \cdot \mathrm{M}^{23}$ $E^{2} S^{2} \cdot M^{2}$ $\mathrm{E}^{2} \mathrm{~S}^{2} \cdot \mathrm{M}^{3}$ $\mathrm{E}^{2} \mathrm{~S}^{3}$ .... $\mathbf{M}^{2}$. $\cdots \mathrm{M}^{2}$ . $M^{3}$. $\mathrm{E}^{2} \cdot \mathrm{M}^{3}$ ... $\mathbf{M}^{2}$. ... $M^{3}$. $E^{2} . M^{2}$. $s^{2}$. $\mathrm{S}^{2}$. $S^{2} \ldots$ $\mathrm{S}^{2}$. . $\mathrm{M}^{2}$. $\dot{\mathrm{S}} \dot{ }$ ... $M^{2}$. $\dot{E}^{2} \cdot \dot{\mathbf{M}^{4}}$ $\cdots \dot{M}^{2+}$ $\mathrm{S}^{2} \cdot \mathrm{M}^{2}$. $E^{2} \cdot M^{2}$. $E^{2}$. $M^{2}$. ... $\mathrm{M}^{2}$. … $\mathrm{E}^{2} \cdot \mathrm{F}^{3} \mathrm{M}^{2}$ $\mathbf{E}^{2} \cdot \mathbf{F}^{3}$. \begin{tabular}{l|l|l|l|l|l|l|l|l|l|l} 
&
\end{tabular}

$$
\text { . }
$$

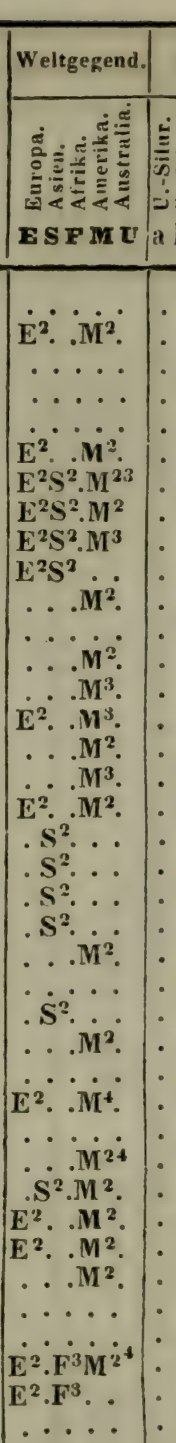

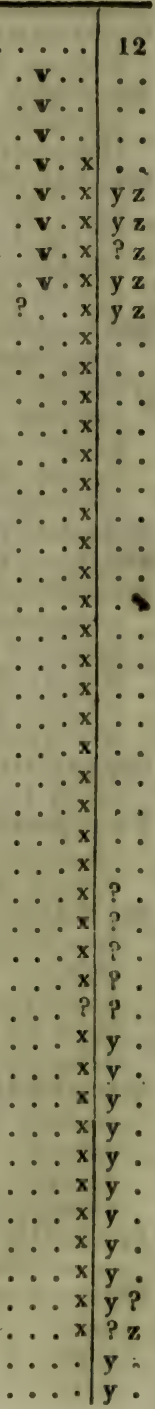




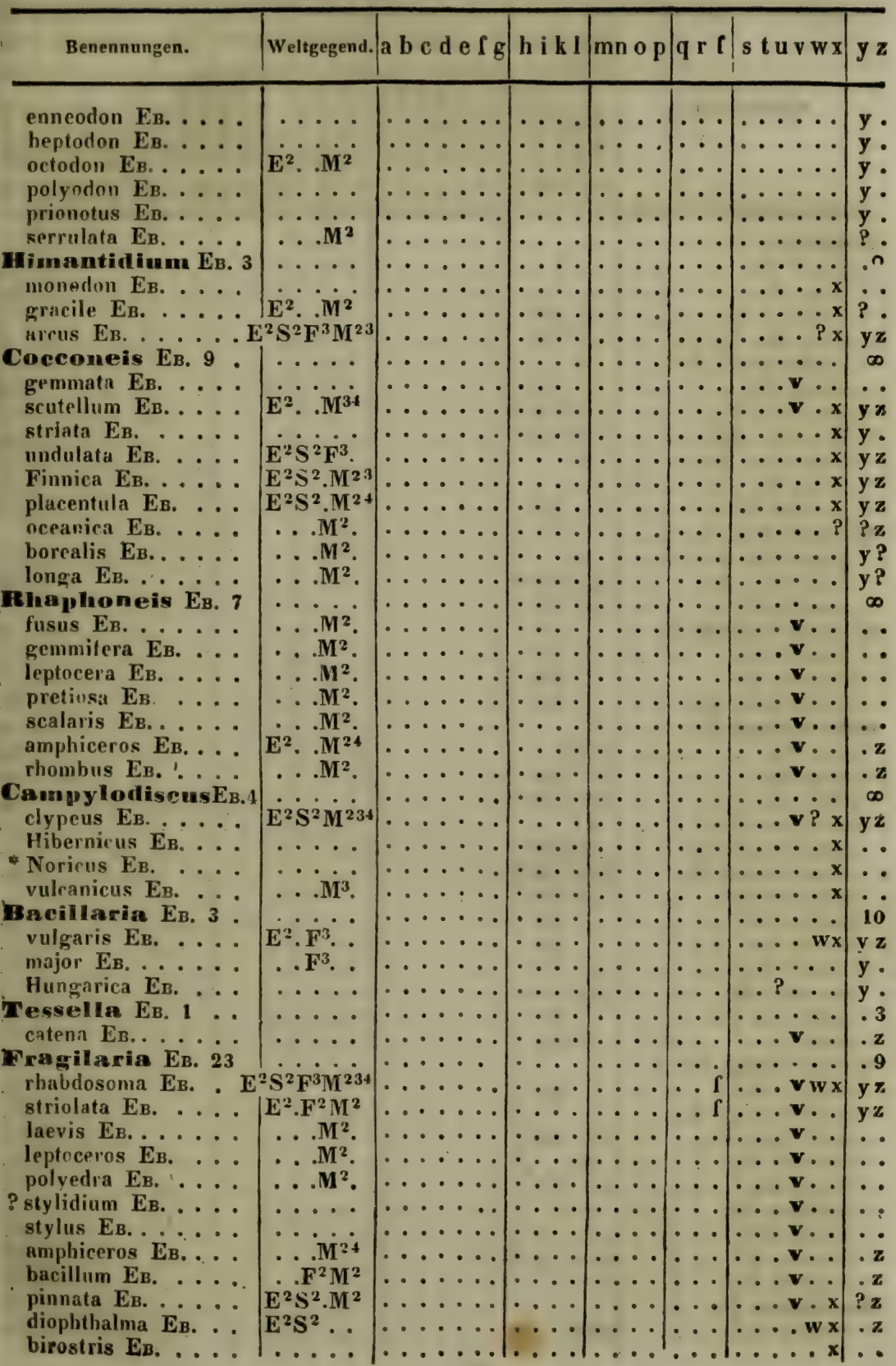




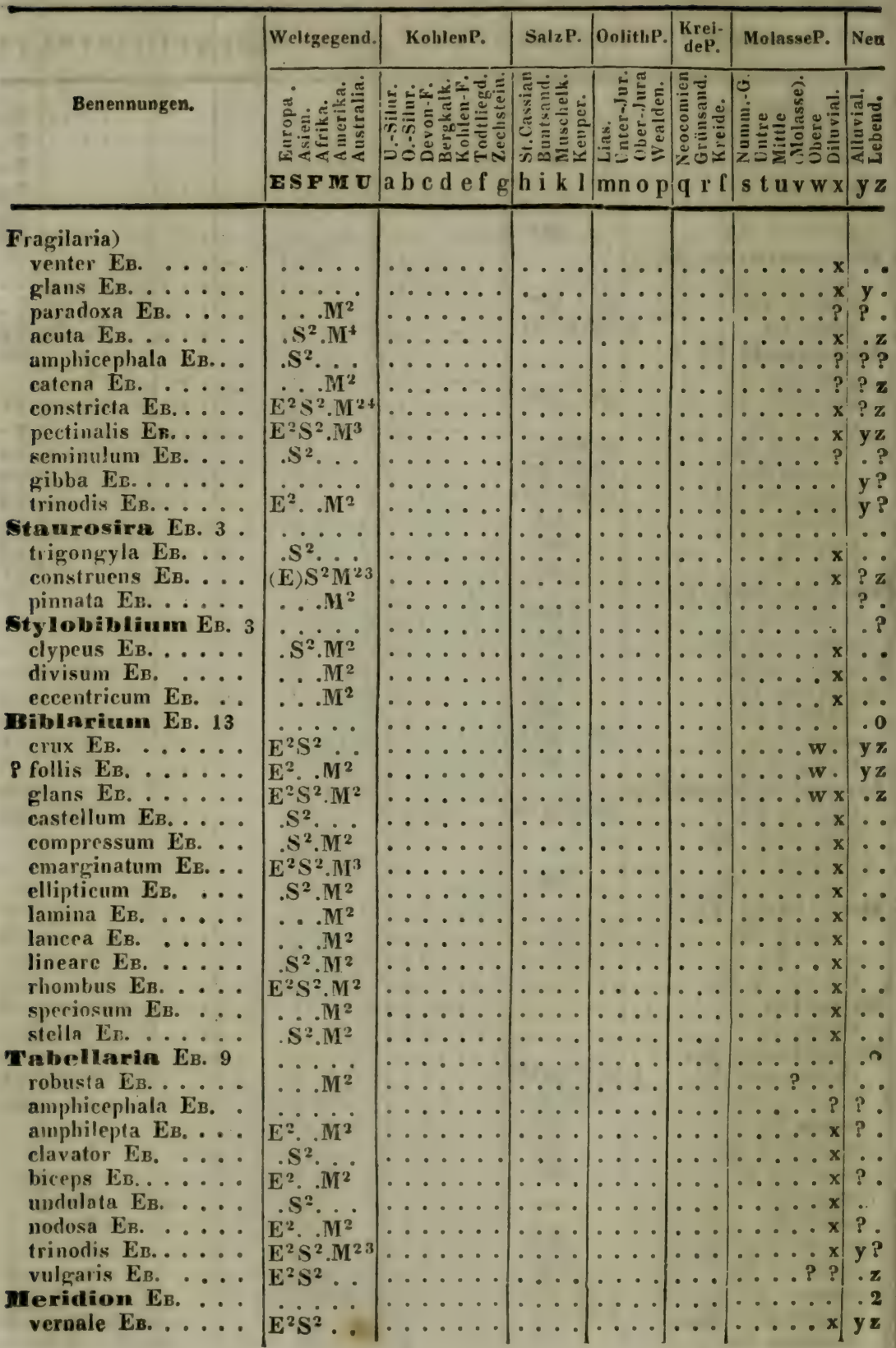




\begin{tabular}{|c|c|c|c|c|c|c|c|}
\hline Bent & nd. & f $g$ & k 1 & m & $q \times<$ & $\mid \mathrm{w} x$ & $y z$ \\
\hline 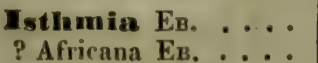 & & • & - & $\cdots$ & $\cdots$ & $\cdots \cdots$ & 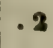 \\
\hline $\begin{array}{l}\text { PAfricana Eв. . : } \\
\text { Denticella Eв. } 5 \text {. }\end{array}$ & - & 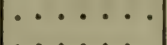 & $\cdots$ & 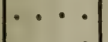 & $\cdots$ & $\cdots \mathbf{v} \cdot \cdot$ & $\ddot{m}$ \\
\hline 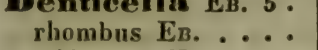 & $\because \dot{M}^{2}$ & & $\cdots$ & $\left|\begin{array}{ll}\cdots \\
\cdots \\
\cdots\end{array}\right|$ & $\cdots$ & $\because \cdots \mathbf{v}$ & $\infty$ \\
\hline tridentata EB.... & .. $M^{2}$ & $\ldots \ldots$ & $\ldots$ & $\ldots \ldots$ & $\cdots$ & $\cdots \mathbf{v}$ & \\
\hline Ев. . . & $\ldots \mathrm{M}^{2}$ & $\ldots \ldots$ & $\cdots$ & $\ldots$ & ... & $\ldots \mathbf{v} \ldots$ & \\
\hline a Eв. ... &. $\mathbf{F}^{2}$ & $\ldots \ldots$ & $\cdots$ & $\cdots$ & $\cdots$ & $\ldots \mathbf{v} \ldots$ & - \\
\hline$\ddot{F} \cdot$ & & $\cdot$ & $\cdots$ & & & $\ldots \mathbf{v} \ldots$ & \\
\hline $\begin{array}{l}1 \text { phis Ев. } 5 \\
\text { a EB. }\end{array}$ & - & $\cdots$ & $\cdots$ & $\cdots \cdots$ & $\cdots$ & $\cdots \cdots$ & $\infty$ \\
\hline $\begin{array}{l}\text { Tuna } \\
\text { tride }\end{array}$ & $\cdots \mathrm{F}^{2}$ & $\cdots \cdots$ & $\cdots \cdots$ & $\cdots \cdots$ & $\cdots \cdot$ & $\cdots \mathbf{v} \cdots$ & - \\
\hline Eв. . & $\because M^{2}$ & $\cdots$ & & $\cdots$ & $\cdots$ & $\cdots \mathbf{v} \cdots$ & • \\
\hline tata $\dot{\mathbf{E}}_{B}$ & $\mathrm{E}^{2} \cdot \mathrm{F}^{2} \mathrm{M}^{2}$ & $\cdots \cdots \cdots$ & $\cdots$ & $\cdots \cdots$ & $\cdots$ & $\begin{array}{l}\cdots \mathbf{v} \cdots \\
\cdots \mathbf{v} \cdots\end{array}$ & \\
\hline pulchella Eв. . . . & $\ldots$ & $\ldots \ldots$ & $\ldots$ & $\ldots$ & ... & $\begin{array}{l}\cdots \mathbf{v} \cdots \\
\cdots \mathbf{v} \ldots\end{array}$ & \\
\hline Anaulus EB. $1 \ldots$ & & $\ldots \ldots$ & $\ldots$ & $\ldots$ & $\ldots$ & $\begin{array}{ll}\cdots \mathbf{v} \\
\cdots\end{array}$ & $\cdot \mathbf{z}$ \\
\hline diseus Eв. . & ... $\mathrm{M}^{2}$ & $\ldots \ldots$ & $\cdots \cdot$ & $\ldots$ & & $\ddot{\cdots} \cdots$ & - $n$ \\
\hline Synedra Eв. $12 \ldots$ & & $\ldots \ldots$ & . & $\ldots$ & & v $\cdots$ & $\ddot{\infty}$ \\
\hline B....... E & $S^{2} F^{2} M^{23}$ & $\ldots \ldots$ & & & & ? & $\infty$ \\
\hline Ев. ..... & $\ldots \mathrm{M}^{2}$ & $\cdots$ & $\cdots \cdot$ & $\ldots$ & $\cdots$ & $\cdots \mathbf{v}$ & \\
\hline . & $\mathrm{F}^{2} \mathrm{M}^{2}$ & $\ldots \ldots$ & $\cdots \cdot \cdot$ & $\ldots$ & $\ldots$ & $\ldots \mathbf{v}$ & \\
\hline ris Ев. & $\mathrm{E}^{2} \mathrm{~S}^{2} \cdot \mathrm{M}^{3}$ & $\ldots \ldots$ & $\cdots \cdot$ & $\ldots$ & $\cdots$ & $\ldots \ldots \mathrm{w} x$ & . $x$ \\
\hline Ев. . . . . & $\mathrm{E}^{2} \mathrm{~S}^{2} \ldots$ & $\cdots$ & $\cdots \cdots$ & $\ldots$ & $\ldots$ & $\ldots \ldots x$ & - \\
\hline atı Ев. .... &. $\mathrm{S}^{2} \ldots$ & $\ldots$ & $\cdots \cdot$ & $\cdots$ & $\cdots$ & $\ldots \ldots x$ & $\cdots$ \\
\hline elegans Ев. . . . . . &.$S^{2}$. & $\ldots \ldots$ & $\cdots \cdot \cdot$ & $\cdots \cdot$ & $\cdots$ & $\ldots \ldots x \mid$ & $\cdots$ \\
\hline а Ев...... & $\mathrm{E}^{2} \cdot \mathrm{M}^{2}$ & $\ldots \ldots$ & $\cdots \cdot$ & $\cdots \cdot$ & $\cdots$ & $\ldots \ldots x$ & $y z$ \\
\hline$\cdots$ & $\mathbf{E}^{2} \mathbf{S}^{2} \ldots$ & $\ldots \ldots$ & $\cdots$ & $\cdots \cdot$ & $\cdots$ & $\ldots \ldots x$ & .8 \\
\hline$\cdots \cdot$ & $? \ldots \dot{U}_{T^{3}}$ & $\ldots \ldots$ & $\cdots \cdots$ & $\cdots \cdot$ & $\cdots$ & $\cdots \cdots$ & $\mathbf{y} \cdot$ \\
\hline$\cdots$ & $\cdots \mathbf{U}^{3}$ & $\cdots \cdot$ & $\cdots$ & $\cdots$ & $\cdots$ & $\cdots \cdots$ & ? \\
\hline$\dot{2}$ & $\ldots U$ & $\cdots$ & $\cdots$ & $\cdot \cdot$ & $\cdots$ & $\cdots \cdots$ & . \\
\hline $\begin{array}{l}\text { phenia EB. } 2 \\
\text { EB. . . . . . }\end{array}$ & & $\cdots$ & $\cdots$ & $\cdots$ & $\cdots$ & $\cdots \cdots$ & . \\
\hline 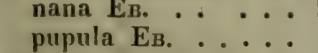 & & . & $\cdots \cdots$ & $\cdot 1$ & $\cdots$ & 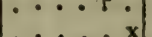 & \\
\hline phala EB. 1 & & ... & $\ldots$ & $\ldots .$. & $\cdots$ & $\cdots \cdots \cdots$ & . 0 \\
\hline s Ев. & $E^{2} S^{2}$ & . & $\cdots \cdots$ & $\ldots$. & $\cdots$ & $\ldots \ldots x$ & \\
\hline na Eв.21 & & & $\cdots \cdots$ & $\cdots \cdots$ & $\cdot 1$ & $\ldots \ldots$ & $\ddot{\infty}$ \\
\hline & $E^{2} \cdot M^{234}$ & & $\cdots \cdots$ & & $\cdots$ & $\ldots v ? \mathrm{x}$ & $y z$ \\
\hline EB. . & $\mathrm{E}^{2} \cdot . \mathrm{M}^{2}$ & ...... & $\cdots$ & . & .. & $\ldots \mathbf{v} \ldots$ & $y \mathbf{z}$ \\
\hline Ев. & 2 & • & $\cdots$ & $\cdots \cdots$ & $\cdots$ & $\ldots \mathbf{v}$ &.$\%$ \\
\hline$\cdots$ & $I^{23}$ & $I^{\circ}$ & $\cdots$ & • & $\cdots$ & $\ldots \ldots \mathrm{w}$ & ? \\
\hline й Ев. . . . & $E^{2} \cdot M^{2}$ & . & $\cdots$ & & $\cdots$ & $\cdots \cdot x$ & $\cdots$ \\
\hline Ев. .... & - & & & & & - & \\
\hline ? palea Eв. ...... & & & • & • & $\cdots$ & $\ldots \ldots x$ & . \\
\hline & $M^{3}$ & & $\cdots$ & & $\cdots$ & $\ldots \ldots x$ & . \\
\hline$\cdots$ & $\cdots$ & & & & . & $|\cdots \ldots x|$ & \\
\hline Ев. . &.$M^{23}$ & & & & 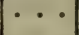 & $\cdots x$ & ?. \\
\hline$\therefore$ & $M^{2}$ & & $\cdots$ & $\cdots$ & - & 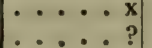 & y. \\
\hline$\cdots$ & $\cdots$ & & & & $\bullet$ & . & ? \\
\hline & & & & & & . & ? \\
\hline & & & & & . & - & $y ?$ \\
\hline act & & & & & & . & $y z$ \\
\hline sug & & & & & & $\cdots \cdots$ & \\
\hline
\end{tabular}




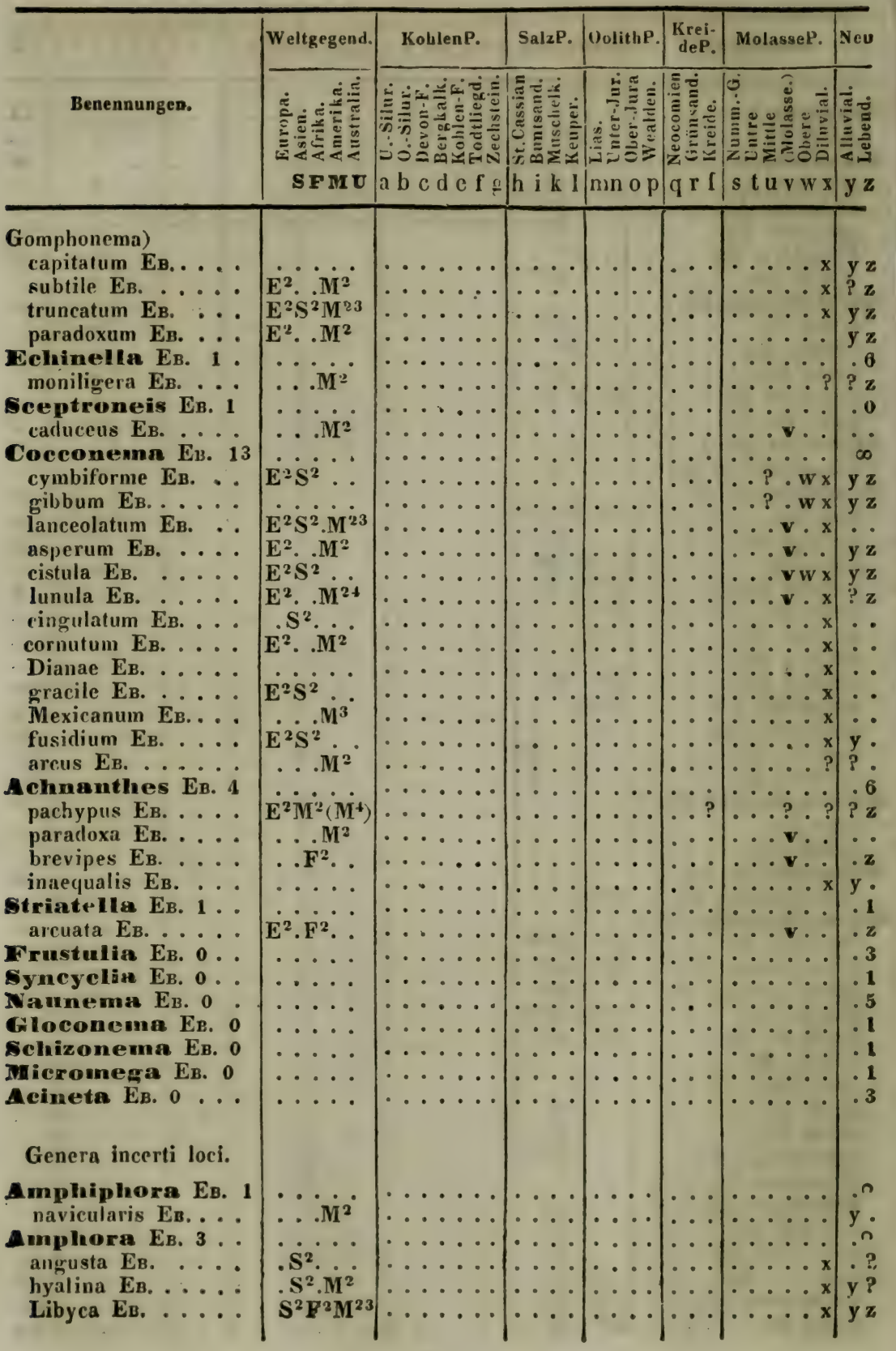




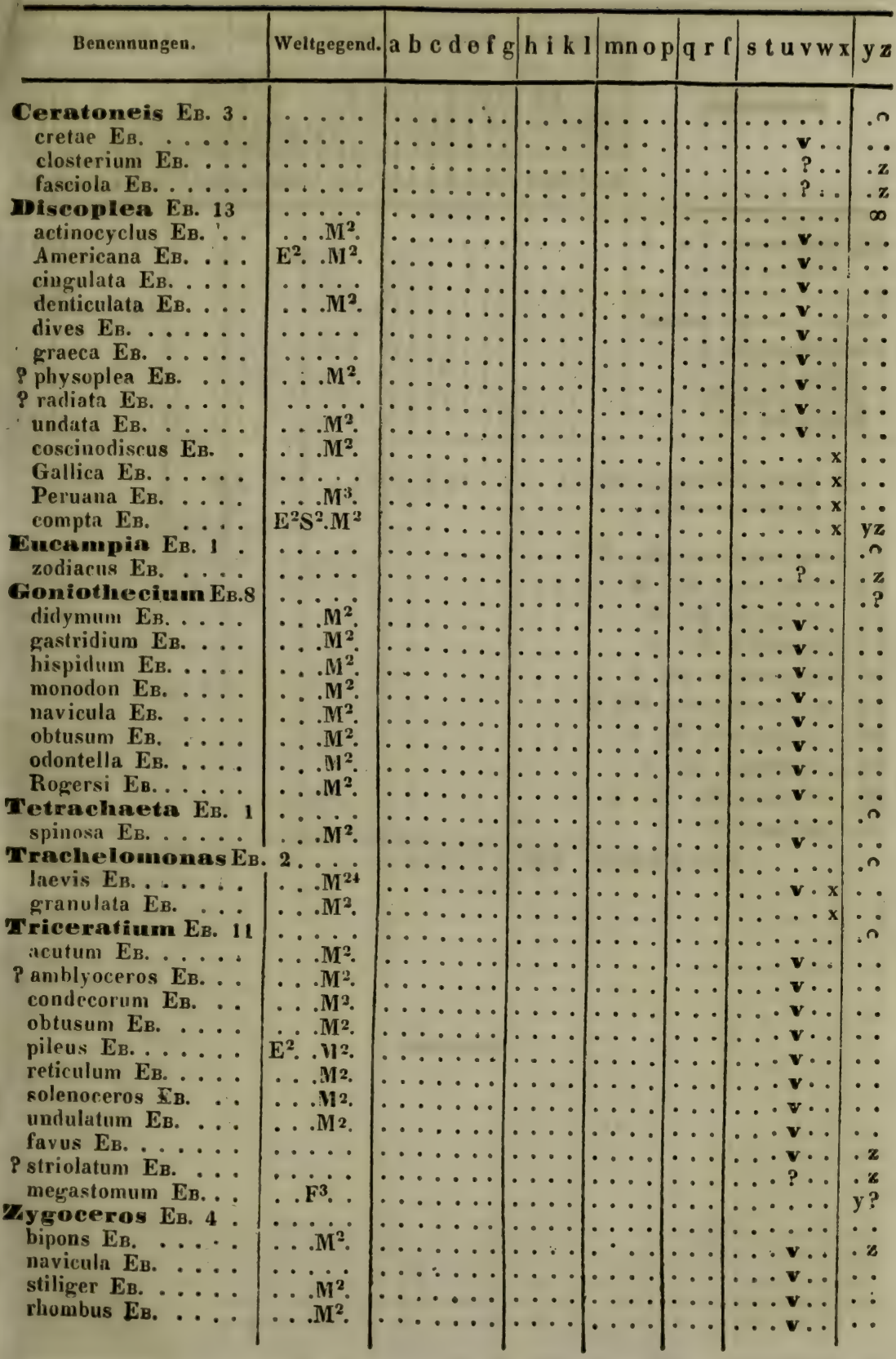




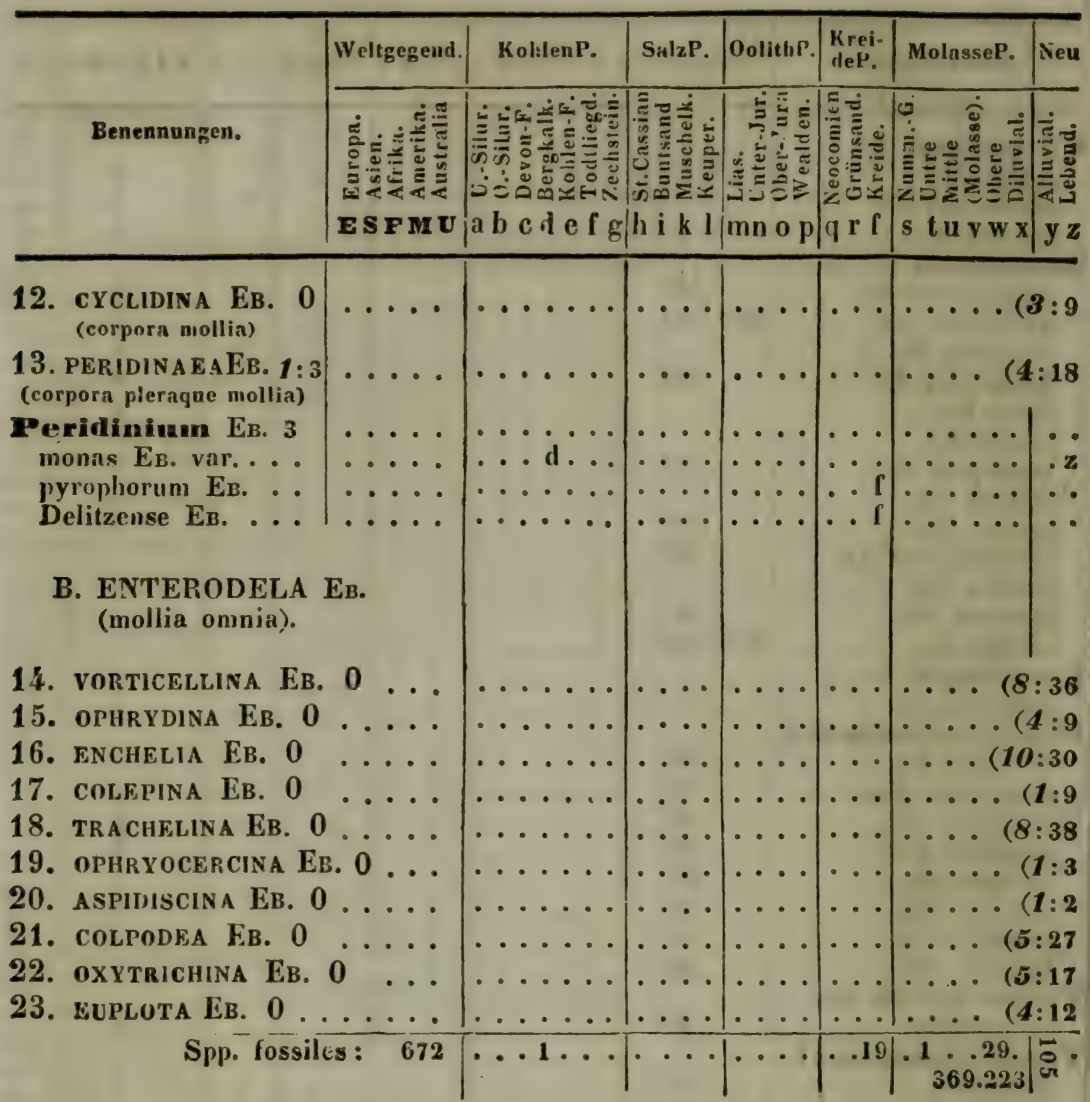




\begin{tabular}{|c|c|c|c|c|c|c|c|}
\hline & Weltgegend. & KohlenP. & SalzP. & OolitliP. & $\begin{array}{l}\text { Krei- } \\
\text { deP. }\end{array}$ & MolasseP. & Neu \\
\hline Benennungen. & 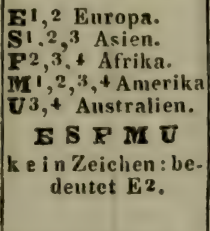 & 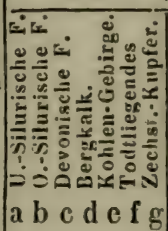 & 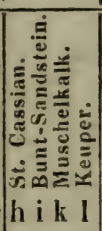 & 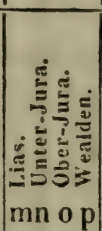 & 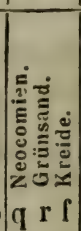 & 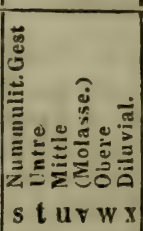 & 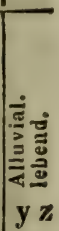 \\
\hline
\end{tabular}

\section{Cl. IV. POLYPI L., Polypen.}

\section{A. Polythalamia !}

(Asiphonoitlea DeH.; Foraminifera d ORB.; Rhizopoda Dus.; Trematophora; Bryozoorum pars EB.)

1. monosomatia Ев.

a. Monostegia D’O. (Miliolina EB.)

? Gromin Es. [lesta carens] 0 universa $\mathbf{D}^{\prime} \mathbf{O} \ldots \mathrm{E}^{2} \cdot \mathrm{F}^{2} \ldots$

\section{b. Stichostegia D'O. (Nodosarina Ëв.)}

CHamdulina D'O $^{\prime} \mathbf{2}$ cylindracea Risuss. laevigata n'0.... Mucronina $\mathrm{D}$ ' 0 . 0 Nodosaria p. 0 . 44 - spp. dulice. † urceolata EB..... t tenuis Mü. .... t Thoa Mü. ......

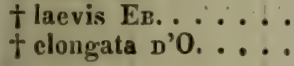
Drbulina $\mathrm{D}^{\prime} \mathrm{O} .1$. $\dot{\mathrm{E}}^{2} \mathrm{~F}^{2} \cdot$

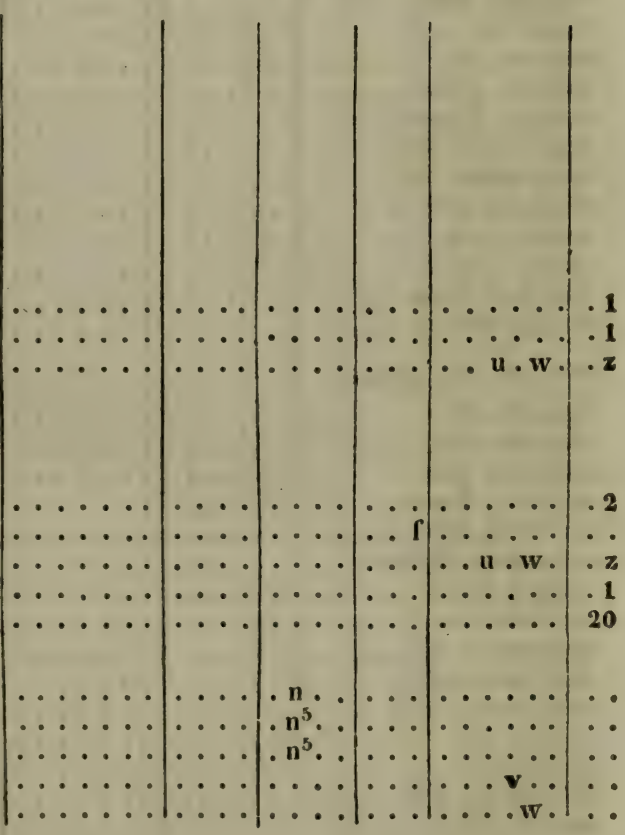

1 Wir haben zwar hier die EnRenrerg'sche Fintheilung und Reihenordnung angenommen, wie er sie in Polge seiner Untersuchungen über einige Thiere in seiner sichrift über die Zusammensetzung der Kreidefelsen mittheilt, haben aber so viel mïglich die D'OrBIGNY'schen Familien und Benenmungen beibehalten und daher EHrensekg's Familien der Asterodiscinen und Frumentarinen, die er dem D'OkBignx'schen Systeme (die zweite selbst nur mit Zweifel) eingeschaltet hatte, wieder ausigeschlossen und zur folgenden Klasse verwiesen. Ausserden sind nur wenige Genera aus einer Familie in die andere versetzt. Die Zahlen lebender Arten sind wur nach D'Orbigny' Tableau (1826) und EhrexberG's "Kreidefelsen" (1839), mithin meistens zu klein angegeben. 


\begin{tabular}{|c|c|c|c|c|c|c|c|}
\hline \multirow[b]{2}{*}{ Benennungen. } & Weltgegend. & KohlenP. & SalzP. & OolithP. & $\begin{array}{c}\text { Krel- } \\
\text { deP. }\end{array}$ & MolasseP. & Neu \\
\hline & 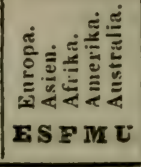 & 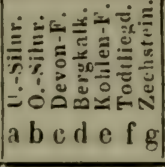 & 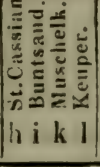 & 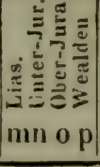 & 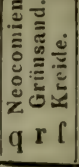 & 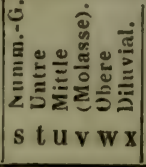 & 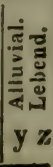 \\
\hline \multicolumn{8}{|l|}{ Nodusaria) } \\
\hline \multicolumn{8}{|c|}{ ** spp. Inngitudinabiler sulculne. } \\
\hline $\begin{array}{l}\text { paucicosta Rok. . } \\
\text { inflata Fuss . . . } \\
\text { tenuicostata Reuss . } \\
\text { paupercula Reuss. } \\
\text { obscura Revss . . } \\
\text { Zippei Reuss . . . }\end{array}$ & $\cdots \cdots$ & $\mid \begin{array}{ccc}\cdots & \cdots & \cdots \\
\cdots & \cdots & \cdots \\
\cdots & \cdots & \cdots \\
\cdots & \cdots & \cdots \\
\cdots & \cdots & \cdots \\
\cdots & \cdots & \cdots\end{array}$ & $\begin{array}{l}\cdots \\
\cdots \\
\cdots \\
\cdots \\
\cdots \\
\cdots\end{array}$ & 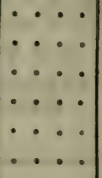 & $\left|\begin{array}{lll}q & \cdots \\
\cdots & f \\
\cdots & f \\
\cdots & f \\
\cdots & f\end{array}\right|$ & 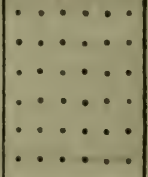 & $\begin{array}{l}\because \\
\therefore \\
\therefore \\
\therefore\end{array}$ \\
\hline $\begin{array}{l}3 \text { frigilis DFr. } \cdots \\
\text { pulchella D'O. }\end{array}$ & & $\ldots \ldots \ldots$ & $\ldots$ & & $\begin{array}{ll}\cdots \\
\cdots & i\end{array}$ & $\ldots \ldots$ & r \\
\hline oblonga D'O. .... & $\cdots \cdots$ & $\ldots \ldots$ & $\cdots \cdots$ & $\cdots$ & $\cdots$ & ...... & $\cdot \mathbf{z}$ \\
\hline $\begin{array}{l}\text { Lamareki n'O. . . } \\
\text { sulcata D'O. . . . }\end{array}$ & $\cdots \cdots$ & $\begin{array}{ll}\cdots \\
\cdots \\
\cdots & \cdots\end{array}$ & $\cdots \cdots$ & $\cdots$ & $\because \cdots$ & $\begin{array}{ll}\cdots \\
\cdots \\
u_{\ldots}\end{array} \ldots$ & $\ddot{z}$ \\
\hline costata g'0..... & $\cdots \cdots$ & $\ldots \ldots$ & $\cdots \cdots$ & $\cdots \cdot \mid$ & $\cdots$ & $\ldots \mathbf{u} \ldots$ & - $\mathbf{z}$ \\
\hline tenclla Erснw. . . & & $\cdots \cdots$ & $\cdots \cdots$ & $\cdots$ & $\cdots$ & . $\mathbf{u} \ldots$ & • \\
\hline Soldanii n'o $\ldots$ & $\cdots \cdots$ & $\ldots \ldots$ & $\cdots$ & $\ldots$ & $\cdots$ & $\therefore \cdots w$ & $\because$ \\
\hline nodosa n’o...... & $\cdots \cdots$ & $\ldots \ldots$ & $\cdots$ & $\cdots$ & $\cdots$ & $\ldots w \cdot$ & - \\
\hline mitida D’O. $^{\prime} \ldots \ldots$ & $\cdots \cdots$ & $\ldots \ldots$ & $\cdots$ & $\cdots$ & $\cdots$ & $\ldots w \cdot$ & • \\
\hline bacillum DFr. . . & $\cdots \cdots$ & $\ldots \ldots$ & $\cdots$ & $\cdots$ & $\cdots$ & $\ldots \ldots w$ & \\
\hline fascia $D^{\prime} 0 . \ldots \ldots$ & $\cdots \cdots$ & $\cdots \cdots$ & $\cdots$ & $\cdots \cdots$ & $\cdots$ & …w. & - $\mathbf{z}$ \\
\hline \multicolumn{8}{|l|}{ *** spp. non sulcritize. } \\
\hline $\begin{array}{l}\text { humilis Ros. } \\
\text { linbata D'O. }\end{array}$ & $\cdots$ & & & $\cdots$ & q & $\ldots .$. & $\cdots$ \\
\hline $\begin{array}{l}\text { linbata D'O. } \\
\text { aculeata EB. }\end{array}$ & - & $\cdots$ & . & $\ldots$ & $\because 1$ & $\cdots \cdots$ & $\cdot$ \\
\hline$\ddot{\cdots}$ & $\therefore$ & $\cdots \cdots \cdots$ & $\cdots \cdots$ & $\cdots \cdots$ & $\because 1$ & $\cdots \cdots \cdots$ & - \\
\hline uss... & $\ldots$ & $\ldots \ldots$ & $\ldots$ & $\ldots$ & $\ldots \mathrm{I}$ & $\ldots \ldots$ & \\
\hline $\begin{array}{l}\text { ulata Reuss } \ldots \\
\text { stricta Reuss } \ldots\end{array}$ & $\cdots \cdot$ & $\ldots \ldots$ & $\cdots$ & $\ldots$ & . . I & $\ldots \ldots$ & - \\
\hline Rauss .... & $\cdots \cdot$ & $\ldots \ldots$ & $\ldots$ & $\ldots$ & $\ldots 1$ & $\ldots \ldots$ & $\cdots$ \\
\hline eula Lк. . . . & $\cdots \cdot$ & $\cdots \cdots$ & $\cdots$ & $\cdots$ & $\cdots$ & $\ldots \mathbf{u} \ldots$ & - $x$ \\
\hline o. . . & $\cdots \cdots$ & $\cdots \cdots$ & $\cdots \cdots$ & •. & $\cdots$ & .. u... & ? \\
\hline A & $\cdots \cdots$ & $\ldots \ldots$ & $\cdots \cdots$ & $\cdots$ & $\cdots$ & ..... & - \\
\hline $\begin{array}{lll}a & D^{\prime} 0 .\end{array}$ & - & $\cdots, \cdots$ & $\cdots \cdots$ & $\cdots \cdot$ & $\cdots$ & ..u. u. w. & \\
\hline$\ldots$ & .. & $\cdots \cdots \cdots$ & $\cdots \cdot$ & $\cdots \cdot$ & $\cdots$ & $\cdots \mathbf{v}, \cdot$ & \\
\hline$\therefore$ & $\cdots$ & $\cdots \cdots \cdots$ & $\cdots \cdots$ & $\cdots$ & $\cdots$ & $\cdots w w^{\cdots}$ & \\
\hline IL. . . . & $\ldots$ & $\ldots \ldots$ & $\ldots$ & $\cdots$. & $\ldots$ & .... & \\
\hline & $\ldots$. & $\ldots \ldots$ & ... & $\cdots \cdot$ & $\cdots$ & 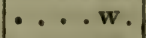 & \\
\hline [?] D'0. . & $\cdots$ & $\ldots \ldots \ldots$ & $\ldots$ & $\cdots \cdot$ & $\cdots$ & ....w & \\
\hline$\cdots$ & $\cdots \cdots$ & $\cdots \cdots$ & $\ldots$ & $\cdots \cdot$ & $\cdots \cdot$ & ....w. & \\
\hline or & $\cdots \cdots$ & $\ldots \ldots$ & $\ldots$ & $\cdots \cdot$ & & .... w. & \\
\hline a d'0.. & $\cdots \cdot$ & $\cdots \cdots \cdots$ & $\cdots$ & $\cdots \cdot$ & $\cdots$ & $\cdots \cdots \cdots$ & .0 \\
\hline 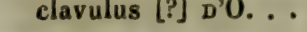 & $\cdots \cdot$ & . & $\cdots$ & $\cdots$ & 1 & $\cdot t \ldots$. & \\
\hline
\end{tabular}




\begin{tabular}{|c|c|c|c|c|c|c|c|}
\hline Benc & gend. & $|a b c d e f g|$ & k l & $\mid \mathrm{mn}$ op & $q \times r$ & st t & $\mathbf{y}$ \\
\hline $\begin{array}{l}\text { Dentalina D'O. } 29 \\
\text { * spp. non striatue. }\end{array}$ & $\cdots$ & $\cdot$ & & $1^{\circ}$ & $\cdots$ & $\cdot \cdots \cdots$ & .8 \\
\hline linearis $R O B$ & $\cdots$ & $\cdots \ldots$ & $\cdots$ & $\cdots$ & $\mid q \cdots$ & $\cdots \cdots$ & - \\
\hline & • & & $\cdot$ & $\cdots \cdot$ & $\ldots \boldsymbol{f}^{\prime}$ & $\ldots \ldots$ & $\cdots$ \\
\hline $\begin{array}{l}\text { B } \\
\mathbf{a}\end{array}$ & $\cdots \cdots$ & $\because$ & $\because$ & $\left|\begin{array}{l}\cdots \\
\cdots\end{array}\right|$ & $\begin{array}{lll}\cdots & 1 \\
\cdots & 1\end{array}$ & $\mid \begin{array}{c}\cdots \\
\cdots \\
\cdots\end{array}$ & \\
\hline . & $\ldots .$. & $\ldots \ldots$ & $\ldots$. & $\ldots$ & $\therefore \mathrm{f}$ & $\ldots \ldots$ & \\
\hline$\cdots$ & $\cdots \cdots$ & $\cdots \cdots$ & $\cdots \cdots$ & $\cdots \cdot$ & $\left|\begin{array}{ll}\cdots & 1 \\
c\end{array}\right|$ & $\cdots \cdots$ & \\
\hline an & . & $\bullet$ & $\left|\begin{array}{ll}\cdots \\
\cdots \\
\cdots\end{array}\right|$ & $\cdots$ & $\left|\begin{array}{cc}\cdots & 1 \\
\cdots & 1\end{array}\right|$ & $\cdots$ & \\
\hline ... & .. & $\ldots \ldots$ & $\cdots$ & $\cdots$ & $\therefore$ i & $\ldots \ldots$ & \\
\hline$\ldots \ldots$ & . & $\ldots \ldots$ & $\ldots$ & $\cdots$ & .. 1 & $\ldots \ldots$ & \\
\hline$\cdots$ & & $\ldots \ldots$ & $\cdots$ & $\cdots \cdot$ & . . I & $\ldots \ldots$ & \\
\hline$\cdots$ & $\cdots \cdots$ & $\cdots \cdots$ & $\cdots$ & $\cdots \cdots$ & $\cdots 1$ & $\cdots \cdots$ & \\
\hline$\cdots$ & $\cdots \cdots$ & $\cdots \cdots$ & $\cdots \cdots$ & $\cdots$ & $\ldots r$ & $\cdots \mathbf{n} \ldots$ & .8 \\
\hline$\cdots$ & & $\cdots \cdots \cdots$ & $\cdots \cdots$ & $\cdots \cdot$ & $\cdots$ & $\ldots \ldots w$ & - \\
\hline \multirow{2}{*}{\multicolumn{8}{|c|}{ ** spp. longriludinuliter striutae. }} \\
\hline & & & & . &.$r f$ & & \\
\hline & 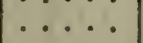 & & & $\ldots$ & $\therefore$. 1 & & \\
\hline & & $\ldots$ &. & $\ldots$ & $\therefore i$ & $\ldots$ & \\
\hline 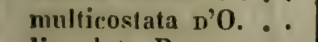 & & $\ldots \ldots$ & $\ldots$ & $\cdots$ & . . & $\ldots$ & - \\
\hline$\cdots$ & & $\ldots \ldots$ & $\ldots$ & $\ldots$ & .. $\mathrm{r}$ & $\ldots$ & . \\
\hline s .. & . & $\ldots \ldots$ & . & $\cdots$ & ... & $\ldots$ & 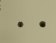 \\
\hline$\cdots$ & - & $\ldots$ & $\cdots$ & $\cdots \cdot 1$ & $\cdots$ & $\ldots \mathbf{u} \ldots$ & \\
\hline 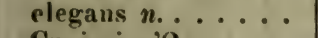 & $\cdots$ & $\ldots \ldots$ & $\cdots$ & . & $\cdot 1$ & ..u. $\mathrm{u} \cdot \mathrm{w} \cdot$ & \\
\hline . & $\cdots \cdot$ & $\ldots \ldots$ & $\cdots \cdot$ & $\cdots$ & - & ..... & . $\mathrm{z}$ \\
\hline . & - & $\cdots \cdots$ & $\cdots \cdot$ & $\cdots \cdots$ & $\cdots$ & $\ldots w$ & . \\
\hline . & $\cdot$ & $\cdots$ & $\cdot \cdot$ & 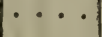 & 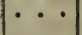 & . . w w & \\
\hline cornicul & - & . & & - & 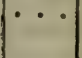 & . . w w & $\cdots$ \\
\hline - & & & & & & $\ldots \mathrm{u} \ldots$ & \\
\hline & & ... & & 1. & & . . u ... & \\
\hline 10 & $\cdots$ & $\ldots \ldots$ & $\ldots$ & & • & $\ldots \ldots$ & .3 \\
\hline & & $\ldots$ & & $\cdot n^{4} \ldots$ & & & - \\
\hline & . & $\ldots \ldots$ & $\ldots$ & ... & $\cdots$ & . & \\
\hline & - & $\cdot$ & $\ldots \cdot$ & $\cdots \cdot$ & $\cdots$ & . u $\mathbf{u}$ & - \\
\hline$s p$. & & $\cdots$ & $\cdots \cdot$ & $\ldots$ & $\ldots$ & 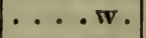 & - \\
\hline & & $\ldots$ & $\cdots$ & $\cdots$ & $\cdots$ & ....v. & $\theta^{\circ}$ \\
\hline . & . & $\ldots$ & $\cdot$ & $\cdots$ & & $\ldots \mathrm{w} \cdot \mid$ & • \\
\hline & $\cdots$ & & . & & & ....w. & \\
\hline • & & & $\cdot$ & $\cdot$ & . & $\cdots \cdots \mathbf{w}$ & \\
\hline$\cdots$ & $\dot{\mathrm{r}}^{\circ}$ & $\cdots$ & - & $\cdot$ & $\cdots$ & $\cdots$ & \\
\hline & & & & & & ...w. & \\
\hline ria $D_{F I}$ & 41 & $\cdots$ & $\cdot$ & & & $\cdots$ & .2 \\
\hline$\cdot$ & & & & & & $\cdots$ & \\
\hline 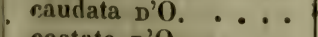 & & & & -0 & & $\cdots$ & \\
\hline & $\cdots$ & 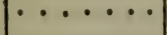 & & $\cdots \cdot$ & & $\cdots \cdot$ & 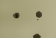 \\
\hline ompressa D'O. & $\cdots \cdots$ & $\cdots$ & $\ldots$ & $\ldots$ & & $\ldots$ & $=$ \\
\hline
\end{tabular}




\begin{tabular}{|c|c|c|c|c|c|c|c|}
\hline Ben & tgegend. & a b c d e $f g$ & k 1 & p & ? & W X & . \\
\hline $\mathbf{E}$ & & & & & $\ldots \mathrm{f}$ & & \\
\hline N. . . & $\ldots$ & $\ldots \ldots$ & $\ldots$. & $\cdots$ & $\cdots$ & $\ldots w^{\prime}$ & \\
\hline a Rов. ... & $\cdots \cdots$ & $\cdots \cdots$ & $\cdots$ & $\cdots \cdots$ & $\cdots$ & $\cdots \cdots w$ & \\
\hline o. & $\cdots \cdots$ & $\cdots \cdots$ & $\cdots$ & $\cdots$ & - & $\cdots w^{\prime}$ & \\
\hline$\ddot{D_{F R}} \dot{ }$ i7 & $\ldots$ & $\cdots$ & $\cdots$ & & & $\cdots \cdots w$ & \\
\hline $\begin{array}{l}\text { Planeulari } \\
\text { f arcuata Mü. }\end{array}$ & $\cdots \cdot \cdot$ & $\cdots \cdots \cdots$ & $\cdots \cdots$ & & $\cdots$ & $\cdots \cdots$ & \\
\hline$\dot{.}$ & $\cdots \cdot$ & $\cdots \cdots$ & $\cdots \cdot$ & $\cdot n^{5} \cdots$ & $\cdots$ & $\ldots \ldots$ & \\
\hline$\cdots$ & $\cdots \cdots$ & $\cdots \cdots$ & $\cdots$ & $\cdot u^{3} \ldots$ & $\cdots$ & $\ldots \ldots$ & \\
\hline$\cdots$ & $\cdots$ & $\cdots \cdots$ & $\cdots$ & ..? & $\cdots$ & $\cdots \cdots$ & \\
\hline$\cdots$ & $\cdots$ & $\cdots$ & $\cdots \cdots$ & $\cdot ? \cdot$ & $\cdots$ & $\cdots \cdots$ & \\
\hline . & & $\cdots$ & & & : & $\cdots$ & \\
\hline$\cdots$ & & $\ldots$ & & $\cdot$ & 9 & $\cdots$ & \\
\hline$\cdots$ & & & & - & - & $\cdots$ & \\
\hline$\cdots$ & & & & & & $\because$ & \\
\hline$\cdots \cdot$ & & & & $\cdot$ & 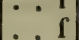 & $\because$ & \\
\hline$\cdots$ & & $\because$ & . & - & $\because \mathrm{f}$ & $\cdots$ & \\
\hline$\therefore$ & 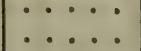 & .. & & $\cdots$ & $\therefore$ & $\ldots w$ & \\
\hline$\cdots$ & $\ldots$. & $\ldots \ldots$ & & $\ldots$ & $\therefore$ & $\ldots w$ & \\
\hline HIL. ... & $\cdots \cdot$ & $\ldots \ldots$ & $\ldots$ & $\ldots$ & $\ldots$ & ....w. & \\
\hline ris Phil. & $\cdots \cdots$ & $\ldots \ldots$ & $\ldots$ & $\ldots$ & $\ldots$ & $\ldots w$ & \\
\hline . & $\cdots \cdots$ & $\ldots \ldots$ & $\ldots$ & $\ldots$ & ... & $\ldots w_{0}$ & \\
\hline$\cdots$ & & $\cdots$ & $\ldots$ & $\cdots$ & $\cdots$ & $\ldots \ldots w$ & $\cdots$ \\
\hline a D'0.22 & . & $\cdots$ & $\cdots \cdots$ & & $\cdots$ & $\ldots \ldots$ & 10 \\
\hline$\cdots \cdot$ & & $\cdots$ & &.$n^{5} \ldots$ & $\cdots$ & $\ldots \ldots$ & \\
\hline •... & $\cdots \cdots$ & $\cdots$ & $\cdots \cdots$ & $\ldots$ & & $\cdots$ & \\
\hline$\cdots \cdot$ & $\cdots$ & $\cdots$ & $\cdots$ & $\ldots$. & - r I & $\ldots \ldots$ & \\
\hline$\cdots$ & $\cdot \cdot$ & $\cdots$ & $\cdots \cdot$ & $\cdots \cdots$ & - r I & $\cdots \cdots$ & \\
\hline$\cdots$ & •. & $\cdots$ & $\cdots \cdot$ & $\cdots \cdots$ & . . I & $\cdots$ & \\
\hline$\cdots$ & $\cdots$ & $\cdot$ & . & - & . . I & $\cdots$ & \\
\hline s... & & & • & $\bullet$ & & $\cdots$ & \\
\hline$\cdots \cdot$ & & & $\cdots$ & & c & $\cdots$ & \\
\hline$\cdots$ & & & $\cdots$ & $\cdots$ & $\therefore \rho$ & $\because$ & \\
\hline $\begin{array}{l}\cdots \\
\cdots\end{array}$ & •• & & $\cdots$ & $\cdots$ & $\therefore$ i & $\ldots$ & \\
\hline … & .. & & ... & .... & . I & $\ldots \ldots$ & \\
\hline ... & .... & .. & $\cdots$ & $\ldots$ & $\ldots$ & $\ldots u \ldots$ & \\
\hline ... & .... & . . & . . & ... & $\cdots$ & . u. . & \\
\hline$\cdots$ & $\ldots$ & $\cdots$ & ... & ... & ... & ....w. & \\
\hline$\cdots$ & $\cdots \cdot$ & . & $\cdots$ & $\ldots$ & & $\ldots w$ & \\
\hline$\cdots$ & $\cdots$ & . & $\cdots$ & $\cdots$ & & . w . & \\
\hline ? a & - & . & $\cdots \cdots$ & $\ldots$ & . & $\ldots w^{\prime}$ & \\
\hline а Рнік. & .... & $\cdots$ & $\cdots$ & $\cdots$ & . & $\ldots w_{0}$ & \\
\hline$\cdots$ & $\cdots \cdots$ & $\cdots$ & $\cdots \cdots$ & $\ldots$ & . & ....w & \\
\hline$\cdots$ & $\cdots \cdots$ & $\cdots$ & $\cdots \cdot$ & $\cdots \cdots$ & & $\cdots \cdots w$ & \\
\hline & - & & & & & 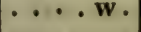 & .2 \\
\hline o. 4. & ・ & - & & & & $\cdots \cdots$ & \\
\hline & - & & & & & & \\
\hline & 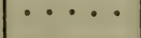 & & & & & & \\
\hline 8 & 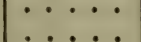 & & & $\cdots$ & $\because 1$ & & \\
\hline Ce & . & & $\cdots \cdots$ & & $\cdot$ & $\because$ & \\
\hline bina & & & & & & & \\
\hline
\end{tabular}




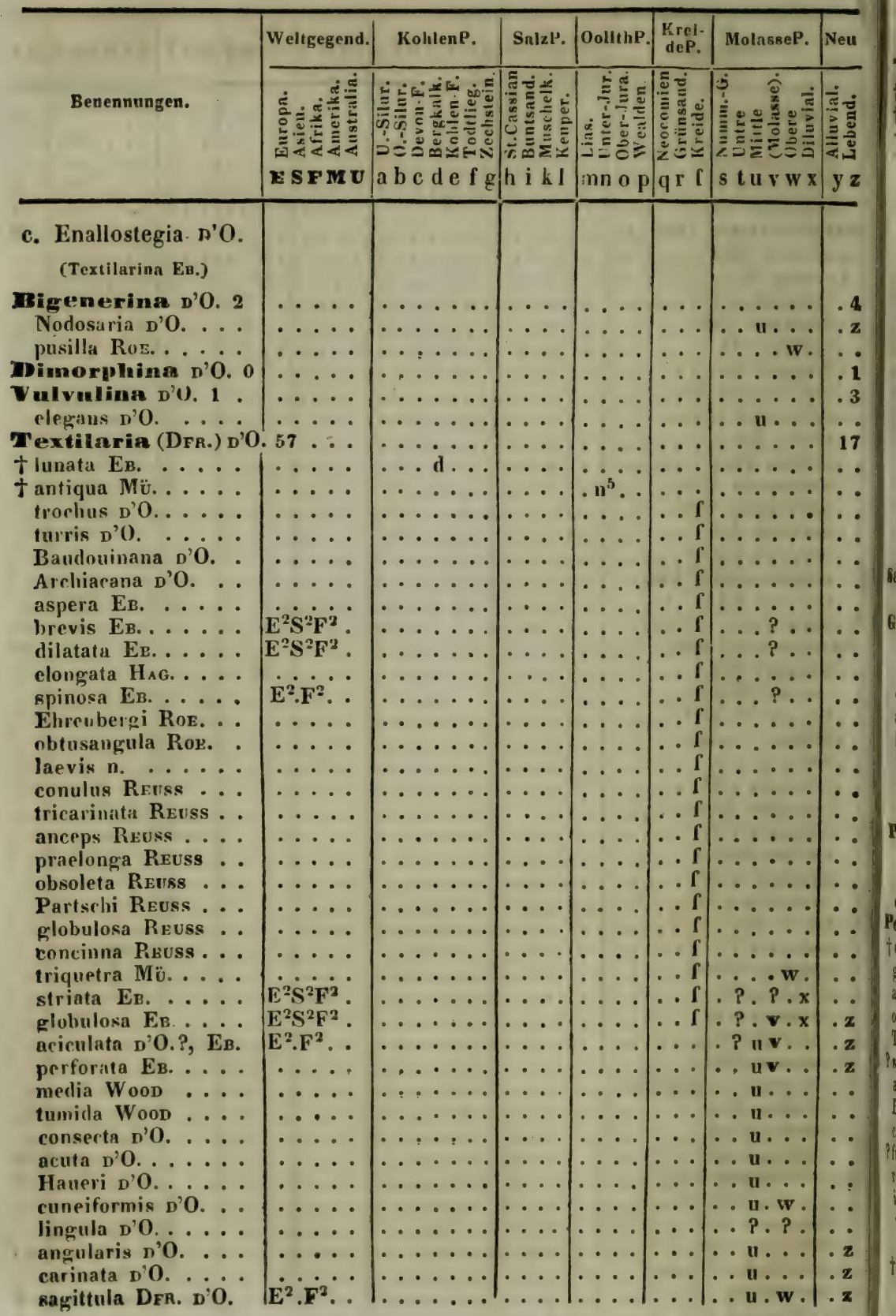




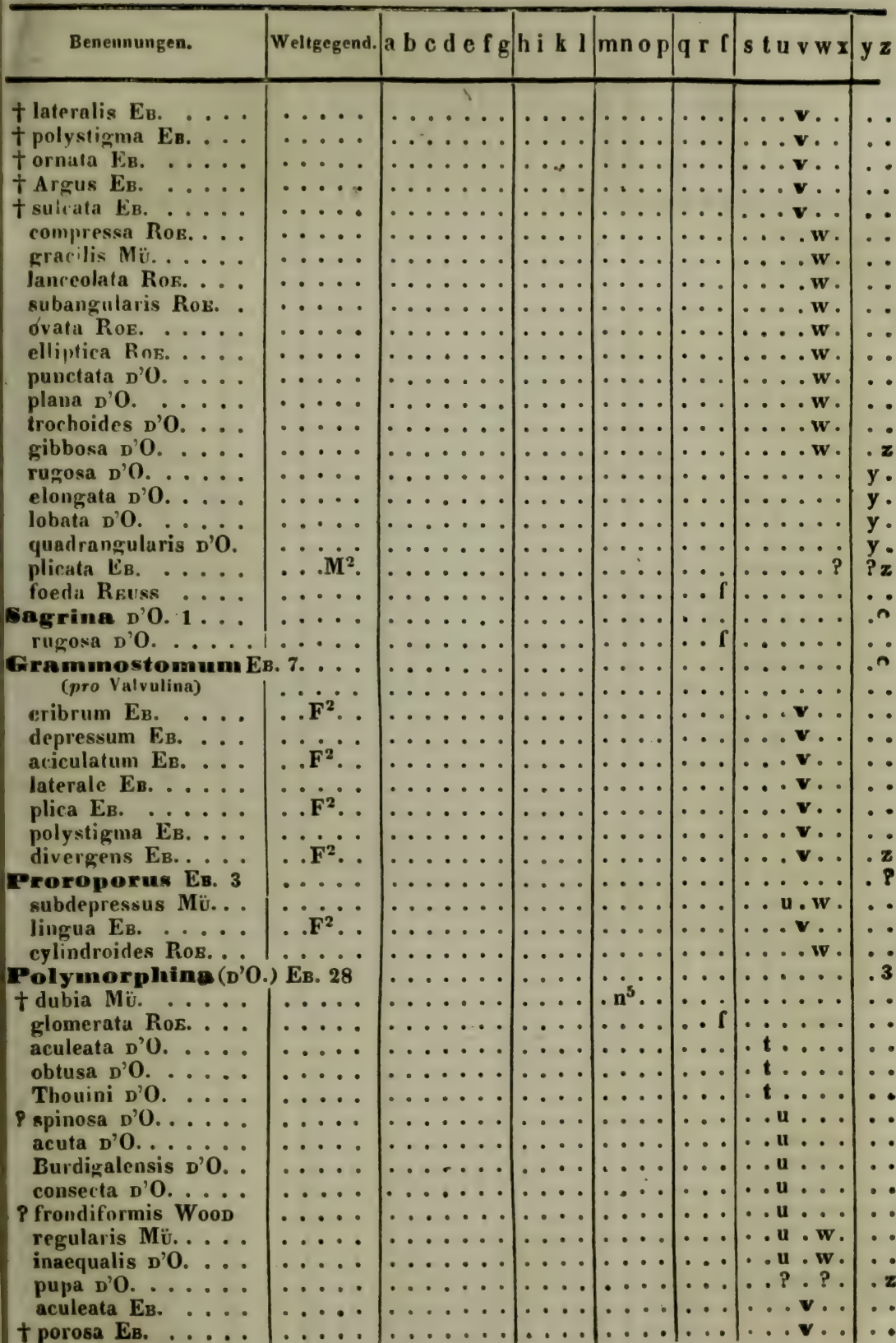




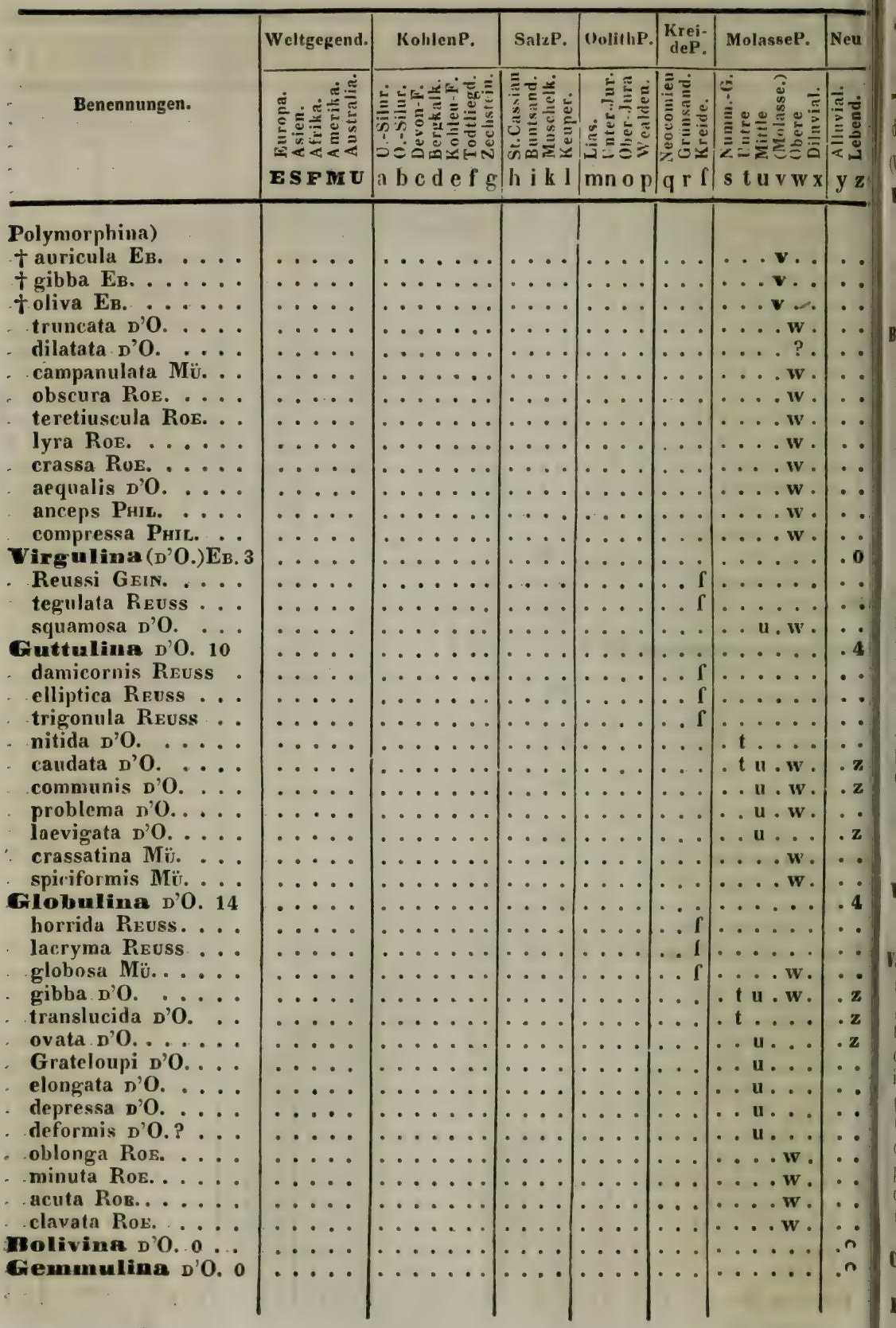




\begin{tabular}{l|l|l|l|l|l|l|}
\hline Benennungen. & Weltgegend. a b c d e f $\mid$ h i k l mn op $\mid$ q r & s t u vw x $\mid$ y z \\
\hline
\end{tabular}

d. Helicostegia D'O. (Uvellina et Rotalina EB.)

Uvigerina D'O. 5 . triangularis $\mathrm{D}^{\prime} \mathrm{O}$. tricarinata n'O. trilobata n'O. pygnaea d'O.

rugosa D'O.

Bulimina D'O. 23 . protea D'O. irregularis $D^{\prime} 0$. obtusa D'O. obliqua D'O. variabilis $D^{\prime} O$. brevis $D^{\prime} O$.

Murchisonana $\mathrm{D} O \dot{O}$. truncata Reuss ovulum Reuss polystropha Reuss intermedia Reuss. subsphaerica Reuss Presil lieuss d'Orbignyi Reuss amphiconica HsG. arcuata b'O.

Ariminensis $\mathrm{D}^{\prime} \mathbf{O}$. punctata $D^{\circ} 0$. costata D'O. echinata D'O. semistriata D'O. cylindriea Ros. uva RoE.

vernemilina n'O. 2 tricarinata D'O. Bronni Reuss

valvulina s'o. 12 spicula Reuss gibbosa D'O. tribullata Hag. quadribullata HiG. ignota DFr. globularis D'O. Gervillei D'O. deformis $D^{\prime} O$. pupa D'O. granularis Mü. parvula Mü.

Colpoplemra Eв. 1 ocellata Eв. .

Porospira EB. 2
princeps $E_{B} . .$.

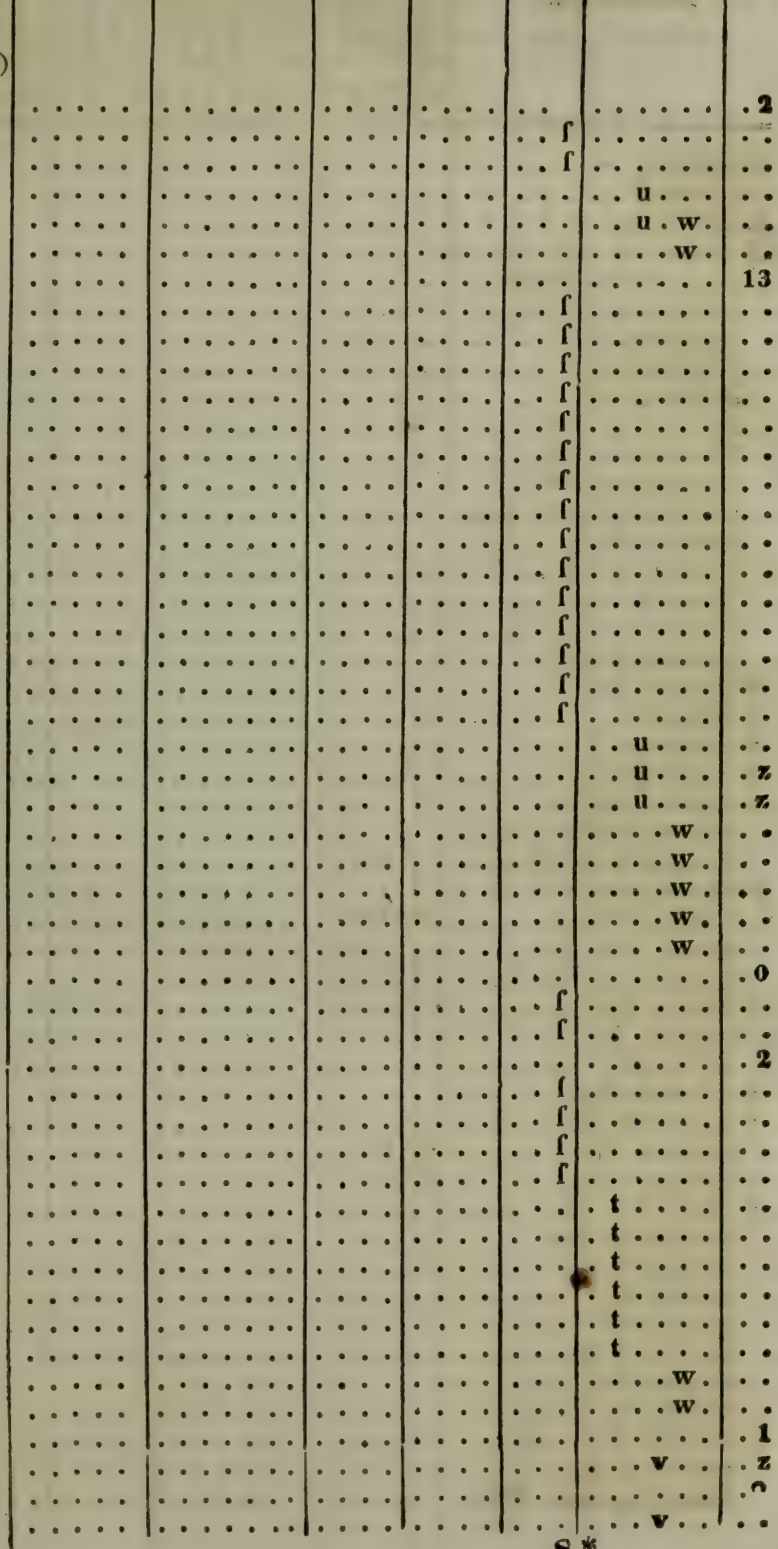




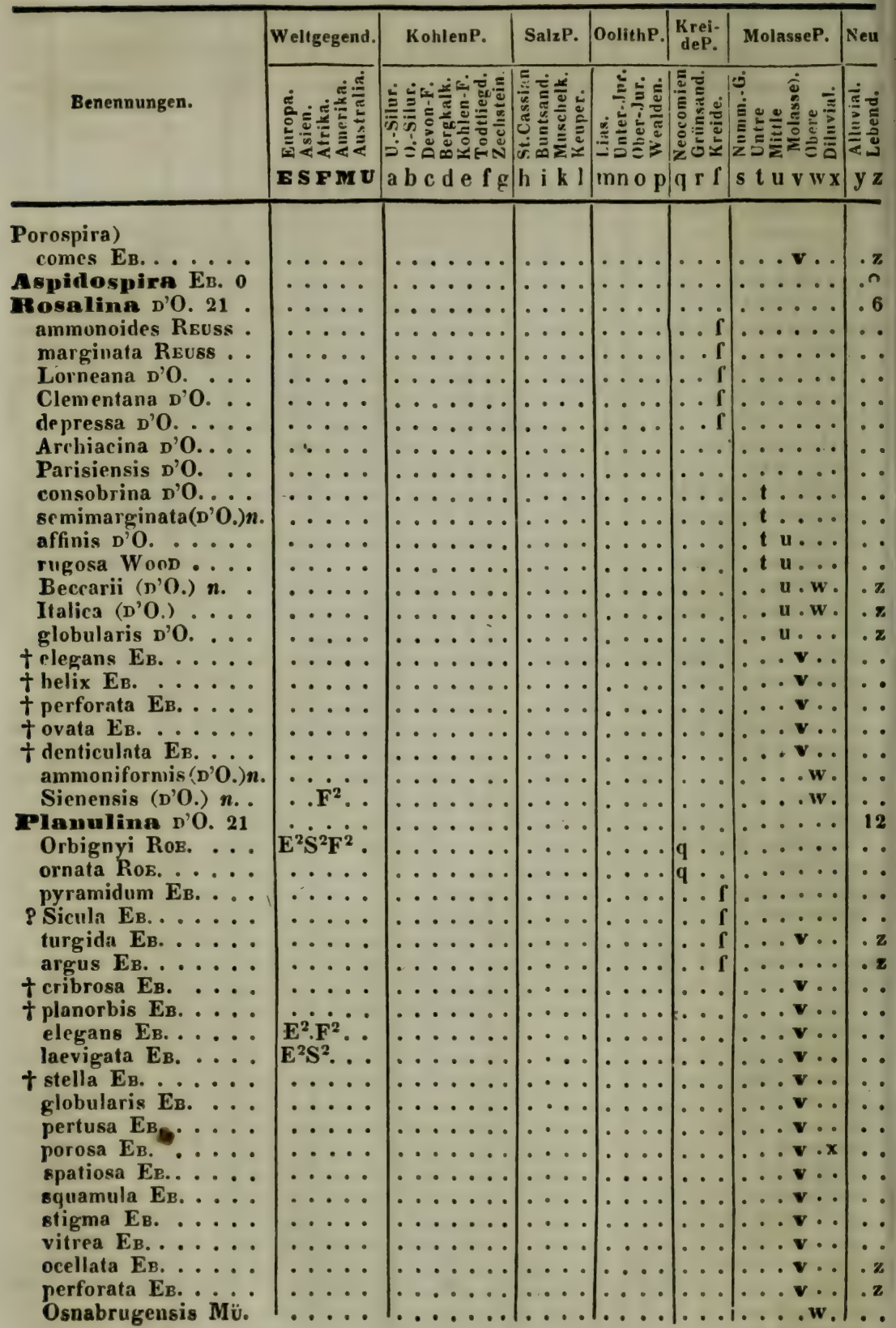




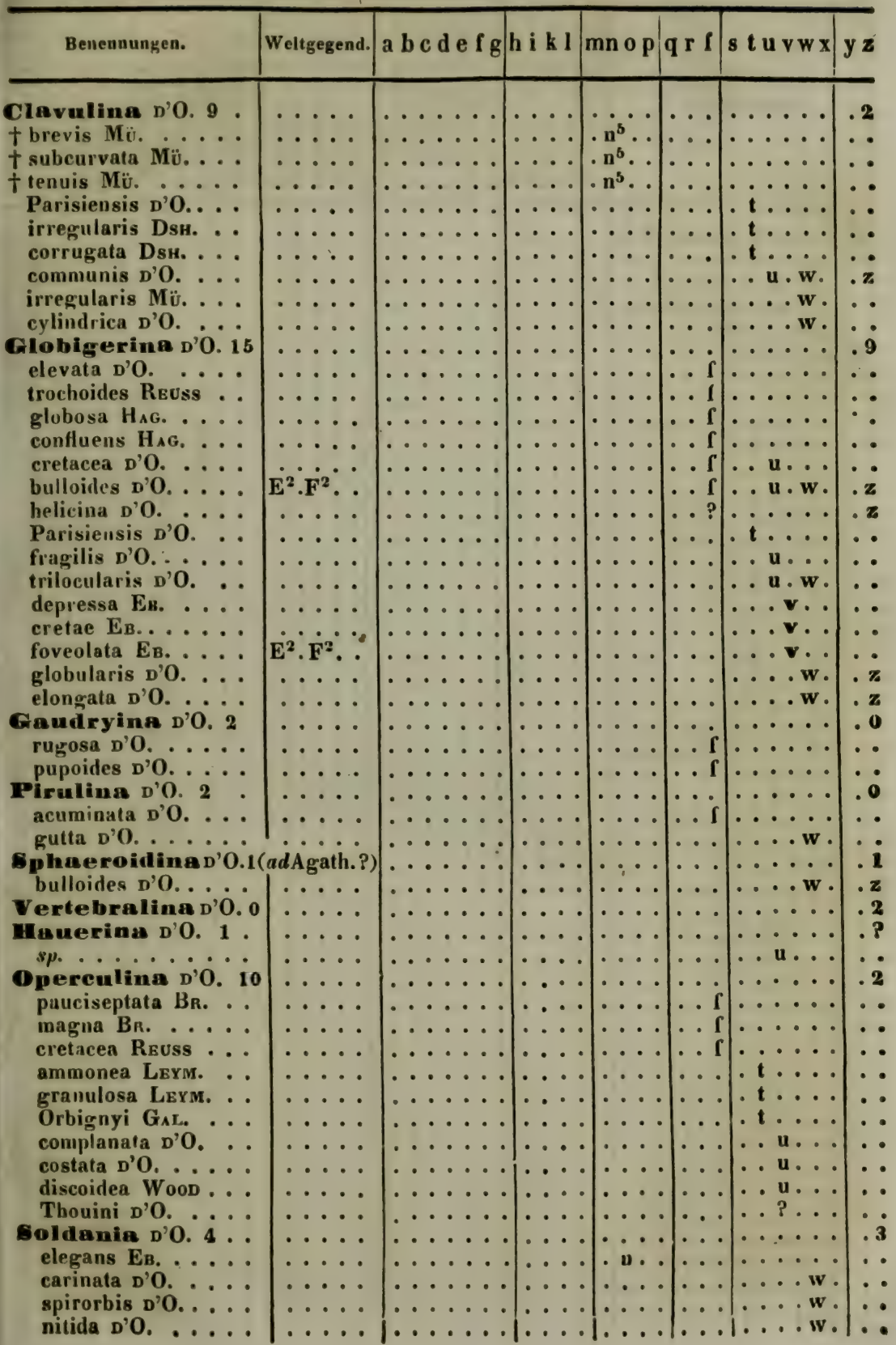




\begin{tabular}{|c|c|c|c|c|c|c|c|}
\hline & gend. & KohlenP. & SalzP. & OolithP. & $\begin{array}{l}\text { Krei- } \\
\text { deP. }\end{array}$ & MolasseP. & Neu \\
\hline Benen & 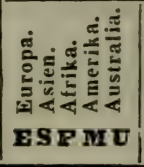 & $\mid$ & 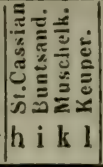 & 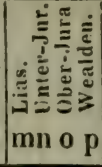 & 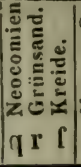 & 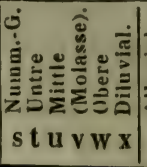 & 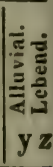 \\
\hline . & $\cdots$ & $\cdots$ & & 1. & & $I^{\circ}$ & .4 \\
\hline & $\cdots$ & $\ldots \ldots$ & $\ldots$ & $\ldots$ & . 1 & $\ldots \ldots$ & \\
\hline & $\cdots \cdot$ & $\cdots$ & $\cdots \cdot$ & $\cdots \cdot$ & . $\mathbf{f}$ & & \\
\hline di & $\cdots$ & $\therefore$ & $\begin{array}{ll}\cdots \\
\cdots \\
\cdots\end{array}$ & $\begin{array}{ll}\cdots \\
\cdots\end{array}$ & .1 & $\mid \begin{array}{cc}\cdots & 0 \\
\cdots & \cdots\end{array}$ & . $z$ \\
\hline lia LK. 70 . & $\ldots \ldots$ & $\ldots \ldots$ & $\ldots$ & $\ldots$ & $\therefore$ & $\cdots \cdots$ & 17 \\
\hline a Es...... & $\ldots$ & $\ldots d \ldots$ & $\cdots$ & $\cdots \cdot$ & $\cdot 1$ & $\ldots \ldots$ & . \\
\hline$\cdots$ & $\cdots \cdot$ & $\cdots \cdots \cdots$ & $\cdots \cdots$ & $\cdot \mathbf{n} \cdot$ & - & $\cdots \cdots$ & \\
\hline - & : & $\because$ & $\cdots$ & $\cdots \cdots$ & $q \cdots$ & $\cdots \cdots$ & $\cdots$ \\
\hline 1 & $\cdots \cdots$ & $\because$ & $\cdots$ & $\mid \cdots \cdots$ & $q \cdot$ & $\cdots$ & $\because$ \\
\hline & ..... & $\therefore$ & $\ldots$ & $\cdots \cdots$ & $\begin{array}{l}9 \\
\cdots\end{array}$ & $\cdots \cdots$ & $\because$ \\
\hline .) & $\ldots$ & $\ldots \ldots$ & $\ldots$ & $\ldots$ & $\therefore \mathrm{f}$ & $\ldots \ldots$ & \\
\hline .). & $\ldots$. & $\ldots \ldots$ & $\ldots$ & $\ldots$ & .. $\mathrm{f}$ & $\ldots \ldots$ & .. \\
\hline$\cdots$ & $\ldots$ & $\ldots \ldots$ & $\cdots \cdot$ & $\ldots$. & $\ldots f$ & $\cdots$ & - \\
\hline . & $\cdots, \cdots$ & $\cdots \cdots$ & $\cdots \cdot$ & $\cdots \cdot$ & $\ldots \mathrm{f}$ & $\cdots$ & \\
\hline EB. $\cdots$ & $\cdots \cdots$ & $\cdots \cdots \cdots$ & $\cdots$ & $\cdots \cdots$ & $\cdots \mathrm{f}$ & $\cdots$ & \\
\hline${ }^{8} \cdots \cdots$ & $\cdots \cdots$ & $\dot{\bullet}$ & 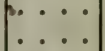 & $\cdots$ & $\cdots f$ & $\because$ & \\
\hline$\because$ & $\ldots \ldots$ & $\therefore$ & $\ldots$ & $\cdots \cdots$ & $\begin{array}{ll}\cdots & 1 \\
\cdots & f\end{array}$ & $\because$ & \\
\hline$\cdots$ & .... & $\ldots \ldots$ & $\ldots$ & $\ldots$ & $\therefore \mathrm{f}$ & $\ldots \ldots$ & \\
\hline$p$ & $\cdots \cdot$ & $\ldots \ldots$ & $\ldots$ & $\ldots$ & . I & $\ldots$ & $\bullet$ \\
\hline o. & $\ldots$ & & $\ldots$ & $\ldots$ & . $\mathrm{f}$ & $\cdots$ & \\
\hline - & $\cdots \cdots$ & - & $\cdots \cdot$ & $\cdots$ & . $\mathrm{f}$ & $\cdots$ & \\
\hline$\cdots$ & $\cdot \cdot$ & $\cdots$ & $\cdots \cdot$ & $\cdots \cdot$ & $\ldots f$ & $\cdots$ & \\
\hline$\cdots$ & $\cdots$ & - & $\cdots \cdot$ & $\cdots \cdots$ & .. f & $\ldots \mathbf{u} \ldots$ & $\mathbf{z}$ \\
\hline$\cdots$ & $\mathbf{E}^{2} \mathbf{S}^{2} \mathbf{F}^{2}$ & & . 1 & $\cdots \cdot$ & .. $\mathrm{f}$ & $\cdots v \cdot$ & $\mathbf{z}$ \\
\hline 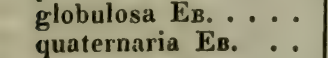 & $\ldots$ & & $\cdots$ & $\cdots$ & $\left|\begin{array}{ll}\cdots \\
\cdots\end{array}\right|$ & $\cdots \cdot \mathbf{v} \cdot \mathbf{x}$ & 8 \\
\hline$\therefore$ & $\ldots \ldots$ & $\cdots \cdots$ & $\cdots \cdots$ & $\cdots$ & $\mid \begin{array}{l}\cdots \\
\cdots\end{array}$ & 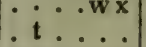 & .2 \\
\hline ‥ & . . • & 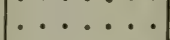 & $\cdots$ & ... & $\ldots$ & t.... & \\
\hline$\ldots$ & $\cdots \cdot$ & $\ldots \ldots$ & $\ldots$ & $\cdots \cdot$ & $\ldots$ &.$t \ldots$ & \\
\hline & $\cdots \cdot$ & $\cdots$ & - & . & 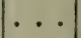 & . t.... & \\
\hline & - & $\cdots$ & - & . & . &.$t \ldots$ & \\
\hline$\cdots$ & - & $\cdots$ & .1 & $\cdots$ & & $\cdot t^{t} \ldots$ & \\
\hline . & : & . & $\because$ & • & & $\cdot t_{t}^{t} \cdots$ & \\
\hline 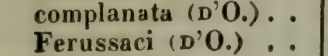 & $\cdots$ & $\ldots$ & & & & $\cdots \cdots$ & \\
\hline $\begin{array}{l}\left(D^{\prime} 0 .\right) \\
0 .)\end{array}$ & $\ldots$ & $\therefore \ldots$ & $\cdots \cdots$ & $\cdots \cdots$ & $\ldots$ & $\because \ldots$ & $\cdots$ \\
\hline$\cdots$ & $\cdots \cdots$ & $\ldots \ldots$ & $\ldots$ & $\ldots$. & . & t $\ldots$ &.$z$ \\
\hline & . & $\ldots \ldots$ & & & & .? ? ... & - \\
\hline D'O & $\cdots \cdot$ & 皮 & & & & . ? ?... & \\
\hline & $\cdots \cdots$ & $\cdots \cdots \cdots$ & $\cdots \cdots$ & $\cdots$ & & $\cdots \mathbf{u} \ldots$ & - \\
\hline & - & $\cdots$ & $\cdots$ & $\cdots$ & & ...... & \\
\hline 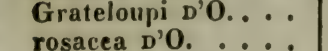 & & & & & & $\because u_{0}$ & \\
\hline 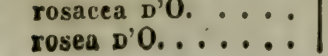 & . & to 20 & & & & $u_{0} u_{0}$ & . \\
\hline
\end{tabular}




\begin{tabular}{|c|c|c|c|c|c|c|c|}
\hline . & end & $f g$ & h i k l & $\mathrm{mn}$ & $\mathbf{r} \mathbf{l}$ & $\mathbf{W} \mathbf{x}$ & . \\
\hline 0.) & • & & & & & $\cdots$ & \\
\hline 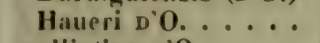 & .... & $\ldots \ldots$ & $\ldots$ & $\ldots$ & $\ldots$ & . u... & - \\
\hline ica n'O. .... & $\cdots \cdots$ & $\ldots \ldots$ & $\cdots$ & $\ldots$ & $\cdots$ & $\ldots$ u... & \\
\hline us D'O. .... & $\cdots \cdot$ & $\cdots \cdots$ & $\cdots$ & $\cdots$ & $\cdots$ & ..u. u. & \\
\hline$\therefore \cdots$ & $\cdots \cdots$ & $\ldots \ldots$ & $\cdots$ & $\cdots$ & & ..u. $\mathbf{u} \cdot \mathbf{w}$ & . \\
\hline B & . & $\ldots$ & & $\ldots$ & 1 & $\mathbf{w} \cdot$ & \\
\hline o. & & . & & $\cdots \cdots$ & 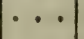 & $\mathbf{w} \cdot$ & \\
\hline . & & $\cdots$ & & $\cdots$ & a & $\cdots$ & . $z$ \\
\hline$\cdots$ & & & & $\bullet$ & . & $w$ & $\cdot \mathbf{z}$ \\
\hline$\cdots$ & & $\cdots \cdots \cdots$ & $\cdots \cdots$ & $\cdots \cdots$ & & $\cdots$ & - $\mathrm{z}$ \\
\hline N & - & $\cdots \cdots$ & $\cdots \cdots$ & $\cdots$ & . & $\cdots$ & . $\mathbf{z}$ \\
\hline c & • & $\cdots$ & $\cdot$ & $\cdots \cdot$ & $\cdots$ & $\cdots$ & yz \\
\hline & $\cdots$ & 0 & & & $\cdots$ & w. & $\cdot \mathbf{z}$ \\
\hline$\cdot$ & & $\cdots$ & $\cdots \cdots$ & $\cdots \cdots$ & $\cdots$ & $\mathbf{w} \cdot$ & $\cdot z$ \\
\hline$\cdots$ & $\cdots \cdot \cdot$ & $\cdots \cdots$ & $\cdots \cdot$ & $\cdots \cdots$ & $\cdots$ & .. & $\cdot \mathbf{z}$ \\
\hline Tis & $\cdots \cdots$ & $\ldots \ldots$ & $\cdots$ & $\cdots \cdot$ & $\cdots$ & w. & $\cdot \mathbf{z}$ \\
\hline EE. & 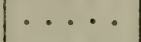 & - & $\cdots$ & $\cdots$ & $\therefore$ & $\cdots$ & - \\
\hline nrae 1 & $\cdot$ & & $\cdots$ &. & - & & - \\
\hline$\cdot$ & & & $\cdot \cdot$ & $\cdots$ & - & $\cdots$ & - \\
\hline . & & & & & - & $\cdots$ & \\
\hline B. $\cdots$ & $\because$ & $\cdots \cdots$ & & : & • & v.. & $\ddot{z}$ \\
\hline Mü. & $\therefore$ & $\cdots \cdots$ & $\because$ & $\cdots \cdots$ & $\therefore$ & $\ldots \ldots$ & $\cdots$ \\
\hline ... & $\cdots \cdots$ & $\ldots \ldots$ & $\cdots$ & $\cdots$ & . & ....w. & 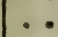 \\
\hline . . & $\ldots \ldots$ & $\ldots \ldots$ & $\cdots$ & $\cdots$ & - & 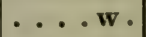 & $\cdots$ \\
\hline . & $\cdots$ & $\cdots$ & $\cdots$ & $\cdots$ & -. & ....w. & $\cdots$ \\
\hline$\cdot$ & - & $\cdots$ & $\cdots$ & $\cdots$ & $\cdots$ & $\ldots \ldots w$ & - \\
\hline . & $\cdots \cdots$ & $\cdots$ & $\cdots$ & $\cdots$ & $\cdots$ & .....w. & - \\
\hline$\cdots$ & $\cdots \cdot$ & $\ldots \ldots$ & $\cdots$ & $\cdots$ & . & ....w. & \\
\hline B. 1 & - • & $\ldots \ldots$ & $\cdots$ & $\cdots \cdot$ & $\cdots$ & $\cdots \cdots$ & .0 \\
\hline$+A$ & $\cdots \mathrm{M}^{2}$ & $\cdots \cdots \cdots$ & $\cdots$ & $\cdot$ & - & $\cdots \mathbf{v} \cdot$ & \\
\hline 'O. 2 & . & $\cdots \cdots$ & $\cdots$ & .1 & . & $\cdots$ & 13 \\
\hline . & - & $1 \cdot$ & $\cdot$ & $\cdot$ & & $\mathbf{W}$ & 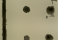 \\
\hline & : & $\because$ & $\cdots$ & $\cdot$ & 1. & . & .0 \\
\hline (LK.) 6 & & & $\because$ & & $\cdots$ & $\ldots \ldots$ & \\
\hline . & $\cdots \cdots$ & $\ldots$ & ... & $\ldots \ldots$ & $\cdots \cdot 1$ & . t... & \\
\hline${ }^{\circ}$ & .... & $\ldots \ldots$ & $\ldots$ & $\ldots$. & $\cdot$ & . t . & \\
\hline . & ... & $\ldots$ & 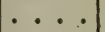 & $\cdots$ & & . t ... & . \\
\hline . & $\ldots \ldots$ & $\ldots \ldots$ & $\ldots$ & $\cdots$ & $\cdots$ & $\ldots \ldots \mathrm{w} x$ & $\mathbf{y} \cdot$ \\
\hline$\cdots$ & . & & $\ldots$ & & $\cdots$ & $\ldots \ldots \mathrm{w} x$ & yz \\
\hline . 1 . & $\ldots \ldots$ & $\ldots \ldots$ & $\cdots$ & $\cdots$ & $0^{\circ}$ & $\cdots \cdots$ &, 2 \\
\hline & $\cdots \cdots$ & $\cdots \cdots \cdots$ & $\cdots \cdots$ & $\cdots$ & & $t \ldots$ & \\
\hline 0.15 & $\ldots$ & $\ldots \ldots$ & & & & $\cdots$ & 0 \\
\hline & $\cdots \cdot$ & $\cdots \cdots$ & $\cdots$ & & & & • \\
\hline & & & & & 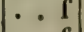 & $\cdots$ & \\
\hline HPT & & & & & & $\cdots \cdots$ & \\
\hline - & $\cdots \cdots$ & & $\cdot$ & & $\cdots f$ & $\ldots \ldots$ & \\
\hline - & 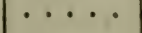 & & $\cdot$ & & & & \\
\hline N & & & & & & & \\
\hline 然 & & & & & f & & \\
\hline & & & & & & & \\
\hline
\end{tabular}




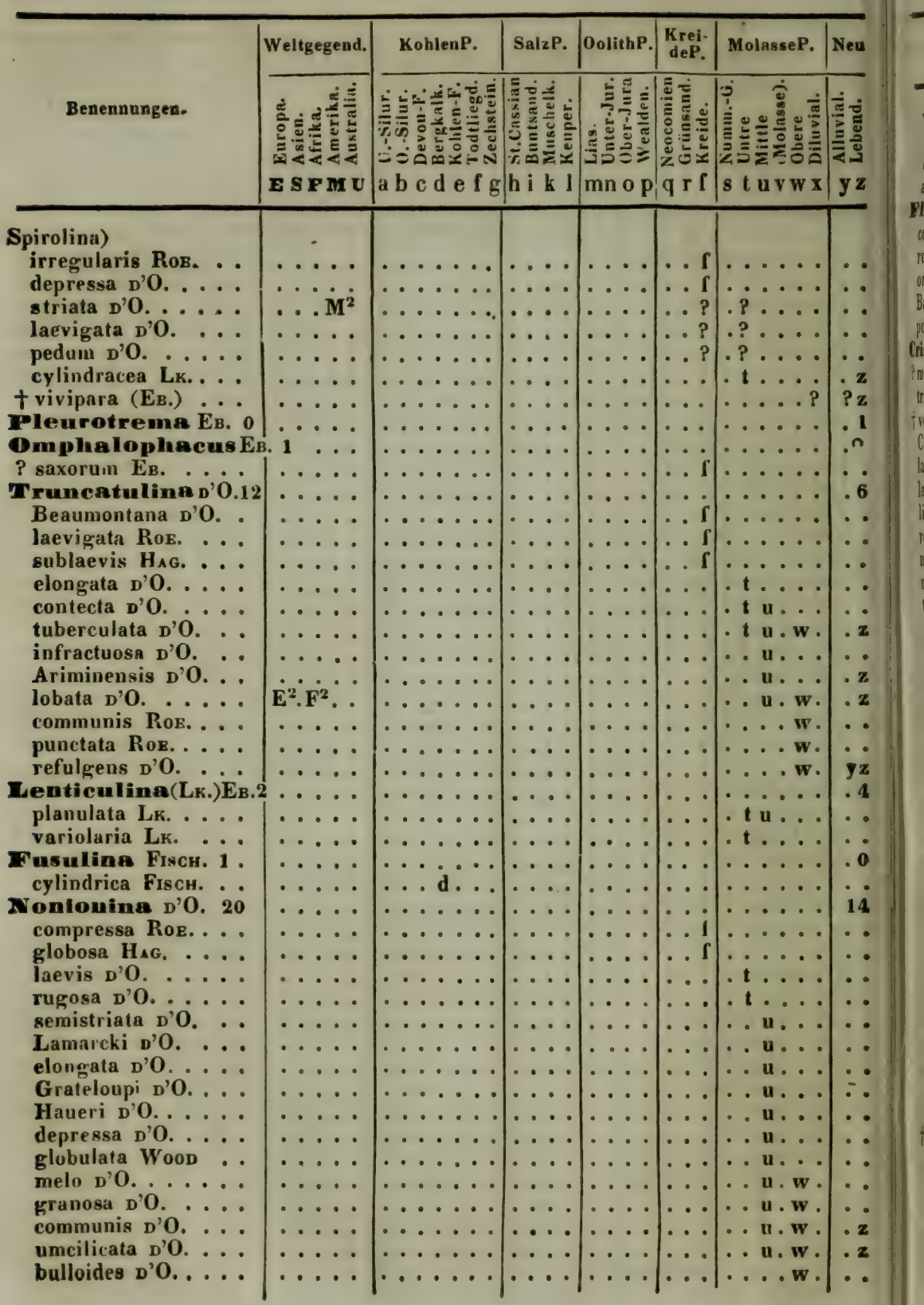




\begin{tabular}{|c|c|c|c|c|c|c|c|}
\hline Benennungen. & WVeltgegend. & a b c d e f g & hi k l & mnop & $q \times f$ & $\mathrm{stu} v \mathrm{w} x$ & $\mathbf{y z}$ \\
\hline$\cdots$ & & & & & & - $\mathbf{w}$. & \\
\hline a RoE...... & $\cdots \cdots$ & $\ldots \ldots$ & $\cdots$ & & $\cdots$ & $\ldots w^{\prime}$ & \\
\hline B............. & $\cdots \cdots$ & $\cdots \cdots$ & $\cdots$ & $\cdots \cdot$ & $\cdots$ & $\cdots \cdot \mathbf{w} \cdot$ & \\
\hline$\ddot{5}$ & $\cdots \cdots$ & & & & & $\cdots \mathbf{w} \mathbf{x}$ & $\cdot \mathbf{z}$ \\
\hline $\begin{array}{l}\text { Ilima D'O. } 5 \\
\text { ta REuss }\end{array}$ & : & $\cdots$ & • & & & $\cdots \cdots$ & $n$ \\
\hline $\begin{array}{l}\text { cordata Rsuss } \ldots \text {. } \\
\text { rugosa D'O. . . . }\end{array}$ & & $\because$ & $\dot{*}$ & & .1 & $\cdots$ & \\
\hline $\begin{array}{l}\text { rugosa D'O. . . . . . } \\
\text { ornata Reuss . . . }\end{array}$ & $\ldots$ & $\because$ & $\mid \begin{array}{c}\cdots \\
\cdots\end{array}$ & . & $\because 1$ & $\left.\mid \begin{array}{c}\cdots \\
\cdots\end{array}\right]$ & \\
\hline Baudouinana D'0. . & $\ldots$ & $\ldots \ldots$ & $\because \cdots$ & $\cdots \cdots$ & $\because \mathrm{f}$ & $\cdots \cdots$ & $\because$ \\
\hline ra D'O. & $\cdots \cdot$ & $\ldots \ldots$ & $\ldots$. & $\cdots \cdot$ & . $\mathrm{f}$ & $\ldots \ldots$ & $\because$ \\
\hline Iaria D'0. 50 & $\cdots \cdot \cdot$ & $\cdots \cdots$ & $\ldots$ & $\ldots$ & $\cdots$ & $\ldots \ldots$ & .9 \\
\hline 8. . . & $\cdots \cdots$ & $\cdots d \ldots$ & $\cdots \cdots$ & $\cdots \cdots$ & $\cdots$ & $\cdots \cdots$ & $\cdots$ \\
\hline cata D' & $\cdots$ & $\cdots$ & $\cdots$ & $\cdot \mathbf{n} \cdot \cdot$ & $\cdots$ & $\cdots$ & \\
\hline $\begin{array}{l}\text { tvenusta M } \\
\text { Cadomensi }\end{array}$ & ・ & $\cdots$ & $\cdots$ & $\because$ & $\cdots$ & $\cdots$ & \\
\hline $\begin{array}{l}\text { Cadomens } \\
\text { laevigrata }\end{array}$ & & $\cdots$ & $\cdots$ & & & $\cdot \cdot$ & \\
\hline $\begin{array}{lll}\text { laevigata } & D^{\prime} O . & \cdots \\
\text { lamellosa } & D^{\prime} O . & \text {. }\end{array}$ & & & $\cdots$ & & 1 & $\cdot$ & \\
\hline $\begin{array}{l}\text { lan } \\
\text { lit }\end{array}$ & & $\cdots$ & $\cdots$ & & $\because \cdots$ & : & \\
\hline$\ddot{0} \cdots \cdots$ & $\dot{\mathrm{E}}^{2} \cdot \dot{\mathrm{M}}^{2}$ & $\ddot{\cdots}$ & 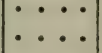 & $\cdots$ & $\mid \begin{array}{ll}\dot{r} & \dot{f}\end{array}$ & $\cdots \cdots$ & \\
\hline$\cdots \cdots$ & $\cdots$ & $\ldots \ldots$ & $\cdots$ & $\ldots$ & . . I & $\ldots \ldots$ & \\
\hline$\therefore \cdot \cdots$ & $\cdots \cdot \cdot$ & $\cdots \cdots \cdots$ & $\cdots$ & $\cdots$ & .. I & $\cdots \cdots$ & • \\
\hline . . & $\cdots \cdot$ & $\cdots \cdots$ & $\cdots$ & $\cdots \cdot$ & .. $\mathbf{f}$ & $\cdots \cdots$ & \\
\hline$\cdots$ & - & $\cdots \cdots \cdots$ & $\cdots$ & $\cdots$ & .. 1 & $\cdots \cdots$ & - \\
\hline$\cdots$ & - $\cdot$ & $\cdots \cdot$ & $\cdots$ & $\cdots \cdot \mid$ & . . . & $\cdots \cdots$ & \\
\hline uss · & & $\cdots$ & $\cdots$ & $\cdot$ & . . I & $\cdot$ & \\
\hline$\cdots \cdot$ & & $\cdots$ & $\cdots$ & - & .1 & • & \\
\hline s8 . & 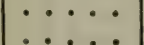 & $\cdots$ & $\cdots$ & $\begin{array}{l}\cdots \\
{ }^{\prime}\end{array}>>$ & $\because 1$ & $\therefore$ & \\
\hline $\begin{array}{l}\text { intermedia Rsuss . } \\
\text { lituola Reuss . . . }\end{array}$ & $\cdots \cdots$ & $\cdots \cdots$ & $\cdots \cdots$ & 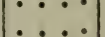 & $\because \mathrm{f}$ & 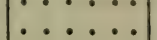 & \\
\hline lobata Reuss .... & $\ldots$ & $\cdots$ & $\cdots$ & $\cdots$ & $\therefore \mathrm{f}$ & 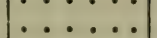 & \\
\hline is Reuss .... & ..... & $\ldots \ldots$ & $\ldots$ & $\ldots$ & . i & $\ldots \ldots$ & \\
\hline exarata HAG. .... & $\ldots$ & $\ldots \ldots$ & $\cdots$. & $\ldots$ & .. I & $\ldots \ldots$ & \\
\hline sta HAG.... & $\ldots$ & $\ldots \ldots$ & $\cdots \cdots$ & $\cdots \cdot$ & . $\mathrm{f}$ & $\ldots \ldots$ & $\cdots$ \\
\hline $\operatorname{cta} H_{A G} \ldots$ & $\ldots$ & $\ldots \ldots$ & $\cdots$ & $\cdots$ & .. & $\ldots \ldots$ & \\
\hline$\cdots$ & $\cdots \cdots$ & $\cdots$ & $\cdots$ & $\cdots \cdots$ & $\cdots \tau$ & $\cdots \cdots$ & - \\
\hline$\cdots$ & - & - & $\cdot$ & $\cdots \cdots$ & - $\mathrm{f}$ & $\cdots \cdots$ & \\
\hline ralina Jouan. . & & $1^{\circ}$ & $\cdots$ & $\cdots \cdots$ & - & $\cdots \cdots$ & \\
\hline$\cdots$ & & - & $\cdots$ & $\cdots \cdot$ & $\cdot$ & $\cdots \mathbf{u} \ldots$ & \\
\hline OD . & & & $\cdot$ & $\cdots$ & $\cdot$ & . u $\ldots$ & \\
\hline$\cdots$ & & & $\cdots$ & $\therefore \cdots$ & $\because$ & . u u & \\
\hline $\begin{array}{l}\text { producta WooD } \\
\text { Osnabrugensis Mü. }\end{array}$ & .. & $\ldots$ & $\cdots \cdots$ & $\cdots$ & $\cdots$ & $\cdots u$ & \\
\hline$\cdots$ & & $\ldots$ & $\ldots$ & ... & . & . u. w. & \\
\hline Vood. & $\ldots .$. & $\ldots$ & $\cdots$ & $\ldots$ & • & $\ldots u \ldots$ &. $\mathrm{z}$ \\
\hline tv & $\cdots$ & $\cdots$ & - & & . & $\ldots \mathbf{v} \ldots$ & . \\
\hline c & .. & 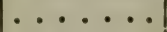 & & & & $\ldots \ldots w$ & 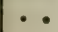 \\
\hline n & & & & & & $\ldots \ldots w$ & \\
\hline$\cdots$ & - & &. & - & & $\cdots \cdot \mathbf{w} \cdot$ & $\cdots$ \\
\hline$\cdots$ & & & & - & & $\cdots \cdot w_{\cdot}$ & $\cdots$ \\
\hline$\cdots$ & - & $\cdots$ & $\cdots \cdots$ & $\cdots \cdot$ & - & $\cdots \cdot \mathbf{w} \cdot$ & $\cdots$ \\
\hline nat & & & & & & w. & $\because$ \\
\hline 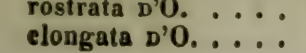 & $\ldots .$. & .. & . & & & $\ldots w_{\text {. }}$ & \\
\hline
\end{tabular}




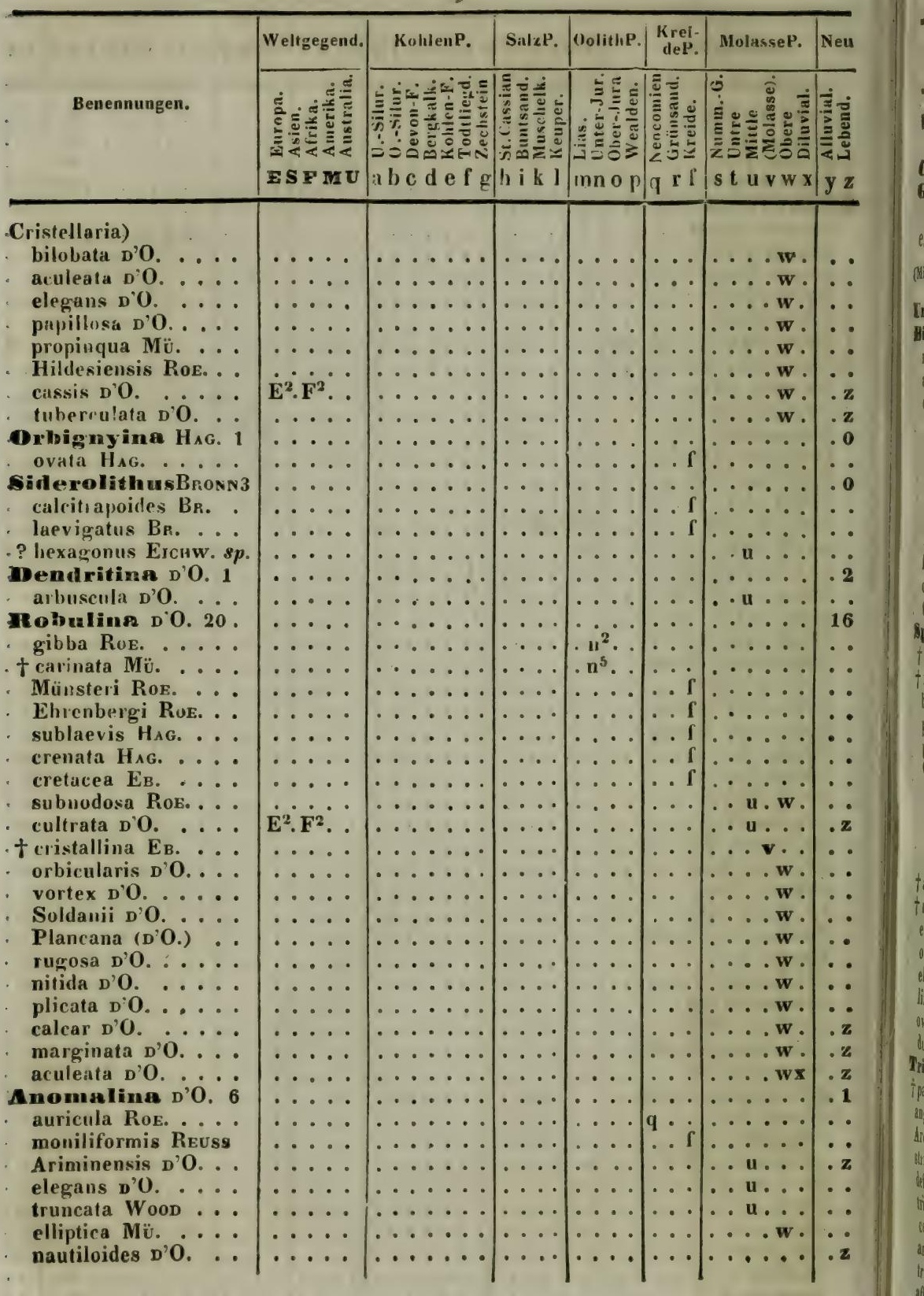




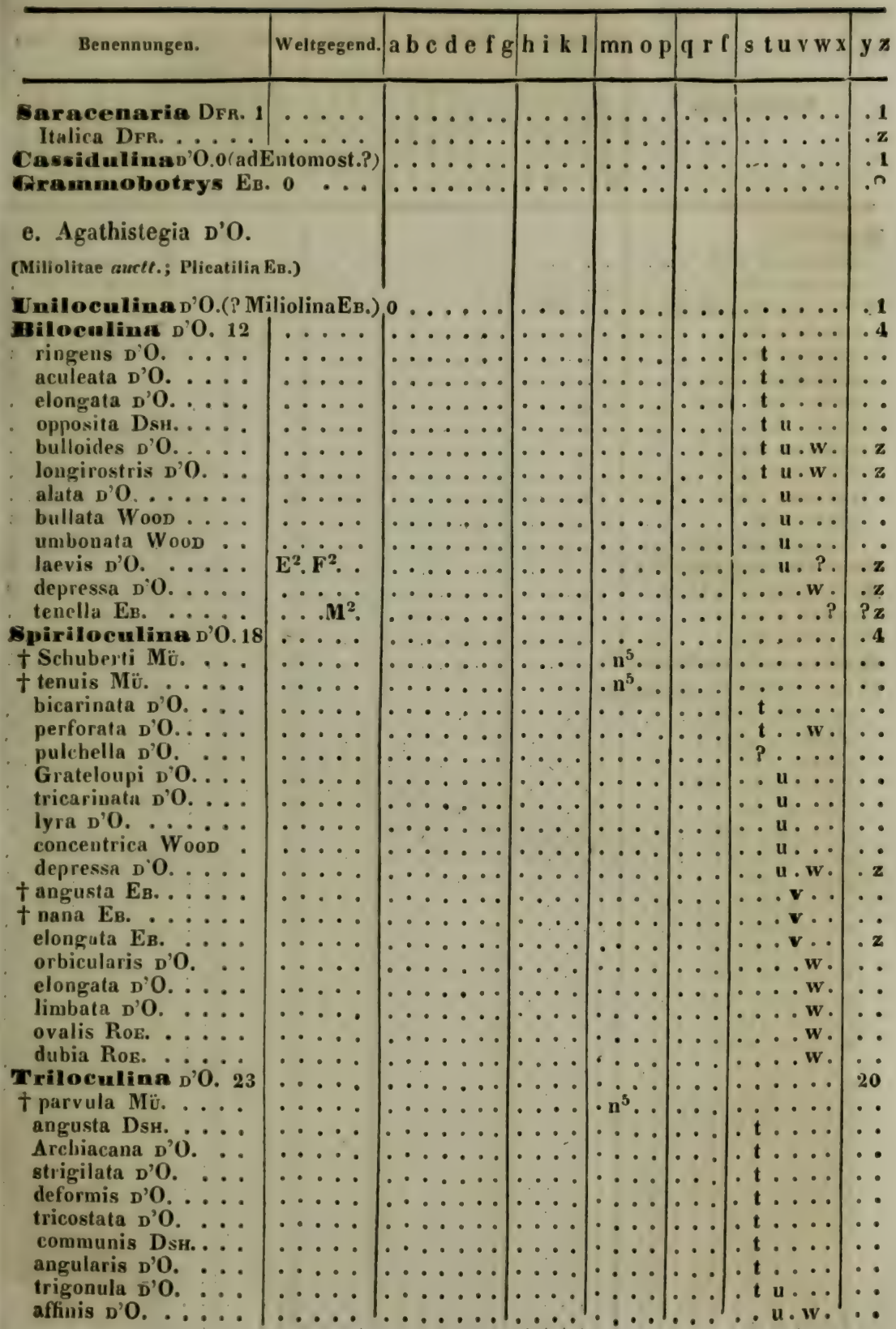




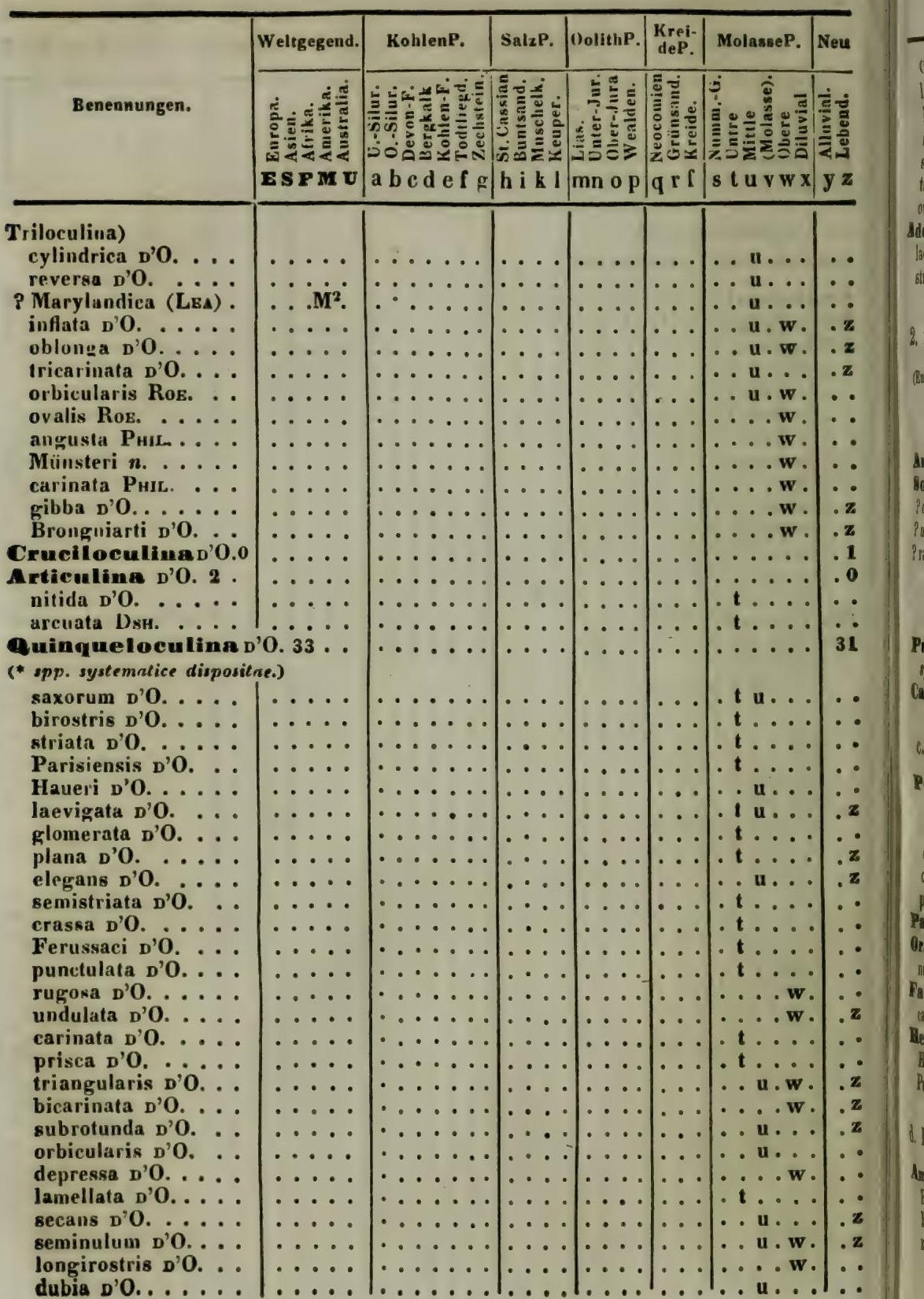




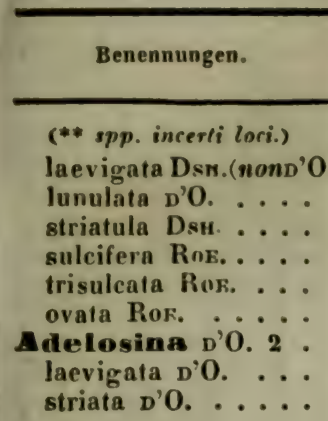

\section{2. polysomatia Eв.}

(Entomostegia aly. v'0.)

a. Soritina EB.

Amplnisorus Eв. 0 Sorites EB. 3 .... ? depressus D'O. sp. . P undatus D'O. sp. . . 9 radiolatus n'0. $s p$. .

b. Uvellina Eв.

Pupina D'O. 1 ... sp. andeina $[?]$ D'0.

c. Helicosorina Eв.

Peneroplis D'0. 5 d'Orbignyi Roe. Fleuriaui (D'O.) opercularis D'0. orbicularia D'O. planata Mr.

Pavomina D'0. 0 Crbiculina $D^{\prime} O .1$ numismalis LK....

Faujasina D'0. 1 . carinata D' 0 . . . .

Iileterosteginà'0.2 Haueri D'O. Puschi Reuss.

d. Helicotrochina EB.

Amphistegina $D^{\prime} 0.4$ Fleuriaui (D'O.). . . Haueri D'O. mammillata $\mathbf{D} O$. \begin{tabular}{|l|l|l|l|l|l|l} 
Weltgegend. a b c d e f g h i k l mn o p & q r f & s t u v w x & y z
\end{tabular} .)

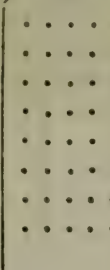

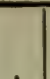

\begin{tabular}{l|l|l|l|l|l|}
\hline & & & \\
\hline
\end{tabular}

$\ldots \ldots$




\begin{tabular}{|c|c|c|c|c|c|c|c|}
\hline & Weltgegend. & KohlenP. & SalzP. & OolithP. & $\begin{array}{l}\text { Krej- } \\
\text { deP. }\end{array}$ & MolasseP. & \\
\hline ennungen. & $\begin{array}{l}\text { EST } \\
\text { ES U }\end{array}$ & $\begin{array}{l}\text { a b c def } \\
\text { a b }\end{array}$ & $\begin{array}{l}0 \\
\text { h i k l }\end{array}$ & $\begin{array}{l}2 \\
\text { mnop }\end{array}$ & 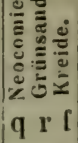 & 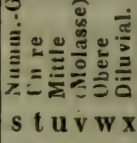 & \\
\hline
\end{tabular}

Amphistegina) vulgaris D'O.

P Asterigerina $D$ O. rosacea D'U.

Polystomella LK. 6 compressa Wood crispa Ік. $_{\text {. }}$ angularis $D^{\prime} \mathbf{O}$. semistriata v'0.... granulosa Ris. . . . strigilata Dsн. ...

Geoponus Ев, 0 .

e. Stichostegia.

Conulina d'O. 0

f. Enallostegia.

Chrysalidina d'0. 1 gradata n'().

Cuncolina D'0. 3 . pavonia D O. ... conica D'O.

Fleuriauana (D'O.).

\section{g. Alveolinea EB.}

Borelis (Mr.) EB. 6 princeps $\mathbf{E}_{\mathrm{B}}$. constricta Ев..... sphaeroidea Es. . . melo FM. $s p$. . . . . bulloides D'O. $8 p$... costulata Eichw. sp.

Alveolina (Bosc) Ë.8 prisca Ев...... cretacea D'ARch. . . elliptira So. $s p . .$. elongata D'O. subpyrenaica LeYm. Bosci DFr. ..... oblonga D'O. .... ovoides D'O. ....

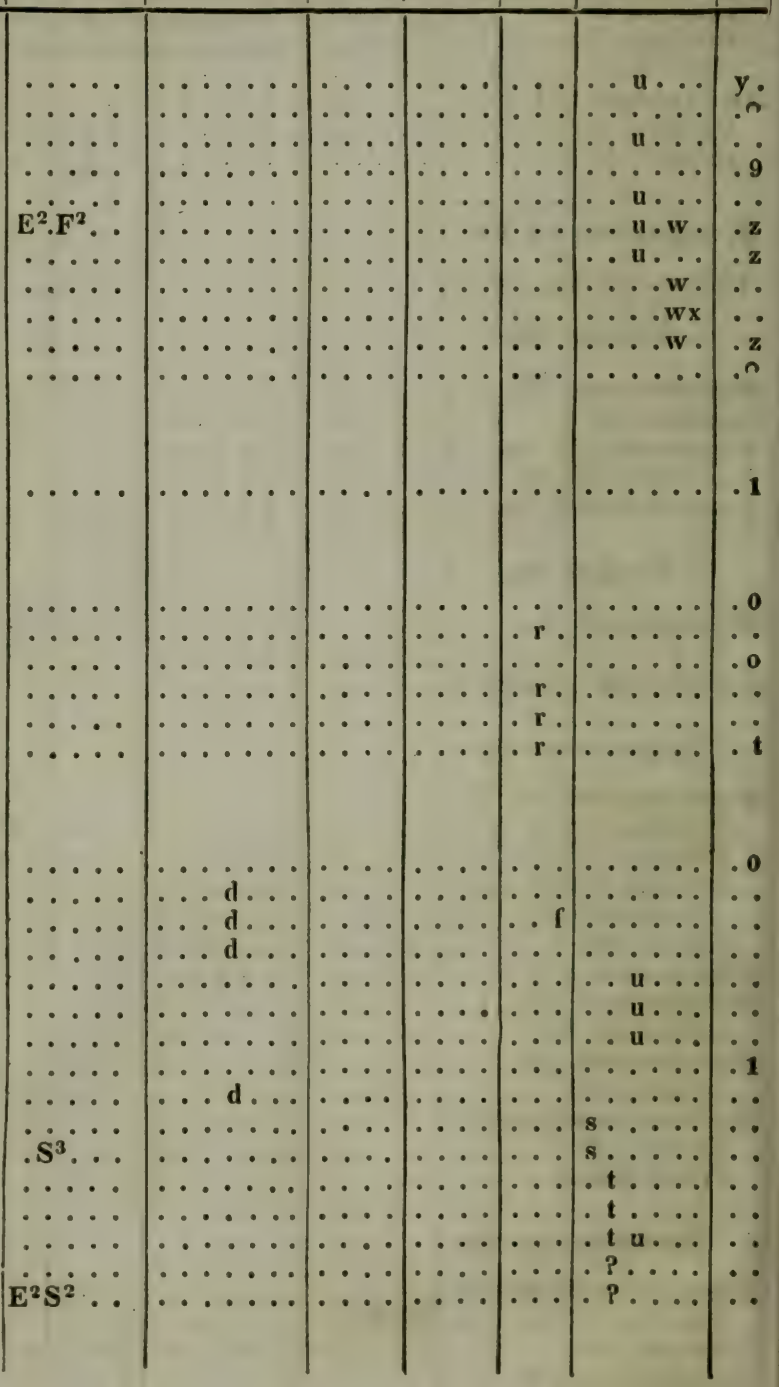




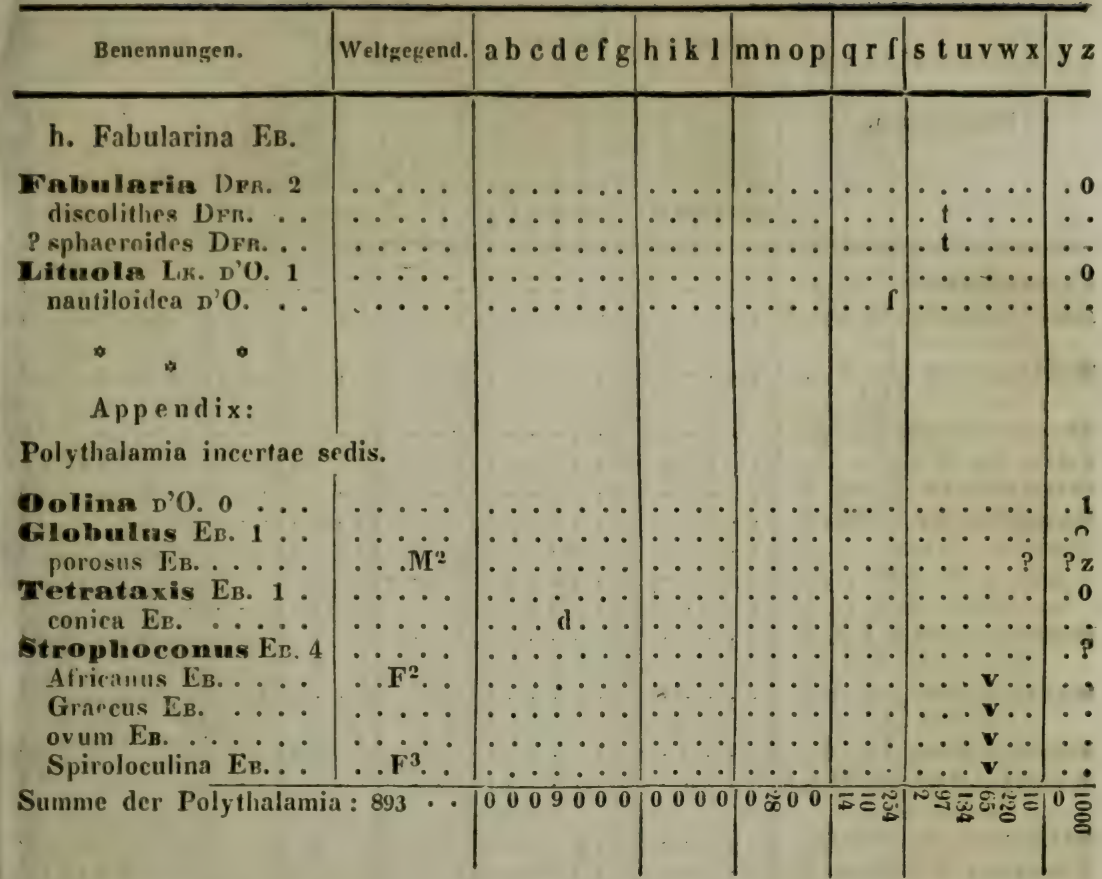

B. BRYOZOA (EB.) RoE. '.

(Bryozoorum pars altera EB.; BLv. ; excl. Anlipathe.)

a. Nuda PLv.

(Corporn omniu mollia.)

Hydra L. 0 etc.

b. Dubia BLv.

(Corporu omnia molliu.)

Pacela lace QG.

Dirnugia Lecrerc o

Aleyonella LK. 0

Plumatella Bosc (Nais Lx.) 0

Cristatella Cuv.

c. Membranacea Buv.

[exclus.: Tubuliporinorum generibus.]

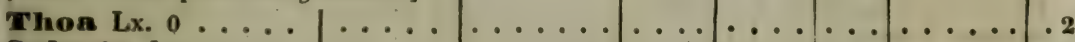

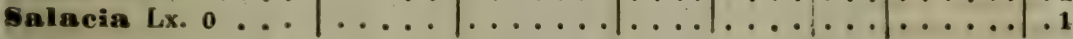

1 Specierum fossilium nummeri solummodo approximativi e Lamarcki editione altera curante Milxe Eidwards resumpti sunt. 
Benennungen.

\begin{tabular}{|c|c|c|c|c|c|c|}
\hline Weltgegend. & KohlenP. & SulzP. & Oolithe. & $\begin{array}{l}\text { Krei- } \\
\text { deP. }\end{array}$ & MolasseP. & Neu \\
\hline $\begin{array}{l}\text { 致 } \\
\text { EST }\end{array}$ & $\begin{array}{l}- \\
\text { a b c }\end{array}$ & 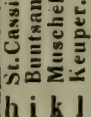 & 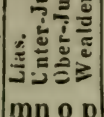 & $\begin{array}{ll}0 \\
0 \\
0\end{array}$ & 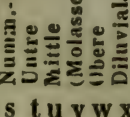 & 造递 \\
\hline
\end{tabular}

Cymodocen Lx. 0

Antenuularia Lk. 0 (Nemertesia Lx.)

Tuliparia Lk. 0 (Liriozoa $L x$ )

Dynamena Lx. 0

Idir Lx. 0 .....

Biseriaria Fr.m. 0

Sertularia Fleim, 3

P antiqua Steiv. .... sericea Mrchn. . . . polyzonias L.? . .

Plumularia LK. 0 (Aglaophenia $\mathbf{L x}$ )

Serialaria Lk. 0 . (Aniathia $\mathrm{L} / \mathrm{x}$.)

Haomedea Lx. 0 .

CampanulariaLk,o (Clythia Lx.)

Silicularia Meyen

Coryna Gintner. 0

Tubularia LK. 0 .

Neoneris $L x$. 0 ..

Tibiana Lк. 0 ....

Aulopora (GF.) EDw serpens (ip.

tubaeformis GF. . . spicata Gr. ..... consimilis Lonsd... conglomerata GF. sarmentacea GF. . . cucullina Мichs. ..

Anguinaxia LK. 0 (Aeten Lx.)

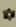
$* \quad *$

Menipaen Lx. 0 . mippothon Lx. 3 . (Catenicella BLv.)

Smithi Morris ... fuberculum Lossd. dentata WooD....

Unicellaria Br.v. o (Eucratea et Alecto Lx.)

Notamia Flem. 0 (Loricaria Lx., Gemmicellaria Buv.)

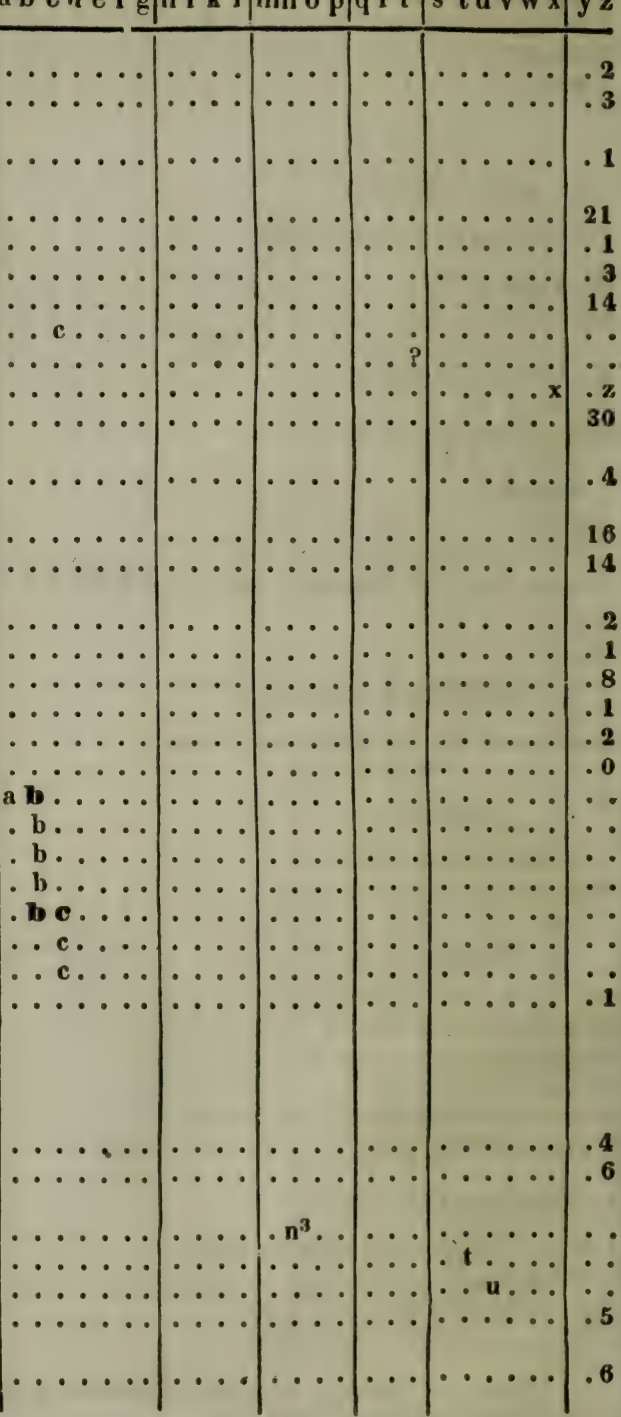


-

(Crisia v. infra) Bicellaria Lx. 0 Acamarchis Lx. 0 Tricellaria FLEM. 0 Caberea Lx. 0 Canda Lx. 0 .... Intricarise DFr. 2 . obscura Porti.. Bajocensis [?] DFr.

Cellaria ( $\mathrm{LK}_{\text {. }}$ Lx. 4 ? elegans STEIng.

?ovata Wood fistulosa Wood gracilis Phic.

Salicornaria Cuv. 6 excavata EDw. fragilis EDw. elegans EDw. affinis EDw. crassa EDw.

Beaumonti EDw.

(GIauconomeGF.) 1 (Salicornaria Cuv.)

disticha GF.

bipinnata Phrzc. pluma MorR. undulata $\mathrm{H}_{\mathrm{Ag}}$. bipunctata $H_{\wedge}$. elliptica $\mathrm{H}_{\wedge \mathrm{G}}$. hexagona Mü. lima $H_{A G}$. piriformis $\mathrm{H}_{\mathbf{A G}}$. prismatica $H_{A G}$ rhombifera Mü. spiralis HaG. trifaux HAG. marginata $M \ddot{v}$. . . .

(Vincularia DFr.) 2 (Salicornaria Cuv.) multangularis Ponte. Bronni Reuss

(Pherusalx, vinfra) Elzerina Ix. 0 Mollia Lx. 0 ....

Flustra (Lк.) Btv. 28 Gottlandica $n$.

Pradiata SteING. ... palmata M'Cox Pparallela PhicL. elegans Mü. antiqua DFr. ornata Reusy

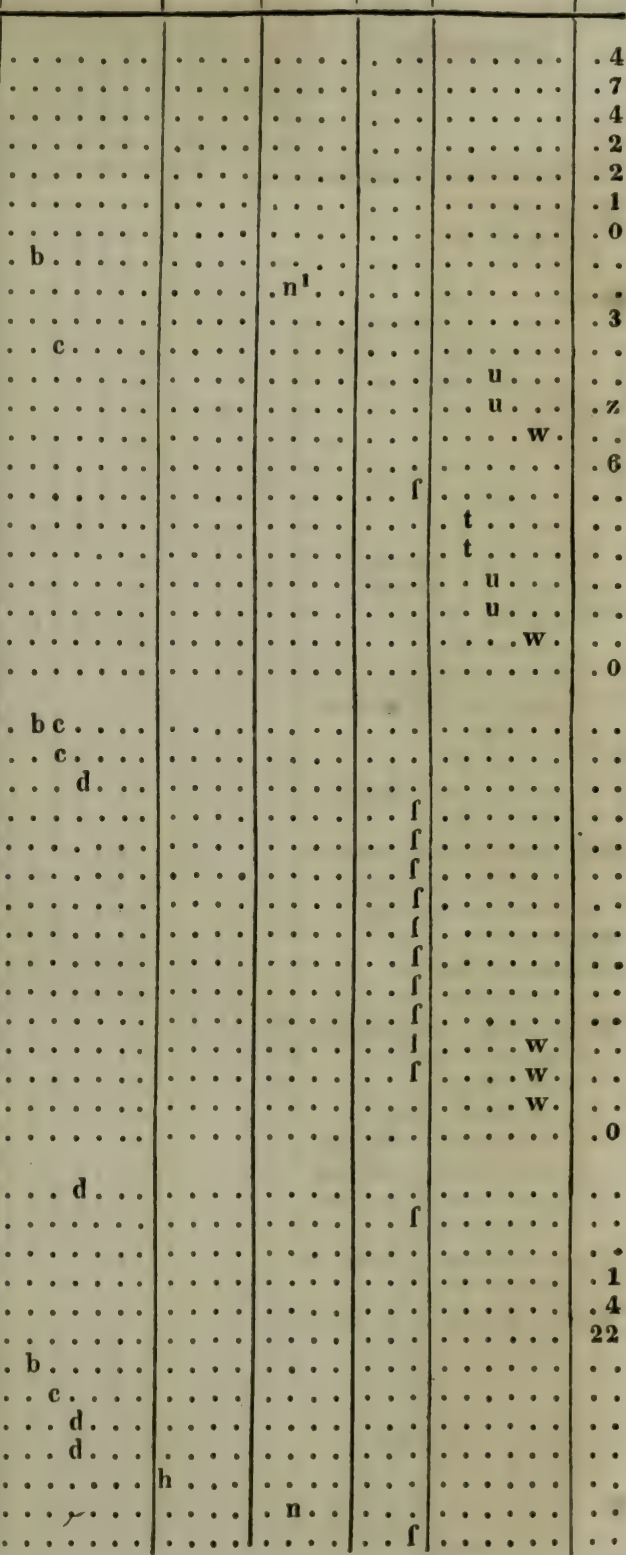




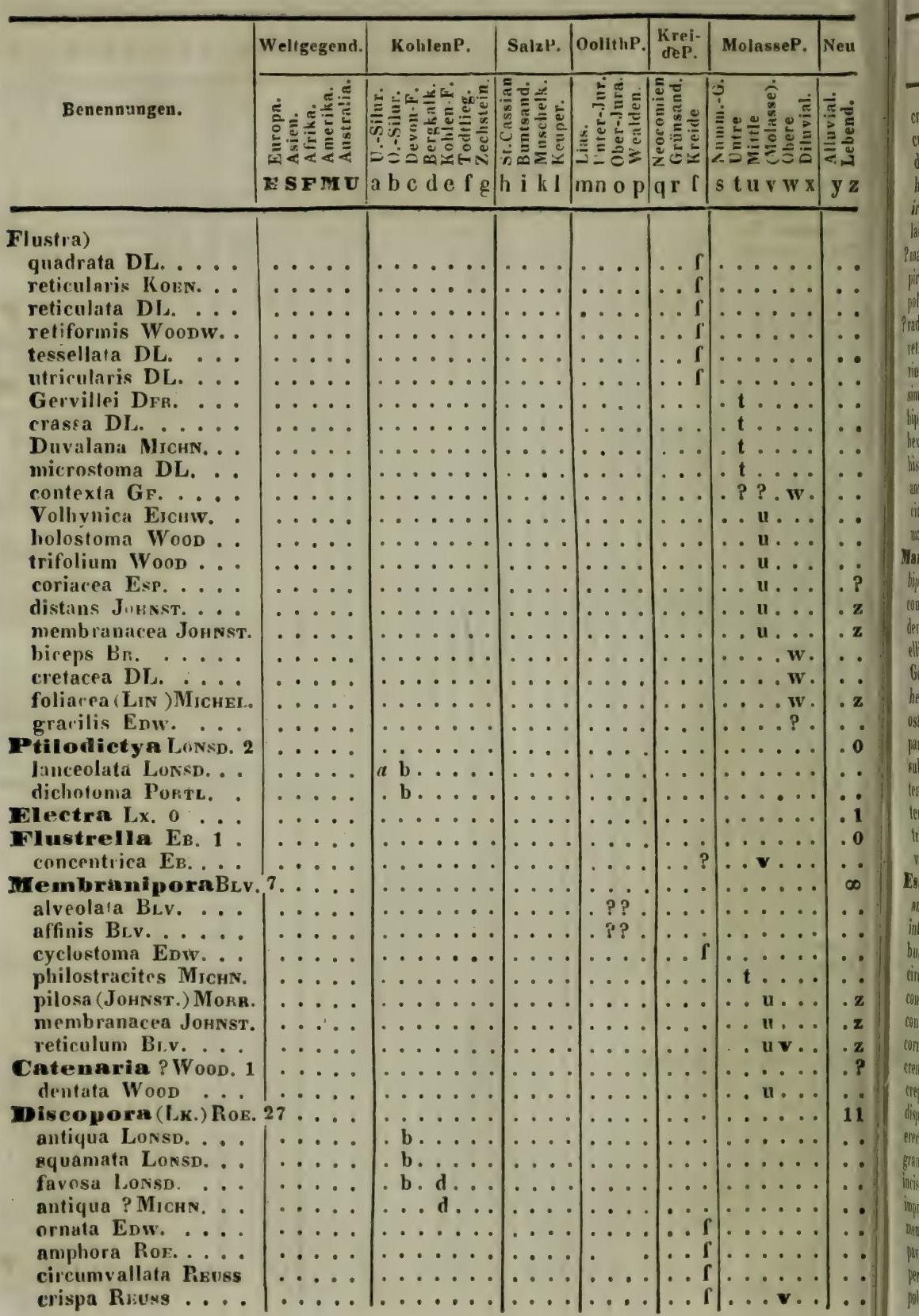




\begin{tabular}{|c|c|c|c|c|c|c|c|}
\hline 1. & nd. & $\mathbf{f g}$ & & $\mathrm{p}$ & I & $\mathbf{W} \mathbf{x}$ & $\mathbf{y}$ \\
\hline & & & & & & & \\
\hline & .... & $\ldots \ldots$ & $\ldots$ & ... & . i & $\ldots \ldots$ & \\
\hline . & $\ldots$. & $\ldots \ldots$ & $\ldots$ & $\ldots$ & . . & $\ldots \ldots$ & \\
\hline Uss ... & $\ldots$ & $\ldots \ldots$ & $\cdots$ & $\ldots$ & $\ldots r$ & $\ldots \ldots$ & \\
\hline aris RoE. . & $\ldots$ & $\ldots \ldots$ & $\cdots \cdot$ & $\cdots$ & & $\ldots \ldots$ & \\
\hline ... & & . . & $\cdots \cdot$ & & & $\ldots \ldots$ & \\
\hline Dw. & . & $\cdots$ & $\cdots \cdot$ & $\ldots$ & $\ldots$ f & $\ldots \ldots$ & \\
\hline & . & & & & & $\cdots \cdots$ & \\
\hline & & & & & $|\cdots T|$ & $\cdots \cdot$ & \\
\hline 7. . . & & & & & $\cdots 1$ & $\cdots$ & \\
\hline$\cdots$ & & & & & $\cdots$ & $\cdots$ & \\
\hline$\cdots$ & $\ddot{\bullet}$ & $\cdots$ & : & & $\mid \begin{array}{ll}\cdots 1 \\
\therefore & 1\end{array}$ & $\cdots$ & \\
\hline & $\because$ & $\because \cdots \cdots$ & 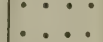 & & $\because 1$ & $\dot{w}$ & \\
\hline$\because$ & $\ldots \ldots$ & $\ldots \ldots$ & $\therefore$ & $\ldots \ldots$ & & $\cdots$ & \\
\hline . & $\cdots \cdot$ & $\ldots \ldots$ & $\ldots$ & $\ldots$ & & $\ldots u \ldots$ & .8 \\
\hline . & $\ldots$ & $\ldots \ldots \ldots$ & $\cdots \cdot$ & $\ldots$ & $\therefore$ & $\ldots w$ & - \\
\hline & . & $\cdots$ & $\cdots \cdot$ & $\ldots$ & $\ldots$ & 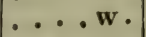 & • \\
\hline$\cdots$ & • & $\cdots$ & $\ldots$ & $\cdots \cdot$ & & $\ldots w_{\cdot}$ & \\
\hline 13 & - & $\ldots \ldots$ & $\cdots$ & $\ldots$. & & $\ldots \ldots$ & $?$ \\
\hline$\cdots$ & • & $\ldots \ldots$ & $\cdots \cdot$ & $\ldots$ & $\ldots f$ & $\ldots \ldots$ & \\
\hline s. & $\cdots$ & $\cdots$ & $\cdots \cdot$ & $\ldots \ldots$ & . & $\ldots \ldots$ & \\
\hline$\cdots$ & - & - & $\cdots$ & $\cdots$ & . & $\cdots \cdots$ & \\
\hline & - & & - & $\cdot$ & . & $\cdot$ ! & \\
\hline$\cdots$ & & & & $\cdot$ & $\cdots$ & • • & \\
\hline$\cdots$ & $\cdots \cdot$ & $\cdots$ & $\cdots$ & $\cdots \cdot$ & - & $\cdot \cdot$ & \\
\hline$\cdots$ & $\cdots \cdot$ & & - & $\cdot$ & $\because$ & $\therefore$ & \\
\hline$\cdots$ & - & - $\bullet$ & $\ddot{m}$ & - & $\begin{array}{ll}\cdots \mathrm{I} \\
\cdots \mathrm{I}\end{array}$ & $\cdots$ & \\
\hline$\dot{.}$ & $\begin{array}{l}\cdots \\
\cdots\end{array}$ & $\therefore \ldots$ & $\ldots$ & ... & $\because \mathrm{f}$ & $\ldots$ & \\
\hline$\therefore$ & $\because \cdots$ & ......... & ... & . & .. I ! & $\ldots \ldots$ & \\
\hline$\cdots$ & $\cdots$ & $\ldots \ldots$ & ... & $\cdots \cdots$ &. $\mathrm{f}$ & $\ldots$ & \\
\hline$\cdots$ & $\ldots$ & $\ldots$ & ... & $\cdots$ &. .1 & $\ldots \ldots$ & \\
\hline nw. 22 & $\ldots$ & $\cdots \cdots$ & ... & $\ldots$ & $\cdots$ & $\ldots \ldots$ & 60 \\
\hline & & . b.. & $\cdots$ & $\cdots$ & & $\cdots \cdots$ & - \\
\hline & & 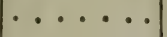 & & & & $\cdots \cdots$ & \\
\hline . & . & . & - & & 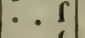 & $\cdots \cdots$ & \\
\hline & $\ldots$ & $\cdots$ & $\cdots$ & $\cdots \cdot$ & r. & $\cdots \cdots$ & \\
\hline$s \quad$ s. & $\ldots$ & ... & $\cdots \cdot$ & $\ldots$ & 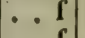 & $\cdots \cdots$ & \\
\hline$\cdots$ & $\cdots$ & 1 & - & . & & $\cdots \cdots$ & \\
\hline$\cdots$ & $\cdots \cdot$ & $\cdots$ & & & & $\cdots \cdots$ & \\
\hline$s \ldots$ & - & & & $\cdot$ & & . • • & \\
\hline$\cdots$ & & - & - & $\cdot$ & & $\cdots$ & \\
\hline s. & & & $\cdots$ & & .1 & $\cdots$ & \\
\hline . & & $\ldots$ & ... & $\ldots$ &.$i$ & $\ldots \ldots$ & \\
\hline & .. & $\ldots$ & ... & $\ldots$. & . I I & $\ldots \ldots$ & \\
\hline & . & $\ldots$ & ... & $\cdots$ & . . $\mathrm{f}$ & $\ldots \ldots$ & \\
\hline Rов. . & $\cdots$ & & - & $\cdot$ & .. I & $\ldots \ldots$ & \\
\hline$\cdots$ & $\cdots$ & . & • & $\cdot$ & & $\cdots \cdot$ & \\
\hline$\because$ & - & 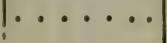 & & $\cdots$ & & & \\
\hline 3 & - & $\theta_{0}$ & a & . & & $\ldots$ & \\
\hline
\end{tabular}




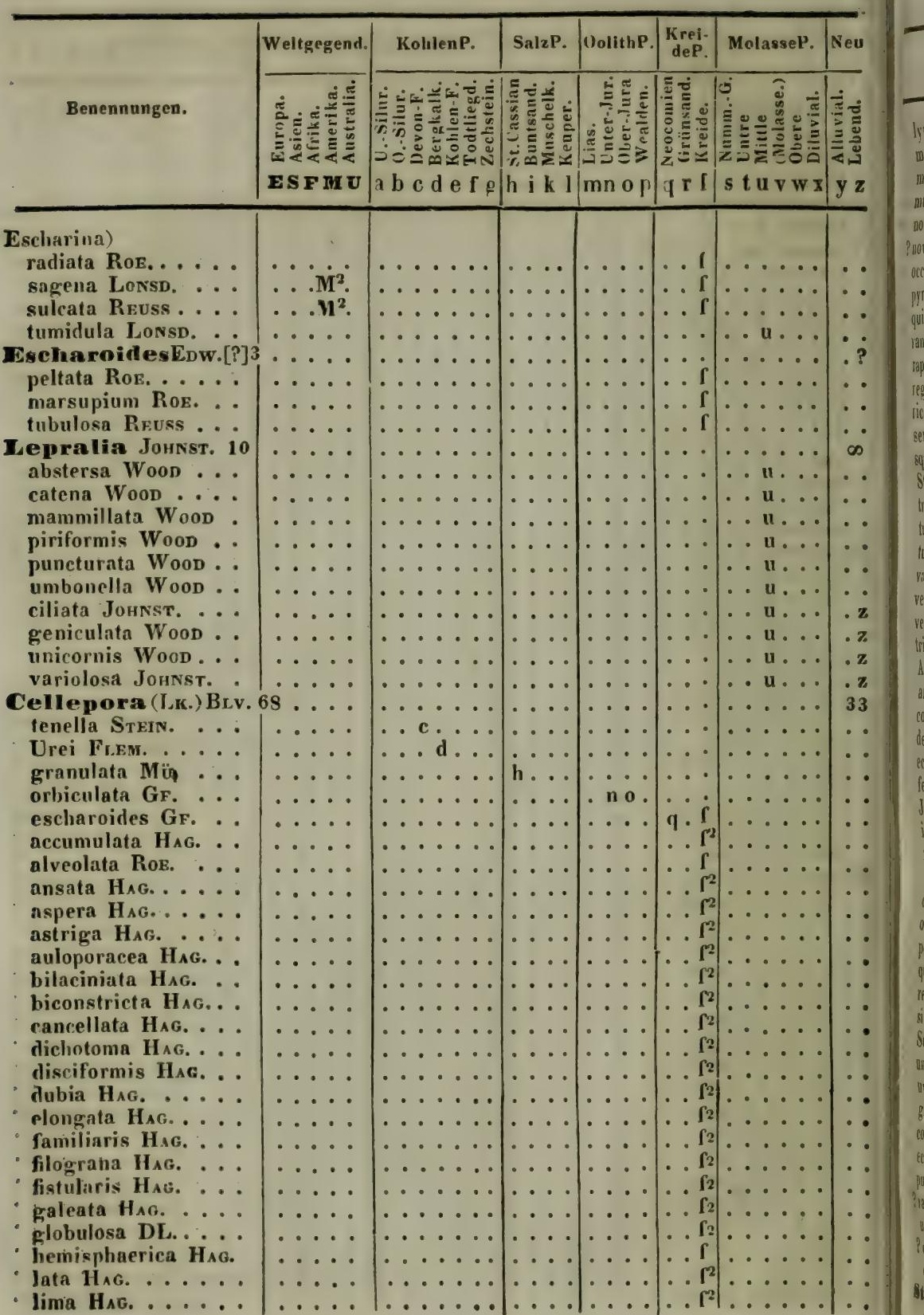




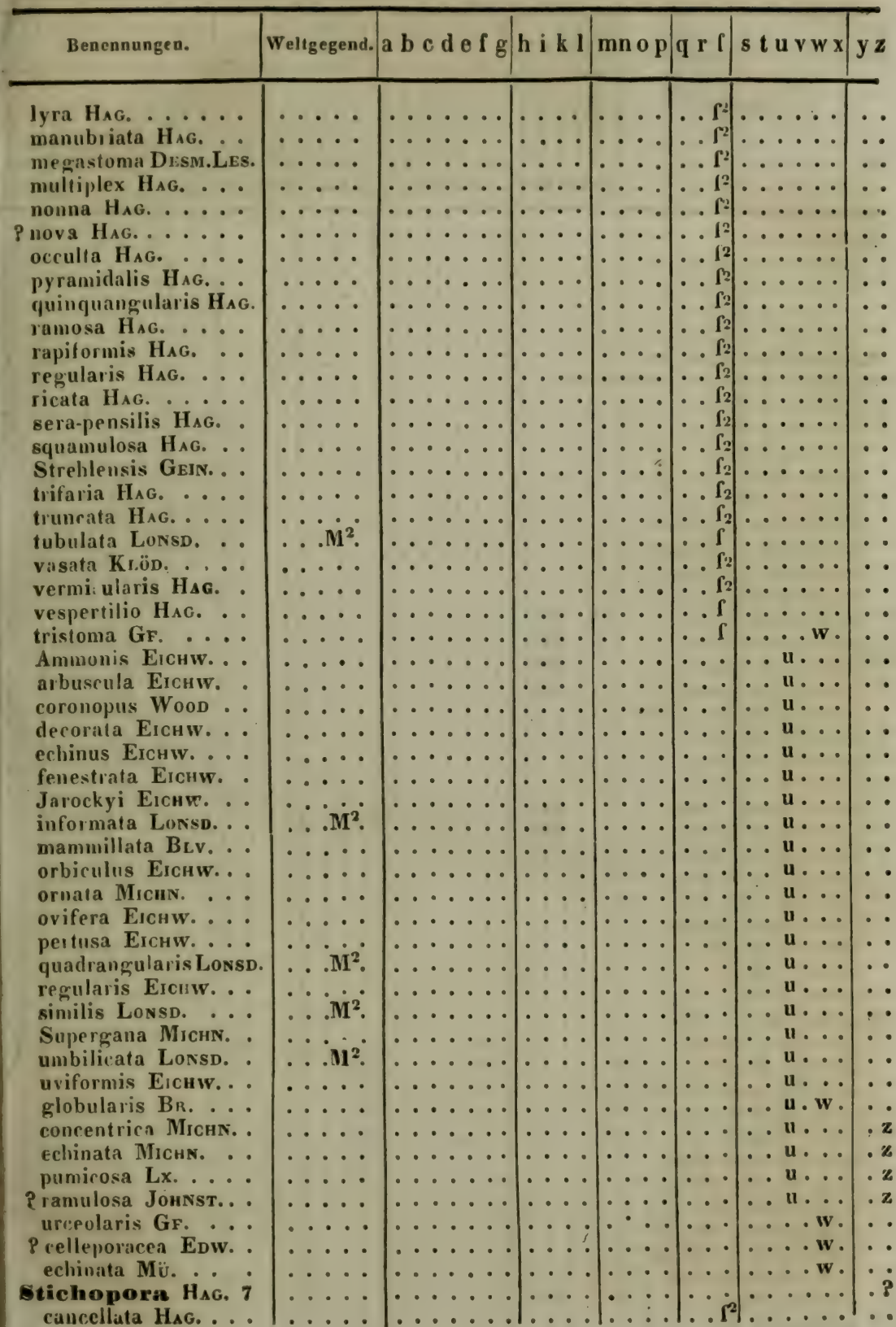




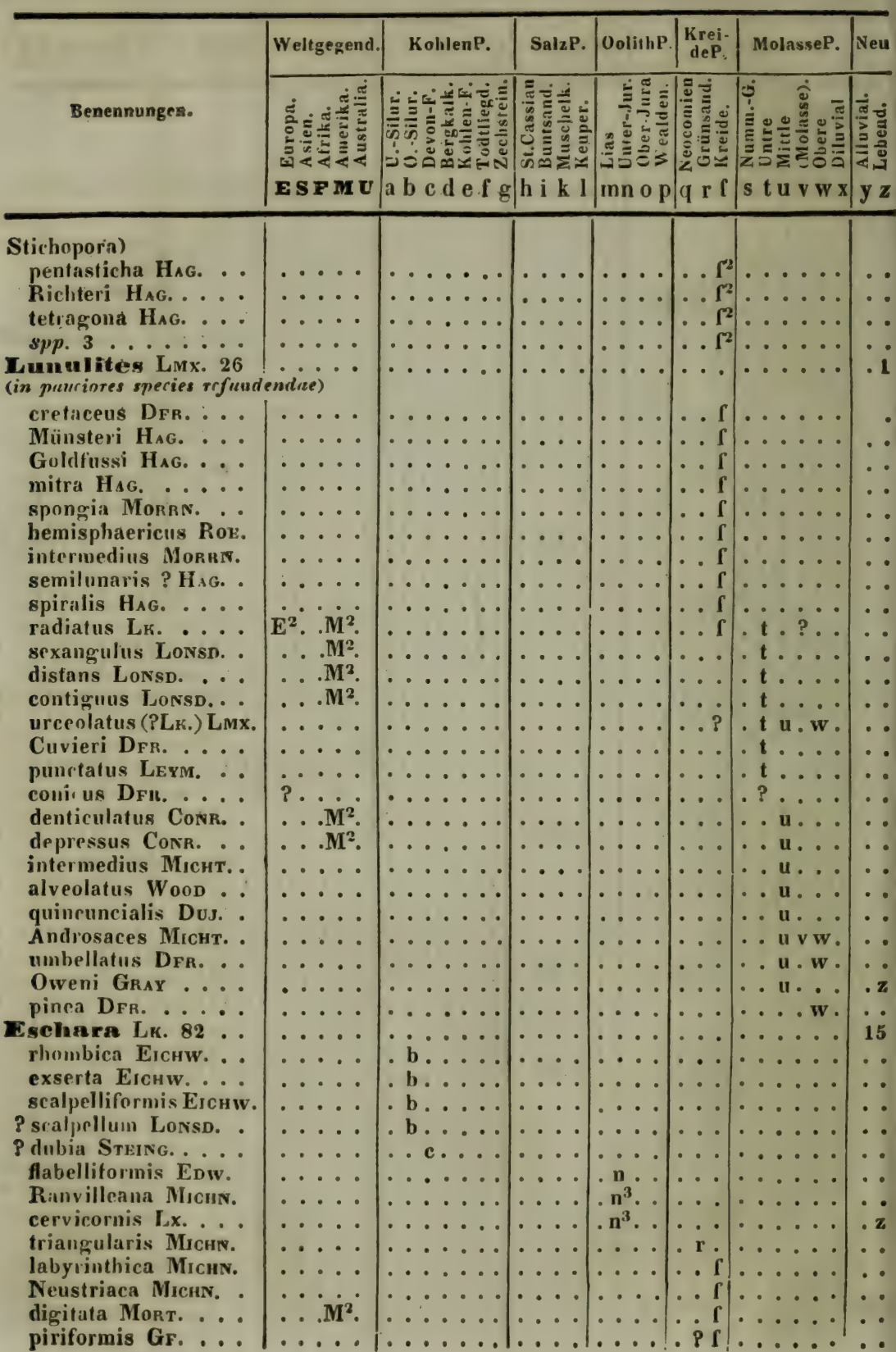




\begin{tabular}{|c|c|c|c|c|c|c|c|}
\hline Bene: & Itgegend. & $\mathrm{f} \mathrm{g}$ & & $\mathrm{mn}$ op & & $W^{\prime} \mathrm{X}$ & \\
\hline ata & & & & $\cdots$ & & $\cdots \cdots$ & \\
\hline cellata GF. . & & $\ldots \ldots$ & $\cdots$ & $\cdots$ & . $\mathrm{f}$ & ...... & \\
\hline ingularis $\left(\mathbf{G F}_{\mathbf{F}}\right)$ En & & $\ldots \ldots$ & $\cdots$ & $\cdots \cdot$ & . ? f f & $\ldots \ldots$ & \\
\hline$\cdots$ & $\ldots$. & $\cdots \ldots$ & $\cdots$ & $\cdots$ & $\ldots 1$ & $\ldots \ldots$ & \\
\hline$\cdots$ & $\cdots \cdot$ & $\cdots$ & $\cdots \cdot$ & $\cdots \cdot$ & $\cdots \mathrm{f}$ & $\cdots \cdots$ & - \\
\hline 7. . & $\cdots$ & & & $\cdots \cdot$ & & $\cdots \cdots$ & \\
\hline ata 1 & & & & & & $\cdots$ & \\
\hline $\begin{array}{l}\text { in } \\
\text { di }\end{array}$ & $\because$ & & & $\therefore$ & 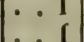 & $\cdots$ & \\
\hline$\because$ & $\ldots$ & $\cdots$ & $\cdots$ & $\cdots \cdots$ & $\begin{array}{ll}\cdots & 1 \\
\cdots & 0\end{array}$ & $\cdots \cdots$ & \\
\hline G. & .... & $\ldots \ldots$ & $\ldots$ & $\ldots$ & . 1 & $\because \ldots$ & \\
\hline$\cdots$ & $\cdots \cdots$ & $\cdots \cdots$ & $\ldots$ & $\cdots$ & $\ldots \Gamma^{2}$ & $\cdots \cdots$ & 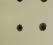 \\
\hline$\cdots$ & $\ldots$ & $\cdots$ & $\ldots$ & $\cdots \cdot$ & . . $\Gamma^{2}$ & $\ldots \ldots$ & \\
\hline$\cdots$ & $\cdots \cdots$ & $\cdots$ & $\cdots$ & $\cdots \cdot$ & & $\cdots$ & \\
\hline G. . & - & $\cdots$ & $\ldots$ & $\cdots \cdots$ & & . & - \\
\hline - & & $\cdots$ & $\cdots$ & $\cdots \cdot$ & & $\cdots$ & - \\
\hline .. & & & & $\cdots \cdot$ & 2 & $\cdots$ & \\
\hline & & & & & & $\cdot$ & \\
\hline - & $\therefore$ & & : & $\left|\begin{array}{c}\cdots \\
\cdots\end{array}\right|$ & & $\because$ & \\
\hline$\therefore$ & $\ldots \ldots$ & $\ldots \ldots$ & $\ldots$ & $\ldots$ & .. $\mathrm{r}^{2}$ & $\therefore \ldots$ & \\
\hline AG. & & $\cdots$ & $\cdots$ & $\cdots \cdot$ & .. $\mathrm{f}^{2}$ & $\ldots \ldots$ & \\
\hline . & . & $\cdots$ & $\cdots$ & $\cdots \cdots$ & ... $\Omega^{2}$ & $\ldots \ldots$ & \\
\hline - & $\cdots$ & $\cdots$ & $\cdots \cdot$ & $\cdots \cdot$ & & $\cdots$ & \\
\hline$\cdots$ & $\cdots$ & $\cdots$ & $\cdots$ & $\cdots$ & & $\ldots \ldots$ & \\
\hline$\cdots$ & $\cdots$ & $\cdots$ & $\cdots$ & $\cdots \cdots$ & & $\cdots \cdots$ & \\
\hline$\cdots$ & $\cdots$ & $\cdots$ & $\cdots$ & $\cdots \cdots$ & - & $\cdots \cdots$ & \\
\hline . & $\because$ & $\because$ & : & $\cdots \cdots$ & $\dot{0}$ & $\because$ & \\
\hline$\cdots$ & $\ldots$ & $\cdots$ & $\ldots$ & $\cdots$ & $\therefore]^{2}$ & 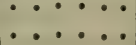 & \\
\hline$\because$ & .... & $\ldots \ldots$ & $\ldots$ & $\ldots$ & ... $\Omega^{2}$ & $\ldots \ldots$ & \\
\hline$A G \quad \therefore$ & .... & $\ldots \ldots$ & $\ldots$ & $\cdots$ & . . $\Gamma^{2}$ & $\ldots \ldots$ & \\
\hline$\cdots$ & $\cdots \cdot$ & $\ldots \ldots$ & $\cdots$ & $\cdots$ & $\left|.8^{2}\right|$ & $\ldots \ldots$ & . \\
\hline & $\ldots$ & $\ldots$ & . & $\cdots$ & $\ldots \Upsilon^{2}$ & $\ldots \ldots$ & \\
\hline G. . . & $\cdots$ & $\cdots$ & . & $\ldots \cdots$ & . . 12 & $\ldots \ldots$ & $\bullet$ \\
\hline HAG. . & $\cdots$ & $\cdots \cdot$ & $\cdots \cdot$ & $\cdots$ & . . 12 & $\ldots \ldots$ & . \\
\hline$\cdots$ & $\ldots$ & . & $\cdots$ & $\cdots$ & & $\ldots \ldots$ & $\cdots$ \\
\hline & $\cdots$ & & & $\cdots$ & & - $\cdot$ & \\
\hline & $\cdots$ & & - & & & $\ldots \ldots$ & \\
\hline a 1 & $\cdots$ & & - & & & $\cdots$ & \\
\hline $\mathbf{R N}$. & $\because$ i & & & $\cdots$ & $\therefore ?$ & 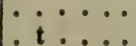 & \\
\hline & $\cdots N$ & & $\cdots$ & $\ldots \ldots$ & $\therefore$ & .1 & \\
\hline ‥ & $\because \mathrm{M}^{2}$ & $\therefore$ & $\ldots$ & .... & $\cdots$ & t... & \\
\hline$\cdots$ & . . $M^{2}$ & . & $\ldots$ & $\cdot$ & $\cdots$ & . t... & - \\
\hline 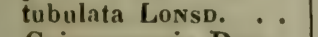 & $\ldots \mathbf{M}^{2}$ & & . & $\cdots \cdot$ & $\cdots$ & . $\ldots$ & . \\
\hline R. . & & & & & $\cdot$ & $\cdots$ & \\
\hline 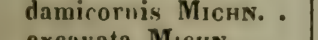 & & & & & & & \\
\hline .. & . & & & & & $\begin{array}{l}\cdots \\
\cdots\end{array}$ & \\
\hline Dw. & $\therefore$ & & & & & & \\
\hline
\end{tabular}




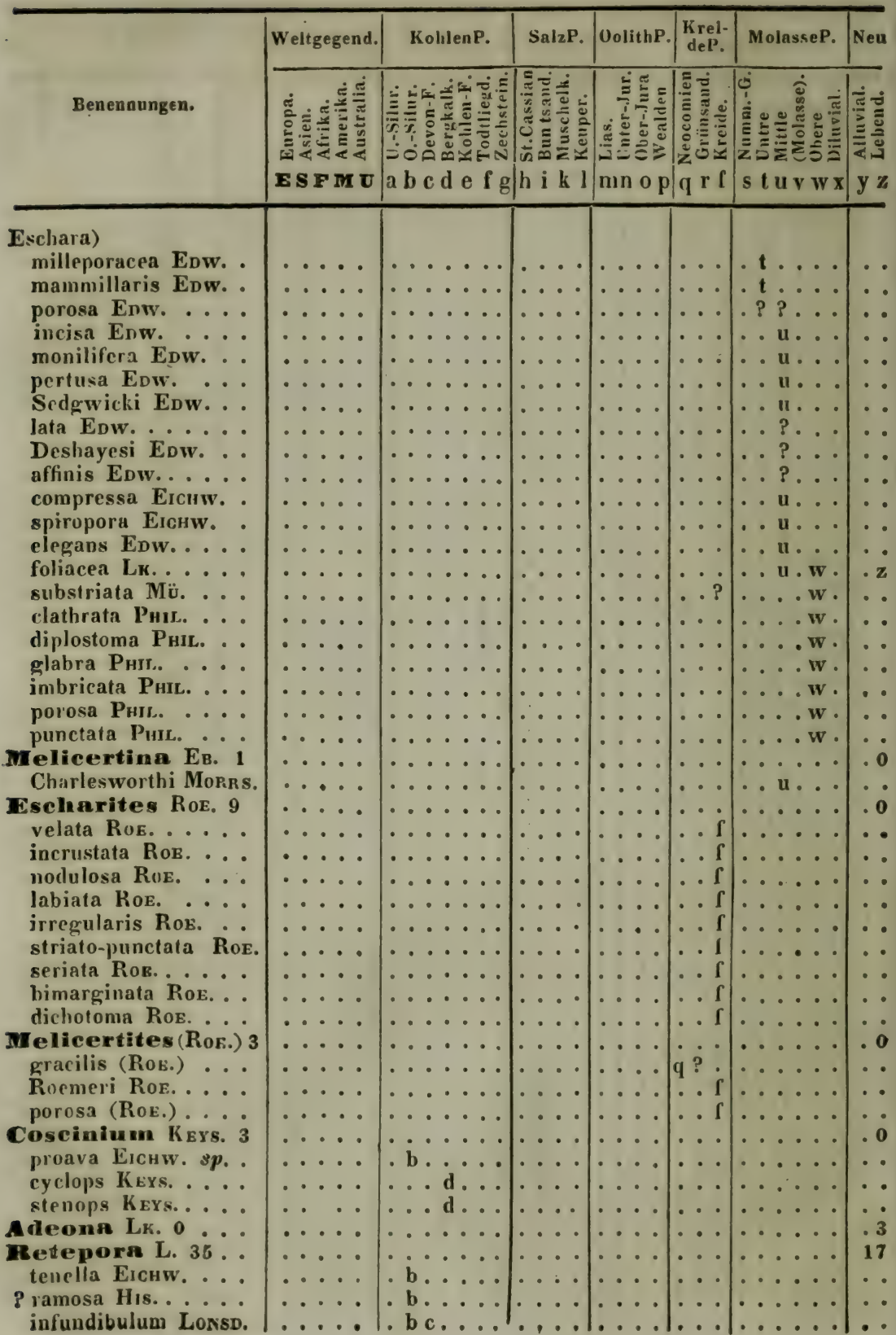




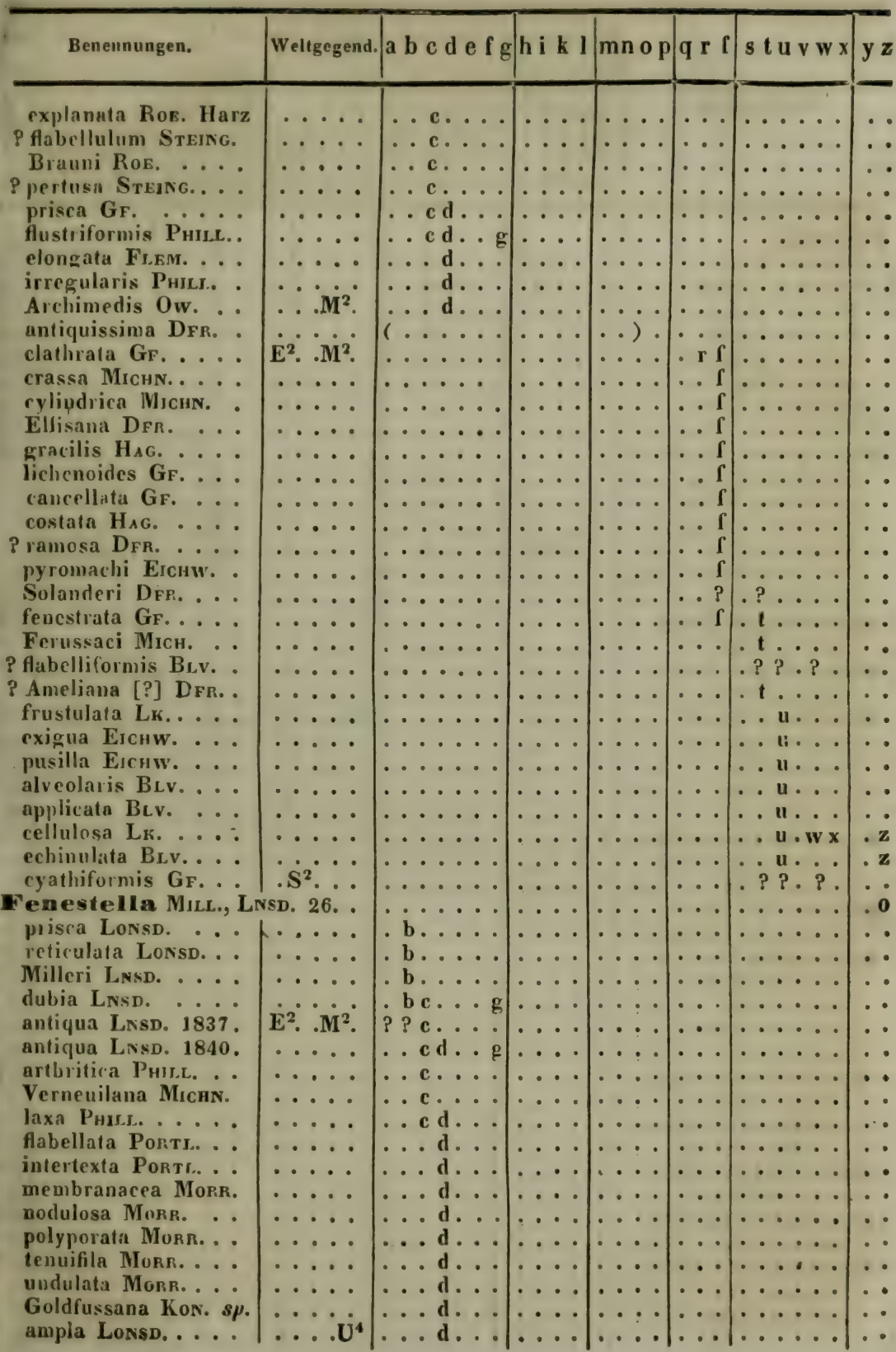




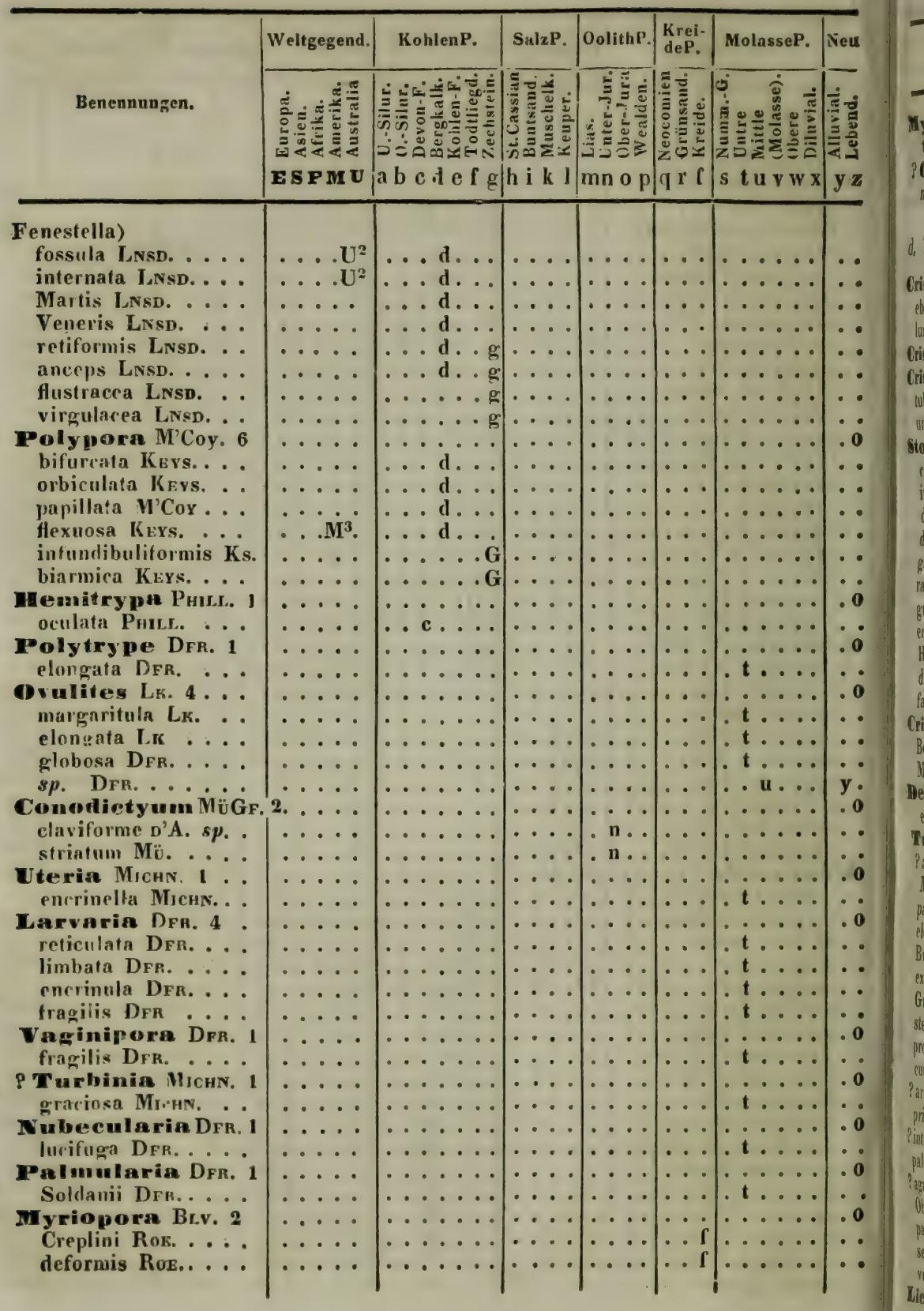


Myriozoon Don. 1 truncatum EB.

PClypeina Michn. marginiporella Micun.

\section{d. Tubuliporina Edw.}

Crisia (Lx.) Fuem. 2 eburnea Lx. .

luxata Fi.em.

Crisidia Edw. 0 ..

Crisoidea Michn. 2 tubiformis Мгсн. undulata Mıснs.

Stomatopora Br. 10 compressa GF. $s p$. intermedia Mü. $s p$. . dichotomu Lx. sp. divaricata Rов. $s p$. . granulata E.pw. sp. ramea Bı.v. $s p$.... gracilis Edw. $s p$. echinata Ros. $s p$. Hagenowi Reuss sp. dispersa Hag. $s p$. fascicularis LYhr.L $s p$.

Criserpia Edw. 2 . Boloniensis Michn. . Michelini Eow.

mendropora Mich. I explicata MínN.

Tubalipora Lк. 19 P arcuata STrin.

Megaera Lwsd. ... parea Row. elegans Mrche. Brongniarti Edw. . explanata Eow... Grignonensis Eow. stelliformis Micun. . proboscidea LNsD. cumulus Erchw.

? arborea Wood primige iria Eгснw.

P intricaria Wuod palmata Woov ? agaricia Wood Obelia Јohnst patina JoHNst. serpens Јонкsт..
verrucaria EDw.

Giehenopora DFr. 8 Cenumana Michs. 


\begin{tabular}{|c|c|c|c|c|c|c|c|}
\hline & Weltgegend. & KohlenP. & Sal_P. & OolithP. & $\left|\begin{array}{c}\text { Krel- } \\
\text { deP. }\end{array}\right|$ & MolasseP. & Neu \\
\hline Benennungen. & 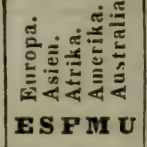 & 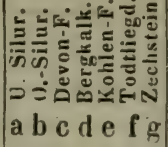 & 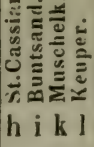 & $\begin{array}{l}x=0 \\
\text { mnop }\end{array}$ & 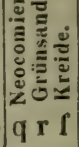 & 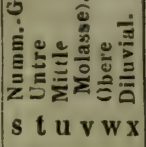 & $\begin{array}{l}3 \\
\mathbf{y} z\end{array}$ \\
\hline
\end{tabular}

Lichenopora) cretacea DFR. cribrosa Reuss Defranceana Micun. crispa Drk. turbinata DFR. tuberosa MichN. mediterranea BLv.

Rubula DFr. Soldanii

Filicella Wood. 1. anguinea Wood

Mornera Lx. 17 .

? crassa LNsD. . . . carinata Reuss Langethali RoE. hippolithus DFr. crispa DFR. opuntia DFR. laev is Evw. retiporarea EDw. radians DFr. striata EDw. decipiens Eicuw. affinis EDw. biseriata PhIL. . . gracilis PhIL. subannulata Phí. elegans Drr.

Cricopora Blv. 13 elegans Buv..... caespitosa Buv....

? dumetosa Buv. tetragona Buv. capillaris Er.v. ablureviata Br.v. straminea Morris verticillata Michn. 2. Tessoni Michn. . culiformis MichN. verticillata Мichn. 1. annulata Reuss... Faujasi BLv.

Idnoner Lx. 15 triquetra Lx. aculeata Micus. tetragona MichN. . disticha BLv.

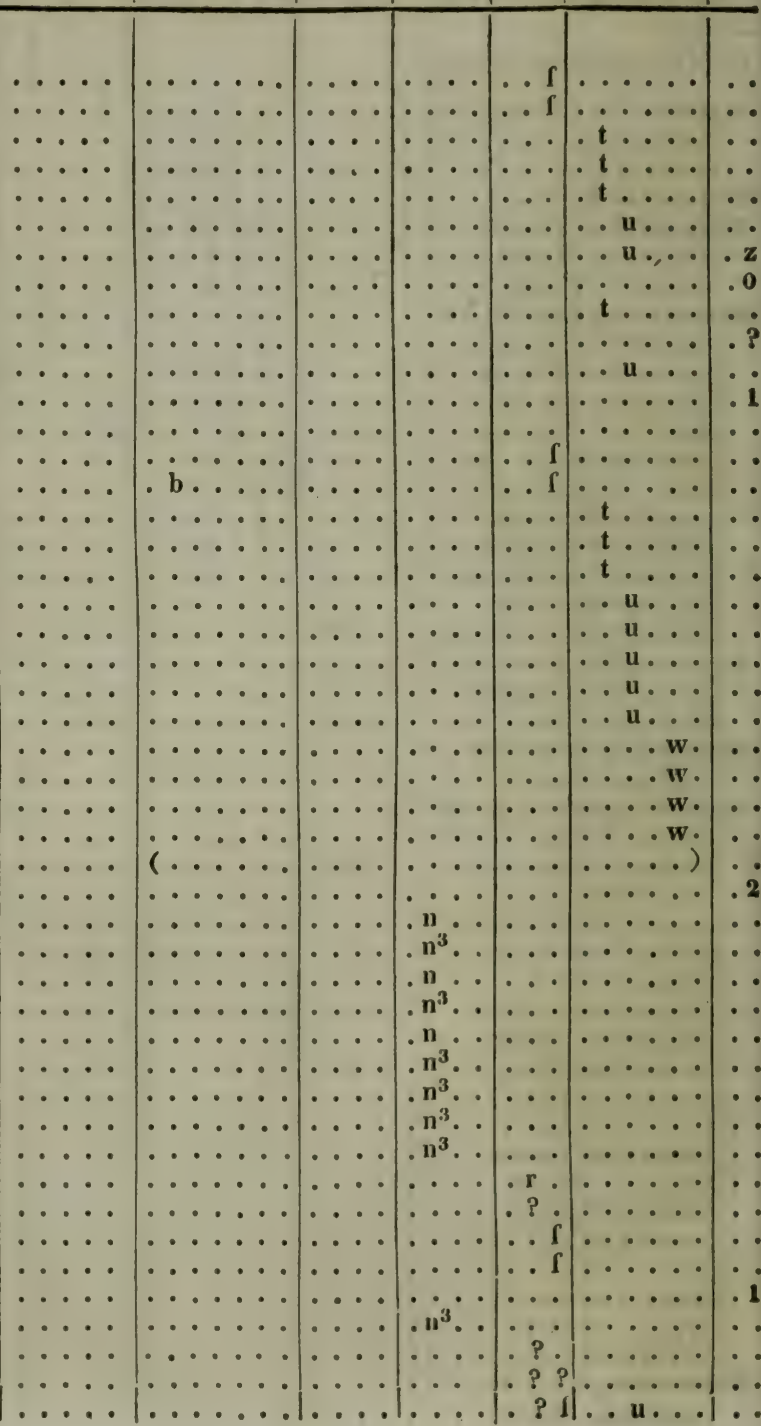




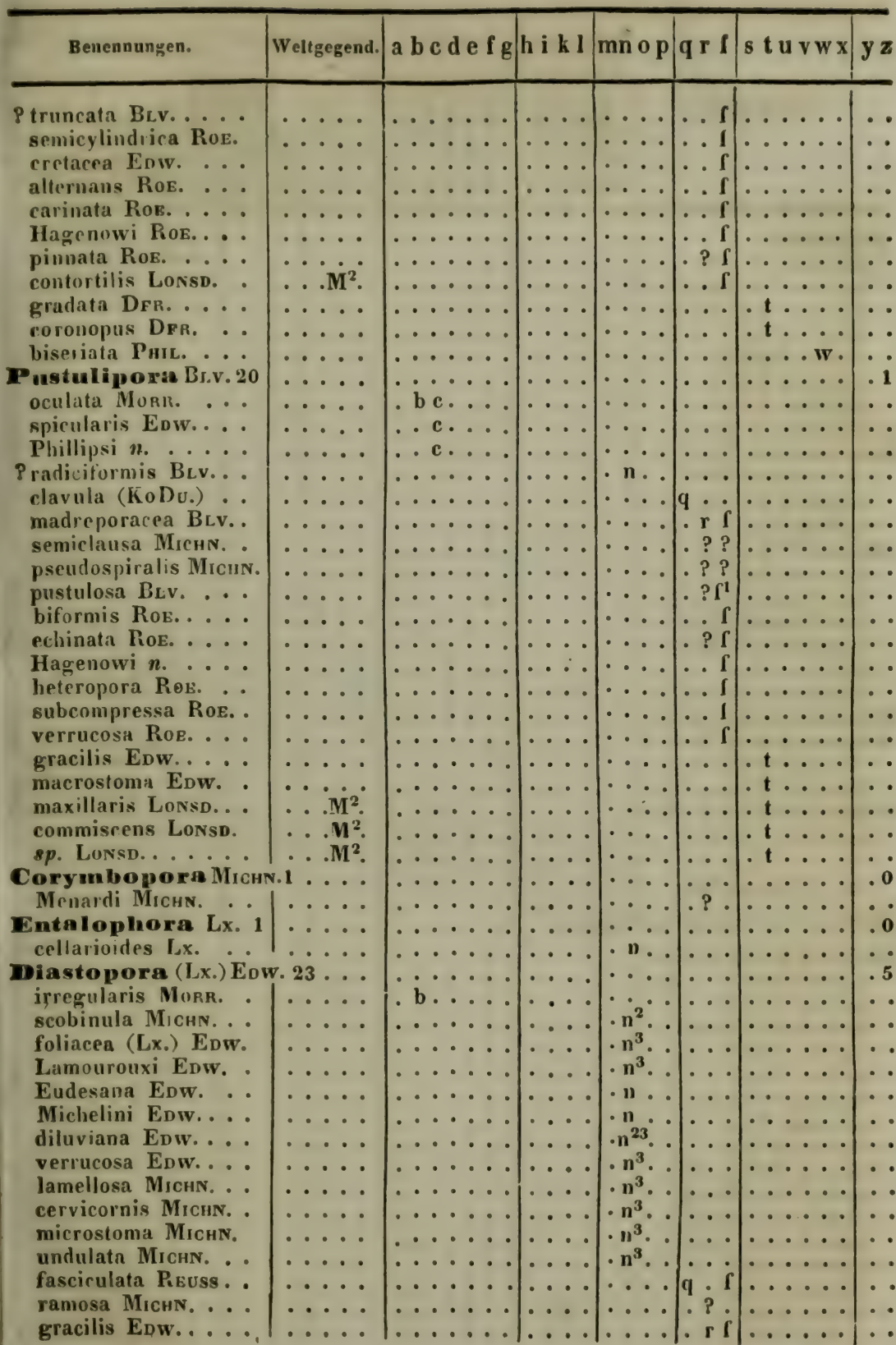




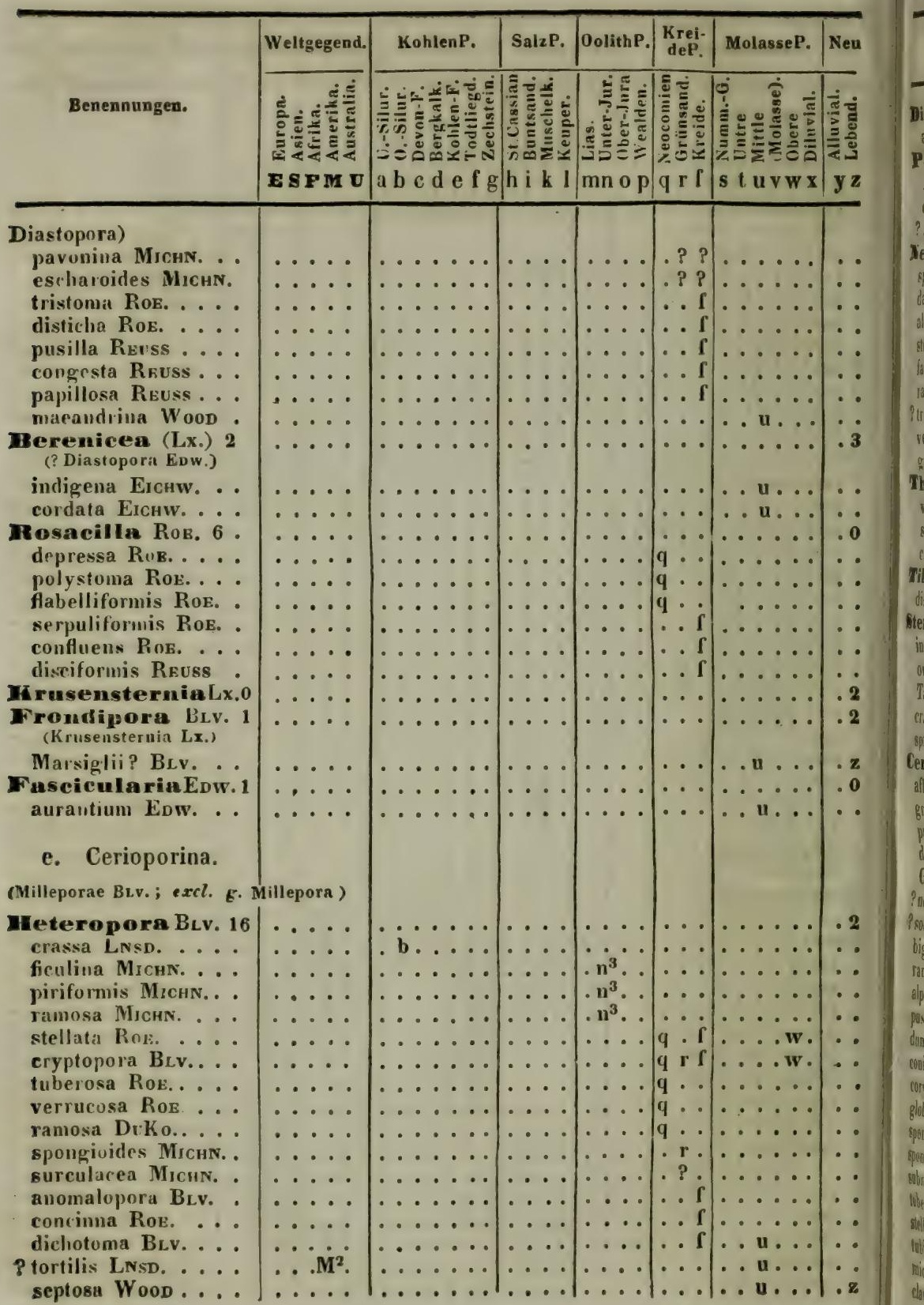




\begin{tabular}{|c|c|c|c|c|c|c|c|}
\hline ene & Itgegend. & $\mathbf{f g}$ & k $\mathbf{l}$ & $\mathrm{op}$ & I & $\mathbf{w} x$ & . \\
\hline . 1 & & & & & & & - 1 \\
\hline$\dot{.}$ & $\cdots \cdot$ & . & & & &.$t \ldots$ & $\because$ \\
\hline $\begin{array}{l}8 \text { DFR. } \\
\text { IS [?] }\end{array}$ & $\cdots \cdot$ & $\cdots$ & & & & $\cdots \cdots$ & .0 \\
\hline $\begin{array}{l}\text { Protaeus [?] } \\
\text { elegans DFk. }\end{array}$ & $\cdots$ & $\cdots$ & . & & $\cdots 1$ & $\cdots$ & \\
\hline ? nitra Br.. & $\ldots \ldots$ & $\cdots \cdots$ & $:$ & $\dot{.}$ & $\because 1$ & $\cdots \cdots$ & \\
\hline Вr. 9. & & $\ldots \ldots$ & & & $\cdots$ & $\ldots \ldots$ & 0 \\
\hline & & $\cdots$ & $\cdots$ & . & $\cdots$ & $\ldots \ldots$ & . \\
\hline & & $\cdots$ & $\cdots$ & .. & $\cdots$ & $\ldots \ldots$ & \\
\hline & & $\cdots$ & $\cdots$ & $\cdot$ & $\cdot$ & $\cdots$ & \\
\hline st & & & $\cdots$ & $\cdot \mathbf{n} \cdots$ & $\cdots$ & $\cdots$ & \\
\hline$\cdots \cdot$ & $\cdots$ & $\cdots$ & $\cdots \cdot$ &.$n \ldots$ & $\cdots$ & $\cdots \cdots$ & \\
\hline$\cdots$ & ・ & $\cdots$ & $\cdots$ & $\cdot 11 \cdot$. & . $\mathrm{f}$ & $\ldots \ldots$ & $\cdots$ \\
\hline 8 trigona & $\cdots$ & $\cdots$ & $\cdots$ & & $\cdot$ & $\ldots \ldots$ & \\
\hline$\therefore$ & & $\cdots$ & $\cdots$ & & 4 & $\cdots \cdots$ & \\
\hline $\begin{aligned} \mathrm{gr} \\
\text { Th }\end{aligned}$ & & $\cdots$ & & & & & \\
\hline Th & & & & & & . & 0 \\
\hline N. & & $\because$ & & & . $\mathrm{f}^{1}$ & . & - \\
\hline N. & : & $\because$ & $\cdots$ & $\cdots$ & .. $\mathrm{f}$ & $\cdots \cdots$ & \\
\hline$\ldots$ & . & .. & $\ldots$. & . & $\ldots$ & $\ldots \ldots$ & 0 \\
\hline . & & & $\cdots$ & . n.. & $\cdots$ & $\ldots \ldots$ & .. \\
\hline 5 & & $\cdots$ & $\cdots$ & $\cdots \cdots$ & $\cdots \cdot$ & $\cdots$ & .0 \\
\hline &. $\mathrm{U}^{4}$ & $\ldots$ & $\cdots$ & $\cdots \cdot$ & $\cdots \cdot$ & $\ldots .$. & $\cdots$ \\
\hline & $\cdots \mathrm{U}^{4}$ & $\ldots d \ldots$ & $\cdots$ & $\cdots \cdot$ & $\cdots$ & $\ldots \ldots$ & ${ }^{\circ}$ \\
\hline NSD. & $\therefore . \mathrm{U}^{4}$ & $\ldots d \ldots$ & $\ldots$ & $\cdots$ & $\cdots$ & $\ldots \ldots$ & \\
\hline - & & $\cdot \cdot \mathrm{g}$ & . & - & $\cdots$ & $\ldots \ldots$ & \\
\hline 81 & & $\cdots g$ & 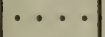 & . & $\cdots$ & $\cdots$ & \\
\hline .) BLv & & $\cdots$ & - & $\cdots$ & $\cdots$ & $\cdots \cdots$ & .0 \\
\hline • & ${ }^{\circ}$ & $\cdots$ & $\cdots$ & $\cdots$ & $\cdots \cdot$ & $\cdots \cdots$ & \\
\hline - & •. & $\cdots \cdots$ & 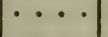 & $\cdots \cdot$ & $\cdots$ & $\cdots \cdots$ & \\
\hline$\cdot \cdot$ & . & $\cdots$ & $\cdot$ & $\cdots$ & $\cdots$ & $\cdot$ & \\
\hline . & - & $\cdots$ & $\cdots \cdots$ & $\cdots$ & $\cdots$ & . & \\
\hline ? no & & 0 & & & $\because$ & $\ddot{0}$ & \\
\hline ? sociata Fise & & $\ldots$.... & & $\ldots$ & $\ldots$ & $\ldots \ldots$ & \\
\hline . . & & $\ldots d \ldots$ & $\ldots$ & $\ldots$ & $\ldots$ & ..... & \\
\hline & ... $\mathbf{M}^{3}$. & ... d... & 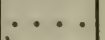 & $\cdots$ & $\cdots$ & $\ldots \ldots$ & \\
\hline & . & $\ldots \ldots$ & & & $\cdots$ & $\ldots \ldots$ & $\cdot$ \\
\hline osa & & $\cdots$ & & $\cdot n^{3} \cdot$ & $\cdots$ & $\ldots \ldots$ & - \\
\hline tosa Michn. .. & & $\cdots$ & $\cdots$ & $\cdot n^{3} \cdot \cdot$ & $\cdots \cdot$ & $\ldots \ldots$ & $\bullet$ \\
\hline$\cdots$ & . & . & . & $\cdot \mathrm{n}^{3} \cdot$ & $\cdots$ & $\ldots \ldots$ & \\
\hline N. . & - & & & . & $\cdots \cdot$ & $\cdots \cdots$ & \\
\hline M & .. & $\cdots$ & & $\cdot n^{3}$ & $\cdot$ & $\cdots \ldots$ & \\
\hline ites $\mathrm{C}$ & - & • & & & & $\cdots \cdots \cdots$ & \\
\hline a 1 & & & & & & $\cdots \cdots$ & \\
\hline OE. & $\cdots$ & & & & & & \\
\hline$\therefore$ & & & & & & $\cdots \cdots$ & \\
\hline & - & 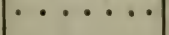 & & & & $\cdots$ & $\cdots$ \\
\hline 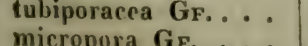 & & & & & & & \\
\hline : & $\because$ & $\because$ & 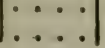 & . & $\mid \begin{array}{ll}q & 0 \\
9 & .\end{array}$ & . & \\
\hline
\end{tabular}




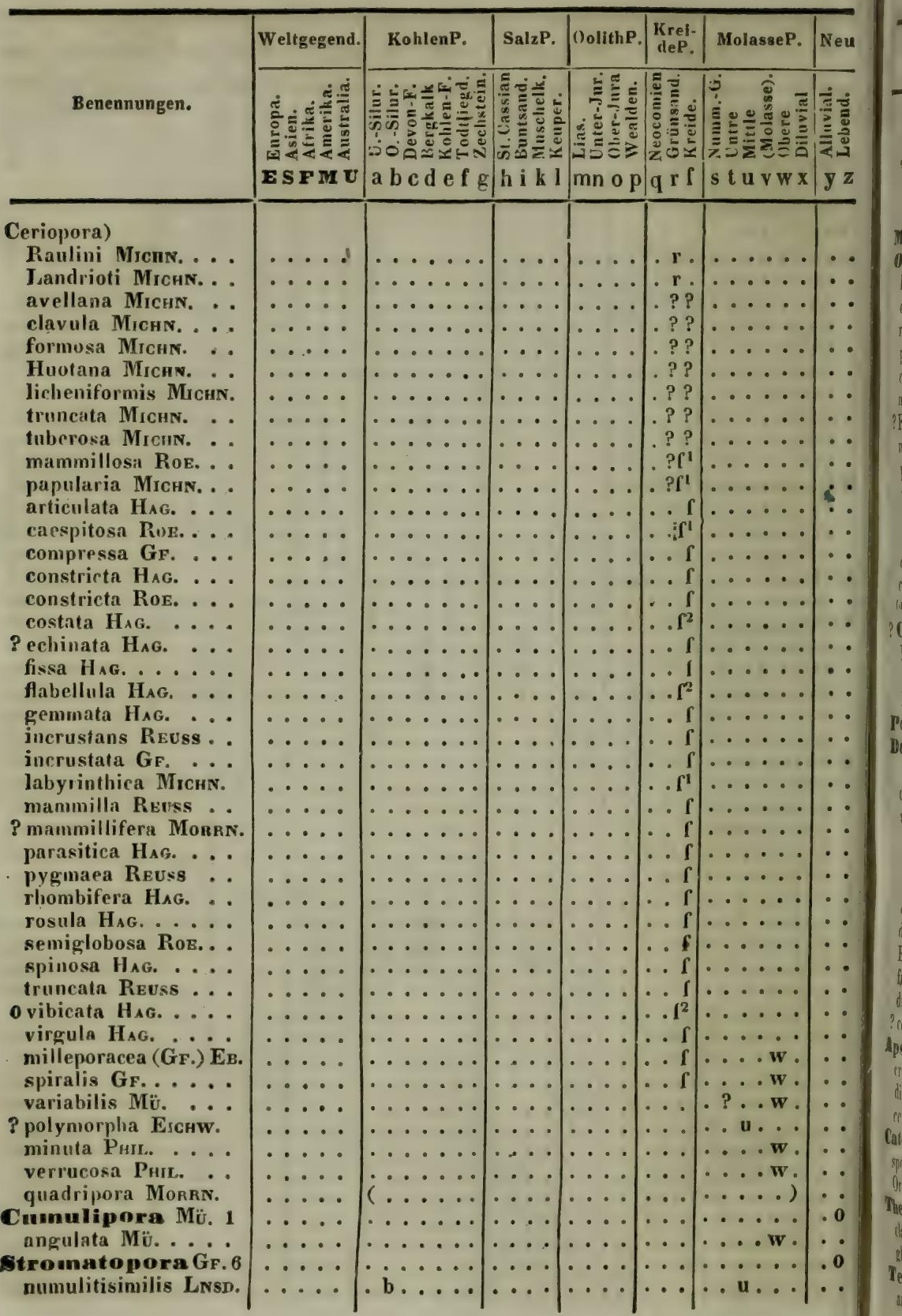




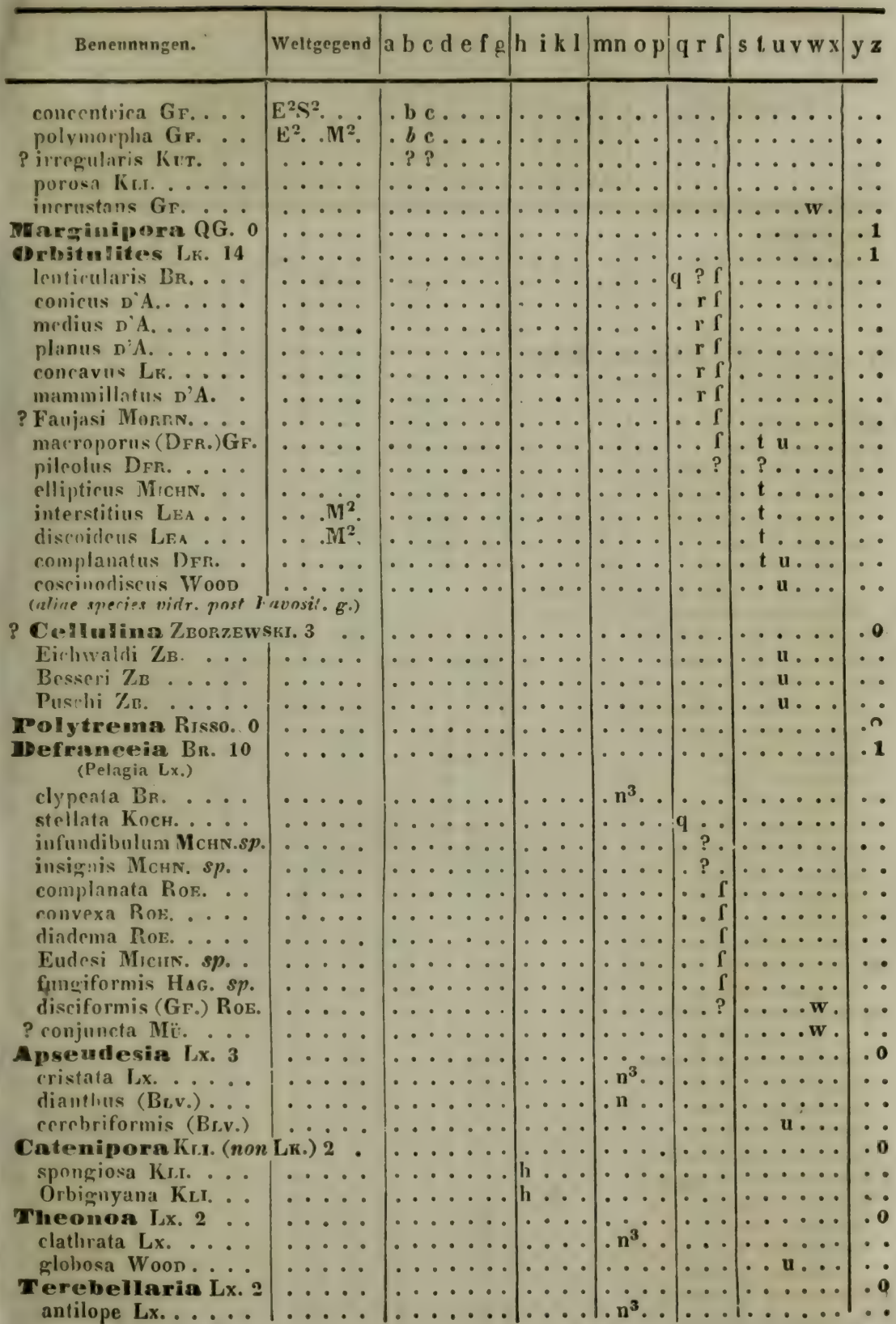




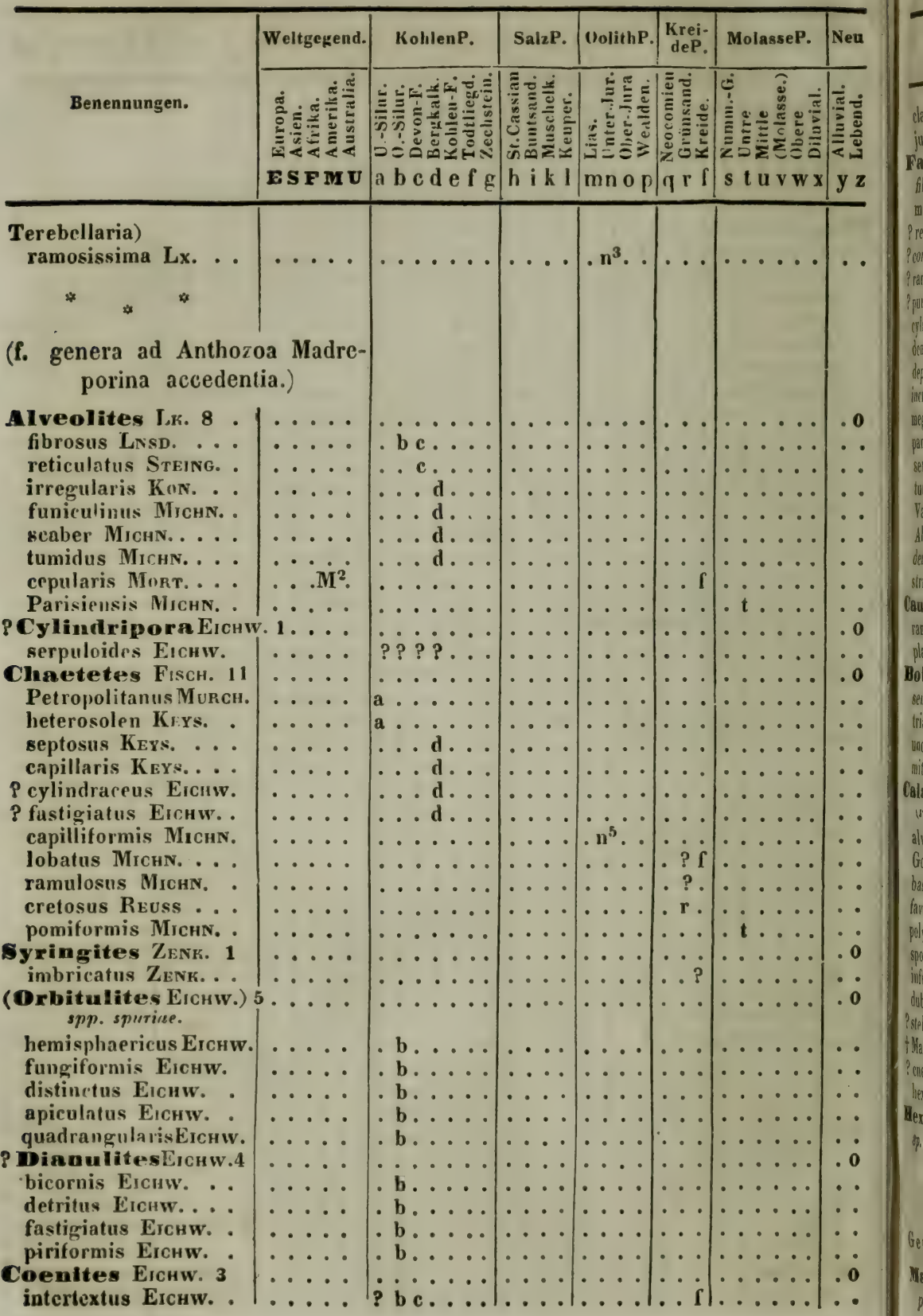


Benennungen.

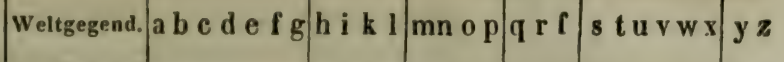

clathratus (STEivg.) juniperinus Еıснw.

Favosites. Lк. 18

fibrosus LNsD.

multiporus LNsD.

P reticulum Eicнw.

$P$ conmunis $\mathbf{L x}$.

P ramosus Brass.

? punctatus Bour. cylindricus Michn. dentiferus Morr. depressus FLEM. incrustans MorR... megastomus MorR. . parasiticus Morr. serialis Pokte. tumidus Morp. Valonensis DFr. Alcyon DrR. demucraticus Risso striatus DFR.

Caunopora Phill. 2 ramosa Phicl. placenta Phicl.

Bolboporites PND. 4 semiglubosus Pand. triangularis $P_{A N D}$. uncinatus Pand... mitralis PAND.

Calrmopora GF, 12 (spp. (ulitis porosis)

alveolaris GF. Gothlandica GF. . . basaltica GF. favosa $\mathrm{GF}$. polymorpha GF. . spongites GF..... infundibulifera GF. dubia Mex.

? stellata Fisch.

+ Mackrothi GEIN.

? cnemidium KLI. hexagonalis Lis м.

Hexaporites $P_{A N D} 1$ sp. (P AND. $_{\text {. }}$

2

\section{$\mathrm{E}^{2} \dot{\mathrm{S}}^{2} \cdot \dot{\mathrm{M}}^{2}$} $E^{2} S^{2} M^{2} U^{4}$ $\mathrm{E}^{2} \cdot \mathrm{M}^{2}$. a b c

\section{.... $\mathbf{M}^{2}$} $E^{2} S^{2} \cdot M^{2}$ $\mathrm{E}^{2} \cdot \mathrm{M}^{2}$. $\mathrm{E}^{2} . \mathrm{M}^{2}$.

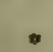

Genera incertae sedis.

Mastrema RAF. 1

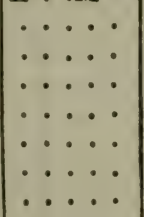
pentagonum RAF..$\ldots \dot{M}^{2}$

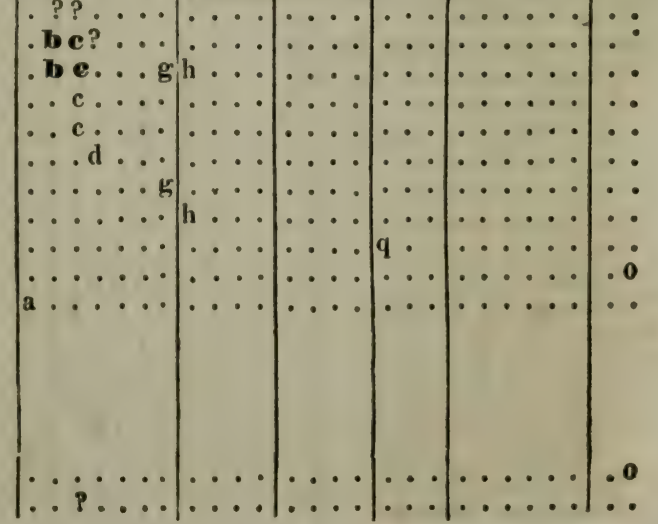




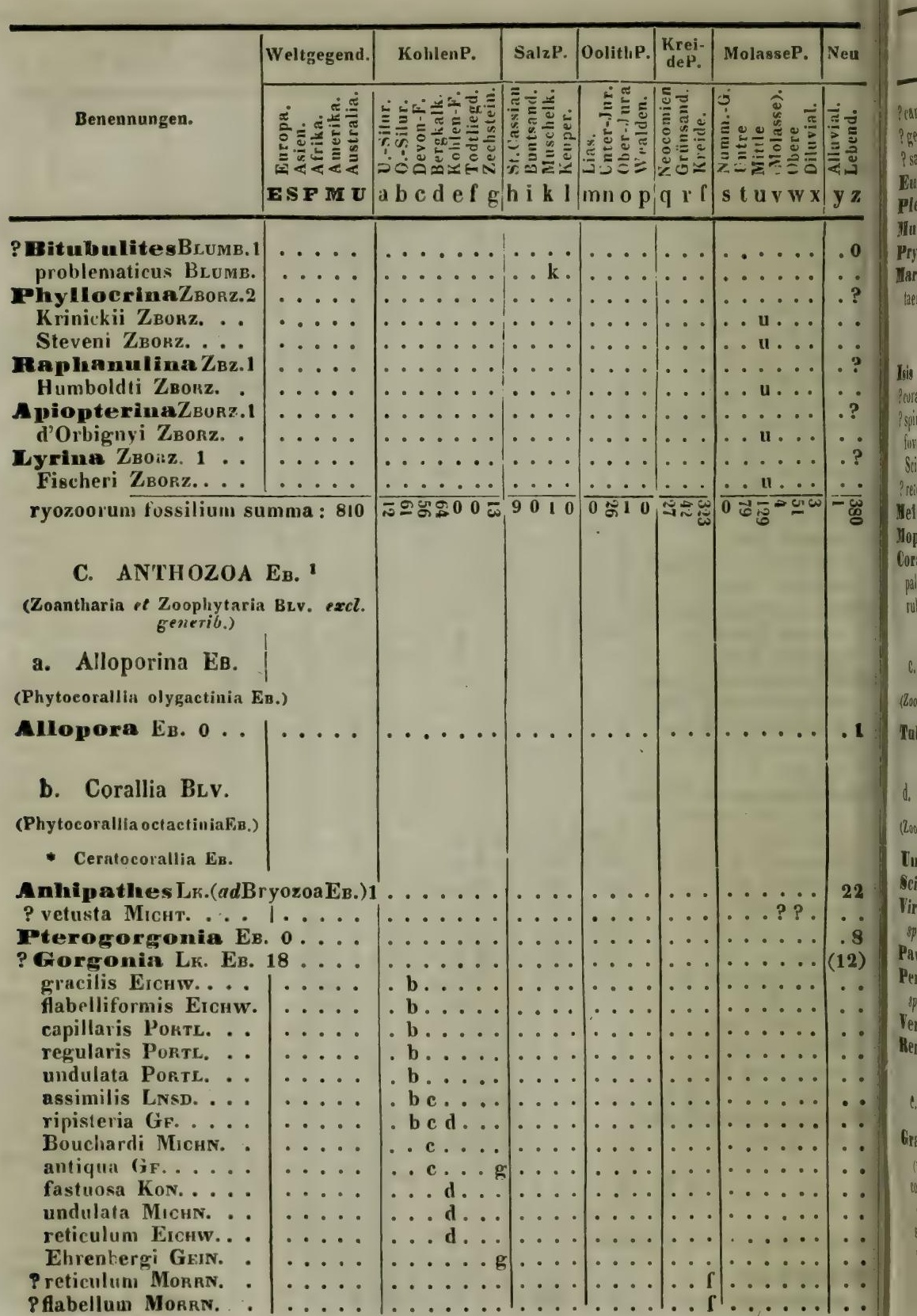

3 Numeros specierum viventium Ehrenbergi liber imprimis praebuit. 


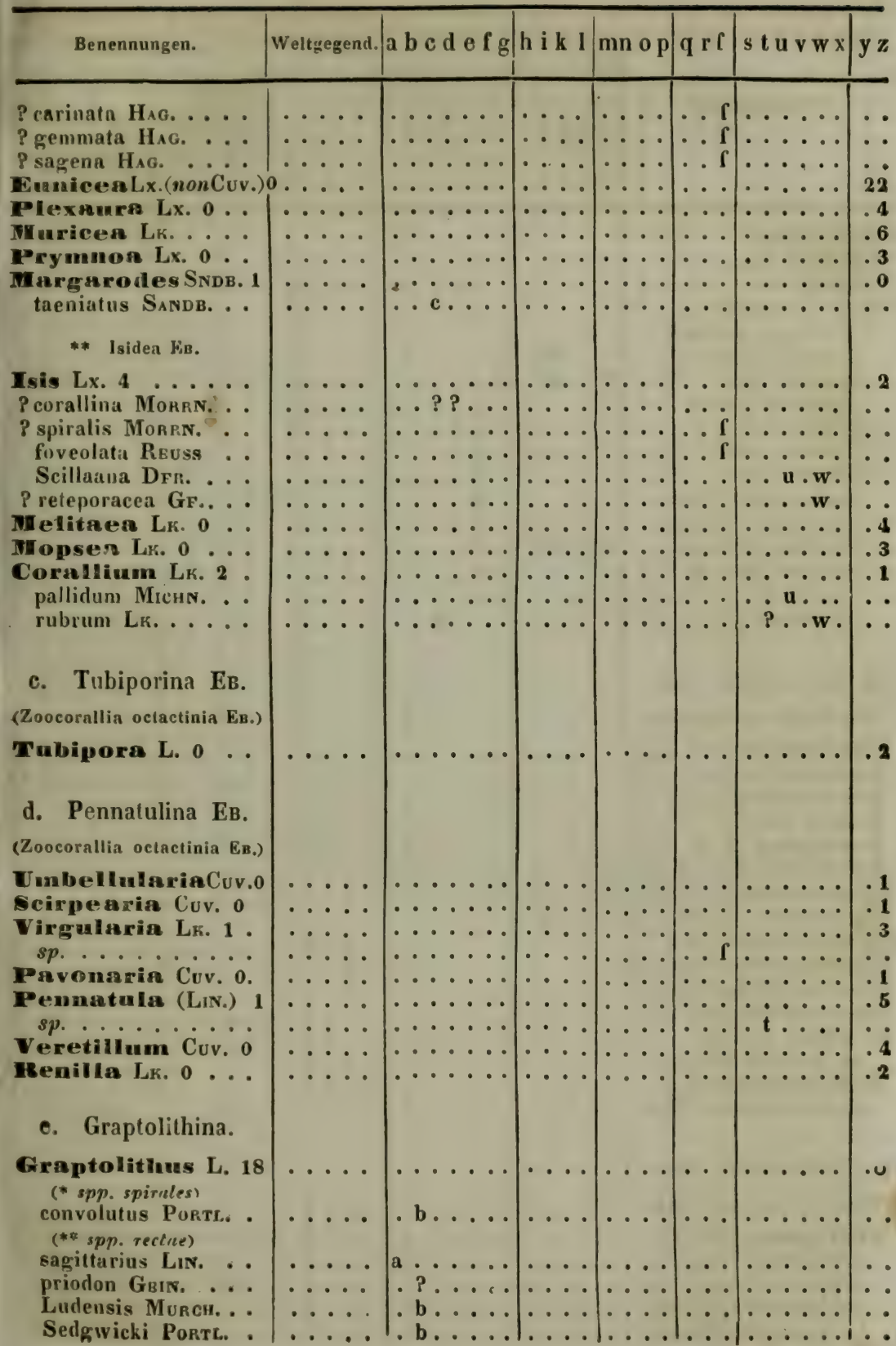




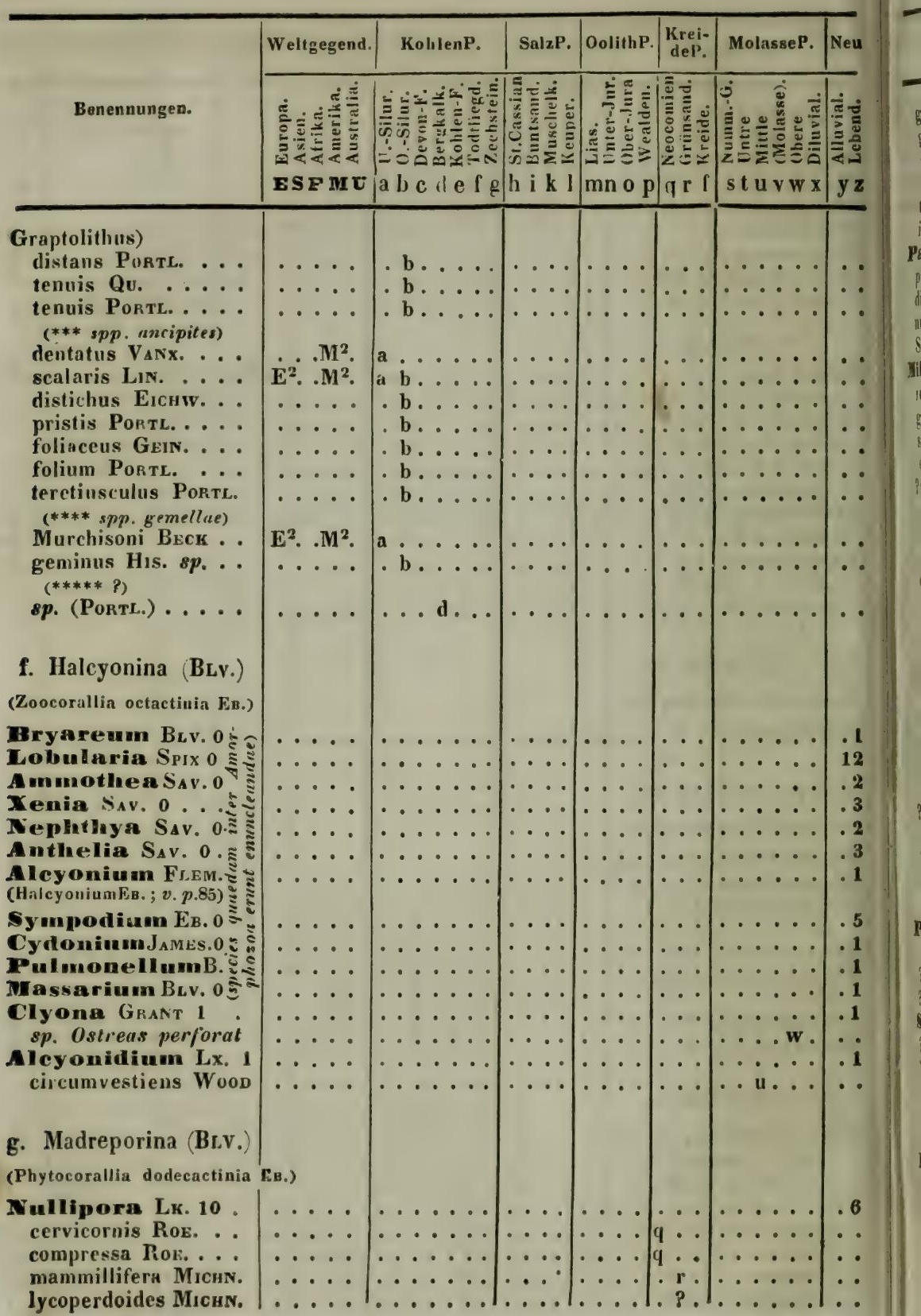


globulus Reuss gracilis Reuss granulosa MichN, palmata Gr. tuberosa Mrchis. informis $\mathrm{L}_{\kappa}$.

Palmipora Blv. 4 polymory ha Roe. dilatata Ros. nuciformis Roв. Solanderi MiснN.

Millepera. Lк.) Eв.23 repens His. gracilis Phrz. similis Phicr. exigua Steing.

Pagariciformis (LK.) DRAPZ interporosa Phile. rhombifera PhiLL. piriformis $\mathbf{L x}$. dispar DFr. spissa DFR. ramosa Flem. Gilberti Mant. elegans DFr. antiqua DFr. compressa Gr. crista-galli Morri. aspera (Lm.) Morren. madreporacea GF.

Pcervicornis Puscu Dekini Morrin.

? foliacea (Bosc) Serr. punctata PHIL. polymoipha FLEM.

Pocillopora (Lx.)Buv. Ë.3 sertifera Micun.

? Solauderi DFR.

Papproximata Eichw.

Seriatopora (LK.)BLV.5

? antiqua DFr.

P cretarea DFr.

? Grignonensis DFr. ? cribraria DFR. subulata (? LK.) SERr.

Porites (LK.) EB. 14 inordinatus Lisp. discoideus LNsD. expatiatus LNsD. patelliformis LNed. tubulatus LNsD. 


\begin{tabular}{|c|c|c|c|c|c|c|c|}
\hline \multirow[b]{2}{*}{ Benennungen. } & Weltgegend. & KohlenP. & SalzP. & Oolithe. & $\begin{array}{c}\text { Krei- } \\
\text { deP. }\end{array}$ & MolatsseP. & Neu \\
\hline & 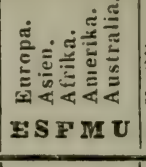 & 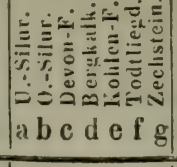 & 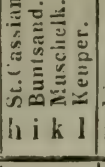 & 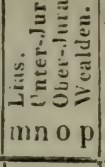 & 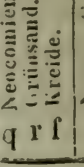 & 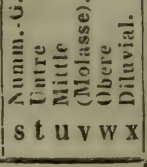 & 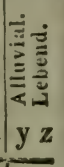 \\
\hline indarnianus & & & & & & & \\
\hline $\begin{array}{l}\text { Swindernianus (Ев.) } \\
\text { Michelini Peuss . . }\end{array}$ & $\begin{array}{l}\cdots \\
\cdots \\
\cdots\end{array}$ & $\begin{array}{l}\cdots \cdots \\
\cdots \cdots \\
\cdots\end{array}$ & $\cdots \cdots$ & $\cdots \cdots$ & $\cdots \dot{f}$ & $\cdots \cdots \cdots$ & $\because$ \\
\hline $\begin{array}{l}\text { Deshayesanus Miснn. } \\
\text { elegans Leym. . . }\end{array}$ & $\cdots$ & $\cdots \cdots$ & $\begin{array}{ll}\cdots \\
\cdots & \cdot \\
\cdots\end{array}$ & $\cdots$ & $\therefore \dot{~}$ & $\begin{array}{l}\cdot t \cdots \\
\cdot t \cdots\end{array}$ & $\because$ \\
\hline aceiosus Eichiv. . . & $\cdots \cdots$ & $\cdots \cdots \cdots$ & $\cdots \cdots$ & $\because \cdots$ & $\cdots$ & $\begin{array}{ll}\cdots \\
\cdots \\
u_{1} & \cdots \\
\end{array}$ & $\therefore$ \\
\hline $\begin{array}{l}\text { glaber Eв. } \\
\text { ? Stromatopora HE. } \\
\text { ? cellulosus FrEM. }\end{array}$ & $\because \mathrm{F}^{1}$ & $(\cdots \cdots \cdots$ & $\cdots \cdots$ & $\cdots$ & $\cdots$ & $\because$ u v. & $\therefore$ \\
\hline $\begin{array}{c}\text { ?cellulosus Fr.EM. } \cdots \\
\text { Comioporre QG. } 0 \\
\text { (Astraeae spp. Eв.) }\end{array}$ & $\cdots \cdots$ & $\cdots \cdots$ & $\cdots \cdots$ & $\cdots$ & $\because \dot{1}$ & $\cdots \cdots$ & $i$ \\
\hline $\begin{array}{c}\text { Mlcrosolewa Lx. } 1 \\
\text { (Madreporae spp. Ев.) }\end{array}$ & $\cdots$ & $\ldots \ldots$ & $\cdots \cdots$ & $\cdots \cdots$ & $\cdots$ & $\ldots \ldots$ & .2 \\
\hline $\begin{array}{l}\text { porosa Lx. } \cdots \cdots \\
\text { Aveopora }(\mathcal{G} .2 \\
\text { (Microsolenae } \operatorname{spp} .2 .)\end{array}$ & $\cdots \cdots$ & $\cdots \cdots$ & $\cdots \cdots$ & $\begin{array}{l}n^{3} \cdots \\
\cdots\end{array}$ & $\cdots$ & $\cdots \cdots$ & $\ddot{4}$ \\
\hline $\begin{array}{l}\text { incrustata MicнN. . } \\
\text { tuberosa Michn. . }\end{array}$ & $\begin{array}{l}\cdots \\
\cdots\end{array}$ & $\cdots \cdots$ & $\mid \cdots$ & $\begin{array}{l}. n^{5} \ldots \\
. n^{5} \cdots\end{array}$ & $\cdots$ & $\cdots$ & 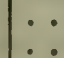 \\
\hline Melioporas BLv, 14 & $\cdots \cdots$ & $\ldots \cdots \cdots$ & $\cdots$ & $\cdots \cdots$ & $\ldots$ & $\ldots \ldots$ & .4 \\
\hline $\begin{array}{l}\text { interstincta } \mathrm{Br} . \ldots \\
\text { niriformis } \mathrm{Brv}\end{array}$ & $\cdots \cdots$ & a b $\quad \ldots .$. & $\cdots \cdot$ & $\cdots \cdots$ & $\cdots$ & $\cdots \cdots$ & $\cdots$ \\
\hline $\begin{array}{l}\text { piriformis Brv } \ldots \\
\text { dubia BLv. . . . }\end{array}$ & $\cdots \cdots$ & $\begin{array}{ll}\cdots & \cdots \\
\cdots & \cdots\end{array}$ & $\begin{array}{ll}\cdots \\
\cdots\end{array}$ & $\because \ddot{?} \dot{0}$ & $\because \cdots$ & $\therefore \cdots$ & $\because$ \\
\hline Blainvilleana МiснN. & $\cdots \cdots$ & $\ldots \ldots$ & $\cdots$ & $\cdots$ & $\cdot \mathbf{r}$ & $\ldots \ldots$ & - \\
\hline $\begin{array}{l}\text { elegans Buv. } \ldots \text {. } \\
\text { sulcata Brv. } \ldots \text {. }\end{array}$ & $\cdots$ & $\begin{array}{ll}\cdots & \cdots \\
\cdots & \cdots\end{array}$ & $\cdots$ & $\cdots \cdots$ & $\because f$ & $\cdots \cdots$ & $\ddot{\bullet}$ \\
\hline deformis Michn. . & $\ldots \ldots$ & $\cdots \cdots$ & $\therefore \cdots$ & $\therefore \cdots$ & $\cdots$ & $i \cdots$ & $\therefore$ \\
\hline $\begin{array}{l}\text { P panicea Blv. } \\
\text { Supergana Michn. }\end{array}$ & $\cdots \cdots$ & $\cdots \cdots \cdots$ & $\cdots$ & $\cdots \cdots$ & $\cdots$ & t $\ldots$ & $\because$ \\
\hline $\begin{array}{l}\text { Supergana MıchN. } \\
\text { plana BLv. } . . . .\end{array}$ & $\ldots \ldots$ & $\ldots \ldots$ & $\cdots$ & $\cdots \cdots$ & $\cdots$ & $\begin{array}{cc}\cdots u & \ldots \\
\cdots \mathrm{u} & \ldots\end{array}$ & $\ddot{\bullet}$ \\
\hline Madrepora(Br.v.Eв.) & $8 . \ldots$ & $\cdots \cdots$ & $\cdots$ & $\cdots \cdots$ & $\cdots$ & $\ldots \ldots$ & .2 \\
\hline $\begin{array}{l}\text { obeliscus Micнs. . } \\
\text { sublatevis Micus. }\end{array}$ & $\cdots \cdots$ & $\cdots \cdots \cdots$ & $\cdots \cdot$ & $\cdot 1^{5} \cdots$ & $\cdots$ & $\cdots \cdots$ & $\cdots$ \\
\hline $\begin{array}{l}\text { aevis Michn. . } \\
\text { villei DFr. . . }\end{array}$ & $\cdots$ & $\ldots \cdots \cdots$ & $\begin{array}{ll}\cdots \\
\cdots \\
\cdots\end{array}$ & $n^{5} \cdots$ & $\cdots$ & $\cdots \cdots \cdots$ & 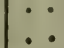 \\
\hline ornata DFR. .... & $\ldots \ldots$ & $\ldots \ldots$ & $\therefore \cdots$ & $\cdots \cdots$ & $\cdots$ & $\ldots \ldots$ & $\cdots$ \\
\hline Solanderi DFr. . . & $\cdots \dot{0}$ & $\ldots \ldots$ & $\cdots \cdot$ & $\cdots$ & $\cdots$ &.$t \ldots$ & - \\
\hline $\begin{array}{l}\text { tubulata LoNsD. . . } \\
\text { exarita Micit. }\end{array}$ & $\therefore M^{2}$ & $\cdots \cdots$ & $\cdots \cdot$ & $\cdots \cdot$ & $\cdots$ & $\ldots$ u... & - \\
\hline $\begin{array}{l}\text { rata Micita . . } \\
\text { andulina Micin. }\end{array}$ & $\cdots \cdots$ & $\ldots \ldots \ldots$ & $\cdots$ & $\cdots \cdots$ & $\cdots$ & $\cdots u, \cdots$ & $\ddot{z}$ \\
\hline $\begin{array}{l}\text { Montipora QG. } 0 \\
\text { (Madreporae spp. Eв.) }\end{array}$ & $\ldots$ & $\ldots \ldots$ & $\cdots$ & $\cdots$ & $\cdots$ & $\because \cdots \cdots$ & .2 \\
\hline $\begin{array}{l}\text { Stylophors.SснwgG.0 } \\
\text { (Madreporae } s p p \text {. Eв.) }\end{array}$ & $\cdots$ & & 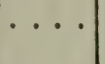 & - & - & $\cdots \cdots$ & .1 \\
\hline $\begin{array}{l}\text { Sideropora BLV. } 1 \\
\text { (Madreporae spp. EB.) }\end{array}$ & $\cdots$ & - & & $\cdots$ & $\cdots$ & $\ldots \ldots$ & .5 \\
\hline 8 sexradiata (EDw.). & 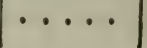 & 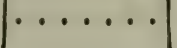 & . & $0 \cdot$ & - & $\cdots$ & $\cdots$ \\
\hline
\end{tabular}




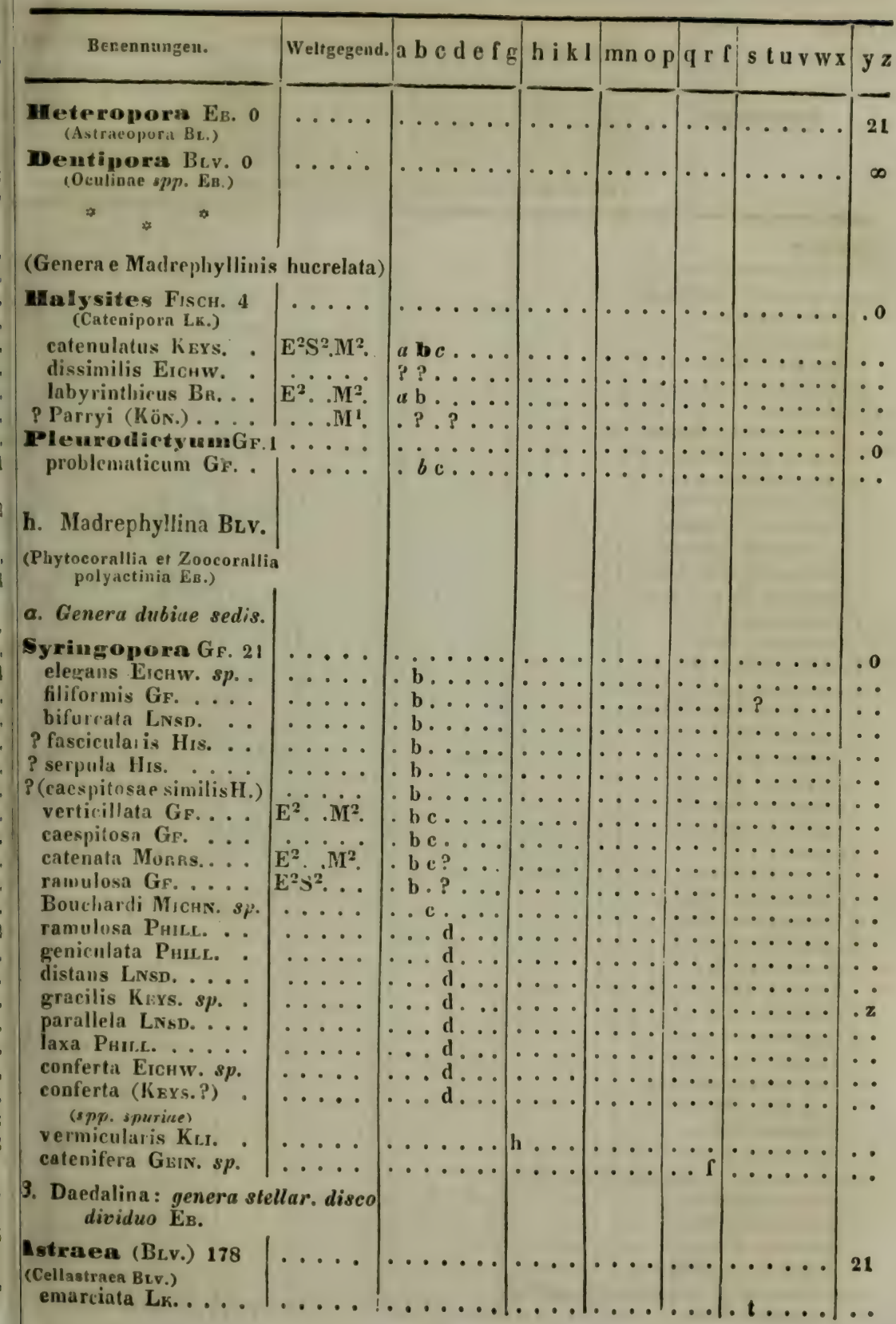




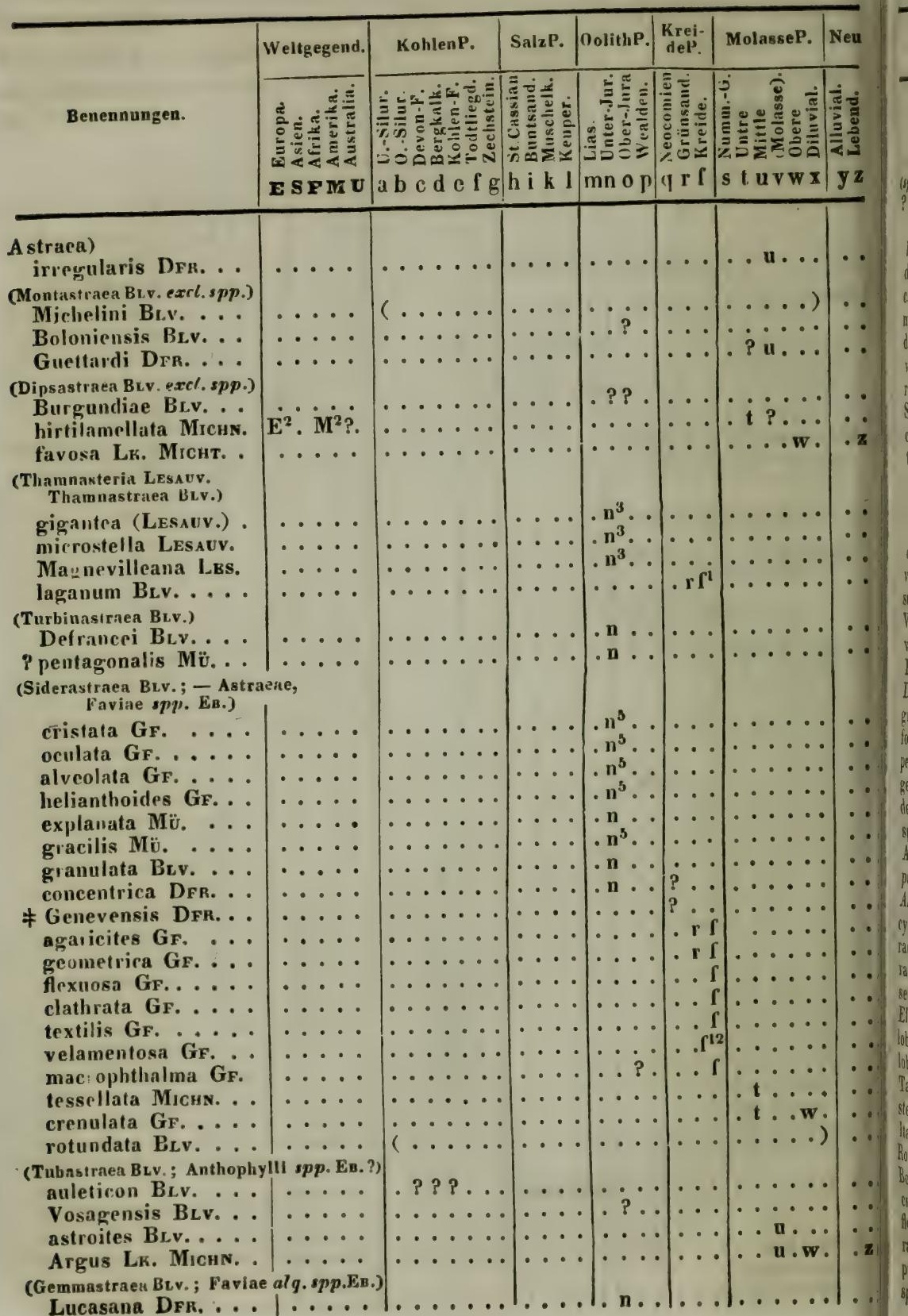




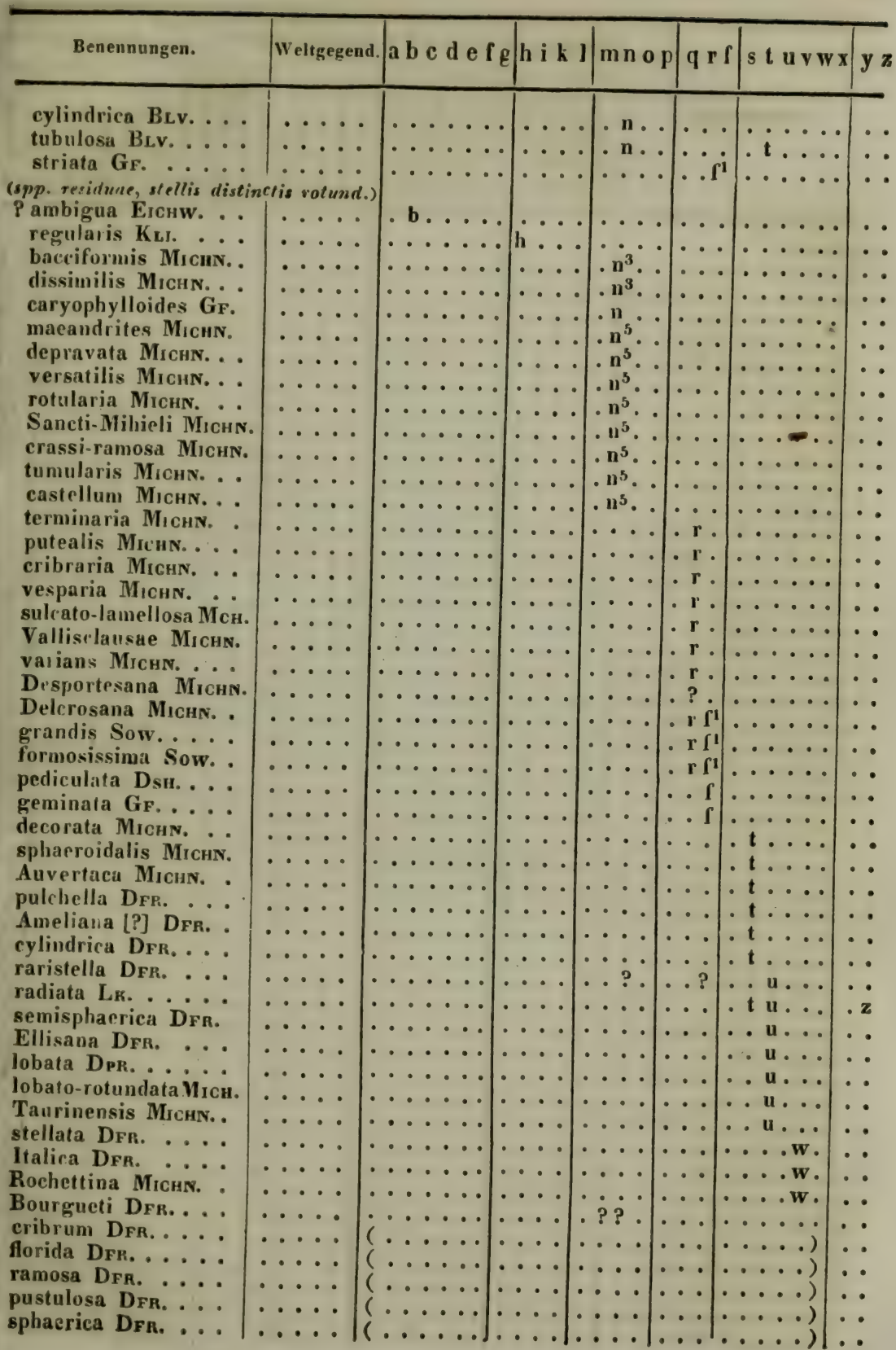




\begin{tabular}{|c|c|c|c|c|c|c|c|}
\hline . & Weltgegend. & KohlenP. & Salzl. & OolithP. & $\mid \begin{array}{c}\text { Krei- } \\
\text { deP. }\end{array}$ & MolasseP. & Neu \\
\hline Benennungen. & 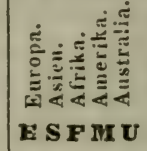 & 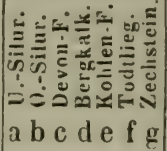 & 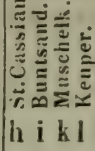 & 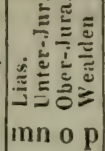 & 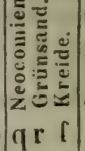 & 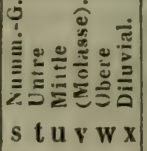 & 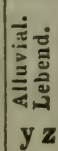 \\
\hline
\end{tabular}

\section{Astraea)}

fuveolata Lx..... ..... (spp. residune stellis contiguis polygon.) Goldfussi KLI. polyggonalis Mrchs. 14 Defrumceana Michn. Lamourouxi Mrchn. limitata Lmx. Cadomensis Mrchn. conrinna Gr. .... Lifoluna Michn. rosacea GF. ...... araneola Мichs. trochiformis Micus. formosa $\mathrm{GF}$. Deluci DFr. conica DFr. ..... cistela DFr. . - ․ lamellistriata MrенN. micraxuna Michn. . lamellosissima MichN. decipiens Micns. . superposita Michs. . reticulata $\mathrm{GF}$. gyrosa GF. angulosa $G \mathrm{~F}$. arachuoides $\mathrm{GF}$. rotula GF...... minuti Gris. rustica DFr. bellula Micнn. ... hystrix DFr. microstella Мıсн. . crispa Mrenn. . . . . polygonalis Michn. 61 plana MichN. diversiformis MichN. ornata Michn..... numisma DFR.

(species vugue) Hennahi LN:D. ... parallela Ros.

? granulata Morron. P corona Morrn. intercellulosa Phicl. irrogularis PorrL.

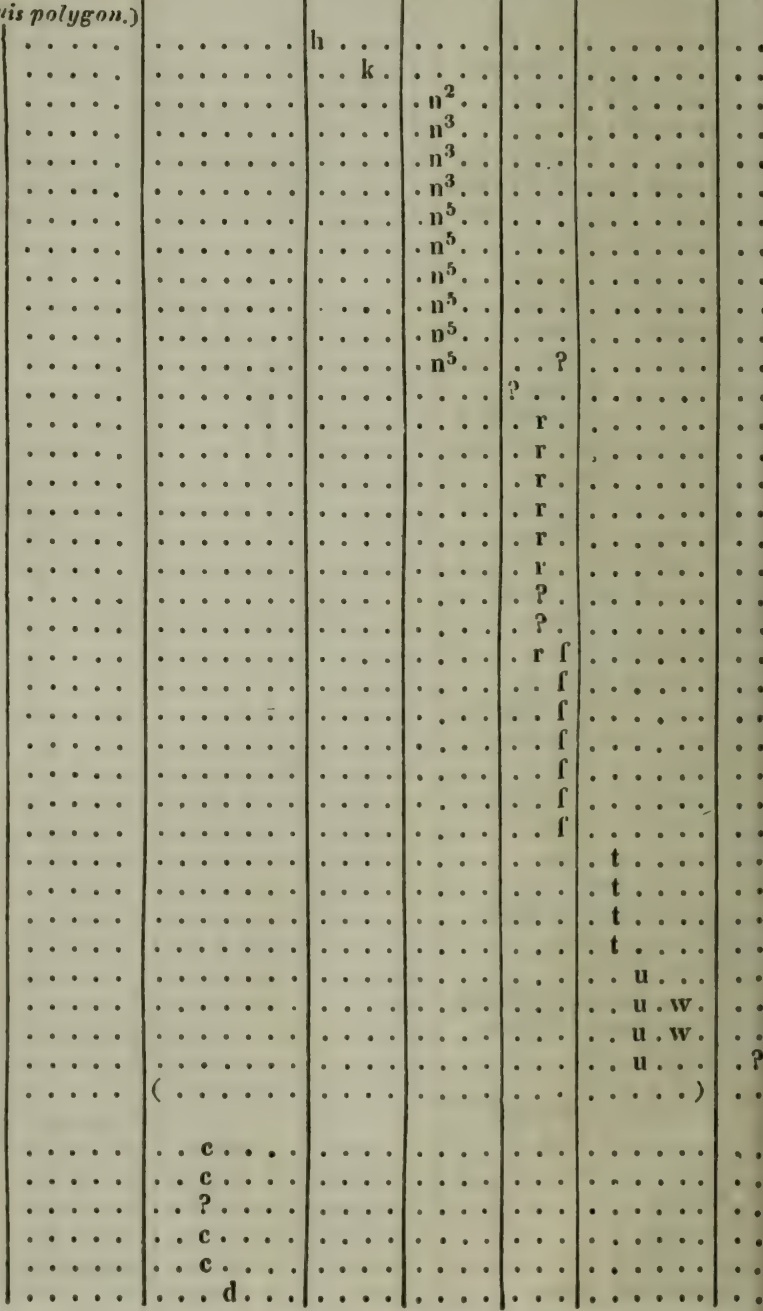




\begin{tabular}{|c|c|c|c|c|c|c|c|}
\hline en & egend. & f $g$ & k 1 & $\mathrm{op}$ & C & $\mathbf{w} \mathbf{x}$ & . \\
\hline ta $T$ & & & & & & & \\
\hline$\cdots$ & $\cdots$ & $\cdots \cdot$ & $\cdots$ & $\cdot n$ & - & $\cdots$ & - \\
\hline . . & . & $\cdots \cdot$ & & 111. & & $\cdots$ & • \\
\hline .. & . & & 1 &.$n^{5}$ & 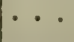 & $\cdots$ & - \\
\hline A & - & - & $\cdot$ & . & & $\cdots \cdots$ & - \\
\hline & & & & : & & $\cdots$ & - \\
\hline$\because \vdots$ & . & . . &. & . & • & $\cdots$ & \\
\hline . & . & $\therefore$ & $\ldots$ &.$n^{5} \ldots$ & $\cdots$ & $\cdots$ & \\
\hline$\therefore$ & $\cdots \cdot$ & . & $\ldots$. &.$n^{5} \ldots$ & $\ldots$ & $\cdots$ & \\
\hline$\cdots$ & $\cdots \cdot$ & $\cdots \cdots$ & $\ldots$ &.$n^{5}$. & $\ldots$ & $\ldots$ & \\
\hline . $\cdot$ & . & $\ldots \cdots$ & $\cdots$ & .115. & $\ldots$ & $\cdots$ & \\
\hline$\cdots$ & & $\cdots$ & $\cdots \cdots$ & $\cdot n^{5} \cdot$ & $\ldots$ & $\ldots \ldots$ & \\
\hline . & - & $\ldots \ldots$ & $\cdots$ &.$n$ & $\cdots$ & $\cdots$ & \\
\hline & 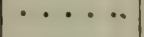 & . . & $\cdots$ &.$n \cdot$ & $\cdots$ & $\cdots$ & $\cdots$ \\
\hline R. & - & & $\cdots \cdot$ & .. 0 & . & $\cdots$ & \\
\hline - $\cdot$ & $\cdots \cdot \cdot$ & $\cdots \cdots \cdots$ & $\cdots$ & $\cdots 0$ & ... & $\cdots$ & \\
\hline . & $\cdots \cdot$ & $\ldots \cdots$ & $\cdots$ & $\cdots$ & & $\cdots$ & \\
\hline$\cdot$ & - & $\cdots \cdots$ & $\cdots$ & $\cdots \cdot$ & & $\cdots$ & \\
\hline .. $\cdot$ & $\cdot \cdot$ & $\cdots$ & $\cdots$ & $\cdots \cdot$ & & $\cdots$ & \\
\hline$\cdots$ & - & $\cdots$ & $\cdots$ & $\cdots \cdots$ & $\cdots r^{2}$ & $\cdots$ & \\
\hline$\cdots$ & & $\cdots$ & - & $\cdot$ & $\cdots 1^{1}$ & $\cdots$ & \\
\hline tit & & & & & $1^{2}$ & & \\
\hline & & & $\because$ & $\cdot$ & $\because 1$ & $\cdots$ & \\
\hline ss & $\cdots \cdots$ & $\ldots$ & $\because$ & $\because$ & $\because 1$ & $\because$ & \\
\hline EUss & $\ldots \ldots$ & $\because$ & $\cdots$ & $\ldots$ & $\because 1$ & $\cdots$ & \\
\hline$\cdot \cdot$ & ... & $\therefore \ldots$ & $\ldots$ & $\ldots$ & $\therefore$ i & $\cdots$ & \\
\hline . & $\ldots$ & $\ldots$ & • • & $\cdots \cdots$ &. .1 & $\ldots$ & \\
\hline$\cdots$ & $\cdots$ & $\ldots \ldots$ & $\ldots$ & $\ldots$ & . . $\mathrm{f}$ & $\ldots$ & \\
\hline . & $\cdots \cdot$ & $\ldots \ldots$ & $\cdots$ & $\ldots$ & $\ldots f$ & $\cdots$ & \\
\hline . . & $\cdots \cdot$ & $\ldots \ldots$ & $\ldots$ & $\cdots$ & $\cdots$ &.$t \ldots$ & - \\
\hline & $\cdots \cdot \cdot$ & $\cdots \cdots$ & $\cdots$ & $\cdots$ & $\cdots$ & $\cdots \ldots$ & \\
\hline 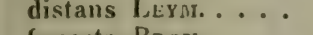 & $\cdots \cdots$ & $\ldots \ldots$ & $\ldots$. & $\cdots$ & $\cdots$ & $\cdots$ & \\
\hline$\cdots$ & & . & & - & $\cdots$ & $\cdot w_{-}$ & \\
\hline . & $\cdot$ & - & & & $\cdot$ & $\cdots$ & \\
\hline & & - & & & & $\cdots$ & \\
\hline Serr. & $\cdots$ & - & & - & - & v... & \\
\hline & $\because$ & & & & $\cdots$ & w. & \\
\hline Lv.) & . & $\ldots$ & . & $\cdots$ & $\cdots$ & ..... & \\
\hline$\cdots$ & & . & $\cdots$ & . & ... &. $\mathbf{w}$ & \\
\hline$\cdots$ & $\cdot \cdot$ & $\ldots$ & $\cdots$ & & $\cdots$ & $\cdots x$ & \\
\hline+ & & & & & & & \\
\hline GF. . & & & & & & & \\
\hline$t$ & & & & & & & \\
\hline$t$ & & & & & & & \\
\hline & & & & & & & \\
\hline $\begin{array}{l}\mathrm{MCH} \\
.\end{array}$ & & .1 & & $\because \mathbf{n} \ldots$ & & & \\
\hline 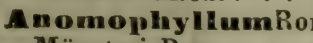 & & & & & $\cdots$ & $\cdots$ & - \\
\hline & & & & & & & \\
\hline
\end{tabular}




\begin{tabular}{|c|c|c|c|c|c|c|c|}
\hline & Weltgegend. & KohlenP. & SulzP. & OolithP. & $\begin{array}{l}\text { Krei- } \\
\text { deP. }\end{array}$ & MolasseP. & Niveu \\
\hline Benennungen. & 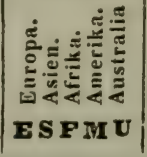 & 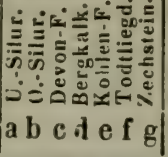 & 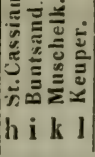 & 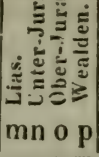 & 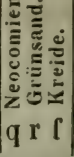 & 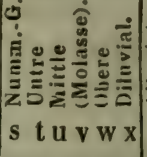 & 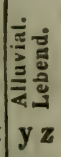 \\
\hline
\end{tabular}

Pavonia (Lк.) Blv. 4 hemisphaerica Michs. maeandrinoidesM $\mathrm{ICHN}_{\text {. }}$ tuberosa GF. irregularis Buv.

TydnophoraFisch.5 (Monticularia Lk.)

Sternbergi Fisch. conferta LNsD. $s p$. . radula Eгснw. cyclostoma PhiLL. microconus (Eв.)

(MonticulariaLK.) 1 maeandrinoides Mcun.

Rhysmotes Fiscr. 1 petiolatus Fiscr.

Tridacophyllia Bu.v.0.

Agraricia (LK.) Buv. 8 ? ramosa $M u ̈$. ramulosa Mrcin. elegans Michs. graciosa Michs. Ludovicina Mrcun. infundibuliformis Mcun

S radiata Risso Apenuinica (Mrcus.)

Polyastra Er. 1 conflu,ns (Ев.)

DictyophylliaBuv.2 ? alternans Fiscr. .. hemisphaerica Br.v.

Nireeand rina LK. 33 Bronni KL.r. labyrinthica Kus. . antiqua Drr. venusta Michn. corrugata Mıсus... Edwardsi VıchN. rastcllima MichN. $\neq$ Raulini MıснN. flamellidentata Місик. montana Michs. Lotharingica (МıснN.) foliacea Thurm. magna ThurM. tenella GF. Soemmeringi Mü.

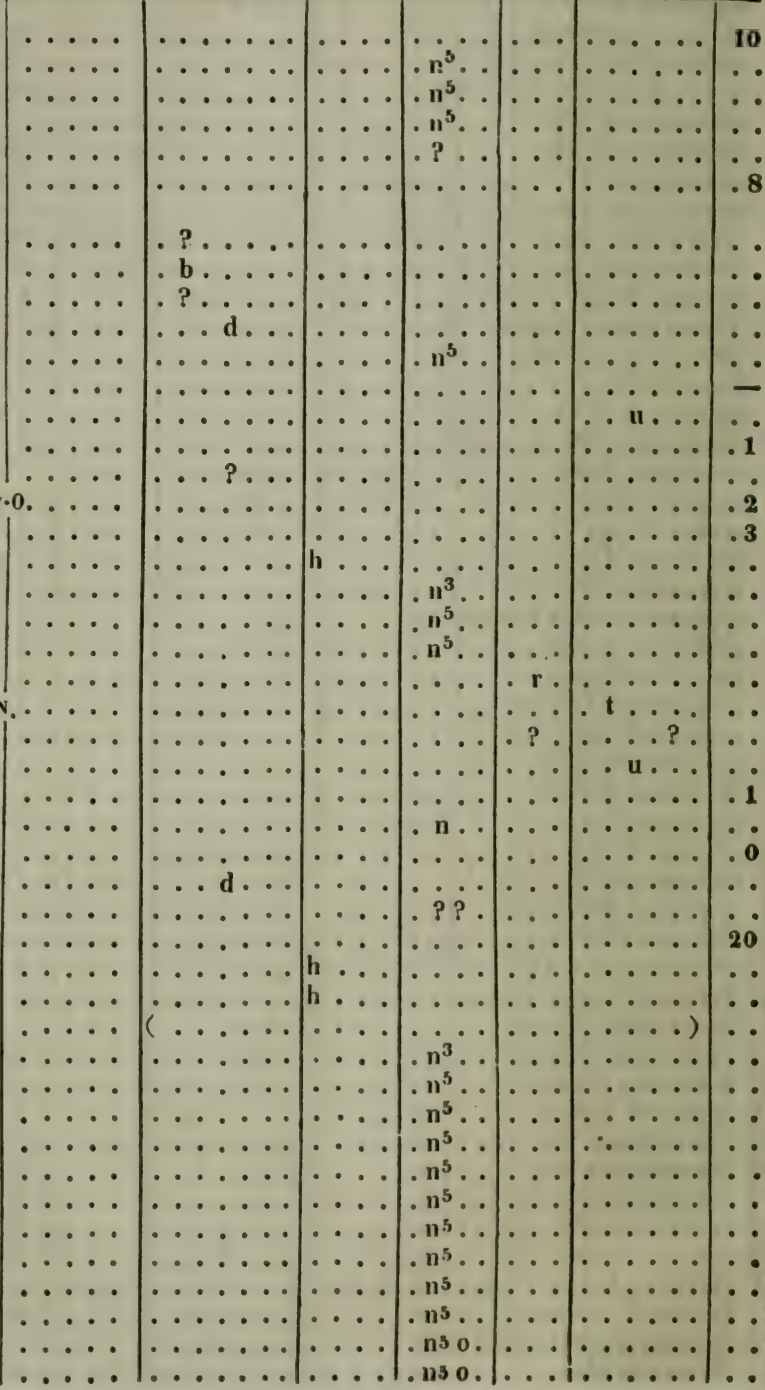




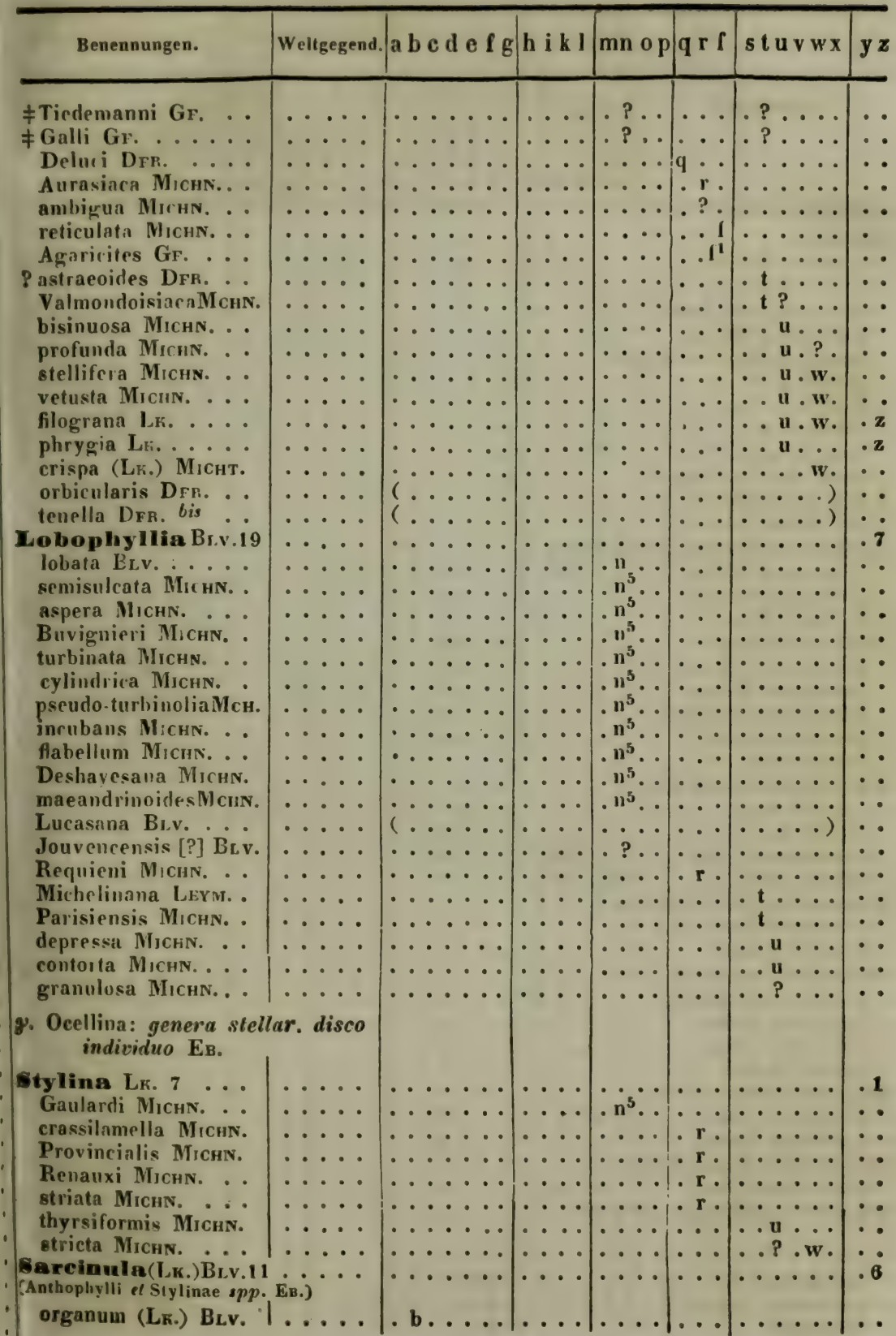




\begin{tabular}{|c|c|c|c|c|c|c|c|}
\hline & Weltgegend. & KohlenP. & SalzP. & Oolithe. & $\begin{array}{l}\text { Krei- } \\
\text { deP, }\end{array}$ & MolasseP. & Neu \\
\hline Benennungen. & 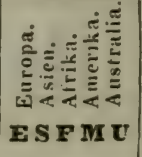 & 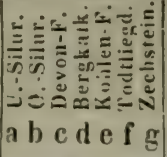 & 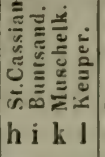 & 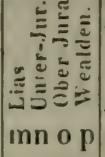 & 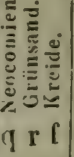 & 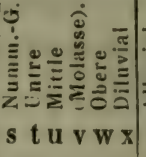 & 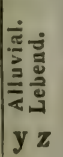 \\
\hline
\end{tabular}

Sarcinula)

Archiaci Mrchs.

favosa Michn.

quincunrivlis Miche.

gratissima Mrcus.

I faveolata (Sor.Ele.) Mrснт.

custata GF.

divaricata BLv.

dubia Brv.

Bonganvillei Br.v.

perfo ata (I.к.) Mrcht.

Anthophyllem 0 . (v. Nomeuclu/nr)

Lithostrotizm LHW alveolatum (GF.) .......

?stellaris (Strin.). striatum Fi.kM.

foi iforme FLEM. marginatum Frem. crenulare Morss. micropliyllum KEYs. regium Morrs.

laeve (GF.)

Columnaria Gr.Roz. 4

antiqua Roe. senilis Kon.

? sexradiuta LNso. sulcata Gr.

(? Cyathophylli spp. EB )

Baltica Schw.

Conimia Vicriv. 4 . cornu-hovis Michs, gigantea Michs. . cylindrica LNsD. patula Michs.

Stylastraea Liso. 2 inconferta LNsD.

vortiralis LNsD.

DiphyphyllumLo.1 concinnum LNed...
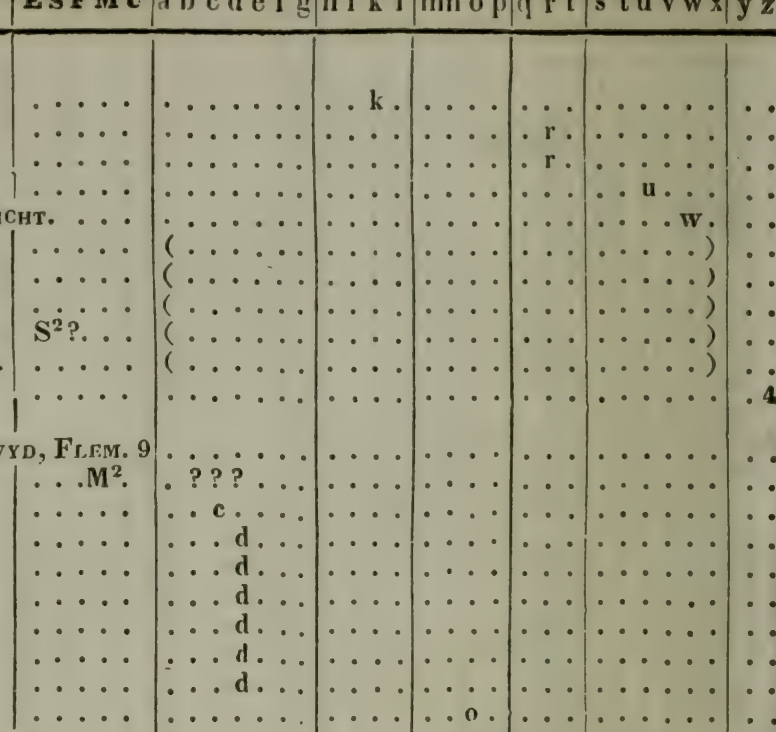

Cyathophylinu (GF.) 46 .

(*)

turbinatum GF. angustum LNsD. Hisingeri

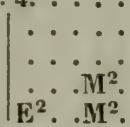

? ? ?

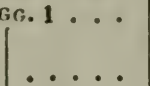

$:$

d.

...d.

$\ldots d$.

$\cdots d$

..d..

$\ldots \ldots$

. d

...d...

...d...

- b .

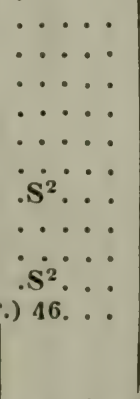

$\therefore$

d...

d.

$\ldots d$.

$\therefore$ d.

...d... ....

a b c d ...

a b ? .... 


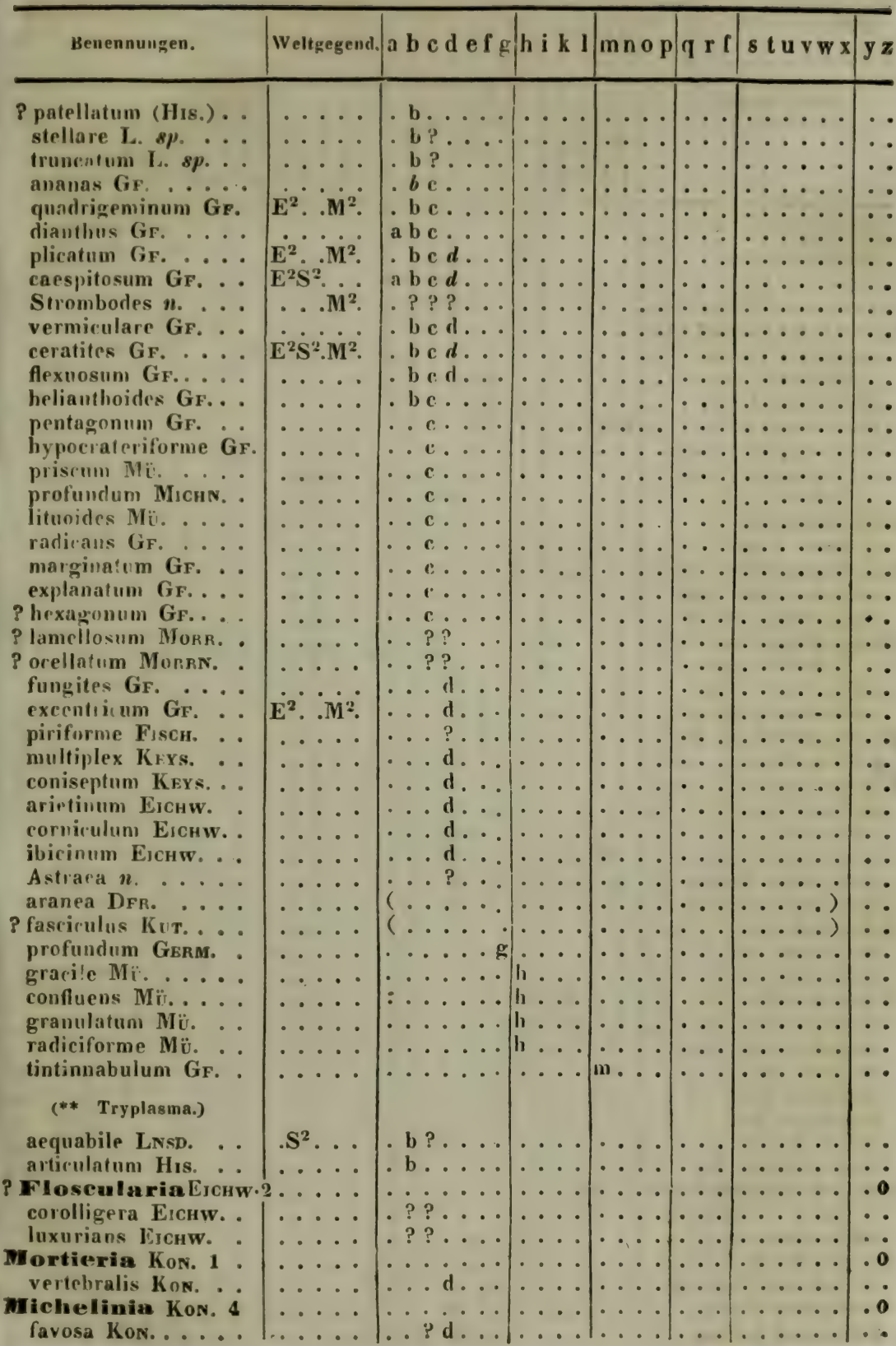




\begin{tabular}{|c|c|c|c|c|c|c|c|}
\hline \multirow[b]{2}{*}{ Benennungen. } & send. & KoblenP. & SalzP. & GolithP. & $\begin{array}{l}\text { Krei- } \\
\text { deP. }\end{array}$ & MolasseP. & Neu \\
\hline & 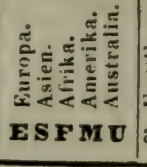 & 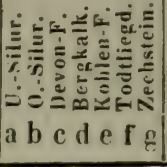 & 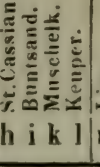 & 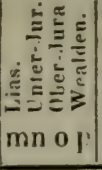 & 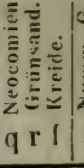 & 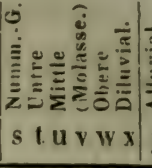 & $\begin{array}{l}\text { y z } \\
\mathrm{y}\end{array}$ \\
\hline \\
\hline $\begin{array}{l}\text { tenuisepta Kon. . . } \\
\text { concinna LNsD. . . } \\
\text { compressa МicнN. . }\end{array}$ & $\cdots$ & $\mid \begin{array}{ll}\ldots & d \ldots \\
\ldots & \text {. } \ldots \\
\ldots & \text {. } \ldots\end{array}$ & $\cdots \cdots$ & $\begin{array}{l}\cdots \\
\cdots \\
\cdots \\
\cdots\end{array}$ & $\cdots$ & $\begin{array}{l}\cdots \cdots \\
\cdots \cdots \\
\cdots \cdots\end{array}$ & $\because \dot{~}$ \\
\hline $\begin{array}{l}\text { CystophyllumLsD. } 7 \\
\text { excavatum Kers. . . }\end{array}$ & $\begin{array}{l}\cdots \cdots \\
\cdots \cdots\end{array}$ & ( & & $\cdots \cdots$ & $\cdots$ & $\cdots \cdots$ & .0 \\
\hline & $\cdots$ & b c. & $\cdots$ & $\cdots$ & $\cdots$ & $\ldots \ldots$ & \\
\hline$\dot{.}$ & $\mathrm{E}^{2} \dot{\mathrm{S}}^{2} \cdots$ & b? & & $\cdots \cdots$ & $\cdots$ & $\cdots \cdots$ & \\
\hline & $\cdots \cdots$ & $\cdots c \ldots$ & $\cdots$ & $\ldots$ & $\cdots$ & $\ldots \ldots$ & \\
\hline & $\cdots$ & $\begin{array}{ll}\cdots & c \\
\cdots & \text { d. }\end{array}$ & & $\cdots$ & $\because \dot{ }$ & $\begin{array}{ll}\cdots & \cdots \\
\cdots & \cdots\end{array}$ & \\
\hline 1 & $\ldots \ldots$ & $\ldots \ldots$ & $\cdots$ & $11^{5} \cdots$ & $\therefore$ & $\cdots \cdots$ & .7 \\
\hline .2 & $\ldots \ldots$ & $\ldots \ldots$ & $\cdots$ & $\cdots$ & $\cdots$ & $\ldots \ldots$ & .3 \\
\hline & $\cdots \cdots$ & $\begin{array}{lll}\cdots & \cdots & \cdots \\
\cdots & \cdots & \cdots\end{array}$ & $\cdots$ & $\mid \begin{array}{cc}.11 & \cdots \\
\cdots & 0\end{array}$ & $\because \dot{r}$ & $\begin{array}{l}\cdots \\
\cdots\end{array}$ & $\cdots$ \\
\hline v.2) & (5) & & . & $\cdots$ & $\cdots$ & $\cdots$ & .5 \\
\hline : & $\begin{array}{l}\cdots \\
\cdots\end{array}$ & & & ... & $\because$ & t. . . & \\
\hline B. 1 & $\cdots$ & - $3^{\circ}$ & $\cdots$ & $\cdots \cdots$ & $\cdots$ & $\cdots u$ & $\because \infty$ \\
\hline & $\cdots \cdot$ & $\ldots d \ldots$ & $\cdots$ & $\cdots$ & $\cdots$ & $\cdots$ & - \\
\hline 16 & $\cdots$ & & . & $\ldots$ & $\cdots$ & $\ldots \ldots$ & .5 \\
\hline & $\cdots$ & . c d . & $\therefore$ & $\begin{array}{cc}\cdots & \cdots \\
\cdots & \cdots\end{array}$ & $\cdots$ & $\cdots \cdots$ & • \\
\hline 3. & $\mathrm{S}^{2} \ldots$ & ...d... & $\ldots$ & $\ldots$ & $\cdots$ & $\ldots \ldots$ & \\
\hline - & $\cdots \cdot$ & $\ldots d \ldots$ & $\ldots$ & $\ldots$ & $\cdots$ & $\cdots \cdots$ & te $>$ \\
\hline & $\cdots \cdot \cdot$ & $\ldots d$. & & & $\cdots$ & $\cdots \cdots$ & 35 \\
\hline$\cdots$ & $\cdots \cdot \cdot$ & - & $\cdots$ & $\cdot 11^{3} \cdot \cdot$ & $\cdots$ & $\cdots \cdots$ & . \\
\hline & & 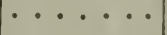 & & $\cdot n^{3} \cdot \cdot \mid$ & - & - & \\
\hline$\cdots$ & 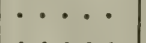 & 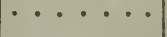 & • & $\cdot n^{3} \cdot \cdot$ & $\cdot$ & . . . & \\
\hline$\therefore$ & $\cdots \mathrm{M}^{2}$ & $\cdots$ & $\cdots$ & $\cdots \cdots$ & $\left|\begin{array}{ll}0 \\
0 \\
0 & \cdots\end{array}\right|$ &.$t \ldots$ & • \\
\hline$\therefore$ & ..... & $\cdots \cdots$ & $\cdots$ & $\begin{array}{c}\cdots \\
\cdots \\
\cdots\end{array}$ & $\cdots$ & t & $\cdots$ \\
\hline Ев. ... & $\cdots \cdot$ & $\ldots \ldots$ & . . & $\ldots$ & $\cdots$ & $\ldots \mathbf{u} \cdot \mathbf{w}$. & $\mathrm{z}$ \\
\hline га Ев. ... & $\ldots \ldots$ & $\ldots \ldots$ & & $\cdots$ & $\cdots$ & $\ldots \mathbf{u} \cdot \mathbf{w}$ & .7 \\
\hline osa Еи. . . . & & & & $\cdots \cdot$ & . . ? & . ? .. w x &.$z$ \\
\hline - & 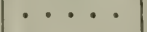 & . & & - & $\cdots$ & $\mid \cdots u, w \times x$ & - $z$ \\
\hline & & & & & $\cdot$ & $\cdots \cdots w$ & - $\mathbf{z}$ \\
\hline on Sch & 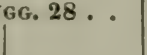 & 1 & . & $\cdots \cdot$ & $\cdots$ & $\cdots \cdots$ & - \\
\hline & & & & - & . & $\cdots \cdots$ & 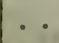 \\
\hline v & & . . & & - • & . 1 & .. & \\
\hline Porte. . & 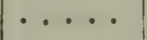 & 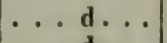 & & • & • $\cdots$ & & \\
\hline annulatum Lossd. & & & & & & & \\
\hline
\end{tabular}


concameratum LNsD. fasciculatum Phicl. costatum LN:D. subdichotomum Mü. sublacve Mï. verticillatum Mü Eunomia Micun. stellariiforme Zn.Nk. Rauracorum Gresr. sociale Roe. (non Phí.) plicatum Gr.

funirulus M:chN. laeve Micux. flabellum IVIcus. articulatum Michs. . Morenuanum Michs. Edwardsi Michn. . pseudo-stylina Micho. irregulare Michs. furca Eichiv. intricatum Micrin. humile Micus. manipulatum Micus.

Caryophyllia Lк. (non Ев.) 39 flexuosa Steing. Lnsd. duplicata Br.v. affinis Br.v. juncea Frem. extinctorium Michis. convexa PHis. retorta MiснN.

Caumonti (I,x.) ... elongata DFr. Moreanana Miche. subcylindrica MichN. dilatata M: СнN. cornuta Michs. ... clavus Mrcun. vasiformis MichN. anuularis Fr.Ex. Cenomana [?] Muchn. striatulata MicuN. globosa MrсHN. Faxöensis Веск affixa Morr. fasciculata(Lк.) Morr. truncala Mıchs. Altavillens's DFr. multistellata GAr. subdichotoma LNso. striata Bi.v.

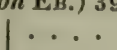

\section{$\mathrm{s}^{2}$}

striata DFr.
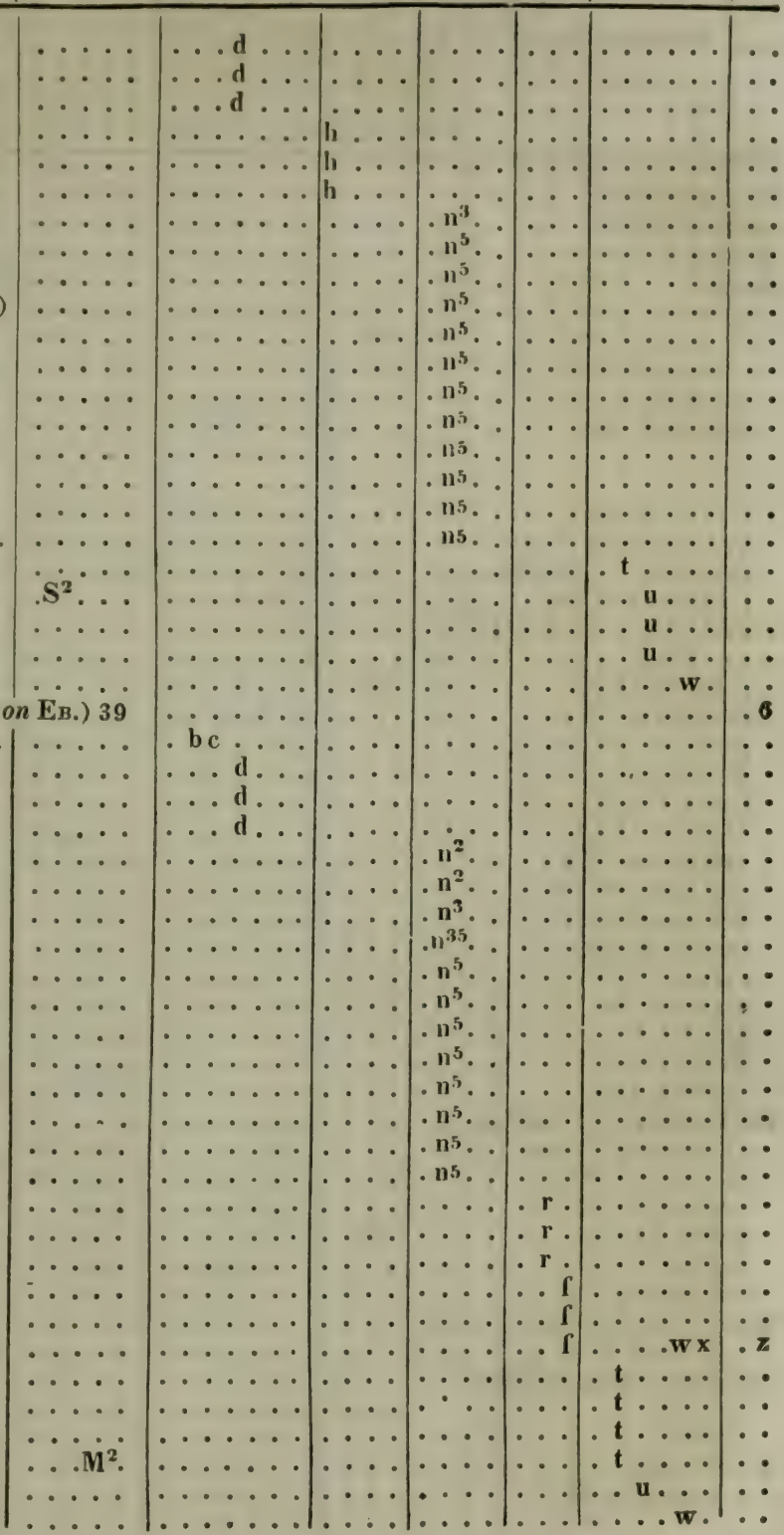


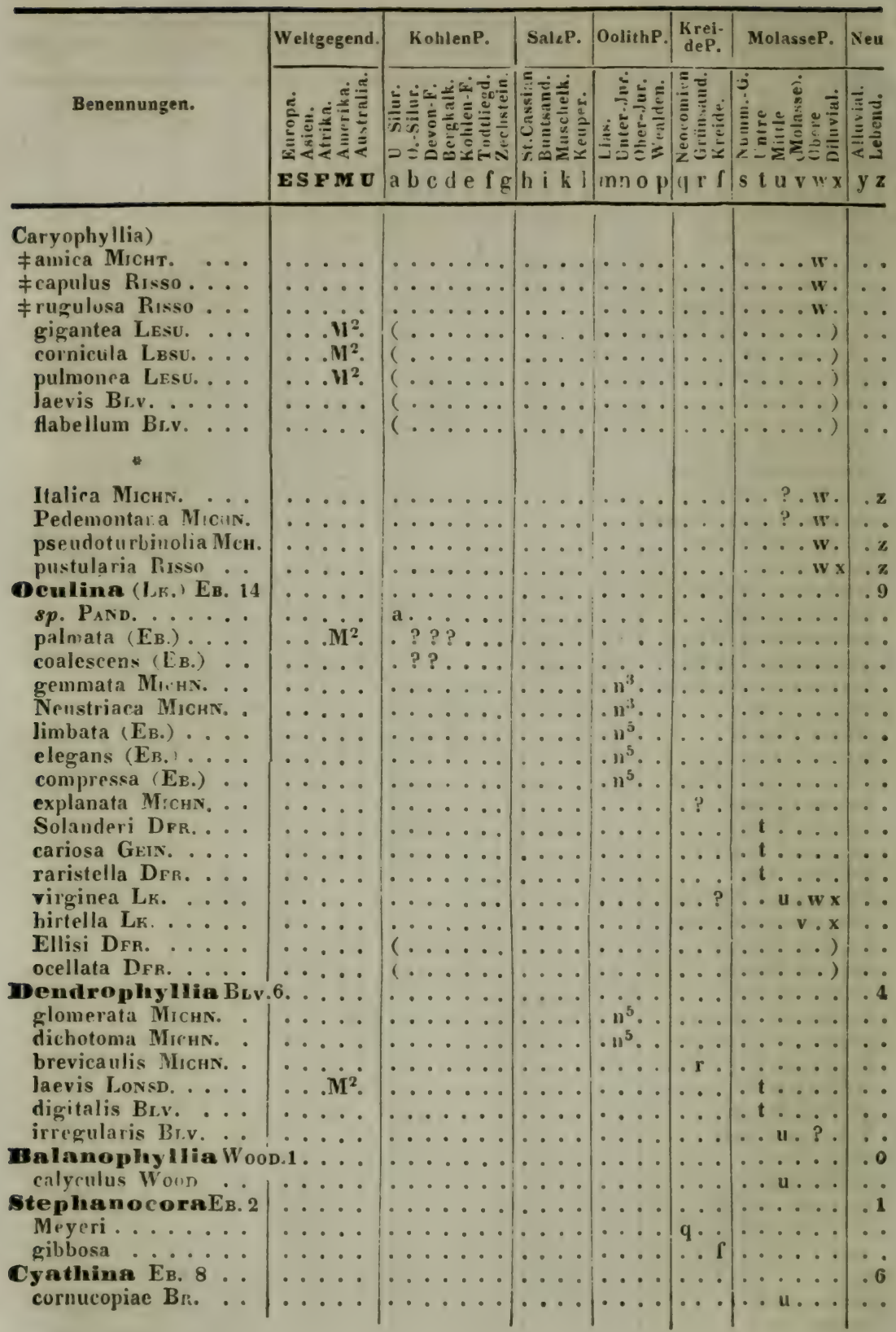




\begin{tabular}{|c|c|c|c|c|c|c|c|}
\hline iungen. & gend. & e $f g$ & h i k l & $\mathrm{mn} 0 \mathrm{p}$ & 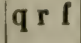 & $8 \mathrm{t}$ & $\mathbf{y}^{2}$ \\
\hline a & & & & & & . u u & \\
\hline . & & $\ldots \ldots$ & $\cdots$ & $\ldots$ & $\ldots$ & $\ldots . . w$ & . \\
\hline$\cdots$ & $\cdots$ & $\ldots \ldots$ & & $\ldots$ & $\cdots$ & $\ldots w_{0}$ & - \\
\hline HIL. . & $\ldots \ldots$ & $\ldots$ & $\ldots$ & $\ldots$ & $\cdots$ & $\ldots \mathbf{w}^{\prime}$ & - \\
\hline$\ldots$ & $e_{0}$ & $\ldots$ & $\cdots$ & $\cdots \cdot$ & $\cdots$ & $\ldots w \cdot$ & - \\
\hline EB. & & & & & $\cdots$ & ....w & $\cdot \mathbf{z}$ \\
\hline c & - & $\cdots \cdots$ & . & & & .....w. &. $\mathbf{z}$ \\
\hline $\operatorname{ltan} E_{B} .2$ & . & $\ldots \ldots$ & $\ldots$ & $\cdots \cdot$ & $\cdots$ & $\ldots \ldots$ & .2 \\
\hline$=$ & $\cdots$ & $\ldots \ldots$ & $\cdots \cdots$ & $\cdots \cdots$ & $\cdots$ & $\cdots \cdots \mathbf{w}$ & . $x$ \\
\hline 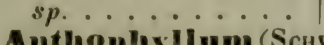 & & $\cdots \cdots$ & $\cdots \cdots$ & $\cdots$ & $\cdots$ & $\mid+\ldots$. & . \\
\hline 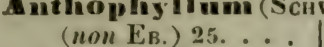 & & & & & & & \\
\hline$\because$ & $\therefore \dot{M}^{2}$ & $? ?$ & & & $\cdots$ & & .5 \\
\hline ... & .... & ..... & $\ldots$ & & . & & $\bullet$ \\
\hline . . & $\ldots$ & $\ldots \ldots$ & h... & & $\ldots$ & $\therefore$ & - \\
\hline HN. . & $\cdots \cdot$ & $\ldots \ldots$ & $\ldots$ & $n^{3}$. & ... & $\ldots \ldots$ & \\
\hline$\cdots$ & $\ldots \ldots$ & $\ldots \ldots$ & $\ldots$ &.$n^{3}$. & $\ldots$ & $\ldots \ldots$ & • \\
\hline . & $\cdots \cdot$ & $\ldots \ldots$ & $\cdots$ &. $\mathbf{n} \ldots$ & $\cdots$ & $\ldots \ldots$ & • \\
\hline . & $\cdots \cdot$ & $\ldots \ldots$ & $\cdots$ & & $\ldots$ & $\ldots \ldots$ & - \\
\hline . & $\cdots \cdot$ & $\cdots \cdots$ & $\ldots$ & . . & $\cdots$ & $\ldots \ldots$ & • \\
\hline . & $\cdots \cdots$ & $\ldots \ldots$ & $\cdots \cdots$ &. $\mathbf{n} \ldots$ & . & $\cdots$ & - \\
\hline$\cdots$ & $\cdots \cdot$ & $\cdots \cdots$ & $\cdots \cdots$ & $\cdot \mathbf{n} \cdot \cdot$ & $\cdots \cdot$ & $\cdots$ & - \\
\hline 8. . & $\cdots \cdot$ & $\cdots \cdots$ & $\cdots \cdot$ &.$n^{5}$. & $\cdots$ & $\cdots$ & - \\
\hline & $\cdots \cdot$ & $\cdots \cdots$ & $\cdots$ & $\ldots$ & $9 \cdot 1$ & . 1 & \\
\hline$\cdot \cdot$ & $\cdots \cdot \cdot$ & $\cdots \cdots \cdots$ & $\cdots \cdots$ & (1 & & $\cdots$ & \\
\hline ? & · & $\cdots \cdots \cdots$ & $\cdots \cdots$ & & ? $?$ & 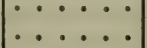 & - \\
\hline 11 & : & $\cdots \cdots$ & $\cdots$ & $\cdots$ & ? & $\ldots \ldots$ & \\
\hline ง. • • & $\therefore$ & $\cdots$ & $\cdots \cdots$ & $\cdots \cdots$ & . ?. & $\ldots \ldots$ & \\
\hline$n$ & $\ldots$ & $\ldots \ldots$ & ... & $\ldots$ & . ? . & $\ldots$ & \\
\hline$\cdots$ & $\ldots$ & $\ldots \ldots$ & $\ldots$ & $\ldots$ & . ? . & $\ldots \ldots$ & \\
\hline & $\cdots$ & $\ldots \ldots$ & $\ldots$. & $\ldots$ & . $\mathrm{I}$ & $\ldots \ldots$ & - \\
\hline & & $\ldots \ldots$ & $\ldots \cdot$ & $\ldots$ & .. I & $\ldots \ldots$ & - \\
\hline - $\cdot$ & $\cdots \cdots$ & $\ldots \ldots$ & $\cdots$ & $\cdots \cdot$ & .. $\mathbf{f}$ & $\cdots$ & $\cdot$ \\
\hline & $\cdots$ & $\cdots$ & & & $\cdot$ & . $\mathbf{t}$ & \\
\hline li & $\cdots$ & . & & & $\cdots \cdot$ & . . u... & - \\
\hline & - & 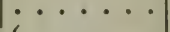 & $\cdots$ & . 1 & $\cdots$ & $\cdots w_{j}$ & - \\
\hline$\cdots$ & & & 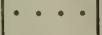 & $\cdots$ & $\cdots \cdot$ & $\mid \cdots \cdots) \mid$ & $\because$ \\
\hline 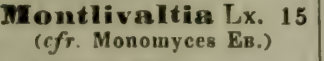 & . & - & 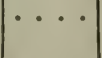 & 10 & . & $\cdots \cdots$ & \\
\hline & & & & $\cdots \cdot$ & $\cdots$ & $\ldots \ldots$ & • \\
\hline$\cdots$ & & & & $\ldots$ & $\cdots$ & $\cdots \cdots \cdot$ & \\
\hline . & & & & $\cdots \cdot$ & $\cdots$ & $\cdots \cdots$ & \\
\hline$\cdots$ & & & & $\cdots$ & $\cdots$ & $\cdots \cdots$ & \\
\hline$\cdots$ & - & & & $\cdots$ & $\cdots$ & $\cdots \ldots$ & \\
\hline . & . & & & $\cdots \cdot$ & $\cdots$ & $\cdots \cdots$ & - \\
\hline U. & & & & & & & \\
\hline$\cdots$ & & & & & & $\cdot$ & \\
\hline 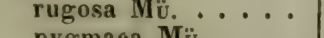 & & & & $\cdots$ & $\cdots$ & $\cdots \cdots$ & \\
\hline$\ldots$ & & & & $\cdots$ & $\cdots$ & $\cdots \cdots$ & - \\
\hline - & & & & & & $\ldots \ldots$ & \\
\hline
\end{tabular}




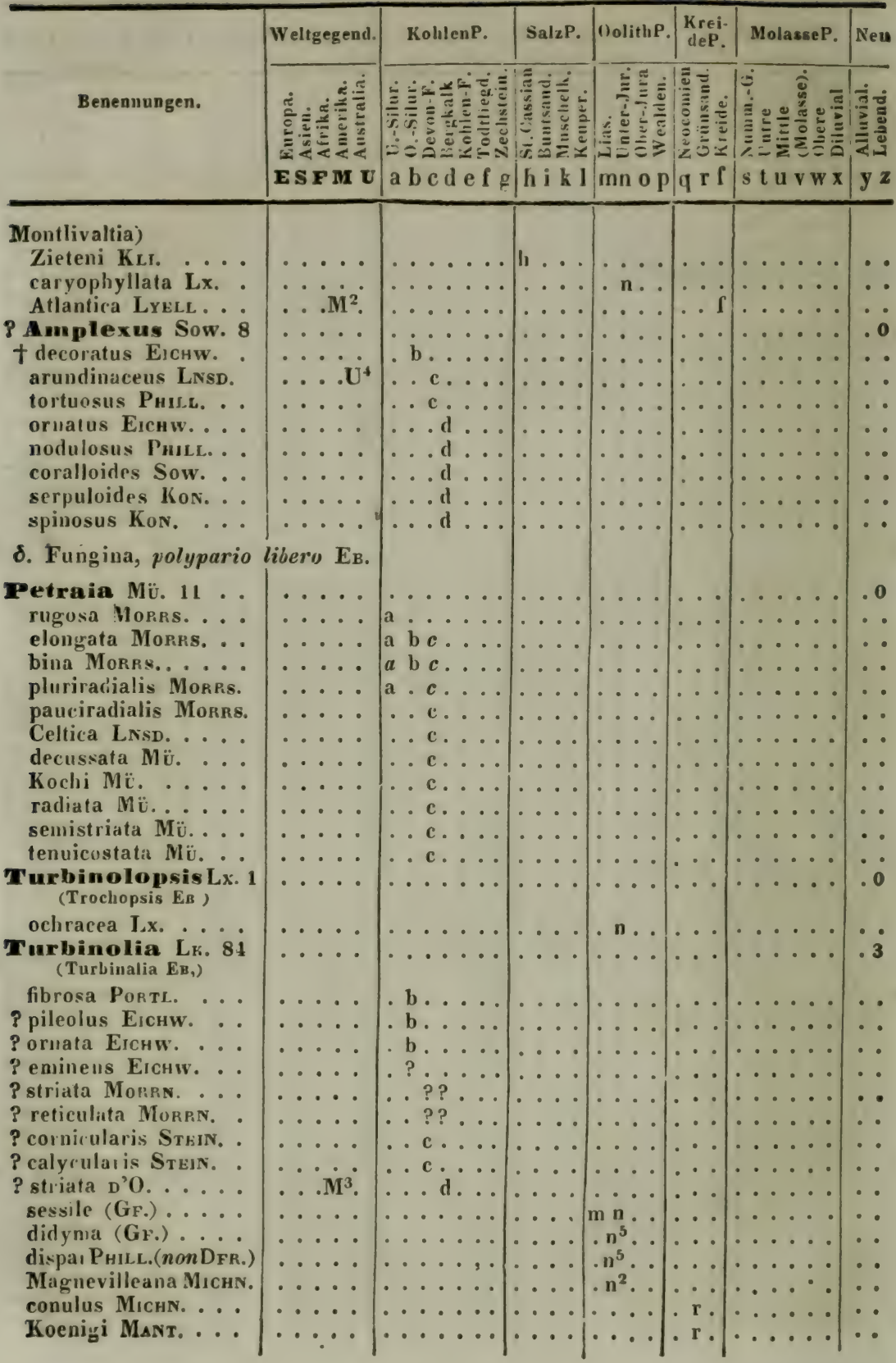




\begin{tabular}{|c|c|c|c|c|c|c|c|}
\hline Beneunungen. & send. & $\mathrm{fg}$ & 1 & $\mathrm{p}$ & ? & $\mathrm{w} \times$ & $\mathbf{y z}$ \\
\hline cuneata Risso ... & 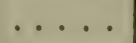 & $\cdots$ & & & & $\cdots$ & \\
\hline compressa Lк. . . & $\ldots \ldots$ & $\ldots \ldots$ & $\ldots$ & $\ldots$ & . $r f^{1}$ & $\ldots \ldots$ & $\cdots$ \\
\hline rudis Micun. .... & $\cdots \cdots$ & $\ldots \ldots$ & $\ldots$. & $\ldots$ & . $\mathbf{r} \mathbf{f}^{\mathbf{1}}$ & $\ldots \ldots$ & \\
\hline ata GF...... & $\cdots \cdot$ & $\ldots \ldots$ & $\cdots$ & $\cdots$ & .? f & $\ldots \ldots$ & \\
\hline w. $\cdot \cdot$ & $\cdots \cdot \cdot$ & $\ldots \ldots$ & $\cdots$ & $\cdots \cdot$ & $\cdots \boldsymbol{I}$ & $\ldots \ldots$ & \\
\hline centralis Ros. ... & - & $\cdots$ & & $\ldots$ & & $\cdots$ & -・ \\
\hline aspera Sow. . . . & $\cdots$ & $\cdots$ & & & $r^{1}$ & $\cdots$ & - \\
\hline is Mort. . . & $\cdots$ & $\cdots \cdot$ & & $\cdots$ & $\cdots \mathbf{f}$ & $\cdots$ & \\
\hline ua $\mathbf{G F}_{\mathrm{F}} \cdots \cdots$ & $\cdot$ & & & $\cdots$ & $\cdot \cdot \rho^{1}$ & & \\
\hline$\cdots$ & $\ddot{\dot{*}}$ & . & & $\cdots$ & 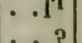 & $\ddot{\mathbf{w}}$ & \\
\hline 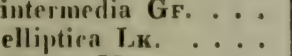 & $\because$ & $\cdots$ & $\cdots \cdots$ & $\cdots$ & $\begin{array}{c}\cdots \\
\cdots\end{array}$ & sit & • \\
\hline Alpina Michn. ... & .... & $\ldots \ldots$ & $\ldots$ & $\ldots$ & $\ldots$ & t.... & \\
\hline . & ... & $\ldots \ldots$ & $\ldots$ & $\cdots$ & $\cdots$ & $\cdots$ & \\
\hline HN. . & ... & $\ldots \ldots$ & $\cdots$ & $\cdots \cdot$ & $\cdots$ & $\cdots$ & \\
\hline BL.L. & $\cdots$ & $\ldots \ldots \ldots$ & $\cdots$ & $\cdots \cdot$ & $\cdots$ & $\cdots$ & \\
\hline & - & $\ldots \ldots$ & $\cdots$ & $\ldots$ & $\cdots$ &. $\mathbf{t} \ldots$. & $\cdot$ \\
\hline IHN. & $\cdots$ & $\ldots \ldots$ & $\ldots$ & $\ldots$ & $\cdots$ &. $\mathbf{t} \ldots$ & \\
\hline No & $\cdots \cdots$ & $\cdots \cdots$ & $\ldots$ & $\cdots \cdot$ & $\cdots$ & . $\mathbf{t} \ldots$. & \\
\hline L.) & $\cdots \cdot$ & $\ldots \ldots$ & $\cdots$ & $\cdots \cdot$ & $\cdots \cdot$ & $\cdots$ & \\
\hline C & - & $\ldots \ldots$ & & $\cdots$ & $\cdot$ & $\cdot \cdot$ & \\
\hline Gi & $\cdots$ & $\cdots \cdots \cdots$ & $\cdots$ & $\cdots \cdot$ & $\cdots$ & $\cdots$ & \\
\hline osa 1 & $\dot{\mathrm{F}}^{2}$ & & & & . & $\cdot$ & \\
\hline$\dot{0}$ & E. & & & & $\cdots$ & $\therefore$ & \\
\hline$\cdots$ & ... & ... & & $\ldots$ & $\therefore$ & $t \cdots w$ & \\
\hline$\therefore$ & .. & $\ldots \ldots$ & $\ldots$ & $\ldots$ & $\ldots$ & t.... & \\
\hline • & . . . M'. & $\ldots \ldots$ & . & $\ldots$ & $\cdots$ &.$t \ldots$. & \\
\hline i LEA & ... $\mathbf{M}^{2}$ & $\ldots \ldots$ & & $\cdots$ & $\cdots$ & . $t$. & \\
\hline LEA & . . $M^{2}$ & $\ldots$ & $\cdots \cdot$ & $\cdots \cdot$ & $\cdots$ & . $t$. & \\
\hline ei LEA & . . $\mathrm{M}^{2}$ & $\ldots$ & $\cdots \cdot$ & $\cdots$ & $\cdots$ &.$t \ldots$ & \\
\hline Dsh. . . . . & ... & $\ldots \ldots$ & $\ldots$ & $\cdots$ & $\cdots$ & ..u... & . \\
\hline - & - & $\ldots \ldots$ & $\cdot$ & $\cdots$ & $\cdots$ & $\ldots \mathbf{u} \cdot \ldots$ & - \\
\hline$\cdot$ & & . & & & - & ..? $\cdot \mathbf{w} \cdot$ & • \\
\hline CHN. & $\cdots \cdot$ & $\cdots$ & & $\cdots$ & $\cdot$ & ..? $\cdot \mathbf{w} \cdot$ & $\cdots$ \\
\hline cl & & & & . & $\cdot$ & $\mathbf{w} \cdot$ & \\
\hline HN. & • & - & & $\cdots \cdot$ & $\cdots$ & $\ldots \mathbf{u} \cdots$ & \\
\hline & & - & & - & & . u u & \\
\hline$\cdots$ & & • & & $\cdot$ & . & $\cdots \mathbf{u} \cdot \ldots$ & \\
\hline$\cdot \cdot$ & & . & $\dot{.}$ & $\begin{array}{ll}\cdots \\
\cdots\end{array}$ & $\because$ & $\cdots w \mathbf{w}$ & \\
\hline costata $\ddot{G}_{F}$. & & & & $\ldots$ & ..? & $\cdots \cdots w$ & r \\
\hline ... & & $\ldots \ldots$ & $\cdots \cdot$ & $\cdots \cdot$ & $\cdots$ & ....w. & - \\
\hline a Rr.... & $\cdots$ & $\ldots \ldots$ & & $\cdots$ & $\cdots$ & $\cdots w^{\prime}$ & - \\
\hline$\cdots$ & - & $\cdots \cdot$ & & 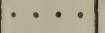 & $\cdots$ & $\cdots \cdot \mathbf{w} \cdot$ & - \\
\hline . & $\cdot$ & $0^{\circ}$ & & 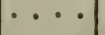 & & $\cdots \cdot \mathbf{w}$ & $\cdots$ \\
\hline sa RI. & - & & & & & $\mathbf{w} \cdot$ & • \\
\hline is Ri. & & & & & & v. & \\
\hline a 1 & & $\ldots$ & .... & $\cdots \cdot \cdot$ & - & & \\
\hline $\begin{array}{l}\text { ta Мiснт. } . \cdots \\
\text { Мiснт. . . }\end{array}$ & - & $\ldots \ldots$ & $\cdots$ & $\cdots$ & $\cdots$ & $\mathbf{w}$ & - \\
\hline pyrami & & & & & & . u. w & \\
\hline
\end{tabular}




\begin{tabular}{|c|c|c|c|c|c|c|c|}
\hline & Weltgegend. & KohlenP. & SalzP. & OolithP. & $\begin{array}{c}\text { Krei- } \\
\text { deP. }\end{array}$ & MolasseP. & Nen \\
\hline Benennungen. & 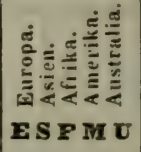 & 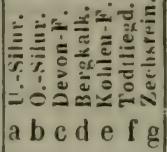 & 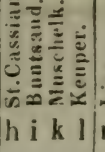 & 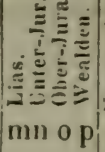 & q $\mathrm{rl}$ & $\begin{array}{l}0 \\
\text { st } t \text { u v w } x\end{array}$ & $\begin{array}{l}0 \\
\mathbf{y} z\end{array}$ \\
\hline
\end{tabular}

\section{Turbinolia)}

Sinensis Мıснт. cornucopiae Micht. praelonga Micht. raricosta Мıснт.

Bellardi MıсnN.

cylindrica Мıснт. .

Bellingheriana Мıнn. versicostata Micrn. fimbriata MıснN. . . acuminata KuT. . . .

P cyathoides LF. caryophyllus LK. . . dubia DFr.

Basorhesi Dfr.

Wndopachys Lnsd. 1 alatum LNsD.

Flabellum Les. 7 costatum BeLLardi .

? cuneiforme LNSD. appendiculatum MснN. avicula. MichN. ... cuneatum MichN.. . Roemeıi Pнir. extensum Michn.

\section{(DiplocteniumGF.2)} = Flabellum. = cordatum Gr. pluma Gr. .

Cyclolithes (LK.) 32 (Cyclolithas Eв.)

- Cyclitae, busi concentrice rugrosa. praeacutus LNsD. lenticulatus Liss. praecox RoE. orbulites (I.x.) ... orbitolites Michn. numismalis (GP.). . deformis МıснN. semiradiatus Buv. semiglobosa Michn. radiatus BLv. clathratus (HAG.). caucellatus BLV. . nlacea Morrn. undulatus BLv. ...

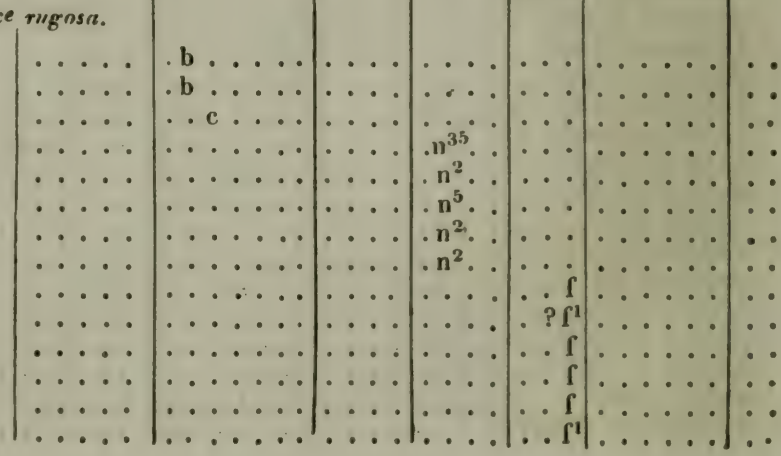




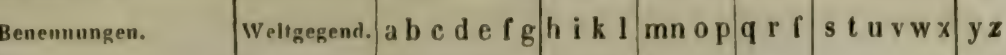

discoidens Biv.... polymorphius (GF.)

? trochoides Nyst

pristatus Bev.

*. Fungiae, busi rudiutu. mactra (BLv.)... stelliferus D'ARch.

Eudesi Michs.

colonula (GF)

semilunatus (Lк.)

Borsoni MichN.

lenticularis (Risso).

agaricoides (Risso)

Paumoten-is (Sтсив.)

complanatus (DFu.)

heterorlitus (DFr.).

titiculalus (DFu.).

*** busi incosnuitr.

nummititoides.ion:

excavatus

StephanophylliaMichn. 3 .

\section{Italica Michs.}

elegans Michn. . .

imperialis Mıchs.

Fungia Кв. $1 \ldots$

? Nicaecusis Мгег. .

Malyglossa En. 0

Polyphylliar EB. 0

Ecmesus Puir. 1 . fungiformis Pusr.

Phyllodes Phic. 1 la iniatus Phr....

\section{i. Zoanthina Eв.}

(Zoocorallia polyatctinia.)

Tughea Ix. 0 . . .

Zhoanthus Cuv. 0 .

NammiliferaLesu.0

Palythoa Lx. 0 ..

Siphonia Park. inter

Limnorea Lx. $\left\{\begin{array}{c}\text { Awor- } \\ \text { phozoa }\end{array}\right.$

Cnemidium GF.

k. Actinina Eb.

(Zoocorallia polyactinia Eв.)

Genera 9-10 mollia

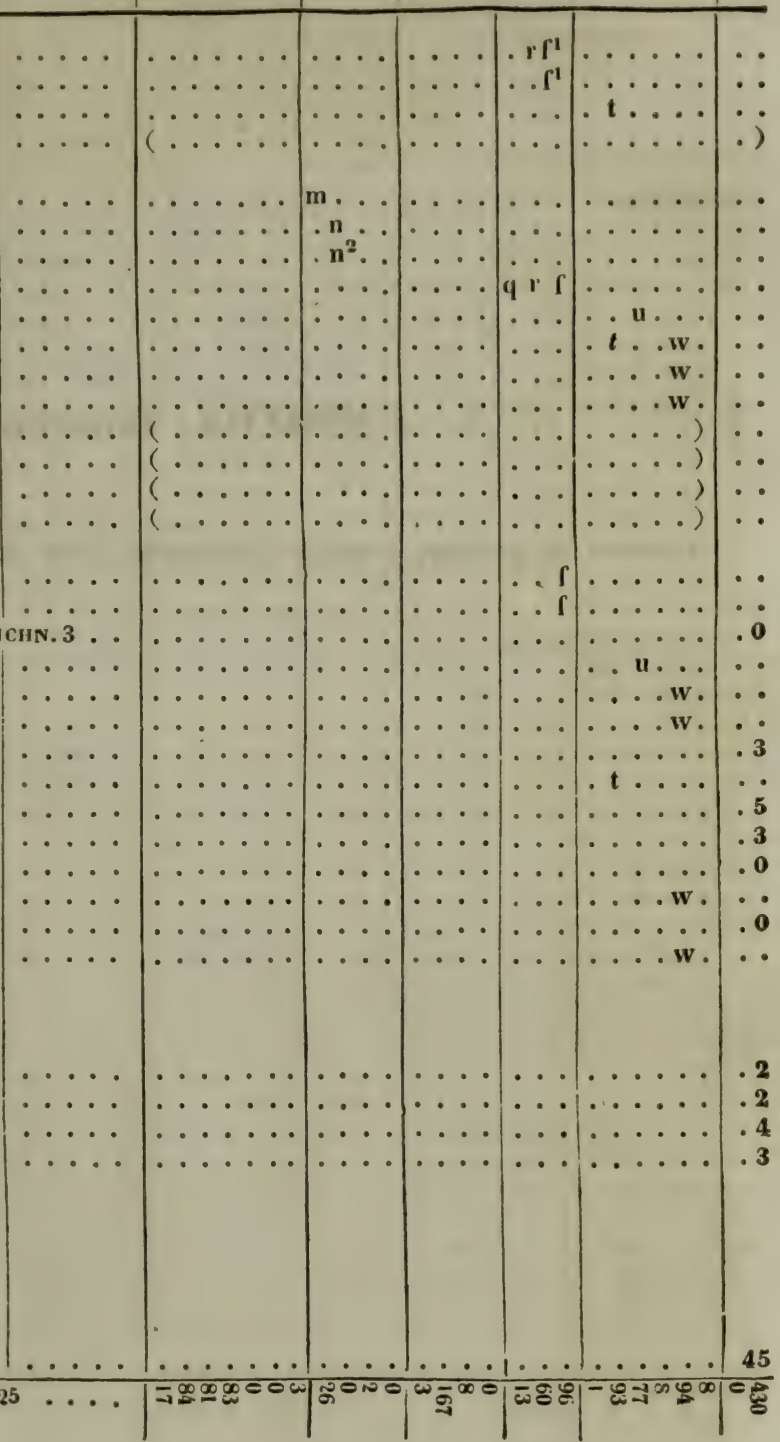

Anthozoorum summa: 825 


\section{Cl. v. ENTOzoA, Binnenwürmer.}

(Corpora in partibus omnibus mollissima, inde nunquam petrificata!) 


\begin{tabular}{|c|c|c|c|c|c|c|c|}
\hline & $W=$ trgegend. & KohlenP. & SalzP. & OolithP. & $\begin{array}{l}\text { Krei- } \\
\text { deP. }\end{array}$ & MulasseP. & Neu \\
\hline Benennungen. & 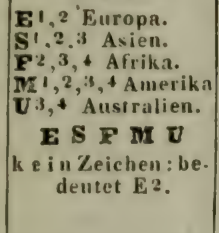 & 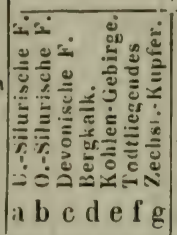 & 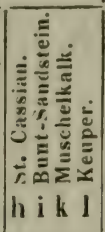 & 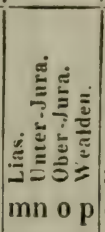 & 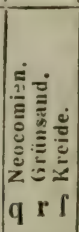 & 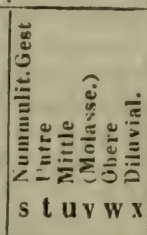 & 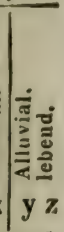 \\
\hline
\end{tabular}

\section{Cl. VI. ACALEPHAE Cuv., Quallen.}

(Arachnodermata BLv.)

(omissı sunt generı viventil uumerosissimu omnin corporibus tntis mollibus.)

Nummulina ER。 ${ }^{1} 21$

? Songuantlie [?] GAs. Mantelli Mort.

Fanjasi $B_{R}$.

stelliata Mü.

? costata Mü.

distans (Dsh.)

irregularis (Dsir.).

rotulata Dsi.

laevigata D’o.

complanata D'O.

globulari $\mathrm{D}$ O.

stabra $L_{K}$.

nummiformis Bran.

? lenticula GaL..... lenticularis BR.

crassa B:.

ephippium Рuser..

Gyzehensis $\boldsymbol{n}$. . .

seminulum (Ев.) . .

celiulosa (Ев.) ...

placentula (Forsi.).

(NumannlitesLk.) 18

= Nummulina EB. =

? sinuosus Ris.

inflatus Ris.

moneta Ris.

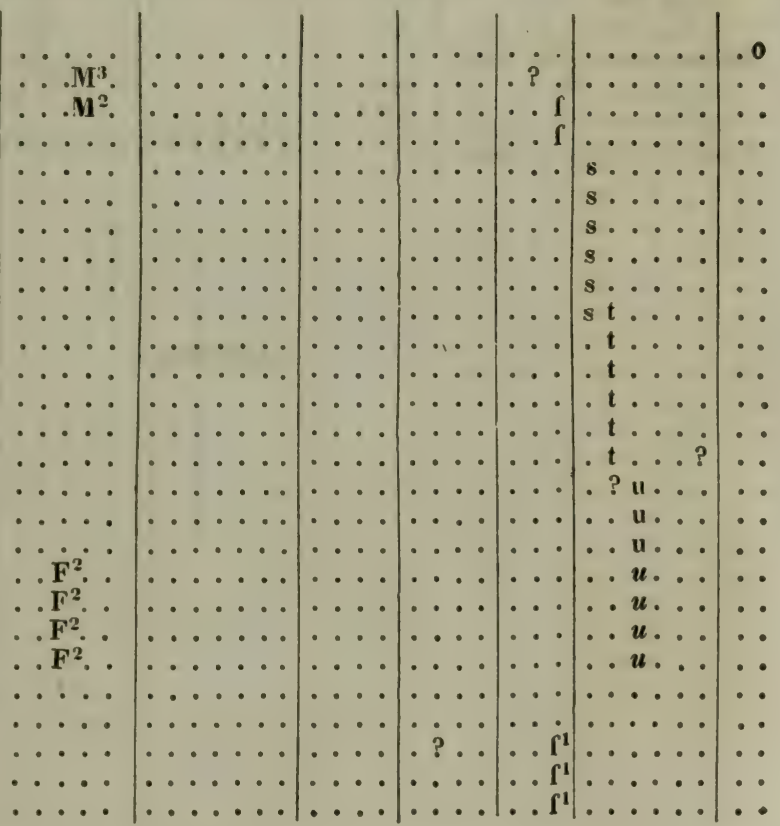




\begin{tabular}{|c|c|c|c|c|c|c|c|}
\hline \multirow[b]{2}{*}{ Benennungen. } & Weltgegend. & KohlenP. & SalzP. & OolithP. & $\begin{array}{l}\text { Krei- } \\
\text { deP. }\end{array}$ & MolasseP. & Net \\
\hline & 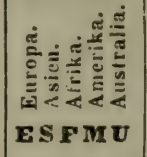 & 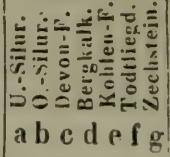 & 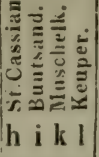 & 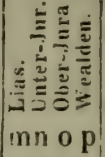 & 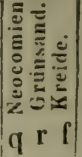 & 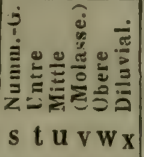 & y z \\
\hline auus $\mathrm{Ris}$ & $\cdots \cdots$ & $\ldots \cdots$ & $\cdots \cdots$ & $\cdots \cdots$ & . $\int^{1}$ & $\bullet \bullet \bullet \bullet$ & \\
\hline us D'A. . . & $\cdots \cdot$ & $\cdots$ & $\cdots \cdot$ & $\ldots$ & ... & s..... & \\
\hline cavus DFr. ... & $\cdots \cdot$ & $\ldots \ldots$ & ... & $\ldots$ & $\cdots$ & $s \ldots$. & \\
\hline crassis Boub. ... & $\cdots \cdots$ & $\ldots \ldots$ & $\cdots$ & $\ldots$ & $\cdots$ & $s \ldots$. & \\
\hline ? exponens So. . . . & $S^{3} \ldots$ & & & & & & \\
\hline $\begin{array}{l}\text { lenticularis Bоoв... } \\
\text { millecaput Borв. . }\end{array}$ & $\cdots \cdots$ & $\begin{array}{l}\cdots \\
\cdots\end{array}$ & $\cdots$ & $\begin{array}{ll}\cdots \\
\cdots \\
\cdots\end{array}$ & $\begin{array}{ll}\cdots \\
\cdots\end{array}$ & 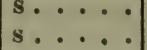 & \\
\hline iceus B & $\cdots \cdots$ & $\cdots \cdots$ & ... & $\cdots \cdot$ & $\cdots$ & s..... & \\
\hline UB. . . & $\cdots \cdots$ & $\cdots \cdots$ & . & $\cdots$ & $\cdots$ & s..... & \\
\hline Ramondi DFr. . . & $\cdots \cdot \cdot$ & $\cdots \cdots$ & $\cdots$ & $\cdots \cdot$ & $\cdots$ & s.... & \\
\hline Ataticus LвYм. . . & & $\cdots \cdots \cdots$ & $\cdots$ & $\cdots \cdot$ & $\cdots$ & t $\cdots$ & \\
\hline $\begin{array}{l}\text { globulus LEYM. . . } \\
\text { lenticula DFr. . . }\end{array}$ & $\cdots$ & $\cdots \cdots$ & $\cdots$ & & $\ldots$ & ? $? \ldots \ldots$ & \\
\hline fragilis Ris. . . . & .... & $\ldots \ldots$ & $\ldots$ & $\ldots$ & $\cdots$ & $\ldots \mathbf{w}^{\prime}$ & \\
\hline Ris. ..... & $\cdots \cdots$ & $\ldots \ldots$ & $\cdots \cdots$ & $\cdots$ & $\cdots$ & $\ldots w \cdot$ & \\
\hline $\begin{array}{l}\text { Yummelaria So. } 2 \\
\text { acuta So. }\end{array}$ & $\dot{S}^{3} \cdots$ & $\cdots \cdots$ & $\ldots$ & $\cdot$ & $\cdots$ & $\cdots \cdots$ & $\mathbf{0}$ \\
\hline a So. $\ldots$ & $S^{3} \cdots$ & $\cdots \cdots$ & 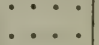 & & & s..... & \\
\hline 耳ycophrys (MF.)So. 2 & $\cdots$ & $\ldots \ldots$ & $\cdots \cdot$ & $\ldots$ & $\cdots$ & $\ldots \ldots$ & .0 \\
\hline $\begin{array}{l}\text { dispansus So. } \\
\text { ephippium So. }\end{array}$ & $\mathrm{S}^{3} \cdots$ & $\cdots \cdots$ & $\cdots$ & $\cdots$ & $\cdots$ & $s \ldots$. & - \\
\hline arus & & 00 & 000 & $\begin{array}{lllll}0 & 0 & 0 & 0\end{array}$ & $\begin{array}{lll}0 & 0 & 6\end{array}$ & 1997 & \\
\hline
\end{tabular}




\begin{tabular}{|c|c|c|c|c|c|c|c|}
\hline \multirow[b]{2}{*}{ Benennungen. } & \multirow{2}{*}{ 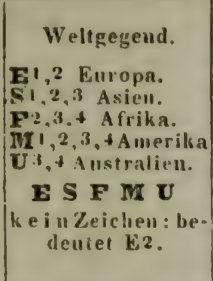 } & KohlenP. & SalzP. & WolithP. & $\begin{array}{c}\text { Krei- } \\
\text { deP. }\end{array}$ & MolasseP. & Neu \\
\hline & & 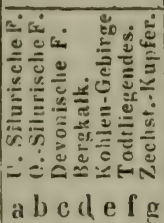 & 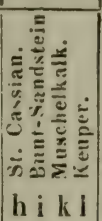 & 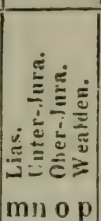 & 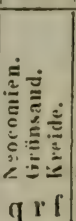 & 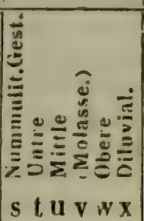 & 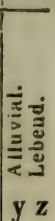 \\
\hline
\end{tabular}

\section{Cl. VII. ECHINODERMATA (LK.) Cuv.}

(Radiaria, Strahlthiere.)

\section{STECLETE INA.}

A. CRINOIDEA (MIr.t.).

1. stylastritaf Martin. (gen. affixu, bruslintu)

a. Poteriocinidae Aust.

Poteriocriuns (Mulf ) A g. 12

Dudleyensis Aust. .

fusiformis RoE.

conicus Phale.

crassus Mut.....

? dactyloides Aust.

impressus Рhic... .

isacobus Arst. . . .

plicatus Auss.

quinquangularis Avst.

radiatus Aust.

rostratus Aust...

tenuis MiLi.

Traxocrinms Рнит.. 8 macrorlactylus Mopis. tuberculatus Morrs. brevidactylus MorR. Egertoni Mork. granulosus Morr. longidactylus Morr. nobilis MORP.... pentagonus Morr.

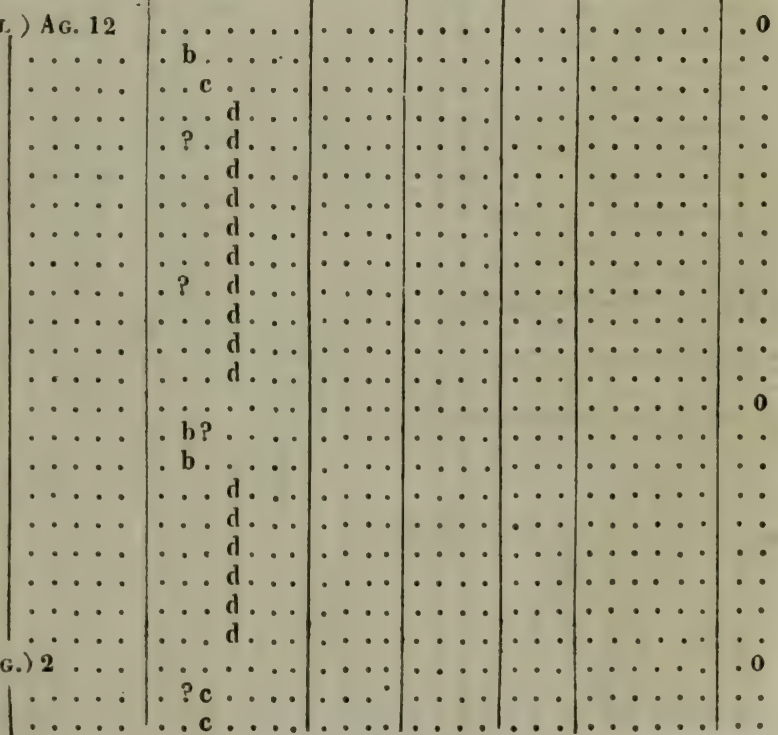

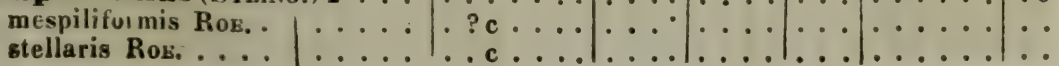




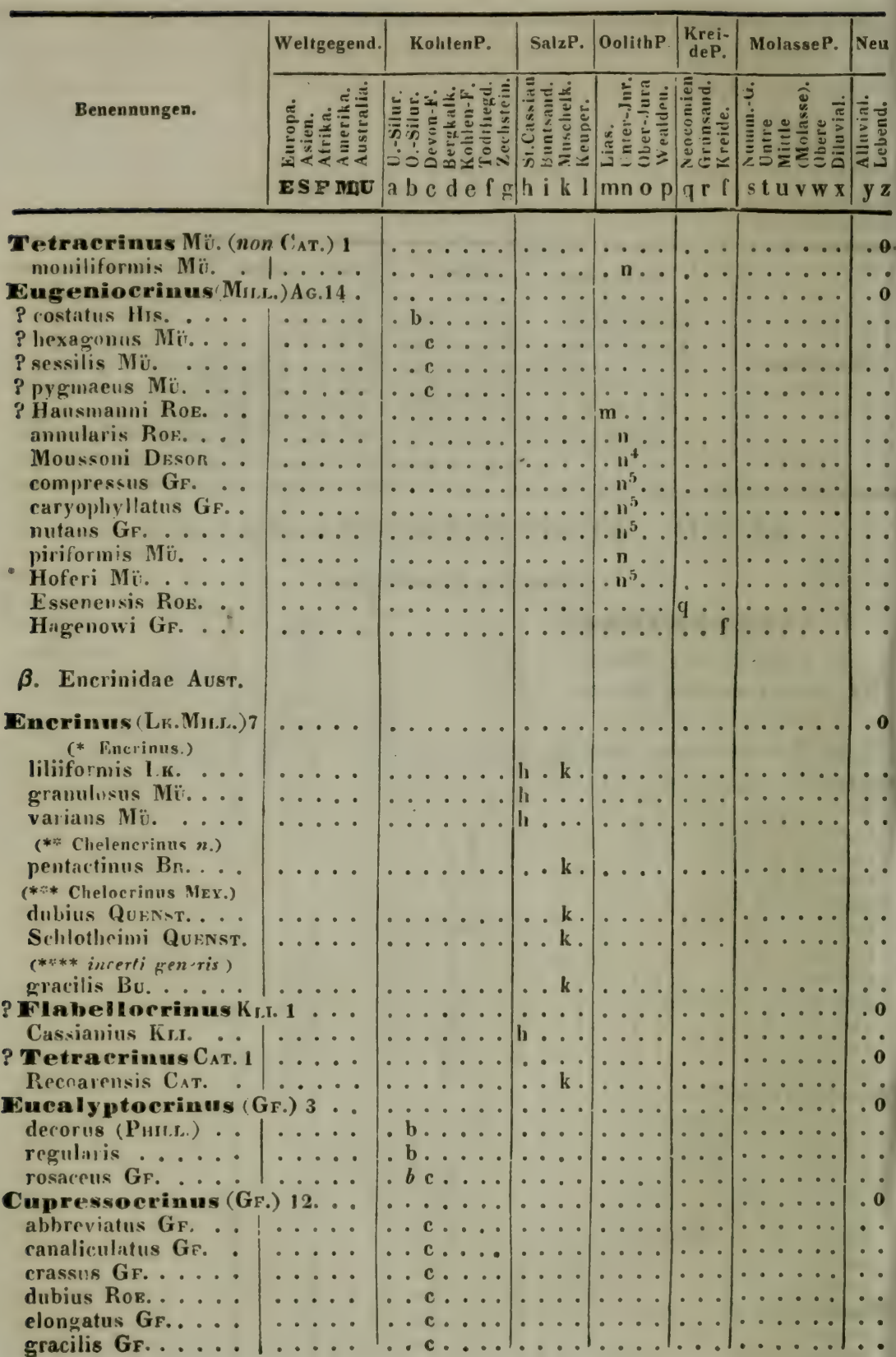




\begin{tabular}{|c|c|c|c|c|c|c|c|}
\hline gen. & send. & $\mathbf{a b}$ & hik l & $\mathbf{o p}$ & f & $\mathbf{w} \mathbf{x}$ & y z \\
\hline & & & & & 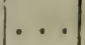 & & \\
\hline us GF. . . & & ..... & $\cdots \cdots$ & $\ldots$ & ... & $\ldots \ldots$ & \\
\hline s Gr.... & $E^{2} \cdot M^{2}$ & $\cdots c \ldots$ & .... & $\ldots$ & $\cdots$ & $\ldots \ldots$ & \\
\hline sendi, KoEN.) & $\ldots \ldots$ & $\cdots ? \ldots$ & -3 & $\cdots$ & $\cdots$ & $\ldots \ldots$ & \\
\hline s Fisch. . & $\cdots \cdot$ & . . ? ... & $\cdots \cdot$ & $\ldots \cdot$ & $\cdots$ & $\ldots \ldots$ & \\
\hline is Eic & $\cdots \cdot$ & . b..... & $\cdots \cdot$ & $\cdots \cdot$ & $\cdots$ & $\ldots \ldots$ & \\
\hline ocrinuss Pun.t. 1 & $\ldots \ldots$ & $\cdots \cdots$ & $\ldots$ & $\cdots \cdot$ & $\cdots$ & $\cdots \cdots$ & .0 \\
\hline Phill. & & $\cdot$ & $\cdots$ & $\cdots$ & $\cdots$ & $\cdots \cdots$ & - \\
\hline rinidae Aust. & & & & & & & \\
\hline Pentacrinus(MuL.)A & . & $\cdots$ & $\cdots$ & $\ldots$ & $\cdots$ & $\cdots \cdots$ & . 1 \\
\hline .. & & ..c & $\cdots$ & $\ldots$ & $\cdots$ & $\ldots \ldots$ & • \\
\hline$\cdots$ & & . & $\cdots \cdots$ & $\cdots$ & $\cdots \cdot$ & $\cdots \cdots$ & \\
\hline$\cdots$ & & & & . & . . & $\cdots \cdot$ & \\
\hline İ̈. . . & & & & $\cdots$ & • & $\cdots$ & \\
\hline$\cdots \cdots$ & $\because$ & & $\begin{array}{l}h \\
h\end{array}$ & $\therefore$ & $\therefore$ & $\cdots$ & \\
\hline u. . . & $\therefore$ & : & h & • & $\cdots$ & $\because$ & \\
\hline stus Ki.I. $\ldots$ & $\dot{.}$ & & & $\mathrm{m}$ & $\cdots$ & $\cdots$ & - \\
\hline Briareus Mili.... & $\dot{.}$ & $\therefore$ & $\cdots$ & $m$. & $\because$ & $\cdots$ & \\
\hline$\therefore \cdots$ & $\dot{0}$ & $\cdots$ & $\ldots$ & 1 & $\because \cdots$ & $\mid \begin{array}{cc}\cdots & \cdots \\
\cdots & \cdots\end{array}$ & \\
\hline i.. & & $\ldots$ & $\ldots$ & $\mathrm{m}$. & $\cdots$ & 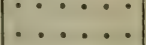 & \\
\hline ... & . & $\ldots$ & $\ldots$ & m. . & ... & $\cdots \cdots$ & \\
\hline . & .... & $\ldots \ldots$ & $\ldots$ & $m \ldots$ & $\ldots$ & $\therefore \ldots$ & \\
\hline . & $\cdots \cdot$ & $\cdots \cdots$ & $\ldots$ & $m u$. & $\ldots$ & $\ldots \ldots$ & \\
\hline e Nir.i. & . & $\ldots$ & $\cdots$ & & $q \ldots$ & $\ldots \ldots$ & \\
\hline & . . & .. & • & $? 1^{4}$. & $r$ & $\ldots \ldots$ & \\
\hline II.T. & - & . & $\ldots$ & ? 1 & $\cdots$ & $\ldots \ldots$ & \\
\hline$\cdots$ & - & . & $\cdots$ & $.11^{2} \cdot$. & $\cdots$ & $\ldots \ldots$ & \\
\hline & & $\cdots$ & $\cdots \cdot$ & $.11^{5} \cdot$. & $\cdots$ & $\ldots \ldots$ & \\
\hline$\cdots$ & $\cdots \cdot \cdot$ & $\ldots \ldots$ & $\cdots \cdot$ & $.11 \ldots$ & $\cdots$ & $\cdots \cdots$ & \\
\hline$\cdots$ & & . & - &. & $\cdots$ & $\cdots \cdots$ & \\
\hline . & $\cdot$ & - & & . $11^{2} \cdot$. & $\cdots$ & $\cdots$ & \\
\hline$\dot{0}$ & 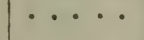 & & . & . $11^{2}$. & $\cdots$ & $\cdots \cdots \cdot$ & \\
\hline DEs. & & $\cdots$ & - &.$n^{4}$. & $\cdots$ & $\cdots$ & \\
\hline$\cdots$ & - & $\cdots$ & $\cdots$ & .1 & $\cdots$ & $\cdots$ & \\
\hline . & - & & & $\cdot 11^{4} \ldots$ & $\cdots$ & $\cdots$ & \\
\hline$\because$ & & & & $\cdot \mathbf{n} \cdots$ & $\dot{q} \cdot \cdots$ & $\ldots \ldots$ & \\
\hline s. & & $\because$ & $\cdots$ & $\cdots$ & $q$ & $\ldots \ldots$ & \\
\hline & & $\ldots$ & - & $\ldots$ & . r & $\ldots \ldots$ & \\
\hline B & . & $\cdots$ & $\cdots \cdot$ & $\ldots$ &. $\mathrm{rr}$ & $\ldots \ldots$ & \\
\hline$\cdots$ & . & $\cdots$ & $\cdots$ & $\ldots$ & $\cdots \Gamma$ & $\ldots \ldots$ & - \\
\hline$\cdots$ & & . & $\cdots$ & $\ldots$ & .. & $\ldots \ldots$ & \\
\hline AG. . & & . & . & $\ldots$ & $\ldots \mathrm{I}$ & $\ldots \ldots$ & \\
\hline & & & & & & $\ldots \ldots$ & \\
\hline s $R_{0}$ & & . & - $\cdot$ & . & $\ldots 1$ & $\cdots$ & \\
\hline Irr. & & & & & & & \\
\hline byi WE & & & & & &.$t$ & \\
\hline ห. 2 & & • & & & $\cdots$ & $\ldots w$ & \\
\hline . & - & & & .1 & . & $\therefore$ & • \\
\hline 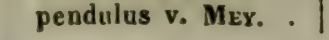 & & & & & & & \\
\hline
\end{tabular}




\begin{tabular}{|c|c|c|c|c|c|c|c|}
\hline & Weltgegend. & KohlenP. & SalzP. & OolithP. & $\begin{array}{l}\text { Krei- } \\
\text { deP. }\end{array}$ & MolasseP. & Neu \\
\hline Benennungen. & 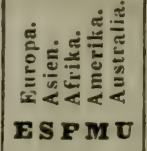 & 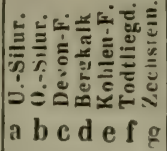 & 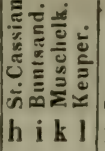 & 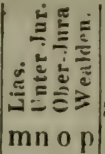 & 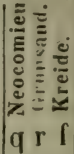 & 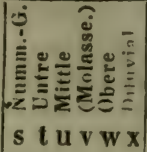 & 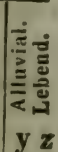 \\
\hline
\end{tabular}

ঠ. Marsupiocrinidae Aust.

Mrarsupiocriums Phel. 1 . caelatus PHit.L.

Crotalocrinus (Aust.) Morr. 1 rugosus Aust.

e. Platycrinidae Aust.

Platycrimus (Mur.) A g. 26.

Buchi Ros.

interscapular is Phice.

ventricosus GF.

hirriglyplicus GF. .

tabulatus GF.

decagonus $\mathrm{Gr}$.

Goldfussi Mü.

brevis Gr.

granuliferus RoE.

exsculptus Gr.

rosaceus Ros.

ornatus GF.....

anaglypticus $\mathbf{G r}$.

rugusus Mice.

elongalus Phicl. .

laevis Mur.r.

antheliontes Aust.

coronatus GF.

granulatus Micr...

microstylus PHicL.

mucronatus Avst.

pileatus Gr.....

spinosus Aust.

striatus Aust. . . .

trigindidactylus Aust.

tuberculatus Mir...

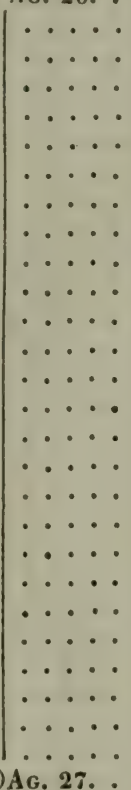

Cyathocrinus( Mill.)Ag. 27.

? corolliferus His.

? pulcher His.

? scrobiculatus His.

capillaris Phis.r.

gonyodactylus Phir.

piriformis PнII..

pinnatus Gr. ...

clathratus Ag.

pentagonus $\mathbf{G F}$.

?decaphyllus Ros...
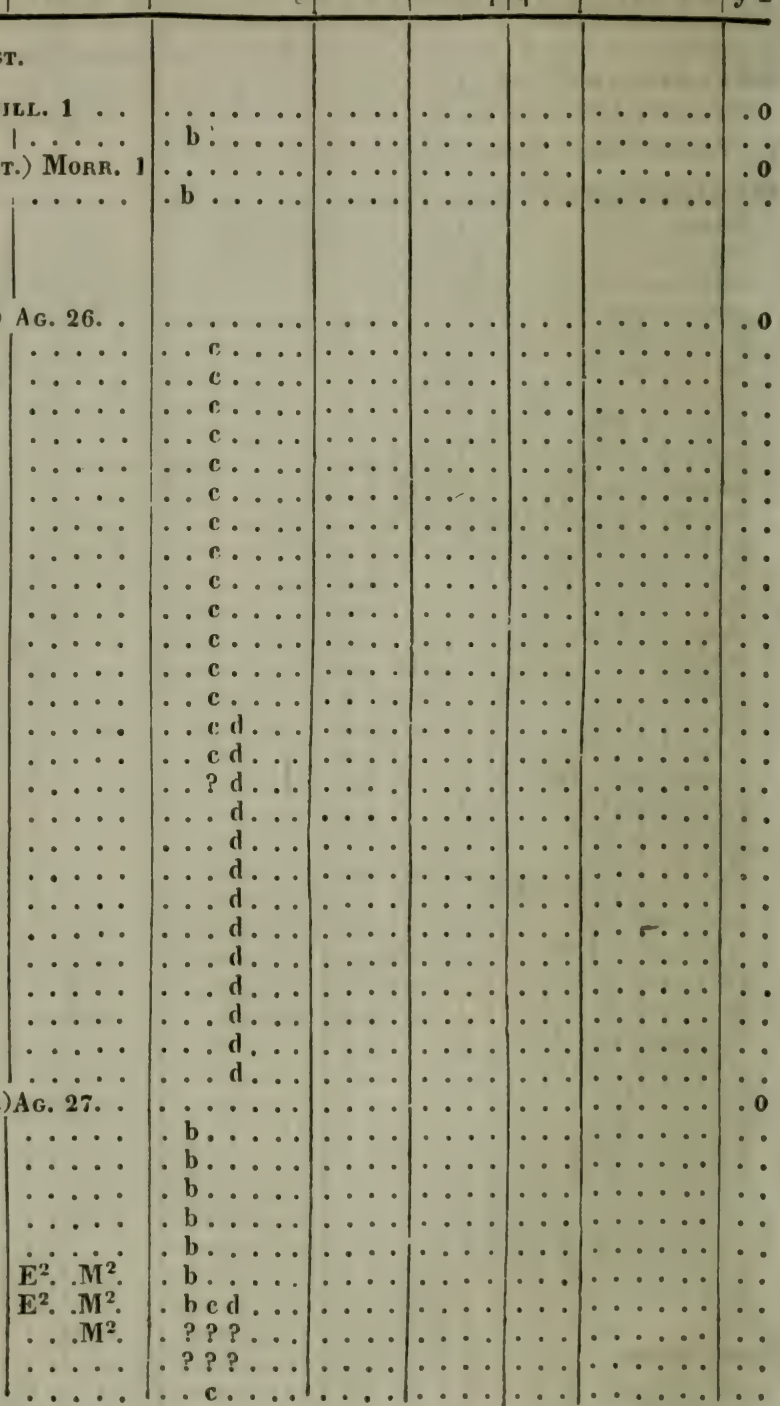


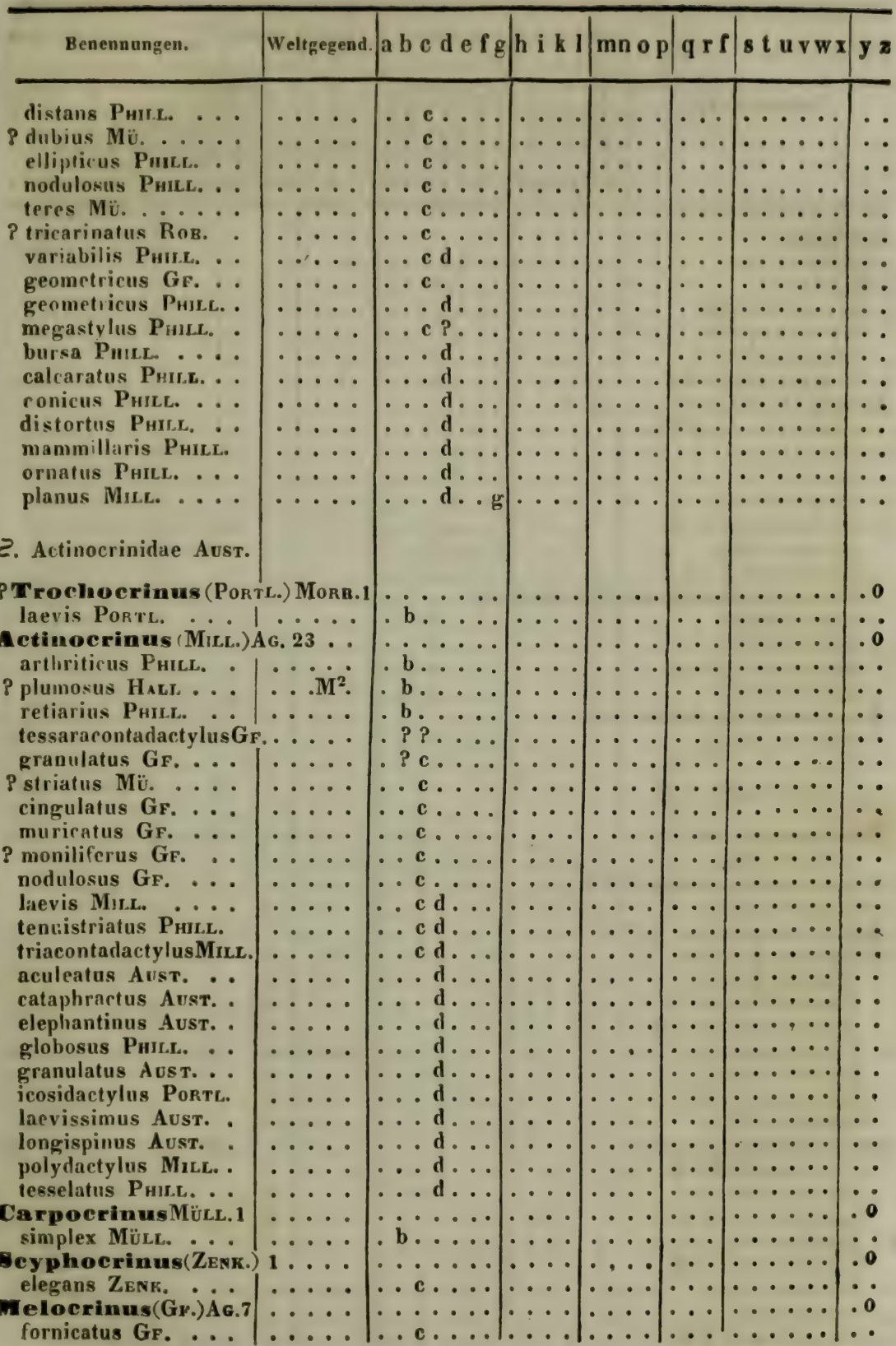




\begin{tabular}{|c|c|c|c|c|c|c|c|}
\hline & Weltgegend. & KohlenP. & SalzP. & OolithP. & $\left|\begin{array}{c}\text { Krei- } \\
\text { deP. }\end{array}\right|$ & MolasseP. & Neu \\
\hline Benennungen. & 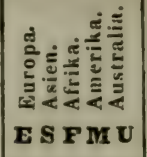 & 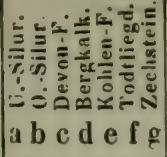 & 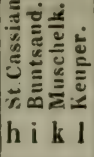 & 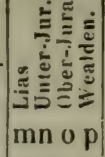 & 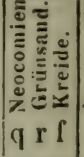 & 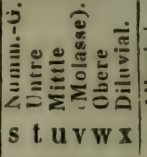 & 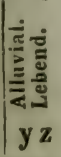 \\
\hline
\end{tabular}

Melocrinus)

gibbosus GF.

laevis GF.

pyranidalis GF.

verrucosus $G_{F}$.

amphora GF.

hieroglyphicus GF.

Rhodoerinus (Mil.. Ag. 7 . quinquangularisMiLI. verus MiLL.

crenatus Gr.

gyratus GP. quinquepartitus GF. costatus Aust.

granulatus Aust. .

$\mid \begin{gathered}\ldots \ldots \\ \cdots \\ \cdots \\ \cdots \\ \cdots \\ \cdots\end{gathered}$

\section{bursa Phile.}

calraratus PhicL. .

mamnillaris Phule. .

simplex Porti.

Tetramerocrinus (Aust.) 1 . formosus Ausx.

ๆ. Apiocrinidae D'O.

MaIanocrinus Ag. 1

subteres DEs. .....

cruettardocrinus $D^{\prime} 0$. dilatatus D'O.

Apiocrinus (Miri)

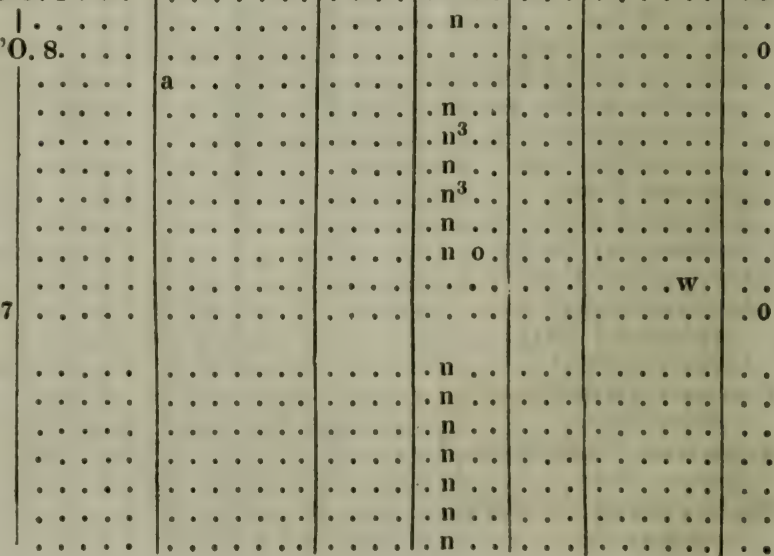

$P$ dipentas Levchts.

elegans D'O.

Meriani Desor ...

Murchisonanus D'O.

Parkinsoni D'O.

similis Desor.

Roissyanus D'O. .

P obscurus Mü.

Millerocrimus d'O.37 (ordine systematico)

simplex D'O.

polydactylus D'O.

gracilis D'O.

Fleuriauanus D'O.

crassus D'O.

elegans D'O.

cupuliformis D'O. 


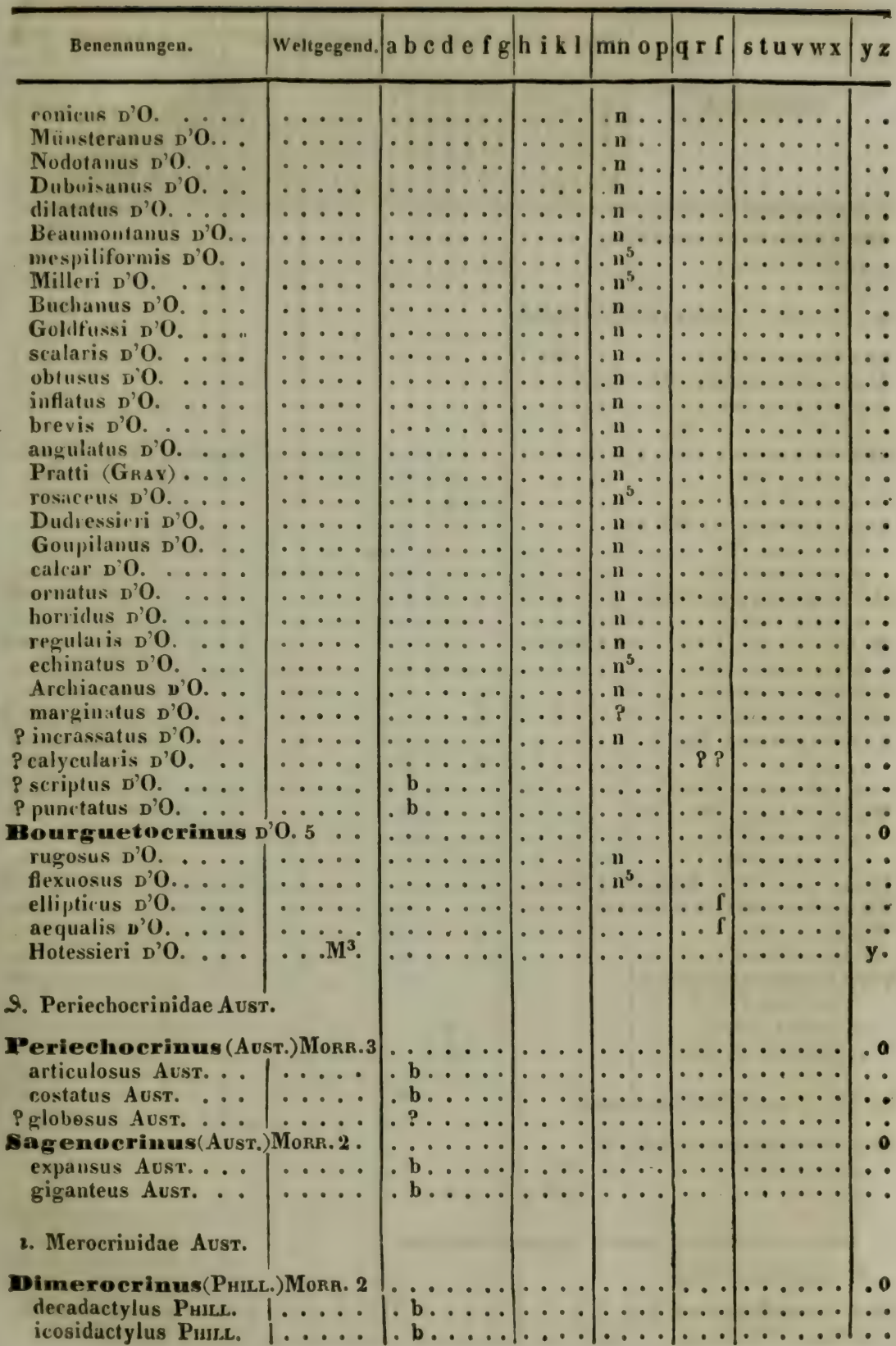




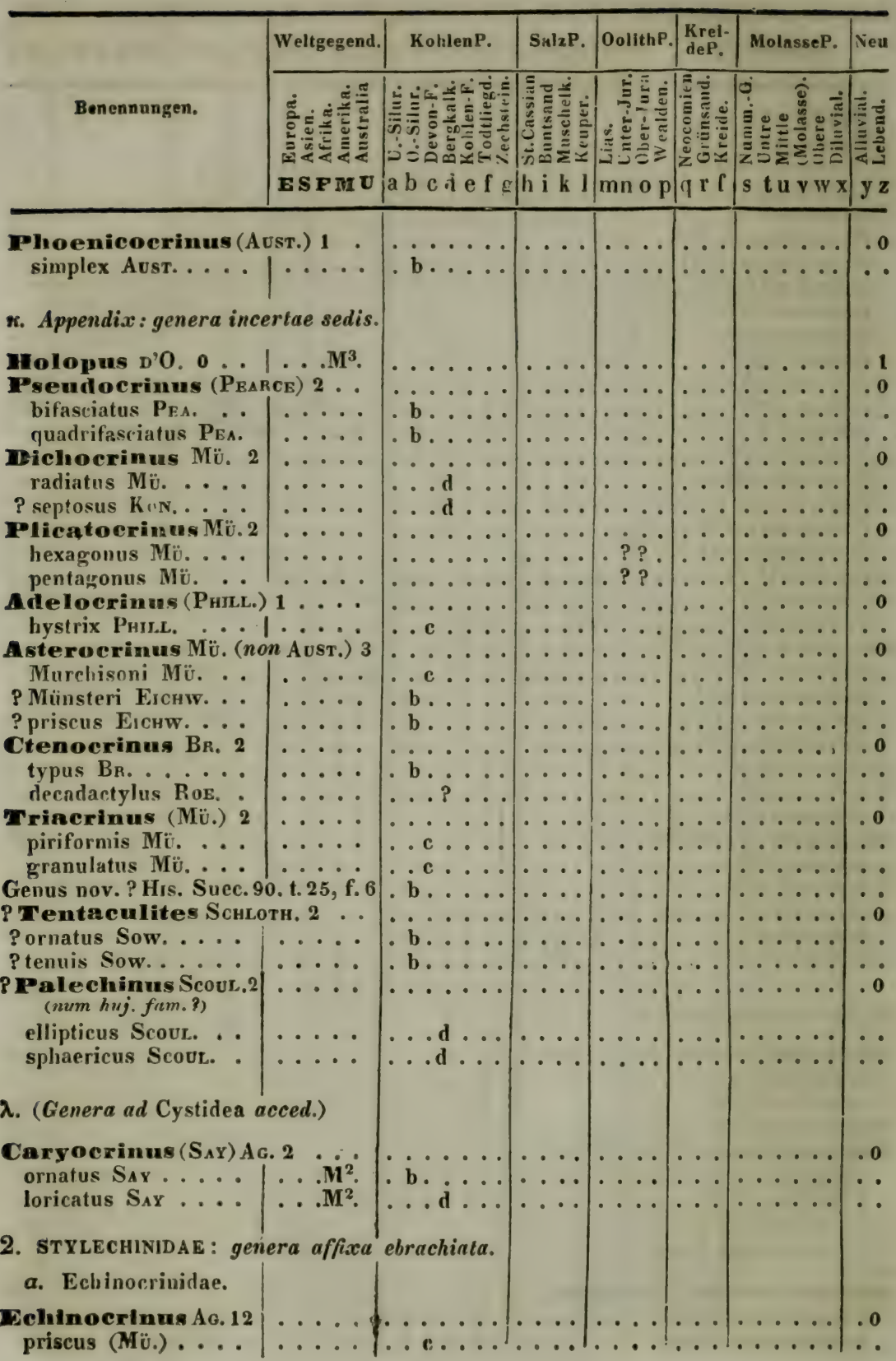




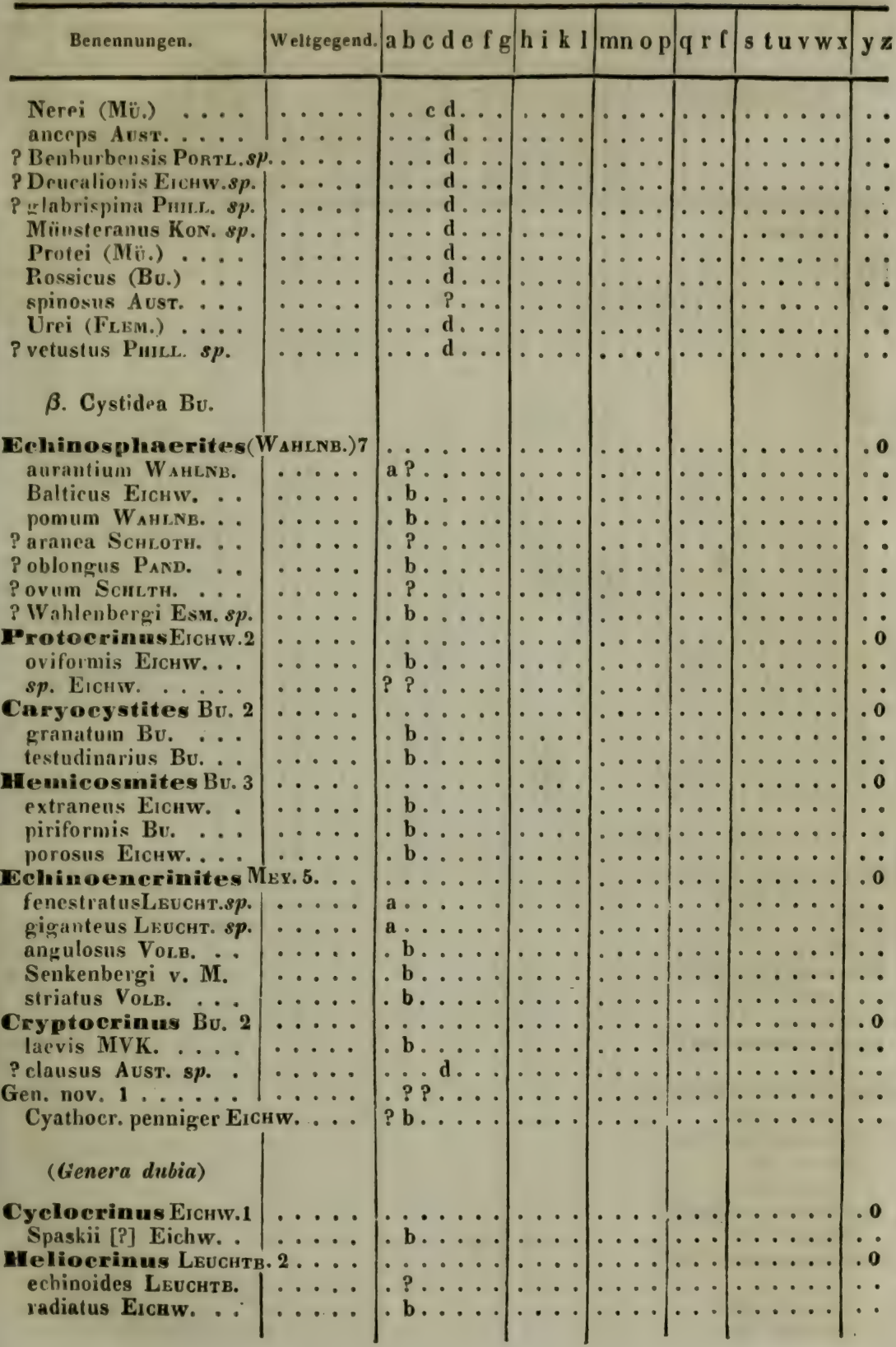




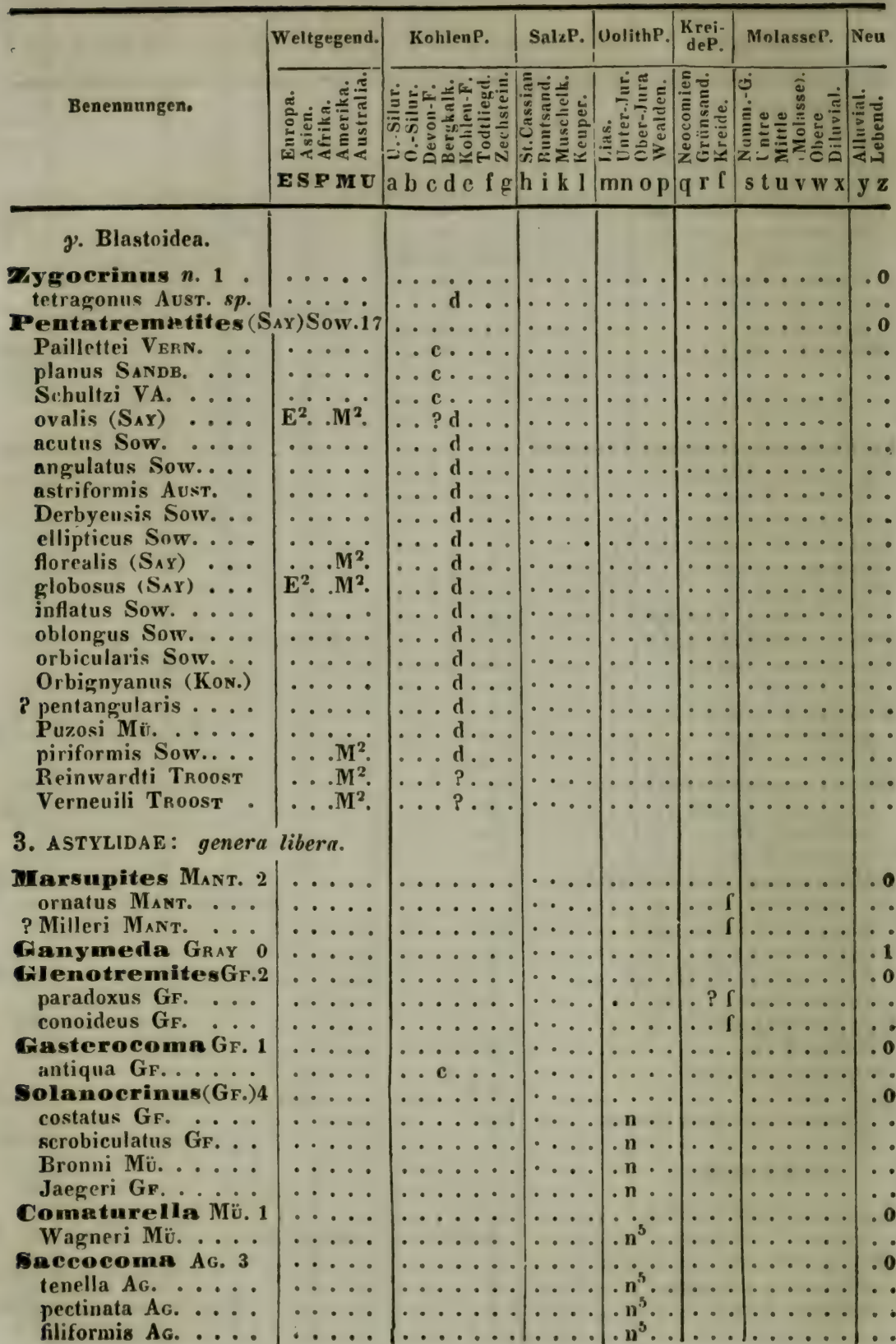




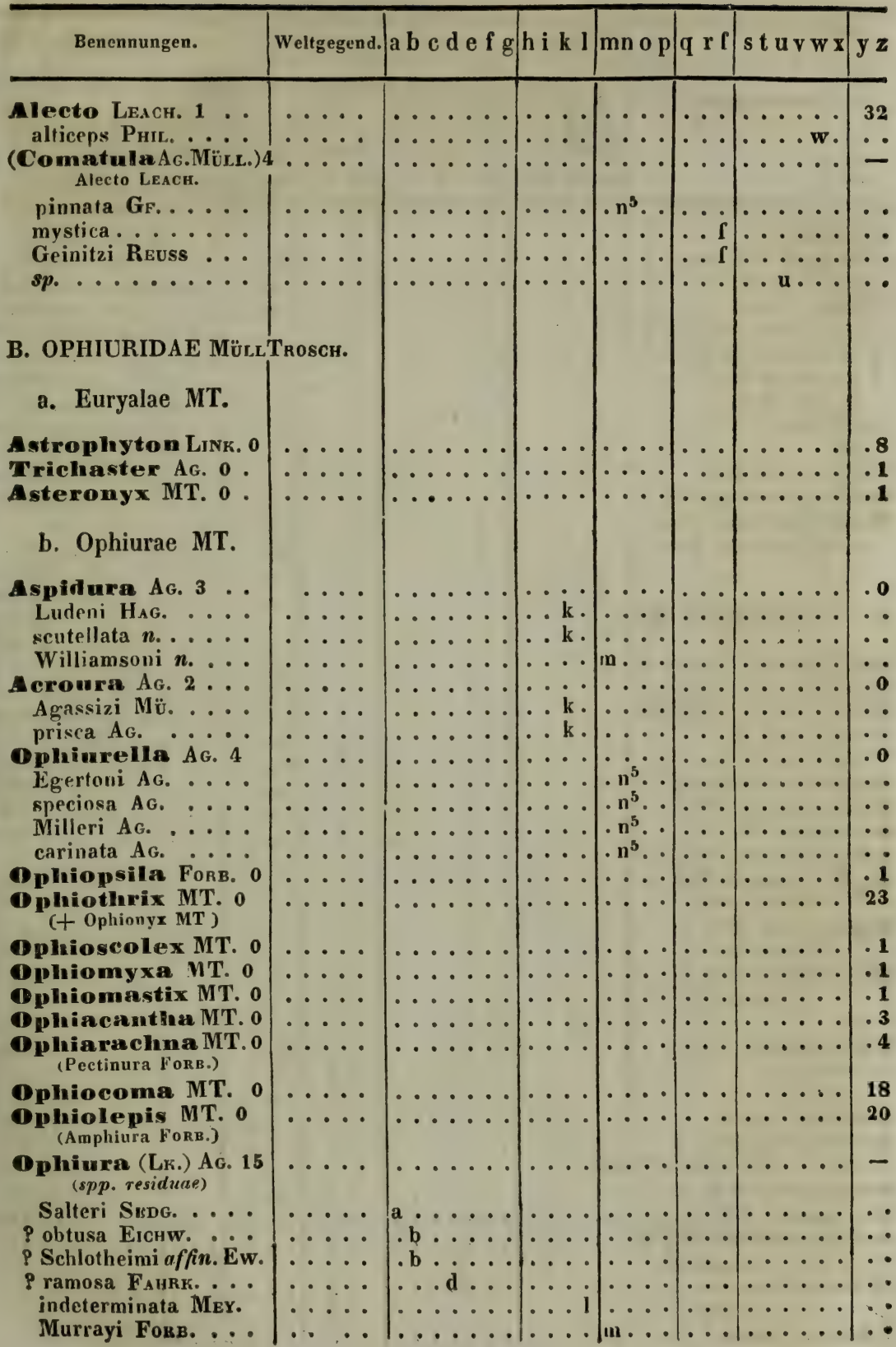




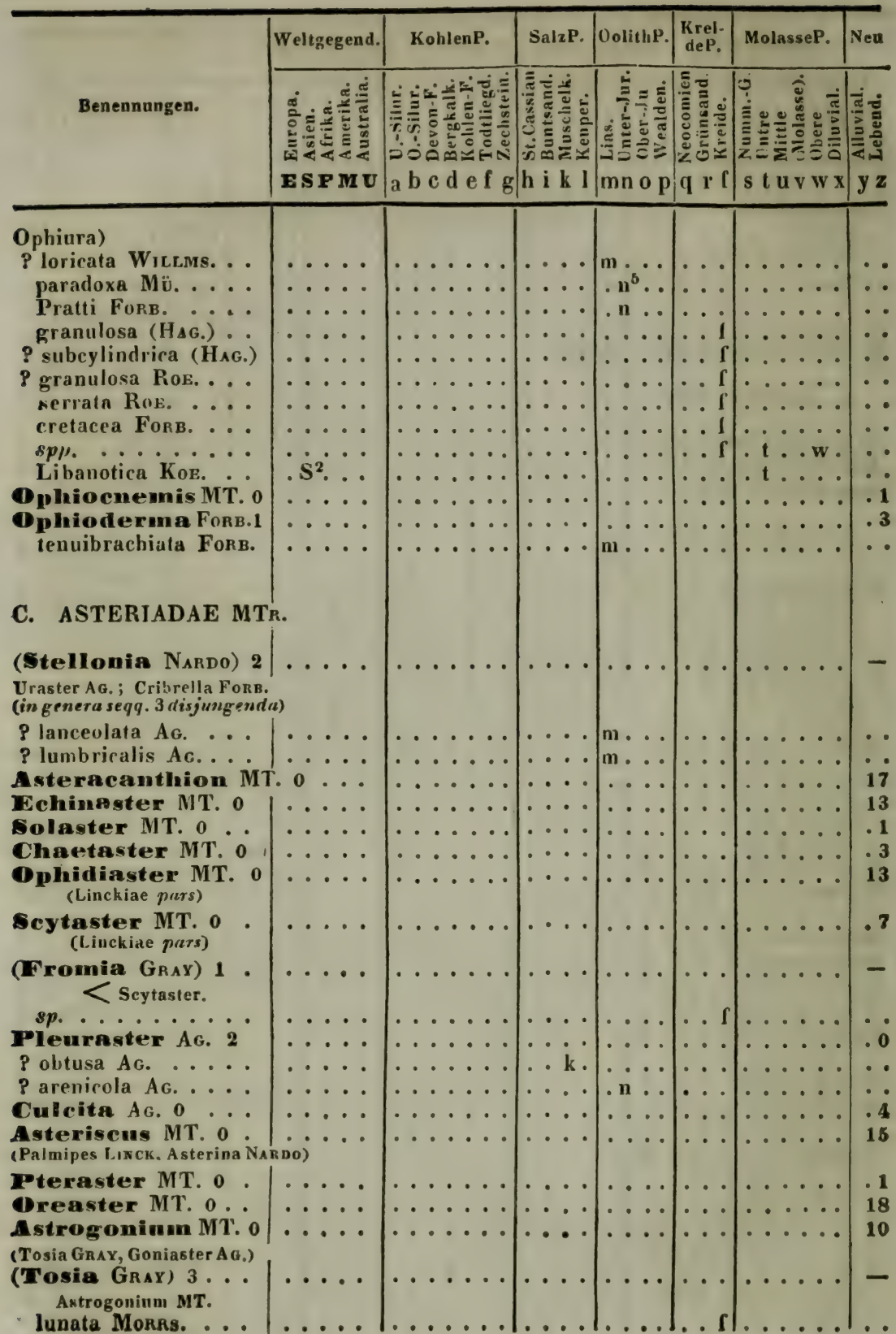




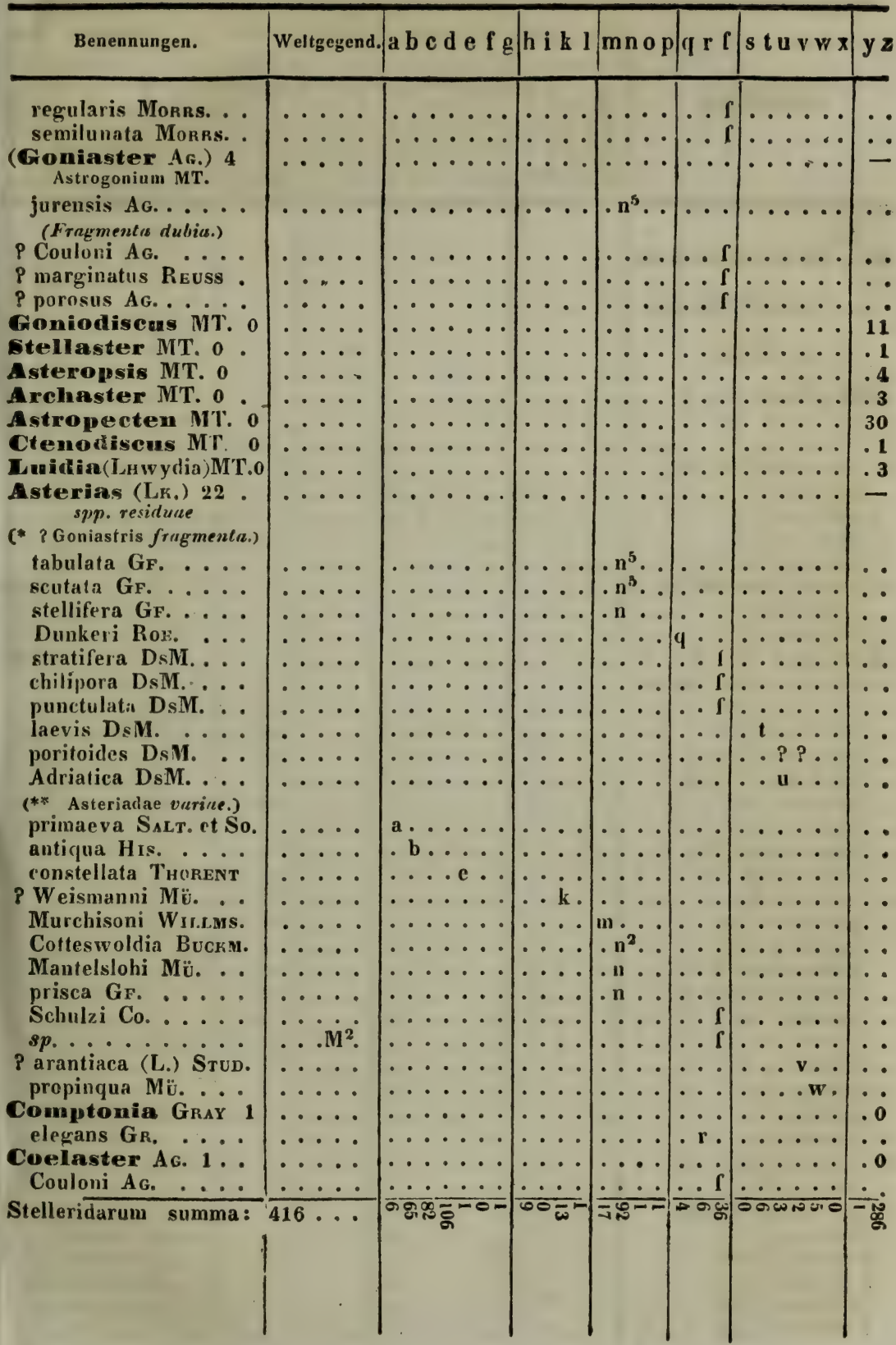




\begin{tabular}{|c|c|c|c|c|c|c|c|}
\hline & Weltgegend. & KolilenP. & SalzP. & OolithP. & \begin{tabular}{|c|} 
Krei- \\
deP.
\end{tabular} & MolasseP. & Neu \\
\hline Benennungen. & 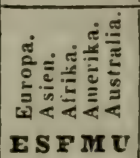 & 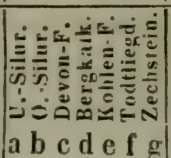 & 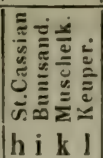 & 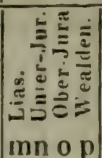 & 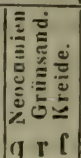 & 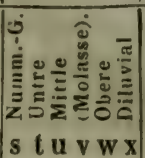 & 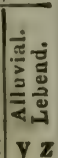 \\
\hline
\end{tabular}

IX. ECIINI DAE.

A. CIDARIDAE Ag.

a. Echini.

Glypticus Afs. 4 .. quercinus AG. nffinis AG. hieroglyphicus Ag. . sp.? Buw в. .....

Conliopsis Ag. 2 .. doma AG.

simplex AG.

Coeloplenrus Ag. 2 equis AG.

radiatus $A G$.

Echinus (L.) AG. 53

* species Agassizi typicue. nodulosus Mü. perlatus Desm. excavatus LESKE bigranularis LK. arenatus LK.

planus Ag.

homocyphus AG. intermedius As.... gyratus Ag. polyporus AG. serratus Ag.

Cadomensis AG. psammophorus Ag. • scrialis AG

laevis Ag. A.... distinctus AG.... fallax Ag. pulcher AG. .... lepidus AG. .... Carantonanus Ag. dubius Ag.

** speries Aliorum.

liasinus $\mathrm{PoE}$.

‡corona Risso

? arenosus Sow.

? nitidus KoEN.

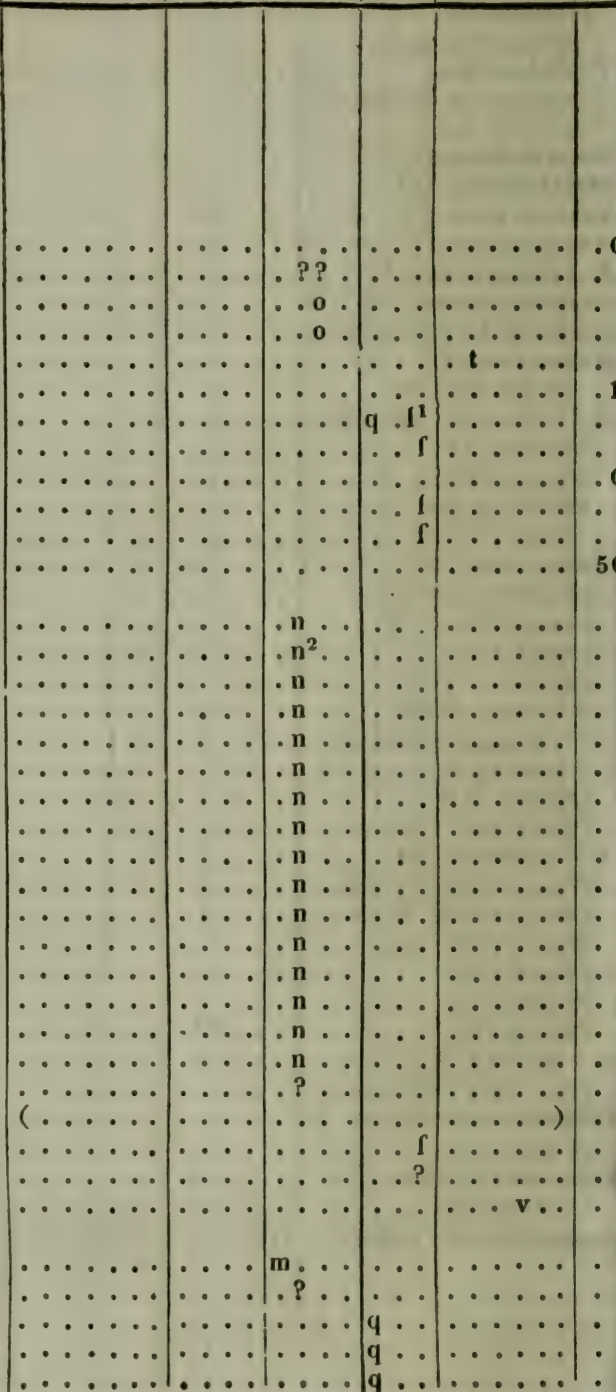




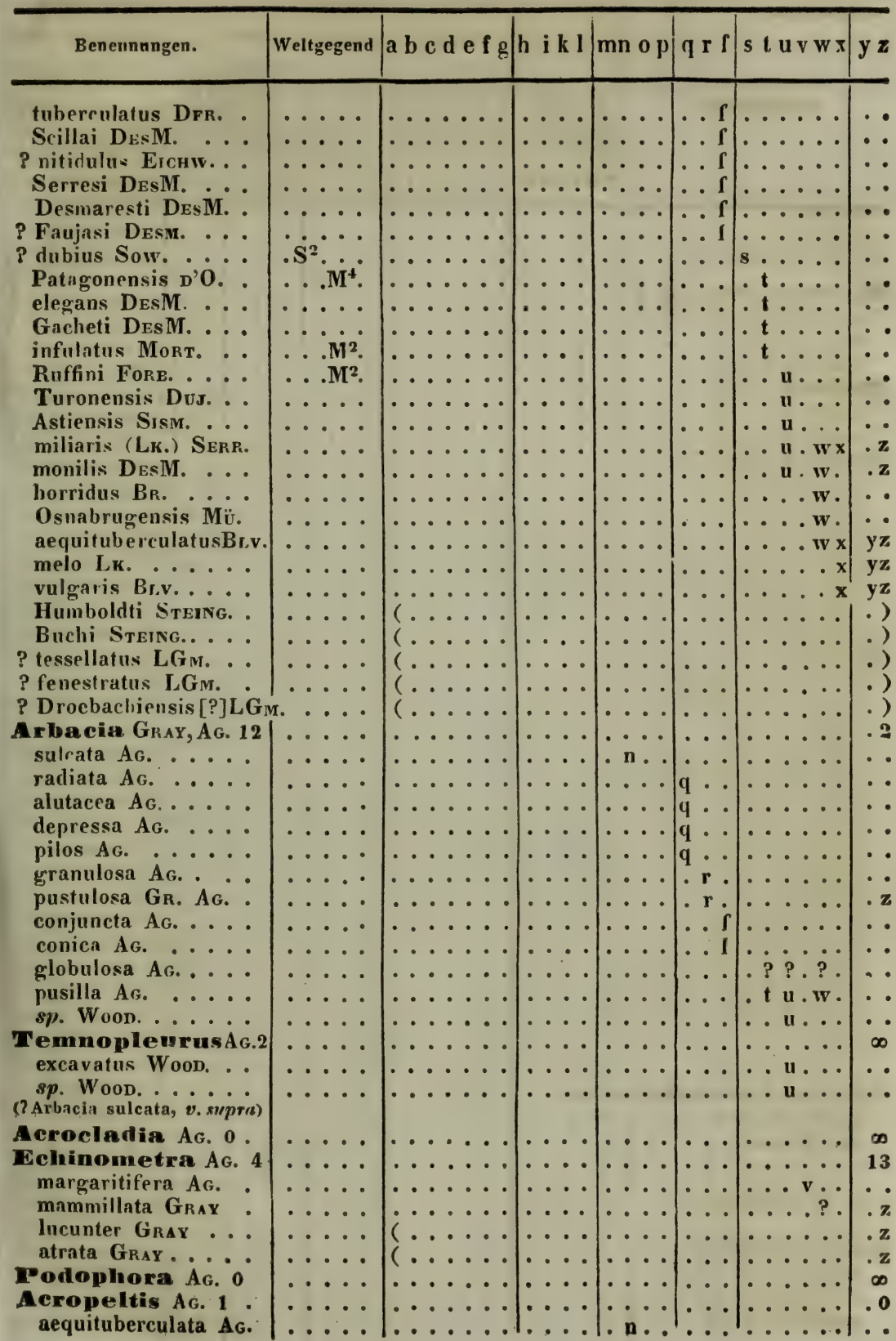




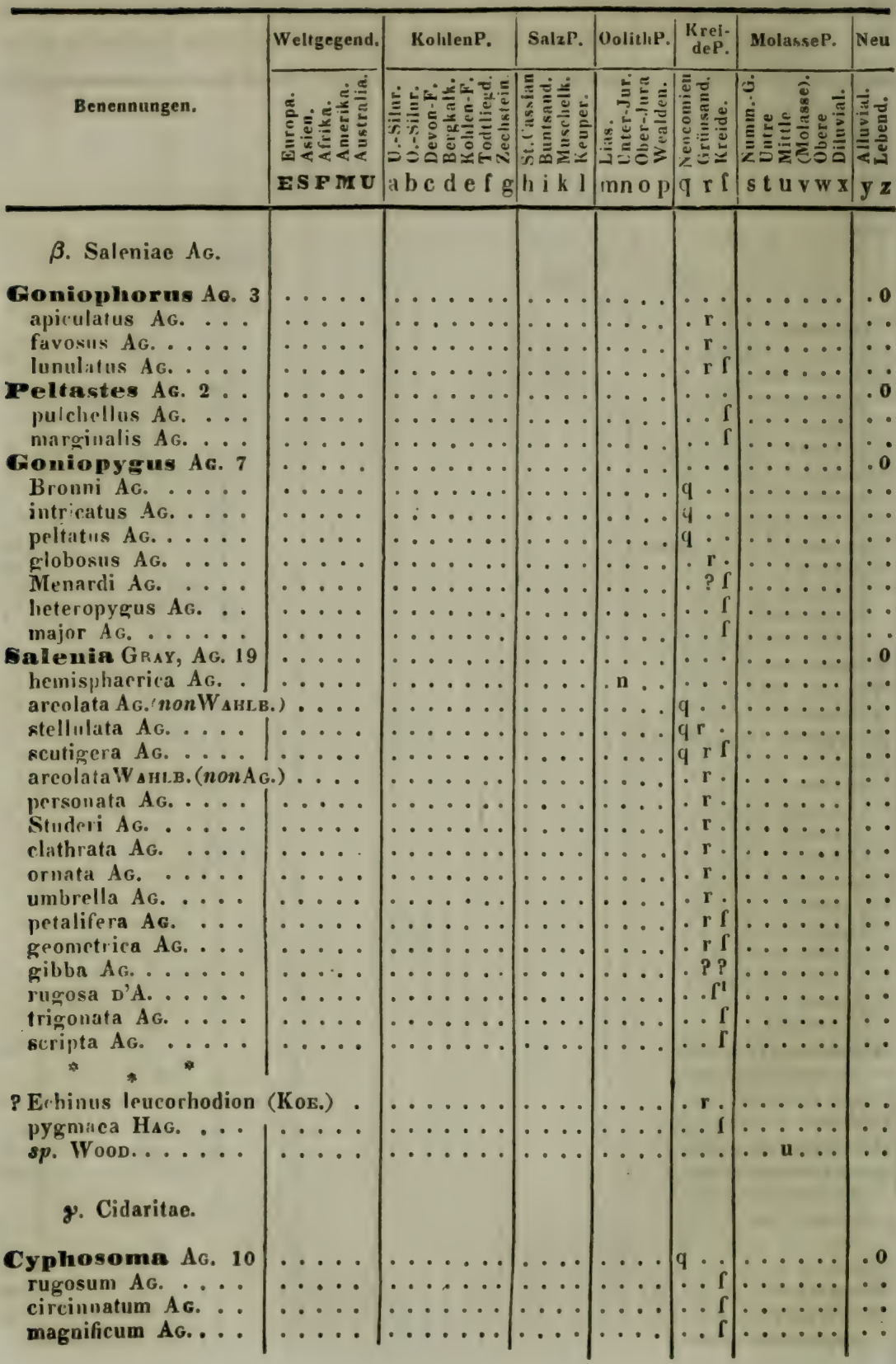




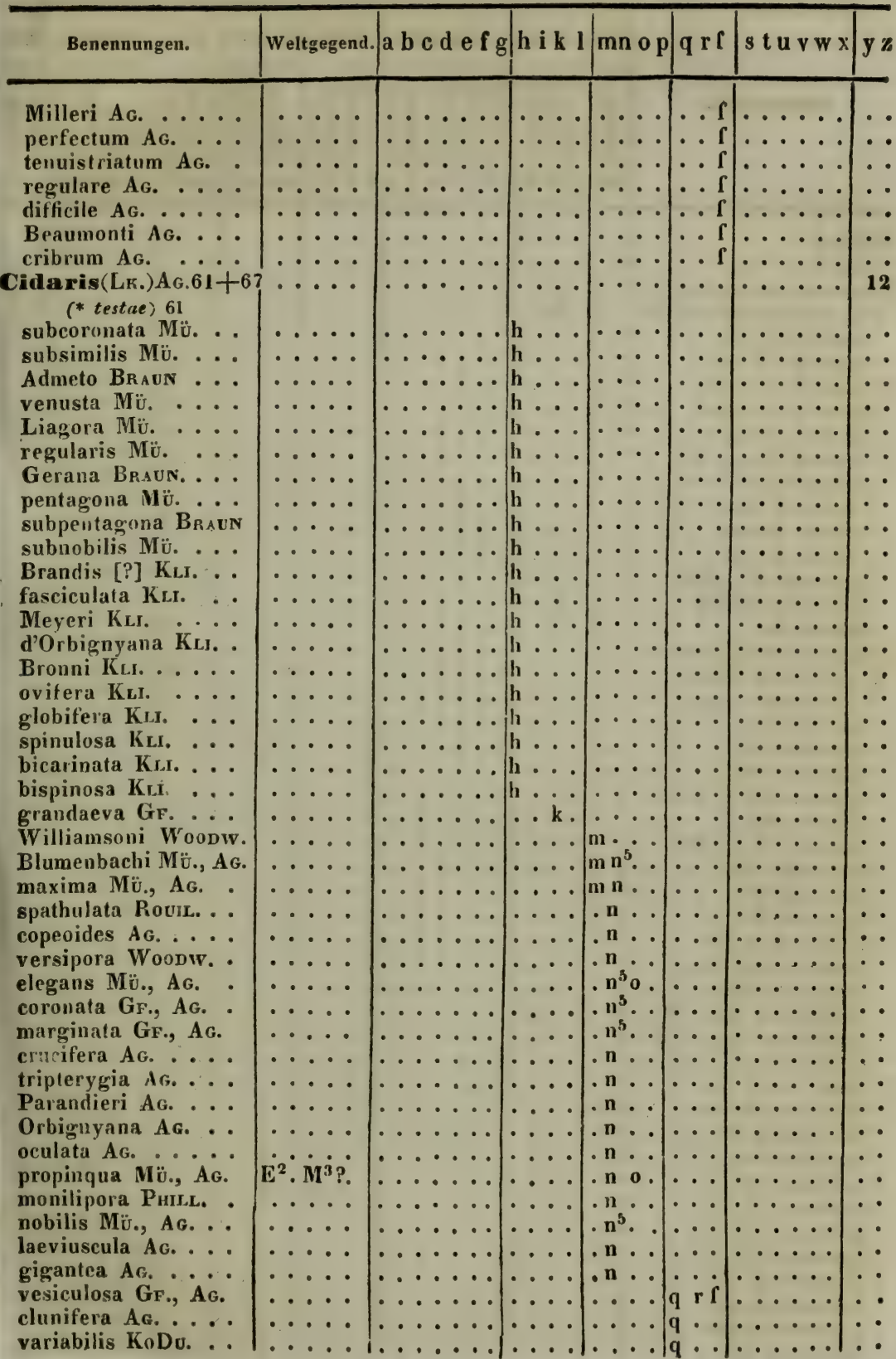




\begin{tabular}{|c|c|c|c|c|c|c|c|}
\hline & Weltgegend. & KohlenP. & Salzl. & OolithP. & \begin{tabular}{|c|}
$\begin{array}{c}\text { Krei- } \\
\text { deP. }\end{array}$ \\
\end{tabular} & MolasseP. I & Neu \\
\hline Benennungen. & 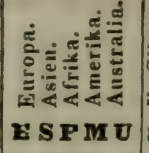 & 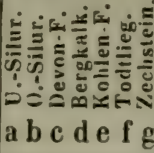 & 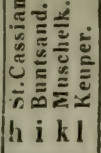 & 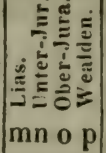 & 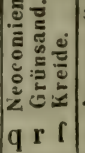 & 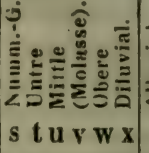 & 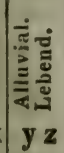 \\
\hline
\end{tabular}

Cidaris)

punctata RoE. mammillata PARK.

? pustulosa Nyst el GaL. pusio Ag.

saxatilis PABK.... regalis Gf., Ag. . . corollaris Leske, Ag. miliaris n'ARCH. . . cretosa Park., Ag. . acrocidaris Pontl. clavigera KoE, AG. armata Reuss.... exigua likuss.... princeps (?) HAG. diatretum Mort. . discus Br.

Avenionensis DesM. ? botryoides KLEIN . . (** acu/ei) 67.

Buchi Mü.

remifera Mü..... biformis Mü.

Hausmanni Wissm. trigona Mü. spiuosa (Ag.) Mü. cinguiata Mü..... flexuosa Mü.

linearis $M \ddot{u}$. semicostata Mü. . scrobiculata Braun. decorata Mü. catenifera (AG.) Mü. baculifera Ag., Mü. dorsata $\mathrm{BR}_{\mathrm{R}} . . .$. . alata (AG.) Mü. . Roemeri Wissm. . Waechteri Wissm. . horrida Mre. .... spinulosa RoE. . . orobus AG.

heteropleura AG. . spathula Ag. pustulifera AG. . . cucumifera AG. . . cinamonea AG. ... filogiana AG.
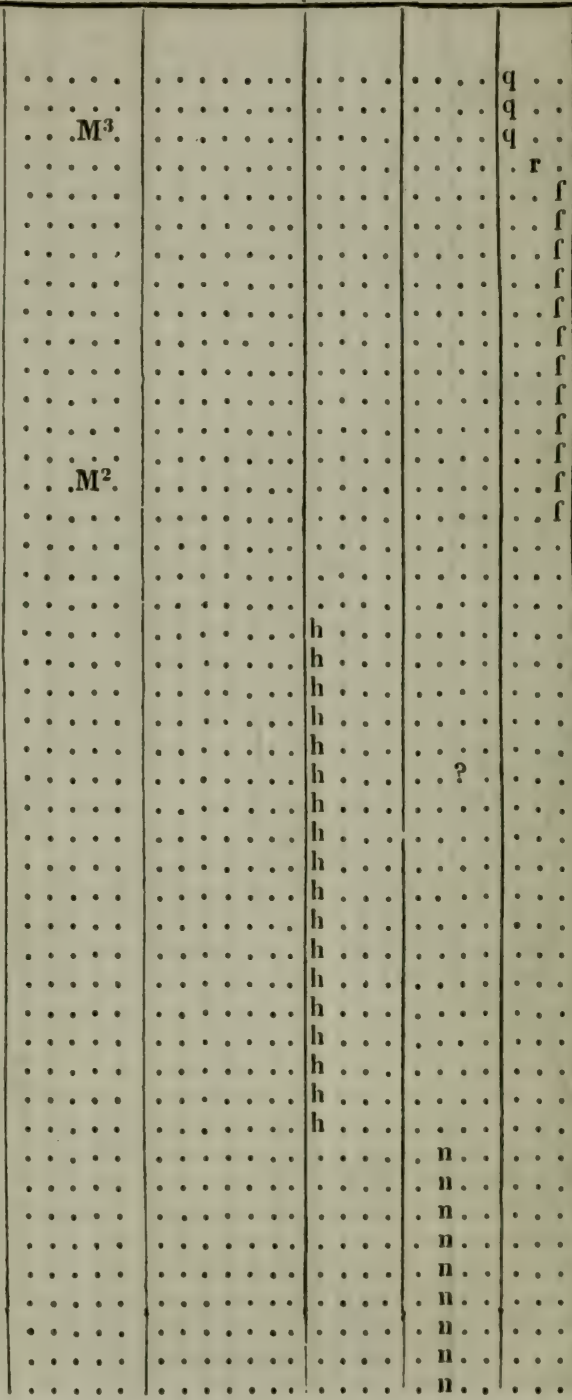


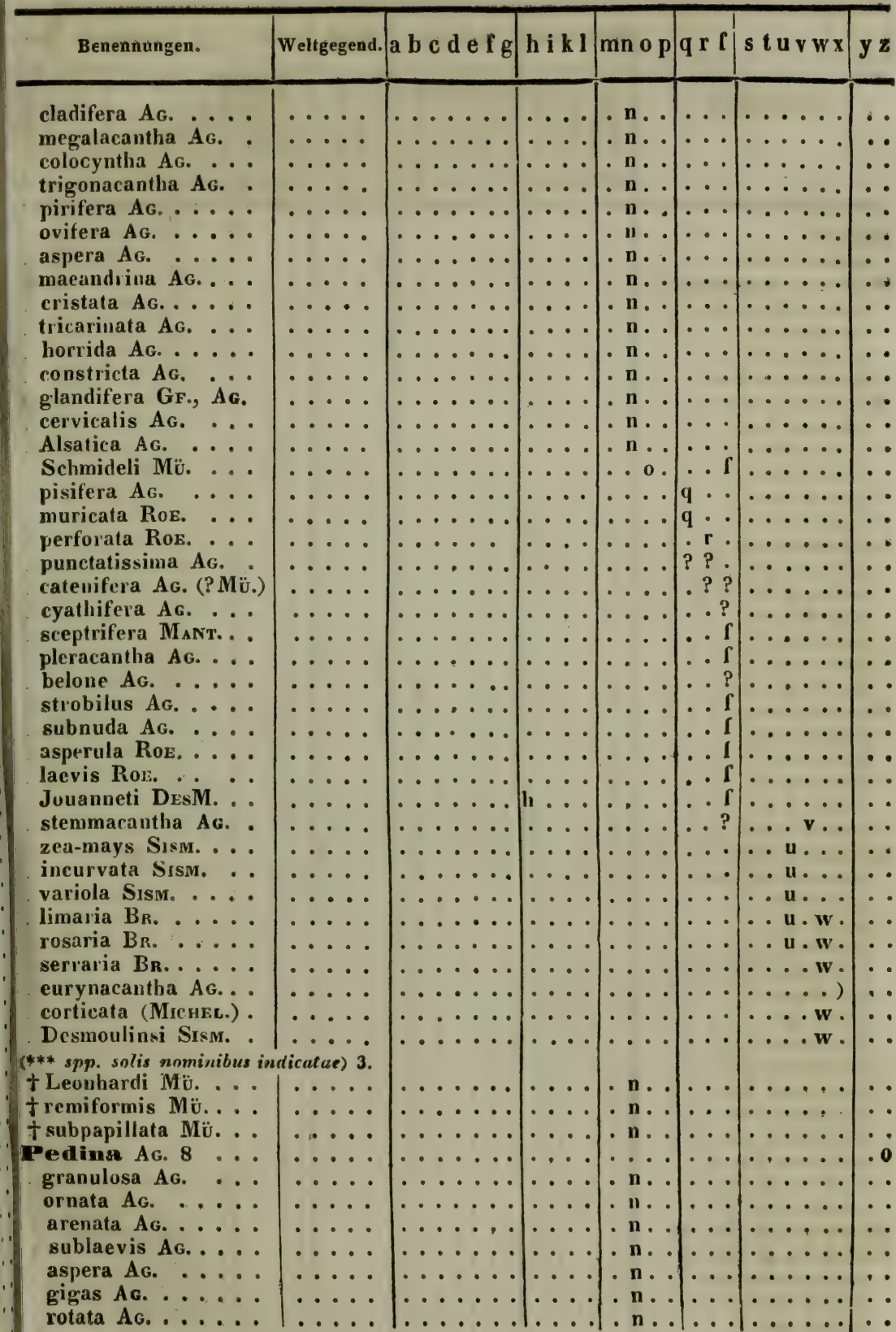




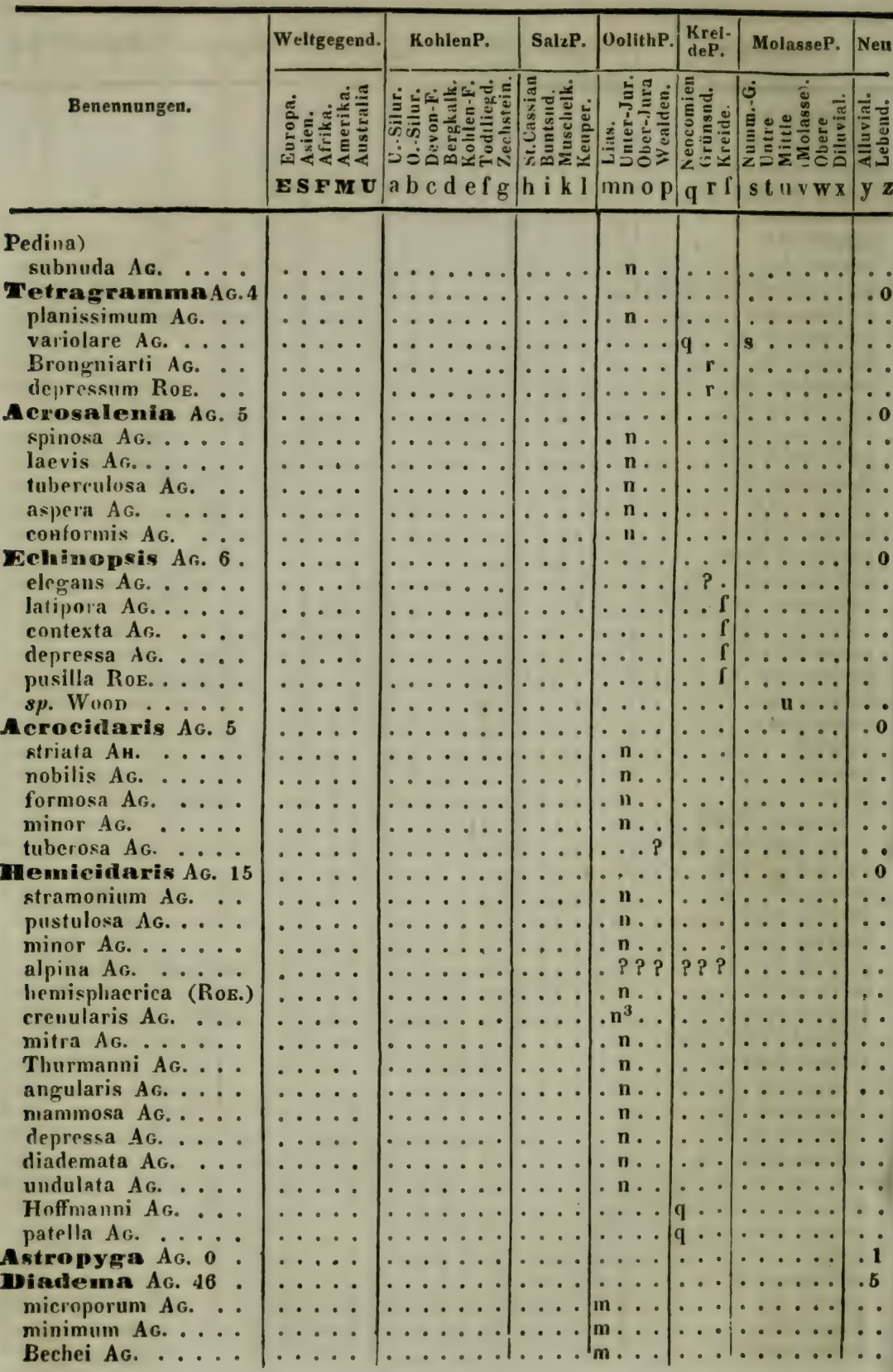




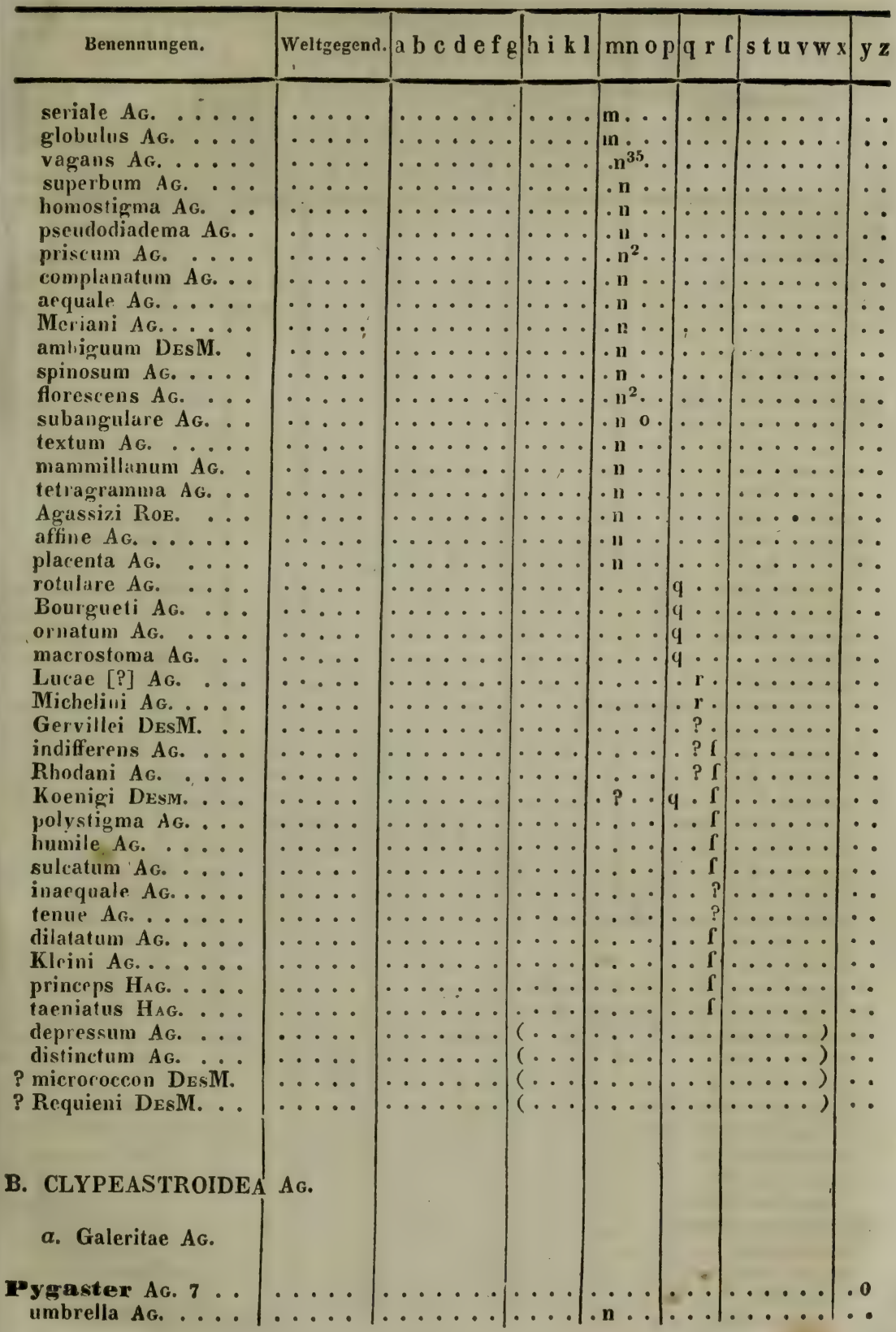




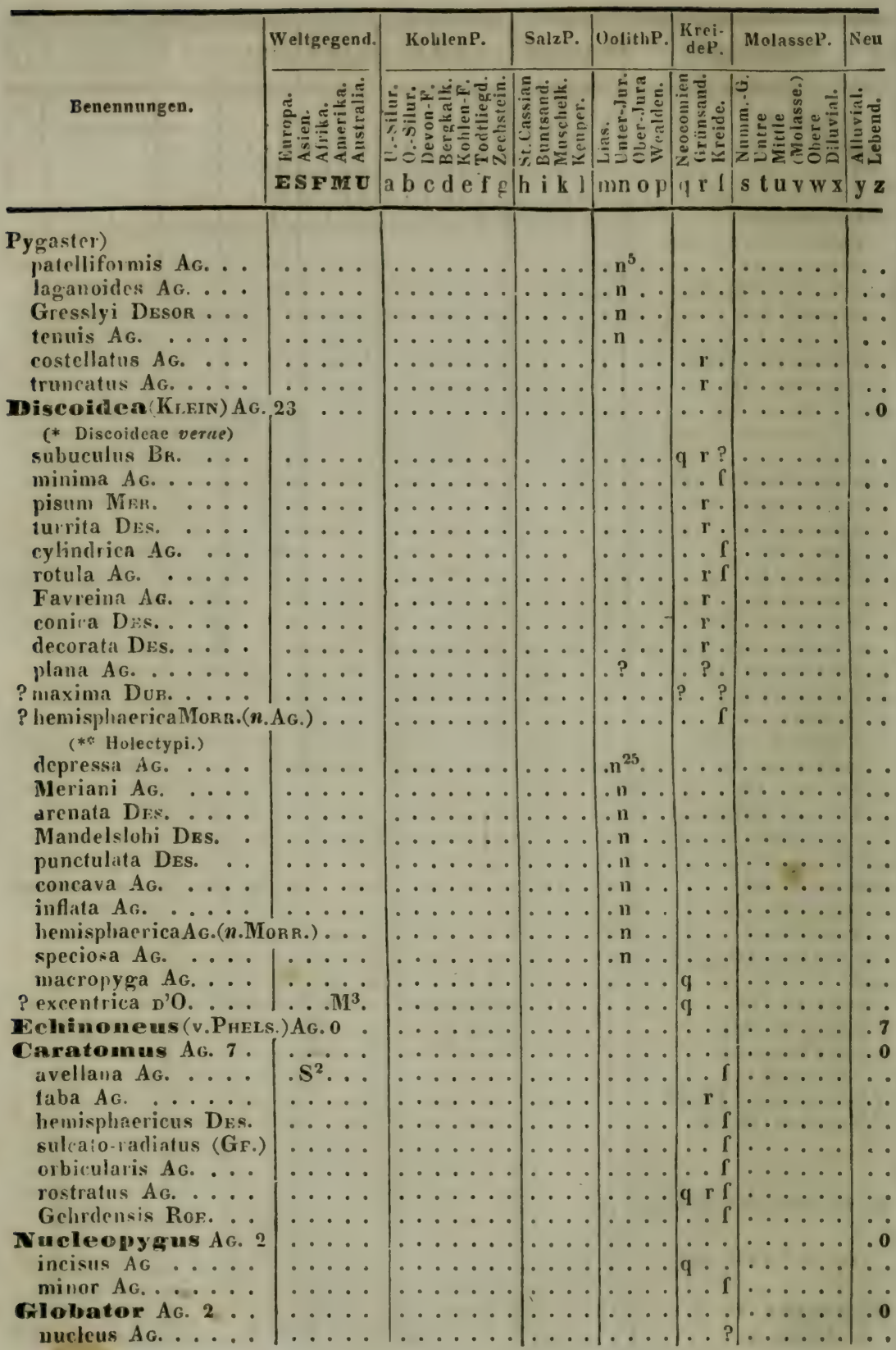




\begin{tabular}{|c|c|c|c|c|c|c|c|}
\hline B & eltgegend. & $\mathbf{a} \mathbf{b} \mathbf{c d}$ & h ik l & $\min 0 p$ & $q \times f$ & $s \quad u v w x$ & $\mathbf{y}$ \\
\hline (DsM.) & & & & & & & \\
\hline Pirima AG. 6 ... & .... & $\ldots \ldots$ & $\cdots$ & ... & & ..... & .0 \\
\hline graea AG. .... & . . . & $\ldots \ldots$ & - $\cdot$ & . . . & & $\ldots .$. & - \\
\hline I. : : & $\cdots \cdot \cdot$ & $\cdots \cdot \cdot \cdot$ & & $\cdots \cdots$ & - $\mathbf{r} \mathbf{f}$ & $\ldots .$. & \\
\hline . . : & $\cdots \cdot \cdot$ & $\cdot \cdot$ & $\cdot$ & $\cdots \cdot$ & $\cdot \underset{p}{r} \mathbf{f}$ & $\cdots \cdots$ & \\
\hline$\dot{A} \cdot \dot{ }$ & $\begin{array}{ll}\cdots \\
\cdots & \bullet\end{array}$ & 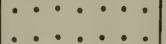 & & . & $\begin{array}{l}\cdot ? \\
.\end{array}$ & $\begin{array}{ll}\cdots \\
\cdots\end{array}$ & \\
\hline M. . & . & $\cdots \cdots \cdots$ & $\cdots$ & $\cdots$ & $\because 1$ & $\cdots \cdots$ & \\
\hline (LF.)Ag. 22 & .... & $\ldots \ldots$ & ... & $\cdots \cdot$ & & $\ldots . .$. & .0 \\
\hline DFR. .... & .... & $\ldots \ldots$ & ... & ... & - r. & $\ldots \ldots$ & - \\
\hline$\dot{1}$ & $\cdots$ & $\cdots \cdots$ & ... & $\cdots \cdot$ & . $\mathbf{r I}$ & $\cdots \cdots$ & \\
\hline is $D^{\prime} A$. & .... & . . . . & & - $\cdot$ &.. $\mathrm{f}$ & $\ldots$ & \\
\hline$\dot{\mathbf{M}}$ & $\cdots \cdot \cdot$ & $\cdots \cdots$ & $\cdot \cdot$ & $\bullet$ & $\cdots \mathrm{f}$ & $\cdots$ & \\
\hline sM. & $\cdots \cdot$ & •・ & & $\dot{.}$ & $\cdots 1$ & - & \\
\hline $\begin{array}{l}\text { LK. } \cdots \cdots \\
\text { AG. } \cdots\end{array}$ & $\begin{array}{l}\cdots \\
\cdots\end{array}$ & $\begin{array}{lll}\cdots & \cdots & \cdots \\
\cdots & \cdots & \cdots\end{array}$ & & & $\begin{array}{ll}\cdots \\
.\end{array}$ & $\because \cdots$ & \\
\hline AG. & ..... & $\cdots \cdots$ & . . . & & $\because \mathrm{I}$ & ... & \\
\hline . & .... & $\ldots \ldots$ & .... & - &. $\mathrm{r}$ & $\cdots$ & \\
\hline . . . & .... & $\ldots \ldots$ & $\ldots$ & $\cdot \cdot$ & . I & $\ldots \ldots$ & \\
\hline G. . & . . . & $\ldots \ldots$ & $\ldots$. & - $\cdot$ & . $\mathrm{f}$ & $\cdots \cdot$ & \\
\hline OR. . & ... & $\ldots \ldots$ & $\cdots \cdot$ & $\cdots$ & . I & $\ldots \ldots$ & \\
\hline laevis Ag. & . . • & 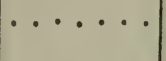 & $\ldots$. & $\ldots$ & .. I & $\cdots$ & - \\
\hline ... & -・ & $\cdots$ & - & $\cdot$ & $\therefore$ I & $\cdots$ & \\
\hline OE. & . . . & $\ldots \ldots$ & 1 & - & . I I & $\therefore$. & \\
\hline E. . . & ... & $\ldots \ldots$ & $\ldots$. & - . & . 1 & $\ldots \cdots$ & \\
\hline . . & $\therefore .$. & $\ldots \ldots$ & $\cdot \cdot$ & . & . $\mathfrak{f}$ & $\cdots \cdots$ & \\
\hline$\therefore \cdot$ & ... & $\ldots \ldots$ & - & - & . $\mathrm{f}$ & $\cdots \cdot$ & \\
\hline siM. & ... & $\ldots \ldots$ & & & $\therefore \mathrm{I}$ & $\ldots$. & \\
\hline ricus CAt. & $\ddot{c} \cdot \cdot \cdot$ & $\cdots \cdot$ & - & - & 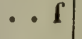 & $\cdots \cdot$ & \\
\hline$\cdots$ &.$S^{3} \ldots$ & ... & - & $\cdot$ & - & $s \ldots \ldots$ & \\
\hline ? D & & 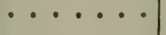 & 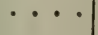 & - & & $\cdots$ & \\
\hline s $A G \cdot 2$ & $\cdots \cdot$ & $\cdots \cdots$ & $\cdots \cdot$ & . • & - & $\cdots \cdots$ & . 0 \\
\hline G. . . & .... & . . & . & $\bullet \mathbf{n} \cdot$ & & $\cdots \cdots$ & - \\
\hline ES. - & $\cdots \cdot \cdot$ & $\cdots \cdots$ & $\cdot$ & . n . & - & $\cdots \cdots$ & $\bullet$ \\
\hline ae $\mathbf{A g}$. & & & & & & & \\
\hline .) $\mathrm{AG}$ & & - • & . & . . & . & $\ldots .$. & .6 \\
\hline chDu. & - $\cdot$ & & & . $\mathbf{1}$. & & $\cdots$ & \\
\hline$\cdots$ & . $\mathrm{M}^{2}$ & . & $\cdot 1$ & - & . $\mathrm{f}$ & $\cdots \cdots$ & \\
\hline r. & . . $\mathbf{M}^{2}$. & $\cdots \cdots$ & 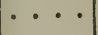 & & - \{ & $\cdots$ & \\
\hline - & $\cdots$ & $\ldots \ldots$ & - & & - $斤$ & $\cdots \cdots$ & - \\
\hline mis His. . & & . . & - & $\cdots \cdot$ & . $\mathrm{f}$ & $\cdots \cdots$ & \\
\hline $\mathbf{S}$ & . . & . & - & $\cdots$ & & $\cdots$. & \\
\hline - &.$S^{3} \cdot$. & •. & $\cdots$ & $\cdots \cdot$ & $\cdots$ & $\cdots$ & • \\
\hline $\mathbf{C}_{\mathrm{A}}$ & ... & $\cdots$ & $\cdots$ & & & - $\mathbf{t}$ & - \\
\hline tii & •. & $\cdots$ & & $\bullet$ & & 16.5 & 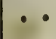 \\
\hline 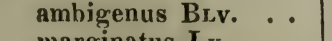 & & $\cdot$ & & & $\bullet$ & $\cdots \mathbf{u} \cdots$ & \\
\hline - $\cdot$ & 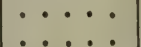 & 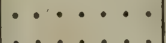 & & - & $\cdot$ & 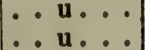 & \\
\hline Turbellianus [?] GRAT. & & & & & & & \\
\hline
\end{tabular}




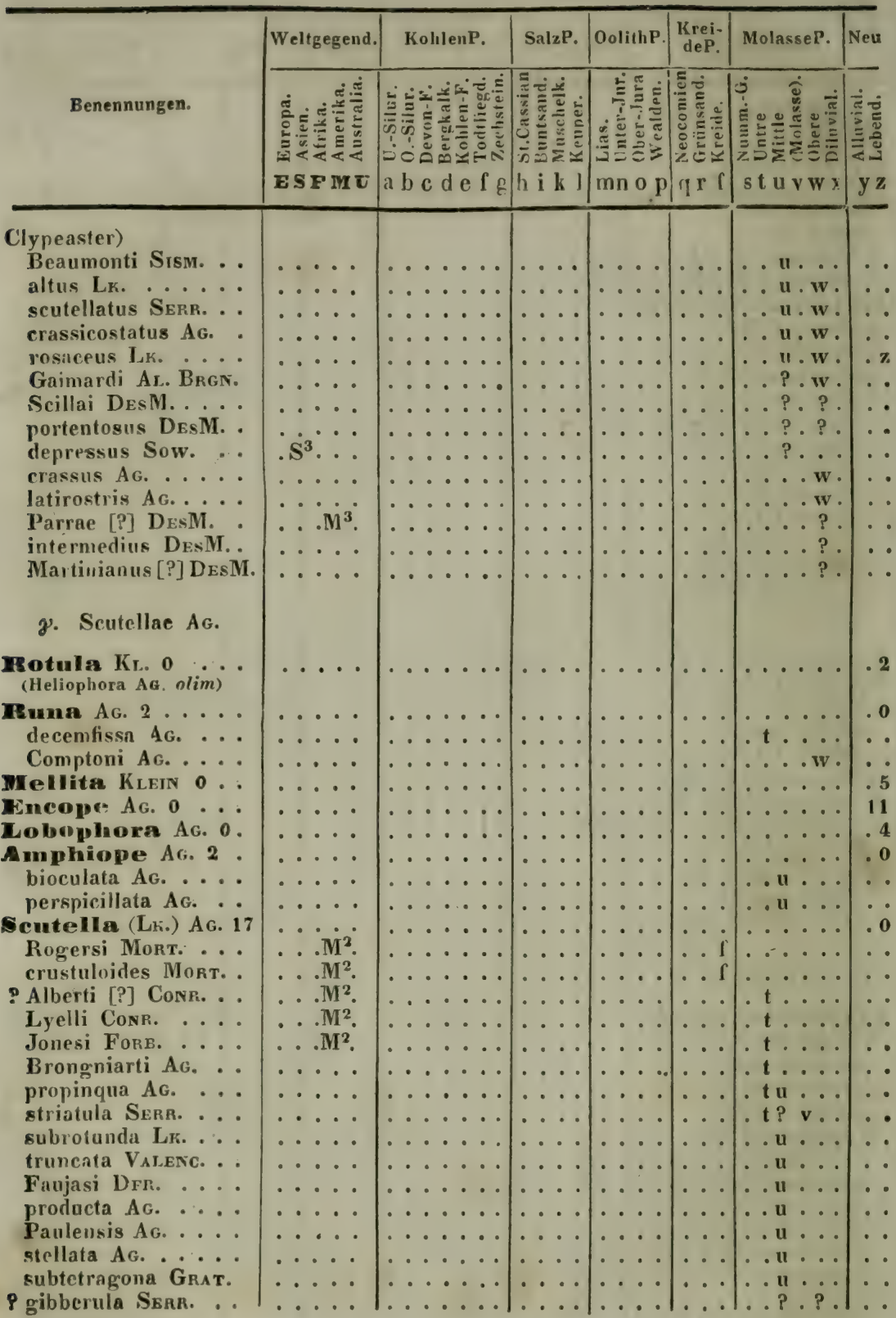




\begin{tabular}{|c|c|c|c|c|c|c|c|}
\hline Bene & end. & a b c & k l & mnop & $q \times 1$ & s t & \\
\hline & & & & & & 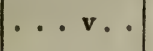 & \\
\hline s v. P & G. 1 & . & $\cdots \cdots$ & $\cdots \cdot$ & & 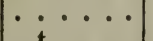 & \\
\hline G. 0 & $\cdots$ & $\cdots \cdots$ & $\cdots$ & $\cdots$ & & $\because$ & \\
\hline Ag. 5. & $\therefore \cdots$ & $\cdots \cdots$ & $\cdots \cdots$ & & $\therefore$ & $\cdots$ & \\
\hline . & $\cdots \cdot$ & $\ldots \ldots$ & $\therefore \cdots$ & $\left|\begin{array}{c}\cdots \\
\cdots\end{array}\right|$ & $\cdots$ & $\ddot{m} \cdots$ & \\
\hline$\cdots$ & $\cdots \cdot$ & $\cdots \cdots$ & $\ldots$ & $\ldots$ & $\therefore$ & $\because \mathrm{t} \ldots$ & \\
\hline G. . . & $\cdots \cdot$ & $\cdots \cdots \cdots$ & $\ldots$ & $\ldots$ & $\ldots$ & $\ldots \ldots$ & \\
\hline$\cdots$ & $\cdots \cdots$ & $\cdots \cdots$ & $\cdots \cdot$ & $\ldots$. & $\ldots$ &.$t \ldots$ & \\
\hline$\cdots$ & $\ldots \ldots$ & $\cdots \cdots$ & $\ldots$. & $\cdots$ & $\cdots$ &. $\mathbf{t} \ldots$ & \\
\hline .. & $\cdots \cdot \cdot$ & $\cdots \cdots \cdots$ & $\cdots$ & $\cdots \cdot$ & . & $\ldots \ldots$ & \\
\hline • & & $\cdots \cdots$ & $\cdots$ & - $n$ & & $\cdots$ & \\
\hline$\cdots$ & $\cdots \dot{\mathbf{M}} \cdot$ & $\cdot$ & . & - ??? & ?? ? & $\cdots \cdots$ & \\
\hline 0. . & $\ldots \mathbf{M}^{3}$ & & & $\cdots \cdots$ & $q \cdots$ & $\cdots \cdots$ & \\
\hline & $\cdots \cdot$ & & $\because$ & $\cdots \cdot$ & $\cdots$ & $\cdots$ & \\
\hline re & ・・ & $\cdots$ & & $\cdots \cdot$ & & $\cdots$ & \\
\hline 3 & 3 . & $\cdots$ & $\because$ & $\cdots \cdot \mid$ & - & • & \\
\hline & & $\because$ & $\cdots$ & $\cdot$ & $\cdots 1$ & $\because$ & \\
\hline . & $\cdots \cdot$ & $\ldots$ & $\cdots \cdots$ & & 11 & & \\
\hline 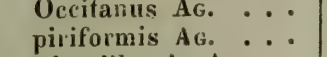 & $\ldots \ldots$ & $\ldots \ldots$ & $\because \cdots$ & & $\begin{array}{ll}\cdots 1 \\
\cdots\end{array}$ & $i t$ & \\
\hline$\cdots$ & $\cdots \cdots$ & $\ldots \ldots$ & $\ldots$ & $\ldots$ & $\therefore$ & t $\ldots$ & \\
\hline$\cdots$ & $\cdots \cdots$ & $\cdots \cdots$ & $\cdots$ & $\cdots$ & $\ldots$ &.$t \ldots$ & \\
\hline$\cdot \cdot$ & $\cdots \cdot$ & $\cdots \cdots$ & $\ldots$ & $\cdots \cdots$ & & . $t$. & \\
\hline$\cdots$ & - & $\cdots$ & $\cdots$ & $\cdots \cdot$ & $\cdots$ & .? . & \\
\hline & . & 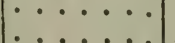 & $\because$ & $\cdots \cdot$ & $\cdots$ & $\cdot \mathrm{t} u \cdot w \cdot$ & \\
\hline$\cdots$ & & • & $\cdots$ & $\cdots \cdot$ & - & $\cdots u$ & \\
\hline$\cdot$ & $\because$ & & $\because$ & $\therefore$ & $\cdots$ & $\ddot{p} \cdot w$ & \\
\hline$\cdots$ & $\therefore$ & $\cdots$ & $\because$ & $\left|\begin{array}{c}\cdots \\
\cdots\end{array}\right|$ & • & .5 & \\
\hline • & . & .... & $\ldots$ & $\cdots \cdots$ & $\cdots$ & $\therefore$ & \\
\hline & $\cdots$ & $\ldots$ & $\ldots$ & $\ldots$ & $\cdots$ & . ? ... & \\
\hline ELI.. 7 & $\cdots$ & - & $\cdots$ & $\cdots$ & $\cdots$ & $\cdots \cdots$ & \\
\hline & & & $\cdots \cdot$ & $\cdots \cdots$ & $\cdots$ & $(\ldots)$. & \\
\hline & & & $\cdots$ & $\cdot \cdot$ & $\cdot 1$ & $\cdots \cdots)$ & \\
\hline & & & & & & $(\cdots \cdots)$ & \\
\hline 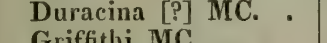 & & ( & . & $\cdot$ & & $\because \cdots \cdots)$ & \\
\hline$\because$ & $\mathrm{S}^{3} \cdots$ & & & & & $\because$ & \\
\hline C. & $\mathbf{S}^{3} \ldots$ & ( . & $\because$ & $\cdots$ & & .) & \\
\hline & & & & & & -5 & \\
\hline & & & & & & & \\
\hline & & & & & & $\cdots$ & \\
\hline & & & & & $\ldots \tau$ & & \\
\hline & $\cdots$ & $\cdot$ & $\therefore \cdot$ & $\cdots$ & $\cdot \cdot$ & $\cdot t \ldots$ & \\
\hline & & & & & & & \\
\hline I. & . & $\cdots$ & & . & & $\ldots w$ & \\
\hline 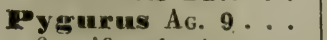 & & & & & & & \\
\hline 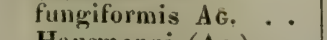 & . & & & & & & \\
\hline & & & & & & & \\
\hline
\end{tabular}




\begin{tabular}{|c|c|c|c|c|c|c|c|}
\hline \multirow[b]{2}{*}{ enennungen. } & eltgegend. & KohlenP. & alzP. & (OolithP. & $\begin{array}{l}\text { Krei- } \\
\text { deP. }\end{array}$ & MolasseP. & Ne \\
\hline & 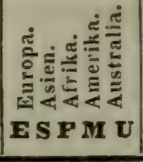 & 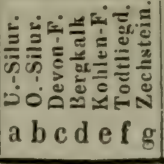 & 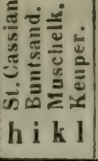 & 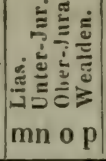 & 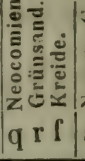 & $\mid$ & \\
\hline \multicolumn{8}{|l|}{ Pygurus) } \\
\hline $\begin{array}{l}\text { AG. } \cdots \\
\text { AG. . . }\end{array}$ & $\cdots$ & & & . n . & & $\cdots \ldots$ & \\
\hline Ag. . & $\ldots$ & $\cdots$ & & $\cdots \cdot$ & $\begin{array}{lll}q & \cdots \\
q & \cdots & \cdot\end{array} \mid$ & $\cdots \cdots$ & \\
\hline$\cdots$ & $\cdots \cdot$ & $\ldots \ldots$ & $\cdots$ & $\ldots$ & $q \cdots$ & $\ldots \ldots$ & \\
\hline$\because$ & $\cdots \cdot$ & $\cdots \ldots$ & $\cdots \cdots$ & $\cdots \cdot$ & $\cdot r \cdot$ & $\ldots \ldots$ & \\
\hline$\dagger$ & $\cdots$ & . & $\cdots \cdots$ & $\cdots \cdot$ & - $r_{j i}$ & $\cdots \cdots$ & \\
\hline AG. 3 & $\cdots \cdots$ & & & $\dot{0}$ & $\ldots 1$ & $\because \cdots$ & \\
\hline$\cdots$ & .... & $\cdots$ & $\ldots$ & $\therefore$ & $\therefore p$ & $\cdots \cdots$ & \\
\hline & $\cdots$ & $\ldots \ldots$ & $\ldots$ & $\cdots$ & $\ldots \mathrm{f}$ & $\cdots \cdots$ & \\
\hline$\cdots$ & $\ldots$ & $\ldots \ldots$ & $\ldots$ & $\ldots$ & $\cdots$ & $\therefore \ldots$ & \\
\hline Ag. 9 & $\cdots \cdots$ & $\ldots \ldots$ & $\cdots$ & $\ldots$ & $\cdots$ & $\ldots \ldots$ & \\
\hline AG. & $\cdots$ & $\ldots \ldots$ & $\cdots$ & $\cdots \cdots$ & ? ? . & $\ldots \ldots$ & \\
\hline A G. . & $\cdots \cdot$ & $\cdots \cdots$ & $\cdots$ & $\cdots \cdot$ & $? \mathbf{r}$ & $\ldots \ldots$ & \\
\hline$\cdots \cdot$ & 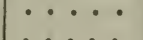 & $\cdots$ & $\cdots$ & $\cdots$ & $\cdot r$ & $\cdots \cdots$ & \\
\hline$\cdots \cdot$ & $\cdots \cdots$ & $\cdots \cdots$ & $\cdots$ & $\cdots \cdot$ &. $\mathbf{r}$ & $\ldots \ldots$ & \\
\hline$\cdots \cdot$ & $\cdots \cdot$ & $\cdots \cdots$ & $\cdots$ & $\cdots \cdot$ & . . 1 & $\ldots \ldots$ & \\
\hline$\cdots \cdot$ & $\cdots \cdot$ & $\cdots$ & $\cdots \cdots$ & $\cdots$ & . ?? & $\cdots \cdots$ & \\
\hline$\cdots$ & $\cdots$ & $\cdots \cdots$ & $\cdots \cdots$ & $\cdots$ & $\cdots$ & $\dot{t} \ddot{w} \dot{w}$ & \\
\hline$\because$ & $\ldots$ & $\cdots$ & $\cdots$ & $\cdots$ & : $: \dot{0}$ & ......... & \\
\hline as (Gra & r) Ag. 32 & $\ldots \ldots$ & $\ldots$ & & $\cdots$ & $\ldots \ldots$ & \\
\hline 3. . & $\cdots \cdots$ & $\cdots$ & $\cdots \cdots$ &.$n^{5}$. & & $\ldots \ldots$ & \\
\hline$\cdots \cdot$ & $\cdots \cdot$ & $\cdots \cdots$ & $\ldots$ & . n . & & ...? & \\
\hline$\cdots$ & $\cdots \cdot$ & $\cdots \cdots$ & $\cdots$ & . n & ? ? & $\cdots \cdots$ & \\
\hline$\cdot \cdot$ & $\cdots$ & - -5 & $\cdots$ & . n . & ?? & $\cdots \cdots$ & \\
\hline c. & $\cdots \cdots$ & $\cdots \cdots$ & $\cdots$ & $\cdots \cdot$ & - & $s \cdots \cdots$ & \\
\hline$\cdots$ & $\cdots$ & $\ldots \ldots$ & $\ldots$ & $\cdots$ & $\cdots$ & $\begin{array}{l}s \\
s\end{array} \ldots$ & \\
\hline$\cdots$ & $\cdots \cdot$ & $\ldots \ldots$ & $\ldots$ & $\cdots \cdot$ & ??? & $\ldots \ldots$ & \\
\hline$\cdots$ & .... & $\ldots \ldots$ & $\cdots$ & $\cdots \cdot$ & . $r$. & $\cdots \cdots$ & \\
\hline$\cdots \cdot$ & $\cdots \cdots$ & $\cdots$ & & 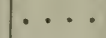 & $? ?$ & ...... & \\
\hline$\cdots \cdot$ & $\cdots \cdots$ & $\cdots$ & $\cdot$ & . & ? ? & $\cdots \cdots$ & \\
\hline & • & & & • & ? & $\cdots$ & \\
\hline 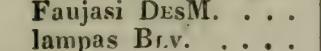 & & - & & - & - 1 & $\cdots$ & \\
\hline$\cdots$ & • • • • & .. & & & . $\int !$ & $\cdot$ & \\
\hline$\cdots$ & $\cdots$ & $\ldots \ldots$ & . & $\ldots$ & $\ldots i$ & $\because$ & \\
\hline catus Ag. $\quad \ldots$ & $\cdots \cdot$ & $\cdots$ & $\cdots$ & $\cdots \cdot$ & $\ldots f$ & $\ldots \ldots$ & \\
\hline Ag. $\cdots$ & $\cdots \cdot$ & $\cdots$ & & & $\cdots \cdot$ &.$t \mathrm{tu} \cdot \mathrm{w} \cdot$ & \\
\hline$\ddot{\cdots}$ & ... & $\cdots$ & & & & 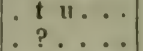 & \\
\hline & $\cdots \cdots$ & ( & ${ }^{\circ}$ & $\cdots$ & $\cdots$ & tu.w. & \\
\hline A. & ${ }^{2} \dot{\mathbf{S}^{3}} \dot{\dot{ }}$ & $u^{\circ}$ & & & & : ? u .w. & \\
\hline & (D) & & & & & & \\
\hline
\end{tabular}




\begin{tabular}{|c|c|c|c|c|c|c|c|}
\hline Bener & d. & $\mathrm{g}$ & & $r$ & [ & $\mathbf{w} \mathrm{x}$ & \\
\hline & & & & & & . & \\
\hline & & e & & $\cdots$ & & $\ldots$ u... & \\
\hline - & $\ldots$ & $\ldots \ldots$ & & $\cdots$ & & ...... & \\
\hline & $\cdots$ & $\ldots \ldots$ & $\cdots \cdot$ & $\cdots \cdots$ & & $\ldots \ldots w \cdot$ & \\
\hline & & & & & & $\ldots . \mathbf{w} \cdot$ & \\
\hline & & & & & & $\cdots w_{j}$ & \\
\hline & & & & & & $\left(\begin{array}{cc}\cdots \\
\cdots\end{array}\right)$ & \\
\hline c. 10 & . & . & $\ldots$ & $\ldots$ & $\cdots$ & $\ldots \ldots$ & \\
\hline & $\ldots \ldots$ & $\ldots .$. & $\cdots$ & $\ldots$ & q.. & $\ldots \ldots$ & \\
\hline & $\cdots \cdot$ & $\ldots \ldots$ & $\cdots$ & $\cdots$ & & $\ldots \ldots$ & \\
\hline & $\cdots \cdots$ & $\cdots \cdot$ & $\cdots$ & $\cdots$ & $\cdots f$ & $\ldots \ldots$ & \\
\hline & & $\ldots \ldots$ & L & $\cdots$ & . . $\mathrm{f}$ & $\cdots$ & \\
\hline & & $\cdots$ & & & & & \\
\hline & & & & & $\cdots$ & $\cdots$ & \\
\hline & & $:$ & & $\left.\mid \begin{array}{c}\cdots \\
\cdots\end{array}\right]$ & $\begin{array}{l}\cdot \\
\dot{0} \\
\dot{*}\end{array}$ & w. & \\
\hline & $\because$ & $\because$ & $\ldots$ & $\ldots$ & $\ldots$ & ‥w & \\
\hline & . &. & & $\ldots$ & ... & ....w & \\
\hline 12 & $\cdots \cdot$ & 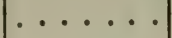 & ・ & $\ldots$ & & $\ldots \ldots$ & \\
\hline & . & $\cdots$ & . & $\cdots \cdot$ & $q$ & $\cdots$ & \\
\hline • & $\cdots$ & $\cdots$ & & $\cdots$ & $q \ldots$ & $\ldots \ldots$ & \\
\hline s Ag. & & $\cdots$ & & $\cdots$ & $q \ldots$ & $\ldots \ldots$ & $\cdot$ \\
\hline$\cdots$ & $\cdots \cdot$ & .... & . & $\cdots$ & & $\ldots \ldots$ & . \\
\hline & $\cdots \cdot$ & $\cdots$ & . & $\cdots$ & $\begin{array}{lll}q & \mathbf{r} & \mathbf{r}\end{array}$ & $\ldots \ldots$ & \\
\hline . & $\cdots$ & - & $\cdots$ & $\cdots$ & $\cdot \mathbf{r} \cdot$ & $\cdots$ & \\
\hline & & . & - & $\cdots$ & $\cdot$ & - $\cdot$ & \\
\hline & $\cdot$ & & & & $\cdots$ & $\cdots$ & \\
\hline 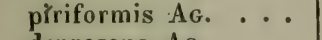 & $\cdots$ &. & & $\mid \begin{array}{l}\cdots \\
\cdots\end{array}$ & $\begin{array}{ll}\cdots & 1 \\
\cdots & ?\end{array}$ & $\therefore$ & \\
\hline$\therefore$ & &. & & $\ldots$ & $\therefore ?$ & 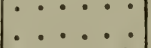 & \\
\hline$\cdots$ & $\therefore$ & .. & & $(\ldots$ & $\cdots$ & $\cdots \cdots$ & \\
\hline 0 & . & $\cdots$ & & $\ldots$ & & $\ldots \ldots$ & \\
\hline$\cdots$ & $\cdots$ & . & . & $\ldots$ & . $\mathrm{r} \mathrm{I}$ & $\ldots \ldots$ & \\
\hline$\cdots$ & . & . & 0.0 & $\ldots$ & ..? & $\ldots \ldots$ & \\
\hline-5 & ... $\mathrm{M}^{2}$. & $\ldots \ldots$ & $\cdot$ & $\ldots$ & . & $\ldots \ldots$ & \\
\hline & $\cdots \cdots$ & $\cdots$ & & $\cdots$ & $?$ & $\cdot \mathbf{t} \cdots \cdot$ & \\
\hline & - & $\cdot$ & & & $\cdot$ & . $t$ & \\
\hline & & & & 1. & & . t & \\
\hline & & & & & $\cdots$ & & \\
\hline$\dot{\text { LK. })} \dot{A G}$ & • & $0^{\circ}$ & & & $\cdots$ & $\therefore \ldots$ & \\
\hline & & & &.$n^{45} \ldots$ & $\cdots$ & $\ldots \ldots$ & \\
\hline & & & & $\cdot \mathbf{n}^{3} \ldots$ & $\cdots$ & $\cdots$ & \\
\hline & $\cdots$ & & &. $\mathbf{n} \cdots$ & & & \\
\hline 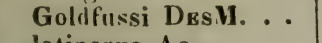 & & & & & & & \\
\hline$x^{-1}$ & & & & & & $\cdot$ & \\
\hline & & & & $\mid \begin{array}{l}n \\
n^{3+5}\end{array}$ & $\cdots$ & $\ldots$ & \\
\hline$\cdots$ & $\cdots$ & & & $.11 \ldots$ & $\cdots$ & $\cdots$ & \\
\hline$\cdots$ & - & & & $\mathbf{n}$ & $\cdots$ & & \\
\hline & & & & & & & \\
\hline
\end{tabular}




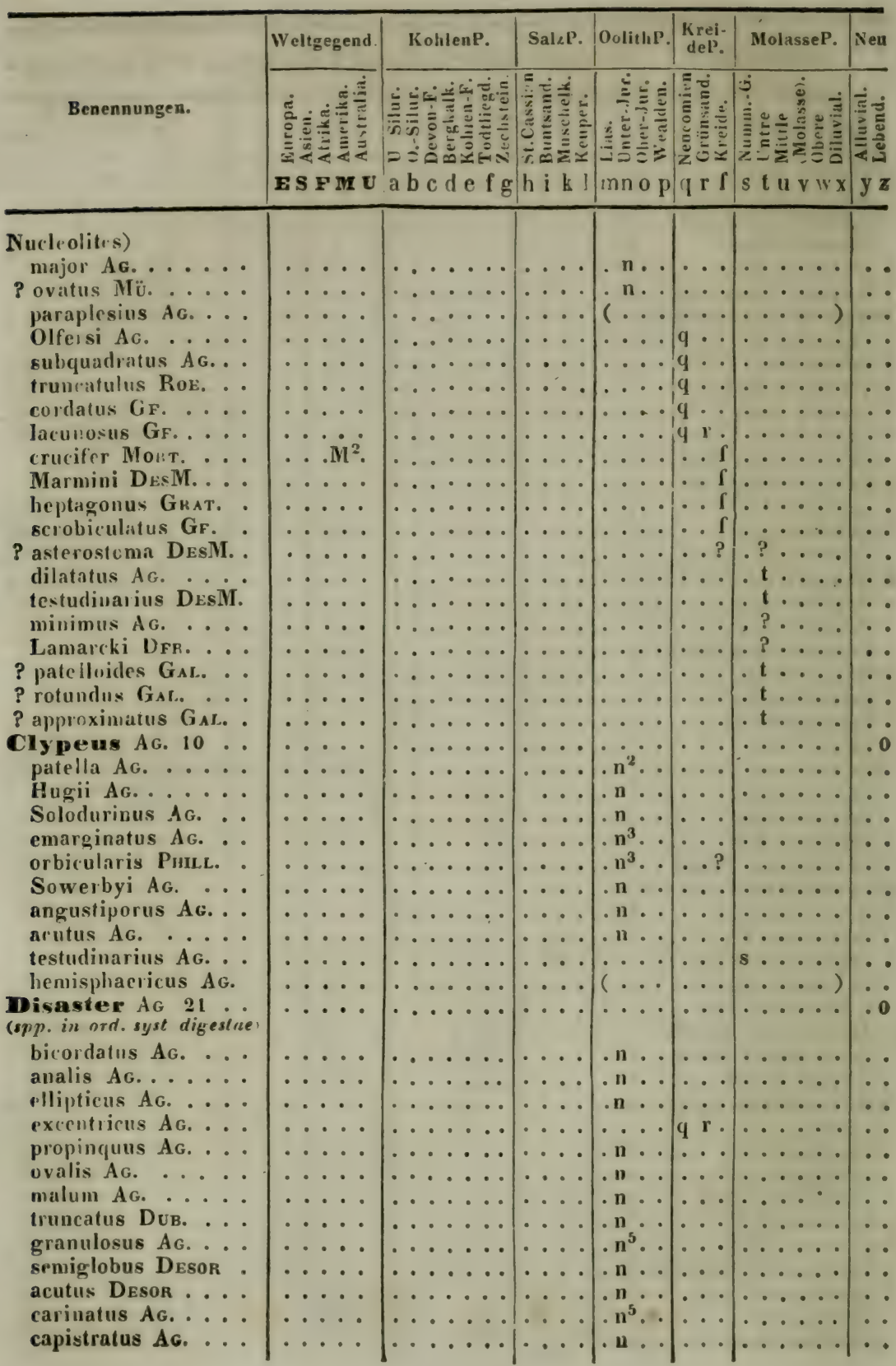


Benennuıgen. IVeltgegend. a b c d e f g h i k l mnop $q$ r l s t u v w x y z

Buchi Desor. ovulum Desor avellana AG.

Eudesi AG. ringens $A G$ Volt $\%$ A G.

? canaliculatus $\dot{A} \mathrm{~s}_{\text {. }}$

Münsteri Desor

Colly rites Desm. 3 (spp. residune)

brissoides DesMI. . heteroclita DesM. trigonata DEsM.

\section{SPATANGOIDEA Ag.}

Erissopsis Ag. 1 elegans Ag. ....

Schizaster Ag. 26 foreatis $A \mathrm{~g}$. eurynotus AG. Goldfussi Ac. stellatus Duв. Corsirus Ag. Graecus Ag. ambularrum AG. cerasus Af. verticalis Ag. aequifissus Ag. cultratus Ag. Studeri Ag. globosus Ac: najor Ag.

Deshayesi AG.

Beaumonti Ag.

Edwardsi Ag.

Diulfensis Dub. Gratelonpi Sisu. intermedius Sism. Agassizi Sısm. Genei Sism. canaliferus Ag. acuminatus $A_{G}$. Borsoni Sisu. ovatus Sism.

Erissus ( $K_{L}$ ) AG. 0 . Gpatangus(KL.)Ag.37 ? ColumbianusNystGaL. elongatus As. tuberculatus AG. subalpinus Risso

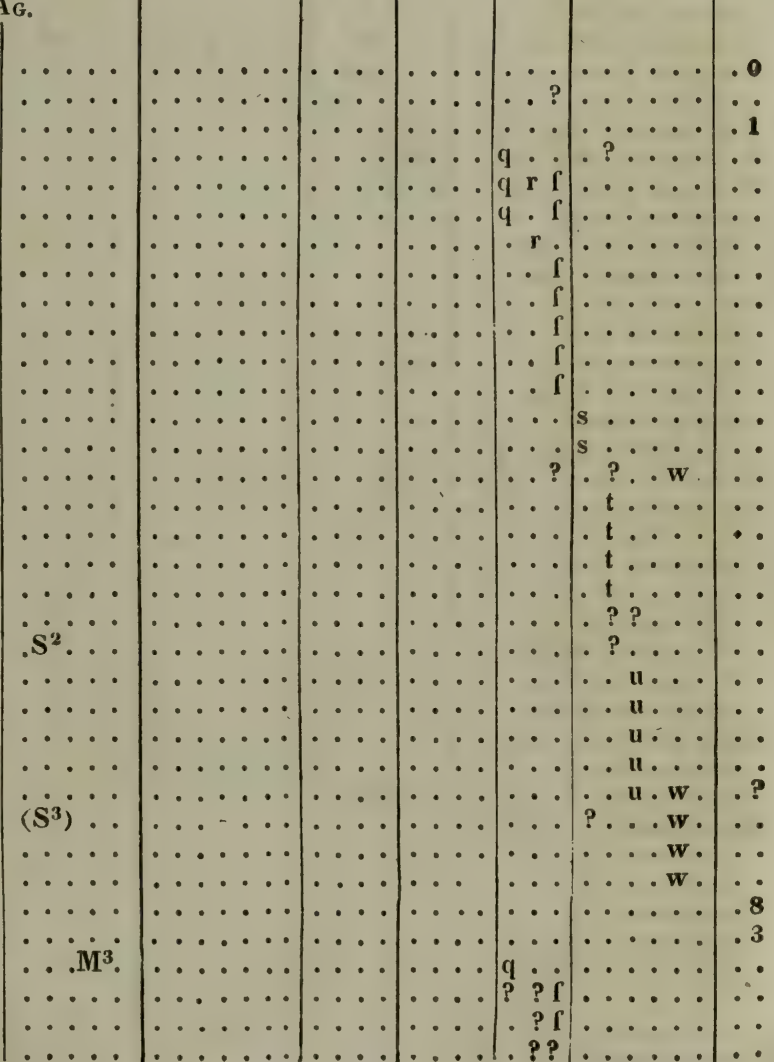




\begin{tabular}{|c|c|c|c|c|c|c|c|}
\hline \multirow[b]{2}{*}{ Benennungen? } & Weltgegend. & KohlenP. & SalzP. & OolithP. & $\begin{array}{r}\text { Krei- } \\
\text { deP. }\end{array}$ & MolasseP. & Nen \\
\hline & 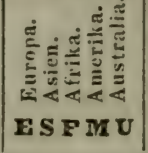 & 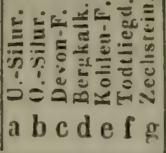 & 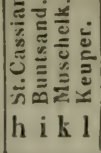 & 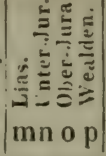 & 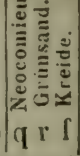 & 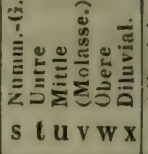 & 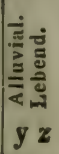 \\
\hline \multicolumn{8}{|l|}{ patangus) } \\
\hline rias A & $\ldots$. & $\ldots \ldots$ & $\ldots$ & $\cdots$ & . . & $\cdots \cdots$ & - \\
\hline P Leskei DEsM. & $\begin{array}{l}\cdots \\
\dot{*}\end{array}$ & $\because$ & : & & $\begin{array}{ll}\cdots f \\
\therefore f\end{array}$ & $\cdots$ & \\
\hline soides LFSKE . & $\ldots$ & $\ldots \ldots$ & $\therefore \cdots$ & $\because$ & $\because 1$ & $\therefore$ & • \\
\hline Kos. & $\ldots \ldots$ & $\ldots \ldots$ & $\ldots \cdot$ & & . I & $\ldots \ldots$ & . \\
\hline c & . & $\cdots \cdots$ & $\cdots \cdot$ & $\cdots \cdot$ & $\cdots f$ & $\cdots$ & - \\
\hline $\begin{array}{l}\text { itaul } \\
\text { us I }\end{array}$ & • & $\therefore$ & $\cdots \cdot$ & & $\cdots 1$ & $\because$ & - \\
\hline $\begin{array}{ll}\text { us } \\
\text { la }\end{array}$ & $\cdots$ & $\ldots$ & $\cdots$ & $\cdots$ & $\therefore i$ & $\mid \begin{array}{c}\cdots \\
\cdots \\
\cdots\end{array}$ & \\
\hline $\begin{array}{l}\text { pillula DesM. } \cdots \\
\text { elatus DrsM. } \ldots \\
\end{array}$ & .... & $\ldots \ldots$ & $\ldots$ & ... & $\therefore \mathrm{f}$ & $\ldots \ldots$ & $\because$ \\
\hline rastatus MoRT. & .. $\mathrm{NI}^{2}$ & $\ldots \ldots$ & $\ldots$. & $\ldots$ &.. $\mathrm{f}$ & $\ldots \ldots$ & \\
\hline a 1 & $\ldots y^{2}$ & $\ldots \ldots$ & $\cdots$ & $\cdots \cdots$ & $\ldots \mathrm{f}$ & .. & : \\
\hline $13 \mathrm{Dr}$ & $\because \dot{B}^{\circ} \cdot$ & $\cdots \cdots$ & $\cdots$ & $\cdots \cdot$ & . . ? & . t u ... & \\
\hline juatus $\$$ &.$S^{3} \ldots$ & $\cdots \cdots$ & - & $\cdots$ & $\cdots$ & $s \ldots$. & \\
\hline P elongatus Sow. .. &.$S^{3} \cdot \cdots$ & . & & & . . & $s \ldots$ & \\
\hline is LAYM. . . . . & $\mathrm{E}^{2} \cdot \mathrm{F}^{2}$ & $\because$ & & & $\cdots$ & $\cdot \mathbf{t} \ldots$ & - \\
\hline$\cdots$ & - & $\cdots \cdots$ & $\cdots$ & $\cdots \cdots$ & $\cdots$ & $\cdot t \cdots$ & \\
\hline Grignonensis Desm. & $\cdots \cdots$ & $\ldots \ldots$ & $\cdots$ & $\cdots \cdots$ & $\cdots$ & $\cdot t \cdots$ & • \\
\hline$\ldots$ & .... & $\ldots \ldots$ & $\ldots$ & $\cdots$ & $\cdots$ & $\begin{array}{lll}t & \cdots & \cdots \\
\text { t } & u & \cdots\end{array}$ & \\
\hline . . . & .... & $\ldots \ldots$ & $\ldots$ & $\ldots$ & $\cdots$ & $\mathrm{t} \ldots$ & \\
\hline is D &.$S^{2} \cdot$. & $\cdots$ & $\cdots \cdots$ & $\ldots$ & ... & . ?.... & \\
\hline I. . . & $\cdots \cdot$ &. & $\cdots \cdots$ & $\ldots \cdot$ & ... & ..... & \\
\hline$\cdots$ & $\cdots \cdot$ & . & $\cdots \cdots$ & $\ldots$ & $\ldots$ & . u.w. & \\
\hline c & $\cdots \cdots$ & $\cdots \cdot$ & - & $\cdots \cdot$ & ... & . . u... & $\mathbf{z}$ \\
\hline$\therefore \cdot$ & $\cdots \cdot$ & $\ldots \ldots$ & $\cdot$ & $\cdots$ & $\cdots$ & $\ldots \mathbf{v} \ldots$ & $\cdots$ \\
\hline$P$ arcuarius $L_{\text {KK. }} \ldots$ & $\cdots \cdot$ & $\cdots$ & . & $\cdots \cdot$ & $\cdots$ & $\cdots \mathbf{v} \cdot \cdot$ & - \\
\hline delphinus & $\cdots$ & 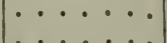 & & $\cdot$ & $\cdot$ & $\cdot ? \cdot \cdot w_{0}$ & 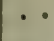 \\
\hline … & $\because \dot{0}$ & .. 1 & & $\cdot$ & $\cdots$ & $\cdot \cdot \cdot \cdot \mathrm{w} \cdot$ & - \\
\hline$\ddot{\cdots}$ & $\ldots$ & $\ldots \ldots$ & & $\because$ & $\cdots$ & $\therefore$. & \\
\hline iM. & $\cdots$ & $\ldots \ldots$ & & $\cdots$ & $\because$ & $\cdots$ & $\because$ \\
\hline$\cdots$ & $\ldots \ldots$ & $0^{\circ}$ & & $\ldots$ & $\cdots$ & $\therefore$ ? & \\
\hline & $\cdots \cdot$ & $\ldots \ldots$ & $\ldots$ & $\ldots$ & $\ldots$ & $\ldots w$ & $y z$ \\
\hline etus AG. 2 & $\cdots$ & $\ldots \ldots$ & & $\ldots$ & ... & $\ldots \ldots$ & .2 \\
\hline i Ag.... & $\because \cdots$ & & & $\cdots$ & . . $\mathrm{f}$ & $\ldots$ & . ? \\
\hline Jus Forb. & $\mathrm{E}^{2} \cdot \mathrm{M}^{2}$ & $\cdots \cdots \cdots$ & & $\cdots$ & $\ldots$ & $\ldots$ u... & - \\
\hline ter Ag. 25 . & $\cdots$ & $\ldots \ldots$ & $\ldots$ & $\cdots$ & $\cdots$ & $\ldots \ldots$ & .2 \\
\hline di AG. ... & $\cdots \cdot$ & $\cdots$ & $\cdots \cdot$ & $\cdots$ & $q r$. & $\cdots \cdots$ & $\cdots$ \\
\hline latus & $\cdots$ & & 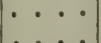 & 1 & . r. & $\cdots$ & $\therefore$ \\
\hline 2. . & .. & & & $\ldots$ & $\left|\begin{array}{r}0 \\
0 \\
r\end{array}\right|$ & $\cdots$ & $\cdots$ \\
\hline $\begin{array}{l}\text { fossarius Morr. } \\
\text { Murchisoni Morr. }\end{array}$ & .... & $\cdots \cdots \cdots$ & & $|\ldots|$ & $r$ & $\ldots \ldots$ & - \\
\hline bufo $A G$ & - & - & & . & $\cdot r$ & $\cdots$ & \\
\hline cor-testudiuarium $\mathrm{AG}$ & . & 1.. & & & & & \\
\hline
\end{tabular}




\begin{tabular}{|c|c|c|c|c|c|c|c|}
\hline Benen & end. & $\mathrm{g}$ & & $\mathbf{p}$ & I & $\mathbf{Y X}$ & \\
\hline . & & & & & & & \\
\hline$\cdots \cdot$ & & & $\cdots$ & $\ldots$. & $\therefore \mathrm{f}$ & $\ldots \ldots$ & \\
\hline$\cdots$ & $\ldots$ & $\ldots$ & $\cdots$ & $\cdots \cdot$ & $\ldots \tau$ & $\ldots \ldots$ & \\
\hline . & $\cdots$ & $\cdots$ & $\cdots \cdot$ & $\cdots \cdot$ & & & \\
\hline b & $\cdots \cdot$ & .. & $\cdots$ & $\cdots$ & f & $\cdots$ & \\
\hline AG. & & & & & & .1 & \\
\hline .. & & & & : & & $\cdots$ & \\
\hline$\ldots$ & $\cdots$ & & $\left|\begin{array}{c}\cdots \\
\cdots\end{array}\right|$ & $\left|\begin{array}{c}\cdots \\
\cdots\end{array}\right|$ & $\because 1$ & 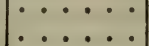 & \\
\hline$\cdots$ & ..... & $\therefore$ & $\ldots$ & $\ldots$ & $\therefore 1$ & $\ldots \ldots$ & \\
\hline$\cdots$ & ... & . & $\ldots$ & $\ldots$ & .. $\mathrm{f}$ & $\ldots \ldots$ & \\
\hline - & $\cdots$ & $\cdots$ & $\cdots \cdot$ & $\ldots$ & - $f$ & $\ldots \ldots$ & \\
\hline $\mathrm{g}$ & 然 & $\because \cdot 1$ & $\cdots \cdot$ & $\cdots$ & $\ldots 1$ & $\ldots \ldots$ & \\
\hline & & & $\cdots$ & $\cdots$ & . . I & $\cdots$ & \\
\hline 1 & & & $\cdots$ & $\cdots$ & & $\cdots \cdot$ & \\
\hline b & . & $\cdots$ & $\cdots$ & $\cdots$ & & .?.... & \\
\hline & & $\cdots$ & $\cdots$ & $\cdots \cdots$ & . & . ? ... & \\
\hline & & & $\cdots$ & $\cdots$ & & $\cdot$ & \\
\hline n & 18. & & . $\cdots$ & . & 1. & & \\
\hline & $i$ & $\therefore$ & $\cdots$ & $\cdots$ & $\because i$ & $\cdots$ & \\
\hline & & . & $\ldots$ & $\ldots$ & - r I & $\cdots$ & \\
\hline$\cdot \cdot$ & . & $\cdots$ & $\ldots$ & $\ldots$ & .. & $\cdots$ & \\
\hline$\cdots$ & $\cdots \cdot \cdot$ & $\cdots \cdots$ & $\cdots$ & $\cdots$ & $\ldots 1$ & $\cdots$ & \\
\hline$\cdots$ & $\cdots \cdot$ & $\cdots \cdot$ & $\cdots$ & $\cdots$ & . . I & $\cdots$ & \\
\hline$\cdots$ & $\cdots$ & $\cdots$ & $\cdots \cdot$ & $\cdots$ & . . I & $\cdots$ & \\
\hline$\therefore$ & & & 烈 & $\cdots$ & - 1 & $\cdot$ & \\
\hline$\because$ & : & • & • & $\because$ & $\because 1$ & .1 & \\
\hline$\cdots$ & .... & $\cdots$ & .... & $\mid \begin{array}{c}\cdots \\
\cdots\end{array}$ & $\therefore 1$ & $\dot{.}$ & \\
\hline . & $\cdots$ & i & 正 & $\ldots$ & $\therefore 1$ & $\therefore$ & \\
\hline$\cdots$ & $\cdots$ & • & & & . I & .. & \\
\hline & $\cdots$ & $\cdots$ & & $\ldots$ & & $\therefore$ & \\
\hline & ( & $\infty$ & & $\cdots$ & $\ldots r$ & $\cdots$ & \\
\hline . . & 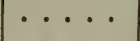 & . & - & - & $\cdots f$ & $\ldots \ldots$ & \\
\hline & & & & 然 & & $\cdots$ & \\
\hline & $\cdot$ & & & • & - 1 & $\cdots$ & \\
\hline$\cdots$ & $\cdot$ & & & & - $\mathrm{f}$ & - & \\
\hline . & & & & & & - & 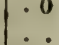 \\
\hline$\cdots$ & $\cdots$ & $\cdots$ & - & $\cdots$ & $q$ & & \\
\hline & & & . & & 9 & $\cdots$ & \\
\hline$\cdots$ & & & $\bullet$ & $\cdots$ & & $\ldots \ldots$ & • \\
\hline & & & & & $q$ & & \\
\hline$\cdots$ & & & & & - & $\ldots \ldots$ & \\
\hline . . & & & & & & & \\
\hline$\cdots$ & & & & & & & • \\
\hline G. & & & & $\ldots$ & - r f & . & \\
\hline$\ldots$ & $\because$ & $\therefore$ & $\cdots \cdots$ & $\ldots$ & $r$ & $\ldots \ldots$ & • \\
\hline$\cdot$ & & & & & $r$ & & $\cdots$ \\
\hline 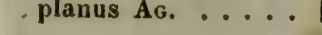 & - & & & & r I & & \\
\hline
\end{tabular}




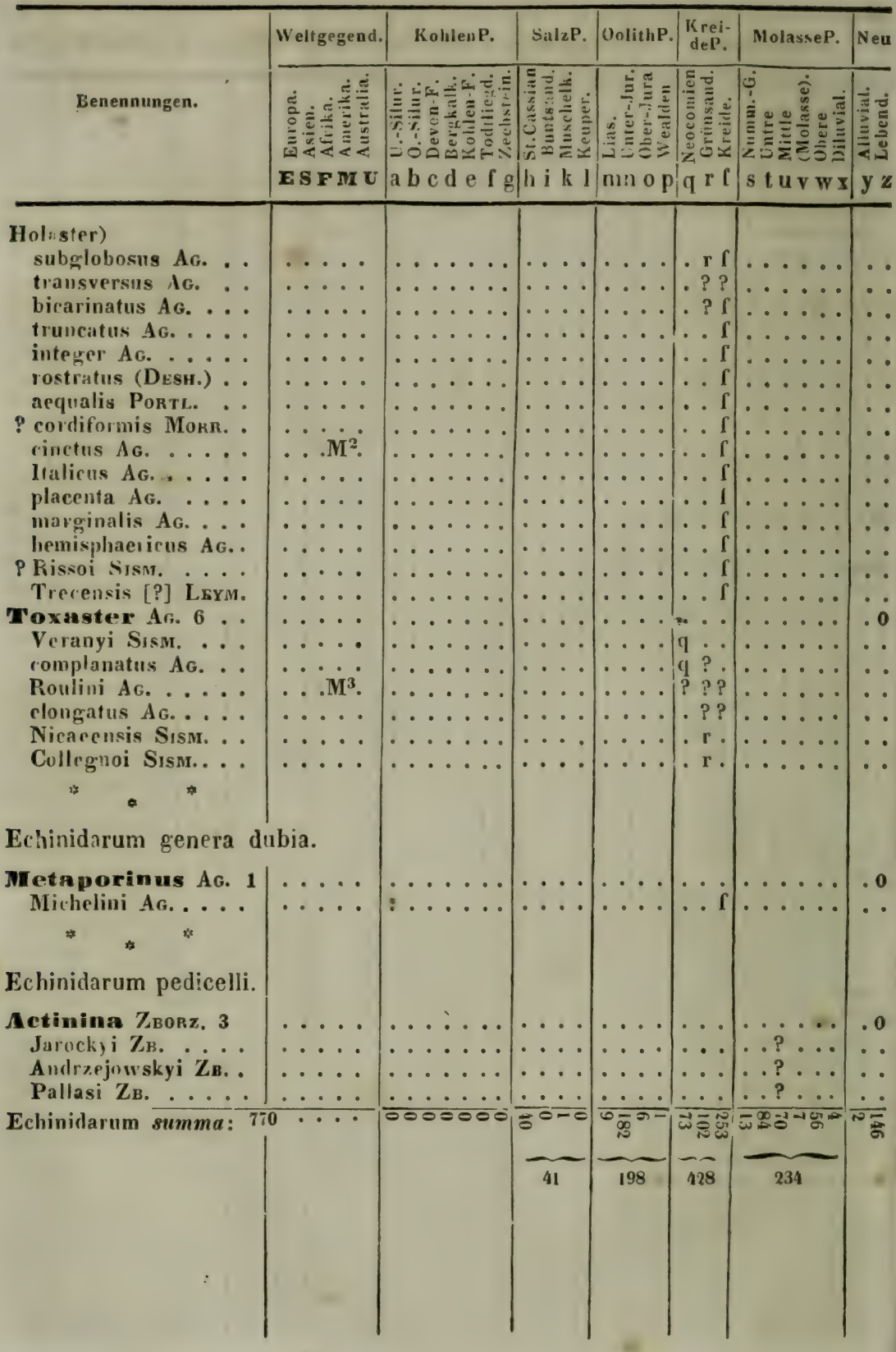




\section{TII. FISTULIEAE.}

(Holothuria Lin.)

Gynapta. 1 ......

SieboldiMü.(pedicelli)

Chirodota Esch. 0

Thyome $\mathrm{O}_{\mathrm{r}} 0 \ldots$ (Mülleria Flem.)

Trepang J J̈̈. 0 . Folothuria (L.) 1

? sp. Rürp. ......

Nulleria JäG. 0 . .

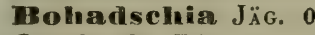

Cuvieria Pŕr. 0 .

Psolus $\mathrm{O}_{\mathrm{k}} 0 \ldots \ldots$

\begin{tabular}{|l|l|l|l|l|l|l}
\hline Weltgegend. & a b c d e f g h i k l & mn op & q r f & s t u vw x & y z
\end{tabular}

Pentacta Gr. 0 ..

(Cucunaria BLv.)

Jinyas Cuv. 0 ...

Fistulidarum summa: $3|\cdots \cdots| 000000000|00000| 02000|000| 01000000 \mid 66$ 


\begin{tabular}{|c|c|c|c|c|c|c|c|}
\hline \multirow{3}{*}{ Beneinungen. } & Weltgegend. & KohlenP. & SalzP. & OolithP. & $\begin{array}{l}\text { Krei- } \\
\text { deP. }\end{array}$ & MolasseP. & Neu \\
\hline & 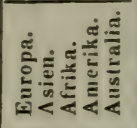 & 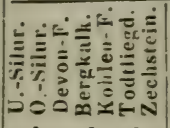 & 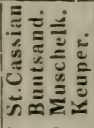 & 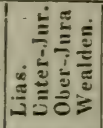 & 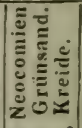 & 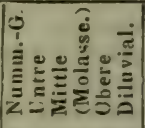 & 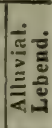 \\
\hline & ESPMU & a b c d e f g & h i k l & mn o p & q $\quad r \quad$ & $s t u v w x$ & $\mathbf{y} z$ \\
\hline
\end{tabular}

Appendix.

\section{PHYTOZOORUM}

\section{genera incertae classis.}

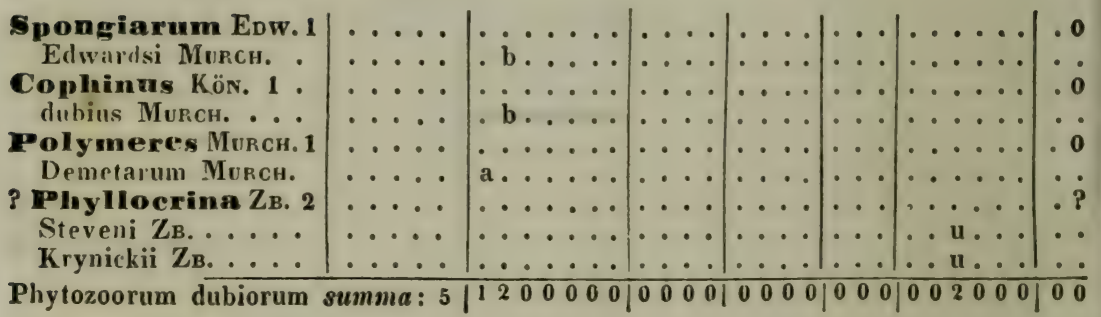




\section{(1) B B D}

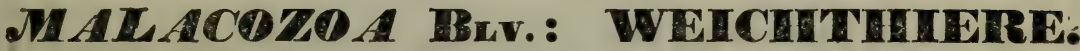

ACEPHALA: Muschelthiere.

CI. VIII. GYMNACEPHALA : Nackt-Muschler.

CI. IX. BRACHIOPODA Cuv.: Arm-Füsser.

CI. $X$. PELECYPODA : Beil-Füsser.

CEPHALOPHORA : Schneckenthiere.

CI. XI. PTEROPODA Cuv. : Flossen-Füsser.

CI. XII. HETEROPODA Lk. : Napf-Füsser.

Cl. XIII. PROTOPODA : Vor-Füsser.

CI. XIV. GASTEROPODA Cuv.: Bauch-Füsser '

CI. XV. CEPHALOPODA Cuv.: Kopf-Füsser.

1 Cuvier verhindet einen Theil der Protopoden (als Tubulibranchia) nnd die Heteropoden Lмк.'s als blosse Ordnungen mit seiner Klasse der Gasteropoden. Wenn sie ihnen aber auch nach der Gesammtheit der Organisation entsprechen, so weichen sie doch ueride in demjenizen Charakter vou ihnen ab. welchen Cuvier selbst als Haupt-Merknal der Gasternpoden bezeichnet und wornach er sie benennt. So lassen sich auch ihre Schalen nicht in die Diagnose der Gasteropoden-Schale" mit einschliessen; sie sind nicht regelmässig spiral mit seitlicher Münduıg: sondern die eiten sind unregelmässig gewunden und die andern ganz regelmässig scheibenfïrmig. symmetrisch. Die Zahlen der lebenden Ârten der einzelnen Geschlechter sind aus A. CATLow's Concholegist's Nomenclator (1845) angegeben. jedoch zum Theil aus den bei der Zoological Society zu London nachlier gehaltenen monographischeu Vorträgen von ReEve u. A. ergänzt, wobei die Summen allerdings noch weit uater der Wahrheit bleilen, indem eine grössere Anzahl von lebenden Arten bereits besclirieben ist. 


\section{Noch einige Zeichen-Erklärungen.}

(Vgl, S. 2 und 74.)

* steht vor den Namen von Arten, welche zwar kurz, aber ungenïgend beschrieben und nicht abgebildet sind und schwer zu enträthscln scyn weiden, oft wahrscheinlich auch schon unter anderen Namen begriffen sind, übrigens oft no.h auf genaucre Erforschung und Priorität Anspruch habeir können.

G (statt g) bedeutet die grosse permische Formation in Russland, da man geglaubt hat, sie enthalte auch fossile Arten aus der Steinkohlen - und aus einipen jüngeren Formationen.

M (statt $\mathrm{m}$ ) bezeichnet die Arten in dem zweifelhaften rothen Kalke des Salzburgischen, welcher reich an Ammoniten ist, oft auch Orthozeratiten, aber nicht Terebratula diphya führt.

$\mathbf{Q}$ (statt $q$ ) deutet Arten aus dem rothen und weissen Ammoniten-Marmor an der Südseite der Alpen an, welcher unter Rudisten-Kalk liegt, Terebratula diphya (oder ähnliche Formen) ohne Orthozeratiten ('nthält und von Catullo als Neocomien, von den meisten andern Geologen als Jurakalk angesehen wird. Er ist mit dem vorigen noch genauer zu vergleichen.

[?] hinter einem Art-Namen: deutet Unsicherheit über seinen Ursprung oder seine richtige Bildung an.

(...) Zwei runde Klammern, welche eine gewisse Anzahl von Rubriken für die Formationen zwischen sich fassen, ohne dass durch pinen Buchstabeu irgend eine solche näher bezeichnet würde, z. B. bei Hornera elegans S. 140, deuten an, dass die Formation, in welcher die fossile Ait vorkommt, innerhalb der von den Klammern angegebenen Grenzen unsicher sey.

Einige Genus-Namen sind zwischen runde Klammern gesetzt, was andentet, dass das so bezcichnete Genus unter ein anderes, gewöhnlich zunärhst vorhergehendes oder sonst näher augedeutetes, piugeordnet oder damit verschmolzen wrorden muss.

Zeigt ein Kursiv-Buchstabe in den Rubriken das geognostische Vorkommen einer Art an, ausser einem Buchstaben aus der giwöhnlichen stehenden Schrift, so ist das durch ersten angedeutete Vorkommen nur ein ausnahmsweises, das andere aber das gewöhnliche.

Eine hiı und wieder beigefügte Hand deutet auf besser verbürgte Fälle hin, wo eine und dieselbe Art in verschiclenen Formationen und FormationsGruppen vorkommt.

Einige der viber Namen, Synonyme oder Formationen ausgedrückten Zweifel rühren daher, dass der Vf. nicht alle benütıten Quellen-Schriften fortdauernd zu seiner Verfügung hatte, mithin nicht alle mit allen verglcichen komnte. Bei möglicher Vergleichung werden diese $Z$ weifel oft leicht zu beseitigen seyn. 


\begin{tabular}{|c|c|c|c|c|c|c|c|}
\hline & W & KohlenP. & SalzP. & OolithP. & $\begin{array}{c}\text { Krei- } \\
\text { deP. }\end{array}$ & MolasseP. & Neu \\
\hline Benennungen. & 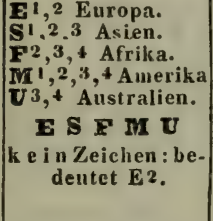 & 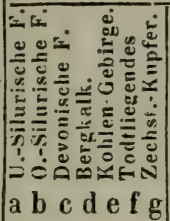 & 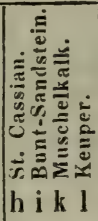 & 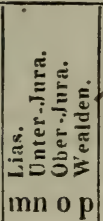 & 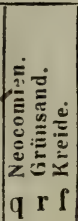 & 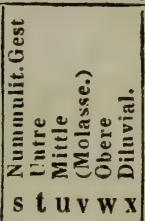 & 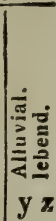 \\
\hline
\end{tabular}

\section{Cl. VIII. GYMNACEPHALA, Nackt-Muscheler.}

(Tunicata Lk.)

[Corporis coriuces el mollia.]

A. THALIADAE.

Thalia Brown 0 .

Salpa Gr. 0 ...

B. PYROSOMATA.

Botryllus Gaertiv, 0 Pyrosoma Pér.o

C. ASUIDIAE.

a. Simplices ....

Roltenia SAv. 0 . Cynthia $S_{A v} 0$... Ciavellima $S_{A v}, 0$ Phallusia Sav. 0 . o. Sacomites Rafin. 1 † granularis RAF. .

b. Aggregatae ....

Diazona $S_{A v} 0 \ldots$ Distomus $S_{A v}, 0$. Sigillina SAv. 0 .. Symoicum LK. 0 . Polyclimum Cuv. o

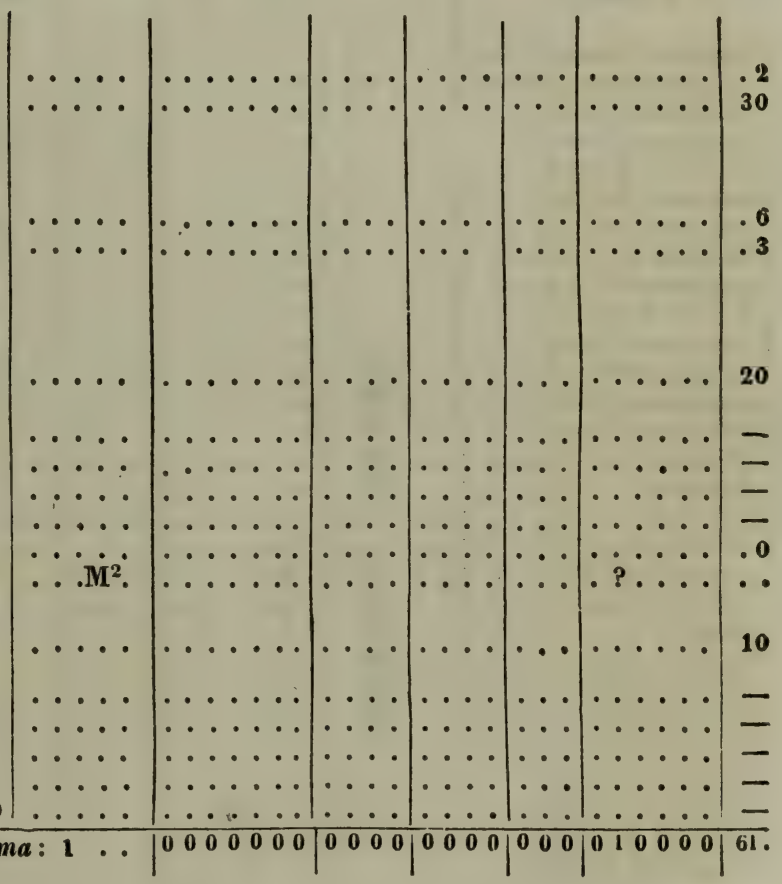

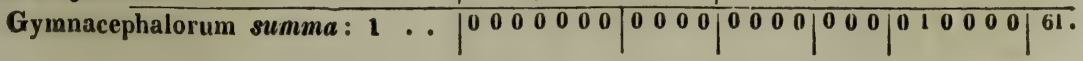




\begin{tabular}{|c|c|c|c|c|c|c|c|}
\hline & Weltgegend. & KohlenP. & SalzP. & UolithP. & $\begin{array}{r}\text { Krei- } \\
\text { deP. }\end{array}$ & MolasseP. & Nen \\
\hline Benennungen. & 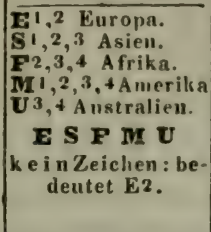 & 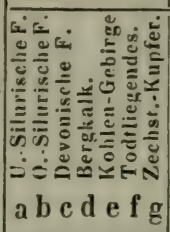 & 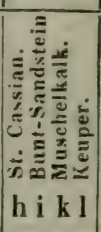 & 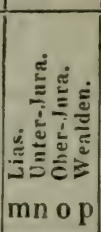 & 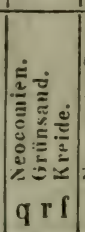 & 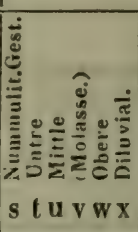 & $\begin{array}{l}\text { y } \\
\mathbf{y}\end{array}$ \\
\hline
\end{tabular}

\section{Cl. IX. BRACHIOPODA Cuv., Arm-Füsser.}

\section{A. GENUINA.}

Dbolus Eichw. 4 antiquissimus Eıснw. siluricus Езснт. Apollinis Eıснw. . . Ingricus Еıснw.

Hingela Lк. 34 .. attenuata So..... longissima Pand... quadrata EıcH w. . . brevis Ponte..... S verrucosa PAND. antiqua Ers. .... acuminata $\mathrm{V}_{\mathrm{ANX}}$. . marginata $\mathrm{D}^{\prime}(0 . .$. . Münsteri D'O. ... dubia D'O. lata So. ....... $P$ striata So. acutirostris $\dot{H}_{A L L} \cdots$ cuneata HaL. $\ldots$ elliptica Hald ... lamellata $\mathrm{H}_{\mathrm{AL} x}$... oblata HaLL .... oblonga $\mathbf{V}_{\mathbf{A N x}}$.... minima So. .....

$P$ cornea So. anatiniformis $\mathbf{P}$ usch elliptica Phiz.... marginata PнгLc... squanitormis Phile.

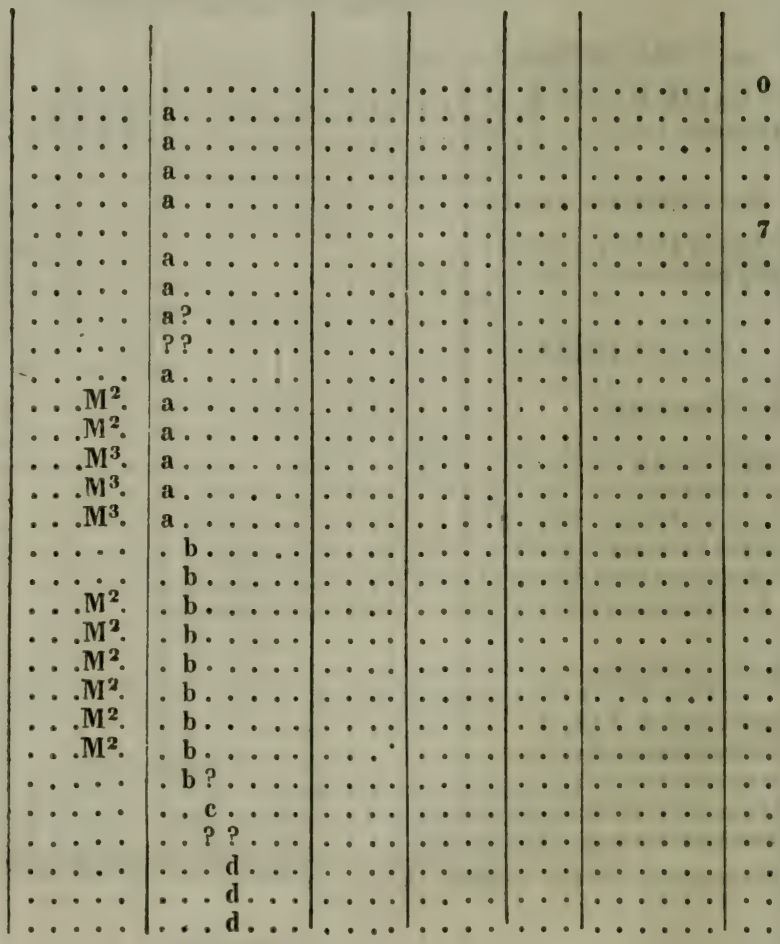




\begin{tabular}{|c|c|c|c|c|c|c|c|}
\hline Benennungen. & Weltgegend. & $\mid a b c d e f g$ & h i $\quad$ k-l & mn op & $q \times r$ & st u v w x & $y \mathbf{z}$ \\
\hline L. $\cdots$ & & 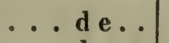 & & & & . & \\
\hline$\cdots$ & $\ldots \ldots$ & ...d..g & $\cdots \cdot$ & $\cdots$ & & $\ldots \ldots$ & \\
\hline inata 1 & $\cdots \cdot \cdot$ & . ? ? ?... & t & $\cdots \cdot$ & $\cdots$ & $\ldots \ldots$ & • \\
\hline$\cdots$ & $\cdots \cdot \cdot$ & $\cdots \cdots \cdots$ & . i k l I & $\cdots$ & .. & $\cdots \cdots$ & - \\
\hline NK. & &. & $\cdots 1$ & $n^{2}$ & & $\cdots \cdots \cdot$ & - \\
\hline $\begin{array}{l}\text { ni PhiLL. } \ldots . . \\
\text { cata So. } . . .\end{array}$ & $\cdots \cdots$ & 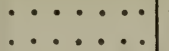 & $\cdots \vdots$ & $n^{2} \cdot$ & & $\cdots \cdots$ & $\bullet$ \\
\hline $\begin{array}{l}\text { truncata So. } \ldots \ldots \\
\text { ovalis? So. . . . }\end{array}$ & $\ldots$ & $\because$ & $\ldots$ & $\cdots$ & $9 \cdot$ & $\cdots$ & - \\
\hline tenuis So. .... & .... & $\ldots \ldots$ & $\cdots$ & $\ldots$ & & $t \ldots$ & - \\
\hline fusca Wood .... & $\cdots \cdot \cdot$ & $\ldots \ldots$ & $\cdots \cdot$ & $\ldots$ & . . . & . u.w. & \\
\hline $\operatorname{taV}$ ERN. 2 & $\ldots$ & $\ldots \ldots$ & $\cdots \cdot$ & $\ldots$ & . . & $\ldots \ldots$ & .0 \\
\hline N. . & - & $\cdots$ & $\cdots \cdot$ & $\ldots \cdot$ & $\cdots$ & $\ldots \ldots$ & . \\
\hline . & $\cdots$ & a.. & $\cdots$ & $\cdots \cdot$ & $\cdots$ & $\cdots$ & \\
\hline Tex & - $\cdot$ & & $\cdots \cdots$ & $\cdots$ & $\cdots$ & $\cdots \cdots$ & 30 \\
\hline spp. & dig & & & & & & \\
\hline (* & & & & & & & \\
\hline $\begin{array}{c}\text { acumi } \\
\alpha .\end{array}$ & & & $\cdots$ & $\ldots$ & . . & $\cdots \cdots$ & $\cdots$ \\
\hline $\begin{array}{l}\text { So. } \\
\text { So. }\end{array}$ & & $\begin{array}{cc}\cdots \\
\cdots \\
\cdots\end{array}$ & $\cdots \cdots$ & $\left|\begin{array}{c}\cdots \\
\cdots\end{array}\right|$ & . & $\begin{array}{l}\cdots \\
\cdots\end{array} \cdots$ & $\because$ \\
\hline - & . & $\ldots$ cd... & $\ldots$ & $\ldots$ & $\cdots$ & $\ldots \ldots$ & \\
\hline & & ... c & $\ldots \ldots$ & $\ldots$ & & $\ldots \ldots$ & \\
\hline . & $\mathbf{E}^{2} \mathbf{S}^{2} \ldots$ & . . c & $\ldots$ & $\cdots$ & $\cdots$ & $\ldots \ldots$ & \\
\hline & $\cdots$ & - & $\cdots$ & $\cdots$ & & $\ldots \ldots$ & • \\
\hline 0. & . . $\mathbf{M}^{3}$ & $\ldots$ c.... & $\cdots$ & $\cdots$ & $\cdots$ & $\cdots \cdots$ & - \\
\hline - & . $\mathrm{M}^{3}$ & $\cdots \mathbf{c} \ldots$ & $\cdots$ & $\cdots$ & . & $\cdots \cdots$ & - \\
\hline L & $\cdots$ & . c c & $\cdots$ & $\ldots$ & $\cdots$ & $\ldots \ldots$ & - \\
\hline . & $\cdots$ & . c c & $\cdots \cdots$ & $\cdots$ & $\cdots$ & $\cdots \cdots \cdots$ & - \\
\hline ง. . & & - & - & $\cdots$ & & $\cdots$ & - \\
\hline v & & $\cdot$ & $\cdots$ & $\cdots \cdots$ & & $\cdot$ & - \\
\hline s & $\dot{ }$ & $\because \mathbf{c} \cdots \dot{G}$ & $\dot{0}$ & $\therefore$ & & 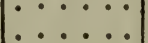 & \\
\hline eKL.1. & & $\cdots \cdots \cdots$ & h... & $\ldots$ & .. & $\ldots \ldots$ & \\
\hline $\begin{array}{l}\text { nnis-AustriaeKLI. } \\
\text { ris KLI. . . . }\end{array}$ & & & h . . . . & $\ldots$ & - & $\ldots \ldots$ & \\
\hline - & . & $\cdots$ & h... & . . & • & $\ldots \ldots$ & \\
\hline & & $\cdots$ & h $\ldots$ & $\ldots$ & . & $\ldots \ldots$ & . \\
\hline - & & $\cdots$ & h ... & $\cdots$ & - & $\cdots \cdots$ & \\
\hline$\cdots$ & & $\cdot$ & $\cdots$ & m.. & $\cdots \cdot$ & $\cdots \cdots$ & \\
\hline • • & - $\cdot$ & & $\cdots \cdots$ & $\mathbf{m} \mathbf{n} \cdot$ & $\cdots \cdot$ & $\cdots \cdots$ & \\
\hline - & & & & . . & & $\cdots$ & \\
\hline . $\cdots$ & & - & $\cdots \cdots$ & $\mathrm{m} n \ldots$ & $\cdots$ & $\cdots \cdots \cdots$ & \\
\hline 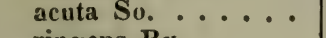 & 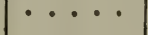 & - & $\cdot$ & $m n \cdots$ & & $\cdots$ & \\
\hline & & - 1 & $\cdots$ & $\cdot 1$ & & $\cdots \cdots$ & \\
\hline .) & - & & $\cdots$ & $\left|\begin{array}{l}n \\
n^{4}\end{array}\right|$ & $\cdots \cdot \boldsymbol{l}_{2}-2$ & $\cdots \cdots$ & 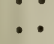 \\
\hline anni Voctz . & $\cdots \cdot$ & $\cdots$ & $\cdots$ & $\cdot \mathbf{n} \cdot \cdot$ & .. & $\ldots \ldots$ & - \\
\hline $\mathrm{SCH}$ & & $\cdots$ & $\cdots \cdots$ & $\cdot \mathbf{n} \cdots$ & & $\cdots$ & \\
\hline$\cdots \cdots$ & & $\cdots$ & $\cdots \cdots$ & $\cdots \cdot$ & $q$ & $\cdots \cdots \cdot$ & \\
\hline$\cdots \cdots$ & • & & & & & $\because$ & - \\
\hline (s) & & - & & & & & \\
\hline dentata Еıснw. & & & & & & & \\
\hline
\end{tabular}




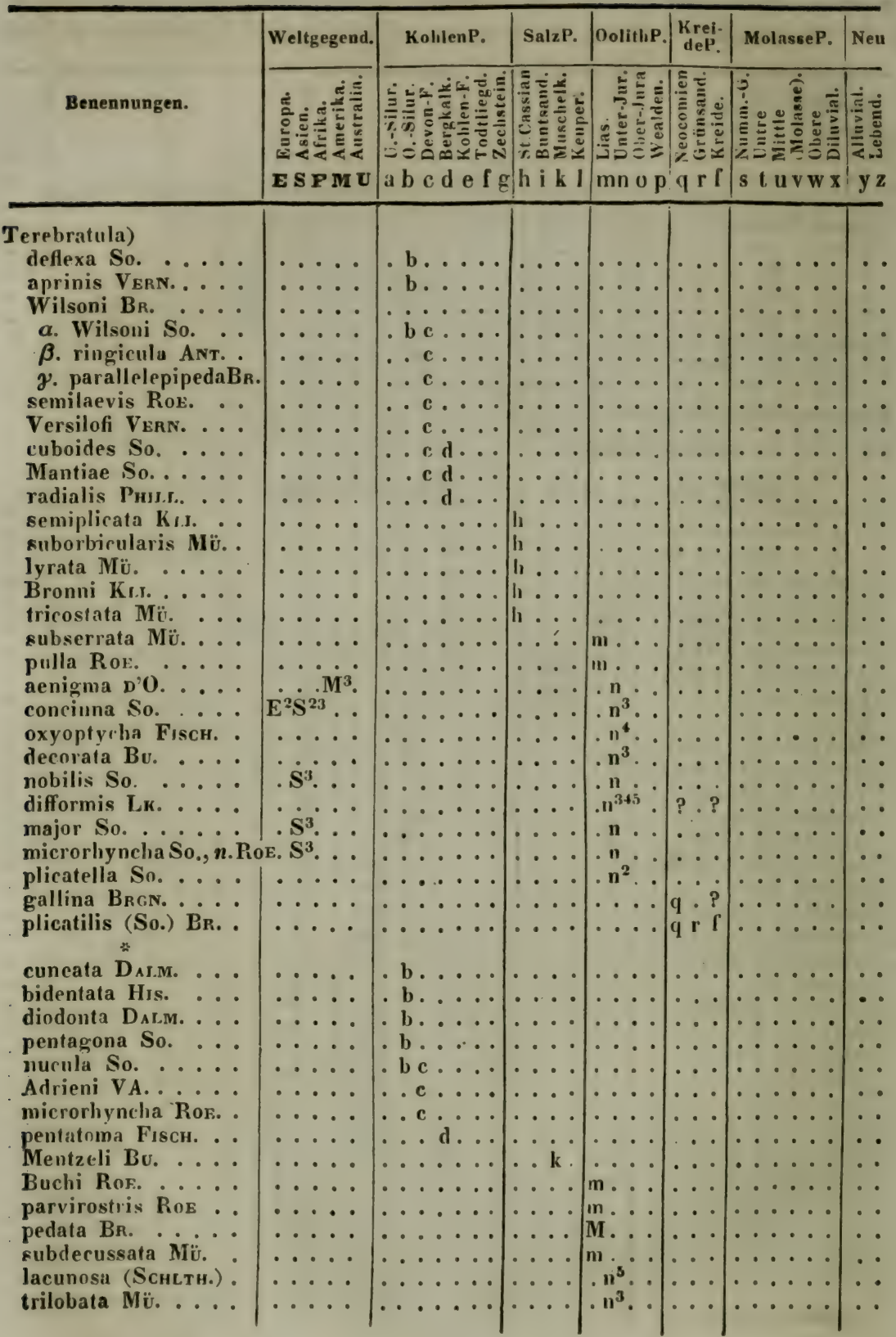




\begin{tabular}{|c|c|c|c|c|c|c|c|}
\hline Benennungen. & Weltgegend. & $\mid a b c d$ e $f g$ & h i k l & $\mathrm{mn}$ op & $\mathbf{q} \mathbf{r} \mathbf{f}$ & st uvw $x$ & $y z$ \\
\hline rostrata So. : . . & & & & . $\mathrm{n}$. & & $\cdots$ & \\
\hline$\beta$. difformis LEYM. & & & & & $q \mathbf{r} \cdot$ & $\cdots \cdots$ & - \\
\hline $\begin{array}{l}\text { acuticosła HeиL. . } \\
\text { lentiformis RoE. }\end{array}$ & $\cdots \cdots$ & $\cdots \cdots$ & $\cdots \cdots$ & $\cdot n^{3} \ldots$ & $\cdots$ & $\cdots \cdots$ & • \\
\hline & $\cdots \cdots$ & $\cdots \cdots$ & $\cdots \cdots$ & n & $\cdots$ & $\cdots \cdots \cdots$ & $\because$ \\
\hline depressa ZiET. . . & $\cdots$ & $\therefore \ldots \ldots$ & $\cdots \cdots$ & $: n^{5} \cdots$ & $\therefore$ & $\cdots \cdots$ & $\because$ \\
\hline peregrina Bu. ... & $\cdots \cdot$ & $\ldots \ldots$ & $\cdots$ & $\ldots$. & $q \cdots$ & $\ldots \ldots$ & $\therefore$ \\
\hline paucicosta Roe. ... & $\cdots \cdot \cdot$ & $\ldots \ldots$ & $\ldots$ & $\ldots$. & $q \cdots$ & $\ldots \ldots$ & . \\
\hline multiformis RoE. . . & & $\ldots \ldots$ & $\cdots \cdot$ & & $q \mathbf{r} \cdot$ & $\ldots \ldots$ & - \\
\hline biangularis Dsн... & - & $\cdots \cdots$ & $\cdots \cdot$ & $\cdots \cdot$ & $q_{1}^{1} r$ & $\cdots \cdots$ & $\cdots$ \\
\hline A. . & 0 & $\cdots$ & & & $q^{2} \mathbf{r}$ & $\cdots \cdots$ & - \\
\hline Mantellana So. ... & $\cdot$ & $\cdots$ & $\cdots \cdot$ & $\cdots \cdot$ & . . $\mathrm{f}$ & $\cdots \cdots$ & - \\
\hline $\begin{array}{c}\text { limbata (Schlth.) } \\
\text { (** Dichntomite.) }\end{array}$ & & $\cdots$ & $\cdots \cdot$ & $\cdots \cdot$ & . $\mathrm{f}$ & $\cdots \ldots$ & $\cdot \cdot$ \\
\hline reticulari & $E^{2} S^{2} \cdot M^{2}$ & $\mathbf{a} \mathbf{b} \mathbf{c} \ldots \ldots$ & $\cdots$ & $\cdots$ & $\cdots$ & $\ldots \ldots$ & \\
\hline HL. . & $\mathbf{E}^{2} \mathbf{S}^{2} \cdot \mathbf{M}^{2}$ & & $\cdots \cdots$ & $\cdots \cdots$ & $\cdots$ & $\cdots \cdots$ & - \\
\hline marginalis DALM. - & $\cdots$ & $\cdots$ & $\cdots \cdot$ & $\cdots \cdot$ & $\cdots$ & $\cdots \cdots \cdots$ & - \\
\hline$\cdots$ & $\cdots$ & b..... & $\cdots \cdots$ & $\cdots \cdots$ & $\cdots$ & $\cdots \cdots \cdots$ & - \\
\hline$\cdots$ & $\cdots$ & . b. & $\cdots \cdots$ & $\because$ & $\cdots$ & $\because \cdots \cdots$ & • \\
\hline $\begin{array}{l}\text { emisulcata SALT. } \\
\text { revirostris So. . }\end{array}$ & & $\cdots \cdots$ & $\cdots \cdots$ & $\cdots$ & $\because \cdots$ & $\because \cdots \cdots$ & $\because$ \\
\hline$\cdots$ & $\cdots$ & $\ldots$ c.... & $\cdots \cdot$ & $\cdots \cdot$ & $\cdots$ & $\ldots \ldots$ & $\ldots$ \\
\hline o... & & . b.. & $\ldots$ & $\cdots$ & $\cdot$ & $\cdots \cdots$ & $\cdots$ \\
\hline laris PhrLL. . & $\ddot{\mathbf{s}_{2}} \cdot \cdot$ & . c c & $\cdots \cdot$ & $\cdots$ & $\cdots$ & $\cdots \cdots$ & - $\bullet$ \\
\hline IS VERN. . & $\cdot S_{2}^{2} \cdot \cdot$ & $\cdots$ & $\cdot \cdot$ & $\cdots$ & - & $\cdots \cdots$ & • \\
\hline Alinensis [?] VerN. . & $\cdot S^{2} \cdot \cdot \cdot$ & ・c... & - & - & $\cdots$ & $\cdots \cdots$ & $\bullet$ \\
\hline$\cdots$ & $\begin{array}{l}\cdots \cdots \\
\cdots \\
\cdots\end{array}$ & $\begin{array}{ll}\cdots \\
\cdots \\
\cdots\end{array}$ & $\because$ & $\cdots \cdots$ & $\because \cdots$ & $\cdots \cdots \cdots$ & • \\
\hline$\therefore$ & $\therefore$ & $\therefore$ c.... & $\ldots$ & $\ldots$ & $\cdots$ & $\cdots \cdots$ & $\because$ \\
\hline rpentina Kon. . . & $\ldots$ & ... d... & $\ldots$ & $\ldots$ & $\cdots$ & $\ldots \ldots$ & $\because$ \\
\hline gona KoDv. & $\cdots$ & $\cdots \cdots$ & & & $\cdots \cdot$ & $\ldots \ldots$ & $\therefore$ \\
\hline aris So. ... & $\cdots \cdots$ & $\cdots$ & & $m n^{3}$. & $\cdots$ & $\ldots \ldots$ & - \\
\hline$\therefore$ & $\cdots \cdot \cdot$ & $\cdots$ & $\cdots$ & $\cdot n^{23} \cdot \cdot$ & $\cdots$ & $\ldots \ldots$ & $\cdots$ \\
\hline osa (Schl.).. & $\cdots \cdot$ & $\cdots$ & $\cdots \cdot \cdot$ & $\left|\cdot n_{5} \cdot\right|$ & $\cdot \cdot$ & $\ldots \ldots$ & - \\
\hline L.) • & & & & $\cdot n^{5}$. & $\cdots$ & $\cdots \cdots$ & • \\
\hline$\cdots$ & - & - & $\cdots$ & $\cdots \cdot$ & $q \cdots$ & $\cdots \cdots \cdots$ & • \\
\hline$\dot{j}$ & & $\cdots$ & $\cdots$ & $\cdots$ & & $\cdots \cdots \cdots$ & • \\
\hline (Sснц.) & - & $\cdots$ & & & $\mid q-1$ & $\cdots \cdots \cdots$ & • \\
\hline n LK. . . . • & $\cdots \cdot$ & $\cdots$ & $\cdots$ & & 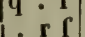 & $\cdots \cdots \cdots$ & \\
\hline a So. & - & $\cdots \cdots$ & $\cdots$ & $\ldots$ & r f & $\cdots \cdots \cdots$ & $\because$ \\
\hline ncei Brign. & $\cdots \cdots$ & - & & $\cdots \cdots$ &. $\mathbf{r} 1$ &.$t \ldots$ & \\
\hline ris RoE. . . & .... & . . & $\cdots$ & $\cdots \cdots$ & . . $\mathbf{f}$ & $\ldots \ldots$ & • • \\
\hline (Sснгт.). & & $\cdots$ & $\cdots \cdot$ & $\cdots \cdot$ & . . $\mathrm{f}$ & $\ldots \ldots$ & ・ \\
\hline Santonensis D'A. . & $\cdots$ & $\cdots$ & $\therefore$. & $\cdots \cdot$ & $\ldots \mathrm{f}^{2}$ & $\ldots \ldots$ & $\cdots$ \\
\hline is (Schi..) . . & & - $\cdot$ & $\cdots \cdot$ & $\cdots \cdot$ & $\cdots \mathbf{f}$ & $\ldots \ldots$ & • \\
\hline$\cdots \cdots$ & - & - $\cdot$ & $\cdots \cdot$ & $\cdots \cdot$ & .. f 1 & $\cdots \cdots$ & \\
\hline & $2^{\circ}$ & $\cdots$ & $\cdots \cdot$ & $\cdots \cdot$ & $\cdots \mathrm{r}$ & $\cdots \cdots$ & \\
\hline Inuxemana LYELL & . & . & . & $\cdots$ & & $\cdots$ & \\
\hline . & ? & $\cdots \cdot$ & $\cdots \cdot \cdot$ & $\cdots$ & . . I I & $\cdots w x$ & $\ddot{z}$ \\
\hline truncata LL. .... & & & & & |. & $\ldots \cdot \mathbf{w}$ & $\cdot 2$ \\
\hline
\end{tabular}




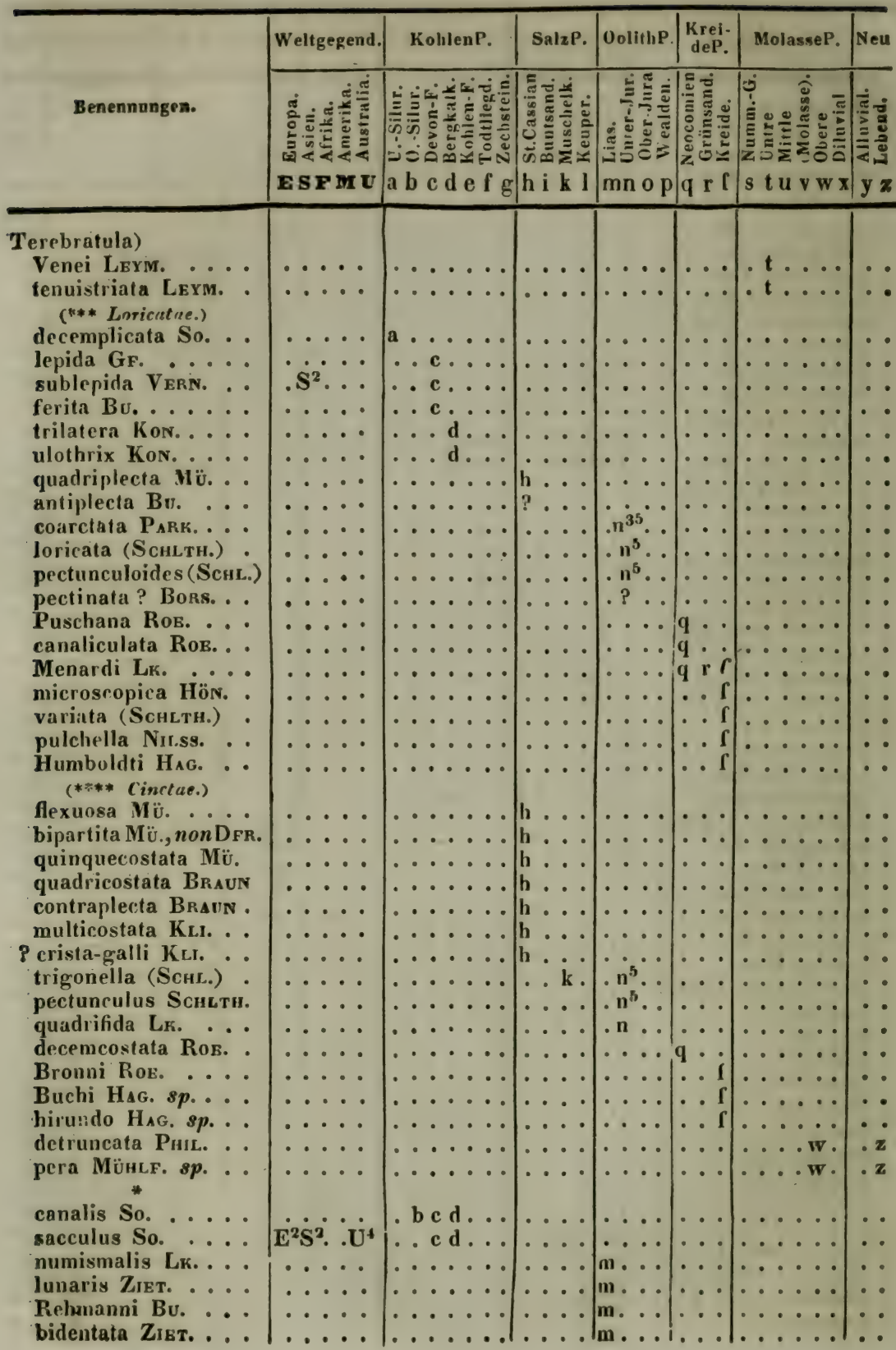




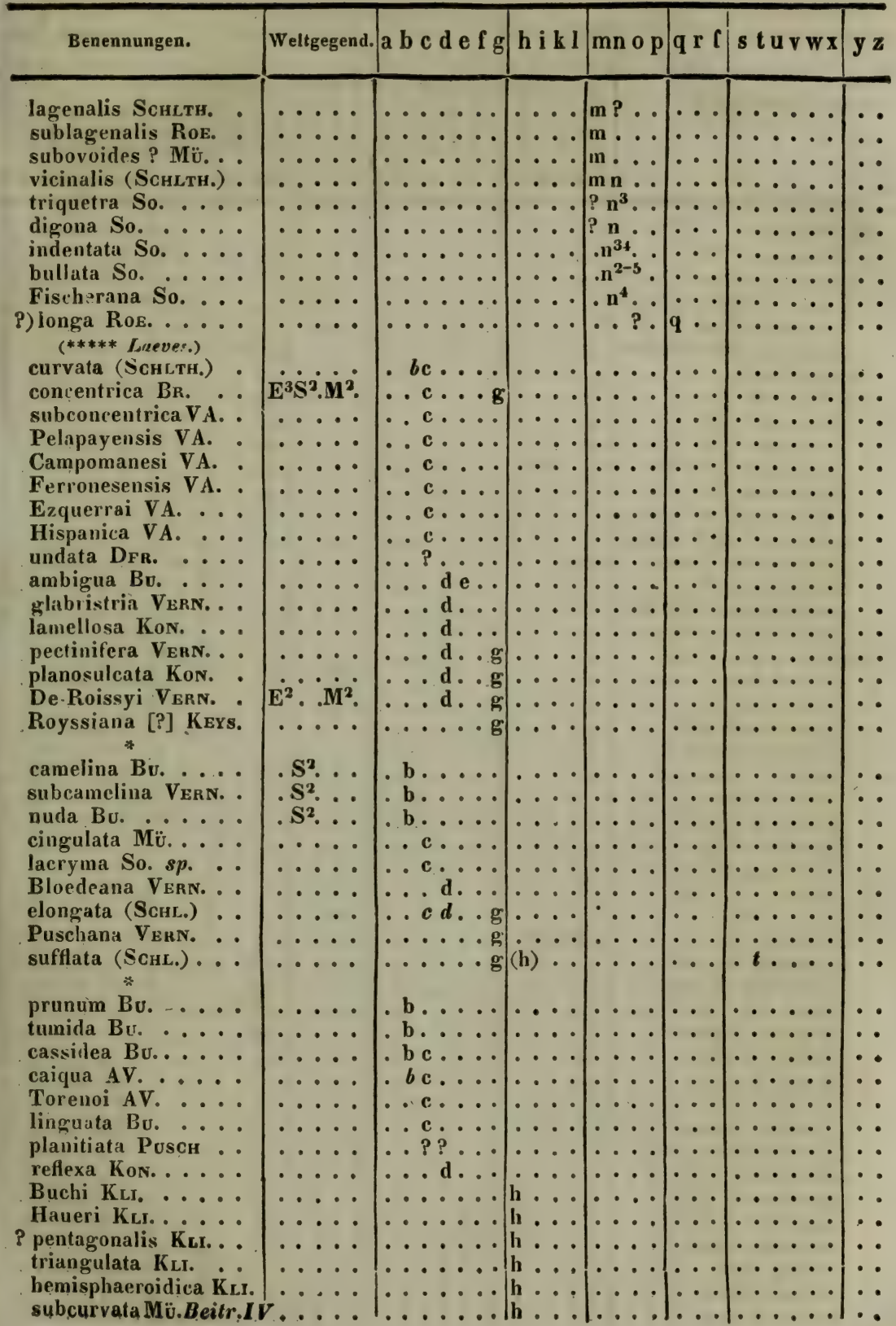




\begin{tabular}{|c|c|c|c|c|c|c|c|}
\hline & Weltgegend. & KohlenP. & SalzP. & OolithP. & $\begin{array}{l}\text { Krei- } \\
\text { deP. }\end{array}$ & MolasseP. & Neu \\
\hline Benennungen. & 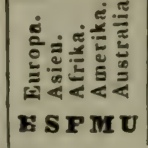 & 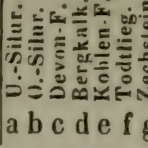 & 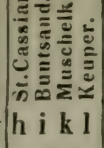 & 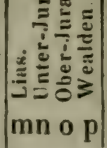 & 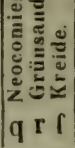 & 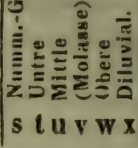 & 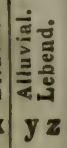 \\
\hline
\end{tabular}

Terebratula)

Wissmanni Mü.

$\boldsymbol{8}$ aequalis Kı.r. vulgaris (Sснгтн.)

amygdala Cᄉt.

ornithocephala(So.)Bv

$?$ ascia Gird.

orbiculata Ros.

telragona Ros.

Royerana D'O.

Ignaciana [?] D'O.

intermedia So.

humeralis Ros. . . .

pseudojurensis LEYM. subtrilobata Dsw.

lengirostris Nisss.

seniglobosa (So.) Bu. carnea (DFr.) Br. . curvirostris NiLss. ovoides So.

Nerviensis b'A. ...

Tornacensis D'A.

undulata Рosch ... incurva (Sснцтн.) incisa Münt. ....

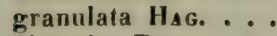
bipartita DFr.

vitrea Lк. . . . . . .

biplicata So.

biplicata St.... Stroganofi Vrikn.

Kleini Lк.

tetragona Pusch -

Sowerbyi Hag. . . .

Fittoni Hag.

Montolearensis Leym. grandis (BLumB.). praemarginata KLs. . subangusta $M u ̈$.

angusta $\mathrm{Bv}$.

pala Bv.

perovalis Ros.

arcuata RoE.

hippopus Ros.

Becksi Roz.

\section{.}

$E^{2} S^{3} \cdot M^{2}$

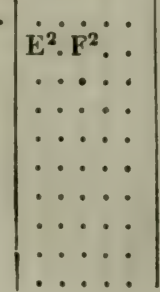




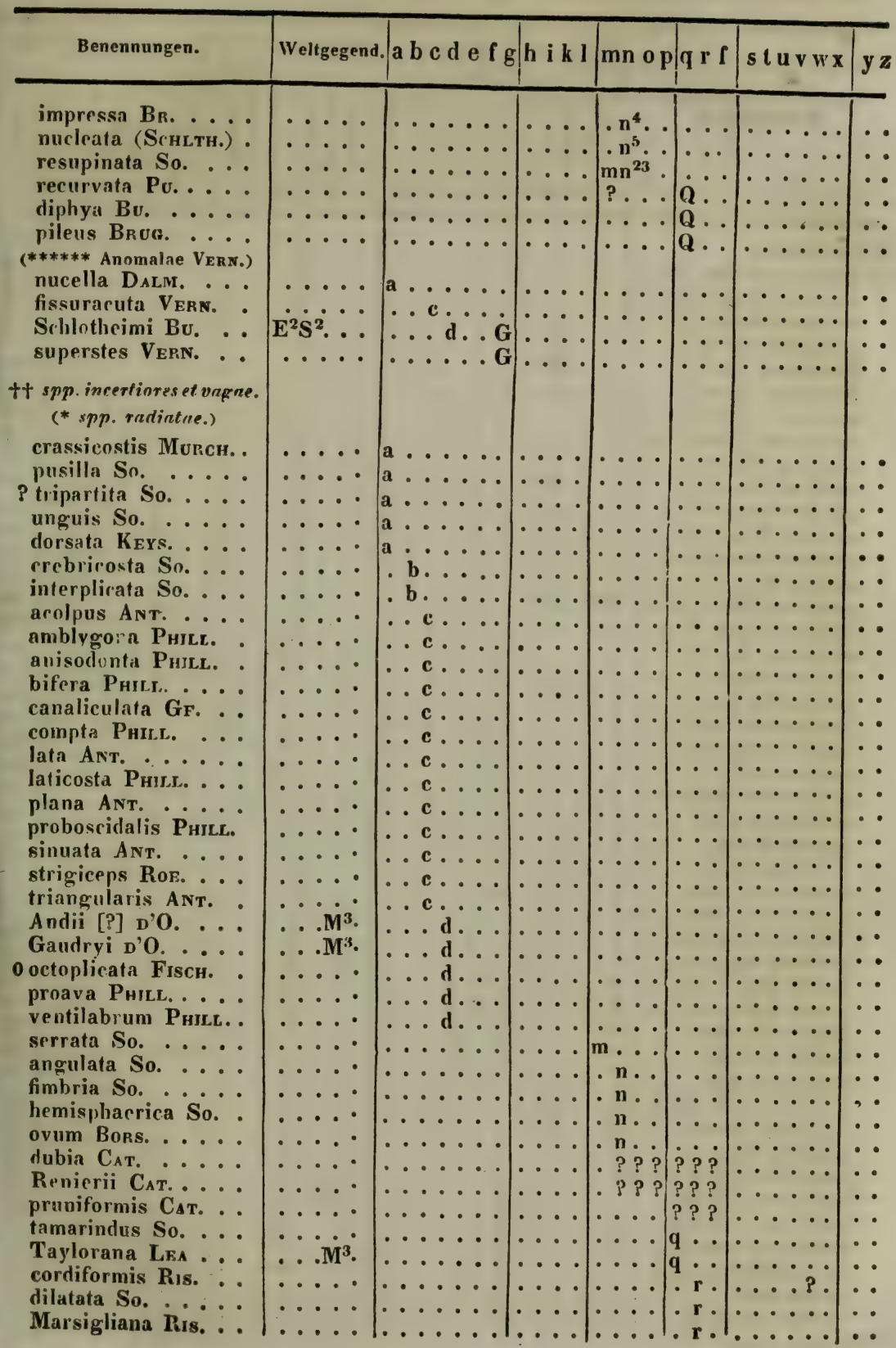




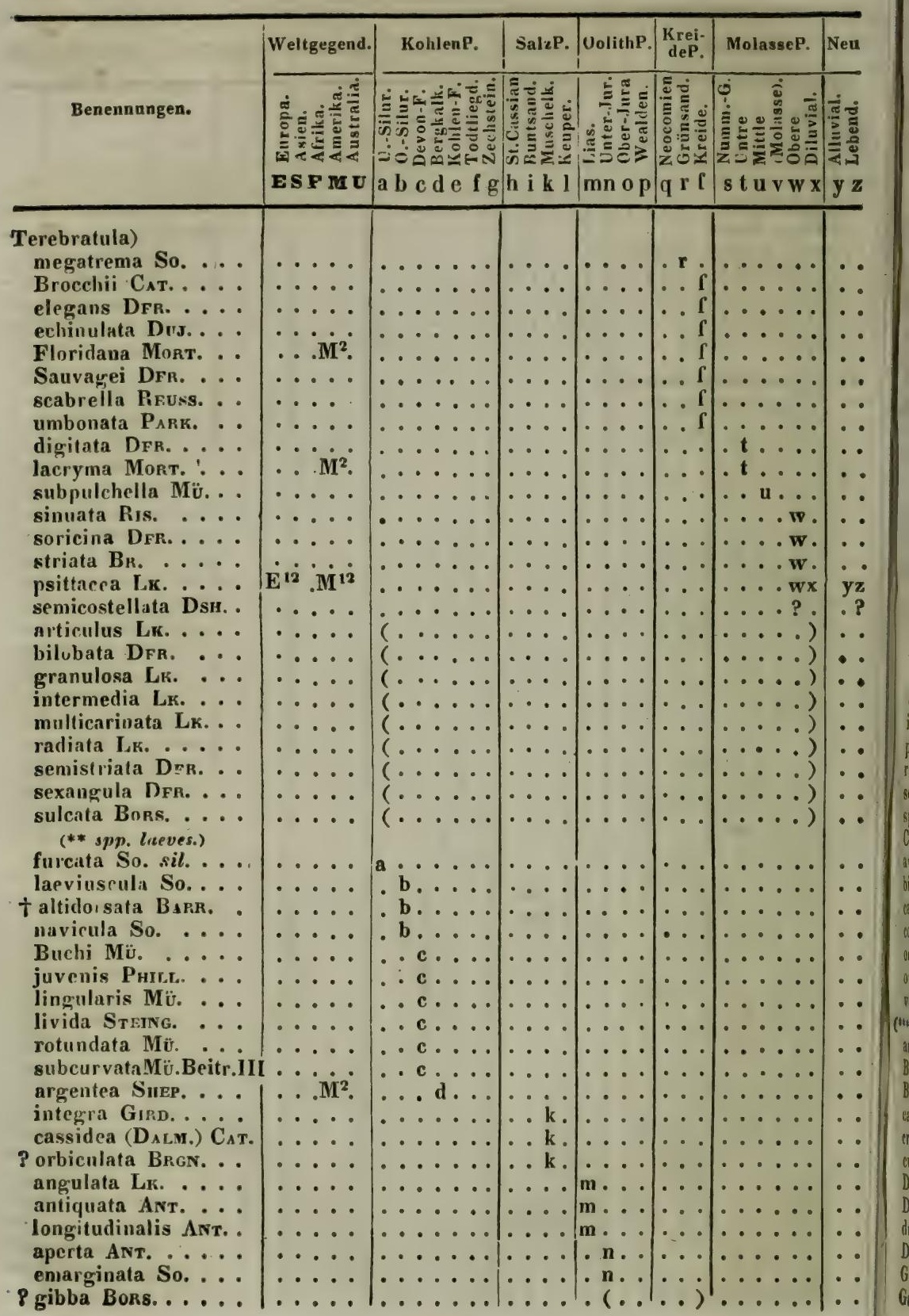




\begin{tabular}{|c|c|c|c|c|c|c|c|}
\hline Benennungen. & Weltgegend. & $\mid a$ b c d e f $g$ & h i k l & $\mathrm{mn} 0 \mathrm{p}$ & q r r & s t u v w x & $\mathbf{y}$ \\
\hline pectoralis Roe. . . & & 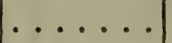 & & & & $\cdots \cdots$ & \\
\hline Poeyana LEs .... & ... $\mathbf{M}^{3}$ & $\ldots \ldots$ & $\ldots$ & $\ldots$ & & $\ldots \ldots$ & . \\
\hline o..... & $\ldots \ldots$ & $\ldots \ldots$ & $\ldots$ & $\ldots$ & & $\ldots \ldots$ & $\cdots$ \\
\hline$\cdots$ & $\cdots \cdots$ & $\ldots \ldots$ & $\ldots$ & $\ldots$ & ? ? & $\ldots \ldots$ & \\
\hline lentoidea LeYm. - & $\ldots$ & $\ldots \ldots$ & $\cdots \cdot$ & $\cdots$ & . $\mathrm{r}$ & $\ldots \ldots$ & - \\
\hline Aberdeenia Ris. . & $\cdots \cdot \cdot$ & $\cdots \cdots$ & $\cdots \cdot$ & $\cdots \cdot$ & $\cdots$ & $\cdots \cdots$ & - \\
\hline Beudantia Ris. ... & & & $\cdots \cdots$ & $\cdots \cdot$ & $\cdots f^{1}$ & $\cdots \cdots$ & - \\
\hline Colonnai Rrs..... & : & $\cdots$ & $\cdots \cdot$ & $\cdots \cdot$ & $\cdots \frac{1}{\cdots}$ & $\cdots \cdots \cdot$ & - \\
\hline$\cdots$ & $\ddot{\cdots} \cdot \vec{x}$ & $\cdots \cdots \cdots$ & $\left|\begin{array}{c}\cdots \\
\cdots\end{array}\right|$ & $\mid \cdots \cdots$ & $\mid \begin{array}{ll}\cdots \\
\ldots\end{array}$ & $\cdots \cdots$ & - \\
\hline $\begin{array}{l}\text { heteroclita Drr. . } \\
\text { lima DFr. . . . . }\end{array}$ & $\begin{array}{l}\cdots \cdots \\
\cdots \cdots\end{array}$ & $\ldots \ldots$ & $\ldots$ & $\cdots \cdots$ & $\because \mathrm{f}$ & $\cdots \cdots$ & - \\
\hline Morlandia Ris.... & $\cdots \cdot$ & $\ldots \ldots$ & $\ldots$ & $\ldots$ & $\therefore \mathrm{f}$ & $\ldots \ldots$ & . \\
\hline$\cdots$ & $\cdots$ & $\ldots \ldots$ & $\cdots \cdot$ & $\ldots$ & $\ldots$ I & $\ldots \ldots$ & - \\
\hline phaseolina LK. . . & $\cdots \cdot$ & $\ldots \ldots$ & $\cdots$ & $\ldots$ & .. 1 & $\ldots \ldots$ & - \\
\hline$\cdots \cdot$ & $\cdots \cdots$ & $\cdots$ & $\cdots$ & $\ldots$. & $\ldots 1$ & $\ldots \ldots$ & - \\
\hline Is. . . & $\cdots \cdot$ & $\ldots \ldots$ & $\cdots \cdot$ & $\cdots \cdot$ & . $f^{1}$ & $\ldots \ldots$ & - \\
\hline$\cdots$ & $\cdots \cdot \cdot$ & $\ldots \ldots \cdot$ & $\cdots \cdot$ & $\cdots \cdot$ & . I & $\cdots \cdots$ & \\
\hline Irss. . . & $\cdots \cdots$ & $\cdots \cdots$ & $\cdots \cdot$ & $\cdots \cdot$ & $\cdots 1$ & $\cdots \cdots$ & - \\
\hline ECK . & & $\cdots$ & $\cdots \cdot$ & $\cdots \cdot$ & . & $\cdots \cdots \cdot$ & • \\
\hline$\cdots$ & $\cdots \cdots$ & $\because$ & $\cdots \cdots$ & $\cdots \cdots$ & $\because$ & $\mid \begin{array}{l}\cdot t \\
. t\end{array} \cdots$ & - \\
\hline LYنLL & $\because \dot{M}^{2}$ & $\cdots \cdots$ & $\cdots \cdots$ & $\cdots \cdots$ & $\because$ & 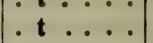 & - \\
\hline $\begin{array}{l}\text { ngtunensisLyeld } \\
\text { Еiсиw. . . . }\end{array}$ & $\ldots$ & $\ldots \ldots$ & $\ldots$. & $\ldots$ & $\ldots$ & . u... & $\dot{0}$ \\
\hline planata DFr. . & $\ldots \ldots$ & $\ldots \ldots \ldots$ & $\ldots$ & $\ldots$ & ... & $\ldots w$. & - \\
\hline ta Рнгк. . . . & $\ldots \ldots$ & $\cdots \cdots \cdots$ & $\ldots \ldots$ & $\cdots \cdot 1$ & $\cdots$ & $\ldots . w$ & $\cdot$ \\
\hline a Рніс. . . . & $\ddot{n} \cdots$ & $\ldots \ldots$ & $\cdots \cdot$ & $\cdots \cdot$ & $\cdots$ & $\ldots . . w$ & - \\
\hline inflexa Dsн...... & $E^{2} \cdot F^{2}$ & $\ldots \ldots$ & $\cdots \cdot$ & $\cdots \cdots$ & $\cdots$ & $\ldots . w$ & - \\
\hline pusilla Pнic. .... & $\ldots \ldots$ & $\ldots \ldots$ & $\ldots$ & $\cdots$ & . . & $\ldots w^{\prime}$ & - \\
\hline rescisa DFR. ... & $\cdots \cdots$ & $\cdots \cdots \cdots$ & $\cdots \cdot$ & $\cdots \cdots$ & $\cdots$ & ....w & - \\
\hline a Pнiz. .... & $\cdots \cdots$ & $\cdots \cdots \cdots$ & $\cdots \cdots$ & $\cdots \cdot$ & $\cdots$ & ....w & • \\
\hline noidea Рнгц. . • & $\cdots \ddot{x}$ & $\cdots$ & $\cdots$ & $\cdots \cdots$ & - & $\cdots \cdots \mathbf{w} \cdot$ & 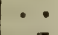 \\
\hline Chilensis D'O. $\cdots$ & $\cdots \mathrm{M}^{4}$ & $\cdots$ & 1. & $\cdots \cdot$ & . & $\cdots \cdots x$ & $\cdot \mathbf{z}$ \\
\hline Ilana DFr. ... & $\cdots \cdots$ & - 1 & $\cdot$ & $\cdots \cdots$ & & $\cdots \cdots)$ & - \\
\hline birostris LK. . . . & $\cdots \cdots$ & $\dot{.}$ & $\cdots$ & $\cdots$ & ... & $\cdots \cdots$ & • \\
\hline Lк. . . . . . & … & $(\ldots$ & $\cdots$ & $\ldots$ & $\ldots$ & $\ldots .)$. & . \\
\hline ulta Ant. . . & $\ldots$. & $(\ldots$ & $J^{\circ}$ & $\cdots$ & ... & $\ldots \ldots)$ & - \\
\hline Lк. .... & ..... & & & $\cdots \cdot \mid$ & $\cdots$ & $\mid \cdots \cdots) \mid$ & $\cdots$ \\
\hline öD. . . & & & & $\cdots \cdots$ & & $\mid \cdots \cdots) \mid$ & - \\
\hline $\begin{array}{l}\text { natcae) } \\
\cdots\end{array}$ & & & & & & & \\
\hline A. ... & $\cdots \cdot \cdot$ & & & & & & \\
\hline$\cdots$ & $\cdots \cdot \cdot$ & $\cdots$ & - & $\cdots \cdot \mid$ & & - & - \\
\hline$\cdots$ & & & & & & & \\
\hline $\begin{array}{l}\mathbf{c} \\
\mathbf{c}\end{array}$ & & & & & & & \\
\hline $\begin{array}{l}\text { cra } \\
\text { De }\end{array}$ & & & & & . $[1]$ & $\because$ & \\
\hline$\therefore$ & • & & $\cdots$ & $\ldots \ldots$ & $\therefore i$ & - & - \\
\hline ( relig.) & . & & & $\cdots \cdot$ & & & \\
\hline$\cdots$ & - & & $\cdots \cdot \cdot$ & 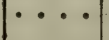 & & & \\
\hline $\begin{array}{l}\text { Gravesi D'A. } \cdots \\
\text { Gussignisensis } \text { D'A. }^{\circ}\end{array}$ & $\ldots$ & & & & & & \\
\hline
\end{tabular}




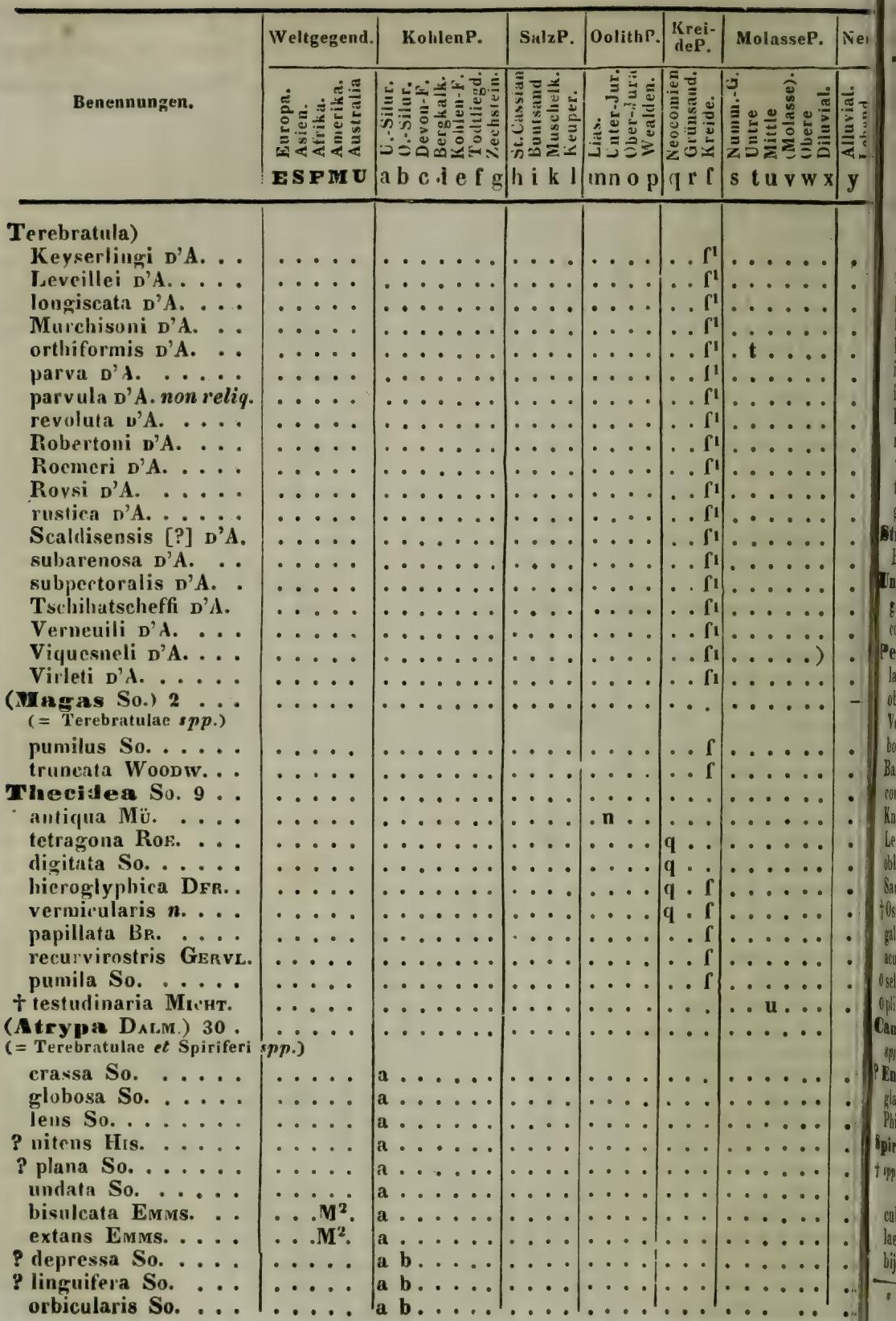




\begin{tabular}{|c|c|c|c|c|c|c|c|}
\hline Benennungen. & d. & $g$ & 1 & $\mathrm{p}$ & 0 & $N \mathbf{x}$ & \\
\hline ersa Portl. • & & & 陌 & ・ * & $\cdots$ & & \\
\hline$\cdots$ & & $\ldots$ & $\ldots$ & $\ldots$ & $\ldots$ & $\ldots \ldots$ & \\
\hline$\cdots$ & $\cdots \cdot$ & $\cdots$ & & $\cdots$ & $\ldots$ & $\ldots \ldots$ & \\
\hline$\cdots$ & $\cdots$ & $\cdots$ & $\cdots \cdot$ & $\cdots$ & $\cdots$ & $\ldots \ldots$ & \\
\hline$\cdots$ & $\cdots$ & & $\cdots$ & 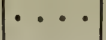 & $\cdots$ & $\ldots \ldots$ & \\
\hline$\therefore$ & $\begin{array}{l}\cdots M^{2} \\
\cdots M^{2}\end{array}$ & $\begin{array}{l}\text { b } \\
\text { b }\end{array}$ & $\cdots$ & & $\cdot$ & .0 & \\
\hline . & $\therefore M^{2}$ & b & $\cdots \cdots$ & & $\ldots$ & & \\
\hline$\cdots$ & $\ldots \mathbf{M}^{2}$ &. & $\ldots$ & $\ldots$ & $\therefore$ & $\cdots \cdots \cdots$ & \\
\hline . & $\ldots \mathbf{M}^{2}$ & .1 & $\ldots$ & $\cdots$ & $\cdots$ & $\ldots \ldots$ & \\
\hline$\cdots$ & $\cdots \cdot$ & $\cdots$ & $\ldots$ & $\cdots$ & $\cdots$ & $\cdots \cdots$ & \\
\hline$\cdots$ & $\cdots \cdots$ & $\cdots$ & $\cdots$ & $\cdots$ & $\cdots \cdot$ & $\cdots \cdots$ & \\
\hline$\cdots$ & $\cdots \cdots$ & $\cdots$ & $\cdots$ & $\cdots \cdots$ & $\cdots$ & $\cdots \cdots$ & \\
\hline$\cdots$ & & $\cdots$ & $\cdots$ & $\cdots \cdots$ & $\cdots$ & $\cdots \cdots$ & \\
\hline$\cdots$ & & & $\cdots$ & $\cdots \cdots$ & $\cdots$ & $\cdots$ & \\
\hline & & & $\begin{array}{ll}\cdots & \cdots \\
\cdots & \cdots\end{array}$ & $\left|\begin{array}{cc}\cdots \\
\cdots\end{array}\right|$ & $:$ & $\because$ & \\
\hline s $s$ & & $\cdots$ & $\cdots \cdots$ & $\ldots$ & $\cdots$ & $\cdots$ & \\
\hline tr & & $\ldots d \ldots$ & $\ldots$ & $\ldots$. & $\ldots$ & $\therefore \ldots$ & \\
\hline asDr & R. 1 & $\ldots$ & $\ldots$ & $\cdots \cdot$ & $\cdots$ & $\ldots \ldots$ & \\
\hline - & $\cdots$ & $\cdots$ & $\cdots$ & $\cdots$ & $\cdots$ & $\ldots \ldots$ & \\
\hline . & $\ldots$. & $\cdots$ & $\cdots$ & $\cdots$ & $\cdots$ & $\ldots \ldots$ & \\
\hline . & $\cdots$ & $\cdots$ & $\cdots$ & $\cdots$ & $\cdots$ & $\cdots \cdots$ & \\
\hline .15 & $\dot{ }$ & . & $\cdots$ & - & - & $\cdots$ & \\
\hline .15 & & & 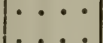 & • & $\cdot 1$ & 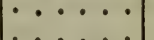 & \\
\hline $\begin{array}{l}\text { la } \\
\text { o }\end{array}$ & & & 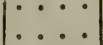 & - & - & 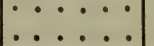 & \\
\hline . & & $a$ & . & $\ldots$. & . & $\ldots \ldots$ & \\
\hline & &. & $\cdots \cdot$ & . & $\cdots$ & $\ldots \ldots$ & \\
\hline N. . &.$S^{2}$. & . b & $\cdots$ & - & $\cdots$ & . & \\
\hline . & & & $\cdot \cdot$ & . & $\cdot$ & $\cdot$ & \\
\hline . & $\mathbf{E}^{2} \cdot . \mathbf{M}^{2}$ & . & $\cdots$ & $\cdots$ & $\cdots$ & - & \\
\hline$\cdot \cdot$ & - & 1. & $\cdot$ & $\cdots$ & . 1 & $\cdots \cdot \cdot$ & \\
\hline & . & & & - & $\cdot$ & $\cdots$ & \\
\hline . & - & & $\cdot$ & - & $\cdots$ & $\cdots$ & \\
\hline t 0 & $2^{\circ}$ & & $\cdot$ & $\cdots$ & $\cdot$ & $\cdots \cdot$ & \\
\hline & & & & $\cdot$ & - & & \\
\hline$\cdot$ & & & & $\because$ & . 1 & 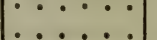 & \\
\hline$\because$ & & $\cdots$ & $\ldots$ & & & $\therefore$ & \\
\hline Kin & & $\cdots$ & $\cdots$ & & & & \\
\hline & & & $\ldots)$ & ... & .. & $\ldots \ldots$ & \\
\hline 1. 2 & $\cdots$ & & & $\cdots$ & . . & $\ldots \ldots$ & . 0 \\
\hline & & & & $\cdots$ & . & $\cdots$ & \\
\hline Sḉн. . . & & & & & - & $\cdots$ & $\bullet$ \\
\hline pirifer So. 157 . & $\because$ & $\cdots$ & $\cdot$ & $\cdots \cdot$ & . & $\cdots$ & $\cdot 0$ \\
\hline 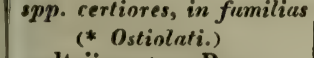 & & & & & & & \\
\hline OB. : & & & & & & & \\
\hline & & & & & & & \\
\hline
\end{tabular}

* spp. puncto pruefixo notatue plicis dichotomi; gaudent, omnes e strutis carboniferii. 


\begin{tabular}{|c|c|c|c|c|c|c|c|}
\hline \multirow[b]{2}{*}{ Benennungen. } & Weltgegend. & KohlenP. & SalzP. & OolithP. & $\begin{array}{r}\text { Krei- } \\
\text { deP. }\end{array}$ & MolasseP. & Neu \\
\hline & 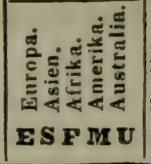 & 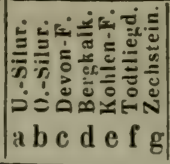 & 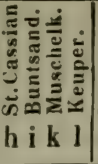 & 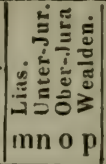 & 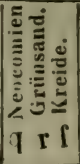 & 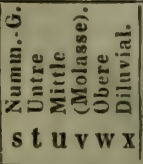 & 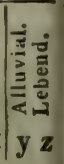 \\
\hline \multicolumn{8}{|l|}{ Spirifer) } \\
\hline ccopt & $E^{2} S^{3} F^{+} M^{3}$ & $\cdots$ & $\cdots$ & $\ldots \cdot$ & $\cdots \cdot$ & $\cdots \cdots$ & \\
\hline Pellicoi VA. . . & $\begin{array}{cc}\cdots \\
\cdots\end{array}$ & $\cdots$ & $\cdots \cdots$ & $\cdots \cdots$ & $\cdots$ & $\cdots \cdots \cdots$ & \\
\hline$-5+2>+2$ & ...... & $\ldots d \ldots$ & $\ldots$ & $\ldots$ & $\cdots$ & $\ldots \ldots$ & \\
\hline CoN. & & $\ldots d \ldots$ & $\ldots$ & $\ldots$ & $\ldots$ & $\ldots \ldots$ & \\
\hline . &.$S^{2} \ldots$ & . b. & $\ldots$ & $\ldots$. & $\ldots$ & $\ldots \ldots$ & \\
\hline LBM. . & $\ldots$ & $\ldots ? d \ldots$ & $\ldots \ldots$ & $\ldots$ & $\ldots$ & $\ldots \ldots$ & \\
\hline - undulatus So. ... & $\cdots \cdot$ & $\ldots d \ldots g$ & $\ldots$ & $\ldots$ & $\cdots$ & $\ldots \ldots$ & \\
\hline o. ..... & $\cdots \cdot \cdot$ & $\ldots d \ldots$ & $\cdots$ & $\cdots$ & $\cdots$ & $\ldots \ldots$ & \\
\hline S SAI & $\cdots \cdot \cdot$ & $a \quad b \ldots .$. & $\cdots \cdots$ & $\cdots$ & $\cdots$ & $\ldots \ldots$ & \\
\hline fragilis $\mathrm{Bt}$ & $\cdots \cdots$ & $\cdots \cdots$ & . . k. & $\cdots \cdot$ & $\cdots$ & $\ldots \ldots$ & \\
\hline Bronnanus KoN. . . & $\cdots \cdot$ & $\cdots d \ldots$ & $\cdots \cdots$ & $\cdots \cdots$ & $\cdots$ & $\cdots \cdots$ & \\
\hline us Bu. & $\cdots$ & $\because \mathrm{d} \cdot \mathrm{g}$ & $\cdots \cdots$ & . 1 & $\cdots$ & $\cdots \cdots$ & \\
\hline $\begin{array}{l}\text { cri: } \\
\text { acu }\end{array}$ & $\cdots \cdots$ & $\cdots c \mathrm{~d} \cdot \cdots$ & $\cdots \cdots$ & $\cdots \cdots$ & $\cdots$ & $\cdots \cdots \cdots$ & \\
\hline $\begin{array}{l}\text { acutus FLEM. . . } \\
\text { heteroclitus Bu. }\end{array}$ & $\ldots$ & $\ldots$ c d ... & $\cdots \cdots$ & $\cdots$ & $\cdots$ & $\cdots \cdots \cdots$ & \\
\hline is Bv. . & .... & . bc .... & $\ldots$ & $\cdots$ & $\cdots$ & $\ldots \ldots$ & \\
\hline z & & & & & & & \\
\hline sulcatus 1 & $\cdots \cdots$ & ? ? .... & ... & $\cdots$ & $\cdots$ & $\ldots \ldots$ & $\cdots$ \\
\hline muralis Veriv. ... & $\cdots$ & $\ldots e \ldots$ & $\cdots \cdot$ & $\cdots$ & $\cdots$ & $\cdots \cdots$ & \\
\hline VERN, & $\cdots \dot{\mathbf{M}}^{3}$ & . c & $\cdots \cdots$ & $\cdots \cdot \mid$ & $\cdots \cdot$ & $\cdots \cdots$ & \\
\hline '0. & $\because M^{3}$ & - c c & $\cdots$ & - & $\cdot$ & $\cdots \cdots$ & \\
\hline Quichu & $\therefore n^{\circ}$ & 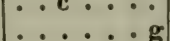 & $\cdots$ & $\cdots \cdots$ & $\cdots$ & $\cdots \cdots \cdots$ & \\
\hline calceola KLI. . . . & & $\cdots$ & h.... & $\cdots$ & $\cdots$ & $\ldots \ldots$ & \\
\hline \multicolumn{2}{|c|}{ MaximilianiLeuchtenbergensisKuI } & $\ldots$ & h... & $\ldots$ & ... & $\ldots \ldots$ & $\cdots$ \\
\hline (**) inerturati, & $F^{2}$ & 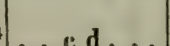 & & & & & \\
\hline - ap & $\ldots \ldots$ & {$\left[\begin{array}{ccc}\cdots & 0 & \cdots \\
\cdots & 0 & \cdots\end{array}\right.$} & $\cdots \cdots$ & $\cdots \cdots$ & $\cdots$ & $\cdots \cdots$ & \\
\hline calcaratus So. . . & .... & . c d... & $\ldots$ & ... & ... & $\ldots \ldots$ & \\
\hline ardi VerN. . . & & ..... & $\ldots$ & $\ldots$ & ... & $\ldots \ldots$ & - \\
\hline Glinkaanus Vers. &.$S^{2} \ldots$ & ...... & $\ldots$ & $\ldots$ & $\ldots$ & $\ldots \ldots$ & \\
\hline us Bu. .. & $\cdots$ & $\ldots \mathrm{c} \ldots .$. & $\cdots$ & $\ldots$ & $\cdots$ & $\ldots \ldots$ & \\
\hline squensis VeRN. . & & $\ldots d \ldots$ & $\cdots$ & $\ldots$. & $\cdots$ & $\ldots \ldots$ & $\cdots$ \\
\hline cus Kon. . & & 25 & $\cdots \cdots$ & $\cdots \cdots$ & $\cdots$ & $\cdots \ldots$ & \\
\hline So..... & $\cdots \dot{\mathbf{x}^{\circ}}$ & $\cdots d \ldots$ & $\cdots \cdots$ & $\cdots \cdot$ & $\cdots \cdot$ & $\cdots \cdots$ & \\
\hline - & $\dot{\mathrm{F}}^{2} \dot{\mathrm{S}}^{2} \mathrm{M}^{3}$ & - d & $\cdots$ & $\cdots$ & $\cdot$ & $\cdots \cdots$ & \\
\hline$\cdots$ & $\mathbf{E}^{2} \cdot \mathbf{M}^{3}$ & $\begin{array}{l}\text { a } \\
\text { d }\end{array}$ & $\cdot$ & & $\therefore$ & $\cdots$ & \\
\hline $\begin{array}{l}\text { atus VERs. } \\
\text { ellosus Kon. }\end{array}$ & ... & $\ldots d \ldots$ & $\ldots$ & $\cdots \cdot$ & $\cdots$ & $\ldots \ldots$ & 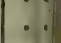 \\
\hline - Tasmaniensis MorR. & . $U^{4}$ & $\ldots d \ldots$ & $\cdots \cdot$ & $\cdots \cdot$ & $\cdots$ & $\ldots \ldots$ & . \\
\hline Ihaui Bu. . . . . & $\mathbf{E}^{1} \ldots$ &.$d$. & $\cdots$ & $\cdots \cdot \cdot$ & $\cdots$ & $\cdots \cdots$ & - \\
\hline isi Ktı. . . . . & $\cdots$ & $\bullet$ & h $\cdot$ & $\cdots \cdots$ & $\cdots$ & $\cdots \cdots$ & - \\
\hline$\underset{*}{\text { Buchi KLI. }} \cdots \cdots$ & & & th & & $\cdots \cdot$ & $\cdots \cdots$ & \\
\hline$u m$ & & & & & & $\cdots \cdots$ & \\
\hline
\end{tabular}




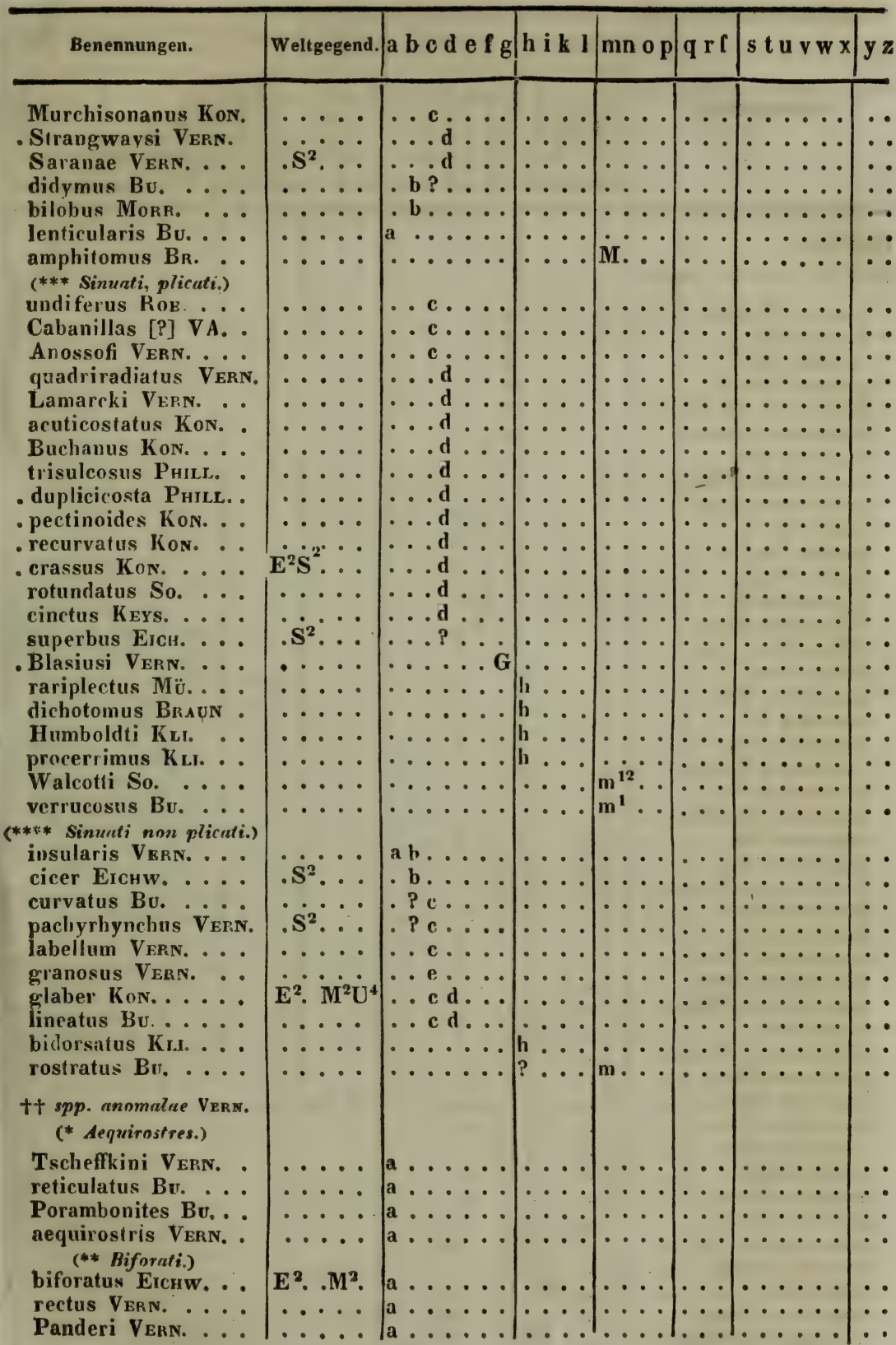




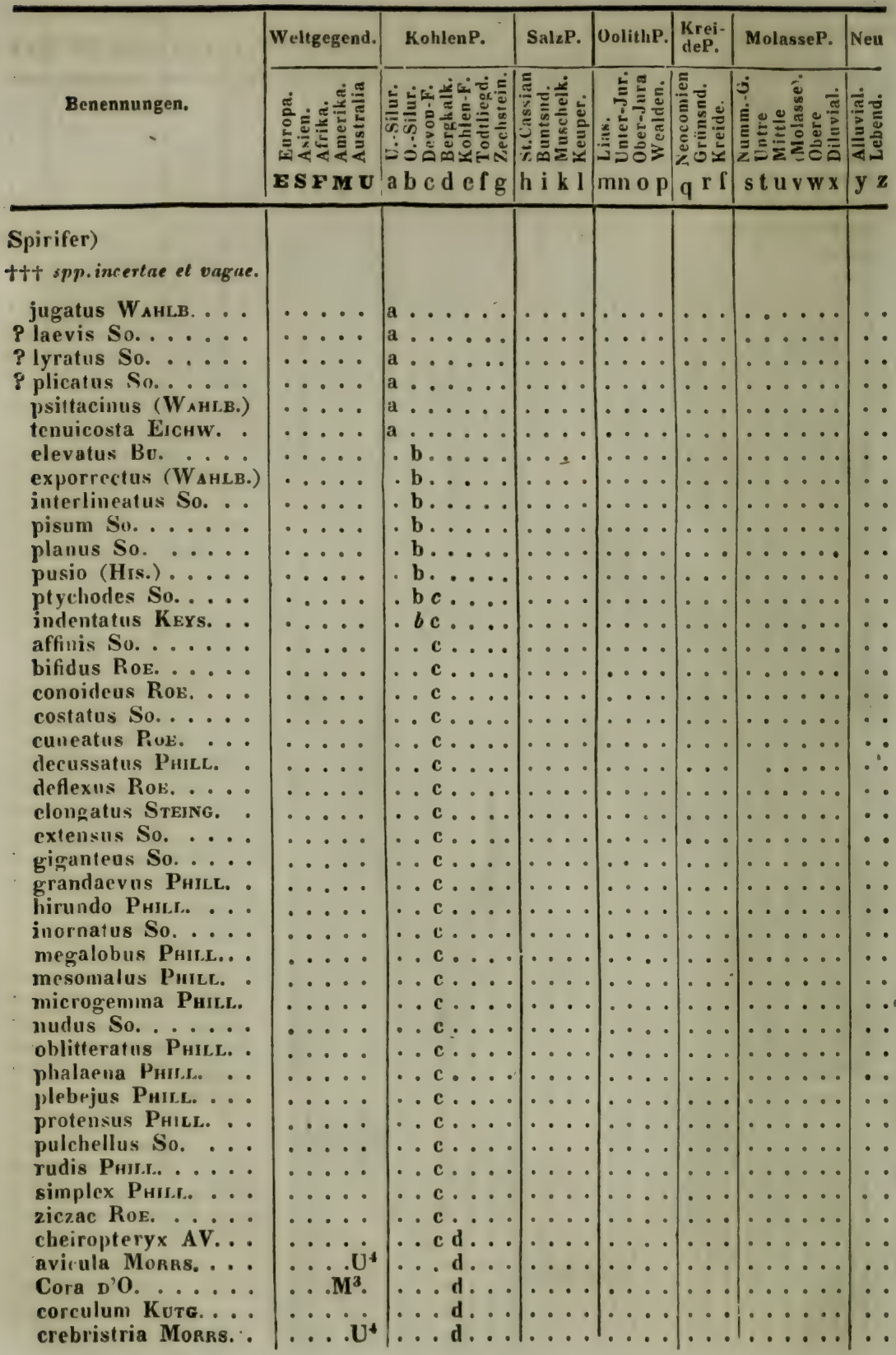




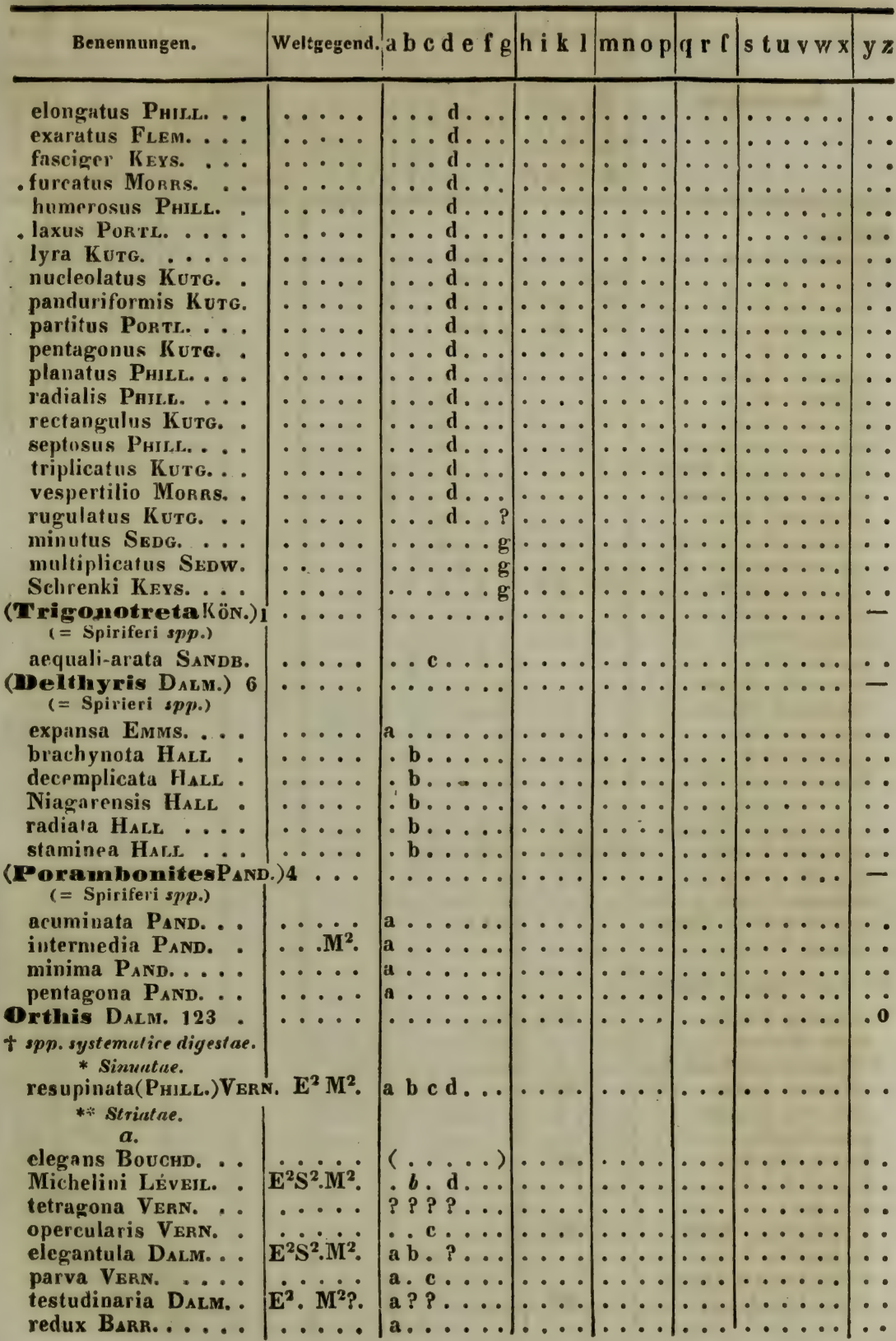




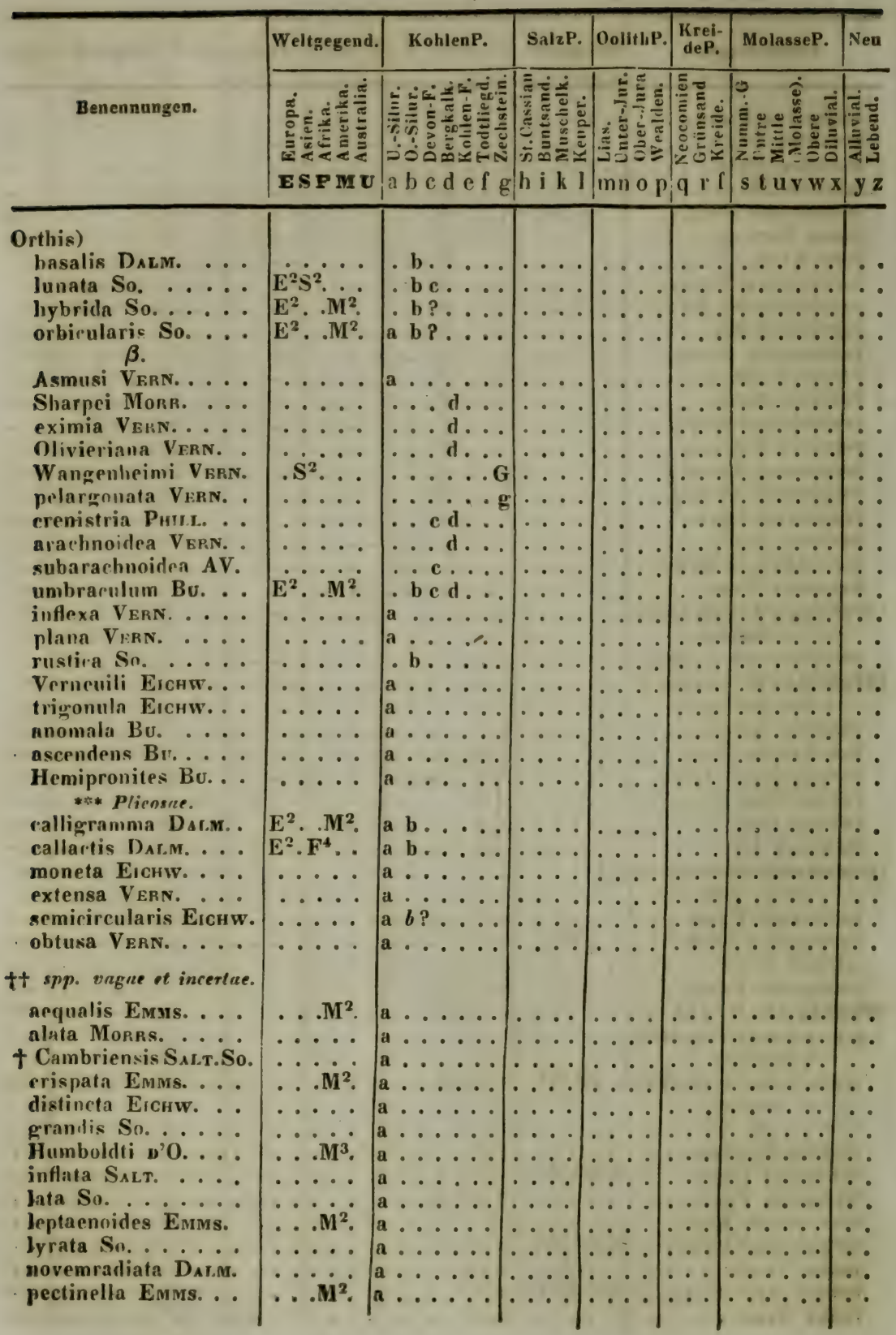




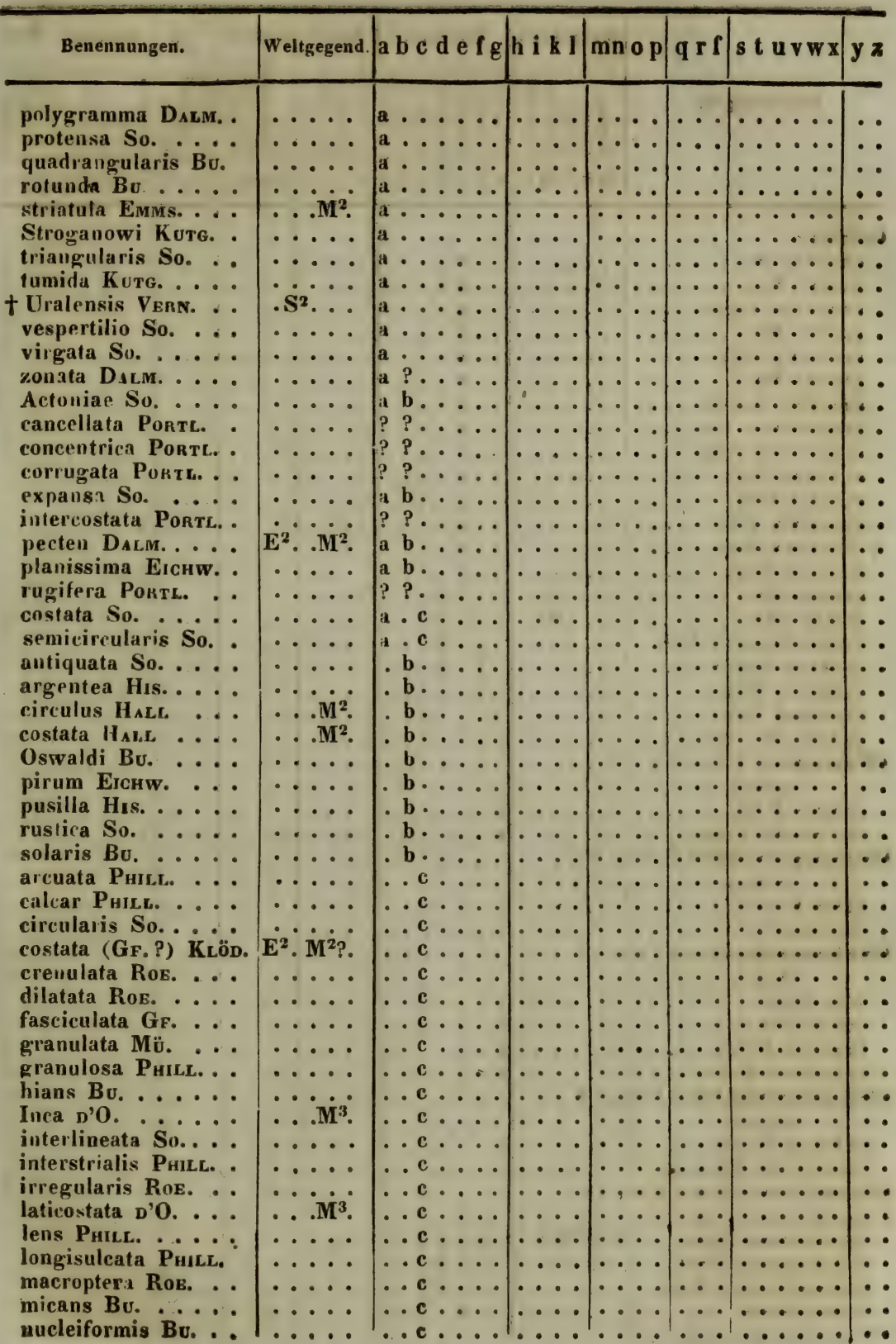




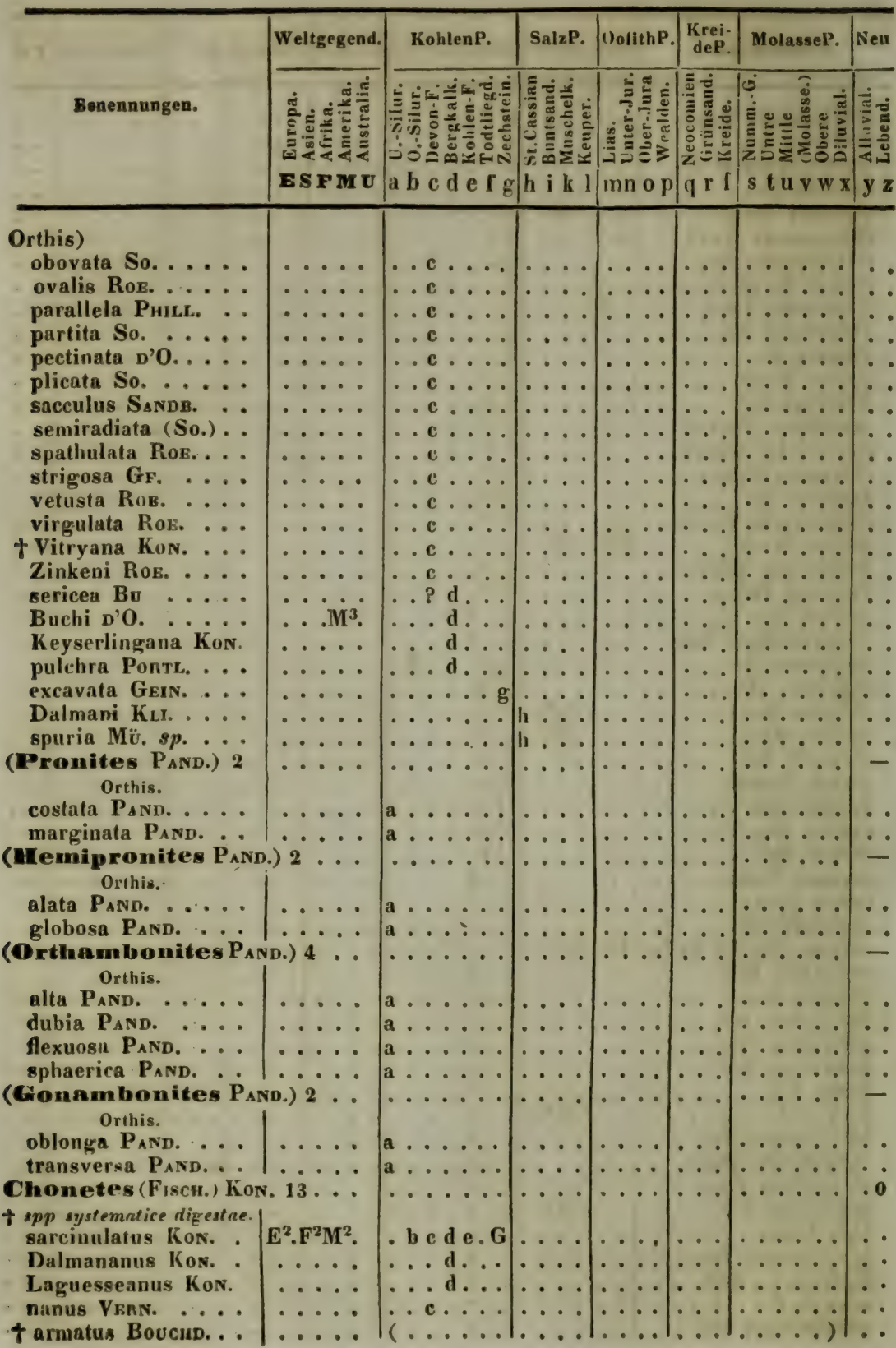




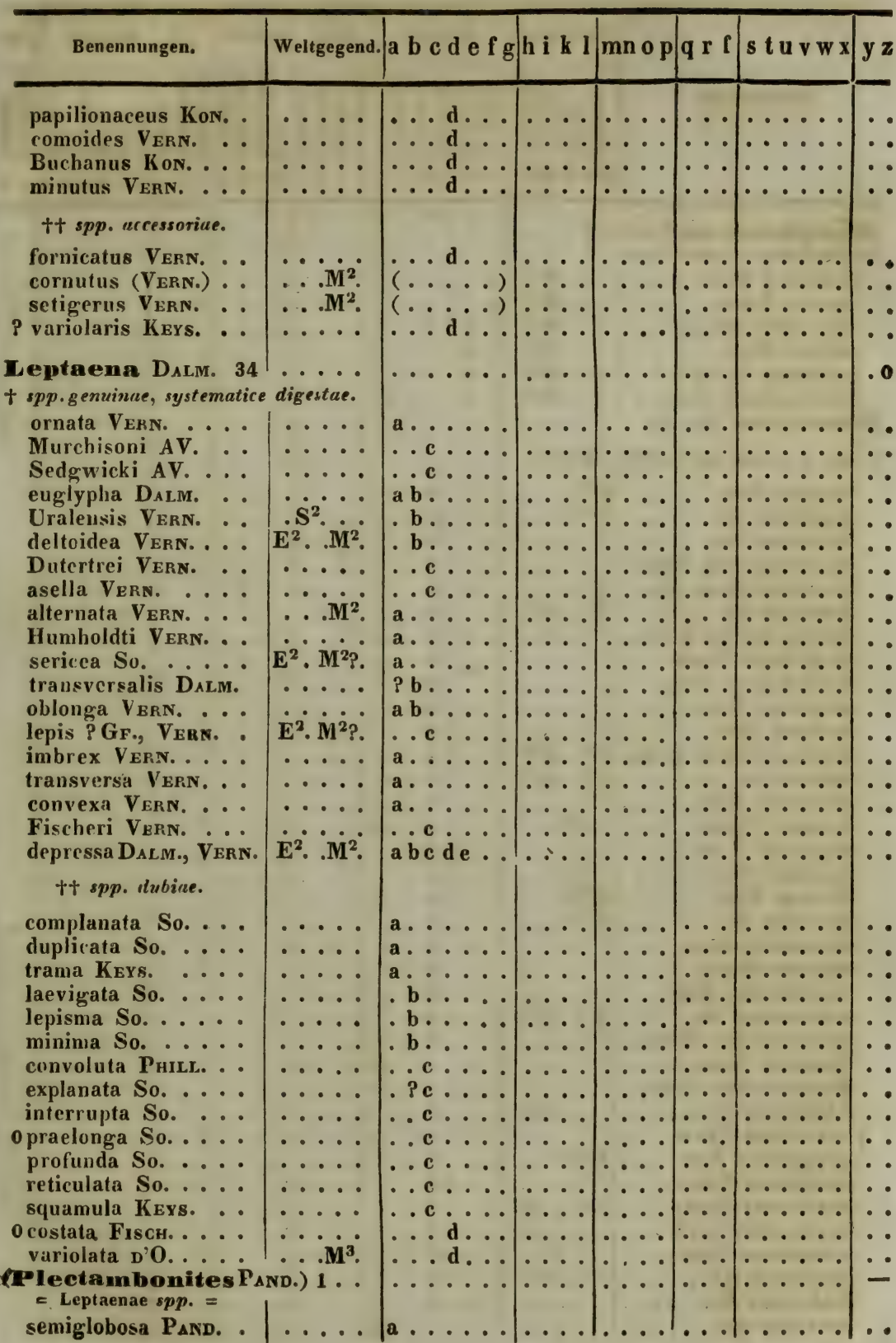




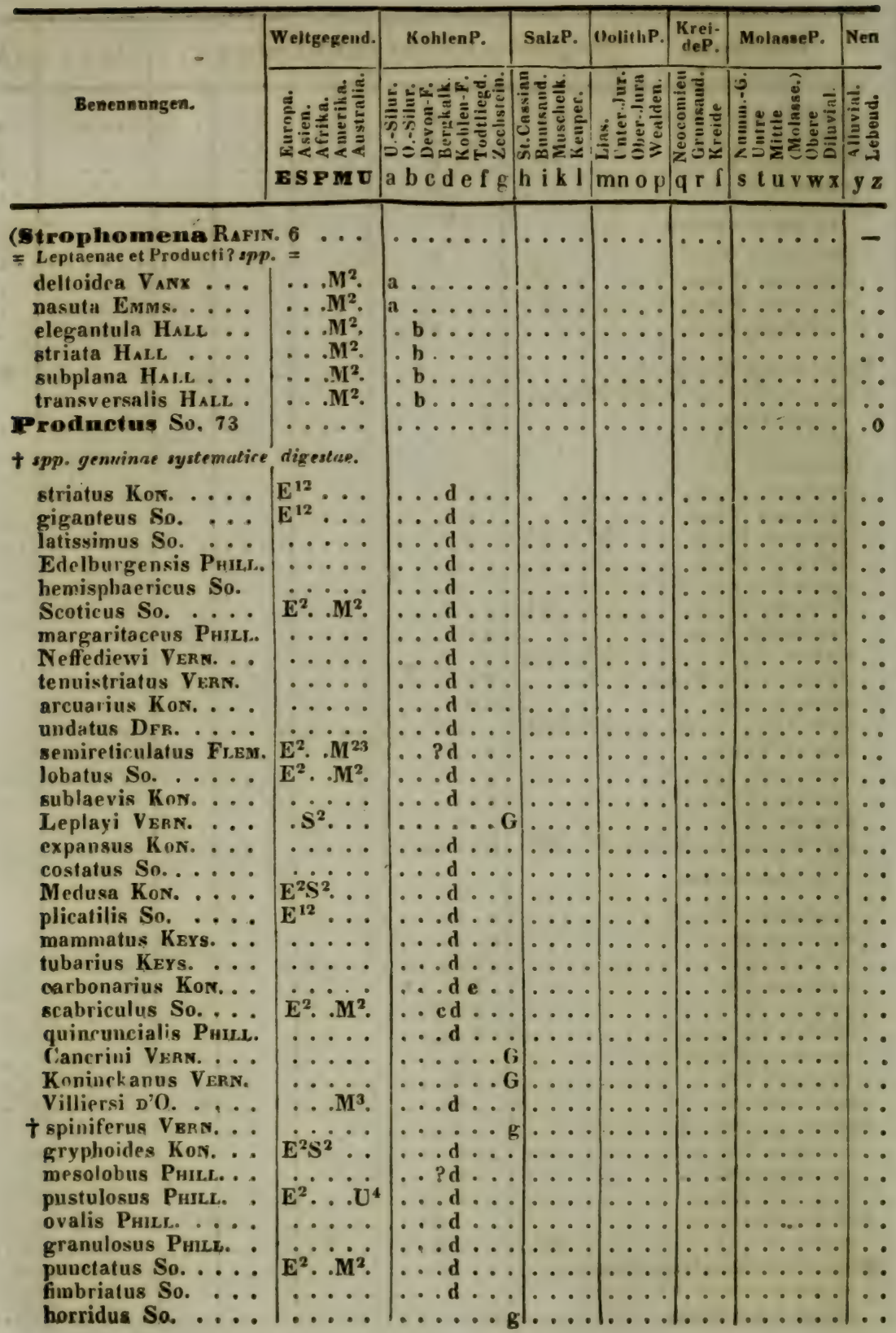




\begin{tabular}{|c|c|c|c|c|c|c|c|}
\hline Bene & d. & $\mathrm{g} \mid$ & & $\mathbf{p}$ & I & $\mathbf{v} \mathbf{x}$ & \\
\hline ho & & $\cdot \mathbf{G}$ & . & $\cdots$ & $\cdots$ & $\cdots \cdots \cdots$ & \\
\hline $\begin{array}{r}H_{u} \\
+\mathrm{M}\end{array}$ & $\mathbf{E}^{2} \cdot \mathbf{M}^{3}$ & . & & & & 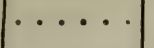 & \\
\hline сн. & $\begin{array}{l}\cdots \\
\cdots\end{array}$ & $\begin{array}{l}. \mathrm{g} \\
. .\end{array}$ & $\cdots \cdots$ & $\cdots \cdots$ & - & $\cdots \cdots$ & \\
\hline pe & & $\ldots$ & $\ldots$ & $\ldots$ & $\cdots$ & $\cdots \cdots$ & \\
\hline & & & $\cdots$ & $\cdots$ & $\cdots$ & $\cdots \cdots$ & \\
\hline & & $\begin{array}{l}\cdot ? c d \\
\text {. c }\end{array}$ & $\cdots \cdots$ & $\begin{array}{ll}\cdots \\
\cdots\end{array}$ & & $\because$ & \\
\hline & & $\ldots d \ldots$ & $\cdots$ & $\cdots$ & $\therefore$ & $\ldots \ldots$ & \\
\hline & - & $\cdots$ & $\cdots \cdots$ & $\cdots$ & $\cdots$ & $\ldots \ldots$ & \\
\hline & & & h. & $\cdots \cdot$ & $\cdots$ & $\cdots$ & \\
\hline ue. & & & & & & & \\
\hline & & & $\begin{array}{ll}\cdots \\
\cdots\end{array}$ & & & $\ldots \ldots$ & \\
\hline & $\mathrm{E}^{2} \mathrm{~S}^{2} \ldots$ & .6 & $\cdots \cdot$ & $\ldots$ & $\cdots$ & $\ldots \ldots$ & \\
\hline U. .. & $\ldots$ & $\ldots$ & $\cdots$ & $\cdots \cdot$ & $\cdots$ & $\ldots \ldots$ & \\
\hline$\cdots$ & 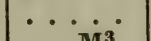 & $\cdots$ & $\cdots \cdots$ & $\cdots \cdots$ & $\cdot$ & $\cdots \cdots$ & \\
\hline -. & . . $\mathrm{M}^{3}$. & $\cdots$ & $\cdots \cdots$ & $\cdots \cdots$ & $\cdots$ & $\cdots \cdots$ & \\
\hline . & $\cdots$ & $\cdots$ & $\cdots$ & $\cdots \cdots$ & $\cdots$ & $\cdots \cdots$ & \\
\hline & $\cdots$ & $\cdots$ & $\cdots \cdot$ & $\cdots \cdot$ & $\cdots$ & $\cdots \cdots$ & \\
\hline . & $\ddot{\bullet}$ & & $\left|\begin{array}{l}\cdots \\
\cdots \\
\cdots\end{array}\right|$ & . & $\because$ & $\cdots$ & \\
\hline 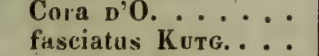 & $\ldots$ & $\cdots d \ldots$ & 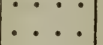 & $\cdots \cdots$ & $\because \cdots$ & $\because$ & \\
\hline$\cdots$ & $\mathrm{E}^{2} \cdot \mathrm{M}^{3}$ & $\ldots d \ldots$ & $\ldots$. & $\ldots$ & $\ldots$ & $\cdots \cdots$ & \\
\hline & & $\cdots$ & $\cdots$ & $\cdots \cdot$ & $\cdots$ & $\ldots \ldots$ & \\
\hline . & $\cdots$ & $\cdots$ & $\cdots$ & $\ldots$ & $\cdots$ & $\ldots \ldots$ & \\
\hline . & $\cdots$ & $\cdots$ & $\cdots$ & $\cdots \cdots$ & $\cdots$ & $\ldots \ldots$ & \\
\hline$\cdot$ & $\cdots$ & & $\cdots$ & $\cdots \cdots$ & $\cdots$ & $\cdots \cdots$ & \\
\hline$\cdots$ & & $\cdots$ & $\cdots$ & $\cdots \cdots$ & $\cdots$ & $\cdots \cdots$ & \\
\hline$\dot{.}$ & $\because$ & $\cdots$ & $\because$ & $\left|\begin{array}{c}\cdots \\
\cdots \\
\cdots\end{array}\right|$ & $\cdots$ & $\because$ & \\
\hline$\therefore$ & ... $\mathrm{M}^{3}$ & $\ldots d \ldots$ & $\ldots$ & $\ldots$ & $\ldots$ & $\ldots \ldots$ & • \\
\hline & . & $\cdots$ & $\cdots$ & $\ldots$ & $\cdots$ & $\ldots \ldots$ & . \\
\hline & $\cdots$ & & $\cdots \cdot$ & $\cdots$ & . & $\ldots \ldots$ & \\
\hline & & & 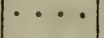 & $\cdot$ & . & $\cdots \cdots$ & \\
\hline & & & 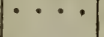 & $\cdots$ & • & $\cdots \cdots$ & \\
\hline w. & & & & & & $\cdots \cdots$ & • \\
\hline$\cdot$ & $\cdots$ & & $\cdots$ & $\ldots$ & ... & $\ldots \ldots$ & • \\
\hline & & & $\cdots \cdot$ & $\cdots \cdot$ & $\cdots$ & $\ldots \ldots$ & - \\
\hline & & & & & $\cdots$ & $\ldots \ldots$ & \\
\hline Duniontana Kov. . . & $\cdots \cdots$ & & & & ‥ & & \\
\hline \multicolumn{2}{|c|}{ Brachiopod. genuin. summa: 952} & 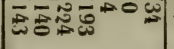 & $0-6 n$ & $\omega^{20}=$ & $\overrightarrow{\mathrm{w}} \overline{\mathrm{E}}$ & $-\frac{2}{2}-0,0$ & $0 \mathscr{A}^{2}$ \\
\hline ? & & & & & & & \\
\hline & & & . & & & $\cdots$ & .7 \\
\hline & & & & & & $\cdot$ & - \\
\hline L. • & & & & & $\cdots$ & $\because$ & . \\
\hline- & & & & & & & \\
\hline
\end{tabular}




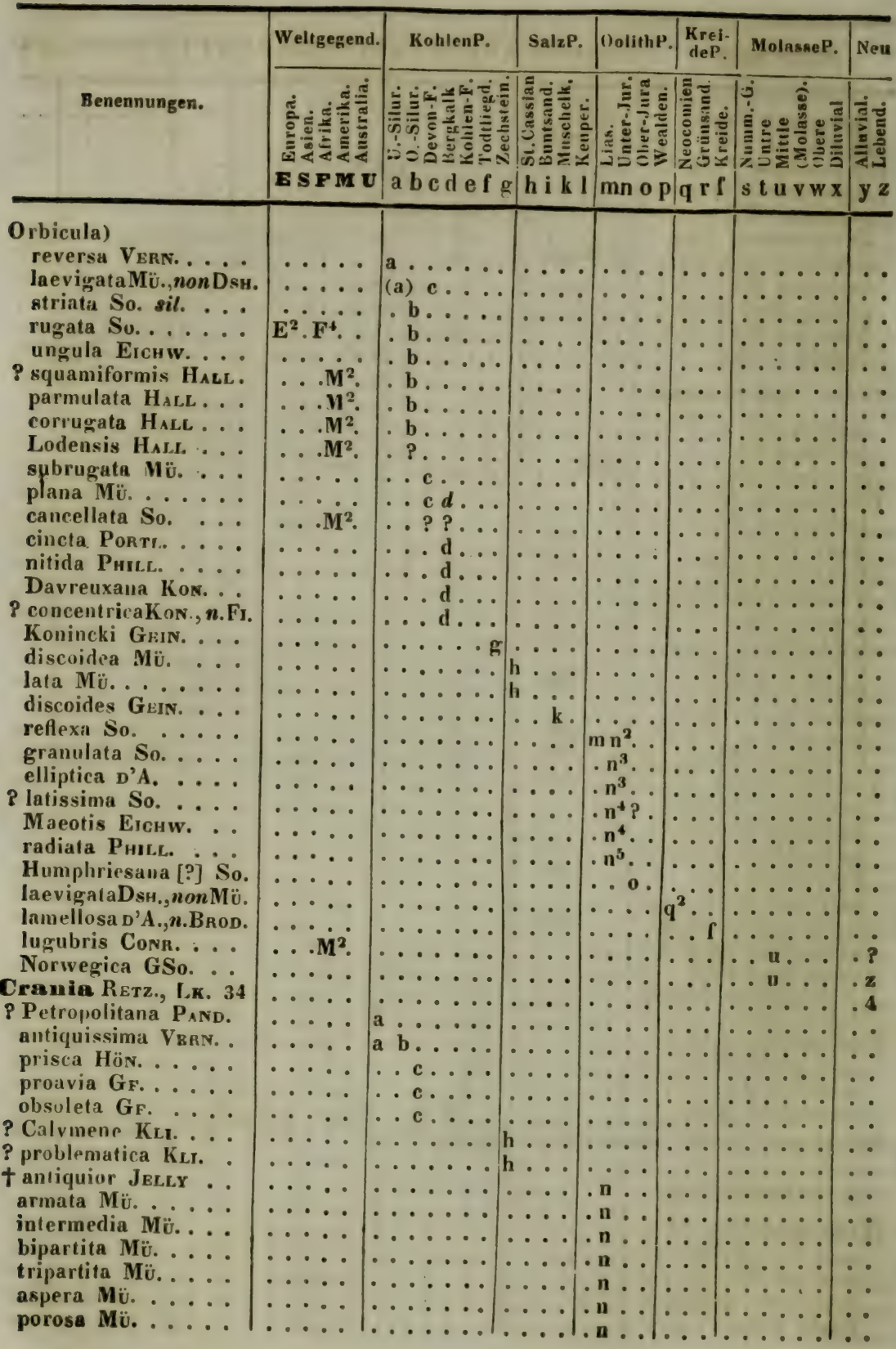




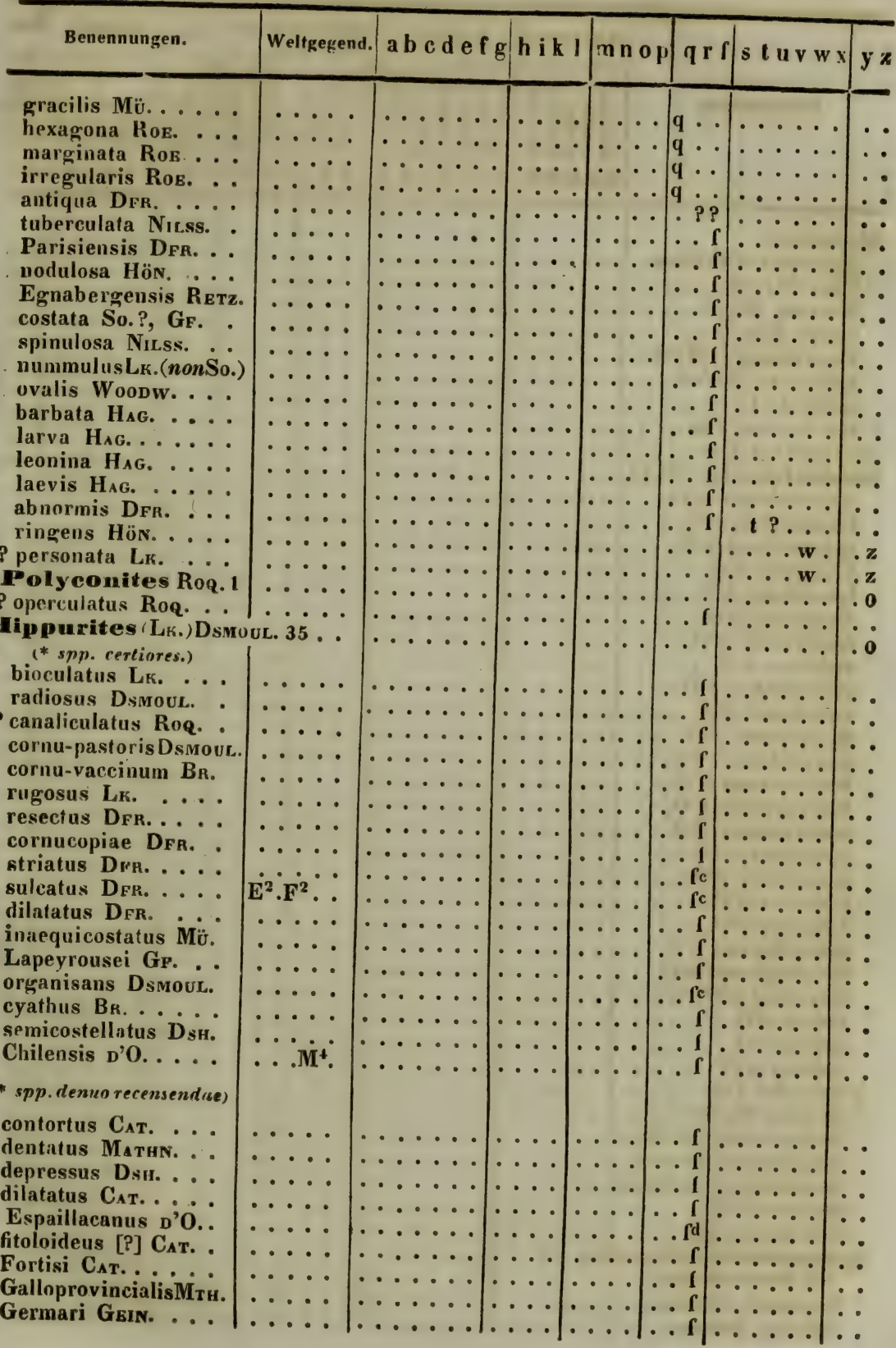




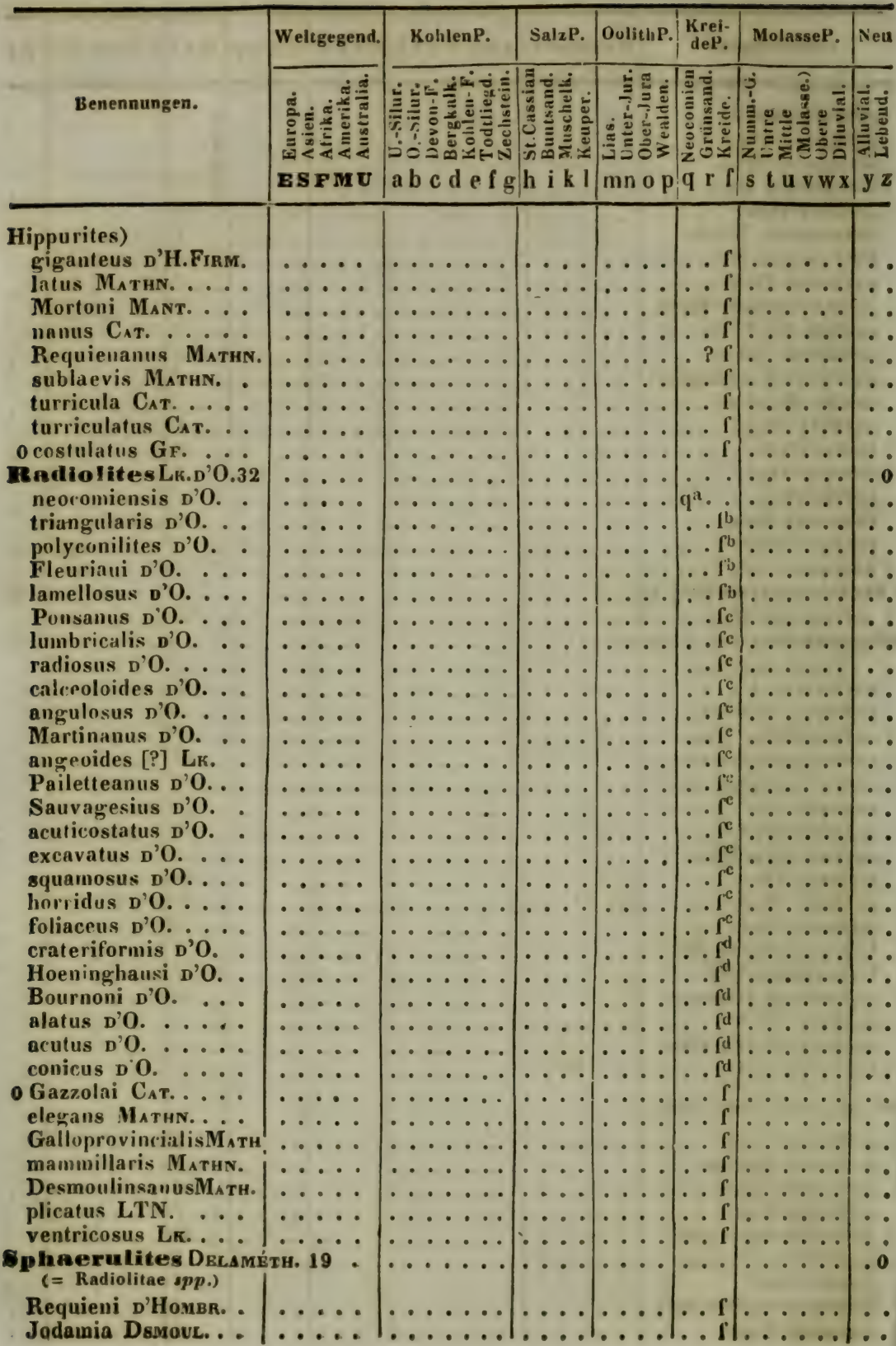




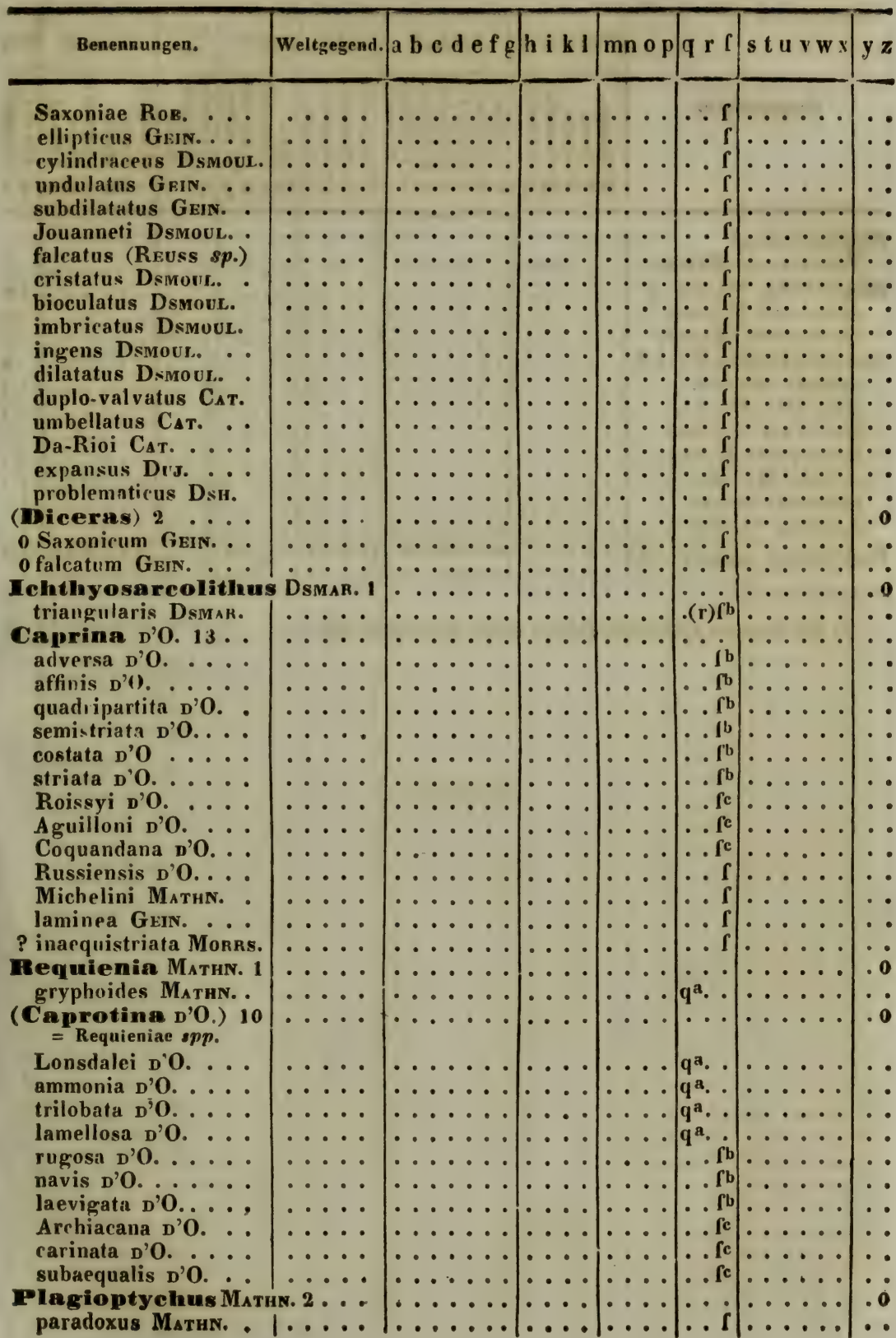




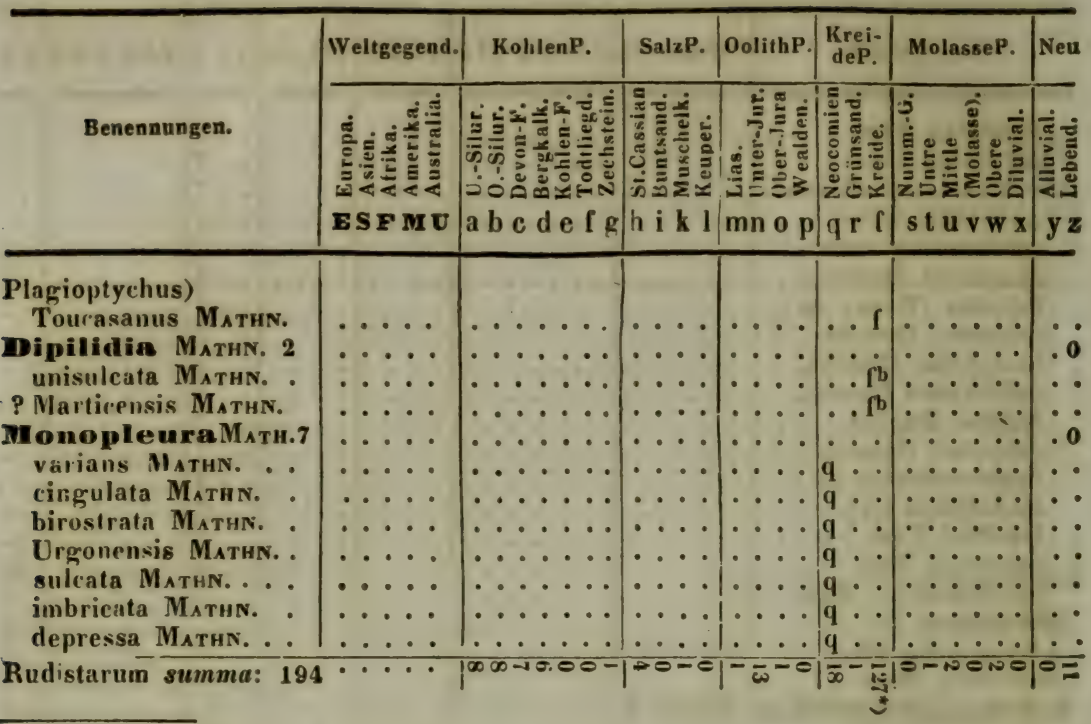

*) Hic numerus synonymis rejectis valde reducendus erit. 


\begin{tabular}{|c|c|c|c|c|c|c|c|}
\hline & Weltgegend. & KohlenP. & SalzP. & UolithP. & $\begin{array}{l}\text { Krei- } \\
\text { deP. }\end{array}$ & MolasseP. & Neu \\
\hline Benennungen. & 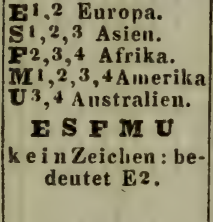 & 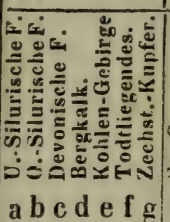 & 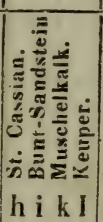 & 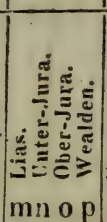 & 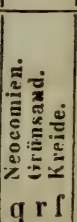 & 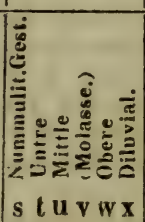 & 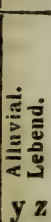 \\
\hline
\end{tabular}

\section{Cl. X. PELECYPOI)A, Beilfüsser.}

(Conchifera Lк., Lamellibranchia Bцv., Elatobranchia Мкв.)

\section{MONOMYA Lr. (emend.), Einmuskeler.}

\section{(= INTEGRIPALLIATA. $=)$}

a. Ostracea.

Anomia L. 30 ... jurensis MORRs. . semistriata BEAN .. convexa So.

laevigata So. .... radiata So.

\section{costulata Roe.}

lamellosa Roe. sinistrorsa SEnR. .. exscissa Reuss ... subradiata Reuss argentaria MoRx. tellinoides Мокт... truncata GeIN. ... tenuistriata DsH... t orbicularis DsM. . † profunda DsM. † dubia Dsh...... ? rugosa Nrst .... ephippium L. . . . undulata LGm. . . . costata Br. margaritacea Pol. . asperella PhIL. . . . orbiculata Brocc.
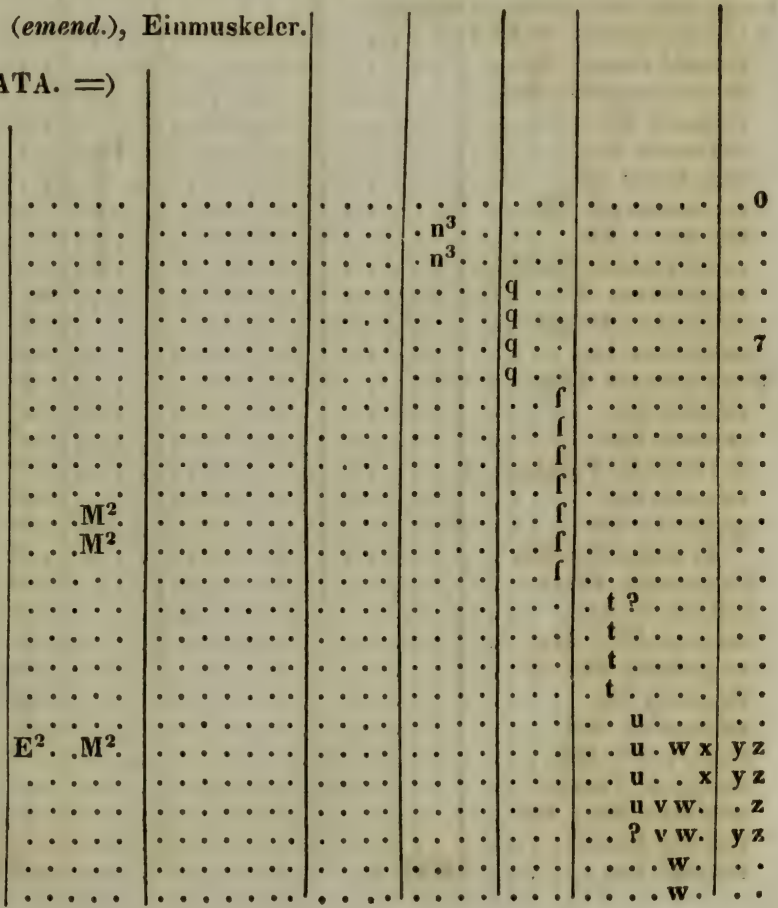


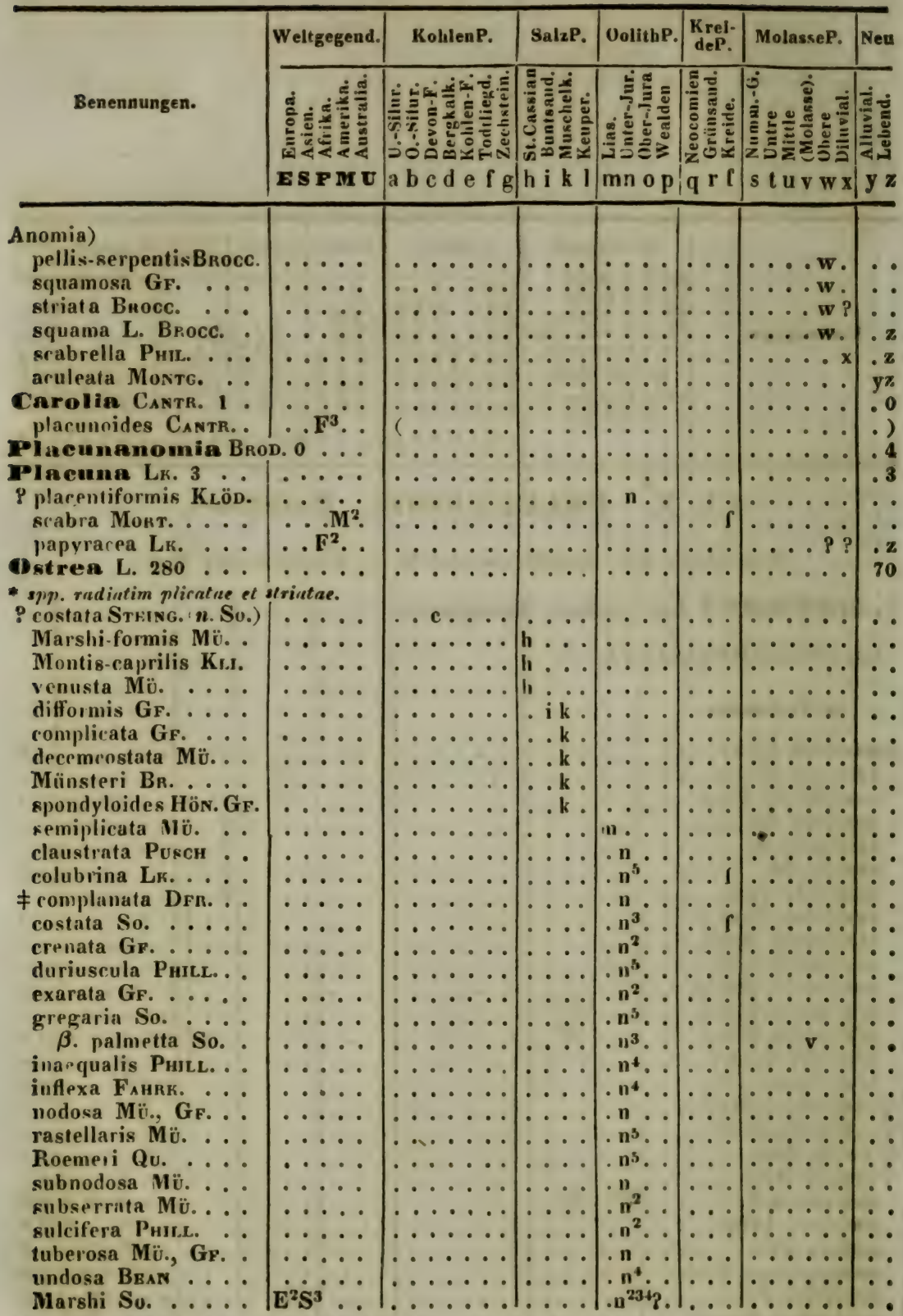




\begin{tabular}{|c|c|c|c|c|c|c|c|}
\hline & egend. & f $g$ & h i k l & $\mathbf{p}$ & $|q \times 0|$ & $\mathrm{st}$ & \\
\hline & & & & o. & & & \\
\hline 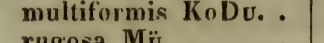 & & & & . 0 & & & \\
\hline . & - & $\cdots \cdots$ & & . 0 . & & $\because$ & \\
\hline 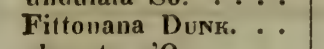 & . & & & & & & \\
\hline & $\ldots \mathbf{M}^{\mathbf{3}}$ & . & $\ldots$ & $\ldots$ & $9 \cdot \cdots$ & $\ldots \ldots$ & \\
\hline & $\mathrm{M}^{3} \cdot$ & & $\cdots \cdot$ & & & $\ldots \ldots$ & \\
\hline & $\ldots \mathbf{M}^{3}$ & & & & & $\cdots \cdots$ & \\
\hline & $\cdots$ & & & & & $\cdots \cdots$ & \\
\hline & $\because$ & & $\bullet$ & & & $\cdots$ & \\
\hline & & & - & $\cdots \cdots$ & & $\cdots$ & \\
\hline . & & & &. & & $\cdots$ & \\
\hline$\ldots$ & & $\because$ & $\mid \begin{array}{c}\cdots \\
\cdots\end{array}$ & & & $\cdots$ & \\
\hline DFr. & & .. & $\ldots$ & $\mid \begin{array}{ll}\cdots & \cdots \\
\cdots & \cdots\end{array}$ & & $\cdots \cdots$ & \\
\hline FR.. & & & $\ldots$ & $\cdots$ & . $r i$ & $\ldots \ldots$ & \\
\hline$\cdots$ & - & & & $\ldots$ & - ri & $\ldots \ldots$ & \\
\hline$\cdots$ & $\cdots$ & $\cdots$ & $\cdots \cdot$ & $\ldots$ & $\cdots 1$ & $\cdots$ & \\
\hline$\cdots$ & - $\dot{M}^{\circ}$ & $\cdots$ & $\cdots \cdot$ & $\cdots \cdots$ & . . I & $\ldots \ldots$ & \\
\hline$\cdots$ & $\ldots M^{2}$ & & $\cdots \cdot$ & $\cdots$ & $\ldots f$ & $\cdots$ & \\
\hline$\cdots \cdot$ & $\cdots$ & $\cdots \cdots$ & $\cdots$ & $\cdots \cdot$ & & $\cdots \cdots$ & \\
\hline er & $\cdots$ & - & $\cdot \cdot$ & $\cdots$ & .. f & $\cdots \cdots$ & \\
\hline . . & $\cdot$ & $\cdots$ & $\cdots$ & $\cdots$ & {$\left[\begin{array}{cc}\cdots \\
0\end{array}\right]$} & $\cdots$ & \\
\hline & & & - & $\cdots$ & & $\cdots$ & \\
\hline$\cdots$ & & & - & $\cdot 1$ & & . & \\
\hline $\begin{array}{l}\cdots \\
\cdots\end{array}$ & & & & $\because \cdots$ & $\begin{array}{lll}\cdots & 1 \\
\cdots & f\end{array}$ & $\cdots$ & \\
\hline$\ddot{\cdots}$ & $\therefore \mathrm{M}^{2}$ & & $\because$ & $\because \cdots$ & $\therefore$ f & $\cdots$ & \\
\hline … & . & $\therefore$ & •. & $\ldots \ldots$ & . . $\mathrm{f}$ & $\ldots$ & \\
\hline ... & . $\mathbf{M}^{2}$ & .. & $\cdots$ & $\ldots \ldots$ & . . $\mathrm{l}$ & $\ldots \ldots$ & \\
\hline$\cdots$ & $\cdots$ & $\cdots$ & $\ldots$. & $\ldots$ & . . ? & $\ldots \ldots$ & \\
\hline$\cdots$ & $\cdot \cdot$ & $\cdots$ & $\cdots \cdots$ & $\cdots$ & $\ldots 1$ & $\ldots \ldots$ & \\
\hline & $\cdots \cdots$ & $\ldots \ldots$ & - & $\cdots$ & & $\ldots \ldots$ & \\
\hline FR.) & & • & & $\cdots$ & . . 1 & $\cdots \cdots$ & \\
\hline$\cdots$ & . & $\cdot$ & $\cdots$ & & $\because 1$ & & \\
\hline$\cdots$ & & & & & .? & $t ? \ldots$ & \\
\hline - & & & & & $\cdots$ & • & \\
\hline$\therefore$ & $\dot{\mathrm{M}}^{4}$ & & & & & & \\
\hline - & & & & $\cdots \cdots$ & $\ldots$ &.$t$ & \\
\hline$\therefore$ & & $\cdots$ & $\ldots$ & $\ldots$. & . & .. & \\
\hline . & & $\cdots$ & $\cdot$ & & $\cdot$ & . $t$ & \\
\hline is & & & & $\cdots$ & & . t $\ldots$ & \\
\hline 1 & & & 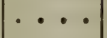 & $\cdots$ & $\cdots$ & $\cdot \mathbf{t} \cdots$ & \\
\hline$\cdots \cdot$ & $\cdots$ & $\bullet^{\circ}$ & $\cdots \cdots$ & & - & $\cdot t \cdots$ & \\
\hline$\cdots$ & & & & & & & \\
\hline & & & & & & & \\
\hline & & & & & & & \\
\hline • & - & $\cdots \cdots \cdots$ & $\cdots \cdot$ & $\cdots \cdot$ & $\ldots$ & .. & \\
\hline$\therefore$ & & $x^{2}=0$ & & $\cdots$ & $\cdots \cdot$ & . $t$ & \\
\hline 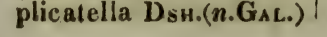 & $\ldots$ & 我 & . & $1 . \cdots$ & & & \\
\hline
\end{tabular}




\begin{tabular}{|c|c|c|c|c|c|c|c|}
\hline \multirow[b]{2}{*}{ Benennungen. } & Weltgegend. & KohlenP. & ialzP. & OolithP. & $\begin{array}{l}\text { Krei- } \\
\text { deP. }\end{array}$ & MolasseP. & \\
\hline & 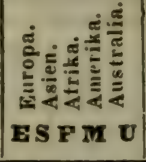 & 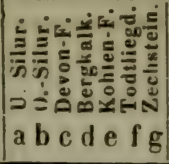 & 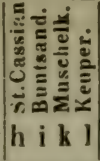 & 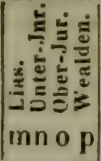 & 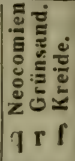 & 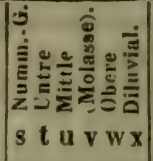 & \\
\hline \multicolumn{8}{|l|}{ strea) } \\
\hline$\cdots$ & $\cdots \cdots$ & $\cdots \cdots$ & $\cdots \cdot$ & $\ldots$ & $\cdots$ & . t... & \\
\hline a GF. & $\dot{\mathrm{E}}^{2} \cdot \dot{\mathrm{M}}^{2}$ & & $\cdots \cdot$ & $\cdots \cdot \mid$ & & . $\ldots .$. & \\
\hline ula LK. . . . & $\cdots$ & • • • • & $\cdots$ & $\left|\begin{array}{c}\cdots \\
\cdots \\
\cdots\end{array}\right|$ & $\cdots$ & 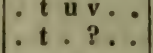 & \\
\hline s. & $\dot{F}^{2} \dot{S}^{3} \cdots$ & $\cdots \cdots$ & $\ldots$ & $\ldots$. & $\cdots$ & . u v v . & \\
\hline a Lк. . . . . & $\begin{array}{l}\mathrm{E}^{2} \mathrm{~S}^{3} \cdot \dot{\mathrm{M}^{2}} \\
\mathrm{E}^{2} \cdot \mathrm{M}^{2}\end{array}$ & $\cdots \cdots$ & $\cdots \cdot$ & $\ldots$. & $\cdots$ & . t u. ? . & \\
\hline ......... & & $\cdots \cdots$ & $\cdots$ & $\cdots \cdots$ & $\cdots$ & . t u. ? . & \\
\hline$\cdots$ & $\mathrm{S}^{3} \cdots$ & $\cdots$ & $\cdots \cdots$ & $\cdots \cdots$ & $\because \cdots$ & . t u v w x & \\
\hline$\cdots$ &. $\mathrm{S}^{3} \ldots$ & $\ldots \cdots$ & $\cdots$ & $\cdots$ & $\ldots$ & . $\begin{array}{l}\text { ? } \ldots \\
\ldots\end{array}$ & \\
\hline &.$S^{3} \ldots$ & $\ldots \ldots$ & $\ldots$ & $\ldots$ & $\ldots$ & $\therefore$. & \\
\hline$\cdots$ & $\ldots$ & $\cdots \cdots$ & $\ldots \ldots$ & $\ldots$ & $\cdots$ & $\mid \begin{array}{ccc}\cdots & \cdots & \cdots \\
\cdots & \text { u. } & \ldots\end{array}$ & \\
\hline 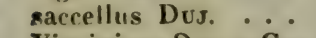 & 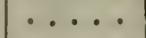 & $\ldots \ldots$ & $\ldots$ & $\ldots \ldots$ & $\ldots$ & $\therefore$ u. & \\
\hline ,n.Giм. & & $\cdots \cdots$ & $\ldots$ & $\ldots$ & $\cdots$ & $\ldots u$ & \\
\hline$\cdots$ & $\therefore M^{2}$. & $\cdots \cdots$ & $\cdots \cdots$ & $\cdots$ & $\cdots$ & . u.w. & \\
\hline$\therefore \cdots$ & $\mathbf{E}^{2}\left(\mathbf{F}^{3}\right)$ & $\cdots$ & $\cdots \cdots$ & $\cdots \cdots$ & $\cdots$ & ..u. w. & \\
\hline и.,n.Dsis. & $\dot{\mathrm{E}}^{2} \cdot \dot{\text { (I }}$ & $\cdots \cdots$ & $\cdots \cdots$ & $\cdots$ & $\cdots$ & . . u v v w. & \\
\hline $\begin{array}{l}\text {,n.Dsh. } \\
\text { ERR. . }\end{array}$ & $\begin{array}{c}\mathbf{E}^{2} \cdot\left(\mathrm{M}^{2}\right) \\
\ldots .\end{array}$ & $\ldots \ldots$ & $\cdots \cdots$ & $\cdots$ & $\cdots$ & . . u v v w & \\
\hline ERR. & $\cdots$ & $\ldots \ldots$ & $\cdots$ & $\cdots \cdot \mid$ & $\cdots$ & 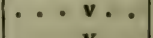 & \\
\hline$\therefore$ & $\cdots \cdot$ & $\ldots \ldots$ & $\cdots$ & $\cdots \cdots$ & $\cdots$ & $\cdots \mathbf{v} \cdots$ & \\
\hline$\cdots$ & . & $\cdots$ & $\ldots$ & $\cdots \cdots$ & $\because \cdots$ & $\begin{array}{ll}\cdots & \mathbf{v} \\
\cdots & \mathbf{v}\end{array}$ & \\
\hline HN. . & .. & . & ... & $\ldots$ & $\ldots$ & ... v. & \\
\hline$\cdots$ & . & $\cdots$ & $\cdots$ & $\ldots$ & $\ldots$ & $\ldots v \ldots$ & \\
\hline$\cdots$ & $\cdots$ & $\cdots \cdots \cdots$ & $\ldots$ & $\ldots$. & $\cdots$ & $\ldots v$ & \\
\hline$\cdots$ & .... & $\cdots \cdots$ & $\ldots$ & $\cdots$ & $\cdots$ & $\ldots v$ & \\
\hline$\cdots$ & 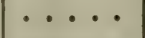 & $\cdots$ & $\cdots$ & $\cdots$ & $\cdots$ & $\ldots \mathbf{v}_{\ldots}$ & \\
\hline$\cdots$ & . & $\cdots$ & • & $\cdots$ & $\cdots$ & $\ldots v$ & \\
\hline$\cdots$ & & $\cdots$ & $\cdots$ & $\cdots \cdots$ & $\cdots$ & ...v. & \\
\hline R. . & $\cdot$ & $\cdots \cdot$ & $\cdots \cdot$ & $\cdots$ & $\cdots$ & $s \ldots \mathbf{v} \cdot \cdot$ & \\
\hline$\cdots$ & & $\cdots$ & • & $\cdots$ & $\cdots$ & $\ldots ? \ldots$ & \\
\hline$\cdots$ & & $\cdots$ & & .1 & $\cdots$ & $\cdots v \mathbf{v} \mathbf{w}$ & \\
\hline$\ddot{\cdots}$ & & $\because$ & $\cdot$ & $\cdots$ & $\cdots$ & $\cdots \cdots w$ & \\
\hline$\cdots$ & $\therefore$ & $\cdots \cdots \cdots$ & $\cdots$ & $\cdots$ & $\cdots$ & $\cdots w$ & \\
\hline снт. & $\cdots$ & $\ldots \ldots$ & & & $\cdots$ & $\cdots w$ & \\
\hline$\ldots$ & $\cdots$ & $\cdots \cdots$ & $\cdots$. & $\ldots$ & $\ldots$ & ....w. & \\
\hline RR. . & & $\cdots \cdots$ & $\cdots \cdot$ & . & . & ...w w & \\
\hline c. .. & $\cdots \cdot$ & $\cdots \cdots$ & $\cdots \cdot$ & $\cdots \cdots$ & $\cdots$ & $\cdots \cdots \mathbf{w} \mathbf{x}$ & \\
\hline$\cdots$ & $\cdot$ & $\cdots$ & 1 & $\cdot$ & $\cdots$ & $\cdots w_{2}$ & \\
\hline H. . & & & & & & $\cdots \cdots$ & \\
\hline $\begin{array}{l}\neq f \\
\neq 1\end{array}$ & & & & & 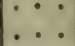 & $\cdots \cdots)$ & \\
\hline LK. . & & & & $\ldots$ & & $\ldots$ & \\
\hline . & & & & 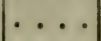 & $\cdots$ & .... & \\
\hline$\cdots \cdot$ & & 年 & r & & $\cdots$ & $\ldots .)$. & \\
\hline
\end{tabular}




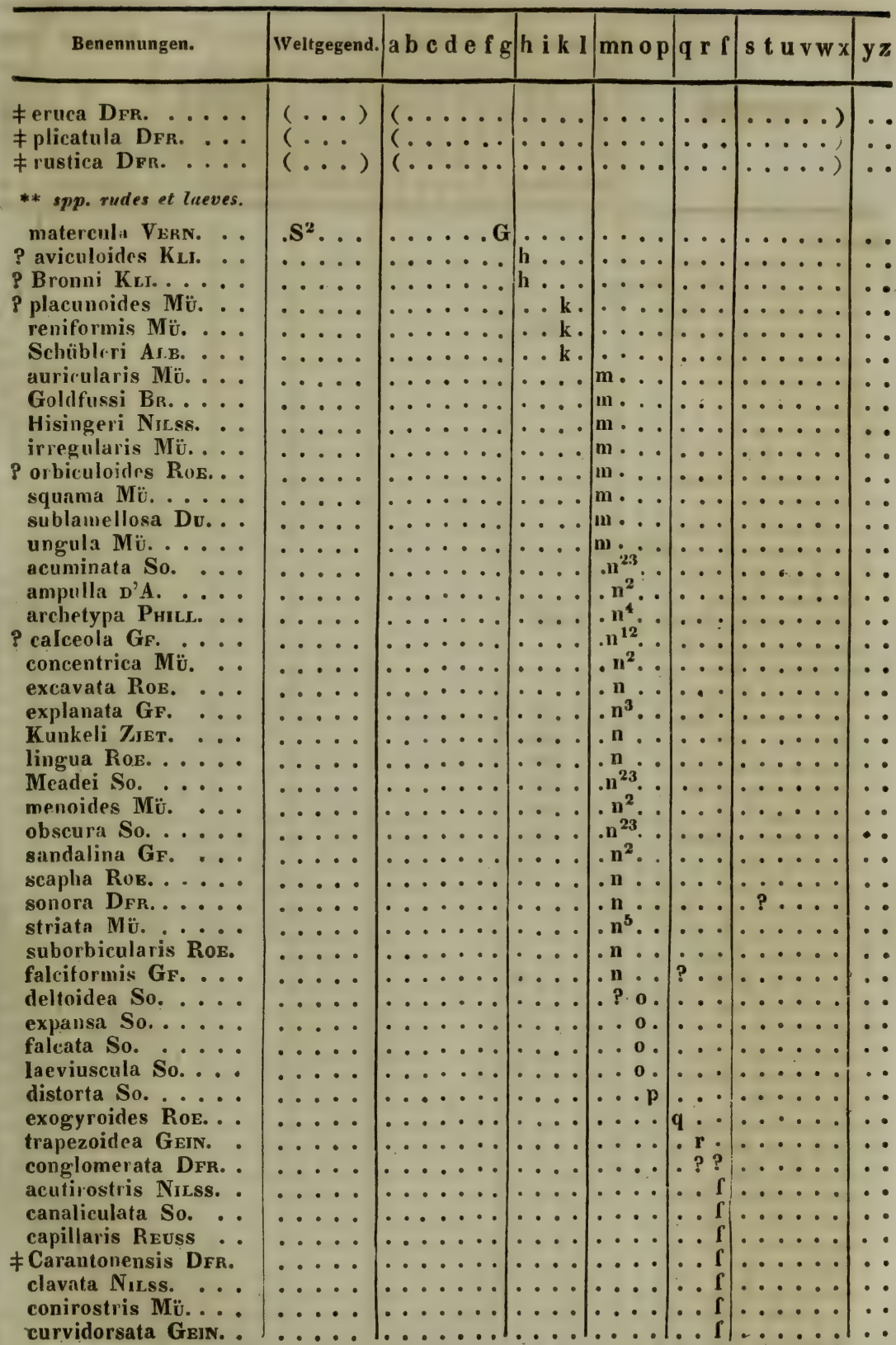




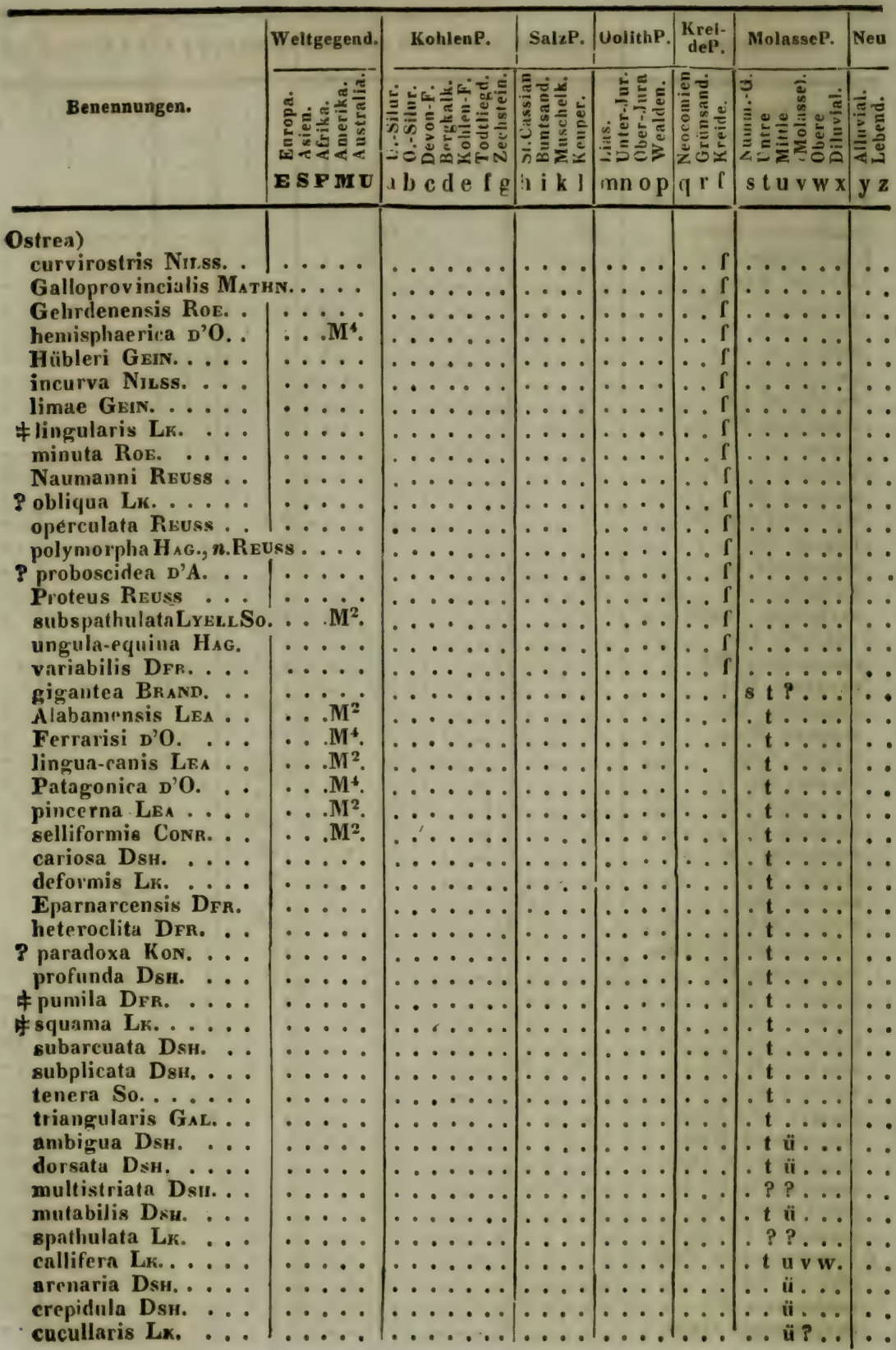




\begin{tabular}{|c|c|c|c|c|c|c|c|}
\hline Bene & & $g$ & & $\mathbf{p}$ & ( ) & $\mathrm{x}$ & . \\
\hline & & & & & & & \\
\hline$\cdots$ & & & $\sigma_{1}+2$ & & & . . ӥ... & \\
\hline$\cdots$ & $\cdots$ & $\ldots \ldots \ldots$ & $\ldots$. & $\ldots$ & $\cdots$ & . ii ... & \\
\hline . : : & $\cdots$ & $\ldots \ldots$ & $\cdots$ & $\ldots$ & $\cdots$ & .. ï. & \\
\hline 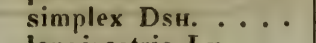 & & $\cdots$ & $\cdots \cdot$ & $\cdots$ & $\cdots$ & $\cdots$ & \\
\hline & $\ldots \mathbf{M}^{2}$ & & & & & & \\
\hline & & & & & & $\cdots$ & \\
\hline - & & $\because$ & $\cdots \cdots$ & $\cdots$ & $\cdots$ & $\cdots$ & \\
\hline . & & & & $\ldots$ & & $w$ & \\
\hline$\ldots$ & . & $\cdots$ & $\ldots$ & $\ldots$ & $\cdots$ & w. & \\
\hline . & $\cdots$ & $\cdots$ & $\cdots$ & $\cdots \cdot$ & $\cdots$ & $\cdots$ & \\
\hline$\cdots$ & $\cdots$ & $\cdots$ & $\cdot \cdot$ & $\ldots$ & $\cdots$ & $\cdots$ & \\
\hline . . & & & & & & & \\
\hline$\cdots$ & & & & & $\cdot$ & $\cdots$ & \\
\hline & &. & & & &. & \\
\hline $\begin{array}{l}\text { ERP. } \\
\ldots\end{array}$ & .... & $\cdots$ & $\because \cdots$ & $\begin{array}{ll}\cdots \\
\cdots \\
\cdots\end{array}$ & $\cdots$ & $\therefore$ & \\
\hline$\because \cdots$ & $\ldots$ & $\ldots \ldots$ & $\ldots$ & $\ldots$. & $\ldots$ & $\ldots \mathrm{v}$ & \\
\hline$\therefore$ & $\cdots$ & $\ldots \ldots$ & $\cdots$ & $\ldots$ & $\cdot \cdot 1$ & $\ldots \mathbf{v} \ldots$ & \\
\hline . . & $\cdots$ & $\cdots \cdots$ & $\cdot \cdot$ & $\ldots$ & $\cdots$ & . & \\
\hline . & $\cdots$ & $\cdots$ & $\cdot \cdot$ & - & $\cdots$ & $\ldots \mathbf{v} \ldots$ & \\
\hline$\cdots$ & $\cdots$ & $\cdots$ & $\cdots$ & $\ldots$ & $\cdots$ & $\ldots \mathbf{v} \ldots$ & \\
\hline$\cdots$ & $\cdots$ & $\cdots$ & $\cdots$ & $\cdots$ & - & $\ldots v_{\ldots}$ & \\
\hline . & . & $\cdots$ & $\cdot \cdot$ & $\ldots$ & - & $\cdot$ & \\
\hline & . & $\cdots$ & $\cdots$ & $\cdots$ & - & $\cdots$ & \\
\hline DFR. & $\cdots$ & $\cdots$ & $\cdots$ & - & - & $v \cdot$. & \\
\hline . & $\cdots$ & $\cdots$ & ... & . & - & $\ldots v_{v}$ & \\
\hline$\cdots$ & $\because$ & $\cdots$ & $\cdots$ & & $\because$ & $\cdots \mathbf{v} \cdots$ & \\
\hline . & $\cdots$ & $\cdots$ & & $\cdots$ & . . & $\therefore$ & \\
\hline $\begin{array}{l}\text { n.RIS. } \\
\text { ERRR. }\end{array}$ & $\therefore$ & $\therefore$ & : & $\because$ & $\therefore$ & $\because$ & \\
\hline & & $\ldots \ldots$ & $\ldots$ & $\therefore \ldots$ & $\therefore$ & . $w \cdot$ & \\
\hline$\ldots$ & .... & $\ldots \ldots$ & $\ldots$ & $\ldots$ & & $\ldots \ldots w$ & \\
\hline n.Sснг. & & $\ldots .$. & $\cdots$ & $\ldots$ & - & $\ldots .$. & \\
\hline & $\cdots \cdot$ & $\cdots \cdots$ & $\cdots$ & & & .... w . & \\
\hline . & • & $\cdots$ & $\cdots$ & & & . w w & \\
\hline & - & . & & & & $\ldots \ldots \mathrm{w} x$ & \\
\hline & $\cdots$ & 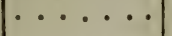 & 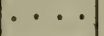 & 1 & 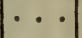 & $\ldots \ldots \mathrm{w} \times$ & - \\
\hline$\cdots$ & & . & & . & $\ldots$ & $\ldots w_{0}$ & - \\
\hline & r & $\cdots$ & ${ }^{\circ}$ & & $\cdots$ & . w . & - \\
\hline & & & & & & - & \\
\hline & 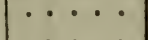 & & & & & $\cdots \cdot x$ & \\
\hline & $1:$ & & & & & . & \\
\hline & & & & & & ) & \\
\hline LK. . & - & & & & $\cdots$ & ․ & \\
\hline 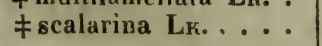 & 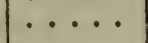 & $1 \cdots$ & $\cdots$ & $\ldots$ & $\cdots$ & $\ldots \ldots)$ & \\
\hline & & & & & & & \\
\hline & 2 & - & & & & & \\
\hline
\end{tabular}




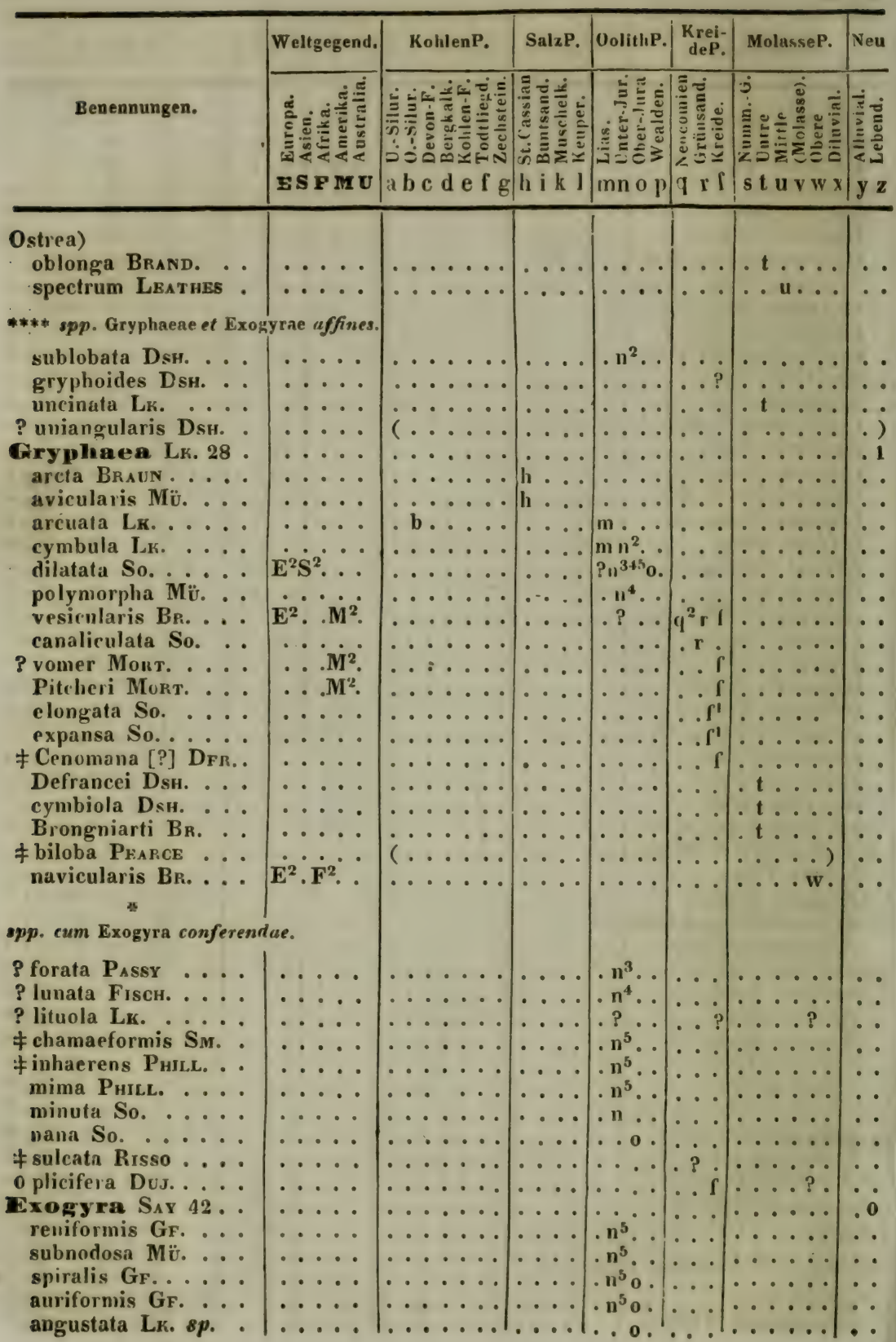




\begin{tabular}{|c|c|c|c|c|c|c|c|}
\hline & end. & $\mathrm{f} g$ & h i k l & $\mathrm{p}$ & $q \times \mathbf{r}$ & $\mathbf{w} \mathbf{x}$ & \\
\hline & & & & & & & \\
\hline . . & $\therefore$ & & - & .00 & & $\ldots \ldots$ & \\
\hline$\cdots$ & $\ddot{\cdots}$ & $\because$ & $\cdots \cdot$ & $\cdots 0$ & - & $\cdots \cdots$ & \\
\hline$\ldots$ & $\cdots \cdots$ & $\cdots$ & $\cdots$ & $\cdots 0$ & $\bullet$ & $\cdots$ & \\
\hline - & $\cdots$ & $\cdots$ & $\left|\begin{array}{ll}\cdots & \cdots \\
\cdots & \cdots\end{array}\right|$ & $\mid \begin{array}{cc}\cdots & p \\
\cdots & p\end{array}$ & & $\therefore$ & \\
\hline ). & $\cdots$ & $\therefore$ & $\ldots$. & $\because \cdots$ & & 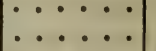 & \\
\hline . & $\cdots$ & $\cdots$ & $\cdots \cdot$ & $\ldots$. & $q \ldots$ & $\ldots \ldots$ & \\
\hline$\cdots$ & & $\cdots \cdots \cdots$ & $\ldots$ & $\ldots$. & & $\ldots \ldots$ & \\
\hline & $\cdots$ & $\cdots$ & $\cdots$ & $\ldots$ & & $\ldots \ldots$ & \\
\hline 0. & & $\cdots$ & $\cdots$ & $\ldots \cdot$ & & $\cdots$ & \\
\hline - & $\cdots \cdot$ & $\cdots$ & $\cdots \cdot$ & $\cdots \cdot c$ & & $\cdots$ & \\
\hline & $\cdots$ & . & $\cdots$ & $\cdots$ & & $\cdots$ & \\
\hline & $\dot{\mathrm{E}}^{2} \dot{\mathrm{M}^{3}}$ & $\cdot$ & .1 & & & & \\
\hline . & $\ldots$ & $\cdots$ & $\mid \begin{array}{ll}\cdots \\
\cdots\end{array}$ & $\begin{array}{ll}\cdots \\
\cdots\end{array}$ & & $\cdots$ & \\
\hline$\therefore$ & $\cdots \cdot$ & $\ldots$ & $\ldots$ & $\ldots$ & $? \mathrm{r}$ & . ?.. P. & \\
\hline . & & $\cdots$ & $\cdots$ & $\ldots$. & $q \times r$ & $\ldots \ldots$ & \\
\hline . & - & $\ldots$ & $\ldots$ & $\ldots$ & . & $\cdots$ & \\
\hline$\cdots$ & $\mathbf{E}^{2} \mathbf{S}^{2} \ldots$ & $\cdots \cdot$ & $\cdots$ & $\cdots$ & - $\mathbf{r} \mathbf{l}^{\mathbf{1}}$ & $\cdots$ & \\
\hline$\cdots$ & $\cdots$ & $\cdots$ & $\cdots \cdot$ & $\cdots \cdot$ & . $r f$ & $\ldots \ldots$ & \\
\hline$\cdots$ & $\cdots \cdot \cdot$ & $\cdots \cdots$ & $\cdots \cdots$ & $\cdots \cdot$ & . $r f$ & $\ldots \ldots$ & \\
\hline$\cdots$ & $\cdots \cdot \cdot$ & $\cdots \cdot$ & $\cdots$ & $\cdots \cdot$ & - $\mathrm{rr}$ & $\cdots \cdots$ & \\
\hline$\cdots$ & $F^{2}$ & $\cdots$ & $\cdots \cdots$ & $\cdots \cdots$ & . I & $\cdots \cdots$ & \\
\hline$\cdots$ & $\mathbf{E}^{2}$. & ... & $\cdots \cdot$ & $\cdots$ & $\cdots f$ & $\cdots$ & \\
\hline - & • & . & $\cdots$ & $\because$ & $\because 1$ & $\cdots$ & \\
\hline . & - & • & $\cdots \cdots$ & $\cdots$ & $\begin{array}{c}1 \\
f^{1}\end{array}$ & $\dot{.}$ & \\
\hline - & $\ldots$ & : & $\therefore \cdots$ & $\ldots$ & $\because \mathrm{f}$ & D & \\
\hline$\because$ & ... & . & $\ldots$ & $\ldots$ & $\therefore$ i & : & \\
\hline$\therefore$ & $\cdots \cdot$ & $\cdots$ & $\cdots$ & $\ldots$ & . . $\mathrm{f}$ & $\cdots \cdots$ & \\
\hline$\ldots$ & - * & $\cdots$ & $\cdots \cdots$ & $\cdots$ & . . $\mathrm{f}$ & $\cdots \cdots$ & \\
\hline$\cdots$ & $\ldots \mathrm{M}^{3}$ & $\cdots \cdot$ & $\cdots \cdot$ & $\cdots$ & $\ldots \mathrm{f}$ & $\ldots \ldots$ & \\
\hline . & $\cdots$ & $\cdots$ & $\cdots$ & $\cdots \cdot$ & $\mid \cdots f$ & $\ldots \ldots$ & \\
\hline . & $\cdots \cdots$ & $\cdots$ & $\cdots$ & $\cdots$ & & $\cdots \cdots$ & \\
\hline (2) & $\cdots$ & $\cdots$ & $\cdots$ & - & $\cdots f$ & - & \\
\hline & - & $\cdot$ & - & - & $\ldots \mathrm{f}$ & $\cdot$ & \\
\hline $\begin{array}{l}\text { sCH.) } 2 \\
=\end{array}$ & & 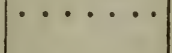 & $\cdots$ & $\cdots$ & $\cdots$ & $\cdots$ & \\
\hline & & • & - & & - $f$ & $\ldots \ldots$ & \\
\hline assus 1 & & & $\cdot$ & $\cdots$ & $\cdots \mathrm{f}$ & $\cdots$ & \\
\hline & & & & & & & \\
\hline & & & & & & ${ }^{\circ}$ & \\
\hline & & & & & & & \\
\hline & & & & $\mathbf{m} \mathcal{\gamma}$. & $\cdots \cdot$ & $\because \cdots$ & \\
\hline & •・ & • • & $\cdots$ & $n$ & $\cdots$ & $\cdots \cdots$ & \\
\hline$\cdots$ & r & & & & $\cdots$ & $\cdots$ & \\
\hline$\cdots$ & • • • & & & $u^{3} 0$ & & $\ldots \ldots$ & \\
\hline
\end{tabular}




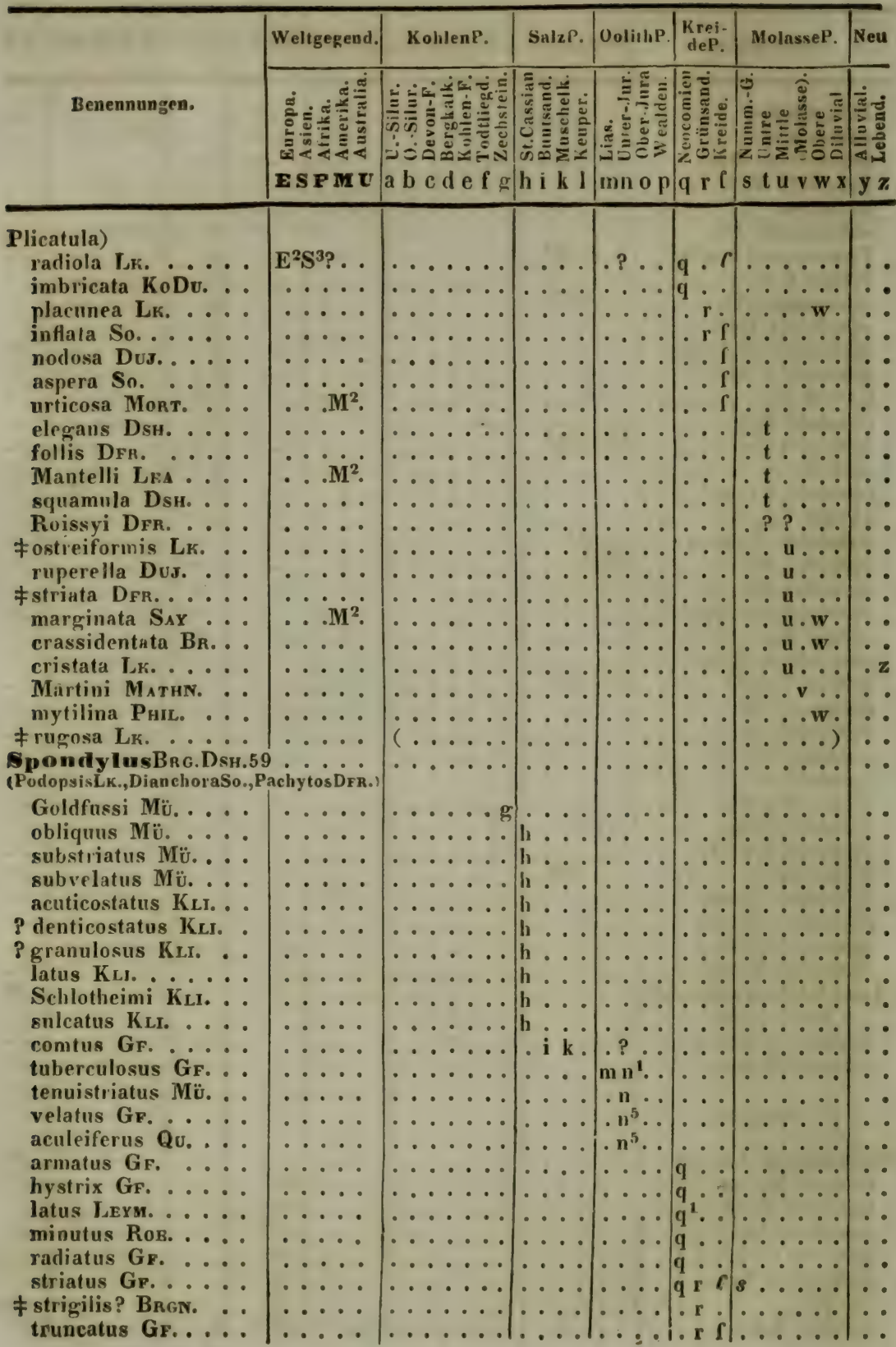




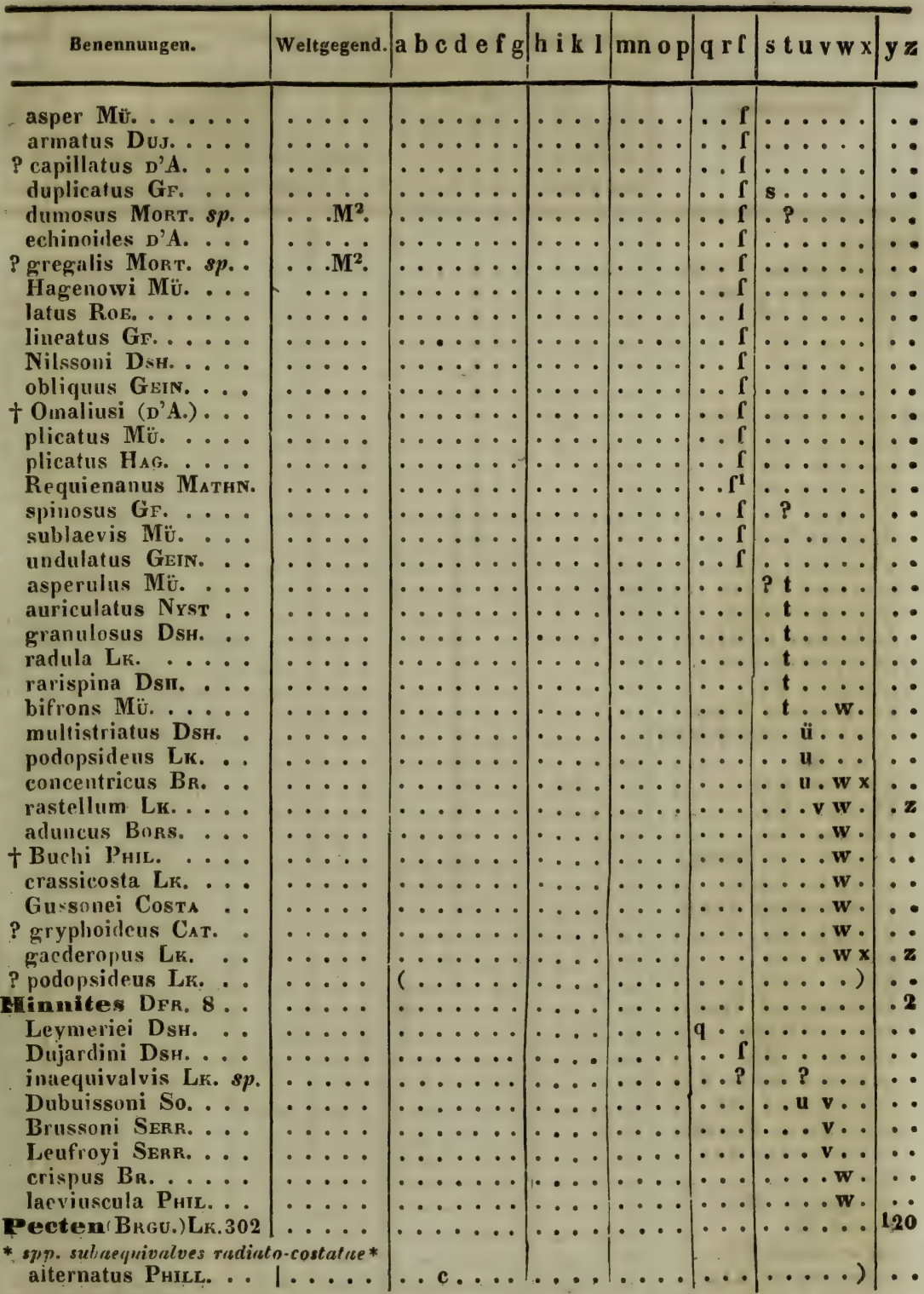

* Numeri speciebus quiluusdım prnefixi fumilius indicant ulteriores a RosMrR in speciebus subatquivalvibus radialo-costrutis distinclus; sunt sr.

$2=A r c u$ at $i:$ radiis extrorsum urcualis ;

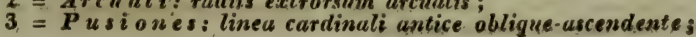




\begin{tabular}{|c|c|c|c|c|c|c|c|}
\hline Benennungen. & 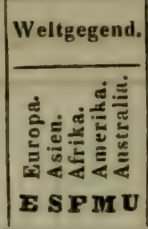 & 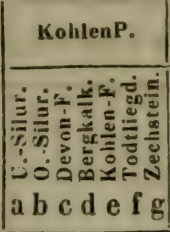 & 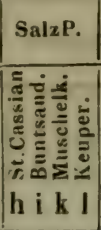 & $\frac{\mid \text { OolithP. }}{\mid}$ & 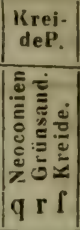 & 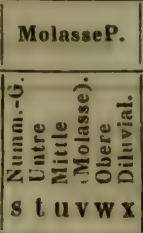 & 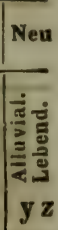 \\
\hline 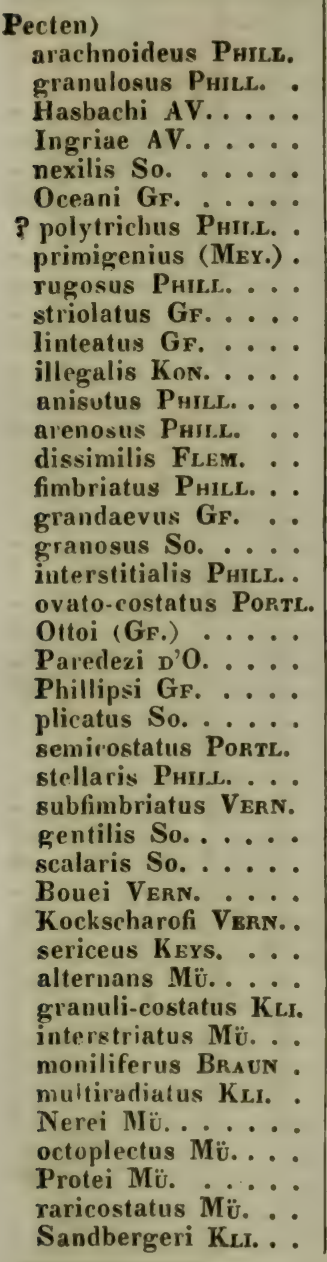 & 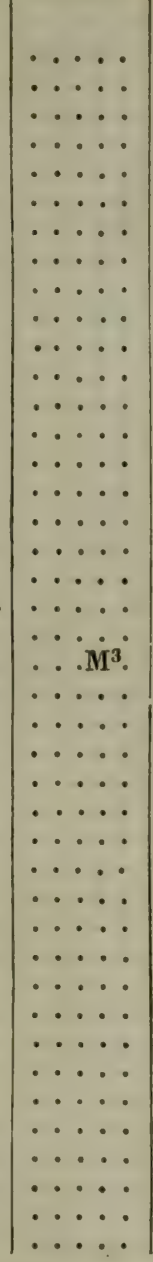 & 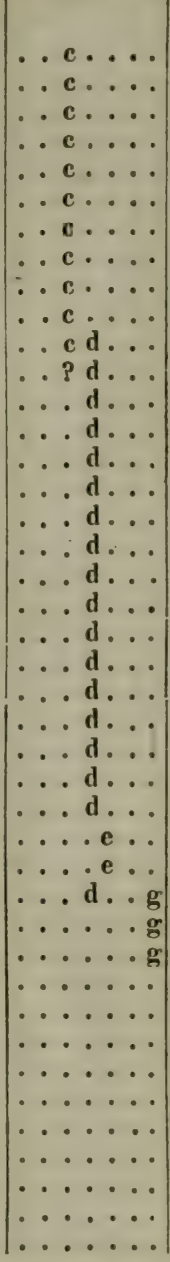 & 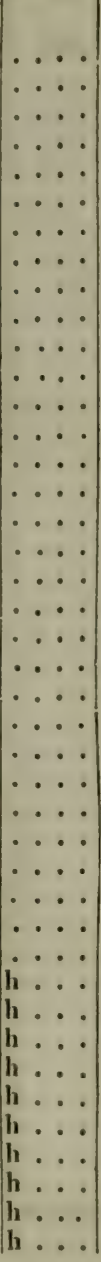 & 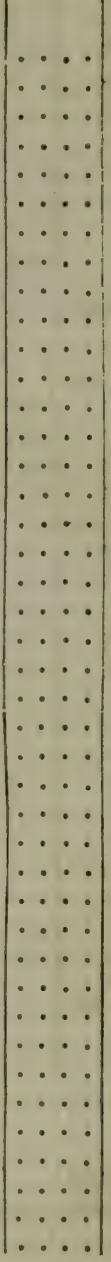 & 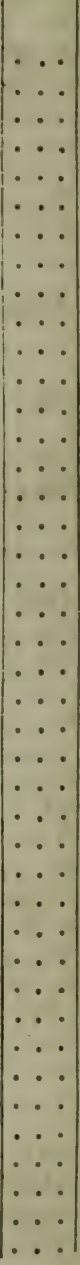 & 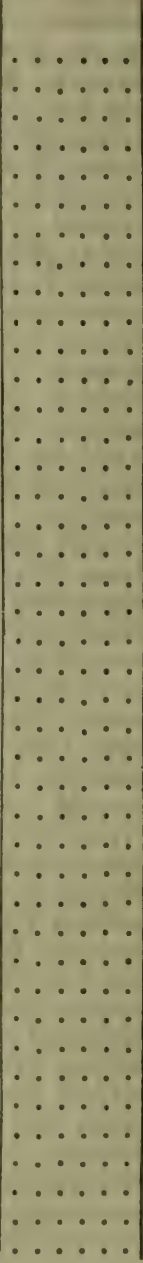 & 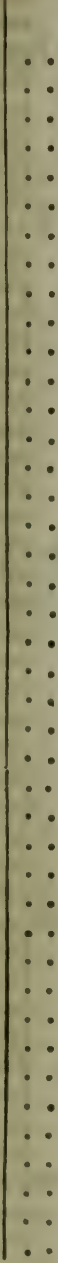 \\
\hline
\end{tabular}

$4=$ rslandicoid ae: radiis alternis brevioribus

$5=P l i c n t i:$ radiis $5-13$, walpne sinistrue ulternis debilioribus, dextrue geminis;

6 = Tranquebarini: vadiis simplicibus subueynulibus; ungulo cardinali-postico recto.

$\mathbf{z}=$ Opercularini: item, sed angulo valde ob/uso. 


\begin{tabular}{|c|c|c|c|c|c|c|c|}
\hline Bene & send & $a b c$ & k 1 & $\mathrm{~m}$ & $q r f$ & s I , & \\
\hline & & & & & & & \\
\hline & & & & & & $\ldots \ldots$ & \\
\hline & & & $\cdots \mathbf{k}$ & & & $\ldots$ & \\
\hline & & . . & & & & i & \\
\hline & $\because$ & $\cdots$ & & & & $\ldots \ldots$ & \\
\hline$\therefore$ & $\ldots$ & & & $\ln \gamma^{2}$ & & $\ldots \ldots$ & \\
\hline HN. & $\ldots$ & $\cdots \cdots$ & & $m^{1}$ & $\cdots$ & $\ldots \ldots$ & \\
\hline . . & $\cdot$ & . & & $\mathrm{m}$ & - & $\ldots \ldots$ & \\
\hline . & & & & & . & $\ldots .$. & \\
\hline$\cdots$ & - & & & & $\cdots$ & $\ldots \ldots$ & \\
\hline$\cdots$ & • & & & & $\cdots$ & $\cdots \cdots$ & \\
\hline$\cdots$ & . & 1 & & & $\cdots$ & $\ldots \ldots$ & \\
\hline 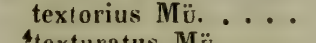 & 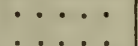 & t & & & $\cdots$ & $\cdots \cdots$ & \\
\hline & & - 1 & & & - & $\cdots$ & \\
\hline DFr. . & & & & & - & $\cdots$ & \\
\hline $\begin{array}{l}\text { DFr... } \\
\ldots \ldots\end{array}$ & $\bullet$ & $\therefore$ & $\begin{array}{c}\cdots \\
\cdots\end{array}$ & & $\therefore$ & $\because$ & \\
\hline OE. & & & $\ldots$. & & $\because$ & $\because \cdots \cdots$ & \\
\hline$\cdots$ & . & & $\cdot$ & & . & $\ldots \ldots$ & \\
\hline$\therefore$ & $\cdots \cdot$ & . & & . . & $\cdots$ & $\ldots \ldots$ & \\
\hline Bi. & $\cdots$ & & & & $\cdots$ & $\cdots \cdots$ & \\
\hline 0 & $\cdots$ & ・ & $\cdot$ & & $\cdots$ & $\cdots \cdots$ & \\
\hline & & & & & . & $\cdots$ & \\
\hline So. & & & & in & $\therefore$ & $\because$ & \\
\hline OE. ..... & . & $\ldots$ & $\ldots$. & . & $\cdots$ & $\ldots \ldots$ & \\
\hline & & & &.$n^{2} \ldots$ & $\ldots$ & .... & \\
\hline$\cdots$ & & $\cdots$ & &.$n \ldots$ & $\cdots$ & $\ldots \ldots$ & \\
\hline$\cdots$ & . & $\cdots$ & $\cdots$ & . & $\cdots$ & $\ldots \ldots$ & \\
\hline$\cdots$ & . & - & & $\cdot$ & $\cdots$ & $\ldots \ldots$ & \\
\hline$\cdots$ & & & & & $\cdots$ & $\cdots$ & \\
\hline 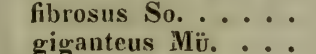 & & & & & • • & $\cdots$ & \\
\hline$\neq q$ & & $\because \cdots$ & & & $\because$ & $\because$ & \\
\hline$\cdots$ & & & & & $\because$ & & \\
\hline L. & & . &. & & $\because$ & & \\
\hline & $\cdots$ & $\ldots \ldots$ & .. & .1 & $\cdots$ & $\cdots$ & \\
\hline & & & & & $\cdots$ & - & \\
\hline$\neq 1$ & & & & & $\ldots$ & $\ldots \ldots$ & \\
\hline$\cdots$ & $\ldots \ldots$ & . & & .n3. & $\ldots$ & $\ldots \ldots$ & \\
\hline & $\cdots$ & $\cdots$ & & & $\cdots$ & $\cdots$ & \\
\hline . & $\cdots$ & $\cdots$ & .. & $. \mathrm{n}+.$. & $\cdots$ & $\cdots \cdots$ & \\
\hline$\cdots$ & & 1. & & & $\cdots$ & $\cdots$ & \\
\hline . & & & & & & . & \\
\hline & & & & $\mid \begin{array}{l}1 \\
.1\end{array}$ & . & $\therefore$ & \\
\hline & & & - &.$n$ & $\cdots$ & $\cdots$ & \\
\hline$\cdots$ & & $1 \cdots$ & & . $\mathbf{n}^{5}$ & $\ldots$ & & \\
\hline & $\cdots \cdots$ & $\cdots$ & 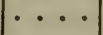 & . $\mathbf{0}$ & . & $\ldots$ & \\
\hline • & . & - & & & 9. & $\cdots$ & \\
\hline & & & & & & & \\
\hline
\end{tabular}




\begin{tabular}{|c|c|c|c|c|c|c|c|}
\hline \multirow[b]{2}{*}{ Benennungen. } & Weltgegend. & KohlenP. & SalzP. & OolithP. & $\begin{array}{l}\text { Krei- } \\
\text { deP. }\end{array}$ & MolasseP. & Neu \\
\hline & 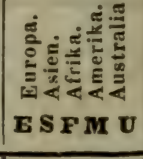 & 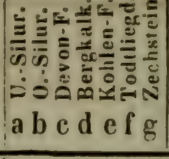 & 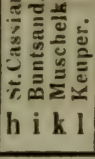 & $\begin{array}{l}20 \\
\text { mn o p }\end{array}$ & 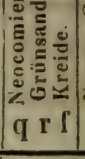 & 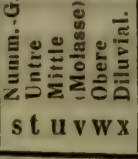 & 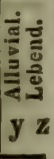 \\
\hline \multicolumn{8}{|l|}{ Pecten) } \\
\hline So & & & &.$n^{3} o$ & $\cdots$ & $\ldots \ldots$ & \\
\hline$\because$ & & & $\cdots \cdot$ & $\cdots 0$ & & $\cdots \cdot$ & - \\
\hline${ }^{3}$ crispus Roe. $\cdots \cdots$ & $\therefore \cdots$ & 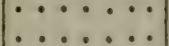 & $\cdots$ & $\because 0$ & & $\cdots \cdots$ & : \\
\hline lineato-costafus Rов. & $\ldots$ & $\ldots \ldots$ & $\ldots$ & $\cdots$ & $q \cdots$ & $\ldots \ldots$ & $\because$ \\
\hline tatus Ros. & $\cdots \cdot$ & $\ldots \ldots$ & $\cdots$ & $\cdots$ & q.. & $\ldots \ldots$ & • \\
\hline subarticulatus Ros. & $\cdots \cdots$ & $\cdots \cdots$ & $\cdots \cdots$ & $\ldots$ & 9. & $\cdots \cdots$ & • \\
\hline Voltzi LE & $\cdots \cdot \cdot$ & $\cdots \cdots \cdots$ & $\cdots$ & $\cdots \cdot$ & $q^{1} \cdot$ & $\cdots \cdots$ & - \\
\hline Goldfussi LEYM. . & $\cdots \cdot$ & $\cdots \cdots \cdots$ & & $\cdots \cdots$ & 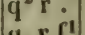 & $\cdots \cdots$ & - \\
\hline$\cdots \cdots$ & $\cdots \cdots$ & $\cdots \cdots \cdots$ & & $\cdots$ & {$\left[\begin{array}{lll}4 & 1 & 1 \\
0 & 0 & 0\end{array}\right]$} & $\cdots \cdots$ & $\bullet$ \\
\hline $\begin{array}{l}{ }^{3} \text { comans RoE. } \ldots \\
\text { hispidus GF. . . . }\end{array}$ & $\ldots .$. & $\ldots \ldots$ & $\cdots \cdots$ & $\cdots \cdots$ & $\left|\begin{array}{ccc}4 & 0 & 1 \\
9 & \cdot & f\end{array}\right|$ & $\cdots \cdots$ & $\because$ \\
\hline${ }^{4}$ nitidus Mant $_{\text {an }} \ldots$ & $\ldots$ & $\ldots \ldots$ & $\ldots$ & $\ldots$ & $q \cdot r$ & $\ldots \ldots$ & . \\
\hline${ }^{3}$ obliquus So. ... & $\cdots \cdots$ & $\ldots \ldots$ & $\cdots$ & $\ldots$ & $q \cdot \mathrm{f}$ & $\ldots \ldots$ & - \\
\hline o. . . & $\cdots \cdot \cdot$ & $\cdots \cdots$ & $\cdots \cdots$ & $\cdots$ & . $\mathbf{r} \cdot$ & $\cdots \cdots$ & - \\
\hline Bdecemcostatus Mü.. & $\cdots \cdot \cdot$ & $\ldots \ldots$ & $\cdots \cdots$ & $\cdots \cdots$ & $\cdot \mathbf{r} \cdot$ & $\cdots \ldots$ & • \\
\hline и. . & • & $\because \cdots \cdots$ & $\cdots \cdot$ & $\cdots \cdot$ & . r & $\cdots \cdots$ & • \\
\hline lineatus NIr.ss. . & & $\cdots \cdots$ & $\cdots \cdot$ & $\cdots$ & . $r$ & $\cdots \cdots$ & $\bullet$ \\
\hline$\cdots \cdots$ & $\ddot{\cdots} \dot{0}$ & $\cdots \cdots \cdots$ & $\cdots$ & $\cdots \cdots$ & $r$ & $\cdots \cdots$ & $\bullet$ \\
\hline $\begin{array}{l}\text { tus GF...... } \\
\text { buryanus So. }\end{array}$ & $\cdots$ & $\cdots \cdots \cdots$ & $\cdots$ & $\cdots$ & . $\mathbf{r}$ & $\cdots \cdots$ & $\dot{\bullet}$ \\
\hline $\begin{array}{l}\text { uryanus So. } \\
\text { atus Gein. }\end{array}$ & .... & $\ldots \ldots$ & ... & $\ldots$ & . $\mathrm{r} r$ & $\ldots \ldots$ & $\because$ \\
\hline So...... & $\cdots \cdots$ & $\ldots \ldots$ & $\cdots \cdot$ & $\ldots$ & . ? f & $\ldots \ldots$ & 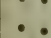 \\
\hline${ }^{5}$ Dujardini Roz. . . & $\ldots \ldots$ & $\ldots \ldots$ & $\cdots$ & $\ldots$ & $\cdot r f$ & $\ldots \ldots$ & . \\
\hline GF.... & .... & $\ldots \ldots$ & $\cdots \cdots$ & $\ldots$ & $\ldots 1$ & $\ldots \ldots$ & - \\
\hline$\cdots$ & $\cdots \cdots$ & $\ldots \ldots$ & $\cdots$ & $\ldots$ & . I ! & $\ldots \ldots$ & - \\
\hline ¥Aldrovandii DFr. & - . . & $\cdots \cdots$ & $\cdots \cdot$ & . & $\cdots ?$ & $\ldots \ldots$ & - \\
\hline ipes Mort. . . . & ... $M^{2}$ & $\cdots \cdots \cdots$ & $\cdots \cdots$ & $\cdots \cdot$ & $\cdots 1$ & $\cdots \cdots$ & - \\
\hline$\because \cdot$ & $\cdots \cdots$ & $\cdots \cdots$ & $\cdots \cdot$ & $\cdots \cdot$ & $\cdots 1$ & $\cdots \cdots$ & - \\
\hline$\cdots$ & $\ddot{\cdots} \cdot \cdot$ & $\because$ & . & $\cdots$ & $\cdots 1$ & $\cdots \cdots$ & - \\
\hline $\begin{array}{l}{ }^{2} \text { concentrice-punctatus } \\
\text { craticula VorT. . . }\end{array}$ & ... $\dot{M}^{2}$. & $\cdots \cdots$ & $\cdots$ & & $\because 1$ & $\cdots \cdots$ & $\ddot{0}$ \\
\hline${ }^{2}$ curvatus Gein. ... & .... & .. & $\cdots \cdots$ & - & .. I & $\ldots \ldots$ & . \\
\hline dentatus Nusss. . . . & $\cdots \cdots$ & $\ldots \ldots$ & $\ldots$ & $\cdots \cdot$ & $\ldots f$ & $\ldots \ldots$ & - \\
\hline denticulatus Hag. & $\cdots \cdot$ & $\cdots \cdots$ & $\cdots \cdot$ & $\cdots$ & $\cdots \int$ & $\ldots \ldots$ & $\cdots$ \\
\hline${ }^{6}$ depressus Mü. . . & $\cdots \cdot$ & $\cdots \cdots$ & $\cdots \cdot$ & $\cdots$ & $\cdots \tau$ & $\ldots \ldots$ & . \\
\hline ‡ discors LK. $\ldots$... & $\cdots \cdot$ & $\cdots \cdots \cdots$ & $\cdots \cdot$ & $\cdots$ & 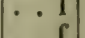 & $\cdots \cdots$ & - \\
\hline atus Reuss. & - & $\cdots \cdots$ & $\cdots \cdot$ & & $\cdots 1$ & $\cdots \cdots$ & - \\
\hline $\begin{array}{l}\neq \text { elongatus LK. } \ldots \ldots \\
\text { exrisus Pusch }\end{array}$ & $\because$ & $\cdots$ & & & $\therefore 1$ & $\cdots$ & \\
\hline $\begin{array}{l}\text { exrisus } \\
{ }^{3} \text { Faujasi DFr. } \ldots\end{array}$ & .... & $\ldots \ldots$ & - . & $\cdots$ & $\therefore 1$ & $\ldots \ldots$ & $\cdots$ \\
\hline lissimus DFR. . & $\cdots \cdot$ & $\ldots \ldots$ & $\cdots$ & $\cdots$ & . $\mathrm{f}$ & $\ldots \ldots$ & 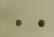 \\
\hline 1 & & & & & . $\mathrm{T}$ & $\cdots \cdots$ & \\
\hline キinflexus Hag. . . . & $\cdots$ & $\cdots \cdots \cdots$ & $\cdots \cdot$ & - & $\cdots 1$ & $\cdots \cdots \cdots$ & \\
\hline $\begin{array}{c}\text { ₹ Leonhardi HAG. } \ldots \\
\text { tmiscellus Mü. } \ldots\end{array}$ & $\ldots$. & $\cdots \cdots$ & 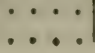 & & $\left|\begin{array}{l}\cdots \\
\cdots\end{array}\right|$ & . & - \\
\hline
\end{tabular}




\begin{tabular}{|c|c|c|c|c|c|c|c|}
\hline Benenunngen. & Weltgegend. & $|a b c d e f g|$ & 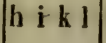 & mnop & q r & st uv $\mathrm{w} x$ & у 8 \\
\hline costatus NiLss. & & & & & .1 & $\cdots$ & \\
\hline so-costatus Hag. & $\ldots$ & & & & . . 1 & $\ldots \ldots$ & * \\
\hline perplanus Мовт. . & . . $\mathrm{M}^{2}$. & $\ldots \ldots$ & $\cdot$ & - & . . I & $\ldots \ldots$ & • \\
\hline ‡ phaseolus $\mathrm{LK}_{\mathrm{K}}$. & $\cdots \cdots$ & $\cdots \cdots$ & $\ldots$. & & .. $f$ & $\ldots \ldots$ & - \\
\hline Poulsoni Mort. . & $\cdot \cdot \mathbf{M}^{2}$ & $\cdots \cdot$ & $\cdots \cdot$ & & $\cdots \tau$ & $\cdots \cdots$ & - \\
\hline${ }^{4}$ pulchellus Nirss. . & $\cdots \cdots$ & . & 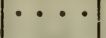 & & $\cdots 1$ & $\cdots \cdots$ & - \\
\hline Puzosanus Mathn. • & $\therefore$ & $\cdots$ & . & - & $\cdots 1$ & $\cdots \cdots$ & • \\
\hline rarispinus Revss . & $\because$ & & - & & $\therefore 1$ & $\cdots$ & $\dot{0}$ \\
\hline $\begin{array}{l}\text { sectus GF. } \\
\text { septemplicatus Nirss. }\end{array}$ & $\cdots \cdots$ & $\ldots \ldots$ & & & $\therefore$ i & $\cdots$ & $\because$ \\
\hline punctatus Mü. & .... & $\ldots \ldots$ & $\ldots$ & $\ldots$ & $\therefore 1$ & $\ldots \ldots$ & $\therefore$ \\
\hline${ }^{3}$ serratus Nicss. . . . & $\ldots \ldots$ & $\ldots \ldots$ & $\cdots \cdot$ & $\ldots \ldots$ & . . & $\ldots \ldots$ & . \\
\hline sexcostatus Woodw. & $\cdots$ & $\ldots \ldots$ & $\ldots$. & $\ldots$ & . . i & $\ldots \ldots$ & . \\
\hline EUSS & & $\ldots \ldots$ & $\cdots \cdot$ & $\ldots$ & . . $\mathrm{f}$ & $\ldots \ldots$ & - \\
\hline Mü. . . . . . & ... & $\ldots \ldots$ & $\cdots \cdot 1$ & $\ldots$ & .. $\mathrm{f}$ & $\ldots \ldots$ & - \\
\hline HAG. & $\cdots \cdots$ & $\cdots \ldots$ & $\cdots \cdot$ & $\cdots \cdot$ & & $\ldots \ldots$ & • \\
\hline rutus LK. . . . & $\cdots$ & $\cdots \cdots \cdots$ & $\cdots$ & & & $\cdots \cdots$ & • \\
\hline NiLss. .. & & • & $\cdots$ & $\cdot$ & $\cdots f$ & $\cdots \cdots$ & - \\
\hline latus MÜ. . & & & & & & - & 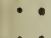 \\
\hline$\dot{\mathrm{C}_{\mathrm{N}}} \cdot$ & $\therefore$ & $\cdots$ & - & & $\cdots 1$ & $\cdots$ & : \\
\hline GF. . & $\because$ & $\ldots \ldots$ & • & •. & $\because 1$ & $\cdots$ & $\dot{0}$ \\
\hline $\begin{array}{l}\text { Ftrisulcus HAG. } \ldots \\
\text { variabilis } \text { HAG. }_{\text {AG. }}\end{array}$ & $\therefore$ & $\ldots \ldots$ & - • & . & . i & $\ldots$ & $\therefore$ \\
\hline$\ldots$ & ... $\mathbf{M}^{2}$. & $\ldots \ldots$ & $\cdots$ & $\cdots$ & $\ldots i$ & $\ldots \ldots$ & . \\
\hline$\ldots$ & $\cdots$ & $\ldots \ldots$ & $\cdots$ & & $\ldots r$ & $\ldots \ldots$ & . \\
\hline $\mathrm{H}_{\mathrm{AG}}$ & $\because \dot{0}$ & $\ldots \ldots$ & $\cdots$ & . & $\ldots \mathrm{f}$ & $\ldots \ldots$ & - \\
\hline So... &.$S^{3}$. & $\ldots \ldots$ & $\cdots$ & $1^{\circ}$ & $\ldots$ & $s \ldots$. & • \\
\hline$\because \cdots$ & $\cdots$ & $\cdots \cdot$ & - & $\cdot 1$ & $\cdots$ & $s t \ldots$ & - \\
\hline Iü. • & $\cdots$ & $\cdots \cdot$ & . & $\cdots$ & $\cdots$ & s.... & - \\
\hline$\cdots$ & . $\mathrm{M}^{2}$ & $\cdots$ & $\cdots$ & $\cdot$ & - & $\cdot \mathbf{t} \ldots$ & • \\
\hline$\cdots$ & - & $\cdot \bullet$ & $\cdot$ & • & & $\cdot t \ldots$ & - \\
\hline . & $\cdots$ & - & & & & $\cdots$ & - \\
\hline a. & $\therefore M^{3}$ & 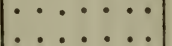 & $\cdots \cdots$ & & $\cdots$ & .1 & : \\
\hline \#Gervillei D & $\cdots$ & $\ldots \ldots$ & $\cdots \cdot$ & $\ldots$ & $\ldots$ & $\ldots \ldots$ & - \\
\hline Hoeninghausi DFr. & & & $\cdots \cdot 1$ & $\ldots$ & $\ldots$ &.$t \ldots$ & . \\
\hline sus Mort. & ... $\mathbf{M}^{2}$ & - & . & & • &. $\mathrm{t} \ldots$ & \\
\hline & $\cdots \cdot$ & - & - & $\cdots$ & - &. $\mathrm{t} \ldots$. & - \\
\hline SH. & & $\cdots$ & $\cdots$ & $\cdots \cdots$ & $\cdots$ & $\cdot \mathbf{t} \ldots$ & - \\
\hline us Dsh. • & & ・ & 0. & $\cdots \cdots$ & . &.$t \ldots$ & - \\
\hline$\cdots \cdot$ & $\cdots$ & . & $\cdots$ & $\cdots \cdots$ & $\cdots$ &.$t \ldots$ & \\
\hline o. . . & $\cdots$ & & & & & . $t$ & \\
\hline D'O. & . . M & . & & $\cdots$ & & $x_{2} \ldots$ & \\
\hline$\cdots$ & $\cdot$ & - & - & & $\cdot$ & $8 \mathrm{t} \cdots$ & \\
\hline$\ddot{x}$ & & & & & & $\begin{array}{ll}0 \\
0 \\
t\end{array}$ & \\
\hline Yst & $\cdots \cdots$ & $\cdots \cdots$ & $\cdots \cdots$ & $\cdots \cdots$ & $\cdots$ & t $\ldots$ & \\
\hline $\begin{array}{l}\text { Impartitus Ds } \\
\text { ‡Beauvoisi DF }\end{array}$ & $\cdots \mathrm{M}^{2}$ & $\ldots \ldots$ & $\cdots \cdot$ & $\ldots$ & $\cdots$ & . ? ?... & \\
\hline . & $\cdots$ & $\ldots \ldots$ & $\cdots \cdots$ & $\cdots \cdot$ & $\cdots$ & $. t u .$. & \\
\hline Dsн. & • & - & $\theta^{\circ}$ & $\cdots$ & & . t ... & \\
\hline$\cdots$ & $\ldots$ & & & & & " & \\
\hline$\cdots \cdots$ & & & & & & & \\
\hline
\end{tabular}




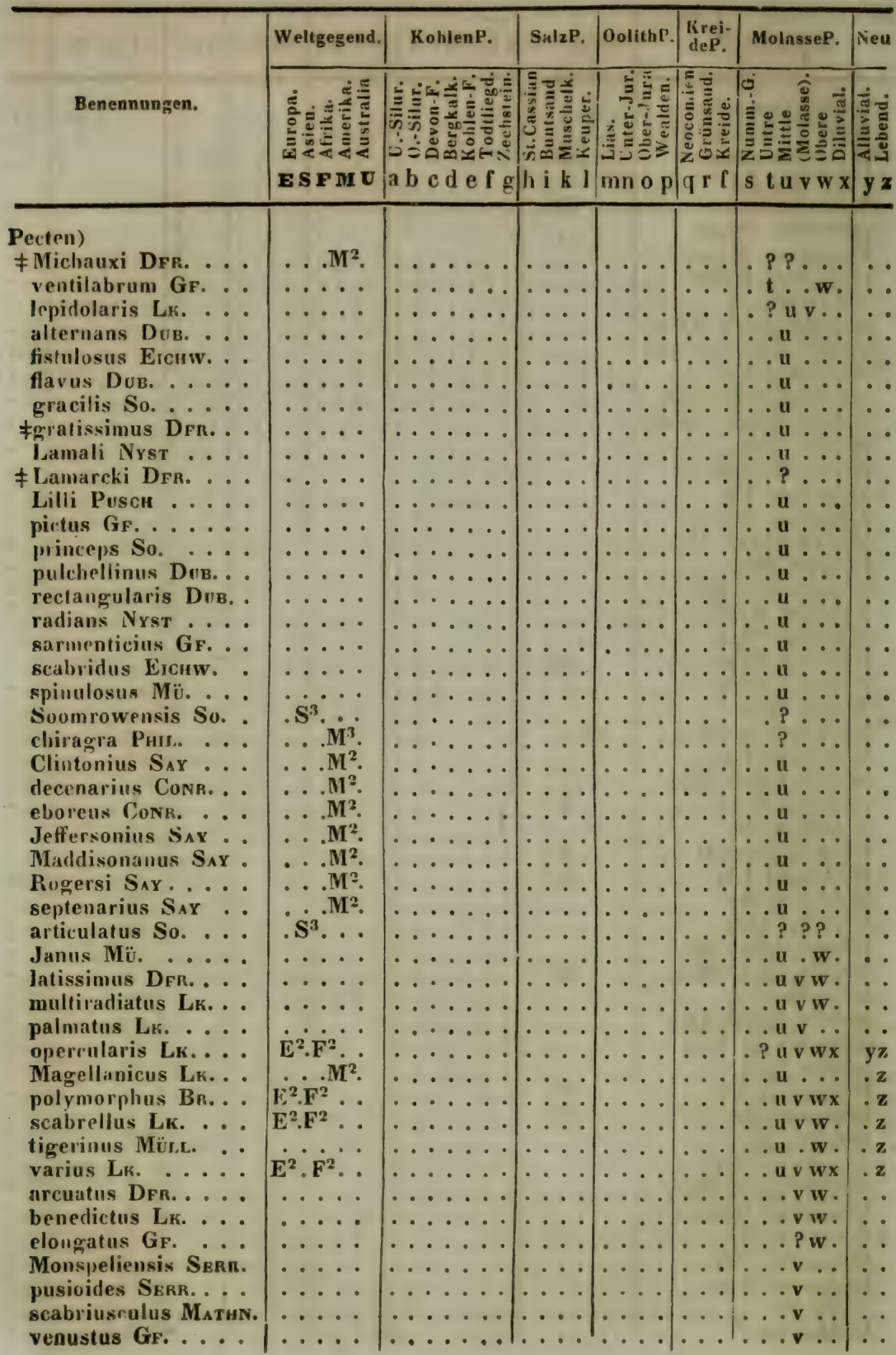




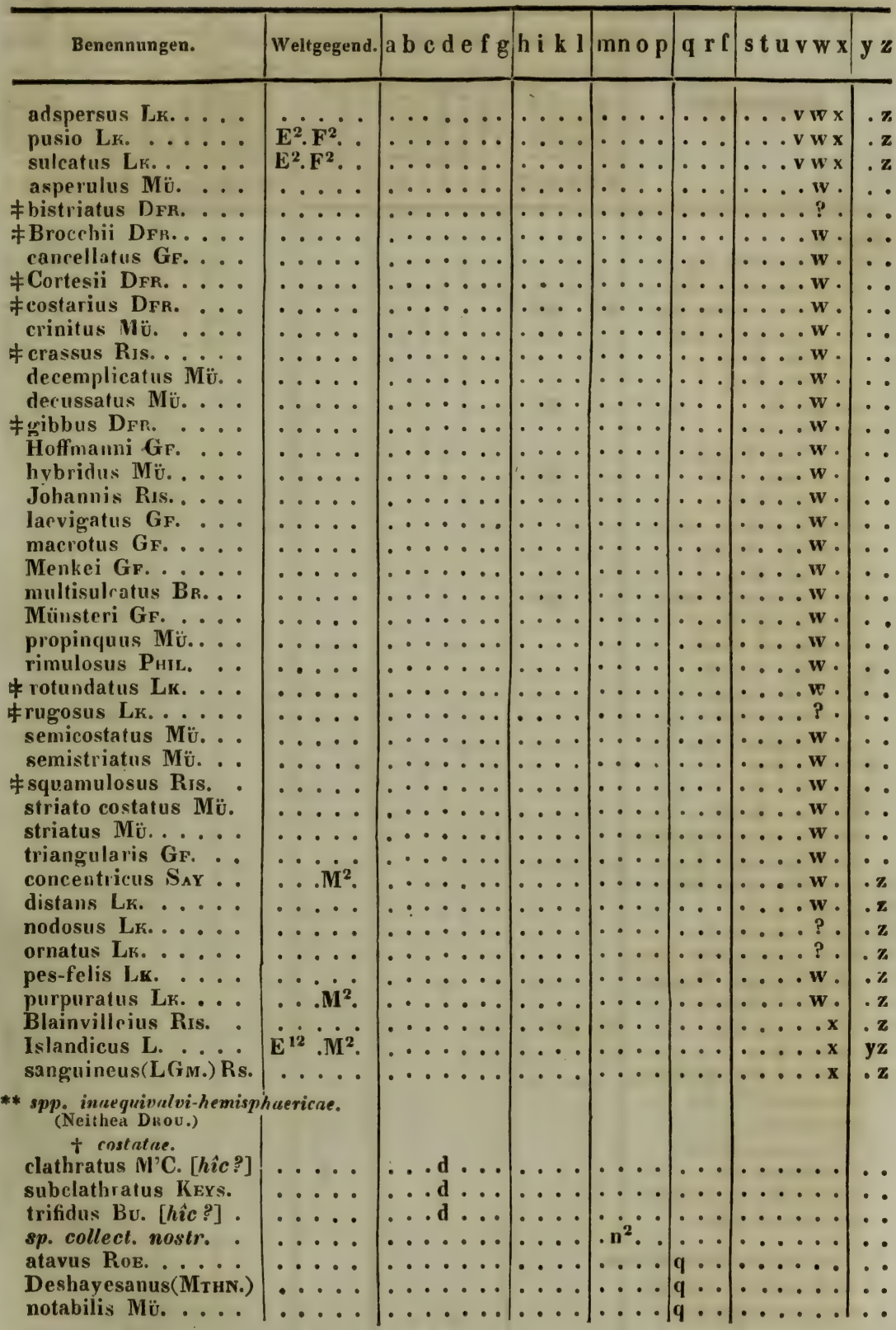




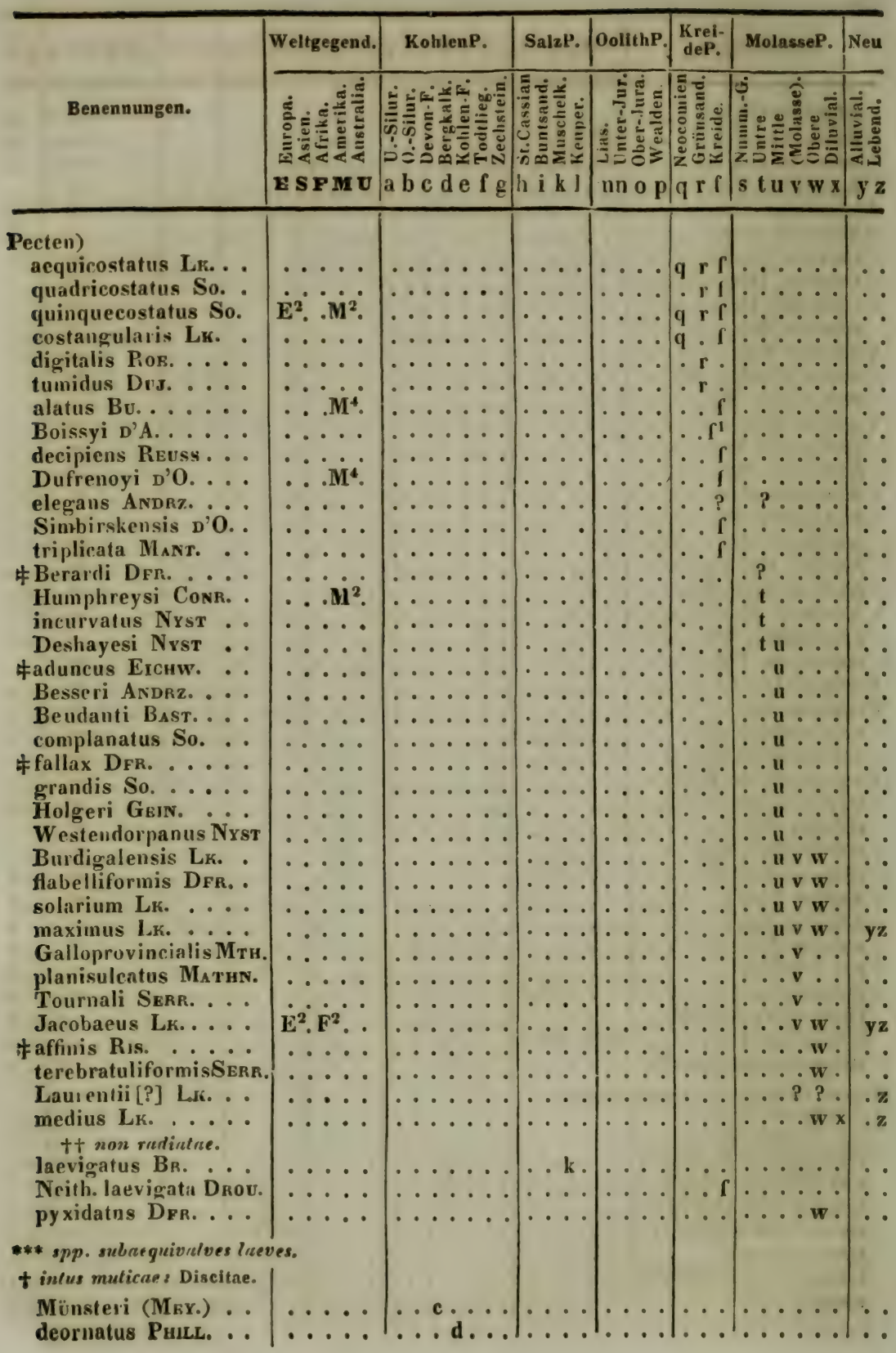




\begin{tabular}{|c|c|c|c|c|c|c|c|}
\hline & d. & g & 1 & o p & $q \mathbf{r} l \mid$ & $\mathrm{w} \mathbf{x}$ & \\
\hline & & & & & & & \\
\hline Noae Eicuw. & $\ldots$ & & & & $\cdots$ & $\cdots \cdots$ & \\
\hline Sibericus VERN. . & $\cdots \cdots$ & $\ldots d \ldots$ & $\cdots$ & - & $\ldots$ & $\ldots \ldots$ & \\
\hline Valdaicus VerN. . & $\cdots \cdots$ & $\ldots d \ldots$ & & & $\cdots$ & $\ldots \ldots$ & \\
\hline Mü. .... & $\cdots \cdots$ & $\ldots . . . g$ & & & $\cdots$ & . & \\
\hline$\cdots$ & & $\cdots$ & & & $\cdots$ & $\cdots$ & \\
\hline$\therefore$ & & & $\left|\mathrm{h}: \mathbf{i}_{\mathbf{k}}\right|$ & $\because$ & $\cdots$ & $\cdots \cdots$ & \\
\hline$\therefore$ & $\cdots \cdots$ & $\because$ & 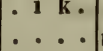 & $\dot{m} a$ & $\begin{array}{ll}\cdots \\
\cdots\end{array}$ & $\cdots$ & \\
\hline$\cdots$ & $\ldots \ldots$ & $\ldots \ldots$ & $\cdots \cdots$ & $\min \ldots$ & $\cdots$ & $\ldots \ldots$ & \\
\hline$\ldots$ & $\ldots \ldots$ & $\ldots \ldots$ & $\ldots$ & $m \ldots$. & ... & $\cdots \cdots$ & \\
\hline$\therefore$ & .. & $\cdots$ & $\ldots$ & & $\cdots$ & $\ldots \ldots$ & \\
\hline$i c$ ?] & $\cdots$ & $\cdots$ & $\cdots \cdot$ & & $\cdots$ & $\ldots \ldots$ & \\
\hline . & 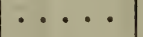 & $\cdots$ & $\cdots \cdot 1$ & mi & $\cdots$ & $\cdots$ & \\
\hline - & & $\cdots$ & $\cdots \cdot$ & & - & - & \\
\hline . & & & $\cdots$ & 1. & & $\cdots$ & \\
\hline$\ddot{H L L .}_{\dot{F}_{1}}$ & • & & $\cdots$ & $n$ & & $\because$ & \\
\hline HL... $\mathbf{F}_{1}$ & $\mathrm{~S}^{3} \ldots$ & & $\cdots \cdots$ & $\mid \begin{array}{l}\mathrm{n}^{-} \cdot \\
\mathrm{n} \cdot\end{array}$ & ・. & $\because$ & \\
\hline$\therefore$ & & & $\ldots$ & . ? . & ... & $\therefore$ & \\
\hline . & . & & $\ldots$ &. $\mathrm{n} \cdot$ & ... & $\ldots$ & \\
\hline ... & $\cdots$ & .. & $\cdots \cdots$ & .? & & $\ldots \ldots$ & \\
\hline . & $\cdots \cdot$ & $\cdots$ & $\cdots$ &. $\mathbf{n} \ldots$ & $\cdots$ & .. & \\
\hline ข.. & $\cdots$ & $\cdots$ & $\cdots \cdot$ & $\ldots 0$ & $\cdots$ & $\ldots$ & \\
\hline$\cdots$ & & & $\cdots \cdot$ & $\ldots 0$ & $\cdots$ & $\ldots$ & \\
\hline .. & & & $\cdots$ & 1.0. & & $\ldots$ & \\
\hline . . & & & & 1..? & $q$ & $\cdots$ & \\
\hline . & & & $\cdots$ & 3 & $r^{1}$ & $\cdots$ & \\
\hline$\cdots$ & - & & . 1 & $\cdots \cdot$ & $\begin{array}{lll}4 & \boldsymbol{r} & \mathbf{1}\end{array} \mid$ & $\cdots$ & \\
\hline . . & $\cdot$ & & $\cdots 1$ & $\cdots \cdot$ & $\mid \begin{array}{lll}-r & 1\end{array}$ & $\cdots \cdots$ & \\
\hline G. - & $\cdots$ & & 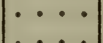 & $\cdots \cdot$ & $\cdots \boldsymbol{f}^{1}$ & 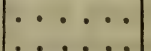 & \\
\hline$\cdots$ & 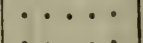 & & $:$ & $\cdots 1$ & $\begin{array}{ll}\cdots & ? \\
\cdots & f\end{array} \mid$ & 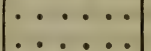 & \\
\hline - & $\dot{0}$ & & .. & $\cdots \cdots$ & $\therefore \mathrm{f}$ & $\cdots$ & \\
\hline$\cdots$ & $\therefore$ & . & . & $\cdots$ & . . i & $\ldots$ & \\
\hline$\cdots$ & $\cdots$ & & $\ldots \ldots$ & - $\cdot$ & $\ldots f$ & $\ldots \ldots$ & \\
\hline .... & $\ldots$ & & .... & ... & $\ldots i$ & $\ldots \ldots$ & \\
\hline & $\cdots$ & & $\ldots$ & .. & $\cdots \mathrm{f}$ & $\ldots \ldots$ & \\
\hline 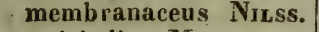 & $\cdots$ & & & - & $\cdots f$ & $\ldots \ldots$ & \\
\hline & & & & - & & $\ldots$ & \\
\hline THN. & & & & $\cdots \cdots$ & $\cdots f$ & $\cdots$ & \\
\hline . & & & $\cdots$ & $\cdots$ & $\ldots r$ & $\cdots$ & \\
\hline & $\cdots$ & & & & $\ldots I$ & & \\
\hline Uे. . & & & & & $\cdots$ &. $\mathrm{t}$ & \\
\hline$\cdots$ & & & & & $\cdots$ & t & \\
\hline$\cdots$ & & & & & $\cdots$ & . $t$ & \\
\hline$h \hat{c} c$ ?] & 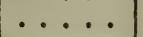 & & & & $\ldots$ & $\cdots$ & \\
\hline • & $\cdots$ & & t & & $\cdots$ & . . u & \\
\hline & & & & & & $\cdots \mathbf{u}$ & \\
\hline byaliuus РнuL. & & & & & & & \\
\hline
\end{tabular}




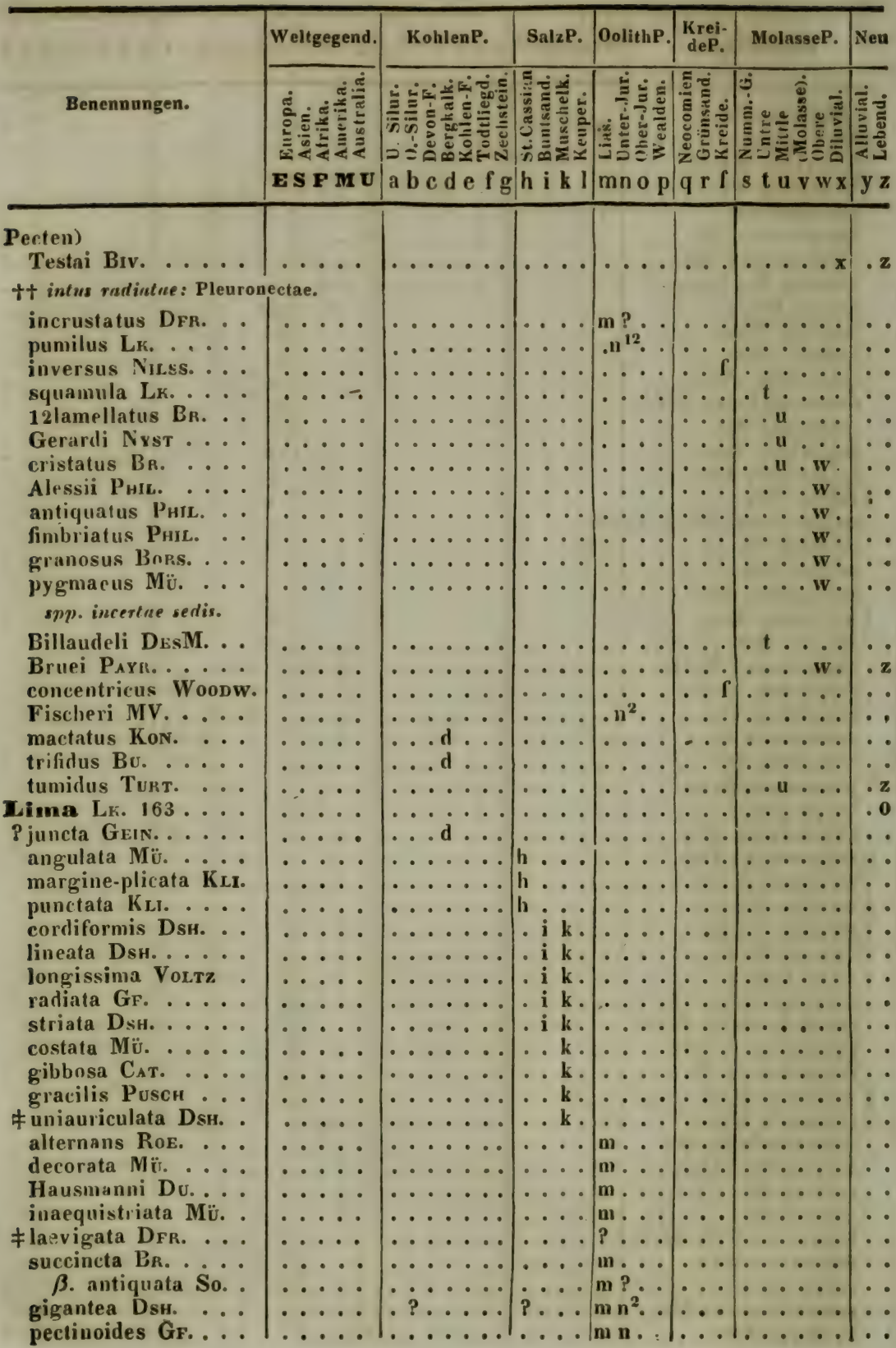


abrupta Gr. aciculata MÜ.

‡alata Pov. antiqua a Mü.

cardiiformis So. sp. consobrina d'O. costulata Pos. decorata Fromн. duplicata GiF. elougata MÜ. pxarata GF. fimbriata Fromi.

\# fragilis Roe. gibbosa So. glabra $G_{F}$.

\# grandis Roe. interstincta Puicl. sp. larviuscula GF. f lingulata Dsh. \# lunularis Desh. lyrata Mü. ninuta Roe. notata Gr. obseura Dsh. ovalis GF. pertiniformis ZiET. Phillipsi d'O. rigida $G_{F}$. rigidula Phir.s. sp. scabrosa Mü. semicircularis GF. semilunaris GF. striatula Mü. * striatula RoE. ₹ subantiquata RoE. substriata Mü. sulcata Mü. tegulata Mü tenuistriata $\dot{M} \ddot{\text {. }}$.

? terebratularis DFR. transversa Dsh. tumidescens Fromu. \# tumida Roe. theteromorpha DsLG. * t sulcata Dsc.g. †variabilis: Dscg. t radista Dscg. t punctata Dsug.

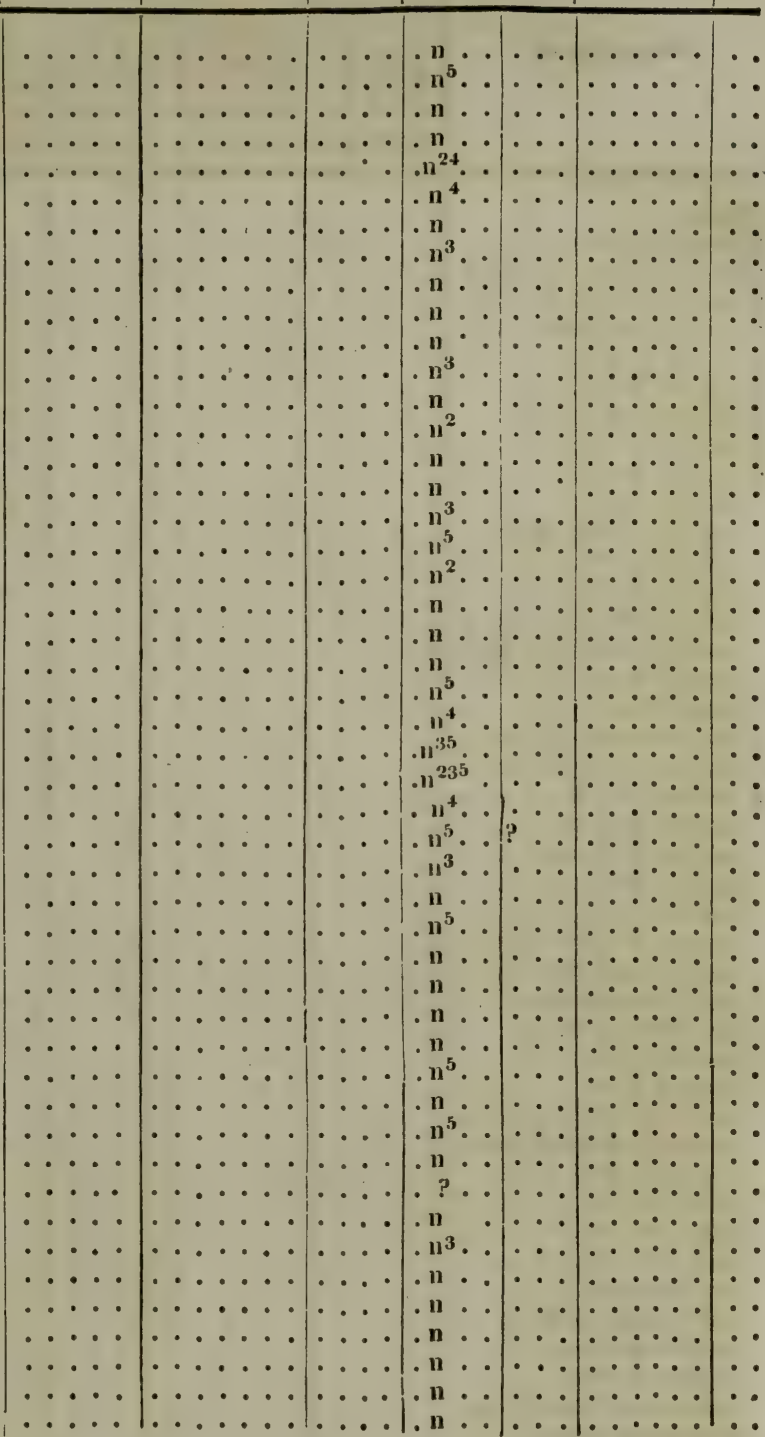

* spp. Deslongchampsnnus numerosas, nobis non nisi nominibus cognitas, hic systematice dispositas serius descriptionibus et figuris illustraties fore sperumus. 


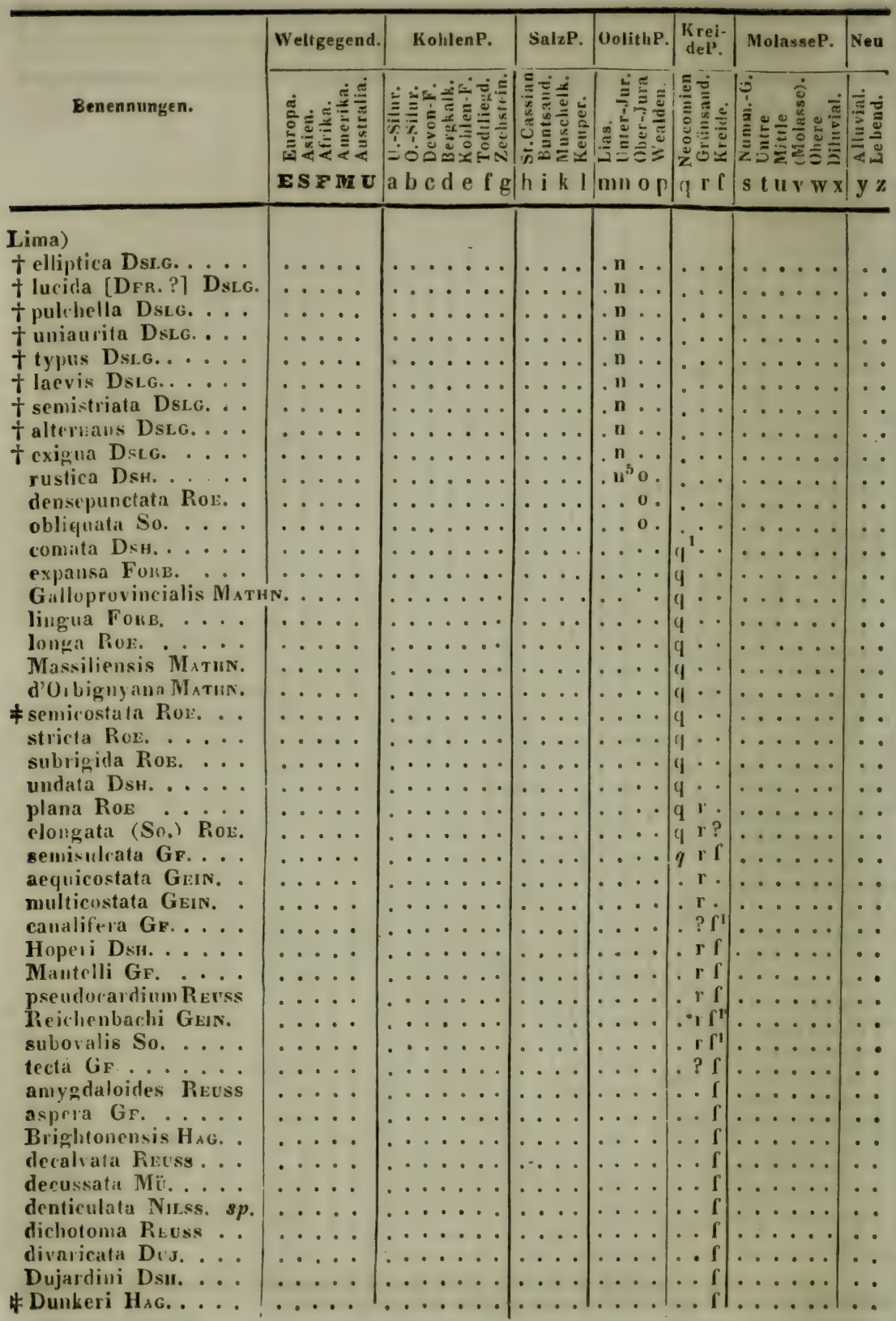




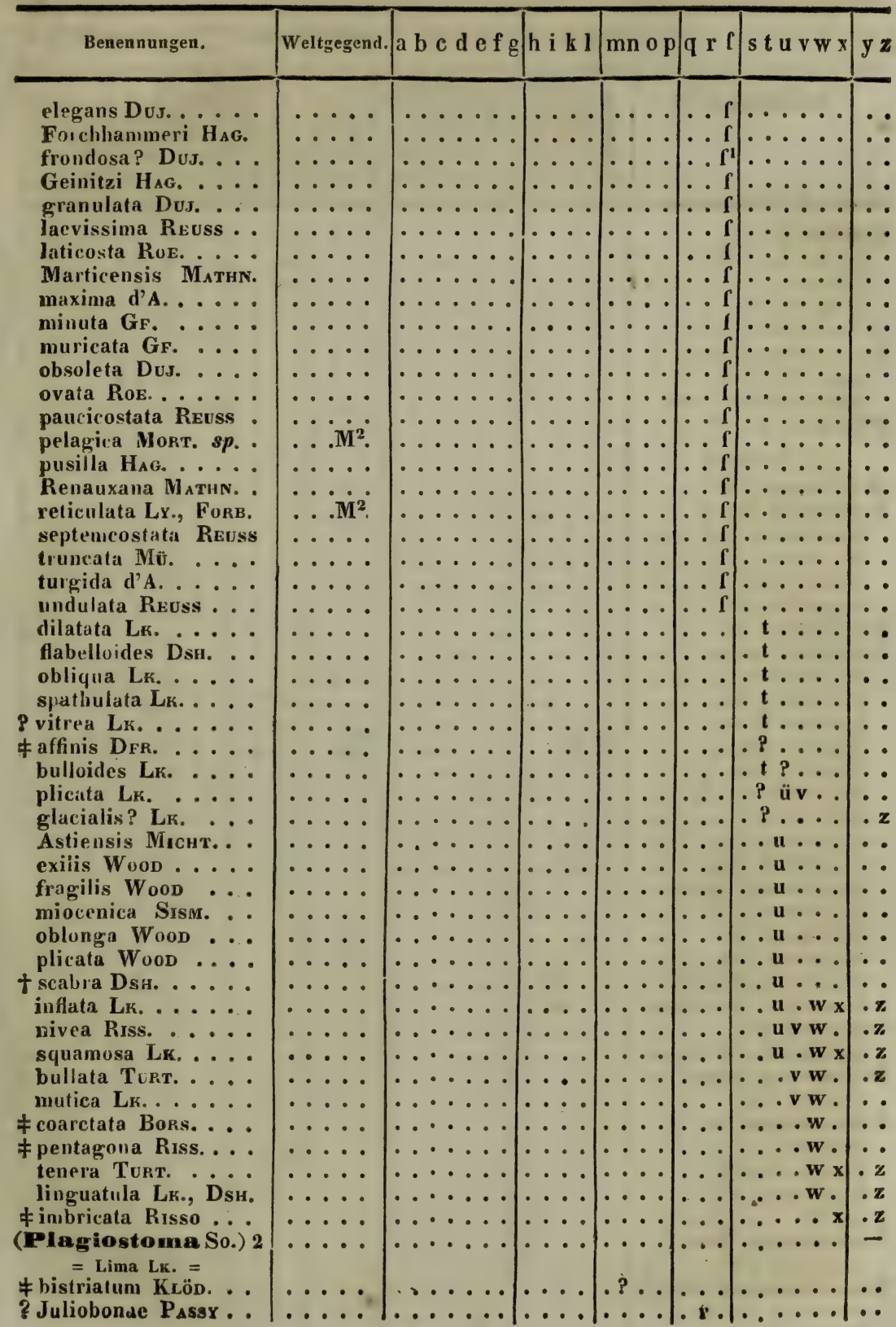




\begin{tabular}{|c|c|c|c|c|c|c|c|}
\hline & Weltgegend. & KohlenP. & SalzP. & GolithP. & $\begin{array}{r}\text { Krei- } \\
\text { deP. }\end{array}$ & MolasseP. & Nell \\
\hline Benennungen. & 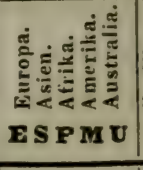 & 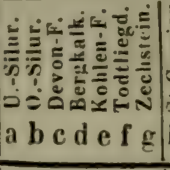 & 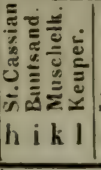 & 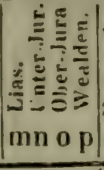 & 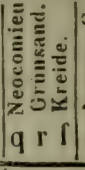 & 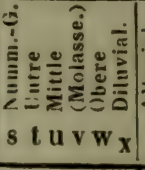 & 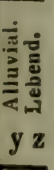 \\
\hline 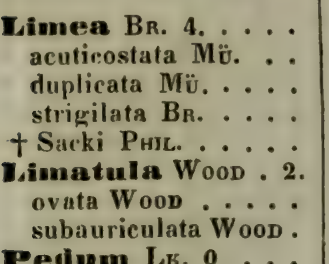 & $\begin{array}{l}\cdots \cdots \\
\cdots \cdots \\
\cdots \\
\cdots \\
\cdots \\
\cdots \\
\cdots\end{array}$ & $\left|\begin{array}{ccc}\cdots & \cdots & \cdots \\
\cdots & \cdots & \cdots \\
\cdots & \cdots & \cdots \\
\cdots & \cdots & \cdots \\
\cdots & \cdots & \cdots \\
\cdots & \cdots & \cdots \\
\cdots & \cdots & \cdots \\
\cdots & \cdots\end{array}\right|$ & $\mid \begin{array}{c}\cdots \\
\cdots \\
\cdots \\
\cdots \\
\cdots \\
\cdots \\
\cdots \\
\cdots\end{array}$ & $\left|\begin{array}{cc}\cdots & \cdots \\
\cdots & \cdots \\
\cdots & \cdots \\
\cdots & \cdots \\
\cdots & \cdots \\
\cdots & \cdots\end{array}\right|$ & $\begin{array}{l}\cdots \\
\cdots \\
\cdots \\
\cdots \\
\cdots \\
\cdots\end{array}$ & $\mid \begin{array}{l}\cdots \cdots \\
\cdots \cdots \\
\cdots \\
\cdots \\
\cdots \\
\cdots \\
\cdots u \\
\cdots u \\
\cdots\end{array}$ & $\begin{array}{l}.0 \\
\cdots\end{array}$ \\
\hline $\begin{array}{r}\text { Pediom LK } \\
\text { भ. Mall }\end{array}$ & $\cdots \cdots$ & $\ldots \ldots$ & $\ldots$ & $\cdots \cdots$ & $\cdots$ & $\cdots \cdots$ & .1 \\
\hline $\begin{array}{l}\text { eus L. } 0 . \ldots \\
\text { ella LK. 4. . } \\
\text { onensis DUJ. . } \\
\text { BwrB. . . . . } \\
\text { ta MÜ. . . . . } \\
\text { rdita LK. . . . }\end{array}$ & $\begin{array}{l}\cdots \cdots \\
\cdots \cdots \\
\cdots \\
\cdots \\
\cdots \\
\cdots\end{array}$ & $\mid \begin{array}{ccc}\cdots & \cdots & \cdots \\
\cdots & \cdots & \cdots \\
\cdots & \cdots & \cdots \\
\cdots & \cdots & \cdots \\
\cdots & \cdots & \cdots \\
\cdots & \cdots\end{array}$ & $\mid \begin{array}{c}\cdots \\
\cdots \\
\cdots \\
\cdots \\
\cdots \\
\cdots \\
\cdots\end{array}$ & $\begin{array}{ll}\cdots & \cdots \\
\cdots & \cdots \\
\cdots & \\
\cdots & \cdots \\
\cdots & \end{array}$ & $\begin{array}{ll}\cdots & \\
\cdots & f \\
\cdots & f \\
\cdots & f\end{array}$ & 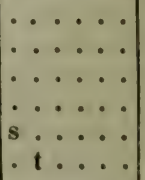 & $\begin{array}{r}.6 \\
.6 \\
.\end{array}$ \\
\hline 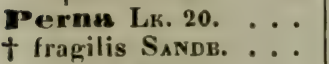 & $\cdots$ & $\cdots$ & & $\therefore \cdots$ & $\cdots$ & $\mid \begin{array}{cc}\cdots & \ldots \\
\ldots & \ldots\end{array}$ & 16 \\
\hline $\begin{array}{l}\text { fragilis SANDB. . . . } \\
\text { velusi GF. . . . } \\
\text { mytiloides LK. . . . }\end{array}$ & $\cdots$ & $\cdots \cdots$ & $\begin{array}{ccc}\cdots & i \\
\cdots & & i\end{array}$ & $\begin{array}{lll}23 & \cdots \\
11^{23} & \cdots\end{array}$ & & 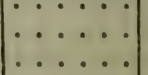 & $\begin{array}{l}\cdots \\
\dot{\bullet}\end{array}$ \\
\hline $\begin{array}{l}\text { i Rourc. . . } \\
\text { a So...... }\end{array}$ & $\mathrm{E}^{2} \mathrm{~S}^{2} ?$ & $\cdots$ & $\ldots$ & $\begin{array}{l}. \\
. ? 0 \\
. ?\end{array}$ & $\ddot{r}$ & $\mid \begin{array}{cc}\ldots & \ldots \\
\cdots & \ldots\end{array}$ & $\because$ \\
\hline Dsн. .... & $\cdots \cdots$ & $\ldots \ldots$ & $\cdots$ & $\cdots \cdot$ & $q^{1} \cdot$. & $\ldots \ldots$ & $\because$ \\
\hline s. & $\cdots$ & $\begin{array}{ll}\cdots \\
\cdots\end{array}$ & $\cdots$ & $\cdots \cdots$ & $q \cdots$ & 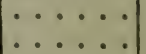 & . \\
\hline EIN. . . & $\ldots \ldots$ & $\ldots \ldots$ & $\cdots$ & $\cdots$ &.$r \mathrm{f}$ & $\ldots \ldots$ & $\cdots$ \\
\hline Hi... & $\cdots \cdots$ & $\cdots \cdots$ & $\cdots$ & $\cdots$ & $\begin{array}{cc}\cdots \\
\cdots & f\end{array}$ & $\left|\begin{array}{cc}\cdots & \cdots \\
\cdots & \cdots\end{array}\right|$ & $\cdots$ \\
\hline $\begin{array}{l}\text { THN. . } \\
\text { EUSS . }\end{array}$ & .... & $\ldots \ldots$ & $\ldots$ & $\cdots \cdot$ & $\ldots \mathrm{f}$ & $\ldots \ldots$ & $\ddot{*}$ \\
\hline i So..... & $\cdots \cdots$ & $\ldots \ldots$ & $\cdots$ & $\cdots \cdot 1$ & $\cdots$ &.$t \ldots$ & .. \\
\hline $\begin{array}{c}\text { Dsн. } \\
\text { udi D' }\end{array}$ & $\cdots \dot{M^{4}}$ & $\ldots \ldots$ & $\cdots \cdot$ & $\cdots \cdot$ & $\cdots$ & $\cdot t \ldots$ & $\cdots$ \\
\hline D'O. . & $\cdots \mathrm{M}^{\mathbf{4}}$ & $\cdots \cdots$ & $\cdots$ & $\cdots \cdots$ & $\cdots$ & $\cdot t \ldots$ & 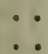 \\
\hline .. & $\cdots$ & $\ldots \ldots$ & & $\ldots$ & $\cdots$ & u.w. & $\therefore$ \\
\hline Bast. & $\cdots$ & $\cdots \cdots$ & $\ldots$ & $\cdots$ & $\cdots$ & $\ldots ? \ldots$ & $\mathrm{z}$ \\
\hline $\begin{array}{l}\text { es Bertr. } 1 . \\
\text { DFR. . . . }\end{array}$ & $\cdots \cdots$ & $\cdots \cdots$ & & & $\cdots$ & $\cdots \cdots$ & .0 \\
\hline Lк. & $\cdots$ & ・・・ & & $\cdot n^{2} \cdot$ & $\ldots$ & $\ldots \ldots$ & .8 \\
\hline & $\ldots \ldots$ & . & & $\mathrm{m} \ldots$ & $\cdots$ & $\ldots \ldots$ & $\ldots$ \\
\hline$\cdots \cdot$ & $\cdots \cdot$ & & & . n. & & $\cdots \cdots$ & \\
\hline$\cdots$ & $\cdots \cdot$ & $\cdots \cdot$ & & . n. & - & $\cdots \cdots$ & - \\
\hline 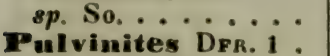 & $\cdots \cdots$ & $\cdots$ & & $\cdots$ & & $\cdots \cdots$ & $\ddot{0}$ \\
\hline$\cdots$ & $\cdots$ & & & $\ldots$. & $\because i$ & $\cdots \cdots$ & $\begin{array}{c}.0 \\
. .\end{array}$ \\
\hline RK.53 & $\bullet \bullet \bullet \cdot$ & ; & & $\cdots$ & $\cdots$ & $\ldots \ldots$ & .0 \\
\hline & $\cdots$ & & & & & & .: \\
\hline & & & & & & & \\
\hline
\end{tabular}




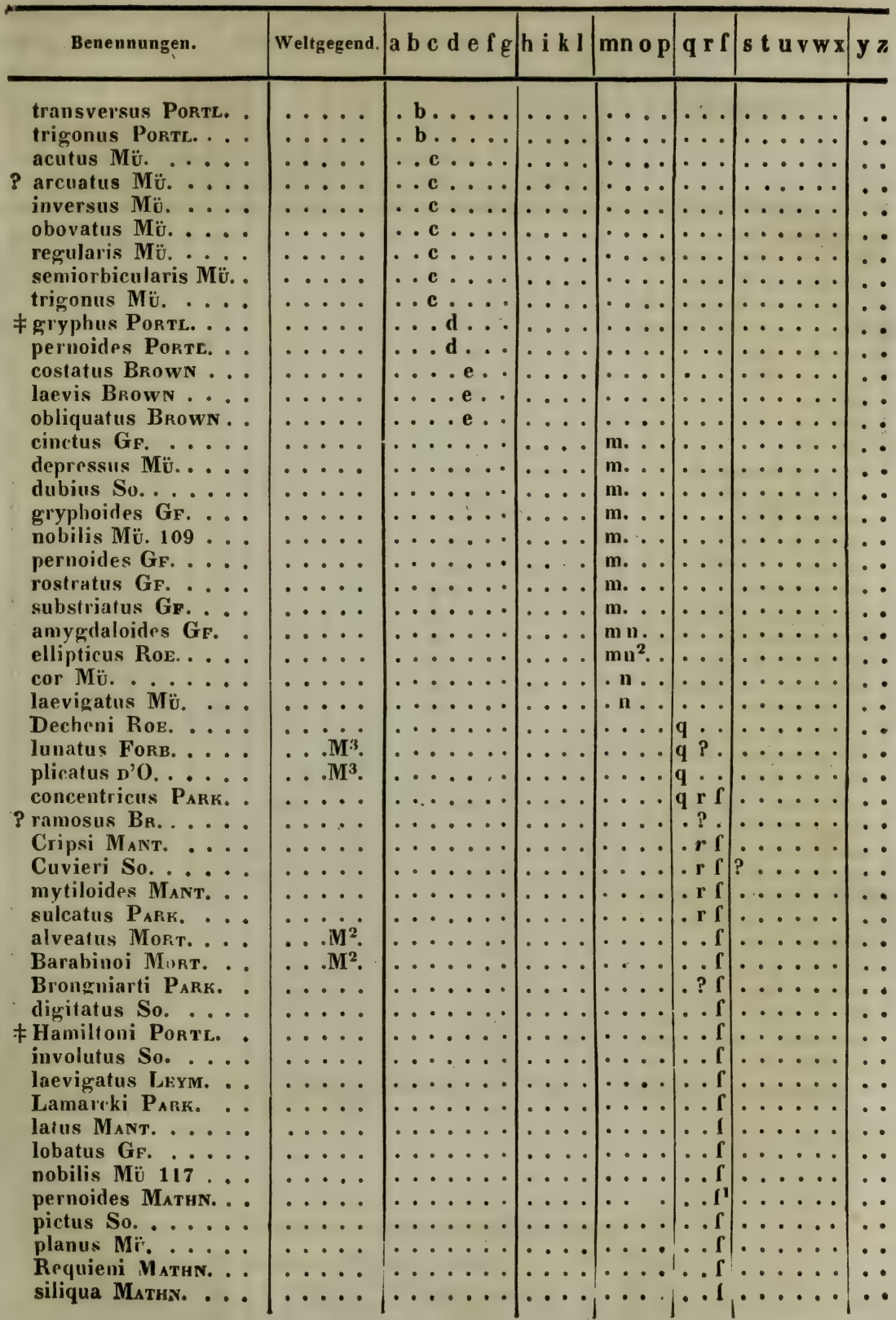




\begin{tabular}{|c|c|c|c|c|c|c|c|}
\hline \multirow[b]{2}{*}{ Benennungen. } & Weltgegend. & KohlenP. & SalzP. & OolithP. & $\begin{array}{l}\text { Krei- } \\
\text { deP. }\end{array}$ & MolasseP. & Neu \\
\hline & 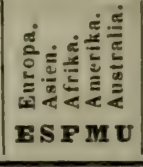 & 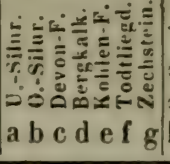 & 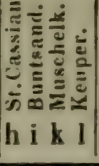 & 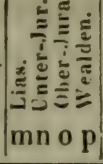 & 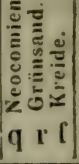 & 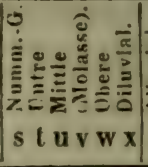 & 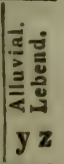 \\
\hline BRGN, 2 . & . & $\cdots$ & $\cdots$ & $\cdots$ & $1^{\circ}$ & $\cdots \cdots$ & .0 \\
\hline nis $\mathrm{Muc}_{\mathrm{u}}$ & $\cdots$ & • & $\cdots \cdot$ & $\cdots \cdots$ & $\cdot r$ & $\cdots \cdots$ & 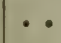 \\
\hline$\neq 1$ & $\cdots \cdot$ & $\cdots \cdots$ & $\cdots \cdot$ & $\cdots$ & .. I & $\cdots \cdots$ & 0 \\
\hline $\begin{array}{l}\text { Posidonomy: } \\
\text { P venusta } M \ddot{\text {. }} \text {. }\end{array}$ & • & I. & $\because$ & $\cdots$ & $\because \cdots$ & $\cdots \cdots$ & .0 \\
\hline $\begin{array}{l}? \text { ven } \\
? \text { cos }\end{array}$ & $\cdots \cdots$ & . c.... & $\ldots$ & $\cdots$ & $\cdots$ & $\cdots \cdots$ & - \\
\hline $\begin{array}{l}\text { ? costata Mĭ. } \ldots \ldots \\
\text { elegans Mü. . . . . }\end{array}$ & $\ldots .$. & $\ldots \mathbf{c} \ldots$ & $\ldots$. & $\ldots$. & ... & $\cdots \cdots$ & $\cdot$ \\
\hline grandis Mü. . . . . . & $\cdots \cdot$ & . c.... & $\cdots$ & $\cdots \cdot$ & $\cdots$ & $\ldots \ldots$ & - \\
\hline$\cdots \cdots$ & $\cdots \cdot$ & . c... & $\cdots$ & . & $\cdots$ & $\ldots \ldots$ & 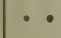 \\
\hline Br... & $\cdots \cdot$ & $\ldots c . .$. & $\cdots \cdots$ & $\cdots \cdot$ & $\cdots$ & $\cdots \cdots$ & - \\
\hline$\cdots$ & $\cdots \cdot \cdot$ & . c.... & $\cdots \cdots$ & $\cdots \cdot$ & $\cdots$ & $\cdots \cdots \cdots$ & • \\
\hline$\cdots \cdot$ & $\cdots$ & $\cdots c$ & $\cdots$ & $\cdots \cdot$ & $\cdots$ & $\cdots \cdots$ & $\bullet$ \\
\hline Mâta Mû. . . . & $\cdots \cdots$ & . c & $\cdots \cdots$ & $\cdots \cdots$ & $\cdots$ & $\cdots \cdots$ & •・ \\
\hline R. ..... & $\ldots \ldots$ & $\ldots c d \ldots$ & $\ldots$ & 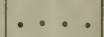 & . . & $\ldots \ldots$ & $\bullet$ \\
\hline • & $\cdots \cdot$ & $\ldots d \ldots$ & $\cdots$ & $\cdots$ & $\ldots$ & $\ldots \ldots$ & -・ \\
\hline hemisphaerica KoN. & $\cdots \ldots$ & ...d... & $\cdots \cdot \mid$ & $\cdots$ & $\cdots$ & $\cdots \cdots$ & 泟 \\
\hline Rs. . . & $\cdots \cdot \cdot$ & ... d... & $\cdots$ & $\cdots \cdot$ & $\cdots$ & $\cdots \cdots$ & \\
\hline RRs. & $\cdots \cdot \cdot$ & $\ldots d \ldots$ & $\cdots \cdots$ & $\cdots \cdots$ & $\cdots$ & $\cdots \cdots$ & $\bullet$ \\
\hline$\cdots$ & $\cdots \cdots$ & $\ldots d \ldots$ & $\cdot$ & - & $\cdot$ & $\cdots \cdots \cdot$ & \\
\hline Wh. . . & 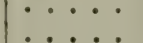 & : & h... & $\cdot$ & $\cdots$ & $\cdots \cdots$ & \\
\hline $\begin{array}{l}\text { enensis Wissm. } \\
\text { Br. ...... }\end{array}$ & $\ldots$ & $\cdots \cdots$ & $\mathrm{i} \mathbf{k i}$ & $\cdots$ & $\because$ & $\cdots \cdots \cdots$ & \\
\hline aris Briun . & $\ldots$. & $\ldots \ldots$ & $\cdots$ & $m \ldots$ & . . & $\ldots \ldots$ & . \\
\hline ala Mü. . . . & $\cdots \cdot$ & & & $.11^{5}$. & $\cdots$ & $\ldots \ldots$ & - \\
\hline canaliculata Mü. & $\cdots \cdots$ & & & $\cdot n^{5} \cdot$ & $\cdots$ & $\cdots \cdots$ & 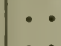 \\
\hline nia Br.anlea.) & $\cdots$ & $\cdot$ & $\cdots$ & $\begin{array}{l}\cdots \\
\cdots\end{array}$ & $\cdots$ & $\cdots \cdots$ & .. \\
\hline & & & & & & & \\
\hline P socialis C & $\cdots$ & &.$\dot{k}$ & . & $\cdots$ & $\cdots \cdots$ & \\
\hline$\cdots$ & $\ldots .$. & . & $\ldots$ & m... & $\ldots$ & $\ldots \ldots$ & \\
\hline$\cdots$ & $\cdots \cdot$ & . & $\ldots$ &. $\mathbf{n}$ & $\cdots$ & $\ldots \ldots$ & 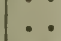 \\
\hline s. . . . & $\cdots \cdot$ & . & $\cdots$ & . n . & $\cdots$ & $\ldots$. & $\bullet$ \\
\hline socialis Mü. ..... & .... & $\ldots$ & $\cdots \cdot$ &. $\mathrm{n}$. & & & \\
\hline Monomyo & & & & & $10=0$ & व $\approx 1$ & \\
\hline
\end{tabular}

Corrigenda: in ultima linea verticali ( $\mathrm{z}$ ):

p. 237, linea 6 (Anomia) pro 0 legendum est 20

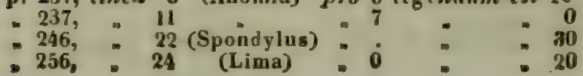




\begin{tabular}{|l|l|l|l|l|l|l|l|}
\hline Benenaungen. & Weltgegend. & a b c d e f g & h i k l & mn o p & q r f & s t. u v w x & y z \\
\hline
\end{tabular}

\section{MIVIYA.}

A. HETEROMYA, Ungleichmuskeler *

\section{(Monomya Lk. pars.) \\ = INTEGRIPALLIATA =}

a Aviculina.

Gervilleia Drr. 34

Felongata Ports. inconspicua Phicl.

laminosa Phicl. squamosa Pric... minor Brown ...

? tumida King $s p$. Gein. angulata $\ddot{M}_{\ddot{v}}$. angusta Mï. intermedia Mü. Johannis-Austriae Ku socialis QU.

Hagenowi Du. ? acuta So.

\# angustata Ros. aviculoides $Z_{\text {IET. }}$ [non Su.].

Bronni KoDu. costatula Dis.g. glabrata Du. lanceolata Mü. monotis Dsig. pernoides DsLg. siliqua Dsı.g. .... tortuosa Qu. sp. Q0. aviculoides So. obtusa Roz. siliquaria Mü. tetragona Ros. solpnoides DFr. linguloides Fo R. . . dentata Krauss . . . Renauxana MathN. . triloba Roe.

Mygina Korv. 3 . Goldfussana ..... lame!losa virgula .

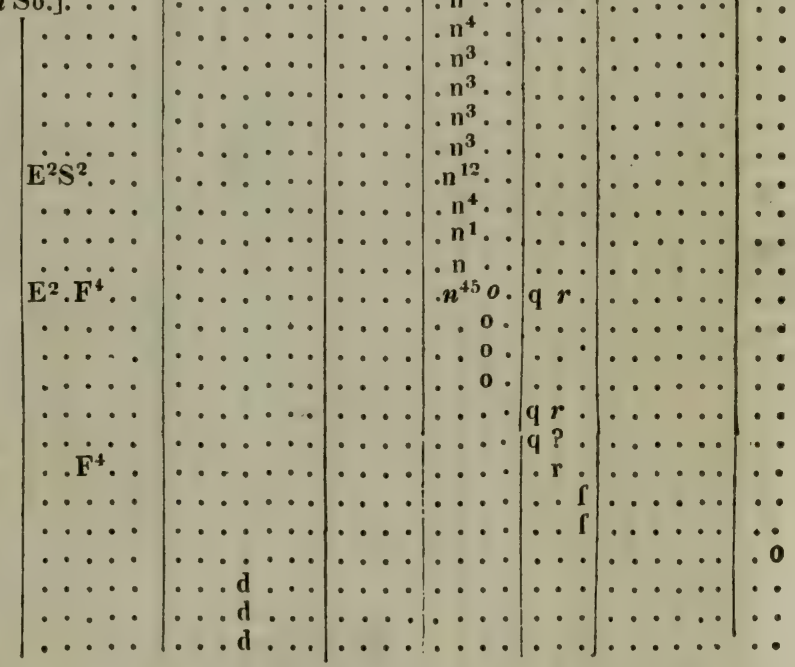

* Character e musculis innequnlilus sumtus non omniǹn constans, ef inde valnre debilis est; unmine indicuto coujunctu domus generu olim Monomyis LK. adscrip/a, quie tumen hine inde muspulos magis aeguales in speciebus quibusdam subuequiluteralibus offerunt. 


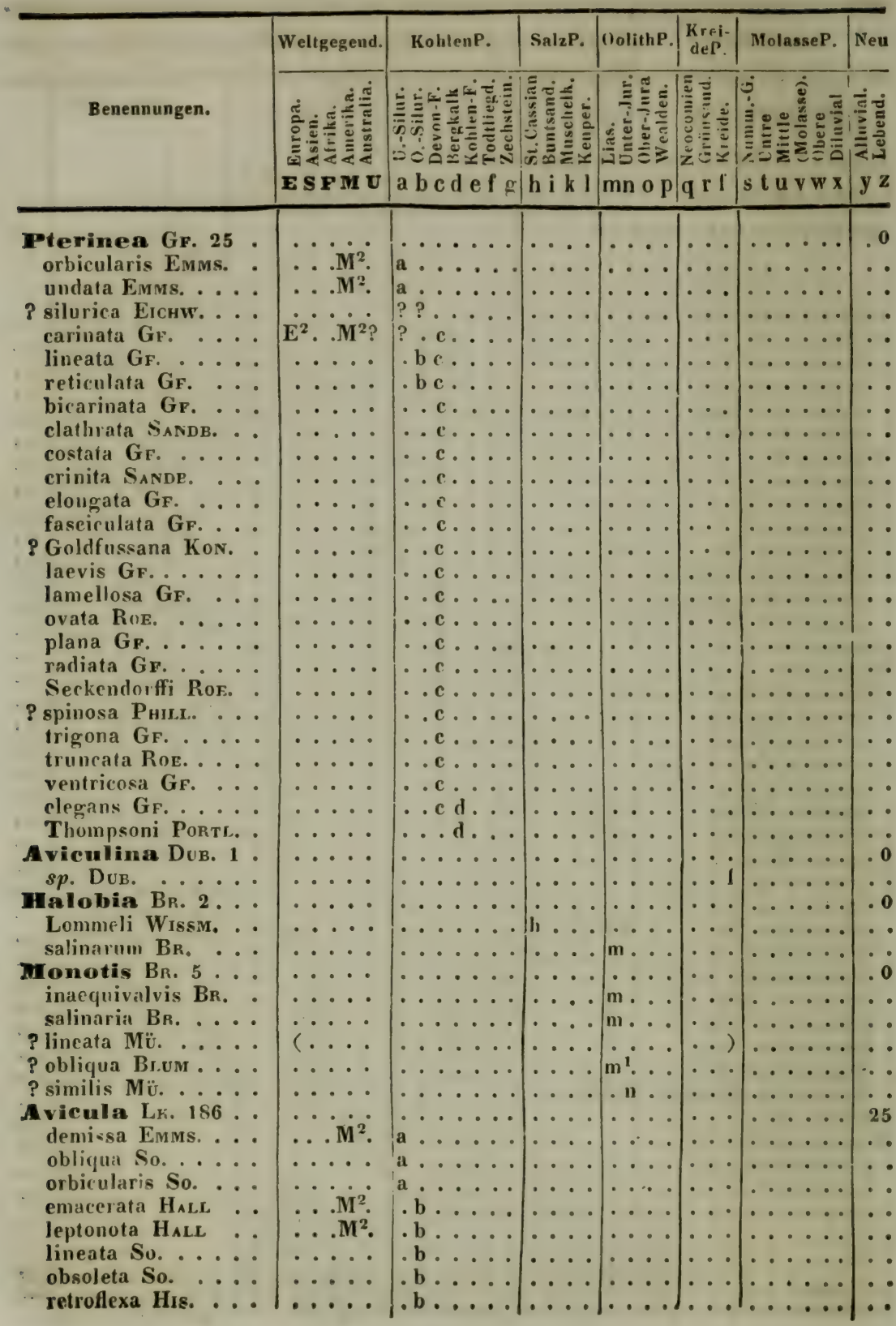




\begin{tabular}{|c|c|c|c|c|c|c|c|}
\hline Benennungen. & Welt! & $\mathrm{g}$ & hik l & mn op & $q \times f$ & $w \mathbf{x}$ & y $z$ \\
\hline antiqua GF. t. 160. & & - & & $\theta^{\circ}$ & & & \\
\hline Neptuni Gr. ... & $\ldots \ldots$ & b c.... & $\ldots$ & $\ldots$ & $\cdots$ & $\ldots \ldots$ & $\therefore$ \\
\hline aculeata GF. ... & $\cdots \cdots$ & $\ldots \mathbf{c} \cdot \ldots$ & $\ldots$ & $\ldots$ & $\cdots$ & $\ldots \ldots$ & - \\
\hline anisota PHic..... & $\ldots \ldots$ & $\ldots \mathbf{c} \ldots$ & $\cdots \cdot$ & $\cdots$ & $\cdots$ & $\ldots \ldots$ & - \\
\hline arcana KEYs. … & $\cdots \cdots$ & $\cdots c \ldots$ & $\cdots \cdot$ & $\cdots \cdots$ & $\cdots$ & $\cdots \cdots$ & • \\
\hline cancellata PHII & $\ldots$. & $\cdots \cdots$ & $\cdots$ & $\cdots \cdot$ & $\cdots$ & $\cdots \ldots$ & ete \\
\hline Damnoniensis So. . & & $\cdots$ & $\cdots \cdot$ & $\cdots \cdot$ & $\cdot \cdot$ & $\cdots \cdots$ & • \\
\hline elongata Mü. . . . . & 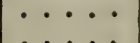 & $\therefore$ & $\cdots \cdots$ & $\cdots \cdot$ & $\cdots$ & $\cdots \cdots$ & • \\
\hline$\cdots$ & $\therefore$ & $\begin{array}{lll}\cdots & \cdots & \cdots \\
\cdots & c & \cdots\end{array}$ & $\cdots \cdots$ & $\mid \cdots \cdots$ & $\cdots$ & $\cdots \cdots \cdots$ & - \\
\hline $\begin{array}{l}\text { eximia MVK. . . . } \\
\text { gibbosa Mü. . . . }\end{array}$ & $\cdots$ & $\therefore$ c.... & $\ldots$ & $\ldots$ & $\ldots$ & $\cdots \cdots$ & • \\
\hline Goldfussi d'A....... & $\ldots \ldots$ & $\ldots c \ldots$ & $\ldots$ & $\cdots$ & $\ldots$ & $\ldots \ldots$ & $\dot{\bullet}$ \\
\hline inflata $M \ddot{U} . \ldots$. & $\cdots \cdot$ & $\ldots c \ldots$ & $\ldots$ & $\cdots$ & $\cdots$ & $\ldots \ldots$ & $\dot{\bullet}$ \\
\hline Jugleri Roe. & $\cdots \cdot$ & ....... & $\cdots \cdots$ & $\cdots$ & $\cdots$ & $\ldots \ldots$ & - \\
\hline Kahlebergensis Roz. & $\cdots$ & $\ldots \mathbf{c} \ldots$ & $\ldots$ & $\cdots$ & $\cdots$ & $\ldots \ldots$ & . \\
\hline$\cdots$ & $\ldots$ & $\ldots$ c... & $\ldots$ & $\cdots$ & $\cdots$ & $\ldots \ldots$ & .. \\
\hline$\cdots$ & $\ldots$. & $\ldots \mathbf{c} \cdot \ldots$ & $\cdots \cdot$ & $\cdots$ & $\cdots$ & $\ldots \ldots$ & 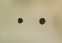 \\
\hline$\cdots$ & $\ldots$. & $\cdots \mathbf{c} \ldots$ & $\cdots \cdot$ & $\cdots$ & $\cdots$ & $\cdots \cdots$ & \\
\hline lematica Mï. & & c.... & $\cdots \cdots$ & $\cdots \cdot$ & $\cdots$ & $\cdots \cdots$ & - \\
\hline ta Mü.. & & c... & $\cdots \cdot$ & $\cdots \cdot$ & $\cdots$ & $\cdots \cdots$ & • \\
\hline o. . & $\cdots \cdot \cdot$ & $\cdots$ & $\cdots \cdots$ & $\cdots \cdots$ & $\cdots$ & $\cdots \cdots$ & - \\
\hline Peticulata PніLl. . . & $\cdots \cdot \cdot$ & $\cdots$ & $\cdots \cdots$ & $\cdots \cdot$ & $\cdots$ & $\cdots \cdots$ & 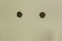 \\
\hline rudis Phine & $\cdots \cdot$ & $\cdots$ & $\cdots$ & $\cdots \cdot$ & $\cdots$ & $\cdots \cdots$ & - \\
\hline$\ddot{\circ}$ & & $\because$ & $\cdots$ & $\cdots \cdot$ & $\cdots$ & $\cdots \cdots$ & - \\
\hline $\begin{array}{l}\text { Siturni Gr. . . . } \\
\text { ? semialata Mü. }\end{array}$ & & $\cdots$ & $\cdots$ & $\cdots \cdot$ & $\because$ & $\dot{.}$ & \\
\hline $\begin{array}{l}\text { P semialata Mü. } \dot{\text { Mü. }} \\
\text { semiausiculata }\end{array}$ & $\cdots$ & $\because c$ & $\begin{array}{ll}\cdots \\
\cdots \\
\cdots\end{array}$ & $\mid \cdots \cdots$ & $\because$ & $\cdots \cdots$ & $\bullet$ \\
\hline diata So. ... & ... & $\ldots \mathbf{c} \ldots$ & ... & $\ldots$ & $\cdots$ & $\ldots \ldots$ & • \\
\hline RAUN . & $\cdots \cdots$ & $\ldots c \ldots$ & $\cdots \cdot$ & $\cdots$ & $\cdots$ & $\cdots \cdots$ & - \\
\hline$\cdot$ & $\cdots \cdot$ & $\cdots$ & $\ldots$ & $\cdots$ & $\cdots$ & $\cdots \cdots$ & \\
\hline Woerthi VE & $\cdots \cdot$ & $\cdots$ & $\cdots$ & $\cdots$ & - & $\cdots \cdots$ & \\
\hline$\cdots$ & & - & . & $\cdot$ & $\cdots$ & - & \\
\hline ana Kon. - & $\cdot$ & 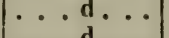 & $\cdots$ & 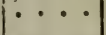 & $\cdots$ & $\cdots$ & \\
\hline Buchana Kov. ... & $\cdots$ & $\cdots$ & $\cdots$ & $\cdots$ & . . & . & • \\
\hline • $\cdot$ & & & $\cdot \cdot$ & & $\cdots$ & 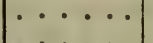 & \\
\hline Dumontana & & $\cdots$ & $\cdots \cdot$ & $\cdots$ & • & $\cdots$ & • \\
\hline a Kon. ... & & 1 & $\cdots$ & $\cdots$ & $\cdots$ & $\cdots \cdots \cdots$ & \\
\hline $\begin{array}{l}\text { lepida GF. . . . } \\
\text { lunulata Kon. . . }\end{array}$ & & $\cdots$ & $\mid \begin{array}{c}\cdots \\
\cdots\end{array}$ & $\cdots \cdots$ & $\cdots$ & $\cdots \cdots \cdots$ & . \\
\hline $\begin{array}{l}\text { lunulata KoN. . . . } \\
\text { magnifica Kon. . . }\end{array}$ & $\therefore$ & $\ldots d \ldots$ & $\ldots$ & $\ldots$ & $\ldots$ & $\ldots \ldots$ & \\
\hline$\ldots$ & $\because$ & $\ldots d \ldots$ & $\ldots$ & $\ldots$ & $\ldots$ & $\ldots \ldots$ & \\
\hline Nystana Kon..... & $\cdots$ & $\cdots$ & . & $\cdots \cdot$ & $\cdots$ & $\cdots \cdots$ & \\
\hline . & . & & $\cdots$ & $\cdots$ & . & & \\
\hline radula Ko & $\cdots$ & & $\cdots \cdots$ & $\cdots$ & $\cdots$ & $\cdots$ & \\
\hline simplex Kor & - & $\cdots$ & $\cdots$ & $\cdot \cdot$ & $\cdots$ & & \\
\hline lata I & & : & $\cdot$ & $\cdots$ & $\cdots$ & $\cdots$ & \\
\hline s & & . & $\cdots \cdot$ & & & & $\therefore$ \\
\hline tumida B & $\cdots \cdots$ & $\cdots$ & $\cdots \cdots \cdot$ & $\cdots$ & ... & $\ldots$ & \\
\hline $\mathrm{N}$ & $\ldots$ & $\Theta_{-1}+2$ & $-\theta_{0}$ & $\ldots$ & $\cdots$ & $\ldots$ & \\
\hline $\begin{array}{l}\text { papyracea GF. . . } \\
\text { modiolaris So. }\end{array}$ & ... & & & & & & \\
\hline
\end{tabular}




\begin{tabular}{|c|c|c|c|c|c|c|c|}
\hline \multirow[b]{2}{*}{ Benennungen. } & Weltgegend. & KohlenP. & SalzP. & OolithP. & $\begin{array}{l}\text { rei- } \\
\text { eeP. }\end{array}$ & MolasseP. & \\
\hline & 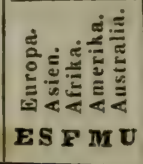 & 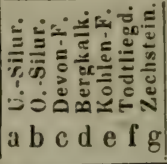 & 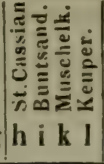 & 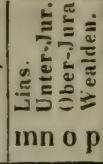 & 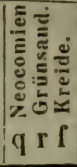 & $\mid$ & \\
\hline \multicolumn{8}{|l|}{ icul } \\
\hline & & & $\cdots \cdots$ & $\cdots$ & $\cdots$ & 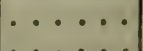 & \\
\hline$\cdots$ & $\cdots$ & $\begin{array}{ll}\cdots \\
\cdots\end{array}$ & & $\cdots$ & $\ldots$ & & \\
\hline$\cdots$ & $\cdots$ & $\ldots \ldots f \cdot$ & - & $\cdots$ & $\cdots$ & $\ldots \ldots$ & \\
\hline . & $\cdots$ & $\cdots \cdots f$ & ( & $\cdots$ & $\cdots$ & $\cdots \cdots$ & \\
\hline$\therefore$ & $\therefore 2^{\circ}$ & 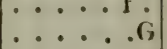 & & $\cdots$ & $\cdots$ & $\cdots$ & \\
\hline K. . &.$S^{2} \ldots$ & $\ldots . . G$ & $\ldots$ & $\ldots$ & $\ldots$ & $\ldots \ldots$ & \\
\hline$\cdots$ &.$S^{2} \ldots$ & $\ldots \ldots \cdot G$ & $\cdots$ & $\cdots$ & $\cdots$ & $\cdots \cdots$ & \\
\hline I. $\ldots$ & 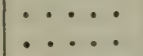 & $\begin{array}{lll}\cdots & \cdots g \\
\cdots & \cdots & g\end{array}$ & & $\begin{array}{ll}\cdots \\
\cdots\end{array}$ & $\begin{array}{l}\cdots \\
\cdots\end{array}$ & $\cdots \cdots$ & \\
\hline F. . . & $\therefore$ & $\ldots \ldots$ & & $\ldots$ & $\cdots$ & $\ldots \ldots$ & \\
\hline . & & & & $\cdots \cdots$ & $\cdots$ & $\cdots \cdots$ & \\
\hline$\cdots$ & - & $\cdots$ & $\mid \begin{array}{ll}h & \cdots \\
h & \cdots\end{array}$ & $\begin{array}{ll}\cdots \\
\cdots\end{array}$ & 1. & $\cdots \cdots$ & \\
\hline$\cdots$ & $\cdots \cdot$ & $\ldots \ldots$ & & $\ldots$ & $\cdots$ & $\ldots \ldots$ & \\
\hline$\cdots$ & $\cdots \cdots$ & $\ldots \ldots$ & & $\cdots$ & $\cdots$ & $\ldots \ldots$ & \\
\hline$\cdots$ & $\cdots \cdot \cdot$ & - & & $\cdots \cdots$ & $\cdots$ & $\cdots$ & \\
\hline 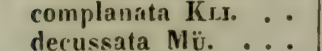 & $\cdots$ & $\ldots$ & & $\cdots \cdot$ & $\cdots$ & $\ldots \ldots$ & \\
\hline 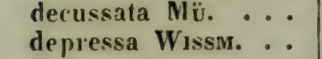 & $\cdots$ & : & & $\cdots$ & 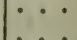 & $\cdots \cdots$ & \\
\hline$\therefore$ & .... & $\cdots \cdots$ & & $\cdots$ & • & $\cdots \cdots$ & \\
\hline$\dot{*}$ & $\cdots \cdot$ & $\cdots$ & h: & $\cdots$ & $\because$ & $\begin{array}{c}\cdots \\
\cdots\end{array} \cdots$ & \\
\hline M. & $\cdots$ & $\ldots$ & h. & $\cdots$ & $\cdots$ & $\cdots \cdots$ & \\
\hline$\cdot$ & $\cdots$ & .. & & $\ldots$ & $\ldots$ & $\cdots \cdots$ & \\
\hline$\cdots$ & $\cdots \cdot$ & $\ldots$ & & $\cdots$ & $\cdots$ & $\ldots \ldots$ & \\
\hline • & $\cdots \cdot$ & $\ldots$ & h. & $\cdots$ & $\cdots$ & $\ldots \ldots$ & \\
\hline$\cdots$ & $\cdots \cdot \cdot$ & $\ldots$ & h. & $\cdots$ & $\cdots$ & $\ldots \ldots$ & \\
\hline$\cdots$ & $\cdots \cdot$ & $\ldots$ & h. & $\cdots \cdots$ & $\cdots$ & $\ldots \ldots$ & \\
\hline$\cdots$ & $\cdots$ & 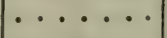 & h & $\cdots \cdot$ & - & $\cdots \cdots$ & \\
\hline$\cdots$ & $\cdots$ & & & $\cdots \cdot$ & - & $\cdots \cdots$ & \\
\hline$\therefore$ & . & & & $\cdots$ & - & $\cdots \cdots$ & \\
\hline sis. & . & $\cdots$ & & & & $\dot{0}$ & \\
\hline & 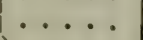 & $\ldots$ & . i . & ... & $\because$ & $\cdots$ & \\
\hline Gisn. & (1) & $\ldots \ldots$ & . i . & $\cdots$ & $\cdots$ & $\cdots \cdots$ & \\
\hline$\cdots$ & $\cdots \cdots$ & . & . $\mathrm{i}$ & $\cdots$ & $\cdots$ & $\ldots \ldots$ & \\
\hline$\cdots$ & $\cdots$ & $\ldots$ & . $\mathrm{i}$ & $\cdots \cdot$ & $\ldots$ & $\ldots \ldots$ & \\
\hline & $\cdots$ & $\ldots \ldots$ & - $\mathrm{i}$ & $\cdots$ & .. & $\ldots \ldots$ & \\
\hline . . & & & . i k I & $\cdots$ & & $\ldots \ldots$ & \\
\hline$\cdots$ & . & & $h \cdot \mathrm{k}$ & $\cdots$ & - & $\ldots \ldots$ & \\
\hline K & $\cdots \cdot$ & $\cdots \cdots$ & & $\cdots \cdot$ & & $\cdots \cdots$ & \\
\hline & . & & & $\cdots$ & & $\cdots \cdots$ & \\
\hline$\ldots$ & - & • • • & $\cdots 1$ & & & .. & \\
\hline & & & & & & & \\
\hline
\end{tabular}




\begin{tabular}{|c|c|c|c|c|c|c|c|}
\hline Be & nd. & $a b c$ & h i k l & $\mathrm{mn} o \mathrm{p}$ & q $\times 1$ & $\mathbf{s} \mathbf{t} \mathbf{u}$ & 12 \\
\hline - & & & & & & & \\
\hline olata So..... & .... & - & $\therefore$ & in... & . & ....... & \\
\hline tata RoE. . . & ... & $\cdots \cdots$ & $\cdots \cdot$ & m $\cdots$ & $\ldots$ & $\cdots \cdot \cdot$ & \\
\hline Ia ZiET. . & .... & . . & $\cdots \cdot$ & $\therefore$ & $\cdots \cdot$ & $\cdots \cdots$ & \\
\hline $\begin{array}{l}\text { So. } \ldots \ldots \\
\text { Mü. } \ldots . .\end{array}$ & • • • & - & $\begin{array}{ll}\cdots \\
\cdots & \cdots\end{array}$ & $\cdot$ & . & . & \\
\hline valvis So. . & $\cdots$ & $\begin{array}{l}\cdots \\
\cdots\end{array}$ & $\cdots \cdots$ & $m n^{234}$ & & $\begin{array}{ll}\cdots \\
\cdots \cdots\end{array}$ & \\
\hline ta Bн. ... & $\mathbf{E}^{2} \mathbf{S}^{2} \ldots$ & $\ldots \ldots$ & ... 1 & $\mathrm{~m}$ ? ... & ... & $\cdots \cdots$ & \\
\hline is Phill. & & $\cdots \cdots$ & $\cdots \cdot$ & $n^{3} \cdot \cdots$ & $\cdots$ & $\cdots \cdot$ & \\
\hline .... & $\cdots \cdot \cdot$ & $\cdots \cdots$ & $\cdots$ & $\left|\begin{array}{ll}\mathbf{n} & \cdots \\
\mathbf{n} & \cdots\end{array}\right|$ & & $\cdots \cdots$ & \\
\hline$\dot{\mathbf{0}} \cdot \dot{ }$ & $\begin{array}{l}\cdots \cdot \cdot \\
\cdots\end{array}$ & $\left|\begin{array}{lll}\cdots & \cdots & \cdots \\
\cdots & \cdots & \cdots\end{array}\right|$ & $\cdot$ & $n^{4} \cdot$ & $\begin{array}{l}\cdots \\
\cdots\end{array}$ & . $\cdot \cdots$ & \\
\hline a BEAN & & $\ldots \ldots$ & & $n^{5} \cdot \cdot$ & . & $\ldots \cdot$ & \\
\hline ... & .... & $\cdots \cdots$ & $\ldots$ & $\cdot \mathbf{u}^{5} \cdot \cdot$ & - & $\ldots \cdot$ & \\
\hline s. $\cdot \cdot$ & $\cdots \cdot \cdot$ & $\cdots \cdots$ & $\cdots$ & . n . & $\cdots$ & $\cdots \cdots$ & \\
\hline$\cdots \cdot$ & $\cdots \cdot \cdot$ & $\cdots \cdots$ & (2) & .11. & $\cdots$ & ..... & \\
\hline$\cdot \cdot \cdot$ & $\cdots \cdots$ & $\ldots \cdots$ & $\cdots \cdot$ & n $\cdot$ & $\cdots$ & $\cdots \cdots$ & \\
\hline OE. & $\cdots \cdot \cdot$ & $\cdots \cdots$ & ... & n . & $\cdots$ & $\cdots \cdots$ & \\
\hline $\begin{array}{l}\text { eri Rr. } \\
\text { GF. . . . }\end{array}$ & $\begin{array}{l}\cdots \\
\cdots \\
\cdots\end{array}$ & $\begin{array}{l}\cdots \\
\cdots\end{array}$ & $\begin{array}{lll}\cdots & \cdots & \\
. & . & \cdot\end{array} \mid$ & $\begin{array}{l}\mathbf{n} \cdot \cdot \\
\mathbf{n} .\end{array}$ & $\begin{array}{ll}\cdots \\
\cdots\end{array}$ & … & \\
\hline IF. & .... & . . . . & & $.1 n^{5}$ & $\cdots$ & $\cdots \cdots$ & \\
\hline ntricum & $\cdots \cdot \cdot$ & $\cdots \cdots$ & $\ldots$. & $m \ldots$ & . . & $\ldots \cdots$ & \\
\hline 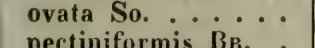 & $\cdots \cdot \cdot$ & $\cdots \cdot$ & &.$n^{3}$. & $\cdots$ & $\cdots \cdot \cdots$ & \\
\hline 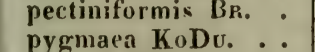 & $\cdots \cdot$ & $\cdots$ & & $\mathrm{n}^{5}$ & $\cdot \cdot$ & $\cdots \cdots$ & \\
\hline 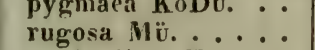 & $\begin{array}{l}\cdots \cdot \\
\cdot \cdots\end{array}$ & . & & n & & $\begin{array}{l}\cdot \cdots \\
\cdots\end{array}$ & \\
\hline Fisch. & .... & .... & & $n^{4}$. & & . & \\
\hline E. & $\cdots \cdots$ & $\ldots \ldots$ & . . & . 11.. & . . & . & \\
\hline a Ya.B. & $\cdots \cdot$ & $\ldots \ldots$ & . . . & $.11^{5}$. & $\cdots$ & $\ldots \ldots$ & \\
\hline Ou. . & $\cdots \cdot \cdot$ & $\cdots \cdot$ & - & . $1^{5}$. & $\cdots$ & $\cdots \cdots$ & \\
\hline$\cdots$ & 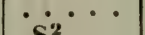 & 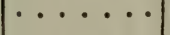 & - & $n^{4}$. & $\cdots$ & $\because \dot{*} \cdot$ & \\
\hline • & $\mathrm{S}^{2} \cdot \cdot$ & ( & . & . 11 . & & 1. ? & \\
\hline U. . & $\cdots \cdot \cdot$ & $\cdot$ & - & n o & & - & \\
\hline U. & $\begin{array}{ll}\cdots \\
\cdots\end{array}$ & . & $\begin{array}{ll}\cdots \\
\cdots & \cdots\end{array}$ & $\begin{array}{l}\cdots 0 \\
\cdots\end{array}$ & & . & \\
\hline & ... & $\ldots \ldots$ & ... & $\cdots p$ & ... & - & \\
\hline THN). & $\cdots \cdot \cdot$ & $\cdots \cdots$ & & $\cdots \cdot$ & 4 & $\cdots \cdot$ & \\
\hline$\cdots$ & $\cdots \cdot \cdot$ & .. & 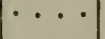 & $\cdot \cdots$ & $q \ldots$ & $\cdots$ & \\
\hline j.) & $\begin{array}{l}\cdots \\
\cdots \\
\cdots\end{array}$ & & & & 9 & - & \\
\hline 0.) & .... & & & & & $\begin{array}{l}\cdots \\
\cdots\end{array}$ & \\
\hline . . & .... & $\cdots$ & & & & & \\
\hline . & $\cdots \cdot$ & $\ldots \ldots$ & & $\cdots \cdot$ & q & $\cdots \cdot$ & \\
\hline ana M MTHN. & $\cdots \cdot \cdot$ & $\cdots \cdots$ & & $\cdots \cdot$ & 9 & - & \\
\hline 8 & $\cdots \cdot$ & & & & $q$ & - & \\
\hline LS. & $\cdots \cdot$ & $\cdots$ & & & - 1 & - & \\
\hline 0. & . . • & -・ & & & $\mid \begin{array}{lll}\cdot & \mathbf{r} & \cdot \\
\cdot & \mathbf{r} & .\end{array}$ & .. & \\
\hline • $\quad \cdot$ & $\cdots$ &. & & . & . r. & - & \\
\hline suss. & 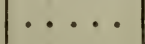 & $\ldots \ldots$ & & ... & - $\mathbf{r}$. & & \\
\hline ata Ge & 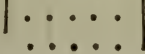 & 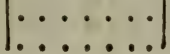 & 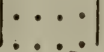 & & $\mid \begin{array}{l}\mathbf{1} \\
\cdot \int^{1}\end{array}$ & - $\cdot$ & \\
\hline
\end{tabular}




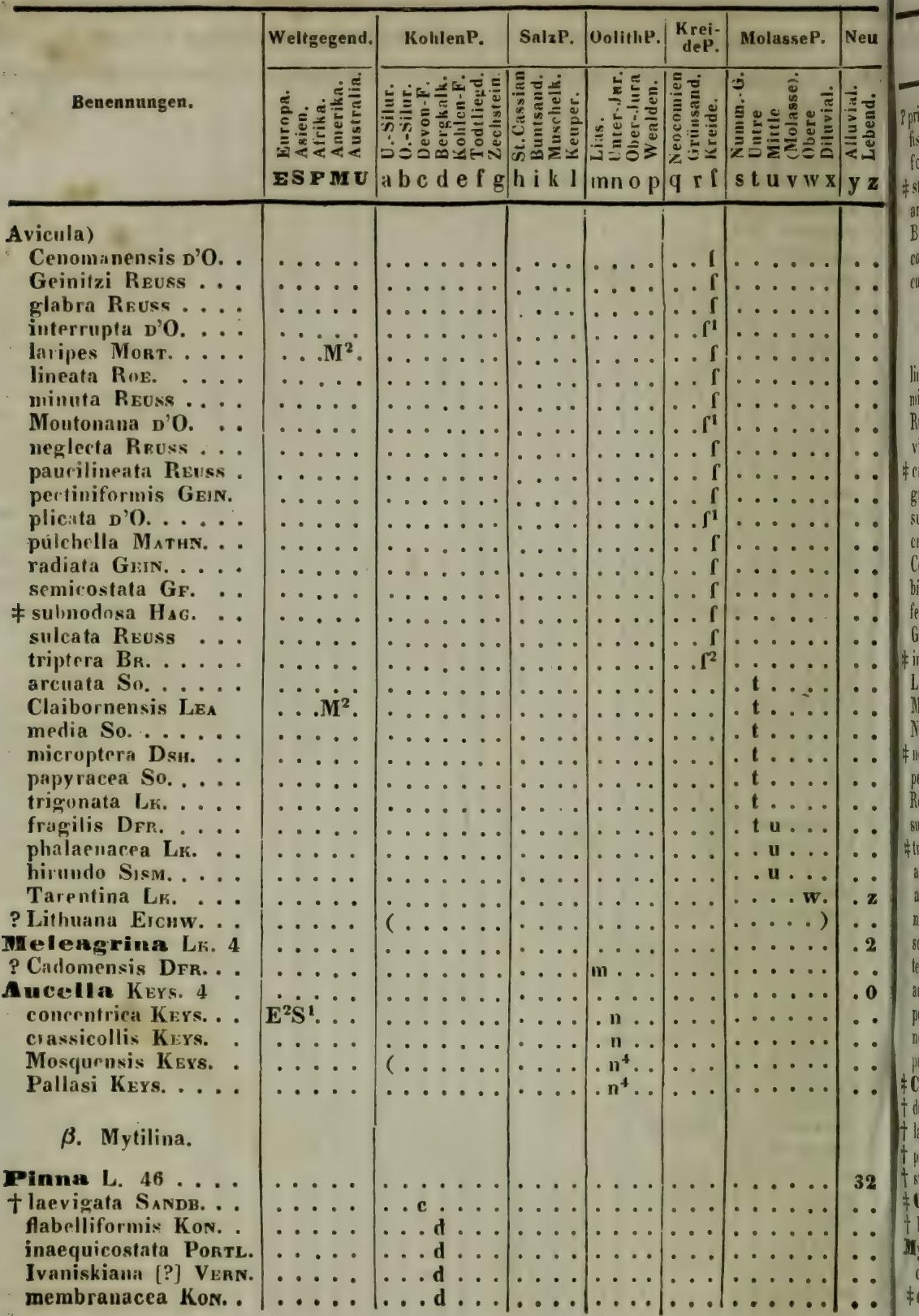




\begin{tabular}{|c|c|c|c|c|c|c|c|}
\hline n. & & & 1 & & $q \mathbf{r}$ & $\mathbf{w} \mathbf{x}$ & yz \\
\hline са Mü. & . & $\cdots \cdots \cdot g$ & & & & $\ldots \ldots$ & \\
\hline fissa $\mathrm{GF}_{0} \ldots \ldots$ & $\cdots \cdots$ & $\ldots \ldots$ & & $\mathbf{m} \ldots$ & 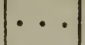 & $\ldots \ldots$ & \\
\hline folium YAB. .... & $\cdots \cdots$ & $\cdots \cdots$ & $\cdots$ & $\operatorname{ma} a$. & & $\cdots \cdots$ & \\
\hline \# striata Drr. . . . . & $\cdots \cdots$ & $\cdots \cdots \cdots$ & $\cdots \cdots$ & In $\cdot 925^{\circ}$ & $\cdots$ & $\cdots \cdots \cdot$ & \\
\hline la Dsн. ... & $\cdots \cdot$ & $\cdots \cdots \cdots$ & & $\mid \begin{array}{l}11 \\
030 \\
.11^{2}\end{array}$ & .. & $\cdots \cdots$ & $\cdot$ \\
\hline $\mathrm{hi} \mathrm{Kol}$ & & & $\cdots$ & $\left|\begin{array}{ll}0112 \\
.11 & \ldots\end{array}\right|$ & & $\cdots \cdots$ & \\
\hline$\cdots$ & $\because$ & $\because$ & $\cdots$ & $n^{3}$ & $\therefore$ & $\because \cdots$ & \\
\hline So.... & ... & $\ldots \ldots$ & $\ldots$ & $\cdot n^{5} \cdot$ & ... & $\ldots \ldots$ & $\because$ \\
\hline$\cdots$ & $\ldots \ldots$ & $\ldots \ldots$ & $\cdots$ & $\cdot \mathbf{n} \cdot$. & $\cdots$ & $\ldots \ldots$ & 政 \\
\hline lü. ... & $\cdots \cdots$ & $\ldots \ldots$ & $\ldots$ & . n . & . . & $\ldots \ldots$ & \\
\hline$\cdots$ & $\cdots \cdots$ & $\cdots \cdots$ & $\cdots \cdots$ & $\cdot n_{24} \cdot$ & $\cdots$ & $\cdots \cdots$ & \\
\hline (2) & $\cdots \cdot$ & $\cdots \cdots$ & $\cdots \cdot \mid$ & $\left|\cdot 11^{24} \cdot\right|$ & $\cdots$ & $\cdots \cdots \cdot$ & \\
\hline$\therefore$ & - & $\cdots$ & $\cdots$ & $\cdot \mathrm{n}^{2} \cdots$ & & $\cdots$ & \\
\hline 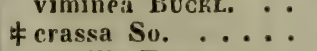 & $\because$ & & $\cdots$ & 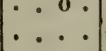 & & $\cdots$ & \\
\hline$\ldots$ & $\ldots \ldots$ & $\cdots$ & $\ldots$ & $\ldots$ & $q$. & $\cdots$ & \\
\hline . . . & $\ldots$ & ... & . & $\ldots$ & $4^{1}$. & $\ldots$ & \\
\hline$\cdots$ & $\cdots$ & $\ldots \ldots$ & $\cdots$ & $\cdots$ & $4 r$. & $=\ldots .$. & $I^{\circ}$ \\
\hline$\ldots$ & $\cdots \cdot$ & $\ldots \ldots$ & $\cdots$ & $\ldots$ & $\cdot r_{i}$ & $\ldots \ldots$ & \\
\hline v. . & $\cdots \cdot$ & $\ldots \ldots$ & $\cdots$ & $\cdots \cdot$ & - . $\mathbf{f}^{\mathbf{1}}$ & $\cdots$ & \\
\hline$\cdots$ & . & $\ldots \ldots$ & $\cdots$ & $\cdots$ & & $\cdots$ & \\
\hline$\ldots$ & & $\cdots$ & & . $\cdot$ & & $\cdots$ & \\
\hline * in & & - & & & & $\cdots$ & \\
\hline$\therefore$ & : & & & & & - 1 & \\
\hline$\cdots$ & $\because \cdots$ & $\cdots$ & & $\cdots$ & $\ldots f$ & $\because$ & \\
\hline \# & ... & 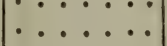 & $\therefore$ & $\cdots \cdots$ & $\cdots \int$ & $\therefore$ & \\
\hline THN. & $\cdots$ & $\ldots \ldots$ & . & . & . $\mathrm{f}^{\mathrm{i}}$ & $\cdots$ & \\
\hline . & $\cdots$ & $\ldots \ldots$ & . & $\cdots$ & . $f^{1}$ & $\ldots$ & \\
\hline . & $\cdots \cdot \cdot$ & $\ldots \ldots$ & $\ldots$ & $\ldots$ & $\ldots \mathbf{T}$ & $\ldots \ldots$ & \\
\hline IAG. . . & $\cdots$ & $\cdots \cdots$ & $\ldots$ & $\cdots$ & $\ldots \mathrm{f}$ & $\ldots \ldots$ & - \\
\hline$\cdots$ & $\cdots \cdot \cdot$ & $\ldots \ldots$ & $\ldots$ & $\cdots$ & $\cdots$ & . t..? & \\
\hline$\cdots$ & $\cdots \cdots$ & $\ldots \ldots$ & $\cdots$ & $\cdots$ & $\cdots$ & . $t \ldots$ & \\
\hline$\cdots$ & $\cdots \cdot$ & $\cdots$ & $\cdots$ & $\cdots$ & $\cdot$ & . t? ... & \\
\hline HIL. & $\cdots \cdot$ & $\cdots$ & $\cdot \cdot$ & & . & $\cdots 1$ & \\
\hline 1 & - & $\cdot$ & 1. & $\cdots$ & - & $\cdots$ & - \\
\hline & & . & •. & •... & - & $\because$ & \\
\hline Dsн. & & $\because \cdots$ & $\therefore \cdots$ & $\cdots$ & $\cdots$ & $\because \cdots$ & $\cdot 2$ \\
\hline$\cdots$ & $\cdots$ & $\ldots \ldots$ & $\ldots$ & $\cdots$ & . . & $\ldots \ldots x$ & . \\
\hline F. 4 . & - & $\cdots$ & 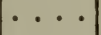 & & &. & - $c$ \\
\hline & $\cdots$ & & & & & & \\
\hline . & $\cdots M^{2}$ & . & & & & $\cdot$ & \\
\hline$\because$ & $\therefore$ & & & & & $\cdots$ & \\
\hline$\because \dot{ }$ & $\ddot{0}$ & , . & $\cdots$ & $\ldots$ & $\cdots$ & $\cdots$ & \\
\hline$\therefore$ & . $\mathbf{M}^{2}$ & $\ldots \ldots$ & $\cdots$ & $\ldots$ & $\cdots$ & $.9 \ldots$ & \\
\hline $193 \cdots$ & $\cdots$ & & & & & $\cdots$ & 50 \\
\hline $\begin{array}{l}\mathbf{P O}_{0} \\
\text { is E }\end{array}$ & & o & & & & $\cdot \cdot$ & \\
\hline
\end{tabular}


Benennungen.

\begin{tabular}{|c|c|c|c|c|c|c|}
\hline Welt & KohlenP. & SalzP. & OolithP. & $\left|\begin{array}{c}\text { Krei- } \\
\text { deP. }\end{array}\right|$ & MolasseP. & Neu \\
\hline 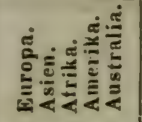 & 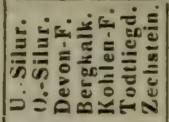 & 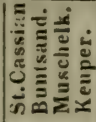 & 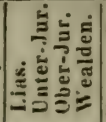 & 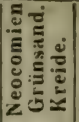 & 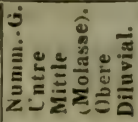 & 列 \\
\hline $\mathbf{E} \mathbf{S}$ & a b c & h i k l & $|\mathrm{mn} 0 \mathrm{p}|$ & $q \mathbf{r} \mathbf{r}$ & $s t u v w x$ & yz \\
\hline
\end{tabular}

Mytilus)

⿰㇒一ncrassatus Eichw. . * planus Eıchw. semirugatus Port. 4 antiquus GF. 168 . costatus MÜ.

Damnoniensis $\ddot{P}_{H}$. irregularis $\mathrm{Mü}$.

Nerei Mü.

obliquus $\mathrm{Mü.}$

priscus GF.

radiatus Mü.

substıiatus Mü.

subsulcatus Mü.

cuspidatus Mü. ...

fragilis Eıchw. ...

pypmaeus GF.

veteratus GF.

crassus Fi.em.

triangularis So.

Hausmanui GF.

t septiferus Kino

\$ squamosus So.

Kr. . . . . .

Maximiliani-Leuchtenbergensis KLI. . . . . . .

Münsteri KLr.

8 praeacutus Kor.

pyymaeus Mü. ...

scalaris Kr.r.

vetustus Gr. . . .

Beaumonti Vern.

aeutus Roe. .....

coralliophagus MER.

cuncatus PHir.L....

curvatus KLöD. . . .

falcatus $M \ddot{\text { ü. }}$

furcatus Mö.

gibbosus Рusch ... lineolatus Puscr ..

9 minutus ZiET.

parvus Roe.

o perneides Roe.

P planus KLöD.

striatus GF....

substriatus Mü.

sulcatus $\mathbf{G r}$.

.
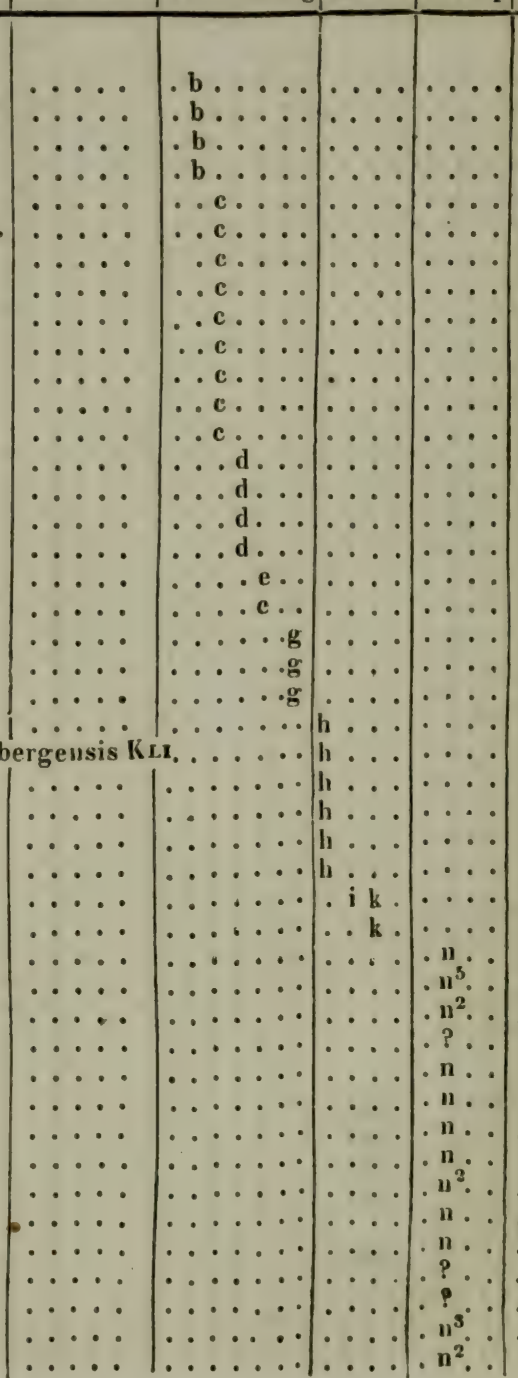


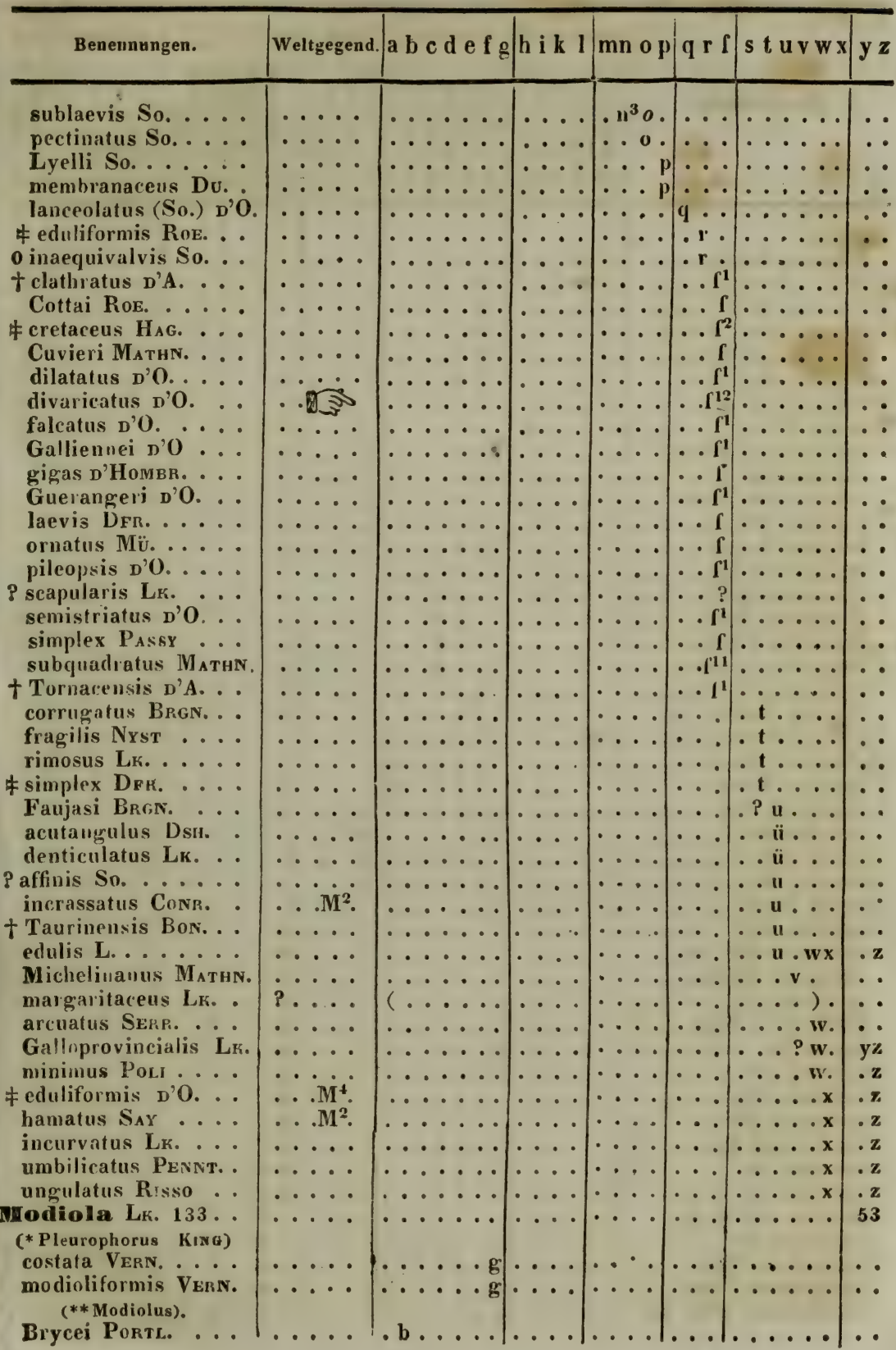




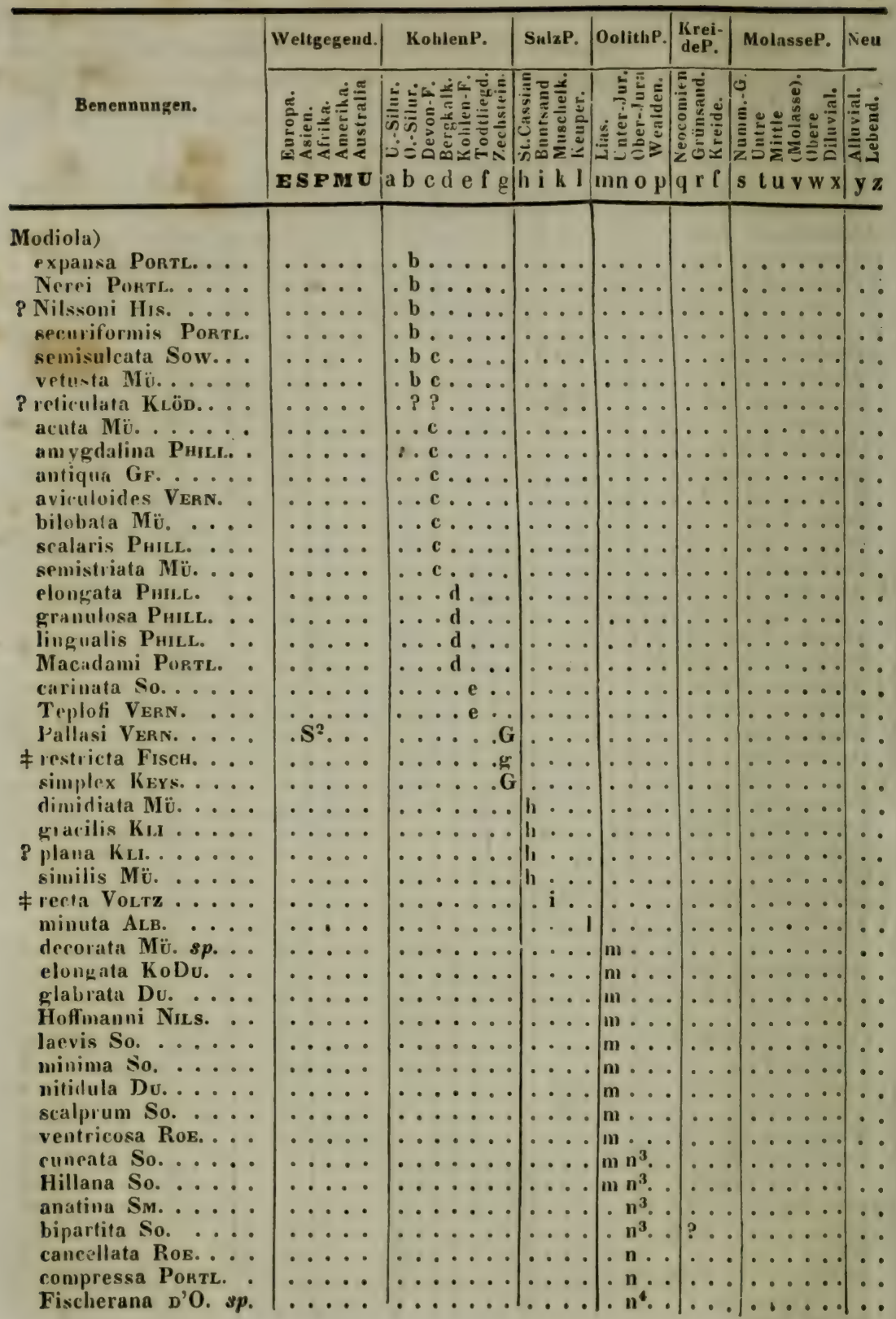




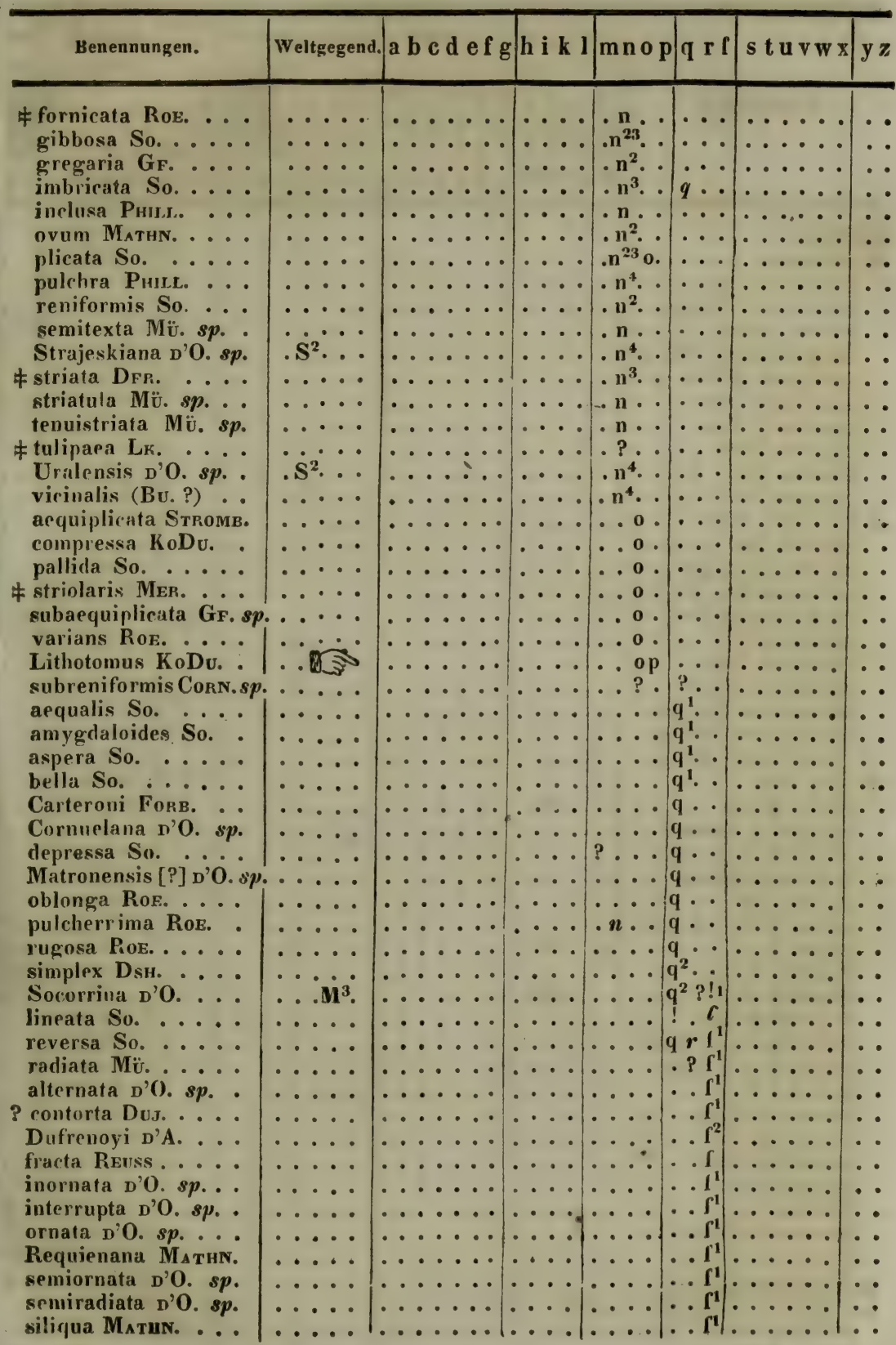




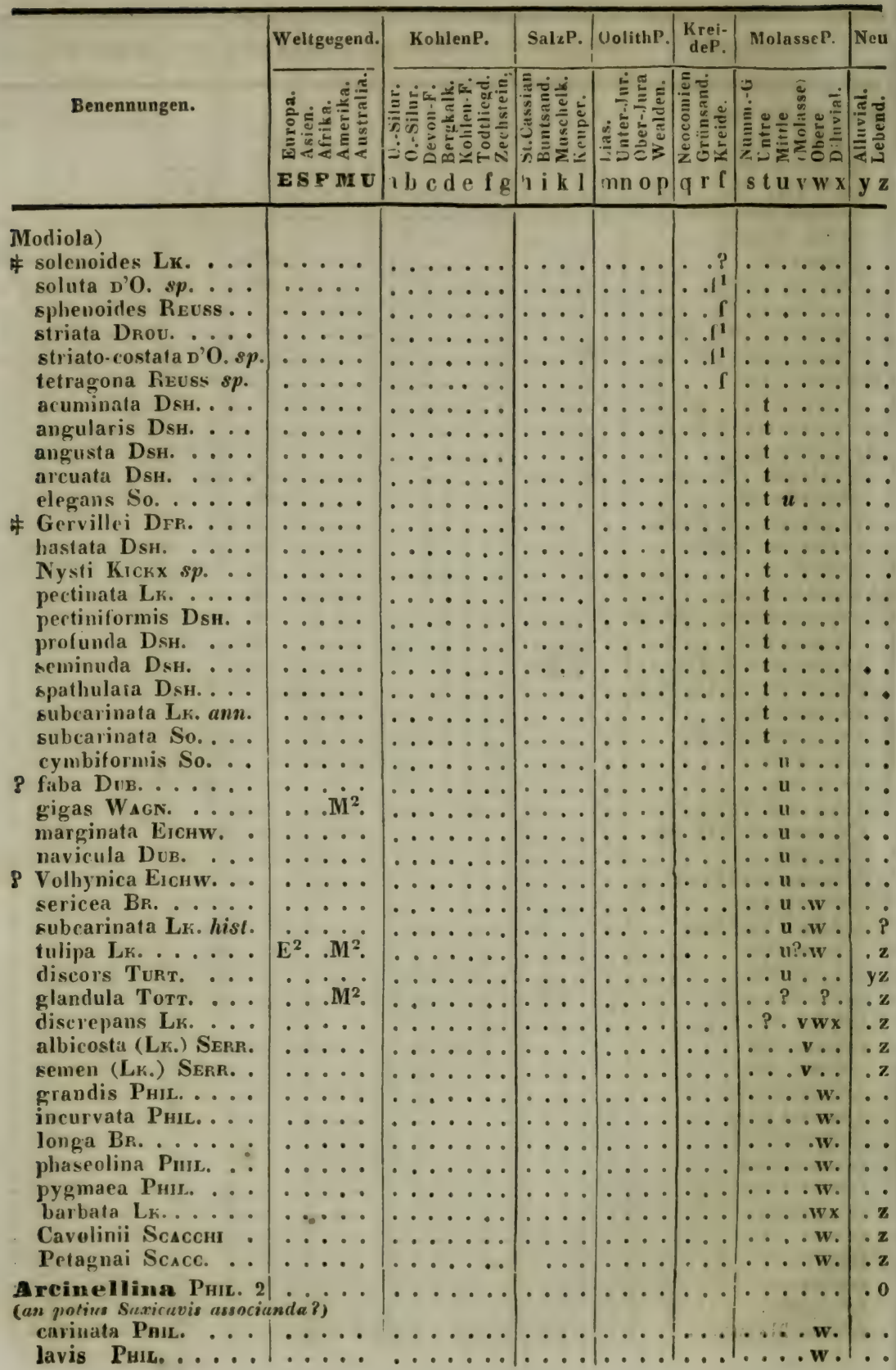




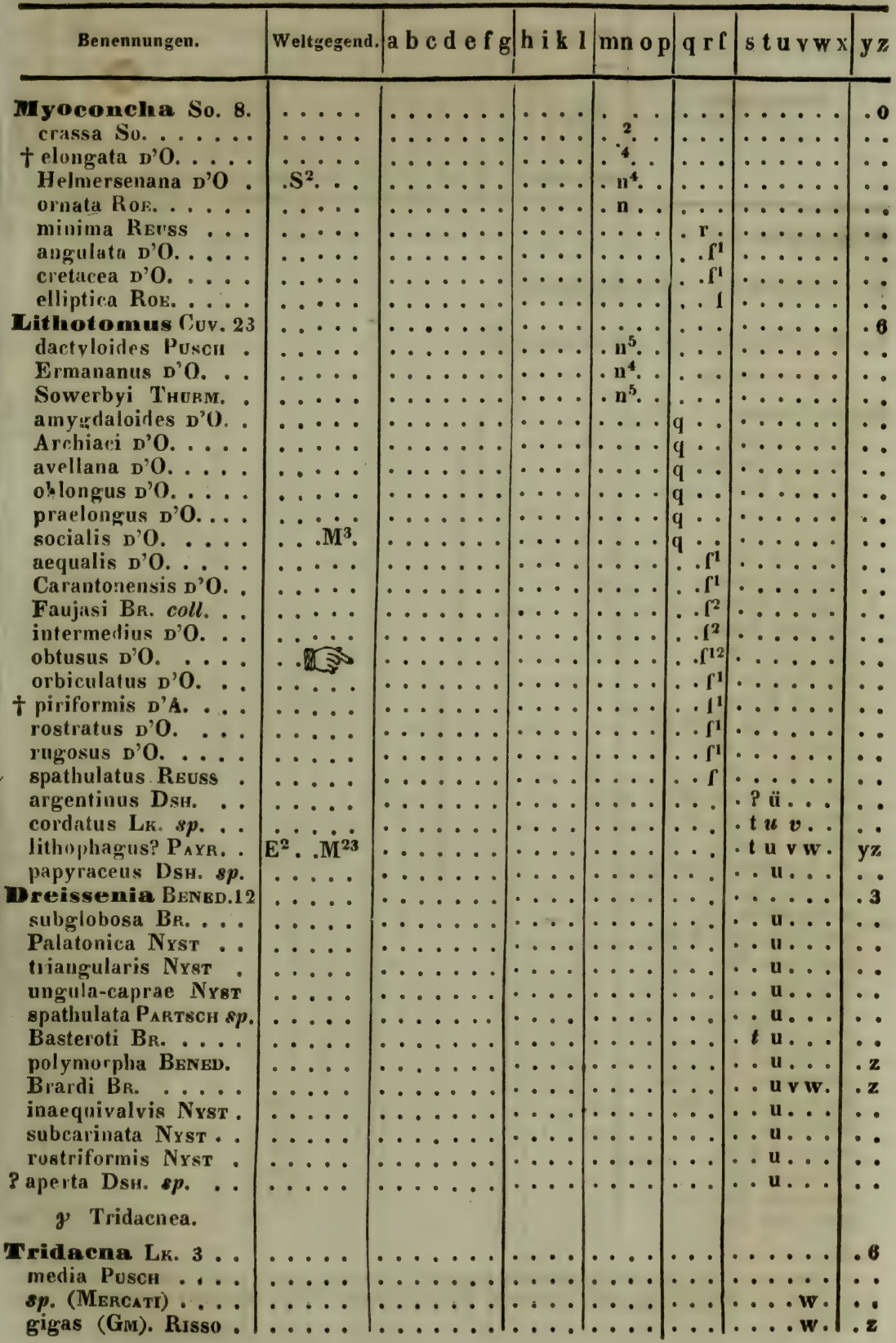




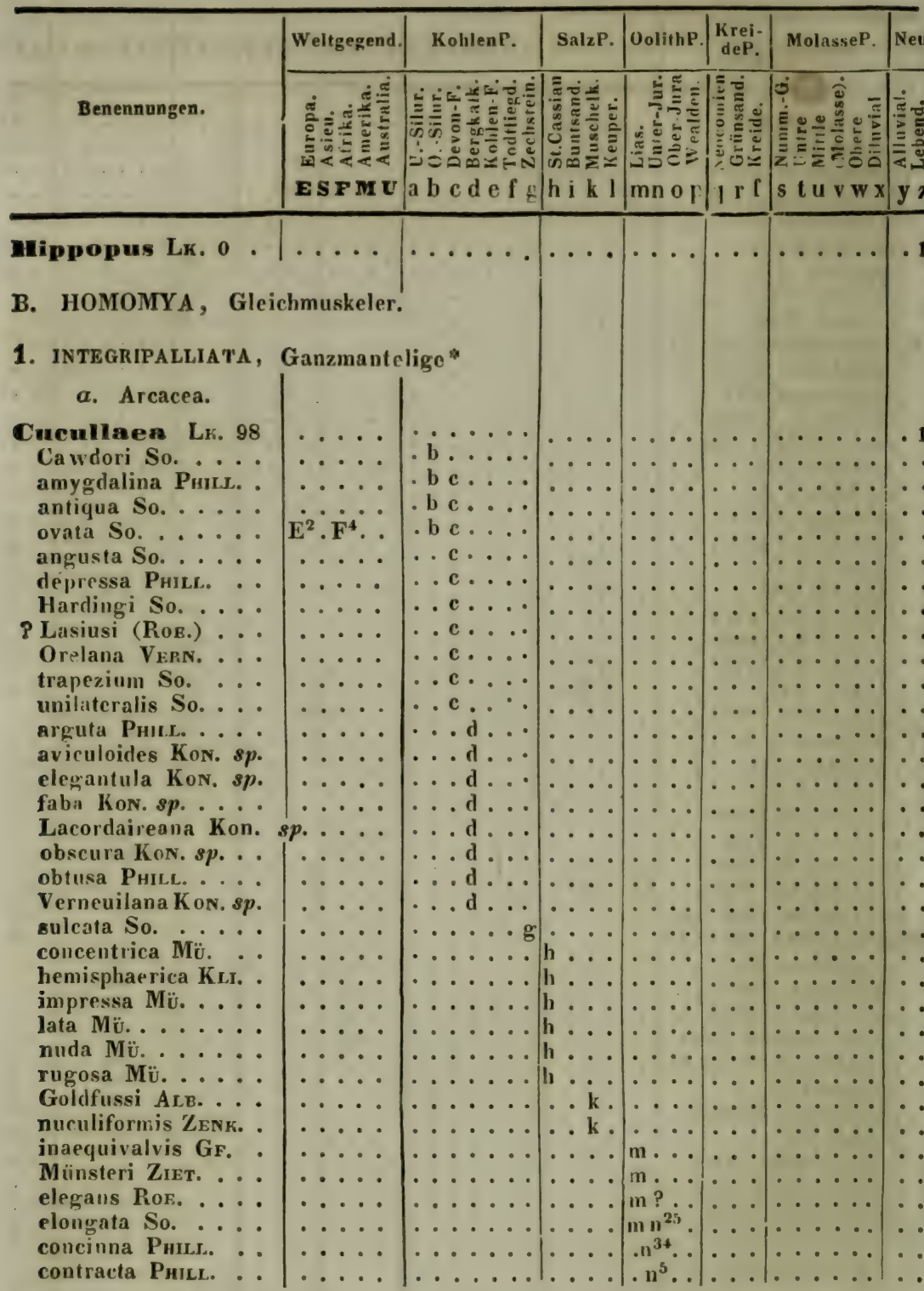

* Nuculne, Cyrente el Cypricurdine singulue qunedum species pullii umlitum sinu parvo emarginatum hubent, siquidem generum illorum speries lnuge plurimat pullium intogrum priselient. 


\begin{tabular}{|c|c|c|c|c|c|c|c|}
\hline Dene? & & & & $p$ & & & \\
\hline & & & & & & & \\
\hline & & & & & & $\ldots \ldots$ & \\
\hline$\cdots$ & $\cdots \cdots$ & & $\cdots$ & & & $\cdots$ & \\
\hline$\because$ & $\cdots$ & $\cdots \cdots$ & $\cdots \cdots$ & & $\cdot$ & $\cdots$ & \\
\hline . & . & a & $\cdots \cdots$ & . & $\cdots$ & $\cdots$ & \\
\hline H & & & & & $\cdots$ & $\cdots$ & \\
\hline $\begin{array}{l}\text { it } \\
\text { li }\end{array}$ & & & & & & & \\
\hline $\begin{array}{l}\text { li } \\
\text { m }\end{array}$ & & • & . & . & & .. & \\
\hline So. & & $\therefore$ & &.$n^{23} \cdots$ & $\cdots$ & $\cdots$ & \\
\hline ... & $\ldots$ & $\cdots \cdots$ & $\cdots$ & . n .. & $\ldots$ & $\ldots$ & \\
\hline$\cdots$ & $\cdots \cdots$ & $\cdots \cdots$ & $\cdots \cdot$ & $\mid \cdot \mathbf{n} \cdot$. & $\cdots$ & $\ldots \ldots$ & \\
\hline . & - & $\cdots \cdots$ & $\cdots$ &. $\mathrm{n}^{5} \cdot$ & $\cdots$ & $\ldots .$. & \\
\hline$\cdots$ & & . & $\cdots$ & & $\cdots$ & $\ldots \ldots$ & \\
\hline . & & 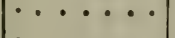 & $\cdots$ & & $\cdots$ & $\cdots$ & \\
\hline$\cdots \cdot$ & $\cdots$ & $\cdot$ & $\cdots$ & & $\cdots$ & $\cdots \cdots \cdot$ & \\
\hline$\cdots$ & $\cdots$ & $\cdots$ & $\cdots$ & & $\cdots$ & $\cdots \cdots$ & \\
\hline$s p$. & . & $\ddot{\ddot{ }}$ & $\cdots$ & $\cdots$ & $\cdots$ & $\cdots \cdots$ & \\
\hline . & & & $\cdots$ & & $\cdots$ & $\cdots$ & \\
\hline & $\because$ & $\cdots$ & $\cdots$ & $\mid \begin{array}{c}n \\
n^{5}\end{array} \cdots$ & 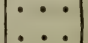 & $\because$ & \\
\hline$\therefore$ & .... & $\ldots \ldots \ldots$ & $\cdots$ & $\therefore 0$ & $\cdots$ & & \\
\hline & $\cdots$ & $\ldots \ldots$ & $\ldots$ & . 0. & $\ldots$ & $\ldots \ldots$ & \\
\hline & $\therefore$ & $\cdots$ & $\cdots$ & . . 0 & & $\ldots \ldots$ & \\
\hline & $\cdots$ & $\ldots \ldots$ & & $\ldots$ & q? & $\ldots$ & \\
\hline$s p$. & $\cdots \cdot \cdot$ & $\cdots \cdots \cdot$ & 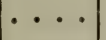 & $\ldots . c$ & $q^{1} \cdots$ & $\ldots \ldots$ & \\
\hline$s p$ & & $\cdot$ & $\cdot$ & 0 & $q^{1} \cdots$ & $\ldots \ldots$ & \\
\hline$\therefore$ & - & - & & & & $\cdots \cdots$ & \\
\hline ss . & • & - & - & $\cdots$ & $q^{2} \ldots$ & $\cdots$ & \\
\hline & ? & & & - & & $\cdots$ & \\
\hline $\begin{array}{l}\text { YM. } \\
\cdots\end{array}$ & ? & $\cdots$ & & & 9 & $\cdots \cdots$ & \\
\hline .. & & & & & & $\cdots \cdots \cdots$ & \\
\hline & & . & & & & $\cdots$ & \\
\hline $\mathrm{P}$ & $\cdots$ & & & & & & \\
\hline$s$ & .... & $\cdots$ & - & $\ldots$ & $q^{1} \ldots$ & $\cdots \cdots$ & \\
\hline o. . . & $\cdots M^{3}$ & $\ldots \ldots$ & $\cdots \cdot$ & $\ldots$. & $q \ldots$ & $\ldots \ldots$ & \\
\hline & $\ldots$ & $\cdots \cdot$ & - & $\cdots$ & $q^{r}$. & $\cdots \ldots$ & \\
\hline o. . & $\cdots$ & $\cdots$ & $\cdots \cdots$ & - & $q \mathbf{r}$. & $\ldots \ldots$ & \\
\hline$\cdots$ & $\cdots \cdot$ & $\cdots \cdots \cdots$ & $\cdots \cdot$ & - & $\cdot r$ & $\cdots \cdot \cdots$ & \\
\hline$\cdots$ & $\cdots$ & $\ldots \ldots$ & $\cdots \cdot$ & $\cdots$ & - $\mathbf{r}$. & $\ldots \ldots$ & \\
\hline$\cdots$ & & 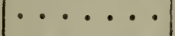 & & $\cdots$ &.$P$. & $\cdots$ & • \\
\hline$\cdots$ & $\cdots$ & $\cdots \cdots$ & $\cdots \cdot$ & $\cdots$ & . $\mathbf{r} \cdot$ & $\cdots$ & \\
\hline$\cdots$ & $\cdots$ & $\cdots \cdots \cdots$ & $\cdots$ & $\cdots$ & $\cdot \mathbf{r}$. & $\cdots \cdots$ & \\
\hline & & . & & $\cdots$ & - $r$ & $\cdots \cdots \cdot$ & \\
\hline & & & & & $\begin{array}{rll}-r & 1 \\
r & c\end{array}$ & $\cdots$ & \\
\hline & & & & & $\because \mathrm{r} f$ & & \\
\hline a & & & & $\cdots \cdot$ & $\ldots 1$ & & \\
\hline & 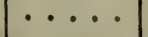 & 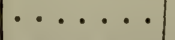 & & & - . $f^{1}$ & $\cdots \ldots$ & \\
\hline$[$ arceana [?] D'O & & & & & & & \\
\hline
\end{tabular}




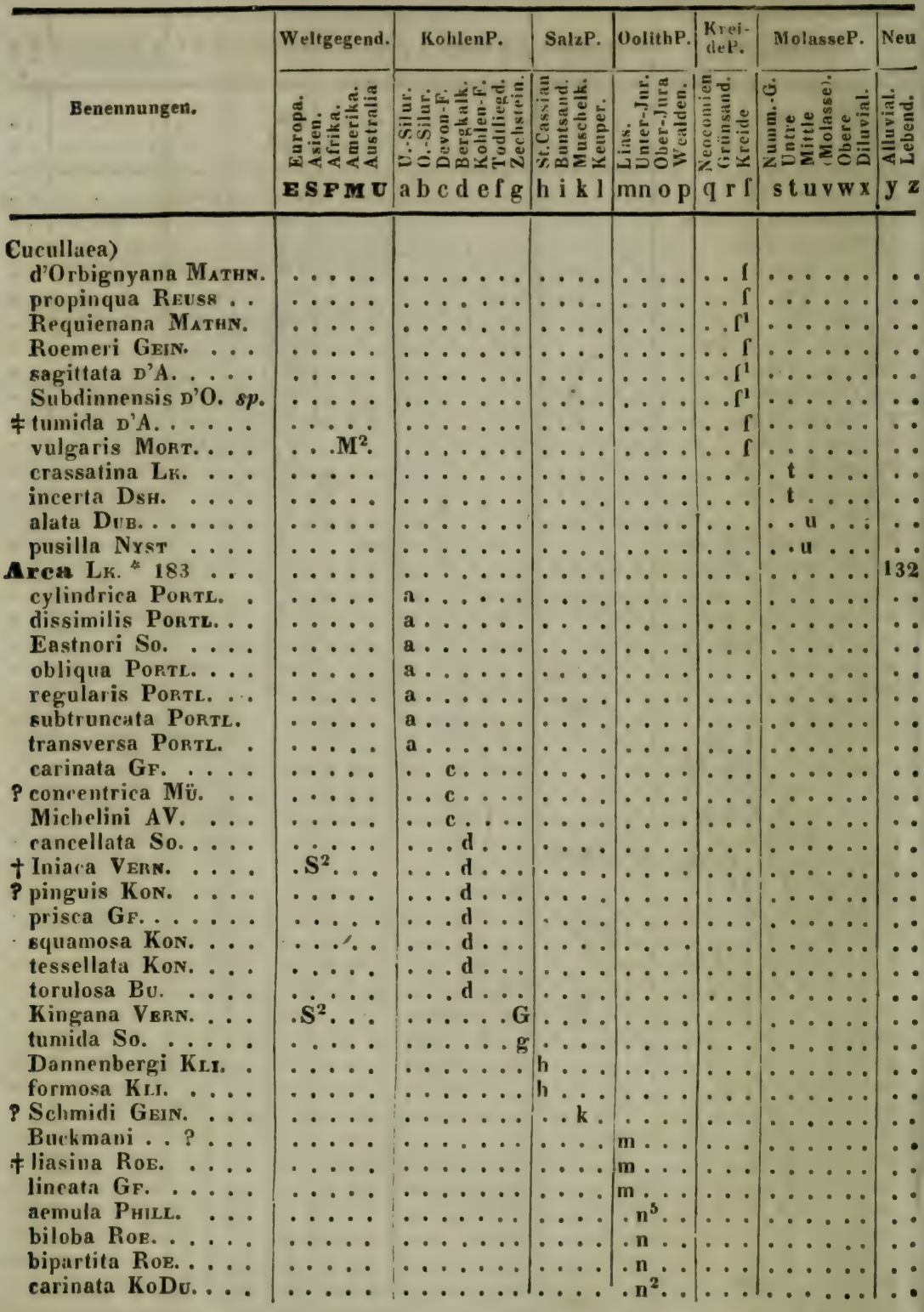

* Numerosa in hoc genere interrogationis signa (3) species indicant forte ad Curullaerm referendus. 


\begin{tabular}{|c|c|c|c|c|c|c|c|}
\hline Bene & end. & $a b c d e f g$ & h i k l & $\mathrm{p}$ & $q \mathrm{r} r$ & $w x$ & $y$ \\
\hline$\cdots$ & $5^{2} \cdot \cdots$ & & 然 & . $\mathrm{u}^{4}$ & & $\cdots \cdots$ & \\
\hline f decussata Roe..... & ... & $\ldots \ldots$ & $\cdots \cdot$ & . n . & & $\ldots \ldots$ & \\
\hline elongata So. .... & $\ldots$ & $\ldots \ldots$ & $\cdots$ &.$n \ldots$ & $\cdots$ & $\ldots \ldots$ & \\
\hline fracta $G_{F} \ldots \ldots$ & $\cdots \cdot$ & $\cdots \cdots$ & $\cdots \cdot$ & . $\mathbf{n}$ & $\cdots$ & $\ldots \ldots$ & \\
\hline imperialis RoE. & ... & $\cdots \cdots \cdot$ & $\cdots \cdot$ & . $n$ & $\cdots$ & $\cdots \cdots$ & \\
\hline I: & & & & & & $\cdots \cdots$ & - \\
\hline$\cdots$ & - & $\cdots$ & $\cdots$ & $a^{\circ}$ & e & $\cdots$ & \\
\hline . $\cdot$ & $\cdots \cdots$ & 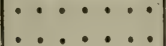 & $\cdots \cdots$ & $\mid \begin{array}{l}\cdot n^{4} \cdots \\
. n^{3} \ldots\end{array}$ & $\cdots$ & $\cdots \cdots$ & : \\
\hline $\begin{array}{l}\text { chra So. } \\
\text { adrisulcata So. }\end{array}$ & $\begin{array}{l}\cdots \cdots \\
\cdots \cdots\end{array}$ & $\ldots \ldots$ & $\cdots \cdots$ & $n^{5} \cdots$ & $\cdots$ & $\cdots \cdots$ & \\
\hline o..... &.$S^{2} \ldots$ & $\ldots \ldots$ & $\ldots \cdot$ &. $\mathrm{n}^{4} \ldots$ & $\cdots$ & $\ldots \ldots$ & \\
\hline & .... & $\ldots \ldots$ & $\ldots \ldots$ &.$n^{5} \ldots$ & $\cdots$ & $\ldots \ldots$ & \\
\hline . ... & $\cdots \cdot$ & $\ldots \ldots$ & $\ldots \ldots$ &.$n^{5} \ldots$ & $\cdots$ & $\ldots \ldots$ & \\
\hline lata KoDu. & . & $\cdots$ & $\cdots \cdot$ & $\ldots 0$ & $\cdots$ & $\ldots \ldots$ & . \\
\hline HN. . & $\cdots$ & - & $\cdots \cdot$ & $\ldots$ & $q \ldots$ & $\ldots \ldots$ & \\
\hline O. ... & & $\cdots$ & $\cdots \cdot$ & & $q^{1} \ldots$ & $\ldots \ldots$ & \\
\hline Bu. $\cdots$ & $\cdots$ & $\ldots \ldots$ & $\cdots \cdot$ & $\cdots$ & & $\ldots \ldots$ & \\
\hline ? rostellata Bu. . . & . $\mathbf{M}^{3}$ & $\ldots \ldots$ & $\cdots \cdot$ & $\cdots \cdots$ & & $\cdots \cdots$ & \\
\hline Roe.... & $\ddot{\mathrm{F}^{i}} \cdot$ & - & $\cdots$ & $\cdots$ & & $\cdots \cdots$ & \\
\hline ss & & & $\cdots$ & . & ? & $\cdots$ & \\
\hline (0.) $\cdots$ & & & $\because$ & $\cdots \cdots$ & - $\mathbf{r}$ & $\cdots \cdots$ & \\
\hline $\begin{array}{l}\text { gardana } D^{\prime} 0 . \\
\text { undata So. . . . }\end{array}$ & & $\because$ & $\cdots \cdots$ & $\cdots \cdots$ & . $r$ & $\cdots \cdots \cdots$ & \\
\hline .. & . & $\ldots \ldots$ & $\cdots \cdot$ & $\ldots$ & . $r i$ & $\ldots \ldots$ & \\
\hline Reuss . & $\ldots \ldots$ & $\ldots \ldots$ & $\ldots$ & $\ldots$ & . $\mathrm{r} \mathrm{f}$ & $\ldots \ldots$ & \\
\hline$\cdots$ & $\cdots \cdot$ & $\ldots \ldots$ & $\cdots \cdots$ & $\cdots$ & $\ldots f$ & $\ldots \ldots$ & - \\
\hline$\cdots$ & . & $\ldots \ldots$ & $\ldots$. & $\cdots$ & $\ldots \mathbf{f}^{1}$ & $\ldots \ldots$ & • \\
\hline$\cdots$ & - & . & $\cdots \cdot$ & $\cdots$ & .. $\mathrm{f}$ & $\ldots \ldots$ & - \\
\hline . . . & - & $\ldots$ & $\cdots$ & $\cdots$ & - $f^{1}$ & $\cdots \cdots$ & \\
\hline uss . & $\cdot$ & $\ldots$ & $\cdots$ & $\cdots \cdots$ & .. & - & \\
\hline$\cdots$ & - & $\cdots \cdots$ & $\cdots$ & $\cdots \cdots$ & $\cdot r^{2}$ & $\cdots \cdot \cdots$ & - \\
\hline Reuss. & • & - & - & $\cdot$ & $\left.\begin{array}{c}1 \\
1 \\
1\end{array}\right]$ & $\cdots$ & \\
\hline ? echinata D'O. . . & & - & $\cdots$ & . 1 & $\left|\begin{array}{ll}\cdots & 1 \\
0 & 1\end{array}\right|$ & $\cdots$ & \\
\hline$\cdots \cdots$ & & $\cdots$ & $\because$ & & $\ell^{1}$ & $\because 1$ & \\
\hline $\begin{array}{l}\text { Gallenne1 D } \\
\text { Galloprovine }\end{array}$ & v. & $\ldots$ & .... & $\ldots$ & . $\Gamma^{1}$ & $\ldots$ & \\
\hline$\cdots$ & $\ldots \ldots$ & $\ldots \ldots$ & $\ldots$ & $\ldots$ & $\ldots \tau$ & $\ldots \ldots$ & \\
\hline . . & . & $\ldots \ldots$ & $\ldots$ & $\ldots$ & . $\mathbf{l}^{1}$ & $\ldots \ldots$ & \\
\hline reri D'O. . . & $\cdots$ & $\ldots \ldots$ & $\ldots$ & $\ldots$ & $\ldots \boldsymbol{f}^{1}$ & $\ldots \ldots$ & \\
\hline a Reuss ... & & $\ldots \ldots$ & $\ldots$ & ... & . $\mathrm{r}$ & $\ldots \ldots$ & \\
\hline pta D'A. . . . & $\ldots \ldots$ & $\ldots \cdots$ & $\cdots \cdot$ & $\cdots$ & $\cdots f$ & $\ldots \ldots$ & \\
\hline a Reuss $\cdots$ & - & $\ldots \ldots$ & $\cdots \cdot$ & $\cdots$ & - $f$ & $\cdots \cdots$ & \\
\hline$\cdots$ & - & $\ldots \ldots$ & . $\cdot$ & & $\boldsymbol{f}^{\mathbf{l}}$ & $\cdots \cdots \cdots$ & \\
\hline 0. & & $\cdots$ & & & $1^{1}$ & $\cdots$ & \\
\hline I & & & & & & $\because 1$ & \\
\hline $\mathrm{Ma}_{2}$ & & $\cdots$ & $\cdots$ & & . $\Gamma^{\top}$ & $\cdots$ & \\
\hline $\begin{array}{l}\text { Ma } \\
\text { mir }\end{array}$ & $\cdots \cdots$ & $\ldots \ldots$ & $\cdots$ & $\cdots$ & . . $1^{2}$ & $\ldots \ldots$ & • \\
\hline $0 . \quad \ldots$ & & $\ldots \ldots$ & & & $\ldots f^{1}$ & $\cdots \cdot$ & \\
\hline$\because \cdots$ & - & ... & & & $\ldots \Gamma^{1}$ & $\cdots$ & - \\
\hline is $D^{\prime} O$. . & - & $\cdots \cdots$ & & & & $\because$ & - \\
\hline & & - & & & & $\cdots$ & \\
\hline
\end{tabular}




\begin{tabular}{|c|c|c|c|c|c|c|c|}
\hline \multirow[b]{2}{*}{ Benennungen. } & Weltgegend. & KohlenP. & SałzP. & Oolithe. & $\begin{array}{l}\text { Krei- } \\
\text { deP. }\end{array}$ & MolasseP. & Neu \\
\hline & 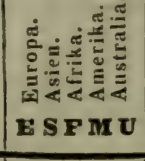 & 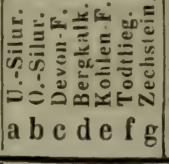 & 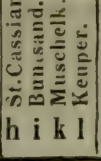 & 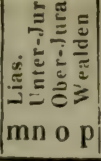 & 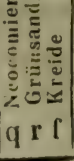 & $\mid \begin{array}{ll} & \\
& \end{array}$ & 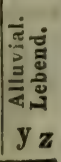 \\
\hline ca) & & & & & & & \\
\hline 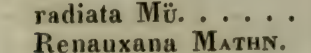 & & & & & & $\because$ & \\
\hline $\begin{array}{l}\text { Renauxana MAтHN. } \\
\text { Requienana D'O. . }\end{array}$ & $\ddot{\cdots}$ & $\cdots \cdots \cdots$ & $\cdots$ & $\cdots$ & $\cdots 1^{1}$ & $\cdots \cdots$ & : \\
\hline$\cdots$ & $\ldots \mathbf{M}^{2}$ & $\ldots \ldots$ & ... & $\ldots$ & $\cdots i$ & $\ldots \ldots$ & r \\
\hline$a\left(D^{\prime} O.\right)$ & $\cdots \cdot$ & $\cdots \cdots$ & $\ldots$ & $\cdots$ & $\cdots l^{2}$ & $\ldots \ldots$ & $\cdots$ \\
\hline$\cdots$ & $\cdots \cdot$ & $\cdots \cdots$ & $\cdots$ & $\cdots$ & $\ldots \rho^{1}$ & $\cdots \cdots$ & - \\
\hline ata $F$ & $\cdots \cdot$ & $\cdots \ldots$ & $\cdots$ & $\cdots$ & $\ldots f^{2}$ & $\cdots \cdots$ & - \\
\hline leata $N$ & $\cdots$ & $\cdots \cdots \cdots$ & $\cdots \cdot$ & $\cdots$ & $\cdots f_{11}^{\prime}$ & $\cdots \cdots$ & $\cdots$ \\
\hline$\cdots$ & $\cdots \cdots$ & $\cdots \cdots \cdots$ & $\cdots \cdots$ & $\cdots \cdots$ & $\because t^{2}$ & $\cdots \cdots$ & $\because$ \\
\hline$\cdots$ & $\cdots \cdots$ & 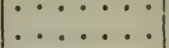 & $\cdots \cdots$ & $\cdots$ & . 10 & $\cdots \cdots$ & $\because$ \\
\hline $\begin{array}{l}\text { subacuta So. } \because \cdots \\
\text { Tailburgensis D'O. }\end{array}$ & $\ldots \ldots$ & $\ldots \ldots$ & $\ldots$ & $\ldots$ & .. $\int^{1}$ & $\ldots \ldots$ & $\because$ \\
\hline tenuistriata Mü. .. & $\cdots \cdot$ & $\ldots \ldots$ & $\ldots$ & $\ldots$ & $\ldots f$ & $\ldots \ldots$ & $\therefore$ \\
\hline ta Reuss ... & $\cdots \cdots$ & $\ldots \ldots \ldots$ & $\cdots \cdot$ & $\ldots$. & .. $\mathrm{f}$ & $\ldots \ldots$ & - \\
\hline $0 . \cdots \cdots$ & $\ldots \ldots$ & $\ldots \ldots$ & $\cdots \cdot \mid$ & $\cdots \cdot$ & $\ldots 1^{2}$ & $\ldots \ldots$ & \\
\hline dineusis [?] D'O. & $\because \dot{s}^{\circ} \cdot$ & $\cdots \cdots \cdot$ & $\cdots$ & $\cdots \cdot$ & ... $f^{\prime}$ & $\ldots \ldots$ & 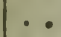 \\
\hline hybrida So. . . . . &. $\mathrm{S}^{3} \ldots$ & $\cdots \cdots$ & $\cdots \cdots$ & $\ldots$. & $\cdots$ & s..... & - \\
\hline$\because \cdots \cdot$ & $\cdots \cdot$ & $\ldots \ldots$ & $\cdots$ & $\cdots \cdot$ & $\cdots$ & $\cdot \ldots .$. & - \\
\hline eulata So. . . & 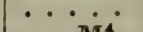 & $\cdots \cdots \cdots$ & $\cdots \cdot \cdot$ & $\cdots$ & $\cdots$ & $\cdot \mathrm{t} \ldots$. & - \\
\hline $0 \ldots \ldots$ & ..... $M^{4}$ & $\ldots \ldots$ & $\cdots$ & $\cdots \cdot$ & $\cdots$ & . t .... & - \\
\hline LK. & $\cdots \dot{\mathrm{M}^{\circ}}$ & $\cdots$ & $\cdots \cdots$ & $\cdots \cdot$ & $\cdots$ & . t.... & - \\
\hline Bonplandana D & $\because$. $^{\circ}$ & • & - & $\cdots$ & $\cdots$ & at $\ldots$ & $\because$ \\
\hline$\cdots$ & $\therefore \dot{M}^{2}$ & $\because$ & $\ldots$ & $\cdots$ & $\ldots$ & t . . . & : \\
\hline R. . & 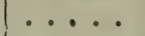 & $\ldots \ldots$ & $\ldots$ & $\ldots$ & $\ldots$ & . t... & \\
\hline $\begin{array}{l}\text { ea Dsh. . . } \\
\text { So. ..... }\end{array}$ & $\ldots \ldots$ & $\ldots \ldots$ & $\cdots$ & $\cdots$ & $\ldots$ & $|. t \ldots|$ & \\
\hline Duchasteli Dsh. .. & $\cdots \cdot$ & $\ldots \ldots \ldots$ & $\ldots$ & $\cdots \cdots$ & . & . t . . . & . \\
\hline$\cdots$ & $\cdots \cdot \cdot$ & $\cdots \cdots$ & $\cdots$ & $\cdots \cdot$ & . & $\cdot \ldots$ & . \\
\hline ana Dsh. .... & $\cdots \cdot \cdot$ & $\ldots \ldots$ & $\cdots$ & $\ldots$ & $\cdots$ & $\cdot t \ldots$ & . \\
\hline globulosa Dsн. . . . & $\cdots \cdots$ & $\ldots \ldots$ & $\cdots$ & $\cdots \cdot$ & $\cdots$ & $\cdot t \ldots$ & -. \\
\hline nulosa Dsн. . . . & $\cdots \cdots$ & $\cdots \cdots$ & $\cdots \cdots$ & $\cdots \cdot$ & $\cdots$ & 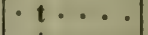 & \\
\hline So...... & $\cdots \cdot$ & $\cdots \cdots$ & $\cdots \cdot$ & $\cdots \cdot \cdot$ & - & $\cdot t \ldots$ & \\
\hline pta Lk. ... & $\cdots \cdots$ & $\cdots \cdot \cdot$ & . & $\cdots \cdots$ & $\cdots$ & $\cdot \mathbf{t} \cdots$ & - \\
\hline late-sulcata Nrst.. & & & - & •. & & - t $\cdots$ & \\
\hline Lyelli Dsн. … & $\bullet$ & • • • & $\bullet$ & $\cdots \cdots$ & ・・ & t $\cdots$ & \\
\hline Itistriata KoN. . . & $\dot{0}$ & - & $\therefore$ & $\cdots$ & & t. & $\dot{0}$ \\
\hline $\begin{array}{l}\text { mitens so. } \\
\text { obliquaria Dsh. } \cdots\end{array}$ & $\begin{array}{l}\cdots \cdots \\
\cdots \cdots\end{array}$ & $\cdots$ & $\because \cdots$ & $\cdots$ & $\cdots$ & $\ldots$ & $\therefore$ \\
\hline Pandorae Brgn. . . & $\cdots \cdot \cdot$ & $\ldots$ & $\ldots$ & $\ldots$ & . & $\cdot \mathbf{t} \ldots$ & - \\
\hline planicosta Dsн. ... & $\cdots \cdots$ & $\cdots \cdots$ & . & $\cdots \cdot$ & - & . t... & - \\
\hline$\cdots$ & $\cdots \cdot \cdot$ & $\cdots \cdots \cdots$ & $\cdots$ & $\cdots \cdot$ & $\cdots$ & $\cdot \ldots$ & $\cdots$ \\
\hline .... & $\cdots$ & $\cdots$ & & 1 & $\cdot$ & - t $\ldots$ & - \\
\hline Roncana BR. & $\because$ & & $\cdots$ & & $\because$ & t $\ldots$ & $\cdots$ \\
\hline rhomboidella & . & $\cdots \cdots \cdots$ & ... & .... & $\ldots$ & t $\ldots$ & 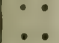 \\
\hline alcicosta Nyst .. & & & & & & . t... & .. \\
\hline
\end{tabular}




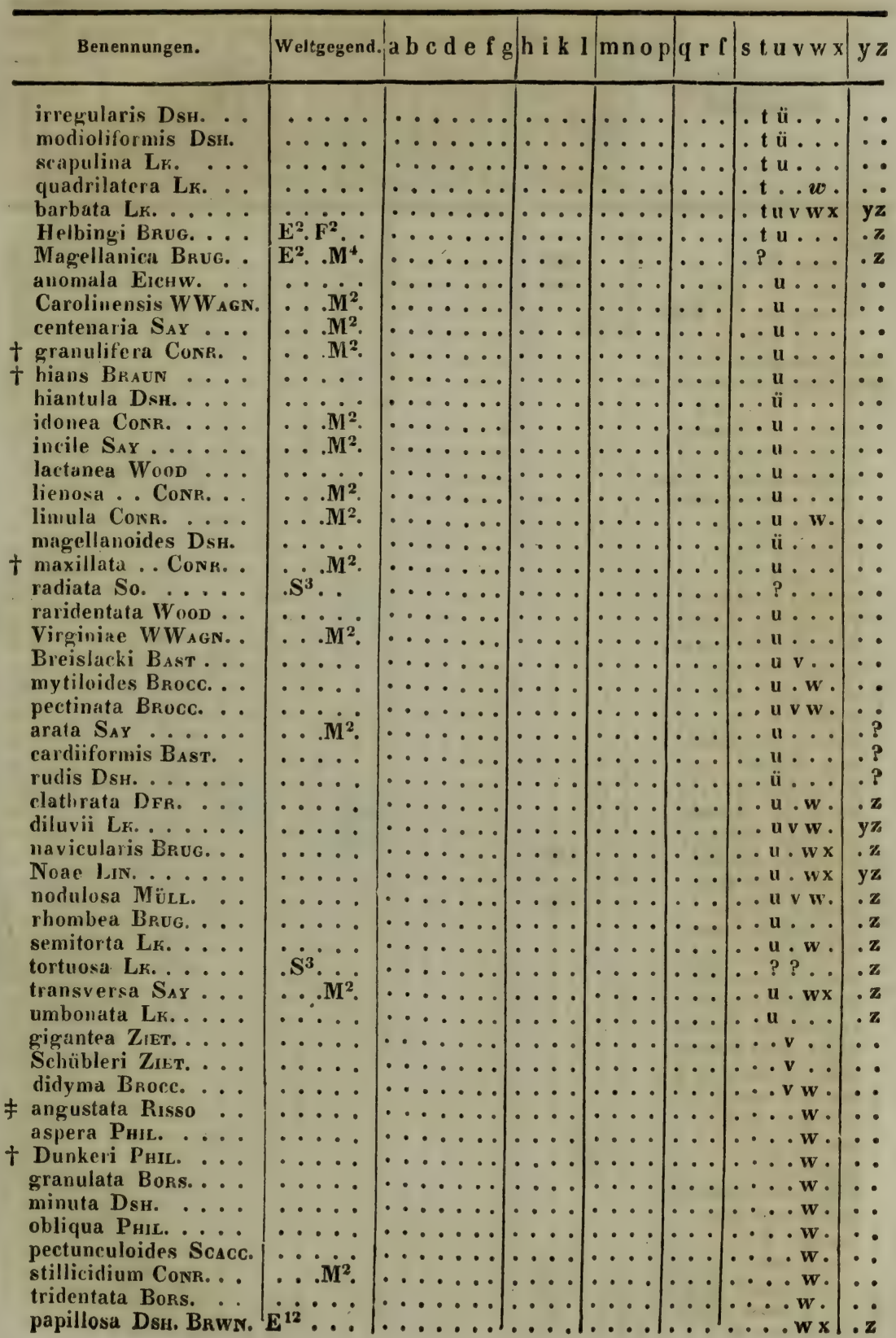




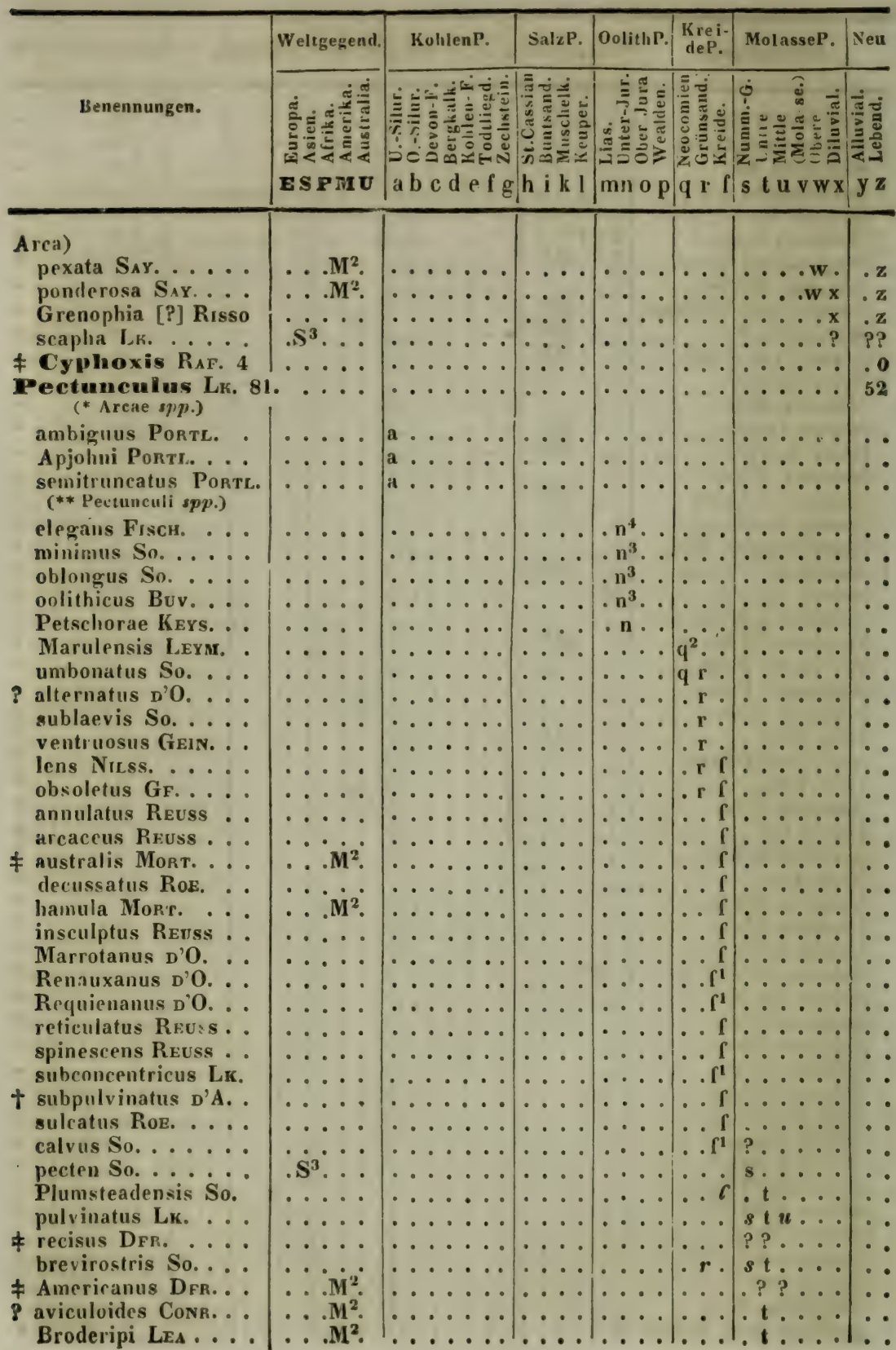




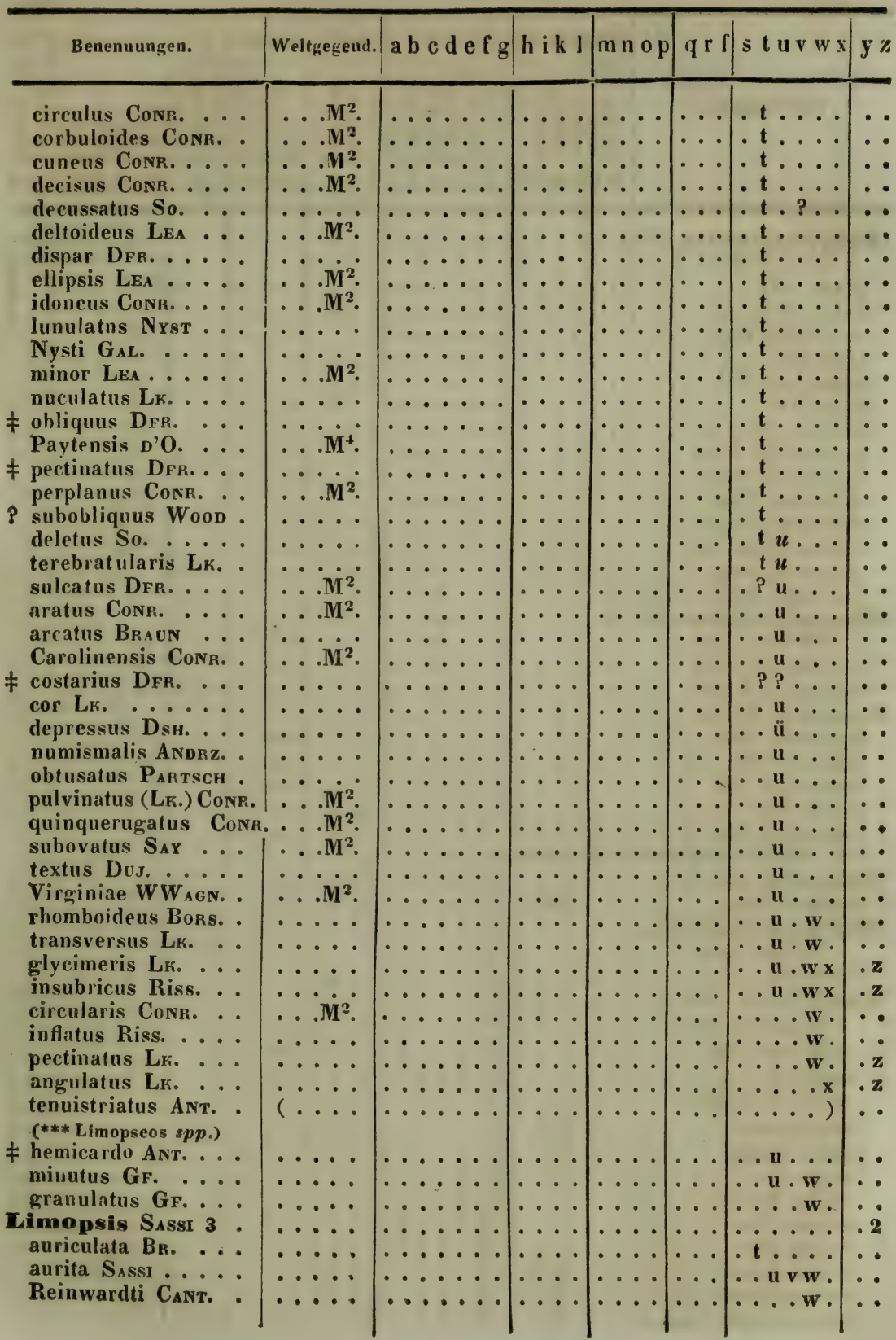




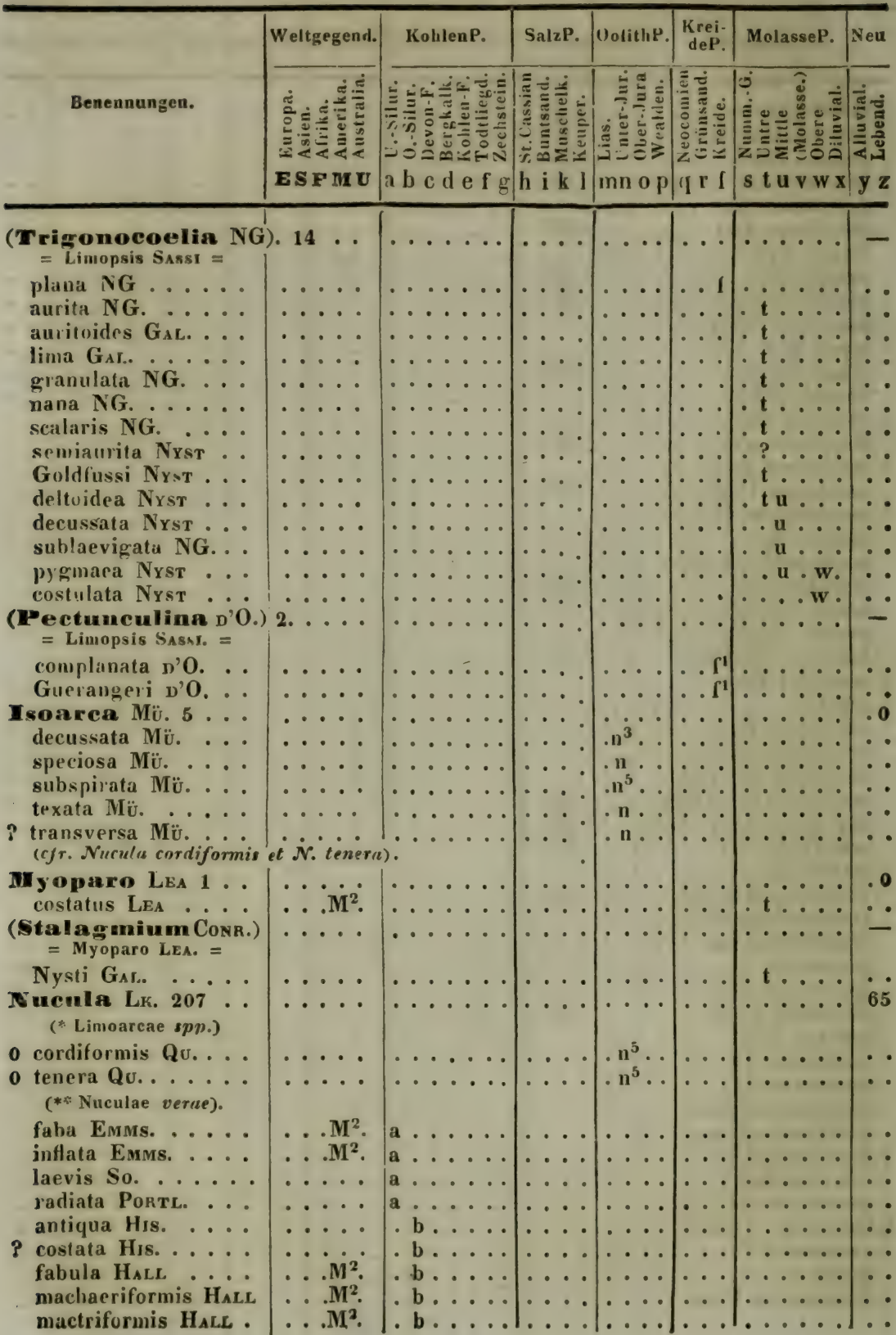




\begin{tabular}{|c|c|c|c|c|c|c|c|}
\hline 1. & d. & $g$ & $h$ & p & 〔 & $\mathbf{w} \mathbf{x}$ & \\
\hline ol & $1{ }^{2}$. & & & & & $\cdots$ & \\
\hline & $\ldots$ & & & $\mid \cdots \cdots$ & ( & $\ldots \ldots$ & \\
\hline A & $\cdots$ & . c... & & $\cdots$ & & $\ldots \ldots$ & \\
\hline I & $\cdots$ & $\ldots$ c... & $\cdots$ & $\ldots$ & & $\ldots \ldots$ & \\
\hline fo & $\cdots$ & $\cdots \cdot$ & $\cdots \cdot$ & $\cdots \cdot$ & $\cdots$ & $\ldots \ldots$ & \\
\hline & & & & $\cdots \cdot$ & .. & & \\
\hline & & & & & & & \\
\hline & & $\because$ & & & & & \\
\hline (1. & • • • & 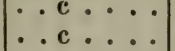 & $\cdots$ & $\dot{.}$ & $\therefore$ & $\therefore$ & \\
\hline$\cdots$ & .... & $\therefore$ c . . . & $\cdots$ & $\left|\begin{array}{l}\cdots \\
\cdots\end{array}\right|$ & $\because \cdot$ & $:$ & \\
\hline$\cdots$ & $\cdots$ & $\ldots \mathbf{c} \ldots$ & $\ldots$ & $\ldots \ldots$ & $\because$ & $\cdots$ & \\
\hline ... & $\ldots \ldots$ & $c \ldots$ & $\cdots$ & $\ldots$ & $\ldots$ & $\ldots \ldots$ & \\
\hline o & $M^{2}$. & $\cdots$ & $\cdots$ & $\ldots$ & $\ldots$ & ... & \\
\hline$p$ & $\cdots$ & - & & $\cdots$ & • & $\cdots$ & \\
\hline $\mathbf{P}$ & - & $\cdots \mathbf{c} \cdot \cdots$ & & $\ldots$ & $\cdots$ & $\ldots \ldots$ & \\
\hline . & $\cdots$ & $\cdots \mathbf{c} \cdot \cdots$ & $\cdots \cdot$ & $\ldots$ & - & $\ldots \ldots$ & \\
\hline$\cdots$ & $\cdots$ & $\cdots$ & $\cdots \cdot$ & $\ldots$ & . & $\cdots$ & \\
\hline 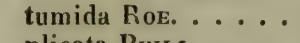 & & $\cdots$ & $\cdots \cdot$ & $\ldots$ & - & $\cdots$ & \\
\hline & & - & . & $\cdots$ & . & $\cdots$ & \\
\hline & 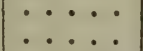 & $\begin{array}{l}\cdots \\
\cdots ?\end{array}$ & & $\therefore$ & e. & - & \\
\hline w. & & $\therefore$ d... & $\cdots$ & $\left|\begin{array}{c}\cdots \\
\cdots\end{array}\right|$ & $\cdots$ & $\therefore$ & \\
\hline w. & & $\ldots d \ldots$ & $\ldots$ & $\ldots \ldots$ & $\cdots$ & $\cdots$ & \\
\hline & $\cdots$ & ... & $\cdots$ &.$\cdots$ & $\cdots$ & ... & \\
\hline & $\cdots$ & . & $\cdots$ & $\ldots$ & $\cdots$ & $\ldots \ldots$ & \\
\hline & . & • & $\cdots$ & $\ldots$ & . & $\ldots \ldots$ & \\
\hline - & $\cdots$ & $\cdot 0$ & $\cdots$ & $\ldots$ & $\cdots \cdot$ & $\ldots \ldots$ & \\
\hline . & $\cdots \cdot \cdot$ & & & $\cdots$ & $\cdots \cdot$ & $\ldots \ldots$ & \\
\hline$\cdots$ & $\cdots \cdot$ & • & . & $\cdots$ & $\cdots$ & $\ldots \ldots$ & \\
\hline . & $\cdots$ & • & $\cdots$ & $\ldots$ & $\cdots$ & $\ldots \ldots$ & \\
\hline$\cdots$ & $\cdots \cdot$ & & . & $\cdots$ & $\cdot \cdot$ & $\ldots \ldots$ & \\
\hline$\because$ & : & e. & & $\cdots$ & $\cdots$ & $\cdots \cdots$ & \\
\hline v. & $\dot{S^{2}} \dot{\cdots}$ & $\because G$ & $\cdots$ & $\ldots$ & $\ldots$ & $\begin{array}{ll}\cdots & \cdots \\
\cdots & \cdots\end{array}$ & \\
\hline$\therefore$ & .... & .. & $\cdots$ & $\ldots$ & $\therefore$ & $\ldots \ldots$ & \\
\hline s. . & .... & $\ldots \ldots G$ & & $\ldots$ & $\ldots$ & $\ldots \ldots$ & \\
\hline & & & & $\ldots$ & - & $\ldots \ldots$ & \\
\hline . & & & & & $\ldots$ & $\ldots \ldots$ & \\
\hline & & & & $\ldots \ldots$ & $\cdots$ & $\ldots \ldots$ & \\
\hline ... & • & & & & $\cdots$ & $\cdots$ & \\
\hline & & & & . & $\cdots$ & $\ldots \ldots$ & \\
\hline & & & & & $\cdots$ & $\ldots \ldots$ & \\
\hline$\cdots$ & & & & $\cdots$ & $\because$ & $\cdots \cdots$ & \\
\hline & & & & $\cdots$ & $\cdots$ & $\cdots \cdots$ & \\
\hline & & & & & & 1 & \\
\hline & & & & & & & \\
\hline$\cdots$ & & & & & $\cdots$ & & \\
\hline u & • & & & $\cdots$ & $\cdots$ & $\ldots \ldots$ & \\
\hline $\begin{array}{l}\text { cunea } \\
\text { Müns }\end{array}$ & & & & & & & \\
\hline & & & & & & & \\
\hline
\end{tabular}




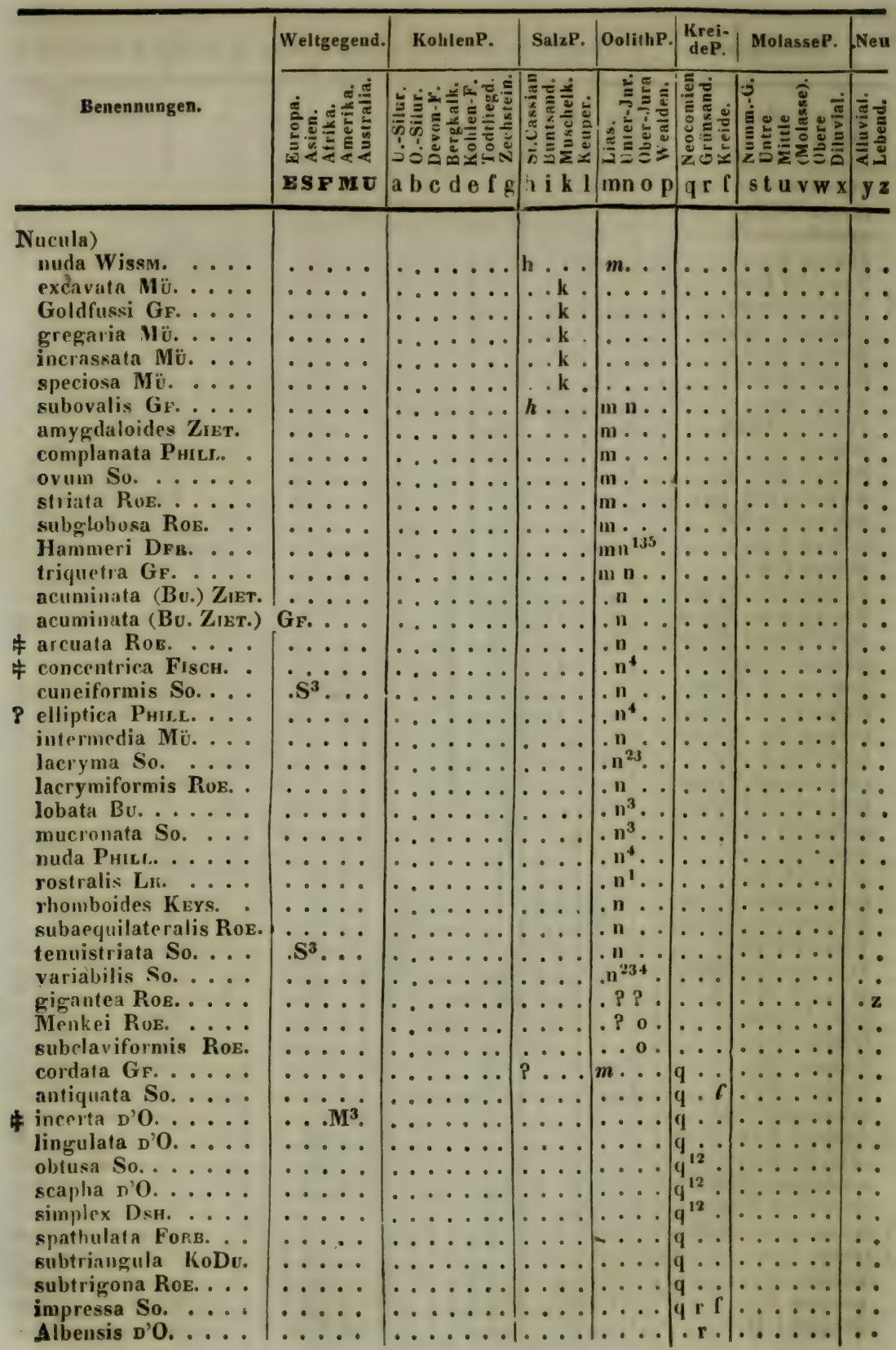




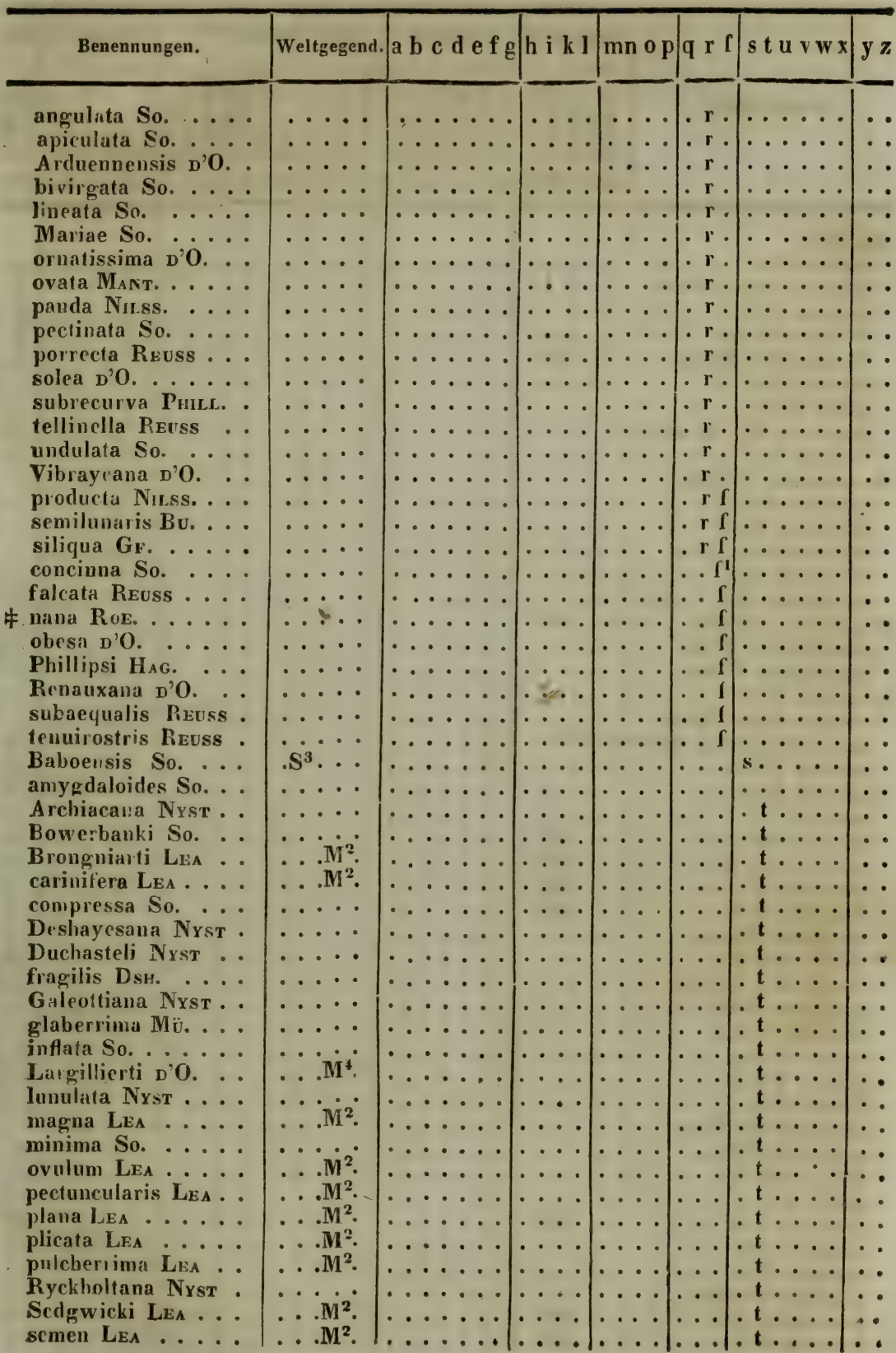




\begin{tabular}{|c|c|c|c|c|c|c|c|}
\hline \multirow[b]{2}{*}{ Benennungen. } & Weltgegend. & KolılenP. & SalzP. & OolithP. & $\mid \begin{array}{l}\text { Krei- } \\
\text { deP. }\end{array}$ & MolasseP. & Neu \\
\hline & 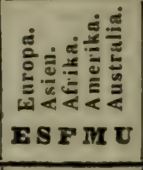 & 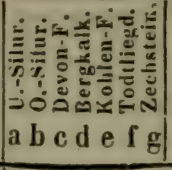 & 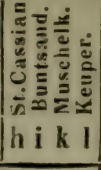 & 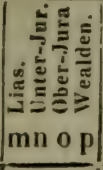 & 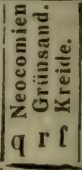 & 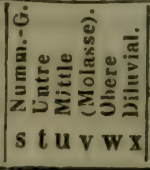 & $\mid$ \\
\hline \multicolumn{8}{|l|}{ Nucula) } \\
\hline similis So. ..... & $\cdots$ & - & $\cdots \cdots$ & $\cdots \cdots$ & $\cdots$ & . t.. & - \\
\hline subtransversa Nyst & $\cdots \cdots$ & $-a^{2}$ & & $\cdots$ & & $\cdot t^{t} \cdot \ldots$ & \\
\hline $\begin{array}{l}\text { trigona So. . . . . } \\
\text { Wetherelli So. } \ldots\end{array}$ & $\cdots$ & $\begin{array}{lll}\cdots & \cdots \\
\cdots & \cdots & \cdots\end{array}$ & $\cdots \cdots$ & $\cdots$ & . & . $t \ldots$. & - \\
\hline Portlandica Нıтснс. & $\therefore \dot{M}^{2}$ & $\mid \begin{array}{c}\cdots \\
\cdots\end{array} \cdots$ & $\left(\begin{array}{c}\cdots \\
\cdots\end{array}\right.$ & $\cdots \cdots$ & $\begin{array}{ll}\cdots \\
\cdots\end{array}$ & 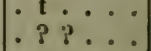 & $\because$ \\
\hline striata Lk. . . . . & $\cdots \cdots$ & $\ldots \ldots$ & $\ldots$ & $\ldots$ & $\ldots$ & 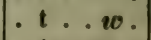 & . \\
\hline pella SERR. • & $\cdots \cdots$ & $\ldots \ldots$ & $\cdots \cdots$ & $\cdots$ & $\cdots$ & . $t \mathrm{u} \cdot \mathrm{w} x$ & - $\mathrm{z}$ \\
\hline pygmaea Mü. et varr. & $\cdots \cdots$ & $\ldots \ldots$ & $\cdots$ & $\ldots$ & . . & $\mid \cdot t \mathrm{u} \cdot \mathrm{w} \times$ &.$\%$ \\
\hline sulenta Br. ..... & $\cdots \cdot$ & $\ldots \cdots$ & ... & $\cdots \cdot$ & $\cdots \cdot$ & $|\cdot t \ldots w \cdot|$ & $\cdot x$ \\
\hline F acuminuta Eıснw. . & $\cdots \cdots$ & $\cdots \cdots$ & $\cdots \cdots$ & $\cdots \cdot$ & $\cdots$ & $\cdots \mathbf{u} \cdots$ & $\cdots$ \\
\hline concava BR. . & $\cdots \cdots$ & $\left.\mid \begin{array}{c}\cdots \\
\cdots\end{array}\right]$ & $\cdots \cdots$ & $\cdots \cdots$ & $\cdots$ & & $\because$ \\
\hline $\begin{array}{l}\text { depressa Nyst . . } \\
\text { Haesendonki Nyst. }\end{array}$ & $\begin{array}{l}\cdots \\
\cdots \cdots\end{array}$ & $\cdots \cdots$ & $\cdots \cdots$ & $\cdots$ & $\cdots$ & 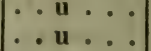 & 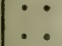 \\
\hline laevigata So. .... & $\cdots \cdot \cdot$ & $\ldots \ldots$ & $\ldots$ & $\ldots$ & ... & $\ldots$ u... & . \\
\hline lancrolata So..... & - . & $\ldots \ldots$ & $\ldots$ & $\cdots \cdot \cdot$ & $\cdots$ & $\ldots u \ldots$ & $\cdots$ \\
\hline f obliqua . . Conr. . . & $\ldots \mathbf{M}^{2}$ & $\ldots \ldots$ & $\ldots \therefore$ & $\cdots \cdot$ & $\cdots$ & $\ldots u \ldots$ & - \\
\hline $\begin{array}{l}\text { senistriata Wood } \\
\text { trigronula Wood. }\end{array}$ & $\cdots \cdot$ & $\cdots \cdots \cdots$ & $\cdots \cdot$ & $\cdots \cdot$ & $\cdots$ & $\ldots u \ldots$ & $\cdots$ \\
\hline $\begin{array}{l}\text { trigronula Wood .. } \\
\text { \# undita DFr. . . }\end{array}$ & $\cdots \cdot$ & $\cdots \cdots$ & $\cdots$ & $\cdots \cdot$ & $\cdots$ & $\cdots \mathbf{u} \cdots$ & $\cdot \bullet$ \\
\hline $\begin{array}{l}\text { \# undata DFR. } \text { Westendorpi Nyst } \\
\text { We }\end{array}$ & -. & $\cdots$ & $\cdots \cdots$ & $\cdots \cdots$ & $\cdots$ & 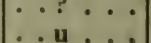 & $\cdots$ \\
\hline $\begin{array}{l}\text { Westendorpi Nyst } \\
\text { margaritacea IsK. (pars) }\end{array}$ & $\mathbf{E}^{2} \cdot \mathbf{F}^{2}$ & $\ldots \ldots$ & $\ldots$ & $\ldots$. & $\ldots$ & . u .w x & $\ddot{z}$ \\
\hline minuta F & $\ldots$ & $\ldots \ldots$ & $\ldots$ & $\cdots$ & $\cdots \cdot$ & . . u . . x &. $\mathrm{z}$ \\
\hline minuta DFr. ... & $\cdots \cdot$ & $\ldots \ldots$ & $\ldots$ & $\cdots \cdot$ & $\cdots$ & . u .w . . &. $\mathrm{z}$ \\
\hline rostrita Lк. . . . . & $\cdots \cdots$ & $\ldots \ldots$ & $\cdots \cdot$ & $\ldots$ & $\cdots \cdot 1$ & . $. \mathrm{u} \cdot \mathrm{w} \times \mathrm{x}$ & $\cdot \mathbf{z}$ \\
\hline$\cdots$ & . $\mathbf{M}^{2}$ & $\ldots \ldots$ & $\cdots$ & $\cdots$ & $\cdots$ & ..u $\cdot \mathbf{w} \mathbf{x}$ & .7 \\
\hline concentrica SAY... & . $\mathbf{M}^{2}$ & $\cdots \cdots$ & $\cdots$ & $\cdots \cdot$ & $\cdots$ & $\ldots u \ldots$ & $\cdot \mathrm{z}$ \\
\hline laevis $S_{A Y} \ldots$. & $\cdots M^{2}$ & $\cdots \cdots$ & $\cdots \cdot \mid$ & $\cdots \cdots$ & $\cdots$ & $\mid \cdots \mathbf{u} \cdot \mathbf{x}$ & $\cdot z$ \\
\hline limatula $S_{\mathbf{A Y}} \ldots$. & $\cdots \mathbf{M}^{2}$ & $\cdots \cdots \cdots$ & $\cdots \cdots$ & $\cdots \cdots \mid$ & $\cdots$ & $\cdots u \cdot x$ & $\cdot \mathrm{z}$ \\
\hline PHILL. . & $\cdots \cdots$ & $\cdots \cdots \cdots$ & $\cdots \cdots$ & $\cdots \cdots$ & $\cdots$ & ... v w w & - \\
\hline Nicobarica Lk. . . & $\cdots \cdots$ & $\ldots \cdots$ & $\cdots \cdots$ & $|\cdots \cdot|$ & $\cdots$ & ... v v & $\cdot \mathbf{z}$ \\
\hline Cobboldiae [?] So. . & $\cdots \cdots$ & $\cdots \cdots$ & $\cdots \cdots$ & $\cdots \cdots$ & $\cdots$ & $\cdots \cdots w$ & - \\
\hline decipiens Pниц. . & $\cdots \cdots$ & $\cdots \cdots$ & $\cdots \cdot$ & $\cdots \cdots$ & $\cdots$ & $\cdots \cdots w$ & - \\
\hline dilatata Pнісь. ... & $\cdots \cdot$ & $\ldots \ldots$ & $\cdots \cdots$ & $\cdots \cdots$ & $\cdots$ & $\cdots w_{\cdot}$ & - \\
\hline excisa Phus. .... & $\cdots \cdot$ & $\ldots \ldots$ & $\cdots$ & $\cdots \cdot$ & $\cdots$ & $\cdots \cdots w \cdot$ & - $\cdot$ \\
\hline glabra Рніск. . . . & $\cdots \cdots$ & $\cdots \cdots \cdots$ & $\cdots \cdot$ & $\cdots \cdots$ & $\cdots$ & $\cdots \cdots \mathbf{v}$ & - \\
\hline ida DFR & $\cdots \cdot$ & $\cdots \cdots \cdots$ & $\cdots$ & $\cdots \cdot$ & $\cdots$ & $\cdots \cdots w \cdot$ & - \\
\hline ongoides & $\cdot \cdot$ & $\cdots$ & & $\cdots$ & . 1 & $\cdots \cdots w$ & \\
\hline pellucida Phile. . & $\therefore \cdots$ & $\mid \cdots \cdots \cdots$ & $\cdots \cdots$ & $\cdots \cdots$ & $\cdots$ & $\cdots \cdots w$ & - \\
\hline Placentina LK. . . & $\cdots \cdot$ & $\ldots \ldots$ & $\ldots$. & $\ldots$ & $\cdots$ & $\ldots w$ & \\
\hline $\begin{array}{l}\text { pusio PHIIL. } \\
\text { proxima } S_{A Y} \ldots\end{array}$ & ... $\mathrm{M}^{2}$. & $\cdots \cdots$ & $\cdots \cdot$ & $\cdots \cdot$ & $\ldots$ & $\ldots \ldots$. . . & .2 \\
\hline tenuis TURT. .... & $\cdots$ & $\ldots \ldots$ & $\cdots \cdots$ & $\cdots \cdot \mid$ & $\ldots$ & $\ldots \ldots \mathbf{w} \times \mathbf{x}$ &. $\mathrm{z}$ \\
\hline ia $\mathrm{S}$ & .... & $\ldots \ldots$ & $\cdots \cdot \cdot$ & $\cdots \cdot$ & $\cdots$ & $\ldots \ldots x$ & - \\
\hline is Woopw. . & ・・ & $\cdots \cdots \cdots$ & $\cdots$ & $\cdots \cdot 1$ & $\cdots$ & $\cdots \cdot x$ & . z \\
\hline lanceolata So. . . & .....M & $\cdots \ldots \ldots$ & $\cdots$ & - & -5 & $\cdots \cdots x$ & $\mathrm{z}$ \\
\hline Puelcha D'0. . . . & ...M+ & & & & & ... $x$ & $\mathbf{z}$ \\
\hline
\end{tabular}




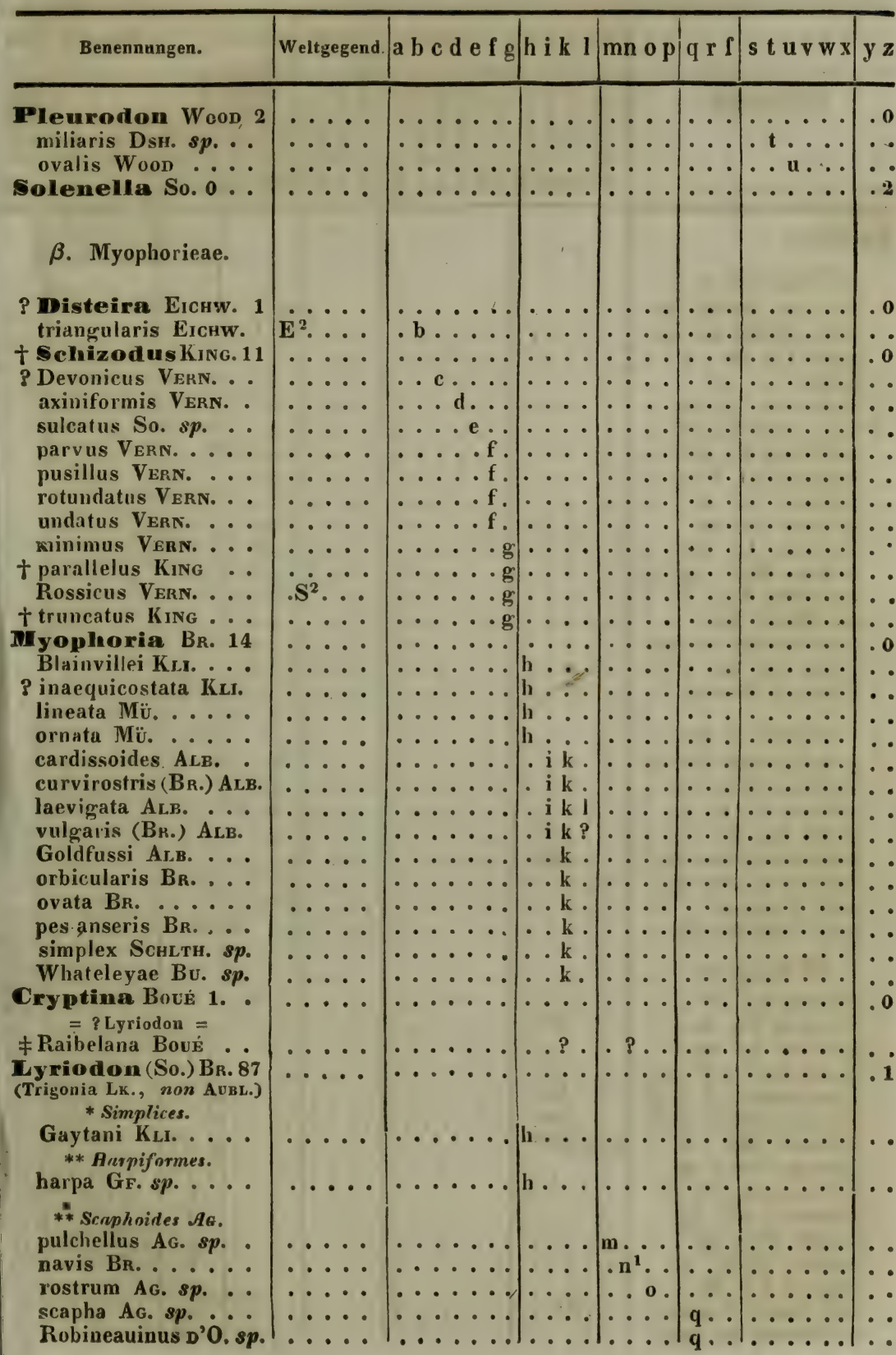




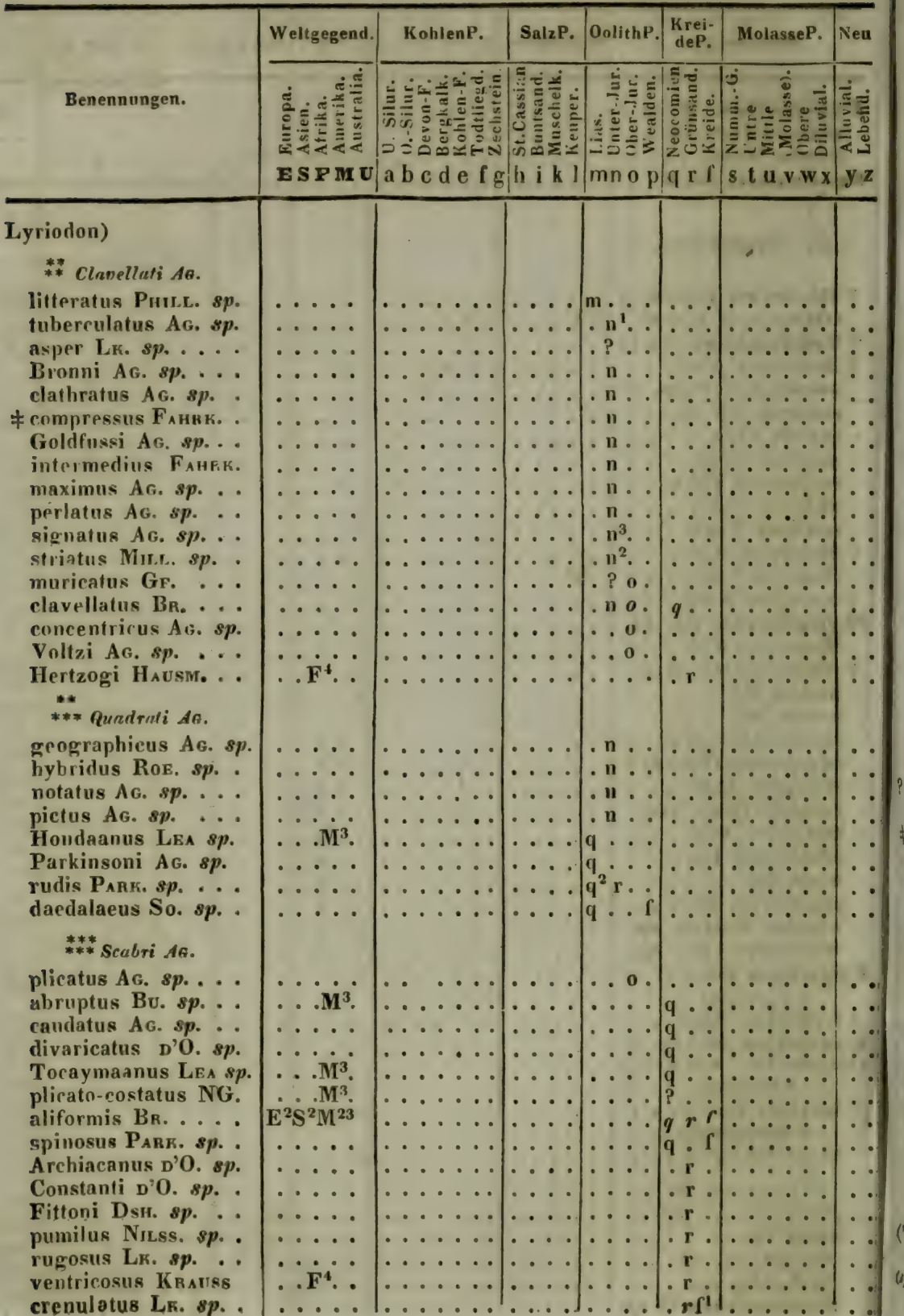




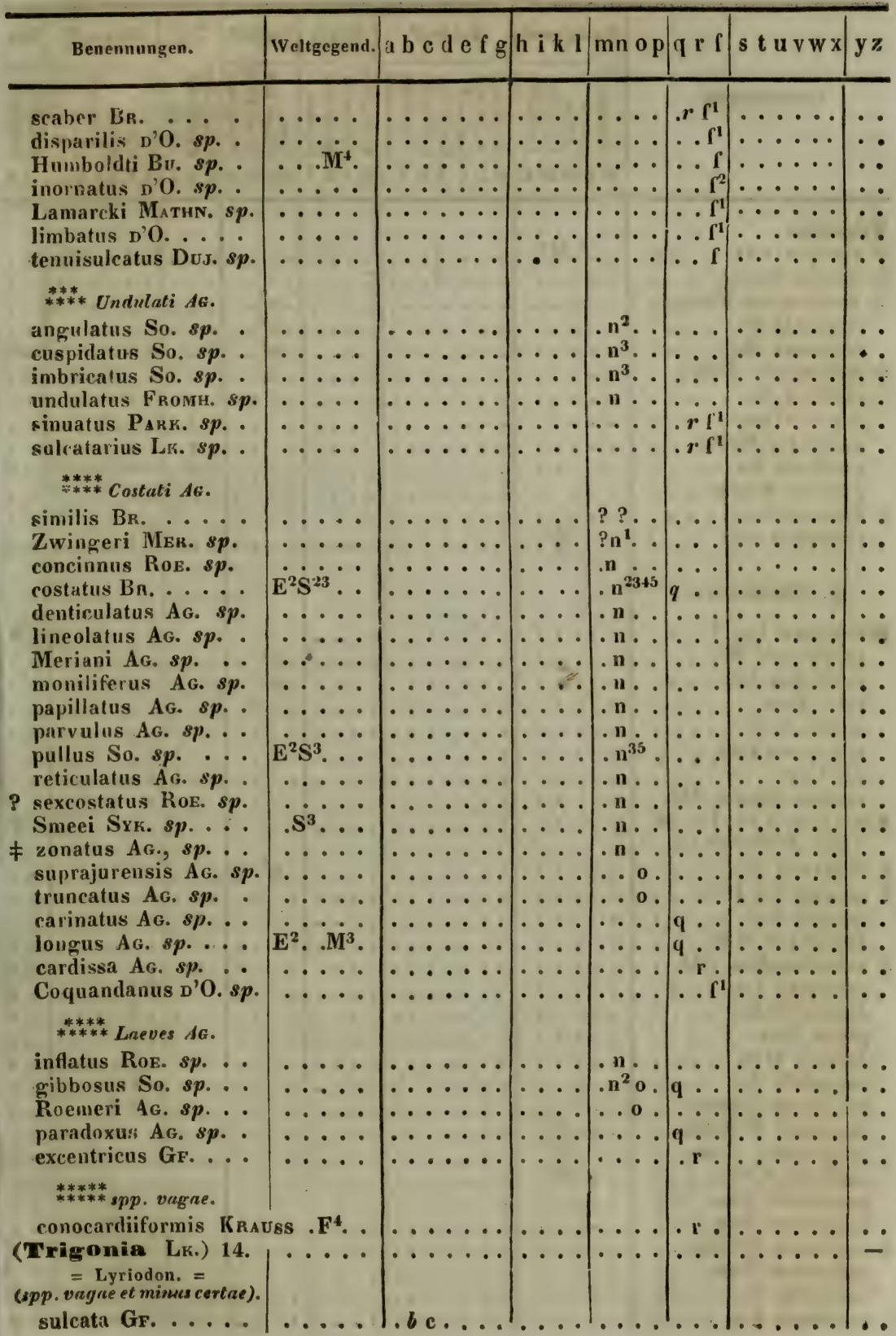




\begin{tabular}{|c|c|c|c|c|c|c|c|}
\hline & Weltgegend. & KohlenP. & SalzP. & OolithP. & $\begin{array}{c}\text { Krei- } \\
\text { deP. }\end{array}$ & MolasseP. & Neu \\
\hline Benennungen. & 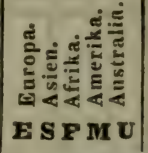 & 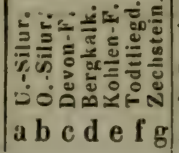 & 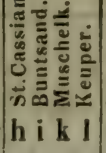 & 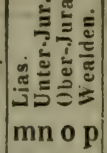 & 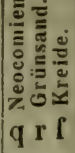 & 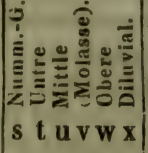 & 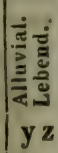 \\
\hline
\end{tabular}

Trigonia)

? antiqua D'O.

₹ incerta Ports.

\# conjungens PhiL.. . duplicata So.

impressa So.

\% subtrigona Pusch incurva So.

Buchi GeIn. pulchella Reuss quadrata So. parvula Reuss

F flexuosa LK. Hanetana D'O.

\section{y. Chamaceae.}

Diceras LK. speciosum Mü.... arietinum Lк. . . . . Luci DrR....... minus Dsh. ..... sinistrum Dsh. $s p$. Сat. . . . . sublamellosum Mü.

Chama Lin. LK. 26 "spp. dextrorsue [?].

Münsteri GF.

geometrica Ros. costata RoE. ..... semiplana RoE. .

生 suborbiculata $D^{\prime} \mathbf{O}$. calcarata $\mathbf{L}$. dissimilis Br. ....

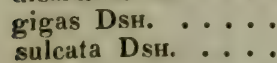
sulcata Dsн. .... lamellosa Chems. Agassizi WWAGN. congregata ConR. corticosa Conr. ... ponderosa Dsн. ...

* squamosa Eichw. . . substriata Dsн. . . . asperella $\mathrm{L}$ K. crenulata LK.

$\ldots \mathrm{M}^{3} \quad \ldots$. $\ldots . \ldots$

....

.....

$\ldots$

$\cdots \cdots$

...

$\ldots$

$\cdots \dot{M}^{4}$

... $M^{4}$.

$\ldots$

....

...

....

.....

…

$\ldots \ldots$

$\ldots$

$\cdots$

…

$\ldots$

...

$\ldots$

…

. $\dot{M}^{2}$

$\therefore M^{2}$

$\ldots M^{2}$

.....

...

...

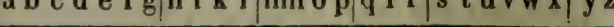

$\ldots$

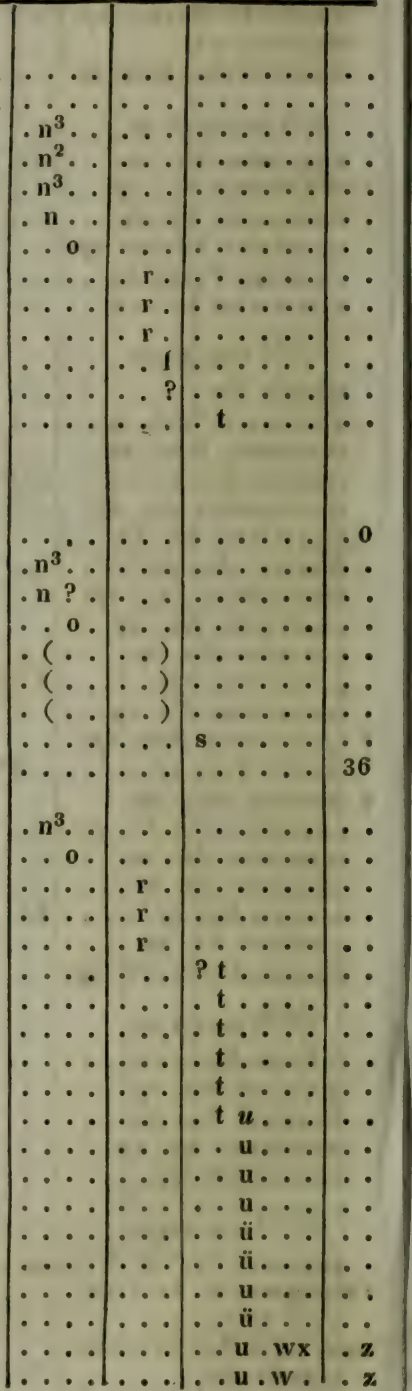




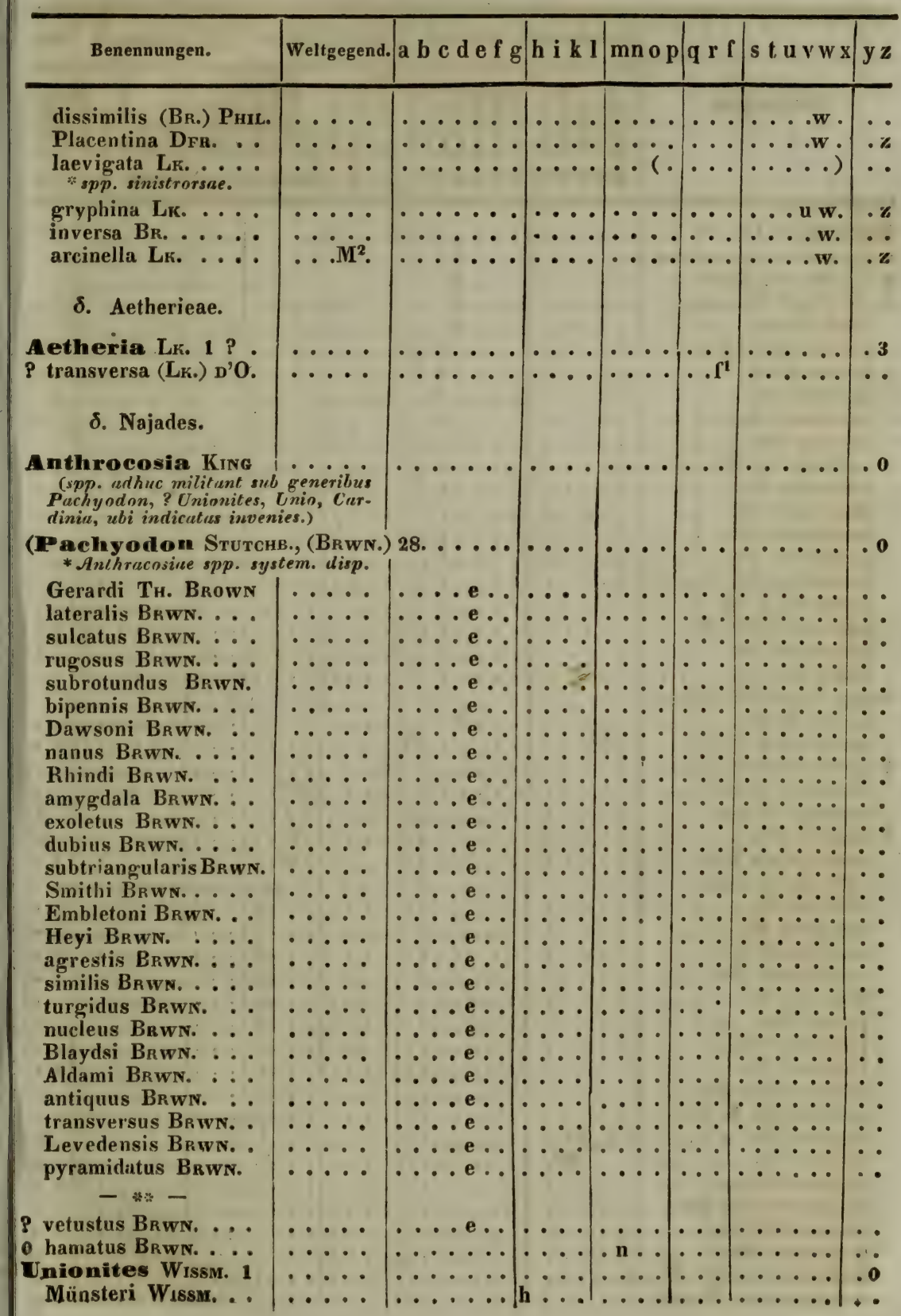




\begin{tabular}{|c|c|c|c|c|c|c|c|}
\hline & Weltgegend. & KohlenP. & SalzY. & OolithP. & $\begin{array}{l}\text { Krei- } \\
\text { deP. }\end{array}$ & MolasseP. & Neu \\
\hline Benennungen. & 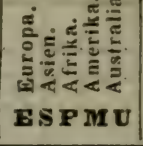 & 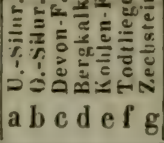 & $\begin{array}{ll} \\
0 \\
0 \\
0\end{array}$ & 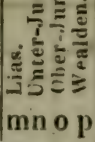 & 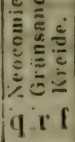 & $\begin{array}{l}0 \\
\text { s t u v w x }\end{array}$ & 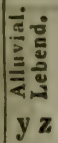 \\
\hline
\end{tabular}

Wnio Brg. LF. 59 ..

* spp.? Anthracosiae Krxg.

\& nuriformis Hгвв. .

\# Phillipsi Willms.

Urei FLem.

abbreviatus GF. .

acutus Dum.

Ansticei So.

aquilinus So.....

atratus GF. ....

carbonarius BR. .

centralis So. ....

dolabratus So.

modiolaris So.

parallelus So.

phaseolus So.

robustus J. So. ...

subconstrictus So.

tellinarius GF.

uniformis GF.

*u spp. dubine (?Curdinice?)

lacvis Erchw. . . . . umbonatus Fisch.

$\rho$ problematicus KI.I. . distortus Bean ... minutus Pusch ...

? striatus Mü.

subtrigonus Dsн. . .

*** spp. genuinue. aduncus So...... antiquus So..... compressus So. ... cordiformis So.... Mantelli So. planus RoE. ...... porrectus So. Roemeri Du. subporrectus Ros. subsinuatus $K \circ D v$. Martinii So. D'O. diluvii D'O. alpinus Mathin. . Bosquanus Mathn. . Cuvieri Mathn. ... flabellatus Gr.

Galloprovincialis Mathi.

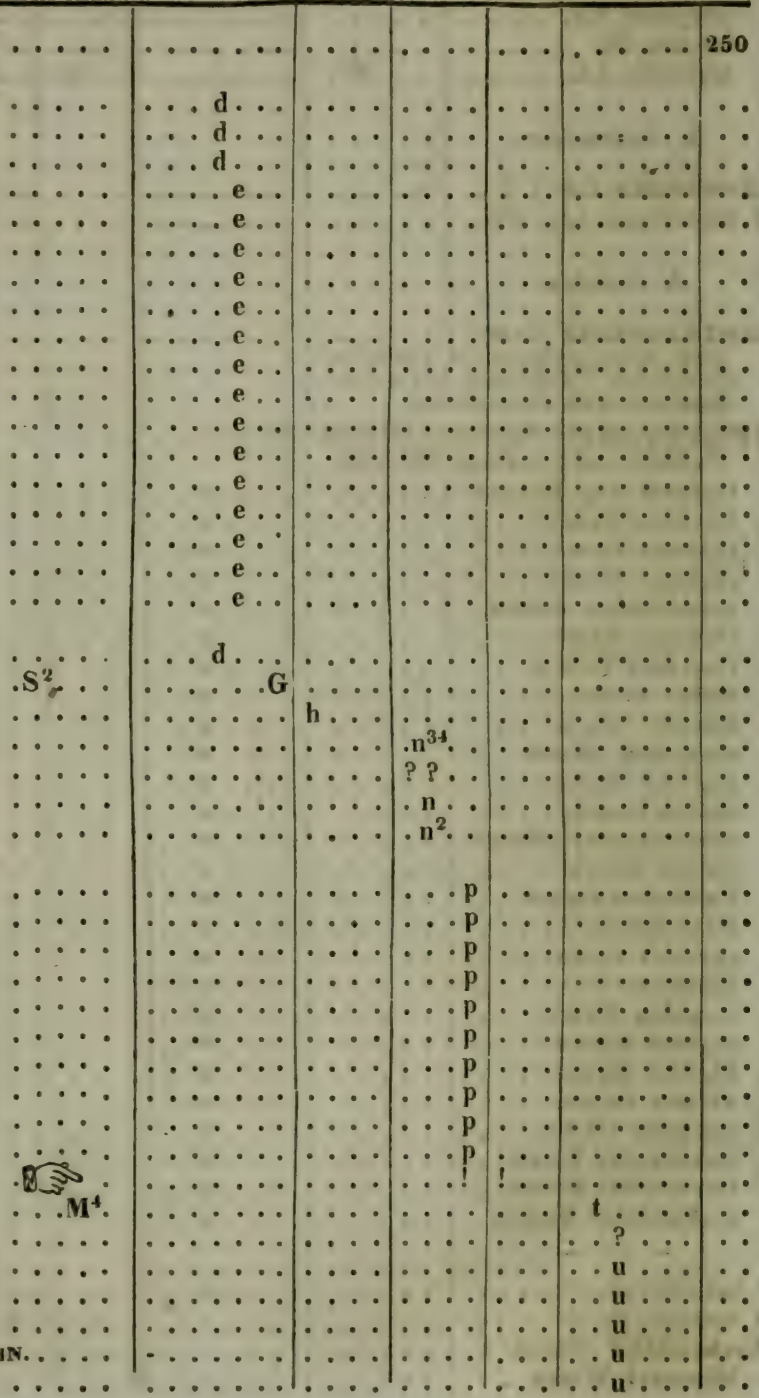
Gardanneusis Mathis. 


\begin{tabular}{|c|c|c|c|c|c|c|c|}
\hline Ben & end. & $\mathrm{f} g$ & 1 & p & 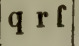 & $\mathbf{W} \mathbf{x}$ & $\mathbf{y}$ \\
\hline enis & & 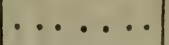 & & $\bullet$ & - & $\cdots \mathbf{u} \cdots$ & \\
\hline ATHN. & - & $\ldots \ldots$ & $\cdots \cdot$ & . & $\cdot$ & $\cdots \mathbf{u} \cdots$ & \\
\hline Toulouzani Matun. & $\cdots$ & $\cdots$ & $\cdots \cdots$ & $\cdots \cdot$ & $\cdot$ & $\cdots u \cdots$ & \\
\hline ventricosus Bouil. . & & $\cdots$ & - & $\cdots \cdot$ & $\cdots$ & $\cdots$ & \\
\hline $\begin{array}{l}\text { concentricus GF. . } \\
\text { costatus GF. . . }\end{array}$ & $\begin{array}{l}\cdots \cdots \\
\cdots\end{array}$ & $\begin{array}{lll}\cdots & \cdots & \cdots \\
\cdots & \cdots & \cdots\end{array}$ & $\cdots$ & $\mid \cdots \cdots$ & $\because$ & 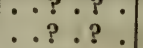 & \\
\hline $\begin{array}{l}\text { costatus GF. } \\
\text { Deccanensis So. . }\end{array}$ & $\mathrm{S}^{3} \cdots$ & $\ldots \ldots$ & $\ldots$ & $\ldots \ldots$ & $\cdots$ & $\cdots$ v. & \\
\hline Lavateri Mü. . . . . & $\cdots$ & $\ldots \ldots$ & $\cdots$ & $\ldots \ldots$ & $\cdots$ & $\ldots v \ldots$ & \\
\hline F. ... & $\cdots$ & $\ldots \ldots$ & $\cdots$ & $\cdots \cdots$ & $\cdots$ & ... v. & \\
\hline ... &.$S^{3} \ldots$ & $\cdots \cdot$ & $\cdots \cdot$ & $\cdots$ & $\cdots \cdot$ & $\ldots \mathbf{v} \ldots$ & \\
\hline rmis Bra & $\cdots \cdot \cdot$ & $\cdots \cdots$ & $\cdots \cdots$ & $\cdots \cdot$ & $\cdots$ & $\ldots \ldots x$ & \\
\hline Batavus Pfeiff. .. & $\cdots \cdot$ & $\ldots \ldots$ & $\cdots \cdot$ & $\cdots \cdot$ & $\cdots$ & $\cdots \ldots x$ & $\mathbf{z}$ \\
\hline$\cdots$ & $\cdots \cdot \cdot$ & $\cdots \cdots \cdots$ & $\cdots \cdot$ & $\cdots \cdot$ & $\cdots \cdot$ & $\ldots \ldots x$ & $y z$ \\
\hline$\cdots$ & $\cdots \cdot$ & $\cdots \cdots \cdots$ & $\cdots \cdot$ & $\cdots \cdot$ & $\cdots \cdot$ & $\cdots \cdots$ & $y z$ \\
\hline$\cdots$ & $\cdots$ & $\cdots$ & $\cdots \cdot$ & $\cdots$ & $\cdots$ & $\cdots \cdots$ & $y z$ \\
\hline . 1 & $\therefore$ & $\cdots$ & & $\cdots \cdots$ & $\cdots$ & $\cdots \cdots$ & $\mathbf{Z}$ \\
\hline Ma & 1 . & $\cdots$ & $\cdots$ & $\cdots \cdots$ & $\cdots$ & $\cdots$ & 20 \\
\hline . & $\cdot$ & $\cdots \cdots \cdots$ & $\cdots \cdots$ & $\cdots P \mid$ & $\cdots$ & $\cdots \cdots \cdots$ & \\
\hline $\mathbf{A n}$ & $\cdots \cdot \cdot$ & $\cdots \cdots \cdots$ & $\cdots \cdots$ & $\cdots \cdots$ & $\cdots$ & $\cdots \cdots \cdots$ & $70^{-}$ \\
\hline$\cdots$ & $\cdots$ & $\cdots$ & $\cdots$ & $\cdots \cdot$ & $\cdots$ & $\cdots \cdots$ & \\
\hline Hw. & & $\cdots$ & $\cdots \cdots$ & $\cdots$ & $\because$ & $\cdots \cdots$ & • \\
\hline C & & $\begin{array}{l}\cdots \\
\ldots\end{array}$ & $\cdots \cdots$ & $\cdots \cdots$ & $\because$ & $\cdots$ & • \\
\hline ol & & $\because$ & $\because$ & $\cdots$ & $\because 1$ & $\cdots$ & \\
\hline $\begin{array}{l}\text { Aquensis } M_{A} \\
\text { anatinoides }\end{array}$ & $\therefore$ & $\ldots$ & $\ldots$ & $\cdots$ & $\therefore$ & $\cdots$ & \\
\hline$\cdots$ & $\cdots$ & $\ldots \ldots$ & $\ldots$ & $\ldots$ & $\ldots$ & $\ldots w$ & \\
\hline (L.) Mukr. & $\ldots$ & $\ldots \ldots$ & $\ldots$ & $\ldots$ & $\ldots$ & $\ldots \ldots$ & $\dot{y z}$ \\
\hline LK. $0 \ldots$ & $\ldots \ldots$ & $\ldots \ldots \ldots$ & $\cdots$ & $\cdots \cdots$ & $\cdots$ & $\ldots \ldots$ & .6 \\
\hline D'O. 0 & $\ldots \ldots$ & $\ldots \ldots$ & 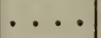 & - & ... & $\ldots \ldots$ & .2 \\
\hline Castalia LK. 0 .. & $\ldots$ & $\ldots \ldots$ & $\cdots$ & $\cdots \cdot$ & $\cdots$ & $\ldots \ldots$ & .1 \\
\hline Ilyria LK. 0 . . & & $\cdots \cdots \cdots$ & $\cdots \cdot$ & & $\cdots$ & $\cdots \cdots$ & .4 \\
\hline$\varepsilon$. & & & & & & & \\
\hline G. 1 & • & $\cdots \cdot$ & $\cdots$ & D. & - 1 & $\cdots$ & 0 \\
\hline$\cdots$ & $\cdot$ & $\cdots$ & & m... & $\cdots$ & $\ldots \ldots$ & - \\
\hline $\begin{array}{c}\text { Cardin } \\
* \operatorname{spp} .\end{array}$ & & $\cdot$ & - & $\cdot 1$ & $\cdots$ & $\cdots \cdots \cdots$ & .0 \\
\hline KeYs. & $\cdots$ & $\ldots d \ldots$ & - & • & $\cdots$ & - & \\
\hline s. • & $\cdots$ & d... & • & $\cdots \cdots$ & $\cdots \cdot$ & $\cdots \cdots$ & \\
\hline$\cdots$ & $\cdots \cdot$ & $\ldots$. & . & $\cdots$ & $\cdots$ & $\cdots \cdots$ & \\
\hline$\cdots$ & $\cdots \cdots$ & $\ldots \ldots \mathbf{e}$ & $1^{\circ}$ & - & $\cdots$ & $\cdots \cdots \cdots$ & \\
\hline$\cdots$ & & $\cdots \cdot \mathbf{e} \cdots$ & 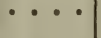 & & - 1 & $\cdots \cdots \cdot$ & - \\
\hline lis $\mathrm{K}$ & $\cdot \cdot$ & e. & & & & $\cdots$ & \\
\hline . . • & & & & m. & $\cdots$ & & \\
\hline Ag. ... & & $\cdots$ & ${ }^{\circ}$ & . $\mathbf{n}$. & $\cdots$ & $\cdots \cdots$ & . \\
\hline AG. .. & & $\cdots$ & 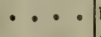 & $\mathbf{m}$. & - $\cdot$ & $\cdots \cdot \cdot$ & \\
\hline RR. . . & & $\cdots \cdots$ & & m . & - & $\cdots \cdots$ & - \\
\hline R. $\quad \cdots$ & $\cdots$ & . & 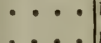 & m. & $\cdot$ & $\cdots \cdots$ & 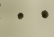 \\
\hline & $\cdots$ & $r^{2}$ & & & & $\cdots$ & \\
\hline
\end{tabular}




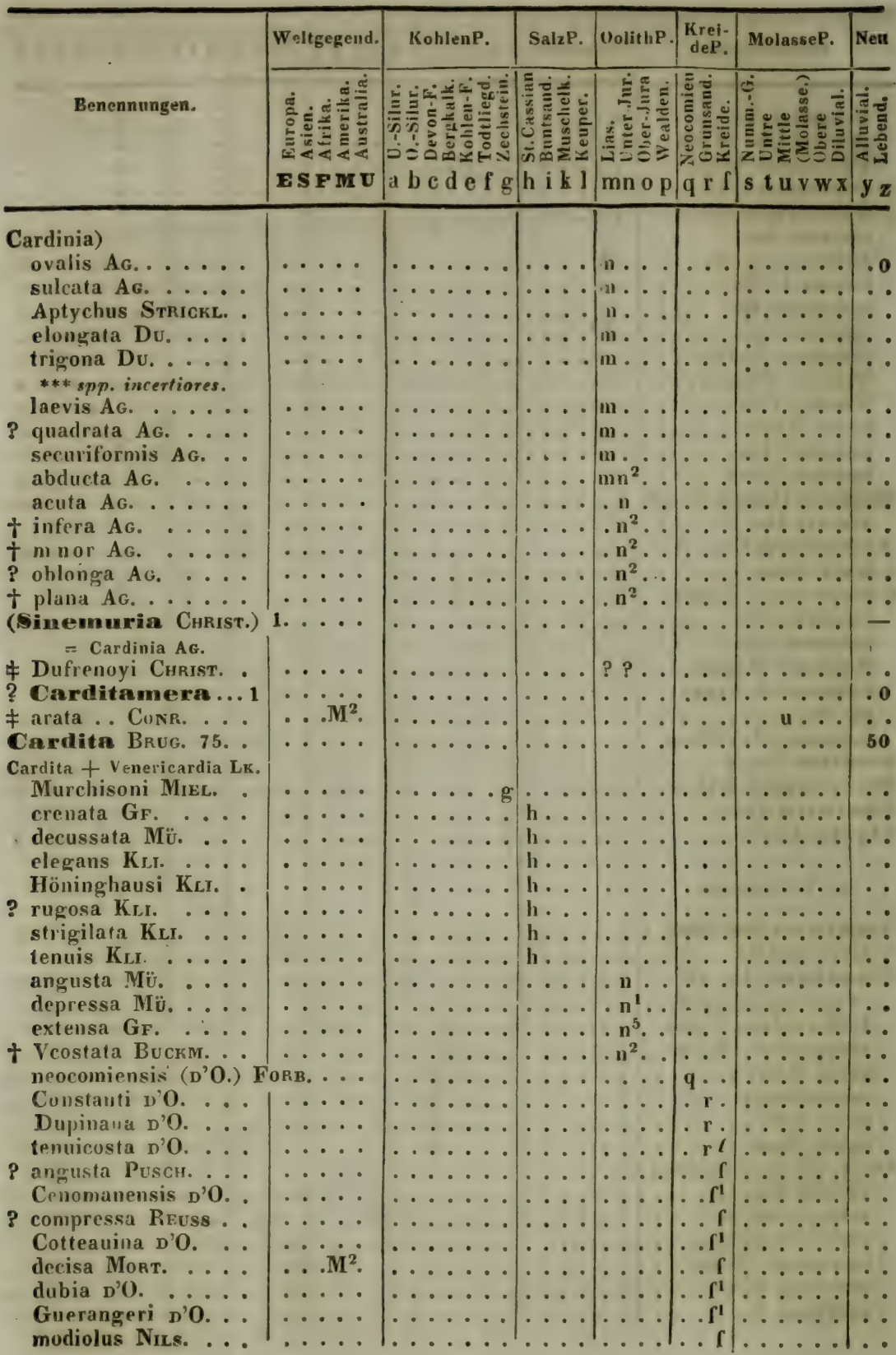




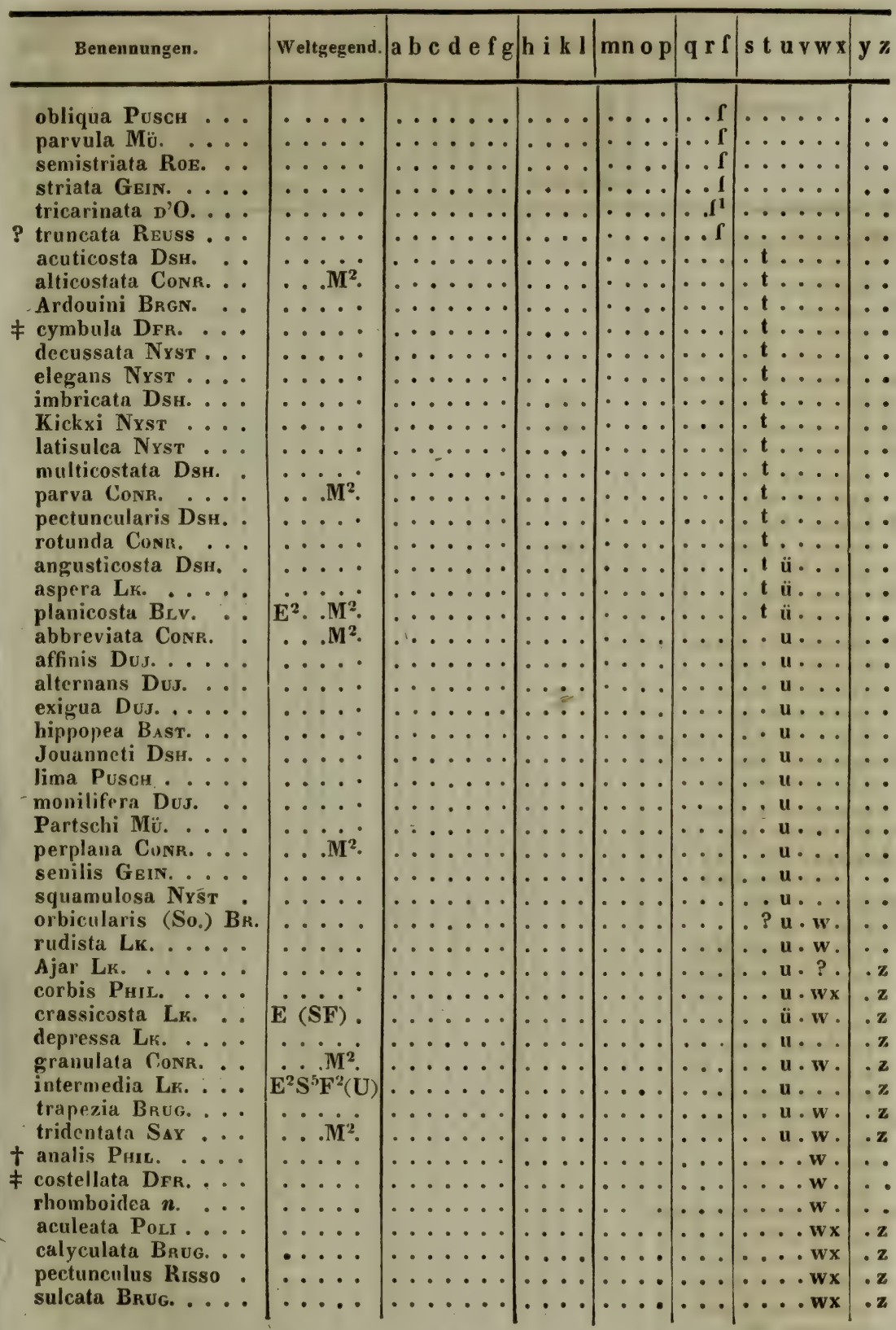




\begin{tabular}{|c|c|c|c|c|c|c|c|}
\hline est & Weltgegend. & KohlenP. & Salze. & OolithP. & $\begin{array}{r}\text { Krei- } \\
\text { deP. }\end{array}$ & MolasseP. & Neu \\
\hline Benennungen. & 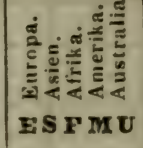 & 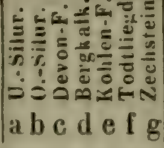 & 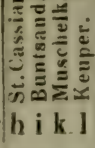 & 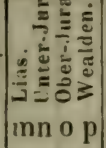 & 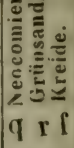 & 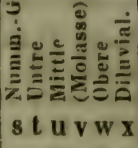 & 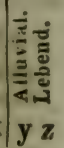 \\
\hline
\end{tabular}

(Vemericardia $\left.L_{K}\right), 24$

= Cardita Lk. =

Goldfussi ALb.

8 crenata Cᄉt.

aculeata Dsh.

asperula Dsн.

\# Brongniarti Mant.

+ Blandingi Lsa ...

\# Carolinensis DFr.

\# concentrica Lк. . . . deltuidea So.

globusa So.

P Laurae Brgn. . . . . minuta Leym. mitis LK. ......

\# spissa Drr. ..... squamosa LK

\# subrotunda DFR. . . trigona Leym. vicinalis Lkym. . . . cor-avium LK. imbricata Lk. (var).

₹ sportella DFr. annulata Pusch ... complanata Dsh. .

* laevicosta Lr. ...

Astarte So. 134 (Crassina Lk.)

?. Neptuni Mü. cincta Gr. rhomboidalis Kon. acutimaryo Ros. arealis Roe. complanata Roe. rhumbea RoE. striato-sulcata RoE. subcarinata Mü.

* tetragona Portc.. Voltzi GF.

elegans PhicL.

Buchana D'0.

\# Burgomontana VERN. carinata Phill. compressa So. cordifornis Dsh. crassitesta RoE. depressa Mü.

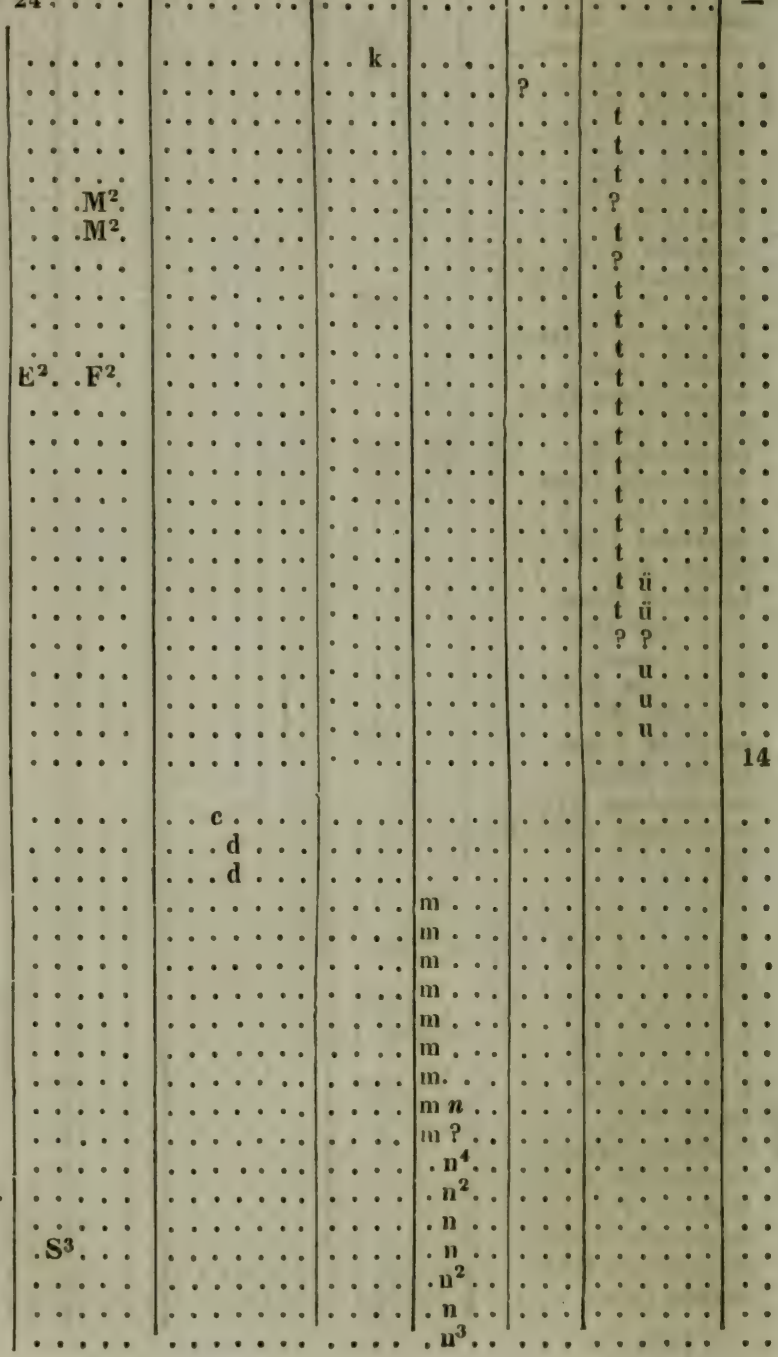




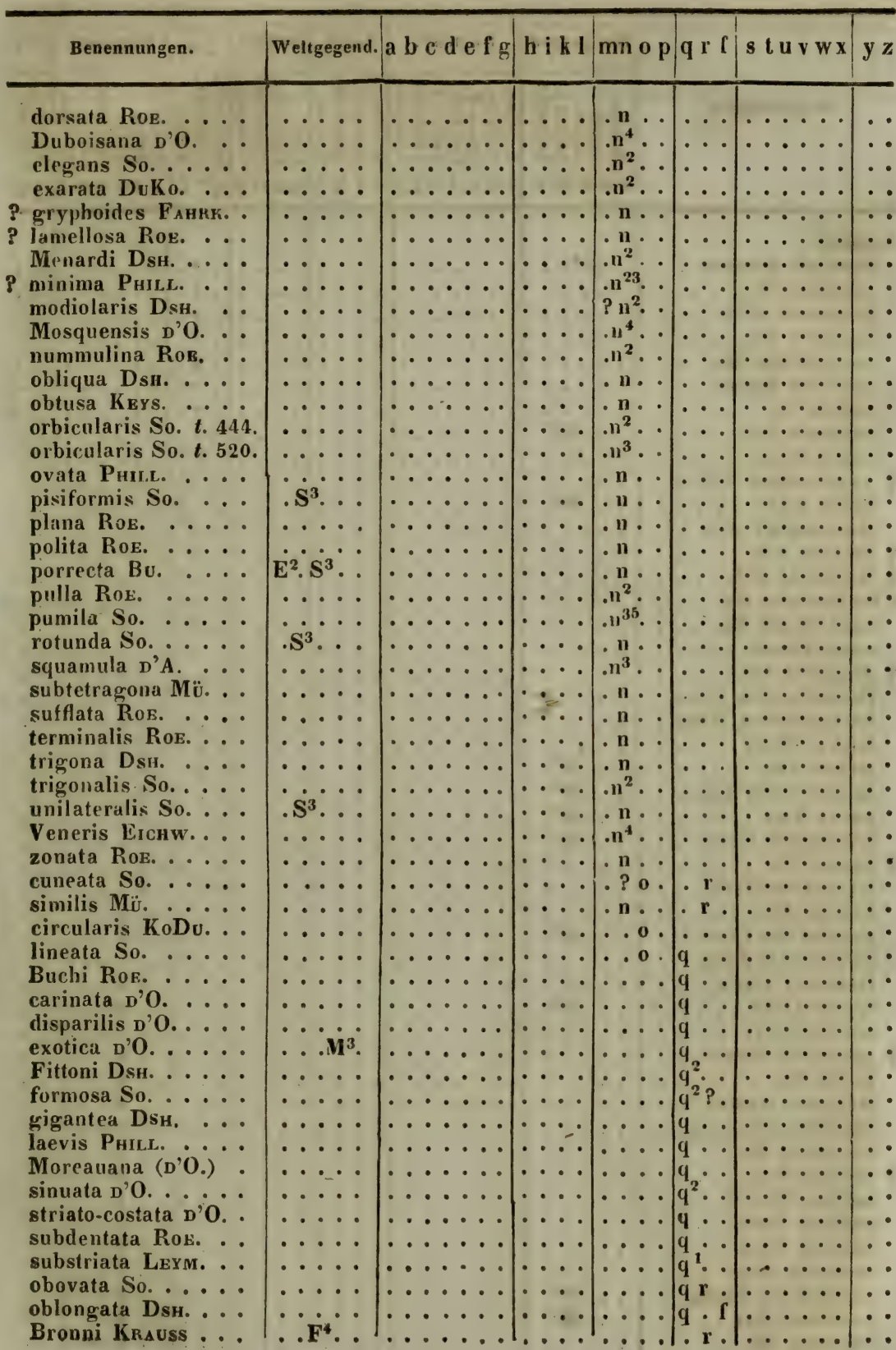




\begin{tabular}{|c|c|c|c|c|c|c|c|}
\hline \multirow[b]{2}{*}{ Senennungen. } & eltgegend. & cohlenP. & alze. & OolithP. & $\begin{array}{l}\text { Krei- } \\
\text { deP. }\end{array}$ & MolnsseP. & \\
\hline & 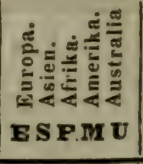 & 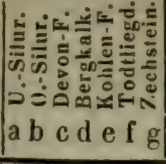 & 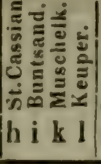 & 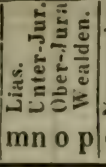 & 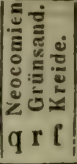 & 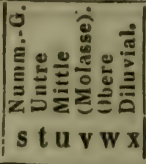 & \\
\hline \multirow{2}{*}{\multicolumn{8}{|c|}{$\begin{array}{l}\text { Astarte) } \\
\text { capensis Krauss .. }\end{array}$}} \\
\hline & . $\mathrm{F}^{4} \ldots$ & - & & & r . & $\cdots$ & \\
\hline Dupinana n'o. ... & $\cdots \cdots$ & 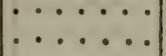 & - & & & $\cdots$ & \\
\hline impolita So. .... & $\cdots \cdots$ & $\cdots \cdots$ & $\cdots$ & . & $\mathbf{r}$ & $\cdots \cdots$ & \\
\hline multistriata So. . & $\cdots \cdots$ & $\cdots \cdots \cdots$ & $\cdots \cdot$ & $\cdots \cdots$ & $\cdot \mathbf{r} \cdot$ & $\cdots \cdots$ & \\
\hline & $\cdots \cdots$ & $\ldots \cdots$ & $\cdots \cdot$ & $\cdot 0$ & . $\mathbf{r}$. & $\cdots \cdots$ & \\
\hline $\begin{array}{l}\text { striata So. } \ldots \ldots \\
\text { truncata Bu. } \ldots \ldots\end{array}$ & $\cdots \dot{M}^{3}$ & $\cdots \cdots$ & $\cdots \cdot$ & & $\dot{r} \cdot$ & $\cdots \cdots$ & \\
\hline acuta Reuss . . . . & $\ldots \ldots$ & $\cdots \cdots \cdots$ & & . & $\begin{array}{l}.6 \mathrm{i} \\
? \mathrm{r}\end{array}$ & $\dot{0}$ & \\
\hline des D'A... & - . & $\ldots$. & t & . & . . $f^{1}$ & . & \\
\hline$\cdots$ & $\cdots \mathrm{M}^{4}$ & $\cdots \cdots$ & $\cdots \cdot$ & $\cdots \cdot$ & $\ldots \mathbf{I}$ & $\cdots$ & \\
\hline$\cdots$ & $\cdots$ & $\cdots \cdot$ & & $\cdots \cdot$ & .. $\mathbf{f}^{\mathbf{t}}$ & $\ldots \ldots$ & \\
\hline$\cdots$ & & 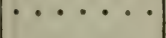 & & $\cdots \cdot$ & . . . $\mathbf{f}^{1}$ & $\ldots \ldots$ & \\
\hline$\cdots$ & $\cdots \cdot \cdot$ & $\cdots \cdots$ & 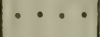 & &.. $\mathrm{f}$ & $\cdots \ldots$ & \\
\hline$\cdots$ & $\cdots \cdots$ & $\cdots \cdots \cdot$ & $\cdots \cdot$ & $\cdots$ & $\cdots f$ & $\cdots \cdots$ & \\
\hline$\because$ & - & $\cdots \cdots$ & $\cdots$ & $\cdots \cdot$ & $\cdots I^{1}$ & $\cdots \cdots \cdots$ & \\
\hline$\therefore$ & $\cdots \cdots$ & $\cdots \cdots$ & $\because$ & & $\begin{array}{c}\cdots 1 \\
\cdots\end{array}$ & $\cdots \cdots$ & \\
\hline YST & .... & $\ldots \ldots$ & $\ldots$ & $\ldots$ & $\ldots$ & 0 & \\
\hline a Nyst. & $\ldots$ & $\ldots \ldots \ldots$ & $\ldots$ & $\ldots$ & $\ldots$ & t $\ldots$. & \\
\hline ST $\ldots$ & $\cdots$ & $\cdots \cdots$ & $\cdots$ & $\cdots$ & $\cdots$ &.$t \ldots$ & \\
\hline$\cdots$ & $\ldots \mathbf{M}^{2}$ & $\cdots$ & $\cdots$ & $\cdots$ & $\cdots$ &.$t \ldots$ & \\
\hline a LEA .. & $\cdots$ & $\cdots \ldots \ldots$ & $\cdots$ & $\cdots \cdots$ & $\cdots$ & $\cdot \mathbf{t} \ldots$ & \\
\hline Conr. . . & $\cdots$ & $\cdots \cdots \cdots$ & $\cdots$ & $\cdots \cdots$ & $\cdots \cdot$ & $\cdot \mathbf{t} \cdots$ & \\
\hline$\cdots$ & $\cdots$ & $\cdots \cdot$ & •. & $\cdots$ & $\cdots$ & $\cdot t \cdots$ & \\
\hline Yst. . . & $\cdots$ & $:$ & $\cdots \cdots$ & $\cdots$ & $\cdots$ & $\left|\begin{array}{ccc}t & \cdots & \cdots \\
. t & \ldots & \cdots\end{array}\right|$ & \\
\hline $\begin{array}{l}\text { YST } \ldots \\
\ldots \ldots\end{array}$ & $\ldots$ & $\ldots \ldots$ & $\ldots$. & $\ldots$ & ... & . u ... & \\
\hline a. . & ... $\mathbf{M}^{2}$ & $\cdots \cdots$ & $\cdots$ & $\ldots$ & $\cdots$ & $\ldots u \ldots$ & \\
\hline$\cdots$ & $\cdots \cdot$ & $\cdots \cdots$ & $\ldots$ & $\cdots$ & $\cdots$ & . и ... & \\
\hline $\mathbf{T} \ldots$ & $\cdots$ & $\cdots \cdots$ & $\cdots$ & - & $\cdot$ & $\ldots \mathbf{u} \ldots$ & \\
\hline$\cdots \cdot$ & $\cdots$ & $\cdots \cdots$ & $\cdots \cdot$ & $\cdots$ & $\cdots$ & $\cdots \mathbf{u} \ldots$ & • \\
\hline$\cdots$ & & $\cdots$ & $\cdots$ & - & $\cdots$ & $\cdots \mathbf{u} \cdots$ & \\
\hline$\cdots$ & & & & - & . 1 & $\cdots u, \ldots$ & \\
\hline & &. & - • & $\cdots \cdot 1$ & $\because$ & u. & \\
\hline & $\cdot$ & 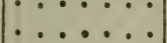 & $\cdots$ & $\cdot$ & $\because$ & ? & \\
\hline$\cdots$ & $\ldots .$. & $\cdots$ & . & $\cdots \cdots$ & $\cdots$ & $\ldots$ u... & \\
\hline$\cdots$ & & $\cdots$ & . . & $\cdots$ & $\cdots$ & . . u . . & \\
\hline$\cdots$ & - & 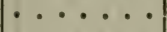 & $\cdots \cdots$ & $\cdots \cdots$ & $\cdots$ & . u . . & - \\
\hline & & •. & & $\cdots$ & $\cdots$ & . u. . . & \\
\hline 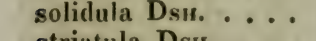 & & 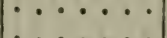 & • & $\cdots$ & ・・ & $\cdots u_{p}$ & \\
\hline$B_{1}$ & & & ..1 & . & $\cdots \bullet$ & .?... & \\
\hline$\cdots$ & $\cdot$ & & & & $\cdots$ & $\therefore$ u. . & - \\
\hline 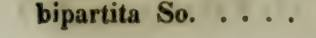 & & & & & & & \\
\hline
\end{tabular}




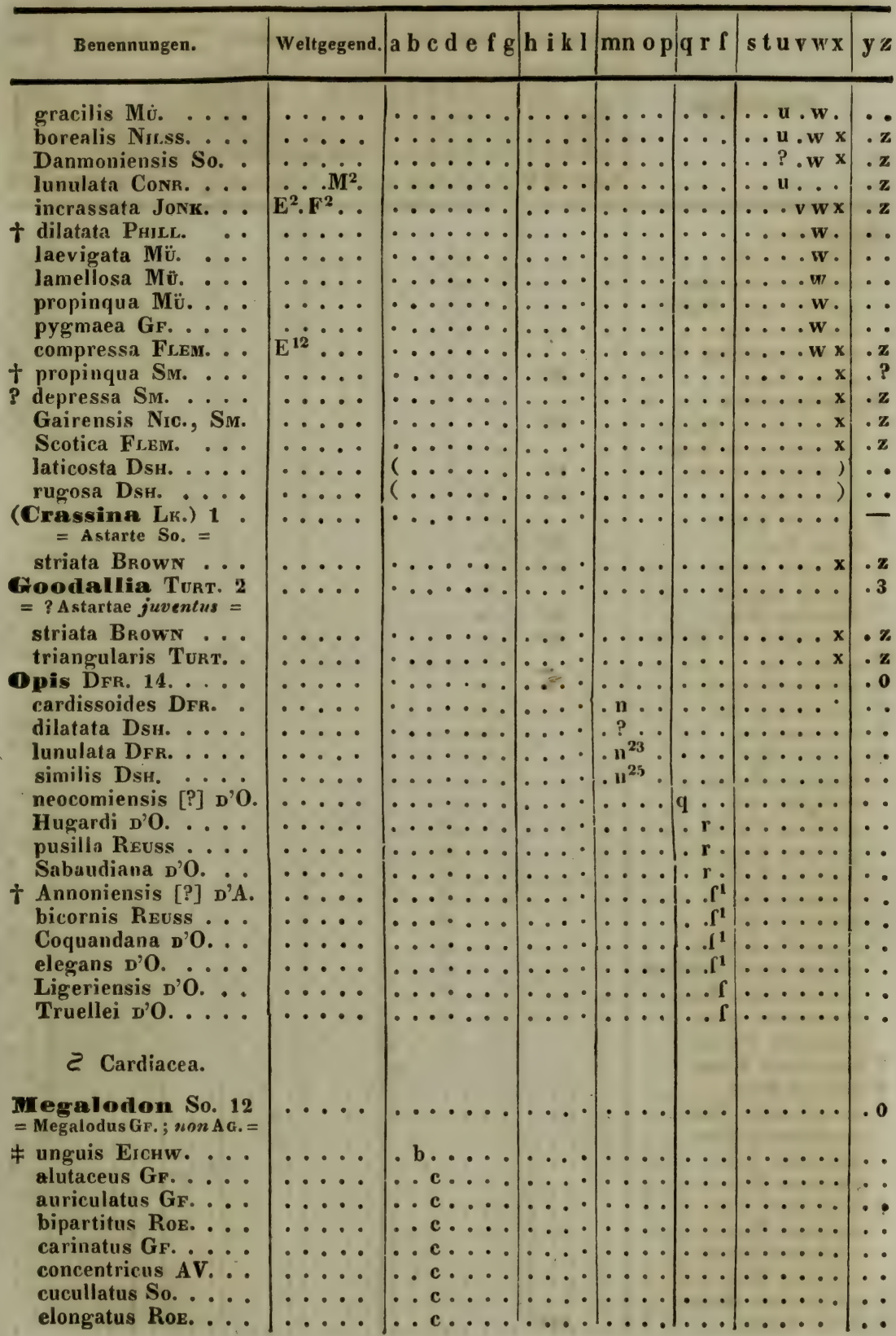




\begin{tabular}{|c|c|c|c|c|c|c|c|}
\hline & Weltgegend. & KohlenP. & SalzP. & OolithP. & $\begin{array}{c}\text { Krei- } \\
\text { deP. }\end{array}$ & MolasseP. 1 & Neu \\
\hline Benennungen. & 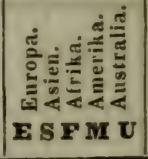 & 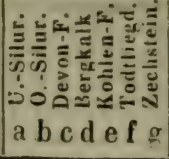 & 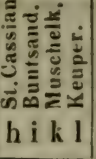 & 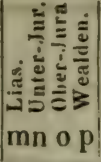 & 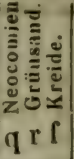 & 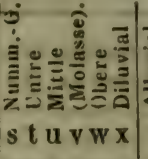 & 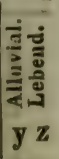 \\
\hline don) & & & & & & & \\
\hline & & & & & .. & $\cdots \cdots$ & \\
\hline $\begin{array}{l}\text { rhomboideus GF. . } \\
\text { suboblongus VERN. }\end{array}$ & $\cdots \cdots$ & 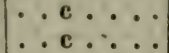 & & $\cdots$ & $\because$ & $\cdots \cdots \cdots$ & \\
\hline trumratus GF. . . . & $\therefore \cdots$ & . c ... & $\cdots \cdots$ & $\cdots$ & $\cdots$ & $\ldots \ldots$ & \\
\hline ? Drthonatha HaLt & 1.... & & & $\ldots$ & $\cdots$ & $\ldots \ldots$ & .0 \\
\hline . & $\therefore \mathbf{M}^{2}$ & b.... & & $\cdots \cdot \mid$ & $\cdots$ & $\cdots \cdots$ & \\
\hline lia LK, 41 & $\cdots \dot{M}^{2}$ & $\cdots \cdots$ & $\cdots$ & $\cdots$ & $\cdots$ & $\cdots \cdots$ & 13 \\
\hline $\mathbf{s} \mathbf{E}_{\text {? }}$ & $\because M^{2}$ & $\cdots$ & $\cdots$ & $\cdots \cdots$ & $\cdots$ & $\cdots \cdots$ & $\cdots$ \\
\hline $\begin{array}{l}\text { iyesana VERN. } \\
\text { laris EmMs. . . }\end{array}$ & $\cdots \dot{M}^{2}$ & $\cdots \cdots$ & $\cdots \cdots$ & $\cdots \cdots$ & $\cdots$ & $\cdots \cdots$ & $\cdots$ \\
\hline Har.L......... & $\therefore \mathrm{M}^{2}$ & b......... & $\ldots$ & $\ldots$ & $\ldots$ & $\ldots \ldots$ & $\bullet$ \\
\hline L. ... & $\cdots$ & ? .... & ... & $\ldots$ & $\cdots$ & $\ldots \ldots$ & : \\
\hline$\cdots$ & $\cdots \mathrm{M}^{2}$ & . b..... & $\cdots$ & $\ldots$ & $\cdots$ & $\ldots \ldots$ & . \\
\hline sta Hacr ... & $\ldots \mathrm{N}^{2}$ & $\cdot b \ldots$ & $\cdots$ & $\cdots$ & $\cdots$ & $\ldots \ldots$ & - \\
\hline$\cdot \cdots$ & - ・. & b.... & $\cdots \cdot$ & $\cdots$ & $\cdots$ & $\cdots \cdots$ & - \\
\hline eta HALL … & $\cdots M^{2}$ & 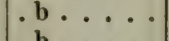 & $\cdots$ & $\cdots$ & $\cdots$ & $\cdots \cdots$ & $\cdots$ \\
\hline $\begin{array}{l}\text { orthonata [?] HaLL } \\
\text { retusa So. . . . . }\end{array}$ & $\cdots N^{2}$ & $\cdots \cdots$ & $\cdots$ & $\cdots \cdots$ & $\cdots$ & $\cdots \cdots$ & $\cdot \cdot$ \\
\hline \# silurira Еichw. . & • & b. . . & $\cdots \cdots$ & $\ldots$ & $\because$ & $\cdots \cdots$ & - \\
\hline les So. ... & $\ldots .$. & b.... & $\ldots$ & $\ldots$ & $\ldots$ & $\ldots \ldots$ & \\
\hline essa So. .... & .... &. $\mathbf{b} \mathbf{c} \ldots$ & $\cdots \cdot$ & $\cdots \cdots$ & .. & $\ldots \ldots$ & \\
\hline ? undata So. ..... & $\cdots$ & . b c ... & $\cdots$ & $\ldots$ & $\ldots$ & $\ldots \ldots$ & $\cdots$ \\
\hline lea Phild. . . . & $\cdots$ & . c... & $\cdots \cdots$ & $\cdots$ & . & $\ldots \ldots$ & - \\
\hline$\cdots \cdot$ & $\cdots$ & $\cdots \mathbf{c} \cdot \cdots$ & $\cdots \cdot$ & $\cdots \cdots$ & . & $\cdots \cdots$ & \\
\hline glabrata Phrce. . . . & ... & $\cdots d \ldots$ & ・・ & $\cdots$ & $\cdots$ & $\cdots \cdots$ & - $\cdot$ \\
\hline globosa Ko & $\cdot$ & $\cdots d \cdots$ & 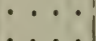 & $\cdots \cdots$ & . & $\cdots \cdots$ & $\bullet$ \\
\hline$\cdots$ & • & $\begin{array}{ll}\cdots d \\
\cdots\end{array}$ & $\because$ & $\therefore$ & 1. & $\cdots \cdots$ & : \\
\hline$\cdots$ & $\ldots$. & $\ldots d \ldots$ & $\ldots$ & $\cdots \cdots$ & $\therefore$ & $\cdots \cdots$ & . \\
\hline 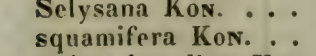 & & $\ldots d \ldots$ & $\cdots \cdot$ & $\ldots$ & $\ldots$ & $\therefore \ldots$ & $\ddot{\dot{*}}$ \\
\hline a KoN. & . & $\ldots d \ldots$ & $\ldots \ldots$ & $\ldots$. & . . & $\ldots \ldots$ & \\
\hline on. . & & $\ldots d \ldots$ & $\cdots$ & . . & . & $\ldots \ldots$ & \\
\hline . . & $\cdots$ & $\ldots d \ldots$ & $\cdots$ & - & $\cdots$ & .... & \\
\hline ta Porte. . . & $\ldots \ldots$ & $\ldots d \ldots$ & - & . . & $\cdots$ & $\ldots \ldots$ & • \\
\hline nata KEYS. . & $\ldots \ldots$ & $\ldots \ldots g$ & & & $\ldots$ & $\ldots \ldots$ & - \\
\hline$\cdots$ & & $\ldots \ldots$ & & & $\ldots$ & $\ldots \ldots$ & - \\
\hline н. . & & $\cdots$ & & $\cdot n^{2} \cdot \cdot$ & . & $\ldots \ldots$ & \\
\hline ormis Dsh. .. & & •... & & $\cdot n^{2} \cdot \cdot$ & & $\cdots \cdots$ & $\cdots$ \\
\hline Fori.... & & $\because$ & & & & $\cdots \cdots$ & - \\
\hline & $\cdots \cdots$ & 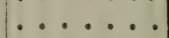 & $\cdots$ & $\cdots$ & . $f^{\prime}$ & • & \\
\hline ca & . & .... & $\cdots$ & $\ldots$ & $\ldots$ & $i$ & . \\
\hline ז. . . . & & & & $\ldots$ & . &.$t \ldots$ & \\
\hline Dsh. . . . & & - & - & $\cdots \cdot$ & & $\begin{array}{l}. t \\
. t\end{array}$ & \\
\hline & & & & & & 31 & \\
\hline
\end{tabular}




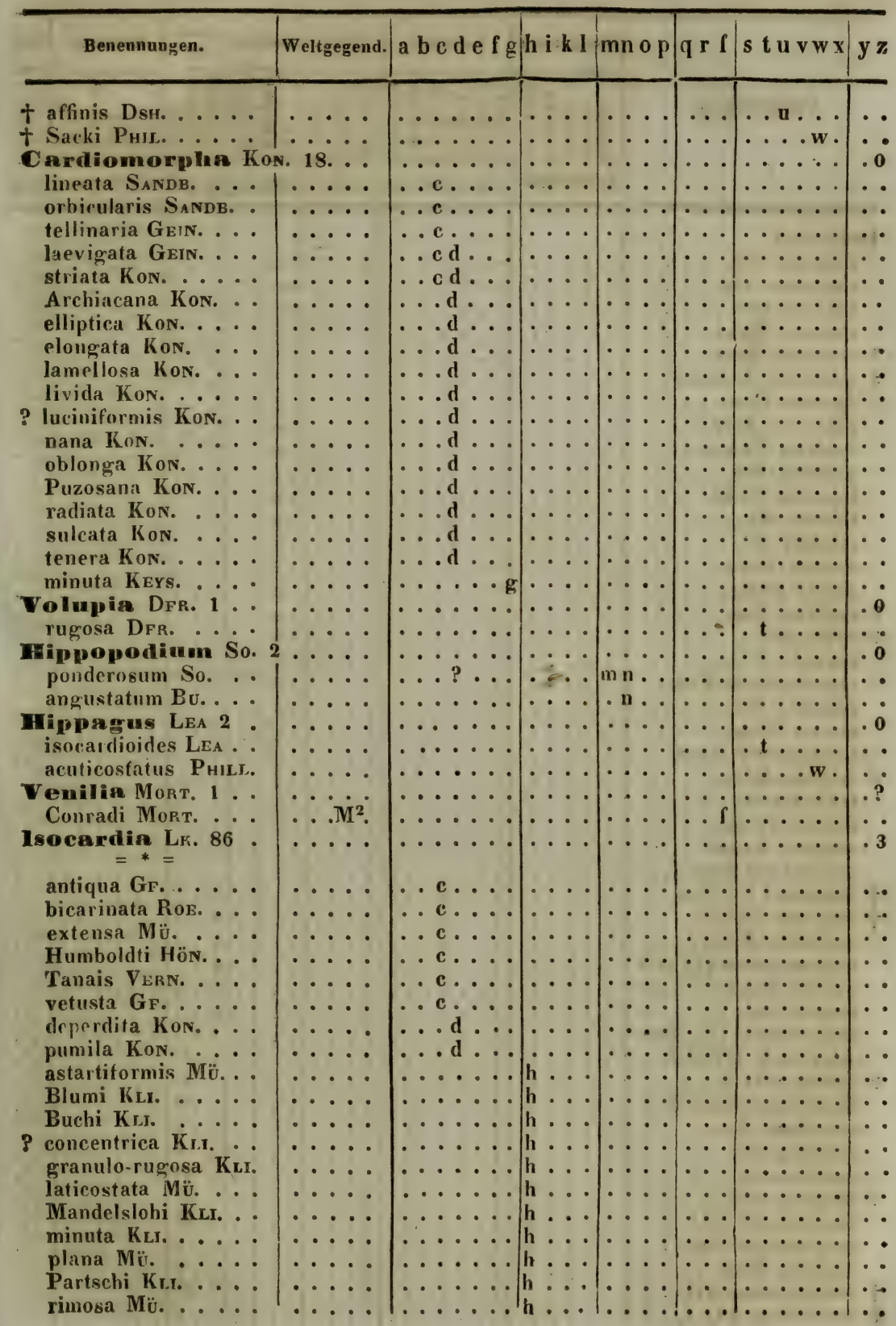




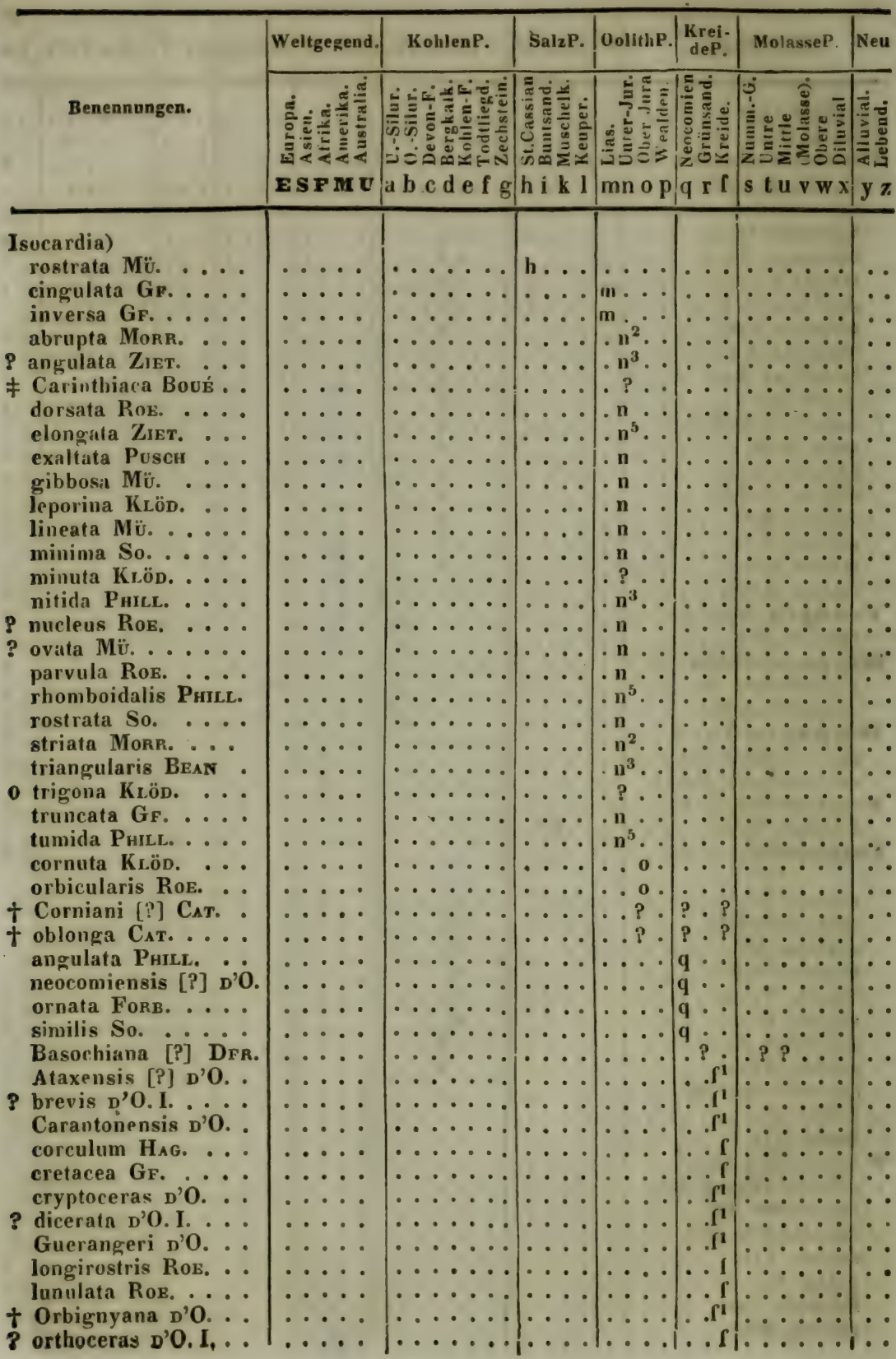




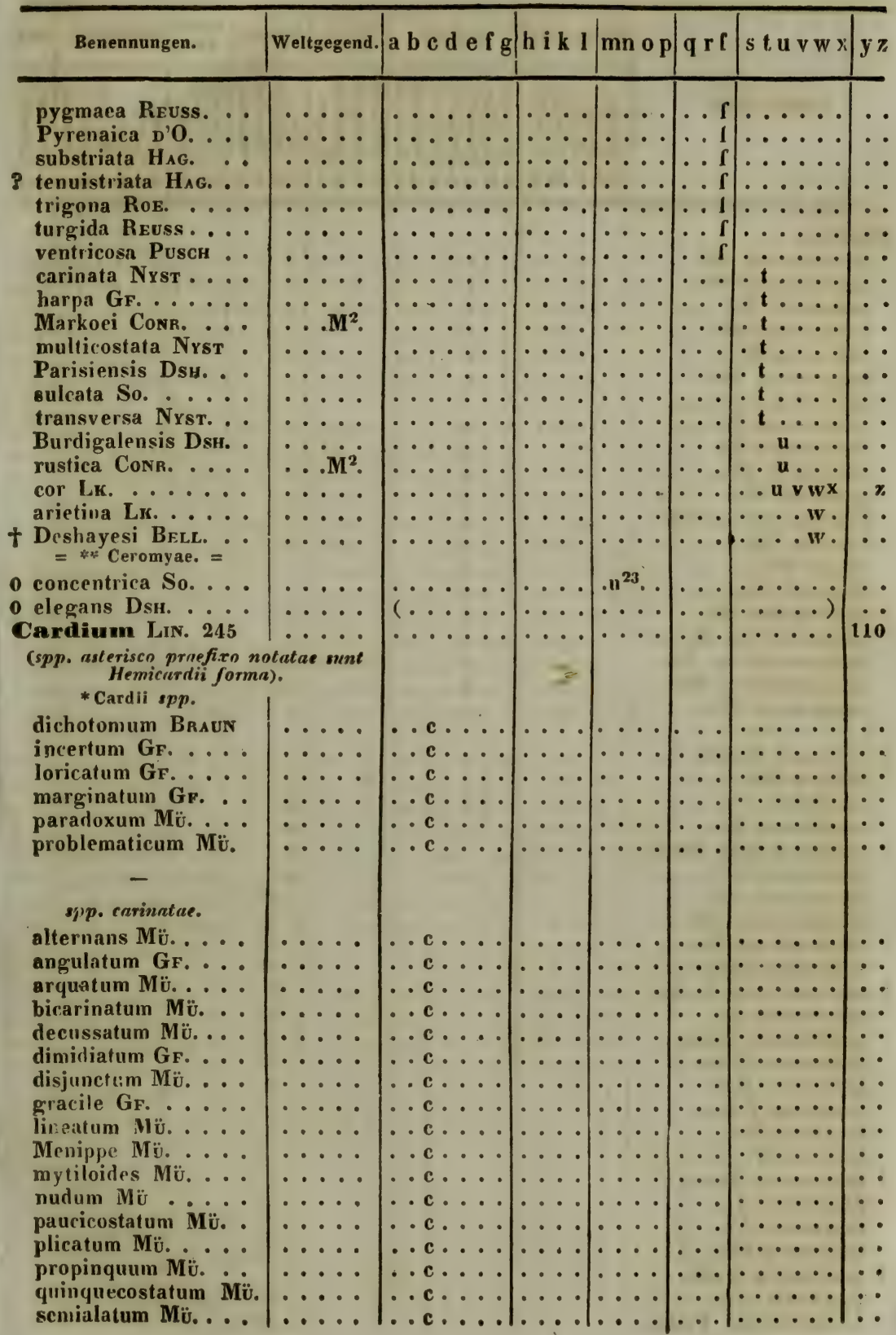




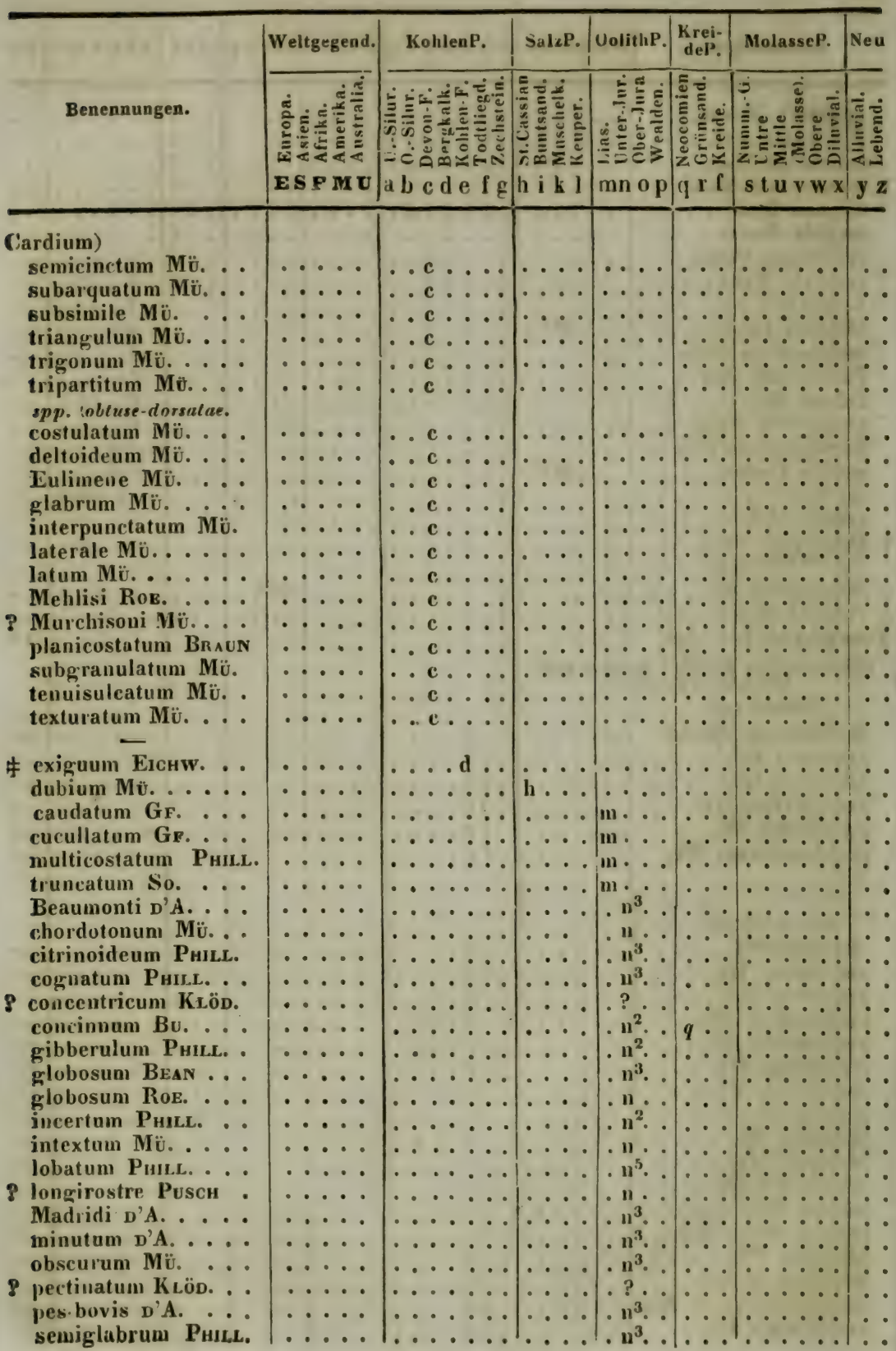




\begin{tabular}{|c|c|c|c|c|c|c|c|}
\hline n. & end. & $a b c$ & h i k l & mn op & $\mathbf{r} \boldsymbol{l}$ & $\mathbf{w} \times$ & \\
\hline 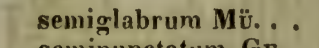 & $\cdots$ & 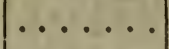 & & 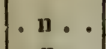 & & 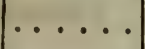 & \\
\hline$s$ & $\ldots$ & $\cdots$ & . & - & & $\cdots$ & \\
\hline $\begin{array}{l}4 \\
t\end{array}$ & $\cdots$ & & & & & $\cdots$ & \\
\hline $\begin{array}{l}\text { LOD. } \cdots \cdots \\
\text { ROE. } \cdots\end{array}$ & $\ddot{\cdots}$ & & & $\left|\begin{array}{lll}0 & n & 0 \\
1 & 0 & 0\end{array}\right|$ & & $\cdots$ & \\
\hline$\ldots$ & $\ldots$. & $\cdots$ & $\cdots$ & $\therefore 0$ & & $\mid \begin{array}{c}\cdots \\
\cdots\end{array}$ & \\
\hline i Fonв. . . . . & $\ldots \ldots$ & $\cdots$ & $\cdots$ & $\ldots$. & & $\ldots \ldots$ & \\
\hline di Forb. & & $\cdots$ & $\ldots$ & $\ldots$. & & $\ldots \ldots$ & \\
\hline [?] D'O. & ... $\mathbf{M}^{3}$. & $\cdots$ & $\cdots \cdots$ & & $q$ & $\ldots \ldots$ & \\
\hline '. & $\cdots \cdot$ & $\cdots \cdots$ & $\ldots$ & & q & $\ldots \ldots$ & \\
\hline 0. & $\cdots \cdot$ & $\cdots \cdots \cdots$ & $\cdots$ & & $q$ & $\cdots$ & \\
\hline$\because \cdots$ & & 0 & & & & $\cdots$ & \\
\hline O. . & & & & & & $\cdots$ & \\
\hline$\cdots$ & & & $\cdot$ & & & $\cdots \cdots$ & \\
\hline$\dot{\rho} \cdot$ & & ; & $\cdots \cdot$ & $\cdots \cdots$ & & $\cdots \cdots$ & \\
\hline$\cdots$ & . & $\dot{1}$ & $\cdots \cdots$ & $\cdots$ & & $\cdot \cdot$ & \\
\hline B. & $\cdots \cdots$ & $\cdots \cdots \cdots$ & $\mid \cdots \cdots$ & $\cdots \cdots$ & & $\because$ & \\
\hline M. & $\ldots$ & $\ldots \ldots$ & $\cdots$ & $\ldots$ & $q^{12}$ & $\because$ & \\
\hline & $\ldots$ & $\ldots \ldots$ & $\ldots$ & $\ldots$ & 1. $\mathrm{r}$ & $\therefore$ & \\
\hline$\cdots$ & $\cdots$ & $\cdots$ & $\ldots$ & $\ldots$ & $\cdot r \cdot$ & $\ldots \ldots$ & \\
\hline . $\cdot$ & - & $\cdots \cdots \cdots$ & $\ldots$ & $\ldots$. & . $\mathbf{r} \cdot$ & $\ldots \ldots$ & \\
\hline$\cdots$ & $\cdots \cdots$ & $\cdots \cdots$ & $\ldots$ & $\cdots \cdot$ & . & $\cdots$ & \\
\hline$\cdots$ & $\cdot$ & $\cdots \cdots \cdots$ & $\cdots$ & $\cdots \cdots$ & & . & \\
\hline . & $\cdots$ & $\cdots \cdot \cdot$ & $\cdots \cdots$ & $\cdots \cdot \mid$ & & $\cdot \cdot$ & \\
\hline & • & . 1 & $1 \cdot$ & & & $\cdot$ & \\
\hline So. . & $\cdots$ & $\cdot$ & $\cdot$ & $\cdot 1$ & & $\cdot$ & \\
\hline$\cdots$ & $\cdots$ & . & 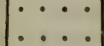 & $:$ & & $\dot{1}$ & \\
\hline$\because$ & $\cdots \cdots$ & $\therefore$ & $\ldots$ & & $\therefore$ f & $\cdots$ & \\
\hline$\therefore$ & $\ldots \ldots$ & $\cdot$ & $\cdots$ &. & $\therefore$ i & $\therefore$ & \\
\hline . . & $\ldots \ldots$ & $\cdots \cdots$ & $\ldots$ & & .17 &. & \\
\hline & ... & - & $\ldots$ & . & . . $\boldsymbol{r}^{1}$ & . & \\
\hline 0. . & $\ldots$ & $\cdots \cdots$ & $\cdots \cdot$ & $\cdot 1$ & $\ldots \mathbf{f}^{\mathbf{1}}$ & . . & \\
\hline$\cdots$ & $\ldots$ & 0 & $\cdots \cdots$ & . & & $\ldots \ldots$ & \\
\hline . . & - & $\cdots$ & $\cdots$ & $\cdots$ & & $\cdots$ & \\
\hline & $\cdots \cdots$ & ${ }^{\circ}$ & $\cdots$ & & & $\cdots$ & \\
\hline$\cdots$ & $\cdots \cdots$ & 0 & $\cdots$ & & & $\cdots \cdots$ & \\
\hline$\cdots$ & 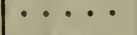 & $\cdot$ & $\cdots$ & $\cdot 1$ & & $\cdots$ & \\
\hline & $\cdots \cdot$ & $\cdots \cdot$ & $\cdots$ & . . . & & 1. & \\
\hline & $\cdots$ & $\cdots$ & - & & f & • & \\
\hline 7. • & & & & & f & & \\
\hline . . & $\cdots \cdots$ & $\cdots \cdots$ & $\ldots$ & $\therefore \ldots$ & f & $\therefore$ & \\
\hline$\cdots$ & .... & & $\cdots$ & & & . & \\
\hline & $\cdots \cdots$ & & & . & $\ldots \Gamma^{1}$ & $\cdots$ & \\
\hline . . & $\cdots$ & & . & 0 & $\ldots f$ & $\ldots \ldots$ & \\
\hline & & & & 0 & $\ldots \rho$ & $\cdots$ & \\
\hline & & . & & $\cdots \cdots$ & & & \\
\hline DU. & $\cdots \cdots$ & & & & & - & \\
\hline$\because$ & $\cdots \cdot$ & 5 & $\cdots$ & . & $\cdots 1$ & $2-5$ & \\
\hline & & & & & & & \\
\hline
\end{tabular}




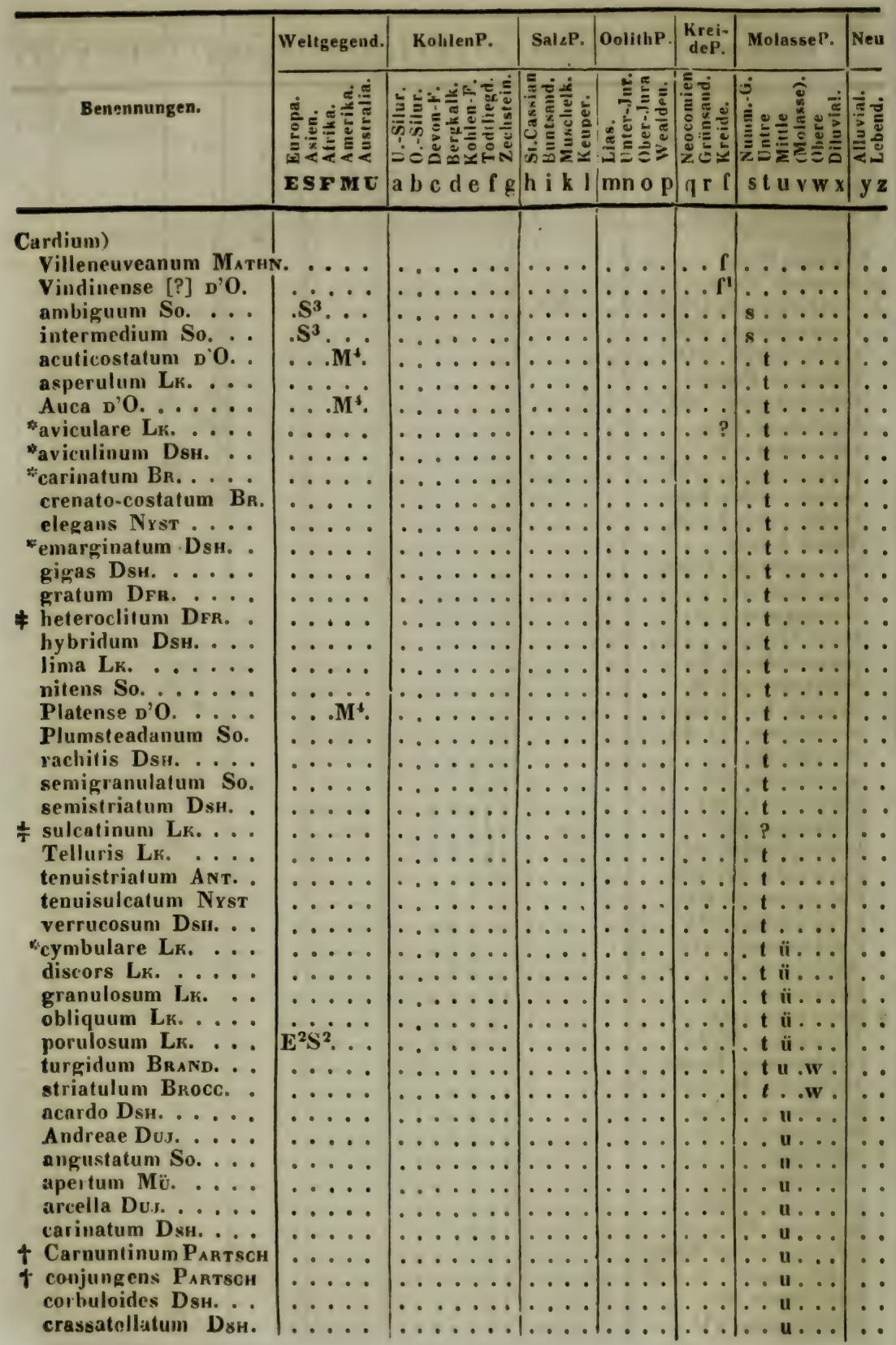




\begin{tabular}{|c|c|c|c|c|c|c|c|}
\hline Benennungen. & Weltgegend. & e f $\mathrm{g}$ & $\mid$ h $\quad$ i k l l & $\mathrm{mn} 0 \mathrm{p}$ & q r r & st uv w $x$ & yz \\
\hline 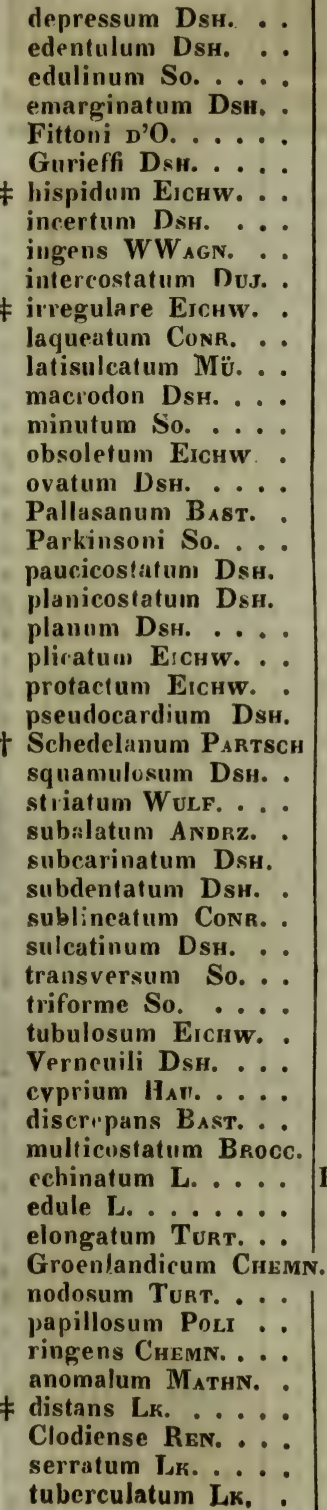 & 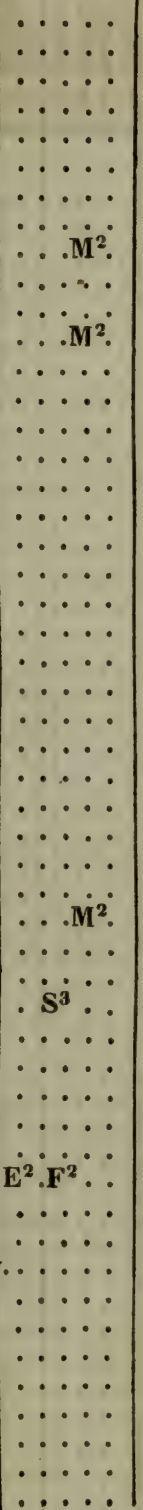 & 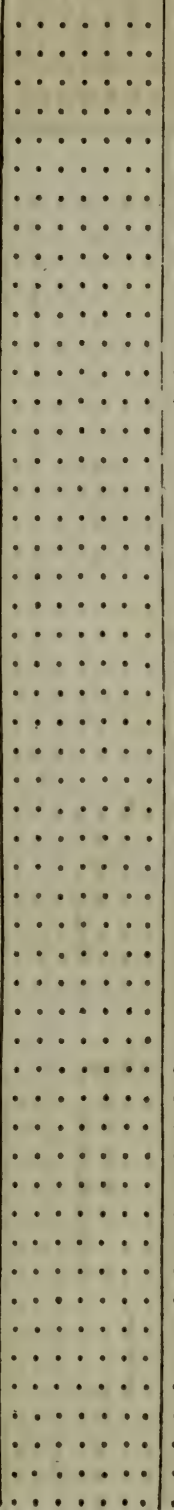 & 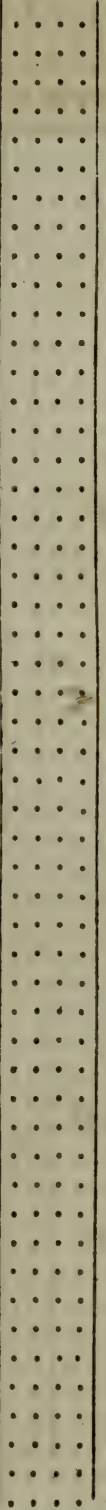 & 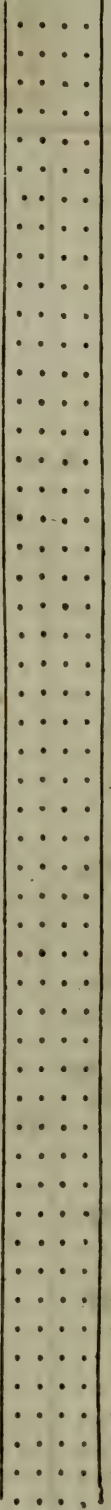 & 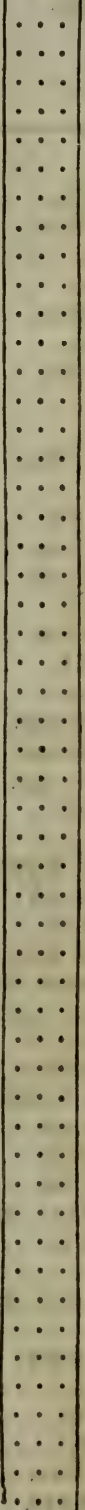 & 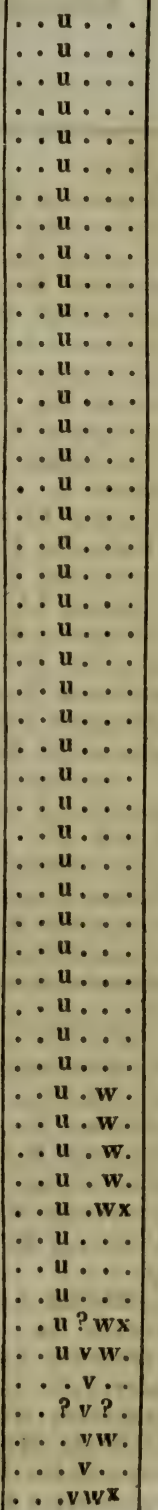 & 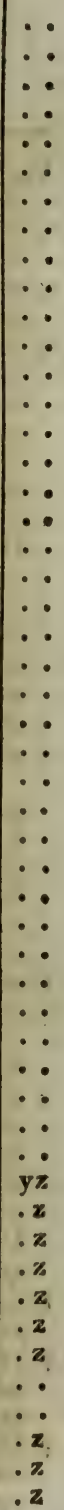 \\
\hline
\end{tabular}




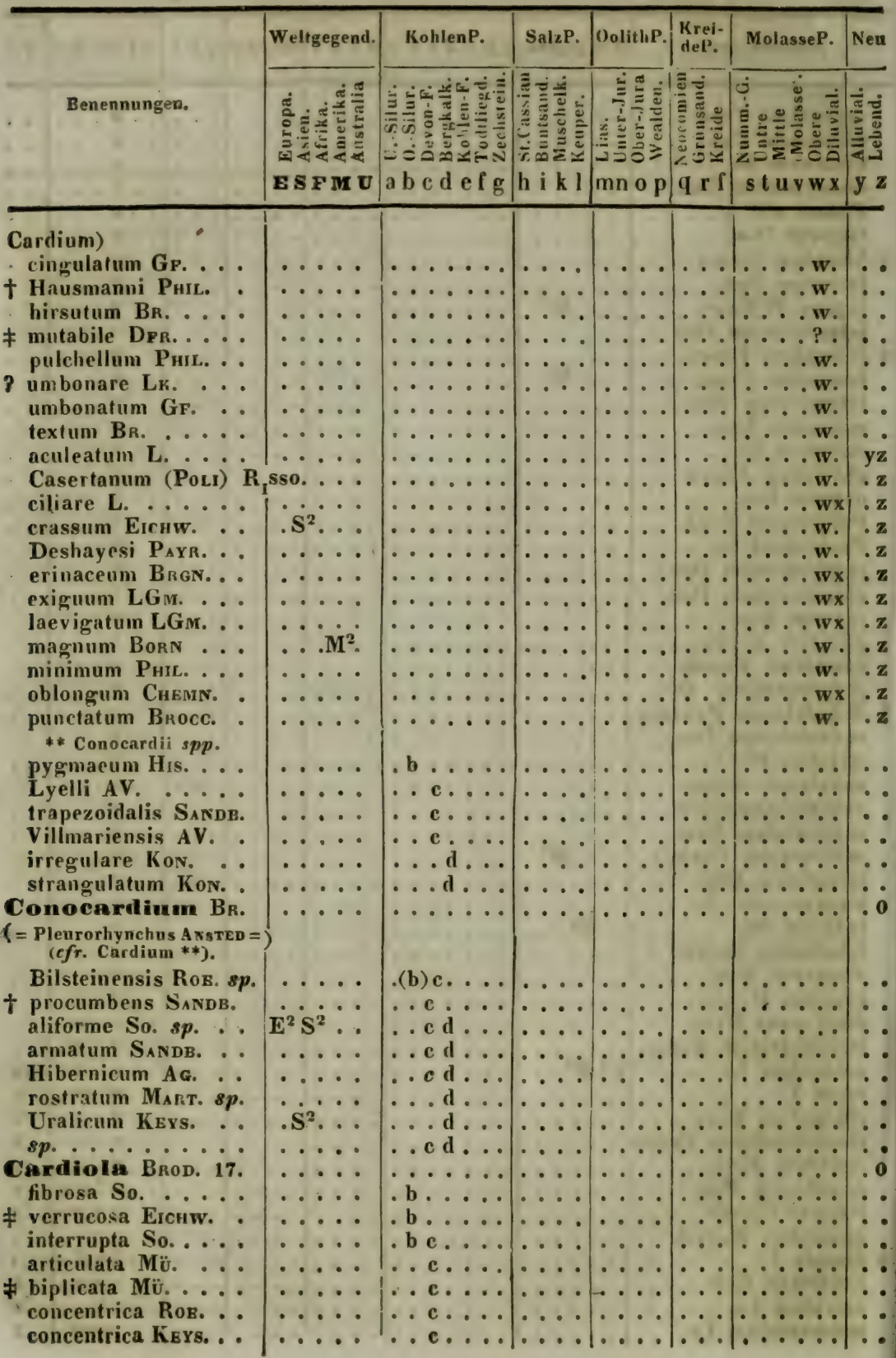


Benennungen. $\quad$ Weltgegend. $\mid$ a b c d e f g h ik l m m op $|q r f| s$ t u v w s $\mid$ y

? dichotoma $\mathrm{Mü}$ duplicata $M \ddot{\text { ü. }}$ elegans $M \ddot{0}$.

intermedia Mü.

- retrostriata KEYs. sinuesa $M \ddot{\text {. }}$.

spuria Mü.

subdecussata Mǘ.

tegulata Mö.

tenuistriata KExs.

Eumulicardium Mü. 10 carpomorphum DaLM. $s p$. canaliferun Mü. excrescens Mü. inaequicostatum Mü. ovatum Mü.

Partschi Mü. piriforme Mü. procrescens Mü. ... semistriatum Mü. . . tetragonum $\mathbf{M u ̈ .}$

\section{$\eta$ Cycladina.}

Cyclas Lr. 34. . . . ? rugosa Du. media So.

Buchi Do...... Brongniarti KoDu. faba Mü.

gibbosa So. Jugieri Du. major So.

? membranacea So. parva So. subqradiata So. subtrigona Du. .... laevigata Dsw. Aquae-Sextiae So. Brongniartina MathN. concinna So. Coquandana $\mathrm{M}_{\mathrm{ATHN}}$ cuncata So. Galloprovincialis Matuv Gardannensis MathN. Gargasensis MathN. gibbosa So.

? globus DuB. numismalis MathN. pisum Mathis.

? triangularis DuB. cornea Lk.

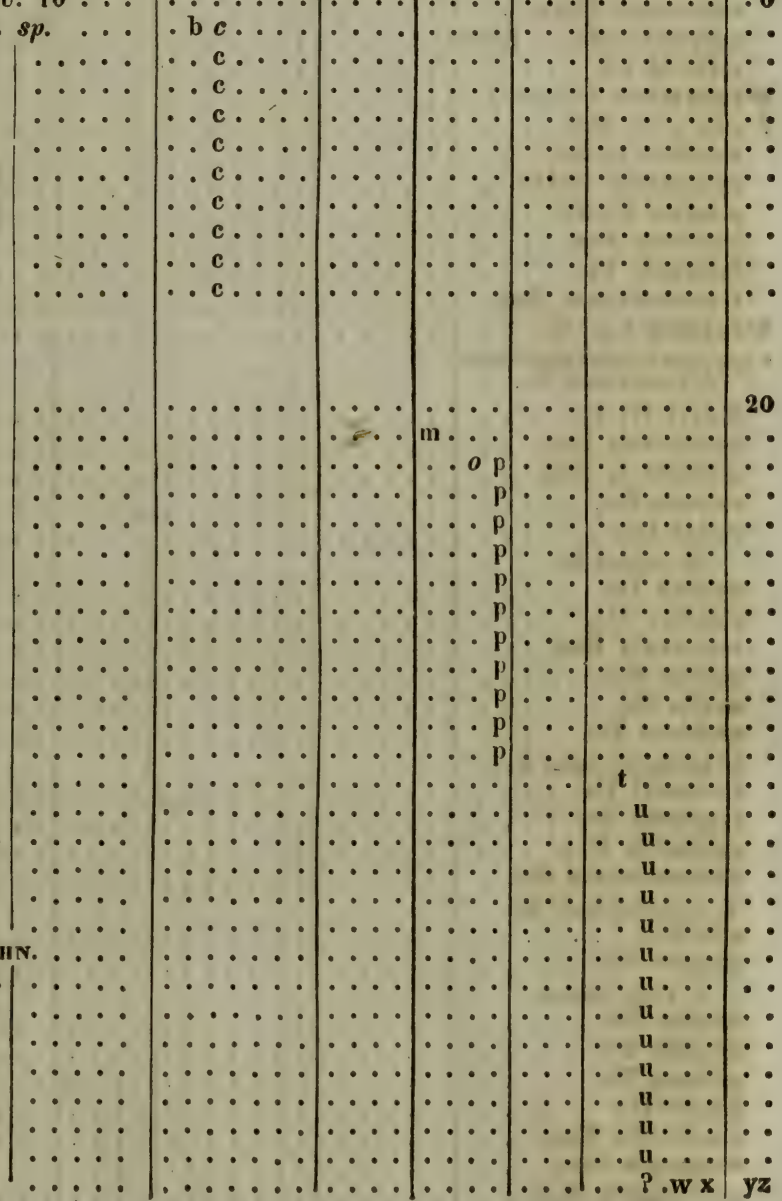




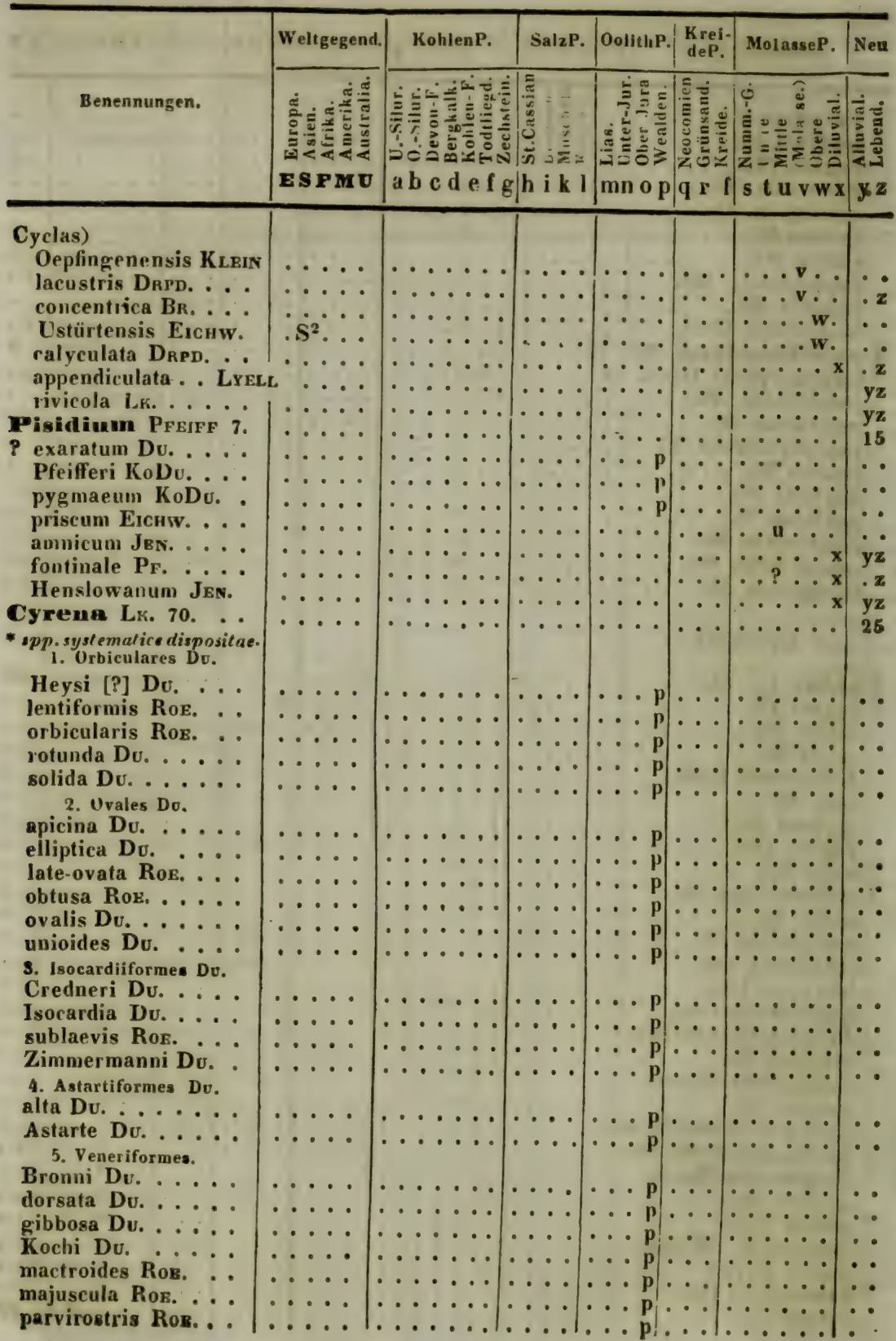




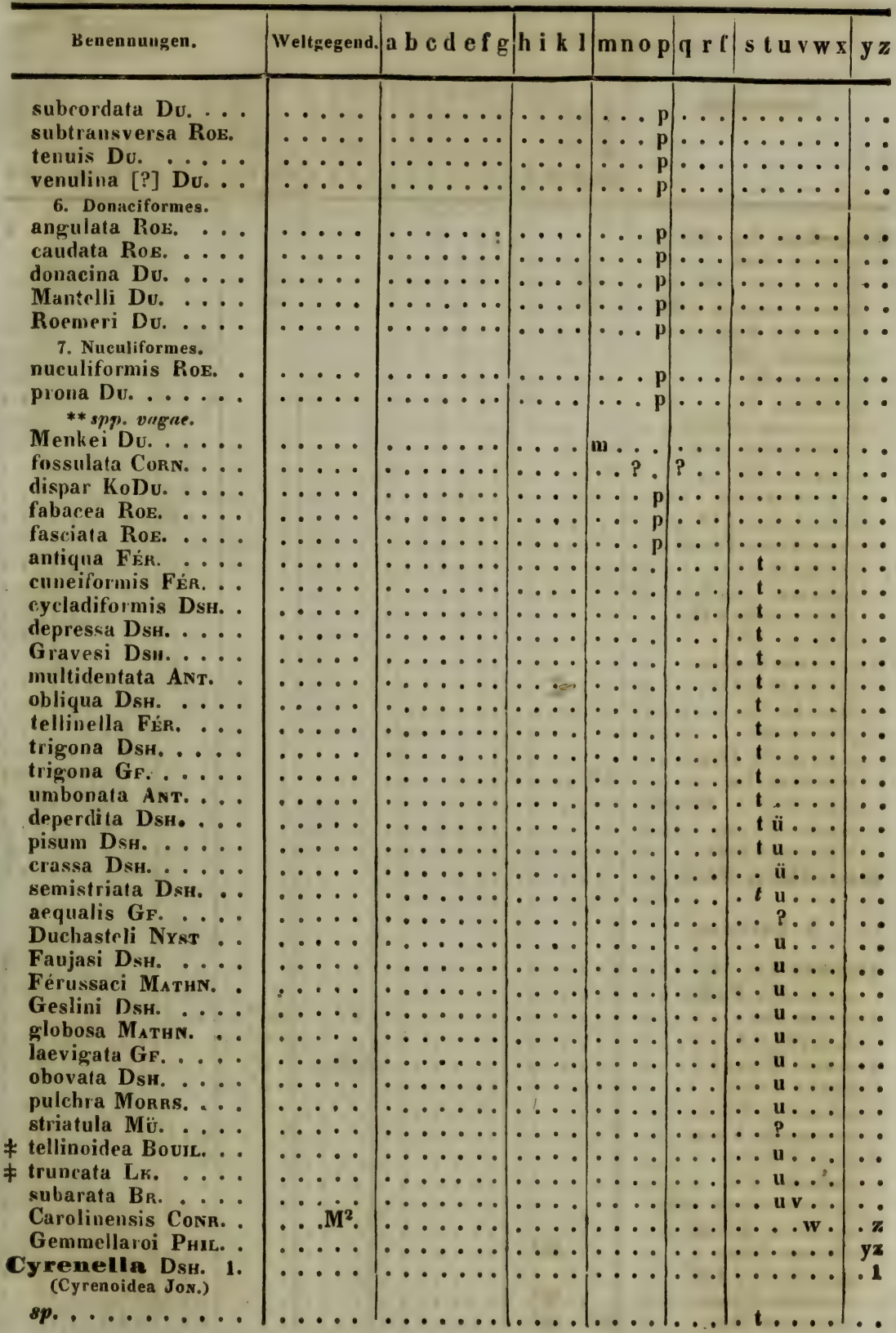




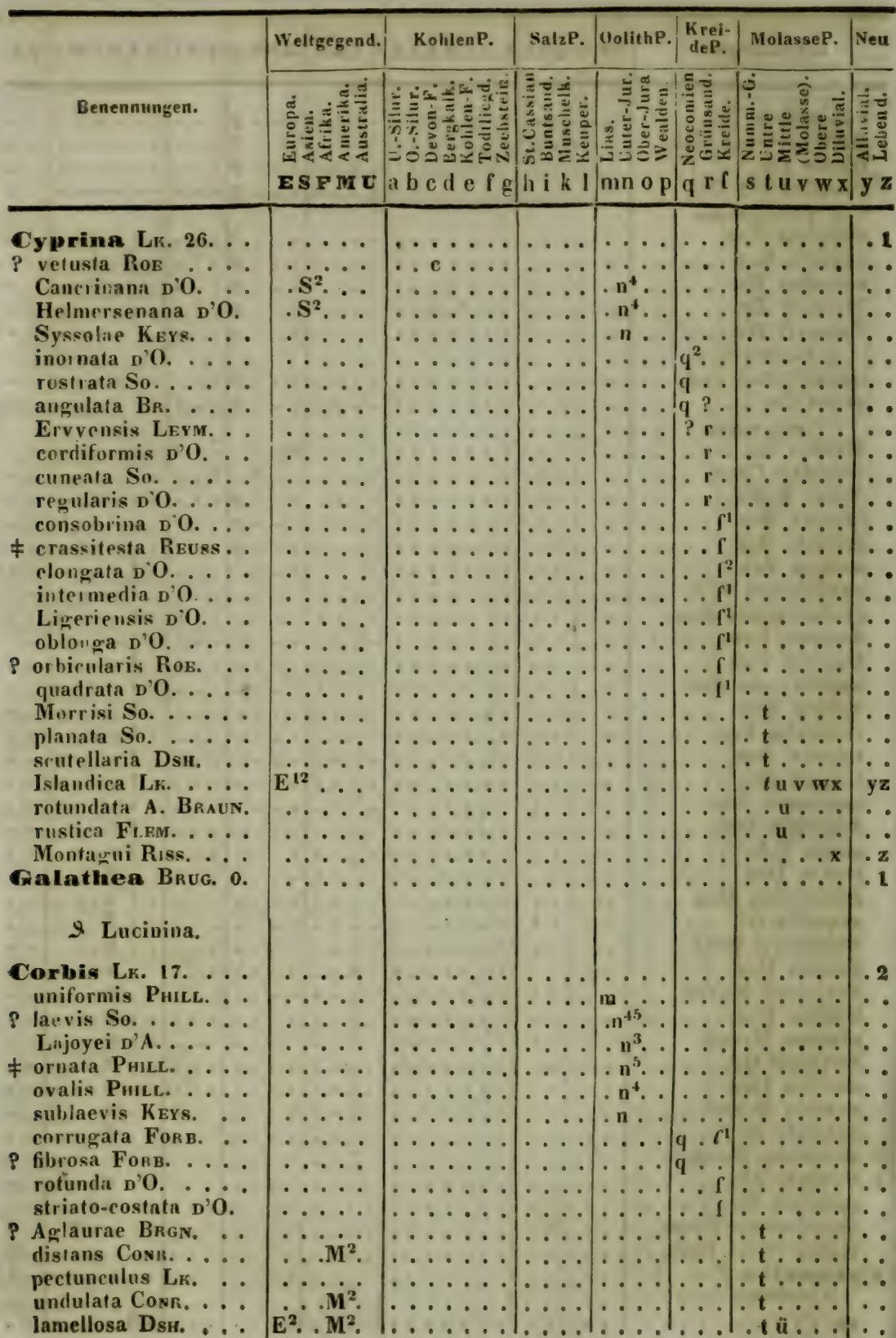




\begin{tabular}{|c|c|c|c|c|c|c|c|c|}
\hline Bene & & gend. & $\mathrm{fg}$ & i $\mathbf{k} 1$ & $\mathrm{mr}$ & $q \times 1$ & st & \\
\hline - & & & $\cdots \cdots$ & & & & - & \\
\hline Sedgwicki Nyst & .. & $\cdots \cdots$ & .. & - & - & $\cdots$ & $\mathbf{w}$. & \\
\hline 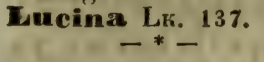 & $\cdots$ & $\cdots$ & $\cdots \cdots$ & $\cdots \cdot$ & $\cdots \cdots$ & $\cdots$ & $\ldots \ldots$ & \\
\hline prisea & . & $\cdots$ & $\cdots$ & $\ldots \ldots$ & $\ldots$ & & $\cdots \cdots$ & \\
\hline & & : & $\because$ & $\cdots \cdots$ & $\cdots$ & . & $\cdots$ & \\
\hline & & - & $\mid \begin{array}{ccc}\cdots & \cdots \\
\cdots & \cdots & \cdots\end{array}$ & 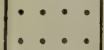 & $\because$ & 1. & $\ddot{\circ}$ & \\
\hline & $\therefore$ & ..... & $\therefore$ c . . . & $\ldots \ldots$ & $\cdots \cdots$ & $\cdots$ & $\therefore$ & \\
\hline ORTL. & . & $\cdots \cdots$ & $\ldots d \ldots$ & $\ldots$ & $\ldots$ & $\ldots$ & $\cdots \cdots$ & \\
\hline Рнizl. & & & $\ldots d \ldots$ & $\ldots$ & $\ldots$ & $\ldots$ & $\ldots \ldots$ & \\
\hline . . & &.$S^{2} \ldots$ & $\ldots . . . \mathrm{g}$ & $\cdots$ & $\ldots$ & $\ldots$ & $\ldots \ldots$ & \\
\hline 1. - & . & $\ldots$ & $\ldots \ldots$ & & $\ldots$ & $\cdots$ & $\ldots \ldots$ & \\
\hline di & & $\cdots \cdot$ & $\cdots$ & h ... & $\cdots \cdot$ & $\cdots$ & $\ldots \ldots$ & \\
\hline & & . . & $\cdots$ & $\cdots \cdot$ & nı... & $\cdots$ & $\ldots \ldots$ & \\
\hline pl & & & & $\cdots$ & m n . . & $\cdots$ & $\cdots$ & \\
\hline$s p$ & $\cdots$ & & - & $\cdots \cdot$ & $\cdot n_{3} \cdot$ & $\cdots$ & $\cdots$ & \\
\hline & & $\dot{S}^{2}$ & $\cdots$ & $\cdots \cdot$ & $\cdot 1^{3} \cdot \cdot$ & $\cdots$ & $\cdots \cdots$ & \\
\hline & & $\because$ & $\because$ & $\cdots \cdots$ & $\cdots$ & $\cdots$ & $\cdots \cdots$ & \\
\hline · & $\because$ & & $\because$ & $\cdots \cdots$ & $\left|\begin{array}{l}n \\
n \\
n^{5}\end{array}\right|$ & 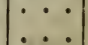 & $\cdots \cdots$ & \\
\hline & & & $\therefore$ & $\ldots$ & $n^{23} \ldots$ & $\begin{array}{ll}\cdots \\
\cdots\end{array}$ & $\cdots$ & \\
\hline & &.$S^{2} \ldots$ & $\cdots$ & $\ldots$ &.$n^{4} \ldots$ & $\cdots$ & $\ldots \ldots$ & \\
\hline & $\cdot$ & $\ldots$ & $\cdots$ & $\cdots$ & $\therefore$ & $\cdots$ & $\cdots \cdots$ & $0^{\circ}$ \\
\hline h & & $\cdot$ & $\cdots$ & $1 . \approx$ & $.11^{4} \cdot$ & $\cdots$ & $\cdots \cdots$ & \\
\hline & &.$S^{2} \cdot \cdot$ & $\ldots \ldots$ & $\cdots \cdot$ & $\cdot n^{4} \cdot$ & $\cdots$ & $\cdots \cdots$ & \\
\hline$\cdots$ & & $\cdots \cdots$ & $\cdots \cdots \cdots$ & $\cdots \cdots$ & $\cdot n \cdots$ & $\cdots$ & $\cdots \cdots$ & \\
\hline . & & . & $\cdots$ & $\cdots \cdot$ & $\cdots$ & $\cdots$ & $\cdots \cdots$ & . \\
\hline & . & 0 & .. & $\because \cdots$ & $\begin{array}{l}n \\
n^{3}\end{array}$ & • • & $\cdots$ & \\
\hline $\begin{array}{l}\mathbf{0} \\
\mathbf{P}\end{array}$ & & $\mathrm{S}^{2} \ldots$ & & $\ldots$ & 114 & $\cdots \cdot$ & $\therefore$ & \\
\hline & & $\ldots$ & & $\cdots$ & . $\mathbf{n} \cdot$ & $\because$ & $\cdots$ & \\
\hline & & - & . & . . & .?? & $\cdots$ & $\ldots \ldots$ & \\
\hline & $\because$ & 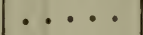 & $\cdots$ & $\cdots$ & . 0 & - & $\ldots \ldots$ & \\
\hline & & & $\cdots$ & $\ldots$ & . 0 & • & $\cdots \cdots$ & \\
\hline & & - & . & $\cdots$ & $\cdots$ & 9 & $\ldots \ldots$ & \\
\hline I & . & . & $\ldots \ldots$ & $\cdots$ & $\ldots$ & & $\cdots \cdots$ & - \\
\hline & & 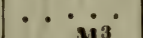 & $\cdots$ & $\cdot \cdot$ & & & $\cdots \cdots$ & - \\
\hline & & & $\cdots$ & $\cdots \cdot$ & $\cdots$ & & $\cdots \cdots$ & 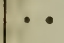 \\
\hline & & & $\cdots$ & $\cdots \cdot$ & $\cdots \cdot$ & & $\cdots \cdots$ & • \\
\hline & & & & & & & $\cdots \cdots$ & $\cdot$ \\
\hline & & & & & $\cdots$ & $\mathbf{r}$. & $\cdots \cdots \cdots$ & \\
\hline 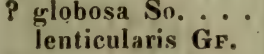 & & & $\because$ & $\cdots$ & $\because \cdots$ & $\mathbf{r}$ & $\because \cdots$ & \\
\hline$\bullet^{-}$ & & .. & $\ldots \ldots$ & $\cdots \cdots$ & $\ldots$ & $r$ & $\because$ & \\
\hline & & & ... & & $\cdots$ & - $r$ & .. & \\
\hline & & & & & & ? & & \\
\hline & & & & & & - $\mathbf{r}_{\dot{i}}$ & $\cdots$ & \\
\hline & & & & & & & & \\
\hline & & & & & & $\ldots \mathbf{f}^{\mathbf{1}}$ & & \\
\hline arse & & & & & & $\therefore \mathrm{r}$ & • . I & \\
\hline
\end{tabular}




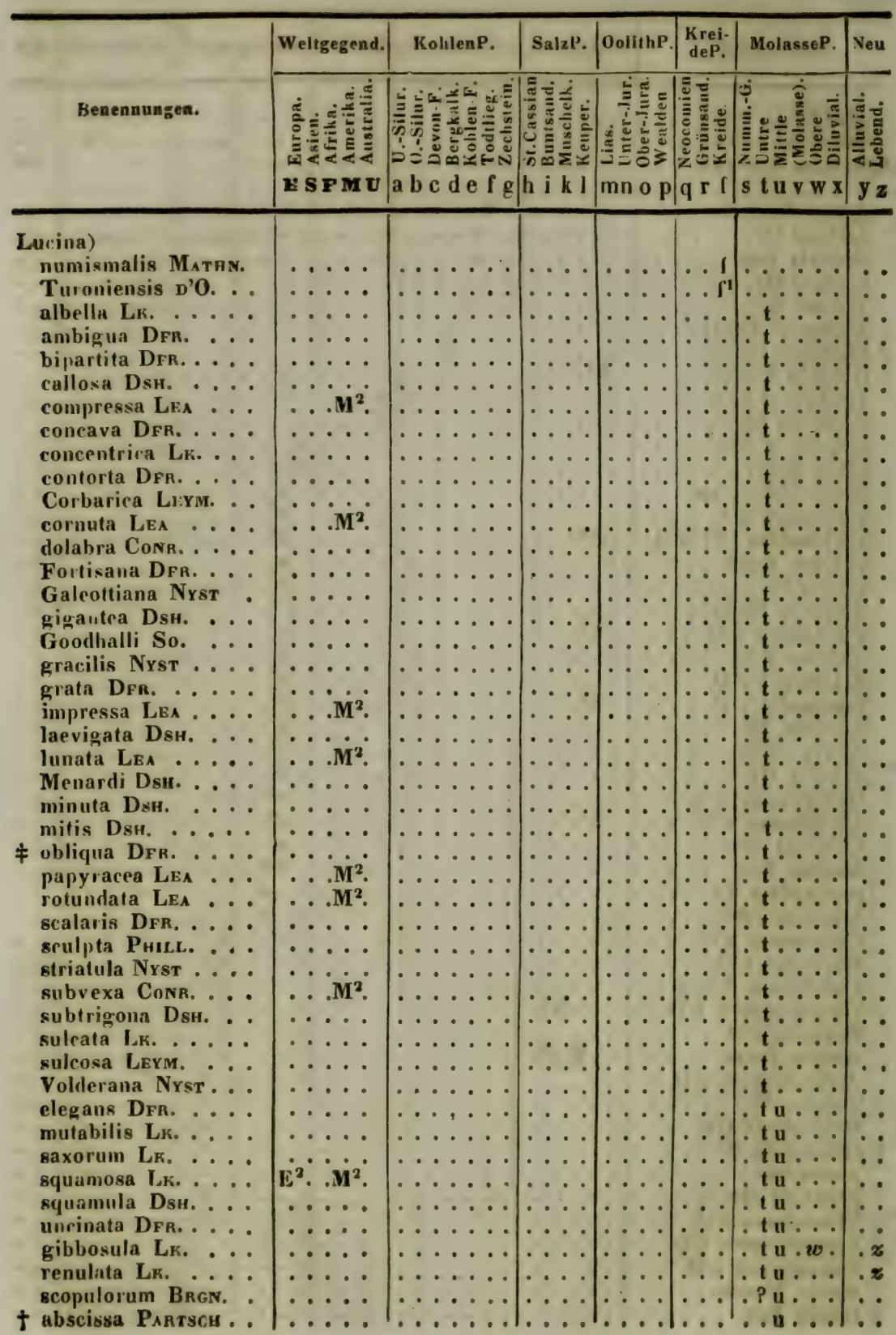




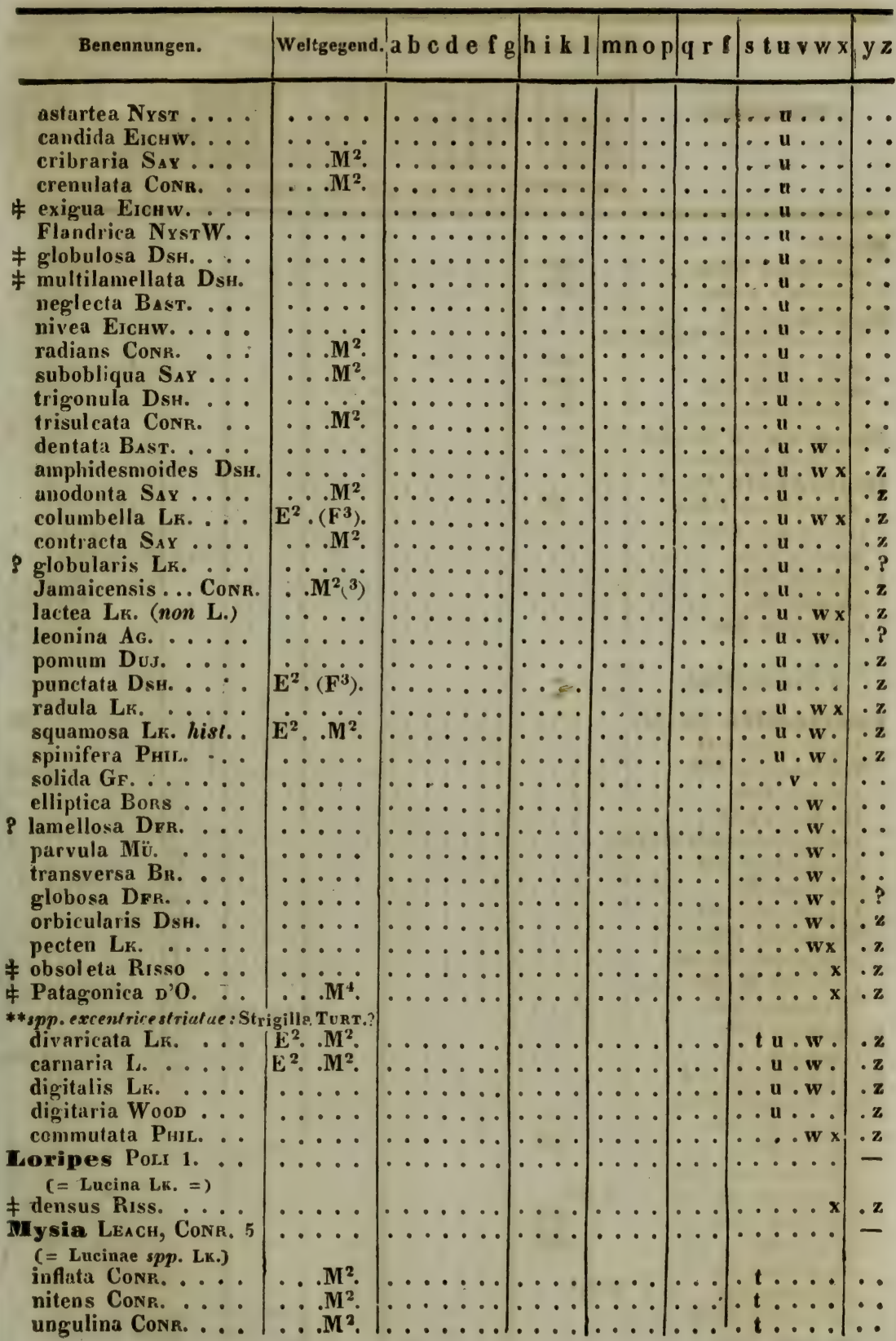




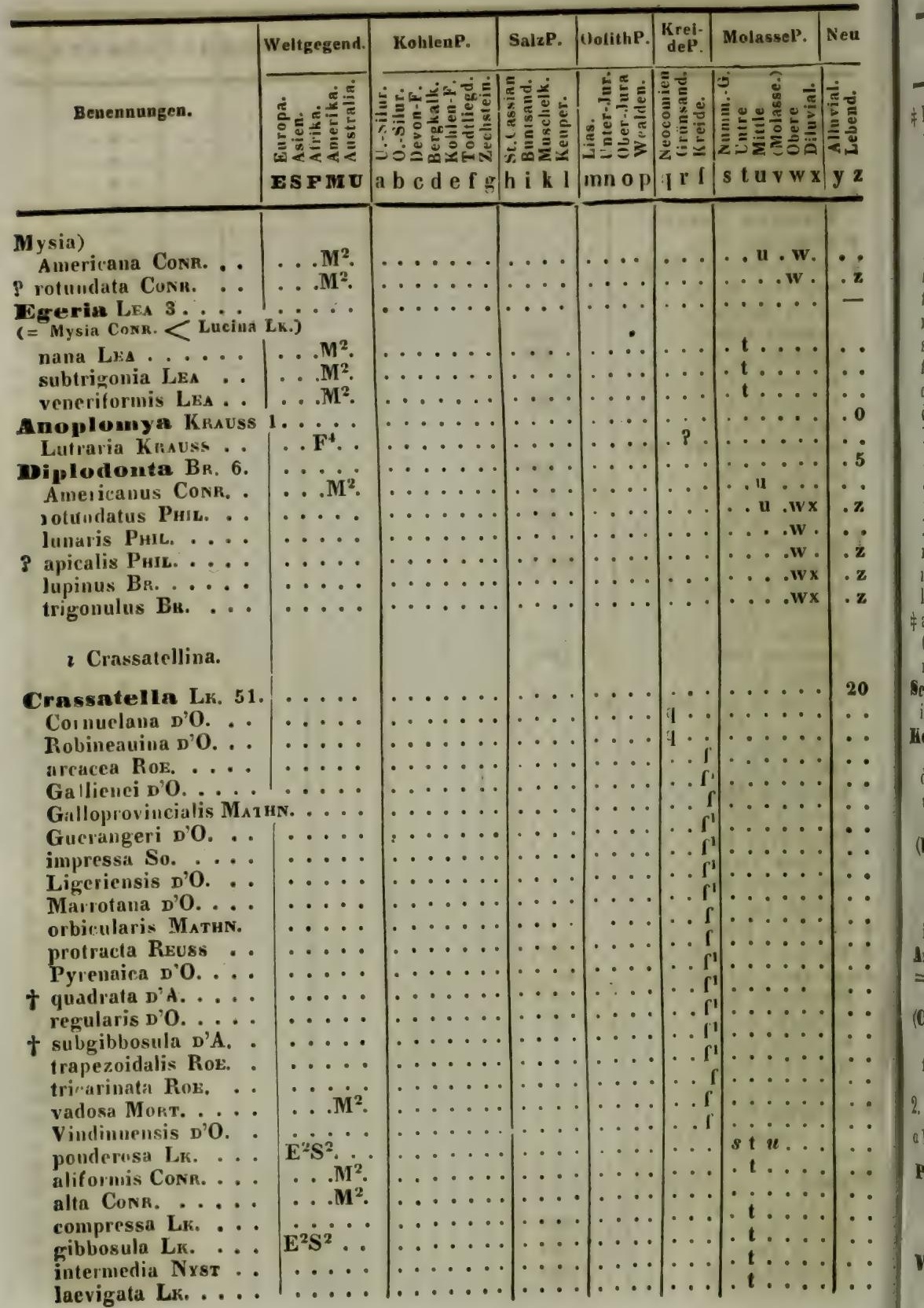


‡ Landinensis Nyst. minima LEYM. plicata So. protexta Conr. scutellaı ia Dsh. securis LEYM. sulcata So. sulcata 1 LK. tenuistriata Dsu. .. rostrata DsH. sinuosa Ds. triangulat is $\mathrm{LK}_{\mathrm{K}}$. conciuna EıcHW. dissita Eıchw. Podolica Eichw. sinuata $L_{K}$. striatula $\mathbf{L K}$. truncata Pusch ... Marylandica Conr. melina ConR. undulata $S_{A Y}$ latissima $\mathrm{LK}$.

中 affiuis NystW. Caspia Erснw. minuta PHIL.

Scacchia Pril. 1. inversa PhiL.

Tellia Turt. 3 (Bornia Phill.) dubia Wood . suborbicularis TURT. ferruginosa Mokrs.

(Eornia Phil. 3.) = Kellia TURT. = complanata PHIL. corbuloides P'HIL. inflata PHiL.

Axinus So (pars) 1 Weltgegend. a b c d e f g bi k l mn o p q r f s t u v w x y z

Cryptodon Turt, Ptychina Puic. angulatus So. (Cryptodon Turit.) 1
= Axinus So.

flexuosus Turt.

2. EMARgINATO-PALliata. a Protocardidae Reuss

Protocaralia BeYr. 1 Hillana BEYK.

$\beta$ Venerina.

Venus (L.) LK. etc. 166 $\mathrm{M}^{2}$ $\dot{E}^{2} \dot{S}^{2}$ ....

$\ldots \ldots$

…

$\cdots$

$\ldots$

$\cdots$

$\because \mathrm{M}^{2}$

$\therefore M^{2}$

$\ldots M^{2}$

$\dot{\mathbf{S}^{2}} \cdot$

$\mathrm{S}^{2}$

-

$\frac{1}{1 . \cdots \cdot 1}$




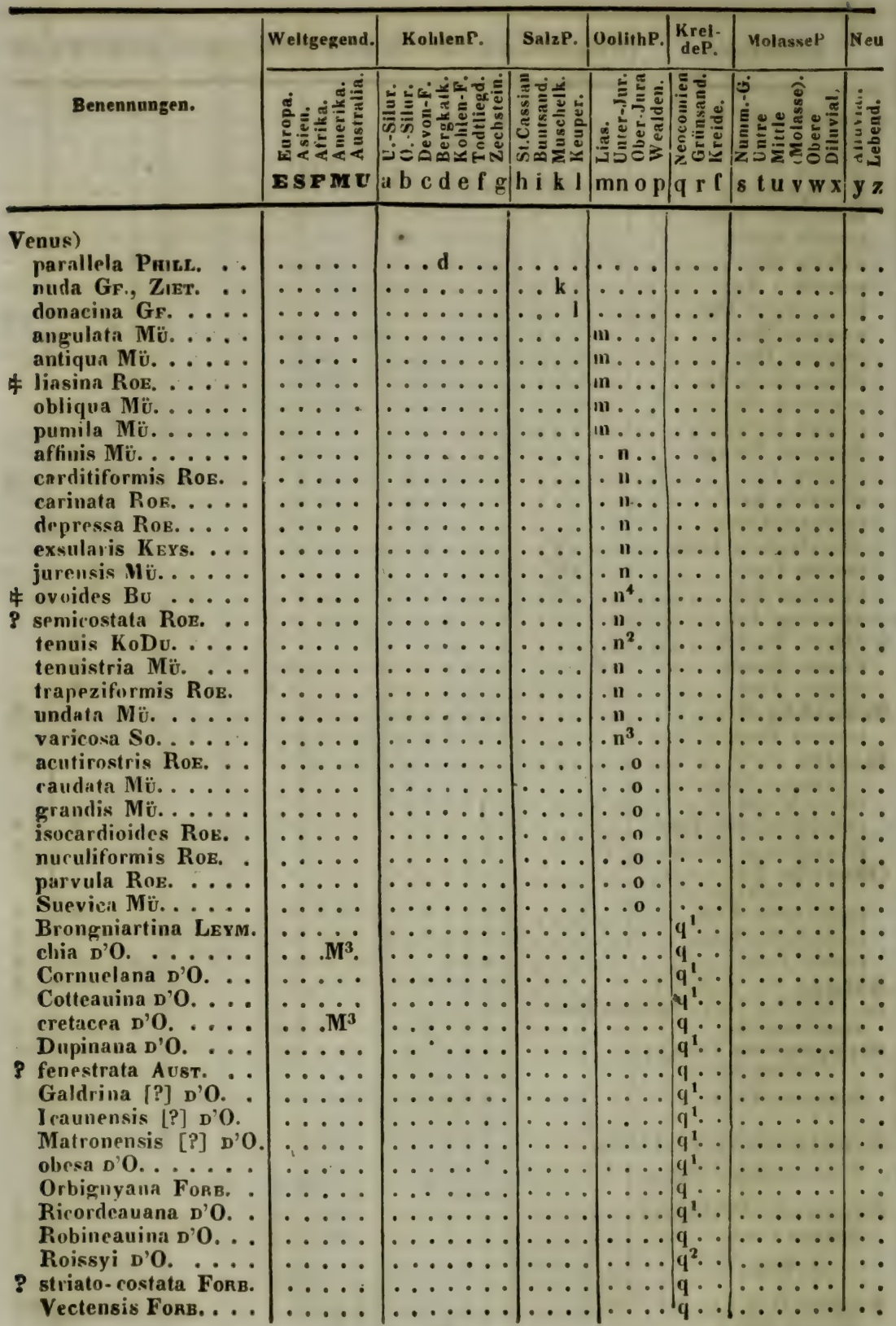




\begin{tabular}{|c|c|c|c|c|c|c|c|c|}
\hline & Ben & Weltgegend. & $d \in f g$ & h i k l & op & $q \times$ I & s t & $\mathbf{y} z$ \\
\hline & D'0. & & & & ........ & $q^{12}$ & 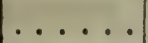 & \\
\hline & Wassyensis (D'O.) & $\ldots \ldots$ & $\ldots \ldots$ & $\ldots$ & $\ldots$ & $q^{2}$. & $\ldots \ldots$ & . \\
\hline & Galloprovincialis MAT & & $\ldots \ldots$ & $\ldots$ & $\ldots$ & $\mathbf{q}$. & $\ldots \ldots$ & - \\
\hline & is So. & $\cdots \cdot \cdot$ & $\ldots \ldots$ & $\ldots$ & $\cdots \cdots$ & $q ?$ & $\ldots \ldots$ & . \\
\hline & So. .... & $\cdots \cdot$ & $\ldots \ldots$ & $\cdots$ & $\ldots$ & q r r & $\ldots \ldots$ & - \\
\hline & Alpagina $\mathbf{C}_{\mathbf{A x}} \ldots$ & - & $\cdots \cdots$ & ... & $\ldots$. & ?? ? & $\cdots \cdots$ & - \\
\hline & Bavarica Mü. ..... & - & $\cdots \cdots$ & $\cdots \cdot$ & $\cdots \cdot$ &.$r$ & $\cdots \cdots$ & - \\
\hline & immersa So...... & & - & ... & $\ldots$. & . $\mathbf{r}^{\circ}$ & $\cdots \cdot$ & - \\
\hline & lata Ros. ..... & $\cdots$ & $\cdots$ & $\cdots$ & $\cdots$ & . ?? & $\cdots \cdots \cdots$ & - \\
\hline & Mü. . . & $\cdots$ & $\cdots$ & . $\cdots$ & $\cdots \cdot$ & . r . & $\cdots$ & - \\
\hline & $\cdots$ & $\therefore$ & . & $\bullet$ & $\cdots \cdots$ & . r . & $\cdots$ & $\cdot$ \\
\hline & truncata Rev & $\ldots \ldots$ & $\because$ & $\therefore$ & $\left|\begin{array}{c}\cdots \\
\cdots\end{array}\right|$ & r if & $\cdots$ & • \\
\hline & laminosa Reuss ... & ...... & $\ldots \ldots$ & $\ldots \ldots$ & $\cdots$ & 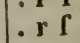 & $\ldots \ldots$ & $\therefore$ \\
\hline & REUSS .. & .... & $\ldots \ldots$ & $\ldots$ & $\ldots$ & . ri & $\ldots \ldots$ & $\therefore$ \\
\hline & $\cdots$ & .... & $\ldots \ldots$ & $\ldots$ & $\ldots$. & . $\mathrm{r} \mathbf{f}^{1}$ & $\ldots \ldots$ & . \\
\hline & Archiacana D'C & $\cdots \cdot$ & $\ldots \ldots$ & ... & $\ldots$ & . . $\Gamma^{2}$ & $\ldots \ldots$ & . \\
\hline & MathN. .. & $\ldots \ldots$ & $\cdots \cdots$ & $\cdots \cdots$ & $\cdots$ & . I & $\ldots \ldots$ & - \\
\hline & $\cdots$ & $\cdots \cdot \cdot$ & $\cdots$ & • & $\ldots$ & . I & $\ldots \ldots$ & • \\
\hline & $\cdots$ & $\cdots \cdot \cdot$ & $\ldots \ldots$ & $\cdots \cdot$ & $\cdots$ & . . I & $\ldots \ldots$ & • \\
\hline & . & $\cdots \cdot$ & $\ldots \ldots$ & $\cdots \cdot$ & $\cdots$ & . . I & $\ldots \ldots$ & - \\
\hline & $\cdots$ & $\cdots$ & $\cdots$ & . & $\cdots$ & . . I & $\ldots \ldots$ & • \\
\hline & gibbosa $\mathbf{M}$ & $\cdot \cdot$ & $\cdots$ & $\cdots$ & $\cdots \cdots$ & $\cdot 1$ & $\cdots \cdots$ & . \\
\hline & granum $M_{A}$ & $\cdots$ & $\cdots$ & $\cdot \cdot 1$ & $\cdots \cdots$ & $\cdots 1$ & $\cdots \cdots \cdot$ & • \\
\hline & $\cdots$ & - & & $\cdots$ & $\cdots \cdots$ & $\cdots 1$ & $\cdots$ & - \\
\hline & L: & & & .1 & $\cdots$ & 1 1 & $\cdots$ & - \\
\hline & Lamarcki M & & & & $\because$ & $\cdot f^{1}$ & $\cdots$ & - \\
\hline & Martiniana [?] MathN. & $\ldots \ldots$ & $\because$ & & $\cdots \cdots$ & . . 1 & $\therefore$ & \\
\hline & & $\ldots$ & - & & . & ... $\mathbf{f}^{1}$ & $\ldots$ & \\
\hline & D’O. & $\ldots$ & $\ldots$ & ... & - & $\ldots f^{1}$ & $\ldots \ldots$ & \\
\hline & - & $\ldots$ & $\ldots \ldots$ & - & $\ldots$ & $\ldots \Omega^{2}$ & $\ldots \ldots$ & . \\
\hline & 5. . & $\cdots$ & $\cdots \cdots$ & & $\ldots$ & . . $\mathrm{f}$ & 1. & - \\
\hline & N. . . & $\cdots$ & $\cdots$ & $\cdots$ & $\ldots$ & $\cdots l^{2}$ & . & \\
\hline & A & $\cdots \mathrm{M}^{4}$ & - & & $\cdots$ & . & $1 . t$. & \\
\hline & $\cdots$ & . . $\mathbf{M}^{\mathbf{4}}$ & $\cdots$ & $1^{\circ}$ & - & - &.$t \ldots$ & . \\
\hline & $\cdots$ & $\ldots M^{*}$ & $\ldots \ldots$ & - & - & $\cdots$ & $\cdot \cdot t$ & \\
\hline & a Dpr. . . & $1 \ldots \cdots$ & $\cdot$ & $I^{\circ}$ & . & & . $t$. & \\
\hline & de & syn. $\dot{\mathrm{M}^{4}}$ & $\cdots$ & & $\cdots$ & & - $t$ & \\
\hline & $\cdots$ & $\cdots$ & $\cdot$ & & & & . $\mathrm{t}$ & \\
\hline & ides Dsн. . . . & $\cdots \cdot \cdot$ & $\cdots$ & - & - & & - & \\
\hline$\gamma$ & $\begin{array}{l}\text { Maura BRGN. } \cdots \\
\text { Morrisi So. }\end{array}$ & - & $\cdots$ & 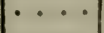 & $\cdots$ & & t & \\
\hline & Münsteri D'O & & ・・ & & & &.$t$ & \\
\hline & DFR. & ... • & 1. & & & & . $t$ & \\
\hline & ol & & & & & & . $t$. & \\
\hline & na D'O. & .... $\mathrm{M}^{4}$. & $\cdots \cdot$ & $\cdots$ & & & . $\mathrm{t}$. & \\
\hline & Proserpina BrgN. . & $\cdots \cdots$ & - & $\cdots$ & • & $\cdots \cdot$ & $\cdot \mathrm{t} \cdots$ & \\
\hline & P & & & $\cdots$ & . & $\cdots$ & - t & \\
\hline & $\begin{array}{l}\mathbf{r e} \\
\mathrm{R}\end{array}$ & & & & & $\ldots$ & . $\mathrm{t}$ & . \\
\hline & scobinellata LK. . . . & & 1. & & & & $\because \ldots$ & \\
\hline
\end{tabular}




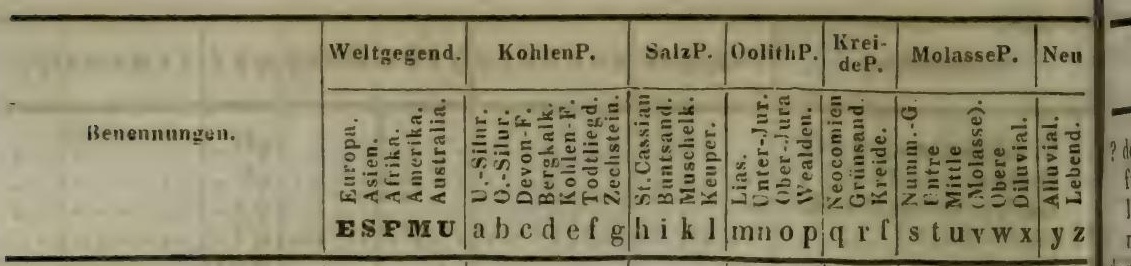

\section{Venus)}

? subpyrenaica Leys. subrugosa $\mathrm{BR}$. tenuis Dsh.

texta LF. turgidula Dsh. Basteroti Dsh.

? chionoides Nyst solida DsH.

\section{aratina L..} cancellata So.

* clathrata Dus. .... cortinaria Cone. coturnix Dus. : . deformis $S_{A X} \ldots .$. dissita Erchw. ...

? gibbosa So....... granosa So. ..... gregaria Partsci

\$ incrassata Егснш. modesta Dus. non-seripta So. obtusa So.

Paphia2 Lк. (non viv.) Rileyi Conr. rudis Dus.

千 squamigera Еichw. striatella Nyst

‡ subrotunda DFR. tricuspis Eichw. vetula Bast.

Brocchii Dsh. rotundata (?L.) Brocc. casina $\mathrm{L}$. corbis LK. decussata $\mathrm{L}$. laeta Pour ........

$\mp$ Levantina . Bous mercenaria Lк. . . . ovata Montg. plicata Gm. senilis Brocc. verrucosa $\mathbf{G m}$.

† augula [?] SERR... intermedia SERR. pullustra Matian.

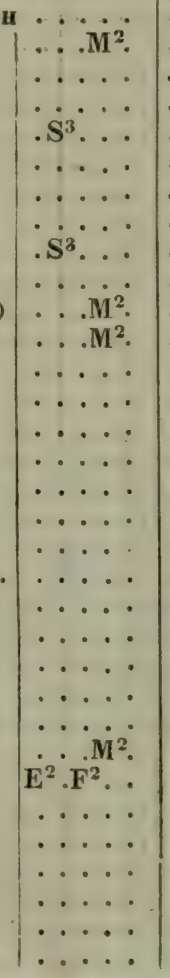

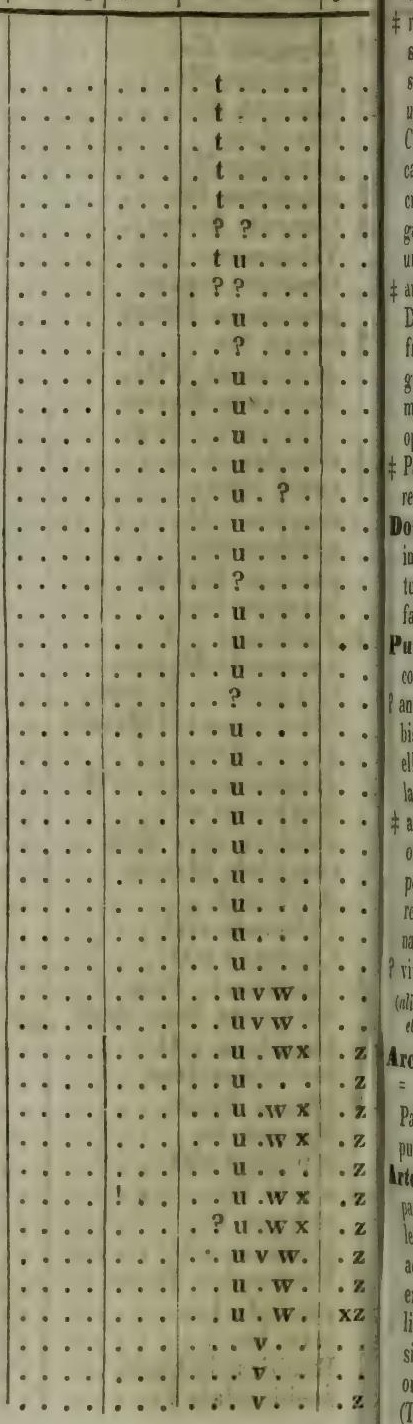




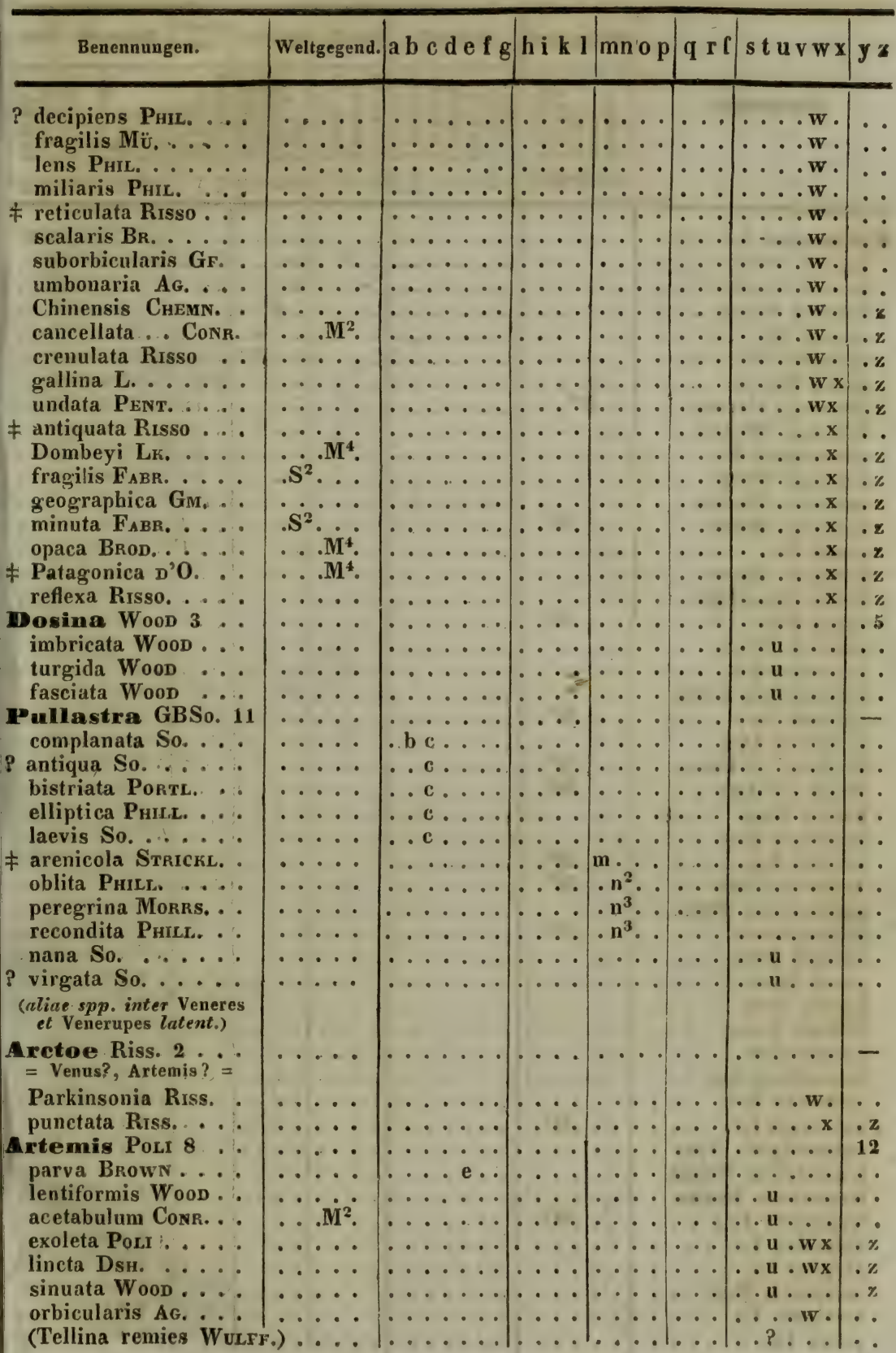




\begin{tabular}{|c|c|c|c|c|c|c|c|}
\hline \multirow[b]{2}{*}{ Benennungen. } & Weltgegend. & KohlenP. & SalzP. & OolithP. & $\begin{array}{r}\text { Krei- } \\
\text { deP. }\end{array}$ & MolanseP. & Nau \\
\hline & 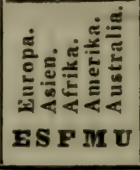 & 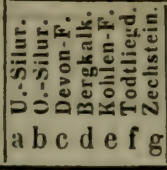 & 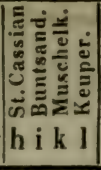 & 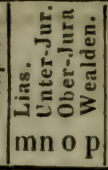 & 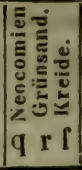 & 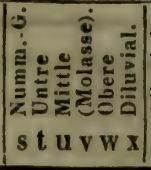 & 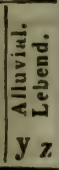 \\
\hline ytherea LK. 81 . & $\cdots \cdot$ & $\cdots$ & $\cdots$ & $\cdot 1$ & $\cdots$ & $\ldots \ldots$ & 130 \\
\hline cornea Voltz.... & $\ldots$ & $\ldots \ldots$ & &. & $\therefore$ & $\ldots \ldots$ & $\ldots$ \\
\hline lucinia Voltz .... & $\ldots$. & $\ldots \ldots$ & $\ldots$ & & $\ldots$ & $\ldots \ldots$ & $\therefore$ \\
\hline dolabra Phill.... & $\ldots$ & $\ldots \ldots$ & $\cdots$ &.$n^{3} \ldots$ & ... & $\ldots \ldots$ & - \\
\hline rugrosa So...... & $\cdots \cdots$ & $\ldots \ldots$ & $\cdots$ & $\cdots 0$. & $\ldots$ & $\ldots \ldots$ & - \\
\hline idea Mï & $\cdots \cdots$ & $\ldots \ldots$ & $\cdots$ & ..? ? & & $\cdots \cdots$ & - \\
\hline$\cdots$ & $\cdots \dot{n}^{4}$ & $\ldots \ldots$ & $\cdots$ & & $q ?$. & $\cdots \cdots$ & • \\
\hline Herizogi Hausm. . &. $\mathrm{F}^{4} \cdot$ & $\cdots \cdots$ & $\cdots \cdots$ & $\cdots \cdots$ & . $\mathbf{r}$. & $\ldots \ldots$ & - \\
\hline lineolata Morrs. . & $\cdots \cdot$ & $\ldots \ldots$ & $\cdots \cdots$ & $\cdots \cdots$ & . r. & $\cdots \cdots$ & - \\
\hline nda So.... & $\cdots$ & $\cdots \cdots$ & & $\cdot \cdot$ & $\cdot r$ & $\cdots \cdots$ & - \\
\hline truncata Morrs. . & $\cdots \cdots$ & $\cdots \cdots \cdots$ & $\cdots \cdots$ & $\because \cdots$ & $\int_{0}^{r} r \mathbf{f}^{1}$ & $\cdots \cdots$ & $\bullet$ \\
\hline caperata Morrs. . & $\cdots \dot{M}^{2}$ & $\cdots \cdots$ & $\begin{array}{ll}\cdots \\
\cdots\end{array} \mid$ & $\cdots$ & .1 & $\cdots \cdots$ & $\therefore$ \\
\hline $\begin{array}{l}\text { excavata MorT. . } \\
\text { plana Gr. . . . }\end{array}$ & $\ldots \ldots$ & $\ldots \ldots$ & $\ldots$ & $\ldots$ & . . $\mathbf{r}^{1}$ & $\ldots \ldots$ & $\because$ \\
\hline $\begin{array}{l}\text { plana GF. . . . } \\
\text { elongata Rouss . }\end{array}$ & $\ldots$ & $\ldots \ldots$ & $\cdots \cdots$ & $\cdots$ & $\ldots f$ & $\ldots \ldots$ & \\
\hline Bellovacina Dsh. . . & ... & $\ldots \ldots$ & $\cdots \cdot$ & $\cdots$ & $\cdots$ &.$t \ldots$ & - \\
\hline$\ldots$. & $\ldots \mathbf{M}^{2}$ & $\ldots \ldots$ & $\cdots \cdots$ & $\cdots \cdots$ & & . t... & $\cdots$ \\
\hline vexa Brgn. ... & $\ldots$ & $\ldots \ldots$ & $\ldots$. & $\cdots \cdots$ & $\cdots$ & . t ... & - \\
\hline corbulina Lk. . . . & $\ldots$ & $\ldots \ldots$ & $\cdots \cdots$ & $\cdots$ & - & . t.... & - \\
\hline Custugensis LEYM. . & $\cdots$ & $\ldots \ldots$ & $\ldots$ & $\cdots$ & . & $\mid . t \ldots$ & $\cdots$ \\
\hline$\cdots$ & ... $\mathbf{M}^{2}$ & $\ldots \ldots$ & $\cdots$ & $\cdots$ & $\cdots$ & $\cdot t \ldots$ & $\cdots$ \\
\hline osa Dsr. ... & . . $\dot{2}^{\circ}$ & $\ldots \ldots$ & $\cdots$ & $\cdots$ & $\cdot \cdot$ & $\cdot \mathbf{t} \cdots$ & - \\
\hline Hydana [?] Conr. . & . $M^{2}$ & $\ldots \ldots$ & ... & $\cdots$ & . & $\cdot \mathbf{t} \ldots$. & - \\
\hline Hydii [?] LeA ... & . $M^{2}$ & $\cdots \cdots$ & $\cdots$ & $\cdots \cdot$ & $\cdots$ & 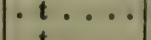 & \\
\hline Kickxi Nrst . . & $\cdots$ & $\cdots \cdots$ & $\cdots \cdot \mid$ & $\cdots$ & $\cdots \cdot$ & 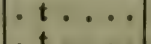 & $\cdots$ \\
\hline lunularia DsH. . . & $\cdots \dot{M}^{2}$ & $\begin{array}{c}\cdots \cdots \\
\cdots \cdots\end{array}$ & $\cdots \cdots$ & $\cdots \cdots$ & $\cdots$ & $\mid \begin{array}{c}t \\
t^{\prime}\end{array}$ & $\because$ \\
\hline $\operatorname{minima}_{\text {Mertoni }}$ LEA $\ldots \ldots$ & $\because M^{2}$ & $\mid \begin{array}{lll}\cdots & \cdots & \cdots \\
\cdots & \cdots & \cdots\end{array}$ & $\cdots \cdots$ & $\cdots$ & $\left|\begin{array}{c}\cdots \\
\cdots\end{array}\right|$ & $\left|\begin{array}{ccc}t & \cdots \\
. t & \cdots & \cdots\end{array}\right|$ & $\because$ \\
\hline $\begin{array}{l}\text { Mortoni Cons. . . } \\
\text { multisulcata Dsh... }\end{array}$ & $\cdots$ & $\ldots \ldots$ & $\ldots$ & $\ldots$ & $\ldots$ & 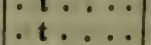 & $\because$ \\
\hline Nuttali Conr. . . . & $\ldots \mathbf{M}^{2}$ & $\ldots \ldots$ & $\cdots$ & $\cdots$ & $\ldots$ & . $\ldots$. & . \\
\hline Dsh. .... & $\cdots \cdots$ & $\ldots \ldots$ & $\cdots \cdot$ & $\cdots \cdot$ & $\cdots$ & . t ... & - $\cdot$ \\
\hline$\ldots \cdot$ & $\cdots \cdots$ & $\ldots \ldots \ldots$ & $\cdots \cdots$ & $\cdots \cdots$ & ... & $\cdot t \ldots$ & $\cdots$ \\
\hline . & - & $\cdots \cdots$ & & $\cdots$ & - & $\cdot \mathbf{t} \ldots$ & \\
\hline bica Leym. . . . . & $\cdots \cdots$ & $\cdots \cdots \cdots$ & $\cdots \cdots$ & $\cdots$ & $\cdots$ & $\cdot t \cdots$ & - \\
\hline$\cdots \cdots$ & $\cdot$ & $\cdots \cdots$ & $\cdots$ & $\cdots$ & $\cdots$ & t $\ldots$ & \\
\hline ulcata Lk. . . & $\because \cdots$ & $\cdots \cdots$ & $\cdots \cdots$ & $\cdots \cdot$ & $\cdots$ & t $\ldots ?$ & \\
\hline $\begin{array}{r}\text { striatula Dsh. } \cdots \text {. } \\
\text { o subcrassa LEa. . . }\end{array}$ & $\because \dot{M}^{2}$ & $\mid \cdots \cdots$ & $\because \cdots$ & $\cdots$ & $\because$ & $\begin{array}{l}\cdots \\
. t\end{array} \ldots$ & $\cdots$ \\
\hline $\begin{array}{l}\text { crassa LEA } \cdots \cdots \\
\text { laevigata NYsT }\end{array}$ & $\ldots$ & $\ldots \ldots$ & $\ldots$ & $\cdots$ & $\therefore$ & t $\ldots$ & . \\
\hline tenuistriata Morr8. & $\cdots$ & $\ldots \ldots$ & $\ldots$ & $\cdots$ & $\cdots$ & $\cdot t \ldots$ & $\cdots$ \\
\hline sa Mor.rs. . & $\cdots$ & $\ldots \ldots \ldots$ & $\cdots \cdots$ & $\cdots \cdots$ & $\cdots$ & $\cdot \mathbf{t} \cdots \cdot$ & - \\
\hline ata LEA & . . $\mathbf{M}^{2}$ & $\cdots \cdots$ & $\cdots \cdots$ & $\cdots \cdot$ & $\cdots$ & . t .... & - \\
\hline$\cdots$ & $\cdots \cdots$ & $\cdots \cdots \cdots$ & $\cdots \cdots$ & $\cdots \cdots$ & $\cdots$ & . t ถี... & \\
\hline & ${ }^{\circ}$ & $\cdots \cdots$ & $\cdots \cdot$ & $\cdots$ & $\cdots \cdot$ & - t & $\because$ \\
\hline $\begin{array}{l}\text { rassata DsH. . . } \\
\text { vigata } \text { LK. }_{0} \ldots\end{array}$ & $\ldots$ & $\cdots$ & $\cdots$ & $\cdots$ & 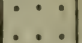 & t ü.... & : \\
\hline $\begin{array}{l}\text { laevigata LK. . . . } \\
\text { polita LK. . . . . }\end{array}$ & $\cdots$ & $\ldots \ldots$ & & & ... & . t ü... & $\cdots$ \\
\hline
\end{tabular}




\begin{tabular}{|c|c|c|c|c|c|c|c|}
\hline en & gegend. & a bc d e $f g$ & 1 & $\mathbf{p}$ & $q \times 1$ & $\mathbf{W} \mathbf{x}$ & \\
\hline & & & & & - & . & \\
\hline a & $\cdots \cdot$ & 然 & $\cdots$ & 1 & $\cdots$ & u... & \\
\hline$\cdots$ & $\cdots \cdot$ & $\cdots \cdots$ & • & .. & $\cdots$ & $\cdots w$. & \\
\hline$\dot{\mathrm{SH}}$ & $\ddot{\cdots} \cdot$ & $\because \cdots$ & $\cdots$ & $\because$ & $\cdots$ & ? & \\
\hline .. & $\therefore$ & $\ldots \ldots$ & $\because \cdots$ & $\cdots \cdots$ & $\cdots$ &.$w$ & \\
\hline R. . & M $\mathrm{M}^{2}$ & $\ldots \ldots$ & $\ldots$ & $\ldots$ & $\ldots$ & & \\
\hline s. & $\ldots$. & $\ldots \ldots \ldots$ & $\ldots$ & $\ldots$ & $\ldots$ & $\begin{array}{ll}\cdots & \ldots \\
\cdots & \text { u. }\end{array}$ & \\
\hline . & $\ldots$. & $\ldots \ldots$ & $\ldots$ & $\ldots$ & $\ldots$ & $\begin{array}{ll}\cdots & \ldots \\
\cdots & \text { u. }\end{array}$ & \\
\hline$\cdots$ & $\cdots \cdot$ & $\ldots \ldots$ & $\ldots$ & $\cdots \cdot$ & $\ldots$ & $\because u \ldots$ & \\
\hline EST. & . . & $\ldots \ldots$ & $\cdots \cdot$ & $\cdots \cdot$ & $\ldots$ & $\therefore$ u. . & \\
\hline R. . & $\ldots \mathrm{M}^{2}$ & $\cdots$ & $\ldots$ & $\cdots$ & $\cdots$ & ..... & \\
\hline$\therefore=$ & . . $\mathbf{M}^{2}$ & $\cdots$ & $\ldots$ & $\cdots$ & $\cdots$ & $\ldots$ u... & \\
\hline$\cdots$ & - $\mathbf{M}^{2}$ & $\cdots$ & $\cdots$ & $\cdots \cdot$ & $\cdots$ & $\ldots \mathbf{u} \ldots$ & \\
\hline$\cdots$ & $\ldots \mathbf{M}^{2}$ & $\cdots$ & $\cdots$ & $\cdots \cdot$ & $\cdots$ & ... u... & \\
\hline$\cdots$ & $\cdots \cdot$ & $\ddot{.}$ & $\cdots$ & $\cdots \cdot$ & $\cdots$ & . & \\
\hline$\cdots$ & $\cdots$ & $\because$ & $\cdots$ & $\cdots \cdot$ & $\cdots$ & $\cdots$ & \\
\hline$\cdots$ & & $\mid \begin{array}{c}\cdots \\
\cdots\end{array} \cdots$ & $\cdots$ & $: 1$ & $\cdots$ & $\ldots 1$ & \\
\hline G. . . & $\therefore$ & $\ldots \ldots$ & $\ldots$ & $\cdots$ & $\cdots$ & $\therefore 1$ & \\
\hline$\therefore$ & $\cdots \cdot$ & $\ldots \ldots$ & $\ldots$ & $\ldots$ & $\ldots$ & $\ldots$ & \\
\hline .?) $G_{F}$. & $\cdots \cdots$ & $\cdots$ & $\cdots$ & $\ldots$ & $\cdots$ & v.. & \\
\hline$\cdots$ & - & $\cdots$ & $\cdots$ & $\cdots$ & $\cdots$ & ..u v v $\mathbf{v} x$ & \\
\hline$\dot{*} \cdot$ & $\mathrm{F}^{2} \cdot \mathrm{M}^{2}$ & $\cdot \cdot$ & $\cdots \cdots$ & $\cdots$ & $\cdots$ & ....... & \\
\hline к. . . & $E^{2} \cdot F^{2} \cdot$ & $\cdots$ & $\cdots$ & $\cdots$ & $\cdots$ & . 1 & \\
\hline$\cdots$ & . & $\cdots$ & $\cdots \cdots$ & $\cdots \cdot$ & & . & \\
\hline$\cdots$ & 政: & $\cdots$ & $\cdots \cdots$ & $\cdots$ & $\cdot$ & $\mathbf{x}$ & \\
\hline$\because$ & • & $\cdots$ & $\cdots \cdots$ & $\cdots$ & $\cdots$ & • & \\
\hline$\because$ & . $\cdot$ & $\cdots$ & $\cdots \cdots$ & $\cdot$ & $\cdots$ & $\nabla \cdot$ & \\
\hline 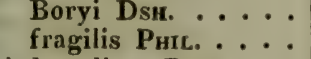 & $\because$ & $\because$ & $\cdots \cdots$ & $\cdots$ & $\cdots$ & v. & \\
\hline R. . . & ... & $\ldots \ldots$ & $\ldots$ & $\cdots \cdots$ & $\because$ & $\because$ & \\
\hline$\ldots$ & & $\cdots$ & $\ldots$ & $\ldots$ & $\ldots$ & $\cdots$ & \\
\hline$\cdots$ & $\cdots \mathbf{M}^{2}$ & $\cdots$ & & $\ldots$ & $\cdots$ & ....w. & \\
\hline$\cdots$ & $\cdots \cdot$ & $\cdots$ & • & $\cdots \cdot$ & $\cdots$ & $\cdots, w x$ & \\
\hline$\cdots$ & • & $\cdots$ & $\cdots \cdot$ & $\cdots$ & $\cdots$ & $\ldots . . x$ & \\
\hline 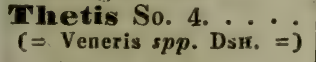 & $\cdots$ & $\cdots \cdots$ & $\cdots$ & $\cdots \cdot$ & $\cdots$ & $\cdots \cdots$ & \\
\hline$\cdots$ & - & $\cdot$ & & & & $\cdots$ & \\
\hline & & & & & & $\cdots$ & \\
\hline & & & & & & $\cdots \cdots$ & \\
\hline 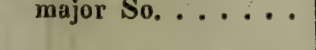 & & & & & & $\cdots$ & \\
\hline z & & & & & & & \\
\hline & & & & & & & \\
\hline & & & & & & $\cdots \cdots$ & \\
\hline & & $\ldots \ldots$ & & & & - & \\
\hline$\cdots$ & 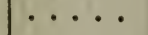 & $\cdots$ & $\cdots$ & & & $\cdots \cdots$ & \\
\hline B. . & & $\cdot 1$ & . & & & - & \\
\hline 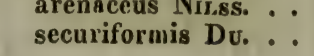 & & & & & & & \\
\hline
\end{tabular}




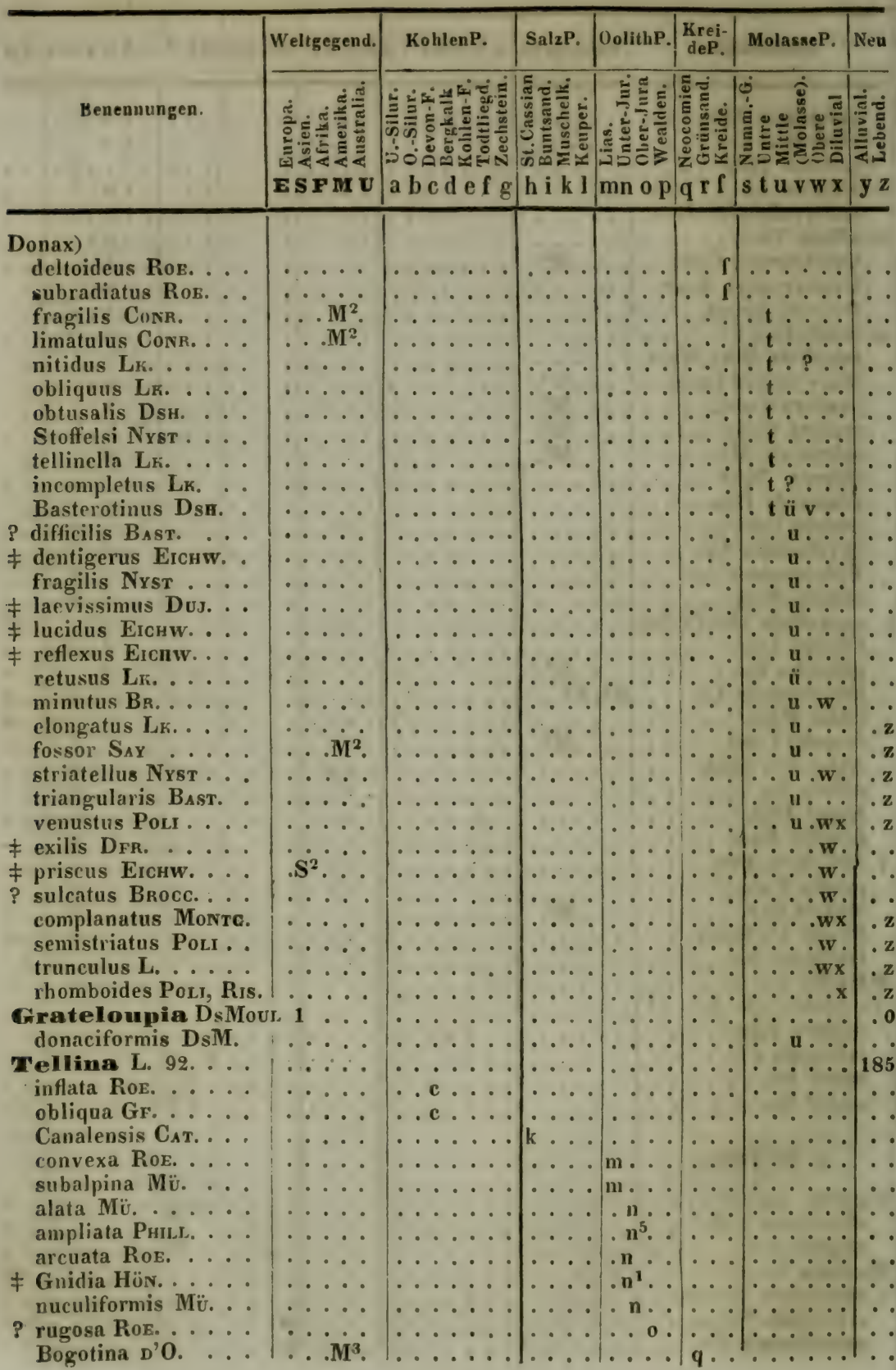




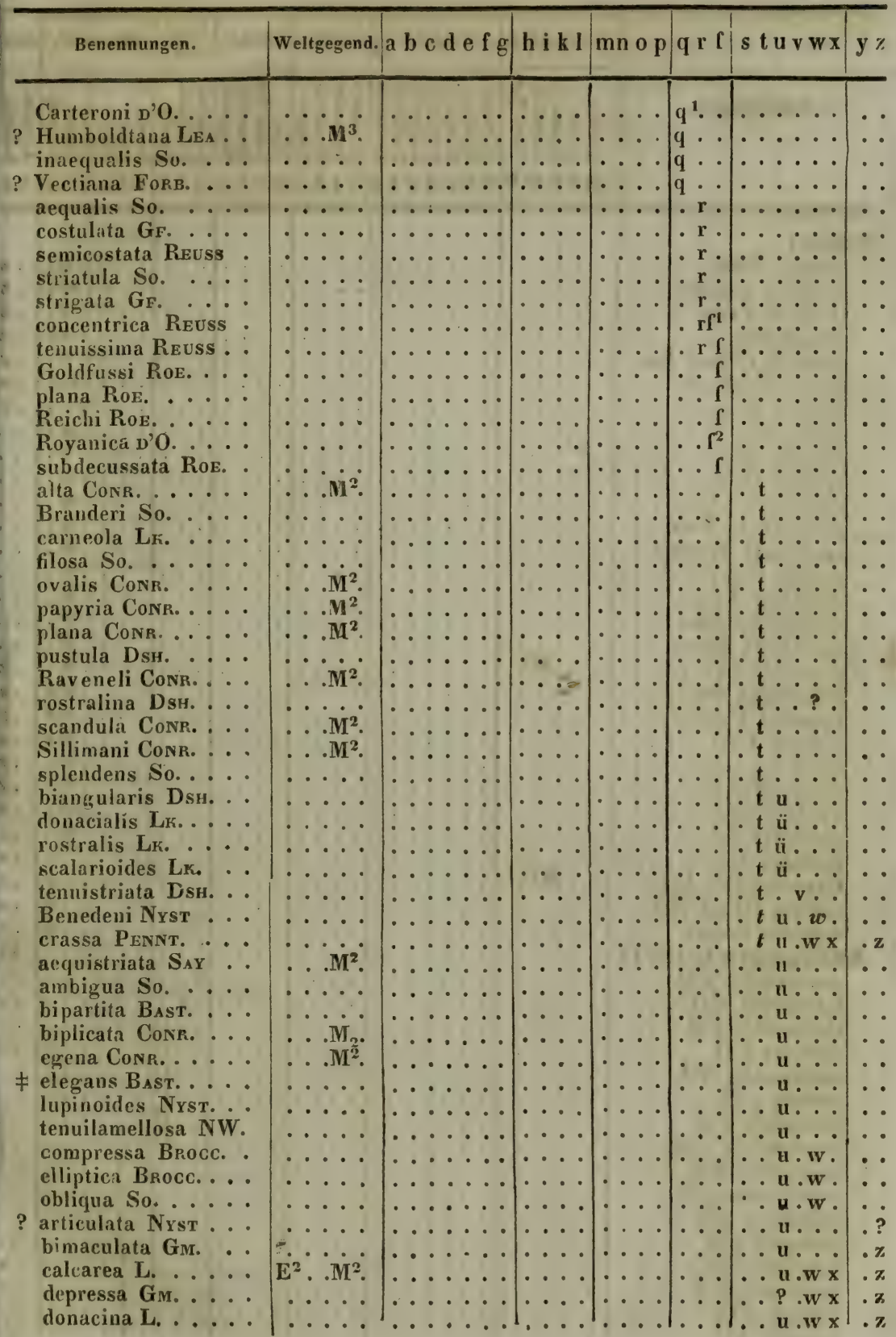




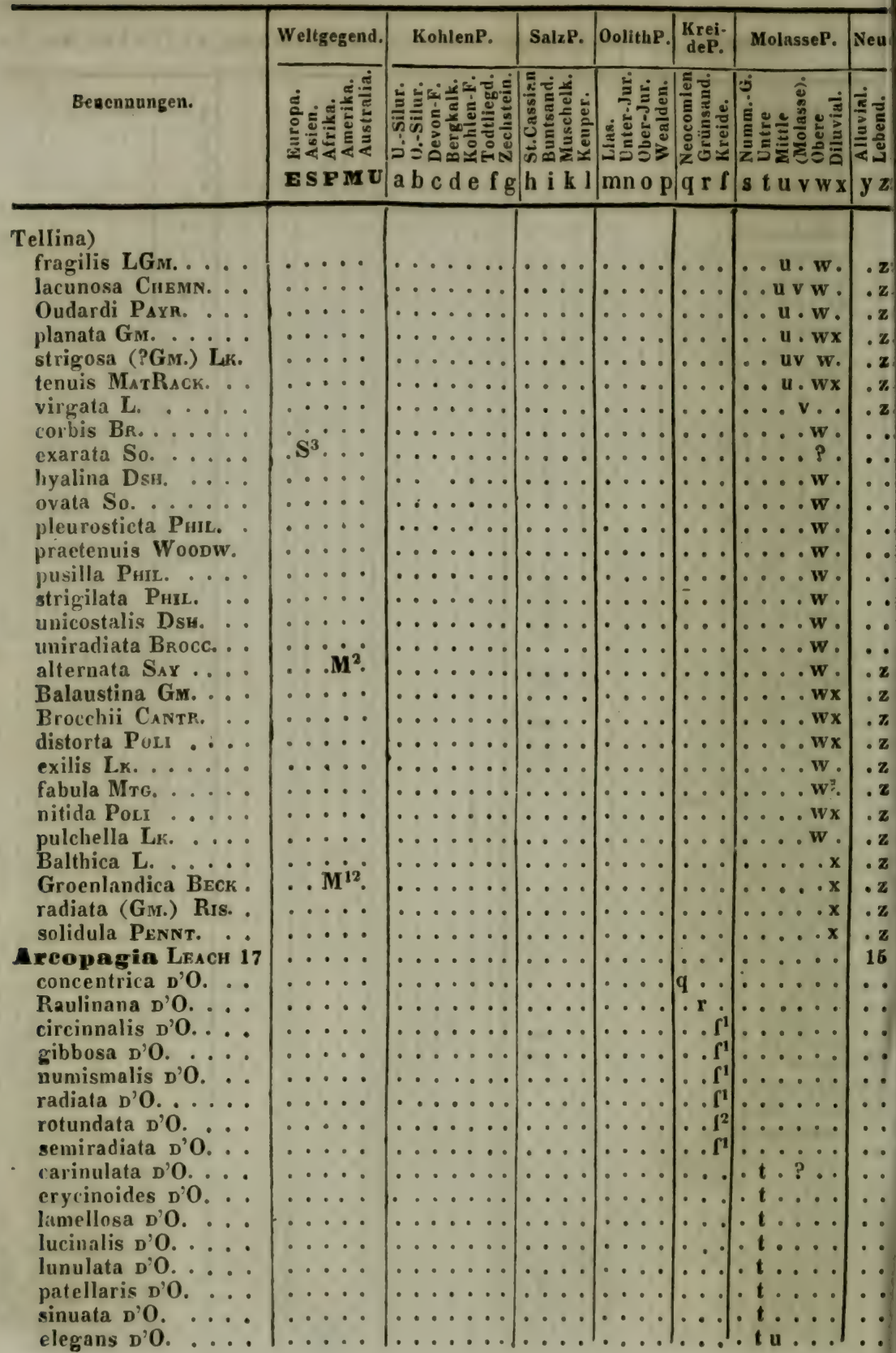




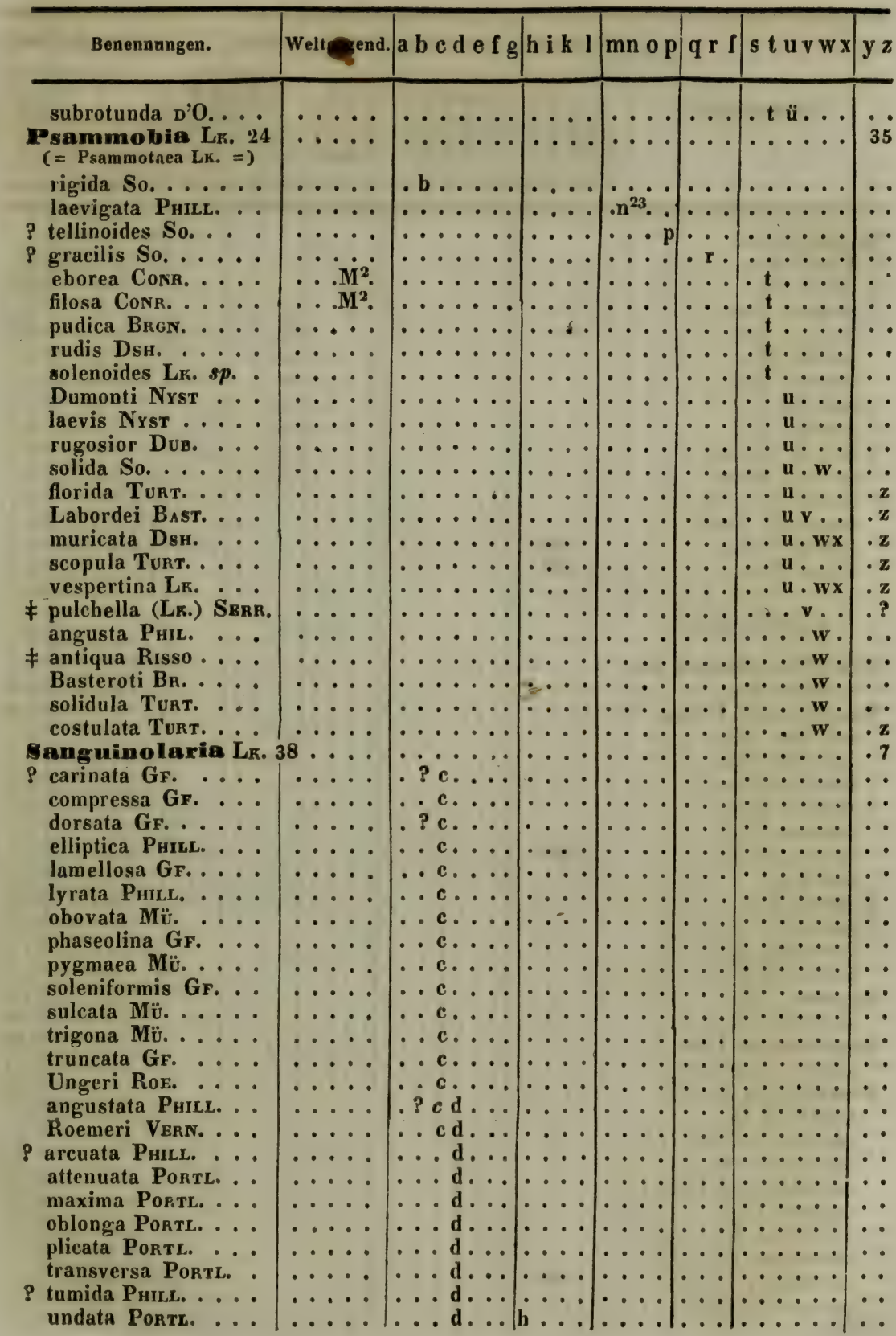




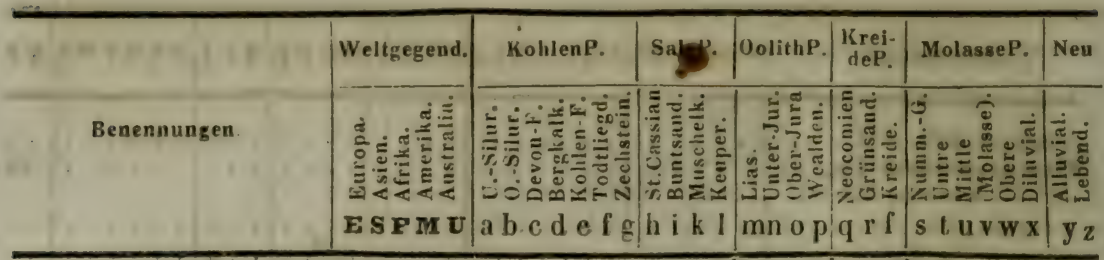

Sanguinolaria)

? Alpina Mü.

\#. Okeni M̈̈.

elegans Paicr.

Neptuni Mü.

pusilla Mü.

vetusta PHIL.L.

lata $\mathrm{M} \ddot{\text { u. }}$

gracilis $\mathrm{Mü.}$

† obtusa Вuckm.

parvula Bean

o Hollowaysi So

Lamarcki Dsh.

lusoria ConR.

fusca Conr.

\section{$\delta$ Petricolina:}

Coralliophaga Buv. 1. . . . dactylus BR.

Generupis Fleur., Lr. 11 corallina Mer. oolithica MER. Brocehii DFr. globosa Dsu. striatula Dsw. subvexa Conr. lrus $\mathrm{LK}_{\mathrm{K}}$.

\# Italica DFr.

‡ parasita DFR. pernarum Bow. perfurans Lk. . . . .

Agina TURT. 1 purpurea TurT ...

Byssomya Cuv. 1. ? petricoloides LEA . . Petricola LK. 18. lamellosa $\mathrm{GF}_{\mathrm{F}}$. canaliculata So. nuciformis So. coralliophaga Dsн. elegans Dsh.

\# variabilis Dsh. . .

? abbreviata Dus. ... centenaria Conr. peregrina BAst.

Lri. 11 .

(1)




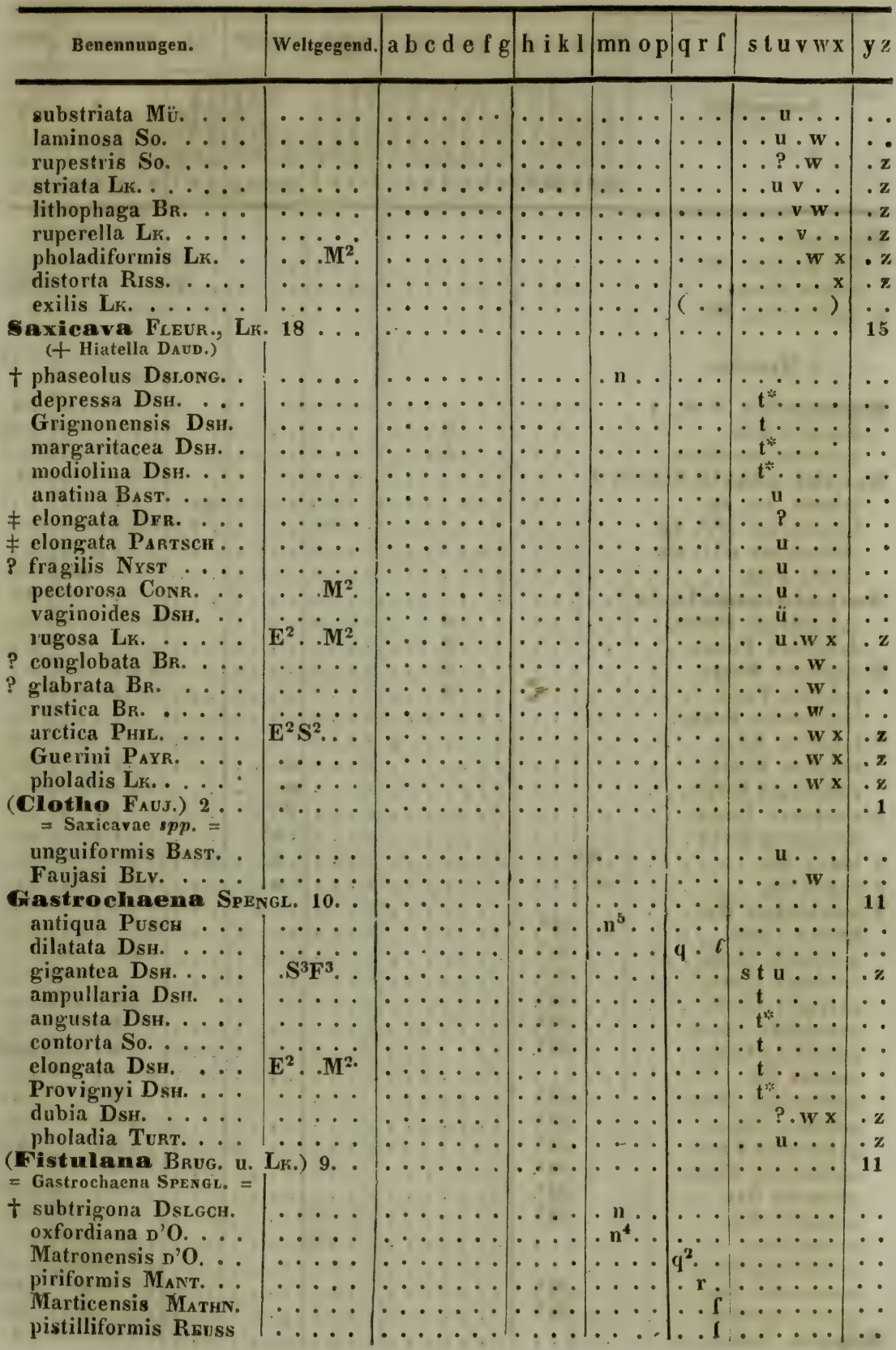




\begin{tabular}{|c|c|c|c|c|c|c|c|}
\hline 68 & Weltgegend. & KohlenP. & SaluP. & WolithP & $\begin{array}{l}\text { Krei- } \\
\text { deP. }\end{array}$ & MolasseP. & Nell \\
\hline \multirow[t]{3}{*}{ Benenuungen } & 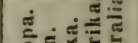 & 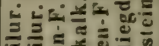 & 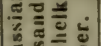 & & & & \\
\hline & 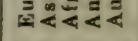 & ธดดัดับับ & 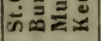 & 离 & 品 & 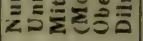 & \\
\hline & ES F M U & a b c d e f $g$ & $\mid \mathrm{h}$ i k l $\mid$ & $|\mathrm{mn} o \mathrm{p}|$ & $q \times 1$ & $s t u v w x$ & $y z$ \\
\hline
\end{tabular}

Fistulana)

Royanensis D'O.

tenuis Pesuss

† lumbricalis $\mathbf{M} \ddot{\text {. }}$.

$\varepsilon$ Mactrina.

Edmondia Kon, 2

Josepha KoN.

unioniformis KoN.

Serobieularia Schum.

minuta AG. $s p$.

Clementinu D'O. sp phaseolina D'O. $8 p$.

tenuis Pur......

Amphidesma Lk. 30 axiniformis Pontr. . deltoidea Pontu. .

† depressa PortL. pristina VERN. carbonaria Porte.

? lunulata KEYs. elliptica KoDu. compressa KoDu.

\# congener PhiLl. . . decussata BEAN

? hians So.

? ovalis So.

$P$ tenuistriata So. limosa Conr. profunda Cons. tellinula Conr. carinata CoNr. constricta CosR. . . lamellosa Conr. . . . minuta So. nuculoides Conr. protexta CoNr. prismatica FLEM. subobliqua CoNr. subovata $S_{\text {AY }}$ aequalis $S_{A Y}$ ovata Dsh. subtrigona DSH. inaequalis $\mathrm{S}_{\mathbf{A X}} \ldots \mathrm{M}^{2}$. transversa $S_{\Delta Y} \ldots \ldots M^{2}$.

Ligula Montc. 2.

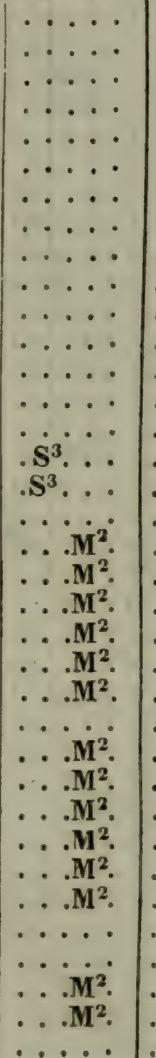




\begin{tabular}{|c|c|c|c|c|c|c|c|}
\hline B & gend. : & f g & 1 & mnop & q $\times 1$ & $w x$ & $y z$ \\
\hline $\begin{array}{l}\text { donaciformis Nyst . } \\
\text { alba Nyst...... } \\
\text { Donacilla LK. 8. } \\
\text { (Mesodema Dsh.; ? Taras R, }\end{array}$ & $\dot{.}$ & $\therefore$ & $\mid \begin{array}{c}\cdots \\
\cdots \\
\cdots\end{array}$ & $\cdots$ & $\begin{array}{c}\cdots \\
\cdots \\
\cdots\end{array} \mid$ & 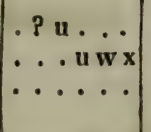 & $\begin{array}{r}1 \% \\
78\end{array}$ \\
\hline 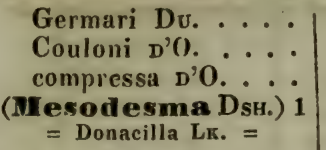 & $\begin{array}{l}\cdots \cdots \\
\cdots \\
\cdots \\
\cdots\end{array}$ & $\begin{array}{lll}\cdots & \cdots & \cdots \\
\cdots & \cdots & \cdots \\
\cdots & \cdots & \cdots \\
\cdots & \cdots & \cdots \\
\cdots\end{array}$ & $\dot{\cdot} \cdot \dot{ }$ & $\left|\begin{array}{cc}m & \cdots \\
\cdots & \cdots \\
\cdots & \cdots \\
\cdots\end{array}\right|$ & $\begin{array}{c}\cdots \\
q \cdot \\
\cdots \\
\cdots\end{array}$ & $\mid \begin{array}{ll}\cdots & \cdots \\
\cdots & \cdots \\
\cdots & \cdots \\
\cdots\end{array}$ & $\because$ \\
\hline 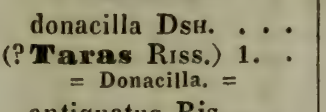 & $\cdots \cdots$ & $\because$ & : & $\because$ & $\cdots$ & $\begin{array}{l}\cdot w \\
\cdots\end{array}$ & z \\
\hline $\begin{array}{l}\cdots \\
5 . \cdots\end{array}$ & $\begin{array}{l}\cdots \\
\cdots\end{array}$ & & $\ddot{*}$ & & $\begin{array}{l}\cdots \\
\cdots\end{array}$ & $\begin{array}{c}\ldots w \\
\ldots \ldots \\
\ldots\end{array}$ & 3 \\
\hline$\cdots$ & $\ldots$ & $\cdots c \ldots$ & $\ldots$ & $\cdots \cdot$ & $\cdots$ & $\ldots \ldots$ & $\cdots$ \\
\hline $\begin{array}{l}\text { aea Mü. } \ldots \ldots \\
\text { a Mü. } \ldots \ldots\end{array}$ & $\cdots \cdots$ & $\begin{array}{ll}\cdots & \cdots \\
\cdots & \cdots\end{array}$ & 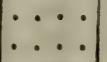 & $\cdots \cdots$ & $\cdots$ & $\cdots \cdots \cdots$ & $\because$ \\
\hline$\dot{\cdots}$ & $\begin{array}{l}\ldots \mathrm{M}^{2} \\
\cdots \mathrm{M}^{2}\end{array}$ & & $\ldots \ldots$ & $\ldots \cdots$ & 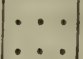 & $\left|\begin{array}{lll} & t & \ldots \\
. t & \ldots & \ldots\end{array}\right|$ & $\ddot{\circ}$ \\
\hline . & .... & $\ldots \ldots$ & $\cdots$ & $\ldots \ldots$ & $\cdots$ & $\ldots$ & $\therefore$ \\
\hline$\cdots$ & $\cdots \cdots$ & $\cdots \cdots \cdots$ & $\cdots$ & $\ldots$ & $\cdots$ & $\cdot t \cdot \ldots$ & - \\
\hline$\cdots$ & $\therefore$ & & $\cdots \cdot$ & $\cdots$ & & $\cdots$ & $\because$ \\
\hline$\cdots$ & $\ldots$ & $\because$ & & $\cdots$ & $\therefore$ & 0 & $\because$ \\
\hline$\cdots$ & $\cdots \cdot$ & $\cdots$ & $\ldots$ & $\cdots \cdot$ & $\cdots$ &.$t \ldots$ & $\cdots$ \\
\hline NR. & $\cdots \dot{M}^{2}$ & & $\cdots \cdot$ & $\cdots \cdots$ & $\cdots$ & $\begin{array}{l}. t \\
. t\end{array}$ & $\because$ \\
\hline$\cdots$ & $\cdots$ & $\ldots \ldots \ldots$ & - $\cdot$ & $\cdots$ & $\therefore$ & t $\ldots$ & $\because$ \\
\hline$\cdots$ & $\cdots \cdots$ & $\cdots$ & . & $\cdots$ & $\cdots$ &.$t \ldots$ & $\cdots$ \\
\hline$\cdots$ & $\cdots \cdots$ & $\cdots \cdot$ & $\cdots$ & $\cdots$ & $\cdots$ & $\cdots$ & - \\
\hline EST. & & & & 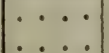 & . &.$t^{*} u \ldots$ & .. \\
\hline $\begin{array}{l}\text { NYSTW EST. } \\
\text { NYAT } \ldots\end{array}$ & $\therefore$ & $\therefore$ & 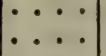 & $\cdots \cdots$ & $\because \cdots$ & $\begin{array}{ll}\cdots & \ldots \\
\cdots & \ldots\end{array}$ & $\cdots$ \\
\hline$\cdots$ & $\cdots \cdots$ & $\cdots$ & $\cdots \cdot$ & $\ldots$ & $\cdots$ & ..... u. & . \\
\hline cc. $\cdot$ & $\cdots \cdot \cdot$ & $\cdots$ & $\cdot$ & $\ldots$ & $\cdots \cdot$ & ....w w & - $\cdot$ \\
\hline & $\cdots$ & $\cdots$ & $\cdots \cdots$ & $\cdots \cdot$ & $\cdots \cdot$ & $\cdots$ & - \\
\hline$\cdots$ & $\cdot \cdot$ & $\cdots$ & $\cdots$ & $\cdots$ & $\cdots$ & $\cdots \cdot$ & \\
\hline 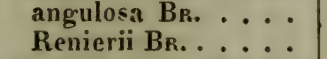 & - & · & 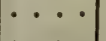 & $\cdot$ & $\cdots \cdot$ & 8. & $\%$ \\
\hline$\Rightarrow$ & $\cdots$ & & $\cdots \cdots$ & 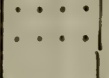 & 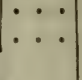 & $\cdots \cdots \mathbf{w}$ & .2 \\
\hline 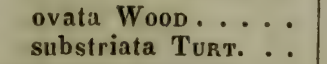 & $\cdots$ & & & $\because$ & $\cdots$ & $\begin{array}{l}\ldots \text { u. . } \\
\ldots \text { u. . }\end{array}$ & $\ddot{z}$ \\
\hline $\begin{array}{l}\operatorname{ming}_{\text {DsM. }} R_{\Delta N} 3 \\
\end{array}$ & & $\dot{0}$ & $\cdots \cdot$ & $\cdots$ & $\cdots$ & $\cdots \cdots$ & .1 \\
\hline $\begin{array}{l}\text { Va } \\
\text { mi }\end{array}$ & $\ldots \mathrm{M}^{2}$ & & & & & $\cdots$ & \\
\hline 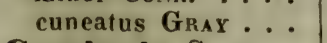 & $\ldots \mathrm{M}^{2}$ & & & $\cdots \cdots$ & $\cdots \cdot$ & ...wx & $\cdot \mathrm{z}$ \\
\hline a So. 0 .. & . & & & $\cdots$ & $\cdots \cdot$ & $\cdots$ & .6 \\
\hline L L. $53 \ldots$. & $\cdots$ & & & $\cdots \cdots$ & $\cdots \cdot$ & $\cdots$ & 60 \\
\hline trigona RoE, . . . & .. & . & & 1. n. . & & I... & \\
\hline
\end{tabular}




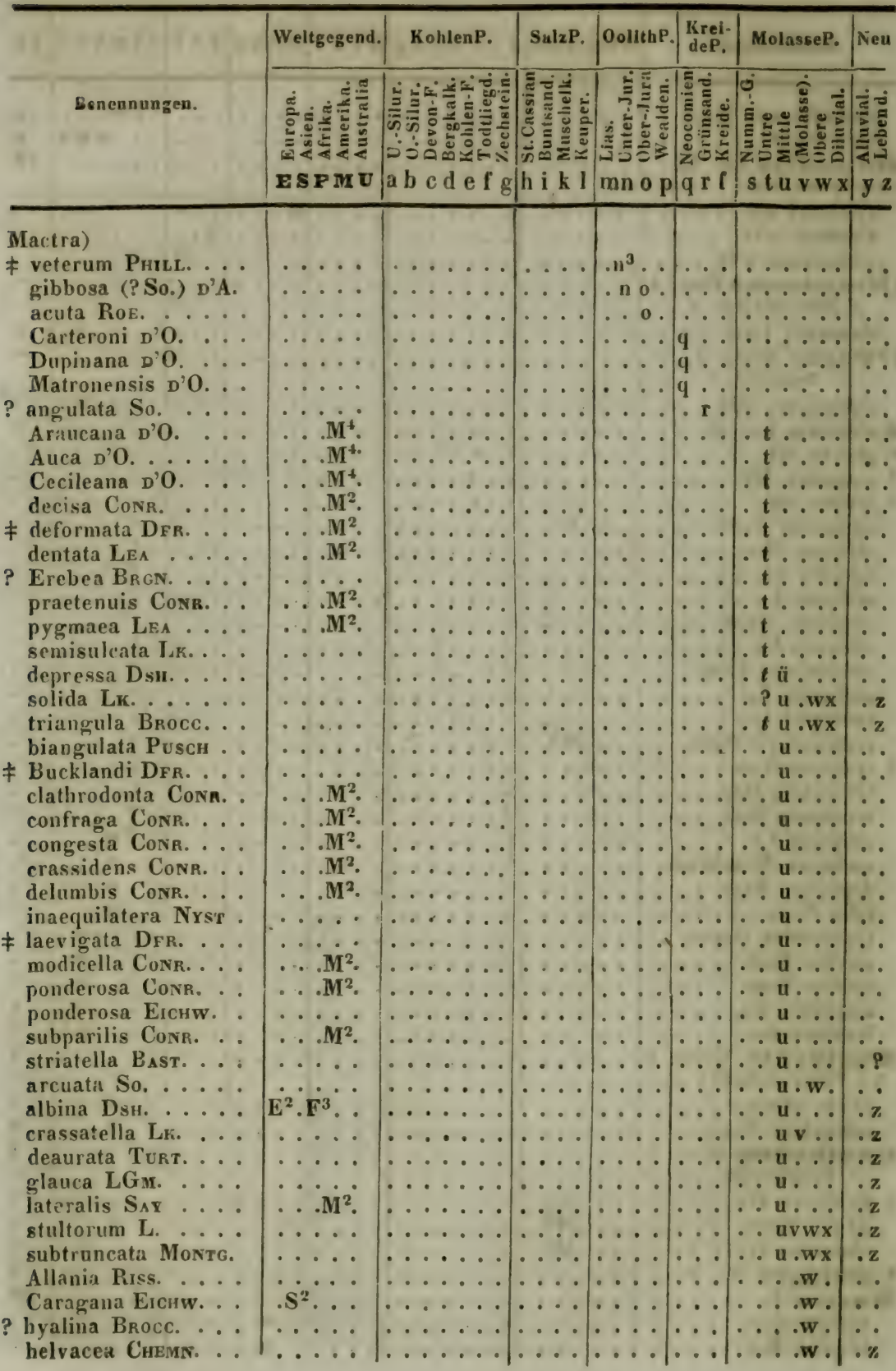




\begin{tabular}{|c|c|c|c|c|c|c|c|}
\hline Beneunungen, & gend. & e $f \mathrm{~g}$ & k 1 & $\mathrm{p}$ & $q \times f$ & & \\
\hline so & $\ldots M^{2}$ & $\cdots \cdots$ & & & $\cdots$ & ......... & \\
\hline & ... $\mathrm{N}^{2}$. & $\ldots \ldots$ & & $\cdots$ & $\ldots$ & ...w. & \\
\hline & $\cdots \cdots$ & $\ldots \ldots$ & & $\cdots$ & $\cdots$ & $\ldots w \mathbf{w}$ & \\
\hline 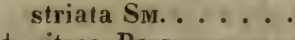 & $\cdots \cdot$ & $\ldots \ldots$ & $\cdots$ & $\cdots \cdot$ & $\cdots$ & $\ldots \ldots x$ & \\
\hline & $\cdots \cdot$ & $\ldots \ldots$ & $\cdots$ & $\ldots$. & $\ldots$ & $\ldots \ldots x$ & \\
\hline 1 & $\cdots$ & $\cdots$ & $\cdots$ & $\cdots$ & $\cdots$ & $\ldots \ldots$ & \\
\hline $\mathbf{T}$ & & $\because$ & & $\cdots$ & $\cdots$ & & \\
\hline unt & & $\cdots \mathrm{d} \cdots$ & & & & $\cdots$ & \\
\hline $\begin{array}{l}\mathrm{p} \\
\mathrm{o}\end{array}$ & $\begin{array}{l}\cdots \\
\cdots\end{array}$ & $\cdots$ & $\cdots$ & ?. & & & \\
\hline RoE. . . & $\ldots \ldots$ & $\ldots \ldots$ & $\cdots \cdots$ & 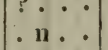 & $\cdots$ & & \\
\hline Phili.. & $\ldots$ & $\ldots \ldots$ & $\ldots$ & . n. . & ... & $\ldots$ & \\
\hline .. & $\cdots \cdot$ & $\ldots \ldots$ & $\ldots$ & $\ldots 0$ & $\cdots$ & $\cdots$ & \\
\hline HN. & $\ldots$ & $\ldots \ldots$ & $\ldots$ & $\cdots \cdot$ & $q \ldots$ & $\ldots \ldots$ & \\
\hline$\cdots$ & - • & $\cdots \cdots$ & $\ldots$ & $\ldots$ & . r. & & \\
\hline la & $\cdots$ & $\cdots \cdots$ & $\ldots$ & $\cdots$ & $\cdots$ & . $t$ & \\
\hline & $\cdots$ & $\cdots \cdots \cdots$ & $\cdots$ & $\cdots$ & $\cdots$ & t... & \\
\hline a & $\because$ & $\cdots \cdots$ & $\cdots \cdots$ & - & $\cdots$ & . u u & \\
\hline & $\begin{array}{l}\cdots \\
\cdots \\
\cdots\end{array}$ & $\cdots \cdots$ & $\cdots$ & $\cdots$ & $\cdots$ & $\cdots u \ldots$ & \\
\hline $\begin{array}{l}\text { cl } \\
\text { la }\end{array}$ & $\because$ & $\cdots$ & $\because \cdots$ & $\cdots \cdots$ & $\because$ & $\cdots \mathbf{u} \cdot w$. & \\
\hline $\begin{array}{l}\text { la } \\
\mathrm{ca}\end{array}$ & $\mathrm{M}^{2}$ & $\ldots \ldots$ & $\ldots \ldots$ & $\cdots \cdots$ & $\because$ & $\cdots y$ & \\
\hline$\therefore$ & $\cdots \cdots$ & $\ldots \ldots$ & $\ldots$ & $\ldots$ & $\cdots$ & $\begin{array}{ll}\cdots u \\
\cdots y\end{array}$ & \\
\hline$\cdots$ & $\cdots$ & $\cdots \cdots \cdots$ & $\cdots \cdots$ & $\ldots$ & $\cdots$ & $\because u$ & \\
\hline & $\mathbf{E}^{2}\left(\mathbf{S}^{3}\right)$ & $\cdots \cdots \cdots$ & $\cdots$ & $\cdots$ & $\cdots$ & . u.w. & \\
\hline . & $\cdots \cdots$ & $\cdots \cdots \cdots$ & $\cdots \cdot$ & $\cdot$ & $\cdots$ & $\cdots$ & \\
\hline $\mathrm{cos}-\mathrm{s}-\mathrm{s}$ & $\cdots \cdot \cdot$ & $\cdots \cdots \cdots$ & $\cdots$ & & $\cdots \cdot$ & $\cdots \nabla$ & \\
\hline & $\cdots$ & • & 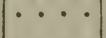 & & $\cdots$ & $\because \mathbf{v} \cdots$ & \\
\hline C & $\cdots$ & • & • & & $\cdots$ & $\cdot \mathbf{w} \cdot$ & \\
\hline & & - & & & & . $x$ & \\
\hline & & & & & & & \\
\hline & & & & & - 1 & $\cdots \cdots$ & \\
\hline & - & 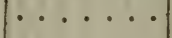 & & $\cdot n^{4}$ & & $\cdots$ & \\
\hline & $\cdots$ & - $\cdot$ & $\cdots$ & $\cdots 0$. & $q$ & $\cdots \cdots$ & \\
\hline & $\cdots$ & i. & • 1 &. & $\begin{array}{lll}q & \cdots \\
q^{1} & \cdots\end{array}$ & - & \\
\hline & $\cdots \cdots$ & . $\cdots$ & - & $\cdots \cdots$ & {$\left[\begin{array}{ll}4 & 0 \\
\cdots & i\end{array}\right.$} & & \\
\hline & $\cdots \cdot$ & $\ldots \ldots$ & . & $\cdots$ & $\ldots$ &.$t$. & \\
\hline & $\cdots \cdot$ & & & $\ldots$. & $\cdots$ & $\ldots \mathrm{u}$ & \\
\hline & C. 0 & & $\cdots$ & $\cdots$ & $\cdots$ & $\cdots u$ & \\
\hline & $\cdots \cdot$ & $\cdots$ & & 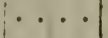 & $\ldots$ & ..u. & \\
\hline & $\cdots \cdot$ & 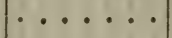 & $\therefore$ & $\cdots \cdot$ & $\cdots$ & $\ldots . . . w$ & • \\
\hline & - & & & $\cdots \cdot$ & $\cdots$ & $\cdots$ & - \\
\hline & $\bullet$ & & & - & $\cdots$ & ....w. & - $z$ \\
\hline $\begin{array}{c}\mathbf{C} \\
=\end{array}$ & & & & & $\cdots$ & $\cdots \cdots$ & \\
\hline & $\cdots$ & & & . n. & $\cdots$ & $\cdots \cdots$ & - \\
\hline & $\cdots$ & & $\cdots$ & $\cdot n \cdot$ & $\cdots$ & $\cdots$ & t. \\
\hline & 0. & & & $\mathbf{n}$. & & & . \\
\hline
\end{tabular}




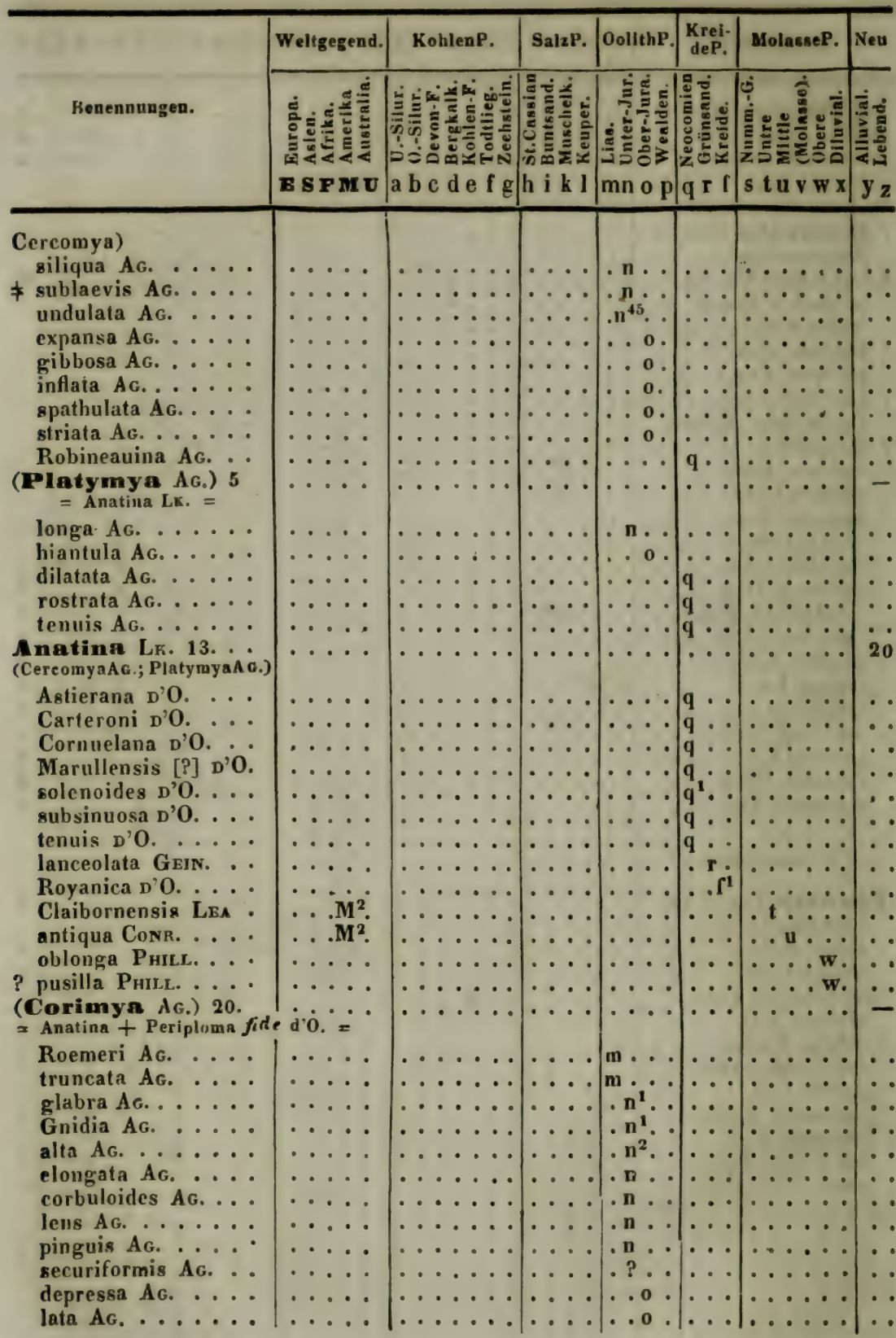




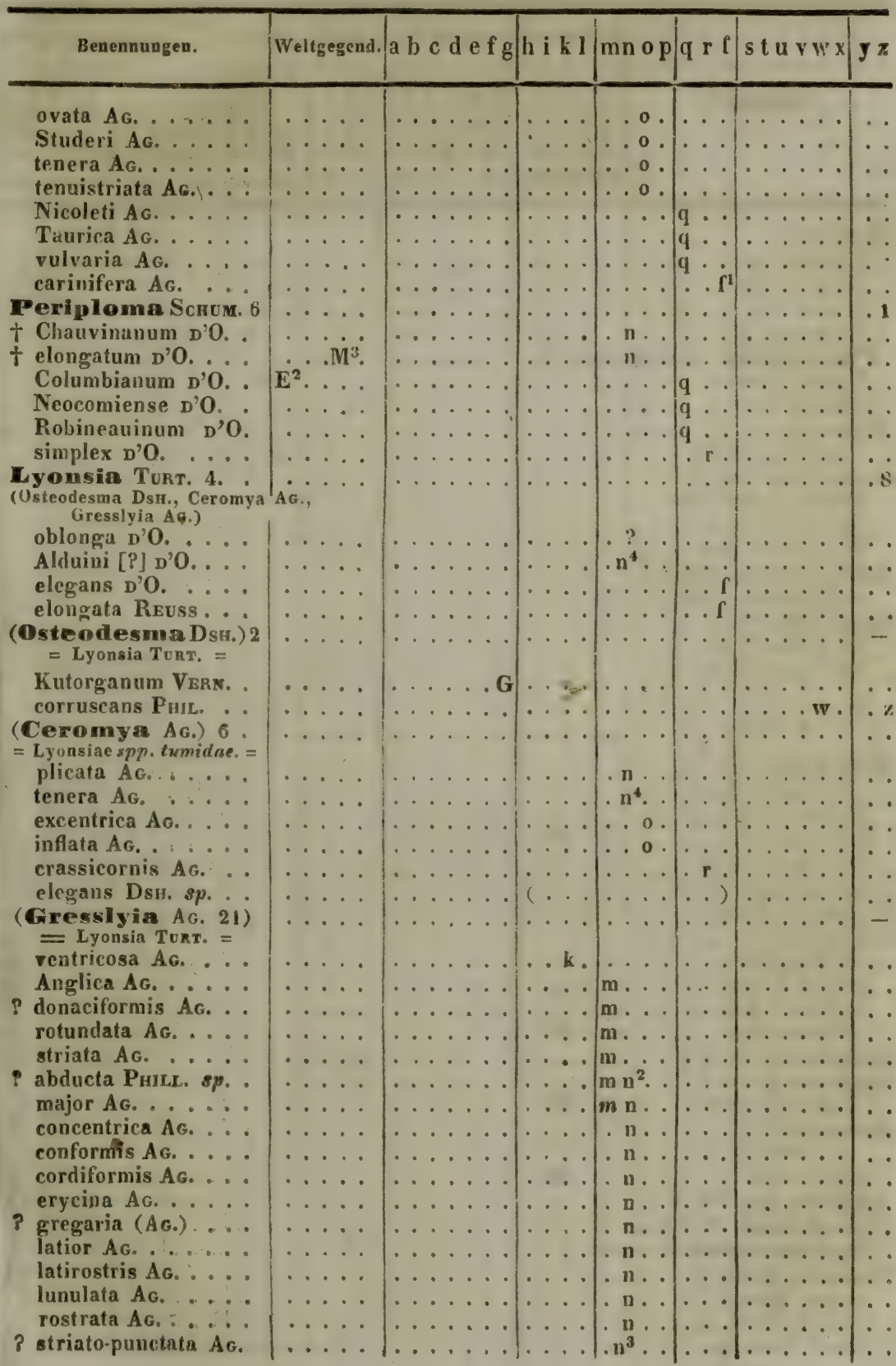




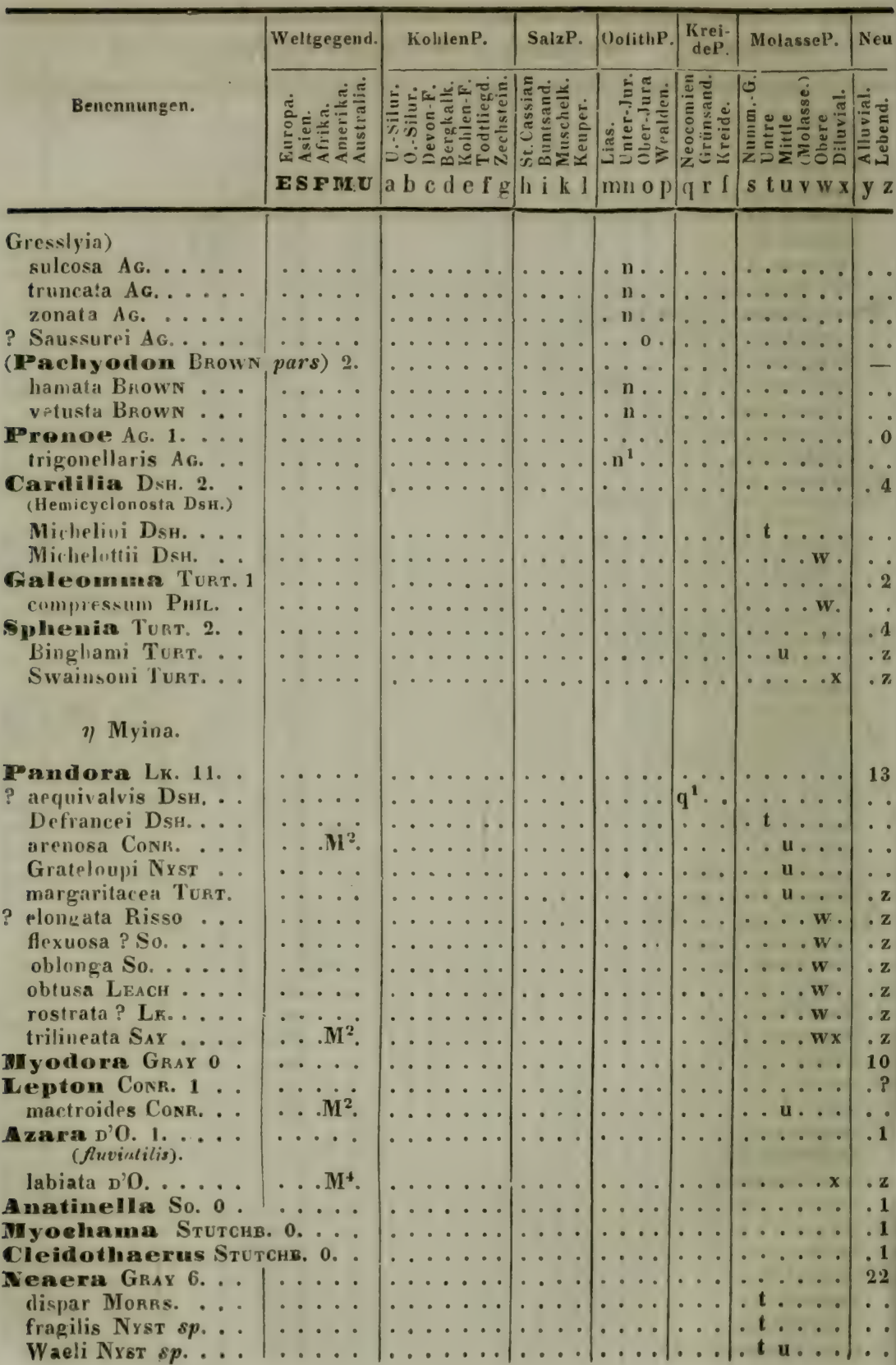




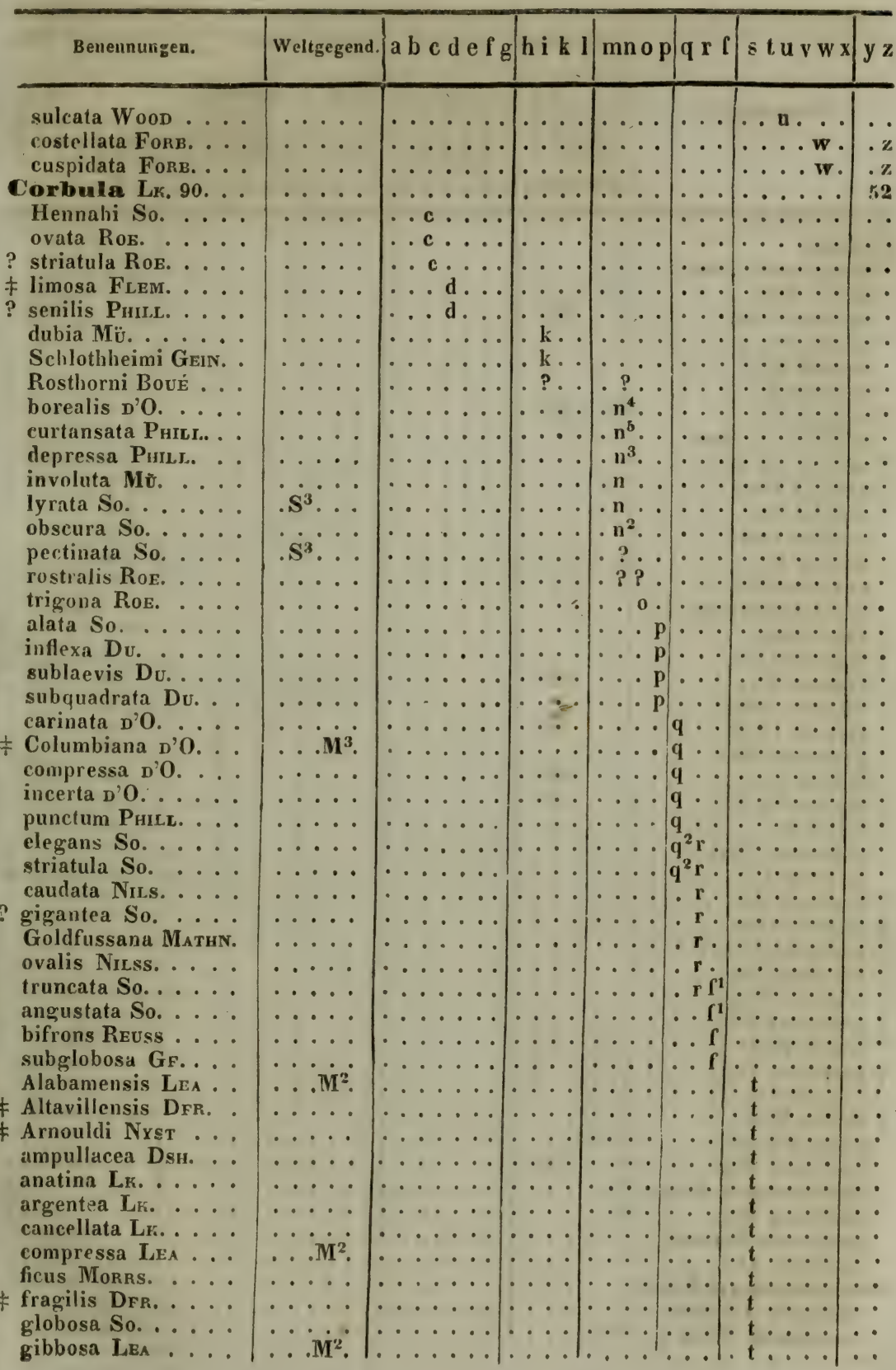




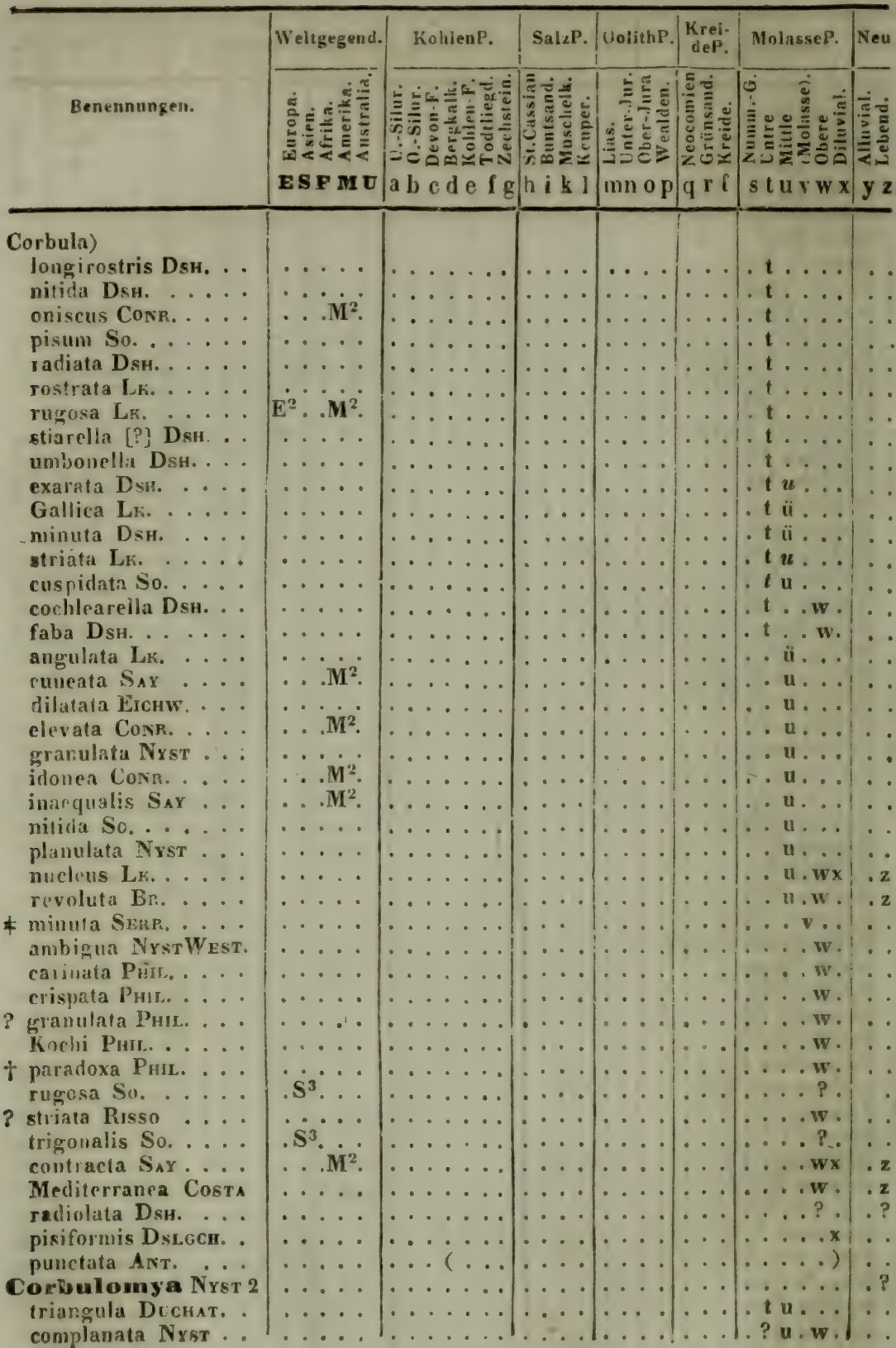




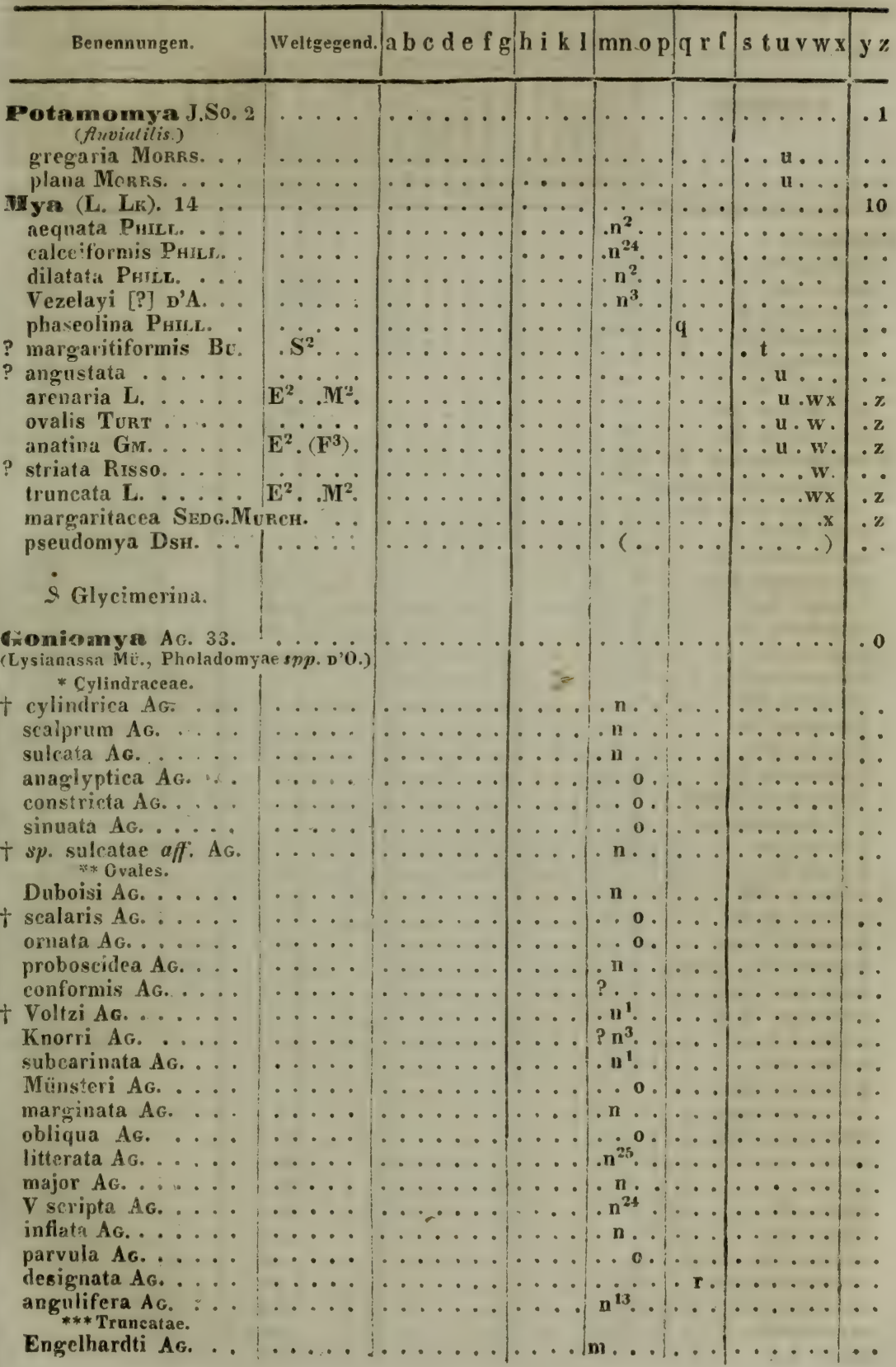




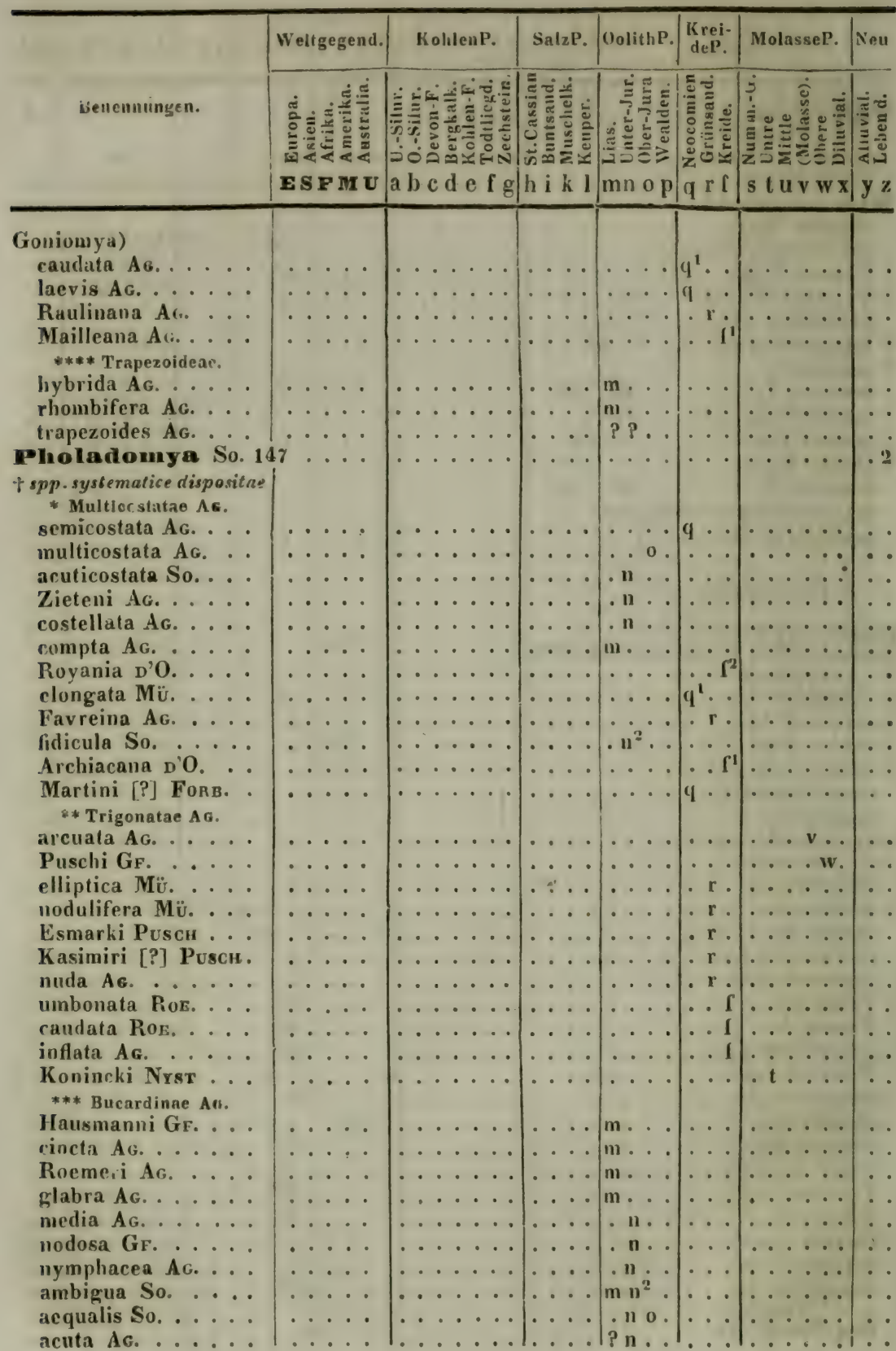




\begin{tabular}{|c|c|c|c|c|c|c|c|}
\hline Benennungen. & end. & $a b c d$ e $f g$ & h i k l & $\mathrm{mnop}$ & $q \times f$ & $w \times$ & $y \%$ \\
\hline exaltat & & & & $\cdot n^{3}$. & & $\cdots$ & \\
\hline decussata Phill. . & 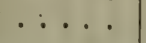 & $\ldots \ldots$ & ... & $\cdots$ & . $\mathrm{f}$ & $\ldots \ldots$ & \\
\hline ‡ alternans Ros.... & $\cdots \cdot$ & $\ldots \ldots$ & $\cdots$ & & $\cdot \cdot \mathrm{I}$ & $\ldots \ldots$ & \\
\hline Murchisoni So.... & $\cdots \cdots$ & $\ldots \ldots$ & $\cdots$ & $\cdot n^{3}$ & $\cdots$ & $\ldots \ldots$ & \\
\hline Heraulti Ag. .... & $\cdots \cdots$ & $\cdots \cdots \cdot$ & $\cdots$ & $\cdot \mathrm{n}^{2} \cdot \cdot$ & $\cdots$ & $\cdots \cdots$ & - \\
\hline bucardium AG. ... & $\cdots \cdot$ & $\cdots \cdots \cdots$ & $\cdots \cdot$ & . n . & $\cdots$ & $\cdots \cdots$ & \\
\hline reticulata $A$ & - & .. & $\cdots \cdot$ & . $\mathbf{n}$ & $\cdots$ & $\cdots \cdots$ & $\cdots$ \\
\hline$\cdots \cdots$ & $\ddot{3}$ & $\cdots \cdots \cdots$ & $\cdots$ & $\mid \begin{array}{c}n \\
m n^{2}\end{array}$ & $\cdots$ & $\cdots \cdots$ & - \\
\hline So. . . . & $\ldots$ & $\ldots \ldots$ & $\cdots$ & $.11 \cdots$ & $\ldots$ & $\cdots \cdots \cdots$ & $\because$ \\
\hline $\begin{array}{l}\text { crassa AG. . . . . } \\
\text { lyrata So. . . . }\end{array}$ & $\ldots$ & ......... & $\ldots$ & $\mathrm{m} \mathrm{n} .$. & $\ldots$ & $\cdots \cdots$ & $\ddot{\dot{v}}$ \\
\hline $\begin{array}{l}\text { lyrata So. . . . . } \\
\text { pruducta So. . . }\end{array}$ & .... & $\ldots \ldots$ & $\ldots$ & m n & $\cdots$ & $\ldots \ldots$ & $\ddot{0}$ \\
\hline ... & .... & $\ldots \ldots$ & $\ldots$ & . $n^{2}$. & $\cdots$ & $\ldots \ldots$ & \\
\hline$\cdots$ & ... & $\cdots \cdots \cdot$ & $\cdots$ & . n . & $\cdots$ & $\ldots \ldots$ & \\
\hline$\cdots$ & $\ldots \ldots$ & $\cdots \cdots$ & $\cdots$ & $\cdot \mathbf{n} \ldots$ & $\cdots$ & $\ldots \ldots$ & \\
\hline$\cdots \cdot$ & $\ldots$ & $\cdots \cdots \cdot$ & $\cdots$ & . n . & $\cdots$ & $\ldots \ldots$ & . \\
\hline M. . & $\ldots$ & $\cdots \cdots \cdot$ & $\cdots$ & . n . & $\ldots$ & $\ldots \ldots$ & . \\
\hline s. ... & $\ldots .$. & $\cdots \cdots$ & $\cdots$ & . . o . & . . & $\ldots \ldots$ & 1 \\
\hline$\cdots \cdot \cdot$ & $\ldots . \cdot$ & $\cdots \cdots \cdot$ & $\cdots$ & ..o. & $\cdots$ & $\ldots \ldots$ & 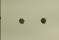 \\
\hline$\ldots$ & $\cdots \cdot \cdot$ & $\cdots \cdots \cdot$ & $\cdots$ & . 0 & $\cdots$ & $\cdots \cdots$ & - \\
\hline$\cdots$ & $\cdots \cdot \cdot$ & $\cdots \cdots \cdots$ & $\cdots \cdots$ & .00 & $\cdots \cdot$ & $\cdots \cdots$ & • \\
\hline OE. . . & 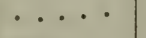 & $\cdots$ & $\cdots \cdot$ & $\cdots 0$ & $\cdots$ & $\cdots$ & \\
\hline$\cdots \cdot \cdot$ & $\cdots$ & $\cdots$ & $\cdots \cdot$ & $\cdots 0$ & $\cdots$ & $\cdots$ & - \\
\hline$\cdots \cdot$ & $\cdots$ & $\cdots$ & $\cdots \cdot \mid$ & $\cdots 0$ & $\cdots$ & $\cdots \cdots$ & - \\
\hline$\cdots$ & $\because$ & $\cdots$ & $\cdots$ & $\mid \begin{aligned} \cdots \\
0 \\
0\end{aligned}$ & $\cdots$ & $\cdots \cdots$ & • \\
\hline truncata AG. . . . & $\cdots$ & $\ldots \ldots$ & $\cdots \cdots$ & $\therefore 0$ & $\therefore$ & $\cdots \cdots$ & $\cdots$ \\
\hline$\cdots \cdot$ & .... & $\ldots \ldots$ & $\ldots$ & ... & .. & $\ldots \ldots$ & . \\
\hline . . & ... & $\ldots \ldots$ & $\ldots$ & $\ldots 0$. & $\cdots$ & $\ldots \ldots$ & - \\
\hline$\cdots$ & . . . & $\ldots \ldots$ & $\ldots$. & . o. & $\cdots$ & $\ldots \ldots$ & - \\
\hline ntrica Ros. . . & ... & $\ldots \ldots$ & $\ldots$. & . n. & $\cdots$ & $\ldots \ldots$ & . \\
\hline$\cdots$ & ... & $\ldots$ & $\cdots \cdot$ &. $\mathrm{n}$. & $\cdots$ & $\ldots \ldots$ & - \\
\hline$\cdots \cdot$ & $\ldots$ & $\ldots$ & $\cdots \cdot$ & . ? . & $\cdot$. & . & - \\
\hline$\cdots$ & $\ldots$ & $\cdots$ & $\cdots$ & . . o. & $\cdots$ & $\ldots \ldots$ & . \\
\hline$\cdots$ & $\cdot$ & $\cdots$ & - & $m \mathrm{n}$. & $\cdots$ & $\cdots$ & - \\
\hline$\cdots$ & $\cdot$ & $\cdots \cdots$ & $\cdots \cdots$ & . n.. & $\cdot$ & $\cdots$ & - \\
\hline o. • & & & $\cdots$ & $\cdots$ & - & $\cdot t^{t}$ & . \\
\hline$a \|$ & & & | & $\ddot{p}$ & $\ddot{p}$ & $\cdot$ & \\
\hline $\begin{array}{l}\text { Escheri AG. } \\
\text { decorata } H\end{array}$ & & & & m. & & $\cdots \cdots$ & \\
\hline I. & $\cdots$ & $\cdots$ & $\cdots \cdot$ & n & $\cdots$ & $\ldots$ & \\
\hline 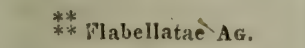 & & & & & & & \\
\hline to & & & & . 0 . & . . & & \\
\hline$\cdots$ & $\ldots$. & . . & & . 0 . & ... & $\ldots$ & \\
\hline 0 & $\cdots$ & $\ldots$ & 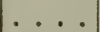 & .. 0 . & $\cdots$ & $\ldots \ldots$ & • \\
\hline A & $\cdots$ & $\cdots$ & , & - $\mathbf{n}$ & $\cdots$ & $\ldots \ldots$ & \\
\hline & & & & & $\cdots$ & & \\
\hline fl & & & & $\mathbf{n}$ & & $\cdots$ & \\
\hline 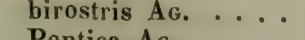 & & & & n & & & \\
\hline$\cdots$ & & & & & & & \\
\hline
\end{tabular}




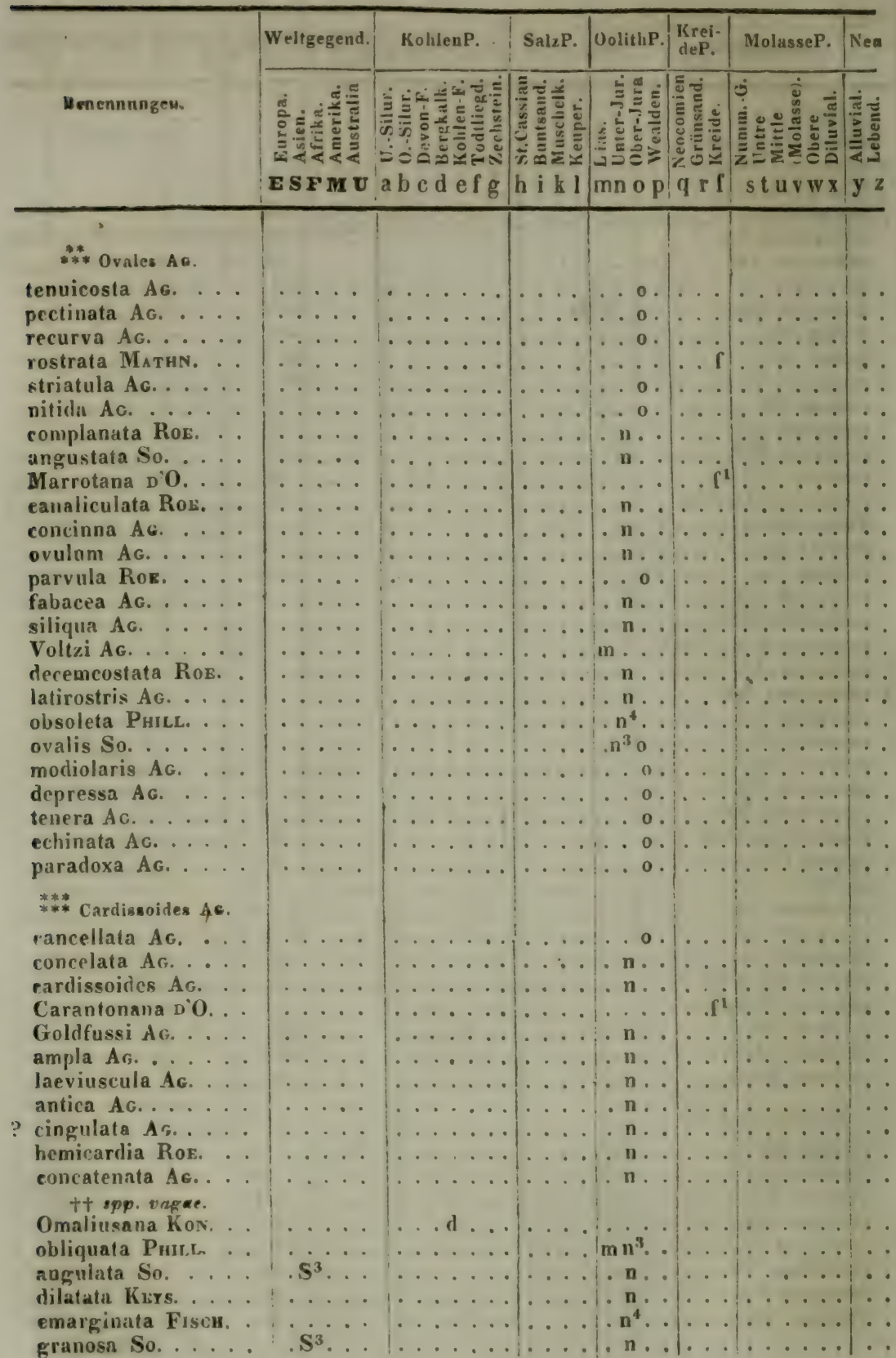




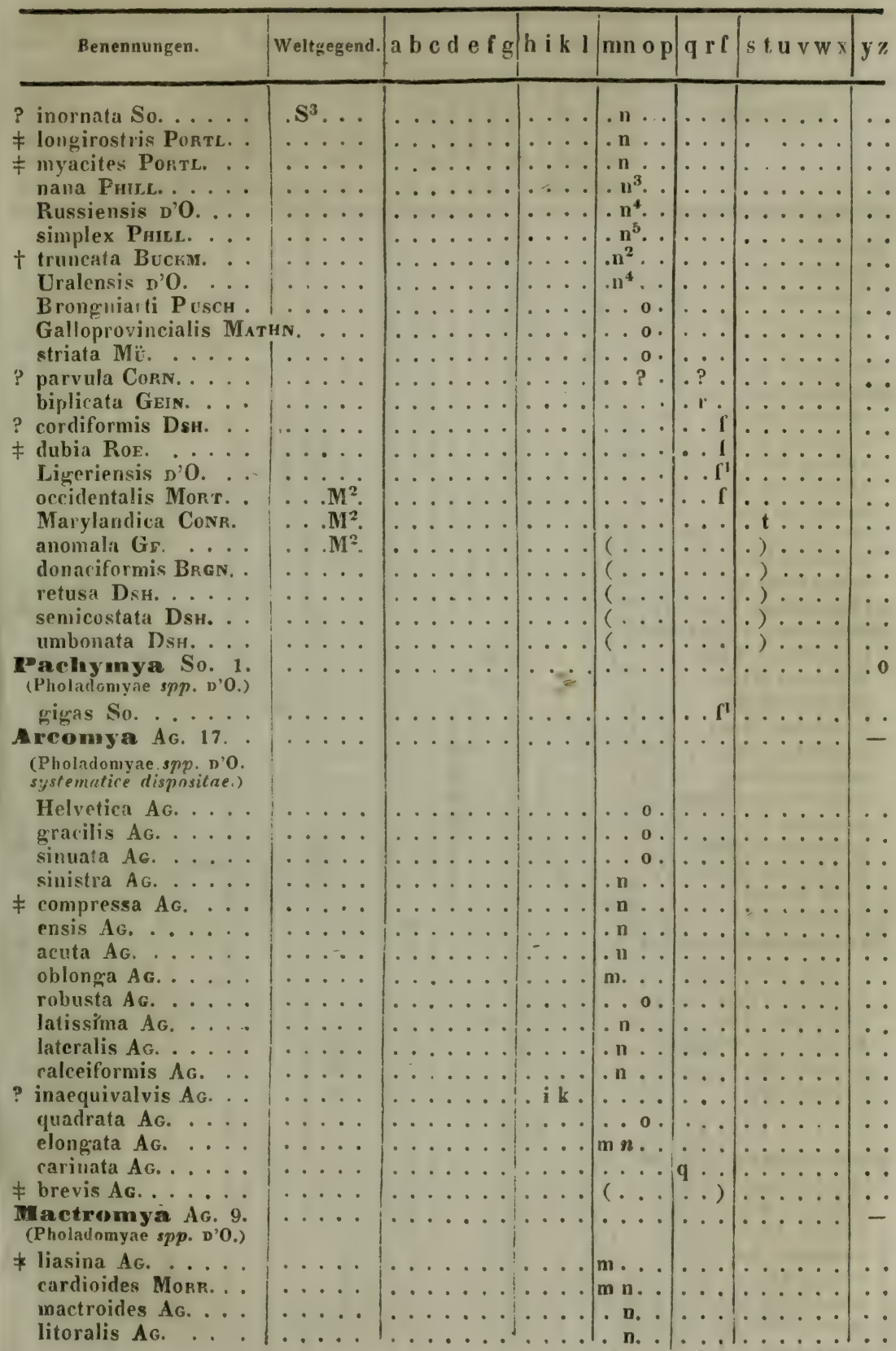




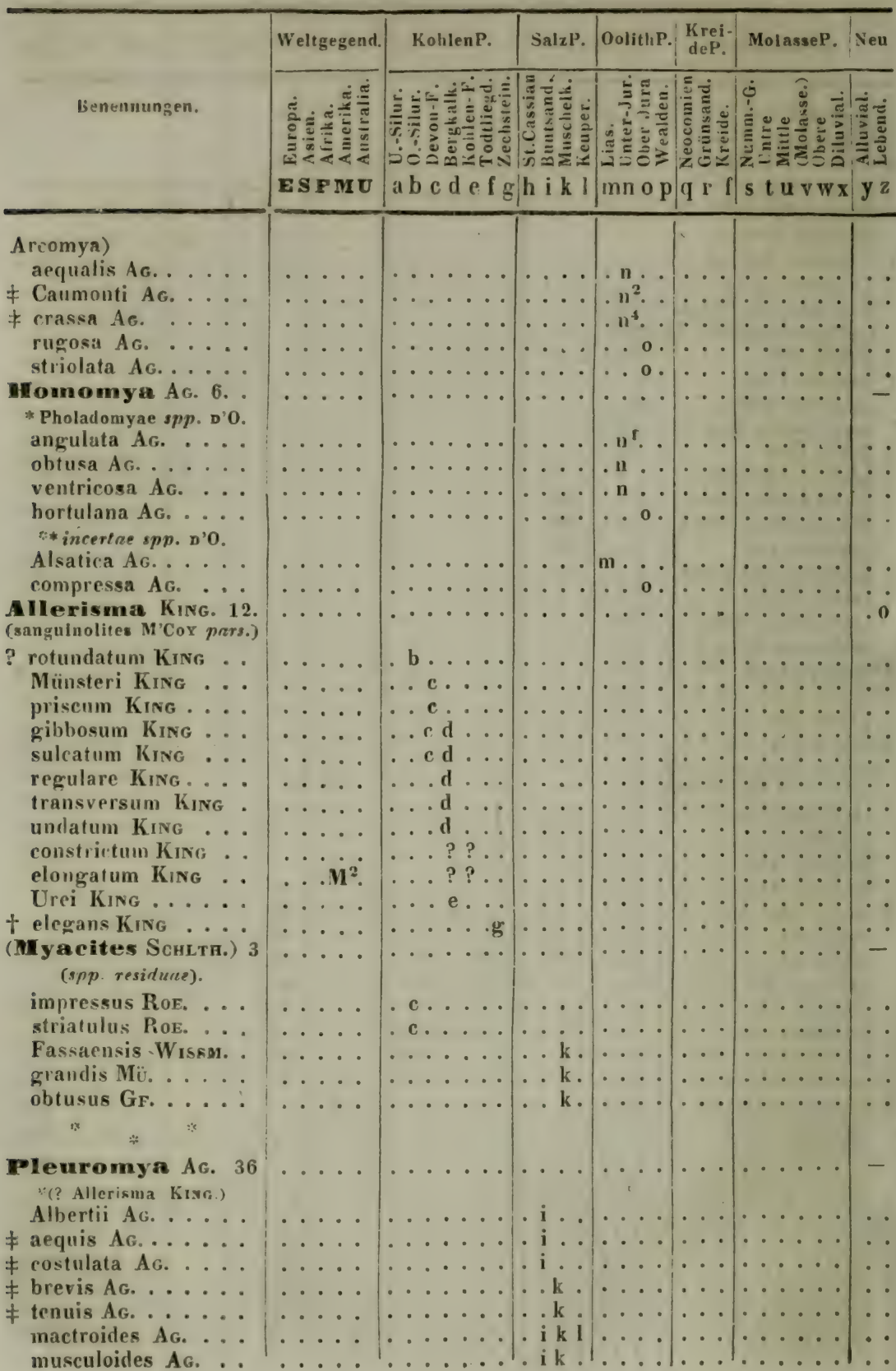




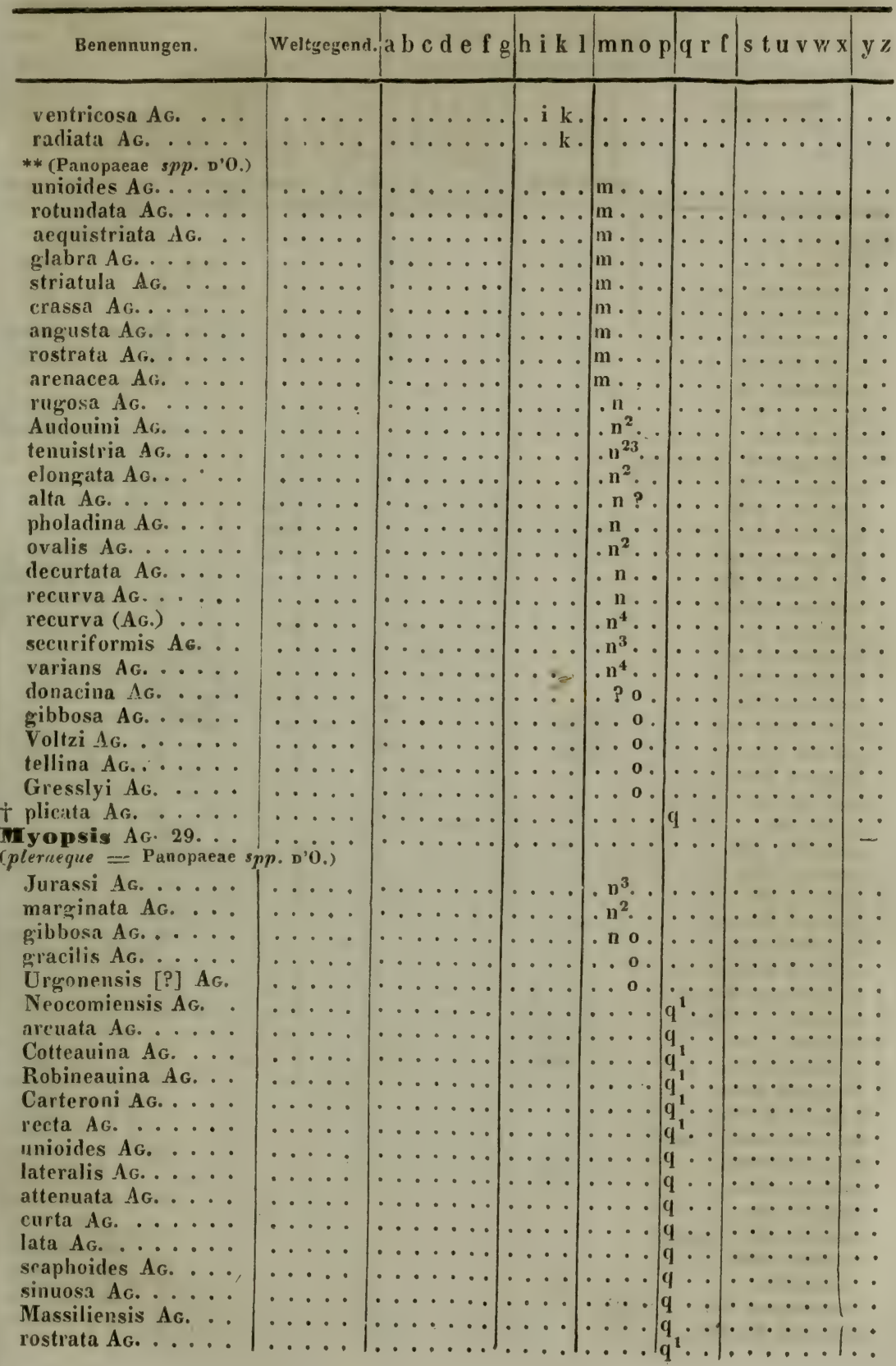




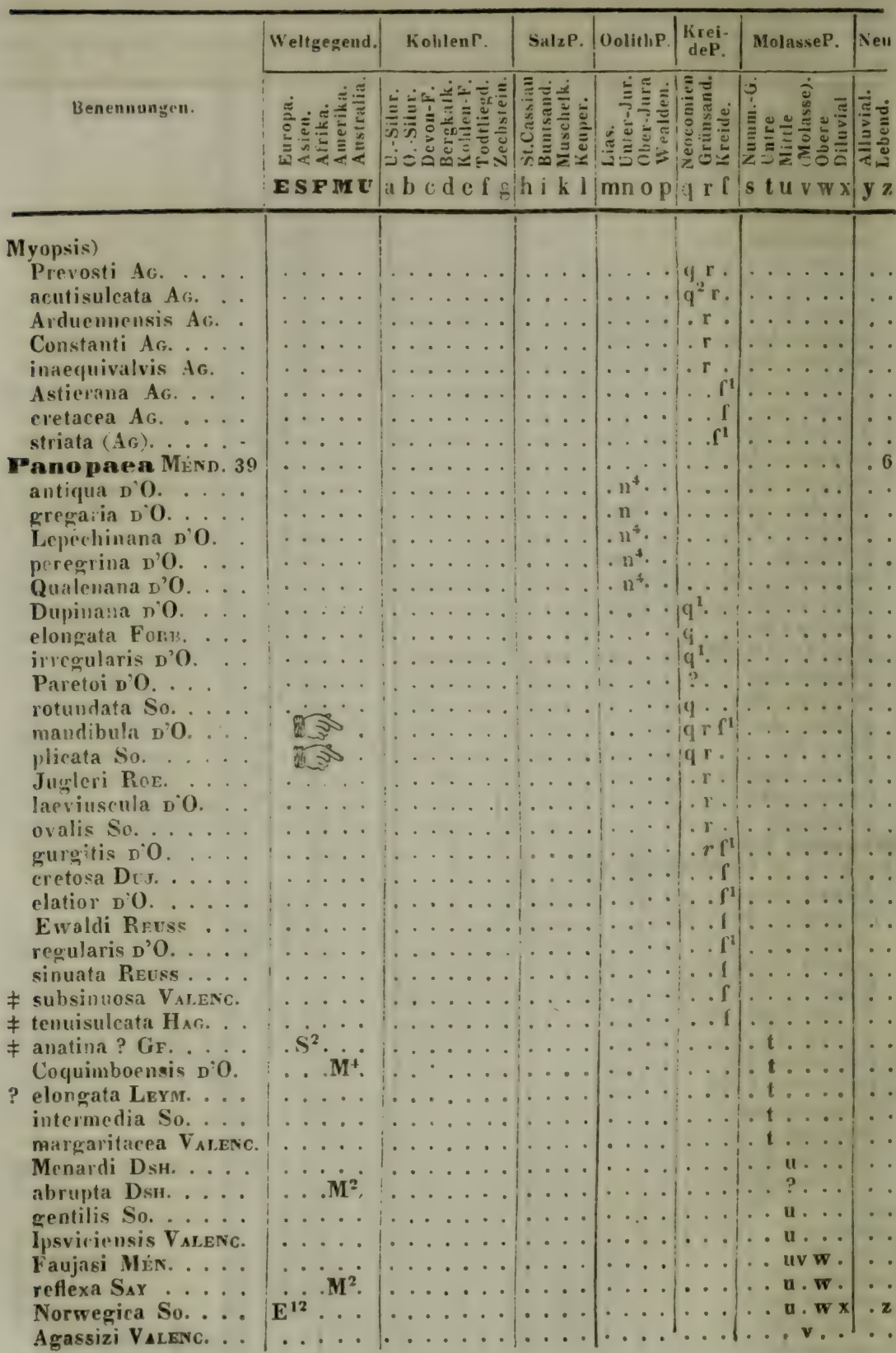


X. PELECYPODA, 11. DIMYA, B. HOMOMYA, 2. EMARGINATO-PALliata. 349?

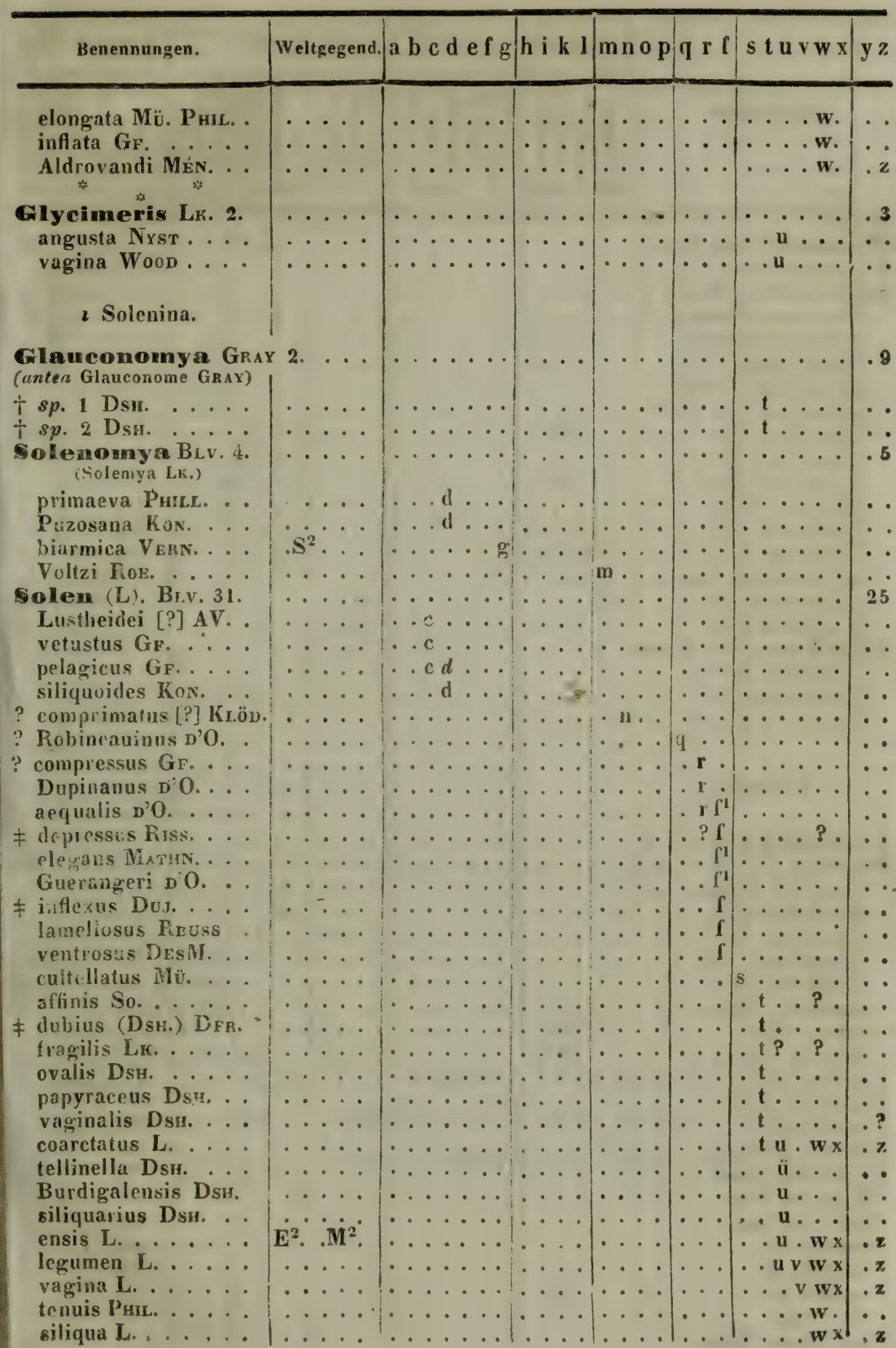




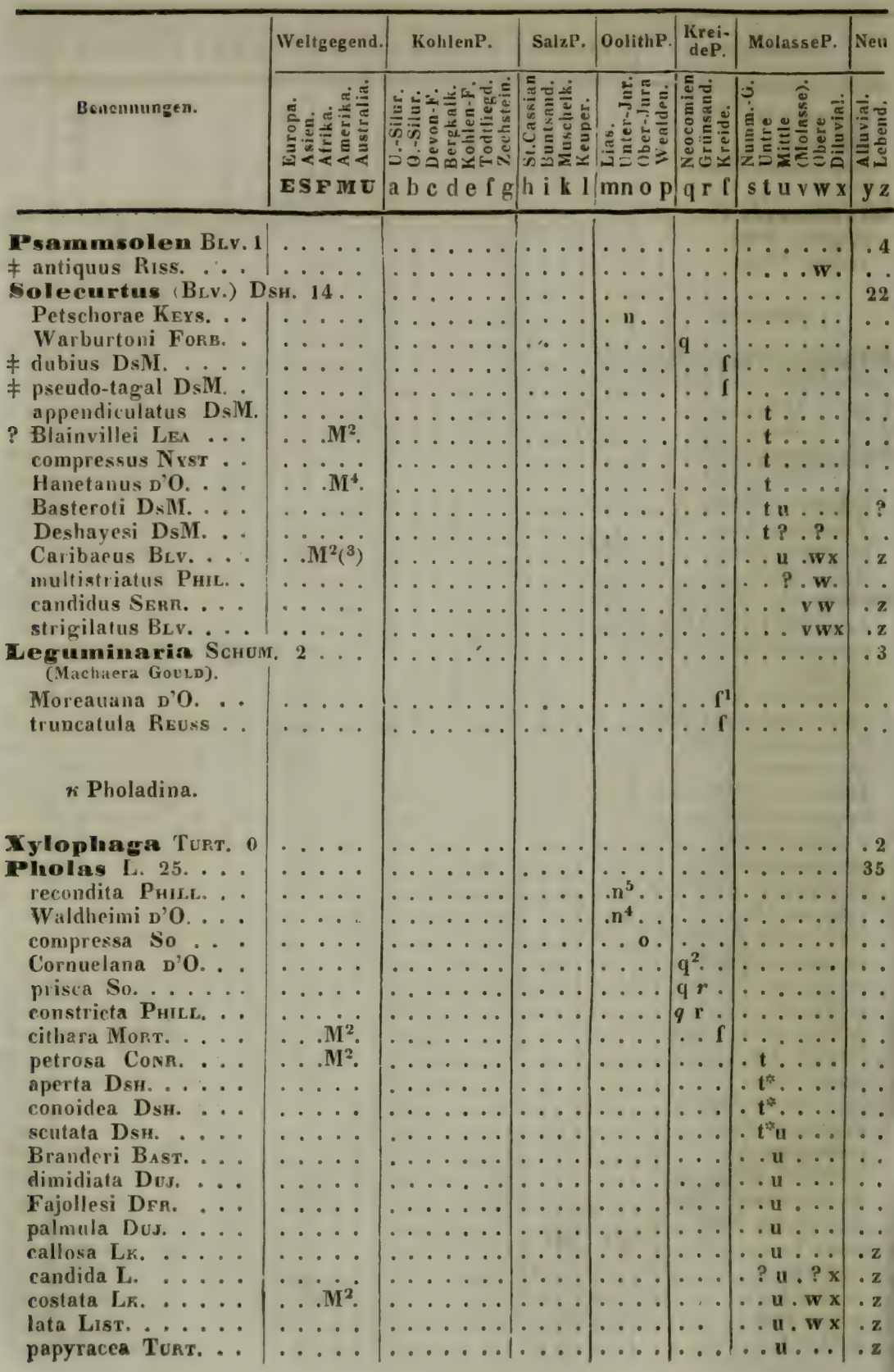




\begin{tabular}{|c|c|c|c|c|c|c|c|}
\hline Benen & Weltgegend. & $\mid a b c d e f g$ & h $\quad \mathrm{i} \quad \mathrm{k} \quad \mathrm{l}$ & $\mid \mathrm{mn} \quad 0 \mathrm{p}$ & f & $\mathrm{wx}$ & $y \%$ \\
\hline pusilla & $\cdots \cdots$ & $\cdots$ & $\cdots$ & $\cdots \cdots$ & $\cdots$ & . . u vw. & . ? \\
\hline$M_{\text {ATHN. . }}$ & $\ldots \ldots$ & $\ldots \ldots$ & $\cdots$ & $\ldots$ & $\ldots$ & ... v. . & - \\
\hline$\cdots$ & $\ldots$ & $\ldots \ldots$ & $\cdots$ & $\cdots$ & $\cdots$ & $\ldots w$ & $\cdots$ \\
\hline Vibonensis PнiL. .. & $\ldots \ldots$ & $\ldots \ldots$ & $\cdots \cdot$ & $\ldots$ & $\ldots$ & $\ldots w$. & . \\
\hline dactylus L. & $\ldots$. & $\ldots \ldots$ & $\cdots$ & $\cdots$ & & $\ldots w$. & $\%$ \\
\hline $\begin{array}{l}\text { Douannetia DsM. } 1 \\
=\text { Pholadis sp. Dsr. }=\end{array}$ & $\cdots \cdot$ & & $\cdots \cdot$ & $\cdots$ & $\cdots$ & $\ldots \ldots$ & .1 \\
\hline senicaudata DsM. . & $\cdots$ & $\cdots$ & .... & $\cdots \cdots$ & $u^{\circ}$ & . u u w. & \\
\hline Teredo L. 19. & $\ldots$. & $\ldots \ldots$ & $\ldots$ & $\ldots$ & $\cdots$ & $\ldots \ldots$ & 10 \\
\hline ? dentata Ros. . . . & $\cdots \cdot \cdot$ & $\ldots \ldots$ & $\ldots$ & $\ldots$ & & $\ldots \ldots$ & $\cdots$ \\
\hline$\cdots \cdots$ & $\cdots \cdot \cdot$ & $\ldots \ldots$ & $\ldots \cdot$ & $\ldots$ & $q \cdots$ & $\ldots \ldots$ & . \\
\hline Buvign. & $\cdots \cdot$ & $\cdots \cdots$ & $\cdots \cdot$ & $\cdots \cdot$ & . $\mathrm{r}$ & $\ldots \ldots$ & $\cdot \cdot$ \\
\hline ? amphisbaeua So... & $\ldots$ & $\ldots \ldots$ & $\ldots$ & $\ldots$ & $\mid \cdot f$ & $\ldots \ldots$ & - \\
\hline ? annularis PARK. & $\cdots \cdot$ & $\cdots \cdots$ & $\cdots \cdot$ & $\cdots \cdot$ & . . I & $\cdots \cdots$ & $\cdots$ \\
\hline Faujasi n. . .... & $\cdots \cdots$ & $\cdots \cdot$ & $\cdots \cdot$ & $\cdots$ & $\left|\cdot f^{2}\right|$ & $\cdots \cdots$ & - \\
\hline $\begin{array}{l}\text { Requienana MathN. } \\
\text { tibialis MoRT. . . }\end{array}$ & $\because \dot{M^{2}}$ & $\cdots$ & $\cdots \cdots$ & $\cdots$ & $\left|\begin{array}{ll}1 \\
\cdots \\
0\end{array}\right|$ & $\therefore$ & $\because$ \\
\hline $\begin{array}{l}\text { MORT. } \ldots \\
\text { SH. . . . . }\end{array}$ & $\begin{array}{ll}\cdots \\
\cdots \cdots\end{array}$ & $\cdots \cdots$ & $\cdots$ & $\cdots \cdots$ & $\left|\begin{array}{ll}\cdots \\
\cdots 1\end{array}\right|$ & $\begin{array}{c}\cdots \\
\cdots\end{array}$ & $\because$ \\
\hline $\begin{array}{l}\text { ₹ sp. Dsh. } \cdots \cdots \\
\text { anlenautae So. } \cdots\end{array}$ & $\ldots \ldots$ & $\ldots$ & $\ldots$ & $\ldots$. & $\cdots$ & t.... & $\cdots$ \\
\hline$\neq$ Burtini Dsн. .... & $\ldots$ & $\cdots$ & $\ldots$ & $\ldots$ & $\ldots$ & t.... & . \\
\hline \# fimbriata DFr. ... & $\cdots \cdots$ & $\ldots \ldots$ & $\ldots$ & $\ldots$ & $\ldots$ & t.... & - \\
\hline . . & $\ldots \mathbf{M}^{2}$ & $\cdots$ & $\cdots$ & $\cdots$ & $\cdots$ &. $\mathrm{t} \ldots$. & -. \\
\hline Tournali Leym. . . . & $\cdots \cdot$ & $\cdots$ & $\ldots$ & $\ldots$ & $\ldots$ & t.... & • \\
\hline$\neq s p$. Dsн. . . . . . & $\cdots \cdot$ & $\cdots$ & $\ldots$. & $\ldots$. & $\ldots$ & . t .... & -・ \\
\hline$\neq s p$. Dsн. . . . . . . & $\cdots \cdot$ & $\cdots$ & $\ldots=$ & $\ldots$ & $\ldots$ &. $\mathrm{t} \ldots$. & • \\
\hline$\ddagger s p$. Dsн. . . . . . . & $\cdots \cdot \cdot$ & $\cdots$ & $\ldots$ & $\cdots$ & $\cdots$ &. $\mathbf{t} \ldots$ & - \\
\hline 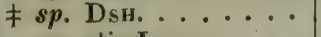 & $\cdots \cdot \cdot$ & $\cdots$ & $\cdots$ & $\ldots$ & $\cdots$ & . t $\ldots$. & $\bullet$ \\
\hline navalis L....... & $\because \cdots \cdot$ & . & $\cdots$ & $\cdots$ & $\cdots$ & . . u .w x & $\cdot \mathrm{z}$ \\
\hline $\begin{array}{l}\text { (Teredolithes LEYM } \\
\text { = Teredinum excavationes }=\end{array}$ & & & & $\cdots$ & $\cdots$ & $\cdots$ & - \\
\hline$\cdots$ & $\cdots \cdots$ & & $\ldots$ & . & & $\cdots$ & \\
\hline na $L_{L K} \quad 3 \ldots$ & & & $\cdots$ & $\cdots$ & & . & $\mathbf{0}$ \\
\hline o clavata RoE. .... & $\cdots \cdot$ & $\cdots$ & $\cdots$ & $\cdots \cdot$ & . . I & . . & - \\
\hline$s p$. Dsн. . . . . . & & - & $\cdot$ & $\cdots$ & . $\cdot \mathbf{I}^{1}$ & & - \\
\hline personata LK. . . & $\cdots \cdots$ & $\cdots$ & & $\cdot$ & .. & $\cdot \ldots \ldots$ & : \\
\hline Septaria RumpF, LK, & $4 \ldots$ & $\cdots$ & & $\mid \cdots \cdots$ & $\cdot$ & $\cdots \cdots$ & .2 \\
\hline $\begin{array}{l}\text { f cylindiacea SERr. } \\
\text { tuberculosas SERr. }\end{array}$ & $\ddot{\cdots}$ & $\therefore$ & $\cdots \cdots$ & $\mid \begin{array}{l}\cdots \\
\cdots \\
\cdots\end{array}$ & $\cdots$ & $\left|\begin{array}{c}\cdots w \\
\cdots \cdots w\end{array}\right|$ & • \\
\hline$\cdots$ &.$S^{3} \ldots$ & & & $\ldots$ & . : & $\ldots \ldots \mathrm{wx}$ &. $\mathbf{z}$ \\
\hline Mediterranea MATHN. I & …. & & & & & 1....w. & $z$ \\
\hline \multicolumn{2}{|c|}{ Emarginato-palliator. summa 1120} & oण हुज़ & & 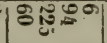 & $\left|\overline{\mathcal{C}^{\prime}} \overline{\bar{\alpha}} \bar{\alpha}\right|$ & $\mid 10$ & ্ֶ: \\
\hline \multicolumn{2}{|l|}{ Integripalliator. summa } & - & $-98 \div 1=$ & $\mid 8 \underbrace{\infty}_{-1}=$ & $\mid$ & - & 0 \\
\hline \multicolumn{2}{|l|}{ Homomyorum summa } & 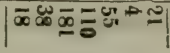 & F & & & & $\bar{\partial}$ \\
\hline
\end{tabular}




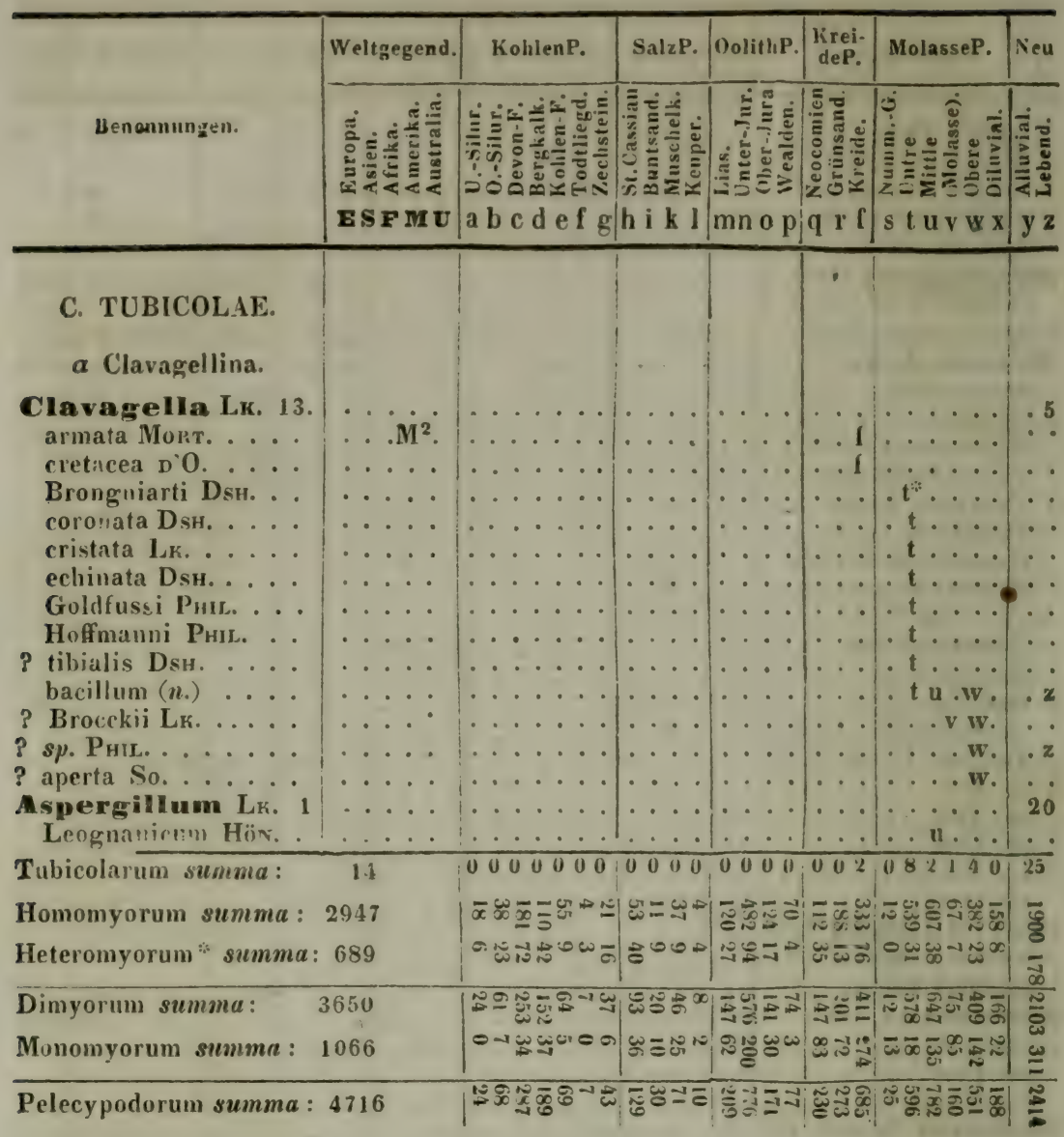

* harc summa p. 276 contrahenda fuit. 


\begin{tabular}{|c|c|c|c|c|c|c|c|}
\hline & Weltgegend. & KohlenP. & SalzP. & OolithP. & \begin{tabular}{|l|}
$\begin{array}{l}\text { Krei- } \\
\text { deP. }\end{array}$ \\
\end{tabular} & MolasseP. & $\mathrm{Neu}$ \\
\hline Benenuunger. & $\begin{array}{c}\mathbf{E} 1,2 \text { Europa. } \\
\mathbf{S} 1,2,3 \text { Asien. } \\
\mathbf{F}^{2}, 3,4 \text { Afrika. } \\
\mathbf{M} \mathbf{1}, 2,3,4 \text { Amerika } \\
\mathbf{U} 3,4 \text { A ustralien. } \\
\mathbf{E} \mathbf{S} \mathbf{F} \mathbf{M} \\
\text { keinZeichen : be- } \\
\text { deutet E2. }\end{array}$ & 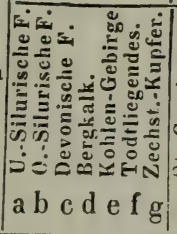 & 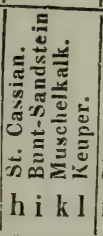 & 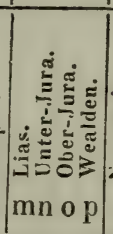 & 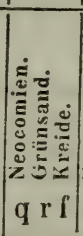 & 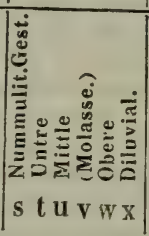 & 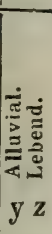 \\
\hline
\end{tabular}

\section{Cl. XI. PTERopOda Cuv. Flossenfüsser.}

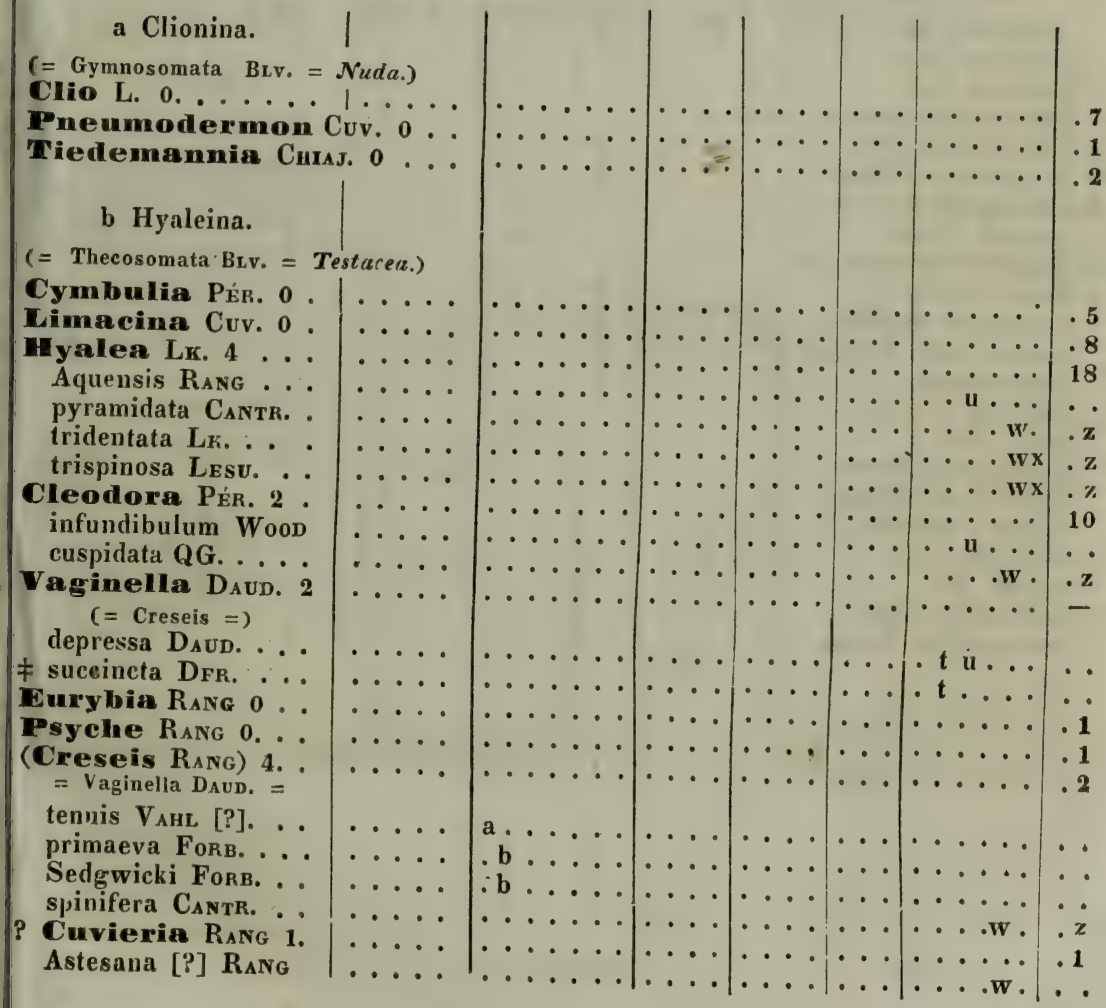




\begin{tabular}{|c|c|c|c|c|c|c|c|}
\hline & Weltgegend. & KohlenP. & SalzP. & OolithP. & $\begin{array}{l}\text { Krei- } \\
\text { deP. }\end{array}$ & MolasseP. & Neu \\
\hline Benennumgen. & 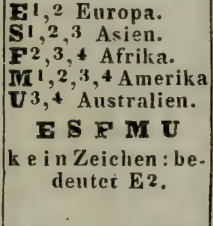 & 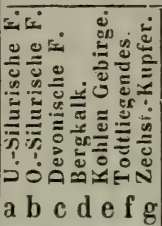 & 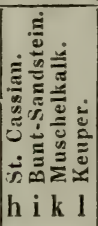 & 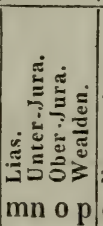 & 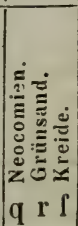 & 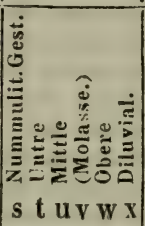 & 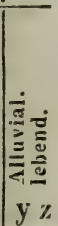 \\
\hline
\end{tabular}

\section{XII. HETEROPODA Cuv. Napffüsser.}

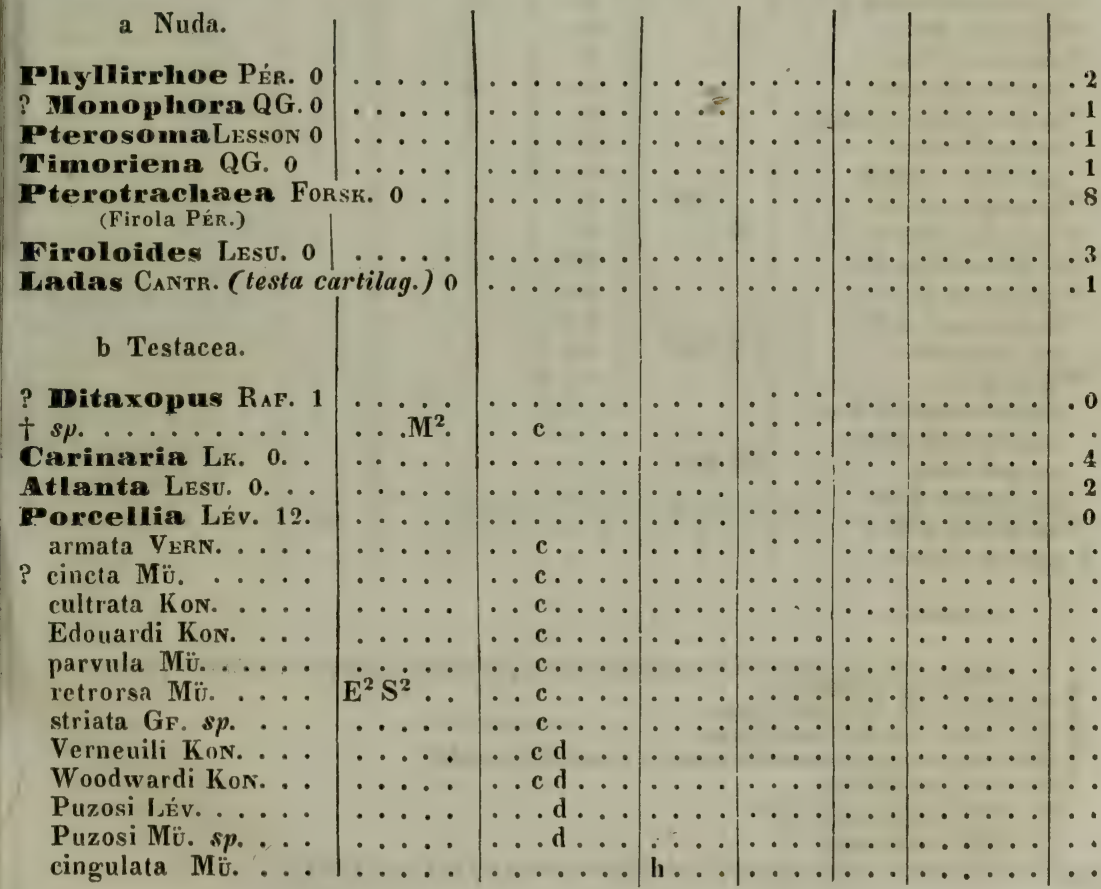




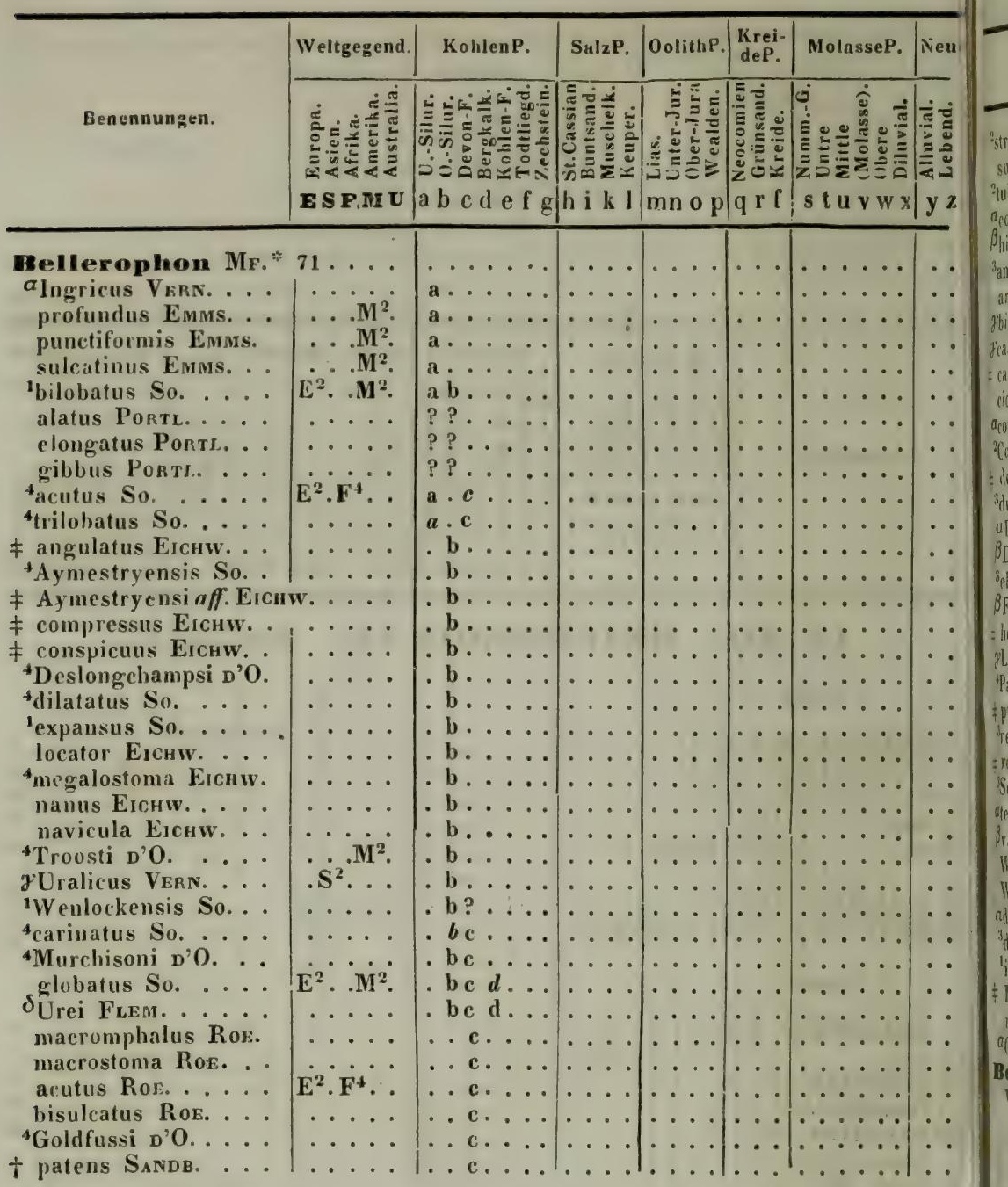

* quoud sectiones generis ub uncloribus variis propositus, litterue nominibus praefixue

a spp. curinutus umbilicutus;

$\beta$ spp. curinutus non umblilicatas,

Y spp. dorso sulcutus umbiliruicus,

S spp. dorso-suldutus exumbilicatus, - et numeri pruefixi

1spp. non umbilicutus,

2spp. umbilici loco impressus,

3spp. anguste umbiliculus,

tspp. late umbilicatus,

significant; omnes autem species in similes sectiones referre non licuit. 


\begin{tabular}{|c|c|c|c|c|c|c|c|}
\hline en. & eltgegend. & $\mathrm{f} g$ & h i $k$ l & $\mathrm{mn} \circ \mathrm{p}$ & $q \mathbf{r}[$ & $\mathbf{w} \mathbf{x}$ & \\
\hline & & & & & & 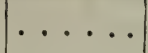 & \\
\hline & & & & & & & \\
\hline$\cdots$ & $\dot{E}^{2} \cdot \dot{M}^{2}$ & ? ? & $\begin{array}{ll}\cdots \\
\cdots\end{array}$ & $\cdots$ & $\cdots$ & $\cdots$ & \\
\hline$\cdots$ & $\ldots$ & e.. & $\ldots$ & $\ldots$ & $\cdots$ & $\ldots \ldots$ & \\
\hline$\cdots$ & $\ldots$ & & $\cdots$ & $\ldots$ & $\cdot$ & $\ldots \ldots$ & \\
\hline & $\cdots \cdot$ & $\cdots$ & $\cdots$ & $\cdots \cdot$ & $\cdots$ & $\cdots$ & \\
\hline$\gamma$ & $\cdots \cdot$ & $\cdots$ & $\cdots$ & $\cdots \cdot$ & $\cdots$ & . & \\
\hline 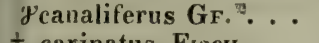 & $\cdot$ & & & . & & - & \\
\hline .. & - & $\cdots d \ldots$ & $\left|\begin{array}{ll}\cdots & \cdots \\
\cdots & \cdots\end{array}\right|$ & & & . & \\
\hline$\cdots$ & $\mathrm{E}^{2} \cdot \mathrm{M}^{2}$ & $\ldots d \ldots$ & $\cdots$ & $\ldots$ & $\cdots$ & $\ldots \ldots$ & \\
\hline$\cdots$ & $\ldots$ & $\cdots$ & $\cdots$ & $\cdots$ & $\cdots$ & $\ldots \ldots$ & \\
\hline r. . & . . & & $\cdots$ & $\cdots \cdot$ & $\cdots$ & $\cdots \cdots$ & \\
\hline $\begin{array}{l}3 \\
{ }^{3} d\end{array}$ & $\because$ & $\cdots$ & $\cdots$ & $\cdots$ & . & $\cdots$ & \\
\hline . & $\cdots$ & $\cdots$ & $\cdots$ & $\cdots$ & • • & $\cdots$ & \\
\hline $\begin{array}{l}\beta \\
3 \mathrm{e}\end{array}$ & .... & $\ldots$ & $\ldots$ & $\ldots$ & $\ldots$ & 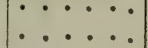 & \\
\hline${ }^{3}$ & & . & . & $\ldots$ & $\cdots$ & $\ldots \ldots$ & \\
\hline . & . & . . & 1 & . & • & $\ldots$ & \\
\hline N. . & $\cdot$ & & $\ldots$ & $\cdots$ & $\cdots$ & $\ldots \ldots$ & \\
\hline & . & & $\cdots$ & $\bullet$ & $\cdot$ & $\cdots \cdots$ & \\
\hline$\cdot$ & $\cdots \cdot$ & $\cdots$ & - & $\cdot \cdot$ & $\cdot$ & $\cdots \cdots$ & \\
\hline $\begin{array}{r}{ }^{3} \mathrm{re} \\
\neq \mathrm{rr}\end{array}$ & $\because$ & & & $\cdots$ & - & • & \\
\hline$\neq_{3 S}^{r}$ & $\dot{0}$ & $\because d \quad \cdots$ & $\cdots$ & i. & - & $\cdots \cdots$ & \\
\hline ate & . . & $\ldots d \ldots$ & $\ldots$ & $\cdots$ & $\ldots$ & $\cdots$ & \\
\hline$\beta_{\mathrm{v}}$ & . & $\ldots d \ldots$ & $\cdots \cdots$ & $\cdots$ & $\cdots$ & . & \\
\hline Kon.) & . & d... & $\cdots \cdot$ & $\cdots$ & $\ldots$ & $\cdots \cdots$ & \\
\hline & & & . & $\cdots$ & . & $\ldots \ldots$ & \\
\hline$x_{\mathrm{dd}}$ & & e.. & . & $\cdots$ & $\cdots$ & $\cdots \cdots$ & \\
\hline${ }^{3} d^{\prime}$ & & . & $\cdots$ & $\cdots$ & $1^{\circ}$ & $\cdots \cdots$ & \\
\hline int & & $\cdot$ & $\cdots$ & - & 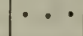 & $\cdots \cdots$ & \\
\hline$\cdot \cdot$ & & • & 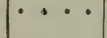 & $\cdot$ & & - & \\
\hline$a_{0}^{n}$ & . & & & - & & & \\
\hline '0.1 & 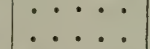 & & & & & $\cdots$ & \\
\hline Vibr & & & & & $\mathbf{r}$ & & \\
\hline & & $=0$ & & & & & $\tilde{\omega}$ \\
\hline
\end{tabular}




\begin{tabular}{|c|c|c|c|c|c|c|c|}
\hline & Weltgegend. & KohlenP. & SalzP. & OolithP. & $\begin{array}{l}\text { Krei- } \\
\text { deP. }\end{array}$ & MolasseP. & Neu \\
\hline 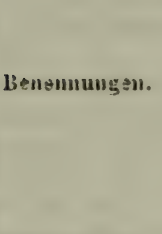 & $\begin{array}{l}\mathbf{E}^{1}, 2 \text { Europa. } \\
\mathbf{S}^{1}, 2^{3} \text { Asien. } \\
\mathbf{F}^{2}, 4 \text { Afrika. } \\
\mathbf{M}^{1}, 3,4 \text { Amerika. } \\
\mathbf{U}^{3}, 4 \text { Australien. } \\
\mathbf{E} \mathbf{S} \mathbf{F} \mathbf{M} \mathbf{U} \\
\text { ke in Zeichen : be- } \\
\text { deutet } \mathbf{E}^{2} \text {. }\end{array}$ & 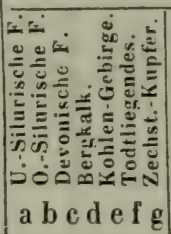 & 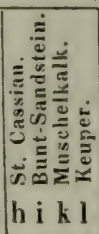 & 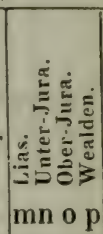 & 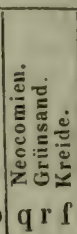 & 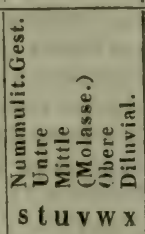 & 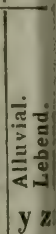 \\
\hline
\end{tabular}

\section{Cl. XIII. PROTOPODA: Vorfüsser.}

\section{CIRTODHEANCHIA WIEgM.}

\section{Dentalium L. 80.}

(† Pyrgopolon Mr.)

- Mosae.

( + Dentalium).

*spp. ungulutue, costatie et striutue.

Saturni HöN.

ornatum KoN.

eanaliculatum KLI. .

decoratum Mü.

tricostatum Gr.

medium So.

decussatum So.

? septangulare FLEm.

0 deforme Lк.

laticostatum Reuss.

polygonum Reuss. quadrangulare Dsr. sexcarinatum Gr.

striatum So.

abbreviatum DsH.

Kickxi Nyst....

0 radicula $\mathbf{L K}_{\mathrm{K}}$.

thalloides Conr. ... pseudo-entalis LK. sulcatum LK. ... semiclausum Nxst.

₹ striatum Erciw. . thallus Conr. ...

Bouei Dsh., ..... fossile LGM. sıxangulum LGm.
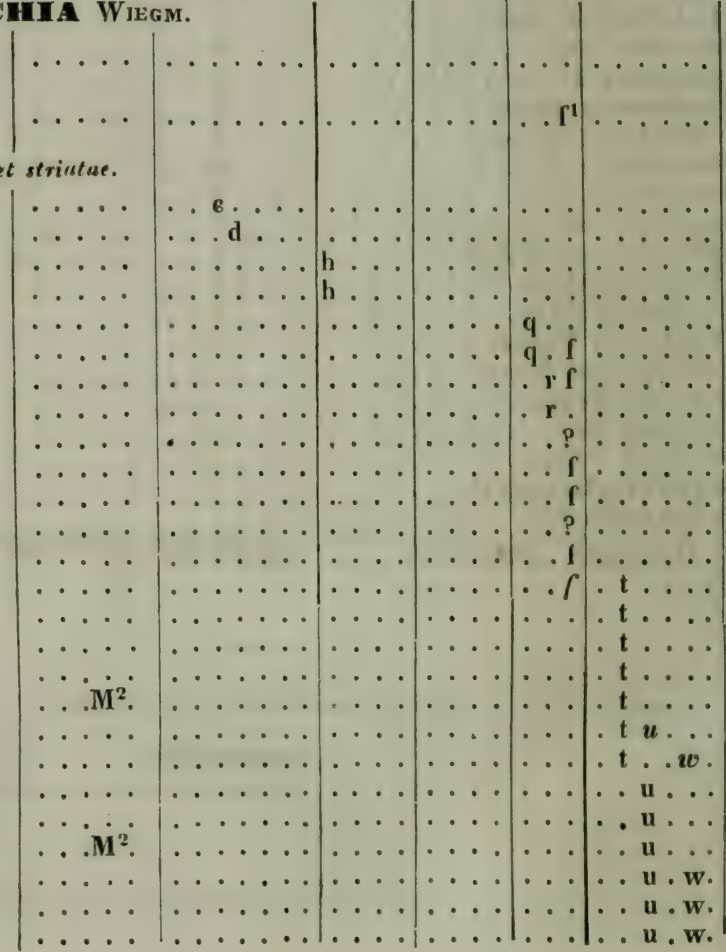


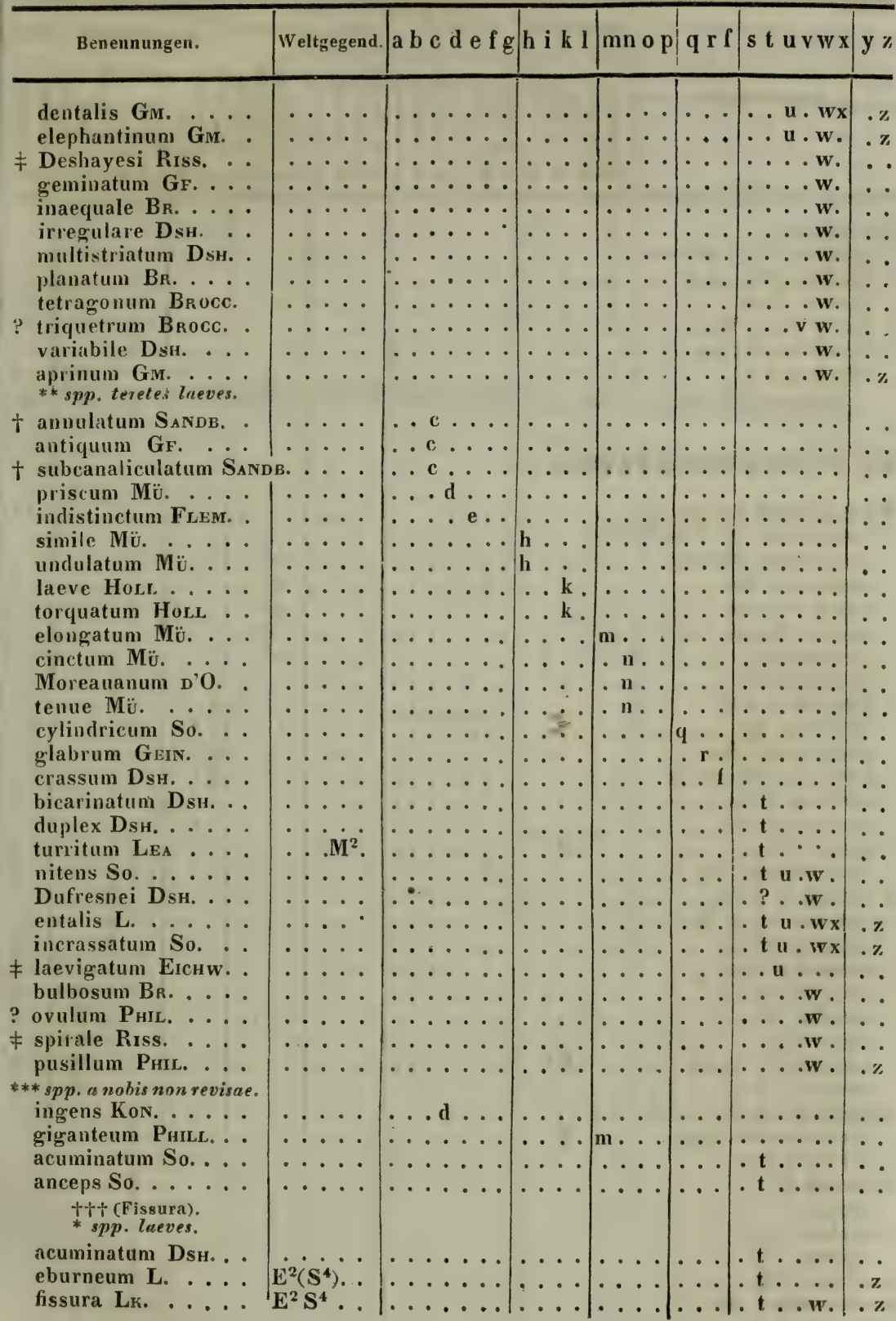




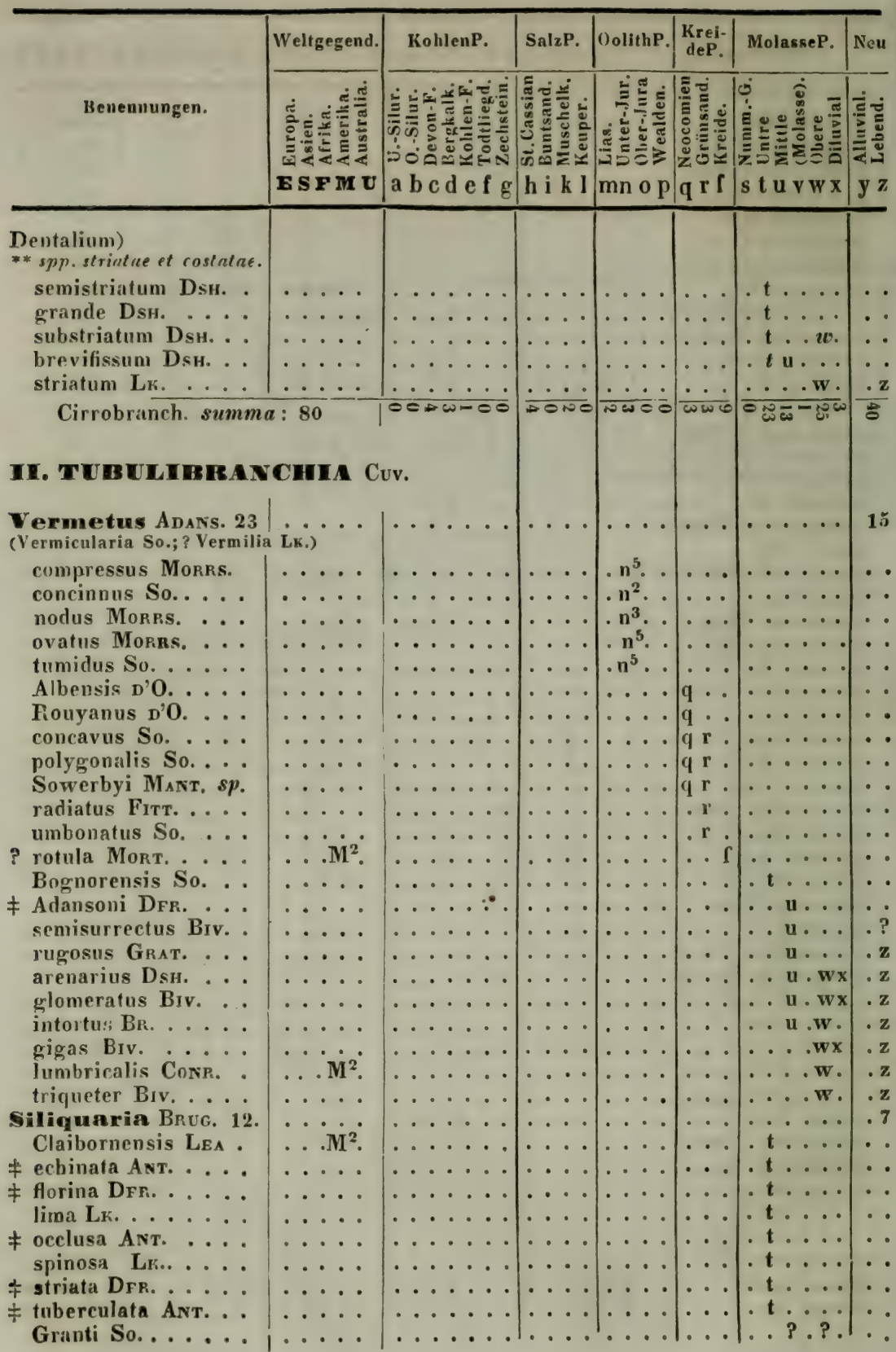

brevifissum Dsн.

iatum $L_{K}$

ermicularia So.; ? Vermilia Lk.)

compressus MorRs.

concinnus So.

tumidus So.

Fouyanus Do.

concavus So.

polygonalis So...

Sowerbyi Mavr, $s p$.

radiatus FitT.

umbonatus So,

semisurrectus Brv.

rugosus Grat. .

arenarius Dsh.

glomeratus Biv.

intortus; $\mathrm{BR}$

gigas Biv.

lumbricalis CoNP.

iliquaria Brug. 12 Claibornensis LEA

* echinata ANT.

florina DFF.

occlusa Avт.

spinosa Lr.

* tuberculata ANT.

Granti So. 


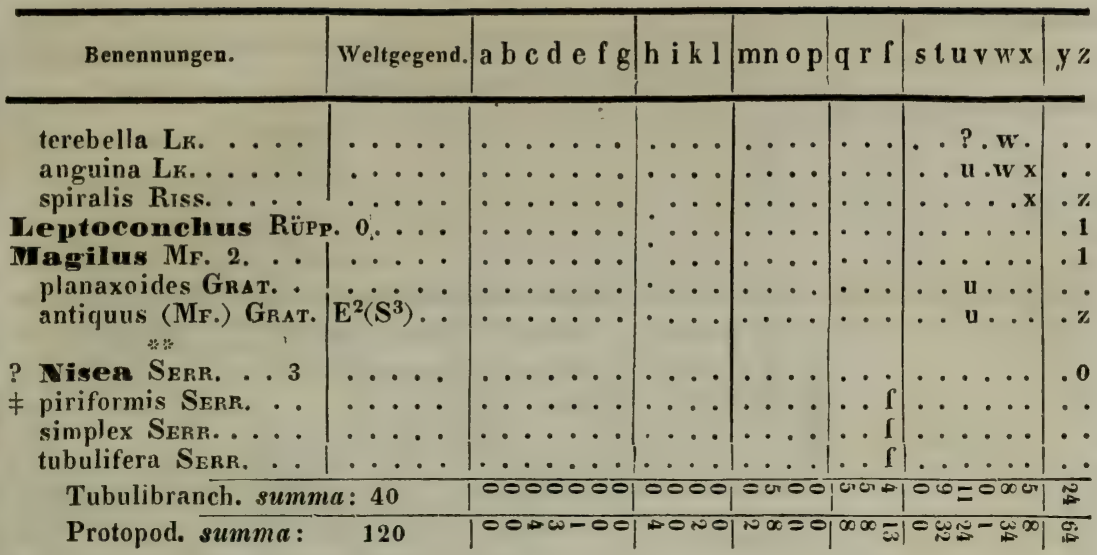




\begin{tabular}{|c|c|c|c|c|c|c|c|}
\hline \multirow{3}{*}{ Benennungen. } & \multirow{3}{*}{ 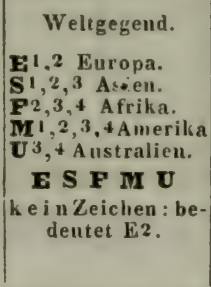 } & KohlenP. & Salz?. & OolithP. & $\begin{array}{l}\text { Krei- } \\
\text { deP. }\end{array}$ & MolasseP. & $\mathbf{N} \in \mathbf{u}$ \\
\hline & & 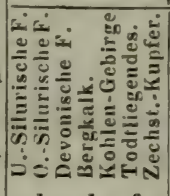 & 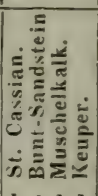 & 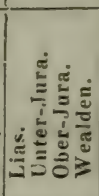 & 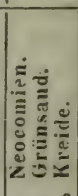 & 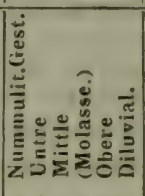 & 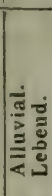 \\
\hline & & $\mid a b c d e f g$ & h i k l & $\mathrm{mn} 0 \mathrm{p}$ & q r I & s t u v w x & y z \\
\hline
\end{tabular}

\section{Cl. XIV. GASTERoPODA Cuv. Bauchfüsser.}

(exl. Heteropodis et Protopodis.)

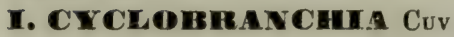

Chitonellus Lr. 0 .

Cliton L. 30. cordiformis $\mathrm{S}_{\text {ANDB. }}$.

+ fasciatus SANDB. . subgranosus SANDB. priscus Mü. .....

$\beta$ Sindbergeranus Rхскн. concentricus Kov.

? cordifer Kon.

Eburonicus Ryckн. gemmatus Kon. . . . Legiacus Rx.

Mempiscus [?] Ry. . Mosensis Ry.

Nervicanus [?] Rx. . Scaldianus [?] Rro. Sluceanus [?] Rx. . Tornacicola [i? RY. Turnacinus [?] Rx. Viseticola [?] Ry. . . sp. King.

\section{? Cottai Gein.} antiquus Conr.... Grignonensis Dsu. . $\leftarrow$ angulosus Wood . . $\dagger$ arcuarius Wood.. + strigilatus Wood

† tenuisculptus Wood fascicularis $\mathbf{L}$.

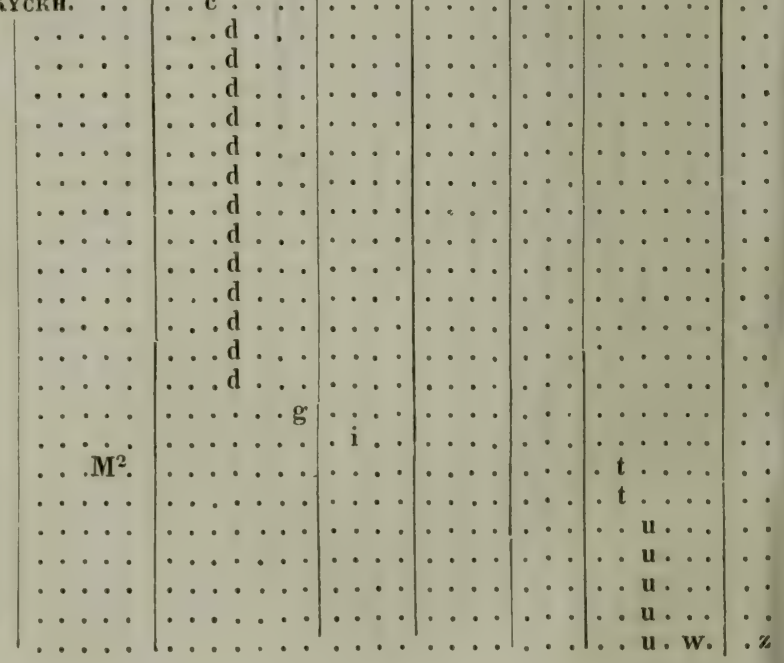




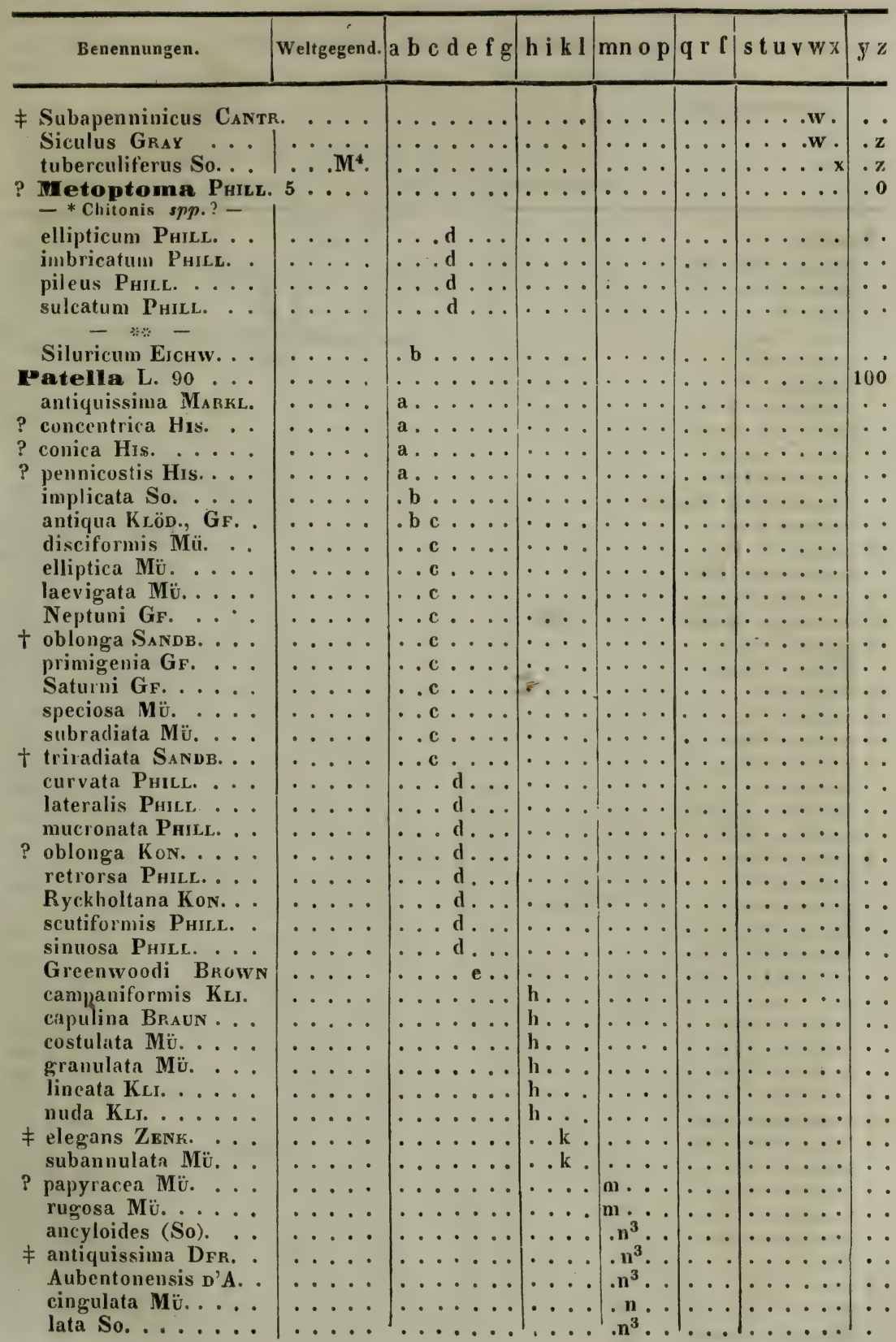




\begin{tabular}{|c|c|c|c|c|c|c|c|}
\hline Benennungen & 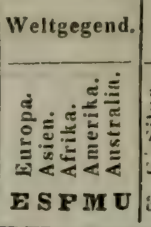 & 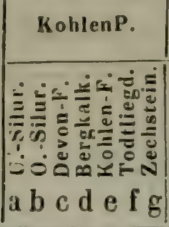 & 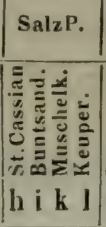 & 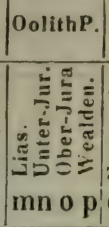 & 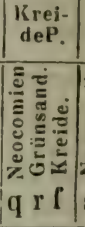 & 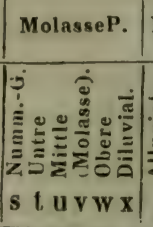 & 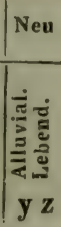 \\
\hline 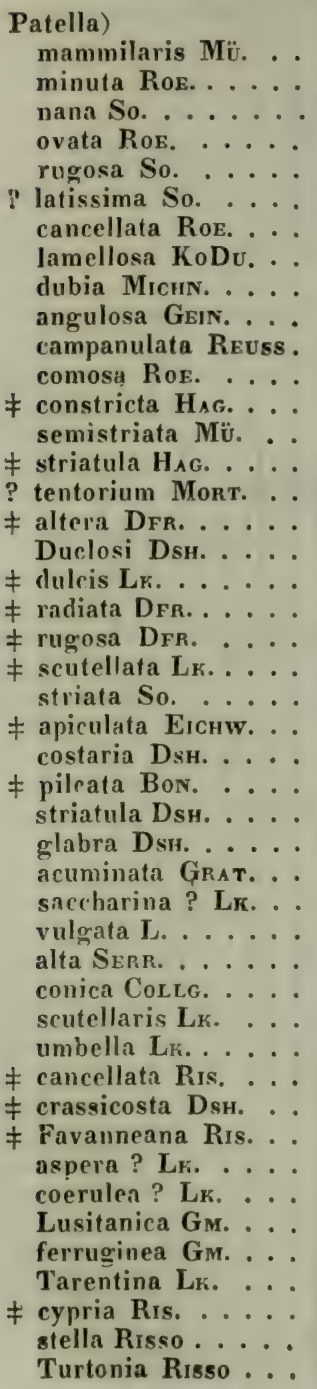 & 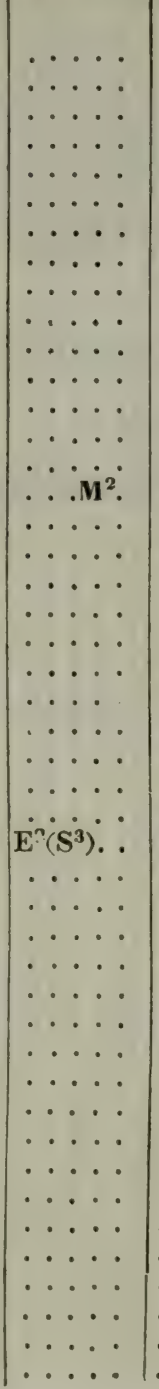 & 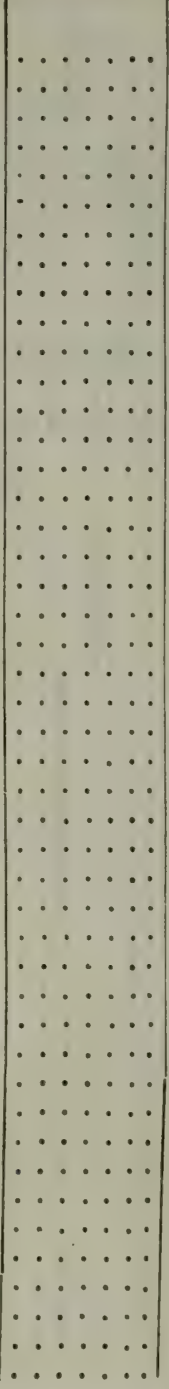 & 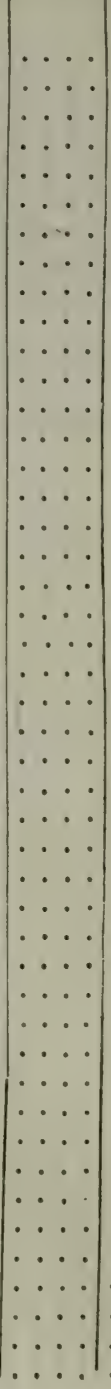 & 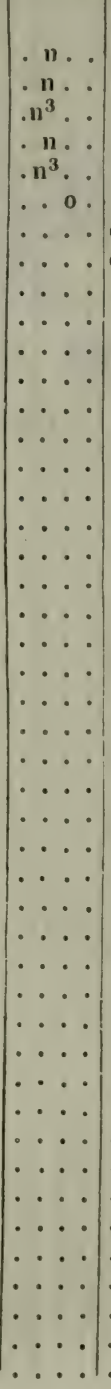 & 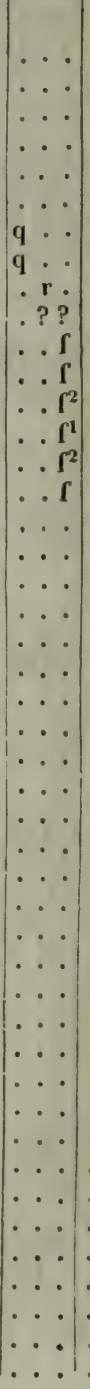 & 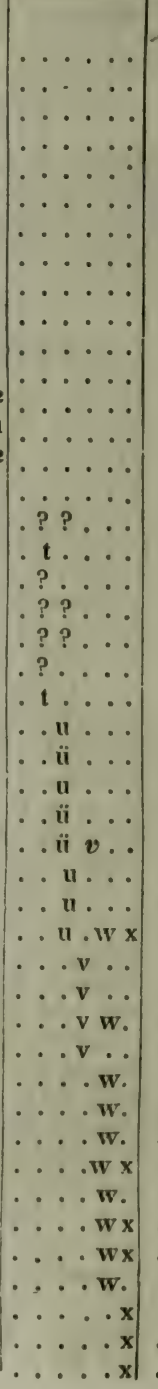 & 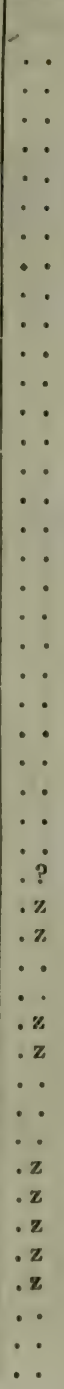 \\
\hline
\end{tabular}




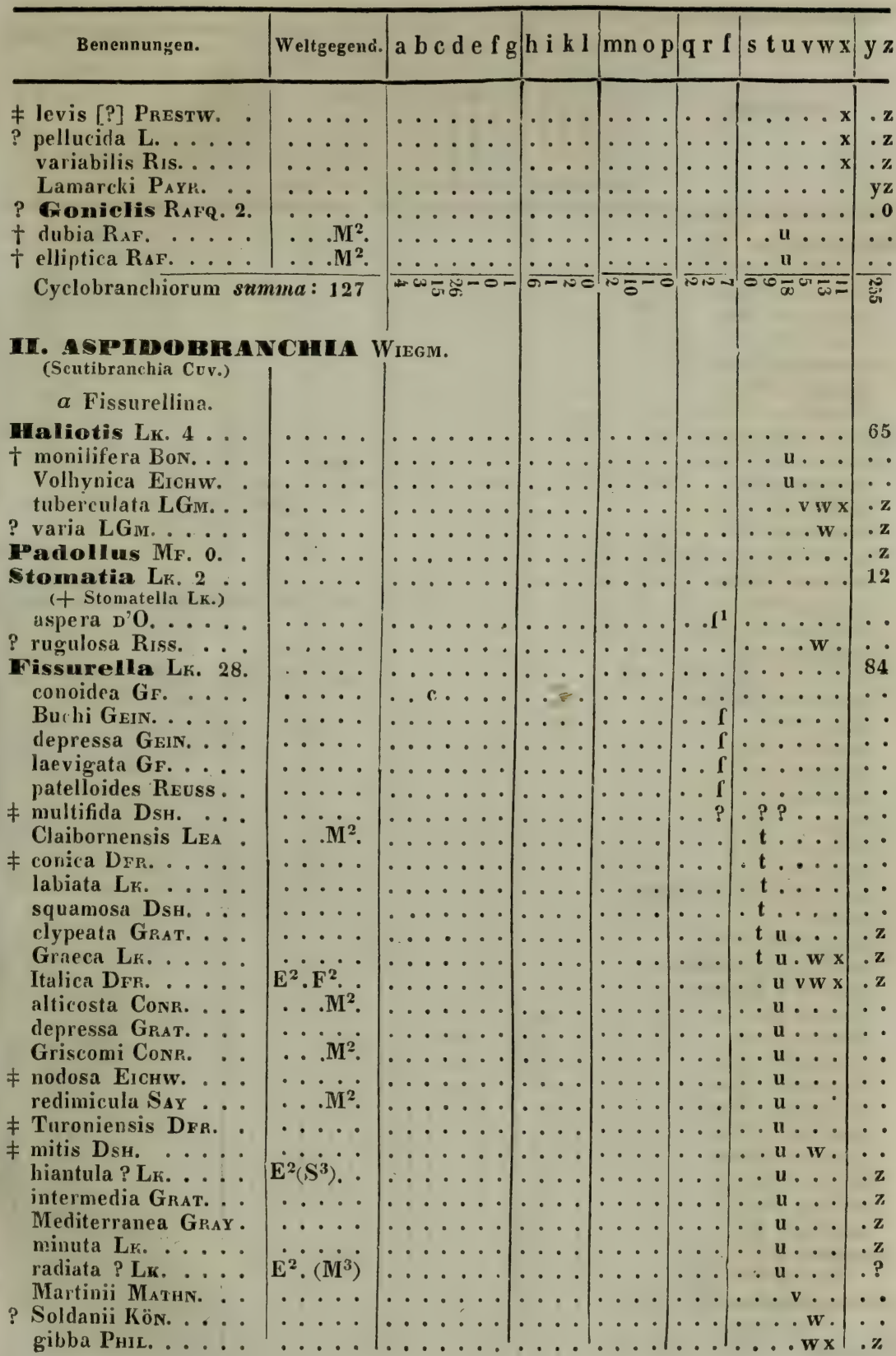




\begin{tabular}{|c|c|c|c|c|c|c|c|}
\hline \multirow{3}{*}{ Benennungen. } & Weltgegend. & KohlenP. & SalzP. & OolithP. & $\begin{array}{c}\text { Krei- } \\
\text { deP. }\end{array}$ & MolazseP. & Neu \\
\hline & 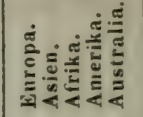 & 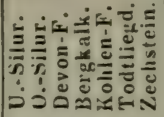 & 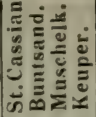 & 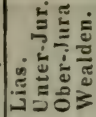 & 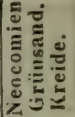 & 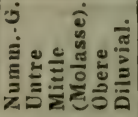 & 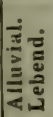 \\
\hline & ES F MU & a b c d e f $g$ & ll i k l l & $\mathrm{mn}$ op & $q \times \mathbf{r}$ & s t u v w $x$ & $y z$ \\
\hline
\end{tabular}

Timularia DFr, 3 (Fissurellite pulli?) clathrata Morrs. Blainvillei $\mathrm{BR}$. fragilis $\mathrm{B}_{\mathrm{r}}$.

Cemoria Leach 2. ? equestris Riss.

Noachina Chems. $s p$.

Emarginula LFi. 33 Goldfussi Roe. decussata Mü. scalaris So. tricajinata So. neocomiensis [?] D'O. carinata Reuss cretosa Dus.

Guerangeri D'O. pelagica D'O. Sanctae-Catharinae D'O

? cancellata Porti... arata Conr. . clathrata Dsw. clypeata $\mathrm{L}$. costata LK. elegans DFr.

\# clongata DFr. radiola LK. clathratiformis Eichw. crassa So. fenestrella Duв. Grateloupi BeL Мich. punctura Wood. Schlotheimi $n$. fissura Flem. . squamata Grat. decussata Phis. punctulata Phic. cancellata Purc. elongata Costa pileolus Micho. solidula Costa papillosa Riss.

Scutus MF. 0 (Pharmophorus LK.)

(Prarmophorus LK). 3 = Scutus Mr. = angustus Dsh.

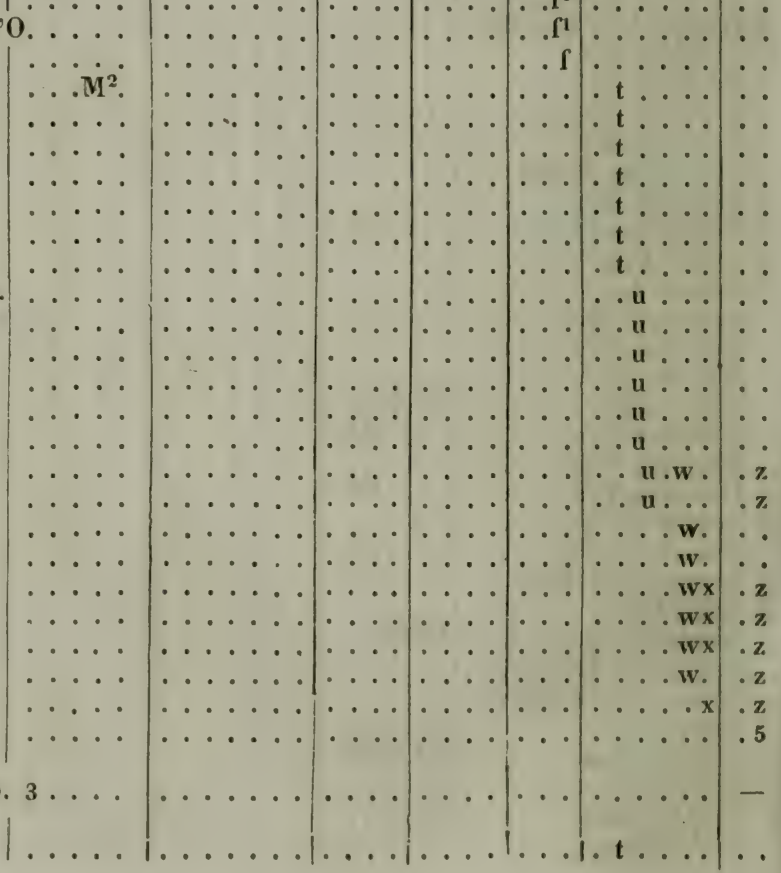




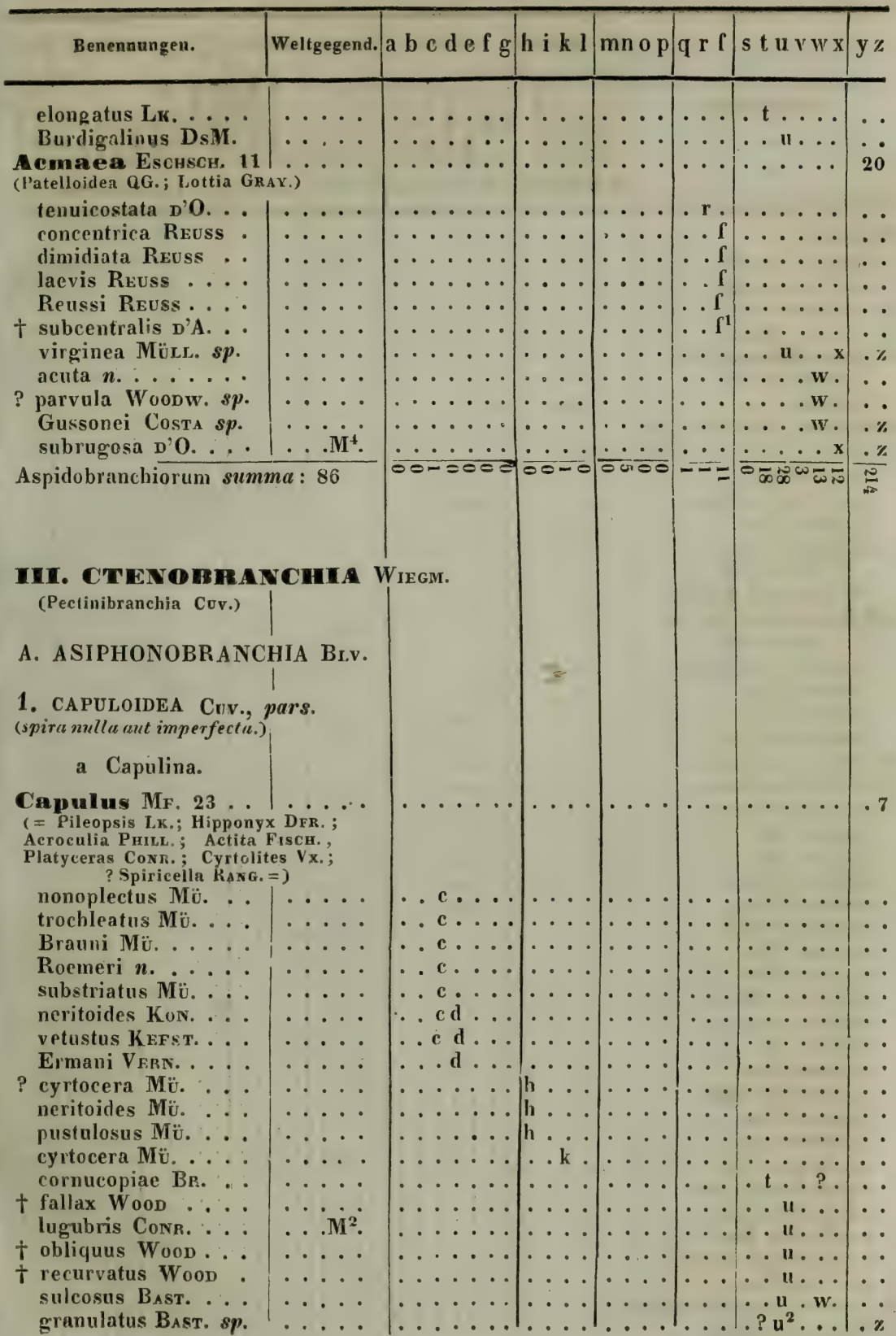




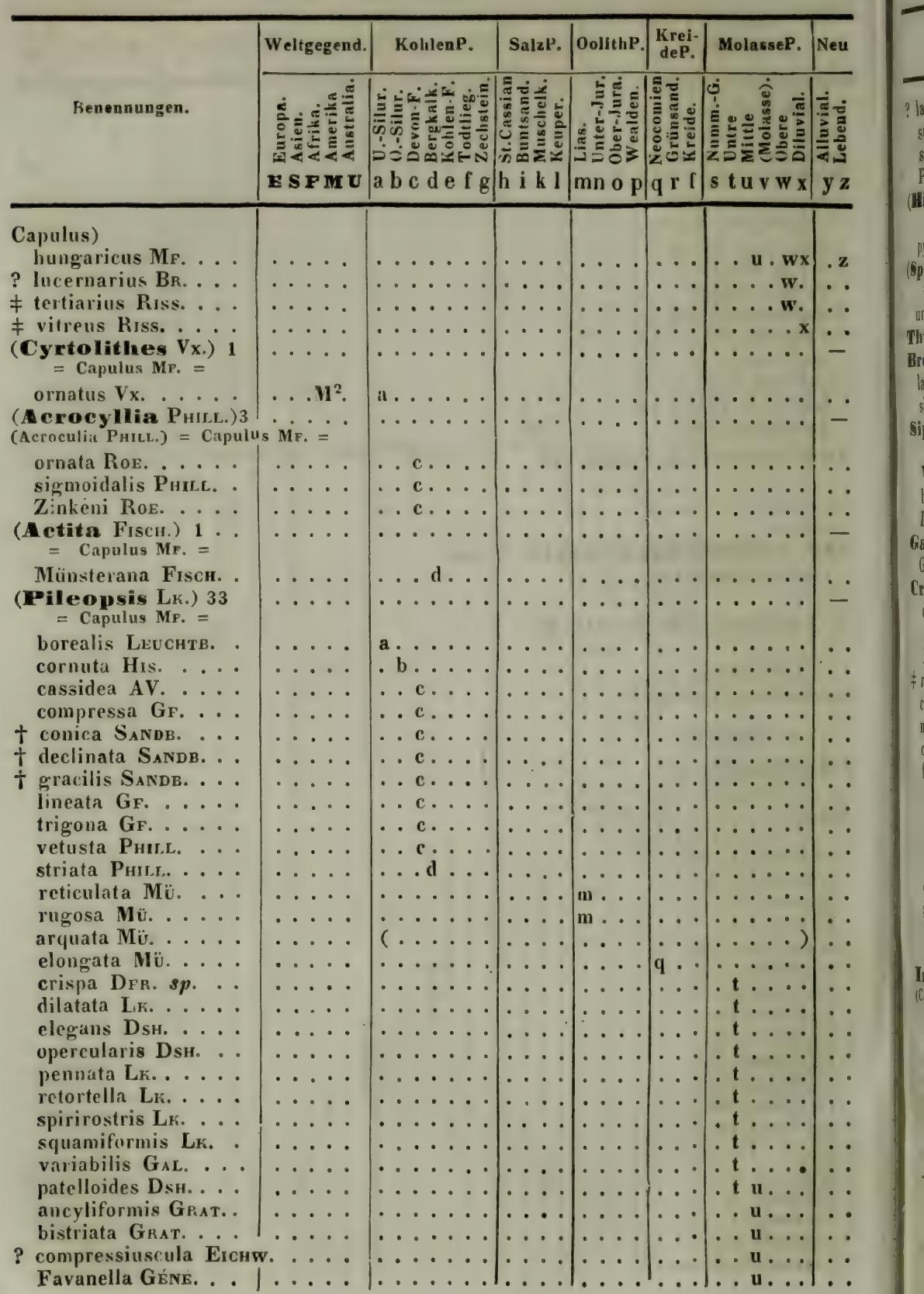


XIV. GASTEROPODA, III. CTENOBRANCHIA, A. ASIPHONOBRANCHIA. 369

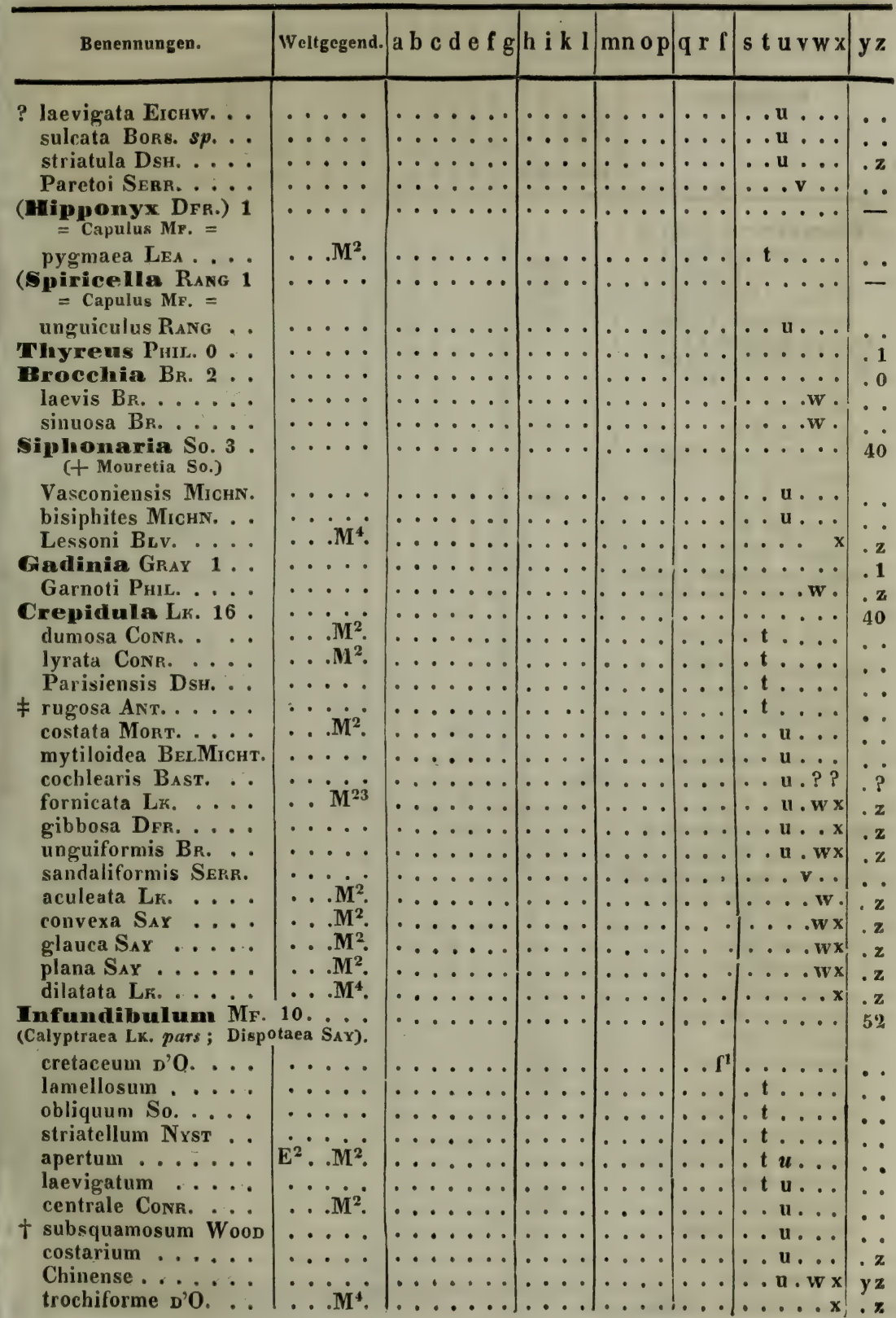




\begin{tabular}{|c|c|c|c|c|c|c|c|}
\hline & Weltgegend. & KohlenP. & SalzP. & OolithP & $\begin{array}{c}\text { Krei- } \\
\text { deP. }\end{array}$ & MolasseP. & Neu \\
\hline \multirow[t]{2}{*}{ Benennungen } & 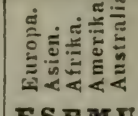 & 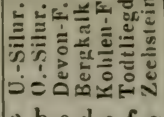 & 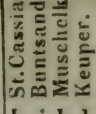 & 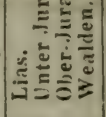 & 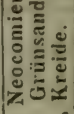 & 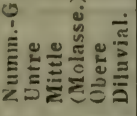 & 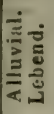 \\
\hline & ESF $\mathbf{M} \mathbf{U}$ & a bc def $g$ & h i k l & $\mathrm{mn}$ op & $q \mathrm{r}$ I & st u v w x & y z \\
\hline
\end{tabular}

(Dispotnea $\left.S_{A Y}\right) 3$ $=$ Calyptraea LK. = constricta Conr. . dumosa Conr. multilineata Conr.

Calyptraea Lr. 8 ( pars = Infundibulum Mr.) $=$ crassiuscula Grat. depressa LK. grandis FÉR.

+ Gualtieriana Géxí. ornata BAST. costata SAY $_{\text {A }}$ deformis LK. rugrosa Bors.

Calypeopsis Less. 0 (? Infundibulum Mr.)

\section{b. Sigaretina.}

Coriocella Brv. 1 perspicua Phic.

velutina $L_{R}, 3$. . (Galericulun kRows)

t capuloides Woop laevigata FLBm. .... elongata ForB.

Marsenia Leach 1 depressa Wood...

(Sigaretus ADs.) LK.16 = Cryptostoma BL. = furcatus $G_{F}$. rugosus GF.

? Urhtae Krys. .... carinatus $\mathbf{M u ̈ .}$ tenuicinclus Ku.

f apertus ANr. arctatus Conn. bilix Corp. declivis ConR. pellucidus Dsh. . . canaliculatus So... politus Dsr. . . . . . fragilis Conr..... depressus Grat. . . haliotoideus LR... elegans PhiL.

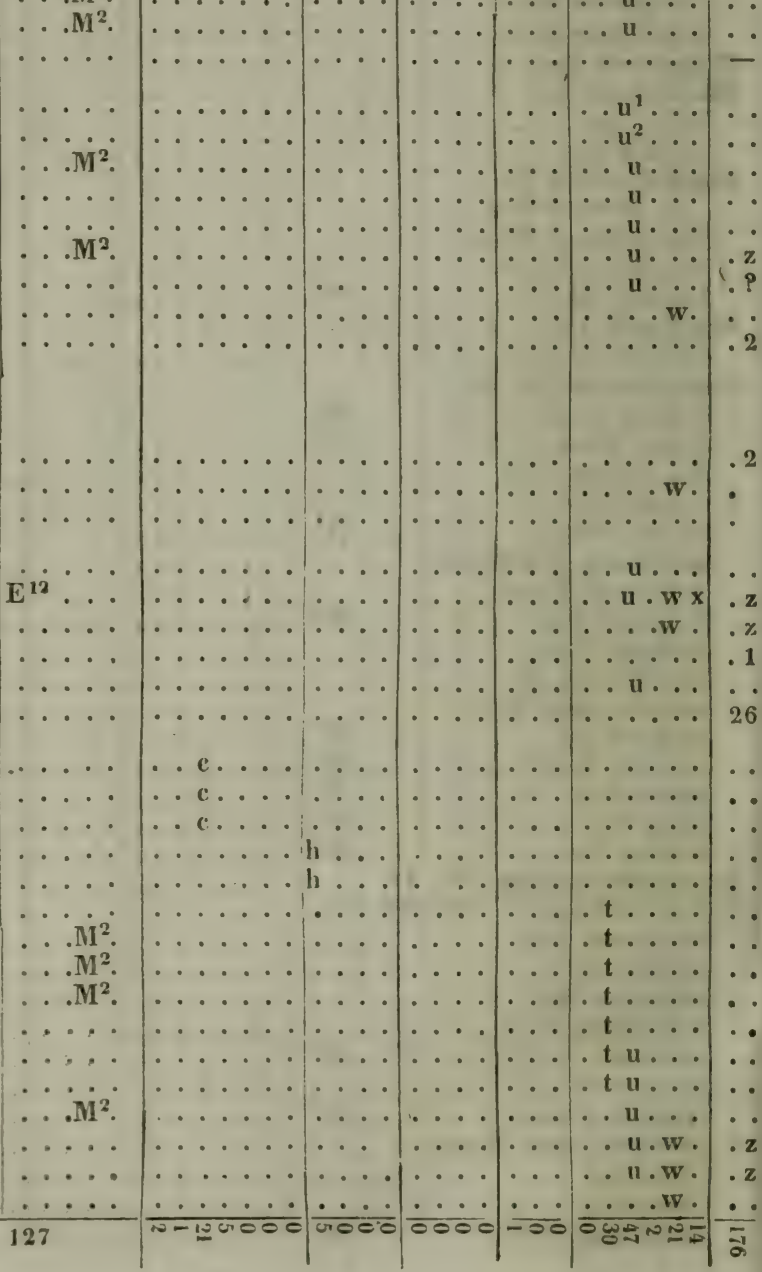


2. Trocholdea Cuv. (apertura integra).

a Naticina.

Natica LK. 219.

() Euspira AG.)

+ ampullacea Eichw.

₹ irregularis Eichw.

キ nodosa Eıchw.

parva So.

\# prisca Erchw.

antiqua ? Mü., GF.

effossa Gr.

excêntrica Roe.

harpula So. sp.

inflata Ros. .

? margaritifera $\dot{A} \dot{V}$.

marginata Roe. meridionalis Phicl.

nexicosta Phill.

t obtusa SANDB. protogaea GF. striatella SANDB. subcostata AV. vetusta So. Antisiensis [?] $D$ 'O. auricularis Gr. buccinoides D'O.

Dione Erchw.

Mariae VerN. Omaliusana (Kor.) Althausi KLI. angusta Mü.

Becksi KLI.

Cassiania Wissm.

Catulloi KLr.

Deshayesi KLr. ... elongata Mü. globosa KLI. gracilis KLI.

Haidingeri KLI. . hieroglypha KL.. impressa Mü. inaequiplicata KLr. Landgrebei Krr. . maculosa KLI. Mandelslohi KII. neritacea Mü. neritina MÜ. Oeynhauseni Ǩr.. ovata K.I. Weltgegend. a b c d e f g h i $k$ l mn op q $\mathrm{r}$ l s t u v w x y z

.

\begin{tabular}{l|l|l|l|l|l|l|l|}
\hline &
\end{tabular}




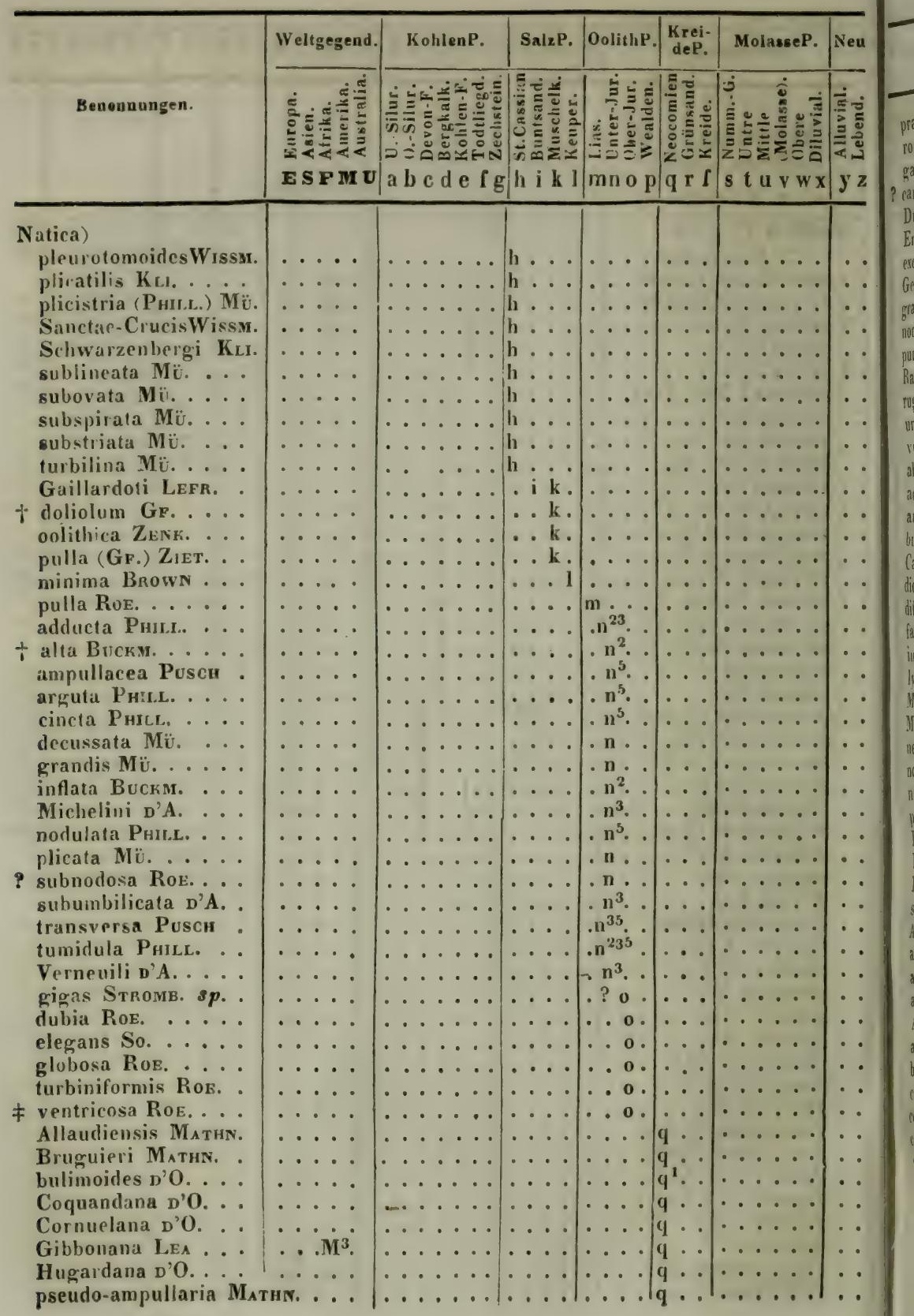




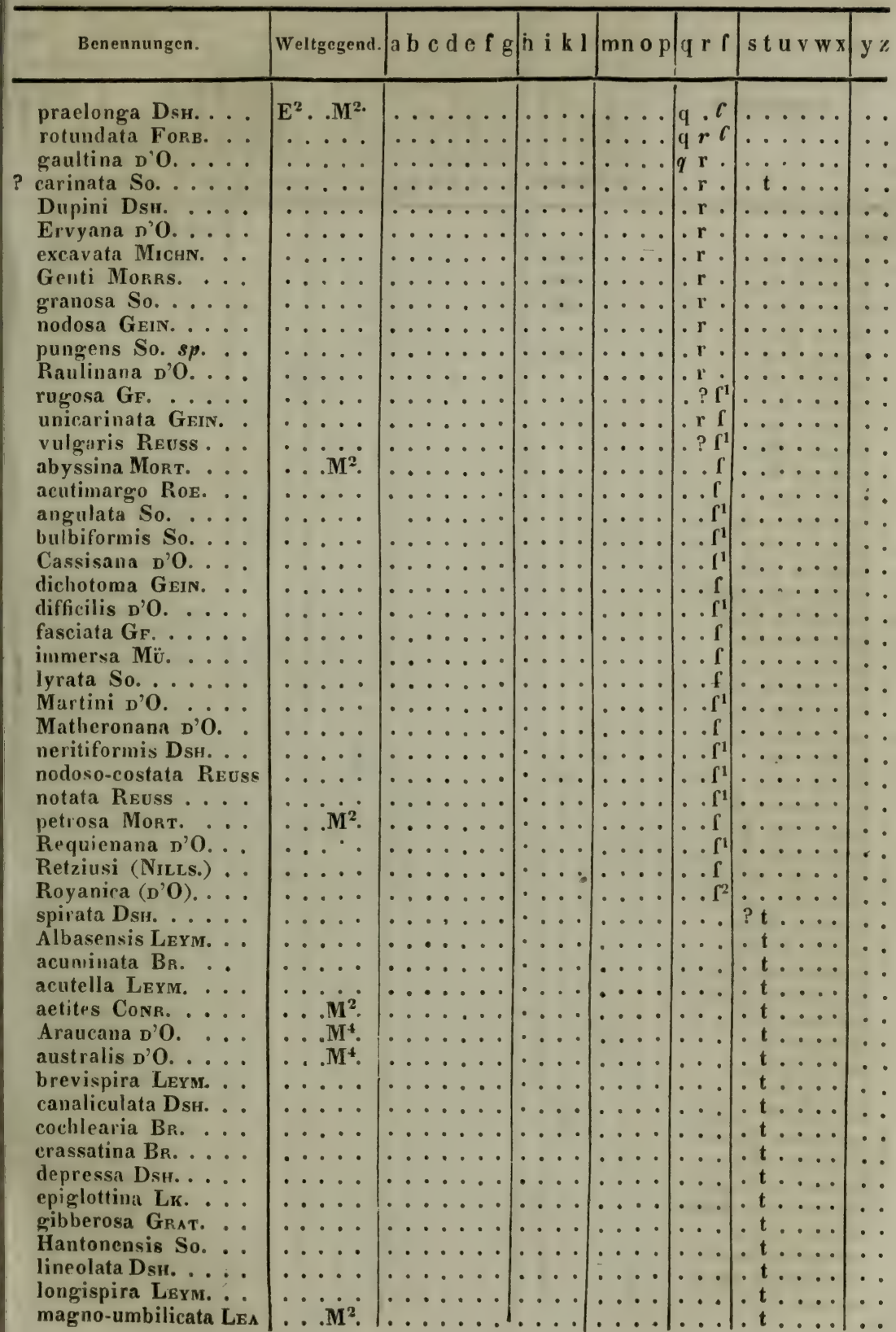




\begin{tabular}{|c|c|c|c|c|c|c|c|}
\hline & Veltgegend. & KohlenP. & SalzP. & OolithP. & $\begin{array}{l}\text { rei- } \\
\text { eP. }\end{array}$ & MolasseP. & Neu \\
\hline Bensnnungen. & 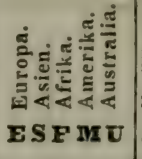 & 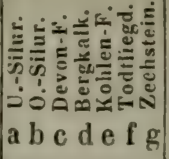 & 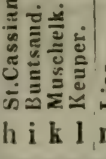 & 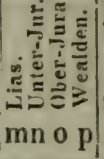 & 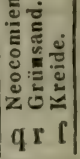 & 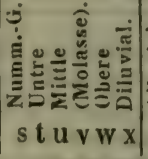 & 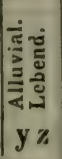 \\
\hline 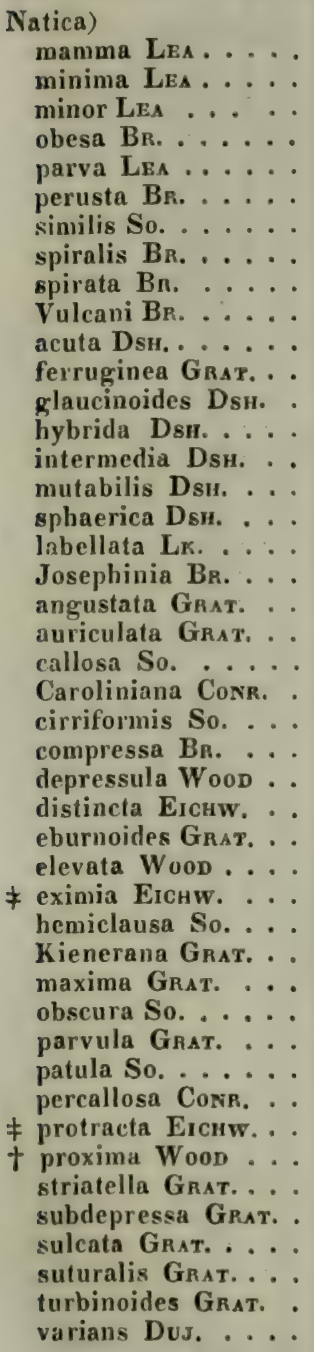 & 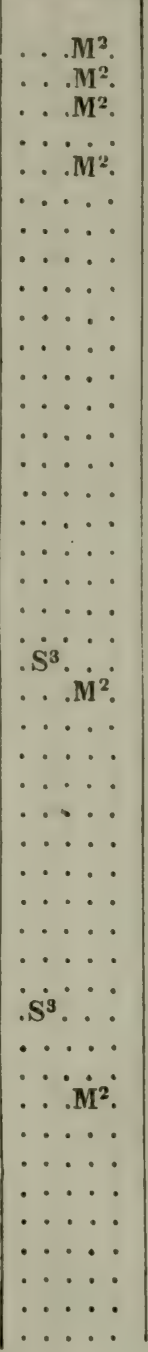 & 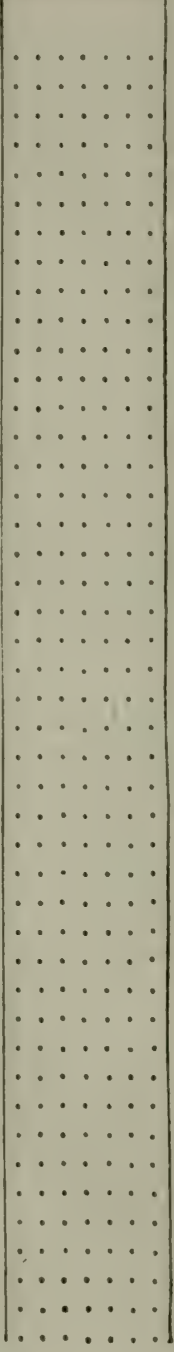 & 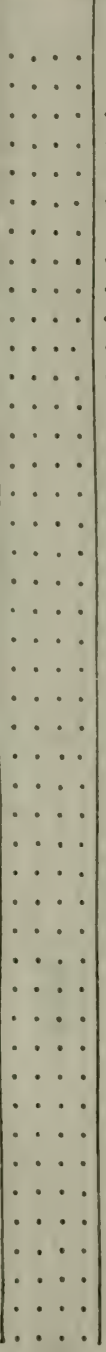 & 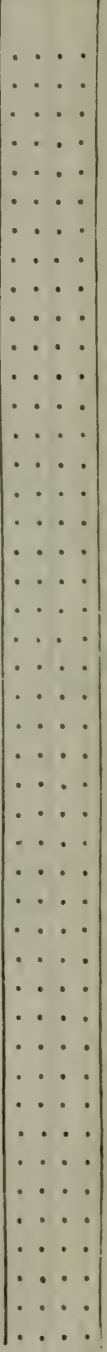 & 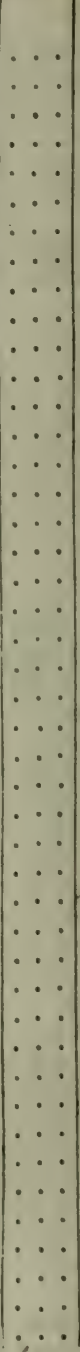 & 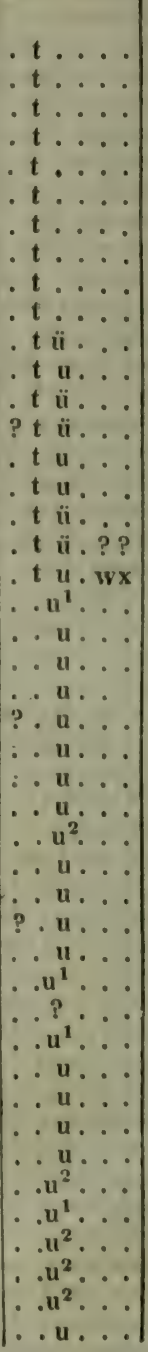 & $\begin{array}{l}\cdots \\
\cdots \\
\cdots \\
\cdots \\
\cdots \\
\therefore \\
\therefore\end{array}$ \\
\hline
\end{tabular}




\begin{tabular}{|c|c|c|c|c|c|c|c|}
\hline Benemin & end. & $\mathrm{fg}$ & h & $p$ & c) & $|x|$ & \\
\hline . & $\cdots$ & & • & $\cdots$ & & 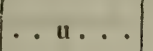 & \\
\hline$n$ & $\cdots \cdots$ & . & ... & $\cdot$ & $\cdots$ & . u... & \\
\hline$\ldots$ & $\therefore$ & $\cdots$ & & & & $w$ & \\
\hline$\cdots$ & $\cdots$ & $\begin{array}{lll}\cdots & \cdots & \cdots \\
\cdots & \cdots & \cdots\end{array}$ & $\mid \cdots \cdots$ & $\mid \begin{array}{ll}\cdots \\
\cdots\end{array}$ & $\cdots$ & $\begin{array}{l}\cdots u \cdot \cdots \\
\cdots u . w .\end{array}$ & \\
\hline . & $E^{2} \ldots M^{12}$ & $\ldots \ldots$ & $\cdots$ & $\cdots$ & $\ldots$ & $\therefore$ u. x & \\
\hline$\because$ & $\ldots$ & $\ldots \ldots$ & $\ldots$ & $\ldots$ & $\cdots$ & $\ldots 1$ & \\
\hline$\therefore$ & $\cdots \dot{2}^{\circ}$ & $\cdots \cdots$ & $\cdots$ & $\cdots$ & $\cdots$ & $\mathbf{w} x$ & \\
\hline & . & $\cdots \cdots$ & $\cdots$ & $\cdots$ & $\cdots$ & $\cdots x$ & \\
\hline G & $\cdots$ & $\because$ & $\cdots$ & $\cdots$ & $\cdots$ & $\mathbf{w} \cdot$ & \\
\hline it & $\therefore M^{2}$ & & & $\cdots$ & & $\dot{x}$ & \\
\hline it & $\mathbf{E}^{2}\left(\mathbf{S}^{3}\right)$ & $\cdots$ & $\begin{array}{ll}\cdots \\
\cdots \\
\cdots\end{array}$ & $\left|\begin{array}{c}\cdots \\
\cdots \\
\cdots\end{array}\right|$ & $\cdots$ & $\mid \begin{array}{lll}\cdots & ? \\
\cdots & \text { u } & \ldots \\
\cdots\end{array}$ & \\
\hline 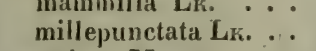 & $\mathrm{E}^{2} \cdot \mathrm{M}^{2}$ & .. & $\ldots$ & $\ldots$ & $\ldots$ & $\dot{w} x$ & \\
\hline$\cdots$ & & $\cdots$ & $\cdots$ & $\ldots$. & $\cdots$ & & \\
\hline$\cdots$ & & $\ldots \ldots$ & $\cdots$ & $\cdots$ & $\cdots$ & w. & \\
\hline$\cdots$ & . & $\ldots \ldots$ & $\cdots \cdot$ & $\ldots$ & $\cdots$ & $\cdots$ & \\
\hline$\cdots$ & & $\cdots \cdots$ & $\cdots \cdot$ & $\cdots$ & $\cdots$ & $\cdots$ & \\
\hline $\begin{array}{l}\text { tec } \\
\text { un }\end{array}$ & • & . & $\cdots$ & $\cdots$ & $\cdots$ & $\cdots$ & \\
\hline un & & & & - & $\cdots$ & $\cdot$ & \\
\hline us & & & & • & I & 8 & \\
\hline$\cdots$ & & $\cdots$ & & $\cdots$ & $\ddot{m}$ & $\begin{array}{l}\mathbf{x} \\
\mathbf{x}\end{array}$ & \\
\hline$\cdots$ & . & $\ldots \ldots$ & $\ldots \ldots$ & $\cdots$ & $\cdots$ & $\cdots$ & \\
\hline i Payr. & & . & & $\ldots$ & $\ldots$ & ... & \\
\hline & & $\cdots$ & & $\because$ & $\cdots$ & .... w. & \\
\hline$\cdots$ & • & $\ldots$ & $\cdots$ & . & $\cdots$ & $\ldots \ldots x$ & \\
\hline & . & & $\cdot$ & $\cdots$ & $\cdots$ & $|\ldots \ldots x|$ & \\
\hline . & & $\cdots$ & - & $\cdots$ & $\cdots$ & $\ldots \ldots x$ & \\
\hline$\cdots$ & 4 & * & $\cdots$ & $\cdots$ & $\cdots$ & $\cdots \cdots x$ & \\
\hline$\cdots$ & & . & $\cdots$ & $\cdots$ & $\cdots$ & $\cdots \cdots x$ & \\
\hline $\begin{array}{l}\text { spp. } \\
\text { us }\end{array}$ & & & & & $\cdots$ & . & \\
\hline$\cdots$ & & . & & - & $\cdots$ & 1. & \\
\hline & & $\ldots c \ldots$ & • & $\therefore \ldots$ & $\ldots$ & $\ldots \ldots$ & \\
\hline 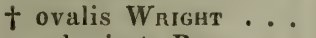 & $\cdots$ & $\ldots d \ldots$ & - & $\cdots$ & $\cdots$ & $\ldots \ldots$ & \\
\hline$\cdots$ & $\cdots$ & 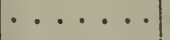 & - & . n . . & & $\ldots \ldots$ & \\
\hline T. • & . & & & $\cdots$ & $\cdot r$ & $\cdots$ & \\
\hline & $\cdot \cdot$ & 1 & $\cdots$ & & & $t$. & \\
\hline • & . & - & - & & & - t t & \\
\hline & & & & & & t $t$ & \\
\hline & $\because$ & $\cdots$ & .... & & $\ldots$ & $\begin{array}{l}0 \\
0 \\
0\end{array}$ & \\
\hline$\cdots$ & & $\ldots$ & $\ldots$ & $\cdots$ & ... & $\therefore u$ & \\
\hline . & & & & $\ldots$ & $\cdots$ & . . u... & \\
\hline & & & & & & $\ldots w$. & \\
\hline d & & & & & & $\ldots \ldots$. & \\
\hline & & & & & & & \\
\hline & & & & & & & \\
\hline 10 & $\cdots$ & 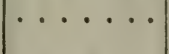 & - & & $\cdots$ & $\cdots$ & \\
\hline Hokrs. … & $\cdots$ & $\cdots$ & & & & & \\
\hline
\end{tabular}


Xiv. GASTEROPODA, III. CTENOBRANCHIA, A. ASIPMONOBR ANCHIA.

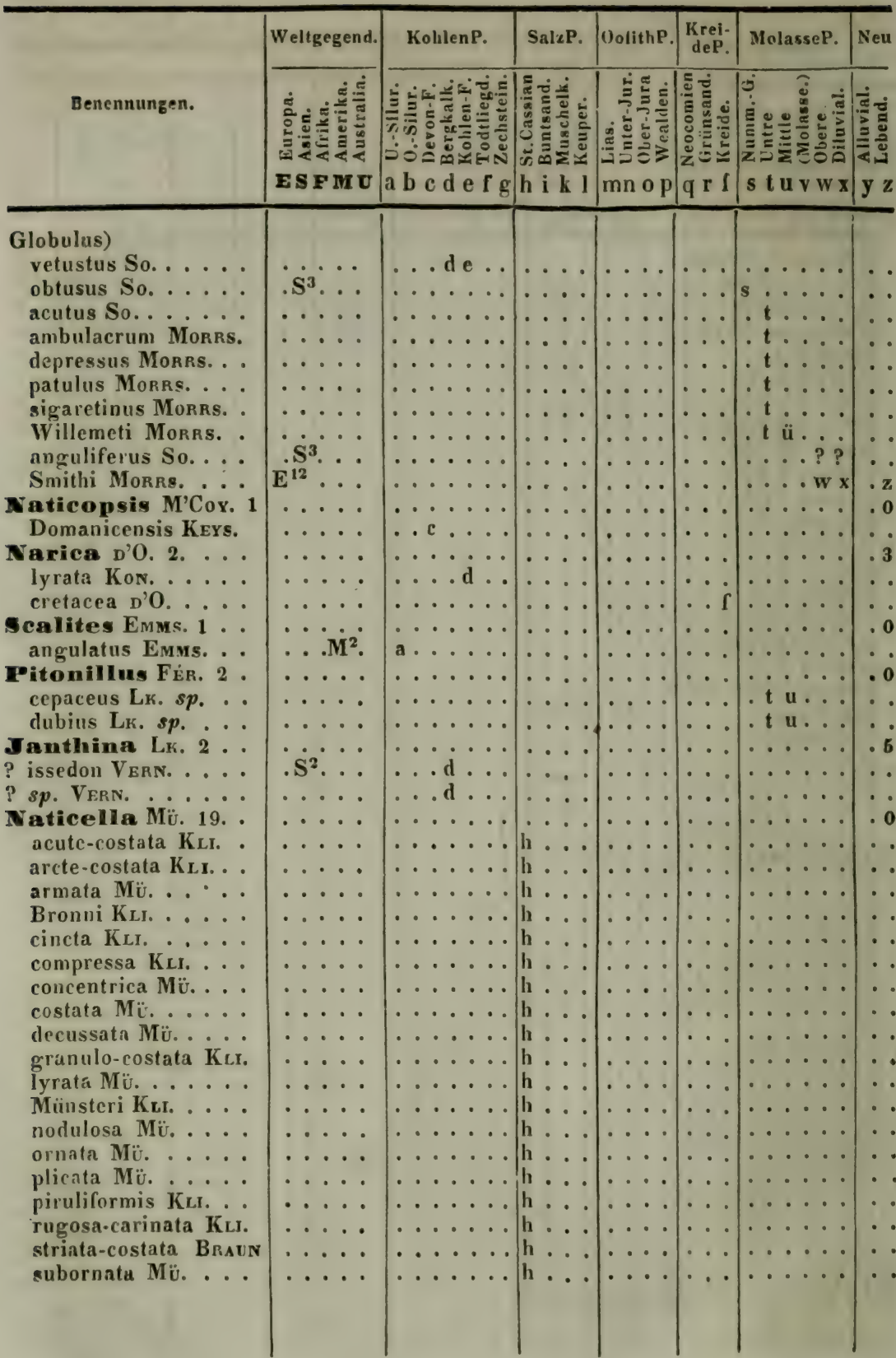


Xiv. Gasteropora, III. CTENobranchia, A. Asiphonobranchia. 377

\begin{tabular}{|c|c|c|c|c|c|c|c|}
\hline Bene & ent. & $\mathrm{a} b \mathrm{c}$ & h i k 1 & $\mathrm{mn}$ op & 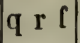 & stuvw & \\
\hline & & & & & & & \\
\hline (non & Mü.) 1 . & $\cdots$ & $\therefore$ & $\cdots$ & & $\cdot$ & \\
\hline & $\cdots \cdot$ & $\cdots \cdots \cdots$ & $\cdots$ & $\cdots \cdot$ & $\cdots$ & $\cdot t \ldots$ & \\
\hline De: & .... & $\cdots \cdots \cdots$ & $\cdots \cdot$ & $\cdots$ & $\ldots$ & $\cdots$ & \\
\hline $\begin{aligned} P i \\
\text { er }\end{aligned}$ & . & & & $\cdots$ & & $\cdots$ & \\
\hline 7 & $\cdots$ & 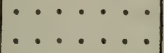 & $\left|\begin{array}{l}\cdots \\
\cdots \\
\cdots\end{array}\right|$ & $\mid \begin{array}{cc}\cdots \\
\cdots\end{array}$ & $\cdots$ & $\cdots$ & \\
\hline$\dot{0} \dot{0}$ & & $\ldots$ & $\cdots$ & $\cdots$ & $\ddot{q} \cdot$ & $\cdots$ & \\
\hline U. & & $\cdots$ & $\ldots$. & $\ldots$ & 9. r $^{\mathrm{i}}$ & $\ldots \ldots$ & \\
\hline - & $\cdots \cdot$ & $\cdot$ & $\cdots$ & $\cdots \cdot$ & . . $\boldsymbol{I}^{\mathbf{1}}$ & $\ldots \ldots$ & \\
\hline$\cdots$ & $\cdots \cdots$ & $\cdots$ & $\cdots$ & $\cdots$ & . . $\mathbf{f}^{\mathbf{1}}$ & $\cdots \cdots$ & \\
\hline . & $\dot{\mathrm{E}}^{2} \dot{\mathbf{S}^{3}} \overrightarrow{0}$ & $\cdots$ & $\cdots$ & $\cdots$ & $\cdots$ & $\cdots \cdots$ & \\
\hline$\dot{0}$ & & . 1 & $\cdots$ & & $\ldots$ & $\cdots$ & \\
\hline & & & & & & T 20.0 & \\
\hline S. & $\cdots$ & & $\cdots$ & $\ldots$ & $\cdots$ & $\cdots \cdots$ & \\
\hline$\cdots$ & $\cdots \cdots$ & $\cdots$ & $\cdots$ & $\cdots \cdot$ & $\cdots$ & $\cdots \cdots$ & \\
\hline$\cdots$ & $\because$ & $\cdots$ & $\cdots$ & - & $\cdots$ & $\cdots \cdot$ & \\
\hline$\dot{.}$ & $\cdots \cdots$ & $\cdots$ & $\cdots$ & $\cdots$ & t & $\cdots$ & \\
\hline . & .... & . . & $\cdots$ & $\mid \begin{array}{c}\cdots \\
\cdots\end{array}$ & $\cdots$ & $\because$ & \\
\hline . & ... & . & $\ldots$. & $\ldots$. & $\ldots$ & $\ldots \ldots$ & \\
\hline .. & $\cdots$ & $\cdots$ & $\ldots$ & $\ldots$ & $\cdots$ & $\ldots \ldots$ & \\
\hline v. . . & $\ldots \ldots$ & $\ldots d \ldots$ & $\ldots$ & $\cdots$ & $\cdots$ & $\ldots \ldots$ & \\
\hline$\ldots$ & .... & $\ldots$ d... & $\ldots$ & $\cdots$ & $\cdots$ & $\cdots$ & \\
\hline . . . & $\ldots$ & & t & . & • & $\cdots \cdots$ & \\
\hline$\cdots \cdot$ & $\cdots \cdot$ & $\cdot \cdot$ & h & $\cdots \cdot$ & . & $\ldots \ldots$ & \\
\hline$\cdots$ & $\cdots \cdot$ & - & h... & $\cdots$ & - & $\cdots \cdot \cdot$ & \\
\hline$\cdot \ldots$ & $\cdots$ & $\cdots$ & $\cdots$ & $\cdots$ & - $\cdot$ & $\cdots \cdots$ & \\
\hline$\cdots$ & $\cdots \cdot$ & $\cdots$ & $\cdots$ & . n... & $\cdot \cdot$ & $\ldots \ldots$ & \\
\hline$\ddot{\cdots}$ & • & & $\cdots$ & $\cdot$ & . & $\cdots \cdot \cdot$ & \\
\hline$\cdots$ & $\cdots \cdots$ & $:$ & $\cdots$ & $\cdots$ & $\cdots$ & $\cdots$ & \\
\hline Rिов. & $\ldots$ & $\because$ & $\cdots$ & $\mid \begin{array}{cc}\cdots & 0 \\
0 & 0\end{array}$ & $\cdots$ & $\cdots \cdots$ & \\
\hline$\ldots$ & $\ldots$ & $\ldots \ldots$ & $\ldots$ & .0. & & $\ldots \ldots$ & \\
\hline & $\cdots \cdot$ & $\cdots$ & $\ldots$ & $\ldots$. & . . $\mathbf{I}^{\mathbf{1}}$ & $\ldots \ldots$ & \\
\hline T. . . & $\ldots \ldots$ & $\cdots$ & $\ldots$ & $\cdots \cdots$ & $\ldots \mathrm{f}$ & $\ldots \ldots$ & \\
\hline$\cdots$ & $\cdots \cdot$ & $\ldots \ldots$ & $\ldots$ & $\cdots$ & $\ldots$ & . t... & \\
\hline & & $\ldots \ldots$ & $\ldots$ & $\cdots \cdot$ & . $\cdot$ &.$t \ldots$ & \\
\hline$\cdots \cdot$ & $\cdots$ & . & $\cdots$ & & &. $\mathrm{t} \ldots$. & \\
\hline & & & $\ldots$ & $\cdots \cdots$ & $\cdots$ & . $t$. & \\
\hline$\cdots$ & $\ldots \ldots$ & . & $\ldots$ & $\cdots \cdot$ & $\cdots$ &.$t \ldots$. & \\
\hline$\cdots$ & & & & $\cdots \cdots$ & $\cdots$ & $\cdot$ & \\
\hline$\cdots$ & & & $\cdots$ & $\cdot$ & & $\cdot$ & \\
\hline 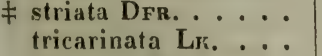 & & & & $\because$ & & $\begin{array}{l}. t \\
. t\end{array}$ & \\
\hline$\cdots$ & $\ldots$. & & $\ldots$ & $\cdots \cdot \cdot$ & $\cdots 1$ & $t u^{2}$ & \\
\hline$\cdots$ & $\cdots$ & & & 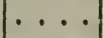 & 1 & $\ldots \mathbf{u}$ & \\
\hline$\because$ & & & & & & & \\
\hline$\ldots \ldots$ & $\ldots$ & $\ldots \ldots$ & $\ldots$ & & & $\begin{array}{l}u \\
u u^{2} \ldots\end{array}$ & \\
\hline
\end{tabular}




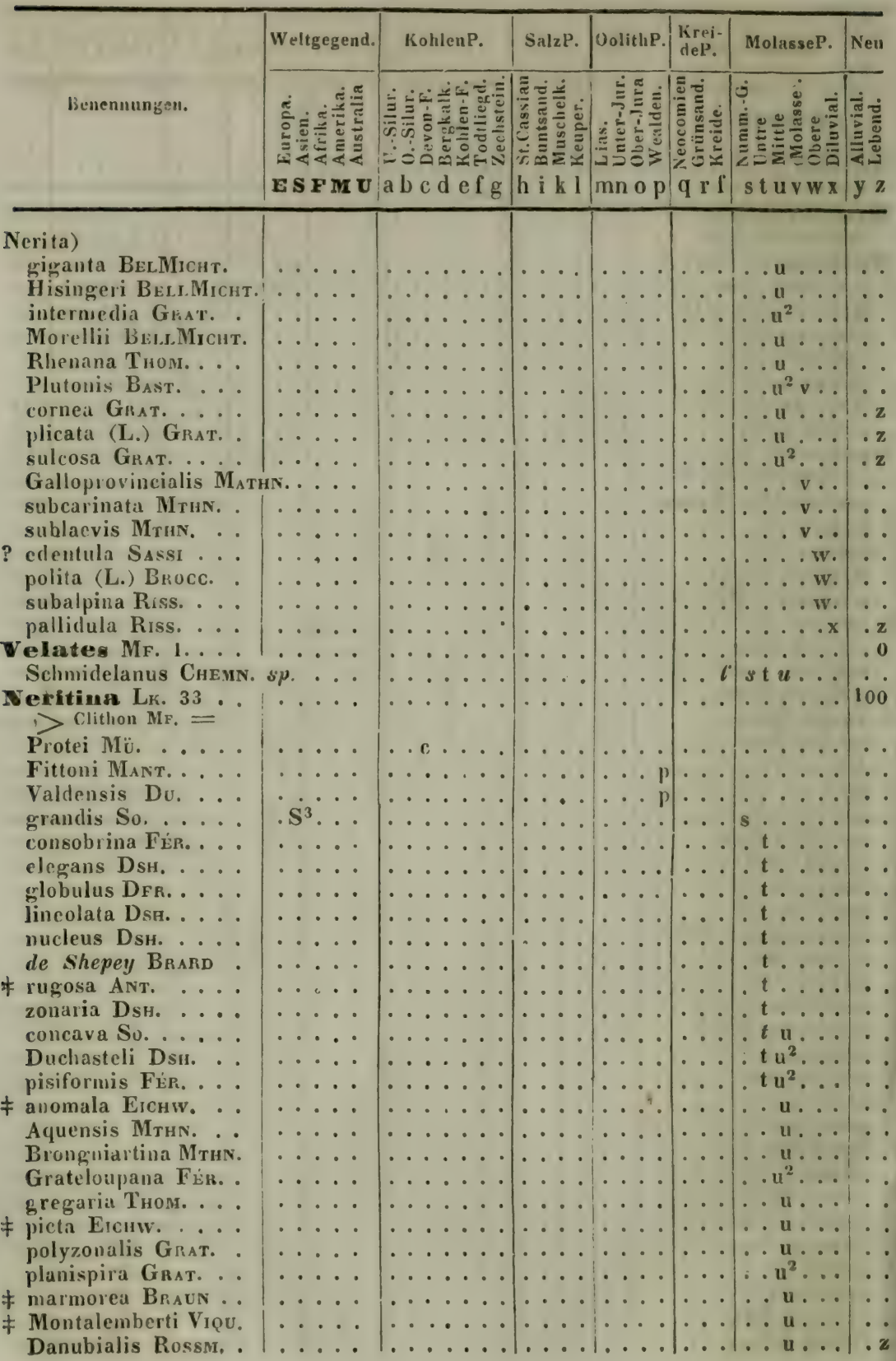




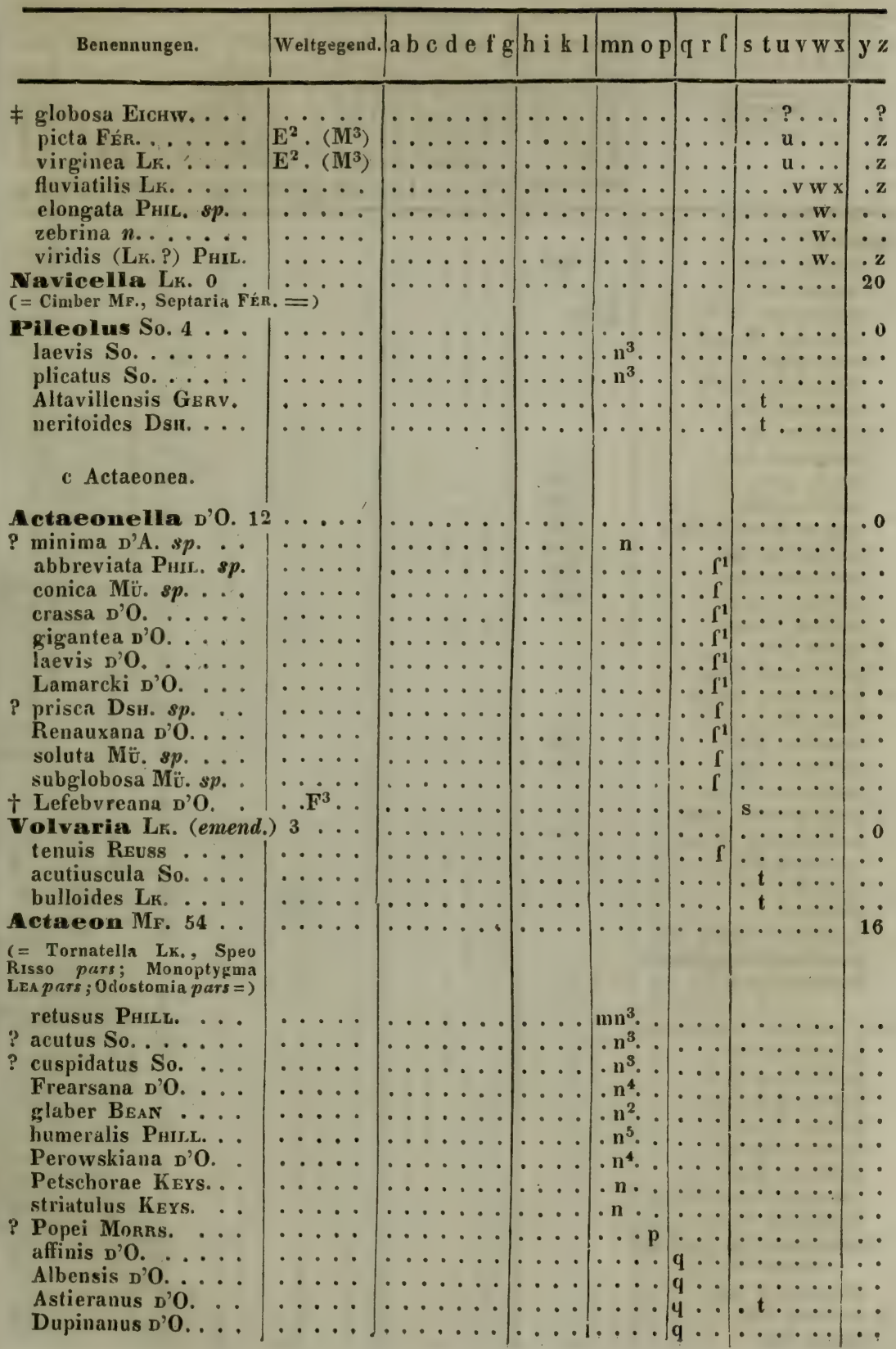




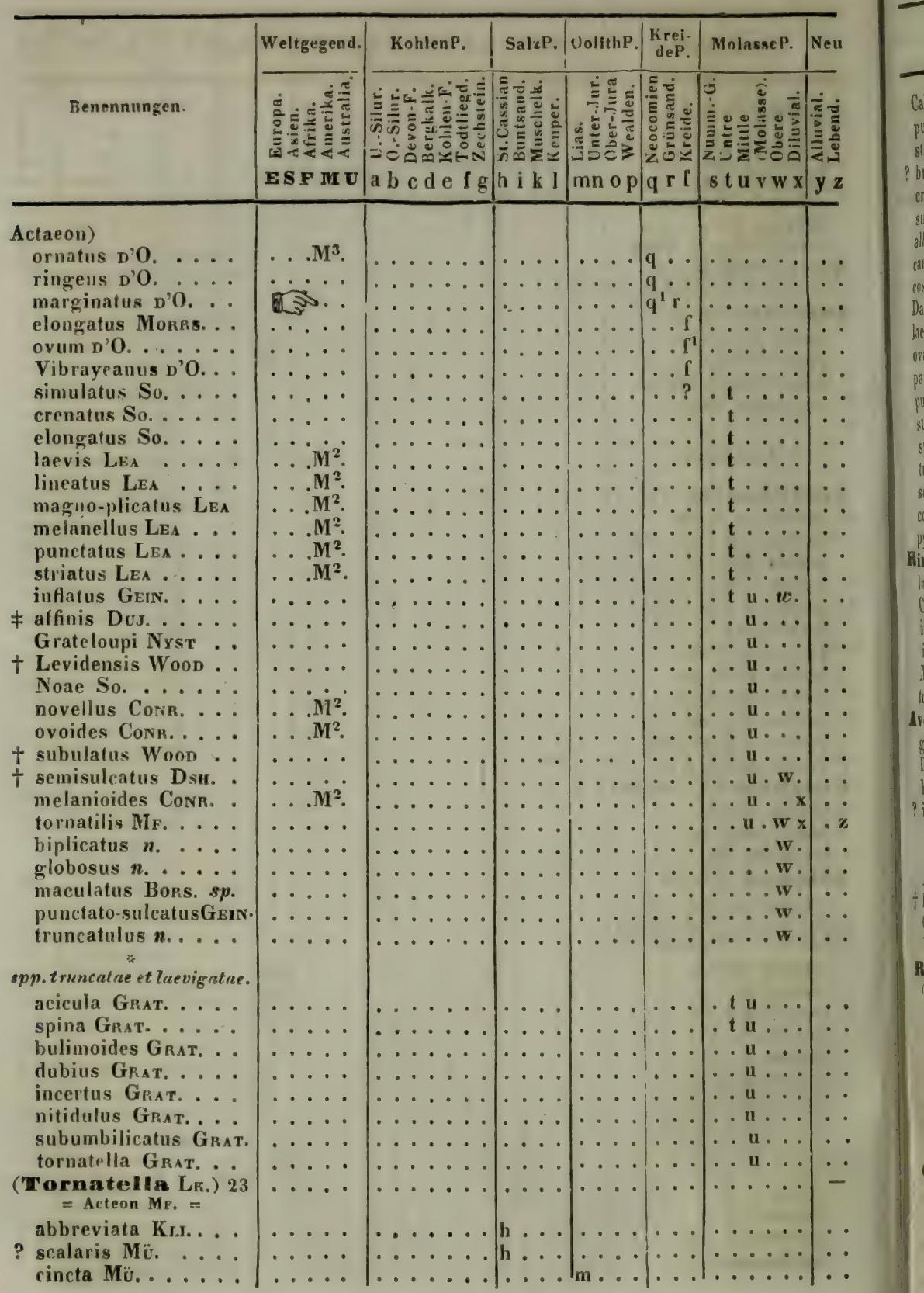




\begin{tabular}{|c|c|c|c|c|c|c|c|}
\hline gen. & nd. & $\mathrm{fg}$ & k 1 & $\mathrm{op}$ & $q \times l$ & $\mathrm{w} \times$ & \\
\hline & & & & & & & \\
\hline & & & $-x_{0}$ &.$n^{2} \ldots$ & . & $\ldots \ldots$ & \\
\hline$\cdots$ & - & $\ldots .$. & $\cdots \cdot$ & n... & & $\ldots \ldots$ & \\
\hline bullata MorT. ... & . $\mathbf{M}^{2}$ & $\ldots \ldots$ & $\ldots$ & $\cdots$ & ... 1 & $\cdots \cdots$ & \\
\hline cretacea Pusch ... & $\cdots \cdot$ & $\cdots \cdots$ & $\cdots$ & $\cdots \cdot$ &.. $\mathrm{f}$ & $\cdots \cdots$ & \\
\hline$\cdots \cdot$ & $\cdot \cdot$ & $\cdots \cdots$ & $\cdots$ & $\cdots \cdot$ & $\cdots \cdot$ & $\cdot$ t $\mathbf{~ u} \ldots$ & \\
\hline$\cdot \cdots$ & $\therefore$ & $\therefore$ & $\cdots$ & $\cdots$ & $\cdots$ & $\cdot t \cdot v \ldots$ & \\
\hline T. $\cdots$ & $\cdots \cdots$ & $\begin{array}{lll}\cdots & \cdots & \cdots \\
\cdots & \cdots & \cdots\end{array}$ & $\left|\begin{array}{l}\cdots \\
\cdots \\
\cdots\end{array}\right|$ & $\therefore$ & $|\cdots|$ & $\left|\begin{array}{ll}\cdots \\
\cdots \\
\cdots & \ldots\end{array}\right|$ & \\
\hline $\begin{array}{l}\text { a Dus. } \cdots \\
\text { i Bast. } \cdots\end{array}$ & .... & $\ldots \ldots$ & $\cdots \cdots$ & $\cdots$ & $\cdots$ & $\cdots$ & \\
\hline $\begin{array}{l}\text { Dargelasi Bast. } \ldots \\
\text { laevigata Grat. . . }\end{array}$ & $\cdots$ & $\ldots \ldots$ & $\ldots$ & $\ldots$ & $\ldots$ & $\therefore$ u. . & \\
\hline$\cdots$ & $\cdots \cdot$ & $\ldots \ldots$ & $\ldots$ & $\ldots$ & ... & . . u. . & \\
\hline$\cdots$ & $\cdots \cdot$ & $\ldots \ldots$ & $\ldots$ & $\ldots$ & $\cdots$ & . u. . & \\
\hline . . & $\cdots$ & $\ldots \ldots$ & $\cdots$ & $\cdots \cdot$ & $\cdots$ & ..... & \\
\hline . & $\cdots$ & $\cdots \ldots \ldots$ & $\cdots \cdots$ & $\cdots$ & $\cdots$ & $\ldots$ u... & \\
\hline r. . & $\cdots \cdots$ & $\cdots \cdot$ & D... & $\cdots$ & $\cdots$ & $\ldots$ u... & \\
\hline$\cdots$ & $\cdots \cdot$ & $\ldots \ldots$ & $\cdots$ & $\cdots$ & $\cdots$ & $\ldots \mathbf{u} \ldots$ & \\
\hline . . . & $\cdots$ & $\ldots \ldots$ & $\cdots \cdot$ & $\ldots$ & & ..u.w. & \\
\hline$\cdots$ & - & $\ldots \ldots$ & $\cdots$ & $\cdots$ & - & $\ldots u, w x$ & \\
\hline . & - & $\cdots$ & & $\cdots$ & . & ....w. & \\
\hline D’0. 6 & & $\cdots$ & . & $\cdots$ & & $\ldots \ldots$ & \\
\hline$\because \cdot \cdots$ & Das. & $\ldots \ldots$ & $\cdots$ & $\cdots$ & $q^{1} r$ & $\cdots \cdots$ & \\
\hline$\cdot \quad \cdot$ & $\cdots$ & $\ldots \ldots$ & $\cdots \cdot$ & $\cdots \cdot$ & . 1. & $\ldots \ldots$ & \\
\hline$\therefore$ & $\cdots$ & $\cdots$ & $\cdots$ & $\cdots$ & - $r$ & $\cdots \cdots$ & \\
\hline . & . & $\cdots$ & 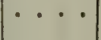 & $\cdots$ & - $\begin{array}{rl}\mathrm{r} & \mathrm{I} \\
\mathrm{C}\end{array}$ & $\cdots \cdots$ & \\
\hline - & & $\therefore$ & 然 & $\cdots$ & ...1 & $\cdots$ & \\
\hline$\dot{0}$ & & $\cdots$ & $\cdots$ & $\cdot$ & . & . $\tau \ldots$ & \\
\hline $\begin{array}{l}10 \\
\cdots\end{array}$ & & & & $\mid \begin{array}{c}\cdots \\
\cdots\end{array}$ & $q^{1} \cdots$ & $\cdots \cdots$ & \\
\hline$\cdots$ & . & $\ldots \ldots$ & 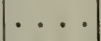 & $\ldots$ & . $\mathrm{r}$. & $\ldots$ & \\
\hline ). . & . & $\ldots \ldots$ & $\cdots$ & $\cdots$ & $\cdot \mathbf{r} \cdot$ & $\ldots \ldots$ & \\
\hline . . . & $\cdots \cdot$ & $\because \cdots \cdots$ & $\cdots \cdot$ & $\cdots$ & - $\mathbf{r} \cdot$ & $\ldots \ldots$ & \\
\hline & & ${ }^{\circ}$ & - & & . & $\cdots$ & \\
\hline$\cdots$ & 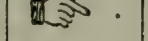 & 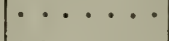 & - & . & - r r 1 & 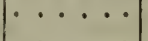 & \\
\hline . . . & $\cdots$ & - & & & 1 & 1 & \\
\hline$\cdots \cdot$ & & - 1 & & & $\mid \begin{array}{ll}0 & 1 \\
0 & 1\end{array}$ & - & \\
\hline$\ldots$ & & & & & . $\mathrm{C}^{2}$ & & \\
\hline 7 & $\cdot$ & $\ldots \ldots$ & $\therefore$ & • & $\cdots$ & $\cdots \cdots$ & \\
\hline & & & & & & & \\
\hline$\cdot$ & - & & • & $\cdots \cdot$ & ... & . u u... & \\
\hline$\cdots$ & & & & & . & $\cdots u$. & \\
\hline & & & & & $\cdots$ & . u vw. & \\
\hline$\therefore$ & & $\cdots$ & $\cdots$ & . & $\ldots$ & . u ... & \\
\hline & 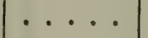 & $\cdots$ & . . & $\ldots$ & $\ldots$ & ....w. & \\
\hline & & $\ldots . .$. & & $\cdots$ & . & ....w w & \\
\hline 0.4 & & & & & & $\cdots$ & \\
\hline & & & & & $\because$ & & \\
\hline$\pi$ & & & $\cdot$ & & $\cdots$ & $l^{2}$ & \\
\hline . . . & 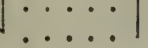 & 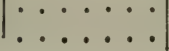 & & 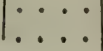 & $\cdots$ & & \\
\hline
\end{tabular}




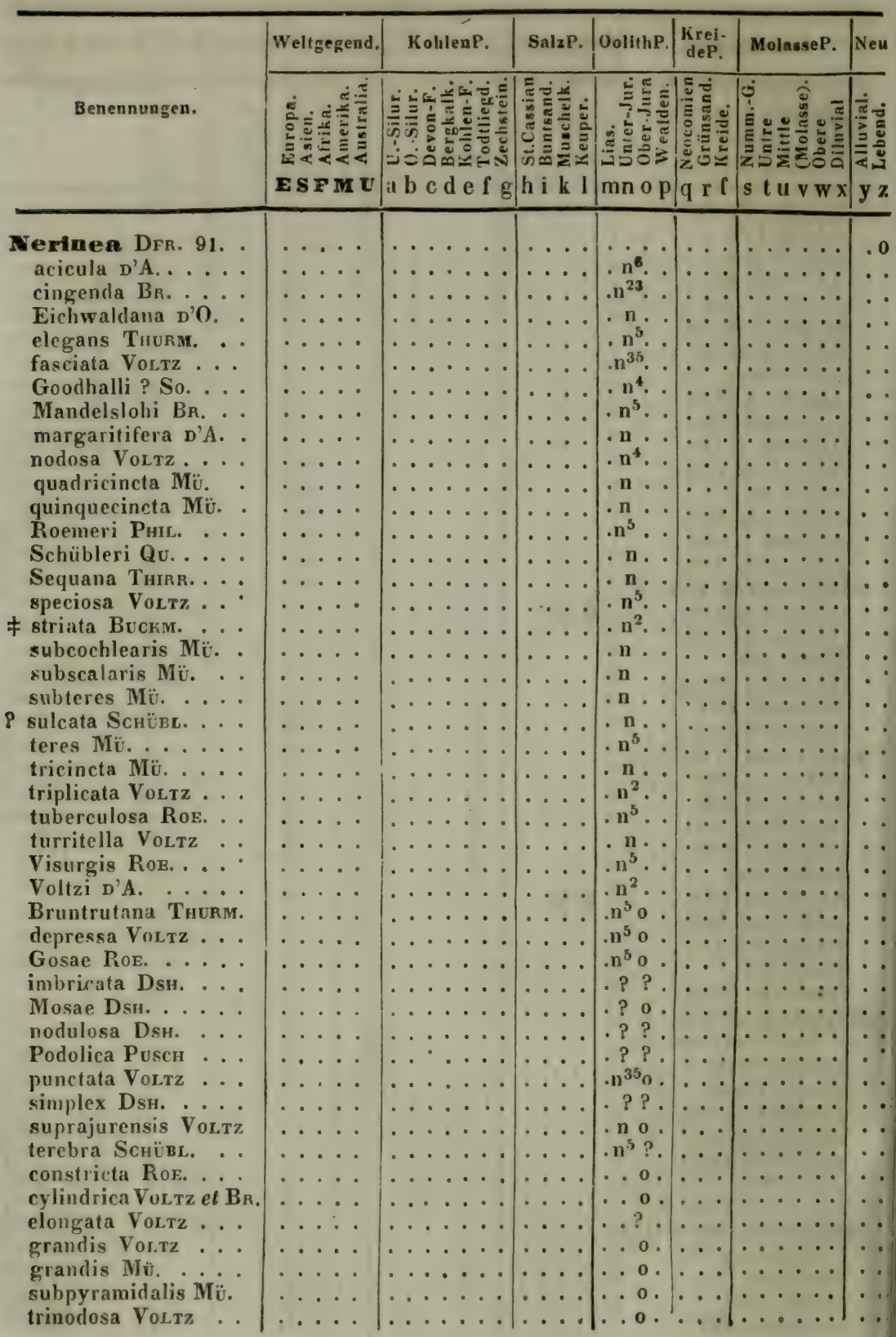


Benennungen. $\quad$ Weltgegend. a b c d e f g h i k l mnop q r f s t u v w x y z

Archimedis D'O

bifurcata D'O.

Carteroni D'O.

Chamousseti D'O.

Coquandana D'O.

Dupinana D'O.

gigantea Frrm.

lobata D'O.

Matronensis [?] D'O.

Renauxana D'O.

Royerana D'O.

Borsoni Br.

longissima Reuss

Aunisana D'O.

ampla Mü.

Baugai D'O.

bisulcata D'A.

brevis FIRM.

Bronni Mü.

Buchi Kefst. $s p$.

cincta Mü.

crenata Mü.

dubia D'A.

flexuosa So.

Fleurinuana D'O.

Geinitzi GF.

granulata Mü.

incavata $\mathrm{Br}$.

involuta $B_{R}$.

Marrotana D'O.

monilifera $\mathbf{D}^{\prime} \mathbf{O}$.

nobilis $\mathbf{M} \ddot{\mathrm{U}}$

Pailletteana b'O.

pauperata D'O.

Perigordina D'O.

Prevesti D'A.

pulchella D'O.

pyramidalis $M \ddot{U}$.

regularis $\mathrm{D}^{\prime} \mathrm{O}$

Requienana D'0.

Royanana D'O.

subaequalis n'O. trochiformis FIRM.

turritellaris $\mathrm{Mü}$.

Ugauxana D'O. unicarinata Morrs.

Pyramidella Lr. 14 canaliculata D'O. carinata Reuss. cancellata NYsT elongata Fír. larvata Conr.

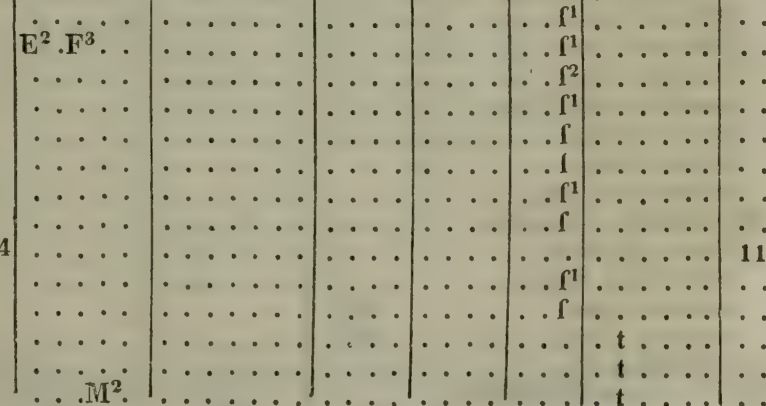




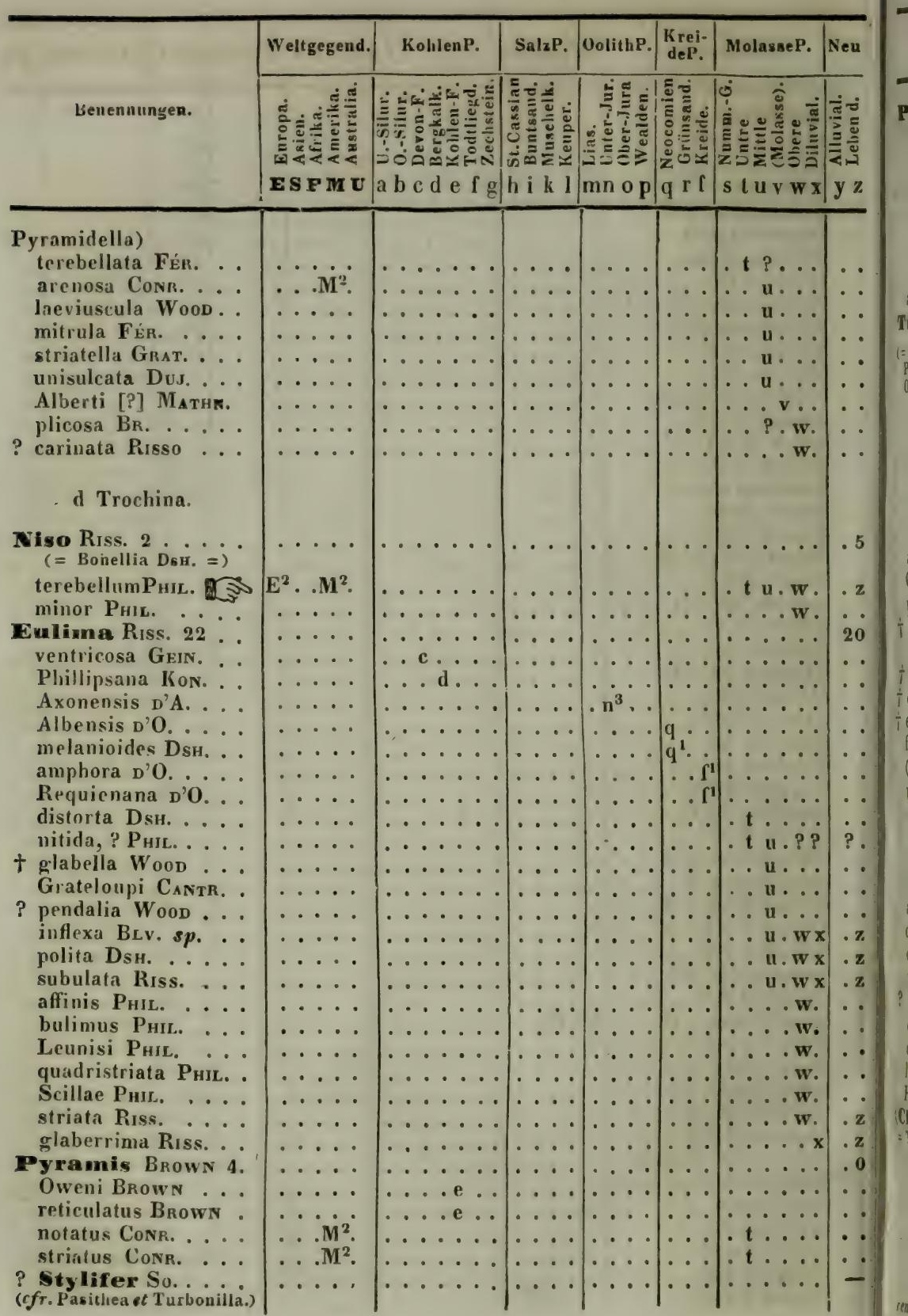




\begin{tabular}{|c|c|c|c|c|c|c|c|}
\hline a. & nd. & $\mathrm{g}$ & 1 & p & & $w \mathrm{x}$ & y \\
\hline 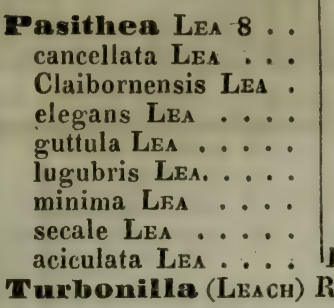 & $\begin{array}{l}\cdots \mathrm{M}^{2} \\
\cdots \mathrm{M}^{2} \\
\cdots \mathrm{M}^{2} \\
\cdots \mathrm{M}^{2} \\
\cdots \mathrm{M}^{2} \\
\cdots \mathrm{M}^{2} \\
\mathrm{M}^{2} \\
\text { Risso } \mathrm{M}^{2} \\
\mathbf{B u}^{2}\end{array}$ & 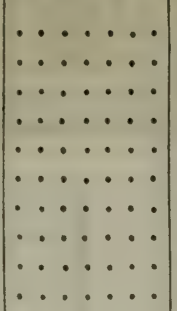 & $\mid \begin{array}{cc}\cdots \cdots \\
\cdots \\
\cdots \\
\cdots \\
\cdots \\
\cdots \\
\cdots \\
\cdots \\
\cdots \\
\cdots \\
\cdots\end{array}$ & $\begin{array}{ll}\cdots \cdots \\
\cdots \\
\cdots \cdots \\
\cdots \\
\cdots \\
\cdots \\
\cdots \\
\cdots \\
\cdots \\
\cdots \\
\cdots\end{array}$ & $\mid$\begin{tabular}{c|}
$\cdots$ \\
$\cdots$ \\
$\cdots$ \\
$\cdots$ \\
$\cdots$ \\
$\cdots$ \\
$\cdots$ \\
$\cdots$ \\
$\cdots$
\end{tabular} & 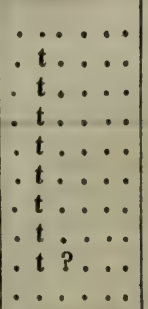 & $\begin{array}{l}10 \\
\therefore \\
\therefore \\
\therefore \\
\therefore \\
30\end{array}$ \\
\hline $\begin{array}{l}\text { 0. ' Loxor } \\
\text {; Pyrgise } \\
\text { b. ; ? Styli }\end{array}$ & & 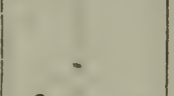 & & & & & \\
\hline 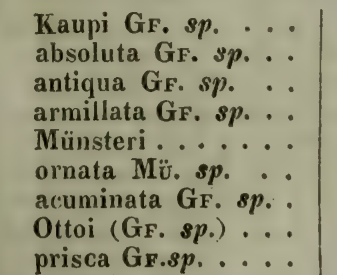 & $\begin{array}{l}\cdots \cdots \\
\cdots \cdots \\
\cdots \\
\cdots \\
\cdots \\
\cdots \\
\cdots \\
\cdots \\
\cdots \\
\cdots\end{array}$ & 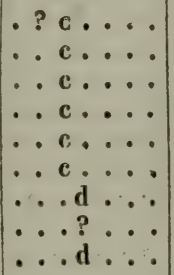 & $\begin{array}{cc}\cdots & \cdots \\
\cdots & \cdots \\
\cdots & \cdots \\
\cdots & \cdots \\
\cdots & \cdots \\
\cdots & \cdots \\
\cdots & \cdots \\
\cdots & \cdots\end{array}$ & $\begin{array}{cc}\cdots & \cdots \\
\cdots & \cdots \\
\cdots & \cdots \\
\cdots & \cdots \\
\cdots & \cdots \\
\cdots & \cdots \\
\cdots & \cdots \\
\cdots & \cdots\end{array}$ & $\begin{array}{c}\cdots \\
\cdots \\
\cdots \\
\cdots \\
\cdots \\
\cdots \\
\cdots \\
\cdots\end{array}$ & 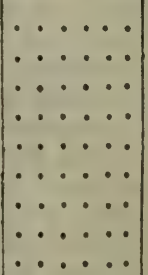 & $\begin{array}{l}\cdot \\
: \\
: \\
\end{array}$ \\
\hline ODAT. & $\therefore \cdots$ & $\cdots \cdots \cdots$ & & $\cdots \cdots$ & & $\therefore$ u. & \\
\hline OOD. & $\ddot{\cdots} \cdot \dot{ }$ & $\begin{array}{llll}\cdots & \cdots & \cdots \\
\cdots & \cdots & \cdots \\
\cdots & \cdots & \cdots\end{array}$ & $\cdots$ & $\begin{array}{ll}\cdots \\
\cdots \\
\cdots & \cdots \\
\cdots\end{array}$ & $\begin{array}{c}\cdots \\
\cdots \\
\cdots\end{array}$ & $\left|\begin{array}{ccc}\cdots & \mathbf{u} & \ldots \\
\cdots & \mathbf{u} & \cdots \\
\cdots & \mathbf{u} & \ldots\end{array}\right|$ & \\
\hline $\begin{array}{l}\text { D. . } \\
\dot{s p .})\end{array}$ & $\begin{array}{l}\cdots \\
\cdots \\
\cdots\end{array}$ & $\begin{array}{lll}\cdots & \cdots & \cdots \\
\cdots & \cdots & \cdots \\
\cdots & \cdots\end{array}$ & $\begin{array}{l}\cdots \\
\cdots \\
\cdots \\
\cdots\end{array}$ & $\left|\begin{array}{ll}\cdots & \cdots \\
\cdots & \cdots \\
\cdots & \cdots\end{array}\right|$ & $\begin{array}{l}\cdots \\
\cdots \\
\cdots \\
\cdots\end{array}$ & $\left|\begin{array}{ccc}\cdots & & \cdots \\
\cdots & \text { u. } & \ldots \\
\cdots & \text { u. } & \cdots\end{array}\right|$ & \\
\hline $\begin{array}{l}\text { uricula Grat } \\
\text { GRAT } s p .\end{array}$ & $s p . \ldots$ & $\begin{array}{ll}\cdots & \cdots \\
\cdots & \cdots\end{array}$ & $\cdots \cdots$ & $\cdots \cdots$ & $\cdots$ & $\begin{array}{l}\ldots \text { u. . } \\
\ldots \text { u. }\end{array}$ & \\
\hline \begin{tabular}{ll|} 
AT & $s p$. \\
OOD & $\ldots$
\end{tabular} & $\ldots \ldots$ & $\cdots \cdots$ & $\cdots$ & $\cdots$ & $\cdots$ & $\because \begin{array}{ll}\cdots \\
\cdots\end{array}$ & \\
\hline T. $s p$ & $\cdots \cdots$ & $\begin{array}{ll}\cdots \\
\cdots\end{array}$ & $\left|\begin{array}{l}\cdots \\
\cdots \\
\cdots\end{array}\right|$ & & & $\begin{array}{ll}\cdots & \mathbf{u} \\
\cdots & \mathbf{u}\end{array}$ & \\
\hline & & $\cdots \cdots$ & $\cdots \cdot$ & & $\ldots$ & $\mathbf{w}$ &.$z$ \\
\hline OD .. & & $\begin{array}{lll}\cdots & \cdots & \cdots \\
\cdots & \cdots & \cdots\end{array}$ & & $\cdots \cdots$ & & $\cdots$ & - $z$ \\
\hline Risso ... & . & $\ldots \ldots$ & . & & ... & $\ldots 1$ & . $\mathrm{z}$ \\
\hline D..... & $\cdots$ & $\cdots \cdots$ & $\cdots \cdot$ & $\cdots \cdot$ & $\ldots$ & $\cdots 1$ & $x$ \\
\hline sso .... & $\cdots$ & $\cdots \cdots \cdots$ & $\cdots \cdots$ & $\cdots$ & … & $\begin{array}{c}\cdots w \\
\cdots \cdots w \\
\cdots\end{array}$ & \\
\hline - & $\because$ & $\ldots \ldots$ & & $\cdots$ & & .... w. & \\
\hline 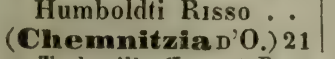 & & & & & & & \\
\hline & & & & & & & \\
\hline arbonaria KoN. . . & & & & & & & \\
\hline
\end{tabular}

* Huec genera includunt animalia proboscidifera, inde cum Ampultarin ad Buecinoiden removenderunt, quando a reliquis satis distingui et e testa recognosci poterunt. 


\begin{tabular}{|c|c|c|c|c|c|c|c|}
\hline \multirow{2}{*}{ Lencsinungea. } & Weltgegend. & KohienP. & Sal «P. & OolithP. & $\begin{array}{l}\text { Krei- } \\
\text { deP. }\end{array}$ & MolasseP. & Neu \\
\hline & 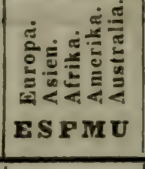 & 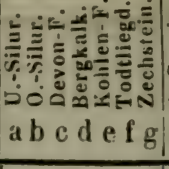 & $\begin{array}{l}0 \\
\text { h i k l }\end{array}$ & 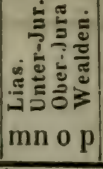 & 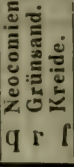 & 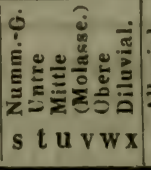 & 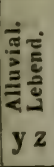 \\
\hline \multicolumn{8}{|l|}{ Chemnitzia) } \\
\hline conetro & . & $\ldots d \ldots$ & & & • & $\ldots \ldots$ & . \\
\hline elongata 1 & $\because$ & $\begin{array}{ll}\cdots d & . \\
\ldots d & .\end{array}$ & & & & $\cdots \cdots$ & . \\
\hline & $\ldots$ & $\cdots$ & $\cdots$ & $\because$ & $\cdots$ & $\cdots \cdots$ & ・ \\
\hline $\mathbf{L}$ & $\cdots$ & $\ldots d \ldots$ & ... & $\ldots$ & $\cdots$ & $\ldots \ldots$ & . \\
\hline KON. & & ... & $\ldots$ & $\ldots$ & $\ldots$ & $\ldots \ldots$ & . \\
\hline . & $\cdots$ & ... & $\cdots \cdot$ & $\cdots \cdot$ & $\cdots$ & $\ldots \ldots$ & 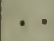 \\
\hline 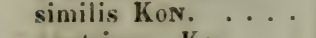 & $\cdots$ & $\ldots d \ldots$ & $\ldots$ & $\ldots$. & $\cdots$ & $\ldots \ldots$ & 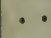 \\
\hline $\mathrm{v}$ & … & $\ldots d \ldots$ & $\cdots$ & $\cdots \cdot$ & $\cdots$ & $\ldots \ldots$ & sis \\
\hline$\because$ & $\cdots M^{3}$ & $\cdots \cdots$ & - ? ? & $\cdots \cdot$ & $\cdots$ & $\cdots \cdots$ & - \\
\hline D'O. . & $\ldots$ & $\cdots$ & $\cdots$ & . $11 .$. & $\cdots$ & $\cdots \cdots$ & - \\
\hline . . & & $\ldots \ldots$ & $\ldots$ & $\ldots$ & ? . & $\cdots$ & 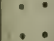 \\
\hline & - ख्य. & $\ldots$ & ... & $\ldots$. & . $r \int^{1}$ & $\ldots \ldots$ & ・ \\
\hline$\cdots$ & ... & $\ldots$ & $\cdots$ & $\ldots$ & $\ldots \Gamma^{1}$ & $\ldots \ldots$ & - \\
\hline $0 . \cdots$ & . & $\ldots \ldots$ & $\ldots$ & $\ldots$ & $\ldots \boldsymbol{I}^{1}$ & $\ldots \ldots$ & - \\
\hline el & $\cdots \cdot \cdot$ & $\cdots \cdots$ & $\ldots$ & $\cdots \cdot$ & $\cdots$ & $\ldots \cdot \mathbf{w} \cdot$ & - \\
\hline$\cdots$ & $\cdots \ldots$ & $\ldots \cdots$ & $\ldots$ & $\ldots$ & $\cdots$ & ...w & - \\
\hline L. . : & $\cdots$ & $\cdots \cdots$ & $\cdots$ & $\cdots \cdots$ & $\cdots$ & …w. & $\cdots$ \\
\hline \multicolumn{2}{|l|}{$\begin{array}{l}\text { (EoxonemaPhiLI.) } 13 \text {. } \\
=\text { Turbonilla (Leach) Rirs. = }\end{array}$} & & $\cdots$ & $\cdots \cdots$ & $\cdots$ & $\cdots \cdots$ & - \\
\hline ? obscurum Por & $\ldots \cdot$ & . b.. & . $\cdot$ & $\cdots \cdot$ & $\cdots$ & $\cdots \cdots$ & - \\
\hline$\cdots$ & $\cdots \cdots$ & . bc .... & $\cdots \cdots$ & $\cdots$ & - & $\cdots \cdots$ & - \\
\hline$\cdots$ & $\cdots \cdot$ & $\ldots \mathbf{c} \ldots$ & $\cdots \cdot$ & $\cdots \cdot$ & . & $\cdots \cdots$ & - \\
\hline Pнiц.. & 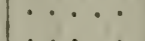 & 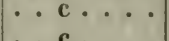 & $\cdots$ & $\cdots \cdots$ & $\cdot$ & $\cdots \cdots$ & $\because$ \\
\hline$\cdots$ & $\cdots$ & $\begin{array}{lll}\cdots & \ldots & \cdots \\
\cdots & \ldots & \ldots\end{array}$ & $\cdots$ & $\cdots \cdots$ & $\because$ & 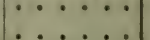 & - \\
\hline eritum Phics. . & . . . & . c... & $\ldots$ & $\ldots$ & $\ldots$ & $\ldots \ldots$ & $\therefore$ \\
\hline PhiLL. . & $\cdots$ & . . c... & $\ldots$ & $\ldots$ & . & $\ldots \ldots$ & - \\
\hline & & ... & $\cdots$ & $\ldots$ & $\cdots$ & $\therefore \ldots$ & $\cdots$ \\
\hline . . & & . . cd. & $\cdots \cdot$ & $\cdots$ & . . & $\ldots \ldots$ & 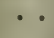 \\
\hline $\mathbf{P H}_{\mathbf{H}}$ & & $\cdot c d .$. & $\cdots \cdot$ & $\cdots$ & $\cdot$ & $\because: \cdots$ & \\
\hline RTL. & & $\cdots \cdot d$ & $\cdots \cdot$ & $\cdots$ & $\cdots$ & $\cdots \cdots$ & . \\
\hline (Melania LK. pars) & 11... & $\cdots$ & $\cdots$ & $\ldots$ & $\cdots$ & $\cdots \cdots$ & - \\
\hline distr & in & & & & & & \\
\hline & & $e$ & $\cdots$ & $\cdots \cdots$ & $\cdots$ & $\cdots \cdots$ & - \\
\hline${ }^{\circ}$ & $\bullet$ & ..c. & $\cdots$ & $\cdots$ & $\cdots$ & $\ldots \ldots$ & $\cdots$ \\
\hline GF. . & . & $\cdots \mathbf{c}$ & $\cdots$ & $\cdots$ & . & $\cdots \cdots$ & $\cdots$ \\
\hline tricmeta nove & & . c . & & & 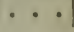 & $\cdots \cdots$ & \\
\hline
\end{tabular}
censel.

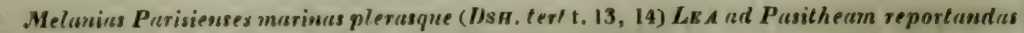




\begin{tabular}{|c|c|c|c|c|c|c|c|}
\hline Benennungen. & Weltgegend. & $a b c$ & h i k l & $\mathrm{mn} 0 \mathrm{p}$ & qre & stu & $y x$ \\
\hline Kielcensis Рúsch . . & $\cdots$ & . .?? . . & $\cdots$ & $\cdots$ & & $\cdots \cdots$ & \\
\hline abbreviata KLI. - . & $\ldots$. & $\ldots \ldots$ & h... & $\cdots$ & $\cdots$ & $\ldots \ldots$ & 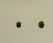 \\
\hline acute-striata KLI. . . & $\ldots \ldots$ & $\ldots \ldots$. & h... & $\ldots$ & $\cdots$ & $\ldots \ldots$ & . \\
\hline Albertii KLI. .... & $\ldots$ & $\ldots \ldots \ldots$ & h... & $\cdots \cdot$ & $\cdots$ & $\ldots \ldots$ & - \\
\hline angusta Mü. . . . & $\ldots$ & $\ldots \ldots$ & h... & $\cdots \cdot$ & $\cdots \cdot$ & $\cdots \cdots$ & - \\
\hline des KLI. & $\cdots$ & & . & $\cdots \cdot$ & $\cdots \cdot$ & $\cdots \cdots$ & $\cdots$ \\
\hline Brongniarti Krr. . & $\cdots \cdots$ & $\cdots$ & $\cdots$ & $\cdots \cdot$ & $\cdots$ & $\cdots \cdots$ & - \\
\hline$\cdots$ & $\cdot$ & & $\cdot$ & $\cdots \cdot$ & $\cdots \cdot$ & $\cdots \cdots \cdots$ & $\cdot \cdot$ \\
\hline LI. . . & $\cdots \cdots$ & $\cdots$ & $\cdot \cdot$ & $\cdots \cdots$ & $\cdots$ & $\cdots \cdots$ & - \\
\hline$\therefore \cdots$ & $\begin{array}{l}\cdots \cdots \\
\cdots\end{array}$ & $\cdots \cdots \cdots$ & $\therefore$ & $\mid \cdots$ & 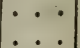 & & $\because$ \\
\hline & $\begin{array}{l}\cdots \\
\cdots\end{array}$ & $\ldots \ldots \ldots$ & h... & $\cdots$ & $\cdots$ & $\mid \cdots \cdots$ & $\because$ \\
\hline $\begin{array}{l}\text { concentrica Mü. . } \\
\text { conica Mü. . . . }\end{array}$ & $\ldots$ & $\ldots \ldots$ & h... & $\ldots$. & $\ldots$ & $\ldots \ldots$ & $\because$ \\
\hline crassa Mü. ..... & ... & $\ldots \ldots$ & . & $\ldots$. & $\cdots$ & $\ldots \ldots$ & . \\
\hline eri Kri. . . . & $\ldots \ldots$ & $\ldots \ldots$ & h ... & $\cdots$ & $\ldots$ & $\ldots \ldots$ & . \\
\hline falcifera KLI. . . . & $\ldots$ & $\ldots \ldots \ldots$ & h ... & $\ldots$. & $\ldots$ & $\ldots \ldots$ & - \\
\hline formosa Kul. . . . & $\ldots$ & $\ldots \ldots .$. & h... & $\cdots$ & $\cdots$ & $\ldots \ldots$ & - \\
\hline fusiformis Mü. . . . & $\ldots$ & $\ldots \ldots \ldots$ & h ... & $\cdots$ & $\cdots$ & $\ldots \ldots$ & - \\
\hline İ̈. . . . & $\cdots \cdot$ & $\cdots \cdots \cdot 1$ & h... & $\ldots$ & $\cdots$ & $\ldots \ldots$ & - \\
\hline wi KLI. . . . & $\ldots$ & $\ldots \ldots \ldots$ & h... & $\ldots$ & $\cdots$ & $\ldots \ldots$ & - \\
\hline i KLI. . . . . . & $\ldots$ & $\cdots$ & $\cdot$ & $\cdots$ & $\cdots$ & $\ldots \ldots$ & - \\
\hline Hauslabi KuI. . . . . & . & $\cdots \cdot$ & b... & $\cdots \cdot$ & $\cdots \cdot$ & $\cdot \cdot$ & - $\cdot$ \\
\hline KLI. $\cdots$ & $\cdot \cdot$ & $\cdots$ & h. & $\cdots \cdot$ & $\cdots$ & $\cdots$ & - \\
\hline a Mü. . & · & 1 & & $\cdots$ & $\cdots$ & $\because$ & - \\
\hline Mü.... & $\therefore$ &. i & & $\cdots \cdots$ & $\cdots$ & $\because \cdots$ & $\because$ \\
\hline ata $\dot{K}_{\text {LI. }} \cdots$ & $\cdots \cdots$ & $\cdots$ & $h \ldots$ & $\left|\begin{array}{ll}\cdots \\
\cdots\end{array}\right|$ & $\cdots$ & $\begin{array}{ll}\cdots \\
\cdots\end{array} \cdots$ & $\because$ \\
\hline ima Mü. . . . & ... & $\ldots$ & h... & $\ldots$ & $\ldots$ & $\ldots \ldots$ & $\cdots$ \\
\hline x. . . & $\ldots \ldots$ & $\ldots$ & h. & $\ldots$ & $\cdots$ & $\ldots \ldots$ & . \\
\hline Mü. . & $\ldots \ldots$ & $\ldots$. & & $\ldots$ & $\cdots$ & $\ldots \ldots$ & . \\
\hline$\ldots$. & $\ldots \ldots$ & $\ldots \ldots \ldots$ & 11. & $\cdots$ & $\cdots$ & $\ldots \ldots$ & - \\
\hline a Mü. . . . . & $\cdots$ & $\cdots$ & . & $\ldots$ & $\ldots$ & $\ldots \ldots$ & $\cdots$ \\
\hline ta BR. & - & & & $\cdots$ & $\cdots$ & $\ldots \ldots$ & $\cdots$ \\
\hline$\cdots$ & $\cdots \cdot$ & . . & $\mathrm{n}$ & $\cdots$ & $\cdots$ & $\cdots \cdots$ & - \\
\hline paludinaris Mü. . . & $\ldots \ldots$ & $\ldots$ & & $\cdots$ & $\cdots$ & $\cdots$ & - \\
\hline Partschi KL.I. . . . . & $\cdots \cdot$ & $\ldots \ldots$ & & $\because \cdots$ & $\cdots$ & $\cdots \cdots$ & - \\
\hline$\cdots$ & $\cdots \cdot \cdot$ & ... & & $\cdots$ & $\cdots$ & $\cdots$ & $\cdot \cdot$ \\
\hline$\cdots$ & $\cdots \cdots$ & . & . & $\ldots$ & $\cdots$ & $\cdots$ & $\cdot \cdot$ \\
\hline ningeri KLI. . & $\cdots$ & & & $\cdots$ & $\cdots$ & $\because$ & $\cdots$ \\
\hline$\because \cdots$ & $\cdot$ & $\cdot$ & . & $\cdots \cdot$ & $\cdots$ & $\because$ & $\because$ \\
\hline ormis Mǘ. $\cdots$ & $\cdot$ & - & & $\cdots$ & & $\therefore$ & $\because$ \\
\hline . & $\because$ & $\cdots$ & h... & $\cdots$ & $\ldots$ & $\ldots \ldots$ & $\ldots$ \\
\hline ri KLI. . . . . & $\ldots$ & . . & . & ... & $\cdots$ & $\ldots \ldots$ & - \\
\hline st & $\cdots$ & $\cdots$ & & $\cdots \cdot$ & $\cdots$ & $\cdots \cdots$ & - \\
\hline . & . & & & $\cdot$ & $\cdots$ & $\cdots \cdots$ & $\cdots$ \\
\hline concentrica $\mathbf{M} \ddot{u}$. & & & & & $\cdots$ & $\cdots$ & • \\
\hline I. . . & & & & $\cdots$ & & $\cdot$ & \\
\hline subovata M & $\cdots$ & . & & $\cdots$ & & $\because$ & $\therefore$ \\
\hline $\begin{array}{l}\text { subscalaris } 1 \\
\text { subtorilis } \mathbf{M}\end{array}$ & • & 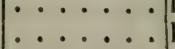 & & & • • & $\cdots \cdots$ & • \\
\hline & . & & & & & & \\
\hline
\end{tabular}




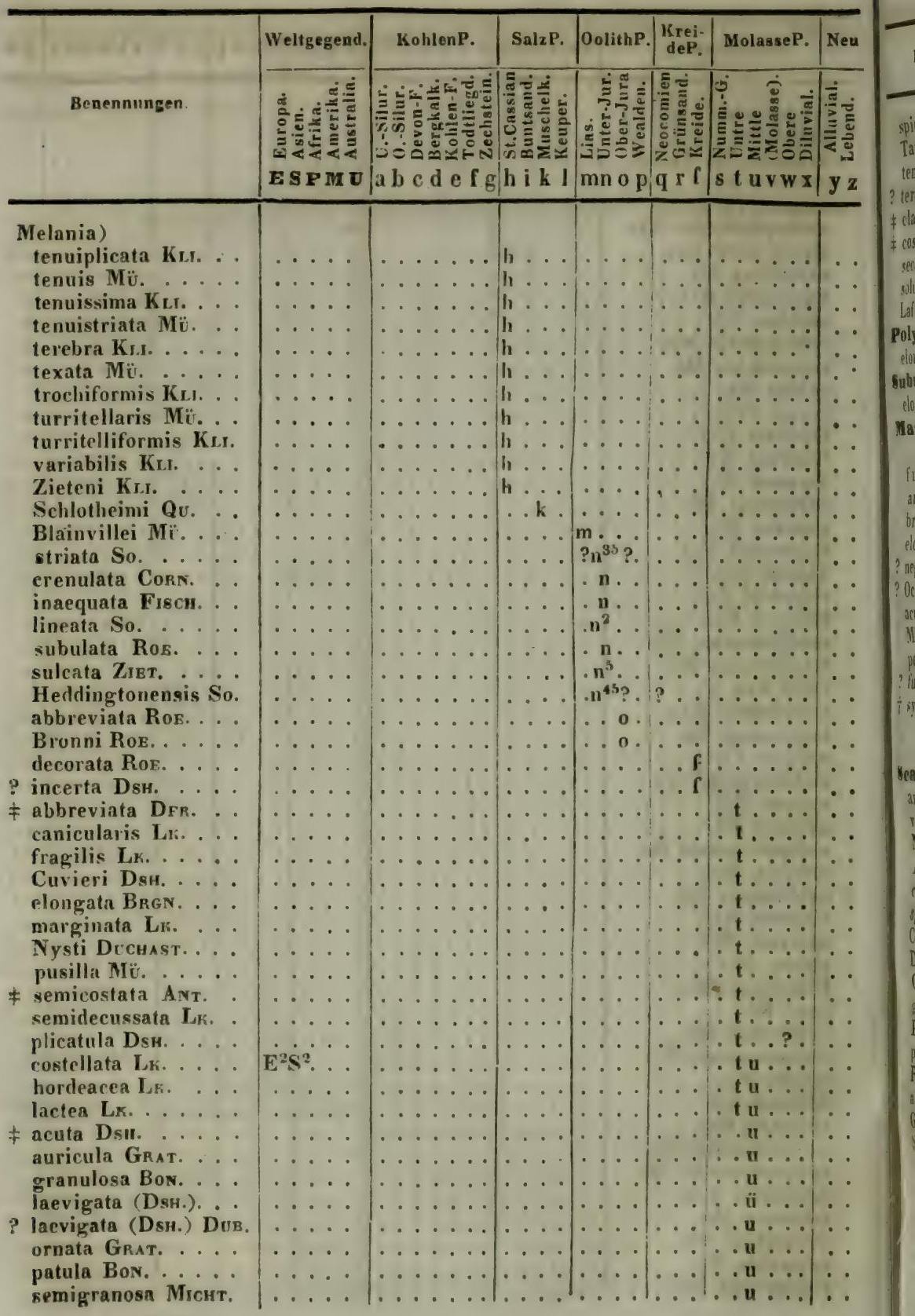




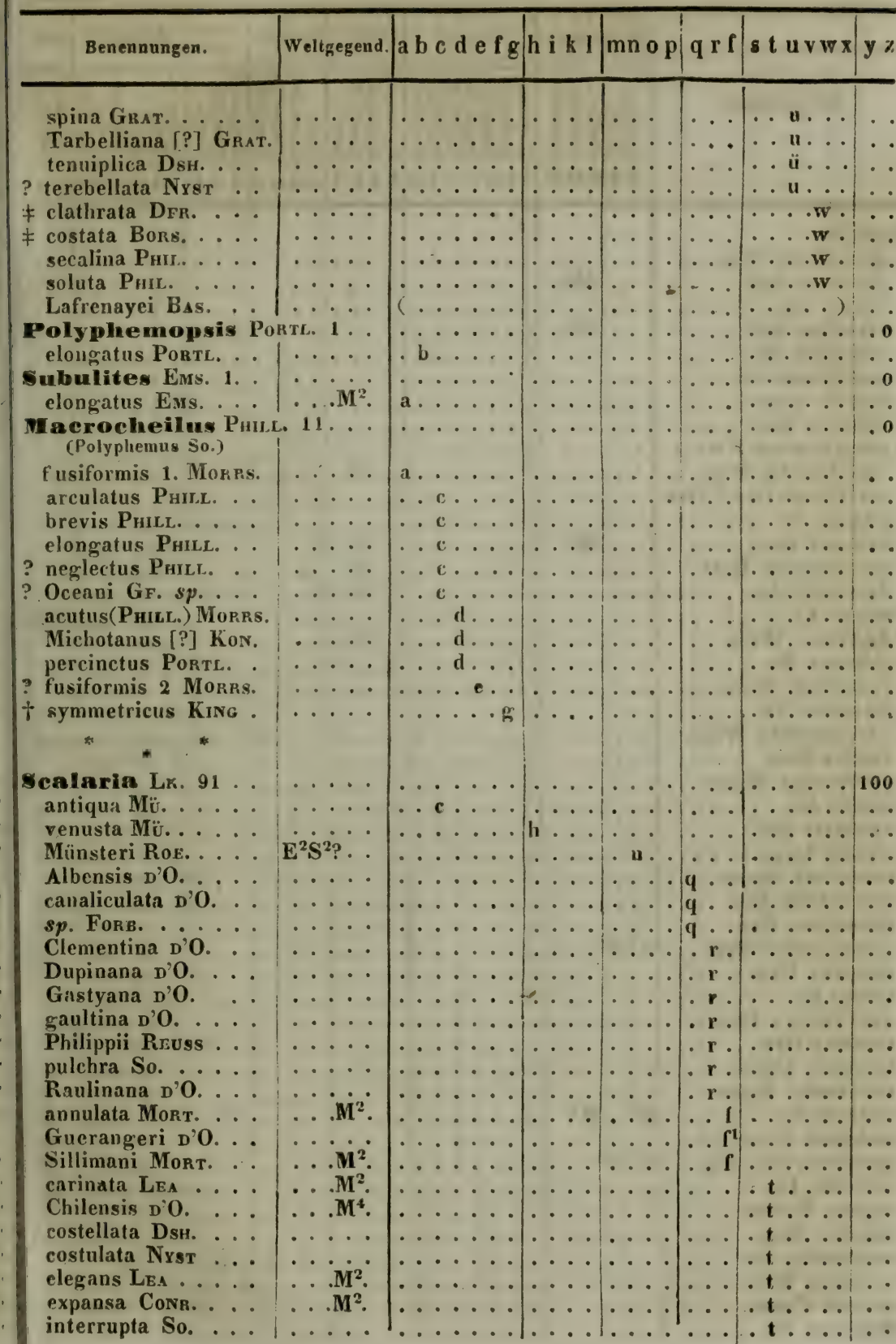




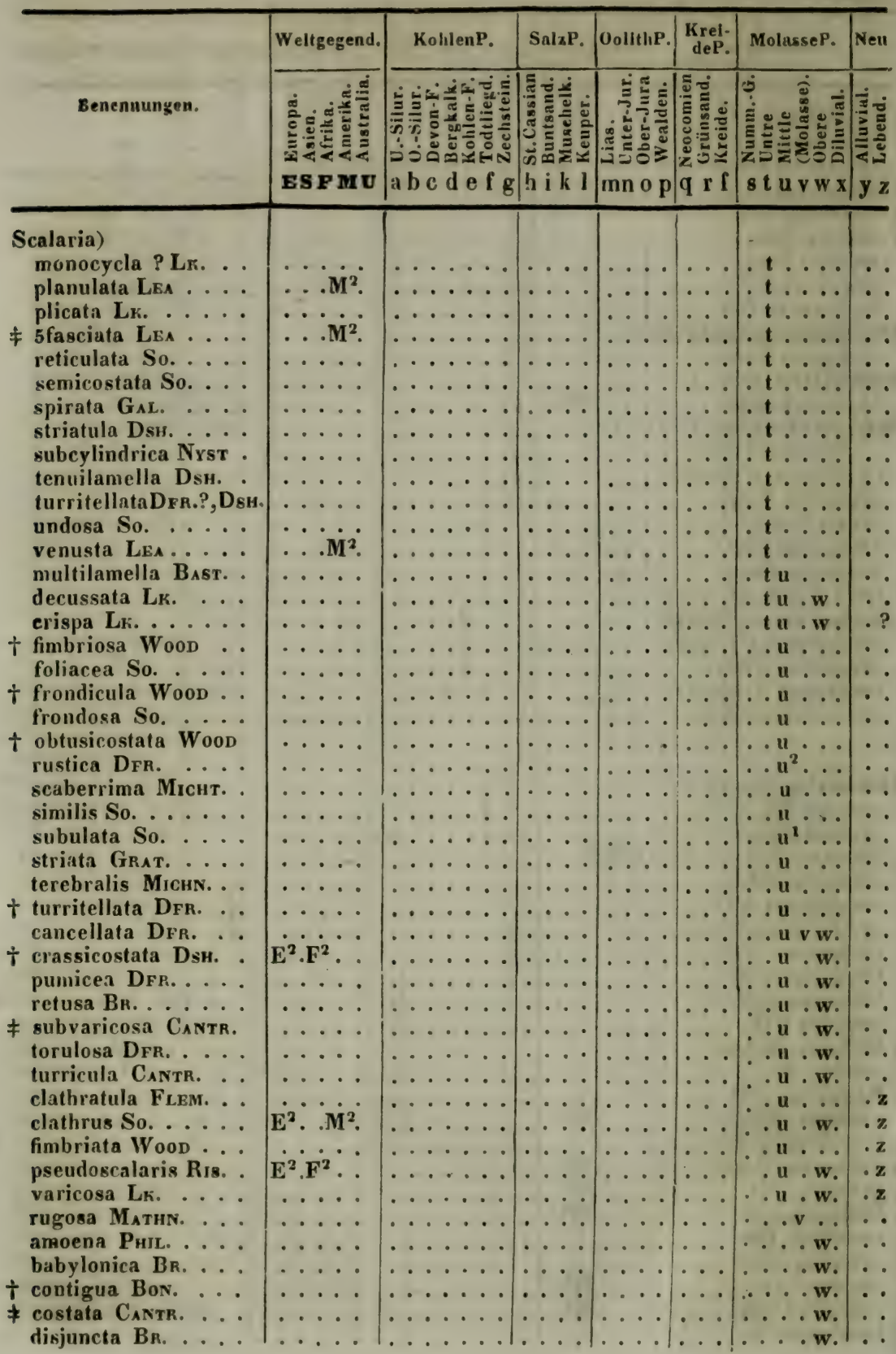




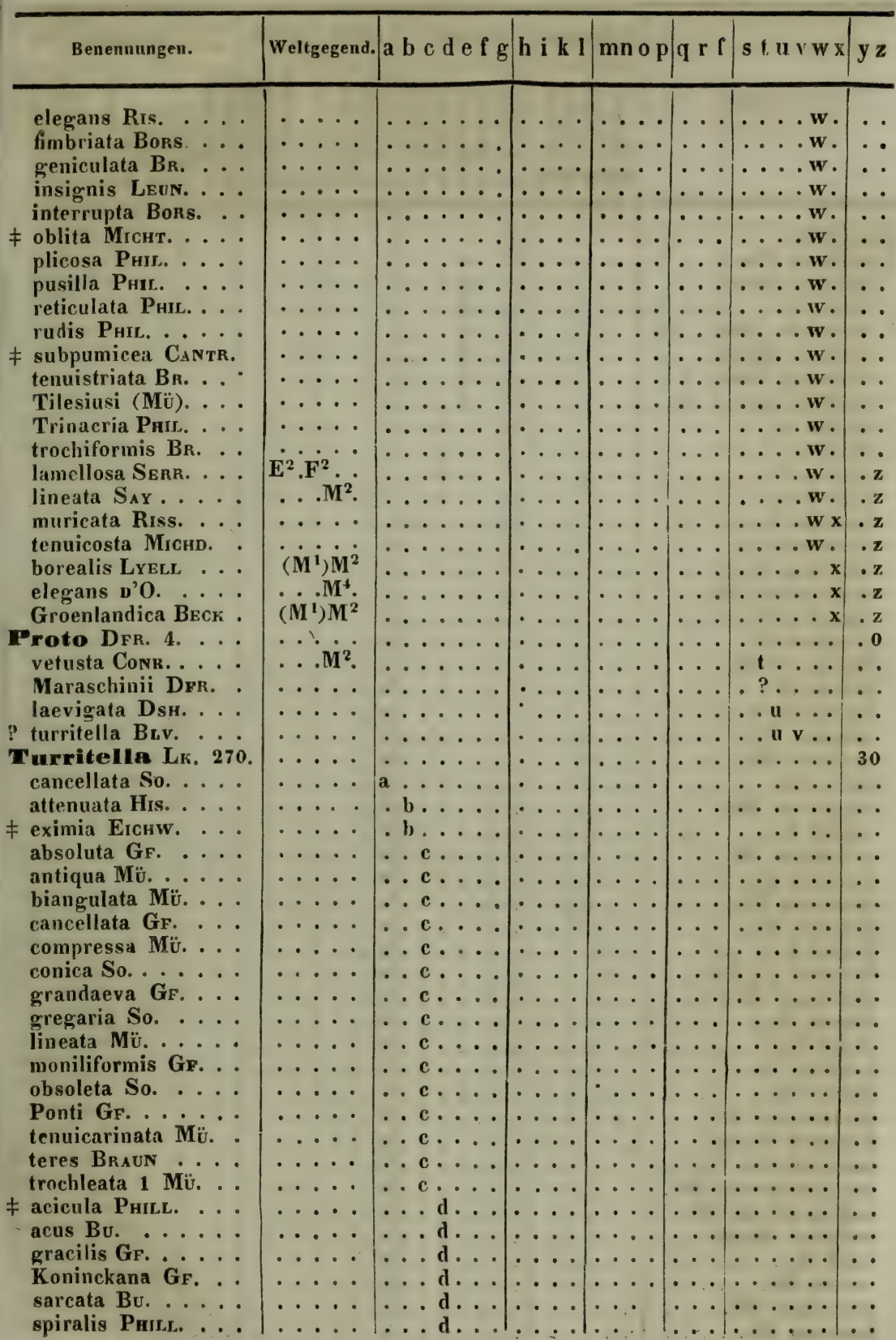




\begin{tabular}{|c|c|c|c|c|c|c|c|}
\hline & Weltgegend. & KohlenP. & SalzP. & nolithP. & $\begin{array}{c}\text { Krei- } \\
\text { deP. }\end{array}$ & MolasseP. & Neu \\
\hline lienennumgen. & 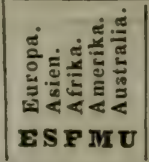 & 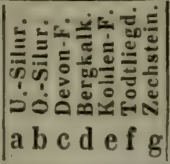 & 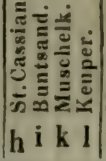 & 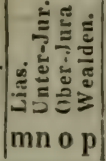 & 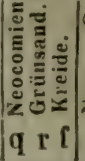 & 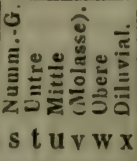 & $\mid \begin{array}{l}2 \\
y z \\
\frac{2}{4}\end{array}$ \\
\hline
\end{tabular}

Turritella)

? sulcifera Portr. suturalis PhiLL. tenuis GF.

? tenuistria Phill. tricincta Morks. triserialis Phick. turbinato-conica Mü.

? clavata So. elongata Flem. minima So.

Urei FLem.

biarmica KuTG. acuticosta KLI. Amalthea KLr. arcte-costata Mü. armata $M \ddot{u}$. binodosa $M \ddot{U}$. bipunctata Mü. Bolina Mü.

Bucklandi KLr. carinata Mü. cochleata Mü. colon Mü. compressa Mü. conica KLI. cylindrica Mü. decorata KLI. decussata Mü..... flexuosa Mü.

Fuchsi Kur.

Gaytani [?] Kiv Goldfussi Ku.

Haueri KLI.

Hehli KLI.

hybrida Mü.

Jaegeri KLI. .

Lommeli Wrssm. margaritifera Mü. margine-nodosa $\mathbf{M}_{\dot{v}}$. nodoso-plicata $M \ddot{\text {. }}$. nodulosa Braun nuda Kt.I. ornata Mü. perarmata Mü. punctata $\mathrm{M} \ddot{\mathrm{C}}$

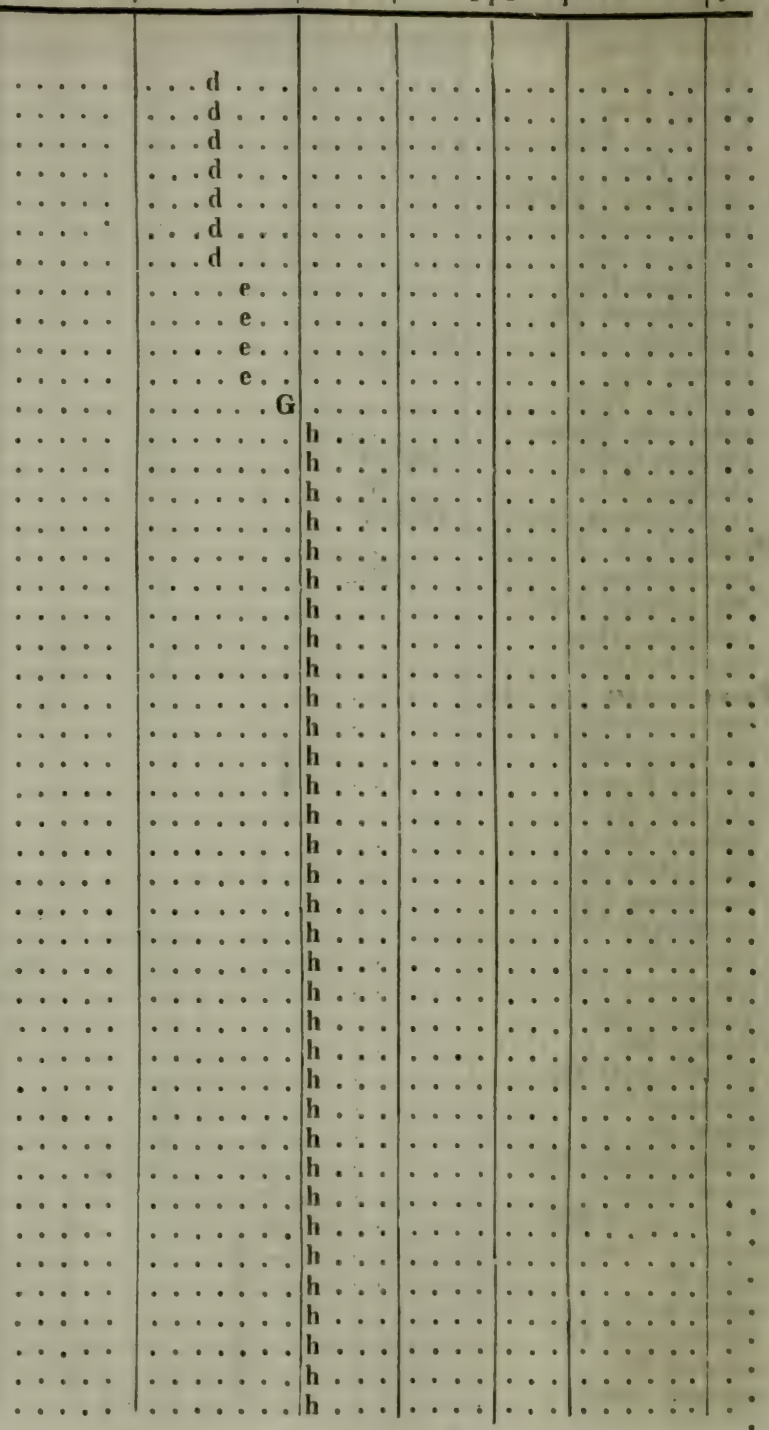




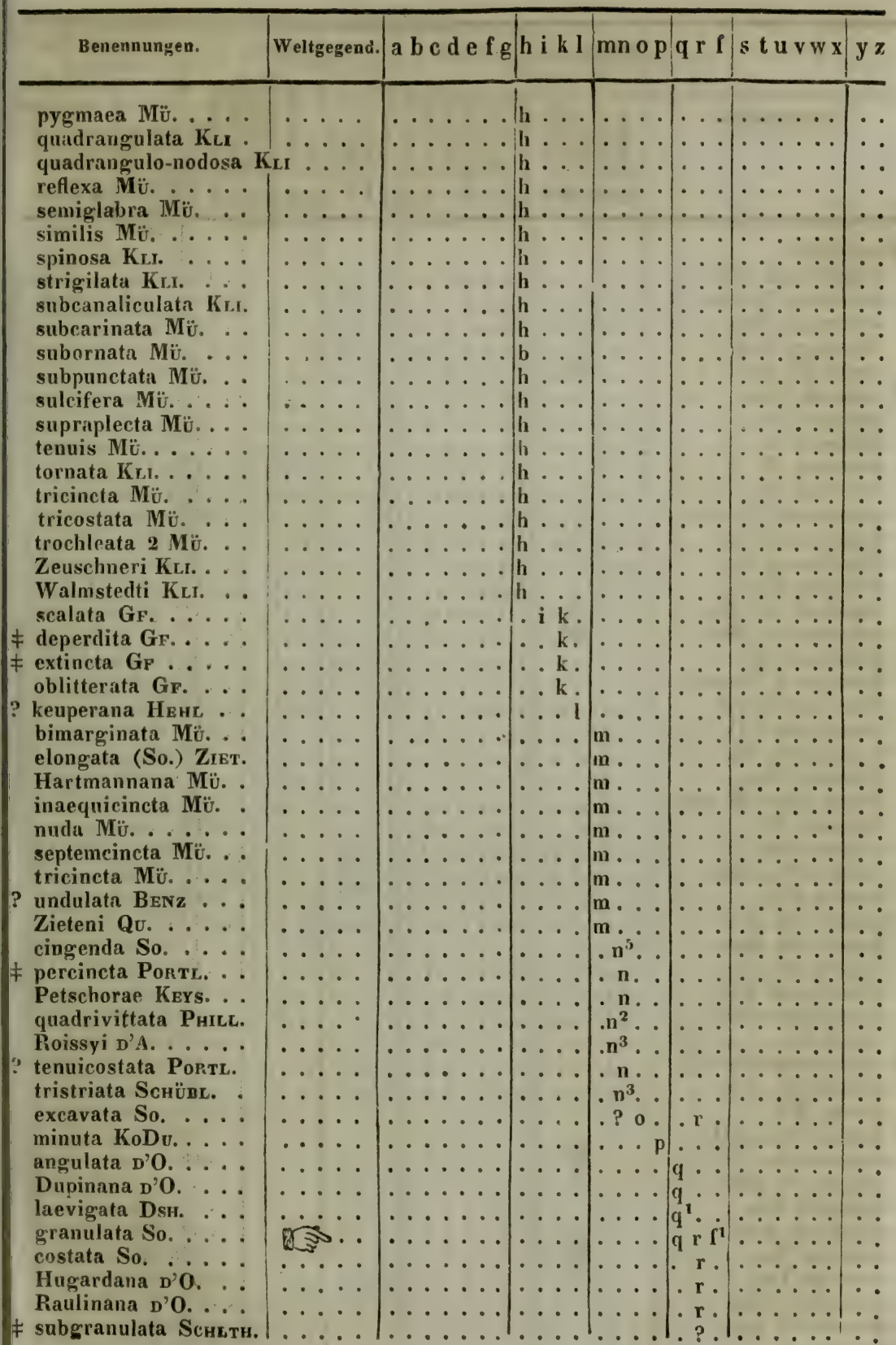




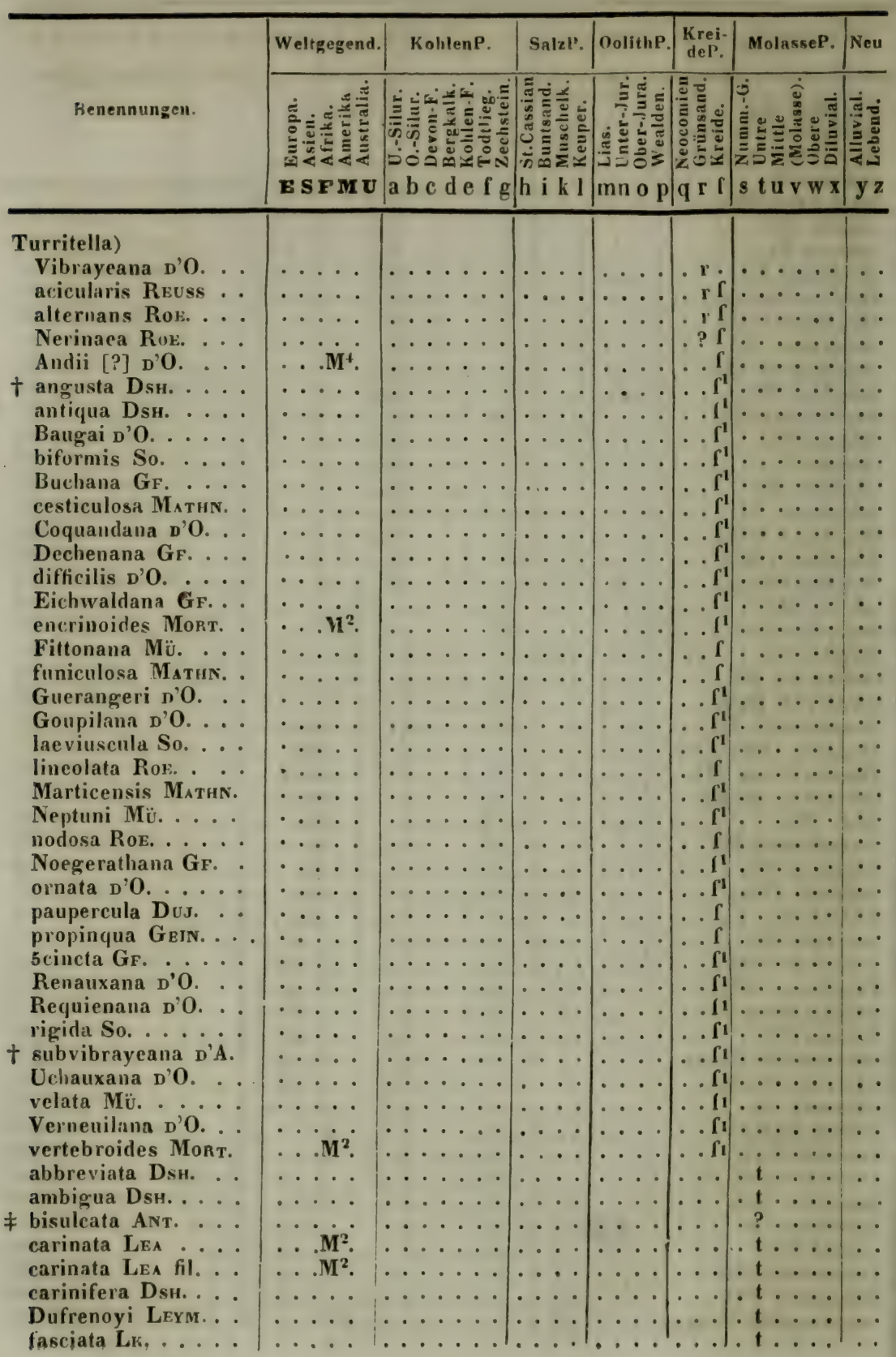




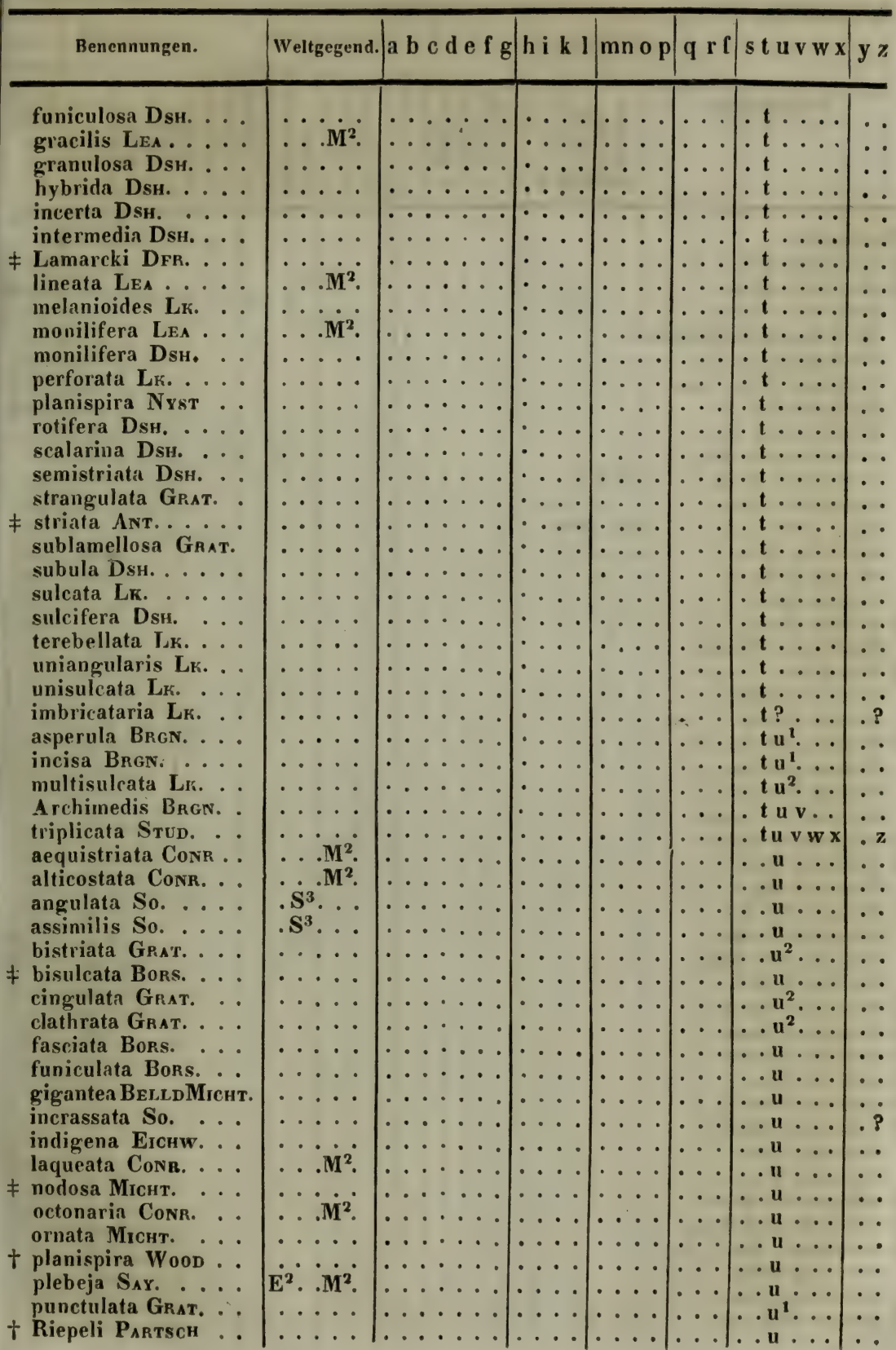




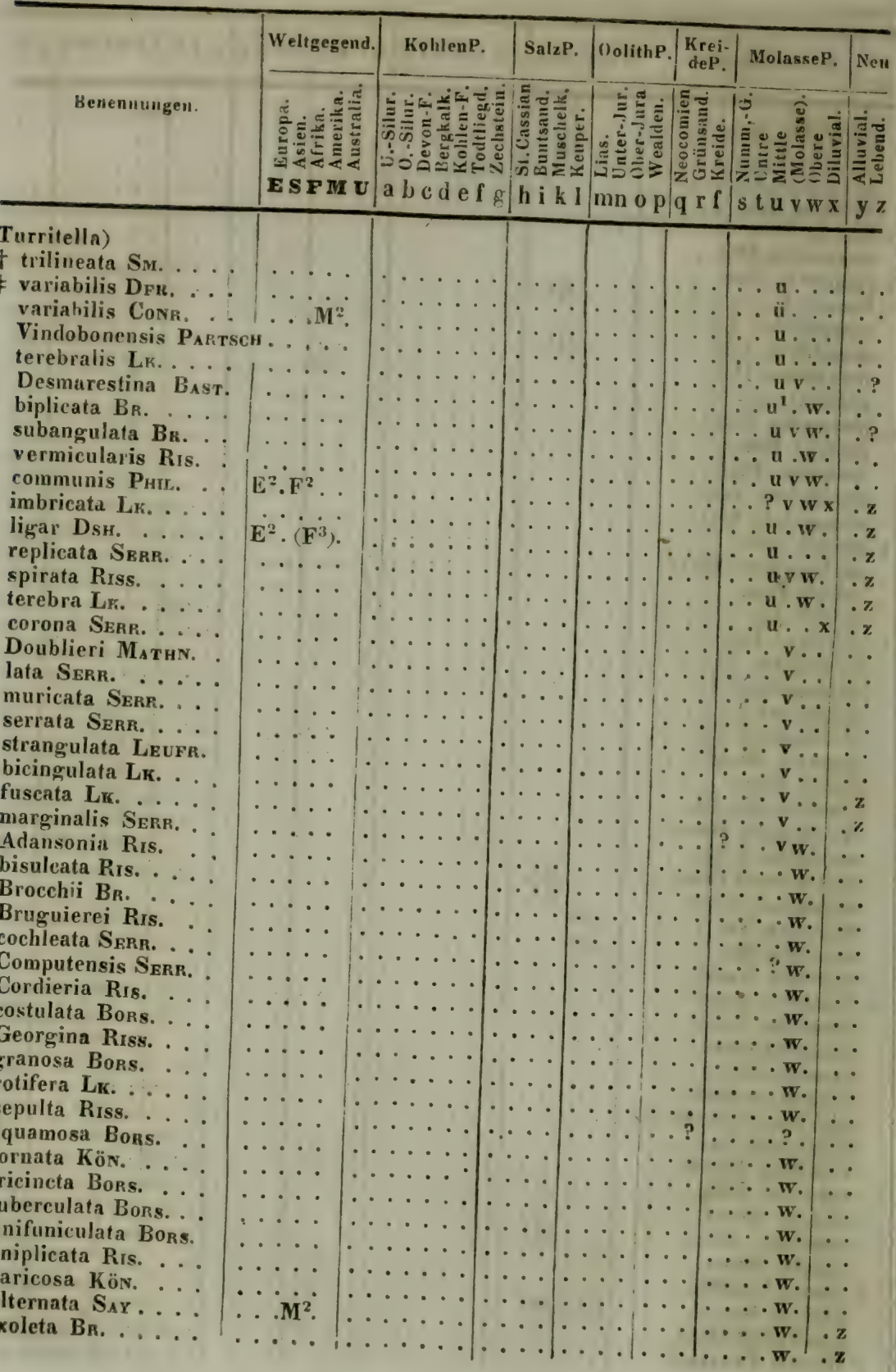




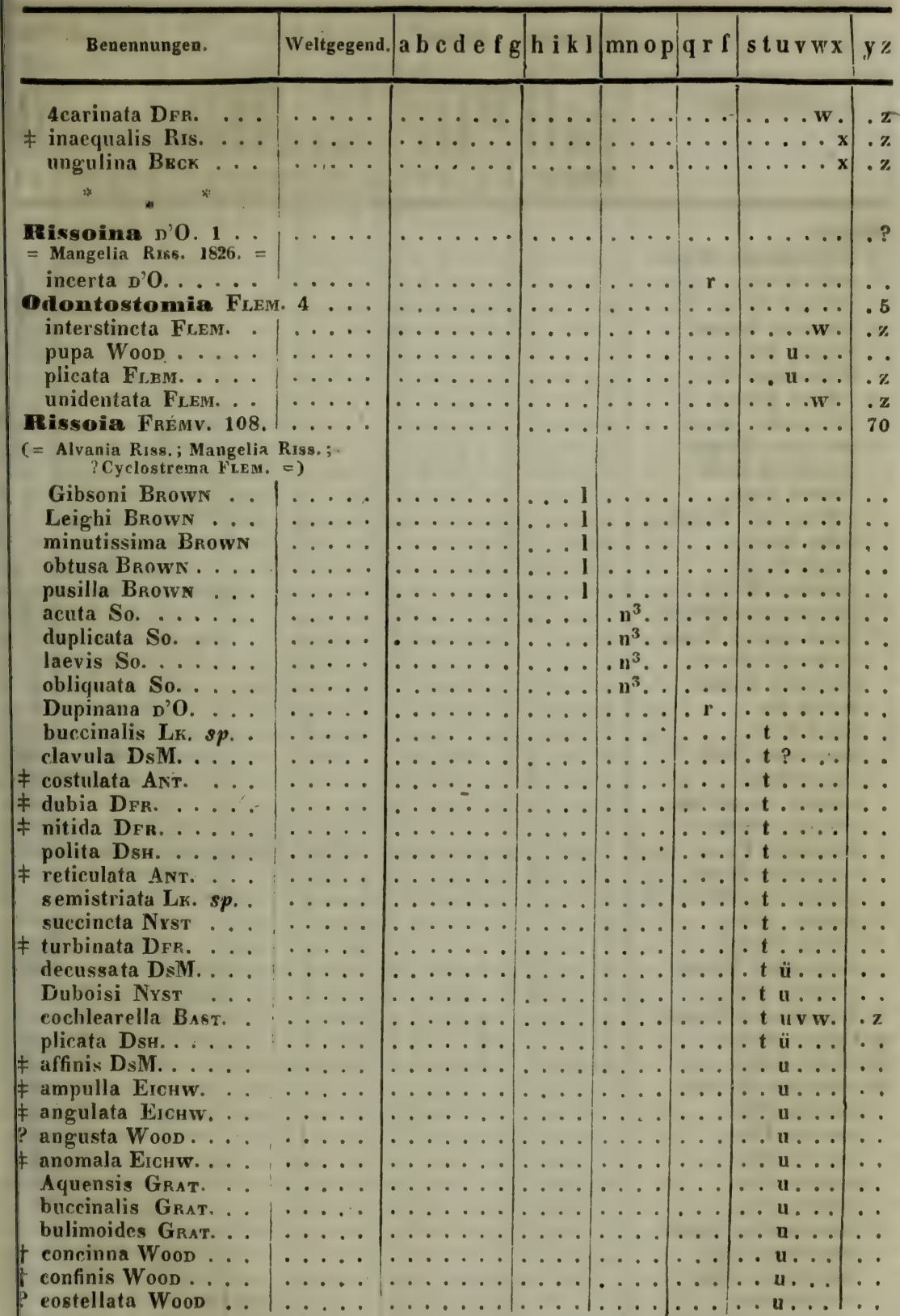




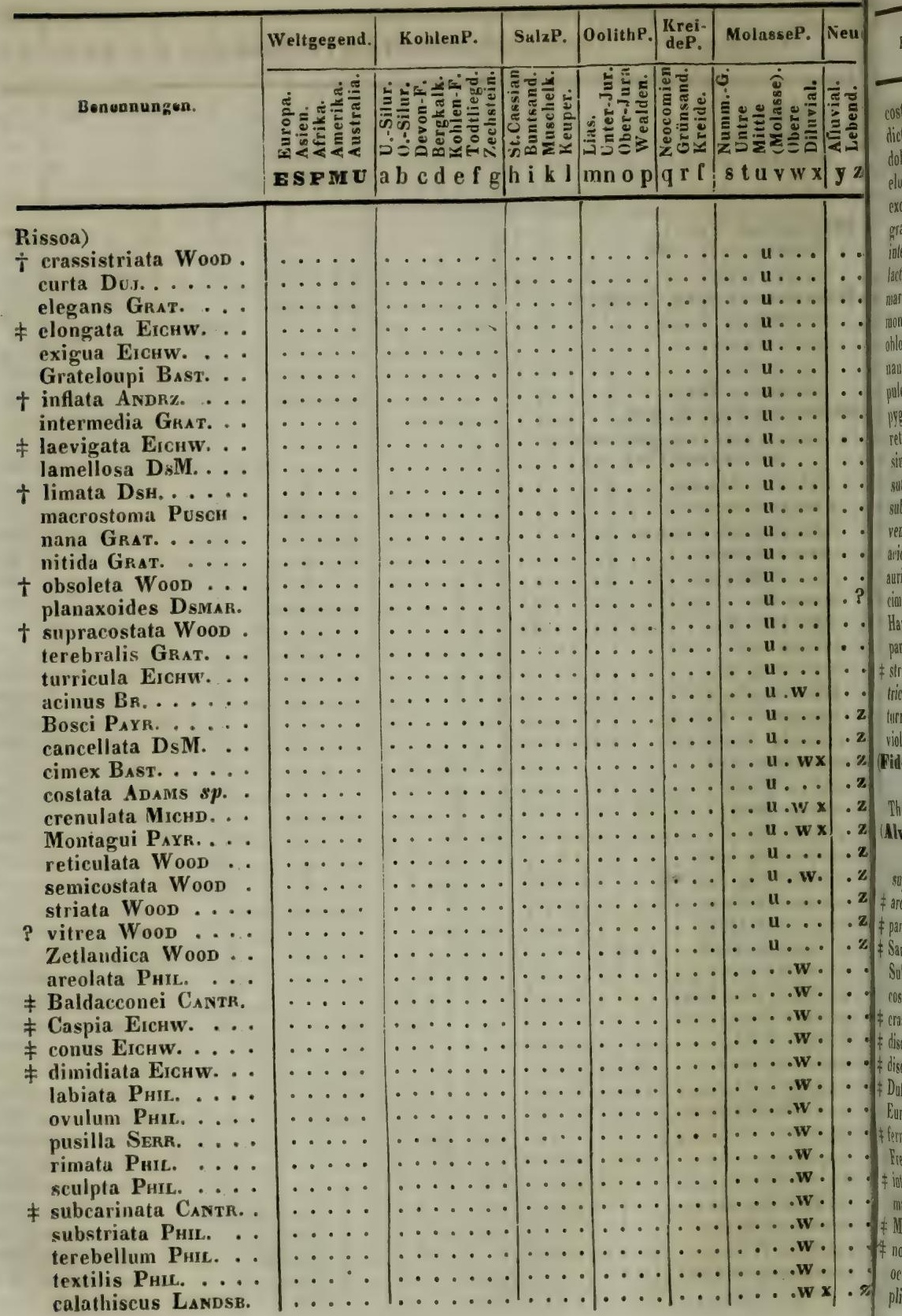




\begin{tabular}{|c|c|c|c|c|c|c|c|}
\hline Bene & end. & $\mathbf{f} g$ & hik I & $\mathbf{o p}$ & q r f $s$ & & \\
\hline & & & & & & . & \\
\hline & . & & & . . & 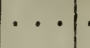 & $\cdots w$ & \\
\hline olum Pнiz. . . & $\cdots$ & . & & ... & . & $\cdots w$ & \\
\hline $\begin{array}{l}\text { gata Phil. . . } \\
\text { vata PHIL. . . }\end{array}$ & $\begin{array}{l}\cdots \cdots \\
\cdots\end{array}$ & $\cdots$ & 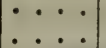 & ... & $\ldots$ & $\cdots \cdot \mathbf{w}$ & \\
\hline$\cdots$ & $\cdots$ & $\begin{array}{ll}\cdots & \cdots \\
\cdots & \cdots\end{array}$ & $\cdots$ & . & $\ddot{\cdots} \cdot \mid$ & $\cdots w_{0}$ & \\
\hline$\cdots$ & $\ldots \ldots$ & $\ldots \ldots$ & $\ldots \ldots$ & $\cdots$ & $\cdots$ & $\begin{array}{l}\cdots \cdots w \\
\cdots w\end{array}$ & .2 \\
\hline$\therefore$ & $\ldots$ & $\cdots$ & $\ldots$ & $\ldots$ & $\cdots$ & $\begin{array}{l}\cdots \\
\cdots \\
\cdots\end{array}$ & $2+2>$ \\
\hline$\cdots$ & $\cdots \cdot$ & $\cdots$ & $\cdots$ & $\ldots$ & $\cdots$ & …w. & \\
\hline . & $\cdots$ & . & $\cdots \cdot$ & $\cdots$ & $\cdots$ & $\cdots w$ & \\
\hline$\therefore$ & . & & $\cdots$ & $\cdots$ & $\cdots$ & $\ldots w \mathbf{w x}$ & . \\
\hline$\cdots$ & $\cdots$ & & $\cdots$ & $\cdots$ & $\cdots$ & $\cdots w^{r}$ & • \\
\hline$\therefore$ & $\because$ & $\cdots$ & - & $\cdots$ & . & $\cdots w$ & \\
\hline$\stackrel{.}{\cdots}$ & $\begin{array}{l}\cdots \\
\cdots\end{array}$ & $\ldots$ & $\cdots \cdots$ & $\cdots$ & . & $\cdots \cdots w$ & \\
\hline$\ddot{*}$ & $\ldots \ldots$ & $\ldots$ & $\cdots$ & $\ddot{\cdots}$ & $\cdots$ & $\begin{array}{l}\cdot \mathbf{w} \text {. } \\
\cdot \mathbf{w} \text {. }\end{array}$ & \\
\hline$\cdots$ & $\ldots$. & $\ldots$ & $\ldots$ & $\ldots$ & $\cdots$ & $\begin{array}{l}\cdots w^{w} \\
\cdots \cdots w\end{array}$ & $\cdot$ \\
\hline OOD & $\cdots$ & $\cdots$ & $\ldots$ & $\ldots$ & $\because$ & $\begin{array}{l}\cdots \cdot w \cdot \\
\cdots \cdots w\end{array}$ & 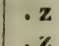 \\
\hline & $\cdots$ & $\cdots$ & $\ldots$ & $\ldots$ & $\cdots$ & $\cdots \cdots w$ & $\because 2$ \\
\hline & $\cdots \cdot$ & $\cdots$ & $\ldots$ & $\ldots$ & $\ldots$ & ..... & .2 \\
\hline . & $\cdots \cdot$ & $\cdots$ & $\cdots$ & $\cdots \cdot$ & $\cdots$ & $\ldots x$ & .2 \\
\hline & $\cdots$ & - $\cdot$ & .. & $\cdots \cdot$ & $\cdots$ & $\cdots \cdot x$ & .2 \\
\hline . & • & $\cdots \cdot$ & $\cdots$ & $\ldots$ & $\cdots$ & $\cdots \cdot x$ & .2 \\
\hline$\cdots$ & $\begin{array}{r}\cdot \\
\dot{*}\end{array}$ & $\therefore$ & $\cdots \cdot$ & $\cdots \cdot \mid$ & $\cdots$ & $\cdots \cdot x$ & .2 \\
\hline$\cdots$ & & 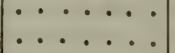 & $\cdots \cdot$ & $\cdots$ & - & $\cdot x$ & $\cdot 2$ \\
\hline$\cdots$ & $\begin{array}{l}\cdots \\
\cdots\end{array}$ & $\begin{array}{l}\cdots \\
\cdots\end{array}$ & $\cdots \cdot$ & $\cdots$ & $\cdot \cdot$ & $\cdot \mathbf{x}$ & . 2 \\
\hline$\cdots$ & $\ldots$ & $\cdots$ & $\cdots \cdot$ & $\cdots \cdot$ & $\cdots$ & $\cdot x$ & $\cdot 7$ \\
\hline 1. & . . . & $\because$ & - & $\cdots$ & ‥ t & $\cdot \mathbf{x}$ & $\cdot 2$ \\
\hline & & & & . & •. & & - \\
\hline & $\cdots$ & & & 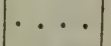 & & - $x$ & \\
\hline , Riss & & & & & $1^{\circ}$ & & - \\
\hline D . & $\cdot$ & $\cdot$ & . & - & $\cdots$ & - & - • \\
\hline$\cdots$ & $\cdots$ & $\cdots$ & $\cdots$ & $\cdots$ & - $\cdot$ & & - \\
\hline & $\cdots \cdots$ & $\cdot$ & $\cdots \cdot$ & $\cdots$ & - & $\cdot x$ & - \\
\hline$\cdots$ & $\cdots \cdots$ & $\cdot$ & - & $\cdots$ & - $\cdot$ & $\cdot x$ & \\
\hline$\cdots$ & $\cdots \cdots$ & . & $\cdots$ & $\cdot$ & & $x$ & \\
\hline & $\cdot$ & & . & $\cdot$ & . & $x$ & 7 \\
\hline & - & & & $\cdot$ & $\cdots$ & $x$ & \\
\hline & & & & • & - $\cdot$ & $\begin{array}{l}\cdot x \\
x \\
x\end{array}$ & \\
\hline$\cdots$ & $\cdots$ & $\cdots$ & $\cdots \cdot$ & & $\therefore$ & $\begin{array}{l}\cdot x \\
x\end{array}$ & 7 \\
\hline & .... & $\cdots$ & $\ldots$. & $\ldots$ & $\cdots$ & $\cdots x$ & \\
\hline & $\cdots$ & $\cdots$ & ... & ... & .. & $\ldots x$ & .2 \\
\hline s. . & $\cdots$ & & $\cdots$ & $\cdots$ & . & ... & 2 \\
\hline & . & $\cdots$ & $\theta^{\circ}$ & & $\cdots$ & $\ldots . . x$ & .2 \\
\hline & $\cdots$ & $\because$ & 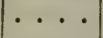 & $0^{\circ}$ & . & $\cdots \cdot x$ & \\
\hline & $\cdots$ & $\cdots \cdot$ & $\cdots \cdot$ & $\cdots$ & • $\cdot$ & $\cdots \ldots x$ & \\
\hline & - & . & $\cdots$ & $\cdots$ & $\cdots$ & $\cdots \cdot x$ & \\
\hline & & & $e^{-1}$ & : & $\cdots$ & $\cdots$ & \\
\hline
\end{tabular}




\begin{tabular}{|c|c|c|c|c|c|c|c|}
\hline & Weltgegend. & KohlenP. & SalzP. & OolithP. & \begin{tabular}{|c|} 
Krei- \\
deP.
\end{tabular} & MolasseP. & Neu \\
\hline Benennungen. & 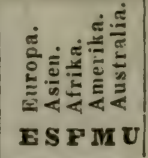 & 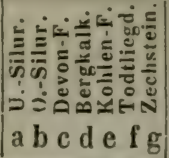 & 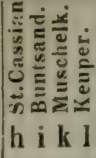 & 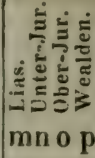 & 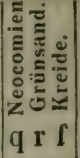 & 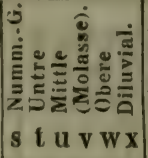 & 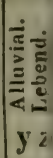 \\
\hline
\end{tabular}

Alvania)

† pyranidata Riss. .

‡ reticulata Riss. ...

* verrucosa Riss.

Cingula Erem, 2. . $=$ ? Rissoia $=$

cirgilla FuRM.

ventricosa FLEM.

Trumcatella Riss. 1 truncatula PHiL.

Cacuma Turt. 1 . vincta Turt.

Pliasianema Wood * lineolata Wood ... sulcata Wood

Plnasianella Lr. 29 (= Tricolia Riss. =) gigas Eıchw.

₹ prisca EICHw fusiformis Gr. neritoidea GF.

† striatella SNon. subclathrata RoE. . . Münsteri Wissm

? $s p$. Gait.L. . . . . cincta Phill.

Leymeriei D'A. neocomensis [?] D'O Ervyana D'O. formosa So. pusilla So. striata So. gaultina D'O. .... jineolata Reuss .. supracretacea D 0 .

* laevis DFr. . . . . \# princeps DrR. senistriata $\mathrm{LK}_{\mathrm{K}}$ turbinoides LK. . . pullus Payr. spirata GRaT. varicosa Grat. speciosa Phic. laevis SERR.

? Basterotina Br. intermedia Scacc.

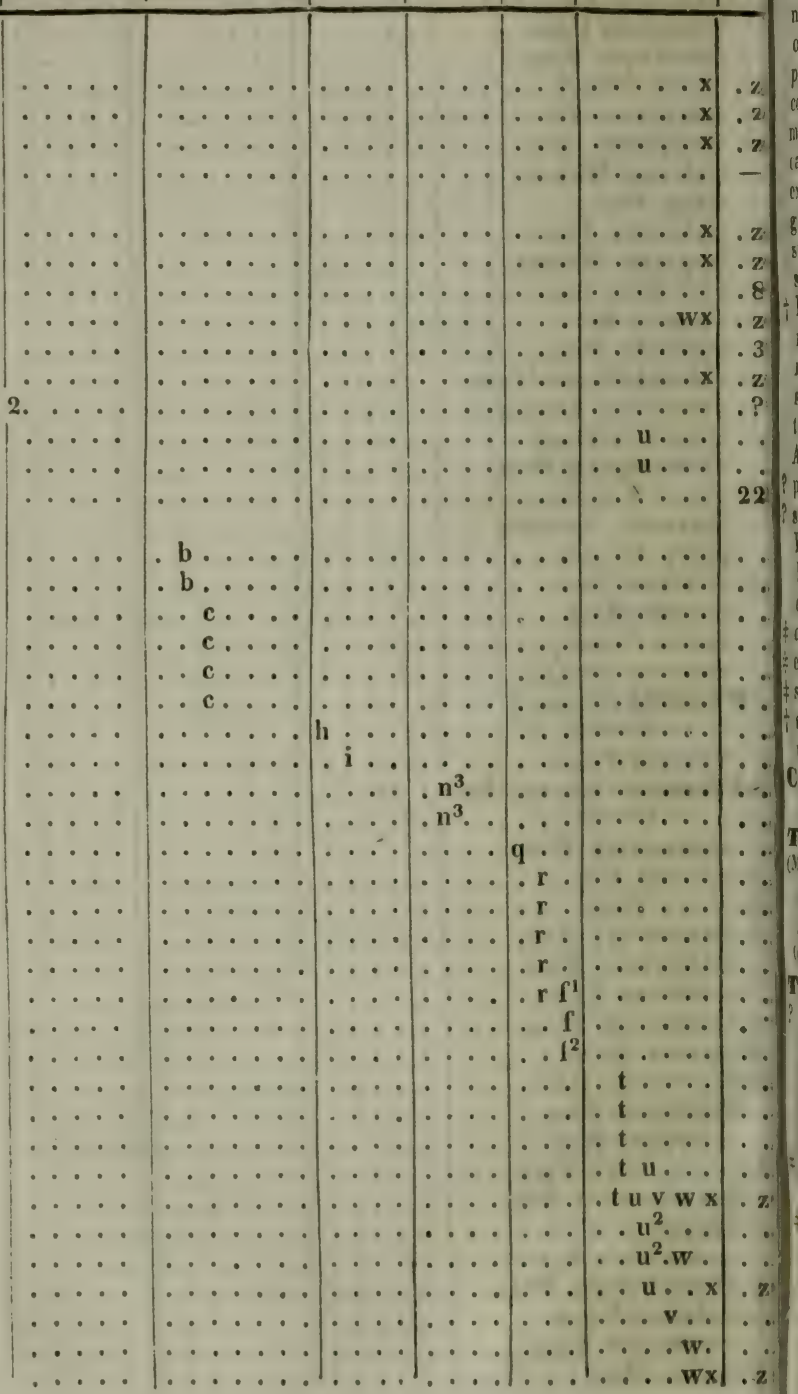


Iitorina FÉR. 30. striaţella So. Lacordaireana KoN. solida Kon.

obscura So. muricoides Dsh. ornata Morrs. punctura BEAN ... conica So. monilifera So. carinata Monss. extensa Morrs. gracilis So. sulcata Nicss. sculpta Reuss. Roissyi D'A. melanioides Dsh. multisulcata Dsh. sulcata Morrs. tricostalis Dsw. Alberti [?] Dus. .... phasianelloides Woon suboperta Wood Prevostina Dsh. litorea FẺr. elongata WooD cancellata CANtr. elegantissima CANTR. submutica Cantr. striata Dsr. ulvae Brown Cyelora Hale 1. minuta HaLL.

\section{' Tuba LEA 2.}

Meleagris Coxr; nou LeA. striata LBA sulcata LEa ( $f r$. Turbo sculptus So.) TuxboL.[restrict.] 245 Petropolitanus Pand. Pupawa Pand. Pryceac So. bicarinatus His. conallii So. lineola Eгснw. parvulus Haxl siluricus Еiснw. sulcifer Eichw. trimarginatus Eıснw. carinatus So. cirrosus So. striatus His.

.

Weltgegend a b e d e f $g$ h i k l mn op q r f s t u v w x y z

$\dot{\mathrm{E}}^{2} \mathbf{S}^{3}$

$1 \cdots$

60

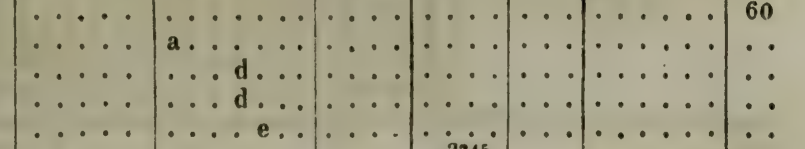

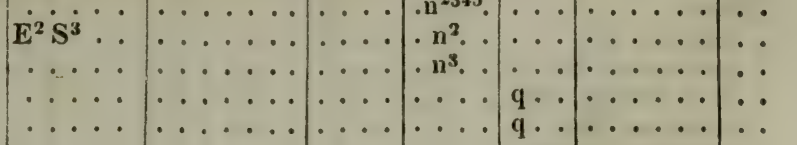

$\therefore$

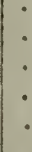

.

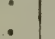




\begin{tabular}{|c|c|c|c|c|c|c|c|}
\hline & IVeltgegend. & Ko $h l \in n P$. & SalzP. & OolithP. & $\begin{array}{c}\text { Krei-i- } \\
\text { deP. }\end{array}$ & MolasseP. & Neu \\
\hline Dimennungen. & 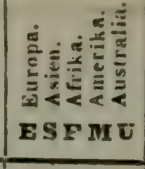 & 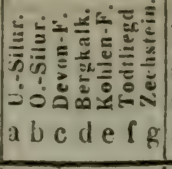 & 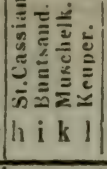 & 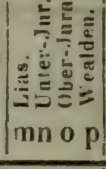 & 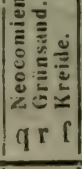 & 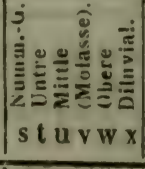 & 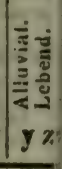 \\
\hline 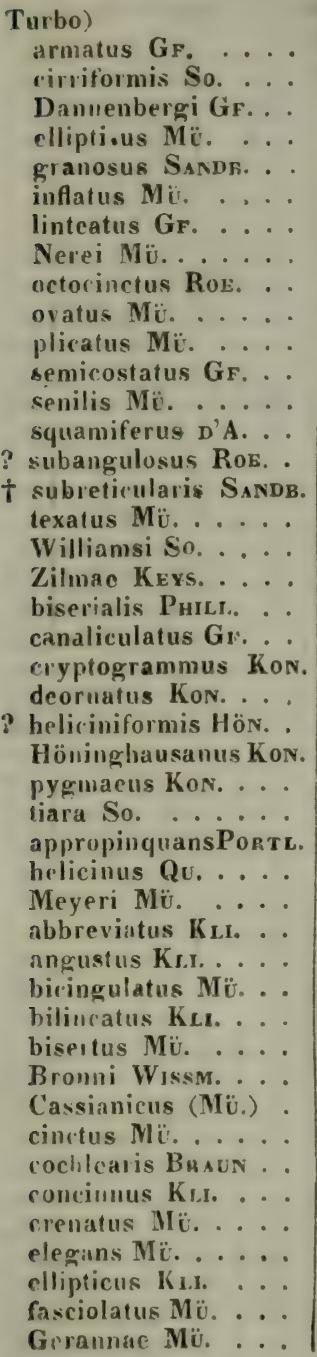 & 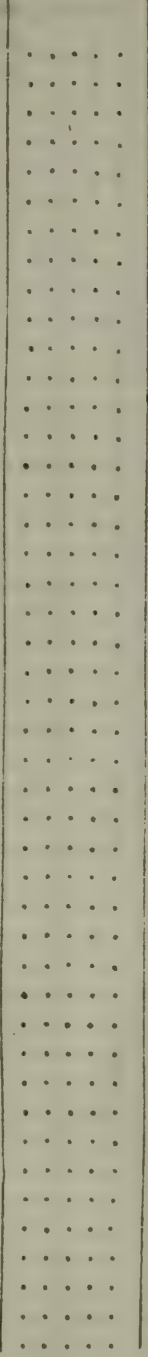 & 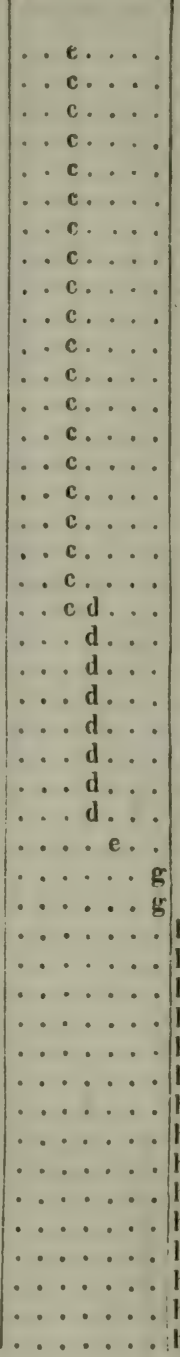 & 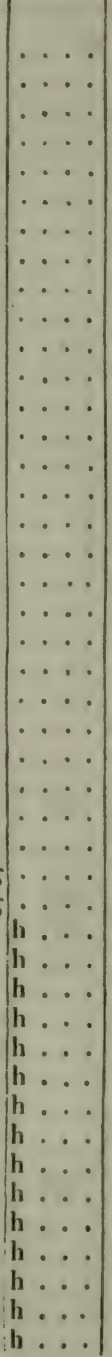 & 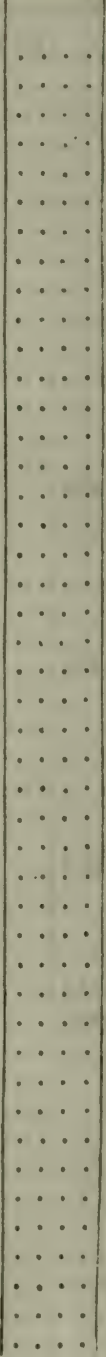 & 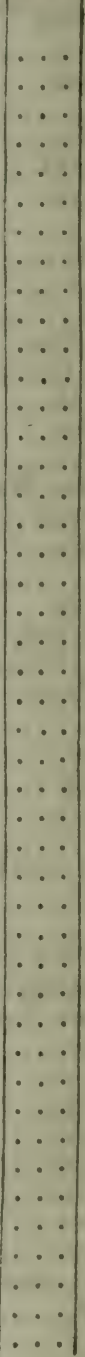 & 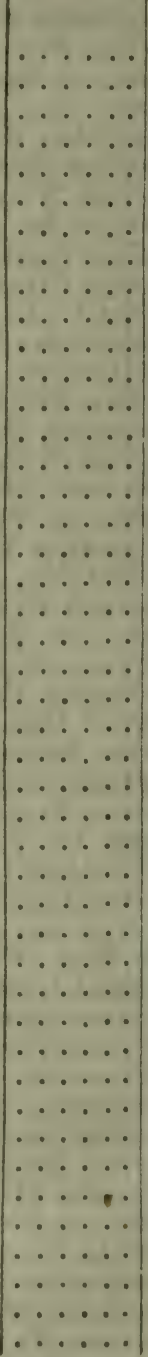 & $\begin{array}{l}\vdots \\
\vdots \\
\vdots \\
\vdots \\
\vdots \\
\vdots \\
\vdots \\
\vdots \\
\vdots \\
\vdots \\
\vdots \\
\vdots \\
\vdots \\
\vdots\end{array}$ \\
\hline
\end{tabular}




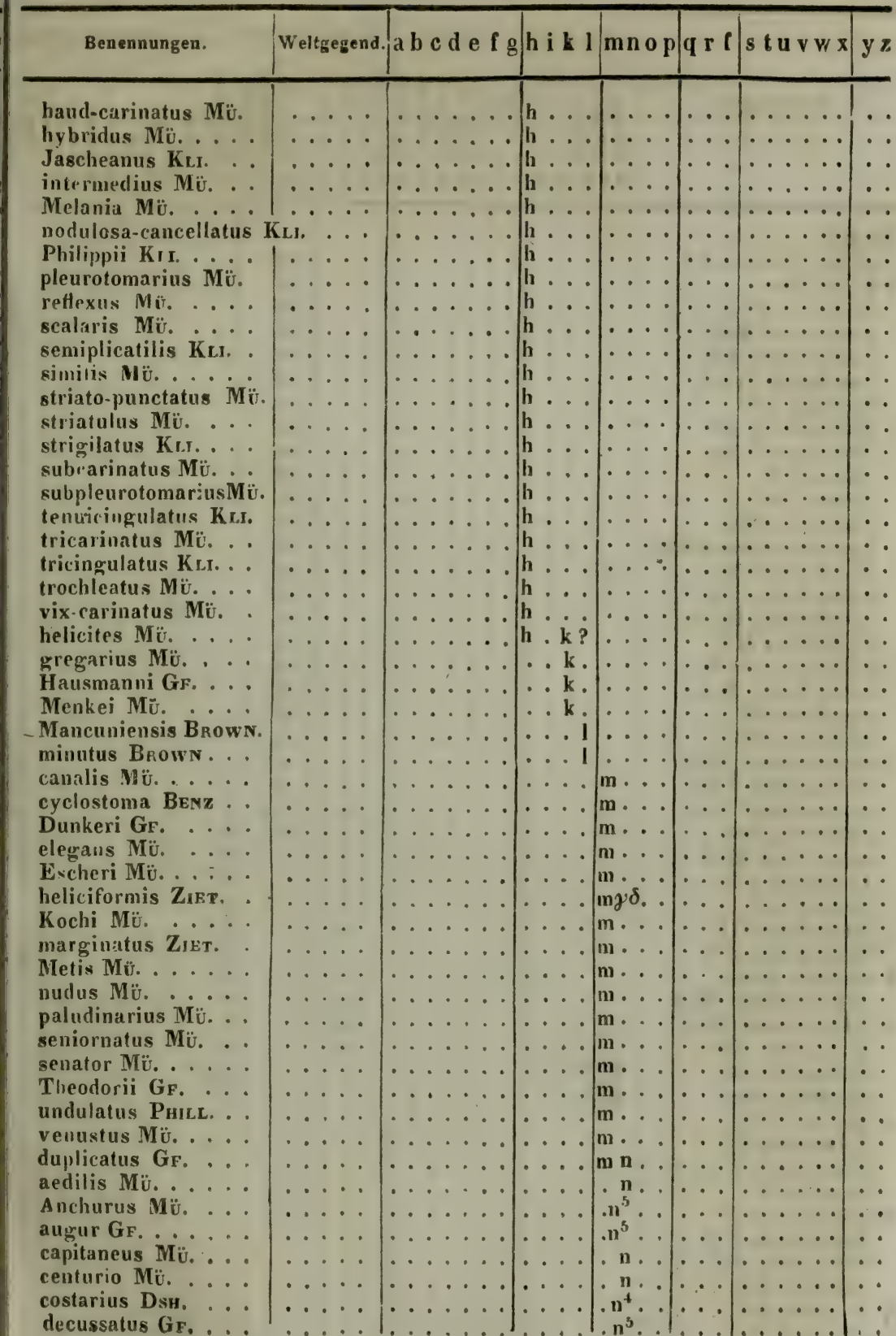




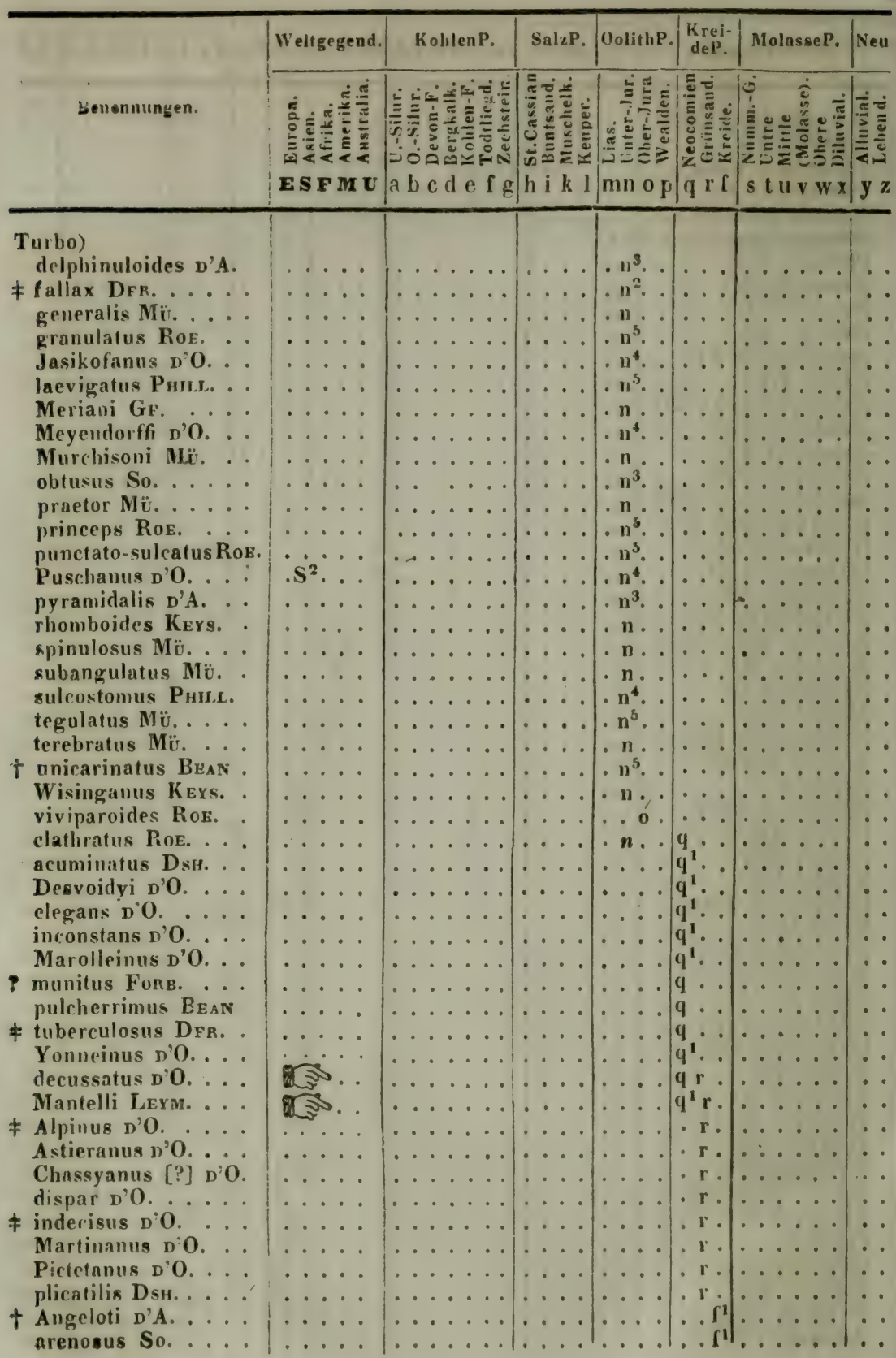




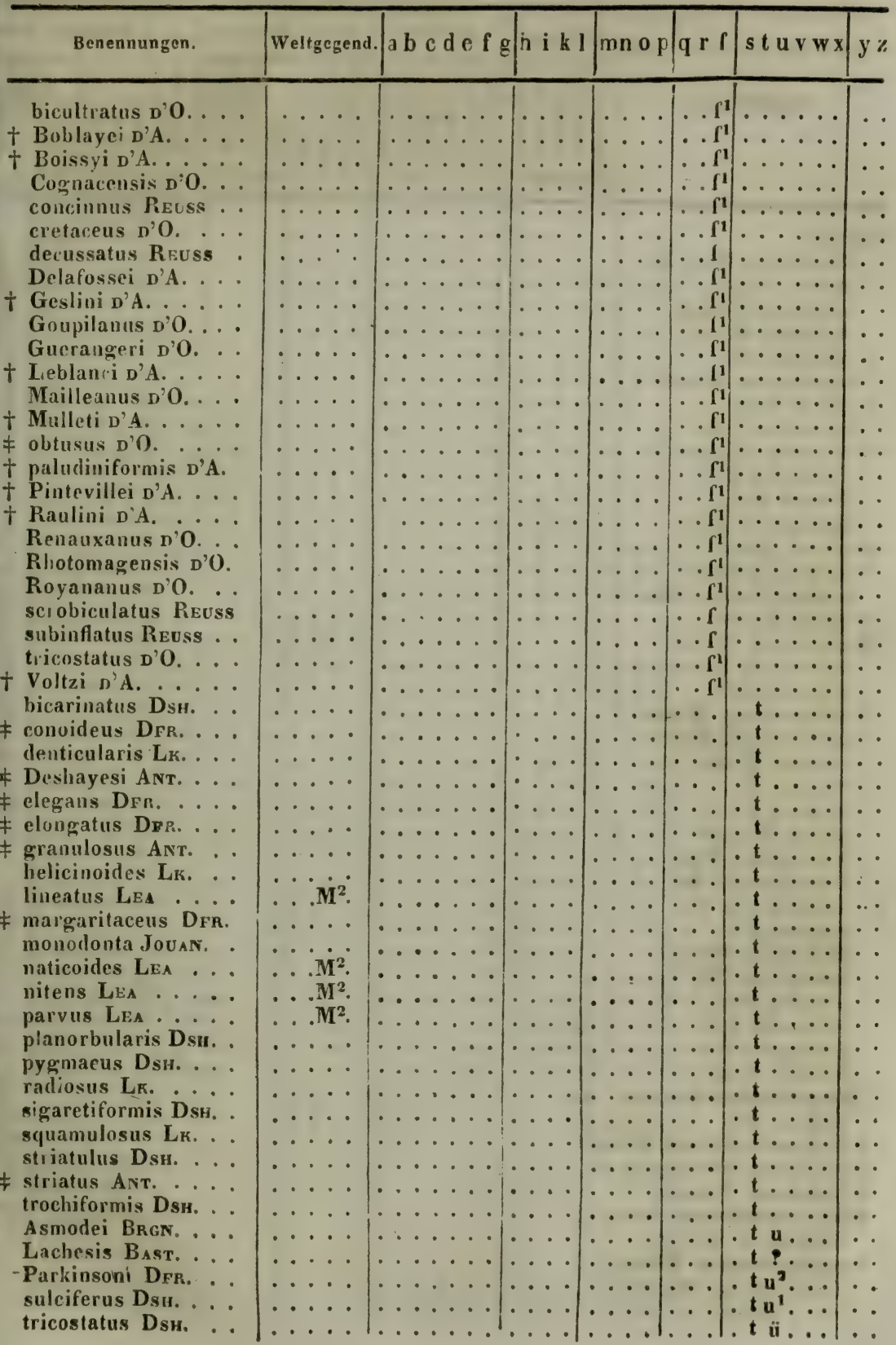




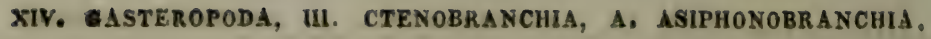

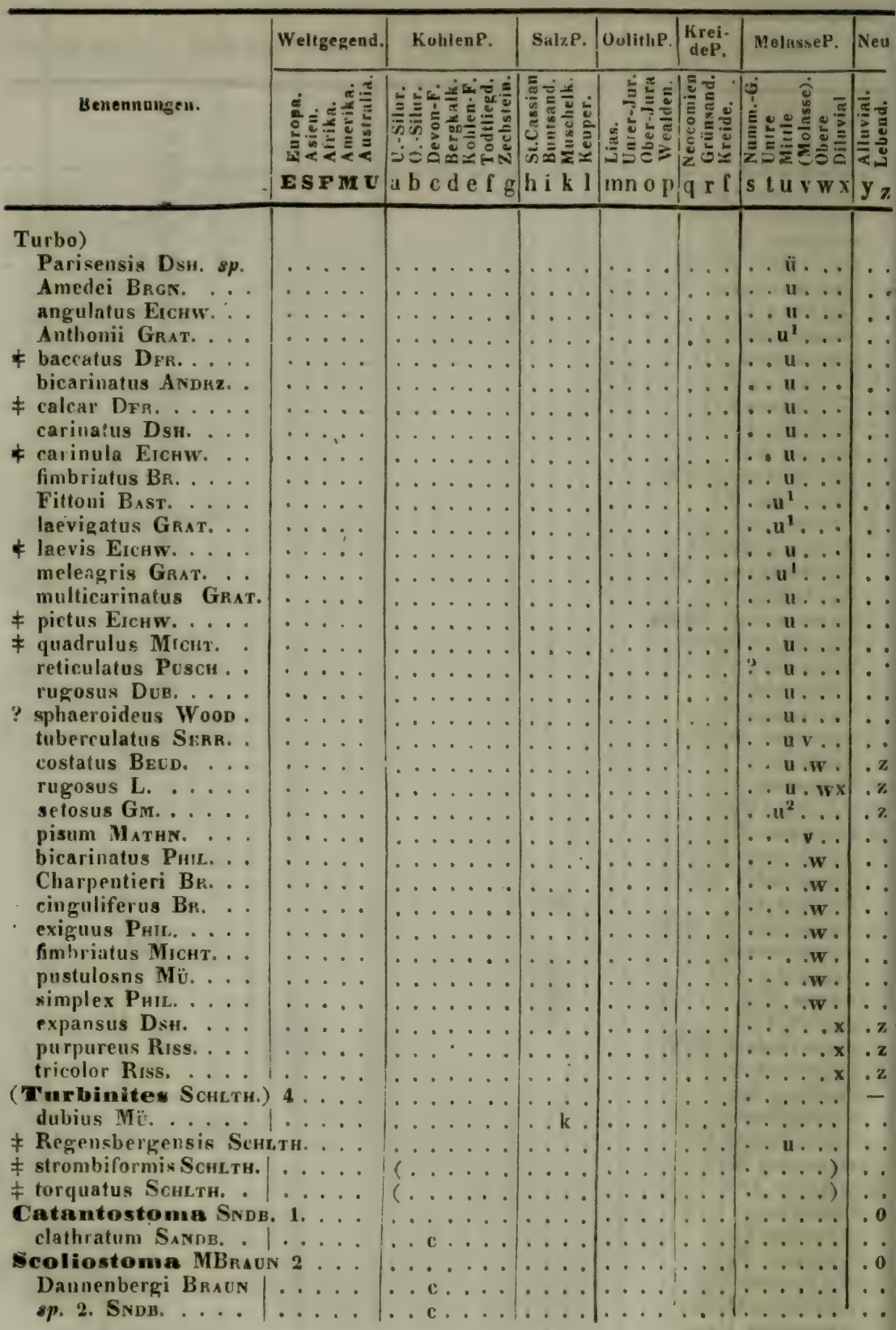




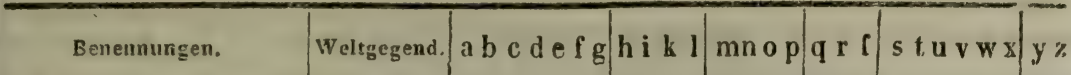

Delphinuta Lk. 53 Leushardi GF. ... t nodosa SNDB. biarnata Kr.r.

? cancellata KLI.

? laevigata Mü.

lincata KLI.

plana KLr.

Vernenili KLr.

† lacvigata D'O.

corunata FL.EM.

funata Gr.

funiculata Gr.

gibbosa THorent. jurensis (MÜ. sp.)

Dupinana D'O.

† Bounardi D'A. curonata RoE.

P lapidosa Mort. biangulata Dsh. calcar $\mathrm{LK}_{\mathrm{K}}$ canalifera LK. conica Lr. . depressa LEA

\# Gervillei DFr. Regleyana Dsu.

t spirorbis Grat. spiruloides DsH. striata Lr. turbinoides LK.

‡ varia DFr. Warnei DFr. callifera Dsu. lima LK. marginata $\mathbf{L K}$. scobina Bast. granulata Grat.

t lyra Conr. Perrisi [?] Grat.

o pyramidata Grat. rutelliformis Gr.AT. striata BeLLMicht. trigonostoma BAST.

$\neq$ Brouni PHIL.

? carinata $P$ HIL. crispula Phil. dubia Phic.

? elegantula Рнгr.

? minima Puz. nitens Phic.

? scabricula PHic. suturalis $\mathbf{P}_{\text {HiL. }}$

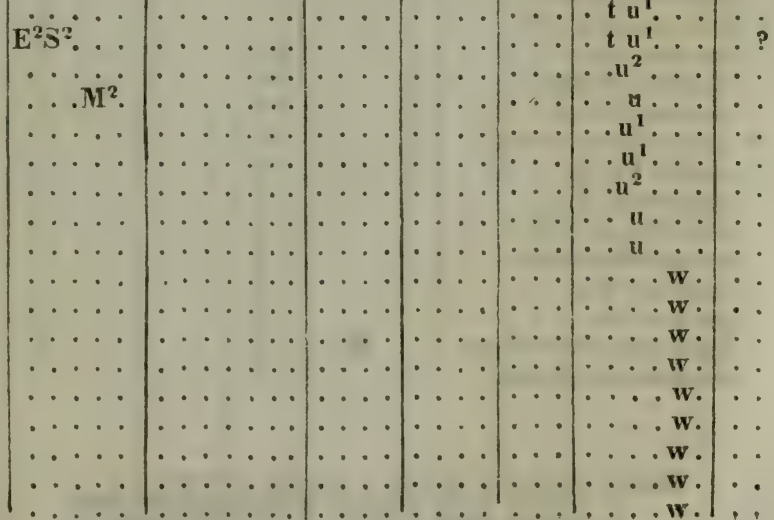




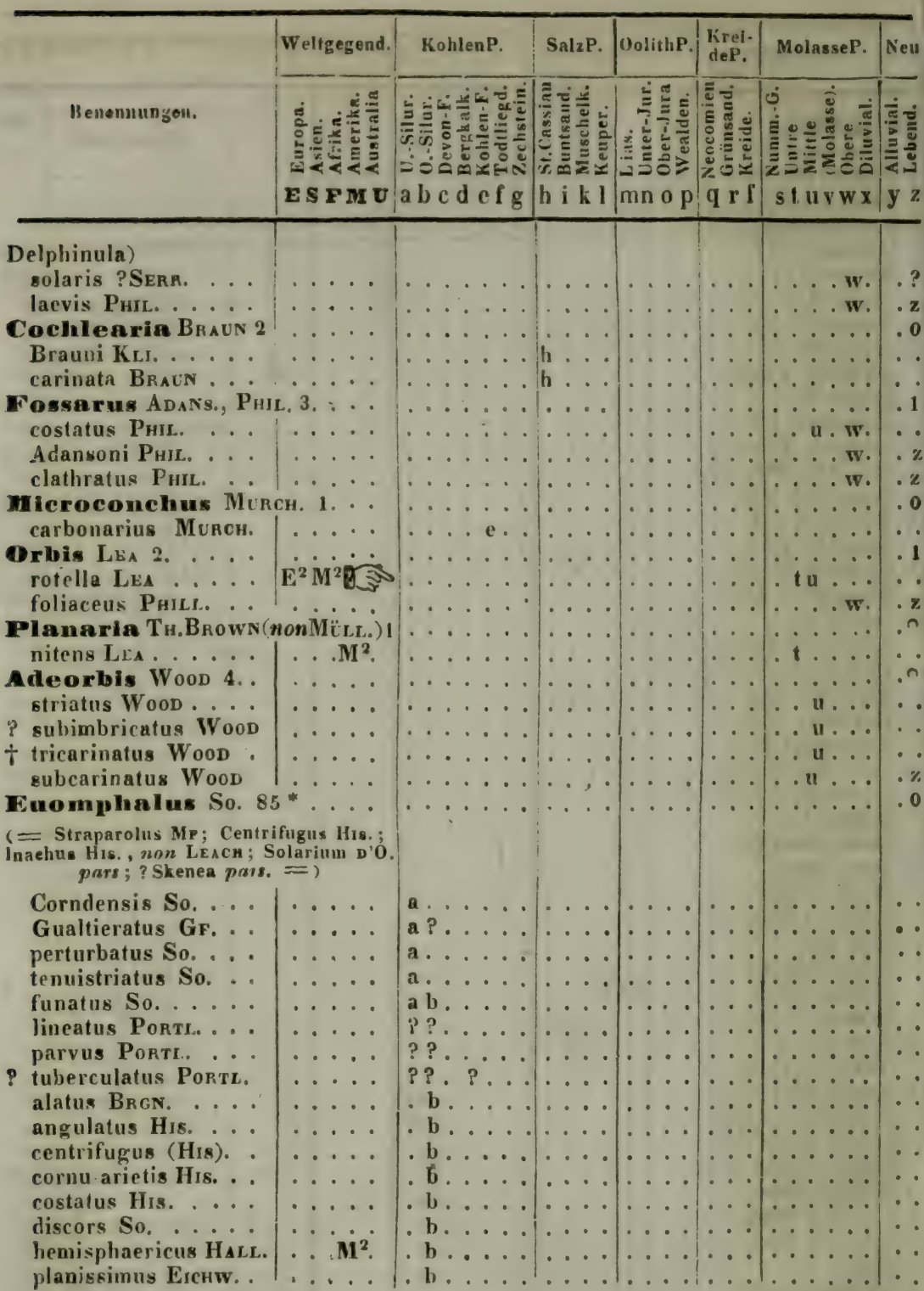

* Numeri nominibus specierum praefixi indicant

1: formam testre planam: Srhisostomnillei KoN.

2: formam testae conoideam: Cirroidei KON. 


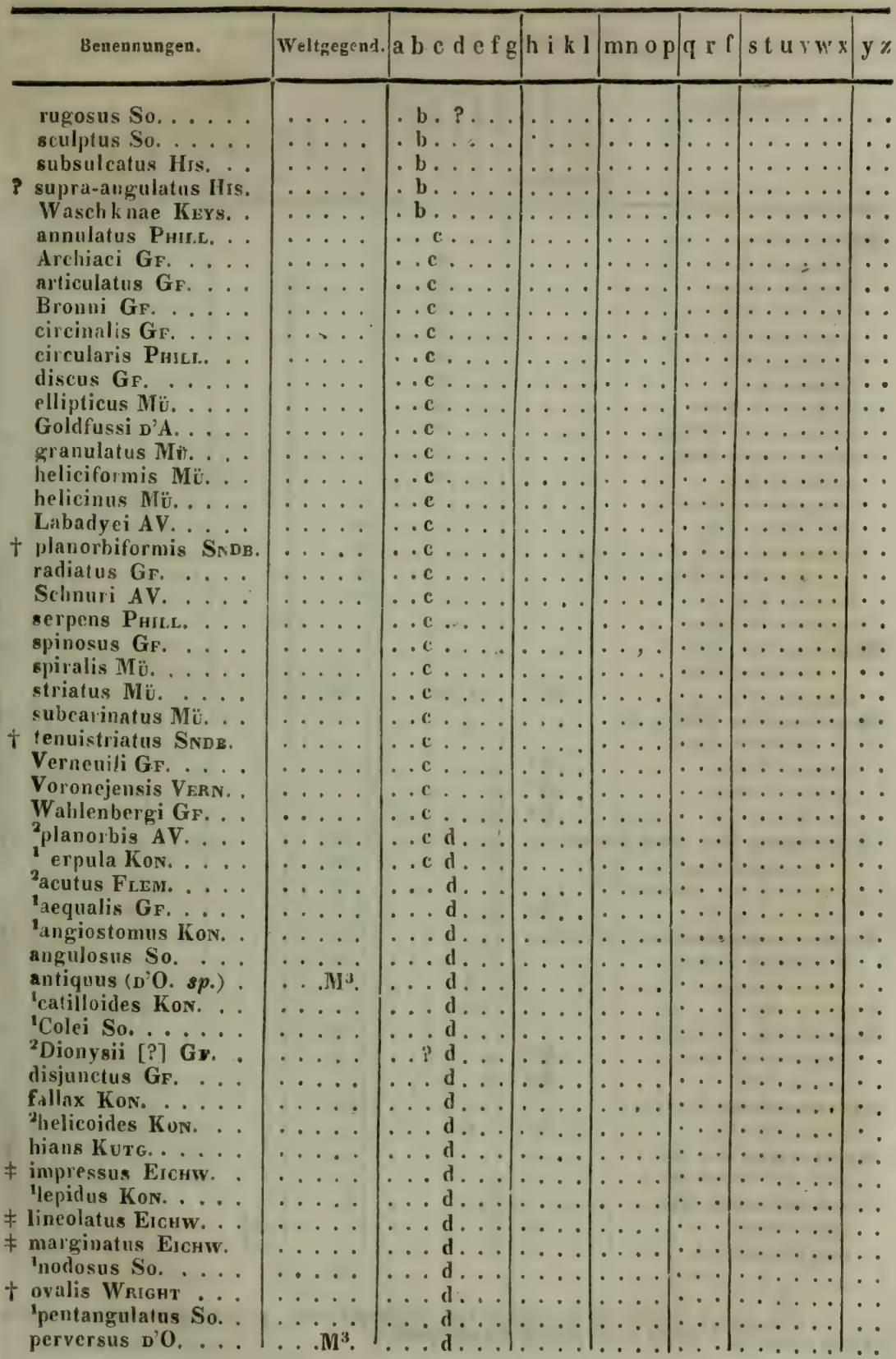




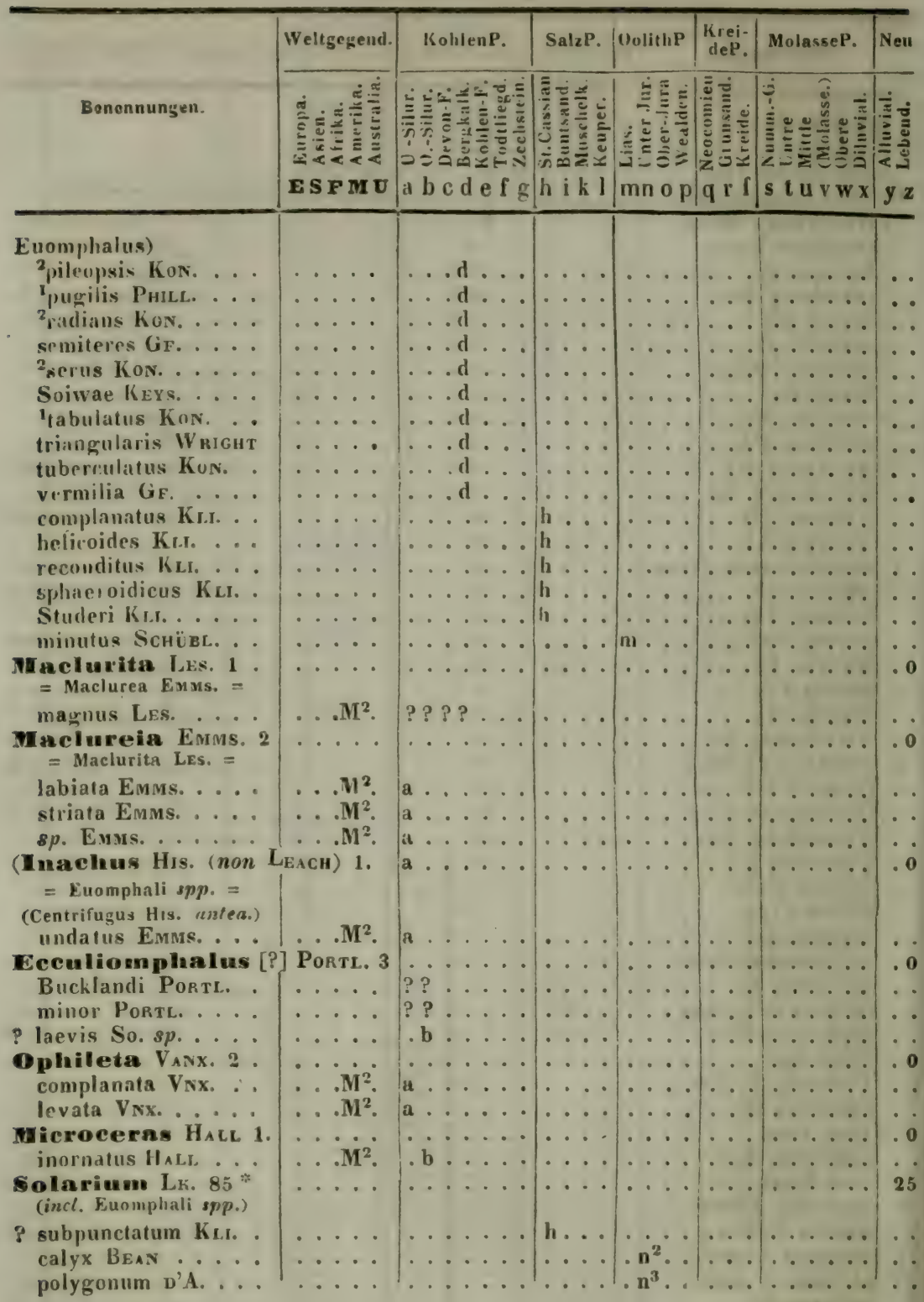

* Cum genere hoc multrs Euomphali spp. conjungit D'O., quas praefixo numero 1 nolu vimus; numerus 2 unlem verus indicul Solarii species. 
P Benstedi ForiB Carcitanense MThN.

${ }^{1}$ Dupinanum D'O.

${ }^{2}$ minimum Fork.

${ }^{2}$ neocomiense [?] D'O

tubulatum PhicL.

${ }^{2}$ Albense D'O.

${ }^{2}$ Astieranum v'O.

1 irroides D'O. conoideum So.

'dentatum D'O.

'dilatatum n'0.

'granosum D'O.

F granulatum So.

'Martinanum D'O.

"moniliferum Місн. ornatum So.

deremestatum Bv. angulatum Reuss 'Guerangeri D' 0 . quarlratum So, 'scalare D'O.

+ Thirrianum D'A. anoenum CoNr. antrosum Conr. bilineatum LEA bistriatum Dsu. cancellatum Conr. discoideum So. Dumonti Nrst elaboratum Conr. elegans LEA exacuum Conr. grande NYst granulatum LEA Henrici [?] LEA marginatum Dsh.

? Nysti Grat. patulum Lk. . simplex Leys.

? suleatum LK. syrtalis Conr.

\# tricarinstum DFr. trochiforme Dsh. umbrosum BrgN. plicatulum DsH. plicatum Lk. spiratum LK. stramineum Phic. affine So. conoideum Grat. delphinulum Grat. 


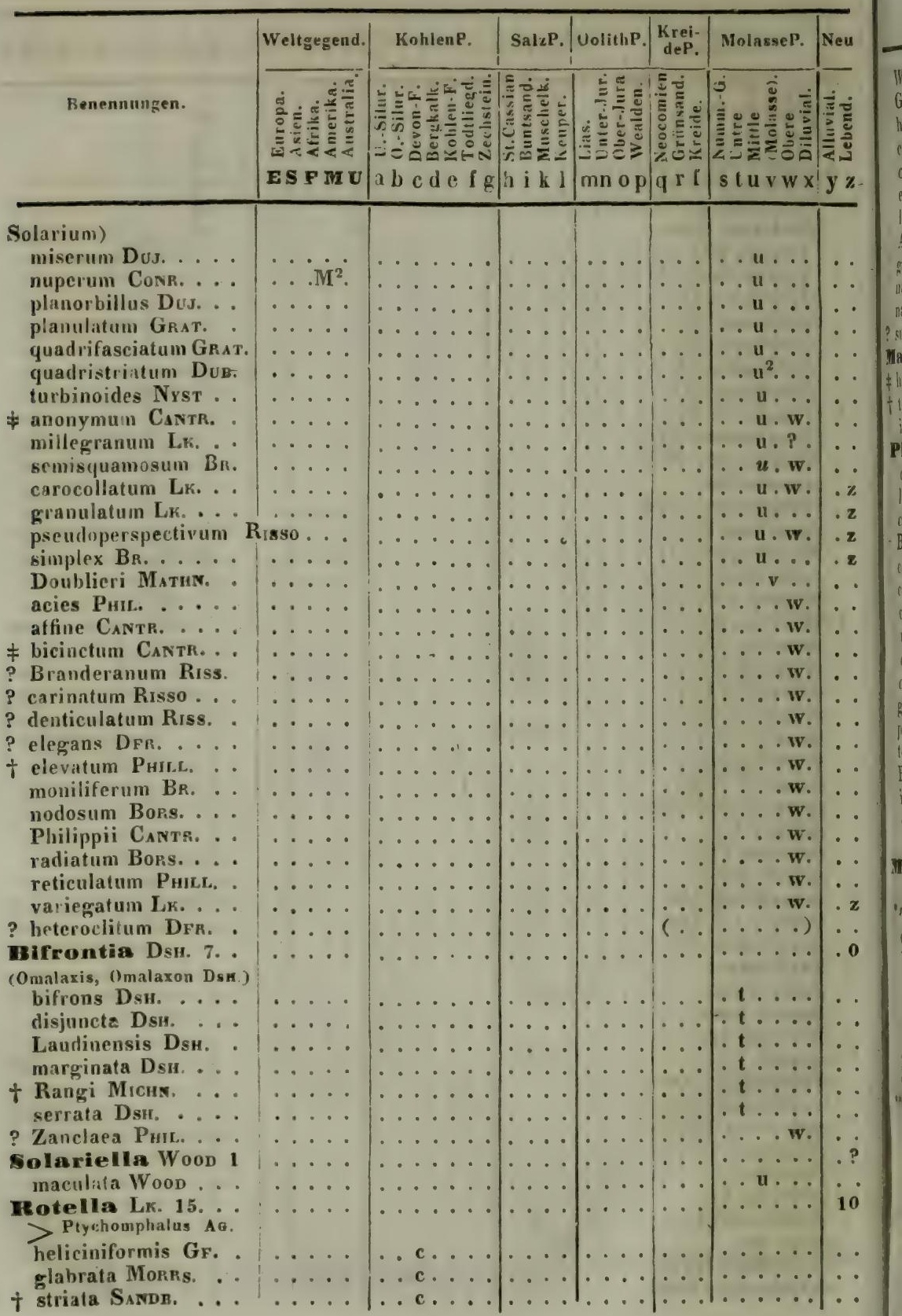




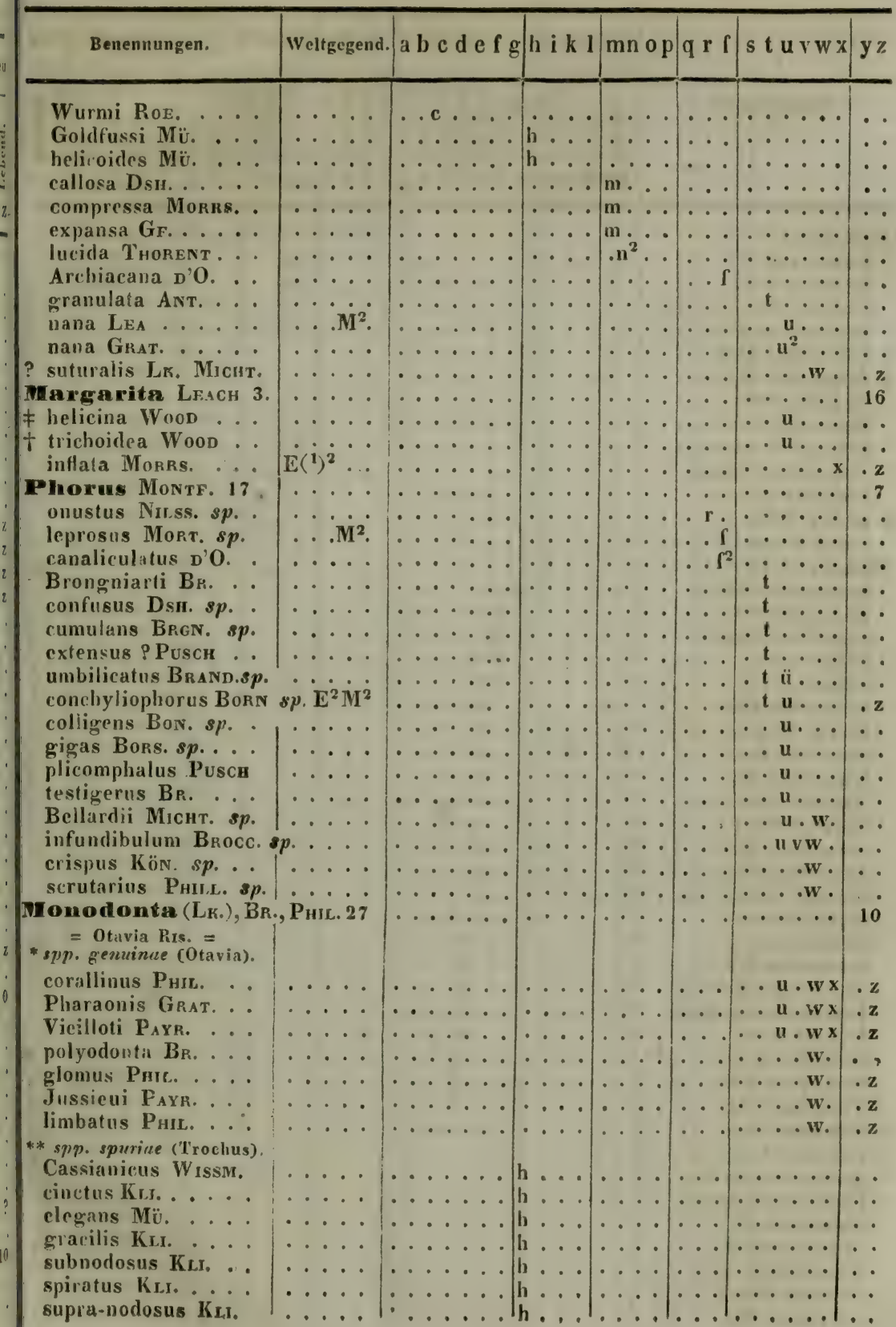




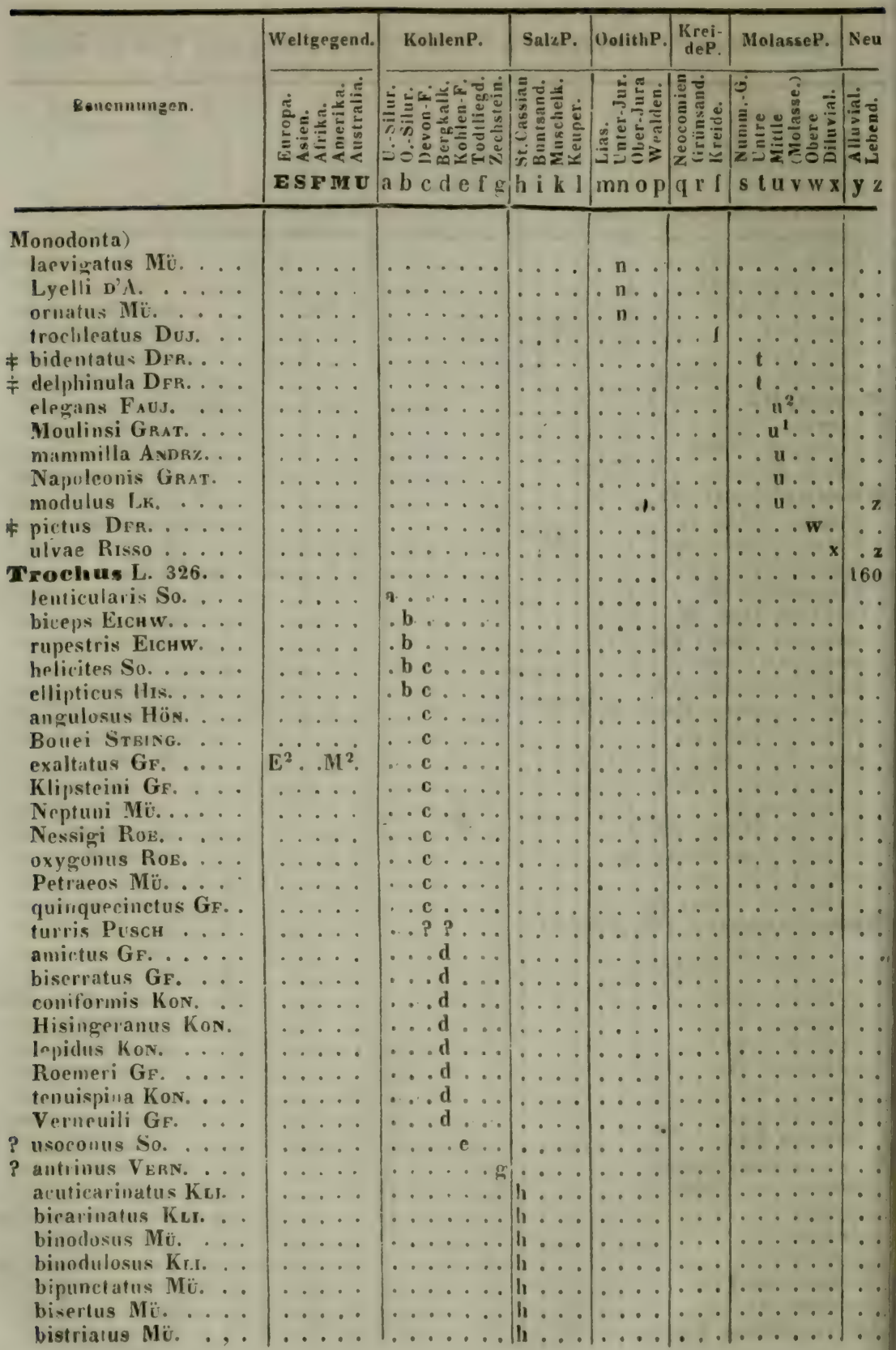




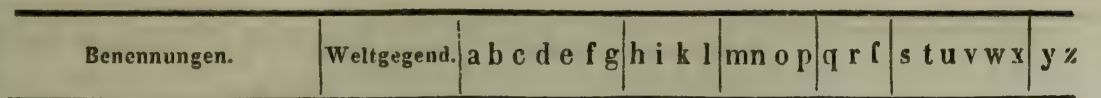

Brauni GF

Caumonti KLI.

Deslongschampsi KLr.

interruptus KI.I.

laticostatus Mü.

Maximiliani-Leuchtenbergensisk nudus Mü.

ornatus KLI.

pyramidalis $\mathrm{M} \ddot{\mathrm{U}}$.

quadrangulo-nodosus KLI.

quadrilineatus Kr...

semipunctatus $\mathrm{M} \ddot{\text {. }}$.

strigilatus $K_{L}$.

subconcavus $\mathrm{MÜ}_{\mathrm{U}}$.

subcostatus Mü.

subdecussatus Mü.

subglaber Mü.

subpunctatus K L.I.

tricarinatus $K_{L J}$.

tristriatus Mü.

verrucosus Mü.

Zinkeni KLr.

echinatus KLöp.

+ depressus Willis.

Doris $M \ddot{u}$.

Fischeri Mü.

flexuosus $M$ ü.

gracilis KoDU.

helicinoides Ros.

imbricatus So.

nudus Mü.

princeps Ko $\dot{\mathbf{D}} \dot{\mathrm{U}}$.

4 cosıatus Mü.

Schübleri ZIET.

Sedgwicki Mü.

Sowerbyi Mü.

subimbriratus KoDu.

subsulcatus Mü.

Thetis Mü.

turrifornis $\mathrm{KoDU}$.

umbilicatus KoDu. acute-carinatus $\mathrm{Mü}$. aequilineatus $\mathrm{Mü.}$ anaglypticus Mü:

Anceu. Mü...

angulat(1-plicatus $\dot{M}$ ü. angulatus So.

angulatus $\mathbf{M u ̈ .}$

biarmatus $\mathrm{M} \ddot{\mathrm{i}}$.

binodosus Mü.

bisertus Phic.

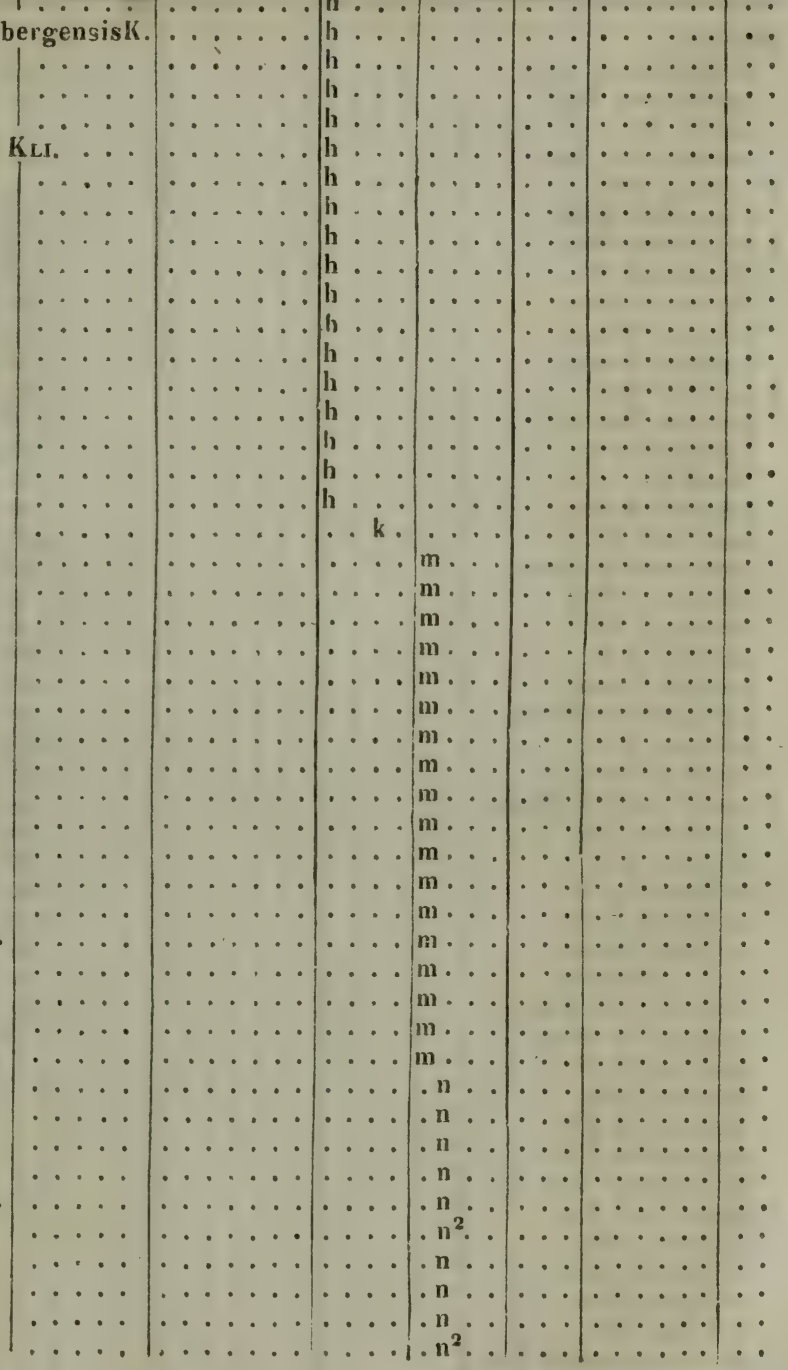




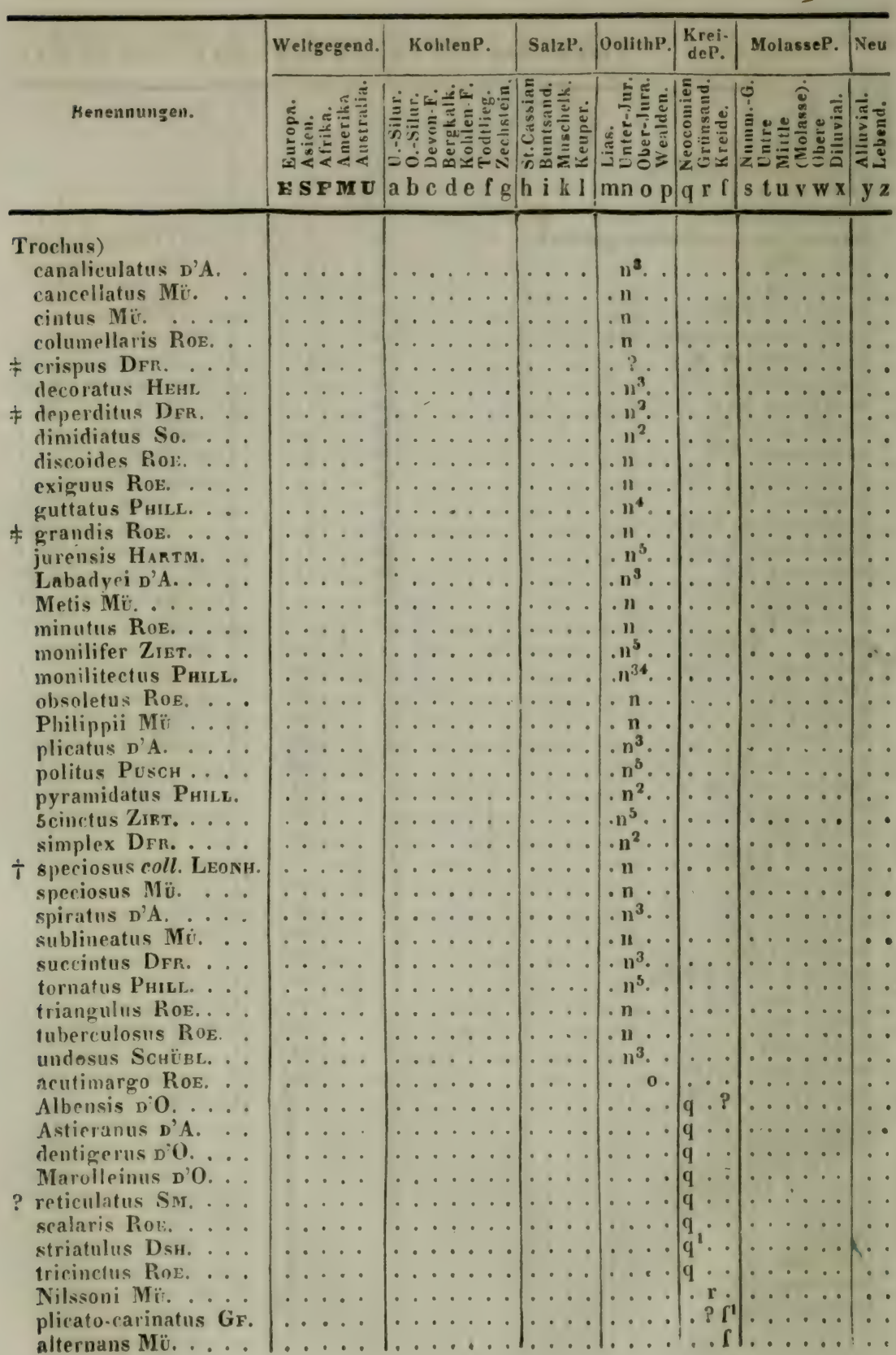




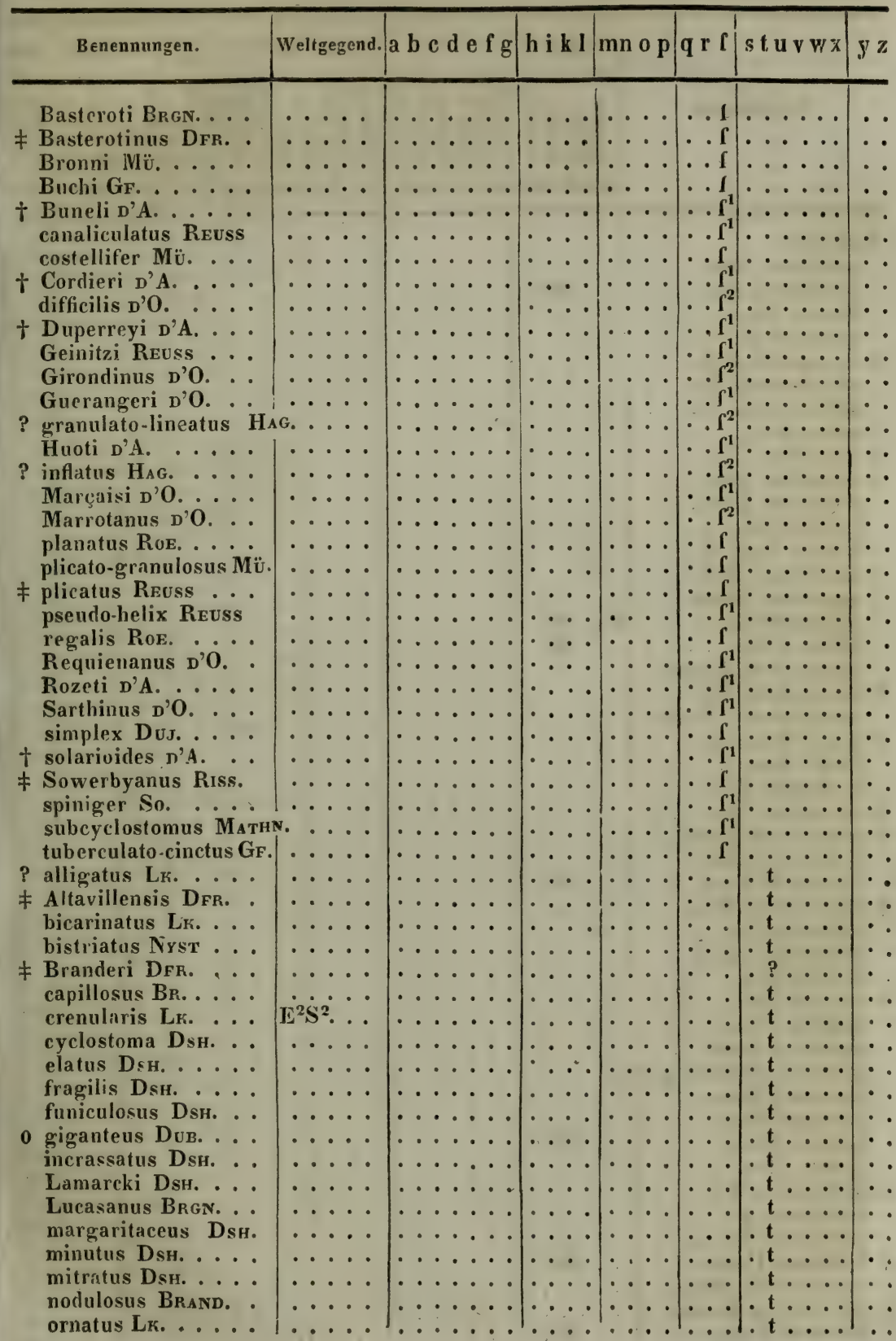




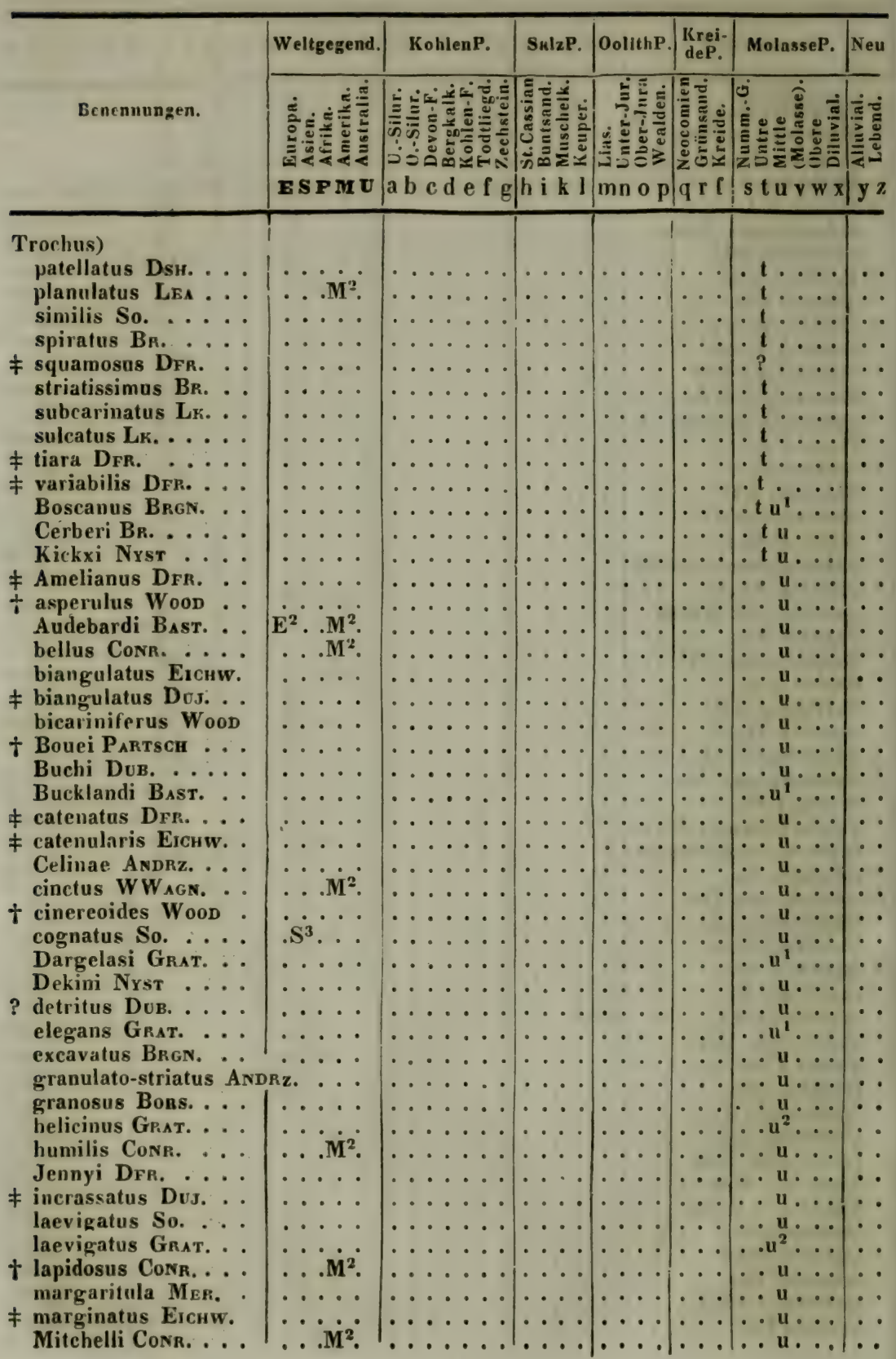




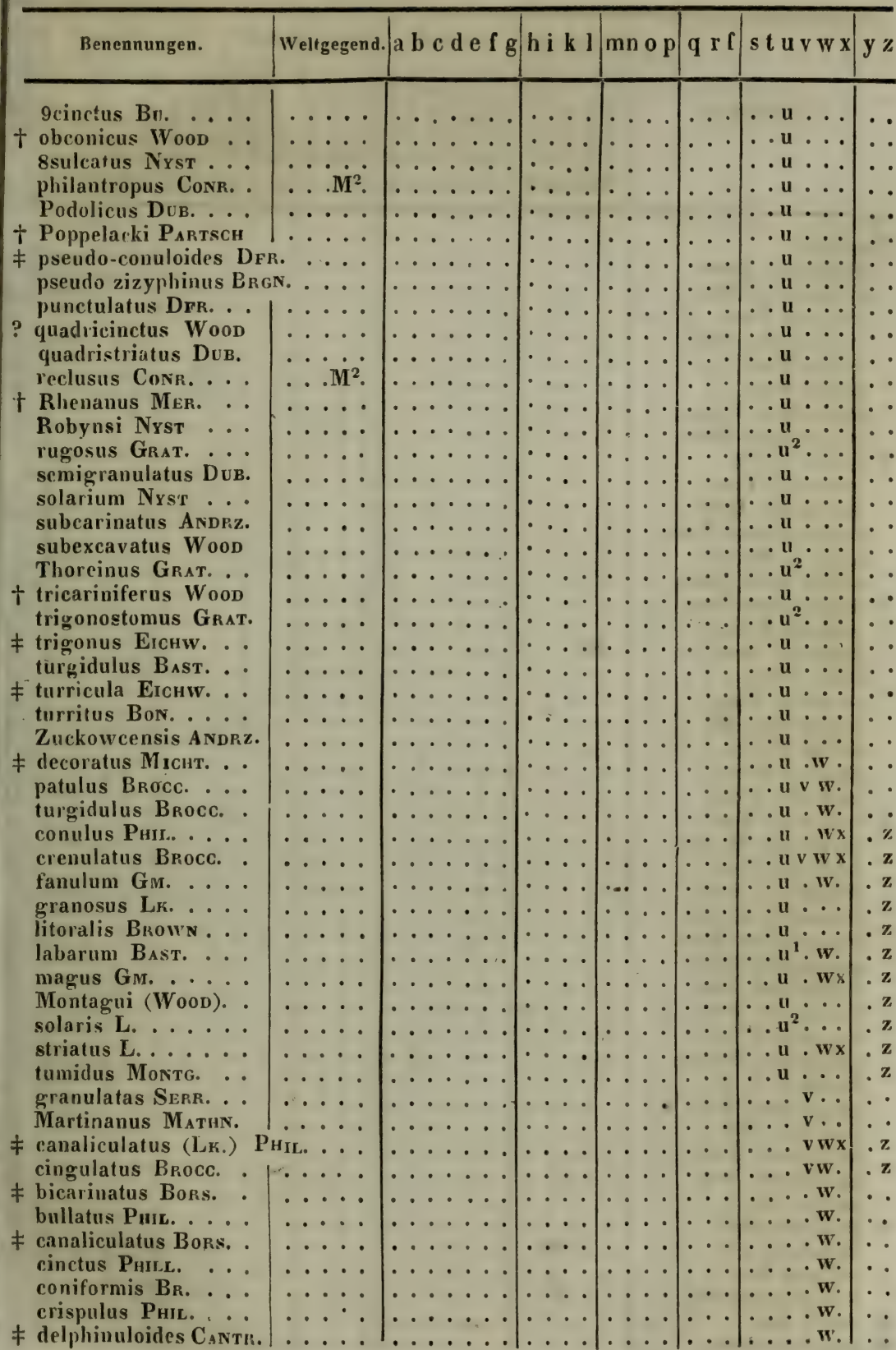




\begin{tabular}{|c|c|c|c|c|c|c|c|}
\hline & Weltgegend. & KohlenP. & Salz.P. & OolithP. & $\begin{array}{c}\begin{array}{c}\text { Krei- } \\
\text { deP. }\end{array}\end{array}$ & MolasseP. & Nell \\
\hline Sencnuunyen. & 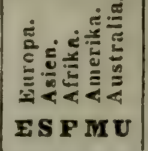 & 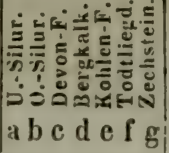 & 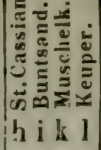 & 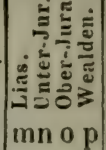 & 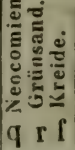 & 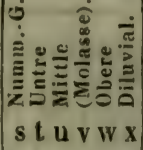 & 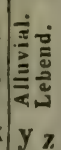 \\
\hline
\end{tabular}

Trochus)

depressus Bонs. elegantulus Pris. elevatus Pнiц. euomphalus Puic. exilis PHiL. filosus PhiL. funiculatus Bors. gemmulatus РнiL.

‡ Genei Cantr. glabratus PhiL. imbricatus Bors. marginulatus PHIL. miliaris Brocc. . . millegranus РнгL. $\dagger$ nitidissimus Pнге.

‡ nodosus Bors. obscurus Dsн. Ottoi Phic. parvulus Phil. 4cingulatus Br. . . Scillai Cantr. sulcatus Broce. . . . suturalis Phiz.

? tuberosus Ris. vorticosus Brocc.. Adansoni PhIL. articulatus PнгL. cinerarius FLEM. fragarioides PHIL. granulatus Bors Guttadauri PHIL. laevigatus PHIL. Laugieri PAYr. . obliquatus Brocc. Richardi PHIL. sanguineus FÉR. A. strigosus GM. turbinatus Borv umbilicaris $\mathrm{GM}_{\text {M. }}$. varius $\mathrm{G}_{\mathrm{M}}$.

\$ Dumerili Riss. luctuosus D'O.

$\ddagger$ miliaris Rrs.

Patagonicus d 0 . ₹ tenuis Ris.

₹ undulatus Riss.

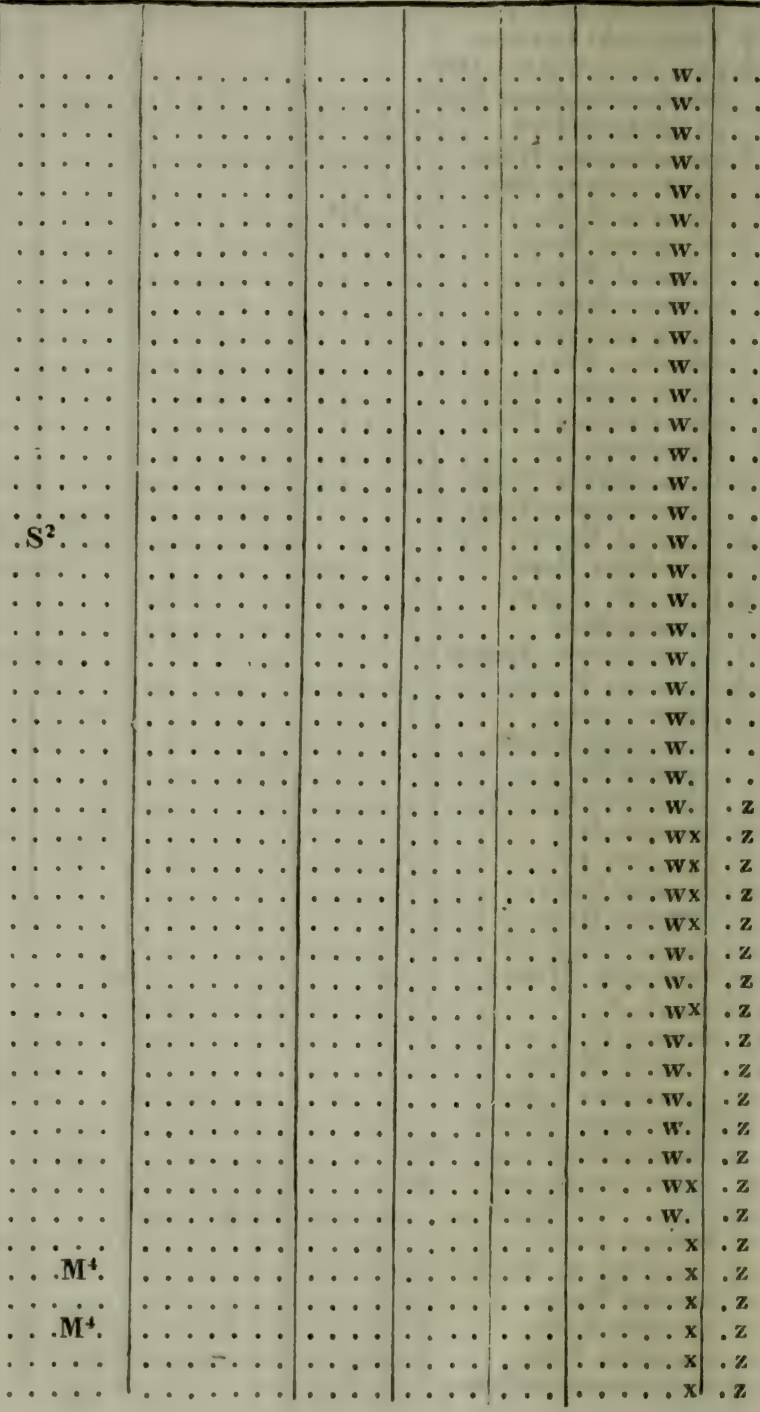




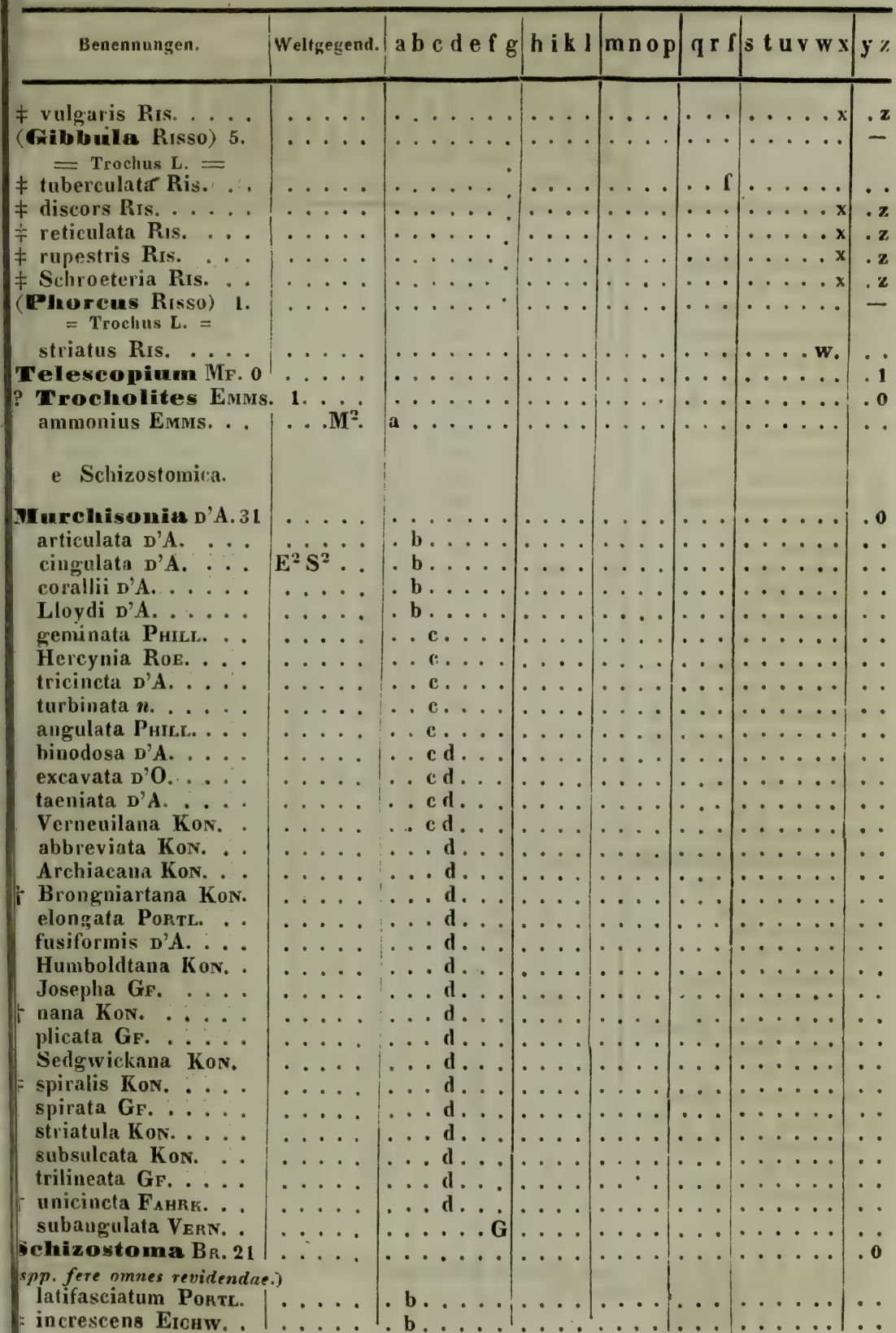




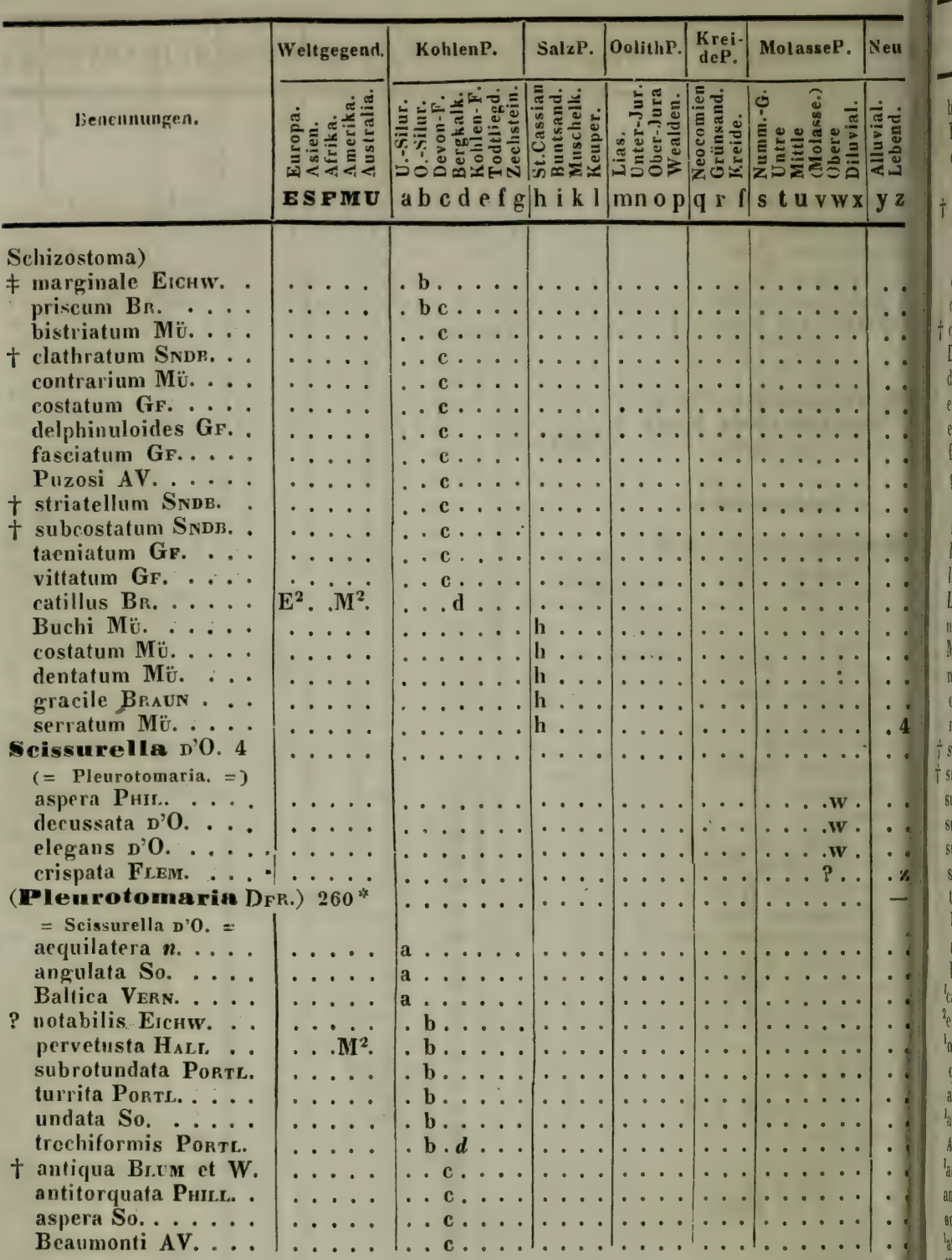

* D'O. et Kon. in duas fumilins distingmunt species abip-sis descriptus, quae numeris nominibus pruefixis indicantur, scil.
1) Ornutue
2) Globosue. 


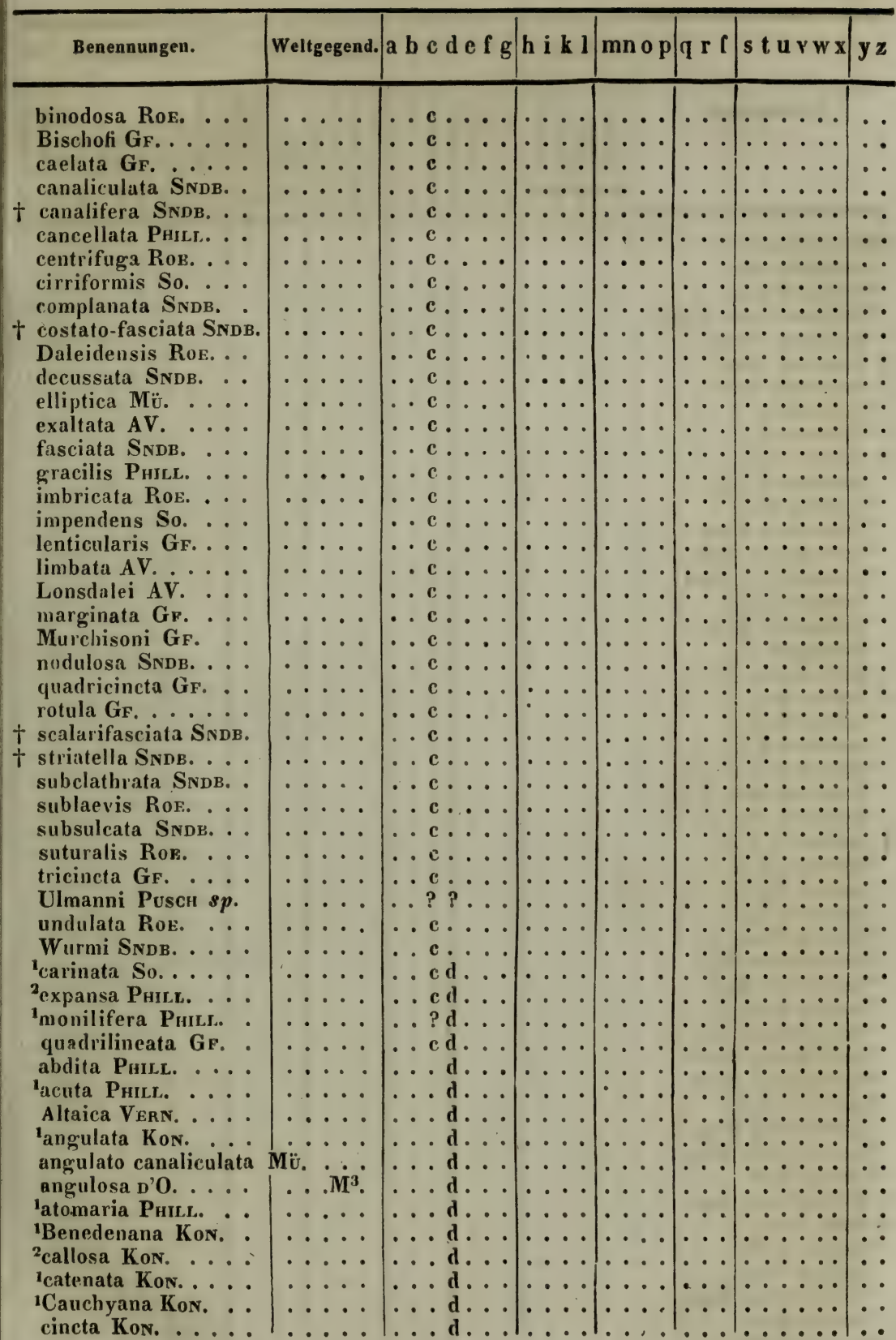




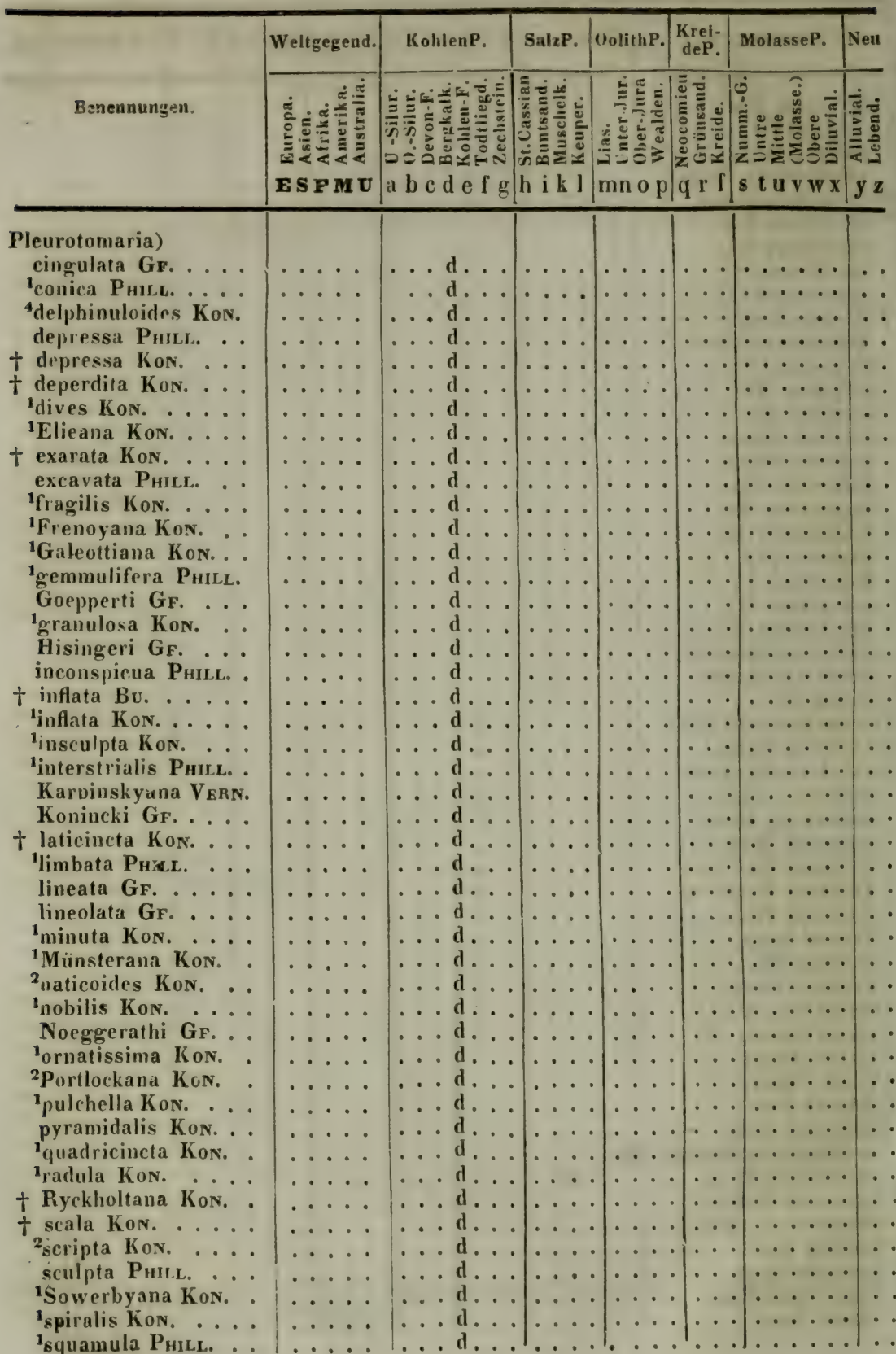




\begin{tabular}{|c|c|c|c|c|c|c|c|}
\hline a. & ad. & a & & $\mathrm{p}$ & I & $\mathrm{Vx}$ & $\mathrm{y}$ \\
\hline strialis & & 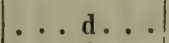 & & & & 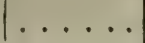 & \\
\hline ... & $\ldots \ldots$ & ....... & 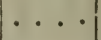 & - & $\cdots$ & $\ldots \ldots$ & $\cdots$ \\
\hline$\cdots$ & $\ldots$ & $\ldots d \ldots$ & $\cdots$ & $\cdots$ & . & $\ldots \ldots$ & 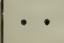 \\
\hline - & $\ldots$ & $\ldots d \ldots$ & & $\cdots$ & $\cdots$ & $\cdots \cdots$ & \\
\hline tilis & $\cdots$ & $\ldots \mathrm{d} \cdot \ldots$ & $\cdots \cdots$ & $\cdots \cdot$ & $\cdots$ & $\cdots \cdots$ & \\
\hline . & $\cdots$ & . & $\cdots$ & $\cdots \cdot$ & . & $\cdots \cdots$ & \\
\hline$u$ & & & & & & $\cdots$ & \\
\hline $\mathbf{U}$ & & & & & & $\cdots$ & \\
\hline $\begin{array}{l}1_{v a} \\
1_{y i}\end{array}$ & $\therefore$ & & & $\cdots$ & . & $\cdots$ & \\
\hline $\begin{array}{l}\text { N. } \ldots \\
\ldots\end{array}$ & $\cdots \cdots$ & $\ldots \mathrm{d} \ldots$ & $\ldots$ & $\cdots$ & $\cdots$ & $\cdots \cdots$ & \\
\hline$\cdots$ & $\cdots \cdot$ & $\ldots d \ldots$ & $\ldots$ & $\ldots$ & $\cdots$ & $\ldots \ldots$ & . \\
\hline$\cdots$ & $\cdots \cdot$ & $\cdots \cdots$ & $\cdots$ & $\cdots \cdot$ & $\cdot$ & $\cdots \cdots$ & \\
\hline$\cdots$ & $\ldots$ & $\ldots \ldots \mathrm{G}$ & $\cdots \cdot$ & $\cdots \cdots$ & $\cdots$ & $\ldots \ldots$ & \\
\hline .. & $\cdots \cdots$ & $\cdots \cdots$ & h $\cdots$ & $\cdots$ & $\cdots$ & $\cdots$ & \\
\hline$\cdots$ & & & & $\cdots$ & . & $\cdots$ & \\
\hline . & & & & 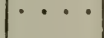 & & $\cdots$ & \\
\hline$\cdots$ & & & & $\cdots$ & - & $\cdots$ & \\
\hline$\because$ & $\begin{array}{l}\cdots \cdots \\
\cdots\end{array}$ & & $h$ & 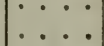 & $\cdots$ & $\cdots$ & \\
\hline$\cdots$ & $\ldots$ & $\cdots$ & h ... & $\ldots$ & . . & $\ldots \ldots$ & \\
\hline .. & $\cdots \cdot$ & $\ldots$ & h... & $\ldots$ & $\cdots$ & $\ldots \ldots$ & \\
\hline & $\cdots \cdot$ & ... & & $\cdots$ & . & $\ldots \ldots$ & . \\
\hline . & $\ldots$ & . & & $\cdots$ & $\cdots$ & $\ldots .$. & • \\
\hline . & $\cdots \cdots$ & & h. . & $\ldots$ & $\cdots$ & $\ldots \ldots$ & . \\
\hline lata $\mathrm{K}$ & & & h & $\cdots$ & . & $\ldots \ldots$ & . \\
\hline$\cdots$ & $\cdots \cdot$ & . & 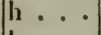 & $\cdots$ & . & $\cdots \cdots$ & • \\
\hline$\cdots$ & $\cdots \cdots$ & . & . & $\cdots$ & . & $\cdots \cdots$ & 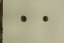 \\
\hline . & $\cdots \cdot$ & & in & $\cdots \cdot$ & $\cdot \cdot$ & $\cdots \cdots$ & 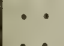 \\
\hline$\cdots$ & - & & & $\cdots \cdot$ & - & $\cdots$ & \\
\hline$\cdots$ & & & & $\cdots$ & $\because$ & $\cdots \cdots$ & 。 \\
\hline 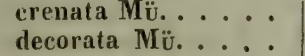 & $\cdots$ & & & $\cdots$ & $\cdots$ & $\cdots$ & \\
\hline$\therefore$ & ... & $\ldots \ldots$ & h ... & $\ldots$ & $\cdots$ & $\ldots \ldots$ & $\therefore$ \\
\hline & $\cdots \cdot$ & . & & $\cdots$ & $\cdots$ & $\ldots \ldots$ & . \\
\hline $\mathrm{e} K_{L I}$ & $\cdots \cdot \cdot$ & . & & $\cdots \cdot$ & $\cdots$ & $\cdots \cdots$ & . \\
\hline & $\cdots \cdots$ & . & $\cdots$ & $\cdots$ & $\cdots$ & $\cdots \cdots$ & \\
\hline & & & & & . & $\cdots$ & \\
\hline$\ddot{\cdots}$ & & & & ${ }^{\circ}$ & $\cdots$ & $\ldots \ldots$ & \\
\hline$\ldots$ & $\cdots$ & $\ldots$ & & . . & $\cdots$ & $\ldots \ldots$ & \\
\hline & $\cdot \cdot$ & - & & - & . & $\ldots \ldots$ & \\
\hline & & 1 & & & . & $\cdots \cdots$ & th \\
\hline & & & & & - & $\cdots$ & 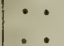 \\
\hline & & & & & & $\cdots$ & 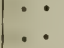 \\
\hline$K_{L}$ & & $\cdots$ & $\mathrm{h}$. & $\cdots$ & $\cdots$ & $\cdots \cdots$ & • \\
\hline 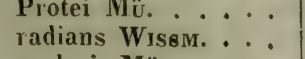 & & & & . & $\cdots$ & $\ldots \ldots$ & $\cdot$ \\
\hline & & & & & . & $\ldots \ldots$ & \\
\hline & & & & & & $\cdot \cdot$ & \\
\hline , & 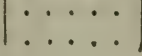 & 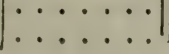 & & & & $\therefore$ & \\
\hline
\end{tabular}




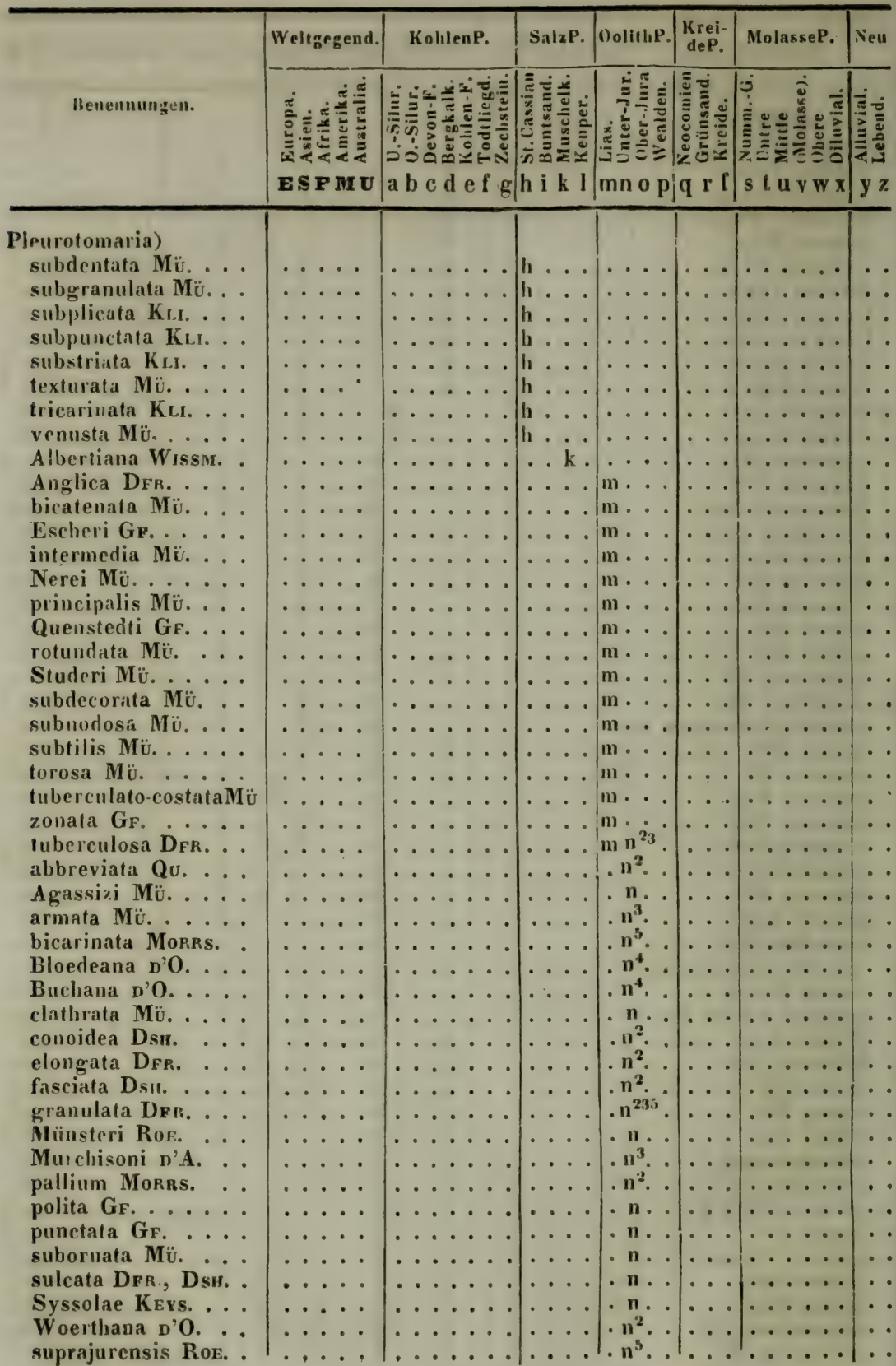




\begin{tabular}{|c|c|c|c|c|c|c|c|c|}
\hline & Benennungen. & d. & $a b$ & 1 & p & $q \times r$ & $\mathbf{x}$ & \\
\hline & . & & & & & & 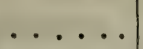 & \\
\hline & $\cdots$ & ... & $\ldots \ldots$ & $\ldots$ & $\ldots$. & $q \ldots$ & $\ldots \ldots$ & \\
\hline & $\cdots$ & $\ldots$ & $\ldots \ldots$ & $\ldots$. & $\ldots$. & q... & $\ldots \ldots$ & \\
\hline & $0 . \ldots$ & $\cdots$ & $\ldots \ldots$ & $\cdots \cdots$ & .... & & $\ldots \ldots$ & \\
\hline & THN. . & $\ldots$ & $\ldots \ldots$ & $\cdots \cdot$ & $\ldots$. & $q \ldots$ & $\ldots \ldots$ & \\
\hline & .. & $\ldots$ & $\cdots$ & $\cdots$ & & & $\cdots$ & \\
\hline & $\therefore$ & $\cdots$ & $\cdot$ & $\cdots \cdot$ & & & $\cdots$ & \\
\hline & $\ddot{D^{\prime}} \dot{0}$. & $\begin{array}{l}\cdots \\
\cdots \\
\cdots\end{array}$ & $\because$ & $\cdots$ & & & 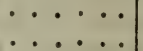 & \\
\hline & . & $\ldots$ & $\therefore$ & $\ldots$ & $\cdots$ & $4 \ldots$ & $\cdots \cdots$ & \\
\hline & $\cdots$ & $\cdots \cdots$ & $\ldots$ & $\ldots$. & $\ldots$ & $9 .$. & $\therefore \ldots$ & \\
\hline & ).. & $\cdots \cdots$ & $\cdots$ & $\cdots$ & $\ldots$ & & $\ldots \ldots$ & \\
\hline & . . & $\cdots$ & $\cdots$ & $\cdots$ & $\ldots$ & $q r$. & $\ldots \ldots$ & \\
\hline & $\cdots$ & $\cdots \cdot$ & $\cdots$ & $\cdots$ & $\cdots$ & . r. & $\ldots \ldots$ & \\
\hline & . & $\cdots$ & $\cdots$ & $\cdots$ & $\cdots$ & $\cdot \mathbf{r} \cdot$ & $\ldots \ldots$ & \\
\hline & di & & $\cdots$ & $\cdots$ & $\cdot$ & $\cdot \mathbf{r} \cdot$ & $\ldots \ldots$ & \\
\hline & $\underline{G}$ & & $\cdots$ & & & . $\mathbf{r}$. & $\cdots$ & \\
\hline & $\ldots$ & $\cdots$ & $\therefore$ & $\cdots \cdots$ & 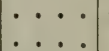 & $\begin{array}{ll}\cdot r \\
. & r\end{array}$ & $\cdots$ & \\
\hline & & & $\ldots$ & $\ldots$ & $\cdots \cdots$ & 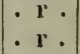 & $\ddot{\cdots}$ & \\
\hline & . & $\ldots$ & . . . & .... & $\ldots$ & $\begin{array}{l}\mathrm{r} . \\
\mathrm{e} r\end{array}$ & $\begin{array}{l}\cdots \\
\cdots\end{array}$ & \\
\hline & $\ldots$ & $\ldots$ & $\cdots$ & 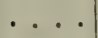 & . & $r$ & $\because$ & \\
\hline & 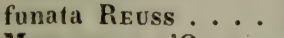 & & $\ldots \ldots$ & • & $\cdots$ & ? ? 11 & 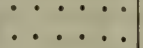 & \\
\hline & $\therefore$ & $\cdot$ & $\cdots \cdots$ & 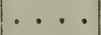 & $\cdots$ & - r f 1 & $\therefore$ & \\
\hline & & & $\cdot$ & & $\cdots$ & . ? [1 & $\ldots \ldots$ & \\
\hline & 'O. & & . & - & $\cdots$ & $\ldots[1$ & ... & \\
\hline & $\cdots$ & . & 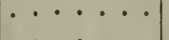 & . . & $\cdots$ & . . $[1$ & . & \\
\hline & .. & & $\cdots$ & • & $\cdots$ & . . I & $\cdots$ & \\
\hline & D & $\cdot$ & & & & $\cdots[1$ & 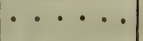 & \\
\hline & $\begin{array}{l}E \\
\text { fo }\end{array}$ & & . & & & $\cdots$ & $\cdot$ & \\
\hline & $F$ & & .... & & . & $\begin{array}{ll}\cdots 1 \\
011\end{array}$ & - & \\
\hline & & & $\cdots$ & & $\because$ & $\begin{array}{ll}\cdots \\
\cdots \\
f_{1}\end{array}$ & • & \\
\hline & & & $\ldots$ & & $\dot{0}$ & $\begin{array}{ll}\cdots & 1 \\
0 & f_{1}\end{array}$ & & \\
\hline & $\ldots$ & & ... & & ... & $f_{1}$ & & \\
\hline & . & $\ldots$ & $\ldots$ & $\cdots$ & $\ldots$ & $\ldots f_{1}$ & $\cdots$ & \\
\hline & $\cdots$ & ... & $\ldots$ & $\cdots$ & . . & $\ldots 1_{1}$ & $\therefore$ & \\
\hline & $\cdots$ & & & . & . & $\ldots \boldsymbol{\tau}_{2}$ & & \\
\hline & . . & & 1... & • & & $\ldots \Gamma_{1}$ & & \\
\hline & $\cdots$ & . . . & ... & $\cdots$ & . & $\cdots f_{1}$ & . & \\
\hline & & & & - & & $\cdots f_{1}$ & & \\
\hline & R & & & & & & & \\
\hline & $\mathbf{R}$ & • & $\cdots$ & & & & & \\
\hline & . & & . . & & & & & \\
\hline & & & $\cdots$ & $\cdots \cdot$ & . & & & \\
\hline & & & $\ldots$ & ... & $\cdots$ & - & & \\
\hline & & & & & & . . 12 & & \\
\hline & . & • & & & & $\ldots \Omega_{1}$ & & \\
\hline & & $\cdots$ & $\cdots \cdots \cdots$ & $\cdot$ & 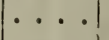 & $\cdots$ & . $t$ & \\
\hline & & & 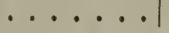 & & $\cdot$ & & . & \\
\hline
\end{tabular}




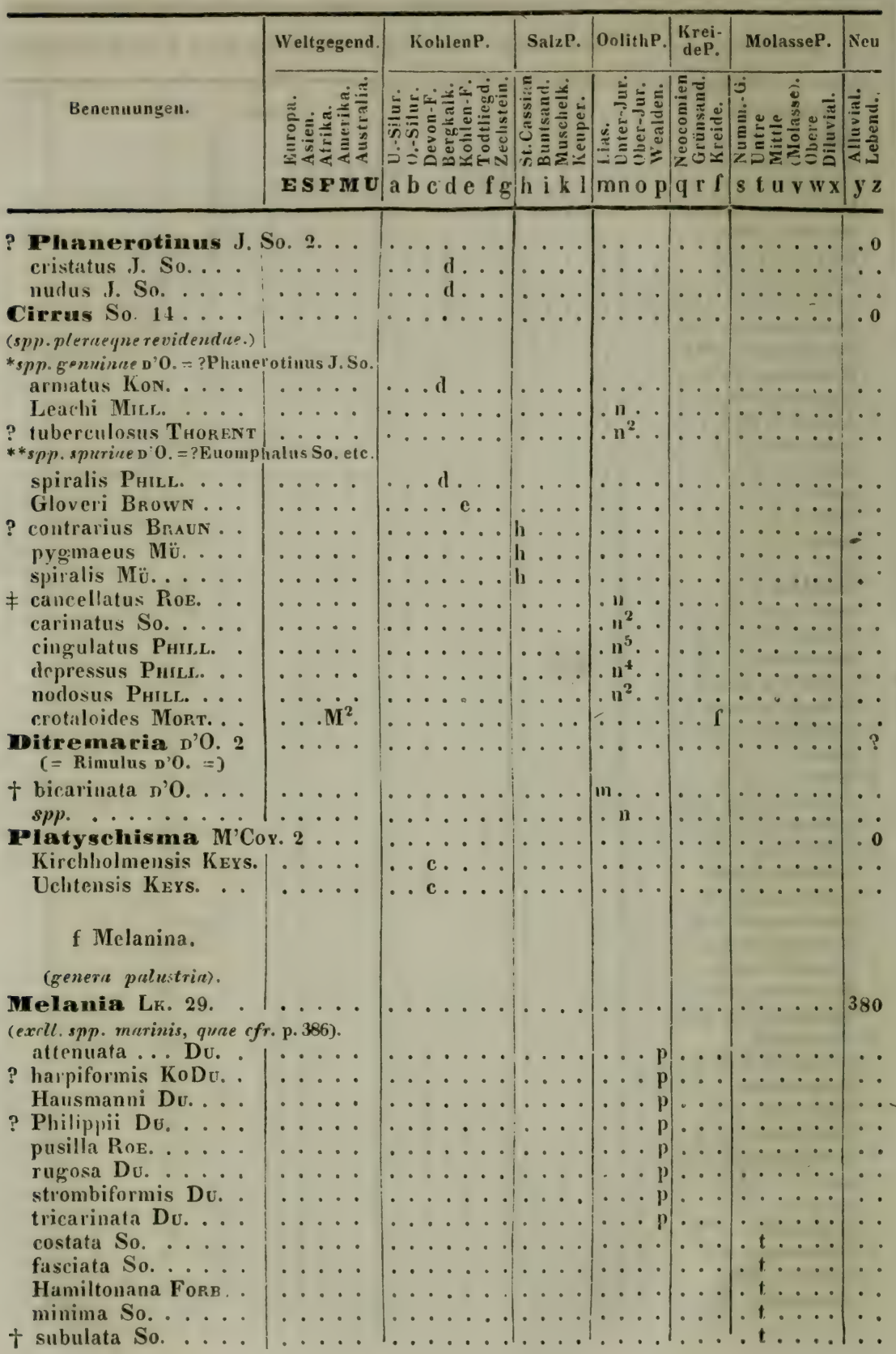




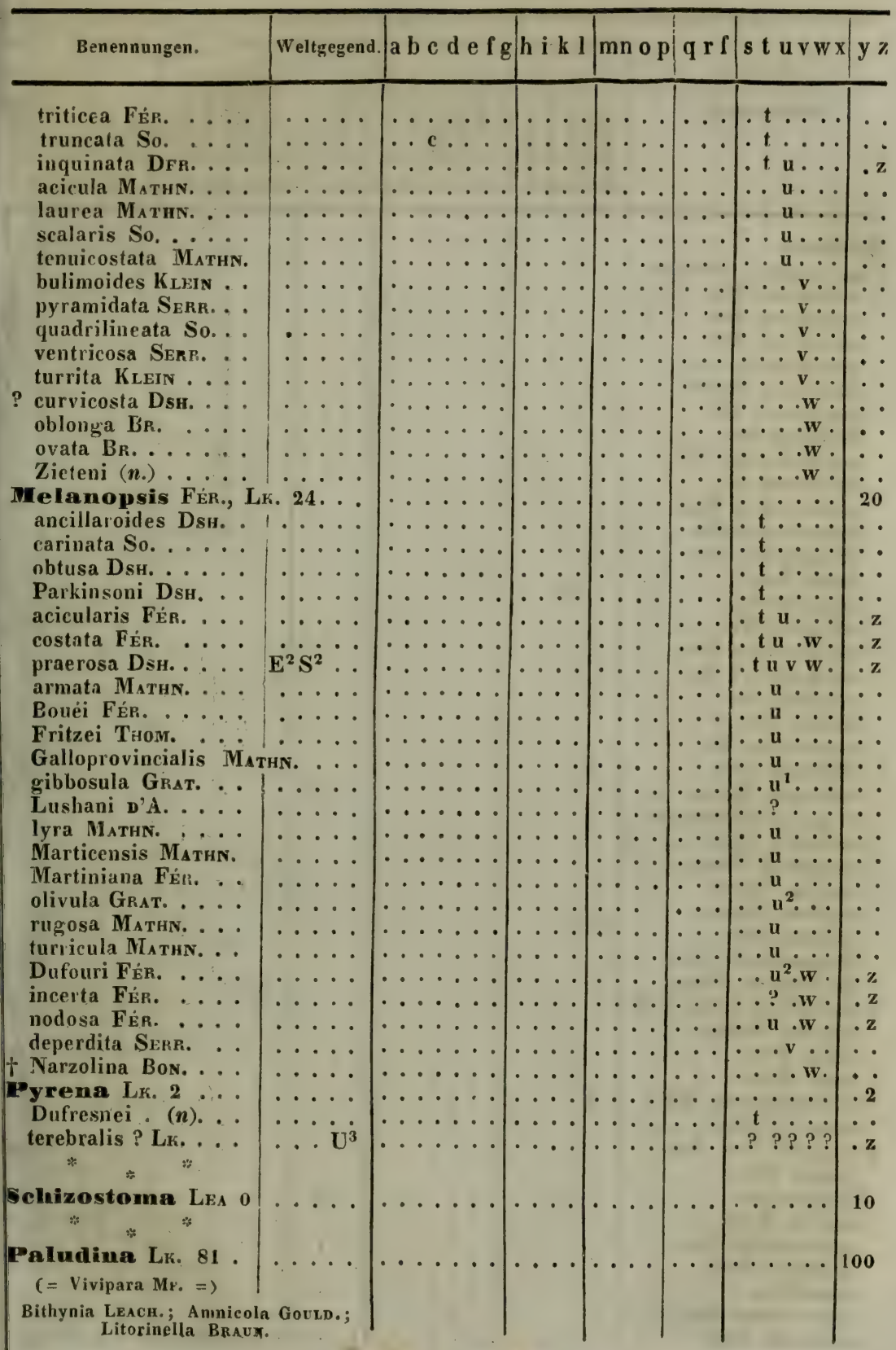




\begin{tabular}{|c|c|c|c|c|c|c|c|}
\hline & Weltgegend. & KohlenP. & SalzP. & OolithP. & $\begin{array}{c}\text { Krei- } \\
\text { deP. }\end{array}$ & MolasseP. & Neu \\
\hline Benenuungen. & 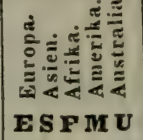 & 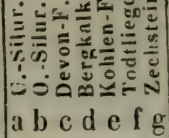 & 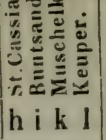 & 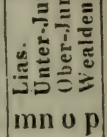 & 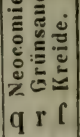 & 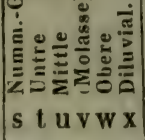 & 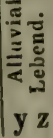 \\
\hline
\end{tabular}

Paludina)

*spp. majores: ? Vivipara.

? acuminata So.

acuminata Du.

carinifera So.

elongata So.

fluviorum MaNT. . .

Hagenowi Dv.

Roemeri Du.

scalariformis Du.

Schusteri Rok.

subangulata Roв. . .

Sussexensis So.

Desnoyersi Dsı. . .

lenta Dsir.

unicolor Sw.

vivipara DRP. . . . .

achatinoides Dsu.

viviparoides BR. . .

achatina Lr.

mobilis KLEIN ....

empullacea Br.

clathrata DsH. . . . .

concinna Murrs.

** spp. indifferentiores.

† ambigua PrÉv. . . angulosa Morrs. .. aspera MiснD. aspersa Mrснд. ... atomus Dsн. .... Draparnaudi NYsT. . Duchasteli (Nyst). .

\# indistincta DFr., FÉr. minuta Morrs.

Nysti Bors.

pupa Nyst . . . . . Stricklandana Forb. subulata Dsh. ....

\# virgula DFr. Fer. . conica Prév.

Desmaresti pusilla Bast.

pyramidalis DsH. . . globulus Dsh. . . . . nana Dsh.

macrostoma Dsh. . . striatula Dsh.

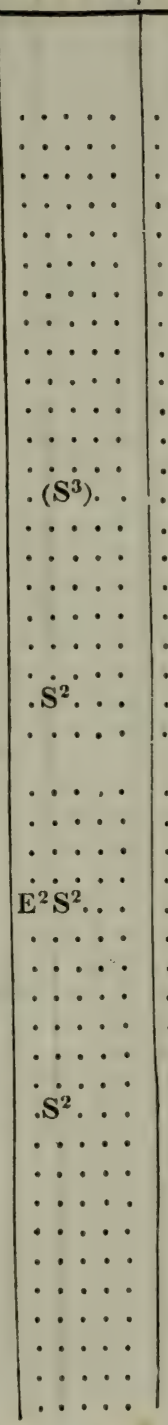


pygmaea Dsir. terebra Dsн. abbreviata Grat. affinis $F_{E} \dot{R}$.

\# Arvernensis Huot . Beaumontana MTHN. Bosquana Mathe. cingulata MATHN.

- denticulata DsMous. Deshayesana Mathn. dilatata Eichw. Dubuissoni Bourl. incerta BouiL. minutissima Grat. ovata Bovic. planata DUB, $s p$. regularis BoviL. rotundata (Pusch). striatella Grat. Viquesneli D'A. diaphana BoviL. Brardi Serr.

Deccanensis So. Helvetica Drr. brevis Serr. exigua Eichw. melanioides Dsh. minuta SERr. Triton Eichw. rubens MENKE similis $\mathrm{Br}$. tentaculata Dsh. variabilis Eicнw. obsoleta Woodw. ventricosa LEAcH. sp. marginata Мгер.

Troscheli ... Al. Braun

Hitorineila Al. Braun) 3 . . = Hydrobia HARTM. = pp. e Paludina requirendue).

inflata $n$. acuta A. Braun . . . PrIudestrina) D'O. = Hydrobia HARTM. = australis D'O.

Valvata 0 . Mǘu. io I,enpoldi Bors. multiformis $\mathrm{BU}$. piscinalis F́́R. cristata Mür.L. spirorbis . . A. Braun

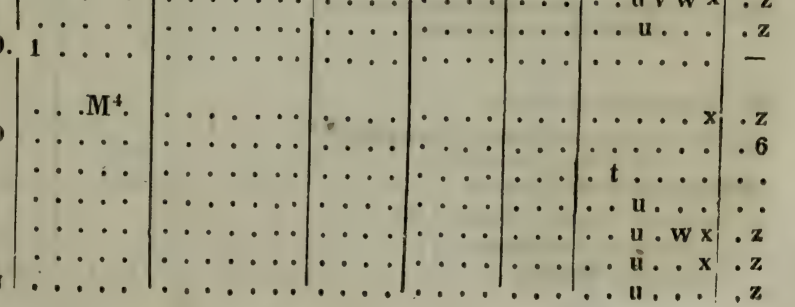




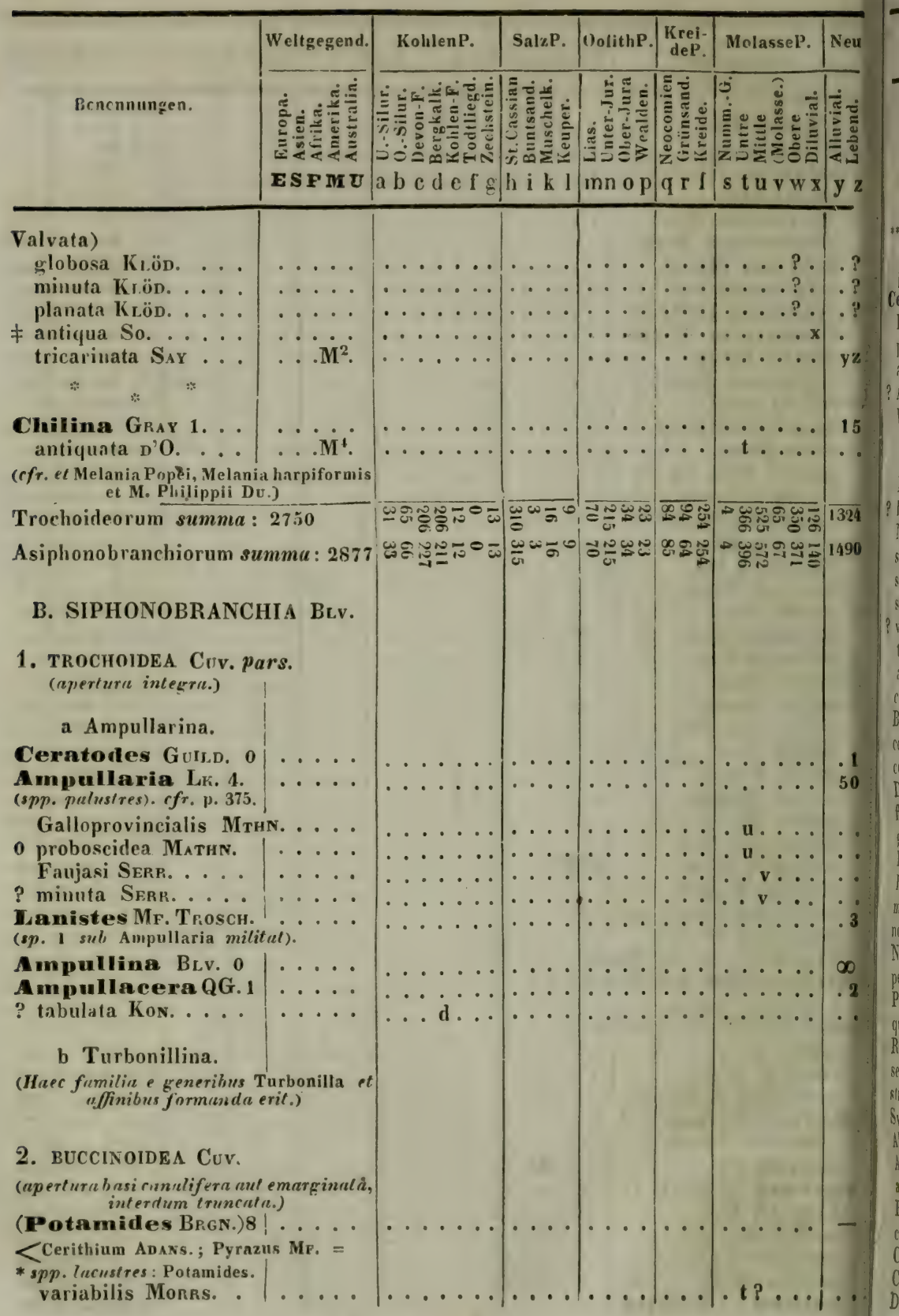




\begin{tabular}{|c|c|c|c|c|c|c|c|}
\hline Benennungen. & id. & g & h & $n$ & & $\mathbf{w} x$ & $y^{2}$ \\
\hline & & & & & & - u & \\
\hline$\cdots$ & $\cdots$ & $\cdots \cdots$ & $\cdots$ & - & $\cdots$ & . u... & \\
\hline cki BFGN. . & $\cdots \cdot$ & $\cdots \cdots \cdot$ & $\cdots \cdot$ & $\cdots \cdot$ & $\cdots$ & $\cdots$ ü... & \\
\hline$\therefore$ & . & $\cdots \cdots \cdot$ & $\cdots \cdot$ & & $\cdots$ & . . u... & • \\
\hline ithium. & $\cdots$ & $\cdots \cdots \cdots$ & - & $\cdots$ & $\cdots$ & $\cdots u$ & \\
\hline & $\because$ & $\cdots \cdot$ & $\cdots \cdot$ & $\cdots \cdots$ & $\cdots$ & . . u ... & \\
\hline plicat & & $\ldots \ldots$ & $\cdots \cdot$ & $\ldots$ & $\cdots$ & .. u... & \\
\hline Cerithium Adans. LF & K. 352,. & $\ldots \ldots$ & $\cdots \cdot$ & $\ldots$. & $\cdots$ & $\ldots \ldots$ & 90 \\
\hline eni VERN. • &. $\mathrm{S}^{2} \cdot \cdot$ & $\cdot b \cdots$ & $\cdots \cdot$ & $\cdots \cdot$ & . . & $\ldots \ldots$ & - \\
\hline$\cdots$ & $\cdots \cdot$ & $\ldots d \ldots$ & $\cdots \cdots$ & $\cdots \cdots$ & $\cdots$ & $\cdots \cdots$ & \\
\hline$\cdots$ & $\cdots \cdot$ & $\cdots$ & $h$ h.... & $\cdots \cdot$ & $\cdots$ & $\cdots \cdots$ & \\
\hline$\cdot \cdots$ & - & $\cdots$ & ha & $\cdots \cdot \cdot$ & $\cdots$ & $\cdots \cdots$ & - \\
\hline$\cdots$ & & & & & $\cdots$ & $\cdots \cdots$ & \\
\hline x. . & $\cdots \cdots$ & $\therefore$ & h. & $\cdots \cdots$ & $\cdots$ & $\cdots \cdots$ & • \\
\hline$\cdots$ & $\cdots \cdots$ & $\cdots \cdots \cdots$ & $h$ & $\ldots$ & $\cdots$ & $\cdots \cdots$ & \\
\hline . & $\begin{array}{l}\cdots \\
\cdots \\
\cdots\end{array}$ & $\ldots \ldots$ & h... & $\ldots$ & $\ldots$ & $\ldots \ldots$ & \\
\hline LI. & $\ldots$ & $\ldots \ldots$ & $h \ldots$ & $\cdots$ & $\ldots$ & $\ldots \ldots$ & \\
\hline$\therefore$ & .... & $\ldots \ldots$ & $\cdots$ & $\cdots \cdot$ & $\ldots$ & $\ldots \ldots$ & \\
\hline Mü. & $\ldots$ & $\ldots \ldots$ & & $\cdots \cdot$ & $\cdots$ & $\ldots \ldots$ & \\
\hline atum $\mathrm{K}_{\mathrm{I}}$ & 1. ... & $\ldots \ldots$ & h & $\cdots \cdots$ & $\cdots$ & $\ldots \ldots$ & \\
\hline Ker. . & $\cdots$ & $\ldots \ldots$ & h... & $\cdots \cdots$ & $\cdots$ & $\ldots \ldots$ & \\
\hline . & $\cdots \cdot$ & $\ldots \ldots$ & $\cdots \cdot$ & & $\cdots$ & $\ldots \ldots$ & \\
\hline$\cdots$ & $\ldots$ & $\ldots \ldots$ & $\cdot$ & $? \mathrm{n}^{23}$ & $\cdots$ & $\ldots \ldots$ & • \\
\hline$\cdots$ & $\cdots \cdots$ & $\ldots \ldots$ & $\cdot 1$ & $\cdot n^{2} \cdot \cdot$ & $\cdots$ & $\cdots \cdots$ & \\
\hline$\cdots$ & $\cdots$ & $\cdots \cdots$ & $\ldots$... & $\cdot \mathrm{n}^{3} \cdot \cdot$ & $\cdots$ & $\cdots \cdots$ & \\
\hline$\cdots$ & $\cdots \cdot$ & $\cdots \cdots \cdots$ & $\cdots$ & . n. . & $\cdots$ & $\cdots \cdots$ & \\
\hline . & - & I & & $\cdot \cdot$ & $\cdots$ & $\cdots \cdots$ & \\
\hline i D'A. . . . & & 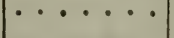 & $\cdots$ &. $\mathrm{n}^{\circ} \cdot \cdot$ & $\cdots$ & $\cdots \cdots$ & \\
\hline tum ${ }_{M i}$ & İ̈. & $\therefore$ & $\cdots$ & . n . & $\cdots$ & $\begin{array}{ll}\cdots & \cdots \\
\cdots & \ldots\end{array}$ & \\
\hline $\begin{array}{c}\mathrm{M} \\
\ldots i\end{array}$ & .... & $\ldots \ldots$ & & $n^{n} \cdots$ & $\cdots$ & $\cdots \cdots$ & \\
\hline GF. & . . & $\ldots \ldots$ & $\ldots$ & n... & $\ldots$ & $\cdots \cdots$ & \\
\hline Mi & . & $\ldots \ldots$ & $\ldots$. & . n.. & $\cdots$ & $\ldots$ & \\
\hline IÜ. & $\ldots$. & $\ldots \ldots$ & $\ldots$. & . n.. & $\cdots$ & $\ldots \ldots$ & \\
\hline & $\ldots \ldots$ & & $\ldots \ldots$ &. $\mathrm{n}^{3}$ & $\ldots$ & $\ldots \ldots$ & . \\
\hline & $\ldots$ & $\ldots \ldots$ & &.$n^{3}$. & $\cdots$ & $\ldots \ldots$ & \\
\hline$\therefore$ & $\cdots \cdots$ & $\ldots \ldots$ & $\cdots$ &.$n^{3}$ & $\cdots$ & $\ldots \ldots$ & - \\
\hline & $\cdots \cdots$ & $\ldots \ldots \ldots$ & $\cdots \cdot$ & $\cdot n_{\cdot} \cdot$ & $\cdots$ & $\ldots \ldots$ & \\
\hline : & $\cdots \cdot$ & $\ldots \ldots$ & - &.$n^{25} ?$ & $\cdots$ & $\cdots \cdots$ & \\
\hline & - & $\cdots$ & & $\frac{n}{n^{3}} \cdots$ & $\cdots \cdot$ & $\ldots \ldots$ & - \\
\hline & & & & $\cdot n^{\circ} \cdots$ & & $\cdots$ & : \\
\hline 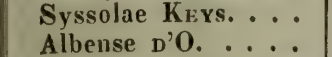 & $\cdots$ & $\ldots$ & $\cdots \cdots$ & $\cdots$ & q & $\ldots$ & \\
\hline$\cdots$ & $\ldots$ & $\ldots \ldots$ & $\ldots$ & $\ldots$ & q $\ldots$ & ....... & \\
\hline & .. & $\ldots \ldots$ & $\ldots$ & $\ldots$ & $q$ & $\ldots \ldots$ & $\ldots$ \\
\hline . & . $\mathbf{M}^{3}$. & $\ldots \ldots$ & & & & $\ldots \ldots$ & . \\
\hline AL. & $\cdots \mathbf{M}^{3}$ & $\cdots$ & & & ??? & & \\
\hline & $\cdots \cdot \cdot$ & $\cdots$ & & $\cdots$ & $q$ & $\cdots$ & \\
\hline C & $\cdots \cdot \cdot$ & & & & & $\cdots \cdot$ & \\
\hline & & & & & & & \\
\hline
\end{tabular}




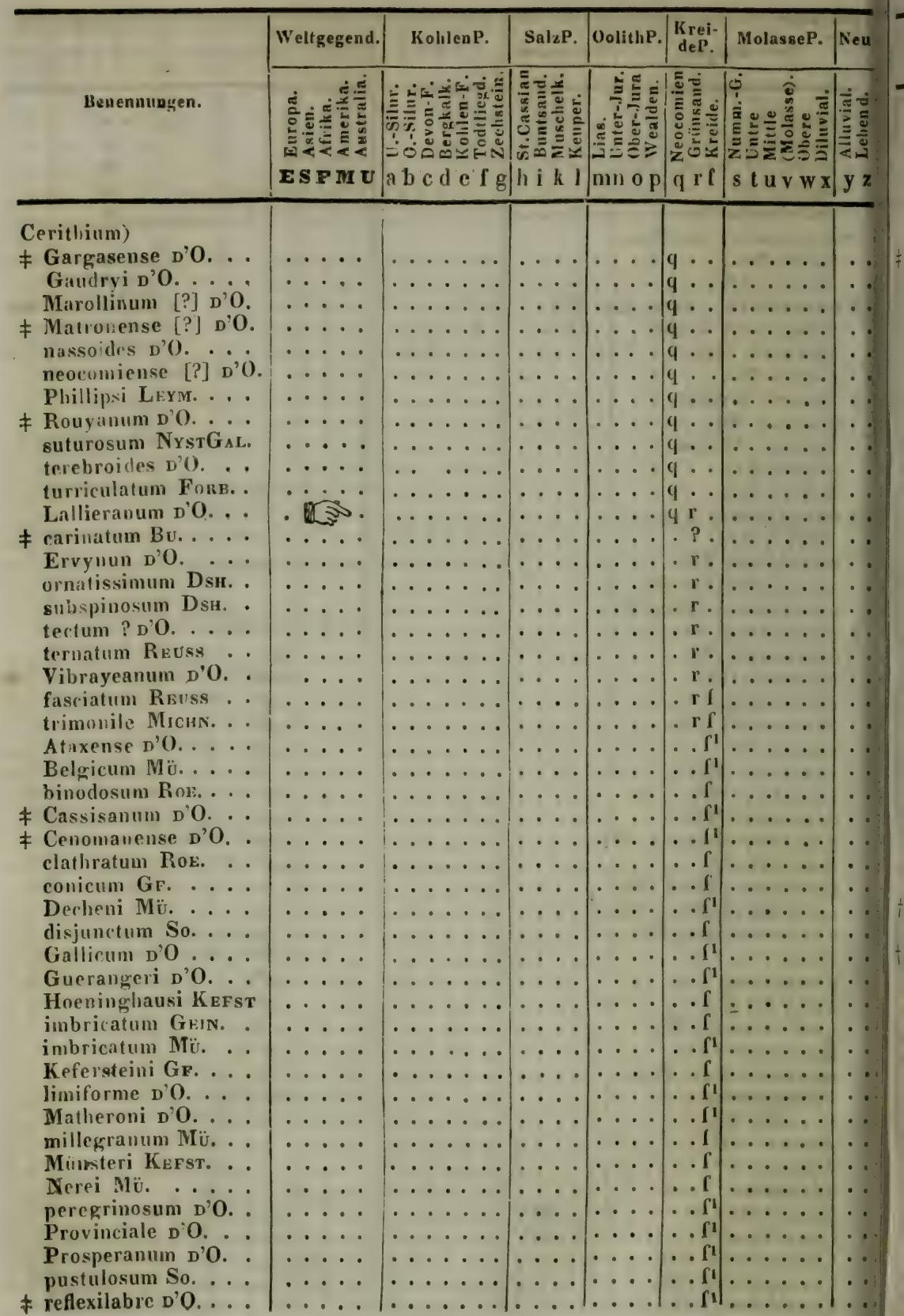




\begin{tabular}{|c|c|c|c|c|c|c|c|c|}
\hline & Ben & Weltgegend. & $\mathbf{a} \mathbf{b} c$ & h i k I & $\operatorname{mn} 0 \mathrm{p}$ & $q \times 1$ & $\mathbf{s} \mathbf{t} \mathbf{u} \mathrm{v} \mathbf{w} \mid$ & $y$ \\
\hline & m) & & $\cdots \cdot \cdot$ & & $\cdot \cdots \cdot$ & . . $\int^{1}$ & $\cdots \cdots$ & \\
\hline & 0. & $\cdots$ & $\cdots$ & $\cdots \cdot$ & 1 & . . $\boldsymbol{f}^{1}$ & ..... & \\
\hline & o.... & . . & . . & ... & $\cdots \cdot$ & . . $\Gamma^{1}$ & $\cdots \cdots$ & \\
\hline & RoE. . & $\cdots \cdot \cdot$ & $\ldots \ldots$ & $\cdot \cdot \cdot$ & $\cdot \cdot \cdot$ & . $f$ & $\cdots \cdots$ & \\
\hline & Ü. · & $\cdots \cdot \cdot$ & $\ldots . .$. & $\cdots \cdot$ & $\cdot \cdot \cdot$ & $\cdot f$ & $\cdots \cdots$ & \\
\hline & m Reuss & $\cdots \cdot \cdot$ & $\cdots \cdot$ & $\cdots \cdot$ & $\cdots \cdot$ & . $\mathrm{f}$ & $\ldots \ldots$ & \\
\hline & $\ldots$. & $\cdots \cdot \cdot$ & $\cdots \cdots \cdot$ & $\cdots \cdot$ & $\cdots \cdot$ & $\cdot \cdot \mathbf{I}^{\mathbf{1}}$ & $\cdots \cdots$ & \\
\hline & $\cdots$ & $\cdots \cdot \cdot$ & $\cdots \cdots \cdot$ & $\cdot \cdot \cdot$ & $\cdot \cdot \cdot$ & $\cdot$ & . t $\cdots$ & \\
\hline & $\begin{array}{l}\text {. } \\
\text { i. }\end{array}$ & $\cdots \cdot \cdot$ & $\cdots \cdot \cdots$ & ... & $\cdots$ & & $\cdot t^{t} \cdots \cdots$ & \\
\hline & $\begin{array}{l}\text { basense LEYM. . } \\
\text { igatum Dsh. . }\end{array}$ & $\cdot \cdot \cdot$ & $\cdots \cdot \cdot \cdot$ & $\cdots \cdot$ & $\cdot \cdot \cdot$ & - & $\cdot t \cdots$ & \\
\hline & $\begin{array}{l}\text { igatum Dsн. . } \\
\text { lernans Dsн. . }\end{array}$ & $\bullet \cdot \cdot$ & - $\cdot$ & •. • • & $\cdots \cdot$ & $\cdots$ & $\cdot t \cdots$ & \\
\hline & $\begin{array}{l}\text { ns Dsh... } \\
\text { atum Br... }\end{array}$ & $\cdots \cdot$ & $\cdots \cdots \cdots$ & & $\cdots \cdot$ & & $\cdot \mathrm{t} \cdots \cdots$ & \\
\hline & $\begin{array}{l}\mathrm{Um}_{\mathrm{m}} \mathrm{BR} . \ldots \\
\mathrm{L} \text {. . . . }\end{array}$ & . . . . & to 10 & $\cdots \cdot$ & $\cdots \cdot$ & $\cdots$ & $\cdots t \cdots$ & \\
\hline & $\begin{array}{l}\text { Lк. } \\
\text { atum Brin. }\end{array}$ & . . & -. & & $\cdot \cdot \cdot$ & & $\begin{array}{lll}\mathrm{t} & \cdots \\
\mathrm{t} & \cdots & \cdots\end{array}$ & \\
\hline & $\begin{array}{l}\text { tum BRGN. } \\
\text { Dsh. ... }\end{array}$ & •. • • & • & & $\left|\begin{array}{lll}\cdots & \cdots & \cdot \\
\cdots & \cdots & \end{array}\right|$ & & $\begin{array}{l}.1 \\
. t\end{array}$ & \\
\hline & Dsн..... & .... & $\ldots$ & & . . . & & $\begin{array}{lll}\cdots & \cdots & \cdots \\
. & \mathrm{t} & \cdots\end{array}$ & \\
\hline & f. . & .... & ...... & $\ldots$. & ... & $\ldots$ & t.... & \\
\hline & SH. . & $\cdots \cdot \cdot$ & 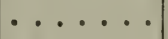 & ... & ... & $\cdots$ & t $\ldots$ & \\
\hline & . . & $\cdots \cdot$ & $\cdots \cdots$ & $\cdots$ & $\cdots$ & . . & . & \\
\hline & K. • & $\cdots \cdot$ & $\cdots \cdots$ & $\cdots \cdot$ & $\cdots \cdot$ & $\cdots$ & . t ... & \\
\hline & - & $\cdots \cdot \cdot$ & $\cdots \cdots$ & & $\cdots$ & . . & . $\mathrm{t} \ldots$ & \\
\hline & •. & $\cdots \cdot \cdot$ & $\cdots \cdots$ & 0 & $\cdots$ & & $\cdot t_{t}^{t} \cdots$ & \\
\hline & $\dot{\mathrm{Br}} \cdot \overrightarrow{.}$ & $\begin{array}{l}\cdots \cdot \cdots \\
\cdot \cdots \cdot\end{array}$ & $\cdots \cdots \cdots$ & & $\cdots$ & & 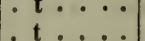 & \\
\hline & BR. . & .... & $\begin{array}{l}\bullet \cdot \\
.\end{array} \cdot \cdot \cdot \cdot$ & & 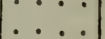 & &. $\mathrm{t}$ & \\
\hline & LF. . . . & . . . & ...... & ... & ... & & . & \\
\hline & LK. & $\cdots \cdot \cdot$ & $\ldots \ldots$ & $\cdots \cdot$ & $\cdots$ & $\cdots$ & . & \\
\hline & LK. ... & $\cdots \cdot$ & $\cdots \cdots$ & $\cdots$ & $\ldots$ & $\cdots$ & . $t \ldots$ & \\
\hline & Dsh. . & $\cdots \cdot$ & $\ldots \ldots$ & $\cdot \cdot$ & $\cdots \cdot$ & $\cdots$ &.$t \ldots$ & \\
\hline & Dsн. . . & $\cdots \cdot$ & $\ldots \cdots$ & $\cdots \cdots$ & $\ldots$ & $\cdots$ & $\ldots t \ldots$ & \\
\hline & Dsн. $\cdots$ & $\cdots \cdot \cdot$ & $\cdots \cdots$ & $\cdots$ & $\cdots$ & & t $\ldots$. & \\
\hline & piae So. . & $\cdots \cdot$ & $\cdots \cdots$ & 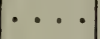 & $\cdots \cdot \cdot$ & &. $\mathbf{t} \ldots$ & \\
\hline & Dsн. .. & $\cdots \cdot \cdot$ & $\cdots \cdots$ & $\cdots$ & $\cdots \cdot$ & - & $\cdot t \cdots$ & \\
\hline & DFR. ... & $\cdots$ & - & ${ }^{2}$ & . & & . $t$ & \\
\hline & um LK. ... & $\cdots \cdot$ & $\cdots \cdots \cdot$ & $\cdots$ & $\cdots \cdot$ & - & $\cdots$ & \\
\hline & um DFR. ... & $\cdot \cdot$ & $\cdots \cdots$ & 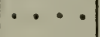 & $\cdots \cdot$ & & . $t$ & \\
\hline & $\begin{array}{l}\text { DFR. } \ldots \\
\mathbf{m} \mathbf{L}_{\text {K. }} \ldots\end{array}$ & - & $\cdot$ & & - & & . $t$ & \\
\hline & 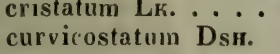 & $\dot{\cdots}$ & & & . & & $\therefore t$ & \\
\hline & sH. . . & . . . & ...... & $\cdot$ & ... & & $\ldots t \ldots$ & \\
\hline & ONR. . . & . $\mathrm{M}^{2}$ & $\cdots \cdot \cdot \cdot \cdot$ & & $\cdots$ & & . $\mathbf{t}$ & \\
\hline & m DFr. . . & $\cdots \cdot \cdot$ & - & - & $\bullet \cdot$ & &.$t \ldots$ & \\
\hline & cei Dsh. . . & & - $\bullet$ & & & & . t & \\
\hline & DFP & - & - & 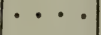 & -・ & & . $t$ & \\
\hline & atum LK. . . & $\cdots \cdot$ & $\cdot$ & & • & - & . $t \ldots$ & \\
\hline & um LEYM. & $\cdots \cdot \cdot$ & $\cdot \cdot \cdot$ & & & & - $t$ & \\
\hline & $\cdots \cdot$ & - & $\cdots \cdot$ & & & & . $t$ & \\
\hline & $\begin{array}{l}\text { i Dsh. . . } \\
\text { es Ls. . . }\end{array}$ & . . & $\begin{array}{l}\cdots \\
\cdots\end{array}$ & . $\cdot$ & & & . $t$ & \\
\hline & DSH. . & - $\cdot$ & •...... & • & & & . $t$ & \\
\hline & legans & & & & & & & \\
\hline
\end{tabular}




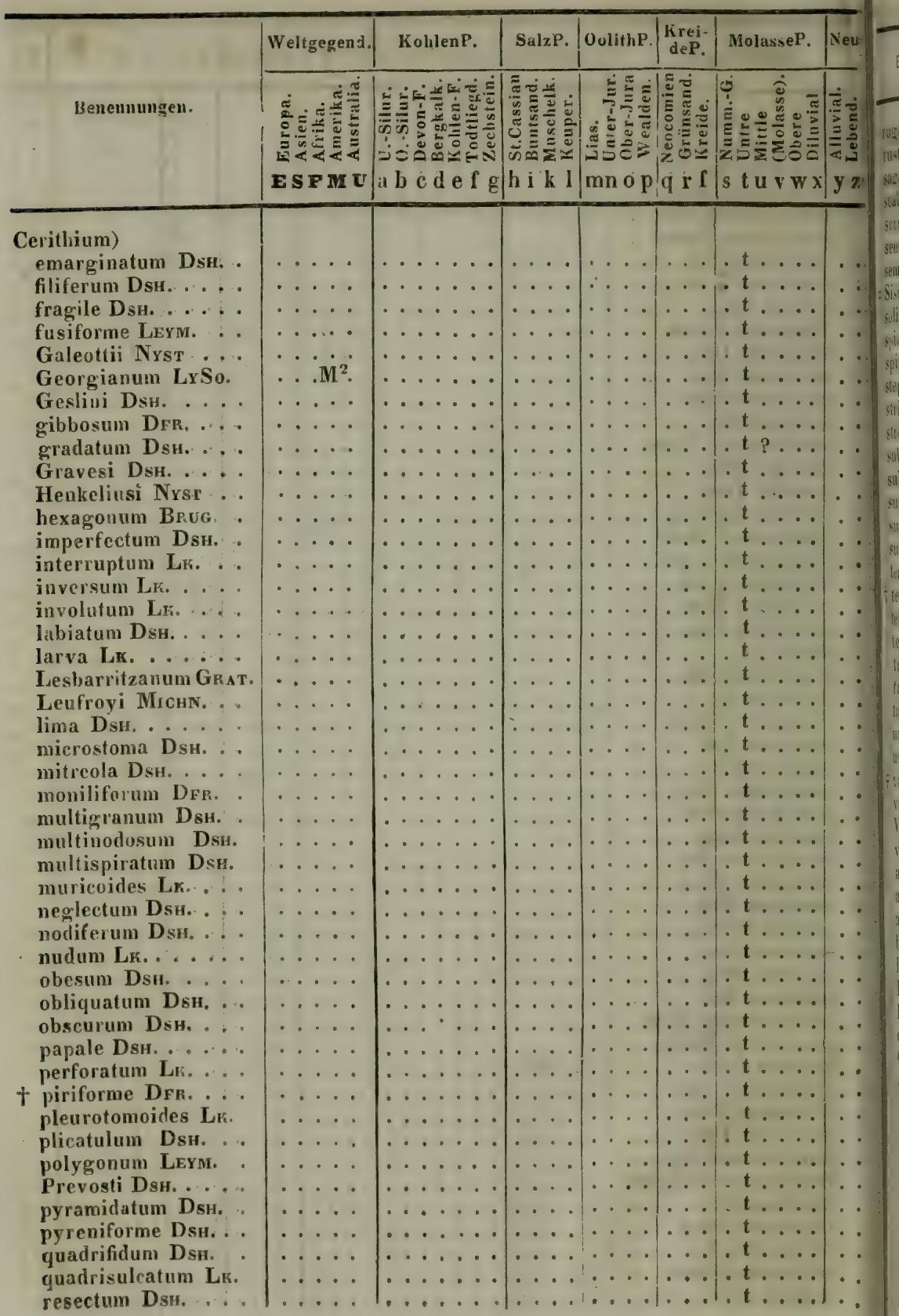


rugosum Lk. rusticum DsH. sagenula Conr. scalaroides DsH. scruposum Dsh. semicoronatum Lk. semicostatum Dsh, Sismondai Micut. solitarium Conr. spinosum Dsh. spirattim Lri. . stephanophorum Ds. striatum Dsr. stroppus BrgN. subcanaliculatumDsu. subpunctatum Dsh. subpyrenaicum Liум. substriatum LK. subula Dsh. tenue Dsh.

t terebra DFr. terebrale LK. textile Dsu. trochleare Lк. turbinatum Dsh. turris Dsh. undosum Brgrs. unisulcatum $\mathbf{L}$.

F yaricosum Ant. variculosum Nrst Venei Leys. ventricosum Dsh. ampullosum Brgev. angulosum LK. angustum DsH. baccatum DFr. Blainvillei DFr.

Bouei Dsh. Brocchii Dsh. calcaratum BrgN. Charpentieri Bast. concavum Dsh. crenatulatum DsH. diaboli BrgN. giganteum LK. globulosum Dsh. lamellosum Brug. lapiduın LK. plicatum Lr. Roissyi Dsh. scabrum Lk. serratum Brug. 


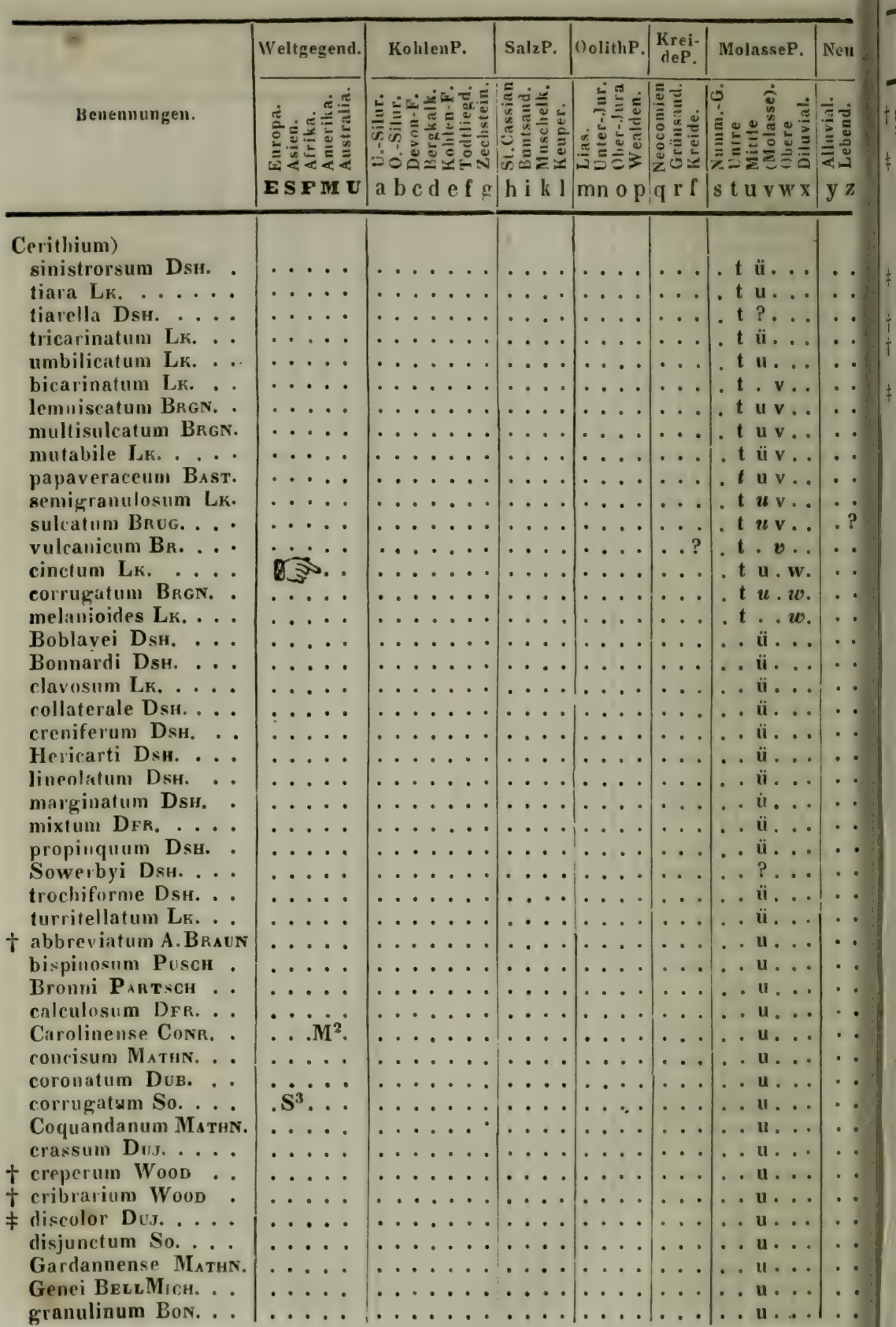


† granosum Wood irregulare Dub.

* laevigatum Erchw. lacvissimum GF. Laurae MathN. lignitarum Еiснw. lineolatum So.

f pulchellum Dus. Provinciale Mathr.

† pseudo-obeliscus Grat.

† punctulum Wood pupiforme Bast.

F resectum DFR. rude So. scalare MATHN. salmo Bast.

Taurinum BectMrch. tiara (LK.) Dus. tuberosum Grat. turritella So. uuilineatum Conr. Zeuschneri Pusch costatum Bops. crenatum DFr. margaritaceum Brign. pictum DFr. punctatum Woodw. adversum Wood di:ocatum SAY Mediterraneum Dsh. perversum LK. scabrum Dsh. semigranosum LK. terebella Br. tuberculare Wood trilineatum РнгL. Basteroti Sisrr. multigranulatum Serr t prismaticum (BRGN.) turbinatum SERR . angrustum DsH.

本 assimile Riss.

Basteroti DsH. bicinctum Riss. bitorquatum Phis. Borsonanum ? Riss. Calabrum Phic.

\# Favannei Riss. Graecuin Dsh.

† granosum Boris. \# laevigatum Serr. † laevum PHIL.

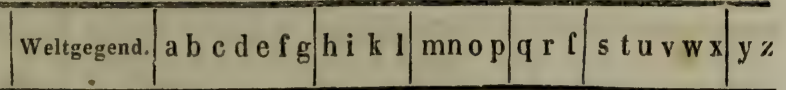

.

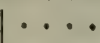

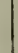

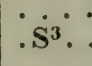

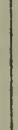

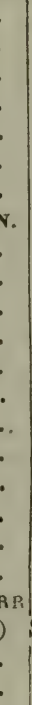

$\ldots$
$\ldots$
$\cdots$
$\cdots$
$\cdots$
$\cdots$
$\cdots$

$\ldots$
c
c
$\cdots$
$\cdots$
$\cdots$
$\therefore$
$\cdots$




\begin{tabular}{|c|c|c|c|c|c|c|c|}
\hline & Weltgegend. & KolilenP. & SalzP. & OolithP. & $\begin{array}{l}\text { Krei- } \\
\text { deP. }\end{array}$ & MolasseP. & Neu \\
\hline Benennungen. & 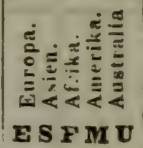 & 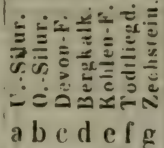 & 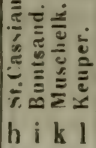 & 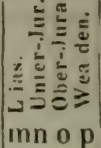 & 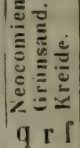 & 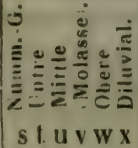 & 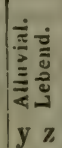 \\
\hline
\end{tabular}

\section{Cerithium )}

lineatum Bors.

F Magnani Riss.

nodosum Bors.

₹ plicatulum Riss.

$\neq 4-5$ cinctum Bors. sinistratum NYst.

₹ tuberculatum SERr.

f turritum Bors. varicosum DFR.

₹ vulgatulum Dsh. . bicinctum BR.

lacteum PhiL. manmillatum Riss. vulgatumi Brug. costulatum Risso

Triforis Dsh. 1. . . plicatus Dsh.

\section{b Strombina.}

Chenopus Phrl. 11. () Aporrhais DaCosta.) Philippii KoDo. spinosus Mü. .... subpunctatus Qu. cingulatus KoDv... strombiforuis KoDu. Buchi Mü.

Sowerbyi Pur. pes pelrcani PHIL.

i decussitus PHIL. . paradoxus Phill. . . occideutalis BEcK . aline spp. e Rostellaria. requirendice.

Rostellaria Lr. 76.

(Rostellaria et Chenopus PHIL. et Hippo crenes Mr.)

t sntiqua Gr.

+ detrita GF. gracilis $M \ddot{u}$. nodosa $\mathrm{Mü.}$. tenuistria Mü. bispinesa Phill.

† bisulcata WiLLMs. caudata Ror.
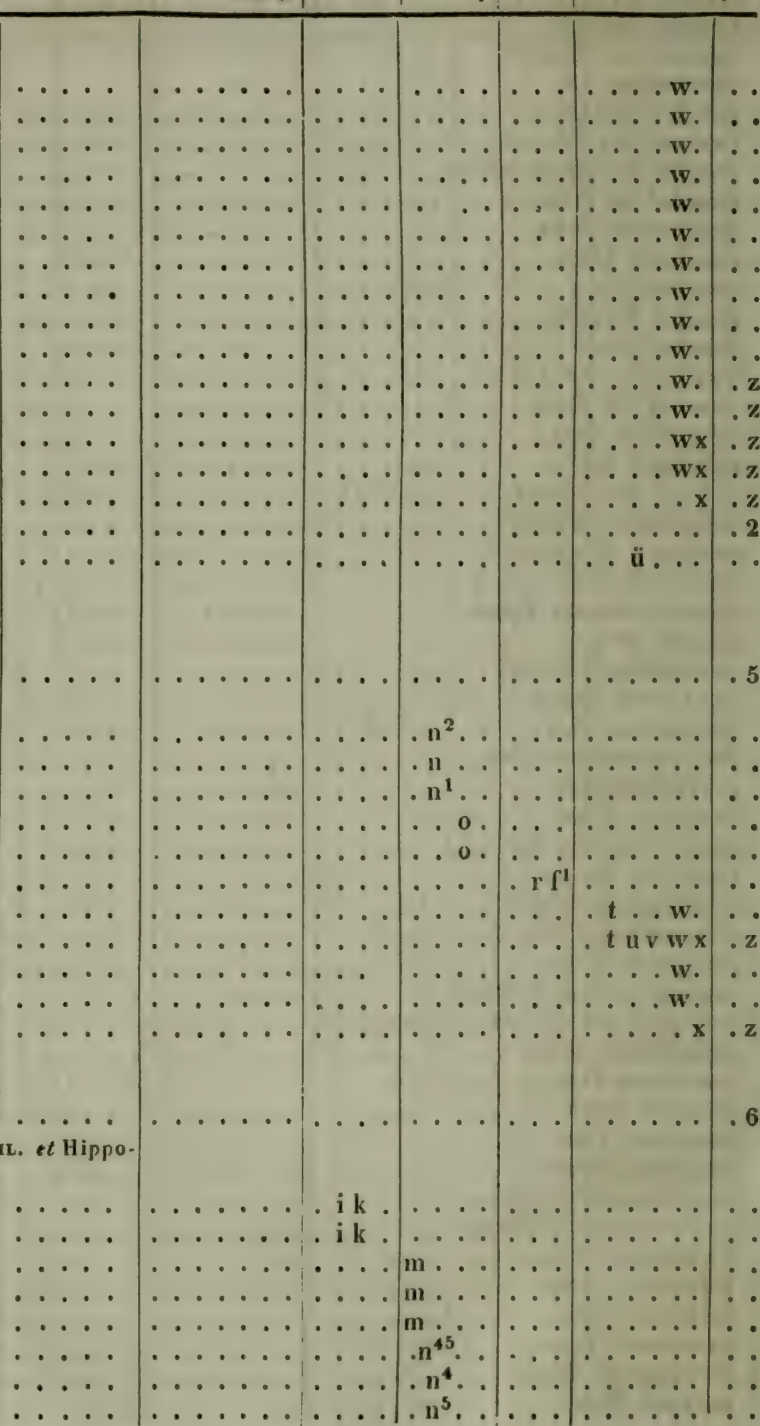


\begin{tabular}{|c|c|c|c|c|c|c|c|}
\hline Benennungen. & Weltgegend. & $a b c d e f g$ & h i k l 1 & $\mathrm{mn}$ o $\mathrm{p}$ & $q \quad r \quad[$ & s t u v $\mathbf{x}$ & $y^{2}$ \\
\hline ( & & & & $\cdot 11 \cdot$ & & $\cdots \cdots$ & \\
\hline$\ldots$ & $\cdots \cdots$ & $\cdots$ & & $.1^{23}$ & & $\ldots \ldots$ & \\
\hline a 1 & $\cdots \cdots$ & $\cdots$ & $\cdots$ & . $\mathrm{n}^{5} \cdot$ & $\cdots$ & $\cdots \cdots$ & \\
\hline is 1 & $\cdots$ & $\cdots$ & & $\cdot n^{2} \cdot$. & $\cdots \cdot$ & $\cdots \cdots$ & \\
\hline$\cdots$ & $\cdots \cdots$ & $\cdots$ & & 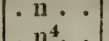 & & $\cdots \cdots$ & \\
\hline$\ldots$ & $\cdots$ & 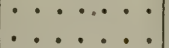 & & $\left|\begin{array}{ll}n^{4} & \cdot \\
0 n^{4} & .\end{array}\right|$ & $\cdots$ & $\therefore$ & \\
\hline $\begin{array}{l}\text { trifida BEA } \\
\text { nodifera K }\end{array}$ & ..... & $\cdots \cdots \cdots$ & $\cdots$ & ... & $\because \cdots$ & $\cdots \cdots$ & \\
\hline$A l_{p}$ pina D'C & . … & $\ldots \ldots$ & $\ldots$ & $\ldots$. & $q \ldots$ & $\ldots \ldots$ & \\
\hline$\cdots$ & . . $\mathrm{M}^{3}$. & $\cdots \cdots$ & $\ldots$ & $\ldots$. & $q \cdots$ & $\ldots \ldots$ & \\
\hline$\cdots$ & . . M M & $\ldots \ldots$ & $\cdots \cdot$ & $\ldots . c$ & & $\ldots \ldots$ & \\
\hline$\cdots$ & $\cdots$ & $\cdots \cdots \cdots$ & $\cdots$ & $\ldots . c$ & & $\ldots \ldots$ & \\
\hline iata Dsн. ... . & ..... & $\ldots \ldots$ & $\cdots \cdot$ & & $4 \cdot$ & $\ldots \ldots$ & . \\
\hline aulti D'O. . & . . $\mathrm{M}^{3}$ & $\cdots$ & $\cdots \cdot$ & $\cdots \cdot d$ & $q$ & $\ldots \ldots$ & . \\
\hline$\ldots$ & $\cdots \cdot$ & $\cdots$ & $\cdots \cdots$ & $\cdots \cdot$ & $q$ & $\ldots \ldots \cdot$ & - \\
\hline$\cdots$ & $\cdots \cdot$ & $\cdots \cdots$ & $\cdots \cdot$ & $\ldots$. & $q$ & $\ldots \ldots$ & \\
\hline$\cdots$ & $\cdots \cdots$ & $\cdots$ & $\cdots$ & $\ldots$. & q. & $\ldots \ldots$ & ${ }^{\circ}$ \\
\hline$\cdots$ & $\cdots \cdot$ & $\cdots$ & $\cdots \cdot$ & $\ldots . c$ & & $\cdots \cdots$ & \\
\hline$\cdots$ & $\cdots \cdot$ & $\cdot$ & $\cdots$ & & & $\cdots \cdots$ & \\
\hline r. . & & & & . & & $\cdots \cdots$ & \\
\hline$\cdots$ & $\cdots \cdots$ & - & $\cdot$ & $\cdots \cdot$ & - r. & $\cdots \cdots$ & \\
\hline $\begin{array}{l}\text { carinata N } \\
\text { elongata }\end{array}$ & $\begin{array}{l}\cdots \cdots \\
\cdots \cdots\end{array}$ & $\cdots$ & $\cdots \cdots$ & $\mid \begin{array}{c}\cdots \\
\cdots\end{array}$ & $\left|\begin{array}{ll}\cdot r & r \\
0 & r\end{array}\right|$ & $\cdots \cdots \cdots$ & \\
\hline $\begin{array}{l}\text { elongata so. } \\
\text { inflata PAssy }\end{array}$ & $\ldots$ & $\ldots \ldots$ & $\ldots$ & $\cdots$ & $\mid \begin{array}{ll}0 & \mathrm{r} \\
\mathrm{r} & \mathrm{r}\end{array}$ & $\cdots \cdots \cdots$ & \\
\hline stoma So. & $\ldots$ & $\cdots$ & $\ldots$ & $\ldots$ & . r. & $\ldots \ldots$ & \\
\hline & $\ldots \ldots$ & $\ldots$ & $\cdots$ & $\ldots$ & . $r \cdot$ & $\ldots \ldots$ & \\
\hline$\cdots$ & $\cdots \cdots$ & $\ldots \ldots$ & $\cdots$ & $\ldots$ & . $r$ & $\ldots \ldots$ & \\
\hline USCH . & $\cdots \cdot$ & $\cdots$ & $\cdots$ & $\ldots$ & . $\mathrm{rI}^{\mathbf{1}}$ & $\ldots \ldots$ & $\cdots$ \\
\hline$\cdots$ & $\cdots \cdot$ & $\ldots$ & $\ldots$ & $\ldots$ & . $r ?$ & $\ldots \ldots$ & . \\
\hline inella $N$ & . . . & $\cdots$ & & ... & - r. I & $\ldots \ldots$ & - \\
\hline$\cdots$ & .... $\mathrm{M}^{2}$ & $\cdots \cdots$ & $\cdots$ & $\cdots \cdot$ & $\ldots \uparrow$ & $\ldots \ldots$ & \\
\hline$\cdots$ & $\cdots \cdots$ & . & $\cdots$ & $\ldots$. & - . r & $\ldots \ldots$ & - \\
\hline$\cdots$ & $\cdots$ & - & $\cdots$ & $\cdots \cdot \mid$ & & $\cdots$ & \\
\hline EUSS .. & . & & $\cdots \cdots$ & $\cdot$ & & $\cdots \cdots$ & \\
\hline$\cdots \cdot$ & $\cdots \cdot \cdot$ & . & $\cdots$ & $\cdots \cdots$ & & $\cdots \cdot$ & \\
\hline$\cdots$ & $\cdots \cdot$ & & $\cdots$ & $\cdots$ & & $\cdots$ & \\
\hline$\therefore$ & $\cdots$ & 1. & $\cdots$ & $\ldots$ & & $\cdots$ & \\
\hline$\cdots$ & - & - & & . & & . & \\
\hline EUSS . & $\because$ & & & & & & \\
\hline $\begin{array}{l}\cdots \\
\cdots\end{array}$ & $\ldots$ & $\cdots$ & $\ldots \ldots$ & $\cdots \cdots$ & $\ldots 1$ & $\cdots$ & \\
\hline GF. . & .... & ... & $\ldots$ & $\ldots$ & $\ldots i$ & . & \\
\hline ata n'O. . . & . ... & $\cdots$ & . & $\ldots \ldots$ & $\ldots \int^{1}$ & . & \\
\hline a Mort. ... & . . $\mathbf{M}^{2}$ & & $\cdots$ & $\ldots$. & $\cdots 1$ & & \\
\hline & $\ldots \ldots$ & & . & $\cdots$ & & & \\
\hline & $\cdots \cdot$ & & & & & & \\
\hline nana D'O. . & $\cdots \cdot \cdot$ & & & & & & \\
\hline lotheimi Roe. . & $\cdots \cdots$ & & - & & & $\cdots$ & \\
\hline 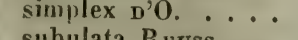 & - & & $\cdots$ & - & & $\cdots$ & \\
\hline$F$ & & & 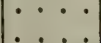 & & & $\cdots$ & ( \\
\hline & & & & & & & \\
\hline
\end{tabular}




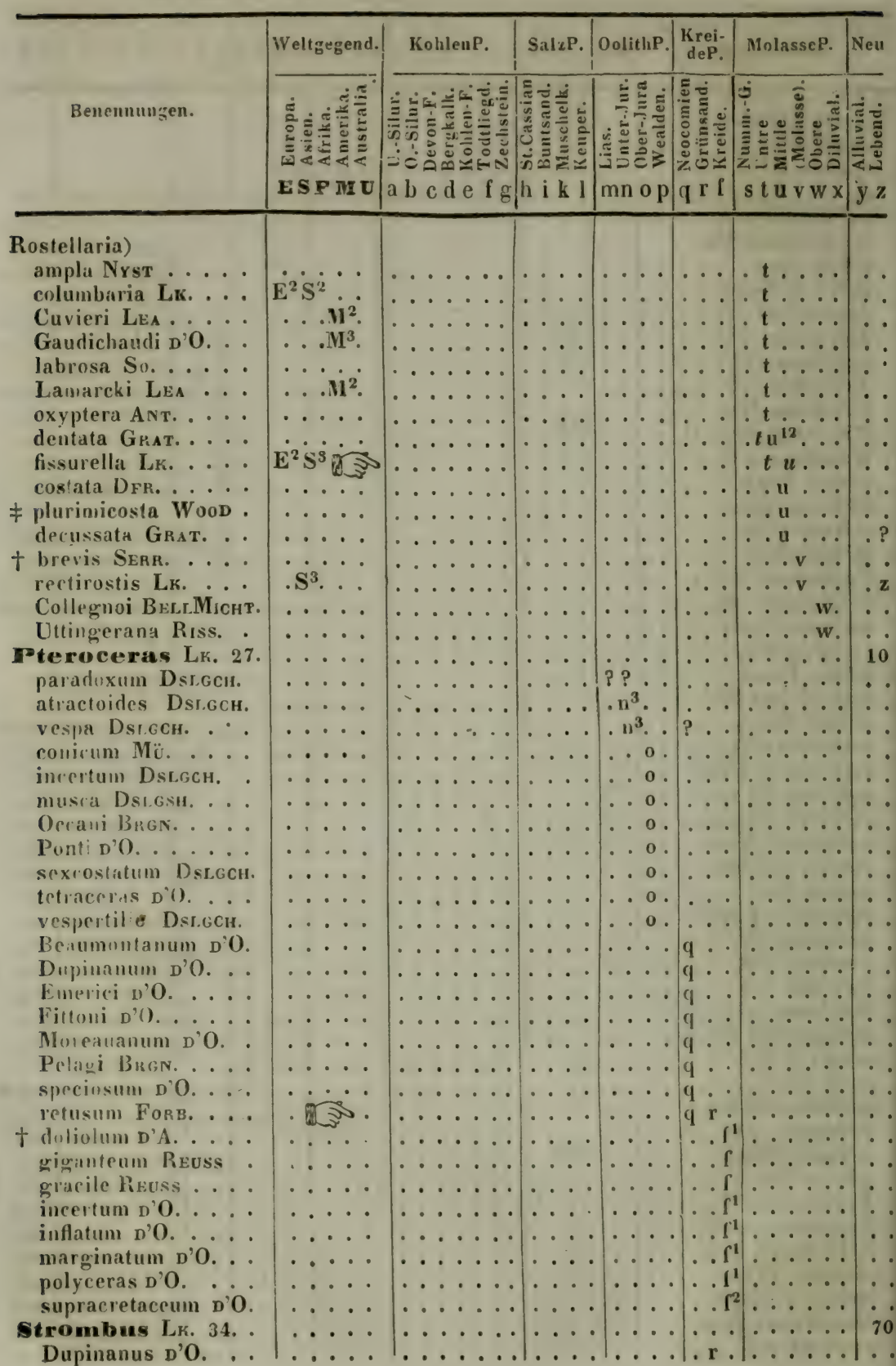




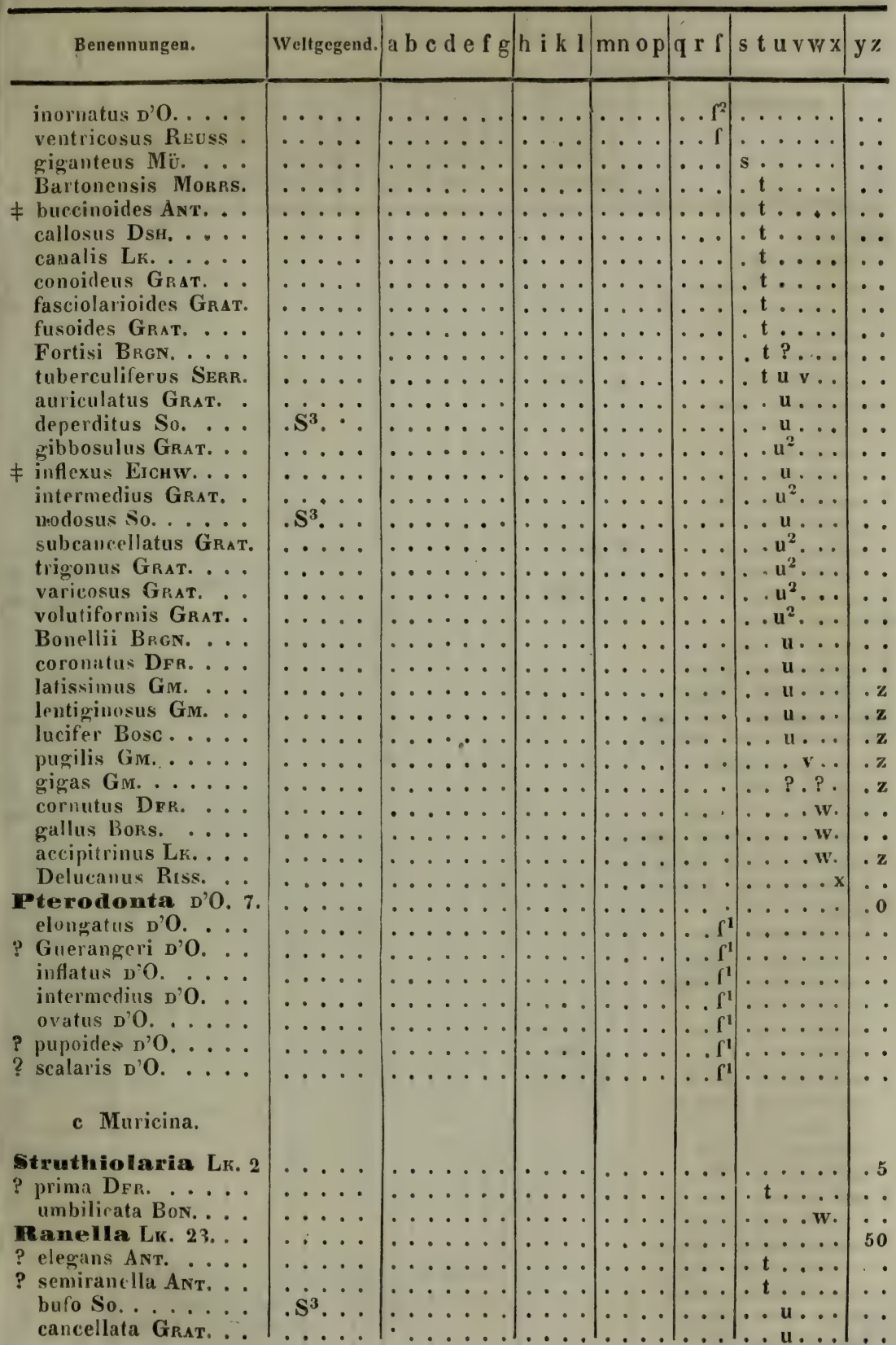




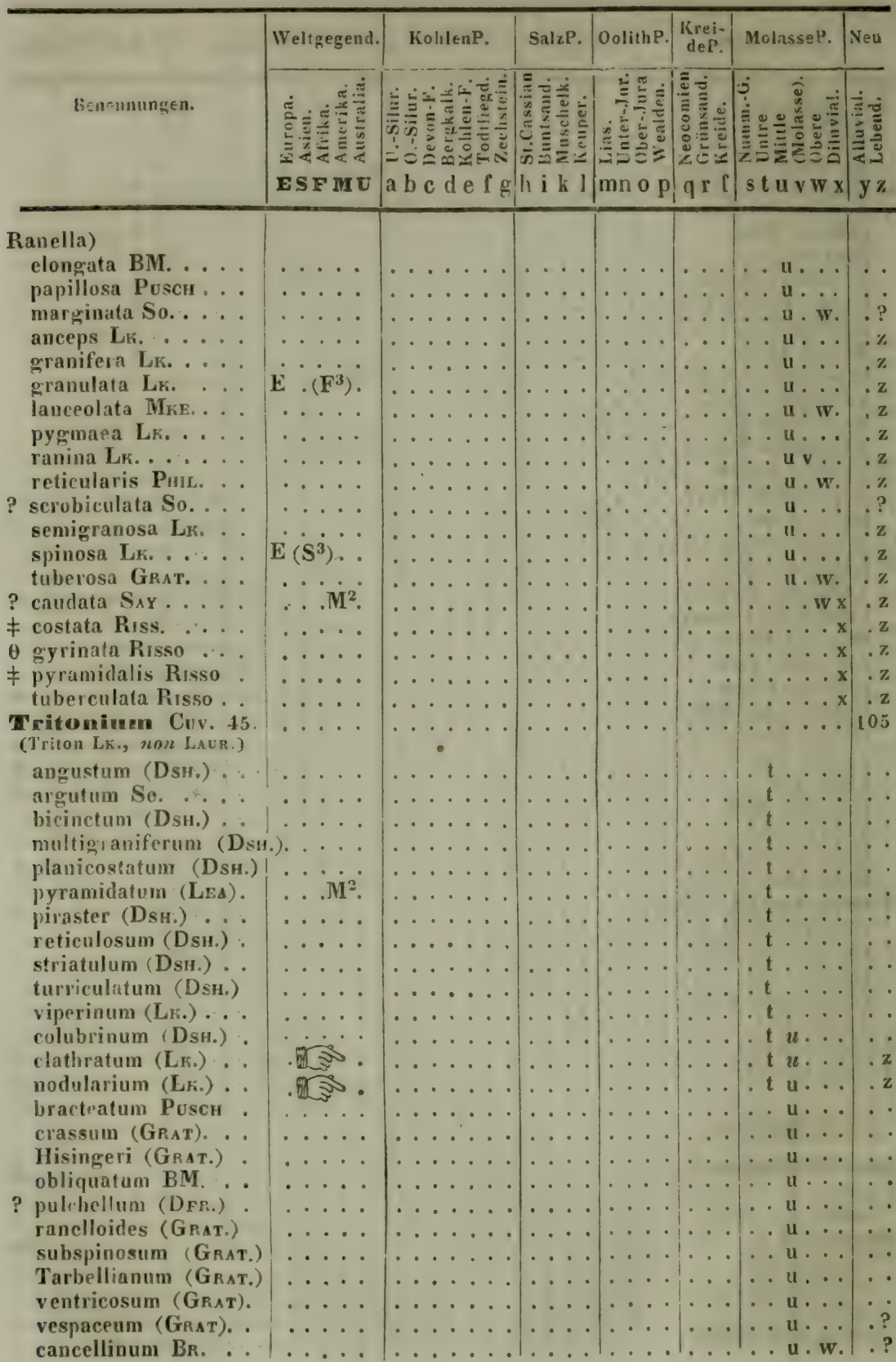




\begin{tabular}{|c|c|c|c|c|c|c|c|}
\hline Bener & Weltgegeñd. & $a b c d e f g$ & k 1 & $m n o p$ & $q \times f$ & $s \operatorname{tuvw} x$ & y z \\
\hline anus &.$\left(\mathbf{S}^{3}\right) \ldots$ & . & $\cdots$ & $\cdots$ & $\cdots$ & . . & . $\mathrm{z}$ \\
\hline к.) & $\ldots$ & $\ldots$ & $\ldots$ & $\cdots$ & $\cdots$ & . . u vw x & . $z$ \\
\hline$\therefore$ & $\ldots \ldots$ & $\ldots \ldots$ & $\cdots \cdots$ & $\ldots$ & $\ldots$ & . . u vw x &. $\mathrm{z}$ \\
\hline leucostoma var. Рusch & $\cdots$ & $\ldots \ldots$ & $\cdots$ & $\ldots$ & $\cdots$ & $\ldots \mathrm{u} \ldots \mathrm{x}$ &.$z$ \\
\hline nodiferum BR. & $\ldots \ldots$ & $\ldots \ldots$ & $\cdots \cdot$ & $\ldots \therefore$ & $\cdots$ & $\ldots \mathbf{u} \cdot \mathbf{w}$ & .7 \\
\hline nctum Pris... & $\ldots$ & $\ldots \ldots$ & $\cdots$ & $\cdots \cdots$ & $\cdots$ & $\cdot \mathrm{u} \cdot \mathbf{w}$ & $\cdot z$ \\
\hline ilosum Bon. . & $\cdots \cdots$ & $\cdots \cdots \cdot$ & $\cdots \cdot$ & $\cdots \cdot$ & $\cdots$ & $\cdots u \cdot \mathbf{w}$ & $\cdot \mathrm{z}$ \\
\hline 3.) & $\cdots \cdots$ & $\cdots \cdots \cdots$ & $\cdots \cdot$ & $\cdots \cdots$ & $\cdots$ & $\ldots \mathbf{v} \ldots$ & \\
\hline omum L & $\cdots \cdot \cdot$ & $\cdots \cdots \cdot$ & $\cdots \cdot$ & $\cdots \cdot$ & $\cdots$ & ... v . & $\cdot z$ \\
\hline (LK.) SERR. & $\cdots$ & $\cdots \cdots$ & $\cdots$ & $\cdots \cdots$ & $\cdots$ & $\ldots \mathbf{v} \ldots$ & $\cdot z$ \\
\hline inicum $\mathrm{BR}$. . & $\cdots \cdots$ & $\cdots \cdots$ & $\cdots$ & $\cdots$ & $\cdots$ & $\cdots w_{0}$ & - \\
\hline Br...... & $\cdots \cdots$ & $\cdots \cdots$ & $\cdots$ & $\cdots \cdot$ & $\cdots$ & $\cdots w$ & - \\
\hline Bir. . & $\cdots \cdot$ & $\cdots \cdots$ & $\cdots$ & $\cdots \cdots$ & $\cdots$ & $\cdots \cdots w$ & - \\
\hline nodosum B & $\cdots$ & $\cdots \cdots$ & $\cdots \cdot$ & $\cdots \cdot$ & $\cdots$ & 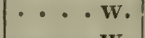 & - \\
\hline rugosum $P$ & $\cdots$ & $\cdots \cdots \cdots$ & $\cdots$ & $\cdots \cdots$ & $\cdots$ & $\cdots \cdots w$ & $\cdot$ \\
\hline$\cdots$ & $\cdots$ & $\cdots$ & $\cdots$ & $\cdots$ & $\cdots$ & $\cdots \cdots \mathbf{w}$ & - \\
\hline BR. . & $\therefore$ & $\cdots$ & $\cdots \dot{0}$ & $\cdots$ & $\because \cdots$ & $\left|\begin{array}{cc}\cdots w \\
\cdots & w\end{array}\right|$ & $\ddot{z}$ \\
\hline $\begin{array}{l}\text { scrol } \\
\text { cutac }\end{array}$ & & $\ldots \ldots$ & $\ldots$. & $\ldots$ & ... & $\ldots \ldots x$ & $\begin{array}{l}\cdot z \\
. z\end{array}$ \\
\hline EI.L. & $\dot{M}^{2}\left(M^{1} E^{2}\right)$ & $\ldots \ldots$ & $\cdots \cdot$ & $\cdots$ & $\ldots$ & $\ldots . x$ & $\mathrm{z}$ \\
\hline (Riss.) & $\cdots$ & $\ldots \ldots$ & $\cdots \cdot$ & $\cdots$ & $\cdots$ & $\ldots \ldots x$ & $\cdot \mathrm{z}$ \\
\hline is $\mathrm{Mr} .8 . \ldots$ & - . : & $\ldots \ldots \cdot$ & $\cdots \cdot$ & . & $\cdots$ & $\ldots \ldots$ & .8 \\
\hline gracilis Conr. . . . & . $M^{2}$ & $\cdots \cdots$ & $\cdots \cdot$ & $\cdots \cdots$ & $\cdots$ & . t .... & . \\
\hline ticus Morrs. . . & $\ldots$. & $\ldots \ldots$ & $\cdots \cdot$ & $\ldots$ & $\ldots$ &.$t \ldots$ & . \\
\hline . . . & $\cdots \cdot$ & $\ldots \ldots$ & $\ldots$ & $\cdots \cdot$ & $\cdots$ & . $t \mathrm{u} \cdot \mathrm{w}$ & . \\
\hline cc. $s p$. & $\cdots$ & $\ldots \ldots$ & $\cdots \cdot$ & . & $\cdots$ & . t u $\cdot \mathrm{w}$ & . \\
\hline$\cdots$ & $\cdots$ & $\cdots \cdots$ & $\ldots$ & ( & $\cdots$ & . t u ... & . $\mathrm{z}$ \\
\hline sta Conr. . & . . $\mathrm{M}^{2}$ & $\cdots \cdots \cdots$ & $\cdots \cdot$ & & $\cdots$ & $\cdots \mathbf{u} \cdots$ & - \\
\hline lus PhiL. . . & $\cdots$ & $\cdots \cdots \cdots$ & $\cdot$ & 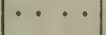 & $\cdots$ & . ?...w. & $\cdots$ \\
\hline erus $n . \cdots$ & - & $\cdots$ & $0^{\circ}$ & $\cdots$ & $\cdots$ & $\cdots \cdots w$ & $\dot{1}^{2}$ \\
\hline Murex (L.) LK. * 180 & $\dot{*}$ & $\because$ & $\cdots$ & $\cdots$ & $\cdots$ & $\cdots$ & 210 \\
\hline irick. & 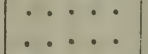 & : & $\cdots$ & . $n^{5}$. & $\cdots$ & $\cdots$ & \\
\hline $\begin{array}{l}\text { isis Puill. } \\
\text { es Pusch . }\end{array}$ & $\therefore$ & $\ldots \ldots$ & $\cdots$ & & $\cdots$ & $\cdots \cdots$ & \\
\hline rostellariaeformis Bu. & $\ldots$ & $\ldots \ldots$ & $\cdots$ &.$n^{5}$. & $\ldots$ & $\ldots \ldots$ & . \\
\hline$\cdots$ & & $\cdots$ & $\cdots \cdots$ & . $\mathrm{n}^{4}$. & $\cdots$ & $\ldots \ldots$ & - $\cdot$ \\
\hline calcar So. & .... & $\cdots \cdots$ & $\cdots \cdots$ & $\cdots$ & . r. & $\cdots$ & - \\
\hline${ }^{2}$ bicostatus Dsн. . . . & - & $\cdots \cdot$ & $\cdots \cdot$ & - & $\cdots$ & - $t$ & - \\
\hline bispinosus So. . . & & $\cdot$ & & & •. & $t \cdots$ & \\
\hline \# ${ }^{3}$ bisulcatus ANT. .. & $\cdots$ & & ( & & $\cdots$ & . t. & \\
\hline${ }^{3}$ calcitrapa LK. . . & $\begin{array}{l}\cdots \\
\cdots \\
\cdots\end{array}$ & . . & $\cdots$ & $\cdots$ & $\ldots$ & t. & \\
\hline ostatus Dsh. & $\begin{array}{ll}\cdots \\
\cdots\end{array}$ & $\ldots \ldots$ & $\ldots$ & $\cdots$ & $\ldots$ & . t... & \\
\hline${ }^{3}$ crispus Lk. . . . . & $\ldots$ & & & & $\cdots$ & - t . & . \\
\hline efossus So. .... & $\cdots$ & & & & & $1 . t \ldots 1$ & \\
\hline
\end{tabular}

* Sectiones Lramarkanae tres indiruntur numeris praefixis : 1, 2, 3, se. 1. spp. longi- et recti-cuudatue

2. ipp. trivaricosae

3. spp. multivaricosae. 


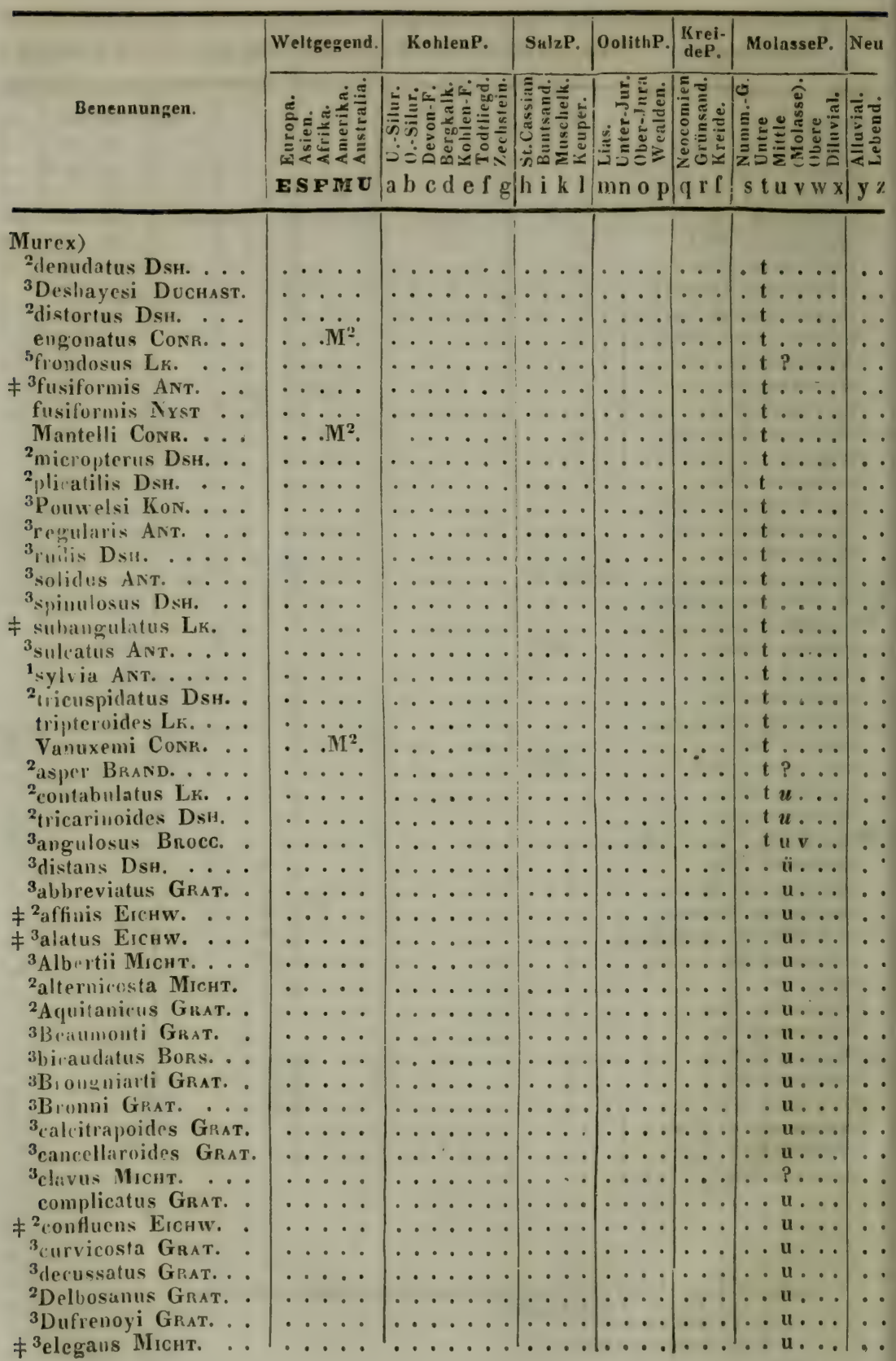




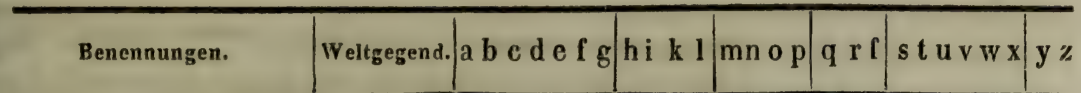

excisus Grat.

3 exiguus Dus.

${ }^{3}$ filosus GÉNÉ

$\neq^{3}$ finbriatus DFr.

$\neq{ }^{3}$ funiculatus DrR.

3Genei BeLLMich. 2graniferus Mrснт. ${ }^{3}$ granuliferus Grat.

₹ 3 gravidus Dus.

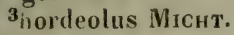

$3_{\text {inflatus Brocc. }}$

intercisus Micut

3 labrosus Bon.

${ }^{2}$ Lamarcki Grat.

latilabris Mıнт BecL.

$\uparrow$ mitellus GéNé

3 modifejus Micht

\# ${ }^{3}$ notatus Eichw.

${ }^{3}$ oblongus Grat.

ornatus Grat.

${ }^{3}$ pirulatus Bon.

$3_{\text {pomum Pusch }}$

\# ${ }^{3}$ pulcher DFr.

3 quadrifons Grat.

\# 3reticulatus DFr.

3rudis Bors.

\# ${ }^{3}$ rusticus DFr.

${ }^{3}$ Serlgwicki Mıснт.

$\neq^{3}$ striarformis Micht.

${ }^{2}$ Sowerbyi Mгст.

3'Taurinensis MIснт. tortuosus So.

o tortuosus Bors.

triacanthus Gri.

${ }^{8}$ trifascialis GRAT.

${ }^{2}$ trifrons Grat.

2tripterns Grat.

3Tritonum Grat.

3trunculoides Pusch .

${ }^{3}$ Turonensis Dus.

ivaricosissimus Bon.

† torularius Lr. . . . .

umbrifer CoNr.

${ }^{3}$ polymorphus Brocc.

1 spinir osta $\mathrm{Br}$.

3 angularis LK.

'brandaris $\mathbf{L}$.

${ }^{3}$ cristatus Brocc.

${ }^{2}$ elougatus LK.

2 crinaceus $\mathbf{L}$.

soranurius LF.

3hippocastanum

L.

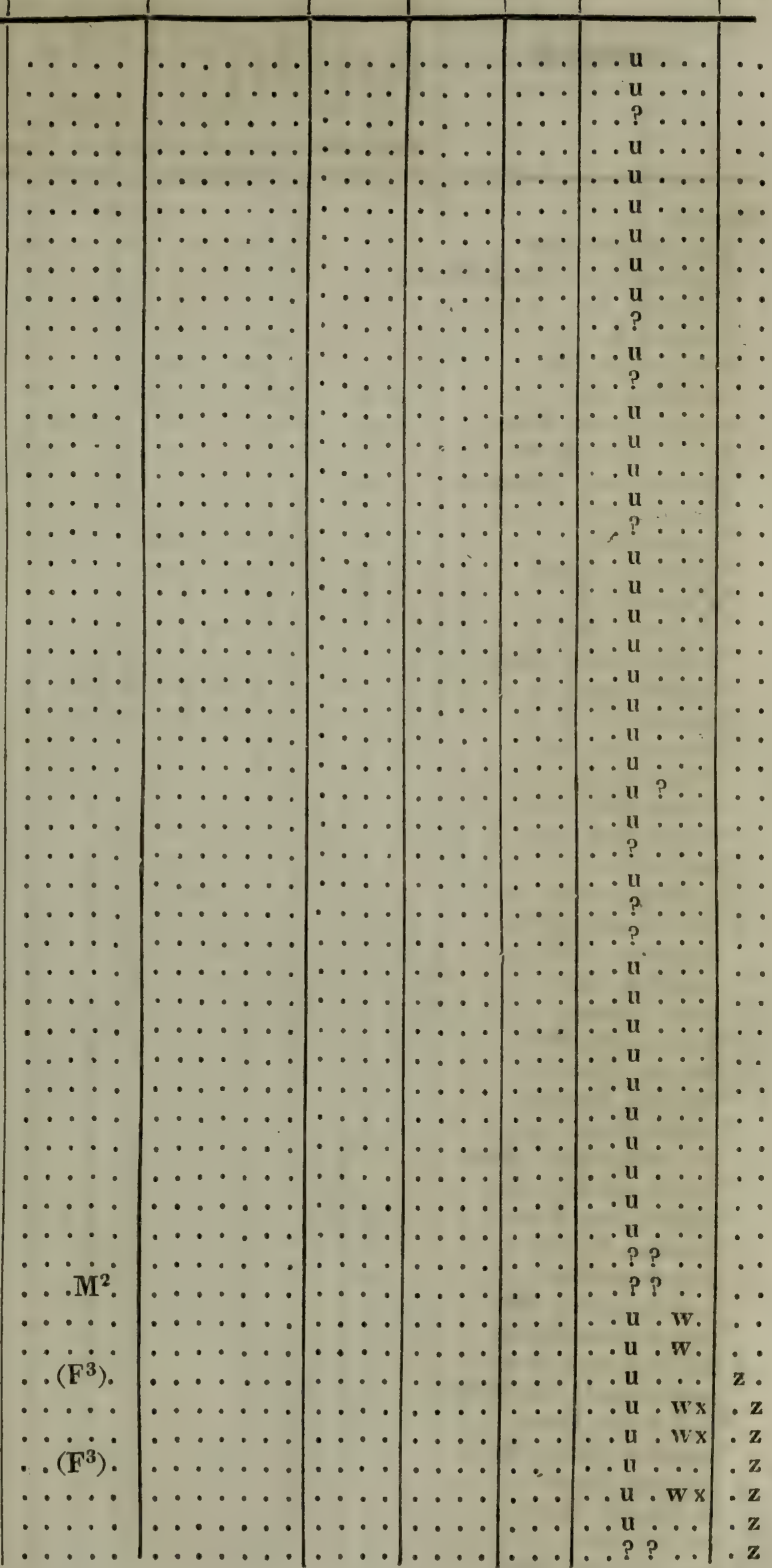




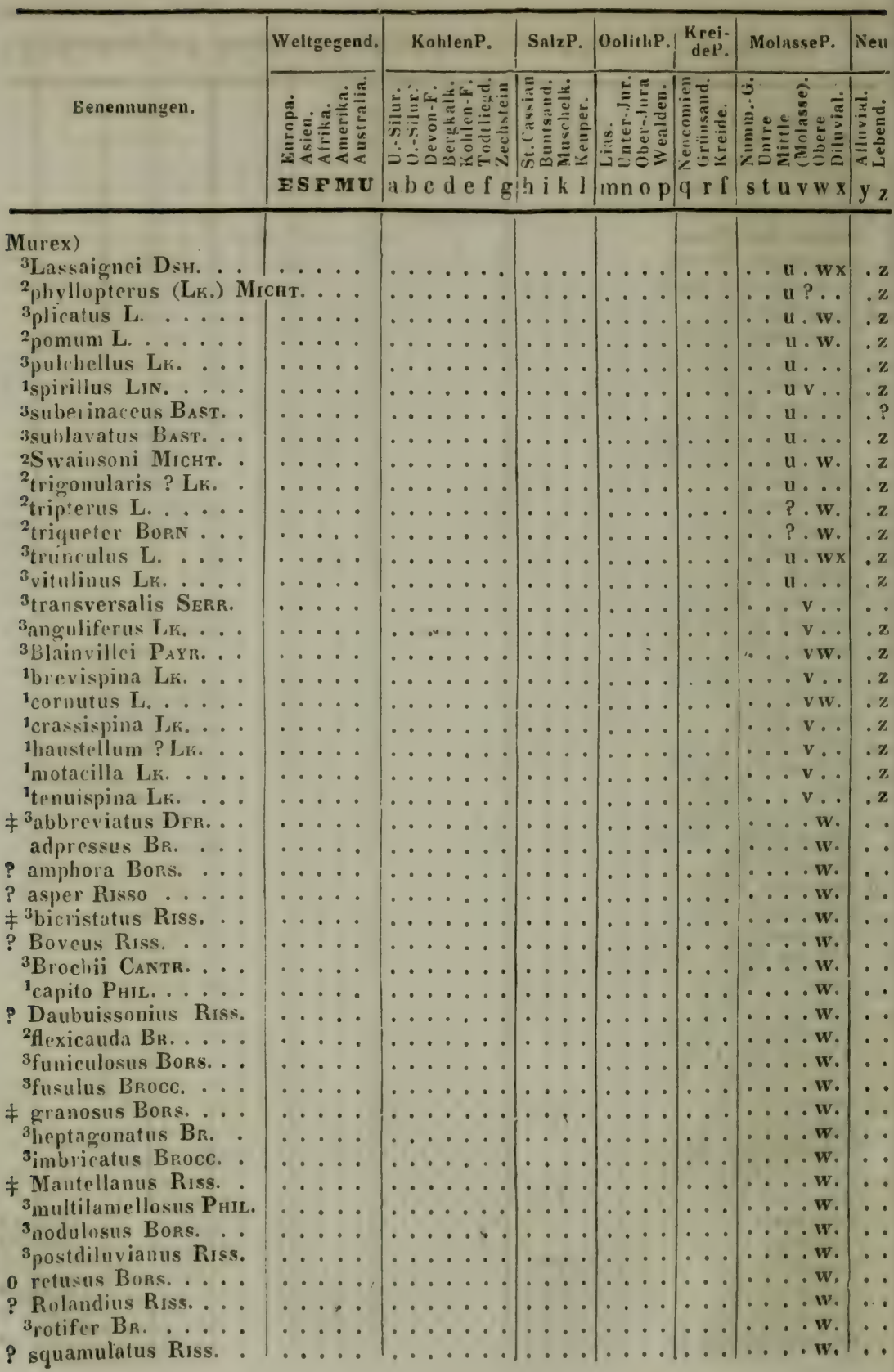




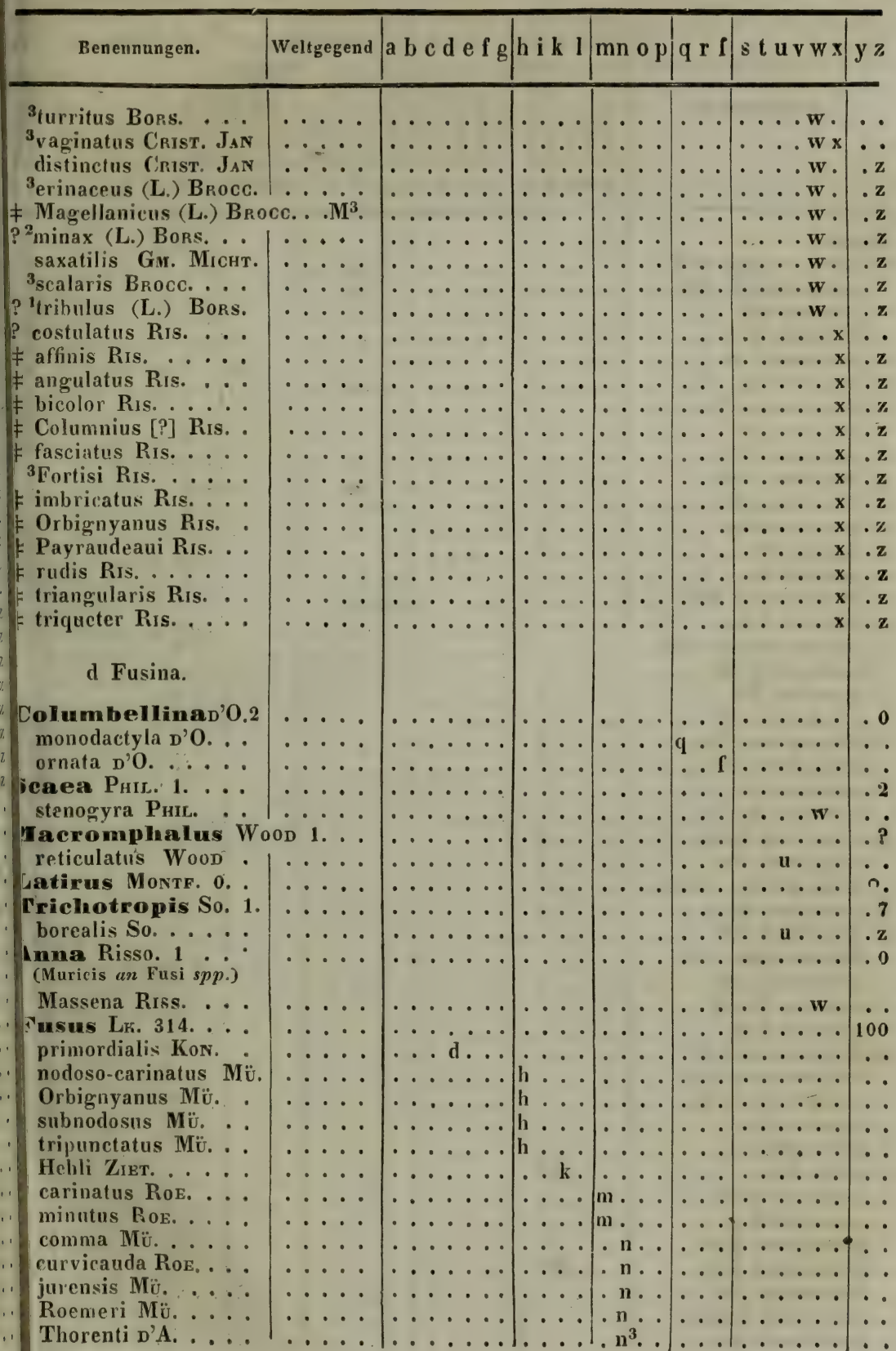




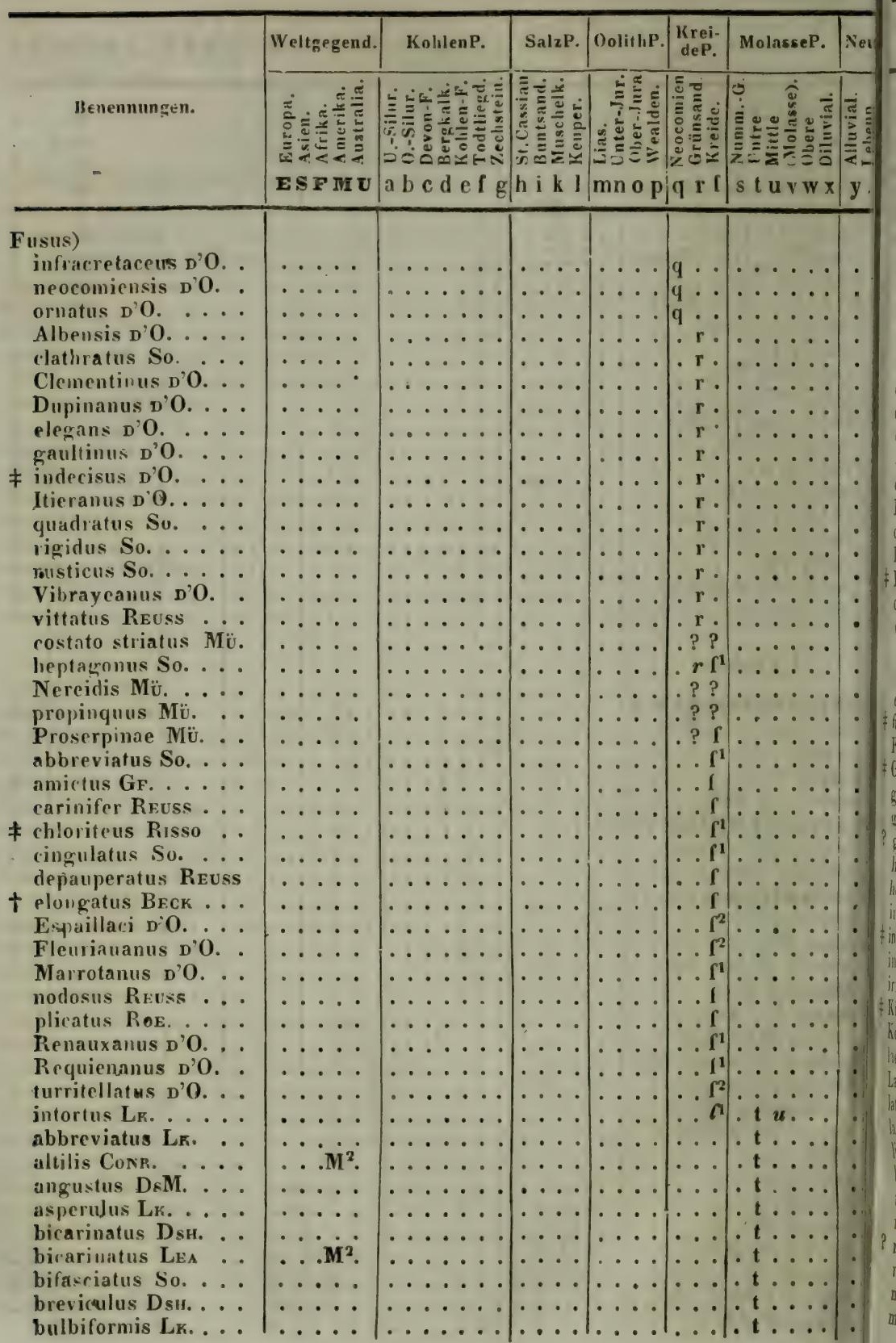




\begin{tabular}{|c|c|c|c|c|c|c|c|c|}
\hline & en. & end. & a b c d e $f g$ & 1 & $m n \circ p$ & $q \times 1$ & s $t u v w x$ & $\mathbf{y}$ \\
\hline & RS. & & & & & & $\cdots$ & \\
\hline & . & $\cdots$ & $\ldots \ldots$ & . & $\cdots$ & $\cdots$ & . t . . & \\
\hline & . & . ・ & $\cdots$ & $\cdots$ & $\ldots$ & t & . t... & \\
\hline & $\cdots$ & $\cdots \mathrm{M}^{*}$ & $\cdots$ & $\cdots$ & - & $\cdots$ &.$t \ldots$ & \\
\hline & o. . . & $\cdots \cdot$ & t & $\cdots$ & $\cdots \cdot$ & $\cdots$ & $\cdot \mathrm{t} \ldots$ & \\
\hline & . & $\cdots \cdots$ & . & $\cdots$ & $\cdots$ & $\cdots$ & $\cdot \mathbf{t} \ldots$. & \\
\hline & $\cdots$ & $\because \dot{M}^{2}$ & 1 & $\cdots$ & $\cdots \cdot$ & $\cdots$ & $\cdots$ & \\
\hline & $\cdots$ & $\because \mathbf{M}^{2}$ & 2 & $\cdots$ & $\cdots$ & $\cdots$ & $\cdots$ & \\
\hline & $\therefore$ & $\begin{array}{l}\ldots \mathrm{M}^{2} \\
\ldots \\
\cdots\end{array}$ & - & $\cdots$ & $\begin{array}{ll}\cdots \\
\cdots \\
\cdots\end{array}$ & $\cdots$ & $\because$ & \\
\hline & 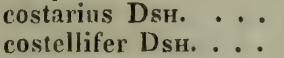 & & & $\mid \begin{array}{c}\cdots \\
\cdots \\
\cdots\end{array}$ & $\begin{array}{ll}\cdots & \cdots \\
\cdots & \cdots\end{array}$ & & $\cdots$ & \\
\hline & 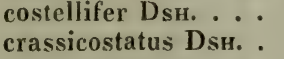 & & $\cdots$ & $\cdots \cdots$ & $\cdots$ & $\cdots$ & $\left|\begin{array}{lll}0 & \cdots & \cdots \\
0 & \mathrm{t} & \cdots\end{array}\right|$ & \\
\hline & EA & ... $\mathbf{M}^{2}$ & $\therefore$ & $\ldots$ & $\ldots$ & $\ldots$ & $\ldots$ & \\
\hline & $\cdots$ & & $\cdots$ & $\ldots$ & $\ldots$ & $\cdots$ &.$t \ldots$ & \\
\hline & $\cdots$ & & . & $\ldots$ & $\ldots$ & $\cdots$ & $\cdots$ & \\
\hline & $\cdot$ & $\cdots$ & $\cdots$ & $\cdots$ & $\cdots$ & $\cdots$ & $\cdots$ & \\
\hline & 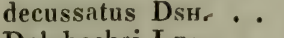 & . & $\cdots \cdots$ & $\cdots$ & $\cdots$ & $\cdots$ & $\cdots$ & \\
\hline & D & & $\cdots \cdots \cdot$ & $\cdots \cdot$ & $\cdots \cdot \mid$ & $\cdots \cdot$ & $\cdots$ & \\
\hline & d & & $\ldots$ & $\cdots$ & . & & $\cdots$ & \\
\hline & D & & & $\cdot$ & - & & $\cdots$ & \\
\hline & • & $\therefore \dot{M^{4}}$ & & $\because$ & $\mid \begin{array}{c}\cdots \\
\cdots\end{array}$ & $\cdots$ & & \\
\hline & $\cdots$ & $\ldots$ & $\therefore$ & $\cdots$ & $\cdots$ & $\cdots$ & $\left|\begin{array}{ccc}1 & \cdots & \cdots \\
. t & \ldots & \cdots\end{array}\right|$ & \\
\hline & $\cdots$ & & . & $\cdots$ & $\ldots$ & $\ldots$ &.$t \ldots$ & \\
\hline & . & . & . & $\cdots$ & $\ldots$ & $\ldots$ & $\cdots$ & \\
\hline & $\cdots$ & $\cdots$ & $\ldots \ldots$ & $\cdots$ & $\cdots$ & $\cdots$ & . $t$ & \\
\hline & $\cdots$ & $\cdots$ & $\ldots \ldots$ & $\cdots$ & $\cdots \cdot$ & $\cdots$ & $\cdot \mathrm{t}$ & \\
\hline & & $\ldots \mathbf{M}^{2}$ & . & . & $\cdots \cdot$ & $\cdots$ &.$t \ldots$ & \\
\hline & $\cdots$ & $\ldots$ & . . & . & $\cdots \cdot$ & $\cdots$ &.$t \ldots$ & \\
\hline & $\cdots$ & & 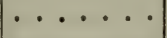 & $\cdot$ & $\ldots$. & $\cdots$ & . 1 & \\
\hline & & $\cdots$ & $\cdots \cdots$ & $\cdots$ & $\ldots$. & $\cdots$ & $\cdot \cdot$ & \\
\hline & H. & $\cdots$ & $\cdots$ & $\cdot$ & $\cdots \cdot$ & $\cdot$ & $\cdots$ & \\
\hline & & & & $\cdot$ & $\cdots$ & $\cdot \cdot$ & . & \\
\hline & & & & - & $\cdots$ & $\cdot$ & . $t$ & \\
\hline & & & & & . & $\cdot$ & $\cdots$ & \\
\hline & $\cdots$ & : & & & $\cdot$ & $\cdot$ & $\cdot t$ & \\
\hline & s. & $\cdots \dot{M}^{2}$ & & $\cdots \cdots$ & $\cdots \cdots$ & $\cdots$ & $\therefore$ & \\
\hline & . & ... & & $\cdots$ & $\ldots$ & $\cdots$ & t & \\
\hline & & & & & $\ldots$ & $\cdots$ & . $t$ & \\
\hline & & & & & . & $\cdots$ & & \\
\hline & La & & & & $\cdot$ & $\cdots$ & & \\
\hline & la & & 1 & & & $\cdots \cdot$ & . $t$ & \\
\hline & la & & & & & $\cdot \cdot$ & - $\mathbf{t}$ & \\
\hline & & & & & & - & $\cdot$ & \\
\hline & & & $\ldots$ & $\cdots \cdot$ & $\cdots \cdot$ & $\cdots$ & $\cdot \cdot$ & \\
\hline & m & & & & . & $\cdot \cdot$ & & \\
\hline & Scur & & & & & • & & \\
\hline & Schlti & & & & & & & \\
\hline & & ...1 & & $\ldots$ & . & . & 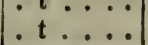 & \\
\hline & $m$ & & & & & & & \\
\hline
\end{tabular}




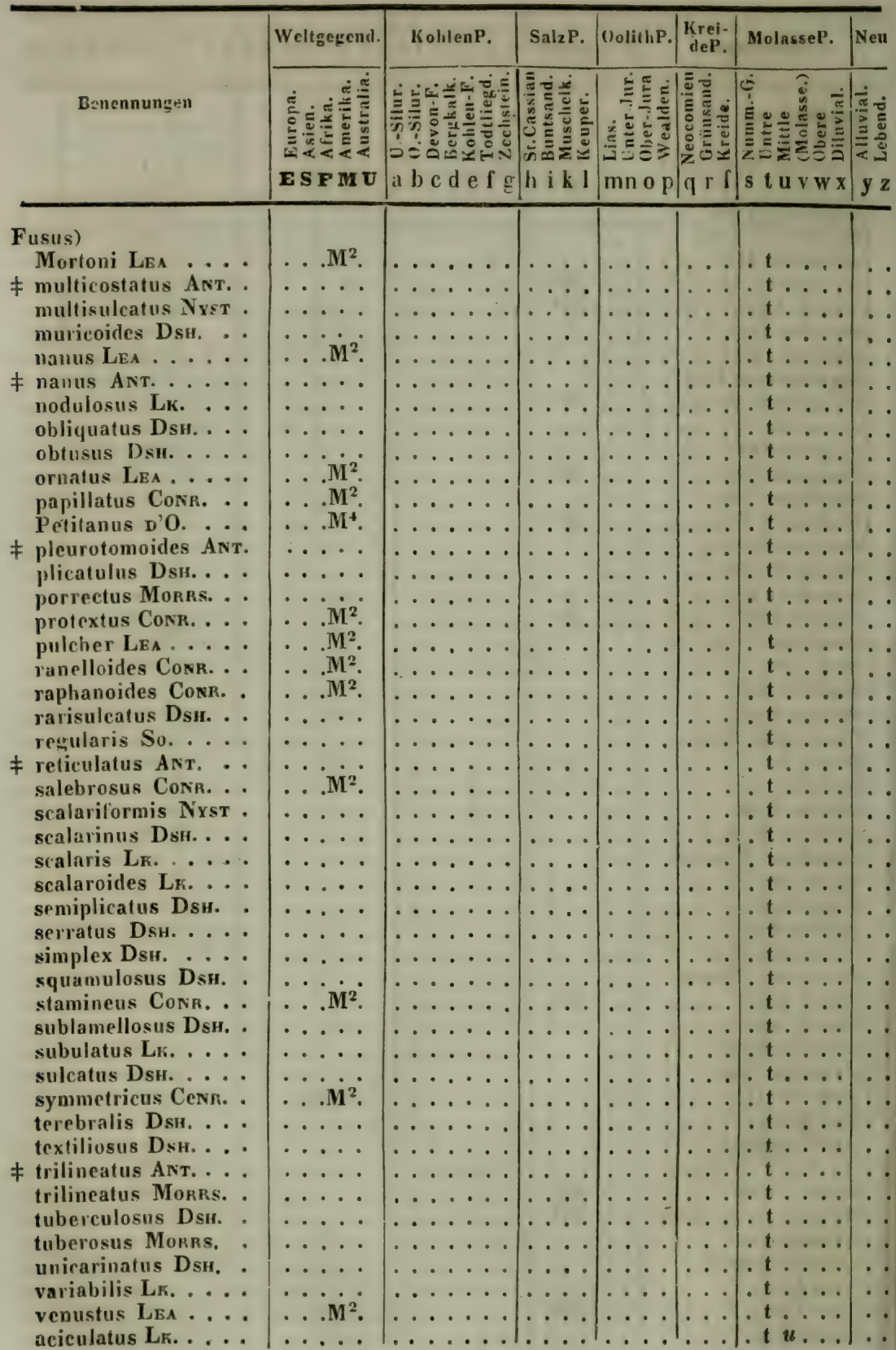




\begin{tabular}{|c|c|c|c|c|c|c|c|}
\hline Benennungen. & id. & $\mathrm{f} g$ & k l & $p$ & 〔 & $\mathrm{x}$ & 5 \\
\hline$\cdots$ & & $\cdots$ & & $\cdots \cdot$ & & . t u ... & \\
\hline$\ldots .$. & $\cdots \cdot \cdot$ & $\ldots \ldots$ & $\ldots$ & $\cdots$ & $\cdots$ & . t ü ... & \\
\hline$\cdots$ & $\cdots \cdots$ & $\ldots \ldots$ & $\ldots$ & $\cdots \cdots$ & $\cdots$ &.$t u \ldots$ & \\
\hline$\cdots$ & $\cdots \cdot$ & $\ldots \ldots$ & $\ldots$ & $\cdots \cdot$ & $\cdots$ &. $\mathrm{t} u \ldots$ & \\
\hline K. . . & $\cdots \cdot$ & $\ldots \ldots$ & $\cdots$ & $\cdots \cdot$ & $\cdots$ & . t $u \ldots$ & \\
\hline gatus LK. . . . & $\cdots \cdots$ & $\ldots \ldots$ & $\cdots$ & $\cdots \cdot$ & $\cdots$ & $\cdot \operatorname{t~} u \ldots$ & \\
\hline Iongaevus Lк. . . . & $\cdots$ & $\ldots 1$ & $\cdots \cdots$ & ... & $\cdots$ & $\cdot \operatorname{tu} \ldots$ & \\
\hline$\cdots \cdots$ & & $\cdots$ & $\cdots \cdots$ & $\cdots \cdot$ & $\cdots$ & $\cdot \operatorname{tg} u \ldots$ & \\
\hline Lк. . . & $\cdots$ & $\cdots$ & $\cdots \cdots$ & $\cdots$ & $\cdots$ & $\cdot$ t ü... & \\
\hline$\cdots \cdot$ & $\dot{\bullet}$ & $\therefore$ & $\mid \cdots \cdots$ & $\cdots$ & $\cdots$ & t t ii $\cdots$ & \\
\hline$\cdots$ & $\begin{array}{l}\cdots \\
\cdots \\
\cdots\end{array}$ & $\cdots \cdots \cdots$ & : & $\left|\begin{array}{ll}\cdots \\
\cdots & \cdots\end{array}\right|$ & $\cdots$ & $\left|\begin{array}{llll}0 & \mathrm{t} & \ldots & \ldots \\
. \mathrm{t} u & \ldots\end{array}\right|$ & \\
\hline$\cdots$ & ..... & $\ldots \ldots$ & $\cdots$ & $\ldots$ & $\cdots$ & $t u \cdot w$ & .? \\
\hline$\cdots$ & $\cdots \cdots$ & $\ldots \ldots$ & $\ldots$ & $\ldots$ & $\ldots$ &.$t \ldots v$ & \\
\hline . . & $\cdots \cdots$ & $\cdots \cdots$ & $\ldots$ & $\ldots$ & $\cdots$ & $\ldots u \ldots$ & \\
\hline LLI & $\cdots \cdot$ & $\cdots \cdots$ & $\ldots$ & $\cdots \cdot$ & $\cdots$ & $\ldots$ и... & \\
\hline . & . & $\cdots \cdots$ & $\ldots$ & $\cdots$ & $\cdots$ & $\ldots u \ldots$ & \\
\hline$\neq \mathbf{a}$ & $\cdots \cdot$ & $\cdots \cdots$ & $\cdots$ & $\ldots \cdot$ & $\cdots$ & $\ldots \mathbf{u} \ldots$ & \\
\hline a & $\cdots \cdot \cdot$ & $\cdots \cdots$ & $\cdots$ & $\cdots \cdot$ & $\cdots$ & $\cdots u$ & \\
\hline . & $\ldots \ldots$ & $\cdots \cdots$ & $\cdots$ & $\cdots \cdot$ & $\cdots$ & $\ldots u \ldots$ & \\
\hline At & - & $\cdots \cdot$ & . & $\cdots$ & $\cdots$ & $\cdots u, \cdots$ & \\
\hline 末 At & $\cdots \cdot$ & $\cdots \cdots \cdot \cdot$ & $\cdots \cdots$ & $\cdots \cdot$ & $\cdots$ & $\cdots u$ & \\
\hline$+b$ & $\cdots \cdot$ & $\cdots \cdots$ & $\cdots$ & $\cdots \cdot$ & $\cdots \cdot$ & . u . . & \\
\hline . & $\cdots \cdot$ & $\cdots \cdots$ & $\cdots$ & $\cdots \cdot$ & $\cdots$ & $\cdots u \ldots$ & \\
\hline г. . & - & $\cdots \cdots \cdot$ & $\cdots \cdot$ & $\cdots \cdot$ & $\cdots$ & $\cdots u, \ldots$ & \\
\hline RAT. & . & $\cdots$ & $\cdots \cdots$ & $\cdots \cdot$ & $\cdots$ & $\cdots u \cdots$ & \\
\hline 1. . & $\cdots \cdot \cdot$ & $\cdot$ & $\cdots$ & $\cdot \cdot$ & $\cdots$ & $\cdots \mathbf{u} \cdot \ldots$ & \\
\hline$\cdots \cdot$ & $\cdots \cdots$ & $\cdot \cdot$ & . . & $\cdots$ & $\cdots$ & . u . . & \\
\hline r. $\cdots$. & - & $\dot{.}$ & . & $\cdots$ & $\because$ & $\cdots$ и . & \\
\hline $\begin{array}{l}\text { Duj. } \ldots \ldots \\
\text { Grat. } \ldots\end{array}$ & : & $\because$ & $\therefore$ & $\cdots$ & $\cdots$ & 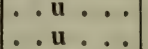 & \\
\hline$B_{R} \ldots$ & $\cdots \cdot \cdot$ & $\ldots \ldots$ & $\ldots$ & $\ldots$ & $\cdots$ & $\ldots$ & \\
\hline ... & .... & $\ldots \ldots$ & $\ldots$ & $\ldots$ & ... & . u ... & \\
\hline OOD. & •. • • & $\ldots \ldots$ & $1^{\circ}$ & $\ldots$ & ... & . u ... & \\
\hline YRAT. . & $\cdots$ & $\ldots \ldots$ & $\ldots$ & $\cdots \cdot$ & .. & $\ldots u \ldots$ & \\
\hline r. . & $\cdots \cdot$ & $\ldots \ldots$ & $\cdots \cdot$ & $\cdots \cdot$ & $\cdots$ & $\ldots \mathbf{u} \ldots$ & • \\
\hline$\cdots$ & $\cdots \cdot$ & $\ldots \ldots$ & $\cdots$ & $\cdots \cdot$ & $\cdots$ & $\ldots u \ldots$ & \\
\hline$\cdots$ & - & - & & $\cdots \cdots$ & & $\ldots u \ldots$ & \\
\hline Wood .... & - & $\cdots \cdots$ & . & $\cdots \cdot$ & $\cdots$ & $\cdots u \ldots$ & \\
\hline$\ldots$ & & $\cdots$ & . & $\cdots \cdot 1$ & • & $\cdots u \ldots$ & \\
\hline AT. & $\cdots \cdot$ & & t & & - & $\cdots u$ & \\
\hline r. $\cdot$ & $\cdots \cdot \cdot$ & & & $\cdots \cdot$ & & $\cdots$ & \\
\hline$H \ldots$ & • & & & $\cdots$ & & . & \\
\hline fragilis BONELLI & . $\mathbf{M}^{2}$. & & & & $\cdots$ & $\ldots$ & \\
\hline ENÉ $\ldots$ & $\ldots$ & $\ldots \ldots$ & $\cdots$ & ... & $\cdots \cdot$ & . u . . & \\
\hline s Gené .... & & .... & & . . & & $\ldots u \ldots$ & \\
\hline † gracilior Wood .. & & & & & & $\ldots u \ldots$ & \\
\hline ? granosus So. .... &. $\mathrm{S}^{3}$. & & . & . . & - & $\ldots \mathrm{u} \ldots$ & \\
\hline hexagonus So. . . &. $\mathrm{S}^{3} \ldots$ & & & & 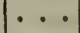 & $\cdots u$ & \\
\hline † Hössi Part & $\cdots \cdots$ & $\cdots$ & $\cdots$ & & - & $\cdots u \ldots$ & - \\
\hline & & & & & & & \\
\hline
\end{tabular}




\begin{tabular}{|c|c|c|c|c|c|c|c|}
\hline \multirow[b]{2}{*}{ Benennungen. } & Weltgegend. & KohlenP. & SalzP. & OolithP. & $\begin{array}{l}\text { Krei- } \\
\text { deP. }\end{array}$ & MolasseP. & Neu \\
\hline & 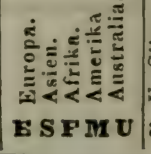 & 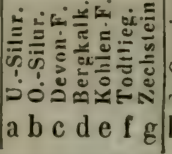 & 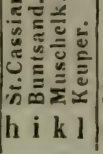 & 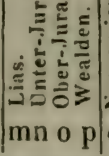 & 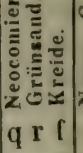 & 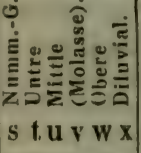 & 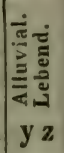 \\
\hline & & & & & & & \\
\hline 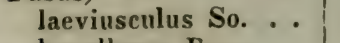 &. $\mathrm{S}^{3} \ldots$ & $\cdots$ & $\cdots \cdots$ & $\cdots \cdot$ & $\cdots$ & $\cdot \cdot \mathrm{u}$ & $\cdots$ \\
\hline s. . . & $\cdots \cdots$ & $\ldots \ldots$ & $\cdots \cdot$ & $\ldots$. & $\cdot 1$ & $\ldots u$ & 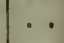 \\
\hline ง. . . & $\cdots \cdot$ & $\cdots \cdots$ & $\cdots$ & $\cdots \cdot$ & $\cdots$ & $\cdots u$ & \\
\hline$\cdots$ & $\cdots \cdots$ & $\cdots \cdots$ & $\cdots \cdot$ & $\cdots \cdot$ & $\cdots$ & $\ldots$ u $\ldots$ & . \\
\hline T. . & $\cdots$ & $\cdots \cdots$ & $\cdots$ & $\cdots \cdot$ & 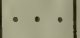 & $\cdots \mathbf{u}$ & . \\
\hline r. & $\cdots$ & $\cdots \cdots \cdot$ & $\cdots \cdots$ & $\cdots \cdot$ & $\cdots$ & $\cdots u$ & \\
\hline $\begin{array}{l}\text { IT. } \\
\cdots\end{array}$ & $\cdots \cdots$ & : & $\therefore$ & - & $\cdots$ & $\because u$ & \\
\hline * nassoides Grat. . & $\ldots$ & $\ldots \ldots$ & $\ldots$ & $\cdots$ & $\cdots$ & $\therefore u$ & \\
\hline$\cdots$ & 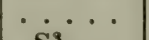 & $\ldots \ldots$ & $\ldots$ & $\ldots$ & ... & $\ldots u \ldots$ & \\
\hline . & $\cdot \mathbf{S}^{3} \ldots$ & $\ldots \ldots$ & $\ldots$ & $\ldots$ & $\ldots$ & $\ldots u \ldots$ & \\
\hline . & $\cdots$ & $\ldots \ldots$ & $\ldots$. & $\cdots \cdot \mid$ & $\cdots$ & $\ldots u \ldots$ & \\
\hline ICHT. & $\cdots \cdot$ & $\ldots \ldots$ & $\cdots$ & $\cdots \cdot$ & $\cdots$ & . . u & \\
\hline$\cdots$ & $\cdots \dot{M i}^{\circ}$ & $\cdots \cdots \cdot$ & $\cdots \cdot$ & $\cdots \cdot$ & $\cdots$ & $\ldots u \ldots$ & \\
\hline $\begin{array}{l}\text { parilis CoNR } \\
\pm \text { paululus Wor }\end{array}$ & $\cdots M^{2}$ & $\cdots \cdots \cdot$ & $\cdots \cdot$ & $\cdots$ & $\cdots$ & $\cdots u$ & \\
\hline $\begin{array}{c}\neq \text { paululus WoOD } \\
\text { punilus LEA } \ldots\end{array}$ & $\cdots \dot{M}^{2}$ & $\begin{array}{lll}\cdots & \cdots & \cdots \\
\cdots & \cdots & \cdots\end{array}$ & $\cdots$ & $\cdots$ & $\cdots$ & $\because u$ & . \\
\hline LeMichт. & $\cdots \cdot$ & $\ldots \ldots$ & $\ldots$ & ... & $\ldots$ & $\ldots u \ldots$ & \\
\hline & $\cdots \cdot$ & $\ldots \cdots$ & $\cdots$ & ... & $\cdots$ & $\ldots \mathbf{u} \ldots$ & \\
\hline ICHT & $\cdots \cdot$ & $\ldots \cdots \cdots$ & $\cdots$ & . $\cdot$ & $\ldots$ & $\ldots \mathrm{u} \ldots$ & \\
\hline rho & $\cdots$ & $\ldots \cdots$ & $\cdots$ & $\cdots$ & $\ldots$ & $\ldots \mathrm{u} \ldots$ & \\
\hline . & $\cdots \mathbf{M}^{2}$. & $\cdots \cdot$ & $\cdots \cdot$ & $\cdots$ & $1^{\circ}$ & . . u & \\
\hline Grat. & $\cdots \cdot$ & $\cdots \cdots \cdots$ & $\cdots \cdot$ & . $\cdot$ & $\cdots$ & $\cdot . \mathrm{u}$ & \\
\hline t scalaris $P_{A}$ & $\cdots \cdot$ & $\ldots \ldots$ & $\cdots \cdot$ & $\cdots$ & & $\cdots u$ & \\
\hline$\cdots$ & $\cdots \cdot$ & $\cdots \cdots$ & $\cdots \cdot$ & $\cdots \cdot$ & $\cdots$ & $\cdots u$ & \\
\hline $\operatorname{sim}_{\text {Sicn }}$ & $\cdots$ & $\cdots \cdots \cdot$ & $\cdots \cdot$ & $\cdots \cdot$ & . . & $\cdots u$ & • \\
\hline $\begin{array}{l}\text { Sisk } \\
\text { stro }\end{array}$ & $\cdots$ & $\therefore \cdots$ & : & • & & $\therefore u$ & \\
\hline (.). & . $\mathrm{M}^{2}$ & $\ldots \ldots$ & $\ldots$ & 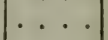 & $\cdots$ & $\therefore \mathrm{u}$ & $\bullet$ \\
\hline ... & $\ldots \ldots$ & $\ldots \ldots$ & ... & $\ldots$ & $\cdots$ & $\ldots \mathrm{u}$ & . \\
\hline sublaevis Pusch .. & & $\ldots \ldots$ & . . & $\cdots \cdot$ & . . &.. $\mathrm{Ul}$ & \\
\hline$\cdots$ & .. $\mathrm{M}^{2}$. & $\ldots \ldots$ & $\cdots$ & $\cdots \cdot$ & $\cdots$ &.. $\mathrm{u}$ & \\
\hline ELLI. & .. & $\ldots \ldots$ &. & $\cdots \cdot$ & & $\cdots u$ & \\
\hline . . . & . . I & $\cdots \cdots$ & & & & $\cdots " 11$ & \\
\hline Grat. . . & $\cdots \cdots$ & $\cdots \cdots \cdots$ & & - $\cdot$ & $\cdots$ & $\because u$ & \\
\hline 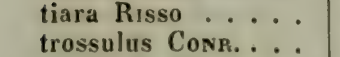 & $\ldots \dot{M}^{2}$ & $\cdots$ & $\cdots$ & $\cdots$ & . . & $\because u$ & \\
\hline $\begin{array}{ll}30 \\
3\end{array}$ & . . . & . . . . & $\ldots$ & $\ldots$ & & $\therefore u$ & \\
\hline$\cdots$ & ... & ......... & $\ldots$ & $\ldots$ & $\cdots$ &.. $\mathrm{u}$ & \\
\hline $\begin{array}{l}\text { S Grat. } \\
\text { Grat. }\end{array}$ & ... & $\ldots \ldots$ & $\cdots$ & ... & . . & . . u & \\
\hline ckneri PaRtsch & & $\ldots$ & & & &.. $\mathrm{u}$ & \\
\hline$\cdots$ & $\cdots$ & $\ldots$ & & & & $\ldots u . w$ & \\
\hline cla & . & $\cdots$ & & & $\cdots$ & $\ldots n \cdot w$ & \\
\hline V & $\cdots$ & $\ldots$ & & $\cdots$ & $\cdots \cdot$ & . . u $\cdot \mathbf{w}$ & - \\
\hline & & $\cdots$ & & $\cdots$ & - & . . u . w . & \\
\hline & & & & & & I. u . . w . & \\
\hline
\end{tabular}




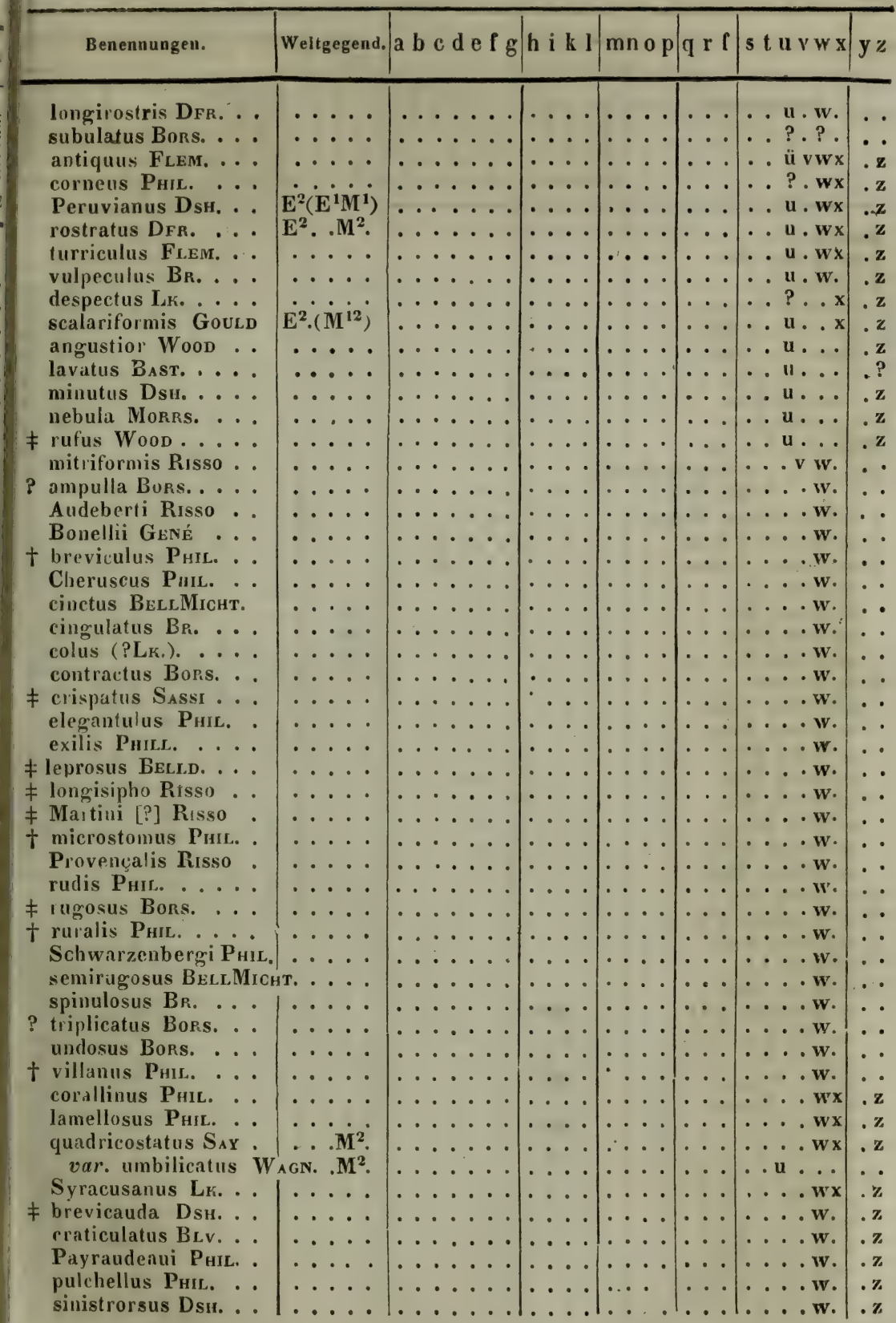




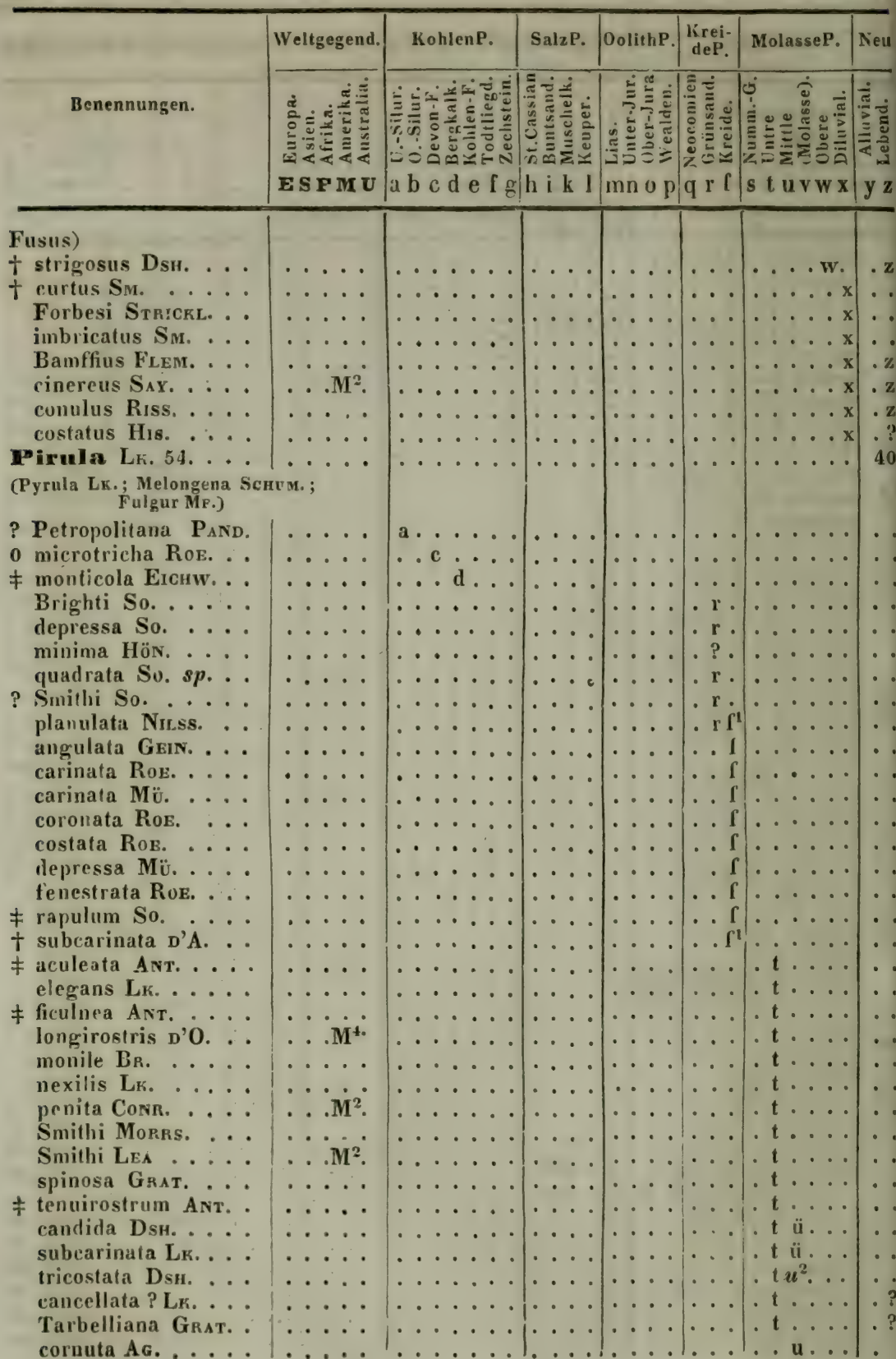




\begin{tabular}{|c|c|c|c|c|c|c|c|}
\hline Benen & end. & $a b c$ & hik l & $\mathrm{mnop}$ & qr I & $\mathrm{x}$ & y z \\
\hline t1 GPA & & & & & & $\cdot \cdots u^{2}$. & \\
\hline Lainei Bast. & . . in & $\ldots \ldots$ & $\cdots \cdot$ & $\cdots \cdot$ & $\cdots$ & $\ldots u^{2} \ldots$ & \\
\hline Nausemondi WWaGN. & ... $\mathbf{M}^{2}$ & $\ldots \ldots$ & $\cdots \cdot$ & $\cdots \cdot$ & $\cdots$ & $\ldots \mathrm{u} \ldots$ & \\
\hline † striatula Dsh. . . . & $\cdots \cdots$ & $\ldots \ldots$ & $\cdots \cdots$ & $\cdots \cdot$ & $\cdots$ & . . и... & \\
\hline AT. . & $\cdots \cdot$ & $\cdots \cdots \cdots$ & $\cdots \cdots$ & $\cdots \cdot$ & $\cdots$ & $\cdots u^{1} \cdot \cdots$ & \\
\hline$\cdots$ & $\therefore$ & $\cdots \cdots$ & $\cdots \cdots$ & $\cdots \cdots$ & $\cdots$ & $\cdots u_{2} \cdots$ & • \\
\hline canaliculata LK. & $\cdots \dot{M}^{2}$ & $\begin{array}{lll}\cdots & \cdots & \cdots \\
\cdots & \cdots & \cdots\end{array}$ & $\cdots$ & $\begin{array}{ll}\cdots \\
\cdots \\
\cdots\end{array}$ & $\cdots$ & $\mid \begin{array}{c}\cdots \\
\cdots\end{array}$ & $y z$ \\
\hline$\ldots \ldots$ & $\mathrm{E}^{2} \cdot \mathrm{M}^{2}$. & $\ldots \ldots$ & $\ldots$ & $\ldots$ & ... & $\ldots u x$ & $y z$ \\
\hline K. . . & $\because \dot{s}^{\prime} \cdot$ & $\cdots \cdots$ & $\cdots \cdots$ & $\cdots$ & $\cdots$ & $\cdots \mathbf{u} \cdot \mathbf{w} \cdot$ & $\mathrm{z}$ \\
\hline$\cdots$ & ). & $\cdots \cdots \cdots$ & $\cdots \cdot$ & $\cdots$ & $\cdots$ & $\cdots u^{1} \ldots$ & $\cdot \mathrm{z}$ \\
\hline$\cdots$ & $\because$ & $\therefore$ & $\cdots \cdots$ & $\cdots \cdot \mid$ & $\cdots$ & $\cdots u \cdot w_{0}$ & $\cdot \mathbf{z}$ \\
\hline$\cdots$ & $\cdot$ & $\cdots$ & $\cdots \cdots$ & $\cdots$ & $\cdots$ & . u u &. $\mathbf{z}$ \\
\hline : & . & $\therefore$ & $\cdots$ & $\cdots$ & $\cdots$ & . u u v w. & $\cdot \mathrm{z}$ \\
\hline $\begin{array}{l}\text { SERR. . . } \\
\text { alis SERR. }\end{array}$ & $\ddot{\dot{m}}$ & $\cdots \cdots$ & $\cdots$ & $\cdots$ & $\cdots$ & $\cdots \mathbf{v} \ldots$ & - \\
\hline Bors .... & ..... & $\ldots \ldots$ & $\ldots$ & $\cdots$ & $\cdots$ & $\cdots v \ldots$ & • \\
\hline s. . . . & $\cdots \cdot$ & $\ldots \ldots$ & $\ldots$ & $\cdots$ & $\begin{array}{l}\cdots \\
\cdots \\
\cdots\end{array}$ & $\begin{array}{c}\cdots w \\
\ldots w \\
\cdots\end{array}$ & • \\
\hline Phil. . & $\cdots$ & $\ldots \ldots$ & $\cdots$ & .... & $\therefore$ & $\begin{array}{cc}\cdots \\
\cdots \\
\cdots\end{array}$ & - \\
\hline$\cdots$ & $\cdots$ & $\cdots \cdots$ & $\cdots$ & $\cdots$ & $\cdots$ & $\ldots \ldots$ & - \\
\hline $\begin{array}{l}= \\
\text { NR. . . }\end{array}$ & ... $\mathbf{M}^{2}$. & & & 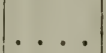 & 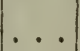 & $\ldots u \ldots$ & \\
\hline inci & . . $\mathrm{M}^{2}$ & . & $\ldots$ & $\cdots$ & $\cdots$ & $\therefore$ u. . & \\
\hline ConR. & $\ldots . M^{2}$ & $\cdots \cdot$ & $\cdots \cdots$ & $\cdots$ & $\cdots$ & . . u. . & \\
\hline Plemro & $\therefore \cdots$ & $\cdots \cdots$ & $\cdots$ & $\cdots$ & $\cdots$ & $\ldots \ldots$ & 370 \\
\hline Clava & & & & & & & \\
\hline$\cdots$ & & & & $\cdots$ & . & $\ldots \ldots$ & \\
\hline LI. . & & $\cdots$ & & $\ldots$ & - & $\ldots \ldots$ & \\
\hline [ü.... & & $\cdots$ & h ... & $\ldots$ & $\cdots$ & $\ldots \ldots$ & \\
\hline mis So. . . & $\cdots$ & $\cdots \cdot$ & $\cdots$ & $\cdots$ & . . $\mathbf{r}^{\prime}$ & $\ldots \ldots$ & . \\
\hline$\cdots$ & - & $\cdots \cdots$ & $\cdots$ & $\cdots$ & $\Gamma^{1}$ & $\ldots \ldots$ & \\
\hline GF. ... & - & $\cdots \cdot$ & $\cdots \cdot$ & $\cdots$ & $\cdot \cdot f$ & $\cdots \cdots$ & \\
\hline$\cdot \cdots$ & $\cdots$ & $\cdots$ & $\cdots$ & $\cdots$ & $\cdots \int^{1}$ & $\cdots \cdots$ & \\
\hline$\cdots$ & & $\cdots$ & $\cdots \cdots$ & $\cdots \cdots$ & $\cdot \cdot \Gamma^{1}$ & $\cdots \cdots$ & \\
\hline$\cdots$ & & $\cdots$ & $\cdots$ & $\cdots \cdots$ & $\cdots$ & $s ; \cdots$ & \\
\hline$\cdots$ & $\cdots$ & $\cdots$ & - & $\cdots$ & - & $\cdots$ & \\
\hline$\cdots$ & $\cdots \dot{M}^{2}$ & $\left.\mid \begin{array}{c}\cdots \\
\cdots\end{array}\right]$ & $\cdots$ & $\begin{array}{c}\cdots \\
\cdots\end{array}$ & $\cdots$ & $\begin{array}{lll}1 & \cdots & \cdots \\
. \mathrm{t} & \cdots & \cdots\end{array}$ & \\
\hline I. . . & . . & $\cdots \cdots$ & $\cdots$ & $\ldots$ & $\cdots$ &.$t \ldots$ & \\
\hline na D'o..... & . . $M^{+}$ & & $\cdots$ & $\cdots$ & $\cdots$ &.$t \ldots$ & \\
\hline$\cdots$ & $\cdots$ & . & $\cdots \cdot$ & $\cdots$ & $\cdots$ & $\cdot \mathrm{t} \ldots$. & \\
\hline$\cdots$ & $\cdots$ & $\cdots$ & $\cdots$ & ... & $\cdot$ & . t . . . & \\
\hline nti LEA. . . & $\cdots \cdot 1$ & $\cdots$ & & 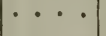 & - & $\cdots \cdots$ & \\
\hline a LK. & $\cdots$ & $\cdots$ & - & - & • & $\cdot \mathrm{t} \cdots$ & \\
\hline a ConR... & ...M & & & & & $\cdot t \cdots$ & \\
\hline . . & . . . & $\cdots$ & & & & $\begin{array}{l}t \\
\mathrm{t}\end{array} \ldots$ & \\
\hline $\begin{array}{l}\text { Mei A } \\
\text { ti } \mathrm{Ny}^{-}\end{array}$ & $\cdots$ & . & . & & & $\begin{array}{l}\cdot \mathrm{t} \\
\mathrm{t}\end{array} \ldots$ & \\
\hline da Dsh. . . & & & & & $\ldots$ & t... & \\
\hline 461. & $\cdots$ & ${ }^{\circ}$ & $\cdots \cdots$ & $\cdots$ & $\cdots$ & . t . . . & \\
\hline 191. & $\cdots$ & 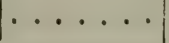 & $\cdot$ & $\cdots$ & $\cdots$ & & \\
\hline & & 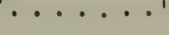 & 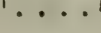 & & & & \\
\hline
\end{tabular}




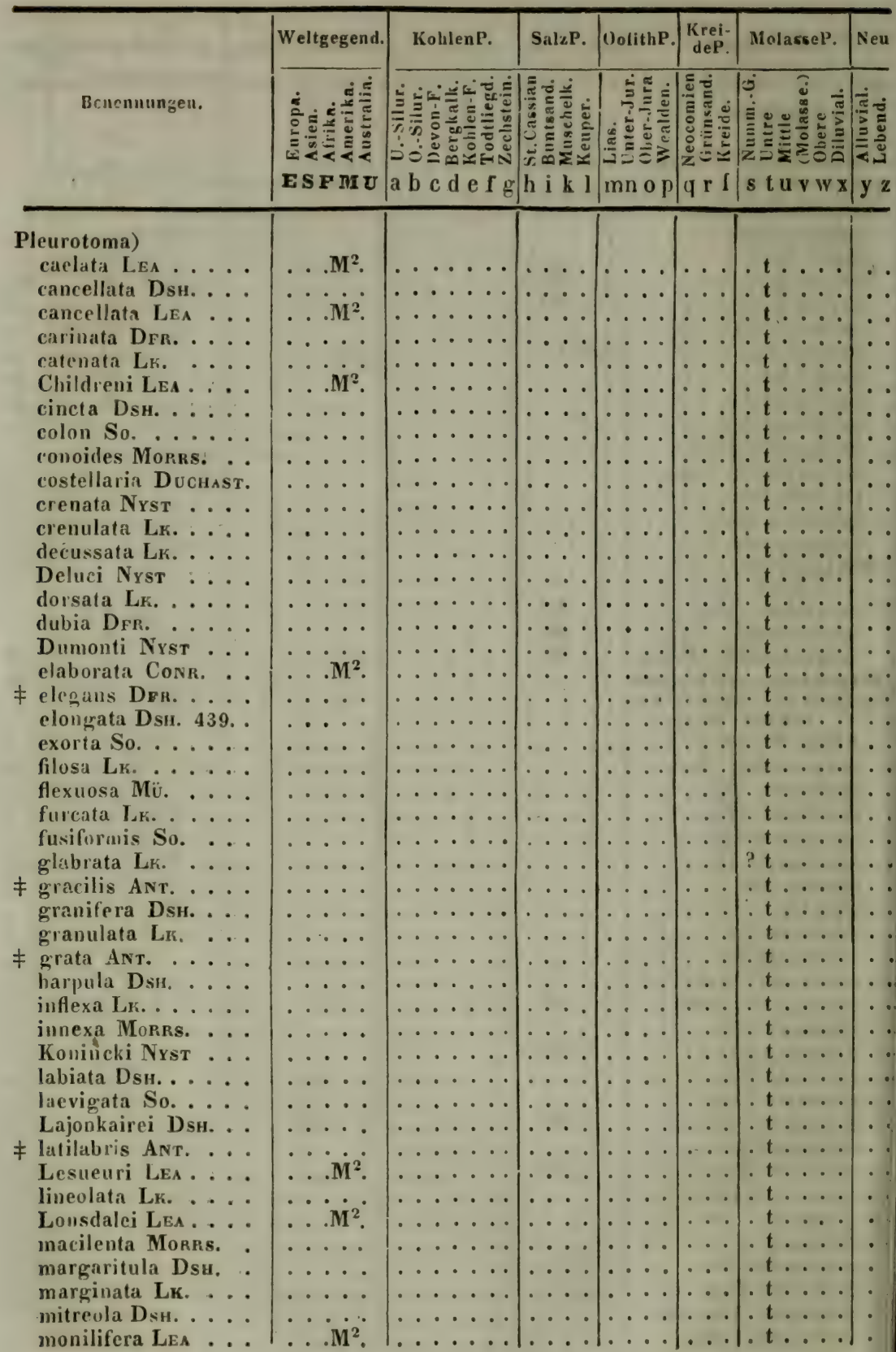




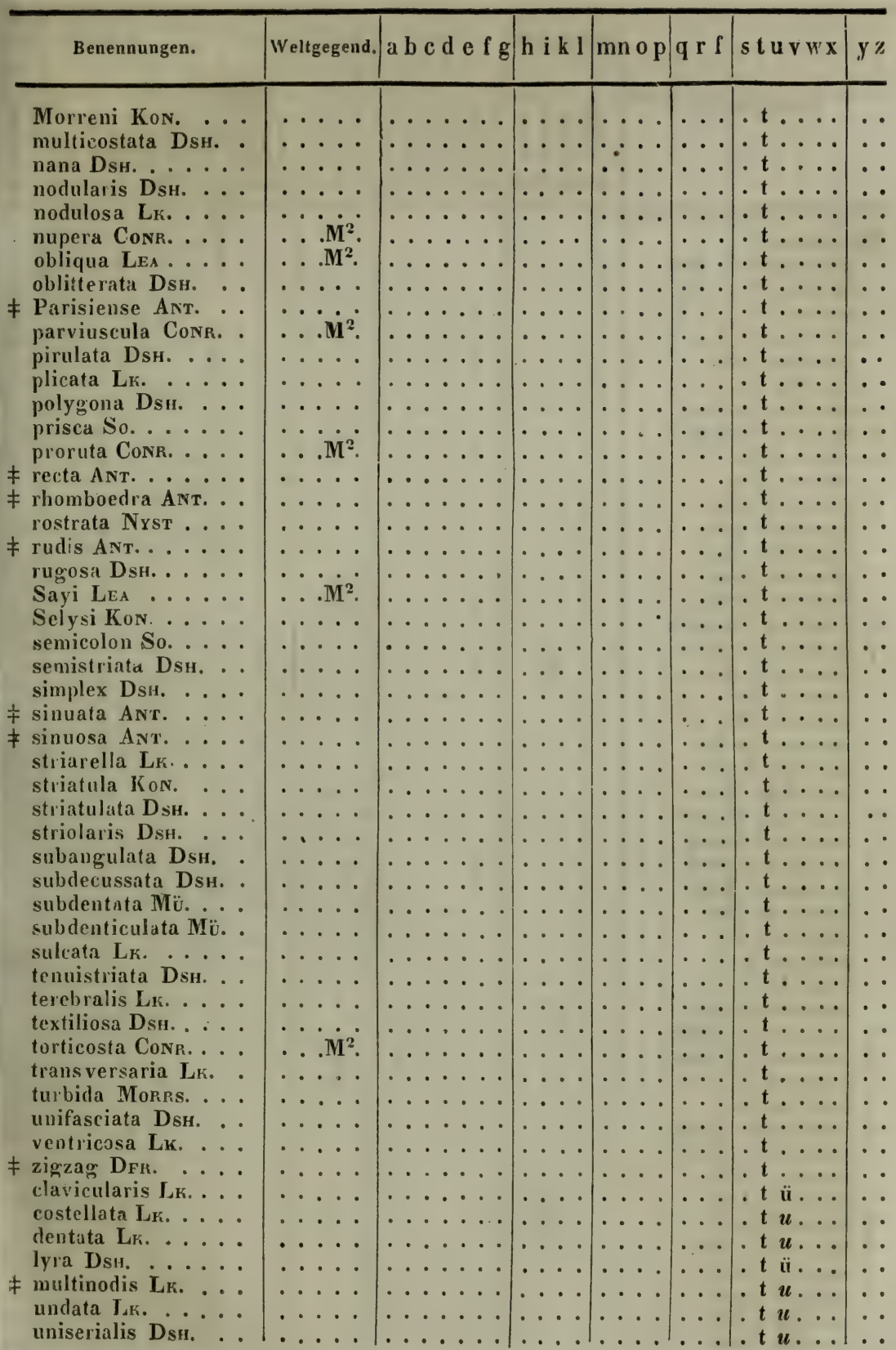




\begin{tabular}{|c|c|c|c|c|c|c|c|}
\hline Bener & 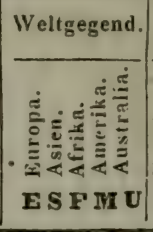 & 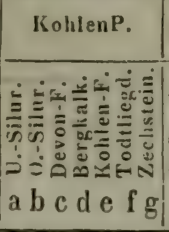 & 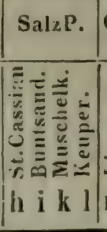 & 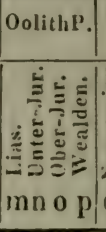 & 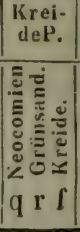 & 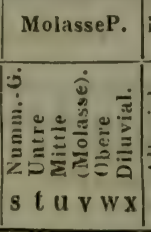 & 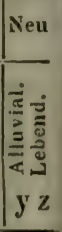 \\
\hline 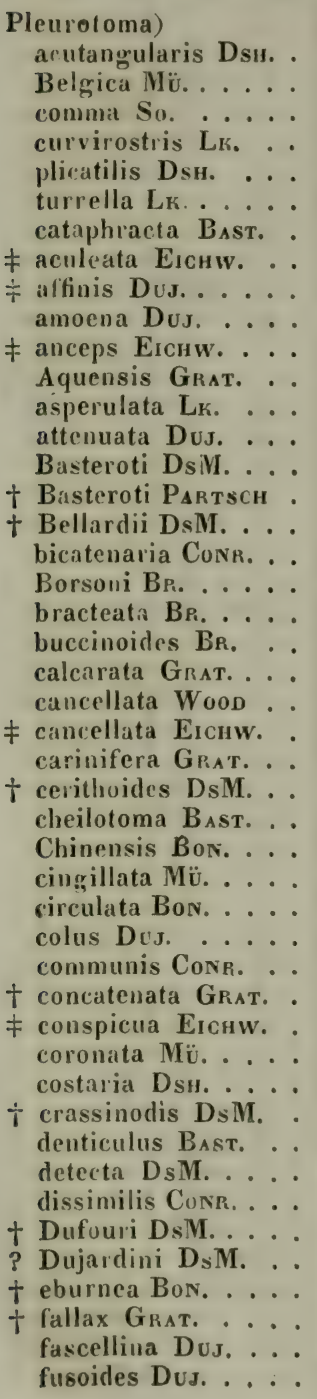 & 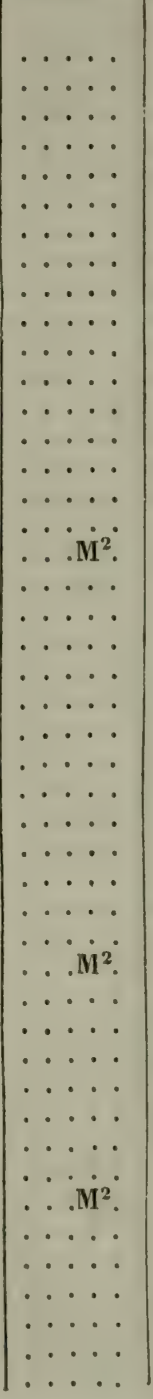 & $\begin{array}{l}\cdots \\
\cdots \\
\cdots \\
\cdots \\
\cdots \\
\cdots \\
\cdots \\
\cdots \\
\cdots \\
\cdots \\
\cdots \\
\cdots \\
\cdots \\
\cdots \\
\cdots \\
\cdots \\
\cdots \\
\cdots \\
\cdots\end{array}$ & 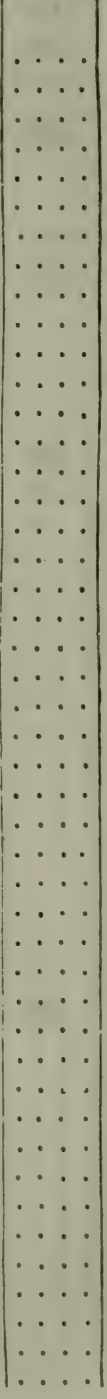 & 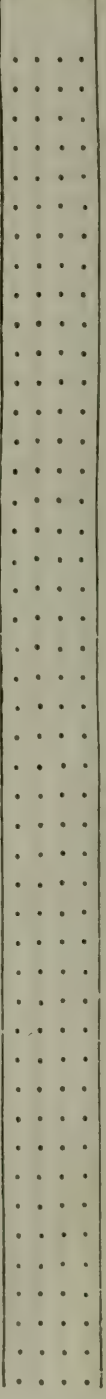 & 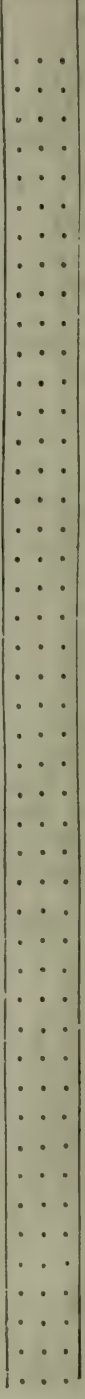 & 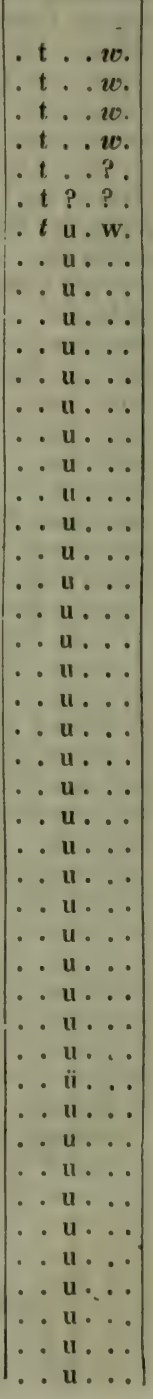 & $\begin{array}{l}\cdots \\
\cdots \\
\cdots\end{array}$ \\
\hline
\end{tabular}




\begin{tabular}{|c|c|c|c|c|c|c|c|c|}
\hline & en & ltgegend. & $\mathrm{fg}$ & h $\mathrm{i}$ k 1 & $\mathrm{mn}$ op & q $\quad r$ r & s t t & $y$ \\
\hline & & & & & & & $\cdot \mathrm{u} \cdot$ & \\
\hline & т. . & $\cdots$ & $\begin{array}{ll}\cdots \\
\cdots\end{array}$ & & & & $\cdots$ & \\
\hline & $\therefore$ & $\cdots \cdots$ & $\cdots \cdots \cdots$ & $\cdots$ & $\cdots$ & . . & $\left|\begin{array}{ccc}\cdots & \cdots & \cdots \\
\cdots & \ldots & \cdots\end{array}\right|$ & \\
\hline & Mü. & $\cdots \cdots$ & $\ldots \ldots \ldots$ & $\ldots$ & $\cdots$ & $\cdots$ & $\ldots$ u... & \\
\hline & . . . & $\cdots \cdot$ & $\cdots \cdots$ & $\cdots \cdot$ & $\cdots \cdot$ & $\cdots$ & $\ldots u, \ldots$ & \\
\hline & $\cdots$ & $\cdots \dot{M^{2}}$ & $\cdots \cdots$ & $\cdots \cdot$ & $\cdots \cdot$ & $\cdots$ & $\cdots u, \ldots$ & \\
\hline & in & $\therefore$ & $\cdots$ & & & 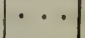 & . & \\
\hline & I $\cdots$ & & $\cdots$ & . & & & $\therefore$ & \\
\hline & $\cdots$ & $\ldots$. & $\ldots \ldots \ldots$ & $\ldots$ & $\cdots$ & $\begin{array}{ll}\cdots \\
\cdots \\
\cdots\end{array} \mid$ & 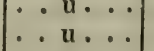 & \\
\hline & $\cdots$ & $\ldots$ & $\ldots \ldots$ & $\ldots$ & $\ldots$ & ... & $\ldots$... & \\
\hline & $\cdots$ & $\cdots \cdot \dot{0}$ & $\cdots \cdot$ & $\ldots$ & $\ldots$ & $\cdots$ & $\ldots u \ldots$ & \\
\hline & & . $\mathrm{M}^{2}$ & $\cdots$ & $\cdots$ & $\cdots$ & $\cdots$ & $\ldots$ и... & \\
\hline & AT. . & $\cdots \cdots$ & $\cdots$ & $\cdots$ & $\cdots$ & $\cdots$ & $\ldots \mathrm{u} \ldots$ & \\
\hline & AT. & $\cdots \cdots$ & 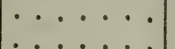 & $\cdots$ & $\cdots \cdot$ & $\cdots$ & $\ldots \mathbf{u} \ldots$ & \\
\hline & - & : & $\cdots$ & $\cdots$ & $\cdots \cdot$ & $\cdots$ & . u . . . & \\
\hline & $\cdot$ & & $\cdots$ & & $\cdots \cdot$ & $\cdots$ & $\cdots$ & \\
\hline & - & & $\cdots$ & & $\mid \cdots \cdots$ & & $\therefore u$ & \\
\hline & $\mathrm{p}$ & ... $\mathrm{M}^{2}$ & $\therefore$ & $\cdots$ & $\cdots$ & $\cdots$ & $\cdots u$ & \\
\hline & CHT & $\cdots$ & $\cdots \cdots \cdots$ & $\cdots \cdot$ & $\cdots$ & $\ldots$ & $\ldots \mathbf{u} \ldots$ & \\
\hline & $\cdots$ & $\cdots$ & $\ldots \ldots$ & $\ldots$. & $\ldots$ & $\cdots$ & $\ldots u$ & \\
\hline & & $\cdot$ & . & $\cdots$ & $\ldots$ & $\ldots$ & . u ... & \\
\hline & - & $\cdots$ & $\cdots \cdots$ & . & $\cdots \cdot$ & $\cdots$ & . u ... & \\
\hline & $\cdots$ & & $\cdots$ & $\cdot \cdot$ & $\cdots \cdot$ & $\cdots$ & .... & \\
\hline & 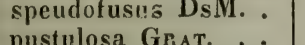 & $\cdots$ & 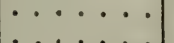 & - & $\cdots \cdot$ & $\cdots$ & $\cdots n$ & \\
\hline & $\cdots$ & $\therefore \mathrm{M}^{2}$ & $\cdots$ & & $\cdots \cdots$ & $\cdots$ & $\begin{array}{ll}\cdots & \ldots \\
\ldots & \ldots\end{array}$ & \\
\hline & $\therefore$ & . . $\mathrm{M}^{2}$ & $\cdot$ & $\cdots \cdot$ & $\therefore$ & $\cdots$ & $\therefore u$ & \\
\hline & $\cdots$ & . & $\cdots$ & $\cdots$ & . & $\ldots$ & $\ldots u \ldots$ & \\
\hline & снт. & $\cdot$ & $\cdots$ & $\cdots$ & $\ldots$ & $\cdots$ & $\ldots$ u... & \\
\hline & $\cdots$ & . & $\cdots$ & & $\cdots$ & $\cdots$ & $\ldots$ u... & \\
\hline & $\mathrm{CH}$. & $\cdots$ & $\cdots$ & & $\cdots$ & $\cdots \cdot$ & ... u. & \\
\hline & Mic & & $\cdots$ & & . . . & $\cdots$ & $\ldots \mathrm{u}$ & \\
\hline & $\mathrm{CH}$ & . & $0^{\circ}$ & & 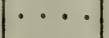 & $\cdot 1$ & $\cdots u$ & \\
\hline & $\cdots$ & & & & $\cdots$ & & $\therefore u$ & \\
\hline & 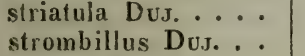 & & • & & - & $\cdots$ & $\ldots$ u. & \\
\hline & $\therefore$ & $\therefore$ & $\because$ & $\cdot$ & $\cdots$ & • & $\therefore$ и. & \\
\hline & . . & . . $\mathrm{M}^{2}$ & $\ldots$ & $\cdot 1$ & . & |. & $\ldots \mathbf{u} \ldots$ & \\
\hline & H . & $\cdots \cdot$ & & & $\cdots$ & $\ldots$ & $\ldots$ a... & - \\
\hline & $00 \mathrm{D}$ & . & $\cdots$ & & $\cdots$ & $\ldots$ & $\ldots$ u. . & . \\
\hline & $\cdot \cdot$ & $\cdots$ & . . & & $\cdots$ & $\cdots$ & $\ldots \mathbf{u}, \ldots$ & - \\
\hline & & $\cdot$ & $\cdots$ & & $\cdots$ & $\cdots$ & $\cdots \mathbf{u}, \ldots$ & \\
\hline & $\cdot$ & $\because$ & & & & $\cdots$ & $\therefore$ & \\
\hline & $\cdot$ & .. & . $\cdots$ & & $\cdots$ & $\cdots$ & . u v. & \\
\hline & & & $\cdots$ & & $\cdots$ & $\cdots$ & . u $\cdot \mathbf{w}$ & $\cdots$ \\
\hline & & & & & $\cdot \cdot$ & $\cdots$ & & $\cdots$ \\
\hline & $\dot{.}$ & & & & & & $\cdot$ & \\
\hline
\end{tabular}




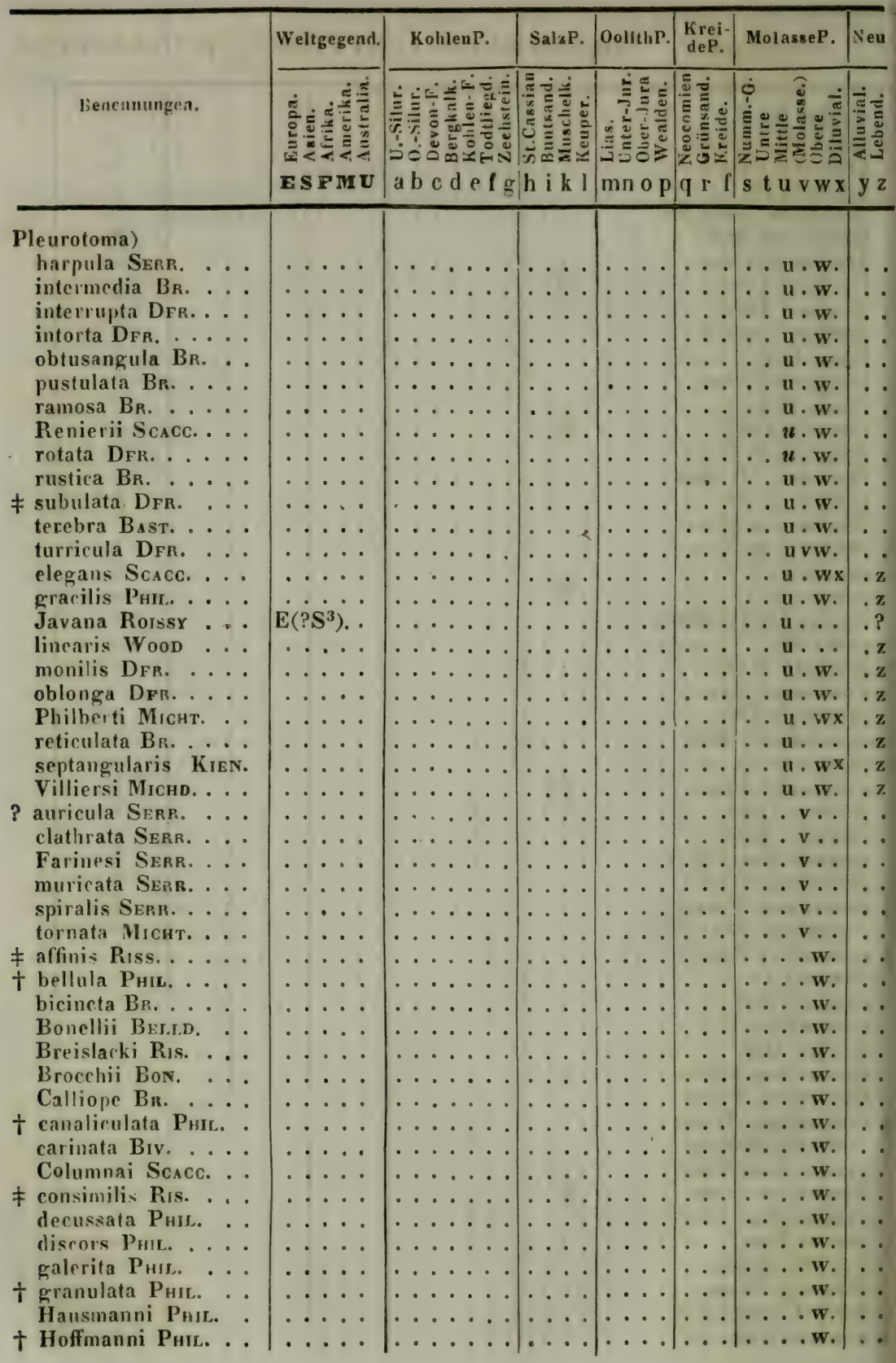




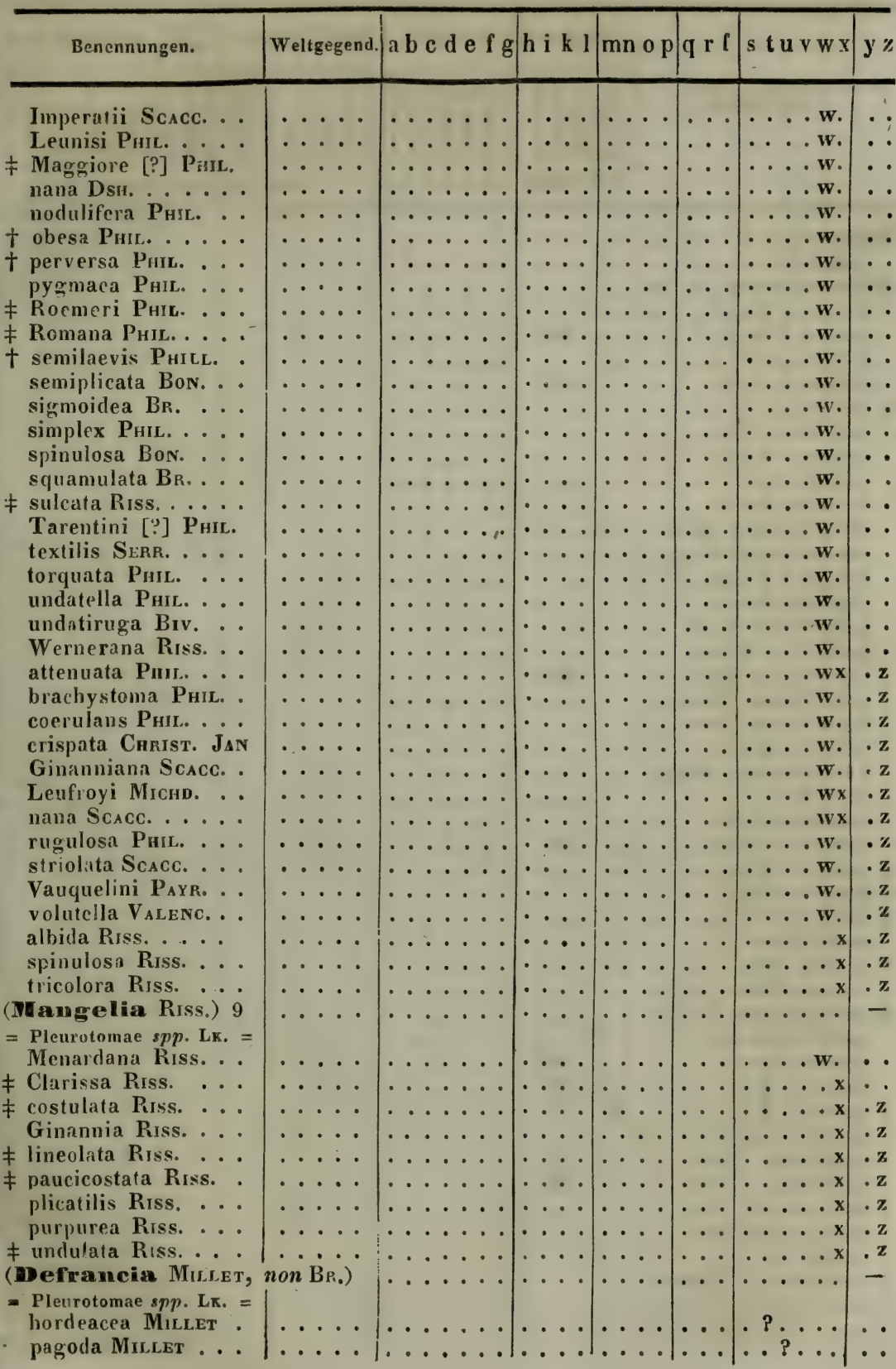


Worsonia Belld, 1. prima Ber.to.

Fasciolaria LK. 29 Roemeri Reuss elevata Reuss. funiculosa Dsh. . . . plicata LEA.

\# ponderosa ANT. uniplicata DFr. aculeata Grat. costati BoN.

fusoides Grat. Michelottiana Grat. nassaeformis Grat. Polonica Pusch pirulina Grat. polygonata Grat. punctifera Grat. rhomboidea Conr. subcarinata Grat. Tarbelliana Grat. tuberosa Grat. Valenciennesi Grat. Afra Grat. clandestina BLv. mutabilis Conr.

$\neq$ nodifera Dus. fimbriata Br.

† fusiformis Рнгс. fusus PhIL.

? pusilla Phin. lignaria PuIL.

Turbinella LK, 28 bulbiformis So... bolaris Conr. fusoides LEA

\# gracilis Ant. piruliformis NYsT piruloides ConR. praetenuis ConR.

$\neq$ semicoslata ANT. Parisiensis Dsu. aflinis So

$\neq$ Basteroti BeI.L.Mich. buccinoides Grat. calicellata Grat.

\begin{tabular}{|c|c|c|c|c|c|c|}
\hline Weltgegend. & KohlenP. & SalzP. & OolithP. & $\left|\begin{array}{c}\text { Krei- } \\
\text { deP. }\end{array}\right|$ & MolasseP. & $\mathrm{Ne}$ \\
\hline 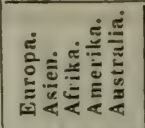 & 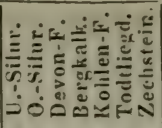 & 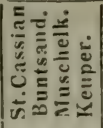 & 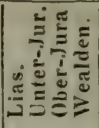 & 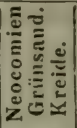 & 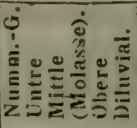 & 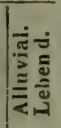 \\
\hline $\mathbf{E S} \mathbf{F} \mathbf{U}$ & a b c d c f g & h i k l & $\mathrm{mn} o \mathrm{p}$ & 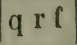 & s t u v w $x$ & y z \\
\hline
\end{tabular}

9

(

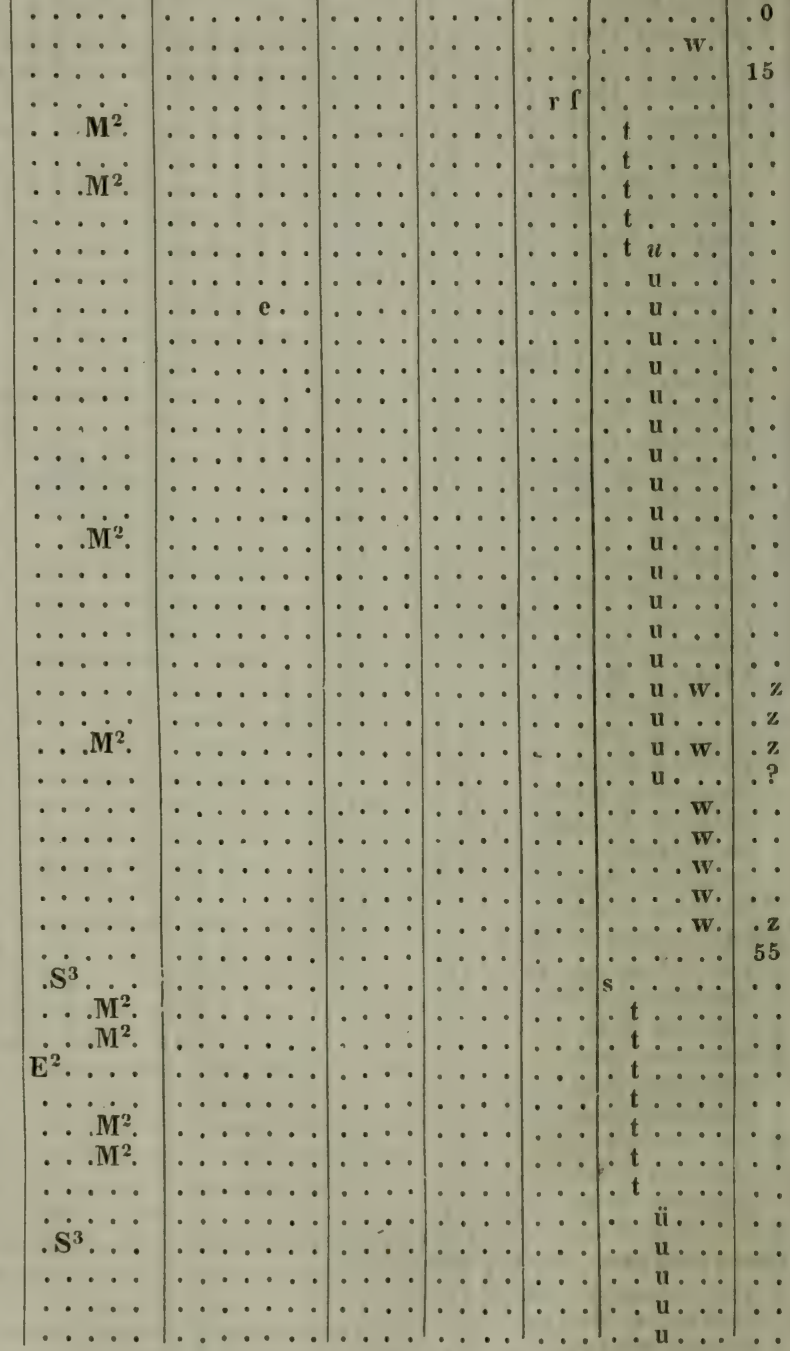




\begin{tabular}{|c|c|c|c|c|c|c|c|}
\hline hungen. & gend. & e $f \mathrm{~g}$ & l 1 & $\mathrm{p}$ & f & $x$ & $y \%$ \\
\hline$\cdots \cdots$ & $\cdots$ & $\cdots$ & & $\cdots \cdot$ & . . & . & \\
\hline roclita Grat. . & $\cdots \cdots$ & $\ldots \ldots$ & $\cdots \cdot$ & $\cdots \cdot$ & ... & . u . . & . \\
\hline labellum Bos. . . . & $\cdots \cdots$ & $\ldots \ldots$ & $\cdots \cdot$ & $\cdots \cdot$ & $\cdots$ & . u . . . & - \\
\hline Lynchi Bast. . . . & $\cdots \cdots$ & $\ldots \ldots$ & $\cdots \cdot$ & $\cdots \cdots$ & $\cdots$ & $\ldots u .$. & $\cdots$ \\
\hline AT. & $\cdots \cdots$ & $\cdots \cdots \cdots$ & $\cdots \cdot$ & $\cdots$ & $\cdots$ & $\cdots u, \ldots$ & $\cdots$ \\
\hline$\cdots$ & $\cdots \cdots$ & $\cdots \cdots$ & $\cdots \cdot$ & $\cdots$ & $\cdots$ & . . u . . & $\cdots$ \\
\hline · $\cdot$ & $\cdots \cdots$ & $\cdots \cdots$ & $\cdots \cdot$ & $\cdots \cdot$ & $\cdots$ & . . u. . . & $\cdots$ \\
\hline$\cdots$ & $\cdots \cdot$ & $\cdots \cdot$ & $\cdots \cdot$ & $\cdots \cdot$ & $\cdots$ & $\cdots$ & • \\
\hline$\cdots$ & $\cdots$ & $\cdots \cdots$ & $\cdots \cdots$ & $\cdots \cdot$ & $\cdots$ & $\cdots$ & \\
\hline$\cdots$ & : & $\dot{v}$ & . & $\cdots$ & $\therefore$ & $\cdots$ & : \\
\hline$\cdots \cdots$ & $\cdots \cdots$ & $\cdots \cdots \cdots$ & $\left|\begin{array}{c}\cdots \\
\cdots\end{array}\right|$ & $\cdots$ & $\cdots$ & $\left(\begin{array}{cc}\cdots & \cdots \\
\cdots & \text { u. }\end{array}\right.$ & $\begin{array}{l}. z \\
. z\end{array}$ \\
\hline LK. . . & $\cdots$ & $\ldots \ldots$ & $\ldots$ & $\ldots$ & $\cdots$ & $\ldots \mathbf{u} \mathbf{v} \ldots$ &. $\mathrm{z}$ \\
\hline$\cdots$ & $\cdots \cdots$ & $\cdots \cdots$ & $\cdots \cdot$ & $\cdots \cdot$ & $\cdots$ & ....w. & \\
\hline$\cdots$ & $\cdots \cdots$ & $\ldots \ldots$ & 1 & . & $\cdots$ & $\cdots x$ & \\
\hline e 1 & & & & & & & \\
\hline a Lк. 70 & $\cdots$ & $\cdots \cdots \cdots$ & $\cdots$ & & $\cdots$ & $\cdots$ & 80 \\
\hline$\cdots$ & $\cdots$ & $\ldots \ldots$ & $\ldots$ & $\cdots$ & $\ldots$ & . t.... & . \\
\hline$\Lambda \cdots$ & $\cdot \mathrm{M}^{2}$ & $\cdots \cdots \cdots$ & $\cdots$ & $\cdots \cdot$ & $\cdots$ & $\cdot \mathbf{t} \cdots \cdot$ & - \\
\hline$\cdots$ & $\cdots$ & $\cdots \cdots$ & $\cdots \cdots$ & $\cdots \cdot$ & $\cdots$ & t $\ldots$. & - \\
\hline$\cdots$ & $\cdots$ & $\cdots$ & $\cdots$ & $\cdots$ & - & $\begin{array}{l}\mathrm{t} \\
\mathrm{t}\end{array} \ldots$ & . \\
\hline$\cdots$ & $\begin{array}{l}\cdots \\
\cdots\end{array}$ & $\cdots$ & $\cdots \cdots$ & $\cdots$ & $\cdots$ & $\begin{array}{l}\mathrm{t} \cdots \\
\mathrm{t} \cdots\end{array}$ & \\
\hline$\therefore$ & $\cdots$ & $\cdots \cdots$ & $\cdots$ & $\cdots \cdots$ & $\therefore$ & $\because \ldots$ & \\
\hline$\cdots$ & . . . & $\ldots \ldots$ & $\ldots$ & $\ldots$. & $\cdots$ & $\ldots t \ldots$ & \\
\hline A . & . . $\mathrm{M}^{2}$ & $\ldots \ldots$ & $\ldots$ & $\ldots$ & ... & t $\ldots$ & \\
\hline$\cdots$ & $\ldots \mathbf{M}^{2}$ & $\ldots \ldots$ & $\ldots$ & $\cdots$ & $\cdots$ & . $\mathrm{t}$. & \\
\hline$\cdots$ & $\cdots \cdot$ & $\cdots \cdots \cdots$ & $\ldots$ & . & $\cdots$ & . $\mathrm{t} \ldots$ & \\
\hline$\cdots$ & .. $\mathbf{M}^{2}$ & $\cdots \cdots$ & $\cdots$ & - & $\cdots$ & . t . . & • \\
\hline LEA . & $\cdots \mathbf{M}^{2}$ & $\cdots \cdots$ & $\cdots$ & $\cdot$ & $\cdots$ & . t $\ldots$ & \\
\hline $0 . \cdots$ & $\cdots \cdots$ & $\cdots$ & $\cdots \cdot$ & $\cdots \cdots$ & $\cdots$ & t $\ldots$. & \\
\hline Dsh. . . & … & $\ldots$ & $\cdots$ & : & $\cdots$ & $\cdots$ & \\
\hline$\cdots$ & $\because$ & $\begin{array}{lll}\cdots & \cdots & \cdots \\
\cdots & \cdots & \cdots\end{array}$ & &. & $\cdots$ & t $\dot{t}^{2} \cdots$ & \\
\hline a. . . & & $\cdots$ & & $\cdots$ & $\cdots$ & $\begin{array}{l}\mathrm{t} u \\
\mathrm{t} u\end{array}$ & \\
\hline$\ddot{\cdots}$ & $\therefore$ & $\ldots \ldots$ & & : & . . & ? и... & \\
\hline L. & .... & $\ldots \ldots$ & $\ldots$ & . & . & $\ldots u^{1} \ldots$ & \\
\hline$\cdots$ & ... $\mathbf{M}^{2}$ & $\ldots \ldots$ & $\ldots$ & $\ldots$ & ... & $\cdots u \ldots$ & - \\
\hline a WW WGN. . & ... $\mathrm{M}^{2}$. & $\ldots \ldots$ & $\cdots$ & $\ldots$ & $\cdots$ & $\ldots u \ldots$ & - \\
\hline Boncllii BerL. . . & $\cdots \cdot$ & $\ldots \ldots$ & $\ldots$ & $\cdots \cdot$ & . . & $\cdot \ldots u \ldots$ & . \\
\hline Bromni BEt & $\cdots \cdot$ & $\ldots \ldots$ & & $\cdots$ & ... & $\cdot . . u \ldots$ & - \\
\hline IFa Bast. ... & $\cdots \cdots$ & $\ldots \ldots$ & $\cdots$ & $\cdots \cdot$ & . . & $\ldots u \ldots$ & \\
\hline$\cdots$ & $\cdots \cdots$ & $\cdots \cdots$ & $\cdots$ & $\cdots \cdots$ & ... & $\cdots u \ldots$ & \\
\hline citharella Pusch... & $\cdots \cdots$ & $\cdots \cdot$ & & $\cdots \cdot$ & $\cdots$ & . u . . & \\
\hline $\begin{array}{l}\text { t comrinna Woud } \\
\text { crassicosta Be }\end{array}$ & : & $\cdots$ & & $\cdot$ & $\cdots$ & $\cdot$ u & \\
\hline $\begin{array}{l}\text { cras } \\
\text { Des! }\end{array}$ & $\because$ & & & & $\therefore$ & $\cdot .1$ & \\
\hline Desh & $\ldots$ & $\therefore$ & - & & . & $\cdots u^{2} \cdots$ & \\
\hline Geslini BAS & $\cdots$ & & & & - & $\cdots \mathrm{u}^{2} \ldots$ & \\
\hline & & & & & & 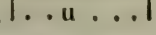 & \\
\hline
\end{tabular}




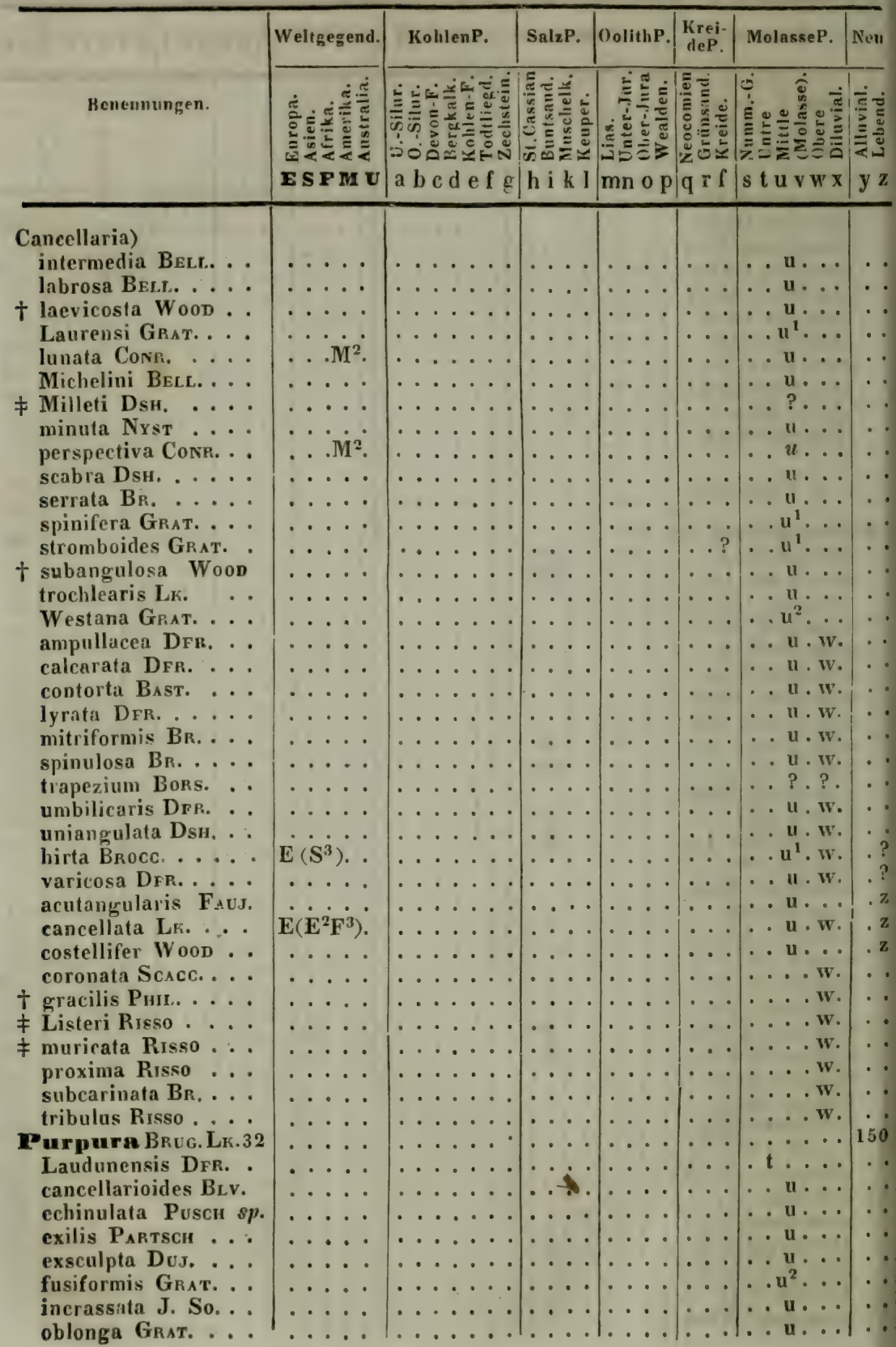




\begin{tabular}{|c|c|c|c|c|c|c|c|}
\hline Beneunungen. & Weltgegend. & a b c d e f g & hi k l & $\mathrm{mnop}$ & $q \times r \mid$ & $s t u v w x$ & y z \\
\hline pleurotomoides Grat. & & . & & . & $\cdots$ & $\left|\cdot u^{2} \ldots\right|$ & \\
\hline briuscula Grat. . & $\ldots$ & $\ldots \ldots$ & $\cdots$ & $\ldots$ & $\cdots$ & $\ldots$.... & \\
\hline tetragona BLv. ... & $\cdots$. & $\ldots \ldots$ & $\cdots$ & $\ldots$ & $\cdots$ & $\ldots$ u... & . \\
\hline torulosa Grat. ... & $\cdots$ & $\ldots \ldots$ & $\cdots$ & $\ldots$ & $\cdots$ & $\cdots u^{2} \ldots$ & \\
\hline haemastoma LK. . . & $\dot{\mathrm{E}}^{2} \dot{\mathrm{M}^{2}}$ & $\cdots \cdots$ & $\cdots$ & $\cdots \cdot$ & $\cdots$ & $\cdots \mathbf{u} \cdot \mathbf{w} \cdot$ & $\cdot \mathbf{z}$ \\
\hline $\begin{array}{l}\text { lapillus LK. } \\
\text { plicata LK. }\end{array}$ & $\begin{array}{l}\mathrm{E}^{2} \cdot \mathbf{M}^{2} . \\
\mathrm{E}\left(\mathbf{S}^{3}\right) .\end{array}$ & $\cdots \cdots \cdots$ & $\cdots$ & $\cdots \cdot$ & $\cdots$ & $\cdots \mathbf{u}, \mathbf{w} \mathbf{x}$ & - $z$ \\
\hline textilosa Lk. & $\mathbf{E} \ldots\left(\mathbf{U}^{4}\right)$ & $\cdots \cdots \cdots$ & $\cdots \cdot$ & $\cdots \cdots$ & $\cdots$ & 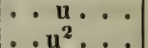 & $\cdot z$ \\
\hline torulosa Grat. ... & $\ldots \ldots$ & $\ldots \ldots$ & . & 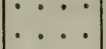 & $\because$ & $\cdots u^{2} \cdots$ & $\begin{array}{l}\cdot z \\
. z\end{array}$ \\
\hline Martinii M MTHN. . & $\ldots \ldots$ & $\ldots \ldots$ & $\cdots$ & $\ldots$ & .. & $\ldots v \cdots$ & $\cdots$ \\
\hline bicostalis LK. .... & . & $\cdots \cdots$ & $\cdots \cdot$ & $\ldots \cdot \mid$ & $\cdots$ & $\ldots \mathbf{v} \ldots$ &. $\mathrm{z}$ \\
\hline LF. . . . . . & ・ & $\ldots$ & $\cdots$ & $\ldots$ & $\cdots$ & $\therefore \mathbf{v} \ldots$ & $x$ \\
\hline pum PhrL. . . & • & - & $\cdot \cdot$ & $\cdots$ & $\cdots$ & $\ldots w^{\prime}$ & . \\
\hline HT. . & $\cdots \cdot \cdot$ & $\cdots \cdots$ & $\cdots \cdot$ & $\cdots \cdot$ & $\cdots$ & $\ldots \ldots w$ & - \\
\hline lineolata Riss. ... & $\cdots \cdot$ & $\cdots \cdots$ & $\cdots \cdot \cdot$ & $\ldots$ & $\cdots$ & $\ldots \ldots w \cdot$ & - \\
\hline striolata BR. ... & & $\cdots \cdots$ & $\cdots$ & $\cdots$ & $\cdots$ & $\cdots, w^{\prime}$ & 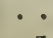 \\
\hline trochlea LK. . . . . & $\cdot$ & $\cdots \cdots$ & $\cdots \cdot$ & $\cdots$ & $\cdots$ & $\cdots \cdots w^{\prime}$ & $\cdot \mathrm{z}$ \\
\hline glabra Riss. · & & & $\cdot$ & & 1.. & $\cdots \cdots x$ & - $\cdot$ \\
\hline $\begin{array}{l}\text { Rafinesqueia Riss. } \\
\text { chocolata Buv. . . }\end{array}$ & $\therefore \dot{M}^{4}$ & $\cdots$ & $\cdots \cdots$ & $\cdots \cdots$ & $\cdots$ & $\cdots \cdots x$ & $\because$ \\
\hline $\begin{array}{l}\text { chocolata BLv. } \cdots \\
\text { corniculata Riss. . }\end{array}$ & $\ldots$. & $\ldots \ldots$ & $\cdots$ & $\mid \cdots \cdots$ & $\because \cdots$ & $\because \cdots x$ & $\cdot \begin{array}{l}\mathrm{z} \\
\mathrm{z}\end{array}$ \\
\hline \# costulata Riss. . . . & .... & $\ldots \ldots$ & $\ldots$ & $\cdots \cdots$ & $\cdots$ & $\cdots \cdots x$ & z \\
\hline cki Riss. . . . & .... & .... & $\ldots$ & $\cdots$ & $\therefore$ & $\ldots \ldots x$ & $\mathrm{z}$ \\
\hline variegata Ris & $\cdots \cdots$ & $\ldots \ldots$ & $\cdots$ & $\ldots$ & $\cdots$ & $\ldots \ldots x$ & $\mathrm{z}$ \\
\hline Concliolepas $L_{K}, 1$ & $\cdots \cdot$ & $\ldots \ldots$ & $\cdots$ & $\ldots$. & $\ldots$ & $\ldots \ldots$ & .1 \\
\hline LK. . . . & $\cdots$ & $\cdots$ & $\cdots \cdot$ & $\ldots$ & $\cdots$ & $\ldots \ldots x$ & yz \\
\hline Nonoceros Lk. 8. & $\cdots$ & $\cdots$ & $\cdots$ & $\ldots$ & $\cdots$ & $\ldots \ldots$ & 20 \\
\hline$\cdot \cdots$ & . . . M $\mathrm{M}^{4}$ & $\cdot \cdot$ & $\cdots$ & $\ldots$ & $\cdots$ & - $t$ & - \\
\hline Blain & ... $\mathbb{M}^{+}$. & $\cdots \cdot$ & $\cdots \cdot$ & $\cdots$ & $\cdots$ & - $\mathrm{t}$. & \\
\hline ol & • & ${ }^{\circ}$ & $\cdots$ & $\cdots \cdot$ & - & $\cdot \mathrm{t}$ & - \\
\hline $\begin{array}{l}\text { semicostatus DsH. } s p \text {. } \\
\text { sulcatus LEA . . . }\end{array}$ & $M^{2}$. & & - & $\cdots$ & & $\cdot-t$ & $\cdot$ \\
\hline is LEA .... & $\therefore \mathbf{M}^{2}$ & & $\cdots$ & : & $\cdots$ & $\begin{array}{l}\cdot t \\
. t\end{array}$ & 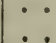 \\
\hline $\begin{array}{l}\text { vetustus Conr. . . } \\
\text { depressus Br. . . }\end{array}$ & $\ldots$ & $\ldots \ldots$ & $\ldots$ & $\cdots \cdots$ & $\left|\begin{array}{ll}\cdots \\
\cdots\end{array}\right|$ & . & \\
\hline thus Br. . . & $\cdots \cdot$ & $\cdots \cdots$ & $\ldots$. & $\ldots$ & ... & $\ldots .$. & \\
\hline $\begin{array}{l}\left.\text { (Ricinuta } L_{K_{0}}\right){ }^{3} \text {. } \\
=\text { Sistrum } M_{F_{0}}=\end{array}$ & $\cdots \cdots$ & 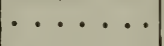 & $\cdots \cdot$ & ... & $\cdots$ & $\ldots \ldots$ & 12 \\
\hline T. . . . & & & & & & $\cdots u^{2}$ & \\
\hline . & $\because$ & & & & $\cdots$ & $\cdots u^{2} \ldots$ & $\dot{z}$ \\
\hline morus LK. & $\cdots \cdot$ & $\cdots$ & & & ... & $\ldots \mathbf{u} \ldots$ & . $z$ \\
\hline Columbella LK. 9 & & & & & & $\ldots \ldots$ & 200 \\
\hline F laeviguta & $\cdots \cdot$ & & & & $\cdots$ & $\cdot \mathrm{t} \ldots$. & - \\
\hline filosa DuJ. & & - & & & $\cdots$ & . u... & \\
\hline ‡ marginata BELLMich. & & $\cdots$ & $\cdots \cdots$ & $\cdots \cdot$ & $\cdots$ & $\cdots \mathbf{u} \cdot \cdots$ & \\
\hline $\begin{array}{l}\text { sulcata Wood .... } \\
\text { semipunctata LK. . }\end{array}$ & $\dot{\mathrm{E}} \cdot\left(\mathrm{F}^{3}\right)$ & & $\cdot$ & & $\cdots$ & $\cdots \mathrm{u}$ & 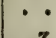 \\
\hline Grecoi PhIL. .... & $\ldots$ & $\because$ & . & $\cdots \cdot$ & 1 & $\cdots u \cdot \cdots$ & $z$ \\
\hline ? subulata Sism, .... & .... & $\ldots$ & & & & $\cdots \cdots w$ & \\
\hline$\cdots$ & $\cdots$ & $\ldots$ & & & & $\cdots w x$ & . $z$ \\
\hline iss. . . . & $\cdots$ & $\cdots$ & & & $\cdots$ & $\ldots \ldots x$ & $\mathrm{z}$ \\
\hline $\begin{array}{c}\text { Oniscia So. 3. . . } \\
\text { cithara So. }\end{array}$ & 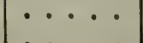 & $\cdots \cdot$ & ${ }^{\circ}$ & & $\cdot 1$ & . & .5 \\
\hline & & & & & & & \\
\hline
\end{tabular}




\begin{tabular}{|c|c|c|c|c|c|c|c|}
\hline & Weltgegend. & KolilenP. & SalzP. & OolithP. & $\begin{array}{c}\text { Krei-i } \\
\text { deP. }\end{array}$ & MolasseP. & Nell \\
\hline Senennungeน. & 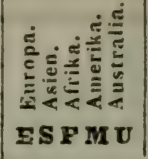 & 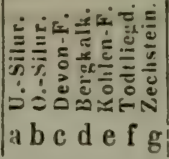 & 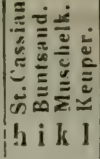 & 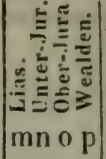 & 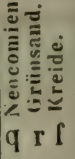 & 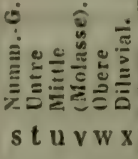 & 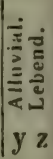 \\
\hline
\end{tabular}

Oniscia)

verrucosa Bon.

? Alicia Riss.

\section{f Cassidina.}

Cassis Lr. 36.

o Eparcyensis D'A.

brevicostata Conr.

cancellata Lr.

harpiformis Lr.

nupera Dsh.

'Taiti Conk.

variabilis BecL. Мich. elegans Grat. calantica Dsw.

t caclata Cons.

Hodgei Cosr. . . . .

incrassata Grat.

intermedia Grat. . . mammillaris Grat. .

† nodulifera PARTsch sculpta So.

cypraeiformis Bors.

Rondeleti Bast.

areola LK.

† bisulcata DsH.

crumena LK.

diadema Drr.

flammea LK.

granulosa LK.

rufa Lk. . . . . . .

saburon Lr.

plicata DFr.

+ atfinis PhiL.

f canaliculata Riss.

fasciata Bore.

Germari PнiL.

₹ gibba Rrss.

Saussurea Riss.

₹ striolata Riss.

† sulcata Riss.

undulata Priz.

Morio MF. 7.

flexuosus Br.

Apnaeae Br.

striatus $B R$.

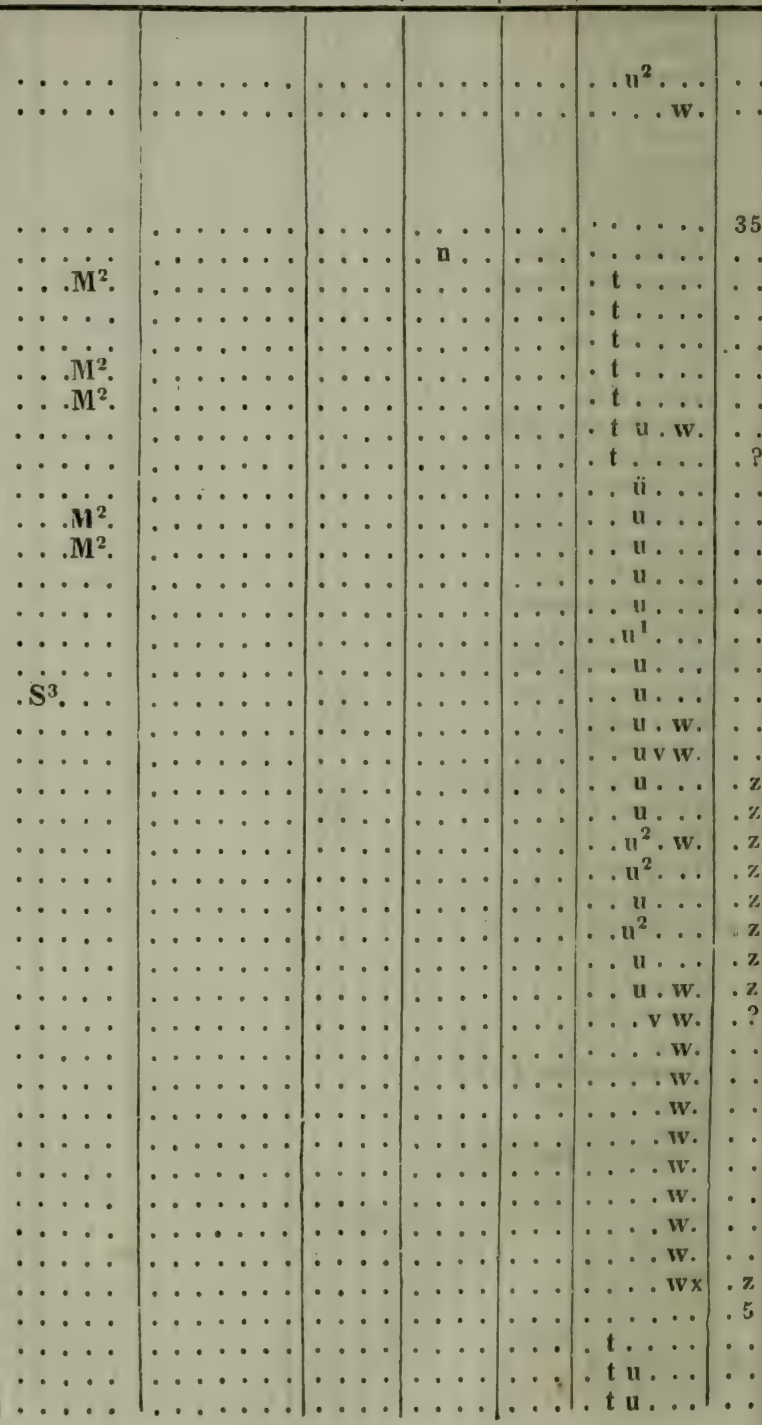




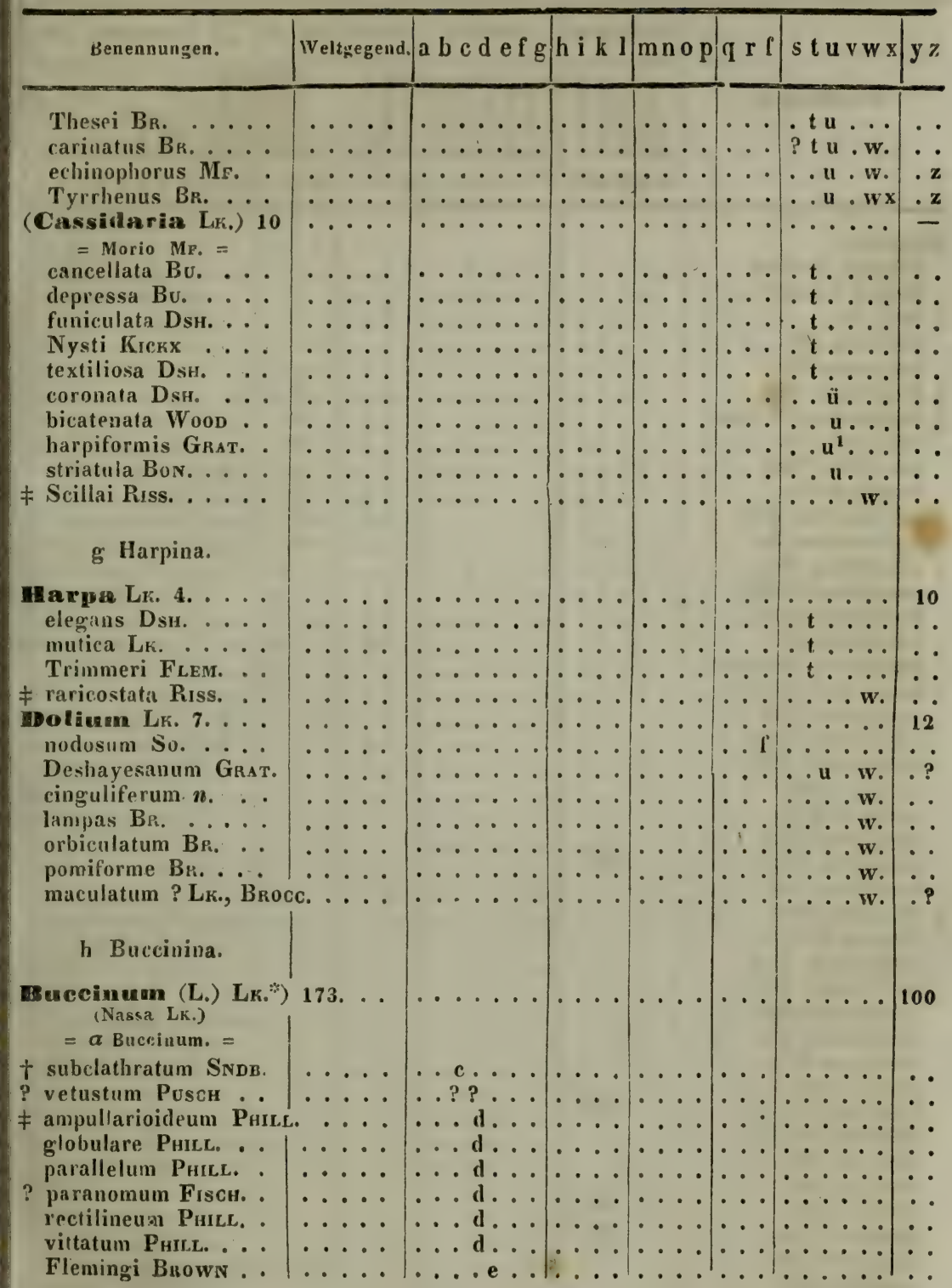

* Spp. nesterisco pruefivo insignilse Nussae habilum prae se ferentes sub hoc nomine jann milituverant; munerus I indicnt spp. verticraliler costains, numerus 2 monstrat spp. ul limo anfractu ecostatas, lat ves aut solum spiratiler slriatus. 


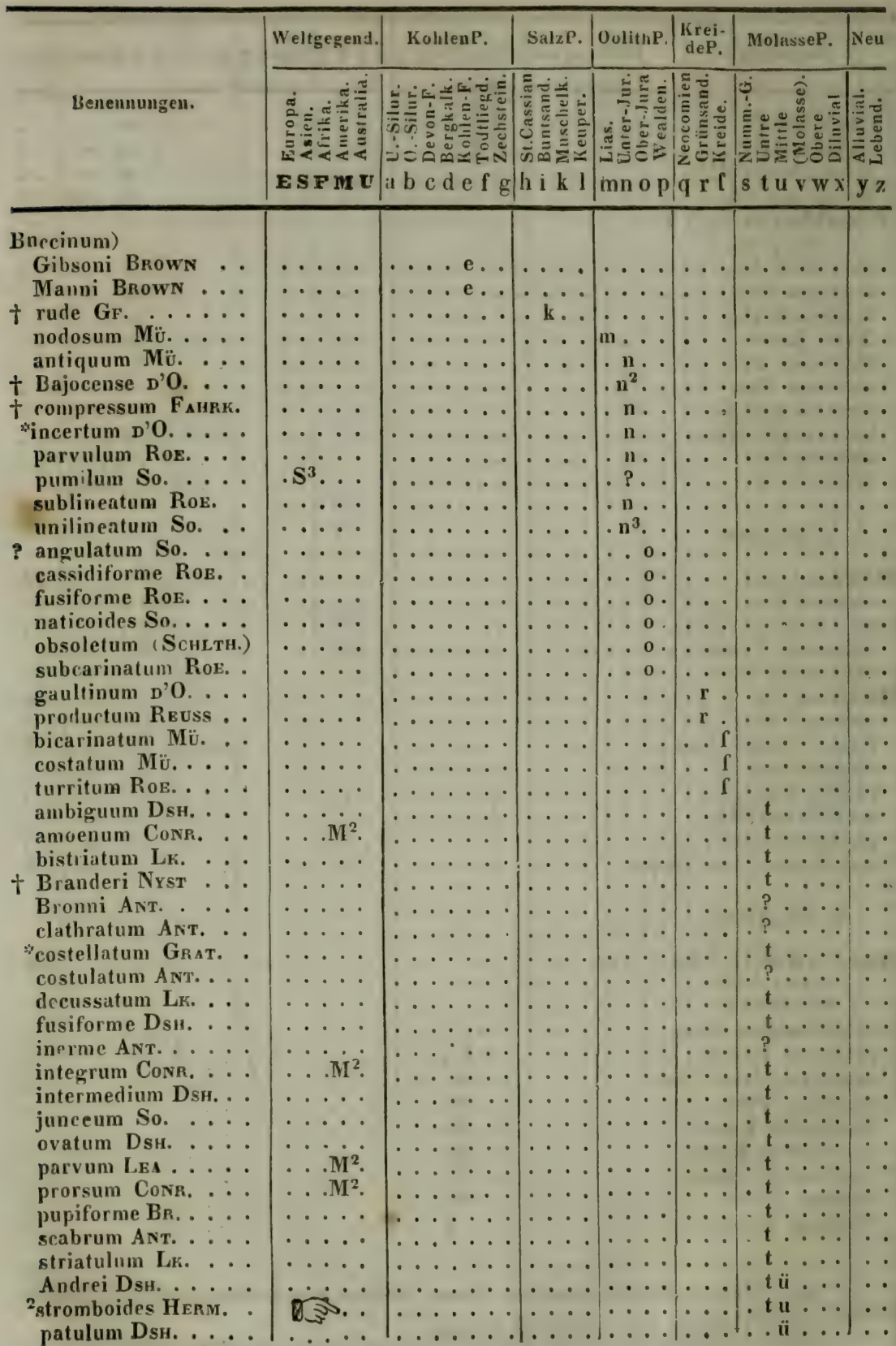




\begin{tabular}{l|l|l|l|l|l|l|l|}
\hline Benennungen. & Weltgegend. a b c d e f gi k l & mn o p & q r f & s t u vw x & y \% \\
\hline
\end{tabular}

truncatum Dsis.

raltile Conr. . . .

'ancillariacformis Grat. aratum $S_{A X}$

+ Badense Paptscir

Cassidaria Br.

1 contortum Dus.

'costulatum Eichiw. crassum Nyst.

1curtum Dus.

Dalei So.

*elegaus So.

*elongatum (So.) Nrst. 1: gibbosuum Grat.

*"granulare Miснт. intercisum GÉné interruptum Conr.

1/aqueatum Conr.

1*lineolatum Grat.

₹ minutum Miснт.

imirabile Bast.

2mitreola Bast. multirugatum Conr. 2papyraceum Grat.

${ }^{2}$ phasianclloides Grit. 2: planaxiforme Grat. 1 porcinum Conr.

"propinquam Sio.

$\neq 1$ pulchellum Dus.

+ ringens Bov.

1 rissoides Grat.

+ Rosthorni Partscir.

? rusticum (?L.) Pusch

${ }_{1}$ substramineum Grat.

'Tarbellicum Grat.

*terebrale Grat.

† Tritonium Partsch

1 turbinellus Brocc.

${ }^{1}$ Turonense Dus.

${ }^{2}$ Veneris FavJ.

${ }^{2}$ ventricosum Grat.

${ }^{2}$ baccatum BAsT. .

* conglobatum (Brocc.)

${ }^{2 *}$ conus $\mathrm{Br}$.

1 flexuosum Brocc.

* granulatum So.

1. musivum Broce.

2 semistriatum Brocc.

1"serratum Brocc.

$\neq$ tessellatum Bon.

Ascanias Brug.

"*clathratum BORN .

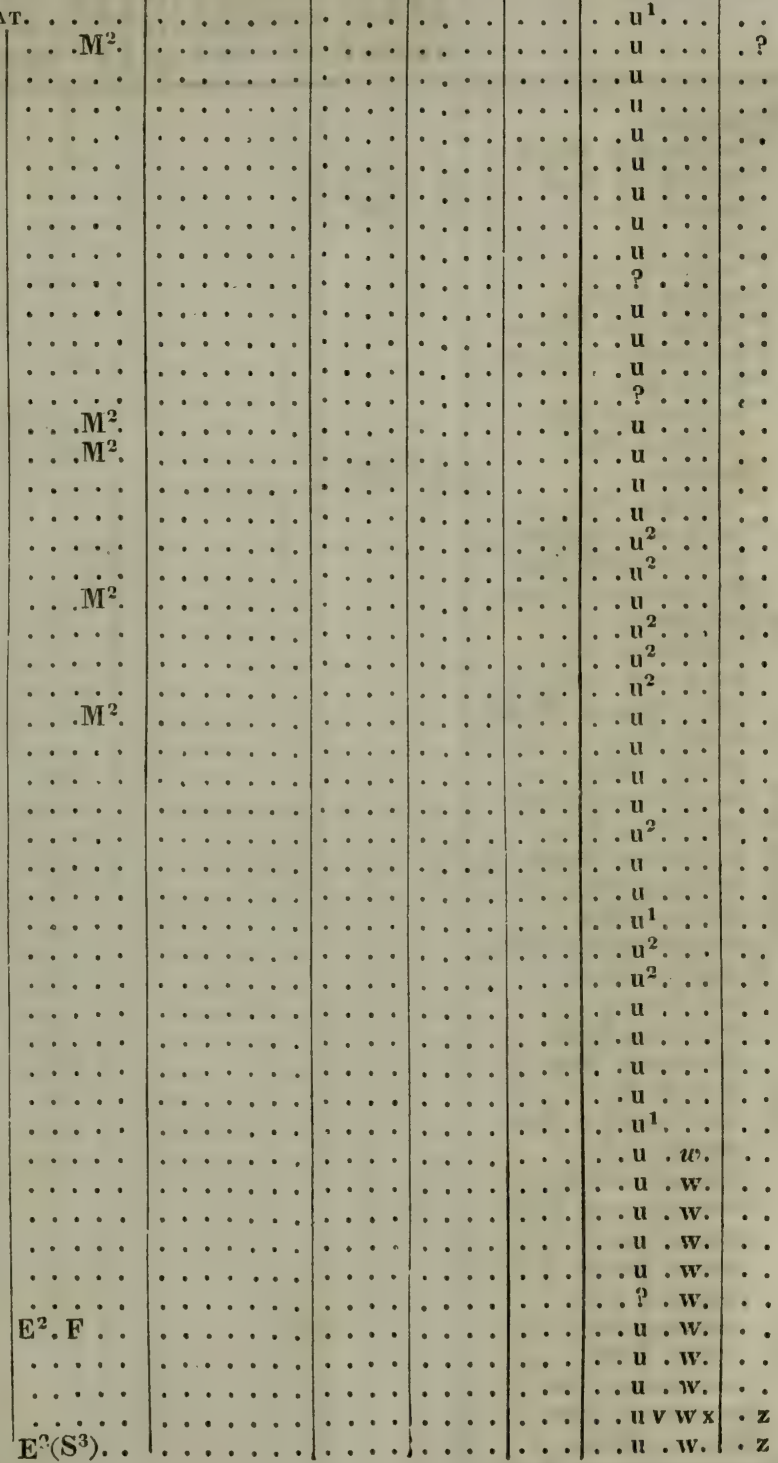




\begin{tabular}{|c|c|c|c|c|c|c|c|}
\hline \multirow[b]{2}{*}{ Benenu ungen. } & We & . & ulzP. & OolithP. & $\begin{array}{l}\text { rei- } \\
\text { leP. }\end{array}$ & MolasseP. & $\mathrm{Neu}$ \\
\hline & 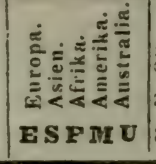 & 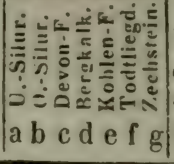 & 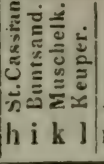 & 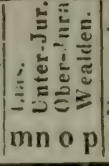 & 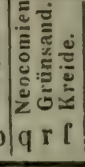 & 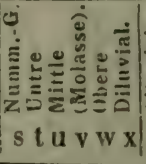 & 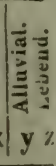 \\
\hline \multirow{2}{*}{\multicolumn{8}{|c|}{$\begin{array}{l}\text { Buccinum) } \\
{ }^{2 \Rightarrow} \text { corniculum Otivi }\end{array}$}} \\
\hline & & & & & & . .?. . w. & \\
\hline "Desnoyersi Dsu. . . & $\mathrm{E} \cdot\left(\mathbf{F}^{3}\right)$ & $\cdots \cdot$ & $\cdots$ & $\cdot$ & $\ldots$ & $\ldots \mathrm{u} \ldots$ & $\mathrm{z}$ \\
\hline$S_{A Y} \ldots$ & $\ldots \mathrm{M}^{2}$ & $\ldots \ldots$ & $\ldots$ & $\cdots \cdot$ & $\ldots$ & . u ... & $\mathrm{z}$ \\
\hline$\cdots$ & $\cdots$ & $\cdots \cdots$ & $\cdots \cdots$ & $\cdots \cdots$ & $\cdots$ & . . u v .. & $\cdot \mathbf{z}$ \\
\hline L. . . & & & $\cdots \cdot$ & $\cdots \cdots$ & $\cdots$ & . u $\cdot \mathrm{wx}$ & $\cdot \mathrm{z}$ \\
\hline Bносс. & $\cdots$ & $\cdots$ & $\cdots$ & $\cdots \cdot$ & $\cdots$ & $\cdot u^{2} v \ldots$ & $\cdot z$ \\
\hline $\begin{array}{l}\text { Вносс. } \\
\text { m Broce. }\end{array}$ & $\cdots$ & $\cdots \cdots \cdots$ & $\cdots$ & $\cdots$ & $\cdots \cdot$ & . u, $\mathbf{w} \cdot$ & - $\mathbf{z}$ \\
\hline . & $\ldots$ & $\ldots \ldots$ & $\cdots$ & $\cdots$ & $\cdots$ & $\cdots u \cdot \mathbf{w} x$ & $\cdot \mathbf{z}$ \\
\hline latum Gm. . . & .... & $\ldots \ldots$ & $\ldots$ & $\cdots$ & $\cdots$ & $\mid \begin{array}{cc}0 \cdot \mathrm{w} \\
\cdots u \cdot w \mathrm{x}\end{array}$ & $\cdot 2$ \\
\hline HIL. . . & & $\ldots \ldots$ & $\cdots \cdot$ & $\ldots$ & $\cdots$ & 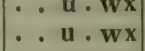 &.$z$ \\
\hline & $\left(\mathbf{E}^{12} \mathbf{M}^{1}\right)$ & $\ldots \ldots$ & $\cdots \cdots$ & $\ldots$ & $\ldots$ & . u.wx &.$z$ \\
\hline$\cdots$ & $\cdots \cdot \cdot$ & $\ldots \ldots$ & $\cdots$ & $\therefore \ldots$ & $\cdots$ & ..u.w. & . $\mathrm{z}$ \\
\hline SERR. . & $\cdots$ & $\ldots \ldots$ & $\cdots$ & $\ldots$. & $\ldots$ & $\ldots v \ldots$ & . \\
\hline & & . & $\cdots$ & $\ldots$ & $\cdots$ & $\ldots v \ldots$ & $\cdots$ \\
\hline iN. & $\cdot$ & $\cdots$ & $\cdots$ & $\ldots$ & $\cdots$ & 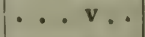 & 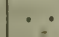 \\
\hline$\neq^{1} p$ & . & $\cdots$ & $\cdots$ & $\ldots$ & $\cdots$ & $\ldots \mathbf{v} \ldots$ & $\because$ \\
\hline$\therefore$ & - & $\cdots$ & $\cdots \cdots$ & $\cdots \cdots$ & $\cdots$ & $\cdots \mathbf{v} \ldots$ & - ? \\
\hline olivareum ?Lк. & • & & & - & $\cdots$ & $\cdots \mathbf{v} \ldots$ & ? \\
\hline affinis Piss. . . . . & & $\cdots$ & $\because$ & ... & $\cdots$ & …w. & $\because$ \\
\hline & $\cdots$ & ..... & $\ldots$ & . . & $\ldots$ & $\ldots w$ & $\dot{.}$ \\
\hline Bors. & . & $\ldots \ldots$ & $\ldots$ & ... & $\ldots$ & $\ldots w$ & . \\
\hline † & & $\ldots$ & $\cdots$ & ... & ... & $\ldots \ldots$ & $\therefore$ \\
\hline catum Risso.. & . & $\ldots \ldots$ & . & ... & $\cdots$ & $\ldots \ldots \mathbf{w}$ & . \\
\hline PHIL. ... & $\cdots \cdot$ & $\ldots \ldots$ & $\cdots$ & $\cdots$ & $\cdots$ & $\ldots \ldots w$ & - \\
\hline$\neq \mathbf{e l}$ & $\cdots \cdot$ & $\ldots \ldots$ & $\cdots$ & $\cdots \cdot$ & - & $\ldots w \cdot$ & • \\
\hline ISso & 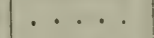 & ${ }^{\circ}$ & & . & -. & $\cdots \cdot \mathbf{w}$ & - \\
\hline Rocc. . . & - & $1^{\circ}$ & & - & $\cdot$ & $\cdots \cdot \mathbf{w} \cdot$ & - \\
\hline $\begin{array}{l}\text { Phis. } \\
\text { orme Bors. . . }\end{array}$ & 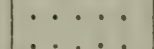 & & & & $\cdots$ & $\mid \cdots w$ & • \\
\hline N. . . & & . & & $\cdots$ & $\ldots$ & $\ldots$ w & • \\
\hline$\cdots$ & . & $\ldots$ & 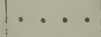 & . & . & $\ldots w$ & . \\
\hline$t_{2} i_{1}$ & - & $\cdots$ & • & $\cdots$ & . & $\ldots w$ & - \\
\hline 21 & - & - & - & $\cdots$ & $\cdots$ & $\ldots w$ & - \\
\hline$n_{11}^{n}$ & & & & & .. & ....w. & - \\
\hline${ }^{1} \mathbf{P}$ & & $\cdots$ & & $\cdots$ & . & $\cdots \cdots w_{0}$ & - \\
\hline Riss. & & $\ldots$ & & . & & $\cdots \cdots w \cdot$ & $\cdot$ \\
\hline II Riss. & .. & $\ldots$ & & & & $\begin{array}{l}\cdots \cdots \mathrm{w} \\
\cdots \cdots \mathrm{w}\end{array}$ & • \\
\hline BoN. & & $\ldots$ & 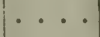 & $\cdots$ & . & $\ldots w$ & . \\
\hline 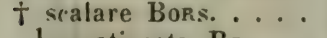 & ... & $\ldots$ & & - & . & $\cdots w$ & · \\
\hline 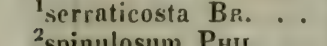 & $\cdots$ & $\cdots \cdot$ & & & & $\cdots \cdot w_{\cdot}$ & $\cdot$ \\
\hline $\begin{array}{l}\text { Phic. } \\
\text { PhIL }\end{array}$ & - & $\cdots$ & & & - & $\ldots w^{\prime}$ & $\cdots$ \\
\hline subcoronatuin $\mathrm{P}_{\text {HIL }}$ & & & & & & ...w. & \\
\hline
\end{tabular}




\begin{tabular}{|c|c|c|c|c|c|c|c|}
\hline ngen. & end. & $f g$ & 1 & $p$ & 〔 & $w x$ & $y z$ \\
\hline 1*arcularium Li. & E $(\mathrm{S}$ & & & & & & \\
\hline onR..... & . . $\mathrm{M}^{2}$ & $\ldots \ldots$ & $\ldots$ & $\ldots$ & ... & .... $\mathrm{w}$ &. $\mathrm{z}$ \\
\hline 'coriugatum Brocc. & $\ldots$ & $\ldots \ldots$ & $\ldots$ & $\cdots \cdot$ & $\cdots$ & $\ldots w$ & $\cdot \mathrm{z}$ \\
\hline I P PYYR. - . & $\cdots \cdots$ & $\ldots \ldots$ & $\cdots$ & $\cdots$ & $\cdots$ & $\ldots \mathrm{wx}$ & .2 \\
\hline im L. ... & $\ldots$ & $\ldots \ldots$ & $\ldots$ & $\ldots$ & $\cdots$ & $\ldots w$ & .2 \\
\hline${ }^{1}$ minimum Montg. . & $\ldots$ & $\cdots \cdots \cdot$ & $\cdots$ & $\cdots \cdot$ & $\cdot \cdot$ & $\ldots \ldots$ & .7 \\
\hline${ }^{2}$ minus Phicl. ... & $\cdots \cdot$ & $\cdots \cdots$ & $\cdots \cdots$ & $\cdots \cdots$ & $\cdots$ & $\cdots \cdots \mathrm{wx}$ & - $z$ \\
\hline 1verrurosum Brocc. & $\cdots \cdot \cdot$ & $\cdots \cdots \cdot$ & $\cdots \cdots$ & $\cdots \cdot$ & $\cdots$ & $\ldots w$ & $\cdot \mathrm{z}$ \\
\hline n Riss. .. & $\cdots$ & $\ldots \ldots$ & $\cdots$ & $\cdots$ & $\cdots$ & $\cdots \cdots x$ & $\cdots$ \\
\hline um His. . & $\cdots \cdot$ & $\ldots \ldots$ & $\ldots$ & $\cdots \cdots$ & $\cdots$ & 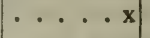 & $\sin 20$ \\
\hline costulosum Riss. .. & $\ldots$ & $\ldots \cdots$ & $\cdots$ & $\cdots \cdot$ & $\cdots$ & $\cdots \cdots x$ & \\
\hline proximum Ris & $\cdots \cdots$ & $\cdots \cdots$ & $\cdots$ & $\cdots$ & $\cdots$ & $\cdots \cdots x$ & - \\
\hline Iss. . & - نंध & $\cdots \cdots \cdots$ & $\cdots \cdots$ & $\cdots \cdot \cdot$ & $\cdots$ & $\cdots \cdots x$ & \\
\hline m Cuemis. . & $\cdots$ & ... & $\cdots$ & $\cdots \cdot$ & $\cdots$ & $\cdots \cdots x$ & $\cdot \mathbf{z}$ \\
\hline $\begin{array}{l}\text { 'Folineae Pнí. . . } \\
\text { glaciale Gм. . . }\end{array}$ & $\left(\dot{\mathrm{E}}^{2}\right) \dot{\mathrm{S}}^{2}$ & $\begin{array}{l}\cdots \\
\cdots \\
\cdots\end{array}$ & $\cdots$ & $\ddot{\cdots}$ & $\because$ & 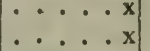 & $\begin{array}{l}. \mathrm{z} \\
. \mathrm{z}\end{array}$ \\
\hline 'globulosum KIEN. & ........ & $\ldots \ldots$ & $\cdots \cdots$ & $(\cdots)$ & $\ldots$ & $\ldots \ldots x$ & .2 \\
\hline $\begin{array}{l}\text { striatum So. } \cdots \\
\beta \text { Gastridium Forz. }\end{array}$ & $\cdots \cdots$ & L & $\cdots$ & $\cdots \cdot$ & $\cdots$ & $\cdots \cdots \mathbf{x}$ & .? \\
\hline Dsh. . . & $\cdots$ & $\ldots \ldots$ & & & . & - $t$ & \\
\hline tiara Dsн...... & & $\ldots$ & ... & $\cdots$ & . &.$t \ldots$ & $\cdots$ \\
\hline (Nassa LK.) $24 \ldots$ & $\ldots$. & $\cdots$ & ... & $\cdots$ & . & $\ldots \ldots$ & 70 \\
\hline $\begin{array}{l}\text { uccinum LK. }= \\
\text { llata So. . . }\end{array}$ & & & & & & . 0 & \\
\hline$\ldots$ & $\therefore$ & $\ldots \ldots$ & & $\cdots$ & . 1 & $\cdots \cdots$ & \\
\hline$\ldots \ldots$ & . & $\ldots \ldots$ & $\ldots$ & $\ldots$ & . . $\boldsymbol{f}^{1}$ & $\therefore \cdots$ & \\
\hline ata So...... & $\cdots$ & $\ldots \ldots$ & . & $\ldots$ & $\ldots \int^{1}$ & $\ldots \ldots$ & 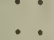 \\
\hline$\ldots \ldots$ & . . $M^{2}$ & $\ldots \ldots$ & . & $\cdots$ & $\ldots$ & t.... & $\therefore$ \\
\hline$\ldots .$. & $\ldots \ldots$ & $\ldots \ldots \ldots$ & $\ldots$ & $\ldots$ & $\cdots$ &.$t u \ldots$ & . \\
\hline D... & .... & $\ldots \ldots$ & $\ldots$ & $\cdots$ & ... & . u. . & $\cdots$ \\
\hline rella Wood . & . . . & $\ldots \ldots$ & $\ldots$ & $\ldots$ & .. & . u... & . \\
\hline a Conr. . . . & .. $\mathbf{M}^{2}$ & $\cdots \cdots$ & $\ldots$ & $\cdots$ & - & . u... & . \\
\hline OOD. & $\cdots$ & $\ldots \ldots$ & . & - . & $\cdots$ & . . и... & - \\
\hline $00 D \ldots$ & . . & $\ldots \ldots$ & 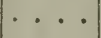 & $\cdots$ & . & . u. . & - \\
\hline ata Conr.... & . . $M^{2}$ & $\cdots \cdots$ & I & $\cdots$ & $\cdots$ & . . u... & • \\
\hline riata Bons. . & $\cdots$ & $\cdots \cdot$ & $\cdots \cdot$ & . $\cdot$ & $\cdot$ & . u. . . & - \\
\hline lata Grat. . & $\ldots \dot{M}^{2}$ & • & . . & . & & . и... & $\cdot \mathbf{z}$ \\
\hline $\mathrm{S}_{A Y^{\prime}} \cdots \cdots$ & $\begin{array}{l}\cdots M^{2} \\
\cdots M^{2}\end{array}$ & $\ldots \ldots$ & $\cdots$ & $\ldots$. & & $\mid \begin{array}{cc}\ldots u \\
\therefore u \\
\therefore\end{array}$ & $\cdot{ }^{\cdot z}$ \\
\hline $\begin{array}{l}\operatorname{ata} \mathbb{S}_{A Y} \ldots \\
\operatorname{ata} \mathrm{S}_{A Y} \ldots\end{array}$ & . . $\mathrm{M}^{2}$. & $\ldots \ldots$ & $\ldots$ & ... & $\cdots$ & 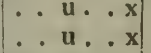 &.$z$ \\
\hline iora Bons. . . & $\ldots$ & $\ldots \ldots$ & $\cdots$ & $\ldots$ & .. & $\ldots w$. & $\cdots$ \\
\hline iformis Bors. . . & $\cdots \cdot$ & $\ldots \ldots$ & & - & & ... . พ. & - \\
\hline s. . . & $\ldots$ & $\ldots \ldots$ & $\ldots$ & $\cdots \cdots$ & $f^{\circ}$ & ... w w & . \\
\hline$\cdots$ & - & $\cdots \cdot \cdot$ & & . & & $\cdots \cdots$ & - \\
\hline a Bors. . . & & $\cdot$ & & & & .... w & $\cdots$ \\
\hline $\begin{array}{l}s \\
t_{1}\end{array}$ & & $\ldots$ & & & & $\cdots \cdots w$ & $\because$ \\
\hline Rs. & $\cdots \cdots$ & $\ldots \ldots$ & & $\cdots \cdots$ & $\cdots$ & $\left|\begin{array}{c}\cdots w \\
\cdots \cdots x\end{array}\right|$ & $\dot{p}$ \\
\hline СKL. . . & ..... & ... & & . & $\cdots$ & $\ldots \ldots x$ & \\
\hline Cyclope Risso 1. . & $\cdots$ & & & & $\cdots$ & $\ldots \ldots$ & .1 \\
\hline $\begin{array}{r}\text { neritea BR. } \\
\text { Eione Risso } \\
2 .\end{array}$ & 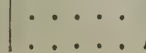 & 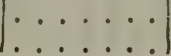 & & & & $\mid \begin{array}{c}\ldots \mathrm{wx} \\
\ldots \ldots\end{array}$ & in \\
\hline
\end{tabular}




\begin{tabular}{|c|c|c|c|c|c|c|c|}
\hline & Weltgegend. & KohlenP. & SalzP. & OolithP. & $\begin{array}{l}\text { Krei- } \\
\text { deP. }\end{array}$ & MolasseP. & Neu \\
\hline Benemuй:'и. & 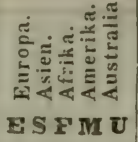 & 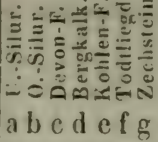 & 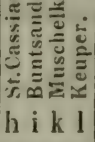 & 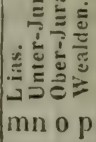 & 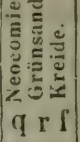 & $\begin{array}{l}\text { bo } \\
\text { st u v w X }\end{array}$ & 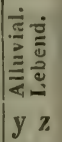 \\
\hline
\end{tabular}

Eione)

inflata Riss.

sulcata Riss.

LeudolivaSivains. 2 (Gastridiuu ForB.)

obtusa So.

aliue spp. sub Buccino lutent.

Eburna LK. 3 .

Brugadina Grat. . . spirata Lrí. glibrata PARK.

Litiopa Rang. 1 papillosa Wood.

Planaxis LK., Riss. 20 $-\alpha-$

‡ multisulcatus Мгст. striatus Grat. ... punctatus Grat.

$$
-\beta \text { - }
$$

0 imbricatus Rrss. mammillatus Riss.

o proxinus Prss.

\# lacvigatus Riss.

\# affiuis Riss. .....

0 Desmarestanus Riss.

₹ Donatianus Riss.

₹ Elfordianus [?] Piss.

- Fichtelanus Riss.

₹ Loques [?] Rrs

0 minutus Rrss.

F Mollanus Riss.

‡ riparius Riss.

\# rosaceus Riss.

0 tenuis Riss. .

o torulosus Riss.

0 trifasciatus Risso..

Terebra ${ }^{*}$ A D. LK. 35. granulata PHiL.. melanioides PHILL.

\# mitriformis Phizl. vittata Morrs. [non LK.]

Portlandica So. minuta NG. coronata So. constricta LEA

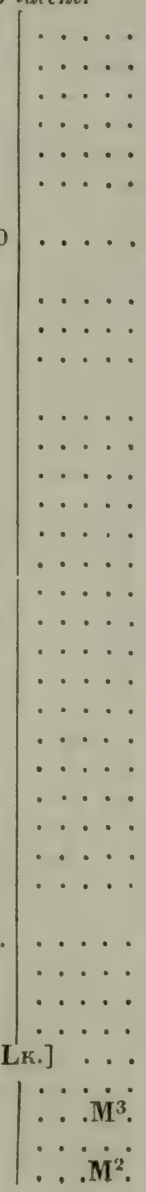


costala LEA laevigata ANT. multiplicata LEA perlata ConR. Vulcani BuGN. plicatula Lr. Basteroti Grat. bistriata Grat. canalis Wood costulata Bors. heterostropha WooD inversa NYsT Lamarcki DrR. melaniana Grat. modesta Trist. murina BAsT. striata BAst. reticulata So. simplex Conr. acuminata Bors. acuminata Grat. fuscata Br. pertusa Bast. strigilata LK. subulata Lr. costata Bors. dislocata Conr.

i Volutina.

olenta LK. 97. clatlirata Peuss acuta So. ambiurua MANT. antiqua Brod. deperdita GF. elongata n'0. Gasparini D'O. Guerangeri D'O. Lahayesi D'O. piruloides MathN. Renauxana $D^{\prime} \mathbf{O}$. Requienana D'O. acuta DFr. alfuis Brocc. ambigua $\mathrm{L}_{\mathrm{K}}$. angusta DsH. athileta So. bicorona Lr. Branderi DFr. bulbula LK. cingulata NysT cithara Lr.

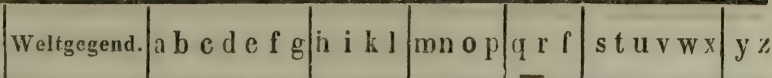

. $\mathrm{M}^{2}$.

. $\mathrm{M}^{2}$

$\mathrm{NI}^{2}$.

$\mathrm{M}^{2}$ 
Benenกแกตั.

\begin{tabular}{|c|c|c|c|c|c|c|}
\hline Weltgegend. & KohlenP. & SalıP. & OolithP. & $\begin{array}{c}\text { Krei- } \\
\text { deP. }\end{array}$ & MolasseP. & Neu \\
\hline 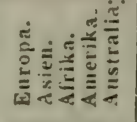 & 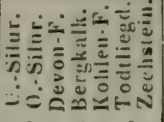 & 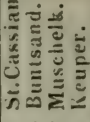 & 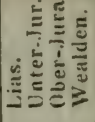 & $\begin{array}{ll} \\
0\end{array}$ & 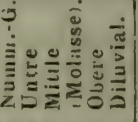 & 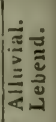 \\
\hline ESF $\mathrm{MU}$ & $a b c d e f g$ & h i k l & mn op & $q \times$ ? & st u v w x & $y z$ \\
\hline
\end{tabular}

Volufa)

costata Brand.

denudata So.

depauperata So.

digitalina LK.

dubia LEA.

plevata So.

\# fusiformis DrR. geminata So .

\# het. roclita LK.

labrella $\mathrm{Lr}$.

lincolata Dsh.

luctator So.

Jyra Lri.

₹ mammosa DFr.

mitrata Dsh.

mitreola LK.

mixta Nyst-

multistriata $\dot{\mathrm{D}}_{\mathrm{SH}}$.

muricina Lk.

mutita Dsh.

nodosa So.

Parkiusoni LE

pertusa Swains.

petrosa CONr.

? pirta DrR.

plicatella Dsr.

prisca (LyelL).

protensa So.

Sayana Conr.

scularis So.

? semigranosa NYST semiplicata Nyst simplex Dsн. spinosa LK.

striata LEA .

suspensa So.

suturalis Nyst

torulosa Dis.

tricorona So.

trisulcata Dsh.

turgidula Dsh.

variculosa $\mathrm{LK}$.

ventricosa DFR.

Wetherelli So.

† crassicosta Dsh.

crenulata LK. 


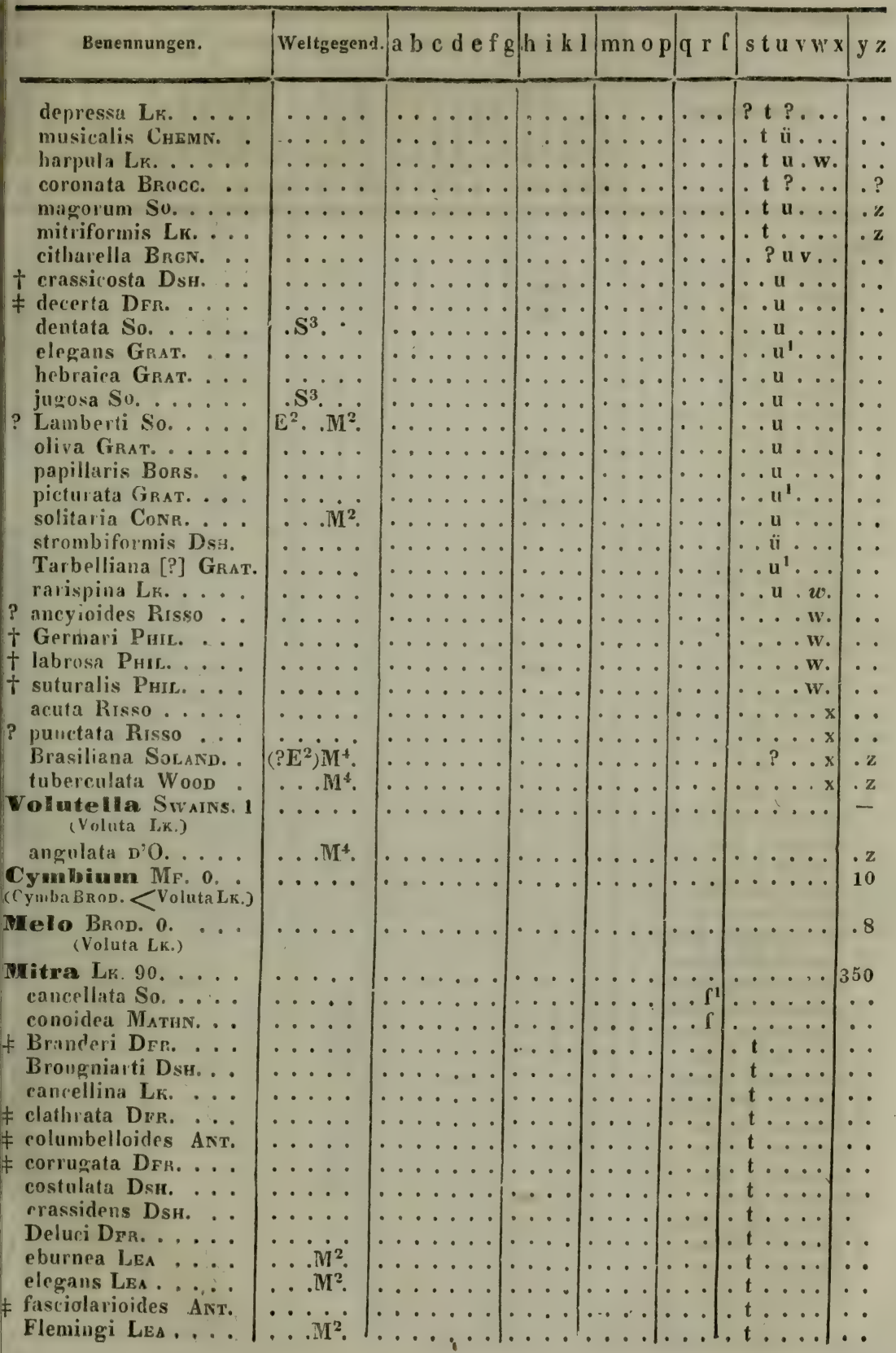




\begin{tabular}{|c|c|c|c|c|c|c|c|}
\hline & Weltgegend. & KohlenP. & SalzP. & OolithP. & $\begin{array}{c}\text { Krei- } \\
\text { deP. }\end{array} \mid$ & MelasseP. & $\mathbf{N}$ \\
\hline Bencnnungen. & 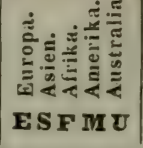 & 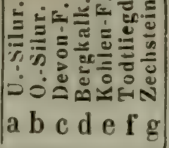 & 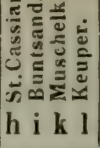 & 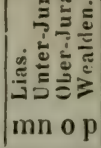 & 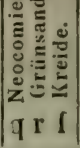 & 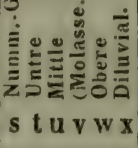 & \\
\hline
\end{tabular}

Mitra)

fusellina Lr.

fusoides LEA

gracilis LEA

graniformis LK.

Jabratula LK.

labrosa Dsh.

laevissima Grat.

Lajoyei Dsh.

lineata LEA

marginata J K.

minima LeA.

mixta Lr.

monodonta LK.

obliquata Dsr.

pactilis Conr.

Parisiensis Dsh.

parva So.

perexilis Conp.
plicatella $\mathrm{LF}$.

pumila So.

raricosta Lr.

scabra So.

‡ vulpina AnT.

crebricosta LK. ...

elongata LK.

mutica $\mathrm{LF}_{\mathrm{i}}$.

subplicata Dsh.

terebellum $\mathrm{L}$.

Bourguetana Grat. .

t cancellata Bonel. clavatularis Grat.

t columbellata Dsh. cupressina DFr. decussata Dus. Dufresnci Bast.

+ elegans Partsch eburnea Grat. fusiformis So. nassoides Grat.

? nodosa Bors. oliviformis Dus.

₹ pliciferit Wood pupa Dus. rissnides Grat. striola Bon. subcylindrica Dus. .

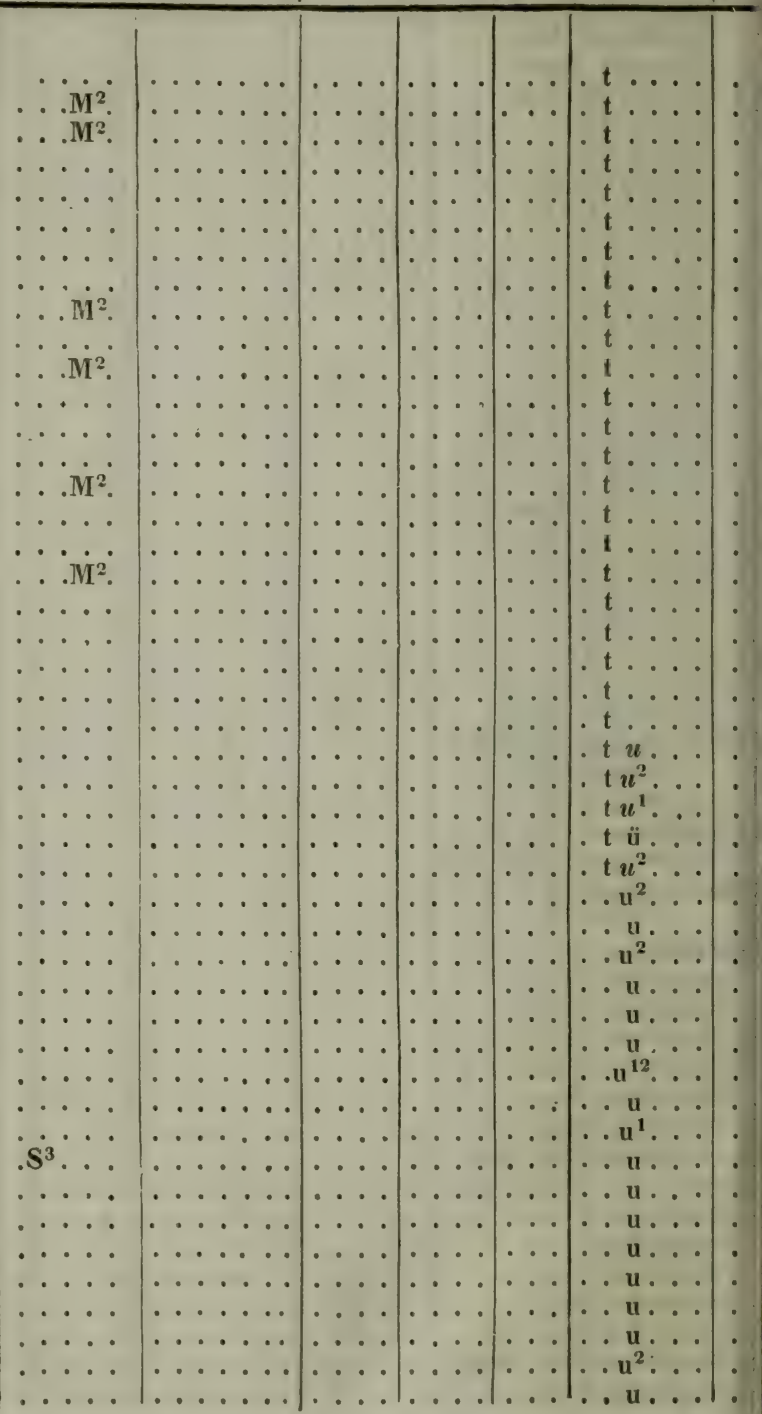




\section{.}

Benennungen.

Weltgegend. $\mid$ a b c d e f g h i k l mn op q r f $\mid$ s t u v w x y z

subulata (? Brocc.) Gipat. tenuistria Dus. turgiclula Grat. ventricosa Grat. scrobiculata DFr. fusiformis Risso pyramidella $\mathrm{Lr}$. striatula Grat.

† biplicata $\mathbf{P}_{\text {HLL. }}$.

₹ Leonardiana [?] Risso obsoleta Br.

† pseudopapalis Micht.

t pupa Bon.

t rugosa Phic.

? scalariformis Bors.

t simplex PhiL. columbellaria Scacc. lutescens Lr. tricolor Gr. $s p$.

$\mp$ Adolphia [?] Risso.

\# Boruana Risso ...

F ventricosa Risso burcinoidea Risso

f corniculum Risso.. costulata Risso.

+ inflata Risso

F litoralis Risso

\# media Risso:

F punctulata Risso

(Mitrella Risso) 3.

Mitrae et Buccini spp. L.K. costulata Risso

F laevigata Risso

flamminea Risso

Irarginella Lк. 32 ovulata LK. angystoma Dsh.

biplicata LEA

columba LEA constricta Conr. crassilabris CoNr. dentifera $\mathbf{L}$. hordeola Dsh. humerosa Conf. incurva LEa larvata Conr. nitidula Dsh. pineum Br. plicata LEA semen LEA eburnea $L_{\kappa}$. phaseolus Brign.

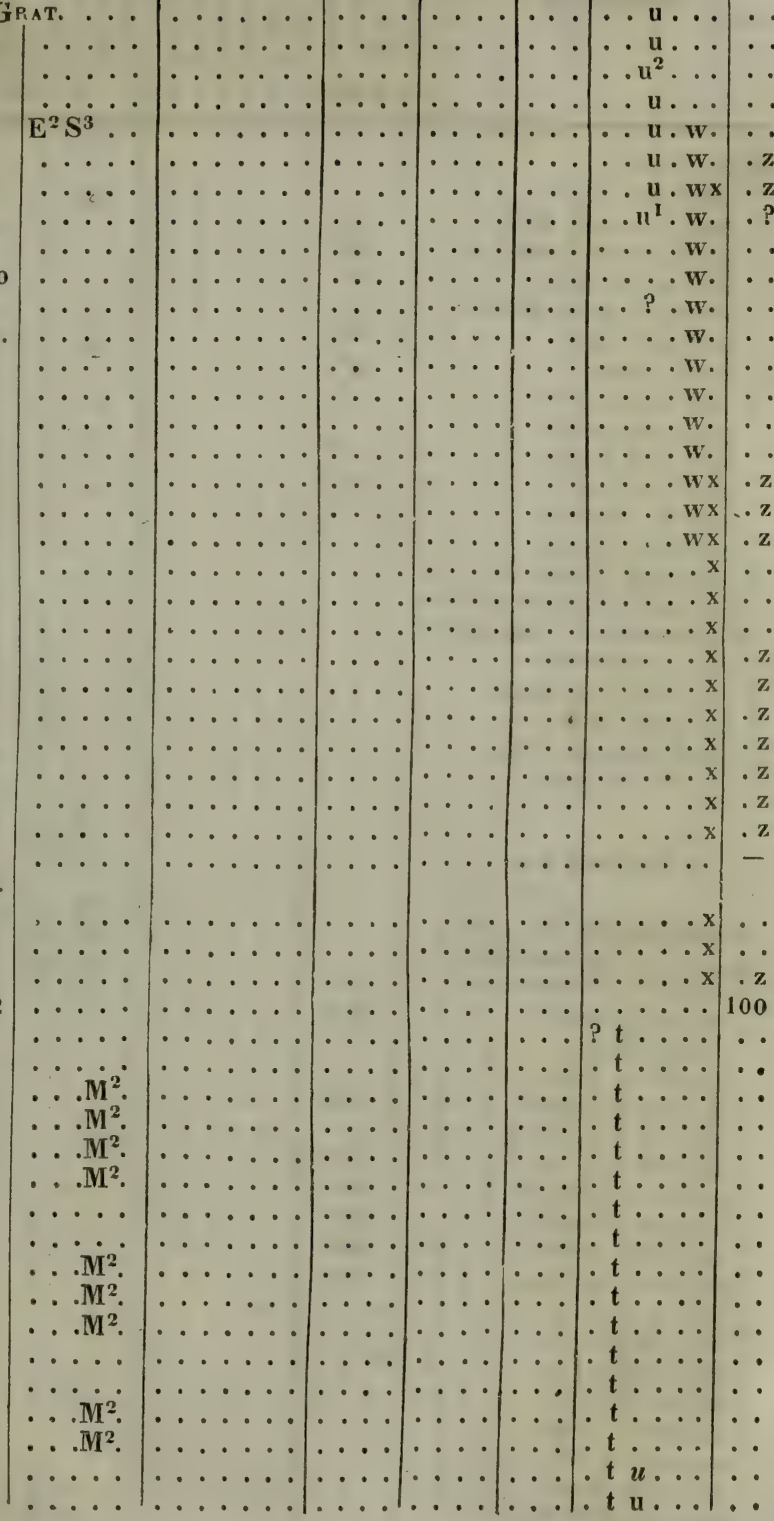




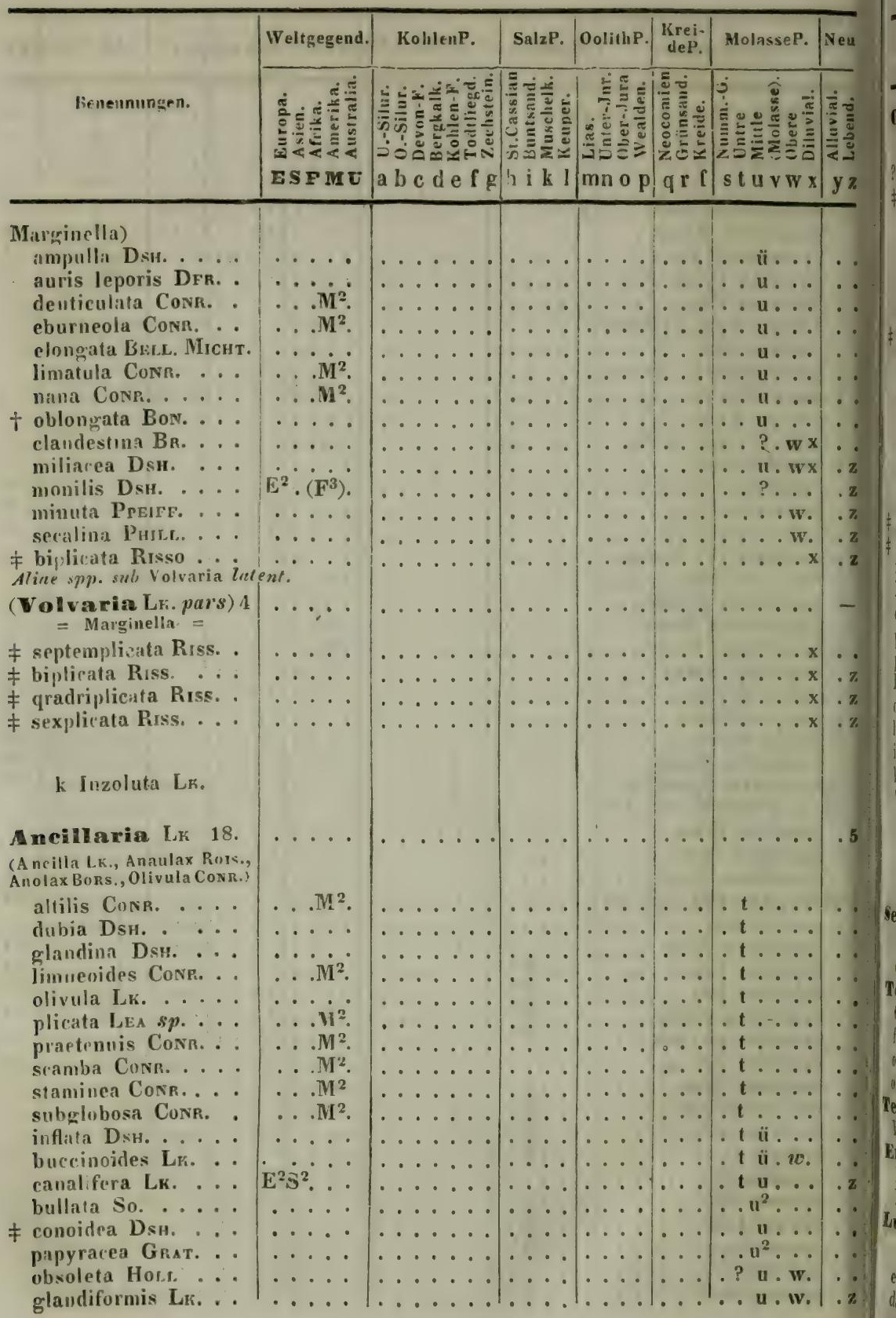




\begin{tabular}{|c|c|c|c|c|c|c|c|}
\hline Benennun & di. & a b & & p & & $\mathbf{w} \mathbf{x}$ & $y$ \\
\hline $\begin{array}{l}\text { Liva L } \\
>^{0 l i v a}\end{array}$ & $\cdots$ & $0^{\circ}$ & & $\left.\right|^{\circ}$ & & $\cdots$ & 12 \\
\hline$\cdots$ & & & h & & $\cdots$ & • $\cdot$ & \\
\hline ONR. & $\ddot{\mathbf{M}^{2}}$ & $\dot{0}$ & . & : & & l & \\
\hline s . & . & . & $\ldots$ & $\ldots$ & $\therefore$ & $\ldots$ & \\
\hline$\cdots$ & & $\cdots$ & $\cdots \cdot$ & $\cdots \cdot$ & $\cdots$ & . t $\ldots$ & \\
\hline$\cdots$ & & $\cdots$ & & & & $\cdots$ & \\
\hline$\cdots$ & & & & $\cdots \cdot$ & $\cdots$ & $\cdots$ & \\
\hline$\cdots$ & & & & ・ & $\cdots \cdot$ & $\cdots$ & \\
\hline 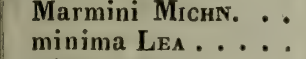 & $\cdots \dot{M}^{2}$ & $\ddot{m}$ & & $\cdots$ & $\cdots$ & $\cdots$ & \\
\hline$\therefore$ & .... & $\dot{.}$ & $\left|\begin{array}{c}\cdots \\
\cdots \\
\cdots\end{array}\right|$ & $\cdots$ & $\cdots$ & $\cdots$ & \\
\hline$\therefore$ & . & $\ldots \ldots$ & $\ldots$ & & $\because$ & $\cdots$ & \\
\hline . & $\cdots$ & $\ldots \ldots$ & $\ldots$ & $\ldots$ & $\cdots$ & $\mid \begin{array}{l}0 \\
0\end{array} \ldots$ & \\
\hline$\cdots$ & $\cdots \cdots$ & $\ldots \ldots$ & $\ldots$ & $\ldots$ & . . & t $\ldots \ldots$ & \\
\hline & $\mathrm{M}^{4}$ & $\ldots \ldots$ & $\ldots$ & $\ldots$ & ... & t.... & \\
\hline . & . & . & $\ldots$ & $\ldots .$. & $\ldots$ &. $\mathrm{t} \ldots$ & \\
\hline$\cdots$ & & $\cdots \cdot$ & $\ldots$ & $\cdots$ & $\ldots$ & . t .... & \\
\hline$\cdots$ & E. & $\cdots \cdots$ & $\cdots$ & $\cdots$ & $\cdots$ & $\mid . t \mathrm{u} \ldots$ & $\sigma_{n}$ \\
\hline$\cdots$ & & $\cdots \cdots$ & $\ldots$ & $\cdots \cdot$ & $\ldots$ & $\cdots$ & .? \\
\hline$\cdots$ & & $\because$ & $\mid \begin{array}{c}\cdots \\
\cdots\end{array}$ & $\mid \begin{array}{cc}\cdots \\
\cdots\end{array}$ & $\ddot{\cdots}$ & $\because \begin{array}{ll}\cdots \\
\cdots\end{array}$ & \\
\hline . & $\dot{S}^{3} \ldots$ & . & - & . & $\cdots$ & $\ldots u \ldots$ & \\
\hline . & -1 & $\cdots$ & $\ldots$ & $\cdots$ & . & w. & .? \\
\hline & & $\ldots \ldots$ & $\cdots$ & $\cdots \cdot$ & $\ldots$ & $2 .$. & $\cdot \mathrm{z}$ \\
\hline$\cdots$ & & $\ldots \cdots$ & $\cdots$ & $\cdots \cdot$ & $\ldots$ & ....... & . $z$ \\
\hline & & • & . & $\cdots$ & 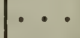 & $\ldots$.... & . ? \\
\hline & $\mathrm{U}^{2}$. & - & $\cdots$ & $\cdots$ & $\cdots$ & ..u. u. w. & . $\mathrm{z}$ \\
\hline$\cdots$ & - & - & $\cdots$ & . & $\cdots$ & $\ldots u \ldots$ & . $z$ \\
\hline & & $\therefore$ & & . & . & $\cdots \mathbf{u} \ldots$ & . ? \\
\hline$\dot{.}$ & $I^{2}$ & $\because$ & $\cdots$ & 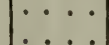 & $\cdot \cdot$ & ... u... & . $\mathrm{z}$ \\
\hline N. & $\mathrm{M}^{4}$. & $\ldots \ldots$ & $\therefore$ & $\therefore$ & - & $\cdots \cdots x$ & $\mathrm{z}$ \\
\hline N. & . & $\ldots \ldots$ & $\cdots$ & $\mid \begin{array}{c}\cdots \\
\cdots\end{array}$ & $\cdots$ & $\mid \begin{array}{cc}\cdots & \cdots \\
\cdots & \cdots\end{array}$ & $\begin{array}{l}. z \\
.1\end{array}$ \\
\hline & & & & & & & \\
\hline & & & & & & . t ... & \\
\hline & . & $\cdots$ & - & & . & $\cdots$ & .2 \\
\hline & $\cdots$ & $\cdots$ & & $\cdots$ & $\cdots$ & & $\cdot$ \\
\hline & & - & & $\cdot \cdot$ & & . & s \\
\hline $\begin{array}{l}0 \\
0\end{array}$ & & & & & & $\left|\begin{array}{ccc}\mathrm{t} & \ldots & \cdots \\
\cdots & \mathrm{u} & \ldots\end{array}\right|$ & \\
\hline SYI & M. 1. & $\cdots$ & $\cdots$ & $\cdots$ & $\cdots$ & $\ldots \ldots$ & .0 \\
\hline & & $\ldots$ & $\ldots$ & $\ldots$ & $\ldots$ & . t... & \\
\hline . & . & ... & ... & $\ldots$ & ... & $\ldots$ & .8 \\
\hline & & $\cdots$ & & & $\cdot 1$ & . $u \cdot w \mathbf{w}$ & . $z$ \\
\hline & & $\ldots \ldots$ & & & $\cdots$ & . u... & - $x$ \\
\hline $\begin{array}{l}2 . \\
\text { LkK.) }\end{array}$ & - & $\ldots \ldots$ & - & $\cdots$ & $\cdots \cdot$ & . $\cdots$ & - \\
\hline & & & & & • & . 1 & $\bullet$ \\
\hline
\end{tabular}




\begin{tabular}{|c|c|c|c|c|c|c|c|c|}
\hline $\mathrm{t}$ & $1+16$ & Weltgegend. & KohlenP. & SalzP. & OolithP. & $\begin{array}{c}\text { Krei- } \\
\text { deP. }\end{array}$ & MolasseP. & Neu \\
\hline 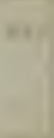 & Kenennuหg gat. & 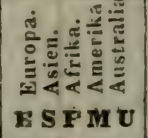 & 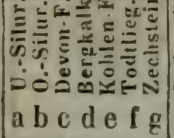 & 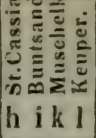 & 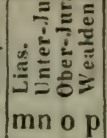 & 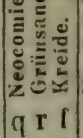 & 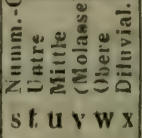 & 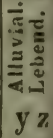 \\
\hline
\end{tabular}

Trivia Grax 11. = Cypraeae spp. Lik. affinis WooD

+ Angliae Wood avellana Grax Duclosana Grax

t globulosa Wood.. retusa Gray

+ testudinella WooD coccinella Guar Europara Gnax dimitiata $n$. sphaericulata Gray

Cypraea (L.) Lк. 77 (incll. Triviae spp. el Luponia GraY)

* spp. utrinque transversim sulcatue. = ? Trivia GRAX = crenata Dsh.

Lamarcki Dsw. oryza Lא. $\mid \begin{gathered}\cdots \\ \mathrm{E}^{2}\left(\mathbf{F}^{3}\right)\end{gathered}$ pulex Soland...... Trivia Grax nucleus LGr. pustulita Lr. .... staphylea LGm.

*k*spp. lneves (et residuree) bullaria Schlth. . Marticensis $\mathrm{M}_{\mathrm{ATnN}}$. spirata $\mathrm{S}$ снг,тн. depressa So.

$\neq$ antiqua LK. columbaria Lx. exerta Dsh.

$\neq$ gibbosa ANT. Levesquei Dsh. media Dsh.

₹ ruderalis $\mathbf{L K}_{\mathrm{K}}$. rugosa Grat.

\# spirata Grat. subrostrata GRAY truncata Br. angystoma Dsн. amygdalum Broce. Brocchii Dsh. inflata $\mathbf{L}_{K}$.

- ambigua Lк. amygdalina Grat.

* Andegawensis DFR. annularia BRGN.

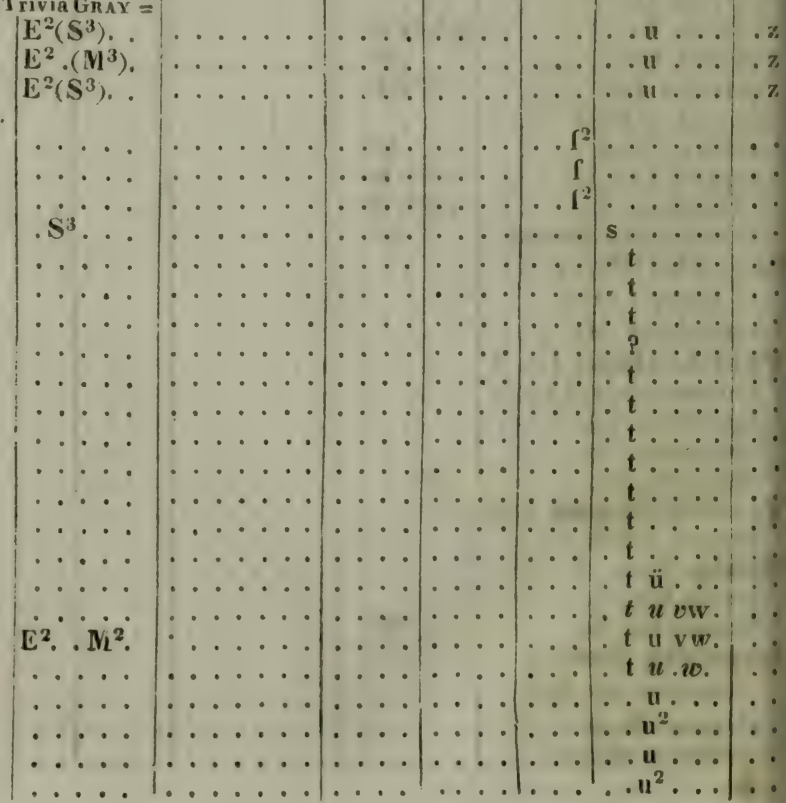




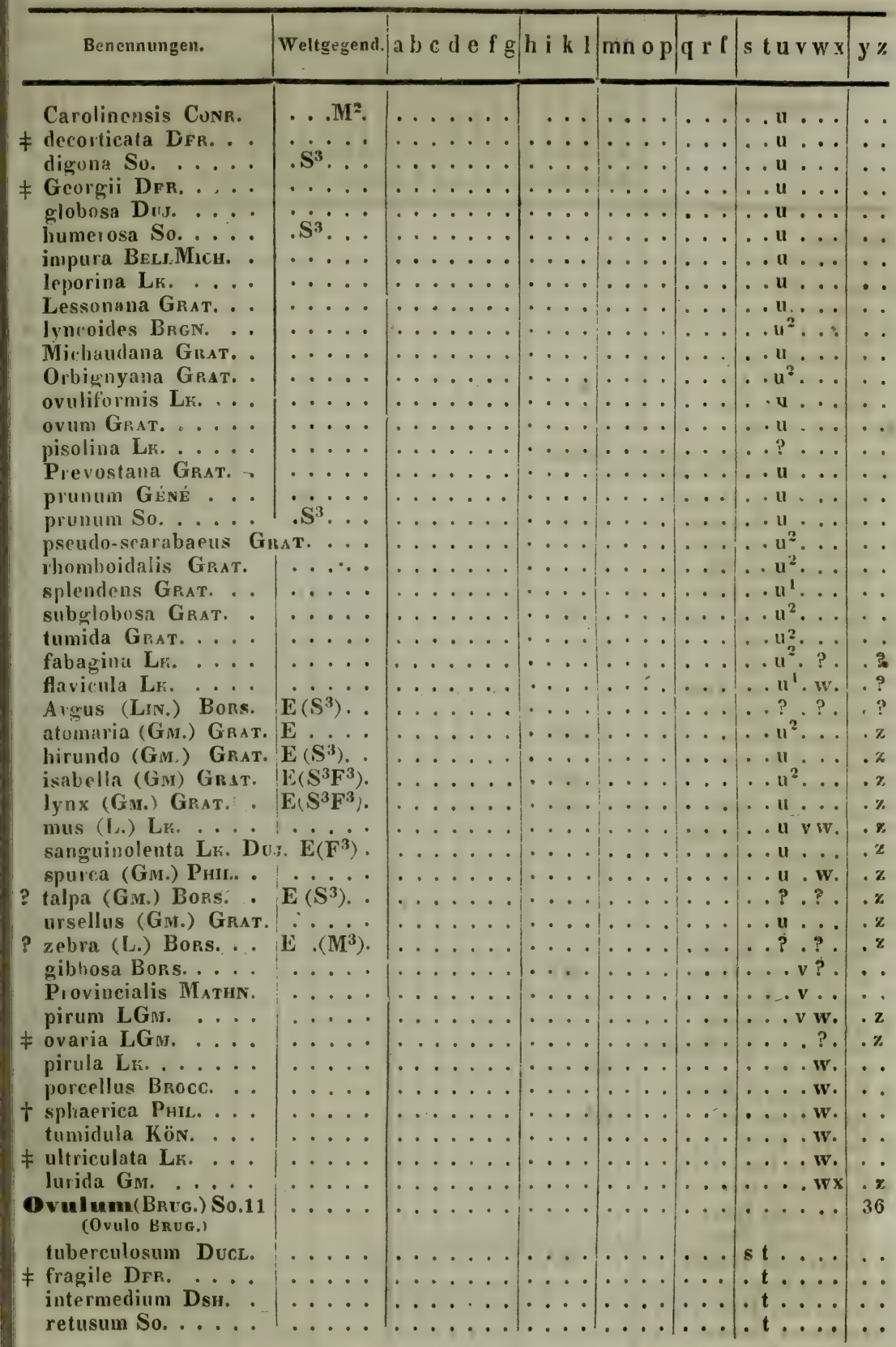




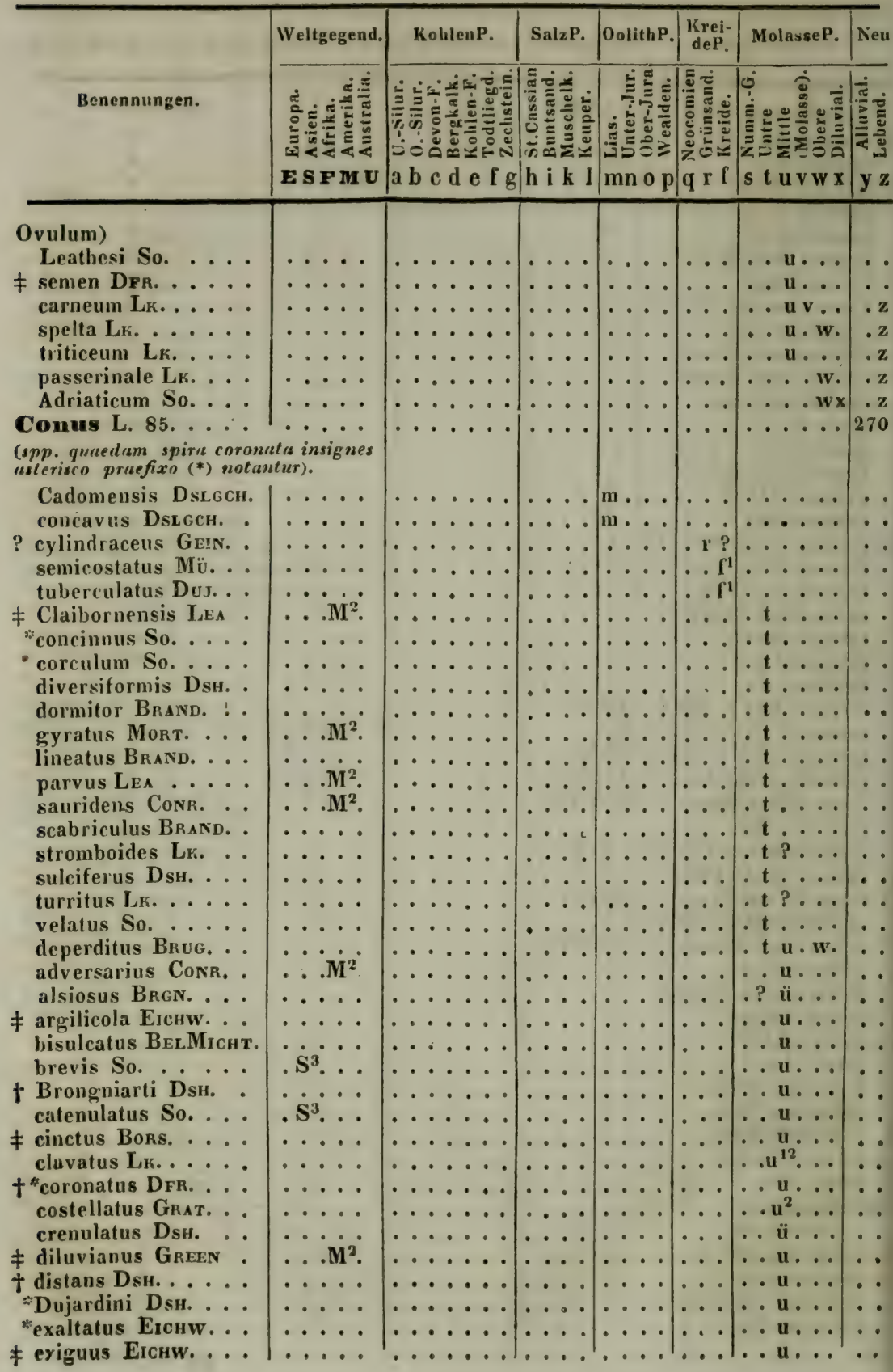


Benennungen. Weltgegend. a b c d e f g h i k l $\mid$ mnop q r f s t u v w x y \% granuliferus Grat. marginatus So.

\# Marylandicus Green militaris So.

$\neq$ parvus Bors. pseudo-litteratus Grat. pseudo-textile Grat. raristriatus BELLMichт strombellus Grat. Tarbellianus [?] Grat. trigonulus Grat.. zonarius GraT. acuminatus Bors. Aldrovandi Broce. avellana Lri.

Baldichieri [?] Bors. betulinoides LK. intermedius LK. ventricosus $\mathrm{Br}$.

$\neq$ lineatus Bors.

figulinus (Lк.).

*imperialis (GM.) Bं. $\mathbf{E}\left(\mathbf{S}^{3}\right)$. informis (LK.) Bors. $\mathbf{E} .\left(\mathbf{M}^{3}\right)$. maculosus Grat.

Mediterraneus Brug.

"Nicobaricus (Lк.) Grat. $\mathbf{E}\left(\mathbf{S}^{3}\right)$. *nosturnus (Lr.) Grat. $\mid \mathbf{E}\left(\mathbf{S}^{3}\right)$.

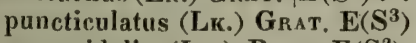
pyramidalis (Lк.) Bors. $\mathbf{E}\left(\mathbf{S}^{3}\right)$. striatulus Brocc. tessellatus (Brug.) Grat. $\mathbf{E}\left(\dot{\mathbf{S}}^{\mathbf{3}}\right)$ virgo (GM.). Bors. . $\mid \mathbf{E}\left(\mathbf{S}^{3}\right)$.

*Marticensis MAThN. antediluvianus Brug. Brocchii Br. canaliculatus Broce. F coloratus DFr.

₹ demissus Phir. elongatus Bors. fulminans $B R$. fusus Bors. . . granularis Bons.

+ Pedemontanus DFr. pelagicus Brocc... semisulcatus Br. glaucus (L.) Bors. . textile (Gm.) BM.

f corona Riss. ‡ postdiluvianus Riss. Siphonobranchiorum summa: 2404 


\begin{tabular}{|c|c|c|c|c|c|c|c|}
\hline & Weltgegend. & KohlenP. & SalzP. & WolithP. & $\begin{array}{c}\text { Krei- } \\
\text { deP. }\end{array}$ & MolasseP. & Neu \\
\hline B. nennungeu & 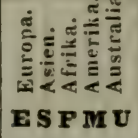 & 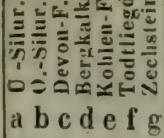 & 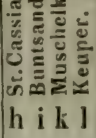 & 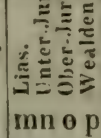 & 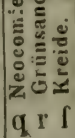 & 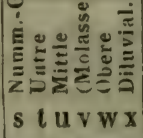 & 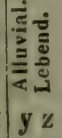 \\
\hline
\end{tabular}

\section{POMATOBIRANCWIAA WIEGM.}

(Tectibranchia Cuv.; Monopleurobranchia

Buv, ; Crypsibranchia Ag. et Pomato. branchia AG.)

1. ACERA LK, (Cuv.)

a Genera testa munitu.

Halia Riss. 1. ... stercus-pulicum . . .

Bullina Fér. 7. . (Alicula kichw.)

crassa Br. . . . . . spirata....... Okeni Erasw. sp. Wetherilii Grat... Ustürtensis Егснw. canaliculata $\mathrm{S}_{\boldsymbol{A} Y}$... secalina FER. . . . nlice spp. athuc sul) Bulla mililunt.

EEulla (L.) FéR 70 . (Scaphander MF,)

* spp. spuriace spirre exserla = Bullina FÉR. =

? oliviformis KoDu. spirata Roe. subquadrata RoE. striatella Lk. . . . . nana Woop..... mamnillata PHiL. terebelluides Phiz.

** spp. genvinue spira in. clusu, el incertue.

elongata Phic.. ... . Hildesiensis RoE. . . suprajurensis RoE. . undulata Bean ... Mantellana So.... Mortoni Lyell Fons. ambigua $D^{\prime} O$..... attenuata So. .... conica Dsh. 


\section{Benennungen.}

\begin{tabular}{|l|l|l|l|l|l|l|}
\hline Weltgegend. a b c d e f $g \mid$ h i k l & mn op & q r l & s t u v w x & y \%
\end{tabular}

coronata Lk. cylindroides DsH. Dekayi LEA filosa So. globulus Dsh. Hilairei LEA . ovulata Lri. plicata Dsu. semistriata Dsh. Sowerbyi Nyst angystoma Dsh. Brugnierei Dsh. constricta So. conulus Dsh. elliptica So. miruta Dsh. lackis DFr. lignaria $\mathrm{L}$. miliaris Brocc. Bronni Mrr. cancellata Grat. concinna Woop crassatina Grat. キ elongata Егснw. fallax Grat. .

† inflata Erchw. labrella Fón. marginata Grat. plícatula Grat. quadrata Wood

0 sculpta Woup Tarbelliana Grat. ventrosa $W_{0 O D}$ acuminata Brug. Brocchii $\boldsymbol{n}$. convoluta Broce. cornea $\mathrm{LK}_{\mathrm{K}}$. cylindracea Moneg. truncata Montg. truncatula Brug. utrieultis Brocc. subumbilicata M MTHN. hydatis Gu.

† apicina PHIL.

† dilatata Pнis. elongata $\mathrm{B}_{\mathbf{R}}$,

\# hyalina FÉr. intermedia PhiL. laevis ArMag. lineata PHIL. retusa Pusl. teretiuscula Purr.

$\dot{M}^{2}$.

$\mathrm{M}^{2}$.

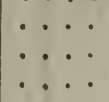

$\cdots$

$\cdots \cdots$

$\mathrm{S}^{i 3}$

$\mathrm{E}^{12}$

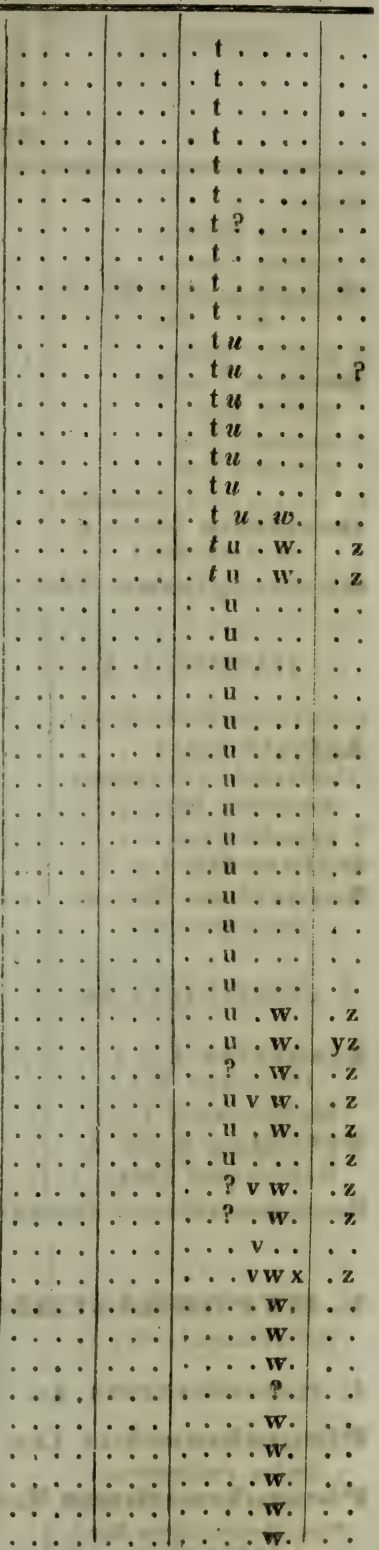




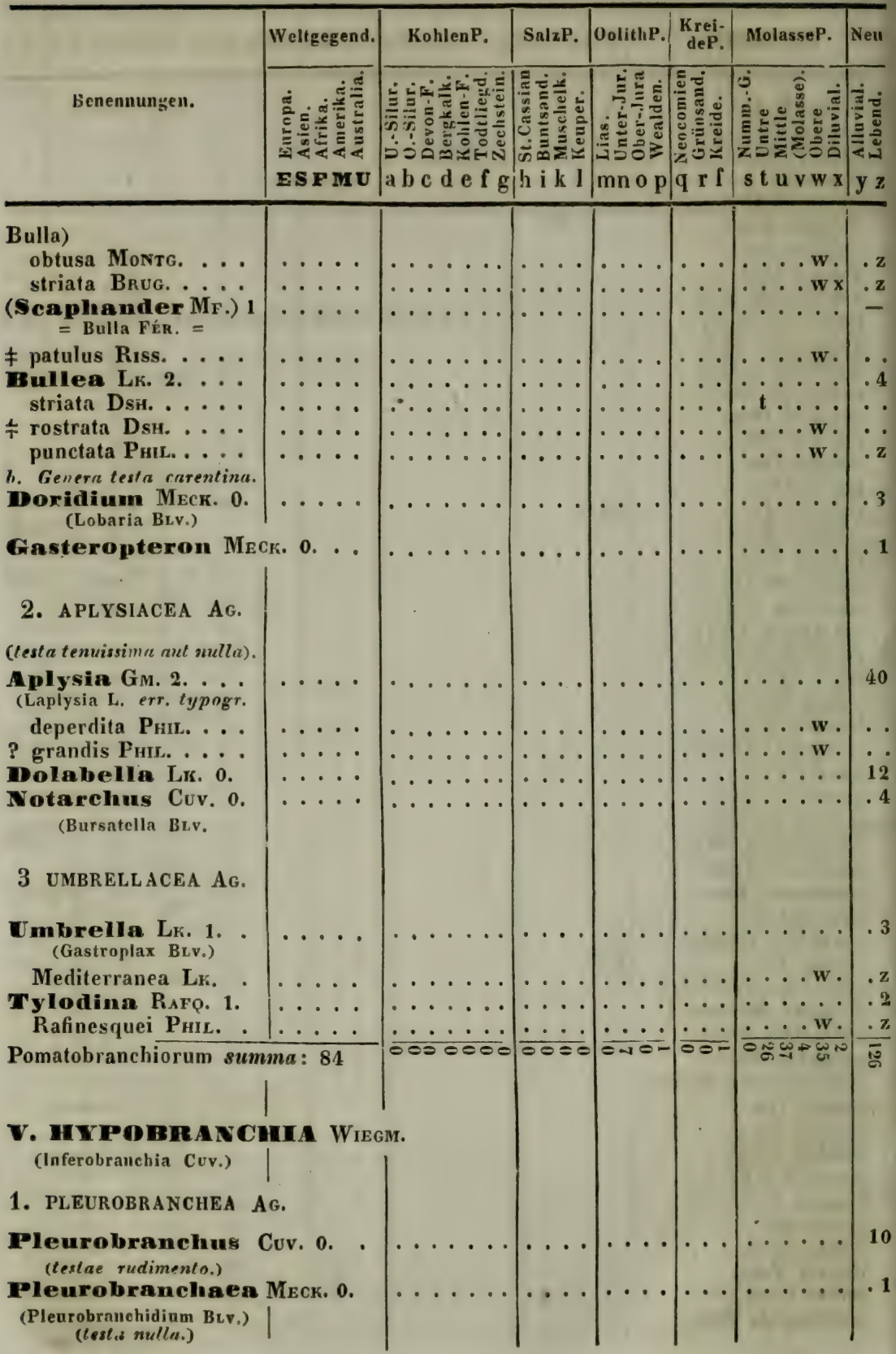




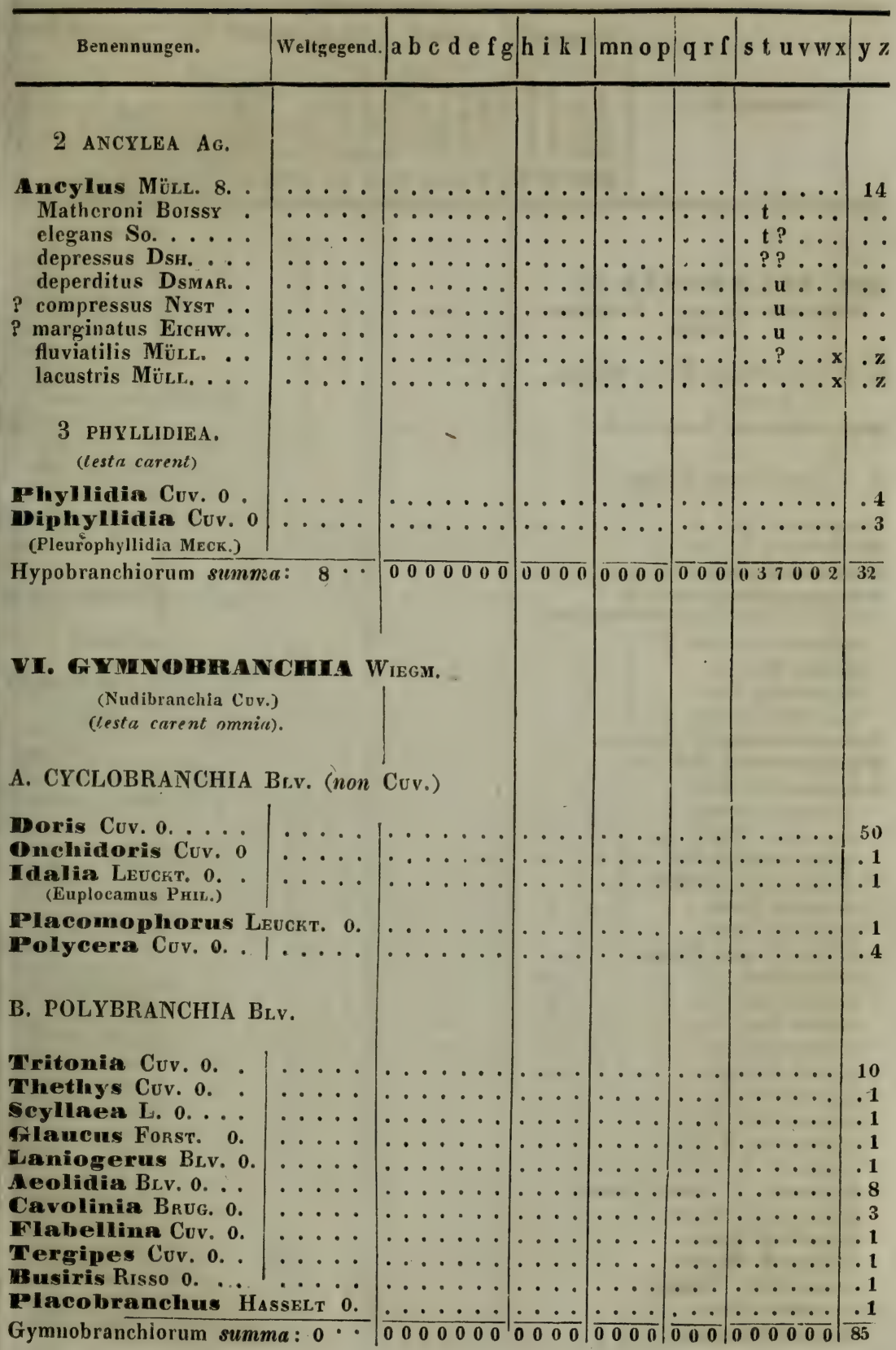




\begin{tabular}{|c|c|c|c|c|c|c|c|}
\hline & Weltgegend. & KohlenP. & Sal $/ \mathrm{P}$. & OolithP. & \begin{tabular}{|c|} 
Krei- \\
dcP.
\end{tabular} & MolasseP. & Neu \\
\hline Benenuungen. & 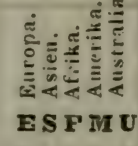 & 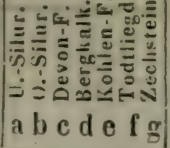 & 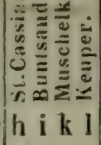 & 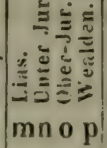 & 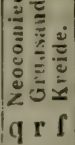 & 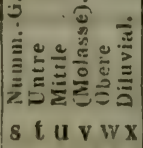 & 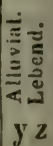 \\
\hline
\end{tabular}

mit. PUEmonatea Cuv.

(Pulnobranchia Buv.; Coelopuoeal SCHwEIG.)

A. AMPHIP NEUSTIA WIEgm. (Nuda.)

\section{Grehidium Bucuan, 0 ;...}

B. OPERCULATA Fír.

Pupina Vign. 0.

Melicina $L$ r. 0 . .

Cilontostoma D'O.0

Ferussacise laner. 4.

(Ferussina Grax, ; Strophostoma Dsh.)

anostomaeformis [?]

striata BR.

tricarinata Br.

lapicida Leufr.

Stegrengtoma TuOSUH.

Cyclostona LK. 41

0 keuperiana Нвн.

Arnoudi Michd.

conoidea Borssy ... cornu-pastoris LK, .

? eleqantilites Bośbé elongata SERr. . . . exravata Serr. . . . heliciniformis Borssy inflata Dsh.

\# macrostoma Ls. mumia LK. planorbula

f plicata Veren. spiruloides $\mathrm{I}$. . . . clegans Dripd.... abbreviata MATHN. Aquensis MithN. .. bisuleata (? $\mathbf{Z}_{\text {IET. }}$ ) Tном. . . bulimoides MАтнN. cancellata Grat. Coquandi MathN. crassilabris MathN.

\% decussata Bos. disjuneta Mavнv.

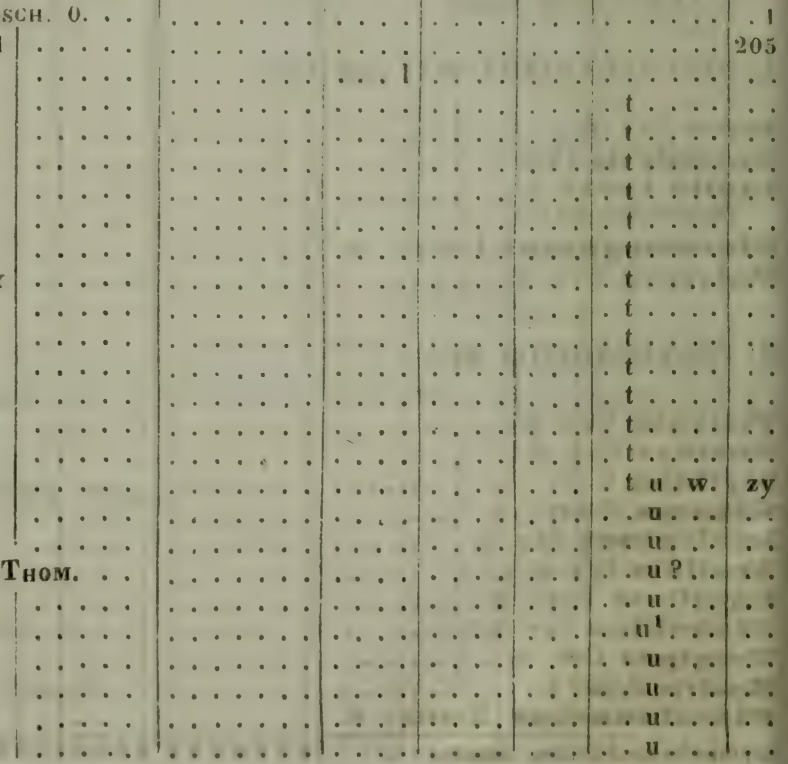


Benennungen. Weltgegend. a b c d e f g h i k l mn op q r f s t u v w x y z

dolicim Thens.

F gregaria Br. heliciformis Mathn. labellum Tном.

Lemani Bast.

Luneli MATHs. microstonia Dsh. solarium MathN. truncata Briad \# subcarinata Michт. bisulcata ZieT. glabra Schüвs. Draparnaudi Mathon. ? delicata PHIL. Serresana Mithr. + affinis Riss. maculatum DPPD.

Nematura Buss. 1. fossilis So.

C. HYDROPHILA FÉR.

a Limnaeaces.

Tanorbis Mür.L. 62

o vetustus Creds.

Jugleri Du.

sp. So.

ammonitiformis $\mathrm{S}$ crassis Serp.

cylindricus So. depressus Nyst inflatus Dsh. inversus Dsh. laevigatus DsH. nitidulus $\mathrm{LK}_{\mathrm{r}}$. obtusus So. planulatus Dsir. planulatus Serr. . Sowerbyi Bp.

Sparnacensis Dsh. Spratti Fons. subangulatus Lr. subanģulatus Dsh. cornu Brgen. euomphalus So. hemistoma So. lens Brgn. Prevostinus Brian. rotundatus BRGN. subovatus DsH. ‡ aunulatus Bovil.

(1)

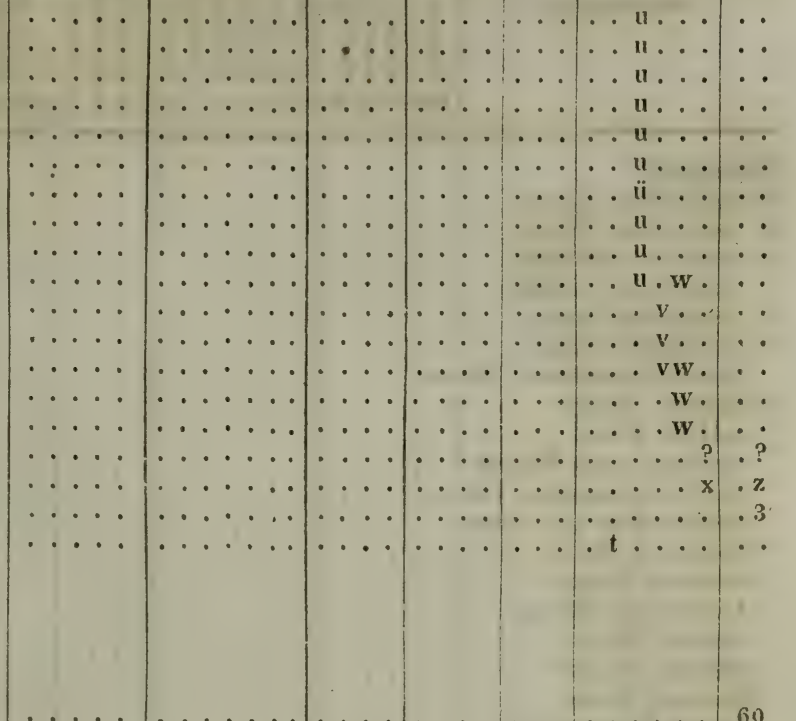




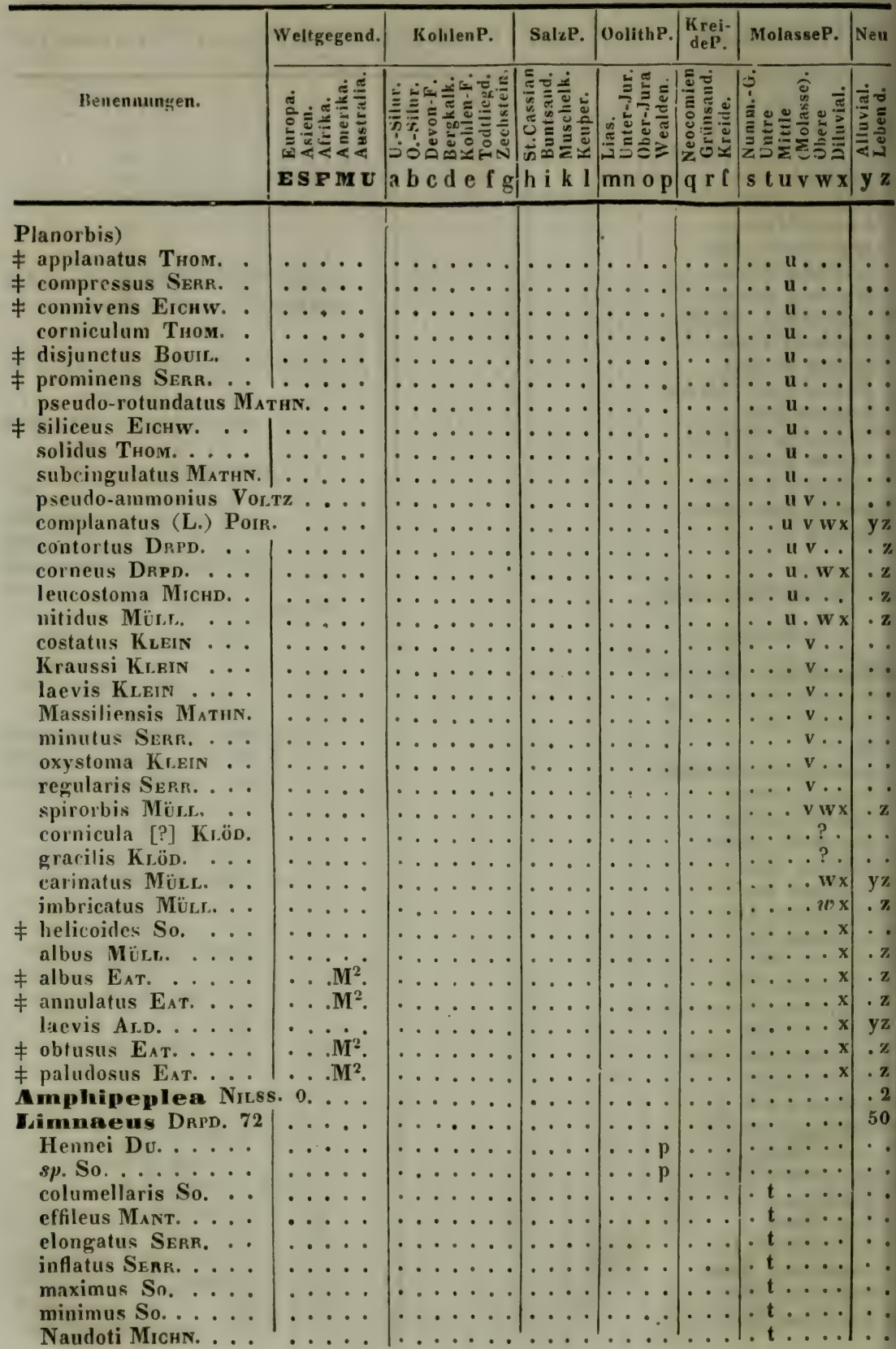




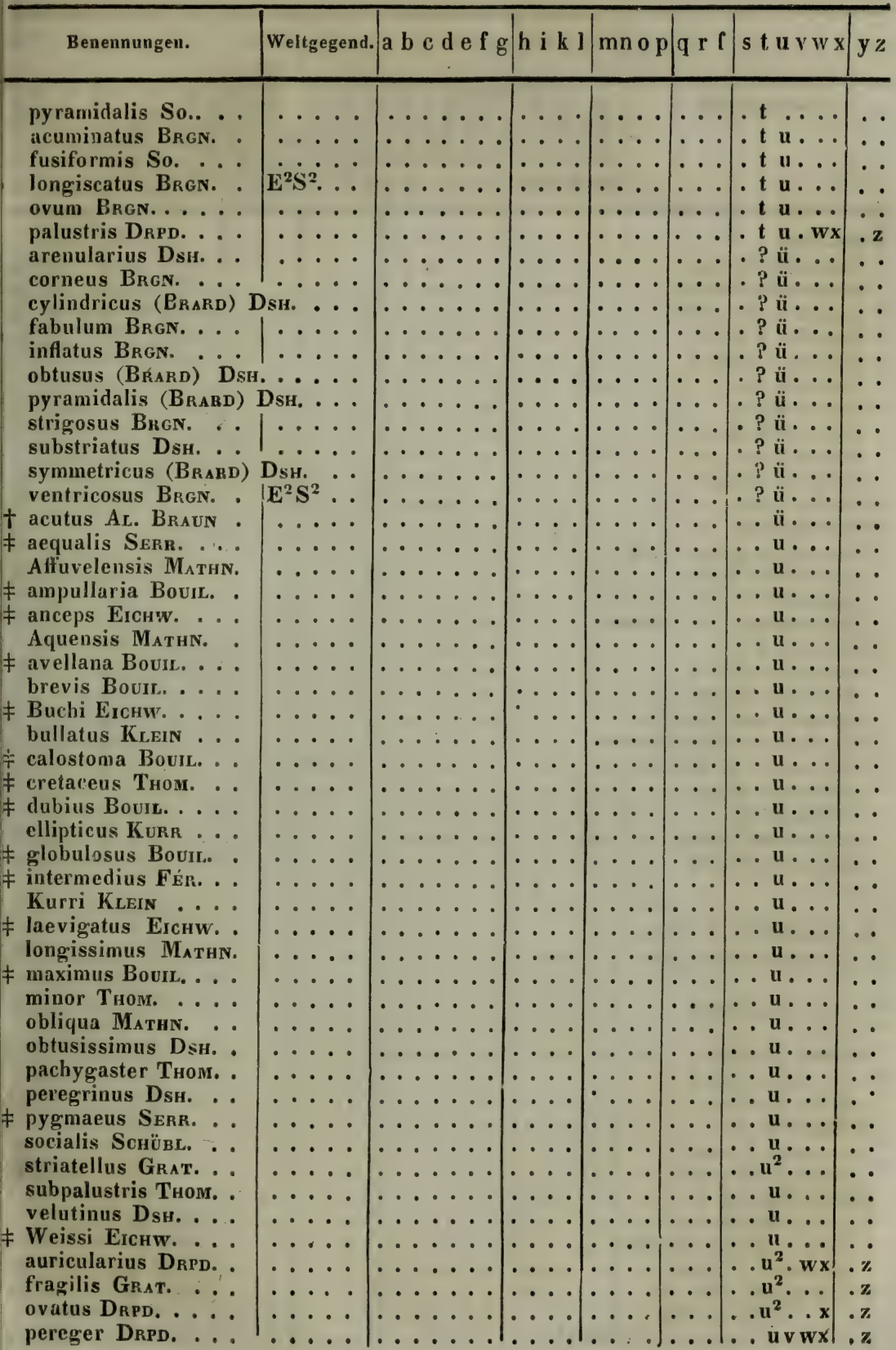




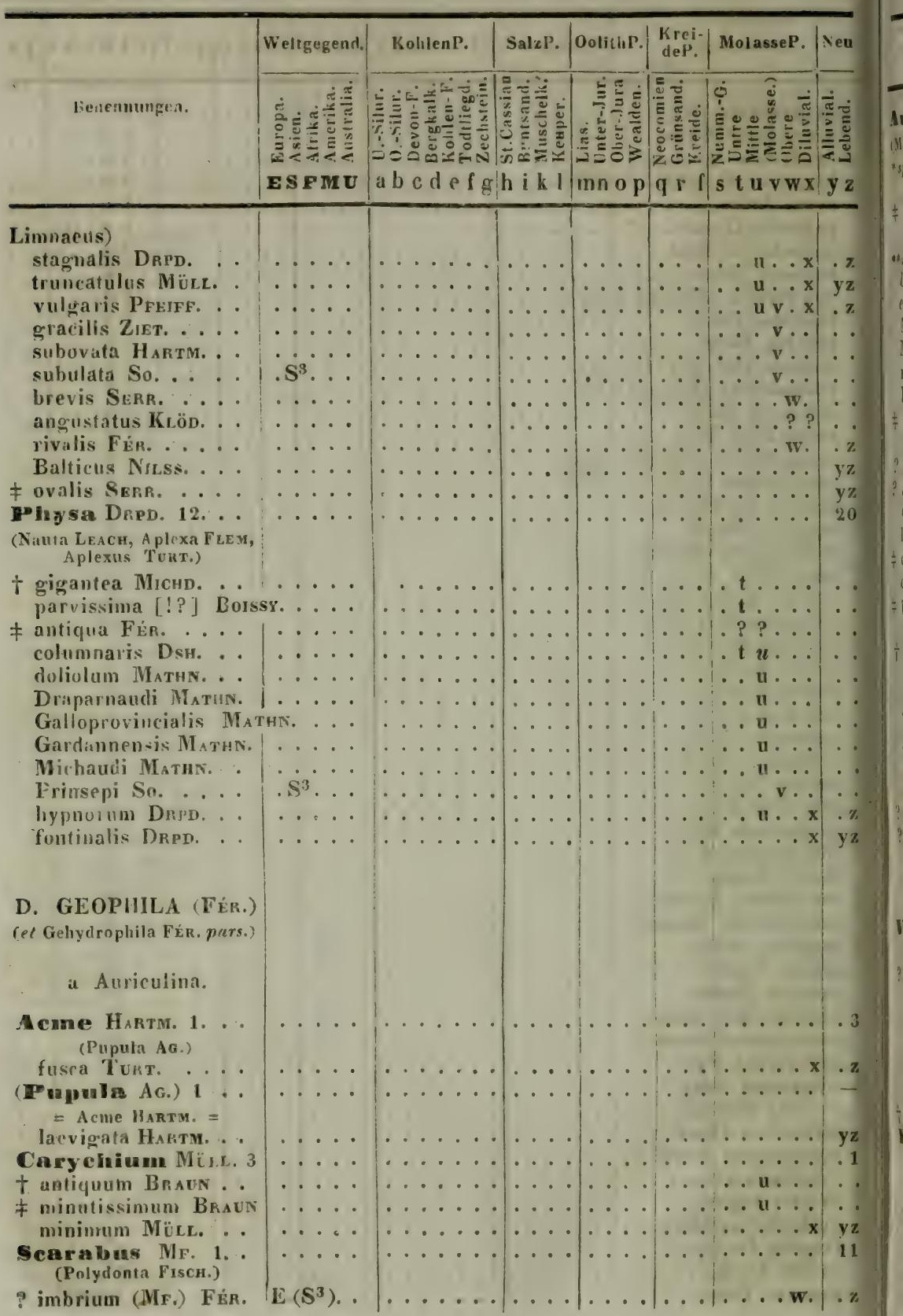


A aricula LK. 28.

(Melamp̧us MF,; Conovula LK.)

* spp. spurice, marinuse (Actaeonidae).

obsoletil PHII L.

‡ striata RoE. decurtata So.

**spp. gensinue terrestres? bimarginata DsH. edentula F́́r. Michelini Borssy Michaudi Borssy miliaris Dsh. Remiensis Bolssy

\# striata ANT. . . citharella Dsh.

? hordeola Lr.

? miliola L.k. ovata LK. biplicata Grat.

₹ oblonga Dsu. ovata Mathn.

$\neq$ pisolina Dsu. Requieni Matun.

† reticulata Woov umbilicata DsH. pyramidalis So. Turonensis Dsh. Judac Lri. myosotis DrPD. myotis Serr.

? gracilis PHL.

? subcylindrica Phit.

\section{b Helicea.}

Vertiggo MüLL. 8. autivertigo Michd.

? muscorum Bouir. pygmaea Fér. edentula Stud. pusilia TurT. substriata TurT. Venetzi Charip. † striolata Braun Pupa Drpo. 34. Archiaci Borssy . . . columellaris Muchd. oviformis Miснd. palangula Bors. Remiensis Bors. Rillyensis Bors. sinuata Michd.

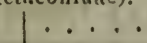




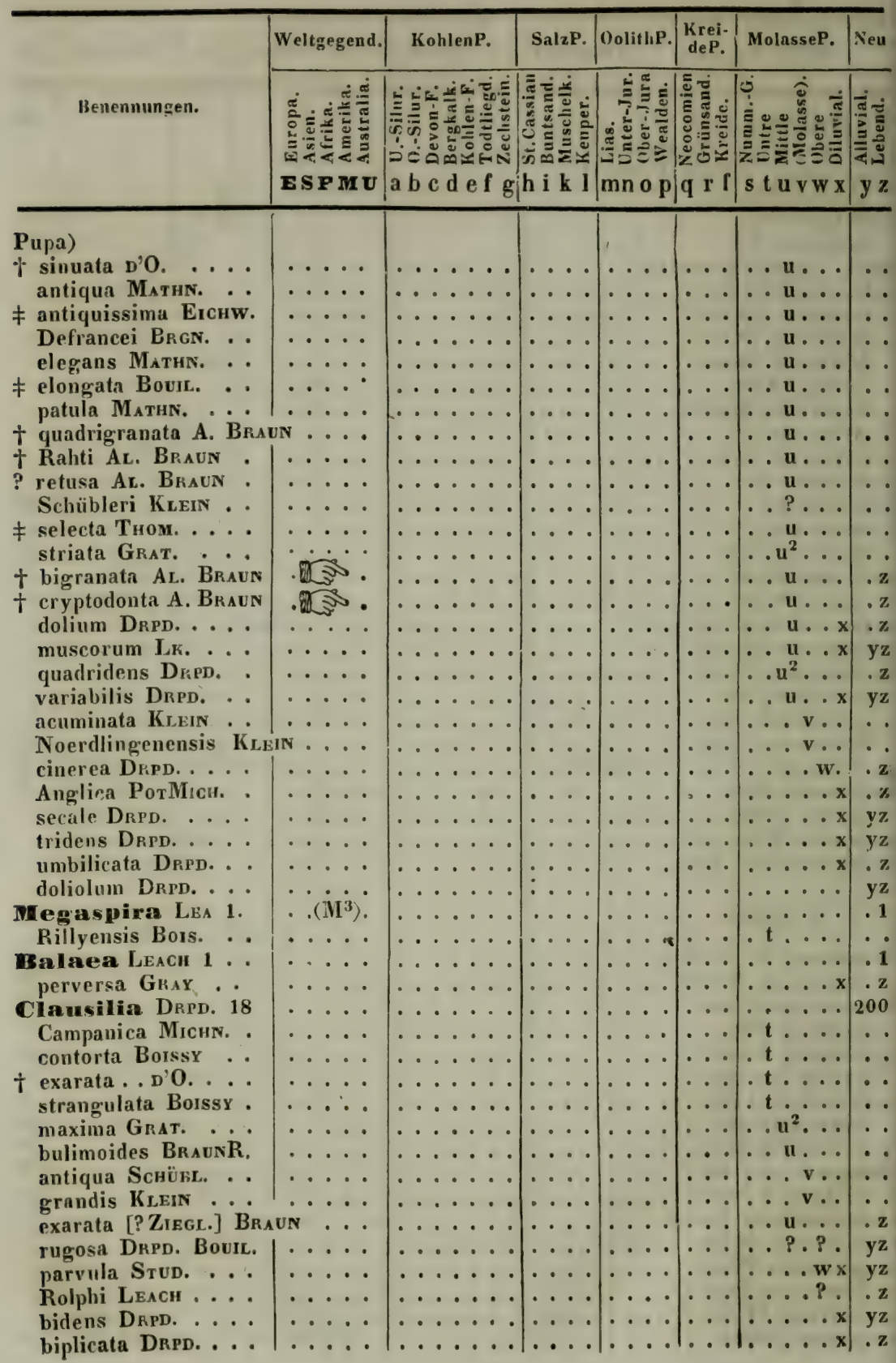




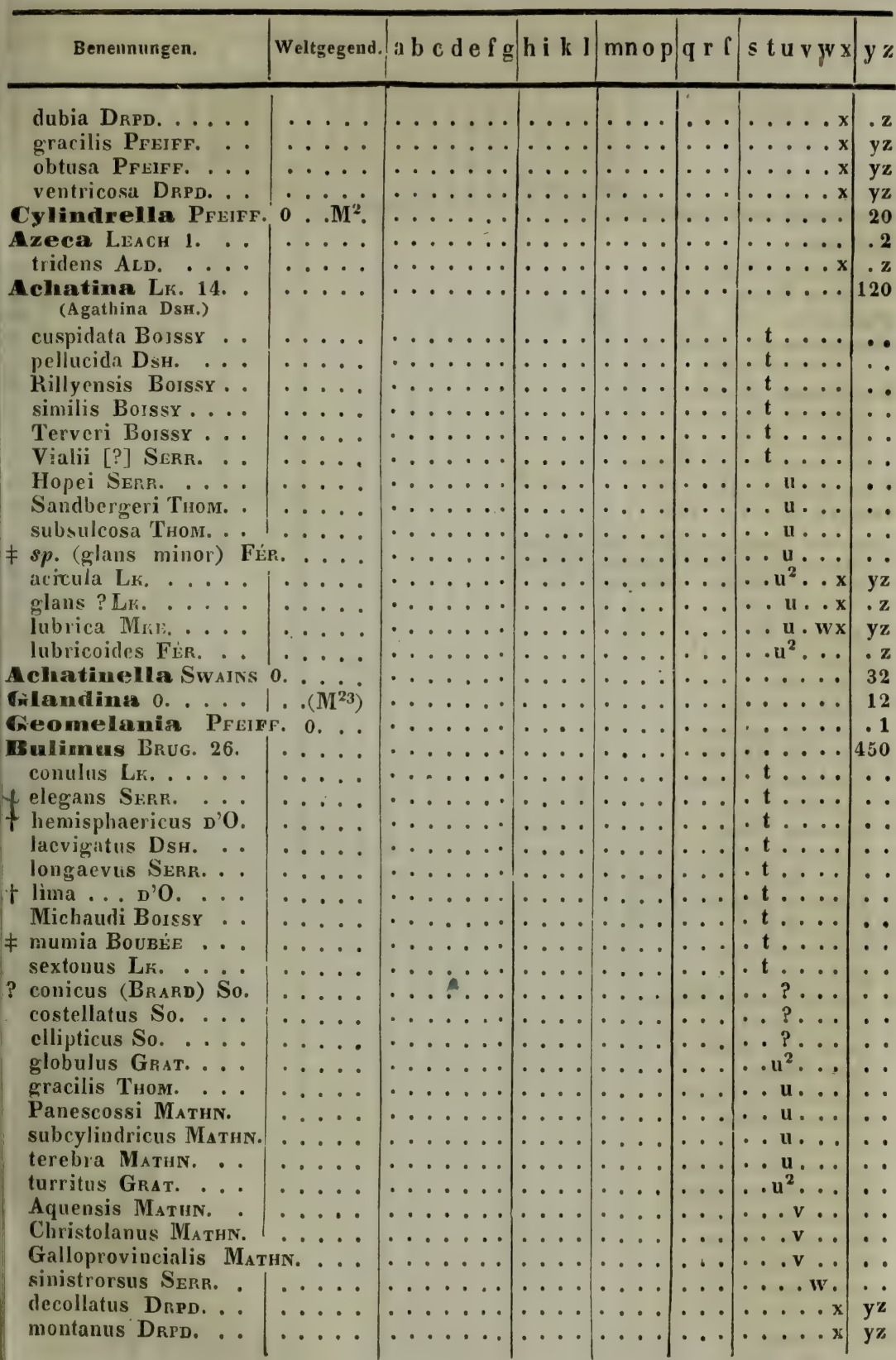




\begin{tabular}{|c|c|c|c|c|c|c|c|}
\hline & Weltgegend. & KohlenP. & SalzP. & OolithP. & $\left|\begin{array}{l}\text { Krei- } \\
\text { deP. }\end{array}\right|$ & MolasseP. & Nell \\
\hline Benennแngยู. & 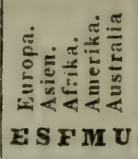 & 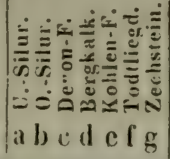 & 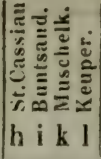 & 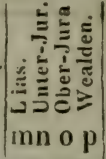 & 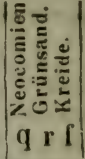 & 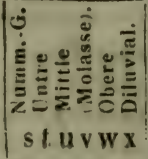 & 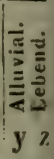 \\
\hline
\end{tabular}

\section{Bulimus)}

obscurus DRPD. acutus DRPD. ....

Partula Fér. 0. .

Anostoma Fiscr. o. (Tomogeres MF.)

Lychmus Marha. 3. ellipticus Matun.

Matheroni Reon.

Urgonensis [?] MaThN.

Streptaxis Gray 0.

Melix (L.) Lк. 191.

(Caracolus, Zonites; elr. Mr. Carocolla LK; TebaL:ach; Helicella, Holigogenaetc. (Fér.)

Riss.; Chilotrema, Cantharaeus Riss.

* spp. spuriue.

jurensis Mü.

pisum Roe.

pusilla Roz.

laevis Pusch.

** spp. gènuinue.

† Arnoudi Мiсну.

Boubéeana SerR cinctites SErr.

? damnata Bros.

Droneti Borssy

dubia Dsh.

Dumasi Borssy

Ferranti Dsw.

Geslini Borssy

globosa So. . ....

\# hemisphacrica Mrchd.

? laevis Mant.

lapicidites Boub.

luna Micud.

nemoralites Serr.

obtusata SERr.

olla SER.

pyramidalis SER. serpentinites Bous.

Desmarestina Brgn. Moroguesi Brgn. . . Ramondi Brgn. . . . cara Bolssy affivis Tном

₹ alba BourL. alloides Тном.

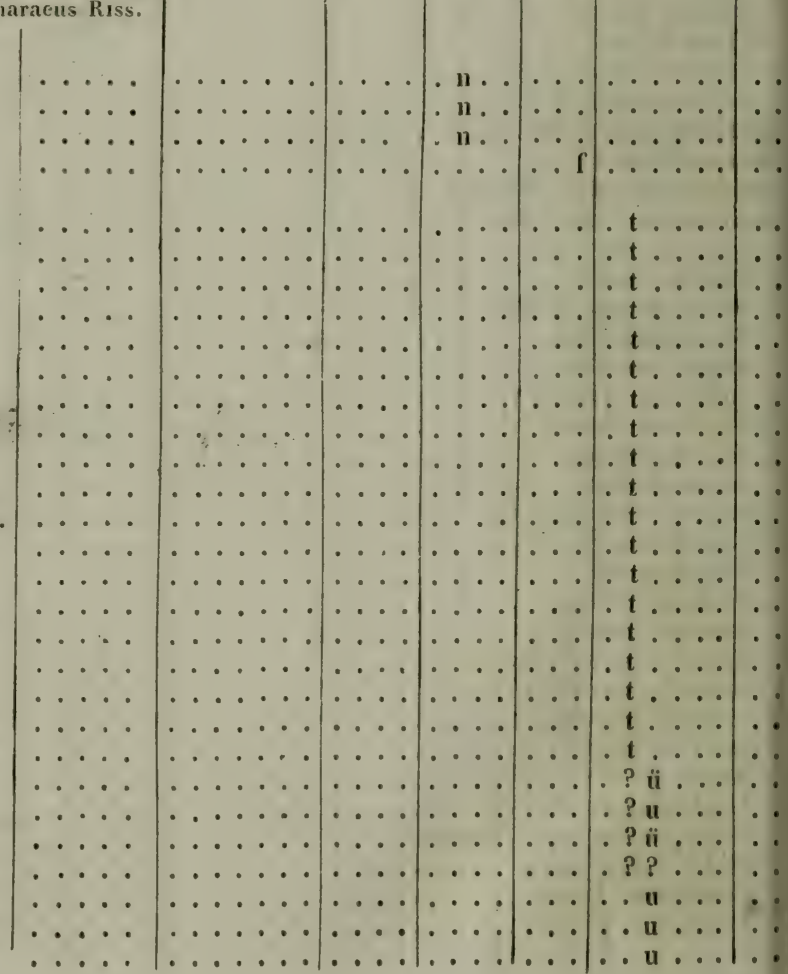




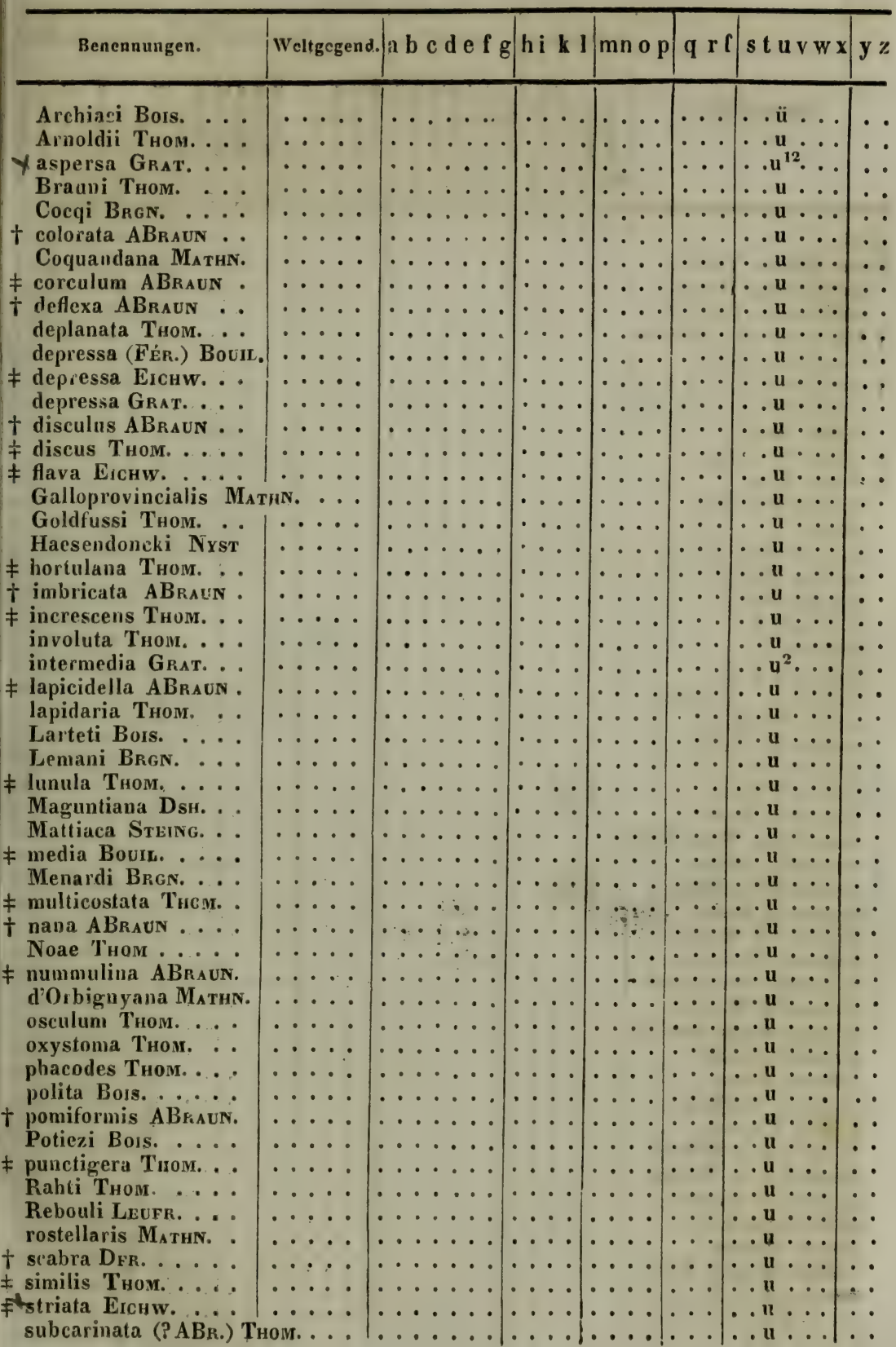




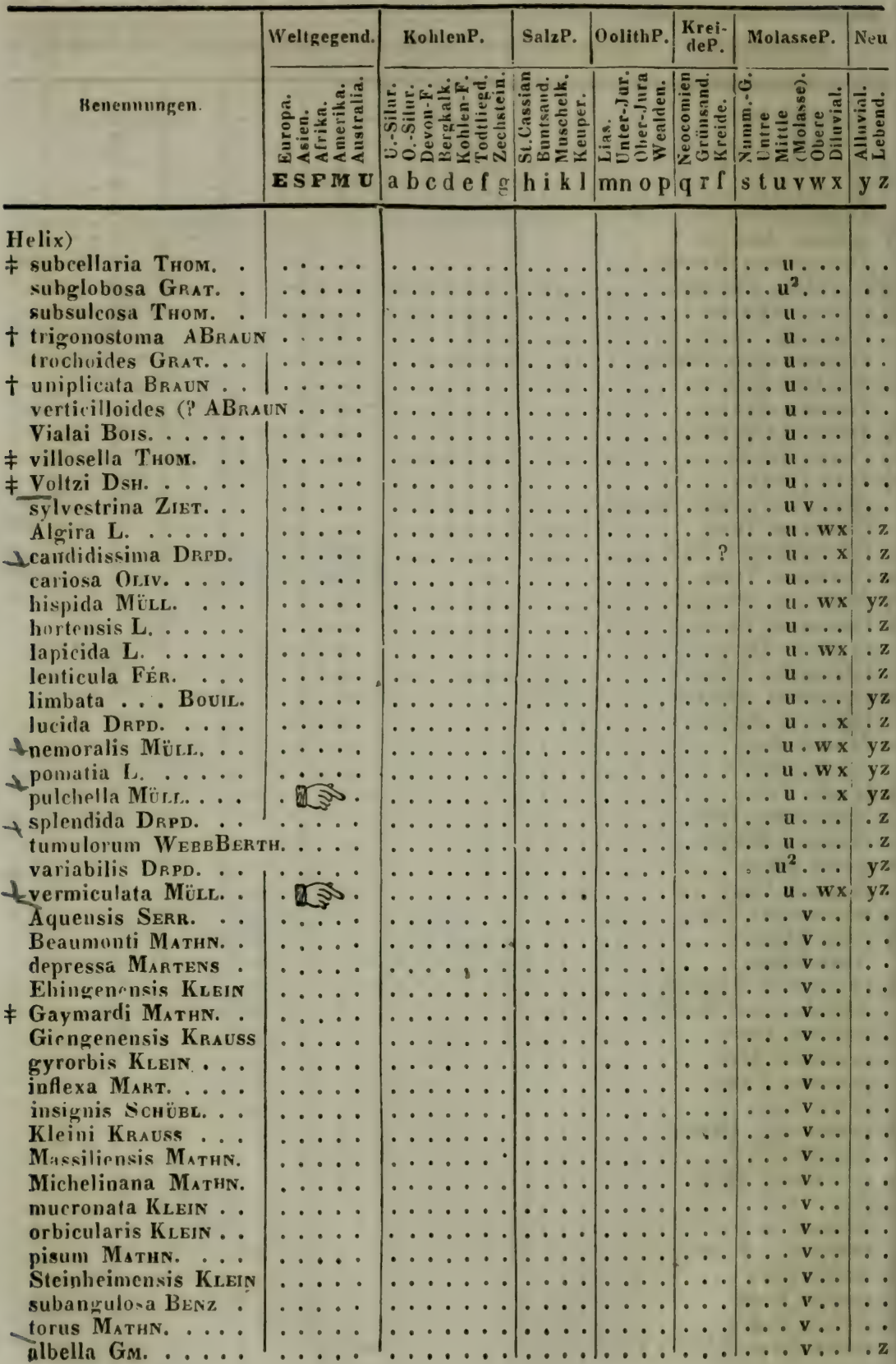


\begin{tabular}{l|l|l|l|l|l|l|l} 
Benennungen. & Weltgegend. a b e d e f g h i k l mn o p & q r l & s t u v w s & y z
\end{tabular}

† acqualis Serr.

† carinata SERr.

Christoli Mathn.

t complanata Ser.R.

† conoideiformis Serr.

t convexa Serr.

Deucalionis Erchw.

† Draparnaudi Serr.

Dufrenoyi Mathn.

$\dagger$ grandis Serr.

$\dagger$ minufa SERr.

† pêrspectiva SERR.

† planorbiformis SERR. pseudo-conspurcata

† rhomboidea Serr.

F sepulta Мгст.

† Sigiensis [?] SERr. sphaeroidea PhiL.

† spiralis SerR.

₹ vermicularia Bon. aspersa Mülc. caelatura FÉr. caespitum Mürc. .. conica DRPD..... cornea DRPD. Mazzullii JANCR. . plebeja DrPD. striata ... SERr. acieformis KLEIN . conoidea So.

submarginalis KLEIN

$\dagger$ alba ABraun aculeata Mǘc. ... alliaria MüLL. arbustorum $\mathrm{L}$. bidentata ABarun Capensis Preiff. . cellaria MüLL. costulata $\mathrm{Z}_{\text {IEGL. }}$ crystallina DRPD. ericetorum Müцc. excavata Bean fruticum DRPD. fulva MüLL. incarnata MüLL. lamellata TURT. montana Srun. nitens Michd. nitida Mürc. nitidosa FÉr. nitidula Dripd. obvoluta MüLL.

$\mathrm{M}_{\text {ATHN. . }}$

I

$\mathrm{E}^{2} \cdot \mathrm{F}^{2} \cdot\left(\mathrm{F}^{3}\right)$

.

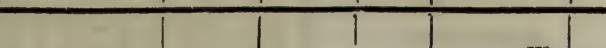

$\ldots \ldots|\ldots| \ldots|\ldots| \ldots|\ldots| \ldots \mid$. $\ldots \ldots \ldots+\cdots$

.

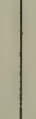

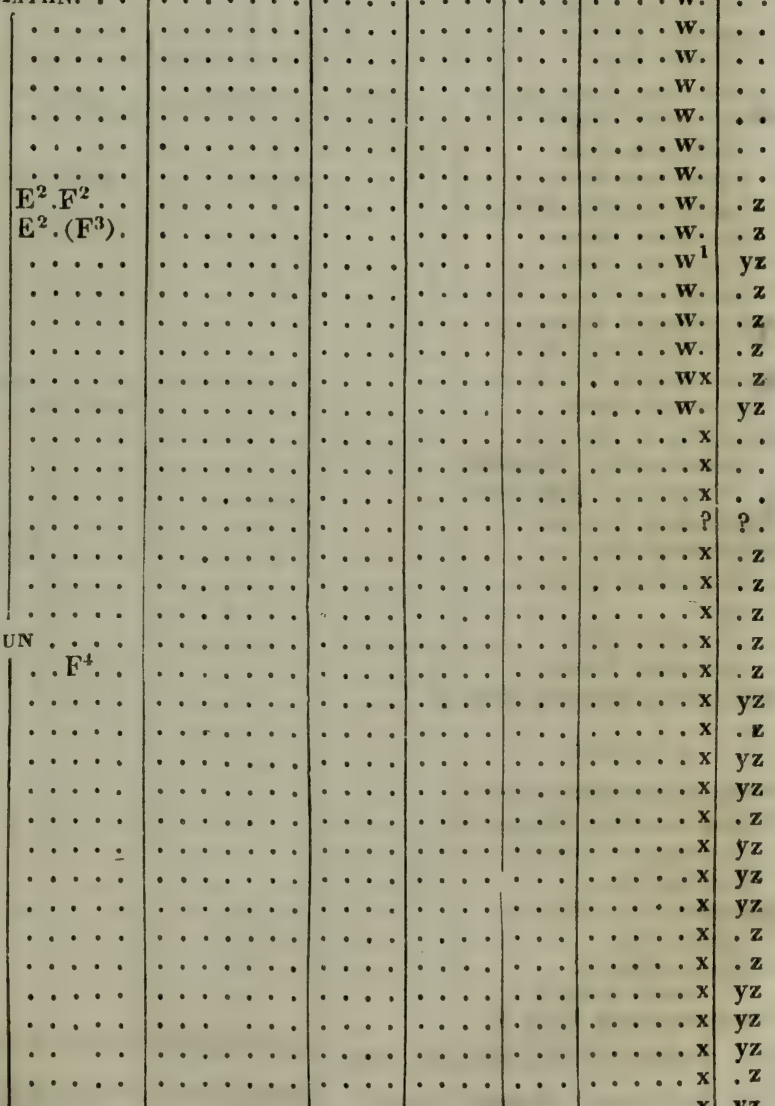




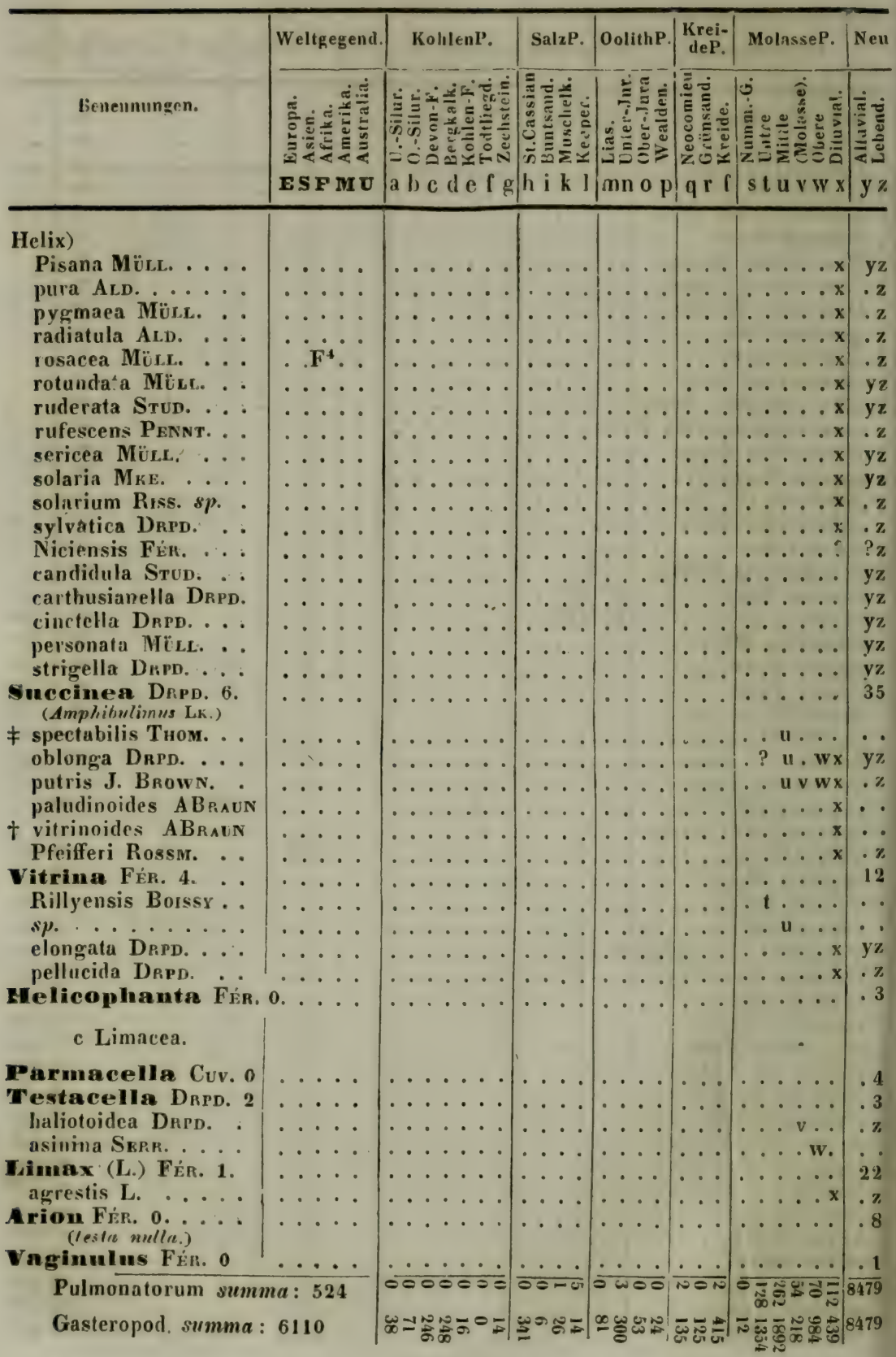




\begin{tabular}{|c|c|c|c|c|c|c|c|}
\hline & Weltgegend. & KohlenP. & SalzP. & OolithP. & $\begin{array}{l}\text { Krei- } \\
\text { deP. }\end{array}$ & MolasseP. & $\mathbf{N} \in \mathbf{u}$ \\
\hline Benemungen, & 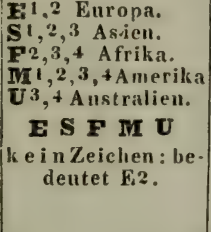 & 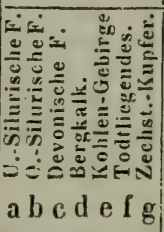 & 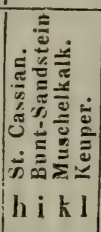 & 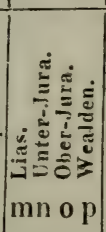 & 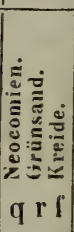 & 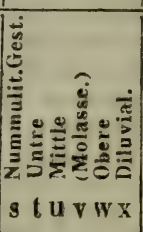 & 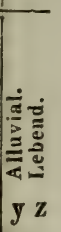 \\
\hline
\end{tabular}

\section{Cl. XV. CEPHALOPODA Cuv.: Kopffïsser.}

I. THTRABEA CHIA Ow., Vierkiemer.

a Ammonitina.

Wactrites G. SANDE. 2 † subconicus SNDb. † sp. 2. SNDe.

Goniatites DeH. 194

(Ellipsolithes So.; Nautellipsites PARK.) a Bactrites SvDr.

Schlothein:i Qu.

$\beta$ Goniatites.

(*Ioko dorsuli simplivi.)

? angustiseptatus Mü. auris Qu.

Beaumonti $\mathrm{M} \ddot{\text { Ü. }}$

Becheri Mü.

biferus Phili..

biimpressus $\mathrm{Bu}$.

Bronni Mü.

Buchi AV.

canalifer I ü. . . .

ceratitoile $\mathrm{Bu}$.

clymeniifa mis Mü. compressy, AV.

$\doteqdot$ Cottai $\mathrm{Mi}$

cucullatus Bu.

Dannenb rgi $A V$.

divisus ü.

falcifer Iü.

globosu Mú.

Hateri Mü.

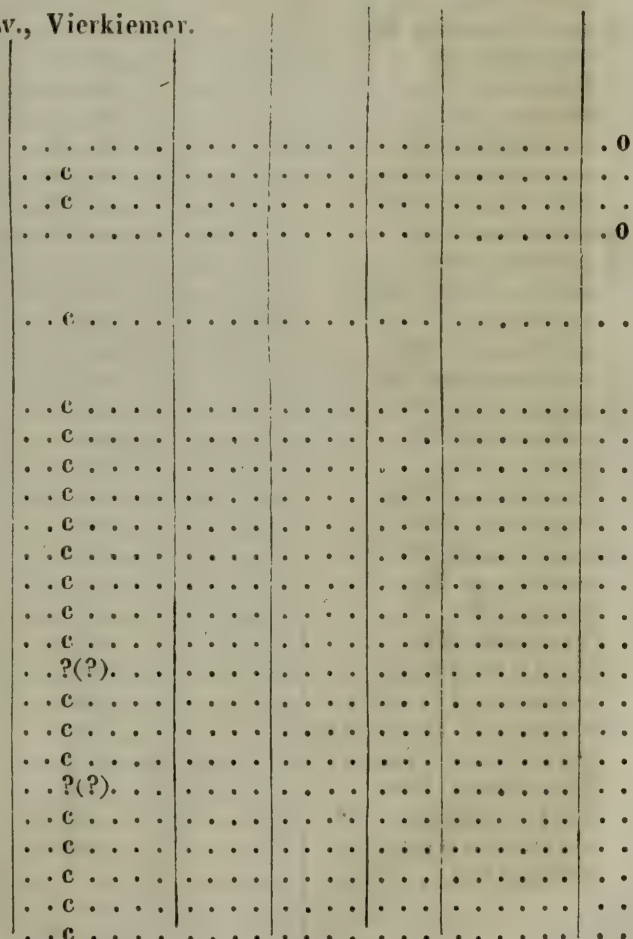




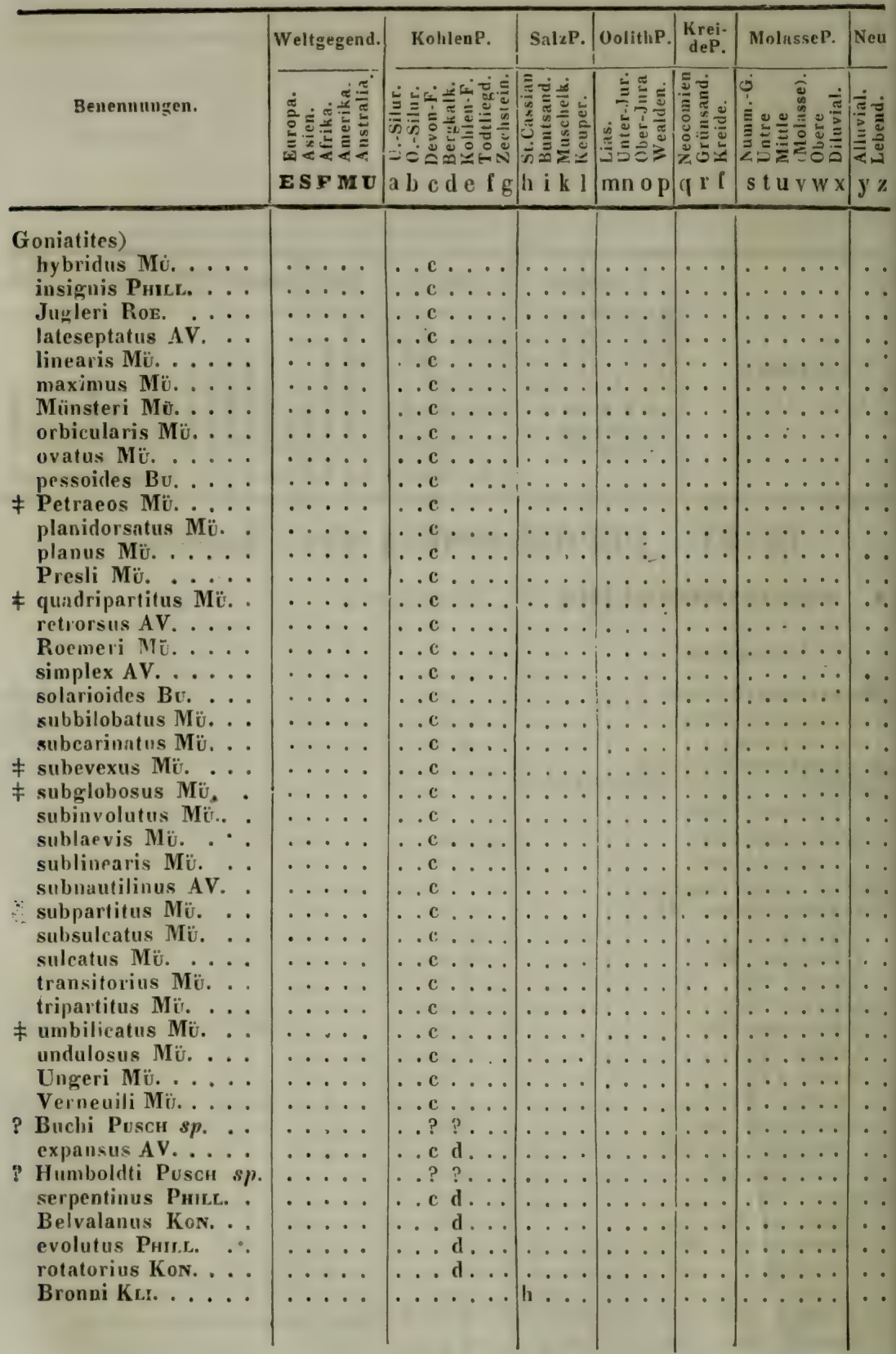




\begin{tabular}{|c|c|c|c|c|c|c|c|}
\hline Benenn & d. & $f \mathrm{~g}$ & & $\mathrm{p}$ & 〔 & $\mathrm{WX}$ & $y^{7}$ \\
\hline \multicolumn{8}{|c|}{$\begin{array}{c}\text { (** lobo dorsuli ambiguo simplici an } \\
\text { trilubulato) }\end{array}$} \\
\hline angustus $\mathrm{Mü} . . .$. & & . c . & $\cdots$ & $\cdots$ & $\cdots$ & $\cdots \cdots$ & \\
\hline oostatus AV. .... & $\therefore \ldots$ & . c . . & & $\cdots \cdot$ & $\cdots$ & $\ldots \ldots$ & \\
\hline intermedius Mü. . . & $\cdots \cdot$ & . c... & $\cdots \cdot$ & $\cdots \cdot$ & . & $\cdots \cdots$ & \\
\hline speciosus Mü. ... & $\cdots \cdot \cdot$ & . c... & $\cdots \cdot$ & $\cdots \cdot$ & $\cdots$ & $\cdots \cdots$ & \\
\hline spurius Mü. .. · & $\cdot \cdot$ & $\cdots c \ldots$ & $\cdots \cdot$ & $\cdots \cdot$ & $\cdots$ & $\cdots \cdots$ & \\
\hline subarmatus $M$ Ü. . . & . & $\cdots \cdot \dot{m}$ & & $\cdots \cdot$ & $\cdots$ & $\cdots \cdots$ & \\
\hline \multirow{2}{*}{\multicolumn{8}{|c|}{ ("*** lobo diorscli trilobulato) }} \\
\hline & $\cdots$ & . . c... & & & & & \\
\hline $\begin{array}{l}\text { acutus MU. } \cdots \text {. } \\
\text { aequabilis BEYR. } s p \text {. }\end{array}$ & ... & . c... & $\ldots$. & $\cdots \cdot$ & $\ldots$ & $\ldots \ldots$ & \\
\hline . . & $\cdots \cdot$ & $\ldots \mathbf{c} \ldots$ & $\cdots \cdots$ & $\ldots$ & $\cdots$ & $\ldots \ldots$ & \\
\hline$\cdots \cdot$ & $\ldots \ldots$ & $\ldots c \ldots$ & $\ldots$ & $\ldots$ & $\cdots$ & $\ldots \ldots$ & \\
\hline rs. . . & $\cdots \cdots$ & ...... & $\cdots \cdots$ & $\cdots$ & $\cdots$ & $\ldots \ldots$ & \\
\hline Bucklandi Mü. .... & $\cdots \cdot \cdot$ & $\ldots c \ldots$ & $\cdots \cdot$ & $\cdots$ & $\cdots$ & $\ldots \ldots$ & \\
\hline ormis AV. & $\cdots \cdot \cdot$ & $\ldots c \ldots$ & $\cdots \cdot$ & $\cdots \cdot$ & $\cdots$ & $\ldots \ldots$ & \\
\hline$\cdot \cdot \cdot$ & $\cdots \cdot \cdot$ & $\ldots \mathbf{c} \ldots$ & $\cdots \cdot$ & $\cdots \cdot$ & $\cdots$ & $\ldots \ldots$ & \\
\hline$\checkmark \cdots$ & $\cdots \cdots$ & $\ldots \mathbf{c} \ldots$ & $\cdots \cdot$ & $\cdots \cdot$ & $\cdots$ & $\ldots \ldots$ & \\
\hline$\cdots$ & $\cdots \cdot \cdot$ & $\ldots c \ldots$ & $\cdots \cdot$ & $\cdots \cdot$ & $\cdots$ & $\cdots \cdots$ & \\
\hline Hoeninghausi BR. . & $\cdots \cdots$ & $\cdots c \ldots$ & $\cdots \cdot$ & $\cdots \cdot$ & $\cdots$ & $\cdots \cdots$ & \\
\hline tus AV. . & $\cdots$ & $\cdots \mathbf{c} \cdots \cdot$ & $\cdots \cdots$ & $\cdots \cdot$ & $\cdots$ & $\cdots \cdots$ & \\
\hline OE. . - & $\cdots$ & $\because \mathrm{c}$ & $\cdots \cdot$ & $\cdots \cdot \cdot$ & $\cdots$ & $\cdots \cdots$ & \\
\hline$\ddot{x}$ & $\cdots \cdots$ & $\because \mathrm{c}$ & $\cdots \cdots$ & $\cdots \cdots$ & $\therefore$ & $\cdots \cdots \cdots$ & \\
\hline $\begin{array}{l}\text { strangulatus KE } \\
\text { Uchtensis KEYS }\end{array}$ & $\cdots \cdots$ & $\therefore$ c.... & $\mid \begin{array}{c}\cdots \\
\cdots\end{array}$ & $\mid \begin{array}{c}\cdots \\
\cdots\end{array}$ & $\cdots$ & $\cdots \cdots \cdots$ & \\
\hline excavatus PhicL. . & $\cdots \cdot$ & $\ldots c d \ldots$ & $\begin{array}{ll}\cdots \\
\cdots\end{array}$ & $\cdots$ & $\ldots$ & $\ldots \ldots$ & \\
\hline DEH. ... & $\cdots \cdot$ & ..c d... & $\ldots$ & $\ldots$ & $\ldots$ & $\ldots \ldots$ & \\
\hline salis Pниц, . . & . . . & $\ldots d \ldots$ & $\ldots$ & $\ldots$ & $\ldots$ & $\ldots \ldots$ & \\
\hline$\cdots$ & $\cdots \cdot$ & $\ldots d \ldots$ & $\ldots$ & $\cdots, \cdot$ & $\cdots$ & $\ldots \ldots$ & \\
\hline na Pintl. . . . & $\cdots \cdot$ & $\ldots d \ldots$ & $\ldots$ & $\ldots$ & $\cdots$ & $\ldots \ldots$ & \\
\hline licatus Kon, $s p$. & $\cdot \cdot \cdot \cdot$ &.$d \ldots$. & $\cdots \cdot$ & $\ldots \cdot$ & $\cdots$ & $\ldots \ldots$ & \\
\hline bus Phicl. . & $\cdots$ & $\ldots d \ldots$ & $\cdots \cdot$ & $\cdots \cdot$ & $\cdots$ & $\ldots \ldots$ & \\
\hline icatus $P$ & $\cdots$ & $\ldots d \ldots$ & $\cdots \cdots$ & $\cdot \cdot$ & $\cdots$ & $\cdots \cdots$ & \\
\hline ruptus Kow. . & $\cdots$ & $\cdots$ & $\cdots \cdots$ & $\cdots \cdot$ & $\cdots$ & $\cdots \cdots$ & \\
\hline L. · & - & a... & $\cdots \cdot$ & $\cdots \cdot \cdot$ & $\cdots$ & $\cdots \cdots$ & \\
\hline bus $\mathbf{P}$ & & $\cdots$ & & $\cdot$ & $\cdots$ & $\cdots \cdots$ & \\
\hline abilis $\mathbf{P H}_{\mathrm{H}}$ & $\cdots$ & $\ldots d \ldots$ & & $\because$ & $\therefore$ & $\begin{array}{cc}\cdots & \cdots \\
\cdots & \cdots\end{array}$ & \\
\hline $\begin{array}{l}\text { usus PHILL. } \cdots \\
\text { cilobus PhiL.. }\end{array}$ & $\cdots$ & $\therefore d \ldots$ & & $\cdots$ & $\cdots$ & 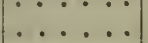 & \\
\hline obus Phicl. & $\ldots$ & $\ldots d \ldots$ & & & . & $\ldots \ldots$ & \\
\hline . & $\ldots$ & ...d.. & & $\ldots$ & $\ldots$ & $\ldots \ldots$ & \\
\hline olobus Parze. & $\cdots \cdot$ & . d. & $\cdots$ & $\cdots$ & $\ldots$ & $\ldots \ldots$ & \\
\hline ca Phick. . . . & 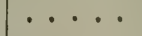 & ... d... & $\cdots$ & $\ldots$ & $\ldots$ & $\ldots \ldots$ & \\
\hline iger Phir.L. . . . & & ...d... & $\cdots$ & $\cdots$ & $\cdots$ & $\ldots \ldots$ & \\
\hline Kon. & $E^{2} S^{2} \ldots$ & $\ldots \mathrm{de}$ e. & & & $\cdots$ & $\ldots \ldots$ & \\
\hline $\begin{array}{l}\text { ticulatus PHILL. · } \\
\text { shaericus DEH }\end{array}$ & $\dot{\mathrm{E}}^{2} \dot{\mathrm{M}}^{2}$ & . de.. & & $\cdots \cdot$ & $\cdots$ & $\cdots \cdots$ & \\
\hline on. . . . & ( & . & & $\cdots$ & $\cdots$ & $\cdot$ & \\
\hline Is VERn. & $. \mathrm{S}^{2}, \ldots$ & - & & & . . & $\cdots$ & \\
\hline$\cdots \cdots$ & & e & - & $\cdots \cdot$ & $\cdots$ & $\cdots \cdots$ & \\
\hline & & & & & & & \\
\hline
\end{tabular}




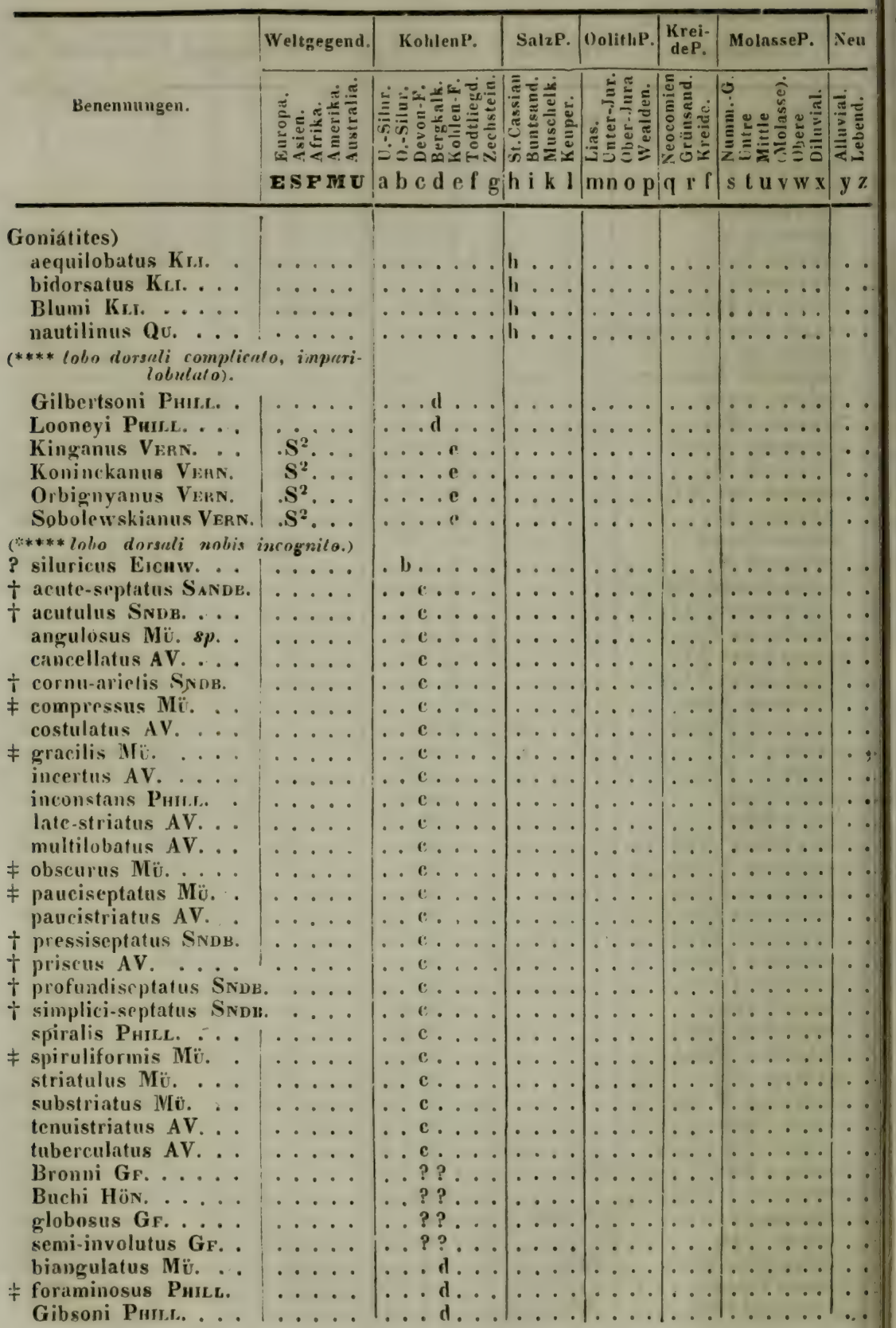




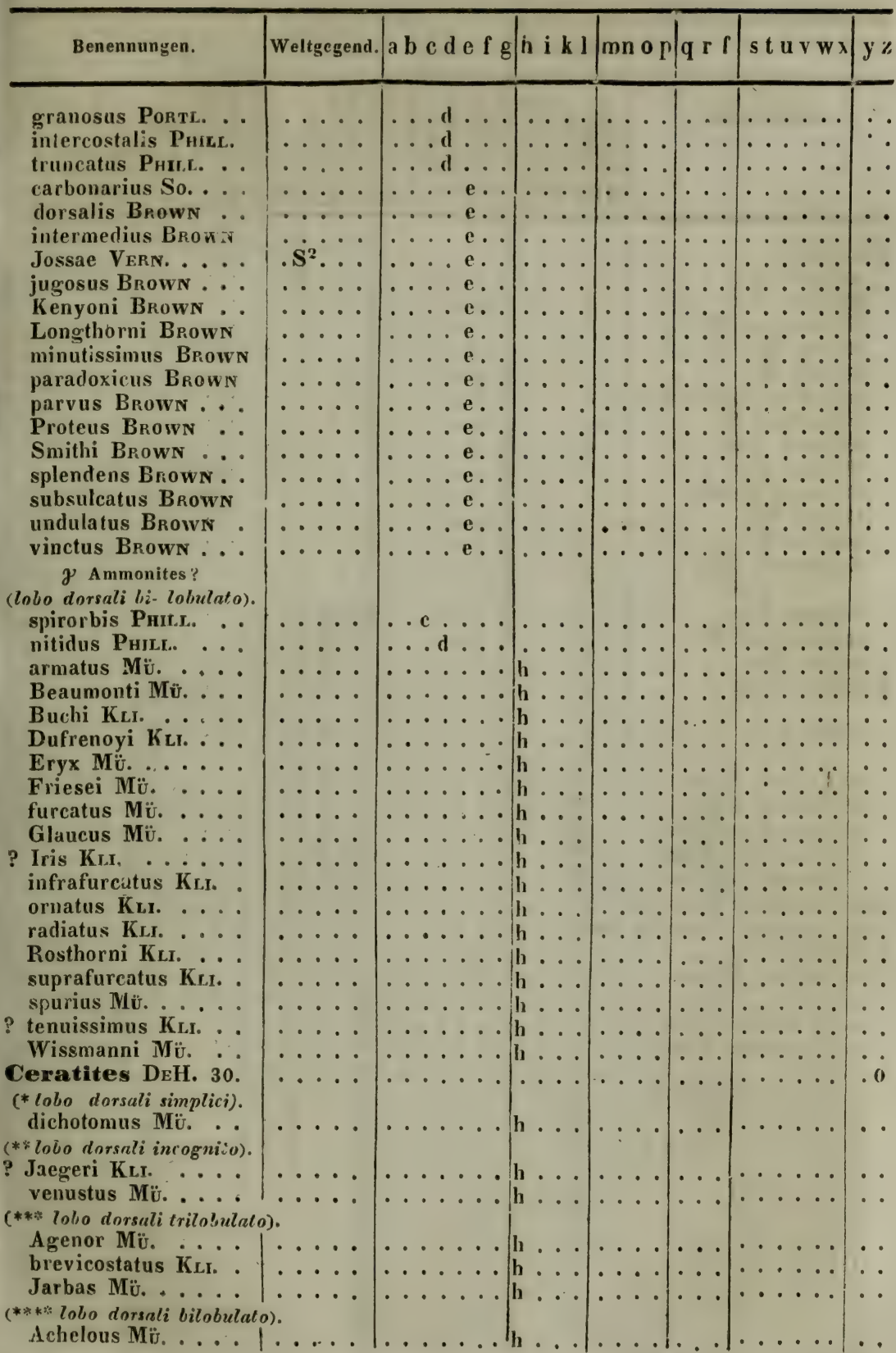




\begin{tabular}{|c|c|c|c|c|c|c|c|}
\hline & Weltgegend. & KohlenP. & SulzP. & OolithP. & $\begin{array}{l}\text { Krei- } \\
\text { deP. }\end{array}$ & MolasseP. & Neu \\
\hline Benenuนngen. & 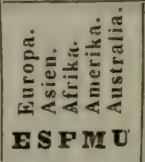 & 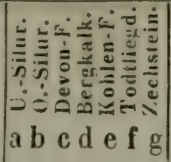 & 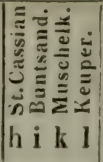 & 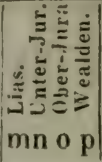 & 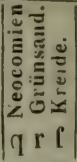 & 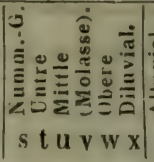 & $\begin{array}{l}\text { y } \\
\mathbf{y}\end{array}$ \\
\hline
\end{tabular}

\section{Ceratites)}

Agassizi KLI.

Basileus Mü.

bipunctatus Mü.

Boetus Mü.

Busiris Mü.

infundibuliformis Mü.

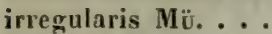

Karsteni KLI.

Meriani KLr. .

Münsteri Wıssm.

Oceani Mü.

sulcifer Mü.

Zeuschneri KLI.

nodosus DEH.

semipartitus MÜ

enodis Qu.

‡ cinctus DEH.

\section{Buchi Ziet.}

Bogdoanus VERN.

Eichwaldi Keys. euomphalus Keys. Hedenströmi Kers.

Middendorfi KEYs.

Ammomites DEH. 523

* spp. nondum in fumilius relutne.

? Dalmani His.

? comnunis Eichw.

? Acis $\mathrm{M} \ddot{\mathrm{U}}$.

acute-costatus Kr.I. aequinodosus $\mathrm{KLr}$. Aon Mü.

armato-cingulatus Kr.J. bicarinatus $\mathrm{M} \ddot{\mathrm{u}}$. bidenticulatus KLI. .

? Bouéi KLr.

Brotheus Mü.

? cingulatus KLr.

Credneri KLI.

Decheni KLI.

furcatus $\mathrm{Mü}$.

Gaytani [?] Kir.

Goldfussi KLr. . .

granulose-striatus KLI.

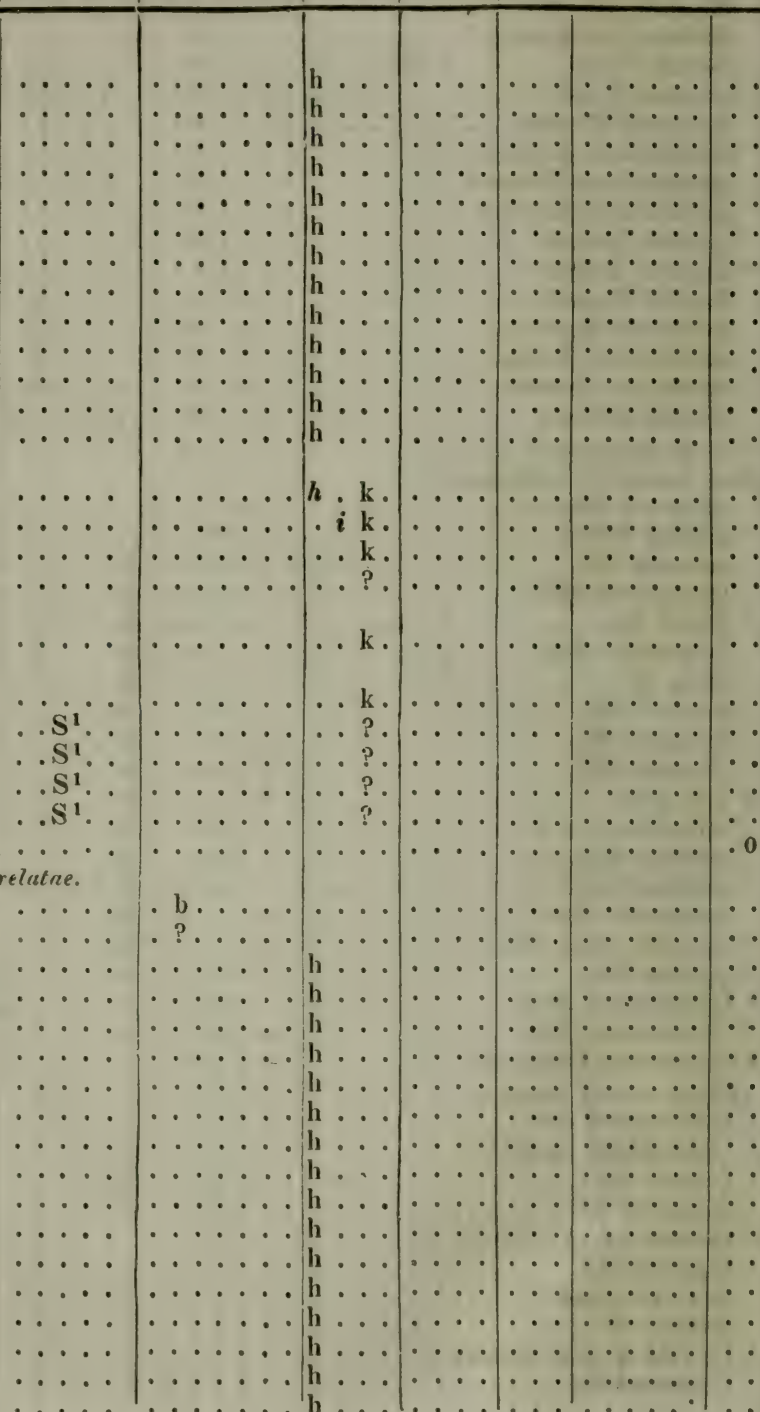




\begin{tabular}{|c|c|c|c|c|c|c|c|c|}
\hline & gen. & eltgegend. & $a b$ & 1 & $p$ & r I & $w x$ & $y$ \\
\hline & & & & & & & & \\
\hline & $\mathbf{K}_{\text {L. }}$. & & & & & $\cdots$ & $\ldots \ldots$ & \\
\hline & labis & $\cdots$ & $\ldots \ldots$ & & $\ldots$ & $\cdots$ & $\ldots \ldots$ & \\
\hline & . & $\ldots$ & $\ldots \ldots$ & h $\ldots$ & $\cdots$ & $\cdots$ & $\ldots \ldots$ & \\
\hline & $\cdots$ & $\cdots \cdot$ & $\cdots \cdots \cdots$ & $h \ldots$ & $\cdots \cdot$ & $\cdots$ & $\ldots \ldots$ & \\
\hline & $\cdots$ & $\cdots$ & $\cdots \cdots$ & & $\cdots$ & $\cdots$ & $\cdots \cdots$ & \\
\hline & Max & & $\cdots \cdots \cdots$ & & $\cdots$ & & $\cdots \cdots$ & \\
\hline & Mey & & & $h$ & & & $\cdots \cdots$ & \\
\hline & $\begin{array}{l}\text { mi } \\
\text { no }\end{array}$ & $\begin{array}{l}\cdots \\
\cdots \\
\cdots\end{array}$ & $\begin{array}{lll}\cdots & \cdots & \cdots \\
\cdots & \cdots & \cdots\end{array}$ & $\mid \begin{array}{l}\mathrm{h} \\
\mathrm{h}\end{array}$ & & - & 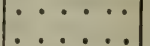 & \\
\hline & KLI. & $\ldots \ldots$ & $\because$ & $h \div$ & $\ldots \ldots$ & $\cdots$ & 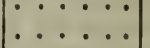 & \\
\hline & . & $\ldots$ & $\cdots \cdots$ & h . . & $\cdots \cdot$ & $\cdots$ & $\ldots \ldots$ & \\
\hline & $s \mathrm{Br} . \ldots$ & $\cdots \cdot$ & $\cdots \cdots$ & h .. & $\cdots \cdot$ & $\cdots$ & $\ldots \ldots$ & \\
\hline & i. . . . & $\cdots \cdots$ & $\cdots \cdots$ & h. & $\cdots \cdot$ & $\cdots$ & $\ldots \ldots$ & \\
\hline & • . • & $\cdots \cdot$ & $\cdots \cdots \cdots$ & h $\ldots$ & $\cdots \cdot$ & $\cdots$ & $\ldots \ldots$ & \\
\hline & Is KLI. & $\cdots \cdot$ & $\cdots$ & $h \cdot \cdots$ & $\cdots$ & $\cdots$ & $\cdots \cdots$ & \\
\hline & $\dot{\mathrm{K}} \dot{\overrightarrow{\mathrm{V}}}$ & $\begin{array}{l}\cdots \\
\cdots\end{array}$ & & & & $\cdots$ & $\cdots \cdots$ & \\
\hline & LI. & $\dot{\cdots}$ & & $h \cdots$ & $\begin{array}{ll}\cdots \\
\cdots \\
\cdots\end{array}$ & & $\cdots \cdots$ & \\
\hline & I. $\ldots$ & .... & $\ldots$ & h... & $\ldots$ & $\cdots$ & $\cdots \cdots$ & \\
\hline & $\therefore$ & .... & $\ldots \ldots$ & $h \ldots$ & $\ldots$ & $\ldots$ & $\because \ldots$ & \\
\hline & KLI. & $\ldots$ & $\ldots \ldots$ & h... & $\ldots$ & $\ldots$ & $\ldots \ldots$ & \\
\hline & $\cdots$ & $\cdots \cdot$ & $\ldots$ & $\ldots$. & & $\cdots$ & $\ldots \ldots$ & \\
\hline & $\cdots$ & $\ldots \ldots$ & $\ldots \ldots$ & $\cdots$ & द... & $\cdot \cdot$ & $\ldots \ldots$ & . \\
\hline & HILL. & $\cdots \cdots$ & $\ldots$ & $\cdots$ & $m \ldots$ & $\cdots$ & $\ldots \ldots$ & \\
\hline & . & $\cdots$ & $\cdots$ & $\ldots$ & M. . . & $\cdots$ & $\ldots \ldots$ & $\cdots$ \\
\hline & HII.L. . & $\cdots$ & $\cdots$ & $\ldots$ & $\mathrm{m}_{\mathrm{i}} \cdot$. & $\cdots$ & $\ldots \ldots$ & . \\
\hline & $\cdots$ & $\cdots \cdot$ & $\cdots$ & $\cdots$ & $\mathbf{M}^{\mathbf{1}} \ldots$ & $\cdots$ & $\ldots \ldots$ & $\cdots$ \\
\hline & - & $\cdots \cdot$ & $\ldots \ldots$ & $\cdot \cdot$ & m... & $\cdots$ & $\ldots \ldots$ & \\
\hline & O. . & $\cdots \cdots$ & $\cdots$ & ${ }^{\circ}$ & $\beta$. & $\cdots$ & $\ldots \ldots$ & - \\
\hline & ). . & $\cdots \cdots$ & $\cdots$ & $\cdots$ & $\begin{array}{l}\gamma \\
\rho\end{array}$. & $\cdots$ & $\cdots \cdots$ & - \\
\hline & $\cdots$ & $\ddot{*}$ & & $\therefore$ & Mi & $\cdots$ & $\cdots$ & : \\
\hline & $\cdots$ & $\cdots$ & $\cdots$ & $\left.\mid \begin{array}{ll}\cdots \\
\cdots\end{array}\right]$ & $\mathbf{M}^{1} \ldots$ & 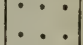 & $\begin{array}{l}\cdots \\
\cdots\end{array}$ & $\cdots$ \\
\hline & $\ddot{v}$ & ... & $\ldots \ldots$ & $\ldots$ & $\mathbf{m} \ldots$ & $\ldots$ & $\therefore \ldots$ & 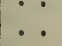 \\
\hline & .. & $\cdots \cdot$ & $\ldots \ldots$ & $\cdots$ & & $\cdots$ & $\ldots \ldots$ & $\cdots$ \\
\hline & $\cdots \cdot$ & $\cdots \cdot$ & $\cdots$ & & $\mathrm{M}^{1}$ & $\cdots$ & $\cdots \cdots$ & •• \\
\hline & ). . . & $\cdots$ & $\cdots \cdots$ & . & & $\cdots$ & $\cdots \cdots$ & \\
\hline & & . & & & $\mathbf{M}^{\mathbf{1}}$ & • & $\cdots$ & \\
\hline & $\cdots \cdot$ & - & $\cdot$ & & $\cdot$ & $\cdots$ & $\cdots \cdots$ & - \\
\hline & $\cdots$ & & & & 1 & , & $\cdots$ & \\
\hline & $\cdots$ & & & & & • & $\cdot 1$ & \\
\hline & $\cdots$ & & & & & & & \\
\hline & iLL. & $\therefore$ & $\cdots$ & $\ldots$ & m. . & $\cdots$ & 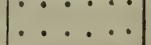 & $\cdots$ \\
\hline & $\cdots$ & ... & $\cdots$ & & y... & $\ldots$ & $\ldots . .$. & $\cdots$ \\
\hline & & . & & & M. & & $\cdots$ & : . \\
\hline & & & & & & & & \\
\hline & Guidonii So. & & & & & $\cdots$ & $\cdots$ & • \\
\hline & $\begin{array}{l}\text { hemisculptus PHILL. } \\
\text { heterogeneus } \mathrm{Y}_{\mathrm{A}} \mathrm{B} \text {. }\end{array}$ & $\cdots$ & $\cdots$ & & $\mathbf{m}$. & $\cdots$ & $\because$ & 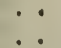 \\
\hline & 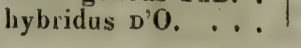 & & & & & & & \\
\hline
\end{tabular}




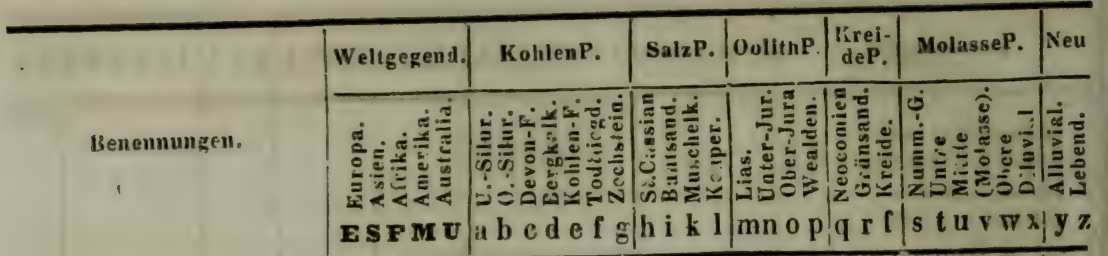

Ammonites)

‡ impendens $\mathbf{Y} A B$

† inornatus WiLlms. Johnstoni So. . . . laevigatus So. lenticulariś Bu.

Ioscbomei So.

Macdonnelli Porits.

Metternichi Hav.

Mimatensis $\mathrm{D}^{\prime} \mathrm{O}$.

₹ multilobatus Br.

† Murleyi Вuскм.

₹ neojurensis Qv. nitescens $Y_{A} B$. ... obliquatus $\mathbf{Y}_{\mathbf{A B}}$. Phillipsi So. planorbis So. .... Raquinanus D'O. Sampsoni Porte. Sedgwicki Buck. stella So. subcarinatus Puicc. Theodosia Dsu. . tornalus BR. trapezoidalis So. variabilis $\mathbf{D}^{\prime} \mathrm{O}$. ventricosus So. vittatus $Y_{A} B$. Sutherlandiae So. arbustiger D'O.

† Baugieri D'O.

bifrons Phill.

biflexuosus D'O. Bouchardanus (D'O.)

Brigthi Pratt Cadomensis DFR. calvus So.

Caumonti D'O. columnatus Fiscr. complanatus ZIET. dorsalis ? LK. Edouardanus D'O [?] Elephantious So.

+ extra-armatus D'O. fascicularis (D'O.) Cat, fissus So. fornix So.

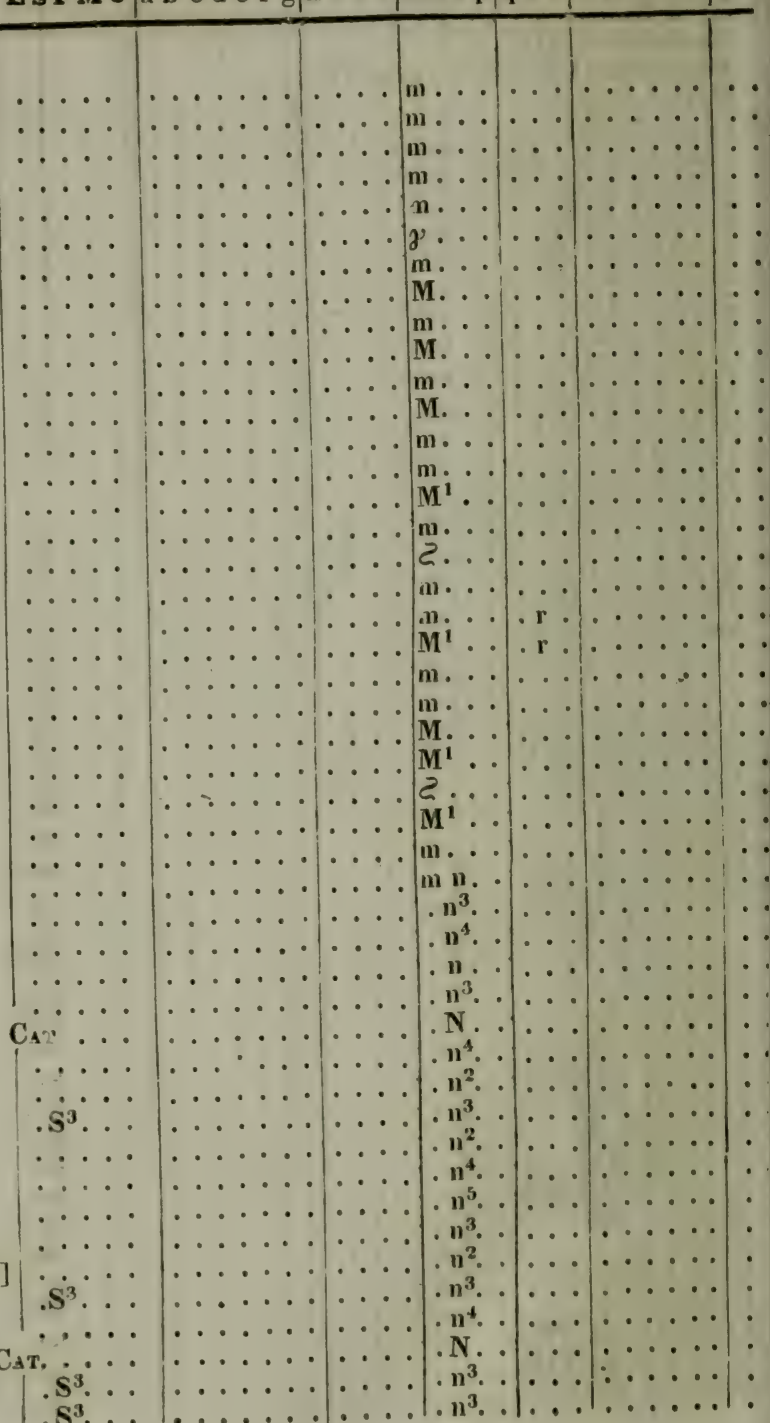




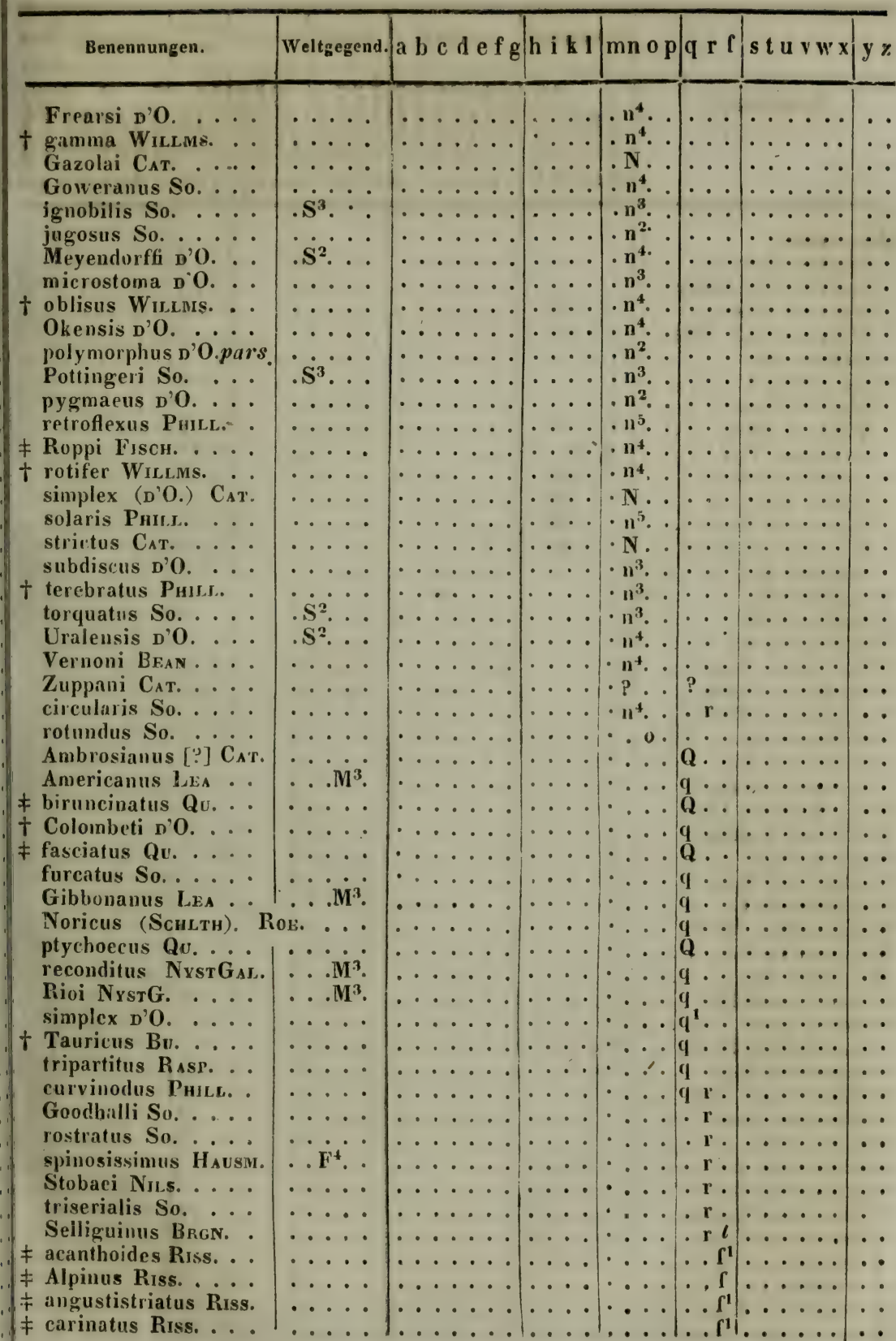




\begin{tabular}{|c|c|c|c|c|c|c|c|}
\hline Benenn & 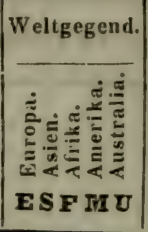 & 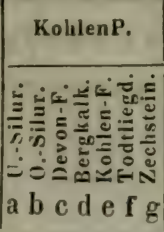 & 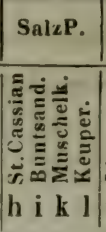 & 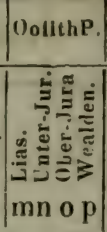 & 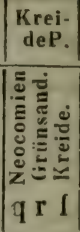 & 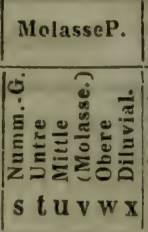 & 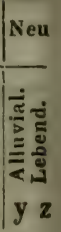 \\
\hline 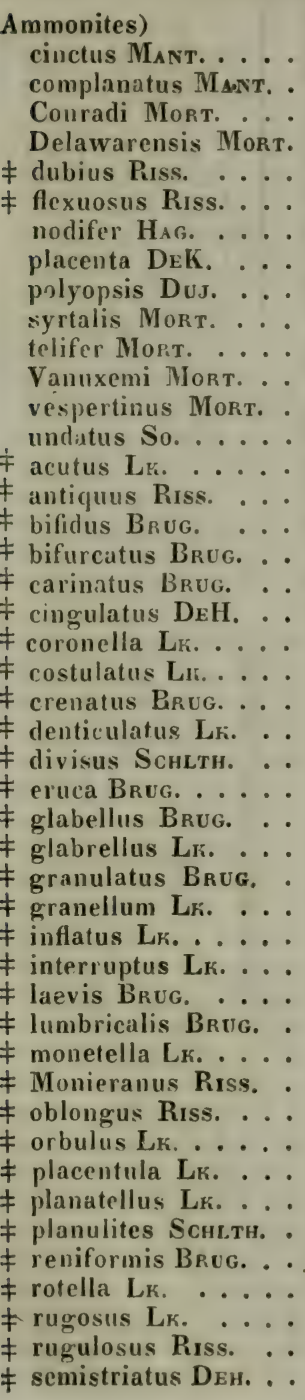 & 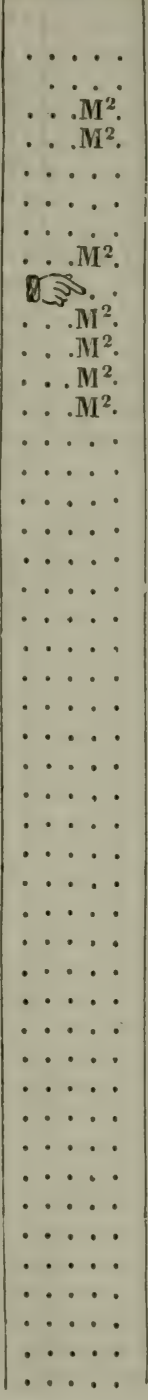 & 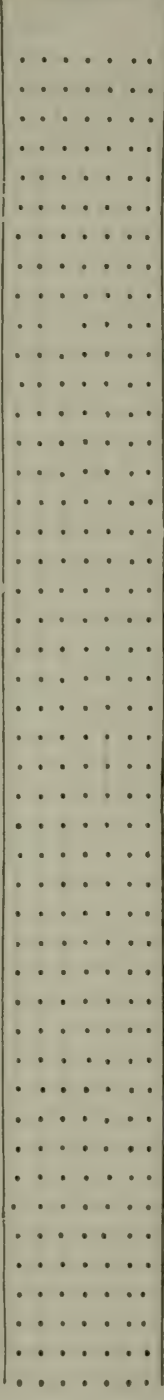 & 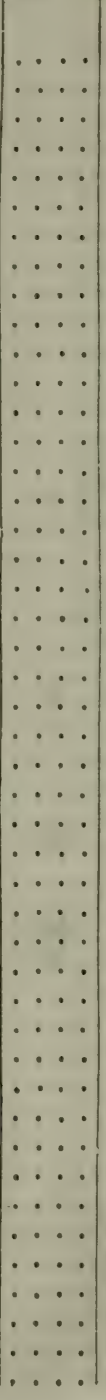 & 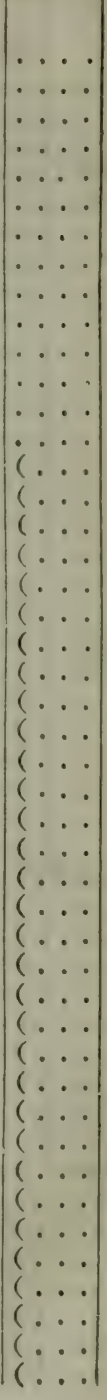 & 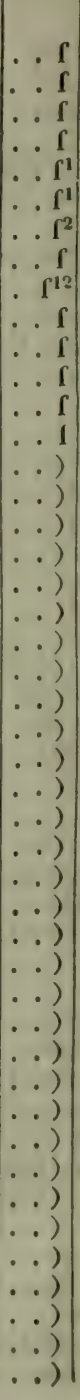 & 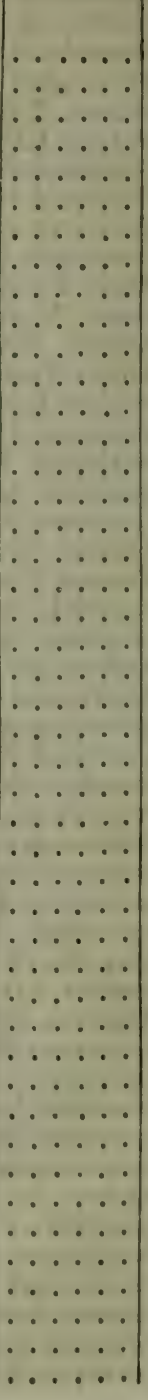 & 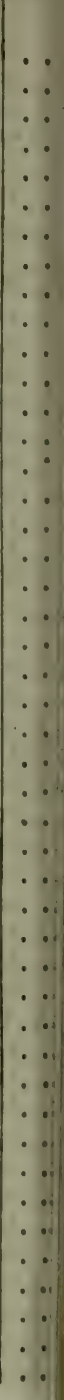 \\
\hline
\end{tabular}




\begin{tabular}{|c|c|c|c|c|c|c|c|}
\hline Bene & eltgegend. & $\mathrm{f} g$ & k l & $\mathrm{p}$ & q r l & stuvw $\mathrm{x}$ & $y z$ \\
\hline 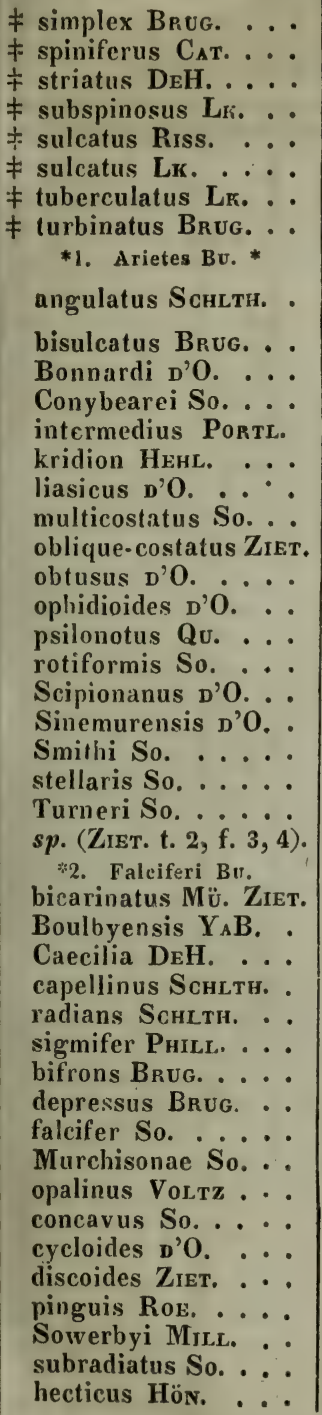 & 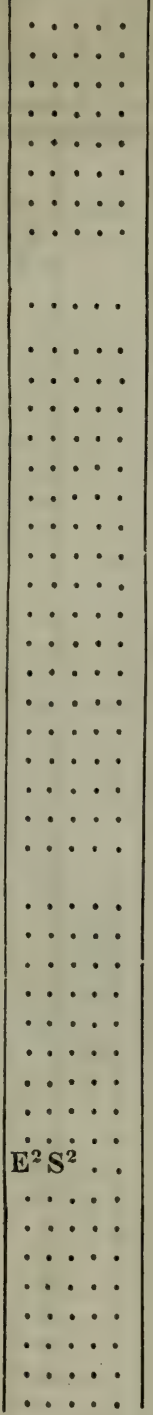 & 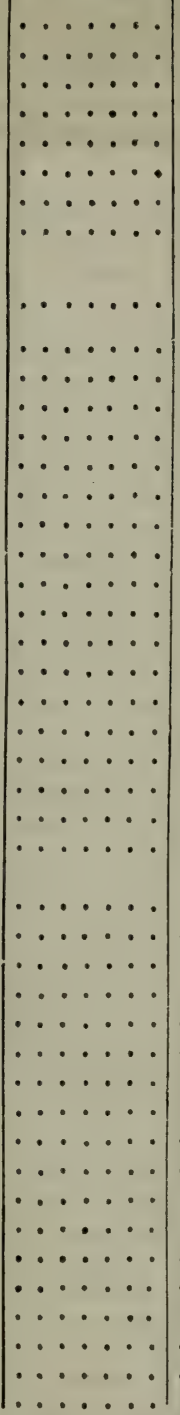 & 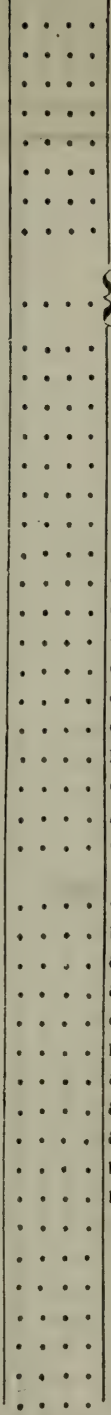 & 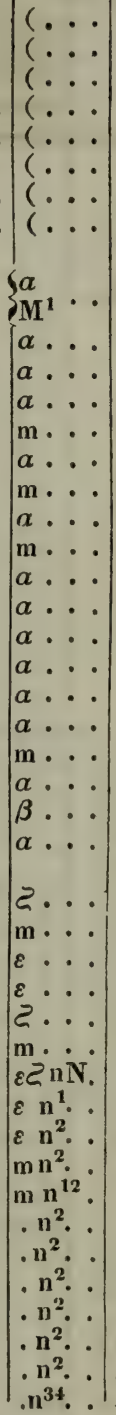 & 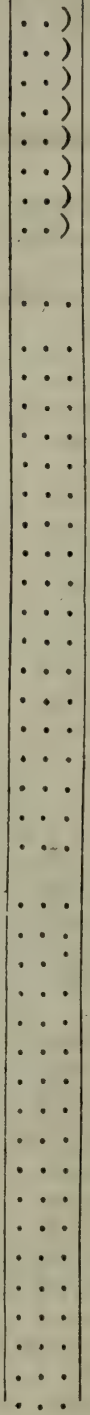 & 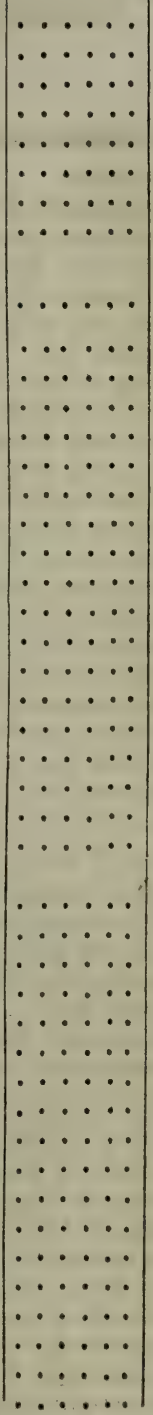 & $\begin{array}{l}\because \\
\because \\
\because \\
\because \\
\because\end{array}$ \\
\hline
\end{tabular}

A. psilonotus Qv. et A. angulatus ScrLxu, carina dorsali carent. 


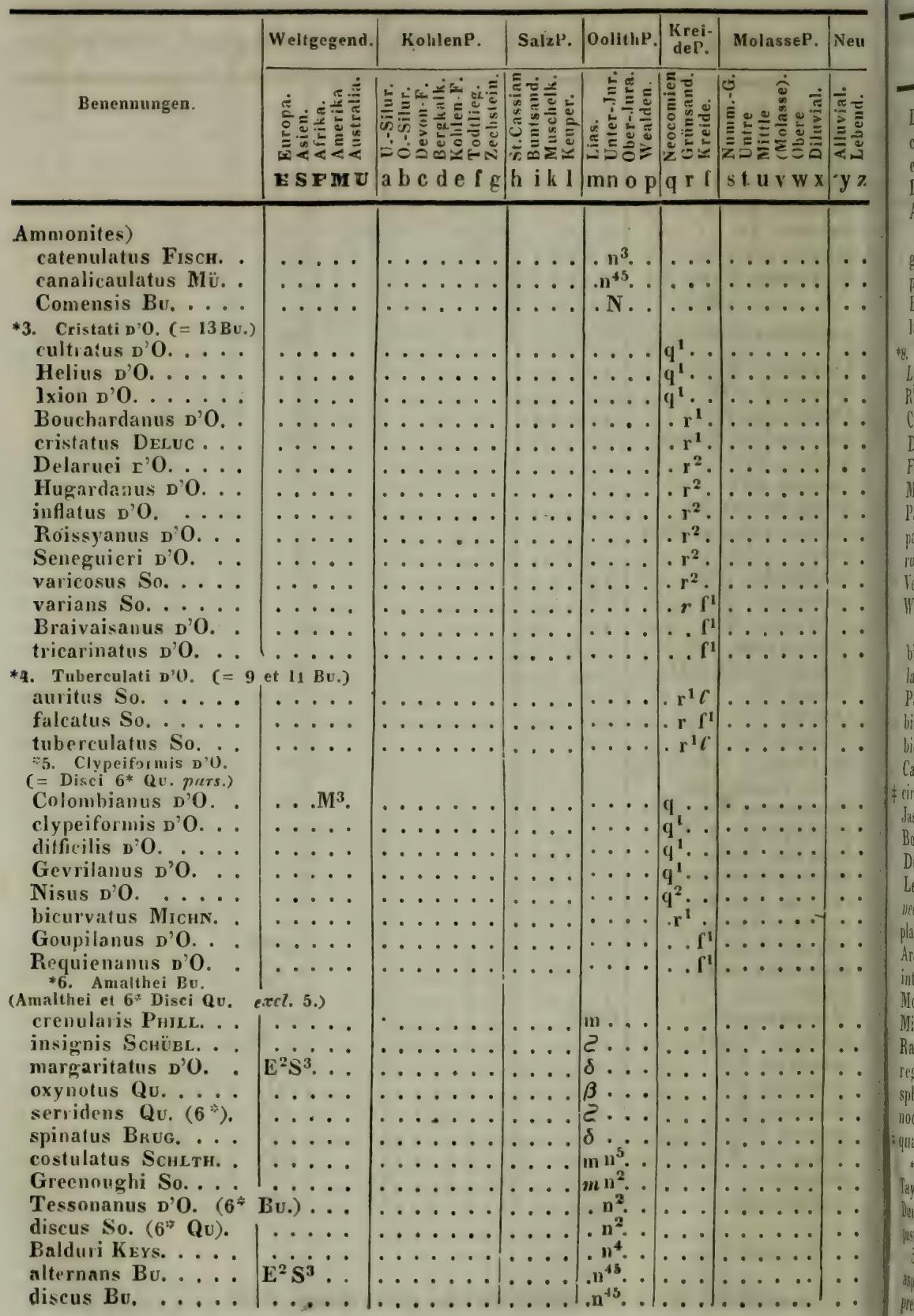




\begin{tabular}{|c|c|c|c|c|c|c|c|}
\hline Ben & send. & $a b c d$ e $f g$ & h i k l & $\mathrm{mn}$ op & 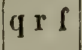 & $\operatorname{stuvw}$ & $y^{2}$ \\
\hline rti So. ... & & & &.$n^{45} \cdot \cdot$ & - & $\cdots$ & \\
\hline datus So. .... & $\mathrm{E}^{2} \mathrm{~S}^{3} \ldots$ & $\ldots \ldots$ & $\ldots \ldots$ &.$n^{45} ?$ & $\ldots$ & $\ldots \ldots$ & 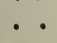 \\
\hline excavatus So..... & $\ldots$ & $\ldots \ldots$ & $\ldots$ & $\ldots 0$ & $\cdots$ & $\ldots \ldots$ & - \\
\hline Leachi So...... & $\cdots \cdot$ & $\ldots \ldots$ & $\ldots$ & $\cdots 0$. & $\cdots$ & $\ldots \ldots$ & 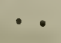 \\
\hline $\begin{array}{l}\text { Aequatorialis Bu. } \\
\text { *7. Pulchelli D'O }\end{array}$ & $\therefore \mathbf{M}^{3}$ & $\ldots \ldots$ & $\cdots$ & $\cdots \cdot$ & $q \cdot \cdot$ & $\ldots \ldots$ & - \\
\hline galeatus Bu. ... & $\ldots \mathbf{M}^{3}$. & - & $\cdots \cdots$ & & & $\cdots \cdots \cdots$ & - \\
\hline $0 . \ldots$ & $\cdots \cdots$ & $\ldots \ldots$ & $\ldots$ & & $q^{1} \cdot$. & $\ldots \ldots$ & 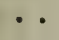 \\
\hline Brottanus D'O. ... & $\cdots \cdot \cdot$ & $\cdots \cdots$ & $\ldots$ & $\ldots$ & $\mathrm{r}^{2}$ & $\ldots \ldots$ & · \\
\hline Itieranus D'O. . . . & $\cdots \cdots$ & $\ldots \ldots$. & $\cdots$ & $\cdots$ & $\cdot \mathrm{r}^{2} \cdot$ & $\ldots \ldots$ & - \\
\hline S D'0. $\quad(=$ & $13 \mathrm{Bv})$. & & & & & & \\
\hline Lyelli I & $\dot{E^{2}} \dot{S}$ & 0.0 & $\cdots$ & $\cdots \cdot$ & $\cdot \mathrm{r}^{2} \cdot$ & $\cdots \cdots$ & $\cdots$ \\
\hline & & & & & - $r r_{1}^{1}$ & $\because$ & $\cdots$ \\
\hline $\begin{array}{l}\text { olinus DO. } 0 \text {. } \\
\text { verianus [?] D'O. }\end{array}$ & $\begin{array}{l}\cdots \\
\cdots \\
\cdots\end{array}$ & $\because$ & $\left|\begin{array}{c}\cdots \\
\cdots\end{array}\right|$ & $\because$ & $\because \begin{array}{c}r^{1} \\
\because c^{1}\end{array} \mid$ & $\cdots \cdots$ & $\because$ \\
\hline Fleuriauanus D'O. & $\ldots$ & $\cdots \cdots \cdots$ & $\cdots$ & $\left|\begin{array}{c}\cdots \\
\cdots\end{array}\right|$ & $\mid \begin{array}{cc}\cdots & 1 \\
\cdots & r^{1}\end{array}$ & $\cdots \cdots \cdots$ & $\because$ \\
\hline$\cdots$ & $\cdots \cdot$ & $\ldots \ldots$ & ... & $\ldots$ & . $? \int^{1}$ & $\ldots \ldots$ & $\therefore$ \\
\hline s n'O. & $\cdots \cdot$ & $\ldots \ldots$ & $\ldots \ldots$ & $\ldots$ & . . $1^{1}$ & $\ldots \ldots$ & $\theta_{-1}+2$ \\
\hline$\cdots$ & $\ldots \ldots$ & $\ldots \ldots$ & $\cdots$ & $\cdots$ & $\ldots 1^{1}$ & $\ldots \ldots$ & . \\
\hline$\cdots$ & $\cdots \cdot$ & $\cdots \cdots$ & $\cdots \cdots$ & $\cdots \cdot$ & $\cdots \boldsymbol{f}^{\mathbf{1}}$ & $\ldots \ldots$ & . \\
\hline Verneuilan & $\cdots \cdot$ & $\cdots \cdots$ & $\cdots \cdots$ & $\cdots \cdot$ & $.1^{1}$ & $\ldots \ldots$ & . \\
\hline $\begin{array}{r}\text { Woolgari I } \\
* 9 \text {. Denta }\end{array}$ & $\cdots \cdot$ & & . & $\ldots$ & $\ldots \mathbf{I}^{1}$ & $\cdots \cdots$ & $\cdots$ \\
\hline - & • & & & M & & $\cdots$ & $\cdots$ \\
\hline и. . & $\ldots$. & $\ldots \ldots$ & $\ldots$ & & $\cdots$ & $\ldots \ldots$ & . \\
\hline$\cdots$ & $\cdots$ & $\ldots \ldots$ & $\ldots$ & $? n^{234} ?$ & $\cdot$ & $\ldots \ldots$ & $\cdots$ \\
\hline S STAHL $_{\text {Ta }}$ & $\cdots$ & ..... & $\cdots$ &.$n^{4} \cdot \cdot$ & $\cdot$ & $\ldots \ldots$ & - \\
\hline$\cdots$ & $\ldots \ldots$ & $\ldots \ldots$ & $\cdots$ & $\cdot \mathrm{n}^{4}$ & • & $\ldots \ldots$ & .. \\
\hline Calloviensis So. . & & $\ldots \ldots$ & $\cdots$ & $\cdot \mathrm{n}^{4}$ & $\cdot$ & $\ldots \ldots$ & - \\
\hline umtentus Bu. . &.$S^{2} \ldots$ & $\cdots \cdots$ & $\cdots \cdots$ & - $\mathrm{n}^{4}$. & $\cdot$ & $\ldots \ldots$ & - \\
\hline Jason Bu. . . . . & $\therefore \quad \therefore$ & $\cdots \cdots \cdot$ & $\cdots$ & $\cdot n^{4}$ & $\cdots \cdot$ & $\cdots \cdots$ & $\cdots$ \\
\hline Bogoiensis ForB. : & $\because \mathrm{M}^{3}$. & $\cdot$ & & . & $q_{2}$ & $\cdots$ & • \\
\hline Dufrenoyi D'0. [11 & Qu.] · & - & & $\cdots$ & & $\cdots \cdots$ & \\
\hline sis $\mathrm{D}^{\prime} \mathrm{O} \cdot \dot{\text { i1 }}$ & $1 \mathrm{Qu}] .$. & $\dot{.}$ & & $\dot{\bullet} \cdot \overrightarrow{\mid}$ & & $\cdots \cdots \cdots$ & $\therefore$ \\
\hline . . & . $\mathrm{M}^{3}$ & ... & & $\ldots$ & $q$ & $\ldots \ldots$ & - \\
\hline . & $\cdots \cdots$ & $=$ & & $\cdot$ & & $\ldots \ldots$ & • \\
\hline - $\cdot$ & • & $\cdot$ & & $\cdots \cdot$ & $\cdot \mathrm{r}^{2} \cdot$ & $\cdots \cdots$ & \\
\hline$\cdots$ & : & . & & & & $\cdots \cdots$ & \\
\hline dlinanus $D$ & & & & & & $\cdots$ & $\because$ \\
\hline Alinanus $\mathrm{D}$ & $\because$ & & & $\dot{.}$ & $\mathrm{r}^{1}$ & $\cdots$ & \\
\hline$\therefore$ & $\cdots$ & $\cdots$ & $\ldots$ & $\ldots$ &.$r^{1}$ & $\ldots \ldots$ & . \\
\hline atus D'O. & .... & $\ldots \ldots$ & & & $\mathrm{r}^{1}$ & $\ldots \ldots$ & . \\
\hline $\begin{array}{c}\text { quadriseriatus DEH. } \\
* 10 \text {. Ornati Bu. }\end{array}$ & & 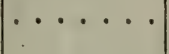 & 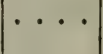 & $(: \ldots$ & $\cdots)$ & $\cdots \cdots$ & $\cdots$ \\
\hline Taylori So & & - & $\cdots \cdot \cdot$ & & $\cdots$ & & $\cdots$ \\
\hline $\mathrm{ni} S$ & & $\cdots \cdots \cdots$ & $\cdots \cdot$ & $\mathrm{n}^{4} \ldots$ & & $\cdots \cdots$ & . \\
\hline atus I & $\cdots$ & $\cdots$ & 0 & $\cdot n^{4} \cdot$ & $\cdots$ & $\cdots \cdots$ & • \\
\hline $\operatorname{Tr}$ & 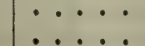 & 0 & . & . & $a^{1}$ & $\cdots$ & $\cdots$ \\
\hline & & & & & & & \\
\hline
\end{tabular}




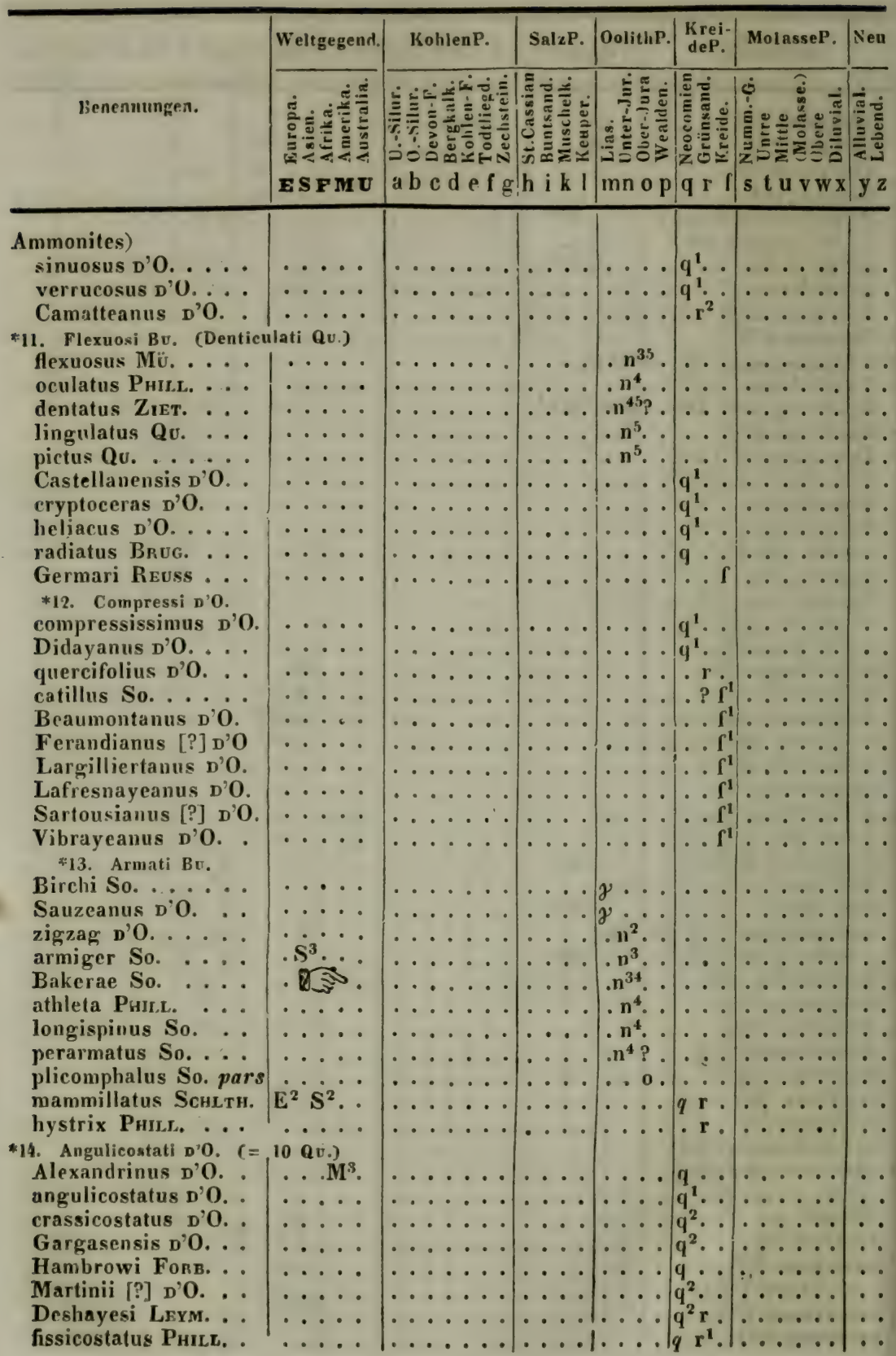




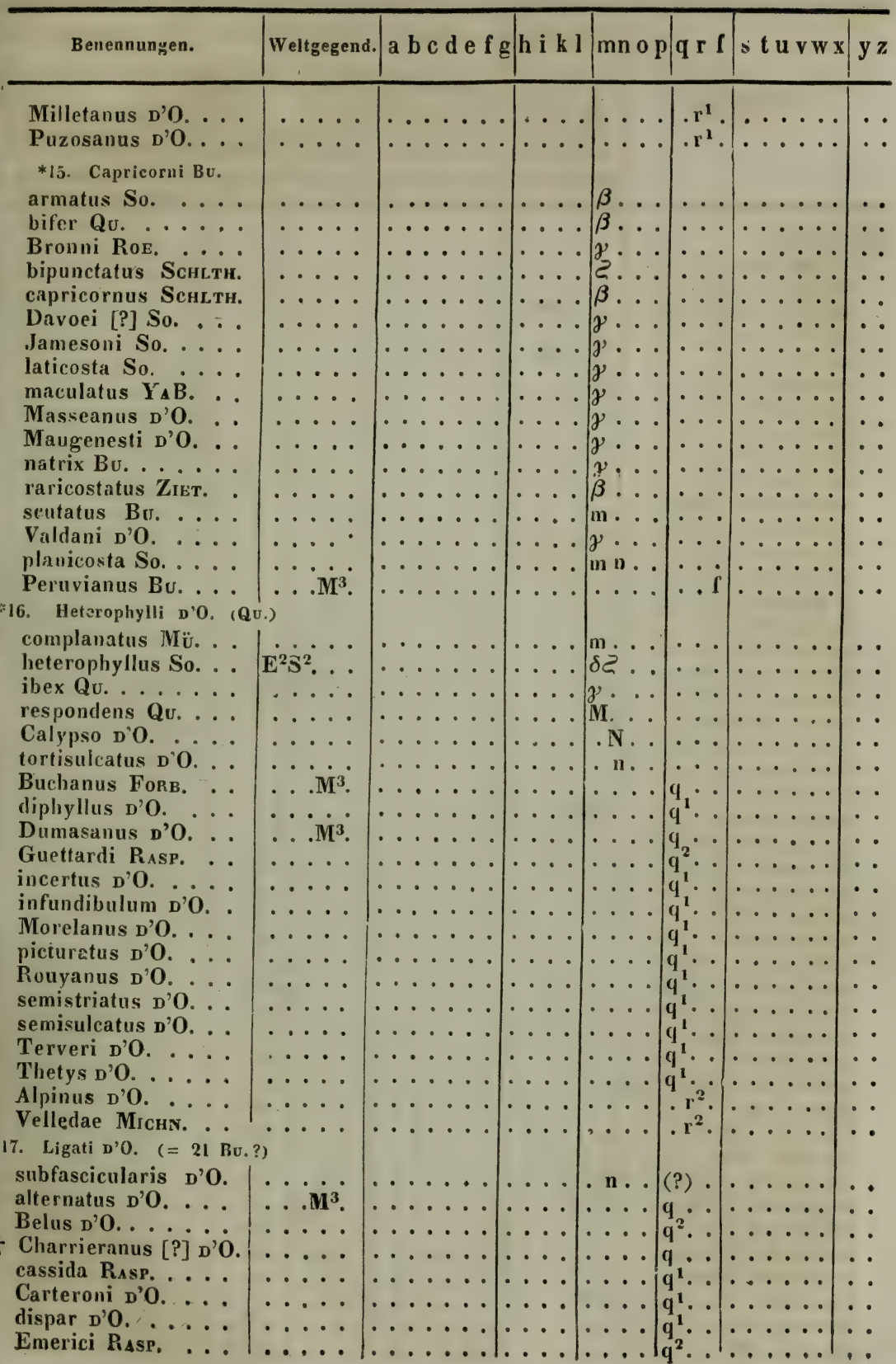




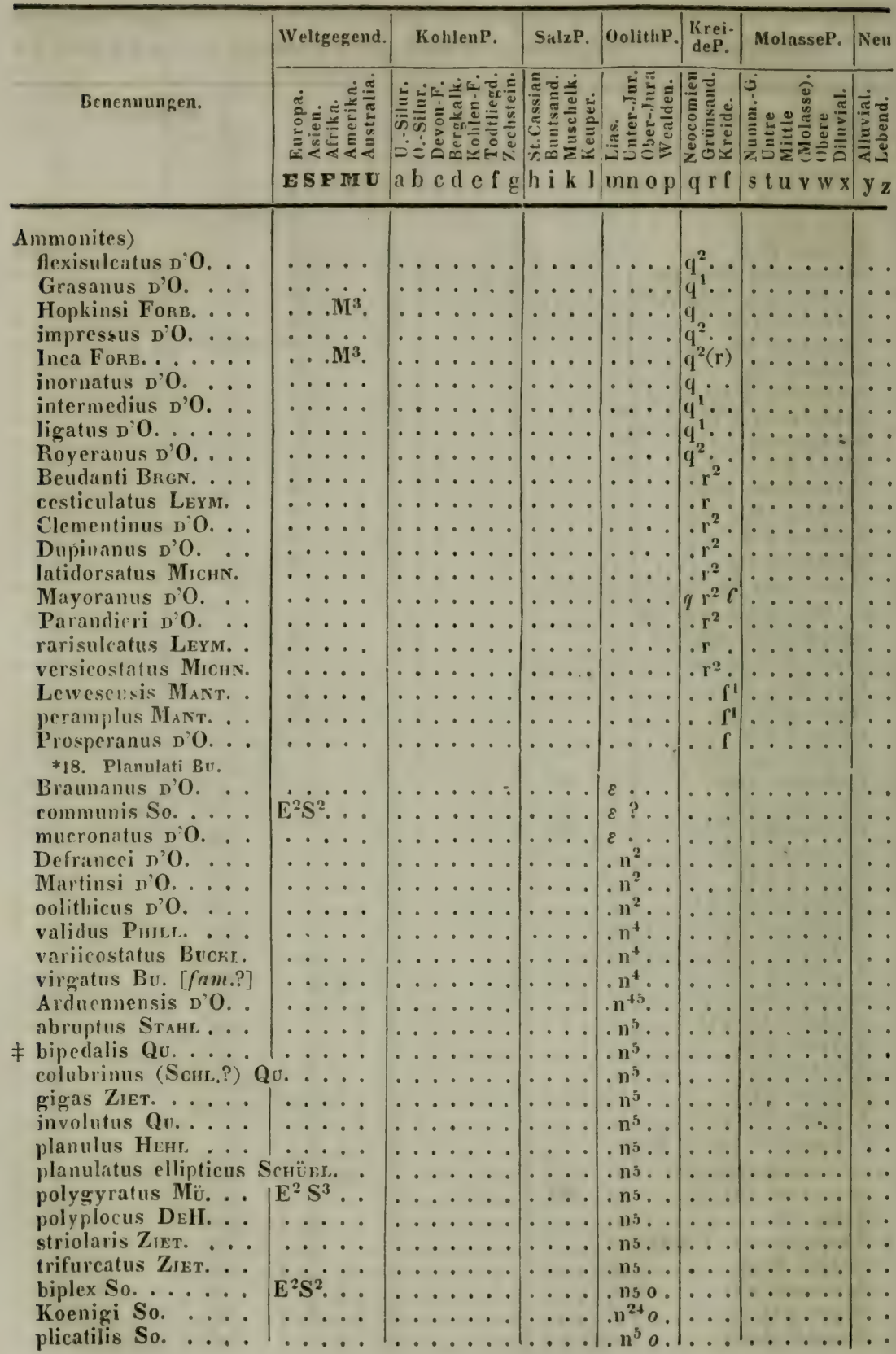




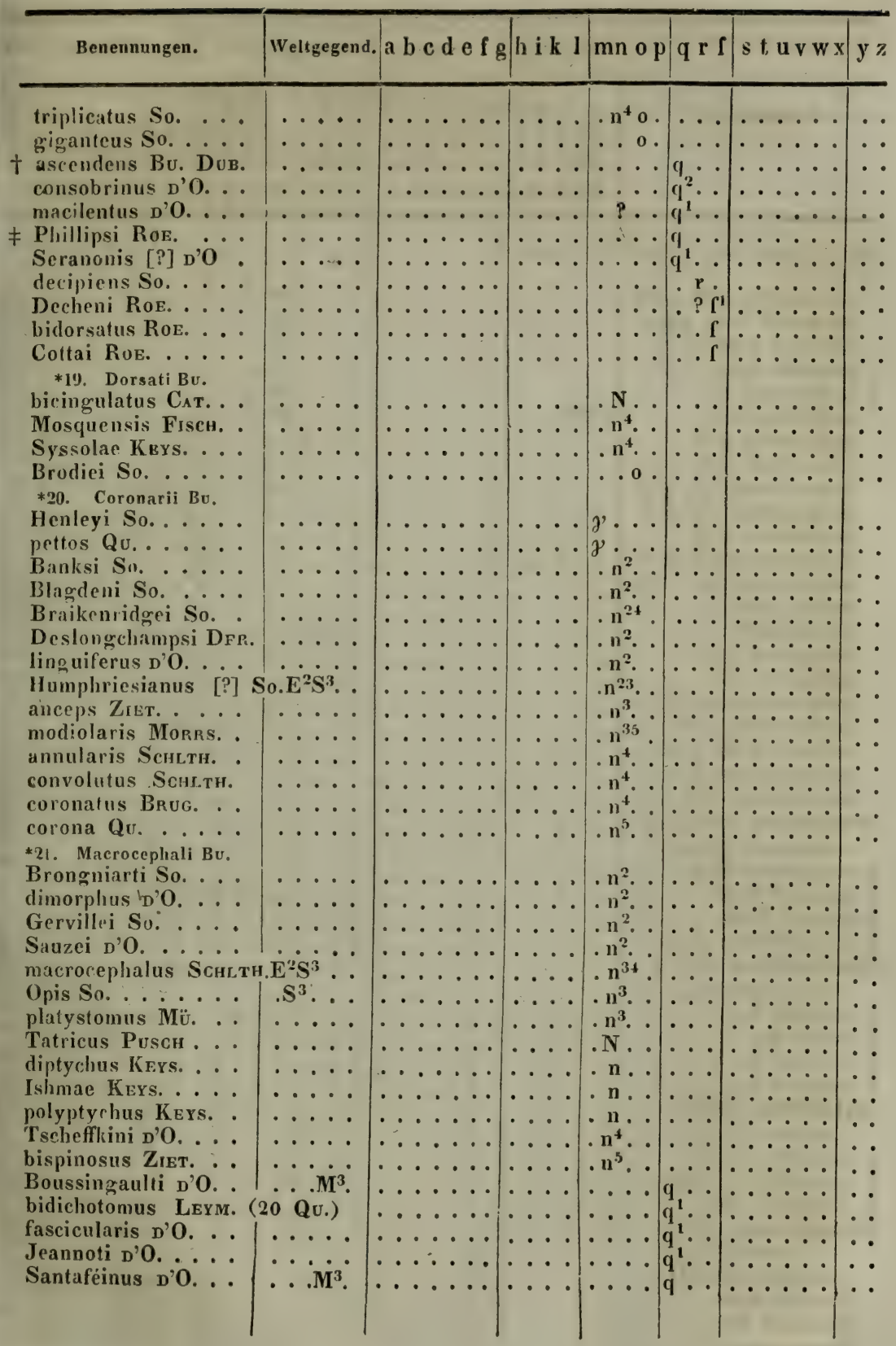




\begin{tabular}{|c|c|c|c|c|c|c|c|}
\hline & Weltgegend. & KohlenP. & SalzP. & OolithP. & $\left|\begin{array}{c}\text { Krei- } \\
\text { deP. }\end{array}\right|$ & MolasseP. & Neu \\
\hline Benennungen. & 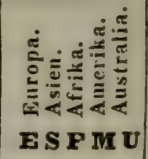 & 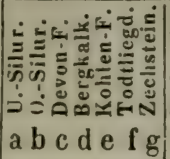 & 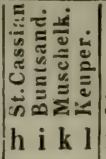 & 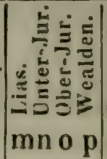 & 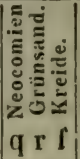 & 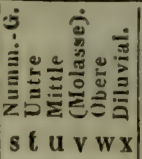 & 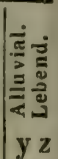 \\
\hline
\end{tabular}

*22. Fimbriati d'0. (Lineati Qu.; fum. $15 \mathrm{Bv}$.)

cornucopiae D'O. pars Germaini D'O. pars. hircinus ScuLth. jurensis $\mathrm{Z}_{\mathrm{IET}}$.

\# ncojurensis Qu.

fimbriatus So. .

Eudesanus D'O.

Linneanus D'O.

Pictaviensis [?] D'O. flexicostatus PuIL.

Duvalanus D'O.

Honnoratanus D'O. . inaequicostatus D'O. Juilleti D'O.

lepidus D'O.

Matheroni D'O. ophiurus d'O. quadrisulcatus $\mathbf{D}^{\prime} \mathbf{O}$. recticostatus D'O. subfimbriatus D'O. striato-sulcatus $\mathrm{D}^{\prime} \mathrm{O}$. strangulatus D'O.

(PIanitea DeH.) 4.

= Anmonites Brvg. = \# cingulatus Deн. . . \# costatus DEH.

F Listeri DeH.

F tenuicostatus DEH.

(Orbulites LK.) 4.

= Ammonites Brug. = \# dorsalis LK.

\# laevis LK.

$\neq$ striatus LK.

f undosus $\mathbf{L K}$.

(Globites DeH.) 3.

Immonites BRUG. = ₹ fasciatus DEH.

$\neq$ granuliferus DEH.

f undulatus DEH.

Crioceras (Lév.)

$=$ ? Tropaeun So. $=$

Cornuelanum D'O.

Emerici LÉv.

Fourneti Duv.

Puzosanum D'O.

1

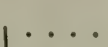

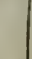




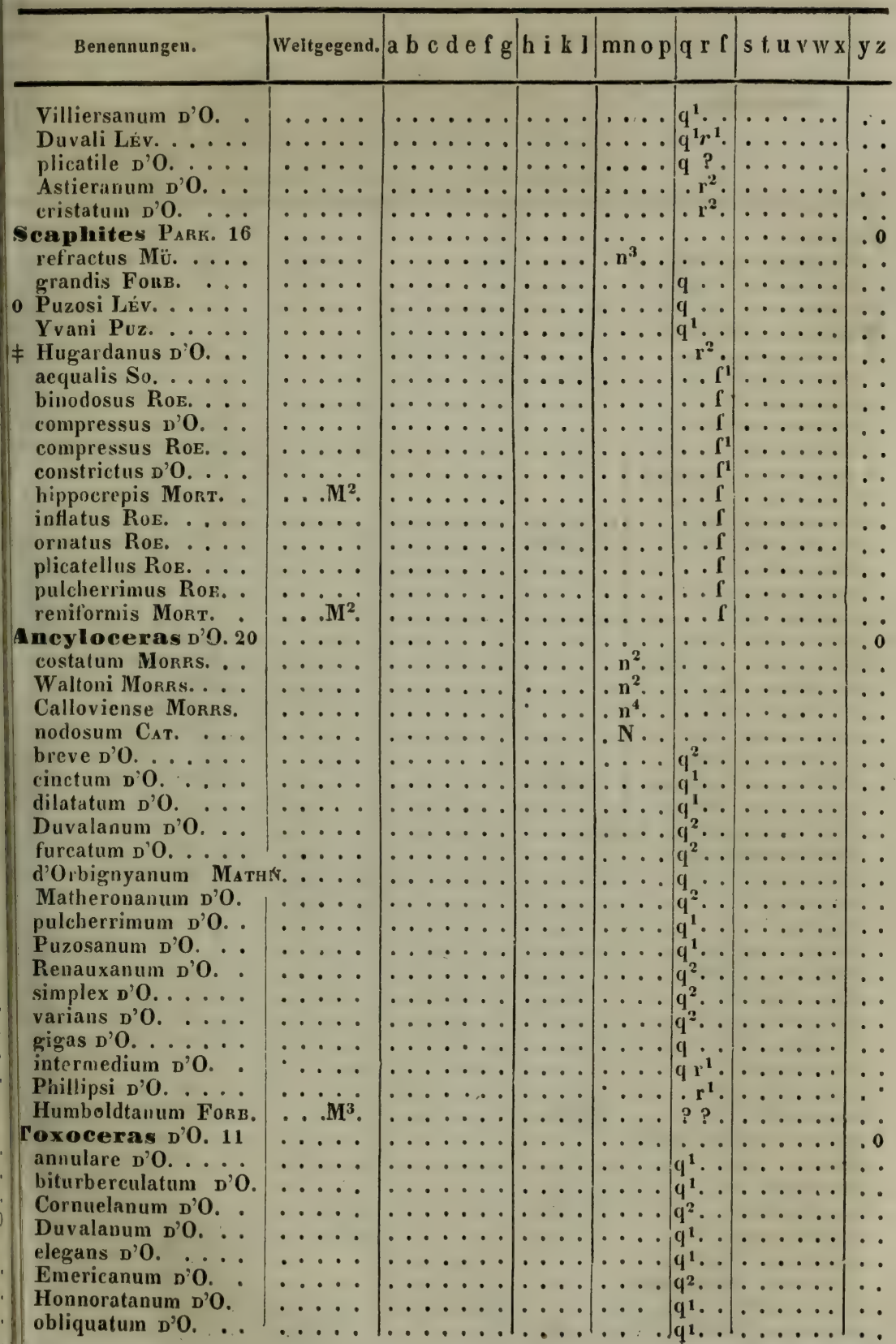




\begin{tabular}{|c|c|c|c|c|c|c|c|}
\hline & Weltgegend. & KohlenP. & SalzP. & OolithP. & $\begin{array}{l}\text { Krei- } \\
\text { deP. }\end{array}$ & MolasseP. & Neu \\
\hline Benennungen. & 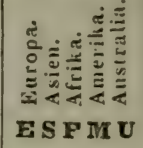 & 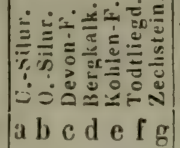 & 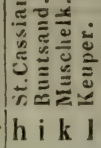 & 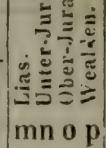 & 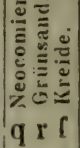 & 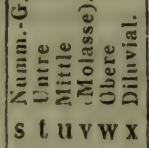 & 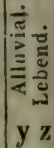 \\
\hline
\end{tabular}

Toxoceras)

Requienanum D'O.

Royeranum D'O.

? gricile D'O.

Hamites PARK. 45.

* Crioceratitae spp. nodosus So..... spinulosus So. tuberculatus So. . turgidus So.

* Hamitac spp. gensinue. biplicatus Roe. decurrens RoE. Degenhardti Bu. . dissimilis D'O. Emericanus D'O. incertus D'O. Labatii [?] Cat: . oblique-costatus Roe.

₹ parallelus Dris.

Royeranus D'O. semicinctus Roß... subuodosus RoE. d'Orbiguyanus Fors. varicostatus Phrr.L. alterne-tuberculatus LEYM. ... Bouchardanus D'O. canteriatus Brgv. elegans n'O. flexuosus D'O intermedius So. Parkinsoni BrigN. punctatus D'O. Raulinanus D'O. rotundus d 0 .

Sablieri D'O.

virgulitus Brgr. armatus So. attenuatus (So.) D'O. Roemeri Geiri. alternans GEIN. arculus Mort. .... cylindraceus D' 0 . ellipticus? Mant. giganteus Dsmar. $s p$. simplex D'O. torquatus MORT. trabeatus Mort.
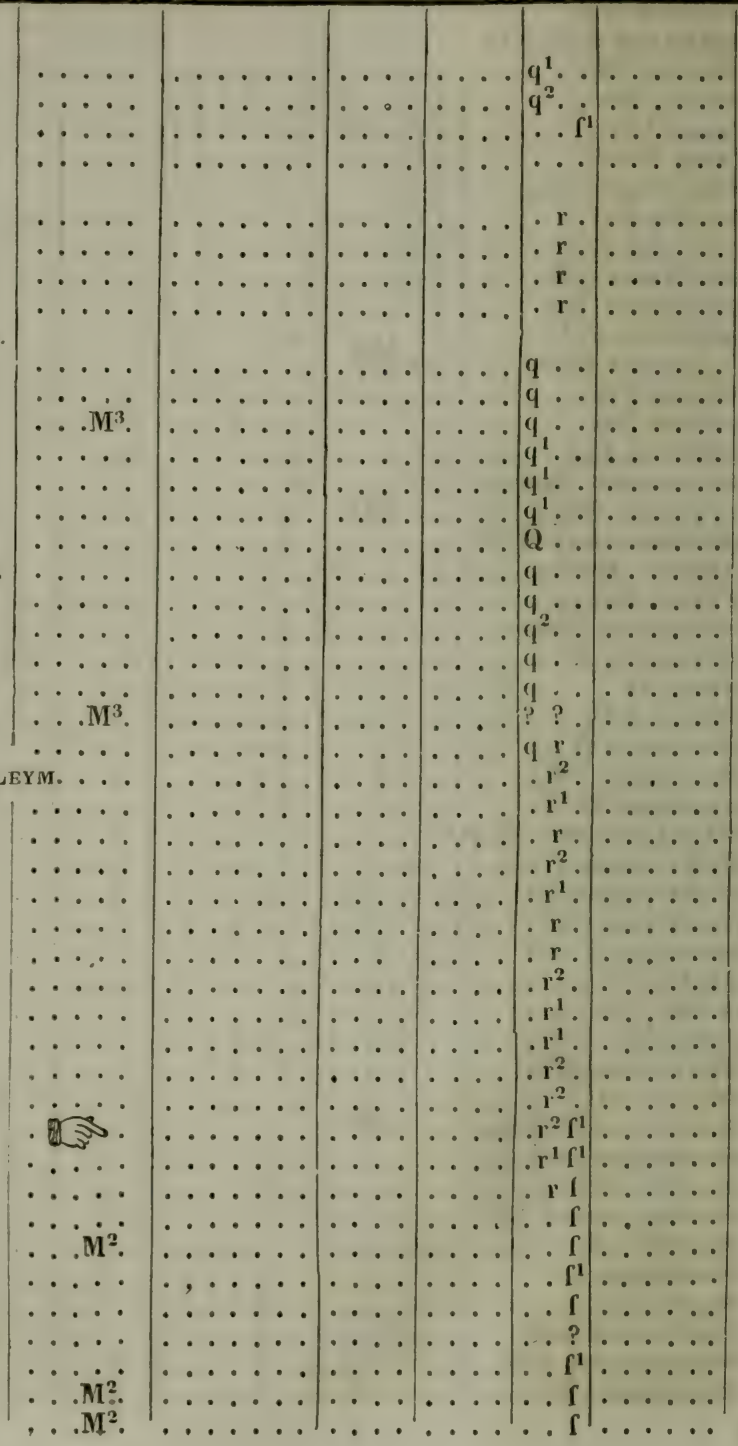


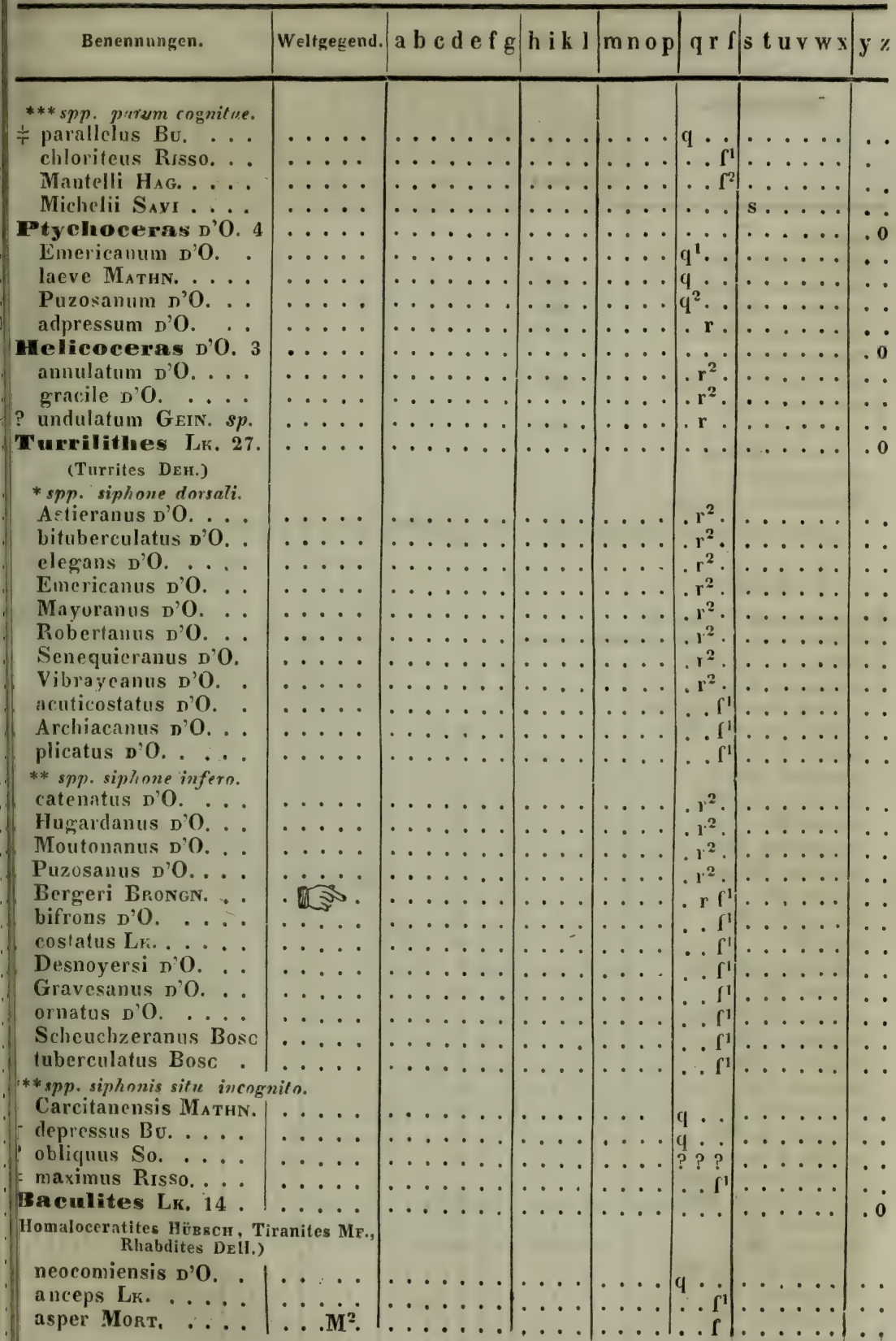




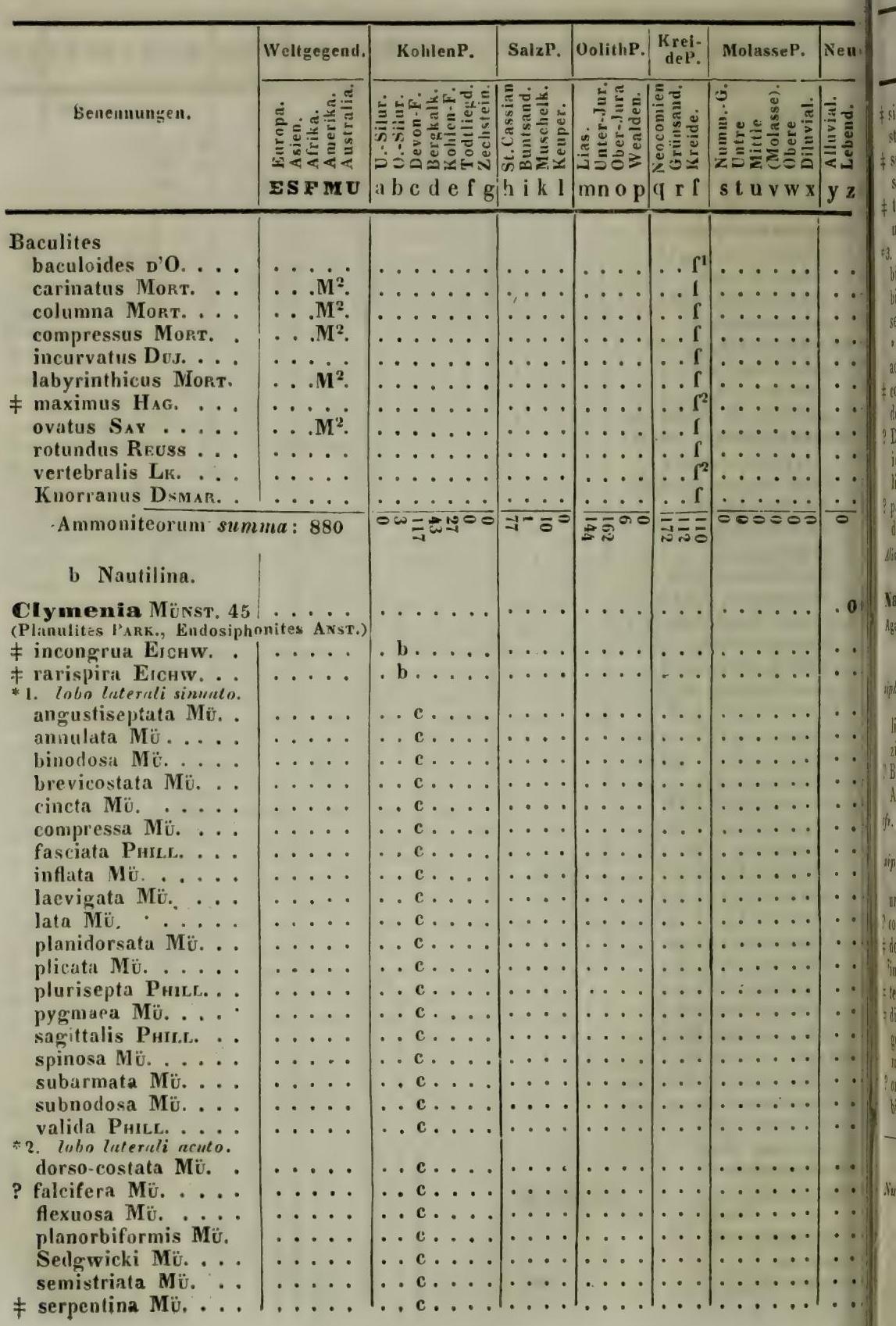




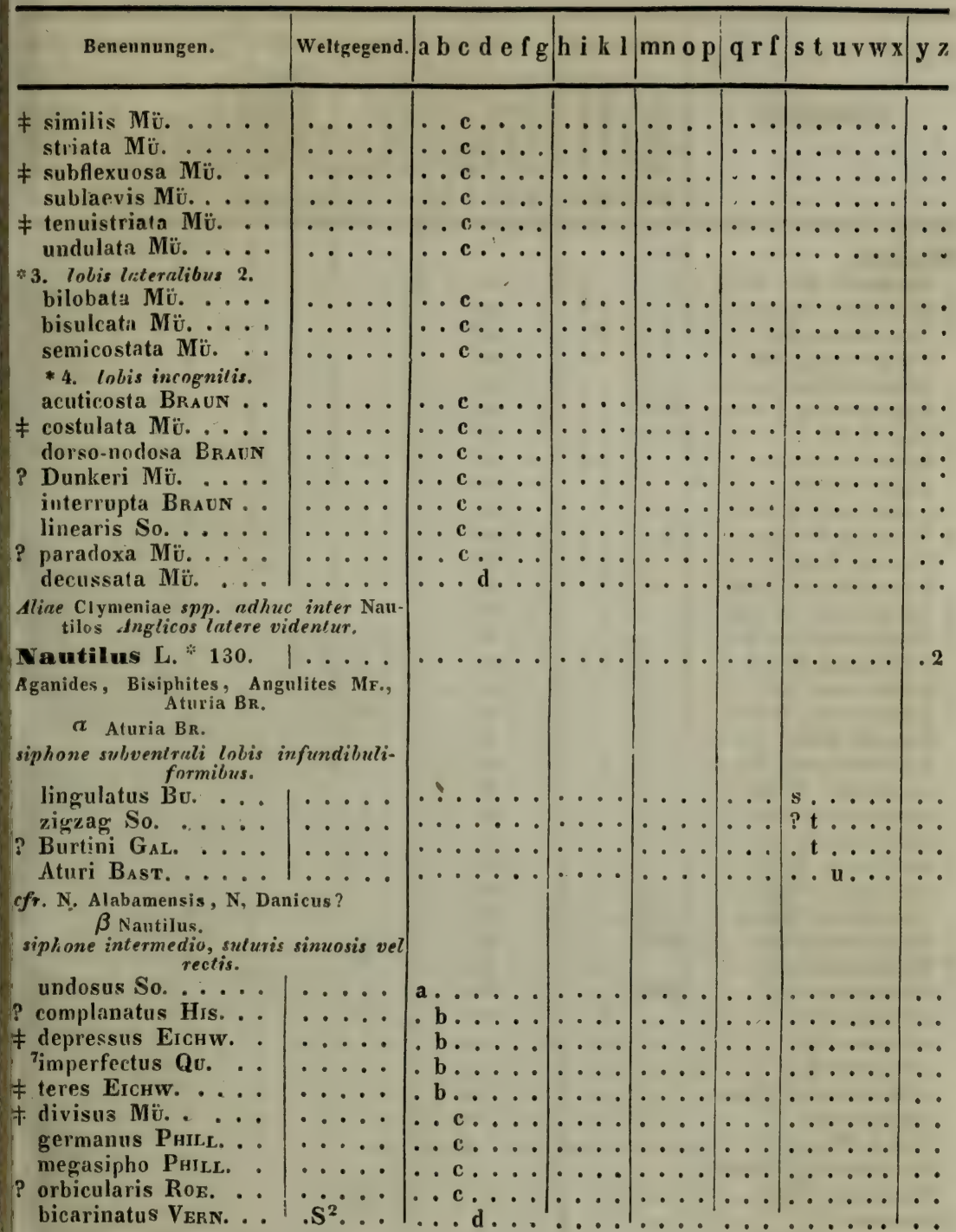

* Sex generis sectiones distinguit Qu., sed paucas species tantum in sectiones suas refert; Vumeris 1-7 indicantur sectiones hae:

1 A turia Br., quam a seguente removendam censemus, $t$

3 Undulati Qu.

4 Simpliees Qu.

5 Bisiphites Qu.

6 Moniliferi Qv.

7 Imperfecti Qv. 
Benยกแนกแูยก.

\begin{tabular}{|c|c|c|c|c|c|c|}
\hline Weltgegend. & KolulenP. & SalzP. & OolithP. & $\mid \begin{array}{c}\mathrm{Krei}_{\mathrm{rei}} \\
\mathrm{deP} .\end{array}$ & MolasseP. & Neu \\
\hline 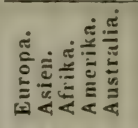 & 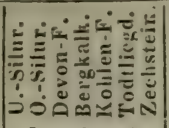 & 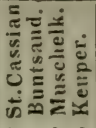 & 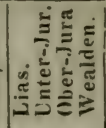 & | & 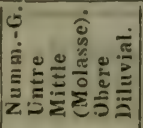 & 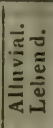 \\
\hline ES F M U & $\mid a \mathbf{b} c d$ e $f g \mid$ & h i k l & $\mathrm{mn}$ op & $q \times 1$ & $|s t u v w x|$ & $\mathbf{y z}$ \\
\hline
\end{tabular}

\section{Nautilus)}

bilobatus So.

† carinatus Елснw.

${ }^{7}$ cariniferus So.

'complanatus So.

costalis Phizr. cyclostomus Paile.

'discus So.

dorsalis Phicl.

globatus So.

goniolobus РнILL.

† hesperis Erchw.

'ingens Martin

Leveilléanus Kon.

$\neq$ marginatus FLEM.

${ }^{7}$ multicarinatus So.

oxystomus P'HILL.

pentagonus So.

perplanatus PontL.

pinguis KoN.

planidorsatus Pontr. quadratus Frem. subsulcatus Puick. : ${ }^{7}$ sulcatus So.

₹ sulciferus PhiLe. tetragonus PHILL.

Tscheffkini VERN. tuberculatus So.

? Woodwardi So. clitellarius So. . stygialis Kon. . . . . armatus So. ..... concavus So. endosiphonus Phill. falcatus So.

Luidi MART. $_{\text {A }}$

Ficieslebeni Gein.

${ }^{6}$ bidorsatus $B_{R}$.

$\ddagger^{6}$ nodosus Mü. annularis Phile. astacoides $Y_{A} B$.

sintermedius So. inornatus $D^{\prime} O$. latidorsatus v'O.

₹ mesodicus Qu. semistriatus D'O. striatus So.

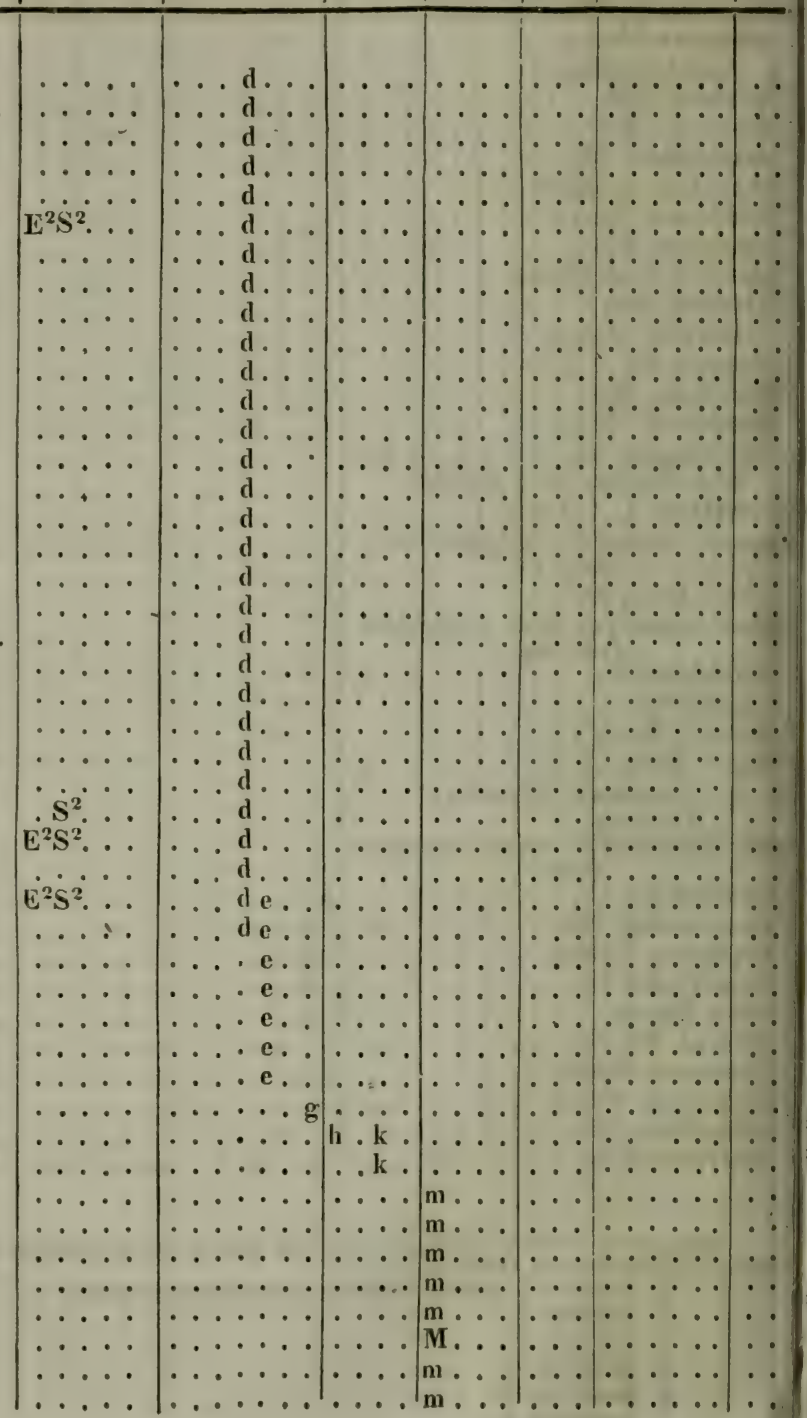




\begin{tabular}{|c|c|c|c|c|c|c|c|}
\hline Benennungen. & Weltgegend. & a b c d e f $g$ & h i k 1 r & $\mathrm{mn}$ op & $q \times 1$ & st u v w x & $8 \%$ \\
\hline & $\cdots \cdot \cdot$ & & & & & & \\
\hline $0 \ldots$ & $\cdots \cdots$ & & $\cdots \cdot$ & $\cdot n^{3} \cdot \cdot$ & $\cdots$ & $\cdots \cdots$ & \\
\hline$\cdots \cdots$ & $\cdots \cdot \cdot$ & $\cdots \cdots$ & & $\cdot \mathrm{n}^{2} \cdot \cdot$ & $\cdots \cdot$ & $\cdots \cdots$ & \\
\hline $\begin{array}{l}\text { excavatus } \\
\text { giganteus }\end{array}$ & $\begin{array}{l}\cdots \\
\cdots\end{array}$ & $\cdots$ & & $\cdot n^{2}$ & $\cdots$ & $\cdots \cdots$ & - \\
\hline o. & $\cdots \cdots$ & $\cdots \cdots \cdots$ & & $\mid \cdot n^{45} \cdot$ & $\cdots$ & $\therefore \cdots$ & - \\
\hline $\begin{array}{l}\text { granulosus D'O. . . } \\
\text { hexagonus So. . . }\end{array}$ & $\ldots$ & $\cdots \cdots \cdots$ & $\mid \cdots \cdots$ & $\left|\begin{array}{l}\cdot n^{4} \cdots \\
\cdot n^{4} \ldots\end{array}\right|$ & $\cdots$ & $\cdots \cdots$ & ․ \\
\hline${ }^{4}$ lineatus So. .... & $\cdots \cdot$ & $\ldots \ldots$ & $\ldots$ & $\cdot 1^{2} \cdot$. & $\ldots$ & $\ldots \ldots$ & $\ddot{\bullet}$ \\
\hline lineatus (So.) Ziet. & ... & $\ldots \ldots$ & $\ldots$. & $\cdot n^{2} \cdot \cdot$ & $\ldots$ & $\ldots \ldots$ & . \\
\hline So. ..... & $\cdots \cdot$ & $\cdots$ & $\ldots$. & $\cdot \mathrm{n}^{2} \cdot \cdot$ & $\cdots$ & $\ldots \ldots$ & - \\
\hline polygonalis So. .. & $\cdots \cdot$ & -1 & $\cdots \cdot$ & $\cdot n^{2} \cdot$ & $\cdots$ & $\cdots \cdots$ & - \\
\hline${ }^{2}$ sinuatus So. .... & $\cdots \cdot$ & $\cdots \cdot$ & $\cdots \cdot$ &. $\mathrm{n}^{25} \cdot \cdot$ & $\cdots$ & $\ldots \ldots$ & - \\
\hline OE. $\cdots$ & $\cdots \cdots$ & $\cdots$ & $\cdots$ & $\ldots 0$. & $\cdot \cdot \cdot$ & $\cdots \cdots$ & $\cdot \cdot$ \\
\hline ' 0. & $\cdots \cdots$ & $\cdots$ & $\cdots \cdot$ & . . 0 & $\cdots$ & $\cdots \cdots$ & $\cdots$ \\
\hline in & $\cdots$ & & & $\cdot .0$ & $\cdots \cdot$ & $\cdots \cdots$ & $\cdots$ \\
\hline $\begin{array}{l}\text { D’O. . } \\
\text { ? ScHLtH. }\end{array}$ & $\therefore \cdots$ & & $\cdots \cdots$ & $\because 0^{0}$ & $\cdots \dot{i}$ & $\cdots \cdots$ & $\cdots$ \\
\hline $\begin{array}{l}\text { uratus ? ScHLTH. } \\
\text { ites MF. . . }\end{array}$ & $\cdots$ & $\cdots$ & $\left|\begin{array}{c}\cdots \\
\cdots\end{array}\right|$ & 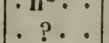 & ?.? & $\cdots \cdots$ & $\therefore$ \\
\hline$\ldots$ & $\cdots \cdot \cdot$ & . & $\ldots$ & $\ldots$. & $q \ldots$ & $\ldots \ldots$ & $\because$ \\
\hline elegans D'O. & $\cdots \cdot$ & $\cdots$ & $\cdots$ & $\ldots$ & $q .$. & $\ldots \ldots$ & \\
\hline$\ldots .$. & & - & $\cdots$ & $\cdot$ & & $\ldots \ldots$ & $\therefore$ \\
\hline${ }^{3}$ radiatus So. . & $E^{2} S^{2} \sqrt{3}$ & $\cdots \cdots$ & $\cdots \cdot$ & $\cdots$ & $q r 1^{1}$ & $\cdots \cdots$ & $\therefore$ \\
\hline${ }^{3}$ arc & $\ldots$ & $\cdots \cdots \cdot$ & $\cdots$ & $\ldots \cdot$ & . $\mathrm{r}$ & $\ldots \ldots$ & - \\
\hline D'O. . & $\cdots \cdot$ & $\cdots$ & $\cdots \cdots$ & $\cdots \cdot$ & . r. & $\ldots \ldots$ & - \\
\hline $0 .$. & $\ldots$ & $\ldots \ldots$ & $\cdots$ & $\ldots$ & $|r \cdot|$ & $\cdots \cdots$ & - \\
\hline$\cdots$ & $\cdots \cdots$ & $\ldots \ldots$ & $\cdots$ & $\cdots$ & - $r$. & $\cdots \cdots \cdots$ & • \\
\hline${ }^{4}$ simplex So. . . . . & $\cdots \cdot \cdot$ & $\cdots$ & $\cdots$ & $\cdots \cdot$ & - $\begin{array}{l}3 \\
0\end{array}$ & $\cdots \cdots$ & - $\cdot$ \\
\hline$\ddot{M} \cdot \cdot$ & $\cdots \dot{\mathrm{M}}^{\circ}$ & & $\Gamma^{\circ}$ & - & (? : 1 & $\cdots \cdots$ & - \\
\hline ensis Mont. & $\cdots M^{2}$ & & & $\cdots$ & $\cdots 1$ & $\because$ & - \\
\hline . & $\therefore$ & & & & $\cdots r^{1}$ & $\because$ & \\
\hline sso. & . . & - 1 & $\because$ & & {$\left[\begin{array}{ll}1 & 1 \\
1 & 1\end{array}\right]$} & $\cdots \cdots$ & \\
\hline Schlth. . & $\cdots$ & $\because$ & & $\cdots$ & $\therefore$ i & $\ldots \ldots$ & \\
\hline RT. . . & . $\mathbf{M}^{2}$ & .... & • & $\cdots$ & $\therefore i$ & $\ldots \ldots$ & \\
\hline sanus D'C & $0 .$. & $\ldots \ldots \ldots$ & $\ldots$ & ... & $\ldots f^{1}$ & $\ldots \ldots$ & - \\
\hline koanus D'O. . & . . M & . . & $\ldots$ & $\ldots$. & $\ldots 1$ & $\ldots \ldots$ & . \\
\hline$\cdots$ & $\cdots \cdot$ & $\ldots$ & . . & ... & $\ldots f$ & $\ldots \ldots$ & - \\
\hline Aus D'O. . & $\cdots$ & 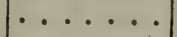 & $\cdots$ & . & . . $\mathrm{l}^{1}$ & $\ldots \ldots$ & - \\
\hline$\cdots$ & $\ldots$ &. & $\ldots$ & - & $\ldots 1$ & $\cdots$ & . \\
\hline lae & $\cdots$ & $\cdots$ & . & & . $1^{1}$ & $\cdots \cdots$ & - \\
\hline lliertanus D'O. & $\cdots \cdot \cdot$ & $\cdots$ & $\cdot$ & & - . $\Gamma^{\prime}$ & $\cdots \cdots$ & $\cdots$ \\
\hline D'O. . & $\cdots \cdots$ & $\cdot 1$ & $\cdots$ & - & 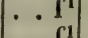 & $\because$ & $\cdots$ \\
\hline Monfortanus Riss. & - & & & &. $\mathrm{I}^{2}$ & . & \\
\hline $\begin{array}{l}\text { perl } \\
\text { pset }\end{array}$ & - $M^{2}$. & & & & & . & \\
\hline $\begin{array}{l}\text { p: } \\
\text { p) }\end{array}$ & & . &. & $\cdots$ & $\cdots \mathrm{r}^{1}$ & & $\cdots$ \\
\hline 0 . . & & 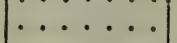 & $\cdots \cdot$ & $\cdots$ & . . $\mathrm{f}^{1}$ &. & \\
\hline . & & & & $\cdots$ & . . $\boldsymbol{1}^{1}$ & $\cdots \ldots$ & \\
\hline - & $\cdots \cdots$ & o & $\cdots$ & $\cdots$ & $\cdots$ & $s \cdot \cdot$ & \\
\hline 4 inpnerialis & $\cdots \cdot$ & $\cdots$ & $\cdots$ & $\cdots$ & & t : & \\
\hline $\begin{array}{l}{ }^{4} \text { imperialis So. } \\
\text { Lamarcki Ds. }\end{array}$ & $\cdots \cdots$ & - & : & $\cdots \cdot$ & .1 & $t ?$ & . \\
\hline
\end{tabular}




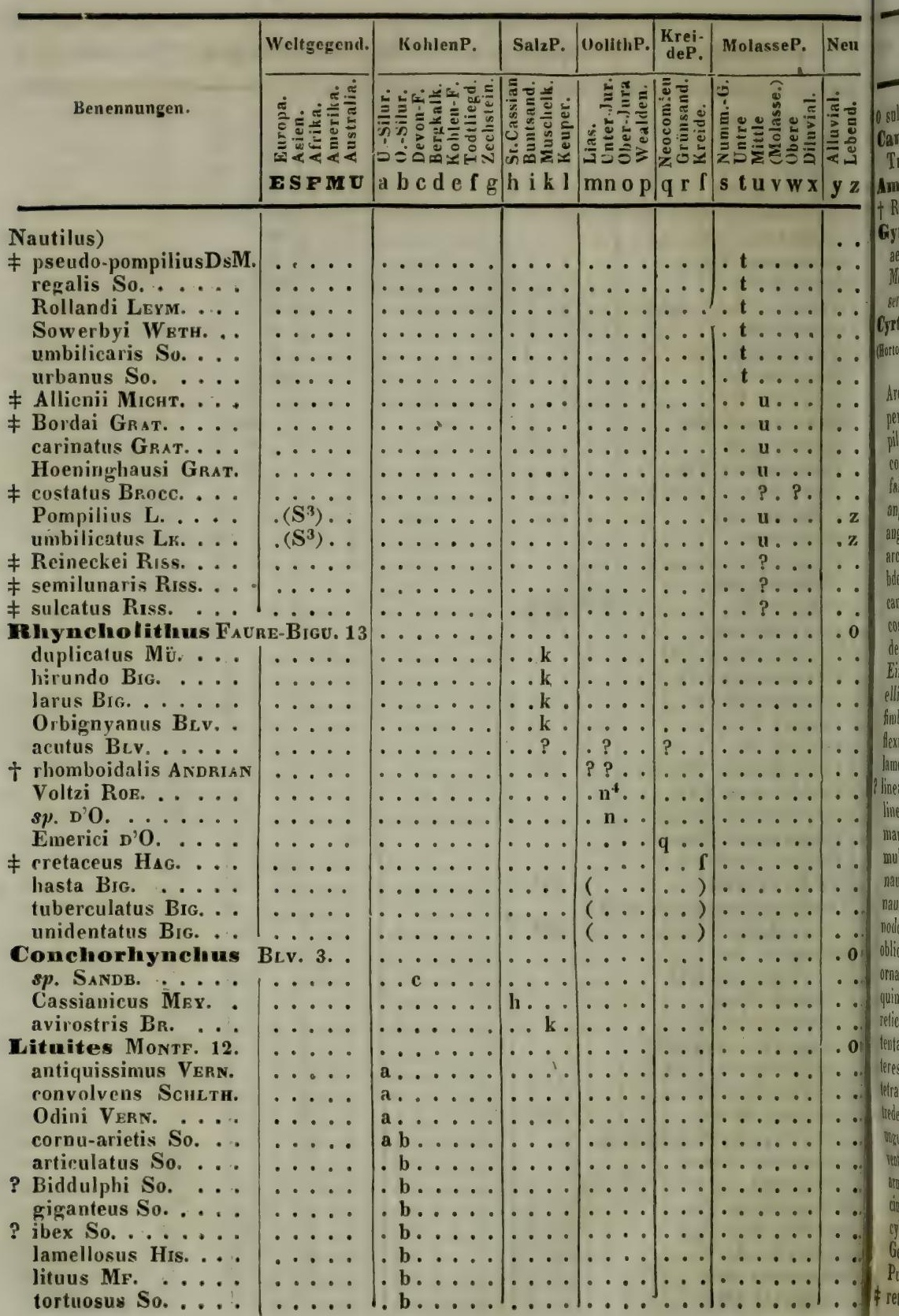


Benennungen. Weltgegend. a b c d e f g h i k l mn op q r l s t u v w s y z

b sulcatus Risso. $1 \ldots \ldots$ Cameroceras Emms. 1. Trentonense Emms.

$\dot{M}^{2}$. Amblyceras GLock - Rittbergense GLock. Gyrocerag KoN. 3. aegocerus Kon. .. Meyeranum KoN. serratum Kon.

\section{Cyrtocerns GF. 44}

Hortolus Mr., ?Amimonus Mr. Campulites Dsh.)

Archiaci Vern. perfectum WhLB. $s p$. pilosum Emms. convolvens MF. falcatum Eichw. angulatum $\mathrm{Mü.}$ angustiseptatum $\dot{M} \ddot{\text {. }}$ arcuatum VERN. bdellalites Phicz. cancellatum Ros. costatum Mü. depressum GF. Eifelense AV. ellipsoideum SNDB. fimbriatum Phill. flexuosum AV. lamellosum AV.

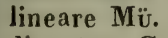
lineatum GF. marginale Phill. multistriatum RoE. nautiloides $\mathbf{A V}$. nautiloideum Phic.. nodosum Phic. obliquatum Phick. ornatum GF. quindecimale Phics. reticulatum PhiLl. tentaculatum Mü. teres Roe. tetragonum AV. tredecimale PhILL. ungulatum Mü. ventricosum RoE. armatum Phice. . cinctum Mü. cylindricum FahRK. Gesneri Kon. Puzosanum Kon. reticulatum KoN.

|

\begin{tabular}{|c|c|c|c|c|c|}
\hline & 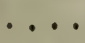 & ??.. & . ? ? & $\cdots \cdots$ & \\
\hline & $\cdots$ & $\cdots$ & $\cdots$ & $\cdots \cdots$ & .0 \\
\hline 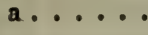 & $\cdots$ & $\ldots$ & $\cdots$ & $\cdots \cdots$ & $\cdots$ \\
\hline & $\cdots$ & $\cdots$ & $\cdots$ & $\cdots \cdots$ & .0 \\
\hline . c ... & $\cdots \cdots$ & $\cdots$ & $\cdots$ & $\cdots \cdots$ & - \\
\hline$\cdots \cdots$ & $\cdots \cdot$ & $\cdots \cdots$ & $\cdots$ & $\cdots \cdots$, & .0 \\
\hline$\because \mathrm{d}$ & $\cdots \cdots$ & $\cdots$ & $\cdots$ & $\cdots \cdots$ & • \\
\hline$\therefore d$ & $\cdots$ & $\cdots$ & $\because \cdots$ & $\cdots$ & \\
\hline
\end{tabular}

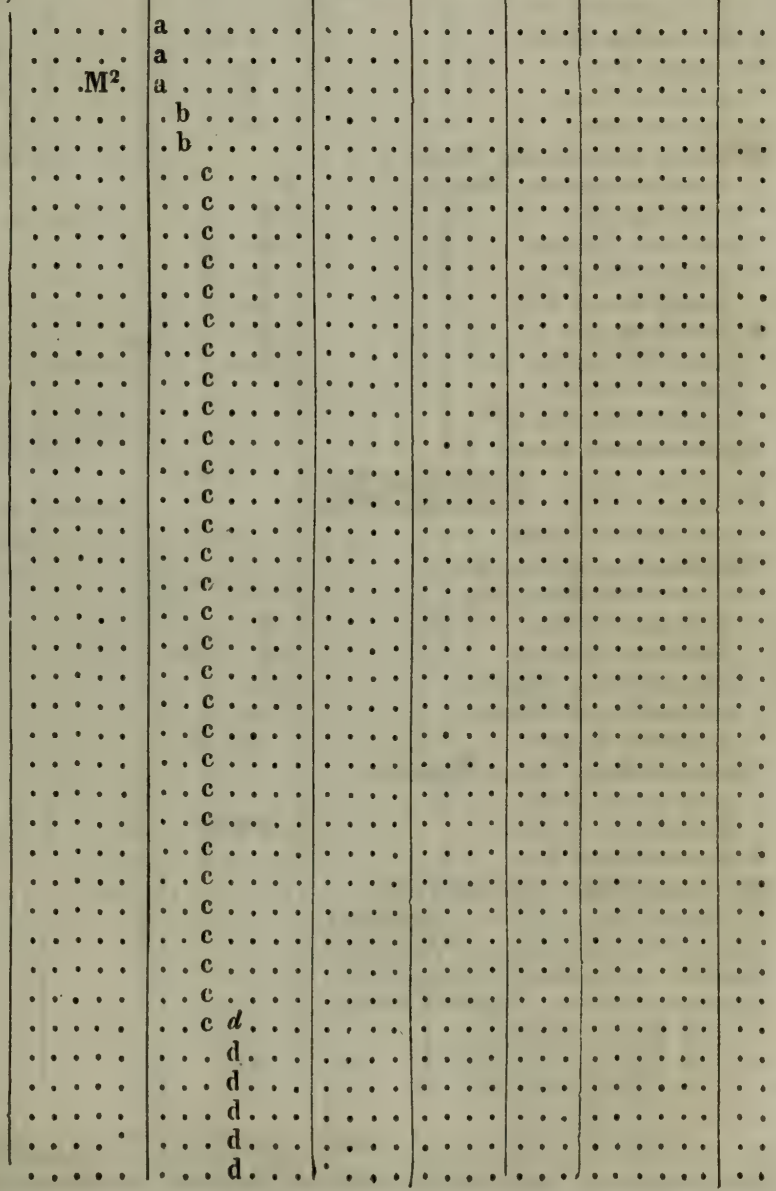




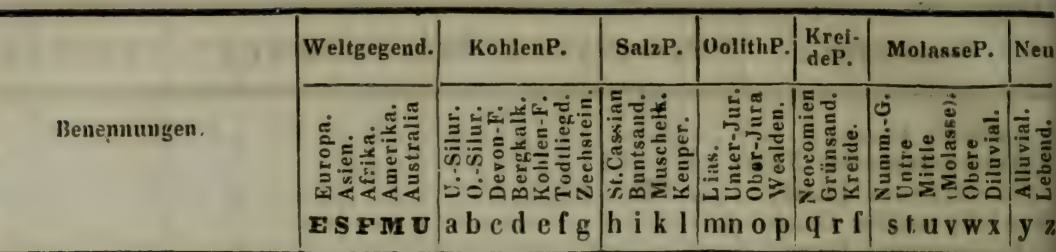

Cyrtoceras)

rugosum KoN.

tesselatum Kon. $\therefore$

unguis KoN.

Verneuilanum Kon.

(Spixala[LK.]GF.,Roe. [nanLk.]) $=$ ? Cyrtoceras Gir. $=$ arcuatella SNDB.

\# costata GF.

† gracilis SNDB.

o sulcata Roz.

Phragmoceras Brod. 9... inaequiseptatum Portl. .... arcuatum So. : ...

? conicum Erchw.

$\neq$ conulus Erchw. . . . compressum So...

? nautileum So. .... . ventricosum So. . . Brateri Mü. subventricosum $\mathrm{AV}$.

Apioceras Fisch. 1. ..... (Conllites Puscr, Gomphoceras So., Bodboceras Fisch., Poterioceras M'Coy) trochoides FAHRT.

(Comphoceras So
= Apioceras Fiscr, $=$ = Apioceras Fisch. $=$ Eichwaldi Vern. . . piriforme MORrs. . . subpiriforme PontL. subfusiforme ?PontL. sulcatum Vern.

(Conilithes (Lж.) Púsch), 2. . $=$ Apioceras Fisch. : Kielcensis Рusch ... ..... ? pyramidatus $L_{k} . \ldots \ldots$ Drthoceras (BreYN) $153 . .$. (Molossus Mr.; Melin, Sannionites Troost; Huronia Bigsiy, Ormoceras STockes, Actinoceras, Conoceras Br., Coleoceras Pontz., Hyoliches Eichw.) I siphone centrali

2 siphone subcentrall.

3 siphone intermedio.
4 siphone marginali.

* Siphonis situs non in omnibus specielus tam certe definiri potest, ut non dubius hrerent inter situs duos vicinos, e. gr. inter centrulem et subcentrulem, inter intermedium et marginalem elc.; tamen jam incertior haec distinctio interdum ad recognitionem ducere potest. 


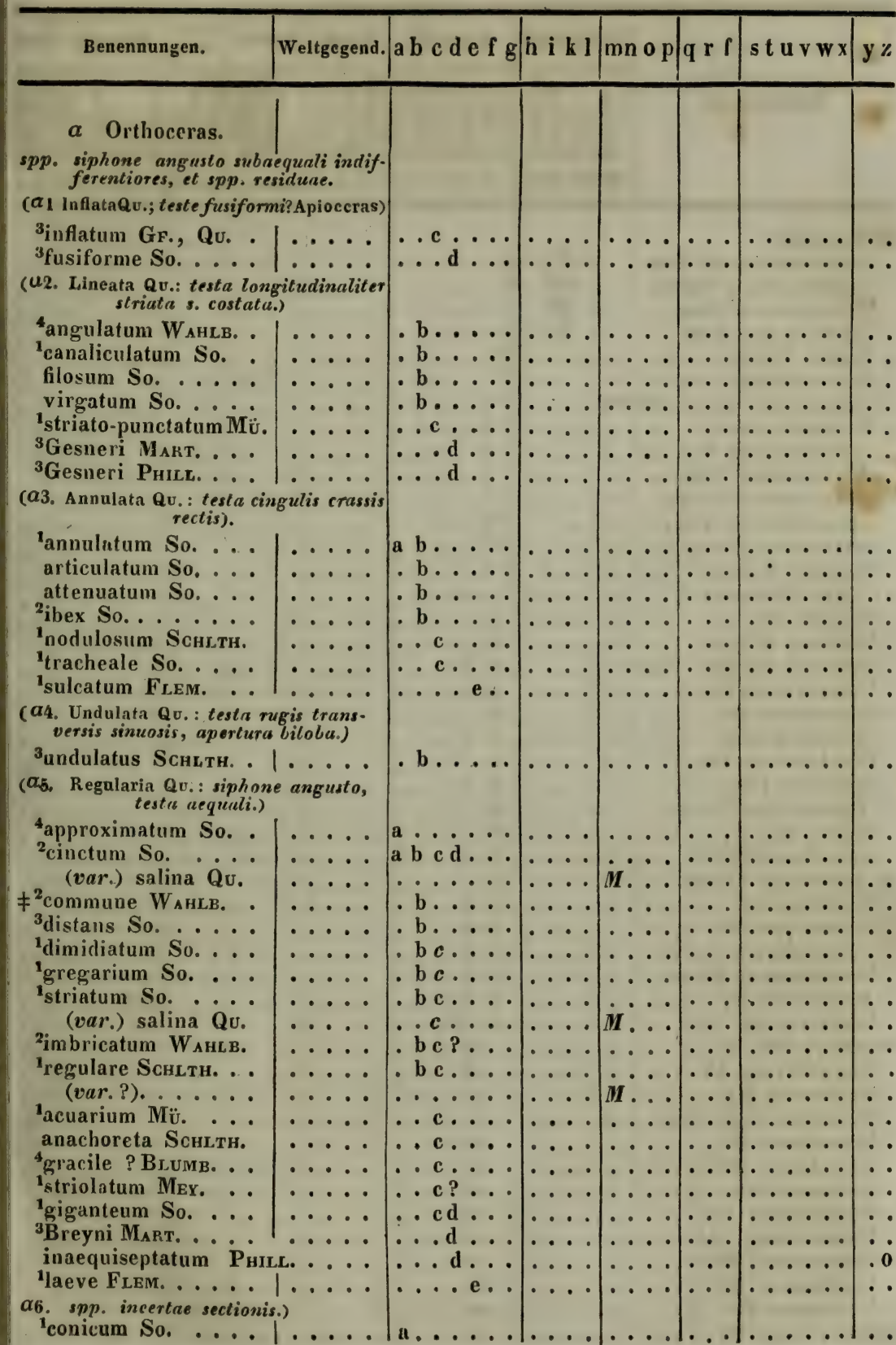




\begin{tabular}{|c|c|c|c|c|c|c|c|c|}
\hline 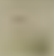 & 78 & Weltgegend & KohlenP. & SalzP. & OolithP. & $\begin{array}{l}\text { Krei- } \\
\text { deP. }\end{array}$ & MolasseP. & Neu \\
\hline & Benennungen. & 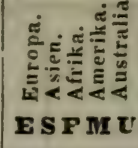 & 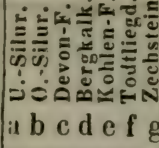 & 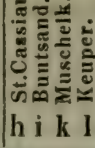 & 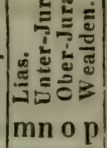 & 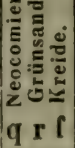 & 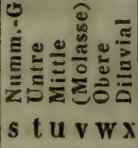 & $\mid \begin{array}{l}\equiv \\
\text { y } \\
\text { y } \\
0\end{array}$ \\
\hline
\end{tabular}

Orthoceras)

multicameratum Ems. multilineatum Eмms. primigenium $\mathbf{V}_{\text {ANx. . }}$ Trentonense Ems. tenuis WaнLB. . breviconicum Porti. ${ }^{3}$ complanato-septum Porti

incertum Porti. perannulatum Ponte. subarcuatum Ponts. subcostatum Forti. subundulatum Porte. triangulare Pontu.

'bacillus Еiснw. var. lineatum.

${ }^{2}$ Brighti So.

iconicum His. elongato cinctum $\mathrm{P}_{\mathrm{R}}$ ? excentricum So. 1 fimbriatum So. gracile Porti.

aMocktreense So. slineafum His. Pomeroense Porte. 4telum Eichw. 1tumidum Porte.

₹ vertebrale Еіснш. 1 bullatum So. calamiteun Mü. ${ }^{2}$ semipartitum So.... 'tenuiciuctum Porta. tubicinella So. scirculare So.

P4anceps Mü.

sanguliferum $\mathrm{AV} \cdot$ 1 carinatum Mü. ${ }^{3}$ crebriseptatum RoE. conoideum Mi. 'costulatum Mü. crassum Roe. cylindricum So. ${ }^{4} D$ annenbergi AV. 'decussatum MÜ. 'duplicatum Mü. Iellipticum Mü. \# excepticum Gr.

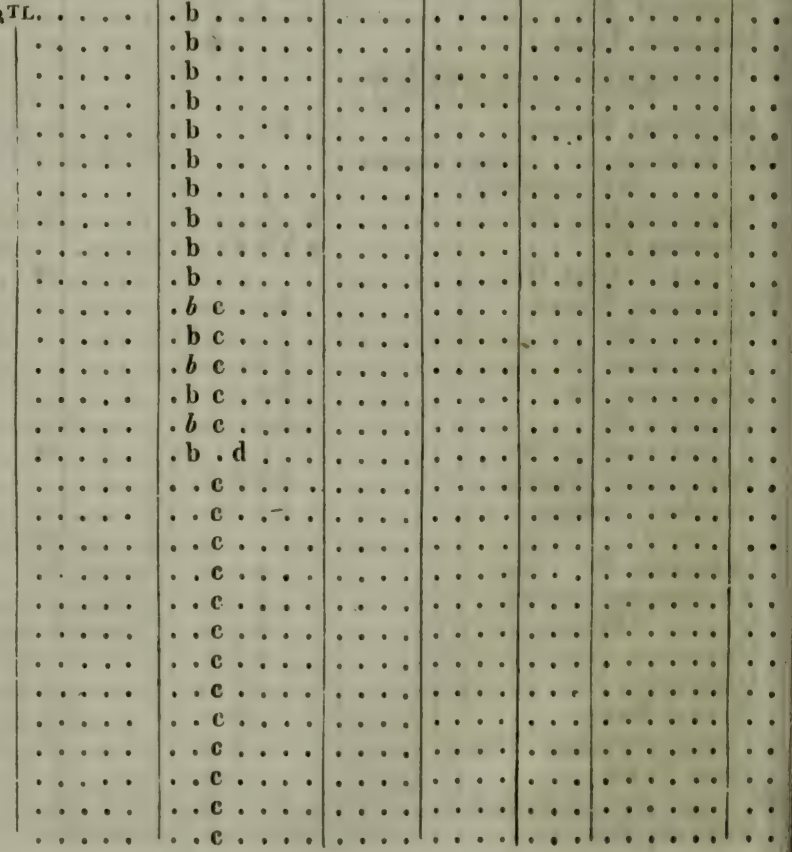




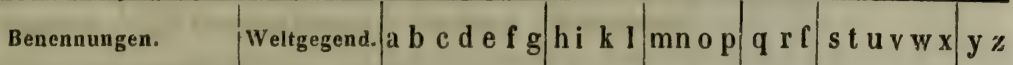

granulatum Mü. interruptum Mü. 3Jovellani AV.

'irregulare Mü. Iaterale Рниц. pal. ${ }^{4}$ paradoxum BraUN punctatum Mü. 1 semiplicatum Mü. . 'speciosum Mü. striatulum Mü. striatulum So.

(var.) salinum $\mathbf{Q u}$. 'subannulare Mü. 'subflexuosum Mü. tsubtrochleatum Mü. tentaculare Phic.. 'tenuistriatum Mü. . ₹ 'torquatum Mü. 3triangulare $\mathrm{AV}$. 3venustum $M \ddot{\text {. }}$. 2virgatum (So.) RoE. 4Wissenbachi AV. icylindraceum So. 1 Goldfussanum KoN. slincolatum Phicl. laffine Pontr. augulare FLEM. ₹ ${ }^{\star}$ arcuatum PhILL. $\neq 1$ calamus Kon. tconquestum KoN. dentaloideum PhiLc. dactyliophorum KoN. $\ddagger^{2}$ filiferum Phill.

Frearsi Vern.

3lineale KoN.

${ }^{3}$ Martinianum [?] KoN. 3Münsteranum KoN. 2ovale Phicc. ${ }^{3}$ paradoxicum So. . \#'Polyphemus Fisch. ${ }^{4}$ pygmaeum Kov. ${ }_{3}$ reticulatum PhiLl. \# scalare Gr.

${ }^{4}$ Steinhaueri So.

${ }^{2}$ subcanaliculatum Kon. ${ }^{3}$ subcentrale KoN. 4sulcatum Fisch. 3undulatum So. 3anceps Kon. attenuatum FLEm. 'cylindraceum Ferm. ${ }^{3}$ dilatatum KoN. 


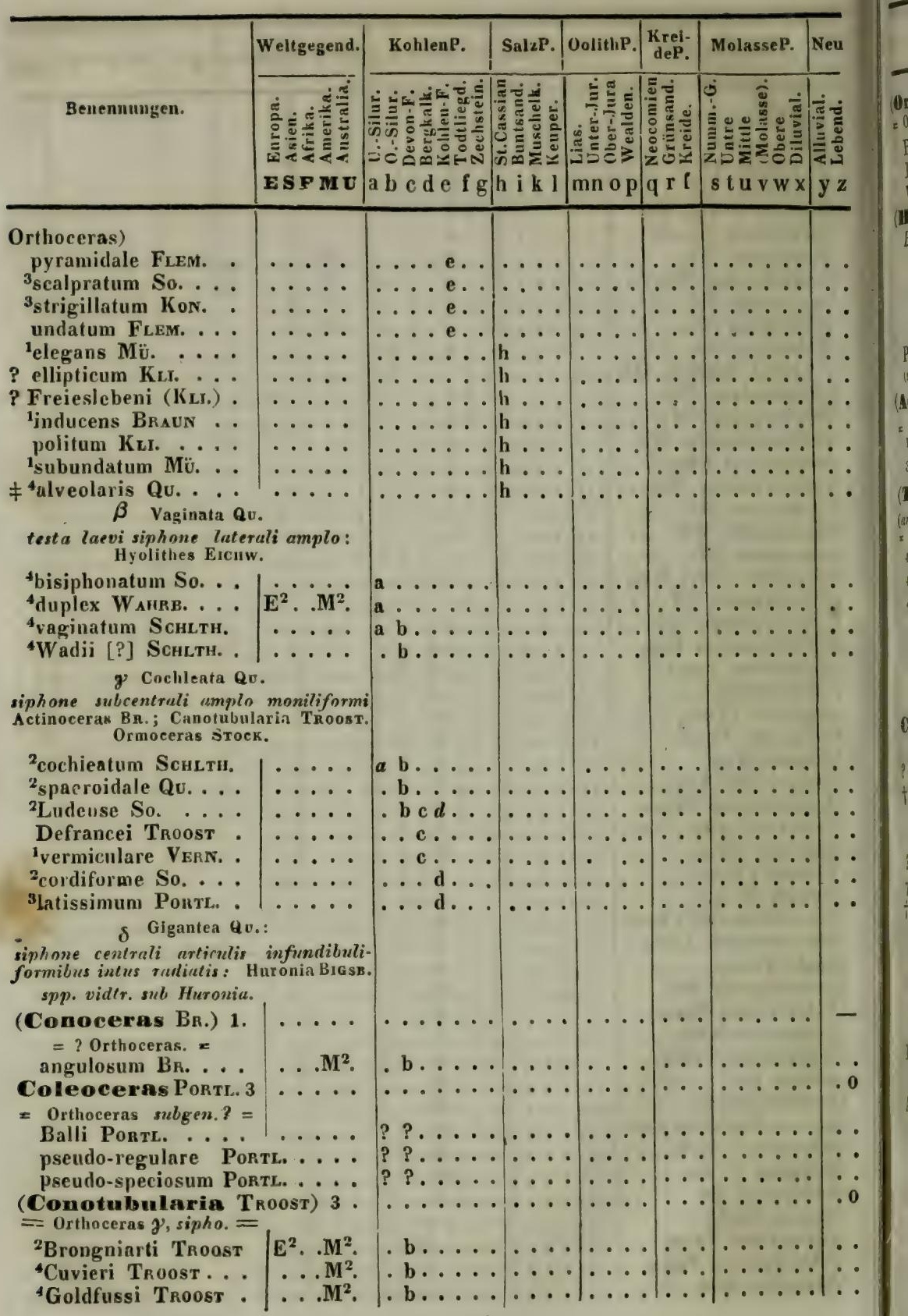




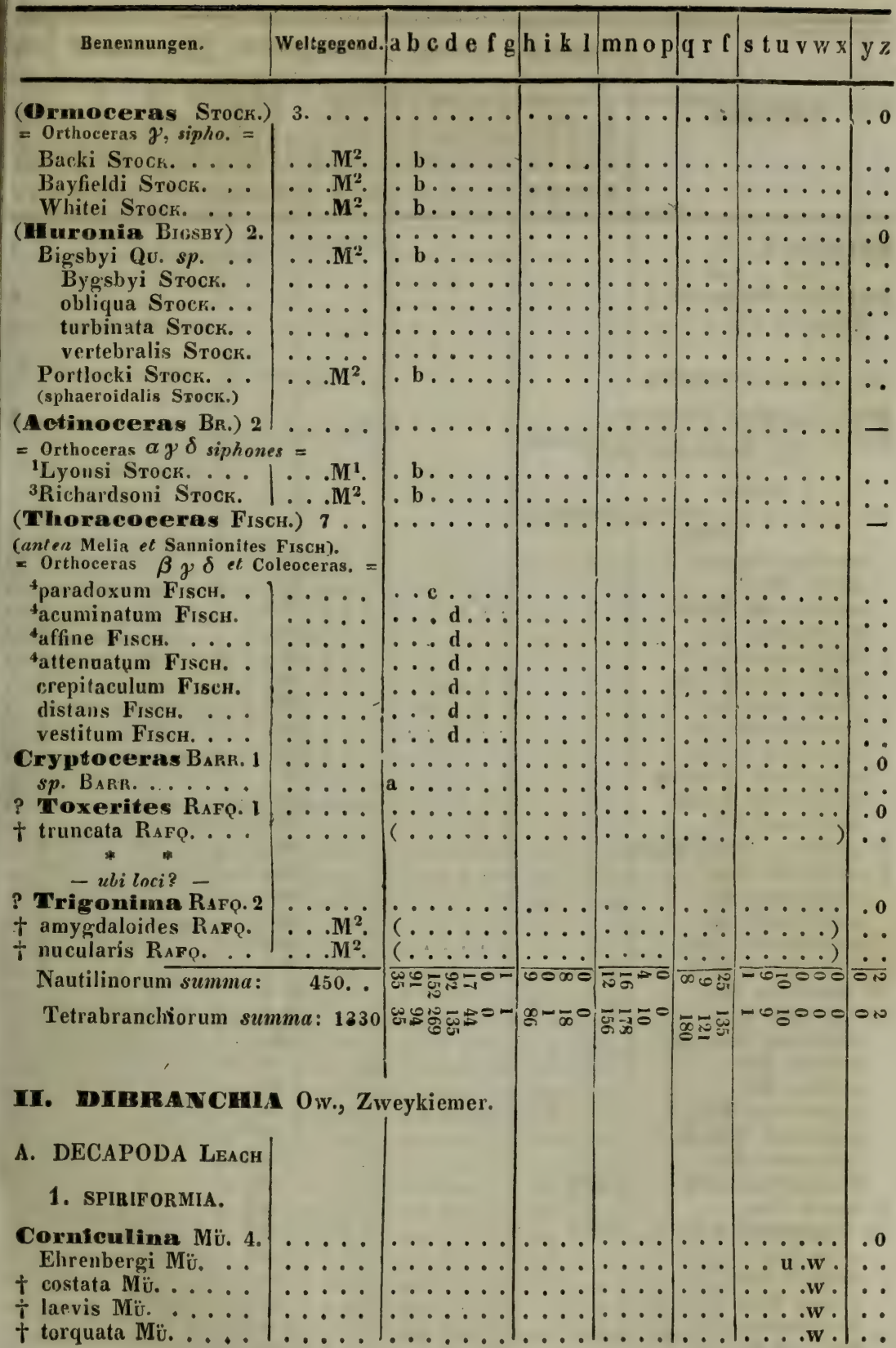




\begin{tabular}{|c|c|c|c|c|c|c|c|}
\hline \multirow{3}{*}{ Benennungen. } & Weltgegend. & KohlenP. & SalzP. & OolithP. & $\begin{array}{l}\text { Krei- } \\
\text { deP. }\end{array}$ & MolasseP. & Neu \\
\hline & 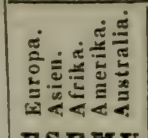 & 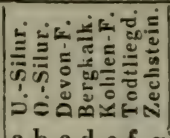 & 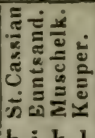 & 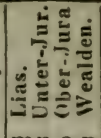 & 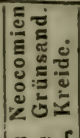 & 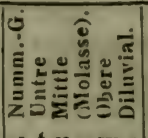 & 可施 \\
\hline & ES F M U & $|a b c d e f g|$ & h i k l & mnop & $q \times \Gamma$ & $|\mathrm{stuvw} x|$ & $y z$ \\
\hline
\end{tabular}

Spirula Lr. 0.

(efr, p. 530)

Epirulirostra d'0, 1

Bellardii D'O.

2. BELEMNOMORPHA.

Belemnites Еннн, 98. . . . . (Belemnosepia Bucku. pars, Actinocamax Mill., Pseudobelus Blv.)

a Acoeli.

(*1. Aeuarii : sulco lineisgue lateralibus nullis).

acuarius Schlth. . acutus MiLL. breviformis VoLTz . brevirostris D'O. Bruguieranus D'O. . compressus Stahl . compressus BLv. . Fournelanus D'O. . . impressus Voutz . . irregularis Schlth. macroconus KuRr Nodotanus D'O. . . . * ornithocephalusTreon. \# striatulus RoE. umbilicatus Brv. . . unisulcatus Br.v. . . abbreviatus Mice. . cylindricus Bev. .

(?) fistulosus BLV. . .

(?) obtusus Blv. . . tripartitus Sснцтн. . borealis D'O.

conulus Mü. excentricus BLv. . . giganteus Вснцтн. . inaequalis RoE. Kirghisensis D'O. \# laevis Roe.

(?) magnificus D'O. meta Buv. .....

$\neq$ Milleri Dsh. ovatus Bev.

Panderanus D'O. Prevosti Dsh.

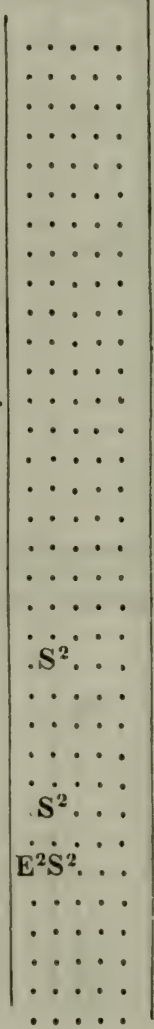

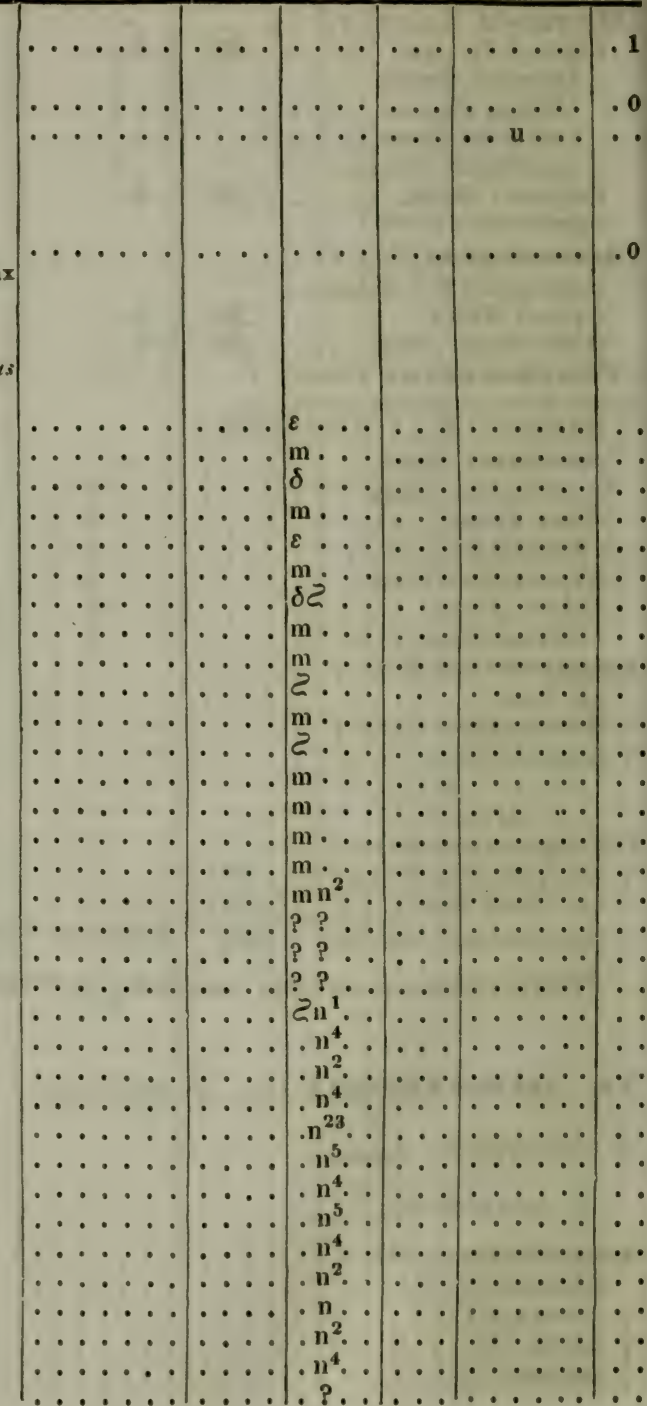




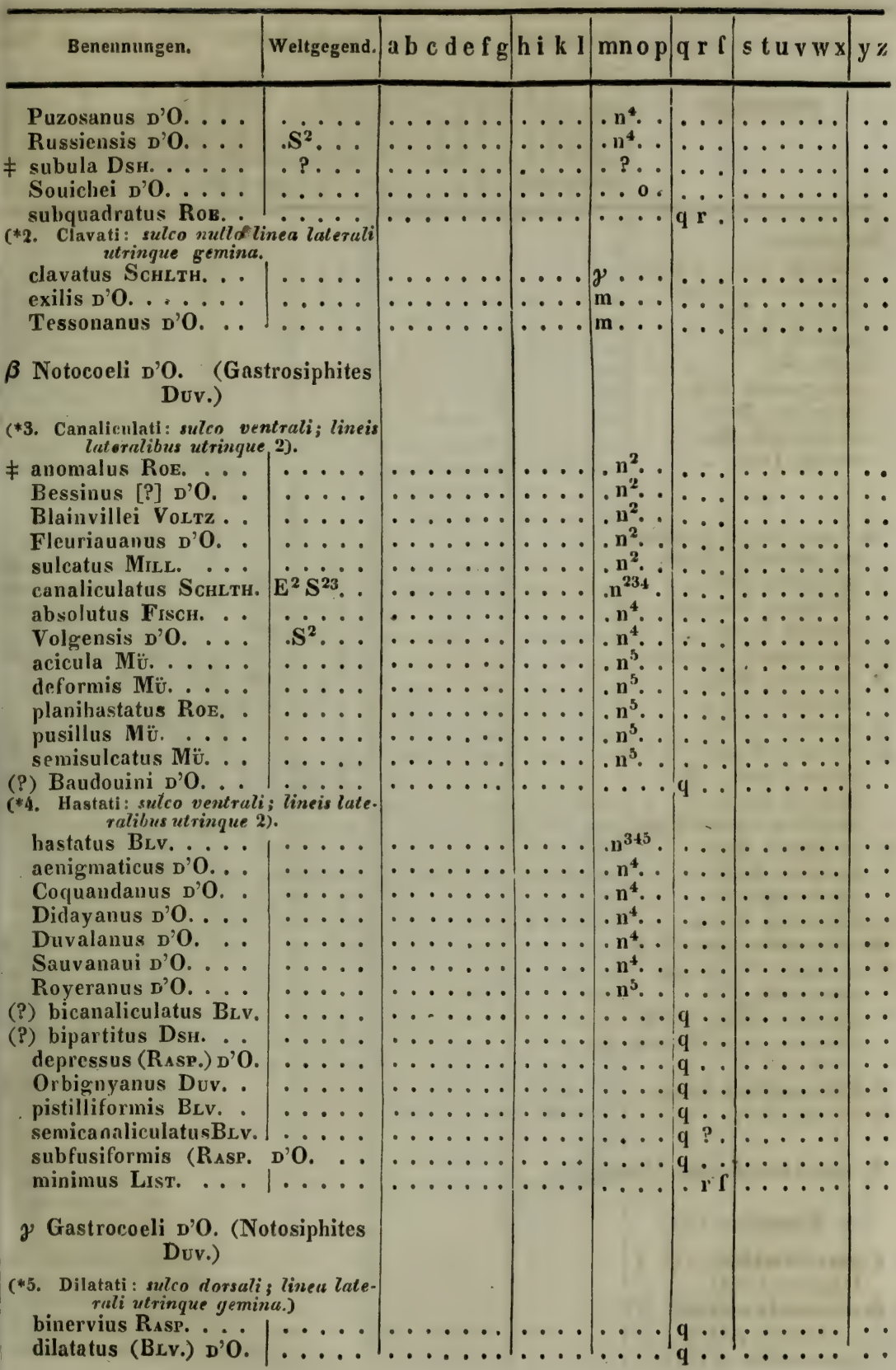




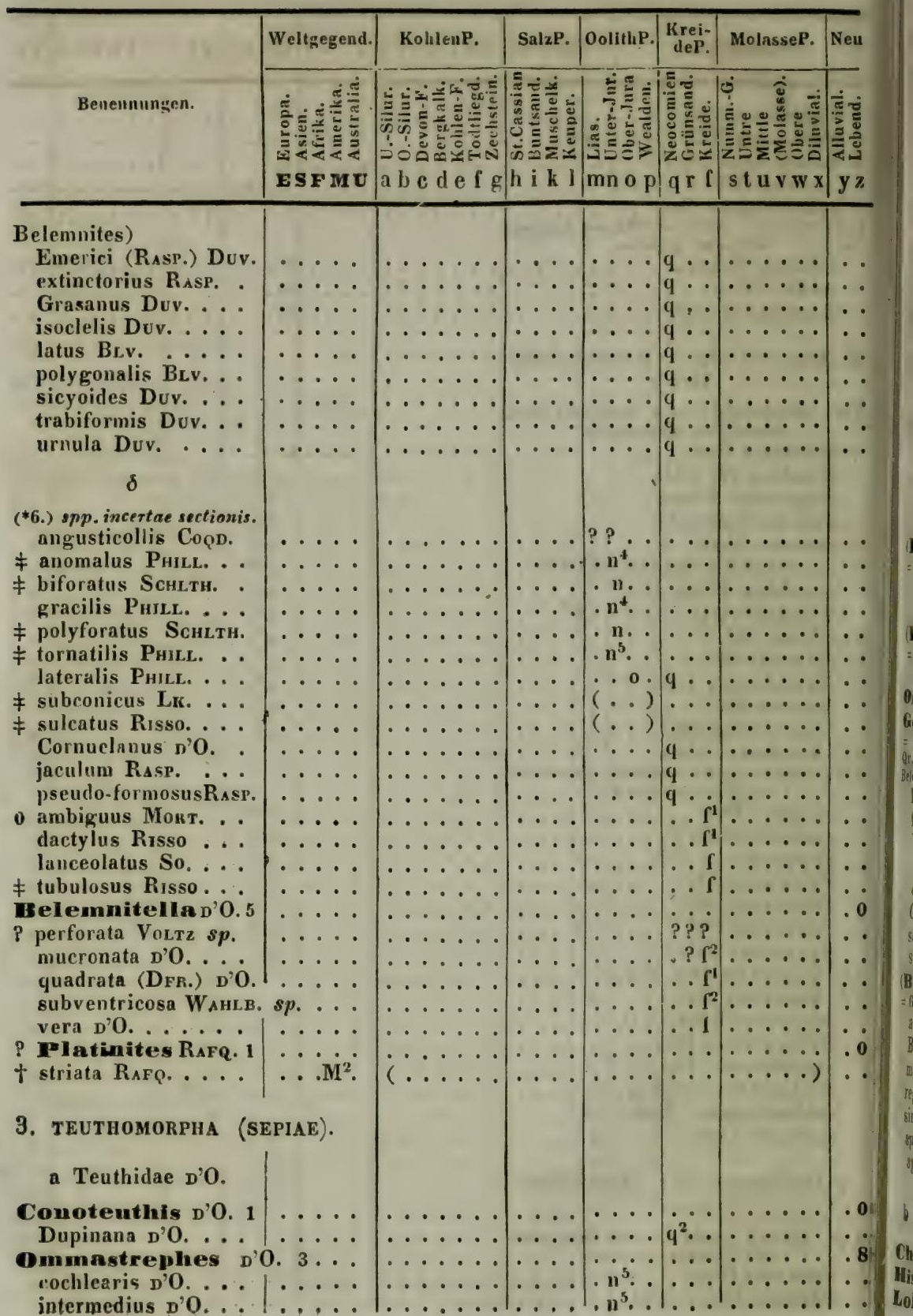




\begin{tabular}{|c|c|c|c|c|c|c|c|}
\hline Benennungen. & d. & $\mathbf{a} \mathbf{b}$ & 1 & p) & $q \times l$ & $\mathbf{w} \mathbf{x}$ & \\
\hline \multirow{2}{*}{\multicolumn{2}{|c|}{ 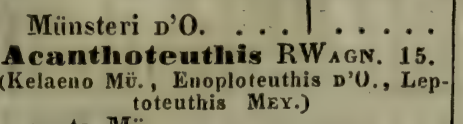 }} & & & • & $\cdots$ & & \\
\hline & & & & & $\cdots$ & & \\
\hline 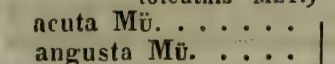 & & $\cdots \cdots$ & $\cdots \cdot$ & $\cdot n^{5} \cdot \cdot$ & $\cdots$ & $\cdots \cdots$ & \\
\hline$\cdots$ & & $\cdots \cdots \cdots$ & & $\begin{array}{l}\cdot n^{3} \cdot \cdots \\
\cdot n^{5} \cdot .\end{array}$ & $\cdots$ & $\mid \begin{array}{c}\cdots \\
\cdots \\
\cdots\end{array}$ & \\
\hline$\ldots$ & & & &.$n^{3} \cdots$ & $\therefore$ & $\cdots$ & \\
\hline$\cdots$ & & & & $\therefore$ & $\cdots$ & $\cdots$ & \\
\hline $\begin{array}{l}\text { i Mü. . . } \\
\text { Mü. . . }\end{array}$ & & $\cdots$ & & & $\cdots$ & $\cdots$ & \\
\hline$\cdots$ & $\begin{array}{ll}\cdots \\
\cdots\end{array}$ & $\because$ & & $\cdot \cdot$ & $\cdots$ & $\cdots$ & \\
\hline$\therefore$ & .... & $\ldots \ldots$ & $\ddot{\cdots} \cdot$ & $\left|\begin{array}{l}\cdot n^{0} \\
. n^{5} \ldots\end{array}\right|$ & $\cdots$ & $\because$ & \\
\hline$\ldots$ & $\cdots$ & $\ldots \ldots$. & $\ldots$ & $n^{5} \cdot$ & $\therefore$ & $\mid \begin{array}{c}\cdots \\
\cdots \\
\cdots\end{array}$ & \\
\hline$\cdots$ & $\cdots \cdots$ & $\cdots$ & $\ldots$ & . $n^{5}$ & ... & $\ldots \ldots$ & \\
\hline$s p$ & & $\cdot \cdot$ & $\ldots$ &.$n^{5}$. & $\ldots$ & $\therefore$ & \\
\hline$\cdots$ & $\cdot$ & $\cdot \cdot$ & $\ldots$ & . $n^{5}$. & $\cdots$ & . & \\
\hline$\because$ & $\ddot{\dot{*}}$ & $\because$ & $\ldots$ & $\cdot \mathbf{n}^{5} \cdot$ & $\cdots$ & $\cdots$ & \\
\hline$\ddot{\circ}$ & $\because$ & •. & $\cdots$ &. $\mathrm{n}^{5} 0$. & $\cdots$ & & \\
\hline in: $=$ & $\cdot$ & $\cdots$ & $\cdots \cdot$ & $\cdots \cdot$ & $\cdots$ & . & \\
\hline$\cdots$ & & $\cdots$ & . & . 1 & $\cdots$ & . . & \\
\hline \multirow{3}{*}{\multicolumn{2}{|c|}{ 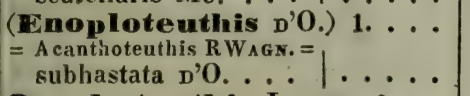 }} & & & . & - & . & \\
\hline & & & - & - & - & . & \\
\hline & & & &.$n^{5} \ldots$ & & & \\
\hline \multirow{4}{*}{\multicolumn{2}{|c|}{ 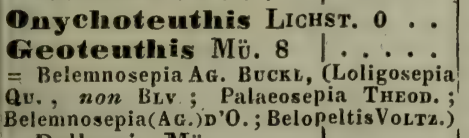 }} & & & & $\cdot$ & $\cdots$ & \\
\hline & & $\ldots$ & $\ldots$ & $\ldots$ & $\ldots$ & $\therefore$ & \\
\hline & & & & & & & \\
\hline & & & & & & & \\
\hline$\cdots$ & $\cdots$ & $\cdots$ & 1 & m & $\cdots$ & . & \\
\hline$\cdots$ & $\cdot$ & & 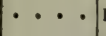 & $\cdots$ & $\cdots$ & $\cdots$ & \\
\hline$\cdots$ & $\cdot$ & & & $\cdots$ & $\cdots$ & $\cdots \cdots$ & \\
\hline & $\cdot$ & & 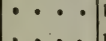 & $\cdots$ & $\cdots \cdot$ & $\cdots \cdots \cdots$ & \\
\hline$\because$ & $\because$ & & & & & $\cdots$ & \\
\hline$\ldots$ & & & & & $\therefore$ & $\because$ & \\
\hline & .... & & & & $\cdots$ & $\cdots$ & \\
\hline \multirow{2}{*}{\multicolumn{2}{|c|}{$\begin{array}{l}\text { (Belopeltis VoLTz) } 7 \\
=\text { Geoteuthis Mü.; Belemnosepia AG. }\end{array}$}} & & & . & & $\cdots$ & \\
\hline & & & & & & - & \\
\hline - & ・ & & & & & & \\
\hline - & - & & & n & $\cdots$ & & \\
\hline & & & & & $\cdots$ & $\cdots$ & \\
\hline & & & & & $\cdots$ & $\cdots$ & \\
\hline & & & & & $\cdots$ & $\cdots \cdots$ & \\
\hline $\begin{array}{l}s p . V \\
s p . V\end{array}$ & & & & & & & \\
\hline & & & & & & & \\
\hline \multicolumn{8}{|l|}{ b Loligopsidae D'O. } \\
\hline & & & 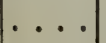 & & $\cdots$ & & \\
\hline 0 & • & . & & & & & \\
\hline
\end{tabular}




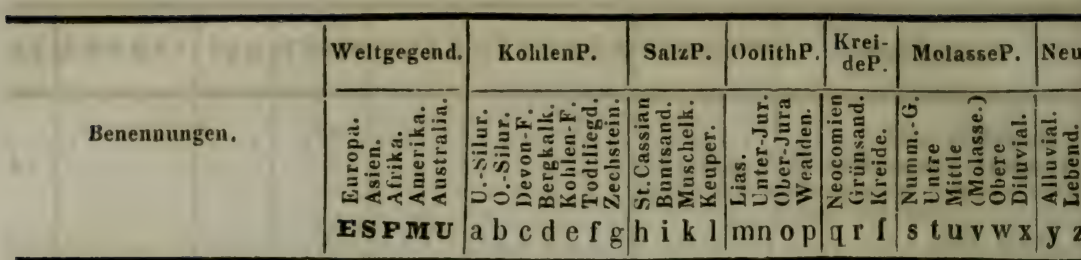

c Loligina.

P Aptychus Mex. 42

(Trigonellites Park, Tellinites Schlth., Lepadites GER M., Ichthy osiagones BouRD., Münsteria DsLgch.

a sectio incertis. vetustus $\mathbf{A V}$.

? antiquus Gr. .

Gallienneauus d'o.

$\uparrow$ striato-punctatus Volтz antiquatus CoQp. politus Coen.

Gravesanns D'O. . $\beta$ Cornei.

elasma MEY. vugulosus Voltz.. striato-laevis VoLTz cuneatus Vortz... praelongus VoLT' $\gamma$ Imbricali. bullatus Mex.... latifrons VoLtz . . . ovatus MEY. speciosus VoLtz Theodosia Dsh. lamellosus VoLtz... depressus Voltz... ₹ elegans Voutz... ₹ elongatus VoLTz Grasi Voltz liamellosus Mü.

₹ Meyeri Voltz profundus VoLtz . \# Provençalis Voltz: $\doteqdot$ punctatus VoLTz.. Didayi Coop. ..... radians Coep.

Seranoni Copd. . . . cretaceus Mü. $\delta$ Cellulosi. acutus Mü. \# heteropora VoLtz . ₹ Thurmanni VoLtz Beaumonti Coed. ‡ latissimus Voltz
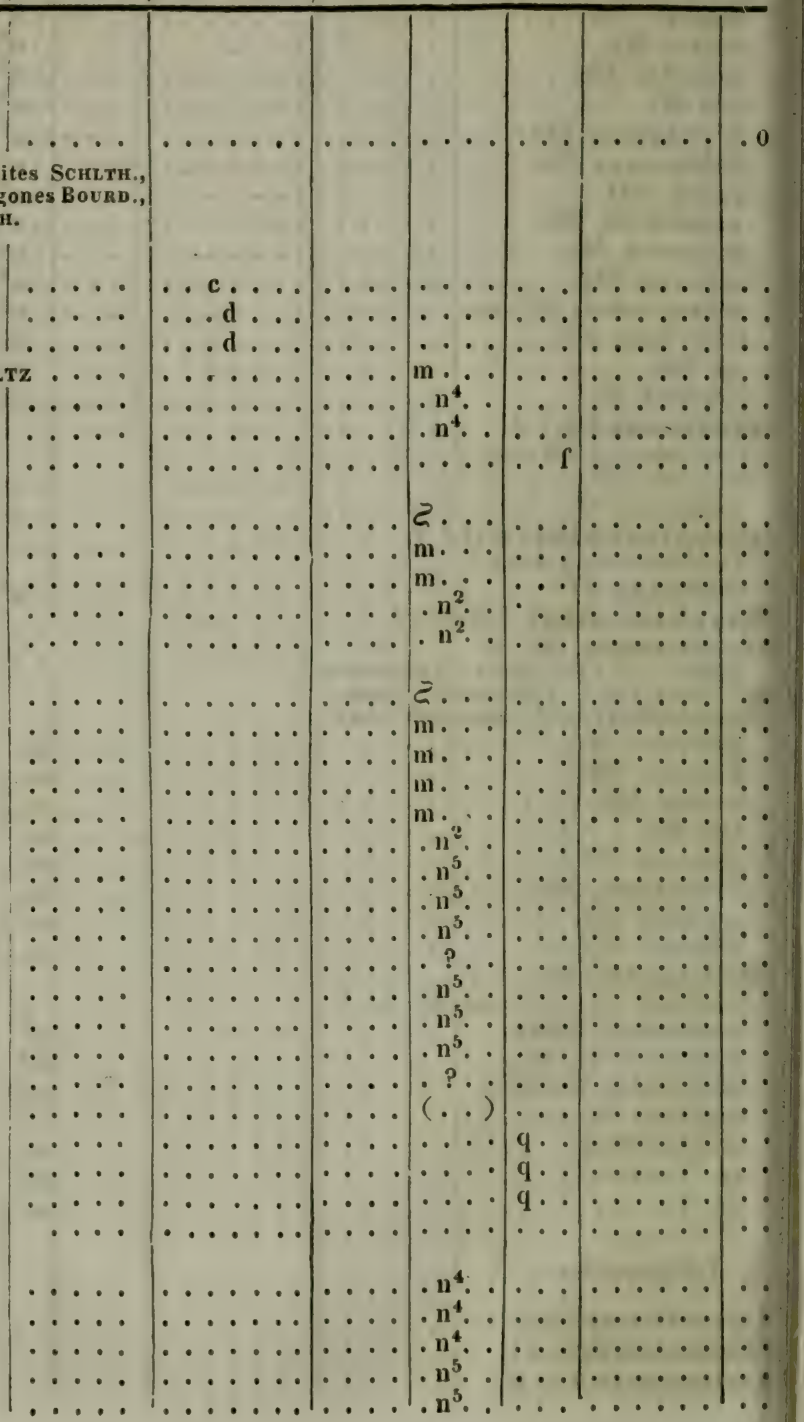


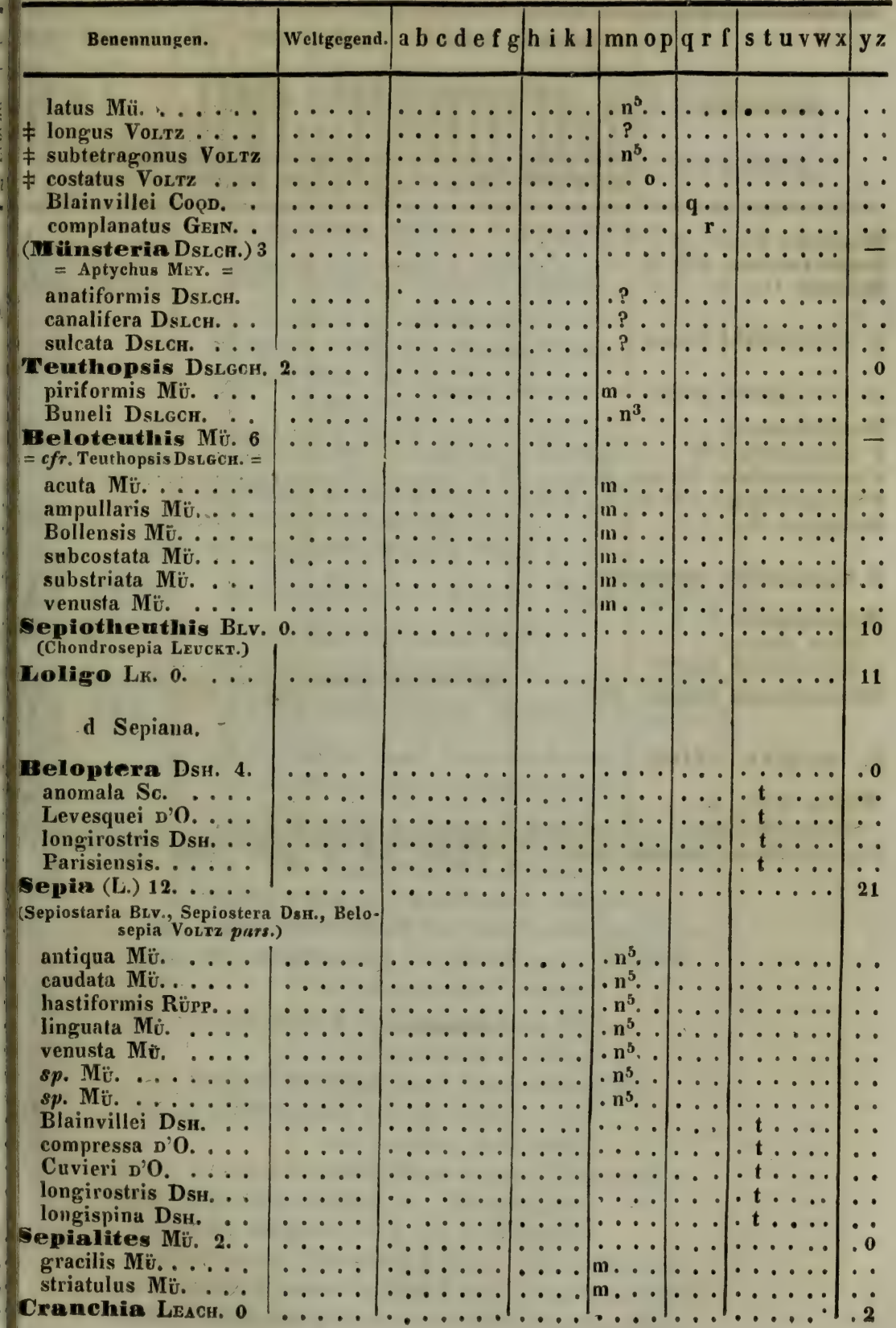




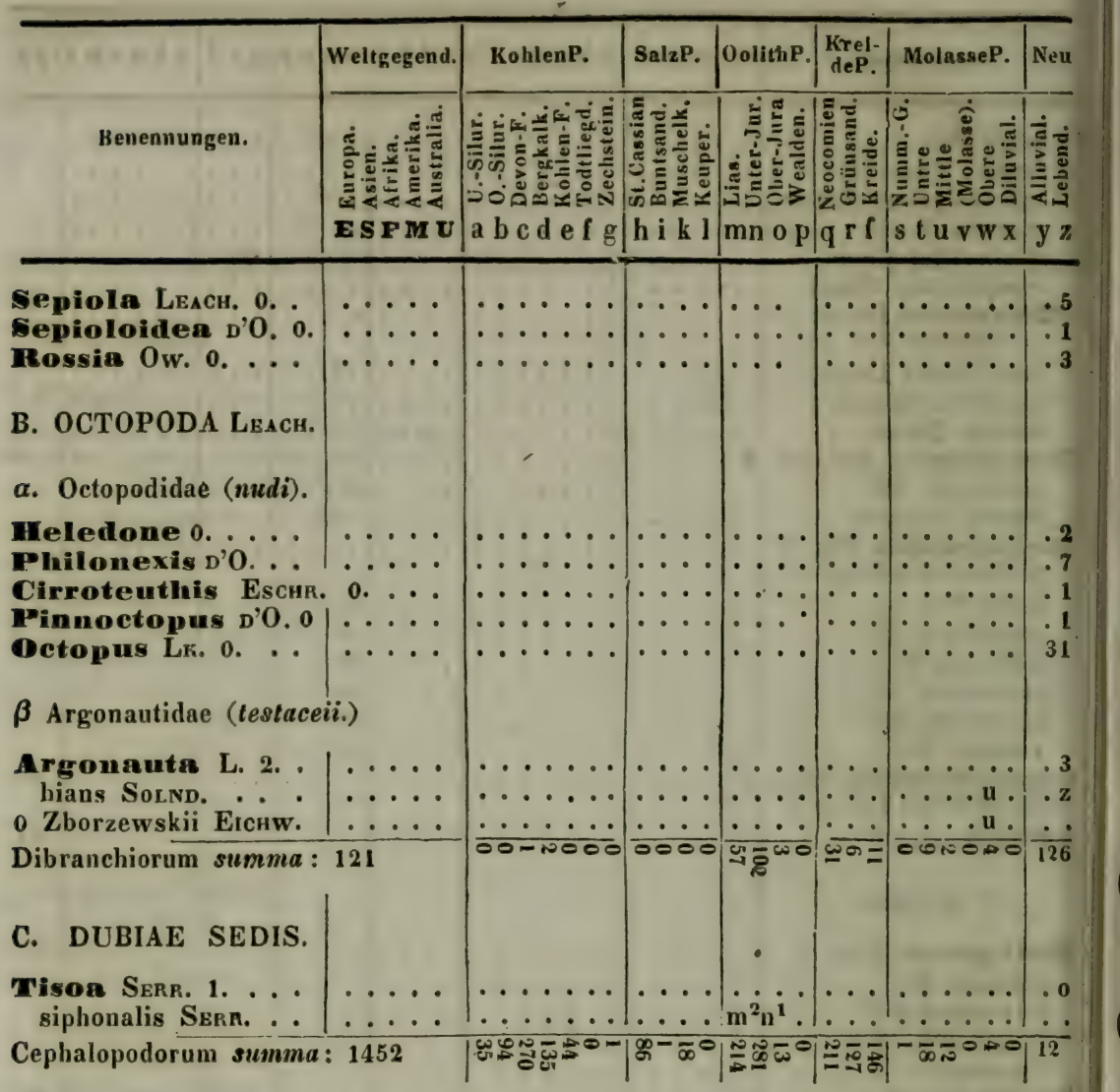


(4)

\section{ENTOMOZOA : TERBTHIERE.}

(INSECTA SENSU AMPLISSIMO).

Cl. XVI. VERMES : Frey-Würmer.

A. ROtatoria Eв.: Ruderthiere.

B. TURBELLARIA Eв. : Strudel-Würmer.

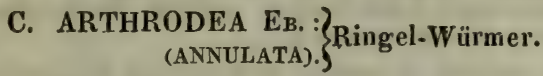

Cl. XVII. CRUSTACEA LTR.: Kruster.

I. Chrferpedin Burm. : Ranken-Füsser.

II. ENTOMUOSTRACA MürL.

HIX, MaLAC@STRACA MüLL.

CI. XVIII. MYRIAPODA LTR.: Tausend-Füsse.

A. GNATHOGENA Brand: Kauer.

B. PULMONARIA Ltr.: Sauger.

Cl. XIX. ARACHNOIDEA Koch.: Spinnen.

A. TRACHEARIA Lrr.: Tracheen-Spinne.

B. FULMONARIA Ltr.: Lungen-Spinnen.

CI. XX. HEXAPODA Ltr. : Kerfe (im engern Sinne).

* HAUSTELLATA PTILOTA.

T. DIPTERA L. : Zweiflügeler, Mücken.

IX. HEPIOPTERA L.: Schuppenflügler, Falter.

III. HEMIPTERA L.: Halbflügeler, Wanzen.

** HAUSTELLATA APTERA.

Iv. SUCTORT DeGrer: Sauger, Flöhe. *** MAXILLATA APTERA.

v. ThYSANURA Ltr. : Fransenschwänzer.

VI. ANOPLURA LEAGH : Läuse.

**** MAXILLATA PTILOTA.

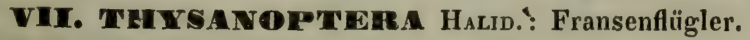

vII. ORTOP'TERA L.: Geradflügeler.

IX. NEUROPTERA L. : Netzflügeler.

X. STREPSIPTERA Kirby : Fächerflügeler.

X. HYMENor'Tena $L$ : : Hautflügeler, Immen.

XI. COLEOPTER L. : Scheideflügeler, Käfer. 


\section{Fernere Zeichen-Wrklärungen.}

(Vrgl. SS. 2, 74, 208).

† Von den mit $†$ bezeichneten Namen sind nur diejenigen in's systematische Verzeichniss aufgenommen worden, bei welchen Aussicht vorhanden war, dass sie von ihreın Autor noch vollständiger mittelst Beschreibung und $\mathrm{Ab}$ bildung bekannt gemacht werden würden.

(?) Ein dem Art-Namen in runder Klammer vorgesetztes Fragzeichen bedeutet Zweifel in die Sektion des Genus, wo er steht.

Die Zeichen der geographischen Fundorte in runden Klammern eingeschlossen bedeuten die abweichende Heimath der lebenden Form derselben Art. Zuweilen ist auch die Heimath eines ganzen (lebenden) Geschlechtes durch ein Zeichen hinter dem Geschlechts-Namen angegeben.

$a^{1},{ }^{2}, b^{1},{ }^{2},{ }^{3}$ sind die Unterabtheilungen der böhmischen Silur-Formation nach BARRANDE.

M $^{1}$ bedeutet die Schichten des Coregna-Berges bei la Speraia, Ammoniten und Orthozeratiten enthaltend.

m $\alpha \beta \gamma \delta$ (die Quenstedr'schen Unterabtheilungen des Lias) werden wegen Mangels an Raum durch ein blosses $\alpha \beta \gamma \delta$ in der Spalte $\mathbf{m}$ ausgedrückt.

N. Noch ehe mehr als 2-3mal von dem Zeichen Q (S. 208) Gebrauch gemacht werden konnte, erfahren wir durch v. Bucr's und DB ZIGNo's (ZGN.) Untersuchungen, dass die von Catulco der durch Terebratula diphya bezeichneten Formation Q zugeschriebenen Ammoniten etc. theils bekannte NeocomienPetrefakten sind und dann dem wirklichen Biancone oder Majolica-Marmor $=q$, theils aber dem rothen Ammoniten-Kalke angehören, einer Jura-Formation die wir vorläufig mit $\mathbf{N}$ bezeichnen, da sie keine Orthozeratiten wip $\mathbf{M}$ onthält, vom Lias durch eine Reihe von Jura-Schichten getrennt ist, Jura-Petrefakten aus verschiedenen Niveaus darbietet, aber $\mathbf{M}$ sehr ähulich ist.

$\mathrm{t}^{*}($ statt $\mathrm{t})=$ Valmondois.

$\mathrm{U}=$ die Gypse von Aix mit Fischen, welche früher blos mit u bezeichnet werden sollten.

$\mathbf{u}^{1}, \mathbf{u}^{2}=$ die blauen und gelben Schichten von Bordeaux nach Grateloup.

$\mathbf{v}^{\mathbf{1}}=$ Bernstein-Insekten.

! in einer Formations-Rubrike, statt des gewöhnlichen Buchstabens angebracht, bezeichnet hie und da eine für die entsprechende Formation vorzugsweise bezeichnende Art. 


\begin{tabular}{|c|c|c|c|c|c|c|c|}
\hline \multirow{3}{*}{ Benennungen. } & \multirow{3}{*}{$\begin{array}{c}\text { Weltgegend. } \\
\text { E1,2 Europa. } \\
\text { S1,2,3 Asien. } \\
\mathbf{F}^{2}, 3,4 \text { Afrika. } \\
\text { M 1,2,3,4 Amerika } \\
\mathbf{U} 3,4 \text { A ustralien. } \\
\text { E S F M U } \\
\text { k e in Zeichen: be- } \\
\text { deutet E2. }\end{array}$} & KohlenP. & SalzP. & OolithP.| & \begin{tabular}{|c|} 
Krei- \\
deP.
\end{tabular} & MolasseP. & $N \in u$ \\
\hline & & 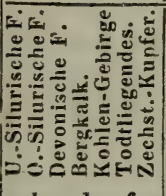 & 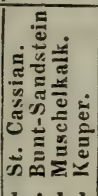 & 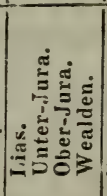 & 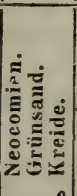 & 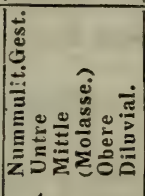 & 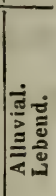 \\
\hline & & a b c d e f g & $h$ i k l & $\mathrm{mn}$ op & $q \times 1$ & s t u v w X & y z \\
\hline
\end{tabular}

\section{Cl. XVI. VERMES, Frey-Würmer.}

(Annulata, Annellides auctorum.)

\section{ROTATTRIA Eв.}

(Rüderthiere. Infusoria, pars.)

(corpore mirroscopico molli.)

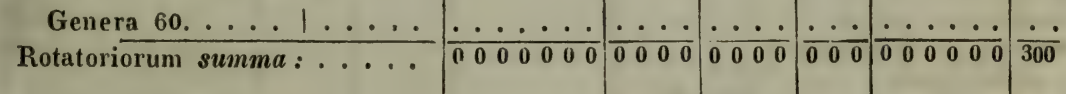

II. TURERTEAMEA EB. (Strudelwürmer.)

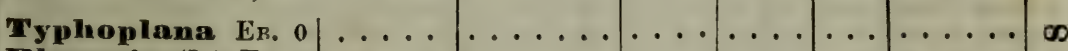

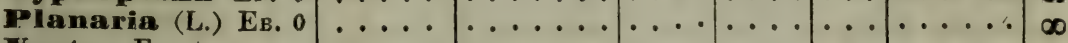
Vortex Ев. $0 . \ldots \ldots \ldots \ldots \ldots \ldots \ldots$ Derostoma Eв. $0 . \quad \ldots \ldots . . \ldots \ldots \ldots \ldots$

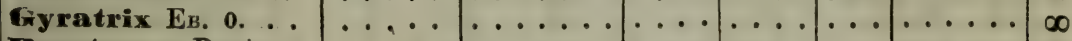

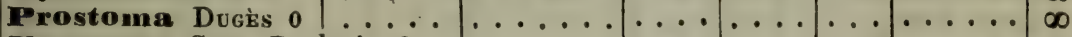

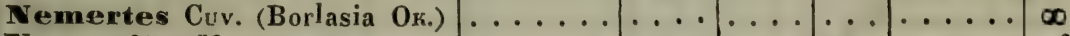

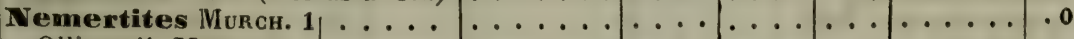

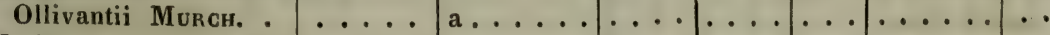

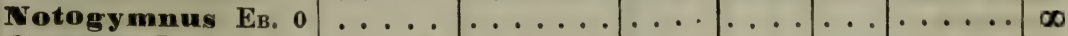

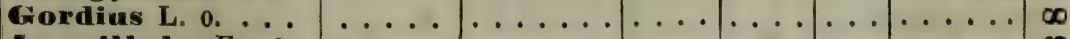

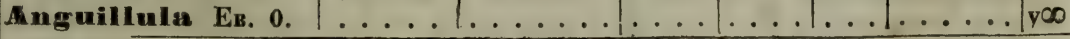

Turbellariorum summa: $\ldots \ldots \mid$\begin{tabular}{llllll|llll|llll|lll|llllllll}
\hline & 0 & 0 & 0 & 0 & 0 & 0 & 0 & 0 & 0 & 0 & 0 & 0 & 0 & 0 & 0 & 0 & 0 & 0 & 0 & 0 & 0 & 0 & 0 & 70
\end{tabular} 
Beneanungen

\begin{tabular}{|c|c|c|c|c|c|c|}
\hline Weltgegend. & KohlenP. & Salsp. & OolithP. & $\begin{array}{l}\text { Krel- } \\
\text { deP. }\end{array}$ & MolasseP. & Neu \\
\hline . & 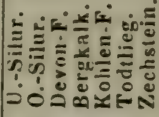 & 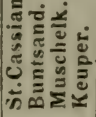 & 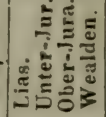 & 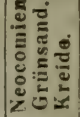 & 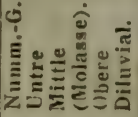 & 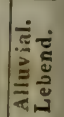 \\
\hline ES S M U & $\mathrm{a} b \mathrm{~b} d \mathrm{e} \mathrm{g}$ & h i k l & $\mathrm{mn}$ op & q r 1 & st u v w x & $y^{2}$ \\
\hline
\end{tabular}

ITT. ARTHROGE A E.

A. APODA. (nuda)

Genera multa viventia speciebus

\section{CHAETOPODA Bev.}

\section{Terricolae Cuv.}

Genèra multa viventia speciebus Tubifex LF. 1 ? antiquus PLIEN.

\section{2. tubicolae Cuv.}

Arenicola Lr. 0 . . Clymene SAv. 0. . Terebella Cuv. 1. lapilloides Mü. ...

Pectinaria Lr. 0 . Ampinitrite Lr. 0 . Sabella Cuv. 0. . . Ditrypa BERKELEY 4 plana Fore. ...... gadus Lyel. .... polita Woon .... subulata BrkL. ...

Spirorbis LK. 33. . Lewisi Sow. . . . . tenuis Murch. ... ammonius EDw. . . † gracilis Sandb. ... omphalodes Edw. minutus Porte. Valvata EDw. .... complanatus Mü. . . planorbiformis EDw. rotula EDw.

conulus anfractus Evw. lituitis DEFR... subearinatus EDw. . conoideus Lr.
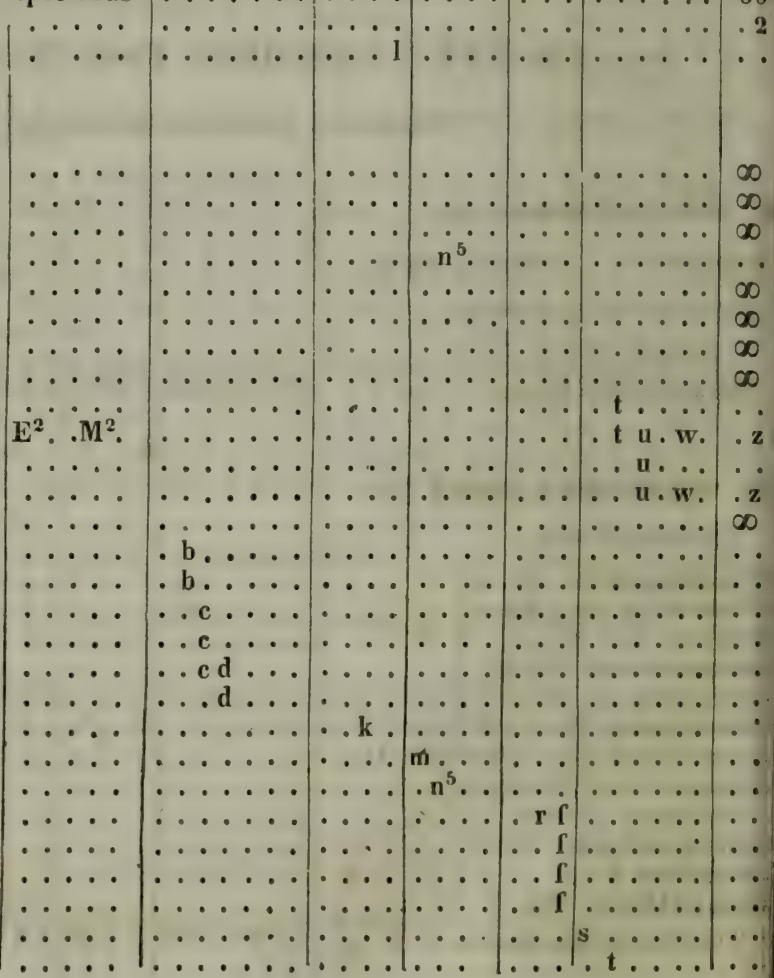


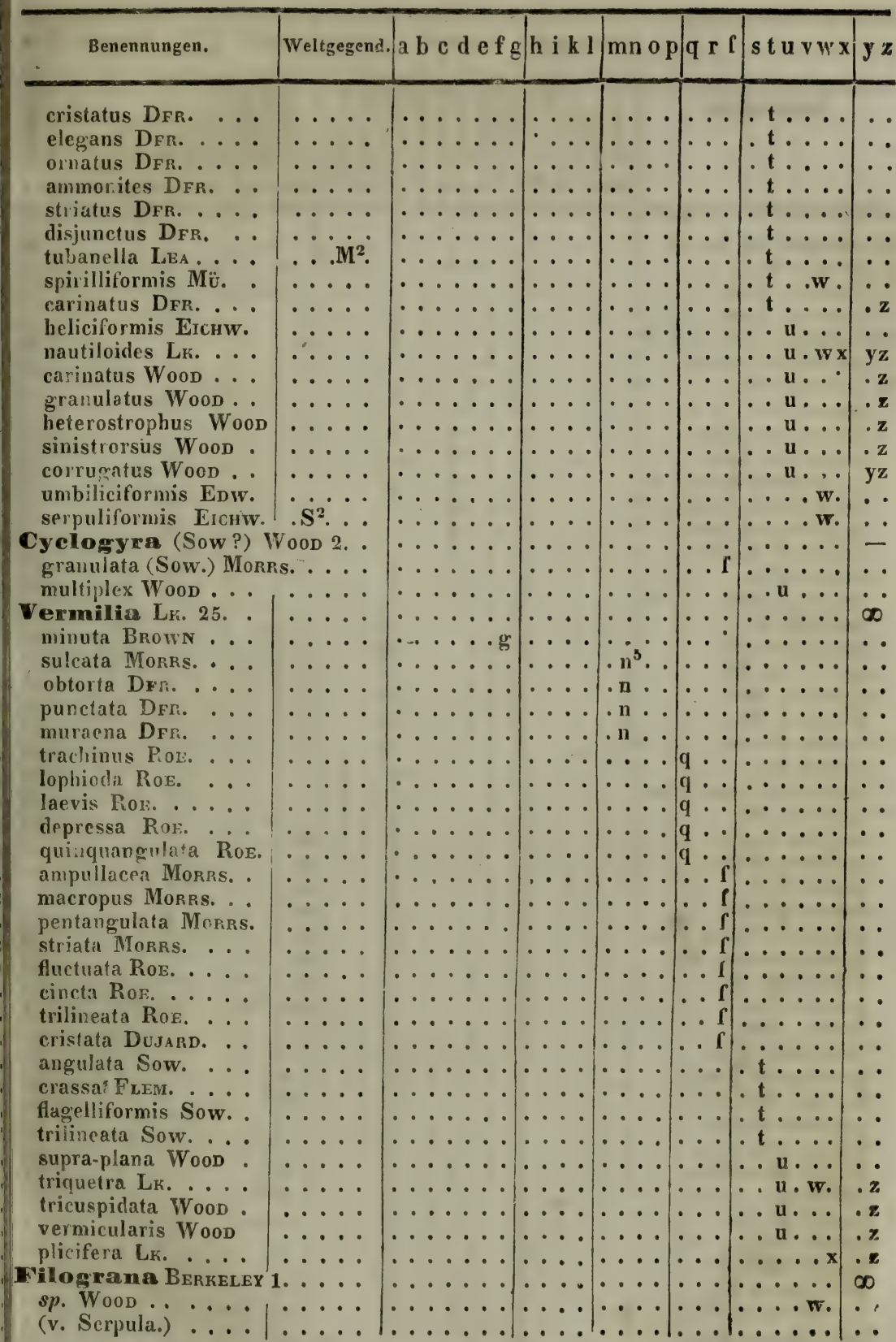




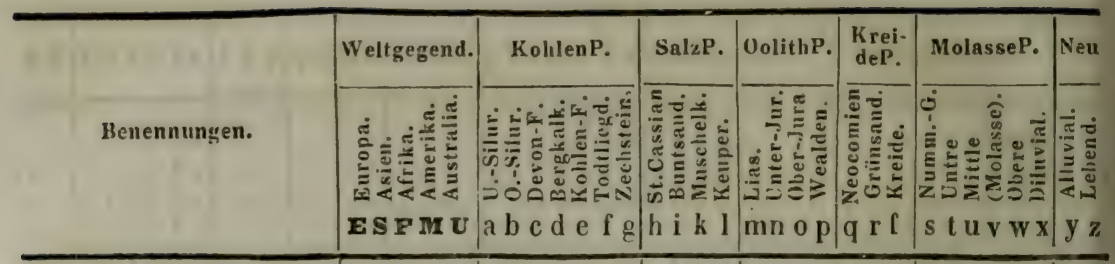

Galeolaria Lr. 3.

? socialis Br. prolifera Eow. angulosa Roz.

Serpula (L.) LK. 195 umbilicata Schlth.

? epithonia Gr. lithuus His. claviformis Kon. compressa Sow. Archimedis Kon. . subannulata Porte. Sowerbyana Kov. subcincta Porte. . . antiqua DFr.

Vallotina DrR. carinula Mü. canalifera Mü. ... monilifera M̈̈. . . . pygmaea $\mathrm{MÜ.}$ Geranae Mü. lineata KLIPST. valvata $\mathbf{G F}_{F}$. serpentina Sснм.Schl ? colubrina Mü. capitata PHir.L. capillaris Roe. stricta RoE. : tricristata Mü. quinque-cristata $M \ddot{\text { Ü. }}$ quinquesulcata Mü. circinnalis Mü. grandis $\mathbf{G F}_{\mathrm{F}}$. Warnii DFr. circinnata DFR. intestinalis PHILL. lacerata PHII.L. quadrata Phicl. triangulata Sow. vertebralis Sow. limax GF. conformis GF. limata Mü. plicatilis Mü. spirolinites Mü. pentagona Gr. quadrilatera GF.

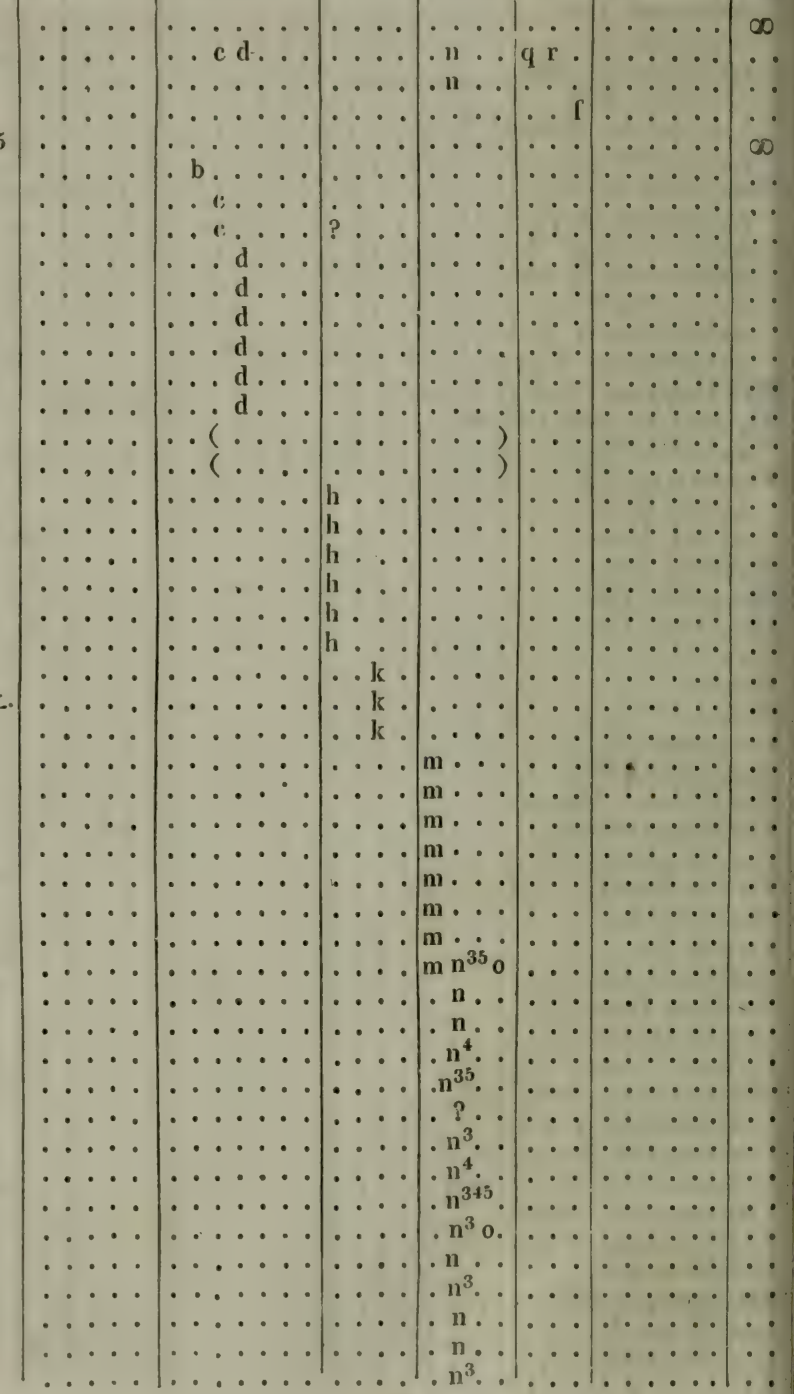




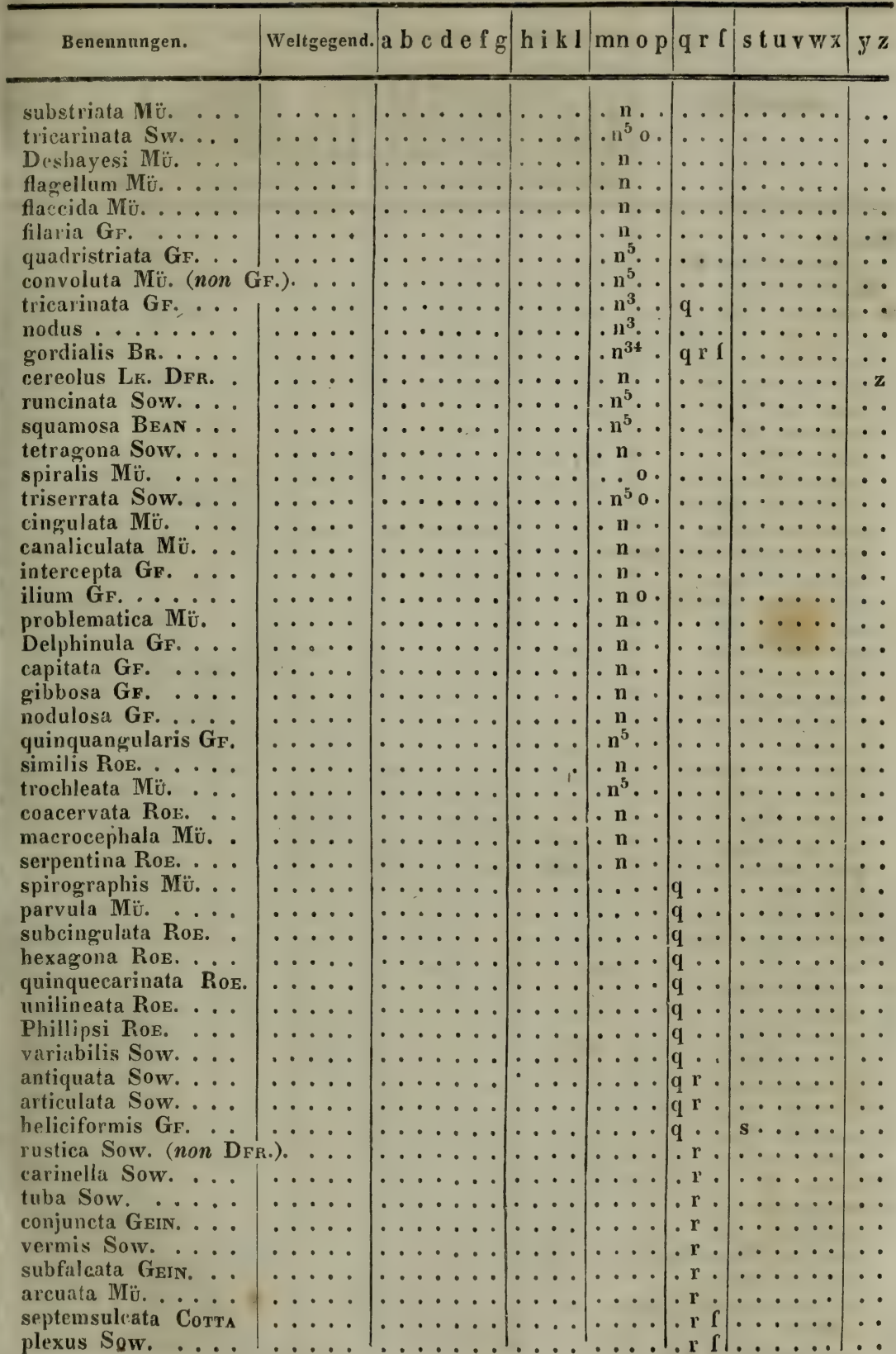




\begin{tabular}{|c|c|c|c|c|c|c|c|}
\hline \multirow{3}{*}{ benesumugen. } & Weltgegend. & KohlenP. & SalıP. & OolithP. & $\begin{array}{l}\text { Krei- } \\
\text { deP. }\end{array}$ & MolasseP. & Neu \\
\hline & 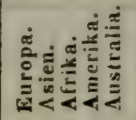 & 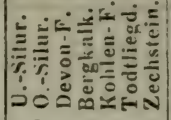 & 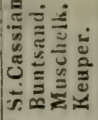 & 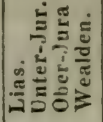 & 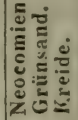 & 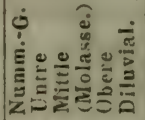 & 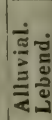 \\
\hline & ESFM & $a b c d e f g$ & h i k l & mn op & $q \times 1$ & $s t u v w x$ & y z \\
\hline
\end{tabular}

\section{Serpula)}

quadricarinata $\mathrm{Mü.}$ lituola Ley.

† sulcataria $\mathrm{L}$ A.

limax DFr. (non Sow.)

lumbricus DFr.

carinata Woopw.

pustulosa Gin.

contracta WooD.

obtusa Sow.

plana Woonw.

subinvoluta Reuss

bipartita Reuss . .

unisulcata Sow?, Morrs. . :

vortex Woonw...

draconocephala GF.

subtorquata Mü.

sexangularis Mü. . .

sexsulcata Mü.

Noeggerathi Mü...

spinulosa Reuss

crecta GF.

Leonhardi Reuss

subrugosa Mü.

vibicata Mü.

barbata Morr.

onyx (MorT).....

filosa Dus.

‡ aspera $H_{\text {AG. }}$.

trochiformis HaG.

conica $\mathrm{H}_{\mathrm{Ag}}$.

‡ umbilicata HaG.

\# caudata HAG. $_{\text {. }}$.

‡ pyomaea HAG.

Bardensis Hag.

* granulosa HaG.

implicata HAG.

₹ maeandra HAG.

canteriata HaG.

F costata HAG.

F heptagona Hag.

intermedia RoE.

quadrangularis RoB.

annulata Reuss. . .

gastrochaenoides LEYM.

Richardi Leym.

quadrangularis Lא.
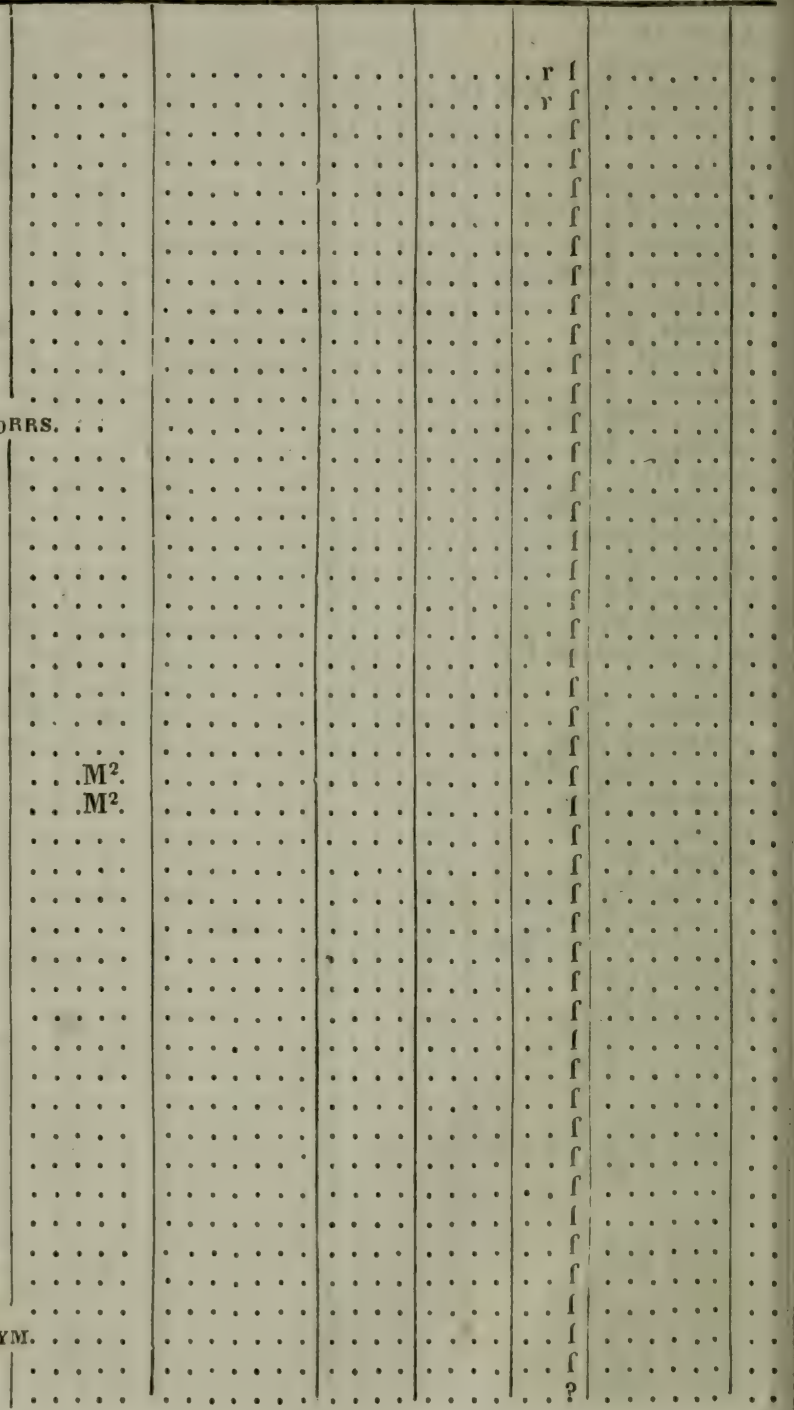


\begin{tabular}{|c|c|c|c|c|c|c|c|}
\hline & nd. & $\mathrm{g}$ & b i k l l & $\mathrm{p}$ & l & $\mathbf{w} \mathbf{x}$ & \\
\hline & & & & & & $8 t \ldots$ & \\
\hline & 5 & $\cdots \cdots \cdots$ & $\cdots$ & $\cdots \cdot$ & & & \\
\hline 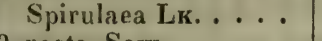 & $\cdots \cdot$ & $\cdots \cdots$ & $\cdots$ & $\cdots \cdot$ & & st $u \ldots$ & \\
\hline$y$ & ... & . & $\cdots \cdot$ & $\cdots$ & $\cdots$ & $\cdots$ & \\
\hline & $\cdots \cdot$ & & & $\cdots \cdot$ & & $\cdots$ & \\
\hline & . & & & & $\because$ & $\cdots$ & \\
\hline$\cdots$ & $\dot{\cdots} \cdot$ & $\cdots$ & $\cdots \cdots$ & $\ddot{\cdots} \cdot \mid$ & $\begin{array}{ll}\cdots \\
\cdots \\
\cdots\end{array}$ & $\therefore$ & \\
\hline . & $\ldots$ & $\cdots \cdots$ & $\cdots$ & $\cdots$ & $\cdots$ & . t... & \\
\hline . & $\cdots \cdot$ & $\cdots$ & $\ldots$. & $\cdots$ & $\cdots$ & . t .... & \\
\hline & $\cdots \cdots$ & $\ldots \ldots$ & $\cdots$ & $\ldots$ & $\cdots$ & $\cdots$ & \\
\hline R. . & $\cdots \cdot \cdot$ & $\ldots \ldots$ & $\cdots \cdot$ & $\cdots$ & $\cdots$ & $\cdots$ & \\
\hline$\therefore$ & . & - & $\cdot \cdot$ & $\cdots \cdot$ & $\cdots$ & $\cdots$ & \\
\hline & & & $\cdots$ & $\cdot$ & . & $\cdots$ & \\
\hline ... & & & & & - & $\cdots$ & \\
\hline$\dot{i o n} \dot{S}_{0}$ & .). & & 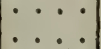 & $\left|\begin{array}{c}\cdots \\
\cdots\end{array}\right|$ & $\cdots$ & $\cdots$ & \\
\hline $\begin{array}{l}\text { (non So } \\
\ldots . .\end{array}$ & . & ... & $\ldots$ & $\ldots$ & $\ldots$ & $\cdots$ & \\
\hline$\ldots$ & $\ldots \ldots$ & $\cdots$ & $\cdots$ & $\ldots$ & $\cdots$ & $\cdots$ & \\
\hline$\cdots$ & $\cdots \cdots$ & $\ldots \ldots$ & $\ldots$ & $\ldots$ & $\cdots$ & & \\
\hline$\cdots$ & $\cdots \cdots$ & $\ldots \ldots$ & $\cdots \cdot \mid$ & $\cdots$ & $\cdots$ & $\cdots$ & \\
\hline$\cdot$ & $\cdots \cdot \cdot$ & $\cdots \cdots$ & $\cdots$ & $\cdots \cdot$ & $\cdots$ & . & \\
\hline$\cdots$ & 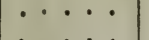 & - & $\cdots$ & $\cdots \cdot$ & $\cdots$ & $\cdots$ & \\
\hline & $\therefore$ & $:$ & - & $\cdots$ & $\cdots$ & $\ddot{*}$ & \\
\hline D DFr. & $\because$ & . & $\because \cdots \cdots$ & $\mid \begin{array}{c}\cdots \\
\cdots\end{array}$ & $\because \cdots$ & $\cdots$ & \\
\hline . & . . $\mathbf{M}^{2}$ & $\cdots$ & $\ldots$ & $\ldots$ & $\ldots$ & . t ... & \\
\hline AL. & $\ldots$ & & $\ldots$ & $\cdots$ & $\ldots$ & . t .... & • \\
\hline & . . & $\ldots$ & $\ldots$ & $\cdots$ & $\cdots$ & . t ... & • \\
\hline - $\cdot$ & $\ldots \mathrm{M}^{2}$ & $\cdots$ & $\cdots$ & $\cdots$ & $\cdots$ & . & - \\
\hline к. . & $\cdots \cdot \cdot$ & $\cdots$ & $\cdots$ & $\cdot$ & $\cdots$ & & • \\
\hline & $\cdots$ & - & $\cdot$ & - & - & & 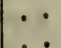 \\
\hline$\dot{0}$ & $\because$ & $\cdots$ & & & $\cdots$ & w. & \\
\hline$\therefore$ & .... & $\therefore$ & $\ldots \ldots$ & $\therefore$ & $\cdots$ & $\therefore t$ & \\
\hline$\cdots$ & $\cdots$ & $\cdots$ & $\ldots$ & $\cdots$ & $\cdots$ & . u. . . & .7 \\
\hline & & . & $\cdots \cdot$ & $\cdots \cdot$ & $\cdots$ & . u . . . & • \\
\hline 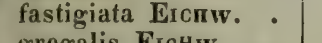 & $\cdots$ & $\cdot$ & $\cdots \cdot$ & $\cdots \cdot$ & $\cdots$ & . u. . & - \\
\hline 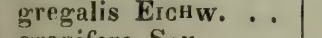 & $\cdots$ & . & & . & $\cdots$ & . & - \\
\hline & $\cdots$ & . & $\cdot$ & • & $\cdots$ & . u & - \\
\hline & - & $\cdot$ & $\cdot$ & $\therefore$ & $\cdots$ & $\cdots$ & - $z$ \\
\hline 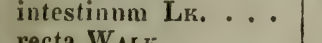 & & & & & ・. & - & $.2>>>3$ \\
\hline & & & & • & $\because$ & $\cdots$ & .2 \\
\hline$\cdots$ & $\therefore$ & . & . & $\cdots \cdot$ & $\therefore$ & $\cdots$ & $\cdots$ \\
\hline$\therefore$ & $\cdots$ & . & $\cdots$ & $\cdots$ & $\ldots$ & . & \\
\hline Ü. . & $\cdots$ & & & & ... & $\ldots$ & \\
\hline n & & & & & - & $\cdots$ & \\
\hline M & & & & $\cdots \cdot$ & $\cdots$ & $\cdots \cdots$ & - \\
\hline 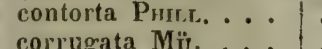 & - & & & - & $\cdots$ & $\cdots$ & i \\
\hline . & & & & 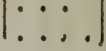 & & 8. & \\
\hline
\end{tabular}




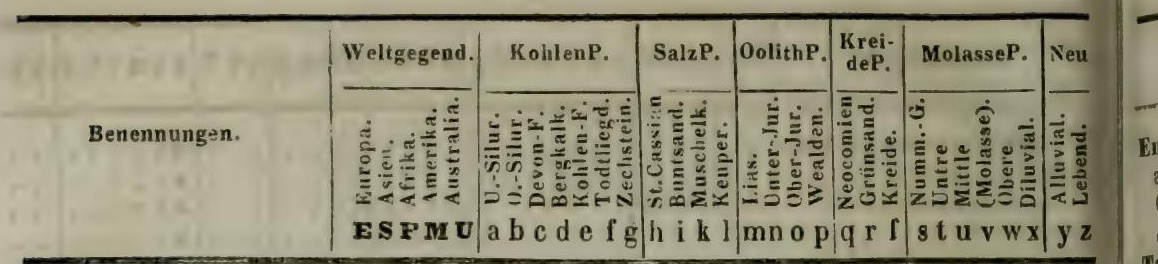

Serpula)

chorda Risso . . . echinata Gr. Brocc. filograna Lк. .... vermicularis LK. ... rupestris Riss.

? cornucopiae Riss.

Serpularia Mü. 2 bicrenata Mü. ... . crenata Mü.

Serpulith es Murch.1 longissimus Мurch.

Spiroglyphus $M^{\prime} \mathrm{Cox}, 1$. . . marginatus M'Cox.

\section{ANTENNATA LK.}

(Fühler-Würmer).

(Genera viventia numerosa omittuntur.

Nereis Cuv. 0. . . .

Nereites Murch. 2. Cambrensis Murch. Sedrwicki Murcu.

Leodice Sav. 1. . sp. Morrs. . . . .

Aphrodite Cuv. 1. $s p$. Porte.

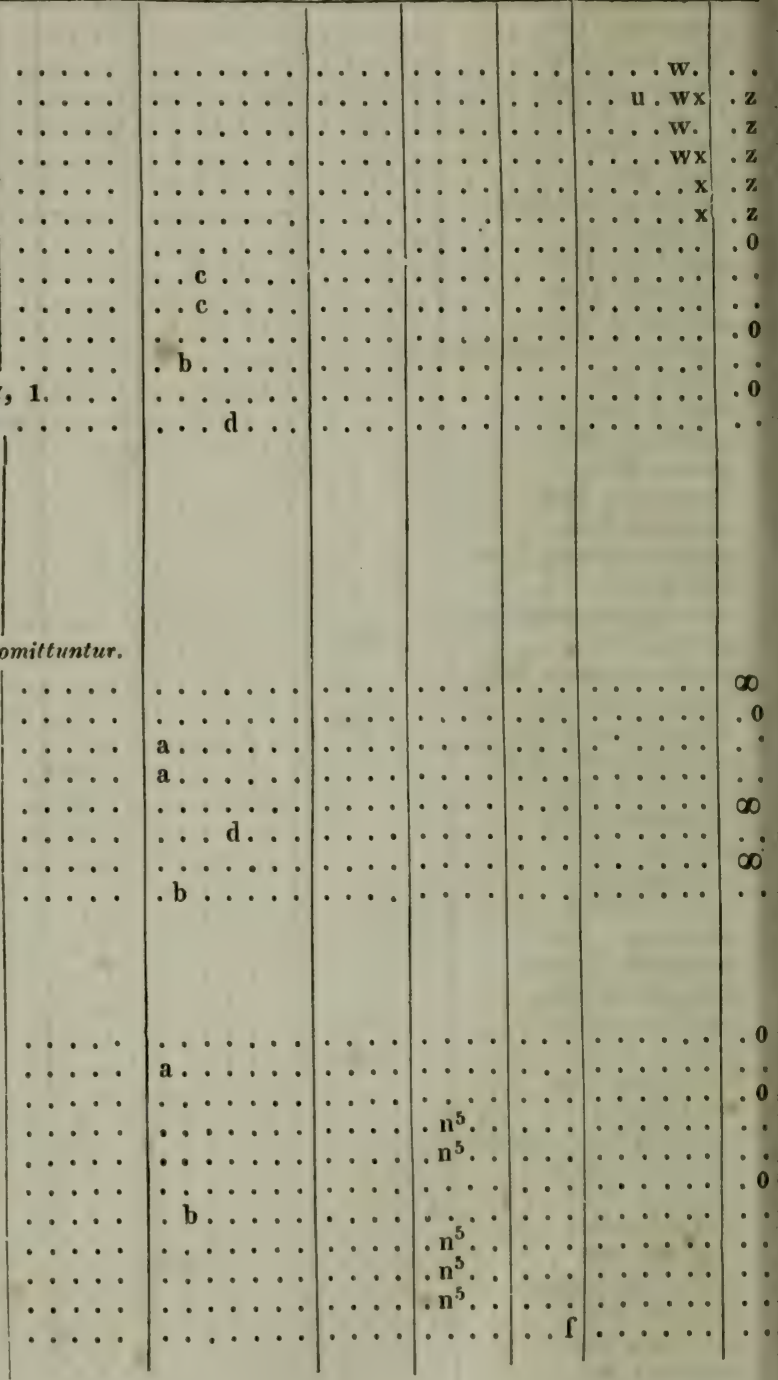

(Genera dubiae sedis.)

IIrrianites Murcu, 1 Macleayi Моrсн. ...

Mrirudella Mü. 2. . angusta Mü.

tenuis Mü.

Lumbricaria $\dot{M}$. 5 ? antiqua Portr.

? gordialis Mü. corjugata $M$ Ü. filaria Mü.

? gregaria Ponte

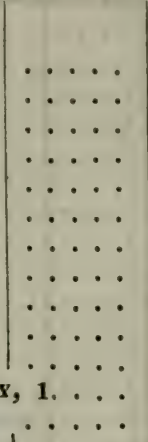




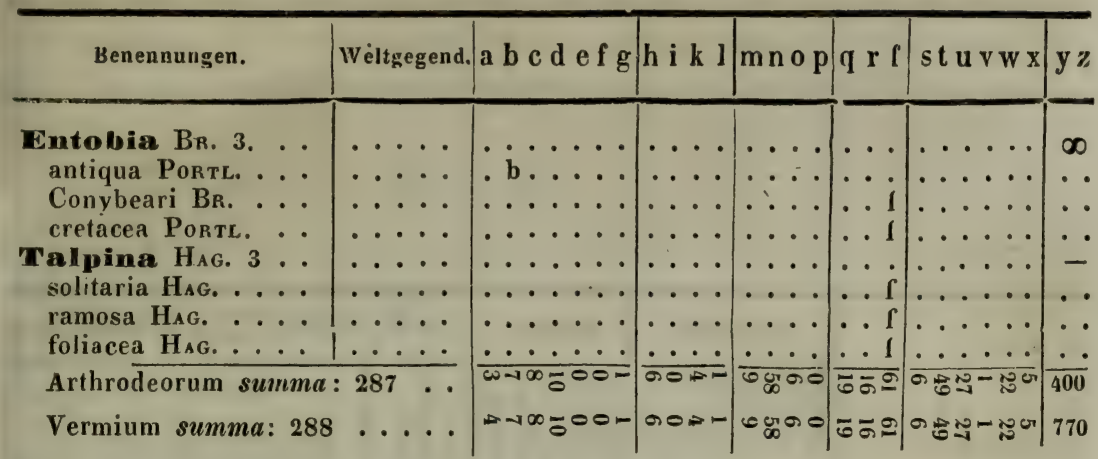




\begin{tabular}{|c|c|c|c|c|c|c|c|}
\hline \multirow{3}{*}{ benenumyell. } & \multirow{3}{*}{$\begin{array}{c}\text { Weltgegend. } \\
\mathbf{E}^{1,2} \text { Europa. } \\
\mathbf{S}^{1}, 2,3 \text { Asien. } \\
\mathbf{F}^{2}, 3,4 \text { Afrika. } \\
\mathbf{M}^{1}, 2,3, \mathbf{4} \text { Amerika. } \\
\mathbf{U}^{3}, 4 \text { Australien. } \\
\mathbf{E} \mathbf{S} \mathbf{F} \mathbf{M} \mathbf{U} \\
\text { ke in Zeishen : be- } \\
\text { deutet } \mathbf{E}^{2} \text {. }\end{array}$} & KohlenP. & SalzP. & OolithP. & $\begin{array}{c}\text { Krei- } \\
\text { deP. }\end{array}$ & MolasseP. & Neu \\
\hline & & 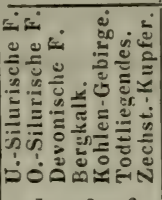 & 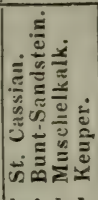 & 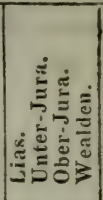 & 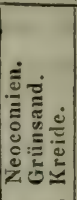 & 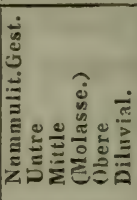 & 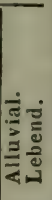 \\
\hline & & a bcdef $g$ & h i k l & $\operatorname{mn} 0 \mathrm{p}$ & $q \times \mathbf{I}$ & s t u vw $\mathrm{x}$ & y $z$ \\
\hline
\end{tabular}

\section{Cl. XVII. CRUSTACEA : Krusten-Kerfe.}

\section{CIIRERPETAR BurM.}

\section{A. BaLANIDAE Gray}

Tubicinella Lk. 1

maxima MORRN.

Diadema (Scuvir) (Polylepas Grax) bifidum Br. . . . . . vulgare Schum.

Coronula LK. 0 . . (Astrolepas GRAY)

Cremsia Lench 1. . sp. DsM.

Clisia Savgn., Leach 1 verruca So.....

Oelnthosia Ravz. 1 Stroemia Ranz.

Pyrgonna Savg. 1. (Adua Leaca)

† undatum Miciт. . sulcatum PHIL. ..

Asemus Ranz. 0 ..

Conia Blv. 0. ... Eluminius LEach, 0 . Acasta Leach, 1 . Montagui Lrach . .

Clntliamal us $R_{A N z .2}$ giganteus Phill. . stellatus RAnz. ... ....

IBalanus (Brug.) Ranz, 42.. 0 carbonarius PEтzH.

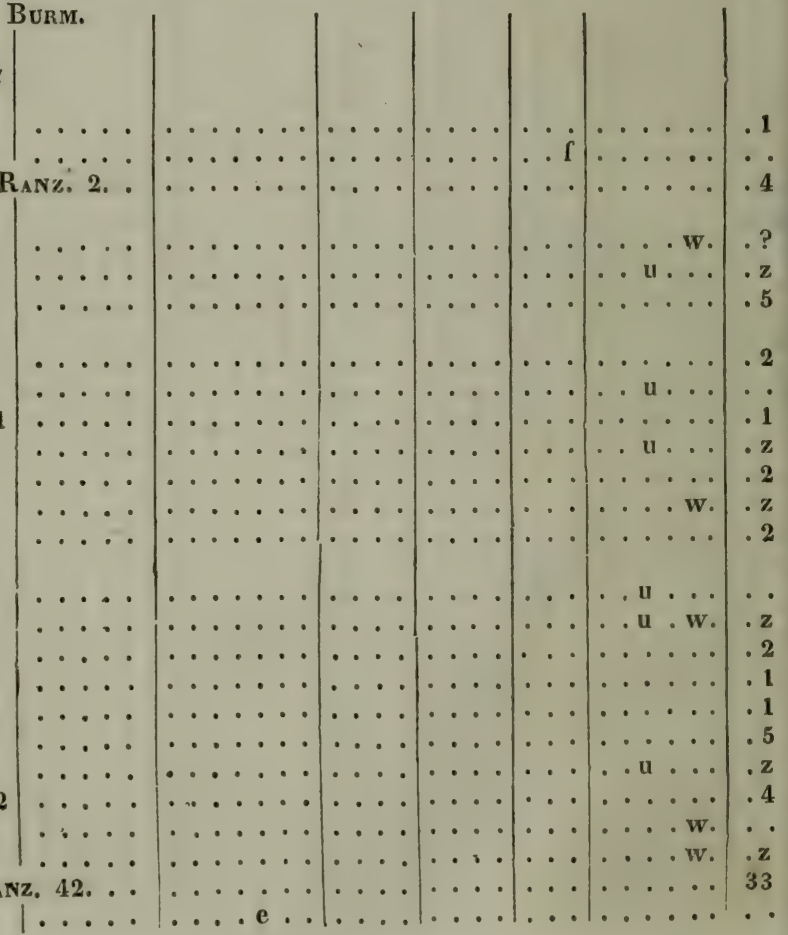




\begin{tabular}{|c|c|c|c|c|c|c|c|}
\hline Bener & Weltgegend. & $|a b c d e f g|$ & hik l & $\mathrm{mnop}$ & $q \times f$ & w $\mathrm{x}$ & $\mathbf{y} \%$ \\
\hline GF. & & & . $\mathrm{k}$. & & & & \\
\hline Ostrearum Con & $\ldots M^{2}$ & $\ldots \ldots$ & $\ldots$ & $\cdots$ & ... & le $t$ & \\
\hline peregrinus Mopr. . & . . $\mathrm{M}^{2}$ & $\ldots \ldots$ & $\ldots$ & ... & ... & . $t$. & \\
\hline circinnatus DFr. . . & $\ldots$. & $\ldots \ldots$ & $\cdots$ & $\ldots$ & ... & . ? ?... & \\
\hline ‡ communis DFr. . . . & $\ldots \ldots$ & $\ldots \ldots$ & $\cdots$ & $\ldots$ & $\cdots$ & . $\mathrm{t} \ddot{\mathrm{u}} \cdot \boldsymbol{v}$. & .? \\
\hline$\neq$ pustula Drr. . . . & . . . & $\ldots \ldots$ & $\ldots$ & ... & . . & . ? ? ?. & - \\
\hline Finchi Lefa .... & ... $M^{2}$ & $\ldots \ldots$ & $\ldots$ & $\ldots$ & $\cdots$ & $\ldots \mathbf{u} \ldots$ & 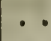 \\
\hline \# Holgeri Gein. . . . . & $\ldots \ldots$ & $\ldots \ldots$ & $\ldots \ldots$ & $\ldots$ & ... & $\ldots u \ldots$ & $\bullet$ \\
\hline Rogers & $\ldots \mathrm{M}^{2}$ & $\ldots \ldots$ & $\ldots$ & $\cdots \cdot$ & $\cdots$ & $\ldots$ и... & 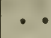 \\
\hline sagittatus LEA & $\dot{s}^{3} \cdot$ & . & $\cdots$ & $\cdots$ & ... & $\cdots \mathbf{u} \cdots$ & - \\
\hline $\begin{array}{l}\text { sublaevis So. .... } \\
\text { balanoides Ranz. . }\end{array}$ & $\therefore \sqrt{3}$ & $\cdots$ & $\cdots$ & $\mid \begin{array}{c}\cdots \\
\cdots\end{array}$ & $\cdots$ & $\left|\begin{array}{cc}\cdots \\
\cdots \mathrm{u}, \mathrm{w}\end{array}\right|$ & . $\mathrm{z}$ \\
\hline$\neq$ costatus MTG. . . . & ..... & $\ldots \ldots$ & $\ldots$ & .... & $\cdots$ & ?.? & $\mathrm{z}$ \\
\hline punctatus MT . . . & .... & $\ldots \ldots$ & $\ldots$ & $\ldots$ & ... & ..?.?. & .7 \\
\hline rugosus Moris. . & $\ldots .$. & $\ldots \ldots$ & $\ldots$ & ... & ... & $\ldots u \ldots$ &. $\mathrm{z}$ \\
\hline$\cdots$ & .... & $\ldots \cdots$ & $\ldots$ & $\cdots \cdot$ & ... & ..u. w. & yz \\
\hline LK. & ... & $\ldots \ldots$ & $\ldots$ & $\cdots$ & ... & ..u. u. w. & yz \\
\hline FF. . . & $\cdots \cdots$ & $\ldots \ldots$ & $\cdots$ & $\ldots$ & $\ldots$ & . . ? w. & $\cdot$ \\
\hline$\cdots$ & $\cdots \cdot$ & $\cdots \cdots$ & $\cdots$ & $\cdots \cdot$ & $\cdots \cdot$ & $\ldots \mathbf{v} \ldots$ & • \\
\hline$\cdots$ & $\cdots \cdots$ & $\cdots$ & $\cdots$ & $\cdots \cdot$ & $\cdots$ & $\cdots \cdots w_{0}$ & • \\
\hline $\mathrm{cri}$ & $\cdot$ & $\cdots$ & $\cdots \cdots$ & $\cdots \cdots$ & $\cdots$ & $\cdots \cdots w_{0}$ & - \\
\hline lati & $\cdots$ & $\cdots$ & $\cdots$ & $\cdots$ & $\cdots$ & $\cdots \cdots w_{0}$ & $\cdot$ \\
\hline Br... & $\cdots \cdots$ & $\ldots$ & $\cdots$ & 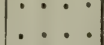 & $\cdots$ & anw & \\
\hline $\begin{array}{l}\text { pietus } 1 \mathrm{H} . \cdots \cdots \\
\text { plicarius } \mathrm{Br} .\end{array}$ & .... & $\ldots$ & $\ldots$ & $\ldots$ & $\cdots$ & .... & $\cdots$ \\
\hline SM. . . . & $\ldots \ldots$ & $\ldots \ldots$ & $\ldots$ & $\ldots$. & $\ldots$ & $\ldots w$ & $\cdots$ \\
\hline . & .... & $\ldots \ldots$ & $\ldots$. & $\ldots$ & $\ldots$ & $\ldots w_{\text {. }}$ & . \\
\hline U. . . & .... & $\cdots$ & $\ldots$ & $\cdots$ & $\ldots$ & $\ldots w_{.}$ & . \\
\hline rhombicus Br. . . & $\ldots \ldots$ & . & . & $\cdots \cdot$ & $\ldots$ & $\ldots \ldots w$ & . \\
\hline$\neq$ squamosus DFR. . & $\ldots$ & . & - & $\cdots \cdot$ & ... & $\cdots w_{0}$ & . \\
\hline stcllaris Br. $\ldots$. & $\cdots$ & $\ldots \ldots$ & $\ldots$ & $\cdots$ & $\ldots$ & 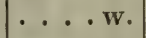 & -・ \\
\hline \# striatus DFr. . . . & $\ldots$ & $\cdots \cdot$ & $\ldots$ & $\cdots$ & $\cdots$ & $\ldots w_{0}$ & . \\
\hline tertiarius DFR. . . & $\cdot$ & & . & $\cdots$ & ... & $\cdots w$ & \\
\hline tessellatus So. & & 0 & . & $\cdots \cdot$ & - & $\cdots w \cdot$ & \\
\hline .. & $\therefore$ & $\cdot$ & : & $\therefore$ & . 1 & $\cdots \cdots w_{0}$ & $\because$ \\
\hline Lк. . & ... & $\cdots \cdots$ & $\cdots$ & & . & $\ldots w_{\text {w. }}$ & $\cdot z$ \\
\hline $\begin{array}{l}\text { patus BRUG. } \\
\text { udraceus Lr. - }\end{array}$ & .... & $\ldots$ & $\ldots$ & $\cdots$ & & $\cdots w$ & $\cdot z$ \\
\hline BRUG. . & & 1 & ... & $\ldots$ & $\therefore$ & $\therefore w$ &.$z$ \\
\hline semiplicatus Lr. . . & . & $\ldots \ldots$ & $\ldots$ & $\ldots$ & $\therefore$ & $\therefore w$ &. $\mathrm{z}$ \\
\hline$\therefore \therefore$ & . & $\ldots$ & $\ldots$ & . . & . & $\ldots w$. &. $\mathrm{z}$ \\
\hline ovulari & $\cdots \cdots$ & $\ldots \ldots$ & 1 & & 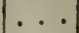 & $\ldots \ldots x$ &. $\mathrm{z}$ \\
\hline Uddewallensis $\mathbf{L}$. . & $\mathrm{E}^{2} \ldots \mathrm{M}^{2}$ & & & & ․ & $\mid \ldots \ldots x$ &. $\mathrm{z}$ \\
\hline So. 0. & . . & & & . & & $\ldots \ldots$ & .9 \\
\hline Catc & $\cdots \cdot$ & $\ldots \ldots$ & $\cdots$ & . & & $\ldots \ldots$ & .1 \\
\hline B. LEPADINA. & & & & & & & \\
\hline Lor & & $\cdots$ & & - & & & .0 \\
\hline mri & & & & & & . & $\ddot{i}$ \\
\hline$-\infty$ & & & & & & & .1 \\
\hline
\end{tabular}


Benennungen.

\begin{tabular}{|c|c|c|c|c|c|c|}
\hline Welt & KohlenP. & SalzP. & WolithP. & $\left|\begin{array}{r}\text { Krei- } \\
\text { deP. }\end{array}\right|$ & MolasseP. & Nen \\
\hline 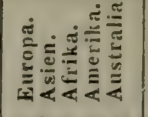 & 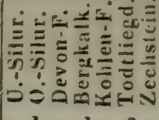 & $\mid$ & 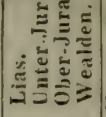 & 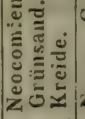 & 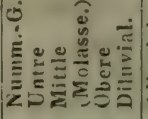 & 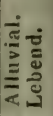 \\
\hline $\mathbf{E S} \mathbf{M U}$ & a b c d e $f g$ & $\mid \begin{array}{llll}h & \mathrm{i} & \mathrm{k} & \mathrm{l}\end{array}$ & $\mid \mathrm{mn}$ op $\mid$ & $|q r d|$ & st u v w x & y z \\
\hline
\end{tabular}

Lithotrya So. 0 . (Litholepas Bev pars.)

Tetralasmis Cuv. o (Ibla Gray.)

Smilium Gray 0.

Scalpellum Leaci, 1 (Polylepas BLy. pars)

† magnum Woon ...

Pollicipes Lr. 29. (Pentalepas Buv. purs.) oolithicus Buckm. radiatus $\mathrm{KoD}$ U. planulatus MorRs. . concinnus Morrs.

Bronni Roe.

Hausmanni DuKo. . radiatus So. radiatus So. laevis So. maximus So. rigidus So. angustatus Gers. asper RoE. conicus Reuss dorsatus Beck ... glaber RoE. gracilis Roz. medius Steenstr. 4carinatus Reuss.. solidulus Steenstr. sulcatus So. uncinatus Roв.... undulatus STEENSTR. validus Sternstr. spathulatus [? So.] .

t antiquus Mrcht. carinatus Purr.. radiatus $\mathbf{M u}$. reflexus So.

Anutifera Gray, 3 (pro Anatifa Brug.) cretae Steenster. Nilssoni Steenstr. . turgida Steenstr.

Cineras Leach? Dtion Leach Pamina Gray 0 . 


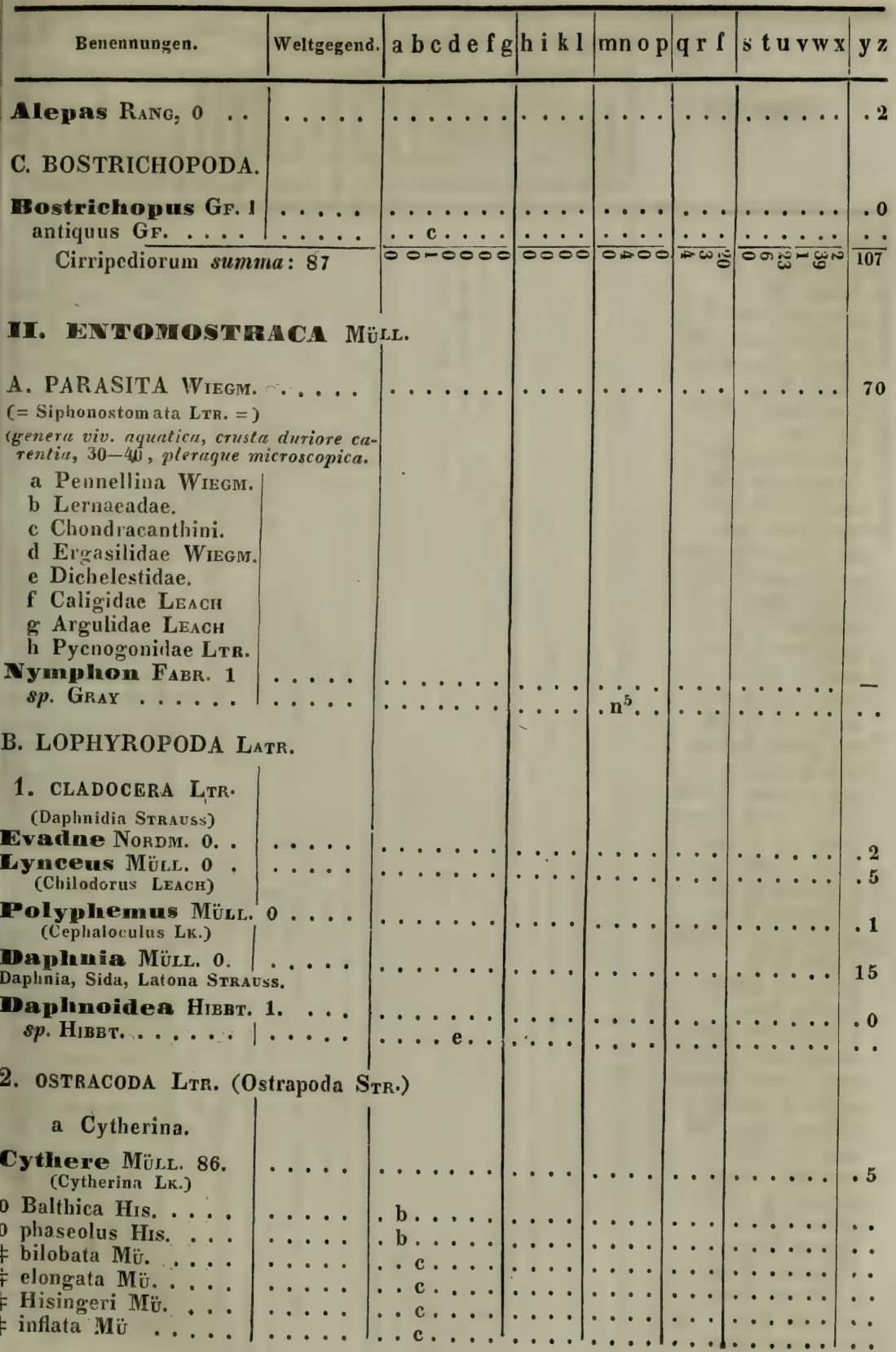




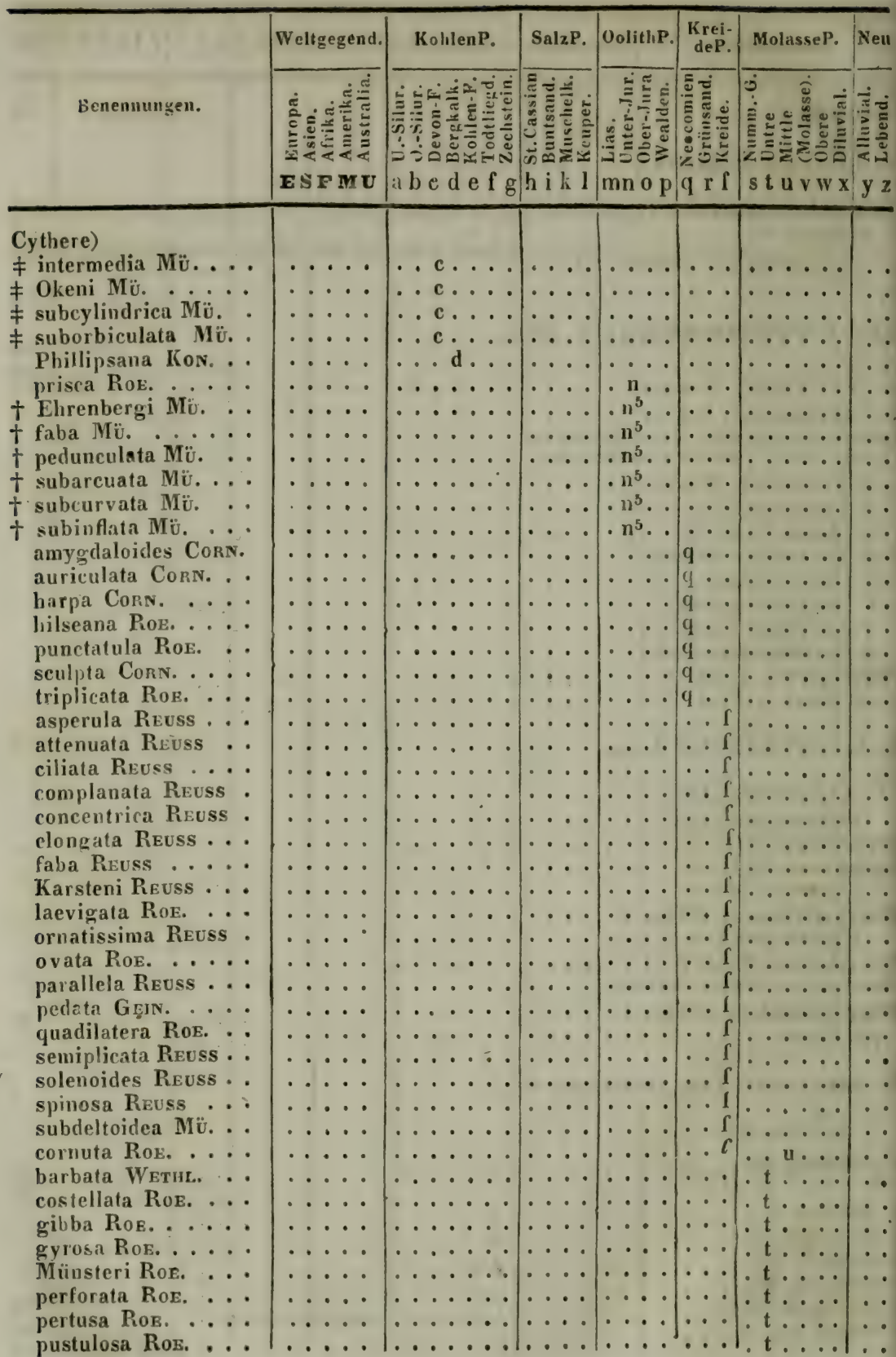




\begin{tabular}{|c|c|c|c|c|c|c|c|}
\hline Benennungen. & Weltgegend. & $a b c d$ e $f g$ & h i k l & $\mathrm{mn}$ op & $q \times 1$ & $\mathrm{st}, \mathrm{uv} \mathrm{w} \mathbf{x}$ & y z \\
\hline ctata RoE. & $\cdots$ & & & & $\cdots$ & & \\
\hline$\ldots$ & $\ldots \ldots$ & $\ldots$ & $\ldots$ & $\ldots$ & $\cdots$ & . $t ? . ?$ & - \\
\hline Mülleıi Mü. . & .... & $\ldots$ & $\ldots$ & $\ldots$. & $\cdots$ & . ? u.w. & . \\
\hline subdelt oidea Mü. . & $\ldots \cdot$ & $\ldots \ldots$ & $\cdots$ & $\ldots$ & $\ldots$ & . $t$ ? ? & . \\
\hline compressa $\mathrm{HAU}_{\mathrm{AU}} \ldots$ & $\cdots \cdot \cdot$ & $\ldots \ldots$ & $\cdots \cdot$ & $\cdots \cdots$ & $\cdots$ & . . u ... & - \\
\hline Haucri Ros. . . . . & $\cdots \cdot \cdot$ & $\ldots \cdots$ & $\cdots$ & $\cdots \cdot$ & $\cdots$ & $\ldots u \ldots$ & - \\
\hline laevis RoE. & $\cdots \cdot \cdot$ & $\cdots \cdots$ & $\cdots \cdot$ & $\cdots \cdot$ & $\cdots$ & $\ldots u \ldots$ & - \\
\hline subdeltoidea $\mathrm{H}_{\mathrm{AU}}$. & $\cdots \cdot \cdot$ & $\cdots$ & $\cdots \cdot$ & $\cdots \cdot$ & $\cdots$ & . . u ... & - \\
\hline Edwardsi Roe. . . & 0 & $\cdots$ & . . . & $\cdots \cdot$ & $\cdots \cdot$ & $\cdots u \cdot \mathbf{w}$ & - \\
\hline$\cdots$ & $\cdots \cdots$ & $\therefore$ & $\cdots$ & $\cdots$ & $\cdots$ & $\cdots u \cdot w$ & • \\
\hline ... & & $\ddot{m}$ & $\cdots$ & $\cdots$ & $\because \because$ & $\cdot u \cdot \mathbf{u}$ & - \\
\hline C. & $\cdots \cdots$ & $\cdots \cdots \cdots$ & $\cdots$ & $\cdots \cdots$ & $\because \cdots$ & $\cdots u \cdot w$ & $\cdots$ \\
\hline $\begin{array}{l}\text { aciculata Roe. . . } \\
\text { arcuata Mü. . . . }\end{array}$ & $\ldots \ldots$ & $\cdots \cdots$ & $\cdots$ & $\cdots$ & $\because \cdots$ & $\begin{array}{l}\cdots \cdots \mathbf{w} \\
\cdots \cdots \mathbf{w}\end{array}$ & - \\
\hline $\begin{array}{l}\text { arcuata Mü. . . . } \\
\text { carinata RoE. . . . }\end{array}$ & .... & $\ldots \ldots$ & $\cdots$ & $\cdots$ & $\therefore$ & 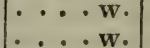 & - \\
\hline & $\ldots \ldots$ & $\ldots \ldots$ & $\ldots$ & $\ldots$ & $\ldots$ & & - \\
\hline $\begin{array}{l}\text { compressa Mü. } \ldots \\
\text { coronata Roe. . . }\end{array}$ & $\ldots \ldots$ & $\ldots \ldots$ & $\ldots$ & $\ldots$ & $\ldots$ & $\begin{array}{l}\cdots w \\
\cdots \cdots \mathbf{w} \\
\cdots \cdots\end{array}$ & - \\
\hline PoE. . . . . & $\cdots \cdot \cdot$ & $\ldots \ldots$ & $\cdots$ & $\cdots \cdot$ & $\cdots$ & ....w. & $\therefore$ \\
\hline Ü. . . & $\cdots \cdots$ & $\ldots \ldots$ & $\cdots$ & $\cdots \cdots$ & $\cdots$ & $\ldots \cdot \mathbf{w}$ & . \\
\hline Jurine Mü. . . . . & $\cdots \cdot \cdot$ & $\ldots \ldots$ & $\cdots$ & $\cdots \cdot$ & $\cdots$ & $\ldots w_{\text {. }}$ & $\cdots$ \\
\hline linearis Roe. . . . & $\cdots \cdots$ & $\ldots \ldots$ & $\cdots$ & $\cdots \cdot$ & $\cdots$ & $\cdots \cdot \mathbf{w}$ & - \\
\hline lineolata Roe. ... & $\cdots \cdot$ & $\cdots \cdots \cdot$ & $\cdots$ & $\cdots \cdot$ & $\cdots$ & $\cdots \cdot w_{\cdot}$ & $\cdots$ \\
\hline lunata RoE, & • & $\cdots$ & $\cdots$ & $\cdots \cdot$ & $\cdots$ & $\cdots$ & $\cdots$ \\
\hline plicata N & • & $\cdots \cdots$ & $\cdots$ & $\cdots \cdot$ & $\cdots$ & $\cdots \cdots w$ & $\cdots$ \\
\hline $\begin{array}{l}\mathrm{ru} \\
\text { su }\end{array}$ & $\cdots \cdots$ & $\cdots \cdots \cdots$ & $\cdots \cdots$ & $\cdots \cdot$ & $\cdots$ & $\cdots \cdots \mathbf{w}$ & $\cdots$ \\
\hline $\begin{array}{l}\text { subovata MÜ. } \cdots \\
\text { subradiosa Roe. }\end{array}$ & $\ldots$ & $\because \vdots$ & $\because \cdots$ & $\cdots$ & $\cdots$ & anw & $\because$ \\
\hline ventrosa PhiL. . . . & .... & $\ldots \ldots$ & $\ldots$ & $\cdots \cdots$ & $\ldots$ & $\ldots w$ & $\because$ \\
\hline Cурris Mü. $21 \ldots$. & $\cdots \cdot$ & $\ldots \ldots$ & $\ldots$ & $\ldots$ & ... & $\ldots \ldots$ & $2 \dot{25}$ \\
\hline spp. Phulc. & $\ldots$ & $. \operatorname{cd} 0 . .$. & ... & $\ldots$. & $\cdots$ & $\ldots \ldots$ & . \\
\hline subrecta Pontr. . . & $\ldots \ldots$ & $\ldots d \ldots$ & $\cdots$ & $\cdots \cdots$ & $\cdots$ & $\ldots \ldots$ & . \\
\hline arcuata BEA & $\cdots \cdot \cdot$ & $\ldots . .$. & $\cdots$ & $\cdots$ & $\cdots$ & $\ldots \ldots$ & . \\
\hline inflata Murc & & $\ldots e . .$. & $\cdots$ & $\cdots$ & $\cdots$ & $\ldots \ldots$ & $\cdots$ \\
\hline digalensis $\mathbf{I}$ & B. & $\ldots$.... & $\cdots \cdot$ & $\cdots \cdots$ & $\cdots$ & $\ldots \ldots$ & - \\
\hline liasica Bror & $\cdots$ & $\cdots$ & $\cdot \cdot$ & $m \ldots$ & $\cdots$ & $\cdots \cdots$ & - \\
\hline SAN . & & $\cdots \cdots$ & $\cdots$ & $\cdot n^{3} \cdot \cdot$ & $\cdots \cdot$ & $\cdots \cdots$ & - \\
\hline osa So. ... & $\cdots$ & $\cdots \cdots$ & $\cdots$ & $\cdots p$ & $\cdots$ & $\cdots \cdots$ & - \\
\hline$\cdots \cdot$ & . & $\cdots \cdots \cdots$ & $\cdots$ & $\cdots p$ & $\cdots$ & $\cdots \cdots$ & \\
\hline$\cdots \cdot$ & - & $\cdots$ & $\cdot \cdot$ & $\cdots p$ & $\cdot$ & $\cdots \cdots$ & \\
\hline ormis DU. . & & . & $\therefore$ & ・.pp & . & $\cdots \cdots$ & \\
\hline si & 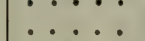 & $\cdot 1$ & & $\cdots p$ & $\cdots$ & $\therefore$ & \\
\hline netata Ros. & . & ... & .... & $\therefore p$ & $\cdots$ & $\ldots \ldots$ & .. \\
\hline rculata So... & - & $\cdots \ldots$ & $\cdots \cdot$ & $\cdots p$ & - & $\cdots$ & \\
\hline Valdensis So. ... & - & 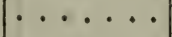 & $\cdots \cdot \cdot$ & $\cdots p$ & $\cdots$ & & \\
\hline$p$ & & $\cdots$ & $\cdots \cdot$ & $\cdot \cdot$ & & . $t$. & \\
\hline fa & - & $\cdots \cdot \div$ & $\cdot$ & $\cdots \cdot$ & $\cdots$ & .? ?... & \\
\hline $\begin{array}{l}\text { cymindrica s } \\
\text { subglobosa }\end{array}$ & & - : & & & $\cdots$ & $\cdots v \cdots$ & \\
\hline . & & & & $\dot{.}$ & & $\cdots v \cdots$ & $\ddot{y z}$ \\
\hline Уy & & & & & $\ldots$ & $\ldots \ldots$ & $y=$ \\
\hline & & & & & & & \\
\hline
\end{tabular}




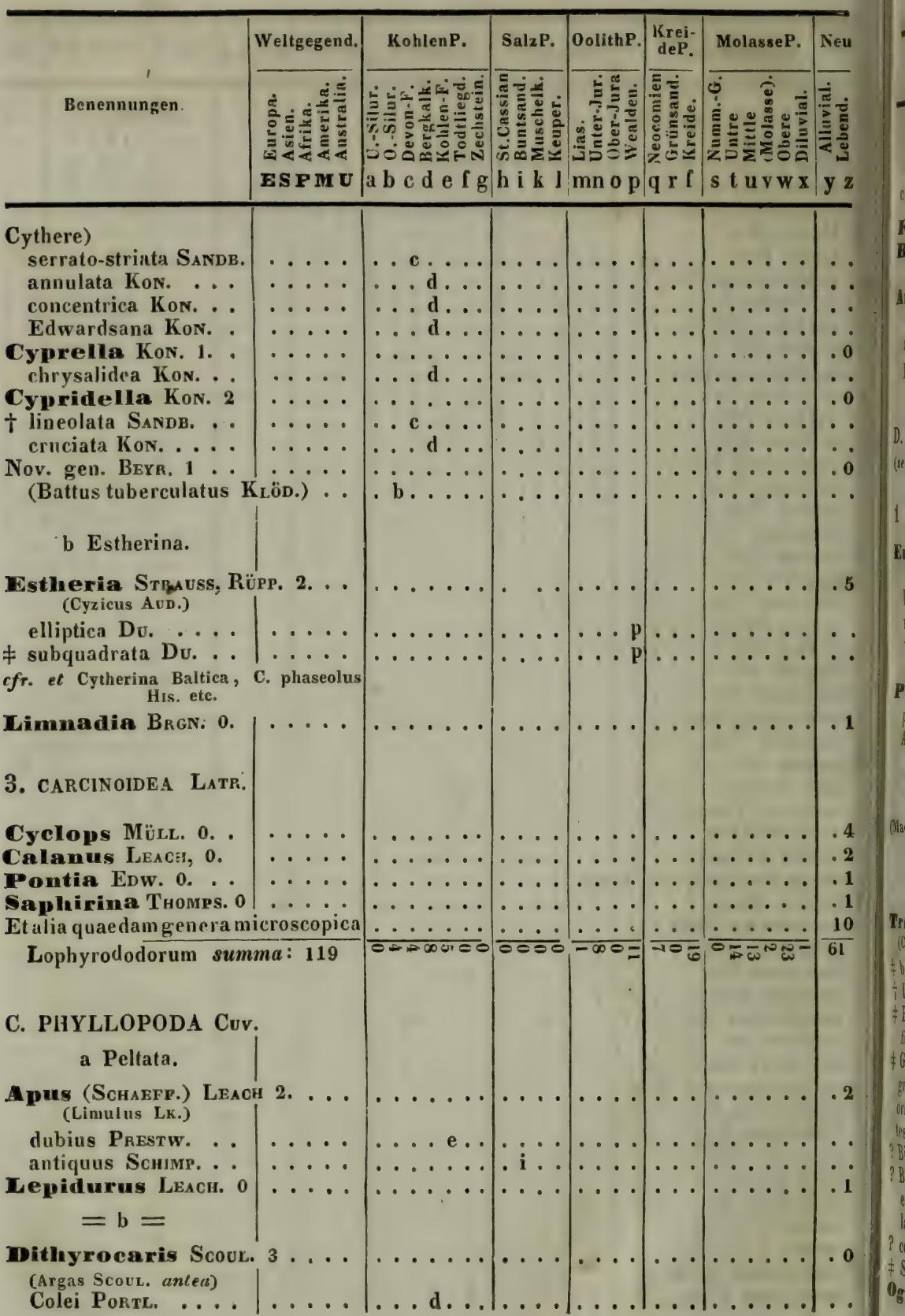




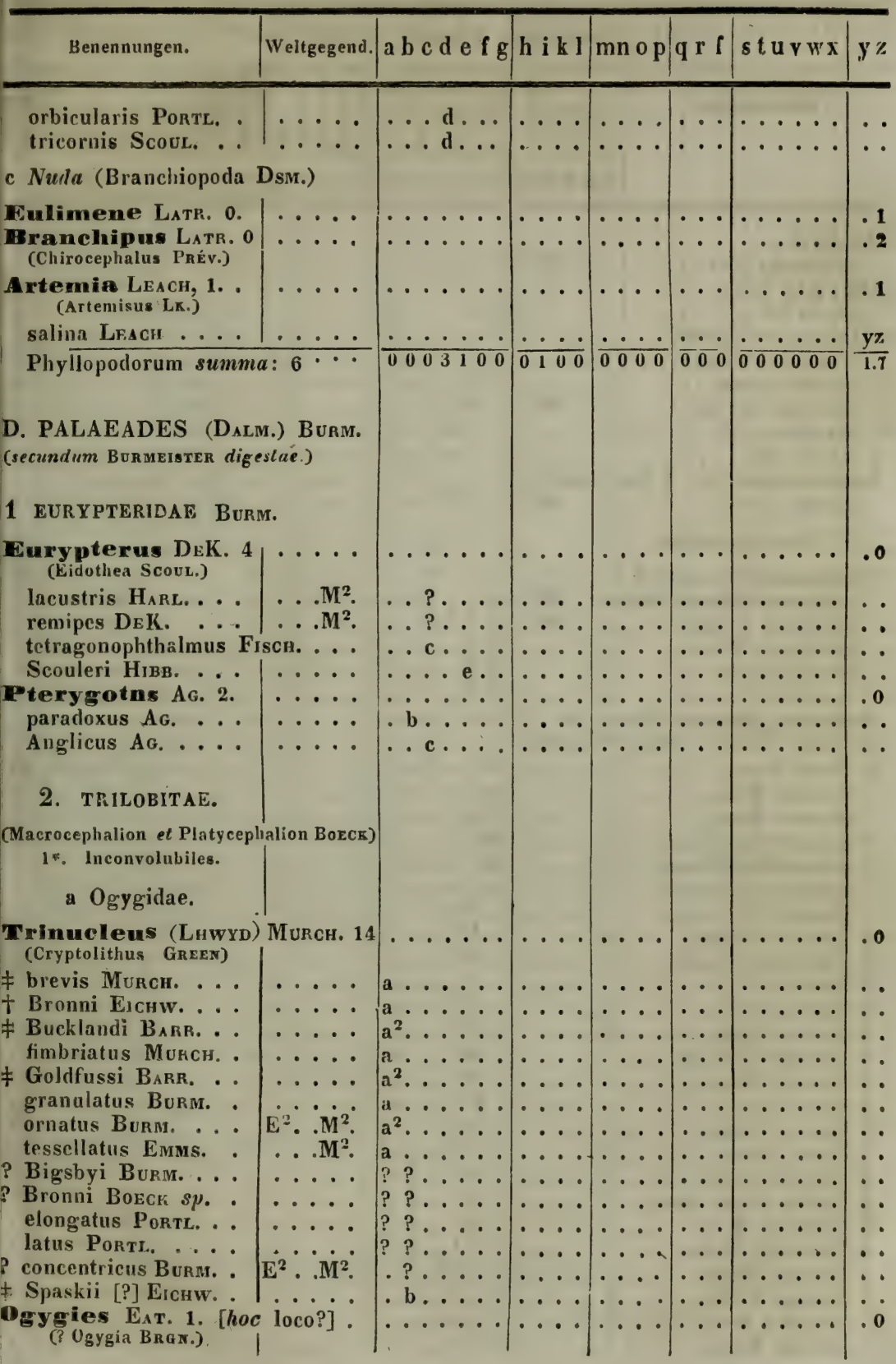




\begin{tabular}{|c|c|c|c|c|c|c|c|}
\hline & Weltgegend & KohlenP. & SulzP. & OolithP & $\begin{array}{c}\text { Krei } \\
\text { del' }\end{array}$ & MolnsseP. & Neu \\
\hline Benennungen. & 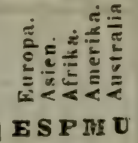 & 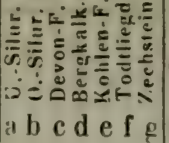 & 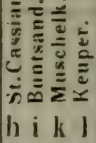 & 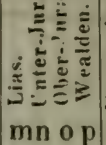 & 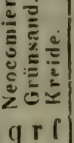 & 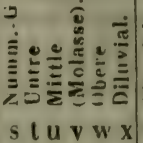 & 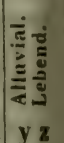 \\
\hline
\end{tabular}

Ogyeirs)

latissimus E^t. ... ...... ${ }^{2}$

Dgygia Bugn. 7. .

Buchi Gr.

? Desmaresti Bran.

Guetlardi Brgn. .

$?$ asellus Bupm.

? Sillimani Brgn. . .

? grandaeva GF.

? pusilla GF.

Nuttainia EAT 2 [hoc loco?] sparsa Eıt. ...........

b. Odontopleuridae.

Ceraurus Green, 5. pleurexauthemus Gress. . $\dot{M}^{2}$. r rosotus LuCKE.... . . M ${ }^{2}$. globiceps Porta. . acicularis GF.

lyra GF.

Odente ............... (Acidaspis Murcu., Anthes fif, pars)

\# Buchi Barr. cornuta BEYR. inermis BEYR.

\# Keiserlingi BARR.

₹ primordialis BARR. vesiculusa BEYR. Brighti GF. ..... \# derelicta BARR. ... ₹ Dufienoyi BaRr... ? forficula

\# Hörnesi BarR.

F laverta Barr.

* Larerla Bara... $\neq$ minut: $\mathrm{B} A$ Вак... F mira BАвк. mutira Emmr. ovata Enuk.

\# Prevosti Barr. ...

₹ subterarmata BARR.

\# Lirormis Banr.

\# Verucuili BARR.

† crenata EnmR. ...

? denlatia BEYR. .... elliptira Buкм ... racliata BEXR.

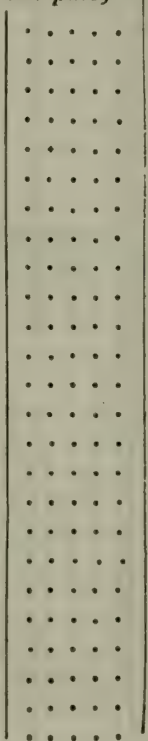

$a^{2} \ldots$ a

$a^{2}$

$a^{2}$

$a$

$b^{3}$

$b$

. b

$b^{2}$

$b^{2}$

$b^{12}$

. b

b

$b^{2}$

.$b^{\prime}$

. $b^{1}$

......

.

(1)




\begin{tabular}{|c|c|c|c|c|c|c|c|}
\hline Ben & end. & $\mid a b c d$ e $f g$ & h i $\quad$ k l l & $\mathrm{mn}$ o p & $q \times f$ & st uvw & $y$ \\
\hline \multicolumn{2}{|c|}{$\begin{array}{l}\text { Staurocephalus BARR. } 1 \ldots \\
\text { sp. Trochurus speciosus BEYR. exl. } \\
\text { pygidio. }\end{array}$} & $\cdot b$ & & & $\cdots$ & - & .0 \\
\hline \multicolumn{2}{|c|}{$\underset{\text { (Trochurus Beyr., }}{\text { Argygidium.) }} \cdots \cdots$} & $\cdots \cdots$ & $\cdots \cdots$ & $\cdots$ & $\cdots$ & $\cdots \cdots$ & .0 \\
\hline $\begin{array}{l}\text { speciosus BEYn. . . } \\
\text { Anglicus BEYR. . . } \\
\text { armatus GF. . . . } \\
\text { c Brontidae. }\end{array}$ & $\mid \begin{array}{l}\cdots \\
\cdots \\
\cdots\end{array}$ & $\left|\begin{array}{ccc}b^{1} \ldots & \ldots \\
\cdot b & \ldots & \cdot \\
\cdots & c & \cdots\end{array}\right|$ & $\begin{array}{c}\cdots \\
\cdots \\
\cdots \\
\cdots\end{array}$ & $\mid \begin{array}{c}\cdots \\
\cdots \\
\cdots\end{array}$ & $\cdots$ & 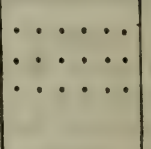 & : \\
\hline Frointe & & $\cdots \cdot$ & $\cdots$ & $\cdots \cdot$ & $\cdots$ & $\cdots \cdots$ & .0 \\
\hline$\therefore$ & $\because$ & a & : & $\cdots \cdots$ & $\cdots$ & $\cdots$ & $\dot{*}$ \\
\hline $\begin{array}{l}\text { BEYR. } \\
\text { BARR. } \\
\text { ps BARR. }\end{array}$ & $\begin{array}{l}\dot{\bullet} \\
\dot{\bullet}\end{array}$ & $\begin{array}{l}? ? \\
\cdot b^{1} \\
. b^{2}\end{array}$ & $\mid \begin{array}{c}\cdots \\
\cdots \\
\cdots\end{array}$ & $\mid \begin{array}{c}\cdots \\
\cdots \\
\cdots\end{array}$ & $\begin{array}{l}\cdots \\
\cdots\end{array}$ & $\left|\begin{array}{ccc}\cdots & \cdots & \cdots \\
\cdots & \cdots & \cdots \\
\cdots & \ldots & \ldots\end{array}\right|$ & $\therefore$ \\
\hline rti BARR. & ... & $\cdots$ & & $\cdots$ & $\cdots$ & $\ldots \ldots$ & $\cdots$ \\
\hline R. . & & $\mid \begin{array}{ll}\cdot b^{2} \ldots & \cdots \\
b^{2} & \cdots\end{array}$ & $\cdots$ & $\mid \begin{array}{c}\cdots \\
\cdots\end{array}$ & $\cdots$ & $\cdots \cdots$ & \\
\hline . & . &.$b^{3} \ldots \ldots$ & $\ldots$ & $\ldots$ & $\cdots$ & $\ldots \ldots$ & . \\
\hline R. . . & $\cdots \cdots$ & $\cdot b^{1} \cdots \cdots$ & $\cdots$ & $\cdots \cdot$ & $\cdots$ & $\cdots \cdots$ & $\cdot \cdot$ \\
\hline$\cdots$ & & $\left|\begin{array}{lll}b_{1}^{2} & \cdots & \cdots \\
b^{2} & \cdots & \cdots\end{array}\right|$ & & $\left|\begin{array}{ll}\cdots & \cdots \\
\cdots & \cdots\end{array}\right|$ & & & \\
\hline$\ldots$ & . & $b^{3} \ldots \ldots$ & $\ldots$ & $\ldots$ & $\ldots$ & $\ldots \ldots$ & \\
\hline R. . & $\cdots$ &.$b^{3} \ldots$. & $\cdots$ & $\cdots \cdot$ & $\cdots$ & $\ldots \ldots$ & \\
\hline P̈HILL. $\cdots$ & $\because$ & $\cdots \cdots$ & - & $\cdot$ & $\therefore$ & $\cdots \cdots$ & \\
\hline$\cdots$ & $\begin{array}{l}\cdots \\
\cdots\end{array}$ & $\cdots$ & $\cdots \cdots$ & $\cdots \cdots$ & $\cdots$ & $\cdots \cdots \cdots$ & \\
\hline $\begin{array}{l}\text { F Zippei } \\
{ }_{3} \text { sp. B B }\end{array}$ & & ?..... & $\ldots$. & $\cdots$ & $\ldots$ & $\ldots \ldots$ & \\
\hline$\neq^{3} \operatorname{sp} . \mathrm{BE}$ & & . b..... & $\cdots \cdot$ & $\cdots \cdot$ & $\cdots$ & $\ldots \ldots$ & - $\cdot$ \\
\hline$\cdots \cdot$ & $\cdots$ & $\cdots$ & & $\therefore$ & $\because$ & $\cdots \cdots$ & \\
\hline $\begin{array}{l}\cdots \\
\cdots \\
\cdots\end{array}$ & & $\cdots$ & $\ldots$ & $\cdots \cdots$ & $\because$ & $\cdots$ & \\
\hline r AV. & & $\cdots$ & $\ldots$ & $\ldots$ & $\ldots$ & $\cdots \cdots$ & \\
\hline 0 glabratus Ros. . . . & & $\ldots$ & $\cdots \cdot$ & $\ldots$. & $\ldots$ & $\ldots \ldots$ & 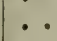 \\
\hline us BEYR. • & & $\cdots$ & . & $\cdots$ & $\cdots$ & $\ldots \ldots$ & \\
\hline$\cdots$ & & $\cdots \cdot$ & $\cdots$ & $\cdots$ & $\cdots$ & & \\
\hline$\cdots$ & & & & $\cdots$ & $\cdots$ & $\cdots$ & \\
\hline $\begin{array}{l}\text { 3scaber GF. } \\
\text { 2subradiatus } \mathrm{Mu} .\end{array}$ & & c.... & & & & $\cdots$ & \\
\hline $\begin{array}{l}\text { 3granulatus GF. . . } \\
\text { d Olenidae. }\end{array}$ & & $\cdots$ & & ${ }^{\circ}$ & $\cdots$ & $\cdots$ & \\
\hline BurM. . . & 1 & & $\cdots$ & . & . & a & $\begin{array}{l}.0 \\
.\end{array}$ \\
\hline
\end{tabular}

* Species in sequentes generis sectiones distribuntur:

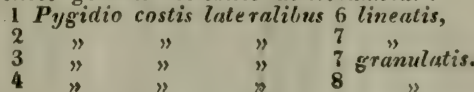




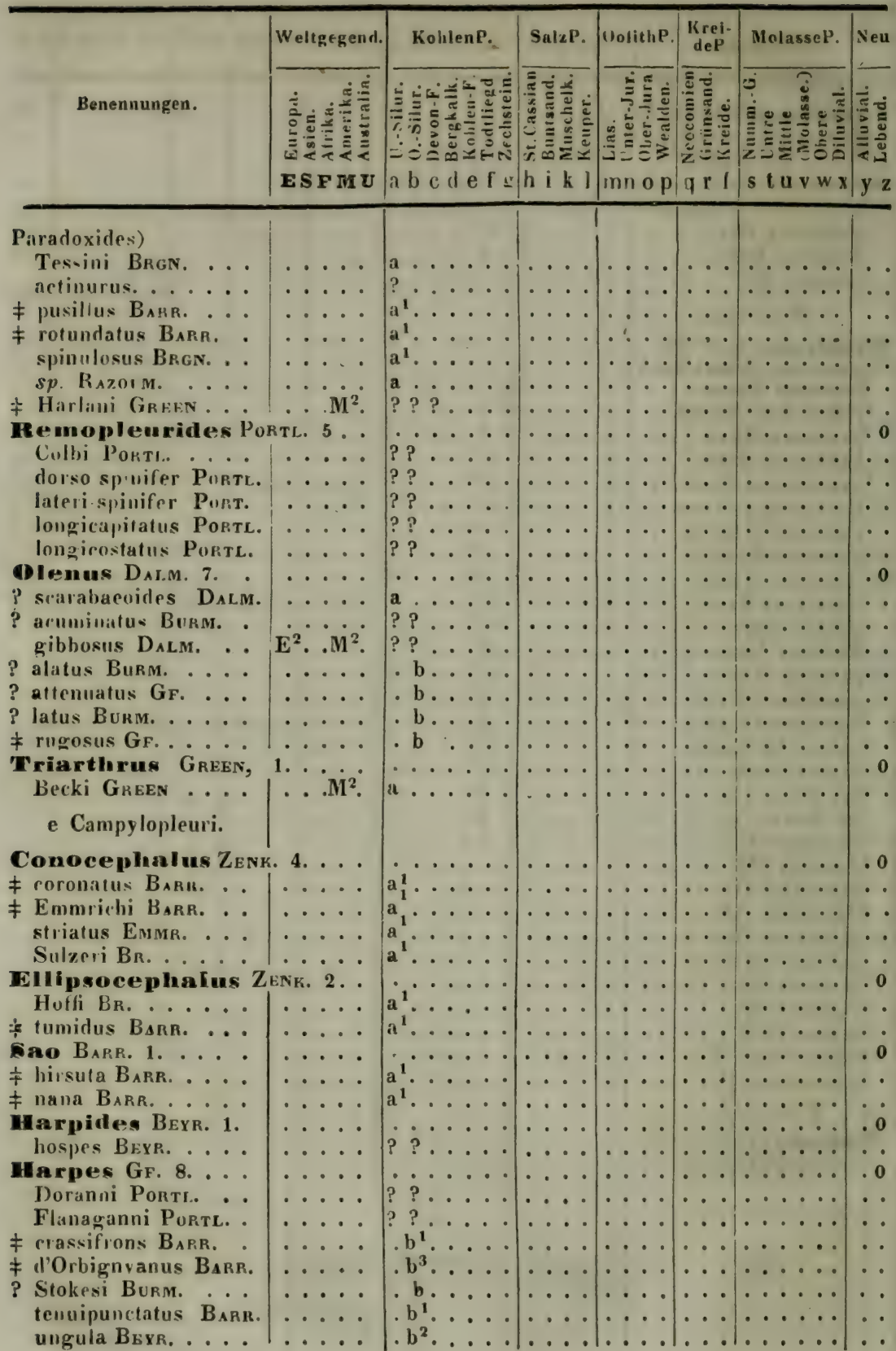




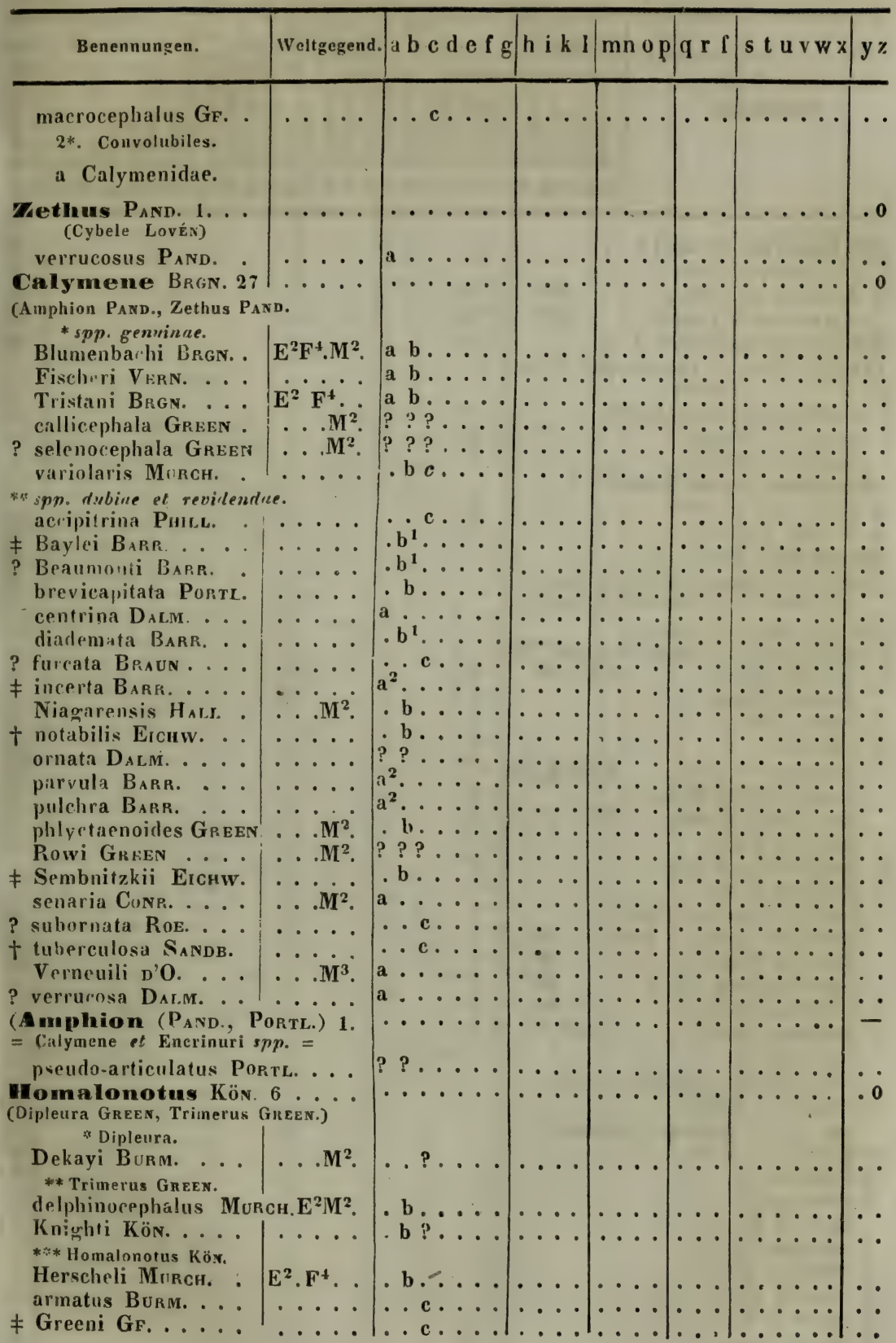




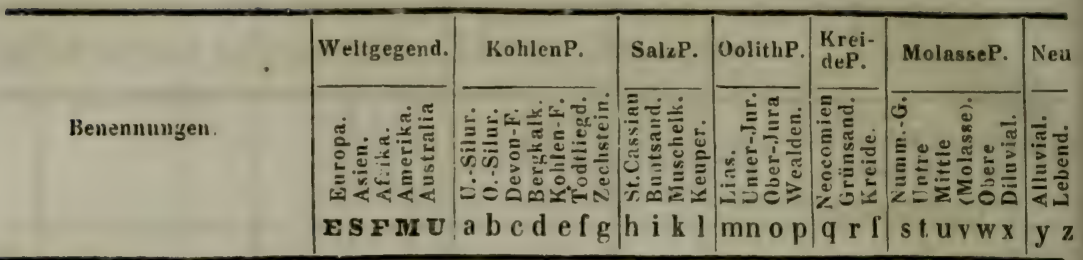

(Trimerus GREEN) 2 = Homalonotus KöN. =

‡ Jacksoni Green.. . . platypleurus Gresiv

Encrinurus Emur. 3

(Amphion Pasd et Cryptonymus Erchw. purtim.)

multisegmentatus Eммг. .... punctatus Emmr.

rugosus Еммr.

(Cryptonymus Eichw.) 2 . ₹ parallelus Eichw.

₹ Wörthi Eichw. . .

Peltura et Pleuracanthus Edw., Phacops et Acaste Gr., Dalmania Eмur.)

*spp. genuinue et certinres. !

con ophthalma(EmmR.)BuRM. $\mathbf{E}^{2} \mathbf{M}^{2}$

caudata Burm.

dentata Burm.

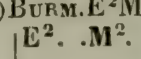

? elliptifrons GF, :

? extensa.

Hausmanni Evir. macrophtalma Burn. mucionata EmMr.

proavia Lмм.

protuberans EмmR.

sclerops EmmR.

truncato-caudata Porte.

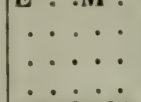

- b

? ?

? ?

$\mathrm{E}^{2} \mathrm{M}^{2} \mathrm{~W}$

anchiops (EмmR.) Burm. $\dot{M}^{2}$. microps GreEN. odontocephala Burm.

? selenura Green latifrons Burm. punctata ...... $\mathrm{E}^{2} \cdot \dot{\mathrm{M}}^{2}$. retundifrons (Еamr.) Burm. stellifera Burm.

*spp. minus cournilae el revidendae.

‡ Deshayesi Barr.

₹ dubia BARR. ....

₹ elongata BsRR. .

Hawlei BАнг....

₹ parabola BARR.

₹ Phillipsi BARR.

\# socialis BARR.

‡ solita ia BARR.

$\frac{1}{7}$ plicatn SARs. $s p$.

$\ldots \ldots$
$\cdots$
$\cdots$
$\cdots$
$\cdots$
$\cdots$

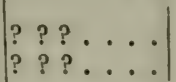

.$b^{23}$

$\ldots \ldots \ldots \ldots$

(a)

$a^{2}$.

$\mathrm{b}^{3}$

$a^{2}:$

? ? ?

? ? ?

$\mathrm{M}^{2}$.

$\mathrm{E}^{2} \mathrm{M}^{2}, 3 ?$

? ? ?

$\ldots$

? $b$ c .

a

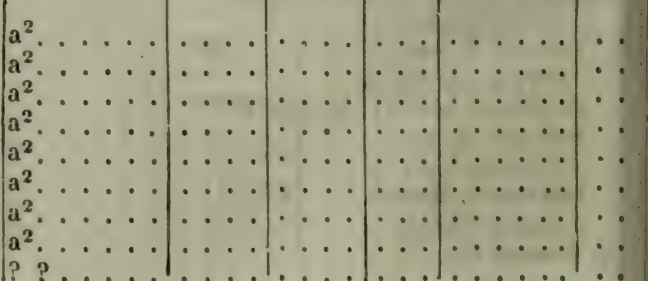




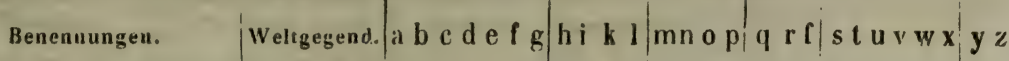

† breviceps BARR.

F Bromini Barr.

F bullicens BarR.

F foecunda Barr.

F intermedia BARR.

F Glockeri BarR.

F laevi:ata BAPR.

F Reussi Barr.

F spinitera BARR.

F trapeziceps $B_{A R R}$

Dalmani Porte.

Jamesi Portc.

Murchisoni Ponte.

\# scabra GF.

\# cryptephthalina EMmr.

Cryphaeus Grean, 2 Buothi Gr. collitelus Gr.

Plillipsia Portr. 9 Bronguiarti Kos. Derbyensis Kon. Eichwaldi Vern. gemmulifera Kon. Jonesi Porte. Maccoyi Portl. pustulata KoN. truncata Keys.

Uralica Vires.

Griffithides Porti. 5 globiceps Porti. longiceps Ports. longispinus PORTL. platyceps Porte.

Cyphaspis Burm. 4 \# Burmeisteri Barr.

\# Cerberus BarR.

F depressa Barr. ceratophthalmus BEYR.

Proętus String. 21 (Gerastos GF.; Aeonia Burm.)

₹ complanatus BARR. concinnus Lov.

\# decorus BARR.

$\neq$ discretus Bakr.

o elegantulus Lov. erraticus

₹ fallax Barr.

? inaequirostatus BarR. F intermedius BARR.

\# gracilis BARR. F lepidus Barr. \# Loveni Bark.

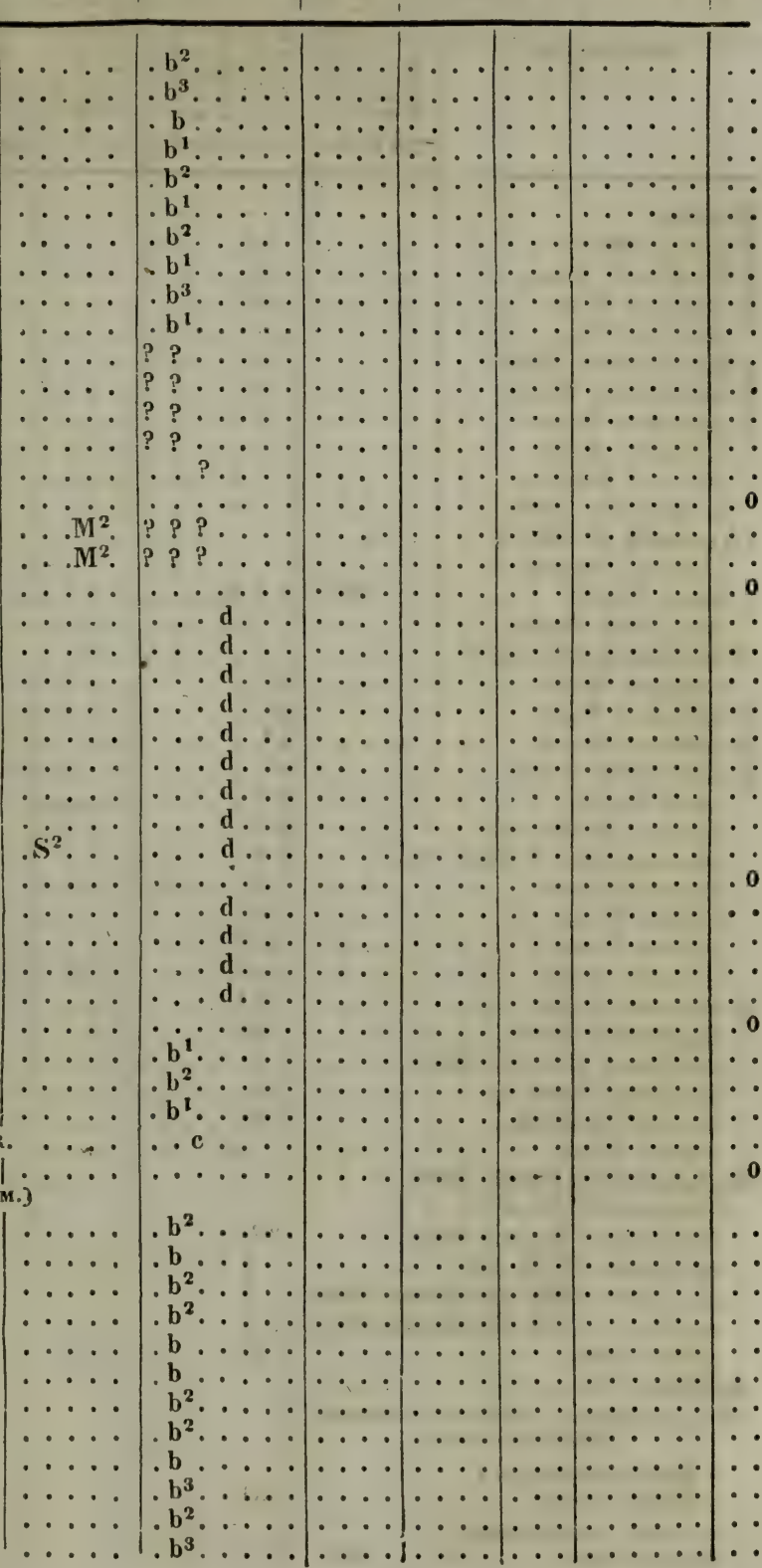




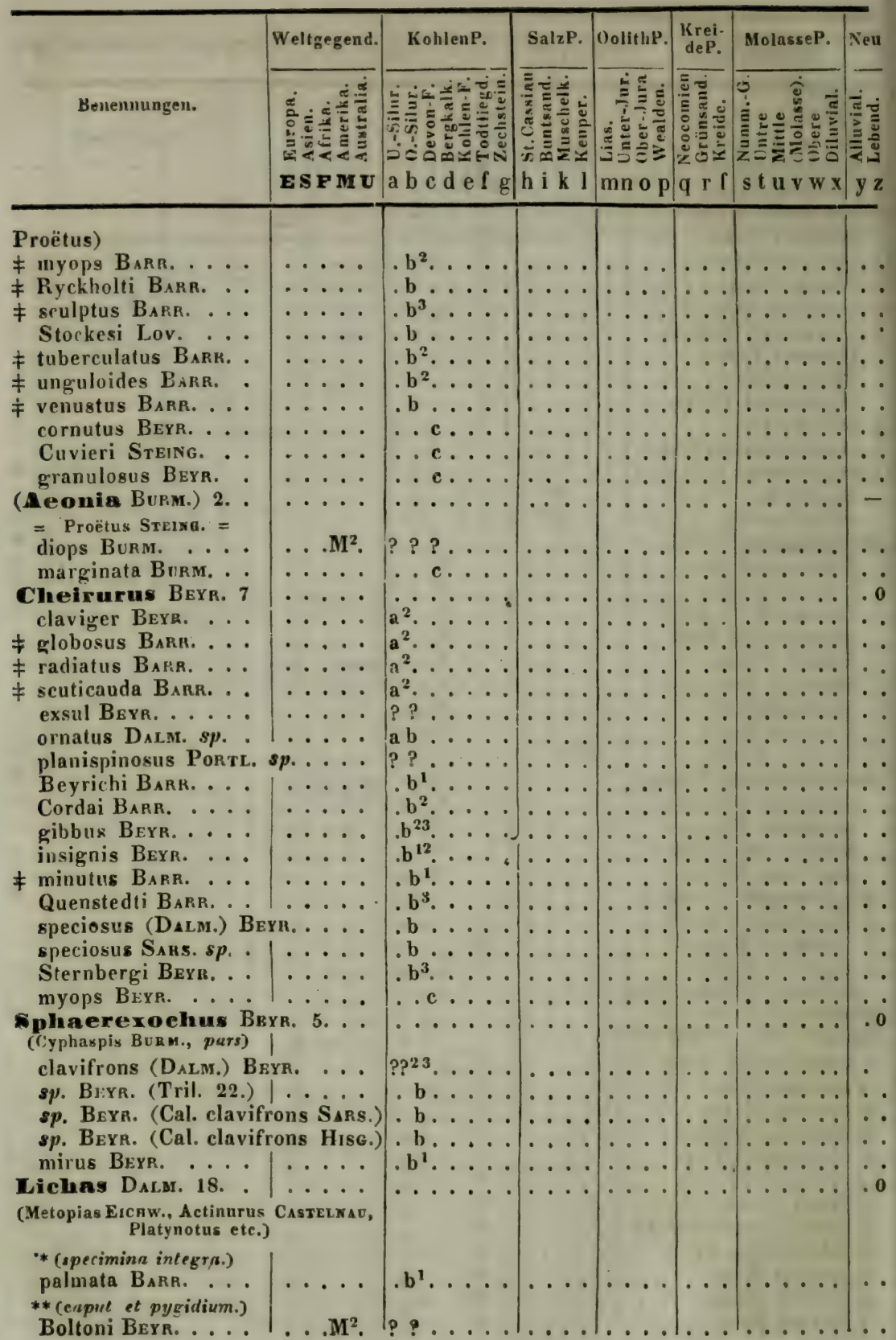




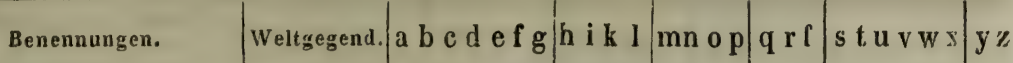

Hibernica Beyr. . .

\# propinqua BarR. . scabra (BEYR.) BARr. *** (capifa.)

coniceps LevchtB. sp. angusta BEYr.

? aries Eıснw. sp. . . Hübneri Егснш. . . pachyrrhina $\mathrm{D}_{\Lambda \mathbf{L} \text { M. }} \boldsymbol{s p}$.

\# porosa BARr. . . .

? simplex Barr. ... verrucosa Eicuw. $s p$

**** (pygidir.)

laciniata DaI M. . . . cicatricosa Lov. dissidens BEYR.

† Haueri BARR. sp. Ber (laciniatus $\cdots \cdots \cdot b^{2}$

Ceratocephala WARDER, is [?hoc loco] ceralepta Äктн. ... . . . M ${ }^{2}$. goniata WARD. .............

\section{b Asaphidae.}

HIaénus (Dalm.) Burm. $10 \ldots$ (Illaenus,Bumastus Murch., Cryptonymus EIcHw., pars.)

* (Illaenus) crassicauda Datm. . giganteus Burm. ** (Bumastus Murch.) Barriensis Burm. ***: incertue sectinnis. Bowmani SaltSu. cornutus Pand. . . . Centaurus Dalm. . quadrato-candatus Porte.

Trentonensis Eммs:

**** segmentis trunci 8 [pro 9-10.]

Hisingeri BAPR. .

Wahlenbergi BarR.

(Bumastus MURCH.)2 = Illaenus =

? Franconicus $\mathrm{M} \ddot{u}$. . Trentonensis Emms.

Arehegonus Burir. 2 *(Dysplanus Burm.) centrotus Burm.

** (Archegonus.) aequalis Burm.

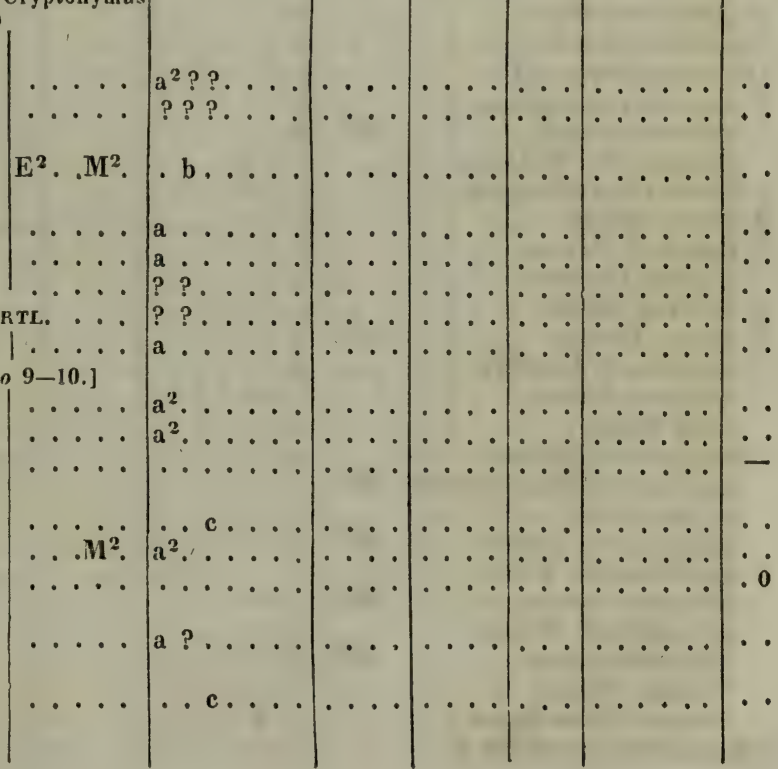


Benenunngen.

\begin{tabular}{|c|c|c|c|c|c|c|}
\hline Weltg & KohlenP. & SalzP. & OolithP. & $\begin{array}{l}\text { Krei- } \\
\text { deP. }\end{array}$ & MolasseP. & Neu \\
\hline 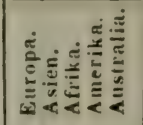 & 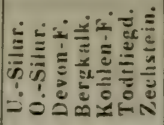 & 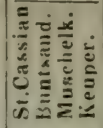 & 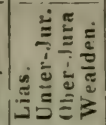 & 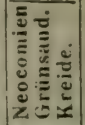 & 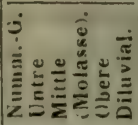 & 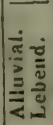 \\
\hline ESF MU & a b c d e $\int g$ & h i k l & $\mathrm{mn}$ op & $9 r$ & stuvw $x$ & $y \%$ \\
\hline
\end{tabular}

Asaphus (Brgn.) Burm. 39. .

'Nileas Dися.; = Sympliysurus Gr.,

"Henicrypturus Green.; = Cryptonymus Eicuw. (purs); 3isutelus DeK.)

*spp. genninue certiores.

iarmadillo Darm. .

$\neq^{1}$ Bouchardii BARR.

(?) ${ }^{2}$ Corndensis Murch.

зсусlops BURm. ...

2expansus DALM. .

2extenuatus $D_{A L M}$.

3 frontalis DALM.

?'berós Dacm.

† ingens BARR.

laeviceps DALM.

‡ marginatus BarR.

${ }^{3}$ megistos Buru.

₹ nubilis BaRR.

palpebrosus DaLm.

${ }^{3}$ platycephalus $\mathrm{S}_{\text {Tock. }}$

$\neq$ platynotus DALM. .

2raniceps DALM. ...

(?) ${ }^{2}$ subtyrannus AV.

** spp. denun exuminundue Boliviensis D' 0 . . .

Brongniarti DsLgch.

brevicaudatus DsLgch.

? brevis GreeN ... Cawdori Murch. . centron Leuchtв. . .

‡ devexus Erchw.

$\neq$ diurus GreEN.... hyorrhinus Lеvентв. latifrons РонтL.

latus Pand.

longicauda LeuchtB.

megalophthalmus Troost . $\mathbf{M}^{2}$.

myops KöN.

polypleurus Gresin.

quadrilimbus Phill.

sclenurus GreEN ..

subcaudatus Murch.

Trimblei GreEN .

Vulcani Murcu.

Vulcani affinis Eicнw.

(Gymphysurus GF.3

|

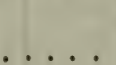

$\cdots$
$\cdots$
$\cdots$
$\cdots$
$\mathrm{M}^{2}$

$E^{2} . M^{2}$.

$\ldots$...

.....

....

$\mathrm{M}^{2}$

..... $a^{2}$.

$\mathrm{E}^{2} \cdot \mathrm{M}^{2}$

....

a.......

b'....

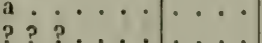

a $b$...

a

a............

$a^{2}$

? b.

b.

$\cdots$

a

a.

$\mathrm{M}^{3}$.

...

...

$\cdots$

$\mathrm{M}^{2}$

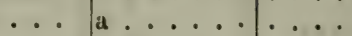

(

a b.

a

b ?

a.

b.

? ?....

a....

b.....

? ?

? ? ?

$\ldots \mathrm{M}^{2}$

.. $\mathrm{M}^{2}$

b b

?

a.

= Asaplius. $=$

… 


\begin{tabular}{|c|c|c|c|c|c|c|c|}
\hline Beneunungen. & Weltgegend. & a b c def $g$ & hi $\mathrm{i} \quad \mathrm{l}$ & mnop & $q r i$ & s tuvw & $y z$ \\
\hline
\end{tabular}

intermedius Gr. laevis GF. ... ob!ongatus GF.

(Nileus Dalm.) 2 $=$ Asaphi subgenus = depressus GF. nauus Levchtb.

(Cryptonyma Eichw.) 5 $=$ Asaphi subgenus $=$ I acuminatus GF. limbatus GF. parallelus Eıciw. striatus GF.

Wörthi Eıchw.

(Tsotelus Dек.) 5. . = Asaphi subgeraus = arcuatus Popres. intermedius Ports. maximus LockE ovatus PortL. rectifroms Ports. .

Ampyx Da Lir. 7. . baccatus Porte.

? incertus DsLgCh. mammillatus SARS . nasutus DaLM.

† Portlocki BaPR. rostratus SARS Sarsi Porte.

\section{c Agnostidue.}

\section{Agnostus Brgn. 7.} (Battus DaLm.)

Boecki Eichw.

0 gigas GF.

0 granum GF. laevigatus GF. pisiformis BRGN. pusillus SARS . spiniger GF.

(Battus DaLm.) 9. = Agnostus Brax. =

$\neq$ affinis BARR.

₹ bibullatus Barr.

F cumeifer BaRr.

₹ granulatus BARR. integer BEYR. nudus Beyr.

F Orion Barr. rex BARK.. tardus BARR.

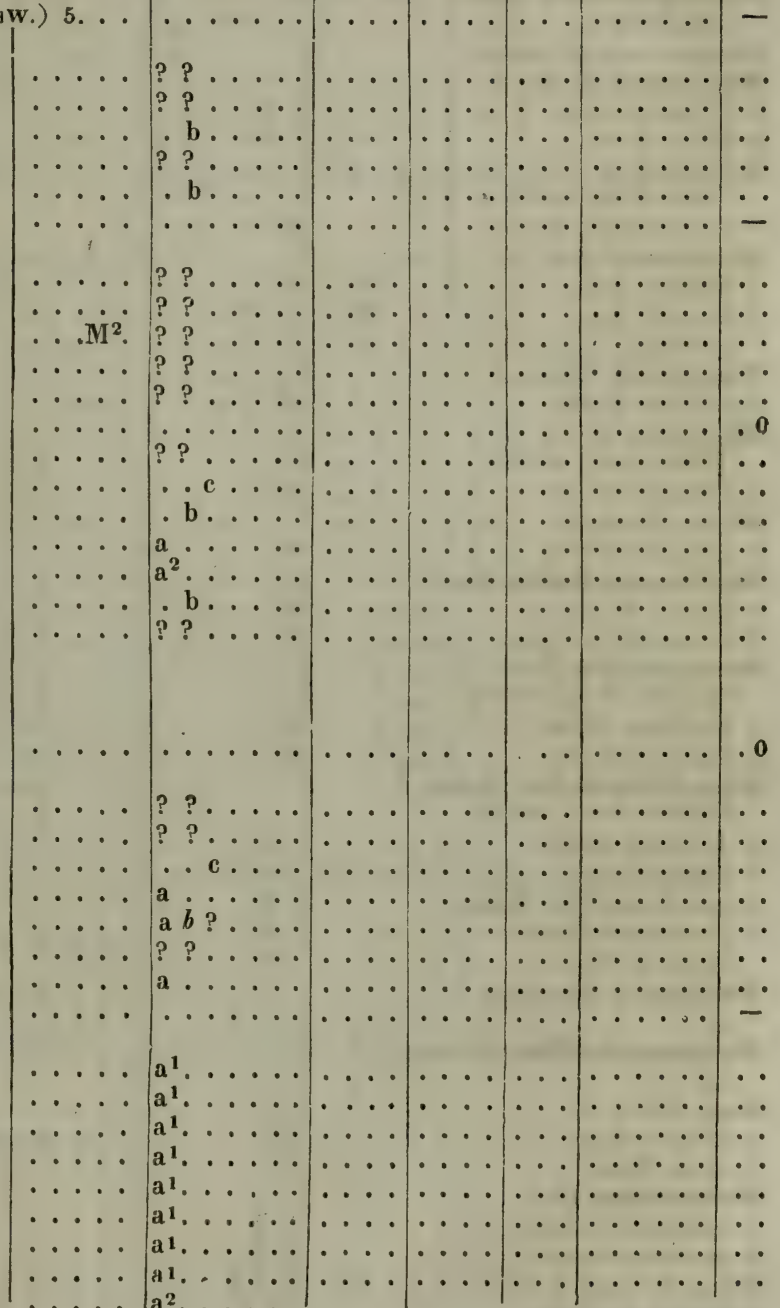




\begin{tabular}{|c|c|c|c|c|c|c|c|}
\hline & W eltgegend. & KohlenP. & SalzP. & OolithP. & $\begin{array}{l}\text { Krei- } \\
\text { deP. }\end{array}$ & MolasseP. & Ne \\
\hline Benennungen. & 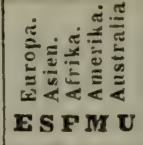 & 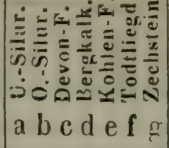 & 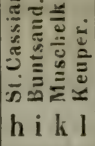 & 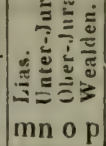 & 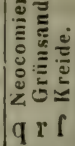 & 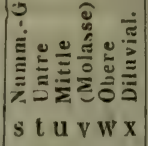 & $\begin{array}{l}4 \\
y\end{array}$ \\
\hline
\end{tabular}

Cyclus Kon. 2

- Brongniartanus KoN. radialis KoN.

3 *Genera incertae familiae

Arethusa Barr. 2.

F Konincki BARR.

‡ nitida BarR.

Arionides Barr. 1 . Arion BARr. unter.

\# ceticephalus BARR.

Hilobites RAF. 3.

+ lobatus RAF.

t lunatus RaF.

sp. DEK.

Capleyra Barr. 1 .

\# radians $B A R R$.

Dionide Barr. 1. (Dione Barr. inter.)

‡ formosi BARR.

Egle Barr. 1.

F rediviva Barr.

mydrocephalus BARR. 1

\# carens BARr.

f saturnoides Barr.

Romadella BARR. 2

(Monadina BarR. antert)

\# distincta BARR.

\# oinicron BARR.

DPagetleonides Ban

(Phaëton BARR, interi).

\# Archiaci BARR.

? latens BapR.

F membranaceus $\dot{B}_{\mathrm{AR} R}$

P planicauda Barr.

striatus BARR.

Polieres Rounult, 1

si).

Prionocheilus Rouaurt. 1 .

sp.

Sao Barr. 2.

\# hirsuta BARr. ....

F nana BARr.

Trilobites 17.

\# angustatus SAks

f armatus Boeck

t cephaleurya Rafo. .

\# decipiens BarR.

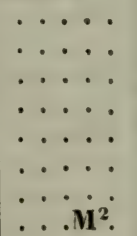

$\mathrm{M}^{2}$.

$a^{1}$
$?$
$?$
$?$
$a^{2}$
$\cdot$
$a^{2}$
$a^{2}$ 


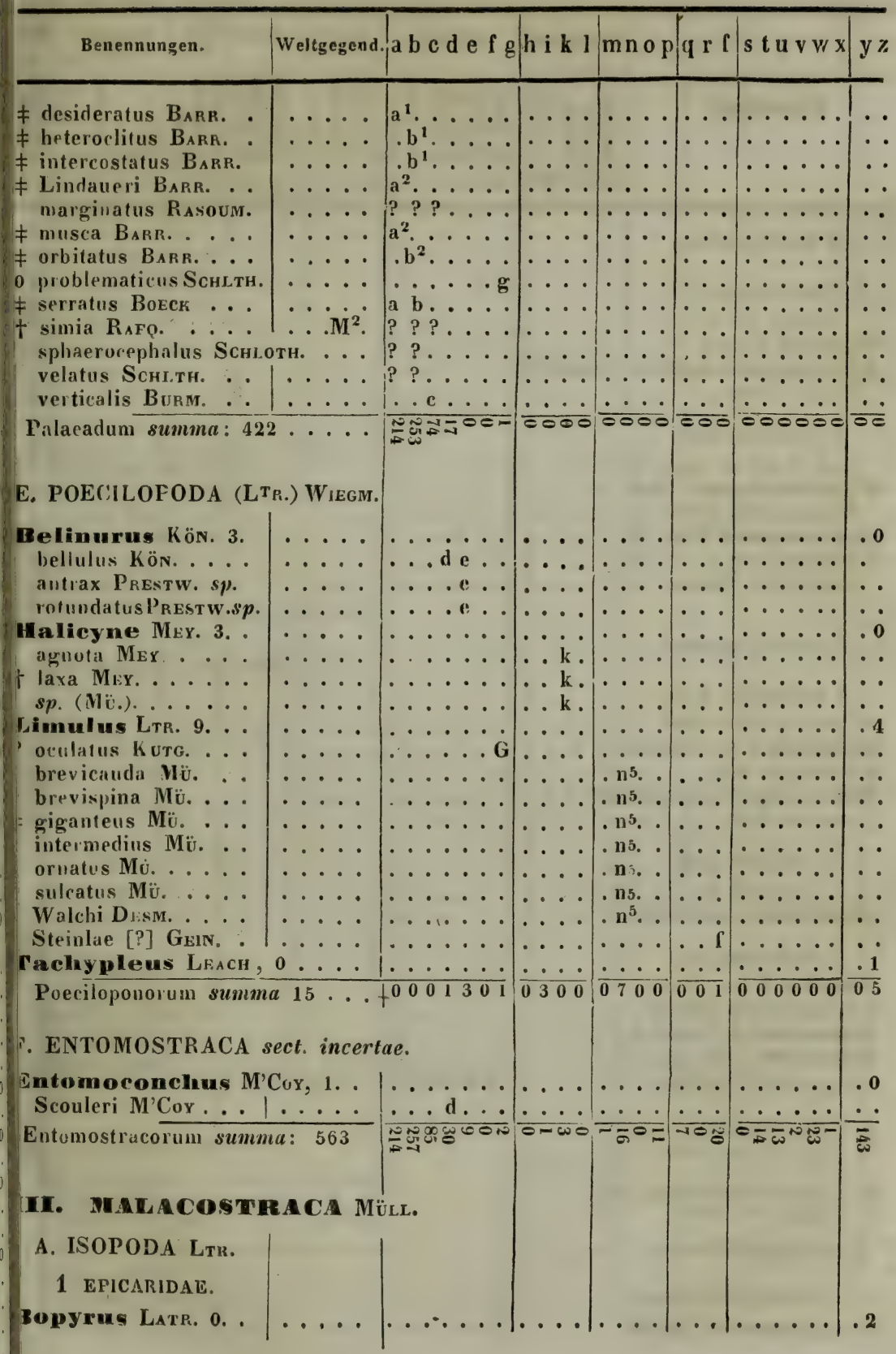




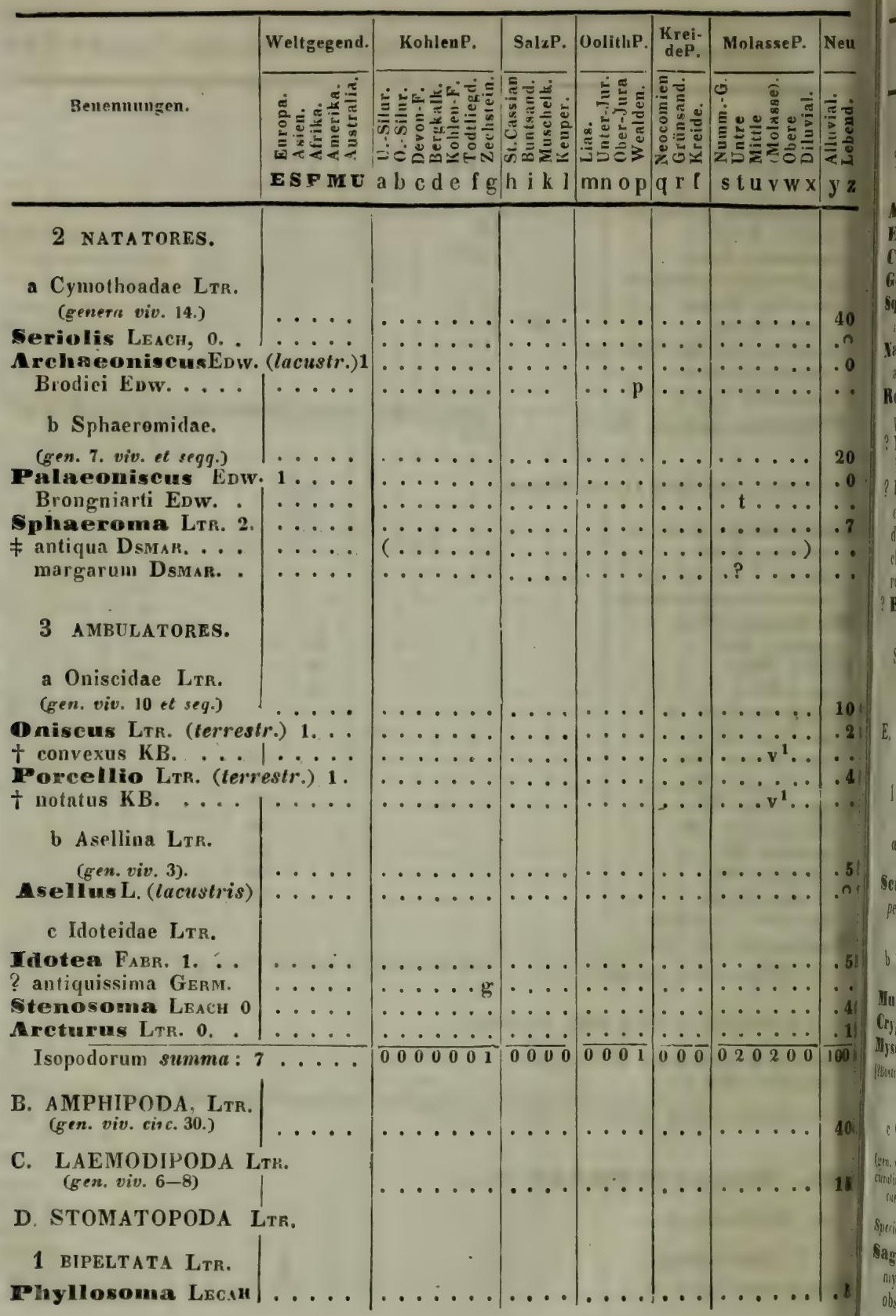




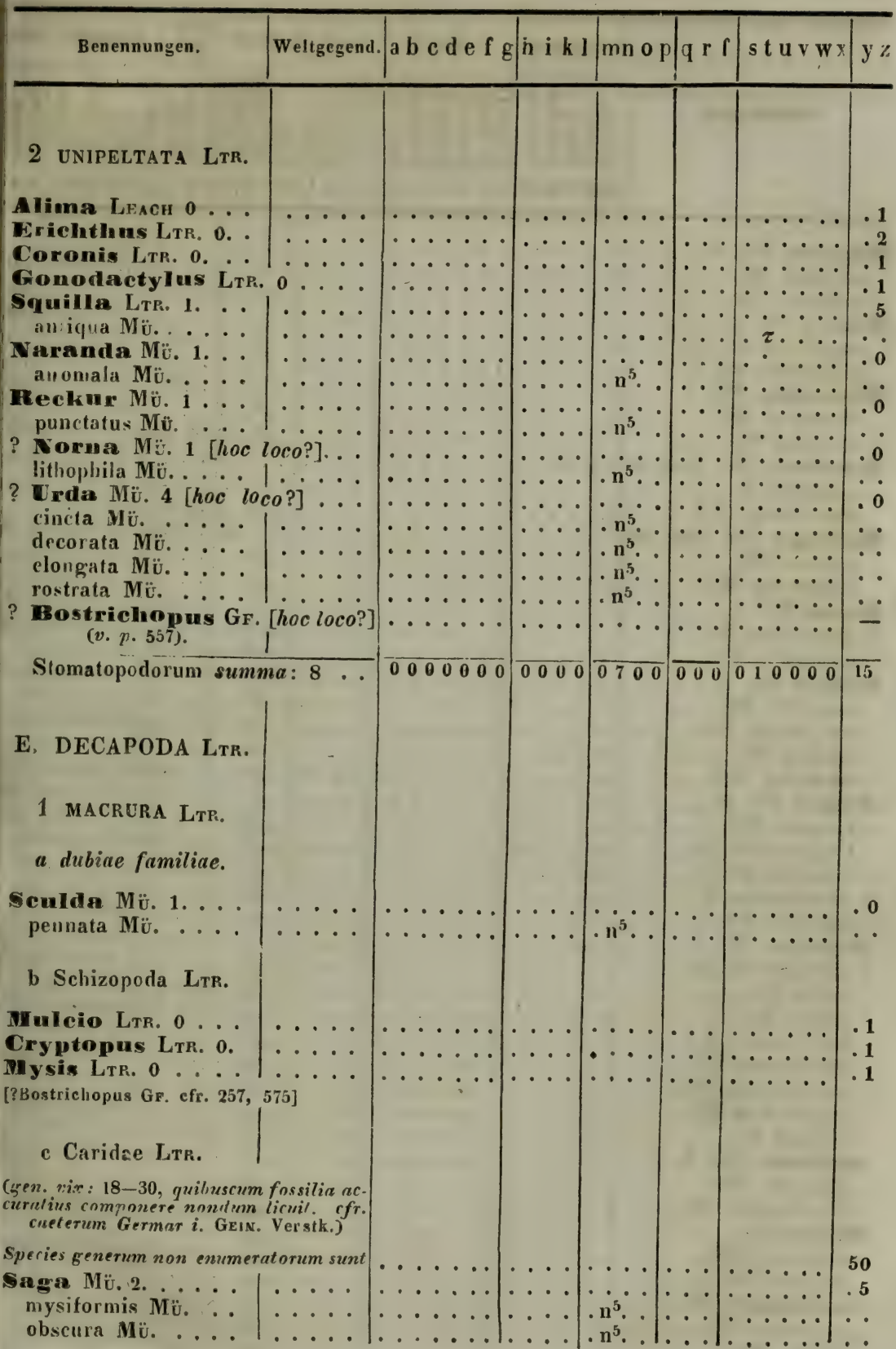




\begin{tabular}{|c|c|c|c|c|c|c|c|}
\hline & Weltgegend. & KolilenP. & SalzP. & OulithP. & $\begin{array}{c}\text { Krei- } \\
\text { deP. }\end{array}$ & MolasseP. & Neu \\
\hline Benениииgен. & 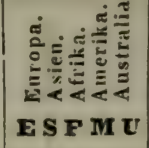 & 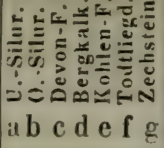 & 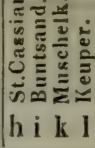 & 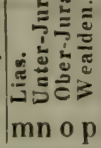 & 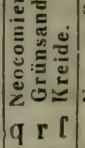 & 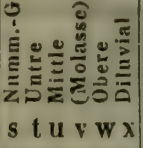 & 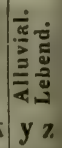 \\
\hline
\end{tabular}

IR auna Mü. 2 . angusta Mü.

multipes Mü.

Elder Mü. 2. unguiculatus Mï. ungulatus Nïü.

Inculla Mü. 2 . breviceps Mũ. nicoides $M u ̈$.

Bombur Mü. [hoc loco?] 2. angustus Mü. complicatus Mü.

Mefriga Mü. [hoc loco?] 2 serrata Mü.

Dusa Mü. 2. denticulata Mü.... monocera Mü.

Udora Mü. 4. . . . . angulata Mü. brevispina $\mathrm{MÜ \text {. }}$ cordata Mü. rarispina $\mathrm{MÜ̈}$.

Aeger Mü. 5. . . . . elegans Mü longirostris $\mathrm{M} \ddot{\mathrm{U}}$ spinipes Mü.

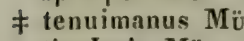
tipuIaris $M \ddot{v}$.

Koelga Mü. 8. curvirostris Mü. dubia Mü. gibba Mü. laevirostris $\dot{M} \ddot{\text {. }}$ quadridens Mü. quinquedens $M$ i septendens Mü. tridens Mü.

Drobna Mü, 2. deformis Mü. . Haeberleini Mü.

mylgia Mü. hexodon Mü. spinosa Mü.

Antrimpos Mü. 2. angustus Mü. bidens Mü.
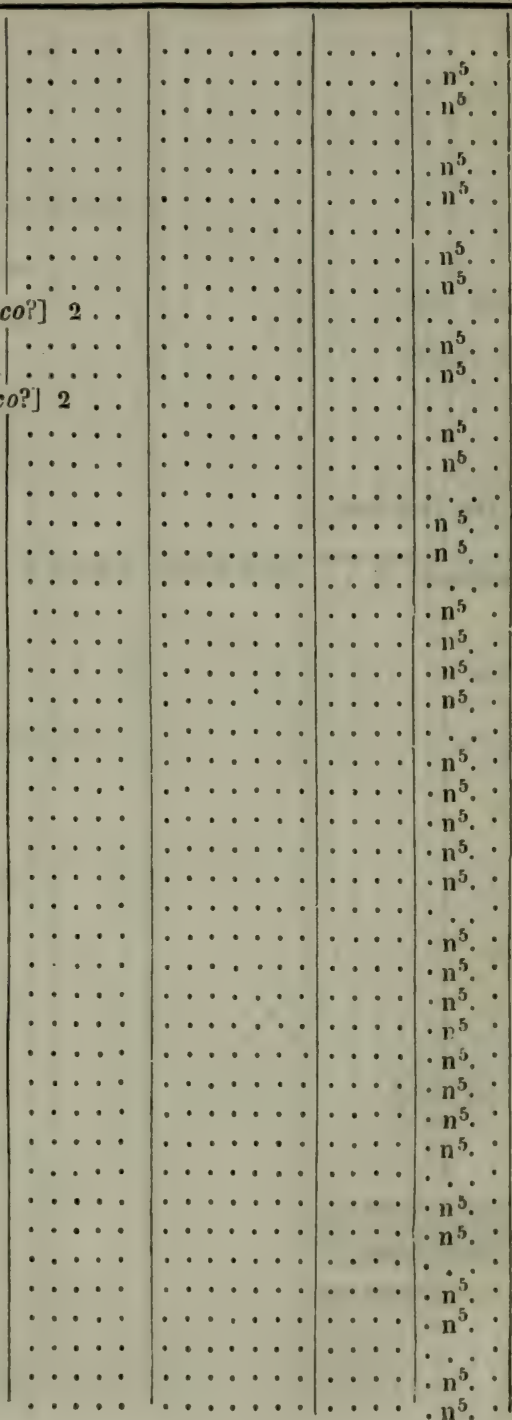


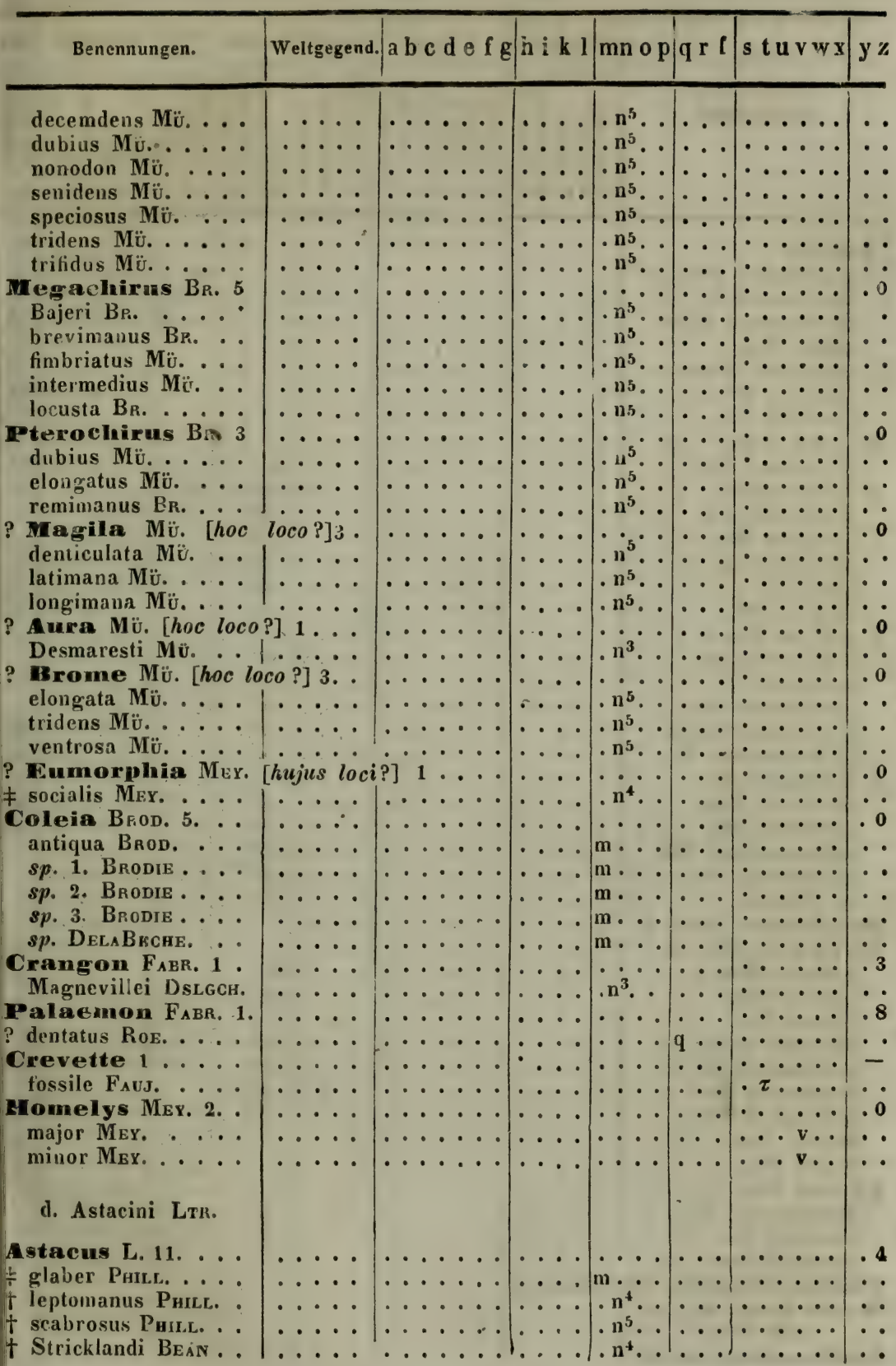




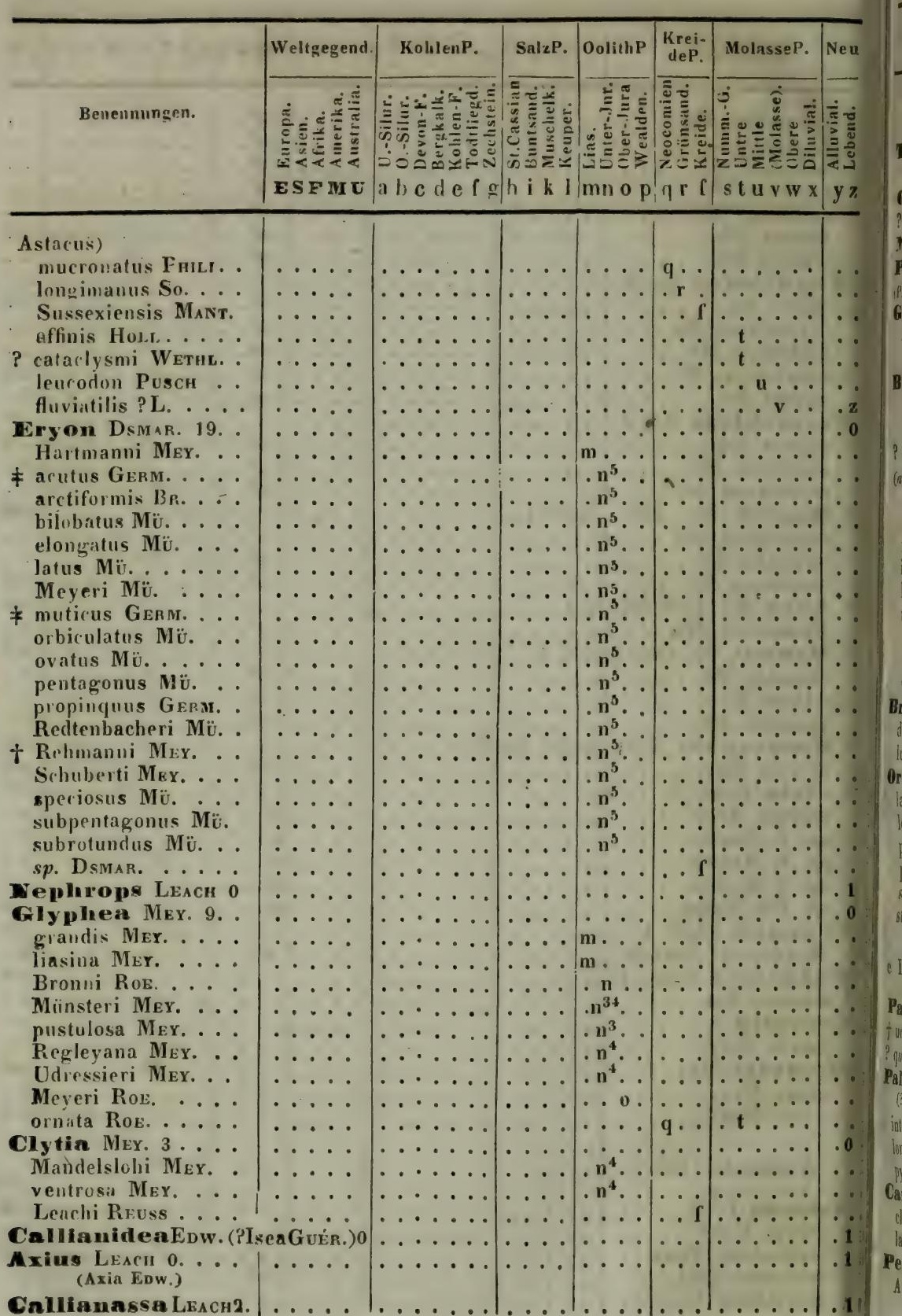




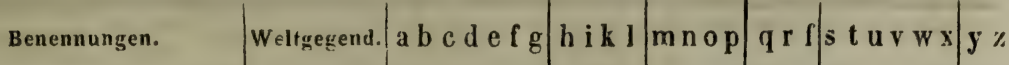

antiqua $\mathrm{OT}_{\mathrm{T}} \mathrm{T}$

Faujasi EDw.

Thalassina Ltr. 1

antiqua BeL.L.

Gebia Leach 1. .

? obscira Mex.

Megalopus Leach 0

Porcellama LK. 0

(Pisidia, Hexapus Leach, Monolepis Say)

Galathea Fabr. 2.

audax MEY. .

antiqua Riss.

Bolima Mü. 2

angusta $M \ddot{u}$.

pustulosa Mü.

? Eryma Mey. 9.

(an ad Caridas potius referenda?)

crassula Mü. sp.

elongata Mü. sp. .

fuciformis $\mathrm{SchL}$ тн. $s p$.

intermedia Mü. sp.

laevigata Mü. sp. .

minuta Sснцтн. $s p$.

modestiformis Sснцтн. $s p$.

Velthcimi Mü. sp. verrucosa Mù. $s p$.

Erisa Mü. 2.

dubia Mü.

lucida Mü.

Orphnea Mü. 6 . laevigata $\mathbf{M} \ddot{\text { ü. }}$ longimana Mü. ... pseudo-scyllarus Mü. pygmaea Mü. squamosa Mü. striata Mü.
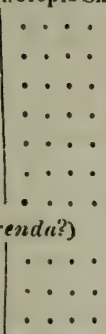

striata

c Locustini (Palinuridae.)

Palinurus Mü. 2 . † uncinatus Phicl. . ? quadricornis (FBR.)

Palinuxima Mü. 3. (? Palinurus FBR.)

intermedia $\mathbf{1}$ ü.

longipes $\mathrm{M} \ddot{\text {. }}$.

pygmaea Mü.

Cancrinos Mü. 2 : clavipes Mü.

latipes Mü.

Pemphix Mex. 2 . Aibertii MEY.
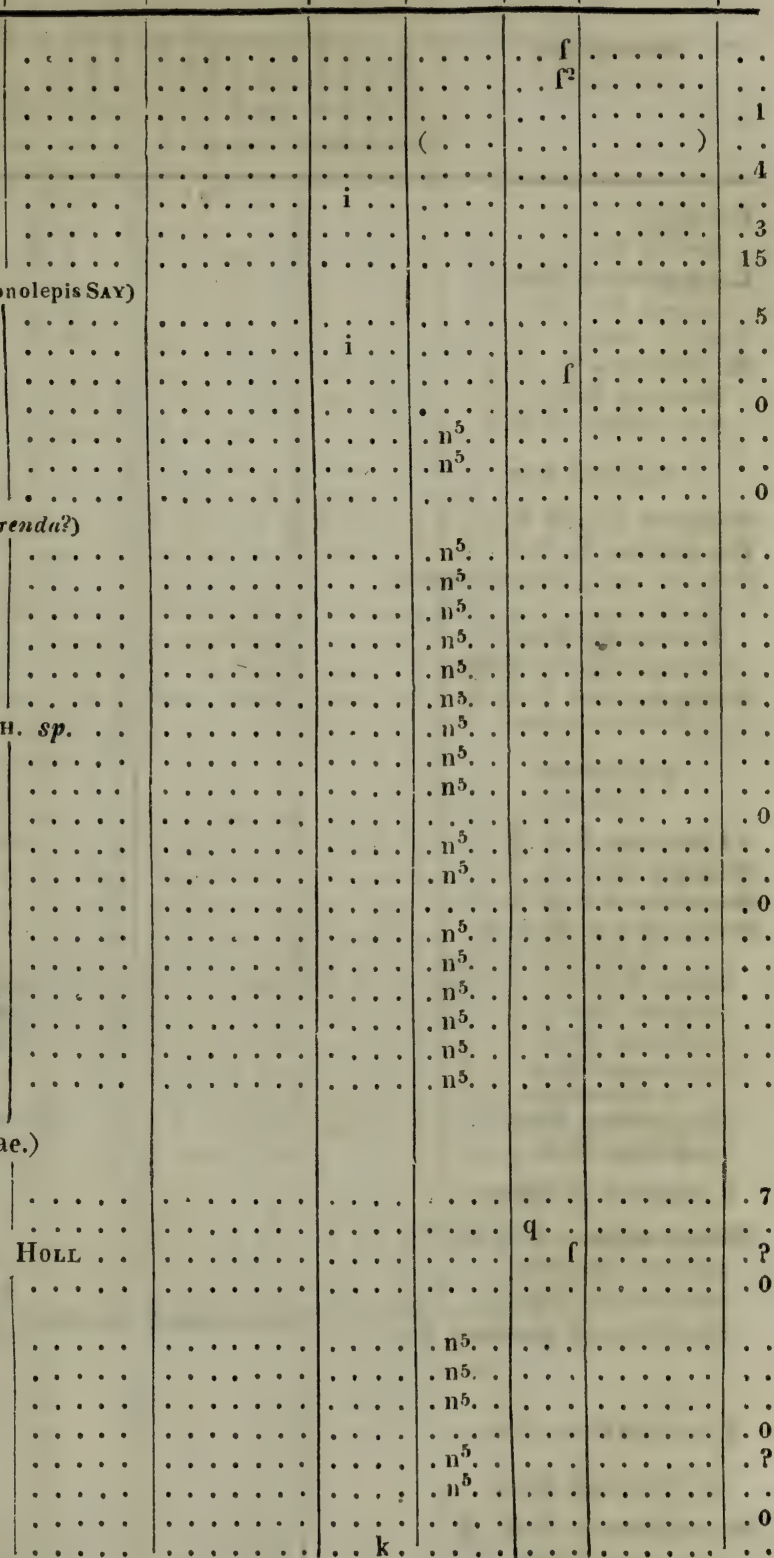


\begin{tabular}{|c|c|c|c|c|c|c|c|}
\hline & Weltgegend. & KohlenP. & Salzl'. & OolithP. & $\begin{array}{l}\text { Krei- } \\
\text { deP. }\end{array}$ & MolasseP. & Neu \\
\hline Benennu ngen. & 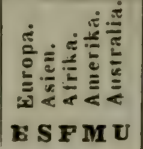 & 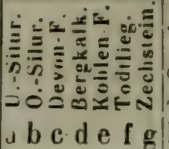 & 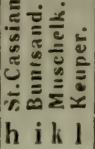 & 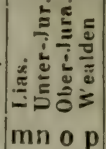 & 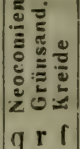 & 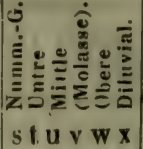 & 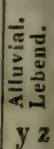 \\
\hline
\end{tabular}

Pewphix)

Sueuri MEY. . . . .

Hitogaster MEY. 2. obtusa MEY, 2. ... sp. 2 Ив. . . .

Scyilarus L. 2. . . Mantelii Dsm. tuberculatus ? Kös.

f Pagurini LTR.

Birgus Leach 0. . .

Pogurus L. 3. ....

(Cenobita Ltr., Cancellus Edw.)

? $s p$. Roв. . . . . .

Desmarestanus Serr

Prophylax LTR. 0 (Glaucothoe EDw.)

g Hippidae. (Anomura)

Alounea Frr. 0 . Hippa Fbr. 0.... Temipes Ltr. 0 .. Prosopon Mey. 6. * Prosopon.

hebes Mer....... simplex MEY. spinosum M6x. ... tuberosum MEY. ** Pithonoton. marginatum MEX. rostratum MEx.

h incertae familiae.

(Macrurites Schцтн.) 2 ... pusillus Scruth. squillarius Sснцтн.

Macrurorum summa: $162 \ldots$ 2 BRAchyura Ltr. a Notopoda Ltr.

Dronilites Eow. 2. pustulosus Reuss

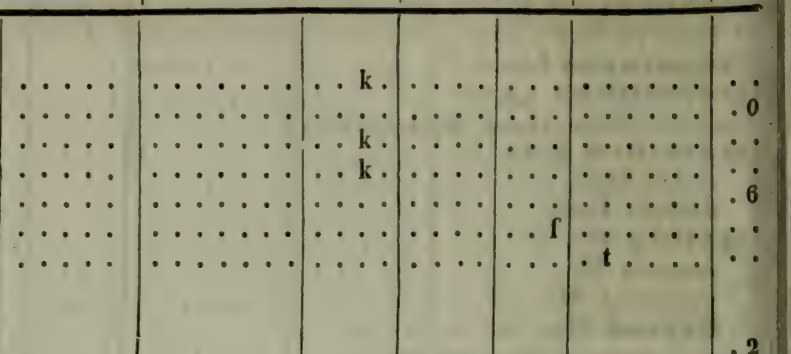




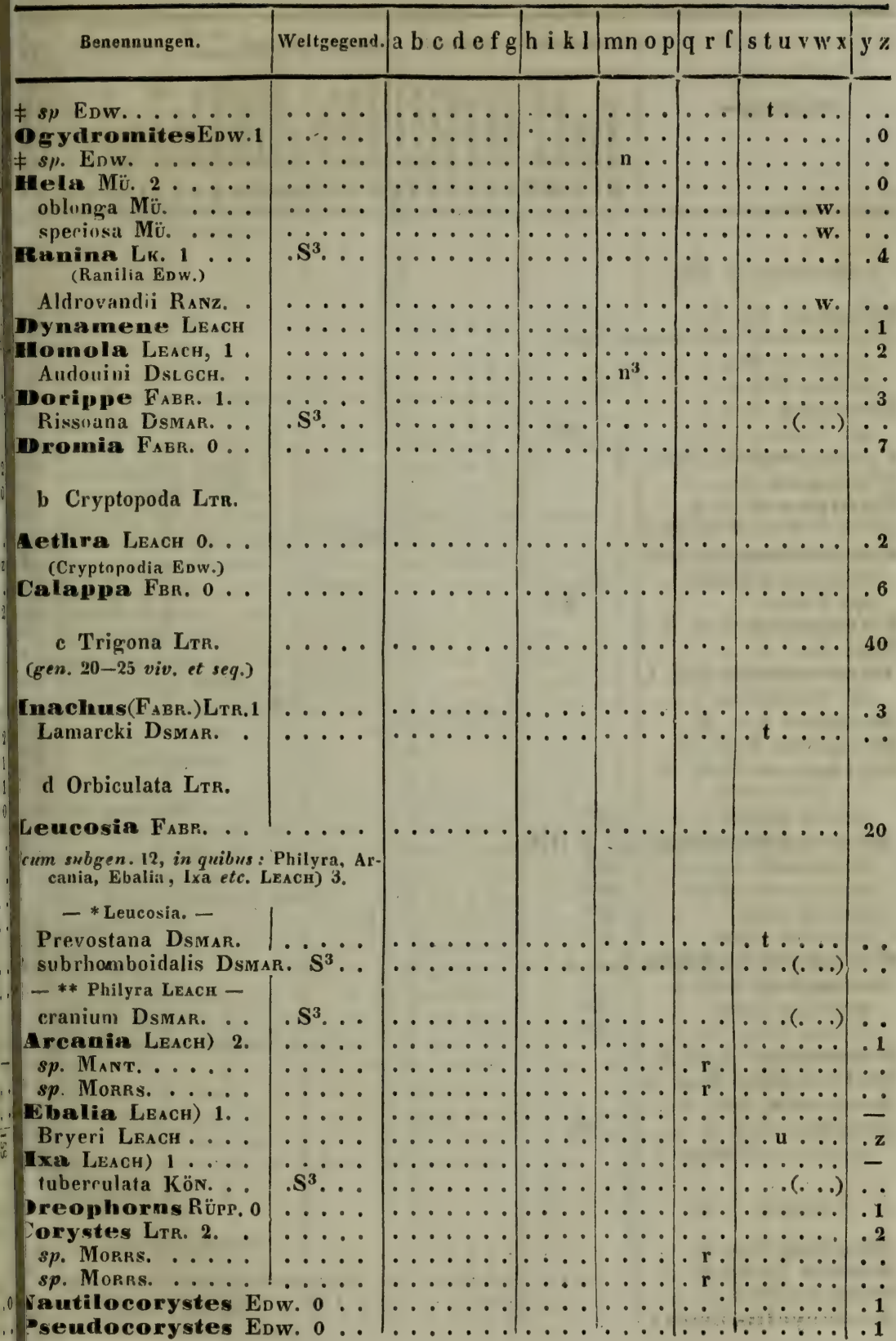




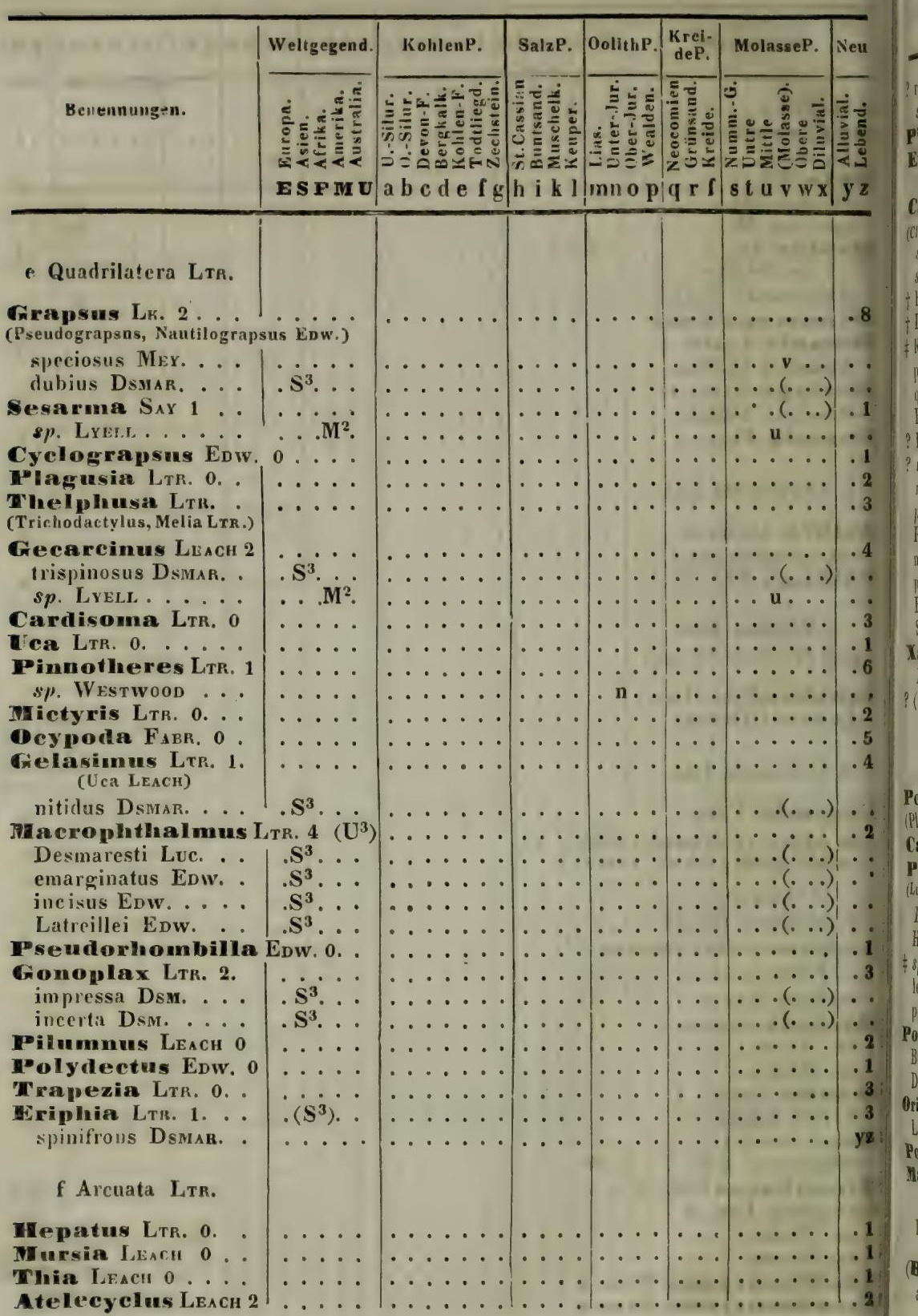




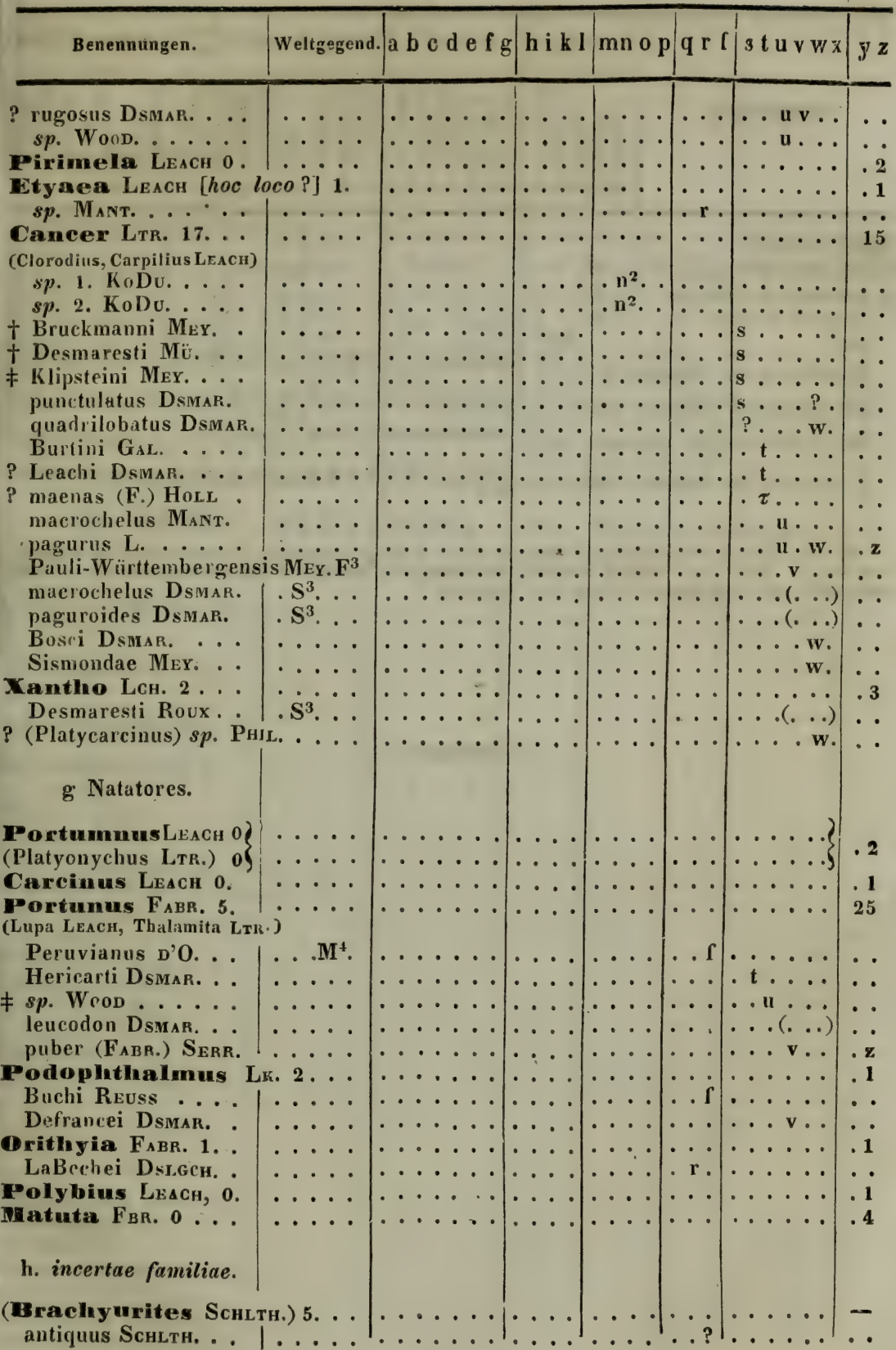




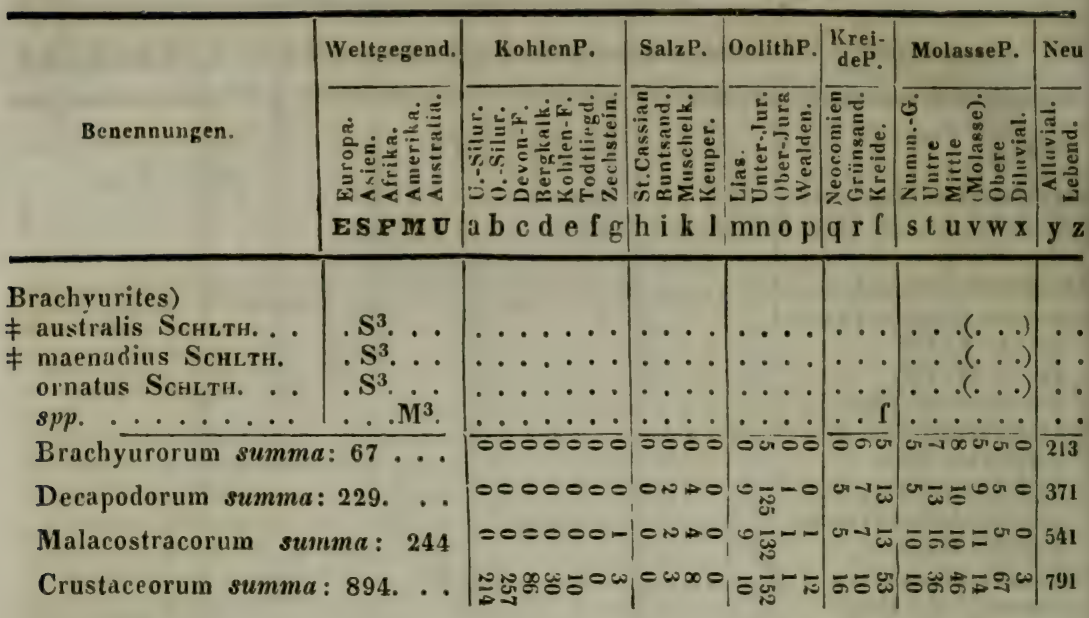

Species indiratae viventes sunt certinres solne et magis cognitae; desunt aline plus minusve numernsut. Jum Munn $1833 \mathrm{R}$. WAGXER Crustucentum viventium specierum uumerum $=1600$ indicavil, qui hodie itaque $=2000$ esse videretur? 


\begin{tabular}{|c|c|c|c|c|c|c|c|}
\hline & Weltgegend. & KohlenP. & SalzP. & OolithP. & $\begin{array}{l}\text { Krei- } \\
\text { deP. }\end{array}$ & MolasseP. & Neu \\
\hline Benennungen. & 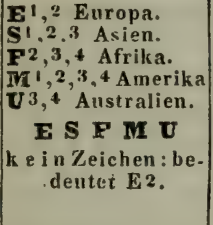 & 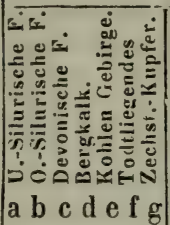 & 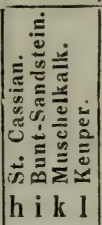 & 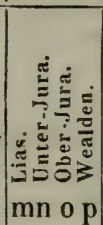 & 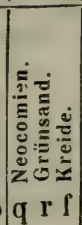 & 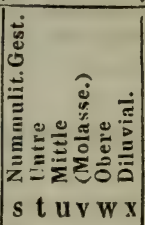 & 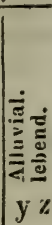 \\
\hline
\end{tabular}

\section{Cl. XVIII. MYRIAPODA LTr., Tausendfüsse.}

\section{(Genera non fossilia pleruque omittunlur.)}

\section{A. GNATHOGENA Brandt.}

1. CHILOPODA LTR.

a Scolopendridae. $\underset{\text { (Scutigera Lk.) }}{\operatorname{Cermatia}} \mathbf{3}$.

Illigeri KB. . . . . .

Leachi KB.

? sp. (araneoidae aff.) HoLL

Fcolopendra (L.) Leach 2.

? sp. Sснцтн..............

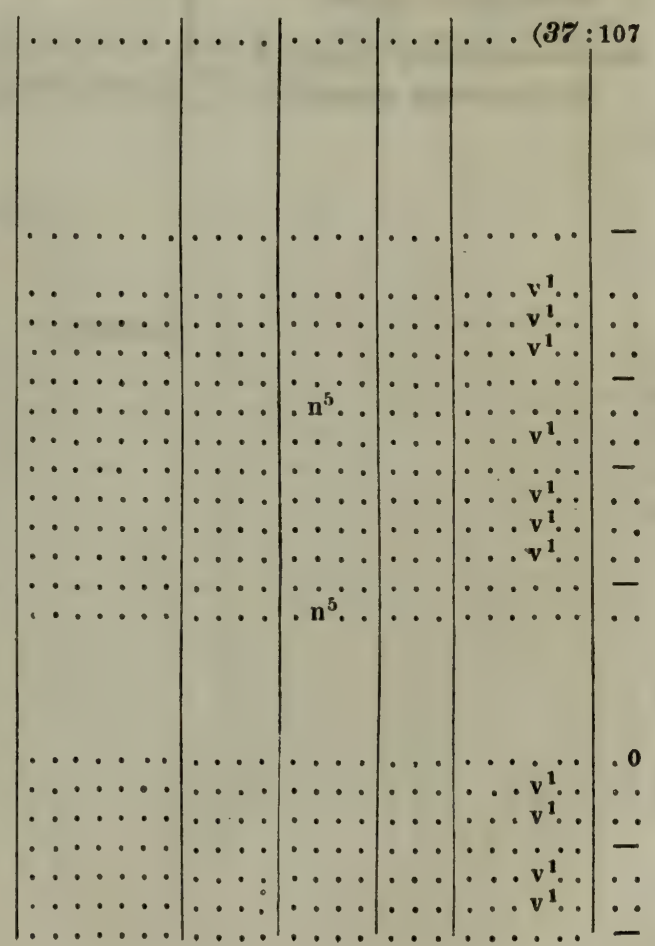



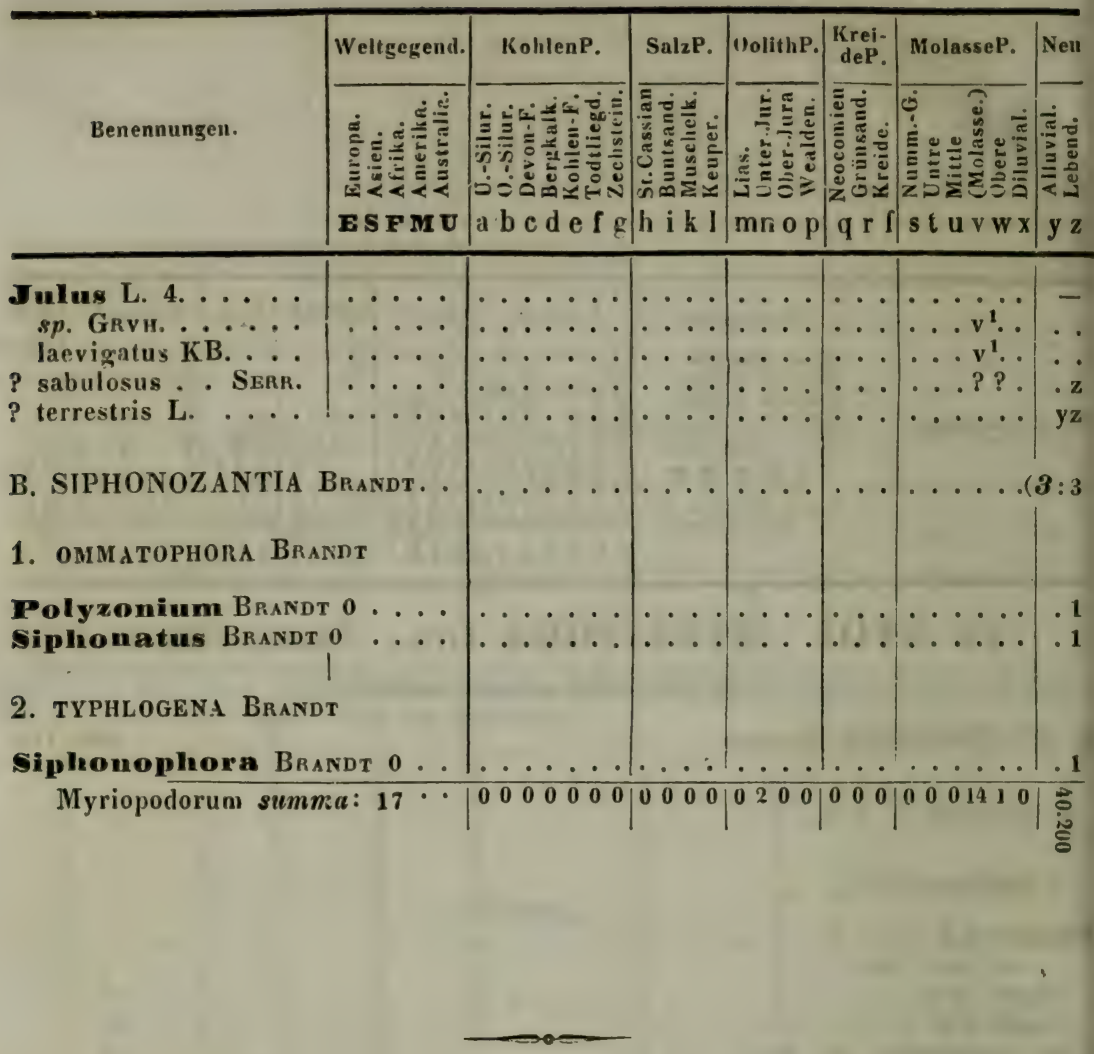


\begin{tabular}{|c|c|c|c|c|c|c|c|}
\hline \multirow[b]{2}{*}{ Benennungen. } & \multirow[b]{2}{*}{ 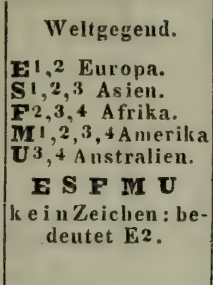 } & KohlenP. & SalzP. & OolithP. & $\begin{array}{l}\text { Krei- } \\
\text { deP. }\end{array}$ & MolassuP. & $N \in u$ \\
\hline & & 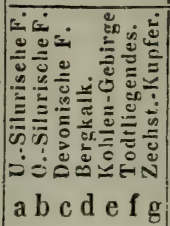 & 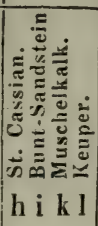 & 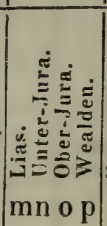 & 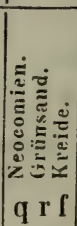 & 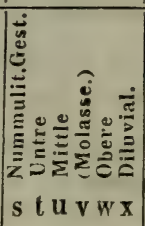 & 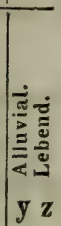 \\
\hline
\end{tabular}

\section{Cl. XIX. ARACHNOIDEA Косн.: Spinnen-Kerfe**}

(Enumeratio generum non fossilium plerumque omittitur.

\section{A. TRACHEARIA Ltr.}

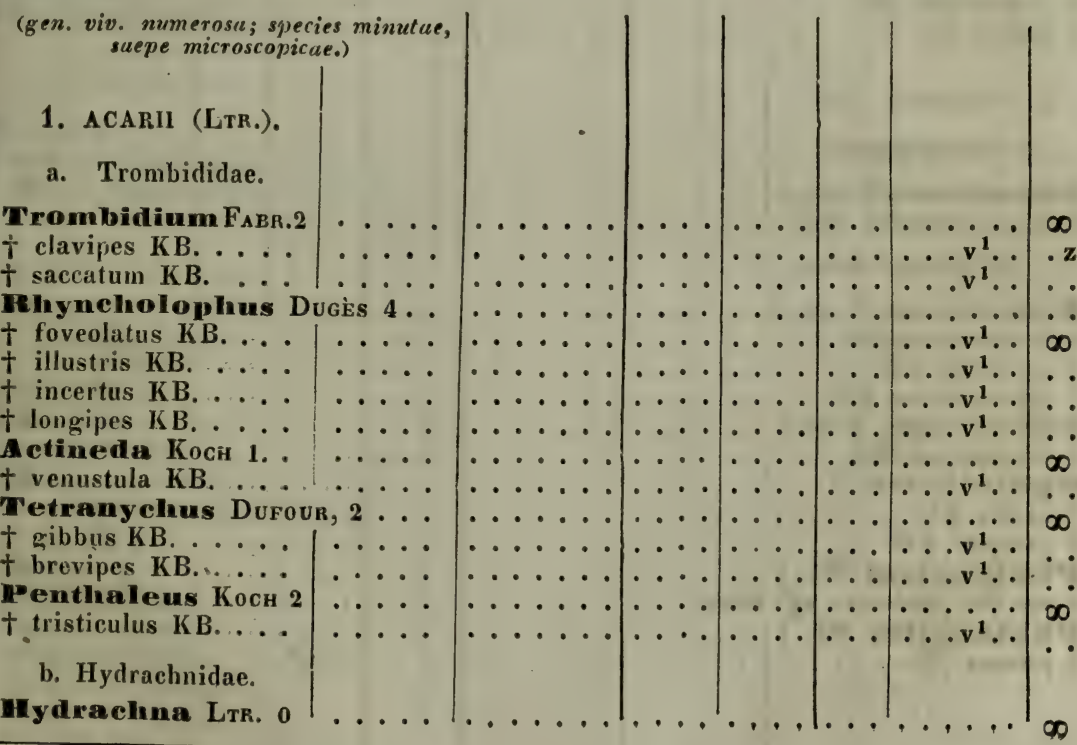

* cfr. Jb. $1845,871 \mathrm{ff}$, 


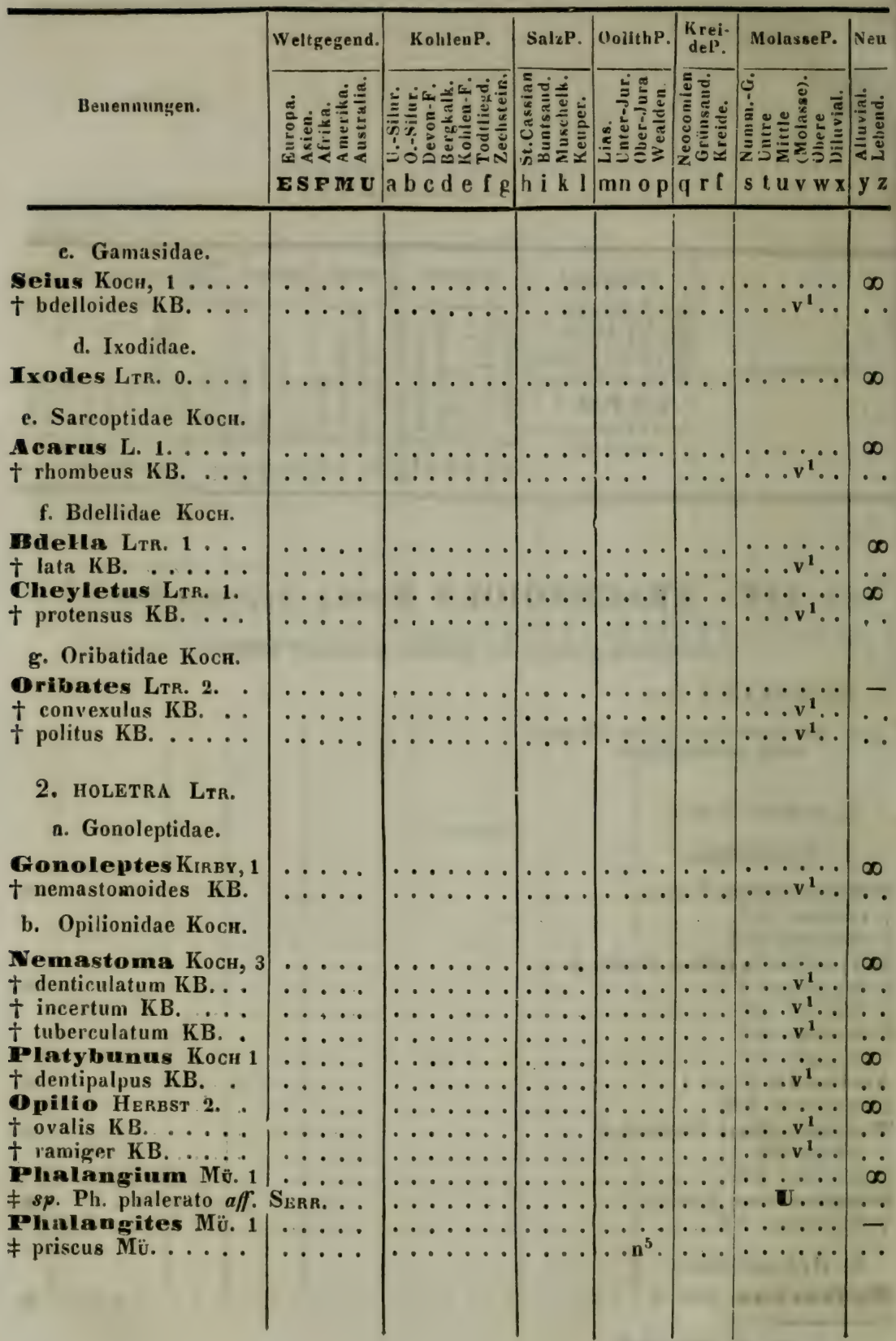




\section{PSEUDOSCORPII (LTR.).}

\section{Microlabis CoRda 1 \\ Sternbergi Corda.}

Chelifer Lench, 4

† sp. Gray......

† Hemprichi KB. . . .

† Ehrenbergi KB. .

† Klemani KB.

Gbisium Leach 1 . † Rathkei KB.

Weltgegend. a b c d e f g h i k I mn op q q I s t u vw x y z

Galeodes Otiv. 0.

Tracheariorum summa : 32

\section{B. PULMONARIA Ltr.}

1. PEDIPALPI LTR.

a Phrynidae.

Phrymus Ltr. 1 . ‡sp. Sern. ……

b. Scorpionidae.

Scorpio L. 0..... ....

Cyclophthalmus CoRda, 1. senior CoRda

\section{ARANEAE LTR.}

a Attidae Косн.

Attus W ALCK. 0 . Leda KB

$\dagger$ promissa KB.

Plidippus KB. 9.

$\dagger$ fasciatus KB.

+ formosus KB.

$\dagger$ frenatus KB.

$\dagger$ gibberulus KB.

$\dagger$ impressus KB.

$\dagger$ marginatus KB.

$\dagger$ melanocephalus KB.

† paul!ulus KB.

$\dagger$ pusillus KB.

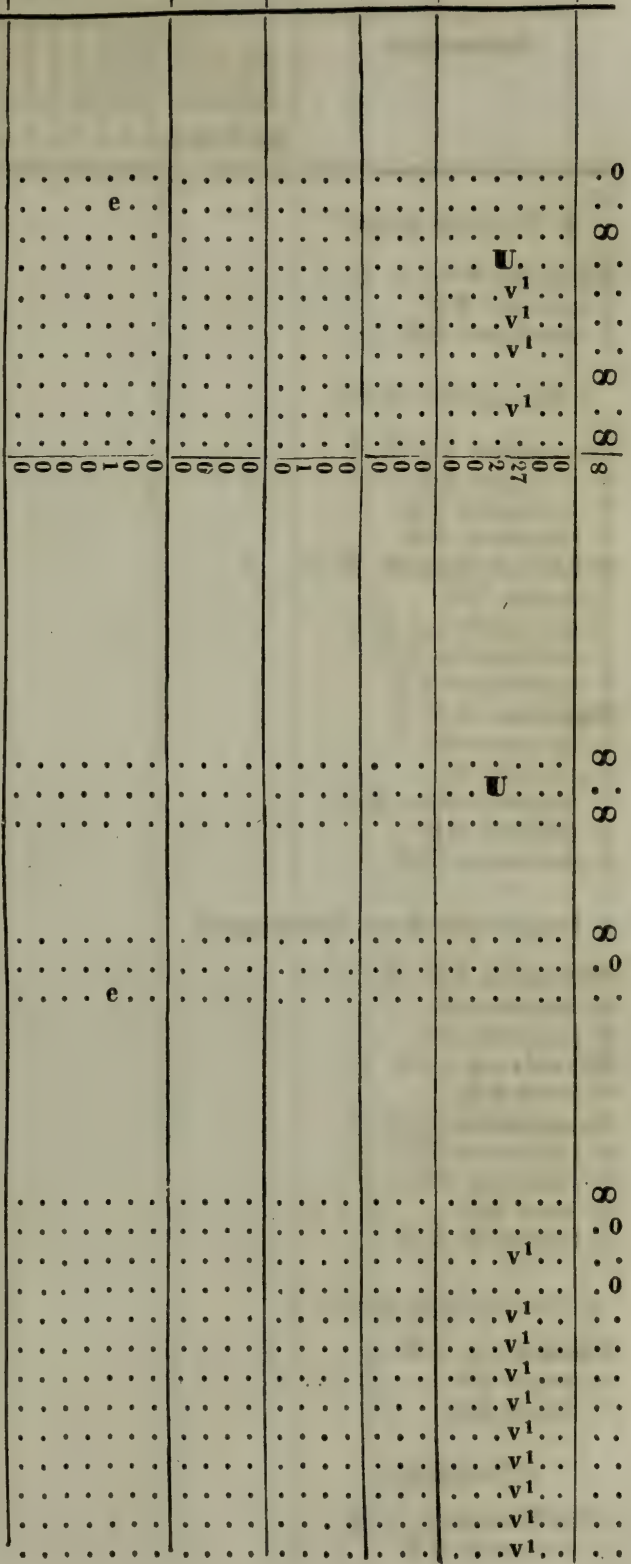




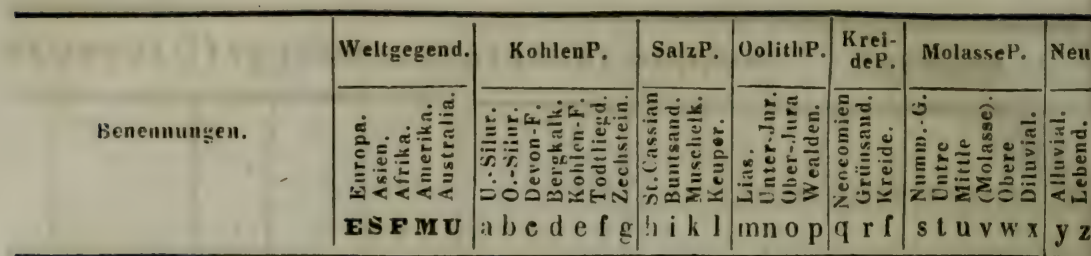

b Eresidae Косн.

Eresus WАгск. 2. . $\dagger$ curtipes KB. . ... $t$ monachus KB.

c Thomisidae Косн.

Deypeta Leach 3. . † crassipes KB. . . . . t decumana KB. ... t triguttata KB.

Philodromus W $\dot{W}_{A L}$ † dubius KB.

† microcephalus KB. .

† spinimanus KB.

† squamiger KB. . . .

Syphux KB. 5. . . . $\dagger$ fuliginosus KB. . . . † gracilis KB.

t megacephalus KB. + radiatus $\mathrm{KB}$.

t thoracicus KB.

d Dysderidae Косн (Tetrapnoa)

Therea KB. 2. . . † hispida KB. . ... t petiolata KB..... ibysdera LTr. 1 . . t tersa KB.

segestria Ltr. 4. . † cylindrica KB. . . . † elongata KB.

$\dagger$ nana KB.

† tomentosa KB.

e Eriodontidae Косн.

Sosybius KB. 2. . . $\dagger$ major KB. ...... † minor KB. .....

f Drassidae.

Clubioma LTr. 6. . t attenuata KB.

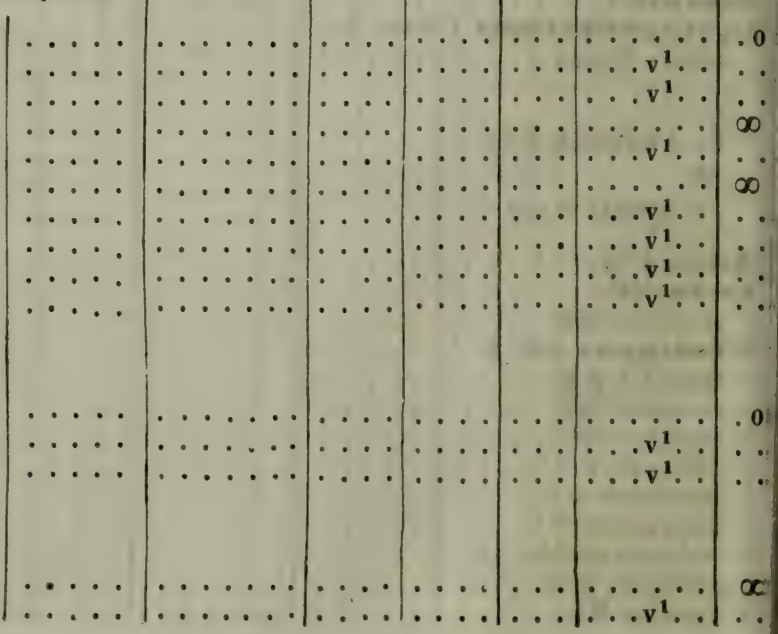




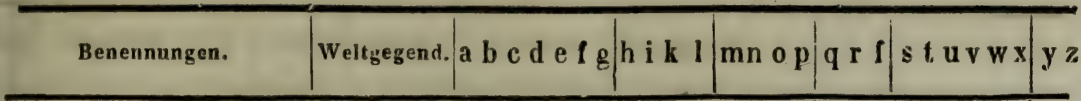

+ lanata KB.

$\dagger$ nicrophthalma $\mathrm{KB}$.

† pubescens KB.

+ sericea KB.

† tomentosa KB.

Anyphrena Sundew. 1.

† fuscrata KB.

Nh acaria Косн. 1 .

† procera KB.

melanophora Koch

t concinna KB.

$\dagger$ mundata KB

+ nobilis KB.

† regalis KB.

Pinytonissa

† affinis KB.

$\dagger$ sericea KB.

Amaurobins Косн 2

† faustus KB.

$\dagger$ rimosus KB.

g Agelenidae Косн.

Thyelia KB. 8, . .

t anomala KB.

t convexa KB.

† fossula KB.

$\dagger$ marginata $\mathrm{K} B$.

t pallida KB.

$\dagger$ scotina KB.

+ tristis KB.

$t$ villosa KB.

Mersilia Sav. 1 .

+ miranda KB.

Textrix Венскш. 1 .

† funesta KB.

$\uparrow$ lineata KB.

Agelena WALCK. 1 t tabida KB.

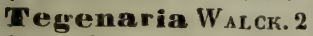

$\dagger$ gracilipes KB.

† obscura KB.

h Therididae Косн.

Clythia KB. 1. . .

t alma KB.

Mizalia KB. 4 ...

globosa KB.

pilosula KB.

punctulata KB.

rostrata KB.

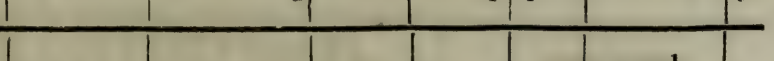




\begin{tabular}{|c|c|c|c|c|c|c|c|}
\hline 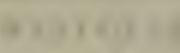 & Weltgegend. & KohlenP. & SalzP. & OolithP. & $\begin{array}{l}\text { Krel- } \\
\text { deP. }\end{array}$ & MolasseP. & Neu \\
\hline Benenuungen. & 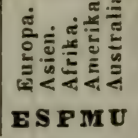 & $\begin{array}{l}3 \\
a b c d e f g \\
0 \\
0 \\
0\end{array}$ & 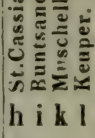 & $\begin{array}{l}2 \\
\text { mn op }\end{array}$ & 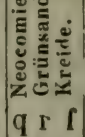 & 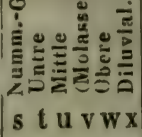 & 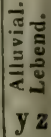 \\
\hline
\end{tabular}

Linyphia Latr. 2.

† cheiracantha KB. .

† oblonga KB.

$\dagger$ infulatus KB.

$\dagger$ molybdinus KB.

† regularis KB.

Erigone Sav. 1. .

† stigmatosa KB.

Theridium $W$ ALCK. 7

$\dagger$ alutaceum KB.

+ desertum KB.

† granulatum KB.

† hirtum KB.

t ovale KB.

† ovatum KB.

† simplex KB.

Его Косн 2.

† setulosa KB.

CIya KB. 1.

$\dagger$ lugubris KB.

Flegia KB. 1. ...

† longimana KB.

i Mithracidae Косн.

Androgeus KB. 2. † militaris KB. .... † triqueter KB. .

k Epeiridae Косн.

V7illa Косн 3

$\dagger$ gracilis KB.

+ porrecta KB.

t veterana KB.

crea KB.

t epeiroides KB.

† obscura KB.

1 Archaeidae KB.

Arehaea KB. 3 † conica KB.

† laevigata KB.

† paradoxa KB.

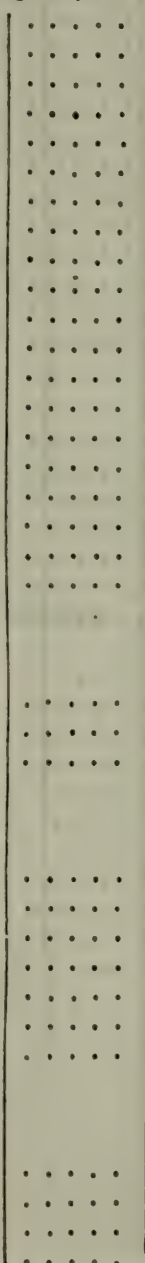




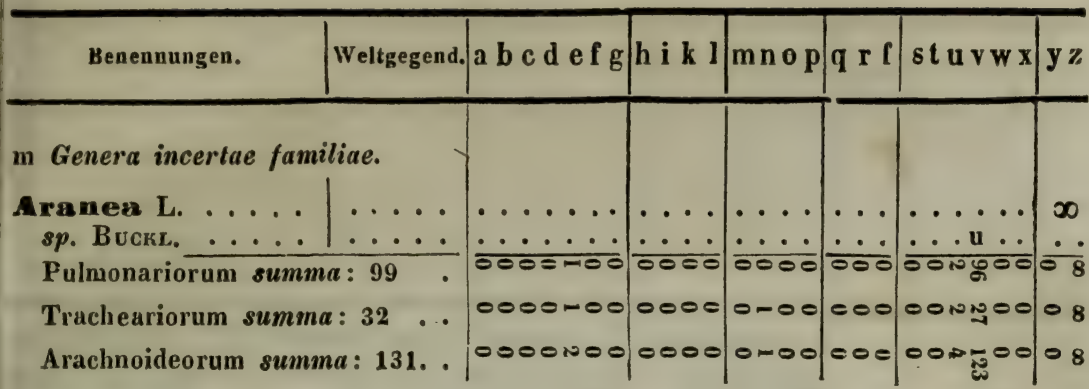




\begin{tabular}{|c|c|c|c|c|c|c|c|}
\hline & & KohlenP. & SalzP. & OolithP. & $\begin{array}{l}\text { Krel- } \\
\text { deP. }\end{array}$ & MolasseP. & Neu \\
\hline benemmungel. & $\begin{array}{l}\mathbf{E}^{1}, 2 \text { Europa. } \\
\mathbf{S}^{1,2}, 3 \text { Asien. } \\
\mathbf{F}^{2}, 3 \text { Afrika. } \\
\mathbf{M}^{1}, 2,3, \mathbf{4} \text { Amerika. } \\
\mathbf{U}^{3}, 4 \text { Australien. } \\
\mathbf{E} \mathbf{S} \mathbf{F} \mathbf{M} \mathbf{U} \\
\text { kein Zeichen: be- } \\
\text { dentet } \mathbf{E}^{2} \text {. }\end{array}$ & 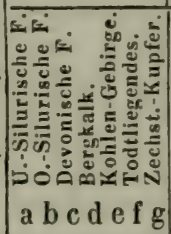 & 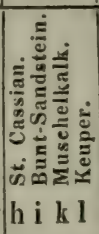 & 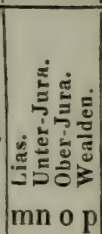 & 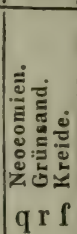 & 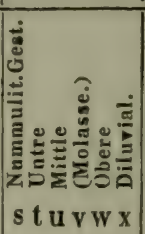 & 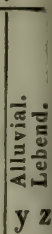 \\
\hline
\end{tabular}

Cl. XX. HEXAPODA (n.), Sechsfüsser, Kerfe $\boldsymbol{c}$. str.

I. DTPTERA L. : Zweiflügler, Mücken *

1. PUPIPARA LTR.

a Hippoboscidae.

2. ATHericera Ltr.

a Phoridae s. Trineurae.

Phora (Latr.) Meig.1 † spp. LB.

b Muscidae MeIG.

Mrusca (L.) Meig. 3 lithophila Mü. . . . † spp. DFr. Serr. . $\left.\begin{array}{l}\text { Nov. gen. LB. } \\ \text { Nov. gen. LB. }\end{array}\right\}+5$

Nov. gen. LB.
Nov. gen. LB. (Anthomyina) † 3 Gehthera LTR, 1. \$ sp. Serr.

c Lonchopteridae.

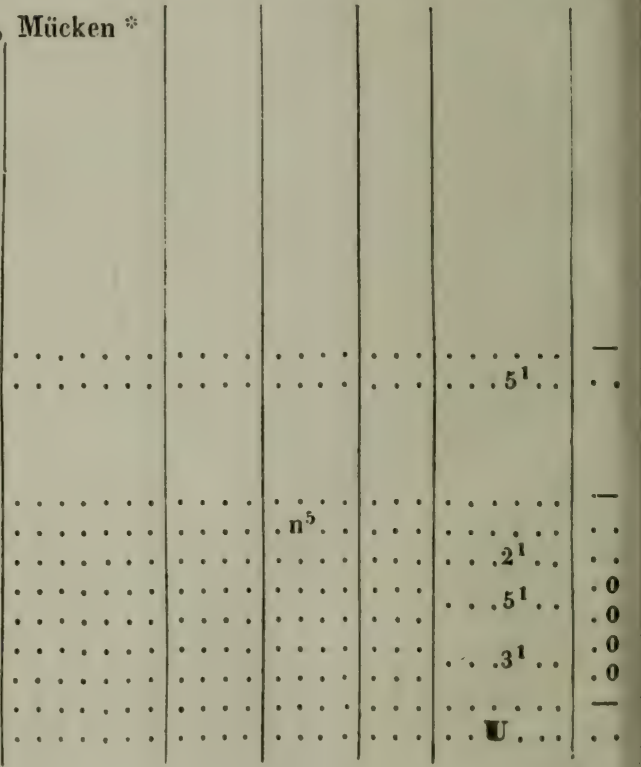

* Specierum nondum nominibus insiguilanum soli numeri (litlernrum loco) in column quuque formutioni geolngicue respondente indicantur. Hi numeri, quundo littere vi loco ponvontur, hor modn $2^{1}$, $3^{i}$ etc. redduntur, ut formutio respondens genlogicu ab aliis ejusdem columnue ( $\bullet$ et $v$ ), ubi simplices numeri 1,2 inveniuntur, diynosci possit. 
d Conopidae.

e Stomoxidae.

f Oestridae.

g Syrphidae.

Syrphus MeIg. 0 . . Melophilus Meig. 1 ? primarius Germ. . . Aphritis LTR. 0 . . $\left.\begin{array}{l}\text { Nov. gen. LB. } \\ \text { Nov. gen. LB. }\end{array}\right\}+6$

3 Notacantha Ltr.

a Stratomyidae.

Dxycera Meig. 1. \#sp. Serr. . . . . . Nemotelus Geoffr. 1 † sp. parva Serr.

Sargus (Frr.) Meig. 1 $\neq s p$. SERr.

Nov. gen. Curt. 1. . sp. Curt.

\section{b Xylophagidae.}

Xylophagus MeIG. 1 ‡sp.X. atri vicinaSERR. Electra LB. 1. ... $\dagger s p$. LB. ..... Chrysothemis LB. 1 † sp. LB.

Nov. gen. 1.

\section{c Midasidae.}

Thereva LTR. 1. † sp. LB.

\section{TABANII.}

a Tabanidae.

Tabanus L. 1 ... . . . . キ sp. mediocr. nigra SERR. . . . Silvius Meig. 1... † $s$. LB.

\section{TANYSTOMATA.}

a Asilidac.

Asilicus Germ. 1. . lithophilus Germ.
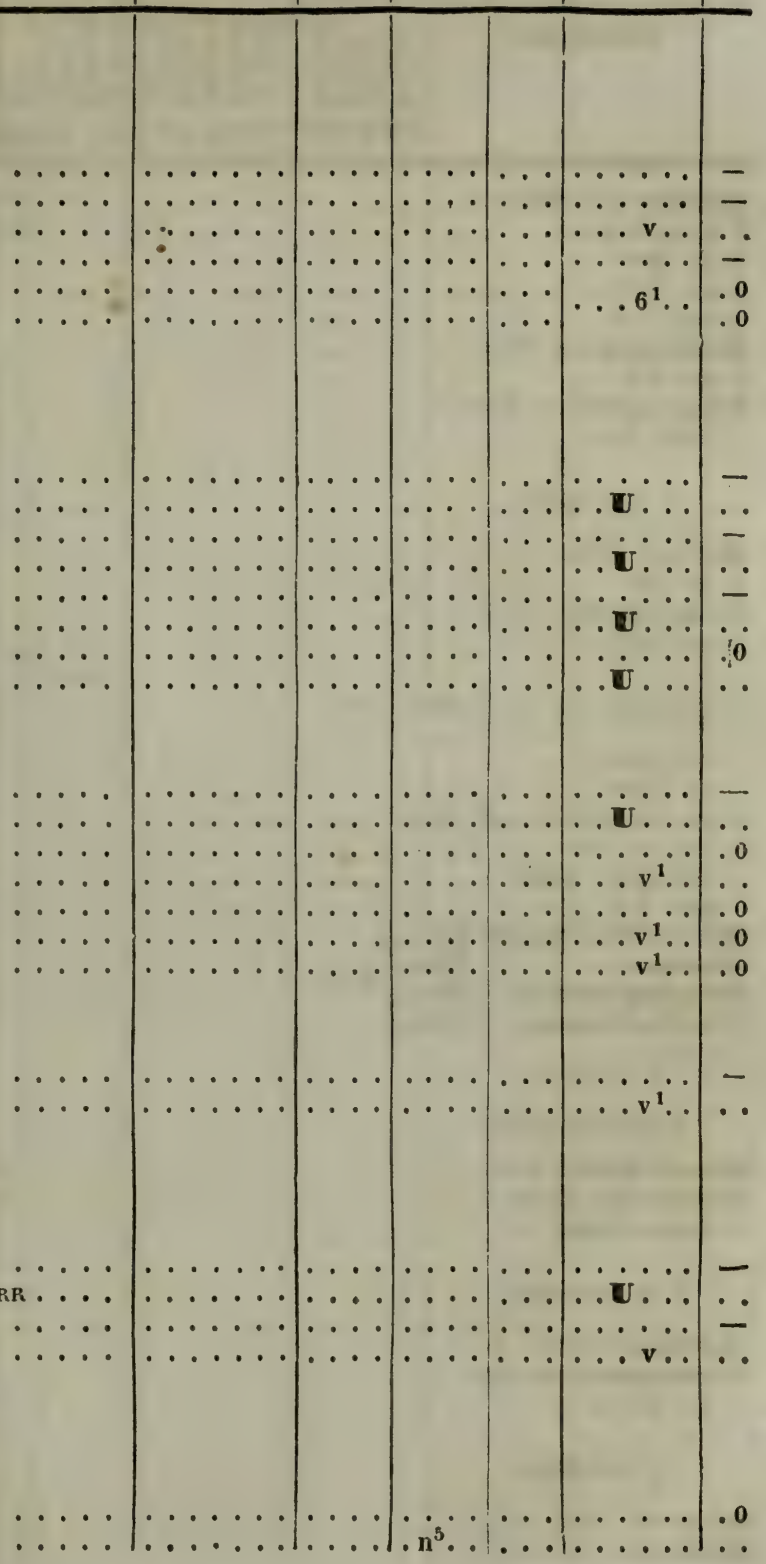


\begin{tabular}{|c|c|c|c|c|c|c|c|}
\hline & Weltgegend. & KohlenP. & SalzP. & OolithP. & $\begin{array}{l}\text { Krei- } \\
\text { deP. }\end{array}$ & MolasseP. & $\mathbf{N}$ \\
\hline Kenenmungen. & 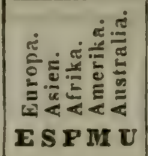 & 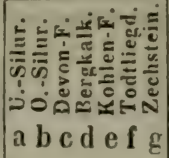 & 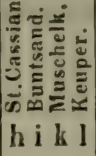 & 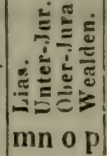 & 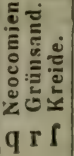 & 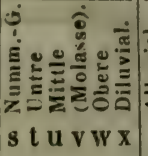 & $\begin{array}{l}\bar{\alpha} \\
\text { y } z\end{array}$ \\
\hline
\end{tabular}

Asilus (L.) Meı́. 5. ? ignotus Brod. .... ‡ sp. SERP.

$\neq s p$. SERR

† spp. LB.

Dasypogón Mва́, 1 † $s p$. LB.

Ceptogaster Meig.1 Helli Ung.

b Hybotidae.

Mybos Meig. 1 . . . † $s p$. LB.

Leptopeza Mậ. 1 † sp. LB.

c Enpidae (et Tachydromidac).

$s p$. BRoD.

$s p$. Curt.

‡ sp. tessellatae aff. SerR.

† spp. 7 Curt. carbonum Germ. spp. LB.

Thamphomyi

Gioma

Brachystoma

Tachydroma

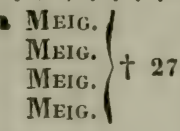

d Acroceri.

e Bombyliidae.

Bombylius Ltr. Phthiria Mejg. 1. dubia Germ.

f Anthracidae.

Anthrax Scop. 0. Nemestrinus LTR, 1 † $s p$. Serr.

g Leptidae.

$\left.\begin{array}{ll}\text { Leptis } & \text { LB. }+ \\ \text { Atherix } & \text { LB. }+\end{array}\right\}$
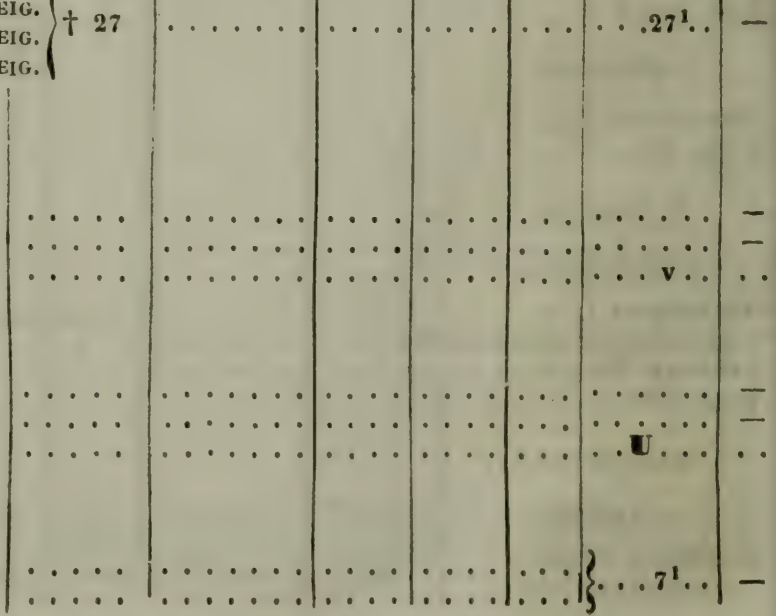


\begin{tabular}{l|l|l|l|l|l|l|l|}
\hline Benennungen. & Weltgegend. a b c d e f g & h i k l & mn o p & q r f & s t u v w x & y \% \\
\hline
\end{tabular}

h Scenopidac.

i Dolichopidae.

Porphyrops Meig. LB. +

$\begin{array}{ll}\text { Nedeterus } & \text { Mrig. LB. }+ \\ \text { Chrysotus } & 40 \\ \text { Merc. LB. }+\end{array}$

k Platypezidae.

1 Pipunculina.

Pipunculus LTr. 1 $\dagger s p$. LB.

6. NEMOCERATA.

a Tipulina.

spp. non nominatae BRoD.

$=\mathrm{a}^{2}$ Floricolae. $=$

Rhyphus LTR. $3+$ priscus Brop.

† spp. I.B.

Dilophas MEIG. $3+$

‡sp. D. marginati vicina SERR.

† $s p$. Serr.

† spp. LB.

Plecia Wiedr. 2 +

† spp. LB.

Simulia Meig. 3 + humida Brop.

† spp. LB

Scatouse Groprr. 3. $\neq s p$. alis fuscis Serr.

† spp. LB.

$=a^{2}$ Terricolae. $=$

Tipula(L.) Meig. $2+$ † spp. LB.

Adetus LB. 2 +.

$+s p p$. LB.

Nov. gen. LB.

Nov. gen. LB.

Nov. gen. LB.

Tanysphyra LB. $1+$

Nov. gen. LB. $1+$.

Trichoneara LB. 1

Nov. gen. LB. 1 †. .

Macrochile LB.

Rhamphidia MEIG. $1+$

Toxorhina LB. 1 十

Styringia LB. $1+$

Cylindrotoma $\mathrm{MI}_{\mathrm{A}} \mathrm{C} .1+$.

A nisomera MeIg. $1+$

Dixa Mnig. $1+$.

$=a^{3}$ Fungicolae. $=$

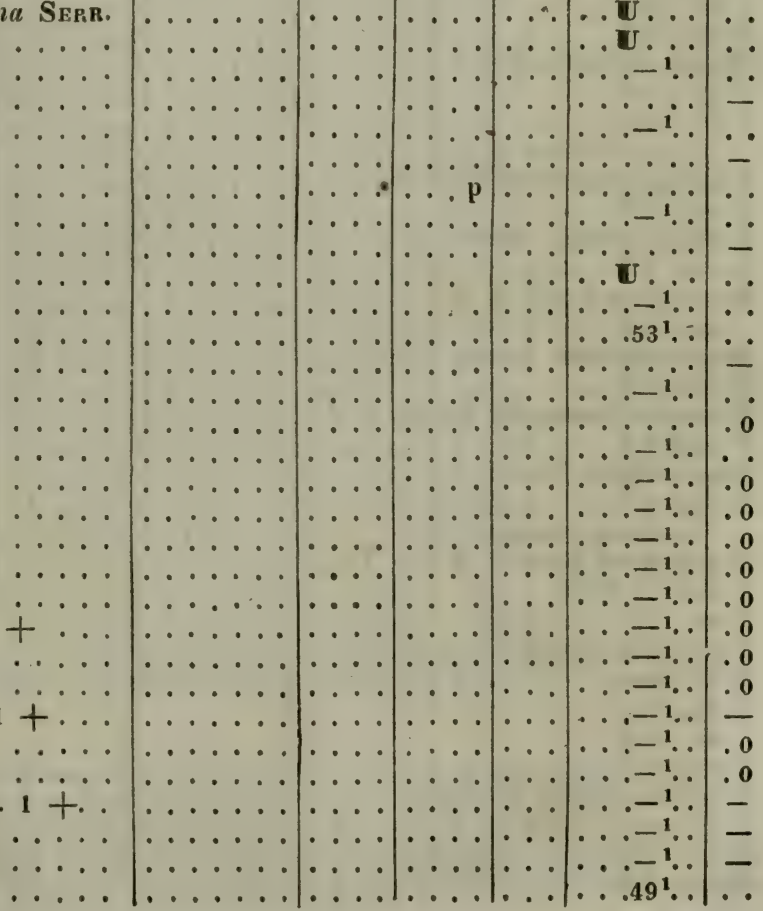




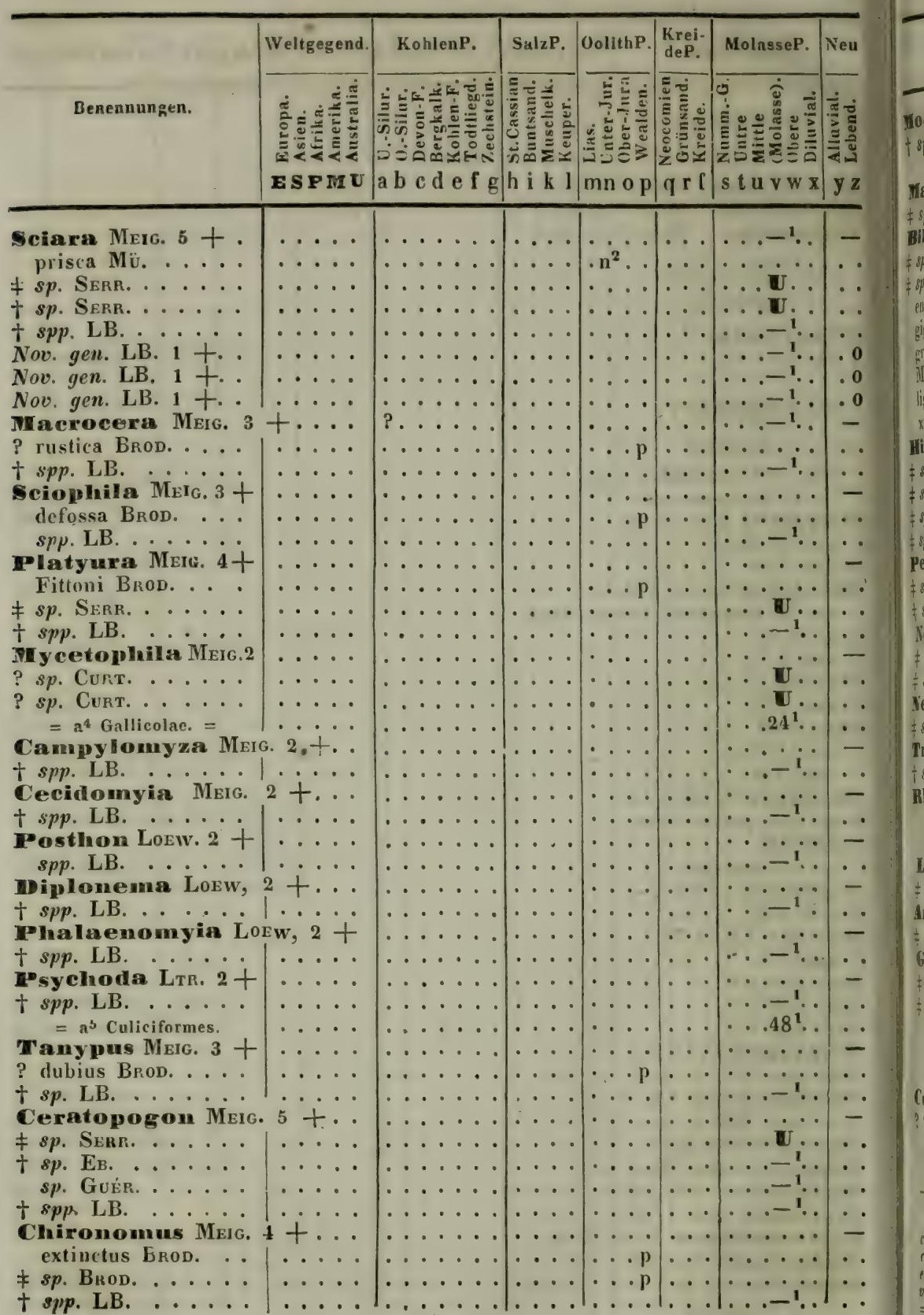




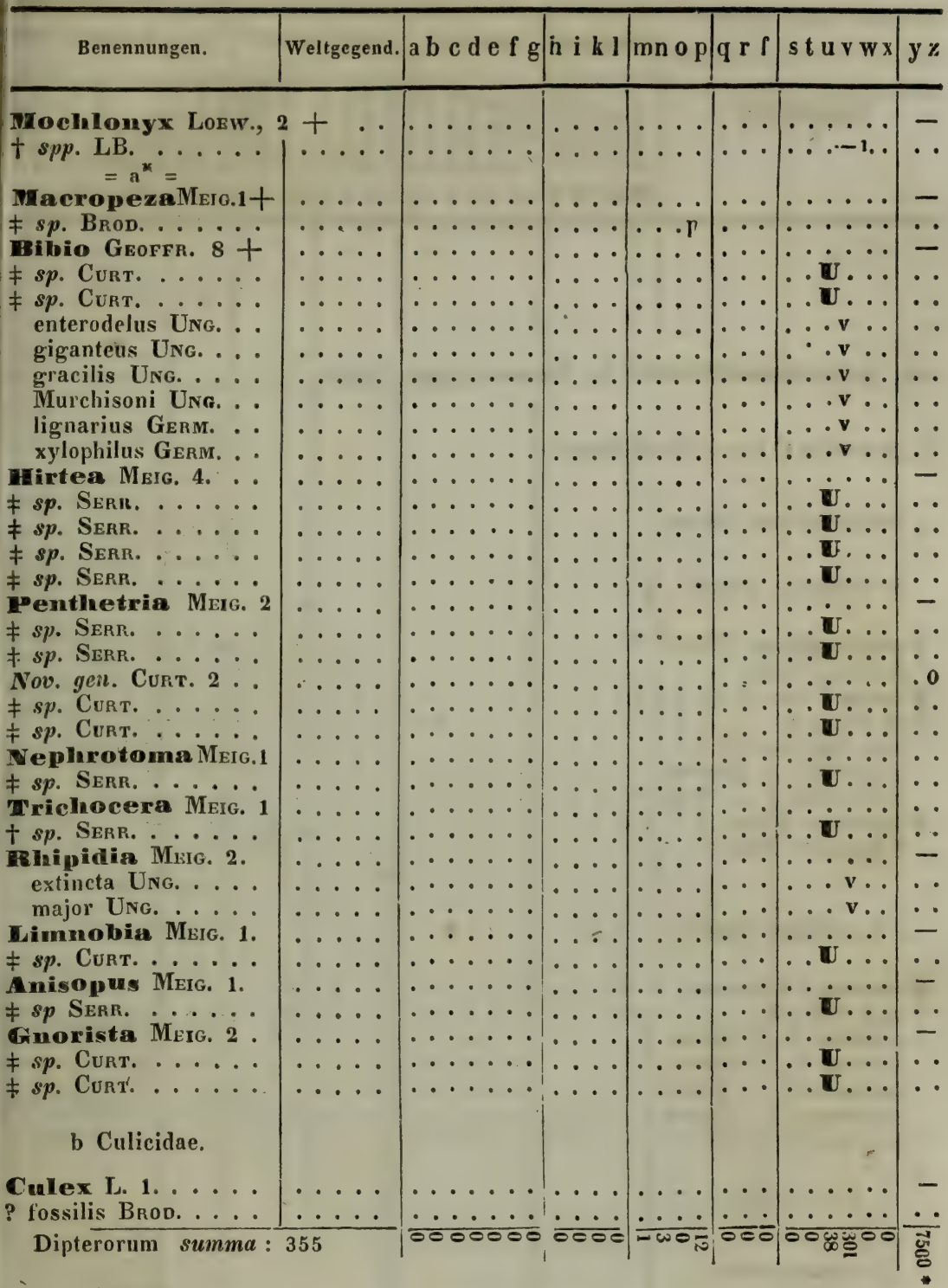

* Meritissimus MeIgex jam anno 1838 species vivenles Europreas 4500, exoticus 2300 indicavit at mullue uline praeserlim Eurnpuene ab hoc tempore descriptue sunt. Qunm autem celeberrimus ROSER anno 1840 in snlo regno Wiirtlembergico species Diplerorum circa 2200, i. e. numerum fere aegulem atque Coleopterorum iljidum nunc cognitorum in catulog consignaverit, tolus Dipterorum viventum numerus, si Coleopterorum rutione ubique idem essel, jum hodi" $=30,000$ uestimandus Joret. Observationibus autem, aequatorem versus Colenpterorum numerum valde augeri, Dipterorum forte dominui certiores facti sumus. 


\begin{tabular}{|c|c|c|c|c|c|c|c|}
\hline & Weltgegend. & KohlenP. & SalzP. & OolithP. & $\begin{array}{c}\text { Krei- } \\
\text { deP. }\end{array}$ & MolasseP. & Neu \\
\hline Benennumgen. & 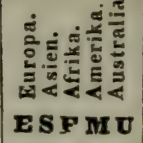 & 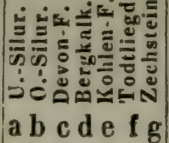 & 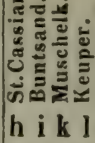 & 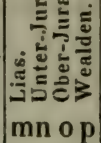 & 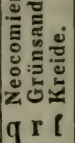 & 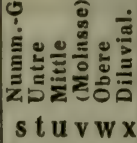 & 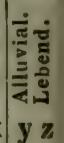 \\
\hline
\end{tabular}

\section{LERIDOFTERA L., Schmetterlinge, Falter.}

1 nocturna Ltr., Nacht-Falter.

a Pterophoridae Zelcer

b Tineidae Leach

Ypsolophus (FARR.) 1... insignis Ger..

Tinea FABR. 4 十. ? $\boldsymbol{s}$ S Sterne. ....

† spp. Grave. .... culmella?... Gravh. pellionella ?... GravH....

\section{Tineites Germ. 1.} lithophilus GrRm.

c Tortricidae STEPH.

Tortrix TreitschKe 5 十. . . † spp. Gravh.

arcuana ?... GRvн. falcana?... GRvн. Lecheana? ... Grvн. urticana ?...Grve.

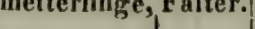


g Ceruridae Ltr.

h Bombycidae.

Dombyx Schrank, 1 ? $s p$. SERR.

i Hepialidae Frr.

2. Crepuscularia Ltr., Abend-Falter.

a Zygaenidae LEACH.

Sesia $F_{A B r .} 2 \ldots$ † sp. SERR.

$\neq s p$. SERR.

Zygaena $F_{A} \ldots$ $\neq s p$. SERr. . . . . .

b Sphingidae LEach.

sphinx (L.) 3 . . . Schröteri Gern. . . atavus CHARP. ..... $s p$. Bernt.
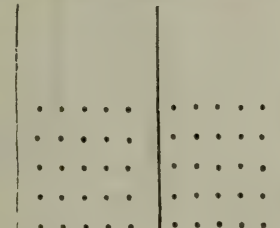

3. DiURNa Lrr., Tag-Falter.

a Hesperidae.

b Papilionidae.

Satyrus LTR. 1. . . $\neq s p$. Serr. . . . . Papilio (L.) LTR. . 祡

Lepidopteri larva SENDEL. . . . Lepidopterorum summa 22

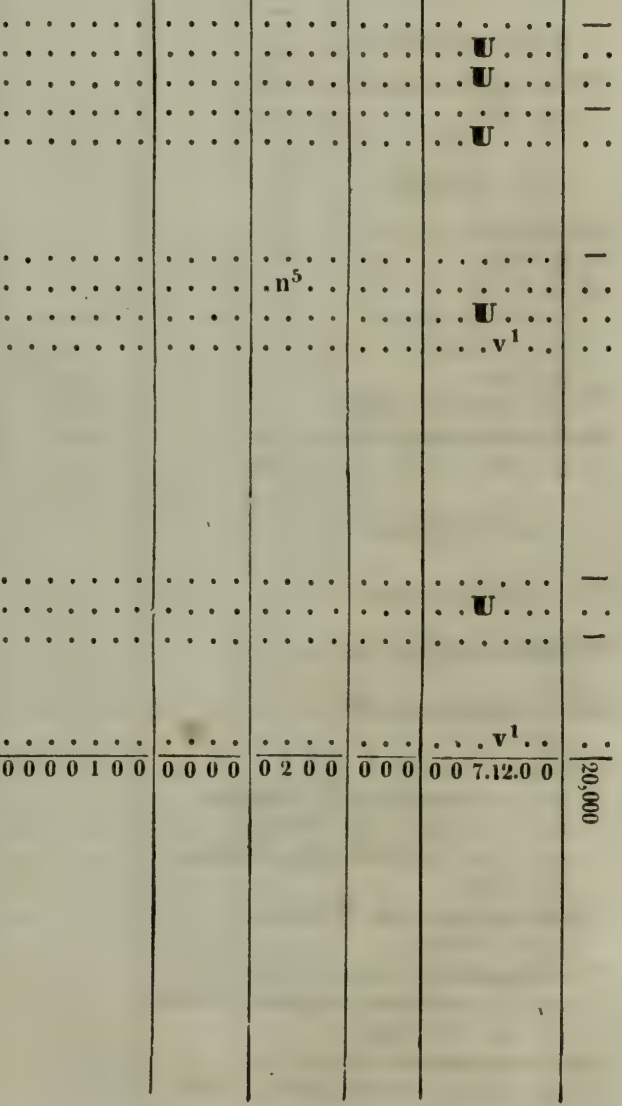


Benennunge॥

\begin{tabular}{|c|c|c|c|c|c|c|}
\hline elt & KolilenP. & SalzP. & OolithP. & $\begin{array}{l}\text { Krei- } \\
\text { deP. }\end{array}$ & MolasseP. & Neu \\
\hline 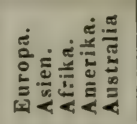 & 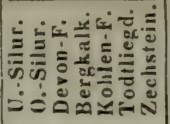 & 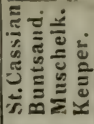 & 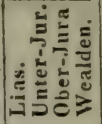 & 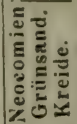 & 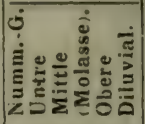 & 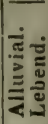 \\
\hline $\mathbf{E} \mathbf{S} \mathbf{Y} \mathbf{U}$ & a b c d e f g & h i k l & mn op & q r I & st. uv w X & y $z$ \\
\hline
\end{tabular}

III. MEvi PTERA L., Halbflügler, Wanzen.

(sec, disposilinnem melhodic IUT RTMEISTERT.)

1. HOMOPTERA.

a Coccina Burm.

Monophlebus Leach $3+\ldots$ spp. GB.

b Aphidina.

Lachnus Illig. 1 . sp. GB.

Aphis L. 7 .....

Valdensis Bron.

?' plana Brod.

$s p$. Curt.

spp. Schilla, GB. .

Schizoneura Hartg. $1 \ldots$... $s p$. GB.

c Psyllodes.

d Cicadellina.

Typhiocyba Germ. 2 十. .

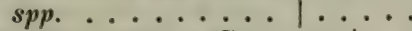
Bythoscopus Germ. $2+\ldots$

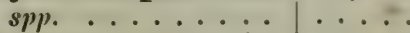

Jassus Germ. $5+\ldots$

$s p$. J. atomario aff. Schillg. sp. J. lineato aff. Schillg. . . $s p$. J. unifasciato $a f f$. Schrllg. spp. GB.

Bitomoptera Germ. 1. ... dubia Genm. .... I .... Tettigonia (Ltr.) Germ. 2. . $\neq s p$. F. violaceae magnitud. SERH. ‡sp. (parva) SERR . . . . . . Aphophora Germ. 2. . . . . ‡sp. A. spumariae simill. Curt. † sp (larva) Schillg. | . . . .

|




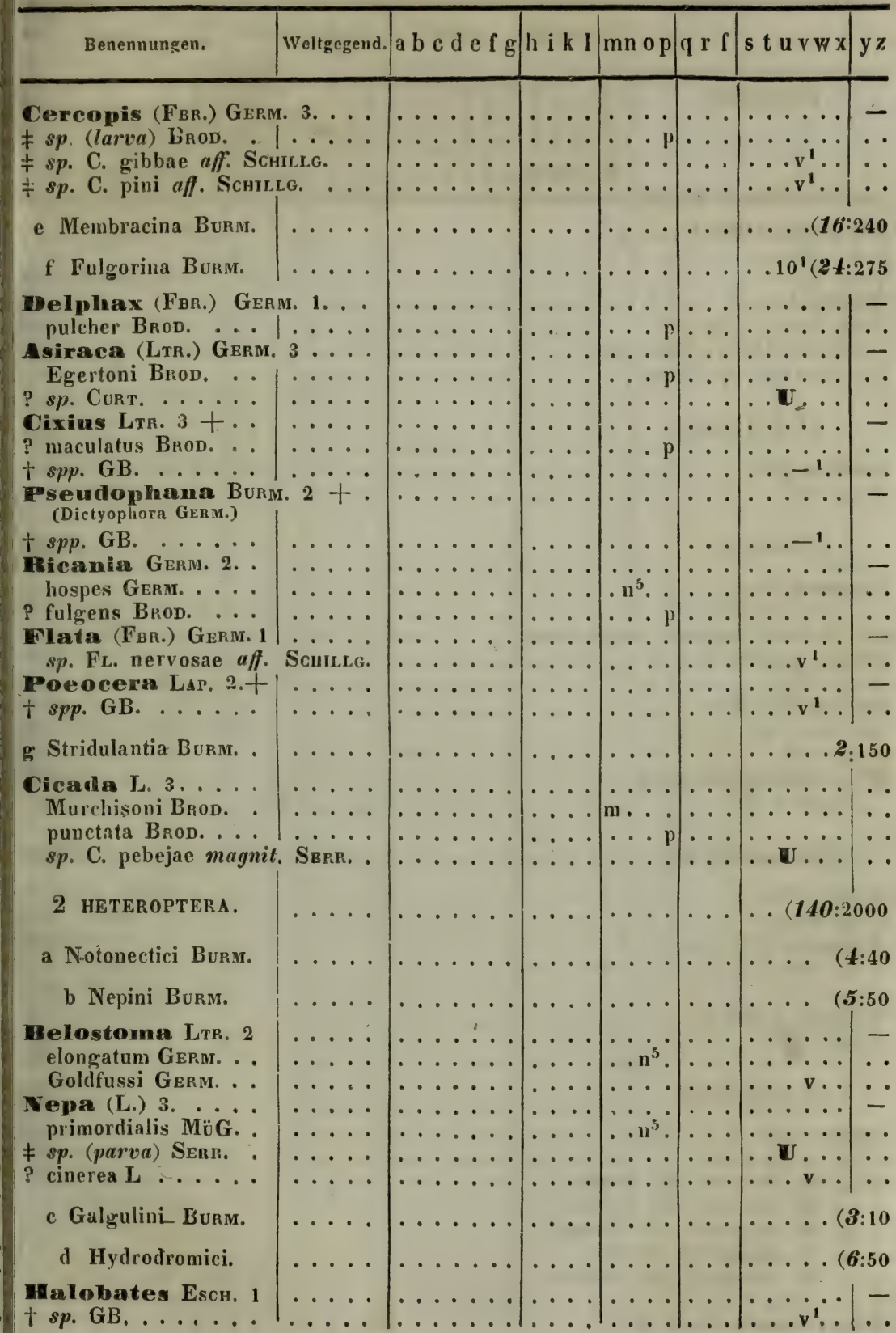




\begin{tabular}{|c|c|c|c|c|c|c|c|}
\hline & Weltgegend. & KohlenP. & SalzP. & OolithP. & $\begin{array}{l}\text { Krei- } \\
\text { deP. }\end{array}$ & MolasseP. & Neu \\
\hline Benemungen. & 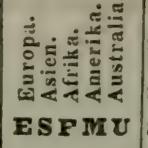 & 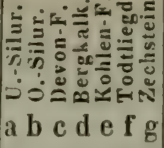 & 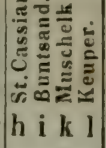 & 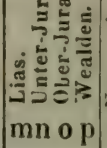 & 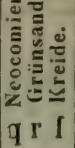 & 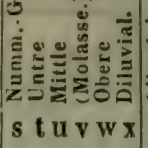 & 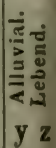 \\
\hline
\end{tabular}

Hydrometra FABR. 2.

$\neq s p$. BroD.

$\dagger s p$. GB.

Velia $L T R$.

† $s p$. BRoD.

currens (Lrri.) SERr.

$? \neq$ (Gerris) $s p$. Sern.

e Riparii Burm.

Salda Frr, 1 $\dagger s p$. GB.

f Reduvini.

Emesa Frr. 1 (Ploiaria Scop.)

sp. Ploiariae medioer. magn. SerR Platymeris LAP. 1 $\dagger s p$. GB.

Reduvius FBR. 1 .

Nabis $L_{T R}$. 1

$\dagger s p$. GB.

Pygolampis Germ.1

gigantea $M \ddot{\text {. }}$

g. Membranacei LTR.

Syrtis Fbr. 1

$\neq s p$. SerR.

Aradus (FBr.) 3. . $\neq s p$. SERR.

$\neq s p p$. GB.

Tingis FABR. 2 . .

$\neq s p p$. GB.

h Capsini Buru.

Miris Frr. 6.

‡ sp. parva CuRT.

₹ sp. M. Tanaceti aff. Schicg.

$\neq s p$. M. Tanaceti aff. Schilg.

$\neq s p$. M. rufipenni aft. Schilg.

$\neq s p$. M. populi aff. Schilg. .

$\neq s p$. M. campestri aff. Schilg.

$\neq s p$. dubia

Phytocoris FALL. 0 


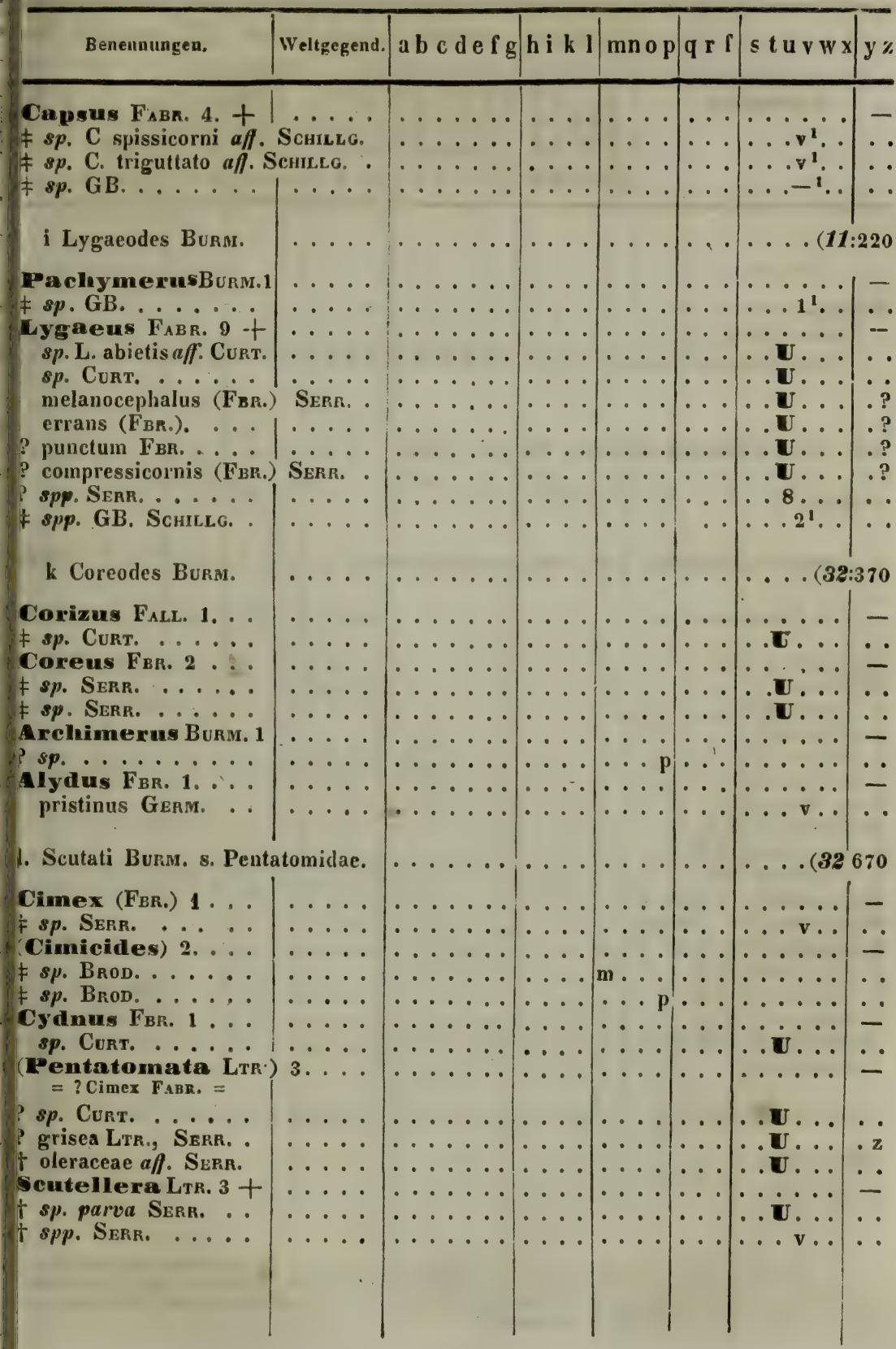




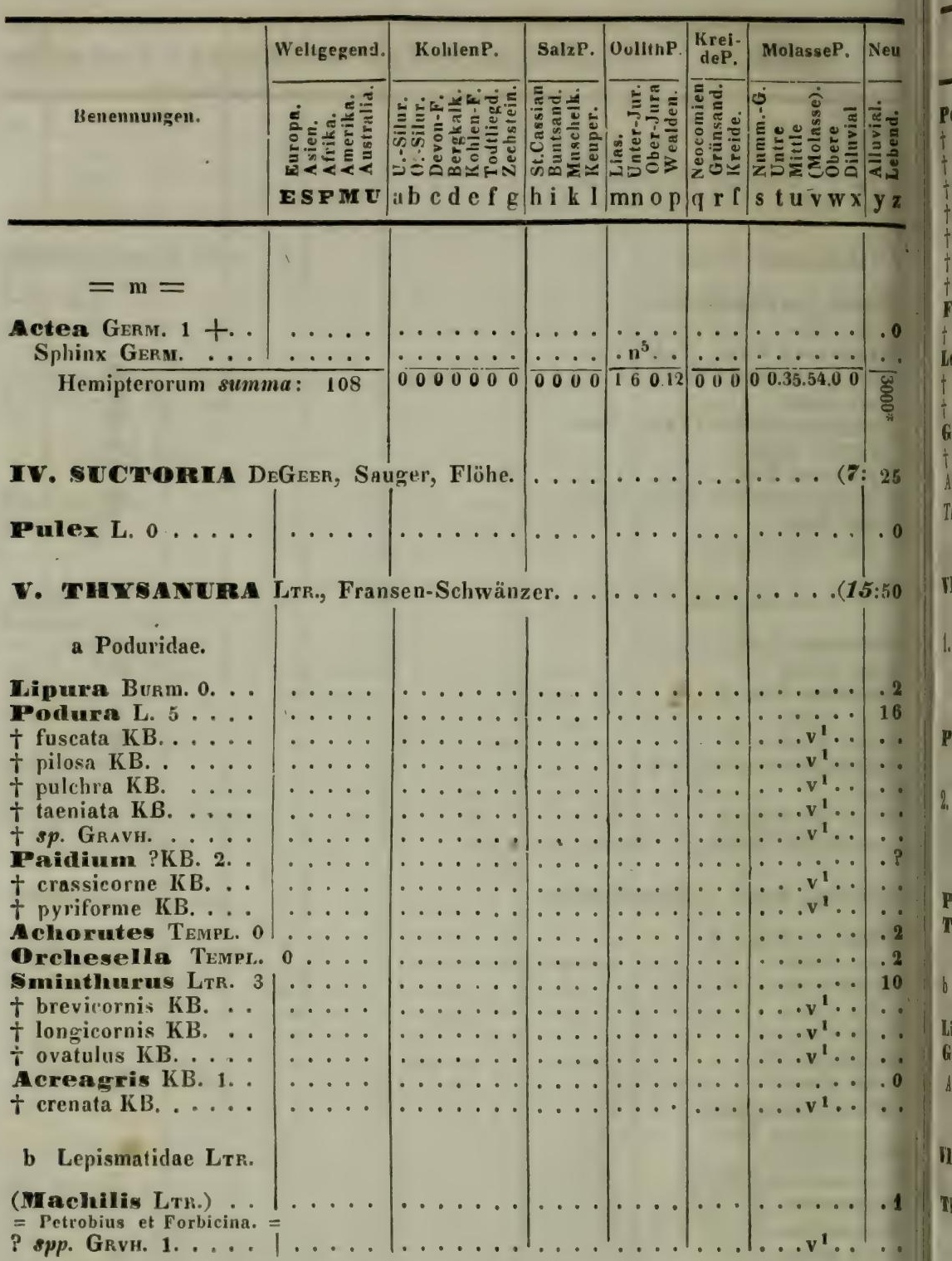

* Hic specierum viven/ium numerus additione specierum a BURMEIsTER in omnibus suis generibus ant descripturum aut indicaturum ortus est; vera nutem specierum congitavim summ hoc numero multo major, forte $=5000$ aestimandı erit, licet reliquae species difficilius scuepe ad sur cenera in illo systemale referratur. 


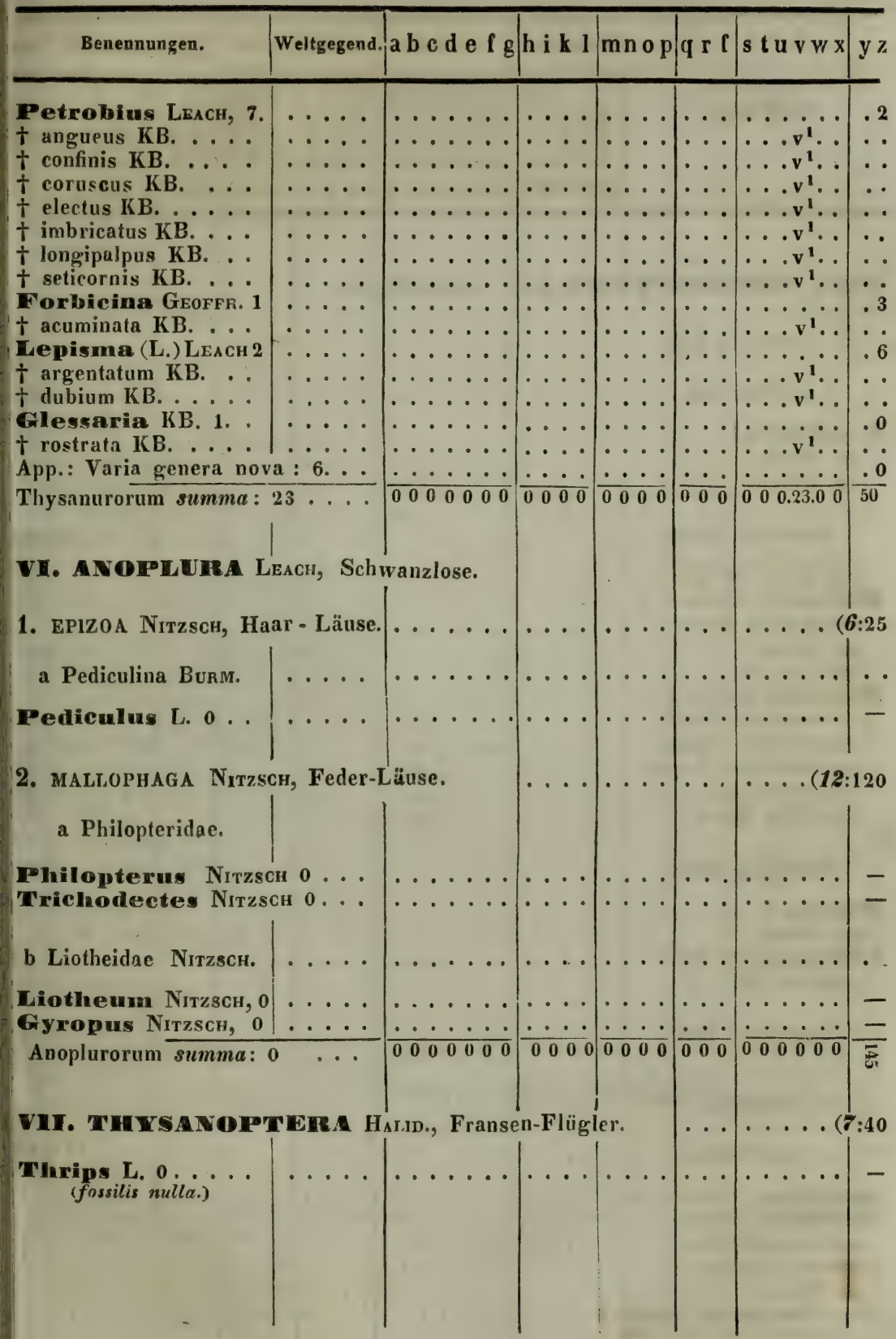


Benemungen.

\begin{tabular}{|c|c|c|c|c|c|c|}
\hline Weltgegend. & KohlenP. & SalzP. & OolithP. & $\begin{array}{l}\text { Krei- } \\
\text { deP. }\end{array}$ & MolasseP. & Neu \\
\hline 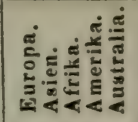 & 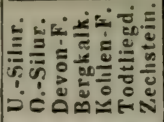 & 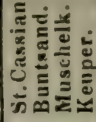 & 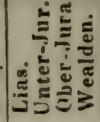 & 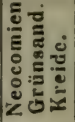 & 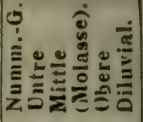 & 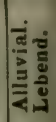 \\
\hline ESPMU & $a b c d e f g$ & h i k l & mnop & $q \mathrm{rr}$ & st u vw $x$ & yz \\
\hline
\end{tabular}

vil. On'Thor'Tena Ltr., Gerad-Fliggler.

1. CURSORIA Ltr.

a Labiduridae.

Forficula L. . . . . sp. SerR. . . . . . sp. Gravh.

b Blattidae Steph.

spp BROD. $2+$ IIatta (L.) Burm. $4+$ Stricklandi BRod. sp. Brod. . . . . . spp. Gravh.

Blattina Ger M. 4 . (Dictyopteris Rost) anaglyptica GERM. . anthracophila GERM. didyma Germ. flabellata Germ.

\section{c Mantodea Burx.}

Mrantis L. 1. . . . sp. Serr. … Chresmoda Mü. 1. obscura Mü. ... .

c Phasmodea Burm.

2. saltatoria Ltr.

a Acridiodea.

Acridium Geofrr. 0 Acridites Germ. 1. carbonatus Germ.

Gryllidae StEPHS. 1. . sp. Brod. ......
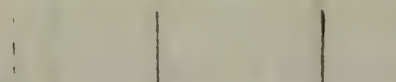

$\mathbf{x} / \mathbf{y}$ 


\begin{tabular}{|c|c|c|c|c|c|c|c|}
\hline Benen & Weltgegend. & $a b c d$ e $f g$ & h i k l & $\mathrm{mn}$ op & $q \times \uparrow$ & $\mathrm{stu}$ & \\
\hline \multicolumn{2}{|c|}{ coerulescens ? FBr. SERr. } & $\cdots \cdot$ & $\cdots$ & $\cdots$ & & $\ldots \mathbf{U} \ldots$ & \\
\hline $\begin{array}{l}s p p . \text { GRAVH. } \ldots \\
s p . \text { EB. . . . }\end{array}$ & .... & $\ldots \ldots$ & $\cdots$ & $\ldots$ & $\cdots$ & $\cdots v^{1} \cdots$ & \\
\hline $\begin{array}{l}s p . \text { EB. } \\
\text { Fryllites GER }\end{array}$ & $\ddot{\cdots}$ & $\cdots \cdots$ & $\cdots \cdots$ & $\mid \begin{array}{c}\cdots \\
\cdots \cdots\end{array}$ & $\cdots$ & $\cdots$ & \\
\hline$\cdots$ & $\ldots$. & $\ldots \ldots \ldots$ & $\ldots$ & $\cdot 1 n^{3} \cdot$ & $\cdots$ & $\ldots \ldots$ & \\
\hline $\begin{array}{l}\text { Dedipoda LTr. } 1 . \\
\text { melanosticta Charp. }\end{array}$ & $\cdots \cdots$ & $\cdots \cdots \cdots$ & $\cdots \cdots$ & 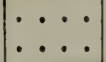 & $\cdots$ & $\because \cdots$ & \\
\hline b Locustina Burm. & $\cdots$ & $\cdot \cdot$ & $\cdots \cdot \mid$ & $\cdots \cdots$ & $\cdots$ & \multicolumn{2}{|c|}{$\ldots(35: 145$} \\
\hline \multicolumn{2}{|c|}{ Phaneroptera LTR. $1 \ldots$} & $\cdots$ & $\cdots \cdots$ & & $\cdots$ & - & \\
\hline Germari M̄è. . . . . & $\cdots \cdot \cdot$ & $\ldots \ldots$ & $\cdots$ & $\cdot n^{5} \cdot \cdot$ & $\cdots$ & $\ldots \ldots$ & \\
\hline 2. & $\cdots \cdot \cdot$ & $\cdots \cdots$ & $\cdots \cdots$ & $\cdots \cdot$ & $\cdots$ & $\cdots \cdots$ & \\
\hline$\cdots$ & $\cdots \cdot \cdot$ & $\cdots \cdots \cdot$ & $\cdots \cdots$ & $\cdots \cdot$ & $\cdots$ & . U. . & \\
\hline$\cdots$ & $\cdots \cdot \cdot$ & $\cdots \cdot \cdot$ & $\cdots \cdots$ & $\cdots \cdot$ & $\cdots$ & $\cdots \mathbf{v} \cdots$ & \\
\hline v. 2. & $\cdots$ & $\cdots \cdots$ & $\cdots \cdots$ & $\left|\cdot \dot{n}^{5} \cdot\right|$ & $\cdots$ & $\cdots$ & \\
\hline Mü Mü. $\ldots$ & $\cdots \cdots$ & $\cdots \cdots \cdots$ & $\cdots \cdots$ & $\mid \begin{array}{ll}n^{3} \\
n^{5} \cdots\end{array}$ & $\cdots$ & $\cdots \cdots$ & \\
\hline \multicolumn{2}{|c|}{ Achetina (Gryllodea Burm). . . } & & $\ldots$ & $\ldots$ & . . & \multicolumn{2}{|c|}{$\ldots \ldots(5: 50$} \\
\hline & P. 6 & - & $\cdots$ & 1. & $\cdots$ & $\cdots$ & \\
\hline & & & & & & & \\
\hline & & & & $\dot{0}$ & & $\therefore$ u. & \\
\hline ) SkrR. & . $\ldots$ & $\cdots \cdots$ & $\cdots$ & $\ldots$ & $\cdots$ & . . ש... & \\
\hline$\cdot 1$ & & $\cdots \cdot \cdot$ & $\cdots$ & $\ldots \cdot$ & $\cdots$ & . . U. . & \\
\hline & & $\cdots$ & $\cdots$ & $\cdots \cdot$ & $\cdots$ & $\ldots \mathbf{U}, \ldots$ & \\
\hline$\cdot 2$ & $\therefore$ & $\therefore$ & $\cdots$ & $\cdots \cdots$ & $\cdots$ & $\cdots v \cdots$ & \\
\hline 2 & $\therefore$ & $\therefore$ & $\ldots$ & $\because$ & $\because \cdots$ & $\cdots$ & \\
\hline & & & $\ldots$ & $\ldots$ & $\ldots$ & $\ldots \mathbf{U} . .$. & \\
\hline $\mathbf{x y a}$ & $\cdots \cdot \cdot$ & . & $\cdots$ & $\ldots$ & $\ldots$ & & \\
\hline$s p . \mathrm{X}$ & & & & $\ldots$ & $\cdots \cdot$ & $\cdot$ & \\
\hline I Pseudoperlidae Pict. & •• & . & $0^{\circ}$ & $\cdots$ & $\cdots$ & $\ldots \ldots$ & \\
\hline Paeu & - & & . & & •・ & & \\
\hline sp. PB. : - & & & & & & & \\
\hline Orthopter & & 0500 & $1 \begin{array}{llll}0 & 0 & 0 & 0\end{array}$ & 3 & & 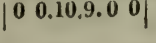 & \\
\hline
\end{tabular}

* Quod jam de Hemipterorum numero $p .605$ observavimus, id quoque ad Orthoptern referendum est. Specierum cognitarum numerus 1000 corte excedit. 


\begin{tabular}{|c|c|c|c|c|c|c|c|}
\hline & Woltgegend. & KolilenP. & SalzP. & OolithP. & $\begin{array}{c}\text { Krei- } \\
\text { deP. }\end{array}$ & MolasseP. & Neu \\
\hline Benenuนทัตеม. & 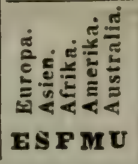 & 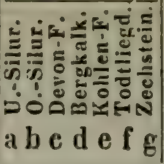 & 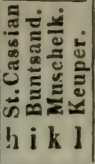 & 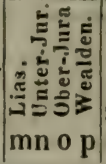 & 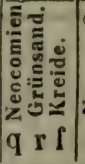 & 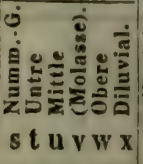 & 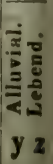 \\
\hline
\end{tabular}

IX. NEUROPTRRA L. Netzflügler.

1. corrodentia Burm.

(Planipennium LTR. pars)

a Termitida Ltr.

Termes L. 6

? graudnevus Brod. pristinus Снавт.

$s p p$. PB.

spp. Serr.

Nov, gen. Ouchamofy, 1 $s p$. Очен.

b Embiidae Burm.

Embia Ltr. 1. . . sp. PB.

e ConiopterygidaeBurm. d Psocidae Stephs.

Poveus LтR. $6+$. t spp. PB.

† spp. GRavu.

2. subulicornia Ltr.

a Ephemeridae Sтернs.

Daëtis LeAch 1... $s p$. PB.

Palingenia Buni, sp. PB. ......

Ephemera (L.) $3+$ sp. Brod.

sp. BRoD, . . .

Potamanthes PB. 1 sp. Р'B.

b Libellulina STEPHs.

Arion (Frr.) Burm. 5 ( 2$)$

Buckmani Brod. . sp. Снав.

|




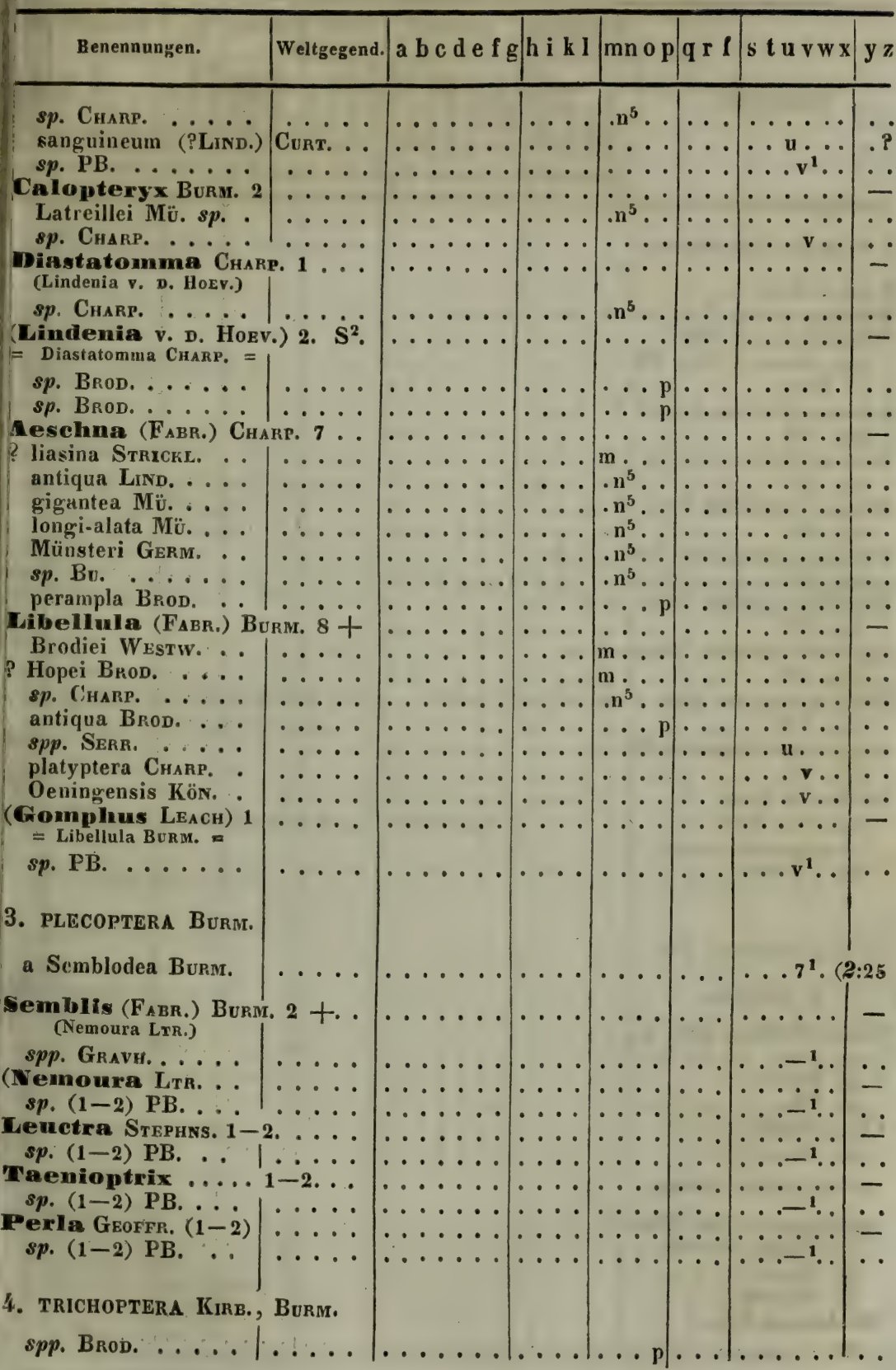




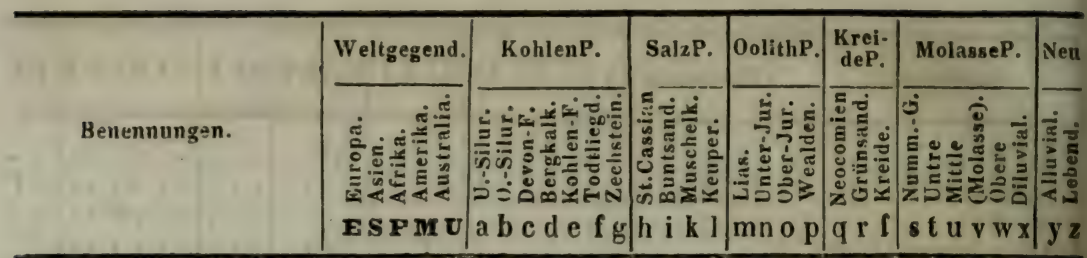

a Phryganeidae Stepus.

‡ sp. 1 BroD.

Phryganea $\left(\dot{L}_{0}\right) \dot{L}_{\mathrm{T}} \dot{4}_{\dot{4}} \dot{+}$ Mombachana Hös. . . . . . . . spp. Gravh., Eв., PB.

sp. Веск …..............

Limnophilus LEAch $2 .+\ldots$ spp. $\mathrm{PB}$.

Normonia Curt. 2 + spp. PB.

rhyacophila Pict. $\dot{t}$. spp. РB. ............ Polycentropus Curt. $2+$. spp. PB.

hydropsyche Pict. $2+$. spp. PB.

Aphelocheira STEPH. $2+$. spp. PB.

Fsychomyia Ltr. $2+\ldots$ spp. PB.

Amphientomum $\mathrm{PB} .+\ldots$ spp. PB.

Indusia Bosc, 1 . . tubulosa Bosc

- Leptoceridue STEPH. sp. 1 BROD.

5. PJANIPENNIA LTR.

a Sialidae.

Chaulioder LTr. 3 ? sp. ВRоD.

? sp. Brod. sp. PB.

Corydalis Ltr. 1 . sp. Murch.

b Panorpidae SтEPH.

Drthophlebia Wrstw. 3. . communis Westw. $s p$. BROD. sp. Westw.

Eittacus LTR, $i$. $s p$.
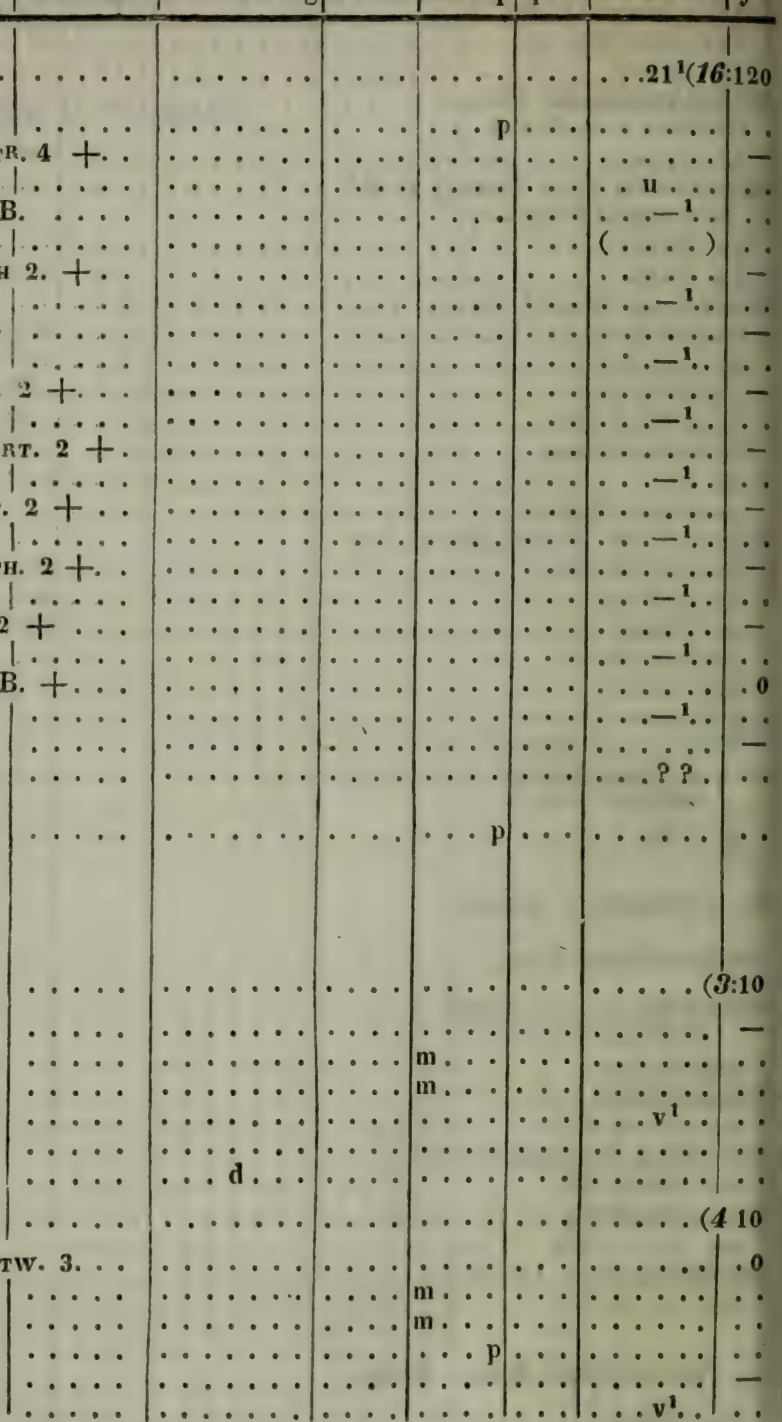


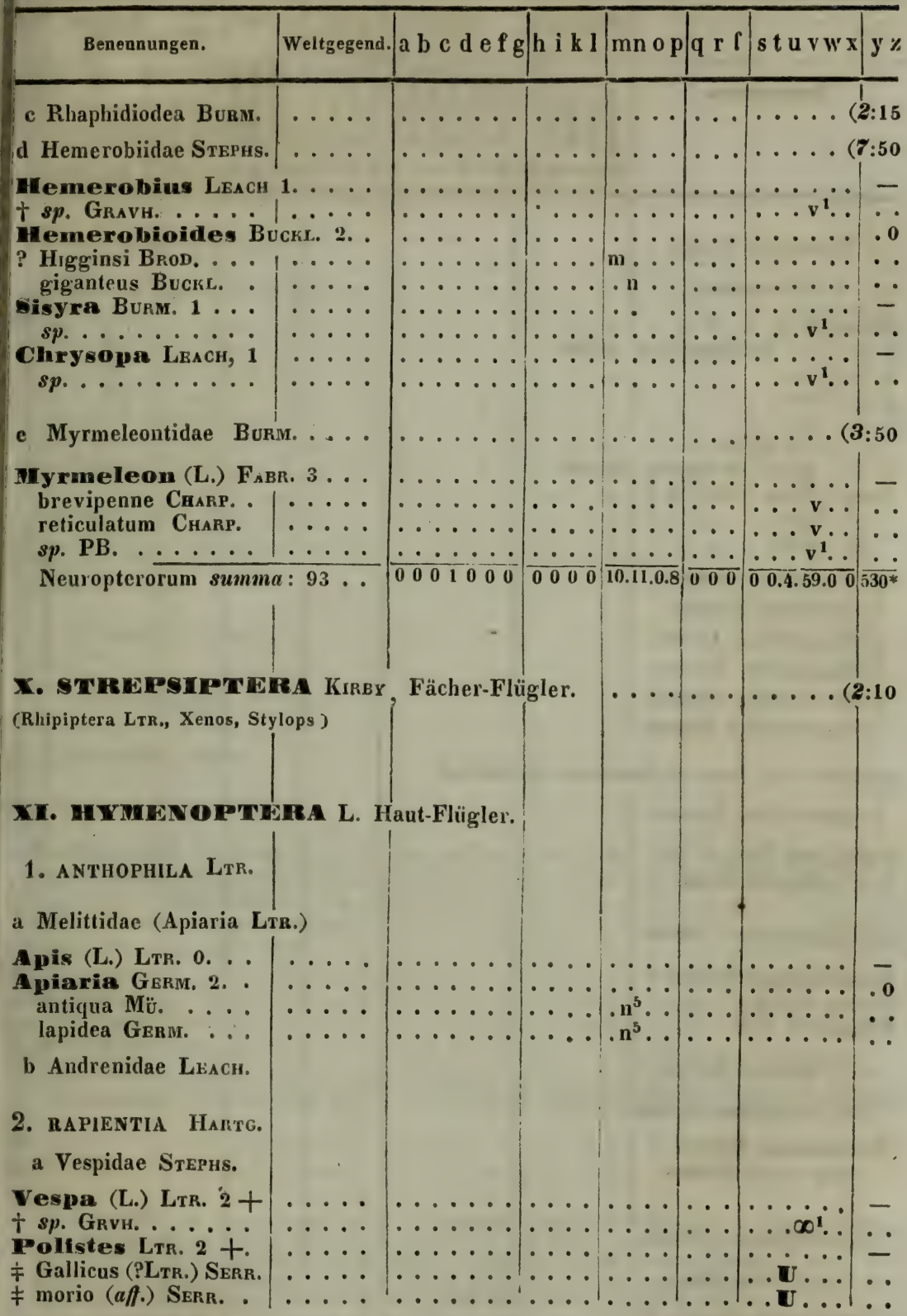

a Melittidae (Apiaria Ltr.)

A pis (L.) Ltr. 0. . antiqua $M \ddot{\text {. }}$ lapidea Gerir.

Andrenidae Leach.

rapientia Harto.

Vespidae STEPHs,

Vespa (L.) Ltr. 2 + Polfstes Ltr. 2 + † Gallicus (?LTr.) SERr. ‡ morio (aff.) SerR.

De hac specierum viventium summa idem, quod ad Hemiptera et Orthoptera pp. 605 et 609 , observandum est. 


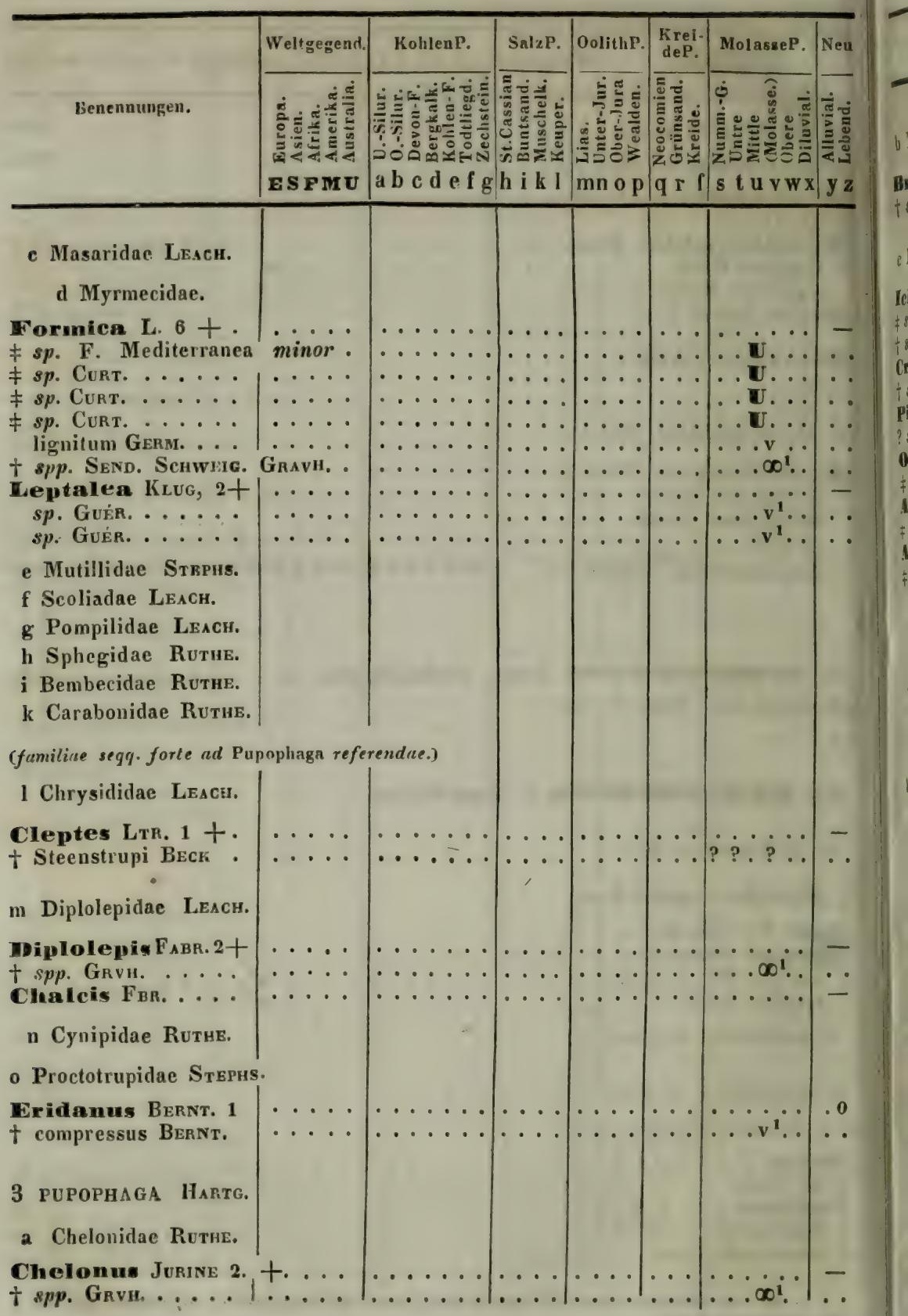




\begin{tabular}{l|l|l|l|l|l|l|l|}
\hline Benennungen. & Weltgegend. a b c d e f g h i k I & mn o p & q r l & s t u v w x & y z \\
\hline
\end{tabular}

b Braconidae Rutus.

Eracon FABR. 2 † † spp. Gravh.

c Ichneumonidae Leach

Tehneumon (L.) Grvi, 3 十.

$\neq s p$. Serr. . . . . .

† spp. Gravh. . . .

Cryptum Fabr. 2 + † spp. Grvi. ..... Pimpla FBr. 1. . ? $s p$. Cunt.

Dphion FaBr. 1. . $\neq s p$. Serr. ..... Agathis LTR. 1... $\neq s p$. Serr. ....... Anomalon Jupine, 1 † sp. SErr.

d Evaniadae Lesch.

4. rhytophaga Hartg.

\section{a Sirecidae SснӥғF.}

b Tenthredinidae LEACH

Tenthredo L. 6 +

‡sp. (Selandria) Curt.

$\neq s p$. (T. viridis magn.

$\neq s p$. (T. viridi major) SERR. . .

† sp. (T. ro sae aff.) Serr. . . .

† spp. Grave. ....

Hylotoma LTr. 1 .

cineracea Charp. . .

Pteronus Jur. 1. .

† sp. mediocris SERR.

Hymenopterorum summa $65^{*}$. $|0000000| 0000|0200| 000|00.13 .50 .00| 5000$

* Numeri omnes incerti sunt. Hymenopterorum individus succino inclusa tot esse, yuot Neuropterorum usserit BERENDT (Bernst. 1, 53), unde numerus specierum fere aequalis esse videtur. Neuroplerorum nutem species habet. 48. Certe tamen species 50 inter plurn genera, quam quae GRAVENHORST indicuvernt, distribuendue erunt. Species viventes non mimus numerose, quim Dipterorum esse possunt, unde 5000 a veritute adhuc multum recederent. 


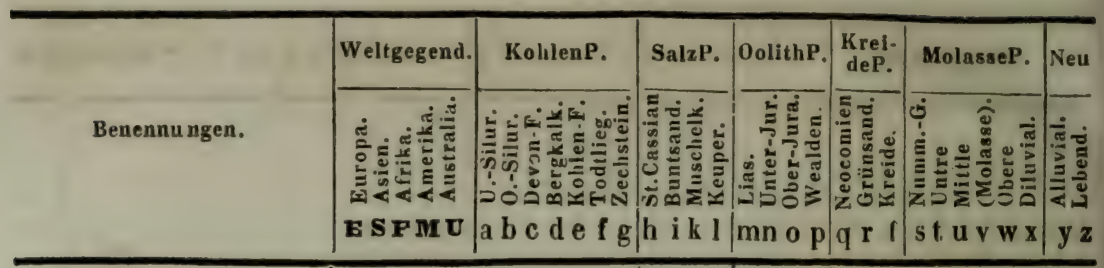

XII. COL EOPTERA L., Käfer. (secund. syst. LATREILlEx in Cuv. regne
A. TRIMERA LTR.
a Fungicolae Lrr.

Lycoperdina ILTR. 1 $s p$. Bernt.

b Coccinellina LrR. ‡sp. Brod. ...... † spp. 2 gen. indet. Coccinella (L.) 9 . Wittsi Brod. .... ? protogaeae GerM. . Andromeda HeER . Hesione Heer ... † spp. 5 BeRnt. ... Seymnus Kuglv. 1. † sp. Bernt.....

e Pselaphidae Herbst. Pselaphus Herest 4 † spp. 4 BERNT. ... Bryaxis Kugla. 1 . $\dagger s p$. Bernt.

Euplectus LEACH, 2. † spp. 2 Bernt. ...

\section{B. TETRAMERA LTR.}

1. CYCLICA † Ltr. a Phalacridae Leach (Clavipalpi LTf.) Phalacrus PAYk. 5 † spp. 5 BERNT. ... b. Chrysomelidae Lfach $s p$. BroD. ? $s p$. BROD

? sp. BroD. † spp. 3 BROD.

† spp. 8 gen. indet. BerNT.
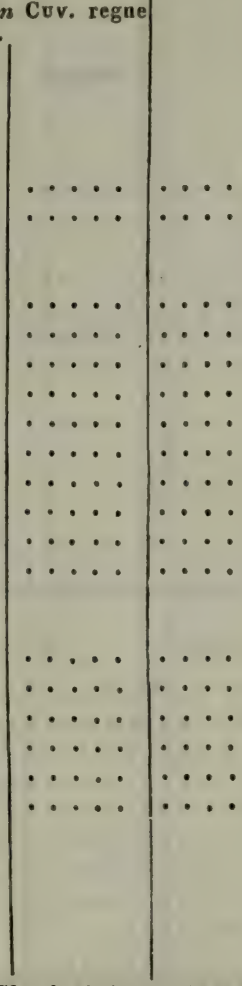


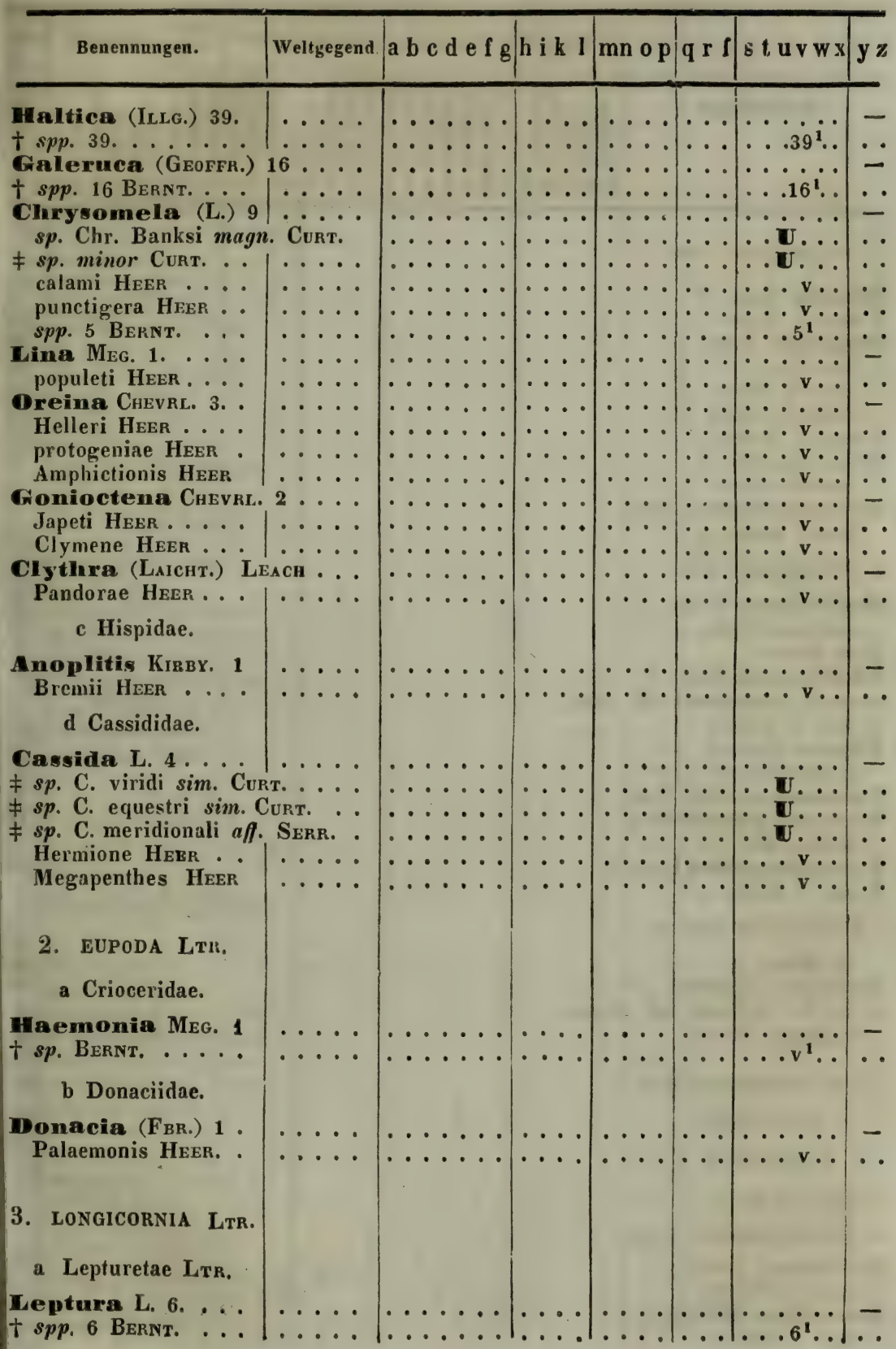




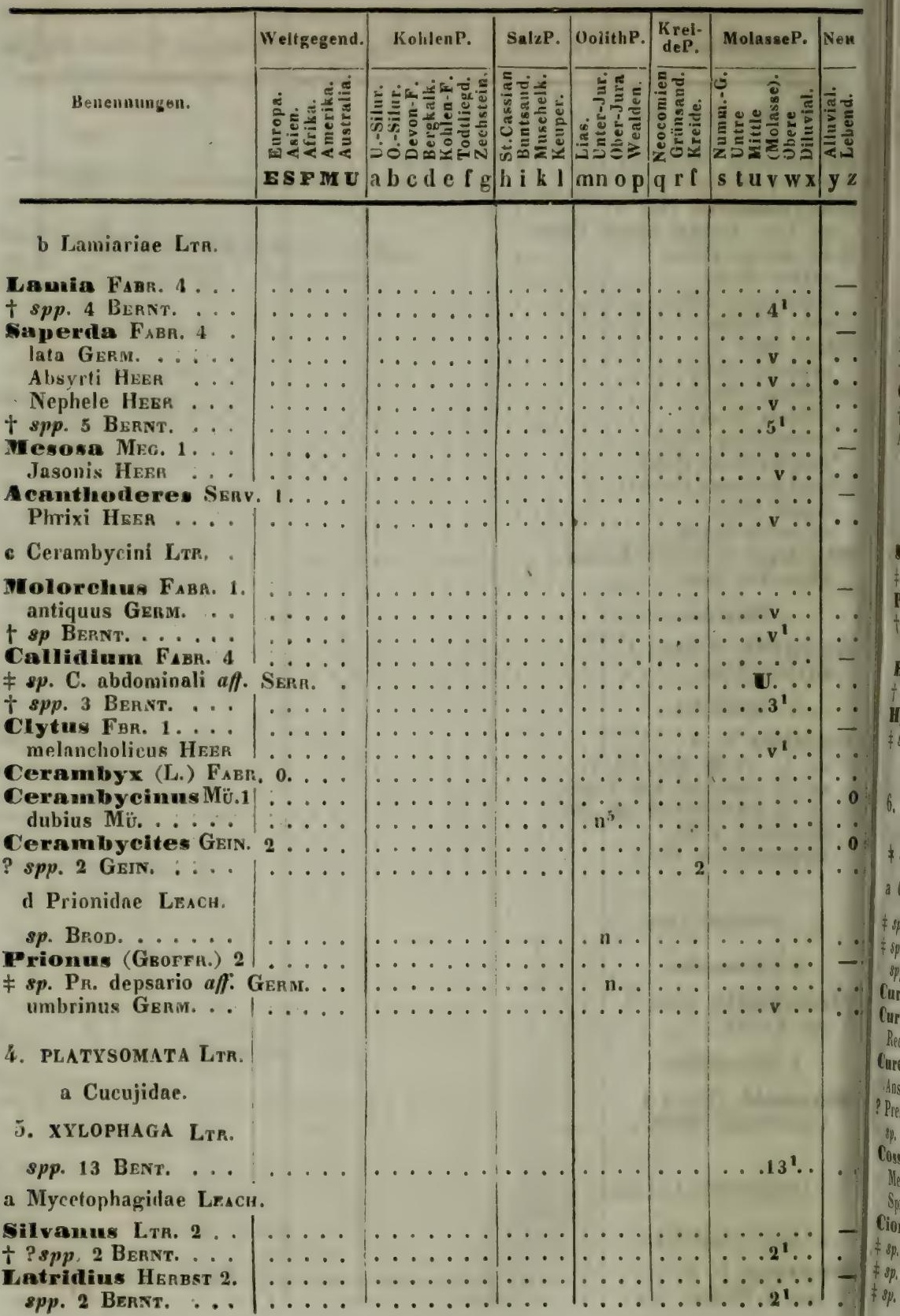




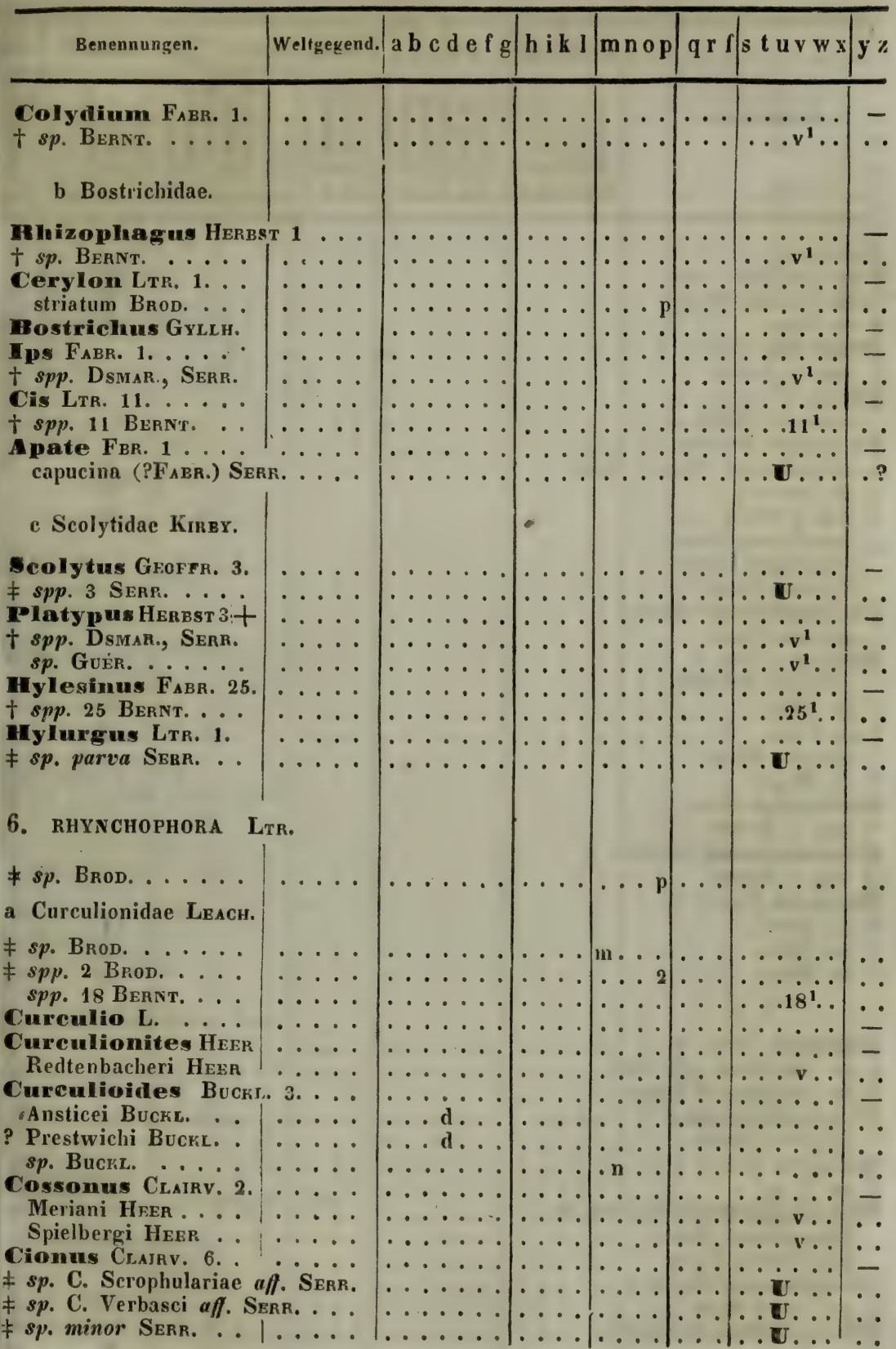




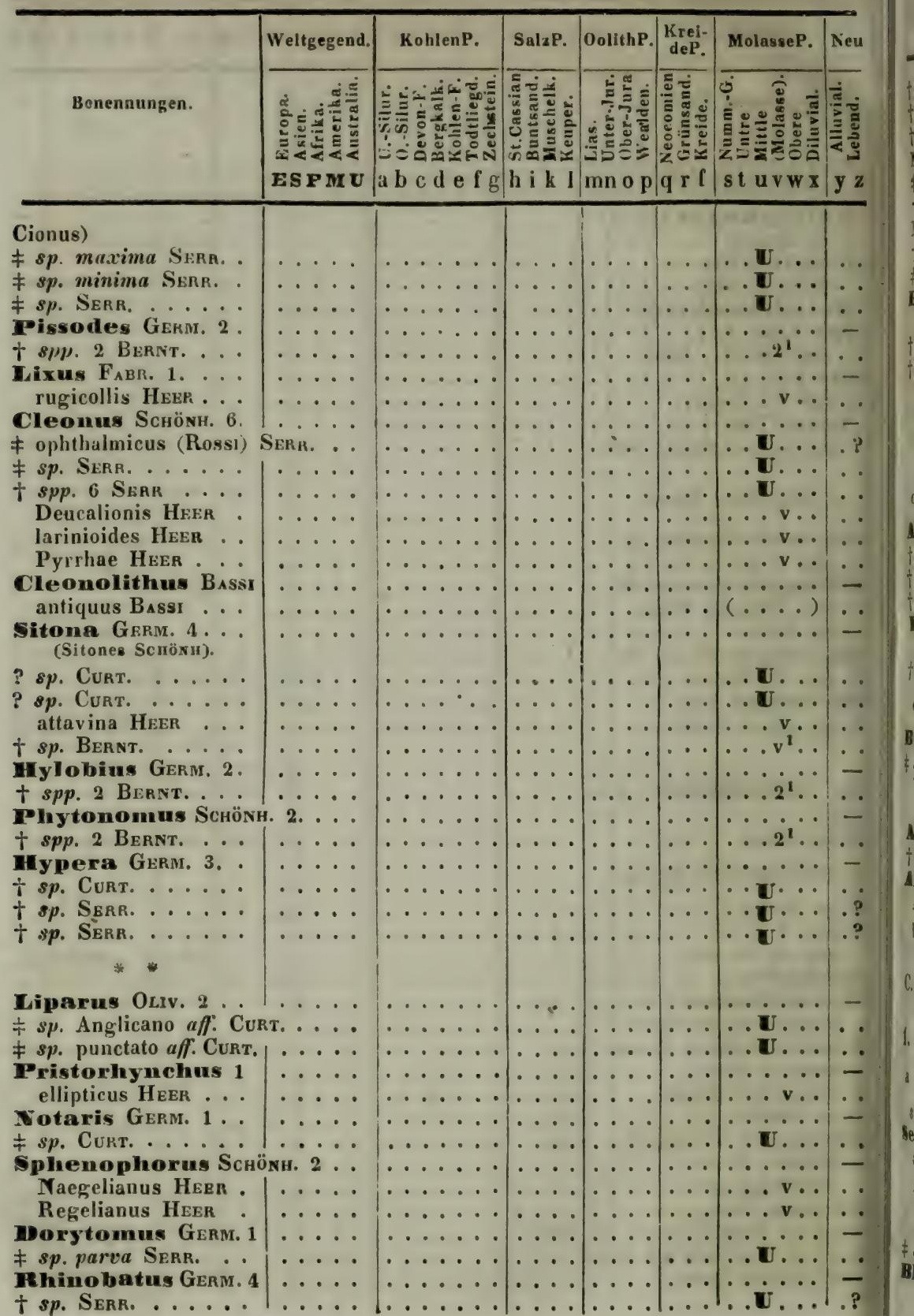




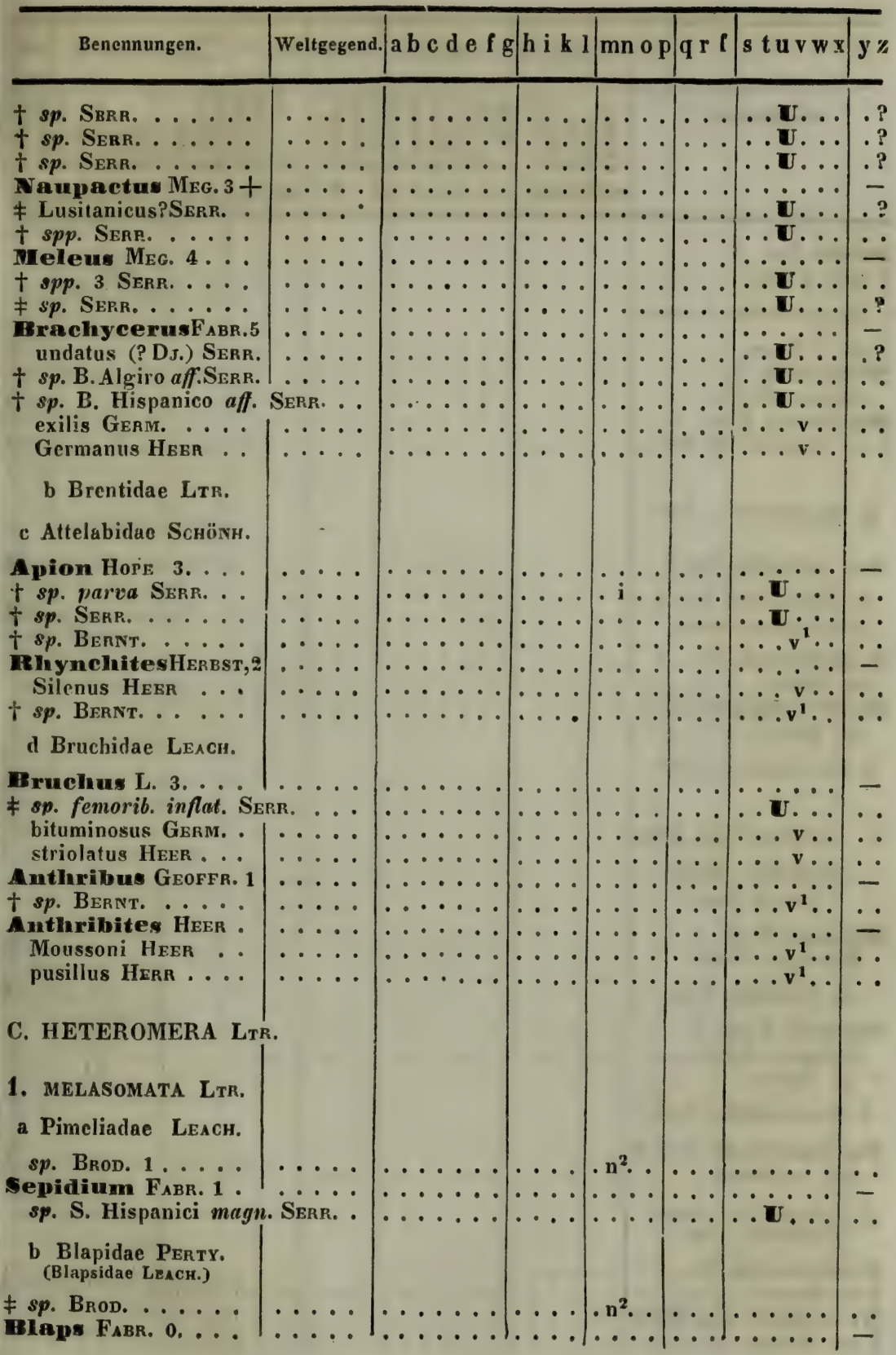




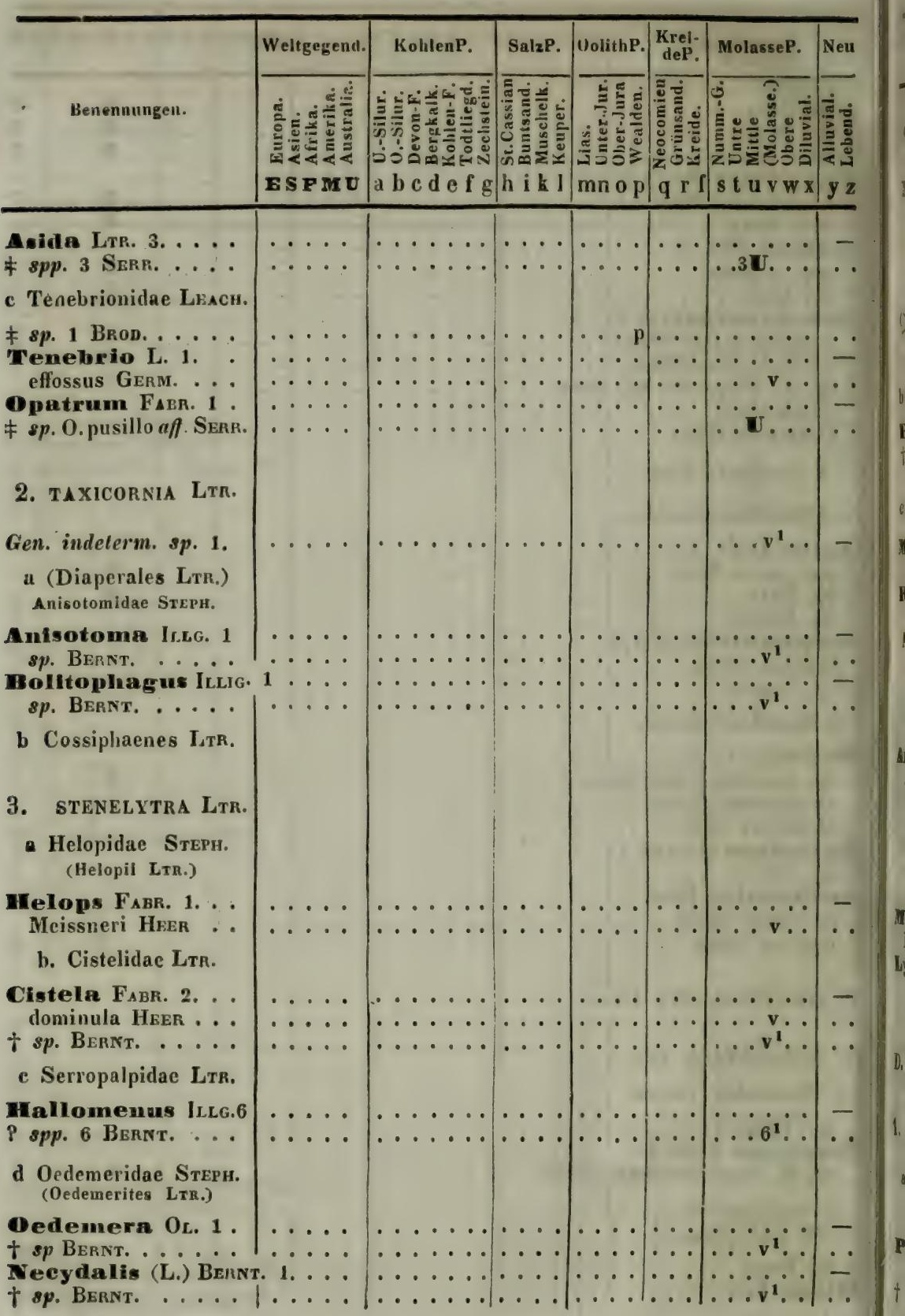




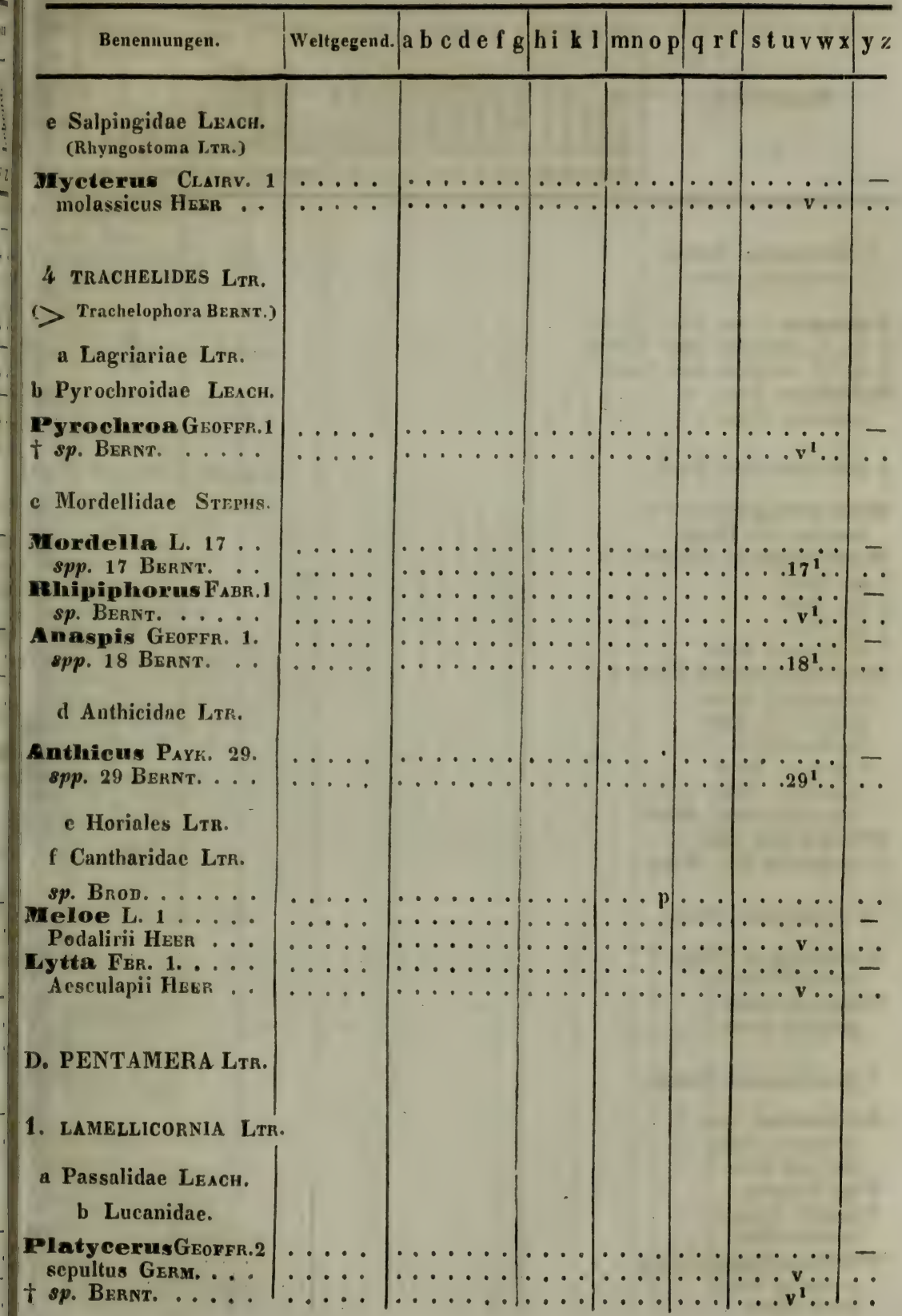




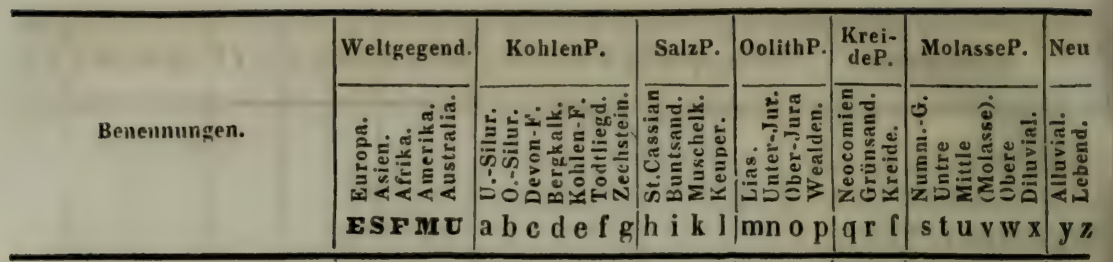

e Cetoniadae Kirby. (Melitophili LTR.)

Cetonia Fabr. 2 . . \& sp. C. hirtellae sim. C.... $\neq s p$. C. strictae sim. Curt. ...

Trichius FABR. 1. amoenus HeER . .

d Melolonthidae MacL.

Rhizotrogus LTR. 1

longimanus HeER.

Melolontha (Grorfr)

Brop.

? $s p$. BROD.

$+s p$. Serr.
$+s p$. SERr.

Greithana HeER

Melolonthites HBER

aciculatus HEER

deperditus HEER

Kollari HeER . . . .

Lavateri HEER

obsoletus HEER

Parschluganus HEER

Pachypus DEJ. 1 .

† excavatus (L.) Serr.

c Dynastidae MıcL.

Geotrupes Fabr. 1 vetustus Gram. . . .

Coprologus .... i gracilis Hegr . . . .

f Scarabaeidae LEACH.

Aphodius Illg 3. . antiquus Hesr... Meyeri Heer . . . .

$\neq s p$. LANDGR.

Copris Geoffr. 1. lunaris FBr. ...

Dnthophagus Lir.2 ovatulus HeER

urus HEER . . . . .
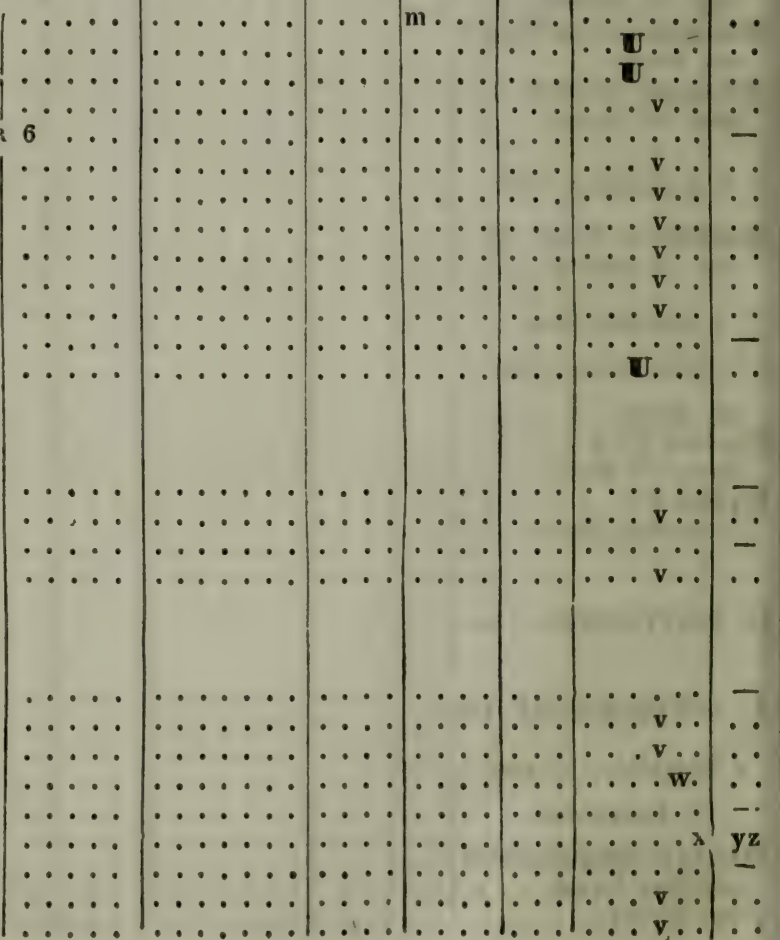


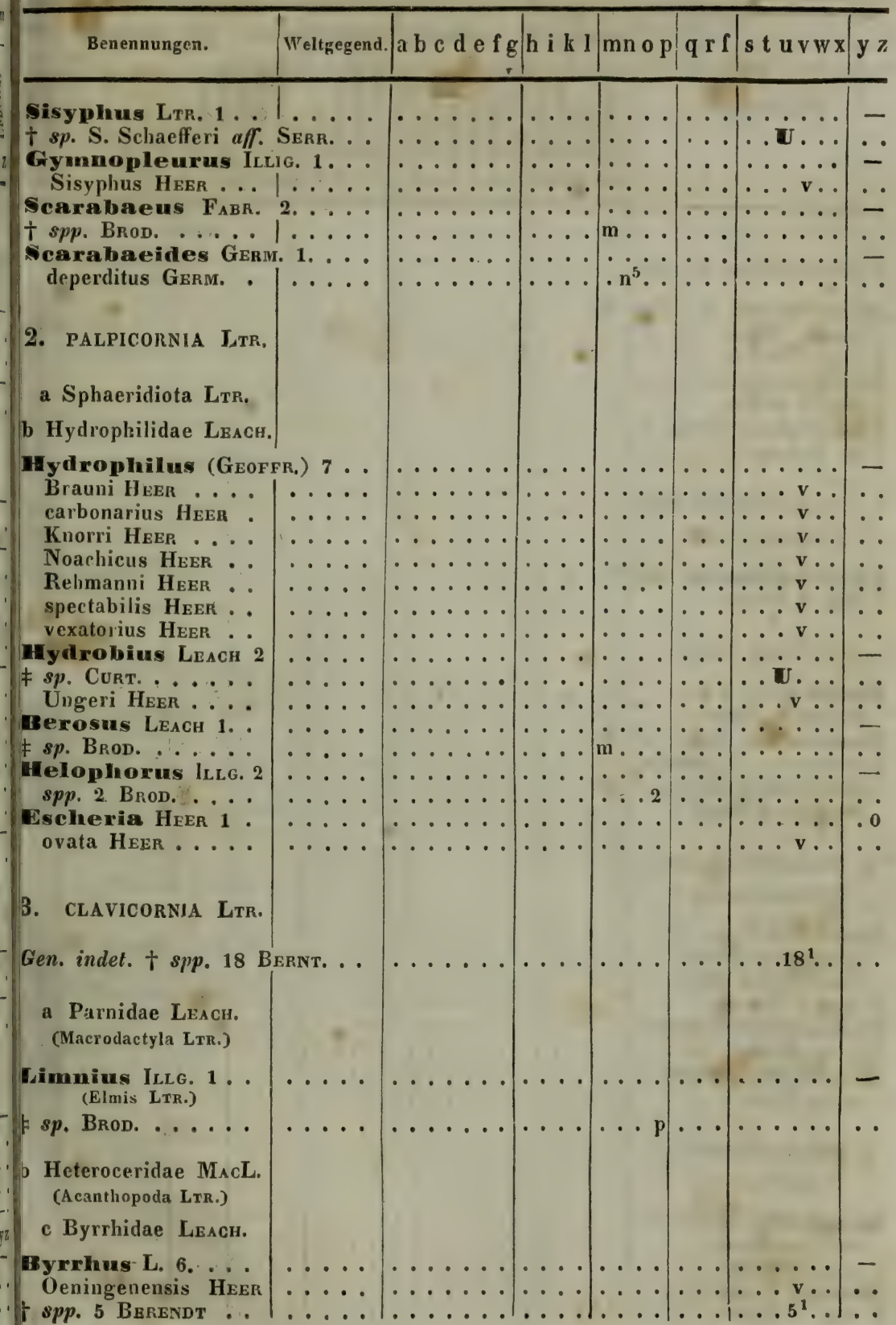




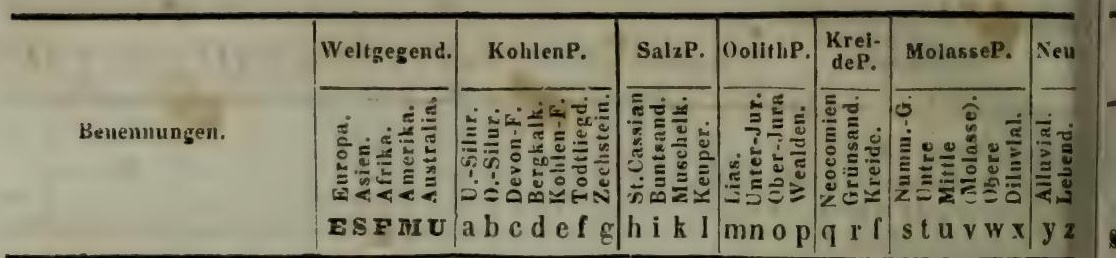

d Dermestidae Lеach.

Dermestes (L.) FBR. 4. pauper HeER .......... $\dagger$ spp. 3 BERNT. . . . .

Anthrenus Geofer. 3 † spp. a BERNT.

Limnichus ZiegiL. 1 † sp. Bkint.

e Cryptophagidae Kıвьу.

(Engidites LTR.)

Cryptophagus Hernst, 9. † spp. 9 BERNT.

f Peltidac Kifiby.

Peltis Illg. 1 . . . . tricostata HeER . . .

Trogosita (OLv.) 3 t coerulea... SERr. tenebrioides Germ. Koellikeri HБВR .

g Nitidulidae Lеach. (Nitidulariae LTR.)

Sitidala FABR. 7. . melanaria HeER . . Radobojana Herr. † spp. 5 BenNT. ... Strongyius Herest, 1 ? + sp. BERNT.

Amphotis ERichs. 1 bella HeER. . . . . .

h Scaphidiidac HeER. (Scaphidites LTR.)

Seaphidium (OL.) 3 deletum HeEr.... $\dagger s p p .2$ BenNT. . . . Catops PАYK. 3. . t spp. 3 BERNT.
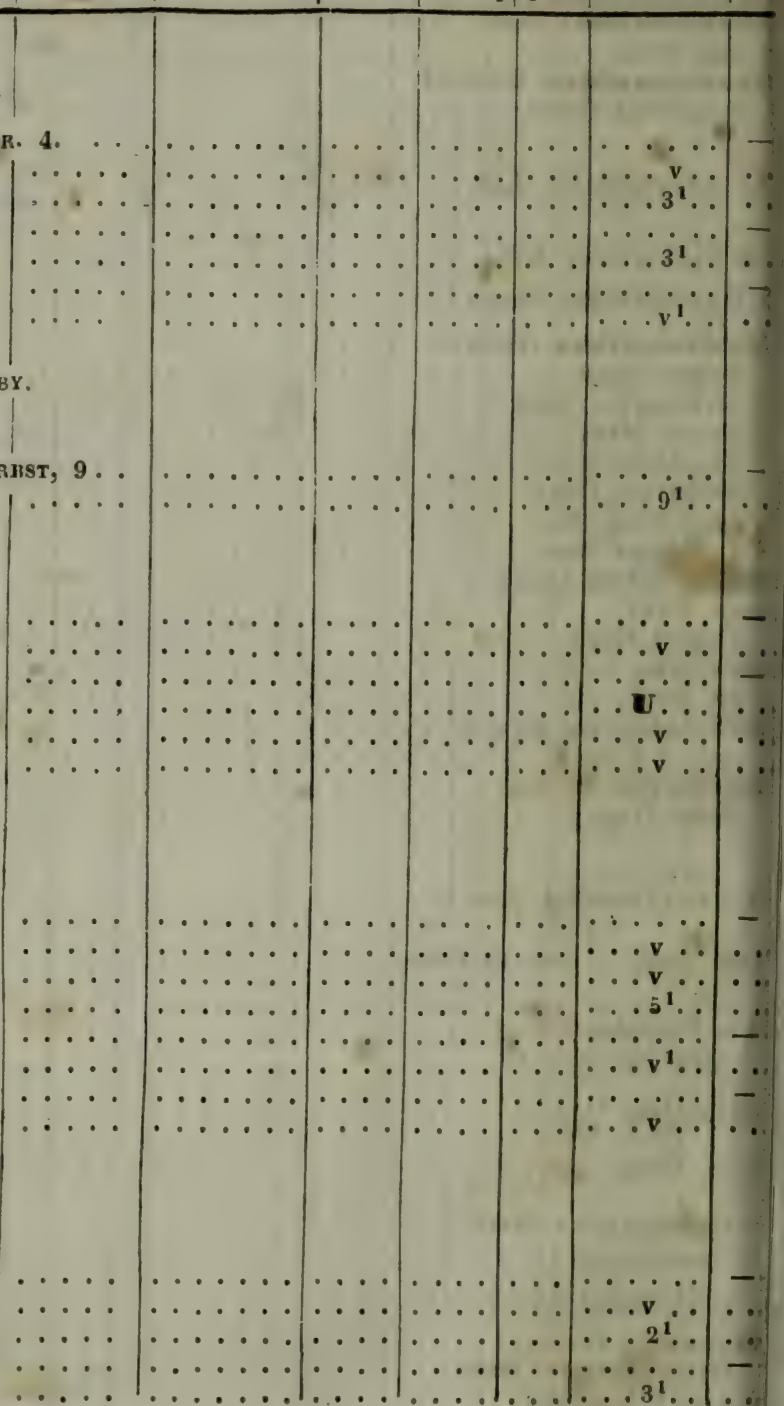


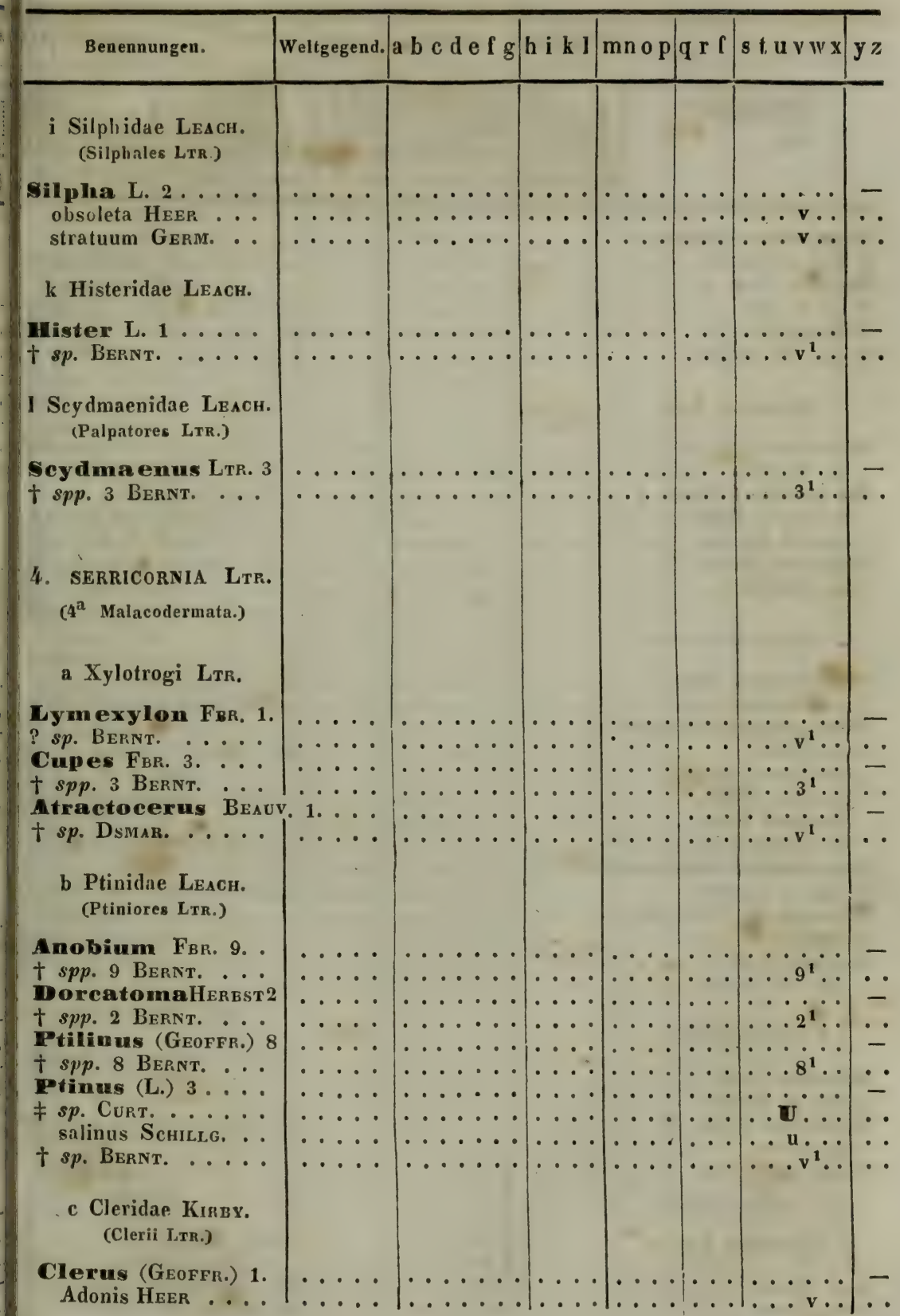




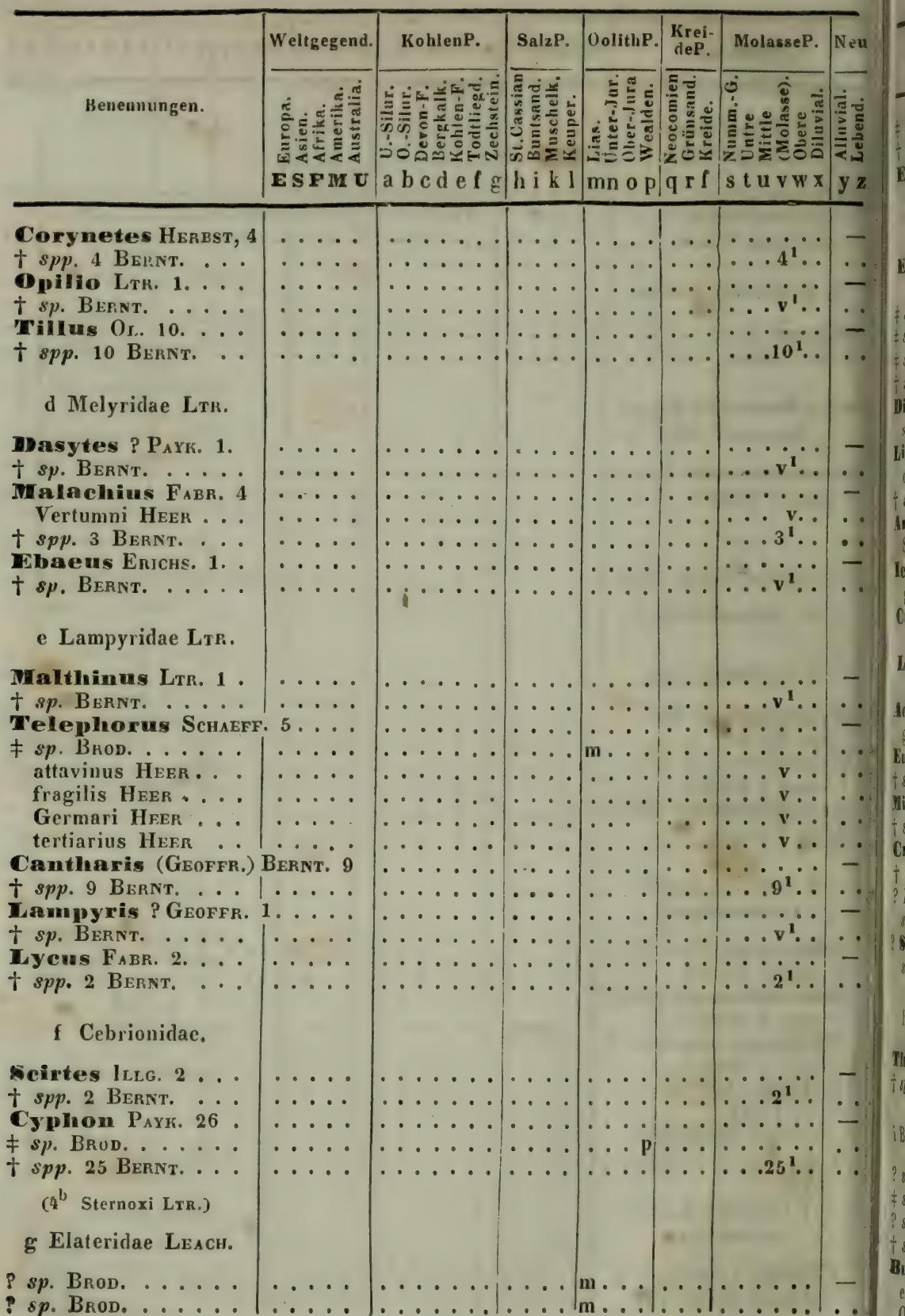




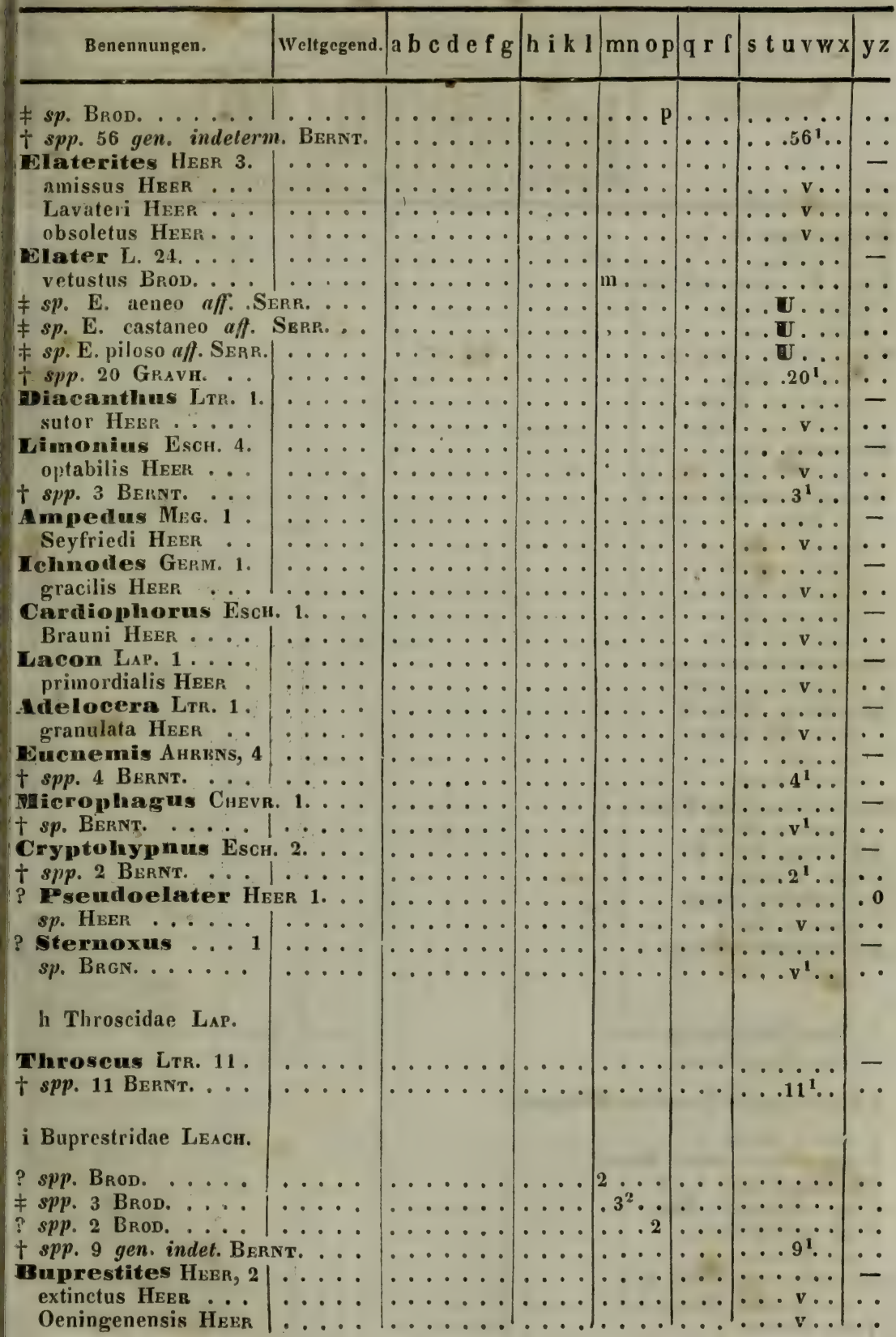




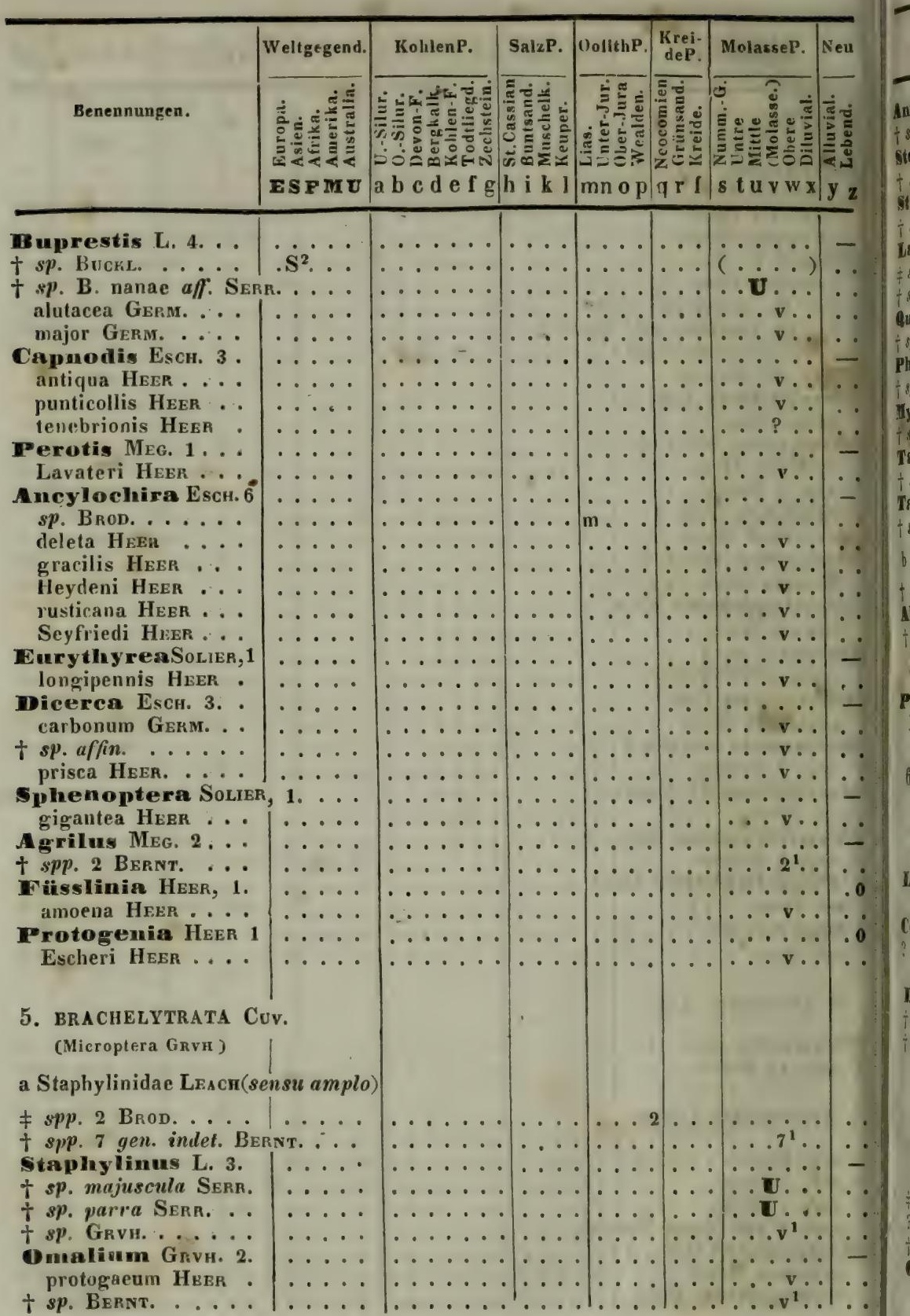




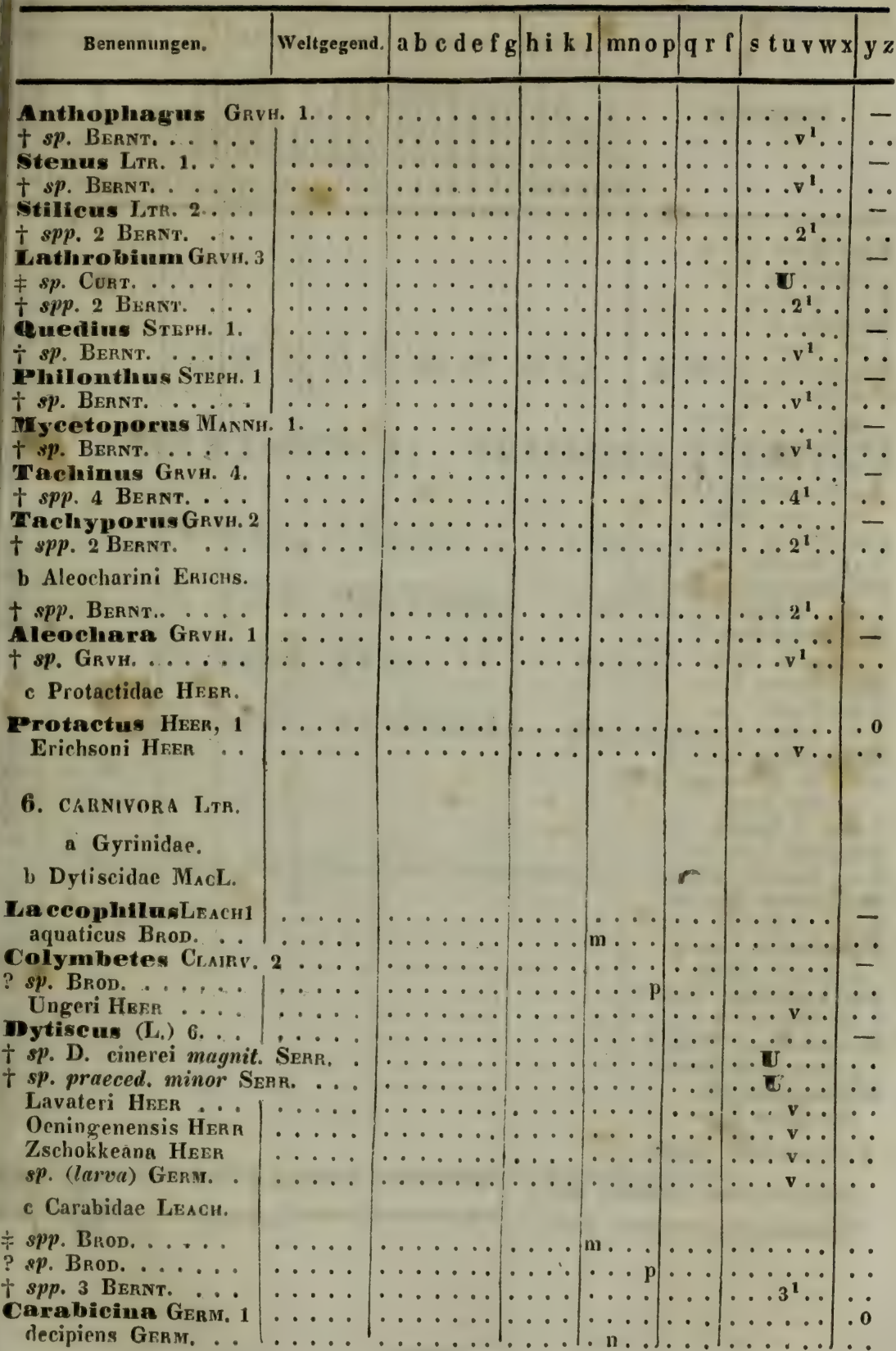




\begin{tabular}{|c|c|c|c|c|c|c|c|}
\hline & Weltgegent. & KohlenP. & SalzP. & OolithP. & $\begin{array}{c}\text { Krei- } \\
\text { deP. }\end{array}$ & MolasseP. & Neu \\
\hline Benennunge». & 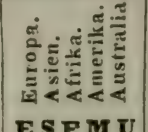 & 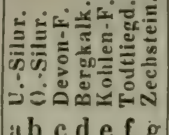 & 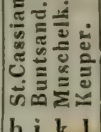 & 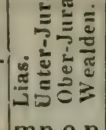 & 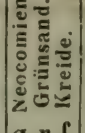 & 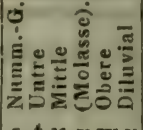 & 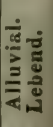 \\
\hline & ESF M U & $|a b c d e f g|$ & h i k l & mn op & 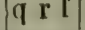 & $\mid s$ t u v w $x$ & У $z$ \\
\hline
\end{tabular}

Carabus (L.) 2. . . elongatus Brod.

$\dagger s p$. Gravh. .....

Nebria Ltr. 1 ...

$+s p$. Berint.

Hadister Clairv. 2. debilis HEEP prodromus HEEn

ChIaenius Bon. 1 . $\dagger s p$. BERNT. . . . orphanus HeEr... Calathus Bon. 1. . † sp. Bernt. .....

Pterostichus Bon. 2 † $s p p .2$ BERNT.

Argutor MEg. 1. . antiquus Heer... Dphonus Ziegl. 2. $\neq s p$. Curt. ? sp. LYELL

Harpalus LTR. 6 .

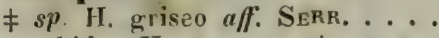
tabidus Heer . . . . $\dagger s p p .4$ BERNT.

Clivina Ltr. 9 . . t $s p$ BERNT.

Dromius Bon. 9.. † spp. 9 Bertit. . .

Cymindis LTR. 1. pulchella HeER.

Polystichus Bon, 1 † sp. BERNT.

Grachinus WEB, 1 primordialis HeER .

Genopterus ... 1 laevigatus HEER

d Cicindelidae Leach.

Coleopterorum summa: 847 .

Hexapodorum summa: 1551.

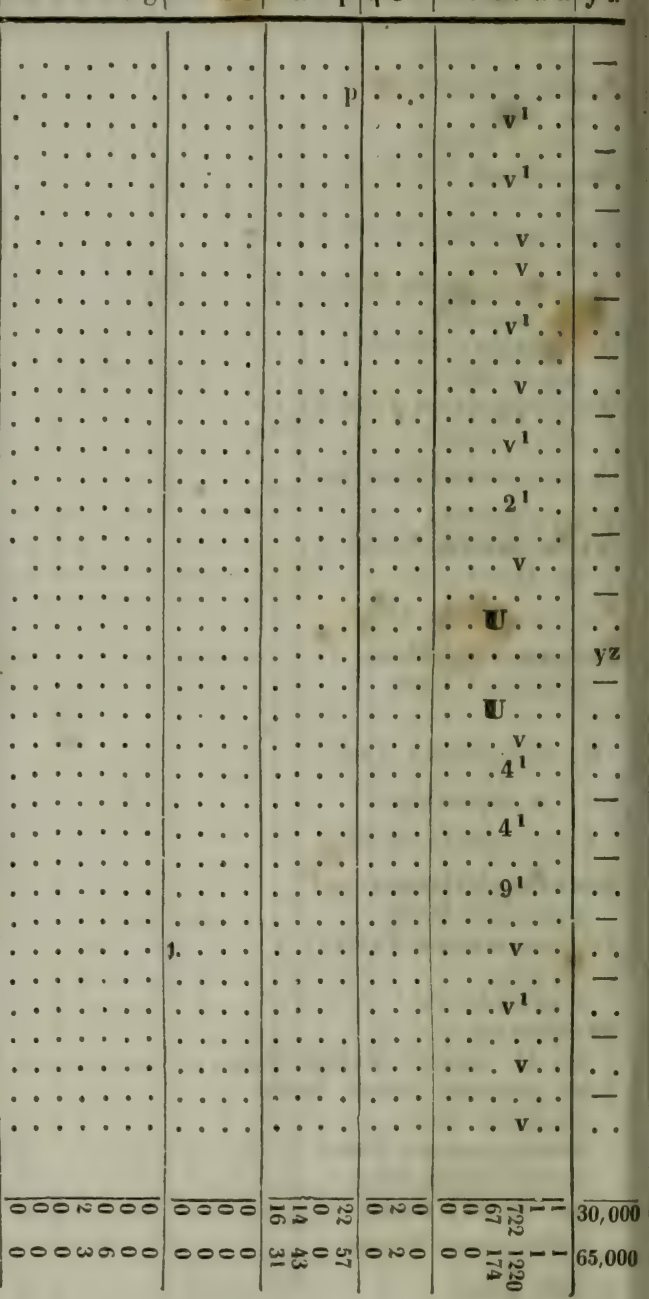




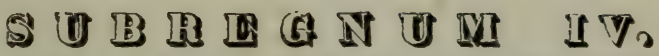

\section{SPONID YLOZOA : WIRBELTHIERE.}

CI. XXI. PISCES : Fische.

Cl. XXII. REP'TILIA : Lurche.

Cl. XXIII. AVES : Vögel.

CI. XXIV. MAMMALIA : Säugthiere *

* Harum clussium trium $(X X I I-X X I V)$ conspectus auctor est H. V. MRYKR. 



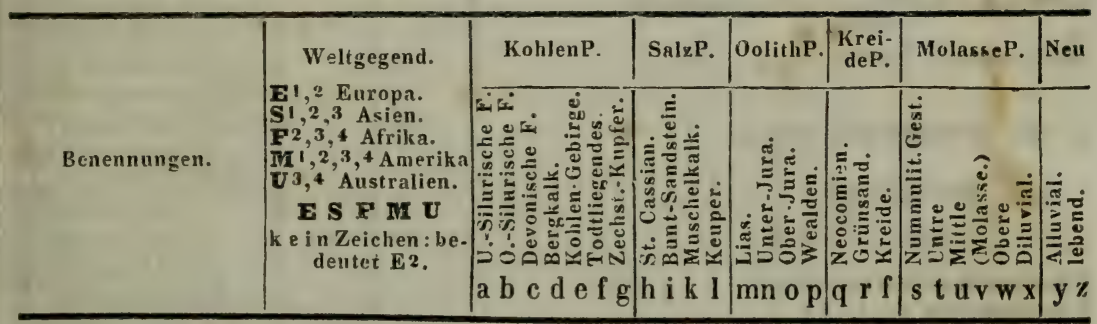

\section{Cl. XXI. PISCES : Fische.}

I. I EPTACA DATI MüLL. (Placoides AG. pars. 1. , Cartilaginei).

a Amphioxidae.

AmphioxusYarReLL

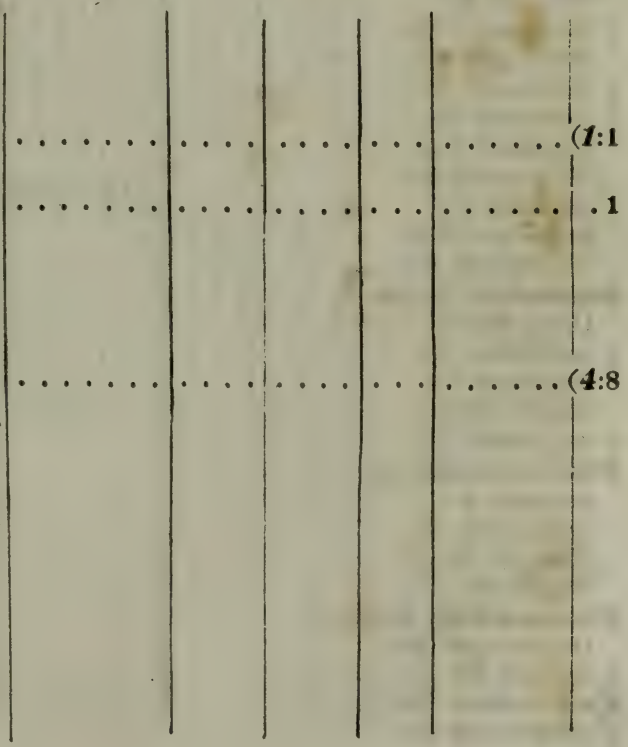

A. HYPEROTRETI MÜLL.

a Myxioidae Mürs.

B. HYPEROARTII MüLL.

a Petromyzidae MüLL. 


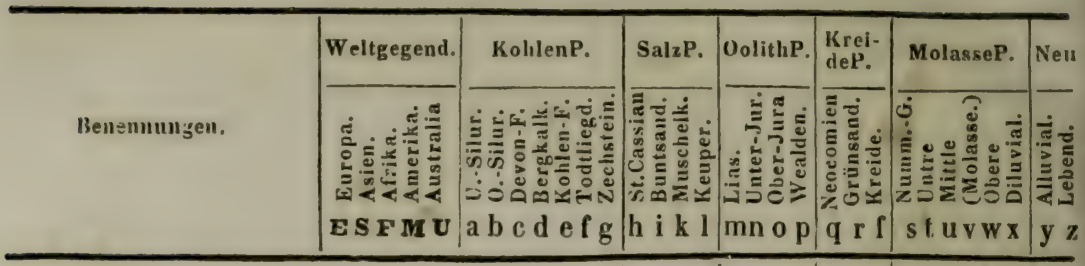

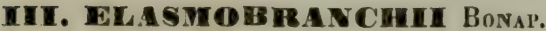

(Selachii Arist.; Placoides pars 3. AG. Cartiluginei dentibus, aculeis et squam is nsseis)

(Generu omnir enumernutur.)

A. HOLOCEPHALI Mǘt.

a Chimerilae Ag.

* Denles.

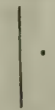

Callorhymchus Gron.

Chimaera (L.), Ag. | . .

(Ischyodon, Ganodus Eg.,Psittacodon A G.)

Ischyodon EG. 12

Johnsoni Ag.....

\# emarginatus Er. . .

Tessoni EG.

$\div$ Beaumonti Eg. . . .

Bucklandi EG.

‡ Dufrenoyi EG. ...

\# Dutertrei Eg. ....

Egertoni EG.

Townsendi Eg. ... .

Agassizi Eg......

brevirostris Ag.

Helveticus EG. ....

Ganodus Egert. 5. Colei Buckr. . . .

$\neq$ curvidens Eg.

neglectus EG....

Oweni BuckL. ...

₹ rugulosus EG.

Psittacodon AG, 4 falcatus Ac. ..... psittacinus Ag. ... . Mantelli ВuскL.

Sedgwicki Á.

Elasmodus Egert,

Greenoughi AG.

‡ Hunteri $\mathbf{E}_{\mathrm{G}} . . . .$.

Psaliodus Eg. 1. .

f compressus AG.

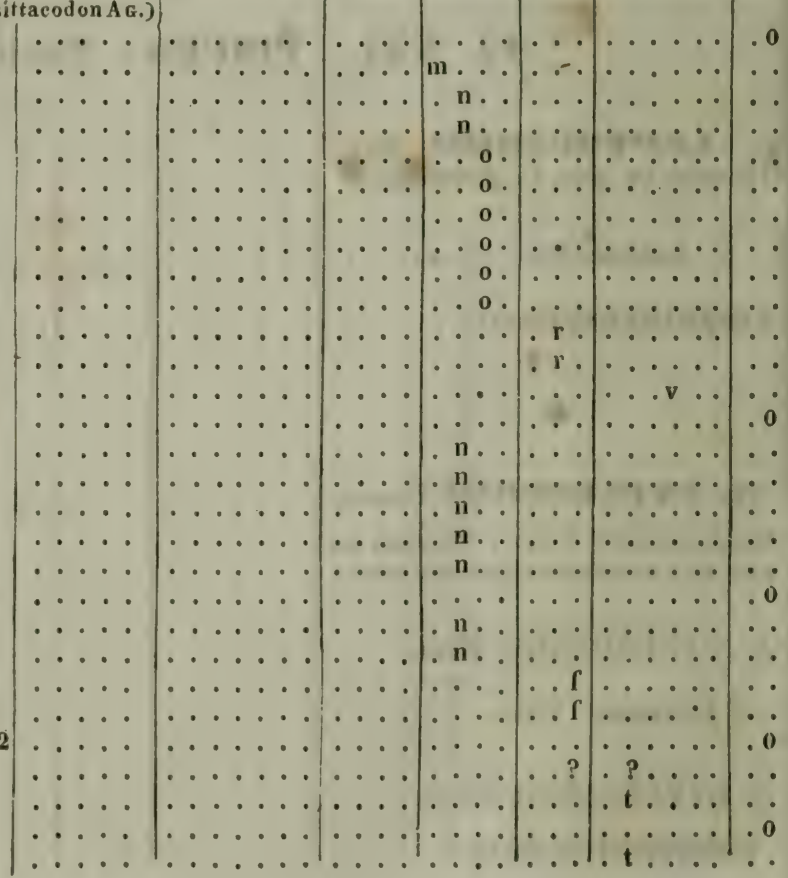




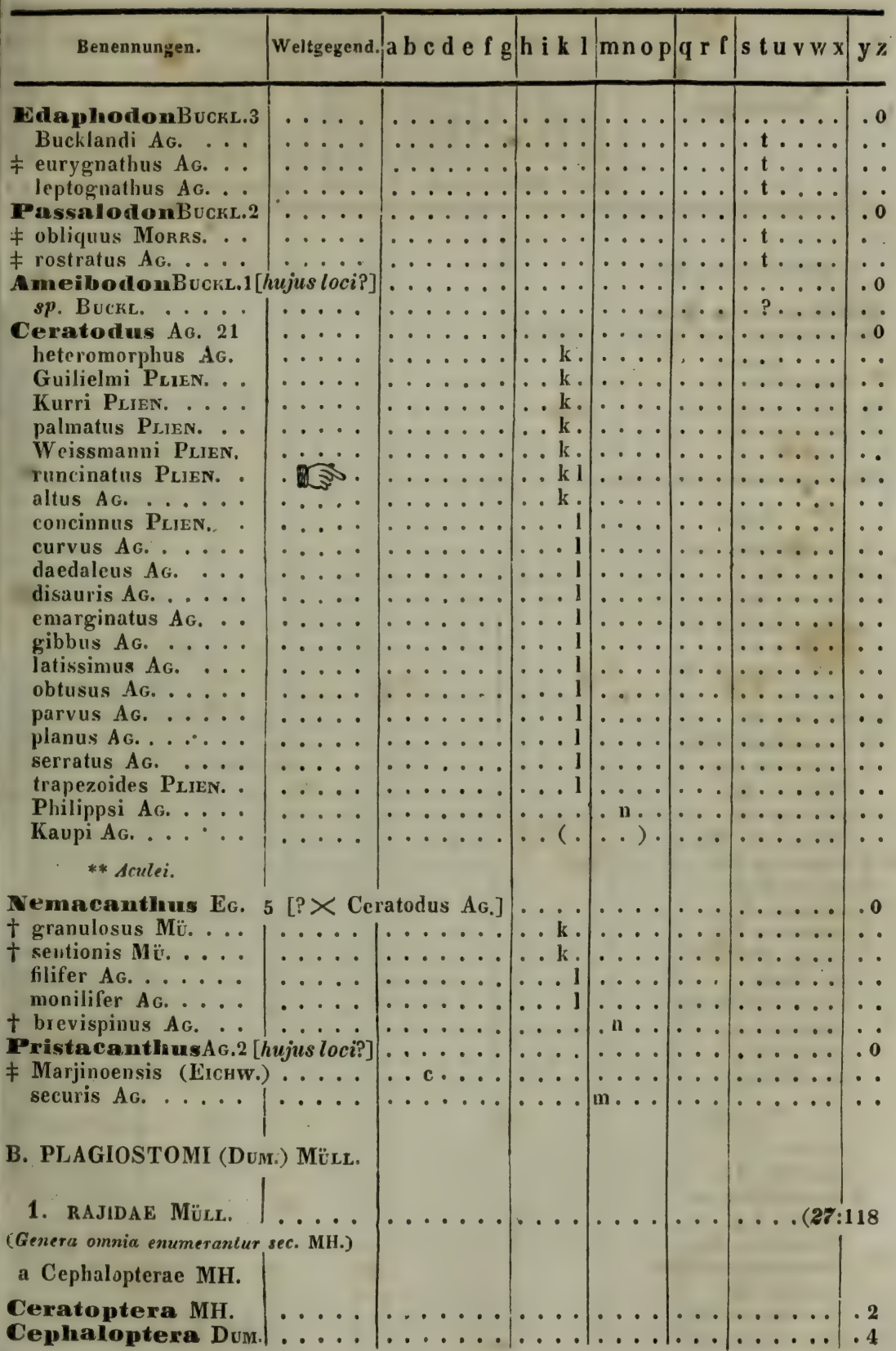




\begin{tabular}{|c|c|c|c|c|c|c|c|}
\hline & Weltgegend. & KohlenP. & SalzP. & OolithP. & $\begin{array}{l}\text { Krei- } \\
\text { deP. }\end{array}$ & MolasseP. & Neu \\
\hline Bonеиииияеn. & 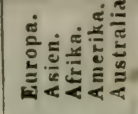 & 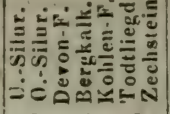 & 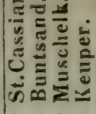 & 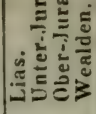 & 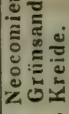 & 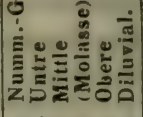 & 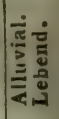 \\
\hline- & ESFIMU & $a b c d e f g$ & h i k l & mn op & $q \times 1$ & s t u vw & y z \\
\hline
\end{tabular}

\section{b Myliobatides MH.}

\section{Thimoptera Kuhr}

Aetobatis (BLv.) Ag. 4 Dentes.

irregularis AG. ...

\# subarcuatus $A$ g. . .

₹ arcuatus AG.

sulcatus Ag.

Myliobatis $(D \cdot \cdots$

\section{( $\left(\operatorname{Sin}^{3}\right)$.}

- Dentes.

₹ Bronguiarti AG.

₹ Colei Ao.

+Dixoni Ag.

goniopleurus $A G$...

gyratus AG.

heteropleurus AG.

jugalis AG.

f nitidus Ac ....

₹ pressidens Miry.

₹ punctatus AG. . . . . Regleyi Ac.

F striatus Ac.

Toliapicus AG. ...

F angustus AG.

$\neq$ laevis Mry.

₹ serratus MEY.

† speciosus $\mathbf{M}_{\ddot{U}}$.

Testai PHI..

sp. РніL.

micropleurus AG. .

Stockesi Ag.

suturalis AG.

$s p$. Fisch. .

**Aculei.

acutus $A G$.

lateralis Ag.

₹ marginalis AG.

Oweni Ag.

Toliapicus Ag. canaliculatus MEx. gracilis Mü.

Haidingeri Mü.

Sterubergi Ag.

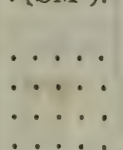

v. 32. 


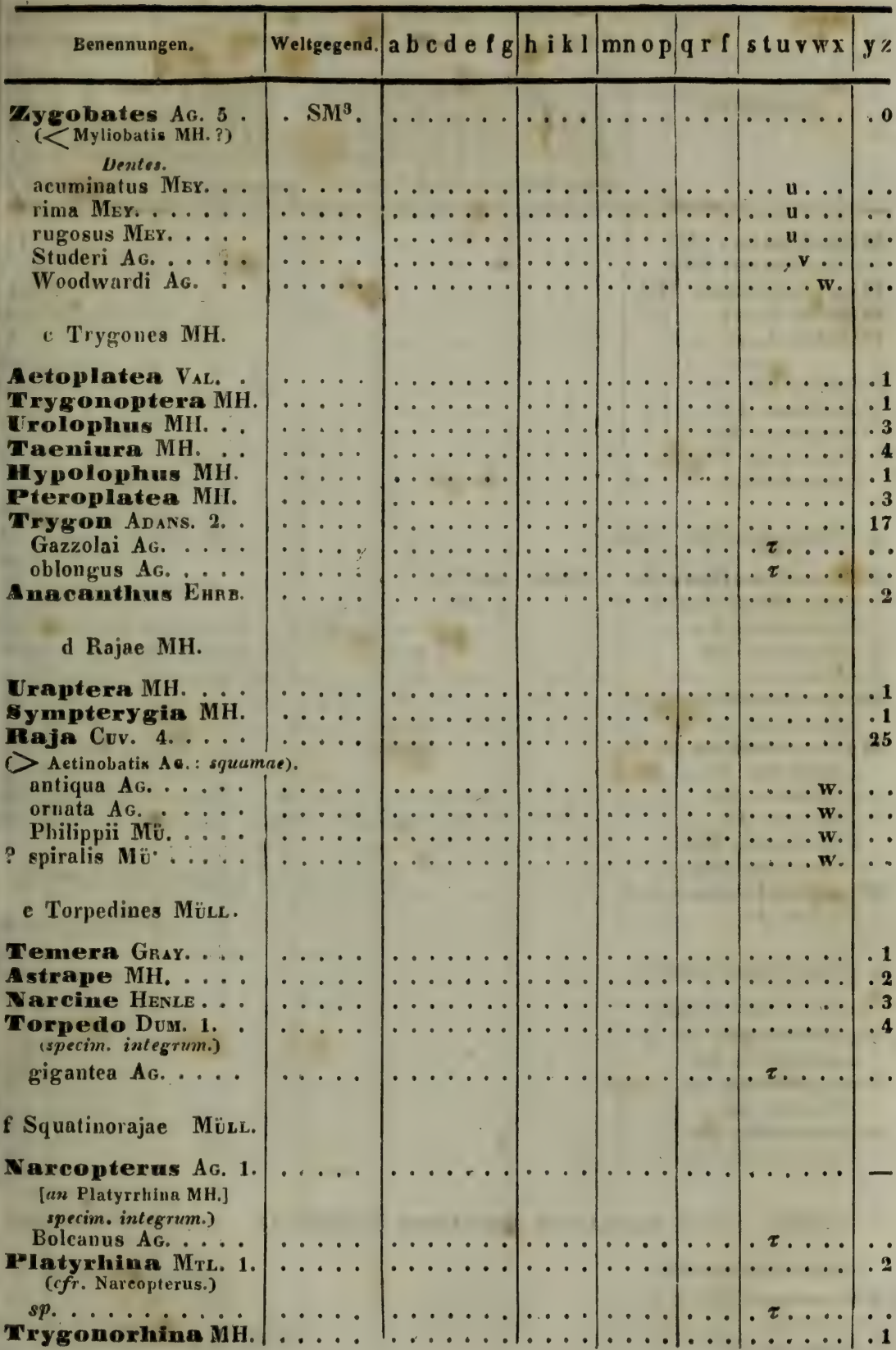




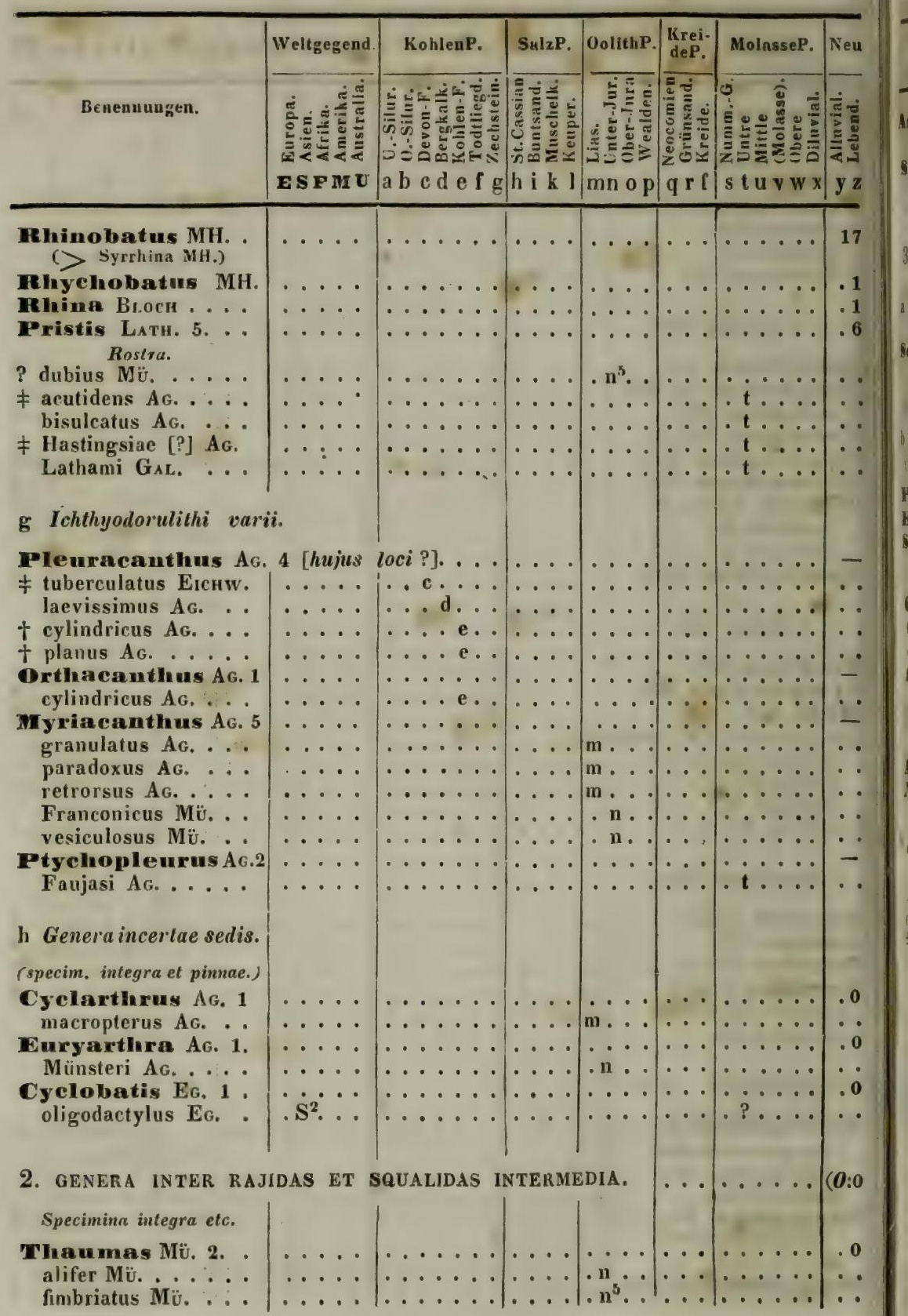


\begin{tabular}{l|l|l|l|l|l|l|l} 
Benenungen. & Weltgegend. & a b c d e f g & hi k l & mn o p & q r f & s t u v w x & y
\end{tabular}

Asterodermus Ag. 1 platypterus AG. . .

Sqaloraja RILEY, 1 dolichognathus RIL.

3. squalidae Ag. (Mült.)

a Squatinae MH. (Dentes cavi.)

Squatina Dum. 2. .

Mülleri Reuss

b Centrinae Ag. (Dentes cavi).

(Scymni et Spinaces MH.)

Pristiophorus $\mathrm{MH}$.

Echinorhinus BLv.

Seymmus Cuv. ....

Centroseyllium MH.

Centrophorus $\mathrm{MH}$.

Centrina Cuv. . . .

major AG.

marginatus $A G . .$. rotundatus Reuss

Acrnthiss Bonap. .

Nustelus Cuv. [ab AG. hic refertur; cfr. p. 644].

c Notidani MH. (Dentes pleni serrati).

\section{Notidanus Cuv. 11}

(Hexanchus, Heptanchus RaF.; Dentes.)

$\neq s p$. Mü.

contrarius Mü.

Hügelia [?] Mừ.

Münsteri Ag.

microdon AG.

pectinatus AG.

serratissimus AG. : .

griseus (?Cuv.) GaL.

recurvus $A G$.

biserratus Mü. . . .

primigenius AG.

d Rhinodontes MH.

Thinodon Sm.

(Cestraciones MH.; vidr. 4.)

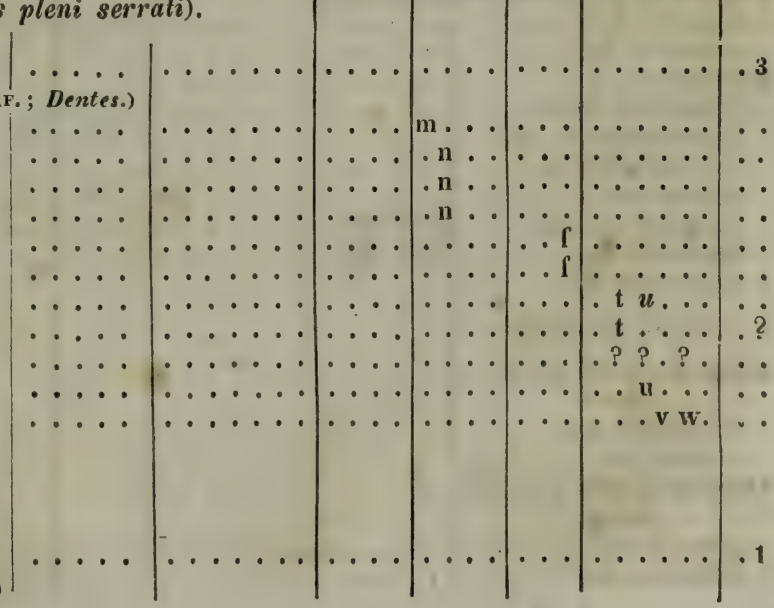

41 (1847) 


\begin{tabular}{|c|c|c|c|c|c|c|c|}
\hline \multirow{3}{*}{ Beneunungen. } & Weltgegend. & KohlenP. & SalzP. & OolithP. & $\begin{array}{l}\text { Krei- } \\
\text { deP. }\end{array}$ & MolnsseP. & Neu \\
\hline & 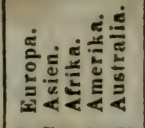 & 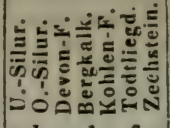 & 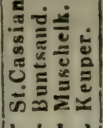 & 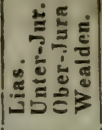 & 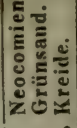 & 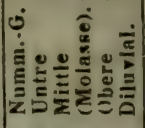 & 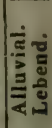 \\
\hline & ESF M U & $|a b c d e f g|$ & $\mid \mathrm{h}$ i k li & $\mathrm{mn} 0 \mathrm{p}$ & $q \times 1$ & s t u v w $x$ & y $z$ \\
\hline
\end{tabular}

\section{e Alopeciae MH.}

Alopias RaF.

f Lamnodei MH. (Dentes pleni.)

= Dentes serrati.

CarcharodonSr.18

subauriculátus Ag. .

lanceolatus AG. ...

angustidens AG.

desauris AG.

súbserratus Af.

Toliapicus Ag.

Escheri Ac;

leptopodon AG.

megalotis AG. .....

auriculatus AG. ...

heterodon Ag.

turgidus AG.

megalodon $A \mathrm{~s}$.

rectidens AG. ....

productus Ag. ...

semiserratus AG. .

sulcidens AG.

Gityphis Ag. 2 [num dentes pleni? hastalis Ag. ungulata MÜ.

Corax Ag. 7 . . . . heterodon Reuss. appendiculatus AG. obliquus Reuss... planus AG. . . . . . pristodentus AG. Egertoni $A$. .

‡ pygmaeus Mü.

- Dontes integerrimi.

Sphenodus AG. 2 . Dentes.

longidens AG.

planus Ag.

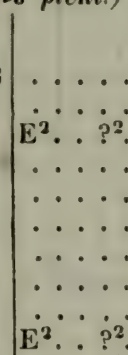

$\mathrm{E}^{2} \cdots ?^{2}$

....

$\dot{\mathrm{E}^{2}} \cdot \dot{\mathrm{M}^{2}}$

$\mathrm{E}^{2} \cdot \dot{\mathrm{M}}^{2}$

.....

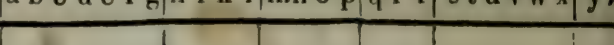

Ddontaspis AG., MH. 13 . . . Dentes.

gracilis AG.

subulata $A G$.

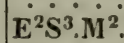

$\mathrm{E}^{2} \cdot \mathrm{M}^{2}$

$\mathrm{L}^{2} \cdot \mathrm{M}^{2}$

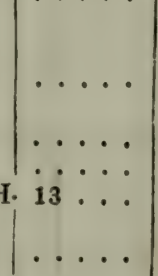


Benennungen.

\begin{tabular}{|l|l|l|l|l|l|l|l|l|l} 
Weltgegend. a b c d e f g h i k l mnop & q r l st u vw x & y z
\end{tabular}

rhaphiodon Ag. constricta EGT. oxyprion EGT. Bronni Ag. verticalis $A G$. Hopei AG. acutissima AG. duplex AG. pygmaca Mü. contortidens Ac. dubia Ag.

Lamna Cuv. 13 Dentes.

acuninata AG. complanata EG. ...

? Mantelli Ag. ..... plicata Ag. plicatella Reuss sigmoides EG. undulata Reuss . . . compressa Ag. elegans AG. . ..... cornubica (Cuv.) $\mathbf{G}_{\Delta L}$. crassidens $A G$. cuspidata Ag. denticulata Ag.... Dxyrhina Ag. 19 . (Meristodion AG.: Dentes) paradoxa AG. . . . subinflata AG.

Zippri AG.

Mantelli Ag. .... acuminata Revss . . angustidens Reriss . heteromorpha Reuss triangularis EG. . hastalis AG. retroflexa Ag. . . . . xiphodon AG. .... crassa Ag.

trigonodon AG.... $E^{2} . M^{2}$. $\mathrm{S}^{2}$ $\mathrm{S}^{2}$.

$\ldots$

$\cdots$

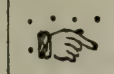

…

$\mathrm{I}^{2} \cdot \mathrm{M}^{2}$. . $\mathrm{S}^{2}$

... $M^{2}$

... $\mathrm{M}^{2}$

$\mathrm{S}^{2}$.

leptodon Ag.

Desori Ag. plicatilis AG. quadrans AG. Numida $V_{\text {ALENC. }} .$. minuta AG.

Gelache Cuv. 1. . . Dentes maximus ('Cuv.) GaL. Dtodus A G. 24

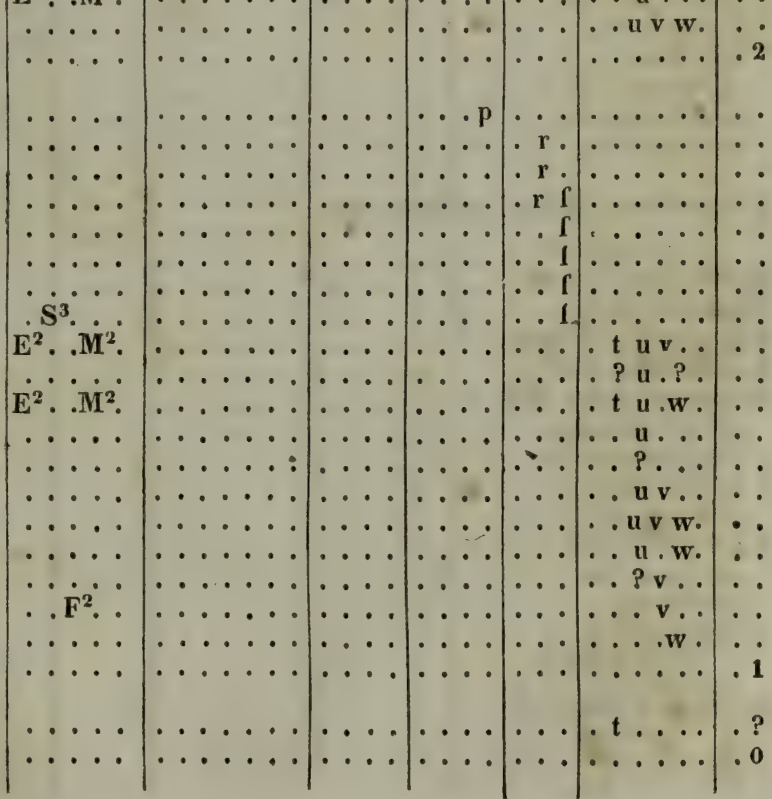




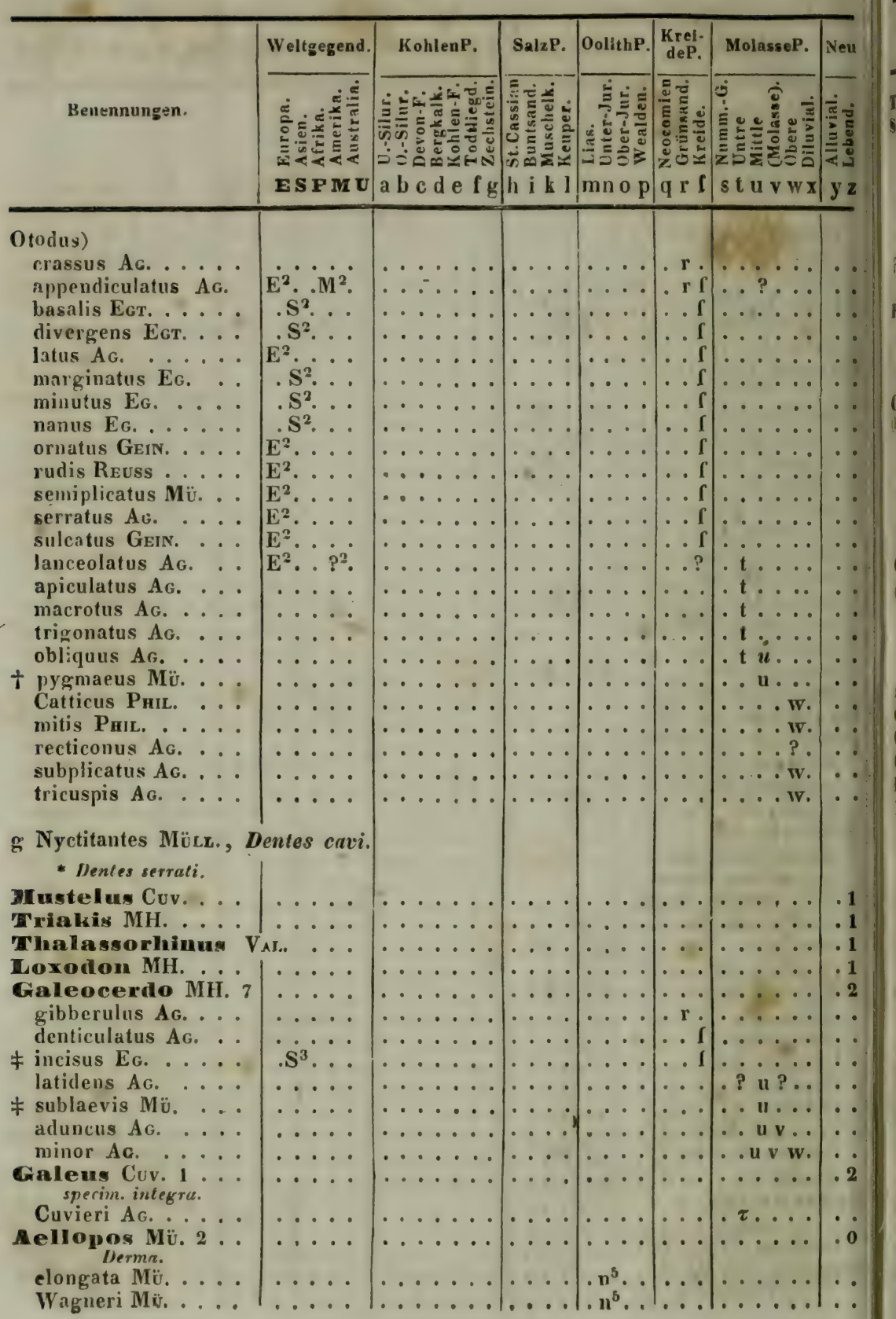




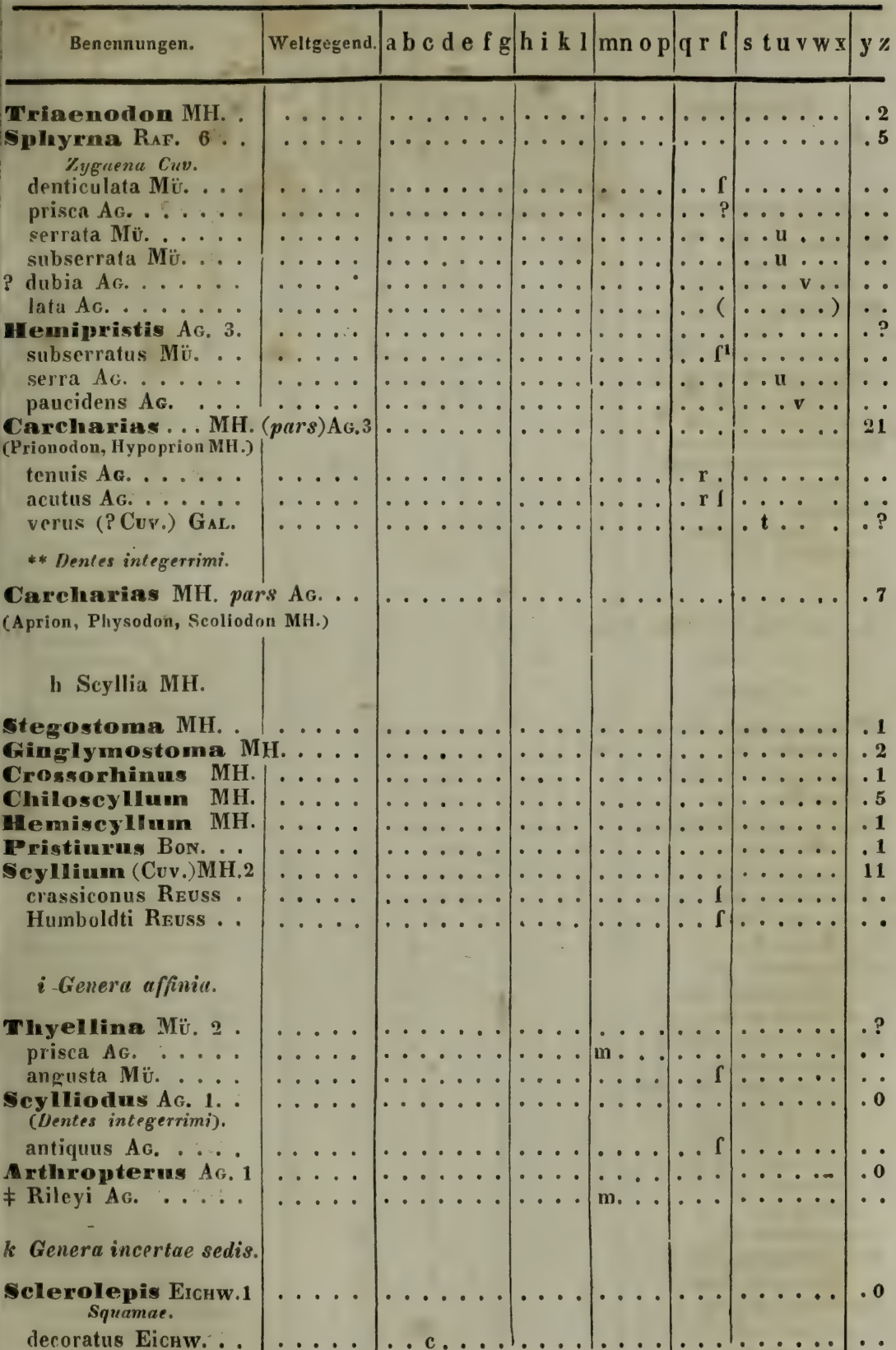




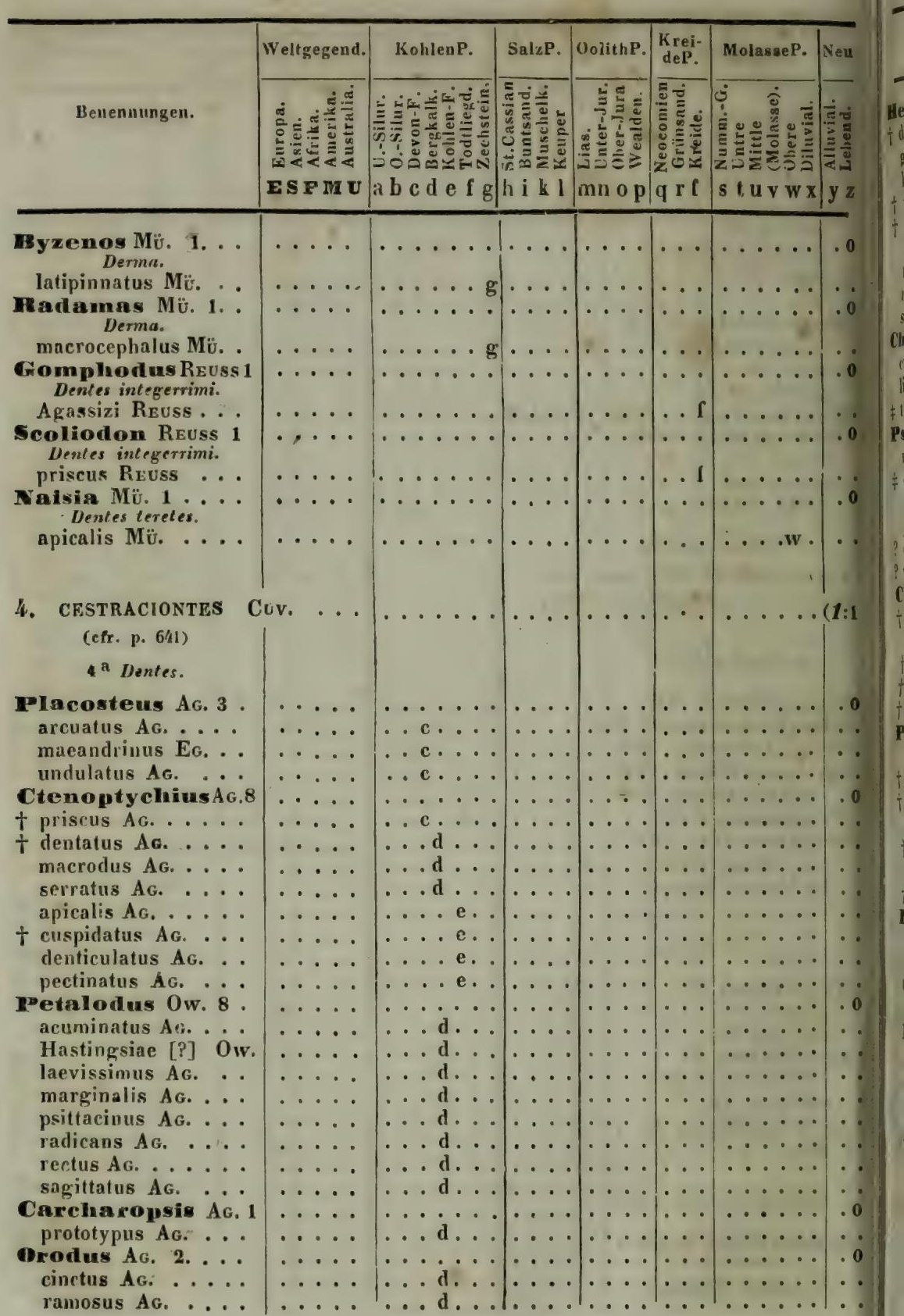




\begin{tabular}{|c|c|c|c|c|c|}
\hline Benennungen. & Weltgegend & a b c d e f g h i k l & $\mathrm{mn}$ op & $q \times r$ & $s t, u v w x y z$ \\
\hline
\end{tabular}

re lodus Ag. 9.

† didymus $\boldsymbol{\Lambda}$ G. . gibberulus AG. laevissimus Ag.

† mammillaris Ag.

$\dagger$ planus Ag. subteres AG. turgidus $A_{G}$. mitratus AG. simplex Ag.

Chomatodus Ag. 3. cinctus AG. linearis AG.

$\neq$ truncatus Ag.

Prammodus Ag. 6. rugosus AG.

† cornutus AG. obtusus Ag. porosus AG.

? orbicularis Plien.

? punctatus RoE.

Cochliodus Ag. 5 .

$\dagger$ acutus AG. contortus $A G$.

† magnus Ag.

† oblongus AG.

$\dagger$ striatus Ag.

Poecilodus Ag. 7. Jonesi Ag.

+ obliquus Ag.

$\dagger$ parallelus Ag.

Rossicus Keys.

t sublaevis AG. transversus Ag.

$t$ angustus $A G$.

Plemrodes AG. 2. affinis AG.

Rankinei AG.

Campodus Kon. 1 Agassizanus Kon.

Ianassa Mü. 4 angulata $M \ddot{\text { ü }}$ bituminosa Mü. Dictea Mü. Humboldti $\dot{M} \ddot{\text {. }}$

Bictea Mü. 1 striata Mü.

Strophodus AG. 17

‡ angustus Mü. arcuatus Mü. angustissimus $\mathbf{A G}$. elytra AG.

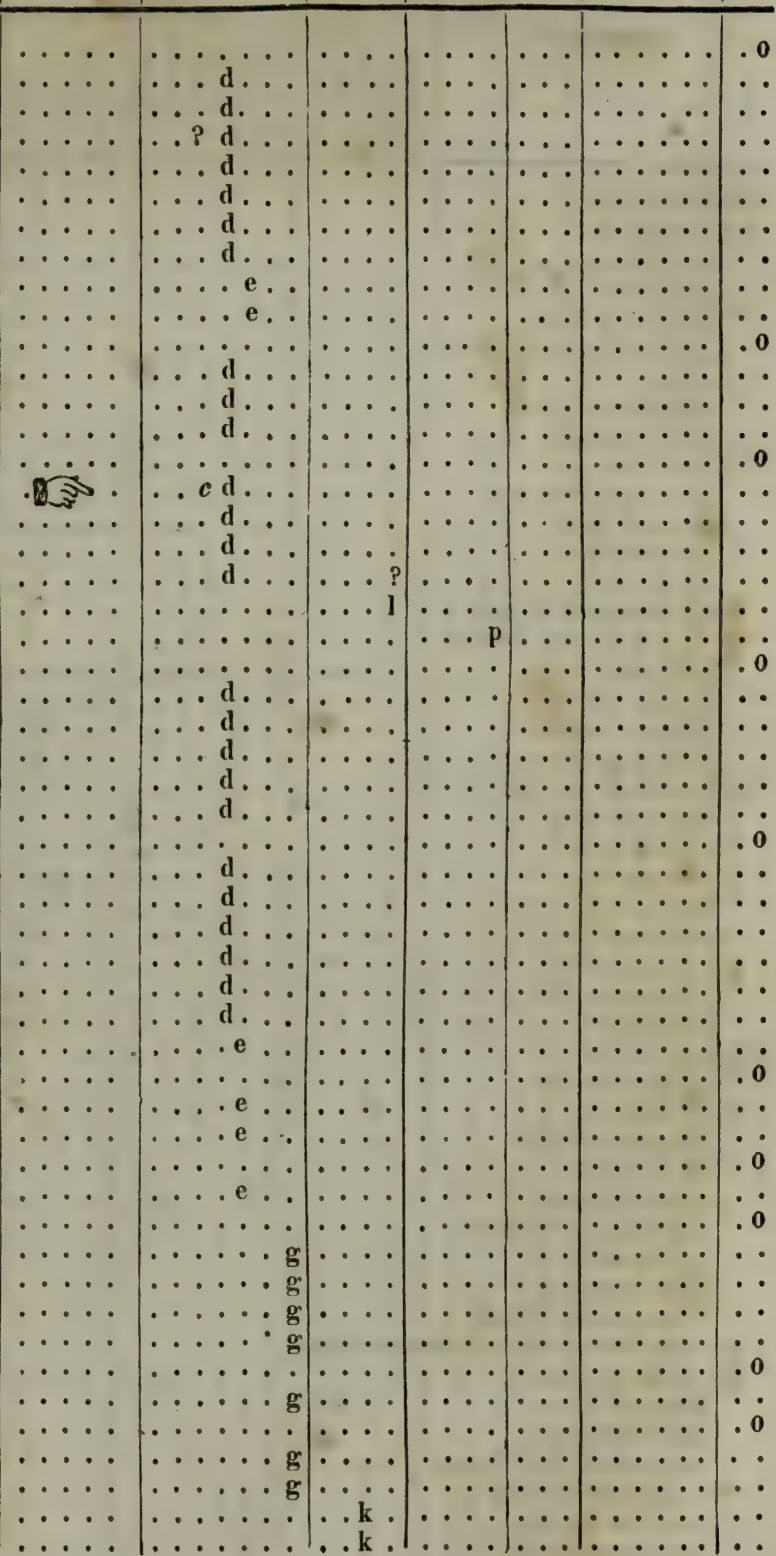




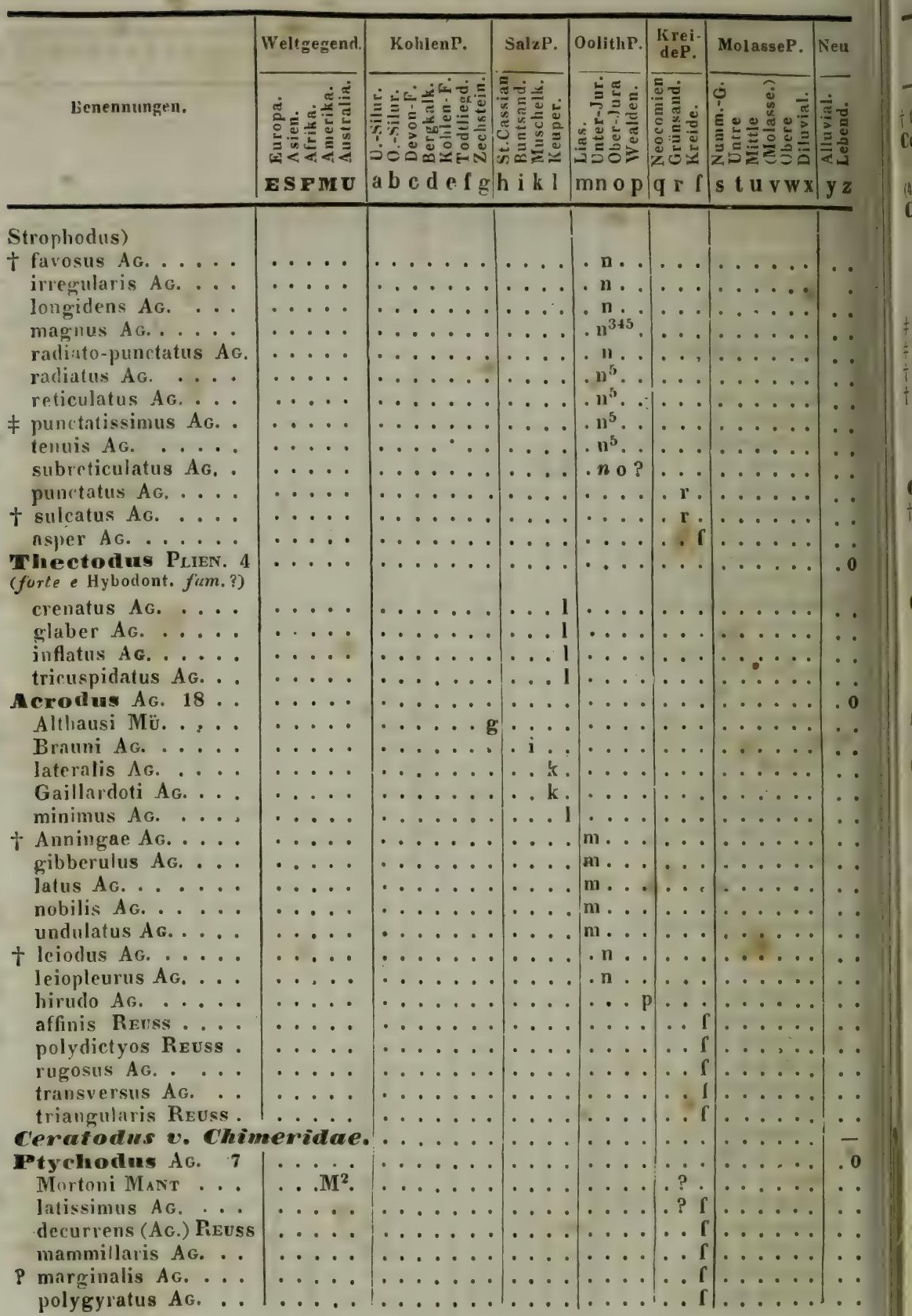




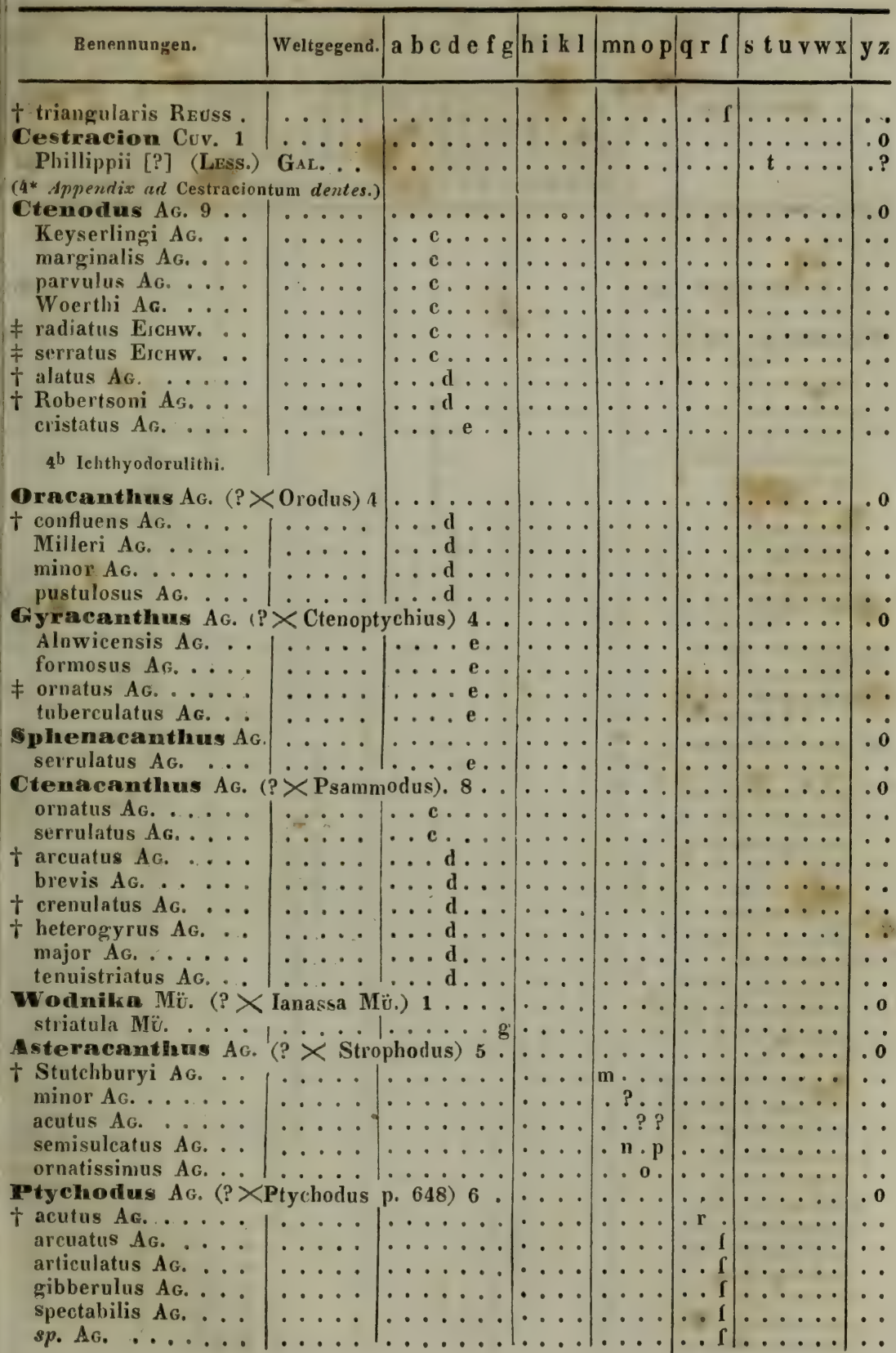




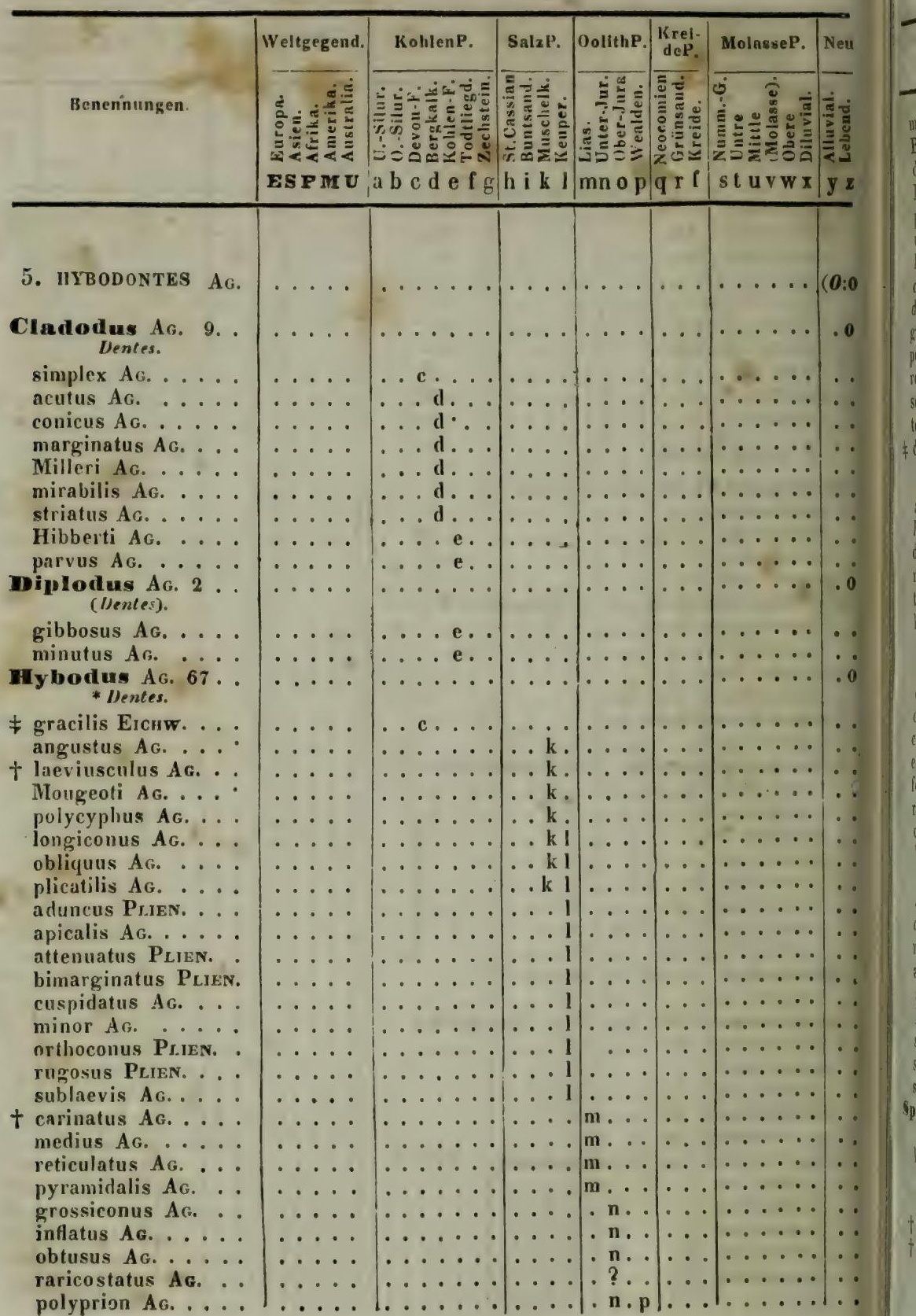


minimus AG.

Bassanus Eg.

dubius Af.

Lawsoni Duff.

pusillus Du.

undulatus Ag.

Bronni Reuss.

cristatus Reuss

dispar Reuss

gracilis Reuss

polyptychus Reuss

regularis Reuss

scrratus Reuss

tenuissimus Reuss

‡ dubius Mü.

\section{** Aculei.}

angulatus $M \ddot{\text { Ü. }}$

hexagonus Mü. ...

dimidiatus Ag.

major AG.

tenuis Ag. . .....

keuperinus Мurch. Strick..

minor AG.

laeviusculus $A G$.

carinatus AG.

crassispinus AG.

curtus Ag.

ensatus AG.

formosus $A G$.

reticulatus AG.

crassus AG. . . . .

leptodus Ag.

pleiodus AG.

apicalis AG.

dorsalis $A G$.

marginalis AG.

acutus AG.

strictus AG.

Fittoni Dv.

striatulus AG.

subcarinatus $\hat{A G}$.

sulcatus AG.

Gphenonchus AG. 5 (Dentes).

hamatus AG.

elongatus AG.

Martini Ross.

† $s p$. AG.

† sp. AG.

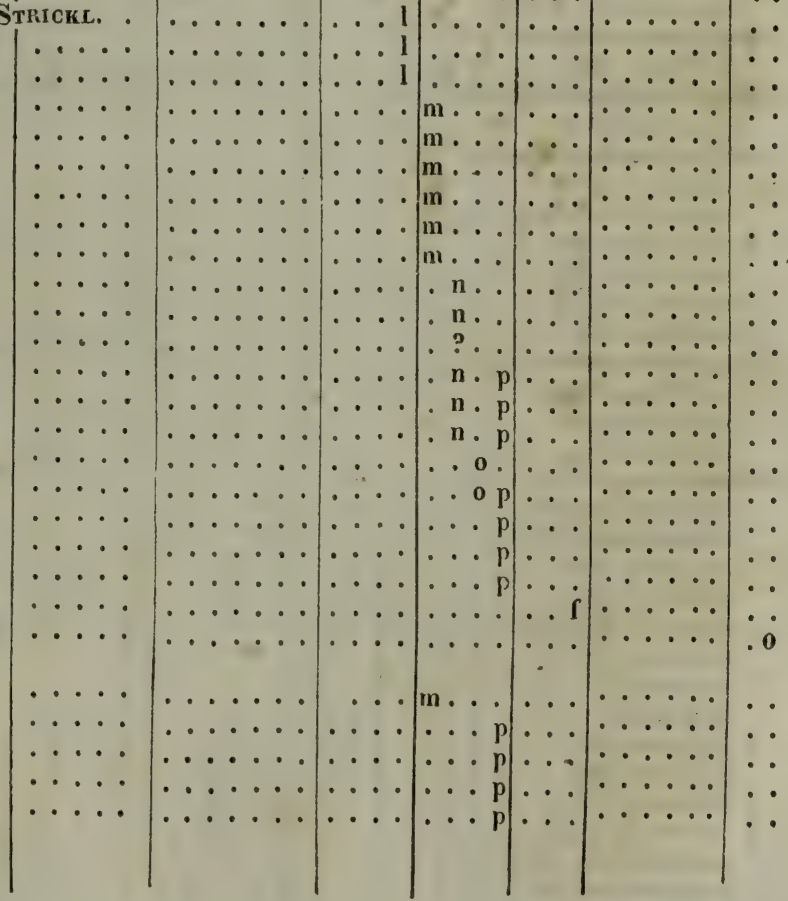




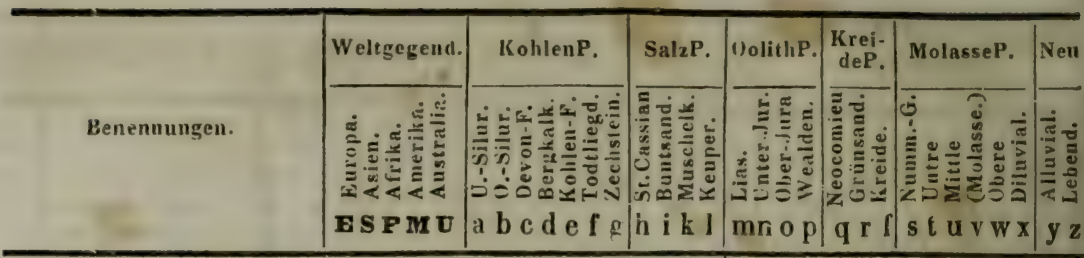

Appeindix:

generum incertarum fumiliarum.

(plerique Ichthyodorulithi)

Thelodus Ag. 1 .

parvidens Ag.

Delerodus Ag. 1. .

parvidens $A g$.

Plectrodus Ac. 2 .

mirabilis AG.

† pleiopristis $A_{G}$.

Spliagodus $A G$. 1 . pristodontus AG.

Dinerncanthas KEYs. 1 . , concentricus KEYs.

NHo macantlins AG.1 arcuatus Ac.

Faplacanthus I $_{\text {i. }} 1$ marginalis Af.

Odontreanthus $A$ crenatus Ag. heterodon AG.

Narcodes AG. 1 . pustulifer Ag.

Naulas A c. 1 sulcatus Ag.

IByssacanthus A G. 3

₹ arcuatus AG. crenulatus AG. laevis Ag.

Onchus AG, 14 Murchisoni AG. tenuistriatus $A C$ arcuatus $\mathrm{Ac}$. arenatus $A \mathrm{f}$.

‡ dilatatus Eicrw. heterogyrus $A \mathrm{G}$. semistriatus $A$ s. sublaevis AG.

+ falcatus $A G$. hamatus Ac.

+ plicatus $A_{G}$

+ rectus Ag. sulcatus AG..... semistriatus Ac.
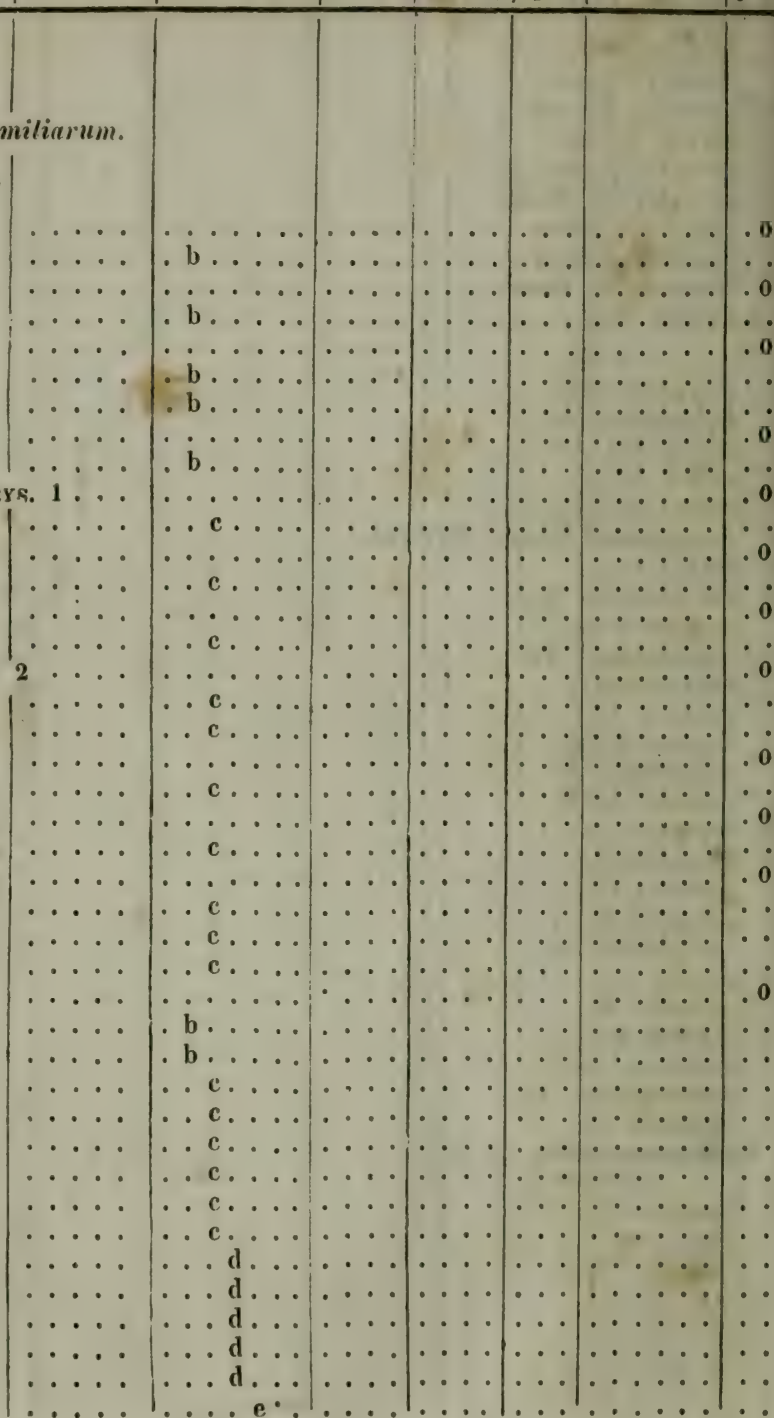


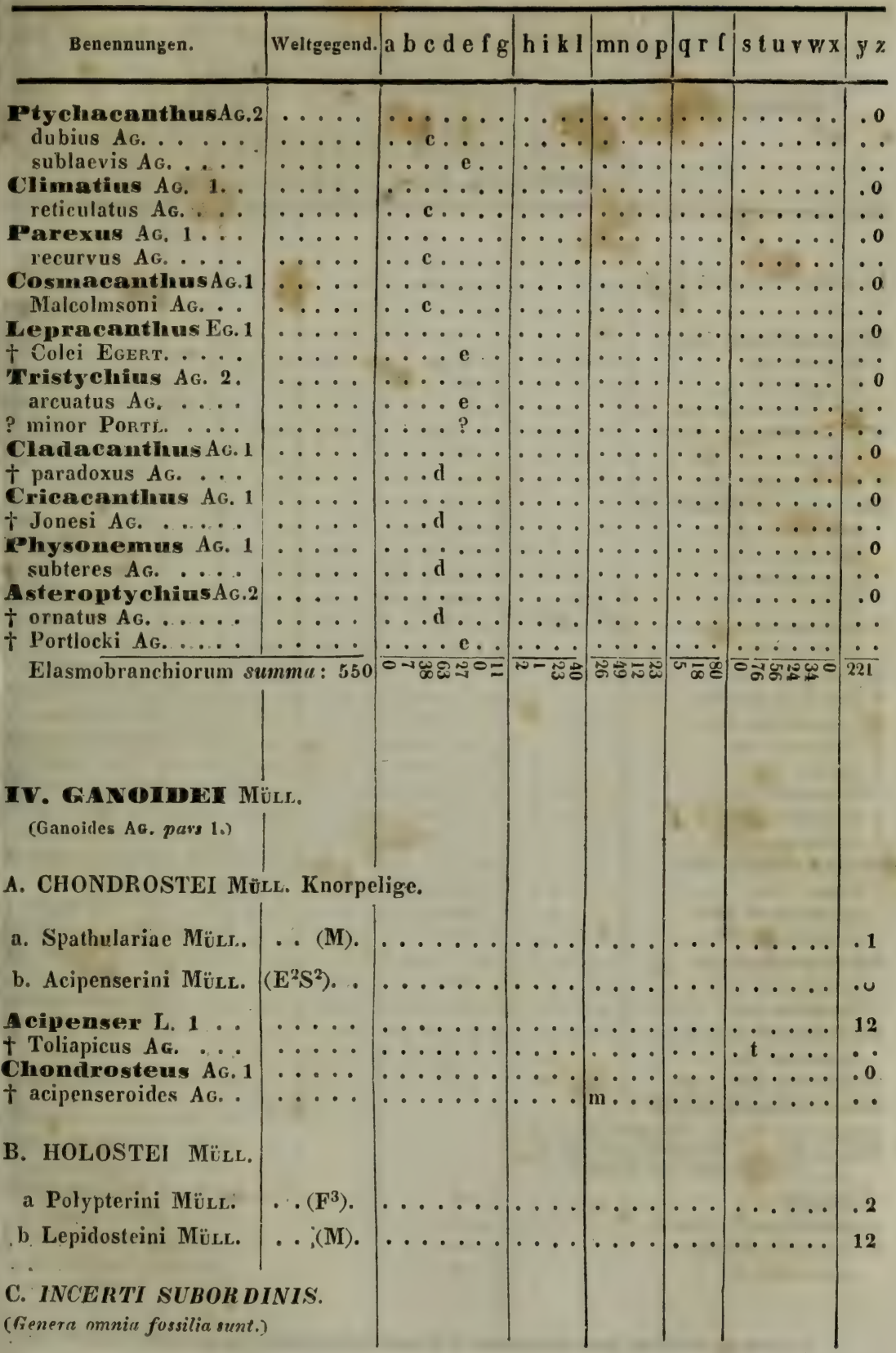




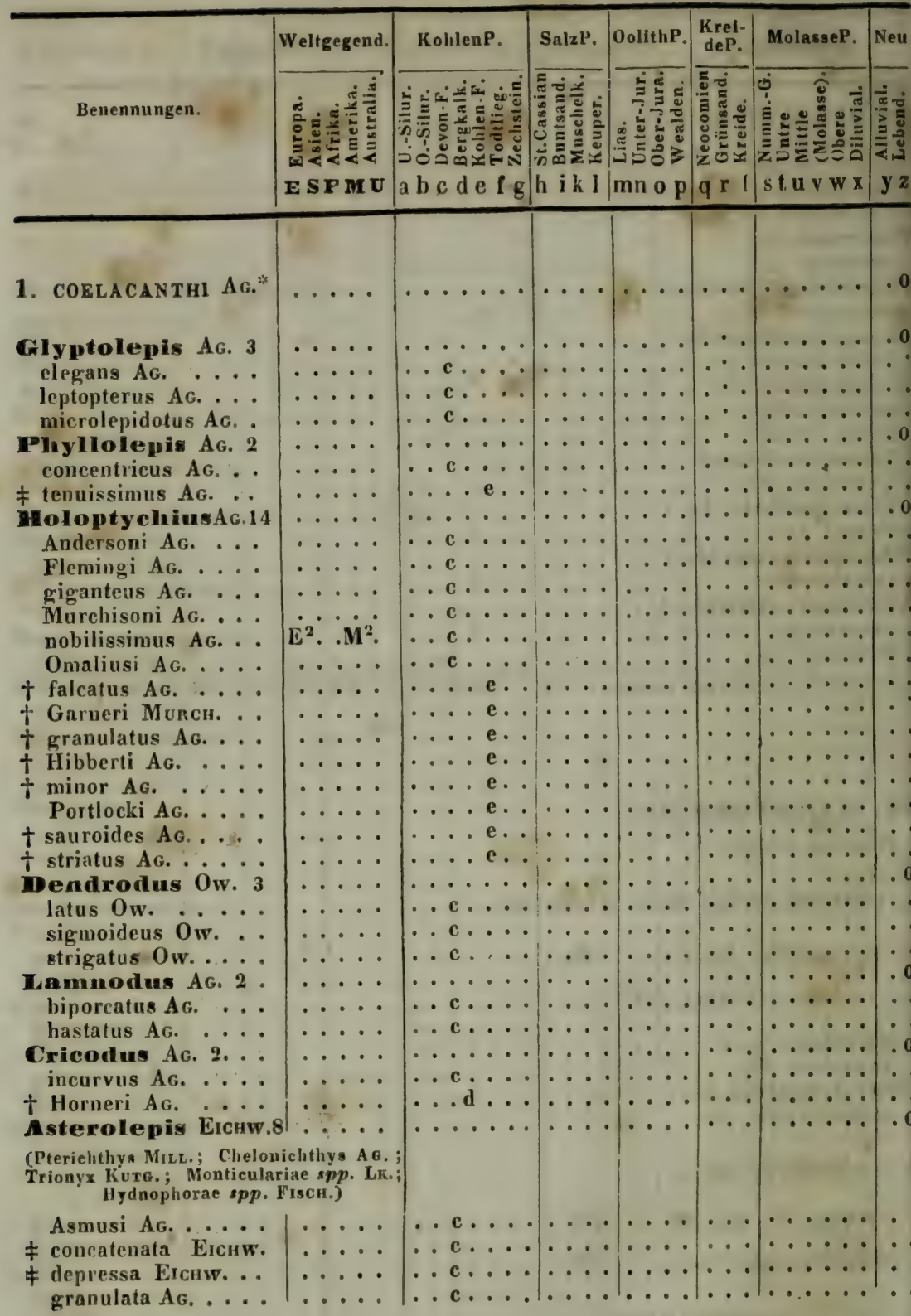




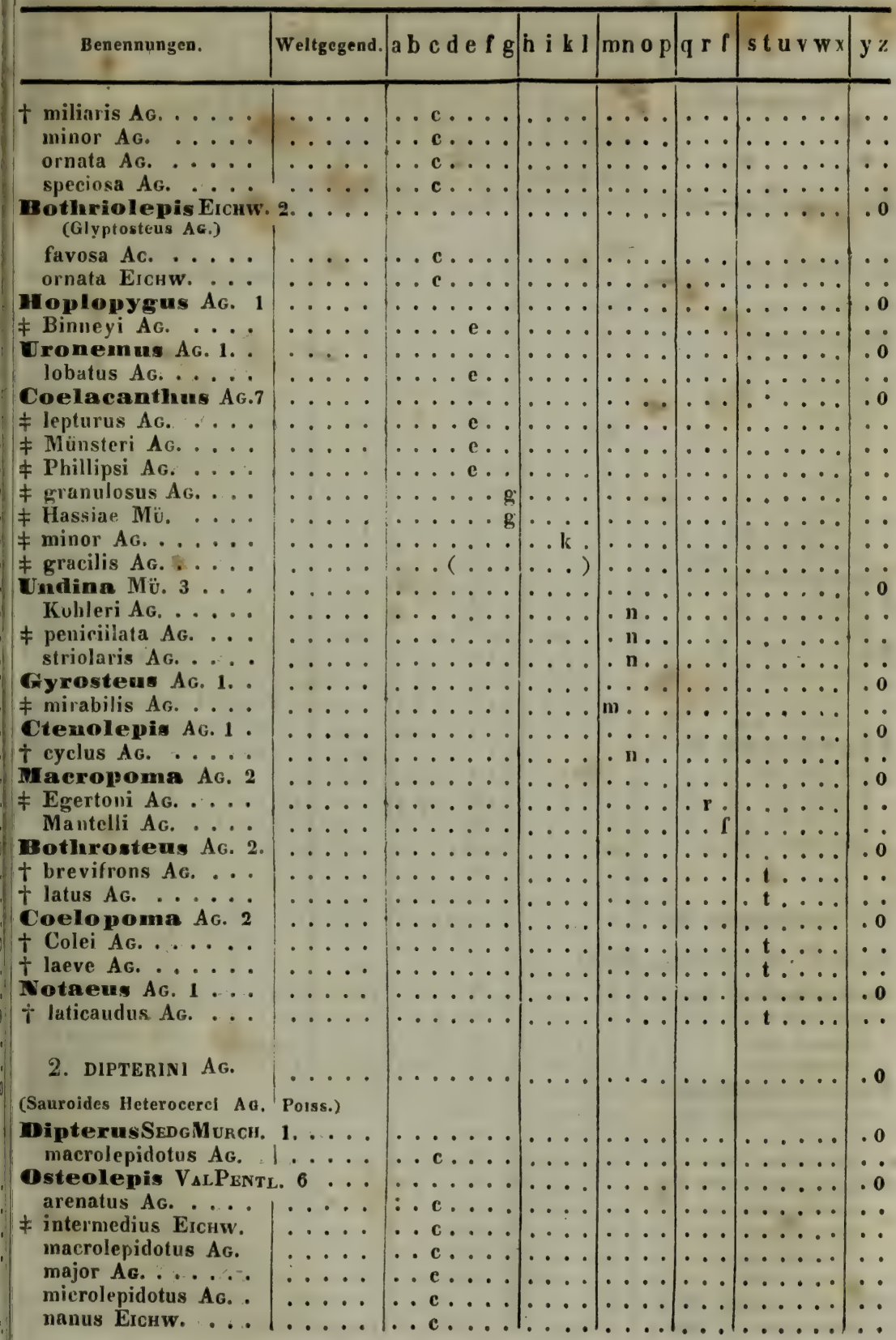




\begin{tabular}{|c|c|c|c|c|c|c|c|}
\hline & Weltgegend. & KohlenP. & SalzP. & OolithP. & $\begin{array}{r}\text { Krei-i } \\
\text { deP. }\end{array}$ & MolasseP. & $\mathrm{Ne}$ \\
\hline Benennungent. & 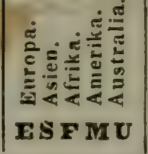 & 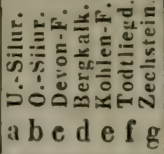 & 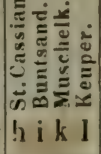 & 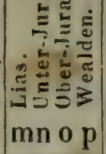 & 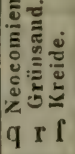 & 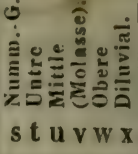 & \\
\hline
\end{tabular}

\section{CEPHALASPIDES Ag.}

(Lepidoides Heterocerci AG. Porss.)

\section{Pterichthys Ag. 9}

cancriformis AG. . cellulosus PAND. cornutus AG. latus Ag. major Ag. Milleri Ag. oblongus $A G$. productus $A G$.

testudinarius AG.

Pampliractus Ag. 2

P Andersoni Ag. hydrophilus AG.

Coccostens Ag. 3 . cuspidatus AG, ... decipiens AG. . . . . oblongus AG. ....

Cephalaspis AG. 4. Lewisi Ag. Lloydi $\mathbf{A}$ G. Lyelli AG. rostratus $A G$.

\section{ACANTHOdEI Ag.}

(Lepidoides Heteroceri AG. Po16s.)

Acanthodes Ag. 3 . pusillus A G. ..... Bronni Ag. sulcatus Ag.

Cheiracanthus $A G$. microlepidotus AG. . minor AG.

Murchisoni Ag.

Diplacanthug Ag. 4 crassispinus Ag. .. longispinus AG. striatulus AG. .... striatus Af.

Cheirolepis Ag. 5 Cumingiae [?] AG.

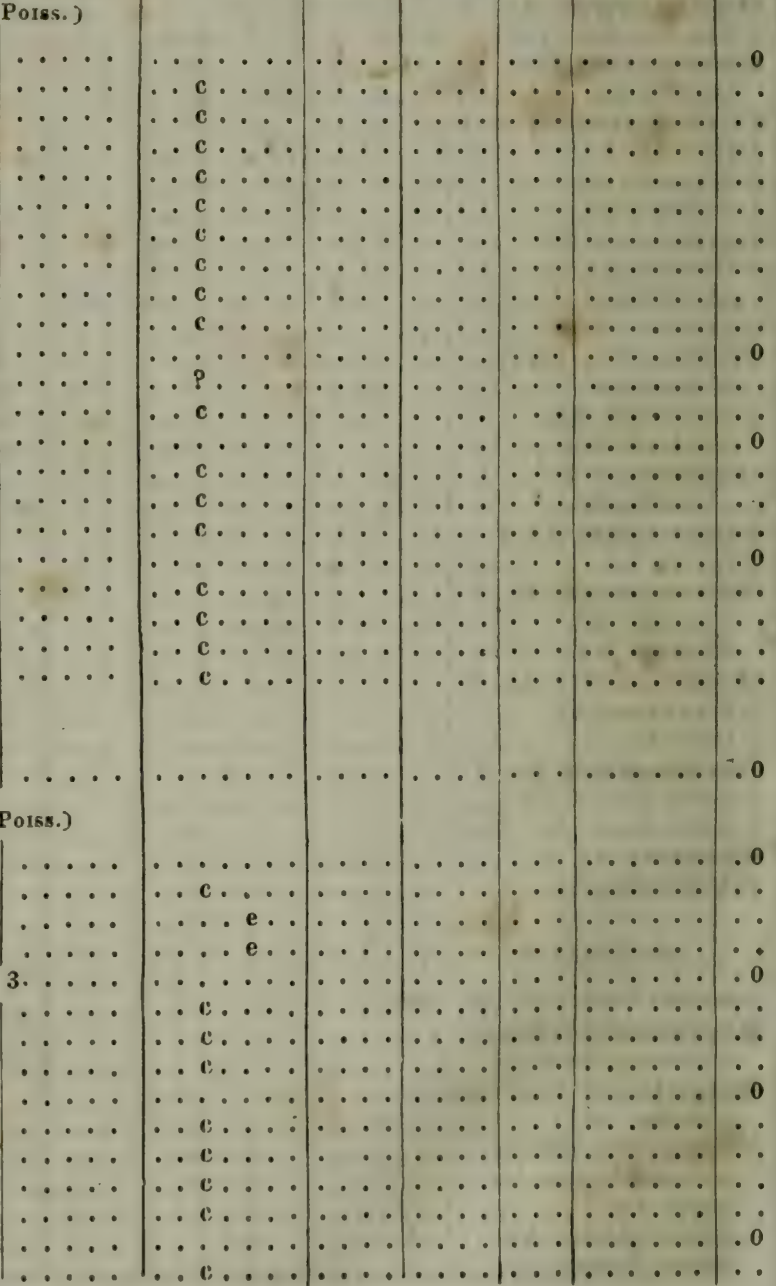




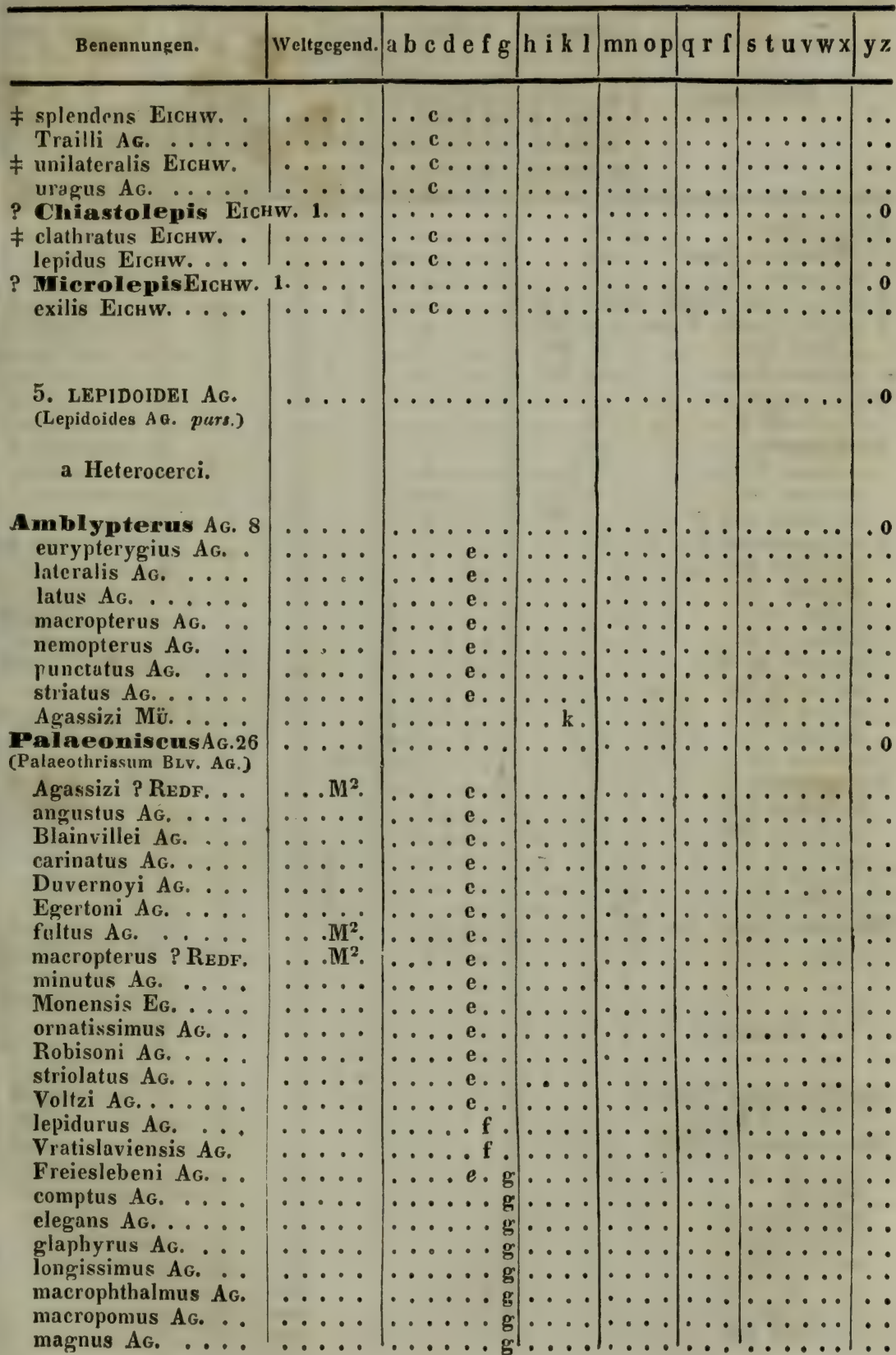




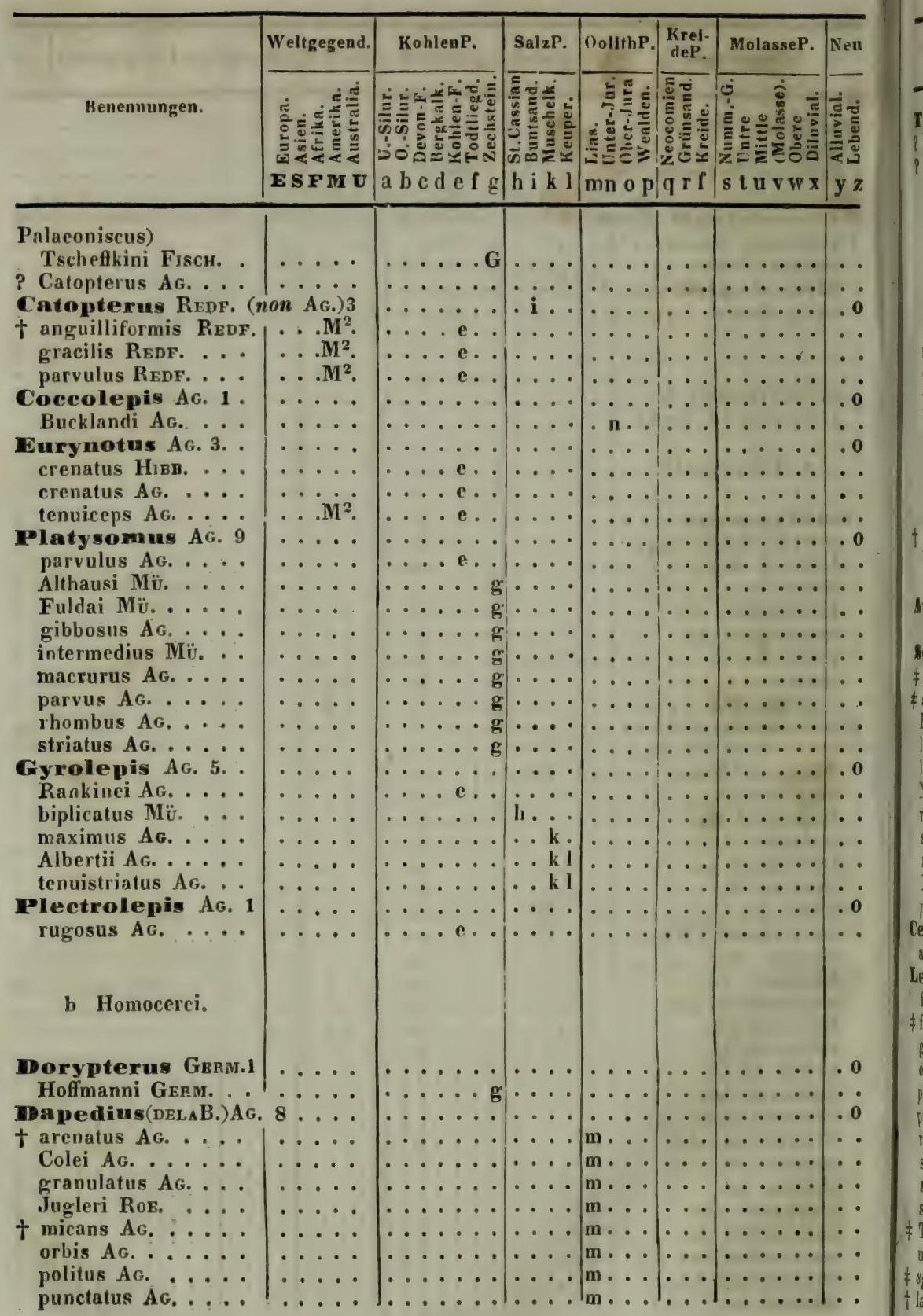




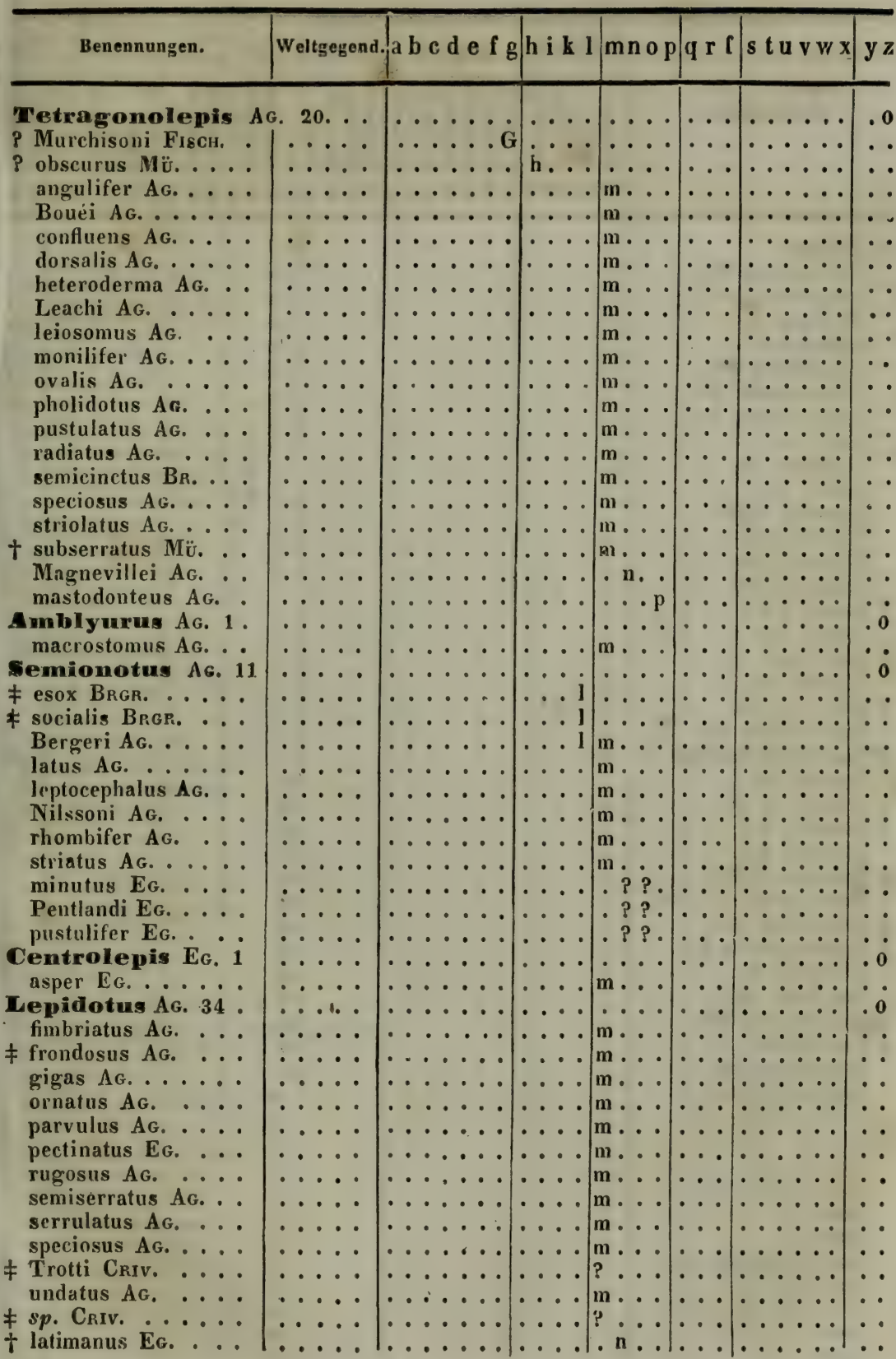




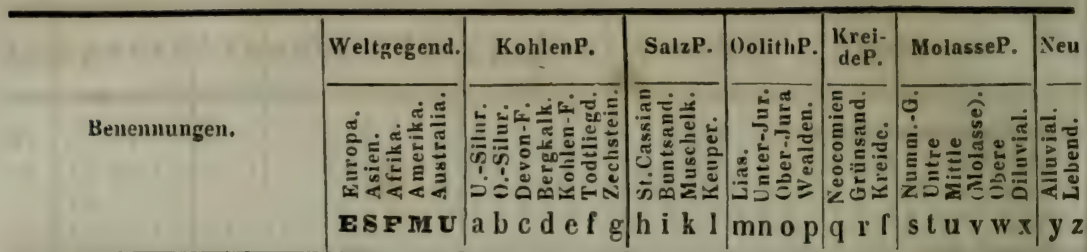

Lepidotus)

macrochirus EG. . .

notopterus AG.

oblongus AG.

radiatus $A G$.

subundatus Mü.

tuberculatus AG.

unguiculatus Ag. .

laevis Ag.

palliatus Ag.

minor AG. .

Agassizi RoE.

Fittoni Ag.

Mantelli Ag.

Roemeri Du.

† Cottai Ag.

Virleti Ag.

† punctatus AG.

striatus AG.

† temnurus AG.

Maximiliani AG.

Pholidophora:

Bechei AG.

† crenulatus EG.

₹ dorsalis Ag. .

furcatus AG.

† Hartmanni EG.

Hastingsiae [?] Ag.

\# latiusculus AG.

+ leptocephalus AG.

linbatus Ag.

onychius AG.

† pachysomus EG.

₹ pusillus AG.

Stricklandi AG....

angustus AG.

Flesheri Ag.

8 dubius

gracilis AG.

intermedius AG. .

latimanus AG.

latus AG.

macrocephalus AG. .

‡ maximus AG. micronyx AG. .... microps AG. minor $A G$.

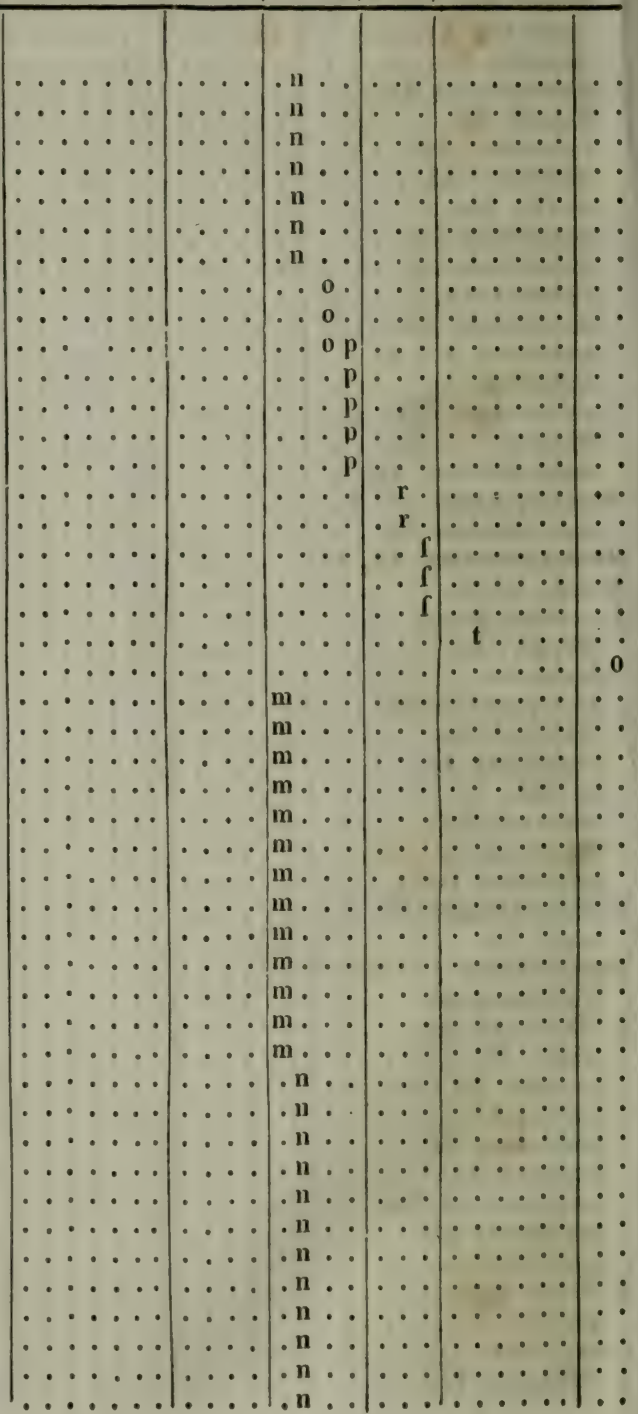


‡ radians AG.

\$ radiato-punctatus AG. striolaris AG.

\% Taxis [?] Ag. tenuiserratus AG. ₹ uraeoides $A \mathrm{G}$.

+ fusiformis $A G$. ornatus AG.

Aethalion Mǘ. 6 angustissimus

$\neq$ angustus Mü.

₹ inflatus Mü.

₹ parvus Mü.

₹ subovatus $\mathrm{M} \ddot{0}$.

$\neq$ tenuis Mü.

Nothosomus AG. 2.

₹ octostygius AG.

₹ laevissimus Ag.

Dphiopsis Ag. 4 .. Münsteri AG. .... procerus AG. dorsalis AG. penicillatus $\mathrm{AG}$.

Natagogus AG. 4 . denticulatus AG.

Zieteni Ag.

latior Ag.

Peutlandi Ag.

Propterus Ag. 2 . microstomus AG... ‡ serratus AG.

6. sauroidei Ag.

(Sauroides AG. pars.)

a Heterocerci.

Diplopterus $\lambda G .6$. [num hoc loco, un in Dipteriis collocandus?].

$\ddagger$ affinis Ag.

$\neq$ arenaceus Eichw.

\# borealis AG.

macrocephalus $\mathrm{AG}_{\mathrm{G}}$. carbonarius Ag.

Robertsoni AG.

GIyptopomus Ag. 1 minor AG.

Vegalichthys AG. 4 $\dagger$ priscus $A G$.

† Fischeri Еichw.

.




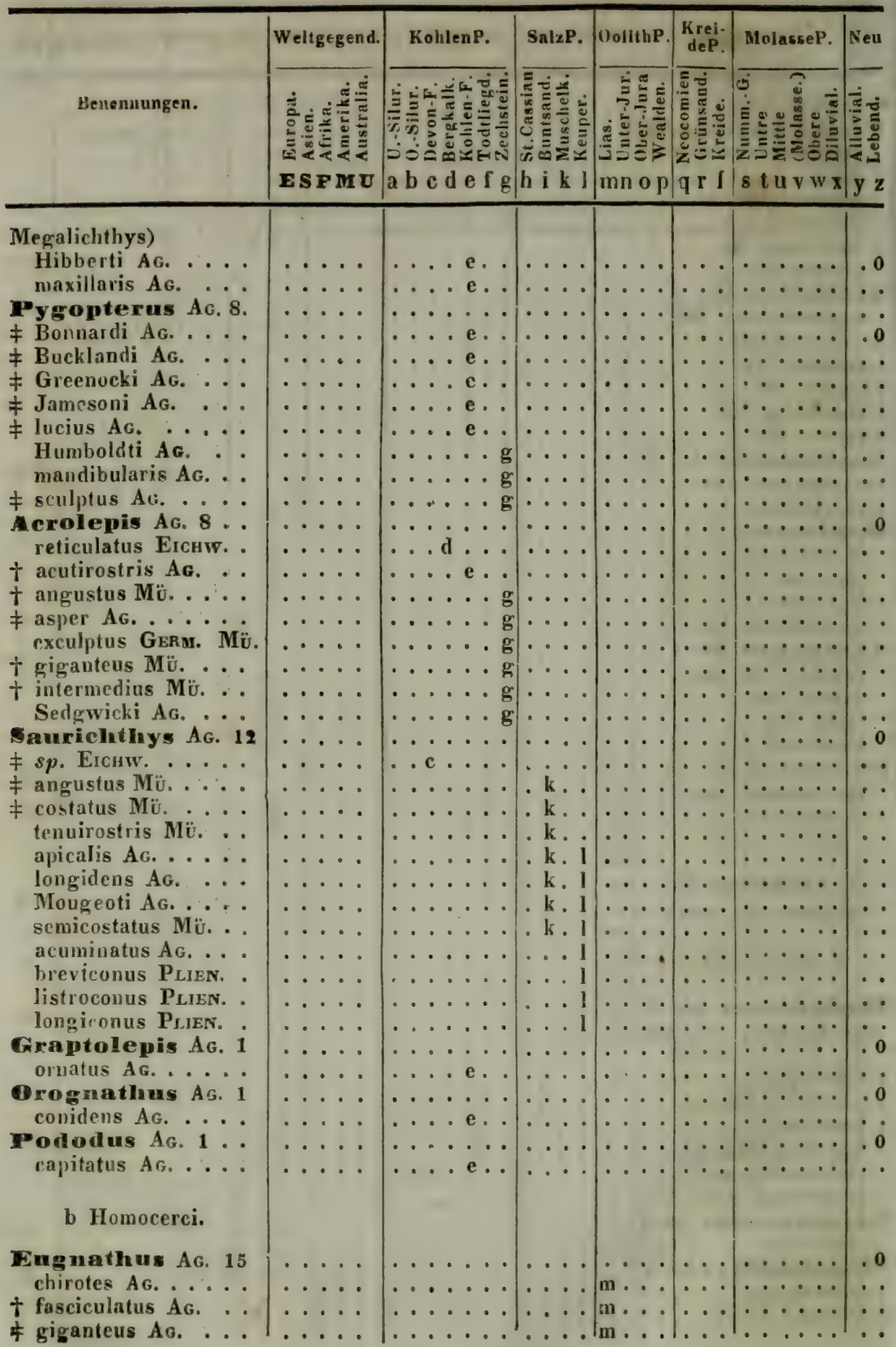




\begin{tabular}{|c|c|c|c|c|c|c|c|}
\hline Ben & end. & $a$ & & n & $q \times f$ & $\mathbf{w} \mathbf{x}$ & $y z$ \\
\hline$\cdots$ & $\cdots \cdots$ & 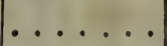 & & $\mathbf{m} \cdot \ldots$ & & & \\
\hline ris AG. . & .... & $\ldots \ldots$ & $\ldots$ & $\mathrm{m} \ldots$ & 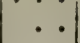 & $\ldots \ldots$ & \\
\hline$\ldots$ & $\cdots \cdots$ & $\ldots \ldots$ & $\ldots$ & $\mathrm{m} \ldots$ & ... & $\ldots \ldots$ & \\
\hline Ag.... & $\cdots \cdot$ & $\cdots \cdots$ & $\cdots \cdot$ & $\mathrm{m} \ldots$ & $\cdots$ & $\ldots \ldots$ & \\
\hline G. $\ldots$. & $\cdots \cdots$ & $\ldots \ldots$ & $\cdots \cdot$ & $m \cdots$ & $\cdots$ & $\ldots \ldots$ & \\
\hline o. . . & $\cdots \cdots$ & $\cdots \cdots$ & & m... & $\cdots$ & $\cdots \cdots$ & \\
\hline Philpo & & & & m $\cdots$ & $\cdots$ & $\cdots \cdots$ & $\cdots$ \\
\hline$\cdots \cdots$ & $\cdots$ & t & & $\mathrm{m} \cdots$ & $\cdots$ & $\cdots \cdots$ & \\
\hline culus AG. . & $\cdots \cdots$ & $\cdots \cdots \cdots$ & & $\mathrm{m}_{\mathrm{m}} \ldots$ & $\therefore$ & $\cdots \cdots \cdots$ & \\
\hline$\cdots \cdots$ & $\begin{array}{l}\cdots \\
\cdots\end{array}$ & $\cdots \cdots$ & $\cdots$ & & $\begin{array}{ll}\cdots \\
\cdots\end{array}$ & $\cdots \cdots \cdots$ & \\
\hline $\begin{array}{l}\text { tenuidens AG. } \ldots \\
\text { microlepidotus AG. }\end{array}$ & $\ldots$ & $\ldots \ldots$ & $\ldots$ &. $\mathrm{n} \ldots$ & $\ldots$ & $\cdots \cdots$ & - \\
\hline Conodus AG. 1. . & $\ldots .$. & $\ldots \ldots$ & $\ldots$ & $\ldots$ & $\ldots$ & $\ldots \ldots$ & $\ddot{0}$ \\
\hline$\ldots$ & $\ldots$ & $\ldots \ldots$ & & m... & $\cdots$ & $\ldots \ldots$ & $\therefore$ \\
\hline Ptycholepis AG. 1 & $\ldots$. & $\ldots \ldots$ & $\ldots$ & $\ldots$ & $\ldots$ & $\ldots \ldots$ & .0 \\
\hline$\ldots$ & $\cdots$ & $\cdots$ & $\cdots$ & $\mathbf{m} \cdots$ & $\ldots$ & $\ldots \ldots$ & . \\
\hline $18 .$. & $\cdots \cdot$ & $\cdots \cdots$ & $\cdots$ & $\ldots$ & $\cdots$ & $\ldots \ldots$ & .0 \\
\hline$\cdots$ & $\cdots$ & $\ldots \ldots$ & & m... & $\cdots$ & $\ldots \ldots$ & • \\
\hline . . & $\ldots$ & $\ldots \ldots$ & & $\mathrm{m} \ldots$ & $\cdots$ & $\ldots \ldots$ & \\
\hline Ag. . & $\ldots \ldots$ & $\ldots \ldots$ & $\cdots$ &. $\mathbf{n} \cdot$ & $\cdots$ & $\ldots \ldots$ & \\
\hline Mü. . & $\cdots \cdots$ & $\cdots$ & $\cdots$ &. $\mathbf{n} \cdot$. & $\cdots$ & $\cdots \cdot$ & \\
\hline$\cdots$ & $\cdots$ & $\cdots$ & $\cdots$ & $.11 \cdot$. & $\cdots$ & $\cdots$ & \\
\hline$\cdots$ & $\cdots \cdots$ & $\cdots$ & $\cdots$ &. $\mathrm{n} \cdot$. & $\cdot \cdot$ & $\cdots$ & \\
\hline : & $\cdots$ & $\cdots \cdots$ & $\cdots$ & . n .. & $\cdot$ & $\cdots$ & \\
\hline Iü. . . & $\cdots \cdots$ & 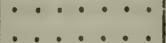 & $\because$ & $\cdot 11 \cdots$ & . 1 & 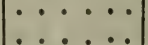 & \\
\hline$\dot{A} G \ldots$ & ..... & $\ldots \ldots$ & $\cdots \cdots$ & $\mid \begin{array}{ll}0 & \cdots \\
.1 & \cdots\end{array}$ & $\cdots$ & $\ldots \ldots$ & \\
\hline ... & .... & 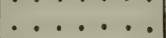 & $\ldots$ & . n .. & $\cdots$ & $\ldots \ldots$ & \\
\hline is Ag. ..... & .... & $\ldots \ldots$ & $\ldots$ & .11. & $\ldots$ & $\ldots \ldots$ & - \\
\hline rus $\mathbf{A G} .$. & $\ldots$ & $\ldots \ldots$ & $\ldots$ &. $\mathbf{n} \cdot$ & $\cdots$ & $\ldots \ldots$ & . \\
\hline$\cdots$ & $\cdots \cdot$ & $\cdots \cdots$ & $\cdots$ &. $\mathbf{n} \cdot$. & $\cdots$ & $\ldots \ldots$ & 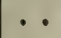 \\
\hline 3. . . & $\cdots \cdots$ & $\cdots \cdots$ & . &.$n \ldots$ & $\cdots$ & $\ldots \ldots$ & 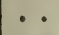 \\
\hline$\ldots$ & $\ldots$ & $\ldots \ldots$ & & $\cdot n \ldots$ & $\cdots$ & $\ldots \ldots$ & \\
\hline$\cdots$ & $\ldots$ & $\cdots$ & $\cdot$ & $\ldots 0$ & $\cdot$ & $\cdots \cdots$ & \\
\hline & $\cdots$ & I & & $\cdots \cdots$ & $\cdot 1$ & $\cdots$ & \\
\hline ws A G. 15 & $\cdots \cdot$ & . & - & $\cdots \cdot$ & . 1 & $\cdots$ & \\
\hline$\cdots$ & $\cdots \cdots$ & & & $\begin{array}{l}\mathrm{m} . \\
\mathrm{m} .\end{array}$ & $\therefore:$ & 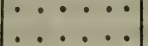 & \\
\hline . . $\therefore$ & . & $\ldots \ldots$ & & $\mathrm{m} \ldots$ & ... & $\ldots \ldots$ & • \\
\hline . . . & $\ldots$ & $\ldots \ldots$ & & $m \ldots$ & $\cdots$ & $\ldots \ldots$ & \\
\hline . $\ldots$ & ... & $\ldots$ & & $\mathrm{m}$. & $\cdots$ & $\ldots \ldots$ & . \\
\hline AG. ... & . & $\cdots$ & & m. & $\cdots$ & $\cdots$ & \\
\hline 18 & & $\cdot$ & & & $\cdots$ & . . & \\
\hline lep & & & & m. & & $\bullet$ & \\
\hline & $\therefore$ & . & & $\mathrm{m}$. & & $\cdot$ & \\
\hline 3. & $\therefore$ & $\ldots \ldots$ & & ${ }^{m} \cdot \mathrm{n}$ & & $\ddot{*}$ & • \\
\hline 0 & . & $\cdots$ & &.$n$ & & & \\
\hline & $\cdots$ & $\cdots$ & & . $n$ & & & \\
\hline & $\cdots$ & ... & $\cdot$. &. $\mathrm{n}$ & - & - & \\
\hline & ${ }^{\circ}$ & & & & & & \\
\hline
\end{tabular}




\begin{tabular}{|c|c|c|c|c|c|c|c|}
\hline \multirow{3}{*}{ Benennungen. } & Weltgegend. & KohlenP. & SalaP. & OulithP. & $\begin{array}{l}\text { Krei- } \\
\text { deP. }\end{array}$ & MolasseP. & Neu \\
\hline & 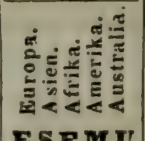 & 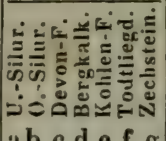 & 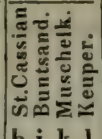 & 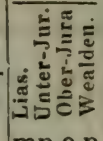 & 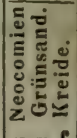 & 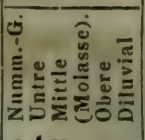 & 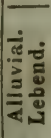 \\
\hline & $\mathbf{E} \mathbf{S} \mathbf{M} \mathbf{U}$ & $a b c d e f g$ & h i k l & $\mathrm{mnop}$ & 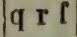 & $|s \quad u v w x|$ & $\mathbf{y} z$ \\
\hline
\end{tabular}

Amblysemius AG. 1 + gracilis Ag.

Sauropsis Ag. 3

\# latus Ag.

longimanus.

$t$ mordax.

Thrissonotus AG. 1

+ Colei Ag.

Thrissops Ag. 7... cephalus Ag. .... formosus $A G$. intermedius AG.

₹ mesogaster AG. micropodius Ag.

\# salmoneus Ac.

₹ subovatus Ag.

Dxygonius Ag. 1 . tenuis $A G \ldots$

Teptolepis Ag. 21.

₹ Bronni Á.

* caudalis Ag.

† filipennis AG. . .

₹ Jägeri Ag. . . . . .

† longus AG. .....

$\dagger$ tenellus AG. .

‡ contractus AG. ... crassus AG.

\& Davilai Ag. dubius Ag.

Knorri AG.

+ latus Ag. macrolepidotus AG. .

\# macrophthalmus EG.

† paucispondylus AG. polyspondylus AG.

† pusillus AG. sprattiformis AG. Voithi Ag.

Brodiei AG. ...... Bronus

$$
\text { * * }
$$

Aspidorlnymelnus

† Anglicus Ag.

† Walchneri Ag. acutirosris $A_{G}$. enodus EG.
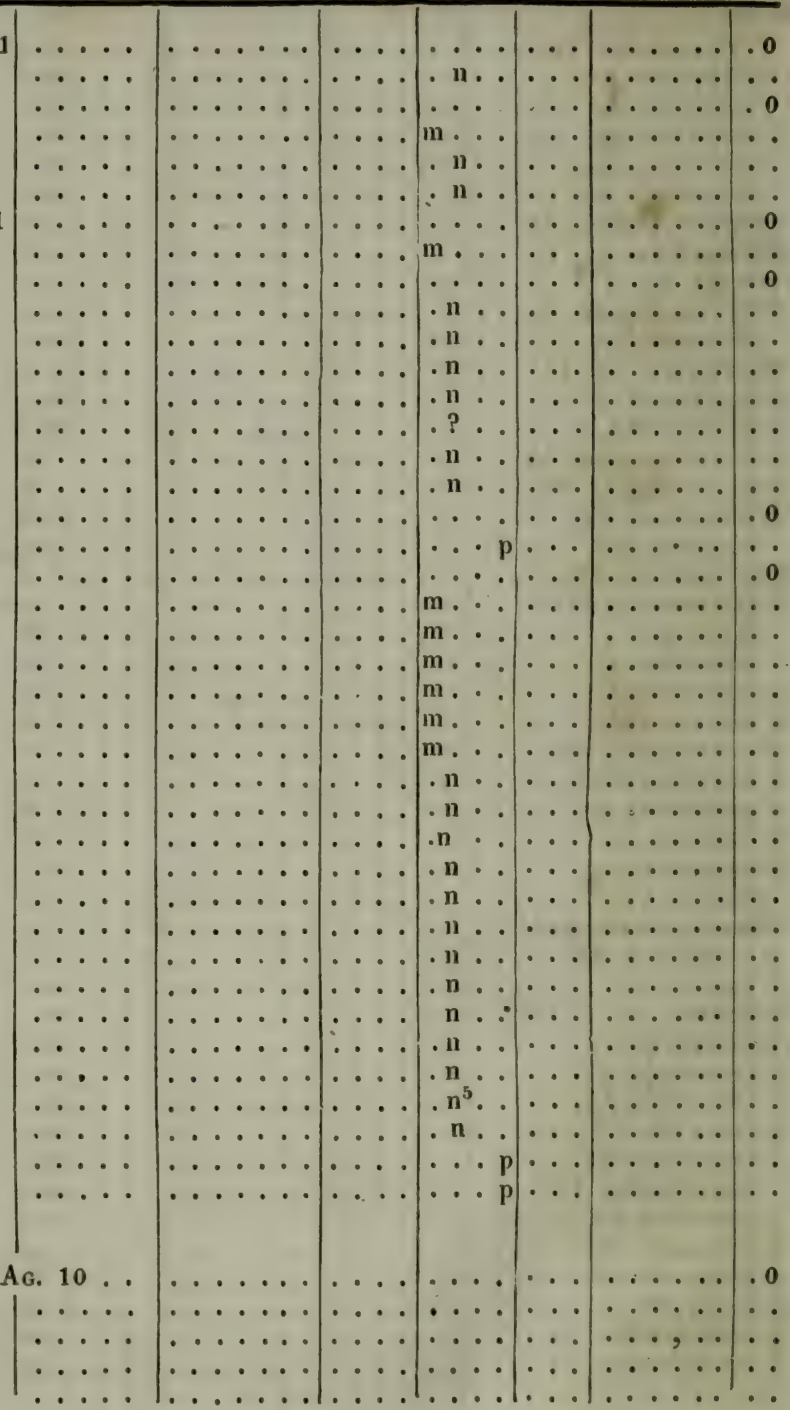


\begin{tabular}{|c|c|c|c|c|c|c|c|}
\hline Benennungen. & Weltgegend. & $a b c d e f g$ & h i k l & $\mathrm{mn}$ op & $q \times f$ & $\operatorname{stuvw} x$ & $y \%$ \\
\hline
\end{tabular}

F Iepturus Ag.

$\neq$ longissimus Mü.

₹ mandibularis AG. ornatus EG. speciosus AG. Comptoni As.

Belonostomus A $_{G} 9$ acutus Ag.

† Anningae AG....

\# Kochi Mü.

+ leptosteus $\dot{A G}$ Münsteri AG. sphyraenoides AG.

₹ subulatus Ag.

₹ tenuirostris AG.

$\neq$ ventralis AG.

Saurostomus AG. 2 esocinus Ag. sp. AG,

Ceramurus Eg. 1. macrocephalus EG.

Megalurus $A G .4$. brevicostatus AG. . . elongatus $\mathrm{M} \ddot{\text {. }}$. lepidotus $A G$. parvus Mü.

Mracrosemius $\dot{A}_{G}$. 2 rostratus AG.

$\dagger$ brevirostris Ag.

Platygnathus $\dot{A g} .2_{2}$ (num ad Heterocercos referendus?) Jamesoni Ag.
paucidens AG.

? Tibys Mü. polypterus $\mathbf{M}$ Ü. ...

\section{PYCNODONTES AG.}

\section{CTobulodus Mü.} elegans Mü.

Pycnodus Ag. 42 .

$\dagger$ priscus Ag.

+ biserialis AG. Bucklandi Ag. didymus AG. + discoides AG. † gracilis Mü. 


\begin{tabular}{|c|c|c|c|c|c|c|c|}
\hline \multirow{3}{*}{ Beñnuüyen } & Weltgegend. & KohlenP. & SalzP. & OolithP. & $\begin{array}{l}\text { Krei- } \\
\text { deP. }\end{array}$ & MolasseP. & Neu \\
\hline & 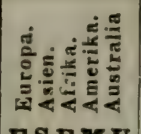 & 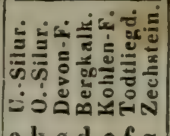 & 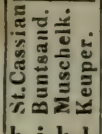 & 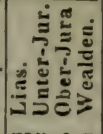 & 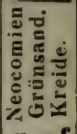 & 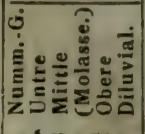 & 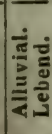 \\
\hline & E S F M U & $a b c d e f g$ & h i k l & mn op & $q \times$ I & st. uvw $\mathrm{x}$ & $y z$ \\
\hline
\end{tabular}

Pycnodus) granulatus $\mathrm{Mü}$ Jugleri Mü.

$\div$ latirostris AG. minutus Mü.

t obtusus Ag. ovalis Ag.

$\dagger$ parvis Ag. Preussi Mü. rugulosus $A G$.

$\dagger$ tristigius Ac. umbonatus A6.... gigas AG.

Ticoleti Ag. rhombus AG. Hugii A 6 .

$\dagger$ latidens AG.

† minor Ros.

Mantelli AG.

+ Couloni AG.

* Hartlebeni Roe.

+ minor AG. complanatus (Ag.) Reviss angustus $A G$.

+ elongatus AG.

† latior Ag.

+ marginalis AG. rhomboidalis Reuss rostratus Reuss... scrobiculatus Reuss semilunaris Reuss subclavatus AG. subdeltoideus AG. orbicularis AG. platessus AG... Toliapicus Ac.

Sphaerodus Ag. 29 minimus Ag. annularis Ag.

$P$ microdon AG

‡ hybridus $\mathrm{Mü}$

? minor AG.

‡ subannularis Mü.

₹ submammillaris Mü.

₹ subradiatus Mö.

₹ tetragonus Mü.

|
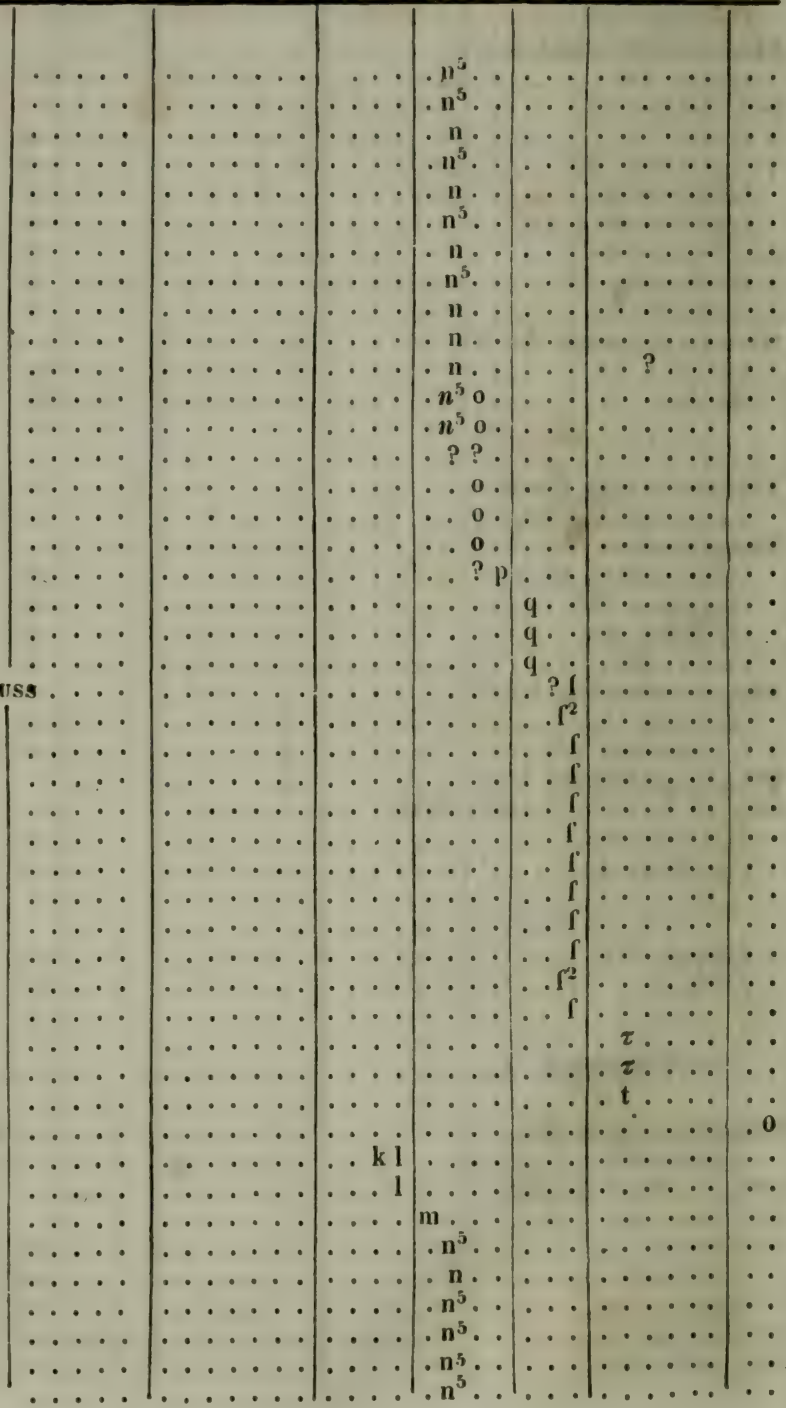
\begin{tabular}{l|l|l|l|l|l|l|l|l|l}
\hline Benennungen. & Weltgegend. & a b c d e f g h i k l mn o p & q r f & s t u v w s & y
\end{tabular}

gigas AG.

‡ cylindricoides Roe. semiglobosus Du. Neocomiensis [?] AG. mitrula Ag.

crassus Ag. rugulosus Eg.

\# tenuis Reuss cinctus AG.

₹ cingulatus $\mathrm{Mü}$. pygmaeus Mü.

₹ subtruncatus $M \ddot{\text { ü. }}$ depressus AG. lens Ag. parvus AG. irregularis Ac. truncatus AG. conicus Ag. discus AG.

? oculus-serpentis $\hat{A G}_{\mathrm{G}}$.

Placodus AG. 5 . impressus AG. Andriani Mü. gigas $\mathrm{Mü}$

Münsteri Mü. rostratus Mü.

Colobodus AG. 1 . Hogardi Ag.

Microdon As. 8. ₹ abdominalis Ag. analis AG. elegans $A G$. hexagonus $A_{G}$ notabilis Mü.

+ platurus AG. trigonus $A G$. radistus Ag.

Scrobodus Mü. $i$ subovatus Mü.

Gyronchus Ag. 1 . oblongus Ag.

Gyrodus Ac. 30

$t$ analis fig.

† circularis Ag. Cuvieri Ag. frontatus AG.

† gibbosus Mü. gracilis Mü.

₹ laticauda Mü. macrophthalma Ag.

$\dagger$ macropterus AG.

₹ maeandrinus Mü.

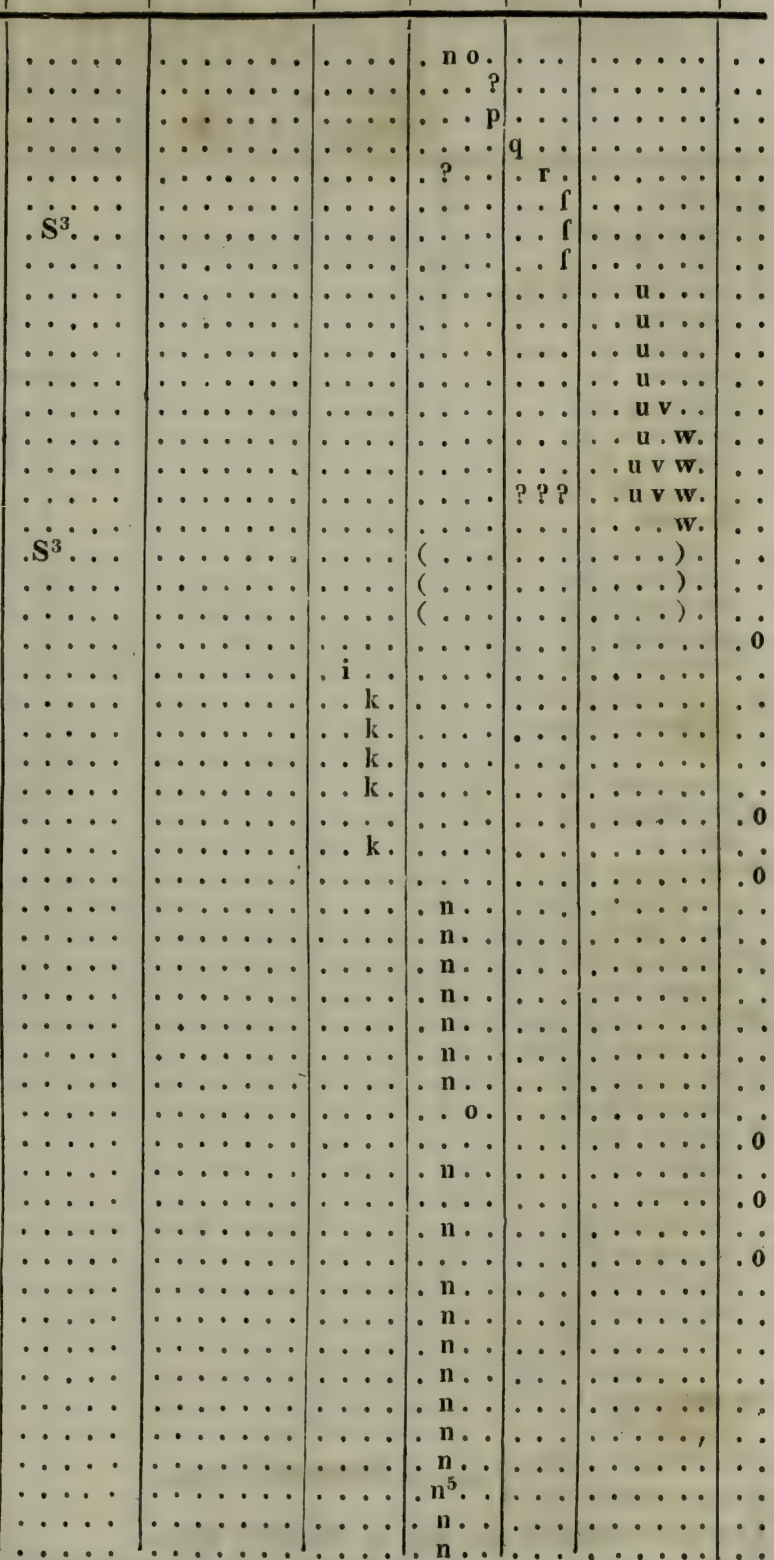




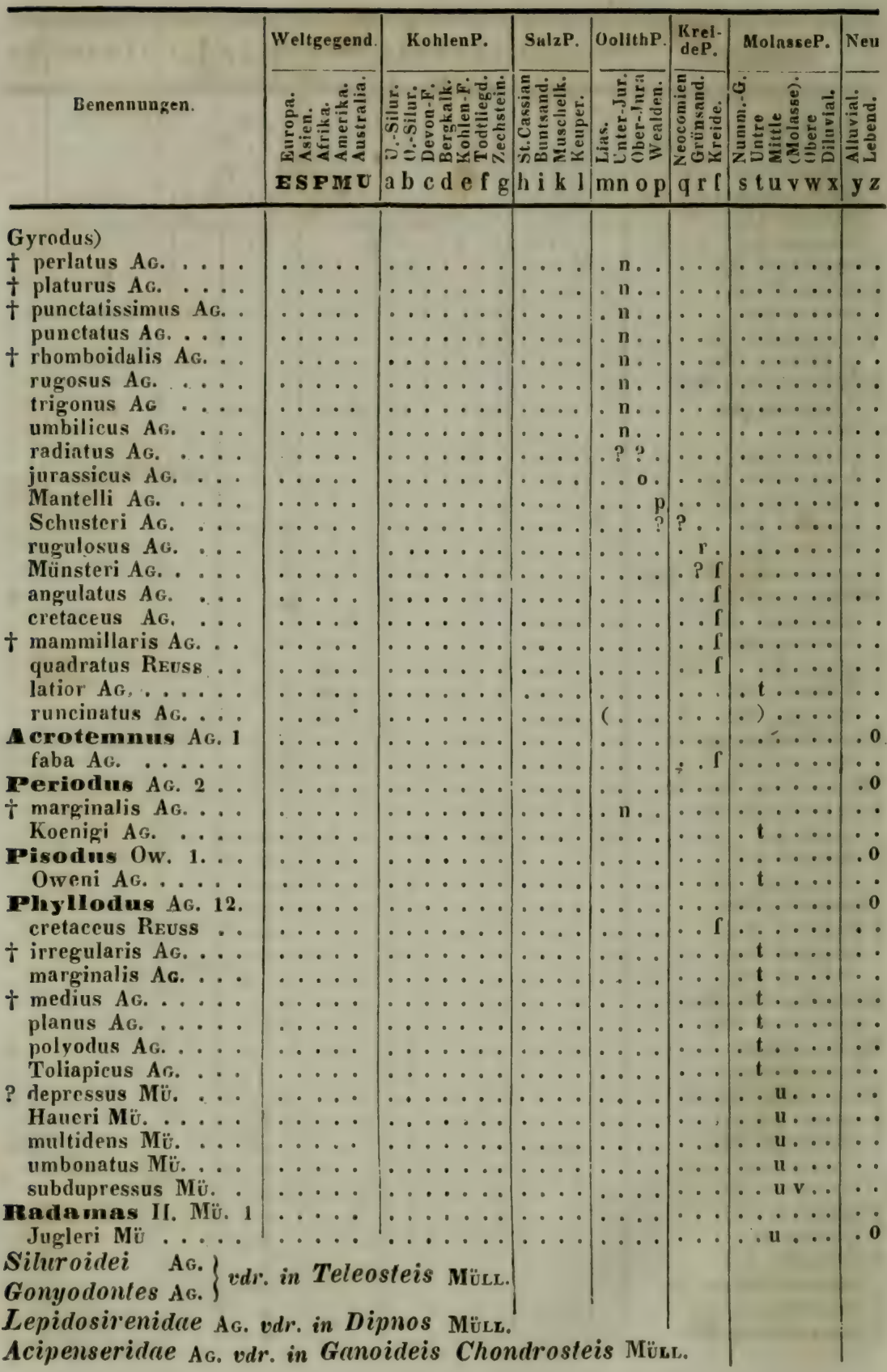




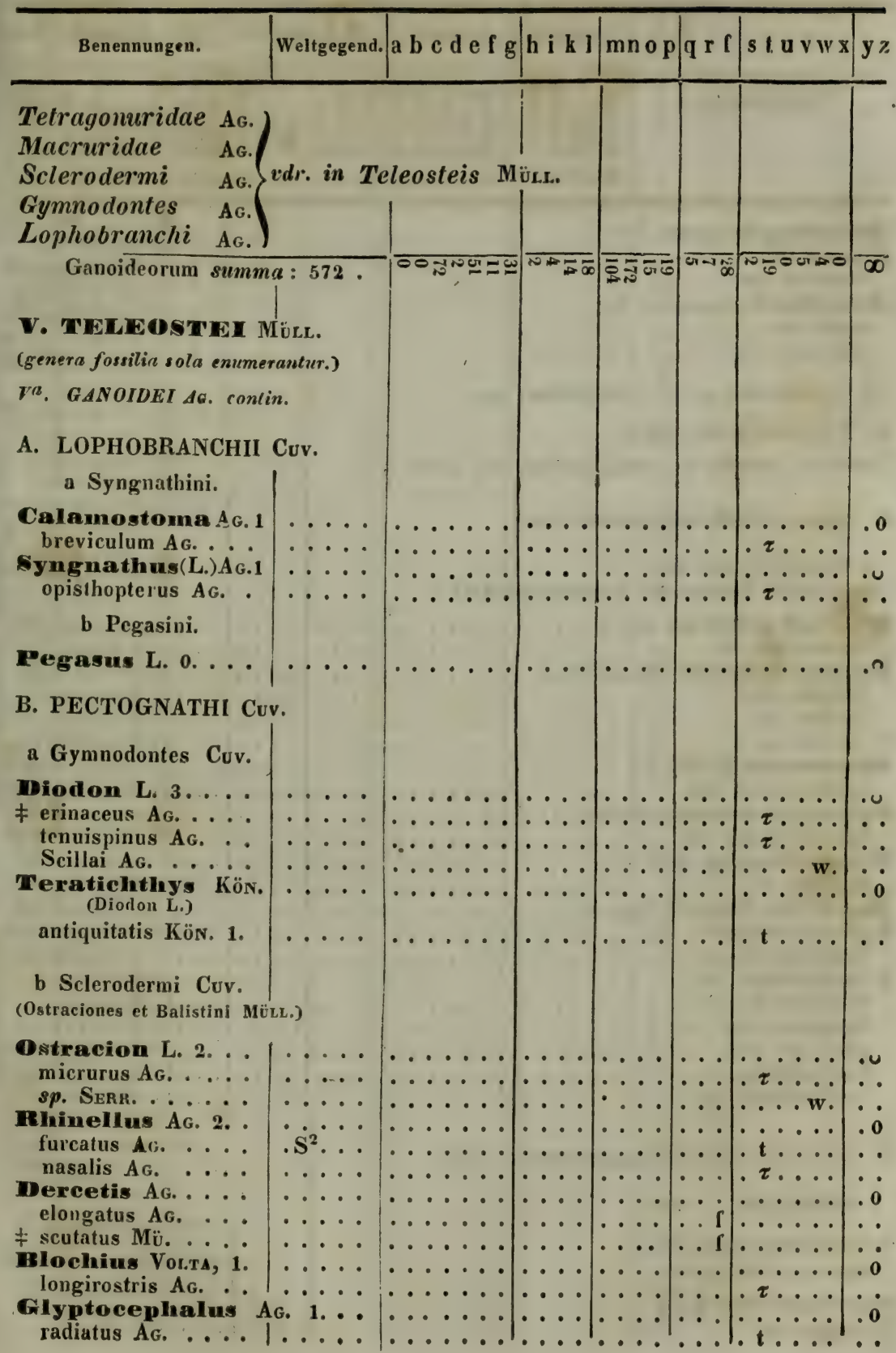




\begin{tabular}{|c|c|c|c|c|c|c|c|}
\hline & Weltgegend. & KohlenP. & SalzP. & OollthP. & \begin{tabular}{|c|} 
Krei- \\
deP.
\end{tabular} \mid & MolasseP. & Neu \\
\hline Benenumugen. & 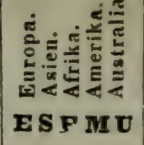 & 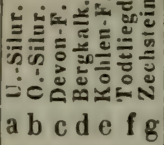 & 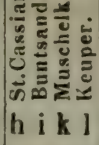 & 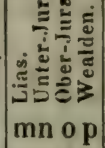 & 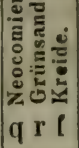 & 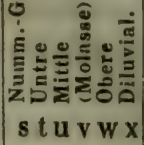 & 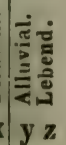 \\
\hline
\end{tabular}

Acanthopleurus AG. 2 ...

\# brevis EG. .

serratus Ac. . . . $1 . .$.

Acanthoderma $A G, 2 \ldots$

ovale Ag.

spinosum $A$ g.

$V^{b}$. CYCLOIDEI ot VE. CTENOIDEI AG.

C. Physostomi Müll.

(Omnes = Cycloldei Aq.; Malacopterygli Cuv. pars.)

1. malacopterygi apodes Cuv.

a Anguilliformes AG.

(Muraenoidei, Symbranehii et Gymnostini MürL.)

Rhynchorhinus AG. 1. . .

† branchialis Ao. . . ! .....

Ceptocephalus (Gron.)Ag.3 gracilis AG. . . . . . . . medius Af. .....

t taenia Ag.

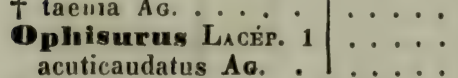

Splnagebranchus $B_{\mathrm{LOCH}}, 1$ formosissimus AG.

Enchelyopus AG.1 tigrinus AG.

Anguilla (Thuns.) Cuv. 8. .

t branchiostegalis AG. ..... brevicula AG. ....

t interspinalis AG. .

latispina AG. .... leptoptera AG.

pachyura AG.... ventralis AG. .... multiradialis AG. .

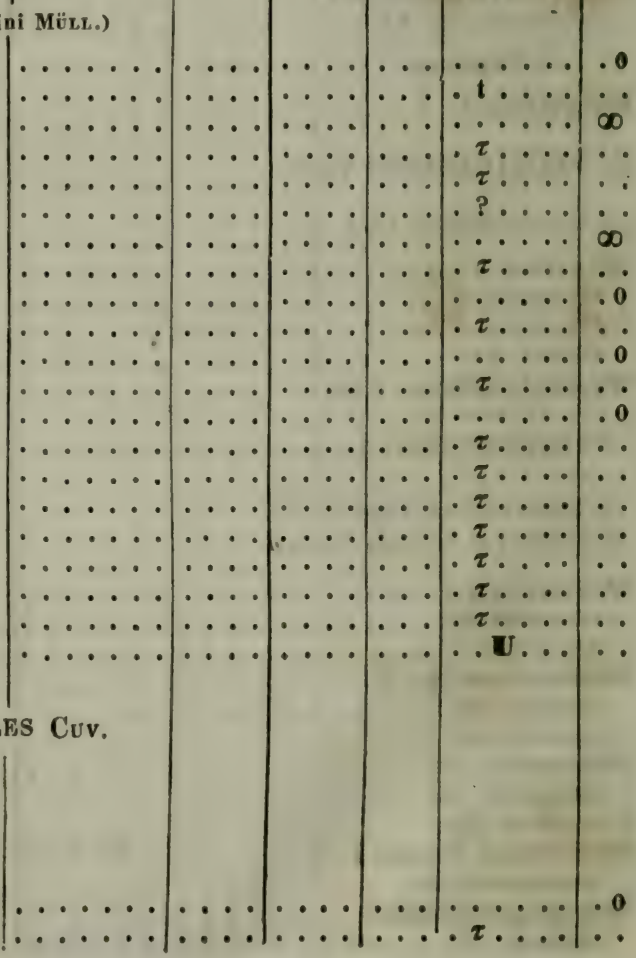

2. Malacopterygil Abdominales Cuv.

a Heteropygii Telck.

b Clupeidae (Cuvv.) MüLL. (Halecoides AG, pars)

Coelogaster Ag. 1 $\dagger$ analis AG.
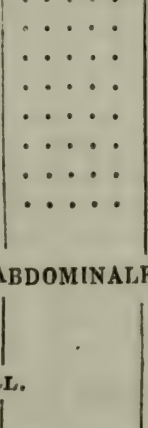


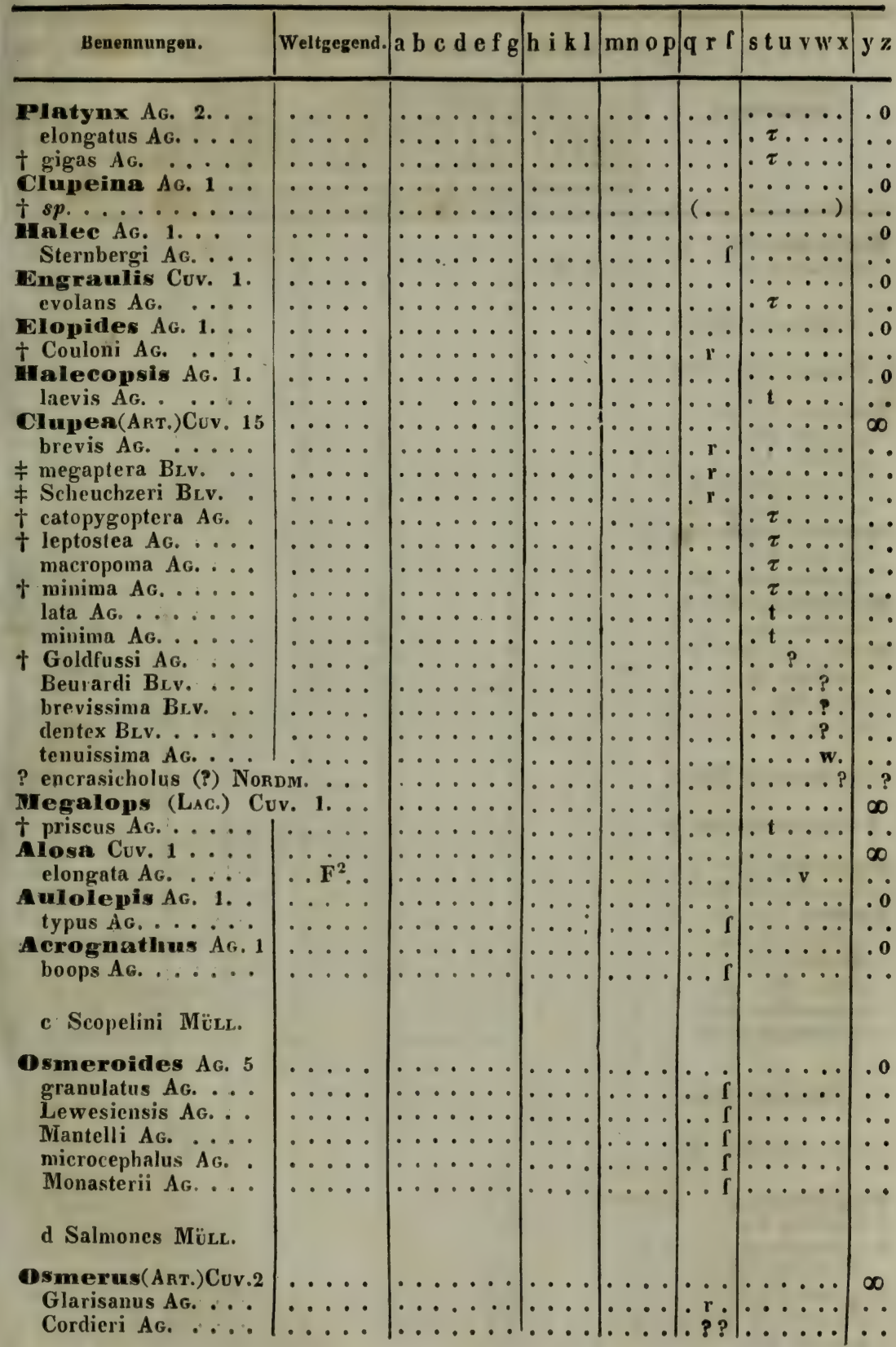




\begin{tabular}{|c|c|c|c|c|c|c|c|}
\hline & Weltgegend. & KohlenP. & SalzP. & OolithP. & $\begin{array}{l}\text { Krei- } \\
\text { deP. }\end{array}$ & MolasseP. & Neu \\
\hline Benennungen. & 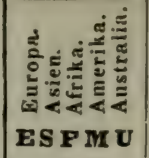 & 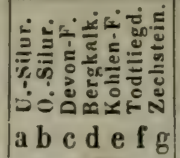 & 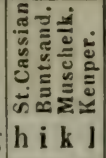 & 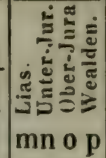 & 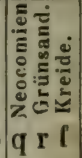 & 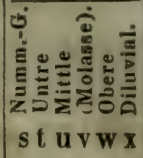 & 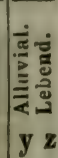 \\
\hline
\end{tabular}

Mallotus Cuv. 1. villosus Cuv.

e Galaxiae Müll.

f Esoces (Cuv.) Mülz.

Istieus Ag. 4 ... gracilis Mü. ..... grandis AG. … macrocephalus AG. . microcephalus AG. .

Sphenolepis Ag. 2. Cuvieri Ag. . . . . . squamosseus AG.

Holosteus AG. 1. . esocinus $A$ g.

Esox (L.) Cuv. 3 ... lepidotus AG. .... Ottoi Ag.

n. $8 p . A$ s.

g Mormyri MüL..

h Cyprinodontes AG

Poecilia Cuv. 1 . . Lametheriei BLv.

Lebias Cuv. 5. . . . cephalotes AG. ... Meyeri Ag. ..... gobio Mü., AG. perpusillus AG. . . crassicaudus AG. . .

\section{i Characini MüLL.}

k Cyprinoides AG.

Thaumaturus Reuss $1 \ldots$ † furcatus Reuss ... $\ldots \ldots$. Cyprinus (L.) Cuv. 1 .... carpio (L.) GaL. . .

Aspius AG. 2 ... .... Brongniarti AG. ..

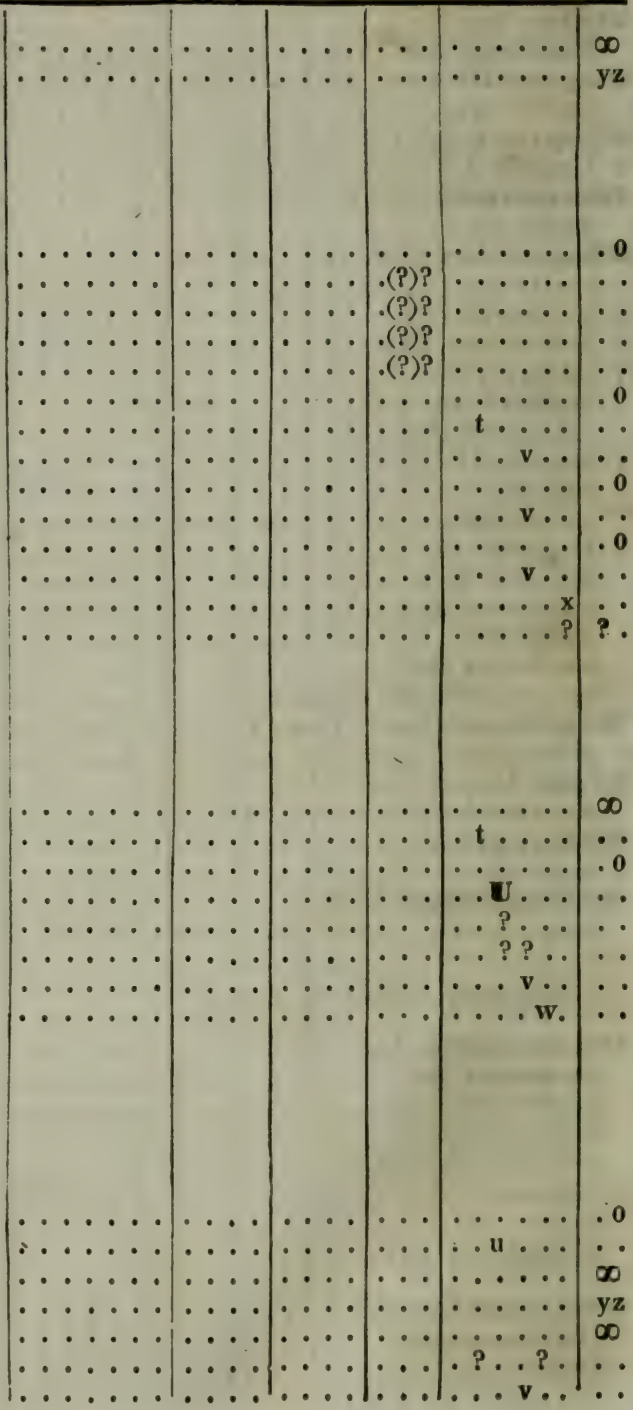




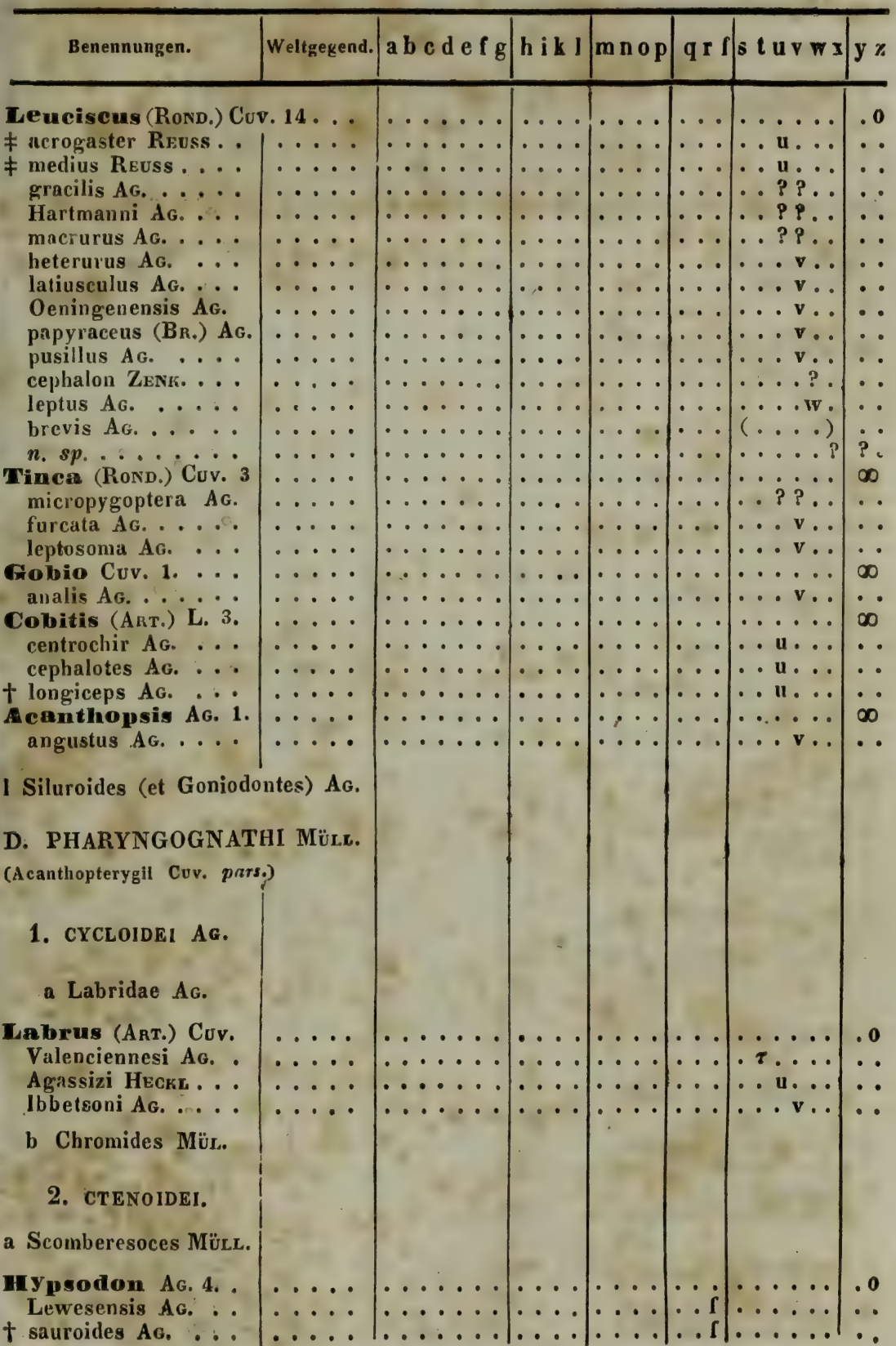

43 (1847) 


\begin{tabular}{|c|c|c|c|c|c|c|c|}
\hline \multirow{3}{*}{ Benennungen. } & Weltgegend. & KohlenP. & SalzP. & OolithP. & $\begin{array}{l}\text { Krei- } \\
\text { deP. }\end{array}$ & MolasseP. & Neu \\
\hline & 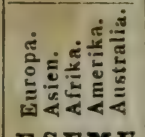 & 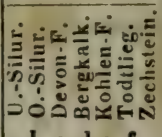 & 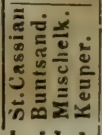 & 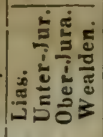 & 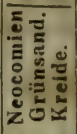 & 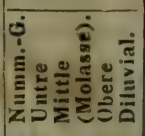 & 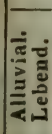 \\
\hline & E S P M U & a b c de $f g$ & h i k l & mn o p & $9 \mathbf{r}$ & s t. u v w $x$ & $\mathrm{y} \mathbf{z}$ \\
\hline
\end{tabular}

Hypsodon)

oblongus AG. .....

Toliapicus AG.

E. ANaCANThiNi Mülz.

Ctenoidei Aa. pars.

a Pleuronectae Cuv.

Pleuronectes (ARt. Lin.) 1 .

? maximus Bl.v. .........

Finombus(LAC.) Ag.1

minimus $A c$.

b Ophidini Mür.L.

c Gadoidei MüLL.

Gadue (Art.) Lin. 1. .... polynemus Fisch. . ....

Ampheristus $\mathrm{A}_{\mathrm{G}} \mathbf{1}^{1} \ldots \ldots$

† Toliapicus Ag. ... .....

Phinocephalus $A G .1$. . .

† planiceps AG. ... . . . .

PrehyeephalusA .1 . . . .

† cristatûs AG. ....

F. ACANTHOPTERI MüLL.

(Aeantopterygii Cuv.; Ctenoidei Ag. plerigue.)

\section{Cycloides Ag.}

a Atherinoides Ag.

Atherina (Ant.) L. 3 macrocephala AG. .

† minutissima AG.

Browni (Gm.) Nordm.

b Pediculati Cuv., Lophioides Ag.

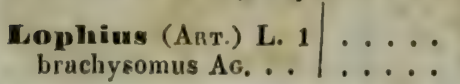

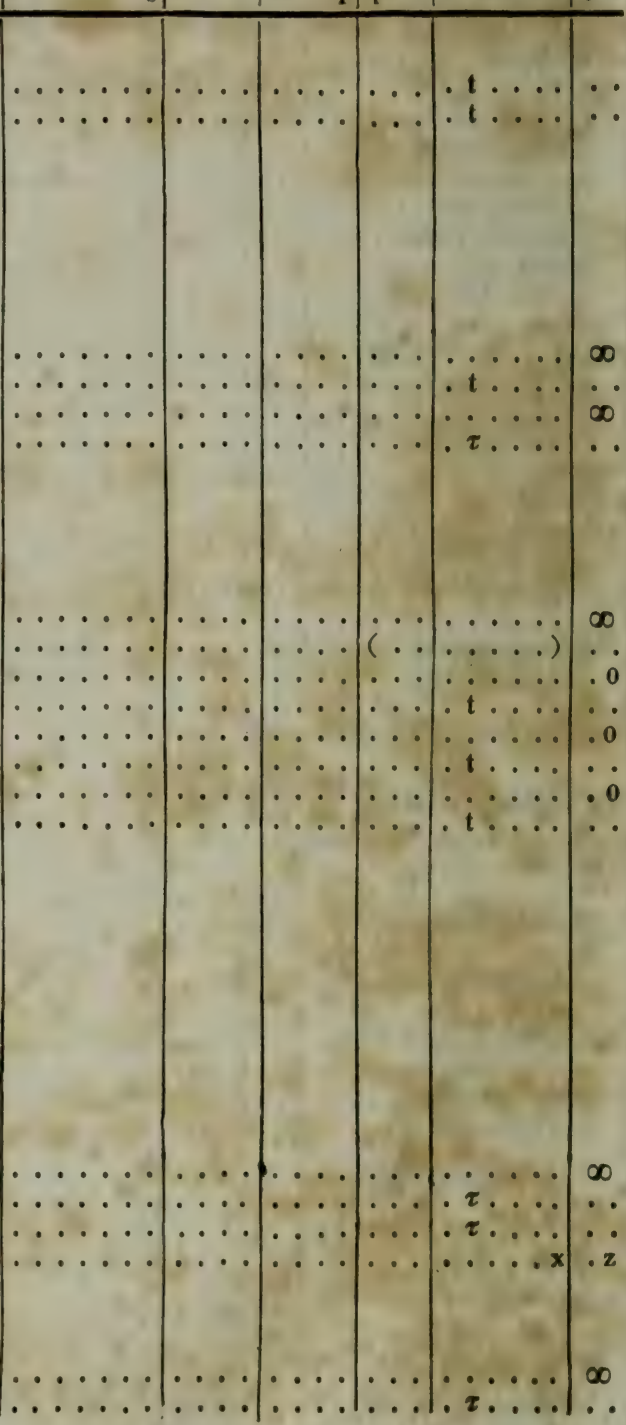




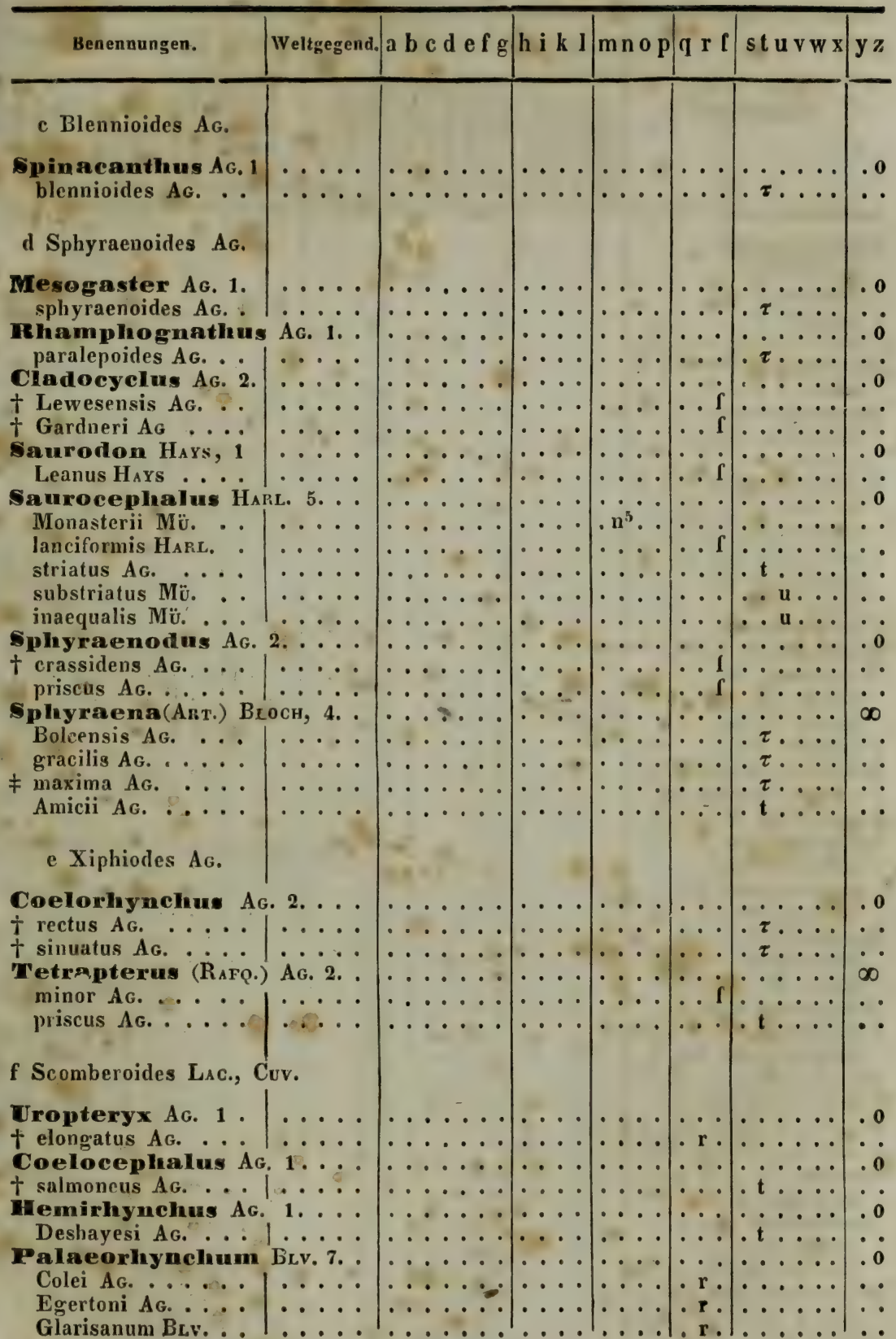




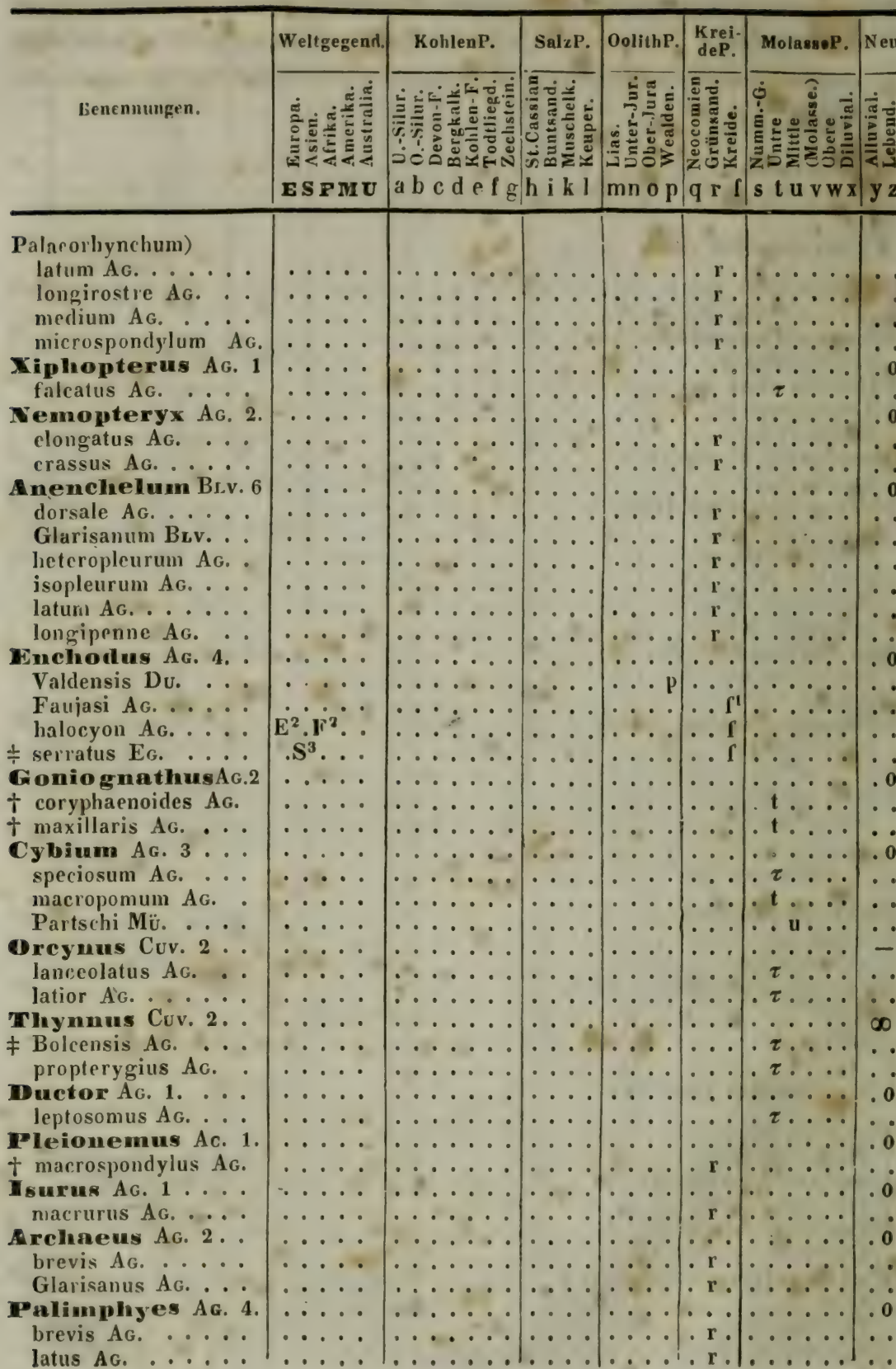




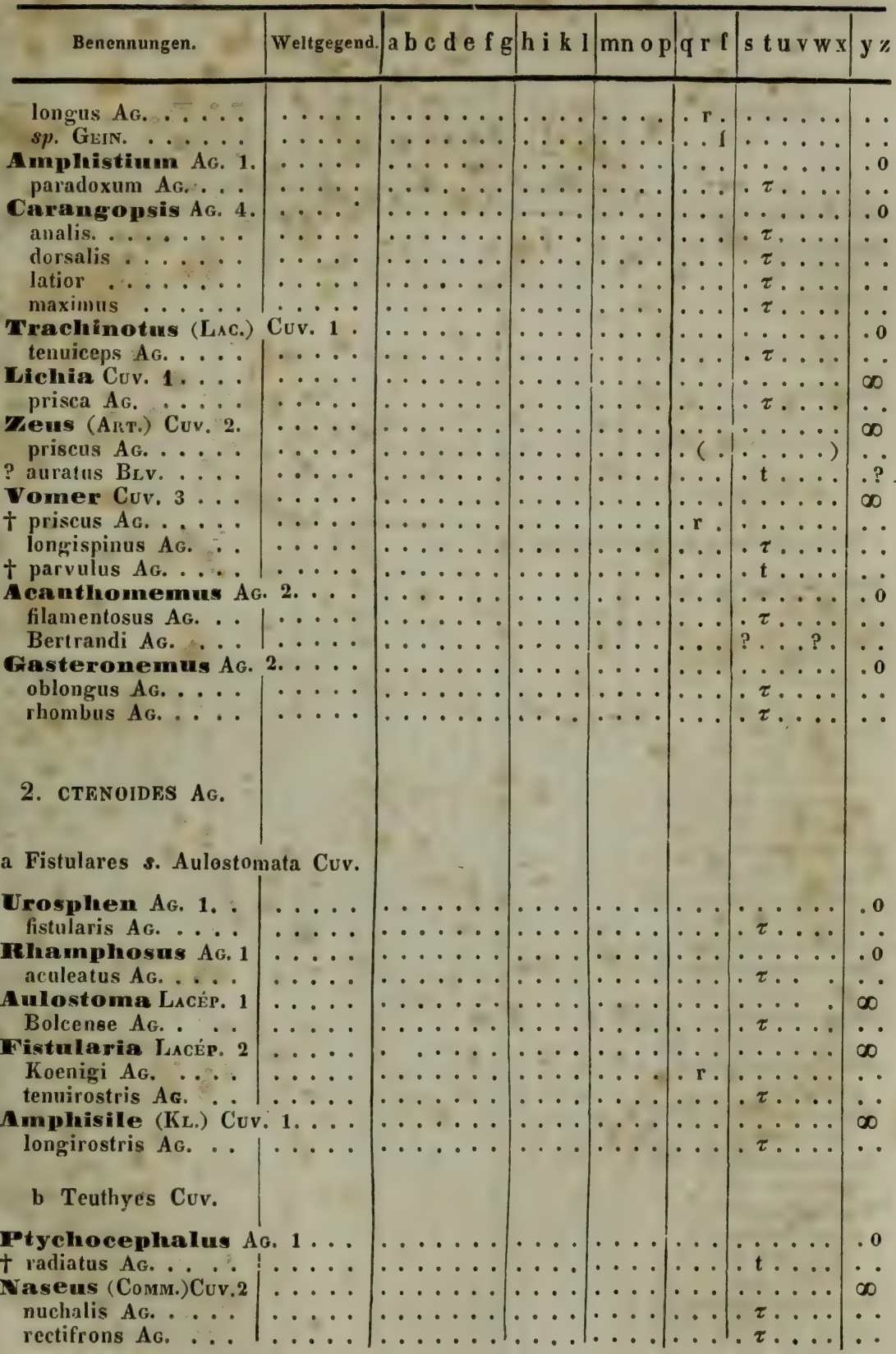




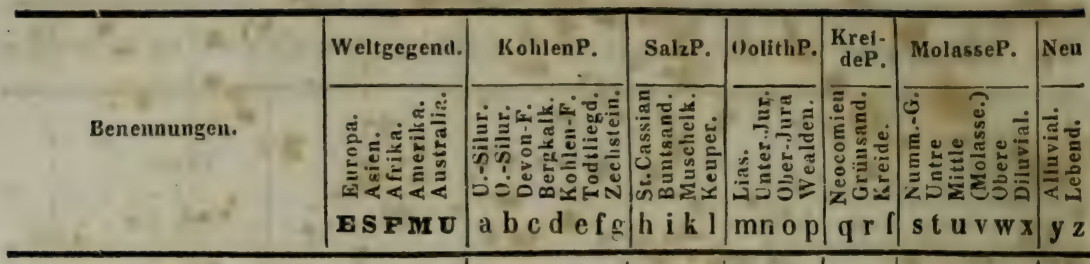

Acamthurus (Forsk.) 2. ovalis AG. tenuis Ag.

\section{c Gubioidei (AG.)}

Gobius (Art.) Cuv. 2 macrurus AG. . ... microcephalus AG.

\section{d Taeniodei.}

e Squamipennes s. Chaetodontes Cuv.

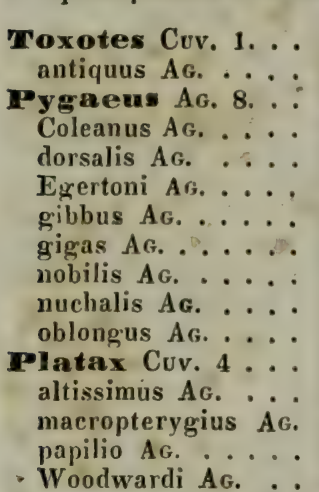

Pomacanthus LACÉr. 1. . subarcuatus AG. . . $\ldots$...

HoIacanthus LAcÉp. 1 microcephalus Ag.

Inacrostoma AG. 1. altum AG.

VZanclus (Сом.), CV.1 brevirostris AG.

Sentoplnagus CV. 1 frontalis AG.

Ephippus Cuv. 3 . Iongipenuis Ag. . oblongus AG. .... Oweni Ag.

Semiophorus Ag. 2 velicans $A G$. velifer AG.

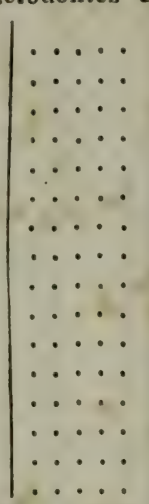


\begin{tabular}{l|l|l|l|l|l|l|l}
\hline Benenuungen. & Weltgegend. & a b c d e f g & hi k l mn o p & q r f & s t u v w x & y z
\end{tabular}

f Mugiloides Civv.

Calmmopleurus Ag. 1 [hoc loco ?] .................... . . . . . .

$\ddagger$ cylindricus Ag.

IIugil (ART.) L. 1 . princeps AG. .... .... $\mathbf{M}^{3}$.

g Labyrinthici (Cuv).

h Sciaenoides Cuv.

Sciaemurus AG. 2. Bowerbanki Ag. crassior Ag.

Ddonteus AG. 1 . sparoides Ag.

Pristipoma Cuv. 1 furcatum Ag.

i Sparoidei Cuv. (et Maenides).

Capitodus Mü. [lüjus loci?].s angustus $\mathbf{M}$ Ü. dubius Mü.

? interruptus Mừ. subtruncatus Mü. truncatus Mü.

Soricidens MÜ. 1 . $s p$. Mü.

Sargus Cuv, $4 \ldots$ Cuvieri Ag. . . . . . Jomnitanus VAL. . . Rusuccuritanus VAL. Sitifensis VAL. .... Sparnodus AG, 5. altivelis Ag. elóngatus AG. . .... macrophthalmus AG. micracanthus $A G$. ovalis AG.

Pagellus Cuv. 2 . microdon Ag. leptosteus A $\cdots$

Dentex Cuv. 6. . . breviceps AG. . . . . . crassispinus AG. leptacanthus $\boldsymbol{A G}$. microdon AG. ventralis AG. Faujasi AG.

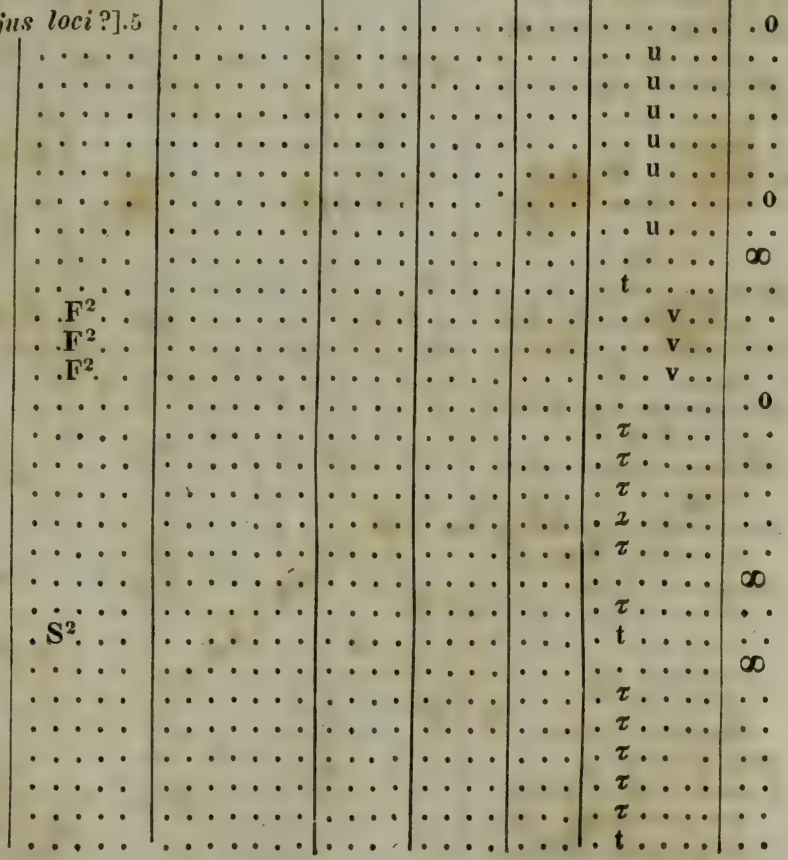




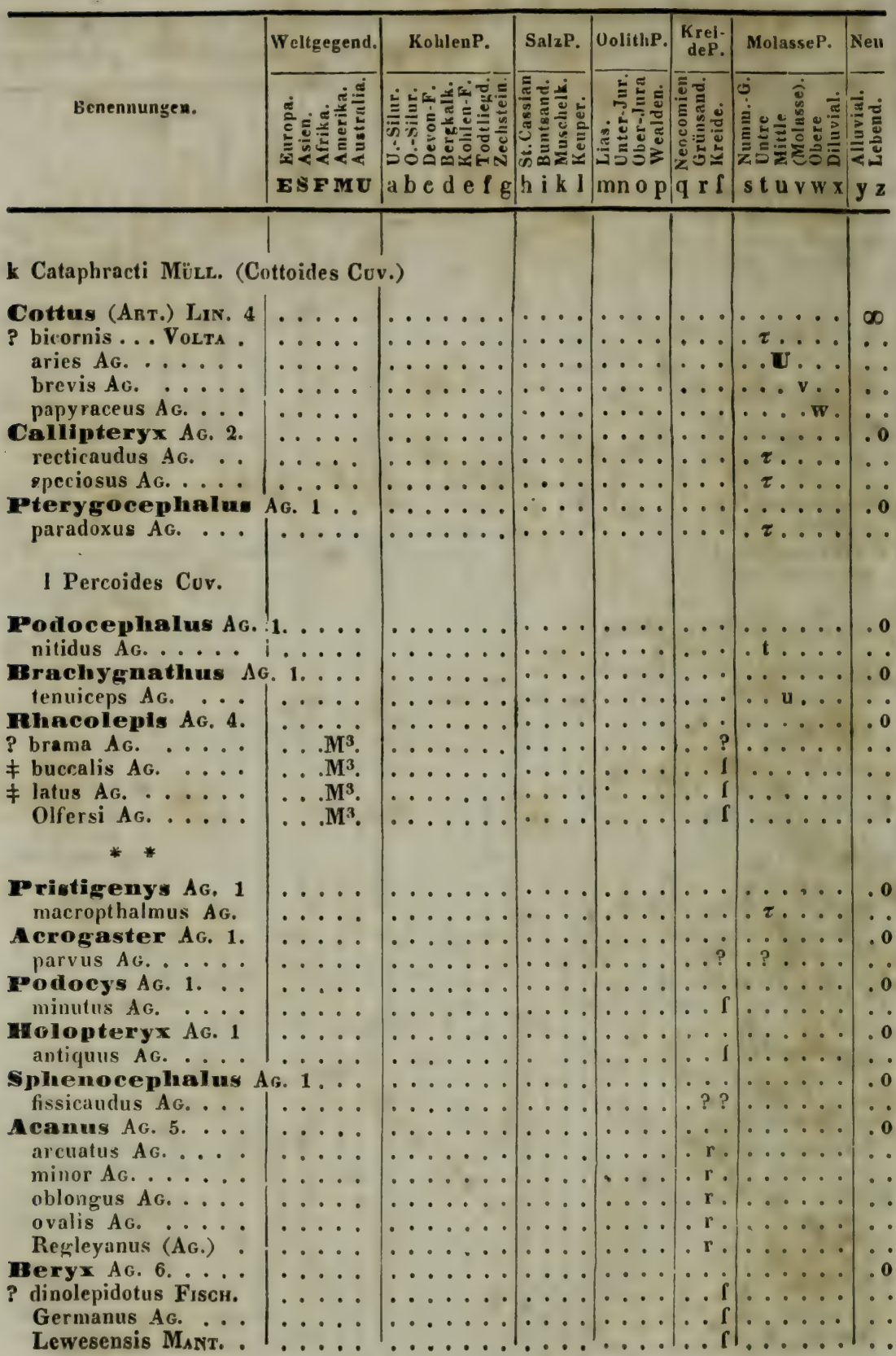




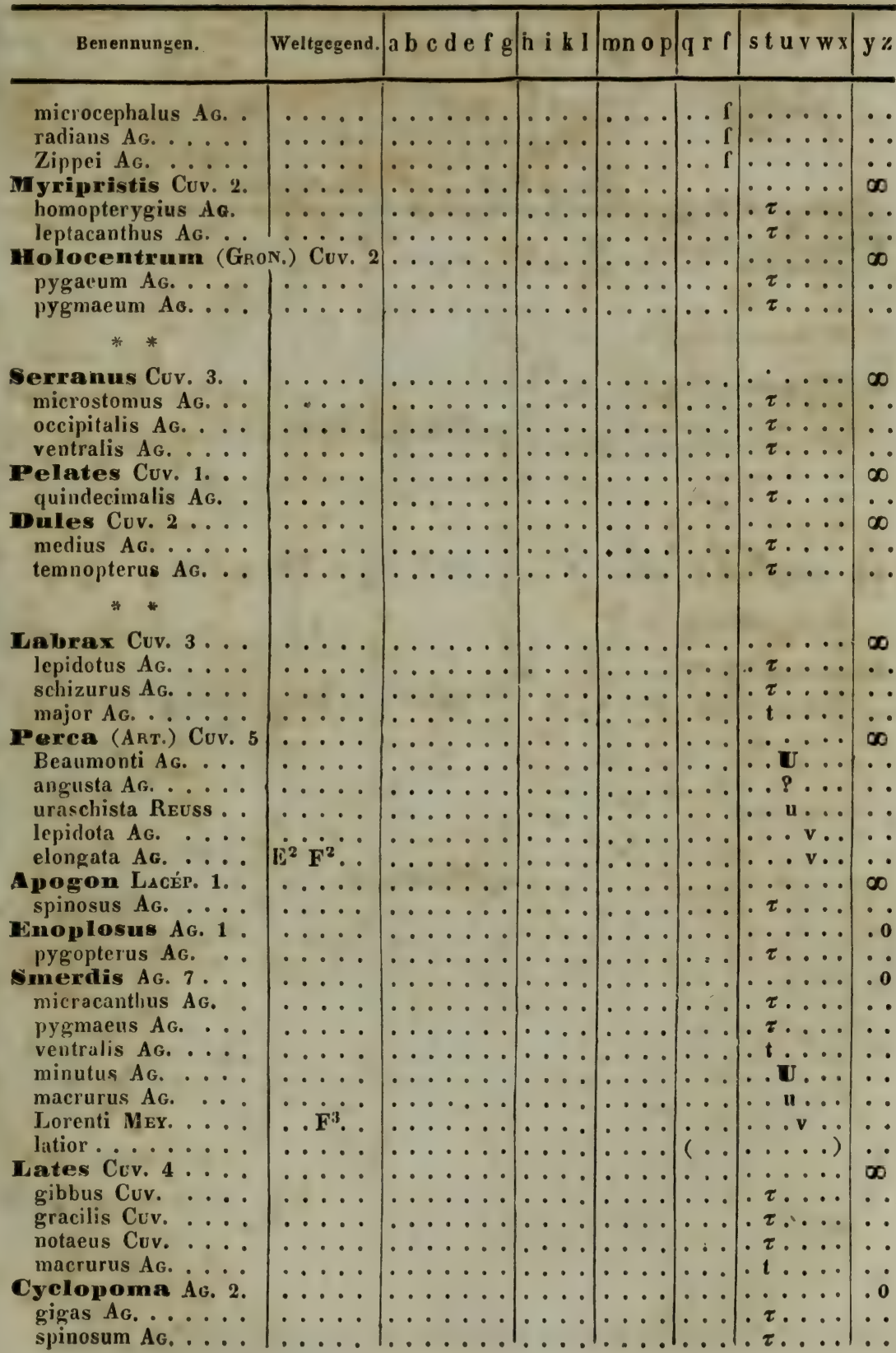




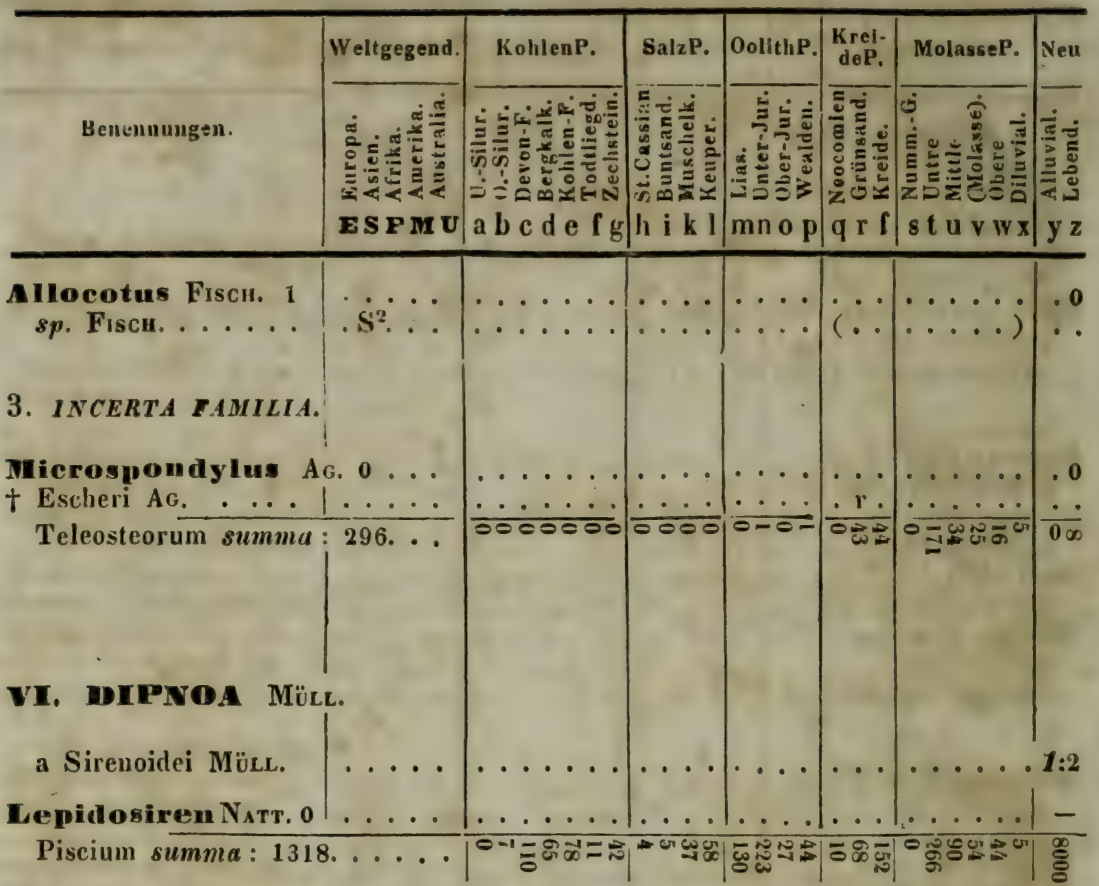




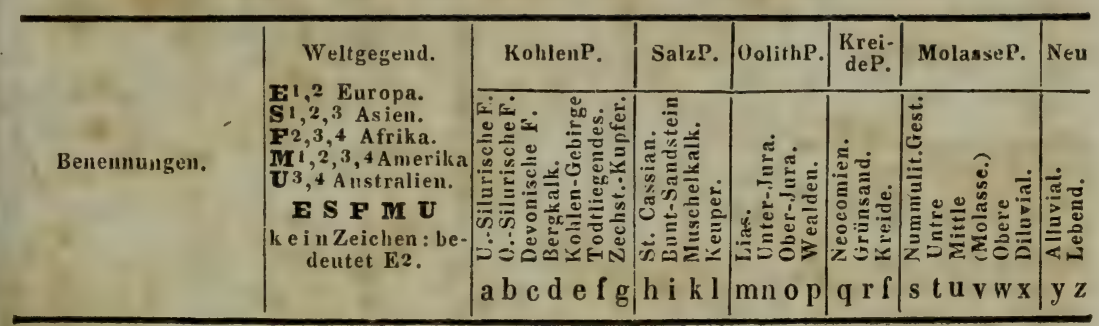

\section{Cl. XXII. REPTILIA : Lurche *}

\section{MATREACHIT BHGN.}

1. BATRACHII INCERTAE SEDIS.

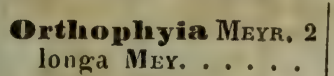
solida-Mey. .....

2. SALAMANDRINAE.

a Tritonides.

Andrias Tscuuni 1. Scheuchzeri Tsch. .

b Tritones.

Triton Laurenti 2. Noachicus GF.... opalinus MBY. ...

c Salamandridae.

Salamandra LiN. $5+\ldots$ ogygia GF...... spp. spp.

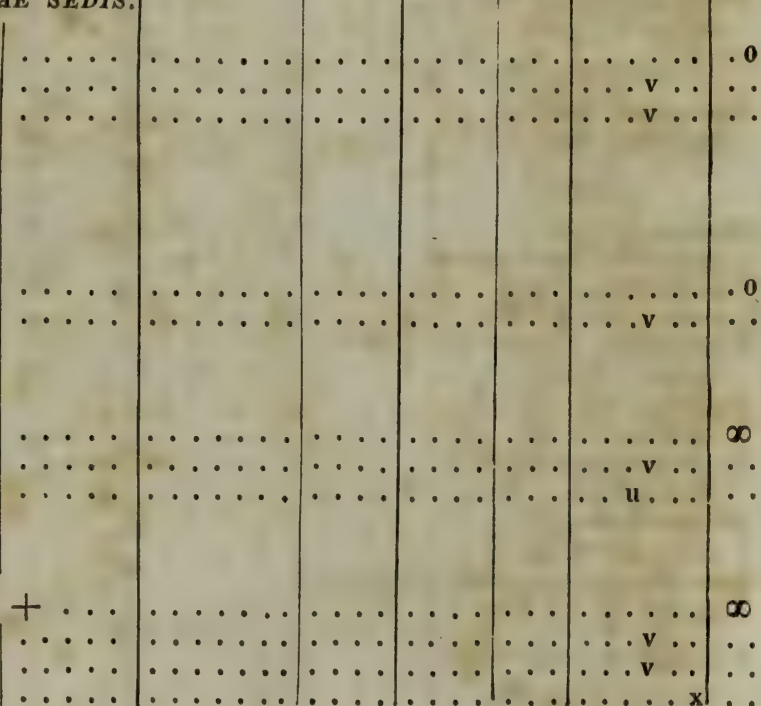

* Reptilium Ichnitue omnes, quns sc. auctor non a pedibus Reptilium ortos esse existimet, in solo Nomenclutore enumerantur. BR. 


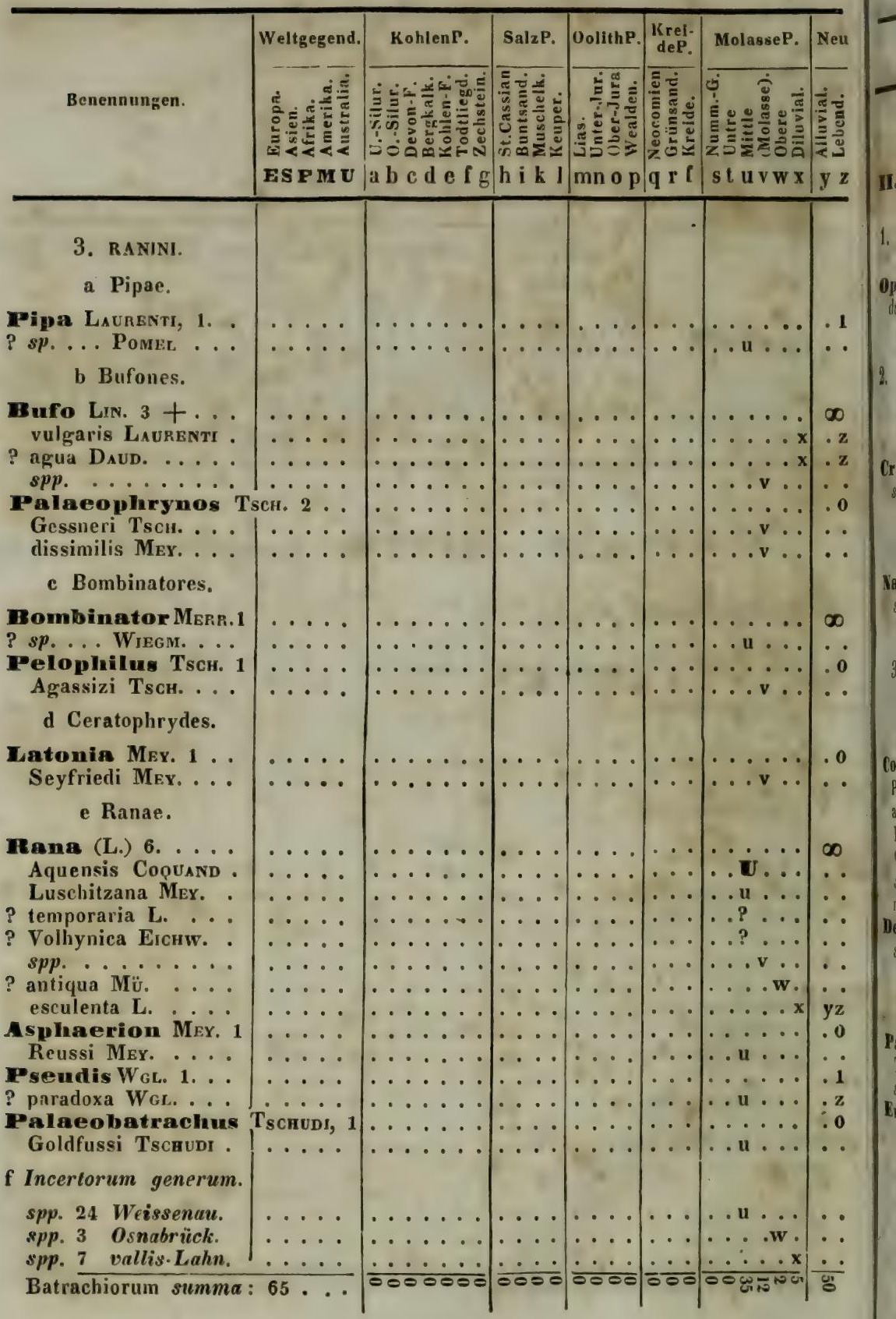




\section{OPMIIII BRgN.}

\section{OPHIDII INCERTAE SEDIS.}

Dphis GF. 1....
dubius GF. ....
2. VENENATI WIEGM.

a Viperinae.

Crotalus L. 1... $s p$. MoRRN.

b Najinac.

Naja Lavr. 1 .... sp. Pomel.....

3. INNOCUI WIEGM.

a Colubrinae.

Coluber (L.) 7 十. Podolicus Mer. ... arcuatus MEY.

Kargi Mex. ..... Oweni Mey.

spp. ........ natrix $L$.

Dendrophis Firz. 1 sp. MorRn.

\section{b Boinac.}

Palaeoplnis $0 w .2$. Toliapicus $0 \mathrm{w}$. sp. Ow............ Eryx Daud. 1 ...

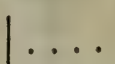
Weltgegend. a b c d e f g h i l l mn o p q r l s t u v w x y z sp. Colder. ..... $\dot{S}^{3}$.

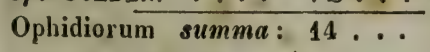

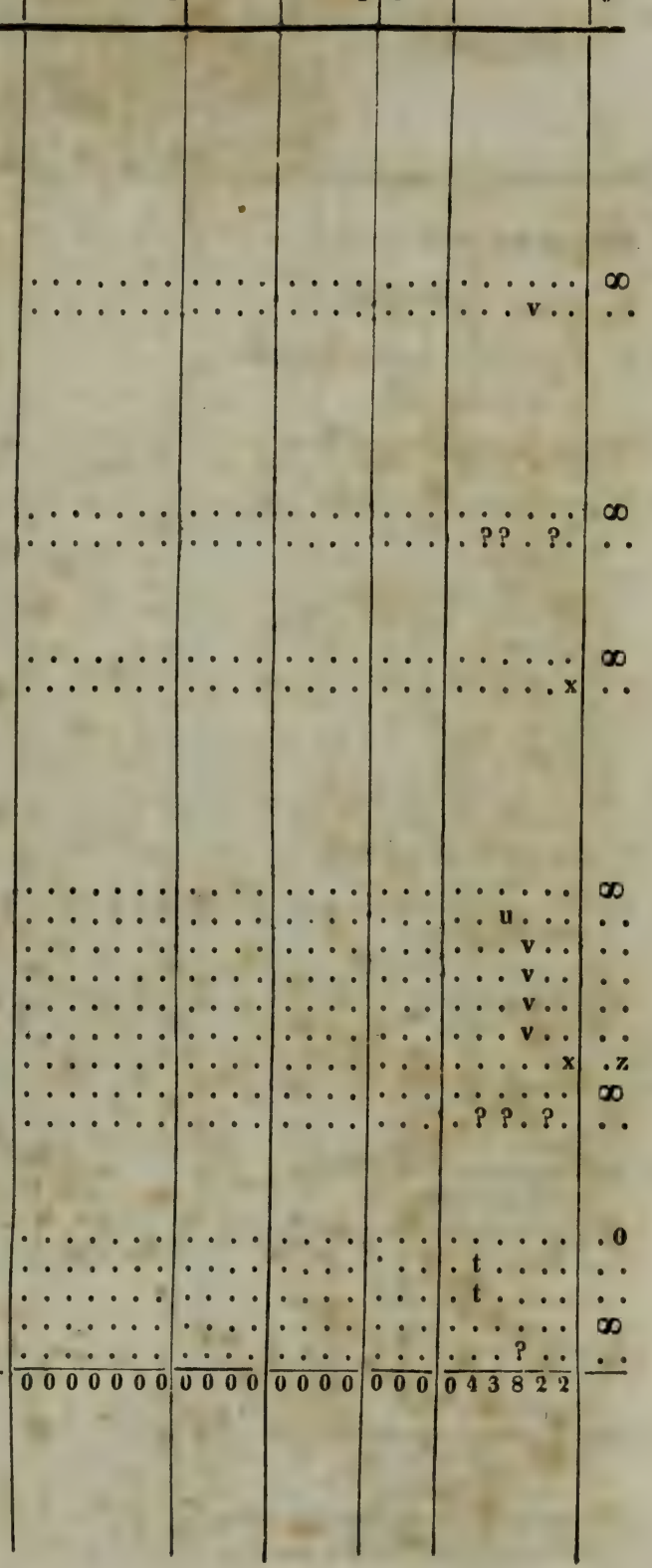




\begin{tabular}{|c|c|c|c|c|c|c|c|}
\hline & Weltgegend. & KohlenP. & SalzP. & OolithP. & $\begin{array}{c}\text { Krei- } \\
\text { deP. }\end{array}$ & MolasseP. & Neu \\
\hline Beneunungen. & 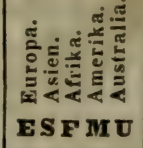 & 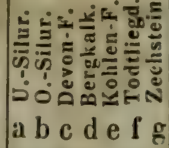 & 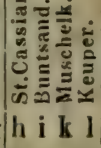 & 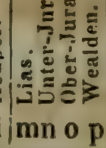 & 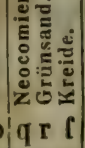 & 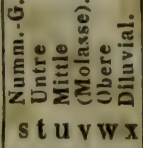 & 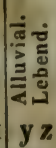 \\
\hline
\end{tabular}

III. SAUTII Brgn.

\section{A. DACTYLOPODES.}

1. Vertebris-non convexis.

a. Tetradactyli.

Macrospondylus MEY. 1 .

Bollensis MEY.

Mystriosaurus $\hat{K}_{\mathrm{A} u P}$

Bronguiarti $\mathrm{Br}_{\mathrm{R}}$.

Chapmani .

Egertoni (non KAUP.)

Laurillardi KAUP .

longipes $\mathrm{BR}$.

Mandelslohi $\mathrm{B}_{\mathrm{R}}$. . .

? Mandelslohi Br. :

Murcki Theod. ...

Schmidti Br. . ...

Senkenberganus MEY.

Tiedemanni Br.

PelagosaurusBr. 1 typus BR.

Stemeosaurus $\mathrm{G}$ erpria. brevirostris $\mathrm{Ow}$.

longirostris MEY.

Teleosaurus GÉfra. 3 asthenodeirus $0 \mathrm{w}$.

Cadomensis Geoffr.

Cadomensis var. Ow.

A eolodon MeY. 1.

priscus MEY.

Pleurosaurus Mey.

Goldfussi Mex.

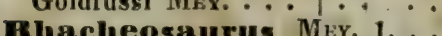
mey. 1... gracilis MEY.

b Pentadactyli.

Protorosaurus MEY. 2. . . .

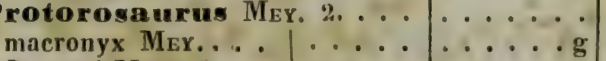
Speneri Mey. . . . .

Homoeosaurus MeY. 2. . . . Neptunius MEY.

Maximiliani MEy.

PoecilopleurumDesionch.2 Bucklandi Deslongch. | . . . .

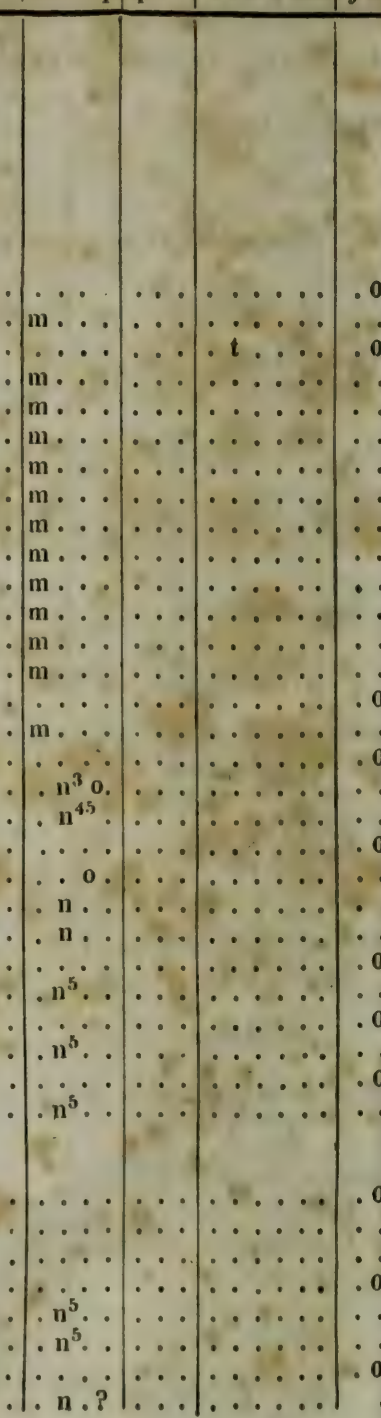




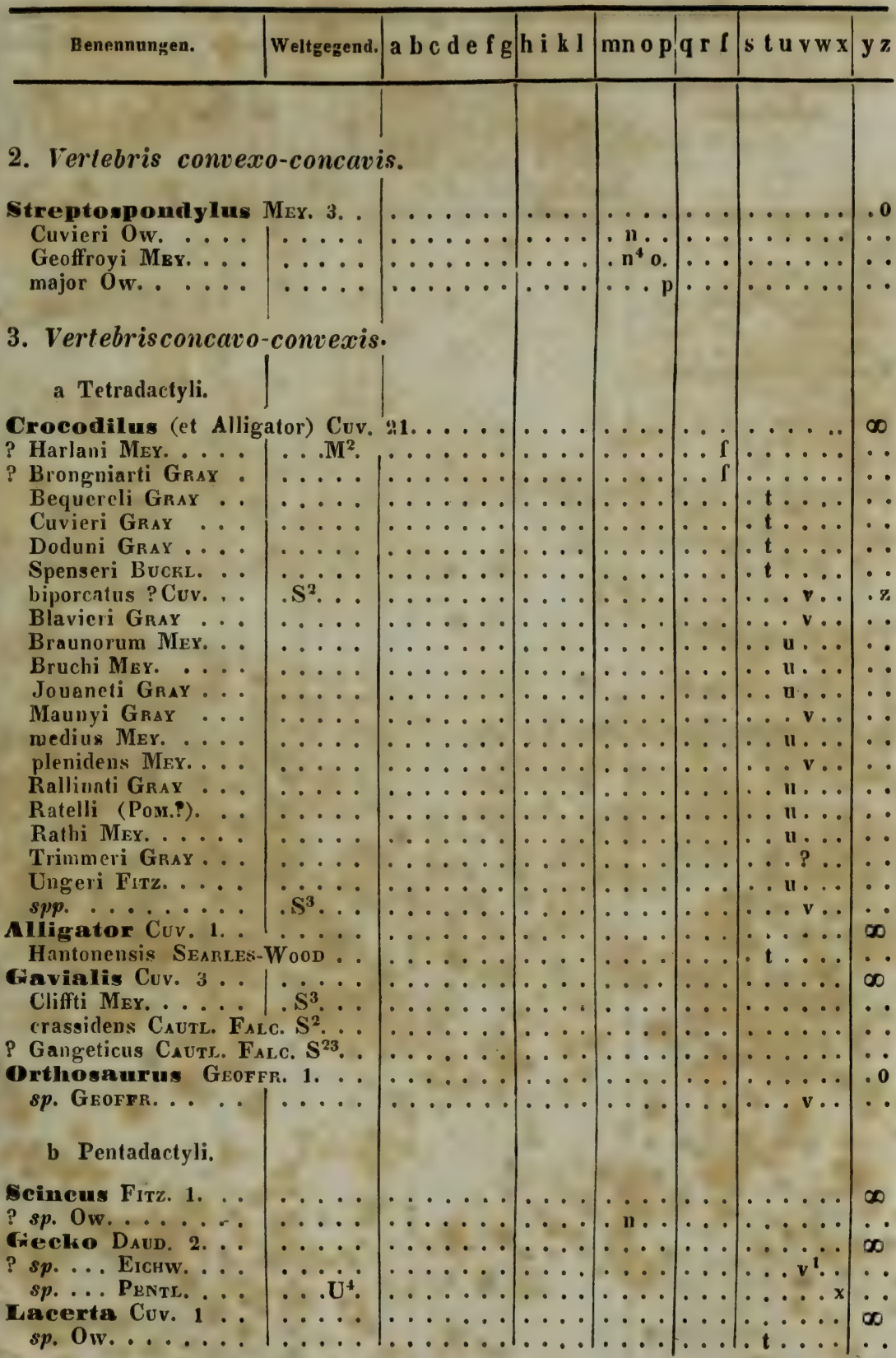




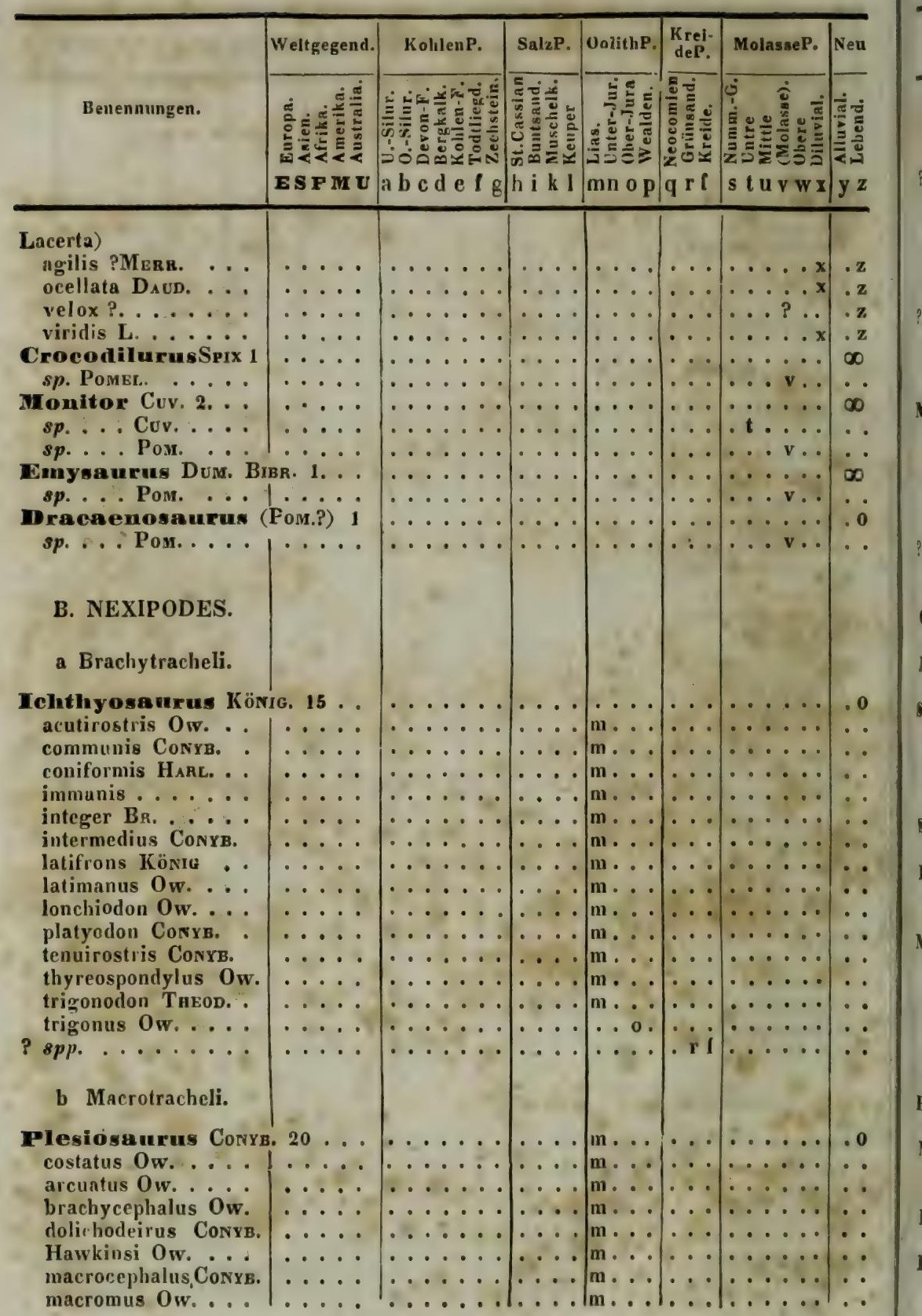




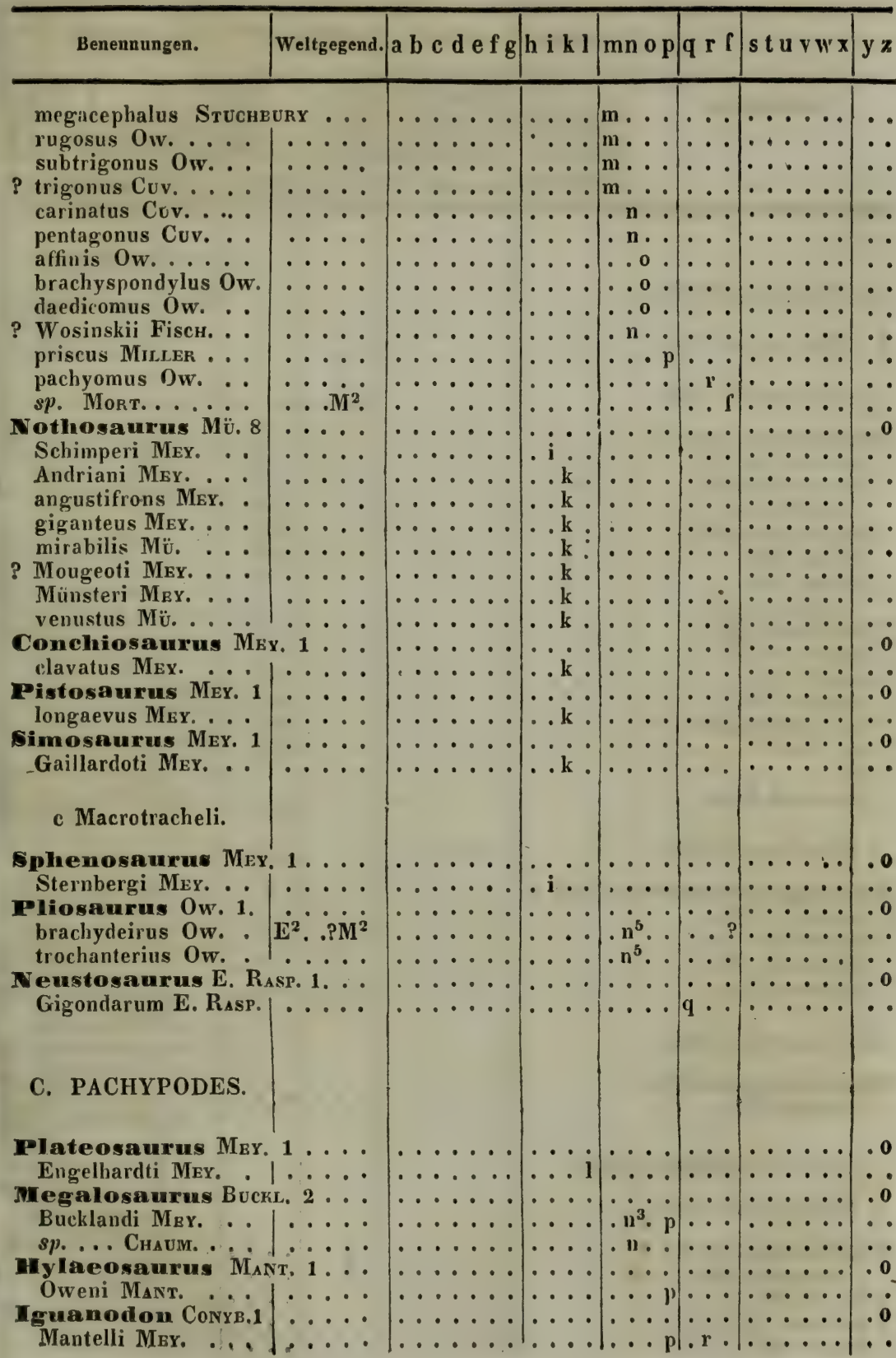




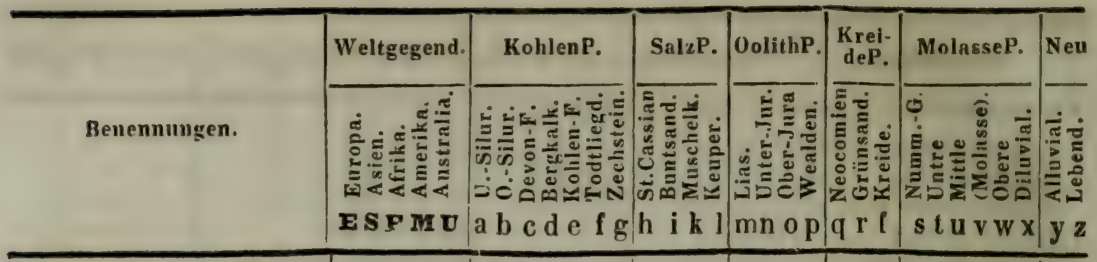

\section{PTERODACTYLI.}

\section{Tetrarthri MEY.}

a Dentirostres Mex.

Pterodactylus. 14 brevirostris Cuv. . . crassirostris GF. . . Kochi WagL. longirostris Cuv. : . medius Mü.

Meyeri Mü. (? Dentirostres.)

? Bucklandi Mer....

? dubius Mü.

? grandis Cuv.

? longipes Mü.

? secundarius MEY.

? $s p$. SpIX .....

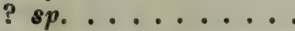

? giganteus BowB.

b Subulirostres Mey.

Rhamphorhynehus MEY, 4 macronyx MEY.

Gemmingi MeY. . longicaudus MEY... Münsteri Mer. . .

\section{Diarthri MEY.}

Drnithopterus Mey. 1. . . . Lavateri MEy.

\section{E. LABYRINTHODONTES.-}

a Mesophthalmi MEY.

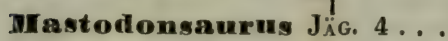

Vaslenensis MEx. . $\mid$.... ? Meyeri Mü.

? Andriani Mü. .... $\ldots$

Jägeri MвY.

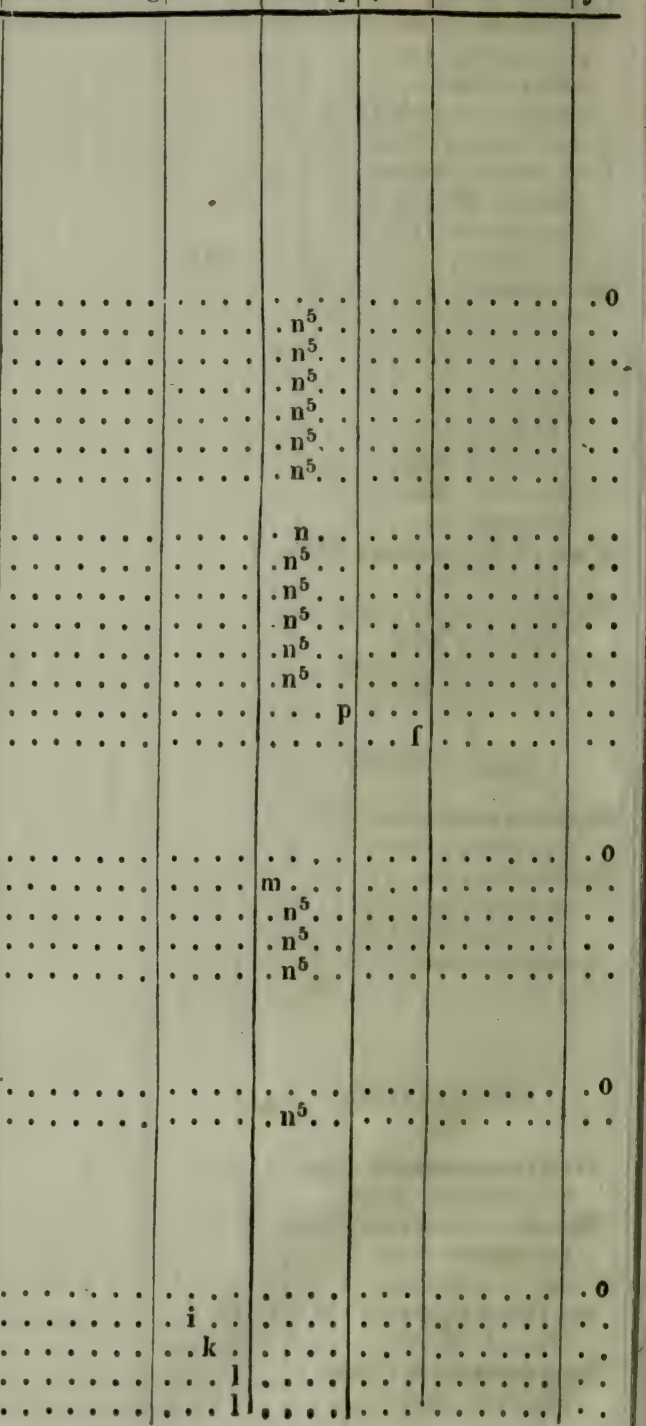




\begin{tabular}{|l|l|l|l|l|l|l|l|}
\hline Benennungen. & Weltgegend. & a b c d e f g & h i k l & mn op & q r f & s t u v w x & y z \\
\hline
\end{tabular}

b Prosthophthalmi Mex.

Metopias Mer. 1 diagnosticus Mry.

c Opisthophthalmi MEY.

Capitosaurus Mü. 2 arenaceus Mü.

robustus MEY.

d Labyrinthodontes incertae sedis.

OdontosaurusMey.1

Volizi Mey.

Trematosnurus Braun 1 .

\section{$s p$.}

Xestorrinytiasiver.1

Perrini Mey.

Labyxinthodon Ow.

Fürstenberganus Mey.

leptognathus $\mathbf{O w}$.

Jägeri Ow. .

pachygnathus Ow.

(Anisopus) scutulatus ventricosus $0 w$.

F. SAURII incertae sedis.

1. Vertebris non convexis : ?Dactylopodes.

pateon MEY. 1 :
pedestris MEY.

Archegosaurus $\dot{G}$.

Decheni GF.

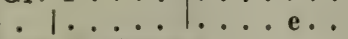

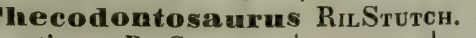

antiquus RiLStutch.

Falacosaurus RiL.2 cylindrodon RiLStutch.

platyodon Ris.Stutch.

relnopalodon Fisch. 2

Murchisoni Fisch. .

Wangenheimi Fisch.

Menodom MEY. 1 .

plicatus MEY.....

Zanclodom PLien. 2 crenatus Plien... laevis PLIEN. ...

Belodon MEY. 1. Plieningeri MEx.

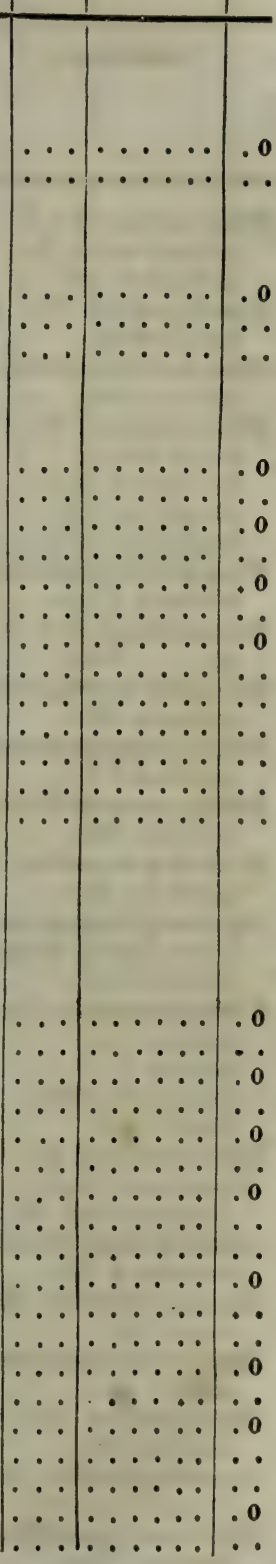




\begin{tabular}{|c|c|c|c|c|c|c|c|}
\hline & Weltgegend. & KohlenP. & SalzP. & OolithP. & $\begin{array}{c}\text { Krei- } \\
\text { deP. }\end{array}$ & MolasseP. & Neu \\
\hline Benemingen. & 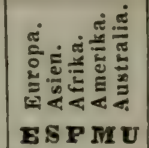 & 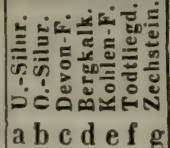 & 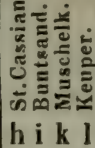 & 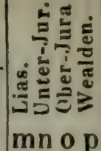 & 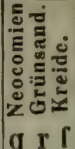 & 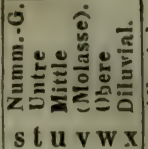 & 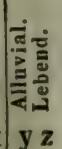 \\
\hline
\end{tabular}

Plnytosaurus J̈̈G. 2 cylindricodon J̈̈̈s. cubicodon $\mathrm{J}_{\mathrm{A} G}$.

Cladyodon 0 w. 1

Lloydi Ow.

Rhynchosaurus $0_{w}, i \ldots$ articeps $0 w$.

Dicynodon Ow, 4

Baini Ow.

Jacerticeps $O_{w}$.

strigiceps $0 \mathrm{w}$.

testudiceps $0 w$.

Termatosaurus Plien. 1. Albertii Plien. .

Rysosteus Ow. 1 sp. . . O $\mathrm{w}$.

GIaphyrorhynchus $\mathrm{MEY}_{\mathrm{r}}$ i Aalensis Mey.

Thaumatosaurus MEY. 1. oolithicus Mex.

Ischy rodom Merian 1 Meriani Mer.

Brachytaenius MEX. perennis MEY.

Spondylosanius Fisch. 2 . Fahrenkobli Fiscr. .

Frearsi Fisch.

Cetiosaurus Ow, 4 medius $0 \mathrm{w}$.

longus $\mathrm{OW}$.

brachyurus 0 w.

brevis $\mathrm{Ow}$.

creosaurus Cuv. 2

? maximus PlieN.

Soenmeringi Mey.

Crnathosaurus MEX. subulatus Mey.

Anguisaurus Mü. 1 bipes Mü.

Machimosaurus Me Hugii MEY.

Sericodon Mex, 2 . Jugleri MEY.

Goniopholis Ow. 1 crassidens $0 w$.

Macrorlnymehus Dunis. 1 . Meyeri Dunk.

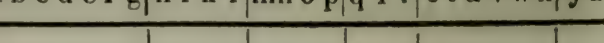




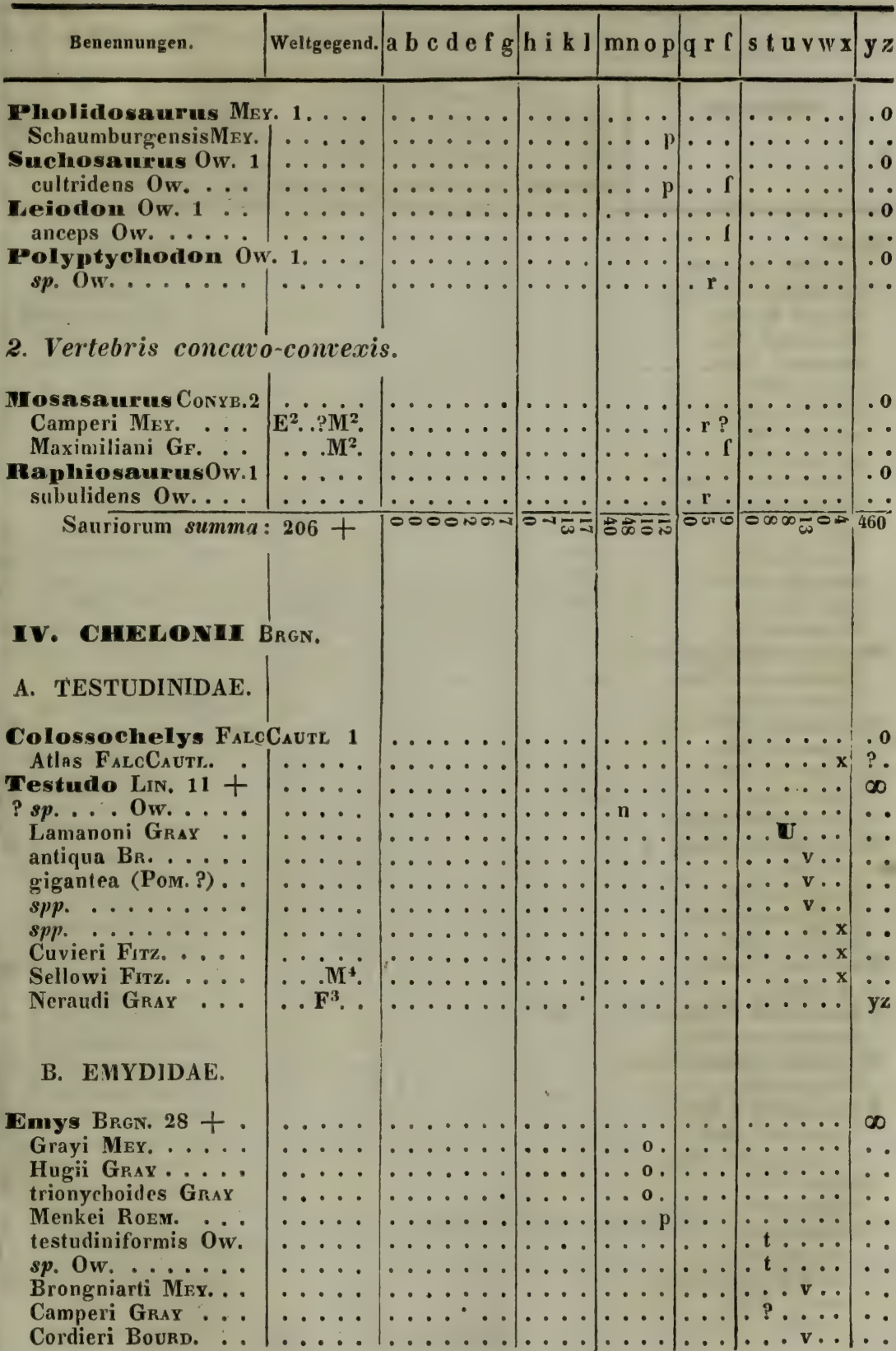




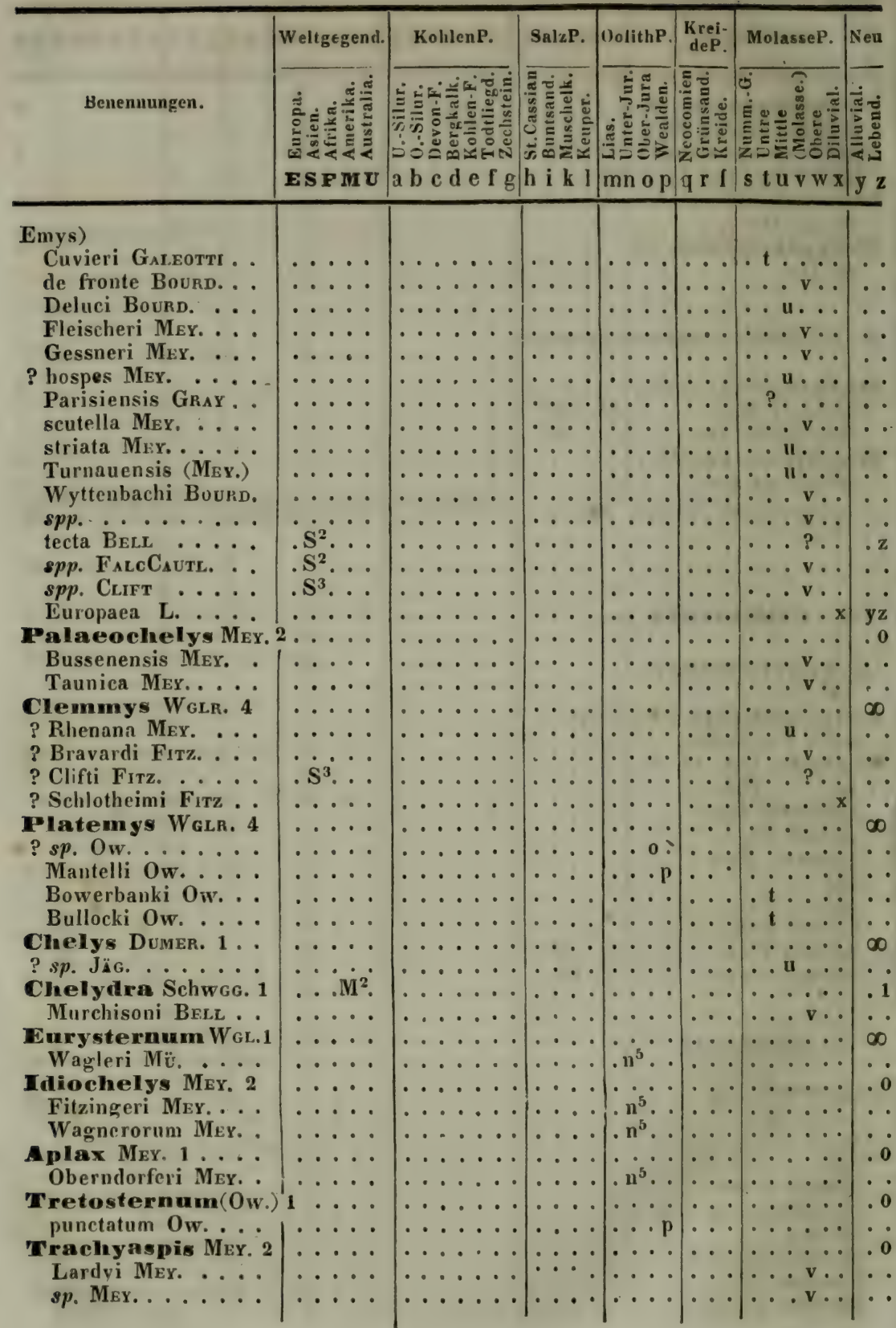




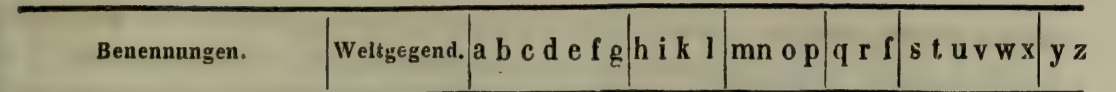

\section{TRIONYCHIDAE.}

Aspidonectes WGLr. 5. . . ? (Trionyx) Áegyptiacus Geofrr. Gergensi MeY. Maunoir [?] MEY.

? Parisiensis Mey. $s p$. Sism.

Trionyx Geofer, 17 inclus. A spidonect. spp.

? sp. Ow.

? sp. Ow.

spp

Doduni Gray

Amansi Gray

Laurillardi Gray.

Lockardi GraY

? Partschi Fıtz.

P Schlotheimi Fitz.

$s p p$.

Clifti Frtz.

clisp. .....

spp. FalcCaUti

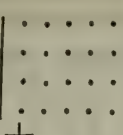

\begin{tabular}{|l|l|l|l|l|l|l|l|l|l}
\hline \\
\hline
\end{tabular}

\section{CHELONIDAE.}

Chelonia Bran. 18. obovata Ow. planiceps Ow. Mantelli Frtz.

Benstedi Mant. . .

Knorri Gray . pulchriceps $0 \mathrm{w}$. Hofmanni GraY Couperi HARL. acutirostris $\mathrm{Ow}$. antiqua Gray breviceps $\mathrm{Ow}$. latiscutata $0 \mathrm{w}$. longiceps $\mathrm{OW}$. planimentum $\mathbf{O}$. subconvexa $0 \mathrm{w}$. subcristata $\mathrm{OW}$. radiata Fiscis. Wagleri Fitz.

sp. Serr DubrJeanj.

Cheloniorum summa: 99. . .

Reptilium summa: 384

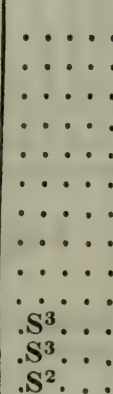

$\mathrm{S}^{2}$. 


\begin{tabular}{|c|c|c|c|c|c|c|c|}
\hline & Weltgegend. & KohlenP. & SalzP. & OolithP. & $\begin{array}{c}\text { Krei- } \\
\text { deP. }\end{array}$ & MolasseP. & Neu \\
\hline benennungen. & $\begin{array}{c}\mathbf{S}^{1,2,3} \text { Asien. } \\
\mathbf{E}^{2,3,4} \text { Afrika. } \\
\mathbf{M}^{1,2,3} \text { Anmerika. } \\
\mathbf{U}^{3}, \mathbf{4} \text { Australien. } \\
\mathbf{E} \mathbf{S} \mathbf{F} \mathbf{M} \mathbf{U} \\
\text { ke i n Zeichen: be- } \\
\text { deutet } \mathbf{E}^{2} \text {. }\end{array}$ & 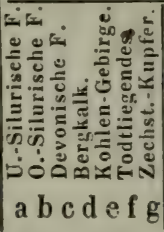 & 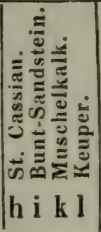 & 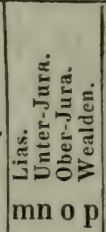 & 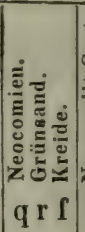 & $\begin{array}{lll} & \\
\text { stu vw x }\end{array}$ & 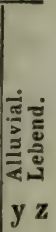 \\
\hline
\end{tabular}

\section{Cl. XXIII. AVES : Vögel.}

Ornithichnites s. d. omnes, quibus sc. auctor aliam originem tribuit, in solo Nomenclatore enumerantur.

\section{T. PA IIMPEDES}

1. LAMELLiRostres.

Mergus Lin. 1 ... Anas MeYrer 2 . . . sponsa L. . . . . . ? tadorna $\mathrm{L} . \quad \ldots \ldots$

Anser Briss. $1 \ldots$ segetum Meyer... Cygrnus Meyer 1.:

? Anas olor Gmel. . .

2. TOTIPALMAF,

Carbo Meyer, 1. . . sp. Cuv. ...... Pelecanus Ill. 1 . $s p$. Cuv. ......

\section{LONGIPENNES.}

Carus Lin. 1. . . . $s p$. Risso . . . . .

Cincoliormis 0 w. 1

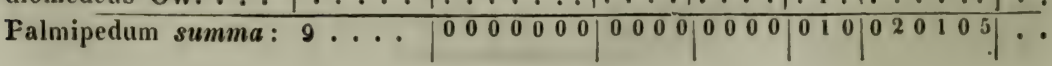




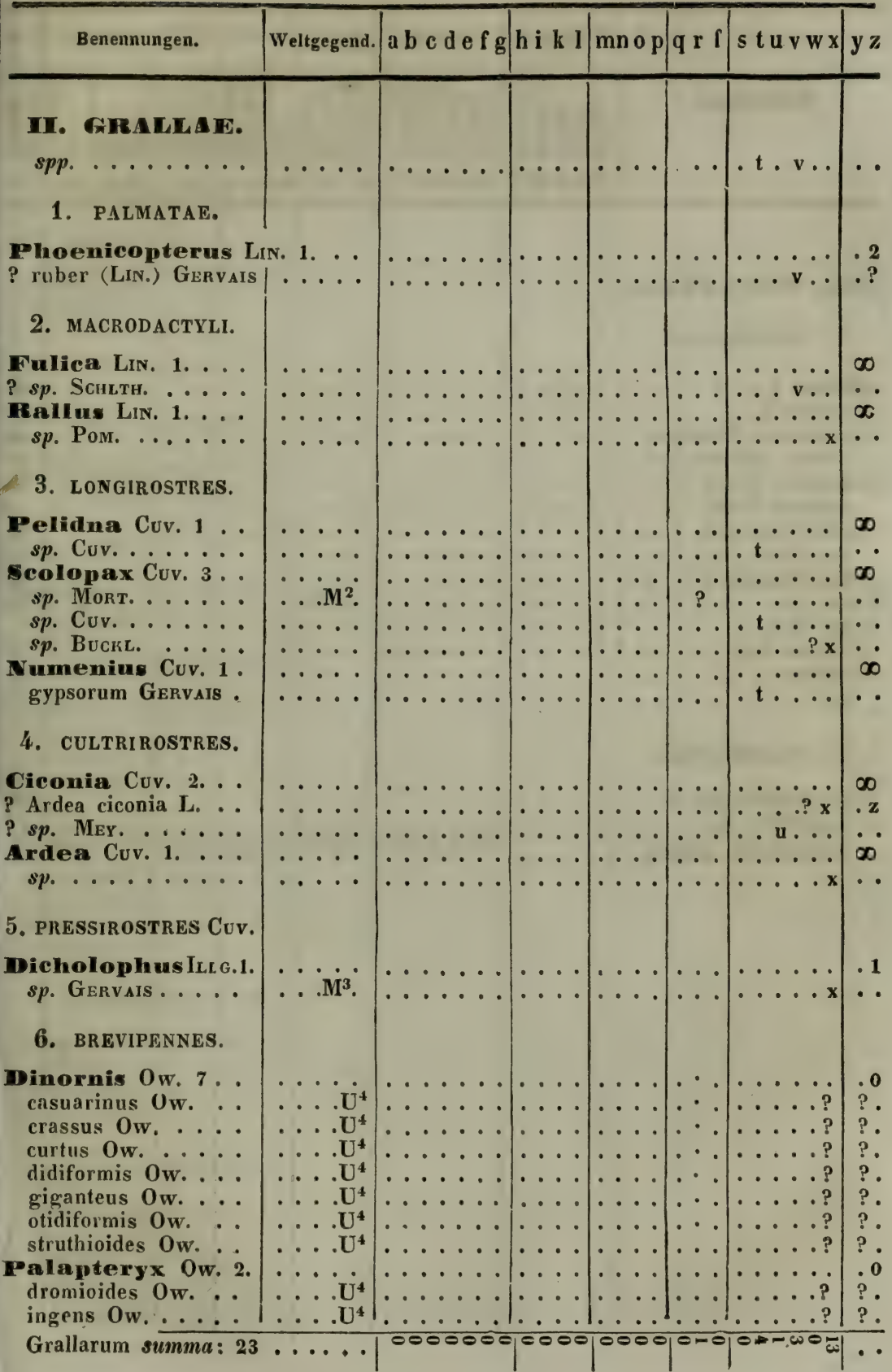


Bellemnungen.

\begin{tabular}{|c|c|c|c|c|c|c|}
\hline Weltgegend. & KohlenP. & SalzP. & OolithP. & $\begin{array}{r}\text { Krei- } \\
\text { deP. }\end{array} \mid$ & MolasseP. & Neu \\
\hline 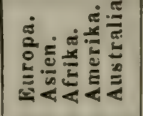 & 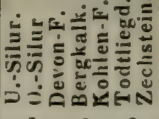 & 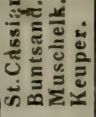 & 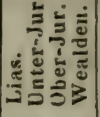 & 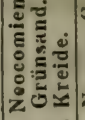 & 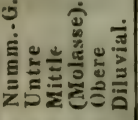 & 这 \\
\hline $\mathbf{E S F} \mathbf{M}$ & e $f$ & h i k l & $\mid \mathrm{mn} 0 \mathrm{p}$ & $|q \times 1|$ & st t & $1 y^{2}$ \\
\hline
\end{tabular}

\section{GALLIVAE。}

1. GALLiNACEAE.

Didus Liv. 1 ... ineptus L. ....

Coturnix Möhrinc, 1 Tetrao coturnix LiN.

Perdix Briss. 1 .. cinereus? Lin. . . . .

Tetrao Liv. 1. . . . sp. Гох. . . . . Phasianus LiN. 3. ? $s p$. Cuv. . . . . . . ? $s p$. MeY. . . . . ? pictus Liv. .....

Gallus 1......

$s p . \ldots \ldots \ldots$

2. COLUMBACEAE.

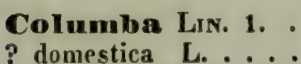
Gallinarum summa:

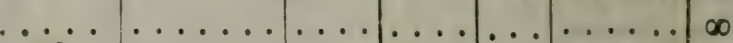

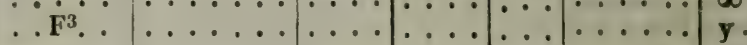

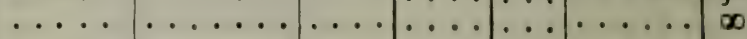

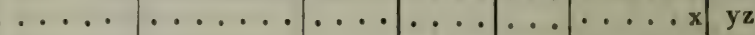

$\ldots \ldots \ldots \ldots \ldots \infty \ldots$

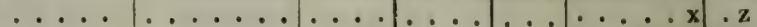

$\ldots \ldots \ldots \ldots \infty \ldots$

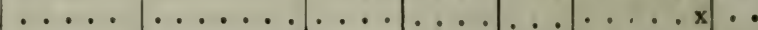

$\ldots \ldots \ldots \ldots \ldots \ldots$

$\ldots \ldots \ldots \ldots \ldots \ldots$

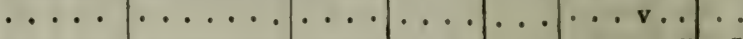

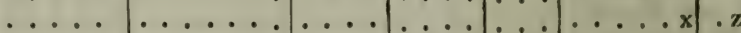

$\ldots \ldots \ldots \ldots \ldots \infty \ldots$

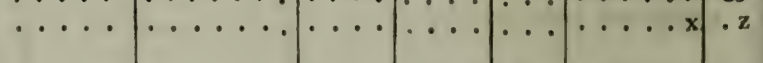

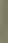




\section{INSWSSTRES.}

d. SCANSORES.

Fittacus Lin. 1. sp. .........

Pieus Liv. 1. martius ? LiN.

\section{B. PASsERES.}

1. Incertae sedis.

Protornis Mer. 1 . Glarniensis Mey.

\section{SYND ACTYLI.}

Malcyornis 0 w. 1 . Toliapica 0 w.

3. conirostres Cuv.

Corvus Lin. 2. ... corax ? Lin. .... cornix LiN.

Sturnus Lin. 1. . sp. . . . . . . .

Loxia Briss. 1... ? sp.

ringilla Liv. 1 . . domestica ? L. ...

A Iauda Lin. 1 ... arvensis ? L. ....

4. Fissirostres Cuv.

Caprimulgus Lin. 1 sp. . . . . . .

Firundo LIN. 1 . . $s p$.

5. dentirostres Cuv.

Motacilla Bechst. 1 sp. . . . . . . .

(

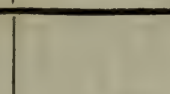

$\cdots \dot{M}^{3}$

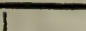




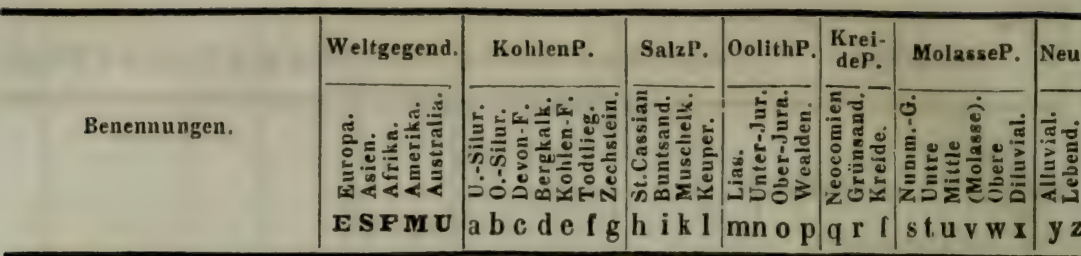

V. ACCIPITEES.

1. NOCTURNI.

Strix LiN. $4 \ldots .$. bubo L........ nyctea ? L.

? sp.

Ulula Cuv. $1 \ldots .$. $s p . \ldots \ldots \ldots$

\section{DIURNI.}

Falco Lin. $6 \ldots$. . Haliaetus ?Lin. ... sp. L. . . . . . pennatus ?Gmes. . . nisus ? Liv. ..... sp. ........

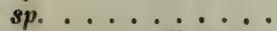

Catharte Cuv. 1. . sp. . . . . . .

VuItur Lis, 1. . . ? cincreus Gmel. . . .

Lithornis 0 w. 1. vulturinus $0 w$.

Accipitrum summa: 14

$\therefore \ldots$

(VI. Variorum Ordinum).

spp. 24 Weissenau. spp. 40 cavernar. Brasil.

spp. 15 cavernar. vallis Lahnthal Avium varior, ordin. summa: 79 Avium summa: 148

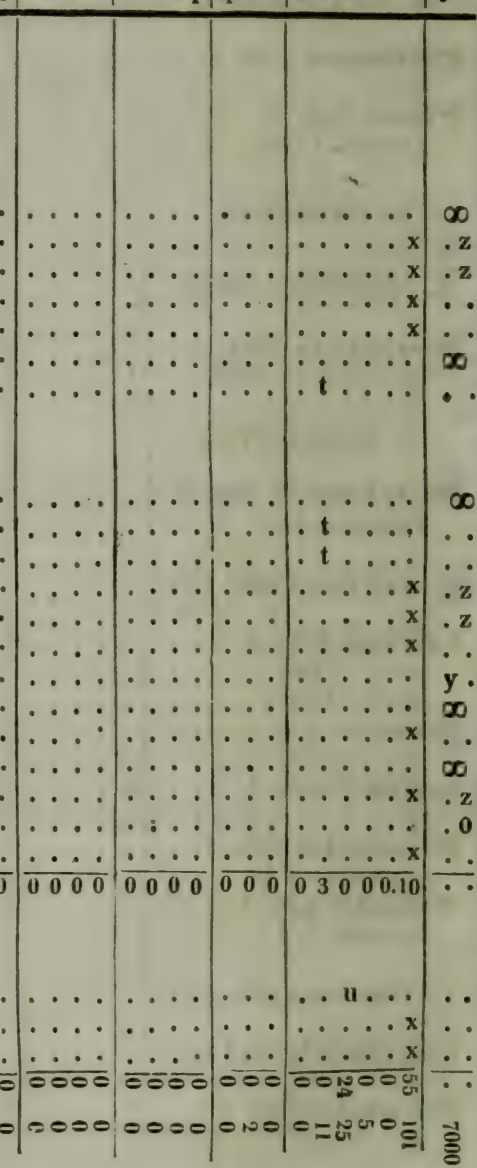




\begin{tabular}{|c|c|c|c|c|c|c|c|}
\hline & Weltgegend. & KohlenP. & SalzP. & OolithP & Krei- $\begin{array}{l}\text { KreP. } \\
\text {. }\end{array}$ & MolasseP. & Neu \\
\hline Bcrennumgen. & 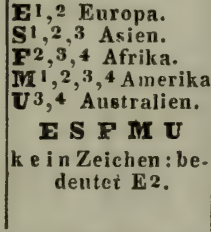 & 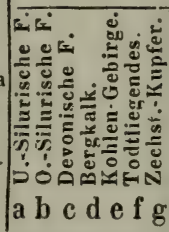 & 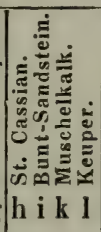 & 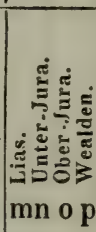 & 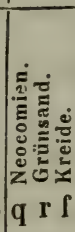 & 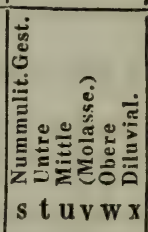 & 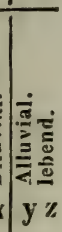 \\
\hline
\end{tabular}

\section{Cl. XXIV. MAMMIFERA: Säugthiere.}

Notandum: Mammalium Ichnites s. d. omnes ab auctore alio modo explicari et inde in Nomenclatore solo nominari.

I. CeTACEA, WaLE.

A. ZOOPHAGA.

a Balaenidae.

Balaena Liv. 4 . . sp. Podgsta $\ldots .$. rp. Ow. mysticetus L. . .

Balaenoptera LACEP. 3 ... Cuvieri Crtv...... sp. Olf........ boops L. .......

Rorqual Cuv. 1 .. sp. BeNEDEN .... Gen. ?, $1 \ldots \ldots \ldots$
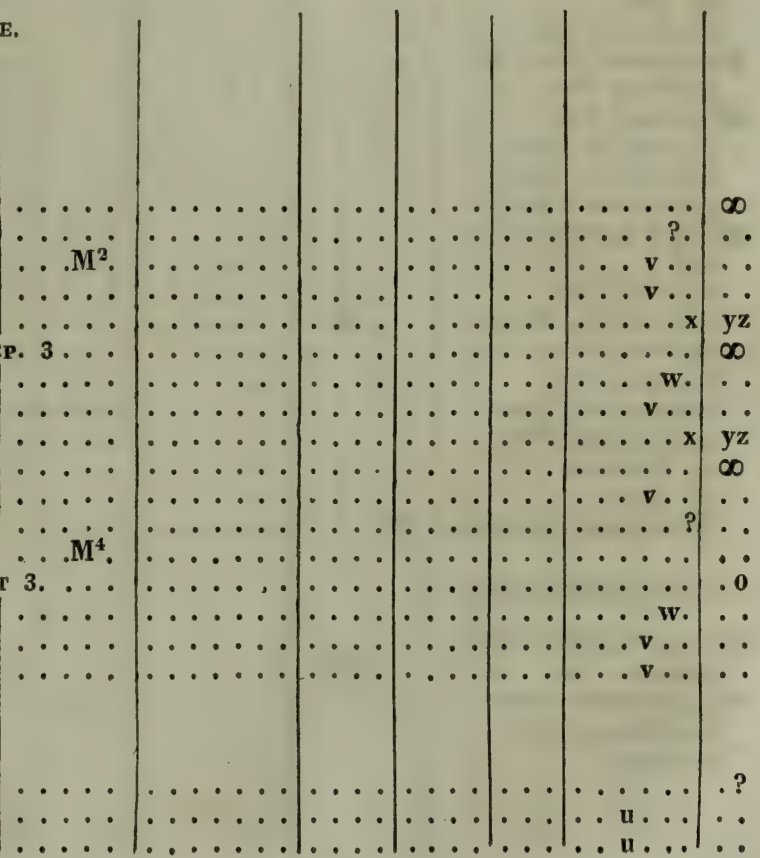

Cherium Brandt 3....

? priscum Brandt .. Rathkei BRANDT

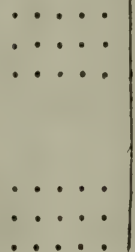

b Balaenodontes.

Balaenodon 0 w. 5 affinis $0 w$. definitus $0 \mathrm{w}$. 


\begin{tabular}{|c|c|c|c|c|c|c|c|}
\hline & Weltgegend. & KohlenP. & SalzP. & OolithP. & \begin{tabular}{|c|}
$\begin{array}{c}\text { Krei- } \\
\text { deP. }\end{array}$ \\
\end{tabular} & MolasseP. & Neu \\
\hline Benennungen. & 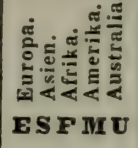 & 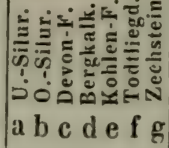 & 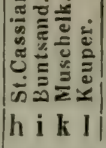 & 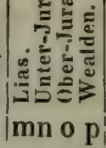 & 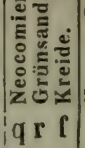 & 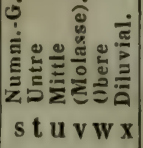 & 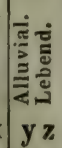 \\
\hline
\end{tabular}

Balaenodon) emarginatus 0 w... gibbosus Ow. physaloides Ow

e Physeteridae.

Pliyseter Lacer. 3. molassicus $\mathbf{J}_{\mathrm{ÄG}}$. $s p$. Ow. macrocep halus $\mathbf{L}$.

d. Delphinidac.

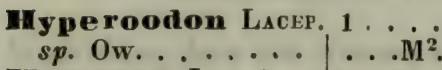
Momodon Lin. 2. $s p$. Ow. monoceros Lin.

Arionius Mey. 1. servatus Mey.

ziphius Cov. 3 cavirostris Cuv. longirostris Cuv. planirostis Cuv.

Delphinus Cuv. $11+$ spp. ....... Brocchii CaIv. ... Calvertensis Hart. Cortesii MEY. Karsteni OLF. longirostris MEY. macrogenius MeY. sp. Podesta .... $s p$. Gratl.

(Phocaena) crosiden $\dot{0} \cdot \cdots$ e Zeuglodontes MEx.

Zueuglodon Ow. 1 cetoides $0 w$.

Squalodon Gratl. 2 Grateloupi MEY. sp. Mex.

$\ldots \ldots$
$\cdots$
$\cdots$
$\cdots$
$\cdots$
$\dot{E}^{2} \cdots$
$\mathbf{M}^{4}$

.

\section{ow....}




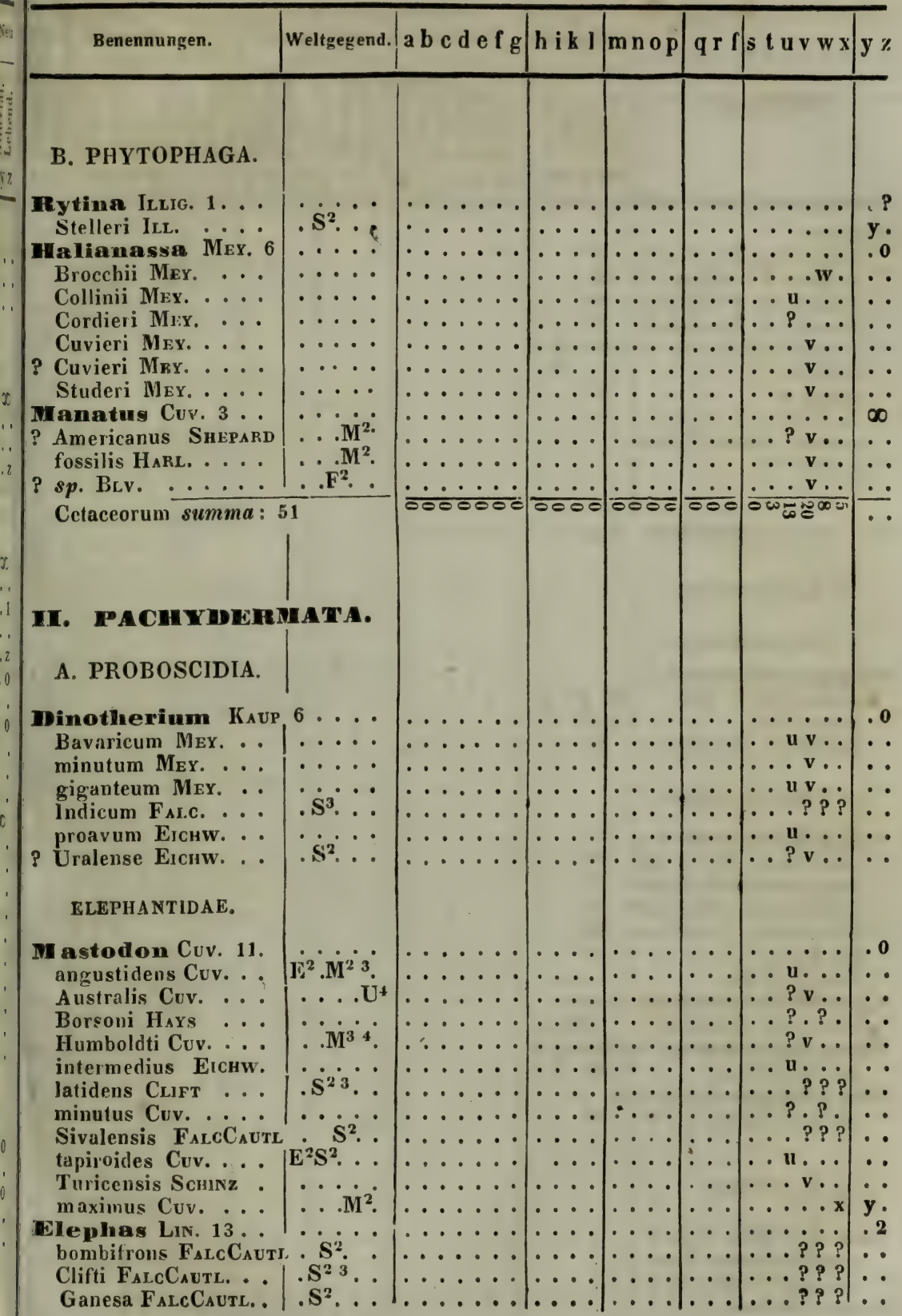




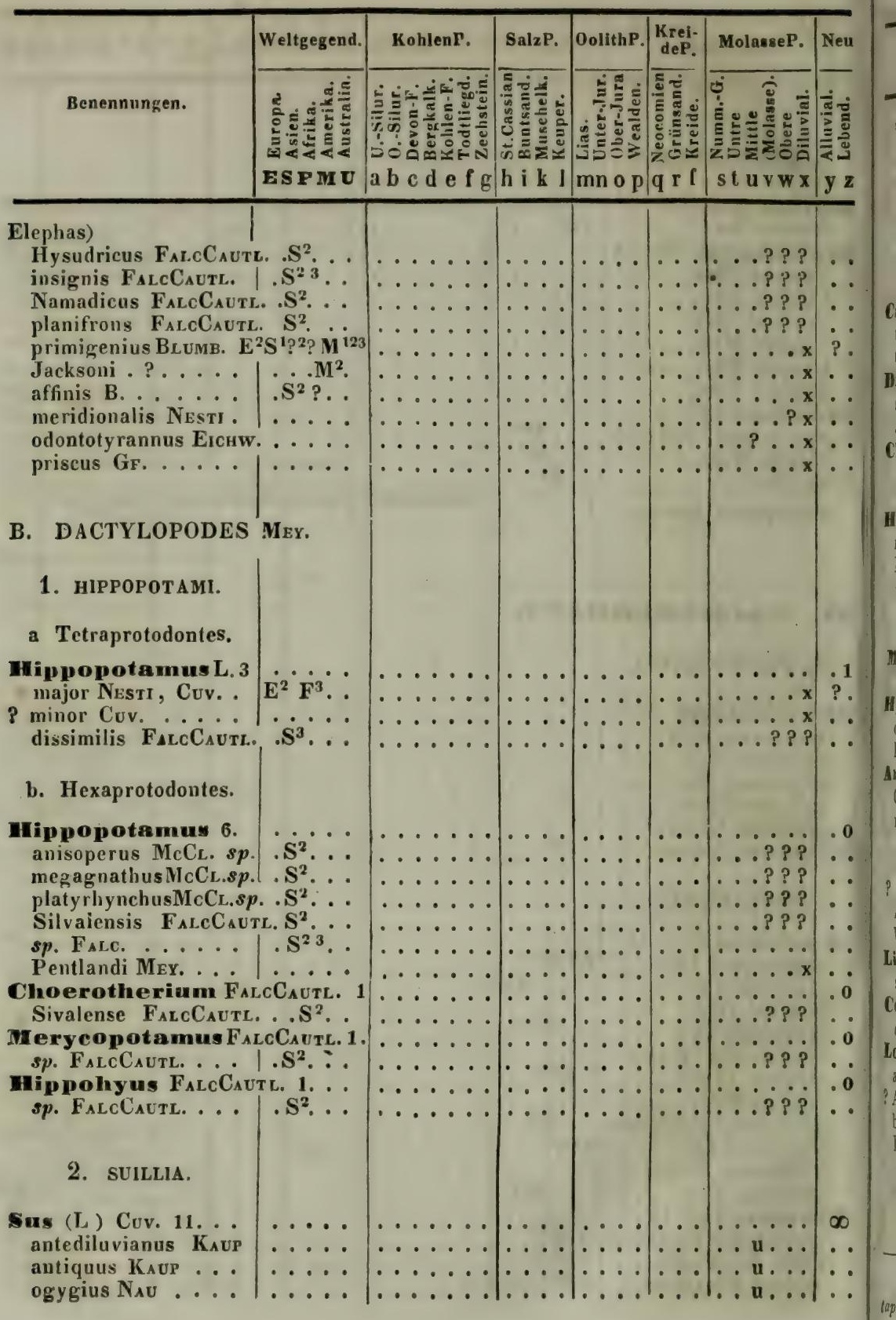




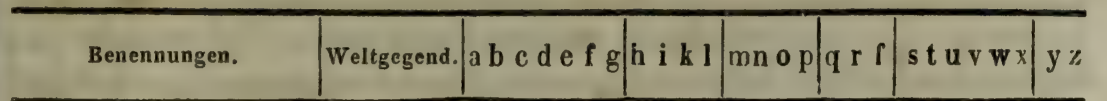

palaeochoerus Kaup

Sivalensis FalcCautL. $\dot{S}^{2}$

Hysudricus Falc $\mathrm{C}_{\text {AUtL. }} . \mathrm{S}^{23} 3^{\circ}$ (Aper) Arvernensis CroizJob. diluvianus Kavp

priscus GF.

priscus SerRDubrJeanj. scrofa Lin.

Calydonius

tener MEY.

trux MEY.

Dicotyles Cuv. $5+$ major Lund ................... spp. 4-5 LUNd .. .... $\mathrm{M}^{3}$.

Choeropotamus Cuv. 2. Matritensis EzQr. Parisiensis Cuv.

Hyotherium MEY, 5 medium MEY.

Meissneri MEY.

sidero-mollassicum majus $\mathrm{J}_{\mathrm{A} G \text {. }}$ sidero-molassicum minus JïG.

Sömmeringi MEY.

Microchoerus Searles-Wood, 1 erinaceus SEARLES-WOOD

Hyracotherium $\mathrm{Ow}_{\mathrm{w}} 2$ cuniculum Ow. leporinum Ow.

Anthracotherium Cuvi 7 . Gergovianum BLv. magnum Cuv. minimum Cuv. minus Cuv. .

P minutum BLv. Silistrense Fente. Velaunum Cuv.

Cistriodon MEY. 1. splendens Mex.

Coryphodon Ow. 1 eocaenus $0 \mathrm{w}$.

Lophiodon Cuv. 15 anthracoideus Buv.

? Aurelianensis batygnathus $0 w$. Buxovillanus communis BLv. * Isselensis medius

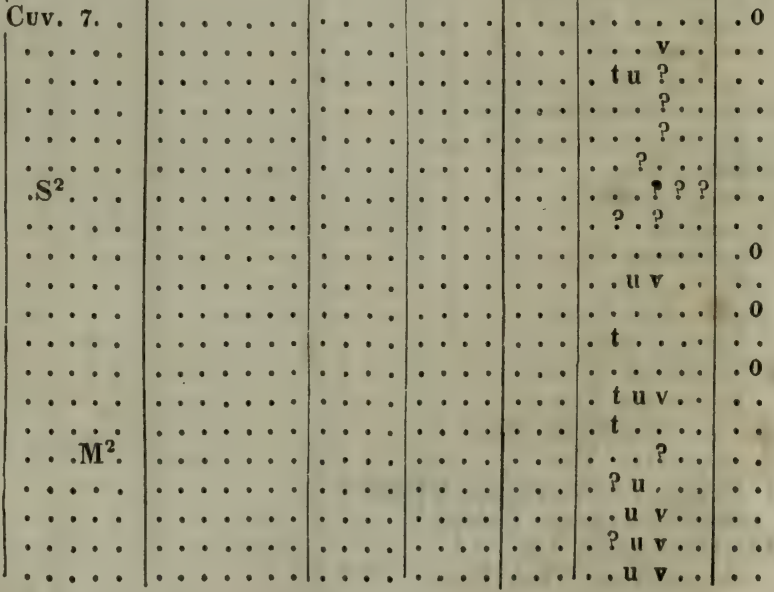

* L. communis BLV., L. Buxovillanus, L. Isselsnsis, L, medius, L. Oecitanicus, $\boldsymbol{L}$. tapiroides et L. Tupirotherioun auctorum. 


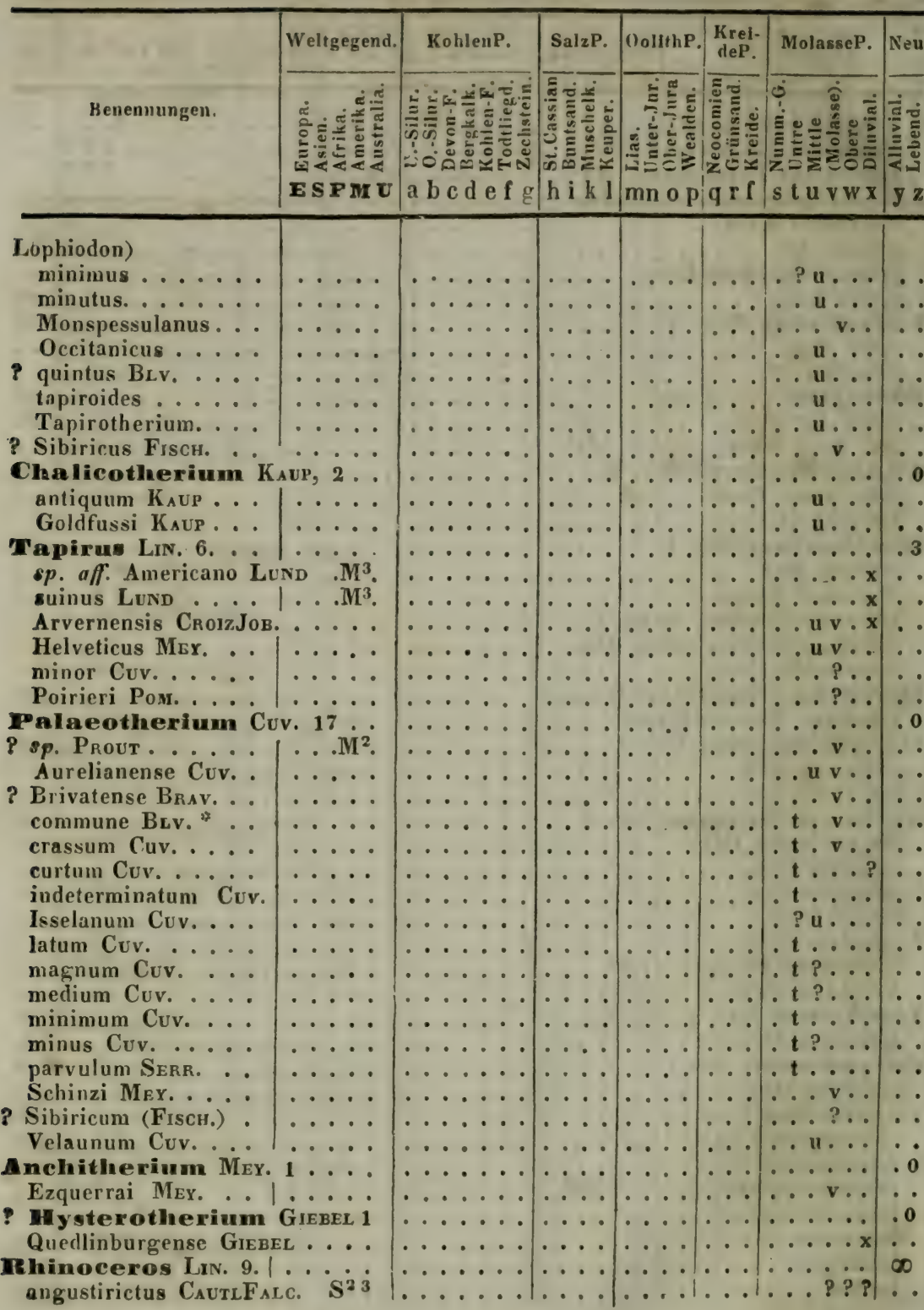

* P. commune BLV., P. crussum, P. curtum, P. Girondicum, $\boldsymbol{P}$. indeterminatum, $\boldsymbol{P}$. latum, $\boldsymbol{P}$. magnum, $\boldsymbol{P}$. mediun et $\boldsymbol{P}$. Velaunum. 


\begin{tabular}{l|l|l|l|l|l|l|}
\hline Benennungen. & Weltgegend. & a b c d e f g h i k l & mn op & q r f & s t u v w x & y \% \\
\hline
\end{tabular}

sp. Cautfalc.

? de Filipii Criv.

Goldfussi KaUP

incisivus Cuv.

minutus Cuv.

? tapirinus Pom.

leptorhinus Cuv.

tichorhinus Cuv.

clacmetheriam

Fiscisch. 2.

Keyserlingi Fisch. $: S^{2}$.

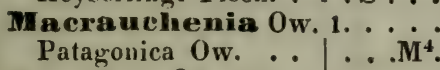

Nesodon Ow. 2
imbricatus Ow. $\ldots$

Sullivani Ow..... $\ldots \mathbf{M}^{4}$

Toxudon Ow. 3.
angustidens Ow. .

Platensis Ow. ...

Paranensis D'O. Lavric. . M4

Anoplotherium Cuv. 3 ... commune Cuv. secundarium Cuv.

Sivalense FAscCAUTL. S $^{2}$.

Dichobune Cuv. 2.| ..... cervina $0 \mathrm{w}$.

leporina Cuv.

xiphodon Cuv. i.

gracile Cuv.

microtherium $\dot{\mathrm{M}}$.

concinnum MEx.

Renggeri MEY.

Tapinodon Mry. 1 Gresslyi MEY.

Adapis Cuv. 1. ... Parisiensis.

\section{SOLIDUNGULA.}

Equas Lin. 8 ... Asinus Lr. $8 \ldots . \ldots$ Caballus Liv.
curvidens Ow. neogaeus Lund. [curvidens?] $\mathrm{M}^{3}$ plicidens $0 \mathrm{w} . . . . . .$. priscus Eıнw.

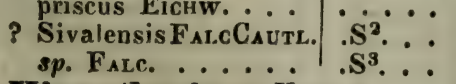

Hippotherium Kavp $1 \ldots$ gracile Kaup .... | ..... Pachydermatum summa: 157

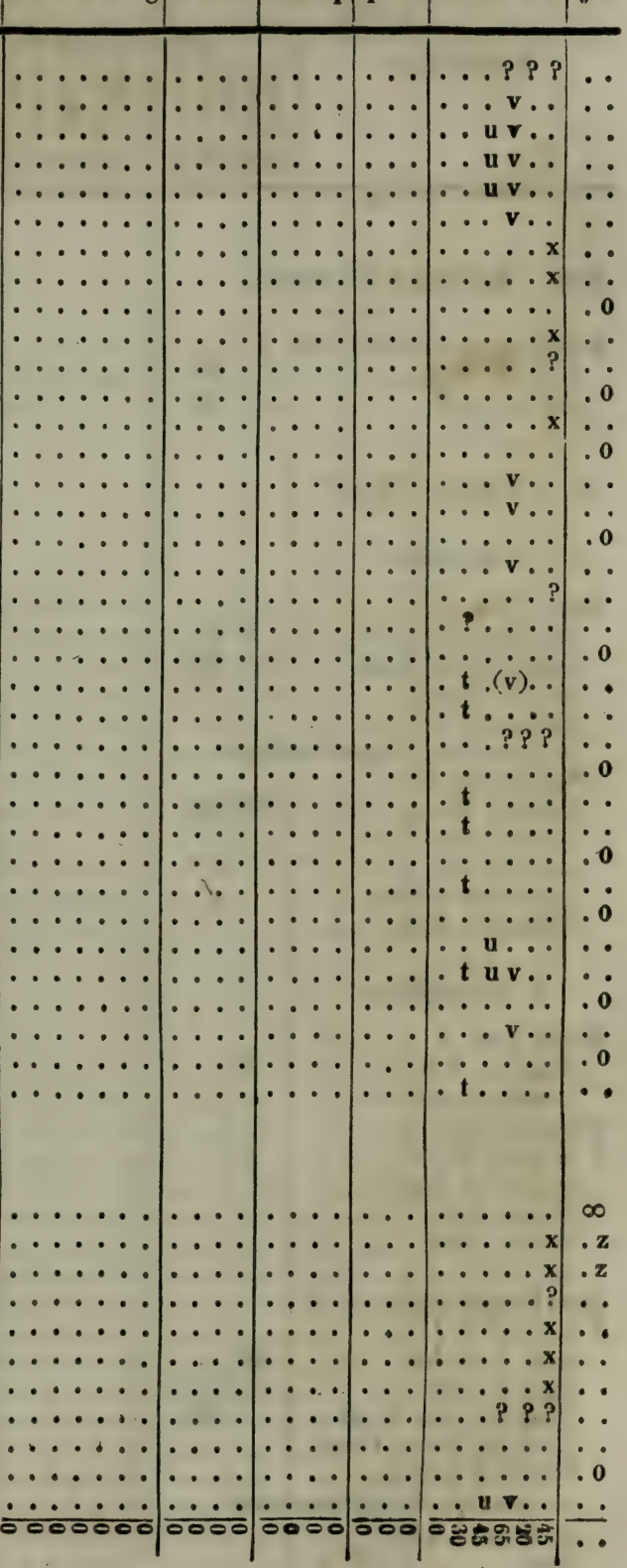




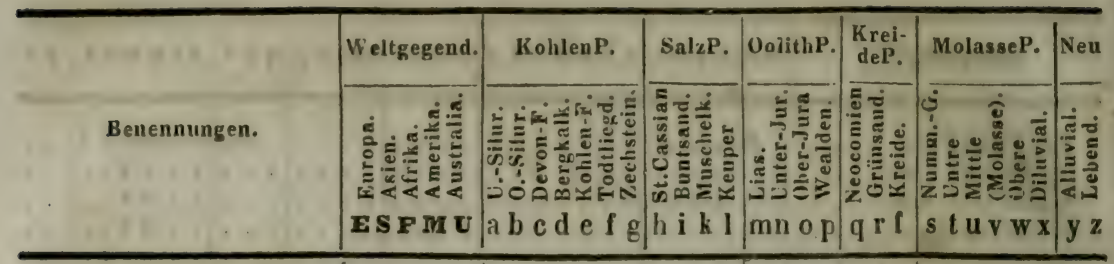

II. IUNIINATA.

Ceptotherium LUND majus Lund .................

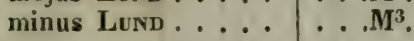

\section{A. COELOCERATI.}

a Bovidae.

Ios Lin. 11 ..... sp. Cautl Falc. . bombifrons HARL. . elatus Crorz. .... giganteus Crorz. . . intermedius SERRDUBRJEANJ. longifrons $\mathrm{OW}$. Pallasi Dekax $\ldots$ primigenius Cuv. priscus Boj. . . : $\therefore$ trochocerus MEY. . . Velaunus Ror.

b Antilopidae.

Dvis Lrs. 2. . . . . . Ammon ? Liv. tragelaphus Cuv...

Capra Lin. 5. . .

? Sakeen Bцyтн.... aegagrus GMbL. . . hircus Lin. . . . . Rozeti Pom.

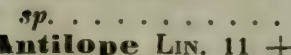
? molassica J̈̈G. ... spp. 2 Clift ... $s p p$. CaUtlFalc. . Maquinensis LuND . Christoli Serr. Pitor.

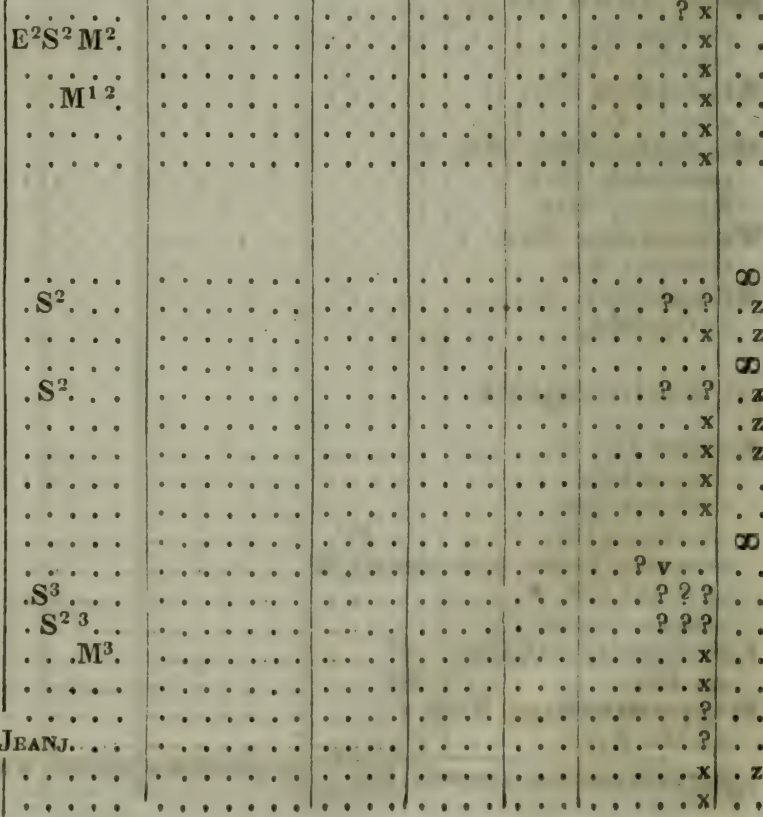
Cordieri Curist. . . recticornis SerrDubrJEanj.... rupicapra Liv, ...
spp. ........ 


\section{B. PYCNOCERATI.}

a Camelopardalidae.

Camelopardalis LiN. 3. affinis FalcCaUtL... $\mid \mathrm{S}^{2}$. sivalensis FatcCaUtL. $\mathrm{S}^{23}$. Biturigum Duvernox $\mid$.

Sivatherium $F_{A L C C} C_{A}$ giganteum FalcCaUte. S $^{2}$.

Bramatherium FALC. 1 Perimense Falc. ....... $\mathrm{S}^{3}$.

b Cervidae.

Cervus L. 58

* Palmati.

Alces Lis.

Dama LiN.

Dama gigunteus Cúv.

Dama Polignacus RoB.

eurycerus ALDrov.

fellinus Fisch

Guettardi Dsmar.

Tarandus LiN. ** Cataglochi.

Bertholdi KaUP haplodon MeY.

lunatus MEY. molassicus Jïc. nanus Kavp. . Partschi Kaup $s p p$. CautrFatc. sp. aff. paludoso Lund . $\mathrm{M}^{3}$ spp. 2 LUND ........... $\mathrm{M}^{3}$. Americanưs HARL. . $\ldots \mathrm{M}^{2}$ antiquus Serr DubrJeanj. Ardei CroizJor. Arvernensis CroizJoв. Borbanicus Croiz... coronatus SERrDubrJeanj. Croizeti Croiz. Cusanus CroizJob. Destremi Christ. diluvianus MEY. Dumasi SerrPitor. elaphus Liv. elaphus Rebouli Christ. Etueriarum CroizJob. Gergovianus CroIz. intermedius SERRDubrJeAnJ.
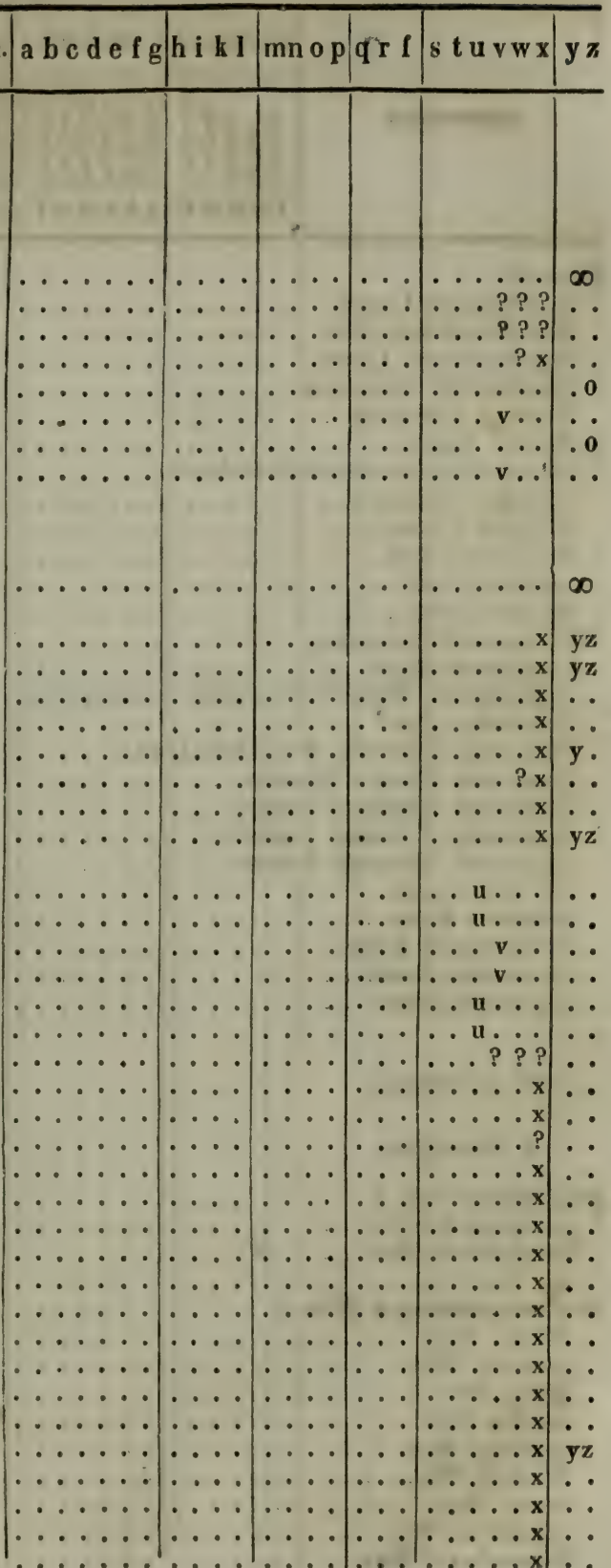


\begin{tabular}{|c|c|c|c|c|c|c|c|c|}
\hline \multirow{3}{*}{ v } & te & Weltgegend. & KohlenP. & SalzP. & OolithP. & $\begin{array}{l}\text { Krei- } \\
\text { deP. }\end{array}$ & MolasseP. & Neu \\
\hline & Bononnungen. & 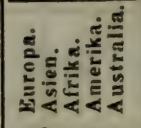 & 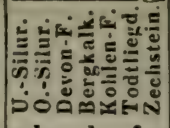 & 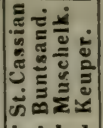 & 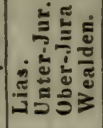 & 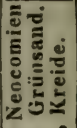 & $\begin{array}{l}\dot{0} \\
\dot{\hat{g}} \\
\mathrm{E}\end{array}$ & 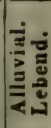 \\
\hline & & ES F MU & abedefg & $\mid h \begin{array}{lll}\mathrm{h} & \mathrm{k} & \mathrm{l}\end{array}$ & $\mathrm{mn} 0 \mathrm{p}$ & q $r i$ & s t u v w x & $\mathbf{y} z$ \\
\hline
\end{tabular}

\section{Cervus)}

Issidorensis Ског\%.

Kirchbergensis J̈̈G.

Neschersensis Croiz.

Pardinensis Crorz.Job.

Perrieri CrolzJob. .

Privati Crovz. ... pseudo-VirginiusSerRDubrJbanJ. ramosus CrorJor. Regardi Croiz. ... Solilhacus Roв. spelaeus Ow. Vialeti Crorz.

*** Anogloehi (Capreolus). curtocerus Kaup. trigonocerus KAUP . Capreolus Lis. Capreolus australis SEBrDubrJe

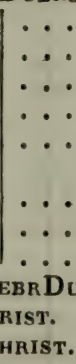

Capreolus Cuvieri Christ.
Capreolus Leufroyi Christ. Capreolus Tolozani CHRist. Capreolus Tournali Christ. Ca** Cervuli. anocerus KAUP... dicranocerus KaUP. dicrocerus LART. . . pygmaeus LART.

\section{ACERATI.}

a Moschidae.

Mosehus LIN. 3. . . ? antiquus KaUP. ...

? Bengalensis Mey. . .

? sp. J̈̈G.

Pa.... $\ldots \ldots$
$\ldots \ldots$
$\ldots \ldots$

$\ldots$
$\ldots$
$\ldots$
$\ldots$
$\ldots$ Bojani MEY. eminens MrT. Kaupi MEx.
medius MEY. ninimus MEY. Nicoleti Mex. minor Msy. Scheuchzeri Msr.

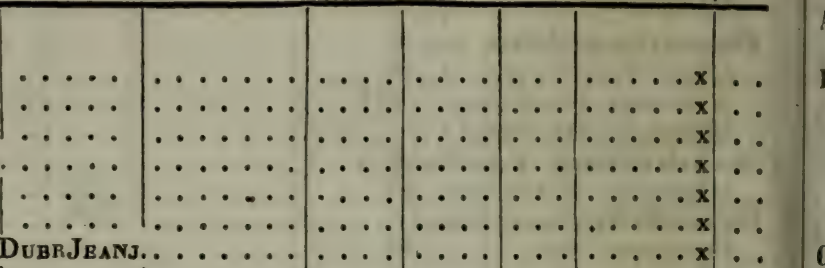

C

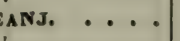


Benennungen. \begin{tabular}{|l|l|l|l|l|l|l|}
\hline Weltgegend. a b c d e f g h i k l mn op & q r l & s t u vw x & y z \\
\hline
\end{tabular}

Drygotherium Mer.

Escheri Mex. . . . . . . .

Dremotherium GEOFFr. 2 :

Feignoui Geofrr. .

nanum Geoffr.

Amphitragulus (Pom.?) 1. . sp. Ром. . . . . ।...

Dorcatherium KaUP, 3 . Guntianum MEY. ..

Naui Kaup ....

Vindobonense МБу.

b Camelidae.

Camelus Cuv. 2. . Sivalensis FalcCautz. $\mathbf{S}^{2} \ldots$

Auchenia ILlg, 3.

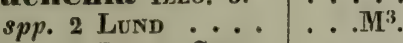
$s p$. aff. Lamae SERr.

Merycotherium Bos. 1...

Sibiricum Bos. . . . | . $\mathrm{S}^{2} \ldots$

Ruminantium summa: 120 . 


\begin{tabular}{|c|c|c|c|c|c|c|c|}
\hline & Weltgegend. & KohlenP. & SalzP. & OolithP. & $\begin{array}{l}\text { Krei- } \\
\text { deP. }\end{array}$ & MolnsseP. & $\mathrm{Neu}$ \\
\hline Benennungen. & 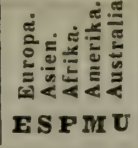 & 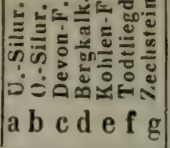 & 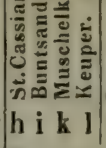 & 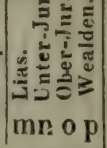 & 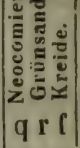 & 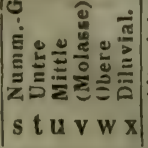 & 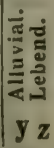 \\
\hline
\end{tabular}

\section{EDENTATA.}

\section{A. MYRMECOPHAGA.}

Myrmecophaga LIN, 2 ... $s p$. aff. jubatae (Liv.) Lund. $M^{3}$ $s p$. aff. tetradactylae (L.) LD. $\mathrm{M}^{3}$ Drycteropus Geoffr. 1. . .

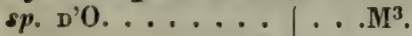

\section{B. DASYPODA.}

Dasypus Liv. 5. . .

? $s p$. BRAv......

sp. aff. Mirim. ... . . $\mathrm{M}^{3}$. $s p$. aff. octocincto LUND . . $\mathrm{M}^{3}$. punctatus Lund ............ sulcatus Lvind .... ....M $\mathbf{M}^{3}$.

Kenurus WagL. 1.

$s p$. aff. nudicaudo LUNe $\mathbf{M}^{3}$.

(Glyptodon Ow.) 4.| . . .

= Chlamydotherium BR. (Hoplophorus LUND) =

clavipes Ow. ..........

ornatus $\mathrm{OW}_{\mathrm{w}} \ldots \ldots . . \mathrm{M}^{4}$.

reticulatus Ow..... . . . $\mathbf{M}^{4}$.

tuberculatus $\mathrm{O}_{\mathrm{w}}$... ..... $\mathrm{M}^{4}$.

(Hingplophorus Lund.) 3. . .

= Chamydotherium $\mathrm{BR}_{\text {. }}=$

(Glyptodon $\mathrm{Ow}$.)

euphractus Lund..

minor LUND .....

Selloi Lund .....

Eeryodon Lund 1.

latidens. . ....

Weterodon LuNn, 1

diversidens. .....

Chlamydotherium Lund [non BR.] $2 \ldots$

Humboldti Lund .. $\mid \ldots \mathbf{M}^{3}$. majus Lund ..... .... $\mathbf{M}^{3}$.

Pachytherium Luvd, 1 ... magnum.

$\mathrm{M}^{3}$.

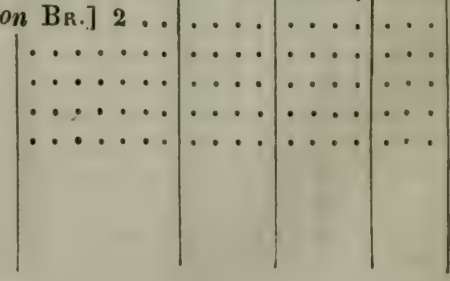




\begin{tabular}{l|l|l|l|l|l|l|l|l}
\hline Benennungen. & Weltgegend. & a b c d e f g h i k l & mn o p & q r l & s t u v w & y \% \\
\hline
\end{tabular}

\section{BRADYPODA.}

MegatheriumCúv.2 Cuvieri Desmar. .. $\ldots \mathbf{M}^{24}$ Laurillardi LuNd ... ....M $\mathbf{M}^{3}$.

Megalon yx Jeffers. 1. . . . Jeffersoni HARL. ... . . $\mathbf{M}^{234}$. Mylodon Ow. 3 .. Darwini 0 w. ............. Harlani Ow. .............. robustus $\mathrm{Ow} . \ldots . . . \mathrm{M}^{4}$.

Scelidotherium 0 w. $4 \ldots$ Bucklandi $\mathrm{O} w . \ldots \ldots \mathrm{M}^{3}$. Cuvieri Ow. .... ...... $\mathbf{M}^{3}$. leptocephalum 0 w. . . . . $\mathbf{M}^{4}$. minutum $0 \mathrm{w} . . . . . \mathrm{M}^{3}$.

Platyonyx Luvd, 4 Agassiri Lund ... Blainvillei Lund . . Brongnia ti Lund. Oweni Lund ....

Sphenodon Lund, 1 $s p$. LuND

. $\mathrm{M}^{3}$ $\ldots \mathrm{M}^{3}$ $\therefore \mathrm{N}^{3}$ $\therefore \mathrm{M}^{3}$

Coelodon Lund, 2. Kaupi Lund .... $\ldots \mathrm{M}^{4}$. Maquinensis Lund . ...M $M^{3}$.

Denotherium Lund, $1 \ldots$ gigas Lund .....

Macrotherium LART. 1. . . giganteum.

Edentatorum summa: $40 \ldots$

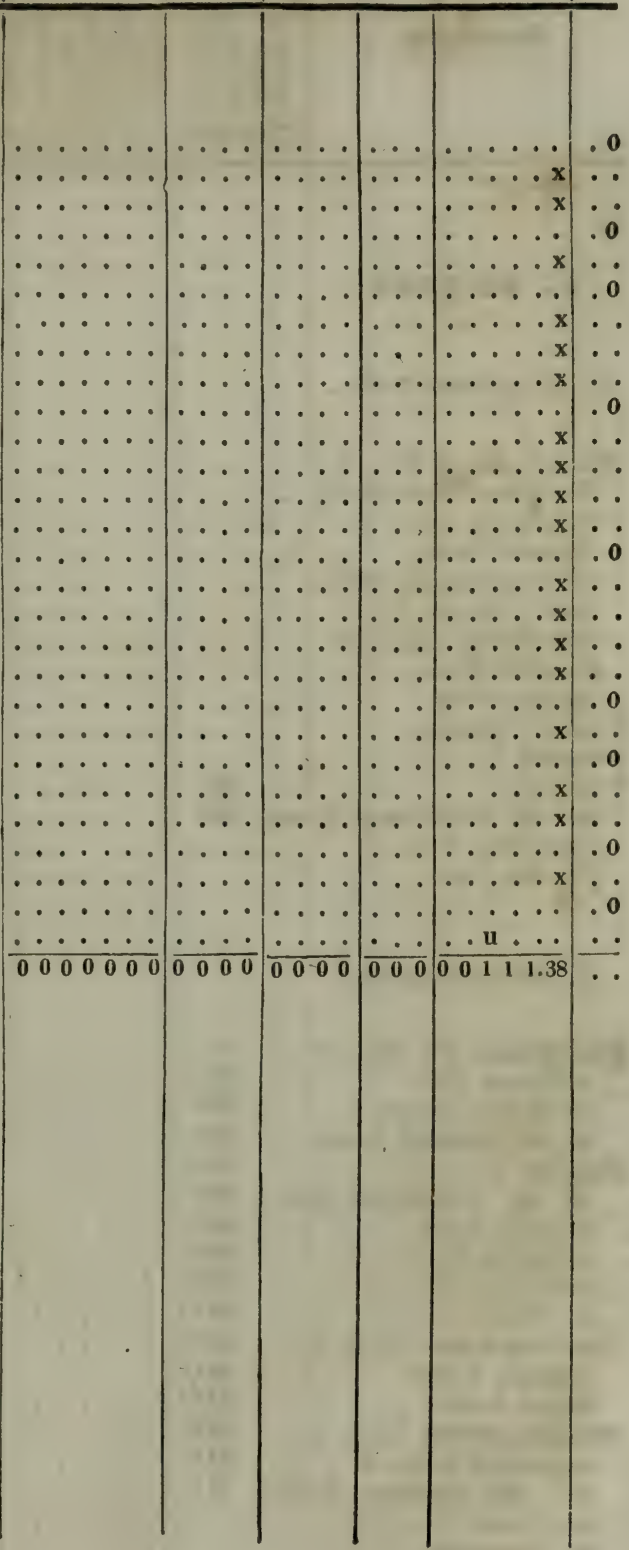




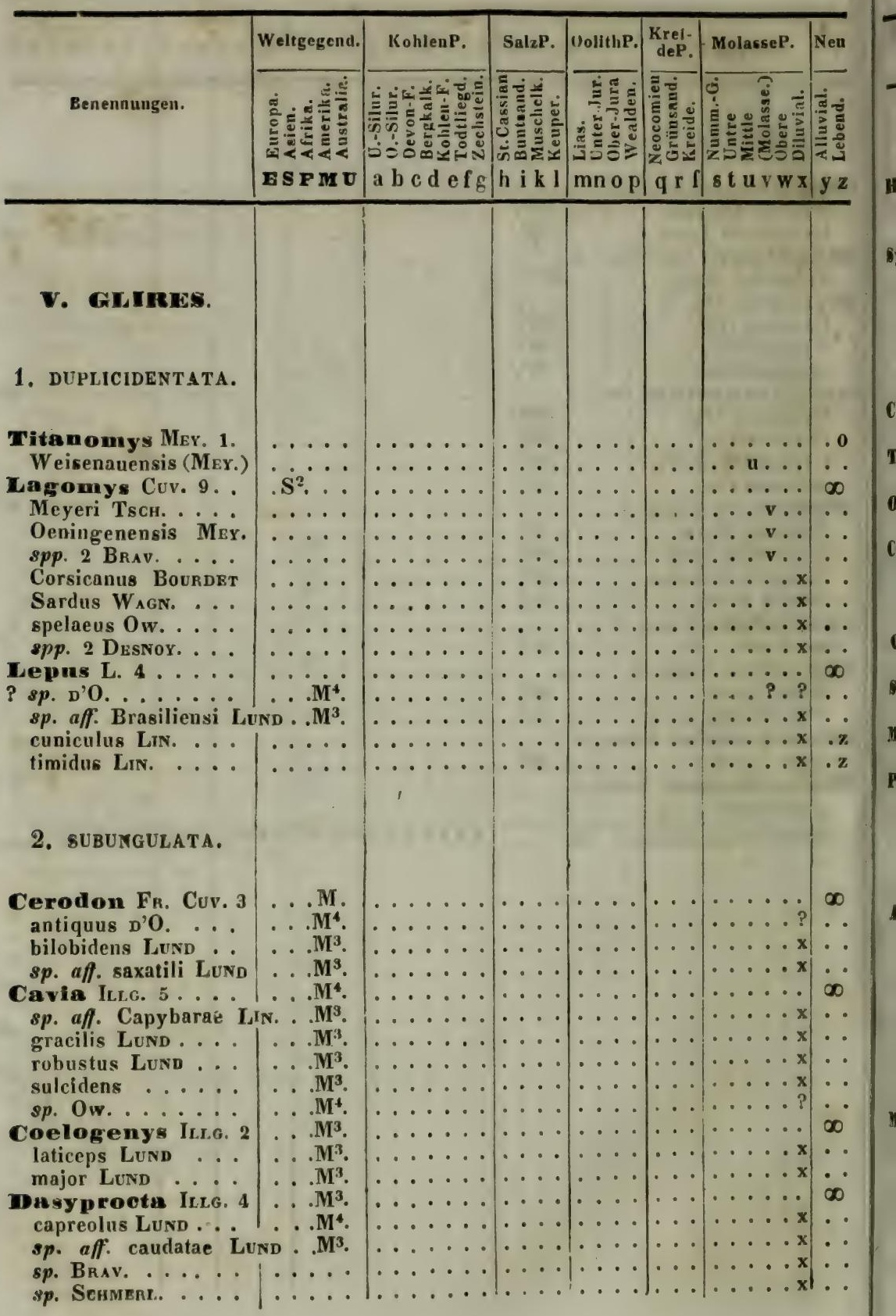


\begin{tabular}{l|l|l|l|l|l|l|l|l|}
\hline Benennungen. & Weltgegend. a b c d e f g & h i k l & mn o p & q r l & st u vw x & y $~$ \\
\hline
\end{tabular}

\section{HYSTRICINA.}

Myetrix Lin. 2

sp. CauthFale. . .

$s p$. Cuv. ........

Bymetheres Fr.Cuv.2

dubia Lund .

magna LuND

\begin{tabular}{|l|l|l|l|}
\hline &
\end{tabular}

\section{Castorina.}

\section{Castor Liv. 1}

Europaeus Ow.

Trogontherium Fisch. 1 . Cuvieri Fisch.

Dsteopera Harl. 1. platycephala HARL.

Chalicomys KaUP, 3

Jägeri Kaup

minutus MeY.

Dmegadon Pom. 1 sp. PeM.

Steneotherium Gropre sp. Geoffr.

Myopotamus Commers. 1.M $\mathrm{M}^{3}$ antiquus Lund ...

Palaeomys Kaup, 1 castoroides KaUP .

\section{MURINA.}

Arvicola Lacep. $9+$ sp. Cuv. ....... $s p$. Ноғм.

sp. Ow.

amphibia. arvalis SALL. pratensis. terrestris.

spp.

Mus Liv. $16+$ spp. Cautr. Falc.

** Hesperomys WATERr. aquaticus Lund debilis Lund aff: expulso Lund aff. fossorio Lund . aff. lasiuro Lund

…

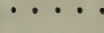

$\cdots \cdots$

…

...

…

$\therefore \cdots$

$\mathrm{S}^{2}$.

$\ldots \dot{M}$

... $\mathrm{M}^{3}$

$\ldots \mathrm{M}^{3}$

$\mathrm{M}^{3}$

$\ldots \mathbf{M}^{3}$.

$\ldots \mathrm{M}^{3}$. 


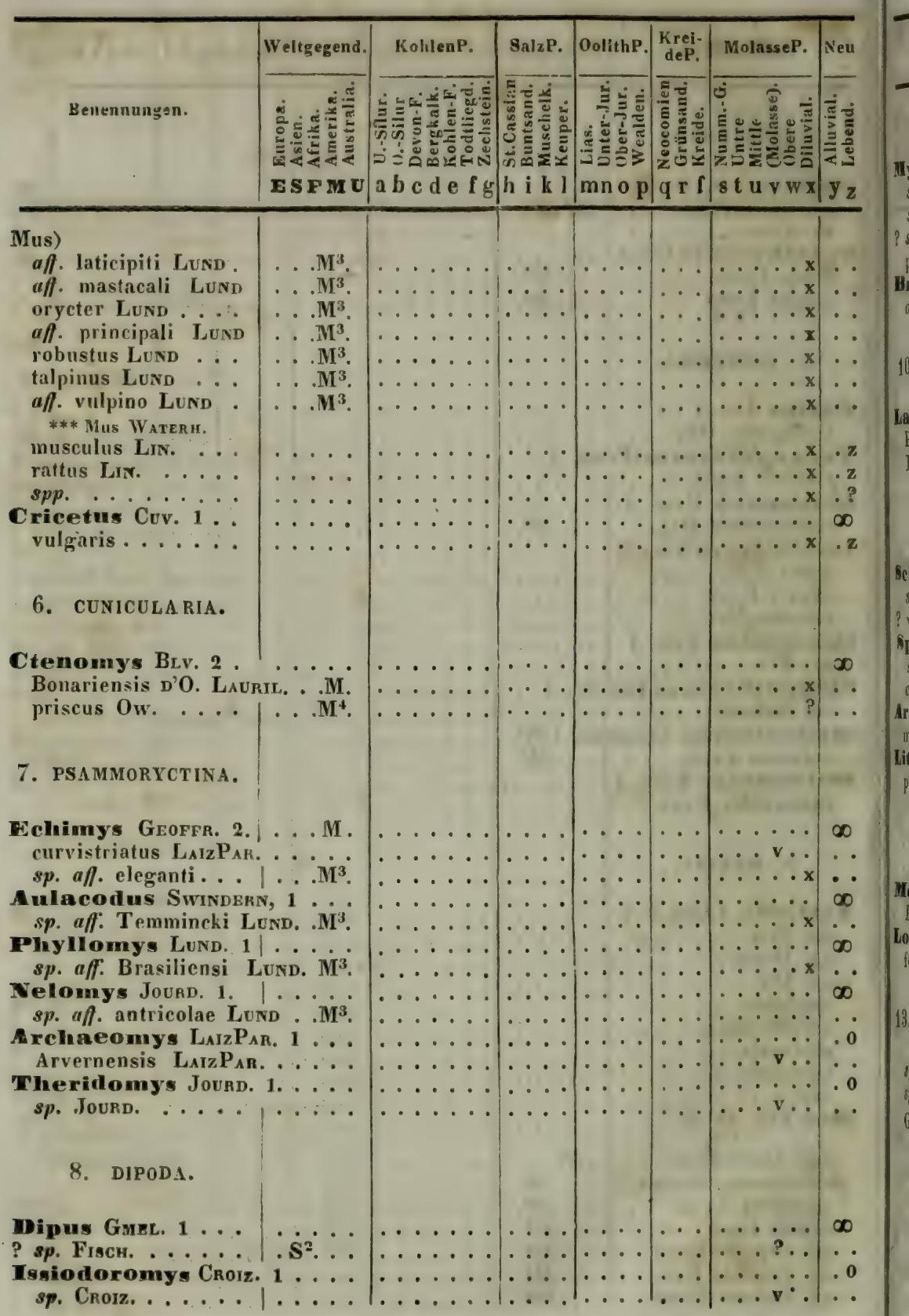




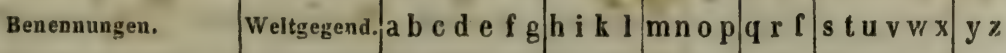

9. MYOXINA.

Myoxus Gmer . 5 . . spp. 2 Cuv. .....

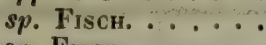
? $s p$. Fisch. . . . . . . priscus SchMERL. . ornatus MEX. ...

10. CHINCHILLIDEA.

LagostomusBroок.2 . . . M Patagoniensis D'O., LAURIL.M ${ }^{4}$. Brasiliensis LuNd . . . . $\mathbf{M}^{3}$.

\section{SCIURINA.}

Sciurus vulgaris $\mathbf{L}$.

Gpermophilus Fr.Cuv. 2 .. speciosus MeY..... citillus

Aretomys Schreb. 1 marmotta Schreb.

Lithomys Mey, 1. . parvulus Mex. ...

12. ORYCTERINA.

Megamys d'O.LauriL. 1 Patagoniensis D'O. LavriL. $\mathrm{M}^{\mathbf{4}}$ Conchophorus Lund, 1 .... fossilis Lund ..................

\section{Genera incerlae familiae.} sp. $\mathrm{OW}_{\mathrm{w}} \ldots \ldots \ldots . . . \mathrm{M}^{4}$ sp. Pentl. $\ldots \ldots . \mid \ldots \mathrm{U}^{4}$ Glir. surnma: 97

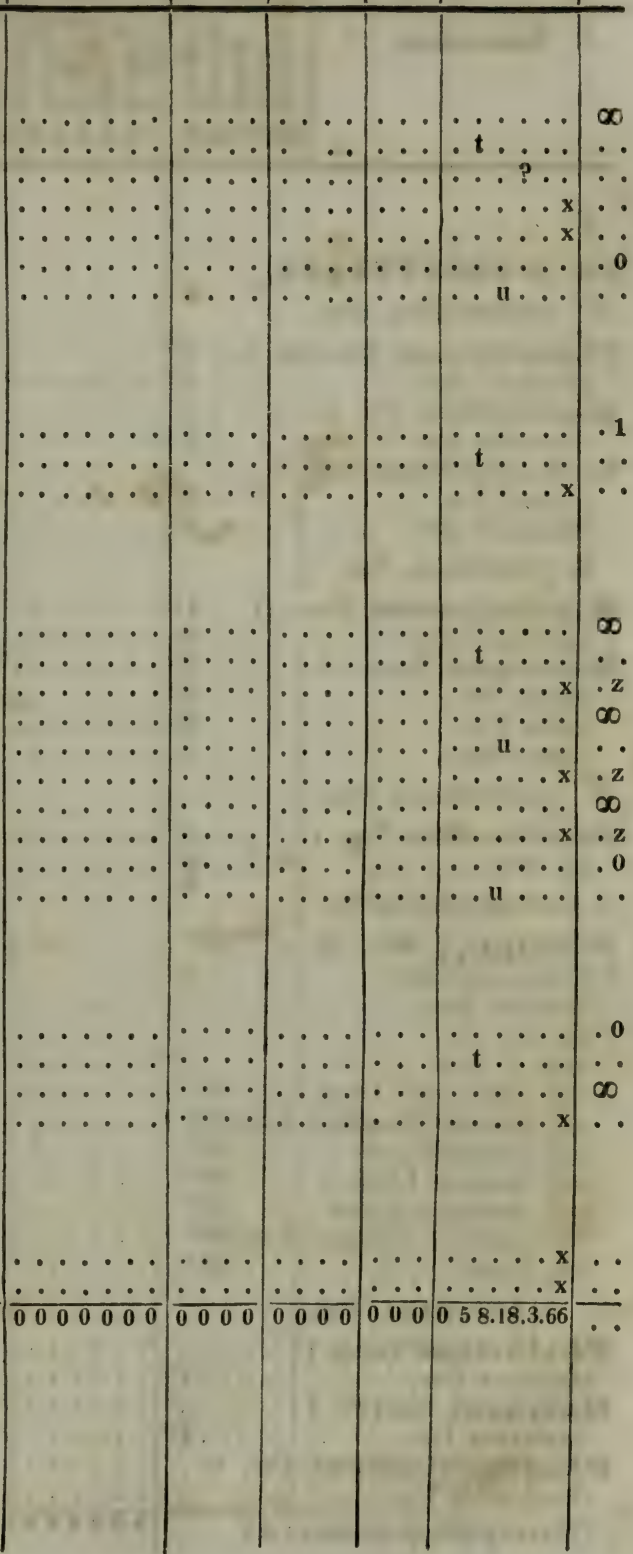




\begin{tabular}{|c|c|c|c|c|c|c|c|}
\hline & Weltgegend. & KohlenP. & SalzP. & OollthP. & $\left|\begin{array}{c}\text { Krei- } \\
\text { deP. }\end{array}\right|$ & MolasseP. & Neu \\
\hline Benonnungen. & 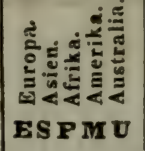 & 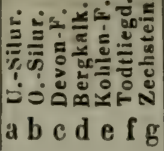 & 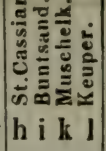 & 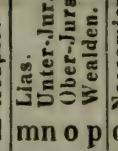 & 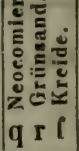 & 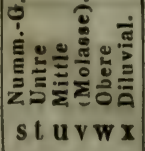 & 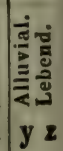 \\
\hline
\end{tabular}

VI. MARSUTITATA.

1. RHIZOPHAGA OW.

Phascolomys Geofrr. 1. . U Mitchelli Ow. ... . ... U U

Diprotodon 0 w. 1 .

Australis Ow. ........ $\mathrm{U}^{4}$

Nothotherium $0 w .2 \ldots$ inerne Ow............ $U^{4}$ Mitchelli Ow.

2. Pоернаga Ow.

rypsiprymnus ILLIG. 1. . U4 spelaeus $\mathrm{O}_{\mathrm{w}} \ldots \ldots \ldots \mathrm{U}^{4}$

Macropus SHaw. 3. .... Ut affinis $\mathrm{Ow} . \ldots \ldots \mathrm{U}^{4}$ Atlas Ow. .......... U Titan Ow. .....

3. CARPOPHAGA Ow.

Phalangista Cuv. 1 sp. Ow.

4. ENTOMOPHAGA OW.

Didelphys Cuv, 12.

? Colchesteri Ow.

Cuvieri Mer.

8 sp.

spp. 2 Рoм.

aff. albiventri LoND

...... $\mathbf{M}^{3}$.

aff. aurictae (PR. MAx) LUNDM ${ }^{3}$.

aff. eleganti LuND . $\ldots \mathrm{M}^{3}$.

$a f f$. incanae LUNd . $\ldots \mathbf{M}^{3}$.

aff. myosurae LuNd ... $\mathbf{M}^{3}$.

aff. pusillae (Dsmar.) Lund $M^{3}$.

sp. LUND ...................

5. SARCOPHAGA Ow.

Thylacinus Tемм. 1 spelaeus Ow. .....

Dasyurus Geofrr, 1

laniarius 0 w.

Phascolotherium 0 w. 1 .

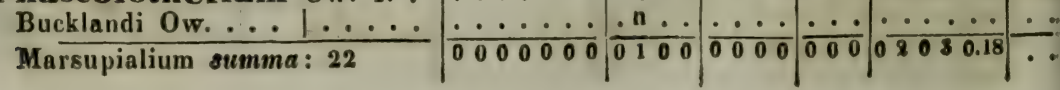


\begin{tabular}{l|l|l|l|l|l|l} 
Benennungen. & Weltgegend. & a b c d e f g h i k l & mn op & q r f & s t u v w x & y z
\end{tabular}

\section{CATR NIUTEA.}

\section{A. PINNIPEDIA.}

Trichechus Lin. 3. Rosmarus LiN. $s p$. Ow.

$\mathbf{M}^{2}$

? sp.

Cystophora Nirs. ? proboscidea Nirs.

Dtaria Péron. 1 . ? fossilis Keferst. . Phoca Lin. 3 . . . . ambigua Mü. rugidens MrY.

Viennensis antiqua BLv. .... Pachyodon MEX. mirabilis Mex.

\section{B. CARNIVORA.}

\section{FELIDAE.}

Felis Liv. $38 \ldots$ aff. concolori (Lin.) LuND. $\dot{\mathrm{M}}^{3}$. exilis Lund ............. $\mathbf{M}^{3}$. aff. macrourae ( $\dot{P}_{R}$ MAx. $)$ L. $\mathbf{M}^{3}$. aff. oncae (LiN.) LuNd $\cdot \mathrm{M}^{3}$. aff. pardali (LiN.) Lund . $\mathbf{M}^{3}$. protopanther LUND . cristata FalcCaUtL. subhimalayana. ... antediluviana KAUP aphanista KAUP. ogygia Kaup ..... palmidens BLv. Pardoides Buv. Pardoides Ow. prisca KauP ? quadridentata Biv. tetraodon BLv. ... sp. Buv. . . . . . sp. BI.v. antiqua Cuv. Arvernensis CrorzJoв. $\mathrm{S}^{2} \ldots$ brevirostris CroizJor. catus LiN. elata Brav.

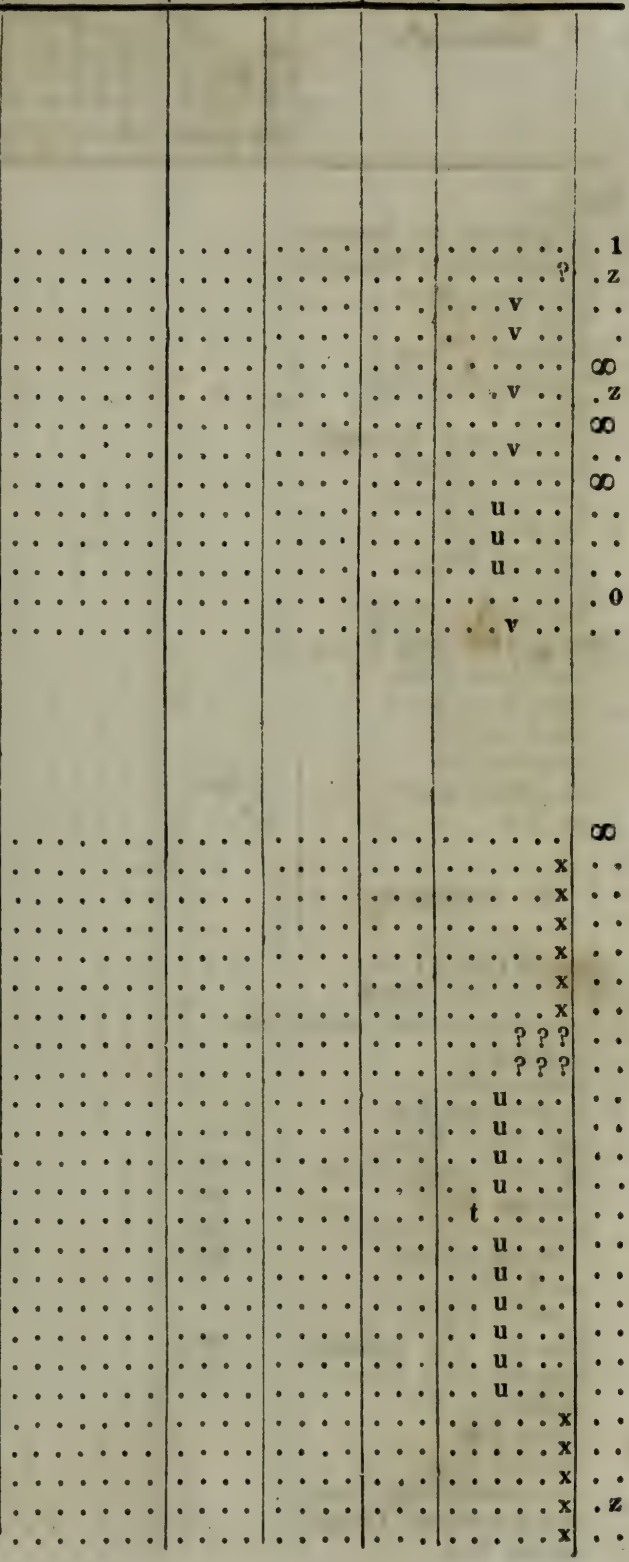




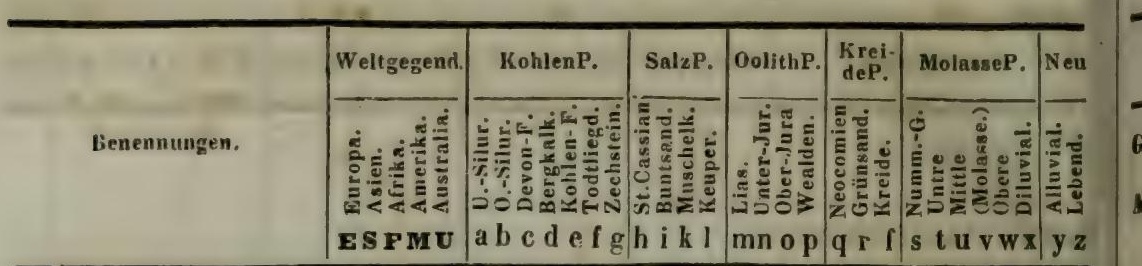

Felis)

Engiholiensis Schmert.

Issiodorensis CROIzJoв.

Juvillaca Brav. .

Leo LiN.

? Leopardus Lis.

leptorhyncha Brav.

Lynx L.

Pardinengis Crorzoв

Perrieri Croiz. prisca Schmerc. .

? Serval Lis.

spelaea GF.

$\cdots$

Velonensis Crorz.

vachairodus KAUP, 6 .

brevidens Pom. cultridens KauP

latidens $\mathrm{Ow}$.

megantereon.

neogaeus 0 w.

? $s p .0 \mathrm{w}$.

\section{CANIDAE.}

Canis Liv. $21+$.

brevirostris CroIz. .

ferreo-jurassicus major JïG. .

gypsorum .....

Parisiensis

? Viverroides Brv.

spp. C CautlFalc.

aff. Havicaudo Lusd

lycodes Lund ....

protalopex LuNd .

robustior LUND ...

aureus LiN.

familiaris Liv.

incertus D'O. LAURIL.

Issiodorensis Croiz.

Juvillacus Brav...

lupus Lis.

medius Brav. ....

megamastoideus Рom. Neschersensis Croiz. vulpes LiN.
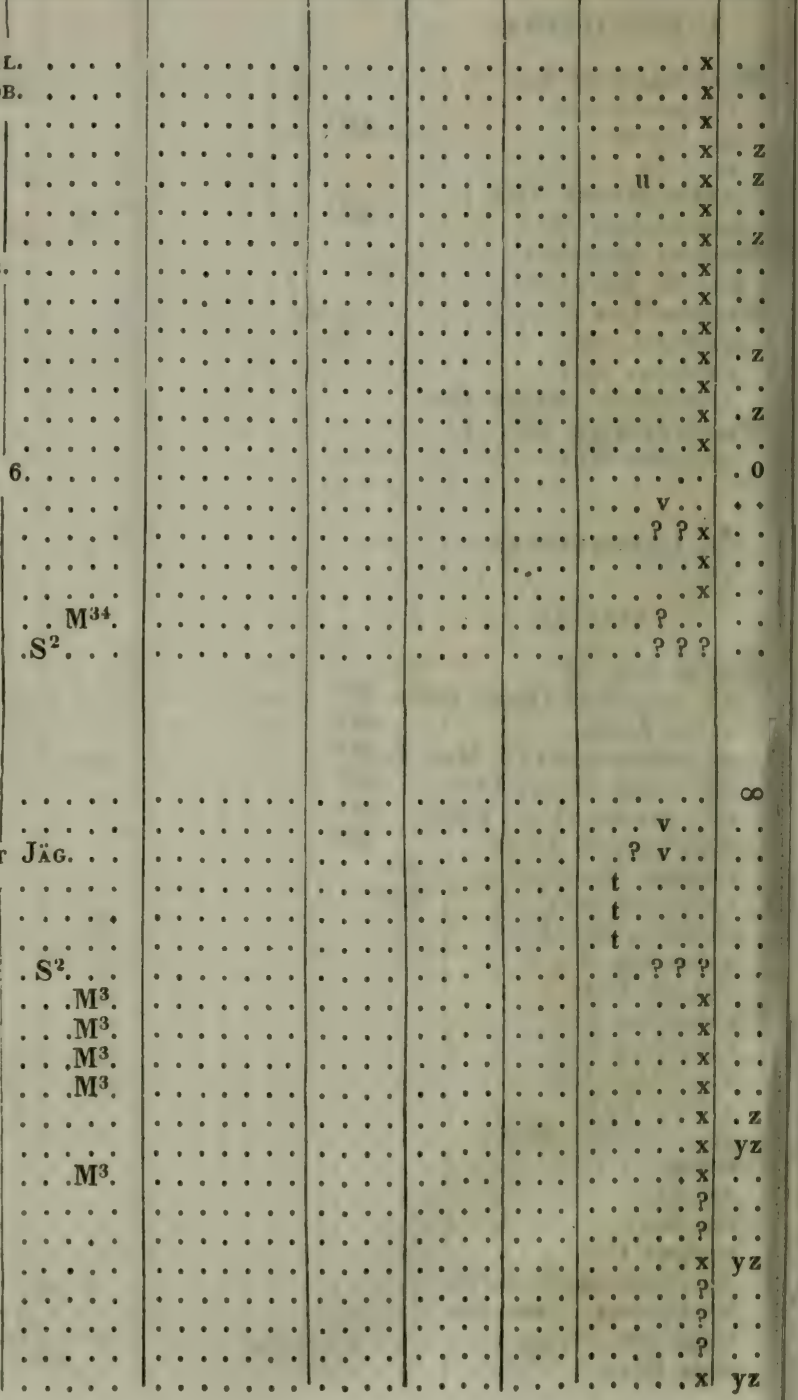


\begin{tabular}{|c|c|c|c|c|c|c|c|}
\hline Bene & Welt & 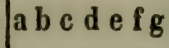 & h i k l & $\mathrm{mn} 0 \mathrm{p}$ & 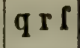 & $\mathbf{W} \mathbf{x}$ & $\mathbf{y} z$ \\
\hline $\begin{array}{c}\text { Galecynus } 0 w .1 \\
\text { palustris . . . . . . }\end{array}$ & & $\cdot$ & & $\cdot$ & $\cdots$ & $\cdots$ & $\mathbf{0}$ \\
\hline 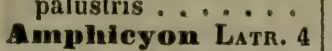 & $\because$ & $\cdots \cdots$ & $\cdots \cdot \mid$ & $\cdots \cdot$ & $\cdots \cdot$ & $\cdots \mathbf{v}_{\cdots}$ & $\therefore$ \\
\hline dominans MEX. . . . & $\begin{array}{ll}\cdots \\
\cdots\end{array}$ & $\cdots \cdots$ & $\cdots \cdot$ & $\cdots \cdots$ & $\cdots$ & $\cdots \cdots$ & .0 \\
\hline Klipsteini MEY. & $\begin{array}{l}\cdots \\
\cdots\end{array}$ & $\cdots \cdots \cdots$ & $\cdots \cdots$ & $\cdots \cdots$ & $\because \cdots$ & 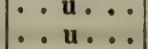 & $\cdot$ \\
\hline major BLv. & $\ldots \ldots$ & $\ldots \ldots$ & $\ldots \ldots$ & $\ldots$ & $\ldots$ & $\because u_{0} \cdots$ & - \\
\hline ? minor Blv. . . . & $\cdots$ & $\ldots \ldots$ & $\ldots$ & $\ldots$ & $\ldots$ & ..u?.. & $\because$ \\
\hline Acanthodon MBY. 1 & $\cdots \cdot$ & $\ldots \ldots$ & $\ldots$ & $\cdots$ & $\cdots$ & $\ldots \ldots$ & .0 \\
\hline i & & $\cdots$ & $\cdots \cdot$ & $\cdots \cdot$ & $\cdots$ & $\ldots u \ldots$ & \\
\hline ND, 2 & $\cdots$ & $\therefore$ & $\cdots \cdot$ & $\cdots \cdot$ & $\cdots$ & $\cdots \cdots$ & .0 \\
\hline UND & $\because \cdots$ & $\because$ & $\cdots \cdot$ & $\cdots \cdot$ & $\cdots$ & $\cdots \cdots x$ & • \\
\hline 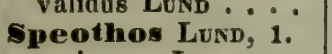 & $\because$ & $\cdots \cdots$ & $\mid \cdots \cdots$ & $\cdots \cdots$ & $\cdots$ & $\mid \begin{array}{c}\cdots \\
\cdots \\
\cdots\end{array}$ & $\ddot{0}$ \\
\hline is Lund .. & ... $\mathrm{M}^{3}$ & $\ldots \ldots$ & $\ldots$ & $\ldots$ & $\ldots$ & $\ldots \ldots \mathrm{x}$ & .0 \\
\hline I.v. 1 & . & $\ldots \ldots$ & $\cdots$ & $\cdots \cdot$ & $\cdots$ & $\ldots \ldots$ & i \\
\hline - & & $\cdots \cdot$ & $\cdots \cdot$ & $\cdots \cdot$ & $\cdots \cdot$ & .?. v... & . \\
\hline Pterodon BLv. 2 . & & $\cdots \cdot$ & $\cdots \cdot$ & $\cdots \cdot$ & $\cdot \cdots$ & $\cdots \cdots \cdot$ & .0 \\
\hline nsis Blv. . & & ・. & - & & $\cdots$ & $\cdot \mathbf{t} \cdots \cdot$ & . \\
\hline Gervals : & …. & $\cdots$ & $\cdots \cdot$ & $\cdots \cdot$ & $\cdots \cdot$ & $\cdots \nabla \cdot \cdot$ & $\cdots$ \\
\hline IOn CaUtLFaL & LC. $s_{2}^{1} \cdots$ & $\therefore$ & $\cdots \cdot$ & $\cdots \cdot$ & $\cdots$ & $\cdots \cdots$ & $\cdot 0$ \\
\hline Sil & $\cdots S^{2} \cdots$ & & $\cdots \cdot$ & $\cdots \cdots$ & $\cdots \cdot$ & $?$ & 0 \\
\hline $\begin{array}{l}\text { n Kaup } \\
\text { p. }\end{array}$ & i. & $\ddot{\cdots}$ & $\cdots \cdots$ & $\cdots \cdots$ & $\cdots$ & $\because$ & $\cdot 0$ \\
\hline MEх. 1 & $\cdots \cdots$ & $\ldots$ & $\cdots \cdots$ & $\ddot{\cdots}$ & $\cdots$ & $\because \ldots \ldots$ & $\ddot{0}$ \\
\hline HIEY: & .... & $\ldots$ & $\ldots$ & $\ldots$ & ... & $\ldots \mathbf{v} \ldots$ & . \\
\hline Wyaenodon LAIzPAR & R. 2 ... & $\ldots \ldots$ & $\ldots$ & $\ldots$ & $\cdots$ & $\ldots \ldots$ & .0 \\
\hline hus Dujar & & - & $\cdots$ & $\cdots$ & $\cdots$ & ... $\mathbf{v} \ldots$ & - \\
\hline \& LAIzPar & & $\cdots \cdot$ & $\cdots$ & $\cdots$ & $\cdots$ & .... $\mathbf{v} \ldots$ & $\bullet$ \\
\hline exium BLv. & 1. . & $\cdots \cdot$ & $\cdots \cdot$ & $\cdots \cdot$ & $\cdots$ & $\cdots$ & .0 \\
\hline LV. • • & & & $\cdots \cdot$ & $\cdots \cdot$ & $\cdots$ & $\mid+\ldots$. & - \\
\hline 3 & & & & & & & \\
\hline Hy & & & & & - & & .3 \\
\hline Arvernensis BravCro1z & zJos. . . & $\cdots \cdots \cdots$ & . & $\ldots$ & $\cdots$ & $\ldots . . x$ & - \\
\hline Arvernensis ? Italica I & BLv. .. & $\ldots \ldots$ & $\ldots$ & $\ldots$ & $\cdots$ & $\ldots \ldots x$ & - \\
\hline d & & $\ldots \cdots$ & $\cdots$ & $\cdots \cdot$ & $\cdots$ & $\cdots \cdot \cdot x$ & - \\
\hline pario & & $\cdots \cdot$ & $\cdots \cdot$ & $\cdots \cdot$ & $\cdots$ & ..? $\cdot x$ & - \\
\hline rieri BRAyC & $\boldsymbol{J E}_{\mathbf{E}}$ & & $\cdots \cdots$ & $\cdot \cdot$ & $\cdots$ & $: \cdots \times x$ & $\cdot \cdot$ \\
\hline IIJJOH & & - $\bullet$ & $\cdot$ & - $\cdot$ & $\because$ & $\begin{array}{cl}\cdots x \\
\cdots x\end{array}$ & \\
\hline s & $\mathbf{E}^{2} \mathbf{S}^{2} \ldots$ & $\cdots \cdots$ & $\ldots$ & $\cdots \cdot$ & $\because$ & $\because \cdots x$ & \\
\hline & & & ... & & $\cdots$ & $\cdots x$ & \\
\hline Sivalensis BArDDUR. & & & 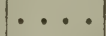 & & $\cdots$ & $? ?$ & - $\cdot$ \\
\hline Faleotherium WAG & SN. 1. & 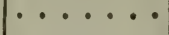 & - $\cdot$ & $\cdots$ & - & $\cdots$ & 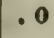 \\
\hline & & & & & & . v.. & $\bullet$ \\
\hline etis Buv. 1 & - & & $\cdots \cdots$ & $\cdots \cdots$ & $\cdots$ & $\cdots \cdots$ & $\cdot 0$ \\
\hline vi & & & $\cdots$ & & $\cdots$ & ... v & $\ddot{m}$ \\
\hline Genetta L & & & & & & $\ldots$ & $\begin{array}{l}\infty \\
. \mathrm{z}\end{array}$ \\
\hline$\cdots$ & $\ldots .$. & $\cdots \cdot$ & $\cdots \cdots$ & $\cdots$ & $\therefore$ & $\therefore$ v... & • \\
\hline & & & & & & & \\
\hline
\end{tabular}

$46(1847,1849)$ 


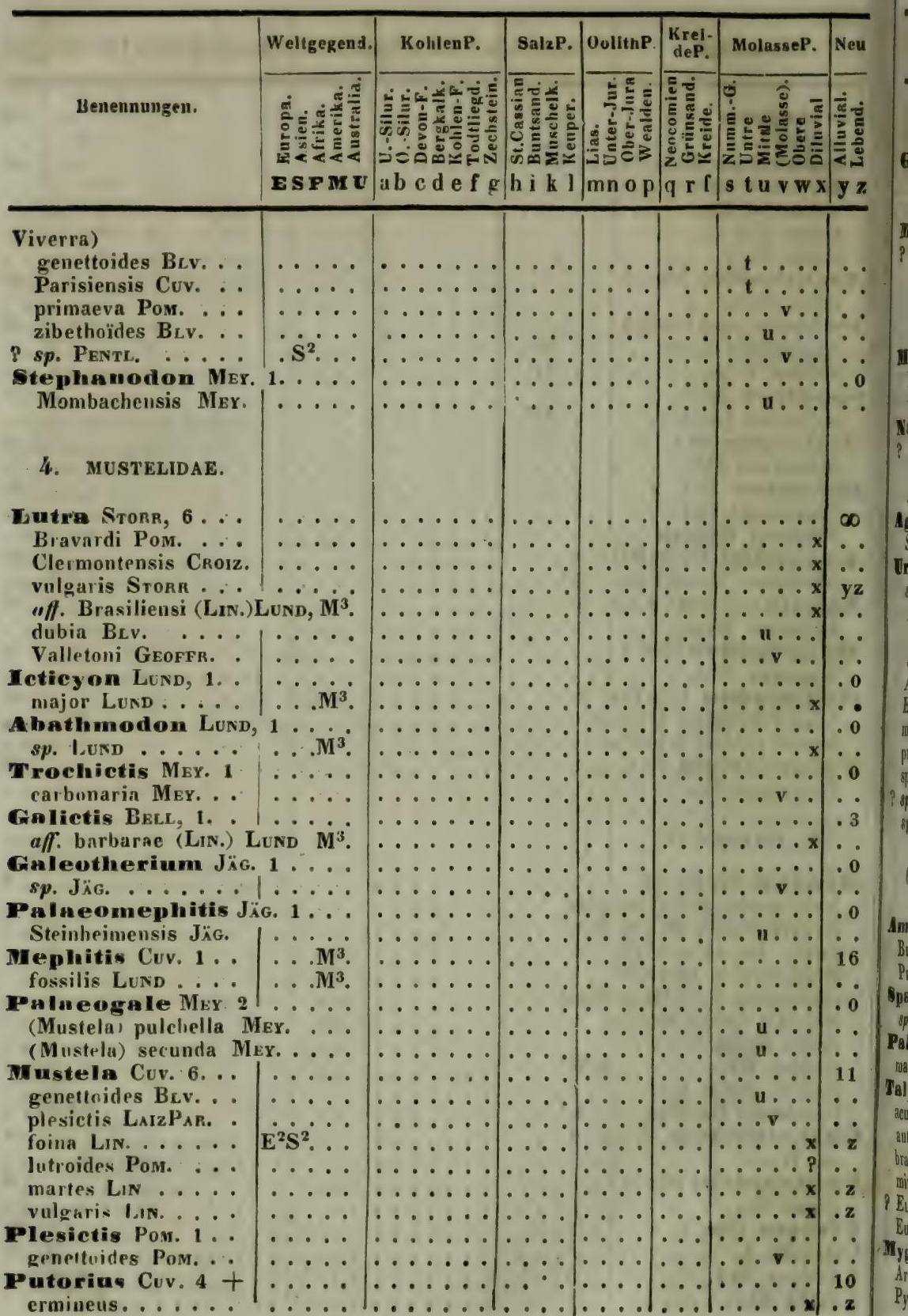




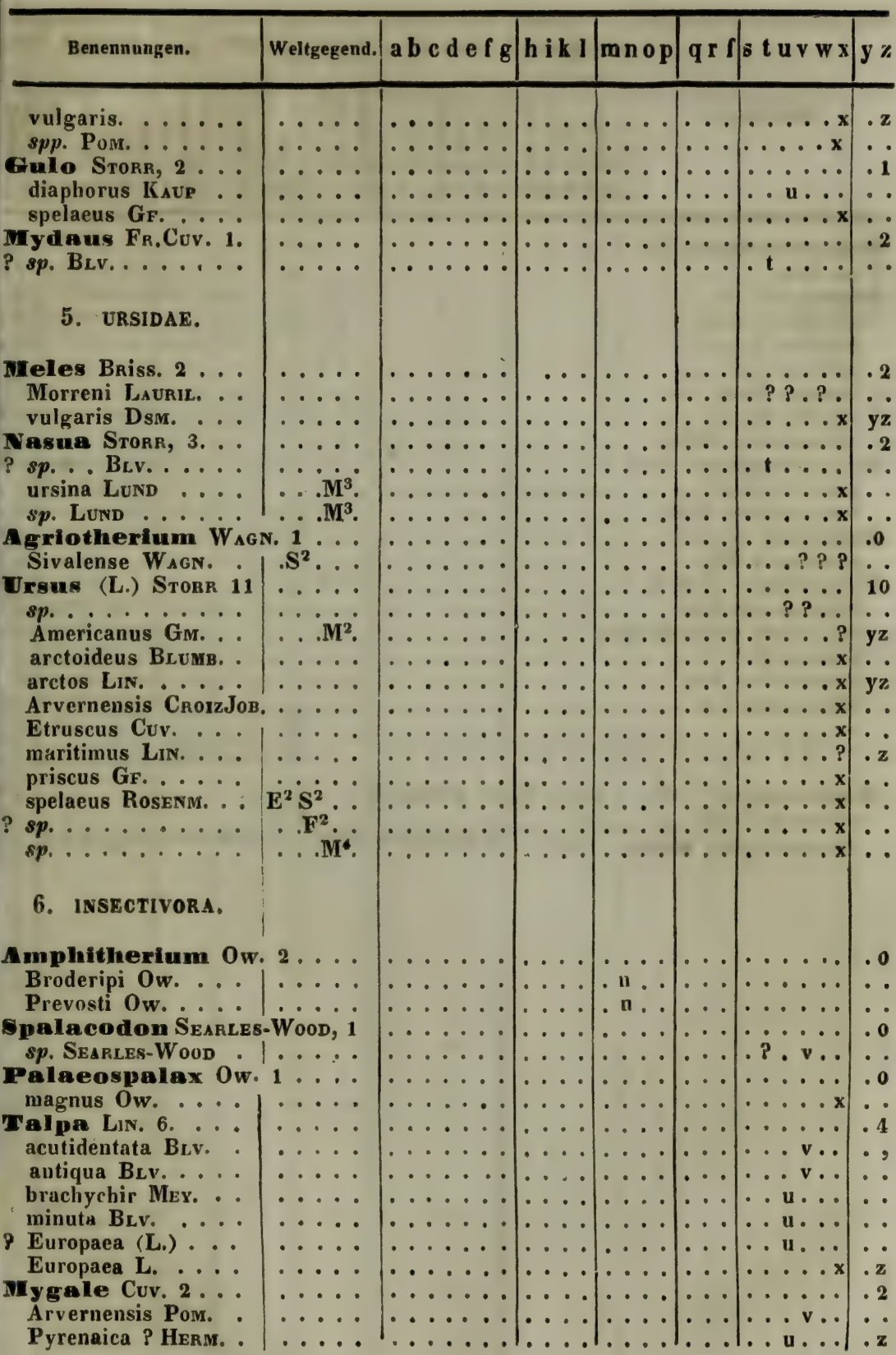




\begin{tabular}{|c|c|c|c|c|c|c|c|}
\hline & Weltgegend. & KohlenP. & SalzP. & oolithP. & $\begin{array}{l}\text { Krei- } \\
\text { deP. }\end{array}$ & MolasseP. & $\mathrm{N}$ \\
\hline Benennungen. & 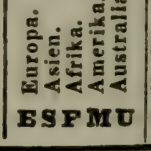 & a bc def f & $\begin{array}{l}\mid \\
\text { h i k l } \\
\text { his }\end{array}$ & 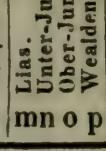 & 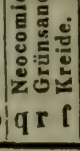 & 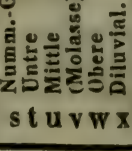 & 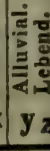 \\
\hline
\end{tabular}

Macroseelides $S_{M}$. 1 . . F.

$\ldots \ldots \ldots \ldots \ldots$

$\ldots \ldots \ldots \ldots \ldots$

giganteus Croiz.

pusillus MEy.

araneus Lin.

fodiens Gmbe.

remifer Gropr...

tetragonurus HerM.

Dxygomphius MEx

frequens $\mathrm{MEr}$.

leptognathus Mex. .

Dimylus MEY. 1 . . paradoxus MEY. ..

Erinaceus Liv. 5.

Arvernensis BLv.

(Centetes) antiquus Buv.

priscus MeY,

soricinoides Brv.

Europaeus Liv. . .

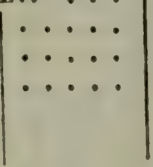

Microlestes PLIEN. 1 [num hujus ordinis?]

antiquus Plien.

Carnivororum summa: 186. .

\section{(2.}

$\ldots 1$

......... . 0 


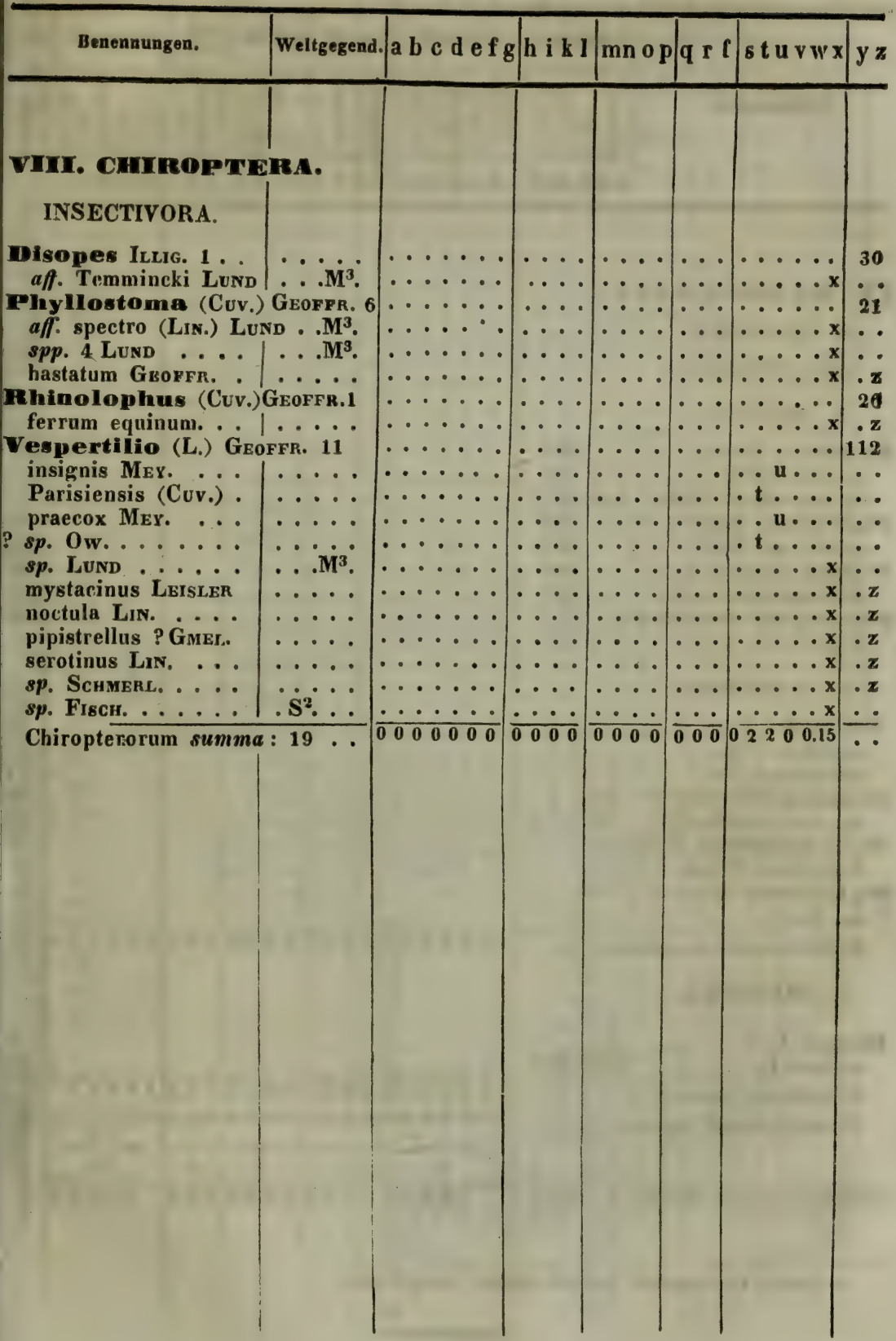




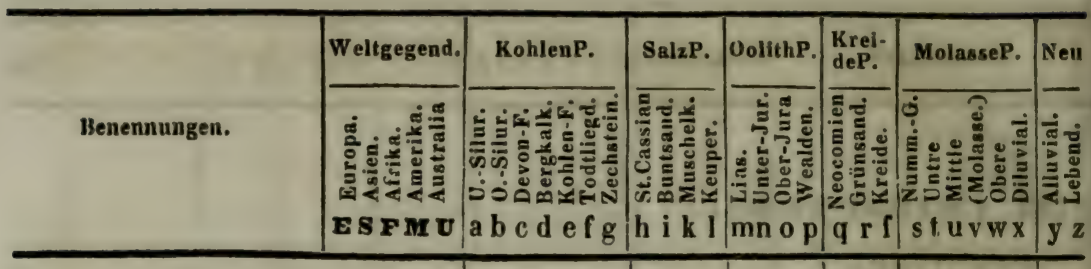

\section{QUATIUMANA.}

\section{A. PROSIMII.}

B. SIMIAE.

1. HESPERINI.

Iacchus GEOFFr。 2 ...( $\left.\mathbf{M}^{3}\right)$ grandis LuND ..... . . M $\mathbf{M}^{3}$. aff. penicillato (Geop.) LND. $M^{3}$.

Callithrix Grofrer. 1 . . ( $\left.\mathbf{M}^{3}\right)$ primaevus Lund ... ...... $\mathbf{M}^{3}$.

Cebus Erxleb. 1 .. ... (M $\left.\mathbf{M}^{3}\right)$ macrognathus LUND $\ldots \mathbf{M}^{3}$.

Protopithecus Lend 1 .

Brasiliensis LuND . . . $\mathrm{M}^{3}$.

\section{ANATOLINI.}

Macaeus Cuv, 2 . eocaenus $0 \mathrm{w}$. pliocaenus Ow.

Semnopithecus Fr. Cuv. 1 subhimalayanus MEx. | . S ${ }^{2}$. .

Mesopithecus WAGN. 1 ... Pentelicus WA $\mathrm{AN}$.

Hylobutes Ilulg. i $\ddot{S}^{3} \ldots$

? $s p$. KaUp ...........

Quadrumanorum summa: 10

\section{IIMA NA}

$\underset{\text { sapiens } L, \ldots \ldots}{\mathbf{E}^{2} \dot{S}^{2} \dot{M}^{23}}$ Bimanorum summa: $1 \ldots \ldots$ Mammiferorum summa: 708..

Spondylozoorum summa ${ }^{1}$ ): 2558

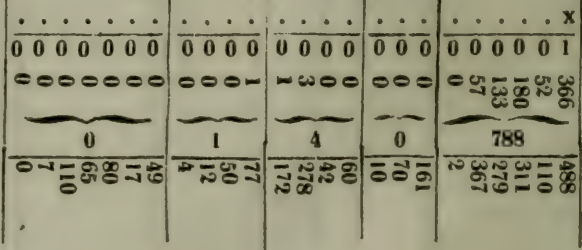

.
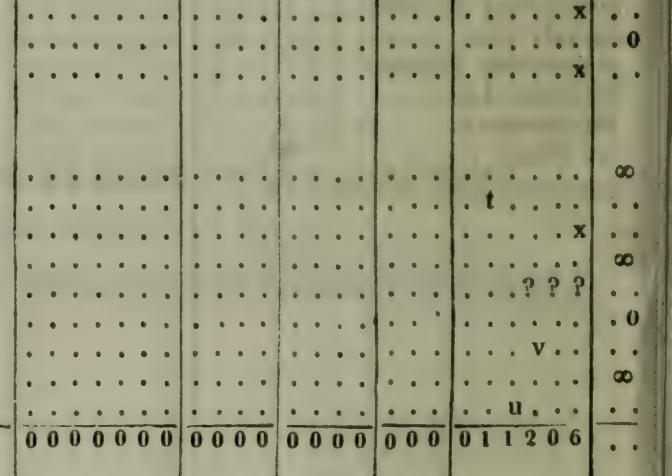

5) Summa ex emendatis Piscium alisque numeris orta. 


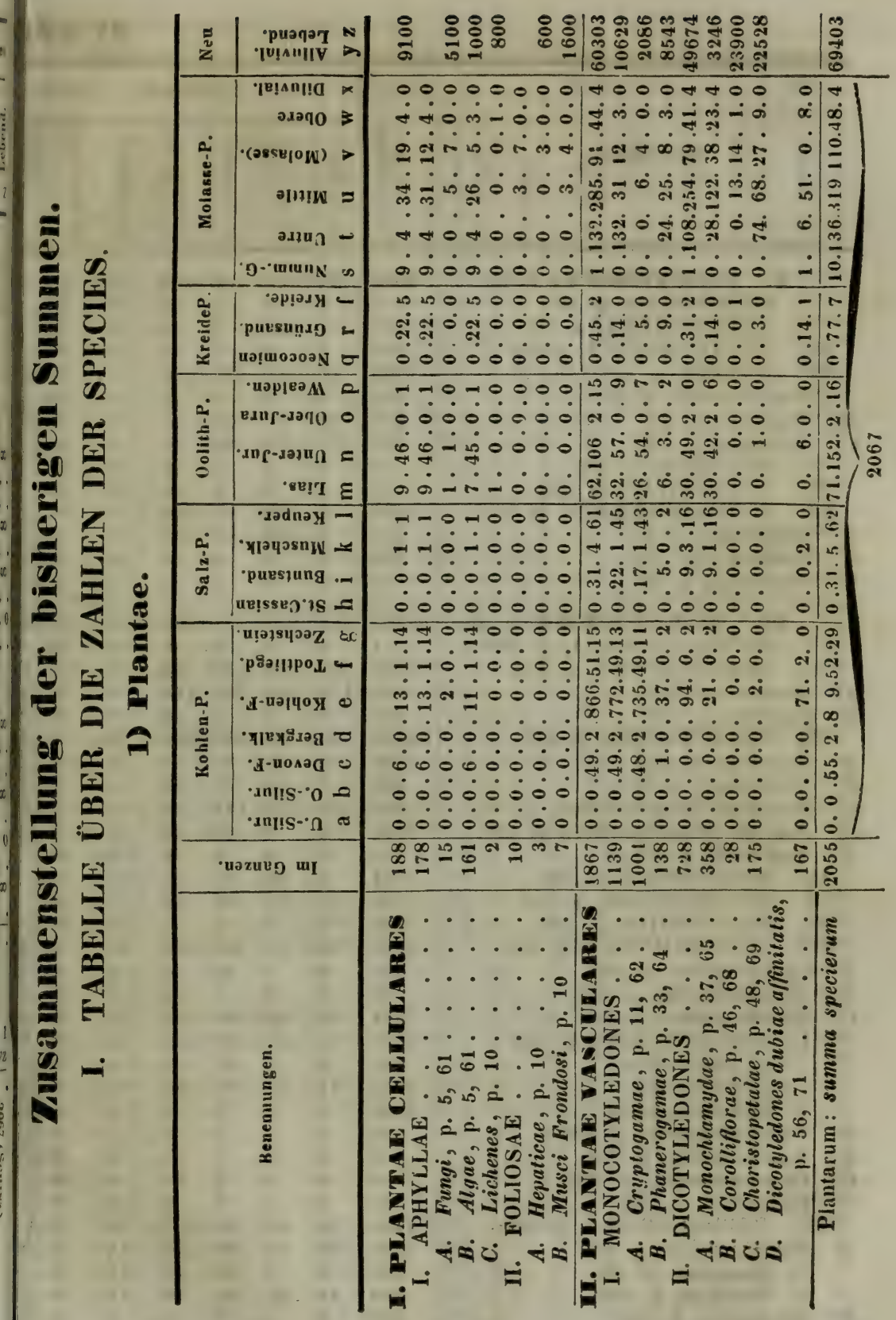


2) Ani-

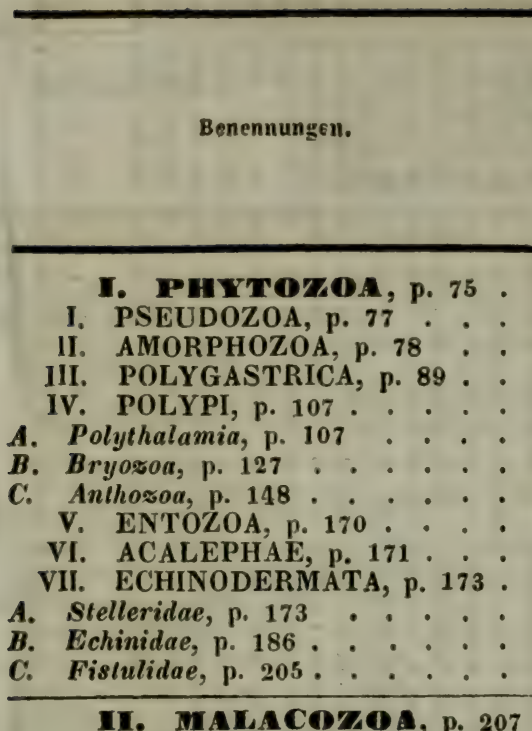

I. GYMNACEPHALA, p. 209 .

II. BRACHIOPODA, p. 210 .

A. Genuina, p. 210 . . . . .

B. Rudistae, p. 231 . . . . . .

III. PELECYPODA, p. 237 .

A. Monomya, p. 237 . . . .

B. Heteromya, p. 263 . • .

C. Homomya Integripalliata, p, 276

D. Homom. Sinuatopalliata, p. 319 .

E. Tubicolae, p. 352 .

IV. PTEROPODA, p. 355 . . .

V. HETEROPODA, p. 355 .

VI. PROTOPODA, p, 358 .

VII. GASTEROPODA, p. 362

A. Cyclobranchia, p. 362 . . .

B. Aspidobranchia, p. 365 . . . .

C. Ctenobranchia, p. 432 . . .

1) Asiphonobranchia, p. 432 .

a. Capuloidea .

b. Trochoidea .

2) Siphonobranchia .

D. Pomatobranchia, p. 486

E. Hypobranchia, p. 488

F. Gymnobranchia, p. 489

G. Pulmonata, p. 490

VIII. CEPHALOPODA, p. 503

A. Tetrabranchia, p. 503...

1) Ammonea, p. 503

2) Nautilina, p. 324

B. Dibranchia, p. 535

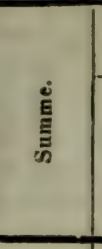

4895

2
461

672

2528

893

810

825

0
43

1189

416

770

13885

1

1146

952

194
4836

1066

686

1950

1120

14

41

85

120

6110

127

86

5281

2877

127

2750

2404

84

8
0
524

1546

1330

880

450

216
Kohlen-P.

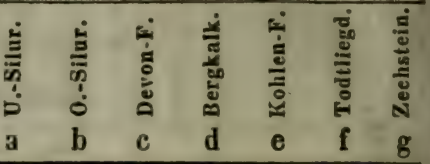

$36.223 .228 \cdot 263$. 1. 0.17

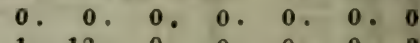

1. 13 . 9. 0 .

0. 0.1 .0 .000

$29.145 \cdot 137,156$. 0 . 0.16

0. 0. 0.09 . 0. 0.0

12. 61. 56. 64. 0. 0. 13

17. 84. 81.83. 0. 0. 3

0.0 .0 .0 .0 .0 .0

0. 0.0 . 0 . 0 . 0 . 0 . 0

6.65.82.106. 1. 0. 1

6. 65. 82.106. 1. 0.1

0 . 0 . 0 . 0 . 0 . 0 . 0

0 . 0 . 0 . 0 . 0 . 0 . 0

$260.416 \cdot 979 \cdot 809 \cdot 143,7 \cdot 94$

o. 0.0 . 0 . 0.0 .0

$151,148 \cdot 131 \cdot 199 \cdot 4 \cdot 0.35$

143.140 .224 .193 . 4. 0. 34

8. 8. 7. 6. 0. 0. 1

$25 \cdot 69 \cdot 287 \cdot 186 \cdot 70,7 \cdot 48$

0. 7. 34, 37, 5. 0. 6

7. 24. 72, 39, 10, 3. 17

18. $33,141,77,52,4 \cdot 17$

0. 5. 40, 33. 3. 0. 4

0. 0. 0. 0. 0. 0. 0

1. $10,13.1$. 1. 0. 0

10. 24, 28, 35, 7, 0, 0

0 . 0.4 3. 1.0 .0

38. $71,246,248,16,0,14$,

4. 3. 15. 26, 1. 0. 1

0. 0. 1. 0. 0. 0.0

$34.68 \cdot 230,222,15$. 0.13

33. $66.227,211 \cdot 12$. 0,13

2. 1. 21 . 5. 0. 0. 0

31. 65.206.206, 12, 0.13

1. 2. 3, 11, 3, 0, 0 30

o. 0 . 0 . 0 . 0 . 0.0 .0

0. 0.0 . 0 . 0.0 . 0

0.0 .0 .0 .0 .0 .000

0. 0.0 .0 .0 .0 .0 .0$.

$35 \cdot 94.270 \cdot 137,44,0$. 1186

$35.94 .269,135$. 44. 0. 1 86 .

0. 3.117, 43.27. 0. 0 77

35. $91.152 .92,17.0 .11$

0. 0. 1. 2. 0. 0. 0 


\begin{tabular}{|c|c|c|c|c|c|c|c|c|c|c|c|c|c|c|c|}
\hline \multicolumn{2}{|l|}{ Salz-P. } & \multicolumn{4}{|c|}{ Oolith-P. } & \multicolumn{3}{|c|}{ Krelde-P. } & \multicolumn{6}{|c|}{ Molasse-P. } & \multirow{2}{*}{ 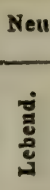 } \\
\hline 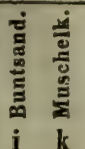 & 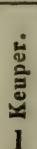 & $\frac{\dot{m}}{3}$ & 㟧 & 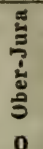 & 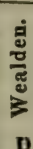 & 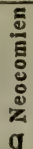 & 范 & $\frac{\dot{0}}{0}$ & 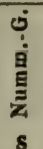 & $\stackrel{\Xi}{\Xi}$ & 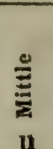 & 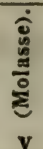 & $\begin{array}{l}\text { 巳ँ } \\
\text { ठิ } \\
\mathbf{w}\end{array}$ & 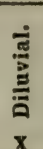 & \\
\hline
\end{tabular}

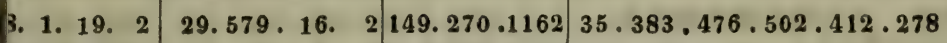

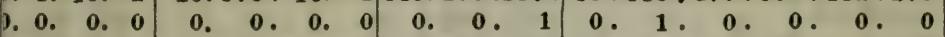

1. 1. 2. 1 0. $81,0.0018 .50,180$, 12.6 .47 .9 .30

4818

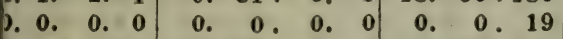

0. 1. 0.369 .29 .223

5. 0. 3. 0 3.221. 9. 0 54.112.673

$3.269 .390 \cdot 77.365 \cdot 21$

500

0. 0.0

0. 28. 0. 0 14. 10.254

2. $97.184,65.220 \cdot 10$

1810

7. 0.1 . 0 0. 26 . 1. 0 27, 42,323

0. $79,129,4,51,3$

1000

3. 0.2 2. 0 3. 167.8 . 0 13. 60.96

1. 93, 77, 8, 94,8

430

o. 0 . 0.0

\begin{tabular}{llll|llll}
0. & 0.0. & 0 & 0. & 0 & 0
\end{tabular}

0. 0.0 . 0 . 0 . 0 . 0

1500

0. 0.0 .00

26.276 . 7. (2) 77.108.289

$13,91,73,9,61,4$

9. 0. 13. 1 17. 92 . 1. (1) 4. 6. 36 0. 6. 3. 2.5 . 0

498

286

0. 0. 1. 0 9.182, 6. (1) 73.102.253 13.84, 70, 7. 56. 4

146

\begin{tabular}{lllllllllllllllll}
0.0. & 0. & 0 & 0. & 2. & 0. & 0 & 0. & 0. & 0 & 0 & 1 & 0. & 0. & 0. & 0 \\
\hline
\end{tabular}

3.38.109. 26533.1455 .242 .102751 .566 .150039 .2125 .2725 .783 .1609 .642

66

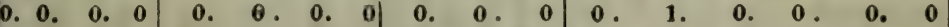

11482

3. 1. 10. 2 24. 80.3 .3161 .26 .227

9. 1. 9. 2 23. 67,2 . 1 43. 25.100

4. 0 . 1. 0 1. 13. 1. 0 18. 1.127

9.30. 71.10212 .786 .173 .77336 .279 .697

6.10. 25. 2 62.200.30. 3 83. 72.274

0.9. 9. 4 30.104.19. 4 41. 19.88

1. 4. 21. 2

60.257 . 30. 64107.111 .225

2. 7. 16.2

60.225 . 94. $6 / 105.77 \cdot 108$

1. 13. 6. $0.23,4$

1. 12. 4. 0. 21. 4

71

48

37

$25,705,783,164,550,189$

13, 118. 135. 85, 142. 22

2413

311

0. 40. 39. 11. 28. $9 \quad 177$

10.307. 327. 37. 170. 70 942

0. 0.0 .0

0. 0.0 . 0 . 0 0. 0.2

2. 232. 286. 30, 212. 88

958

$\begin{array}{lllllllllllllllll}0 . & 0 . & 0 . & 0 & 0 . & 0 . & 0 . & 0 & 0 . & 0 & 0 & 0 & 2 . & 8 . & 0 & 8 . & 2\end{array}$

$\begin{array}{lllllllllllllllll}0 . & 0 . & 0 . & 0 & 0 . & 0 . & 0 . & 0 & 0 & 1 . & 0 & 0 & 0 . & 0 . & 0 & 0 . & 0\end{array}$

25

62

$\begin{array}{lllllllllllllll}\text { 4. } 0.2 . & 0 & 2 . & 8 & 0 . & 0 & 8 . & 8 & 13 & 0 & 32 . & 24 . & 1 & 34 . & 8\end{array}$

1. 6. 26.14

81.300 . 53. $24 \mid 135.125,415$

$12.1354 .1892 .218,984.439$

6. 1. 2. 0

1. 10 . 1. 0 2. 2,7

0. 9. 18. 5. 13. 11

0. 0. 1. 0

0. 5. 0. 0 1. 1 . 11

79. 275 . 52. 23130.122 .395

15. 3. 16.9670 .215 .34 .2385 .64 .254

5. 0.0 .0

0 . 18. 28. 3. 13, 12

12.1170 .1540 .152 .853 .300

4. 396, 572. 67.371 .140

23

64

8673

214

5520

1490

0. 30. 47. 2. 21. $14 \quad 176$

70. $215.34 .2384 .94 .254 \quad 4.366 .525 .65 .350 .126$

1324

8. 774. 968. $85 \cdot 482.160 \quad 3030$

0. 26. 37. 4. 35, 2 126

$\begin{array}{llllllllll}0.2 . & 5 . & 0 & 9 & 60 & 18 . & 0 & 45 & 58.141\end{array}$

$\begin{array}{llllllllllll}0 . & 0 . & 0 . & 0 & 0 . & 7 . & 0 . & 1 & 0 . & 0 & 1\end{array}$

0. 0. 0.0

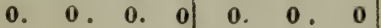

0 . 3. 7. 0. 0. 2

32

0. 0. 0. 0 . 0.0

\begin{tabular}{llllllllllll}
0.0 & 0. & 0 & 0. & 0. & 0. & 0 & 0. & 0.0 & 0 \\
\hline 0.0. & 1. & 5 & 0. & 3. & 0. & 0 & 2. & 0.0 & 2
\end{tabular}

86. 1. 18. 0 214. 281 . 13. 0 211.127.146

186. 1. 18. 0 156. 178 . 10. 0 180.121 . 135

77. 1. 10. 0 144.162 , 6. 0172.112 .110

0. 128. 262. 54. 70,112

85

2441

1. 18. 12. 0. 4. 0 . 128

1. 9. 10. 0. 0. 0 2

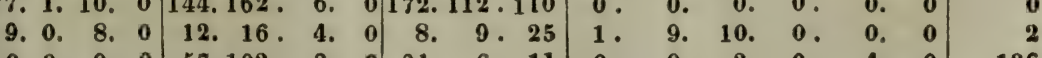

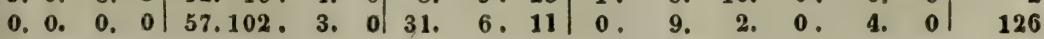


(Fort ileang

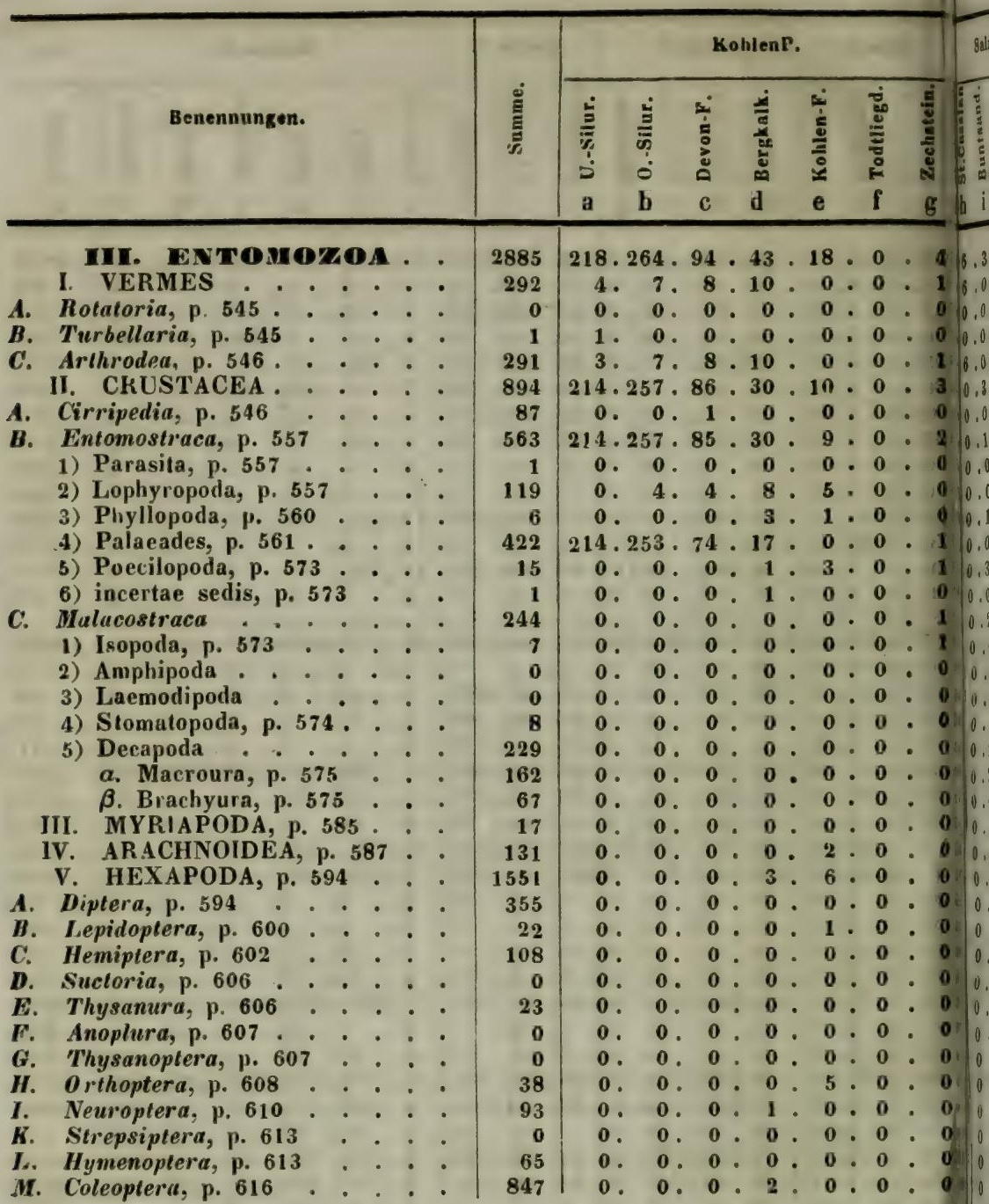




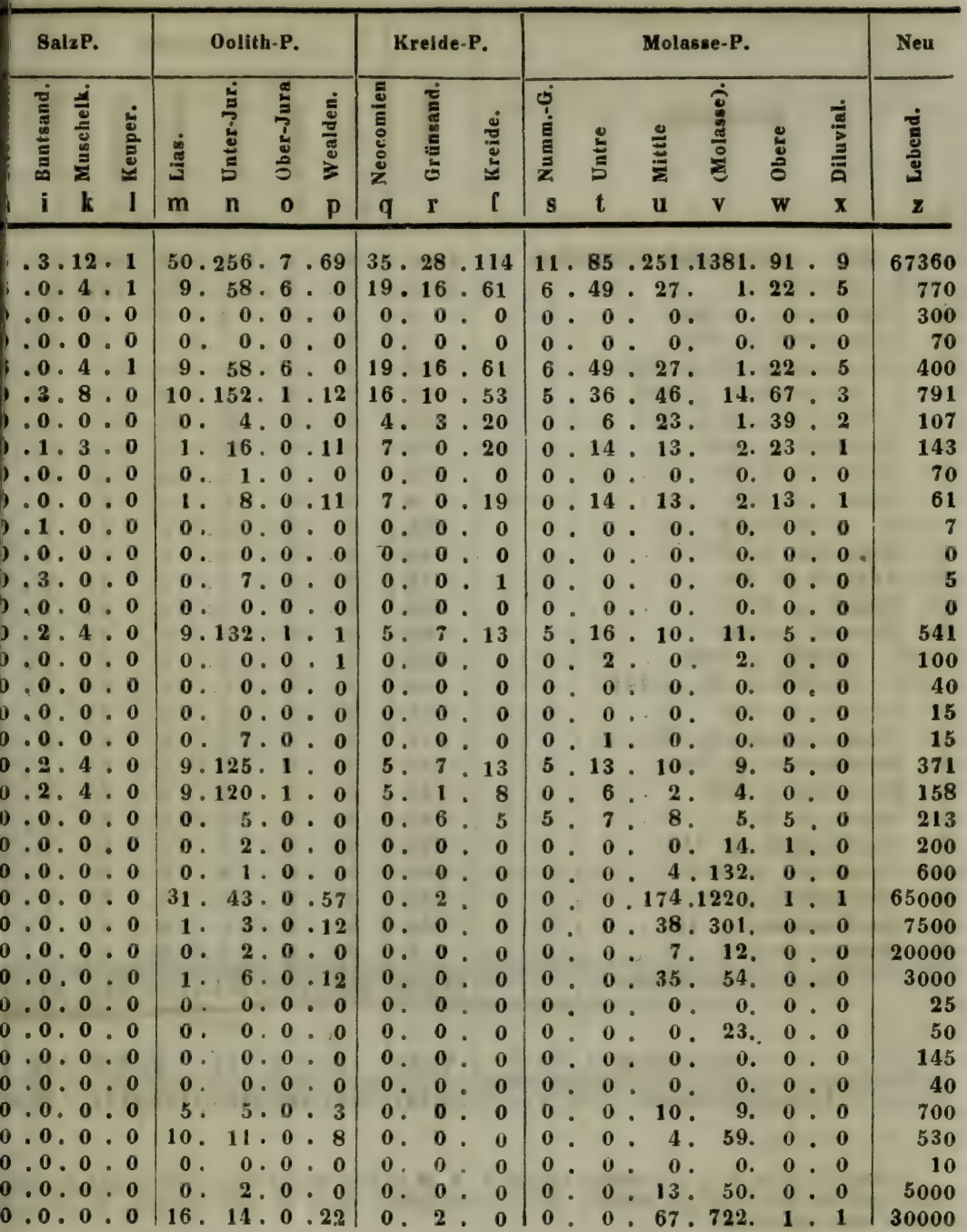


(Fort

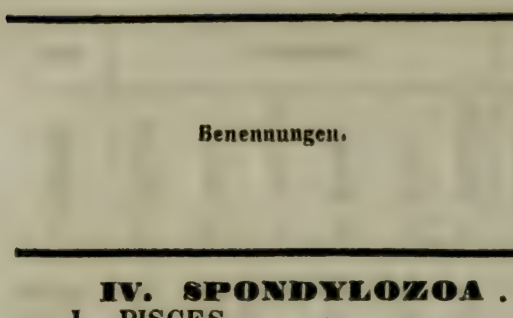

1. PISCES

A. Leptocardii, p. 635 . . . .

B. Cyclostomi, p. 635 . . . .

C. Elasmobranchii, p. 636. . .

1) Holocephali, p. 636

2) Plagiostomi, p. 637 . .

D. Ganoidei, p. 653 . . . . .

E. Teleostei, p. 669 . . . . . .

F. Dipnoi, p. 682 . . . • •

II. REPTILIA

A. Batrachii, p. 683 . . . .

B. Ophidii, p. 685

C. Saurii, p. 686 .

D. Chelonii, p. 693 I) * * •

III. AVES, p. 696 : : :

IV. MAMMALIA, p. 701 . .

A. Cetacen, p. 701 p.
B. Pachydermata, p. $703: \therefore:$
51

2701

1461

146

0
0

550

58

492
572

339

0

384

65
14

14
206

99

148

708

C. Ruminantia, p. 708 . . . . . 120

D. Edentata, p. 712 . . . . . . 40

E. Glires, p. 714 . . . . . . 97

F. Marsupialia, p. 718 . . . . . 22

G. Carnivora, p. 719 . . . . . 186

H. Chiroptera, p. 725 . . . . . 19

I. Quadrumana, p. 726 . . . . . 10

\begin{tabular}{|c|c|c|c|}
\hline & \multicolumn{3}{|c|}{ Kohlen-P } \\
\hline 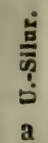 & $\begin{array}{l}\stackrel{\dot{z}}{\bar{y}} \\
\stackrel{0}{0} \\
\text { bे } \\
\text { b }\end{array}$ & 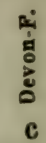 & 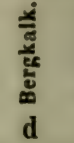 \\
\hline
\end{tabular}

0.7 $1110 \cdot 65 \cdot 80 \cdot 17 \cdot 40$

$0 \cdot 7 \cdot 110 \cdot 65,78,11 \cdot 42$

$0.0 \div 0.0 .000 .0$

0.0 .0 .0 .0 .000

0.7 $, 38,63,27,0,11$

0.0 .9 .0 .0000

$0 \cdot 7,37.63 .27,0.11$

0 . 0 . 72, 2, 51, 11, 31

0 . 0 . 0 . 0 . 0 . 0 .

0. 0 . 0 . 0 . 0 . 0 . 0 .

0.0 .0 .0 .2 .6 .7

0.0 .0 .0 . 0.0 . 0

0. 0 . 0 . 0 . 0 . 0 . 0

$0 \div 0 \div 0.0 .2 .6 .7$

0 . 0 . 0 . 0 . 0 . 0 . 0

$0.0 .0 .0 .0 . ? .00$

$0 \div 0 \div 0.000 \div 3: 0$

0 . 0 . 0. 0. 0. 0. 0

0. 0.0 . 0 . 0 . 0 . 0

$\begin{array}{lllllll}0 & 0 & 0 & 0 & 0 & 0 & 0 \\ 0 & 0 & 0 & 0 & 0 & 0 & 0\end{array}$

$0: 0: 0.00 .000$

$0 \div 0 \div 0 \div 0 \div 0 \div 0 \div 0$

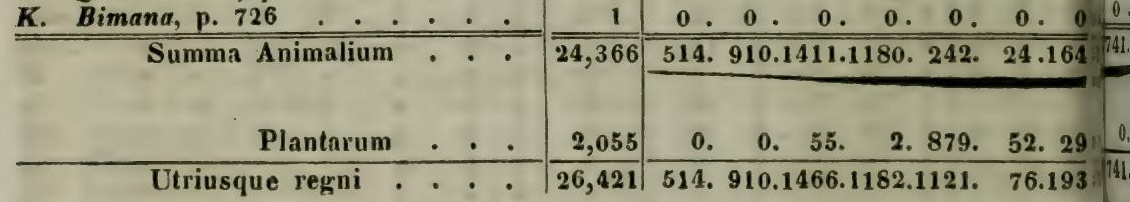

0 . 0 . 0 . 0 . 0 . 0 .

$\begin{array}{llllll}0 & 0 & 0 . & 0.0 & 0 & 0\end{array}$

$10 \begin{array}{llllllll} & 0 & 0 & 0 & 0 . & 0 . & 0 . & 0 . \\ & 0 & 0 . & 0 . & 0 . & 0 . & 0 .\end{array}$

1) Diese 3 Nulle in q, r, c sollten heissen 1, 9, 10 und in diesem Sinne alle darauf gestützten Zählungen und Berechmungen geändert werden; der Fehler wurde zu spät bemerkt, ist jedoch im Ganzen sonst unerheblich. 
laung.)

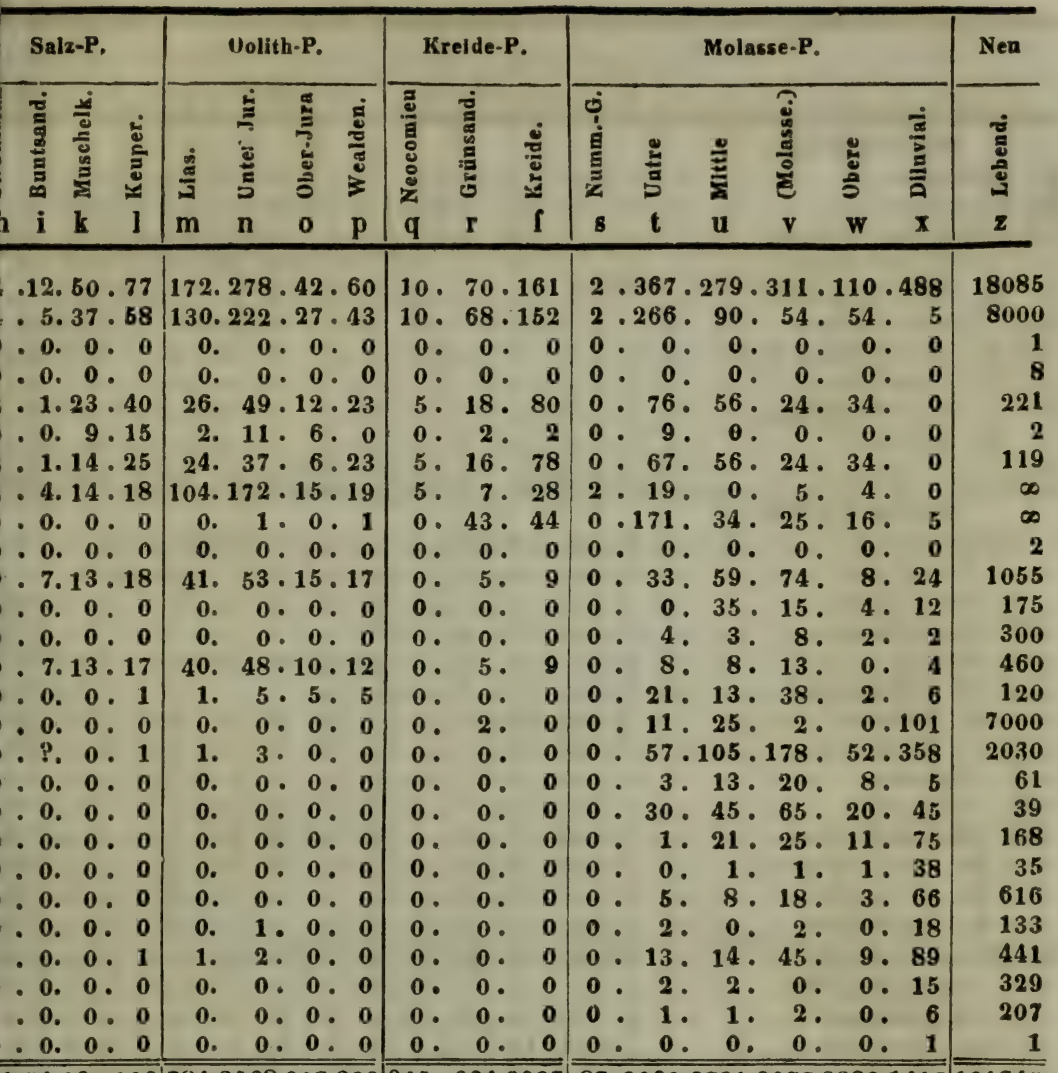

\begin{tabular}{|l|l|l|l|l|}
\hline$\overline{41.54 .190 .106}$ & $\mathbf{7 8 4 . 2 5 6 8 . 3 0 7 . 2 3 3}$ & $\overline{945.934 .2937}$ & $\overline{87.2960 .3721 .2977 .2222 .1417}$ & $\overline{101745}$ \\
\hline
\end{tabular} 27628

\begin{tabular}{ll|ll|lll|lll|l}
0.31. & 5.62 & 71. 132. & 2. 16 & 0. & 77. & 7 & 10. 136. 319. 110. & 48. & 4 & 69403 \\
\hline
\end{tabular} $\overline{41.85 .195 .168} \overline{855.2720 .309 .249} \overline{945.1011 .2944} \mid \frac{10.3096 .4040 .3087 .2270 .1421}{171148}$ 
II. TABELLE ÜBER DIE

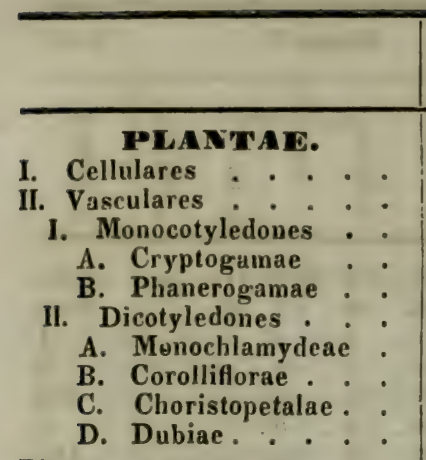

Plantarum summae

\section{ANIMALIA.}

1. PHYTOZOA

I. Pseudozoa

II. Amorphozoa

1II. Polygastrica

IV. Polypi
A. Polythalami
B. Bryozoa
C. Anthozoa .

V. Entozoa

VI. Acalephae

VII. Echinodermata .
A. Stelleridae
B. Echinidae
C. Fistulidae

II. MALACOZOA .

I. Gymnacephala

II. Brachiopoda
A. Genuina

B. Rudistae .

III. Pelecypoda
A. Monomya
B. Heteromya
C. Integripalliata
D. Sinuatopalliata
E. Tubicolae

IV. Pteropoda

V. Heteropoda .

Vi. Protopoda

VII. Gasteropoda
A. Cyclobranchia
B. Aspidobranchia
C. Ctenobranchia

1) Asiphonobranchia 2) Siphonobranchia

D. Pomatobranchia

E. Hypobranchia .

F. Gymnobranchia

G. Pulmonata .

VIII. Cephalopoda
A. Tetrabranchia .

B. Dibranchia .

\begin{tabular}{|c|c|c|c|c|c|c|c|c|c|}
\hline \multicolumn{9}{|c|}{1.} & II. \\
\hline & b & c & d & $\mathbf{e}$ & f & g & $\mathrm{a}-\mathrm{g}$ & I. & h i $k$ I h-l Il \\
\hline
\end{tabular}

-. -. 2. -. 8. 1. 2. 13. 8-.-. 1. 1. 2 .

- - $19.2 .113 .14 .15,163.116-.15 .3 .25 .43 .3$ 一. - 19. 2.101.13.12. 147.101 一.10. 1.19. 30. 2.8 .2

- - 1 18. 2. 85.13.10.128. 84 - 7. 1.16. 24. 2 .

一. 一. 1. - 16.-. 2. 19. $17-$ 3.-. 3. 6.

- - - - - 12. 1. 3. 16. 15 - 5. 2. 6. 13. 1 . 1

- - - - - 10.- 2. 12. $12-$ 5. 1. 6. 12.

-. -. -. -. -..... -. - -....... -. -..

-. - - - 1.- 1. 1 - 1 . - - -

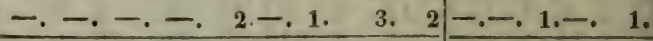

0 . 0.21 . 2.121.15.17. 176.124 0.15. 4.26.45. 3

20. 88. 68. 59. 1. 1. 6. 243.146 24. 1.12, 2. 39. 3/.

-. -. - - . -.... -. - -....... -

1. 8. 5. - - - - 14. 11 6. 1. 2. 1. 10.

- - - - 1. -... 1. 1. 1 - - - - - -

13. 47. 45. 39. - - 6. 150.82 14. - 3.- 17.116

一. 一. 一. 7. -...-. 7. 7 -....... -

6. 24. 22. 11. - - 4.67 .38 6. 6 . 1. -7 .

7. 23. 23. 21. - - 2. 76. 37 8., $2 .-10$

一. -. -. -. -..... -. - - -...... -.

一. -. - - - - - . - - - -....... -

6. 33. 18, $19.1 .(1)-78.52$ 4.- 7. 1. 12

6. 33. 18. 19. 1.(1)-. 78. 52 3., 6. 1. 10.

-. -. - . - ...... -. - 1.-. 1., 2 .

-. - - - - - - - - - . - - - - - - - - - -

44. 62. 94. 90. 35. 4.33. 362.14963 .20 .44 .14 .141 .

11. $\overrightarrow{13}, \overrightarrow{13}, \overrightarrow{12}, \overrightarrow{5 .}, \overline{7}, \overline{61}, \overline{18}$ 6. 1. 3. 1. 11.

9. 11. 12. 11 . 5.- 6 . 54. 16 4. 1. 2. 1. 8 .

2. 2. 1. 1. - . 1. 7. 2 2. $-1 .-3$

5. 18. 35. 33, $13,4.18,126,5124.13 .23,7,67$.

-. 2. 5. 4. 2. - 2. 15.66 6. 5. 6. 1. 18.

2. 4. 5. 7. 4. 1. 5. 28. 7 6. 5. 4. 2. 17 .

3. 9. 15. 14. 5. 1. 8. 55, 22 11. 1. 6. 2. 20.

- 3. 10. 8. 2. 2. 3. 28, 16 1. 2. 7. 2. 12.

-. -. -. - . -..... -. - -....... -.

1. 4. 3. 1. - - $-9,5-,-,-$,

1. 1. 3. 3. 1.-., 9. 3 1.-.,- 1 .

1. 1. 1. 1. 1., - 5. 1 1. - 1., 2 .

18. 16. 30. 31. 11. - 7. 113. 54 25. 5.13. 5. 48 .

1. 1. 2. 3. 1.- 1. 9. 3 1. 1. 1., 3.

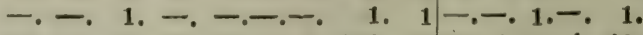
17. 15. 27. 28, $10,-, 6,103.5024 .4 .10$. 4. 42 . 16. 14. 25. 23. 9.- $6.93,43$ 21. 3. 7. 4.35 . 1. 1. 2. 5, 1.-. 10.7 . $3.1 .3 .-7$. -. -. -. -. -.... - -. - -....... -. -. -. -. -.... -. - -...... - - - - - - - - -. - -... 1. 1. 2 7. 9. 9. 9. 4. - 1. 39.17 6. 1. 4. 1. 12. 7. 9. 9. 8. 3.- 1. 37. 16 6. 1. 4. 1.12. 


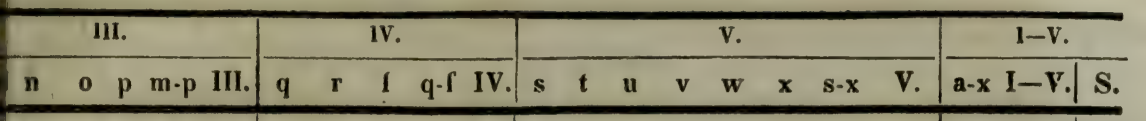

$.15 .-2.22 \cdot 18-.9 \cdot 4 \cdot 13.12$ . $39 \cdot 1.10 \cdot 75 \cdot 57-.23 \cdot 1 \cdot 24 \cdot 24$ $25 .-5.48 .39$ $22 .-3.41 .32$ 3.-. 2.7.7 14.1. $5 \cdot 27 \cdot 18$ 11. 1. $5.24 \cdot 15$ 1.:- - - - - $2 .-, 2.2$ $54 \cdot 1 \cdot 12 \cdot 97 \cdot 75$

122. $8 .-144.125$ - - - - - - - 1 10. 1

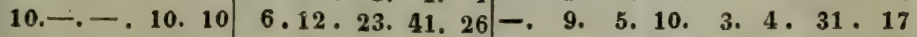
-...-. - - - - - . 7. 7. 7 -. 一. 2. 63. 14.32.111. 80 68. 5. - 77. 7028.42 .100 .170 .105 3. 79.110. 37. 81.16.326.164 14. 1. - . 15. $14 \quad 8$. 8. 41.57. 38 2. 24. 45. 29. 43. 8.151.67 24. $1 .-25.2413 .16 .37 .66 .44-28.37 .4 .16 .3 .88 .56$ 30. 3. - . 37. 32 7.18.22. 47. 27 1. 27. 28. 4. 22. 5. 87. 41

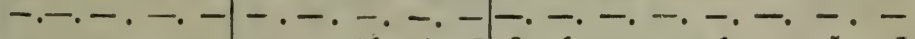

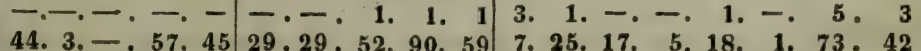
19. 1. - 27. 21 3. 2. 12. 17. 15 - 3.2 . 1.4 .5 .11 .6 23. 2. - 28. $2226.27,40,73.44$ 7. 21.15 .4 . 13. 1. 61.35 2.-. - 2. 2 -. -. -. - - - 1. - - - - - 1.1 132.66. $27.303 .157 \quad 116.101 .146 .363 .181$ 25.199.218.93.209.146.890.301

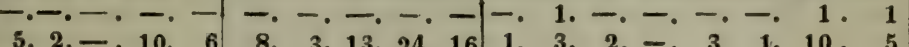

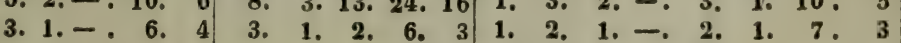
2. $1 .-$ 4. 2 5. 2. $11.18 .13-$ 1. 1. $-1.1 .-3.2$ 74.43. 13.175 .85 61. 53. 68.182. 83 13. 77. 85. 41. 85. 54.355.113 14. 7. 3. 34. 15 11. 11. 15. 37. 15 4. 9. 9. 7. 11.5 .45 .12

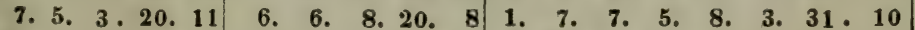
20.13. 4. 49. 25 16. 13. 18. 47. 19 6. 28. 26. 15. 24. 17.116. 35 33.18. 3. 72. 34 28, 23. 26. 77. 40 2. 32. 41. 13. 41, 29. 158. 54

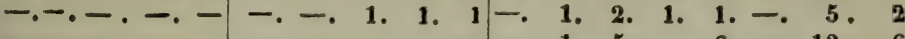
-. - - - - - - - - -1 - $1.5 .-6.612 .6$

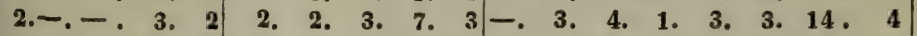
38.16. 14, 86. 48 31. 31. 52.114. 62 9.111.116, 51.111. 88, 486. 166 1. 1. - 3. 1 1. 1. 1. 3. 1 0. 2. 3. 1. 2. 2. 10.3

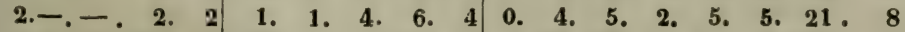
33.15 .11 . 76. 41 28, 29. 44.101. 54 9. 88. 89. 35. 84. 62.367.123 24. 9. 5. 50. 31 17. 18. 29.64. 35 4. 50. 47. 17. 44. 31. 193. 67 9. 6. 6. 26. 10 11. 11. 15. 37. 19 5. 38. 42. 18. 40.31.174. 56

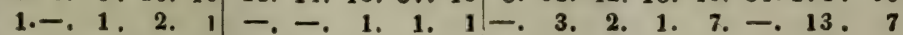
$-.-.-1-$ - - - - - - - -. 1. 1. -. - 1. 3. 1

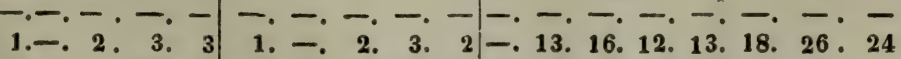
13. 5. - 29. 16 14. 11. 10. 35. 16 2. 3. 6. - $1 ., 12.6$ 5. 2. - 11. 6 11. 9. 8. 28. 12 2. 2. 2. - - - -6.6

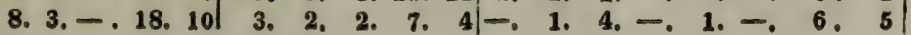

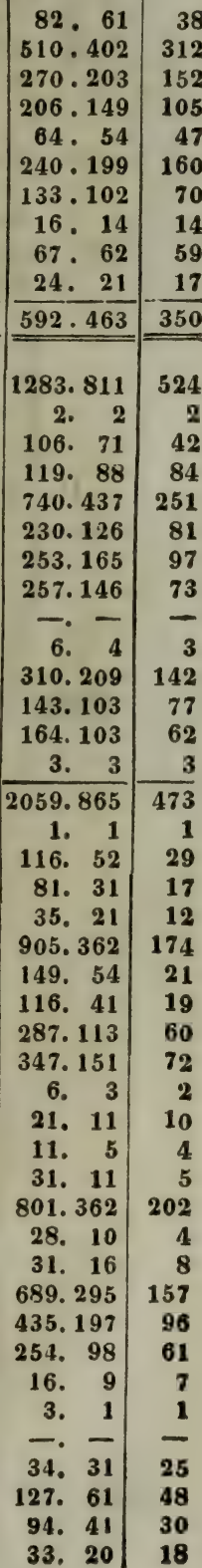




\begin{tabular}{l}
\hline \\
\cline { 2 - 5 }
\end{tabular}


tzung.)

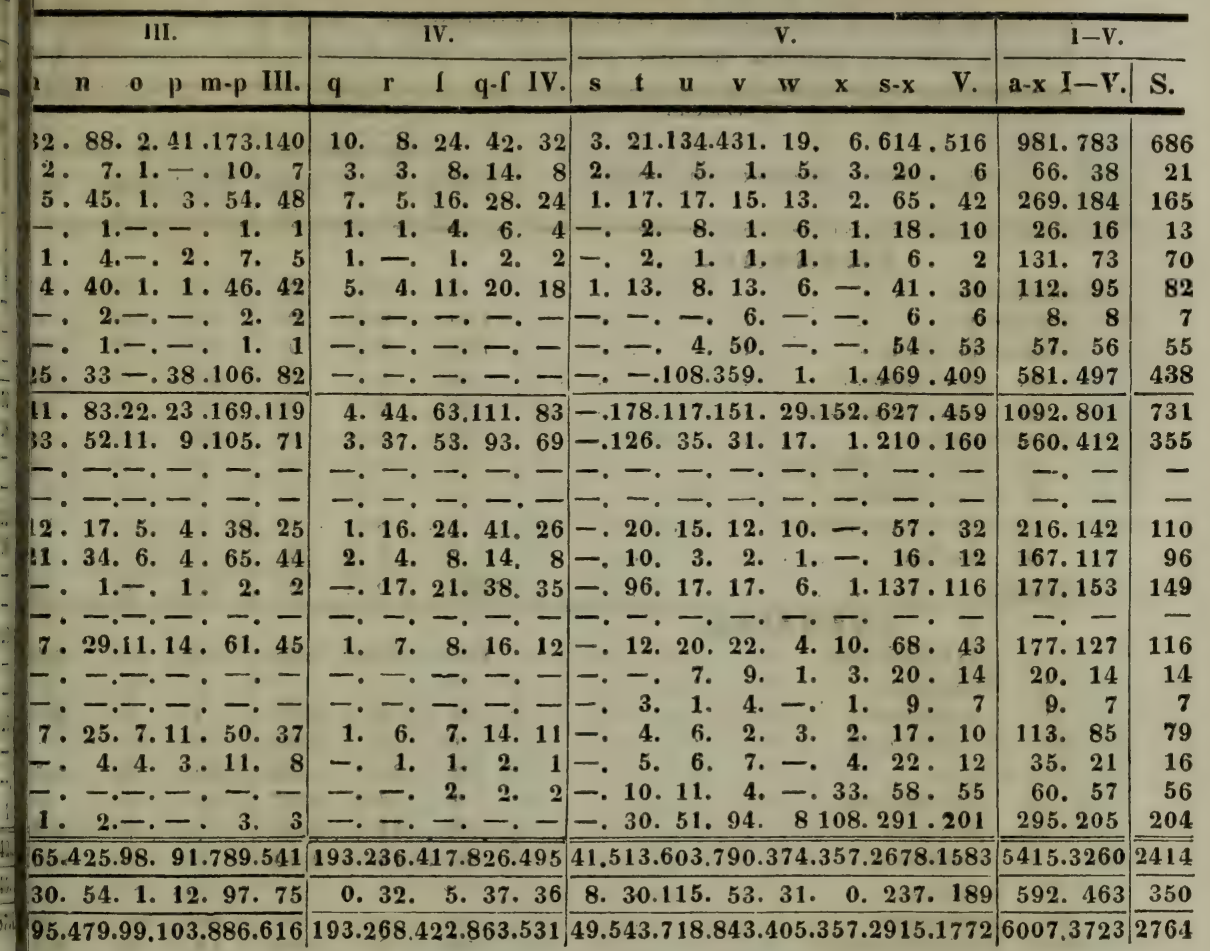




\section{TABELLE: VERHÄLTNISSE DER:0}

(Lebende Genera, welche eine Periode überspringen, sind in dieser nicht uit- $z$ Genera würden gezählt werden müssen. - Viele Genera sind eigentlich noch bet

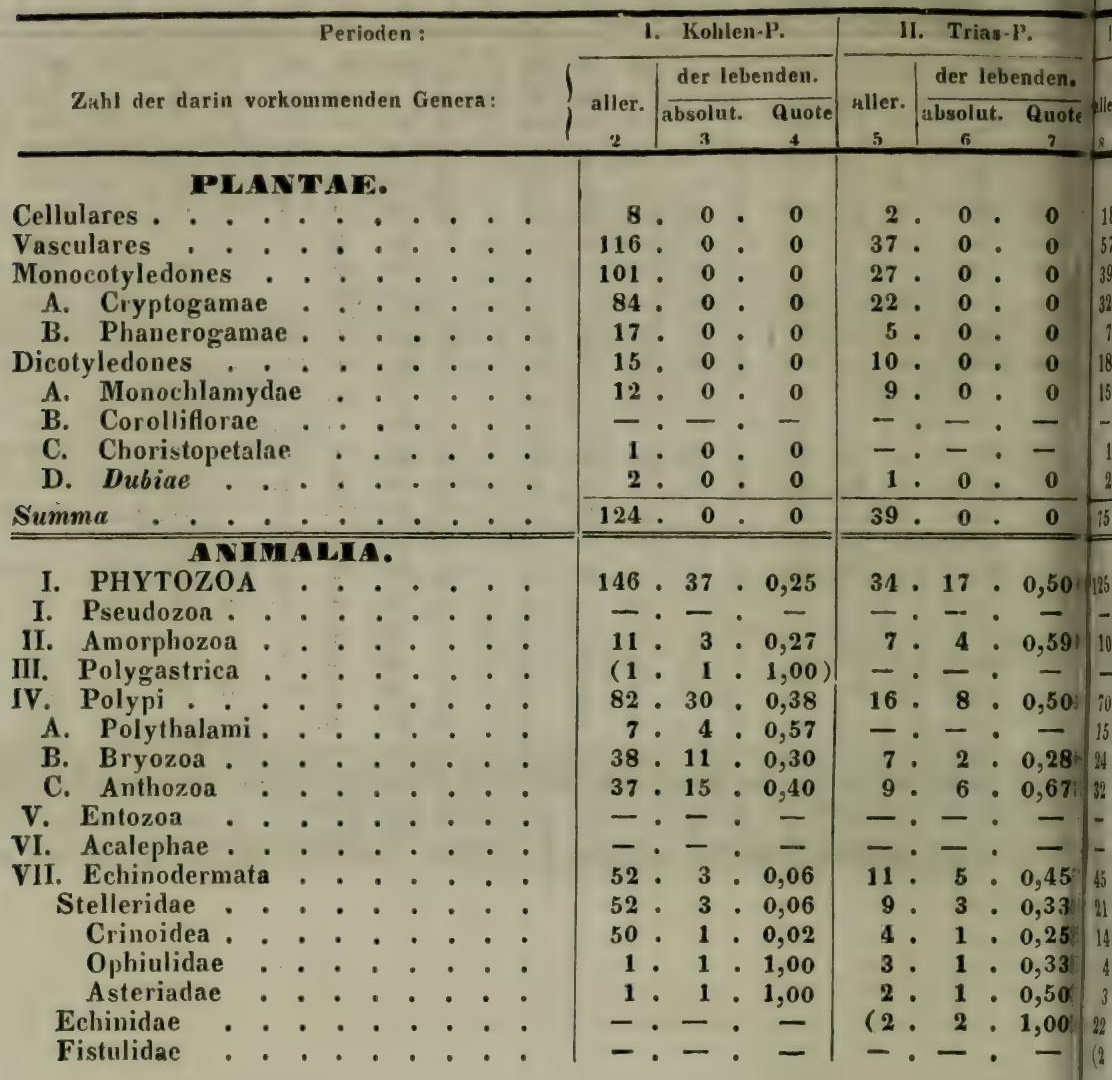




\section{DSSILEN GENERA ZU DEN LEBENDEN.}

zählt, sonst würde ihre $Z_{a h l}$ grösser ausfallen; aber auch die überspringenden fossilen end, aber im Fossil-Zustande durch andere Namen angedeutet bei Pflanzen, Krustern etc.

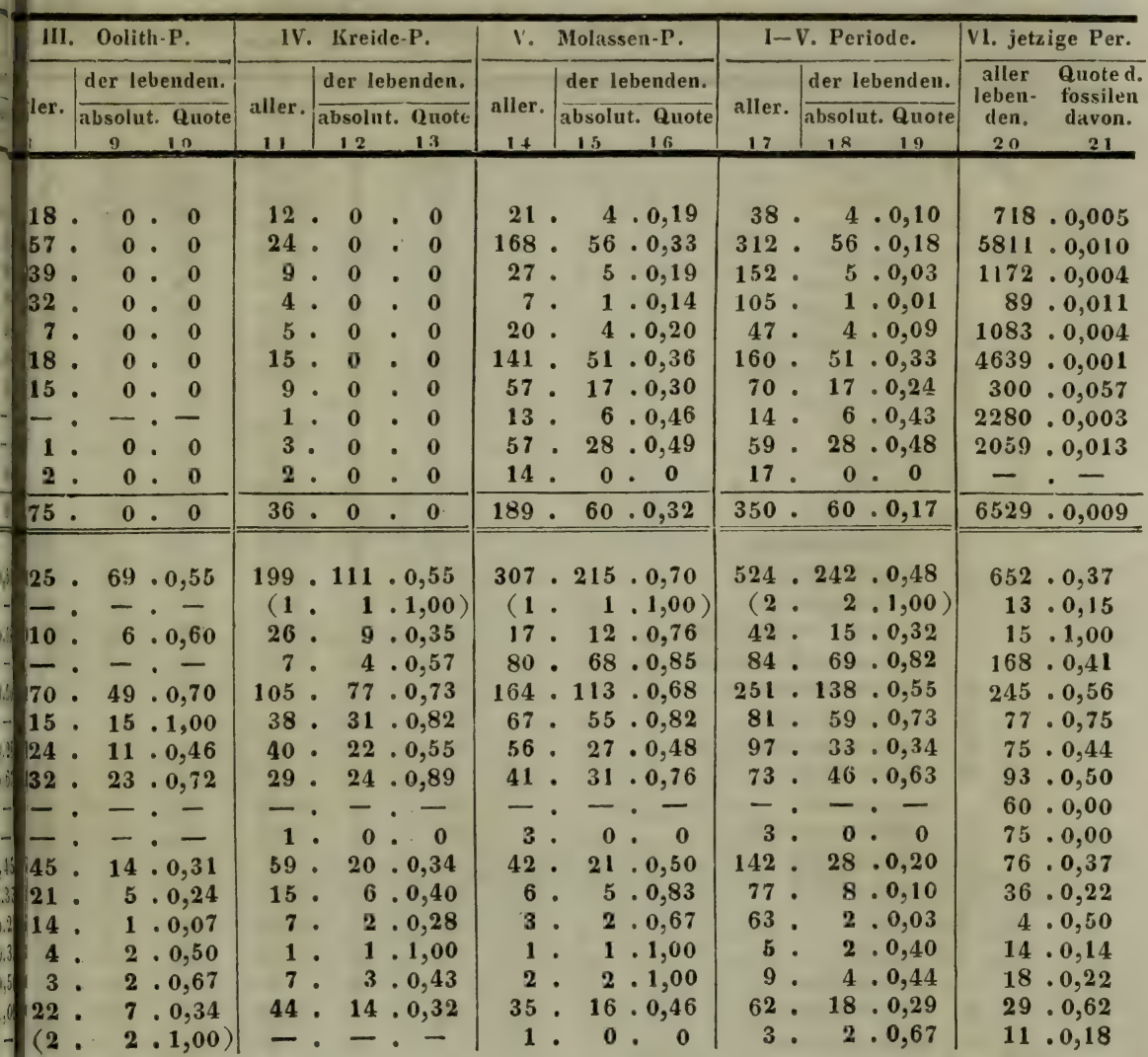


(For

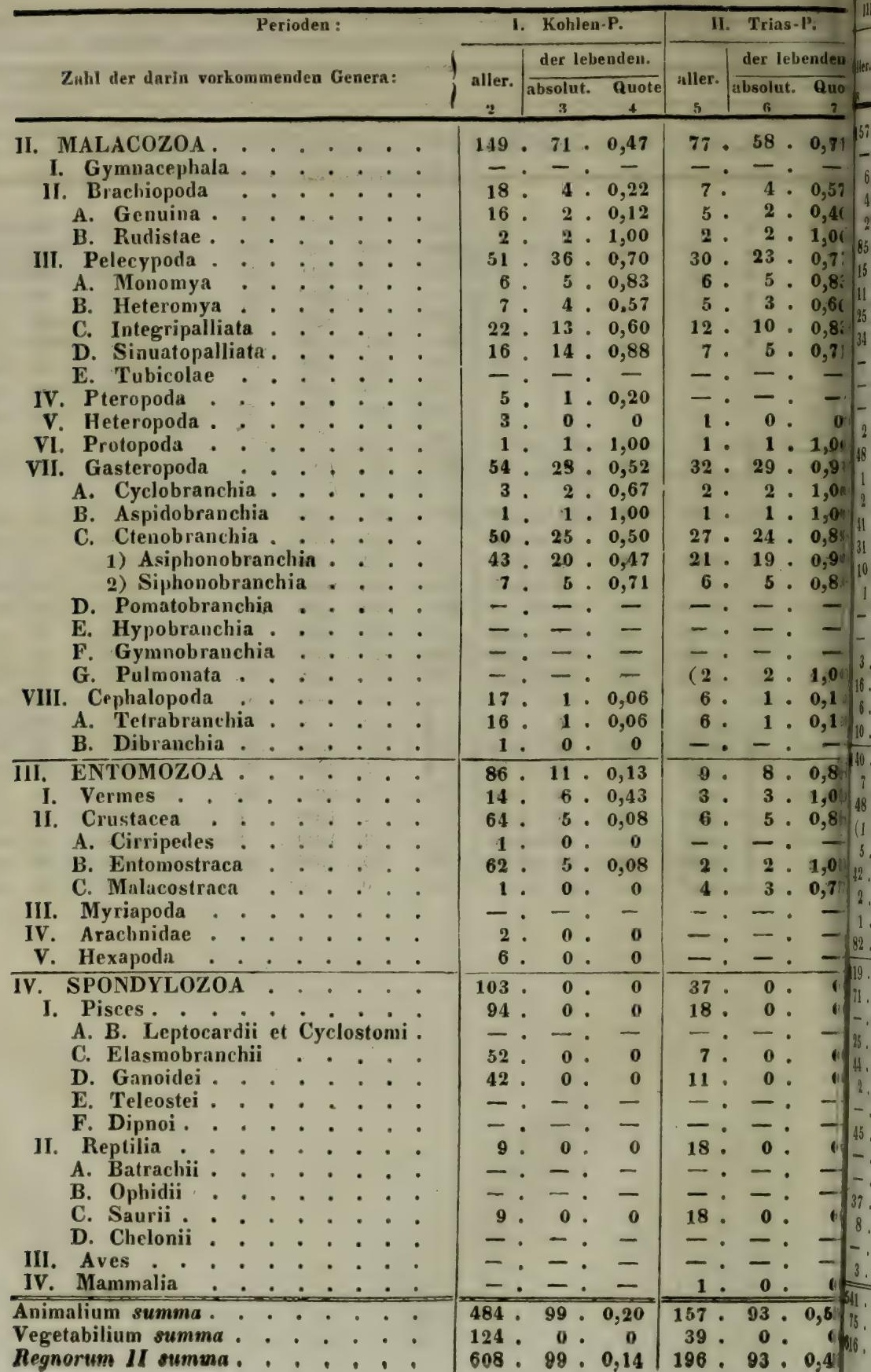




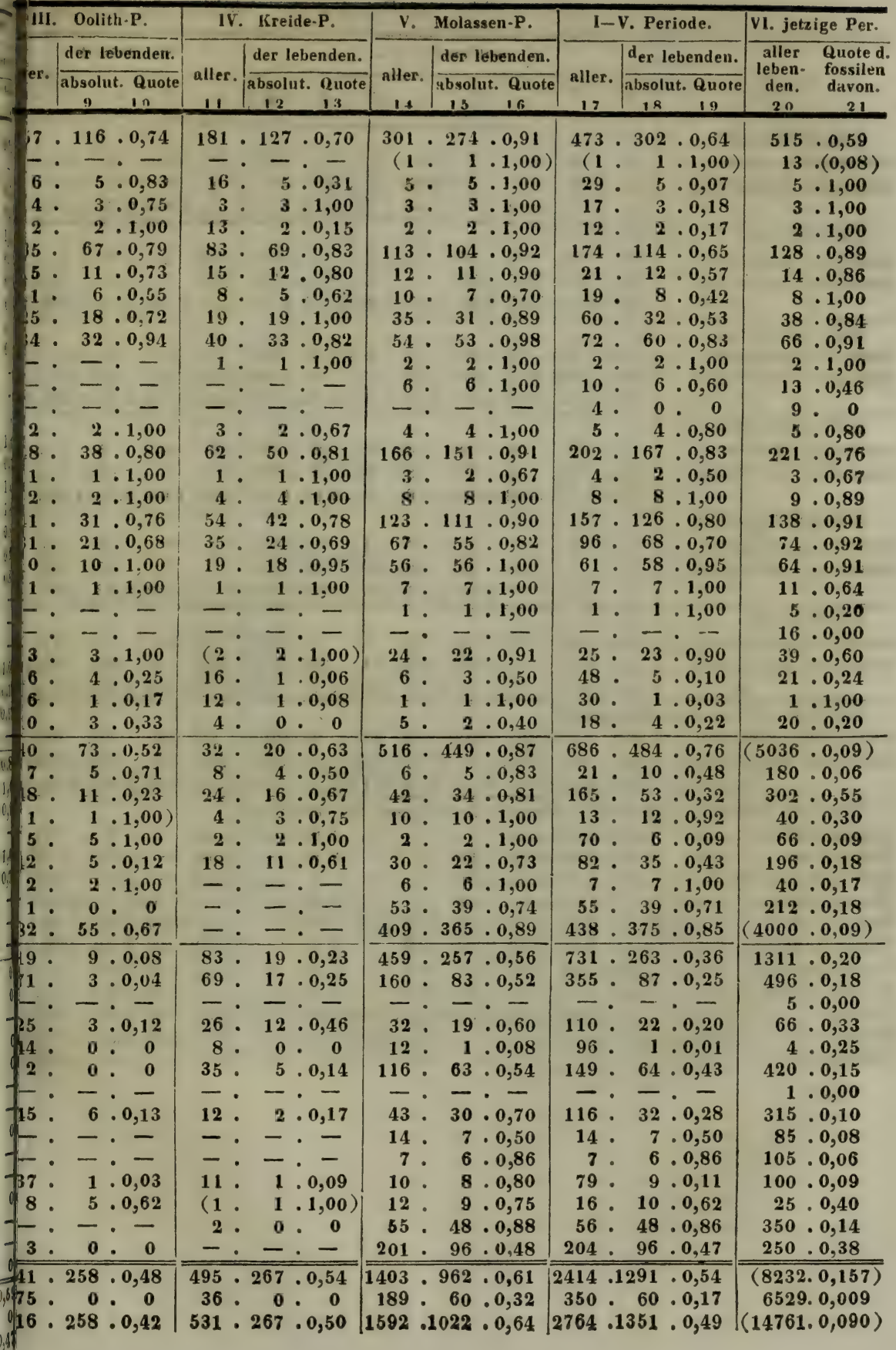




\section{TABELLE : ÜBER DAS ZAHLEN}

(a-x enthält das Resultat der Addition der Genera alle fubri

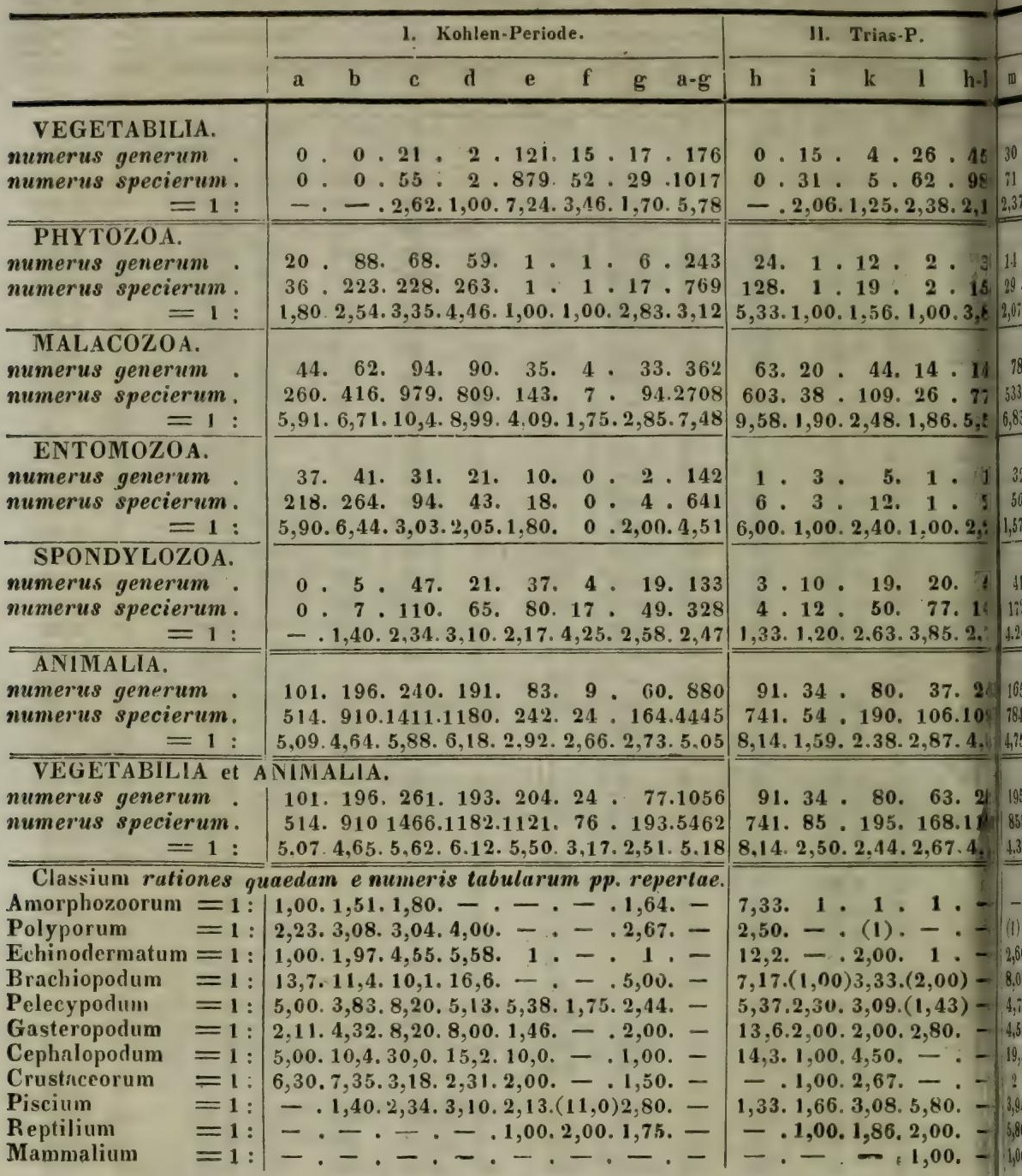




\section{ERHÄLTNISS DER GENERA MIT DEN SPECIES.}

ubriken a bis $\mathbf{x} ; \mathbf{I}-\mathbf{V}$ das der Perioden $\mathbf{I}$ bis $\mathbf{V}$; $\mathbf{S}$. die wahre Anzahl.)

\begin{tabular}{|c|c|c|c|}
\hline III. Ooli & IV. & a.P. & $\mathbf{I}-\mathbf{V}$ \\
\hline p o-p & $q-\Gamma$ & $s-x$ & $a-x \quad I-V$ \\
\hline 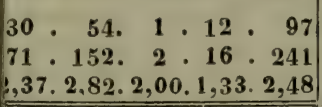 & $\begin{array}{r}0 \cdot 32 \cdot 5 \cdot 37 \\
0 \cdot 77 \cdot 7 \cdot 84 \\
-.2,41 \cdot 1,40 \cdot 2,33 \\
\end{array}$ & 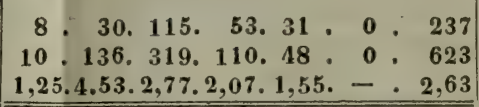 & $\begin{array}{l}592 \cdot 463 \\
-\cdot \frac{1}{3,47 \cdot 4,44}\end{array}$ \\
\hline $\begin{array}{r}122.8 \cdot 2 \cdot 144 \\
579.16: 2 \cdot 626 \\
07.4,74.2,00 \cdot 1,00.4,34 \\
\end{array}$ & $\begin{array}{r}\text { 63. } 83 . \\
\text { 149. } 270.1 \\
2,36.3,25.6\end{array}$ & 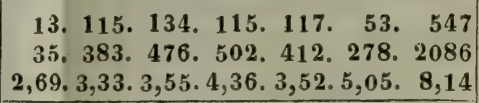 & $\frac{1283.811}{-\cdot \frac{1}{3,81} \cdot 6,04}$ \\
\hline $\begin{array}{lr}\text { 78. 132. } 66 . & 2 \\
33.1455 .242 . & 10 \\
83.11,0.3,66.3,7 \\
\end{array}$ & $\begin{array}{r}116.101 \\
751.566 \\
6,47.5,60 \\
\end{array}$ & $\begin{array}{rrrr}25.199 .218 . & 93.209 . & 146 . & 890 \\
39.2125 .2725 . & 783.1609 . & 642 . & 7281 \\
1,56.10,7.12,5.8,45.7,70.4,40 . & 8,18 \\
\end{array}$ & $\frac{2059 \cdot 8}{6,74 \cdot 16}$ \\
\hline $\begin{array}{rrrrr}32 . & 88 . & 2 . & 41 . & 173 \\
\text { 30. } & 256 . & 7 & 69 . & 382 \\
\text { 57. } & 2,91.3,50.1,68.2,21 \\
\end{array}$ & $\begin{array}{rrrr}10 . & 8 . & 24 . & 42 \\
35 . & 28 . & 114 . & 177 \\
3,50 . & 3,50 . & 4,75.4,21 \\
\end{array}$ & 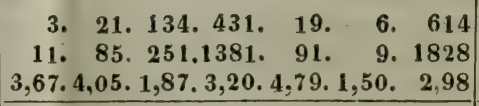 & $\begin{array}{l}981 \cdot 783 \\
-\cdot \frac{7}{2.96} \cdot 3,68\end{array}$ \\
\hline 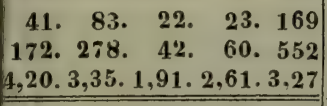 & \begin{tabular}{rrrr|}
4. & 44. & 63. & 111 \\
10. & 70. & 161. & 231 \\
$2,50$. & $1,59.2 .55 .2,08$ \\
\end{tabular} & 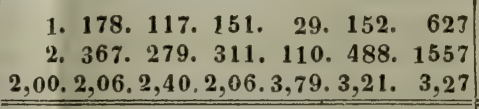 & 1092. 801 \\
\hline $\begin{array}{rrr}5.425 . & 98 . & 91.789 \\
44.2568 . & 307 . & 233.3892 \\
75.6,04.3,14.2,56.4,92 \\
\end{array}$ & $\begin{array}{r}945.934 \\
4,90.3,95 \\
\end{array}$ & $\begin{array}{r}41.513 .603 \\
87.2960 .3721 .2 \\
2,12.5,77.6,17.3 \\
\end{array}$ & $\begin{array}{r}5415.3260 .2 \\
-.0 .24 \\
4,50.7,47 .\end{array}$ \\
\hline $\begin{array}{l}95.479 . \\
55.2720 .30 \\
38.5,68,3,1 \\
\end{array}$ & $\begin{array}{r}193.268 .422 .863 \\
945.1011 .2944 .4900 \\
4,90.3,77.6,98.5,68 \\
\end{array}$ & $\begin{array}{r}49.543 .718 \\
97.3096 .404 \\
1,98.5,65.5,6 \\
\end{array}$ & $\begin{array}{r}6007.3723 .2 \\
-.70 .7,00.26 \\
\end{array}$ \\
\hline 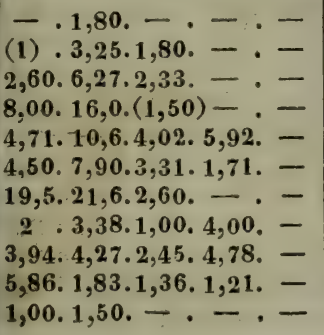 & $\begin{array}{l}3,00 \cdot 4,17 \cdot 7,83 .- \\
1,93 \cdot 2,67 \cdot 6,73 .- \\
2,65 \cdot 3,72 \cdot 5,56 .- \\
7,62 \cdot 8,67 \cdot 17,4 .- \\
5,51 \cdot 5,26 \cdot 10,2 .- \\
4,36 \cdot 4,03 \cdot 7,98 .- \\
15,1 \cdot 11,5 \cdot 14,6 .- \\
2,28 \cdot 2,00 \cdot 3,31 .- \\
3,33 \cdot 1,83 \cdot 2,87 .- \\
(1,00 \cdot 1,00) 1,00 .-\end{array}$ & $\begin{array}{l}4,70 \cdot 3,00 \cdot 7,50 .- \\
2,08 \cdot 4,51 \cdot 1,31 .- \\
1,80 \cdot 3,39 \cdot 4,00 .- \\
-\cdot 7,67 \cdot(4,00)- \\
4,00 \cdot 6,54 \cdot 3,50 .- \\
4,27 \cdot 8,86 \cdot 4,99 .- \\
-.4,00 \cdot-.- \\
1,00 \cdot 5,15 \cdot 1,50 .- \\
1,74,3,18 \cdot(5,00)- \\
5,37 \cdot 2,00 \cdot 2,40 .- \\
0.1,89.6,50.3,32 .-\end{array}$ & $\begin{array}{l}50 \\
, 78 . \\
1,5 \\
2,0 \\
3,3 \\
6,9 \\
5,4 \\
90 \\
55 \\
0 . \\
02 \\
69\end{array}$ \\
\hline
\end{tabular}


V. TABELLE DER GEOGRAPHISCHEN $A R B$

\begin{tabular}{|c|c|c|c|c|c|c|c|c|c|}
\hline \multirow{2}{*}{$\frac{\text { Welttheile: }}{\text { Zonen : }}$} & \multicolumn{3}{|c|}{ Furopa. } & \multicolumn{3}{|c|}{ Asien. } & \multicolumn{3}{|c|}{ Afrika. } \\
\hline & 1 & & 2 & 1 & 2 & 3 & 2 & 3 & 4 \\
\hline Genera und Spezies : & g. $s p$. & g. & sp. & g. sp. & g. sp. & g. sp. & g. sp. & g. sp. & g. $\mathbf{s p .}$ \\
\hline
\end{tabular}

I. PLANTAE . . .

Cellulares aphyllae. foliosae.

Monocot. eryptogamae phanerogamae.

Dicot. monochlamydeae

" corolliflorae . : :

I. PHYTOZOA.
Pseudozoa . . . . .

Amorphozoa : . . .

Polygastrica . . . . .

Polypi Polythalami . .

Bryozoa, Anthozoa .
Entozoa

Acalephae . : : :

Echinodermata . . . .

II. MALACOZOA .

Gymnacephala . . . .

Brachiopoda . . . .

Pelecypoda . . . .

Pteropoda . . . . . .

Heteropoda . . . . .

Protopoda . . . . . .

\begin{tabular}{l} 
Gasteropoda \\
Cephalopoda : \\
\hline
\end{tabular}

III. ENTOMOZOA .

Vermes . . . . . .

Crustacen . . . . . .

Myriapoda . . . :

Arachnoidea . . . .

$\frac{\text { Hexapoda }}{\text { IV. SPONDYLOZOA }}$

Pisces . . . . .

Reptilia
Mves

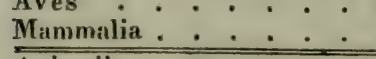

Animulia $\dot{\mathrm{v}} \cdot \dot{v^{\prime}} \cdot$

Animalia et Vegetabilia .

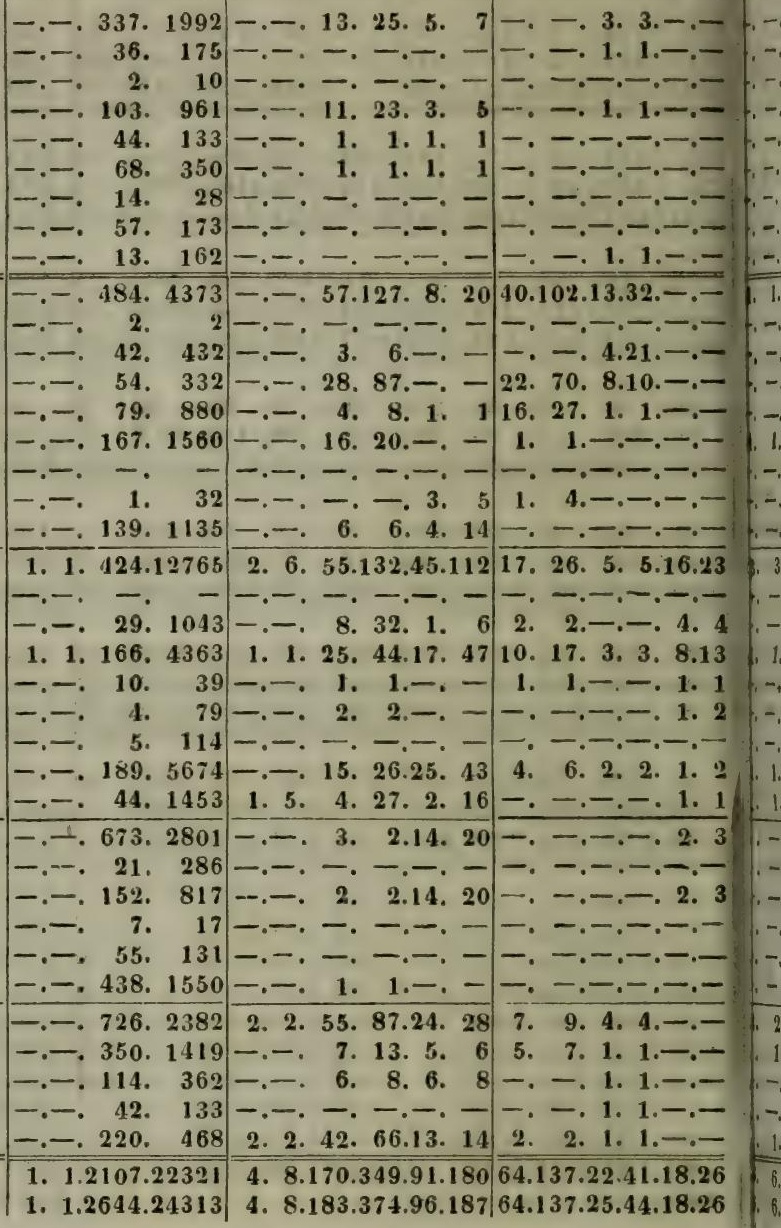


RBREITUNG DER GENERA UND SPECIES.

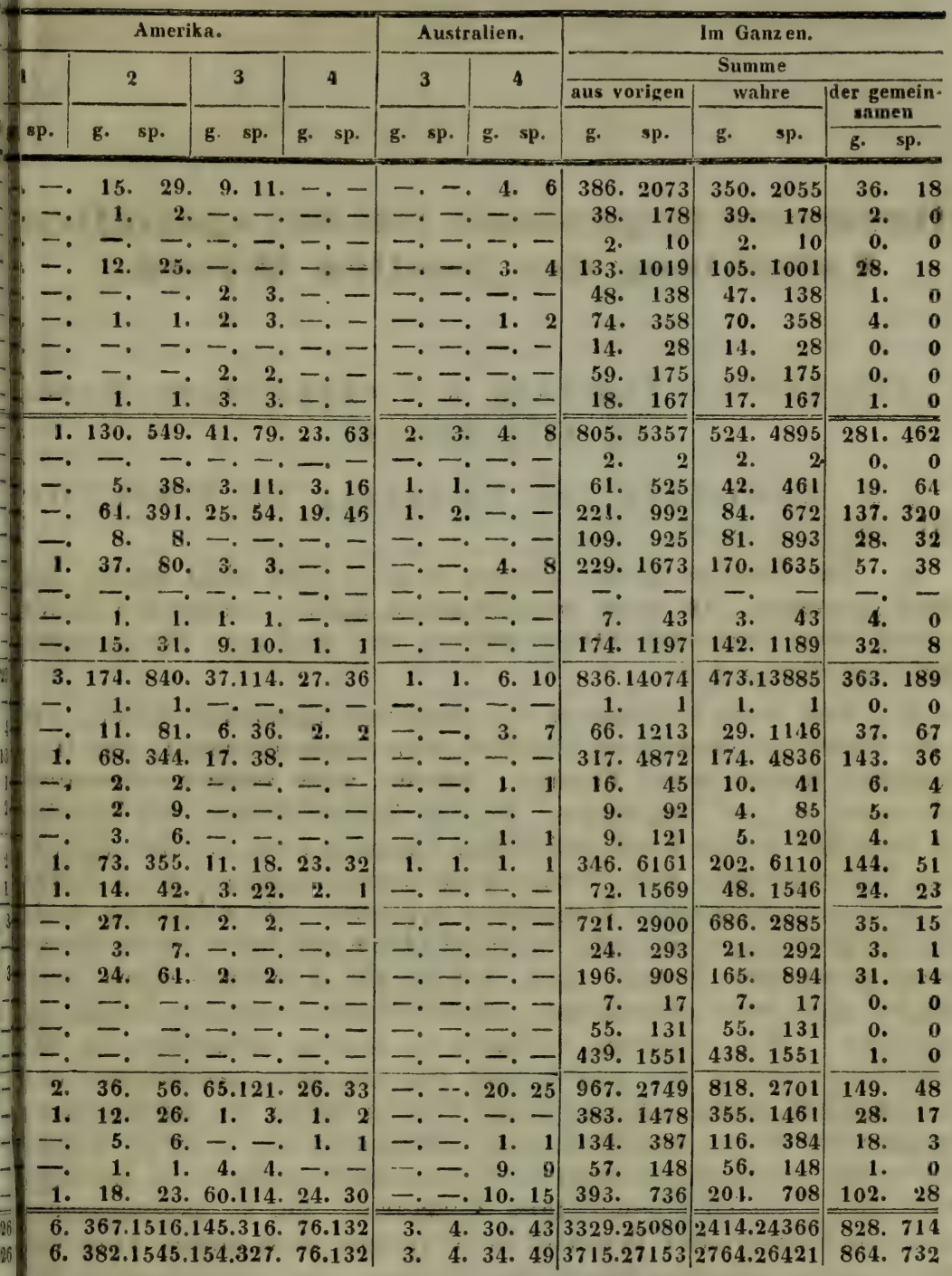




\section{Algemeine Ergebnisse aus der speziellen Aufzählung der fossilen Wesen.}

\section{A. Allmähliche Änderung der organischen Wesen im Allgemeinen.}

§. 2. Entstehung und Untergang der Arten, Geschlechter u. s. w.

A. Die fossilen Arten, Genera u. s. w. sind nicht immer dieselben gewesen. Zu ganz verschiedenen Zeiten entstanden haben sie sich sehr ungleicher Dauer erfreut und sind zum Theile schon wieder untergegangen.

B. Das erste Erscheinen der ältesten Organismen-Arten, wie es die frühesten silurischen Erd-Schichten schon andeuten, das Hinzukommen immer nener Formen, welche neue Genera, Familien, Ordnungen u. s. w. begründen, bis zur letzten Grenze der TertiärZeit lässt sich weder durch freiwillige Zeugung noch ans der allmählichen Umwandelung einer Form in die andere während einer Reihe von aufeinanderfolgenden Zeugungen erklären; wir erblicken darin nicht die Wirkung einer noch fortdauernden Kraft der in ihrem Gange einmal geregelten Natır, noch würde die Annahme einer einst thätigen aber jetzt erloschenen solchen Natur-Kraft zur Erklärung hinreichen. Wir erkennen vielmehr in diesem Auftreten, in der Verbindungs-Weise der gleichzeitig mit einander bestehenden und der allmählich aufeinanderfolgenden Wesen wie in der wunderbaren Organisation der so manchfaltigen Lebenwesen und in ihrer Anpassung an die jedesmaligen äusseren Lebens-Bedingnisse eine durchgeführte Idee, ein so planmässiges Verfahren, ein so angemessenes Ineinandergreifen aller Wechsel-Bedingungen, dass dieses Alles wie jedes Einzelne nur eben sowohl die Wirkung einer unbegrenzten Allmacht wie die Anordnung einer unbegreiflichen Weisheit seyn kann; kein zufälliges Entstehen und keine Zeugung hat stattgefunden, sondern ursprüngliches, absichtliches, planmässiges $\mathrm{E} r \mathrm{~s} \mathrm{ch}$ a f f e n durch einen unbedingt selbstständigen, einen in Dauer, Allmacht und Weisheit unbegrenzten $\mathbf{S} \mathrm{c}$ h öp $\mathrm{er}$, der in alles Geschaffene sogleich die 
Nothwendigkeit des individuellen Unterganges mit der Fähigkeit des verjüngten Wiederauftretens in anderer Individualität und hiedurch des fortdauernden Bestehens im ewigen Wechsel und Vergehen legte.

Über das Unzureichende einer Ableitung der organischen Wesen von einer geringeren Anzahl ursprünglicher Art-Formen - mit Ausnahme etwa einer geringen Anzahl für Arten angesehener konstanter Varietäten - einer Generatio aequivoca der niederen und höheren, so wie einer Ableitung der höheren Organismen von den niederen durch allmähliche höhere Entwickelung und Steigerung der Organisation nacheinanderfolgender Geschlechter vom Infusorium an bis zum Elephanten und Ochsen haben wir uns schon in der Geschichte der Natur, Bd. II, ausgesprochen,

C. Die schon früher mehrmals angeregte Streitfrage, ob alle Individuen der Thier- und Pflanzen-Arten nur von je einem Individuum oder einem Stamm-Paare herrührten, oder ob jede Spezies gleich anfangs durch mehre Individuen zugleich vertreten gewesen seye, müssen wir in letztem Sinne entscheiden; daher denn auch jede Spezies sogleich auf einer grösseren Fläche und sogar auf verschiedenen von einander entfernten, aber in ihren Leben-bedingenden Verhältnissen gleichen Flächen in grössrer Anzahl zugleich, ja sogar unter denselben gleichen Bedingungen in verschiedenen Zeiten neu auftreten konnten.

a. In allen Fällen würde man, die wenigen Hermaphroditen oder durch Theilung und Sprossung sich vermehrenden Formen der niedersten Klassen aus. genommen, als Anfang jeder Spezies doch wenigstens 2 Individuen, gerade ein männliches und ein weibliches, annehmen müssen. Diese Nothwendigkeit zugestanden, sehen wir nicht ein, welche Einwendung man gegen die Annahme von gleichzeitig geschaffenen uranfünglichen $3-6-12$ und mehr Individuen einer Art machen könne. Wenn aber weiter ein Paar Rinder z. B. nach einigen Monaten erst im Stande ist ein Junges zur Welt zu bringen und diesem frühestens binnen Jahresfrist ein zweites beizufügen, das selbst frühestens erst 2 Jahre später fortpflanzungsfähig wird, daher erst binnen 4 Jahren das erste Paar als durch ein neues ersetzt betrachtet werden kann, so müsste es einem einzigen Paare oft unmöglich werden in der Nähe grosser Raubthiere ihre Spezies fortzupflanzen, diese müsste sogleich wieder untergehen! Mögen nun auch die grossen Raub-Säugthiere im Allgemeinen später als die grossen Herbivoren geschaffen worden seyn, immer hat es doch noch eine ansehnliche Zahl der letzten gegeben, die noch mit den Raubthieren zugleich erschienen sind. Und so auch in andern Klassen.

b. Geben wir aber zu, dass eine Spezies uranfänglich aus verschiedenen gleichzeitigen und beisammenlebenden oder entfernt zerstreuten oder successiv aufgetretenen Stamm-Paaren entsprossen seyn köune, so verliert der Begriff "Spezies" jene starre Festheit, die er ausserdem besitzen würde. Wir können dann gewisse uranfängliche Verschiedenheiten innerhalb einer Spezies nicht ausschliessen. Vielleicht können wir unsere Ansicht durch ein Beispiel deutlicher machen: Es gibt gewisse Helix-Arten, welche nur sehr wenig, aber sehr beständig von einander verschieden sind ( $z$. B. Helix hortensis und $H$. sylvestris); ja wir finden vielleicht in diesem Geschlechte die einander ähnlichsten wirklich verschiedenen Spezies. Setzen wir nun die Summe aller spezifischen Merkmale einer jeden dieser ähnlichsten Arten $=1,00$, so betragen jene, welche die ähnlichsten 2 derselben von einander unterscheiden, vielleicht nur 0,05. Es könnte aber ein Stamm-Paar gegeben haben, dessen Verschiedenheit von anderen Paaren gar nur 0,01 gewesen wäre: bildete diess Paar nun noch eine eigene Art? Jene Differenz $=0,01$ könnte bei den Nachkommen entweder durchaus beständig oder 
wieder schwankend in Ait und Maas seyn. In beiden Fallen Könnten früher odter später Nachkommen dieses Paares' sich fruchtbar mit solchen der andern so ähnliehen Paare verbinden; dann würde jene Differenz nur noch die einer Varietät seyn. Oder diese Verbindung erfolgt nicht, obschon beide Formen beisammen leben: dann werden wir dieselben als 2 Arten unterscheiden, sofern wir nämlich von der Beständigkeit jener wenn auch kleineren Differenz uns überzeugten; konnten wir Letztes nur aus Mangel an günstiger Gelegenheit nicht, so werden wir sie mit Unresht vermengen. Oder endlich die Verbindung erfolgte wirklich nicht, weil beiderlei Formen durch Zeit oder Raum von cinander getrennt gelebt haben, dann fehlen uns die Mittel einer definitiven Entacheidung; und da in der jetzigen Lebenwelt Spezies, wclche nur un 0,01 ihrer spezifischen Merkmnle brharrlich von einander verschieden sind, nicht oder nur schr selten? vorkommen oder anerkannt sind, so würde man der Analogie nach nicht anders können, als jene so wenig abweichenden Formen in eine Spezies verbinden.

D. Den allmählichen Untergang der Arten hat man 1) bald blos zufälligen Ereignissen, ") bald der periodisch fortschreitenden Abkühlung der Erd-Oberfläche und dem abnehmenden Kohlensäureund mithin relativ zumehmenden Sauerstoff-Gehalt der Luft zugeschrieben, bald 3) von der Beendigung der jeder Art so wie dem Individuum zugemessenen Lebens-Daner (BRocch, Lyezt, R. Owen, v. MeYer) herzuleiten gesucht, welche Einwirkungen und Ansichten in der Geschichte der Natur (Band II, 29 ff., 508) ausführlicher erörtert worden sind. Die fortwährende Entstehung neuer Formen scheint uns auf eiue Veränderung der Lebens-Bedingnisse der ErdOberfläche hinzuweisen, die mithin den alten Formen nicht mehr zusagen konnten, daher wir auch in diesen Veränderten LebensBedingnissen früherer Zeit vorzngsweise den Grund des Untergangs der Arten finden.

a. Was die Ansicht von Broccur betrifft, so beruht sie auf einer Theorie, die sich weder widerlegen noch streng erweisen laisst. Sie hat dadurch und in dem Naase an Geltung gewonuen, als gegen die 2 Vorstellungen von der einstigen Höhe des Klima's der Erde und dem Reichthum des Kohlensäure-Gehaltes der Atmosphäre mehr Einwendungen erhoben wurden; sie würde nur dadurch erwiesen werden können, dass wir entweder analuge Erfahrungen in der jetzigen Schöpfung machten, was indessen noch nicht geschehen ist, - oder etwa dass wir das Ungenuigende der übrigen Ursachen zeigten.

Die dritt: Annahme, dass jeder Spezies, wie dem Individuum, ihr Alter an. erschaffen seye, mithin von innerer Bedingung abliange, und dass sie solches nicht zu überleben vermöge, würde sich nur in Verbindung mit der Ammahme einer fortdauernden planmässigen Schöpfung neuer Arten denken lassen, welche die entstehenden Lücken wieder angemessen ausfïlten. Ohne dipse neue Annahme stïnde nicht nur cin allmähliches Aussterben oder -- bei unplanmässiger Wiederersetzung der vergangenen Arten - cin dem Glcichgewichte verderbliches Missverhältniss der Formen in Aussicht, sondern es würde die dritte Annahme nothwendig und schnell zur ersten und zweiten, d. h. zur Bedingung des Aussterbens der Arten durch ä ussere Ursachen - das Entstehen und Vergehen bedingender Pflanzen- und Thier-Arten - führen.

b. Die allmählichen Veränderungen der Lebens-Bedingungen auf der Oberfäche der Erde könnten vielleicht wohl zu der Erklärung der Erscheinung des allmählichen Erlöschens der Orğamismen-Arten hinreichen, wenn man nicht eine einzelne derselben hervorhebt, sondern alle (Koblensäure-Gehalt, Wärme-Höhe, 
Differenzirung der klimatischen Zonen, T'mwandelung einer Insel-Welt in Kontinente, Übergang von insularem mildem in excessives Klima, Salz-Gehalt des Meeres u. dgl. m.) zusammenfasst, so weit man sie als stattgefunden oder als einander gegenseitig bedingend nachweisen kann (Gesch. d. Nat. II, 42-61 und 505-508), in welch' letzter Beziehung insbesondere nicht vergessen werden darf, wie sehr das Entstehen und Vergehen gewisser Pflanzen und niederen Thiere wieder beziehungsweise das von auderen niederen und höheren Thicren bedingt u. dgl. m. Ja die neuen Formen mögen zun Theile selbst mit den alten, obschon beziehungsweise verwandten, unverträglich gewesen seyn, wie $z$. B. die Ratte (Mus rattus) überall verschwunden ist, wo die Wanderratte (M. decumanus) überhand genommen hat. Könnten wir aber das Zusammentreffen der Veränderung allgemeiner Lebens-Bedingungen a uf der Erd-Oberfläche mit Veränderungen der Flora und Fauna nachweisen, so wäre dadurch wenigstens eine grosse Wahrscheinlichkeit für diese Ansicht gewonnen. Nun können wir aber z. B., abgesehen von der Abkühlungs-Hypothese an sich, die stattgefundene Wärme-Abnahme und ihro: Abstufungen nicht unmittelbar, sondern eben nur etwa aus den Veränderungen der Thier - und Pfanzen-Welt beweisen, die doch erst durch jene erklärt werden sollen, so dass sich Beides wohl gut zu einer Theorie vereinigen, aber nicht gegenseitig beweisen lässt. Hat die Abkühlung der Erde Schuld, welche die Tropen-Gegenden weniger als die Pole betroffen hat, so müssten hier die Veränderungen in der organischen Welt bedeutender als dort gewesen seyn, wie Das wirklich der Fall ist.

c. Leichter allerdings lässt sich das Zusammentreffen mehr örtlicher und zufällig scheinender Ereignisse, mechanischer Veränderungen der Erde, grosser Gebirgs-Hebungen, Abtrocknung grosser Wasser-Flächen, Überschwemmung weiter Festländer u. s. w. mit dem Verschwinden einer grossen Anzahl Arten nachweisen, wogegen sich aber eben die Örtlichkeit der Erscheinung einwenden lässt, die zwar wohl Individuen vieler Arten zugleich, aber nur in seltenen Fällen die Existonz einer ganzen Spezies bedrohen kann.

d. $\mathrm{Da}$ die neu entstehenden Pflanzen- und Thier-Formen van den friberen immer mebr oder weniger abwichen und so allmäblich z.u einer ganz anderen Gestaltung und Vertheilung des Thier- und Pflanzen-Reiches führten, wie sich das einstweilen aus dem Enumerator ersehen lässt, so war đer periodische Schöpfungs-Plan jedenfalls immer etwas geäadent und in Ganzen nach ciner gewissen Richtung voranschrcitend. Wenu es sich nun blas danum gehandelt hätte, die allmählich entstehenden Lücken wieder auszufüllen, so wünde eine fortdauernde Thätigkeit der alten Schöpfungs-Kraft in Wiederherstellung der alten und bloss durch ihr Alter erloschenen Arten genügt haben. Die Veränderung in den Erzeugnissen dieser Schöpfungs-Kraft aber scheint uns cine hinreichende Induktion zur Annahme zu bieten, dass die äusseren Lebens-Bedingungen auf der Erd-Oberfäche sich selbst fortwährend veränderten und somit auch nicht mehr den alten, sondern vur wieder neuen Formen zusagen konnten.

e. Unger stellt in Bezug uuf die fossilen Pflanzen die Ansicht auf, dass in früherer Zeit [immer? bis?, oder wenigstens in der Miocän-Zeit] die Tenperatur auf der Erde überall eine gleichmässige gewesen seye, und dass da, wo diese Temperatur sich erhalten, auch der Pflanzen-Typus bis jetzt derselbe geblieben seye; wo aber das Klima sich geändert, da seyen dic alten Arten nicht ausgewandert, sondern seyen allmählich zu Grunde gegangen und durch andere von abweichendem Typus allmählich ersetzt worden ').

E. Wiu haben aber überall ausser dem absoluten Erseheinen und Verschwinden - Schöpfung und Aussterben - der Arten, Sip-

1) Jahrb. $1848,607-508$. 
pen u. s. w. auch noch das beziehungsweise, das örtliche Erscheinen und Verschwinden - Aus - und Einwanderung - zu unterscheiden, welches uns oft, so lange wir die ganze allmähliche oder gleichzeitige geographische Verbreitung der Arten u. s. w. noch nicht kennen, wohl als absolutes erscheinen kann.

F. Diese neuen Untersuchungen der statischen Paläontologie, welche in folgenden $\$ S$. mitgetheilt werden, sind Ergebnisse noch sehr unvollkommener Hülfsquellen, deren Mängel wir später zu beleuchten Gelegenheit finden werden. Indessen geben sie wenigstens ein Bild der Wissenschaft, das mit dem jetzigen Zeitpunkte abschliesst, und dürften in soferne immer einigen Werth behalten, weun einst auch Vieles verbessert und ergänzt seyn wird, wovon wir jetzt nur sehr unvollkommene Kunde besitzen. Ein erster Versuch der Art, der folglich noch weit unvollkommener seyn muss, ist 1831 mitgetheilt worden ${ }^{1}$ ).

\section{3. Dauer der Arten.}

A. Wir können die Dauer früher bestandener wie jetzt lebender Arten im Allgemeiuen wie im Besonderen nicht in einer absoluten Anzahl von Jahren angeben; wir können die der ersten nur relativ nach der Anzahl der Gebirgs-Schichten bezeichnen, worin sie vorkommen. Und so finden wir in der That, dass, während ein sehr grosser Teil der Arten sich nur in einzelnen Gebirgs-Schichten einfindet, andere durch zwei, drei und mehr derselben hindurchreichen und selbst in die Schichten anderer Formationen, zuweilen sogar anderer Perioden übergehen.

Wir werden uns beschränken, die unten folgenden Beispiele aus dem Enumerator zu entuehmen; in später erschienenen Schriften ist aber ihre Anzahl noch erheblich vergrössert worden. Einige frühere Zahlen-Zusammenstellungen über diesen Gegenstand findet man in der Lethaea, wie in Jahrbuch, dabei Manches gestützt auf später berichtigte Bestimmungen ${ }^{2}$ ).

B. Wir dürften nicht nöthig haben Beweise anzuführen für den gewöhnlichsten Fall des Vorkomınens in zwei oder mehren Schichten einer Formation, wie wir sie (unter Einschaltung einiger Rubriken für zweifelhafte und örtliche Gebilde) im Enumeratur angenommen haben, zumal wir so viele Belege für eine weit längere Dauer anzuführen im Stande sind.

C. Das Vorkommen einer Art in zwei aufeinanderfolgenden Formationen ist nach den bisherigen Untersuchungen nicht eben selten, wenn wir auch zugeben, dass ein nicht ganz unbedeutender Theil der aus unserem Enumerator ersichtlichen Fälle auf unrichtiger Be-

1) Brons, Italiens Tertiär-Gebilde und deren organische Einschlüsse, Heidel berg, 176 SS. $8^{0}$.

2) Jahrb. 1839, 734, 735; 1841, 796, 797, u. a.; 1842, 82-84. 
stimmung der Arten oder der Formationen zu beruhen scheint ${ }^{1}$ ). Sie kommen bei allen Formationen vor.

Indem wir daher durch blosse Zitate auf die weniger verbürgten Beispiele und auf den Enumerator im Allgemeinen verweisen, heben wir die nach Art und Formation verlässigsten Fälle, wic die im Enumerator durch cine bezeich. neten u. e. a. aus und werden im Folgenden noch einen Theil der sichersten durch ein ! bemerklich machen, die Formationen aber der Kürze wegen nur mit den im Enumerator gebrauchten Buchstaben ausdrücken. So kommen vor in den Formationen :

a + b: Aulopora serpens, Heliopora interstincla, Halysites catenulatus, H. labyrinthicus, Cyathophyllum-Arten, - Terebratula reticularis, Orthis elegantula, O. calligramma, 0 . callactes, Leptaena imbrex, Orthis pecten, 0 . sinuata, 0 . biforata $u$. a.; Crania antiquissima, viele Trilobiten, wobei Calymene Blumenbachi ${ }^{2}$ ) und viele andere Spezies, die unangemessen wäre alle zu nennen, da sie sich im Enumerator so leicht überblicken lassen und in der Regel nicht bestritten sind.

b + e: Aulopora conglomerata und A. tubaeformis, Glauconome disticha, - Al. veolites fibrosus, Calamopora 5 Arten, wobei C. Gothlandica ${ }^{3}$ ), Syringopora 2-3 Arten, Cyathophyllum-Arten, - Terebratula nucula, T. reticularis, T. Wilsoni, T. sphaerica, T. aspera, Pentamerus galeatus ${ }^{4}$ ), Orthis lunata, Spirifer ptychodes, Leptaena lata, L. Uralensis, Cucullaea antiqua, Bellerophon carinatus, B. globatus u. s. w. Auch diese Arten sieht man zahlreich im Enumerator angedeutet; allein viele stehen auch auf die Auctorität von Phillips dort, welcher in seinen Palaeozoic Fossils einen Thril der nämlichen Schichten mit ihren organischen Resten dem Devon-Kalke beizuzäblen scheint, die er in seinem Werke über Yorkshire zur Devon-Formation rechnete (vgl. S. 4). WeAver zählte 1859 im Corker Kalke 7 eigenthümliche, 24 silurische Arten und 17 aus der Kohlen-Formation ${ }^{5}$ ), also b 24 e $17 \mathrm{~d}$; doch beruhen nach DE Verneull jene 24 grossentheils auf falschen Bestimmungen ${ }^{6}$ ) und Äusserungen aus finer Zeit, wo man die devonische Formation noch nicht anerkannte. Später zählt Murcison selbst unter 375 Arten 338 b 10 c 27 auf, so dass der Devon-Sandstein über $1 / 4$ seiner Arten aus dem Silur-Gesteine hätte.

d: Cyathophyllum. und manche andere Arten wirbelloser Thiere; - wie Platycrinus laevis, Orthis umbraculum ${ }^{7}$ ), Spirifer glaber, Sp. lineatus, Melania rugifera; aber auch einen Fisch! Psammodus rugosus führt Agassiz selbst in beiden Formationen an.

d + : Goniatites diadema, G. reticulatus, G. sphaericus, Nautilus stygialis u. e. a., obschon hier eine See - mit einer Süsswasser-Formation in Verbindung steht.

e + f: Hier fehlen die gemeinsamen Arten, weil - Süsswasser-oder Brackwasser-Formation, f zwar Meeres-Niederschlag, aber als Sandstein. Gebilde an fossilen Resten ausserordentlich arm ist.

1) So auch viele der von D'Archisc zusammengestellten Fälle, Jahrb. 1841, 796-798.

7) Jahrb. 1849, 126, 127, 128.

3) M'Cox kann nach der sorgfältigsten Untersuchung die Art der Eifel (c) nicht von der des Irischen Kohlen-Gebirges (d) unterscheiden. Ann. nathist. $1819, b$, III, 134.

4) Jahrb. 1849, $125 \mathrm{ff}$.

b) Das. 1840, 242 .

e) Das. 1841, 767 .

7) Das. 1841, 775. 
$\boldsymbol{r}+\boldsymbol{g}$ : Aus dem letzten Grunde können wir nur $\mathbf{5}$ diesen zwei Formatianen F und zustehende Spezies nach G sisiтz nambaft machen: Orthothrix lamellosa, Productus horridus, Terebratula Schlotheimi, Cardita Murchisoni, Caulerpites selaginoides ${ }^{1}$ ); aber manche kommen in $g$ und den älteren Kalk-Schichten b, eder and e gemeinsam vor, wovon später die Rede seyn wird.

+ In: Avicula antiqua.

h [die St. Cassianer Formation ${ }^{2}$ )] sollte nach Münster und Wissmanv unter 42 ' Arten 12 mit dem Kohlen-Gebirge, 10 mit der Trias, 11 mit Lias und 3 mit Jura gemein haben ${ }^{3}$ ).

1) Das. 1848, 505 .

2) weleher wir bisher die Stelle h gegeben, entspricht dem oberen Theil des Muschelkalk-Gebildes (k) nach Maasgabe folgender St. Cassianer Arten (vgl. Jahrb 1848, 54-55), wie insbesondere L. v. Bucr dargethan hat.

Spirifer rostratus acutus

Terebratula Mentzeli

Terebratula trigonella

Dadocrinus graeilis

Ammonites modestus

Gervillia socialis

Terebratula vulgaris

Ammonites nodosus

Encrinites liliiformis

Posidenemya Clarai.

3) Die von Münstsa als schon anderwärts bekannt bezeichneten Arten sind folgende (wobei die mit einem * in die Rubriken eingetragenen Arten identisch, die mit + nur analog sind und Ziffern I, II, III das Kohlen-, Triasund Oolith-Gebirge, die zwei Spalten unter III aber Lias und Jura bedeuten).

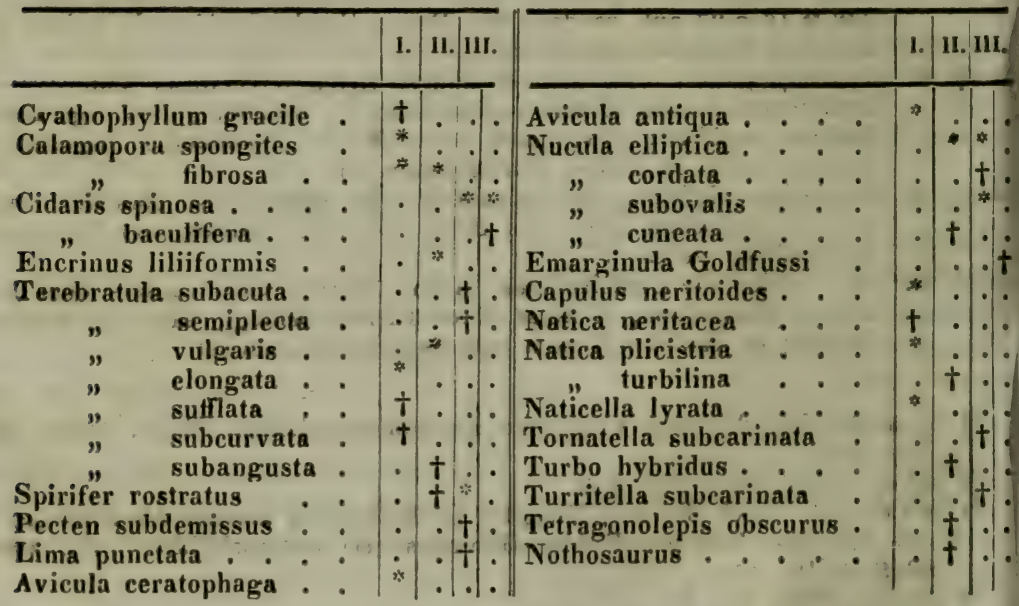

In diesem Verzeichniss sind einige Bestimmungen allerdings einer wiederholten Prüfung bedürftig. - Einige Ammoniten des Cassianer Kalkes finden sich nach v. HAURR im Muschel-Marmor von Raibel (A. Gaytani), 
5 + i: haben in der That keine Arten gemein, was zum Theile der ArtenArmuth des unteren Theiles der Buntsandstein-Formation (i) zuzuschreiben, zum Theil aber auch von einer wirklichen grösseren hier vorhandenen Kluft lierzuleiten ist.

1 + k: Pecten discites, Lima-Arten, Mytilus vetustus, Pleuromya ventricosa, Natica Gaillardoti, Turritella scalata, - Ceratites semipartitus, u. a.

$\mathbf{k}+1$ : Ceratodus runcinatus, Gyrolepis Albertii, G. tenuistriatus, einige Saurichthys-Arten, Sphaerodus minimus, - Zanclodon crenatus, u. a.

I $+\mathbf{m}$ : Keuper und Lias: haben Nucula Münsteri, Clathropteris meniscioides BRGN. und vielleicht noch einige andere Pflanzen-Arten mit einander gemeinsam. (Durch Einschaltung der St. Cassianer Formation und der rothen Ammoniten-Marmore zwischen beiden Gebilden würde die Zahl der gemeinsamen Arten noch grösser werden.)

n: Nilssonia compta, Zamites Bechei, - Terebratula furcillata, T. vicinalis, mehre Pecten-Arten, Lima gigantea, Avicula inaequivalvis und A. echinata, Nucula triquetra, !Pholadomya deltoidea, $\mathbf{P h}$. obliquata und eine grosse Anzahl anderer Arten, die man im Enumerator bald überblickt ${ }^{1}$ ); dann Ainmonites tatricus, A. heterophyllus u. a. ${ }^{2}$ ),

o: Cellepora orbiculata, Ostrea solitaria, Exogyra spiralis, E. auriformis, Plicatula tubifera, Lima rustica, Myopsis gibbosa, - Pleurotomaria reticulata, - Pycnodus Nicoleti, P. gigas, - Streptospondylus Geoffroyi [?], Cetiosaurus longus.

$+\mathbf{p}$ : Cyclas media So.

$+\mathbf{p}$ : !Modiola lithotomus nach den Autoren Koch und Dunker selbst. Unter

im opalisirenden Muschel-Marmor von Bleiberg in Kärnthen (A. Johannis Austriae und A. Jarbas), am Ovir-Berge bei Klagenfurt, zu Hall im nördlichen Tyrol, im Kalke der Wochein wie im rothen Ammoniten-Kalke von Berchlesgaden und Hallein, von Aussee, Hallstutt (Jahrb. 1847, 105), Dürrenberg (und la Speszia?') wieder, von welchen Lokalitäten aber der Dür. renberg nach LiLL auf dem Sandstein von Werfen rubt, der nach unserer Nachweisung Lias-Sandstein ist. Eben so liegt der Ammoniten-Kalk von Adneth (eine jedoch nach HAUER an Arten abweichende Bildung) auf Gebirgen mit Petrefakten-Formen des Lias. In den Ammoniten des MuschelMarmors hat D'Orbigny Arten des Oxford-Thones und des Kelloway-rocks erkannt (QUENSTEDT glaubte sogar an Neocomien-Ammoniten). KLipsteiN meinte unter den St. Cassianer Schichten Lias-ähnliche Schiefer mit Ammonites cordatus und darunter Muschelkalk mit Ammonites nodosus annehmen zu müssen. An anderen Orten fehlt der erste. Was nun die Arten betriff, welche identisch im Cassianer und im Cephalopoden-oder Ammoniten-Kalke vorkommen sollen, so müssen wir eine Revision ihrer Bestimmungen abwarten. Wahrscheinlich kommen zu Hallstatt $\mathrm{u}$. a. der genaunten Orte Schichten verschiedenen Alters vor, wie voN Hausn a. a. 0 . andeutet und der vorhin zitirte Dadocrinus vermuthen lässt. Vgl. Jahrb. 1842, 123; 1844, 328, 791; 1846, 818; 1847, 631; 1848, 44, 105, $109,136,373,714,715,716$.

1) Über die Verbreitung der fossilen Arten in den untergeordneten Gliedern von $\mathbf{m}$ ist nur noch in einem Theile unseres Enumerators Auskunft gegeben durch die dem $\mathbf{n}$ beigefügten Zeichen. Dass auch hier noch die Arten aus einer Schicht in die anderen übergehen, gesteht D'OrBIGNy ausnahmsweise zu und erkennen WiLliamson im Jahrb. 1813 (Collectan.), Rominger im Jahrb. 1846, 293 ff. u. A. an.

2) BayLe im Jahrb. 1849, 498. 
den Fischen Lepidotus minor und Hybodas strictus; damn sollen einige Hybodus-Arten und Asteracanthus semisulcatus aus $\mathbf{n}$ herüber kowmen; doch dürfte die Formation noch zu bestätigen seyn.

$\boldsymbol{p}+\boldsymbol{q}$ : scheinen anfangs ausser durch ! Unio Martini keine Gemeinschaft zu zeigen, da $\boldsymbol{p}$ eine Süsswasser -, q eine Mreres-Formation ist; aber auf Wight wechsellagern die Süsswasser-Schichten von $\mathbf{p}$ wiederholt mit den meerischen von $\boldsymbol{q}$, ehe diese Formation herrschend wird '), obschon ältere meerische Glieder der Oolithen-Periode manche Arten mit der Kreide-Periode gemein haben, die wir vachher unter E. aufzählen werden.

- r: Die Versteinerungen der 3 Kreide-Formationen sind in Ostindien und Sũd-Amerika nicht mehr in derselben Weise gruppirt und vergesell. schaftet, wie in Europa ${ }^{2}$ ); ja in Europa selbst enthalten die Schichten von Fir und im Sixt-Thale ein merkwürdiges Gemenge von solchen Kouchylien-Arten, welche sonst in der chloritischen Kreide vorkommen, mit denen des Gault ( $\mathbf{r}$ mit $\mathbf{x})$, Picter ${ }^{3}$ ).

a + r: Manon peziza, ! Cucullaea striatella ${ }^{4}$ ), Lueina sculpta, Corbula elegans, C. striatula, !Panopaea mandibula, !P. plicata, :Actaeon marginatus, !Ringinella lachryma, !Turritella granulata, !Turbo decussatus, ! Turbo Mantelli, ! Cerithium Lallieranum, ! Pteroceras refusum, Ammonites de. narius ${ }^{5}$ ) u. v. $a$.

$\mathbf{r}+\mathbf{r}$ : Manon peziza, !Mytilus divaricatus, ! Arca bifida, A. trapezoidea, ! Panopaea mandibula, ! Avellana Raulinana, ! Turritella granulata, !Pleurotomaria Moreauana, ! Ammonites latidorsatus, ! A. Mayoranus, ? A. influtus, ! Hamites armatus, !Turrilithes Bergeri, u. v. a. meist nach D'ORBJGri; indessen ist eine gewisse Anzahl der im Enumerator aufgezählten Arten unsicher, weil Deutsche u. a. Geologen die verschiedenen Grünsande zu oft mit einander verwechselt haben, wesshalb manche Spezies in r $\mathbf{r}$ eingetragen worden seyu dürfte, die nur in $\mathbf{r}$ gehört. Andere werden noch an den $\mathrm{zu}+\mathbf{x}$ genannten Stellen zitirt ${ }^{5}$ ).

$\boldsymbol{r}+\boldsymbol{s}(\boldsymbol{\tau})$ : Ostrea lateralix, 0 . vesicularis, Pecten arcuatus $\left.{ }^{6}\right)$. Wir werden später mehre Arten zu nenuen haben, welche in Kreide und jüngern Tertiär-Schichten zugleich vorkommen (vgl. S. 759).

1) Jahrb. 1844, 623 .

2) Das. $1849,116$.

3) Das. 1848,757 .

4) Das. 1848, Collect. 79.

8) Jahrb. 1843, Collect. $81-84$ und $85-94$.

6) Mém. géol. b, II, 189 ff. > Jahrb. 1848, 864. - Die Nummuliten-Gesteine bieten noch immer grosse Schwierigkeiten dar. Es werden folgende unterschieden (Jahrb. 1844, 750, 751, 752; 1848, 73, 361, 366, 494, 587, 623, $713-716,860 \mathrm{ff}$.):

a) in u: über Macigno liegend, von Ewald angegeben. BEYrich erklärt den Nummulitenkalk der Karpathen für tertiär, vielleicht übereinstimmend mit vorigem. Wir haben ihn unseres Wissens nirgends mit s vermengt.

$\boldsymbol{\beta}$ ) in t: das Terrain nummulitique Soisonnais Dr BEAUmont's, das Système Iberien Tallavigne's, welches auf den Ligniten des plastischen Thones ruht und bisher als unterer Theil des Grobkalkes angenommen worden ist. Es enthält viele Säugthier-Reste und zahlreiche Konchylien, wovon ein Theil im jüngeren Eocän-Gebirge, im Grobkalke selbst, einige (wobei Echinopsis elegans) im älteren Nummuliten.Gebirge (5 von 109 Arten von Bos Arros allein am Monte Bolca) und 15-20 Arten auch in der Kreide vorkommen. Es ist das erste post-pyrenäische Sediment-Gestein. Eq 
+ t: Verticillites cretaceus, Guettardia stellata, Spongus ovatus, Frondicularia ornata.

+ t: Crassatella ponderosa, Gastrochaena gigantea, !Velates Sehmidelanus, Serpula siliquariaeformis und S. spirulaea, dann !Echinopsis elegans AG. ${ }^{2}$ ) werden angeführt; indem aber die jüngeren tertiären Num. muliten-Gesteine nun in gleichen Rang mit den Nummuliten-führenden Eocän-Schichten im unteren Theile des Pariser Beckens treten, werden die organischen Berührungs-Punkte viel zahlreicher.

+ u : !Rosalina rugosa, !Lenticulina planulata, !Triloculina trigonula, !Quinqueloculina saxorum; - Lunulites urceolatus, Orbitulites complanatus,

lagert neben den Pysenåen bei Biaril\% (die Echinodermen-Schicht), zu Bos Arros bei Pau, im obern Theil der Corbières, in der Montagne noire.

y) Den Pariser Pisolithen-Kalk, welchen wir bis jetzt unter mitbegriffen, die Mastrichter Schichten und das Terrain Danien, bisher mit $\mathbf{r}$ und mit $\mathbf{r}^{2}$ bezeichnet, möchte ELIE DE BEAvmONT als Äquivalent der vorigen betrachten; sie sollen aber selbst bei Paris, ausser den ersten Sängthier-Resten, nach Heberт's neueren Mittheilungen keine tertiären Arten enthalten, und könnten also nicht mit den vorigen norh eocänen Bildungen vereinigt werden; und doch liegen sie auf eocänem Töpferthon?

8) in : das Terrain nummulitique mediterranéen DB Braumosr's, das Système Alaricien Tallavignes' trägt zu Abesse am Adour den Grobkalk in a b weichender Lagerung, liegt in der Schweils tiber Caprotina-Kalk und unter Fucoiden-Schiefer oder Flysch, in der Brianza über FukoidenFormation mit Fucoides intricatus und Rudisten-Konglomerat, aber unter Fucoiden-Flysch, im Vicentinischen über Scaglia und unter Macigno mit Fucoiden, führt Linsen-förmige Nummuliten und keine Säugthier-Reste. Es ist das letzte antepyrenäische Sediment-Gestein, liegt mitten in den Pyrenäen am Mont Alaric, bildet den unteren Theil der Corbières unter Aufnahme von Rudisten (die selten auch schon in $=\mathbf{r}^{2}$ vorkommen), die unteren Schichten von Biaritz, Kommt an der Süd-Seite des Mont perdu vor, in Navarra, Aragonien, im Sixt-Thale, an den Diablerets, am Col Lauranier, zu Genua, am Monte Bolca und zu Roncis $(=\tau$, wo es nach DE Zigno zwischen Macigno oder Fukoiden-Sandstein oben und Scaglia unten liegt), am Karst, in Istrien, zu Gutaring in Kärnthen, Sonthofen, Kressenberg; - ? in der Krim, in ? Ägypten, in Kleinasien, am Kaukasus, Ararat u. s. w. Da indessen die Nummuliten-Schichten in der Krim und in Ägypten die eocänen Aıten Ovula tuberculosa, Cerithium ?giganteum, Ostrea gigantea var. latissima (wie bei Paris und im Adour-Becken) enthalten ohne Kreide-Versteinerungen aufzunehınen, so scheinen dieselben vielmehr mit $\beta$ verbunden werden zu müssen.

$\varepsilon$ E) EWLD trenut hievon als älter und der Kreide angehörig die Schichten vom Etang de Berre (nicht von Gap) mit kugeligen Nummuliten und Hip. puriten. Wir wissen nicht, welche der unter $\delta$ gestellten Örtlichkeit noch damit verbunden werden müssen, kennen aber solche kugelige Nummuliten auch aus dem Visentinischen. Nach D'Orbigny und DE VerneuIL wären in Europa und Amerika die ächten Nummuliten auf Tertiär-Gebirge beschränkt; während andere ähnliche Körper dieses Gebirges und der Hippuriten-Kreide ein neues Genus Orbitoides bildeten.

己) Nach Zeuschner gäbe es in deu Karpathen noch einen NummulitenDolomit unter Neocomien [?].

') Vgl. Bull. géol. 1848, b, V, 413 ff. $>$ Jahrb. 1848,861 . 
mehre Echinolampas- und Spatangus-Arten; - Pecten-Arten, Avicula trigonata, Trigonocoelia deltoidea, Cardium turgidum, mehre LucinaArten, Crassatella ponderosa und $\mathrm{Cr}$. triangularis, einige CythereaArten, Donax Basterotinus, Arcopagia elegans, Neaera Waeli, Corbula cochlearella, Corbulomya triangula, - Vaginella depressa, Dentalium sulcatum, D. nitens, Sigaretus canaliculatus, S. politus, einige NaticaArten, Pitonillus cepaceus, P. dubius, Nerita Caronis, !Velates Schmidelanus, einige Neritinae, Actaeon inflatus, Melania-Arten, Scalaria-, Turbo-, Turritella-, Delphinula-Arten, ! Orbis rotella, viele CerithiumArten, !Rostellaria fissurella und R. dentata, Strombus tuberculiferus, Arten von Murex, Fusus, Pirula, Pleurotoma, Fasciolaria, Cancellaria, Cassis, Morio, ! Buccinum stronboides, Arten von Marginella, Ancilla. ria, Oliva, Cypraea, Conus, - Bulla, Aurirula, - - Serpula decussata, - - Notidanus serratissimus, Carcharodon Escheri, Odontaspis Hopei, !Lamna elegans nach Agassiz selbst, Oxyrhina hastalis, O. xiphodon, Otodus obliquus; - endlich Anthracotherium magnum und Lophiodon anthracoideus, wo noch die Formationen einer Bestätigung bedürfen möchten, wie auch 2 Palaeotherium-Arten und Microtherium.

u $+\mathbf{w}$ : haben eine sehr grosse Arten-Zahl gemein, vielleicht dic Hälfte der in u vorkommenden Konchylien; wir zitiren daher nur einen Fisch, ! Odontaspis contortidens, welchen Agassiz selbst als gemeinsam bezeichuet.

v: ohnediess theils zu u und theils zu w gehörige Schichten, über deren richtige Stellung sich noch nicht entscheiden lässt. Ob aber die Bernstein. Insekten ( $v^{1}$ ) hier bleiben können oder nebst noch einigen Kouchylien zu t zu rechnen seyn werden, darüber müssen spätere Untersuchungew entscheiden ${ }^{2}$ ).

$\mathbf{w}+\mathbf{z}$ : Auch zwischen diesen beiden Schichten, wie schon zwischen w und $z$, gibt es eine Menge gemeinsamer Arten, die wir hier nicht aufzählen können und von welchen im Allgemeinen unter E. (S. 760) gehandel werden wird.

D. Manche Arten reichen durch drei und mehrere Formationen einer Periode, zuweilen durch eine ganze Periode mit oder ohne Überspringung einzelner Schichten hindurch, obschon auch hier sicher einzelne Angaben auf unsichern Bestimmungen beruhen. So in: Periode:

I: $\mathbf{a}-\mathbf{s}$, und zwar in

a-c: Calamopora alveolaris, C. Gothlandica und eine sehr grosse Anzah anderer.

a-d oder a-e: Cyathophyllum turbinattim, C. caespitosum, Orthis resu pinata, Leptaena depressa ${ }^{2}$, Orthoceras cinctum.

b-d : Orthis umbraculum, Favosites fibrosa, Gorgonia ripisteria, Spirife speciosus.

b-g: Calamopora spongites, Fenestella dubia, ? Gorgonia antiqua ${ }^{3}$ ), Cho netes sarcinulatus.

c-g: Retepora flustriformis, Gorgonia antiqua, Terebratula concentrica, T elongata, Pleurotomaria carinata, Loxonema rugifera.

d-g: Fenestella retiformis, F. anceps, Cyathocrinus planus, Lingula myti loides, Terebratula Schlotheimi, T. pectinifera, T. planosulcata, T

1) Jahrb. 1548, 49, 72。

3) Das. $1849,126,128$

3) Das. 1844, 736. 
elongata, T. De-Roissyi, Productus Cancrini '), Spirifer cristatus, Sp. undulatus, Avicula antiqua.

e-g: Palaeoniscus Freieslebeni, PCalamites Suckowi ${ }^{2}$ ), ?C. gigas, Neuropteris tenuifolia, Lepidodendron elongatum ${ }^{3}$ ).

Aus D'Archiac und de Verneuic's sorgfältigen Vergleichungen und Bestimmungen entnehmen wir folgendes Zahlen - Ergebniss übergreifender Arten $\left.{ }^{4}\right)$ :

\begin{tabular}{|c|c|c|c|c|c|c|c|c|c|c|c|c|c|}
\hline & & & & & Arten. & $(\mathbf{a}) \mathbf{b}$ & bc & c & cd & d & bd & bed & $?$ \\
\hline Fische & - & - & - & - & 78 & 8 & - & 50 & 一 & 20 & - & - & - \\
\hline Kruster . & . & - & - & - & 216 & 135 & 7 & 32 & 2 & 24 & 2 & 1 & 36 \\
\hline Insekten . & . & - & . & - & - & 4 & - & - & - & 4 & - & - & - \\
\hline Anneliden & - & · & - & - & 11 & 4 & - & 5 & - & 2 & - & - & - \\
\hline \multicolumn{14}{|l|}{ Mo!lusken } \\
\hline Heteropoder & . & : & • & : & $\begin{array}{r}448 \\
24\end{array}$ & $\begin{array}{l}82 \\
15\end{array}$ & $\begin{array}{r}10 \\
6\end{array}$ & $\begin{array}{r}199 \\
22\end{array}$ & $\begin{array}{l}6 \\
3\end{array}$ & $\begin{array}{r}168 \\
36\end{array}$ & $\begin{array}{l}2 \\
1\end{array}$ & $\begin{array}{l}2 \\
2\end{array}$ & $\begin{array}{r}22 \\
5\end{array}$ \\
\hline Pteropoden & . & . & . & . & 11 & 6 & 1 & 4 & - & 2 & - & - & - \\
\hline Gasteropod & & $\because$ & & . & 382 & 63 & 7 & 116 & 16 & 225 & 5 & - & 10 \\
\hline Dimyen & & . & • & . & 302 & 49 & 9 & 145 & 5 & 126 & 1 & 2 & 2 \\
\hline Monomyen & . & •. & * & · & 161 & 33 & 3 & 60 & 15 & 77 & 1 & - & - \\
\hline Brachiopod & & . & & . & 568 & 230 & 30 & 182 & 28 & 229 & 7 & 3 & 12 \\
\hline Radiaten & 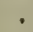 & - & & . & 163 & 42 & 1 & 59 & 10 & 75 & 1 & 3 & 3 \\
\hline Foraminiferer & . & . & & . & - & - & - & $?$ & - & $?$ & - & - & - \\
\hline Polyparien . & - & - & • & - & 260 & 115 & 36 & 107 & 4 & 83 & 1 & 2 & 2 \\
\hline Infusorien & - & & 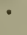 & - & - & - & - & - & - & $?$ & - & 一 & - \\
\hline unbestimmt . & $\cdot$ & $\bullet$ & & • & 30 & 25 & 3 & 3 & - & 1 & 1 & - & 5 \\
\hline im Ganzen . & • & 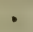 & 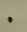 & • & 2698 & 807 & 113 & 984 & 79 & $|1072|$ & 22 & 15 & 97 \\
\hline
\end{tabular}

Nach Murchison, de Verneuic und v. Keyserling wäre allein in Russ. land (ohne Rücksicht auf ausländisches Vorkommen) die Vertheilung der Arten in den älteren Formationen folgende ${ }^{5}$ ):

im Ganzen . . . . $\left|\frac{}{392}\right| \frac{(a) b}{104}\left|\frac{b c}{2}\right| \frac{c}{116}\left|\frac{c d}{5}\right| \frac{d}{122}\left|\frac{d g}{3}\right| \frac{g}{37}\left|\frac{b g}{1}\right| \frac{b c d g}{1}$

II i-I : Lingula tenuissima, Gervilleia socialis, Posidonomya minuta, Avicula subcostata, Myophoria laevigata, Pleuromya mactroides.

III m-p: Hier finden wir zwar keine durch 3 Glieder durchgreifende Art, theils weil a schon selbst eine Gruppe von $3-5$ Formationen in Werthe von i, $\mathbf{k}$ und $\mathbf{l}$ bildet, theils weil o nicht sehr reich an Arten, theils endlich weil $\mathbf{p}$ Brackwasser-Formation ist, also mit den meerischen Gebilden $\mathbf{m}$, $\mathbf{n}$, fast nichts gemein haben kann. Von $\mathbf{m}+\mathbf{n}$ und von $\mathbf{n}+\mathbf{o}$ sind $\mathrm{S}$. 753-754 Beispiele gegeben.

n-p: Megalosaurus Bucklandi und Poecilopleurum?

IV $\boldsymbol{y}$-r: Manon peziza, Heteropora cryptopora, Ceriopora stellata und C. tubiporacea, Orbitulites Ienticularis, Cyclolithes coronula, - Codiopsis doma Ag., Salenia scutigera, Cidaris vesiculosa, Schizaster curynotus, Holaster nodulosus, Terebratula pectiniformis, T. car-

1) Jahrb. 1844, 736 .

2) Das. 184t, 84 .

3) Das. 1814, 735; 1846, 621.

4) Das. 1843, 625 .

5) Das, 1846, 621, wo diese Arten auch benannt sind. 
dium, T. Menardi, T. longirostris, T. semiglobosa, T. carnea, T. curvirostris, Exogyra plieata, Spondylus striatus, Pecten asper, P. aequiradiatus, P. quinquecostatus, P. orbicularis, P. costangularis u. a. (S. 250,254 und 255 des Enumerators), Lima semisulcata, Inoceramus concentricus, Modiola reversa, Lyriodon aliformis und L. spinosus, Astarte oblongata, Venus faba, Panopaea mandibula nach D'OrBigny selbst, Dentalium medium, Turritella granulata theilweise nach D'OREIGNY; Nautilus radiatus, $N$. simplex nach D'Aнснілс U. A; doch wird von diesen Arten bei genauer Revision derselben wie der Formationen, denen sie in einzelnen Örtlichkeiten zugetheilt worden sind, noch ein Theil gestrichen werden müssen, während andere zweifelsohne hinzukommen. $\mathrm{Vgl}$. darüber d'Archiac '), Lexmerie ${ }^{2}$ ), Fr. A. Roemer ${ }^{3}$ ), d'Orbigny ${ }^{4}$ ).

V -x und zwar (s) t-w : !Spiroloculina perforata, - Arbacia pusilla, Cardium striatulum, Corbula faba u. s. w.; von noch weiter hindurch reichenden Arten wird weiter unten S. $770 \mathrm{ff}$. die Rede seyu.

E. Melire Arten reichen aus einer Periode in die andere hinüber. Soferne sie hiebei nur in die zwei Grenz-Formationen eintreten, findet man schon eine Anzahl derselben unter C. genannt. Andere reichen weiter in zwei aneinander grenzende Perioden hinein. Beispiele vom Einen oder dem Andern kommen an der Grenze aller Perioden vor. So in

Perioden:

I - II: Hier scheinen bis jetzt nur für den Fall gemeinsame Arten angezeigt zu seyn, dass das St. Cassianer Gebilde mit der Trias wirklich verbunden werden muss, wie ausser uns auch L. v. Bucн annimmt (vgl. S. 752). - Favosites ramosus und Calamopora spongites aus den silurischen und devonischen Schichten sollen bis in die St. Cassianer Formation heranfreichen.

1I-1II: Hier ist die ausgezeichnete Terebratula trigonella $(\mathbf{k}+\mathbf{n})$ des $\mathbf{M u}$ schelkalks anzuführen, eine Art, welche Anfangs nur aus den Horn. stein-Nieren des oberen Theiles des braunen Jura's bekannt war. Catulese hatte sie im Muschelkalk als $T$. aculeata beschrieben und nach vielen Bemühungen $z u$ Vertheidigung dieser Art sie endlich in 2 Arten getrennt, in die $\mathbf{T}$. aculeata und die wirkliche $\mathbf{T}$. trigonella, die er also hiemit zugab. Indessen sind diese beiden keineswegs so sebr von einander verschieden, als die Varietäten der $T$. trigonella, ler Jura-Formation in verschiedenen Gegenden sind, die gleichwohl auf beiden Seiten durch manchfaltige Zwischen-Formen vermittelt wer. den ${ }^{5}$ ). Gehörte dagegen die St. Cassianer Formation mit zur Trias, so wären unter anderen Nucula Münsteri $(\mathbf{h}, \mathbf{k}, \mathbf{I}, \mathbf{m})$, N. nuda $(\mathbf{h}, \mathbf{m})$ und $\mathbf{N}$. subovalis $(\mathbf{h}, \mathbf{m}, \mathbf{n})$ als Binde-Glieder anzuführen.

III - IV: Oolith und Kreide sind zwar an vielen Orten durch eine Brackwasser. Formation von einander getrennt; dennoch kommen ihnen einige Arten gemeinsam zu. Angefithrt werden in

n-r: ! Terebratula biplicata, Serpulit gordialis.

no-q: Lyriodon clavellatus ${ }^{6}$ ), L. gibbosus.

n-r: Terebratula sella und Ammonites decipiens, D'Archisc ${ }^{7}$ ).

3) Jahrb. 1841, 793.

2) In meinen Collectaneen I, 80.

3) Das. I, $85 \mathrm{ff}$.

4) Das. I, 104.

5) Vergl. der Literatur wegen den Nomenclator p. 1253 und die Lethaea.

6) Jahrb. 1839, 735 .

7) Das. 1841, 796. 
n-q: Serpula tricarinata, Lyriodon costatus.

o-r: Cidaris Schmiedeli.

p-q: ! Unio Martini, sehon oben genannt.

Eine grosse Anzahl von Arten, welche der Oolithen- und der Kreide. Formation gemeinsam wären, stellt ans anderen Autoren D'Arснıсс 1) zusammen; da indessen viele derselben einer erneuerten Bestimmung bedürfen, so übergehen wir sie hier.

IV - V: Kreide- und Tertiär-Periode sind im Grossen schärfer als dic anderen gegen einander abgegrenzt, einerseits durch das plötzliche Verschwinden aller Ammoneen, Belemniten und mehrer Brachiopoden-Genera mit der Kreide und andrerseits durch das Beginnen der Säugthiere in den Tertiär-Bildungen. Dennoch haben wir oben (S. 754) unter $\mathbf{C}+\mathbf{s}$ und $\mathbf{r}+t$ mehre gemeinsame Arten aufgeführt und können noch andere namhaft machen, wenn wir tiefer theils in das Kreide- und theils ins Tertiär-Gebirge hineingreifeu wollen; 80 in

Formationen :

$\boldsymbol{q}+\mathbf{s}$ : Serpula heliciformis; Pecten quinquecustatus ${ }^{2}$ ).

qrf + w: Heteropora stellata und H. cryptopora.

? $\mathbf{r}+$ stw?: Exogyra (Ostrea) lateralis ${ }^{3}$ ).

$\mathbf{r r}+\mathbf{s t}$ : Terebratula Defrancei ${ }^{3}$ ).

rr + : Serpula quadricarinata ${ }^{3}$.

r t : Terebratula tenuistriata LEYM. ${ }^{3}$ ).

rta: Orbitulites macroporus.

r uv: Pyxidicula prisca.

r $+\mathbf{w}$ : Terebratula chrysalis 4), Globulina globosa, Textilaria triquetra.

$\mathbf{r}+\mathbf{x}$ : Textilaria striata.

$\mathbf{r}^{2}+\mathbf{u}$ : Reuss hat 8 Polyparien der Mastrichter Kreide (Terrain danien) unter den 207 Arten des Wiener Beckens wieder erkannt ${ }^{5}$ ).

Die älteren Angaben đes Vorkommens identischer Arten in der Kreide - und Tertiär-Periode findet man gesammelt von D'Archisc 6), bei denen wir jedoch nicht verweilen wollen, weil abermals wenigstens ein Theil davon auf unrichtigen Bestimmungen der Arten oder der Formationen beruhet.

V-VI: Die Tertiär-oder Molassen-Periode hat mit der jetzigen Schöpfung eine sehr grosse Auzahl von Arten gemein. DEsнayes hatte bekanntlich 1831 auf den Grund sehr sorgfältiger Vergleichungen und Bestimmungen hin angenommen, dass die alt-tertiären Schichten 0,03, die mittel-tertiären 0,19 und die ober-tertiären 0,52 ihrer sämmtlichen Konchylien-Arten in đer lebenden Schöpfung wiederfänden "). Wir

1) Jahrb. 1841, 796.

2) Durrenoy in Jahrb. 1844, 751. D'Ornignv frennt jetzt letzte Art in zwei.

$\left.{ }^{3}\right)$ Über diese Exogyra, Terebratula und Serpula vergl. Jahrb. 1844, 755 ; 1845,$240 ; 1848,74$. - Die Guettardia stellata und vielleicht auch Spondylus (Plagiostoma) spinosus aus der weissen Kreide sollen sich in dem Nummuliten-Gebirge von Biaritz nur auf sekundärer Lagerstătte finden. Deshay. > Jahrb. 1845, 241; Michelin Iconogr. Zoophytol. p. vir.

4) Diese Art wird nun wohl nur $\mathbf{r}+t$ gemeinsam seyn, nachdem die Magdeburger Thone sich von höherem Alter ergeben haben (Jahrb. $1848,766)$.

3) Jahrb. 1848, 759 .

6) Das. 1811, 797 .

7) Zahlen, die mit den etwas früher von uns nach unzureichenderen Materialien gefundenen ziemlich übereinstimmteu; - obwohl Desнaves später 
hatten nachgewiesen, wie manchem Wechsel diese Quoten oder Prozente in verschiedenen Lokalitäten unterworfen seyen; eine grosse Anzahl Konchyliologen hat früher und später dieses Vorkommen identischer Arten in der übrigen tertiären und der lebenden Welt anerkannt (Grateloup, Deshayes, Partsch, Nyst, Cantraine '), Renieri, Brocchi, Philippi u. v. A.). Lrei.c hat sodann 5 tertiäre GebirgsGlieder angenommen, nämlich

$$
\begin{aligned}
& \text { eocäne mit : . 0,01-0,02 } \\
& \text { miocäne . . } 0,20-0,30
\end{aligned}
$$

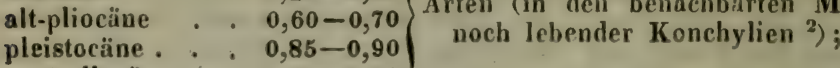

$$
\begin{aligned}
& \text { post-pliocäne : : } 0,99-1,00
\end{aligned}
$$

nachdem nämlich Paı́ıPPI gezeigt, dass in den ober-tertiären Schichten verschiedener Lokalitäten alle Quoten - Abstufungen lebender Arten von 0,56 bis 0,99 vorkommen können. Agassiz' Versuch die spezifische Verschiedenheit der fossilen obcrtertiären Arten von den mit ihnen für identisch gehaltenen lebenden im Einzelnen nachzuweisen, hat uns Gelegenheit gegeben, Diess für eine grosse Anzahl Arten zu widerlegen und ihre Identität mit lebenden zu bestätigen; R. Owen, H. v. MeYer u. A. haben solche Identitäten auch für die Säugthiere dargethan.

a. Der eocänen Arten, welche nach Deshayes auch noch lebend vorkommen sollten, waren unter 1400 untersuchten Spezies 38 , gegen deren einige jedoch er selbst später $\mathbf{Z}$ weifel erhob und sie in mehre Arten trennte. Unter den eocänen Organismen-Arten, welche noch jetzt lebend vorkommen sollen, werden aufgezählt: Guttulina caudata po., die sich auch in den $\mathrm{Z}$ wischenSchichten findet; Globulina gibba D'O. und Truncatulina tuberculata, ebenso; Rotalia gyroidina und Spirolina cylindracea, welche sie überspringen; Biloculina bulloides und B. longirostris, welche wieder dazwischen vorkommt; Quinqueloculina laevigata, ebenso; Qu. plana, überspringend; - Echinocyamus pusillus (in $\mathbf{u} \mathbf{w z}$ ) wach Forbes ${ }^{3}$ ); - Ostrea edulis, andauernd, doch in Varietäten; Arca barbata und A. Helbingi, andauernd; Nucula pella, $N$. pygmaea und N. sulcata, ebenso; Lucina gibbosulit, L. renulata, L. divaricata [?], Tellina crassa [?], Gasterochaena gigantea, Mactra triangula [?], Solen coarctatus [?], Clavagella tibialis [?]: - Dentaliun entalis, D. incrassatum, D. fissura, Fissurella graeca, ! Niso terebellum, Eulima distorta [?], Turritella triplicata, Rissoia cochlearella (varr.), Melanopsis acicularis, M. costata, M. praerosa, Chenopus pes-pelecani (varr.), ! Tritonium clathratum, $\mathrm{Tr}$. nodularium [?], Typhis tubifer, Terebra plicatula [?], Voluta magorum und V. mitriformis, Ancillaria canalifera, !Oliva flammulata, O. Laumontana [?], Bullina spirata, Bulla lignaria, B. miliaris; - - Ditrypa gadus $[\mathbf{t}$ w $\mathbf{z}]$, D. subulata [s u w z] "), Serpula minima und S. proteusa, von welchen Arten freilich gar manche zweifelhaft sind, indem die bis in die jetzige Schöpfung herabreichenden Formen etwas abweichend, öfters als Varietälen, zuweilen aber auch als besondere Arten beschricben worden sind; - PAlanzen, so wie höhere Thiere noch lebeader Arten kenut man in den Eocän.Schichten vicht.

mehre jener eocänen Arten gänzlich von den gleichnamigen lebenden unterschied und D'OrBIGNY das Vorkommen Jebender Arten in eocänen Schichten gavz läugnet, da er sie alle unterscheidbar findet.

1) Jahrb. 1848, 638 .

2) Das. 1848, 738 .

3) Das. 1846,873 .

4) Brons, Italiens Tertiäı-Gebilde S. 169-170.

s) Enumerator p. 546; Jahrb. 1848, 864. 
b. Die miocänen Arten, welche noch lebend vorkommen, sind nach DesHAYES fast 200 unter 1000 Konchylien-Arten und wenn von diesen auch in Folge richtigerer Bestimmungen einzelne abgehen, so kommen in Folge neuer Entdeckungen immer wieder andere hinzu '); fast alle diese Arten sind bereits auch in den pliocänen Schichten bekannt. In der übersichtlichen Tabelle unseres Enumerators wird man diese zahlreichen Arten schnell überblicken, daher ihre Aufzählung hier nicht nothwendig ist. Auch hier kann man mit Bestimmtheit weder Kerbthiere, noch Wirbelthiere, noch Pflanzen lebender Arten nachweisen. Die Insekten von Aix dürften nach HEER alle von den lebenden abweichen.

c. Wir hahen dic Molasse und die Braunkohlen-Bildung mit den BernsteinSchichten zwischen die miocänen und cocänen Schichten geste!lt, weil wir nicht. sicher waren, wohin sie gehörten, indem ihre Wirbelthiere mehr für miocänes, die Weichthiere für pliocänes Alter sprachen und die letzten mithin grösstentheils mit lebenden Arten übereinstimmten. Was aber die übrigen Organismen-Klassen betrifft, so ist höchst merkwürdig, dass die

Pflanzen der Schweitzer u. a. Braunkohlen nach HEer und Göppert,

Pfla nzen des Berusteins nach GöPPERT,

Insekte n der Braunkohle und des Bernsteins nach Germar u. A.,

Insekten von öningen nach O. HeEr

alle von den lebenden spezifisch verschieden sind; wozu wir freilich bemerken müssen, dass GöPPERT eine Anzahl solcher Pflanzen für verschieden ausgibt, weil sie ungeachtet aller Übereinstimmung mit den lebenden hinsichtlich der erhaltenen Fruktifikations- u. a. Theile doch in den nicht erhaltenen Theilen abweichen könnten (vgl. S. 767).

d. Die pliocänen Schichten haben Deshayes's unter 700 Arten über 360 $(0,52)$ lebende Konchylien.Arten geliefert. Ich hatte 1831 deren etwas weniger gefunden, nämlich $0,40^{2}$ ). PHILIPpI weiset nach einzelnen Örtlichkeiten in Italien und Sisilien alle Abstufungen von 0,56 bis 1,00 noch lebender KonchylienArten nach.

In Sicilien:

Ganze 'Zahl be- Noch lebende Arten. kannter Arten. Zahl. Quote.

um Buccheri, Callagirone, Caltaniselta, Castrogiovanni,

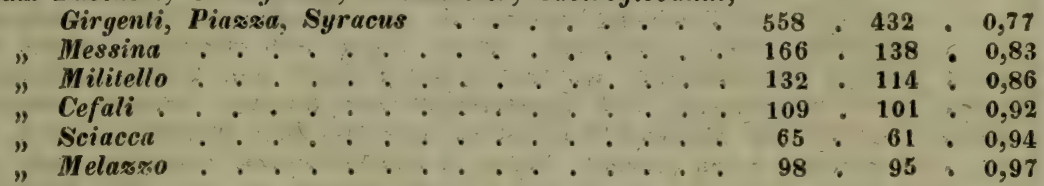

In Calabrien:

zu Cutro zwischen Catansaro und Crotone . . . $69.39 \cdot 0,56$

im Thale des Lamato : . . . . . . . . . . 107 . 67 . 0,60

zu Gravina in Apulien . . . . . . . . 173 . 135 . 0,78

zu Pezso, Messina gegenüber . . . . . . . . . 82 . $67 \cdot 0,82$

zu Carrubare bei Reggio . . . . . . . . . . . $129,115,0,89$

zu Monteleone . . . . . . . . . . . . . . 59 . 54 . 0,92

zu Tarent . . . . . . . . . . . . . . . . 162 . $153.0,94$

auf Ischia . . . . . . . . . . . . . 156 . 154 . 0,99

beim Monte Nuovo . . . . . . . . . . . . . 99 . $99 \div 1,00$

in Posquoli

In Calabrien und Sicilien im Ganzen . . . . . . 576 . 383 . 0,66

1) Wie durch JefFreys im Jahrb. 1849, 496.

2) Bronn, Italiens Tertiär-Gebilde S. 170. 
Darehgehen wir aber das ganze System, so finden wir bei den meisten Klassen, wenigstens der Thiere, ein ähnliches Verhältniss. Von Polygastrica ist eine grosse Anzahl von Arten lebend und pliocän zugleich, letzte besonders aus dem Diluviale vorhanden; obschon man die aus fremden Welttheilen stam. menden Arten sehr oft nur erst im fossilen Zastande kennt und sie lebend erst kemen lernen kann in dem Maase, als man an Ort und Stelle selbst das Mikro. skop anzuwenden im Stande seyn wird. So ist es bei den Foraminiferen (oder Polythalamien), bei den Bryozoen, den Anthozoen; weniger zahlreich sind die noch lebend vorkommenden Spezies unter den Echinodermen, Hexapoden, Myria. poden und Arachniden; wieder etwas häufiger bei den Crustaceen; sie fehlen bei den Fischen und Vögeln, sind dagegen sehr zahlreich bei den Säugethieren. Wir verzichten darauf, darüber Tabellen zusammenzutragen, weil die Vergleichungen sehr zufällige Ergebnisse liefern, welche einestheils von der Art der einschliessenden Gebirge und der Erhaltungs-Fähigkeit der verschiedenen Thier-Klassen, anderntheils von den Ansichten derjenigen Geologen sehr abhängig sind, welche sich mit der Untersuchung dieser Reste vorzugsweise beschäftigt haben. Im Allgemeinen erkenuen wir die meisten noch lebenden Arten in denjenigen Thier-Klassen, welche Meeres-Thiere mit kieseligen und kalkigen Schalen einschliessen, die sich also auch in den manchfaltigsten Meeres-Formationen leicht und kenntlich erhalten (Polygastrica, - Mollusken, Anneliden, Foraminiferen, Crustaceen, Korallen), wobei jedoch jene Gruppen Modifikationen veranlassen, welche nur tropischen Meeren oder früheren Erd-Perioden zusteben (Stylastriten, Ammoneen, Brachiopoden, - Anthozoen); - wir finden an wenigsten von solchen Land- und Luft-Thieren, welche nur durch Zufall in regelmässige Formationen einzeln eingeschlossen zu werden pflegen und noch zufälliger sich darin kenntlich erhalten (Arachniden, Hexapoden, - Vögel). Nur der Mangel noch lebender Fisch-Arten in den Pliocän-Schichten befremdet anfangs mehr; indessen muss man sich erinnern, dass die jüngsten MeeresFormationen der Erhaltung fossiler Fische nicht günstig sind, sondern selbe in ihre einzelnen Wirbel und Gräten auseinanderfallen lassen; andererseits scheint Agassiz, dem wir bis daher die Bestimmung fast aller fossilen Fische verdanken, in einigen Fällen wenigstens die jüngsten (pleistocänen) fossilen FischArten mitunter durch sehr unbedeutende Merkmale von den lebenden getrennt zu haben. Dass aber auch von den höchsten Wirbelthieren, von den Säugthieren sogar viele noch lebende Spezies in den Diluvial-Schichten zusammen mit ausgestorbenen Arten vorkommen, Diess ergibt sich ebenfalls sogleich bei Ansicht nuseres Enumerators, wo an 70 Arten fossil und iebend aufgezählt werden, deren Bestimmung sich grossentheils auf Richard Owen's Autorität grüudet, welcher für England ') unter 40 nen-pliocänen Säugethier-Arten 30 $(0,75)$ noch lebende Arten erkennt. Eben so weiset Corton neuerlich unter 42 Säugethier-Arten bloss der englischen Knochen.Höhlen 20 von noch lebenden Arten nach, die mithin fast 0,50 der Gesammtzahl ausmachen ${ }^{2}$ ). Wir berufen uns auf diese herausgehobenen Beispiele lieber, als auf das aus unserem Enumerator sich ergebende Resultat, weil sie auf dell speciellsten und sorgfältigsten Bestimmungen eines ausgezeichneten Zoologen (OWEN) beruben. Eben so bezeichnend in dieser Hinsicht und zugleich für die Stellung des Gebildes im Systeme selbst sind die Reste im Lösse unter den sogenannten Diluvial-Gebilden, worin AL. Braun 30 Arten Binnen-Konchylien gefunden, mit nur 3 ganz untergegangenen Spezies, während in verschiedenen Gegenden Knochen ausgestorbener Säugthiere aus der letzten Tertiâr-Zeit darin angetroffen worden sind, wic von Elephas primigenius, Rhinoceros tichorhinus,

1) A history of British fossil Mammals and Birds, London 1846; > Jahrb. 1846,632 .

2) Jahrb. 1848, 765. 
PUrsus spelaeus, woraus eben hervorgeht, dass selbst in einem und demselben Gebilde versehiedene Thier-Klassen verschiedenen Gesetzen unterliegen, was sich auch in andern Fällen bestätigt '). Diese ausgestorbenen WirbelthierArten siod dieselben, welche in Italien im Subapenuinen-Gebilde mit SeeKonchylien zusammenliegen, die ebenfalls nur $0,48-0,01$ ausgestorbener Arten enthalten.

e. Übrigens erstrecken sich diese von $\mathbf{s}$ und $\mathbf{t}$ bis in die lebende Schöpfung reichenden Ärten nicht alle über alle Tertiär-Schichten, sondern überspringen bald die eine und bald die andere derselben, so weit nämlich unsere jetzigen Kenntnisse reichen.

F. Einige Arten hat man in drei zusammenhängenden Perioden gefunden. So in :

III, IV, V: Serpula gordialis: nur mitunter etwas in der Grösse verschieden ${ }^{2}$ ).

IV, V, VI ! Terebratula caput-serpentis nach ForeEs $(\mathbf{r}+\mathbf{w x}+\mathbf{z})$, T. truncata $(\boldsymbol{f}+\mathbf{w}+\mathbf{z})$, ! Dentalina communis $(\mathbf{r}+\mathbf{w w}+\mathbf{z})$, ! Planulina turgida Eв. $(\boldsymbol{r}+\mathbf{v}+\mathbf{z})$, ! Globigerina bulloides o'0. $(\boldsymbol{\Gamma}+\mathbf{u} \mathbf{w}+\mathbf{z})$, ?Rotalia umbilicata n'O. ( $+\mathbf{f}+\mathbf{z})$, ! R. perforata; ! R. globulosa EB., Textilaria globulosa $\mathbf{E}_{B}$. $(\mathbf{f}+\mathbf{v x}+\mathbf{z}),-$ ! Fragilaria rhabdosoma $(\mathbf{r}+\mathbf{v} \mathbf{w} \mathbf{x}+\mathbf{y z}), \quad$ ! $\mathbf{r}$. striolata $(\mathbf{r}+\mathbf{v}+\mathbf{y} \mathbf{z})$, ! Gallionella aurichalcea $(\mathbf{C}+\mathbf{v}+\mathbf{y z})$, Spongilla lacustris und Sp. erinaceus EB. $(-\boldsymbol{r}+$ tuvwr $+\mathbf{y z})$, wobei indessen $z u$ bemerken ist, dass die 2 letzten Arten nach isolirten Schwamm-Nadeln bestimmt sind, weiche immerhin eine Unsicherheit über die Art übrig lassen.

G. Wenige Arten werden in zwei verschiedenen Erd-Perioden gefunden, weiche durch eine dritte getrennt werden, worin diese Arten nicht vorkommen. Auch Diess wiederholt sich zu verschiedenen Zeiten; auch hier lassen sich die Bestimmungen in einigen Fällen noch in Zweifel ziehen. So in den:

Perioden

I und III : $(\boldsymbol{d}+\mathbf{m})$ haben nach Ac. Brongniart und Éche ne Braumont zunächst mehre Pflanzen der Steinkohlen-Formation bei Petit-Coeur und am Col de Chardonel ${ }^{3}$ ) gemein, welehe in der Tarantaise in der durch erst noch neuerlich von Sismonds zahlreich aufgefundene ${ }^{4}$ ) Petrefakte wohl charakterisirten Lias-Formation vorkommen; doch wäre vielleicht cine Revision jener Pflanzen-Bestimmungen zu wünschen ${ }^{5}$ ). Diese Pflanzen sind:

1) Jahrb. 1841,$606 ; 1842,588 ; 1843,583$ und Collectancen $62-65 ; 1845$, $356 ; 1847,49,185,365 ; 1848,469$. Wegen der dem Lösse nahe stehenden Kalk. Tuffe vergl. die Collectaneen a. a. O. und Jahrb. 1842, 229, $590 ; 1844,28$.

2) Vergl. Enumerator S. 549, und Jahrb. 1844, 753.

3) Vergl. Annal. d. scienc. nat. 1828, XIV, 113 , und $\mathrm{XV}, 353$; vergl. auch UNGER im Jahrb. 1842, 607.

4) Im Jahrb. 1848, 746 .

5) Bunbury hat so eben durch neue Untersuchungen nicht nur den Charakter der Pflanzen ganz der Steinkohlen-Farmation entsprechend gefunden, sondern auch wenigstens einige Arten dieser Formation mit Sicherheit wieder erkannt, wie Neuropteris flexuosa, Odontopteris Brardi, Pecopteris cyathea, Calamites approximatus, Annularia longifolia. Vergl. Lond. geolog. Quartj. 1849, V, 130-142. 
Calamites canuaeformis.

" approximatus.

" Suckowi BrGN.

". Cisti Brgr.

Stigmaria ficoides.

Asterophyllites equisetiformis BGN.

Volkmannia erosa Ste.

Annularia brevifolia STB.

Sigillaria Brardi Brgs.

" ? tessellata

") ? notata.

Neuropteris alpina Stв.

" gigantea Bron.

" tenuifolia Brgv.
Neuropteris flexwosa BrgN.

" $\quad$ Soreti BrGN.

Odontopteris Brardi BRGN.

obtusa BrgN.

Pecopteris plumosa BrgN.

" Beaumonti Brgn.

" arborescens BRGN.

" polymorpha BRGN.

" pteroides BaGN.

" platyrachis BRGN.

" Pluckeneti BRGN.

Lepidodendron ornatissimum $\mathrm{B}_{\mathrm{GN}}$. ? crenatum.

Ebenso haben wir selbst Posidonomya Becheri der Devon-Formation auch in den Lias-Srhiefern angenommen, da sich beide Formen bis jetzt nur durch die verschiedene Grösse unterscheiden lassen, wesshalb man denn auch die kleinere Lias-Form P. liasina genannt hat.

IV u. VI : Es finden sich im Grünsand und lebend zugleich $(\mathbf{r}+\mathbf{z})$ Arbacia pustulosa AG., sehr ungewiss; dann in weisser Kreide und lebend zugleich $(\mathbf{f}+\mathbf{z})$ Planulina argus Ez., Xanthidium furcatum und $\mathbf{X}$. hirsutum EB.

H. Noch andere Arten sollen in noch grösserer Ausdehnung mit ansehnlichen Unterbrechungen vorkommen. So:

I, III, IV : Galeolaria socialis wird in $\mathbf{c}, \mathbf{n}, \mathbf{q} \mathbf{r}$ angeführt, obgleich daselbst wahrscheinlich nur ähnliche Arten getroffen werden, welche sich bei unmittelbarer Vergleichung wohl unterscheiden liessen.

I u. V: Diplazites enarginatus GöPr., eine Pflanze der Steinkohlen-Formatien, soll nach Unger (a. a. O. 1842.) auch in den Braunkohlen von Parschlug vorkommen (e $+\mathbf{w}$ ). Doch hat UNGER in seiner Synopsis 18.15 die Angabe nicht wiederholt, aber auch eiuen grossen Theil der zuvor erwähnten Tarentaiser Kohlen-Pflanzen nicht für die dortige abweichende Formation (m) aufgeführt, wie sie Göpprat in unseren Enumerator nicht eingetragen hat (s. o.).

I u. VI: Endlich glaubt EnRenberg in einer Quarz-Niere der Kohlen-Formation eine leichte Varietät des lebenden Peridinium monas erkannt zu haben.

1. Zwar kann man gegen die Richtigkeit eines Theiles der Bestimmungen der bisher aufgezählten Arten Zweifel erheben, wie wir selbst mehrfach angedeutet haben. Es gibt jedoch mehre praktische und durch ihre speziellen Untersuchungen höchst achtenswerthe Paläontologen (um die blossen Nachsprecher zu übergehen), welche es aus vorgefassten Theorie'n in Abrede stellen, dass eine Art in zwei verschiedenen Formations-Gliedern oder wenigstens Formationen zugleich primitiv vorkommen könne, und desshalb alle gegentheiligen Angaben als auf solchen unrichtigen Bestimmungen der Arten oder Formationen beruhend ansehen. Immerhin aber gibt es eine Anzahl unantastbarer und sogar vou jenen Gegnern in einem unbewachten Augenblicke oder unter Hinzufügung irgend einer besonderen Erklärung ausdrücklich zugestandener und in allen diesen Fällen von uns mit ! bezeichneter Arten, welche nicht uur in 2, sondern sogar bis in 3 Perioden zu- 
sammenhängend oder mit Überspringung der mitteln verbreitet sind. Wir bestehen hiebei mehr auf Darstellung der Sache, als auf Ausmittelung der Anzahl wirklich vorkommender Fälle, welche nur nach Revision der Bestimmungen und nach monographischer Behandlung aller dieser Arten möglich werden würde.

J. Nach unserer Überzengung, die wir in $\S .19 \varsigma, C, S .750 \mathrm{ff}$. ausgedrückt, kann das Auftreten und sogar das Wiederauftreten identischer Arten in verschiedenen Zeiten, wie es uns praktisch entgegenkömmt, auch theoretisch nicht geläugnet werden; wir kennen weder ein Ereigniss, noch ein allgemeines Naturgesetz, welches die Dauer der Spezies nur auf eine kurze Zeit beschränkte, oder ihre Wiedererschaffung in späterer Zeit unmöglich gemacht hätte. Wir müssen dalıer das Vorkommen identischer Arten in verschiedenen Zeiten zugestehen, wenn wir nicht in Stande sind, sie praktisch zu unterscheiden.

Die entschiedensten und zugleich gewichtigsten Gegner einer Zulassung von einerlei Art in zweierlei Formations-Gliedern oder Formationen und gar Perioden sind Alcide d'Orbigny und unsere Freunde Agassiz und Göpprrt.

Agassiz behauptet überall, dass keine Art in 2 Formationen, geschweige denn in 2 Perioden vorkomme. Er gibt daher anch zwischen der geologischen und der jetzigen Zeit auch nicht eine gemeinsame Art zu und gestattet nur zwischen älteren Perioden einige Ausnahmen, indem er daselbst die Mehrzahl der Identitäten verwirft. So an einer Stelle '): il n'existe point d'identités entre les éspèses fossiles et vivantes, et toutes celles, que l'on admet encore de nos jours, reposent sur des fausses determinations ... II est de même de la plupart des autres identités, que quelques géologues persistent à vouloir admettre; ... il n'existe point de liaison directe au point de vue zoologique entre les differentes époques géologiques, et chaque époque a eu sa faune propre. Die seiner Ansicht widersprechenden Beobachtungen erläutert er aber auf folgende Weise: „dass kein sogenannter Charakter, d. h. kein wahrnehmbares Zeichen „so auffallend seyn kann, um absolut spezifische Unterschiede anzudeuten, aber "auch an sich nie für so gering gehalten werden darf, um absolut auf Identität "hinzuweisen; dass überhaupt Charaktere die Art nicht abmarken, wohl aber "das Gesammtverhalten zur Aussenwelt in allen Umständen des Lebens. Er "zweifle daher nicht daran, dass man dereinst die spexifische Verschiedenheit "der organischen Überreste narh den Umständen ihres Vorkommens wird aus"sprechen müssen, ohne Unterschiede zwischen denselben angeben zu können ${ }^{2}$ )." Diese Worte sind zwar nach unserer eigenen Überzeugung (vgl. S. 747 ff.) nicht ohne inneren Gehalt an Wahrheit; aber wenn man sich erinnert, dass a) auch das Gesammtverhalten zur Aussenwelt bei verschiedenen Individuen piner Art unter verschiedenen Bedingungen so aussernrdentlich weit auseinandertreten kann, dass es nur neue $Z$ weifel erweckt, statt die alten zu schlichten; b) dass dieses Gesammtverhalten im Leben nie als Kriterium an todten Exemplaren anwendbar ist, c) dass bei fossilen Körpern überhaupt kein "Verhalten zur Aussenwelt in allen Unständen des Lebens" mehr stattindet und das früher stattgefundene sich nur allein aus der Natur der Gebirgs-Schicht und aus der Gesellschaft der mitvorkommenden fossilen Arten erschliessen lässt, so muss man

1) Jahrb. 1841, 356 .

2) Im Bulletin de la Société des sciences naturelles de Neuchatel, 1843-1844, p. $107,108$. 
zugestehen, dass die Hülfe, welche solcher Grundsatz dem Palz̈ontologen bei Bestimmung der Art gewähren kaun, cben keine grosse ist; dass er, wenn auch Natur und Augenschein noch so sehr dagegen sprächen, zur Folgerung führen muss: "Gleiche Schichten gleiche Arten, ungleiche Schichten ungleiche Arten", zumal wir ja eben oft die Natur, das Alter der Schichten selbst aus den Arten zu erschliessen genöthigt sind, uns also in einem Ringschlusse bewegen würden. Unter solchen Verhältnissen würde jedenfalls die unbefangenste - nicht einer vorgefossten Meinung angepasste, sondern zu einem endichen Resultat führende - Weise die seyn: Was man spezifisch zu unterscheiden keine Mittel hat, in eine Art zu verbinden; die Erklärung aber des Vorkommens wirklich oder scheinbar identischer Arten in verschiedenen Formationen, wenn man nicht die einfache Thatsache als solche hinnehmen will, mag dana Jeder nach seiner Ansicht versuchen, nicht aber die Thatsache um dieser Ansicht willen verdrehen, oder eiu unbefangenes Resultat von vornherein unmöglich machen. - Aus jenem obigen Gı undsatze erklären sich denı auch die Schwankungen bei Agassiz und seinen Schülern hinsichtlich der Trennung von Individuen zweier Formationen als Arten, obwohl ihnen die Merkmale abgehen ${ }^{3}$ ).

Alcior d'Orbigny hat in der französischen Akadenie wie anderwärts wie. derholt crklärt, dass die untergegangenen Thier-Arten nirgends den Schluss der von ihm aufgestellten Formationen überdauert hätten, und gleichwohl führt er selbst Belege des Gegentheils ein, wo einzelne Arten in andere Formationen übergehen, ja durch zwei Perioden hindurch gehen oder eine nittle liberspringen: Diess ist mit einigen Foraminiferen der Kreide der Fall, die von noch lebenden und tertiär vorkommenden $z u$ unterscheiden unmöglich sey; diese und audere von ihm gelieferten Beispiele werden wir unten aufzählen. Er hat für verschiedene Fälle zugleich verschiedene Erklärungen. Hinsichtlich einiger Cephalopoden der Kreide.Periode nimmt er an, dass sie keineswegs zur Zeit von 2 successiven Formationen gelebt hätten, sondern dass Schaalen ein. zelner Individuen dadurch aus der mitteln in die obere Kreide-Formation tibergegangen seyen, dass solche nach dem Tode des Thieres dureh den Luft-Gehalt ibrer Kammern über dem Wasser gehalten, ohne oder mit Unterbrechung bis zur Zeit des Niederschlages der nächsten Formation auf dem Meere umhergetrieben und endlich mit dieser letzten abgesetzt worden seyen. Wir haben keine Erfahruug darüber, bezweifeln aber doch, dass eine den VerwitterungsEinflüssen ausgesetzte Schaale auch nur einige Jahre lang im Wasser liegen oder damit in Berührung bleiben könne, ohwe sich damit wenigstens so weit zu füllen, um untersinken zu müssen; uuch genügt nach VoLrz schon ein geringer Luftdruck, um Wasser z. B. durch eine frische Spirula-Schaale eindringen zu machen. Natürlich reicht diese Ansicht für einkammerige und zweischatige Konchylien so wie für andere fossile Reste nicht aus. $\mathrm{Da}$ hat sich denn D'OrBigny zur Annahme einer Erklärung genöthigt gesehen, die er mir mündlich vertraut hat, indem er sagte, „wenn eine, seye es auch absolut identische Form von "Organismen, in zwei entfernten Perioden wiederkehrt, so dass man annehmen "muss, sie seye inzwischen erloschen gewesen und nachher neu erschaffen "worden, so ist es für mich eine neue, wenn auch nicht unterscheidbare Art". Wenn wir uun aber nicht wissen, noch je werden beweisen können, ob alle zu je einer Art gezäblten Individuen wirklich von einem, oder ob sie von mehren gleichzeitig nebeneinander erschaffenen Ältern-Paaren abstammen, so werden wir auch nicht behaupten können, dass zwei Gruppen von Individuen, die von einander in keiner Weise unterscheidbar sind, desshalb swei verschiedene Arten bilden müssen, weil sie von zwei nacheinander geschaffenen Ältern-Paaren abstammen. - Auch Qurssted hat sich für die Annahme der Wiedererschaffung von Übergangs-Orthozeratiten-Arten in dem zum Muschelkalk (oder nach ihm selbst gar zum Neocomien) gehörigen Ammoniten-Marmor Süddeutsch-

1) Vergl. Jahrb. 1842, 485 u. a. 
lauds erklärt, da zwischen beiden Bildungs-Zeiten ein so grosser Zwischenraum liegt; vollständige Exemplare indessen werden wahrscheinlich erweisen (v. Haver), dass hier wenigstens diese Hypothese unnöthig ist.

Göppent in Bezug auf die fossilen Pflanzen geht zwar nicht von dem ausgesprochenen Grundsatze aus, dass einerlei Art in zweierlei Formation nicht vorkommen könne; er wagt aber auch bei vollständigcr anatomischer Überein. stimmung zwischen zwei fossilen Hölzern oder zwischen einem fossilen und einem lebenden Holze, oder $\mathrm{zwischen} z$ weierlei solchen Blättern und selbst Blüthen, Fruchtständen u. s. w., soferne er nicht alle Theile der ganzen Pflanze zur Vergleichung mit der ihr ähnlichen Art beisammen hat, die Identität nicht auszusprechen und sieht sich hiedurch genöthigt, fossile Pflanzen.Theile, die er von den entsprechenden lebenden Arten zu unterscheiden nicht im Stande ist, in beiden Fällen streng konsequent genommen: je des einzeln gefundene Pflanzen-Bruchstück, Holz, Blatt, Frucht etc., aus einer oder aus verschiedenen Forma. tionen unter verschicdenen Namen aufzuführen, sey es, dass er dem GenusNamen die Sylbe ites anhängt oder einen ganz neuen bildet ${ }^{1}$ ). Nur indem er willkührlich seinen Grundsatz nicht auch auf solche anwendet, welche innerhalb derselben Formation an verschiedenen Orten gefunden worden sind, gibt er zu erkennen, dass er das Zugeständniss gleicher Arten in verschiedenen Formationen ungerne macht. UNGSR erkennt zwar in solehem Falle die Identität der Sippe, bezweifelt aber die der Arten auch da, wo sich die vorgefundenen Reste nicht vou den Theilen einer lebenden Art unterscheiden lassen ${ }^{2}$ ), während 0 . Hesn die Übereinstimmung tertiärer und lebender Holzarten auch durch Beibehaltung des Namens der letzteu unbefangen eingesteht, obwohl ihm die mit denselben gleichzeitigen fossilen Insekten alle von den lebenden Arten abzuweiehen scheinen ${ }^{3}$ ).

Wenn nun aber zwei Gruppen von organischen Individuen weder praktisch durch spezifische Merkmale unterschieden werden können, noch auch aus deren Auftreten in verschiedenen geologischen Zeiten, wie wir vorhin bemerkten, eine Verschiedenheit der Arten theoretisch gefolgert werden kann und sich endlich sehr oft nicht einmal mit Bestimmtheit entscheiden lässt, ob zwischen dem Vorkommen in zwei verschiedenen Zeiten eine Lücke vorhanden, also die Annahme einer neuen Erschaffung, einer neuen Art nach D'Orвigny, nothwendig ist oder nicht, so halten wir für den bei Weitem angemessensten Weg, alle diese Individuen unter einen Art-Namen zusammenzufassen, sie jedoch in solchem Falle innerhalb desselben durch beigeschlossene Bezeichnung der Formation zu gruppiren und Jedem dann selbst zu überlassen, welche Ansichten er sich über solche identische Arten in verschiedenen Formationen bilden und festhalten wolle, da wir den Vertheidigern entgegengesetzter Ansicht gerne zugestehen, dass es wahr seye, dass dic meisten Theile des Skelettes verschiedener lebenden Fuchs - oder Hasen-Arten, wemn nicht die vollständigen Skelette vorliegen, dass viele lebende Helix-Arten, wemn sie ihre Epidermis verloren haben, viele Finus-Arten, ohne Zapfen, u. s. f. nicht von einander unterschieden werden können. Doch haben auch manche tertiäre Konchylien ihre Farben bewahrt, welche wie in allen Theilen der Form, ja selbst in den Farben mit den lebenden übereinstimmen.

Die Voraussetzung, dass in verschiedenen Formationen alle Arten verschieden seyen, hat allerdings auch das Gute, dass deren Repräsentanten überall mit schärferem Auge verglichen und geprüft werden, als ausserdem, dass wir mehr Formen genauer unterscheiden lernen, welche man vorher bequem in eine

J) Jahrb. 1840, 570;1841, 366 .

2) Jahrb. 1848, 507 .

3) Jahrb. 1846, 213. 
Spezies zusammengeworfen; sie führt aber bei starrêr Durchführung endlich auch dahin, dass sogar wirkliche Varietäten und endlich Individuen-Gruppen als Arten aufgeführt werden und der Begriff der letzten sich gäuzlich auflöst. Daher hat es denn allerdings seine grossen Nachtheile, wie Agassiz in seiner Schrift auseinandersetzt, wenu wirklich verschiedene Arten miteinander verwechselt und verschmolzen werden; wir haben aber ${ }^{1}$ ) dagegen auch jene Nachtheile auseinandergesetzt, welche entstehen, wenn naturwidrig einerlei Art in 3-4 verschiedenen Spezies getrenut wird, und glauben beiden auf dem vorgeschlagenen Wege entgegengehen zu können.

Will jemand aber die Formen verschiedencr Gesteins-Gruppen alle als verschiedene Arten trennen, so ist es gänzlich nur seiner Willkühr überlassen, wie weit er dabei gehen und was er als verschiedene Gesteins-Gruppen betrachten will, da es uun einmal universelle Lïcken, Grenz-Klüfte zwischen den verschiedenen Gebirgs-Bildungen nicht gibt, welche hiebei zu Grunde gelegt werden könnten.

Nach diesen Vorbemerkungen fassen wir nun nochmals die schlagendsten, auf den zuverlässigsten und $z$. Th. gegnerischen Bestimmungen beruhenden Belege zusammen :

1) Agassiz selbst gesteht in seinen früheren Schriften zu das Vorkommen, bei den Fischen, von Psanmodus rugosus in $\mathbf{c}$, d; von Lamna elegans in $\mathbf{t}$, $\mathbf{u}, \mathbf{v}, \mathbf{w}$; von Odontaspis contortidens in $\mathbf{u}, \mathbf{v}$, w; bei den Konchylien das von Lucina (Cytherea) leonina in $u, w$ und hat - gegen seine eigene unbedingte Abläugnung aller tertiär - und - lebend vorkommenden Arten die Übereinstimmung der pliocänen Cytherea chione und Cyprina islandica meiner Samm. lung mit den lebenden dieses Namens müudlich anerkannt (wovon nachler); in einer neueren mit DrsoR gemeinschaftlich herausgegebenen Arbeit über die Echiniten ${ }^{2}$ ), die wir für den Enumerator noch nicht benützen konnten, unterscheidet er eine grössere Menge von Formationen, als wir oben angenommen, und findet darin von 1005 fossilen Arten die folgenden in mehrfachen Formationen vertheilt.

\begin{tabular}{|c|c|c|c|}
\hline & 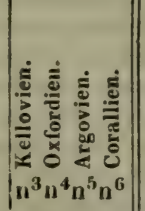 & & 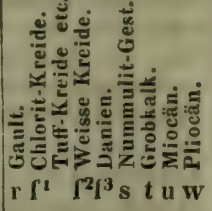 \\
\hline 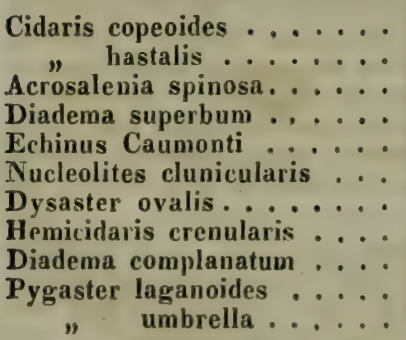 & 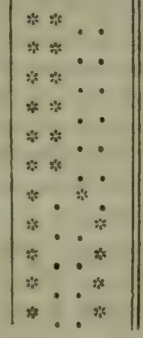 & $\begin{array}{l}\text { Holaster Greenoughi } \\
\text { Micraster cor-anguinum } \\
\text { Cidaris vesiculosa ... } \\
\text { Hemiaster prunella . } \\
\text { Micraster brevis . . . } \\
\text { Echinopsis elegans. . } \\
\text { Cidaris hirta..... }\end{array}$ & $\begin{array}{l}* \ldots \\
\cdots \\
\cdots \\
\ldots\end{array} \ldots$ \\
\hline
\end{tabular}




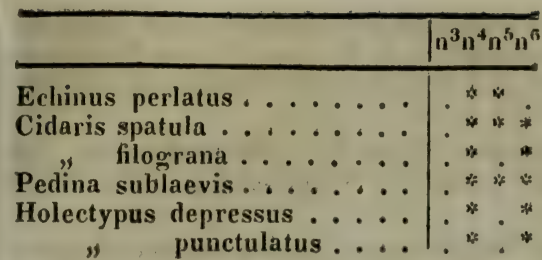

Agassiz, der sonst das Vorkommen verschiedener Arten in versehiedenen Formationen überall so sehr hervorhebt, spricht hier im Texte nirgends darüber; - noch zahlreicher würden aber diese Fälle geworden seyn, wenn er die unter $\mathbf{s}, \mathbf{t}, \mathbf{u}$, wedesmal vereinigten Örtlichkeiten nicht nach den Echiniden allein, sonderin nach ihren sämmtlichen Fossil-Resten in ihre Rubriken richtiger zusammengestellt hätte. Wir müssen indessen noch beifügen, dass a. a. O. Diadema stiperbum und Echinus perlutus nur in der angehängten Ta. belle und nicht im Texte, Cidaris hirta nur im Texte und nicht in der Tabelle in zweierlei Formationen aufgezählt werden.

2) D'Orbigny zitirt selbst Unio Martinii in $\boldsymbol{p}$ und $q$ und Ammonites latidorsatus, A. Mayoranus, A. inflatus, Hamites armatus und Turrilites Bergeri in Gault und chloritischer Kreide zugleich $(\mathbf{r}+\mathbf{r})$ '), wie er das Vorkommen mehrer lebenden Foraminiferen (Dentalina communis und Rotaliua umbilicata) in tertiären Bildungen sowohl als auch in der Pariser Kreide ausdıücklich erklärt ${ }^{2}$ ) und die Dentalina sulcata, Marginulina compressa und Cristellaria rotula der weissen Kreide auch im Grünsande aufzählt $(\boldsymbol{r}+\mathbf{r})$, um 4 anderer Fälle des Vorkommens in oberer weisser Kreide und in der Polypen-reichen Kreide von Tours und Mastricht (Terrain Danien) nicht zu erwähnen ${ }^{3}$ ).

3) Eduard Forees behauptet wach eigenen Untersuchungen und Vergleichungen Terebratula caput-serpentis in $\mathbf{r}, \mathbf{w}, \mathbf{x}, \mathbf{z}$, Echinocyamus pusillus in $\boldsymbol{t}, \mathbf{u}, \mathbf{w}, \mathbf{z}$, Panopaea mandibula (Panopaea obliquá n'O. $=-\mathbf{q}$; P. mandibula $=\mathbf{r}$ ? $\mathbf{r}$ ) in $\mathbf{q}, \mathbf{x}, \mathbf{r}$, Panopaea plicata Só. in $\mathbf{x}, \mathbf{r}$ und a. m. ${ }^{4}$ ).

4) Ehaenberg hat ebenfalls nach eigener gewissenhafter Vergleichung [und nach Ausscheidung einiger von ihm damit vermengten tertiären, miocänen,

1) Paléont. Franc., terr. crét. I, 625.

2) D'OrbignY sagt in den Mémoires de la Société géologique, IV̀, 13 et 32 in Bezug auf diese 2 Arten, nachdem er sie nach Exemplaren der Pariser Kreide beschrieben hat: Dentalina communis n'O. tabl. 89: "Son analogue se rencontre fossile dans les terrains subapennins de l'ltalie [w] et de l'Autriche [u] et vivant dans l'Adriatique. Nous avons compare entre eux plusieurs individus et nous n'avons pas trouvé un seul caraclère qui puisse séparer les échantillons de lu craie de Meudon de ceus: de l'Adriatique." Rotalina umbilicata n'O. tabl. 112: "Commune à Meudon, à St. Germain; elle est rare à Sens et en Angleterre; elle est aussi commune dans les terrains tertiaires de l'Autriche [u]; nous trouvons son analogue vivant à Rimini dans l'Adriatique, et malgré la comparaison minutietse, que nous avons faite, nous n'avons rencontré aucune difference entre les exemplaires vivants et les cxemplaires fossiles."

3) Mémoires de la Sociélé géologique, IV. Diese letzterwähnten Fälle sind: Bulimina obtusa, Textularia turris, Dentalina multicostata und Rotalina Cordierana.

4) Jahrb, 1546, 768, 
Schichten und jener, wo die Formation zweifelhaft seyn kann]') eine nicht unbeträchtliche Anzahl Polygastrica und Foraminifera in Kreide, Tertiär-Bildungen und jetziger Schöpfung zugleich gefunden und einige von D'ORBIGN angegebene Fälle lediglich bestätigt.

5) M'Cox, gewiss ein scharfer Beobachter, kann Favosites Gothlandica aus dem Devon- und Kohlen-Gebirge nach der schärfsten Vergleichung nicht unterscheiden ${ }^{2}$ ).

6) Nach R. Owen reichen nicht nur Megalosaurus Bucklandi und Poecilopleurum Bucklandi aus $n$ nach $p$ hinüber ${ }^{3}$ ), sondern auch eine grosse Menge pliocäner Säugethiere in die jetzige Schöpfung ${ }^{4}$ ). Mastodon angustidens, welches fast allerwärts als miocän gilt, auch in der Molasse vorkommt, ist nach ihm in England, nach Andern in Frankreich zugleich auch pliocän.

7) BAYLE weist ganz neuerlich ${ }^{5}$ ) gegen D'Oreigny nach, dass Ammonites Chalypso von A. Tatricus durchaus nicht verschicden seye, und dass daher diese letzte Art im Ober-Lias, Unteroolith und Oxfordthon, ganz wie Ammonites heterophyllus So. auch vorkomme; er sagt, nur in Folge eiver vorgefussten Theorie könne man behaupten wollen, dass jede Formation bloss eigenthümliche Arten führe; er könne noch mehr Beispiele nachweisen.

8) Leicht dürfte der Leser selbst Gelegenheit finden, dic ächte längliche Terebratula biplicata des obern Jura mit der der Kreide ( in Ameriku) oder die T. trigonella in Muschelkalk und Jura (h und $\mathbf{m}$ ) zu vergleichen.

Alle Botaniker sind zähe gewesen mit dem Zugeständniss, dass fossile Arten noch lpbend vorkommen; doch gesteht Az. Braun, dass sich gewisse Pflanzen-Reste zu Öningen von Isoctes lacustris, Acer campestre und Tilia grandifolia unserer lebenden Flora nicht unterscheiden lassen ${ }^{6}$ ).

So dürfen wir wohl sicher keinen gegründeten Widerspruch mehr erwarten, wenn wir zur Behauptung gelangen, dass es unzweifelhaft feststehe, dass nicht sehr selten Individuen von so übereinstimmender Form, dass sie sich weder als Arten noch als Varietäten unterscheiden lassen und desshalb unter einerlei Art-Namen aufgeführt zu werden verdienen, nicht allein iı zweierlei Formationen, sondern selbst in zweierlei geologischen Perioden vorkonmen. QUenSTELT sagt von den Species, die erst verscliwinden und dann später wieder zum Vorschein kommen, sie "erwachen wieder" ?).

Ist es nun überhaupt als erwiesen anzunehmen, dass dieselbe Art in $2-3$ geologischen Perioden vorkommen könne, so gilt Diess auch für die tertiäre und jetzige Periode, wofür wir schon so viele und gerade einige der am besten verbürgten Belege angeführt haben. Wir würden uns damit vollkonmen beruhigen, wenn nicht ÄGassiz gerade dieses letzte gemeinsame Vorkommen theils in Bulletin de Neuchutel 1813-1844 (p. 70), theils in einer cigenen Schrift ${ }^{8}$ ), wovon indessen nur der erste Theil ersehienen oder wenigstens allein uns

1) Man findet die Literatur zur Oıientirung dartiber nachgewiesen in Jahrb. $1841,729,730 ; 1843$, Collectan. 136,$142 ; 1844,756 ; 1845,239$, 369 (C. Prévost); 1846,104 (Fr. Hoffmann); 1849, 735.

2) Ann. nathist. 1849, b., III., 134 .

3) BR. Collect. 54 .

4) S. 762 unten; im Jahrb. 1846, 632,

b) Im Bullet. géol. b, V, 450. Jahrb. 1849, 498.

6) Jahrb. 1845, 166-173. - ${ }^{7}$ ) Das. 1845, 684 .

8) L. Agassiz: Iconographie des Coquilles terliaires reputées identiques avec des éspéces vivantes ou dans les differens terrains duns l'époque tertiaire, Neuchâtel 1845, $4^{0}$, ausführlicher beurtheilt im Jahrb. 1846, 250-256. 
zugekommen ist, noch bestimmter geläugnet und spezicller zu widerlegen sich bemüht hätte, was auch uns zu einer sorgfältigeren Prüfung dieser letzten Airbeit veranlasste. Agsssiz hat nämlich für seinen $Z$ weck etwa 20 lebende und fossile Arten aus den Geschlechtern Artemis, Venus, Cytherea, Lucina [in welchen allein $110 \mathrm{mal}$ ein-doppeltes Vorkommen seye es in zweierlei TertiärFormationen oder in einer solchen und dem lebenden Zustande behauptet wird] ausgewählt, um daran zu zeigen, dass die ausgewählten fossilen Arten - und so dann, analog zu schliessen, alle übrigen - von den wit ihnen verbundenen lebenden wirklich verschieden seyen. Wir haben indessen a. a. 0 . gegen ihn nachgewiesen,

I. dass 2-3 fossile Arten allerdings nur in Folge nicht stattgefundener Vergleichung mit Original-Exemplaren der lebeuden Arten, auf schlechte Beschreibungen und Abbildungen hin, mit letzten verwechselt worden seyen;

II. dass 5 Arten aus der Gruppe der Venus Brocchii mehr oder we. niger von der Ansicht des Systematikers über den Umfang der Spezies abhängig seyn mögen, zum Theile seh $r$ zweifelhaft seyen und von den Autoren jedenfalls schon lange nicht mehr, wie Ввоссн gethan, mit der lebenden Cyprina Islandica verwechselt werden, also hier nicht mehr als Gegenbeweis dienen;

III. dass, wenn man die fossile Artemis lincta nach Agsssiz in noch so viele Arten trennt, noch immer eine fossile Form von Castell'arquato übrig bleibt, die sich von der lebenden $A$. lincta nicht unterscheiden lässt; dass die fossile Muschel, welche Acassiz für die V. verrocosa der Auctoren hält und zur neuen Art Venus cincta macht, nicht die ächte fossile $V$. verrucosa und dass die ächte subapenninische $V$. verrucosa in der That von der lebenden nicht verschieden ist; - dass die fossile Cytherea chione in bei weitem der Mehrzahl der Exemplare diejenigen Unterscheidungs - Kennzeichen nicht besitzt, welche ihr Agsssiz beilegt, um daraus eine C. laevis zu machen, und dass sie daher ebenfalls von der lebenden in nichts verschieden ist; - dass endlich Cyprina Islandica LK. aus Sizilien, welche Agassiz als wirklichen Repräsentanten der gleichnamigen lebenden Árt anerkennt, in Sicilien nicht, wie er annimmt, in quartären, sondern in wirklich tertiären Schichten mit andern tertiären Arten und ganz übereinstimmend auch in den tertiären (pliocänen) Bildungen von Castell'arquato vorkomme, mithin einen von ihm selbst zugestandenen Fall von Identität [einer tertiären] mit einer Iebenden Art bilde; - - eben so streitet er die Lucina (Cytherea) leonina Bast. zwar der jetzigen Fauna ab, wo man sie nit L. tigerina verwechselt habe, gibt aber ihr Vorkommen wenigstens in 2 tertiären Formationen zu, in der miocänen um Bordeaux und in der pliocänen der Subapenninen. Die im Bulletin de Neuchatel beleuchteten Arten siud aus den Geschlechtern Pirula, Cytherea und Solen, und man kaun von ihnen ungefähr Dasselbe sagen, wie von den obigen: einige derselben (Solen) hatte schon vor ihm DesHAYEs in mehre Arten geschieden; die Scheidung der Cytherea- und PirulaArten ist ebenfalls zum Theil schon von Andern vorgenommen und nach Vergleichung guter Original-Exemplare begründet gefunden worden; über andere aber wird man sich schwer einigen können, weil sich nicht bestimmen lässt, was Art und Varietät ist. Wo aber cinmal $4-5$ dergleichen Fälle einzeln eingestanden werden müssen (was Agassiz bei Cytherea chione und Cyprina Islandica, die ich ibm vorlegte, auch mündlich gethan hat), da ist die Thesis anerkannt und kann es sich nur noch 1 m die Änzahl der Fälle handeln. Bei andern praktischen Paläontologen aber ist unsres Wissens das Vorkommen lebender Arten auch in pliocänen und miocänen Schichten nie in Zweifel ge. zogen worden, wenn sie auch das fossile Vorkommen in zweierlei Formationen 
zu läugnen bemuiht waren; wesshalb wir denn die Thatsache als gänzlich ausser Zweifel gestellt ansehen und nun verlassen können.

K. Da die Dauer der Arten schon im Grossen so ungleich ist, so darf man denı auch im Kleinen nicht erwarten, dass solche Alten, welche gleichzeitig miteinander in einer Schicht auftreten, auch in allen aufeiuanderfolgenden Schichten miteinander wieder erscheinen; sondern die einen werden andauern, wälırend andere aufhören und noch andere längere oder kürzere Zeit aussetzen.

Es können demnach z. B. je 8 versehiedene Konchylien-Arten $(a-h)$ in 6 aufeinanderfolgenden Schichten $(1-6)$ beispielsweise in folgeuder Art vertheilt seyn :

$$
\begin{aligned}
& \text { b.a.....h } \\
& 5 . \text { a ...e.g h } \\
& \text { 4. a bc...gh } \\
& 3 . a b c d \ldots h \\
& 2 \text { a acde... } \\
& \text { 1. a b c d e } f g h
\end{aligned}
$$

und so lassen sich im Einzelnen noch viele andere Kombinationen denken, wie sie auch in Wirklichkeit vorkommen.

Als beobachtetes Beispiel mag man die Verbreitung der Versteinerungen in Kreide und insbesondore den Neocomien-Schichten nehmen, wic sie im Jahrb. 1843, Collect. S. 78, 79, 81-84 und 85-94 mitgetheilt worden. Ein anderes wollen wir aus Reuss' Monographie der fossilen Polyparien des Wiener TertiärBeckens $\left(1848,4^{\circ}\right)$ hiehersetzen, welches 207 Arten enthält, wovon einige bis an den Jura hinab und andere bis an die lebende Schöpfung hinauf reichen:

$$
\frac{\mathrm{n}}{\mathrm{i}}\left|\frac{\mathrm{I}^{2}}{8}\right| \frac{\mathrm{t}}{5}\left|\frac{\mathrm{u}}{207}\right| \frac{\mathrm{v}}{1}\left|\frac{\mathrm{w}}{\mathrm{7}}\right| \frac{\mathrm{z}}{?}
$$

wobei wir indessen doch vermuthen dürfen, dass die Untersuchung vollkommenerer Exemplare etwas abweichende Resultate ergeben würde. - Eine nicht sehr vollständige Zusammenstellung der miocänen Organismen Piemonts, welche Michelotri beschrieben ${ }^{1}$ ), zeigt bei 848 Arten (wovon aber die meisten neu und mithin auf die Gegend beschränkt sind)

$$
\frac{t}{21}\left|\frac{u}{848}\right| \frac{w}{129} \mid \frac{z}{94}
$$

L. Man darf daher als feststehend ansehen, dass A r t e n organischer Wesen zu allenZeiten unasgesetzt entstanden und vergangen sind und dass zukeiner Zeit alle einstigen Thier-und Pflanzen-Arten der Erd-Oberfläche gleichzeitig geschaffen worden oder gleichzeitig untergegangen sind, und wenn je mit irgend einem geologischen Abschnitte der Gebirgs-Bildung ein solches Ereigniss irgendwo zusammenzufallen scheint, indem dort nur sehr wenige Arten aus einer früheren in die spätere Formation hinüberreichen, so ist der Analogie nach als Ursache davon anzunehmen, dass nur eben in dem bis jetzt ïberhanpt untersuchten Theile der Erd-Oberfläche dort ein solcher Abschnitt oder eine solche Lücke besteht,

1) Vergl. Jahrb. 1846, 503. 
sey es, dass schon ursprünglich dieser Theil auf lange Zeit dem Meere enthoben und daher unfähig war die den Übergang vermittelnden Schichten zu bilden und die entsprechenden OrganismenArten darin aufzunehmen, während es an andern Orten geschah, oder dass diese Schichten und Arten sich zwar wirklich absetzten, aber durch spätere Entblössung wieder zerstört worden sind.

Dass aber in der Schichten-Reihe einer Formation jede Schicht einen Theil der Arten in sich aufnimmt, welche in der oder den zunächst vorhergehenden und uachfolgenden vorkommen, ist nicht befremdend, sondern vielmehr den jetzigen Zuständen der Natur entsprechend, wie jede Tiefen-Zone des Ozeans zwar eine bestimmte Fauna hat, die aber zusammengesetzt ist aus Arten, welche ihr theils eigen sind, theils auch in einer oder einigen der nächst höhern und tiefern Regionen vorkommen; füllte sich dieses Meeres-Becken nun durch eine Reilıe von Schichten aus, so müsste jede Schicht ebenfalls ein Gemenge von eigenen Arten mit solchen der Nachbar-Schichten darbieten.

\section{4. Dauer der Sippen.}

A. Nachdem wir einerlei Pflanzen- und Thier-Arten durch mehre Schichten, Formationen und Perioden hindurchreichen gesehen haben, kann die Dauer eines Genus während mehrer entsprechender Zeit-Abschnitte nicht mehr befremden. Indessen ist auch hier noch (neben einigen Ausnahmen) die Regel, dass natürliche Sippen, welclie weder zweifelhafte noch fremde Arten einschliessen, sich nur auf eine oder einige benachbarte solche Abschnitte beschränken, so dass, wenn einerseits Arten-arme Sippen nur in wenigen Formationen vorkommen kömnen, andererseits doch auch die Arten-reichen sehr oft keine weitere Verbreitung besitzen.

So kommen folgende grössere Genera mit der beigefügten Zahl aller oder fast aller fossilen Arten vor in den Formationen :

a: Obolus 4; Siphonotreta 2; Maclureia 3; Ophileta 2; ?Trinucleus 13/14; Paradoxides 8; Conocephalus 4; Ellipsocephalus 2; Sao 2; Illaenus $11 / 12 ; \mathrm{Ag}$. nostus $14 / 16$; Hydrocephalus 2 .

ab: Cheirurus 17.

b: Lituites $8 / 11$; ? Harpes $7 / 8$; Lichas $16 / 18$; Phaëtonides 5.

c: Lunulicardium 10; Scoliostoma 2; Bactrites 2; Clymenia $42 / 45$; Proetus $18 / 21$; Asterolepis 8; Osteolepis 6; Pterichthys 9; Cephalaspis 4; Diplacanthus 4 ; Cheirolepis 5.

ce: Holoptychius 14.

d: Myalina 3; Gyroceras 3; - Phillipsia 9, Griffithides 5; - Petalodus 8; Poecilodus $6 / \%$.

e: Volkmannia 7; Sphenophyllum 13; Annularia 11; Trichomanites 11; Asplenites 10; Aphlebia 8; Lepidodendrou 19; Lepidostrobus 11; Bergeria 7; Ulodendron 10; Trigonocarpum 7; Cardiocarpum 6; - Blattina 4; - Amblypterus 8.

r: Calamitea 4; Medullosa 3; Psaronius 13.

g: Janassa 4: Platysomus $\% / 9$.

h: Montlivaltia 13/15; Naticella 19.

i: Aethophyllum 2; Echinostachys 2; Albertia 4. 
I6: Pemphix 2; Litogaster 2; Ceratodus ${ }^{20} / 21$.

1: Thectodus 4 .

m: ?Cardinia 14; Geoteuthis 15; Koleia 5; - Dapedius 8; Tetragonolepis 16! ${ }_{120}$; Eugnathus 14; - Mystriosaurus 11; Ichthyosaurus 13/15; -- Pterodactylus $\mathrm{P}_{2} / 14$.

n: Halymenites $12 / 13$; Millerocrinus 33, Solanocrinus 4; Hemicidaris $13 / 15$; Clypeus $\% / 10$; Aucella 4 : Urda 4; Koelga 8; Aeger 5; Antrimpos 9; Megachirus 5; Eryon $18 / 19$; Eryma 9; Orphnea 6; Ganodus 5; Aethalion 6; Microdon $7 / 8$.

no: Cercomya 13.

p: Oxygonius 1; Ceramurus 1.

Q : Monopleura 7 ; Toxoceras $10 / 11$.

r: Credneria 7; Helicoceras 3; Anenchelum 6; Aeanus 5.

r: Marginaria 13; Escharites 9; Hippurites 35; Radiolites $31 / 32$; Sphacrulites 19 ; Caprina 13; Pterodonta 7; Baculites 13/1; Belemnitella 5; Ptychodus 7; - Osmeroides 5; Beryx 6.

$\tau$ : Carangopsis 4 ; Pygaeus 8 ; Sparnodus 5.

t: Nipadites 13; Cupressinites 13; Faboidea 25; Leguminosites 18; Scutellina 5 : Bifrontia 6 ; Beloptera 4 :

u: Flabellaria $10 / 14$; Ferussacia 4.

vv: Brocehia 2.

Dann in mehren Formationen der Perioden :

I: Asterophyllites 24; Noeggerathia 10; Stigmaria 7; Sigillaria 71; Knorria 9; Aspidiaria 15; Aulopora 7; Fenestella 26; Favosites 18; Graptolithus 18; Syringopora 21; Poteriocrinus 12; Taxocrinus 8 ; Cupressocrinus 12; Platycrinus 26; Cyathocrinus 27; Actinocrinus 23; - Pentamerus 15; Orthis $121 / 123$; Chonetes 13; Leptaena 34; Productus $71 / 73$; Pterinea 25; Anthracosia 57; Megalodon 12; Cardiomorpha 18; Cardiola 17; Conularia 16; Porcellia 11/12; Bellerophon 71; Macıocheilus 11; Murchisonia 31 ; Goniatites 170/15; Cyrtoceras 44; Phragmoceras 9; Odontopleura 25; Bronteus 30; Calymene 27; Homalonotus 8; Phacops 44: Asaphus 51; - Ctenoptychius 8; Ctenodus 9; Cladodus 9; Onchus 14; Palaeoniscus 26.

II : Encrinus 7; Myophoria 14; Gyrolepis $4 / 3$; Saurichthys $11 / 12$; Placodus 5 ; - Nothosaurus 8; Mastodonsaurus 4; Labyrinthodon 6.

III: Zamites $29 / 30$; Thuites $10 / 11$; Taxites 13; Mactromya 9 ; Homomya 6 ; Pholiduphorus 33; Caturus 18; Parhycormus 15; Thrissops 7; Leptolepis 21; Belonostomus 9; - Plesiosaurus $18 / 20$.

IV: Coeloptychium 15 ; Salenia 18/19; Cyphosoma 10; Galerites 20 ; Ananchytes 8; Holaster ${ }^{27} / 28$; Requienia 11; Ringiuella 4 ; Avellana 10 ; Globiconcha 4; Columbellina 2; Crioceras 12 ; Scaphites 15/16; Hamites 40 ; Turrilithes 27. V: Proto 4; Phyllodus 11/12; Smerdis 7 und fast alle ausgestorbenen SäugethierGenera u. s. w.

Oder in 2 zusammenhängenden Perioden :

I + II : Calamites $49 / 30$; Caulopteris 12 ; - Euomphalus 85.

II + III: Nilssonia 12; - Pleuromya 35 .

III + IV: Discoidea 23; Dysaster 21; Exogyra 42; Myoconcha 8; Diceras 7; Opis 14; Goniomya 33; Myopsis 29; Nerinea 91; ? Ammonites 580 ; Ancyloceras 20 ; Belemnites 98 ; I.epidotus 34 ; Gyrodus 30.

III + VI : Acanthoteuthis 15; Aeschna 7.

IV + V: Nummulina 39; Scutella 17; Pygorhynchus 10; Catopygus 12; Hypnodon 4.

V + VI: Quercus (11); - Actinocyclus 40; Navicula 67; Biloculina 12; Quin- 
queloculina 33; Tubulipora 19; Diplodonta 6; Saxicava 11/18; - Siliquaria 12: - Crepidula 16; Ringicula 7; Melanopsis 24; Valvata 10; Ranella 23; Tritoniun 45; Typhis 8; Murex 17t/180; Fasciolaria ${ }^{28} / 29$; Turbinella 28 ; Cancellaı ia 70 ; Purpura 32; Monoceros 8; Columbella 9; Cassis 35; Morio 17; Mitra ${ }^{8 \times / 90}$; Marginella 32 ; Ancillaria 18; Oliva 32 ; Trivia 11; Ovulum 11; Conus 80 ; Cyclostoma 40 ; Physa 12; Auricula 25; Vertigo 8; Pupa 34; Clausilia 18; Achatina 14; Bulimus 26; Hclix 187; Sucrinea 6; Balanus 42; - Myliobatis 32; Carcharodon 18; Dentex 6; - Trionyx 17; - die noch lebenden Säugthier-Genera etc.

Oder in 3 zusammenhängenden Perioden :

I, II, III : Sphenopteris ${ }^{95} / 96$; Neuropteris ${ }^{63}$;64; Alethopteris 42; Pecopteris $38 / 60 ;-$ Posidonomya 29 ; Orthoceras 153.

II, III, IV : Achilleum 29 ; Tragos 26 ; ? Ammonites 580 ; Rhyncholithus 13 ; Hybo. dus $66 / 67$.

III, IV, V: Nucleolites 30 ; Cricopora 13 ; - Pycnodus $41 / 42$.

IV, V, VI: Dentalina 29; Frondirularia 41; Bulimina 23; Lunulites 26; Echinocyamus 13; Schizaster 26; Spatangus 37 ; Crassatella 51 ; Arcopagia 17; Anatina 13; Teredo 19; Clavagella 13; - Fissurella $27 / 28$; Infundibulum 22; Pyramidella 14; Scalaria 88/91; Phorus 17; Pirula 51; Pleurotoma 302 ; Voluta 97; Cyprella 77; - Odontaspis 13 ; Lamna 13; Oxyrhina 18; Otodus 24; - Crocodilus 21.

Oder in 4 zusammenhängenden Perioden :

I, II, III, IV: Scyphia 118; Inoceramus 53; Gervilleia 34; - Strophodus 17; Acrodus 18.

II, III, IV, V: Sphaerodus 29.

III, IV, V, VI : Cristellaria $49 / 50$; Echinus 53 ; Diadema 46 ; Clypeaster 27 ; Echinolampas 32; Thecidea 9 ; Anomia 30; Plicatula 28; Lithodomus 23; Pectunculus 78; Chama 26; Unio 33; Cyprina 25; Corbis 17; Cytherea 81; Mactra 53; Thracia 12; Pholadomya 147; $\mathbf{P a -}$ nopaea 39 ; Solecurtus 14; Pholas 25; - Vermetus 23; Actaeon 76 ; Cerithium 250; Pteroceras 27; Fusus $308 / 310$; Terebra 34 ; Bulla 70; Vermilia $24 / 25$; Pollicipes 29; Notidanus 11; - CheIonia 18.

Oder in 5 zusammenhängenden Perioden :

I - V: Ceriopora 63 ; Pentacrinus 37.

II - VI: Cidaris 128; Ostrea 278; Lima 163; Perna 20; Lyriodon 100; Emarginula 33 ; Rissoia 108 etc.; Chenopus et Rostellaria 87.

Oder in 2 Wechsel-Perioden, wo indess wahrscheinlich das Genus anch in der $Z$ wischen-Periode existirt hat und nur bis jetzt noch nicht gefunden worden ist.

I + III: Cyclopteris 38 ; Ceratites 42.

I + III, IV: Eugeniacrinus 14; Spirifer 157 (I, II, m).

I + III, IV, V: Cyclolithes 32.

I +.III, IV, V, VI: Caulerpites 36; Chondrites 24; Caryophyllia 39; Turbinolia 84; Pinna 46; Astarte 134; Amphidesma 30; Solen 31;

$1+\mathrm{V}$ : Borelis 6 . Cythere 86.

I + V, VI: Chiton 30.

I, II + V, VI : Capulus 23.

I, II, III + V, VI: Sanguinolaria 38 . 
$\mathrm{III}+\mathrm{V}$ : Rimularia 3 ; Pileolus 4 .

III + V, VI : Cyclas 34; Cyrena 70; Neritina 33; Melania 29; Paludina 81; Planorbis 60; Limnaeus 72 ; Sepia 12 ; Emys 28.

IV + VI: Xanthidium 12; Micraster 25; Hinnites 8 ; Limopsis 19 ; - Spi$\operatorname{nax} 3$,

Oder in 3 Wechsel-Perioden :

$\mathbf{I}+\mathbf{I I I}+$ V, VI: Cypris 21 .

Oder in allen Perioden: Caulerpites, Chondrites (beide doch nicht in der 11. Periode), Sphaerococcites und viele Farnen, welche freilich alle als künstliche Genera erst in ein richtiges Verhältniss mit den lebenden Geschlechtern gesetzt werden müssen; Astraea 178; Maeandrina 33; Lithodendron 28; Antho. phyllum 25 ; Pentacrinus 37 ; Lingula 34; Tercbratula 419 ; Orbieula 35 ; Crania 34 ; ?Spondylus 59; Pecten 302; Avícula 186; Mytilus 193; Modiola 133; Cucullaea 98; Arca 183; Nucala 207; Cypricardia 41; Isocardia 86; Cardium 245; Lucina 137; Venus 166; Tellina 92; Corbula 90; Dentalium 80; - Patella 90 ; - Natica 250 ; Nerita 52 ; Turritella 270; Turbo 245; Trochus 326 ; Pleurotomaria 260; Buccinum 200; - Nautilus 130; - Spirorbis 33; Serpula 198. Manche davon werden aber durch bessere Charakteristik und natürliche Spaltung dieser Arten-reichen Genera verschwinden,

B. Während also die Dauer eines einzelnen Genus durch eine his füuf (und beziehungsweise, mit der jetzigen, 6) Perioden hindurch reichen kann, lässt sich über die mittle $\mathbf{D}$ a u er aller Genera an. geben, dass bei den

$$
\begin{array}{ll}
\text { in unsern } & \text { in unsern } \\
\text { Perioden : } & \text { Formationen : }
\end{array}
$$

Pflanzen die 350 Sippen 463mal und 592mal $=1: 1,32: 1,69$

Thieren die 2501 Sippen $3347 \mathrm{mal}$ und $5415 \mathrm{mal}=1: 1,34: 2,17$

Zusammen die 2851 Sippen $3810 \mathrm{mal}$ und $6007 \mathrm{mal}=1: 1,34: 2,11$

gezählt werden, wenn man die Zahlen der in jeder Periode oder Formation gefundenen Sippen addirt; so dass also im Mittel eine Sippe in $1 \frac{1}{3}$ Perioden und in etwa 1,7 bis 2,2 Formationen gefunden wird; auch mit andern Worten: unter 10 Pflanzen- und Thier-Sippen sind $3-4$, welche noch in eine zweite angrenzende oder entfernte Periode übergehen, bei diesen etwas weiter als bei jenen; auf 100 Pflanzen-, 100 Thier- und 100 gemeinsamen -Sippen sind nahezu beziehungsweise 7, 12 und 11 Sippen, welche in eine zweite unsrer Formationen übergehen coder es gehet jede Sippe noch in 0.7 , oder 1.2 und 1.1 Formation über), wenn man annimmt, dass eine Sippe, welche sicl sogar in eine dritte und vierte Formation fortsetzet, 2-3 der elsten repräsentire; wobei indessen abermals zu erinnern ist, dass unsere Formationen $\mathbf{v}$ und $\mathbf{x}$ später ausfallen werden, in welchem Falle dann das Verhältuiss schwächer erscheinen wird. Die Dauer der Genera verhält sich zu der der Arten (S. 784) = 211 ; 112, also ungefähr $=2: 1$.

Diese Nachweisung ist das Ergebniss aus den Zahlen der obigen Zusammenstellung der Zahlen aller Genera (S. 734), leidet aber an den auch für die Arten angedeuteten Gebrechen (S. 750 und $784 \mathrm{ff}$.). Man wird mit Hülfe jener Tabelle leicht auch die Berechnung machen können über das Verhältniss, welches eintreten würde, wenn man die Formationen $\mathbf{v}, \mathbf{x}$ sogleich 
unter die tibrigen ( $\mathbf{u}$ und $\mathbf{w}$ ) eintheilte; die Dauer wird dann scheinbar kürzer werden, weil das so oft wiederholte Vorkommen in $\mathbf{v}$ und $\mathbf{x}$ wegfiele. Dagegen aber ist zu erinnern, dass die 2 Formationen und Perioden, worin eine Sippe auftritt, nicht immer 2 unmittelbar aneinandergrenzende sind, wodurch also die Dauer wieder länger ausfällt; - so wie dass (nach den vorhergehenden Seiten) zuweilen die Dauer eines Genus durch mehre oder alle Perioden hindurchgehet (3-5faches Vorkommen), wesshalb denn auch die Zahl der mehren Perioden wirklich gemeinsamen Genera im Ganzen geringer bleibt, als oben mit Unterstellung eines blos doppelten Vorkommens gefunden worden ist.

C. Diejenigen Genera, welche durch mehre Formationen oder Perioden hindurchreichen (geologische Verbreitungs-Area), pflegen in jeder derselben durch eine verhältnissmässig gleiche Anzahl von Arten vertreten zu seyn; so dass sie eine Zeit lang in gleicher Dauer behırren und an beiden Enden entweder ganz plötzlich aufhören (so viele sehr scharf begrenzten Genera, aus welchen alle fremden Arten sich leicht ausscheiden lassen: Belemnites, Nerinaea u. s. w.) oder doch sehr schnell in den nächsten 1-2 Formations-Gliedern verschwinden, - zuweilen auch noch einen Anhang von einigen wenigen zweifelhaften Arten in grössrer Entfernung zeigen; während ein allmähliches Zunelımen von einem Anfangs-Punkte nur bis zu einem Culminations-Punkte - Centrum der geologischen VerbreitungsArea - und ein allmähliches Abnehmen von da an bis zum gänzlichen Verschwinden eine mehr ausnahmsweise Erscheinung darstellt. Nur bei solchen Geschlechtern insbesondere, welche ihre grösste Entwickelung in der jetzigen Schöpfung haben, sieht man öfters ein allmählicheres Zunehmen der Arten-Zahl gegen dieselbe hin.

Edward Forbes ist geneigt, überall einen Zentral-Punkt der geologischen Verbreitungs-Area der Sippen anzunehmen ${ }^{1}$ ), den wir indessen - wenn man nicht unter diesem Punkt wieder eine Fläche (Zentral-Area) verstehen will, nur in einigen wenigen Geschlechtern finden können, deren Verbreitung wir, ausser einem Normal-Bilde, durch folgende Figuren am besten versinnlichen zu können glauben, obschon auch unter dieser geringen Auzahl noch 1/4 der Figuren ziemlich dem Normal-Bilde entspricht, indem sie eine Zentral-Area statt eines Zentral-Punktes zeigen. Der Stern deutet die Culminations-Stelle, den ZentralPunkt an.

1) Jahrb, 1848, 754 . 


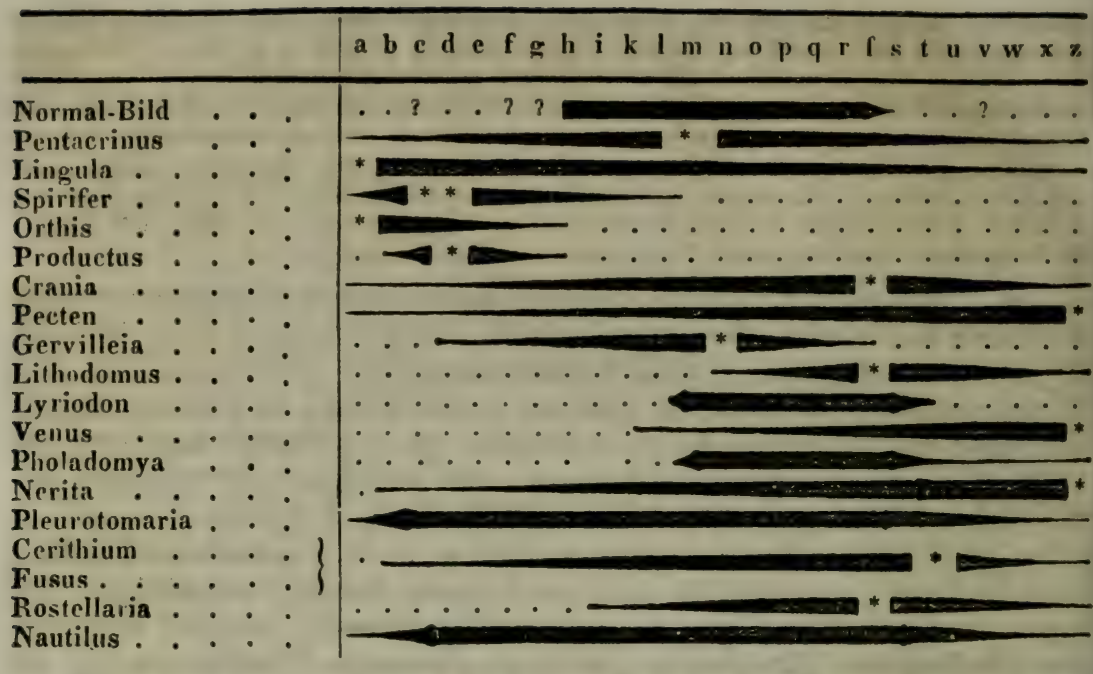

Viele noch in der jetzigen Schöpfung fortlebende grössere Genera zumal aus den Abtheilungen der Homomven und Gasteropoden gewähren durch ihre geologische Verbreitung dieselben Bilder, wie jene sind, die wir für Venus und Nerita gegeben, nur dass sie etwas früher oder später bcginnen.

D. Die periodisch aussetzenden Genera dïrften nur scheiubare Ausnahmen bilden, wenn es sich nämlich darmm handelt, aus ilırer jetzt bekannten geologischen Verbreitung auf ihre einstige geologische Existenz zu schliessen. Ein Theil derselben ist nämlich offeubar aus nicht zusammengehörigen Dingen zusammengesetzt, welche bei genauer Prïfung in verschiedene Genera getrennt werden müssen, wodurch sich jener Widerspruch lösen würde. Ein anderer Theil zeigt nur desswegen eine Unterbrechung, weil ein Theil der in die Mitte seiner geologischen Verbreitung fallendeu Schichten zur Konservirung gerade dieser Reste nicht geeignet war; oder auch weil die in diesen Schichten wirklich vorhandenen Arten nur zufällig noch nicht aufgefunden worden sind. In noch anderen entsteht die Unterbrechung nur daher, dass Meeres - und Süsswasser-Formationen mit einander wechseln, deren jede die Arten der andern ausschliesst.

So fehlen namentlich in der Kreide die Süsswasser-Schichten gäuzlich, während sie kurz zuvor (p) und nachher (in $\mathbf{t}, \mathbf{u}, \mathbf{w}$ ) ansehnlich entwickelt sind. Daher denn auch von den intermittirenden Geschlechtern die unter der Bezeichnung III $+V$, VI eine nicht unbeträchtliche Quote ausmachen (S. 776).

Von den kleineren Geschlechtern haben wir hier keine Notiz genommen, da sich ihre Verbreitung nicht so genan nachweisen lässt; es ist aber wahrscheinlich, dass sie denselben Regelı unterliegt. 
E. Bei den niederen Pflanzen und Thieren kommen Sippen mit viel längrer Dauer als bei den höheren vor. Während nämlich mehre Genera der Meeres - Algen aus der Haupt-Abtheilung der ZellenPflanzen und viele Genera der meerischen Polypen, Weich-Thiere und Ringel-Würmer aus der Haupt-Abtheilung der Wirbel-losen Thiere die ganze Reihe der Formationen durchlaufen, beschränken sich die der Gefäss Pflanzen, der übrigen Kerb-Thiere (ausser den Ringel-Würmern) und sämmtlicher Wirbel-Thiere auf kürzere Zeiträume, so dass die der übrigen Insekten, der Fische und Reptilien fast alle nur während einzelner oder meistens e iner Periode und die der Vögel und Sängthiere während höchstens einer Periode und gewöhnlich nur einer Formation daraus vorkommen, jene ausgeuom. men, welche noch in die lebende Schöpfung übergehen. Wo immer die fossilen Reste noch genügende Merkmale von generischem Werthe mit sich erhalten haben, da wird man auch erwarten dürfen, die Geschlechter künftig mehr und mehr auf eine oder einige wenige Perioden beschränkt zu selıen in dem Masse, als diese Merkmale sorgfältiger geprüft und benützt werden.

Wir haben fast alle diese andasernden Genera auf den vorhergehenden Seiten 775-776 nambaft gemacht und auch die Abstufungen in der Dauer der übrigen ausreichend bezeichnet. Norh weitere Details ergeben dann unsere Tabellen.

Wir sehen jetzt noch manche Brachiopoden- und Anthozoen-Genera weit durch die Perioden-Reihe hindurchreichen; allein die von mehren Autoren neuerlich versuchte Scheidung der ersten in viele Geschlechter, die von Mrn.neEdwards und Haime begonnene Bearbeitung der zweiten führt überall zu dem Resultate der Beschränkung der generischen Typen auf eine geringe PeriodenZahl. Wo aber bei Bearbeitung solcher Gruppen ein hinsichtlich seiner Merkmale indifferenter Rest von Arten in einzelnen Geschlechtern zurückbleibt ( Te. rebratulu), oder wo diese Merkmale in der ganzen Gruppe überhaupt sehr indifferent sind und mit dem inneren organischen Bau der untergegangenen Thiere nur in entfernter Bezichung gestanden ('Turbo, Trochus, Natica etc.), da wird man auch später die weite geologische Verbreitung der Genera andauern sehen.

F. In einer geologisch beschränkten Klasse oder Ordnung von Organismen müssen es auch alle Genera seyn (Säugthiere, Choristopetalae); in einer geologisch ausgedehnten dagegen können auch fast lauter ausgedehnte Genera (Monomya) beisammenstehen, oder sie können aus sehr ausgedehnten und sehr beschränkten Untergruppen (Brachiopoden mit Rudisten und Genuinen) oder aus lauter beschränkten Untergruppen (Pteropoden) zusammengesetzt seyn.

Fälle solcher Art wird man als fernere Belege im Enumerator wie in der Tabelle S. 734 leicht auffinden.

\section{5. Dauer der Familien, Ordnungen und KIassen.}

A. Sogar unter den Familien und Ordunngen der Pflanzen und Thiere bemerkt man noch welche, die, aus einer geringen SippenAnzahl zusammengesetzt, sich auf eine Formation oder eine Periode 
beschränken, während andre einen mehr oder weniger grossen Theil aller durchlaufen. Wir sehen dabei von solchen Gruppen ab, welche offenbar nur sehr unvollkommen bekannt sind (Insekten-Ordunngen und Familien ete.). Die meisten eigenthümlichen Gruppen von Pflanzen und Thieren, welche in andern Perioden nicht vorkommen, enthalten die gegenwärtige und die ilır zunächst angränzenden Perioden, während die ältesten sogar grossentheils bis in die jetzige Zeit heraufreichen. Nur wenige sind beschränkt auf einzelne

Formationen, wie:

e (Thiere): Pisces Dipterini und P. Cephalaspides.

ce (Thiere): Pisces Acanthoidei.

C (Pflanzen): Asterophyllitae, Sigillarieae mit 68/71 Arten; Diploxyleae.

ef (Pflanzen): Psaronieae.

Auf einzelne Perioden beschränkt findet sich schon eine grössere Auzahl von Gruppen; so in

Periode :

I: Stigmarieae: - (Crinoidea) Stylechinidae; - (Crustacea) Palatades; (Pisces) Dipterini; Cephalaspides; Acanthodei.

II: (Reptilia) Labyrinthodontes.

III: (Pisces) Sauroidei homocerci.

IV : (Brachiopoda) Rudistae (verae, excl. 8c. Orbicula el Crania); - (Pisces) $\mathbf{v}: \ldots$ Scopelini.

In zwei aufeinanderfolgenden Formationen finden sich:

I, II : (Pisces) Sauroidei heterocerci.

II, III : . . .

III, IV : (Cephalopoda) Belemnomorpha; - (Reptilia) Pterodactylina.

IV, $\mathbf{V}: \ldots$

V, VI: Hepaticae, Musci und alle Pflanzen-Familien der Dicotyledoues Monochlamydae Amentaceae Jussiev, und alle der Dicotyledones Corolliflorae und D. Choristopetalae, soweit beide fossil vorkommen, mit Ausnahme von 12 Arten in Ganzen. - (Gasteropoda) Hypobranchia: (Gasteropoda Pulmonata) Operculata et Geophila; - (Cephalopoda Dibranchia) Octopoda; - (Pisces) Lophobranchii; Anguilliformes; Cyprinodontes; Cyprinoidei, Labridae; Pleuronectae; Gadoidei; Atherinoidei; Pediculati; Blennioidei; Teuthyes; Gobioidei ; Squamipennes; Sciaenoidei; Sparoidei; Cataphracti; - (Reptilia) Batrachii; Ophidii; Trionychidae.

In dreien dergleichen :

I, II, III : (Pisces) Lepidoidei heterocerci.

II, III, IV: (Reptilia) Saurii Nexipodes et Pachypodes.

IV, V, VI: Spatangoidea ; - Tubicolae; - (Crustacea) Stomatopoda - - (Pisces) Pectognathi, Physostomi (- insbesondere Clupeoidei, Salmones); Pharyngognathi (zumal Scomberesoces, Sphyraenoidei, Xiphioidei, Scomberoidei, Fistulares, Mugiloidei, Percoidei).

Auf vier Perioden vertheilen sich :

I, II, III, IV : (Cephalopoda Tetrabranchia) Ammonitina; (Pisces) Lepidoidei homocerci (mit 1 Ausnahme).

II, III, IV, V: (Pisces) Sauroidei et Pycuodontes.

III, IV, V, VI: Najadeae; - Clypeastroidea; Fistulidae; - Pholadiua ; - Tubu 
libranchia; Siphonobranchia (einige ältere Arten sind zweifelhaft); Pomatobranchia; (Gasteropoda Dibranchia) Decapoda; - (Crustacea) Cirripedia; - Pisces Teleosti (fast nur in IV, V); Chelonii.

Auf fünt Perioden:

I - V: (Pisces) Lepidoidei.

II - VI: Echinidae (Cidaridae); - Aspidobranchia ; - (Crustacea) Decapoda; - (Pisces) Lamnoidei.

In wechselnden Perioden finden sich ein, und zwar in :

I, II + VI: (Crustacea) Phyllopoda.

I, II, IV, V, VI: Smilaceae.

I, III, IV, V, VI: Polythalamia (Foraminifera); Saurii Dactylopodes.

I + III, V, VI : Asphodileae.

III + V, VI: Hydropterides; - (Gasteropoda Pulmonata) Hydrophila.

In allen :

I-VI : Algac; Equisetaceae; Filices; Lycopodiaceae; Gramineae : Palmae; Cycadeae; Coniferae; - Bryozoa; Anthozoa; - (Crinoidea) Stylastritae (fast nur in I-III) et Astylidae; - Monomya; Heteromya; Homomya; Cirrobranchia; - Cyclobranchia; (Ctenobranchia) Asiphonobranchia ; (Crustacea) Lophyropoda et Poecilopoda; - (Pisces) Coelacanthi.

B. Die Dau er der K las s e n ist ebenfalls sehr ungleich, selten zwar auf nur eine Periode beschränkt, wenn anders die ihnen angehörigen Organismen zu einer Erhaltung im fossilen Zustande geeignet sind. Aber keine Klasse gehört der ältern Periode ausschliesslich an ; und alle verlaufen sich entweder in die jetzige, oder beschränken sich ganz auf diese (wie die Entozoen).

Perioden :

V, VI: Dicotyledones Corolliflorae und D. Choristopetalae; - Aves mit 2 Ausnahmen; - Mammalia (mit 3 Ausnahmen).

IV-VI: Pseudozoa; Polygastrica (671/672 Arten); Acalephae; Echinodermata.

Unterbrochen:

$\mathbf{I}+\mathbf{V}, \mathbf{V I}:$ Pteropoda.

In allen Perioden:

I-VI: Plantae cellulares; PI. vasculares monocotyledoneae ; PI. vasculares dicotyledoneae; - Amorphozon: Polypi ; Echinodermata (Stelleridae); Brachiopoda ; Pelecypoda (Monomya et Dimya); Protopoda; Gasteropoda (Cyclobranchia et Ctenobranchia); Cephalopoda (Tetrabranchia Nautilina et Dibranchia); - Vermes; Crustacea (Entomostraca et Malacostraca); - Arachnidae; Hexapoda ; Pisces (Elasmobranchii, Ganoidei); - Reptilia (Saurii).

In den 3 tieferen Unterreichen der Thipre gibt es also viele Klassen, welche selbst so wie ein Theil ihrer Genera die ganze Reihe der Formationen durchlaufen; die Klasse der Fische thut dasselbe mit nur 2 ihrer Ordnungen; ihre Genera reichen nie mehr durch die ganze Perioden-Reihe und sclten aus einer Periode in die andere hinein; ähnlich bei den Reptilien; in den Vögeln und Säugthieren haben wir Spezies (von den Fährten abgesehen), welche kaum 
durch eine Periode hinaus, und Genera, welche selten in zwei Formationen hineinreichen.

C. Die Dauer der Klassen, Ordungen, Familien, Genera und selbst Arten verkürzt sich im Ällgemeinen mit zunehmender Ilöhe ihrer Organisation. Wie verschiedene Unterreiche und Klassen überhaupt in ungleich frühen Perioden beginnen, gewöhulich jedoch bis in die jetzige Periode andauer'n, so ist auch das Alter der verschiedenen Ordnungen, Familien und Geschlechter, die zu einer Klasse gehören, ungleich, aber der Fall weniger selten, dass sie schon vor der Jetztzeit wieder aufhören. Indessen ist hierin ein Unterschied zwischen den unvollkommenen und vollkommenen Pflanzen, zwischeı Wirbel-losen und Wirbel-Thieren.

Bei den PfI a $\mathrm{z}$ en bieten nur die unvollkommenen Gruppen, wie die Zellen-Pflanzen, die kryptogamen Gefäss-Pflanzen und vielleicht selbst die gymnospermen Dikotyledonen, Sippen von läıgster Dauer durch alle Perioden dar. Unter den corollifloren und choristo. petalen Dikotyledonen hat man nur 2-3 Genera in verschiedenen Perioden angegeben.

Bei den Wi r be I-los e n $\mathrm{T}$ h i e re n im Besonderen reichen die 3 Unterreiche von der ersten Periode an durch alle folgenden hindurch, und wo es einzelne ihrer Klassen oder Ordnungen nicht thun, da liaben wir Ursache zu glauben, dass ilıre Reste nur eben entweder ungeeignet gewesen sind zur Erhaltung im Fossil-Zustande, oder dass sie zufällig noch unsrer Aufmerksankeit entgangen sind. Eben so

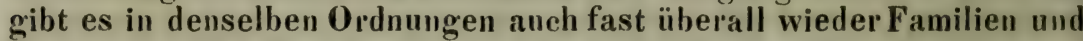
Genera, welche dieselbe Länge haben, wie die Familie, die Ordumng selbst, wahrscheinlich die höher stehenden Luft-Entomozoen und vielleicht die Polypen ausgenommen, wenn letzte in der Weise von MiLne Edwards und Hame, von Lonsdate und M'Coy sorgfältiger bearbeitet seyn werden.

Bei den Wirbel-Thieren aber, deren Dauer überhaupt und in den einzelnen Klassen selbst kürzer ist, haben die einzelnen Ordnungen mitunter eine sehr ungleiche Dauer, die nicht auf blossen Zufälligkeiten beruhen kann. Bei den $F$ is $\mathbf{c}$ he $n$ ist es nur die Ordıung der (plagiostomen) Flasmobranchier, welche gleichsam als Achse, woran sich die übrigen theils später beginnenden, theils früher endigenden Ordnungen und Familien anlehnen, mit einigen Familien (Cestraciontes) durch die ganze Fisch-Zeit von I an bis VI lindurchreicht; weder die kürzer danernden Ganoiden, noch die bloss auf 2 Perioden beschräıkten Teleosti haben eine solche mit der Ordnung gleichlange Familie noch aufzuweisen; aber ein vollstäudig von I bis VI hindurchziehendes Genus ist überall nicht mehr bekannt, indem selbst nur wenige sich durch 3 Perioden hindurch erstrecken. - - Die Dauer der Klasse der Reptili en ist eben so lang, als die der Fische; die vou Anfang bis zu Ende durch alle VI Perioden hindurch sie vertretende Ordnung ist die der Saurier mit den 2 Unterordnungen der 
Krokodilier und Lazertier; aber von den noch lehenden Geschlechtern ist keines, das mit verlässigen Arten weiter als bis in die V. Periode zurückginge. Die Ordnung der Chelonier dagegen hat seit ilırem Beginne in III auch die 3 Haupt-Genera Testudo, Emys und Chelonia erkennen lassen, vorbehaltlich freilich genauerer Bestimmungen, wenn es gelingen wird, ihre Osteologie vollständiger zu erforschen. Die Schlangen und Frösche gehen nicht über die V. Periode zurück. - Die Säugthiere endlich, welche mit 4-5 Ausnahmen alle erst aus der V. Periode stammen, haben aus lebenden Geschlechtern doch nur 1 Cervus- und 2 Myoxus-, 1 Sciurus-, 2 Didelphys-, 1 Felis-, 3 Canis-, 2 Viverra-, 1 ? Midaus-, 1 Nasua-, 2 Vespertilio-Arten und 1 Macacus ans der Eocän-Zeit geboten; nur 11 unsrer vielen jetzigen Genera sind also dort vertreten gewesen. So zieht sich die Dauer der Klasseı, Ordnungen, Familien, Genera und selbst der Arten immer kürzer zusammen, je höher man auf der Leiter der Organisation hinansteigt; sie beschränkt sich iımer mehr auf kürzere, schärfer begrenzte Zeit-Abschnitte, und wo man auffallende Ausnahmen zu sehen glaubt, da darf man in der Regel eine unrichtige Bestimmung, seye es der Organismen selbst oder seye es der sie einschliessenden Formation, vermuthen.

So ist endlich keine höhere Wirbelthier-A rt mit Sicherheit in 2 Perioden bekannt. In zwei Formationen werden zwar noclı einige Arten von Fisch-Zähnen, einige Reptilien, doch diese z. Th. nur zweifelhaft; - bei Säugthieren werden nur 4-5 Fälle angeblicher Gemeinschaft zwischen $t$ und $u$, wo aber wahrscheinlich eine der Gebirgs-Arten unrichtig angegeben ist, und vielleicht nur einer zwischen $\mathbf{u}$ und $\mathbf{w}$ (Mastodon angustidens soll in Europa in $\mathbf{u}$ und $\mathbf{w}$, in Amerika in w liegen) angeführt.

B. Untersuchung über die Zahlen-Verhältnisse im Allgemeinen.

(Paläontologische Statik.)

§. 6. Überhaupt.

A. Eine genaue Vergleichung der Zahlen der zu verschiedenen Zeiten lebenden Thier- und Pflanzen-Formen ist nicht möglich, weil wir 1) nicht bestimmen können, in wie ferme sich die den einzelnen Schichten, Formationen, Perioden entsprechenden Zeit-Abschnitte unter sich gleich verhalten, oder ob nicht der eine derselben 2-3-4mal länger als der andere in gleiche Kategorie gestellte Abschnitt ist; - 2) weil die Mehrzahl der Thiere und Pfanzen, welche zu verschiedenen solchen Zeiten existirt haben, in ungleichem Grade erhaltungsfähig gewesen sind und daher bei eigener Gleich-Zahl eine 
ungleich grosse Anzahl von Rest-Arten uns hinterliessen; - 3) weil eben so die Bildungs-Weise und die Mineral-Natur der in verschie. deuen Zeiten entstandenen Gesteins-Schichten in sehr ungleichem Grade geeignet war, die organischen Reste in sich aufzunehmen und uns zu überliefern; - 4) weil die Anzahl noch nicht auf ihre Arten zurückgeführter Synonyme noch zu gross ist; - 5) weil überhaupt die Zeit unsrer Forschungen noch zu kurz und die bereits erforschte॥ Antheile unserer Erd-Oberfläche noch zu klein sind. Nur durch Vergleichung grösserer Zeit-Abschnitte mit einander, wo sich die Verschiedenheiten in der Gesteins-Natur mehr ausgleichen, Zufälligkeiten aller Art mehr verschwinden, und durch den Ausdruck der Ergebnisse in verglichenen statt in absoluten Zahlen dürfen wir hoffen, einen Theil der gröbsten Irrthümer zu vermeiden.

a) Zuerst müssen wir erinnern, dass durch das oftmálige Vorkommen von einerlei Art in verschiedenen Schichten die Zahlen der fossilen Wesell, welche man durch Addition der einzelnen Rubriken $\mathbf{a}$ bis $\mathbf{x}$ (mit Ausschluss von o oder der Arten, die in die lebende Schöpfung übergehen) erhält, um fast 1/s grösser ausfällt, als wenn man die einzeln autgeführten Namen zusammenzählt. Unser Enumerator führt fast keine Pflanzen in ziveierlei Schichten auf, indem das doppelte Vorkommen meistens nicht eingetragen ist, daher wir der wichtigsten Fälle S. $763-764$ nachträglich erwähnt haben. Wohl aber ist Solches bei den Thieren durchgehends der Fall. Wir erhalten daher

\begin{tabular}{|c|c|c|c|c|c|c|}
\hline & & & $r$ & & on & 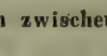 \\
\hline & & & 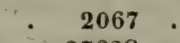 & 12 & 0 & \\
\hline & & & 20020 & 3322 & 1000 & 134 \\
\hline Zusa & en & 26421 & 29695 & 3334 & 100 & $0: 1124$ \\
\hline
\end{tabular}

Daher im Mittel der durch mehrfache Zählung einer Art in den verschie. denen Rubriken entstandene Überschuss (jedoch von dem lebenden Vorkommen abgesehen) $=0,124$ beträgt, wovon indessen wieder eine Quote för fehler hafte Bestimmungen abgelit. Aber im Einzelnen genommen muss bei den Pflanzen Überschuss und Abzählung aus dem angegebenen Grunde kleiner als bei den Thieren bleiben; und unter diesen hat man die Kerbthiere mit Ausnalıme von Trilobiten und Ringelwürmern, die Fische, Reptilien und Vügel nicht oder selten und auch Säugthiere nur wenig in verschiedenen Schichten uder Formationen angegeben. Die Überschüsse und Ausfälle treffen daher vorzugsweise auf die Infusorien, Polyparien, Weichthiere, Ringelwürmer und Trilobiten $(\mathbf{a}+\mathbf{b})$ zusammen. Sie treffen bei weitem mehr in die sehr vervielfältigten und zum Theile nur für unsichere Gesteine aufgestellten oder künttig unter die übrigen einzutheilenden tertiären Rubriken (nämlích fast nur in die gleichzeitige n Rubriken "und $\mathbf{v}$, oder $\mathbf{v}$ und $\mathbf{w}, \mathbf{w}$ und $\mathbf{x}$ ), als in die andern; daher deun auch jene Überschüsse und Quoten keineswegs vollständig tür den Ausdruck des Vorkommens identischer Arten in verschiedenen Formationen genommen werden dürfen.

Ausserdem müssen wir bitten, etwaige kleine Abweichungen in der Zählung derselben Arten unseres Enumerators bei verseliedenen Anlässen zu entsehuldigen, da einige derselben von der Unsicherheit herrühren, die über die geologische Lagerung mancher Arten herrscht, andere aber auf späteren Verbesserungen beruhen.

b) Wir haben schon anderwärts crwälnt, dass man die Zahl der noch in unserem Enumerator eingereilheten Synonyme, die erst in Folge genauer 
Einzeln-Forschungen ihren wahren Spezies zugetheilt werden können, gegen 0,20 schätzen mag, welcher Ausfall aber, freilich in nicht überall entsprechen. der Weise, durch diejenigen Entdeckungen bereits wieder ersetzt ist, die seit Vollendung des Enumerators gemacht worden sind. Da indessen die Pflanzen, nach Göppert's und Unger's monographischen Arbeiten, -- die Infusorien, Polythalamien, Insekten (mit Ausnahme der Ringelwürmer), nach den neuesten Arbeiten von Ehrenberg, d'Orbigny, Berbendt und Burmeister, - die Fische, nach der eben vollendeten Monographie von Agıssiz (wo nur Schuppen, Zähne und Stacheln einer Art zuweilen noch unter verschiedenen Namen stehen), und die 3 höheren Wirbelthier-Klassen nach der sorgfältigen Sichtung Hermans voN Meyer's so ziemlich von ihren bloss synonymen Arten gereinigt sind und diese sich also fast nur bei den Polyparien, Ringelwürmern und Mollusken finden, so mag jene Quote nur für diese anwendbar seyn, und für die oben angeführten 26,420 Arten des Enumerators würden dann nicht viel über 22,00023,000 übrig bleiben, obschon die Fische, Pflanzen u. s. w. keine Verminderung erleiden. Wir müssen bei mehren nachfolgenden Untersuchungen diese ZahlenUnterschiede im Gedächtniss behalten.

c. Unsere paläontologischen Forschungen beschränken sich geographisch auf Europa (und berühren sogar hier nur wenig manche an dessen Grenzen umherliegende Länder, wie Portugal, Spanien, Corsica, Italien, Ungarn und die übrigen untern Donau-Länder, Türkei, Griechenland, einen Theil von Russland), auf den Ural, auf einige kleine Punkte in Ostindien und Neu-Holland, der Nordküste Afrikas und am Kap, auf die Vereinten Staaten und einige Stellen in Südamerika. Man mag daher annehmen, dass die ganze übrige Erd-Oberfläche noch nicht zu $1 / 5-1 / 6-1 / 8$ so genau erforscht ist, als Europa.

d. Diese unvollständige geographische Kenntuiss fällt mit der Kürze der Zeit zusammen, die wir uns erst mit paläontologischen Forschungen beschäftigen. Die ersten wissenschaftlich systematischen Bestimmungen von fossilen Körpern lieferten uns Brander 1766, und LAMARCK seit 1802-1806, beide begünstigt durch die vortreffliche Erhaltung der fossilen Konchylien des eocïnen Pariser-Londoner Beckens; Blumenbach 1803 wenige; Schlotheim 1804, 1816 und 1820 noch sehr ungenügend; Sowerby 1812 und Brocchi 1814; Cuvier 1812 in seinen Ossemens fossiles (deren Theile jedoch schon zuvor einzeln in den Annales du Museum erschienen waren). Der Brander'sthen Arten mögen unsers Wissens 100 seyn. Laмакск wird damals (mit einigen späteren Nachträgen in seiner Histoire naturelle) etwa 600 beschrieben haben; was also mit einigen Pflanzen bei Schlorнerm und einer nicht grossen Anzahl von Wirbelthieren bei Cuvier die ganze Summe unserer Kenntniss in dieser Beziehung ausdrückt zur Zeit, als Sowsrby seine Mineral.Conchology und Brocchi seine Conchologia begannen. Freilich lag ausserdem noch eine Menge von Abbildungen, die man später der Art nach zu erkennen keine Mühe hatte, in mancherlei älteren Werken (von Soldant, Fichrel und Moli, Volta, Denis MonFort, Sendel u. v. A.) zerstreut, aber ohne systematische Namen, ohne zuverlässige Bestimnung. Wenn nun auch die von Schlotнeim gelieferten Beschreibungen und Bezeichnungen der Arten seiner Sammlung durchaus ungenügend und nicht besser waren, als die so vieler Andern, so bot uns doch sein 1820 erschienenes Handbuch zusammen mit den andern genannten Autoren (Sowerby, so weit er damals erschienen war) abermals eine vollständige Übersicht der bis dahin bekannten Arten dar. Die neue sehr bereicherte Ausgabe von Cuvisn erschien unmittelbar darauf (1821-1824); und erst seit dieser Zeit ist die $P_{a}$ läontologie Gegenstand allgemeiner Thätigkeit. Wir wollen bier keine Geschichte derselben liefern, sondern nur einige Data feststellen, um die Zunahme 
der bekannten Arten fosşiler Organismen zu beleuchten. Die Zunahme der bekannten Arten war

bei Pflanzen:

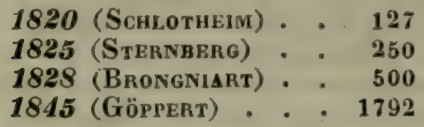

bei Thieren ungefähr:

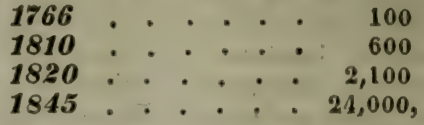

wornach sich also die Zahl der fossilen Pflanzen seit 1800 ungefähr alle 6 Jahre, die der Thiere alle 7 Jahre verdoppelt hätte, obschon Diess bei beiden in den 2 letzten Dezennien, wo die absolute Zahl doch schon sehr beträchtlich, etwas langsamer geschehen wäre als vorher. Ist nun auch ein zunehmendes Steigen in dieser Progression für die Dauer nicht mehr möglich, so würde doch schon eine bleibende stetige Vermehrung der bekannten Arten fossiler Organismen, wie sie auf das letzte Dezennium fiel, in runder Summe zu mindestens 10,000 Arten angenommen, uns nach einem halben Jahrhundert schon wieder die doppelte Anzahl neuer Arten liefern, welche jetzt bekannt ist.

e. Wie sehr die Entstehungs-Weise und Mineral-Natur der FelsArten eines Zeit-Abschnittes auf unsere Kenntniss von der damaligen Fauna und Flora Einfluss haben müssen, mögen einige Beispicle erläutern. Die ausgedehntesten Meeres-Gebilde können uns keine Reste von Landbewohnern bringen; diese finden sich nur an den Küsten-Rändern der ersten und in Süsswasser-Niederschlägen, welche aber gänzlich fehlen bis zur Wealden-Bildung. Gleichwohl hat es schon vor der Zeit der Kohlen-Formation eine Menge Land-Pflanzen gegeben, in deren Blättern Insekten-Larven ihre Gänge ausböhlten; und die Kohlen-Formation hat auch einige Arachniden, Orthopteren, Neuropteren und Käfer selbst geliefert. Insekten vieler Klassen haben daher in jener Zeit schon bestanden; aber es fehlte an Gesteinen, die für ihre Aufnahme und Erhaltung günstig waren. Sulche Gesteine sind äusserst selten; doch haben sich in England einige sehr zarte Lias - und Oolith-Schichten (m, $\mathbf{n}$ ), in Deutschland der Solenhofer Schiefer $\left(\mathbf{n}^{6}\right)$ und in England wieder die Wealden. Formation (P) günstig gezeigt, welche als Gebilde des Süsswassers, so wie die Solenhofer Schiefer als Absätze eines vielleicht nur brackischen, jedenfalls aber kleinen von Land umschlossenen Beckens dem trockenen Lande nahe genug waren, un gelegentlich Insekten von daher aufzunehmen. Dennoch mangelı uns von da an abermals alle Insekten bis in die Miocän-Zeit herunter, wo die Schiefer von Aix, von Radoboy, von Öningen, und der Bernstein uns eine reiche Ausbeute gewähren. Aber auch die Ausbeute in Lias - und OolithSchiefer ist nicht nehr arm, nur des zerstückten Zustandes der fossilen Theile wegen schwer bestimmbar; einzelne Geschlechter sind sogar für eine so beschränkte Örtlichkeit reich zu nennen und beweisen, dass auch die anderen Insekten-Gruppen reich vorhanden gewesen seyn müssen; denn die Libellen sind gefrässige Raub-Insekten, welche andere Insekten im Fluge haschen. Sie setzen ferner, nicht wie die meisten übrigen, nur im ausgrebildeten Zustande trockenes Land als Aufenthalt voraus, sondern auch stagnirendes Süsswasser für ibre Larven-Zustände, da sich unseres Wissens wenigstens ihre Larven nie im Salzwasser auflalten (Ephemera, Agrion, Libellula und verwandte Ge. schlechter); und doch haben wir fast keinen direkten Beweis für das Vorhandenseyn von Süsswasser in und vor dieser Zeit, da man sich die Steinkoh. len-Lagen mit ihren Pflanzen-Resten und Familien, die jetzt dem Süsswasser angehören, noch immer als mit dem Meere in Verbindung gestandene Niederschläge denkt, was auch durch See-Konchylien in denselben an manchen Orten bestätigt wird. Das Unio-ähnliche Muschel-Geschlecht Anthracosia kann als ein ausgustorbenes keinen bestimmten Beweis liefern. 
f. Sehr kleine weiche Thiere haben uns überhaupt keine kenntlichen Reste hinterlassen können: so die Magen-Infusorien ohne Kiesel-Panzer (die gepanzerten machen nur eine verhältnissmässig kleine Familie derselben aus), die Räder-Thierc. - So auch die sehr zerfliesslichen Quallen oder Acalephen nicht, mit Ausuahme der Porpiten. - Eben so wenig die weichen und immer in andern Thieren eingeschlossenen, in diesen nothwendig bald verwesenden Eingeweidewïrmer. Die nackten Weichthiere enthalten mit wenigen Ausnahmen keine Erhaltungs-fähigen Theile (die Limax-Arten und die Sepien nur zum Theil innere Schaalen, letzte mitunter harte Kiefer und an den Armen hornartige Häckchen oder Saugnäpfe); die nackten Ringelw'ürmer und die meisten insbesondere weicheren lnsekten (mit Ausnahme der grösseren kalkschaligen Kruster) setzen wenigstens sehr günstige Verhältnisse voraus, wenn die Hülle ihres Körpers sich kenntlich erhalten soll. Selbst unter den $\mathrm{F}$ ischen sind einige Ordnungen, die Leptocardii (Amphioxus) und Cyclostomi (mit Ausnahme der hartzähnigen), welche kium einen fossilen Rest zu hinterlassen vermögen. Dicse Thiere werden also nicht oder nicht leicht unter den fossilen Arten vorkommen und bei den paläontologischen Untersuchungen einen Ausfall veranlassen nicht nur in der Anzahl der fossilen Arten und in ihrem Zahlen-Verhältniss zu den Arten anderer Gruppen, sondern auch in der Repräsentation gewisser Formen, welche gleichwohl mehr oder weniger sicher schon in früherer Zeit existirt haben. - Eben so werden bei den Pflanzen die Zellen-Pflanzen weit weniger zur Erhaltung im Fossil-Zustande geeignet seyn, als die Holzbündel-haltenden Gewächse: es werden insbesondere die mikroskopischen Formen, die zerfliesslichen Pilze, die Flechten, die Moose, die meisteu Süsswasser-Algen einer früheren Zeit sogar in den günstigsten Verhältnissen selten zu uns gelangen können; nur die Leder-artigen und holzartigharten See-Algen erhalten sich im Fossil-Zustande leichter. Und selbst von den Gefäss-Pflanzen sind nur einzelne Theile, die Stämme (falls sie in frischem unverfaultem Zustande im Gebirge eingeschlossen werden), die Blätter und harte Fruchtschaalen unter günstigen Umständen zur Erhaltung geeignet, die Blüthen, weiche Früchte und die Saamen aber sehr vergänglich. Die Pflanzen leben nur geringentheils, die Thiere schon grösstentheils in den Wassern, aus welchen die einschliessenden Schichten sich absetzen.

g. Für die absolute wie relative Länge der geologischen Zeit$R a ̈ u m e$, die wir mit einander vergleichen wollen, haben wir durchaus keinen Massstab. Man hat solchen in der Mächtigkeit der Schichten gesucht, die indessen überall eine andere ist und nur ganz im Grossen, unter Berücksichtigung aller Welt-Gegenden vielleicht einiges Anbalten wird bieten können. Ein anderer Massstab ist die Menge und die Umgestaltung der ihnen entsprechenden organischen Wesen selbst, von welchem wir aber hier keinen Gebrauch machen können, da wir eben umgekehrt cinen Zeit-Massstab suchen, um damit diese letzten zu messen.

\section{§. 7. Arten-Zahlen.}

A. Vergleicht man die Anzahl der fossilen Pflanzen- und ThierArten überhaupt, so weit solche jetzt bekannt sind, mit der der lebenden, so erscheint die der fossilen im Gauzen allerdings noch beträchtlich kleiner als die der letzten, obschon sie immerhin eine sehr anselunliche Menge ausmachen, wenn man bedenkt, dass ihrem Studium noch kaum ein Drittheil so viel Zeit gewidmet worden ist, als dem der lebenden. Die fossilen Thiere betragen nämlich fast ein Viertel, die fossilen Pflanzen ein Fünfunddreissigstel und beide zusammen über ein Sechstel der lebenden. 


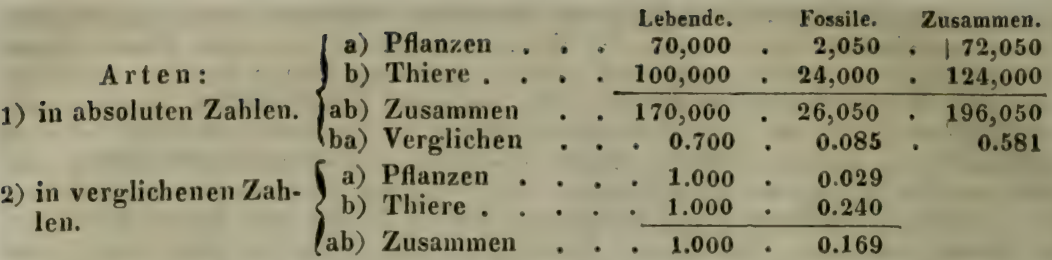

Wir haben dabei die lebenden Pflanzen uur zu 70,000 angenommen, obwohl sie GöPPERT ') auf 80,000 berechnet, wo man aber auch die Thiere höher setzen müsste.

Während also die Zahl der lebend bekannten Thier-Arten der der Pflanzen nahezu gleichkommt (1000: 700), ist die Differenz. der fossilen unter sich achtmal grösser (1000:85), und das Verhältniss beider zusammen ein weit über mittles (1000:581). - Während bei den Thieren von der Zahl der lebenden Arten die der fossilen beinahe ein Viertel beträgt (1000:240), macht sie bei den Pflanzen wenig über den zehnten Theil so viel aus $(1000: 29)$, und ist sie für beide zusammen eine wenig über mittle $(1000: 169)$, $d$. h, sie beträgt über ein Sechstel derselben.

B. Wie schon oben (S. 784) gesagt, ist die mittle Dauer einer fossilen Art = 1,12 unserer Formationen, oder von 100 Arten einer Formation gehen 12 noch in eine andre, doch nicht immer nächste Formation über, durch welch letzten Umstand die Dauer eine noch merklich längere wird; dagegen sind die auch in die lebende Schöpfung übergehenden Arten hiebei nicht mit berechnet.

Wir haben schon oben (S. 784, a) zu dieser Berechnung das Erläuternde bemerkt und verweisen desshalb darauf.

C. Diese Anzahl fossiler Arten hat aber nicht gleichzeitig neben einander bestanden, sondern vertheilt sich auf die ganze geologische Zeit. Will man daher den numerischen Reichthum ehemaliger Bevölkerung mit dem der jetzigen vergleichen, so darf man weder unsre ganze jetzige Fauna und Flora mit der ganzen fossilen noch mit derjenigen fossilen des kleinen Theiles der Erd-Oberfäche, welcher bis jetzt genauer geologisch untersucht ist (S. 755, c), noch auch die lebende eines einzelnen Landes mit der fossilen desselben Landes im Ganzen oder aus einer einzelnen Periode desselben, sondern höchstens aus einer einzelnen Formation neben einander stellen. Solcher Formationen wird man etwa 15 zählen; noch richtiger aber wird man die ganze geologische Zeit als durch einen etwa 30-40maligen Arten-Wechsel (Ärten-Alter wie Menschen-Alter) gemessen sich vorstellen.

- Oft vergleicht man die fossile Fauna und Flora im Ganzen mit der leben. den im Ganzen, was indessen nicht weiter dienen kann, als um einen allgemeinen Begriff von den bisherigen Fortschritten der Paläontologie zu erlangen. Oft vergleicht man die Flora und Fauna der Jetztzeit mit der irgend einer Erd. Periode, welche doch noch immer mehre $(3-4-5)$ allmählich entstehende und vergehende Floren und Faunen in sich enthält. Selbst die Vergleichung unsrer

1) Jahrb. 1845, 408 . 
ganzen jetzigen Flora oder Fauna mit der fossilen irgend eines noch kürzeren geologischen Zeitraumes, dem einer Formation, muss zum Nachtheil der letzten ausfallen, weil wir den Inhalt dieser Formation nicht, wic die jetzige Fauna und Flora, auf allen Theilen der Erd-Oberfäche, in allen Längen und Breiten, sondern nur an einzelnen Stellen kennen, welche in keinem Falle geeignet waren, alle Organismen oder auch nur alle Familien, alle Ordnungen von Organismen uns zu ïberliefern, während andrerseits auch eine solche Formation noch oft mehre Arten in successiven Schichten darbietet, die in keiner derselben zusammentreffen. Allerdings gehen selbst aus einer Formation in die andere einzelne Spezies über, und es stellt daher nicht einmal jede Formation eine ganz neue und selbstständige Organismen.Welt dar, und man würde nur etwa folgende 15 Formationen als Repräsentanten je eines Zeit-Abschnittes betrachten können, der keine erhebliche Zahl von Arten mit den Nachbarn gemein hätte:

a?, b, e, de, fge, ikl, m, $\mathbf{m}^{2}$ (vielleicht $3-4$ fach), $\mathbf{m}$, (p ist bloss Süsswasser-Bildung) $\boldsymbol{q}, \mathbf{r}, \mathbf{s ,}, \mathbf{t}, \mathbf{u v w x}$.

Gehen danu doch noch einzelne Spezies aus einer dieser Formationen in die andre über, so sind deren nur wenige, nach vorigem Paragraphen nur 0,12 , so dass unter 100 Arten noch 12 zwischen jedesmal zweien dieser Formationen gemeinschaftlich wären; eine $Z$ ahl, die noch sehr ansehnlich vermindert wird, wenn man berechnet, dass wir $\mathbf{i}, \mathbf{k}$, zusammengeworfen, $\mathbf{k}$ nur für zwei gerechnet, $\mathbf{u}, \mathbf{v}, \mathbf{w}, \mathbf{x}$ wieder vereinigt haben, wodurch dann fast allen gemeinsamen Arten bis auf vielleicht 200-300 (auf 25,000, also $=0,01$ ) gänzlich ausfallen werden. Aber selbst die noch übrig bleibenden reichen dann in der Regel nicht durch zwei benachbarte Formationen ganz hindurch, sondern nur in deren unmittelbar aneinander grenzenden Schichten hinein. Da indessen andrerseits gewiss eine sehr grosse Anzahl der Arten einer solchen Formation ebenfalls nur durch $1-2$ der ihr untergeordneten Glieder hindurchgehen und daher sogar in jeder Formation noch einen 2-3fachen Wechsel darstellen, was für das Silur-System aus dem Murchison'schen Werke, wie für das Lias- und Oolithen-Gebirge aus Quenstedt's "Grebirge Württembergs", für das Kohlen-Gebirge aus Göppert's Preisschrift über die Steinkohlen-Bildung (Haarlem 1848, $4^{0}$ ) etc. genügend erhellt, so dürfte man die Zahl der successiven Schöpfungen statt auf 15 richtiger auf 30 bis 40 und noch mehr anzusetzen haben $(\mathbf{n}, 2-3 \mathbf{b}, \mathbf{c}, \mathbf{d}, \mathbf{e}$, $\mathbf{f}, \mathbf{s}, \mathbf{i}, \mathbf{k}, \mathbf{I}, 2-3 \mathbf{m}, 5 \mathbf{n}, \mathbf{o}, \mathbf{p}, 2 \mathbf{q}, \mathbf{r}, 3 \mathbf{s}, \mathbf{t}, \mathbf{u}, \mathbf{w}, \mathbf{x})$, wenn man nämlich mehr von einer gemeinschaftlichen Begrenzung der Daucr einer jedesmal grossen Anzahl dieser Arten absehen und nur die Anzahl der wahrscheinlichen Arten-Wechsel im Ganzen etwa so beurtheilen will, wie man die mittle Zahl der Generationen des Menschen, der Menschen-Alter, binnen einer gewissen Zeit-Periode brechnet, obschon jedes Individuum zu einer andern Stunde geboren ist, ein andres Alter erlangt und zu einer andern Stunde stribt, als die übrigen.

D. Ganz örtlich gehaltene Vergleichungen ${ }^{1}$ ) zwischen der jetzigen und allen Abschnitten der geologischen Schöpfung (welche mithin beiderseits gleich kurzen Zeit-Abschnitten entsprechen) führen zur Überzeugung, dass es zu jeder Zeit Gruppen von Pflanzen und Thieren gegeben hat, welche durch nicht minder zahlreiche oder noch zahlreichere Arten als in der jetzigen Schöpfung vertreten waren, und dass daher zu jeder Zeit die Fauna und Flora, so we it sie damals

1) Diese Ansicht und einige darauf gestützte Berechnungen sind schon vor mehren Jahren (Jahrb. 1846, 510) von Agsssiz veröffentlicht worden; aber nicht in Verbindung mit den vorangehenden Sätzen $(C)$; mich hatten meine Arbeiten schon früher darauf geführt. 
schon ihre Repräsentanten hatten, im Allgemeinen wenigstens eben so zahlreich waren, als jetzt, wenn man nämlich den Umfang der früher repräsentirt gewesenen Gruppen nicht weiter ausdehnt, als er in der That nachweisbar ist; wo dann freilich dem Umfange der aus der Beobachtung zu ziehenden Folgerungen immer einige Willkühr beigelegt werden kann.

Die Frage ist nämlich: Wenn die Entomozoa - Crustacea - Malacostraca - Decapoda - Macrura - in einigen Familien seit der Jura-Zeit (solenhofer Schiefer oder Lias) reichlich vertreten gewesen sind, in wie fern sind hiedurch alle Familien überhaupt und in wie ferne sind die Macrura, die Derapoda, die Malacostraca, die Crustacea, die Entomozoa überhaupt seit derselben Zeit als reich vertreten zu betrachten? Man würde freilich die reiche Vertretung bis zu den Familien nachweisen müssen, wenu man nicht an vielen und verschiedenartigen Orten zu einer Zeit eine z. B. gleich zahlreiche Flora beisammen fände als jetzt in diesen Orten von gleicher Ausdehnung; in diesem Falle würde man aber auch ohne Vergleichung bis in die Familien herab obigen Schluss ziehen dürfen.

Wir wollen einige Belege hier aufzählen.

a-e. Es wird für die ältern Formationen schon genügen, wenn wir auf unsre Listen der Plantae vasculares monocotyledones, auf die Anthozocn-Gruppe, auf die der Brachiopoden, der Cephalopoden, der Trilobiten, der GanoidenFische u. s. w. verweisen, um die Überzeugung zu erwecken, dass Fauna und Flora, soferne sie damals üherhaupt vertreten waren, nicht arm gewesen seyen, obschon diese Listen aus verschiedenen Perioden und noch verschiedeneren Lokalitäten zusammengetragen sind.

h. Die St.-Cassianer Bildang mag in die II. Periode oder zu m gehören, jedenfalls besteht sie aus einer nicht grossen Schichten-Reihe auf beschränkter Örtlichkeit, die eines unsrer Arten-Alter nicht überreicht, für welche immer eine Meeres-Fauna aus 700 Spongien-, Korallen-, Echinodermen- und Weichthier-Arten reicher ist, als wir sie in der jetzigen Schöpfung irgendwo auf gleichem Raum zusammenbringen könnten. Insbesondere bietet sie wenigstens 20 Cidaris-Arten dar, während in allen europäischen Mecren jetzt nur 3 Arten vorkommen ") und E. Fopres die Gesammtzahl der im Mittelneer lebenden Echiniden nur auf $12-15$ und die des ägäischen Meeres nur auf 9 angibt ${ }^{2}$ ), während Norwegen deren 13 hat ${ }^{3}$ ) und ganz Europa nicht 40 Arten zäblt.

m. Während man vor dem Lias noch kein Dutzend geflügelter Insekten zusammengebracht hat, bietet uns eine sehr beschränkte Örtlichkeit in England nicht weniger als 24 Genera mit 50 Arten und dabei 4 Libellulinen aus 3 Geschlechtern auf einer Meeresschicht-Fläche dar in einer Gegend, für welche jetzt anf trockenem Lande vielleicht nicht die doppelte Arten-Zahl lebend zusammenzufinden wäre? Diese Insekten aber verzeliren im Larven- wie im reifen Zustande eine grosse Menge andrer, theils in Wasser lebender, theils fliegender Insekten, deren Anwesenheit sie uns also verrathen, anch wenn wir solche nicht finden. - Auch ist die Zahl der Lepidoiden- und Sauroiden-Fische sehr ansehnlich, die man in andern Gegenden Englands in wenigen Steinbrüchen beisammengefunden hat. So haben die Lias-Schiefer-Brïche von Lyme-Regis allein 3 Sippen 21 Arten Elasmobranchier und 18 Sippen 49 Arten Ganoiden

1) Agassiz et Desor Catalogue des Echinodermes, 1847, 142.

$\left.{ }^{2}\right)$ Ann. nathist. 184, XIII, 517.

3) Isis 1848, 534 . 
(deren heutige Vertreter sich auf 4 Sippen 27 Arten im Ganzen beschränken) geliefert.

m. Aus Forest marble des Calvados in den Gemeinden Ranville, Luc, Lebisey und Langrune hat Michelis allein 67 Arten Polyparien und Spongiaren beschrieben, die man jetzt wohl auf keiner Küsten-Strecke von einigen Stunden Länge auffinden würde; da EHrenberg im ganzen Rothen Meere, das doch ein Drittel aller bekaunten Korallenthier-Arten enthält, nicht über 120 Arten zusammen bringen kounte.

$\mathbf{m}^{5}$. Aus dem obern Jura von Streitberg hat GoldFuss 45, von Giengen 17, von Nattheim 8, von Thurnau 7 Arten Spongiarien und Polyparien beschrieben, ohne deren zu gedenken, welche nun noch von schon anderwärts beschriebenen Arten an denselben Orten vorkommen. Im Ganzen aber zählen Hartmans an 80 Arten in Württemberg allein, und Gondfuss und Münster allein 40 ScyphiaArten dieser Formation in Franken und Schwaben auf. Münster. hat der Bayveuther Naturalien-Saınmlung 130 Arten Polyparien und Spongien mit allein 67 Scyphien aus Franken (Streitberg, Muckendorf, Rabenstein) gegeben. Alle diese Arten stammen aber aus einer Gebirgs-Abtheilung, welche weder einer vollen Arten-Dauer $\left(\mathbf{n}^{5}\right)$ entspricht, noch die alleinige Gebirgs-Facies aus dieser Zeit seyn kanı. Vergl. die folgende.

$\mathbf{m}^{5}$. Einer der wichtigsten Fund-Orte ist das Solenhofer-Gebilde, weil es, obschon hinsichtlich seiner Stellung genügend charnkterisirt, doch wieder so eigenthümlich in seinen Fossil-Resten ist, dass man es in seiner ganzen Aus. dehnung und Mächtigkeit sicher als blos örtliche Facies gleichzeitig einer andren Gebirgs-Bildung als Erzeugniss e iner Periode ansehen kann, wo ausser der allmählichen Auffüllung des See-Grundes, der seinen Einfluss auf die dortige Lebenwelt wohl nicht verläugnet haben mag, kaum irgend ein audrer Wechsel cingetreten ist. Diese Örtlichkeit liefert ausser Konchylien manchfalliger Art u. a. auch

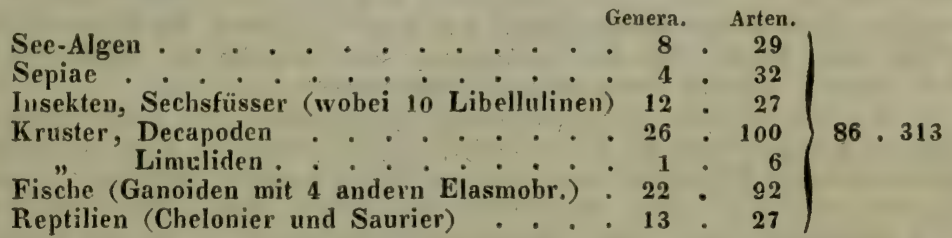

Einen solchen Reichthum an Pflanzen und Thieren aus den genannten Klassen und Ordnungen (deren Arten zudem fast alle sonst nirgends angetroffen werden, als in dieser Örtlichkeit) dürfte man auf einer Fläche von wenigen Quadrat-Meilen jetzt nirgends bcisammen finden, die sechsfïssigen Insekten ausgenommen, welche, dem Wasser fremd, nur um der vielen Libellulinen willen hier mit aufgenommen worden sind.

Niঞsu, dessen See-Thiere seit langen Jahren am sorgfältigsten beobachtet worden sind, hat nach Risso doch nur 105 Sippen mit 310 Arten Fische aller Ordnungen, nach Verany 12 Genera 28 Arten Sepien mit und ohne Schulpen, 72 sehr zerspaltene Genera Crustaceen mit 108 Arten (44 G. 72 A. Decapoden). Chelonier und Saurier sind fast mehr als ganz Europa jetzt liefern könnte.

p. Ähnlich verhält es sich mit dem abgeschlossenen norddeutschen und englischen Becken der Wealden-Formation ')

1) Jahrb. 1846, 856 . 


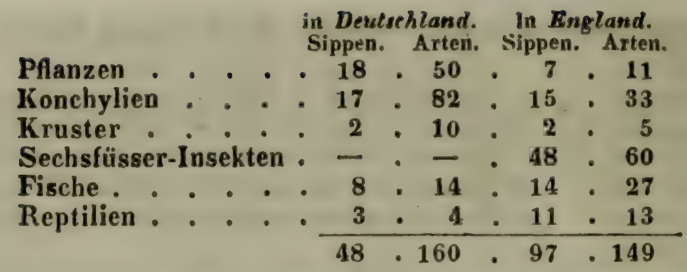

In dem norddeutschen Becken allein erscheint das Genus Cyrena mit 38, d. i. anderthalbmal so viel Arten, als man jetzt über die ganze Erd-Oberfläche lebend zählt, und die Süsswasser-Genera Limnaeus, Planorbis, Paludina, Neritina u. a. zum ersten Male mit ächten Arten. Überall wird es jetzt schwer seyn, ein abgeschlossenes Süsswasser-oder Brackwasser-Becken mit 8 Sippen und 14 Arten oder gar 14 Sippen und 27 Arten Fische zu finden, oder in demselben und seinem Unfange 11 Sippen 13 Arten Reptilien zu entdecken; und doch ist das, was wir aus diesen Becken kennen, keineswegs die ganze Anzahl der darin vorhanden gewesenen Arten. Wegen der Fische vrgl. noch $\mathbf{n}^{5}, \tau$ und $\mathbf{v}$.

$\mathbf{r}^{2}$. Die beschränkte und in ihrem Fels-Gebilde ebenfalls eigenthümliche Örtlichkeit von Masıricht, die man dem sogenannten Système Danien zuzu. schreiben geneigt ist, hat uns, um von ihren merkwärdigen Reptilien (Schildkröten und Sauriern), ihren zahlreichen Konchylien, ihren Krustern und Foraminiferen zu schweigen, 9 Genera mit 19 Arten Radiaten, 4 Genera mit 8 Arten Amorphozoen und 11 Genera mit 51 Arten Polyparien (Anthozoen und Phytozoen) geliefert, die man jetzt nur etwa in tropischen Breiten auf so kleinem Raume beisammen finden würde. Bei Nisza zählt Verany ohne die Holothurien nur 8 Genera mit 23 Arten Radiaten (meist Asterias, aber gar keine Kalk-Polypen) auf.

(\$) $\tau$. Dem Monte Bolca danken wir ausser 13 Arten Fucoiden 71 Genera mit 128 Arten Fische, alle aus der Ordnung der Teleostei, für welche bei Nisঞa nur 93 Genera mit 270 Arten übrig bleiben würden.

t. Aus den eocänen Bildungen heben wir ebenfalls cine lokale SüsswasserFormation heraus, weil dic zur Vergleichung dargebotenen Meeres-Gebilde des Englisch-Pariser Beckens in vertikaler wie in horizontaler Richtung zu unsicher begrenzt sind. Das kleine Süsswasser-Becken von Rilly zählt nicht weniger als 14 Sippen von Land- und Süsswasser-Konchylien mit 39 fast ihm ausschliesslich zustehenden Arten, eine Anzahl, welche auffallender erscheinen muss, wenn man berücksichtigt, dass 8 Sippen und 24 Arten Land-Konchylien darunter sind, die nur mehr zufällig vom Lande her hineingerathen seyn können, und die LandKonchylien der Gegend nur ungenügend vertreten dürften. Eine sorgfältige Durchforschung der Umgegend unsrer Stadt auf 4 Stunden im Unkreise gibt nur 90-100 Arten, wovon etwa die Hälfte gemein, die übrigen mehr oder weniger selten sind '); heisse Gegenden sind nicht reicher an Binnen-Konchylien als gemässigte. - Ebenso weisst MARcbe dr Serres in dem mit vorigem ungefähr gleich-alten, aber durch andre eigene Schnecken-Arten bezeichneten Süsswasser-Gebilde von Castelnaudary, Aude, folgende Fauna nach ${ }^{2}$ ).

$$
\begin{aligned}
& \text { Säugthiere Sippen. Arten. } \\
& \begin{array}{l}
\text { Reptilien } \\
\text { Binnen-Konchylien, fast nur Land-Bewohner: }
\end{array}
\end{aligned}
$$

für welche einzelne Ortlichkeit die Zahl der Reptilien wieder nicht gering ist.

1) Jahrb. 1848, 637 .

2) Jahrb, 1845, 738 . 
u. Die miocănen Seethier-Reste, welche Micwszorry kürzlich $\left.{ }^{1}\right)$ beschrieben hat, belaufen sich auf

\begin{tabular}{|c|c|c|c|c|c|c|}
\hline & & & Sipper & & Arten. & \\
\hline Rhizopoden & & & 8 & - & 19 & \\
\hline Polypen & & . & 33 & . & 103 & \\
\hline Echinoderme & & . & 8 & • & 23 & \\
\hline Kruster : & • & • & 1 & . & 1 & $171: 470$ \\
\hline Cirripeden & $\bullet^{\circ}$ & • & 3 & - & 6 & \\
\hline Annelliden & $\bullet$ & • & 1 & . & 1 & \\
\hline Konchylien & • & . & 117 & . & 587 & \\
\hline
\end{tabular}

Sie sind zwar einer ansehnlichen Mergel-Schichtenfolge bei Turin, Asti, Tortona und selbst dem ziemlich entlegenen Bacedasco im Piacentinischen entnommen, die aber in ihrer Natur und ihren fossilen Resten selır gleichförmig ist und keinen Wechsel der Dinge während ihrer Absetzung andeutet. Nun aber hat dE Gerville ${ }^{2}$ ) auf der ganzen Küste des Manche-Departements nur 28 Genera (im alten Sinne) mit 180 Arten lebender Konchylien und 9 Cirripeden, - Philippr an einem grossen Theile der Sicilischen und Kalabrischen Küste, mit Ausschluss der nackten Arten und Binnen-Konchylien, nur 545 Arten lebender Konchylien und 18 Cirripeden zusammengebracht, so dass die frühere Miocän-Fauna des Meeres keineswegs im Rückstand gegen die jetzige ist.

u. Das Wiener Becken hat bereits 1018 Thier-Arten geliefert, worunter 23 Säugthiere, 65 Fische, 499 Weichthiere, 63 Kruster, 251 Foraminiferen, 153 -207 Polyparien ${ }^{3}$ ), die sich mit denen entsprechender Küsten-Strecken unsrer jetzigen Meere überall messen dürfen und sie oft namentlich an Polyparien selbst in den reichen Tropen-Gegenden übertreffen werden; und doch ist Diess nur die Ausbeute weniger Jahre.

u. Für die mittel-tertiären Schichten wählen wir noch 2 sehr kleine, selbst nur sehr wenig von einander entfernte Stellen bei Wiesbaden und Hochheim, ebenfalls Süsswasser-Bildungen zweifelsohne aus einer sehr kurzen Zeit, von wо Tномё Helix mit 32 Arten und 12 andere Sippen Binnen-Konchylien mit noch 23 fast durchaus neuen Arten beschreibt $\left.{ }^{4}\right)$. Nach AL. Braun bietet Hochheim 57, Wiesbaden 22 Arten Land-Schnecken, wovon ihnen jedoch, ihrer Nähe ungeachtet, nur 8 gemeinschaftlich zustehen. In ganzen, ebenfalls geographisch nicht ausgedehnten miocänen Süsswasser-Becken, das kaum 9 Quadrat-Meilen einnimmt, zählt AL. Braun 74 Arten Land- und 28 Arten Süsswasser- und Brackwasser-Konchylien, zusammen also 102 Arten aus 20 Sippen, oder so viele als man jetzt in derselben Gegend überhaupt aufzutreiben im Stande seyn würde. Denn es bietet dasselbe Rheinbecken von

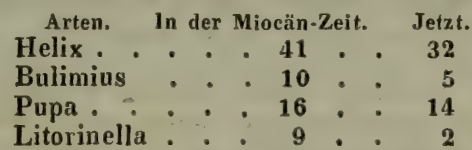

u. s. w. Im Ganzen war einst die Zahl der Land-Konchylien grösser als jetzt und ist jetzt die der Süsswasser-Konchylien grösser als einst, wenn man nämlich die Arten des Brackwassers (Dreissena, die Litorinellen und einige Neritinen, einige Cerithien, Potamiden u. s. w.) nicht mitzählt. Und wenn man nun dabei an einer einzig e $n$ Lagerstätte in demselben Becken von meist grösseren Säugthieren noch 17 Sippen mit 32 Arten allein von Kaup beschrieben findet, ohne die

1) In den Naturkund. Verhandl. van de Maatsch. te Harlem, 1817, b, III, II, 1-408, pl. 1-17.

2) Mém. Soc. Calvad. 1825, 169-224.

3) Jahrb. 1848, 757; 1849, 105.

4) Jahrb. 1845,629 . 
kleineren und anderen Orten dieses Beckens entnommenen Arten bei Herm. v. Mвуек u. s. w. zu rechnen, so unterliegt keinem Zweifel, dass Diess so viel ist, als was jetzt irgend eine eben so grosse Strecke der Erd-Oberfläche bieten kann.

u. Die Sïsswasser-Ablagerung von Sansan in Gers-Departement ist gleichen Alters mit der Mainzer; LARTEt hat darin 76 Säugthier-, 22 Reptilien- und viele Vögel-Arten gefunden, während Deutschland in grosser Ausdehnung genommen jetzt kaum 60 und 30 wilde Arten aus beiden ersten Klassen zählt ' ${ }^{1}$ ).

v. Die eigenthümlichen, einer jedenfalls nur kleinen ungetheilten BildungsZeit angehörigen Molasse-Mergel von öningen haben bis jetzt geliefert :

Pflanzen nach Ac. BrauN ${ }^{2}$ )

Sippen. Arten.

Weichthiere (Süsswasser-Bewohner).

Insekten, vorerst nur die Käfer bestimmt von Oswald

НввR ${ }^{3}$ ). . . . . . . . . . . .

$\cdot \cdot \cdot \cdot \cdot \cdot 13 \cdot, 19$

Reptilien nach H. v. MeYer . . . . . . . . . . . . 12 . 16

Vögel

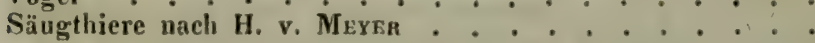

$\frac{x \cdot x}{130+x \cdot 197+x}$

Vou allen diesen Resten sind aber, ausser etwa einigen Pflanzen und In. sekten, nur die Mollusken, Fische und Reptilien an ihrer Stelle, die Reste aller übrigen Organismen nur zufällig in diese Schichten gerathen, mithin jedenfalls nur ein kleiner Theil der durch sie vertretenen Klassen oder Familien. Diese Reptilien aber, ausser den Batrachiern jetzt überhaupt in sehr geringer Anzahl in Europa, treten in grösster Manchfaltigkeit auf und in einer Menge, wie man sie wieder nicht leicht anderwärts beisammen antreffen würde. Von Fischen zählt Hartmanv ${ }^{4}$ ) in allen daran so reichen Flüssen und See'n der Schweils 13 Sippen mit 44 Arten auf, von welchen dem fischreichsten, dem Budensee, 6 Arten fehlen. Für die Flüsse und Teiche der Gegend von Mains hatte Nau ${ }^{5}$ ) nur 10 Sippen mit 33 Arten, für jene von Ulm von Martens ${ }^{6}$ ) 10 (13) Sippen mit 35 Arten. Aber es wïrde unmöglich seyn, zwei Drittheile dieser Arten in einem Teiche oder Flusse beisammen zu finden, wie Das mit den bezeichneten fossilen Arten der Fall ist. Zwischen Karpathen und Pyrenäen kennt man aber bis jetzt schon 17 Sippen mit 42 Arten miocäner Sïsswasser-Fische ${ }^{7}$ ).

Von gleichem Alter und gleicher Bildung mit Öningen ist Parschlug in Steyermark, welches ausser Resten von Mastodon angustidens wie zu Öningen auch 4-5 Insekten-Arten und 67 Sippen Pflanzen mit 140 Arten geliefert hat, aus welchen 19 mit Öningenschen identisch sind. Diese Manchfaltigkeit von Pflanzen- und insbesondere von Baum - und Strauch.Blättern, fast ohne Kräuter, in 2 nahe übereinander liegenden Schichten einer einzigen Fundstätte ist so ansehnlich, dass UNGer ${ }^{8}$ ) annimmt, sie seyen aus cinem weiten Fluss-Gebiete dahin zusammengeschwemmt, wogegen jedoch wieder ihre vortreffliche Erhaltung spricht.

Diese Beispiele, aus ungleichen Zeiten und von verschiedenen Natur-Wesen

1) Jahrb. 1848,725 .

2) Jahrb. 1845, 164.

3) Jahrb. 1846, 161, 721 .

*) Helvetische Ichthyologie, Zürich 1826, $8^{\circ}$.

5) Naturgeschichte der Fische von Mains, 1787, $8^{\circ}$.

6) Reise nach Venedig, I, 46 ff.

J) Jahrb. 1848, 432.

') Dic fossile Flora von Parschlug (aus der Steyermärkschen Zeitschrift, IX, Jahrg.). 
entnommen, dürften genügen um zu zeigen, dass das Pflanzen - und ThierReich, so weit ihre einzelnen Klassen, Familien u. s. w. überhaupt in verschiedenen Zeiten repräsentirt gewesen, in allen Zeit-Abschnitten nicht ärmer als jetzt gewesen sind, wenn auch in chronologischer wie in geographischer Ausdehnung hier das cine und dort das andere Glied des Systemes mehr vorgewaltet haben oder mehr zurückgetreten seyn mag. Dass aber unser Pflanzen - und Thier-Reich nicht in allen Zeit-Abschnitten mit allen ihren jetzigen Familien, Ordnungen, Klassen u. s. w. bestanden haben, dass sie in gewissen Zeiten Gruppen enthielten, die jetzt gänzlich mangeln, Diess geht schon durch einen Blick auf unsern Enumerator hervor, und wird sich in spätern Paragraphen noch weiter verfolgen lassen.

Man könnte noch die Einwendung machen, dass die zahlreichen Arten, welche man in einzelnen solchen Örlichkeiten antrifft, eine grössere Verbreitung besessen hätten als die jetzigen Arten, und dass daher die Gesammtzahl der Arten einstens bei zahlreicherer Individuen-Zahl doch kleiner gewesen seyn könne. Inzwischen spricht keine Beobachtung entschieden dafür, und viele sprechen dagegen.

E. Eine annähernde Berechnung, wie viele Pflanzen und Thiere es vor der jetzigen Schöpfung überhaupt gegeben, liegt um so mehr ausser den Grenzen der Möglichkeit, als seit 30-40 Jahren sogar unsere Berechnungen über die Zahl der noch lebenden Thier - und Pflanzen-Arten auf's Doppelte gestiegen sind und daher auch noch jetzt keinen sichern Boden darbieten können. Gehen wir aber 1) von dem eben erörterten Grundsatze aus, dass die einzelnen Klassen und Ordnungen von Pflanzen und Thieren, so lange sie in mehr als vereinzelten Spuren bestehen (worïber wir das Nöthige vorerst aus dem Enumerator entnehmen), überhaupt jederzeit eben so viele Arten wie jetzt enthalten haben mögen, gleichviel ob hier diese und dort jene untergeordnete Gruppe mehr existirt, stärker vorgewaltet, weiter zurück getreten seye als jetzt; - dass 2 ) die ganze geologische Zeit durch wenigstens 30 Arten-Alter oder Arten-Folgen (S. 789) gemessen werden könne, - und betrachten wir 3 ) die jetzt bekannte Anzahl von Pflanzen - und Thier-Arten als Einheit des Arten-Alters, die bald unter und bald über der früheren Wirklichkeit stehen mag, so würde uns die Rechnung doch ein wenigstens nicht ganz unwahrscheinliches Resultat, nämlich von 500,000 Pflanzen- und 1,500,000 Thier-Arten darbieten. Da in der jetzigen Schöpfung die Kerb-Thiere bei Weitem die zahlreichste Unterabtheilung des Thier-Reiches bilden, so würde eine kleine darin begangene Überschätzung natürlich von den grössten Folgen seyn und vor zu hohen Ansätzen, insbesondere vor einer zu langen Artenfolgen-Reilie warnen müssen, wenn wir nicht einerseits bei aller Ungunst der conservirenden Bedingungen schon in der Kohlen - Formation ausser manchfaltigen Krustazeen auch selbst Insekten-fressende Arachniden, Käfer, Neuropteren und Schmetterlinge angedeutet sähen und wüssten, in welch' enger und nothwendiger Ökonomie-Bezielıung die Insekten zur Pflanzen-Welt stehen, deren frühere Einförmigkeit aber allerdings auch in Anschlag gebracht werden muss, wie es wieder in Bezng auf parasitische Pflanzen und Thiere nöthig ist. 
796

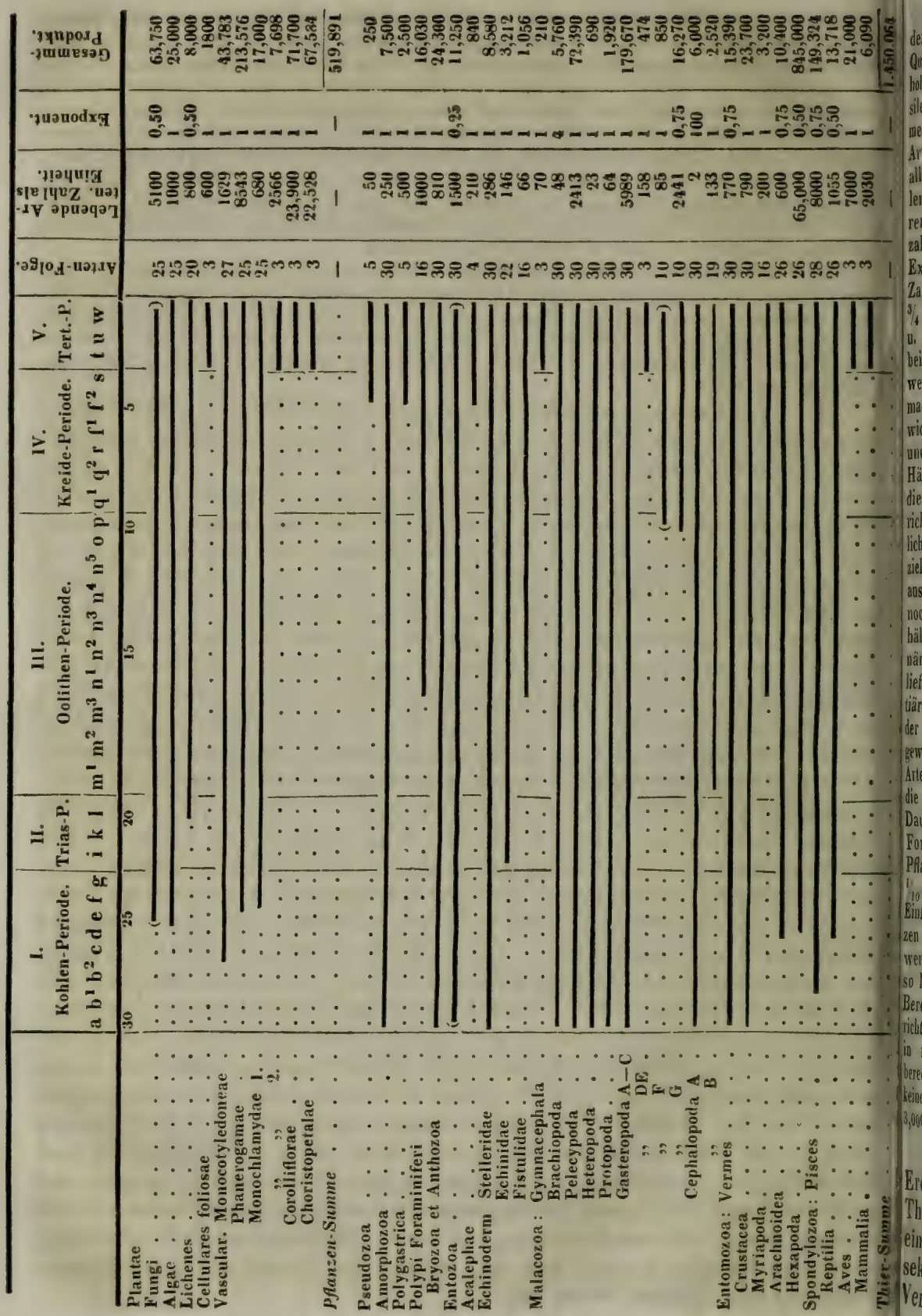


Zu dieser Berechnung nun einige Erläuterungen. Wir haben die Dauer der Klassen, Ordnungen $u$. s. w. in neben stehender Tabelle durch einen Querstrich angedeutet, welcher in Parenthese steht, wenn jene Dauer bei hoher Zersetzlichkeit des Körpers der entsprechenden Wesen nicht aus den fossilen Resten nachweisbar gewesen, sondern aus andern Gründen so angenom. men werden muss. Wir haben in einigen Fällen der Zahl der jetzt lebenden Arten einen Exponenten gegeben, wenn nämlich diese Zahl nicht als Einheit für alle Arten-Alter gelten zu können schien. Dieser Exponent ist bei den Schaalen-Cephalopoden $A=100$, weil wir jetzt nur 2 lebende Arten kennen, wähend die fossilen Cephalopoden bis zum Anfang der Tertiär-Zeit immer sehr ahlreich waren; er ist bei den Brachiopoden $=4$ aus ähnlicher Ursache; beide Exponenten scheinen kein $\mathrm{zu}$ hohes Resultat zu geben, auch wenn man die Zahl der Arten-Alter als zu hoch bestreiten wollte. Der Exponent ist öfters $1 / 4$ in Fällen, wo er für die eine Hälfte der Dauer der Klasse, Ordnung 1. s. $w$. $=1 / 2$, für die andere $=1$ gesetzt werden zu müssen schien. Er ist ei den Schwämmen und Algen und Sechsfüsser-Insekten $=1 / 2$ angenommen, veil diese zum grossen Theile parasitischen Wesen in frühester Zeit keine so nanchfaltigen Pflanzen- und Thier-Formen zur Grundlage ihrer eigenen Entvickelung finden konnten und mithin selbst weniger manchfaltig seyn mussten, and weil man bei den letzten, welche der jetzigen Schöpfung mehr als die Hälfte aller Thier-Species geliefert und daher als einheitlicher Massstab für lie Berechnung der Arten früherer Perioden am ehesten eine wesentliche Unichtigkeit der Zahlen herbeiführen konnten, licber unter als über der Wirkichkeit bleiben wollte, um ein jedenfalls wahrscheinlicheres Resultat zu erielen. Bei den Entozoen eudlich, welche ganz aus Parasiten bestehen, hat man us dem vorhin angegebenen Grunde den Exponenten $=1 / 4$ gesetzt. - Man wird roch die Einwendung machen können, dass wahrscheinlich dasselbe Zahlen-Verältniss zwischen Pflanzen und Thieren immer so bestanden habe, wie jetzt, ämlich $7: 10$, während das obige Resultat das doppelte Verhältniss $=1: 3$ iefert. Indessen ist in der That die Pflanzen-Welt bis zu Anfang der Teriär-Zeit, wo erst die vollkommenen Klassen und mit ihnen die lange Reihe er Kronen-blüthigen Dikotyledonen auftreten, eine bei weitem einförmigere ewesen, als die Thier-Welt, welcher damals nur die zu der Gesammtzahl der irten nur eine geringe Quote liefernden Warmbluter noch fehlten, zumal auch ie Acalephen, Gymnacephalen und nackten Gasteropoden gewiss eine längere pauer besessen, als wir angenommen haben. Die vollkonmenen Pflanzen. cormen, welche erst mit der Tertiär-Zeit auftreten, liefern fast $3 / 4$ des flanzen-Reichs, die mit ihnen beginnenden vollkommenen Thier-Formen nur 10 des ganzen Thier-Reichs! Daher ihr langes Ausbleiben nicht dieselbe införmigkeit in der Fauna veranlasst, wie das spätere Erscheinen jener Pflan. en im Pflanzen-Reiche; allein die Einförmigkeit der Flora hat wenigstens nothvendig eine gewisse Einförmigkeit auch der Insekten-Fauna bewirken müssen, o lange die Dikotyledonen gänzlich fehlten. - Man wird diesen Versuch einer jerechnung wohl da und dort verbessern könneı, hauptsächlich, wenn man ichtigere Zählungen vornimmt, genauere Exponenten wählt, die Ordnungen a mehr Familien theilt um jede Familie genauer nach ihrem Anfange zu erechuen; allein es wird für das Ganze (worauf es uns hier allein ankommt) einen wesentlichen Unterschied machen, ob wir statt obiger Summe gar $, 000,000$ oder nur 1,000,000 Arten erhalten würden.

F. Von diesen $2,000,000$ Arten, welche in früherer Zeit die ird-Oberfläche allmählich bevölkert haben, ist natürlich ein grosser Theil unbedingt unfähig gewesen uns fossile Reste als Denkmäler instiger Existenz zu hinterlassen; ein noch weit grösserer Theil (Inekten, Pflanzen u. s. w.) vermochte Diess nur unter den günstigsten Verhältnissen und auf eine sehr zufällige Weise, daher uns nur eine 
sehr unbedeutende Quote derselben je bekannt werden mag; noch andere waren dazu zwar vollkommen (Konchylien, grössere Kruster, grössere Reptilien und Säugthiere) geschickt, allein die stattindende Erhaltung der Reste aller Thier- und Pflanzen-Arten im fossilen Zustande setzt ein Zusammenwirken von mancherlei Bedingnissen voraus, welches in Wirklichkeit doch nicht überall eintreten kann. Daher vielleicht nur von 0,10 aller früheren Arten, d. h, etwa 200,000 Spezies noch kenntlich erhaltene Reste im Schoose der Erde begraben liegen mögen, und man vielleicht nur $0,05, d$. h. 100,000 Arten darin je zu entdecken hoffen darf. Für einzelne Klassen und Ordnungen würde sich das Verhältniss freilich sehr abweichend stellen.

G. Ein Blick auf die Tabelle I, S. 727 ergibt eine grosse U ngleichheit des Arten-Reichthums dereinzelnen For$\mathrm{m}$ ationen, welche theils eine ursprüngliche und theils von der Fähigkeit der Gestein-Arten abhängige seyn kann, die fossilen Reste in kenntlichem Zustande zu bewahren, wobei wir aber nicht länger zu verweilen gedenken. Wichtiger ist die Ungleichheit des ArtenReichthums in den einzelnen Perioden, wo wir durch Addition der Zahlen aller Formationen fïr

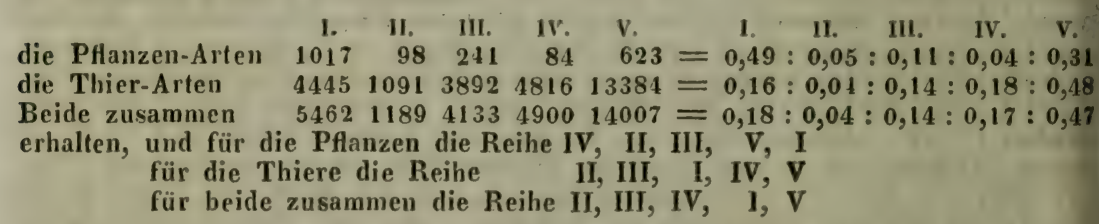

gewinnen, worin die Beträchtlichkeit der Unterschiede in den ArtenZahlen uns auf grosse Ungleichheiten in der Länge der Perioden oder in dem Reichthume ihrer Schöpfungen schliessen lassen würde, wenn wir uicht sähen, dass Pflanzen- und Thier-Welt sehr auffallend verschiedene ja fast entgegengesetzte Verhältnisse liefern, welche doch in der Natur höchst wahrscheinlich nicht stattgefunden haben, sondern nur eine Folge der grossen Ungleichheit des Vermögens der Gesteine in den verschiedenen Formationen ist, die verschiedenartigen organischen Reste zu erhalten. So sind alle Kreide- und Trias-Gesteine offenbar sehr ungeeignet für die Erhaltung der Vegetabilien; die in dieser Hinsicht so günstige Steinkohlen-Bildung war beendigt, aber die Manchfaltigkeit der dikotyledonischen Gewächse noch nicht vorhanden, welche in der V. Periode gewiss grösser war, als in der Steinkohlen-Bildung, aber wegen Mangels eines eben so vortrefflichen Ansammlangs- und Erhaltungs-M̄ittels uns gleichwohl weit weniger fossile Reste zu überliefern vermochte, als jene erste Flora, der wir die Hälfte aller fossilen Pflanzen-Arten danken. Die Kreide enthält kein zur Erhaltung der Pflanzen geeignetes Gestein und insbesondere keine Land- und Süsswasser-Gebilde. Die Trias-Periode war übrigens im Ganzen weitaus die ärmste, wie die Tertiär- 
Periode die reichste an Arten, namentlich an Thier-Arten, wovon sie uns fast die Hälfte der ganzen Anzahl geliefert hat, was wir, wie später noch zu zeigen, nicht ganz weder dem Zufalle noch einer etwa längeren Dauer dieser Periode zuschreiben können. - Wegen der Einzelu-Verhältnisse in den Unter-Abtheilungen beider Reiche verweisen wir auf die Tabellen I. und IV.

§. 8. Zahlen der Sippen (überhaupt und der gemeinsamen insbesondere, Tabelle II, S. 734).

A. Die Anzahl der Sippen lebender wie fossiler Organismen lässt sich weniger genau in Ziffern ausdrücken, als die der Arten, da sie nicht nur wie diese in Folge stets neuer Entdeckungen, sondern auch in Folge fortwährender Theilung der alten Sippen im Verhältnisse einer sorgfältigeren Untersuchung in unausgesetzter $\mathrm{Zu}$ nahme begriffen ist. Auch bietet die Verschiedenheit der Ansichten iber den Umfang, welcher den Sippen überhaupt oder denen einer gewissen Klasse, Ordnung u. s. w. zu geben, einen ferneren Grund grosser Unsicherheit dar. Ferner sind manche Sippen unter mehrachen Namen aufgestellt worden, die wir im Enumerator, um Vervielfältigung der Arten-Benennungen zu vermeiden, grösstentheils beipehalten mussten, und sind endlich viele Sippen nur für einzelne Theile : Früchte, Blätter, Stämme, Zähne, Schuppen, Stacheln erichtet worden, die, wenn man diese Theile in richtige Verbindung zu einer Pflanze oder einem Thiere zu bringen wüsste, auf eine geingere Anzahl zurückgeführt werden würden, als man jetzt noch zu hun in Stande ist. Daher Alles, was wir über die Anzahl der ossilen Genera im Allgemeinen sagen können, sogar für den Augenjlick selbst, wo wir Solches thun, nur als eine ungefähre Angabe be. rachtet werden darf, wenn gleich wir in der Tabelle, worin wir die Zahlen-Verhältnisse zusammengestellt haben, und auf welche wir hinsichtlich der Einzelheiten verweisen müssen (S. 734), diese Zahen sehr scharf ausgedrückt erscheinen. Auch wird es wenigstens iützlich seyn, hier nochmals zu wiederholen, dass diese Zahlen nur las ausdrücken, was wir gefunden haben, und nicht das, was eintens vorhanden gewesen, indem sie uns einige der vertreten gewesenen Klassen des Systems reichlich, andre spärlich, noch andre gar icht zurückbringen.

a. So erfahren die Genera der Anthozoen, welche bisher nicht monograhisch bearbeitet worden waren, in diesem Augenblicke eine vielfältige Theiung und Vermehrung durch eine gemeinschaftliche Bearbeitung der lebenden wie der fossilen Formen durch MinNe-Edwards und Haime, wornach sie viel zahlreicher auftreten werden als jetzt. - Von den fossilen Fischen hat man vor 20 Jahren noch kaum 100 Genera gekannt, und von deren Bestimmungen ist furch Agassiz kaum eine oder die andere als richtig beibehalten und eine vielfältig grössere Anzahl ist durch ihn hinzugefügt worden. - Vor 25 Jahren hat man fast noch keine regelmässig umschriebenen Foraminiferen-Geschlechtcr im fossilen noch im lebenden Zustande gehabt, deren Anzahl sich jetzt durch D'OR- 
BIGNy's und Ehrenberg's Arbeiten auf Hunderte beläuft. Ebenso ist es mit den Pflanzen ergangen in Folge der Arbeiten von Strbnberg, Broneviart, Göp. PERT, Unger u. S. w. Überall seit LINNÉ sind die anfänglich aufgestellten Genera des Systemes fortwährend in neue getheilt, überall sind neue Generu für neuc Formen $\mathrm{zwischen}$ die alten eingeschaltet worden.

b. Die Tabelle II, S. 734 enthält die Angabe der Zahlen der Sippen, welche von jeder Familie, Ordnung, Klasse, von jedem Unterreiche, Reiche und end. lich von allen fossilen Wesen im Ganzen bis jetzt bekannt geworden sind, zu. erst in jeder der bisher angenommenen Formationen $\mathbf{a}$ bis $\mathbf{x}$, dann in den $\mathbf{V}$ geologischen Perioden und endlich in allen V Perioden zusammen genommen. Da nun ein Genus gewöhnlich in mehren aufeinanderfolgenden Formationen und selbst Perioden vorkommt, so gibt die Summirung dieser einzelnen Formationen, so wie sie in den Rubriken $\mathbf{a}-\mathbf{g}, \mathbf{h}-\mathbf{I}, \mathbf{m}-\mathbf{p}, \mathbf{q}-\mathbf{f}, \mathbf{s}-\mathbf{x}$ erfolgt ist, eine beträchtlich grössere Auzahl Sippen, als wirklich vorhanden ist, daher dieselben in jeder Periode nochmals durch unmittelbare Zählung gesucht und in eine letzte Spalte jeder Periode, die mit 1, II, III, IV, $\vec{V}$ ïberschrieben ist, eingetrageu sind. Eben so verhält es sich, wenn man die Anzahl der in diesen letzten Spalten aller Perioden 1 bis $\mathbf{V}$ vorhandenen Zahlen zusammenzählt: auch sie fällt noch beträchtlich grösser aus, als wenn man alle Sippen unmittelbar zählt. Daher sind in 3 letzten Spalten dersel. ben Tabelle die Ergebnisse der Zählung der Genera durch Addition der Zahlen aller Formationen (Spalte $\mathbf{a}-\mathbf{x}$ ), durch Addition der Zahlen aller Perioden (Spalte $1-V$ ) und durch unmittelbare Zählung aller Sippen (Spalte $\mathbf{S}=$ Summe) zusammengetragen worden.

c. Verschiedene Zählungen indessen ergeben immer kleine Verschiedenheiten, die sich nicht vermeiden lassen, weil nämlich von einer Anzahl Arteu und somit oft auch der Genera (wenn andere Arten derselben nicht in allen Formationen vorhanden sind) die Formation des Vorkommens unsicher ist, und sie somit entweder ganz ausgelassen, oder in allen möglichen gezählt, oder nur in irgend einer mitteln oder wahrscheinlicheren angenommen werden können; weil viele fossile Vögel- und Insekten-Arten gar nicht in bestimmte Genera, sondern nur in ihre Familien eingetheilt sind; - weil oft die Vertheilung der Arten eines Geschlechts unter mehre synonyme Geschlechts. Namen Schwankungen und Irrungen veranlasset; - oder weil manche Arten zwar unter gewisse Genera eingereihet sind, zu denen sie aber offenbar nicht gehören und welche sie in Formationen andeuten, denen diese Genera in der That fremd sind, wie Dieses auch mitunter ausdrücklich angegeben ist. So auf S. 282 des Enumerators die ältern Arten von Pectunculus im untersilurischen Gestein (n), während das Genus erst in den Oolithen (n) zu beginnen scheint; so S. 498 die älteren Helix-Arten in den Oolithen und der Kreide ( $\mathbf{n}$ und $\mathbf{r}$ ), während das Genus erst in der Tertiär-Zeit aufzutreten scheint; - so die sämmtlichen meerischen Melanien-Arten, welche alle Formationeu von den de vonischen bis $\mathrm{zu}$ den neuesten Gebilden (c bis w) durchlaufen und desshalb S. 386 des Enumerators abgesondert aufgezählt worden sind von den ächten Arten der Süsswasser, welche S. 428 genannt werden und erst mit dem Ende der Oolithen-Periode (in p) beginnen. Obschon wir aber nun wissen, dass jenes keine Melanien sind, auch dass sie grossentheils zu Pasithea, Turbo. nilla u. s. w. gehören, so sind wir doch nicht im Stande, sie sogleich alle richtig einzutheilen und die ihnen entsprechenden Genera jedes in seiner rich. tigen Formation zu zitiren. Und so oder ähnlich verhält es sich in sehr vielen andern Fällen. Ja wir gestehen sogar, die mechanischen Geschäfte der Zählung und Berechnung nicht so oft wiederholt $\mathrm{zu}$ baben, dass nicht kleine Reclonungs-Fehler da und dort mit untergelaufen seyn könnten, weil eine vollkommene Genauigkeit doch unmöglich und auch die möglich-genaueste Angabe von keinem Bestande seyn würde; die auf eine oft wiederholte Zählung und 
Berechnung zum Zwecke einer absolut fehlerfreien Ausführung zu verwendende Zeit hat uns ausser Verhältniss zu stehen geschienen mit dem davon zu erwartenden Nutzen. Daher können, wir wiederholen es, kleine Schwankungen und Abweichungen zwischen den Zahlen verschiedener Tabellen unter sich und den Einzelnheiten des Enumerators wohl vorkommen; doch, wie wir glauben, keine erheblich irrigen.

B. Wir finden auf der Tabelle III, dass es im Ganzen 350 fossile Sippen bei den Pflanzen, 2414 bei den Thieren und folglich 2764 bei beiden zusammen gibt. Vertheilt man diese auf die fünf geologischen Perioden $(I-V)$ und die 24 Formationen $(a-x)$, so erhält man als Mittel

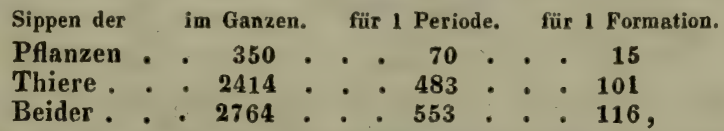

welche nämlich auf jede dieser Abtheilungen kommen würden, wenn keine derselben eine Sippe mit andern gemein hätte, welche Zahlen für die Formationen dann noch um je $1 / 8$ grösser ausfallen würden, wenn man die Formation $f$ ihrer Unbedeutendheit, die Formationen $v$ und $x$ wegen ihrer Gleichzeitigkeit $z u$ und w mit den übrigen vereinigte.

C. Wenn man aber alle Pflanzen- und Thier-Sippen auf solche Weise unmittelbar zusammenzählt, so erhält man kleinere Zahlen $(\alpha)$, als wenn man die in jeder der V Perioden gefundenen summirt und dann die Gesammtzahl durch Addition der Summen der Perioden oder der Formationen zu finden sucht $(\beta)$, oder wenn man eben so gar mit den in allen einzelnen Formationen gefundenen verfährt $(\gamma)$, weil nämlich viele Sippen mehren Formationen und selbst mehren Perioden gemeinsam sind. Und zwar ist das mittle Verhältniss von $\alpha: \beta: \gamma=0,46: 0,62: 1.00$. Denn es ist, wie schon früher angegeben, die Summe der fossilen

$$
\begin{aligned}
& \underset{\text { bei unmittelbarer aus allen aus allen }}{\boldsymbol{\beta}} \stackrel{\boldsymbol{\gamma}}{\mathrm{a}} \\
& \text { Zählung. Perioden. Formationen. } \\
& \text { Pflanzen }=350.463 .592 \\
& \text { Thiere }=2414.3260 .5415 \\
& \text { Beider }=2764.3723 .6007 \\
& \text { d. i. }\left\{\begin{array}{l}
\text { Pflanzen }=100: 132: 169 \\
\text { Thiere }=100: 135: 224 \\
\text { Beider }=100: 135: 217
\end{array}\right\} \text { oder }\left\{\begin{array}{l}
59: 78: 100 \\
45: 60: 100 \\
46: 62: 100
\end{array}\right\} \text { und }\left\{\begin{array}{l}
75: 100 \\
74: 100 \\
74: 100
\end{array}\right.
\end{aligned}
$$

d. h. man wird durchschnittlich die Genera aller einzelnen Formationen bei den Pflanzen auf 0,78 , bei den Thieren auf 0,60 und im Ganzen auf 0,62 ; die aller einzelmen Perioden aber überall auf fast $0,75 \mathrm{zu}$ reduziren haben, um die wahre Anzahl derselben zu finden, ein Verhältniss, das nun freilich bei den einzelnen Gruppen des Systems und für einzelne Perioden und Reihen von Formationen 
noch in manchfaltigen Verhältnissen abändern kann. Auffallend ist bei den Pflanzen, dass diese Reduktion von den Formationen $(\gamma)$ zu den Perioden $(\beta)$ schwächer, von diesen aber zur wah'en Summe $(\alpha)$ fast genau so stark ist, als bei den Thieren. Diese ganze Reduktion, nämlich von $\gamma$ auf $\alpha$, wird am grössten und am kleinsten seyn: das Eine bei solchen Gruppen, welche die Reilıe und Zahl der Formationen am vollständigsten durchlaufen, und das Andere bei jenen, die sich am meisten darin beschränken. So gehen zurück

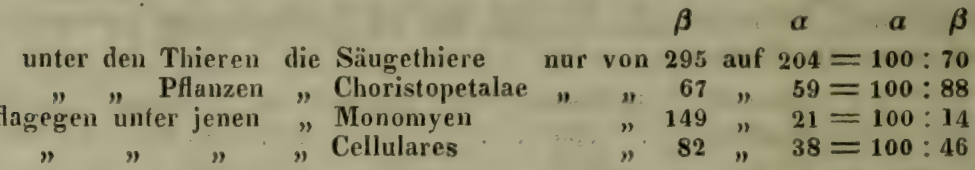

Und da die geologisch ausgedehntesten Gruppen in beiden Reichen den unteren, die beschränktesten den höchsten Klassen angehören, so muss die Reduktion jene am meisten, diese am wenigsten treffen. Bei ganz kleinen Familien oder gar einzelnen Geschlechtern ist das Verhältniss natürlich ein ganz zufälliges und kann zwischen 100:100 und 100:0 wechseln, was man nebst allen weiteren zu wünschenden Einzelnheiten in der angeführten Tabelle ersehen kann.

D. Jede Periode enthält also $3 / 4$ eigene und $1 / 4$ mit andern gemeinsame, jede Formation fast $1 / 2$ eigene und über $1 / 2$ gemeinsaine Sippen. Denn stellt man die Ergebnisse der 2 vorigen Absätze B und $\mathbf{C}$ nicht bloss in Summe, sondern diese Summen $\alpha, \beta, \gamma$ für die eimzelnen Perioden und Formationen, durch je 5 und beziehungsweise 24 getheilt, nebeneinander, so erhält man

a) Zahlen der Sippen, welche in jedem dieser Zeit-Abschnitte überhaupt vorkommen,

b) Zahlen der Sippen, welche jedem dieser Zeit-Abschnitte eigenthïmlich sind,

c) Zahlen der Sippen, die ihm mit anderen gleichwerthigen Abschnitten (mit Ausnahme der jetzigen Schöpfung) gemeinsam zustehen, wie folgt:

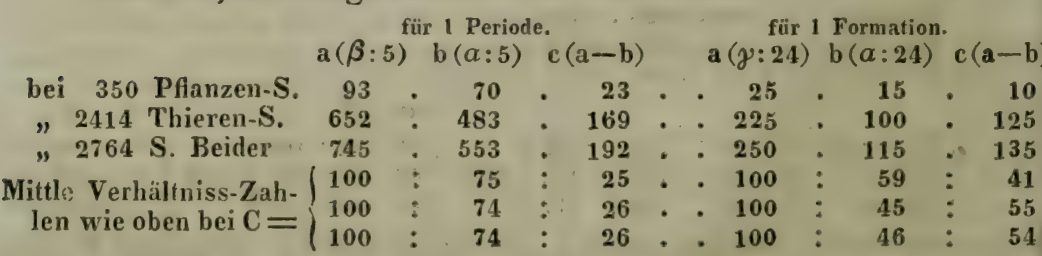

E. Im Allgemeinen scheinen die Genera um so bestimmter auf gewisse Perioden und Formationen beschränkt zu seyn, 1) einer je: höheren Klasse und Ordnung sie in beiden Reichen angehören; die höheren Pflanzen (Dikotyledonen) gehen in dieser Beziehung, merkwürdig genug, den höheren Thieren parallel, u. u.; 2) scheinen die 
der Land- und Süsswasser-Bewohner es mehr, die der See-Bewohner weniger zu seyn. 3) Organismen, deren fossile Überbleibsel der Art sind, dass sich generische Unterschiede leicht daran wahrnehmen lassen, bieten mehr Gelegenheit zur scharfen Unterscheidung der Sippen, die damn wohl eher auch den geologischen Zeit-Abschnitten entsprechen, als jene, deren Reste mehr indifferenter und unwichtiger Art sind (wie die Konchylien und unter diesen namentlich die Trochus-artigen u. e. a. Formen). In den Extremen einzelner Fälle aber können, selbst wenn man nicht zu sehr auf kleine Gruppen eingehen will, welche mehr oder weniger Zufälligkeiten unterliegen, bald fast alle Sippen einer Klasse, Ordnung u. s. w. einer Periode oder einer Formation eigenthümlich, und bald fast alle derselben mit andern gemeinschaftlich zustehen. Erstes ist bei einigen ausgestorbenen Gruppen und bei den höheren Wirbelthieren, Letztes vorzugsweise bei den Weichthieren und insbesondere den Monomyen der Fall.

Die Zählung der Säugthier-Sippen nach den Formationen, nach den Perioden und im Ganzen ergibt die Zahlen 295, 205 und 204, und diese ganze Anzahl, mithin die ganze Klasse ist mit Ausnahme von 4 Sippen auf die tertiäre Periode beschränkt. Lässt man diese 4 bei Seite, so gewährt die Zählung der tertiären Sippen nach Formationen und im Ganzen die Ziffer 291 und 200, folglich nur eine Differenz von 91 , welche aber fast völlig verschwinden wird, wenn man die Formationen $\mathbf{v}$ und $\mathbf{x}$ als zu andern gleichzeitig in diese eintheilt und einige ungenaue Angaben in $t$ und $\mathbf{n}$ genauer prüft. - So sind bei den Crustaceen die ausgestorbenen Trilobiten auf die erste Periode und darin (mit 3 ganz abgesonderten Ausnahmen in d) auf die 3 Formationen a, $\mathbf{b}$, c beschränkt. Davon sind nur einige Sippen zwischen a und b, welche ohnehin nicht scharf geschieden sind, - sehr wenige zwischen b und cemeinsam, obschon die Unsicherheit der Angaben über die Formation des Vorkommens vieler Arten diese Zahlen jetzt noch viel grösser erscheinen lässt, als sie wirklich sind (vgl. den Enumerator S. 561-573). - Bei den Monomyen anderntheils geht die grösste Zahl der Sippen durch alle Perioden und Formationen hindurch, so dass das Verhältniss $=24: 54: 149$ oder fast $1: 3: 7$ wird.

E. Nach der Anzahl der Sippen der Thiere ordnen sich die einzelnen Perioden so:

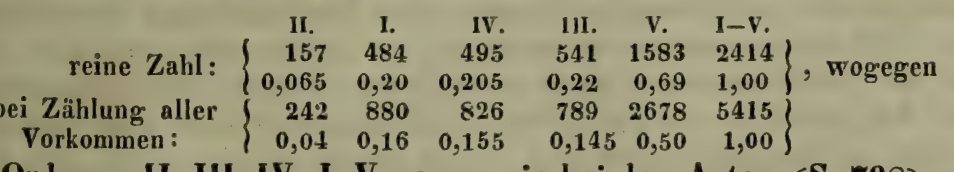

die Ordnung II, III, IV, I, V genau wie bei den Arten (S. 798) werden würde, was sich erklärt, wenn man sich erinnert, wie die zahlreichen Glieder der ersten Periode viele gemeinschaftliche Arten und somit auch Genera haben und hiedurch weiter hinauf rücken, während die III. Periode in mehre Formationen zugleich einschliesst, und die Süsswasser-Formation p mit andern nichts, die arme Formajion o nur wenig gemein hat, wodurch im Verhältniss zu ihrer Arten-Zahl die doppelten Zälılungen seltener werden. - Im Ganzen aber würden sich bei Berücksichtigung bloss der reinen Sippen-Zahl die die einzelnen Perioden ziemlich gleich stellen, mit Ausnahme der II. 
oder Trias-Periode (die im Falle die Formation k zu m versetzt werden müsste, sogar noch über die Hälfte ihrer Arten verlieren würde), welche wirklich einen kürzeren Zeitraum zu umschliessen scheint, als die anderen, da ihr auch einige Formen-Gruppen, welche in der vorhergehenden wie in der folgenden Periode vorkommen, gänzlich oder fast gänzlich fehlen, - und der V. oder Tertiär-Periode, welche alleiı eben so viele Arten enthält, als alle andern zusammengenommen. Ein Theil der Ursache liegt in der trefflichen Erhaltung der Überreste dieser Formation und ihrer leichten Gewinnung und Be. stimmung; ein anderer Theil in dem Auftreten der hohen Wirbelthiere, der Land-Kerbthiere, einiger Malakozoen-Gruppen und der Dikotyledonen, welche in den vorhergehenden Perioden ganz fehlen. Immerhin mag aber noch ausserdem eine beträchtlich grössere Sippen-Zahl für diese Periode übrig bleiben, als für die vorhergehenden, und würde man diese grössere Anzahl einer längeren Dauer der Periode zuschreiben können, wenn nicht die 'Zahl der durch die ganze Periode hindurchreichenden Arten grösser oder wenigstens eben so gross zu seyn schiene, als bei anderen, so dass entweder nur die Aunahme eines grösseren Reichthums an Sippen überhaupt, oder eines allmählicheren Überganges der aufeinanderfolgenden Schöpfungen in einander (etwa in Folge weniger allgemein zerstörender äusserer $\mathbf{U} \mathbf{r}$ sachen, als solche in frühern Zeiteu stattgefunden), oder beide Annahmen zusammen übrig bleiben, wovon die letzte in soferne auch Manches für sich hat, da, wie wir gesehen, mehre Arten sogar aus der Kreide-Zeit bis in die jetzige Schöpfung gelangt sind.

§. 9. Zahl der noch lebend bestehenden Genera. Tabelle III, S. 7:38.

A. Wir haben im vorigen Paragraphen das gemeinsame Vorkommen einer gewissen Anzahl von Geschlechtern in den früheren und in der jetzigen Erd-Periode ausser Acht gelassen, sonst würde die Quote der gemeinsamen Sippen noch beträchtlicher ausgefallen seyn. Wir haben auch nicht versucht, in Zahlen anszudrücken, wie weit die gemeinsamen Sippen einer jeden Periode durchschnittlich in die folgenden und vorhergehenden Perioden hinein und hindurch reichen, wollen Diess aber, da es von grösserem Interesse ist, nun für die noch lebend vorkommenden Genera versuchen. Die Einzelnheiten haben wir in Tabelle III, S. 735 zusammengestellt.

a. Die Zusammenstellung dieser Verhältnisse beruht auf noch mehr Schwierigkeiten, als die der vorigen (S. $784-788$ ), weil nämlich manche Paläontologen, wie mehrfältig erwähnt, fast keine den früheren mit der jetzigen Schöpfung gemeinsamen Genera gelten lassen wollen, andere aber wenigstens für eine nothwendige Vorsicht halten, die fossilen Genera, auch wo sie von den lebenden nicht unterschieden werden können, doch durch eigene Namen zu bezeichnen. So würde sich namentlich ein grosser Unterschied für die Zahl der fossilen Pflanzen-Sippen nach der GöPPERT'schen und nach der Unger'schen Bearbeitung derselben ergeben, da der letzte weit lieber den Namen der lebenden 
Genera beibehält, als erster. Die zahlreichen fossilen Formen werden übrigens von allen Autoren mit eigenen Namen belegt, und doch ist, wie auch GöPPERT in seinen Werke über dieselben nachgewiesen hat, Grund zu glauben, dass, wenn wir ihre Fruktifikationen vollständig kännten, sie grossentheils in die lebenden Genera eingereiht werden könnten.

Wir haben schon im Enumerator 1) theils alle Genera der Pseudozoen, der Amorphozoen, der Polygastrica, Polycystina und Bacillarina, der Polypen (ausser einigen weichen S. 127, 169), der Echinodermen, Malacozoen, Crustaceen (ausser den Parasiten und einem Theile der Malakostraca S. 573 und 575), der Pisces Elasmobranchi und Ganoidei, selbst der nur lebend vorkommenden, vollständig aufgezählt und überall bei den fossil auftretenden Geschlechtern bemerkt, ob sie nur fossil, oder auch lebend, und mit wie viel Arten ungefähr sie in diesem Zustande vorkommen. 2) Desgleichen sind die Zahlen aller lebenden Genera nur mit Übergehung einer erst neulich aufgestellten Anzahl und einiger wenig erheblichen Formen nach den einzelnen Familien aufgeführt bei den Pflanzen, den meisten Polygastrica, bei den Hemiptera bis Neuroptera (S. 602-613), bei den Pisces Leptocardii, Cyclostomi und Dipnoi. 3) Dagegen sind die lebenden Genera nur theilweise angegeben bei den Würmern (S. 546 -553, wo insbesondere die übrigen Angaben bei den Rotatorien, Apoden und Antennaten ausfielen); und sind 4) bloss die fossilen Genera aufgezählt, bei den meisten Entomozoen, den Pisces Teleosti, den Reptilien, Vögeln und Säugthieren. 5) Ganz übergangen sind die Entozoen und Acalephen, da es unter ihnen keine noch bestehenden Genera mit fossilen Arten gibt. Auf der Tabelle III aber sind theils jene Sonder-Zahlen in grössere Sammel-Zahlen vereinigt, theils die nöthigen Zahlen, welche dort noch fehlten, ergänzt. Nur eine Zahl indessen, freilich die beträchtlichste von allen, die der lebeuden Sippen sechsfüssiger Insekten, ist auf eine blosse Schätzung hin mit 4000 angesetzt worden, da bei Ungleichheit der Bearbeitung dieser grossen Abtheilung auch eine sorgfältige Zählung, so weit sie nämlich möglich ist, zu keinem verlässigeren Resultate führen würde. Übrigens ist diese Zahl eher zu klein, als zu gross.

B. Unter 2764 im fossilen Zustande bekannt gewordenen Geschlechteru kommen, so weit man hei den erwähnten Schwierigkeiten der Zählung urtheilen kann, 1351, oder 0,49 der Gesammtzahl, auch noch im lebenden Zustande vor; die übrige grössere Hälfte = 0,51 mit 1413 Geschlechtern würde also ausgestorben seyn. Bei den Thieren ist das Gesammt-Verhältniss $=\mathbf{0 , 5 4}$, bei den Pflanzen 0,17; bei den 4 Unterreichen der ersten in aufsteigender Ordnung $=0,48$, $0,64,0,76,0,36$, während für kleinere Abtheilungen des Systemes alle Verhältnisse zwischen ausgestorbenen und noch lehenden Genera vorkommen können von $1: 0$ bis $0: 1$, oder das Verhältniss der einen wie der andern zur Gesammtzahl der Sippen von 0,01 bis 100 .

a. Man wird wegen einiger kleinen Ausfälle etwas weniger als jenen Rest, oder, $=0,50$ in rundem Verhältniss als in ausgestorbenen Genera bestehend betrachten können. Die nicht unbeträchtliche Anzahl von Insekten - und insbesondere Vögel-Arten, deren Genera noch nicht genau bestimmt sind, würden einen Zuwachs der absoluten Zahl wohl auf beiden Seiten verursachen und daher das Verhältniss vielleicht nicht wesentlich ändern.

b. Aus den Gruppen der Myriapoden, Hypobranchier, Pomatobnanchier, Aspidobranchier, Tubicolae und Pseudozoa hat man bisher noch keine ausgestorbenen Genera entdeckt, und das Verhältniss der lebenden zur Gresammtzahl ist .

Bei den Polygastrica, Polythalami, Anthozoa, Fistuliden, Monomyen, Homomyen (Integripalliaten und Sinuatopalliaten), Pteropoden, Protopoden, 
Gasteropoden, und überhaupt den Malakozoen, bei den Cirripeden, Arachnoideen und Hexapoden, überhaupt bei den Entomozoen in Ganzen, bei den Batrachiern, Ophidiern, Cheloniern und Vögeln sind die lebenden Genera über die fossilen vorherrschend, so dass sie Verhältnisse bilden von . . . . . . . . . . . . . . . . . . . . 0,95

bis . . . . . . . . . . . . . . 0,50

Bei allen Pflanzen dagegen, bei den Phytozoen im Ganzen und insbesondere den Amorphozoen und Bryozoen, Stelleriden und Echiniden, bei den Brachiopoden, Heteromyen und Cephalopoden - bei den Vermes, Entomostraca, Malacostraca, - bei den Spondylozoen im Ganzen und namentlich den Fischen, Sauriern und Säugthieren sind die ausgestorbenen Genera vorherrschend; die lebenden sind im Verhältnisse von ... $\left\{\begin{array}{l}0,48 \\ 0,19\end{array}\right.$ und bei den kryptogamischen Monokotyledonen, den Stelleriden, den 4kiemigen Cephalopoden, den Ganoiden selbst wie ... . . . 0,03 u. 0,01

Bei den Trilobiten und Ammoneen (und Rudisten) . . . . . . . 0,00

C. Die Genera früherer Schöpfungen, welche auch bis in die jetzige Schöpfung hineinreichen, verhalten sich zu denen der jetzigen Schöpfung überhaupt wie 1350 zu ungefähr 14760 oder bilden nahezu den zehnten Theil derselben (genauer 0,09 ); bei den Pflanzen verhalten sie sich $=60: 6529$ oder bilden 0,009 der lebenden Gesammtzahl, also nur ein Zehntel der Quote wie bei jenen; bei den Thieren ist das Verhältniss 1291 : 8232 oder sie bilden 0,157 der ganzen lebenden Zahl. Stellen wir die Pflanzen und die 4 Unterreiche in aufsteigender Ordnung untereinander, so erhalten wir

\begin{tabular}{|c|c|c|c|c|c|c|c|c|}
\hline \multicolumn{2}{|c|}{ lebende Genera. } & \multicolumn{5}{|c|}{$\begin{array}{l}\text { lebend und fossil vor- } \\
\text { kommende Genera. }\end{array}$} & \multirow{2}{*}{\multicolumn{2}{|c|}{$\begin{array}{l}\text { verglichene } Z_{\text {ahl. }} \\
\text {. } \quad 0,009\end{array}$}} \\
\hline & 6529 & 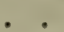 & 然 & 60 & .. & . & & \\
\hline zenthiere & 65 & . & • & 242 & . & & & . 0,37 \\
\hline iere & 515 & . & 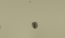 & 302 & . & . & & . $\quad 0,59$ \\
\hline e. & 5000 & . & . & - 484 & . & & & 0,09 \\
\hline belthiere & 1311 & & & . 263 & & & & . 0,20 \\
\hline
\end{tabular}

wobei die Luft-Insekten unter den Kerbthieren ihrer' Weichheit wegen natürlich weniger Mittel zur Unterscheidung eigenthümlicher Genera bieten mussten, als andere Gruppen.

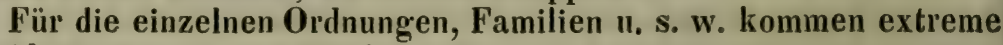
Verhältnisse vor von 0,001 bis 1,00 .

Grar keine fossilen Genera haben geboten die weichen, nackten und z. Th. kleinen Gruppen der Entozoen, Acalephen, Heteropoden, Gymnobranchier, Leptocardier, Cyclostomen und Dipnoen . . . . $=0,00$

Die kleinsten Verhältnisse liefern die Pffanzen, und auch die Unter-| 0,001 Abtheilungen derselben übersteigen 0,013 nur in einem Falle, in dem sie 0,013 sich bei den Monochlamydeen auf 0,057 belaufen ....... 0,057

In den meisten Fällen bleiben die fossilen Genera der Thiere ihrer 0,06 Zahl nach unter der der nicht fossilen zwischen ........ . . 0,49

Sie gleichen der Zahl der nur im lebenden Zustande vorkommenden ? Genera: bei den Anthozoen und Stelleriden. . . . . . . . und überwiegen sie bei den Polythalamien und durch diese bei den Polypen überhaupt, bei den Echiniden, - den Pelecypoden, Protopoden und meisten Gasteropoden (Cyclobranchier, Aspidobranchier, Ctenobran- 0,56 chier, Pomatobranchier und Pulmonaten) und hiedurch bei den Malaco- 0,92 zoen überhaupt, - bei den Crustaceen (durch die Paläaden und Solenhofer. Decapoden) 
Sie bilden sogar grössere Gruppen, die in der lebenden Scböpfung ganz fehlen, wie die Ammoneen, Paläaden (und Rudisten nach Ausschei- 1,00 $^{-}$ dung der nicht zugehörigen Genera)

D. Vergleicht man aber die Zahl aller fossilen Genera mit der

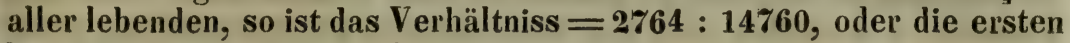
betragen gegen 0,02 der letzten, nämlich
0,0053 bei den Pflanzen.
0,56 bei den Wirbelthieren.
0,80 bei den Pflanzenthieren.
0,29 bei den Thieren überhaupt.
0,92 bei den Weichthieren.
0,19 bei Pflanzen und Thieren zu-
0,14 bei den Kerbthieren. sammen.

In einzelnen extremen Fällen aber kann die Verhältniss-Zahl der fossilen Genera gegen die der lebenden, beide im Ganzen genommen, seyn von $=0: 1$, wie bei den Entozoen, bis $=1$ : 0 , da es für einzelne fossile Gruppen keine lebenden Repräsentanten gibt (Paläaden, Ammoniten, Rudisten), über welche Fälle man durch Vergleichung der Rubriken 17 und 21 in der III. Tabelle umständliche Auskunft erhält.

E. Dieselben Verhältnisse B-D lassen sich nun auch nach den einzelnen geologischen Perioden vergleichen. Obschon Diess mehr in die Geschichte der organischen Welt gehört, wollen wir hier nur bemerken, dass die Verhältnisse aller fossilen zu den fossil-lebenden sind:

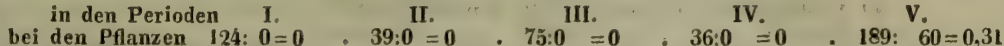

$$
\begin{aligned}
& \text { bei den Thieren } 484: 99=0,20 \cdot 157: 93=0,59 \cdot 541: 258=0,48 \cdot 495: 267=0,54 \cdot 1403: 962=0,61 \\
& \text { bei beiden } \quad 608: 99=0,14: 196: 93=0,47.616: 258=0,42.531: 267=0,50: 1592: 1022=0,64
\end{aligned}
$$

§. 10. Zahlen-Verhältnissder Sippenzu den Arten. Tabelle IV, S. 742.

A. Alle Schwierigkeiten solcher Zahlen-Bestimmungen, wie wir schon oben bei den Arten und bei den Sippen aufgezählt haben, treffen hier vereinigt zusammen; man darf die sich herausstellenden Verhältnisse daher nur als angenäherte Werthe betrachten.

Wir verweisen desshalb auf S. 784 und 804.

\section{B. Das Verhältniss der Arten zu den Sippen ist}

bei del Pflanzen : . . . . . . . . . 5, 57

bei den Thieren $\quad \cdot \quad \cdot 10,10$

bei beiden zusammen $\quad . \quad . \quad . \quad . \quad 0,59$

oder ein Geuus enthält 5,5\%, 10,1 und 9,59 fossile Arten; bei den Pflanzen also nicht $2 / 3$ so viel als bei den Thieren.

Wegen einzelner Unterreiche, Klassen, Ordnungen verweisen wir auf die IV. Tabelle, S. 742. In extremen Fällen kann das Verhältniss für ein Unterreich von 3,30 bis 29,3 gehen, wie bei den Malacozoen; - für eine Klasse bis 30,0 und 32,0 , ja bis 39,5 wie bei den Brachiopoden (die übrigens einer Umarbeitung bedürfen); für noch mehr untergeordnete Gruppen bis zu den Genera herab können die Extreme noch weiter auseinander liegen.

C. Eine nähere Betrachtung der Tabelle IV zeigt uns aber, dass wenn man von einzelnen Ausnahmen und Schwankungen, welche oft 
von der mehr oder weniger sorgfältigen Bearbeitung einer Gruppe abhängen, und hauptsächlich von ganz kleinen und daher meist zufälligen Ziffern absieht, die Arten eines Genus durchschnittlich um so zahlreicher werden, je zahlreicher die Genera einer Ordnung, Klasse u. s. w. selbst sind, und beide nehmen mit einander an absoluter Anzahl zu und ab; die Zahlen-Entwickelung der einen gibt sich im Allgemeinen auch in den andern kund.

Bei den 2 Reichen ist das Verhältniss :

1) Pflanzen . . für 2055 Arten $=5,87$

2) Thiere . . " $24366, "=10,10$

Bei den 4 Unterreichen der Thiere auffallend regelmässig :

1) Spondylozoen für 2701 Arten $=3,70$

2) Entomozoen " $2885,=4,20$

3) Phytozoen . " $4895, "=9,34$

4) Malaco\%oen . " $13885, "=29,3$

Bei den wichtigsten Thier-Klassen mehr schwankend :

1) Mammalia . für 708 Arten $=2,34$

2) Reptilia . . " $384 "=3,31$

3) Pisces . . " $1461, "=4,12$

4) Crustacea . " $894 "=5,36$

5) Polypi .. " $2528 n=10,1\}$ (neu zu bearbeiten.)

6) Amorphozoa " $" 461 "=11,0\}$ (neu zu bearbeiten.)

7) Echinodermata " $1189 "=15,4$

8) Pelecypoda . " $4836 "=27,7$

9) Gasteropoda " $6110,=30,2$

10) Cephalopoda " $1546 "=32,2$

11) Brachiopoda . " $1146, "=39,5$ (neu zu bearbeiten.)

Einzelne Genera können 1-200-300-400 Arten enthalten (Ammonites über 500).

D. Diese Zusammenstellung der Thier-Klassen zeigt ferner :

1) Dass in Klassen u. s. w., die sich auf wenige Perioden beschränken, nicht so viele Arten auf eine Sippe kommen als bei solchen, die - mit ihren Sippen - die ganze Reihe der Formationen durchlaufen (1, 2 im Gegensatz von 8, 9, 10, 11).

2) Dass Familien, deren Reste solcher Art sind, dass sie hinreichende Merkmale bewahren, um darnach den lebenden gleichwerthige Genera aufzustellen, wohin insbesondere die Wirbelthiere mit Ausnahme der Vögel gehören, also das Unterreich der vollkommensten Thiere im Allgemeinen, dann aber auch die Kruster, Echinodermen und Polypen, nicht so viele Arten in einem Geschlecht zu vereinigen pflegen, als andere von einer überbaupt oder doch im Fossil-Zustande indifferenteren Beschaffenheit $(1,2,3,4,5$ zumal nach den neuesten Bearbeitungen und 7 im Gegensatze von 9, 11).

3) Die Arten der Landbewohner (Pflanzen, Insekten, Säugthiere, Reptilien) mögen, weil nur zufällig, nicht in gleichem Ver- 
hältniss zahlreich in die meerischen Erd-Schichten eingeschlossen worden seyn, als die der Seebewohner (fällt dann mit $\mathbf{C}$ zusammen).

4) Bei unvollkommen bearbeiteten Gruppen kann die Arten-Zahl bald kleiner und bald grösser erscheinen, als der Regel entspricht $(5,6,11)$.

5) Manche ausgestorbene Genera oder grösstentheils ausgestorbene Gruppen scheinen, wenigstens im Vergleich zur Dauer ihrer Existenz in vorzüglich zahlreichen Arten vorhanden gewesen zu seyn Cordnungen der Fische, Echinodermen, - daun der Gasteropoden und Brachiopoden).

E. Die Regeln über das Verhältniss der Arten - zu den SippenZahlen gelten zweifelsohne in einem etwas veränderten Massstabe auch zwischen den Arten - und Familien-Zahlen u. s. w.; doch ist diese Abstufung des Systems bis jetzt noch immer eine zu willkührliche gewesen.

C. Gesetze, wornach die organische Welt sich in der geologischen Zeit allmählich zu ihrer jetzigen Beschaffenheit gestaltet hat.

(Die "Gesetze", welche wir hier aufstellen, sind blosse Abstraktionen aus der Summe bisheriger Beobachtungen, - oft nur unvollkommene Induktion, - ohne eine mathematische Nothwendigkeit. Neue Beobachtungen können sie modifiziren oder umstossen.)

\section{a. Durch Zunahme der Kahlen.}

ธ. 11.

A. Die Zahl der anfänglich gleichzeitig nebeneinander bestandenen Klassen, Ordnungen, Familien, Geschlechter, Arten, ist all. mählich immer grösser geworden und zwar, wenn man in 1-2 Fällen, wo eine Thier-Klasse ein oder einige Zwischenglieder überspringt, sie auch in diesen anrechnet, wie folgt (vgl. S. 811):

Pflanzen: Periode: I. II. III. IV. V. I-V. VI.

\begin{tabular}{|c|c|c|c|c|c|c|c|c|}
\hline Klassen 1 ) & & 5 & 5 & 6 & 6 & 10 & 10 & 10 \\
\hline Familien & 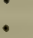 & 22 & 11 & 22 & 17 & 76 & 82 & 276 \\
\hline Genera. & • & 176 & 45 & 97 & 37 & 237 & 350 & 6,529 \\
\hline $\operatorname{Arten}^{2}$ ) & • & . 1017 & 98 & 241 & 84 & 623 & 2055 & 70,000 \\
\hline
\end{tabular}

Thiere :

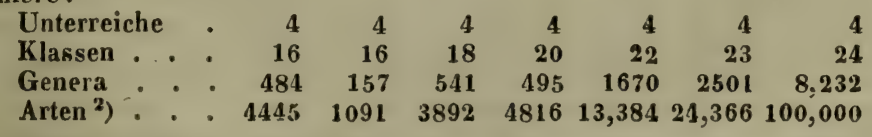

1) In der Arten-Tabelle S. 727, 728 ff. mit grossen römischen Buchstabeu bezeichnet. Eine oder zwei einzelne Arten, welche der Masse einer Klasse in früheren Perioden vorausgingen, hat man nicht berechnet.

3) Die Arten sind hier für die einzelnen Perioden nur durch Addition der Rubriken jeder Periode gefunden. Vergl. $\$$. 3. 
Die Zunahme der Zahlen aller systematischen Kategorie'n zeigt sich in den oberen Abtheilungen oder Haupt-Typen (Unterreichen, Klassen), wo zufüllige Einflüsse sich mehr ausgleichen können, am gleichmässigsten; in den untern, den Geschlechtern und Arten dagegen für die 4 ersten Perioden schwankend, weil, wie schon erwähnt, ihre Länge nicht gleich abgemessen (die der II. Periode offenbar viel kürzer), ihre Gesteine sehr ungleich geschickt sind zur Aufbewahrung fossiler Reste u. s. w. (S. 784); allein sie tritt in der V. Periode gegen die früheren überall bestimmt hervor, und wieder stärker in der VI. (jetzigen) als in der V. - In den Arten der einzelnen Geschlechter u. s. w. würde ein solches Resultat freilich nicht zu erreichen seyn. Zum Theil rührt die Zunahme in den Klassen allerdings daher, dass einige lebende unter denselben (Enthelminthen, Acalephen) überhaupt nicht des fossilen Zustandes fähig sind; - während bei den Ordnungen, Familien und Geschlechtern umgekehrt eine grössere oder kleinere Anzahl, bei den Pflanzen 5 Familien, im fossilen Zustande vorkommen, welche sich nicht bis in die jetzige Zeit erbalten haben, daher auf eine weniger rasche Zunahme der entsprechenden Zahlen hinwirken, ja, da sie hauptsächlich den ältesten Perioden angehören, sogar zur Vergrösserung einiger Zahlen in den frühesten Zeit-Abschnitten im Vergleich zu den späteren mitwirken, welche aber wenigstens in den wirklichen Schöpfungen der ersten Zeit-Perioden - bei gleicher Länge aller - zwcifelsohne nicht stattgefunden hat. Die Pteropoden und Heteropoden der ältesten Periode gehören mit denen der jüngsten, bis zu welchen eine lange Lücke ist, wahrscheinlich gar nicht in eine Klasse zusammen. 
Die Klassen beider Reiche stellen sich so dar:

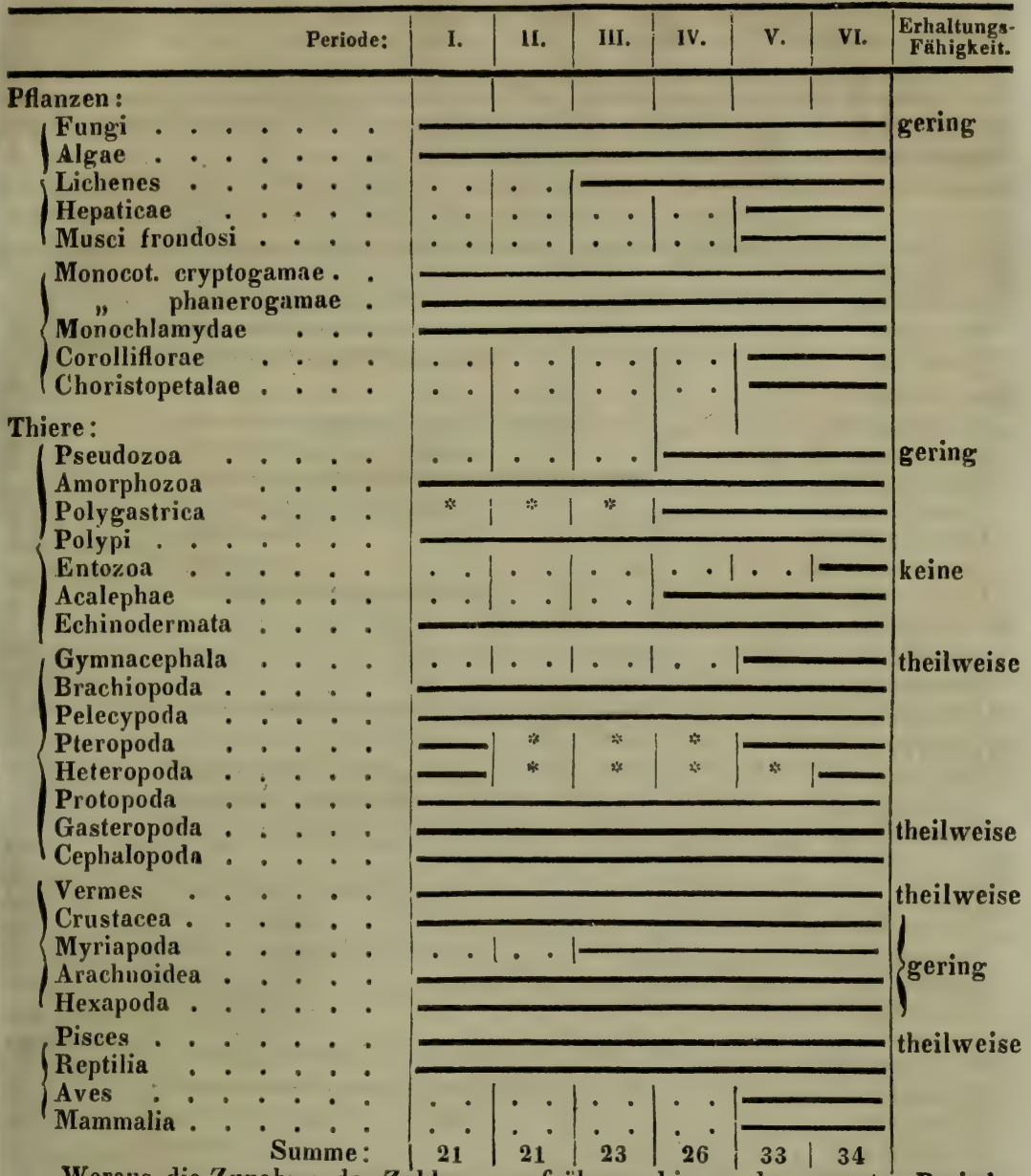

Woraus die Zunahme der Zahlen von früheren bis zu den neuesten Perioden klar hervorgeht, die Sippen- und Arten-Zahlen mögen schwanken, wie sie wollen ; nirgends eine Abnahme!

Wir haben die Ordnungen der Thiere, mit welchen man die Familien der Pflanzen am ehesten gleich setzen kann, oben nicht gezählt, weil man über deren gleichen Rang nicht überall einverstanden ist; doch hindert Diess nicht zu bemerken, wie diese Ordnungen, so wie wir sie einmal angenommen haben (vergl. die Arten-Tabelle I, S. 727) allmählich bei den Echinodermata von 1 auf 3 , bei den Gasteropoda von 2 auf 7 , bei den Vermes von 1 auf 3 , bei den Crustacea von 1-2 auf 3 , bei den Hexapoda von 2-4 auf 12, bei den Pisces von 2 auf 6, bei den Reptilien von 1 auf 4 zunimmt. 
Abnahmen kommen unter den Thier-Ordnungen überhaupt nicht vor, so weit als zuverlässige Bestimmungen reichen; sie treten wohl bei Pflanzen - in Folge schon angedeuteter Einwirkungen, oder in beiden Reichen ein und das andere Mal dadurch ein, dass eine ohnehin spärlich vertretene Gruppe einmal eine Periode ganz $\mathrm{zu}$ überspringen scheint, $d$. $h$. bis jetzt noch nicht aufgefunden worden ist. Spätere Entdeckungen dürften übrigens in den einzelnen Zahlen noch Manches ändern. Die angedeuteten Fälle sind:

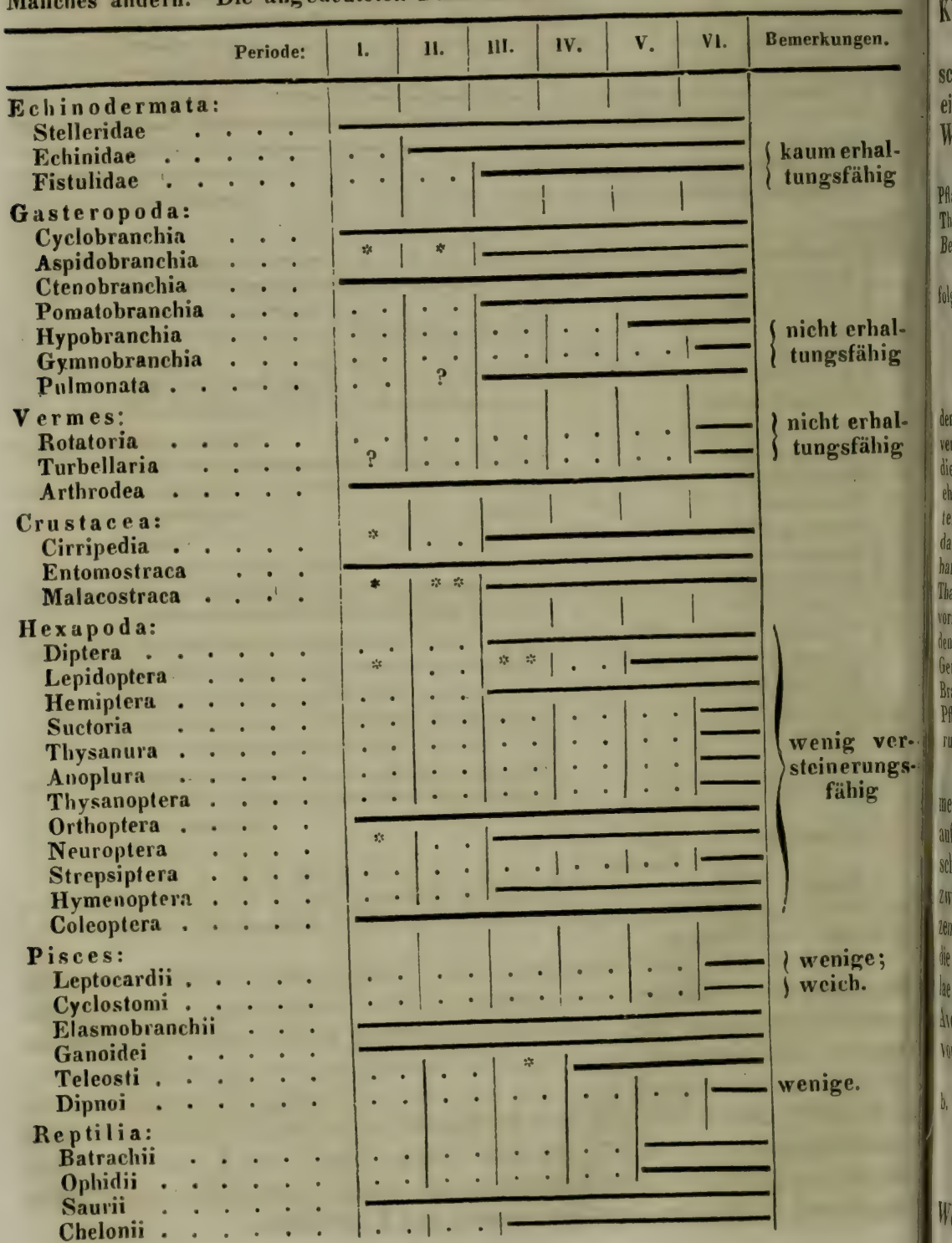


Wie hier mit den Ordnungen, so verhält es sich denn auch mit manchen Unterordnungen u. s. w. (Vergl. die Entomostraca, Malacostraca, Elasmobranchii u. s. w.)

B. Mit der Zunahme der absoluten Zahlen, folglich mit dem Voranschreiten des Schöpfungs-Ganges überhaupt, hat auch das Verhältniss der Arten-Zahlen zu den Sippen-, Familien-, Ordnungs-, Klassen-Zahlen zugenommen.

So ändert sich das Zahlen - Verhältniss der Arten zu den Geschlechtern nach Tabelle IV, S. 742 (wo man die Details für die einzelnen Unterreiche, Klassen u. s. w. beisammen findet) auf folgende Weise allmählich ab.

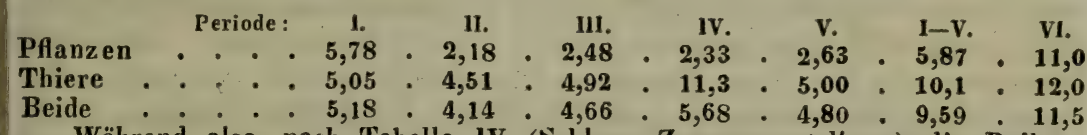

Während also nach Tabelle IV (Schluss-Zusammenstellung) die Reihenfolge der Perioden nach der Zunahme seyn würde bei den

Arten * * • • • • • . II, III, IV, I, V, I-V, VI,

Geschlechtern . . . * II, IV, III, II, V, I-V, VI,

ist sie nach der Proportion beider II, III, V, I, IV, I-V, VI,

Die grösste Arten-Zahl kommt also auf die kleinste Geschlechter-Zahl in der I. und zumal in der IV. Periode; in denjenigen beiden Perioden, wo es verhältnissmässig die meisten und die wenigsten Pflanzen gibt. Man würde dieses Verhältniss nach einem früher ausgesprochenen Gesetze (S. 808) am ehesten da haben erwarten müssen, wo die absoluten Arten-Summen am grössten waren, wie es sich denn auch für die Periode VI und I-V. in Summe, dann für II und selbst noch III bestätigt (die unbedeutend mehr als die IV. hat). Aber in der I. und IV., der Kohlen - und Kreide-Periode, fehlen in der That die vielen kleinen Geschlechter mit nur $1-2$ Spezies, die in den übrigen vorkommen, grösstentheils, und es wirken bei $\mathbf{V}$ vorzüglich die neu auftretenden, an Arten noch armen Säugthier-, Vögel-, Insekten - und DikotyledonenGenera (nach der S. 808 gegebenen Regel), wie der Mangel an Arten-reichen Brachiopoden- und Cephalopoden-Genera, bei I insbesondere die Arten-reichen Pflanzen-, Brachiopoden-, Cephalopoden - und Fisch-Geschlechter auf die Änderung der Reihenfolge ein.

C. Es ist demnach fortwährend die Manchfaltigkeit der Formen im Pflanzen - wie im Thier-Reiche grösser geworden und die auffallendste Zunahme der fossilen Typen der Klassen aus theils schon angedeuteten und theils noch später zu bezeichnenden Ursachen zwischen der Kreide- und Tertiär-Periode erfolgt, wo die der Pflanzen und Thiere zusammengenommen, durch die Musci Frondosi, die Hepaticae, (die Lichenes,) die Corolliflorae, die Choristopetalae, die Acalephae (die Gymnacephala erscheinen kurz vorher), Aves, Mammalia (abgesehen von einigen vereinzelten älteren Arten) von 26 auf 33 steigen.

b. Durch Hinzukommen vollkommner Organismen-Formen.

6. 12. Im Allgemeinen. Pflanzen.

A. Der allmähliche Wechsel in den Formen der organischen Welt wird vermittelt durch das allmähliche Auftreten immer neuer 
Formen-Typen und durch das allmähliche Wiederverschwinden eines Theiles derselben. Die in den ältesten Schichten aufgefundenen organischen Reste, obschon bereits sehr manchfaltig, enthalten doch gewisse höhere Typen der Organisation noch nicht, welche dann vielmehr erst in denen späterer Perioden oder selbst erst in der jetzigen Zeit zum Vorschein gekommen sind. Es lässt sich daher ein Voranschreiten von unvollkommener zu vollkommener Organisation nachweisen, ohne dass jedoch diejenigen tieferen und tiefsten Klassen der Organisationen, die einmal bestanden haben, darüber versclıwänden oder absolut seltener würden; vielmehr nehmen auch sieihrerseits in spätern Perioden an der zunehmenden Manchfaltigkeit der Gestaltung Antheil und bilden zuweilen in ihren eigenen Typen höhere Stufen aus. Die nach längerem oder kürzerem Bestehen allmählich wieder untergehenden Formeu sind keineswegs immer die niedrigsten in ilırer Klasse, Ordnung oder Familie; ihr Erlöschen scheint daher andern Gesetzen zu unterliegen. - Zwar sind der erloschenen Genera mehr als der bis jetzt am Leben gebliebenen (S. S05); aber dennoch sind damit keine löheren systematischen Kategorie'n als aufwärts bis zu den Familien der Pflanzen, und zwar nur der kryptogamischen Monokotyledonen und gymnospermen Dikotyledonen (Asterophyllitae, Psaronieae, Stigmarieae, Sigillarieae, Diploxyleae) und bis zu den Unterordnungen der reptilen Thiere (Rudistae bei richtigerer Eintheilung, Palaeades, Pterodactyli, Labyrinthodontes) ganz, einige Unterordnungen und andre Ordnungen nur beinahe (Crinoidea, Brachiopoda, Cephalopoda, Cestraciontes, Ganoidei) untergegangen, während neue Ordnungen und Klassen von höhrer Organisation dafür zum Vorschein gekommen sind.

B. Wie schon erwähnt worden und später noch weiter ausgeführt werden soll, wäre ein reihenförmiges Auftreten stufenweise immer höher entwickelter Klassen von Organismen nicht möglich gewesen; die gegenseitige Abhängigkeit der tieferen von den höheren ebensowohl als der höheren von den tieferen Formen und die geolegischen Veränderungen der äussern Bedingungen des Lebens verschiedenartiger Klassen würden nicht die einzigen Hindernisse gewesen seyn, die einem so einfachen Entwicklungs-Gange in den Weg getreten wären. Ohnehin ist es dem Systematiker nicht einmal mög. lich, alle Klassen von Organismen in eine einzige Stufen-Reihe zu ordneu, da manche hinsichtlich ihrer Organisation mehr neben-als über-einanderstehen und nicht selten eine Klasse die andre in eineı Beziehung überragt, von der sie in der andern überragt wird. Die Lebens-Weise der Organismen, ihr Verhalten zur Aussemwelt ha aber sicher oft einen mächtigeren Einfluss auf die Ordnung ihres all mählichen Aufrretens und Untergehens geäussert, als die Organisa tions-Höhe vermocht haben würde, wenn diese auch als leitende Ídee zı Grunde gelegen bätte. Man kann daher den Entwicklungs-Gang, in so 
fern er von der einfachen Aufeinanderfolge immer höherer Organisationen abweicht, noch in folgenden Sätzen näher bezeichnen, wenn man von den zur fossilen Erhaltung wenig geeigneten und daher hinsichtlich ihrer geologischen Entwicklung uns offenbar nur unvollständig bekannten Gruppen zunächst ganz absieht :

1) Pflanzen und Thiere sind ungefähr gleichzeitig entstanden.

2) Eine grosseManchfaltigkeit organischer Formen aus beiden Reichen und von sehr verschiedenen, doch nicht den höchsten Stufen der Organisation beider ist als erste Grundlage der organischen Schöpfung schon in der silurischen Zeit vorhanden gewesen. War indessen die Dauer der einzelnen Formations-Zeiten wirklich so lange, wie einige Verhältnisse anzudeuten scheinen (BIScHoF berechnet die der Steinkohlen-Bildung (e) auf nahezu 1,000,000 Jahre), so war auch innerhalb der Silur-Zeit selbst noch hinreichende Frist zu successiver Entwicklung nach allgemeinen Gesetzen.

3) Später sind zu den einmal vorhandenen Rang-Begriffen des Systems beider Reiche (Unterreiche, Klassen, Ordnungen) immer höhere Unterreiche, Klassen oder Ordnungen noch hinzugekommen.

4) Höhere Haupt-Kategorie'n (Unterreiche, Klassen, Ordnungen) sind - mit 1 oder 2 Ausnahmen - einer ganzen gleichwerthigen Kateyorie von niedrigerer Organisation nicht vorangegangen, obwohl ifters eine höhere Klasse vor den Ordnungen, Familien u. s. w. einer liedrigeren Klasse vorangegangen ist; - denn

5) Jede Kategorie jener Art hat sich, sobald sie einmal vorhanlen war, selbstständig auch in sich nach Höhe und Breite weiter entvickelt, schneller oder langsamer als andere gleichwerthige, sie iberholend oder von ihnen überholt (vergl. C u. a.).

6) Die fortschreitende Entwickelung spricht sich aber nicht blos lurch das Hinzukommen höherer Formen zu den früheren unvollommeneren (Zunahme in der Höhe der Formen), sondern auch durch as Überhandnehmen der Zahl dieser höheren Formen in der Geammt-Zahl und gegen die der unvollkommeneren aus (Zunahme in ie Fülle oder Manchfaltigkeit oder dem Reichtlıum der Formen), adem diese letzten entweder langsamer als die ersten an Zahl unehmen, oder sogar an Zahl zurückgehen, oder ganz aufhören, ährend jene noch wachsen.

7) Diese Entwicklung entsprang aus einer allmählichen Umgetaltung nicht der aufeinanderfolgenden Generationen eines gemeinamen Urstammes, sondern der aufeinanderfolgenden neuen Schöfungs-Akte.

8) Erweisliche Zustände der äussern geologischen Lebens-Bedinungen haben in der stufenweise fortschreitenden Entwicklung der rganisation einige Störungen verursacht (s. u. $\$ \$ .14,15)$.

9) Je untergeordneter und kleiner die systematischen Kategorie'n erden, desto mehr scheinbare oder wirkliche. Störungen der anfäng- 
lich angedeuteten Gesetze $(2-5)$ treten bei ihnen ein. Diese lassen sich daher nicht überall bis zu den kleinern Familien herab, noch weniger bis zu den Geschlechtern erkennen. Aber auch bei sehr armen, bei sehr schwierig-erhaltungsfähigen höheren Kategorie'n, deren einstiges Verhalten wir aus den sparsam auf uns gekommenen Resten beurtheilen sollen, wofern diese nicht etwa ganz ausgeblieben sind, dürfen uns Ausnahmen nicht überraschen, die wir berechtigt sind einstweilen für nur scheinbare zu halten.

a. Die Ansicht einer fortschreitenden Entwicklung der Organisation während der geologischen Zeit ist alt. Lamarck und Geoffrox St. Hilark liessen successive Generationen gleicher Urältern sich immer höher und höher gestalten; später sollte jede neue Schöpfung immer höhere Organismen zu denen der früheren hinzufügen.

Agsssiz suchte die Ansicht aufzustellen und bei Fischen und Echinodermen durchzuführen, dass das Thier-Individuum von seinem Embryo-Zustande an bis zu seiner Reife in allen Beziehungen dieselben Stufen der Entwicklung durchlaufe, wie die Thier-Klasse, wozu es gehört, von ihrem ersten planetarischen Erscheinen an bis zum Culminations-Punkt ihrer Ausbildung, und dass man in beiden Fällen dieselben Schöpfungs-Gedanken verfolgen könne; doch bedürfen manche Klassen zu dem Ende zuerst noch einer naturgemässen Klassifikation. Indem wir das Geistreiche dieser Ansicht, den Grad der Wahrheit, welcher in diesen verführerischen Sätzen liegt, und die glückliche Anregung, welche aus ihnen entspringen muss, nicht verkennen, glauben wir doch nicht, dass sie als vorherrschende Grund-Gedanken des Schöpfungs-Planes geltend gemacht werden können, wollen aber versuchen, wie weit sie sich mit obigen Modifikationen durchführen lassen, und behalten uns vor auch andere Sätze in den folgenden Paragraphen damit zu vergleichen.

b. Auch die Theile unsrer jetzigen trocknen Erd-Oberfläche lassen je nach ihrer Grösse eine Abstufung in der Vollkommenheit ihrer Säugthier-Bevölkerung, im Voranschreiten vom Niederen zum Höheren wahrnehmen, die der geologischen Abstufung analog ist, indem hier wie dort nicht reihenweise die ganzen höheren Ordnungen nach ganzen tieferen auftreten, sondern gewisse tiefere Formen aus verschiedenen Klassen den Anfang machen für Stufen höhere Formen, die sich wieder in verschiedene Ordnungen vertheilen. 1) Die kleiner entfernten Inseln der Südsee nähren ausser den mariven Säugthieren ihrer Kü sten nur einige kleine Fledermäuse und Nager, welche erst in späterer Zei eingeschleppt worden seyn mögen, ursprünglich also wahrscheinlich gar keine 2) Neu-Holland und die Nachbar-Inseln besassen bei ihrer Entdeckung nur we nige Nager und viele Beutethiere, wovon die ersten wie es scheint das Trin ken ganz entbehren können, die andern aber in den langer Trockne ausgesetz ten Strichen oft weit nach Wasser zu wandern gezwungen sind, wobei sie ihr Jungen nur in ihren Beuteln mit sich führen können; ihrer Placental-Bildun und ihrer Trächtigkeit nach sind sie die unvollkommensten Säugthiere; Nager un Beutelthiere sind auch dic ersten und einzigen Mammiferen in den Oolither 3) Die Säugthiere Amerika's und besonders Süd-Amerika's stehen auf einer ti feren Stufe als die der alten Welt, wie noch einige Beutelthier-Genera, die viele Edentaten und auch Nager, die Lama's statt der Kameele, der fast gänzlicl Mangel an Wiederkäuern (ausser Hirschen) und Pachydermen (ausser Peca und 2 Tapiren) zumal der grösseren Formen, die Kleinheit der Raubthie: (statt der Löwen, Tiger, Wölfe), die Affen mit seitlichen Nasenlöchern uı 6-7 Backenzähnen (statt 5) wohl erkenuen lassen. 4) Die alte Welt, d grösste der Kontinente, hat nicht nur die zahlreiehste, sondern auch die höcho vollkommenste Säugthier-Fauna. 
C. Im Ganzen zeigt sich im Entwicklungs-Gange der Pflanzen und Thiere (ohne den Menschen) eine grosse Analogie. Bei beiden sind die niedrigsten Klassen schon ganz gleichzeitig vorhanden; eine kleine mittle etwas später auftretende Gruppe entwickelt sich in der geologischen Zeit stärker und nimmt gegen deren Ende wieder ab; die höchsten Klassen erscheinen wenigstens in Masse erst in der Tertiär-Zeit, und es stehen sich parallel

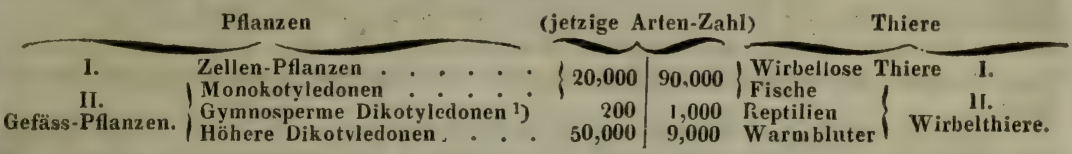

Daraus geht hervor, dass 1) das Pflanzen-Reich gleichzeitig oder noch etwas später als das Thier-Reich beginnend sich, obwohl tiefer stehend als dieses, doch nicht rascher, nicht vor ihm entwickelt hat und weder durch Anfang noch Vollendung dem höheren Reich vorangegangen ist; dass es 2) vielmehr in der Zahl der Klassen, Ordnungen, Geschlechter und Arten bis zu Anfang der Tertiär-Zeit weit gegen dieses zurückgeblieben ist. Denn es machen jene niedrigeren Zellen- und monokotyledonen Gefäss-Pflanzen nur $2 / 7$ im Systeme des ganzen Pfanzen-Reicls, dagegen diese niedrigeren wirbellosen Thiere und Fische $9 / 10$ in dem des Thier-Reichs aus, daher dieses gleich von Anfang an eine weit grössere Manchfaltigkeit der Formen darbieten konnte, als die Pflanzen.

So geht anch in der Folge jede Klasse, Ordnung u. s. w. ihren eigenen Entwicklungs-Gang, und wenn auch eine tiefere Klasse früher oder gleichzeitig mit einer höheren beginnt, so kann jene zu ihren höchsten Ordnungen $u$. s. w. doch später als diese gelangen, oder sogar numerisch zurückgehen, wo diese noch voranschreitet.

1). Schon die erste Frage, ob Pflanzen oder Thiere früher zum Bestehen gerufen worden sind, zeigt uns ein ungefähr gleichzeitiges Auftreten beider Reiche in der I. Periode, wobei jedoch die fossilen Reste der Thiere denen der Pflanzen, die des höhern Reiches denen des tieferen, noch etwas vorausgegangen sind. Die Thiere beginnen in den untersilurischen, die Pflanzen erst in den devonischen Schichten.

In den silurischen Schichten gibt es bereits Pflanzen-Thiere, Weich-Thiere und Fische; aber noch kennt man keine Pflanzen, obschon kaum zu hezweifeln ist, dass Diess bloss in Folge der schwierigeren Erhaltung zumal der unvollkommeneren Pflanzen in den Erd-Schichten so der Fall seye.

1) Ad. Brongniart hat früher die Koniferen und Cycadecn unter dem Namen gymnosperme Phanerogamen zusammengefasst. Was man auch gegen den ersten 'Theil des Namens einwenden nag, wir behalten ihn hier bei, um eine geologisch und botanisch verwandte Gruppe kurz zu bezeichnen. 
Diesclbe Frage lässt sich auch - da das blosse Unbekanntseyn silurischer Pflanzen noch keinen Beweis ihrer Nicht-Existenz in silurischer Zeit licfert - von einer doppelien theoretischen Seite aus exörtern, in wie fern năm. lich die silurischen Thiere ohne Pflanzen athmen und sich nähren konnten. Was die Nalırung betrifft, so leben die unvollkommensten Thierchen von orga. nischer Materie, jeder Art, die in Wasser aufgelöst ist, und setzen also nicht gerade Pflanzen voraus, können aber dann selbst wieder hüheren Thieren, Mollusken u. s, w. zur Nahrung dienen. Die (silurischen) Polygastrica, Polypi, Echinodermata, Malacozoa und Plagiostomen haben wir nicht Ursache für ausschliessliche Pflanzen-Fresser zu halten; die gewöhuliche Nahrung der Thiere dieser Klassen ist eine animalische. Von dieser Seite also würde die Nothwendigkeit eines Auftretens der Pflanzen vor den Thieren nicht bewiesen werden kömneu.

Zwar ist Bischof's Bemerkung zu berücksichtigen, dass selbst die niedersten Thiere und schon die Infusorien überhaupt vorzugsweise aus Protein bestehen und entstehen, das, selbst ein Pflanzen-Produkt, die Grundlage aller thierischen Nahrung seye und ihnen von Pflanzen zubereitet werden müsse, weil Thiere nicht selbst vermögten Stirkstoff-Verbindungen aus deren Elementen herzustellen, wie dagegen Pflauzen keine organischen Stickstoff-Verbindungen, sondern nur unorganische Elemente wie Ammoniak, Koblensäure und Wasser in sich aufzunehmen und daraus ihre Substanz zu bilden vermögen, daher schon das Bestehen von Infusorien das Bestehen von Pflanzen voraussetze ${ }^{1}$ ). Man köunte noch hinzufügen, dass nach $\mathrm{C}$. Scнmid's chemischer Zerlegung wenigsteus die zahlreichen Bacillarieen unter den Infusorien (welche von Vielen schon selbst als Pflanzen angeschen werden) nicht Protein, sondern wie die Pflanzen Cellulose zur Grundlage haben und hiedurch also noch strenger auf Pflanzen-Nahrung angewiesen seyen. - Denkt man sich aber die Thier-Klassen nicht stufenweise nach einander, sondern viele derselben gleichzeitis mit einander entstanden, worauf die Beobachtung eben hinzuweisen scheint, so würde wohl nichts der Ansicht in Wege stehen, dass die finmal vorhandenen Polypen und Muschel-Thiere sich von Infusorien (unter welchen die Räder-Thiere wohl eine mehr animalische Zusammensetzung haben dürften) und die Infusorien wie. der von den verwesenden Resten der Polypen und Muscheln wie ihrer eigenen Klasse nährten. Wir würden daher abermals der Pflanzen nicht nothwendig vor den Thieren bedürfen.

Was das Athmen der silurischen Thiere betriff, in einer Zeit also, wo dic ganze Masse des nachher in der Steinkohlen-Formation niedergeschlagenen Kohlenstoffes noch an den Sauerstoff der Athmosphäre gebunden die Athmungs-Tauglichkeit der letzten für Thicre verminderte, so vermögen einestheils die Infuso. rien wenigstens selbst Kohlensäure zu zerlegen; anderntheils ist der Respirations-Prozess der Polypen, Echinodermen, Mollusken (von den sehr spärlichen Fischen darf man wohl absehen) zweifelsohne auch in einer Athmosphäre möglich, welche reicher als die jetzige an Kohlensäure ist und deren SauerstoffGehalt im Gleichgewicht zu erhalten wieder grosse Massen von Magen-Infusorien selbst durch ihre Zerlegungs-Kraft haben beitraven können.

Indessen ist genug innere Wahrscheinlichkeit für das Entstehen der Pflanzen mit oder vor den Thieren vorhanden und ist die negative Thatsache, dass man Pflanzen-Reste in den silurischen Schichten noch nicht kennt, von so geringem Gegengewichte, dass wir selbst von jenem gleichzeitigen oder früheren Entstehen ïberzeugt sind, wenn wir es auch auf praktischem oder theoretischem Wege noch nicht strenge beweisen können.

\section{E. Aber in der Entwicklnng des Pflanzen-Reiches ${ }^{2}$ ) selbst}

1) G. Bischof im Jahrb. $1848,632$.

2) Hinsichtlich der ältesten, nämlich der Grauwacke-Pflanzen verdient noch: der neuere Aufsatz von Göppsax Beachtung, im Jahrb. 1847, 675 ff. 
zeigt sich ein unverkennbares Fortschreiten vom Tieferen zum Höheren, vom Einfachen zum Zusammengesetzteren, zumal wir nämlich das späte Erscheinen fossiler Reste eines Theiles der niedersten Pflanzen, der zarten Leber- und Laub-Moose wie der Lichenen theils ihrer sch wierigeren Erhaltung, theils anderartigen Ursachen zuschreiben müssen. Hätten sie auch von Anbeginn her in der silurischen Zeit als die ersten Vorboten der Vegetation existirt, wie es wohl möglich ist, wir würden kaum hoffen dürfen noch Spuren von ihnen aufzufinden. Pilze und Algen als alleinige Repräsentanten der Zellen-Pflanzen, (krytogamische wie phanerogamische) Monokotyledonen und jener Theil der monochlamydeen Dikotyledonen, welche zunächst an vorige angrenzend früher als Cycadeen und Polykotyledonen bezeichnet wurden und zusammen Brongniart's gymnosperme Phanerogamen bilden, als Repräsentanten der Gefäss-Pflanzen, erscheinen neben einander erst mit der devonischen Zeit; während alle höheren Dikotyledonen, mithin alle höheren Monochlamydeen, Corolliflorae und Choristopetalae, welche zusammen gegen 50,000 von den 70,000 lebenden Pflanzen-Arten liefern, in der W eise auf die tertiäre Zeit zurückgedrängt erscheinen, dass nur 10 völlig vereinzelte und wohl zum Theil noch der Bestätigung bedïrfende Arten davon bis jetzt in Kohlen-Gebirge, Muschelkalk, Oolithen- und Kreide-Bildungen gefunden worden sind.

Indessen lässt sich unter jenen ältesten Gliedern der Flora, wenn wir von den schwer erlıaltbaren Zellen-Pflanzen ganz absehen, noch eine Abstufung bemerken. Denn wenn schon die monokotyledonischen Gefäss-Pflanzen auch in der II. bis IV. Periode noch die Mehrzahl der Pflanzen-Arten liefern, so sind doch jene nächst-vollkommenen gymnospermen Phanerogamen, die in der heutigen Flora nicht einmal 0,0025 aller Arten ausmachen, in der II. bis IV. Periode ausser ihnen am häufigsten und die übrigen, jetzt so vorwaltenden Dikotyledonen um mehr als das Zehnfache übertreffend. - Zwar werden noch einige dikotyledonische Blätter unbekannter Familie (Crednerien) in der IV. Periode und 73 dikotyledonische Frucht Arten unbekannter Familien in der Kohlen-Periode, 5 in den Oolithen und 9 in der Kreide aufgezählt; allein die Angabe einer so grossen Anzahl Früchte, welche überhaupt weniger leicht erhaltbar sind und daher immer nur eine kleine Quote der vorhanden gewesenen darstellen könnten, in solchen Perioden, wo fast keine Spur von andern dikotyledonischen Pflanzen-Theilen vorhanden ist, darf vorerst hinsichtlich ihrer richtigen Bestimmung noch in Zweifel gezogen werden. Der relativ-numerische Entwicklungs-Gang der Flora vom Tieferen zum Höheren würde sich also durch folgendes Bild ausdrücken lassen. 


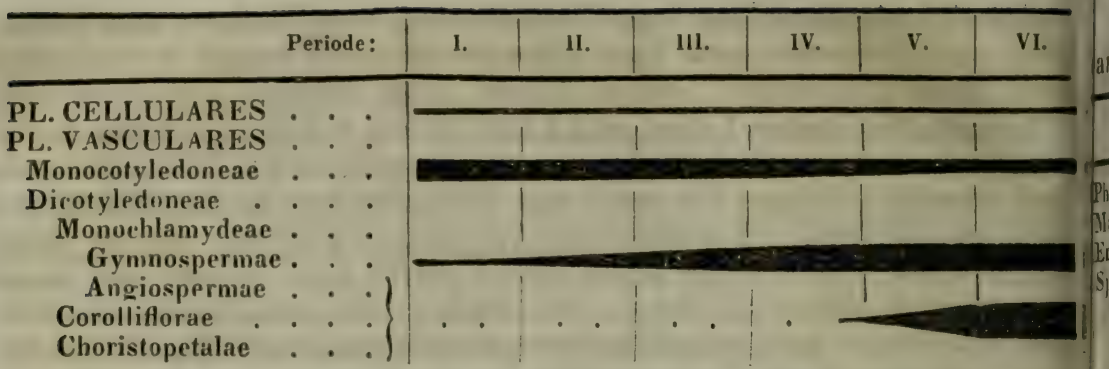

Die Abnahme selbst der Monokotyledonen ist jedoch bloss eine relative in Bezug auf die andern zunehmenden Abtheilungen des Systemes, da man in der Kohlen-Formation gegen 900, aber lebend über 2000 Arten kennt.

F. Das späte Erscheinen der höheren Dikotyledonen (mit Ansnahme näılich der gymnospermen Monochlamydeen) schloss nothwendig auch das der Laubholz.Waldungen in sich, welche vor der Tertiär-Zeit nur durch einzelne Palmen und Cycadeen, durch niedre Farnen-Gebüsche und durch Nadelholz-Wälder ersetzt waren, eine Erscheinung von höchster Wichtigkeit für die klimatischen und unmittelbaren Lebens-Verhältnisse der Thiere.

§. 13. Thiere.

A. Die fortschreitende Entwicklung des Thier-Reiches, dessen Spuren schon vor den Pflanzen-Resten mit der Silur-Zeit reichlich auftreten, das Erscheinen immer höherer Organisation in späterr Zeit-Abschnitten gibt sich bei den Unterreichen und Klassen kund it folgender vierfacher Abstufung.

a) In der Silur-Zeit reicht unsres Wissens die Organisatior noch nicht höher hinauf, als durch die Pflanzen - und Weich-Thier bis zu den Krustazeen, Ringelwürmern und Fischen, von welch letz ten als alleinigen Wirbel-Thieren indessen nur sehr spärliche Rest vorliegen.

b) Die höher stehenden Land-Insekten treten mit (den Land Pflanzen und) den (? Wasser-) Reptilien zusammen erst nach jene in der devonischen Zeit auf.

c) Die warmblütigen Wirbel-Thiere, nämlich Vögel und Säuge Thiere, erscheinen (ausser 4-5 Arten in der Oolith - und Kreide Periude) erst und zwar sogleich in Masse in der Tertiär-Zeit, wi die höheren Dikotyledonen Pflanzen. Jedoch hat man unzweifellıaft Vögel-Fährten und vielleiclıt auch Sängthier-Fährten schon wie ar Fude der Kohlen-Periode im rothen Sandsteine von Massachussett so am Anfang der Trias-Zeit in bunten Sandsteine Deutschlana an mehren Orten häufig gefunden; worüber man jedoch vielleicl erst später genaner wird urtheilen können. 
d) Der Mensch tritt als letztes aller ? organischen Wesen erst in der Grenze der Tertiär- und Jetzt-Zeit hervor.

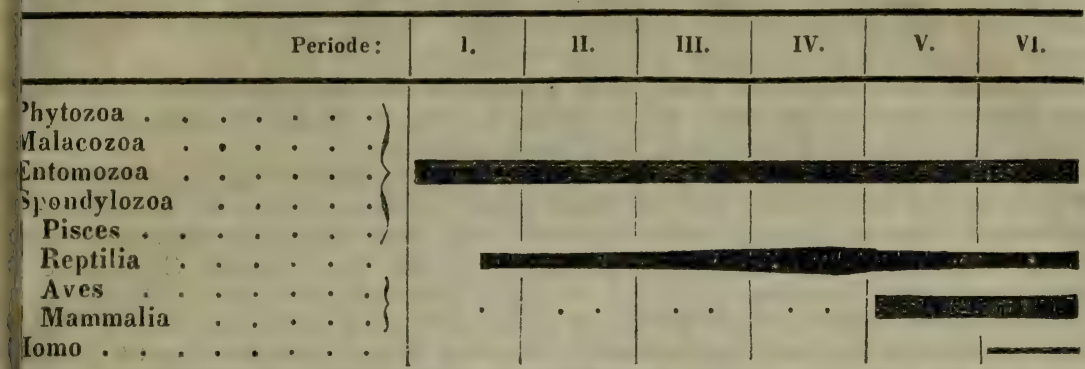

a. Unter den Pflanzen-Thieren können wir, ausser für die Echinoermen keine weiteren Abstufungen angeben. Die Pseudozoen sind zu wenig, ie Polygastrica zu klein und grösstentheils ganz weich, die Polythalami meitens ebenfalls zu klein, die Entozoen und Akalephen als die weichsten aller hiere zu wenig zur Erhaltung geeignet, als dass wir in genetischer Hinsicht uf das spätere Erscheinen ihrer Reste irgend ein Gewicht legen dürften. iei den Weich-Thieren ist der Mangel von den schalenlosen Gymnacephalen nd Gymnobranchiern, von den nur aus wenigen Geschlechtern bestehenden und benfalls zum Theil schalenlosen Tubicoleen, Protopoden, Cyclobranchiern, Aspiobranchiern, Pomatobranchiern und Dibranchiern ebenfalls kein Beweis ihrer Ticht-Existenz in den ersten Formations-Zeiten und Perioden. Wir haben zwar, hne diesen Einwand uns selbst zu machen, auf ihr späteres Erscheinen hingefiesen, wo es sich mehr um die Beachtung der fossilen Reste als um die Ersrschung des Entwicklungs-Ganges der Natur handelte. Wenn sie also weder 'ür noch Gegen beweisen, da wird es logisch seyn, sie den für ihre nächsten erwandten gefundenen Gesetzen unterzuordnen. Bei den Pulmonaten aber 'eten andere Einflüsse hinzu, auf die wir anderwärts zurückkommen. Es sind emnach die Brachiopoden und Pelecypoden, die Mehrzahl der Gasteropoden und er Cephalopoden, welche die Weich-Thiere ganz von Anfang an hauptsächlich ertreten. - Die Kerb-Thiere sind mit Ausnahme der meisten Ringelwürmer nd der Kruster lauter Luftbewohner und konnten daher nur zufällig in die iederschläge des Wassers gerathen; auch ist die kenntliche Erhaltung dieser uftbewohner in den Gesteinen schwierig; ihrem Mangel in den meisten Schichn dürfen wir daher keine Beweis-Kraft für ihre Nicht-Existenz beilegen. So ind auch ein grosser Theil der Ringelwürmer (Rotatorien, Turbellarien und iele Arthroden) nackt; viele Kruster ziemlich weich oder sehr klein. Dienigen Abtheilungen aber dieser 2 Wasser-bewohnenden Klassen, welche härtere alkige Schalen und Gehäuse besitzen, treten gleich in den frühesten silurischen chichten mit gewissen Formen auf, obschon sie mit anderen ebenso erhaltaren erst viel später zum Vorschein kommen. - Die ältesten Fisch-Reste bephen in bloss 7 Arten Flossen-Stacheln (Enumerator p. 652), welche man lagiostomen Elasmobranchiern noch unbestimmter Familien zugeschrieben; und urh sie rühren nur aus den obern silurischen Schichten her. Übrigens lassen ch bei den Fischen noch untergeordnete Abstufungen des Auftretens beeichnen.

b. Indessen hat es Luft-athmende $L$ and-Insekten doch schon zur Zeit er Kohlen-Bildung grgeben. Im Berg-Kalk sind Neuropteren und Ruisselïfer, in der Kohlen-Forination Neuropter-n-Flügel, Skorpionen und Afterkorpionen gefunden worden. Man hat Pflanzen-Blätter aus der Kohlen-Zeit pfunden, welche von misfarbigen Linien durchzogen waren, wie sie die linir-Raupen gewisser Schmetterlinge noch jetzt hervorbringen. - Die in der 
Kohlen-Periode auftretenden Reptilien sind Saurier und zwar nicht mit Ruderfüssen, sondern mit deutlichen frei-beweglichen Zehen, theils den Krokodilen verwandt, welche unter unsern heutigen Sauriern die einzigen Wasser-Thiere sind und selbst im Meere unhersehwimmen, theils unbekannter Familien, aber wahrscheinlich ebenfalls zeitweise Wasser-Thiere, wie jene, da sie anf dem Lande wahrscheinlich nicht eine genügende Menge animalischer Nahrung zu ihrem Unterhalt gefunden haben würden. Hinter diesen Erstlingeu treten die übrigen Reptilien in mehren Zeit-Abstufungen auf.

c. Was die warmblütigen Wirbel-Thiere betrifft: so kennt man von Vögeln in der Kreide-Zeit nur die Reste cines Sperlings-artigen in den Glarner Schiefern, welche jedoch, wie es scheint, von dem Grünsande hinweg in ein etwas höheres Niveau derselben Periode, in das der ältesten NummulitenGesteine veriegt werden müssen; - eines Schnepfen-artigen ebenfalls aus ? Grünsand in Amerilia; und P. Owen hatte nuch einen Albatross-artigen Vogel aus der Kreide Englands angegeben ${ }^{1}$ ), wälırend die angeblichen Vogel-Knochen aus den Wealden wahrscheinlich zu Pterodactylus gehören ${ }^{2}$ ). - Vou Säugthieren kennt man aus der Oolithen-Zeit: die nochmals zu prüfenden Zühne eiues Insektivoren auf der Grenze zwischen Keuper und Lias Deulschlands, dic Kinnlade eines Marsupialen und zweier Insektivoren in den Oolithen (n) Englands $\left.{ }^{3}\right)$. Alles was man sonst an fussilen Resten warmblütiger Wirbel-Thiere kennen gelernt has, geliört der Tertiär.Zeit an, worin diese Reste sogleich mit den ältesten Schichten ( $\boldsymbol{t}, \tau)$ des Pariser Brckens anfireten.

Aber viel älter sind die blossen Fuss-Spuren, Fährten dieser Thiere*). Im rothen Sandsteine in New-Jersey, Connecticut und Massachusetts ${ }^{5}$ ), worin die Fische unscres europäischen Rothliegenden und Magnesia-Kalks vorkommen, hat man Hunderte von Fälirten von 34 verschiedenen Arten in je $2-10$ facher Wicderholung der Fuss-Faare gefunden, vou welchen bei weitem die Mehrzahl ¿weifüssigen hochbeinigen Thieren angehört, deren Füsse in der Grösse jedesmal der Schritt-Write entsprechen und $3-4$ lange in Krallen endigende Zehen besitzen, welche überall genau dieselbe Phalangen-Zahl, die ausschliesslich bei allen unsern Vögeln vorkommt, unterscheiden lassen, nämlich, von der inneren oder Hinter-Zehe angefangen, 2, 3,4 und 5, wie ein mit diesem Gesetz durch. aus unbekannter Bericht.Erstatter in Sil.timan's Journal durch seine Abbildungen nachgewiesen hat. Daher sulche nach unseren jetzigen Kenntnissen auch nur als Ornithichniten oder Dipodichniten den Vügeln augeschrieben werden köunen, womit sich selbst die antänglichen Gegner dieser Ansicht, wie Lyele und R. OWEN, endlich einverstanden erklären mussten. Bei einigen sind bloss 3 Zehen nach vorn, wovon die mittle an längsten, bei anderen ist noch eine aufliegende oder aufstehende Hinterzehe vorhanden; die Länge des Fusses ist $2^{\prime \prime}-19^{\prime \prime}$ und die entsprechende Schritt-Weite dann $3^{\prime \prime}-55^{\prime \prime}$. Die dreizehigen Füsse würden, so weit wir aus der Organisation der jetzigen Vogel-Welt schliessen dürfen, sämmtlich uuf Lauf- und Sumpf-Vögel (Cursores oder Brevipennes und Grallae) oder, in sofern die Hinterzehe nur etwas zu hoch stund und z.l kurz war um sich im Boden abdrücken zn können, noch auf Hühner-Vögel (Gallinae) hinweisen; die vierzehigen könnten aus 5 verschiedenen Ordnungen seyn; doch die Gesellschaft, die Örlichkeit in der sie sich finden, die bekannte Lebens. weise der Vögel, Spuren von Hautsäumen an den Zehen oder wirklich selbst von Schwimmhäuten würden vorzugsweise Sumpf-Vögel (Grallae) undeuten. Die Grösse der Füsse und die Länge des Schrittes sind bei einigen viel beträcht-

1) Jahrb. 1841, $857 ; 1346,638 .{ }^{2}$ ) Jahrb. 1846, 637, 638

3) Enumerator, p. 697, 699, 718, 723, 724.

4) Vrgl. Gesch. d. Natur II, 449 ff. 762 .

Jahrb. 1836, 467; 18.77, 60\% ;841, 739, 856;1843 Collect. 44, 46; $1814,248,635 ; 1845,753 ; 1816,125,126,765^{\text {bis }} ; 1818,880$. 
licher als brim Strauss und weisen auf Vögel von fast doppelter Grösse hin. Andere Vogel-Fährten der Arten sind von Degenhardi im rothen Sandsteine der Provinz Socorro in Mexiko in $5000^{\prime}$ Seehöhe gefunden worden ${ }^{1}$ ). - Diese Vögel gehören daher keineswegs gerade den niedersten Typen an; ja es nehmen die Cursores in mancher organischen Beziehung die erste Stelle ein und morgen als Misch-Typen ( $\$$. 14) gelten.

Mit den Vogel-Fährten von gleichem Alter und zum Theil in gleichen Schichten hat man in Deutschland, England und Nordamerika anch VierfüsserFährten, Tetrapodichniten ${ }^{2}$ ) entdeckt, die nıan grösstentheils einstimmig von Reptilien hergeleitet hat. Doch lässt sich nicht läugnen, dass man einige derselben gewiss versucht wäre Säugthieren zuzuweisen, wenn man überhaupt schon Reste derselben in gleich-alten Gebirgs-Schichten gefunden hätte. So möchte Hırсисоск seinen Tetrapodichnites didactylus aus der Grauwacke NewYorks, der, wie der Name ausdrückt, nur zweizehig ist, einem Säugthiere zuschreiben, das wie ein Känguruh oder Dipus sich nur in weiten Sätzen auf den Hinterfüssen bewegt bätte ${ }^{3}$ ).

Unter den Vierfüsser-Fährten im Bunt-Sandsteine von Hildburghausen ${ }^{4}$ ) möchten wir die des Chirotherium für solche eines Säugthieres haiten, aus Gründen, welche wir schon fruber ${ }^{5}$ ) ausführlich auseinandergesetzt haben. Dann würden auch die Chirotherien- und verwandte Fährten im Neuroth-Sand. stein Englands ${ }^{6}$ ) und die von Kıng als Thenaropus beschriebenen Fährten im Sandsteine des Steinkohlen-Gebirges zu Greensburg in Pensylvanien ${ }^{7}$ ) dahin gehören. Doch gestehen wir, dass diese frei von allen vorgefassteu Meinungen gewomnene Ansicht nicht so fest erwiesen ist, dass wir darauf eine Folgerung bauen möchten, die so tief in den Schöpfungs-Plan eingreifen würde.

Selbst weun aber Vögel und Säugthiere im Bunten oder im Rothen Sand. steine nachgewiesen würden, so würden sie einestheils noch den Reptilien und Fischen wicht an Alter gleichstehen, anderntheils aber ihrer Hauptmasse nach immer der Tertiär-Zeit verbleiben.

d. Die Geschichte der fossilen Menschen-Kuochen, die Bchauptungen und Widerlegungen ibrer Ächtheit, d. h. ihrer Abstammung aus der Zeit, wo auch andere jetzt untergegangene Thiere noch lebten und vielleicht noch nicht alle jetzt lebenden Thiere existirten, ist viel zu lang und in viel zu zahlreichen, ja in Hunderten von Werken zerstreut, als dass wir uns entschliessen möchten, sie vollständig hier abzuhandeln. Da wir ohnediess unter K (S. 871) nochmals darauf zurückkommen müssen, so genügt es hier, einstweilen das Resultat mitzutheilen, dass nach dem Erscheinen der ersten ältesten Menschen-Reste in den Schichten der Erd-Rinde keine neuen Organismen-Arten mehr geschaffen worden sind, der Mensch also wie das höchste so auch das letzte Geschöpf zu seyn scheint.

B. Auch das allmähliche Auftreten der Ordnungen einer Klasse, der Familien einer Ordnung u. s. w. deutet oft anf eine stufenweise höhere Eıtwicklung ihrer Organisation hin; aber je untergeordneter die systematischen Kategorie'n sind, desto weniger tritt es an ihnen

1) Jahrb. 1840, 458.

$\left.{ }^{2}\right)$ Vrgl. Gesch. d. Natur II, 460 ff., 761 ; Jahrb. 1835, $230,233,322,327$; $1836,110,122 ; 1837,110-112,122,243,244,379 ; 1339,136,491$, $492 ; 1841,265,455,556 ; 1842,125,215,239,246,450: 1843,501$, $705 ; 1846,1,125 ; 1847,382,383$.

3) Jahrb. 1837, 602. - 4) Jahrb. 1840, 556.

$\left.{ }^{5}\right)$ In der Geschichte der Natur II, 460 ff.; Jahrb. 1835, 1836, 1842, u. a. 0 .

$\left.{ }^{6}\right)$ Jahrb. 1843, 501 u. a. $-{ }^{7}$ ) Jahrb. 1846, 763 und 1847, 383. 
hervor, indem alsdann theils andere Momente zu grossen Einfluss gewinnen, theils die Merkmale höherer und niedrigerer Organisation sich zu manchfaltig durchkreutzen und zu vielfältig sich wieder aufwiegen. Wir werden daher in der Folge nur solche systematische Gruppen der näheren Betrachtung unterziehen, welche einerseits eine hinreichende systematische Gliederung zeigen, um in solcher Hinsicht eine genügende Beurtheilung zu gestatten, andrerseits aber auch eine so ungleiche chronologische Vertheilung wahrnehmen lassen, um dieselbe mit jenen Gliederungen in Parallele zu setzen.

C. Bei den P h y to z o e n wird man nur hinsichtlich der Polypen bemerken dürfen, dass unter den Polythalamien (welche im Enumerator verkehrt angeordnet sind, mit den höchsten statt niedersten Formen beginnend) die höher stehenden Monosomaten mehr vorzugsweise der jetzigen, die tieferen Polysomaten mehr der frïheren Zeit angehören; - dass eben so die tiefer stehenden Authozoen in der frühesten, die den Mollusken sich nähernden Bryozoen in den späteren Perioden etwas mehr vorzuwalten strebten. - Bei den Ech i n odermen müssen wir die Fistuliden als weiche Thiere mit nur wenigen kalkigen Elementen gauz ausser Acht lassen, da sie wenig geeignet sind, ihre Anwesenheit, wenn sie in alten Perioden stattgefunden hat, zu verrathen; dagegen bieten uns die übrigen Echinodermen, die Stelleriden und Echiniden treffliche Gelegenheit dazu. Die Stelleriden und Echiniden stellen folgendes Bild geologischer Verbreitung dar:

\begin{tabular}{|c|c|c|c|c|c|c|c|c|c|}
\hline & & Per & iode : & I. & II. & 111. & IV. & v. & VI. \\
\hline \multirow{6}{*}{ 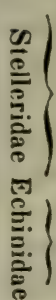 } & $\begin{array}{l}\text { Crinoidea } \\
\text { Stylastritae }\end{array}$ & : & & & & & & & \\
\hline & Stylechinidia & & . . & $=$ & & & & & \\
\hline & $\begin{array}{l}\text { Astylidae } \\
\text { Ophiuridae }\end{array}$ & · & · $\cdot$ & - & & & & & \\
\hline & Asteriadae & & .. & - . & & & & & $\operatorname{sen}$ \\
\hline & $\begin{array}{l}\text { Cidaridae } \\
\text { Clypeastroidea }\end{array}$ & $\dot{.}$ & $\therefore$ & & & & & & \\
\hline & Spatangoidea & · & . $\cdot$ & & & & & & \\
\hline
\end{tabular}
Echiniden anf.

Die tiefere Ordnung der Stelleriden tritt vor der höheren der

Die Stelleriden selbst beginnen mit den Krinoideen, welche ihrerseits die niedersten unter ihnen sind durch die Anheftung des Thieres (wie im Larven-Zustande der Comatula) mittelst eines Stieles in den meisten Sippen, durch den Mangel der Augen, wie er öfters bei wenig beweglichen Thieren vorkommt, durch die ansehnlichen Arm-Anhänge ohne wesentlichere innere Organe, durch deren oft sehr komplizirte Vertheilung, welche zu endloser Wiederholung gleichwerthiger Organe fülırt u. s. w. Sie sind daher, mit den chenfalls angewachsenen aber mehr konzentrirten Stylechiniden, nicht nur die frühesten 
(mit 2-3 Ophiura-Arten), sondern auch massenhaft vorhanden schon mit dem Beginne der organischen Schöpfung. Erst später folgen die Astyliden, welche im Jugend-, im Larven-Zustande wie jene durch einen Stiel festgelieftet, sonst nicht wesentlich verschieden sind, daher man die Krinoiden als beständige Astyliden-(Comatula-) Larven bezeichuen kann.

Die Ophiuriden und Asteriaden stehen entschieden höher als vorige, weil sie frei sind, weil die Vielzähligkeit identischer Theile (Arme) in ihren Verästelungen mehr und mehr verschwindet und die Arm-Anhänge sich selbst zu Buchten der Eingeweide-Höhle erheben; sie treten daher auch als Unterordunngen später auf als die Unterordnung der Krinoiden, wenn gleich sie den höheren Krinoiden (Comatula) noch etwas vorhergehen.

Die Echiniden stehen höher als die Stelleriden durch Konzentrirung des Körpers und seiner Eingeweide-Höhlen, Fixirung der Ordnung, der Zahl !nnd Bestimmung der eiuzelnen Täfelchen der Körper-Wand, und Übergang der noiden in die sphenoide Forn. - In den Erd-Schichten nun gehen die runden, einfach ooiden Formen den länglichen sphenoiden im Ganzen wie im Einzelnen voran. Am tiefsten stehen die Cidariden, wovon schon mehre Arten im eigentlichen Muschelkalk vorkommen ${ }^{1}$ ), durch ihre regelmässige Ooid-Form, wie sie den Pflanzen zusteht; die Spatangoiden stehen von den Cidariden am weitesten entfernt und mithin in dieser Beziehung am höchsten; denn jene haben zentralen Mund und zentralen After einander entgegengesetzt und alle Theile radial und fünfählig um dieselben gelagert; die Clypeastroiden haben wur noch einen zentralen Mund und exzentrischen hintern After, und ihre runderen Formen (Clypeus, Holectypus) treten vor den länglichen (Echinolampus, Galerites, Pirina) auf; bei den Spatangoiden sind beide Öffnungen exzentrisch und ist die sphenoide Thier-Form vollkommen hergestellt: sie erscheinen daher auch am spätesten von allen.

Die Ordnung der Fistuliden kennt man nicht früher, als in den Solenhofer-Schiefern.

D. Unter den Malakozoe" haben wir\% 2 Haupt-Abtheilungen zn unterscheiden, die Acephala und die Cephalophora, welche gleich früh beginnen und bis heute andauern. In Bezug auf diese müssen wir bemerken, dass die absoluten Zahlen der ersten überhaupt im fossilen wie im lebenden Zustande viel kleiner sind, als die der letzten, daher auch bei der Vergleichung noch da zurückstehen, wo sie verhältnissmässig stärker sich entwickeln. Wir müssen Diess daher bei der Betrachtung berücksiclitigen, werden auch die nacktleibigen so wie die kleineren Klassen (Gymnacephala, Pteropoda, Heteropoda, Protopoda) ausschliessen, da sie kein nennenswerthes Resultat darbieten. Wir erhalten dann zwar lauter von Anfang bis zu Eude durchführende Klassen, aber doch mit verschiedenen Modifikationen. Die

) Jahrb. 18t7, 576 . 
Zahlen ihrer Genera und Arten sind folgende, wenn man die Arten durch Addition der Formations-Rubriken berechnet.

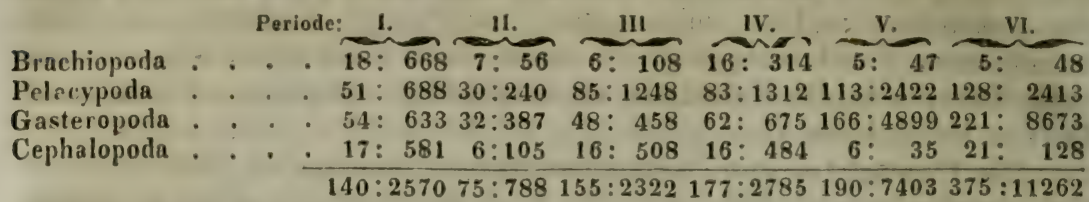

Da diese Klassen nach den Graden ihrer höheren Organisation nacheinanderfolgen, so ergibt sich ein Fortschreiten zur höheren $\mathrm{O}_{\text {r- }}$ granisation in aufeinander folgenden Perioden leicht. Am niedersten stehen die Brachiopoden; sie nehmen in jeder der späteren Perioden bis in die jetzige Schöpfung nicht nur relativ, sondern auch an absoInter Anzahl ab, obschon sie in der Kreide durch die Rudisten eine vorübergehende Verstärkung erhalten, deren Organisation jedoch viel zu hypothetisch ist, als dass man anf ihr spätes Auftreten irgend einen Schluss gründen dïrfte. - Ihnen folgen die Pelecypoden, in allen Perioden in vielleicht, vergleichungsweise zn deren Länge, ziemlich gleichbleibender Anzahl; ihr höchster Entwicklungs-Punkt fällt in die Tertiär.Zeit, gegen welche sie jetzt schon wieder ansehnlich zurückstehen. Ihre beiden Haupt-Abtheilungen Monomyen und Dimyen beginnen zwar gleichzeitig und dauern bis zur jetzigen Schöpfung, lassen aber dennoch ein verschiedenes Entwicklungs-Verhältniss unterscheiden, das wir nur durch die Zahlen der Genera ausdrücken wollen:

$\begin{array}{lrrrrrr}\text { Perioden: } & \text { I. } & 11 . & \text { III. } & \text { IV. } & \text { V. } & \text { VI. } \\ \text { Mononymen } & 6 & 6 & 15 & 15 & 12 & 14 \\ \text { Dimyen } . & 45 & 24 & 70 & 68 & 101 & 114\end{array}$

Die noch ungleichseitigen, fast gleichendigen Monomyen scheinen demuach ihre grösste Zahlen-Entwicklung in der Mittc der geologischen Zeit erreicht zu haben und jetzt anf dem Rückzuge zu seyn, während die an sich viel zahlreicheren höher entwickelten gleichseitigen Dimyen mit entschiedenem Vorn und Hinten in stetiger Zumahme hegriffen sind, so dass die Monomyen in Höhe der Organisation wie der Fintwicklungs-Weise eine mittle. Stelle zwischen Brachiopoden und Dimyen einnehmen. Unter den Dimyen sind dann zuerst wieder die Heteromyen und Integripalliaten gleichmässig durchgreifend, da die höher stehenden Sinuatopalliaten von Anfang bis Ende stärker zunehmen und die freilich armen Tubicolae erst in der IV. - V. Periode erscheinen.

Noch höher stehen anerkannter Weise die Gasteropoden, welche zwar in der III. und IV. Periode gegen vorige zurückbleiben, (was zum Theil in der Beschaffenheit des See-Grundes seine Ursache haben kaun,) aber sich in der V. und VI. Periode zur doppelten und vierfachen Überzahl emporschwingen. Diese Zunahme rührt von den siphonobranchen Ctenobranchiern und den Pulmonaten ${ }^{1}$ ) her, von

1) Die Augabe von Pulmonaten vor der Wealden-Bildung und in der Kreide. Periode beruht $\mathrm{zw}$ eifelsohne auf unrichtigen Bestimmungen. 
welchen vor der Kreide nur wenige Vorlänfer vorhanden sind, die Haupt-Entwicklung aber in die Tertiär-Zeit fällt; auch stellt man jene gewöhnlich, diese Luft-athmende Gruppe immer höher als die asiphonobranchen Ctenobranchier, so dass sich, wenn man die zum Theil wohl unrichtig zusammengesetzten, ibbrigens doch keine sehr wesentliche Ausnahme zeigenden ärmeren Unterabtheilungen übergeht, auch hier folgendes Bild gestaltet:

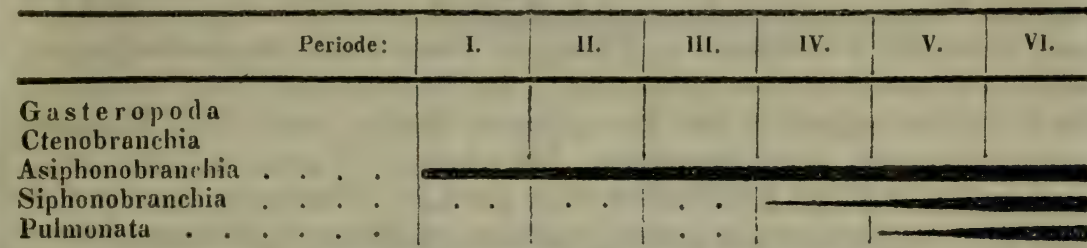

Anders verhält es sich mit den Cephalopoden, die man als die höchsten Malakozoen betrachtet, und welehe gleichwohl, wie die Brachiopoden, von der Kreide an sich rasch vermindern und folglich als erste Ausuahme von einem Gesetze erscheinen, das wir bisher immer bestätigt gefundeı haben. Man könute die Frage aufwerfen, ob die Ähnlichkeit der fossilen gekammerten Schaalen mit denen unserer wenigen lebenden Nantilen und Spirulen genüge, um zu beweisen, dass jene ebenfalls in die Klasse der Cephalopoden gehören? Es würde dieser $Z$ weifel von grossem Gewicht seyn, wenn diese Ausnahme die einzige bliebe. In sich selbst aber leisten die Cephalopoden dem allgemeinen Gesetze wieder Genüge, wenı man die tetrabranchen Ammoneen, was freilich hypothetisch ist, als unter den tetrabranchen Nautileen stehend betrachten und beide vielleicht selbst als 2 den Dibranchiern gleichwerthige Ordnungen ansehen darf, über welchen jedenfalls die letzten in der Organisation stehen. Ihre Verbreitung ist, wie das untenstehende Bild zeigt, der Abstufung ihrer Organisations.Höhe ganz entsprechend mit Ausnahme der kleinen und schlecht zusammengesetzten Gruppe Spiriformia, den wahrscheinlich niedersten Dekapoden vor der Verbreitung der Oktopoden.

\begin{tabular}{|c|c|c|c|c|c|c|c|}
\hline & Periode: & 1. & 11. & 111. & IV. & v. & VI. \\
\hline \multicolumn{8}{|l|}{ Tetrabranchia } \\
\hline $\begin{array}{c}\text { Ammonea } \\
\text { Nautilea }\end{array}$ & \\
\hline \multicolumn{8}{|l|}{$\begin{array}{c}\text { Decapoda ... . } \\
\text { Spiriformia }\end{array}$} \\
\hline \multicolumn{8}{|c|}{ Belemomorpha } \\
\hline $\begin{array}{l}\text { Teuthomorpha } \\
\text { Octopoda }\end{array}$ & $\therefore$ & & & & & & \\
\hline
\end{tabular}

Im Übrigen dürfen wir glauben, die geologische Verbreitung bei Weitem der meisten Malakozoen, derjenigen näılich, welche mit einer derbern Kalk-Schaale versehen sind und im Wasser wohnen, 
woraus sich die Fossil-Reste umschliessenden Erd-Schichten unmittelbar absetzten (also aller mit Ausnahme der wenigen Landbewohner, der wenigen nackten und sehr düunschaligen Formen, auf die wir schon oben hingewiesen), am genauesten zu kennen; ein jeder Rest ist in der Regel auch mehr als bei den meisten übrigen Thieren genïgend zur Bestimmung von Genus und Art.

E. Die Entomozoen zerfallen zunächst ebenfalls in 2 Gruppen, die Wasser-athmenden und die Luftathmenden, - jene mit Kiemen, diese mit Luftröhren und Lungen. Dass unter jenen die Ringelwürmer hinsichtlich der Athmungs-, der Bewegungs-, der Sinnes-, der Fortpflanzungs-Organe die niederste Stelle unter allen Entomozoen einnehmen, ist unbestritten. Die übrigen Wasser-Athmer aber, die Kruster, werden gewöhnlich über die Luft-athmenden Kerbthiere gestellt, was übrigens in jedem Systeme zu Missverhältnissen führt; denn in der That stellen sie eine Formen-Reihe dar, in welcher die Lernäen, jedenfalls die unvollkommensten Fuss-Kerbthiere, mit eingeschlossen sind und eine lange Stufenfolge höherer Organisationen erst allmählich zu den Brachyuren unter den Decapoden führt, wegen deren man den Krustern die höchste Stelle unter den Kerbthieren angewiesen hat: ihr durch Verkürzung konzentrirtes Bauchmark, ihre zahlreicheren und ausgebildeteren Sinnes-Organe, ihr vollkommeneres Gefäss-System sind nebst Anderem die Ursache dazı. Aber es lassen sich auch Gründe gegen diese Stellung selbst bei den Dekapoden geltend machen. Ihre Kiemen-Respiration entspricht der des Larven-Zustandes vieler Luft-athmenden Insekten, während einige andere Kruster sogar eines eigenen Respirations-Organes entbehren ; die grössere Anzahl gleichwerthiger Theile sowohl bei den Kauwerkzeugen als den äusseren Bewegungs-Organen (Füssen) gelten sonst überall als Merkmale tieferer Organisation; auch das ansehnliche Abdomen, ohne anderes inneres Organ als den Darmkanal selbst bei den Dekapoden, spricht nicht für eine höhere Stellung, wie auch aus dem Gegensatze zwischen Makruren und höheren Bachyuren hervorgeht; sehr viele andere Kruster aber stehen offenbar auf tieferer Stufe, als die Luftathmer, deren Gebisse, Bewegungs-Organe und zum Theil Lungensäcke ihnen eine ansehnliche Stellung sichern.

So bilden also die Entomozoen etwa zwei nebeneinander laufende Reihen, worin die Wasserthiere wenigstens in mehrfacher Hinsicht tiefer als die Lufthewohner stehen, wenn sie diese auch in anderer Beziehung zum Theile überragen; diese Wasser-Bewohner nun, Ringelwürmer wie Kruster, treten etwas früher als die Lufthewohner auf, doch ist der Zeit-Unterschied nicht gross; jene trifft man mit ihren beiden Hauptformen schon in den älteren Silur-Gesteinen häufig; diese treten erst von der Kohlen Zeit an sparsam anf, woran indessen die schwierige Erhaltung ilırer hornartigen Hüllen und das fremde Wohn-Element im Vergleiche zur leichten Erhaltbarkeit der kalkigen Krusten und Röhren der Wasser-Bewohner Schuld seyn mag. 
a. Die Wasser-Bew o h ner geben folgendes Bild ihrer geologischen Entwicklung.

\begin{tabular}{|c|c|c|c|c|c|c|c|}
\hline & Periode: & 1. & II. & III. & IV. & v. & VI. \\
\hline \multirow{7}{*}{$\begin{array}{c}\text { Vermes } \\
\text { Crustacea } \\
\text { Cirripedes } \\
\text { Entomostraca } \\
\text { Malacostraca } \\
\text { Isopoda } \\
\text { Stomatopoda } \\
\text { Decapoda } \\
\text { Macrura } \\
\text { Brachyura }\end{array}$} & . & \multirow{3}{*}{ ? } & \multirow{3}{*}{$?$} & & & \\
\hline & . . & & & & & \\
\hline & $: \vdots$ & & & & & & \\
\hline & $\cdot \cdot \cdot$ & & & & & & \\
\hline & $\therefore \quad$. & & & & & & \\
\hline & & ? & & & & & \\
\hline &.. & & & & & & \\
\hline
\end{tabular}

Die Würmer durchziehen ziemlich gleichmässig die ganze Formationen-Reihe; allein da es mehr kleine, weiche und nackte, als grosse und behäuste Formen unter ihuen gibt, dürfen wir nicht hoffen, das wahre Verbältniss ihrer geognostischen Verbreitung zu erkennen. Ähnlich rerhält es sich bei den Krustern mit den angeheftet lebenden Cirripeden, die theils weich- und theils sehr dünn-schalig sind: das Vorkommen ihrer Reste in den 2 ersten Perioden ist in der That problematisch. Höher stehen die Entomostraca, und da sie in aufsteigender Reihe die ersten sind, welche (mit Ausnahme jedoch der kleinen und weichen Lernäen oder Parasita, dic wir meistens kaum hoffen dürfen je zu entdecken) harte leicht erhaltbare Krusten besitzen, so begegnen wir ihnen auch in grosser Zahl sogleich in den silurischen Schichten. Die auf die erste Periode beschränkte Unterordnung der Paläaden besitzt aber gewisse Charaktere (vgl. den folgenden Paragraphen), wodurch sie unter die übrigen Entomostraca hinabsinken, welche durch alle Perioden bis in die Zetztzeit hindurchreichen. Später als die Entomostraca, erst in der Trias, beginnen endlich die Malacostraca, welche die höchsten Kruster-Formen in sich, und zwar unter den brachyuren Dekapoden, einschliessen. Die Abtheilungen der Isopoden und Phyllopoden sind zu arm und zum Theile auch zu klein, als dass wir hoffen dürften, ihr wahres Entwicklungs-Verhältniss zu erforschen. Die reichen, grossen und hartschaligen Dekapoden aber zerfallen in die tieferen Makruren und die höheren Brachyuren, welche letzten denn auch wieder beträchtlich später als die Makruren in den Oolithen und etwas reicher in der Kreide auftreten, ihre Entwicklungs-Höhe aber erst in der Jetztzeit erreichen ${ }^{1}$ ).

b. Hinsichtlich der L u f $\mathrm{t}-\mathrm{B}$ ew oh $\mathrm{n}$ e r dürfen wir ihrer schon erwähnten hornartigen Hüllen wegen nicht erwarten, ihren geognostischen Entwicklungs-Gang richtig zu erforschen. Wir beschränken uns daher, hier zu erinnern, dass die Rüsselkäfer des Bergkalkes Pflanzenfresser, die Skorpionen der Steinkohlen-Formation Thier-

1) Wir haben kürzlich hingedeutet auf Makruren, die schon im Kohlen-Gebirge vorkommen; doch sind Diess wohl Amphipoden? - Vgl. Jahrb. 1848, 125. 
fresser sind und bereits die Existenz einer grösseren Anzahl anderer kleiner Insekten voraussetzen, von welchen wir wirkliche Überbleibsel wohl nie finden werden. Das später häufigere Erscheinen ihrer Reste ist Folge vorzüglich günstiger Erhaltungs-Mittel; aber auch das obenerwähnte Auftreten fast der ganzen dikotyledonen Flora erst in der Tertiär-Zeit muss die Zahl der Pflanzenfresser in allen vorhergehenden Perioden sehr beschränkt und hiedurch anch wieder hemmend auf die Entwicklung der Raub-Insekten zurückgewirkt haben. Die Myriapoden sind zu wenig zahlreich, als dass ihr Mangel in den ältesten Perioden befremden könnte; doch erscheinen sie schon in den Oolithen.

F. In der Klasse der $\mathbf{F}$ is $\mathbf{c}$ he bestehen die 2 ersten Ordnungen, die Leptocardii und Cyclostomi uur aus wenigen (5) Geschlechtern; sie sind unbeschuppt, haben blosse Knorpel statt der Knochen, und nur ein Theil der letztgenannten besitzt harte Zähne, die einzigen Theile, welche erhaltungsfähig wären. Auch die letzte Ordunng, die der Dipnoen, ist unbeschuppt und nur von einem Geschlechte mit 2 Arten gebildet. Diese müssen wir daher unbeachtet lassen und unsere Betrachtung anf die übrigen 3 Ordnungen beschränken. Ihre geologische Verbreitung kann durch folgendes Bild ausgedrückt werden, worin die der ersten Periode entsprechenden Formen nach Agassiz ${ }^{1}$ ) das Eubryo-Alter der vollkommenen Fisch-Organismen vertreten.

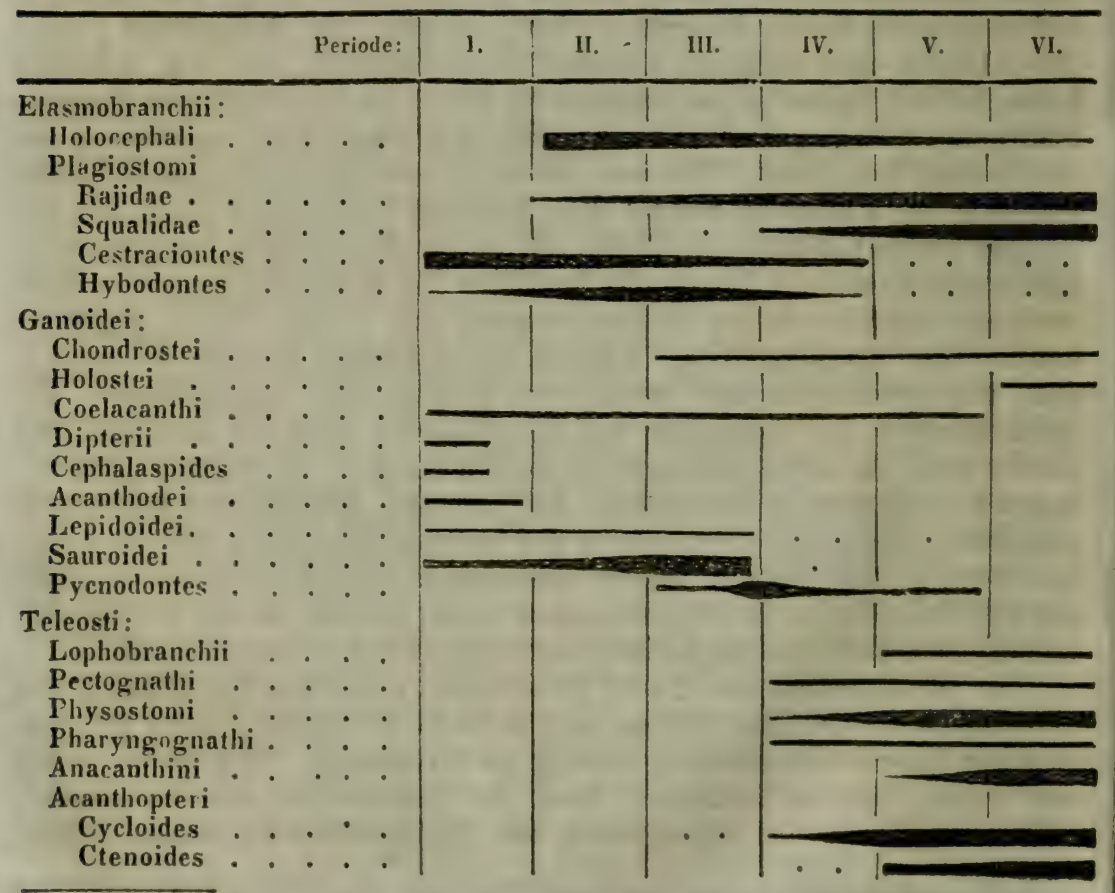

2) Jahrb. 1846, 508-512. 
Die El as mobra n chier (Placoiden AG.) stehen unter den angeführten 3 Ordnungen anf der niedersten Stufe durch ihr nur knorpeliges, dem des Knochenfisch-Embryos an Konsistenz ähnliches Skelett, ihre grosse Anzahl grosser und doch gleichwerthiger Zähne, ihre ebenfalls zahlreichen und dabei mit der Körper-Decke verwachsenen, nicht freien Kiemen, ihı heterocerkes Schwanz-Ende: Merkmale, wodurch sie sich theils den Fisch-Embryonen und theils den so unvollkommenen Cyclostomen enge anschliessen; - und so beginut mit ihnen auch die geologische Entwicklung der Fische schon in den oberen SilurSchichten, wo sie uns freilich nur durch einige vielleicht noch problematische Flossen-Stacheln verkündigt werden. Sie setzen durch alle Perioden bis in die jetzige fort, obschon es, ausser den Rajiden, verschiedene Unterordnungen sind, welche in den älteren und in den jüngerenPerioden vorkommen. Sie enthalten mehre ansgestorbene Familien.

Die Organisation der $\mathbf{G}$ anoiden kennen wir im lebenden $\mathrm{Zu}$ stande nur durch die Störe und die Genera Lepidosteus und Polypterus, denen vielleicht noch 1-2 andere zugeselit werden müssen. JoH. MüLLER stellt sie wegen ihrer weniger zahlieichen freien Kiemen und ihres wenigstens in den noch lebenden Formen zum Theile knöchernen Skelettes u. s. w. über die vorigen, wie sie auch im äusseren Habitus ein Bindeglied zwischen diesen und den ächten Knochen-Fischen oder Teleosti abgeben. Agassiz scheint sie Anfangs wenigstens tiefer gehalten zu haben, wofür die grössere Anzahl gleichwerthiger Klappen in dem Herzarterien-Stamme, wie andererseits ihr frülieres fast völliges Aufhören, während die Elasmobranchier jetzt noch reichlich fortdauern, zu sprechen schien. Er machte darauf aufmerksam, dass 1) auch hier in Folge der noch unvollkommenen Skelett-Bildung die Wirbel meistens gänzlich fehlen und am Kopfe, wo im Embryo Anfangs nur die äusseren Knochen vorhanden sind und der eigentliche Hirnkasten sich erst später bildet, dieser öfters zeitlebens knorpelig bleibt nnd im fossilen Zustande durch keine Spur angedeutet ist, wofür denn zum Ersatze ein Haut-Skelett aus harten steinartigen Schuppen sich vorfindet, das freilich der Embryo nicht besitzt; dass 2) die Körper-umsäumenden oder zahlreichen Flossen, welche bei vielen derselben vorkommen, den ersten individuellen Entwicklungs-Ständen der ächten Knochen-Fische entsprechen, wo im EmbryoZustande fast der ganze Körper von Kopf bis After mit einer vertikalen Flosse umgeben seye, die sich später spalte und durch immer breiter werdende Lücken theile, stellenweise verkümmere, aber in den erhalten bleibenden Theilen Flossen-Strahlen bekomme, wie in der Devon-Zeit die Dipterini, Glyptolepis und wahrscheinlich Platyguathus unter den Coelacanthi, Diplacanthus unter den Acanthodiern doppelte und unter sich fast zusammenhängende Rücken- und AfterFlossen (ein Charakter, der sich freilich auch selbst bei den KnochenFischen einige Male wiederholt) zeitlebens behalten; - er zeigt 
3) wie die Fisch-Embryonen auch der Knochen-Fische eine heterocerke Schwanz-Bildung haben, die erst später in die den Knochen-Fischen eigene homocerke Bildung übergehe, während jene bei den lebenden Stören und bei fast allen unter den Jura-Schichten gefundenen Ganoiden ebenso wie bei den Elasmobranchiern bleibend seye; 4) bei allen Fischen der Devon-Formation ist ferner der Kopf breit und flach, vorn wie abgestutzt, daher fast nie im Profil siclithar; das Maul offen, halbbogenförmig, die Angen meist weit nach den Seiten hinabgedrängt, - und ähnliche Formen finden sich auch bei den Embryonen selbst derjenigen unserer Fische, welche später zusammengedrückt, langund spitz-schuäbelig sind; 5) die Cölakanthen zeichnen sich noch durch tief gefaltete Dentine ilırer Zähne ans, tiefer als sonst irgendwo im Thier-Reiche; es scheint ebenfalls ein Charakter grösserer Unvollkommenheit?

Die fossilen Ganoiden, schon zahlreich, beginnen nur wenig später als die Elasmobranchi in der Devon-Formation und zeigen in der ersten Periode sogleich ihre höchste Entwicklung, in so fern 3 ihrer 9 Unterordnungen auf diese allein, ja 2 derselben von der fremdartigsten Bildung auf die Devon-Formation beschränkt sind und 3 andere von da an bis in die III. und selbst V. Periode fortsetzen; 2 beginnen erst mit den Oolithen und reichen die eine bis in die TertiärZeit, die andere nur kleine bis in die jetzige Periode herïber; eine andere kleine gehört dieser ausschliessend an. Obschon also die Ganoiden etwas später als die Elasmobranchier zu beginuen scheinen, so sind sie doch andererseits durch ihr sogleich massenhaftes Auftreten und früheres fast völliges Erlöschen wieder mehr als jene als vorweltliche Fische zu betrachten.

Bei weitem die grösste Zahl der Fische überhaupt, mehr als 0,95 derselben bilden die ächten Knochen-Fische oder Tele osti (Ctenoiden und Cycloiden), welche jedenfalls die vollkommensten unter den 3 Ordnungen sind; sie erscheinen daher auch viel später als jene 2 andern, auffallend genug die Entwicklung der höheren Pflanzen und Thiere nochmals bei den Fischen wiederholend, in mässiger Manchfaltigkeit in der Kreide-, in vollkommener Entwicklung aber erst in der Molasse-Periode. Nur von den Familien der Siluroiden, der Chersobaten, der Discoboli und der Gadoiden hat man fossile Repräsentanten bisher noch nicht gefunden.

Die vollkommenste aller 6 Ordnungen, die der Dipnoen, wenn man sich auf sie berufen dürfte, würde noch später, in der jetzigen. Periode zum Vorschein kommen.

G. In der Klasse der Reptilien ist nur eine der 4 Ordnungen ansehnlich entwickelt, wie folgende Darstellung ergibt, worin die Gruppen etwas abweichend vom Enumerator mehr nach den Systemen füı die lebenden Reptilien geordnet sind. 


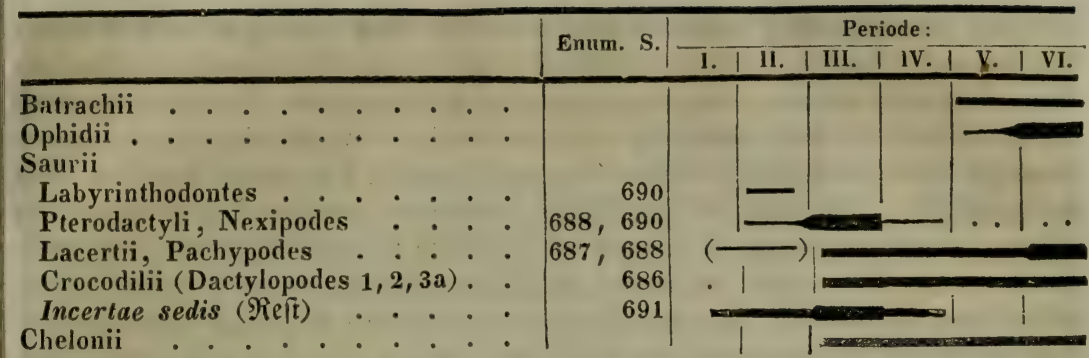

Zunächst dürfen wir auf das frühere oder spätere Auftreten der Sch l a n gen-Reste (Ophidii) keinen sehr grossen Werth legen, weil sie fast alle fern von Wasser und dessen ruhigen Niederschlägen in trockenen Gegeuden wohnen und ihre feinen Schädel-Knochen und Wirbel sich zur Erhaltung wenig eignen; andere Knochen haben sie nicht. - Fast eben so ist es mit den Knochen der B atrachier; doch besitzen diese verhältnissmäsig starke Extremitäten, wohnen an und in dem Wasser, werden also viel leichter in dessen Niederschlägen eingeschlossen. Da sie die niedersten Reptilien sind, so würde man nach der bisherigen Regel sie auch schon in den ältesten Schichten erwarten dürfen. A ber sie sind̉ Süsswasser-Bewohner und können folglich nicht früher als die Süsswasser-Niederschläge auftreten. Wären übrigens, wie R. Owen will, die Labyrinthodonten ihre ersten Repräsentanten mit Krokodil-Charakteren, die sich statt der SüsswasserRänder die See-Küsten zum Aufenthalte gewählt lıätten, so würde dennoch auch in Bezug auf sie der ersten Regel ziemlich genügt seyn, da sie in i beginnen. Unter den übrigen $\mathrm{S}$ a urie rn finden wir die ältesten Reptilien überhaupt, und zwar hauptsächlich unter den Geschlechtern von unbestimmter Verwandtschaft (hauptsächlich Lacertier), wozu sich bald auch Protorosaurus gesellt, welcher manche Beziehungen der Organisation mit den Krokodiliern hat.

Unter denjenigen $S$ a u ri er n, welche bestimmtere Gruppen bilden, treten die Nexipoden am frühesten auf, wie sie denn auclı durch ihre Lebensweise, ganz im Wasser, durch ilıre Bewegungs Organe, ihre bikonkaven Wirbel eine nähere Verwandtschaft mit Batrachiern und Fischen und mit den Ei-Zuständen hölserer Reptilien beurkunden; die Pterodactyli (welche Manche übrigens auch für Ruder- und folglich W asser-Thiere angesehen) stehen ihnen in mancher Hinsicht, wie namentlich in der Wirbel-Bildung nahe, würden jedoch in Lebens- und Bewegungs-Weise wesentlich verschieden gewesen seyn. Dagegen haben beide auch wesentliche Beziehungen zu den Krokodiliern und ist die systematische Stellung derselben schwierig. Die Lazertier, weiche unseren jetzigen entsprechen, sind S. 687 des Enumerators unter der Aufschrift Pentadactyli verzeichnet $(\mathbf{n}-\mathbf{z})$; dazu aber kommen nach R. OWen als vorweltliche Formen noch die Scink- 
artigen Pachypoden oder Dinosaurier S. 689 (n-r), wic Thecodontosaurus und Palaeosaurus S. 691 (g), dann Phytosaurus, Cladyodon, Rhynchosaurus, Raphiosaurus, - Cetiosaurus, Leiosaurus, PoJyptychodon S. 692-693 (f-C), wodurch die Erstreckung dieser Gruppe also vom alten rothen Sandsteine (f) bis in die heutige Zeit herabreicht. Sie überbieten daher die vorhergehenden Gruppen an Alter, obschon sie im Systeme als höhere Organisationen erst nach ihnen aufgeführt sind, scheinen mithin die Regel zu verletzen. Aber alle diese Reptilien, von den Labyrinthodonten an, sind so ausserordentliche Bildungen und ihre Verwandtschaft durchkreutzt sich doch wieder so vielfältig, dass ihre Stellung im Systeme unsicher und dass durch ihre Aufnahme die bisherigen Charaktere der Ordnungen und Familien gänzlich verändert werden. Namentlich sind dabei riesige Wasser-Thiere, dergleichen man lebend unter den Lacertiern nur e in Miniaturbild kennt. Auch sind unter den Saurii incertae sedis noch andere so eigenthümliche Gestalten übrig, dass wir noch nicht sagen können, vor welchen der bisherigen Gruppen die ältesten dieser Geschlechter vorangehen müssteı. - Die älteren Krokodilier S. 686 mit Suchosaurus S. 693 des Enumerators, wie ähnlich sie anch mitunter den lieutigen gewesen seyn mögen, nähern sich den Nexipoden und Fischen gleichwohl durch einen meerischen Aufenthalt und bikonkave Wirbel und besitzen hohle Zähne; die stärkere Umpanzerung (und das Vorwalten gepanzerter Reptilien überhaupt) in den Wealden u. s. w. entspricht dem Vorwalten der ganoiden Fische in älteren Formationen; sie sind, wenn ihnen noch einige Geschlechter aus den Genera incertae sedis (und vielleicht Archegosaurus selbst ${ }^{1}$ )), obwohl er sich den Lazertiern nähert, beigesellt werden, gleichzeitig mit den Lazertiern, mit welchen sie auch, abnehmend, bis in die jetzige Schöpfung hereinreichen, während diese zunehmen, stehen aber ïberhaupt in der Zahnfügung u. a. Theilen der Schädel-Bildung den Säugthieren näher als die übrigen Saurier. - Übrigens gestehen wir, dass die Nachweisung des Gesetzes einer fortschreitend höheren Entwickelung bei den Sauriern die grössten Schwierigkeiten findet, wenn man sie nicht als Keim.Formen der warmblütigen Wirbelthiere hervorheben will, wesshalb wir hinsichtlich ihrer noch auf den folgenden Paragraphen S. 844 verweisen.

Die Chelonier endlich, grossentheils ebenfalls Wasserthiere, treten in der Mitte der Perioden-Reihe auf; in mancher Hinsicht höher als die Saurier stehend cobschon sie ihre systematische Stellung auch zun Theile der Absicht verdanken, durch sie nicht die manchfaltigen Übergänge von den Ophidiern zu den Sauriern zu unterbrechen) entsprechen sie dadurch der allgemeinen Regel. Da die lebenden Genera zum Theil nur Süsswasser- und Land-Bewohner einschliessen, so würde man wenigstens diese noch später habeu e!warten dürfen;

3) Jabrb. 1847, 401. 
aber wahrscheinlich waren sie ehedem, gleich andern Wasser-Bewohnern, mit den Meeres-Küsten vertrauter als jetzt.

H. Auf die nähere Betrachtung des Entwicklungs-Ganges der Vög el müssen wir verzichten, da wir zu wenig von ilıen kennen.

Vgl. die Artikel Ornithichnites und Ornithoidichnites im Nomenclator, der übrigens - da H. v. MEYER die Vogel-Fährten nicht als solche anerkennt, welche desshalb im Enumerator wie im Nomenclator ganz von ihm übergangen worden waren, erst unmittelbar vor dem Abdrucke ausgearbeitet weder an Arten noch an Synonymen ganz vollständig ist.

1. Die $S a ̈ u g t h$ ier-Reste erscheinen, mit 4 Ausnahmen in den Oolithen, alle erst in der Tertiär-Zeit. Die Frist ihrer Entwickelung ist zu kurz, zu sehr zusammengedrängt, als dass deren Gang so klar hervortreten köınte. Jene ältesten Reste in der Grenz-Breccie zwischen Keuper und Lias werden einem sehr ungenau bekannten Insektivoren oder vielleicht richtiger Beutelthiere (Microlestes), die in den Stonesfielder Schiefern 2 Insektivoren (Amphitherium) und einem Bentelthiere zugeschrieben, welche jedoch $R$. 0 wen alle 3 noch den insektivoren Beutelthieren beizählt ${ }^{1}$ ); nun stehen aber die Beutelthiere unter allen Säugthieren den Eier-legenden Wirbelthieren wenigstens in den Fortpflanzungs-Organen, in der Becken-Bildung, zum Theil im Schulter-Gerüste u. s. w. am nächsten, mithin ain tiefsten. In der eocänen Tertiär-Periode treten abermals einige Beutelthiere und Cetaceen, Pachydermen in Masse, dann einige wenige Wiederkäuer und Nager - bis daher also die niedersten Ordnungen - auf, zu welchen sich dann nothwendig auch einige Raubthiere gesellen, auch einige Fledermäuse hinzukommen. Die mittel-tertiären Schichten liefern hauptsächlich Reste noch von Cetaceen und Pachydermen, dann in zunehmender Anzahl Wiederkäuer, Raubthiere und Insektenfresser. Die ober-tertiären Schichten ergïnzen nicht nur die zuerst genannten Ordnungen mehr und mehr in Geschlechtern, sondern zeichnen sich hauptsächlich durch ihren Reichthum an Ruminanten, Edentaten, Nagern, Marsupialen, Raubthieren, Fledermäusen und Quadrumanen aus. Ein Aufsteigen von tieferen zu höheren Organisationen ist also im Ganzen auch hier deutlich ausgesprochen; nur zwei Erscheinungen wirken störend, das massenhafte Auftreten der Bentelthiere und Edentaten erst in den oberen und die Auffindung eines Quadrumanen schon in den unteren Tertiär-Schichten. Jenes erste findet z. Th. seine Erklärung einfach in den zufällig sehr günstigen Verhältnissen, durch welche Neu-Holland und SüdAmerika vermochten uns die Reste ihrer letzten Bevölkerungen, die den jetzigen schon höchst ähulich waren, reichlich zu überliefern; das Erscheinen eines eocänen Affen aber in Europa ist eine vereinzelte Ausnahme, wie die einzelıen Säugthiere und höheren Dikotyledonen in den Oolithen u. a. m.

1) Jahrb. 1846,632 . 
Vergl. übrigens, was in vorigen Paragraphen S. 816 über die Beziehung. der Säugthier-Welt zu den Kontinenten gesagt ist.

K. Die letzte Erscheinung aus den organischen Reichen ist der Mensch, der höchste aller Organismen und daher der Schluss-Punkt der Schöpfung. Während das Pflanzen-Reich mit aufsteigender Entwicklung sich in seinen vollkommensten Formen, den Dikotyledonen, am breitesten entfaltet, zieht sich das Thierreich von breiter Basis aus gegen seine höchste Klasse, die der Säıgthiere, immer näher zusammen und konzentrirt sich im Menschen - hier bloss als Organismus betrachtet - in seinem höchsten und letzten Gipfel-Punkte. Zwar hat es schon vor dem Menschen viele Thier-Arten gegeben, die noch jetzt mit ihm existiren; aber es ist nicht erwiesen, dass er mit einer der ausgestorbenen Arten (so ferne sie nicht erst von ihm selbst neuerlich ausgerottet worden) zusammengelebthabe, und noch weniger', dass eine solche erst nach ihm geschaften worden seye. Von bloss zoologischem Stand-Punkte aus könnte daher der Mensch als zuletzt erschaffenes Geschöpf überhaupt eben so wohl den Schluss der V. Periode als Ende der Schöpfung bilden, - wie als erstes und einziges intelligentes Geschöpf (in höherem Sinne) die VI. Periode eröffnen, eine Stelle, die wir ihm theils als ganz neuem Begriff erschaffener Wesen und theils seiner geologischen Wichtigkeit wegen von Anfang her angewiesen haben.

a. Wir haben bei verschiedenen Gelegenheiten angeführt, wie verschiedene Tertiär-Schichten, in welchen eine $S$ pur von Menschen sicher noch nicht vorkommt, 3-20-50 und mehr Prozent Thiere von noch jetzt lebenden Arten enthalten; die also schon vor dem Menschen vorhanden waren und mit bezie. hungsweise 50-80-97 Prozent jetzt ausgestorbener Arten zusammengelebt haben, ehe er erschien.

b. Die Frage aber, ob der Mensch mit solchen Arten noch zusammengelebt habe, welche jetzt durch geologische Wirkungen allmählich erloschen sind, ist oft und weitläufig behandelt und bald bejahet und bald verneinet worden; die darauf bezügliche Literatur ist schon für sich allein eine so $\mathrm{um}$. fangreiche '), dass wir durch vollständige literarische Erörterung der Frage die uns gesteckten Grenzen weit überschreiten müssten. Wir wollen hier nicht bei den Fällen verweilen, wo man zufällige Sandstein - und KalksteinFormen ganz unorganischen Ursprungs, oder Salamander-Skelette, Elephanten-Zähne u. dgl. von Menschen abgeleitet oder in den unförmigen Schädeln Blödsinniger die Repräsentanten einer untergegangenen gewaltigen MenschenRasse zu erkennen geglaubt hat; wir beschränken uns auf die Erörterung

1) Die Literatur des Jahrbuches und den dort ausgezogenen Schriften findet man in den Jahrgängen 1817, XI, 240;1830,105,107, 108, 363, 365, 365,$370 ; 1831,40 ; 1832,350,350,351,351,461 ; 1833,39,370,496$, $590,592,596,599,600 ; 1834,103,106,370,494 ; 1335,241,247$, $497,498,722 ; 1837,108,366,545,621,723 ; 1838,165,606,719$; 1839,$124 ; 1840,341 ; 1841,497,502,606 ; 1843,118 ; 1844$, $107,502,869 ; 1845,370,371,376,377,627 ; 1848,106,107$; Br. Collect. 7, 7, 10, 16, 17. - Journ. de Phys. XCII, 227; - Cuv. discours prélim. (1825) 131; - Bucks. Reliq. diluv. und Geol. I, 103, 602; - Kotzebub's Reise III, 31; - Annal. scienc. nat. 1824, 1II, 138. - Isis 1828, XXI, 481; - v. MeYer, Paläol. $117 \mathrm{ff}$. 
wirklicher Menschen-Reste. Über die Mehrzahl derselben sind die Untersuchungen so weit gediehen, dass wir sie leicht abweisen können; bei den andren ist eine Schwierigkeit vorhanden zu beweisen, dass die beisammenliegenden Thier- und Menschen-Reste sich beide noch auf primitiver Lagerstätte befinden. Wo ein unbefangener Beurtheiler geneigt ist, dieses letzte Verhältniss anzunehmen, da wird eine skeptische Kritik immer noch im Stande seyn, neue $\mathrm{Zweifel}$ darüber zu erregen, bis man nicht Menschen-Reste unmittelbar unter einem noch zusammenliegenden Skelette einer ausgestorbenen ThierArt gefunden hat, da nämlich im Falle einer sekundären Ảblagerung dieses letzte seine Lage unmöglich hätte behaupten können, den Fall des Ausschmel. zens aus einem Eis-Blocke etwa ausgenommen.

1) Ein Theil der fossilen Menschen-Knochen liegt primitiv nur in Alluvial-Bildungen; so viele, die aus Torf bekannt geworden ${ }^{1}$ ); die MenschenSkelette aus dem noch in Fortbildung begriffenen Meeres-Kalk auf la Quadeloupe $^{2}$ ); die Menschen-Knochen in jugendlichem Travertino oder Kalk-Tuff zu Martres-de-Veyre im Allier-Thale ${ }^{3}$ ); die Menschen-Skelette im alluvialen Muschel-Sande vielleicht absichtlich begraben zu St. Michel-en-Lherm in der Vendée ${ }^{4}$ ); das Menschen-Skelett im gehobenen alluvialen Muschel-Sand wäh. rend seiner Bildung eingeschlossen zu Newton-Head in Waterford ${ }^{5}$ ); so mehre Menschen-Skelette mit dem Lande fremder Schädel-Form in wahrscheinlich seit 5000 Jahren in Hebung begriffenen Muschelsand-Lagern mit noch natürlicher Schichtung in Skandinavien $\left.\left(58^{0} 25^{\prime} \mathrm{N} . \mathrm{Br}\right)^{6}\right)$.

2) Manche Menschen-Reste liegen in Srhichten und selbst harten Gesteinen, deren Alter sich nicht nachweisen lässt. So die in einem Kalk-Block zu Alais im Gard-Departement gefundenen Menschen-Gebeine ${ }^{7}$ ). So ein Theil der Knochen-Lagen in basaltischer Asche, Trümmern und Breccien zwischen Puy und Clermont in Auvergne ${ }^{8}$ ).

3) Einige liegen zum Theil noch als ganze Skelette primitiv in diluvialen oder tertiären Schichten, sind aber absichtlich oder zufällig von Men. schen in denselben begraben worden. Sie sind zuweilen sogar später noch von Stalaktiten bedeckt worden. So im Knochen-Lehme mehrer französischen und englischen Knochen-Höhlen ${ }^{9}$ ).

4) Zuweilen liegen Menschen. Knochen mit solchen von ausgestorbenen Thieren in einem Boden zusammen, welcher nebst ihnen durch Wasser-Strömungen vou seiner Stelle entführt, umgeschüttet, auf neuer Lagerstätte wieder abgesetzt worden ist.

Dieser Fall ist ganz offenbar eingetreten in den Lütticher Knochen-Höhlen, durch welche zum Theil die Bäche noch fliessen, welche die Verschüttung bewirkt haben ${ }^{10}$ ).

Dieselbe Ansicht ist zweifelsohne auch die zulässigste für die Höhlen in Süd-Frankreich zu Biøe im Aude-Departement ${ }^{11}$, zu Fausan und Césaras im Herault-Departement, zu Pondre und Souvignargue im Gard-Departement ${ }^{12}$ ), zu Nabrigas im Losère-Departement ${ }^{13}$ ) u. v. a., wo man oft noch, wenn auch nicht mehr diese Bäche, doch deutlich ihre Spuren wahrnimmt ${ }^{14}$ ).

1) Jahrb. 1834, $370 ; 1835,722 ; 1838,606 ; 1839,124 ; 1841,501$.

2) Jahrb. 1837, 723 u. a. - 3) Jahrb. 1830, 361 .

4) Jahrb. 1838, 719. - 5) Jahrb. 1844, 502.

6) Forhandl. Skandin. Naturforsk. 1844, IV, $93 \mathrm{ff}$. - Isis 1848, $524 \mathrm{ff}$.

7) Jahrb. 1844, 869; 1845, 370, 371.

8) Jahrb. 1845, 376, 377. - 9) Jahrb. 1844, 105-107.

10) Jahrb. 1833, 38, 502-596; 1837, 108 etc.

11) Jahrb. 1830, 105, $107 ; 1831,461$.

12) Jahrb. 1830, 108, 363-366. - ${ }^{13}$ ) Jahrb. 1837, 365.

14) Jahrb. 1838, 496, 600 . 
5) Oder durch Unterwaschung tertiärer Sand - und Thon - Wände sind deren Schichten mit ihrem Knochen-Inhalte über Menschen-Gebeine hergeschüttet worden.

So im Mississippi-Thale bei Natches im Missouri-staate ${ }^{1}$ ).

6) Manche Angaben und Beobachtungen sind nicht genau genug, oder nicht entscheidend; hieher gehören sehr viele Fülle, die man mittelst der obigen Zitate leicht wird verfolgen können, wobei wir aber hier nicht verweilen wollen.

BouÉ berichtet von Menschen-Knochen im Löss zu Aar im Badenschen, die aber doch durch die Thätigkeit eines benachbarten Baches erst später in demselben begraben worden seyn könnten ${ }^{2}$ ).

Razoumowski soll bei Baden in Nieder-österreich Menschen-Knochen und -Schädel mit plattgedrückter Stirne (wie viele Amerikaner haben) in Vermengung mit solchen ausgestorbener Thier-Arten gefunden haben ${ }^{3}$ ).

7) Bemerkenswerther ist noch ein aus Brasilien berichteter Fall. BergDirektor Clavssen näılich, welcher über 100 dortige Höhlen durchforscht und gegen 80 derseiben mit Knochen versehen gefunden hat, entdeckte auch in einer derselben einen grossen Theil des Skelettes von Platonyx Cuvieri wohl erhalten und sogar noch mit den Krallen an den Vorder-Füssen versehen, und zwischen und unter diesen Knochen, un welche die Erde nicht aufgewühlt worden zu seyn schien, Bruchstücke von Töpfer-Waaren, die mit einer dünnen Stalagmiten-Schicht bedeckt waren $\left.{ }^{4}\right)$. Dicser Fall würde alle geforderten Bedingungen des schlagenden Beweises erfüllen, wenn nicht LUND später ${ }^{5}$ ) gegen die Wahrheit des ersten Theiles dieser Mittheilung, die Durchforschung so vieler Höhlen voll Knochen nämlich, Verwahrung cirgelegt hälte, womit wir denn auch auf den zweiten kein Gewicht legen dürfen. Die Nachricht, dass auch Lund daselbst mexikanische Menschen-Schädel, Knochen und Reibsteine im Gemenge mit [einzelnen] Gebeinen ausgestorbener ThierArten gefunden hat, beide in gleicher Art erhalten und versteinert ${ }^{6}$ ), hat nicht die Beweis-Kraft des vorigen Falles: obwohl Lund sich in Folge dieser u. a. Beobachtungen in Brasilien der Ansicht zuneigt, dass die Amerikanische Menschen-Rasse schon gleichzeitig mit einigen jetzt ausgestorbenen Thier-Arten dort gelebt habe ${ }^{7}$ ).

L. Nachdem sich die anfănglich angedeutete Erscheinung des allmählichen und immer häufigeren Auftretens vollkommenerer Organismen-Formen zu den anfangs vorhanden gewesenen im Allgemeinen bestätigt hat, fragen wir nach der Nothwendigkeit dieser Erscheinung, ohne eine solche auffunden zu könneı, weder in äısseren Ursachen, noch in dem Prinzipe selbst. Denn das junge Individuum einer einmal erschaffenen Art muss allerdings nach den der Art einmal eingeprägten Gesetzen von seinem anfangs selır unvollkommenen Zustande an gewisse immer vollkommenere Stufen der Organisation durchlaufen, um endlich auf der höchsten ihın möglichen Stufe anzukommen; bei der Schöpfung aber, die ein neuer Akt der Allmacht und nicht die Folge eines bereits vorhandenen Natur-Gesetzes gewesen zu seyn scheint, war aueh ein allmähliches Fortschreiten von

1) Jahrb. 1848, 106, 107. $-2^{2}$ ) Jahrb. 1830, 363.

3) Jahrb. $1830,363$.

4) Bullet. Acad. Bruxell. VIII, 16. $>$ Jahrb. 184I, 497.

5) Jahrb. 1843, 785. - ${ }^{6}$ ) Jahrb. 184t, $606 ; 1843,118$.

7) Jahrb. 1845, 627 . 
dem Embryonal-Zustande der Klassen bis zu ihrer höchsten Stufe nicht geboten; und es wäre vielleicht ein gleichzeitiges Erschaffen aller Wesen eben so möglich gewesen?

Lamarck nahm an, dass die höheren Pflanzen und Thiere in aufeinanderfolgender Generation aus den znnächst vorangehenden niedrigen Árten, Geschlechtern, Ordnungen, Klassen, durch unmittelbaren Übergang entstanden seyen ${ }^{\mathbf{1}}$ ).

Mulder $\left.{ }^{2}\right)$ glaubt, dass die niedern Pflanzen und Thiere zuerst den "Stoff und die Form" für die höheren wie diese für die böchsten zubereiteh mussten, ehe diese letzten entstehen konnten.

Die Hypothese des ersten ist von den Nafurforschern längst aufgegeben; die des letzten hat in strenger Form wohl nie eine Annahme gefunden, obwohl wir nicht lä̈gnen, dass das Wiesel unter andern den Vogel, der Vogel unter andern das Insekt, disses den Apfelbaum, der eine Humus-Bildung voraussetzt, woran sich nach Umständen Gräser, Farne, Moose betheiligen können u. s. w.; aber von einer Vorbereitung der Form z. B. des Weich-Thieres durch das Infusorium kann doch keine Rede seyn.

Der Grund der stufenweisen Entwicklung, so weit sie nachweisbar ist, wird daber in anderen Verhältnissen zu suchen und sie selbst eine wenigstens theilweise zufällige seyn.

\section{c. Auseinandertreten anfïnglicher Prototype in verschiedene Reihen.} S. 14.

A. Die härteren Theile einstiger Organismen, welche im fossilen Zustande bis auf uns gekommen, sind in der Regel keine sehr wesentlichen: bei den Pflanzen Holz und Blätter, bei den Thieren sind es mit Kalk- oder Kiesel-Erde erfüllte Wohn-Röhren, Schaalen, Schichten und Anliänge der Haut, welche uns über die äussere Gestalt, den Gesammt-Eindruck des Thieres belehren können, oder Knochen und Zähne, welche letzte bei 2-3 Klassen der Wirbel-Thiere allerdings zu den wichtigsten Klassifikations-Mitteln der Genera und Ordnungen benutzt werden. Auch an die zahlreichen Knochen, die ein ganzes Skelett zusammensetzen, lassen sich manche wichtige Betrachtungen über die Organisation ankü̈pfen; aber in den meisten Fällen kennen wir das Skelett nur sehr unvollständig. In alleı diesen Fällen aber erfahren wir doch unmittelbar nichts über die Beschaffenheit der Eruährungs-, Zirkulations - und Athmungs-Organe, nichts über die Fortpflanzungs-Werkzeuge, nichts über das Nerven-System, nichts über Lebensweise und Befähigungen der Thiere; nur die Beschaffenheit der nach aussen gekehrten Bewegungs-Organe fällt uns bei vollständigen Exemplaren in die Augen. In allen übrigen Beziehungen aber müssen wir aus dem Grade der Ähnlichkeit der Beschaffenheit der erhalten gebliebenen Theile mit den analogen Theilen noch lebender Thiere auf den Grad der Ähnlichkeit der verloren gegangenen Theile mit den analogen dieser letzten schliessen, um uns so das vollständige Bild der ersten zu schaffen.

1) Gesch. d, Natur II, 193.

2) Das Streben der Materie nach Harmonie, Braunschweig 1844, $8^{\circ} .>$ Jahrb. 1844, 624 . 
In diesem Ergänzen des Unbekannten mit Hïlfe des Bekannten nach der Analogie jedesmal des ähnlichsten unter den lebenden Wesen, oft aber auch, wo sich ein genügend ähnliches nicht finden liess, mit Zuhülfenahıne der Organisation von zwei, drei oder vier verschiedenen Typen für die einzelıen Theile der Organisation ist uns Cuvier mit glänzendem Beispiele vorangegangen; nicht selten haben spätere Funde das bestätigt, was er aus der Beobachtung anderer Theile früher geschlossen hatte. Doch ist er nicht immer so glücklich gewesen, und wie er selbst mitunter für die verschiedenen Skelett-Theile e i n e r fossilen Art mehre heterogene Typen der jetzigen Schöpfung benützen musste, um das Bild jener ersten zu ergänzen, so konnte er auch in andern Fällen, wo das Skelett noch unvollkommen bekannt war, nicht ahnen, nach welchen heterogenen Typen es zusammengesetzt seye. Noch grösser ist natürlich die Schwierigkeit, wenn aus der Schaale, dem Skelette, den Zähnen anf die Beschaffenheit der weichen Theile der Ernährungs-, Kreislaufs-, Athmungs-, Bewegungsund Empfindungs-Organe, oder aus dem Holz und den Blättern auf die Blüthen und Früchte geschlossen werden sollte; und wir dürfen fast nie hoffen über etwaige Fehlschlüsse in Bezug auf dieselben spätere Berichtigung zu erlangen. So haben denn auch in der That manche fossile Genera, manche kleine Familien vorerst nur eine sehr unsichere Stellung im Systeme erlangen können (Rudisten, Trilobiten, Bellerophon, Conularia ". a. m.), und manche, die wir sicher glauben, mögen am unrechten Platze stehen.

Die Wohn-Röhren, Kalk-Stöcke und Schaaleı der PflanzenThiere und Mollusken bieten uns zu unvollkommene, zu einseitige, mit der wesentlichen Organisation zu wenig verknüpfte AnhaltPunkte dar, und die Kerb-Thiere (mit Ausnahme der Kruster) sind zu schlecht erhalten, als dass sie der Spekulation eine so feste und manchfaltige Grundlage bieten könıten, wie Diess bei dem vollständigen Skelette eines Wirbel-Thieres eintritt, das in hundertfältigen Verhältnissen und Beziehungen mit denen aller anderen verglichen werden und bald hier und bald dort eine theilweise Analogie finden kann ${ }^{1}$ ).

B. Die Betrachtung solcher Verwandtschaften der verschiedenen Theile oder Verhältnisse des Skelettes einer fossilen Art mit denen von ganz verschiedenen lebenden Typen hat $R$. OWEN' ${ }^{2}$ ) zu Feststellung einer Erscheinung bei manchen der ältesten und jetzt meistens beinahe oder ganz a usgestorbenen Typen vorzüglich der WirbelThiere geleitet, welche auch wir hier nicht übergehen dürfen: es ist die Differenzirung der Formen durch ein innres Gesetz, das allmäh-

1) Vergl. darüber H. v. M SYER im Jahrb. 183t, 63.

2) Jahrb. 1843, $239 \mathrm{ff}$; BR. Collect. I, $52 \mathrm{ff}$. 
liche Auseinandertreten ursprünglicher Klassen-Typen in verschiedene Ordnungen, oder ursprünglicher Ordnungs-Typen in verschiedene Familien u.s. w., das Entfalten einer ursprïnglichen Grund-Form in verschiedene Reihen von Formen, die beständige Aussonderung gewisser Charaktere aus einer ursprünglichen Kombination, wonach es oft schwierig erscheinen muss, solche Ur-Typen in unsere jetzigen Klassen, Ordnungen u. s. w. einzureihen, aber auch nicht selten Verbindungs-Glieder zwischen heterogenen Organisationen gefunden werden. So leitet $O$ wen öfters unsere jetzigen organischen Formen-Reilien von solchen ursprünglichen Misch-Typen her, wo Agassiz die Entwicklung eines niedrigen Typus zu höheren Formen nachweisen möchte, obschon auch er in andern Fällen dieselbe Ansicht aufgestellt hat. Wir unsrerseits können jedoch nicht ganz Das in der Erscheinung finden, was man darin zu sehen glaubt. Wir erkennen die unwidersprechliche Thatsache an, dass die meisten Klassen und Ordnungen von Pflanzen und Thieren mit solchen Gruppen beginnen, welche in unseren für die lebende Natur gescliaffenen Systemen entweder nicht oder nur schwach vertreten sind, Gruppen, welche bald etwas tiefer als die lebenden und bald ihnen gleich stehen, aber auch im ersten Falle keineswegs in allen Beziehungen unter diesen zu stehen pflegen, sondern durch einzelne Charaktere oft weit in die höheren hineingreifen. Darin eben können wir aber keine Erscheinung sehen, welche von denen der hentigen Schöpfung abweichend wäre; denn auch hier gibt es kaum eine Klasse, Ordnung, Sippe, die nicht, mag sie hoch oder tief stehen, durch einzelne Organisations-Verhältnisse im ersten Falle an tiefere und im letzten an höhere und in beiden Fällen oft an weit entfernt stehende fremde Gruppen von gleichem Werthe hinanreichte, wobei allerdings der von R. OwEN hervorgehobeue Unterschied zwischen Affinität und Analogie der Organisation nicht übersehen werden darf und benutzt werden kann, eben das von $\mathrm{OWEN}_{\mathrm{W}}$ aufgestellte Gesetz in manchen Fällen zu sichern.

Um ein Beispiel zu wählen, so nimmt man zwar bei den Reptilien folgende Abstufung in der Höhe der Organisation an : Chelonier, Saurier, Ophidier, Batrachier; allein-die verschiedenen Saurier-selbst sind so ungleich in ihrer Organisation, dass die zu ihnen gehörenden Krokodilier in der ZahnBildung u. a. Merkmalen des Schädels sich weit über die andern Reptilien und bis zu den Säugthieren erheben, während bei den über ihnen stehenden Cheloniern die Zähne gänzlich verkümmern und der Respirations-Apparat unvollkommen wird; die tiefer stehenden Scink-artigen Saurier dagegen gehen allmählich in die fusslosen Ophidier über, welche ihrer tiefern Stellung ungeachtet doch ebenfalls noch eine Zahn-Bildung haben, die den Cheloniern fehlt, welche sich vielmehr den Schnabel der Vögel angeeignet haben. Die Batrachier aber, die in der Respiration, der Wirbel-Bildung u. s. w. so tief unter allen anderen Reptilien stehen, dass sie fast nur ihren Fötus-Zustand repräsentiren, haben immerhin vollkommenere Bewegungs - Organe als die Ophidier, während ihre Einathmung und ihr Gebiss sich in einigen Fällen denen der Chelonier nähert. Man würde daher, wenn eine dieser Ordnungen unserer jetzigen Schöpfung ganz fehlte, aber in der I. und II. Periode reichlich repräsentirt und in unserer Schöpfung z. B. durch die Nexipoden er- 


\section{2}

setzt wäre, bei ihrer Einreihung in Systeme in dieselbe Verlegenheit kommen, wie jetzt bei der Einreihung der Nexipoden.

C. Solche Misch-Typen im P f I a n zen-Reich e scheinen die kryptogamischen Monokotyledonen und die gymnospermen Dikotyledonen für das ganze Unterreich der Gefäss-Pflanzen und vielleicht ihre ausgestorbenen Familien für sie selbst zu seyn, da sie, in verhältnissmäsig kleiner Zahl auf der Grenze zwischen den mächtigsten Gruppen des Pflanzen-Reiches und am Anfange der Reihe der vollkomineneren Pflanzen stehend, die heterogensten und oft wieder eigenthümlichsten Bildungen in der Textur des Stammes, seiner Wachsthums-Weise, der Zahl und Form der Kotyledonen, der Art und Zusammensetzung ihrer Blüthen und Früchte mit einander vereinigen.

D. Bei den Phytozoen wären vielleicht die Graptolithen für die Polypen, - die Stylechiniden und Stylastriten für die Echiniden als solche Ausgangs-Formen zu nennen. Wenigstens sagt Agassiz vón den Krinoiden, sie seyen Prototyp und Ausgangs-Punkt der Echinodermen, eine Synthese der ganzen Klasse mit Ausnahme der höher stehenden Holothurien ${ }^{1}$ ). Wir haben die Krinoiden zwar oben S. 825 als die niederste Abtheilung der Echinodermen dargestellt, aber doch reiclıen sie in einzelnen Beziehungen auch zu höheren Stufen hinan, ihr Darmkanal hat eine doppelte äussre Mündung, wie sie selbst vielen Asterien fehlt. Am vollendetsten aber ist der synthetisclie Charakter vielleicht in den Cystideen ${ }^{2}$ ) ansgesprochen, der ältesten und zugleich vergänglichsten Gruppe, die mit deın konzentrirten Körper ebenfalls den Stiel verbindet, aber noch nicht das Radien-weise geordnete Getäfel der anderen, noch die strahlenständigen RespirationsOrgane der ungestielten Gruppen besitzt, sondern diese vielmehr oft zer'strent über der Oberfläche des Körpers zu tragen scheint; ihre Vierzahl erinnert an die viel tiefer stehenden Akalephen.

E. Bei den Mollusken möchten die Brachiopoden für das Unterreich überhaupt, und die Ammoneen (Nanliteen?) für die Cephalopoden jene Stelle vertreten ; für die Pelecypoden und Gasteropoden dürfte es unmöglich seyn eine solche Gruppe zu finden, so dass daraus wohl hervorgeht; dass das Beginnen aller KIassen mit einer solchen Gruppe nicht zu den Schöpfungs-Gedanken gehört habe. Oder sollte man bei den Gasteropoden die Anfangs so zahlreichen Melania-artigen Formen hieher rechnen, Meeres-Bewohner mit der Schaale der Asiphonobranchiaten, aber nach ihren lebenden Verwandten (Pasithea, Turbonilla) u. s. w. zu urtheilen, den Thieren nach wirkliche Siphonobranchiaten?

1) Jahrb. 1846, 506 .

2) Wir ersehen eben aus den neuesten Arbeiten von Agassiz, dass Desor Echinocrinus in Palacocidaris umgetauft und von den Stelleriden wieder zu den Echiniden versetzt hat. 
F. Bei den Krustern würde man die Paläaden und insbesondere die Trilobiten als eine solche Knoten-Gruppe bezeichnen können, welche die ausgezeichnetsten Entomologen seit:30 Jahren bald neben Chiton bei den gasteropoden Mollusken, bald bei den Krustern zu den isopoden Malacostraca oder zu den pöcilopoden oder zu den branchiopoden Entomostraca, und wieder als besondere Ordnung oder Unterordnung zwischen den Amphipoden und Entomostraca, neben den isopoden Malacostraca oder zwischen Phyllopoden und Pöcilopoden gestellt habeı. In der That entwickelt Burmeister über sie dieselben Ansichten, wie Owan über die alten Reptilien. Nachdem er ihre Verwandtschaft mit den Entomostraca in den grossen zusammengesetzten Augen mit bloss glatter (nicht facettirter?) Hornhaut, in dem Mangel eines gemeinsamen Brust-Panzers, wie einer festen Zahl von 5-7 Brust-Ringen und grösserer hartschaliger Fühler und sichtbarer gegliederter Füsse, in dem schildförmig erweiterten Kopfe und KörperRande nachgewiesen, welche eine gewisse habituelle Ähnlichkeit mit den isopoden Malacostraca nicht hindert, zeigt er, dass sie auch vou den Entomostraca wie noch mehr von allen andern Krustern dadurch wesentlich abweichen, dass die Zahlen ihrer Brustkasten-Ringe (in verschiedenen Geschlechtern $=6,8,9,10,11,13$ und endlich 16-20) sich gar nicht auf bestimmte Grund-Zahlen zurückführen lassen, und dass sie mit den einzelnen Unterordnungen der Entomostraca, den Lophyropoden, Phyllopoden und Pöcilopoden sich noch weniger vereinigen lassen, da die letzten durch ihre Grösse, ganze Körper-Eintheilung und die zum Theil starken gegliederten Gangfüsse, deren Hüften die Kiefern vertreten, am weitesten von den Trilobiten abstehen, die ersten aber klein sind, wohl entwickelte Fühler und nur ein einziges oder zwei sehr k leine Augen haben, da die Phyllopoden endlich bei grösserem Körper, umfangreichen Augen, unentwick elt en Fühlern und sch wachen Füssen, wie bei den Trilobiten, einen beharlich eilfgliedrigen Rumpf mit eben so zahlreichen Bewegungs-Organen besitzen (den Kopf dazu gezählt gibt $12=3 \times 4$ Glieder). Burmeister sieht daher in den Paläaden die verschiedenen Eigenschaften gleichwerthiger Gruppen mit einander verschmolzen eine Form ergeben, die in unverarbeitetem Zustande die mancherlei Eigenschaften vereint besitzt, welche heutzutage als sehr wichtige Gruppen-Unterschiede stets von einander gesondert angetroffen werden, und glaubt im Allgemeinen die Verschmelzung verschiedenartiger Typen in eine Form um so grösser und die aus der Auseinanderlegung der typischen Ideen abgeleitete organische Manchfaltigkeit um so geringer, je älter die Organismen waren, welche uns in den Erd-Schichten entgegentreten (was sich indessen gewiss nicht überall nachweisen lässt). Während also die Trilobiten durch ihre schwankenden Zahlen.Verhältnisse, ihre gänzlich verkümmerten Fühler und wahrscheinlich mehr als bei den Phyllopoden verkümmerten Füsse ganz aus der Klasse der Kruster hinaus. 
tretell, zeigen sie doch wieder mit der heutigen Kruster-Klasse, mit der Ordnung der Entomastraca, mit der Unterordnung der Phyllopoden: mehr eine so auffallende Analogie, als innige Verwandtschaft, dass man sie den Entomostraca als Repräsentant für die Gruppe der unbedeckten schildlosen Branchipiden (Branchipus) neben den Phyllopoden als eigene Unterordnung einschalten muss; welche, wenn man sie ihres Kopfschildes wegen zunächst mit der Familie der Apiden (Apus) in Parallele setzt und Eurypterus und die alten Cytherinen (C. balthica und C. phaseolus) als Vertreter der Muschel-schaaligen Estherien mit hinzu zieht, die man dann unter dem Namen der Paläaden zusammenfassen könnte, unsere jetzigen Phyllopoden nicht allein im Ganzen, sondern auch ihren einzelnen 3 Familien nach, als Analoge im Ur-Meere repräsentirt haben würden ${ }^{1}$ ).

Da indessen die (Trilobiten wenigstens unter den) Paläaden durch die unbestinmte Ringel-Zahl des Rumpfes, durch den Mangel der Fühler und die jedenfalls sehr verkümmerten, aber fast noch so gut wie unbekannten, Füsse tiefer als alle übrigen Entomostraca stehen, so nehmen sie in der Schöpfungs-Geschichte die vorderste Stelle mit Recht schon nach der im vorigen Paragraphen entwickelten Regel ein.

G. Bei den F is clı en würden solche Kuoten-Punkte sich durchkreutzender Organisationen in den Ganoiden, und unter diesen hauptsächlich in den Cephalaspiden zu finden seyn, die man bald für Limulus-artige Thiere, bald für riesige Wasser-Käfer gehalten hat. Über die sauroiden Ganoiden sagt Agassiz selbst, dass sie den Sauriern durch die Nähte ihrer Schädel-Knochen, ihre grossen kegelförınigen längsstreifigen Zähne, durch die Anfügung der DornenFortsätze und Rippen an die Wirbel, ihre Schuppen-Bedeckung u.s. w. nahe stehen. lhre Knochen, Zähne und Schuppen sind wenig von einander verschieden und in der That oft schwer unterscheidbar. Agassiz sieht in jenen Sauroiden der Kohlen-Formation gleichsam den Keim, woraus sich später die gewöhnlichen Fische, die Reptilien, die Vögel und Säugthiere durch Differenzirung und theilweise höhere Organisirung entwickelt hätten, unter welchen nach ihnen die Reptilien dieselbe Stelle übernälımen, indem in den Ichthyosauren und Plesiosauren sich die Osteologie der Cetaceen und noch später in den Dinosauriern die der Pachydermen vorbereitete, während die Fische mehr und mehr ächte Fische werden ${ }^{2}$ ).

H. Bei den Reptilien liefern die Saurier mehre solche WurzelFormen divergirender Organisationen. Die ganze Klasse der Reptilien müssten eigentlich die Batrachier eröffnen, zu welchen nach R. Owens Ansicht die Labyrinthodonten gehören. In der That treten sie auch

1) Burmeister, die Organisation der Trilobiten, Berlin 1843, $4^{0}$.

2) Agassiz, Poissons fossiles, I, Indroduct. p. XXXr. 
schon im rothen Sandsteine ( $\left.\mathbf{I}^{1}\right)$ Englands auf. Aber es sind nach ihm die höchsten unter den Batrachiern, Batrachier-Typen mit aufgeimpften Krokodilier-Merkmalen, vorangeschrittene Batrachier oder zurückgebliebene Krokodile, nicht höher entwickelte Fische; Repräsentanten der Krokodile unter den Batrachiern; es sind Batrachier, was den doppelten Occipital-Condylus, den Vomer-artigen Gaumen-Knochen, die Zähne u. s. w. betrifft; aber der Antlitz-Theil des Schädels war' wie beim Krokodil, mit Modifikationen der Kiefer nach der Weise der Batrachier; der Nasen-Kanal, abweichend von diesen, im hintern Theile des Gaumens einmündend, was wieder Einathmungs-Apparat, wohl-ausgebildete Rippen und Fortpflanzungs Weise wie bei den Krok odiliern voraussetzt; dagegen waren ein Theil der Zahn-Bildung, das Episternum und die bikonkaven Wirbel vom Ichthyosaurus; im knöchernen Gaumen Verwandtschaft mit den Lacertiern; in Befestigung und Wechsel der Zähne mit gewissen Fischen, und die OberarmBeine wieder ganz wie bei den Fröschen; im Becken eine Verbindung von Krokodil-und Frosch-Charakteren; Oberschenkel-Beine mächtig und die Tibien viel grösser, wesentlich von der Form wie bei den Hüpf-Beinen der Frösche; die Knochen mit Grübchen und die Haut mit Schuppen wie bei den Krokodilen ${ }^{2}$ ).

Die l chthiosauren haben von den Fischen nur etwa die Locomotions-Organe (Wirbel-Form, Flossen-Füsse), während alle Modifkationen ihres Saurier-Skelettes, welche auf die Athmung, Verdauung und Zeugung Bezug haben (insbesondere die Anatomie des Schädels mit Ausnahme der grossen Zwischenkiefer-Beine, die Zähne, die Einlenkung der Neurapophysen an die Wirbel, der komplizirte Brustbogen, die Brustbeine, die vollständige Schliessung des Bauches durch Rippen u. s. w.) den höchsten Typen der Saurier selbst entsprechen und der knöcherne Augenring mehr der Augen-Bildung bei den Vögeln, als bei den Eidechsen und Fischen angemessen ist. Keine Art von Übergangs-Form kann die Ichthyosauren mit den Fischen verbinden; keine Zwischenform knüpft die Ichthyosauren, in welchen Vogelund Cetaceen-Charaktere auf Saurier-Typus geimpft sind, in älteren oder neueren Perioden an die höheren Chelonier, Vögel oder Cetaceen an ${ }^{3}$ ).

Der Schädel des R hy nchos a urus (aus neuem Roth-Sandsteine $=\mathbf{f}^{\prime}$ ) an der Spitze der Lacertier vereinigt in sich Kennzeichen von

1) Die Labyrinthodonten sind im Enumerator in die Rubrik 1 gestellt worden, vielleicht irrig; denn R. Owen sagt a. a. O. überall, dass die Labyrinthodonten in England im New-red-Sandstone auftreten; der New-redSandstone Englands ist aber sonst $=\mathbf{f}$, wo auch Rhynchosaurus citirt ist; da aber Owev hinzufügt, dass die Reptilien des Magnesian-Kalkes (z) beträchtlich älter seyen, so könnte freilich nur noch gemeint seyn, worin auch die deutsche Art vorkommt.

2) Jahrb. 1843, 239-246; Collectan. 55.

3) R. Owen, in Collectan. S. 55. 
Krokodiliern, Lacertiern, Cheloniern und Vögeln ohne durch Übergänge an andere Geschlechter sich anzuknüpfen; seine bikonkaven Wirbel mit den schiefen Fortsätzen und Rippen-Anlenkungen wie bei den lebenden Eidechsen weichen gleichwohl von denen der Echsen, Schildkröten und Vögel ab. Rhynchosaurus verbindet die Lacertier enger mit den Cheloniern und den Vögeln als irgend eine lebende Form ${ }^{1}$ ).

Zu den sonderbarsten Saurier-Formen gehört Dicy nod on, wovon man schon 4 Arten aus einem alten Sandsteine Süd-Afrika's keunt ${ }^{2}$ ). Sie sind ihrer Gesammt-Bildung nach Lacerten, mit Abweichungen gegen die Krokodilier und Chelonier hin, also ebenfalls in höherer Richtung, und nähern sich dem Rhynchosaurus mehr als den lebenden Formen. Ihr Schädel macht in der Gestalt und durch den Mangel der gewöhulichen Zähne fast den Eindruck eines SchildkrötenSchädels. Auch ist der. Vordertheil der Kinuladen wie bei diesem mit einem hornartigen Überzug versehen. Wie bei Gift-Schlangen ist der Zwischenkiefer zahnlos und einfach und trägt jedes KieferBein nur e i n e $n$ langen spitzen gekrümınten Hauzahn; dieser entspringt unter oder hinter den Augen-Höhlen, legt sich in vorwärts gehender Richtung dicht an den Schädel an und krümmt sich allmählich abwärts, ohne vorn und unten den Schädel sehr zu überragen. Seine mikroskopisclıe Struktur ist wie bei den Krokodiliern mit Abweichungen nach den Raub-Säugthieren. Wie die Schneidezähne der Nager-Säugthiere wuchs er, bei mangelıden Ersatz-Zähnen, beständig aus der Alveole nach, ein den Reptilien ganz fremder Charakter. Die bikonkaven Wirbel deuten auf einen mehr ansschliessenden Aufenthalt im Wasser.

Die Pachypoden oder Dimosaurier, Repräsentanten unsrer Scinke und Lacerten in n-p, zeigen am besten, dass nicht jede Reptilien-Ordnung ihre höchsten Typen in der jetzigen Schöpfung habe. Denn es gibt kein lebendes Reptil, welches diezusammengesetzte scheidezähnige Zahı-Bildung mit verhältnissmäsig so grossen und starken Extremitäten verbindet und das Gewicht des Rumpfes durch Synchondrose oder Anchylose in ein so langes und zusammengesetztes Sacrum unterstützte, wie die Dinosaurier; Megalosaurus und Iguanodon überragen in Organisation und Grösse eben so wohl die Krokodilier (die höchsten lebenden Saurier), welche ihnen vorangehen, als jene, welche ihnen nachfolgen; ja die höchste Blüthe der Reptilien-Klasse ist seit| dem Erlöschen dieser Gruppe in fortwälıender Abuahme. Statt der meist zahlreichen und wichtigen Krokodil-Saurier ist ein Schwarm kleiner Echsen mit einigen mäsigen Krokodilen an der Spitze übrig geblieben, deren Wirbel-Körper sich vorn statt hinten (Streptospondylus) anshöhlen, ohne hiedurch eine Stufe höher zu steigen ${ }^{3}$ ).

1) Jahrb. 1844, 114; Brons Collectan. 54, 55.

2) Jabrb. 18\$5, 255;1846, 876.

3) R. OWEN, in Collectan. S. 55. 
I. In der Klasse der $\mathbf{V}$ ög el liegt der Knoten-Punkt auseinanderlaufender Organisationen offenbar in den ungeflügelten Lauf-Vögeln (Dinornis etc.), obwohl jene Neu-Holländischen, deren Skelette man mit grösserer Genauigkeit kennt, keineswegs die ältesten sind; aber die Grösse und Beschafienheit ihrer Füsse, die Länge ihrer Beine stimmen se ganz mit den freilich weit älteren grossen Vogel-Fährten in dem alten Sandsteine Nord-Amerika's (S. 823, 836) überein, dass R. Owen erst nach Ansicht der ersten nicht nur die letzten mit Überzeugung für Vogel-Fährten anzuerkennen begann, sondern auch eine Hypothese zur Erklärung der Wanderung dieser Familie aus NordAmerika nach Neu-Holland im Verlaufe mehrer Erd-Perioden ersann. Dinornis ${ }^{1}$ ) übertriff't in mehren seiner !) Arten den Strauss bei Weitem mehr noch durch Dicke als durch Länge der Knochen und vereinigt in sich Charaktere von Apteryx, von Struthio, Dromaius, Rhea und Didus. Der Schädel war dem des Didus verwandt, aber im Gehirn weniger entwickelt; der Vogel mithin stupider; sein Bau gedrungen, pachydermal; das Brustbein ohne Kiel und die Langknochen ohne Luft-Kanäle, daher absolute Unfähigkeit zu fliegen; der Lauf nicht so rasch als beim Strauss; die Beine kräftiger zum Scharren der Erde; die Füsse hatten 3 Zehen, gewöhnlich noch mit einer sehr verkümmerteu vierten (wie Apteryx).

Selbst der letzte der ausgetilgten Vögel, der Didus, war mit Albatross, Scharr-Vögeln, Lanf-Vögeln und Geiern zugleich verwandt; keiner Ordnung wollte er sich einfügen; zuletzt hat man ihn zu den Tauben versetzt. Seine Ernährungs-Weise ist ein Räthsel, obschon wir noch Berichte von Reisenden haben, die ihn zu Tausenden gesehen, und obwohl er in Europa in Gefangenschaft gehalten worden ist.

Und die Lauf- und Hühner-Vögel selbst: sind sie die höchsten oder die niedrigsten unter den Vögeln? Gewöhulich weist man ihnen eine mittle Stelle an über den $W$ ad- und $W$ asser-Vögeln, unter den Raub- und Hock-Vögeln. Aber die entwickelteren Genitalien des männlichen Strausses (die übrigens doch ziemlich entwickelt auch bei Wasser-Vögeln vorkommen) und sein geschlossenes Becken haben einige Zoologen veranlasst ihn höher als alle andern zu stellen; in keinem Falle aber scheint von dem späten Dinornis aus sich die ganze Klasse der Vögel erst entwickeln zu können. Doch wie verschieden von ihm mögen - bis auf die Grösse - jene alten Vögel gewesen seyn, welche die Fuss-Spuren im rothen Sandsteine Amerika's hinterlassen haben!

K. Unter den $\mathbf{S}$ ä ugthieren lat man die ältesten in den Oolithen gefundenen Reste lange Zeit von Beutelthieren hergeleitet, was sich später nur theilweise bestätigt hat; jene Thiere sind zu unvollkommen bekannt, und es sind ihrer zu wenige, um jetzt schon in ibnen den Knoten-Punkt nachzuweisen, von welchem die Formen-Reihen der

1) Jahrb. 1813, 334; 1844, 241, 381; 184\%, 379. 
Säugthiere ausgegangen seyn könnten. Nach der Zeit des zahlreicheren Auftretens herrschen Pachydermen in der Eocän-Zeit zwar vor, aber ohne ihrerseits einen so hohen Grad von Durchkreutzung fremdartiger Cliaraktere erblick en zu lassen, wie man ihn später in der Miocän- und Pliocän-Zeit bei Cetotherium, Squalodon und Halianassa unter den Cetaceen (denen jedoch der noch merkwürdigere aber vereinzelte Zeuglodon allerdings vorangeht), bei Dinotherium und Macrauchenia unter den Pachydermen, wo sich die Charaktere der Cetaceen oder der Nager einmengen, bei den Pycnoceraten unter den Ruminanten, bei der ganzen Ordnumg der Edentaten und bei einigen Marsupialen gewahrt, so dass die dadurch bezeichneten Formen sich zwar nicht auf eine bestimmte Ordnung konzentriren, jedoch sich vorzugsweise in die niedrigeren Ordnungen vertheilen.

L. Wir haben gesehen, dass sich bei einigen gerade der ältesten, wichtigsten und formenreichsten Klassen (Pelecypoden und Gasteropoden) solche vielkeimige Grund-Typen für die spätere Entwicklung. mehr auseinander tretender Formen-Reihen nicht nacliweisen lassen; - dass in andern Fällen, wie in mehren Ordnungen der Säugthiere, dieselben wenigstens den Ordnungs-Charakter nicht üherschreiten; dass diese Keim-Formen bald in einer ganzen Klasse zerstreut, bald in eine Ordnung oder Familie zusammengedrängt sind; dass sich eine Noth wendigkeits-Ursache für dieselben nicht nach weisen, sondern nur etwa eine dem Schöpfer willkührlich unterlegte Idee eines für alle Klassen oder Ordnungen gleichmässigen Entwicklungs-Ganges, doch ohne eine gerade diesen Gang bedingende und ihn herbei zwingende Natur-Kraft, annehmen lässt, während die übrigen Natur-Erscheinungen durch gewaltige in der Natur selbst liegende Kräfte in ihrem festen Gange geleitet werden.

Wir möchten demnach die Frage stellen, ob die Erscheinungen, von welchen wir in diesem Paragraphen gehandelt, sich nicht richtiger und klarer durch die Sätze ausdrücken lassen: dass im Allgemeinen die Ähnlichkeit der früheren organischen Formen eines Reiches, Unterreiches, einer Klasse, Ordnung 11 . s. w. mit den jetzigen Formen derselben Gruppen im umgekehrten Verhältniss zu der Zeit wachse, die sie von einander trenut; - dass in dessen Folge manche älteste Formen den Charakter der Klasse oder Ordnung, wozu sie gehör'il, gleichsam durchbrechen, um in andre Klassen hinüberzngehen und so zwei oder drei benachbarte Klassen bei ihrem genetischen Ursprunge mit einander zu verschmelzen; dass es aber kein bestimmtes und für alle Klassen gleiches Mass für das Zurücktreten der ältesten Formen von den jetzigen gebe und dasselbe daher bald sehr gross und bald unbedeutend seyn könne. - Es dürfte sich nachweisen lassen, dass auch manche unsier heutigen Ordnungen z. B., wenn sie nicht lebend, sondern nur in der Urwelt existirten, behufs ihrer Aufnahme in's 
System zuerst eine Erweiterung des Charakters der ihr zustehenden Klasse erheischen würde.

Wäre die Erscheinung auf obige Weise richtig ausgedrückt worden, so würde es auch möglich werden, die Übereinstimmung derselben mit einem später zu bezeichnenden Gesetze, ihren Grund und ihre Nothwendigkeit nachzuweisen (vgl. den folgenden $§ .211$ ).

\section{d. Durch Abnahme der Körper-fr̈öse der Arten.}

§. 15.

A. Es ist eine schon seit längerer Zeit verbreitete Meinung, dass die Thiere der Vorwelt grösser' als die jetzigen gewesen seyen; man hat Dasselbe von manchen Pflanzen behauptet und sogar aus dem ersten Grunde das Zweite als nothwendige Folge dargestellt; indessen ist jene Meinung nur eine theilweise begrüdete.

Man kann näılich als Regel aufstellen, dass zu der Zeit, wo eine Formen-Gruppe von Pflanzen oder Thieren ihrer Zahl nach zu ungewöhulicher Entwicklung gelangt, auch ein Theil der Arten eine ungewöhnliche Grösse erreiche. Es erklärt sich Diess zum Theil schon daraus, dass unter einer grösseren Zahl eben auch leichter sowohl die grössten als die kleinsten Dimensionen vorkommen können, als unter einer kleineren. Diese Regel ist aber nicht ohne Ausnahme, und obschon die grössten Arten sich sehr oft in denjenigen Gruppen finden, worin sich nach $\mathbf{O}_{\text {WEN }}$ Charaktere verschiedener Formen-Reihen vereinigen, so treffen sie doch weder immer mit diesen, noch immer mit den vollkommensten, noch stets mit den unvollkommensten Repräsentanten einer Klasse, Ordnung u. s. w. zusammen; und wo die höchste Zahlen-Entwicklung in die jetzige Schöpfung fällt, da findet man die grössten Arten gewöhnlich auch noch im lebenden Zustande vor. Nicht die frühere oder spätere Zeit an sich war der körperlichen Entwicklung der Organismen günstiger, sondern diejenigen Bedingungen, welche eine reichere Entfaltung der Klasse, der Ordnung herbeiführten, begünstigten auch die Grösse der Arten in ihren Individuen, und als solche kennen wir: warmes, fruchtbares Klima, Reichlichkeit der Nahrung und in manchen Fällen räumliche Ausdehnung des Mediums der Existenz ${ }^{1}$ ). Diese Bedingungen aber hatten nicht für alle Wesen einen gleichen zu- oder ab-nelimenden Gang.

B. Im Pflanzen-Reiche sind zuerst die kryptogamen Monokotyledonen, dann die gymnospermen Phanerogamen, endlich die Dikotyledonen mit doppelten Blumenhüllen herrschende Formen-Gruppen. Und in der That treten auch zuerst während der I. und II. Periode für jene die Equisetaceen mit ihren mächtigen Calamiten, die Farnen mit ihren Riesenstämmen in Protopteris, Caulopteris, Cottaia und Karstenia, die Lycopodiaceen mit ihren verhältnissmässig ungeheuren Lycopoditen, Lepidodendren, Sagenarien, Megaphyten u. s. w, und

1) Gesch. d. Nat. II, 106, 101, 86. 
endlich die zwischen den 2 letzten stehenden ansgestorbenen Familien der Sigillarieen, Stigmarieen und Psaronieen ebenfalls mit ansehnlichen Stämmen auf. Die gymnospermen Phanerogamen der mittlen Erd-Perioden scheinen sowohl als Cycadeen und Diploxyleen wie als Coniferen den jetzigen an Grösse wenigstens nicht nachgestanden zu haben. Die übrigen Monochlamydeen, die corollifloren und choristopetalen Dicotyledonen, welche ihre höchste Entwicklung in der tertiären und noch mehr in der jetzigen Zeit finden, erscheinen in beiden von manchfaltigen Grösse-Abstufungen bis zu derjenigen ansehnlicher Bäume; aber (so viel man weiss) doch erst in der letzten Periode mit der höchsten Grësse-Entwicklung, als Adansonia u. s. w.

C. Bei den PfIanzen-Thieren haben wir nicht Gelegenheit gehabt mächtigere Formen aus den fossilen Resten kennen zu lernen, weder bei den Amorphozoen, noch den Polygastrica, noch bei den polythalamen, bryozoen oder anthozoen Polypen, noch selbst bei den Echinodermen, wo doch die Stelleriden und insbesondere die Crinøiden als eine der reich entwickelten Anfangs-Gruppen der fossilen Formen-Reihen vorzugsweise zu deren Erwartung berechtigten.

D. Anders verhält es sich bei den Weich-Thieren, wo man schon unter den Brachiopoden zur Zeit ihrer höchsten Entwicklung einige untergegangene Genera mit vergleichungsweise grösseren Arten (Productus, Strygocephalus etc.) findet. - Die Pelecypoden und die Gasteropoden aber, welche erst in der jetzigen Periode zur höchsten Ausbildung kommen, bieten hier auch ihre riesigsten Repräsentanten dar, jene in den zu Taufbecken verwendeten Schalen der Tridacna aus der höchsten und jetzt zahlreichsten Abtheilung der Dimyen, diese in den Cassis-, Tritonium- und Pteroceras-Arten aus der ebenfalls höchsten und jetzt zahlreichsten Gruppe der siphonobranchen Ctenobranchier, dann in Bulimus unter den noch höheren Pulmonaten. Aber die Cephalopoden, jetzt noch durch einige ansehnliche NautilusArten repräsentirt, boten zur Zeit der höchsten Blüthe in den Ammoniten des Lias und der Oolithe manche Arten dar von dem doppelten und dreifachen Durchmesser, wie diese jetzigen Nautilen; uid diese Nautilen selbst sind früher vielleicht durch andere Arten ihres eigenen Geschlechts wie durch Orthoceren von $5-\delta^{\prime}$ Länge überboten worden.

a. Nach der von frühe an abnehmenden Ausdehnung der Meere sollte man denken, die Testaceen auch in stets abnehmender Grösse zu finden. Aber theils steht das eben erörterte Gesetz damit in Widerspruch, theils beschränkt sicb die Wirkung dieser Ausdehnung hier wohl mehr nur auf die Individuen, wit aus den Beobachtungen von E. Forbes erhellt, welcher identische Arten im Mittelmeere kleiner fand, als im atlantischen Osean ${ }^{2}$, wic sic auch in der Ost. see kleiner als in der Nordsee sind und die nur noch wenig zahlreichen Arter im schwarsen und kaspischen Meere nach EichwaLD in kleineren Individuen. auftreten, als im Mittelmeere. Doch wirkt im letzten Falle die bloss brackisch Natur des Wassers mit.

1) Jahrb. 1844, 635 . 
b. Eine andere Anwendung wird man machen können, wenn man nicht die össe der Konchylien überhaupt zu verschiedenen Zeiten, sondern die gewisser rdnungen und Familien in gewissen Ländern und Weltgegenden miteinander rgleicht. Da findet man denn in dem Pariser Eacän-Beckeu z. B. die Siphobranchier im Allgemeinen (dabei Ceritherium giganteum) und eine Anzahl derer Genera in einer Grösse vor, wie sie jetzt in keinem europäischen Meere, ohl aber in den tropischen Gewässern Ost- und West-Indiens und der südsee ich anzutreffen sind; und einige südfranæösische Süsswasser-Becken haben find-Konchylien (Bulimus, Megaspira) geliefert, wie sie jetzt nur in Brasilien Id in tropischen Ländern vorkommen. Ebenso einige miocäne Clausilien. Eine cäne Physa ist aber grösser, als die weit zahlreicheren jetzigen Arten. - Al-dings aber zeigen sich die meerischen Konchylien der I. bis IV. Periode aus ch jetzt zahlreichen Ordnungen nicht grösser, als jetzt auch.

E. Die Kerb-Thiere theilen sich, wie wir schon gesehen, in Gruppen, wovon die eine dem Wasser, die andere der Luft angejrt. Unter den Wasser-Bewohnern sind die Würmer gleichmässig hrch alle Perioden verbreitet, daher nirgends durch beträchtliche Irösse ausgezeichnet; die Kruster sind für die Entomostraca am ichsten durch die Paläaden in der I. Periode vertreten, welche denn ch durch $2^{1}-2^{1} / 2^{1}$ lange Arten ${ }^{1}$ ) die noch lebenden Formen in Grösse eit überbieten, bedingungsweise die der kleinen Pöcilopoden-Grupn ausgenommen, welche in der Jura-Zeit etwas häufiger als jetzt ftrat, jetzt aber eine ungefähr noch eben so grosse Art darbietet. ie Malicostraca dagegen, welche ihre grösste Entwicklung in den lekapoden der jetzigen Zeit erlangen, bieten hier auch die grössten rten dar in Palinurus, Astacus, Cancer etc. - Die Luft-Kerfe haben re höchste Ausbildung erst iu der Jetztwelt, und so haben die Erdchichten auch keine solche Riesen-Schmetterlinge und Riesen-Käfer e unsere Tropen-Länder aufzuweisen.

F. Von den W irbe1-Thieren haben unter den Fischen die asmobranchier nach den fossilen Hai-Zähnen zu schliessen in der 'eide- bis Molasse-Zeit sowohl eine grössere Zahl von Arten wie ch grössere Individuen als jetzt geliefert, während die zahlreichen und grössten Teleostei, deren Auftreten erst kurz vor der Terir-Zeit beginnt, in dem jetzigen Meere leben (Thynnus, Anarrhias etc.). Dagegen ist uns nicht bekannt, ob es unter den zahlieichen ssilen Ganoiden eben so grosse Arten gebe als unter den wenigen ch lebenden die Sippen Acipenser und Lepidosteus $\left(6^{\prime}-8^{\prime}\right)$ darbie n? - Die Blïthe-Zeit der Reptilien-Welt fält mit den Sauriern in e II.-IV. Periode, wo dann auch in der That unsere grössten jetzien Krokodile durch die Megalosauren, Hyläosauren, Iguanodonten a. bis ums Dreifache an Länge und um ein Vielfaches an Masse pertroffen werden. Noch grösser würde das Missverhältniss seyn vischen Labyrinthodon und unsern Fröschen, wenn erstes Genus irklich zu den Batrachiern gerechnet werden müsste. - Die peliste Zahl und Manchfaltigkeit der Vogel-Klasse scheint nach

1) Jahrb. 1846, 765 . 
den sparsamen fossilen Vogel-Resten zu schliessen in die jetzige Zeil zu fallen, wo sie durch den Lämmergeyer und Condnr unter den Raub Vögeln, den Schwan unter den Wasser-Vögeln, mehre Reiher unter " den Sumpf-Vögeln und zumal durch die Ordnung der Strauss-artiger Vögel ihre höchste Grösse (bei 9' Höhe) erreichen. Aber in der Di : luvial-Zeit, aus welcher wir so wenige Vogel-Knochen besitzen, gal a es in Neuseeland Dinornis-Arten bis von doppelter Grösse, und die nacl den Modelen mehrer Ordnungen gebildeten Fuss-Spuren in den alter rothen Sandsteinen von Massachuset/s sind zum Theil noch ansehn licher. Es entsteht also die Frage, ob nicht die Vögel schon vie on früher, als wir bisher angenommen, nicht nur existirt sondern auch $\mathrm{zr}^{\mathrm{W} / \mathrm{a}}$ einer in Arten-Zahl und Körper-Grösse höchst bedeutenden Entwick lung gelangt sind? Da übrigens die Dinornen nach OWEN unter alles Vögeln den am meisten pachydermalen Charakter besitzen, wovor man sich einen massigen Körper nicht ausgeschlossen denken kanı so lässt sich hier die Erscheinung aus dem Misch-Typus der älteste Vögel herleiten. - Die tertiären Säugethiere endlich bieten in deo nach allen nöthigen Verbesserungen in der Zusammensetzung de Skeletts noch immer über $\mathbf{7 0}^{\prime}$ langen Zeuglodon für die fleichfres senden Cetaceen, in Dinotherium, Mastodon u. a. für die Pachyder men, in Sivatherium und Bramatherium für die Ruminanten, in zah] reichen Formen für die Edentaten, in Phascolotherium, Nothotheriur u. a. für die Marsupialen, in Amphitherium für die Insektivoren ansehn liche, die jetzige Grössen-Masse weit überschreitende Vorbilder dai

G. Die Grösse wenigstens eines Theiles der Wirbelthiere stell heutzutage aber anch noch im Verhältniss zur Grösse des Kontiner tes oder des Meeres, worin sie wohnen, in der Weise nämlich, das ohıe die kleinsten Maase auszuschliessen, die grössten unter del Säugthieren und wohl auch Reptilien in den grössten Kontinente oder Meeren wohnen. Da wir aber die einstige Grösse der Kontinent nicht kennen, so würden wir eher versucht seyn, dieselbe aus de Grösse ihrer Bewohner zu erschliessen, als den Zusammenhang de letzten mit der Grösse der ersten beweisen zu können.

Der alte Kontinent als der grösste besitzt die grössten Land-Säugthiere i den Elephanten, Nashöruern, Flusspterden, Pferden, Kameelen, Rindern, Löwe Leoparden, Tigern u. s. w.; der neue Kontinent entbehrt die 4 ersten als d grössten gänzlich und kamn auch einen anderweitigen Ersatz für sie nicht bieter-un seine grössten Katzen-Arten sind kleiner als die asiatischen und afrikanische statt der Kameele hat es nur die Lamas; - nur der amerikanische Bison allei kann den Rindern der alten Welt gleich gesetzt werden; die viel kleiner Sunda-Inseln besitzen zwar grosse Thiere, aber sie scheinen solche nur in Folg eines frühern Zusammenhangs mit Asien noch zu haben, zum Theil identist mit den asiatischen Arten. Neuholland und die benachbarten Inseln der Süds haben ausser den selbst sehr mässigen Känguruhs und Beutelhunden nur seł kleine Säugthiere bis zur Grösse der Hauskatze etwa. Auf den noch kleiner und von den Kontinenten entfernten Südsee-Inseln kommen kaum noch Flede mäuse und Mäuse vor. Von den Meeres-Säugthieren leben die grösseren fleisc Wesel fressenden Cetaceen nur im weiten und tiefen Orean und verirren sich nur se bich ten und meist zu ihrem Verderben in die Mittelmeere und tieferen Busen; d 
mnen-Meere haben nur noch etwa Seehunde und Delphine zu Bewohnern gl. S. 816).

Die lebenden Reptilien scheinen einigermassen demselben Gesetze zu gehoren: obwohl auch noch unter dem Einflusse des Klimas zu stehen. Die gröss1 unter ihnen sind die Riesenschlangen, welche wenigstens auf kleinen Inseln cht vorkommen, und die Krokodile, welche an der Einmündung grosser Flüsse - Meer wohnen. Aber den grössten Molch nährt Japan jetzt in seinen Süssassern. - Bei Vögeln und Fischen scheint dieses Gesetz keine Geltung mehr haben: denn mit Ausnahme des afrikanischen und des südamerikanischen rausses wohnen die grossen Strauss-artigen Vögel auf Inseln, der Kasuar auf in grossen Sunda-Inseln, der Emu auf Neuholland, der Apteryx auf Neuseend; der Dudu war auf dem kleinen Bourbon zu Hause gewesen, und die Di|jnis-Reste rühren ebenfalls aus den neuseeländischen Inseln her; auch grosse asser- und Sumpf-Vögel sind auf kleinen Inseln in nicht zu grosser Entferling von Kontinenten nicht selten. - Unter den Fischen übertreffen die Störe id Welse in den Flüssen der alten und die Lepidostei in den See'n der neuen elt fast alle Seefische an Grösse, die räuberischen Haie ausgenommen, welche cht in die Süsswasser übergehen,

Es scheint demnach, dass die grossen Lauf-Vögel in ihrer Grösse eben so enig von der Grösse des von ihnen bewohnten Landes als von der numerihen Entwicklung der ganzen gleichzeitig mit ihnen bestehenden Vogel-Welt hängig sind?

\section{Durch fortschreitende Umbildung des Schöpfungs-Typus nach Massgabe der geologischen Veränderungen in den äussern Lebens-Fedingnissen.}

§. 16. Im Allgemeinen.

A. Wir haben in den zwei vorhergehenden Paragraphen von wei Prinzipien der geologischen Entwicklung der Organismen geundelt; das erste, welches man schon lange angenommen und das seinen jetzigen Modifikationen in Agassiz einen lebhaften Vertreter efunden, ist das der allmählichen Ausbildung von niedrigen und dem ötus-Zustand analogen zu höheren Stufen der Organisation; das pdere, nicht selten mit dem ersten im Widerspruch stehend und insesondere von $\mathbf{R}$. OWEn, von Burmeister u. A. vertheidigt, ist das es Beginnens der Thier-Klassen oder Ordnungen $\mathbf{u}$. s. w. mit solchen ormen der Organisation, in welchen sich die Organisations-Typen erschiedener Gruppen in einer Weise durchkreutzen, wie es in der tzigen Schöpfung nicht mehr der Fall, so dass sich diese älteren epräsentanten ohne eine Änderung oder Erweiterung der Charaktestik unserer jetzigen Klassen dem Systeme nicht einordnen lassen, - und an welche jedesmal mehre unserer jetzigen Formen-Reihen, öhere wie tiefere sich anschliessen. Wir haben gesehen, dass beide rinzipien sich in der That bis zu einem gewissen Grade durchführen issen; dass sie in vielen Fällen und zwar das erste unter gewissen lodifikationen stattfinden, die wir oben näher bezeichnet haben; dass ie aber auch oft einander ausschliessen, so dass bald nur das eine nd bald nur das andere zur Erklärung vorliegender Erscheinungen nwendbar ist, woraus sich schon ein strenger Einwand gegen ihre vesentliche Geltung ergibt; dass es endlich auch Fälle gibt, auf die ich weder das eine noch das andre anwenden lässt. In allen diesen ällen aber wird man ferner nach dem Causal-Zusammenhange, nach 
der bewirkenden Ursache wie nach dem Zwecke fragen, wodurch und warum die Erscheinungen sich nach solchen Prinzipien ordnen sollen, Wenn es uns nun gelingt, jenen Zusammenhang in vielen oder den meisten Fällen nachzuweisen, so wird es zwar in der Regel, wie in so manchen andern Natur-Verhältnissen, unmöglich seyn zn sagen, ob die Ursache um der Folge willen, oder die Folge bloss wegen der Ursache vorhanden ist (ob der Fisch Kiemen haben muss, weil er in Wasser lebt, oder ob er im Wasser leben muss, weil er Kiemen hat): aber wir werden jedenfalls dadurch ein drittes schon in sich wahrscheinliches und nothwendiges Prinzip zur Erklärung der allmählichen genetischen Gestaltung der organischen Welt bis zu ihrem jetzigen Zustande gewinnen, das Prinzip der organis chen E ntwicklung in Übereinstimmungmit den A ussen-Verhältnissen, welches sogar dann, wenn wir seine Geltung nicht sogleich in allen Fällen nachweisen können, seinen Werth überhaupt doeh nicht einbüsst, weil es uns jetzt natürlich nicht mehr vergönnt ist, alle einstigen geologischen Erscheinungen nach ihrem Zusammenhang und alle Wirkungen dieser Erscheinungen unter sich und anf die Gestaltung der organischen Welt zn enträthseln. Es wird genügen das Walten dieses zwar auf äussere Ursachen gegründeten, aber nothwendigen Prinzips im Entwicklungs-Gange der organischen Reiche überhaupt nachzuweisen, um ihm, wemn richt eine ausschliessliche, so doch eine wesentlichere Geltung als den andern nicht auf äussere Nothwendigkeit gegründeten, sondern theils bloss aus der geistreich untersteflten Analogie des Entwieklungs-Ganges der orga nischen Individuen mit den organisehen Reichen hergeleiteten, theils ans Erfahrungs - Bruchstücken zusammengesetzten Prinzipien zu sichern. Ja wir zweifeln nicht daran, dass, wenn wir alle früheren Natur. Verhältnisse genau kennten, dieses Prinzip die alleinige Grundlage aller Erscheinungen bleiben wïrde, wie sich ihm denn auch das der allmählichen Vervollkommnung schon grösstentheils unterordıen lässt.

Auch R. Owev hat dieses Motiv der allmählichen Unbildung der fossilen Organismen erkanmt nud in einigen Bezichargen bei den Reptilien nachzuweisen gesueht ').

B. Welches sind aber diejenigen ä us se re n L e bens-Bedin g un g e $n$, deren Wechsel in der geologischen Zeit von Einfluss auf das organische Lebew war? Nur einige sind uns mit mehr oder weniger $\mathrm{Si}$ cherheit bekannt, andere nur hypothetisch theils ans geologischen und theils aus den organischen Merkmalen selbst erschliessbar und daher nur unsichere Unterlagen wener Schlüsse. Wir fassen unsere in det Geschichte der Natur (Band I) aufgestellten Ausichten, unter Berück. sichtigung einiger neueren Betrachtungen inshesondere nach Bischоғ nochinals zusammen, um diejenigen Momente herauszufinden, welche anf die Entwicklung der organischen Lebens Bedingungen eingewirk

1) Br. Collect. I, 57 . 
haben müssen. Die Erde ist anfangs feurig-flüssig gewesen und hat im Verhältnisse fortschreitender Abkühlung eine starre Rinde gewonnen, worin vor begonnener Verwitterung die Metall-Beimengungen weniger oxydirt und die jetzigen kohlensauren Erden als Silikate ohne Kohlensäure vorhanden, die Wärme höher und von der äussern Bestrahlungs-Wärme fast unabhängig unter allen Zonen nahezu gleich geweseu sind; daher, so lange als nicht die Schwere der Luft die Expansiv-Kraft dieser Wärme überwog, eine weit grössere Menge von Gasen und Dünsten diese Atmosphäre erfüllte, wesentlich vergrösserte, ihren Druck, ihre Dichte und ihre Wärme-Leitung erhöhete. Diese Luft-Masse drang aber durch Risse und Poren immer tiefer in die erkaltende Erd-Kruste ein; der Verwitterungs-Prozess, die Bindung von Sauerstoff der Atmosphäre und theilweise von etwas Wasser durch die sich oxydirenden Metall-Oxydule (Eisen,!Mangan), von Kohlensäure durch die Kalk-und Talk-Erde der bisherigen Kalkerde- und Talkerde-Silikate der krystallinischen Gesteine begann, die Bewegungen der koinprimirten Atmosphäre, später des troptbar flüssig-gewordenen Wassers der Meere und Ströme beförderten das Zerfallen, die Fortführung und Ablagerung an tieferen Stellen der nun oxydirten und kohlengesäuerten Stoffe; die Bildung geschichteter Niederschläge unter dem Einflusse einer noch immer von imnen nachströmenden und in ihnen sich häufenden, örtlich wieder mehr gegen die Oberfläche der Erde vorrückenden Hitze begann lange vor dem Erwachen des organischen Lebens; die ältesten zum Krystallinischen neigenden Petrefakten-freien Schiefer- und Kalk-Steine bildeten sich. Unter dem Einflusse der Kälte des Weltraums konnte übrigens die noch sehr dünne Erd-Kruste bald so kühl (unter $100^{\circ} \mathrm{C}$.) seyn, um wenigstens Pflanzen hervorzubringen, selbst als sie wenige Fuss tiefer noch glühend war; in welchem Falle folglich die Ausströmung von Wärme in die noch immer dichte und daher besser leitende (aber anch höhere) Atmosphäre durch Klüfte, Quellen und Dünste noch beträchtlich seyn konnte. Im Verhältnisse fortschreitender Abkühlung der Oberfläche wie der Atmosphäre trat der Unterschied des ZonenKlimas mehr hervor, und während die Tropen-Gegenden wenig WärmeAbnahme mehr erfuhren, schritt sie nächst den Polen immer weiter. Die fortdauernden Bewegungen des flüssigen Inneren, die Zusammenziehungen des starren Äusseren der Erde bewirkten mehr und mehr Ungleichheiten der Oberfläche; Inseln tauchten aus dem WasserMeere auf, vergrösserten sich allmählich zu buchtigen Kontinenten, worauf See'n und Flüsse entstunden und deren Gebirge höher und hölier emporstiegen und ein auch topographisch verschiedenes Klima annahmen, während das bisher gleichmässiger tiefe ausgedehntere aber seichtere Meer im Verhältniss seines weitern Zurückzuges stellenweise immer tiefer wurde und an den seichteren Küsten manchfaltig in die Kontinente eingriff; die regelmässigen See- und LuftStrömungen stellten sich ein. Das organische Leben begann, zuerst 
im Wasser, und bald auch auf dem Lande. Aller Kohlenstoff, Wasserstoff, Sauerstoff und Stickstoff, dessen die gleichzeitig bestehende Pflanzen- und Thier-Bevölkerung zu ihrer Bildung bedarf, musste der Luft oder dem Wasser jetzt entzogen werden; allen Sauerstoff, den das verwitterte Schutt-Gebirge vorzüglich in seinem Eisen mehr enthält, als das ursprüngliche, woraus es entstanden, - aller Kohlenstoff, den das Kalk- und Dolomit-Gebirge jetzt als Kohlensäure gebunden enthält, - wie alle organische Materie, welche in Form von Bitumen, Stein- und Braun-Kohle u. s. w. zwischen den Gesteinen eingeschlossen ist, wurde allmählich aus der Atmosphäre abgeschieden, worin solche entweder gleichzeitig vorräthig waren, oder wohin sie ebenfalls nur allmählich im Verhältniss ihres Verbrauches aus bekannten und unbekannten Quellen gelangt waren. Die Organismen wurden in dem Maase geschaffen, als jeder Klasse, Ordnung, Familie derselben, als ihrer Menge und Grösse theils die geologischen Bedingungen ihrer Existenz, theils die Wechsel-Bedingungen zwischen jenen Gruppen selbst zusagend wurden.

So ungefähr haben wir uns schon früher (Geschichte der Natur I. und II.) die allmähliche Entwicklung der Bedingungen des organischen Lebens auf der Erd-Oberfläche gedacht; doch wollen wir, durch Fucrs und G. Biscrof ${ }^{1}$ ) neuerlich veranlasst, noch einige erläuternde und beziehungsweise berichtigende $\mathrm{Be}$ merkungen dem Früheren beifügen.

Es ist den Plutonisten von Fucrs u. A. gegen ihre Ansicht eingewendet worden, dass in einer Schmelz-Hitze, wie sie die feuerflüssige Erde voraussetzt, ein für sich bestehender kohlensaurer Kalk nicht denkbar seyn, indem unter solchen Verhältnissen die Kalkerde mit der Kieselerde zu einem Silikate zusammentreten, die Kohlensäure aber in die Luft entweichen würde. Will oder muss man auf diese Ansicht eingehen, gegen welche sich Berzeluus erklärte, so hätte man 1) anzunehmen, die Atmosphäre habe zu einer Zeit allen Kohlenstoff in Form von Kohlensäure enthalten (Gesch. d. Nat. I, 131) und wäre noch viel ausgedehnter, schwerer, dichter, heisser und irrespirabler gewesen, als wir früher angenommen haben, und müsste man sich 2) nach G. Biscноғ zwischen der Entstehung der krystallinische Kiesel-Silikate enthaltenden Gesteine der ErdKruste und dem Beginn des organischen Lebens eine noch längere Periode denken, als wir früher daselbst angedeutet, wo die Verwitterung der plutonischen Massen allmählich so viel jener krystallinischen Gesteine in Sand und Erde verwandelte, um nicht nur den Stoff für fast alle unsre kieselig-sandigen und thonig-erdigen Sediment.Gesteine daraus zu gewinnen, sondern zugleich auch so viel Kalk- und Talk-Erde aus den Silikat-Verbindungen in jenen plutonischen Gesteinen zu befreien und mit Kohlensäure der Atmosphäre zu verbinden, als zer Bildung aller unserer sedimentären Kalk-Gebirge nöthig ist. Kommen aber durch ansehnliche Senkungen des Meeres-Bodens oder der Kontinente solche Schichten kohlensauren Kalkes wieder in grössere Tiefen in dem Bereich der Glühehitze hinab, wo auch jüngere sedimentäre Schiefer sich eben zur Annahme einer krystallinischen Beschaffenheit neigen, da werden die Kalksteine zerlegt, da entstehen die Kohlensäure-Exhalationen in Verbindung mit vulkanischen Erscheinungen, während die freiwerdende Kalkerde durch die die ErdRinde durchziehenden atmosphärischen Wasser entweder Kieselerde zugeführt erhält, welche in jener Hitze sich mit der freien Kalkerde zu verbinden im Stande ist, oder selbst bis zu andern eine solche Verbindung vermittelnden Ört-

1) Lehrbuch der chemischen und physikalischen Geologie I, II, 28 ff., 59 ff. 
lichkeiten entführt wird. Kalkerde und Kohlensäure können sich auf diese Art öfters trennen und verbinden, aber nicht mehr im getrennten Zustande anhäufen, zumal seitdem die Vegetation an der Bindung der letaten mitwirkt.

Einige aus diesen Bemerkungen abzuleitende Folgerungen werden wir in den nächsten Paragraphen mit einschliessen. Wir haben uns übrigens um die hier angedeuteten Zustände jetzt nur in soferne zu kümmern, als sie noch zur Zeit des Beginnens des organischen Lebens vorhanden gewesen seyn können.

C. Weun „alles Lebende aus dem Flïssigen" sich entwickelt, so muss zu einer Zeit, wo ein bei Weitem grösserer Theil der Erd-Oberfläche noch mit Wasser bedeckt war, als jetzt, auch die Gesammtheit der Organismen in Art und Zahl dem Anfange der Entwicklung noch näher gestanden seyn; es müssen die unvollkommeneren vorwaltend gewesen seyn und nur im Verhältnisse der überhandnehmenden Trockne allmählich auch vollkommneren Formen Raum gestattet haben ( $(.207)$. Aber es erklärt sich daraus auch wieder, warum das Thier-Reich früher als das Pflanzen-Reich, das ausser den Fucoiden fast nur Land-Bewohner enthält, in grosser Entfaltung auftreten musste $(\$ .20 \%, D)$, und warum grosse Landthiere erst spät erscheinen konnten $(\$ .210, G)$.

So lange die geologischen Lebens-Bedingungen für die Organismen noch nicht differenzirt, so lange Ozean und Continent, Strand und Meeres-Tiefe, Hochland und Tief-Ebene, Berg und Thal, Salzund Süss-Wasser, Strom und See, Regen und Trockne, Sommer und Winter, Tropen- und Polar-Klima nicht geschieden waren, konnten auch die Organisationen nicht geschieden seyn, welche so ungleichen ausschliesslichen Zuständen entsprechen; daher die Misch-Gebilde am Anfange der verschiedenen Organismen-Reihen ( $\$$. 209).

So lange diese verschiedenartigen Verhältnisse nicht bestunden und wieder in dessen Folge ganze Reihen von organischen Formen nicht bestehen kounten, waren auch jene Organismen unmöglich, welche von letzten abhängen, von ihnen genährt, getragen und geschützt werden; daher die zunehmende Formen-Manchfaltigkeit in den aufeinander folgenden Perioden ( $\$$. 206).

Wir sehen daher, dass die 3 bis jetzt aufgestellten EntwicklungsGesetze selbst in dem vierten, dem der fortschreitenden Umbildung der organischen Welt nach Massgabe der äusseren Bedingungen wurzeln; die ausführlichere Nachweisung wird sich in den folgenden $\mathrm{Pa}$ ragraphen ergeben.

D. Die wichtigsten geologischen Momente, deren Zusammenhang mit dem Entwicklungs-Gange der organischen Welt wir zu prüfen haben, sind also theils solche, welche allerwärts gleichmässig eintraten (1); theils andere, welche eine immer grössere Differenzirung der verschiedenen Gegenden der Erd-Oberfläche zu bewirken strebten, nach Zonen (2) oder mehr zufälligen Grenzen (3-5), jedoch zum Theil abhängig von vorigen (5).

1) Die veränderliche Mischung der Atmosphäre. 

schen Zouen.

2) Die Abkühlung der Erde und die Differenzirung der klimati-

3) Veränderte Ausdelınung und Tiefe des Meeres und Wechsel seiner Strömungen, in Folge der Land-Bildungen.

4) Die Erliebung und das Wachsen der Inseln und Kontinente mit ihren Gebirgen aus dem Meere; ihre Grösse, ihre Alluvionen, Feuchtigkeits-Zustand im Allgemeinen; die Entstehung von Flüssen und Süsswassern anf dem Lande.

5) Wechselseitige Existenz-Bedingung vieler Organismen gegeneinander.

๒. 17. Nach veränderter Mischung und Masse der Atmosphäre.

A. Das organische Leben konnte zwar nicht früher beginnen, als bis die Temperatur der Erd-Kruste wie die der Atmosphäre so weit gesunken waren, dass sie sich mit jenem Leben vertrugen. Diese Temperatur hätte für die starre Erd-Oberfäche schon unter $100^{\circ} \mathrm{C}$. stehen müssen (Gesch. d. Natır II, 43 ff.), ін welchem Falle dann aucli die Atmosphäre nicht mehr heisser seyn konnte, da die Abkühlung von aussen nach innen voranschreitet; allein eine solche Temperatur ist, wie wir an Lava-Strömen sehen, wenige Fuss hoch über feurig-flüssiger Gestein-Masse schon möglich, und somit hätte das organische Lieben schon sehr frühe in der' geologischen Zeit beginneu können, wenn ihm nicht andere Hindernisse im Wege stunden.

Eine Temperatur der Atmosphäre zwischen $100^{\circ} \mathrm{C}$. und der jetzigen würde an sich keine nothwendige Ursache einer von der jetzigen abweichenden Zusammensetzung gewesen seyn, mit Ausnahme eines durch die vermehrte Verdunstung erhöhten Wasser-Gehaltes (welcher durch seinen Druck die Verdunstungs-Fähigkeit des Wassers wieder etwas vermindert hätte).

Aber erweislich ist erst nach Beginn des organischen Lebens aus der Atmosphäre genommen worden

a. aller Stickstoff-Überschuss, welcher in die Bildung der nachherigen Thier- und Pflanzen-Bevölkerung einging, da wir sonst keine Form kennen, in welcher er damals bestanden haben köunte; -

b. aller $\mathrm{K}$ ohle n st of $f$ in Form von Kohlensäure, welcher jetzt 1) zur Bildung der Stein - und Braun-Kohlenlager und der DammErde, 2) der zufälligen kohligen Bestandtheile in fast allen Gebirgs. arten, wie 3) der lebenden Thier- und Pflanzen-Bevölkerung und 4) selbst zur Bildung wenigstens eines Theiles der später abgesetzten kohlensauren Kalksteine verbraucht ist (so weit nämlich es nicht gelingen sollte, diesen Kohlenstoff aus einer andern Quelle abzuleiten, worauf wir sogleich zurückkommen werden);

c. derjenige Sa u er's toff, welcher seitdem zur höheren Oxydation von Eiset- und anch etwas Mangan.Oxydul, zur Verwandlung 
jenes Kohlenstoffs in Kohlensäure und zur Lieferung des nöthigen Uberschusses über den Wasserstoff im Thierreich? verwendet worden ist, wodurch also, wenn alle diese Stoffe gleichzeitig in der früheren Atmosphäre enthalten gewesen wären,

d. auch eine zwar vielfach grössere Höhe und Dichte der A tmosphäre, ein grösserer Druck auf die Unterlagen, eine stärkere Wärmeleitungs-Fähigkeit derselben bewirkt worden wäre, Verhältnisse, die jedoch um so viel geringer ausfallen würden, für einen je grössern Antheil jener Stoffe man einen allmählichen Übergang in die Atmosphäre aus bekannten oder unbekamuten Quellen nach Massgabe ihres stattfindenden Verbrauches unterstellen dürfte.

a. Es wird schwer seyn, die im lebenden Thier-Reiche enthaltene S'tickstoffMenge zu berechnen.

b. Bischof wählt folgende Ansätze, um die Menge des Kohlenstoffs auf der Erd-Oberfläche zu beurtheilen: Die Atmosphäre enthält 0,0006 Kohlensäure mit 2800 Billiouen Pfund Kohlenstoff, woraus man eine noch nicht 1"' dicke Kohlen-Schicht rings um die Erde bilden kömute. 1) Die Saarbrückener Kohlen-Formation stellt eine Kohlen-Masse dar von 8,1 Quadratmeilen und $338 \mathrm{1} / \mathbf{2}^{\prime}$ Mächtigkeit, welche in 90,8 Billionen Pfund Kohle 72,6 Billionen Pfund Kohlenstoff enthält, d. i. 1/41 des in der Atmosphäre enthaltenen Kohlenstoffs auf $1 / 11+322_{2}$ der Erd-Oberfläche. Nun sind aber die westphälischen, belgischen, nordfraızösischen, englischen Kohlen-Becken nicht minder mächtig und liegen nicht sehr weit auseinander. Daher hat Lisbig die SteinkohlenVorräthe der Erd-Oberfläche einst viel zu gering angeschlagen, als er ihreu Kohlen-Gehalt unter den der Atmosphäre setzte (Gesch. d. Nat. II, 51). RoGERs lat den der letzten auf 850,000 Millionen Tonnen und den der Steinkolslen-Lager auf 5 Billionen Tonnen, d. i. 6 mat so viel als in der jetzigen Atmosphäre, berechnet ${ }^{\mathrm{l}}$ ). Wäre aller atmosphärische Sauerstoff einmal mit Kohlenstuff zu Kohlensäure verbunden gewesen, sagt Brscнor, so hätte man auf jeden Quadratfuss Erd-Oberfläche 504 Pfund Sauerstoff, der mit 189 Pfund Kohlenstoff gesättigt werden würde, welcher einer Kohlen-Schicht von $2^{\prime} 3$ Dicke entspräche. 2) $\mathrm{Da}$ aber alle -Sediment-Gesteine ebenfalls kohlige Theile enthalten, solche sogar durch Wasser eingeführt in plutonischen Gesteinen vorkommen und deren Mlenge mit 0,01 nicht zu hoch berechnet ist, so würde man schon bei einer 2 geographische Meilen dicken Sediment-Rinde der Erde ${ }^{2}$ ) allein eine Kohlen - Schicht vou $46^{\prime}$ erhalten, was 20 fach $2^{\prime} 3$ wäre. Über den Betrag der Humus- und Pflanzen-Decke um die Erd-Rinde wie der Thiere vgl. Gesch. d. Nat. I, 127.

c. Wemn aller Kohlenstoff in und auf der Erde, wie er vorhin nachgewiesen worden ist, einmal an $S$ aue rst off gebunden war, so muss jetzt der grösste Theil dieses Sauerstoffs aus der Atmosphäre verschwunden seyn. Zur Bildung des Thier- und Pflanzen-Reichs ist er nicht nöthig gewesen, da diese ihren Sauerstoff-Bedarf durch Zerlegung des Wassers gewinnen; er kann nur verwendet worden seyn zu höherer Oxydirung des Eisen-Oxyduls und etwas Mangan-Oxyduls, welche in vielen plutonischen Gesteinen, Basalten u. s. w., vorkommen, die bis 0,$0 ;-0,12$ Eisenoxydul enthalten. Aller jetzt in der Atmosphäre enthaltene Sauerstoff würde nur hinreichen eine 191' dicke Schicht cines so Eisenoxydul-reichen Basaltes bei der Verwitterung zu oxydiren. Es lässt sich aber leicht ersehen, dass allmählich zur Oxydirung nun zerstörter und in

1) Silurm. Journ. 1844, XLVII, $105>$ Jahrb. 1846, 111.

$\left.{ }^{2}\right)$ Sollte Diess nicht doch als Durchschnitt viel zu hoch angesetzt seyn? Sollten die in der Tiefe begrabenen Granite eben so viele Kohle etc. enthalten, wie die oberflächlichen? 
Erde verwandelter Gebirgs-Massen viel mehr Sauerstoff verwendet worden ist, als die jetzige Atmosphäre noch enthält, so dass also die Atmosphäre einst um den Betrag des verschwundenen Antheils reicher an Sauerstoff, oder um denselben Betrag reicher an Kohlensåure war als jetzt, aber immer ungefähr ihre jetzige Mischung besass, soferne sie den Abgang fortwährend aus bekann. ten oder unbekannten Quellen wieder ersetzen konnte (Візсноғ). Indessen enthält nach Chevandier das Holy an freiem Wasserstoff 0,0144 seines KohlenstoffGehaltes. Rührte nun dieser Wasserstoff von zersetztem Wasser her, dessen Sauerstoff in die Atmosphäre zurückging, so würde dieser $1 /{ }_{23}$ desjenigen betragen, den die Pflanzen von aufgenommener Kohlensäure wieder ausscheiden. Bekanntlich bilden die Pflanzen fortwährend Kohlensäure durch Entziehung von Kohlenstoff in Sauerstoff um, und erzeugen die Thiere fortwährend Kohlensäure aus Sauerstoff. Bei einem gewissen Verhältniss beider zu einander - vielleicht wie es jetzt ist - gleichen sich beide Prozesse aus und die Luft-Mischung bleibt dieselbe. Hätten aber jene einmal vorgeherrscht, so würde sich der Sauerstoff-Gehalt, - hätten es die Thiere, so würde sich der Kohlensäure-Gehalt der Luft fortdauernd vermehrt haben.

d. Denkt man sich diejenige Kohlensäure, welche jetat die Kalk-Formationen der Erd-Rinde wesentlich zusammensetzen hilft, während der Glühe. Zeit der Erde in Gas-Form von deren festem Kern getrennt, weil alle Kalkerde an Kieselerde zu Silikaten gebunden war (im Librador), so konnte die Vereinigung von Kalkerde und Kohlensäure erst im Verhältniss fortschreitender Abkühlung einerseits und Verwitterung jener Silikate andrerseits eintreten, und anfangs zwar lebhafter als nach der Abkühlung bis zu dem dem Organismen-Leben günstigen Grade, wegen der grössern Menge von Kohlensäure, der höhern Temperatur und des stärkern Druckes. Es hätten sich zwischen der plutonischen Zeit und der organischen Schöpfungs-Zeit mit den krystallinischen Schiefern u. s. w. (da es an Zeit und Wasser nicht gebricht) wohl hinreichende Kalk-Schichten bilden können, un alle jene Kohlensäure aus der Atmosphäre aufzunehmen und dieser mithin schon vor dem Beginne des organischen Lebens eine angemessenere Mischung zu geben. Da aber noch unermessliche Kalk-Formationen nachher entstanden sind, so müssen wir fragen, woher nun die Kalkerde und insbesondere die Kohlensäure für sie gekommen seyen, wenn die letzte nicht noch in der Atmosphäre enthalten gewesen war. Die Masse der sedimentären Kalk.Formationen lässt sich zwar nicht bemessen; aber die Annahme einer $1000^{\prime}$ mächtigen Kalk-Schicht um die ganze Lirde scheint wenigstens nicht übertrieben, und diese würde 3529 Procent Kohlensäure, d. i. den 35 fachen Betrag unsrer ganzen Atmosphäre zu ihrer Sättigung erfordern (Візсноғ). Die Kalkerde könnte aus der noch fortdauernden Verwitterung der Kalkerde-Silikate in den plutonischen Gesteinen kommen [obschon uns die Kalk-Formationen zu der sedimentären Sand- und ThonFormation in einem viel zu grossen Verhältniss zu stehen scheinen, um alle nur von der gleichmässigen Zersetzung der Kalksilikat-haltigen plutonischen Gebirgs-Arten herzuleiten]. Die Kohlensäure wird noch fortdauernd an zahllosen Stellen durch Exhalation aus der Tiefe der Erde zu Tage geführt, und dieser Prozess könnte während der ganzen geologischen Zeit fortgewährt und so allmählich die erforderliche Menge geliefert haben, möglicher Weise sogar ohne je den Kohlensäure-Gehalt der Atmosphäre wesentlich zu ändern. Aber woher jene Exhalationen? Wir können sie nur erklären, wenn wir annehmen, dass in der Tiefe der Erde ältere und jüngere Kalk-Schichten durch Senkungen und dgl. in den Bereich der Glühhitze gerathen und dort gebrannt werden. Es wäre demnach möglich, dass alle jetzt an Kalkerde gebundene Kohlensäure schon vor dem Auftreten der Organismen mit derselben vereinigt und in den frühesten Sediment-Kalkgebirgen abgesetzt gewesen und dann entweder durch den Kreislauf kohlensaurer Wasser in der Erd-Rinde allmählich aufgelöst und in neueren Formationen wieder abgesetzt worden wäre, - oder dass durch Brenuen der ältern Kalk-Schichten im Innern der 
Erde deren Kohlensäure fortwährend befreit worden und mit derjenigen Kalkerde zusammengetreten wäre, welche theils durch Verwittern der Kalk. Silikate frei, theils nach dem Brennen ältrer Kalk-Lagen von atmosphärischen Wassern erreicht und aufgelöst wurde, um so allmählich alle unsre neueren Kalk-Formationen zu gestalten. In beiden Fällen hätte die Atmosphäre bei Beginn des organischen Lebens nicht mehr Kohlensäure zu enthalten brauchen, als nöthig war für die Masse-Bildung der ersten Organismen; aber es ist unwahrscheinlich, dass die Natur stets so genaves Mass gehalten, weil beide Erscheinungen durch von einander unabhängige Ursachen bedingt sind; es ist unwahrscheinlich, dass die plutonischen Gesteine beim Verwittern eine so grosse Quote Kalkerde liefern konnten; es ist unwahrscheinlich, dass dieses Kalk-Brennen unter der Erde in solchem Maasse fort und fort dauern soll, da unsre Quellen doch verhältnissmäsig nur sehr wenig Kalkerde aus grosser Tiefe heraufbringen u. s. w., so dass überall das Gegentheil eben so gut möglich ist. Wollen wir indessen ganz darauf verzichten zu erklären, woher die Aushauchungen des kohlensauren Gases rühren, so können wir sogleich uns damit beruhigen zu sagen, dass dieselben von Anfang an bestehen und den Kalken und übrigen Felsarten, dem organischen Leben u. s. w. jederzeit nur eben so viel Gas geliefert haben, als diese brauchten oder verarbeiteten. Wenn wir indessen Alles das zusammenfassen, so scheint uns doch wohl wahrscheinlich, dass die Kohlensäure-Masse der Atmosphäre früher, vielleicht zcitweise, beträchtlicher als jetzt war und sich allmählich vermindert hat. Sehr viel grösser könnte aber ihre Quote seit begonnenem Leben der Organismen nie mehr gewesen seyn, ohne diese zu ersticken und die ganze Schöpfung periodisch zu vertilgen.

B. Die Atmosphäre konnte also vor Anfang des organischen Lebens absolut reicher gewesen seyn an Stickstoff, Sauerstoff, Kohlensäure und Wasser-Dunst; - zunächst also höher, dichter, schwerer, wärme-leitender, dadurch dem vegetativen Leben günstiger. Unmöglich dürfte es aber in diesem Falle seyn zu berechnen, in welchem Grade jedes dieser Elemente zur Masse-Vermehrung der Atmosphäre und somit zu einer Änderung ihrer Mischung beigetragen habe, wenn schon viele Gründe für die Kohlensäure sprechen. Welches aber auch die anfängliche Verschiedenheit der Átmosphäre gewesen, ihr Übergang zur jetzigen Beschaffenheit war zweifelsohne nur ein allmählicher.

Brongniart setzte den anfänglichen Gehalt der Atmosphäre an Kohlensäure 0,05-0,08; nach Rogers hätte sie vor der Kohlen-Zeit die 6fache Menge des jetzigen besessen; Візсноғ gestattet, ohne sich bestimmt auszusprechen, für die Kohlen-Zeit wenigstens einen $100 \mathrm{fachen}$ Gehalt $=0,06$, nimmt aber daneben für die späteren Perioden, im Verhältniss des stattfindenden Verbrauchs, immer neue Aushauchungen aus der Erde an. Man erkennt, die Möglichkeiten sind zu manchfaltig um sich für ein bestimmtes Maas zu entscheiden, und während Alles zur Annahme einer grossen Menge Kohlensäure hindrängt, so steht wieder die Unmöglichkeit eines manchfaltigen organischen Lebens, wie wir es doch angedeutet finden, in einer daran auch nur mäsig reichen Atmosphäre im Wege, zunal wenı man an die Vögel-Fährten im rothen Sandstein denkt!

C. Wenn aber nun die erwähnten Verschiedenheiten auch noch während des Anfangs des organischen Lebens in so geringem Grade wirklich stattgefunden hätten, um das letzte nicht unmöglich zu machen, welches waren die äussersten dieser möglichen Grade? Und welche Folgen für das Leben dürfen wir von denselben erwarten? Welchen Charakter muss der heutigen gegenüber die damalige Organismen-Welt besessen haben? Und in wiefern entspricht sie diesen 
Auforderungen in einem gegen die Jetztzeit immer mehr abweichenden Grade? Auf alle diese Fragen sind wir noch nicht im Stande eine genügende Antwort zı geben; doch Das dürfen wir behaupten, dass schon in der ersten Periode, wo es Land-Pflanzen, Fische, LandReptilien und ? Vögel gegeben, die Mischung der Luft nicht mehr sehr verschieden gewesen seyn kann von der gegenwärtigen.

a. Wir müssen gestehen, dass wir noch viel zu wenig die Ábweichungen in der Mischung der atmosphärischen Luft kennen, welche die verschiedenen Organismen zu entragen im Stande sind. Die englische Wissenschafts-Gesellschaft hat jetzt anf's Neue eine Reihe von Beobachtungen angeorduet, um wenigstens das Wachsthum verschiedener Pflanzen.Formen in einer Luft mit verscliedenem Kohleusäure-Gehalt auszumittelı '). Man hatte unterstellt, dass die an. fänglich grosse Menge von Kohlensäure in der Atmosphäre das Pflanzen-Leben begünstigt, das Leben höhrer Thiere unmöglich gemacht habe; erst durch den Niederschlag des Koblenstoffs sey der Sauerstoff frei geworden, der die Luft zur Respiration höherer Thiere brauchbar machte (obschon wir vorhin gesehen haben, dass dieser schon vordem reichlicher in der Luft gewesen seyn könne, und obschon der Niederschlag des Kohlenstoffs mit der Entwicklung des höheren Thier-Lebens nicht zusammentrifft). Statt also die Entwicklung der Lebenwelt durch die geologischen Erscheinungen zu erläutern, sieht man sich hierin gleich Anfangs in der Nothwendigkeit für diese dort Hülfe zu suchen.

b. Als Typus einer Gegend mit einer dichten, feuchten und warmen Atmosphäre könnten uns vorzugsweise die Ebenen der Sunda-Inseln dienen im Gegensatze zu luftigen Gebirgs-Höhen. Die Vegetation jener Gegenden gegenüber der der letzten ist die aller-üppigste, manchfaltigste, grossartipste, mit Bäumen und Kräutern, mit Palmen- und Baum-Farnen. Ebenso ist die Fauna reich, manchfaltig, aus allen Klassen des Thier-Reichs zusammengesetzt, mit den grössten Reptilien (Krokodilen, Schlangen), Vögeln und Säugthieren. Der grössere Luft-Druck an sich scheint nicht von Einfluss zu seyn (Gesch. d. Nat. Il, 49 -53), da dieselben Arten van Pflanzen in den 7000' hohen Alpen wie in den lappländischen Ebenen, dieselben Individuen der Thiere in der Ebene wie in $10,000^{\prime}$ Höhe ohne Beschwer fortkommen, der Condur sogar sich bis in 24,000' Höhe erhebt, wo die Anstrengung des Flıges wegen Verdünnung der Luft zunimmt, während der Mensch von 12,000' Höhe über bis zu $1200^{\prime}$ unter dem Meere hinabsteigt und arbeitet. Die älteste Fauna und Flora aber zeigen alle jene Verhältnisse nicht (nur die Baum-Farnen stellen sich bald ein als Freunde eines warm-feuchten, vor Allem aber gleichmäsigen Klimas), zweifelsolune weil ihnen Hindernisse andrer Art in den Weg traten oder andre mächtigere Gesetze ihre Entwicklung leiteten (vgl. \$. 212, G); denn dic Manchfaltigkeit und Grösse der Organisationen vegetabilischer wie animalischer Natur sind nicht der Ausgangs-Punkt, sie sind vielmehr das Ende, das Ziel der Veränderung gewesen, welche die organische Schöpfung von ihrem Anfange bis jetzt durchlaufen hat.

c. Eine Atmosphäre relativ reicher an Sauerstoffgas, wir wissen nicht, was sie bewirken würde? - Eine relativ grössere Menge für die Respiration an sich indifferenten Stickgases, welche mithin zunächst bloss eine Verminderung von Sauerstoffgas bedingte, würde den Respirations-Prozess schwäohen und vorzüglich denjenigen Thieren hinderlich seyn, welche einer sehr intensen Respiration bedürfen, mithin vorzugsweise den Vögeln, Säugthieren, welche in der That während der Steinkohlen-Periode noch gänzlich fehlen. Würde übrigens eine durch Stickgas verdünnte Sanerstoff-Luft durch ihre

1) Jahrb. 1848, 876 . 
grössere Masse komprimirt, so könnte gleichwohl dieselbe Sauersoff-Menge in die Lungen gelangen. Indessen gerade das Leben derselben Thier-Arten in verschiedenen Höhen zeigt, dass sie auch hierin nicht allzu empfindlich sind. Das Erscheinen von grossen Vögeln verschiedener Ordnungen, so weit man aus den Fuss-Spuren schliessen kann, schon bald nach der Kohlen-Formation würde beweisen, dass dann auch die Luft nicht mehr reicher an Stickgas war, als jetzt? Stickgas wird nicht unmittelbar von den Thieren, sondern in geringer Menge nur vou den Pflanzen assimilirt und geht mit diesen als Nahrung in die Thiere über; dic ältesten Pflanzen-Formen (Farnen, Lycopodiaceen u. dgl.) scheinen aber keineswegs sehr Stickstoff-reich zu seyn, noch aus einer Stickstoff-reicheren Luft mehr Vortheil ziehen zu können.

d. Aber in einer reichlichern Kohlensäu re sollen nur die Pflanzen besser gedeihen und zwar auch sie nur bei einem Betrage bis von 0,08 in der Sonne, während ihnen im Schatten 0,01 besser zusage; neulich in England angestellte Versuche haben noch keinen Ausschlag in dieser Frage gegeben. Thieren ist eine grössere Menge - von welcher Quantität? - verdenblich und endlich tödtlich, zweifelsohne wieder den lebhaft respirirendeu Lungen-Thieren mehr, als den kaltblütigen Lungen- und Kiemen-Thieren.

e. Eine reichlichere Feuchtigkeit endlich in einer warmen Luft ist allen vegetativen Entwicklungen günstig, wie sie in kalter Luft durch Unterdrücken der Ausdünstung und Schwächung des Somnen-Lichtes durch Nebel-Bildung hinderlich werden würde.

Zu einer Zeit, wo R. Owen die Vogel-Fährten in den alten Formationen Nordamerika's als solche anzuerkennen noch Bedenken trug, äusserte er sich in Bezug auf diesen Gegenstand in folgender Weise ${ }^{\text {3) }}$ : die Reptilien unterscheiden sich von den Vögeln und Säugthieren hauptsächlich durch die geringere Thätigkeit der Respiration, durch die tiefere und einfachere Struktur der Lunge und des Herzens, wodurch sie in ihrer Existenz so zu sagen weniger abhängig vom Samerstoff-Gehalte der Luft wenden. Wenn daher die Geologen und B.otaniker Grund haben zu glauben, dass die Atmosphäre pinst reicher gewesen sey an Kohlensäure, so möchte dann der Anatom a priori schliessen, dass die höchsten Thier-Klassen, welche zum Einathmen eines solehen Mediums geschickt gewesen, nur kaltblütige Fische und Reptilien waren. Auch dürften die Landbewohnenden Reptilien vermöge der geringeren Energie ihrer Muskel-Kontraktionen und noch mehr vermöge der grössern lrritabilität und Fähigkeit fortgesetzter Thätigkeit' der Muskel-Fasern die höchsten Organismen-Arten seyn, welche fähig waren unter einem höheren als dem jetzigen Luft-Drucke zu existiren. In einem solchen gewissermassen dem Wasser sich nähernden Medium mochte wohl auch am ehesten ein kaltblütiges Thier sich in schwerfülligem Fluge über die Oberflärhe erheben können, indem damals eine geringere Miuskel-Thätigkeit hiezu erfordlich war. Sobald aber die Mischung der Atmosphäre sich verbesserte und die Respiration zu verstärken geschickter wurde, und zugleich ihre Dichte und ihr Druck abnahm, so wurde sie auch dem Bau der Vögel angemessener, bei deren Erscheinen [?] in den Wealden die Dinosaurier vorwalteten, bei welchen der Bau des Thoraxes auf eine eben so vollständige Cirkulation schliessen lässt, als sie bei den Krokodilen während ihres Aufenthaltes am Lande stattfindet. (Alle Reptilien nämlich, deren Rippen am vordern Theile des Toraxes durch Knopf und Höcker an Centrum und Neurapophyse der Wirbel angelenkt sind, haben ein Herz mit 2 getrennten Kammern und 2 Vorkammern. Die aneinanderliegenden Aorten, welche aus den 2 Kammern entspringen, kommuniziren durch eine so gestellte Öffuung mit einander, dass diese durch die Sigmoid-Klappen geschlossen wird, wenu das Blut gleichzeitig durch beide ausströmt. Wenn aber das Krokodil längere Zeit unter Wasser verweilt, so erhält die Aorte der linken Kammer durch die oben erwähnte Kommunikation venöses Blut aus den überladenen Höh-

1) Br. Collect. I, 57 . 
len der rechten Seite des Herzens; wenn dagegen auf dem Lande die Athmung in vollster Kraft ist, so geht ein unverdünnter Strom arteriellen Blutes durch die linke Aorte zu Kopf und Vorder-Extremitäten. Da nun die Dinosaurier dieselbe Struktur des Thoraxes besitzen und sie durch ihre Füsse u. s. w. noch mehr zum Aufenthalte auf dem Trocknen angewiesen erscheinen, so hatten sie wahrscheinlich ein vierkammeriges Herz und eine fast so vollständige Zirkulation, als die warmblütigen Thiere. - Endlich möchte man vorauszusagen versucht seyn, dass die ersten Spuren der warmblütigen ThierKlassen, wenn sie im Zeitalter der Reptilien (der verdichteten Atmosphäre) aufträten, in Form solcher kleinen Säugthiere erscheinen mussten, welche jetzt die schwächste Respiration besitzen, wie sich Solches anch an den MarsupialenResten von Stonesfield (m) bestätigt hat.

D. Wir kënnen jedoch diesen Gegenstand nicht ver'assen, ohne nochmals auf die Grösse der gleich anfänglich in die Erd-Schichten niedergelegten Kohlenstoff-Masse aufmerksam zu machen und die ungeheure Länge der Zeit in Betracht zu ziehen, welche zu deren Bildung allein nothwendig gewesen ist, mithin auch für die sonstigen Vorgänge im allmählichen Ausgleichungs-Prozesse einen hinreichenden Spielraum liess. (Bischof.)

Eine Berechnung dieser Zeit wird nur möglich unter den Voraussetzungen, dass 1) die Thätigkeit der Vegetation in Aneignung von Kohlenstoff aus der Atmosphäre, auf dieselbe Quadrat-Fläche berechnet, währeud der SteinkohlenFormation eben so lebhaft als jetzt gewesen seye; 2) dass die Steinkohlen-Lager sich über dieselben Flächen verbreiten, aut welchen einst die Steinkohlen-Pflanzen gewachsen sind; 3 ) dass von diesen Pflanzen nicht mehr verloren gegangen, als der Umwandlungs-Prozess derselben in Kohle nothwendig erheischt (sicher hat indessen auch auf andern Flächen noch eine Vegetation stattgefunden und ist ein grosser Theil der Masse der Einschliessung im Boden ganz entgangen). Nun nimmt a) Liebig ${ }^{1}$ ) an, dass ein mit Pflanzen bewachsener Quadratfuss Land jährlich $1 / 40$ Pfund Kohlenstoff aus der Luft anziehe zu Erzeugung von Pflanzen-Masse; wo dann zu Hervorbringung der S. 859 bezeichneten Steinkohlen-Lager nöthig seyn würde cin Zeitraum von 1,004,177 .Jahren. b) Chevandier berichtet ${ }^{2}$ ), dass ein Wald binnen 100 Jahren so viel Holz erzeuge, als nöthig wäre, seinen Boden, wenn dieses Holz erst in Kohle verwandelt würde, mit einer 7" Par. dicken Kohlen-Lage zu bedecken; darnach wären zur Bildung der Suarbrückener Kohlen-Lager erforderlich 672,788 Jahre.

§. 18. Nach Abkühlung der Erde und Unterscheidung der Klimate.

A. Mag die Erde einmal feurig-flüssig gewesen seyn, oder, wie Andre wollen, allezeit starr in einer heisseren Region des Weltraums, als wo sie sich jetzt bewegt, sich erhitzt haben (Gesch. d. Natur I, 72-73) : alle Physiker und Astronomen behaupten wie die meisten Geologen, dass ihre Rinde eiumal viel heisser als jetzt gewesen und durch sekuläre Abkühlung allmählich bis zu ihrer jetzigen Temperatur gelangt sey. Bevor diese Temperatur unter die des siedenden Wassers herabgekommen, war eine Bevölkerung derselben unmöglich.

1) Agrikult. Chemie 14.

2) Compt. rendus 1844, > ERDM. und $\mathrm{M}_{\mathrm{ARCH}}$. Journ. 1841, XXXI, $441 \mathrm{ff}$. $>$ Jahrb. 1844, 843. 
Wie weit sie etwa schon darunter gelangt gewesen und ob sie nicht sogar schon ilıre jetzige Temperatur besessen, als diese Bevölkerung begann, Das wissen wir nicht, da in der historischen Zeit eine noch messbare Abnahme nicht mehr stattfindet.

B. Wenı nun das Mögliche wirklich stattgefunden und bei Beginn des organischen Lebens die Erd-Rinde noch eine höhere Temperatur besessen hätte, so wäre durch diesen gleichen Zuschuss von Wärme auf der ganzen Erd-Oberfläche und zu allen Jahres-Zeiten der Temperatur-Unterschied zwischen Äquator und Pol, zwischen Sommer und Winter kleiner gewesen als jetzt; das Klima der Erde war dann im Ganzen ein heisseres, von der Sonne weniger abhängiges, in allen Zonen gleicheres, im Sommer und Winter, bei Tag und Nacht gleichmässigeres. Das Gesammt-Klima ist dann allmählich kühler, seine Zonen, die Jahres- und Tages-Zeiten sind ungleicher, die Polar-Gegenden unbewohnbarer geworden in Progressionen, deren Beziehungen zu den verschiedenen spätern Schöpfungs-Perioden und Erd-Formationen uns nicht bekannt sind. - Doch hat man bis zu den Alluvial-Schichten Spuren von Gletschern, Schliff-Flächen, Rund-Höcker, Schrammen u. dgl. irgend welche andre Auzeigen einer anhaltenden Eis-Temperatur in den Fels-Schichten wenigstens noch nicht gefunden. Wir würden aber doch immer nicht bestimmen können, wie weit unter $100^{\circ} \mathrm{C}$. das Maximum der Temperatur stund, als das Leben begann; wir würden jedoch, wenn dieselbe noch beträchtlich hoch war, unterstellen müssen, dass das Leben nächst den Polen angefangen habe und allmählich gegen den Äquator vorgerückt sey.

a. Setzen wir in runden Zahlen die jetzige Temperatur des Äquators $=28^{\circ} \mathrm{C}$, die in einiger Entfernung von den Polen $=1^{\circ} \mathrm{C}$. (im Sommer höher, im Winter tiefer) und nehmen beispielsweise an, die Erde habe bei Beginn des organischen Lebens noch einen Überschuss von $50^{\circ}$ besessen, so würde (abgesehen von einer kleinen Differenz, welche auch dieser Überschuss schon an Polen und Äquator zeigen müsste) die Temperatur

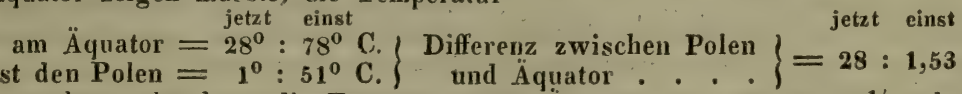

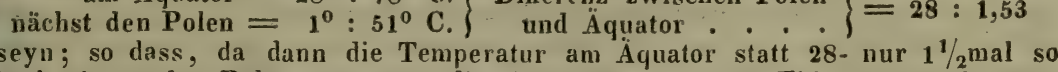
hoch als an den Polen gewesen, dieselben Pflanzen- und Thier-Arten oder wenigstens Sipprn, welchen eine solche Temperatur-Höhe einmal zusagte, fast in allen Breiten vorkommen konnten. Natürlich musste aber ein Land, dessen Temperatur auf $50^{\circ}$ heruntergesunken war, während die des andern noch auf $78^{\circ}$ stand, schon früher oder für eine grössere Anzahl von Wesen bewohnbar seyn, als dieses.

b. Der Mingel an Gletscher-Spuren aus älterer als der Alluvial-Zeit, ein freilich nur negatives Kriterium, welches jeden Tag durch die Erfahrung beseitigt werden kaun, würde andeuten, dass noch in der Tertiär-Zeit die Polar-Gegenden nicht so unbewohubar kalt gewesen seyn können als jetat; doch kennen wir leider, wie es scheint, keine festen tertiären Bildungen in den Polar-Zonen, welche uns dort zwischen ihren S hichten jene Merkmale nur aufbewahrt haben köunten. In wärmer lienenden Gletscher-Gebirgen kommen zwar mancherlei Tertiär-Sihichten vor, aber bis heute überall ohne Spuren von Gletschern in früherer Zeit. 
C. Hätte also das organische Leben wirklich mit jener höheren Temperatur begonnen, so würden wir müglicher Weise die folgenden vier Abstufungen in der geographischen Verbreitung der Organismen, oder jedenfalls die 3 letzen derselben durch ihre organischen Reste angedeutet finden müssen :

1) die Polar-Gegenden bewohnt durch eine tropische Bevölkerung, die Tropen noch unbewohnt;

2) die ganze Erd-Oberfläche bewohnt durch eine Bevölkerung entsprechend der unsrer wärmern Zonen;

3) ebenso, die Bevölkerung jedoch örtlich abweichender nach Mass. gabe unterscheidbarer geographischer Zonen.

4) Die Polar-Zonen fast unbewohut; die beiden andern mit Bewohnern der gemässigten und heissen Zonen und nach diesen Zonen getrennt (die jetzige Vertheilung);

was zu untersuchen und zu bestätigen sowohl durch die Vorfrage, wie die Bewohner kalter, gemässigter und heisser Klimate in allen Klassen des Pflanzen- und Thier-Reiches zu erkeunen seyen, als durch unsre noch völlige Unbekanntschaft mit den organischen Resten verschiedener Erd-Perioden auf ausgedehnten Strecken und selbst ganzen Zonen der Erd-Oberfläche schwierig wird.

a. Wir übergehen hier gänzlich die Unterstellung, dass die Zonen zwar schon früher existirt, aber eine andere Lage als jetzt gehabt haben, weil die Astronomen Diess für unmöglich erklären und wir keine geologischen Anzeigen dafür finden. Auch Gri.Pins Versuch ${ }^{1}$ ) ist nicht gelungen. Wir werden noch später darauf zurïckkommen (S. 871).

b. Die umständliche Nachweisung über die geographische Vertheilung der Organismen in den verschiedenen Perioden der Erde gehört in pinen spätern Abschnitt, welcher jedenfalls auch das vollstïndigere Material für die jetzige Frage liefern wird. Hier handelt es sich nur um die Nachweisbarkeit des glcichen oder ungleichen klimatischen Charakters der jederzeitigen Bewohner der verschiedenen Zonen.

c. Allerdings gibt es eine grosse Anzahl von Geschlechtern wie von Familien (Gesch. d. Nat. II, 247-251), deren lebenden Arten sämmtlich nur einer Zone oder nur benachbarten Theilen zweier unsıer Zonen angehören; aber es gibt auch nicht seltene Ausnahmen und Beispiele des Gregentheils selbst in guten natürlichen Sippen, so dass auf bloss verwandte Arten ein strenger Beweis übereinstimmenden Klimas nicht gegründet werden kann. Wir haben aber noch weiter manche Beispiele aufgeführt, wie sogar gewisse identische Arten in ganz verschiedenem Klima wohnen können (a. a. O. S. 248-249), obwohl diese nur mehr und weniger seltene Ausnahmen bilden.

d. Was ferner unsre Bekanntschaft mit den Organismen der verschiedenen Zonen in verschiedenen Zeiten betrifft, so wird solche durch die beschränkte Verbreitung der verschiedenen Formationen selbst während ganzer Perioden der Erd-Bildung oder doch durch unsre beschränkte Kenntniss derselben, wie durch deren ungleichen Petrefakten-Reichthum sehr gehemmt. So sind Gesteine der I., 1V. und V. Periode zwar in den verschiedensten Welt-Gegenden bekannt; die Trias (II) aber ausser in einem kleinen Theile Europus nur in einer ebenfalls sehr beschränkten Gegend Sibiriens; - die Oolithe (III) durch ganz Europa,

1) Jahrb. 1845, 243. 
n Sibirien und dem wärmeren Festlande Asiens, ausserdem wie es scheint nur auf einer kleinen Stelle Central-Amerikas, welche zugleich in der südlichen Halbkngel der einzige Vertreter der beiden Perioden II und III wäre. Die nördiche kalte Zone repräsentirt nur spärlich die J. und V., die nördliche gemäsigte alle Perioden, die heisse endlich die $I$. (jrdoch 1 . a-c nur in CentralAmerika) und die III. spärlich (auf einem kleinen Fleck ebendaselbst, kaum noch n Indien?), die IV. und V. reichlich; - die südliche gemässigte nur die I., IV. in Afrika und Amerika) und V.; aus der südlich kalten Zone sind gar keine reptunischen Formationen bekannt. Die beiden kalten Zonen haben also nur lutonische und vulkanische Gesteine und ausser wenigen neptunischen Niederchlägen der I. Periude aus der Kohlen- und Permischen ? Zeit gegen den nörd. ichen Pol hin nur einige am Ende der Tertiär.Zeit gehobene Küsten. - Wenn ach ausserdem das eine oder das andere Gestein noch an einer Stelle vorrommt, so vermögen wir es wenigstens nicht aus seinen Petrefakten zu eriennen.

D. Waren in der frühesten Zeit die Polar-Gegenden mit tropishen Bewohnern versehen, die Tropen-Länder aber unbewohnt, wie Diess dem als zuerst möglich unterstellten Temperatur-Zustande der Erde entsprechen würde? Wir kennen ältere und jüngere Silur-, sowie Devon - ? Gesteine in den von D'ORBIGny besuchteu Gegenden des tropischen Amerikas mit charakteristischen Versteinerungen (in ersten Asaphus Boliviensis, Calymene Verneuili, Orthis Humboldti und mehre Lingula-Arten; in letzten Orthis Inca, O. laticostata, Spirifer Boliviensis und Sp. Quichua, Terebratula Antisiensis und T. Peruviana, welche zum Theil anch in andere Gegenden und jüngere Formationen ibergehen, wie Spirifer speciosus). Der Bergkalk aber mit seinen nanchfaltigen Thier- und die Steinkohlen-Formation mit ihren zahleichen Pflanzen-Arten sind in den Tropen-Gegenden Amerikas wie Asiens und seit Kurzem wohl auch der Sunda-Inseln und Afrikas? be. kanut. Dieselben Formationen aber mit ihren charakteristischen Versteinerungen reichen auch, wie wir sogleich näher zeigen werden, bis in die Polar-Gegenden hinein. Die Erd-Oberfläche ist also von Anfang an überall gleichzeitig bewohnbar gewesen; nichts deutet in der Entwicklung der Organismen eine örtlich so hoch gesteigerte Hitze an, welche die Gegend überhaupt unbewohnbar gemacht hätte.

E. Eine bejahende Antwort erhalten wir auf die zweite Frage, ob i m A nf ange der organischen Schöpfung die ganze Erd-Oberfläche eine gleichartige Bevölkerung besessen habe, aus der sich auf eirfe überall gleiche und zwar höhere, tropische, Temperatur schliessen lasse. Eine solche scheint anfangs und wenigstens bis in die Kreide-Periode wirklich bestanden zu lıaben, um erst später nach Massgabe der Zonen sich verschiedenartiger zu gestalten.

a. Alle Gesteine der I. Periode, welche eine so allgemeine Verbreitung von Spitabergen und der Bärrn.Insel an bis nach Neuseeland besitzen, enthalten bekanntlich allerwärts dieselben organischen Formen, und auf weite Strecken hin sogar zahlreiche identische Arten; allein ehe man daraus sogleich auf eine Übereinstimmung des polaren mit dem tropischen Klima scbliesst, muss man sich 
erinnern, dass damals überhaupt die Fauna und zumal die Flora, gegen die jetzige genommen, noch sehr einförmig und die klimatischen Verschiedenheiten des Charakters derselben schon hiedurch in engere Grenzen eingeschlossen waren. Von der andern Seite kann man geltend machen, sowohl dass diese Ein. förmigkeit an sich noch kein über-tropisches Klima beweise, wie dass noch jetzt die Faunen und Floren selbst der Tropen-Zone verschiedener Gegenden wie Amerikas, Afrikas, der Sunda-Inseln und der Inseln des stillen Oreans, bei allem Formen-Reichthume doch immer nur wenige Arten miteinander gemein haben. Die Hälfte der Thier- und Pflanzen-Sippen der I. Periode sind ausgestorben, wodurch sich die Zahl der zu Schlüssen aus der Analogie über das einstige Klima geeigneten Gruppen des Systems wesentlich vermindert. Hienach dürfte es schwer seyn, schon jetzt zu verlässigen Beweis-Mitteln zu gelangen, und wir müssen uns fast darauf beschränken, die Total-Eindrücke der periodischen Bevölkerung in verschiedenen Gegenden miteinander zu vergleichen.

b. (Gleichartigkeit.) Die Fauna und Flora der silurischen und devonischen Zeit, die wir freilich nur aus gemässigten und heissen Gegenden kennen, hat bis jetzt noch keine Art auffallender örtlicher Verschiedenheit dargeboten, mithin auch keine, die von Verschiedenheit des Klimas und der Zonen hergeleitet werden könnte. D'Orbigny sagt von Süd-Amerika, dass die SilurFormation auf Gneiss ruhe und in den unteren 3 Viertheilen keine Versteine. rungen enthalte, im oberen Viertheile aber in einer unermesslichen Ausdehnung Lingula, Calymene und Asaphus in den Europäischen wahestehenden oder selbst identischen Formen beherberge. Die devonischen Sand-Niederschläge bieten Terebratula-, Spirifer- und Orthis-Arten dar, so dass die Schichten ein den Europäischen analoges Ansehen gewinnen 1).

Weit besser übrigens ist uns die organische Welt der Kohlen-Zeit (d, •) aus den verschiedensten $Z$ onen bekannt; sie allein winkt uns aus dem PolarKreise zu. Wir kennen sie von Melville-Island und der West- und Ost-Küste Grönlands im $75^{\circ}-72^{\circ} \mathrm{N}$. Br. durch ganz Nord-und Süd-Amerika bis wieder gegen den $52^{\circ}$ S. B.: von Spitabergen und der Bären-Insel ${ }^{2}$ ) in $78^{\circ}$ und $74^{\circ}$ N. Br. durch ganz Europa und streckenweise am östlichen Theile Afrikas bis Port-Natal hinunter; und ebenso durch Kontinental-Asien (Indien) uiber die Sundu-Inseln bis Neuholland, Vaxdamiensland und Neuseeland in $44^{\circ} \mathrm{S}$. Br. Überall findet man darin dieselben Familien, dieselben Sippen und oft auf weite Strecken hin dieselben Arten von Pflanzen und Thieren wieder. Hat Europa als der am besten bekənnte Weltheil auch eine Anzahl beson. derer Sippen und selbst Familien vor andern voraus, die aber durchaus ohne klimatischen Charakter sind und vorerst auf keine Zonen hinweisen, so werden später einige eigenthümliche Formen auch in andern Gegenden nicht ausbleiben. Was die identischen Arten anbelangt, so ist es zwar richtig, dass sie sich weniger von Norden nach Süden als von Osten nach Westen, also in der Richtung unsrer jetzigen Zonen verfolgen lassen und dass sie selbst aus der nördlichen in die südliche gemässigte Zone übergehen, ohne sich in der heissen darzu. bieten; wovon aber die Ursache wohl zum Theil darin lipgt, dass wir nur erst sebr spärliche organische Reste aus der Kehlen-Formation der heissen Zone kennen, indem fast alle aus den 2 gemässigten abstammen, zum Theile aber immer in einer kleineren Temperatur-Verschiedenheit liegen kann. Wir stellen das Ergebnis der Zählung in Enumerator in folgender Tabelle zusammen:

1) Jahrb, 1848, 866 .

$\left.{ }^{2}\right)$ L. v. Buch, die Bären-Insel $>$ Jahrb. 1847, 506. 


IIIIIIIIIIIIII|-x

- nördliche kalte und gemäasigte Zone.

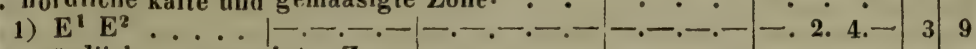

nördliche gemässigte Zone. $\mathrm{E}^{2} \mathrm{M}^{2}$ - .

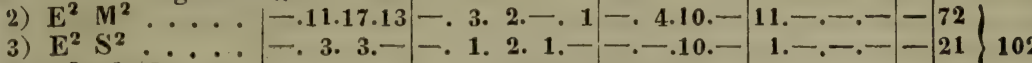

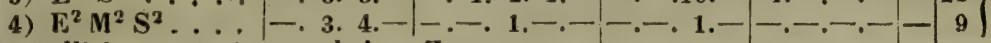

nördliche gemässigte $u$. heisse Zone.

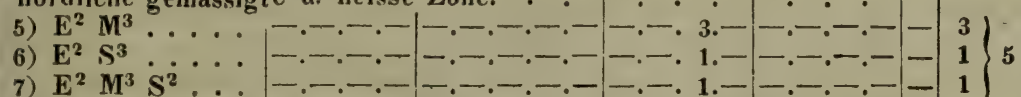

1. nördliche $u$. südliche gemåssigte $Z_{0}$ en.

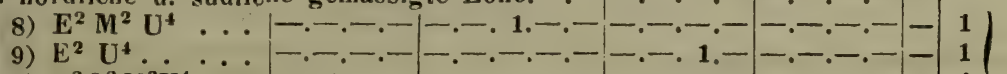

10) $\mathrm{E}^{2} \mathrm{~S}^{2} \mathrm{M}^{2} \mathrm{U}^{4} \cdots-1-1-1-1-1-1-10$

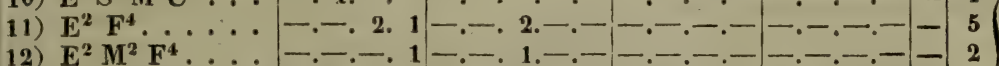

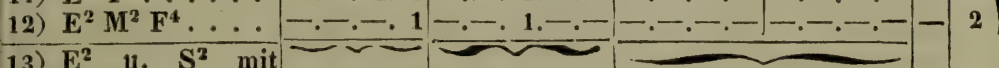

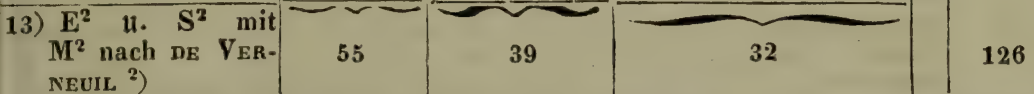

Woraus sich. also ergibt, dass die alten Formationen der I. Periode - die zahlreichsten Virwandtschuften zwischen den Ländern der nördlihen gemäsigten Zone (102), insbesondere zwischen Europa ( $\left.\mathrm{E}^{2}\right)$ und NordImerika $\left(\mathrm{M}^{2}\right)$ wie Asien $\left(\mathrm{S}^{2}\right)$, - dann zwischen diesen und denen der üdlichen Hemisphäre (10) wie Süd-Afrika $\left(\mathrm{F}^{*}\right)$ und Australien ( ${ }^{*}$ ) stattinden, während die nördliche gemäsigte mit der nördlichen kalten nur 9 und hit der heissen nur 5 gemeinsame Arten zählt, wovon wir die Ursache vorin angegeben haben. Nach Göppert's, Bunbury's und DE Verneull's neuesten Tergleichungen (s. die letzte Zeile der Tabelle) sind die Zahlen allein der zwichen Nord-Amerika und Europa mit West-Asien gemeinsamen Thier-Arten och merk!ich zahlreicher, als wir sie aus dem Enumerator entnommen haben. $V$ ir wollen einen Theil der gemeinsamen Organismen aus der Kohlen-Formaion näher ins Auge fassen, welche in verschiedene Zonen und Floren überrehen. Die Steinkohlen-Formation Grönlands und Spitabergens bietet Farnen on Arten, die auch in Grossbritanien vorkommen. Unter den Thier-Resten Spitabergens hat man Productus giganteus bis von 2 "Grösse, Pr. striatus, P. plicatilis, Pr. punctatus, Calamopora polymorpha und Fenestella antiqua rkannt, welche sämmtlich auch auf der Bären-Insel und dann bis herab in die Mitte Europa's vorkommen, von wo ein Theil derselben mit wieder andern Arten der Kohlen-Formation nach andern Welt-Gegenden übergeht, nämlich Productus punctatus nach dem nördlichen, Pr. Humboldti und Pr. Gaudryi ach dem tropischen Ameriku, Pr. semireticulatus nach beiden, Pr. membranaceus nach Nord-Amerika und ? Süd-Afrika, Pr. pustulosus nach Australien. In den Kohlen-führenden Schichten Süd-Amerika's sind die Productus-Arten noch zahlreicher als in Europa (D'OrbigNy). In Gross-Britannien kennt man 300 Pflanzen-Arten in der Kohlen-Formation, worunter 140 Farnen, von welchen 30 auch in andern Europäischen und in Nord-Amerikanischen Ländern vorkommen. Darunter befinden sich 11 zwischen Europa und Nord-Amerika

1) Jahrb. 1846, 505. $-{ }^{2}$ ) Jahrb. 1848, 98-102. 
gemeinsame Kohlen-Pflanzen; allein obschon diese Zahl aus dem von GöpPERT entworfenen Theile des Enumerators entnommen ist, scheint sie noch zu klein zu seyn, du derselbe schon 1839 deren 18 namentlieh aufgezählt hatte ').

Unter 16 aus dem Kohlen-Revir von Tuscaloosa in Alabama $\left(33^{\circ} \mathrm{N}\right.$. Br.) von Lyecr. mitgebrachten fossilen Pflanzen-Arten hat Buneury 9 Arten (also über 0,50) elkannt, die jenes Revier allein mit England u. a. europäischen Ländern gemein bat, welche $15^{\circ}-25^{\circ}$ weiter nördlich liegen; diese Übereinstimmung aber ist grösser, als sie jetzt zwischen irgend 2 Läıdern der Erde besteht, die unter gleichen Meridianen eben so weit aus Süden nach Nor. den auseinanderliegen ${ }^{2}$ ). Durch diese Nachträge sehen wir uns veranlasst, eine vollständigere Liste der zwischen Nord-Amerika und Europa gemeinsanen Kohlen-Pflanzen zu entwerfen, als unser Enumerator gewährt.

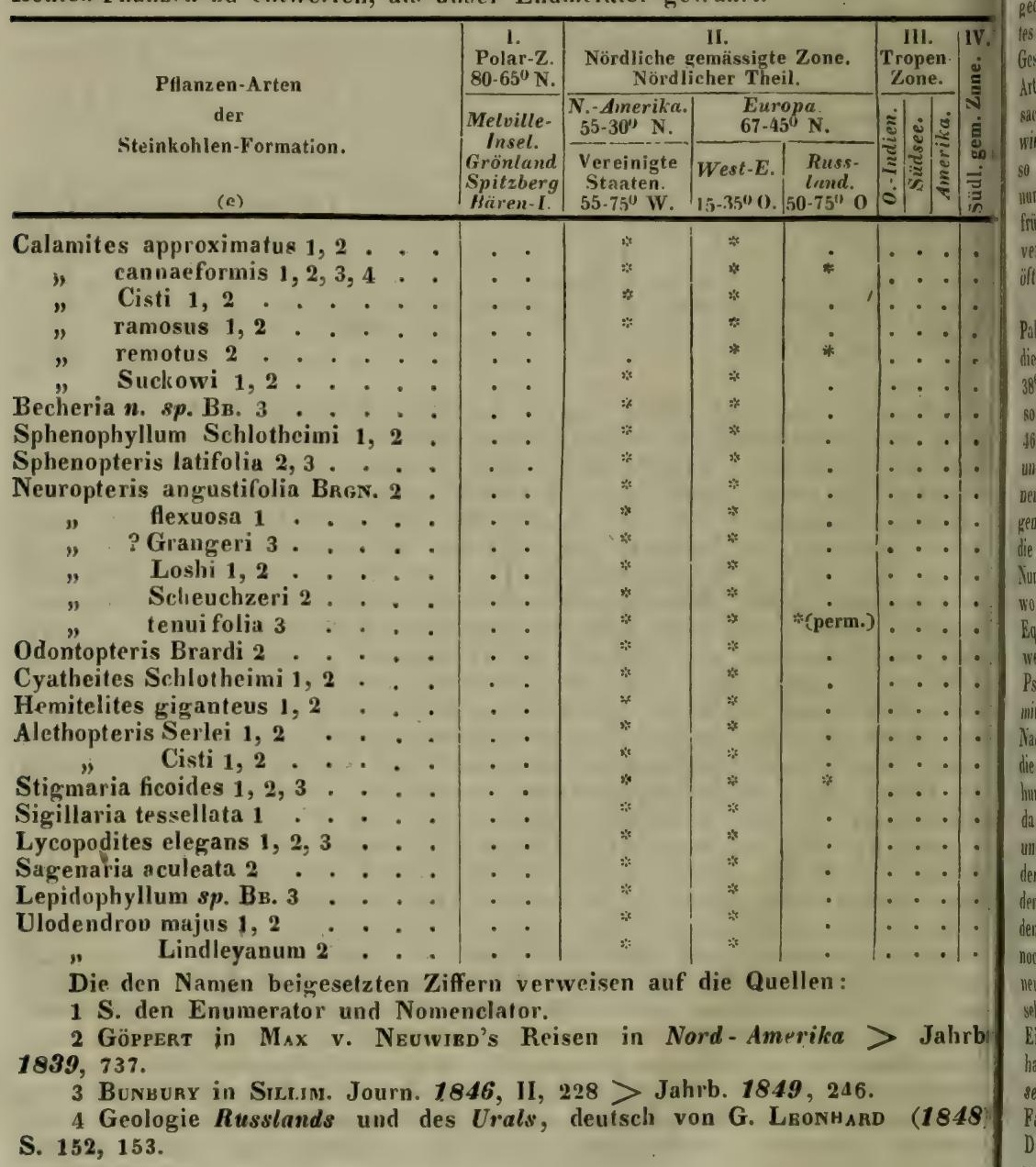

1) Jahrb. $1839,737$.

2) Sillim. Journ. 18t6, b, II, 228-233 > Jahrb. 1849, 246. 
Welches aber inmer der klimatische Charakter wäre, der durch diese thierischen und pflanzlichen Organismen angedeutet wird, so ist es doch unmöglich alle diese alten geologischen Ablagerungen $(\mathbf{a}-\mathbf{c})$ mit ihren gleichartigen organischen Resten in irgend einer die Mitte der Erde in beliebiger Richtung umgürtenden Zone zu vereinigen; denn sie sind in allen Richtungen über die ErdOberfläche verbreitet; es ist also unmöglich sie mit GILPIN als Beweise einer ehemals andern Lage der Tropen-Zone anzuführen ').

c. (K limatischer Charakter.) Da während der I. geologischen Periode die meisten unsrer jetzigen organisehen Formen-Gruppen zum Theil in Folge früher erörterter Ursachen noch fehlten und dagegen die meisten der in der Kohlen-Zeit vorhanden gewesenen Formen jetzt ausgestorben sind, und da eine Menge der jetzt lebenden Geschlechter und selbst Familien eine so ausgedehnte klimatische Verbreitung besitzen, dass sie im Ganzen für cin bestimmtes Klima nichts beweisen (da selbst dann, wenn alle bekannten Arten eines Geschlechtes z. B. der Tropen-Zone angehören, die nächste der dazu entdeckten Arten der gemäsigten Zone zufallen kann), da endlich auch noch andre Ursachen auf die Reihen-Folge der organischen Wesen verschiedener Zeiten einwirkten, deren gesetzlichen Wirkungen ebenfalls zum Theil problematisch sind, so sind wir mit Schlüssen aus der Analogie äusserst beschränkt, so dass wir nur mehr aus dem Gesammt-Charakter der Bevölkerung auf das Klima einer früheren Zeit schliessen können. Wir werden übrigens bei Beurtheilung der verschiedenen Gruppen des besseren Zusammenhanges wegen das Verhalten öfters sogleich in der ganzen geologischen Zeit verfolgen.

(Pflanzen.) Schon in der Geschichte der Natur II, 250 haben wir die Palmen, wovon die äussersten der lebenden Arten nur bis $38^{\circ} \mathrm{Br}$. reichen, die Cycadeen, deren Vorposten an der Süd-Küste Spaniens ebenfalls in $37^{\circ}-$ $38^{\circ} \mathrm{Br}$. stehen, die Piperaceen, Musaceen, Scitamineen (wovon einige eben so weit verpflanzt worden sind), die baumartigen Farnen, welche $23^{\circ} \mathrm{N}$. und $46^{\circ} \mathrm{S}$. nicht überschreiten, die baumartigen Gräser, die grössern Equisetaceen und Lykopodiaceen, dic Mimosen, Cacteen und Melastomen unter die bezeichnendsten Pflanzen-Familien der Tropen-Gegenden gezählt. Von den zuletztgenannten Dikotyledonen-Familien nüssen wir indessen hier absehen, indem die böheren Dikotyledonen zur Kohlen-Zeit überhaupt noch nicht existirt haben. Nun sind aber die vorherrschenden Pflanzen-Formen der Steinkohlen überall, wo man sie findet, und zum Theil noch der Buntsandstein-Formation: grosse Equisetaceen, Farnen (zum Theil baumartig) und Lycopodiaceen, zwischen welchen sich die ganz fossilen Familien der Asterophylliten wie der riesigen Psaronieen, Stigmarieen und Sigillarieen als nächste Verwandte einschalten, mit einem geringeren Verhältnisse von Palmen, Cycadeen, Cannaceen (dis Nachbarn der Musaceen) und einigen Koniferen. Es sind Diess also, bis auf die letzten, genau die Familien, welche oben bezeichnet worden waren; beziehungsweise mit demselben weiteren Charakter der ansehnlichen Grösse, und da in den fossilen Pflanzen-Familien sogar da Baum-Arten vorkommen, wo unsere Tropen-Zone kaum einige Fuss hohe Formen aufweisen kann, so ist der Charakter der Kohlen-Flora nicht allein ein wesentlich tropischer, sondern man würde aus dem fast ausschliesslichen Herrschen dieser bezeichnenden Formen und aus ihrer noch ansehnlicheren Grösse sogar auf ein auch noch heisseres Klima zu schliessen versucht seyn. - Indessen liefern die Farnen weitaus die Mehrzahl der Arten, und doch ist nur ein geringer Theil derselben erweislich baumartig (Protopteris, Caulopteris, Cottaia, Karstenia). Ein solches Vorherrschen der Farnen gross und klein über die andere Flora hat der Botaniker НоокеR jedoch auch ausserhalb der Tropen z. B. auf Neuseeland beobachtet, wo er auf einer nur wenige Acres grossen Fläche 36 Farnen-Arten sammelte, welche dieser Fläche, die ausserdem noch kaum ein Dutzend Kräuter und Bäume enthielt, ein üppiges Aussehen verliehen. Er

1) Jahrb. 1845, 243. 
knüpft an diese Thatsache die ferneren Beobachtungen, 1) dass, wo in ge. mäsigten Klimaten die Farnen und vorzüglich Pecopteris-Arien als Repräselltanten unserer Pteris durch Individuen $\mathrm{Zahl}$ über andere Pflanzen vorwalten, ganze Familien dieser letzten kümmern und fehlen; - 2) dass eine üppige Vegetation vieler Farnen-Arten üher viele Grade der Länge und Breite ausgedehnt ein einförmiges (nicht übermäsig heisses) Klima und eine arme Blüthen Flora andeure, während manche auf den ersten Blick steril scheinende Flächen ohne Farnen in denselben Welt Gegenden eine manchfaltige BlumenFlora besitzen. Als Zeichen eines gemäsigten, feurhten und in allen Jahreszeiten fast gleichartigen Klima's (wie es die westindischen und Südsce-Inseln selbst südwärts von den Tropen haben), sagt Hoorer weiter, habe man, nach der Analogie mit den lebenden, die Faruen der Kohlen-Periode schon lange betrachtet, aber nicht als Beweise einer armen Flora, die sich auch in der Kohlen-Periode offenbar darstelle. Gleichförmiger müsste das Klima der KohlenZeit schon in Folge höherer Temperatur gewesen seyn: an Feuchtigkeit würde es ihm zu einer Zeit nicht gefehlt haben, wo zusammenhängende trorkene Kontinente fohlten; wir haben aber keinen Beweis, dass ein gleichförmiges und feuchtes Klima den lebenden Farnen nicht zusage, wenn es zugleich heiss ist. НоокеR selbst gesteht ein, dass die parenchymatöse Struktur der charakteristischen Pflanzen-Fanilien der Kohlen-Zeit nicht dazu gemacht sey, strenge Winter, wie die auf Melville-Island, Grönland, Bären-Insel und Spiţbergen ') zu ertragen; es müsse daher das Klima dort einst viel milder - das kann in so hoher Breite doch wohl nur "wärmer" bedeuten - gewesen seyn; gälte Diess aber auch für die anderen Örtlichkeiten der Kohlen-Formation in der nördlichen wie in der südlichen Halbkugel, so muss das Klima auf der ganzen Erd-Oberfläche und mithin wohl auch zwischen den Wendekrcisen selbst wärmer gewesen seyn. - Diese charakteristischen Pflanzen-Formen der I. Periode vermindern sich später immer mehr an absoluter wie an verglichener Anzahl; jedoch nehmen die Cycadeen und Palmen mit den Coniferen in der 1I. und III. Periode zu und sind bis in die Mitte der Tertiär-Zeit wenn auch nicht mehr zahlreicher als jetzt, doch bis über die Mitte von Europa herauf weit über die jetzigen Grenzen verbreitet, obschon die übrige Vegetation Mittel-Europa's dann höchstens nur noch der jetzt um einige Grade südlicheren entspricht.

(Pflanzen-Thiere.) Schlüsse aus den klimatischen Beziehungen einzelner Geschlechter lebender Pflanzen-Thiere reichen nicht zurück bis zur I. Periode des organischen Lebens, obschon viele unter ihnen und selbst manche kleine Familien sich theils auf die Tropen-Gegenden beschränken, theils nur his in die Mitte der gemäsigten Zonen hinaufreichen und an der Grenze der kalten Zone die Pfanzen-Thiere überhaupt selten werden. Aber bei den PolypenThieren ist die ganze Abtheilung der Lithophyten, welche hauptsächlich aus den mit Polypen-Stöcken versehenen Geschlechtern der Ordnung der Anthozoen besteht, welchen cinst die S. 146-147 des Enumerators aufgeführten Genera der Alveoliten, Favositen, Chaeteten, Calamoporen u. a. geholfen haben, in Massen zusammengehäuft als Inseln und Riffe, an eine Mecres-Temperatur zwischen $28^{\circ}-23^{\circ}$ gebunden und nur vorübergehend im Winter bis $18^{\circ} \mathrm{C}$. zu ertragen fähig, die sie in ihrer gewöhulicheu Station zwischen 10 und 20 Faden Tiefe finden ${ }^{2}$ ), daher sie nordwärts im rothen Meere und südwärts im stillen Ozean den $28^{\circ}-30^{\circ} \mathrm{Br}$. nicht überschreiten, ja sich an der West-Küste Süd-Amerika's der von Süden kommenden kalten Meeres-Strömungen wegen bis in die Tropen zurückziehen. Fast nur einige Caryophylliden reichen in Gesellschaft der Alcyonien sowohl weiter bis an die Polar-Kreise

1) Wo Calamiten, Sigillarien und Lepidodendren namentlich zitirt werden. (Jahrb. 1847, 507.)

${ }^{2}$ ) Covthouy in Jahrb. 18.19, 767; DANs struclure and classification of $\approx 00$ phytes (Philad. 1816, $4^{\circ}$ ) p. 101-103. 
hinan als tiefer in den Ozean hinab. Manche Arten der Caryophylliden wie Madreporiden kommen als vereinzelte Polypen-Stöcke noch im Mittelmeere vor; und in den Schottischen Meeren sind, der verhältnissmäsig zur gengraphischen Breite milden Temperatur ungearhtet die Stern-Korallen so selten, dass man bis jetzt nur 4 Arten, wovon 2 in pinzigen Exemplaren, gefunden hat, Fleming '). Nun finden wir aber in der Silur- und in der DevonFurmation bis $70^{\circ} \mathrm{N}$. $\mathrm{Br}$. bei Ingloolik in Nord-Amerika wie vielleicht im Ural, weniger wierler in Zechstein, mehr im Sanct-Cassianer Gebirge, sehr reichlich im Unteroolith und noch mehr im Coral-rag, dann wied,r im Grünsand und in der weissen Kreide (terrain danien) solthe Korallen-Riffe bis nach Schweden und England hinauf in ansehnlicher Entwirklung. Zwar scheinen dif Cyathophyllen-, Syringop ren - und Calamoporen-Formen (wie ausserdem die Graptoiithen) keiner unsrer jetzt lebenden Anthozoen-Familien genau zu entsprechen und daher einer strengen Analogie sich nicht zu fügen: sie könnten daher ehen sowohl eisem kühleren als einem no.h heisseren Klima angehören; aber die Mäaudrinen, die Asträen, die Madreporen, von welchen allen sich einzelne Repräsentanten auch schon in der I Periode einstellen, sind wenigstens im Coral-rag (zu Kirkdale in England in $54^{\circ} \mathrm{Br}$.), in der Kreide, ja selbst, wie es scheint, in den ältesten Tertiär-Gesteinen dip herrschenden Formen und sprechen daher dafür, dass nicht nur in diesen Zeiten, sondern noch mehr in der vorhergegangenen devonischen $u$. s. w. ein tropisches oder subtropisches Klima bis nach Schweden und England geherrscht habe. Ja noch in der mittel-tertiären Formation sind die 32 Anthozoen unter 207 Polyparien des Wiener Beckens nach Reuss ${ }^{2}$ ), die 82 Anthozoen unter 103 Polyparien Ober-Italiens nach Міснеготті ${ }^{3}$ ) und zwar die letzten mit zahlreichen Asträen, Mäandrinen, Monticularien, Madreporen durchmengt, wie sie eben noch die neuesten Korallen-Gebäude zusammensetzen, vollkommen geeignet, nach strenger Analogie ein subtropisches Klima bis in die mitteln Breiten von Europa zu erweisen. Das an lebenden Polyparien so reiche rothe Meer hat nur 120 im Ganzen geliefert (S. 791). - Die Polypen unterstützen also wesentlich die Theorie einer einst liöheren Temperatur, und es würden wahrscheinlich die fossilen Stelleriden ebenfalls damit übere̊instimmen, wenn die noch jetzt lebenden Formen zahlreich genug wären, um eine Zone, irgend pin Klima auf breiterer Grundlage verlässig vertreten zu können. Die Polypen sprechen für ein tropisches Klima in dem Wiener-Becken noch während der Miocän-Zeit, entgegen ELIE DE BEaumonts Ansicht, welcher schon in der EocänZeit für das $\mathbf{P}$ ariser-Becken nicht mehr daran glauben will, weil eben die fels. bauenden Korallen dort fehlten ${ }^{4}$ ).

(Weichthiere.) Dic Malacozoen der frühesten Zeiten geben keinen Ausschlag weder für noch gegen die Annahme eines wärmeren Klima's, weil einestheils sich ein klimatischer Gegensatz auch in den jetzigen Weichthier-Familien nur wenig ausspricht und anderntheils diejenigen Gruppen, auf welche als Reprïsentanten wärmerer Meere man sich berufen möchte, nämlich die siphonobranchen Ctenobranchier noch nicht existirt haben. Aber ihr Erscheinen in plötzlich vorwaltender Anzahl in den ältesten Tertiär-Schichten zumal des Pariser - und Londoner-Beckens deutet für jene Zeit-Frist sicher eine höhere Temperatur an, als dieselbe Gegend jetzt besitzt, und Desнayes hat gezeigt, dass die miocänen Konchylien von Bordeaux u. s. w., unter welchen fast zuerst noch lebende Arten auftreten, zum Theil ihre lebenden Identischen an der Westküste Afrika's bis in die Breite von Senegumbien, also in der Gegend des Wendekreises besitzen, ohne eine Einmischung nordischer Formen zu erfahren. - Vielleicht wird man einwenden wollen, dass das Fehlen der Siphonobranchier in den ältesten Schichten eben in Folge eines damals nie-

1) Im Jahrb. 1848, 864. - 2) Jahrb. 1848, 757.

3) Jahrb. 1848, 502. - ${ }^{4}$ ) Jahrb. 1837, 63. 
drigeren Klima's stattfinde. Diess wäre an und für sich möglich, obschon es nit dem bei den Polypen u. s. w. erlangten Resultate im Widerspruch steht: anderntheils haben wir aher gefunden (S. 825), dass ihr anfängliches Fehlen dem aufsteigenden Entwicklungs-Gange entspricht, daher nur nach Be. seitigung der Gesetzlichkeit dieser Erscheinung in jenem vorigen Sinne ausgebeutet werden dürfte. - Anders würde es sich verhalten mit den vierkiemigen Cephalopoden, welche in den frühesten Entwicklungs.Fristen der Organismen - jenem aufsteigenden Entwicklungs-Gange anscheinend zuwider - so häufig gewesen sind; aber ihre jetzt lebenden Repräsentanten, anf $1-2 \mathrm{Nau}$ tilus-Arten beschränkt, sind zu wenig zahlreich, um auf ilır Vorkommen in tropischen Meeren eine feste Analogie für jene reiche Menge untergegangener Formen zu gründen. - Von D'OrbigNy erfahren wir in Bezug auf die jetzige Fauna, dass in beiden Welt-Meeren, welche Süd-Ameriku begrenzen, $95 \mathrm{Ge}$ nera vorkommen, von welchen wegen der grossen orographischen Verschiedenheit der östlichen und westlichen Küste unter gleicher Breite doch nur 45 beiden Küsten gemein, 50 je einer von ihnen eigenthümlich sind, - dass beide Küsten nur 1 Art gemein haben, - dass im atlantischen Orean 12 Arten sich über 19, in Stillen Meere der aus dem Süden kommenden kalten Strö. mungen wegen sogar 15 Arten über 22 Breite-Grade in verschiedenen Zonen ausdehnen ').

(Ke rbthiere.) Die älteste Entomozoen-Welt kennen wir viel zu unvollständig, un aus ihren Resten auf die Beschaffenheit des einstigen Klima's zu schliessen. Nur die Entomostraca unter den Krustazeen würden vielleicht mit ihren Paläaden eine Ausnahme machen, wenn dergleichen noch lebend existirten, um uns Aufschluss zu geben über das ihnen nöthige Klima. Die Pöcilopoden sind zwar in früheren Zeiten fast eben so zahlreich als jetzt vertreten, wo sie in heissen und genäsigten Meeres-Gegenden vorkommen; immerhin aber ist ihre lebende Zahl zu gering, um einen sichern Aufschluss zu gewähren.

(Reptilien.) Reptilien können die niedre Temperatur und den NahrungsMangel unsrer europäischen Winter weder überstehen noch wie die Vägel ihnen durch Wanderung entgehen; sie verfallen daher in der Erde und die Batrachier unter Wasser versenkt in Winterschlaf und sind so in Stande in jmmer abnehmender Grösse und Anzahl der Arten wie der Individuen sich bis in die Breite von Schotlland und Irland (Batrachier) in etwa $55^{\circ} \mathrm{N}$. als äusserste? Grenze hinaufzuzichen. Die Schildkröten bleiben zwar schon weiter südlich zurück, scheinen aber doch in geschichtlicher Zeit noch bis Schonen gereicht zu haben. Aber die Scinke gehören noch gemäsigteren und die grossen Eidechsen-Arten den Tropen-Ländern an; die grossen Krokodile reichen nur in Amerika bis zum $33^{\circ} \mathrm{N}$. Breite (früher mögen sie in Ägypten fast eben su weit gereicht haben), verfallen aber auch hier schon mehre Monate hindurch in Winterschlaf, aus dem man sie bei tiefer Temperatur nicht wecken kann (Gesch. d. Natur, II, 269). Diese ganze Thier-Klasse ist daher jetzt mehr als irgend eine andre an warme und heisse Klimate gebunden; dort erlangt sie die ansehnlichste Grösse; dort allein sicht man die grösseren dieser Thiere (Schildkröten, Krokodile, Echsen, Schlangen) in einer Lebens-Frisehe, von der man sich nach den lebend zu uns gebrachten Exemplaren auch in der wärmsten Jahreszeit keine Vorstellung machen kann. Diese Klasse aber hat, mit Krokodilen und Echsen in der Kohlen-Zeit beginnend, in der Trias-, Lias-, Oolith- und Kreide-Zeit ihre höchste Entwicklung in Manchfaltigkeit eigenthümlicher Organisationen und Mächtigkeit der Individuen erreicht; es ist ummöglich zu glauben, dass diese zum Theil bis über $70-80^{\prime}$ langen Kolosse, die einst über ganz Europa bis in den Ural verbreitet gewesen, sollten einem so rauhen Klima augehört haben, als jetzt

1) Jahrb. 1845,373 . 
dieser Welttheil besityt, wenn man auch den Wasser-Bewohnern unter ihnen gestatten wollte, während des Winters sich auf den Grund zu versenken oder mit ihren mächtigen Rudern wärmeren Gegenden zuzuziehen.

(Sä ugthiere.) Unter den Säugthieren hat man Arten solcher Geschlechter oder Familien, welche heutzutage lediglich auf die Tropen-Länder beschränkt sind, als Eeweise des ehemals heisseren Klima's in denjenigen Ländern gemäsigter Zonen angeführt, wo jetzt ihre Reste gefunden werden, nachdem man einmal von der Ansicht abgegangen war, dass ungeheure Meeres-Ströme ganze Faunen cines Landes bis nach fremden Klimaten fortgeführt und dort niedergelegt haben konnten (Elephanten, Nashorne, Flusspferde, Giraffen, Kameele u. s. w.); die zufällige Entdeckung eines Elephas primigenius noch mit Haut und Haaren zeigte indess, dass manche dieser Thiere ehemals besser als ihre heutigen Geschlechts-Genossen zu Ertragung einer strengen Kälte ausgerüstet seyn konnten, ohne Solclies im Skelett-Bau erkennen zu lassen, wenn man auch zugeben muss, dass die in Eis-Meere begrabenen Elephanten sich auf den jetzt angrenzenden Küsten-Strecken zu nähren nicht im Stande seyn würden und daher jedenfalls durch Flüsse von den sibirischen Gebirgs-Gegenden herab nach dem Meere geführt worden seyn müssen. (Murcuison ${ }^{1}$ ), MidDENDORFF.) Aber doch ist unter den Säugthieren der Hippopotamus, wie es scheint, nicht verträglich mit einer Temperatur, wobei die Flüsse und See'n sich im Winter dick mit Eis belegen; er hat einst über Italien bis England heraufgereicht.

d. (Jüngere Perioden.) Somit haben sich, wenn auch nicht durch alle Pflanzen- und Thier-Klassen hindurch, doch gerade bei denjenigen, wo eine strengere Beurtheilung aus der Analogie an ehesten zulässig ist, überall Gründe ergeben für die Annahme eines ehemals wärmeren Klima's nicht in der ersten Periode allein; sondern auch - zum Theile mit abnehmendem Gewichte in der II., III., IV. und bis in die IVitte der V. Periode. Alle angeführten Belege sprechen dafür, dass noch in der Eocän- und Miocän-Zeit bei uns ein wärmeres, ein subtropisches oder doch zulet»t noch dem mittelmeerischen ähnliches Klima geherrscht habe. Es ist uns wohl bekannt, und wir werden auf der folgenden Seite so wie auch in dem geographischen Theile dieser Arbeit darauf zurückkommen, dass man in den Insekten der Oolithen-Periode, der Bernstein- und Molasse-Zeit, in den mittel-tertiären Pflanzen und einigen andren Organismen-Gruppen der europäischen Gebirgs-Schichten einen solchen subtropischen Charakter nicht hat finden können und höchstens zu Andeutungen eines nur unbedeutend wärmeren Klima's, als das jetzige der gleichen Gegenden ist, gelangen konnte; aber theils beruhen gerade diese Schlüsse auf verhältnissmäsig viel weniger bekannten Gruppen fossiler Organismen, theils könnten sie höchstens die vorhin aufgestellten Schlüsse in etwas schwächen, zumal direkt widersprechende, d. h. ein kälteres Klima als das jetzige andeutende Formen bisher überall nirht gefunden worden sind; wenigstens nicht vor der Pliocän- und zumal Diluvial-Zeit, die uns hier nicht beschättigt. Denn zu den pliocänen Säugthieren wärmerer Klinate gesellen sich allerdings überall viele noch jetzt in der Gegend einheimische Arten von Mammiferen, wie von Muscheln u. s. w; sie machen in der Diluvial-Zeit über die Hälfte der Arten aus.

F. Auch inder zweiten bis vierten Periode deutet noch Alles, wie wir gesehen haben auf ein noch fortdauernd wärmeres und, wie wir aus andern Andeutungen ersehen werden, gleichförmigeres Klima über die ganze Erd-Oberfläche hin. Nur aus den Polar-Zonen haben wir kein Beweis-Mittel; und nur in der Kreide (IV. Periode) sollen Spuren einer zonenweisen Sonderung bereits auftreten?

1) Jahrb, 1848, 597 . 
a. Petrefakten-führende Gebirgs-Glieder der Trias-Periode sind bis jetzt sicher nur in Europa und muthmasslich im angrenzenden Sibirien bekaunt. D'Onbigny zitirt jedoch noch bunte Thone und Sandsteine der Trias ohne bezeichnende Versteinerungen in Süd-Amerika ${ }^{1}$ ).

b. Oolithe kennen wir nur in Europa von Frankreich bis Moskau und jenseits dem Ural, im Himalaya und benachbarten Gegenden Mittel-Asiens ausserhalb des Wendekreises, den sie im Cutch Ostindiens nur berïhren, so dass die klimatische Verschiedenheit dieser Gegenden sowohl als die Menge der organischen Reste selbst zu klein ist, um mit ihrer Hülfe klimatische Zonen Verschiedenheiten zu verfolgen. Was man aus den asiatischen Jura-Gebilden kennt, stinsmt vielmehr theils den Arten nach, die mitunter sehr charakteristische sind, mit den europäischen Ergebnissen uberein, theils ist es wenigstens nicht in der Weise verschieden. um ein anderes, insbesondere heisseres Klima andenten zu können. Im Himalaya werden der identischen Arten viele angeführt; im Cutch an der See-Küste finden wir, abgesehen von den zweifelhaften Arten, nur die puropäische Trigonia costata und Ostrea Marshi genannt ${ }^{2}$ ), wenn anders diese Bestinimungen richtig und nicht die sämmtlichen sekundären Gebilde des Cutch der Kreide-Periode zczuzählen sind?

Einige neue Blicke jedoch eröfnet uns die Insekten-Welt in die damalige Verbreitung der Thiere, die wir uns nicht verbergen dürfen. Was durch BroDIE und WESTwood ${ }^{3}$ ) aus dem englischen Lias bekannt geworden, entspricht nicht sowohl heissen als gemäsigten Klimaten, aber mehr des jetyigen NordAmerika's, als Europa's. - Die Insekten-Sippen Solenhofens sind nach GERMAF nur geringentheils ausgestorbene; es sind in Ganzen keine tropischen Formen, doch weisen sie theils selbst (Picania, Belostomum), theils durch Grösse der Arten (Locusta, Nepa, Pygolampis) auf ein wärmeres Klima hin, etwa wie in Süd-Europa oder Nord-Afrika, einige spezieller auf Amerika (Belostomum, Ricania, zum Theil Pygolampis) ${ }^{4}$ ), überhaupt jedenfalls auf ein wärmeres Klima, als die in Bernstein und Braunkohle ${ }^{5}$ ). - Was man durch Brodue von Wealden-Insekten kennt, fällt durch Kleinheit auf, die nicht zu Gunsten eines heissen Klima's spricht; und wenn darunter Ricania und einige Flïgel fïr ein wärmeres obgleich nicht nothwendig tropisches Klima zeugen, so finden sich dabei wieder Reste von Aphis vor, welche in den Tropen durch grössere Formen ersetzt werden würden. Indessen bleibt unsere Vorstellung von der Insekten.Welt dieser Periode nur sehr unvollkommen.

Was endlich an Jura-Erzeugnissen (III) aus dem tropischen Amerika bekannt geworden, ist nur unbedeutend und theils noch zweifelhaft, D'OrBigny ${ }^{6}$ ).

c. Dass in der Kreide-Periode noch eine höhere Temperatur als jetzt wenigstens in der jetzigen gemäsigten Zone geherrscht, haben wir auf den vorhergehenden Seiten erschlossen aus den Resten der Anthozoen wie der Reptilien, unter welchen Gavial-artige Formen und Dinosaurier in Amerika noch bis $41^{\circ}$, in Europa bis $52^{\circ} \mathrm{N}$. Br. (England) hinaufreichen ${ }^{7}$ ). - Dafür aber, dass die Bewohner der Erd-Oberfläche damals schon zonenweise nach dem geographischen Klima verschieden waren, haben wir (insofern wir die Prüfung sämmtlicher Nummuliten-Gestaine cinem spätren Absatze vorbehalten) noch

1) Jahrb. 1843,866 .

2) Jahrb. 1841, 803. $-{ }^{3}$ ) Jahrb. 1846, 381.

4) Belostomum hat unter 12 lebenden Arten 10 Amerikanische und 2 Afrikanische; Ricania unter 25 Arten 20 Süd-Amerikanische und 5 Ost-Indische, Afrikanische und Neu-Holländische; Pygolampis 2 Brasilische und 2 kleinere Deutsche Arten.

5) Brows Collectun. 125.

6) Jahrb. $1843,866$.

7) Jahrb. 1844, 222; 1847, 122, 381. 
keine sicheren Beweise, wenn wir auch einige Andeutungen nicht übersehen wollen ${ }^{1}$ ).

Die Kreide-Formation herrscht in Spanien, Portugal, vielen Gegenden Frankreichs, Englands, Schwedens, Dänemarks, Belgiens, Deutschlands, Böhmens, der Karpathen Siebenbürgens, der Alpen, der Adriatischen, Sirilischen und Türkischen Länder, des Archipels, Russlands, Mittel-Asiens bis Indien, ? Ägyptens, Süd-Afrika's, Nord- und Süd-Amerika's bis zur Maghellans-Enge; das Neocomien-Meer allein hat von $52^{\circ} \mathrm{N}$. Br. bis zu $54^{\circ} \mathrm{S}$. Br. gereicht. Wir würden also hoffen dürfen, die Spuren einer Zonen-weisen Vertheilung der KreideBevölkerung zu entdecken, wenn sie bestanden hätte.

Dic Quadersandstein-Flora Schlesiens enthält nach Göppert Akotyledonen, Koniferen, Dikotyledonen, Palmen (Flabellaria) und Baum-Farnen (Protopteris), welche zwei letzten für ein vom jetzigen ganz verschiedenes, tropisches Klima sprechen ${ }^{2}$ ). Cords stellt aus 47 ihm bekannten Pflanzen-Arten der KreidePeriode folgende Berechnung über das Klima jener Zeit an ${ }^{3}$ ):

Arten: erfordern jetzt eine

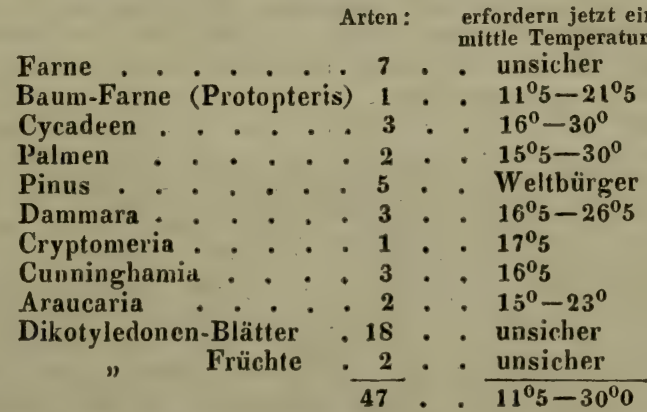

oder als Mittel aus den 15 näher bestimmten $19^{\circ}-20^{\circ}$ [richtiger $20^{\circ} 5$ ], wozu nun noch kommt, dass auch die Dikotyledonen-Blätter durch ihren allgemeinen Habitus und ihren lederartigen Bau an tropische und subtropische Formen, insbesondere Laurineen, Proteaceen, Piperaceen, Styraceen und Melastomaceen, nicht aber an die Blatt-Formen der gemässigten Zone erinnern. Die Pflanzen. Formen der böhmischen Kreide deuten eine Strand-Flora an, wie sie am ähnlichsten jetzt am stillen Orean zwischen $40^{\circ} \mathrm{N}$. und $45^{\circ} \mathrm{S}$. Br. vorkommt.

Hinsichtlich der Foraminiferen hat die Pariser-Kreide, nach $\mathbf{D}^{\prime} \boldsymbol{n}_{\mathrm{RBgNY}}$, die grŏsste Ähnlichkeit mit der jetzigen Fauna des Adriatischen Meeres; nur hier ist wie dort die grosse Menge der Stichostegier und die grosse Zahl von Buliminen-Arten; hier allein kommen noch lebende Frondicularien vor, die in der weissen Kreide so manchfaltig sind; hier endlich finden sich auch die 2 einzigen Arten, die sich noch lebend erhalten haben ${ }^{4}$ ).

Wir theilen ferner eine Zusammenstellung der Zählungen über die Verbreitung der Thier-Reste dieser Periode in Enumerator nebst einigen späteren aus mehren Arheiten von Fopres entuommenen Zusätzen ${ }^{5}$ ) zu derselben mit, wonach sich folgende Übersicht ergibt:

1) D'Orbigny setzt den Anfang klimatischer Verschiedenheiten bei der Bevölkerung der Erd-Oberfäche an den Anfang der oberen Kreide (Jahıb. 1843, 868), ohne indessen eine Parallele mit unsern jetzigen Klimaten zu ziehen.

2) Jahrb. 1842, 252. - 3) Jahrb. 1847, 120.

4) Jahirb. 1842, 369.

5) Jahrb. 1848, 756;1849, 116-118. 


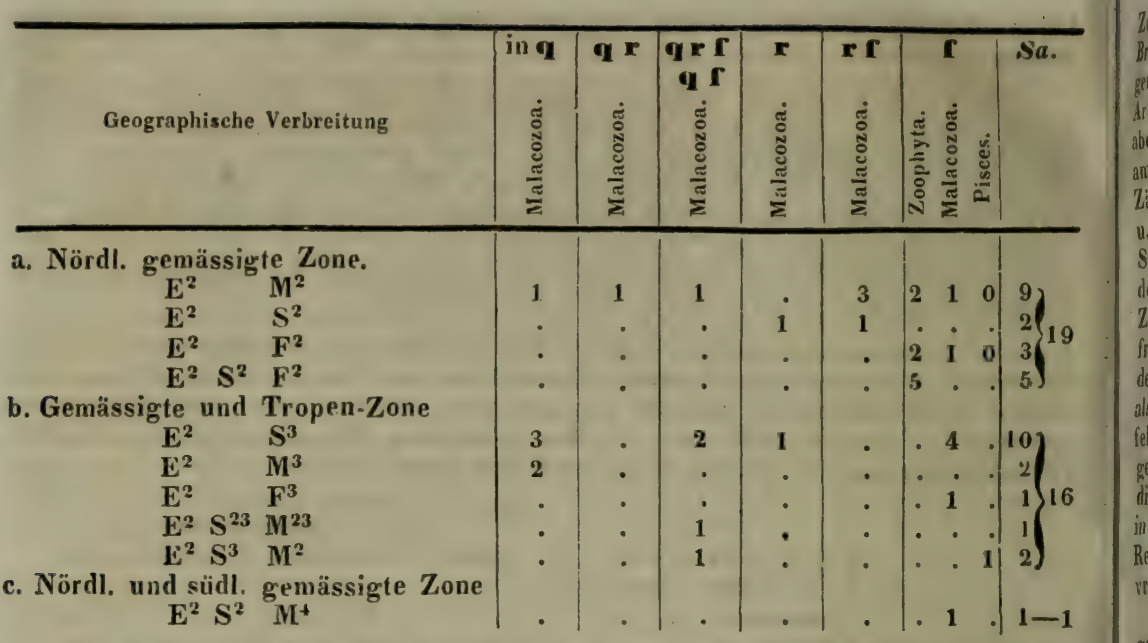

Wir finden also hier (im Vergleich zur ersten Periode S. 869) eine grössere Anzahl von Arten, welche der nördlichen gemässigten mit der tropischen Zone gemein sind, und weniger solche, die sich auch in der südlichen gemässigten wiederholen, weil die Gesteine der Kreide-Periode uns dort in grössrer Ausdehnung bekannt geworden sind, als hier. Die zwischen Europa und dem tropischen Asien gemeinsamen Arten sind hier desshalb sogar zahlreicher, als jene zwischen Europa und dem gemässigten Asien oder Amerika, obschon es zum Theil dieselben sind und die Nord-Amerikanische Kreide uns im Ganzen schon mehr Arten geliefert hat, als die Ostindische. Ausser diesen gemeinsamen Arten gibt es aber in allen verschiedenen Örtlichkeiten noch eine Menge stellvertretender, diesen oder jenen Europäischen Species sehr ähnlicher Arten, die man oft leicht mit ihnen verwech-eln könnte, und welche hier strenge geschieden worden sind. Wenn main nun berücksichtigt, dass dirjenigen KonchylienArten, durch welche das gemässigte Europa mit dem tropischen indien in $13^{0}$ $\mathrm{S}$. Br. übereinstimmt, 12 unter 165, die Fische 1 unter 14 aus letzter Gegend bekannten Arien betiagen; - ja wenn nach D'OrBignx's Versicherung ${ }^{1}$ ) im tropischen Chili und Kolumbien 0,50 der neoromischen Fossilien-Arten mit denen des Pariser Beckens nahe verwandt und 0,20 identi-ch sind - was weit, weit über die in der jetzigen Schöpfung möglichen Verhältnisse ist; wenn selbst das Neocomien der Maghellans-Strasse noch eiue Verwandtschaft zeigt mit dem des Miltelmeerischen beckens ${ }^{2}$ ); wenn man sich endlich der geographischen Verbreitung des Lyriodon aliformis in $\mathbf{E}^{2} \mathrm{~S}^{23} \mathrm{M}^{23}$ erinnert, welche wohl kein jetzt lebendes Mollusk besitzt, und dass ihm mehre andre Arten der Kreide in dieser Hinsicht nahestehen, so ist es unmöglich zu glauben, dass während der KreidePeriode derselbe Unterschied des Klima's zwischen Europa und Ostindien oder dem tropischen Süd-Amerika stattgefunden habe, wie jetzt. In fast norh grösserer geographischer Ausdehnung und in jedenfalls schlagenderen Verhältnissen, als es selbst in der crsten Periode geschehen, scheint es, habe der Beweis eines überall gleichartigen und nicht durch klimatische und andre geographische Einflüsse örtlich wechselnden Organismen-Lebens uns nochmals dargeboten werden sollen, ehe vielleicht selbst noch am Ende der Kreide-Zeit, jedenfalls aber in der Tertiär.

1) Ann. sc. nat. b, XIX, $266>$ Jahrb. 1843, 866 .

${ }^{2}$ ) Forbes rechnet dieselben Arten dem Gault, v. Buch der Kreide zu. 
Zeit solches in seinem vollen Form-Reichthume und seiner nach Längen- und Breiten-Ausdehung abwechselnden Manchfaltigkeit auftretend zu unsren jetzigen Verhältnissen sich anschickt. Zwar besitzt Europa einige Genera und viele Arten vor andren Weltgegenden voraus, wcil es am genauesten bekannt ist, aber die charakteristischen Formen sind allerwärts die nämlichen selbst noch am Ende der Periode; Ostindien bietet uns sehr ähnliche Arten von HaiZähnen, von Ammoniten (28 Arten), von Hamites, Baculites, Ptychoceras u. s. w. dar, wie Europa; nur sind in Folge der weiten Entfernung dieselben Schichten nicht mehr wie in Europa zu erkennen und liegt, was hier grtrennt, dort und in süd-Amerika öfters in einer Schicht vereinigt beisammen; von Zonen keine Spur. Nur dadurch bekommt die Indische Kreide-Fauna ein befremdendes, mehr tertiäres Ansehen, dass sie eine grössere Anzahl Arten aus den Siphonobranchier-Geschlechtern Voluta, Oliva, Cypraea und Murex enthält, als die Europäische Kreide, wo solche indessen, ausser Oliva, auch nicht ganz fehlen. Die Fauna scheint sich hierdurch schon in der Kreide-Zeit der jetzigen Fauna Indiens zu nähern und Forbes nimmt desshalb an, man erkeune in diesen fossilen Resten, dass die genannien Genera ihre Verbreitungs-Centra in Indien besessen und von dort her gegen Europa vorgerückt seyen. [Als Regel würden wir eher eine Ausbreitung gegen den Äquator erwarten müssen, vrgl. S. 867, C.]

d. D'Orbigny hat nicht nur in Amerika eine Theilung der bis dahin allgemein gleichförmigen Fauna in verschiedene Lokal-Faunen erst seit der oberen Kreide (r) angenommen ${ }^{1}$ ), sondern auch das ganze Französische KreideGebilde (IV) in 4 Becken geordnet, die in allen gleichzeitigen Alters-Abstufungen eine gewisse Anzahl von Arten mit einander gemein und andre eigenthümlich haben. Selbst in einem und demselben ehemaligen wie jetzigen Becken würde man in einem Theile, an pinem Rande eine gewisse Zahl von Arten finden, die in den andern nicht vorkommen; wie viel mehr also in verschiedenen Becken, deren jedes entweder abgeschlossen ist von dem andern oder mit andern grösseren Meeren im Zusammenhang ist. Eine klimatische Verschiedenheit, eine $\mathbf{Z}$ onen-artige Vertheilung geht daraus noch nicht hervor. D'OrbigNy hat zwar von 5 Rudisten-Zonen gesprochen, diese jedorh in chronologischem, nicht geographischem Sinne verstanden; sie kommen in verschiedenen Höhen, vom Neocomien bis ins Terrain danien $\left(\mathbf{r}^{3}\right)$ vor $\left.{ }^{2}\right)$. Doch haben mehre Schriftsteller geglaubt auch in letztem Sinne eine Zonen-weise Verbreitung der Rudisten zu erkennen, so dass jene chronologischen Zonen in genau gleichen Formationen nach gewissen geographischen Parallel - Linien verbreitet wären und in den andern fehlten. In der That scheint man aber ausser cinem Hippuriten in südlichsten Theile Nord-Amerika's $\left(31^{0}\right)^{3}$ ) und einem in Chili (H. chilensis*) ausserhalb Algerien, Marokko und Europa noch überhaupt keine Rudisten gefunden zu haben und das Vorkommen aller Rudisten in Europa sich mit mancher Unterbrechung auf einen Strich zu beschrän. ken, worin Lissabon, die Pyrenäen, Marseille, Salsburg, die Ostseite des Adriatischen Meeres und vielleicht noch Cretı liegen, und der eine Breite von $25^{\circ}$ hätte. In der Englischen, Belgischen, Helgoländischen, Sächsischen, Böhmischen und Russischen? Kreide hat man nur einzelne seltene Exemplare von Rudisten entdeckt. D'Archic hat schon seit $1839^{5}$ ) drei von NO. nach SW. ziehende Zonen der Kreide unterschieden, welche vielleicht den Isothermen früherer Zeit entsprächen. Die nördlichste derselben ginge von Schweden und

1) Jahrb. 1843,866 .

2) Jahrb. 1842, 749;1845, 381; ausführlicher in Ann. sc. nat. 1842, XVII, 173-192.

3) Jahrb. 1844, 223.

4) D'Ors. voyage, Paléontol. 105.

s) Jahrb. 1841, 797. 
Dänemark, (?) Belgien, Westphalen durch Sachsen, Preussen, Böhmen, Podolien, Volhynien, Lithauen, Ukraine und Bess-Arabien, Simbirsk und ganz SüdRussland nach dem Kaukasus und dem Kaspischen Meere und gehört wohl mit Ausnahme einiger Bildungen in den Karpathen der oberen oder dritten Gruppe an; sie wird durch viele Arten von Ostrea, Exogyra, Pecten, Lima, Terebratula und Crania charakterisirt. Die mittle Zone geht von Süd-England, Nord-Frankreich und den Ardennen nach Österreich bis in die Krim und wird vorzüglich durch Ammoneen bezeichnet; nimmt aher an der nördlichen und südlichen Grenze auch einige Arten aus den Nachbar-Zonen auf. Die dritte oder sudlichste $Z_{0}$ endlich geht vom Atlantischen Orean bis ans rothe und Kaspische Meer, hat Rudisten fast zum ausschliessenden Eigenthum, ist überfüllt mit Foraminiferen, reich an Fucoiden und oft in harten Kalkstein verwandelt. $Z$ u ihr gehören: Lissabon, Süd-Spanien, Asturien, die Pyrenäen, die Corbières, die Departements Gard, Vaucluse, Bouches-du-Rhône und Var, Mailand, der Komer-See, das Vicentinische und Veronesische, Tyrol, Salsburg, Steyermark und zumal die Nord-Seite der Ost-Alpen, Illyrien, Transsylvanien, Karpathen, Dalmatien, Albanien, Morea, Sicilien, KleinAsien, Libanon, Sinai und in Afrika noch Constantine. Aber 1) jener Verbreitungs-Strich der Kreide überhaupt und der Rudisten insbesondere kreutzt von Gibraltar bis ans rothe Meer, zur Mündung des Indus und dem südlichen Ostindien verlängrert gedacht, die Parallel-Kreise unter dem starken Winkel von $20^{\circ}$ südwärts; die geographischen Zonen und Isothermen müssten daher ehedem eine andere Lage gehabt haben als jetzt und die letzten ausserdem weit stärker unregelmässiger als jetzt vom ersten abgewichen seyn; die Thatsache könnte nur dann einen Werth in geographischer Beziehung erlangen, wenn sie mit andern verwandten in Verbindung träte. 2) D'Анснгс unterscheidet bei jener Darstellung durchaus nicht; was unmittelbare Folye der geographischen Lage, und was mittelbare Folge nämlich des geographischen Hervortretens der yeologischen Schichtung seye; er unterscheidet die dreierlei Kreide-Formationen nicht, welche allerdings zu jener Zeit hinsichtlich ihrer Verbreitung noch nicht so genau unterschieden waren, als sie es jetzt zum Theil in Folge seiner eigenen Forschungen sind. 3) Da es geologische Rudisten-Zonen in allen Höhen des Kreide-Systems gibt, so müssen die Arten zuerst genau unterschieden werden, ehe man von geographischen Rudisten-Zonen sprechen kaun.

e. Dagegen hat Ferd. Roemer die Bemerkung gemacht, dass die Or. ganismen-Arten des Kreide-Systemes in den nördlichen Vereinten Staaten, in Neu-Jersey, ihre Identischen und Analogen in England und dem nördlichen Frankreich wiederfinden, was nicht nur einer klimatischen Verschiedenheit der Zonen sach parallelen, sondern anch bereits noch unseren jelzigen is ot be rmen Linien zu entsprechen scheint, während jene in Missouri und Texas die ihrigen in dem Lmkreise des Mitlelmeeres haben, und ähnliche Beziebungen auch zwischen den beiderseitigen Kreide-Gesteinen selbst bestehen sollen'), obwohl wir bei Lyels sehen, dass er die „Ober-Kreide von Neu-Jersey hinsichtlich ihres lithologischen Charakters am ehesten mit den Europäischen Schichte॥ über den Gatulte vergleichbar funde ${ }^{2}$ ). Die uns aus Neu-Jersey (in $40^{\circ} \mathrm{Br}$.) bekannten, mit Europäischen Arten (in $46^{\circ}-54^{\circ}$ Br.) identischen Spezies sind Belemnites mucronatus, Terebratula biplicata, ! Pecten quinquecostatus, Ostrea vesicularis, Corax pristodontus, Otodus

1) Wir entnehmen diese Nutitz von Bové (Jahrb. 1848, 859), da uns Roemer's Mi theilung darüber entgangen ist, daher wir auch nicht wissen, ob Roemer hiebri eine 'bionologische Verschiedenheit der nördlichen und südlichen Kı eide-Schichten unterstellt oder nicht.

2) Jahrb. $18 \$ 5,720$. 
appendiculatus $^{1}$ ) u. v. a., von welchen jedoch die 2 mit ! bezeichneten Arten auch schon in tropisch-asiatischer Kreide gefunden worden sind. Eine zweite Mosasaurus-Art aus Kreide nordwörts von St. Louis $\left(39^{\circ} \mathrm{Br}\right.$. $)$ stammend repräsentirt die Europäische, welche nördlich vom $50^{\circ} \mathrm{Br}$. vorkommt ${ }^{2}$ ). Es würde also hier wie dort für gleiche Isothermen doch immer noch ein BreiteUnterschied von etwa $10^{\circ}$ stattinden.

f. Gegen eine zur Kreide-Zeit höhere Temperatur Europa's scheinen zu sprechen diejenigen in der obern weissen Kreide vorkommenden PetrefaktenArten, welche noch jetzt in Europa leben (S. 769). Man würde berechtigt seyn, auf sie den erwähnten Schluss zu stützen, wenn nicht andere erheblichere Momente entgegenstünden, oder wenn ihre eigene Anzahl verhältuissmässig beträchtlicher wäre. Wir liaben aber früher gesehen, dass es einzelne MeeresThiere von klimarisch sehr ungleicher Verbreitung gibt.

G. Eine Unterscheidung der Bevölkerung nach geographischen Zonen, mithin eine klimatische Unterscheidung dieser letzten selbst, dïrfte noch kaum mit dem Beginne der Tertiär Zeit in dén Nummuliten- und andern Eocän-Gesteinen $\left.(s, \tau, t)^{3}\right)$, aber deutlich erst in deren Mitte möglich werden, wo die lebenden identischen Spezies schon zahlreicher auftreten, doch das Klima noch etwas wärmer oder wenigstens gemässigter als jetzt gewesen zu seyn scheint. Mit unsren heutigen klimatisch übereinstimmend werden diese Zomen aber erst in der Pliocän-Zeit, indem der Charakter der Organisation und die relativen Zahlen in jeder Zone und in jedem der jetzigen Faunenund Floren-Bezirke schon den jetzigen vollkommen entsprechen und sogar die Arten grösstentheils übereinstimmen.

a) Ein Theil der $\mathbf{N}$ u m m liten. Ges te ine, welche Rudisten enthalten oder damit enger verbunden sind, gehören der KreidePeriode an (so in Marokko, wo der Nummuliten-Kalk zwischen Kalk mit Chama ammonia und Fukoiden-Sandstein liegt; an Etang de Berre, wo die Nummuliten kngelförmig ${ }^{4}$ ), zu Mastricht, wo sie sehr dünne sind); sie haben wir bei der gegenwärtigen Untersuchung auszuschliessen, obschon noch nicht alle in dieser Beziehung mit Sicherheit bestimunt sind $(s)$.

Die jüngeren Nummuliten-Gesteine ${ }^{5}$ ) unterscheidet Ewald in eocäne mit linsenförnigen Nummuliten, Nautilus lingulatus und Pentacrinus didactylus (die 2 folgenden Gruppen, $\tau$ und $t$ ) und in miocäue? über dem Macigno (u?).

1) LYELL im Jahrb. 1845, 720.

2) GoLdFiss im Jahrb. 1847, 122.

3) Jahrb. 1848, 72, 76, 86, 235, 361, 366, 493, 587, 597, 621, 623, 713, $714,715,716,758,764,842,844,859,860,864$. Wir haben oben die mancherlei Nummuliten-Gesteine durch die Buchstaben s, $\tau$, $t$ unterschieden, bemerken aber, dass bei Ausarbeitung des Enumerators wir noch manche unter stellen mussten, welche jetzt einer der andern Abtheilungen zugewiesen werden könuen.

4) Nach DE VERneUL sollen diese Körper keine wirklichen Nummuliten seyn. Jahrb. 1848, 597.

3) Über das Verhältniss zum Pisolithen-Kalk und Terrain Danien $\left(\mathbf{r}^{3}\right)$ vrgl. Bull. géol. 1846, IlI, 1797; Jahrb. 1848, 72, 85, 86, 833. 
Von diesen ersten ist pin Theil, welchen Taliavianss nach dem MontAlaric in den Central-Pyrenäen Système Alaricien, ELIE DB BeumnNT Terrain nummulitique Méditerranéen genaunt, das letzte ante-pyrenäische Grstein, da es noch mit den Pyrenäen gehoben ist, und wird von Elie dB BeaUMoNT desshalb und weil es noch einige $(15-20)$ Mollusken-Arten aus der Kreide enthält, noch zur Kreide-Periode gerechnet, obschon andere Geologen es schon für tertiär betrachten, indem es allerdings auch schon eine Anzahl fossiler Arten mit dem folgenden gemein hat. Dazu gehören ausser den genannten die Glariser-Schiefer (welche in Enumerator unter $\mathbf{r}$ eingereihet sind, dann die Vicentinischen im Val Roncì und an Monte-Bolca $(\tau)$, welche immer über Scaglia und unter Macigno (Fukoiden-Schiefer, Flysch) ruhen. Hier treten zuerst die Knochen-Fische in ihrer ganzen Manchfaltigkeit auf.

Ein anderer Theil, Tallavignes' Système Iberien, de Beaumonts Terrain Soissonais mit Ostrea gigantea etc. (t), liegt an den Räudern der PyrenäenKette auf der Spanischen wie auf der Französischen Seite, im AdourBecken zu Biaritæ bei Bayonne, in der Montagne noire, in den Corbières, an welchen Orten man 108 fossile Arten darin gefunden hat, von denen 3 Arten sonst der Kreide, 38 dem Tertiăr. Gebirge angehören und 67 dem Nummuliten-Gebirge eigen sind. Er ruhet im Pariser Becken auf den Ligniten des plastischen Thones als Basis des Grobkalkes, von welchem wir es auch bisher nicht getrennt haben, findet sich in Belgien u. s. w. Mit Ausnahme einiger Reste in dem unter den genannten Ligniten ruhenden PisolithenKalk finden sich hier die ersten Säugthiere und zwar schon mit grosser Manchfaltigkeit ein.

Die weiter ostwärts liegenden Nummuliten.Gesteine wagen wir noch nicht bestimmt writer $\mathrm{zu}$ scheiden, werden jedoch bei einigen durch Beifügung eines der drei oben gebrauchten Buchstaben die vermuthliche Stellung näher angeben Sie kommen nämlich noch vor in den Hoch- und Nieder-Alpen Frankreichs und am Col di Tende; in den West- und Ost-Alpen, in den Diablerels, am Kressenberg (t); zu Mattsee bei Salsburg, zu Sonthofen, zu Althofen bei Guttaring in Kärnthen; im Wiener-Berken (Bové's tertiärer Korallen-Kalk, t), in Ungarn und Siebenbürgen (? $\mathbf{u})$; am Karste und in Istrien; in Agypten als Pyramid Gesteine (t), in der Krim (t) ${ }^{1}$ ) und dann ostwärts bis zu den Sind-Ländern Indostans, im Himalaya und bis China. Diese Linie geht zwar nicht uberall durch gleiche Breiten, bildet aber in den letztgenannten Gegenden eine Kurve, welche der der heutigen Isotherme entspricht. Ausserdem schpinen Nummuliten-Gesteine nicht vorzukommen, als irn südlichen Theile Nord-Amerika's, wo sie die Lagerstälte des Zeuglodon bilden, aber keine eigentlichen Nummuliten, sondern nur diesen ähnliche Körper einschliessen, welche jedoch küralich Forbes für Orbituliten erklärte und D'ORBigny nun Orbitoiden nennt, da sie den letzten mehr als den ersten verwandt sind.

Indessen geht aus diesen Thatsachen nur hervor: 1) dass die Nummuliten einst in einem Theile des alten Kontinents grewohnt haben, vielleicht sogar darauf beschränkt gewesen sind, der mit dem eben bezeichneten Numnuliten-Strich zusammenfüllt; 2) dass hier auch die geologischen Bedingungen vorhanden waren, unter welchen sich ein Nummuliten-Gesteiu bilden und später erhalten und zu Tage treten konnte; 3) dass jener Streifen einen grossen Theil des alten Kontinents von Osten nach Westen durchzieht. Es ist noch kein Beweis gegeben, dass derselbe in einer nothwendigen Beziehung zu den Parallel-Kreisen oder den Isothermen stehe; dieser würde sich erst dann etwa finden, wenn man nachweisen könnte, dass

1) Vrgl. Jahrb. 1838, 350-354. 
gleichzeitige Äquivalente des Nummuliten-Gesteins in andern Zonen mit andern organischen Resten ebenfalls in Zonen-weiser Verbreitung sich vorfinden. Wenn wir aher z. B. BolÉ's geognostische Erd-Karte zur Hand nehmen, so sehen wir, dass sich die Formationen der verschiedenen Perioden überall vorzugsweise längs der Haupt-Hebungs-Achsen der Kontinente fortziehen, in Amerika also von Norden nach Süden, im alten Kontiuente von Osten nach Westen. In der That ist diese Achse in Europa bis zum Kaspischen Meere auch durch das Streichen der Gebirgs Ketten des Atlas, der Pyrenäen, Alpen, Karpathen, des Balkan und des Kaukasus mit den dazwischen liegenden Meeren, weiterhin durch das des Mimalaya u. s. w. grossartiger als irgend eine andere ausgedrürkt; und mithin ist, wenn diese Gestaltung der Erd-Oberfläche schon von der II., III. Periode her stattfand und während späterer Hebungen anhielt, auch natürlich, duss erst sich die Gebirge parallel dieser Linie ablagerten oder doch in den Verhältuisse, als sie wieder von andren bedeckt wurden, mit ihren Ausgehenden an den Seiten der gehobenen Ketten unbedeckt zu Tage kamen. E's ist ferner ganz begreiflich, dass ein Meer, das anscheinend in ähnlicher Weise wie das jetzige Mittelmeer, aber in viel grossartigerem Masse sich uuf jenem Erdstriche pinst zwischen zwei Kontinenten von Westen nach Osten dahin zog, eine vergleichungsweise ähnliche Bevölkerung in seiner ganzen Erstreckung beherbergt haben könne, ohne dass ein Zonen-Verhältniss davon die Ursacbe war.

Zu den Konchylien-Arten, welche die eocänen Nummuliten- (Orbitoiden-) Gesteine Alabamas (ob nicht schon zu den spätren Eocäı-Gesteinen t gehörig?) mit den Parisern gemeinsam besitzen, gehören Cardita planicosta, Solarium canaliculatum und Niso terebellum ').

Wenn wir indessen die Beweise einer Zonen-artigen Vertheilung der Organismpn über die Erd-Oberfläche in jener Zeit noch nicht zu finden oder anzuerkennen im Stande sind, so wollen wir doch die Untersuchungen über diesen Gegenstand hiedurch nicht abschneiden, sie nicht tür üherflüssigg erklären, sondern wünschen viclnehr lebhaft ihre Fortsetzung auf der so gewonnenen Basis.

Die zahlreichen Fische des Monte-Bolca sind von Agsssiz genau untersucht worden; diese Fisch.Fauna hält nach ihm das Mittel zwischen denen der Kreide und des Grob-Kalks und hat jedenfalls einen tropischen Charakter.

b) Der Süsswasser-Kalk von Rilly bei Reims, zwischen Kreide und plastischem Thon gelegen, enthält nach DE Boissx́ ausser ausgestorbenen Arten solcher Geschlechter, welche jetzt wohl auch in wärmere Gegenden sich ausdehnen, jedoch noch der Gegend entspreclien könuten, und einer der Valvata spirorbis äusserst ähnlichen Spezies (V. Leopoldi), auch eine schöne Art des jetzt (mit der einzigen lebend bekannten Spezies) auf Brasilien beschränkten Genus Megaspira ${ }^{2}$ ).

c) Anilere e cäne Bildungen über den Nummuliten-Gesteinen kennen wir im gemässigten Nord-Amerika in Carolina und Florida, wenn nicht selbst die vorhin erwähnten Orbitoiden-Gesteine Alabamas noch hieher gerechnet werden müssen; — dann in-Süd-Amerika

1) Jahrb. $18+8,587,764$.

$\left.{ }^{2}\right)$ Jahrb. 1848, 637; Mém. gẻol. 1848, b, III, 265-285, pl. 5, 6. 
in weniger erheblicher Erstreckung; in Europa in dem London-ParisBelgischen Becken; seit Kurzem an mehren kleineren durch NordDeutschland zerstreuten Stellen als Fortsetzung der Belgischen, und theilweise vielleicht in Böhmen und Ungarn; daun in der Ukraine, an der Wolga $^{1}$ ) und in Armenien; näher an den Polen als etwa bis zu $55^{\circ} \mathrm{Br}$. ist diese Formation nicht beobachtet worden. - Der Charakter dieses Zeit-Abschnittes beruht darin, dass der Typus der Schöpfung sich in Art und Reichthum der Formen dem der jetzigen überhaupt enger anschliesst, dass aber doch in verschiedenen WeltGegenden die Faumen und Floren überall von einander verschieden sind, wie jetzt in verschiedenen Welttheilen zwischen den Tropen;dass sie im Einzelnen eben so von den jetzigen Floren und Faunen derselben Gegenden abweichen, indem sie nur theilweise identische Genera, aber noch fast keine identischen Arten darbieten; - dass selbst in gemässigten Gegenden der Total-Eindruck noch ein entschieden trop ischer ist, während in der Nähe der Polar-Zone, wenn man eocäne Bildungen dort auffinden könnte, deren Bevölkerung wahrscheinlich mehr Analogie mit derjenigen gemässigter Gegenden überhaupt zeigen würde.

Wir sind nicht sicher, ob die Altsatteler Pflanzen-Reste in diese Forma-1 tion gehören, doch sind sie älter als die Öninger; es sind fast nur Baum-Blätter, die sich bis auf 3-4 Ausnahmen nicht auf lebende Sippen zurückführen lassen. Der Gesammt-Eindruck derselben scheint übrigens PöpPIG'N mit dem der Flora von Süd-Florida übereinzustimmen; ein Palmen-Blatt aus dem LataniaGeschlerht spricht für Peru, ein Aroideen Blatt für Tropen im Allgemeinen; Pinus-Zapfen können überall hindeuten, RossmüssLer ${ }^{2}$ ).

Die Konchylien dieser Formation kennt man aus dem Paris-London-Brüsseler Becken durch Lamarck, Deshayes, Sowerby und Nyst vollständiger, als die irgend einer andren. Die für Tropen-Gegenden so charakteristischen Siphonobranchier-Geschlechter (Conus, Cypraea, Oliva, Voluta, Mitra, Strombus, Rostellaria, Murex, Tritonium, Cerithium etc.) treten theils zum ersten Male, theils wenigstens erstmals mit grossem Arten-Reichthum und auch vorwaltend über die Asiphonobranchier auf, wie sie auch später in Schichten gemässigter Gegenden nie wieder erscheinen; der Arten-Reichthum der Konchylien überhaupt spricht für mehr als tropisches Klima, da man nach DESHAYES in WestEuropa und -Afrika findet ${ }^{3}$ )

$$
\text { lebende }
$$
$\begin{array}{lr}\text { Arten } & \text { fossile } \\ \text { Arten }\end{array}$ in $80^{\circ} \mathrm{N} . \mathrm{Br}$. . . . . $8-10$ in nördlichen Pliocän-Becken . wenige in der Breite des Mittelmeeres. in den Tropen: Senegal, Guinea 600 im mittelmeerischen Pliocän-B.

$900 \mathrm{im} \mathrm{franะösischen} \mathrm{etc.} \mathrm{Miocän-B.} 1000$ das pariser etc. Eocäı-B. ( u. $\tau$ ) 1400 noch wäımer war also.... .

Ja allein die Umgegend von Paris hat auf 2000 Quadrat-Stunden 1200 Spezies geliefert, hauptsächlich eben aus Siphonobranchier- und vorzugsweise tropischen Geschlechtern. Unter den 1400 Arten des Gesammi-Beckens sollten nur $38(0,03)$ noch am Leben seyn, meistens in tropischen Gewässern weit

1) Jahrb. 1844, 85 .

2) Jahrb. 1841, 821 .

3) Coquilles de Paris, II, 769-780; Annal. scienc. nat. 1936, V, $289 \mathrm{ff}$. Juhrb. 1887, 62. 
verbreitet, doch einige zugleich bis in die Nordsee hinaufgehen. Indessen stellt sich das Verhältniss etwas abweichend heraus, nachdem Deshayes selbst neuerlich mit Recht einen Theil jener als identisch bezeichneten fossilen Arten noch für eigene erkannt hat, und weil man sich nicht alle Arten des Pariser-Beckens als gleichzeitig bestehend denken darf.

Dubois DE MontPerreux hat zu Boutschack am Dniepr in der Ukraine 18 und mehr, in Armenien 5 Arten des Pariser Grobkalkes wiedergefunden unter ') einer nicht beträchtlich grösseren Gesammtzahl eocäner Konchylien.

Die eocänen Schichten im südlichen Theile Nord-Amerika's haben mit den Paris-Londoner Schichten zwar einige Konchylien-Arten gemein, doch ist deren Anzahl auffallend klein, selbst noch, wenn man die weite Entfernung in Rechnung zieht; ihre Grösse ist im Allgemeinen weit unbeträchtlicher; die Siphonohranchier stehen sehr zurück; der Charakter ist im Ganzen verschieden, doch nicht näher mit dem einer jetzigen Malakozoen-Fauna vergleichbar. Ob diess Folge klimatischer Versthiedenheit seye, oder ob es sich nur um eine andere Facies derselben Formation handle, müssen spätre Erfahrungen zeigen. Korallen scheinen fast gänzlich zu fehlen (LEA.) - lin südlichen Amerika kommen nach D'O und bieten zu beiden Seiten ungleiche Arten aus fast gleichen Genera dar, woraus aber noch keine mit den Europäischen übereinkommende Art bekannt zu seyn scheint, obsrhon sie ihnen im Habitus wieder näher stehen, als jene Nord-Amerikaner. Wie die 2 Weltmeere zu beiden Seiten Süd-Ameriku's nach D'Orbigny jetzt nur I lebende, beiderseitige quartären Schichten unter bis jetzt bekannten 22 östlichen und 11 westlichen Artell gar keine Art $^{3}$ ) mit einander gemein haben, so scheinen auch schon in der Eocän-Zeit die Mollusken-Faunen zu beiden Seiten der Kordilleren ganz ohne Bcziehung zu einander gewesen zu seyn, was auf eine schon damals vollendete Trennung beider Meere hindeuten würde ${ }^{4}$ ); mit ihnen treten auch in Amerika die ersten Säugthiere auf: Megamys und Toxodon. An eocänen Konchylien hat D'Opisisny von den Tropen an bis nach Patagonien hinab auf der Ost-Seite der Kordilleren 11, im Westen derselben 29 Arten gesammelt, wovon keine Art mehr lebend vorkommt und selbst viele Genera auf den jetzigen Küsten der entsprechenden Gegend ganz fehlen. Die Arten der West-Seite gehören 20 Genera an, wovon 3 (Rostellaria, Monoceros, Pectunculus) zwischen den Tropen in $5^{\circ} \mathrm{S}$. Br. gefunden worden sind, woselbst indessen das erste derselben jetzt nicht mehr lebend vorkommt; von den 17 andern, welche alle aus $30^{\circ}-37^{\circ} \mathrm{S}$. Br. stammen, scheinen nur 4 in andern Arten noch an derseiben Küste zu leben; von 5 wird gesagt, dass sie überhaupt nicht an der Westküste Süd-Amerika's, insbesondere nicht in Chili, Peru und Columbien vorkommen; von den 8 übrigen (Bulla, Natica, Oliva. Fue sus, Pleurotoma, Cardium, Lucina, Arca) mit 12 Arten wird ausdrücklirh erklärt, dass sie an der Westküste Amerika's erst um $17^{\circ}-37^{\circ}$ weiter nordwärts in der Nähe des Äquators leben ${ }^{5}$ ). Hier sind also entschiedene Erzeugnisse eines wärmeren Eocän.Klima's, aber keine Übereinstimmung der Fauna an 2 Küsten eines Kontinentes mehr. - DarwiN ${ }^{6}$ ) hat von den Küsten Chile's, Chiloe's und Patagoniens $\left(23^{\circ}-53^{\circ} \mathrm{S}\right.$. Br. $) 60$ Arten tertiärer Konchylien zurückgebracht, welche zum Theil mit den von D'ORBIGNY gefundenen übereinstimmen

1) Jahrb. 1833,$353 ; 1836,360$.

2) Jahrb. 1843, 867.

3) D'Ori. Voy., Paléont. 163-167.

4) Jahrb. 18\$5, 373.

5) D'Orb. Voy., Paléont. p. $135-140$.

6) Geological observations on South-America, London, $1846,8^{\circ}$, p. 249-264. 
und alle eocän zu seyn schejnen; allein es ist weder eine aus Europa bekannte eocäne Art, noch eine an jenen Küsten oder anderwärts lebende darunter. Die universelle Übereinstimmung der Fauna scheint also in dieser Eocäı-Zeit nicht mehr geherrscht zu haben, wie noch zur Zeit des Neocomien (S. 879), obschon sich eine Zonen-weise klimatische Differenzirung der Formen und ein Gleichbleiben derselben in gleicher Zone noch nicht erkennen lässt.

Die alt-tertiären Fische des Londonthones u. s. w. gehören nach Agassiz grösstentheils zu Familien, welche zwar noch lebend vorkommen, aber ferne von unsien Küsten wohnen'); und aufgeführt werden in dieser Hinsicht insbesondere die fast ganz tropischen Teuthyes, Xiphoilen, Sphyränoiden und die Characinen: dagegen manche ehedem sclten gewesen sind, die jetzt die Meere um England und weiter nordwärts bevölkern. Übrigens sind unter 44 Knuchenfisch-Sippen dennoch nur 4 noch lebende, und zeigen sich trotz dem im Ganzen südlichen Habitus bei den Gadoiden und Labroiden schon Annäherungen an nördlichere Formen. In Ganzen aber stammt 1/3 der Arten aus erloschenen Geschlechtern. Der tropische Charakter dieser Fauna ergibt sich aus den zahlreichen Arten aus solchen Geschlechtern, welche von den Tropen aus jetzt uicht oder kaum mehr bis in unsere Breiten reichen.

Die eocäne Reptilien-Fauna Britanniens hat offenbar noch einen südlichen Charakter: sie besteht nach R. Owen aus Krokodilen, Land-Schildkröten, Trionyx, Chelone und grossen Schlangen. Das Krokodil steht dem $\mathrm{Cr}$. Schlegelii MüLc. vou Borneo an nächsten ${ }^{2}$ ); Land-Schildkröten kommen in Süd-Europa und mit Trionyx in Nord-Afrika und dem wärmeren Theile von Nord-Amerika vor; Chelone gelangt zwar jetzt noch zuweilen an die Britische Küste, gehört aber wärmeren Gegenden des Oreans an; grosse Schlangen sind ein Eigenthum tropischer Länder.

d) Miocäne Fol'mationen, in der Mitte Europa's (Gironde, Touraine, Angers, älterer Crag in England, Aix, Mains, Superga, Tortona, Wien, Siebenbürgen, Podolien, Süd-Russlands etc.), NordAfrika's, Nord-Amerika's bis zu $41^{\circ} \mathrm{N}$. Br. aufwärts und vielleicht Ostindiens bekannt, enthalten schon 0,19-0,40 und mehr Arten lebender Konchylien, welche theils in den benachbarten und theils in wärmeren, nirgends aber in kälteren Meeren noch gefunden werden, lassen hiedurch wie durch den Charakter anch der sie begleitenden ausgestorbenen Arten eine Zinen-weise Temperatur-Verschiedenheit der Erd.Oberfiäche schon erkennen, wornach aber die gemässigte Zone noch durchschnittlich wärmer als jetzt gewesen wäre, womit die Ausdehnung der Korallen bis über den 55. Breite-Grad hinans übereinstimmt (S. S72); während in der Mitteteuropäischen Pflanzen-, Insekten-, Reptilien- und Sängthier Welt dieser Zeit ein südlicherer Charakter nicht mehr so entschieden und höchstens im Betrage von 2-10 Breite-Graden ausgedrückt ist.

Was Europa betrift, so stossen wir hier zuerst auf die organischen Reste des Bernsteins, welche bald über Kunst-Produkten lagern, bald für miocän genommen worden sind (wir hatten sie unter $\mathbf{v}^{\prime}$ gestellt), bald endlich und zwar in neuester Zeit, wo man ausgedehnte eocäne Bildungen in Nord-Deutschland

1) Jahrb. 1847, 125; und Poissons foss., I. Introduct. p. XXVII.

2) BR. Collect. I; 53 . 
gefunden, diesen beigerechnet werden. Die Ins e kten erinnern grösstentheils an Europa, während unter den Vegetabilien die reichlich damit vorkommenden Thujen für ein wärmeres Klima sprechen ${ }^{1}$ ). Zu diesen Insekten werden wir auf S. 888 zurückkommen. Auch in anderen vielleicht jüngeren Braun-Kohlen längs der Ost-See, im Siebengebirge wie im Baireulhischen tragen die InsektenReste narh Germar zum Theil einen südlicheren Charakter als jetzt dem Lande zukommt ${ }^{2}$ ).

Unger ${ }^{3}$ ) sagt ther die fossile Flora von Parschlug, mit welcher das miocäne Mastodon augustidens und Dorcatherium Naui vorgekommen sind und zur relativen Alters-Bestimmung dienen: die Menge von immergrünen Laubhölzern neben solchen mit häutigen Blättern, aber ohne Palmen, deutet auf ein Klima von $12^{\circ}-15^{\circ}$ mittler Jahres-Temperatur (die jetzige ist nur $=9^{\circ} \mathrm{C}$.), was in Europa $45^{\circ}-42^{\circ} \mathrm{N}$. Br. oder den Küsten-Ländern des Mittelmeeres, in Nord-Amerika $43^{\circ}-37^{\circ} \mathrm{Br}$. oder Süll-Virginien u. s. w. entspricht; im Einzelne! aber ist die Verwandtschaft grösser mit dem südlichen Nord-Amerika und Hoch-Mexiko als mit den mittelmeerischen Ländern, was also nicht, wie man gewöhnlich annimmt, ein minder excessives, milderes, - sondern ein im Som. mer heissres, in Winter kältres Klima vorauszusetzen scheint. Einige Arten sind von jetzt lebenden nicht zu unterscheiden. In Bezug auf die fossilen Palmen sagt neuerlich UNGER: dass sie nie zahlreicher gewesen seyen, als in der Eocän- und Miocän-Zeit, da von den 56 bekannten in allen Perioden zerstreuten Arten in erster 17, in letzter 25 vorkommen. Ihre hauptsächlichsten miocänen Fundstätten sind Häring in Tyrol und Radoboj in Kroatien; ihre Begleiter Laurineen (Laurus), Myrireen, Melastomaceen, Leguminosen, Koniferen (Araucarites Goepperti, Cupressitides taxiformis, Thuytides callitrina, Juniperites, Thuya nudicaulis etc.), Amentaceen, Apocyneen, Verbenaceen, Acerinen, Anacardiaceen, Xanthoxyleen, Formen, welche theils den Wendekreisen und theils den mildesten Gegenden ausserhalb derselben entsprechen. Wären dip Palmen auch nicht zahlreicher als jetzt gewesen, wo sie $1 / 200$ aller lebenden Phanerogamen betragen, so sind sie dorh über ihren jetzigen Verbreitungs-Bezirk hinaus viel weiter gegren die Pole gegangen ${ }^{4}$ ). Die damit gleichzeitigen, der untern Süsswasser-Molasse angehörigen zahlreichen Pflanzen-Blätter am hohen Rhonen gehören nar'h 0 . HEER ${ }^{5}$ ) 33 Sippen aus 21 Familien an, von welchen 24 Sippen noch jetzt in dortiger Flora leben, die andern mehr südliche Zonen bewohnen. - Die Pflanzen von Öningen (v), wo Mastodon anzustidens ebenfalls vorkonmt, entsprechen der oberen Süsswasser-Molasse und sind demnach nur wenig jünger als die vorigen, zeigen auch mehre Arten, die ihm mit den beiden andern Fundorten gemeinsam zustehen. Ax. BвAun ${ }^{6}$ ) zählt 32 PflanzenSippen mit 55 Arten auf, worunter 38 Laubhölzer; 19 Sippen sind deutsche, 22 europäische, 10 aussereuropäische, welche sämmtlich nebst einem Theile jener europäischen jetzt in Nord-Amerika, zum Theil aber auch zugleich in SüdAmerika, dann in Nord-Afrika, Mitlel-Asien und Japan einheimisch sind. Die Wetterauer Braunkohle (wenn sie nicht älter als u ist?) deutet durch ihre bekanıten Wallnüsse zunächst auf Nord-Amerika. Die Flora von Aix erinnert durch? Buxus balearica und Thuja? articulata an die Berberey, durch Podocarpus macrophylla und Laurus dulcis an Indien. Es sind die vier einzigen von LiNDeEx näher bestimmten, aber doch noch zweifelhaften Arten von dieser Gegend ${ }^{7}$ ). - Es ist oben schon (S. 873) auseinandergesetzt worden, wie der miocäne Korallen-Reichthum des Wiener-Beckens ebenfalls auf ein südlicheres, fast tropisches Klima hinweise; selbst im Englischen Coralline-Crag scheint noch

I) Jahrb. 1845, 876. - ${ }^{2}$ ) Jahrb. 1846, 212. - 3) Jahrb. 1848, $505 \mathrm{ff}$.

4) Jahrb. 1848, 116. - 5) Jahrb. 1848, 369.

6) Jahrb. $1845,164 \mathrm{ff}$.

7) Jahrb. 1830, 354. 
pine Andeutung darauf zu liegen. - Eben so ist es anerkannt, dass die mincänen Schichten ihre theils identischen und theils analogen Kon ch ylie n-Arten immer in ganz benachbarten oder meist in wärmeren, nie in kälteren Meeren finden: die mitteleuropäischen Miocän-Konchylien also in Mittelmeere und noch mehr am Sene. gal ${ }^{1}$, nicht in der Nord-See. Deshayes versichert $80 \mathrm{gar}{ }^{2}$ ) in den miocänen Becken insbesondere von Bordeaux und Dax fast 200 (190) Arten der tropischen Zone wieder erkannt zu haben, so dass das Verhältniss - Tropen-Zone z : 900 (200) 1000: "Europa - auf beiden Seiten eine fast gleirhe Quote, nämlich $0,22: 0,20$ gemeinsamer Arten ergibt, worunter gerade solche, welche die tropischen Meere von Guinea und Senegal am meisten charakterisiren ${ }^{3}$ ). (Oben waren für u nur 0.19 noch lebender Arten angenommen, weil die Zahl der identischen Arten in Wirklirhkeit 200 nicht ganz erreicht.) Wir kenuen jedoch in westlicheren Berken aurh einige Arten aus dem Mittelmeere. Insbesondere sind unter den Süsswasser-Konchylien von Melania, welches Genus in gleicher Breite nit dem innein Europa jelzt fast nicht mehr vorkommt, einige Arten ganz übereinstimmend mit solchen, die an den Küsten und auf Inseln des Mittelmeeres noch leben. Aber nicht allein diese Identität so vieler tropischen und suhtropischen Arten, sondern auch der erwähnte Arten-Reichthum in jetzt ge-mässigten Breiten spricht für ein ehemals tropisches Klima. - Wir gelangen zur Betrachtung der miocinen Insekten-Welt; und zwar zuerst zu der von Aix. Die ältren Bestimmungen von MARCEL De StrRes 1 . A. sind nach O. Herr sehr unzuverlässig: doch hat Coouand neulich einen Schmetterling aus dem Geschlechte Cyllo voyar noch nit Farben gefunden, dessen Geschlerhts Verwandte im Indischen Arclijpel wohnen 4). Dann die Käfer, zunächst wieder von Öningon, Parschlug, dem hohen Rhomen und vor Radoboji in Kroatien, von welchen 0 . HeER's sorgfältige Untersurhungen 119 Arten nachgewiesen haben: 100 derselben aus 68 Sippen słammen aber allein von Öningen; 51 dieser Sippen leben noch jetzt in der Schweits, 4 sind nicht genau bestimmbar, 5 werden jetzt nicht näher als in Süd-Europa, 1 nur in Nord-Amerika gefunden und 7 sind ausgestorben. Aber auch aus den jetzt noch in der Schweits lebenden Gesthlechtern sind viele zugleich in Süd-Europa, wenige nur in der schweitz und Deutschldand einhrimisch und finden virle Aiten ihre nächsten Verwandten nicht mehr in der Schweitz, sondern nur in Süd-Europa, woraus O. Hrer ${ }^{5}$ ) den Schluss zieht, dass die Öninger Käfer-Fauna den Charakter derienigen des südlichen Europa's oder besser der Zona mediterranea habe, dass aber in diese einige wenige Amerikanische Formen eingestreut seyen. Unter 25 Insekten-Arten aus deutscher Braunkohle (u ?) hat Germar 21 Europäische Sippen und 1 NordAmerikanisches Genus (Belostoma) gefunden. [BERENDT ${ }^{6}$ ) bemerkt über die von uns schon vorhin (S. 886) berührten organischen Reste des Bernsteins: dass 718 der 800 Insekten-Arten von ganz einheimischem (Preussischen), unter den übrigen je ein Theil von Süd-Europäischem, von Nord-Amerikanischem, nur sehr wenige (2?) von hochnordischem oder von tropischem Geschlechts-Typus, mehre jedoch ausgestorben seyen]. - Von den miocänen Fischen Europa's sagt

1) Im Enumerator S. 481 - 485 und anderwärts hahen wir von vielen noch lebenden und zugleich miocänen Arten von Bordeaux nach Grateronp's Bestimmungen das wärmere Vaterland in Parenthese beigesetzt $\left(S^{3} M^{3} F^{3}\right)$, wo sie jetzt noch angetroffen werden.

2) Coquill. de Paris, II, 777.

3) Jahrb. 1837, 62.

4) Jahrb. 1848, 760 .

5) Jahrb. 1847, 163; HEER die Insekten-Fauna von Öningen etc., Leiprig $1847,4^{0}$.

6) Jahrb. 1845,876 . 
Agassiz ): sie gehören meistens Geschlechtern an, welche in gemässigten und tropischen Meeren zugleich heimisch sind (Platax, Carcharodon, Lamia, Mylio. batis); ihre Vergesellschaftungs-Weise aber (ihre relativen Zahlen?) entspricht mehr den letzten als den ersten. Die Öninger Süsswasser-Fische gehören Ge. schlechtern an, welche noch jetzt in der Gegend leben, aber sich zugleich auch weiter südwärts erstrecken; nur eine Art entstammt dem Geschlechte Lebias, das gegenwärtig sich auf Italien, Arabien und hauptsächlich Amerika beschränkt. - Auch die R eptilie n Öningens sprechen nach H. v. MEYER weder entschieden für ein wärmeres noch für ein kälteres Klima; deuten auch einige etwas mehr nach Süden, so scheint Lagomys unter den Säugthieren wieder nach höherem Norden hinzuweisen. Aber das Schwanz-Batrachjer-Gcschlecht Andrias findet seine Familien-Verwandten nor in Jupan und in Nord-Amerika, und Chelydra lebt noch jetzt nur in Nord-Amerika 2). Indessen liegen sowohl der entsprechende Theil von Nord-Amerika als Japan zwischen gleichen Parallelen mit den mittelmeerischen Ländern.

Alle diese Nachweisungen würden also keinen Zweifel darüber lassen, dass in der miltlen Tertiär-Zeit das Klima Europa's wenigstens etwas wäımer als jetıt gewesen seye; zum strengen Beweise aber, dass die ganze Erd-Oberfläche damals wärmpr gewesen, würden freilich auch übereinstimmende Anzeigen aus andern geographisrhen Längen, oder besser aus dem höheren Norden oder aus der südlichen gemässigten Zone nothwendig seyn.

Die an Kirsel-Infusorien reirhen mittel-tertiären Schichten Nord-Amerika's kennt man in Maryland, Tirginien und beiden Carolina in $32^{\circ}-40^{\circ} \mathrm{Br}$. (was Süd-spanien, Sicilien, Griechenlınd und Nord-Afrika entspricht) bis nach Massachusetts in $41^{\circ} \mathrm{Br}$. Eıste ruhen auf eocänen Formationen und biefen narh LYELL ${ }^{3}$ ) (unter 147 Arten) 0,17 Konchylien-Spezies dar, welche an der nahen Küste noch leben, und auch pinige nördlirbere und sudlichere enthalten; 10 dieser Arten kommen auch in Europa lebend und nebst 4 weitren in Europäischen Miocän-Schichten vor; sie wohnten also in der Miocän.Zeit sowohl als jetzt in Europa weiter nordwärts, als sie in Amerika gefunden worden sind; sollten sie in nördlicher vorkommenden Miorän-Lagen Nord-Amerika's nicht vorhanden seyn, so würde man aus dieser Erscheinung allerding auf eine der jetzigen analoge und selbst noch stärkere Krïmmung der Isothermen von Amerikn nach Europa während der Miocän-Zeit schliessen müssen. Unter den 10 Polyparien glaubte LyelL auch 1 mitlel-tertiäı e Art aus Europa, unter den Echiniden 1 aus dem Englichen Crag, dann 5 Arten Fisch-Zähne aus Europäischen Miocänund Molasse-Bildungen zu erkennen. Nach Lonsdale aber enthalten die Polyparien, aus der durch's Mittelmeer ziehenden Parallele von $37^{\circ} \mathrm{Br}$. stammend, keine lebende Art; ihre Geschlechter sind theils allverbreitete, theils mittelmeerische (Lunulites) theils zugleich Bewohner wärmerer Meere, wie Astraea und besonders Anthophyllum, das in rothen Meere vorkommt, oder ausgestorbene, wie Columnaria. davon eine Art die Grösse-Dimensionen der Anthozoen wärmerer Meere besitzt. Die Polyparien scheinên demnach, nach Lonsdate, auf ein mittelmeerisches oder selbst noch wärmeres Klima zu führen, was dann der jetzigen geographischen, oder selbst noch jetzigen isothermalen Lage jener Länder entspräche und auf kein einst im Ganzen wärmeres Klima schliessen liesse. Dagegen kommt Carcharodon productus in Amerika in $41^{\circ} \mathrm{Br}$., in Europa südlicher auf Maltu in $37^{\circ}$ vor. - Mastodon angustidens kommt auch vor, wie in Europa.

e) Aus der pliocänen $\mathrm{Z}$ eit, wo das Pflanzen-und Thier-Reich ihren Formen-Reichthum durch die letzten Schöpfungen vollendet sehen, kennen wir Bildungen aus allen Welt-Gegenden, obwohl oft von

1) Poiss. foss., I, Introd. p. XXVI.

2) Jahrb. 18+6, 635 .

3) Jahrb. 1844, $222 ; 1848,734$. 
unbeträchtlicher Erstrecknng. Am ausgezeichnetsten unter ihnen sind die Subapenninen-Bildungen, der Knochen-fïhrende rothe Crag Englands, gewisse Lagen in Nord-Deutschland und Schichten gehobener Küsten-Strecken fast in allen Kontinenten. Thier-und Pflanzen-Welt besitzen schon völlig ihren jetzigen Charakter, indem sie unter 100 schon 50-70-50-90-95 Arten zählen, welche in denselben Gegenden, zuweilen etwas südlicher, sehr selten nördlicher noch leben. Än den Polar-Kreisen sind die Ablagerungen arm an Arten, wie es die Faunen der Polar-Gegenden noch jetzt sind; in den gemässigteı Gegenden sind beide beträchtlich reicher, in den Tropen sehr reich; daher jedes Becken andre Zahlen und andre Formen aufzuweisen hat, die näher mit der jetzigen Flora und Fanna derGegend als mit denen entfernterer Becken von gleichem Alter übereinstimmen.

a. Die Fauna macht im Ganzen noch immer einen südlichen Eindruck; die grossen Pachydrrmen, die Giraffen, die Affen in mehreren Gegenden Europa's u. a. Erseheinungen tragen dazu bei.

B. In den gemässigten Gegenden Europa's findet man eine Flora, worin die Dikotyledonen und insbesondere Blätter, Hölzer und Fruchttheile unserer Koniferen und Amentaceen vorherrschen; doch mitunter noch mit lirinnerungen an Nord-Amerika (F,üchte von Juglans cinerea in Italien). Die Koralle n. Bildungen ziehen sich in ihre jetzigen Grenzen zurück. Die Konchylien sind den jetzt in der Gegend lebenden Arten ähnlich, einige aber jetzt auf wärmere, sehr wenige (z. B. Cyprina Islandica in Siøilien lebt an den Britischen Küsten) auf höhere Breiten angewiesen, so dass z. B. die Plincän-Bildungen bei Kassel unter 29 noch lebenden Testacepn-Arten nur 7 mit der nahen Nordsee, aber 26 mit dem Mittelmeere und 1 mit ? Senegambien gemein haben, Philipp 1 ${ }^{1}$ ). Die Fische, die Reptilien, die Säugthiere sind wenigsters aus noch in der Gegend lebenden Geschlechtern und oft noch dieselben Arten. Ja es tritt die Übereinstimmung der Faunen mit den jetzigen derselben Länder nicht deutlicher hervor, als in den diluvialen Säugthieren. Wälırend der alte Continent bereits von Pachydermen seine Elephanten, Hippopotamen, Rhinocerosse und Pferde, von Wiederkäuern seine Giraffen, Kameele und Moschus, Ziegen und Schafe, von Fleischfressern seine Hyänen, Löwen, (Bären) zum Theil in ausgestorbenen Geschlechtern, von Affen scine Semnopithecus, von Nagern seine Myoxus, Dipus, Hystrix, Lagomys, von Insectivoren seine Talpa, Myognale, Sorex, Erinaceus ausschliesslich besitzt, zeirhnet sich Amerika wie jetzt an Vögeln durch seinen Dicholophus und seinen Carthartes, an Säugthieren durch seinen überschwenglichen Edentaten-Reichthum aus den Geschlechtern Bradypus, Dasypus, Myrmecophaga, an Schweinen durch seine Dicotyles, an Beutelthieren durch seine Didelphys, an Nagern durch seine Cerodon, Cavia, Coelogenys, Dasyprocta, Synetheres, Osteopera, Myopotanus, Ctenonys, Echimys, Lagostomus, Lonchophorus, an Raubthieren durch seine Galictis, Mephitis. Nasua, an Affen durch seine Jacchus, Callithrix, Cebus aus (S. 712-726 des Enumerators), und besitzt Neu.Holland eine seine jetzige noch weit übertreffende Marsupialen-Manchfaltigkeit ebenfalls von Iebenden wie ausgestorbenen Geschlechtern. Ja es baben diese Länder einen grösseren Reichthum an solchen Formen besessen, als jetzt die ganze Erd.Oberfäche darbieten kann: und die von ihnen gelieferten Dokumente für die Ähnlichkeit des Klima's mit dem heutigen wobei Menge und Grösse der Arten und selbst die Verbreitung mancher

3) Jahrb. $1841,614$. 
Geschlechter noch immer an eine etwas mildere Temperatur erinnern hat um so mehr Werth, als sie auf beiden Hemisphären, in beiden gemässigten Zonen dasselbe Resultat geben, daher auch den Beweis liefern, dass in dirser Tertiär-Zeit die Erd. Axe nicht etwa eine andere Lage besessen haben könne.

$y$. Indessen müssen wir auch an einige Fälle erinnern, welche keine so triftigen Beweise für die einstige Milde des Klima's abzugeben scheinen, als man früher von ihnen angenommen hatte. Wir meinen hauptsächlich die fossilen Nashorne und Elephanten Sibiriens, deren Reste in so unsäglicher Menge nicht nur an der für alle grösseren Herbivoren ganz unbewohubaren Polar-Küste, sondern auch fast noch häufiger auf den Juseln ferno im EisMeer gefunden werden und sirh in Gesellschaft von Rind, Hirsch, Mosrhus, Pferd und wahrscheinlich Megatherium in der Eschscholw-Bai auf der Amerikanischen Seite der Behrings-Strasse in $67^{\circ} \mathrm{Br}$. reichlich wiederfinden ${ }^{1}$ ). Einerseits scheint das dichte und lange Haarkleid, das man an einigen wohlerhaltenen Exemplaren noch gefunden, wie es keines unserer heutigen Parhydermen besitzt, schon auf die Fähigkeit und Bestimmung dieser früheren Thiere hiuzuweisen, ein kälteres Klima als die jetzigen Pachydermen wenigstens vor. übergehend zu ertragen. Andrerseits zeigen die neuesten Mittheilungen v. MiddendorfF's, dass dieselben Schichten, welche noch wohlerhaltene Elephan. ten-Skelette einschliessen, auch Konchylien enthalten, wie sie noch jetzt im nahen Eis. Meere leben, woraus denn folgen würde, dass schon damuls dasselbe Klima wie jetzt in jenen hochnordischen Gegenden geherrscht habe, während jene Pachydermen die weiter südlich gelegenen Horhgegenden bewohuten, von welchen die mächtigen Ströme jener Länder bei ihren periodi. schen Anschwellungen die unbehülflichen Thiere nebst zabllosen NadelholzStämmen - was sie noch jetzt thun - mit sich fortgerissen und dem Meere zugeführt hätten, welches dann diese beiderlei Reste, vielleicht unterstützt und getragen von Eis-Massen, an die Küste geworfen und zwischen den dort lebenden Konchylien begraben hätte. An der Sibirischen wie Amerikanischen Küste der Behrings-Strasse liegen übrigens die Knochen all der erwähuten Thipr-Arten in der Regel nirht mehr Skelett-weise, sondern unordentlich durcheinander in märhtigen Erd-Anschwemmungen, welche vom EisMeere bespült und fortwährend unterwaschen an vielen Orten in Form steiJer Küsten-Wände aufgeschlossen sind; sie sind nach LYELL (a. a. O.) später als das nordische Drift entstanden, und die in ihnen eingeschlossenen ThierArten hätten also die Kälte ïberlebt, welche mit der Fortführung des letzten zusammenfiel? Wir gestehen indessen, dass uns durch jene Erklärung noch nicht alle Erscheinungen ganz klar sind. Dann hat man in Grossbritannien wie in Nord-Amerika einige jugendliche Schichten - alluviale? - aus der „Eis-Zeit" gefunden, welche Konchylien-Arten einscbliessen, die jetzt etwas weiter nordwärts leben; jedoch in - oft vorwaltender Gesellschaft von solchen, die noch in derselben Breite und weiter südlich wolınen, so dass daraus kein Beweis für eine klimatische Änderung im Ganzen gezogen werden kann. So findet sich auch Cyprina Islandica unter Hunderten mittelmeerischer Muschel-Species in den Supenninen-Sthichten Italiens und Sisiliens.

$\delta$. Während wir indessen eine gewisse Analogie zwischen den fossilen Formen Europa's von der Jura-Zeit an bis jetzt und den lebenden Amerika's wahrgenommen, tritt hier in so ferne das Umgekehrte ein, als Amerika einige Reste von pliocänen Pferden, Hippopotamen, Elephanten, Antilopen etc. aufweist, welche seit der Pliocän-Zeit bis jetzt in grosser

1) Jahrb. 1883, 367, 370; 1848, 857; London. Edinb. philos. Mag. 1848, XXIII, 193. 
Häufigkeit dem alten Continente angehörten, noch ein letztes Anzeigen von der anfangs universelleren Verbreitung der Formen. Von der Übereinstimmung der pliocänen mit den jetzt in diesen Gegenden lebenden Arten findet man übrigens im Enumerator wie in den vorhergehenden $\$ \$$. so viele Beispiele aufgezählt, dass wir hier nicht nöthig glauben, länger dabei zu verweilen.

H. Wenn aber ein wärmeres Klima in gemässigten Breiten einstens bis zu Anfang und Mitteder T ertiär-Zeit noch geherrscht hat, so ist zu erwarten, dass nicht nur die eigenthümlichen Formen, sondernauch die reicheren Zahlen wärmerer Gegenden sich in jenen Breiten noch befanden, dass also bis dahin nicht allein dort, sondern in dessen Folge auch auf der ganzen Erd-Oberfläche zusammen genomınen mehr Sippen und Arten derjenigen Klassen, Ordnungen u. s. w., welche zu jener Zeit bereits ihre Vertreter hatten (und diese hatten seit der Tertiär Zeit alle), existirt hahen als jetzt; und Diess bestätigt sich genügend aus den $\mathrm{S}$. $790 \mathrm{ff}$. zusammengestellten Beispielen. Auch diese numerischen Verhältnisse sprechen daher als neuer Beweis für das wärmere Klima; sie sprechen wenigstens von Seiten der Säıgthiere sogar noch in der Pliocän-Zeit dafür, wo wir noch mitunter Beweise einer reicheren Bevölkerung der Gegenden finden, als jetzt.

1. Sichere Beweise von jährlich wiederkehrender oder regelmässig andauernder strenger Kälte, welche Gletscher an den Gehirgs-Ähhängen, schwimmende Eisberge im Meere erzeugt und durch beide die Felsen schranmt, glättet und ritzt und weite Strecken der Polar-Zonen für Pflanzen und Thiere unbewohubar macht, können wir nur seit dem Ende der Diluvial Zeit nachweisen, woselbst diese Erscheinungen in sogar noch ausgedehnterem Grade wenigstens auf einem Theile der polaren Erd-Oberfläche stattgefunden zu haben scheinen als jetzt.

a. Hïten sich schon früher die genannten Erscheinungen so wie seit der Diluvial Zeit eingestellt, so wüıden sie auf Flächen älterer Gesteine, welche mitunter später von neuern Schichten wieder bedeckt worden wären, die genannten Zeichen gerade so zurückgelassen haben wie jetzt. Es würden auf manchen Schichtungs- oder Auflagerungs-Flärhen Fels-Schliffe, Schrammen und Ritzen vorhanden seyn eben so gut, wie die Wellen-Flächen, Fährten u. dgl. m.

b. Indessen besitzen diese Sätze nicht ganz diejenige Beweiskraft, die man ihnen beilegt. Denı nach unsern bisherigen Ergebnissen hätte die Temperatur überall gleichmässig abgenommen; die Gletscher und Eis-Berge müssten von den Polen selbst und von den höchsten Berg-Spitzen aus sich zu bilden begonnen und sich allmählich immer weiter über ihre anfängliche Erstreckung ausgedehnt, folglich die Spuren ihrer frühesten Wirkungen immer weiter selbst bedeckt haben; ja es wäre unmöglich gewesen, dass da, wo ein Gletscher einmal sich zu bilden begonnen hätte, sich neue feste Schichten als Erzeugnisse späterer Perioden auf die früheren Gesteine absetzten, bedingungsweise abgesehen von Moränen und Schutt-Wällen. Nur eben die Zurückziehung der Gletscher von einem Theile der Flächen, die sie bei Beginn der Âlluvial-Zeit eingenommen, hat uns möglich gemacht, ihre unmittelbaren Wirkungen auf die unterliegenden Gesteius-Flächen zu 
studiren. Aber dic schwimmenden Eis-Berge hätten so wie jetzt Blöcke, Schutt und Sand mit sich fortschiffen, in fernen Gegendeu über fremdem, jüngerem Gestein allmählich niederfallen lissen und da, wo sie selbst gestrandet, durch die wechselnde Bewegung des Wellenschlages steigend und sinkend noch eine Zeitlang glättend und ritzend auf die Oberfläche der Ufer-Felsen einwirken können. Auch die Bildung von Furchen wäre denkbar gewesen da, wo diese Eisberge mittelst der an ihrer Unterseite eingefrorenen Felsblöcke bei ihrer Voranbewegung im Meere auf dessen Grund angestreift wären. Die von den Eisbergen entführten und längs deren Wege allmählich sich ablösenden und auf den See-Grund niederfallenden Blöcke pflegen sich von gewissen Punkten oder Linien aus in gleich-oder auseinander-laufenden Streifen zu ordnen, mit der Entfernung von ihrem Ausgangs-Punkte an Menge und Grösse abzunehmen, bergab und bergan selbst da, wo Gletscher nicht aufsteigen hönnten, ohne Unterbrechung fortzusetzen, aus scharfkantigen wie auch zum Theil geglätteten Felstrümmern zu bestehen, sowohl durch die Richtung der Reihen als durch die Art des Gesteines auf den Ausgangs-Punkt zurückzuleiten und durch diese verschiedenen Merkmale zusammengenommen sich von andern Block- und SchuttAnhäufungen zu unterscheiden. Es würde mithin allerdings mögli.h werden, sie zu erkennen, wo sie zwischen älteren Fels-Schichten sich vorfänden, obgleich immer ein Zufall dazu gehören möchte, um uns zu deren Entderkung zu leiten. Bis jetzt konnen wir aus deren Unbekanntschaft mithin nur einen negativen Bew eis nehmen.

c. Bekanntlich hat man die erratischen Phänomene der Eis-Zeit als Beweis einer vor Beginn der jetzigen Periode stattgefundenen grossen TemperaturErniedrigung angeführt. Wir können uns hier nicht nochmals in eine weitläufige Erörterung dieser Erscheinung einlassen und beschränken uns auf die Bemerkung, dass jene Temperatur-Einiedrigung dann jedenfalls nur eben eine einmalige vorübergehende, $k u r z e$, als gleichzeitig und allgemein über die ganze Erde nicht erwiesene und nicht von der inneren Abkühlung der Erde abhängige gewesen seye.

K. Als nur negativen Beweis einer einstig höheren Temperatur der Erd-Oberfläche könnte man den Mangel an eigentlichen TorfMooren sogar noch bis zur Diluvial-Zeit anführen, weil wenigstens unsere Torf-Moore nur in solchen Breiten noch vorkommen, wo ein jährlicher Frost die nengebildete Humus-Säure durch Entziehung ihres Hydrat-Wassers fortwährend unauflöslich macht, oder wo überhaupt eine niedrigere Temperatur der Zersetzung einen Theil des Jahres hindurch Einhalt thut (Gesch. d. Nat. II, 3Ss). Will man sich an diese Erfahrung strenge halten, so würde daraus folgen, dass selbst bis zur Diluvial-Zeit auch im hohen Norden jährlicher Frost nicht stattgefunden liabe.

Indessen scheint es drei wesentliche Bedingungen zu geben, ohne welche Torf-Lager nicht entstehen können: 1) das erwähnte Klima; 2) Süsswasser-Sümpfe; 3) Torf-Pflanzen. Diese letzten bestehen theils aus Kryptogamen (Konferven und Sumpf-Moosen), theils aus Binsen und Riedgräsern, theils endlich aus kronenblüthigen Dikotyledonen - Stauden und -Sträuchern; beim Holz-Torf auch noch aus Nadelhölzern. Jene Dikotyledonen aber haben, wie wir gesehen haben, bis in die Kreide-Periode überhaupt kaum und bis zu Anfang der Tertiär-Zeit nicht in grosser Anzahl existirt; auch Süsswasser-Bildungen sind uns vor dieser Zeit nur spärlich bekannt geworden, wie wir bei andrer Veranlassung zeigten. Welches aber nun die Ursacbe aller dieser Erscheinungen seyn mag, so scheinen doch während der 
Tertiär-Zeit wenigstens alle Bedingungen erfült gewesen zu seyn, die zur Torfmoor-Bildung erforderlich sind, wenn in höheren Breiten ein regelmässiger Winterfrost bereits stattgefunden hätte.

In Gegenden freilich, die von jüngeren Meeren später wieder hoch überfluthet worden sind, würden die schon gebildeten Moore ihrer Leichtigkeit wegen gehoben und zerstört worden seyn. Es könnte also, von eigenthümlich günstigen Verhältnissen abgesehen, nur von jüngeren Meeres-Bildungen unbedeckte, nur unter Binnen-S'huttland, unter Süsswasser-Kalk liegende oder ganz unbedeckte Tertiär-Torf-Lager geben.

Doch! wir erinnern uns des Infusorien-reichen Torf-Lagers tief unter den Fundamenten von Berlin, dessen noch fortpflanzungsfähigen Infusorien-Arten sonst bei Berlin noch nicht beobachtet worden, aber wohl in dem mit Braunkohle und Sandstein wechselnden Lager von Infusorien-Mehl zu Klineken bei Dessau enthalten sind (Gesch. d. Nat. II, 401). Es ist zwar nach den vor uns liegenden Proben noch kein eigentlicher Torf; auch scheint die Frage über das Alter jener Braunkohle noch nicht entschieden; ist es die mit der BernsteinBildung in Verbindung stehende oder eine jüngere? ist es eocäne, miocäne oder gar pliocäne Braunkohle? Jedenfalls scheint Diess der älteste Anfang zur TorfBildung, der Torf aber noch von einer Art zu seyn, deren Bildung an weniger strenge Gesetze geknüpft war.

Die Erörterungen anderer geographischen Fragen, als derjenigen, welche sich unmittelbar auf den allmählichen Temperatur-Wechsel der Erd-Oberfläche beziehen und zu dessen Beweise im Ganzen dienen können, sind einem späteren Abschnitte vorbehalten.

\section{§. 19. Noch fortschreitende Entwicklung der Welt- meere.}

A. Theorie und Erfahrung haben uns bis jetzt in der Ansicht geleitet und wechselweise bestärkt, dass die starre Erd-Oberfläche anfaugs keine grossen Höhen-Unterschiede darbot, indem das Meer ansgedehnter und seichter, die Kontinente weniger zusammenhängend und niedriger waren. Ein tieferes Einsinken des Meeres-Bodens zieht das Meer eben sowohl von den Küsten zurück, als Ansteigen der Inseln und Kontinente es von denselben verdrängt. Das Meer loat also allmählich an Ausdehnung und Zusammenhang ab-, an Tiefe zugenommen. Das wenige niedrige Land war anfangs mehr nur ein Ruhe-Punkt für Luft athmende Küsten- und Meeres-Bewohner, als ein Aufenthalt selbstständiger Landthiere und Pflanzen. Die zwischen den Inseln und kleinen Kontinenten hindurchziehenden Meeres-Arme waren nicht tief; die mit der Rotation der Erde zusammenhängenden Strömungen des Meeres von den Polen gegen den Äquator und unter diesem von Osten nach Westen waren durch vorliegende Kontinente nicht oder nur wenig unterbrochen und gaben daher keine erkältenden oder erwärmenden Ströme in querer Richtung ab; an der Küste bot sich noch nicht der Raum für so viele untereinanderliegende Regionen der Bevölkerung unit abnehmender Temperatur, zunelımendem Druck und manchfaltig wechselnden Boden-Aiten dar, und wegen ihrer zu grossen Tiefe fast ganz unbewolnte Meeres-Striche konnten nicht so häufig und ausgedehnt seyn, wie jetzt. 
Land oder Meer war zwar nicht immer an der nämlichen Stelle, sondern wechselten durch Hebungen und Senkungen des Bodens miteinander ab, wie die manchfaltig unterbrochenen Schichten-Reihen der jetzt aufgetauchten Kontinente beweisen. Während dort ein Land emporstieg, konnte hier ein andres ins Meer versinken; während hier die Tiefen-Stationen der Küste durch Senkung des SeeGrundes sich vervielfältigten, konnte Dasselbe dort mit den HöhenStationen der Berge durch hölıere Hebung derselben geschehen; und überall vervielfältigten sich die Abstufungen des Lebens und Bestehens. Diess hinderte aber nicht das Fortschreiten der Umgestaltung der Erd-Oberfläche als Ganzes genommen in der vorhin angedeuteten gleichförmigen Richtung.

Aber einige Theile derselben haben den bezeichneten Charakter noch theilweise behalten; der ansehnlichste dieser Theile ist die Südsee mit ihren Insel-Gruppen. Dort ist noch auf weite Erstreckung hin der untiefe See-Grund, welcher noch in wechselnder Hebung und Senkung begriffen ist, mit seinen Korallen-Riffen und den niedern wenig unterbrechenden Inseln, und dort herrschen noch jetzt die regelmässigsten Passat-Strömungen.

B. Die nothwendigste Folge der ansgedehnteren, aber unznsammenhängenden Meeres-Erstreckung war das Vorwalten der Wasser- über die Land-Bewohner, nicht sowohl durch die in gleichem Masse mit dem Meere vergrösserte Menge von Meeres-Bewohneru, denen es ebenfalls an manchfaltigen Existenz-Bedingungen noch gebrechen musste, als vielmehr durch die zurïcktretende Anzahl der Landbewohner.

a. Bei den Pflanzen gibt es fast keine Meeres-Bewohner als die Fukoiden und einige Najadeen. Beide sind in verschiedenen geologischen Zeiten vertreten, jene seit der I., diese seit der 111. Periode (Enumerator S. 6 und 34) bekannt, während die allermeisten Land-Pflanzen noch fehlten.

b. Bei den Phytozoen dagegen sind alle Klassen und Ordnungen mit Ausnahme der Polygastrica, welche theils im Süsswasser und theils im Meere vorkommen, dann einzelner Amorphozoen und Polypen, nur Meeres-Bewohner. Während nur die ganz vorherrschenden Meer-bewohnenden Klassen von der I., II. oder III. Periode an in zahlreichen Formen bestehen, scheinen bloss aus äussern zufälligen Gründen die sparsamen und wenig erhaltungsfähigen Pseudozoen, dic mikroskopischen und zur Unterscheidung in ältren Gesteinen nicht geeigneten Polygastrica und die in dieser Bezichung nur wenig mehr begüinstigten (doch seit der Kohlen-Formation d mehrfach angedeuteten) Polythalamien Ausnahmen zu machen, welche indessen nicht gegen den obigen allgemeinen Satz verstossen.

c. Ebenso sind auch alle Malakozoen, die wir in allen Abtheilungen von Anfang an so reichlich vertreten sehen, Meeres-Bewohner, mit Ausnahme nur einiger Pelecypoden- und Gasteropoden-Genera, welche das Land- und Süsswas. ser bewohnen und demnach auch erst am Ende der III. Periode (p) und noch später in $\mathbf{V}$ erscheinen.

d. Unter den Entomozoen sind die Vermes und Crustacea Wasser-, die Arachnidae, Myriapoda und Hexapoda Land-Thiere, indem von den 3 letzten nur einige wenige Genera im reifen oder im Larven-Zustande in Süsswassern vor. 
kommen. Dass jedoch ihr sparsames Erscheinen in den frühesten Perioden nicht aliein von ihrer damaligen Seltenheit, sondern auch von ihrer schwierigen Erhaltbarkeit herrihre, haben wir schon mehrmals angeführt. Die Vermes aber und die Crustacea, so weit sie erhaltbar sind, haben nur einige Genera kleiner Formen in Süsswassern, welche in keiner Weise wesentlich zu einer fossilen Fauna beitragen könnten.

e. Bei den Wirbelthieren endlich sind die Fische ausschliessliche Was. ser-Bewohner, die wir daher auch ganz frühzeitig erscheinen sehen, ohne Repräsentanten bestimmter Sïsswasser-Formen daruuter zu entdecken. Die Reptilien sind theilweise Bewohner des Wassers, doch meistens des süssen; bleibende Meeres-Bewohner sind nur ein Theil der Schildkröten (Chelonia), während Andre wohl in Brackwasser gelebt haben; doch erscheinen sie vor der Mitte der Oolithen-Zeit (o) nicht; die Saurier dagegen reihen sich den ersten Fischen bald (in I.) an, und es scheinen sich den Krokodilen schon frühe einige wenige aber grosse Lacerten, Scinke beizumengen: als Misch-Formen, als Repräsentanten der ersten Lungen-Vertebraten, wenn nicht die Vögel - nach den Fährten zu vermuthen - schon gleichzeitig mit ihnen siud; die Krokodil-artigen Reptilien sind zwar Lungen-Thiere, die gewöhnlich in Flüssen und Binnen-Gewässern wohnen, aber von deren Mündungen an auch ziemlich weit ins Meer hinausgehen; die Lacerten und Scinke sind jetzt nur Land-Bewohner mit Ausnahme des von Darwin erst vor wenigen Jahren auf den Gallopugos-Inseln entdeckten Amblyrhynchus-Geschlechts, welches von den Küsten aus ins Meer hinausschwimmt, un seine Nahrung zu suchen ${ }^{1}$ ). Die ausschlipsslichsten Land-Bewohner-Klassen, neben den Hexapoden, Arachniden und Myriapoden sind die Vöyel und Säugthiere, die wir demnach auch am allerspätesten in Menge erscheinen sehen.

C. Es ist bekannt, dass viele fossile Saurier in der WirbelBildung von den jetzt lebenden abweichen, in so ferne die 2 GelenkFlächen der Wirbelkörper nicht die eine konvex und die andern konkav, sondern beide flach oder konkav sind, wie Diess bei den Fischen (ausser Lepidosteus) und diesen zunächst stehenden Reptilien, nämlich an Batrachiern auch der Fall ist. Man hat desshalb dieses Merkmal anch als einen Beweis niedrigerer Organisation bei denjenigen fossilen Sauriern anführen wollen, wo es vorkommt. Es gehört aber nicht nothwendig tiefer stehenden, sondern mehr den im Wasser lebenden und lebhaft schwimmenden unter den niedern Wirbel.Thieren an und macht die Wirbelsäule biegsamer, beweglicher, geschickter beim Schwimmen, daher auch die mit Flossenfüssen versehenen Ichthyosauren und Plesiosauren bikonkaven Wirbel haben, obwohl dagegen einige schıelzschuppige Fische mit Knochen-Skelett (Lepidosteus etc.) konvex-konkave Wirbel besitzen. Nach R. OWEN kommen von unten nach oben die ersten konvex-konkaven procölischen Wirbel (wie sie ausser Gecknalle lebenden Saurier haben) vor bei Lacertiern in der Kreide (Mosasaurus), bei Krokodiliern in Londonthon, bei Ophidier" in Londonthou. Aber aus dem Uinstande, dass selbst alle Krokodilier (Dactylopoden) vor der Kreide (ausser Streptospondylus) nicht-konvexe Wirbel haben (Enumerator S. 6S6, und Archegosaurus, Goniopholis, Suchosaurus u. a. S. (691-693), so wie, dass 2

1) Сн. Danwin, Journal of Researches, $1845,8^{\circ}, 385$, c. icone. 
ausgestorbene Gruppen von Riesen-Sauriern (Cetiosaurus, Polyptychodon, Mosasaurus und Leiodon S. 692-693), wovon die eine der jetzigen Schöpfung ganz fremd ist, die andere (Pachypoden, Megalosaurus, Hylaeosaurus, Iguanodon, S. 659) in der Organisation zum Theile unsren Scinken näher stehet, ebenfalls mit bikonkaven Wirbelı versehen sind, würde vielleicht hervorgehen, dass dieselben sich anch schwimmend im Meere bewegt haben; doch mögte ein Theil der letzten geschlossene Brackwasser-Busen nicht verlassen haben.

D. War das Meer bei grössrer Verbreitung von geringerer Tiefe, so fehlten ihm in demselben Verhältnisse die ausgedehnten Striche, welche ausser schwebenden Infusorien, Würmern u. dgl. fast gar keine Bewohner haben, indem der Grund zu tief unter $600^{\prime}$ von Pflamzen, unter $1800^{\prime}$ von Thieren nicht bewohnt (Forbes) und daher auch von wandernden Fischen und Raubthieren nicht besucht ist. In der That fältt es auf, dass wir so wenige ruhig aus dem Meere niedergeschlagene Gesteins-Schichten kennen, welche nicht die gewöhnlichen Thier-Reste, Konchylien, Würmer u. s. w. enthielten. Nur die Sand-Schichten machen eine Ausuahme, da sandiger Meeresboden in allen Tiefen, etwa mit Ausnahme von Würmern, unbewohnt zu seyn pflegt. - Wo ausgedehnte Meere nicht zugleich t i ef sind, können grosse Cetaceen nicht leben. - Der Wasser-Druck scheint auf die Verbreitung der Organismen nach der 'Tiefe des Meeres von geringem Einflusse zu seyn; weit wichtiger ist die Wärme, welche, gleich dem Lichte, mit der Tiefe des Ozeans bekanntlich abnimmt und endlich auf $3^{\circ}-2^{\circ} \mathrm{C}$. herabsinkt; - daher in den spätesten geologischen Perioden die meerische Küsten-Bewohner der Polar-Kreise die ihnen nothwendige Temperatur in grossen Tiefen gemässigter Gegenden wiederfinden und eine geographische Verbreitung gewinnen konnten, die ihnen ıächst der Oberfläche unmöglich wäre.

a. Doch ist Diess nicht bei den älteren Schichten allein, sondern auch bei jüngeren der Fall und steht wieder mit der Thatsache im Zusammenhang, dass in sehr tiefen Meeren, welche in Verhältniss ihrer Tiefe auch weit von den Küsten entfernt sind, keine Schichten-Nieders'hläge stattfinden, wenn nicht Kalk-Quellen u. dgl. zu Hülfe kommen, daher Schichien Bildung und organisches Leben durch eine dritte gemeinsame Bedingung mit einander verknüpft sind.

b. Die grossen Cetaceen erscheinen erst mit Beginn der Tertiär-Zeit, obwohl es scheint, dass ausser etwa Knochenfisch-Nahrung und der gleichbleibenden allgemeinen grossen Tiefe das Meer ihnen schon früher alle Bedingungen der Existenz geboten haben müsse.

c. Aber auch untiefe Stellen in den Sandbänken des offnen Meeres sind wegen der Beweglichkeit des Grundes und der Bewegungs-Kraft des Wassers gäuzlich unbewohnt. Eine Tiefe von einigen hundert Fussen auf der hohen See, von $100^{\prime}-120^{\prime}$ in ruhigeren Buchten bieten das reichste Leben dar ${ }^{1}$ ).

1) Davis on tidal currents (Cambridge 1849) p. 125. 
E. Im Ührigen treten die Wirknngen der Verhältnisse der frühesten Meere deutlicher durch den Gegensatz hervor, wenn wir die der sich ausbildenden Kontinente betrachten.

F. Bimnenmeere trennen unsre gegenwärtigen Floren und Land-Faunen nicht, sondern nur ausgedehnte Weltmeere, und selbst bei diesen findet sich an den entgegengesetzten Küsten gewöhnlich eine Anzahl identischer Formen (Gesch. d. Nat. II, 247). Eine noch grössere Übereinstimmung werden wir daher erwarten dürfen in den geologischen Gebilden, die sich in einem kleineren oder ausgedehnteren Meeresbecken einst niedergeschlagen und entweder noch vom Meere selbst oder von jüngeren Bildungen in ihrer Mitte bedeckt, in Folge eingetretener Hebungen jetzt gleich alten Küsten rings um den tiefern Theil desselben zu Tage gehen; - und, in den Resten der m e e$r$ is chen Organismen, welche in den Schichten dieser ehemals entgegengesetzten Küsten aufhewahrt liegen.

So erblicken wir landeinwärts von der Nord- und Süd-Küiste des Mittelmeeres, in Europa wie in Afrika dieselben subapennischen, dieselben Rudisten-reichen Kreide-Bildungen mit gleichen organischen Einschlüssen von Konchylien, Foraminiferen und Infusorien.

Befremden aber müsste die Verschiedenheit der Organismen in dem eocänen Becken, welches ehedem über Nord-Frankveich (Paris), Süd-England (London), Belgien und einen Theil von Nord-Deutschland sich ausbreitete, da man den Reichthum an Konchylien überhaupt und an grossen und schönen Siphonobranchiern insbeson. dere, wie er in der Nähe von Paris stattfindet, in den andern Gegenden dieses nicht sehr giossen Beckens vergeblich sucht. Wir wissen aber, dass auch jetzt in einem und dem nämlichen Becken verschiedene Ursachen, wie Tiefe, Art des Bodens, Art der Ufer, kältre und wärmere Strömungen, Salz- und Kalk-Gehalt u. dgl., grosse Abweichungen in der Bevölkerung hervorrufen können.

๑. 20. Nach fortschreitender Entwicklung der Kontinente.

A. Unter Verhältnissen, wie wir sie im vorigen Paragraphen vorausgesetzt, war das Land anfangs flach, daher mehr als jetzt von Buchte" und Meeres-Armen durclischnitten, mehr in kleine und grosse Inseln getrennt, welche erst später mehr ansteigend in Kontinente zusammen flossen. Hohe Gebirgs-Ketten, Hochebenen u. s. w. waren fast noch ohne Einfluss auf die Wärme- und Feuchtigkeits-Erscheinungen der Atmosphäre, die überall milde und feucht war; SchneeGebirge konnten wenigstens in vielen Gegenden noch nicht abkühlend wirken, wo sie später vorhanden sind ; der Gegensatz zwischen Kontinental- und Küsten Klima, zwischen excessivem und gemässigtem Klima war noch unbedeutend; grelle Witterungs-Wechsel, manche unregelmässige und gewaltsame Luft-Strömungen mangelten; die Entladung und die Ablenkung der Wolken durch ausgedehute Gebirgs . Ketten, die Zusammenhäıfung derselben in den Knoten-Punkten mehrer Ketten fand nicht oder wenig Statt; mächtige Flüsse von ausgedehntem Laufe konnte es nicht geben; vielleicht waren Süsswasser- 
Flüsse und See'n seltener, weil der neue dem Meere entstiegene Bodeu noch nicht überall ausgesüsst war. So war nur erst eime geringe Manchfaltigkeit in den topographisch-klimatischen Verhältnissen des Landes, welche allmählich uicht nur selbst immer mehr zunehmen, sondern auch die Lebens-Bedingungen für die organischen Wesen immer mehr vervielfältigen sollten, wie die Inseln und Kontinente an Ausdehnung wuchsen.

Wir würden jene anfängliche Beschaffenheit des Bodens und des Klimas am ehesten in ebeneren Theilen Gross-Britaniens, in dem milden griechischen Archipel, in Westindien und (von den zum Theil sehr hohen Gebirgen abgesehen) auf den Sunda-Inseln, vorzüglich aber auf den kleinen Inseln in der Süd-See und auf Neuholland noch einigermassen verwirklicht finden. Da, wo die Zustände des Meeres bis jetzt noch fast dieselben geblieben sind, finden wir auch die des Landes wieder: die zahlreichen, grossen und kleinen, meist nur niedrigen und dann stromlosen Inseln mit dem milden, feuchten, in räumlicher und zeitlicher Dauer gleichförmigen Klima, das schon allein einen Theil der Erscheinungen erklären würde, die wir vorhin (\$.18) aus der höheren Ur Temperatur der Erde abgeleitet haben : die einförmige Farnen-Vegetation der ersten Periode (S. 871 c.) in Neuseeland, weiterhin die Baum-Farnen, Cycadeen und Palmen der tropischen InselGruppen, die täglich weit ins Meer hinaus schwimmenden Eidechsen der Gallopagos-Inseln (Amblyrhynchus), die in Knochen-Struktur dem riesigen Megalosaurus ähnlichen grossen Scinke Australiens, welche wie diese mit Araucarien und Cycadeen, mit Clavagellen, Terebrateln, Trigonien und sogar Beutelthieren zusammen vorkommen ${ }^{1}$ ), den Mangel an andern, wenigstens an grössern Säugthieren u. s. w.

a. Vgl. hiezu was $\$ .12$ S. 816 über die Beziehungen der höhern Organisation der jetzigen Säugthiere zur Grösse der Kontinente gesagt ist.

b. Wir haben früher ( $\$$. 17) die Möglichkeit gesetzt, dass die Atmosphäre einst höher, dichter, schwerer, Kohlensäure-reicher gewesen seye, Verhältnisse, auf die wir desshalb hier nicht mehr Rücksicht zu nehmen haben.

B. Die Folgen eines in Dauer und Ausdehnung gleichförmigen milden und feuchten K lim as, ohne Erkältung durch Schnee-Gebirge, mussten sich zunächst in der Vegetation zeigen, und wir haben schon mehrfach gesehen, wie eben eine dominirende Farnen-Flora, BaumFarnen, grosse Lykopodiaceen u. s. w. einem solchen Klima so vorzugsweise entsprechen, dass es schon aus deren Auwesenheit gefolgert werden kann (S. S71). Im Thier-Reiche würde man die Spuren desselben etwa unter den Reptilien am ehesten wahrnehmen.

Der Einfluss des Klimas, soferne dasselbe nicht von der Temperatur-Höhe allein herrührt, zeigt sich bei Wasser-Bewohnern wenig, und daher bei Thieren in Ganzen weniger, als bei Pflanzen, indem die ersten vorherrschend Wasser-Bewohner, und nur in den höhern Unterreichen meistens Land-Bewohner

1) R. OwEN im Jahrb. 1842, 492. 
sind, welche alser theils (Insekten) zu reichlich wiecier verloren gegangen, theils (die 2 höhern Wirbelthier-Klassen) zu spät erschienen sind, um uns Kunde von den frühesten klimatischon Zustïnden der Erde bringen zu köunen. So bleiben uns unter den Thieren nur die Reptilien übrig, eine Klasse, die im Ganzen nur den wärmeren Klimaten entspricht und in diesen theils in trorkner Luft (Eidechsen), theils an und in dem Wasser (Krokodile, Schildkröten, Batrachier) lebt und durch diese letzten der angedeuteten Beschaffenheit des Klimas zwar wohl entspricht, aber in den ältesten Zeiten zu viele fremde Furmen enthält, um uns eine verlässige Botschaft zu gewähren über andre klimatische Beziehungen.

C. Der anfängliche Mangel an trockuem Land und zumal an ausgedehnten Kontinenten (soferne dessen Folgen nicht schon in $B$ mitbegriffen sind) würde sich zuerst in dem verspäteten Auftreten von Landbewohnern überhaupt, welche vorzüglich in Pflanzen bestehen, und nachher in dem Mangel zahlreicher und grosser Säugthiere erkennen lassen, wie wir bereits $\mathbf{S}$. 816 nachgewiesen laben. Das bestätigt sich in der That bis zum Beginne der Tertiär-Zeit, wo es danı gewiss an grösseren Kontinenten nicht mehr gefehlt hat. Wir geben folgende Übersicht des Erscheinens der Binnen-Thiere, woraus die Spärlichkeit derselben vor der Tertiär-Zeit deutlich wird; die Unterbrechung in der Kreide-Zeit rührt von dem bisherigen Mangel an Siisswasser-Niederschlägen aus dieser Zeit her.

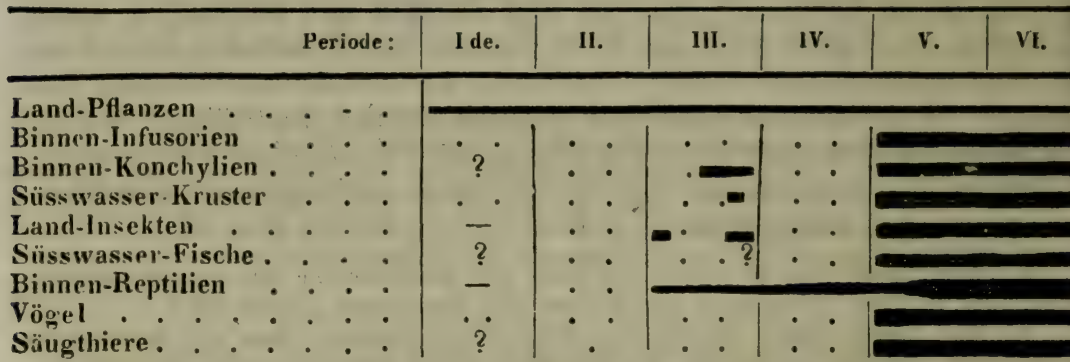

Von Binnen-Konchylien haben wir vor der Wealden-Formation keine verlässige Spur, wenı nicht das ausgestorbene Genus Anthracosia, welches trüher mit Unio verwechselt worden, das letzte auch durch seinen Aufenthalt in Süsswasser vertrat. Auch die Süsswasser-Kruster beginnen mit Cypris in den Wealden; was man früher von diesem Geschlechte angeführt, wird wohl aus dem Meere stammen und ganz oder zum Theil andern Geschlechtern anheim fallen. In manchen Fischen der Kohlen Formation, die mit Pflanzen und Anthracosia vorkommen, könnte man Süsswasser-Bewohner vermuthen, aber nicht beweisen; in den Wealden kommen ebeufalls nur ausgestorbene Fisch-Genera vor mit Arten aus solchen, die sonst im Meere leben. Wie viele von den Reptilien der Kohlen-Formation reine Lilıdthiere, Bewohner von Süsswasser-Rändern und von Mceres-Küsten gewesen, lässt sich noch nicht eutscheiden. Vögel haben in den Gesteinen der 1. Periode nur Fährten hinterlassen.

D. Die Abwesenheit beträchtlicher Gebirgs-Höhen in den ersten Periodeu der organisclien Welt maclite die Zusammenhäufung der Bewohner verschiedenartiger Klimate anf kleinen HorizontalStrecken unmöglich; und in der That finden wir in den pflanzlichen 
Resten der Gesteins-Schichten nichts, was auf eine Zusammenhäıfung im Pflanzen-Reiche hinwiese, sondern erkennen darin nach der Analogie urtheilend nur Bewohner niedrigerer Gegenden; der Mangel an Gebirgs-Höhen ist daher eine Mitursache der geringen Ma nchfaltigkeit in der Flora frühester Zeiten. Hinsichtlich des ThierReiches aber können wir Zeugniss-gebende Überreste nicht erwarten, da der Land-bewohnenden Klassen wenige, die Insekten schlecht erhalten, und die warmblütigen Wirbelthiere zu spät aufgetreten sind. Die riesigen Echsen, die Krokodile und andere Reptilien früberer Zeit waren aber sicher Bewohner der Niederungen.

E. Eine Folge der Erhebung zusammenhängender Gebirgs. Ketten ist die Verschiedenheit der Klimate anf beiden Seiten derselben; entgegengesetzte Expositionen entstehen, warme und kalte, feuchte und trockne, und der Bevölkerung der Niederungen wird der Weg versperrt sich in dieser Richtung weiter auszudehnen und an beiden Seiten in einander zu greifen, wie die Bewohner der Höhen nicht über die Niederungen hinweg andre Gebirge erreichen können. Ausgedehnte Gebirgs-Ketten schieden daher verschiedent F a u n e und F loren auf beständige Weise, sobald eine solehe Verschiedenheit in verschiedenen Welt-Gegenden einmal bestund, d. h. nur von der Kreide- oder ersten Tertiär-Zeit an, falls auch hinrei chend ausgedehnte Ketten schon frühei vorhanden gewesen wären.

Wir dürfen eine sichtliche Wirkung der Gebirgs-Ketten in dieser Beziehung nicht früher als von Ende der Kreide- oder vom Anfange der Tertiär-Leit an erwarten, weil vorher überall eine gleichartige Bevölkernng gelebt zu haben scheint.

So sind in der That nach D'Oraigny die Konchylien-Arten in den EocälıSchichten zu beiden Seiten der Anden unter sich schon ebenso verschieden, als die jetzigen Bewohner des Atlantischen und des Stillen Ozeans es sind, die mur eine gremeinsame Art darbieten ').

Se stimmen nach Enrenberg die mittel- oder ober-tertiären Bildungen im Westen der Rocky-nountains an der Mündung des Columbia in den Stillen Osean hinsichtlich ihrer Kiesel-Infusorien und zumal des Reichthums an BiblariumArten nicht mit denen im Osten desselben Gebirgs, sondern mit jenen im ferne gegenüberliegenden China und Sibirien überein ${ }^{2}$ ).

F. Da wir Süsswas er-Quellen selbst auf den kleinsten und niedrigsteı noch kahlen Korallen-Inseln entstehen sehen, so köu nen wir wohl nicht daran zweifelı, dass es dergleichen anch auf den in der Urzeit zuerst aufgetauchten Land-Strecken gegeben, dass sie sich in Flüsse vereinigt, dass sie Süsswasser-See'n gebildet hahen u. s. w., sohald sie nur den jungfräulichen Boden erst ausgesüsst, ilım erst die Salz-Theile entzogen hatten, welche er aus dem Schoose des Meeres mit emporgebracht hat. So scheint es, habe auch frïlızeitig schon eine Fauna und Flora der Süsswasser bald nach Beginn des

1) Jahrb. 1815, 373. - 2) Berlin. Munatsb. 1815; 63. 
Binnen-Lebens überhaupt entstehen können; doch finden wir uns in dieser Erwartung auffallend getäuscht; Süsswasser-Pflanzen (die überhaupt auch jetzt selten sind) kennen wir erst ans der Tertiär-Zeit, Süsswasser-Thiere seit Mitte und Ende der Oolithen-Periode unter dem Oxford-Thon und in den Wealden.

Es ist schon angeführt, dass unter den Fischen, Muscheln (Anthracosia) und vielleicht selbst kleinen Krustazeen der Kohlen-Formation möglicher Weise Bıackwasser- und Süsswasser-Bewohner seyen; aber beweisen lässt es sich nicht; Dasselbe gilt von den Fischen in den mitteln Oolithen von Brora und in den Wealden, und vielleicht von Schildkröten aus solchen Geschlechtern, die jetzt sich auf Süsswasser beschränken, da solche nicht selten auch in rein meerischen Schichten fossil gefunden worden sind. Aber das zufällige Erscheinen der Libellen in dem meerischen Lias und in den lithographischen Schiefern, deren Larven in Süsswassern gelebt haben müssen, die Cyclas-, Cyrena-, Pla. norbis- und Limnaeus-Arten in Oolithen und Wealden dürften jedenfalls den Beweis liefern, dass von der III. Periode an es an bewohnten Süsswassern nicht gefehlt habe, obschon sich in der Kreide-Zeit deren Spuren wieder verlie. ren und sich hinsichtlich der Wealden noch ibre grosse obwohl jetzt unterbrochen scheinende Ausdehnung (von England bis zum Harse) und das Vorkommen einzelner Meeres Muscheln einwenden lässt, wornach diese Bildung wohl eher nur einem Brackwasser, einem Etang ihren Ursprung verdanken könnte.

Wie schwierig oder vielmehr unmöglich es sey, die $\mathrm{F}$ is che nach Familien und Geschlechtern in See und Süsswasser-Bewohner zu unterscheiden, - wie selbst eine und dieselbe Spezies zuweilen beide Arten des Wassers bewohne, und über ähnliche Verhältnisse in andern Thier-Klassen haben wir das Nöthige auseinandergesetzt in der Gesch. d. Nat. Band II, S. 52-58.

Murchien, Hibbert, Conybeare u. A. haben zwar wiederholt Süsswasser. Schichten schon in der Kohlen-Formation argenommen, Diess aber zu einer Zeit, wo man die fossilen Reste in solcher Beziehung noch nicht so scharf zu beurtheilen wusste, wie jetzt ' $)$. Die angeblichen Schildkröten und Gavial-Zähne sind zu Fisch - Resten, die angeblichen Cyprinoiden. Fische zu Ganoiden, die Unionen zu zweifelhaften Anthracosien, die Süsswasser-Kruster aus dem Cypris. Geschlechte zu meerischen Cytheren und Cypridinen geworden, die Landpflan. zen ohne Beweis-Kraft.

Dagegen hat zuerst RoBERTgon am Brora-Flusse in Southerlandshire das Vorkommen von Süsswasser-Konchylien in Schichten der Oolithe entderkt, welche nach Murchison jedenfalls beträchtlich unter dem Oxford-Tlione, also im Herzen der Oolithe liegen. Die Schichten-Folge ist

f. Kalkige Sandsteine

e. Schiefer und Kohlen einige Fusse märhtig

d. Schiefer mit Süsswasser-Fossilien, etwa $1^{\prime \prime}$

c. Schiefer und Kohlen wis e, $2^{\prime}-3^{\prime}$

b. Thon mit Fossilien, 14"

a. Schiefer mit einigen Pflanzen.

Die Schicht b ergab Schuppen von $2-3$ Lepidotus-Arten, Zähne von PAcrodus minimus und Hybodus minimus Ag., einige Paludina-Arten, $2-3$ ? PernaArten, eine neue Unio-Art, einige Cyrenu-Arten, worunter auch Cyclas media der Wealden, einige Cypris-Arten, verkohlte Pflanzen-Reste; - die Schicht d lieferte Schuppen wie von Lepidotus fimbriatus und Megalurus, einige neue PaludinaArten, zum Theile die vorigen; Cyclas (Cyrena-) Arten 1-2 neue; neue CyprisArten, zum Theil die vorigen; undeutlichen Pflanzen. Da dieselben Fische im

1) Jahrb. 1834, 468-470. 
See-, Süss- und Brack-Wasser vorkommen können, so hat man also in beiden Schichten nur Süsswasser-Organismen, mit Ausnahme der Perna-Arten in den untern, welche noch schärfer untersucht werden müssen ').

Die Wealden-Gebilde sind zu vielfältig bekannt, als dass wir nöthig hâtten, hier noch weitläufig auf sie zurückzukommen. Eine Übersicht ihrer fossilen Reste ist schon S. 791 gegebell. funden.

In der Kreide (IV.) hat man noch durchaus keine Süsswasser-Schicht ge-

G. Süsswasser im Gegensatze des Meeres haben auch Einfluss nicht allein auf das Erscheinen gewisser Sippen, sondern auch auf die Ausbildung gewisser Art- und Individuen-Formen.

Diess ist deutlich bei solchen Ctenobranchiern, die im See- und SüssWasser zugleich leben können, wie Paludina und Neritina. E. Fonses beobachtete auf der Insel $\operatorname{Cos} 3$ über einander folgende Schichten tertiärer Süsswasser-Niederschläge, die aber nach oben brackisch werden und durch welche je eine Art beider Sippen ganz hindurchreicht; in der untersten ist ihre Srhaale glatt und ungefaltet, in der mitteln höher und mit einer starken Furche, in der obersten noch höher und ebenfalls mit einer starken Furche längs der Ümgänge umgeben. Einen gleichen Formen-Wechsel hatte Forbes schon früher an andern lebenden Paludinen- und Neritinen-Arten beobachtet, die dem Wechsel von Süss-, Brack- und See-Wasser ausgesetzt waren.

§. 21. Nach den Wechselbeziehungender Organismen unter sich selbst.

A. Die Organismen stehen in so manchfaltigen Wechselbeziehungen zu einander, einzelne zu einzelnen Reichen, Klassen, Geschlechtern, Arten, dass die Existenz der einen die der andern voraussetzt. Diese Wechselbeziehungen sind entweder mittelbare, indem sie zunächst nur mit der Atmosphäre in Verbindung stehen und die Respirabilität derselben wie das Klima bedingen; oder unmittelbare und dann nährende, beherbergende, schützende u. s. w.

B. In einer mittelbaren Wechselbeziehung, der wichtigsten von allen, steht das gesammte Pflanzen-Reich mit dem gesammten ThierReiche durch den Respirations.Prozess, in soferne jenes der Atmosphäre unausgesetzt den Kohlenstoff zu seiner eignen Ernährung entzielit, welchen dieses der Luft eben so unausgesetzt zuführt (Gesch. d. Nat. II, 466); nur durch diese Wechselthätigkeit bleibt die Luft nährend für die Pflanzen und athembar für die Thiere; in Klimateı, wo die Vegetation im Winter fast ganz ruhet, ist auch das ThierLeben in dieser Zeit weniger thätig (viele Säugthiere und Reptilien schlafen im Winter, viele Vögel wandern aus, die Insekten und Würmer ruhen); vielleicht sind in kalten Klimaten die immergrünen $\mathrm{Na}$ delholz-Wälder bestimmt den Athmungs-Prozess von Seiten der Pflanzen wenigstens theilweise im Winter zu unterhalten, so viel das Bedürfniss des Thier-Reichs erheischt. Vorausgesetzt also, dass die Mischung der Luft von Anfang her so wie jetzt beschaffen gewesen

1) Jahrb. 1844, 623, 624 . 
und ihr durch andre Kräfte mehr Stoffe als jetzt weder zugeführt noch entzogen worden wären, hat das Pflanzen-Leben gleichzeitig mit dem Thier-Leben beginnen müssen, weil die Respiration eines von beiden allein für die Dauer nicht möglich war; war das quantitative Verhältniss der Respiration des Pflanzen- und des Thier-Reiches ein anderes als jetz,t, so hat sich die Luft-Mischung und hiedurch das Verhältniss beider Reiche zu einander mit der Zeît ändern müssen. Wären der Pflanzen relativ weniger als Thiere gewesen, so hätte sich die Kollensäure in der Luft bald in einem für letzte und endlich für beide verderblichen Grade häıfen müssen; wären ihrer mehr geweseı, so konnten sie die Luft allmählich von einem Übermasse der Kohlensäure, die anfänglich existirt hätte, befreien. Da die Atmosphäre aber offenbar auch noch andre Zuflüsse und Verluste gehabt hat, als durch die organischen Wege, so ist es schwierig, bestimmte Folgerungen zu ziehen (vgl. $\$ .17$ ).

C. Eine andre mittelbare, aber ebenfalls sehr mächtige Beziehung tritt ein durch den Einfluss des Pflanzen-Reichs auf W itterung und K I ima, wie wir solchen schon in der Geschichte der Natur (Bd. II, S. 465-496) auseinander gesetzt haben. Insbesondere ist es die Wälder-Vegetation und mehr jene anf Gebirgen als in der Ebene, welche das Klima bedingt, die Temperatur milder und gleich. förmiger, die Feuchtigkeit der Luft stärker, die Regen regelmässiger macht, die Flüsse reichlicher und steter mit Wasser versieht, welches oft entfernte Gegenden zu bewässern bestimmt ist ; sie ist es endlich, welche oft die Stärke und Richtung der herrschenden Winde bedingt, die Schnee-Grenze zurïckdrängt u. s. w. So wirken die Wälder, indem sie die Eigenschaften des Ḱlimas und selbst mitunter des Bodeus bedingen, auch auf das Thier-Leben ein, nicht sowohl indem sie es auf diesem Wege möglich oder unmöglich machen, als indem sie seine Vertheilung und Verbreitung abändern. Die anfänglich vorhanden gewesenen Farmen- und Sigillarien-Wälder scheimen aber ganz andre Wirkungen und die letzten insbesondere eine mehr geologische gehabt zu haben.

a. Wir können zwar mit Bestimmtheit einen ursächlichen Zusanımenhang nicht nachweisen zwischen dem genauern Zusammentreffen der reichen LaubWaldungen sogleich im Beginn der Tertiär-Zeit und der allomemen Verbreitung der Süsswasser-Bildungen, nachdem früher nur in den Weulden (\$. $20 \mathrm{~F})$ ein obwohl grossartiges Beispiel bekannt geworden und sie in der Kreide-Zeit wieder ganz fehlten. Aber es ist so auffallend, dass wir uns der Frage nicht erwehren können, ob man denn vor Entstehung der Wälder, ob man vor der Tertiär-Zeit irgendwo Spuren von Bächen, Flussbetten gefunden, die ja doch gewiss uls mächtige Eindrücke der Erdoherfäche, als Einschnitte in ganze Reihen von Gebirgs-Schichten u. s. w. in vielen Fällen von neueren Schichten bedeckt und so als Denkmäler der füheren Geschichte der Erde viel leichter aufbehalten worden seyn müssten, als Diess mit den sogenannten RegentropfenLöchern, den Fährten u. \&. w. der Fall gewesen ist. Müsste man nicht hier und dort die charakteristischen Geschicbe.Bänke der Flussbetten zwischen den Gcbirgs-Schichten längst cutdeckt haben? Es ist aber uns wenigstens nicht 
bekaunt geworden, dass dergleichen irgendwo nachgewiesen worden seyen. Und wenn solche nun in den ältesten Gebirgs-Schichten wirklich nicht aufgefunden werden könnten, würde es glaublich seyn, dass Flüsse und Ströme einst existirt hätten? Wüıde nicht dieser Mangel dem Mangel an gleichzeitigen Süsswasser-Bildungen, den man als Thatsache betrachten darf, zur Seite stehen? Und doch, ist denn ein Pflanzen- und Thier-Leben überhaupt ohne Regen, ohne Quellen und Flüsse möglich?

b. Vor der Tertiär-Zeit könnte es nur Nadelholz., Palmen-, Lykopodien-, Kalamiten-, Farnen.Wälder gegeben haben; aber obwohl wir fossile Bäume dieser Art kennen, so wissen wir doch nicht, ob sie zusammenhängende Wälder gebildet haben. Dagegen haben wir sichere Anzeigen von Sigillaria-oder Stigmaria-WäIdern') eigenthümlicher Art, welche uur die den Überschwemmungen ansgesetzten Seeküsten bedeckt und ganz andere Wirkungen hervorgebracht zu haben scheinen, als unsre jetzigen trockenen Hochwaldungen. Vielleicht latten sie mit unsren Mangle-Wäldern eine gewisse Analogit und mehr Bedeutung für die Bildung der Erd-Rinde als für die Ernährung des Thier-Reichs und des Pflanzen - Wuchses. Die Sigillarien werden gewöhnlich in die Nähe der Farnen gestellt, haben aber in der innern Textur mit den Koniferen viele Ähnlichkeit. Man hat in den Sand- und Thon-Schichten der Steinhohlen-Gebirge zahlreiche Stämme von Lykopodien, Kalamiten und insbesondere Sigillavien theils liegend und theils - insbesondere diese letzten - aufrecht stehend reftinden, welche $2^{\prime \prime}-2^{\prime}-4^{\prime}-5^{\prime}$ dick waren und, wenn auch nicht mehr in ihrer ganzen Höhe sichtbar, doch öfters $6^{\prime}-10^{\prime}-15^{\prime}$ weit durch verschiedene Schichten hindurch verfolgt werden konnten. Besonders in englischen KohlenWerken fand man sie oft in grösserer Zahl neben einander, zu Wigan an der Jiverpooler Eisenbahn einen ganzen Wald bildend und nn' $8^{\prime}-12^{\prime}$ weit von pinander entfernt. Wo sie nach unten oder nach oben in ein Kohlen-Flötz pindringen, da lässt sich oft ihre Spur nicht mehr weiter verfolgen, während jedoch in andern Fällen es gelungen ist. Wo man aber in Sand-und besonders festem Thon-Gebirge sich dem untern Ende der Stämme nähert, da werden allmählich die charakteristischen Rippen und Narben der Sigillarien undeutlicher, und es entspringt zuletzt ein Kranz von Wurzeln aus dem untern Ende. Obschon dieses in Sandstein-Schichten oft deutlicher hervortritt, so ist es in gewissen feuerfesten an Eisenstein-Nieren reichen schlammigen ThonMassen (die gewöhnlichen Schiefer-Thowe dürften dazu nicht geeignet seyn) mit allen seinen Anhãngen am besten erhalten, wie sich bei sorgfältiger Nachforschung zeigt. Die untern Enden liegen aber, in den Sandsteinen wenigstens, in ungleichen Niveau's. Geht man den daraus entspringenden, fast horizontal ausstrahlenden, bald etwas auf- und bald ab-wärts gerichteten WurzeIn nach, welche anfangs noch undeutlich gefurcht, dann runzelig, nachher rauh von Oberfläche sind, sich wiederholt gabelförmig theilen und über $15^{\prime}-20^{\prime}$ weit verfolgen lassen, so sieht man sie $4^{\prime}-6^{\prime}$ vom Stamme entfernt allmählich in Stigmarien mit den charakteristischen spiral-ständigen Narben und Anhängen übergehen, die man früher als Blatt-Narben und Blätter betrachtet hatte, welche aber fusslange strickförmige zusammengedrückte Wurzeln sind, die jenen Thon $2^{\prime}-4^{\prime}$ tief in allen Richtungen dicht durchziehen ${ }^{2}$ ).

Erst in neuerer Zeit hat man zwischen den Sigillarien auch aufrechte Kalamiten in beträchtlicherer Anzahl gefunden, die aus Quincunx-ständigen

') J. HАพк8наw > Jahrb. 1843, 374, 375, 1844, 871; J. E. Bowman, das. 1843, 375: Barber-Beaumont das. 378; E. W. Binney $>$ Jahrb. 1848, 254, 376; Rich. Brown das. 377; - Hooker in James. Journ. 1849, XLVI, $73-78,174-180$.

2) Dass der innere Bau der Sigillarien von dem der Baum-Farnen und Lepidodendren abweiche und nit Stigmaria einerseits, Cycadeen andrer- 
Narben an den untern Abgliederungen überall ähnliche Würzelchen schief abwärts senden wie Stigmaria. BINNEY fragt daher, ob dip Kalamiten etwa bloss junge Sigillarien seyen, da er jene nie über, diese nie unter $6^{\prime \prime}$ dick gefunden; doch führt Riсн. Brown welche von 2" Dicke an. Zwischen diesen aufrechten Sigillaria- und Kalamiten-Stämmen hat man gewöhnlich auch viele schiefstehende und liegende theils derselben Art und theils von Lepidodendren mit Abdrücken von Farnen-Wedeln, Lepidophyllen, Sphenophyllen u. s. w. gefunden. Auch Koniferen-Stänme scheinen da und dort aus der Nähe herbeigeschwemmt, nicht aber an Ort und Stelle gewachsen zu seyn. Es scheint hienach keinem $\mathbf{Z}$ weifel zu unterliegen, dass hier die Sigillarien noch mittelst ihrer Stigmarien-Wurzeln auf ihrem natürlichen Boden stehen. Nach der Verbreitung der strickartigen Wurzeln zu urtheilen war Thon dieser Boden, einst ein thoniger Schlamm. über welchen während der Vegetation der Sigil. Jarien-Stämme sich Schichten von Kohle und Sand anhäuften und die Basen jener Stämme umgaben. Einmal hat man auf der Fläche zwischen der Thonund der unmittelbar darauf ruhenden Kohlen-Schicht eine grosse Menge Lepido. strobus Früchte gefunden, als ob sie sich dort auf natürlicher Boden-Oberfläche allmählich angesammelt hätten. Die Kohlen-Schicht selbst ist eine Ansammlung der kohligen Rückstiinde aus der Vermoderung der fortwährend absterbenden Theile der während Jahrhunderten hier gediehenen Sigillaria-Wälder: die Humus. Sehicht des Waldbodens, die sich nach Massgabe der fortschreitenden Vermoderung und späterer Austrocknung noch mehr zusammensetzte. Die ganz gleichmissige Dicke aller oft nur je $1^{\prime \prime}$ bis $3^{\prime}$ und viel mehr $\left(20^{\prime}\right)$ haltenden und sich bis über 100 fach wiederbolenden Kohlen-, Thon- und Sandstein-Wechsellager des Kohlen-Gebirgs auf Erstreckungen von mehren Hundert Quadratmeilen wie sie in Belgischen, Englischen und Nordamerikanischen Revieren bekannt sind, ist ohne eine gleichzeitige nivellirende Thätigkeit der Gewässer, und zwar selbst nivellirter See-artiger Gewässer, nicht denkbar. Es kann aber hinsichtlich des Schlammes und Humus das Nivellement dadurch bewirkt worden seyn, dass jene Pflanzen oder wenigstens die Sigillarien unter oder, wenn auch über dem Wasserspiegel wachsend, doch nirht höher gediehen, als dass das Wasser in schlammigem Boden sich überall reich. lich bis zu ihren Wurzeln aufwärts ziehen konute, wie bei unsern Torfnooren. Damit würde aber nur die einmalige Bildung einer Thon- und einer Humus. Lage sich erklären; sollten nun Sandstein-Schichten sich darüber absetzen, so musste die ganze Oberfläche des Bodens gleichmässig unter den See-Spiegel einsinken, damit die See die bereits gebildeten Schichten gleichmässig mit Sand überfluthen konnte bis fast wieder zur Höhe des Spiegels, worauf SchlammNiederschläge und vielleicht während fortdauerndem Sinken eine erneuerte Vegetation folgte, wie vorhin. Nur in Folge eines solchen fortdauernden Sinkens und der Annahme einer Stigmarien-Vegetation in der Höhe des SeeSpiegels selbst lässt sich die hundertfältige Wechsellagerung von Thon, Kohle und Sandstein erklären. Mit dieser Ansicht scheint die sehr grosszellige Textur der Sigillarien in Einklang zu stehen, da solche eben Wasser-Gewächsen zuzukommen pflegt. Die Eisenstein-Nieren urd-Lager, welche in den Kohlen-Gebirgen und insbesondere in dem Thone nicht selten sind, würden den Sumpf-Erzen unsrer Torfmoore einigermassen entsprechen, obschon ihre Bildungsweise abweichend gewesen seyn muss. Wälder dieser Art, auch an Höhe unsren Hochwäldern nicht vergleichbar, haben natürlich in klimatologischer Hinsicht nicht leisten können, was diese letzten; aber ihre geologische Wirkung mag ansehulich gewesen seyn. Die weiten flachen

seits nahe übereinkomme, bestätigt und beschreibt ausführlich AD. BrongNIART in A rchiv. d. mus. d'hist. nat. 1840, I, 405-461, > pl. 25-35 > l'Institut. 1840, 415. 
Mulden von mehr als 40 Stunden Länge und Breite, worin ihre Überreste abgelagert zu seyn pflegen, die ansehnliche Erstreckung seichter Meeres-Becken, beide entsprechen vollkommen dem Bilde, welches wir uns (S. $898 \mathrm{ff}$.) von der Beschaffenheit der Erd-Oberfläche in jener Zeit gemacht haben.

D. Unter den unmittelbaren Wechselbeziehungen der Organismen steht die gegenseitige E rnährung ohen an, womit sich freiwillige und unfreiwillige Beherbergung und Schutz oft verbindet. Wenn man sich erinnert, dass die Pflanzen bis an's Ende der Kreide-Zeit nie mehr als 22 Familien mit 176 Geschlechtern auf einmal aufzuweisen hatten, dass mit der Tertiär-Zeit aber die höhren Dikotyledonen hinzukommen und sich hiedurch, obschon manche ältere Formen ganz verschwinden, die Zahl der uns bis jetzt bekannt gewordenen Familien und Geschlechter sogleich auf 76 und 237 erhob, welche jetzt 276 : 6529 ist (S. $799 \mathrm{ff}$.) und auch in der Tertiär-Zeit gewiss schon fast gleich vollständig existirt hat, so wird man leicht ersehen, dass hiemit erst die Bedingung der Existenz für alle diejenigen Pflanzenfressenden Landthiere gegeben war, welche sich nicht von den rauhen Farnen, Equiseten, Schachtelhalmen, Palmen, Cycadeen und Koniferen mit den zwischen ihnen stehenden ausgestorbenen Familien zu nähren vermochten; denn diese 12 Familien, welche fast die Hälfte aller successiven vor tertiären Pflanzen-Familien betragen, enthalten gegen $2 / 3$ der Geschlechter und $3 / 4$ aller bis dahin vorhandenen Arten in sich, wozu wieder die Farnen allein die Hälfte der Arten lieferten, eine Familie von der sich verhältnissmässig nur wenige Thiere nähren. Wir setzen daher sicher das Verhältniss nicht zu hoch an, wenn in Anbetracht des Vermögens vieler Thiere sich doch von Pflanzen verschiedener Familien zu nähren, wir sagen, dass wenigstens 0,9 aller jetzigen herbivoren Land-Thiere wegen Mangels an Futter-Manchfaltigkeit früher überhaupt nicht bestehen konnten, was dann wieder die Unmöglichkeit der sie verzehrenden Fleischfresser nach sich gezogen haben würde. Wir unterscheiden daher zunächst in dieser Beziehung 1) als Herbivoren, 2) als Verzehrer dieser Herbivoren

1. Pflanzen - Fresser.

Myriapoda und einige Arachnidae tracheariae.

Hexapoda: viele Diptera; alle Lepidoptera, Hemiptera, Orthoptera; die Hälfte der Hymenoptera und fast alle Coleoptera nähren sich von Pflanzen.

Reptilien : fast keine. (also jetzt nur die Krokodile und

Vögel: die Körnerfresser unter den Insessores, die Rasores und einige Grallae.

2. Fleisch-Fresser.

Arachnidac: die meisten; und unter den

Hexapoda: viele Diptera; die meisten Neuroptera; die Hälfte der Hymenoptera, viele Coleoptera (Carnivora, Brachelytrata etc.) leben von andern Insekten.

Reptilien: fast alle Landbewohner.

See-Schildkröten ausgenommen).

Vögel: die Insekten-Fresser unter den Insessores, die meisten Grallae; die Raptores.

(also nur die Palmipedes meistens ausgenommen).

S ä u g-Thiere: herbivore Cetacea; S ä ug-Thi ere: Edendata und MarRuminantia; Pachydermia; Glires; supialia zum Theil; Insectivora und 
Edentata und Marsupialia zum Theil; einige Quadrumana; Carnivora.

Quadrumana meistens.

(also nur die Pinnipedes und ächten Cetacea nebst einigen Fisch-fressenden Raub-Thieren ausgenommen).

Allein es leben nicht allein Thiere einer Klasse von einander (eigentliche Raubthiere), oder höhere Thiere von niedrigeren Thiereı und von Pflanzen, sondern auch umgekehrt niedre von höheren, die sie entweder als lebendige Beute ergreifen (wie manche Raubvögel und theilweise auch die Krokodile Säugthiere), oder als äussere und innere Parasiten (Epizoen und Entozoen) bewohnen, um sich anf ihre Kosten zu nähren; oder sie saugen deren Blut ohne auf ihnen zu wohnen (Hämatopoten), oder verzehren sie nach ihrem Tode (Nekrophagen), oder endlich sie finden ihre Nahrung in deren Abgängen und bringen darin ihre Brut unter (Coprobia).

1. Epizoen:

Viele Luft-Insekten unter den TracheenSpinmen (Zeeken etc.) und den Diptera (Hippoboscidae); die Suctoria, die Anoplura.

2. Entozoen:

fast sämmtliche Enthelminthes; einige Diptera (Östriden) zeitweise.

3. Hämatopoten:

manche Dipteren (Tabanii, Culiciden),

Hemipteren (Acanthia) etc.

E. Wie nun manche Thiere an andre Thiere oder an Pflanzen in soferne gebunden sind, als sie ohne dieselben nicht leben künnen, so sind sie es auch von der andern Seite durch die Nothwendigkeit, dieselben in ihrer numerischen Entwicklung zu beschränken und das erforderliche Gl e i $\mathrm{ch}$ ge w i ch t zwischen den einzelnen Gruppen herzustellen. Wir sehen daher fast in allen Klassen, wo es dergleichen gibt, Herbivoren und Karnivoren nngefähr gleichzeitig auftreten, weil die letzten olıne die ersten nicht bestehen können, die ersten ohne die letzten sich bald bis zum Verhungern vervielfältigen würden.

F. Das Auftreten des Menschen am Ende der SchöpfungsZeit ist eine nothwendige Folge nicht allein des Gesetzes der allmählichen Vervollkommnung der organischen Welt $(\$ .12,13)$, sondern nach der Entwicklung der Glieder beider organischen Reiche in Verhältnisse mit den äussern Existenz-Bedingungen, welches letzte Gesetz, wie wir gesehen haben, die eigentliche Grundlage des ersten ist ( $\varsigma .16, C)$. Der Mensch bedurfte aber nicht allein gleich den Thieren bloss Boden, Luft, Trank und Speise, sondern um sich über die ganze Erde verbreiten zu können, auch der Mittel für Kleidung und Wohnung; er bedurfte um alle seine intellektuellen Fähigkeiten zu entwickeln, um sich zum selbstbewussten Herrn der Schöpfung zu machen, um die Summe seiner Erkenntniss bis zum höchsten Grade zu steigerı, um endlich seinen Mitmenschen Gerechtigkeit und Liebe, um der Gottheit,
III 
um dem höchsten Guten Verehrung zu zollen, - er bedurfte, um aus einem intelluktuellen Wesen zugleich ein moralisches zu werden, des ganzen reichen Füllhorns der Natur, un sich, aus diesem sowohl alle Befriedigungs-Mittel seiner kürperlichen Bedürfnisse wie alle BildungsMittel seines Geistes zu schöpfen, die sich wechselseitig bedingen und heben. Das Erscheinen des Menschen am Ende der Schöpfung ist daher lediglich Folge des Gesetzes der Existenz-Bedingungen; welche indessen an sich nicht ausschliessen, dass etwa noch eine Anzahl von Thieren und Pflanzen gleichzeitig mit ihın geschaffen worden wären: er konnte nicht vor den lefzten, aber er musste uicht uach den letzten der übrigen Organismen erscheinen.

G. Es ist treilich denkbar, dass, wenn die ganze jetzige Organismen-Welt zugleich geschaffen worden wäre, auch der Mensch damit hätte bestehen können; wir wissen also nicht, ans welchem Grunde eine allmähliche wiederholte Schöpfung statt einer einmaligen und vollendeten stattgefunden; können aber auch hier nur die Nothwendigkeit der Wechselwirkung der natürlichen Kräfte unterstellen, da wir im II. Bde. d. Gesch. d. Nat. und später gezeigt haben, welch' mächtigen Einfluss die organische Welt auf die Mischung der Lnft auszuüben im Stande gewesen ist und welchen Antheil sie an der Gestaltung des geschichteten Theiles der Erd-Oberfäclse wirklich genommen hat und noch nimmt.

\section{f. AlImählicher Annüherungs - Fang der Schöpfung zu ihrex jetzigen Beschaffenheit.}

§. 22.

A. Welche Ursachen immer auf die Beschaffenheit der ersten organischen Formen oder auf ihre allmählichen Veränderungen gewirkt haben mögen, wir sehen einen allmählichen Übergang der frühesten Schöpfung zur gegenwärtigen vor uns, der sich bethätigt:

durch ein fortwährendes Hinzutreten neu erschaffener vollkommenerer Formen zu den anfänglich vorhandenen und ein fortwährendes Aussterben eines Theiles der ältern;

durch ein Auseinandertreten anfänglich chaotischer FormenKeime in differenzirte scharf geschiedene Formen-Reihen; daher durch eine Vervielfältigung der Zahl und Manchfaltigkeit der anfänglichen Gestalten, so wie in Verbindung damit das Steigen der zu einem Genus gehörigen Arten-Zahlen im Allgemeinen (\$. 10); durch eiı häufiges Zurückgehen anfänglich gigantischer Masse zu minderen Grössen;

durch ein fortwährendes Anpassen an die allmähliche geologische Umgestaltung,der äusseren Existenz-Bedingungen, welche selbst hinfort als Ursachen der vorher genannten Veränderungs-Gesetze der Organisation erscheinen müssen.

B. Diese Ursachen waren von Anbeginn her thätig, und mit ihnen dauerte von Anbeginn das Hinzutreten neuer vollkommenerer 
Formen zu den alten und das theilweise Aussterben der früheren; aber das Auseinandertreten jener Formen-Keime in neue FormeuReihen begann bei verschiedenen solchen Keimen je nach deren früherem oder späterem Auftreten zwar zu sehr verschiedenen Zeiteı, aber immer wieder nach dem gleichen Gesetze; - die Zeit der Scheitel-Höhe der Zahlen-Reihen wurde durch Beides bedingt; - und die der höchsten Grösse-Steigerung fiel theils mit der höchsten ZahlenEntwickelung und theils mit der höheren Wärme des Klima's u. s. w. zusammen;! jede diese Erscheinungen verfolgte also doch ihren eigen $\mathrm{t} h$ ii $\mathrm{ml}$ ic hen G a ng, den wir noch weiter theils näher beleuchten, theils in Vergleich mit dem der andern betrachten wollen.

C. Der Formen-Wechsel ist bei verschiedenen Gruppen ein selır ungleich rascher. Denn ein Theil der niedrigeren Organismen (Korallen, Infusorien?, Pelecypoden, Gasteropoden u. s. w.) beginnt niclit mit so fremdartigen Gestalten wie die übrigen und hat, um zu den jetzigen Ausdrücken der Form zu gelangen, wenige oder gar keine Veränderungen der Organisation zu durchlaufen. Andern ist dagegen eine sehr lange Formen-A bstufung beschieden; diejenigen unter ihnen, welche zuerst (in Periode I und II) auftreten, haben dazu eine lange Zeit vor sich, während die spätesten Ordnungen (manche Reptilien, die Säugthiere u. s. w.) dieselbe rasch durchwandern müssen. Fremde Gestalten stehen daher von Anfang an neben den bekannten, und noch im Beginue und in der Mitte der Tertiär-Zeit sehen wir neben Wolf und Löwe die abenteuerliclien Erscheinungen des Zeuglodon und des Dinotherium, - ja an deren Ende noch die „aus dem Munde gefallenen" Formen Siid-Amerikanischer Edentaten. Die Molasse enthält nebeı miocänen Land-Säugthieren (Mastodon angustidens etc.) schon eine ganz pliocäne Testaceen-Fauna. Es erstreckt sich Diess bis auf die lebenden Arten fort; denn neben den ausgestorbenen Hyänen und Bären unsrer Höhlen oder den Elephanten, Nashörnern u. s. w. des Lösses liegen oft auch schon in demselben Knochen-Lehm, in derselben Breccie u. s. w. die an der Oberfläche umherkriechenden Helix-Arten.

So sollen in den Süsswasser.Schichten von Mundesley die Fisch-Arten nach Agassız ausgestorben seyn, während die Konchylien noch lebenden Arten angehören ').

So enthält der Löss neben ausgestorbenen Arten von Elephas, Rhinoceros, Ursus nach Alex. Braun unter 97 Konchylien Arten 89 noch lebende und nur 8 jetrt ausgestorbene ${ }^{2}$ ).

So der Süsswasser-Kalk im $I l m$-Thale bei Weimar mit ausgestorbenen Arten von Elephanten, Nashorn, Hyäne (und vielleicht lebenden des Pferdes, Rindes, Hirsches) nur Konchylien lebender Arten, wovon 7 genannt werden ${ }^{3}$ ).

So kommen nach LyeLL ausgestorbene Pferde- und Mastodon-Arten mit SeeKonchylien lebender Spezies zusammen in Süd-Carolina und ausgestorbene Mastodon - und Megatherium-Arten mit vielen ebenfalls noch lebenden

1) BR. Collect. 58. - ${ }^{2}$ ) BR. Collect. 65. $-{ }^{3}$ ) Jahrb. 1847, 313. 
Seekonchylien-Arten in Georgia vor. Mastodon mit Binnen-Konchylien lebender Arten an den Niagara-Fällen in New-York, in Rochester und in Genesee ').

In Sibirien fand von Midpendorf die Mammuth-Reste in Gesellschaft derjenigen Konchylien-drten im Boden eingebettet, welche daselbst noch jetzt das Eismeer bewohnen ( $\$$. 19). haben ${ }^{2}$ ).

Gleiche Bemerkung scheinen auch Horner und Darwiv gemacht zu

Fasst man indessen die Formationen ins Auge, worin das Mammuth vorkommt, und berücksichtigt die Menge der eben darin enthaltenen Säugethiere noch lebender Arten, so verliert diese Erscheinung einen Theil ihres Befremdenden.

D. Die Veränderungen, welche eine Annäherung zur jetzigen Schöpfung bezwecken, erfolgen theils sehr allmählich (die Uimgestaltung und Verminderung ächter Brachiopoden), theils auch sehr plötzlich (Auftreten der Dikotyledonen, der Teleosti etc.) und in gewissen Zeitscheiden nach der einen oder nach der andern Weise, bald mehre miteinander und bald einzeln nacheinander.

E. Die so auffallende, so höchst wichtige Differenzirung der Klimate nach Massgabe der geographischen Zonen in Folge stattgehabter 'Temperatur-Abnahme, wenigstens in so weit sich solche aus der geographischen Vertheilung der organischen Reste erkennen und beurtheilen lässt, fällt ihrer Haupt-Wirkung nach merkwürdiger Weise nicht mit einer der Zeitscheiden zwischen je zweien der aus den erheblichstenGründen von uns angenommenen Perioden zusammen, sonder" mitten in eine solche Periode hinein (vou $t$ bis w).

F. Unter allen geologischen Zeit-Scheiden ist in paläontologischer Hinsicht keine auffallender als die $z$ wischen der Kreide- und TertiärZeit, wenn man solche nur im Grossen und Ganzen berïcksichtigt, wälırend bei Paris u. a. 0 . der Schichten-Übergang zwischen beiderlei Gebirge ein ganz allmählicher ist, wie auch sogar einzelne ThierArten unbezweifelt aus der Kreide-Periode in die tertiäre und bis in die jetzige übergehen. In diesem Zeitpunkt fällt aber zusammen :

1) Das gänzliche und plötzliche Aufhören der bisher so zahlreichen Ammoneen und Belemniten unter den Cephalopoden und der grossen Rudisten unter den Brachiopoden.

2) Das der Cestracionten und Hybodonten und beinahe auch der Ganoiden bei den Fisclien, und das der letzten von den abenteuerlichen Familien bei den Reptilien, welche bis an das Ende der OolithZeit herrschend waren.

3) Die höheren Dikotyledonen-Pflanzen (die Hälfte der Monochlamydeen, alle Corollifloren und Choristopetalen), welche bei weitem die grösste Masse des ganzen Pflanzen-Reiches bilden, erscheinen plötzlich in ganzer Fülle, nachdem nur sehr wenige noch zweifelhafte Vorboten vorhergegangen.

1) Jahrb. 1843, 858, 859. - 2) Jahrb. 1848, 738. 
4) Die Knochen-Fische, bei weitem die Mehrzahl im ganzen System der Fisch-Klasse bildend, erscheinen, nachden allerdings schon in der Kreide-Periode eine Anzahl von Geschlechtern derselben aufgetreten ist, in ihrem ganze" Unfange, wobei zu bemerken, dass gegen das Alter der Glarner (r) Fische Zweifel geweckt worden sind und sie ihre richtigere Stellung wohl in $\tau$ finden werden.

5) Unter den Reptilien treten Batrachier und Schlangen auf.

6) Die höchsten Thiere, die Klassen der (Vögel und) Säugthiere erscheinen von da an in Menge, nachdem zuvor nur 4-5 Arten im Ganzen bekaunt geworden.

7) Gewiss ist auch die grösste Masse der herbivoren Insekte॥ erst um diese Zeit erschienen (S. 907), obwohl wir nicht die Mittel haben, es aus ihren Resten zu beweisen. Der bedingende Zusammenhang zwischen Satz :3 und 6 ist deutlich, der von 3 einerseits mit 4 und 5 andrerseits nicht; auch fällt Letztes nicht so genau zusammen, wie Erstes.

Wenn also so ungeheure Veränderungen in der organische⿰t Welt plötzlieh eintreten und doch einzelne Spezies ihre Existenz uIIzweifelhaft fortbehaupten, wird man an gewaltsam allzerstörende Katastrophen zwischen den verschiedenen Perioden zu glauben gerue verzichten.

G. Auf Verhältnissen ganz anderer Art beruhet die Begrenzung zwischen der tertiären und der jetzigen Zeit. Schon vor ihr hatte die Schöpfungs-Kraft in Bezug auf die bloss physischen Organismen gauz abgeschlossen; mit ihr hörte das Erlöschen der Organismen aus geo. logischen Ursachen auf, der Mensch als intellektueller Organismus mag nun erschaffen seyn mit oder nach den zuletzt erschaffenen physischen Naturen (S. s:3i, K). Dabei ist der Übergang aus der tertiäreı in die jetzige Zeit ein so allmählicher, dass man, ohne das Menschen-Auftreten und das nachherige Erlöschen der Schöpfungs-Kraft zu berücksichtigen, immer versucht seyn würde beide nur als eine Periode, die jetzige nur als die unmittelbare Fortsetzung der tertiären Periode zu betrachten; denn wir kennen nur hier und an keiner alldern Zeit-Scheide den Fall, dass die der Grenze unmittelbar vorangehenden Schichten so grosse Mengen von mit der Folge-Zeit gemeinschaftliche Arten darböten, wie hier, wo wir die Quoten dieser Arten allmählich von $0,02 \mathrm{zu} 0,20-0,50-0,50-0,90-0,95$ u. s. w. wachsen sehen.

H. Wir haben früher gezeigt, dass Pflanzen und Thiere, so weit sie ihren Klassen, Ordnungen, Familien nach vertreten waren, jederzeit eben so reichlich vorhanden gewesen sind, als jetzt (S. $790 \mathrm{ff}$.). Nun waren aber seit der Tertiär-Zeit alle Klassen, Ordnungen u..s. w. vertreten, so dass, wenn auch einzelne kleine Familien, Genera u. s. w.noch fehiten, andre jetzt fehlende ihre Stelle einnahmen ; - 
es stimmt daher nicht nur mit diesem Gesetze überein, sondern ist auch wenigstens durch alle einigermassen vollständiger erhaltenen Klassen, Ordnungen u. s. w. hindurch im Verhältniss dieser Erhaltbarkeit nachweisbar, dass wälırend der ganzen Tertiär-Zeit in den 3 (S. 796) für sie angenommenen Arten-Altern Thier- wie PflanzenReich im Ganzen genommen wenigstens eben so zahlreich waren, als jetzt. Ja in Folge der anfänglich ( $t$ und $u$ ) noch höheren Temperatur waren beide sicher noch beträchtlich zahlreicher als jetzt, wenigstens in denjenigen heissen und gemässigten Breiren, welche man bisher genauer zu untersuchen und wo man die Beweise jener höheren Temperatur zu entdecken Gelegenheit gehabt hat. Die Annäherung der frühesten zur jetzigen Schöpfung fand also in diesem e i $\mathrm{z}$ i g e $\mathrm{n}$ Falle nicht auf dem geradesten Wege Statt; und doch war diese Abweiclınng eiue Folge successiver Ännäherung des Klima's, kombinirt mit der annähernden Zunahme des Formen-Reichthums.

Es dürfte überflüssig seyn, noch mehr Belege für jene Behauptung zu häufen, als S. $792-794$ bereits zusammengestellt sind.

K. Die Steigerung der Lebenwelt bis zur Schöpfung der Menschen musste daher zuerst den numerischen Kulminations-Punkt erreicht gehabt haben und den der Organisation erreichen, ehe sie den absoluten Kulminations-Punkt, den der Schöpfung der Vernunft erreichen kounte. Der Mensch selbst erschien auf den Gipfel der Organisation gehoben; aber das Gewicht der Masse sank vor der Herrschaft des Geistes.

\section{Geographie der fossilen organismen.}

๖. 23. Im Allgemeinen.

A. Die Geographie der fossilen Organismen könnte man in eine stetige oder beharrliche und eine periodische, dann in eine allgemeine und besondere theilen. Die Grundlage der periodischen Geographie wird nach dem bisher Vorgetragenen die Geographie der GebirgsFormationen seyn.

B. Unsere Bemühungen indessen irgend welche zuverlässige, durch alle Perioden hindurchgreifende Züge einer s te tig e n Pflanzen - und Thier-Geographie zu entdecken, sind vergeblich gewesen. Ausser der unserer jetzigen Zonen-Richtung fast entsprechenden Zonen-artigen Vertheilung einiger Kreide-, Nummuliten- und späteren Tertiär-Gesteine, die man in der nördlichen Hemisphäre, nämlich vou Amerika durch das Mittelmeer bis China entdeckt zu haben glaubte (S. 881 ff.), und ausser der Gemeinschaftlichkeit einer gewissen mässigen Arten-Zahl, welche zwischen je zwei benachbarten Perioden überall zu bestehen pflegt (S. 749 ff.), ja in einigen wenigen Fällen in einer und der nämlichen jetzt gemässigt warmen Gegend von der 
weissen Kreide an his in die jetzige Periode sich erstreckt (S. 763, 764), ist in der Pflanzen- und Thier-Geographie verschiedener Zeiteı nichts bleibend gefunden worden.

Diese Arten sind a. a. O. genannt; hier nur Einiges über ihre geographische Verbreitung in verschiedenen Zeiten:

Terebratula caput-serpentis findet sich in der weissen Kreide (r) Englands, Belgiens, Rügens und des Deutschen Festlands, im Subapenninen-Gebilde Siciliens (w) und lebend (z) an den Kästen Niciliens, Skandinaviens wie NordAmerika's (New-York) und, wenn wir nicht irren, auch am Cap der guten Hoffnung.

Dentalina communis und Rotalina umbilicata D'O. kommen fossil in der $\boldsymbol{P a}$ riser (r) Kreide, im Wiener Becken (u), im Subapenninen-Gebilde Italiens (w) und lebend (z) im Mittelmeere vor.

Echinocyamus pusillus findet sich in den cocänen Schichten von Paris (t), in den miocänen der T'ouraine und Englands (u), in den pliocänen Süd-Frankreichs (w) und lebend sowoh! an der Norwegischen und Englischen Küste wie im Golf von Tarent und im Ägäischen Meere, soferne nämlich Fibularia ovulum und F. Tarentina L. nach Fopess nur Varietäten einer Art sind.

Andre noch lebende Arten der Europäischen Tertiär-Schichten gehören theils den diesen Schichten benachbarten Meeren, theils wärmeren und selbst tropischen Klimaten entfernter Meere an. So Niso terebellum in den Gewïssern von Nicobar? - Tritonium clathratum Lk. im Südamerikanischen Orean; - Tritonium nodularium LK. nach Grateloup in Ostindischen Osean; - Ancillaria canalifera Lк. im Indischen Ozean (A. candida?); - Oliva flammulata Lк. am Senegal; so dass diese andre Reibe von Arten nicht nur der obigen gegenüber für ein wärmeres Klima spräche, soudern auch durch die Verschiedenheit ihrer jetzigen Heimath wieder die kleine Übereinstimmung theilweise verwischt, welche zwischen der eocänen und der jetzigen Schöpfung Europa's zu bestehen schien.

C. Die besondere Pflanzen-und Thier-Geographie kann sich immer nur auf einen Zeitraum (Periode, Formation) anf einmal beschränken. Sie kann entweder die Verbreitung der Schöpfung Klasse um Klasse über die Länder verfolgen, oder Land um Land hinsichtlich seiner Schöpfung mit einander vergleichen, so dass in ersten Falle man das Bild der gleichzeitigen Verbreitung jeder einzelnen Klasse и. s. w. über die ganze Erdoberfläche, im andern das Bild der gesammten Schöpfung in jedem einzelnen Lande auf einmal erhält. Die Aneinanderreihung beider Arten von Bildern nach Perioden und Formationen fällt dann der Geschichte der organischen Welt anheim. Die besondere Geographie aus dem ersten oder systematischen Gesichts-Punkte ist speziell in unserem Enumerator schon enthalten; er stellt sie in vertikaler Folge dar (während die verschiedenen Perioden und Formationen in horizontaler Richtung neben einander gereihet sind), was uns erlaubt, hier kurz darüber wegzugehen, indem wir bloss einige Allgemeinheiten hervorheben. Die Betrachtung aus dem zweiten Gesichtspunkte, Zonen-, oder Regionen- oder Länder-weise, ist die eigentliche geographische Behandlung des Gegenstandes und, obschon in unsrem Eummerator im Speziellen ebenfalls schon gegeben, doch noch einer umständlicheren allgemeinen Bearbeitung fïhig, zumal die historische Aneinanderreihung daselbst nicht 
immer dentlich wird. Znerst wollen wir indessen noch einige allgemeine Fragen erläutern, welche mehr auf den ersten Gesichts-Punkt oder auf beide Bezug haben.

Dieser Gegenstand lässt sich durch methodische Zerlegung aller damit ver. knüpften Fragen sehr ausgedehnt und ins Einzelne behandeln; unsre Detail-Beobachtungen aus andern Welt-Gegenden sind aber noch viel zu unvollkommen, als dass sich erhebliche Früchte eines so ins Einzelne gebenden Verfahrens erwarten lirssen.

D. Wie in der jetzigen Schöpfung, so gibt es auch in der fossilen örtlich beschränkte und weit verbreitete Arten und S i p pen. Von den ausgebreiteteren unter ihnen ist oben S. $868 \mathrm{ff}$. bei den Untersuchungen über die Temperatur-Veränderungen der ErdOberfäche u. S. 914 schon mehrfach die Rede gewesen; viele sind genannt, alle sind im Enumerator ihren Verbreitungs-Zonen und Welttheilen nach bezeichnet worden. Während aber gegenwärtig der klimatische Unterschied der Zonen die Verbreitung der Organismen von Norden nach Süden in der Weise beschränkt, dass nur wenige Arten eine Ausdehnung von $20^{\circ}-30^{\circ}-40^{\circ}$ in dieser Richtung besitzen, und während der zweimalige Wechsel von Festland und Weltmeer der Verbreitung in gleichem Klima von Osten nach Westen im Wege steht, so dass mit Ausnahme einer kleinen Quote jeder der 2 grossen Kontinente so wie die grösseren und entlegeneren Inseln und jedes der zwei Weltmeere auch unter gleicher Breite eine um so verschiedenere Flora und Fauna besitzen, einen je grösseren Kreis die Zone um die Erd-Achse beschreibt und je weiter die Kontinente und Weltmeere gegen den Äquator hin und die ersten südlich über denselben hinaus auseinander laufen können; so fand früher die Verbreitung der Arten wenige Hindernisse von Norden nach Süden in der klimatischen Verschiedenheit der Zonen und weniger von Osten nach Westen in der Trennung der Weltmeere durch die mächtigen queerziehenden Kontinente. Nur insoferne wirklich des Landes überhaupt weniger, daher sein Zusammenhang geringer, die Fntfernung der Inseln und kleinen Kontinente von einander im Ganzen grösser gewesen, mussten Land-Bewohner mehr Schwierigkeit gefunden haben, sich von einem Stammpaare und einem k le in e u Scliöpfungs-Zentrum aus, falls dergleichen wirklich existirt hätten, was nicht wahrscheinlich ist, weit auszubreiten, woferne nicht ihre Eier und Samen fein und leicht genug waren durch Wind und Wogen in grosse Ferne geführt zu werden. Auch scheint sich in der That eine ehemals weitre Ausbreitung dieser lełzten sowohl als der Seethiere zu bestätigen (S. 868 ff.), während wir über die eines Theils der frühesten Land-Thiere (Insekten) wegen der Seltenheit und schwierigen Bestimmung ihrer fossilen Reste nicht urtheilen können, ein andrer höher organisirter Theil derselben aber (Reptilien) einst vielleicht eben so wie jetzt überhaupt eine beschränktere Verbreitung als die niederen Organismen im Allgemeinen besessen laben mag. Wie übrigens selbst ein und derselbe Ozean 
oder Kontinent unter gleicher Zone in verschiedenen Gegenden doch eine verschiedene Fanna und Flora hat, so haben solche Verschiedenlıeiten. bei einem gleichförmigeren Klima frïherer Zeit gewiss auch nicht ganz gefehlt, wie die Beobachtung bestätigt.

a. Man würde aus theoretischen Gründen eine weitre Verbreitung erwarten dürfen: (1) überhaupt mehr bei mikroskopischen Organismen und solchen mit mikroskopischen Eiern und Samen, die also im reifen oder im Ei-Zustande von Winden leicht über Land und Meer, über Berg und Thal hinweg getragen werden komten (Iıfusorien, kryptogamische Zellen- und Gefäss-Pflanzen); (2) bri unvollkommen organisirten, noch wenig differinzirten Wesen, welche sich dann auch gegen den Wechsel der äussern Lebensbedingungen indifferenter als die vollkonmueren zu zeigen pflegen, Frost und Trockne überstehen und sich nachlser wieder erholen können (lıfusorien); (3) bei See-Bewohnern (den meisten Infusorien, Polypen, Echinodermen, Mollusken, Krustern, Fischen) im Gegensatze der Binnen-Organismen, weil das Meer nach allen Richtungen mehr Zusammenhang hatte, als das Land; - und unter den letzten mehr bei den beschwingten Lufi-Bewohnern (Vögehn) als den für einen weiten Flug meistens zu schwachen Insckten und den am Boden lebenden Säugthieren und Reptilien: - (4) bei frei-beweglichen Thieren melur (Foraminiferen) als bei festsitzenden (Anthozoen, Bryozoen, Krinoiden), welche dem Einflusse des Witterungs-Wechsels u. s. w. nicht entgehen könuen und mehr für nur eine bestimmte Art der Witterung oder des Klimas geschaffen sind. Wenn nach dieser Theorie die Infusorien überall voranstehen, so fanden wir auch thatsächlich mehr als bei irgend einer andern Klasse ihre vorzugsweise weite Verbreitung oft durch mehre Zonen hindurch in Enumerator bestätigt, freilich nur aus selır junger Zeit, da ihre Reste in älteren Gesteinen nicht leicht mehr kennbar seyn können. Auch bei fossilen Echinodermen, Krustern und Fischen findet sich nicht selten eine beträchtliche Verbreitung ausgedrückt; nicht bei Polypen, Krinoiden, Reptilien, Säugthieren und den überhaupt sehr wenig bekannten Luft-Insekten und Vögreln. Nur Elephas primigenius und Mastodon angustidens unter den Säugthieren machen eine unerwartete Ausnahme, der Mastodon dursh sein Vorkommen in $\mathbf{E}^{2}$ von Russland bis Madrid und in $\mathbf{M}^{2}$ bis $\mathbf{M}^{3}$; der erste duch seine Veıbreitung von Sibirien bis zum heissen Indien, bis Italien und durch ganz Amerika $\left(S^{12} E^{2} M^{123}\right)$. Vielleicht müssen sie aber, wie man auch aus andern Gründen schon vermuthet, in mehre Arten zerlegt werden. Auch Bos Pallasi geht durch die kälteren Theile der 3 nördlichen Welttheile hindurch.

b. Unter den Infusorien besitzen sowohl die meerischen als die Arten der Binnen-Gewässer eine weite Verbreitung, wie man S. 89-106 des Enumerutors rasch übersehen kanı. Unter den letzten wollen wir nur

Himantidium arcus $\mathrm{z}$ in $\mathrm{E}^{2} ; \mathbf{x}$ in $\mathrm{E}^{2} \mathrm{~S}^{2} \mathrm{~F}^{3} \mathrm{M}^{23}$

Pimularia viridis. " " $\mathrm{E}^{2} ; ", \mathrm{E}^{2} \mathrm{M}^{234}$

Navicula fulva : . " " $\mathbf{E}^{2} ; ", " \mathbf{E}^{2} \mathbf{S}^{2} \mathbf{F}^{3} \mathbf{M}^{3}$ erwähnen.

Überhaupt sind dic a. a. $\mathbf{O}$. in $\mathbf{v}$ aufgefundenen Arten gewöhnlich meerische, die in $\mathbf{x}$ eingetragenen Arten Süsswasser-bewohnende Arten.

E. Auf die weite geographische Verbreitung gewisser Arten wirken indessen nicht allein die Verbreitungs-Mittel derselben, welche bei ganzen Klassen und Ordinungen gleich zu seyn pflegen und daher anch eine gleiche Verbreitung aller Arten derselben bewirken konnten, sondern noch mehr die Biegsamkeit derselben in Bezug auf äussere klimatische u. a. Leben-Bedingnisse ein. Diese Biegsamkeit scheint jedoch überall mehr nur einzelnen Spezies zuzustehen, und 
wir sind ausser Stande die Bedingungen bei denselben nachzuweisen, worauf sie beruhen ( $\mathrm{Vgl}$. D, b).

F. In vielen Fällen ist es die Möglichkeit vertikaler topographischer Ausbreitung, welche dem Vermögen weiter horizontaler Ausdelınung zu Hiilfe kommt. Organismen, welche die Ebenen külılerer Gegenden bewohnen, finden das ihnen zusagende Klima auf den Höhen wärmerer wieder'; und solche, welche an der Oberfläche gemässigter Meere wolıneı, können dieselbe Temperatur in den Tiefen heisser Meere, wo die Temperatur bis gegen $2^{0}$ herabsinkt, wiederfinden (Gesch. d. Natur II, 254-261), obwohl mit dem Unterschiede, dass in jenen die 'Temperatur' mit der Jahres- und Tages-Zeit wechselt, in diesen beständig bleibt; mit Orts-Bewegung versehene Wesen können sich aber auch im ersten Falle wenigstens bei anderer Jahreszeit einen Standort von angemessenerer Temperatur aufsuchen und jedenfalls dem raschen Wechsel entgehen. Eine Temperatur von $2^{0} \mathrm{C}$. würde also in allen Klimaten bei hinreichender Tiefe des Meeres anzutreffen und gleiche Arten zu nähren fähig seyn, wenn solche Tiefeu überhaupt noch bewohnt wären; Meere kalter Gegenden aber sind in allen Tiefen gleich kalt, an der Küsten-Oberfläche jedoch im Sommer einer beträclitliclıen Erwärmung fähig, daher Edw. Forbes und Lovén die Beobachtung machen, dass Mollusken und andere SeethierArten, welche nordwärts nächst der Oberfläche des Meeres wohnen, weiter südwärts in grösserer Tiefe vorkommen und, vermöge ihrer Fähigkeit dort manchfaltigen Temperatur-Wechsel zu ertragen, überhaupt diejenigen Arten in sich begreifen, welche zur weitesten geographischen Verbreitung geeignet sind. Schichten, welche mithiu an derselben Stelle in ungleicher Tiefe abgesetzt werden, können in fossilen Arten und deren Charakter sehr ungleich, und solche, welche in grösserer Entférnung von einander in gleicher Höhe entstanden sind, sehr gleich seyn. So wichtig diese Beobachtungen indesseu für die Geographie der heutigen Schöpung sind, so finden sie auf die älteren Schöpfungs-Perioden weniger Anwendung als auf die jüngeren, nachdem wir gefunden zu haben glauben, dass es damals noch keine kalten Zonen mit kalten Meeren wie auch nur weniger hohe und zusammenlängende Gebirge gab.

Auch auf ost-westliche Ausbreitung kömuen sie keinen Einfluss haben; und doch gibt es kaum eine Tebende Pflanzen-oder ThierArt, welche von Natur die ganze Erde in dieser Richtung umgäbe.

Ein Theil der hicher gehörigen Erscheinungen war vor 5 Jahren noch wicht bekannt, als der I1. Theil der Geschichte der Natur, in welchem sie ihre Stelle hätten finden müssen, veröffentlicht wurde.

EDw. Forbss ') und später Lieutenant $S_{P R \wedge T r}{ }^{2}$ ) theilen das Ägäische Meer. seiner Tiefe nach in 8 Regionen, die im Sommer ungefähr folgende Temperaturen nach Celsius haben.

1) Jahrb. 1844, 633, 634; 1848, 116. - 2) Jahrb. 1849, 254. 


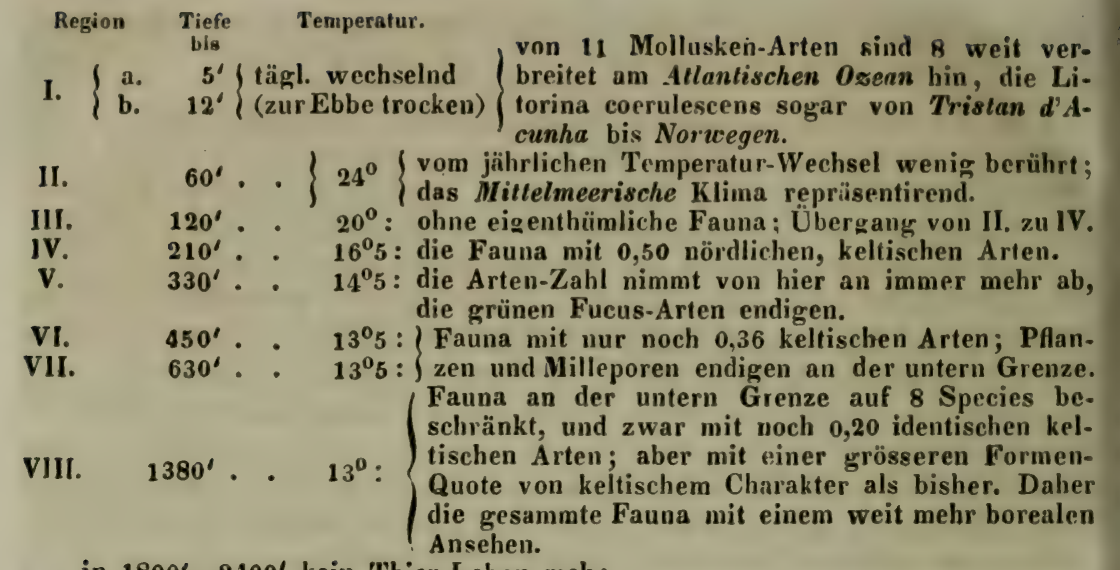

in $1800^{\prime}-2400^{\prime}$ kein 'Thier-Leben mehr.

Auch in den tiefern Regionen gibt es Arten mit ansehnlicher Vertikal-Verbrritung, da Forbes 9 Arten in VI, 17 in V, 2 in VIIl Regionen zugleich aufzalilt, von welchen mehr als die halbe Anzahl, gleich jenen in I einen gewissen Temperatur-Unterschied $z u$ ertragen befäiigt, auch wieder eine weite HorizontalVerbreitung besitzt; denn von 17 Arten, welche V Zonen gemein sind, geht die Hälfte durch die Meerenge von Gibrallar bis in's deutsehe Meer hinauf; von jenen, die IV Zonen gemein sind, lebt $1 / 3$ noch in Allantischen $O_{\text {sean }}$, und von allen, welche nach der Höhe eine noch geringere Ausdehnung haben, wieder 1/3 der Arten. Auch die meisten der mit dem britischen Meere gemeinsamen Arten kommen in grösseren Tiefen vor. Unter ihnen sind auch viele schon in den Tertiär-Schichten enthalten. Aber es sind auch einige subtropische Arten darunter, welche in südlicheren Meeren wahrscheinlich in grösserer Tiefe eben so kühl leben als hier.

Wäre nun das Ägäische Neer $3000^{\prime}$ tief und füllte sich durch Niederschläge auf, so würde man von unten nach oben erhalten: $1000^{\prime}$ Schichten ohne fossile Reste; $620^{\prime}$ (VIII) mit nur 8 Arten von borealem Charnkter; $1270^{\prime}$ (VII-IV) mit vielen keltischen Arten, an Zahl zunehmend; $90^{\prime}$ (III) indifferent; $70^{\prime}$ (II, l b) reich an mittelmeerischen Formen; und doch wäre Alles an einem geographischen Punkte entstanden.

Lovés hat zwischen Gothenburg und Norwegen in $58^{\circ}$ Breite erst in 80 und in 20 Toisen Tiefe dieselben Mollusken-Arten gefunden, welche bei $70^{\circ} \mathrm{Br}$. an der Finnmärkischen Küste schon in 20 Toisen und an der Oberfläche vorkommen ${ }^{1}$ ), wo demnach die höheren Regionen auch viel näher zusammengedrängt sind.

G. Wir finden eine Bestätigung der vorigen Sätze $\mathbf{E}$ und $\mathbf{F}$ auch darin, dass dieselben fossilen Arten, welche die weiteste vertikale, chronologische Erstreckungbesitzen, a uch die weiteste horizontale geographische Verbreitung haben, wie zuerst d'Archic und dF Vernedic ${ }^{2}$ ) machgewiesen haben, und was wir nur durch einige Belege darthun wollen, wälı'end maı die übrigen leicht im Enumerator überblicken ${ }^{3}$ ) kann.

1) Jahrb. 1849, 117. - ${ }^{2}$ ) Jahrb. 1843, 625.

3) Dazu die Nachträge im Jahrb. 1817, 506, 1848, S. 735, 18.19, 98, 116. 
a. Zwar sind in manchen der vor uns liegenden Fälle die fernländischen Formationen zumal der Kreiden-Periode etwas unsicher; auch hat man oft zwischen den aus verschiedenen Gegenden sowohl als aus verschiedenen aufeinander folgenden Formationen stammenden Individuen gewisse. Abweichungen bemerkt und sie desshalb in mehre Varietäten und Arten scheiden wollen, was in manchen Fällen, dic noch auf ungenanern Untersuchungen beruhen, sachgemäss seyn wird; indessen haben in andern Fällen sorgfältige Vergleichungen stattgefunden, welche keine oder keine grösseren Abweichungen dargethan haben, als heutzutage zwischen den Individuen einer Art von verschiedenen Küsten auch bestehen, und oft mit Übergängen zwischen diesen Abweichungen.

b. Die Elementar-Theile der Amorphozoen übergehen wir als zu unzuverlïssig hier absichtlich. Die übrigen Klassen bieten uns, nach Übergehung der minder auffallenden Vorkommnisse, folgende Auslese dar, wobei die nach Art und Formation zuverlässigsten Arten mit ! bezeichnet sind.

Der Nomenklator enthält in der Regel alle Belegstellen für das verschiedenartige im Enumerator aufgezählte Vorkommen der Arten; cinige haben in. dessen nicht mehr nachgetragen werden können, obschon das weitre Vorkommen im Enumerator noch aufgenommen wurde. 
Pf I a z en sind viele geographisch weit verbreitet, aber geologisch beschränkt, S. 871 .

\section{Polygastrica.}

:Flustrella limbata ..... I Amphitetras antediluviana. ! Dictyocha aculeata

! binoculus ...

! Gallionella aurichalcea . !

!

!Coscinodiscus excentricus

!

! spp. $4 \ldots$

! Actinocyclus spp. $11 \ldots$.

!Actinoptychus senarius. distans ............ granulata ... $\mathbf{E}^{2} \mathbf{S}^{2} \mathrm{~F}^{2}$ granulata ...

! Navicula silicula ...... ! fulva ......... $\mathbf{E}^{2} \mathrm{~S}^{2} \mathrm{~F}^{3} \mathbf{M}^{3}$. !Pinnularia didyma $\ldots \mid \mathbf{E}^{2} \mathrm{~F}^{2} \mathbf{M}^{2}$ :Himantidium areus .... SS $^{2} \mathrm{~F}^{3} \mathbf{M}^{23}$ !Cocconeis scutellum . . . $\mathbf{E}^{2} \mathrm{M}^{34} \ldots$ !Campylodiscus clypeus. ES $^{2} \mathbf{M}^{234}$ !Fragilaria rhabdosoma ... ES ${ }^{2} \mathrm{~F}^{3} M^{234}$ ! striolata $\ldots \mathbf{E}^{2} \mathbf{F}^{2} \mathbf{M}^{2}$. ! Synedra ulna etc.

\section{Polypi.}

:Orbulina universa .....

!Textilaria striuta globulosa....

! Globigerina bulloides . . .

!Rotalia globulosa .....

Iunulites radiatus

!Calamopora alveolaris .. $\mathbf{E}^{2} \mathrm{~S}^{2} \mathbf{M}^{2}$. ! Gothlandica . . $\mathbf{E}^{2} \mathrm{~S}^{2} \mathbf{M}^{2} \mathrm{U}^{4}$. Halysites catenulatus $\left.\ldots\right|^{2} \mathbf{M}^{2} \ldots$ Cyathophyllum caespitosum ceratites ....

Malacozoa.

!Terebratula reticularis ... sacculus .... concentrica ... de Roissyi .... biplicata .... biplicata $\cdots \cdots \mathbf{E}^{2} \mathbf{S}^{2}$

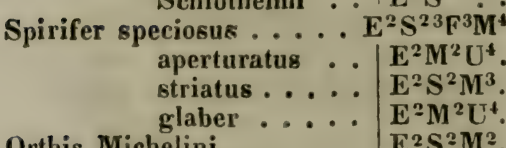

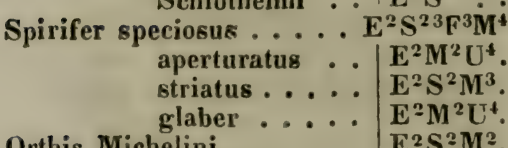

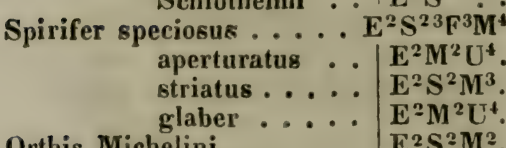

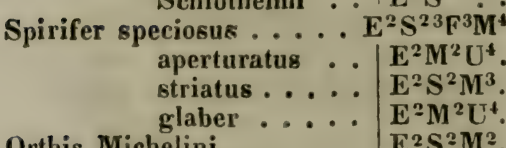
Spirifer speciosus .....
aperturatus
$\begin{array}{r}\text { striatus . . . . } \\ \text { glaber ..... }\end{array}$

$E^{2} S^{2} M^{2}$ $E^{2} S^{2} U^{4}$ $\mathbf{E}^{2} \mathbf{S}^{2} \mathbf{M}^{2}$. $\mathbf{E}^{2} \mathbf{M}^{2}$ $\mathbf{E}^{2} \mathbf{S}^{3} \mathbf{M}^{2}$ Chonetes sarcinulatus $\mathrm{E}^{2} \mathrm{~S}^{2} \mathrm{M}^{2}$ $E^{2} S^{2} M^{2}$

$\mathbf{E}^{2} \mathbf{S}^{2}$ $\mathbf{E}^{2} \mathbf{S}^{2} \mathbf{M}^{2}$.
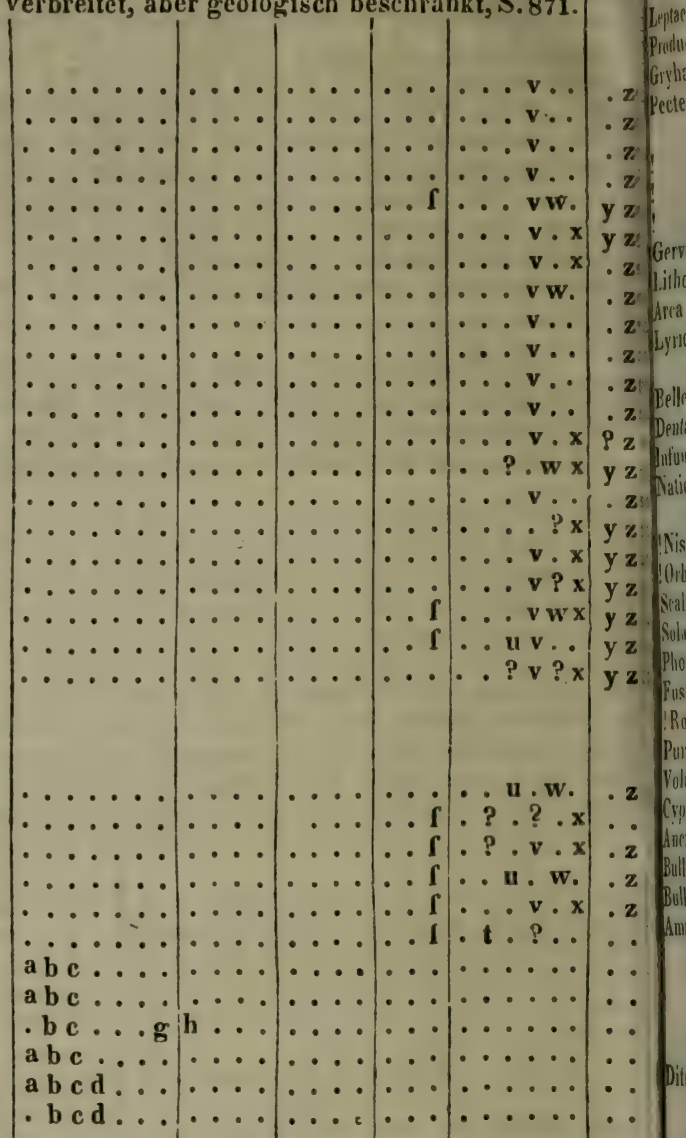

.

.

- .

. . . .

... . .
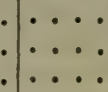

a b c....

b c...g $h$

a bc ....

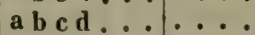

- bcd.
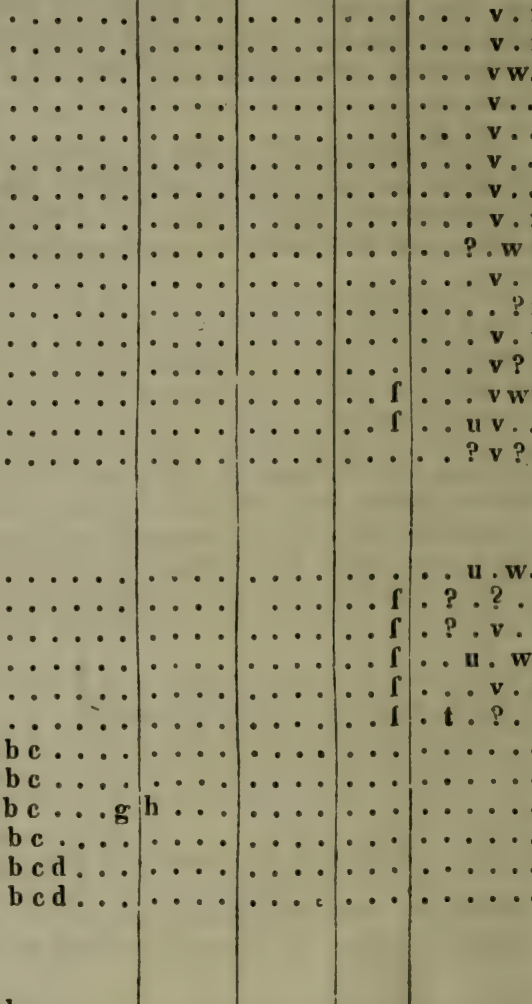

$\ldots v \cdot x$

v $\mathbf{w}$

$\ldots \mathrm{v}$ 


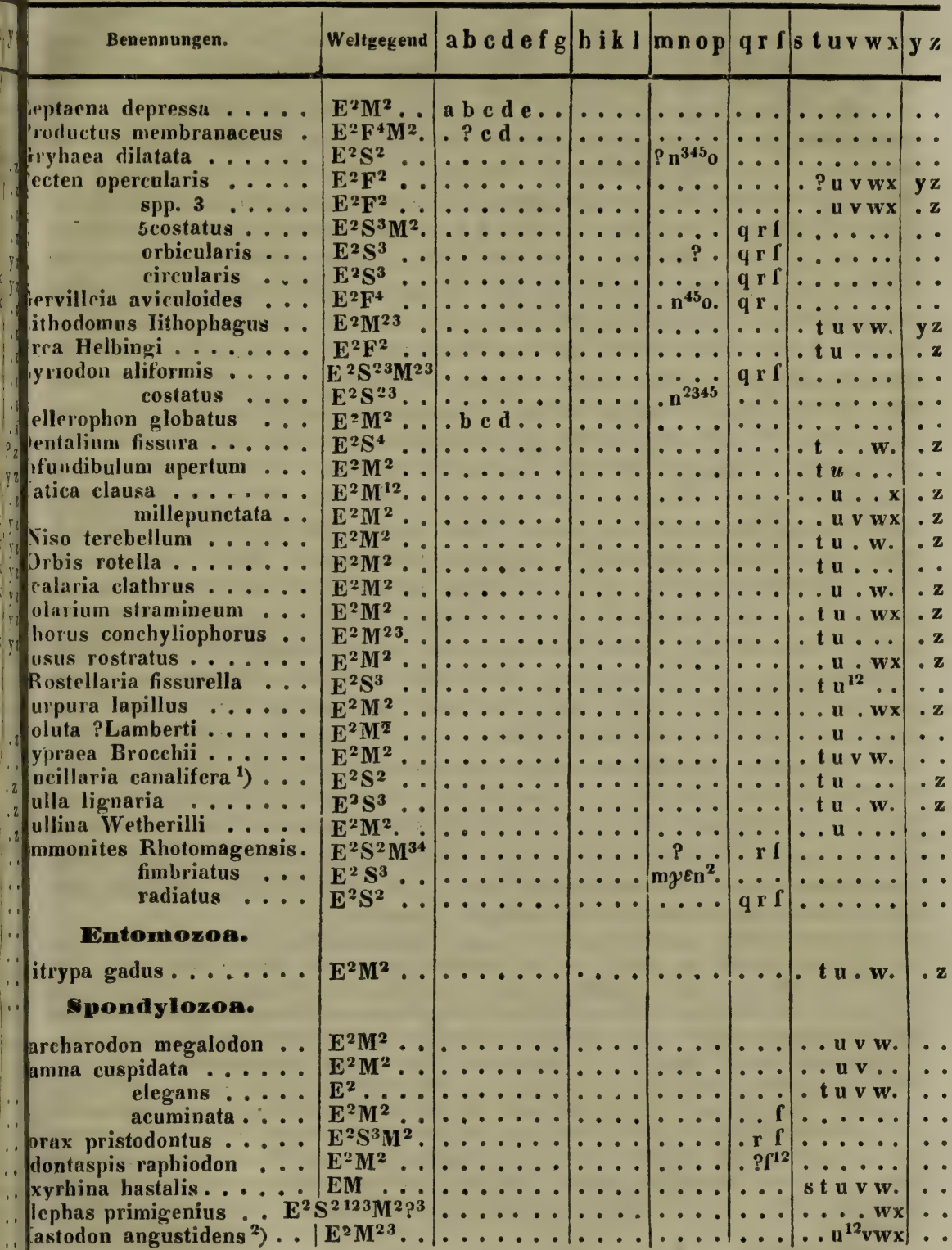

1) Viele andre hauptsächlich miocäne Arten, welche in wärmern Meeren noch lebend vorkommen, stehen im Enumerator S. 430-485 verzeichnet; da ihr jetziges Heimath-Land immer in Parenthese beigesetzt ist, $\mathbf{z}$. B. $\left(\mathbf{F}^{3}\right)$ oder $\left(\mathbf{S}^{3}\right)$ und $\left(\mathbf{M}^{3}\right)$, so sind sie leicht zu überblicken, und wir haben sie der Raum-Ersparniss wegen nicht mehr hier aufrählen wollen.

2) Mastodon angustidens wird nur von R. OwEv in den Pliocän-Schichten (Süss- 
Wir führen hier nicht die zahlreichen Belspiele auf, wo fossile Arten wirbelloser Thiere durch $\mathrm{v} \mathbf{w} \times$ hindurch reichen und dann auch noch eine mehr oder weniger beträchtliche geographische Verbreitung besitzen; man wird sie im Enumerator alle schnell übersehen können.

H. Wenı irgendwo eine Thier-Art nach ihrer Erschaffung anf beschräıktem Raume allmählich zu häufig wurde, da ist sie zweifelsohne nach andern günstigen Gegenden allmählich vorger ü ckt. Auch die manchfaltigen geologischen Veränderungen der Erd-Oberfläche haben oft die Ursache der A us anderung gewisser Orga. nismen aus einer Gegend und der Einwanderung derselben in einer andern seyn müssen, wenn sie hier die alten klimatischen oder andre wesentliche Lebens-Bedingungen wiederfanden, die sie dort verloren lıatten. Diese Wanderung konnte theils von den alten Individuen vollbracht werden, theils durch Entführung ihrer Eier und Larven nach den ihrer Entwicklıng günstiger werdenden Örtlichkeiten erfolgen, möglicher Weise auch durch die Schöpfungs-Kraft bewirkt werden, welche in gleichen Lebens-Bedingungen gleiche oder analoge Arten neu hervorrief. Kleine Abweichungen von den alten Bedingungen kounten in diesem wie in jenem Falle kleine Abweichungen der Formen veranlassen und neue Varietäten alter Arten bilden (Gesch. d. Nat. II, 209 ff. 65 ff.). Wanderungen solcher Art, die wir auch heıtzutage an den Vögelı ${ }^{1}$ ) u. a. Thieren beobachten, sind übrigens grosser Beachtung werth, wenn es sich darum handelt aus der Übereinstimmung einer Ablagerung mit einer andern von bekannter Formation hinsichtlich der in ihmen enthaltenen fossilen Arten das geologische Alter der ersten zu bestimmen. Es köınte nämlich in Folge des späteren Erscheinens ausgewanderter Arten an einem andern Orte leicht als gleichzeitig erscheinen, was ungleichzeitig ist, und müsste die Alters-Bestimmung jedenfalls sehr erschwert werden, wenn nicht jenes Vorrücken so langsam erfolgt wäre, dass die Versetzung derselben Arten in andre Gegenden in der Regel erst in einem späteren Arten-Alter bemerkbar werden konnte.

a. Allmähliche Abkühlung der ganzen Erd-Oberfläche, wodurch ein gemässigteres Klima langsam von den Polen gegen die Tropen heranzog; ein schnellerer Temperatur-Wechsel in Folge von Niveau-Änderungen der bisherigen Regionen des Festlands wie der Meeres-Tiefen; der mittelbare Einfluss, welchen die Entstehung hoher Berge, die sich mit bleibendem Schnee und Eis bedecken, in Gegenden haben müssen, wo dergleichen bisher nicht vorgekommen; oder die Erhebung langer und hoher Bergketten in Richtungen, wo sie warme und kalte, trockene und feuchte Luft-Strömungen abhalten; die Bildung von Kontinenten in solchen Gegenden und Richtungen, wo tropische heisse oder polare kalte Strömungen des Mecres nach Orten von entgegengesetzter Beschaffenheit gelenkt, oder von ihnen abgehalten werden; endlich Wechsel zwischen trocknem Festland und Meer: Diess sind wohI die möglichen Haupt-Ursachen stattgefundener Wanderungen.

wasser-Crag) Englands (Jahrb. 18.16, 632), aber von mehren Schriftstellern in den Diluvial-Bildungen Nord-Amerika's angeführt, an beiden Orten in Gesellschaft von Elephas primigenius und Pferden.

1) ВREHM in Isis 1848, 421. 
b. In Folge solcher Ereignisse sind aber doch inmer nur entweder bloss mehr und weniger örtliche oder, wenn ausgedehntere, so äusserst langsame Erscheinungen bezeichneter Art möglich, dass sie auf die im Enumerator für je zwei aufeinanderfolgende Formationen im Ganzen erzielten Resultate in der Regel keinen wesentlichen Einfluss haben konnten; sie würden sich nur bei Vergleichung sämmtlicher Schichten-Glieder zweier verwandter Formationen mit einander in nicht zu grosser Entfernung unterscheiden lassen.

c. Doch ist eine nicht unbeträchtliche Anzahl von Konchylien bei Bordeaux u. s. w. als miocän bekannt, welche jetzt nicht mehr in so hohen Breiten, sondern am senegal und weiter südlich leben; wir können aber freilich nicht nachweisen, dass sie in Folge der Temperatur-Abnahme aus den höheren Breiten ausgewandert sind, ehe wir nicht am Senegal ebenfalls miocäne Schichten vorfinden werden, worin sie alle oder zum Theile fehlen. Wahrscheinlicher sind sie nur in kühler gewordenen Gegenden ausgestorben?

d. Horner setzt ferner folgenden hypothetischen Fall ') zu Erlâuterung der Möglichkeit des Vorkommens gleicher Arten in ungleichzeitigen Bildungen in Folge von Wanderungen. Es seyen zwei von einander entlegene Gegenden des Ozeans einander gleich in Temperatur, Tiefe und Art des Bodens, so dass sie auch gleiche oder doch einander repräsentirende Konchylien-Arten zu nähren vermögen und zwar solche, welche an und nächst der Küste in mässiger und grössrer Tiefe wohnen und deren Reste allmählich in den Niederschlägen am Boden begraben und einer späteren Zeit aufbewahrt würden. Man denke sich, dass nun durch eintretende Erhebung des See-Grundes die erste Gegend seicht werde und die Bewohner der Tiefe zu Grunde gehen, die der mittlen und obern Stationen dagegen beziehungsweise überhand nelımen, eben so viele andere Arten durch neu entstandene Strömungen herbeigeführt, und dass in den jetzt entstehenden Niederschlägen Konchylien-Reste mit 0,16 ausgestorbener Arten eingeschlossen werden; - man denke sich endlich, dass in der Nähe dieser seichtgewordenen Meeres-Gegend eine so ausgedehnte Hebung des Landes eintrete, dass hohe Gebirge mit ewigem Schnee und Eis sich bilden, die Temperatur herabdrücken, die bisherigen Bewohner gemåssigten Klima's zu Grunde richten und ihre Reste unter dem reichlicher abgeschwemmten Sand und Schlamm begraben, während gleichviele neve Arten kälterer Klimate einwandern, und dass hiebei abermals 0, 16 der anfänglichen Arten aussterben. Wenn nun nach einiger Zeit endlich diese Niederschläge ganz über den Meeres-Spiegel emporgehoben würden und ein freologe sie untersuchte, so würde er zu unterst eine Abtheilung mit 0,68 , darüber eine mit 0,84 und $\mathrm{zu}$ oberst eine mit lauter noch lebenden Arten entdecken und folgern, dass hier alt-pliocäne, neupliocäne und postpliocäne (vergl. S. 760) Schichten übereinander liegen, wenn er nicht weiss, dass die hier ausgestorbenen Arten in andern Gegenden des Ozeans noch leben. Wenn nun in dieser $\mathbf{Z}$ wischenzeit in der zweiten der obeu angenommenen Gegenden des Oreans keine andere Veränderung einträte, als dass sich der Boden mit Konchylien-führenden Niederschlägen auffüllte und endlich eine Strecke davon in's Trockene emporgehoben würde, deren organische Reste jetzt ein Geologe untersuchte und ganz übereinstimmend fände mit den Arten der verschiedenen Tiefen des benachbarten Meeres, so würde er jene Bildungen für postpliocäne erklären, obschon sie gleichzeitig mit den obigen entstanden sind. Es folgt daraus, dass man mittelst der Quoten noch lebender Konchylien-Arten, deren Reste in den Gebirgs-Schichten eingeschlossen sind, allerdings ZeitWechsel unterscheiden kann, wenn sie von Wechseln topographischer und phy.

1) Jahrb. $1848,738$.

2) Geology of South America 105. 
sikalischer Lebens-Bedingungen begleitet sind und nur für eín beschränktes Feld der Erd-Oberfläche, nicht aber für deren ganze Ausdehnung gelten sollen; daher Dakwin ${ }^{1}$ ) schon mit Recht davor gewarut hat, einen Wechsel in den organischen Formen und in der Zeit für unbedingt aneinander geknüpft $\% u$ erachten. Was aber hier in Bezug auf Tertiär- und Jetzt-Zeit gesagt ist, gilt überall an der Grenze zweier Perioden oder Formationen, wo es sich um eine Anzahl gemeinsamer Arten handelt, wie bei Untersuchungen über die Gleichzeitigkeit ältrer Becken. - Ebenso kann ein Klima-Wechsel Organismen-Arten veranlassen aus einer Gegend in die andere zu wandern, welche somit früher in jener und später in dieser ihre Reste den Erd-Schichten überliefern, ohne dass diese Übereinstimmung in den organischen Resten einer Gleichzeitigkeit der Bildungen entfernterer Gegenden entspräche.

e. Denken wir uns ein geologisches Becken, dessen See-Spiegel in oq liegt und dessen 'Tiefe 4 (hundert oder tausend Fuss) beträgt, so dass sich bei $M$ in dessen Mitte die Schichten $m$ n o p q absetzen und es dadurch auffüllen. Allein nachdem sich die Schichten $m$ und $n$ gebildet, beginnen dieselben sich bei $\mathrm{Z}$ zu senken und bei $A$ anzusteigen, so dass nachher das Niveau von o bei $A$ in 0 und bei $Z$ in 4 gelangt; so wird die nun entstehende Schicht 0 bei $Z$ von neuem dieselben Ar-

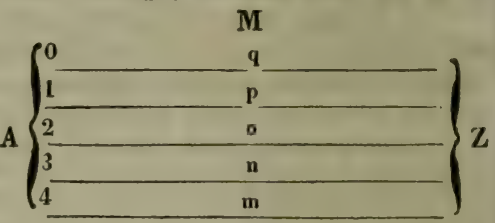
ton aufnehmen können, welche $m$ bereits enthielt, dann etwas näher gegen die Mitte fortfahren dieselben Fossilien zu beherbergen, welche $\mathbf{n}$ daselbst sclion hat, in der Mitte dic ihm schon anfänglich zusagende Bevölkernng erhalten, weiter gegen A hin diejenige aufnehmen, welche bei horizontal gebliebener Lage $p$ erhalten haben würde, und bei $A$ selbst jene empfangen, die q entsprocheu hätten. Ähnlich würde es nun mit $p$ und $q$ weiter gehen; aber eine ncue Hebung oder Senkung, überall gleichmässig oder bei $\mathbf{Z}$ s'hwächer oder stärker als bei A kann den ganzen Plan der Vertheilung der Arten in den Schichten abermals verwirren: eine Folge, welche sich dem Auge nicht überall so klar darlegen wird, weil doch immer jede Höhen-Region auch eisen Theil ihrer Arteu mit der oder den nächstvorhergehenden oder folgenden gemein hat.

I. Bei den Arten und Sippen unsrer heutigen Schöpfung kanı man oft melır oder weniger deutliche $Z$ en tral-Punk te oder - F läch e n unterscheiden, wo dieselben am zahlreichsten, mit den grössten Individuen oder den bezeichnendsten Arten auftreten und von wo aus sie nach allen Richtungen abnehmen und nur zuweilen einzelne Individuen oder Arten noch in weitere Fernen senden. Man wird also ein älnliches Verhalten auch in den früheren Schöpfungen unterstellen dürfen, mögen die Spezies nun nur von einem Älter'ı-Paare oder von ganzen Gruppen derselben abstammen (Gesch. d. Nat. II, 200 -205). Obwohl wir aber oben gesagt haben, dass ein Wechsel der geographischen Verbreitung anf die Ergebnisse in unserem Enumerator keinen erhehlichen Einfluss geübt haben dürften, weil dessen Rubriken nämlich kürzere Zeit-Räume als die der Formationen nicht umfassen, so sind nach allen Anzeigen eben diese Zeit-Räıme doch sehr lange (S. 815 u. a.), so dass innerhalb ihrer Dauer unzählige

') Geology of South-America, 105. 
Wechsel solcher Art vor sich gehen konnten, die wir anch bestätigt finden dürften, wenn es uns vergönnt wäre die vollständige SchichtenFolge einer solchen Formation Glied un Glied und in ihrer ganzen Breiten-Ausdehnung auf ihre fossilen Reste zu durchforschen. Da indessen der geographische Ortswechsel der Arten nicht allein als blosses Vorrücken in Folge einer zu grossen Vervielfältigung der Individuen auf einer und derselben Fläche, sondern auch als Auswanderung in Folge geologischer Ereignisse (S. 92:) stattgefunden hat, welche leicht von einer Seite her nach der andern hin drängen konnten, so würde die Verbreitungs-Weise auch nicht überall eine radiale, sondern mitunter eine lineare, eine einseitig divergirende u. dgl. seyn können. Und ähnlich kann es sich bei manchen Geschlechtern verhalten.

K. Die Vergleichung verschiedener Örtlichkeiten hinsichtlich ihrer geognostischen Verwandtschaft mittelst der ihnen gemeinsam zustehenden Arten in absoluten Zahlen führt zu keinen haltbaren Resultaten, wenn man nicht die ganze Anzahl der Arten kennt, unter welchen sich jene übereinstimmenden befinden. Aber auch wenn man die Verwandtschaft zweier entlegenen nicht zusammenhängenden Orte aus der Übereinstimmung ihrer organischen Reste nach Procenten identischer A rten beurtheilen, wenn man insbesondere ihr relatives Alter darnach untersuchen will, so wird man nur mit grosser Vorsicht verfahren dürfen, weil die theilweise Übereinstimmung oder Abweichung in dieser Beziehung ebensowohl eine Folge der zeitlichen als der räumlichen, der geologischen und der geographischen Verwandtschaft seyn kann. Man wird einerseits sogar bei wirklich ganz gleichzeitiger Bildung nie erwarten dürfen lauter, sondern nur eine gewisse Anzahl solcher identischen Arten zu finden; und es wird andrerseits selbst eine grössre Anzahl solcher identischen Arten, theoretisch genommen, noch kein sicherer Beweis völliger Gleichzeitigkeit seyn, wenn schon in Wirklichkeit der Fall sehr selten vorkommen dürfte, dass zwei ungleichzeitige Bildungen in dieser Beziehung eine grosse Übereinstimmung zeigen, - weil eben messbareren Zeit-Verschiedenheiten anch schon wieder ähnliche Verschiedenheiten in der Organismen Welt üherhaupt entsprechen.

Die mehr oder weniger deutliche Nichtübereinstimmung gleichzeitiger Bildungen hinsichtlich ihrer organischen Einschlüsse kann also abhängen:

1) Von geographisch - klimatischer Verschiedenheit der Orte (nord-sïdliche Entfernung).

2) Von topographischer Verschiedenheit derselben (auf dem Land: Berg und Ebene, Exposition u. s. w., und im Wasser: Süssund See-Wasser; in erstem: Quelle, Bach, Fluss, Teich, See; in letztem Binnen-See, Mittelmeer, Ozean, Hochmeer, Küste, KüstenRegion; eines Beckeus weiter oder beschränkter Zusammenhang mit kälteren oder mit wärmeren Meeren u. s. w.). 
3) Von sehr grosser Entlegenheit auch hei sonst gleichen V'erlıältnissen (da z. B. auch jetzt in einer und derselben Zone, die Arten der nach allen Richtungen zusammenhängende Nordpolar-Länder und -Meere ausgenommen, kaum e in e Spezies rund um die Erde überall vorkommen dürfte und die Äquatorial-Zone allein eine grosse Menge von einander sehr verschiedener Faunen und Floren enthält).

Im ersten Falle kann die Schichten-Gliederung der zwei verglichenen Örtlichkeiten noch ziemlich ähnlich, aber auch sehr unähnlich, in den zwei letzten Fällen muss sie in Folge grosser topographischer Verschiedenheit oder grosser geographischer Entfernung sehr abweichend seyn.

Grössre und kleinre Übereinstimmung ungleichzeitiger Bildungen in ihren organischen Einschlüssen wird nicht bloss von einer klimatischen, topographischen Ähnlichkeit beider bedingt, sondern wohl auch eine Einwanderung der Organismen aus ihrem früheren Wohnort in den späteren vermuthen lassen.

Es wird beutzutage Niemand im Ernste mehr erwarten dieselbe SchichtenFolye in den 3 Formationen der Kreide-Periode in Indien oder in Süd-Amerika zu finden wie in Europa; ja es dürfte von den Schichten selbst abgesehen nicht eimmal wahrscheinlich seyn, ein genaues Äquivalent für die Europäischen Gienzen zwischen diesen 3 Formationen zu entdecken; es ist also in diesem wie schon im ersten Falle unmöglich, selbst wenn überall dieselben Arten vorhanden wären, sie in so entlegenen Gegenden in derselben Reihenfolge, in derselben Gruppirung wiederzufinden, und solange man nicht überall alle Schichten vollständig ausgebeutet hätte, jene vollständige Übereinstimmung in den organischen Resten $7 \mathrm{u}$ entdecken. Was wir indessen hier in Folge allgemeiner Ansicht aussprechen, bestätigt sich in der That nicht nur an so entlegenen Orten, als wir oben angenommen, sondern schon alsdann, wenn wir die verschiedenen gleichzeitigen Kreide-Becken Frankreichs (D'OnBIsNy) ${ }^{1}$ ), die Jura-Schichten in der Schreilz und in U'ürttemberg (Rominger) ${ }^{2}$ ) u. s. w. mit einander vergleichen, in einer über Erwarten auffallenden Weisc.

L. Selbst nach Würdigung aller dieser Verlıältnisse kanı man die Verwandtschaft zweier Orte mit einander durch die Quote der ihnen gemeinschaftlichen Arten nur dadurch genauer ausdrücken,

1) dass man die absoluten Zahlen, welche von beiden Örtlichkeiten zur Vergleichung verfügbar waren, mit angibt, indem sie in demselben Verhältnisse, als sie kleiner werden, auch ein mehr unsicheres und zufälliges Verhältniss darzubieten im Stande sind;

2) dass man die Zahl der identischen Arten auf beiden Seiten mit jenen 2 Gesammtzahlen vergleicht und die Quote derselben ron beiden Seiten angibt, weil diese bei grosser Ungleichheit der Gesammtzahlen beiderseits sehr ungleich ausfallen muss;

3) dass man unter beiden Quoten diejenige vorzugsweise berücksichtigt, welche durch Vergleichung der Źahl der beiden Ört-

1) Paléontologie Française, terrains crétacés, I, 636-643.

$\left.{ }^{2}\right)$ Jahrb. 1846, 293-306. 
lichkeiten gemeinsamen Arten mit der absoluten Zahl der Arten von derjenigen Örtlichkeit hervorgeht, welche die wen iger reiche ist.

a. Ein Beispiel 'mag Diess erläutern : Nach D'OrBigny ${ }^{1}$ ) hahen in Frankreich das "Mittelmeerische" und das "Pariser" untere Neocomien-Becken 9 Cephalopoden-Arten mit einander gemein. Enthielten nun die Becken überhaupt keine grössere Anzahl fossiler Arten, so wäre ihre Identität absolut; enthielte jedes derselben 1000 Arten im Ganzen, so wäre sie fast nur so gross, als wie zwischen 2 verschiedenen aneinander grenzenden Formationen einer Gegend. In der That hat aber das "Pariser" Becken nur 14 fossile Arten geliefert und jene 9 Arten sind mithin fast 2 Drittel, nämlich 0,64 von allen; sie scheinen also eine sehr nahe Verwandtschaft beider Becken auszudrücken; allein das "Mittelmeerische" Becken hat 87 Arten dargeboten und davon betragen jene nämlichen 9 gemeinschaftlichen Arten nur etwas über 0,10, die Verwandtschaft ist demnach sphr klein. Weder die absolute Anzahl der beiden Becken gemeinsamen Arten noch jede der 2 verglichenen Zahlen allein könnte uns in diesem Falle über das wahre Verwandtschafts-Verhältniss aufklären, sondern nur alle zwei nebst den absoluten Gesammtzahlen zusammen vermögen es. Ohne die absoluten Gesammtzahlen der Arten an beiden Orten zu kennen, würde man nicht wissen, ob die 0,64 und 0,10 identischer Arten nur aus der Vergleichung von 2-7 oder von 200-1000 Arten jederseits hervorgegangen sind, was immerhin die Verlässigkeit sehr modifiziren würde; - und nachdem man sie beide kennt, wird man vielleicht anfangs versucht seyn, die aus der grössren Gesammtzahl der reicheren Örtlichkeit gezogene Quote als die verlässigere zu betrachten, weil die kleinere in der That doch sehr klein und daher leichter einer Zufälligkeit ausgesetzt ist. Aber obschon dieser letzte Einwand im Allgemeinen wahr ist, so drücken hier dennoch die aus der kleineren Gesammtzahl gezogenen Prozente identischer Arten das Verhältniss viel richtiger aus, als die aus der grösseren. Denn während die 14 Arten des Pariser Beckens alle mit solchen des Mittelmeerischen identisch seyn und mithin 100 Prozent zu ergeben vermögten, würde das letzte alsdann doch nicht über $14 /{ }_{87}$, das ist 0,16 , mit dem ersten gemeinsam habrn können, nicht mehr nämlich, als das letzte im Ganzen an Arten besitzt; die übrigen ${ }^{73} / 87$ Arten müssten ihm immer eigenthümlich bleiben. Dagegen würde man aber berechtigt seyn aus der Analogie zu unterstellen, dass wenn in dem Pariser Becken ebenfalls 87 Arten gefunden würden, wie in dem Mittelmeerischen, sich auch bei den hinzukommenden 73 Arten dasselbe Verhältniss, wie bei den ersten 14 zeigen und sich so dasselbe Verhältniss wie im Pariser Berken, nämlich 0,64 einstellen würde. - Die zweckmässigste Form die Verwandtschaft bloss zweier Örtichkeiten hinsichtlich der ihnen gemeinsam zustehenden Arten auszudrücken würde also darin bestehen, dass man die Zahl der gemeinsamen Arten zwischen die der zwei absoluten Gesammtzahlen setzte, in obigem Falle mithin etwa so: P 14 (9) $87 \mathrm{M}$, oder wenn man einen verglichenen Ausdruck sogleich dabei haben wollte $P_{14}=0,64(9) 0,10=87 \mathrm{M}$.

Nach D'Orbigny, welcher die chloritische Kreide in 4 verschiedene Becken Franlereichs vertheilt ${ }^{2}$, enthalten dieselben an Cephalopoden die folgenden absoluten Zahlen aller gemeinsamen und eigenen Arten, welchen wir verglichene Zahlen zur Seite setzen:

1) Terrains crél. I, 636.

$\left.{ }^{2}\right)$ Terrains crét. I, 640 . 


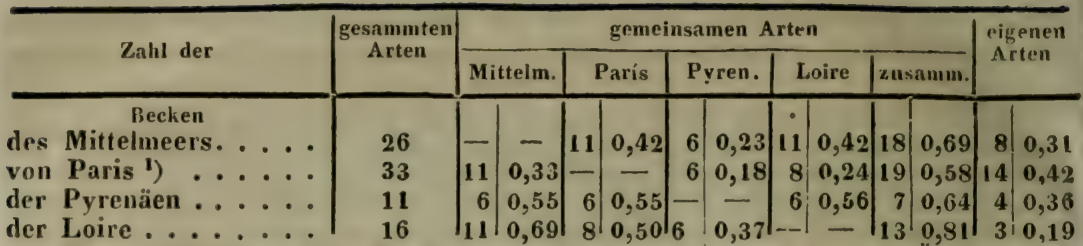

Man sieht, dass hier für je 2 mit einander zu vergleirhende Órtlichkeiten immer 2 verglichene Zahlen vorkommen, und um das richtige VerwandtschaftsVerhältniss zu finden, wird es nach der vorher gegebenen Erläuterung nicht mehr gleichgültig seyn, welche von ihnen man zu Rathe ziehe, sondern man wird sich zu dem Ende in der Regel derjenigen zu bedienen haben, welche vo der kleineren der 2 absoluten Gesammtzahlen der an beiden Örlichkeiten iberhaupt vorkommenden Arten abgeleitet ist. Wenn also das Pariser Becken mit dem der Pyrenäen 6 Arten gemein hat und diese von sämmtlicheu

\section{Arten des Pariser Beckens 0,18}

11 Arten des Pyrenäer Beckens 0,55

betragen, so gibt wieder die letzte Quote das Verwandtschafts-Verhältniss richtiger als die erste an; und so in allen andern Fällen dieser kleinen Tabelle. Die

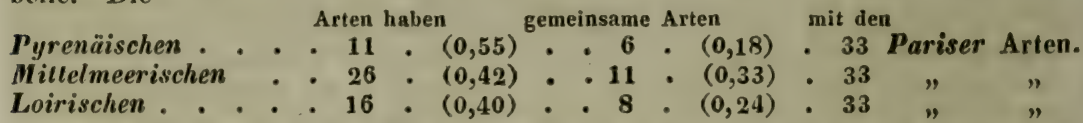

Hier ist die Ordnung der Verwandtschaft mit Paris nicht diese: Mittelmeer-, Loire-, Pyrenäen-Becken, wie aus den absoluten Zahlen gemeinsamer Arten (die gewöhuliche Betrachtungsweise), oder aus deren Vergleichung mit derjenigen Örtlichkeit, welche die meisten Arten besitzt (33), folgen würde: sondern sie ist: Pyrenäen-, Mittelmeer-, Loire-Becken, wie aus deren Vergleichung mit denjenigen Urten hervorgeht, welche am wenigsten Arten enthalten, vorausgesetz. dass diese letzte Zahl nicht so klein $(2,1)$ wird, dass sie als eine blosse Zufälligkeit erscheint.

Numerische Ähnlichkeiten und zumal Verschicdenheiten, welche sich auf diesem Wege zwischen 2 Becken ergeben, sind übrigens insoferne sehr unzuverlässige Fülırer, als sie abhängen a) von der genauen Übereinstimmung der untersuchten Schichten-Folge, b) von deren Entfermung bei jugendlichen Bildungen zumal von Norden nach Süden; c) von Zufälligkeiten des Erhaltenseyns, und d) vom Reichthume der an jedem der beiden Orten gemachten Sammlungen.

b. Was vorhin über die Vergleichung verschiedener Örtlichkeiten einer Formation unter sich gesagt ist, gilt auch da, wo es sich darum handelt zu bestimmen, ob eine zwisclıen 2 Formationen gelegene Schichten-Reihe nach den Summen oder Quoten ihrer mit beiden Formationen gemeinsamen Arten der einen der der andern derselben zugezählt werden solle. Wären $z$. B.

absolute Arten-Zahlen verglichene Zahlen gemeinsamer Arten

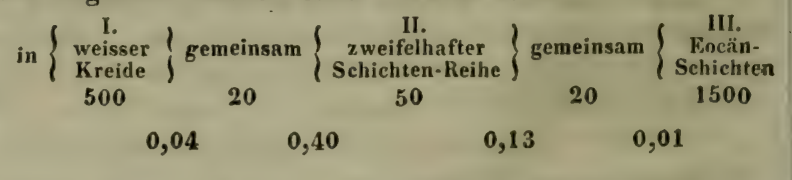

1) Hier ist eine fehlerhafte Angabe in der Originalschrift, da die eignen Arten des Pariser-Beckens und jene, welche es mit allen andern einzeln genommen gemein hat, noch nicht einmal der Summe seiner Arten gleichkommen; wie es nach einer Zählung scheint, müssten 14 statt 4 eigner Art gesetzt werden. 
so wird, was schon für sich leicht verständlich, bei gl e icher Anıahl der Arten, welehe die zweitellatte Srhichten-Reihe mit den 2 angrenzenden Formatiouen gemein hat, die absolut ärmere Forma ion im Übergewi, hre der Verwandtsrhaft seyn, und das Verwandischafts.Verhältniss wird richtiger durch die Quote 0,40 als durch 0,04 ausged üikt werden.

Wird aber die absolute Anzahl wie die Quote der gemeinsamen Arten. Zahlen auf beiden Seiten ungleich, etwa so:

$\begin{array}{lccccc} & \text { 1. } & \text { gemein } & \text { II. } & \text { gemein } & \text { III. } \\ \text { absolute Zahlen } & 500 & 10 & 50 & 40 & 3000 \\ \text { verglichene Zahlen } & & (0,02) & (0,20) & (0,80) & (0,01)\end{array}$

so entscheidet nicht die grössere Anzahl gemeinsamer Arten an sich, sondern die grössere der verglichenen Zahlen, welche durch Zusanmenstellung dieser absoluten Zahlen gemeinsamer Arten mit der gesammten Arten-Zahl der äımeren von den 2 Formatiouen, denen sie gemeinsam sind, hervorgeben; in voranstehendem Falle also würde die Örtlichkeit oder die Schicht II zu III zu schlagen seyn in Folge des grössten der 4 Quotienten $=0,80$, obschon auf derselben Seite auch der kleinste der 4 Quotienten $=0,01 \mathrm{zum}$ Vorschein gekommen ist und bei flüchtiger Auffassung die Ursache werden könnte, dass man II von III zurück zu I verweise, wo die 2 mitteln Quotienten stehen.

M. Was die Ähnlichkeit der Schichten-Entw i ck I ung zwischen zwei verschiedenen Ortlichkeiten eines Beckens oder gar zwischen zwei verschiedenen Becken derselben Formation im Ganzen anbelangt, so stellt d'Archiac folgende Erfahrungs-Sätze auf ${ }^{1}$ ):

„Je mehr die verschiedenen Abtheilungen einer Formation entwickelt sind (wie Diess in der Mitte der Becken zu seyn pflegt), desto schärfer sind auch die zoologischen Charaktere einer jeden ausgesprochell, und desto weniger gemeinsame Arten kommen darin vor," und „im Maase, als die Zahl der Glieder oder Abtheilungen dieser Formation sich vermiıdert (wie es an den Rändern der Becken einzutreten pflegt), mischen sich nicht nur die verschiedenen PetrefaktenArten derselben unter einander, sondern entwickeln sich auch immer mehr neue Arten und selbst neue Geschlechter" [?];

daher sich aus den erlangten Zahılen und andern Verhältnissen erkennen lässt, ob eine geognostische Örtichkeit mitten oder gegen die Grenze des Beckens oder jenseits der letzten (als "A uslieger") abgesetzt worden war, oder ob sie erst durch Entblössung davon getrennt wurde.

Neben den Zahlen-Verhältnissen der Arten darf man die der Individuen nicht übersehen; nur die Arten, welche in zahlreichen Individuen auftreten, sind bezeichnend oder "leitend", die andern für den Geognosten mehr zufällig; an zwei verschiedenen Örtlichkeiten können sich aber zwei Arten hinsichtlich der Menge ihrer Individuen gerade umgekehrt verhalten, was eben nur an Ort und Stelle zu beurtheilen und aus den gewölınlichen Verzeichnissen nicht zu erkennen ist.

N. Von theoretischer Seite würde man zu etwas anderen Ansichten über Wirkungen der topographischen Verschiedenheiten in einem und demselben Becken (vgl. F) gelangen, als sich in den obigen

1) Jahrb. 1841, 793 . 
Erfalırungs-Sätzen (M) ausdrücken, bestimmte Anzeigen von HöhenUnterschieden über dem Meere in den Absätzen unter dem Meere aber kaum erwarten dürfen, sondern nur etwa in solchen SüsswasserGebildeı finden köınen, die auf ungleichen Gebirgs.Höhen selbst - entstanden sind.

Meerische wie Süsswasser-Becken haben gegen ihre Mitte hin eine andre Beschaffenheit als an Rande, und daher die in ihnen entstehenden Niederschläge andere Einschlüsse; gewöhnlich nämlich am Rande im Gegensatze zur Mitte in folgender Weise:

Becken am Rande.

geringere Tiefe (F) . . lebhafter Wellenschlag:

Kies- und Sand-Boden

mit Laich-Stellen : ... mit jährlichem KlimaWechsel .......

Süsswasser an Fluss. mündungen ...... oft Brackwasser binter Schären ........

Gezeiten veranlassen . . Sturm bewirkt ..... Zerstörung früherer Bildungen (H d) erzeugt

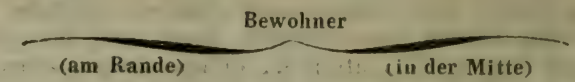

höherer Regionen . . . der Brandung...... des Sandes und Schlam. mes ........ werhselnde nach der Jahres-Zeit ..... eingeschwemmte Binnen-Bewohner ... des Brackwassers ... reichere Verschüttung . gewaltsame Zertrümmerung........ ungeordnete Altersfolge der Tiefe und in geriugerer Zahl. des Hochmeeres. des reinen und KalkWassers. reine Sec-Bewohner. des Salz-Wassers. spärliche Verschüttung. ruhige Erhaltung. geordiete Altersfolge. stete.

O. Von der Existenz be wald e ter $\mathbf{S}$ tre c k e n der Erd-Oberfläche geben uns gewisse Stamm- und Laub-Ablagerungen Kunde. Vou den ganz eigenthümlichen Sigillaria-Wäldern der I. Periode war schon an einem früheren Orte die Rede. In ihnen und später finden wir wohl auch einzelne Koniferen, Palmen und Cycadeen, doch nicht in solcher Menge und Zusammenhäufung, um daraus geschlossene ausgedehnte Waldungen zu folgern. Die jüngeren Kohlen-Lager, welche man in den Lias-, den Jura- und Wealden-Bildungen Englands und Deutschlands und in den Obergrünsand-Gebilden bei $C \ddot{\prime} l n$ kennen gelernt hat, sind sehr vereinzelte und örtlich beschränkte Erscheinungen, die letzten insbesondere blosse Zusammenhäufungen der Trümmer von Kouiferen-Stämmen, die nicht an Ort und Stelle gewachsen sind; die Bildungs-Weise der andern scheint noch nicht aufgefklärt zu seyn. Ansehnliche Lager von dikotyledonischen Laubholz-Bäumen, welche auf grössere Laubholz-Waldungen mit Bestimmtheit schliessen lassen, kommen erst in den Tertiär-Gebirgen vor.

Wir haben diese Verhältnisse schon in anderer Beziehung erörtert, konnten sie aber auch hier nicht ûbergehen. Vgl. das Weitere auf S. 904. 
§. 24. Im Besondern mit Rücksicht auf bestimmte Zone n und Lä nder.

A. Wenn einmal alle Länder, alle Welt-Gegenden, alle Zonen hinsichtlich ihrer organischen Reste genau durchforscht seyn werden, wird es eine anziehende Aufgabe seyn, dieselben hinsichtlich ihrer früheren Faunen und Floren, mit Rücksicht auf die jetzigen, untereinander zu vergleichen; unsere jetzigen Kenntnisse sind aber so bruchstückartig, dass die ausführliche Bearbeitung dieses Gegenstandes noch kein befriedigendes Resultat zu gewähren verspricht, daher wir nur einzelne Skizzen hervorheben.

B. Wir haben schon bei einer andern Veranlassung nachgewiesen, dass die Pflanzen- und Thier-Geographie der ganzen ErdO be rfläch e anfangs überall gleichartig war, mit Beginn der TertiärZeit sich zu differenziren anfing, aber erst in der Mitte dieser Tertiär-Zeit überall einen Charakter annahm, welcher dem der jetzt daselbst einheimischen Flora und Fauna zu entsprechen begann (S. S81). Wenn wir also früher wesentliche geographische Verschiedenheiten noch nicht nachzuweisen im Stande sind, während wir die Annäherung zur jetzigen Beschaffenheit in der miocänen und pliocänen Zeit schon mehrmals erörtert haben, so wird es noch von luteresse seyn, die erwähnte Ungleichheit der Fauna am Anfange der Tertiär-Zeit im Ganzen zu überblicken, so weit als es unsere jetzigen Hülfsmittel erlauben.

a. Man könnte noch immer einen Zweifel erwecken, ob die Fauna und Flora nicht doch schon in der I. Periode sogar einige Zonen-weise Verschiedenheiten unterscheiden lassen, da die Verbreitung der verschiedenen GebirgsFormationen und unsere Kenntniss derselben nach den Zonen noch immer sehr beschränkt ist. In der That scheinen die 2 Tabellen in $\$ .213$ darauf hinzu. weisen, dass wenigstens die Arten der 2 gemässigten Zonen unter sich ähnlicher, als mit denen der Tropen-Zone sind; und wenn wir auch später viele oolithische Arten Europa's in Indien wiederfinden, so rühren diese doch aus dem ausser-tropischen Theile dieses Landes her. Sollte nicht, wenn auch die ErdWärme hoch genug gewesen, um den Unterschied der Zonen.Temperatur zu verwischen, doch die ungleiche Vertheilung des Sonnenlichtes nothwendig einen Unterschied auch der Bevölkerung bewirkt haben? Aber so wahrscheinlich Diess auch ist, so sehen wir doch gerade zwischen der (nördlich) kalten und gemässigten Zone, wo diese Ungleichheit am grössten, weit weniger Verschiedenheit, als zwischen der gemäsigten und heissen Zone; wir sehen Arten und Familien von Pflanzen in die kalte Zone hineinreichen, von denen wir eben so wenig begreifen, wie sie das halbjährige Dunkel ertragen haben, als wir begreifen würden, wie sie jetzt deren Kälte ertragen sollten ').

b. Eine eocäne Fauna kennen wir in Frankreich, England, Belgien, NordDeutschland, vielleicht in Böhmen und Ungarn, an der Wolga, in der Ukraine, in Armenien, dann in südlichen Theile Nord-Amerika's (Alabama) und zu beiden Seiten der Kordilleren in Süd-Amerika von $5^{\circ}$ bis zu $48^{\circ} \mathrm{Br}$. hinab, jedoch hauptsächlich zwischen dem 30. und 41. Breite-Grade. Die zur Vergleichung dienenden fossilen Reste sind nur Konchylien, da ausser dem an bekann.

1) A. de Candoler in Jahrb. 1837, 612. 
ten Säugthieren und insbesondere Pachydermen so reichen Paris-Londoner Becken nur noch an der Ostseite der Cordilleren im $32 .^{\circ}$ und $41 .^{\circ}$ Breite SäugthierReste aus den bisher nur dort gefundenen Genera Megamys und Toxodon vorgekommen sind, ein riesiger Nager und ein Dickhäuter mit Nagezahu-artigen Backen-Zähnen, dessen 2 Geschlechts-verwandte Arten jedoch ebendaselbst in jüngerer Formation lagern; letztes Genus hat wenig nit der jetzigen Säugthier-Fauna süd-Ameriku's gemein; doch steht erstes dem dortigen Geschlechte Lonchophorus nahe. An eocänen Konchylien aber enthält das Pariser Becken 1200 und das ganze Paris-London-Brïsseler Becken uber 1400 Arten, welche um Paris vorzüglich als grosse und schön erhaltene Siphonobranchier in Sand und Kalkstein liegen, während um London Thone herrschen, worin jene Gruppe sehr zurücktritt; in Belgien und Nord-Deutschland hat die eocäne MolluskenFauna mehr Ähulichkeit mit der Englisih-Belgischen als Französischen. Aus der Ukraine hat Duво1s unter einer nicht grossen Anzahl von Eocän-Konchylien wenigstens 5 Pariser Arten mitgebracht '); bei Saratof an der Wolga hat man 5 Arten des London. Thons gefunden ${ }^{2}$ ); in der Krim hat Dubors ebenfalls $5-6$ Paris-Londoner Arten mit Nummuliten und Ostrea gigantea $(s, t)$ angegeben ${ }^{3}$ ); und auch in Armenien scheinen noch von diesen vorzukommen. In Alabama in Nord-Amerilka sind die Siphonobranchier sehr zurückgedrängt, fast alle Konchylien-Arten klein, und unter 250 fossilen Arten zähil LEA ${ }^{4}$ ) einige aus bloss eocänen Geschlechtern Europa's, aber auch nicht eine daselbst vorkonmende eocäne Art mit Sicherheit auf; denn seine Venericardia planicosta, Fusus longaevus und Actaeon lineatus (? Tornatella inflata FÉr.) lassen noch erhebliche $\mathbf{Z}_{\text {weifel }}$ übrig, wie er selbst sagt. Die Ötlichkeit von Fort Washington bei der Stadt Washington und zu Vance's Ferry in Süd-Carolina verlialten sich ähnlich. Nur betrachten wir seine Pasithea umbilicata als die in Europäischen Eocän-Bildungen bis, wie es scheint, in die jetrige Schöpfung vorkommende Niso terebellata. Daher dorh nur eine sehr kleine Zahl gemeinsamer Arten in cocänen Schichten beider Kontinente angrgeben sind, die selbst noch einer genaueren Prüfung und unmittelbaren Vergleichung bedürfen. In südlichen Amerika aber, wo D'ORBıG 40 eocäne Konchylien-Arten sammelte und DARwIN andere zufügte, ist keine weder mit Europa noch mit Nord-Amerika gemeinsame Art, ja selbst unter den von $\mathrm{D}^{\prime} \mathrm{O}_{\mathrm{rBIGNY}}$ aus $32^{\circ}-41^{\circ} \mathrm{S}$. Br. mitgebrachten 24 Konchylien-Arten von der West - und 11 Bivalven-Arten von der Ost-Seite der Anden, welchen ersten DARwin noch 56 andere an den Küsten Chiles bis Patagoniens gesammelte Arten beigefügt hat ${ }^{5}$ ), ist nicht eine einzige Art identisch zwischen Osten und Westen; ihre Genera aber kounmen im jetzigen Amerika grösstentheils nur iunerhalb oder näher bei den Tropen vor, und einige Arten erinnern, wenn sie auch nicht identisch sind, doch sehr an Pariser Formen, wie Rostellaria Gaudichaudi, Fusus difficilis, Venus auca ${ }^{6}$ ) u. a. Bei dem Mangel aller identischen Arten könnte man wohl an der richtigen Alters-Restimmung der süd-Amerikanischen Formation noch zweifeln, obschon das entschiedene Vorherrschen der grossen Siphonobranchier wenigstens auf der Ost-Seite in Verbindung mit dem Mangel aller noch lebenden Arten eine positive Hinweisung auf Eocän-Bildungen enthält, während bei dem Mangel an solchen Arten, die in andern Formationen vorkämen, jener Annahme nichts im Wege steht.

C. a) Europa ist nicht nur der bei Weitem am genauesten durchforschte Welttheil, sondern bei aller Kleinheit seiner Flächen-

1) Jahrb. 1833, 353; 1836, 360. - 2) Jahrb. 1844, 85.

3) Jahrb. 1838, 350. - 4) Contributions to Geology, 1833.

6) Geological observations on South-Amerika, Loudon, 1846, $8^{0}$.

6) d'Onbignx, Voy., Paléontologie. 
Ausdehnung die vollständigste Musterkarte aller geologischen Formationen, die auf der Erd.Oberfläche bekannt sind. Auf dem ganzen übrigen weiten Gebiete derselben ist bis jetzt keine neptunische Bildung bekannt geworden, welche nicht in Europa ebenfalls und zwar bis jetzt noch weiter und ausgedehnter als dort vertreten wäre, während die anderı mehrfach grösseren Welttheile, so weit bisher unsere Kenntnisse reichen, von den Schichten-Reihen und mithin den FossilResten ganzer Formationen, ja ganzer Perioden uns noch keine Proben geboten haben, wie folgende Übersicht zeigt:

Europa hat Formationen . . . . a b c d e f g/h i k l/m n op/q r f $/ \mathrm{s}$ t u v w $x$

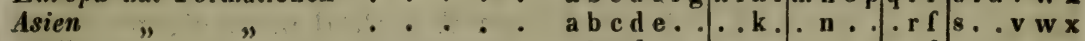

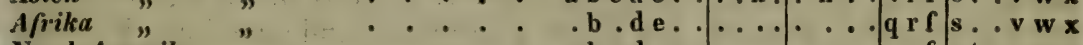
Nord-Amerika " . . . . a bede......... Süd-Amerika " . . . . a b c d e......? .. q rf $\mathrm{s} t ? \mathbf{v} \mathbf{w} \times$ Australien

Noch einige andere Bildungen sind zwar da oder dort angezeigt, aber nicht in erheblicherer Ausdehnung mit Bestimmtheit nachgewiesen oder aus ihren fossilen Organismen mit Gewissheit erkannt worden. Zweifelsohne werden noch viele der vorhandenen Lücken ausgefüllt werden; aber offenbar fehlen andern Wolttheilen gewisse Formationen doch auf viel weitere Strecken als in Eurora. Waren sie dort je vorhanden? und, wenn Diess nicht der Fall, fehlten sie dort in Folge einer langen Erhebung des Landes über das Meer, oder einer langen Versenkung in zu grosse Tiefen eines weiten Ozeans?

b) Demzufolge ist das gemäsigte Europa (das polare hat nur 1 Art des Enumerators dargeboten) so reich an fossilen $\mathrm{Gesch} \mathrm{lech}$ ter'n, dass von allen $\mathbf{2 7 6 4}$ im Enumerator aufgezählten Genera (vgl. die V. Tabelle) 2557 in Europa vorkommen und nur 207 daselbst fehlen. Die verschiedenen Welttheile haben, wenn die in verschiedenen Zonen eines Welttheiles gefundenen Geschlechter und Arten jedesmal als neu gezählt werden, was im Ganzen einen Ïberschuss von 0,29 bei den Geschlechtern und $0,02 S$ bei den Arten gibf, bis jetzt in folgendem Maasstabe Beiträge zu unserer Kenntniss der fossilen Reste geliefert.

$\begin{array}{lrrrrrrr} & \text { Europa } & \text { Asien } & \text { Afrika } & \text { Amerika } & \text { Australien } & \text { Addition } & \begin{array}{c}\text { wirkliche } \\ \text { Summe }\end{array} \\ \text { Genera } & 2644 & 265 & 107 & 618 & 38 & 3672 & 2764 \\ \text { Arten } & 24314 & 569 & 207 & 2010 & 53 & \mathbf{2 7 1 5 3} & \mathbf{2 6 4 2 1}\end{array}$

Diese Genera zeigen folgende geographische Beziehungen zu Europa, wenn man sie bloss nach ihren Zahlen beurtheilt:

a. Ganze Zahl

Genera . 2764

Quoten von 2557 und 718
Quoten von 2764

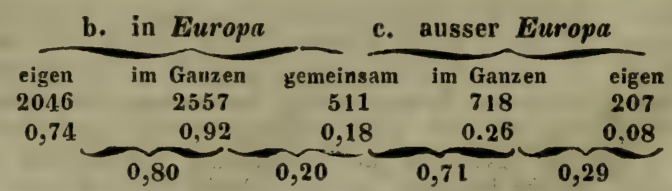

Europa bietet also, lebende und ausgestorbene Sippen auf beiden Seiten zusammengezählt, nicht nur $31 \frac{1}{2} \mathrm{mal}$ so viel Genera dar, als die gesammte übrige Erd-Oberfläche, sondern hat unter dieser 
viel grösseren Menge nicht bloss eine grössere Zahl, sondern eine über $8 / 3$ mal so grosse Quote eigenthümlicher Geschlechter, näınlich 0,80, während die ausser-europäischen Länder nur 0,29 besitzen; somit hat Europa $10 \mathrm{mal}$ so viele eigene Genera, als die ausser-europäischen Länder zusammen, daher es in paläontologischer Hinsicht ungefähr dieselbe Rolle wie in petrographischer spielt. Diese 207 ausser-euro. päischen Welttheilen eigenen Sippen reduziren sich aber, wenn man 1) alle in mehren Weltheilen und Zonen sich wiederholenden nur 1 nal zählt, 2) von denjenigen aus der mitteln und jüngeren TertiärZeit, hauptsächlich Polygastrica, Säugthiere, Vögel und Westindische Hölzer ans $\mathbf{x}$, w und $\mathbf{x}$. wo schon jeder Welttheil seine eigene Fauna und Flora hatte, absieht, und 3) die synonymen und unsichern RafinesQue'schen Genèra unbeachtet lässt, auf kaum 25 Sippen. Alle diese exotischen Sippen zeigen endlich bis zur Mitte der Tertiär-Zeit herein keinen andern eigenthümlichen Charakter, als die Europäischen (vielleicht nur der isolirte Dicynodon des Cap's von ungewissem Alter ansgenommen); man erk ennt daran nirgends mehrfaclie Floren undFaunen, die unter sich auch nur eben so verschieden wären, als die heutigen in einerlei Zonen beisammengelegenen Floren und Faunen sind.

Jene dem Auslande eigenthümlichen 28 Gencra sind: unter den

Pflanzen: Trizygia, Vertebraria $\left(S^{3}, e\right)$, Anomopteris nur fast ganz $\left(S^{2}, \bullet\right)$.

Polyparien: Diphyphyllum (S, $\mathbf{d}^{2}$ ).

Akalephen: Nummularia und Lycophrys $\left(\mathbf{S}^{3}, \mathbf{s}\right)$.

Echinodermen: Caryocrinus (M2, b $\mathbf{d})$, Moulinsia ( $\mathbf{S}^{2}, \boldsymbol{t}$ ?), ? Cyrtoma $\left(\mathbf{S}^{3}\right.$, ?), Scalites, Subulites, Maclureia, Ophileta, Cameroceras $\left(\mathbf{M}^{2} \mathbf{a}\right)$.

Mollusken: Carolia in $\mathbf{F}^{3}$ (?), Orthonatha, Microceras $\left(\mathbf{M}^{2} \mathbf{b}\right)$, Myoparo, Tuba $\left(\mathbf{M}^{3} \mathbf{c}\right)$.

Crustaceen: Triarthrus, Cryphacus, Ceratocephalus, Nuttainia $\left(\mathbf{M}^{2} \mathbf{a}-\mathbf{c}\right)$.

Fische: Catopterus Redf. ( $\left.\mathbf{M}^{2} \mathbf{c}\right)$, Rhacolepis und Calamopleurus $\left(\mathbf{M}^{3} \mathbf{F}\right)$.

Reptilien: Dicynodon $\left(\mathbf{F}^{+} \mathbf{f}\right.$ ?).

Säugthiere: Zeuglodon $\left(\mathbf{M}^{2}, \mathbf{t}\right)$. Megamys $\left(\mathbf{M}^{4}, \mathbf{t}\right)$.

Dieses auffallende Verhältniss scheint übrigens doch nur eine einfache Folge theils der Kenntuiss der grösseren Masse von Formen in Europa, theils des genaueren Studiums derselben zu seyn. Überhaupt legen wir keinen grnssen Werth auf diese Zahlen-Ergebnisse aus einer in Bezug auf die ErdOberflärhe so ungleichmässig erhobenen Zusammenstellung, als unsere bisherige ist.

Wie wir so viel vertranter mit den Europäischen als den exotischen Fossilien sind, so muissen auch alle unsere Vergleichungen von Europa ansgehen. Dieses grenzt aber mit seiner breitesten Seite und allen seinen Klimaten an Asien, so dass es damit die grörste Ähnlichkeit zeigen muss, indem wir bis jetzt eben diesen angrenzenden Theil Asiens auch fast am genauesten kennen, einige beschränkte Örlichkeiten in Indien ausgenommen. Längs der SürdGrenze Europa's liegt A/rika, zwar durch das Mittelmeer davon getrennt; aber es ist kein Zweifel, dass schen von der Kreide-Zeit an die dem Mittel. meere zufallenden Sciten beider Kontincute die Niederschläge eines einzigen gemeinsamen Meeres aufgenommen haben und diher durch das Mittelmeer nicht sowohl getrennt als verbunden erscheinen. Was wir aber aus dem übrigen Afrika kennen, ist zu unbedeutend, un zu einer geographisrhen Vèrgleirhung zu genuigen. - Fin Anderes aber ist es mit Nord-Amerika, welches ebenfalls eine grosse Veru undtschiaft seiner Bildungen und ihrer organischen Einschlïsse mit Europa hit und, wenn auch unter einer Zone, doch so weit 
iber davon entfernt liegt, dass es weder zu Land, wenn hier je ein Zusammen.511. hang bestanden hätte, noch zur See sich an Europa anschliessen konnte. So bilden denn eigentlich nur $\mathbf{M}^{2}, \mathbf{M}^{34}, \mathrm{~S}^{23}$ (mit Ausschluss der Ural-Gegenden), $\mathrm{U}^{3+}$ und $\mathrm{F}^{34}$ solche Erd-Striche, denen wir, wie die Beobachtung allerdings bestätigt, eigene Faunen und Flosen zutrauen dürfen, obwohl wir gerade diese Gegenden fast alle nur schr unvollständig kennen. Da mithin ein wissenschaftliches Ergebuiss nicht zu erwarten, so verzichten wir darauf zu untersuchen, wie viele Genera und Arten diese Welttheile im Einzelnen unter sich und mit Europa gemein haben.

c) Was die A rten anbelangt, so gibt es deren 513 , welche in und ausser Europa zugleich beobachtet worden sind, nämlich 16 Pflanzen (ohne die Kiesel-Theilchen), 250 Phytozoen (meist Infusorien), 203 Malacozoen, 17 Entomozoen (Vermes und Crustacea) und 27 Spondylozoen. Diese stellen sich, wenn man die exotischen Arten in der oben bemerkten Weise addirt, wo im Ganzen (diese 513 mitbegriffen) 732 Arten oder 0,0 :S mehr gezählt werden, als vorhanden sind, so zu den übriggen

a. Ganze Zahl

Arten: 26421

Quoten von 26421

” von 24314 u. 2839

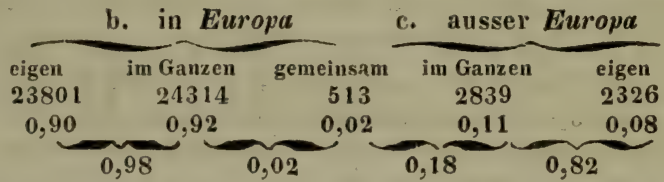

Europa hat mithin über $8 \mathrm{mal}$ so viel Arten als die ganze übrige Welt geliefert, welche ihm mit Ausnahme von nur 2 Prozent alle eigen sind, während die wenigen $(0,11)$ exotischen Arten nur grösstentheils, nämlich mit Ausnahme von 0,18, niclit in Europa vorkommell.

Ein richtiges Bild der einstigen Pflanzen- und Thier-Geographie von Europa liefern uns diese Zahlen aber nicht, weder den übrigen Welttheilen gegenüber, welche, wie eben aus dem Vorhergehenden ersichtlich, noch viel zu wenig durchforscht sind und insbesondere noch keine solche werthvollen TodtenGrüfte, wie Solenhofen, Öningen, der Bernstein u. s. w. in Europa, dargebo ten haben, um uns auch nur einigermaasen den nöthigen Stoff dafür liefern zu können, - noch in seinem Innern, da in den eben verzeichneten Zahlen die Faumen und Floren aller successiven Perioden zusammengefasst sind, und weil unser Enumerator deren geographische Vertheilung nur noch bis zur Unterscheidung der gemäsigten und kalten Zone berücksichtigt, von welchen die letzte bis jetzt bloss 1-2 Arten yeliefert hat. Auch würden wir uns, selbst wenn wir bis ins Einzelnste eingehen wollten, - da sich die örtliche Vertheilung der Fossil-Reste mehr von der zufälligen Erstreckung der untersuchten Gebirgsarten als von den einstigen Wohnorten der Organismen abhängig zeigt - ein genaueres Bild der ehemaligen Pflanzen - und Thier-Geographie Europa's kaum versihaffen künnen, als bereits in den Entwickelungs-Gesetzen (S. $809 \mathrm{ff}$.) ausgedrückt ist, weil die frühesten Faunen und Floren über die ganze Erde gleich waren, die beginnenden Verschiccieuheiten zu Ende der Kreide - und Aufang der Tertiär-Zeit noch nicht an hinreichend vielen Punkten untersucht sind, und von der mitteln Tertiär-Zeit an $(\boldsymbol{u})$ die Faunen und Floren allerwärts sich mit einem etwas wärmeren Charakter sehr rasch den jetzigen annähern. Einige treffende Andeutungen darüber, wie nahe am Eude der Tertiär-Zeit solche den gegenwärtigen schon überall gestanden, haben wir S. $898 \mathrm{ff}$. mitgetheilt. Also 
hauptsächlich nur in der Tertiär-Zelt kann es angemessen seyn, einige paläontologische Verschiedenheiten mehrer Welttheile noch etwas näher zu betrachten.

D. Da die Sä ugthiere ilıre Entwicklıngs.Phasen ganz in der Tertiär-Zeit durchlaufen, daher diese in unsern Tabellen nicht hinreichend gegliedert hervortreten konnten, und da kein Land so genau durchforscht ist, als Europa, so wollen wir versuchen die geographische Verbreitung der Säugthiere in Europa während der Tertiär-Zeit noch spezieller in eine Tabelle zusammenzustellen, wo im Kopfe der $R u$. briken die Unterabtheilung a ausgestorben, exotisch, I noch in Europa lebend bedeutet. 


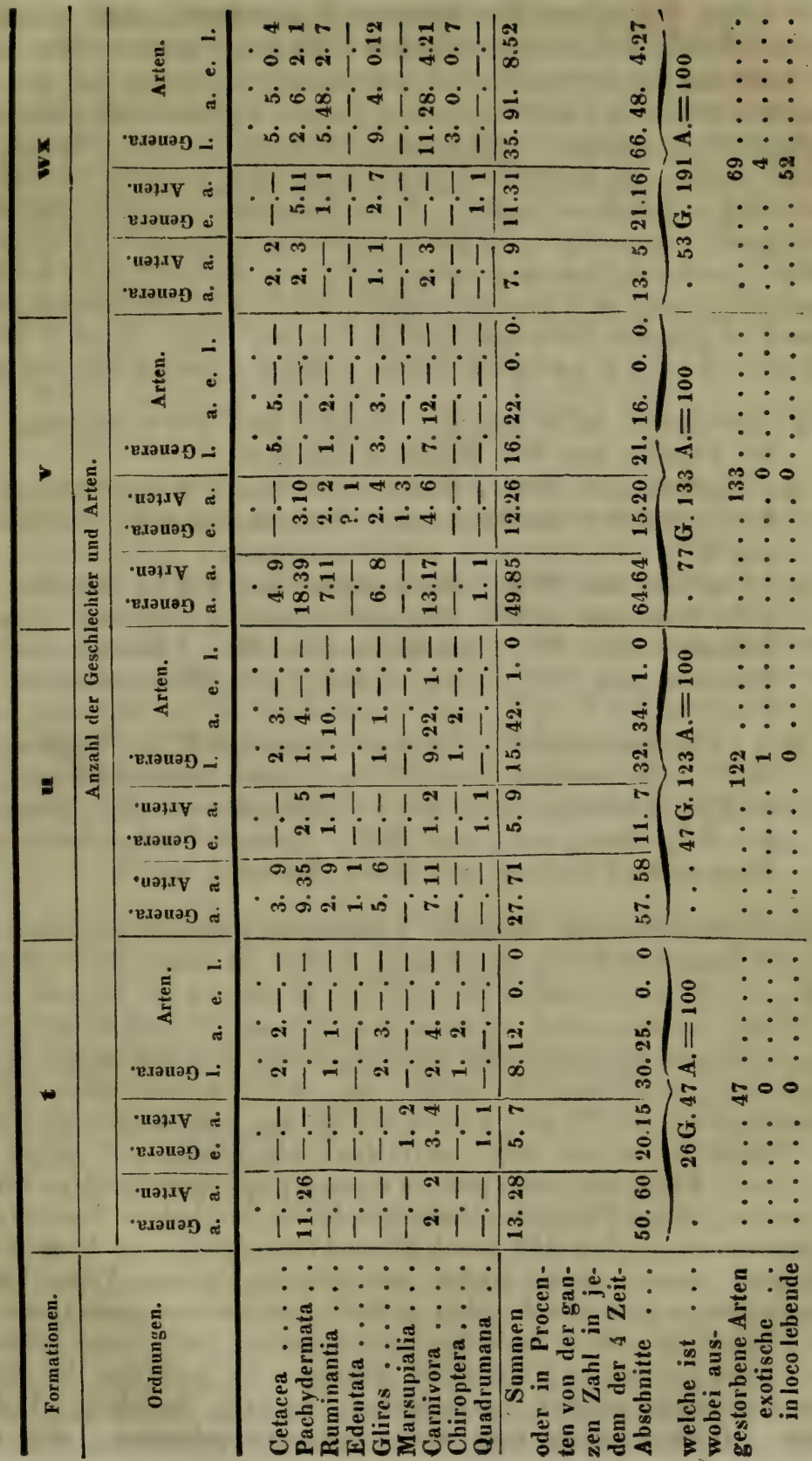


In Folge des späten Erscheinens in der Tertiär-Zeit trägt die Sängthier-Fauma keinen universellen Charakter mehr. Die Europäische ist von der lndischen und Amerikanischen schon im Anfange sehr verschieden, eine eigenthümliche Fanna, die aber doch mehr bloss geographisch als klimatologisch abgeschlossen zu seyn scheint. In der Zeit $t$ sind die Säugthiere überhaupt noch nicht so häıfig und manchfaltig gewesen als später. Die erloschenen Geschlechter kompensiren oder überwiegen die noch lebenden in den ersten Zeiten $\mathbf{t} u$ $\mathbf{v}$ überall, und erst in $\mathbf{w} \mathbf{x}$ werden die letzten plötzlich weit vorherrscliend; hier treten auch die exotischen Geschlechter weit zurück gegen die noch in Europa einheimischen $(1: 3)$, zu denen sie sich bisher fast wie $1: 2-3: 4$ verhalten hatten. Die exotischen Genera sind:

in $\mathbf{t}$ : Didelphys jetzt aus $\mathbf{M}^{3}$, Virerra aus $\mathbf{S}^{3} \mathbf{F}^{3}$, ?Mydaus aus $\mathbf{S}^{3}$, ? Nasua aus $\mathbf{M}^{3}$, Macacus aus $\mathbf{S}^{3}$;

in $\mathbf{n}$ : Tapirus aus $\mathbf{M}^{3} \mathbf{S}^{3}$, Rhinoceros aus $\mathbf{S}^{3} \mathbf{F}^{3}$, Moschus aus $\mathbf{S}^{23}$, (grosse Felis-Arten und) Viverra aus $\mathbf{S}^{3} \mathrm{~F}^{3}$, ? Hylobates aus $\mathbf{S}^{3}$.

in $\mathbf{v}$ : Tapirus aus $\mathbf{M}^{3} \mathbf{S}^{3}$, Rhinoceros ans $\mathbf{S}^{3} \mathbf{F}^{3}$, Moschus aus $\mathbf{S}^{23}$, Ovis aus $\mathbf{S}^{2} \mathbf{F}^{3}$, ? Dasypus aus $\mathbf{M}^{3}$, Lagomys aus $\mathbf{S}^{2}$, Echimys aus $\mathbf{M}^{3}$, Didelphys aus $\mathbf{M}^{3}$, ? Otaria aus $\mathbf{U}^{3}$, ? Hyaena aus $\mathbf{S}^{3} \mathbf{F}^{3}$, Viverra aus $\mathbf{S}^{3} \mathbf{F}^{3}$, ?Macroscelides aus $\mathrm{F}^{2}-{ }^{2}$.

in $\mathbf{w} \times$ : Elephas aus $\mathbf{S}^{3} \mathbf{F}^{3}$, Hippopotamus aus $\mathbf{F}^{234}$; ?Tapirus aus M:S ${ }^{3}$, Rhinoceros aus $\mathbf{S}^{*} \mathbf{F}^{3}$, Equus (und Antilope) aus $\mathbf{S}^{23} \mathbf{F}^{34}$, Camelopardalis aus $\mathbf{F}^{3}$, Auchenia aus $\mathbf{M}^{34}$, Lagomys ans $\mathbf{S}^{2}$; Dasyprocta aus $\mathbf{M}^{34}$, (grosse Felis - Arten und) Hyaena und Viverra aus $\mathbf{S}^{3} \mathbf{F}^{3}$, Macacus aus $\mathbf{S}^{3}$.

Eine nacheinander folgende Veränderung des Charakters der Fauna ist daraus nicht zu entnehmen, iidem zu allen Zeiten Sippen aus $\mathbf{M}^{3}, \mathbf{S}^{3}, \mathbf{F}^{3}$ vorkommen, und nur von $\mathbf{u}$ an, wo die Sippen etwas zahlreicher werden, anch einzelne ans $\mathbf{S}^{2}$ und $F^{2}$ sich beigesellen; die exotischen Genera sind also zu allen Zeiten fast nur tropische und subtropische; unverkennhar deuten sie aber jederzeit eine nähere Verwandtschaft mit $\mathbf{S}^{3}$ und $\mathrm{F}^{3}$ als mit $\mathbf{M}^{3}$ an, indem die anerikanischen Sippen in Europa mehr auffallend als häufig und sicher bestimmt sind. Unter den noch jetzt in Europa einheimischen Geschlechtern reichen einige Cetaceen allerdings bis in den hohen Norden hinauf; insbesondere aber sind durch ihren Arten-Reichthum in $\mathbf{w} \mathbf{X}$ und theils anch schon etwas früher die Genera Cervus, Felis, Canis, Hyaena, Ursus auffallend, ohme jedoch, da sie mit Ansuahme von Hyaena jetzt in allen Welttheilen verbreitet sind, der früheren Fauna einen besondern Charakter beilegen zu können. - Selhst in früher historischer Zeit waren Bären, Löwen, Schakale, Wölfe, Hirsche, Ochsen noch weit über Earopa verbreitet, die sich 
jetzt in einzelne Winkel des Kontinentes oder gar nach Asien zurückgezogen habe॥. Betrachten wir endlich die obigen Zeit-Abschnitte nach der Art ihrer Gesammt-Bevölkerung, so sind in Europa vorzugsweise charakterisirt:

t durch Palaeotherium, Anoplotherium, Dichobune, Xiphodon, Adapis: lauter Pachydermen, alle ausgestorben;

$\mathbf{u}$ durch Squalodon, Hyotherium, Lophiodon, Chalicotherium, Tapirus, Palaeomeryx, Dorcatherium, - Felis, Amphicyon: hauptsächlich Pachydermen, nur 2 Genera lebend.

v durch Halianassa, - Dremotherium, - Lagomys : manchfaltige Ordnungen, z. Th. ausgestorbene Geschlechter.

wx durch Elephas, - Bos, Cervus, - Lagomys, Arvicola, Felis, Canis, Hyaena, Ursus: Sippen aus manchfaltigen Orduungen, alle noch lebend.

E. Wir haben versucht, die Pflanzen- und Thier-Geographie nach den einzelnen Welttheilen und Ländern zu bearbeiten; da wir aber allgemeinere Verschiedenheiten derselben als hinsichtlich einzelner Genera bis in die Mitte der Tertiär-Zeit nicht erlangen konnten und nicht wissen, wie weit selbst diese Abweichungen namentlich bei den Wirbelthieren, deren Einschliessung und Erhaltung im Gestein von so vielen Zufälligkeiten abhängt, nur zufällig sich darbietende seyen, so sind wir davon abgestanden. Das Wichtigste gibt unser Enumerator an; und wer bis anf die Verschiedenheiten einzelner europäischer Länder eingehen will, der kann hinsichtlich der WirbelThiere das Detail bei Giebel und hinsichtlich andrer Gruppen Einiges bei Ander'n finden ${ }^{1}$ ).

\section{E. Chronologie der fossilen Organismen.}

\section{a. Im Allgemeinen.}

๒. 25. zerlegen :

A. Die Chronologie kann die Paläontologie verfolgen und

i) nach der Zeitfolge,

2) nach den Klassen,

3) nach den Weltgegenden.

1) So über Mollusken überhaupt: D'Origny im Jahrb. 1845, 372; QuBnstedt im Jahib. 1840, 253 ; D'OrBigny in Br. Collectan. 96 ; im Jahrb. 1844, 116 ; - über Fisthe: Gieber. Fauna der Vorwelt I, ur, 395-467 (1848) $>$ Jahrb. 1848, 750; - über Vögel: Gieber a. a. O. I, II, 1-40; über Reptilien: Giebec a. a. O. I, II, 1-217 (1847) > Jahrb. 1848, 103 : R. OWEN in Br. Collect. $52 ;-$ über Säugthieı: Giebel a. a. O. I, I, 1-281 (1847); - und úber Wirbelthiere uberhaupt I, III, 407 . 
Sie hat den ersten dieser 3 Gesichtspunkte voranzustellen und kann hierna ch die Klassell- und wieder die Länder-weise Betrachtung zunächst folg en lassen. Die erste Weise wird die wichtigere seyn, die letzte mehr mit der Geographie zusammenfallen. Indessen wïrde durch ein streng methodisches Verfolgen solcher Gliederung die Wissenschaft zu einer Weitläufigkeit geführt werden, welche mit den jetzt schon davon zu erwartenden Resultaten nicht im Verhältnisse steht, und ist daher ein etwas freierer Weg einzuhalten.

B. Eine bisher überall nachgewiesene merkwürdige Thatsache ist die vollkommene Gleichzeitigkeit 1) des Auftretens über die ganze Erd-Oberfläche der verschiedenen Klassen, Ordnungen, Familien, Genera, Spezies der Organismen, 2) des Erlöschens eines Theiles derselbe॥, dann 3) der allmählichen Differenzirung der Floren und Faunen seit Anfang der Tertiär-Zeit und endlich 4) der beginnenden und fortschreitenden Identifizirung der fossilen Genera und Arten mit den noch in der Gegend lebenden.

Manchfaltige Beziehungen haben uns genöthigt, uns schon in früheren Paragraphen mit diesen Erscheinungen zu beschäftigen, daher wir hier darauf verweisen können.

C. Nach welchen allgemeinen Gesetzen die Entwicklung der organischen Welt auf der Erd-Oberfläche stattgefunden habe, ist schon S. 809 ff. auseinandergesetzt. Doch finden anscheinende Abweichungen und Störungen der einzelnen Gesetze statt, 1) in so ferne von den, wie es scheint, $z$. Tlı. von einander unabhängigen Gesetzen öfters mehre mit einander in Konflikt gerathen, wo denn nur eines, und zwar vorzugsweise das der Anfïgung an die Entwicklung der äusseren Lebens-Bedingungen die Oberhand behält; - 2) in so ferne es Verhältnisse und Kombinationen der bedingenden Kräfte gegeben hat, die uns jetzt mehr oder weniger unbekannt sind, und worauf ebenfalls schon gelegentlich hingewiesen worden ist. - Welchen Verlauf aber in dessen Folge die chronologische Entwicklung der Schöpfung 3) im Einzelnen wirklich genommen habe, Diess stellt unser Enumerator in ganzer Vollständigkeit dar. In welcher Beziehung solche endlich zur jetzigen Schichten-Eintheilung stehe, wollen wir in dem spätern Paragraphen 221 nachweisen. Hier noch einige allgemeine Erörterungen.

D. Wir wissen bereits, dass es fossile Arten gibt, welche eine geringe, und andre die eine ausgedehnte geographische, - eben so welche, die eine geringe und andre die eine ausgedehnte geognostische Verbreitung besitzen; - und endlich, dass die geographisch verbreitetsten auch die längste geologische Dauer zu habeu pflegen. Nur solche von grösserer geographischer Verbreitung und nicht zu geringer individueller Frequenz können charakteristisch für diese oder jene 
Schichten-Gruppe seyn ; von der Grösse ihrer gleichzeitigen vertikalen Verbreitung hängt es aber ab, so sie als bezeichnend (bei den Konchylien Leitmuscheln genannt) für eine bestimmte Schicht, eine Schichten-Reihe, eine Formation, eine Periode dienen können, oder wegen vertikaler Überschreitung der Periode ihrer grossen Verbreitung ungeachtet nicht dazu brauchbar sind.

Wir haben schon bei mehren Gelegenheiten angedeutet, dass es undenkbar sey, dass in einem und demselben Zeit-Abschnitte ein e Fels-Schicht oder eine überall gleichbleibende Schichten-Folge sich zusammenhängend oder auch nur nach Massgabe der verschiedenen damals bestandenen Meeres-Becken unterbrochen rund um die ganze Erde abgelagert habe, weil die Erd-Rinde sich nicht überall gleichzeitig gehoben oder gesenkt haben, auf- oder unter-getaucht seyn, das Meer nicht überall gleichzeitig Küste oder Hochmeer, Bucht oder Ozean, in Strömung oder in Ruhe gewesen seyn kann; - es ist aber dann natürlich auch nicht möglich, dass eine und dieselbe Art fossiler Organismen rund um die ganze Erde eine und dieselbe Schicht oder eine und dieselbe Schichten-Reihe charakterisire, weil sie selbst so wenig überall gleichzeitig bestehen als jerre gleichzeitig entstehen konnte.

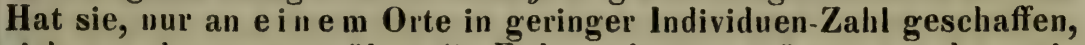
sich von da aus weit über die Erde verbreiten müssen, so kann sie unmöglich überall gleichzeitig auftreten; ist sie an mehren Orten geschaffen worden, so ist zwar der doppelte Fall denkbar, dass Diess an verschiedenen Stellen zu verschiedenen Zeiten, oder dass es überall zu einer Zeit geschehen ist. In keinem Falle wenigstens wird man aber annehmen können, dass sie bei einer einigermassen beträchtlichen geographischen Verbreitung überall ganz gleichzeitig erloschen sey; woraus also folgen würde, dass, abgesehen von der vorhin erwähnten Unmöglichkeit einer allerweitigen vollkommenen Schichten Gleichheit, auch das Entstehen und Erlöschen einer Art in verschiedenen Welt-Gegenden nicht überall absolut dieselben Zeit-Grenzen bezeichnen könne, weun es auch in der Regel einen ungefähren Masstab dafür abgeben mag und analoge Faunen und Floren überall in gleicher Ordnung auf einander gefolgt sind. Und da sich verschiedene Arten in dieser Hinsicht eine jede wieder anders verhalten können, so werden auch die nach einander entstehenden, wie die nach einander vergehenden Arten manchfaltig in einander eingreifen. Wir folgern aus dem Gesagten :

1) dass in einer Schicht verschiedene Organismen-Arten beisammen liegen können, welche theils charakteristisch sind für die einzelne Schicht, theils für eine Schichten-Reihe, für die Formation, oder die ganze Periode, wozu jene Schicht gehört;

2) dass eine solche Art nicht rund um die Erde für dieselbe gleichzeitige Schicht charakteristisch seyn könne, soferne eine solche Schicht nicht existirt; 
3) dass zwischen ihrem Erscheinen und Verschwinden auch nicht ïberall genan derselbe Zeit-Abschnitt liegen müsse, und zwar um so weniger, je weiter die Örtlichkeiten auseinander liegen;

4) dass dieselbe Art in verschiedenen Gegenden hier für eine Periode und dort für eine Formation und noch weiterhin endlich nur für eine Schicht derselben Periode bezeichuend seyn könne; ja es ist möglich, ist gewiss, dass sie in Folge von Wanderungen in verschiedenen Gegenden verschiedene Zeiten und Schichten charakterisiren könne;

5) dass in der Mitte und vielleicht schon zu Anfang der TertiärZeit die ältern Schichten höherer Breiten mehr und weniger übereinstimmen müssen mit jüngern Schichten niedriger Breiten.

Die gewöhulichen Vorstellungen von der festen Verbindung gleicher Arten mit gleichen Schichten an allen Orten des Auftretens müssen nach diesen Sätzen berichtigt werden.

a) Führen wir das Bild von einer von den Polen gegen den Äquator voransrhreitenden Abkühlung weiter aus, so könnte in z. B. 7 aufeinanderfolgenden Formations-Zeiträumen $(\mathbf{r}-\mathbf{z})$, in welchen in den höheren Breiten der zunehmenden Kälte wegen immer wisder eine neue Schöpfung $(\tau-z)$ entstanden und die ältere weiter Tropen-wärts gerürkt wäre, sich die geographische Ver. theilung der successiven Faunen in folgender Weise gestalten:

\begin{tabular}{|c|c|c|c|c|c|c|c|c|c|c|c|}
\hline & r. B & & & & & & & & & & \\
\hline & & & & $\mathbf{r}$ & r & $\mathrm{g}$ & $t$ & $\mathbf{u}$ & $\mathbf{v}$ & $\mathbf{w}$ & $x$ \\
\hline Fauna in & $80^{\circ}$ & : & & $\tau$ & $\mathrm{u}$ & $\mathrm{u}^{1}$ & $\mathbf{v}$ & $\mathrm{v}^{\prime}$ & $\mathbf{w}$ & $w^{\prime}$ & \\
\hline & $70^{\circ}$ & . & . & - & $\tau$ & $\mathbf{u}$ & $u^{\prime}$ & $v$ & $v^{1}$ & $\mathbf{w}$ & $w^{2}$ \\
\hline & $60^{\circ}$ & . & . & - & - & $\tau$ & $\mathrm{u}$ & $u^{\prime}$ & $\mathbf{v}$ & $v^{x}$ & $\mathbf{w}$ \\
\hline & $50^{\circ}$ & . & . & - & - & - & $\tau$ & $\mathbf{u}$ & $u^{1}$ & $\mathbf{v}$ & $v^{\prime}$ \\
\hline & $40^{\circ}$ & . & , & - & - & - & - & $\tau$ & $\vec{u}$ & $u^{\prime}$ & $\mathbf{v}$ \\
\hline & $30^{\circ}$ & . & & - & - & - & - & - & $\tau$ & $\mathbf{n}$ & $u^{\prime}$ \\
\hline & $20^{\circ}$ & . & & - & - & - & - & - & - & $\tau$ & $\mathbf{u}$ \\
\hline
\end{tabular}

An zahlreichen Belegen zu diesem theoretischen Bilde fehlt es uns zwar weil in höheren Breiten ältere Tertiär-Bildungen zu selten sind; doch kommt uns wenigstens einer zu Statten, der für diese Vorstellungs-Weise spricht, wenn auch später andere Werthe eingesetzt werden müssen. - Über ein entgegengesetztes Verhältniss im Norden des alten Kontinents am Anfang der jetzigen Zeit vgl. \$. 27.

Dass die Arten wirklich nicht überall eine gleichlange Dauer besessen, was in einigen früher zitirten Fällen noch immer bloss scheinbar eine Folge ungleicher Erhaltung und Durchforschung der verschiedenen Formationen in verschiedenen Gegenden seyn konnte - geht nämlich deutlich aus dem schon erwähnten Beispiele hervor, dass bei Bordeaux gegen 200 Arten miocäner Konchylien vorkommen, welche jetzt nicht mehr dort oder anderwärts in Europa, wohl aber am Senegal und in Guinea noch leben, mithin in Europa bei $45^{\circ}$ $\mathrm{Br} .=\mathbf{u}$, in Afrika mit $10^{\circ} \mathrm{Br} .=\mathrm{z}$ entspmechen. Es würde interessant seyn an Senegal auch miocäne Schichten zu entdecken, un zu sehen, ob und in welcher Verbiulung dieselben Arten schon in jener Zeit dort gelebt haben (vgl. Satz 5).

b) Das Verhältniss der für mehre Schichten, Formationen etc. bezeichnender Arten kann durch folgendes Beispiel deutlicher gemacht werden: 
enthält nämlich die Formation

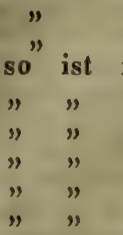

die fossilen Arten $\quad \alpha \beta \gamma$

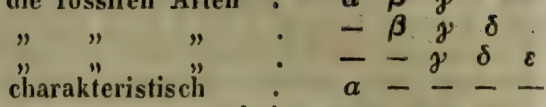

" keine $\quad---$

" $\quad-\quad--\frac{-}{\beta}$

c) So mag Lyriodon alifornis immerhin in Europa wie in Nord- und Süd-Amerika und im gemäsigten und tropischen Asien die Kreide-Periode bezeichnen; aber weder aus der Beschaffenheit der Gebirgs-Schichten, noch aus den sie begleitenden Organismen-Arten hat man bis jetzt nachweisen können, dass es in diesen fernen Welt-Gegenden wie in Europa der Grünsand (Gaulı) oder seine Zeit ist, die sie vertritt; denn das Gebirge hat ein anderes Ansehen, und die begleitenden Konchylien, so weit sie auch in Europa bekannt sind, werden als solche theils aus höheren und theils aus tieferen Schichten be. zeichnet.

So können wir uns auf das S. 927 ff. gegebene Beispiel der Kreide berufen, von welcher in Frankreich 4 Becken bestehen, das Mittelmeerische, das Pariser, das Pyrenäische und das Loire-Becken, davon nur die 2 ersten das Neocomian und den Gault, alle 4 die chloritische Kreide und die 2 mittlen die weisse Kreide enthalten.

So sagt Pictet, dass in der Gegend von Genf auf einer 20 Stunden langen Linie der Gault in $6-7$ Örtlichkeiten vorkomme, welche hinsichtlich ihrer Organismen-Arten und deren Verbreitung ganz mit einander übereinstimmen; nur einige Schichten von Fis und Sixt machen eive Ausnahme, in so fern sie ein merkwürdiges Gemenge von Arten des Gault mit solchen der chloritischen Kreide darbieten ${ }^{\mathbf{1}}$ ).

Das Kreide-Grebirge von Columbien in $3^{0}-7^{0}$ nördl. Br., von Chili und Peru ist nach Maasgabe seiner fossilen Arten gerechnet worden:
1. von v. BuCH ${ }^{2}$ )
zur Kreide
im Allgemeinen
2. von D'ORBIGNY ${ }^{3}$ )
zum Neocomien wegen
3. von E. Forbes ${ }^{4}$ ) zum Gault wegen wegen

Ammonites Rhotoma. gensis $\mathbf{r}^{\mathbf{1}}$.

Neithea $s p .9-\mathbf{r}^{1}$.

Die unter einer nur

kleinen Arten-Zahl ge. funden wurden.

\begin{tabular}{l|c} 
Natica praelonga & - \\
Actaeon affinis. & \\
Cardium peregri. & \\
norsum. & \multirow{2}{*}{ Trigonia Lajoyei. } \\
Exogyra Couloni. & $\Xi$ \\
(? Trigonia aliformis \\
aus r).
\end{tabular}

Trigonia aliformis $\mathbf{r}$. und einigen vicarirenden unter

17 untersuchten Arten, wobei noch ein Ancyloceras für q spricht.

Wobei freilich noch genauer zu untersuchen bleibt, in wieferne diese Arten nicht doch auch in Amerika aus verschiedenen übereinanderliegenden Schichten stammen, was d'Orbigny und E. Forbes nicht anuehmen. Die von Forbes untersuchten und die meisten von D'ORBIGNY beschriebenen Arten stammen aus der Nähe von Sa. Fé de Bogota, die übrigen weiter her. Wir können daher

1) Jahrb. 1848, 757. - 2) Jahrb. 1838, $607 \mathrm{ff}$.

$\left.{ }^{3}\right)$ Voyage, Paléontol. 98-100. Unter 43 Arten hat er 5 identische, und 12 sehr ähnliche mit solchen des Europäischen Neocomiens, 1 mit Arten aus dem Gault verwandte und 6 identische (? Trigonia aliformis) oder meist nur ähnliche der chloritischen Kreide gefunden. Auch ist bemerkenswerth, dass die 5 identischen alle in Pariser und nur eine davon (E. Couloni) zugleich auch im Mittelmeerischen Neocomien-Becken vorkommen.

4) Jahrb. 1848, 756 . 
auf die ungleichen Bestimmungen dieser als eine Formation betrachteten Schichten Zentral-Amerika's noch kein besonderes Gewicht legen. - Zu Pondicherry, Trichinopoly und Verdachellum im südlichen Ostindien kommen ebenfalls KreideGebilde vor, welche an diesen 3 Orten einige Arten gemein haben und daher einer Formation zugezählt werden; aber EGERTon rechnet sie nach den FischResten zur obersten Kreide und findet, dass sie selbst mit den Tertiär.Gebilden eine bestimmte Verwandtschaft haben; E. Foress zählt sie nach den Konchylien zur entschiedenen Kreide, obschon das Auftreten vieler Siphonobranchier ihm ebenfalls einen tertiären Charakter andeutet, der in Europa fremd ist '); aber die in Europa sich wiederholenden Arten sind aus verschiedenen Formationen, nämlich :

aus Neocomien, q. Ammonites Juillieti. n Rouyanus. aus $\mathbf{q}-\mathbf{r}$.

Pecten obliquus (Fокв.)

" 5 costatus " aus Gault, r. Nautilus Clementinus.

Ostreae $s p$.

Lyriodon aliformis. aus $\mathbf{r}, \mathbf{r}$.

Pecten orbicularis (ForB.). aus Kreide $r$.

Corax pristodontus, $\mathbf{r}^{2}$. Enchodus n. sp., $\mathbf{r}^{2}$. POdontaxp. rhaphiodon $\mathbf{r}$. Otodus appendicul. (r) $r$. ? Nautilus laevigatus $\mathbf{r}^{\mathbf{t}}$. Pinna restituta r.

Cardium Hillanum $\mathbf{r}^{\mathbf{1}}$.

Wir wollen die Beispiele nicht bäufen; indessen ist es bekannt, dass es noch nicht gelungen ist z. B. die einzclnen Glieder der Englisch-Nord/ranzosischen Oolithen-Reihe in Deutschland wieder aufzufinden, obwohl die identischen Versteinerungs-Arten dort wie hier ungefähr in derselben Reihen-Folge übereinander liegen, im Einzelnan und Kleinen aber schon in Entfernungen wie von der Schweits bis Württemberg um einige Schichten von der Ordnung abweichen, weiter aufwärts oder tiefer abwärts reichen und daher mit andern Arten hier und dort in Verbindung treten müssen.

E. Mehre Fälle, wie ungleichzeitige Faunen gleich, und gleichzeitige ungleich werden können, sind schon $S .924,942$ u. a. auseinandergesetzt. Doch steht, was dort als möglich angenommen, als so reines Extrem in Wirklichkeit nicht zu erwarten.

F. So wahrscheinlich, so übereinstimmend es indessen mit Erscheinungen der jetzigen Schöpfung wäre, dass einzelne A rten auch vordem vom Zentral. Punkte ihrer Schöpfung aus (S. 942) nach verschiedenen Richtungen gewa ndert seyen, so wenig ist es von der ganzen Bevölkerung eines Landes, eines Meeres, einer Küste, einer KüstenRegion glaublich, dass sie mit oder ohne Aufgebung jenes SchöpfungsPunktes in Masse sich nach irgend einer andern Stelle versetzt und die dortige Bevölkerung verdräıgt habe; wir werden überhaupt nie erwarten dürfen, an zwei von einander entlegeneren Stellen genau dieselbe Bevölkerung ganz und ausschliesslich wieder zu finden, wie wir jetzt anch unter gleicher Breite uirgends ganz derselben Fanna und Flora wieder begegnen. Da es ferner scheint, dass in den früheren Perioden, wo die Wärme der Erde die Verschiedenheit der Klimate noch nicht fühlbar werden liess, auch ein wesentlicher Grund zur allgemeinen Wanderung in einer bestimmten Richtung nicht stattgefunden haben könne und wir mithin von kleinen bloss durch

1) Jahrb. 1848, 116. 
topographische Modifikationen veranlassten Ortswechseln ahgesehen, solche Wanderungen vor der Tertiär-Zeit überhanpt nicht erwarten, da das Vorkommen einzelner Arten zu verschiedenen Zeiten in verschiedenen Gegenden an sich eine stattgefundene Wanderung noch nicht beweist, - endlich da mit Ausnahme einer mässigen Quote die Dauer der Arten sich überhaupt auf eine nicht zu lange SchichtenReihe zu erstrecken pflegt und es in grösseren Entfernungen unmög: lich wird, die Repräsentanten der einzelnen Schichten dieser Reihe wieder aufzufinden, um sich über ein Vorkommen derselben Art in verschiedenen Schichten aussprechen zu können; so müssen unsere speciellen Nachforschungen nach der Wanderung und Verbreitung ler einstigen Bevölkerung der Erde eben so beschränkt als schwierig werden, und es kann kaum überraschen, wenn wir ein genügendes Resultat über etwa stattgefundene Wanderungen der Art nicht erlangen.

a. Wir wollen aus $\$$. $23 \mathbf{G}$, S. 920 diejenigen Beispiele hier zusammentellen, wo 1) Art und Formation als sicherer bestimmt angesehen werden könen, und 2) eine bestimmte Abweichung. zwischen den verschiedenen Weltregenden hinsichtlich der Formation wahrgenommen wird, mit dem Bemerken, lass solche Fälle, wo eine Art z. B, in $\mathrm{E}^{2}$ in eiser längeren FormationenReihe, in $\mathrm{M}^{2}$ aber bloss in einer. mittlen aus diesen. Formationen gefunden vird, nicht belehren könmen, wie übrigens einige Beispiele zeigen werden... Es önnte freilich noch zahllose Wanderungen je innerhalb $\mathbf{f}, \mathbf{t}$ oder $\mathbf{u}$ so wie nnerhalb $\mathrm{E}^{2}$ oder $\mathrm{M}^{2}$ u. s. w. gegehen haben; da aber manche Arten doch uch in entfernten Formationen verschiedener Weltgegenden beobachtet worden ind, so müssten gerade diese letzten Beispiele mehr geeignet seyn, den ervünschteu Aufschluss zu geben, als jene. Die Kiesel-Infusorien wollen wir edoch hier grösstentheils ausschliessen, weil sie der Wind jeden Augenblick in veite Fernen aller Richtungen entführen kann. Darnach bleiben aber nur sehr renige Fälle übrig, die auch ein Resultat nicht ergeben:

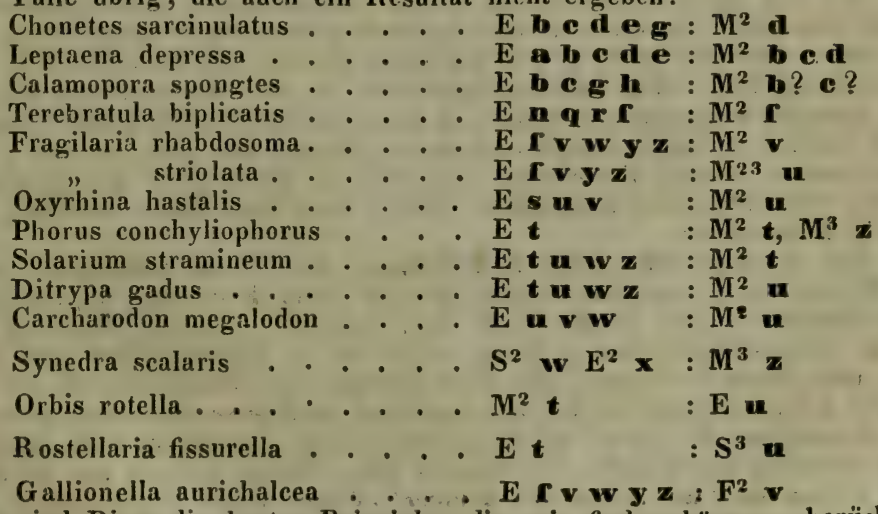

Es sind Diess die besten Beispiele, die wir finden können; berücksichtigt Ian dabei noch, dass die Formationen $G$ und $H$ ausser Europa noch nicht beannt sind, also auch die ersten dieser Arten dort nicht ebenfalls beherbergen önnen, so wird man es nicht versuchen wollen, daraus irgend eine Richtung er Wanderung einer grössern Aiten-Zahl zu irgend einer Zeit zu folgern.

b. Anders verhält es sich mit den S. 430-485 des Enumerators als miocän nd lebend bezeichneten Arten, deren jetzige Heimath daselbst in Parenthese eigefüigt ist. 
Diejenigen von ihnen, welche in der mittel-tertiären Zeit im westlichen Ruropa gewohnt haben, finden sich jetzt in wärmeren tropischen Meeren theils an der Westküste Afrika's als der von West-Europa am wenigsten und gerade südlich entfernten, zahlreicher noch in den tropischen Gewässern Asiens und einige in denen von Ost-Amerika, so dass sich 42 dasplbst aufgezählte Arten so in diese Weltgegenden theilen $S^{3}: F^{3}: M^{3}=26: 13: 3$.

(wozu aber auch 3 Arten aus nördlichen Gegenden kommen, S. 455 und 472). Man dürfte sich wundern, nur halb so viele Arten von den benachbarten Afrikanischen Kästen dabei zu finden, als aus dem fernen Indien; wogegen aber in Betracht zu zielien ist, dass Deshayss, dessen Detail-Untersuchungen wir leider nicht antühren und benützen können, eiue viel grössere Auzahl miocüner Konchylien-Arten, nämlich 190 Arten in noch lebendem Zustande wieder erkannt hat und solche hauptsächlich als Bewohner des Senegals und Guineas bezeichnet, während die obigen Bestimmungen der Asiatischen u. n. Arten alle von Gnaresour herrühren, welchem eine reiche Konchylien-Fauna von der WestKüate Afrika's nicht nu Gebot gestanden huben mag. Aber in allen Fälen werden wir eine stattgefundene Wanderung dieser Arten aus der gemässigten in die tropische Zone nicht beweisen können, so lange wir nicht auch genügende miocáne Ablagerungen im tropischen Afrika und Asien untersucht und dieselben Arten dort in ihnen vermiss t haben werden. Waren sie aber auch dort schon in der Miocän-Zeit vorhanden gewesen, so wären sie in der gemässigten Zone nicht ausgewandert, sondern bloss ausgestorben.

G. Auch der Versuch zu erfahren, ob nicht - im Gegensatze derselben Arten - derselbe $S$ ch ö p fung $\mathrm{s}-\mathrm{T}$ y $\mathrm{p}$ u $\mathrm{s}$ in aufeinander folgenden Zeiten fortgerü ckt seye in einer bestimmten geographischen Richtung, hat uns ein Resultat nicht gegeben.

Da wir bis ans Ende der Kreide- oder an den Anfang der Tertiär-Periode nicht im Stande sind, wesentliche Verschiedenheiten zwischen den Bevölkerungen verschiedener Welt.Gegenden zu entdecken, so fehlen uns bis dahin auch die Mittel nach der Voranbewegung eines denselben entsprechenden SchöpfungsTypus, der sich nicht in identisehen Arten, sondern durch stellvertretende Spezies und identische und stellvertretende Geschlechter. Formen aussprechen würde, zu forschen. Da aber ferner, wie wir oben gezeigt (S. 874, $886 \mathrm{ff}$ ), in der mittel-tertiären Zeit schon annäherungsweise, in der letzt-tertiären aber voll. ständig derselbe Organisations-Typus, ja grossentheils dieselben Arten von Pflanzen und Thieren in jedem Lande einheimisch waren, wie jetzt, so ist ein vergleichungsweise langer Zeit-Raum für etwaige Wanderung der SchöpfungsTypen um die Erte nicht vorhauden. Alles, was man dahin beziehen könnte, besteht in Folgendem:

1) Aus Nord-Amerika nach Neu-Holland. Die Reste zahireicher ungeflügelter Riesen-Vögel aus den Sippen Dinornis und Palapteryx in Neuseelands Diluvial-Schichten, die Kunde von der einstigen Existenz ähnlicher Vögel aus dem Didus-Geschlechte u. s. w. noch vor 2 Jahrhunderten auf Isle de France und deren Nachbarn, die Fuss-Spuren von Riesen-Vögreln im Sandsteine der Kohlen-Formation und des New red im Connecticul-Thale Nord-Amerika's veranlassten R. OWEN zur Hypothese: dass Neuseeland das letate Eude eines wellenartig wandernden Kontinentes sey, dessen Anfong zur permischen Zeit in Connecticut war, von wo demnach dieser Vogel-Typus immer fortbe stehend auf dem Rücken der fortschreitenden Kontinental-Welle allmählich bis Neuseeland gelangt wäre '). R. OwEN schrieb Diess unter dem Eindrucke, wel. chen Danwiv's Theorie von fortwährender Hebung und Senkung wechselnder Felder der Erd-Oberfäche, wie sie in Korallen-Bauten sichtbar werden, aul

1) Jahrb. 18*r, 380. 
ihn machte; er schrieb zu einer Zeit, wo man glaubte dass diese Vögel auf Neuseeland noch lebend vorkämen, also mit denen auf Isle de France noch gleichzeitig existirt hätten; jetzt nachden ihre Knochen der Diluvial-Zeit anheim zu fallen scheinen, müsste man viclmehr Isle de France zum Ende jener Land-Welle machen und diese, sey es in östlicher oder westlicher Richtung, den unbequemen Umweg über Neusecland nehmen lassen; die Strauss-artigen Vögel, welche noch in Süd-Amerika, Afrika, den Sunda-Inseln und Neuholland leben, würden dabei ganz ausser Acht bleiben; endlich ist jene Theorie sehr gewagt, in so ferne man von jener Steinkohlen.Zeit an bis zu Anfang der Tertiär-Zeit von der Beschaffenheit der Vogel-Welt gar nichts weiss, die Existen\% dieser letzten aber doch anerkannt werden muss, wenn man jene zahlreichen und manchfaltigen Eindrücke im Sandstein Connecticuts u. a. einmal unbedingt für Vogel-Fährten erkennt, welche sehon für sich allein einen grossen Reichthum und Manchfaltigkeit von Formen beurkunden.

2) Aus Europa nach Amerika. Die Ahulichkeit mancher Sippen und Familien der Oolithen- bis Tertiär-Periode mit Formen derse!ben Gruppen, wie solche jetzt im wärmeren Nord-Amerika bestehen, ist wiederholt hervorgehoben worden. Man könnte daraus folgern, dass der entsprechende Schöpfungs-Typus aus Europa nach Amerika fortgerückt sey, und zwar zu verschiedenen Zeiteu in mehren aufeinander folgenden Perioden.

m: schon in der Lias-Zeit die Insekten von Gloucestershire, von denen Brodie sugt, dass sie mehr denen des jetzigen Nord-Amerika's als des jetzigen Europa's entsprechen. Doch mag diese Verwandtschaft eine mehr zufällige und wenigstens theilweise negative seyn; deutlich tritt sie erst mit den TertiärSchichten hervor, in

t: der Eocän-Zeit : zuerst durch die Megaspira von Rilly (S. 852, 883) und lurch die Didelphys-Arten in Pariser - und Londoner Eocän-Schichten : die Ge. schlechter sind jenes süd-, dieses mittel- und nord-Amerikanisch; dann durch die Altsattler Pflanzen, wenn sie in diese Formation gehören (S. 884). Auch unter len Bernstein-Insekten erscheinen Nord-Amerikanische Formen (S. 886, 888). Aber die eocäne Mollusken-Fauna Europa's zeigt keine Ähnlichkeit und nụ wenige Analogie mit der jetzt in Nord-Amerika lebenden.

u : in der Miocän-Zeit : zeigen die Wälder und die Insekten der BernsteinSchichten ${ }^{1}$ ) (wenn sie nicht $\mathrm{zu}$ t gehören) wie die Insekten anderer Brauncohlen-Lager an der Ostsee, im Siebengebirge und im Baireuthischen ${ }^{2}$ ), die Flora on Parschlug und von Öuingen Verwandtschaft mit dem jetzigen Nord-Amerika. Parschlug hat 67 Pflanzen-Sippen, wovon über 40 der alten und neuen Welt ungleich, aber nur 3 ausschliesslich der aiten und 10 ausschliesslich der neuen Welt zusteben (Taxodium [?], Liquidambar [?] , Comptonia, Achras, Prinos [?], Nemopanthes, Ceanothus, Smilax, Robinia, Amorpha ${ }^{3}$ ). Unter 32 Öningenschen Sippen sind 22 Europäische und 11 Amerilknische (Comptonia, Carya, Ceanohus, Karwinskia, Negundo, dann minder ausschliessend Taxodium, Juglans, Lifuidambar, Gleditschia, Prinos, Cordia ${ }^{4}$ ). Die Insekten und insbesondere die Reptilien von Öningen (Chelydra, noch jetzt auf Nord-Amerika beschränkt; indrias, als Repräsentant der Kiemen-Batrachier in Nord-Amerika und Japan) uaben cine auffallende Verwandtschaft mit denen des jetrigen Nord-Amerika's. Per im Wiener-Becken neulich ${ }^{5}$ ) gefundene Psephophorus so wie, wenn sich sein Familien-Typus bestätigt, der Bravard'sche Dasypus von Puy de-Dome ${ }^{6}$ ) vürden die süd-Amerikanische Fanilie der Dasypodidae, wie Macrotherium die der Bradipodidae, Archaeomys und Theridomys die der Psammoryctidae in Europa epräsentirt haben. Unter 147 miocänen Konchylien-Arten aber hat Carolina

Meine Collectan. I, S. 869, 876.

2) Germar im Jahrb. 1846,-212. - 3) Jahrb. 1848, 507.

4) A. Braun im Jahrb. 1845, 164.

8) Jahrb. $1847,579$.

6) worin aber DE BLAinville nur einen Biber erkennt, Jahrb. 1840, 118. 
30 noch lebende Arten und unter diesen nur 4 fossile und 4 noch lebende Arten mit den Miocän-Schichteı Europa's gemein 1), welche 8 also von den 1000 miocänell Arten Europa's nicht 0,01 betragen und wegen ungenauer Vergieichung wohl noch tiefer an $\mathrm{Z} a h l$ herabsinken dürften.

w: in der Pliocän.Zeit endlich finden wir noch einige Erinnerungen an Nord-Amerika in der Flora, wie Juglaus cinerea der Subapenninen-Formation, Liriodendron tulipifera var. und ? Platanus occidentalis zu Sinigaglia ${ }^{2}$ ). Indessen sind identische Pflanzen-Arten in beiden Welttheilen (die der Steinkohlen ausgenommen) nur von dieser Zeit an bekannt; und wollte man ein Wandern der Schöpfung von Europa nach Amerika (also von Osten nach Westen) anneh. men, so müsste man dieses Wandern nicht den Arten, sondern dem ganzen Schöpfungs-Typus zuschreiben, welcher nicht an die identischen Arten, Sippen u. s. w. gebunden ist. Doch scheinen die bisher gesammelten Thatsachen zur Annahme einer solchen Wanderung auch nur dieses Typus noch nicht zu berechtigen.

3) Aus Ostindien nach Europa. Die Ostindische Kreide ist weit reicher an grossen siphonobranchen Gasteropoden (zumal Cypraea- und Oliva-Arten) und Calypträen als die Europäische, Amerikanische und Afrikanische, obschon ein Theil der Kreide in den 2 letzten Ländern ebenfalls innerhalb der Wende-Kreise oder dicht an denselben liegt. Die grössern Siphonobranchier gebören jetzt vorzugsweise den tropischen Meeren, hauptsächlich aber dem Ostindischen Orean und der Südsee an; EDw. Fories betrachtet daher Ostindien als den frühesten Schöpfungs-Herd dieser Gruppe, in welchen sie auch bis jetzt vorzugsweise zahlreich geblieben wäre, obschon sie von dort nach verschiedenen Gegenden ausgewandert und vorzüglich in der eocänen Zeit schon reichlich in West. Europa aufgetreten seye. Die Richtung des Fortschrittes wïrde a!so eine westliche seyn, wie in den unter (2) erwähnten Fällen; aber zuglcich cine nordwestliche aus den Tropen heraustretend, um alsbald wieder in dieselben zurückzukehren. Sollte man nicht vielmelir vermuthen dürfen, dass bloss eive Ähulickheit der Meere, des jetzigen und des Kreide-Meeres Ostindiens untereinander, so wie dieser mit dem eocänen Meere Europa's, also eine Übereinstimnung der äussern Lebens-Bedingungen in beiderlei Zeiten und Gegenden die Ähnlichkeit der Faunen hervorgebracht habe? Die Temperatur war in den 3 Fällen ungefähr gleich; setzt man nun auch noch eine Gleichheit des Meeres, hauptsächlich seiner Tiefe, seiner Küsten, seines Grundes, seiner KorallenBauten, seiner Zuflüsse, seiner Bewegungen, so weit Diess alles hauptsächlich für jene Formen bedingend seyn mag, so ist, däucht uns, die Erscheinung besser erklärt, als durch die Abnahme eines fortrückenden Schöpfungs-Typus, der seinen Grund nur in sich selbst trüge.

4) Ans Europa nach Japan. Eine geringe Verwandtschaft des miocänen Europa's mit dem jetzigen Japan drücken Taxodium, Andrias und etwa die zahlreichen Frösche in den Süsswasser-Mergelu der Molasse von Öningen und der Gingko in den ungefähr gleichalten oder etwas jüngeren Gypsen von Sinigaglia aus $^{3}$ ); wollte man also Wanderungen annehmen, so wären diese hier von Westen nach Osten gerichtet.

5) Als aus Europa nach der Sïdsee ausgewandert könnte man die seit der Kohlen-Zeit dort so häufigen Korallen-Riffe, die Beutelthiere der Oolithe und Tertiär-Gypse mit ihren Begleitern (S. 899, A) ansehen; also gewisse Familien.

1) Jahrb. 1848,735 .

2) Wir haben jedoch selbst gegen die sichere Bestimmung dieser 2 Arten Zweifel erhoben (Jahrb. 1830, 118), und GöpPERT hat die letzte derselben weder in Enumerator noch im Nomenclator aufgenommen. Auch steht noch dahin, ob Sinigaglia nicht etwas älter als $\mathbf{w}$, nämlich $=\mathbf{u}^{2}$ ist.

3) Br. Collect. 1, 153 . 
und geringentheils Sippen-Typen, nicht identische Arten. Wir sehen indessen nicht nur die Richtung dieser Wanderung in Widerspruch mit allen übrigen, sondern finden auch die Möglichkeit die Ursache der Erscheinung abermals durch den Wechsel der äussern Lebens-Bedingnisse zu begründen.

H. Viele noch jetzt lebende Familien und Geschlechter haben zur Zeit ihres ersten Auftretens oder bald nachher offenbar eine weitere Verbreitung besessen, als jetzt. Es schliesst sich diese Erscheinung an eine früher erörterte an, wenn schon die höhere Temperatur jetzt oft keinen grossen Einfluss mehr ausüben konnte. Wir können Diess besonders an Landthiereu nachweisen und beschränken uns dabei auf solche Beispiele, wo die fossilen Reste zur Bestimmung der Genera auszureichen scheinen. Mehre der unter $G 2$ aufgezählten Fälle werden später gewiss auch noch hieher zu zählen seyn. Ob dagegen andere Genera eine geringere Verbreitung als jetzt besessen, Diess zu beweisen reichen unsere Mittel noch nicht hin, da sie zu sehr negativer Art sind.

So waren Elephas ${ }^{1}$ ), Hippopotamus ${ }^{2}$ ), Equus ${ }^{3)}$, Antilope ${ }^{3}$ ) anfangs in der alten wie in der neuen Welt vesbreitet, während sie jetzt nur noch der alten angehören. Solche Fälle, wo Säugthier-Genera der neuen Welt einst auch über die alte ausgebreitet gewesen wären, können wir mit Sicherheit jetzt nicht nachweisen.

$$
E^{2} S^{1} S^{2} S^{3} F^{3} M^{1} M^{2} M^{3} M^{4} \quad E^{2} S^{2} S^{3} F^{2} F^{3} F^{4}
$$

Elephas fand sich früher in $E^{2} S^{1} S^{2} S^{3}-M^{1} M^{2} M^{3}-$ jetzt in $-S^{3} F^{2} F^{3}-$

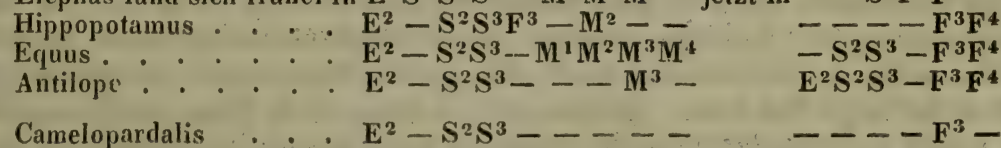

wobei zu erinnern ist, dass Central-Afrika hinsichtlich seiner fossilen Reste noch ganz unbekannt ist und die Ausfüllung dieser Lücke erst von spätern Zeiten zul erwarten steht, dass überhaupt ein Zuwachs neuer Fundorte nicht mehr auf Seiten der lebenden, wohl aber der fossilen Thiere vorauszusehen ist. Auch die bei so grossen Thieren der jetziøen Schöpfung beispiellos weite Verbreitung des Mastodon-(ieschlechtes in $\mathbf{E}^{2} \mathrm{~S}^{23} \mathbf{M}^{234} \mathrm{U}^{3}{ }^{4}$ ) stimmt mit der vorigen Erscheinung überein: ja man muss versucht seyn, zur Zeit der Mastodonten in Australien $\left(\mathrm{U}^{3}\right)$ einen ausgedehnten Kontinent anzunehmen, da der jetzige zu klein schrint (vgl. S. 816, b), um Thiere von solcher Grösse je beherbergt zu haben. Endlich würde sich in Folge weiterer Entdeckung fossiler Reste an neuen Fundstellen in andern Welt-Gegenden gewiss die einstige weitere Verbreitung noch mancher Geschlechter herausstellen.

§. 26. In Bezug auf geologische Gruppirung.

A. Wir haben bisher von den allmählichen Veränderungen im Thier- und Pflanzen-Reiche gesprochen und uns Behufs der chrono-

1) Jahrb. 1841, 739 u. a.

2) Hartan in Br. Collectan. 32; Jahrb. 1848, 598.

3) Wir entrehmen dieses Vorkommen z. Thl. aus dem Jahrb. 1841, 741 ; 1343,$859 ; 1845,626$; im Enumerator ist es nicht ganz angezeigt; selbst in der Eschschol $r$-Bay Nord-Amerika's in $67^{\circ} \mathrm{Br}$. kommen noch Pferde-Reste vor (Lond. Edinb. Magas. 18\$3, XXIII, 193).

4) Jahrb. 1844, 291; 1845, 379, 752. 
logischen Ordnung derselben statt einer Zeit-Eintheilung nach Jahren und Jahrhunderten der Schichten-Eintheilung nach Systemen, Formationen und Gruppen bedient, worin die fossilen Überreste jener einstigen Wesen gefunden werden. Aber dieser Eintheilung selbst, auf frühere Gewohnheiten und Vorschläge gestützt, durch eigene Beobachtungen verbessert, haben wir unserer Seits noch keine Begründung gewidmet, wozu wir indessen hier die geeignete Stelle zu finden glauben.

B. Wenn die organischen Wesen früherer Zeiten allmählich nach einander aufgetreten und eben so allmählich nach einander wieder untergegangen sind, so kann das Auftreten und Verschwinden einzeluer Arten, Genera, Familien u. s. w. keine geologischen Abschnitte bezeichnen. Nur wenn ganze Gruppen von Thieren und Pflanzen in manchfaltigen Geschlechtern entwickelt plötzlich anf der Erd-Oberfäche erschienen, oder eben so ganze Gruppen derselben plötzlich und eine jede auf allen Punkten gleichzeitig verschwunden wăren, könnte man auf geologische Ereignisse schliesseı, welche mit jenem Erscheinen und Verschwinden in ursächlichem Zusammenhange gestanden und zur Begründung geologischer Zeit-Abschnitte geeignet wären. Man hatte früher eine grössere Anzahıl solcher Abschnitte bezeichnet; allein je mehr unsere Kenntnisse sich ansdehnen, an je mehr über die Erd Oberfläche hin zerstreuten Punkten wir die Auflagerung der Gebirgs-Gruppen zu beobacliten Gelegenheit finden, desto mehr verschwinden auch die geologischen und paläontologischen Gebirgs-Marken. Diejenigen, welche noch übrig sind, werden im Laufe fortgesetzter Beobachtungen wohl ebenfalls allmählich verschwinden, da sie auf Beobachtungen nur aus einem oder dem andern Welttheile beruhen. Solche paläontologische Kennzeichen würden nach unsern Tabellen noch folgende seyn, wenn wir die min. der bedeutenden und schlecht umschriebenen Genera und einzelnen Arten übergehen und durch Angabe der entsprechenden Seiten-Zahlen des Enumerator's den Leser in den Stand setzen, das Ausführlichere dort nachzusehen.

C. Die A nfänge können bezeichnen:

I. In der Kohlengebirgs-Periode : den

b: der obersilurischen Schichten: das erste Erscheinen von FischResten.

cs der devonische n Formation: das erste Erscheinen der Pflanzen, and zwar nur in Form von Zellen - und von kryptogamischen GefässPflanzen S. 5-11; das Anftreten des Arten-reichen Geschlechts Cardium S. 305 und Lnnulicardium S. 306; unter den Cephalopoden das Beginnen von Bactrites und Goniatites.

e: der Kohlen-Schichten: das Auftreten der Sippen-reichen Calamitaceen S. 11, und der Filices-Familie S. 14, 62 (letzte mit sehr wenigen devonischen Ausnahmen), wovon mehre Genera den Kohlen ganz eigen sind; das erste Ersrheiven der phanerogamischen Monokotyledonen und der Dikotyledonen, jedoch lediglich aus der Klasse der Monochlamydeen S. 37, 65; Erscheinen der ersten Goniatiten mit getheiltem Dorsal-Lobus und der ersten Saurier-Reste (S. 691). 
f: der Zechstein.Formation (des Todtliegenden $=\mathbf{f}$ ): das erste Auftreten von Vogel-Spuren (Fahrten) in Nord-Amerika.

II. In der Trias-Periode: und zwar den Anfang:

k: des Muschelkalks: der Beginn der Formen-reichen Echiniden-Ordnung S. 186, insbesondere mit dem Genus Cidaris; dann unter den Konchylien der Beginn der Genera Ostrea, Spondylus, Lima, Ceratites, Anmonites; unter den Reptilien das Erscheinen der Nexipoden-Saurier.

III. m: Der 0 olithen-Formation (des Lias): das Erscheinen von Plicatula u. e. a. Genera, so wie der grossen Masse der mantelbuchtigen Muscheln (Pelecypora sinuato-palliata) mit einzelnen ältern Ausnahmen S. $319 \mathrm{ff}$, der Belemniten, der Sepien-artigen Cephalopoden S. 538. Hier beginnen die homocerken Fische, nach einigen älteru Vorläufern, und das massenhaftere Auftreten der Saurier.

n: Des Jura-Gebildes (Unterjura): das erste obwohl noch spärliche Auftreten dir Siphoniferen S. 107, nach einigen (9) Formen des Bergkalks; der Beginn von Echinus und vielen andern Echiniden.Geschlechtern wie Discoidea, Nucleolites, Clypeus, Dysaster u. a. S. 186, 200 u. ff.; - unter den Konchylien das Auftreten des Genus Exogyra S. 244, Pectunculus S. 282, Vermetus S. 360, Nerinea S. 382, der ersten Siphonobranchier mit wenigen älteren Ausnahmen (bei Cerithinm, Strombinea, Fusus u. a.), des Genus Pollicjpes S. 556, Limulus S. 573, der Amphipoden und makrouren Dekapoden (mit einigen Ausnahmen) S. 374, der sechsfïssigen Insekten (mit wenigen Ausnahmen im Kohlen-Gebilde und Lias) S. 594, und vieler Fisch-Genera insbesondere der Chimäriden S. 636 und der Squaliden-Familie S. 641 (die jedoch in der Kreide häufiger werden); der Beginn der Chelonier (S. 693) mit einigen eigenthümlichen Geschlechtern.

- Des Ober-Jura's ....? .

p: Der W ealden : das erste auggesprochenere Erscheinen von Süsswasser. Thieren aus verschiedenen Klassen und meistens noch lebenden Geschlechtern, wie Cyclas, Pisidium, Cyrena, Neritina, Melania, Paludina, Planorbis.

IV. q: Der Kreide.Periode : das Auftreten des Genus Siphonia und verwandter Amorphozoen-Genera S. 81; dann unter den Bryozoen das von Lunulites S. 115 ; unter den Echiniden das fast aller SpatangoidenGenera S. 201; das der Mollusken-Geschlechter Crassatella, Scalaria; das der ersten Fisch-Arten aus noch lebenden Geschlechtern, insbesondere das Auftreten vieler Squaliden S. 641, wie der ächten Knochenfische, Teleostei (S. 669) พ. s. w.

r: Des Grünsandes....?

r: Der Kreide: das Erscheinen der Kiesel-Polygastrica (mit Ausnahme einer Art in der Kohle) S. 89 , während die Mehrzahl allerdings den Tertiär-Gebirgen anheimfällt; das der Nummuliten, wovon jedoch die meisten in die folgende Gruppe s gehören S. 171 und nur Orbituliten und das neue Genus Orbitoides übrig bleiben werden; unter den Konchylien das Erscheinen von Fissurella u. a.

V. : Der Tertiär-Periode (der Nummuliten-Gesteine): das massenhafte Erscheinen der wahren Nummuliten, die damit verwechselten Formen der Kreide abgerechnet.

t: Der E.ocän-Gebilde: das Auftreten der dikotyledonisehen Klasse der Choristopetalae S. 48, mit sehr wenigen (6) früheren Ausnahmen; unter den Echiniden das der Familie der Scutellen ebenso S. 196; unter den Konchylien das Erscheinen von Limopsis?, Siliquaria, Crepidula, Infun- 
dibulum, Sigaretus, vielen Siphoniferen- und Pulmonaten-Genera S. 490, mit wenigen ältern und jüngern Ausnahmen; der Beginn der Balaniden S. 554; unter den Fischen das Auftreten der Rajiden-Familie S. 637 (mit Ausnahme einiger zweifelhaften Genera); unter den Reptilien der Beginn der Batrachier S. 683 ; das erste Auftreten von Vögel-Knochen (ausser Protornis), wogegen die Vogel-Fährten ein viel höheres Alter besitzen (s. o.); endlich das der Säugethiere, denen die Knochen von nur 4 Arten (S. 718, 723, 724) in ältern Schichten vorangehen; - im Allgemeinen aber das erste Auftreten noch jetzt lebender Thier-Arten mit mit 4-5 älteren Ausnahmen.

ui Der Miocän-Gebilde: das Erscheinen der Dikotyledonen-Klasse Corolliflorae S. 46, 68, nach einem ältern Vorläufer im Grünsande: unter den Konchylien das Erscheinen von Dreissenia u. m. a.; insbesondere aber das massenhafte Auftreten woch jetzt letender Arten von Thieren.

v: Der Pliocän-Gebilde: das Erscheinen von bereits 0,50 norh lebender Arten wenigstens unter den Konchylien, Anneliden, Säugthieren u. a. m.

x: Der Diluvial-Gebilde: das Erscheinen der Säugthier-Geschlechter in ihrer jetzigen Verbreitung.

D. Für den Bestand, den Umfang eines geologischen Gebildes würden bezeichnend seyn, nämlich für :

I. a: Das untere Silur-Gebirge: unter den Brachiopoden? Obolus S. 210, Siphonotreta S. 211; unter den Gasteropoden: Maclureia, Ophileta; unter den Trilobiten: Triarthrus, Conocephalus, Ellipsocephalus, Sao, Agnostus u. m. a. S. 572.

ab: Das ganze Silurgebirge: die Familie der Cystideen (mit ciner Ausnahme) S. 181, das Schnecken-Genus Pentamerus, die Trilobiten. Genera Trinucleus (fast nur a), Ceraurus, Paradoxides, Remopleurides, Olenus, Encrinurus, Cheirurus, Sphaerexochus, Lichas, Ceratocephala.

b: Das obere Silur-Gebirge: die Echinodermen-Genera Periechocrinus, Sagenocrinus, Dimerocrinus, Phoenicocrinus, die Trilobiten-Genera Arethusa, Phaetonides: einige noch näher zu bestimmende FischStacheln.

c: Das Devon-Gebirge: dic Pflanzen-Sippe Knorria S. 30, die Brachiopoden-Sippe Stringocephalus S. 221, das Muschel-Genus Megalodon S. 301, die eigenthümlichen Schnecken-Genera Catantostoma und Scoliostoma S. 406, Clymenia mit wenigen Ausnahmen; das KrusterGenus Bostrichopus, mehre Trilobiten-Genera; einige Fisch-Genera S. 652 .

ca: Das Devon- und Kohlen.Gebirge: die Krinoiden-Genera Platycrinus, Pentatrematites S. 182.

d: Den Kohlen-Kalk: das Echinodermen-Genus Echinocrinus, das Phyllopoden-Genus Dithyrocaris S. 560, die Trilobiten-Genera Phillipsia, Griffithides, Cyclus; Spirifer-Arten mit zweitheiliger Strahlung; manche Fisch-Genera $S$. $646 \mathrm{ff}$.

e: Die Steinkohlen-Schichten: die Familie der Asterophylliten S. 13, der Sigillarieen S. 28, 64, der Lykopodiaceen S. 29, 64, mit einigen Ausnahmen; unter den Palmaceen die Sippe Trigonocarpum S. 36 ; die Musaceen S. 37, eiue grössere $Z$ ahl von Sippen unsicherer Stellung S. $58 \mathrm{ff}$ : unter den Muscheln Anthracosia; unter den Pöcilo. poden das Genus Belinurus.

I: Das Todtliegende: Die meisten Psaronieen S. 27, 63. 
g: Den:Zechstein: manche Fische, als Palaeoniscus zum Theil, Platysomus, Acrolepis, die Saurier Protorosaurus, Thecodontosaurus, Palaeosaurus, Rhopalodon S. 686, 691; dic Arten der Permischen Flora stehen denen der Steinkohle so nahe, dass dieselbe nur eine Fortsetzung der Steinkohlen-Flora bildet, während sie keine Verwandtschaft mit der Trias-Flora zeigt ${ }^{1}$ ).

II. Die Trias-Periode: das Genus Myophoria S. 289 ; einige Fisch-Genera, insbesondere Ceratodus und Nemacanthus aus der Chimäriden Familie, daun Thectodus, ein grosser Theil von Hybodus, das Genus Saurichthys S. 662, Placodus S. 667; unter den Sauriern die Labyrinthodonten u. e. a. S. 690 . - Eine neue in Vogesen-Sandstein gefundene Art, L. Fürstenbergianus, bietet daher Ursache den VogesenSandstein nach A lberta, H. v. Meyer ${ }^{2}$ ), Sandberrer u. A. bei der Trias zu belassen, statt ihn mit Murchison der Zechstein-Formation zu verbinden.

i: Den Buntsandstein : aus der Familie der Abietineen die Albertien.

k: Den Muschelkalk: einige Ophiuren-Genera S. 183, das PöcilopodenGenus Halicyna, die Krebse Pemphix und Liogaster, - die SaurierGenera Nothosaurus, Conchiosaurus, Pistosaurus, Simosaurus, alle aus der Macrotrachelen-Gruppe S. 688.

1: Den K euper...?

III. Die Oolithen-Periode: unter den Cycadeen S. 38 die Sippe Zamites, während Pterophyllum und Nilssonia den Keuper enge mit den Oolithen verbinden. Unter den Fischen sind viele Genera den Oolithen ganz eigen.

m: Die Lias-Formation: die Sepien-Genera Geoteuthis S. 539, Be. loteuthis S. 541 ; - manche Fisch-Geschlechter, insbesondere homocerke Lepidoiden, wie Dapedius Tetragonolepis etr.

n: Den Unter-Jura: die Echinodermen-Genera Apiocrinus, Millerocrinus S. 178, Ophiurella S. 183, die Sepien-Genera Ommastrephes, Acanthoteuthis.

o: Den Ober-Jura : einige eigenthümliche Saurier-Genera S. 692.

p: Die Wealden-Formation: mehre Saurier-Geschlechter S. 692.

IV. Die Kreide-Periode: unter den Echiniden die Salenien-Familie S. 188, das Genus Cyphosoma S. 188, Caratomus, Nucleopygus, Globator, Pirina S. 194, Catopygus S. 199; unter den Brachiopoden die ganze Rudisten-Familie, soweit sie ausgestorben S. $233 \mathrm{ff}$, die PlicaceenGeschlechter Ringinella, Avellana, Globiconcha, viele AmmoneenGenera (Crioceras, Scaphites, Ancyloceras mit einigen älteren Ausnahmen, Toxoceras, Hamites, Pt" ${ }^{\prime}$ choceras, Turrilithes, Baculites S. 520 ff.).

r: Den Grünsand: die Cephalopoden-Sippen Helicoceras und Bellerophina; dann viele einzelne Arten der Ammoneen- und Plicaceen-Geschlechter S. $381,511,520 \mathrm{ff}$.

f: Das Kreide-Gebirge: das Krinoiden-Genus Marsupites S. 182, das Echiniden-Genus Galerites S. 195, das Konchylien-Genus Actaeonella u. s. w.

V. t: Die Eocän-Schichten: die Pandaneen S. 35 mit einer Ausnahme; aus der Familie der Cupressineen S. 42 die Sippe Cupressites; aus den Protaceen dis Familie Petrophiloides S. 46; aus den Malvaceen die Familie Hightea S. 51 ; aus den Sapindaceen Cupanoides S. 52; aus den Leguminosen Faboidea, Leguminosites u. a. S. 55; aus den Konchylien Bifrontia u. a.

u: Die Miocän-S chichten : die meisten Palmaceen-Genera (Fasciculites Perfossus, Flabellaria, Phoenicites) S. 36 ; aus der Familie der Cu-

1) Jahrb. 1846, 621, - 2) Jahrb: 1847, 186. 
pressineen S. 42 die Sippen Juniperites, Cupressites, Thuites, ThuioxyJum; die Taxineen S. 44; unter den Konchylien das Genus Ferussacia u. m. a. Das erste Auftreten mancher weichleibigen Insekten u. a. Genera und Familien ist bloss durch das zufällige Vorkommen des Bernsteins bedingt.

w: Die Pliocản-Schichten.... terisiren :

E. Den Schluss gewisser Formations-Zeiten charak-

I. C: Der Devon-Schichten: das Aufhören von Aulopora S. 128, und unter den Echinodermen von Eucalyptocrinus, Cupressocrinus; - unter den Amoneen das von Phragmoceras; unter den Trilobiten von Ogygia, Odontopleura, Arges, Bronteus, Harpes, Calymene, Phacops, Cyphaspis, Proetus, Illaenus, Archegonus, Asaphus, Ampyx u. s. w.

d: Des Bergkalks: unter den Bryozoen das Erlöschen von Favosites S. 147; unter den Anthozoen das von Graptolithus S. 149, Halysites und Syringopora (mit 2 unsichern Ausnahmen) S. 153, Lithostrotium, Columnaria, Caninia, Stylastraea S. 160, Cyathophyllum (mit mehren Ausnahmen), Michelinia, Cystophyllum S. 162, Amplexus S. 166; unter den Echinodermen das der Genera Poteriocrinus, Taxocrinus, Cyathocrinus, Actinocrinus, Melocrinus, Rhodocrinus, Gilbertsocrinus; unter den Brachiopoden das von Leptaena; unter den Pelecypoden von Pterinaea, unter den Gasteropoden von Murchisonia (mit eiuer Ausnahme), unter den Nautilaceen das von Lituites, Gyroceras, Cyrtoceras, Apioceras, ? Orthoceras (mit Ausnahmen); das Erlöschen der Trilobiten.

e: Des Kohlen-Gebildes: das Erlöschen unter den Pteropoden von Conularia, unter den Heteropoden von Porcellia und Bellerophon.

E: Des Zechsteins: das Aufhören von Orthis und Productus (mit nur zwei bis drei Ausnahmen) S. 225.

II. Für die Trias-Gruppe: das Erlöschen von Encrinus.

k: Des Muschelkalkes:...

1: Des Keupers: mit ihm schliesst die Masse der heterocerken Fische, indem (ausser den Squaliden) nur noch einzelne Arten durch die folgenden Bildungen bis in die lebende Schöpfung herab auftreten.

III. m: Der Lias.BiÎng: Das Aufhören von Spirifer u. a.

n: Des Unter-Jura's: ....

- Des Ober-Jura's:....

p: Der Wealden:....

IV. q: Des Neocomiens: ...

r: Des Grünsandes:....

r: Der Kreide: das Aufhören von Exogyra, Nerinaea, aller Ammoneen und Belemniten, wie (unter den Fischen) der Cestracionten S. 646 und Hybodonten S. 650 u. a.; - schon die obere weisse Kreide mit Feuersteinen $\left(\boldsymbol{r}^{2}\right)$ und das Terrain danien enthalten meistens keine Ammoniten mehr, aber noch Belemniten.

v. : Der Nummuliten-Gesteine:

t: Der Eocän.Schichten: Das gänzliche Aufhören der Nummuliten ?

u: Der Miocän-Schichten:....

w: Der Pliocän-Schichten: ....

F. Wir finden also die Grenzen der Bildungs-Perioden, wie man sie bisher nach der Summe der auf einem kleinen Theile der ErdOberfläche beobachteten Thatsachen festgestellt hat und, um irgend eine Eintheilung der Schichten-Folge in der Erd-Rinde zu haben, auch ferner beibehalten mag, ausser dem Aufhören (fast) aller früheren und dem Auftreten von (fast) lauter neuen Spezies, noch durch tol- 
gende Erscheinungen der Klassen, Ordnungen, Familien und Geschlechter aus den organischen Reichen angedeutet :

I : II. Zwischen Kohlen- und Trias-Periode: das Aufhören mehrer bis dahin sehr entwickelter Brachiopoden-Genera, als Orthis, Productus und fast auch Spirifer; das Beginnen der Echiniden-Ordnung; unter den Cephalopoden das der Ceratiten und der Ammoniten im engeren Sinne; unter den Monomyen das erste Erscheinen einer Anzahl später sehr artenreicher Genera; und ähnlich unter den Fischen (Chimäroiden) und den Sauriern, wo die Labyrinthodonten erscheinen.

II : III. Zwischen Trias- und Jura-Periode liegt der Anfang der grossen Masse der mantelbuchtigen Muscheln, dann der Belemniten - und Sepien-artigen Cephalopoden, das Ende vieler heterocerken und der Anfang der homocerken Fische, jedoch mit einigen Ausnahmen; endlich der Anfang vieler Saurier-Formen.

III : IV. Zwischen Jura- und Kreide-Periode: der Beginn der Siphonien, Lunuliten, Spatangoiden, der Salenien-Familie u. a. Echiniden-Genera, der Rudisten, Crassatellen, Scalarien, vieler Pliaceen- nnd Ammoneen-Genera, vieler Squaliden und ächten Knochen-Fische.

IV : V. Zwischen Kreide-und Tertiär-Periode: ist einer der am schärfsten bezeichneten geologischen Abschnitte; es trifft dahin einerseits das Aufhören der Exogyren, Nerinäen, Ammoneen, Belemniten, wie (aus den Fischen) der Cestracionten und Hybodonten; andererseits das massenhafte Erscheinen der Cupressiten, Pandanaceen und der vielen corollifloren und choristopetalen Pflanzen-Formen, der Scutellen-Familie, vieler Siphoniferen - und PnImonaten-Genera (welche Familien bisher im Ganzen nur selır antergeordnet gewesen), wie der Balaniden; unter den Fischen die Rajiden, unter den Reptilien die Schlangen und die Batrachier und die grosse Masse der Säugethiere.

V : VI. Zwischen der Tertiär-und jetzigen Periode ist irgend eine Gränze vielleicht gar nicht $\mathrm{zu}$ bestimmen möglich, wenn man schon eine sehr grosse Zahl jetzt lebender Genera und selbst ganze - der Versteinerung nicht fähige - Ordnungen von Thieren und Pflanzen nennen könnte, die noch vicht in fossilem Zustande gefunden worden sind.

Ungeachtet dieser Resultate ist die Unterscheidung der Perioden durch organische Kennzeichen doch meistens weniger scharf, als wir hier ausgedrückt haben, und es sind meistens doch nur einzelne Genera, welche ganz diesseits oder jenseits der Perioden-Grenzen fallen; selten sind es kleinere Familien; wo Ordnungen oder Klassen angegeben werden, hahen sie fast olıne Ausnahme einzelne Vorläufer 
schon in ältern Schichten, oder Nachzügler in jüngeren. Sehr selten sind die, wie zwischen Kreide - und Tertiär-Periode, wo einige grössere Thier-Gruppen als Nerinäen, Ammoneen, Belemniten, Cestracionten) und Hybodonten plötzlich ganz und ohne eine Ausnahme abschneiden, während andere, wie die Balaniden, die Batrachier und (mit nur \& Ausnahmen) die Säugthier ganz neu auftreten. Eine so scharfe Abgrenzung unsrer Organismen-Gruppen wiederholt sich in der ganzen geologischen Schichten-Reihe nicht mehr. Indessen ist der Ânalogie nach zu erwarten, dass nach genauerer Erforschung der geologischen Verhältnisse über einen grösseren Theil der Erd-Obberfläche auch jene scharfe Begrenzung sich mehr und mehr verwischen werde; obschon wir mit mehren Zoologen und Botanikern hoffen, dass es einer schärfer'n und natürlichen Systematik künftig besser gelingen werde, wenigstens die untergeordneten Genera der meisten Klassen auf einzelne Perioden und Formationen zu beschränken.

Leicht hätten wir überall aus unscrm Enumerator noch einzelne Genera und Spezies zur Charakteristik der versehiedenen Formationen und Perioden den obigen beifügen können; einerseits aber würde uns ein solches Detail weit über die Grenzen unseres jetzigen $Z$ weckes hinausgeführt haben, andrerseits kann Jeder, der Solches wünscht, Diess mit einem Blick über die Tabellen unseres Enumerators sehr schnell nachholen. Hier galt es nur den Gesammteindruck zusammenzufassen.

\section{§. 27. In Bezug auf einzelne Länder.}

Wie überhaupt so können auch in einzelnen Ländern die allmählichen Veränderungen in der Pflanzen- und 'Thier Schöpfung verfolgt werden, ohne aber früher als in der Kreide - oder Tertiär-Zeit ein örtlich eigenthümliches Resultat zu geben. Es würde interessant seyn, Schritt um Schritt zu verfolgen, durch welche Mitglieder der ursprünglich überall gleichförmige Typus der Schöpfung in den jetzt überall örtlich eigenthüılichen während der Tertiär-Zeit übergegangen ist und woher etwa auch die verschiedenen Formen eingewandert sind. Wir enthalten uns des Versuches noch, weil die Reihe und der Zusammenhang der teriären Bildungen doch nur erst in wenigen Ländern etwas genauer bekannt ist und daher ein mit dieser weitläufigen Ausholung im Verhältniss stehendes Resultat noch kaum zu erzielen scheint.

Ein sehr Hypothesen-reiches Feld, auf dem man sich schon vielfach herumgetummelt hat!

Interessante Versuche über die wahrscheinliche Einwanderung der jetzigen Fauna und Flora in England haben EDw. Forbes '), über die der Flora auf den

1) E. Forbes Beziehungen zwischen der Verbreitung der jetzigen Fauna und Flora der brilischen Inseln und den geologischen Veränderungen, welche deren Oberfläche hauptsächlich zur Zeit des nordischen Drifts betroffen haben: im I. Band der Memoirs of the Geological Survey of Great Britain, London 1846, $4^{0}$. 
britischen Inseln bis Island hinauf Martivs ${ }^{2}$ ), über die der Mollusken-Fauna in Skandinuvien LovÉN ${ }^{2}$ ) geliefert.

Lovév gelangt zu folgendem Resultate: Die skandinavische Mollusken-Fauna besteht aus zwei Elementen, aus den germanischen und dem arktischen. Jenes crreicht in Bohus-Lehn und Süd-Norwegen sein Maximum; dieses in Finnmarken; im mittlen Norwegen mengen sich beide. Während der "Post-TertiärPeriode" (Quartär-Periode?) gab es in Skandinavien nur die hochnordische Fauna, wie aus der Untersuchung der gehobenen Konchylien-Lager hervorgeht. Später bat die Fauna der Nordisee einen mehr südlichpn Charakter angenommen, germanische sowohl als arktische Formen sind weiter nordwärts and einige hochnordische sogar in skandinavien ausgestorben, während im deutschen Meere jetzt eine rein germanische Fauna angesiedelt ist. Man kann daher unterscheiden: 1) solche Arten, welche in hohen Norden weniger reich an Individuen sind, als in der Nordsee, und im Mittelmeere ganz fehlen; 2) Hospites: alle mit dem Mittelmeere gemeinsamen Arten; 3) Aborigines: die im hohen Norden vorzugsweise entwickelten. Eine Vergleichung mit andern Gegenden gibt folgende Zahlen-Beziehungen im Ganzen und zwischen den mit Schaalen versehenen Gasteropoden und Acephalen:

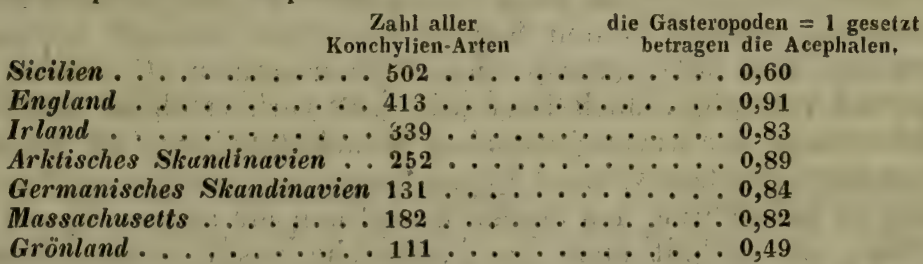

Lovín unterstellt, dass sich die Acephalen zu den Schaalen Gasteropoden im Ganzen $=0,50^{3}$ ) verhalten, wie es in der That in dem ganz von nordischen Strömungen umgebenen Grönland und nahezu auch in Sicilien gefunden wird, während in den mittlen Gegenden, wo beide Faunen sich mischen, das Verhältniss der zählebigeren Acephalen weit mehr vorherrscht, iudem sie nicht nur den andern südlicheren Mollusken voraus eingewandert, sondern auch länger hinter den übrigen nordwärts auswandernden zurückgeblieben sind.

\section{b. Im Resonderen.}

§. 28. In Bezug a f einzelne Reiche, K lassen u. s. w. wissen wir im Allgemeinen bereits, dass beide Reiche in allen Unterabtheilungen immer formenreicher werden, immer mehr der jetzigen Schöpfung sich nähern, anfangs gleichmässig über die Erd-Oberfläche verbreitet seit der Tertiär-Zeit sich nach den jetzigen Gesetzen geographischer Verbreitung zu vertheilen anfangen und sogar schon zum Theil diejenigen Arten zeigen, welche noch jetzt existiren.

A. Die Pflanzen im Besondern sind von Anbeginn an verbreitet. Zwar hat man in den silurischen Schichten noch keine organischen Form-Theile derselben entdeckt; allein die Menge kohliger

1) On the vegetable colonisation of the Britich Islands, Shelland, Feroë and Iceland, in JAMES. Journ. 1849, XL VI, 40-52.

2) Jahrb. 1848, 256.

3) Wir haben mit Ausschluss der Land- und Süsswasser-Bewohner und von etwa 50 Brachiopoden-Arten das Verhältniss auf ungefähr 0,40 berechnet. 
und bituminösèr Bestandtheile gewisser Schiefer u. a. FormationsGlieder lässt nicht an ihrer damaligen Anwesenheit zweifeln. Ja Forснн амmer leitet den Salz-Gehalt der alt-silurischen Alaun-Schiefer Skandinaviens ab von See-Tangen (Ceramites Hisingeri LiEb.), die sie reichlich in sich aufgenommen ${ }^{1}$ ). Es würde in diesem Falle eine der niedrigsten Pflanzen-Klassen und die einzige, welche durchgelıends Meeres-Bewohner enthält, die Flora auf der Erde einleiten, wie sie auch durch alle spätern Formationen bis in die Jetztzeit in der That hindurchreicht. Die übrigen theils kleinen und zarten, theils sehr vergänglichen Zellen-Pflanzen des Landes sind uns nicht in so reichlichen Resten aufbewahrt worden, um daraus ihren Entwicklungsgang zu entziffern. Wir wenden uns zum nächsten Pflanzen-Kreise, den Gefäss-Pflanzen. Was man in den auf die silurischen zunächst folgenden Devon-Bildungen und Kohlen-Kalken zuerst in deutlielier Form fiudet, sind nur (mit einer Ausnahme) kryptogamische Monokotyledonen, welche in den Kohlen-Schiefern, obwohl mit monokotyledonen und mit gymnospermen Plıanerogamen (Cycadeen und Koniferen) vereinigt, doch über alle weit vorherrschend auftreten. Die Abtheilung der kryptogamischen Monokotyledonen ist auch als der zusammengesetzte Wurzel-Typus des ganzen vascularen PflanzenReiches zu betrachten, aus desseu Entfaltung die andern Typen hervorgehen: sie enthält nicht nur zahlreiche ausgestorbene Genera, sondern auch allein mehre erloschene Familien, deren sich bei den phanerogamen Monokotyledonen keine und bei den gymnospermen Phanerogamen nur die der Diploxyleen zwischen Cycadeen und Ko. niferen einfiudet. Weiter bietet uns das ganze Pflanzenreich keine ausgestorbenen Familien melır mit Sicherheit dar. Die genannten 4 Gruppen setzen nun auch bis zu Anfang der Tertiär-Zeit die Flora fast allein zusammen, so jedoch, dass die kryptogamischen Monokotyledonen in Familien, Sippen und Arten absolut wie relativ ab-, die monokotyledonen und die gymnospermen Phanerogamen aber wenigstens relativ zunehmen. Noch lebende Geschlechter mischen sich unter die rein fossilen. Kaum ein halbes Dutzend andrer, angiospermer Dikotyledonen-Arten (ahgesehen nämlich von einer Reihe den Dikotyledonen zugeschriebener Früchte und Saamen) von noch mehr oder weniger zweifelhaftem Charakter kommen in der langen Formationen-Reihe bis zum Ende der Kreide-Periode zum Vorschein; und erst mit dem Beginne der Tertiär-Zeit tritt auch das höhere Di-

1) Jahrb. 1845, 743 und Skand. Förhand. 1844, i. Christiania S. 281. Durch diese Nachweisung wird also berichtigt und beziehungsweise bestätigt, was S. 817 ff. über das ungleichzeitige Erscheinen der ältesten Pflanzen und Thiere gesagt worden ist. Diese Nachweisung ist zwar schon älter als die Niederzeichnung jener Seiten; da uns jedoch die Quelle nicht zugänglich gewesen, so war uns bis nach Abulruck jener Stelle unbekannt ceblieben, dass eine bestimmte Pflanzen-Art in den silurischen Schiefern hat erkannt werden können, ein Ceramites nämlich. 
kotyledonen-Reich, treten die dikoryledonen Kränter, Stauden und Laubhölzer, welche $9 / 10$ unsrer heutigen Flora ausmachen, in grossem Reichthum der Zahlen und in üppiger Manchfaltigkeit der Formen z11mal anf. Nirgends können sich daher die 2 Entwicklungs-Gesetze der organischen Welt, das allmähliche Hinzutreten immer höherer Gruppen zu den tiefern und das Auseinandertreten frühester komplizirter Organisationen in geschiedene Formen-Reihen schöner zeigen, als bei den Pflanzen. Die Abhängigkeit dieser Entwicklung von den äussern Lebens - Bedingungen tritt dagegen weniger hervor, weil theils in der That die Pflanzen unabhängiger als die Thiere sind von den möglichen Modifikationen dieser Bedingungen, theils die ehemalige Beschaffenheit dieser bedingenden Ursachen, so weit sie die Pflanzen angehen, problematischer als für die Thiere ist ${ }^{1}$ ).

Noch lebende Pflanzen-Genera sind bisher nur in der TertiärZeit $(0,32)$ angegeben worden, doch nicht sowohl weil sie früher überhaupt niclit existirten, sondern weil bei der Unvollständigkeit der älteren Fucoiden-, Farnen-, Koniferen, und andern PflanzenTheile die Botaniker vorzogen, die Übereinstimmung nicht mit Bestimmtheit auszusprechen.

B. Bei den Phytozoen müssen wir die Klassen der Pseudozoen, der Entozoen, der Acalephen und die Ordnung der Fistuliden theils wegen ihrer geringen Anzahl und theils wegen ihrer Unfähigkeit zur fossilen Bewahrung ausser Acht lassen; dass aber die En. tozoen bestanden liaben, so lange als es Thiere andrer Klassen gibt, und dass sie mit deren Zahl und Manchfaltigkeit selbst in beider Hinsicht zugenommen haben, dariber kaun kein Zweifel seyn. Die sämmtlichen erhaltungsfälıigen 4 Klassen der Phytozoen, nämlich Amorphozoa, Polygastrica, Polypi und Echinodermata, haben seit und mit der silurischen Zeit bestanden. Ihre noch lebend gebliebenen Genera steigen in den 5 Perioden von 0,25 auf 0,70 . Inzwischen müssen wir auch noch von den Polyg a stric a bemerken, dass sie, obwohl theilweise mit anscheinend sehr erhaltungsfähigen Kieselpanzern versehen, doch ebensowohl in den älteren kompakten Gesteinen durch ihre Kleinheit dem Ange entschwinden, als sie in loser Zusammenhäufung einem Umgestaltungs-Prozesse unterliegen, der sie unkenntlich macht und wahrscheinlich die Masse selbst in kompaktes Kiesel-Gestein, in Halbopal nnd dergleichen verwandelt, wie Ehrenberg's Beobachtungen ${ }^{2}$ ) andeuten. Auch ihre Entwicklungs-

1) Im Augenblicke der letzten Durchsicht dieser Druck-Seite kömmt mir Sнarpe's Beobachtung einer mächtigen untersilurischen Kohlen-Formation bei Oporto zu mit Farnen-Arten, welche denen der gewöhnlichen Steinkohlen-Formation sehr ähnlich, wenn vicht $\approx$. Th. identisch sind (Lond. geol. Quart. Journ. 1849, V, 142-153), wodurch also die Farnen schon mit den ältesten Thieren und jener Tang-Art zugleich auftreten.

7) Jahrb. 184r, 116. 
Gesetze dürfen wir daher noch nicht zu enthüllen hoffen, und es ist auch weniger. Werth darauf zu legen, dass die wenigen vor-tertiären Arten, die man in der Kohlen - "nd in der Kreide-Formation gefunden, eben noch lebenden Geschlechtern angehören sollen. In der Kreide- und Tertiär-Zeit machen die noch lebend erhaltenen Sippen $0,57-0,85$ aus, auch hier ein sehr hohes Verhältniss, welches der weiten geopraphischen Verbreitung entspricht. Die Amorphoz o en würden seit der Zeit ihres Erscheinens bis jetzt an Zahl und Mauchfaltigkeit im Ganzen zugenommen haben, weun man annehmen darf, dass die Gesteine aller Perioden ihrer fossilen Erhaltung eben so günstig gewesen seyen, als der Jura-Kalk und die Kreide; denn in den Tertiär-Schichten sind sie viel spärlicher als in der Kreide und in der jetzigen Welt vorhanden. Ihre noch lebend bestehenden Genera nelimen in den V Perioden von 0,27 auf $0,76 \mathrm{zu}$.

Bei den Polypen sind die Polythalamien, obwohl schon im Kohlen-Kalke beobachtet, doch anfangs nur sparsam vorhanden, nachher in rascher Zunahme, um in der letzten Tertiär- wie in der Jetzt-Welt ihre höchsten Zahlen und Formen.Entwicklung zu finden. Die ältesten Formen sind vorzugsweise polysomatische, welche gewiss unvollkommener als die monosomatischen sind, und unter diesen halten wir die Monostegier und Agathistegier (Milioliten) für die höchsten, die ihrerseits (mit 3 von Münster bezeichneten aber nicht näher beschriebenen Ausnahmen) sich auf die tertiäre und jetzige Zeit beschränkeı. - Die Anthozoen und Bryozoen zeigen eine ungefähr gleiche langsame Zahlen-Zunahme von den ältesten Schichten an, in welchen sie schon ziemlich zahlreich vorkommen, bis in die jetzige Zeit. Bevor diese Klassen richtiger in ihrem Inneren klassifizirt sind, dürfen wir nicht hoffen, den leitenden Faden zu finden, an welchen sich ihr allmähliches Auftreten knüpft; jedoch bemerken wir in der ältesten Schicht ausser den problematischen Graptolithen vorzugsweise die röhrenartigen, im Innern zum Theil mit Querwänden versehenen und oft porenwandigen, durch Einsclıaltung neuer Röhren wachsenden Sippen der Bryozoen, die sich den Anthozoen sehr nähern; dann Anthozoen, deren Röhren mit unvollkommenen Strahlen-Lamellen versehen und durch Quer-Röhren verbunden und, wenn jene vollkommener, wahrscheinlich durch eigenthümliche Vervielfältigungsweise u. s. f. ausgezeichnet sind, worüber uns erst die begonnen monographischen Arbeiten aufklären müssen. Die Formen mit einzelnen grossen vollständigen Sternzellen und mit ästigen Sternlamellen scheinen vorzugsweise den jüngsten Schichten und Zeiten anzugehöı'en ; die ausgebildetsten, die Kalkstock-Iosen Actinien, könnenen keine fossilen Reste darbieten. Die Quote der noch lebend erhaltenen Polypen-Genera steigt von der I. bis V. Periode von 0,40 bis 0,76 , in der Kreide sogar noch höher, uämlich bis 0,89 .

Die Echino dermen und insbesondere Stelleriden sind ebenfalls 
ganz frühzeitige Erscheinungen, deren zahlreichen gestielten Sippen, gleichsam den Jugend-Zustand der ungestielten freien Formen lebensänglich repräsentirend, diesen wieder weit vorangehen, so dass nur wenige Arten der letzten seit der ersten Periode auftreten, wie nur wenige der ersten in die Oolithen- und spätern Zeiten hinüberreichen. Die andere Ordnung, die Echiniden, treten zuerst (1 Art vielleicht schon im Kohlenkalk von Visé?) im Muschelkalk und den Oolithen uf, in älteren Formationen durch die Stylechiniden, wie die Comaeln und Ophiuren durch die Stylastriten angedeutet. Unter ihnen ind wieder die regelmässig radialen Cidariden die ältesten $(\mathbf{k}-\mathbf{z})$, lie weniger regelmässigen Clypeastroideen jünger $(\mathbf{n}-\mathbf{z})$, während ndlich von den sehr vollkommenen sphenoiden Spatangoideen $(\mathbf{q}-\mathbf{z})$ ur eine einzige Art vor $q$ erscheint. Die Quote der noch lebend geliebenen Sippen steigt von 0,06 in der I. Periode bis auf 0,50 in der etzten, ein auffallend kleines Verhältniss, was im Anfang durch das /orherrschen der fast ausgestorbenen Stelleriden und später durch lie sorgfältigen die Sippen sehr weit zerlegenden Arbeiten von lasssiz bedingt ist.

C. Die Malacozoen bieten überall viele noch jetzt lebende ienera dar; ihre Quote im Ganzen nimmt in den V Perioden von 1,47 auf $0,91 \mathrm{zu}$. Sie bestehen ans einer tieferen Haupt-Abtheilung der Kopflosen und einer höheren Abtheilung, den Cephalophoren. Von rsten ist die unterste Klasse der Gymnacephalen oder Tunicaten nicht rhaltungsfähig, und die zweite der Brachiopoden bietet in der noch nmer problematischen Gruppe der Rudisten die eigenthümliche Ercheinung des plötzlichen Auftretens und Verschwindens eines fremdrtigen und zahlreichen Typus innerhalb einer einzigen Periode dar, ;ährend sowohl vor als nach der Kreide keine Spur von ihnen zu entecken ist. Die übrigen Acephalen bilden 3 übereinanderstehende laupt-Gruppen: Brachiopoden, monomye und dimye Pelecypoden. ene, mit wenig ausgebildeten Kiemen, sind fast gleichseitig, meist ngleich-klappig, angeheftet, ohne entschiedenes Vorn und Hinten in er äussern Gestalt, sind von den frühesten Zeiten an reich an Gechlechtern und Arten entwickelt, doch nur mit 0,12 lebender Sippen ersehen, und nehmen fortwährend $a b$ an beiden, bis in der jetzigen chöpfung nur noch 5-6 Sippen mit wenigen Arten übrig bleiben; chon in der Kreide gehören alle Arten noch jetzt lebenden Sippen n; doch werden sich diese Verhältnisse in Folge neuer monographicher Bearbeitungen wesentlich ändern. Die meistens angehefteten nd daher noch ungleich - klappigen einmuskeligen Pelecypoden sind on Anfang her weniger zahlreich, bleiben sich aber unter allmähcher Zunahme doch fast gleich an Menge und Manchfaltigkeit und elbst in der Identität der Geschechter wie in der Quote der mit den och lebenden identischen Sippen, welche, stets ansehnlich, nur von 83 auf 0,90 steigt. Die fast durchgehends freien und gleichseiti- 
gen zweimuskeligen Beilfüsser endlich sind zwar von Anfang her am zahlreichsten, aber auch fortwährend in beständiger starker $\mathrm{Zu}$ nahme, die sie abermals vorzugsweise der höchsten unter ihren Gruppen, den ganzmanteligen (sinuato-palliaten) Sippen verdanken; denn die Tubicolae sind auch in jetziger Schöpfung zu wenig zahlreich, um auf ihr Verhalten einen Werth legen zu können; - sie verlieren nur wenige der früher vorhandenen Geschlechrer. In Ganzen steigt die Quote der mit lebenden identischen Geschlechter der Pelecypoden von 0,70 auf 0,92 .

Wie hei den Kopflosen überall, so zeigt sich auch bei den $\mathrm{K}$ op $\mathrm{f}$ Mollusken im Ganzen ein Voranschreiten von dem Unvollkommneren zum Vollkommneren. Die Pteropoden, Heteropoden und Protopoden sind zum Theile nackt und daher unfähig der Erhaltung; sie sind zugleich zu wenig zahlreich und zum Theil aus zu ungleichartigen oder problematischen Elementen zusammengesetzt, um eine wesentliche Beachtung zu verdienen.

Das Verhalten der Gas te rop ode n hängt fast allein von dem der Ctenobranchier ab, welche die übrigen Ordnungen bei Weitem überwiegen. Sie nehmen stetig an Menge und Manchfaltigkeit zu, aber die tiefer stehenden Asiphonobranchier nur wenig, die Siphonobranchier mächtig, besonders von der Kreide an. Sie gewinnen fortwährend viel mehr an neuen Geschlechtern, als sie durch den $A b$ gang von alten verlieren. Die Quote der noch lebend bekannten Genera erhebt sich fïr alle Gasteropoden von 0,52 auf 0,91 . In den Lungen-Gasteropoden treten die ersten Luft-athmenden Binnen-Bewohner erst am Ende der Oolithen-Periode auf und nehmen von da an rasch an Menge zu.

Die höchste Ordnung der Cephalophoren aber, die C e p h a l op oden verhalten sich den früheren entgegengesetzt; sie lassen kein Fortschreiten zur höheren Entwicklung erkennen. Die Quote noch lebender Genera in den $V$ Perioden nimmt von 0,06 auf $0,50 \mathrm{zu}$. Ihre Tetrabranchier, von Anfang an reichlich vertreten, setzen sich hauptsächlich aus 2 Gruppen zusammen, aus Ammoneen, welche mit sehr einfach gebildeten Kammer-Scheidewänden ihrer Schaalen beginuen, an Zusammensetzung zunehmen, in Oolithen- und KreidePeriode ihre böchisten Arten- und Sippen-Zahlen erlangen und mit der Kreide plötzlich aussterben; die Nautileen dagegen zeigen eine hohe Manchfaltigkeit der Geschlechter und hohen Reichthum an Arten gleich anfänglich, nehmen fortwährend $a b$ und retten sich in die jetzige Schöpfung kaum mit 2 Sippen und 3-4 Arten. Aber die höher stehenden zum Theil nackten und daher in ihrer Entwicklung uns vielleicht nicht vollständig vorliegenden Dibranchier erscheinen wieder auch später als die Tetrabranchier, erst im Lias, mit den fremdartigen Geschlechtern der Belemniten, Geoteuthen, Beloteuthen u. s. w.; Menge und Manchtaltigkeit der Schaalen-Formen nehmen 
zwar nach den Oolithen und der Kreide fortwährend ab; aber in der jetzigen Schöpfung treten die Sippen zahlreicher als zuvor auf, weil hier auch die nackten, wahrscheinlich höchsten Geschlechter sichtbar werden.

D. Die Entomozoen zerfallen in Wasser- und in Luft-Bewohner. Unter den ersten sind die Würmer, so weit sie mit Schaalen versehen, in fast gleichmässiger Entwicklung von Anfang her bis jetzt zu finden, ohme erheblichen Wechsel der Formen. Die Quote der noch lebenden Genera unter ihuen schwankt von 0,43 bis zu 0,83 aufwärts.

Die $\mathrm{Kr}$ uster enthalten in ihren 2 ersten Ordnungen ebenfalls viele theils weiche und theils sehr kleine Formen, die sich im fossilen Zustande nicht mehr auffinden lassen. Die Cirripiden scheinen gleichwohl zuerst in den Oolithen nur spärlich, aber von da an wachsend in Menge und Manchfaltigkeit aufzutreten; vielleicht weil Reste älterer Formen nicht erhaltungsfähig waren. - Entomostraca dagegen beginnen sogleich reichlich mit den Paläaden, einem gemischten Ausgangs-Typus (Prototyp) nicht nur für diese, sondern vielleicht auch die folgende Ordnung, welcher keinenfalls die tiefste Stufe einnimmt; aber diese Paläaden verschwinden schon mit dem Schlusse der ersten Periode völlig. Die bis dahin sie begleitenden Ostracoden ziehen sich durch alle Formationen hindurch; aber ihre und einiger verwandten Gruppen Kleinheit und selbst Weichheit lässt uns nicht annehmen, dass wir ihr einstiges Verhalten aus den fossilen Resten ganz zu enträthseln vermögen; erst in der Jetztwelt ist uns dargeboten sie reiflich zu beobachten; nur die grossen Pöcilopoden treten auch in mitteln Zeiten schon heran, reichlicher als sie jetzt leben. -Die an Vollkommenheit alle vorigen überragenden Malacostraca endlich bestehen theils aus kleinen Formen, von welchen wir noch kaum einen oder den andern Rest im fossilen Zustand erkannt haben, theils und hauptsächlich aber in grösseren Decapoden, welche wieder in tiefere Macruren und hölere Brachyuren zerfallen; jene treten in der zweiten Periode zuerst auf und sind in der dritten schon hoch entwickelt; diese sind in der dritten spärlich und erst in der vierten zahlreicher, um ihren Gipfelpunkt erst mit der jetzigen Schöpfung zu erreichen. Im Ganzen erhebt sich bei den Krustern die Quote noch lebend bekannter Genera, welche anfangs wegen der vorherrschenden Trilobiten nur 0,08 ausmacht, allmählich auf 0,81 .

Wir haben schon mehrfach angedeutet, wie die $\mathrm{L}$ uf $\mathrm{t}$ - I n s e kte n nur zufällig ins Wasser gelangen und in entstehende GesteinSchichten eingeschlossen werden und ihrer Kleinheit und Weichheit wegen nur selten in kenntlichem Zustande bis auf uns gelangen konnten. Wir dürfen kein Gewicht auf die negative Beobachtung legen, dass von den ohnediess nicht zahlreichen Myriopoden die ersten spärlichen Reste in den Oolithen vorkommen. Die Arachniden 
sind wenigstens durch einen Skorpion und einen After-Skorpion"seit der Kohlen-Zeit nachgewiesen und dann gewiss auch schon durch viele andre Formen vertreten gewesen. Eben so haben wir von dieser Zeit her Kenntniss von der Existenz verschiedener andrer Insek. ten-Ordnungen aus der Sechstüsser-K I ass e, die sich dann auch in Lias und Oolith schon viel reichlicher einfinden. A ber bei dem Mangel an allen höheren Pflanzen, wo mithin die ganze Flora noch nicht den dritten Theil ilurer jetzigen Familien entwickelt hatte, muss auch die grösstentheils von Pflanzen lebende Sechsfüsser-Welt bis zum Beginne der Tertiär-Zeit noch immerhin ferne gewesen seyn von ihrer jetzigen Manchfaltigkeit. Die Quote der lebenden Genera erhebt sich bei diesen 2 letzten Orduungen während der V Perioden von 0 auf 0,74 und 0,89 .

E. Spondylozoen kennen wir zwar noch nicht in den untersilurischen Schichten; doch beginnen die Fische in den obersilurischen, die Reptilien in den Kohlen-Gebilden, die Vögel nach ihren Fährten zu schliessen in den darauf folgenden Saridsteinen und die Säugthiere im untern Grenz-Gebilde des Lias oder wenigstens in den mitteln Oolithen; ihr Auftreten folgt daher der Vollkommenheits. Skale.

Unter den Fis che n gibt es drei sehr kleine Ordungen, von welchen die zwei ersten und niedersten überdiess durchaus nur weiche, unbeschuppte Kuorpel-Fische nur zuweilen mit einigen harten Zähnen enthalten, die letzte und zugleich höchste unter allen Fischen bloss aus 1 - 2 lebenden Genera besteht; von diesen allen findet sich nichts Fossiles vor. Die übrigen bilden drei Ordnungen, ihrer zunehmenden Vollkommenheit nach aufgezählt: die knorpeligen Elasmobranchier, die knorpeligen und knochigen Ganoiden und die ganz knochigen Teleostei, welche in gleicher Ordnung auch in den ErdSchichten auftreten, in den unter-silurischen, in den devonischen und in den oolithisehen Schichten, herrschend aber eigentlich erst jene beiden in den obersilurischen, diese in den Glariser Schiefern $(=\mathbf{s}$ ?) und den Tertiär-Schichten. Aber während die Elasmobranchier zahlreich bleiben bis in die jetzige Schöpfung, nehmen die Ganoiden (welche Agassiz anfänglich als Prototyp der Fische betrachtet hatte) bis auf wenige Genera ab (was eine tiefere Stellung vermuthen lassen würde, die aber wenigstens einem Theil der noch lebenden Formen widerspricht); die Teleostei aber, weitaus alle übrigen Fische zusammen überwiegend, nehmen von der Kreide an rasch an Menge zu. Die Unterabtheilungen der Elasmobranchier reihen sich so aneinander; unsichere Genera (b-e),Hybodonten (c-f, tw), Cestracionten (e-f, $\mathbf{t z}$ ), Squaliden und Rajiden(ausser einigen unsichern Geschlechtern in c, a, e, $\mathbf{g}$ mit nur 7 Arten, $=\mathbf{m}-\mathbf{z}$ entsprechend). - Bis auf einige knorpelige Chondrostei (mit wenigen Arten in $\mathbf{m}-\mathbf{z}$ ) und knochige Holostei (in $z$ ) sind wohl durchweg knorpelige und sonstige Ganoi- 
den ganz ausgestorben und auf $\mathbf{c - w}$ vertheilt, aber von $\mathbf{n}$ an in starker Abnahme. Die sämmtlichen (lebenden wie fossilen) Elasmobranchier und meisten Ganoiden sind ungleichschwänzig (heterocerci), so zwar, dass die Gleichschwänzer erst viel später als jene auftreten. Die ungleichschwänzigen Ganoiden, welche sich in verschiedenen Ordnungen allein, in andern mit den gleichschwänzigen zusammen finden, reichen, soweit die Genera hinsichtlich ihrer Schwanz-Bildung bekannt sind, nur bis in 1 am Ende der II. Periode (1 Coccolepis und die Ordnungen Chondrostei und Holostei ausgenommen), während die Homocerci (unter derselben Bedingung und bloss 3 ältere Arteı ausgenommen) erst mit $\mathrm{m}$ am Anfang der III. Periode beginnen. - Alle Teleostei sind homocerci. Ilıen gehören alle verschiedenen weichflossigen und ganzschuppigen Süsswasser-Fische aus rein fossilen Geschlechtern an. Sie lassen ein chronologisches Gesetz ihres Erscheinens nicht mehr weiter unterscheiden. Die Quote der noeh lebenden Fisch-Genera zwischen Anfang und Ende der geologiselien Zeit erhebt sieh von 0 nur auf 0,52 , was theils eine Folge der sorgfältigen Bearbeitung des vorhandenen Materials duroh AGassiz und theils der Seltenheit von Lagerstätten mit mioeänen und zumal mit pliceänen Fischen ist.

Bei den Re ptilien treten die Ordnungen in dieser Folge auf: Saurier, Chelonier, Ophidier und Batrachier: Die Saurier beginnen schon mit e, erreichen den Gipfelpunkt ihrer Entwicklung in der III. Periode und nelimen von dort bis in die jetzige Schöpfung an Grösse und verschiedenartigen Hanpt-Typen ab, an Zahl der Genera und Arten in einigen untergeordneten Typen zu. Die ältesten Formen sind Krokodile und Eidechsen, zuweilen mit Schildkröten-Charakteren; dann gesellen sich in der II. Periode schwimmfüssige und ? Froschähnliche (Labyrinthodonten) Saurier-Gestalten hinzu; in der dritten vermehren sich die Schwimmfüsser, verschwinden die Frosch-ähnlichen und treten die Dactylopoden und riesigen Scinke neu hinzu; in der Kreide erscheinen nur noch wenige Riesen-Echsen, und in den Tertiär-Schichten findet man mit 1-2 Ausnahmen nur die jetzt gewohnten Sippen. Aber eine merkwürdige Erscheinung ist noch, dass die Saurier bis in die Kreide (r) bikonkave und selten konvexkonkave (opisthocoeli), erst in und nach dem Grünsande $(\mathbf{r}, \boldsymbol{C})$ konkavkonvexe Wirbel besitzen. Die Schildkröten beginnen viel später in der Oolithen-Periode, die Schlangen und Batrachier in der Tertiär-Zeit, wenn man nicht, wie R. Owen, die riesigen Labyrinthodonten $(\mathbf{i}-\mathbf{1})$ den Fröschen beizählen will. Die Quote der noch lebenden Reptilien-Geschlechter bewegt sich in den V Perioden von 0 auf 0,70 .

Welcher Art alle die $\mathbf{V}$ ö g e l gewesen, deren Fährten man in den Sandstein-Schichten über der amerikanischen Kohlen-Formation (f) und im Buntsandstein gefunden hat, können wir mit Gewissheit 
nicht sagen; obwohl uns die Grösse vieler unter ilınen und die oft beobachtete Anzahl von 3 bloss vorderen Zehen auf Lauf-Vögel schliessen lässt. Aber damit ist die damalige Vögel-Fauna keineswegs erschöpft. Die ersten Vogel Kuochen kommen ausser 1 Art in dem Grünsand und den Glarner Schiefern erst in der Tertiär-Zeit (t) vor, anfangs ausgestorbene, dann noch bestehende Sippen, die sich den jetzigen des Landes mehr und mehr anschliessen. Die Schwierigkeit aus einzelıen Knochen den ganzen Ban eines Vogels richtig zu deuten, hat uns noch nicht zur näheren Einsicht in die Entwicklungs-Folge der Vogel.Welt gelangen lassen, wie aus demselben Grunde kein Werth darauf zu legen ist, dass in der Tertiär-Zeit 0,8s noch lebend erhaltener Vogel-Genera angegeben werden.

Die $S$ ä $u \mathrm{gth}$ i er e endlich beschränken sich, mit Ausnahme von 3 bis 4 Arten Insektivoren in $\mathbf{1}, \mathbf{m}$ und $\mathbf{n}$, welche theils und viel. leicht alle den Beutelthieren angehören, anf die Tertiär-Zeit. Die Zusammendrängung ihres ersten Auftretens in eine so kurze Zeit erschwert die Erkenntniss ihrer Entwicklungs-Folge; doch sieht man zuerst Cetaceen, Pachydermen, Ruminanten, Nager, Bentelthiere, Raubthiere, Fledermäuse, Affen, mithin ausser den Edentaten alle Ordnungen gleichzeitig schon in t erscheinen; nur stehen die Raubthiere sehr zuriick gegen ihr Verhältniss zu den Herbivoren in der mitteln und spätern Tertiär-Zeit. Die Geschlechter insbesondere der Pachydermen sind anfangs in t meistens $\left({ }^{18} / 30\right)$ der Jetztwelt fremd, einige auf jene Formation beschränkt; sie werden den jetzigen allmählich nicht nur im Ganzen ähnlicher und gleicher, sondern auch insbesondere mit jenen übereinstimmend, welche noch jetzt in der Gegend des Vorkommens der fossilen leben, so dass man in $\mathbf{v} \mathbf{w} \mathbf{x}$ zusammen etwa 0,54 noch lebender Genera findet. Eine verhältnissmässig kleine Zahl ausgestorbener Genera liefern die Carnivoren, die grösste Quote derselben noch in $\mathbf{x}$ die Edentaten. Der Mensch macht entschieden den Schluss, so dass er mit oder nach den letzten unsrer jetzt lebenden Säugthiere auftritt. Wenn die Quote noch lebender Genera für sämmtliche Säugthiere sich in der Tertiär-Zeit nur auf 0,32 erhebt, so liegt die Ursache darin, dass innerhalb dieser Zeit auch noch das erste Auftreten der Säugthiere in Masse, wo mithin noch fast alle Sippen von denen der jetzigen Schöpfung abwichen, dass dahin also der Anfang und das Ende der Quoten-Reihe fällt.

F. Rückschlüsse aus den organischen Erscheinungen a u die Erd-Geschichte.

§. 29.

A. Wir haben in den vorhergehenden Paragraphen ( $\$ .211 \mathrm{ff}$.) allmählich wechselnde Zustände der Erde unterstellt, wie wir solche im I. Bande der Geschichte der Natur aus geologischen Verhält- 
nissen abgeleitet hatten, um mittelst dieser Reihe von Unterstellungen die allmählichen Erscheinungen in der organischen Welt zu erklären. In vielen Fällen ist Diess glücklich gelungen, so dass eben sowohl die Geschichte dieser letzten der Geschichte der unorganischen Erd-Rinde zur Erläuterung und Bestätigung diente, wie umgekehrt. In andern F'ällen hat sich ein direkter Beweis des Einen aus dem Andern nicht ergeben; und in noch andern ist das Ergebniss sogar gegentheilig ausgefallen, obwohl die diessfallsigen Untersuchungen noch nicht als abgeschlossen angesehen werden können. Inzwischen wird es angemessen seyn, die wichtigsten Resultate dieser Art neben einander zu stellen, um sie fernerer Prüfung und Erläuterung zu empfehlen, mögen sie nun bejahend oder verneinend ausgefallen seyn.

B. Die auf die reichlich stattgefundenenKohlenstoff-Niederschläge während der Bildung der vielen Steinkohlen- und BraunkohlenLager und Kohlensauren-Kalk-Gebirge gegründete Annahme eines nach begonnener Schöpfung grösseren Kohlensäure-Gehaltes der Atmosphäre findet, nachdem auch Bıschof die Möglichkeit der Erklärung jener Kohlenstoff - Ansammlungen auf andere Weise dargethan, keine Stütze mehr in der Entwicklung der Organismen; indem vielmehr die Existenz einer ziemlich ansehnlichen Menge von Luft athmenden Thieren und insbesondere, ausihren Fährten zu schliessen, von Vögelı, deren Respiration unter alleu Thieren die lebhafteste ist, schon seit der Steinkohlen-Zeit unverträglich zu seyn scheint mit jener Annahme ( $(.212, C)$. Doch bleiben Untersuchungen über den möglichen Gehalt einer zur Respiration geeigneten Luft an Kohlensäure noch wünschenswerth.

C. Es ist bis jetzt kein Grund anzunehmen, dass die $L$ a ge der E rd - A c h se seit Beginn der Schöpfung sich geändert habe, indem die anfängliche Bevölkerung überall einen so gleichartigen Charakter zeigt, dass man aus ihm auch auf ein gleichartiges Klima schliessen muss, das über die erste Lageder Achse, der Pole oder des Äquators überall keinen Anfschluss gibt. Wo aber in der Tertiär-Zeit aus den organischen Resten Zonen zu unterscheiden möglich wird, da haben sie schon gleiche Lage mit den jetzigen.

Diess würde zwar einen Achsen-Wechsel, wie ihn neulich wieder SavcL und Luввоск unterstellt haben, um den Wechsel der Gebirgs-Formationen zu erklären, wenigstens vor der Tertiär-Zeit nicht widerlegen; aber wir würden dann noch immer in der Verlegenheit bleiben, die einstigen Stellen zweier unbewohnbar gewesenen Polar-Zonen zu finden (falls man nämlich die Annahme einer einstens höheren Temperatur damit beseitigen wollte) und eine neue Er. klärung für den Schichten-Wechsel während der Tertiär-Zeit zu erdenken.

D. Die Annahme einer einstens höheren Temperatur der Erde bestätigt sich durch alle Perioden hindurch bis zum Ende der Tertiär-Zeit so, dass das Klima anfangs überall fast gleich gewe- 
sen, dann allmählich gesunken, aber erst in der Tertiär-Zeit eine Abkühlung von den Polen her bemerkbar geworden wäre $(\S .213$ und S. 936 D). Von einem wellenförmigen Gang dieser Abnahmen, von einer stärkeren Abnahme und Wiederzunahme der Temperatur an der Grenze zweier Perioden, Formationen ete. (Agassiz) finden wir keine Spur.

Wenn sich damit die Gleichbit des Pflanzen- und Thier-Lebens bis in die höchsten Breiten hinauf esklärt, so bleibt doch das Gedeihen entwickelterer Pflanzen-Formen in so hohen Breiten, in denen es einen grossen Theil des Jah. res hindurch an lebhaften Lichte ganz gebricht, immer ein Räthsel, das sich indessen theilweise lösen würde, wemn sich ergeben sollte, dass jene Pflanzen tuberall nur in Kraut-artigen Farnen bestanden hätten?

Inzwischen ist mit Nachweisung der Erscheinung ihre Ursache noch keines* wegs enthüllt.

Da West - Europa jetzt die höchsten Isothermen besitzt, so würden wir Mühe haben, aus bekannten noch jetzt waltenden Ursachen für dasselbe cine einstens noch höhere Temperatur abzuleiten, auch wemn wir uns bis in die Tertiär-Zeit berab seine Gebirge niedriger und weniger ausgedehnt denken wollten; es würde uns wohl nicht gelingen, mit jenen Mitteln das Klima des Senegals nach Bordeaux zu versetzen, wie Solches in der Miocän-Zeit noch stattgefüviden hat. Anders verhält es sich mit der Pliocän-Zeit. Denn wenn wir auch nicht in Zweifel ziehen wollten, dass vor dem Ende dieser Pliocăn-Zeit, wo das Mammont zu Grunde ging, Sibirien, welches jełzt die niedersten Isothermen besitzt, in Folge einer geringeren Erhebung seiner Central-Masse, in Folge einer grösseren Zerstïckelung des Landes durch zahlreiche Meeres-Arme and in Folge vom Sïden heraufdringender See-Strömungen ein Klima besessen haben könne, wie das jetzige West-Europa, in welchem das Mammont zu gleicher Zeit wie in Sibirien lebte: wo aber bleiben dann die weiten Gebirgs-Ketten, von deren hohen Rücken die mächtigen Ströıe entspringeu mussten, welche die zahtreichen Mammont-Kadaver in das Eismeer hinabfïhrten, um sie an dessen Küsten mit Komchylien jetziger Arten des Polar-Meeres (MiddendorfF) wieder abzusetzen? Wenn aber dieses Thier damals dort lebte und die Kälte an der Küste schon damals gross genug war, um seine mächtigen Kadaver sogleich ins Eis einzuschliessen und für immer gegen Zerstörung zu schützen, wie dürfen wir jenen Gegenden ein merklich wärmeres Klina als jetzt zugestehen?

Wir wissen nicht, wie warm in der That die Erd-Oberfläche zur Silur-Zeit noch gewesen sey, als das organische Leben sich zu entwickeln beganı; wir wisseu aber, dass wenn danals die Erde sich in einem eben so kalten Weltraume als jetzt bewegte, die wirkliche Oberfäche der Erde, gleich der einer erst neulich ergossenen Lava, schon sehr kühl, schon nahezu so kühl wie jetzt gewesen seyn kann, wenn auch in geringer Tiefe die Wärme noch sehr ansehnlich und die Temperatur-Zunahme nach unten daher eine viel raschere als jetzt war. Die Wärme-Ausstrahlung durch die trockene Erd-Rinde, der Wärme-Ausfluss in Verbindung mit Quellen und Wasser-Dünsten u. s. w. war dann allerwärts grösser als jetzt und war fähig auch die Teniperatur der Atmosphäre zu erhöhen, und je höher diese aus dem Innern konmende Wärme, je rascher insbesondere die Temperatur-Zunahme nach unten gewesen, desto höher konnte auch derjenige WärmeÜberschuss im Ganzen seyn, den die Erde durch Bestrahlung von der Sonne erhielt; beide stchen zu einander in einem bestimmten Verhältiisse. Jene WärmeZunahme beträgt jetzt 1/30 Grad auf den Meter, jener Überschuss ungrefähr 1/32 Grad.

Nach Erie de Beavmonr's Berechnungen kann zur Zeit der Steinkohlen-Bildung jene Zunahme wohl noch $1 / 3$ Grad, dieser Uberschuss dann aber nicht über $1 / 3$ Grad betragen haben, was also in den Klimaten keine wesentliche Änderung bedingen konnte. Da eine solehe Änderung aber dennoch stattgefunden uod insbesondere 
die Polar-Gegenden kälter geworden sind, so muss man die Ursache davon in begleitenden Umständen suchen: 1) das Polar-Eis existirte in der ältesten Zeit noch nicht; dessen Beseitigung würde noch heut zu Tage die mittle Temperatur des Poles von etwa $-25^{\circ}$ auf $0^{\circ}$ heben. 2) Das Meer dieser Gegenden muss daher eine von unten nach oben weit nehr gleichbleibende Temperatur besessen haben: seine Oberfläche konnte sich zu keiner Jahres-Zeit sehr unter die Temperatur der ganzen Masse abkühlen; es musste sich in den Polar-Gegrenden mit Nebel bedecken, sobald die Sonne unter den Horizont sank. 3) Da die Tempe. ratur nach der Tiefe der Erd-Rinde zehnmal so schnell zunahm, so mussten fast alle Quellen als Thermen erscheinen, die Nachts den Boden in Nebel hülten, die nächtliche und winterliche Wärme. Strahlung unmöglich machten und so den Winter und die Nächte um die Pole erwärmten, ohne in Sommer gegentheilig zu wirken ${ }^{1}$ ). Aber auf die Erscheinung des Elephanten an dem Polar-Meere kann Diess keinen Einfluss mehr gehabt haben, weil damals das Polar-Eis schon existirte.

E. Für die Annahme einer anfänglich weniger beträchtlich en Erhebungder Länder undeineswenigerkontinentalen Zus a m m u ha $\mathrm{nges}$ derselben haben wir in der Beschaffenheit der Floru, in der Menge meerischer Reptilien, in dem Mangel an Flüssen und stehenden Süsswassern bis in die III. Periode und weiter herab eine Bestätigung zu finden geglaubt; aber dieser letzte Mangel ist noch grösser und dauernder, als sich aus jenen Voraussetzungen erwarten liess, so dass er selbst zum Probleme wird.

F. Die Annahme allmählicher Senkungen und Hebungen der Kontinente im Ganzen olne stärkere Schichten. Störungen und ohne gleichzeitige Bildung von hohen Berg-Kämmen findet eine Stütze in den zahllos sich wiederholenden SteinkohlenLagern, deren jedes einzelne nur in der jedesmaligen Nähe des Meeres-Spiegels sich gebildet haben kann und dann eingesunken seyn muss, um einer neuen Schicht gleicher Art Platz zn machen (§. $216 \mathrm{C}, \mathrm{S} .904)$.

G. Die fossilen Reste beweisen vollkommen (was ohne dieselben zu bestätigen schwer seyn würde), dass ein oft wiederholter $\mathbf{S}$ c h i c h. t e n.W e ch s l dadurch stattgefunden, dass senkrecht über derselben Stelle der Erd-Oberfläche bald Luft (Trockene) und bald Wasser, bald Meer- und bald Süss-Wasser, bald ein tiefes und bald ein seichtes Meer, eine hohe See oder eine brandende Küste etc. gewesen seye, wie es ohne beschränktere oder ausgedehntere, langsamere oder schnellere Hebungen und Senkungen des Bodens nicht denkbar ist.

H. Die Frage von der ungleichen Verbreitung der Ge. birgs-Niedersehläge über die Erd-Oberfläche liegt mit der von der Verbreitung der fossilen Reste nahe zusammen. Wir kenneı Reste aus der Trias- und Oolithen-Zeit bis jetzt nur in Europa und einem Theile von Asien; in den übrigen Welt-Gegenden scheinen sie nur höchstens noch eine kleine Verbreitung besitzen zu köunen. Da nun ohne geologische Niederschläge sich die organischen Reste

1) Jahrb. 1837,64 . 
nicht erhalten, Land- und Süsswasser-Bildungen aber in jenen 2 Perioden überhaupt noch nicht vorkommen (s. S. 901), so bleibt zuletzt nur die Frage zu beantworten, wie es komıne, dass, wie eben aus dem Mangel von Trias- und Oolith-Versteinerungen hervorgeht, die meerischen Schichten während jener 2 Perioden ganz fehlen? Lag in jener Zeit die Oberfläche des grössten Theiles der starren Erd-Kugel über dem Meeres-Spiegel, oder zu tief unter demselben in einem weit ausgedehuten Ozean? Theor'etisch ist Erstes wahrscheinlicher.

1. Aus den organischen Resten bestätigt sich vollkommen, was über die I ange Dauer der Bildung unserer Erd-Oberflä c h e (Geschichte der Natur I, 444-446) schon aus geologischen Gründen gesagt worden war ${ }^{1}$ ).

Eine der treffendsten Berechnungen nach G. Вiscноғ haben wir S. 860 und 865 angeführt.

K. Aus der völlig verschiedenen Eocän-Fauna zu beideı Seiten der Cordill e ren $S$ üd-Amerika's scheint hervorzugehen, dass zu Anfang der Tertiär-Periode die Anden schon in ihrer ganzen Längen. Ausdehnung von Norden nach Süden zwei Meere getrennt haben, da auch jetzt nur eine einzige Mollusken-Art bekannt ist, welche an der östlichen und westlichen Küste Süd-Amerika's zugleich lebt; und während die analogen Sängthiere der Eocän-Zeit Europa's in fernen Tropen-Gegenden zu finden, deutet die nahe Übereinstimmung der pliocänen Säugthiere wie übrigen Faunen mit den jetzigen in den gleichen Länderı an, dass die Gesammt-Form der pliocänen Länder und Meere noch den jetzigen entsprechend gewesen seyn müsse ${ }^{2}$ ).

\section{G. Zu s ammenfassung.}

๒. 30.

Setzen wir die Annahme der Geologen von einer einst Kohlensäure-reicheren dichteren höheren und dunstreicheren Atmosphäre, von einer einst höheren Temperatur der Erde, von einer allmählichen Abnahme derselben, von damit verbundener Differenzirung der klimatischen Zonen, von einer grösseren Ausbreitung und Zusammenhang aber geringeren Tiefe der Meere, von einem mehr Insel-artig zertheil-

1) Zu den angeführten Belegen für die beträchtliche Länge der geulogischen Zeit - Räume können jetzt noch andere bezeichnet werden, wie die von LXeLr, wonach seit der Existenz des Mastodon in Amerika (x), nach welcher erst die Niagara-Fälle begannen die Schlucht auszuhölılen, in die sie hinabstïrzen, wenigstens 20,000 Jahre verflosseu sind (Jb. 1844, 608); - dann die Berechnungen G. Brschof's über die Zeit, die eine Achat oder ein mässiger Quarz - oder Baryt-Gang zu seiner Bildung aus Mineral-haltigem Sicker-Wasser brauchte, oder über die Zeit, welche nach dem Abkühlungs. Gesetze seit der Steinkohlen-Formation bis jetzt verflossen seyn muss, und die er auf 9,000,000 Jahre anschlägt (Jb. 18t t, 285-286).

${ }^{2}$ ) R. OWEN, im Athenäum $=$ Ann. nathist. 1816, XVII, 197-200. 
ten Zustande des Festlandes, von minder hohen und ausgedehnten Gebirgs-Zügen, von einer auch in dieser Beziehung gleichförmigeren Witterung und von einem allmählichen, doch mit vielen gewaltsamen Bewegungen der Erd-Rinde in Verbindung stehenden Übergang zum jetzigen ungleicheren, unstäteren, manchfaltigeren Zustande der ErdOberfläche voraus, so finden wir auf Seiten der organischen Welt folgende Bestätigungen, Eı'weiterıngen, Berichtigungen oder Ausflüsse dieser Ansicht als wesentlichste Resultate der vorangehenden ausgedehnten Untersuchungen.

1) Zwischen der Schöpfung der ältesten bekaunten Organismen und jetzt liegt eine Zeit von vielen Millionen Jahren.

2) Die ältesten Organismen kommen schon in den ältesten neptunischen Bildungen vor, in den untersilurischen nämlich, welche unmittelbar auf krystallinischen Gesteinen ruhen.

3) Sie bestehen schon anfänglich in Pflanzen und Thieren verschiedener Klassen zugleich.

4) Die Kohlenstoff Menge vegetabilischen Ursprungs, welche schon in den untersilurischen Bildungen und in der Haupt-Steinkohlen-Formation abgesetzt ist, würde beweisen, dass die Atmosphäre anfänglich viel reicher an Kohlensäure gewesen seye, wenn nicht diese Kohlensäure aus unterirdischen Quellen erst ebenso allmählich in die Atmosphäre übergegangen ist, als die Vegetation den Kohlenstoff (und die Verwitterung der Felsarten zu Erden den Sauerstoff) immer wieder daraus hinwegnahm.

5) Das Erscheinen grosser luftbewohnender Reptilien und Vögel schon in der Steinkohlen-Zeit zeigt, dass dieser Luftreinigungs-Prozess schon damals nicht, oder nicht mehr nöthig war, indem der Kohlensäure-Gehalt der Luft bereits sehr gering war.

6) Die organische Welt zeigt uns anfangs überall und selbst bis in den Polarkreis eine tropische Temperatur, kann uns aber keinen Beweis liefern, dass irgend ein Theil der Erd-Oberfläche jemals eine erheblich höhere Temperatur als jetzt die Tropen-Gegenden besessen habe. (Die Menge und Grossartigkeit der noch später erfolgten mechanischen Bewegungen der Erd-Rinde macht es jedoch wahrscheinlich.)

7) Ein dem tropischen ähuliches, warmes, feuchtes, gleichmäsiges und überall gleiclies Klima ist im Charakter der Lebenwelt bis zur Eocän-Zeit ausgedrïckt.

8) Erst in der Miocän-Zeit erkenmen wir deutlich eine Scheidung der Erd-Bevölkerung in ërtliche Faunen und Floren; erst in der Pliocän-Zeit sind dieselben überall den jetzt an gleichen Orten befindlichen analog und $\mathrm{z}$. Th. gleich, doch immer noch mit einem etwas wärmeren Charakter. Ein Beweis für ein einstiges höheres Klima liegt, ausser den scho⿰ angeführten auch darin, dass alle diejenigen Organismen, welche jetzt nur noch als vereinzelnte Nachzïgler einst mächtig eutwickelter Gruppen erscheinen, den Tropen-Gegenden angehören. So 
Pentacrinus caput Medusae für die Krinởiden, Nautilus und Spirula fïr die Schaalen-Cephalopoden, Cestracion Philippii für die FischFamilie der Cestracionten, Polypterus und Lepidosteus für die Ordnung der Ganoiden, die Krokodile und Scinke für mehre Reptilien-Familien, unsre verhältnissmässig wenigen heutigen Pachydermen (mit Ausnahme bloss des Wildschweines) und Edentaten.

9) Das Zurückstehen der fossilen Reste ganzer Klassen von Land-Bewohnern gegen die der näclist-verwandten See-Bewohner spricht für die Ansicht der Geognosten von einem einst weiteren Zurückstehen des Landes gegen das Meer; - die Bildung der KohlenSchichten für ein langsames Sinken gewisser Stellen der Erd-Oberfläclıe; die gänzliche Verschiedenheit der eocänen Mollusken-Fauna an beiden Seiten der Cordilleren für eine vollständige Trennung beider Ozenne schon in der Eocän-Zeit.

10) Entschiedene Süsswasser-Bildungen treten erst in und nach den Oolithen, allgemein und bezeichnend erst in der Tertiär-Zeit anf.

11) Die Leben-Welt war im Anfang beziehungsweise arm und ist in jeder späteren Zeit fast immer reicher an Klassen, Ordnungen, Familien, Geschlechtern und Arten geworden, als sie zuvor war. Sie hat immer mehr an Reichthom und Manchfaltigkeit zugenommen.

12) Aber es sind zu jeder Zeit auch organisehe Arten verschwunden, obwohl stets in kleinerer Zahl, als entstanden. Die Bevölkerung der Erde ist daher von Zeit zu Zeit eine ganze andre geworden.

13) Die erste wie die spätre Entstehung organischer Wesen, ihre Auswahl und Vergesellschaftung miteinander waren Akte nicht eines unveränderlich bestehenden Natur-Gesetzes, sondern einer weisen Schöpfungs-Macht. Das Vergehen der Arten nach zeitlichen und örtlichen Verhältnissen zeigt gleichfalls von Plan und Absicht.

14) Die Haupt-Typen der Pflanzen und Thiere waren immer die nämliclien wie jetzt, insoferne es keine Kreise und Klassen und nuy wenige Familien von Organismen gibt, welche gäızlich wieder untergegangen wären; doch sind die meisten der anfänglichen Genera verschwunden und alle anfänglichen Arten. Fast nur die spät geschaffenen Genera und die ganz zuletzt geschaffenen miocänen und noch mehr pliocänen Arten sind grossentheils bis auf uns erhalten geblieben.

15) Die verschiedenen Klassen, Familien, Genera, Arten hatten daher eine gegenseitig ungleich lange Dauer, manche Genera, Arten u. s. w. eine $2-3-4-5 \mathrm{mal}$ so lange als andre.

16) Das Auftreten und Vergehen derOrganismen selbst war auch vicht an gewisse Zeit-Abschnitte gebunden; es knnnte zu jeder Zeit in grösserem oder kleinerem Maasstabe erfolgen. Es hat keine Reihe gariz getrennter Schöpfungen existirt. Wenn aber während Hunderttansendein von Jahren an einer Stelle nur wenige oder gar keine Schichten sich absetzen konnten oder selbst durch gewaltsame Bewegurigen der Erd Rinde ein Theil der bereits abgesetzten wieder zerstört wurde, so muss sich wenigstens an dieser Stelle zwischen den 
Resten der Organismen in früheren und späteven Schichten ein rascherer oder gar eil plötzlicher, allgemeinerer oder gänzlicher Wechsel zeigen, als ausserdem (geologische Zeit-A bschnitte). Jenc bedingenden Ursachen können wohl über einen grossen Theil der Erd-Oberfläche hin, aber nie über die ganze Erde zugleich in einerlei Weise wirksam gedacht werden.

17) Wenn mas das, was die Geologen Formationen und Perioden genannt haben, hinsichtlich des Wechsels der Organismen näher prüft, so findet man, dass in einer einzelnen Formation of noch ein a-3maliger und in jeder der V Perioden durchschnittich ein 4-10maliger Wechsel der meisten Arten stattgefunden bat, so dass man wenigstens einen 30maligen durchschnittlichen Arten-Wechsel annehmen kann. Haben dann auch 0,02-0,05-0,10 der jedesmaligen Arten länger gelebt, so ist die Dauer so vieler andern kürzer gewesen.

18) Diejenigen Genera, Familien, Ordnungen der Organismen, welche in irgend einer geologischen Zeit einmal zur Entwicklung gelangt waren, sind in derselben nicht spärlicher repräsentirt gewesen, als sie es heutzutnge sind; eine gleiche Erd-Fläche war an Bewohnerı aus denselben eben so reich als jetzt.

19) Wir kennen jetzt gegen 27000 Arten fossiler Wesen, wovon die Pflanzen nicht 0,10 ausmachen. Nach jener Voraussetzung (17) können wir berechnen, dass es allmählich über 500,000 Pflanzen und 150,000 Thiere, im Ganzen also über 2,000,000 Organismen-Arten gegeben haben müsse, während die jetzige Schöpfung nach der Zahl der jetzt bekannten Arten die reichste von allen bereits 200,000 Arten zählt, worunter die Zahl der Pflanzen der der Thiere viel näher kommt, als (z. Th. in Folge geringerer Erhaltungs-Fähigkeit der P(lanzen) unter den fossilen Resten.

20) Das Grund-Gesetz, wodurch das allmähliche Auftreten der Lebenwelt geleitet worden, war das der jederzeitigen Anpassung derselben an die äusseren geologischen Exisienz-Bedingungen.

21) Je manchfaltiger diese Existenz-Bedingungen in Folge fortschreitender Differenzirung der Meere, Länder und Klimate wurde, desto manchfaltiger wurde auch die Lebenwelt.

22) Man hat die ersten Repräsentanten der einzednen Klassen Ordnungen und Familien von Pflanzen und Thieren, welche auf der Erd. Oberfläche erschienen siud, jeden einzelnen mit einem Typen-Keime verglichen und Prototyp oder wohl angemessener Archetyp* genannt, durch deren Entwicklung und immer weiter schreitende Differenzirung (im Verhältnisse mit der Differenzirung der Existenz-Bedingungen) ihrer Zweige dann die verschiedenen späteren der nämlichen Klasse, Ordnung und Familie entsprechenden Formen-Reihen auseinandertretend hervorgingen. Dieser Vergleich ist indessen bis jetzt keines-

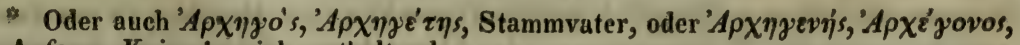
den Anfang, Keim in sich enthaltend. 
wegs überall aiwendbar. Aber die Sache zeigt sich ain auffallendsten und ausgedehntesten bei den kryptogamischen Gefäss-Pflanzen, Krinoiden, Brachiopoden, Trilobiten und Reptilien.

23) Die grössten Arten einer Klasse u. s. w. zeigen sich da, wo diese Klasse und ilır Element am mächtigsten entwickelt ist, daher bald in früheren Schöpfungen und bald in der jetzigen.

24) Es findet ein allmählicher Fortschritt der organischen Welt zum Vollkommneren Statt, grossentheils erweisslich ebenfalls in Folge dafür geeigneter werdender Existenz-Bedingungen : nicht so, als ob anfängliche unvollkommenere Klassen, Ordnungen u. s. w. allmählich durch vollkommnere ersetzt würden, sondern so dass zu den anfänglich vorhandenen immer noch vollkommnere hinzukommen.

25) Doch sind Pflanzen und Thiere gleichzeitig entstanden und haben schon gleich anfangs von beiden mehre niedrigere Kreise und Klassen neben einander existirt. Die ansteigende Entwickelung hat daun wie an einem Strauche stattgefunden, dessen verschiedenen Stämme weiter zuwachsend immer höher und höher Zweige treiben, wovon aber die Ausbildung der höchsten nicht bis zur Vervollständigung der tiefsten zurückgehalten ist, sondern wieder gleichzeitig oder selbst vor einem Theile derselben erfolgen kann; auch sterben wie am zuwachsenden Strauche einzelne untere Zweige, die ihre Existenz-Bedingungen nicht länger finden, früh wieder $a b$, und sind es jährlich (periodisch) immer andere Blätter und Blïthen, welche an diesen Zweigen hängen. Wie der Strauch, so verästelt und verzweigt sich die Lebenwelt mit der Zeit immer feiner, und es fällt die 'grösste Vervielfältigung und Breiten-Ausdehnung immer unter das Niveau der Spitze.

26) Anfangs waren von Pflanzen nur Zellen- und Gefäss-Kryptogamen, monokotyledonische Blüthen-Pflanzen und sogenannte nacktsaamige Exogenen(Cykadeen und Coniferen), von Thieren nur PflanzenThiere, Weichthiere, Kerbthiere und Fische vorhanden; - dann schwanden die Gefäss - Kryptogamen an Zahl und nahmen die nacktsaamigen Exogenen zu, während sich (von einigen Vorläufern in der Kohlen - Zeit abgesehen) den Thieren die Reptilien beigesellten und zu mächtiger Eníwickelung gelangten; - mit der Tertiär-Zeit treten erst die grossen Massen der fruchtsamigen eigentlichen Dikotyledonen oder Exogenen unter den Pflanzen und die Säugthiere unter den Thieren auf; - am Ende dieser Zeit der Mensch. Ähnliche Abstufungen der Vervollkommnung lassen sich auch in den einzelnen Klassen, Ordnungen u. s. w. wahrnehmen.

27) Wir könuen zwar nicht alle Existenz-Bedingungen für die einzelnen Erscheinungen in diesem Fortschreiten zum Vollkommeneren nachweisen; sie beruhen jedoch hauptsächlich in der fortschreitenden Ausbildung der Kontinente, ihres Bodens und ihres KontinentalKlimas aus dem anfänglich einförmigen Wasser-Reiche; in der immer weiter gehenden Differenzirung der klimatischen Lebens-Bedingungen; 
in der z. Th. dadurch bedingten Differenzirung der organischen LebensBedingungen, so ferne das Leben einer Menge von Thier-Formen von der Existenz andrer (zumal dikotyledonischer) Pflanzen- und ThierFormen ablängig ist. Die Masse der Luft-Insekten und der Säugethiere konnte nicht vor den Dikotyledonen, der Mensch nicht vor dem Daseyn der Säugthiere, ja nicht vor der Schöpfung der beiden ganzen Naturreiche ihre Existenz-Bedingungen finden.

2S) Mit den Dikotyledonen kommen auch die Laubholz-Wälder erst in der Tertiär-Zeit zum Vorschein, wahrscheinlich nicht ohne Einfluss auf Quellen-Bildung (wenn gleich gewiss schon früher viele Quellen existirt haben). Daher sind auch die Süsswasser-Bewohner gleich den Land-bewohnendenThieren eine verspätete Erscheinung(10).

29) Indem alie äusseren Existenz - Bedingungen sich den jetzt besteheuden immer mehr näherten, wurde auch die Schöpfung in Formen-Reichthum, Höhe nnd Vertheilung den Organismen der jetzigen immer ähnlicher.

30) Solche Klassen aber, die erst spät ihre Existenz-Bedingungen fanden, hatten, gleichwohl von eben so fremdartigen Ur-'Typen ausgehend, dieselbe Metamorphose der Formen in der kürzeren übrigen Zeit zu durchwandern, wie die früh enstandenen in der längeren. Die noch fremden Formen der ersten treffen daher mit den bereits assimilirten der letzten zusammen (Säugthiere).

31) Geschlechter und Arten haben anfangs (in Folge gleicherer Existenz-Bedingungen) eine viel weitere geographische Verbreitung besessen als jetzt; doch lässt sich diese weite Verbreitung nicht an allen nachweisen, indem es wohl auch so wie jetzt örtlich beschränkte Formen gegeben haben muss.

32) Die geographisch verbreitetsten Geschlechter und Arten pflegen zugleich diejenigen zu seyn, welche die längste geologische Dauer haben, was Beides für einen weiteren Umfang des Vermögens der Anfügung an die äusseren Lebens - Bedingungen (für grössere Lebens Zähigkeit) spricht.

33) Unsere Kenntuisse über die alteGeographie der Organismen sind übrigens noch sehr beschränkt. Denn von den bis jetzt bekannten 26421 Arten fossiler Wesen hat Europa 24314 und das Ausland nur 2839, mithin nur 0,11 geliefert, was auf die einzelnen Welttheile und Perioden vertheilt nur ein sehr schwaches Bild ihrer einstigen Bevölkerung gibt.

34) Doch sind bis zur Mitte der'Tertiär-Zeit herab nur 28 besser begründete exotische Genera bekannt, die nicht auch in Europa vorkämen; und unter diesen ist der Dicynodon aus einem alten Sandsteine in der Nähe des Kaps das einzige Genus, welches vielleicht Anspruch auf Begründung einer eigenen Familie - unter den Reptilien - machen kann. 
35) Für eine eheinals andere Lage der Erd-Axe liegen keine Anzeigen vor,

36) Von einstigen Wanderungen der Erd-Bevölkerung oder einzelner Gruppen und Arten haben wir (wegen 33) auch noch keine sicheren Beweise, sondern bis jetzt nur solche von der Vertilgung gewisser Arten in einem Theile ihres einstigen Verbreitungs-Bezirks. Dahin zählen wir noch die vielen miocänen Konchylien West-Europa's, welche jetzt im tropischen West-Afrika gefunden werden. 


\section{VERBESSERUNGEN}

1. Nomenclator.

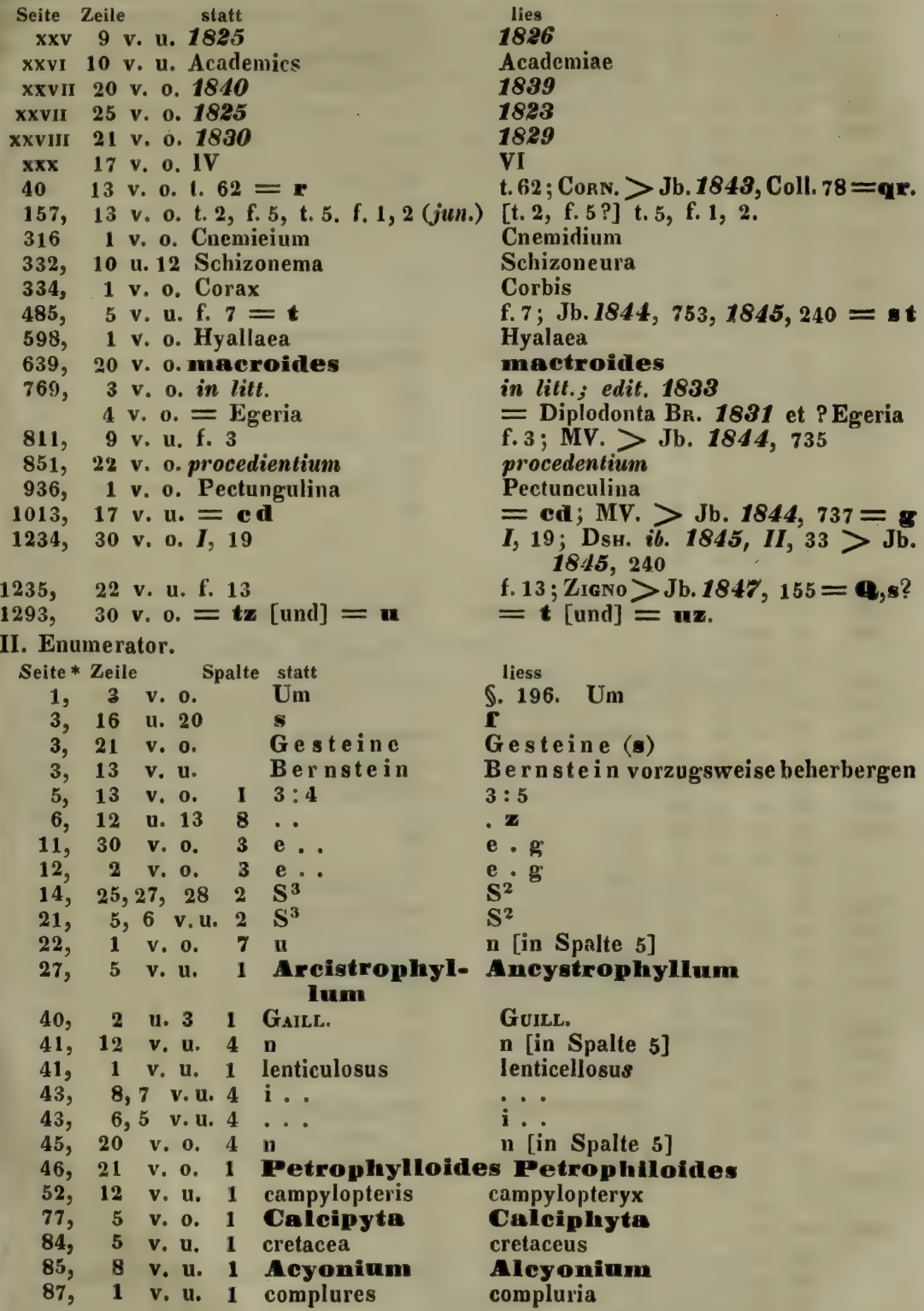

* Die Zeilen sind immer nur vom Kopfe der Tabellen an gezählt. 


\begin{tabular}{|c|c|c|c|c|c|c|c|}
\hline Seite & Zeile & & & palte & statt & lies. & \\
\hline 89 & 3 & v. & & 1 & aliorum & aliarum & \\
\hline 102 & 21 & v. & o. & 8 & 0 & $\infty$ & \\
\hline 107 & 9 & $\mathbf{v}$. & 0. & $\mathbf{I}$ & lesta & testa & \\
\hline 111, & 7 & v. & u. & 1 & Cythaxina & Citharima & \\
\hline 113, & 25 & v. & & 1 & Valvulina & Vulvulina & \\
\hline 123, & 2 & v. & 0. & 7 & $\cdots \cdots$ & $\ldots w^{\prime}$ & \\
\hline \multirow[t]{2}{*}{ 126, } & 21 & v. & 0. & 8 & - t & $\cdots$ & \\
\hline & 25 & v. & 0 . & 6 & $\dot{2} \dot{W}_{\text {OOD }}^{f}$ & Sivicty & 1 \\
\hline $\begin{array}{l}130 \\
138\end{array}$ & $\begin{array}{c}11 \\
2 \mathrm{u}\end{array}$ & $\begin{array}{l}v . \\
3\end{array}$ & u. & 1 & $\begin{array}{l}\text { ?WOOD } \\
\text { U. }^{2}\end{array}$ & $\begin{array}{l}\text { SAVIGNY } \\
\mathrm{U}^{4}\end{array}$ & \\
\hline 140 & 16 & v. 3 & & 3 & . b.... & $\ldots \ldots$ & \\
\hline 144, & 1 & $\mathbf{v}$ & u. & 7 & $\ldots \mathbf{u} \ldots$ & $\cdots \cdots$ & \\
\hline 145, & 4. & v. & o. & 4 & $\cdots$ & h... & \\
\hline 146 & 1 & v. & u. & 6 & $\ldots \boldsymbol{f}$ & $\cdots$ & \\
\hline \multirow[t]{2}{*}{148} & 12 & v. & 0. & 1 & ryozoorum & Bryozoorum. & \\
\hline & 22 & v. & & 1 & Anhipatines & Antipathes & \\
\hline 149, & 8 & v. & u. & 8 & & 0 & \\
\hline 152 & 9 & v. & o. & 2 & $\mathbf{F}^{\mathbf{I}}$ & $F^{2}$ & \\
\hline 153 & 13 & v. & u. & 8 & $\cdot z$ & $\ddot{m}$ & 11 \\
\hline \multirow{2}{*}{ 169, } & 6 & v. & o. & 4 & $\begin{array}{l}\mathbf{m} \\
\mathbf{n}\end{array}$ & $\mathrm{n}$ [in 5. Spalte $]$ & $F$ \\
\hline & & $\begin{array}{l}\text { v. } \\
\text { v. }\end{array}$ & & $\begin{array}{l}4 \\
4\end{array}$ & $\begin{array}{l}n \\
n^{2}\end{array}$ & 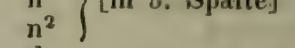 & \\
\hline \multirow[t]{2}{*}{ 175, } & 8 & v. & 0. & 3 & b & d & \\
\hline & 17 & v. & o. & 7 &.$t \ldots$ & $\ldots \ldots$ & \\
\hline 180, & 21 & v. & 0. & 3 & b..... & $\ldots c \ldots$ & \\
\hline 198, & 9 & v. & & 1 & Amplypygus & Amblypysus & \\
\hline 206 & $\begin{array}{c}4 \\
10\end{array}$ & $\begin{array}{l}\text { und } \\
11 \text {, }\end{array}$ & & $\begin{array}{l}1 \\
1\end{array}$ & \} sind & zu streichen; stehen & auf S. 86 ห. 148. \\
\hline 209, & 1 & v. & u. & 8 & 61 & 71 & \\
\hline 220, & 6 & v. & o. & 7 & . t... & $\cdots \cdots$ & \\
\hline 222 & 2 & v. & 0. & 1 & maccopterus & macropterus & \\
\hline 225, & 26 & v. & o. & 1 & Spirieri & Spiriferi & \\
\hline 228, & 5 & v. & u. & 2 & $\mathbf{F}^{2}$ & $\mathbf{S}^{2}$ & \\
\hline 235 & 23 & v. & 0. & 8 & . & 0 & 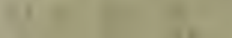 \\
\hline \multirow{2}{*}{ 237, } & 6 & v. & o. & 8 & .0 & $\begin{array}{l}20 \\
\cdots\end{array}$ & 1 \\
\hline & $\begin{array}{l}11 \\
13\end{array}$ & $\mathbf{v}$. & 0. & 8 & $\cdot{ }^{7}$ & $\cdots v, \ldots$ & \\
\hline 238, & $\begin{array}{l}13 \\
26\end{array}$ & v. & $\begin{array}{l}0 . \\
0 .\end{array}$ & 5 & n & $\ddot{m} \cdots v$ & \\
\hline 246 & 22 & $\mathbf{v}$. & o. & 8 & . & 36 & \\
\hline 247, & 2 & v. & 0. & 1 & armatus & asper & \\
\hline \multirow[t]{2}{*}{256} & 14 & v. & u. & 1 & Dsh. & GF. & \\
\hline & 24 & v. & 0 . & 8 & - & 20 & \\
\hline 262, & 16 & v. & 0 . & 4 & $\cdots$ & $m \cdots$ & -8 \\
\hline 268, & 14 & v. & u. & $\mathbf{1}$ & & $a^{2}=2 d_{1}$ & \\
\hline 273, & 15 & v. & u. & 6 & $q^{2} ? 1$ & $q^{2} ? p^{1}$ & \\
\hline 274, & 4 & v. & u. & 1 & Arcinellina & Arcinedia & \\
\hline 275 & 2,3 & v. & u. & 5 & [und] & $\begin{array}{l}\mathrm{n}^{2}[\text { und }] \mathrm{n}^{2} \\
\text { Lithodomus }\end{array}$ & \\
\hline \multirow{2}{*}{ 282, } & 6 & v. & 0. & 1 & 4 & 0 & \\
\hline & & v. & & 8 & 0 & 4 & . \\
\hline \multirow[t]{4}{*}{290} & 26 & v. & 0. & 5 & $q \cdots$ & & \\
\hline & $\begin{array}{l}27 \\
28\end{array}$ & $\begin{array}{l}\text { v. } \\
\text { v. }\end{array}$ & $\begin{array}{l}\text { o. } \\
\text { o. }\end{array}$ & $\begin{array}{l}5 \\
5\end{array}$ & $q_{2} \cdots$ & sind in die 6. Spalte & zu versetzen. \\
\hline & 29 & v. & 0. & 5 & $q \ldots r$ & & \\
\hline & 8 & v. & u. & 6 & $q r f$ & $q \mathrm{r} f$ & \\
\hline \multirow[t]{2}{*}{295} & 18 & v. & u. & 1 & Cardidacea & Cardiacea & \\
\hline & 17 & v. & u. & 5 & .0 & ... & \\
\hline
\end{tabular}




\begin{tabular}{|c|c|c|c|c|c|}
\hline Seite & Zeil & & palt & statt & lies. \\
\hline 297, & 10 & v. u. & 2 & $\mathbf{S}^{5}$ & $\mathbf{S}^{3}$ \\
\hline 352 , & 1 & v. u. & & hare & haec \\
\hline 354 & 8 & v. o. & 7 & mammilanum & mammilianum \\
\hline & $\begin{array}{l}10 \\
33\end{array}$ & v. 0 & 1 & & $\begin{array}{l}\text { mammillanum } \\
\text { NiLss. }\end{array}$ \\
\hline $\begin{array}{l}373, \\
375,\end{array}$ & $\begin{array}{l}33 \\
32\end{array}$ & $\begin{array}{l}\text { v. } 0 . \\
\text { v. } 0 \text {. }\end{array}$ & 1 & $\begin{array}{l}\text { NrLLS. } \\
\text { marinae). }\end{array}$ & $\begin{array}{l}\text { Nisss. } \\
\text { marinae) } \cdot 16\end{array}$ \\
\hline 376, & 15 & v. 0. & 3 & $\ldots d \ldots$ & $\ldots d \ldots$ \\
\hline 377, & 18 & v. 0 . & 3 & . b...d.. & . b.d... \\
\hline 379, & 2 & v. u. & 7 & $p^{t} \cdots \cdots$ & $\cdots \cdots$ \\
\hline 381, & 4 & v. u. & 7 & & \\
\hline 381, & 3 & v. u. & 7 & & sind in die 6. Spalte zu versetzen. \\
\hline $\begin{array}{l}381, \\
381 .\end{array}$ & 2 & $\begin{array}{l}\text { v. u. } \\
\text { v. u. }\end{array}$ & $\begin{array}{l}7 \\
7\end{array}$ & $f^{1}$ & 1 \\
\hline $\begin{array}{l}381, \\
386,\end{array}$ & 14 & v. u. & 3 & $\ldots c d \ldots$ & $\ldots \mathrm{cd} \ldots g$ \\
\hline 429 & 2 & v. 0 . & 3 & $\ldots c_{2} \ldots$ & \\
\hline 430 & 16 & v. 0 . & 2 & $\cdot\left(\mathbf{S}^{3}\right) \ldots$ & $E^{2}\left(S^{3}\right) \ldots$ \\
\hline 432, & 5 & v. u. & 1 & 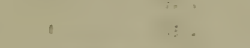 & dazwischen cinzuschalten: a.Cerithiina. \\
\hline $\begin{array}{l}432, \\
445,\end{array}$ & $\begin{array}{l}4 \\
1\end{array}$ & $\begin{array}{l}\text { v. u. } \\
\text { v. } 0 .\end{array}$ & $\begin{array}{l}1 \\
2\end{array}$ & . $\left(\mathrm{S}^{3}\right) \ldots$ & $\mathbf{E}^{2}\left(\mathbf{S}^{3}\right) \ldots$ \\
\hline 447 & 7,4 & v. u. & 2 & $\ldots\left(\mathbf{F}^{3}\right)$ & $\mathbf{E}^{2} \cdot\left(\mathbf{F}^{3}\right)$ \\
\hline 457, & 11 & 140 & 2. & $\cdot\left(\mathbf{S}^{3}\right) \cdot \cdot$ & $\mathbf{E}^{2}\left(\mathbf{S}^{3}\right) \ldots$ \\
\hline 461, & 2 & v. 0 . & 3 & $\cdots c \ldots$ & $\cdots \cdots$ \\
\hline 464, & 11 & v. 0. & 3 & $\cdots \mathrm{e} \cdot$ & 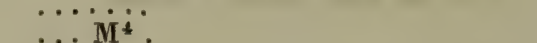 \\
\hline $\begin{array}{l}467 \\
480\end{array}$ & $\begin{array}{l}26 \\
23\end{array}$ & $\begin{array}{l}\text { v. } 0 \text { : } \\
\text { v. } 0 \text {. }\end{array}$ & 2 & Inzoluta & Involuta \\
\hline 502, & 2 & v. u. & 8 & 8479 & . \\
\hline 518 & 16. & $\mathbf{v} \cdot \mathbf{0}$ & 6 & $r^{2}$ & $\mathrm{r}^{2} \mathrm{f}$ \\
\hline 542, & 9. & v. $\theta_{0}$ & 1 & testaceii & testacei \\
\hline 543 , & 15 & v. 0 & & PULMONARIA LT & TR. SIPHONOZANTIA BRNDT. \\
\hline 543 & 17 & v. 0 . & & Spinne & Spinnen. \\
\hline 543 & 22 & v. 0 & & Lepioptera & Lepidoptern \\
\hline 543, & 5 & v. u. & & Drtoptera & ptera \\
\hline $\begin{array}{l}545, \\
547\end{array}$ & $\begin{array}{l}10 \\
22\end{array}$ & $\begin{array}{ll}\text { v. } & 0 . \\
\text { v. } & 0 .\end{array}$ & 1 & Typhopiana & Ty phoplana \\
\hline 548 & 5 & v. 0 . & 1 & $\begin{array}{l}23 \\
195\end{array}$ & 198 \\
\hline 549 , & 11 & v. 0 . & 7 & $\ldots \ldots$ & $3 \ldots \ldots$ \\
\hline 550 , & 2 & v. 0 & 7 & $\therefore$. & $5 \ldots \ldots$ \\
\hline 552, & 11 & v. 0 . & 1 & Serpulithes & Serpulites. \\
\hline 561 , & 1 & v. 0 . & 1 & Cythere & Cypridina \\
\hline 561 , & 12 & v. u. & 1 & Lophyrododorum & Lophyropodortum \\
\hline 563, & 7 & v. u. & 1 & Pa radoxides & Paradoxides \\
\hline 568 & 16 & v. 0 . & 1 & 7 & 17 \\
\hline 572, & $6-8$ & $3 \mathrm{~V} \cdot \mathrm{u}^{\circ}$ & 1 & Poeciloponorum & $\begin{array}{l}\text { ist zu streichen; steht schon S. } 564 \text {. } \\
\text { Poecilopodorum. }\end{array}$ \\
\hline 575, & $\begin{array}{l}9 \\
9\end{array}$ & v. u. & & $\begin{array}{l}\text { Poeciloponorum } \\
257\end{array}$ & $\begin{array}{l}\text { Poec } \\
557\end{array}$ \\
\hline 575 & 7 & v. u. & 1 & vix. & viv. \\
\hline 576 & 6 & v. u. & 1 & Mü. & Mü. 2 \\
\hline 576 , & 3 & v. u. & 1 & 2 & 9 \\
\hline 585 & 3 & v. 0 . & 8 & 107 & 197 \\
\hline 588 & 4 & v. u. & 1 & Mü. & FABR. \\
\hline 591, & 10 & v. 0 . & 2 & 1 & 4 \\
\hline 591, & 16 & v. u. & 1 & 1 & $\begin{array}{l}2 \\
\cdots\end{array}$ \\
\hline $\begin{array}{l}593, \\
596,\end{array}$ & $\begin{array}{r}3 \\
15\end{array}$ & $\begin{array}{l}\text { v. } 0 . \\
\text { v. } 0 .\end{array}$ & $\begin{array}{l}7 \\
1\end{array}$ & & $\begin{array}{l}\text {. u... } \\
\text { dazwischen ist zu setzen }\end{array}$ \\
\hline 596, & 16 & v. 0 . & 1 & & dazwischen ist zu setzen „GmpisL.13" \\
\hline 599, & 9 & v. u. & $\mathbf{5}$ & $\cdots$ & 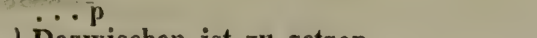 \\
\hline 604, & 17 & V. 0 . & 1 & & azwischen ist zu set? \\
\hline 504, & 18 & v, 0 & 1 & & $\int \quad n s p, 1 \mathrm{~GB}, \ldots \mathrm{v}^{1} \ldots$ \\
\hline
\end{tabular}




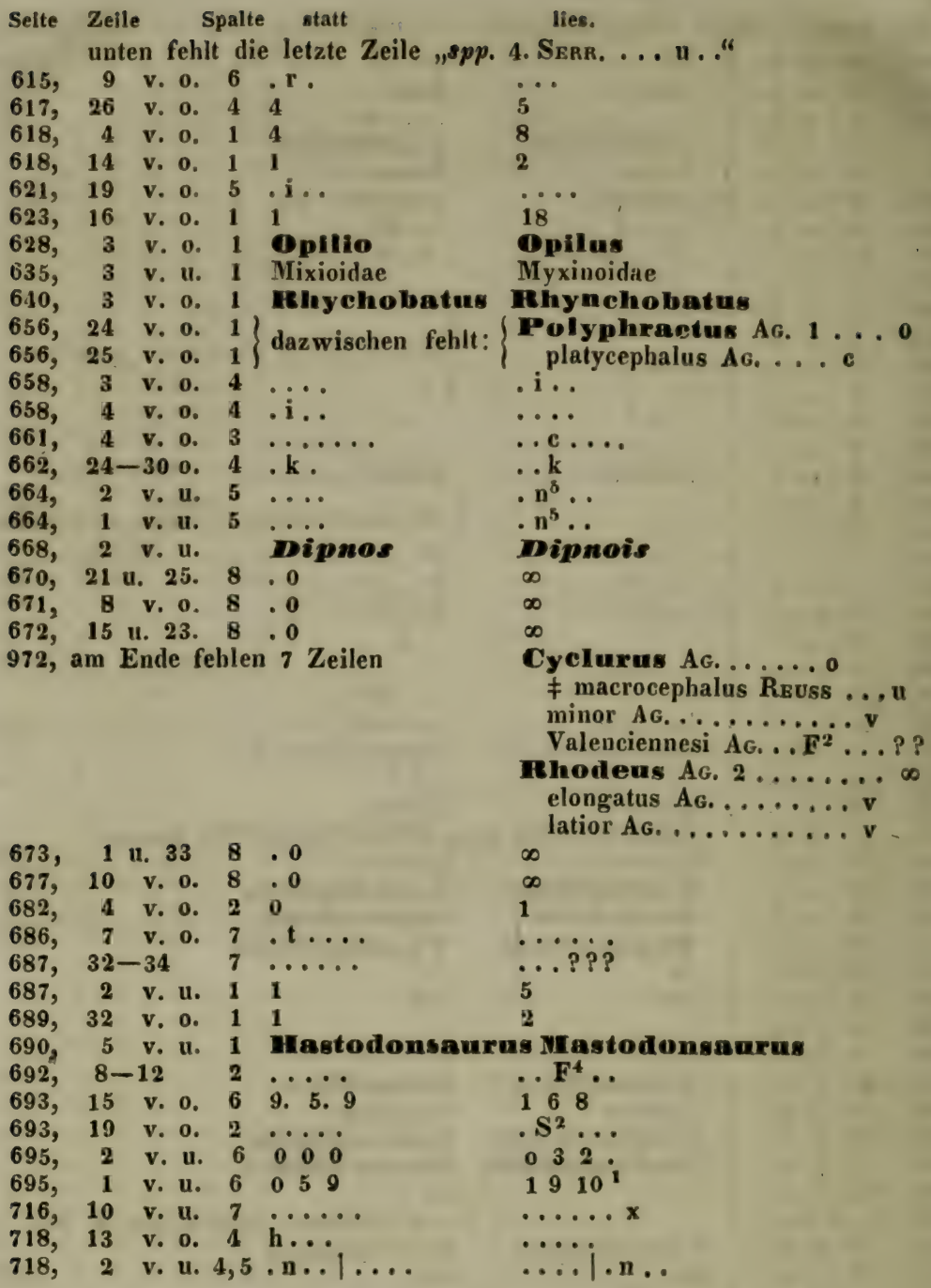

In der Summirung der Arten kommen hin und wieder noch mehre Zählungs-Fehler vor, welche jedoch bei Zusammentragung der Tabellen aut $\mathrm{S}$. $727-745$ berichtigt sind, 'so weit sie uns bekannt geworden waren. 





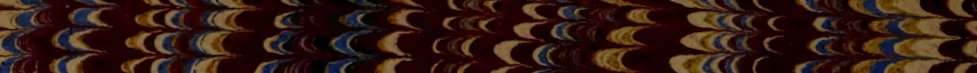

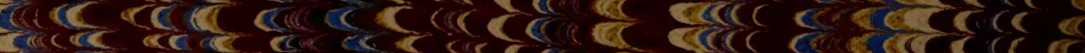

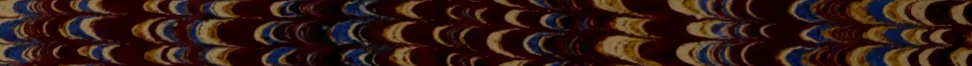

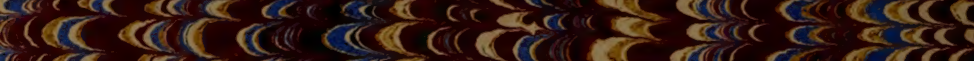

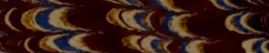

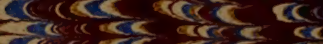

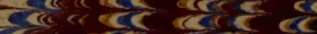

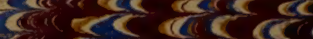
$\operatorname{coc} C \mathrm{CC}$ $\left.\frac{\operatorname{coc}}{\cos } \mathrm{c}\right) \mathrm{CS}$ बला बरे से खे

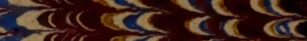

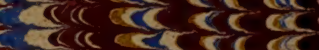

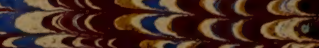

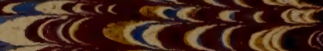
पCC

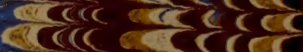

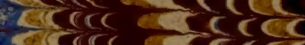
secrect

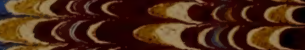
erces asc

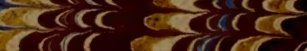

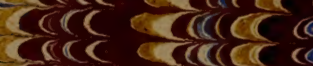

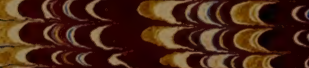

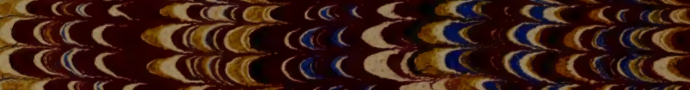

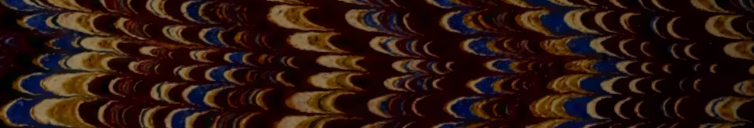

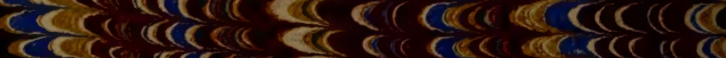

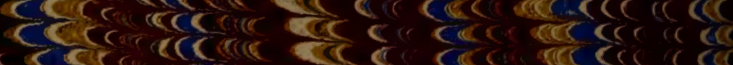

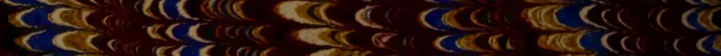

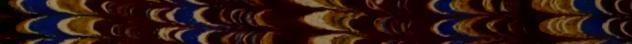

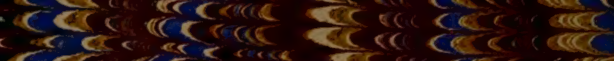

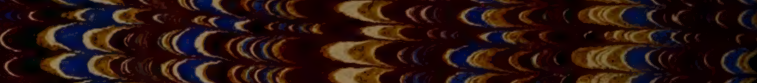

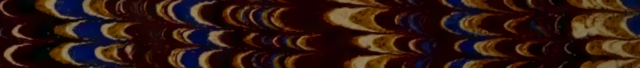

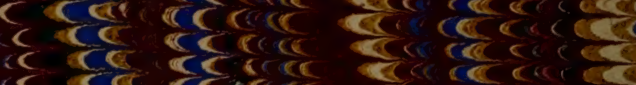

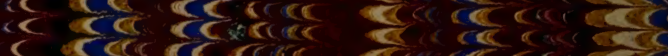

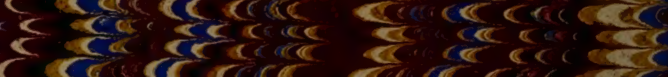

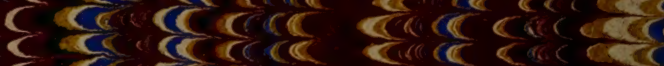
2
2

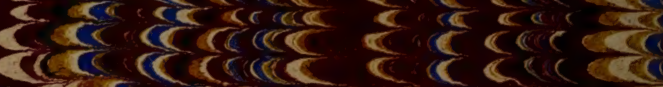

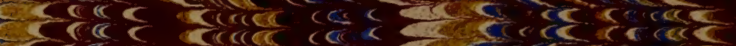

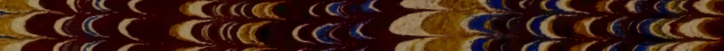

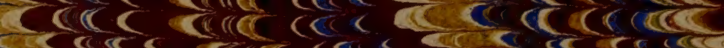

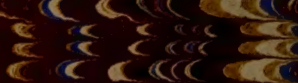

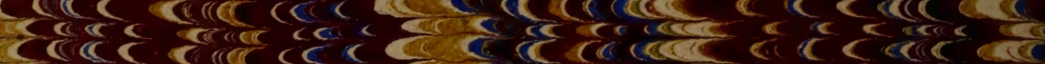

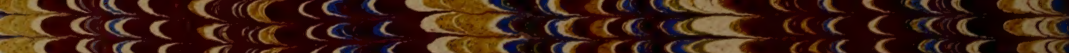

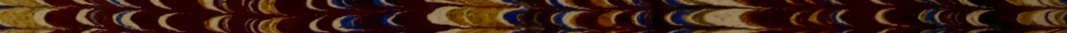

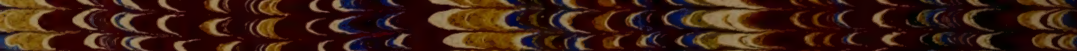

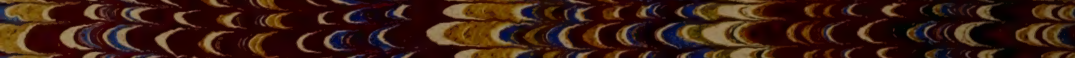

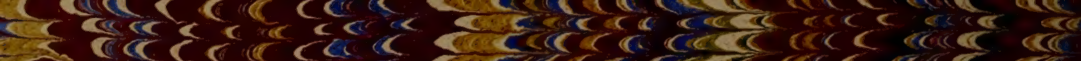

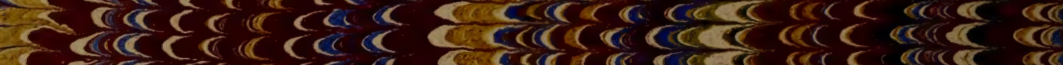

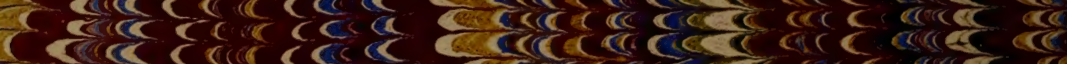

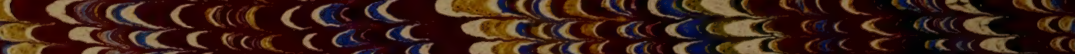

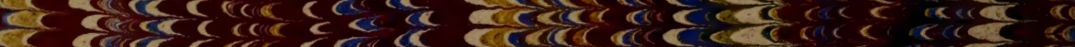

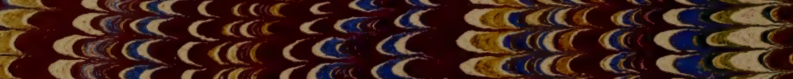

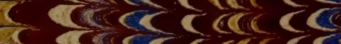
$\sin \cos ^{2} \mathrm{cos}$ $\cos \operatorname{coc} c(2)$

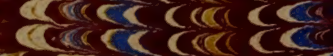

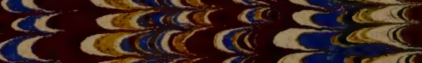

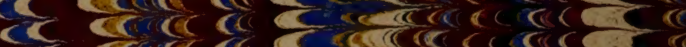

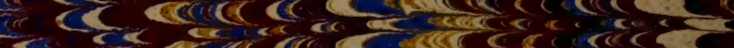

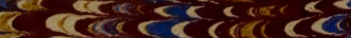
$\operatorname{cocec} c \cos 20$

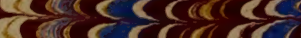
तलि क्ति

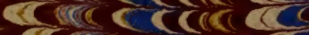
$x \cos c<c a c$

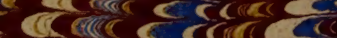

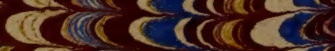

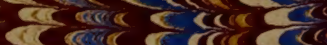

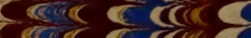

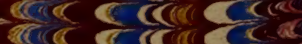

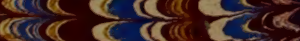
बे

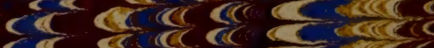
तe बतब बत

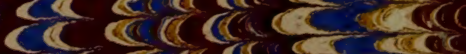

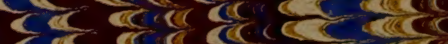

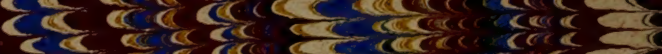

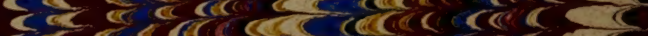

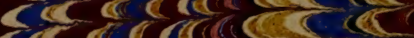

<e esere $\rightarrow \frac{c}{4} \operatorname{coc} c$ $\operatorname{tac} \operatorname{coc}$ ac aces

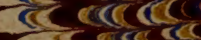

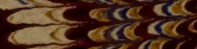
craces $<$ scdera $<\mathrm{accec}$

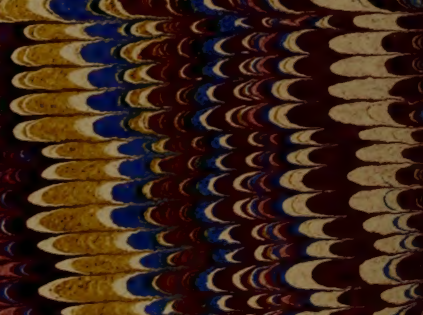

$\frac{\sqrt{a}}{a c}$

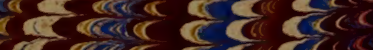

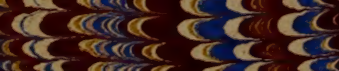




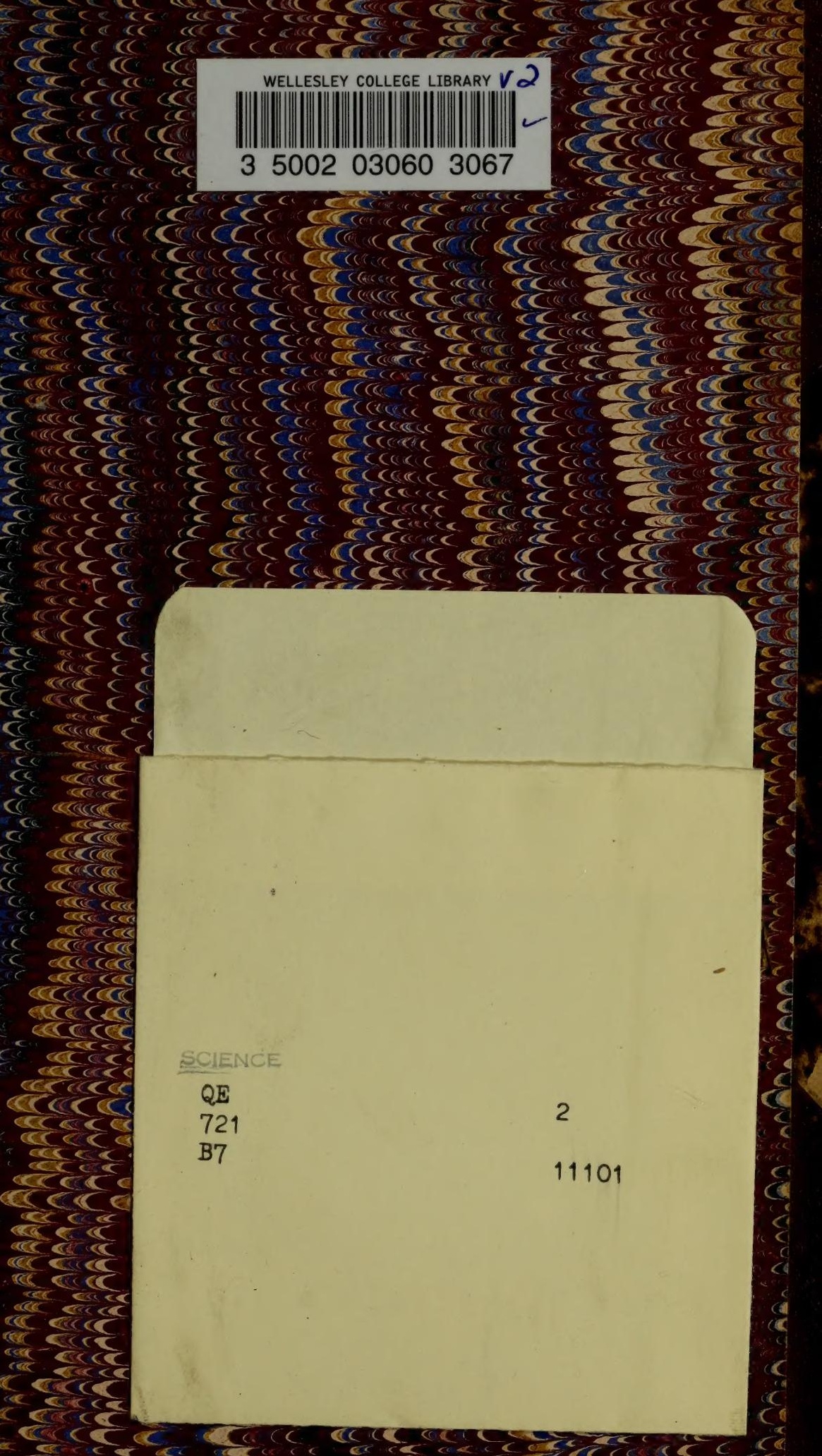




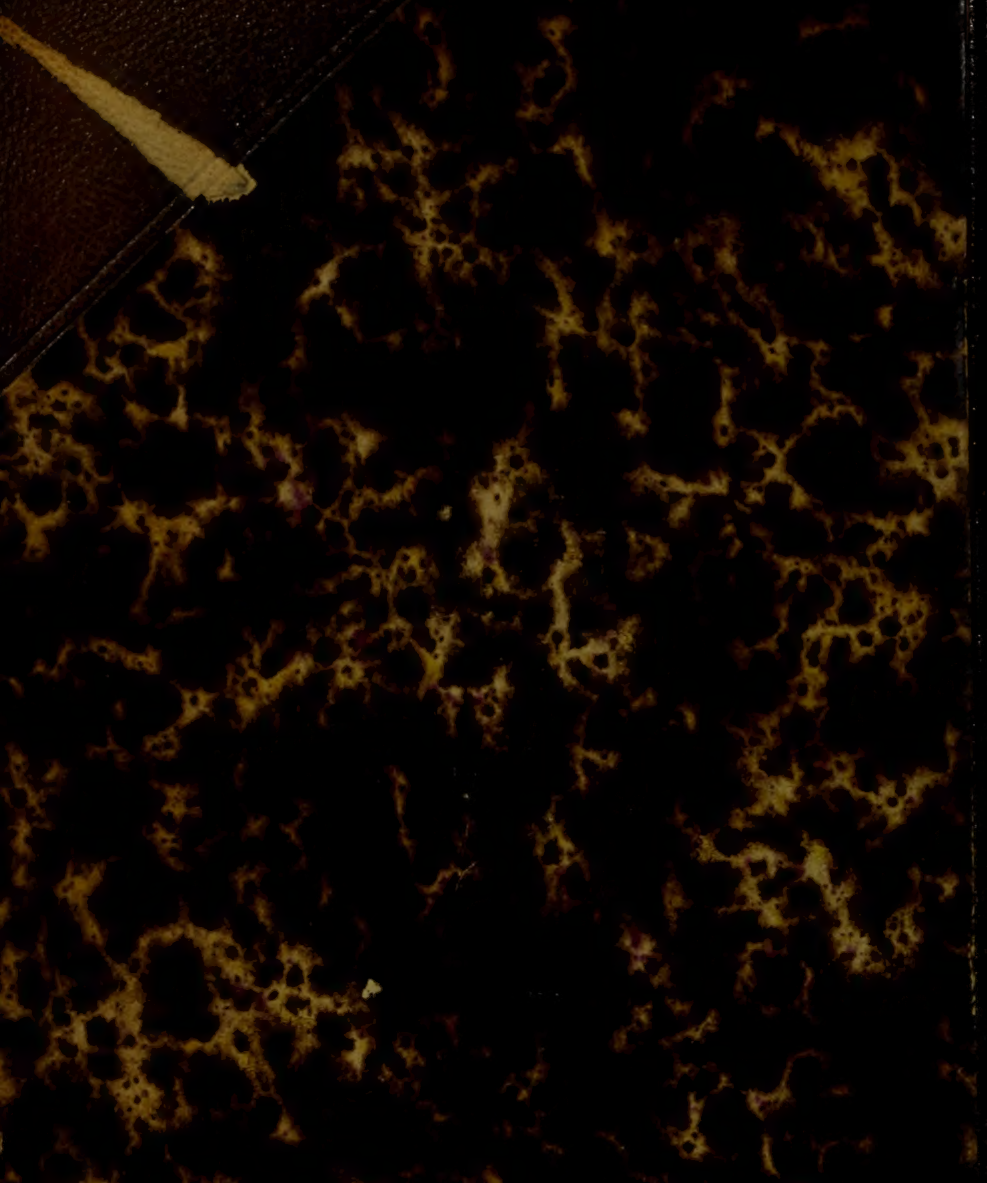

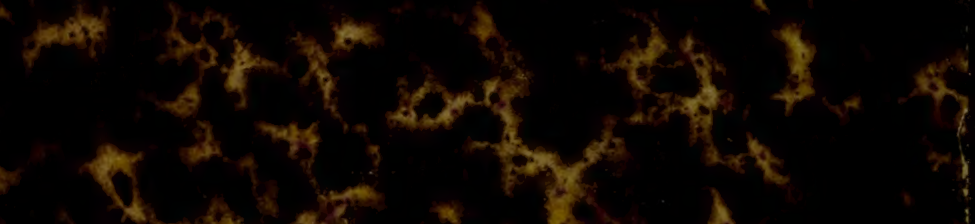

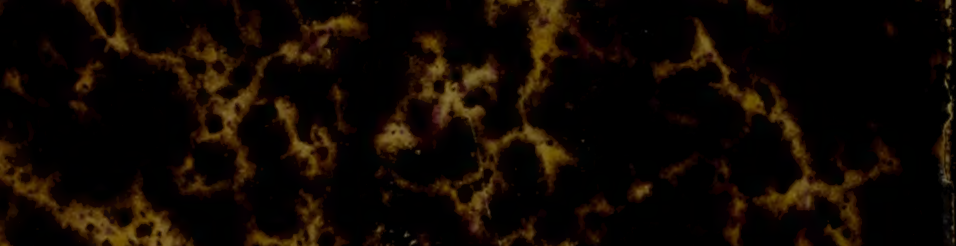

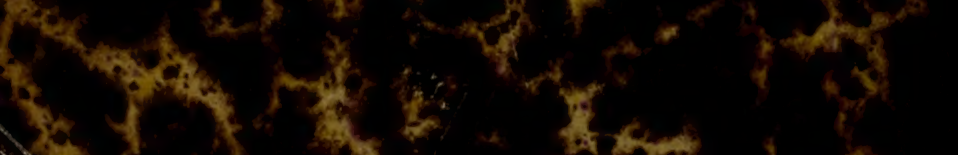

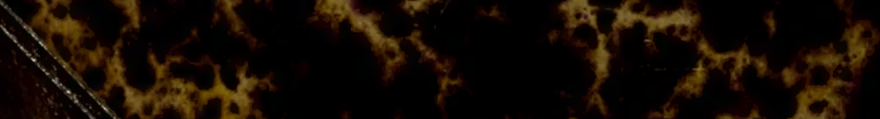

atherat?

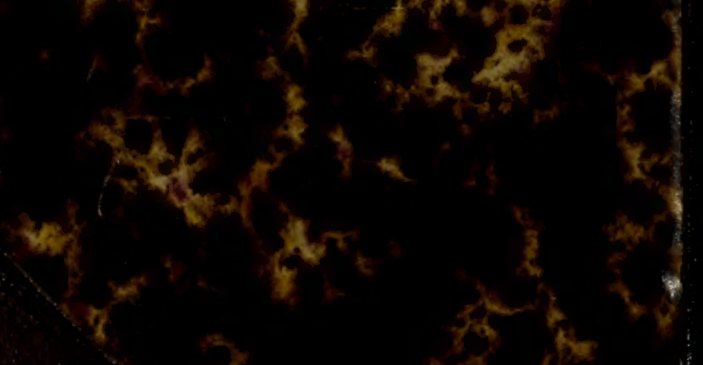

